\title{
THE ZIEGLER POLAR EXPEDITION 1903-1905 SCIENTIFIC RESULTS
}




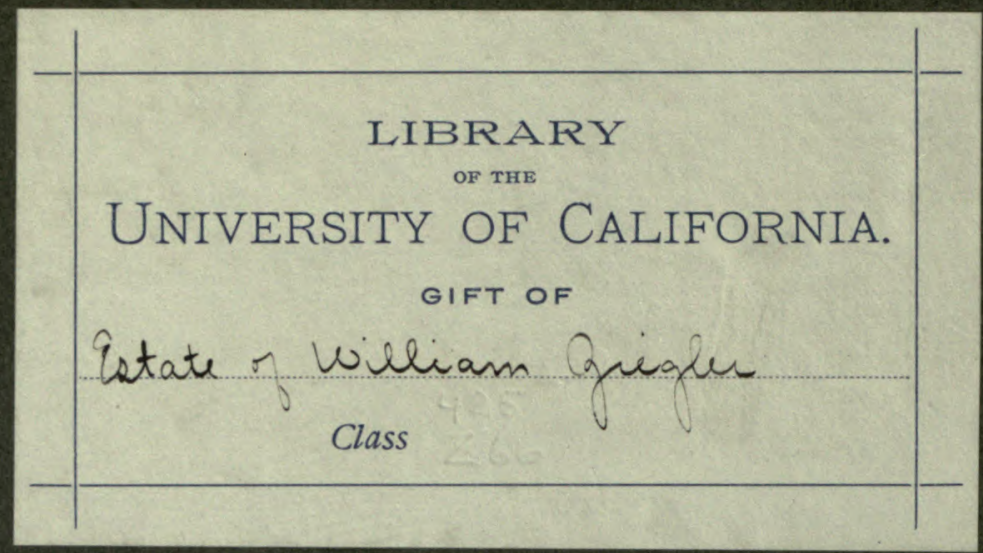

COMPLIMENTS OF THE

ESTATE OF WILLIAM ZIEGLER

NO. 60 LIBERTY STREET

NEW YORK CITY 




\section{THE \\ ZIEGLER \\ POLAR EXPEDITION \\ $1003+205$ \\ WTHONY GHALA, COMBAMBRE \\ SCIENTIFIC RESULTS}

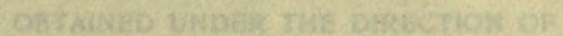

WRLHAR A. PETERS

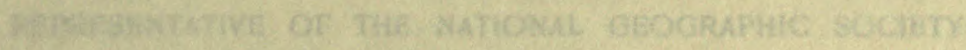

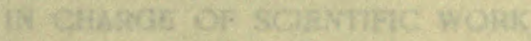

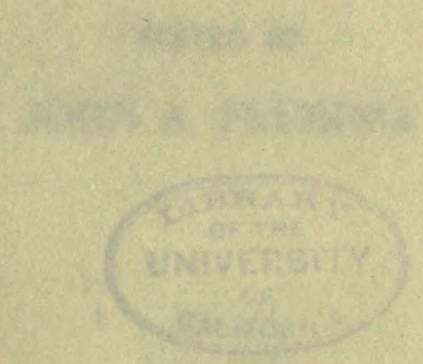

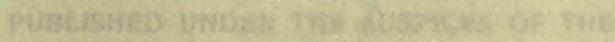

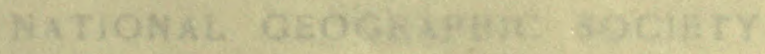

exi the

ESTATE OF WHILAMT ZIEGLER

Wasting regne, b, C.

1907 
THE

ZIEGLER

POLAR EXPEDITION

1903-1905

ANTHONY FIALA, COMMANDER

SCIENTIFIC RESULTS

OBTAINED UNDER THE DIRECTION OF

WILLIAM J. PETERS

REPRESENTATIVE OF THE NATIONAL GEOGRAPHIC SOCIETY

IN CHARGE OF SCIENTIFIC WORK

EDITED BY

JOHN A. FLEMING

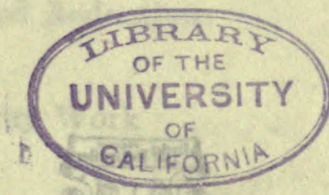

PUBLISHED UNDER THE AUSPICES OF THE

NATIONAL GEOGRAPHIC SOCIETY

BY THE

ESTATE OF WILLIAM ZIEGLER

WASHINGTON, D. C. 


\section{NATIONAL GEOGRAPHIC SOCIETY}

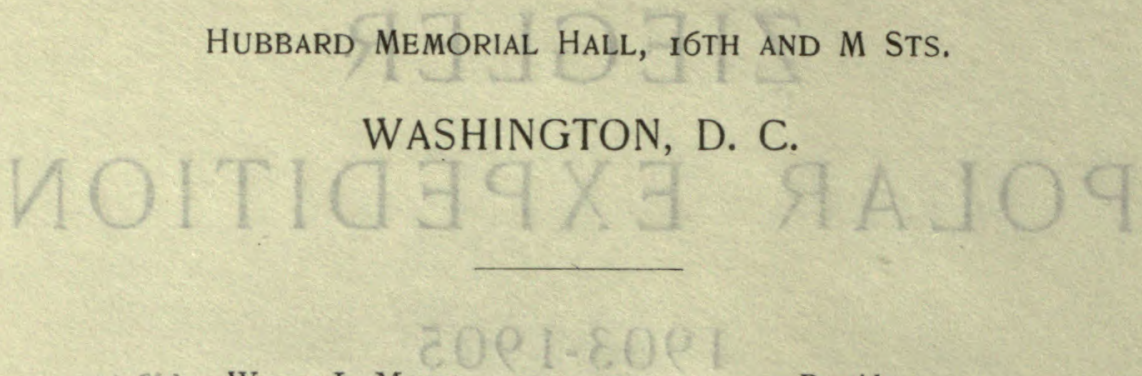

WILLIS L. MooRE......................President

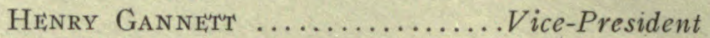

JoHn Joy Ëpson.................... Treasurer

O. P. Austrin......................Secretary

GILBERT H. GROSVENOR................... Editor

Eliza R. SCIDMORE..............Foreign Secretary

F. B. EICHELBERGER...............Asst. Treasurer

JoHn OLIVER LA Gorce..............Asst. Secretary

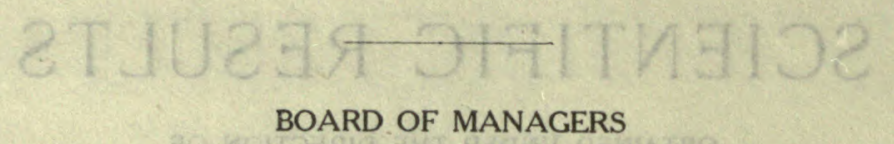

1905-1907

1906-1908

1907-I909

Alexander Graham Beli, O. P. Austin AlfallW heinty F. Blount

Henry Gannetr Charles J. Belil

C. M. CHESTER

J. How ARD GoRE

T. C. ChamberLin

F. V. CoviLLE

A. W. GREELYY

Gilibert H. Grosvenor

GEORGE DAVIDSON

Charless Denby

JOHN JOY EDSON

D. C. Giliman

ANGELIO HeILPRIN

O. H. Tirtmann

DAVID FAIRCHILD

Rudolph Kauffmann

A. J. Henry

WILLIS L. MOORE:

JoHN M. WILSON

C. Hart Merriam

S. N. D. North

\section{DVIMAIF A WHOL}

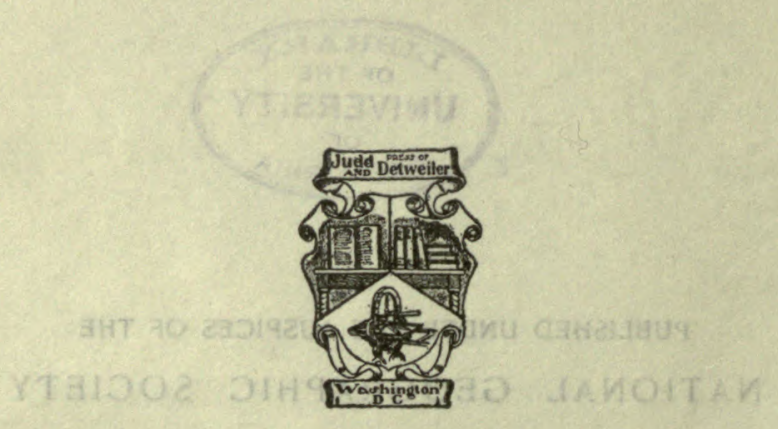




\section{CONTENTS}

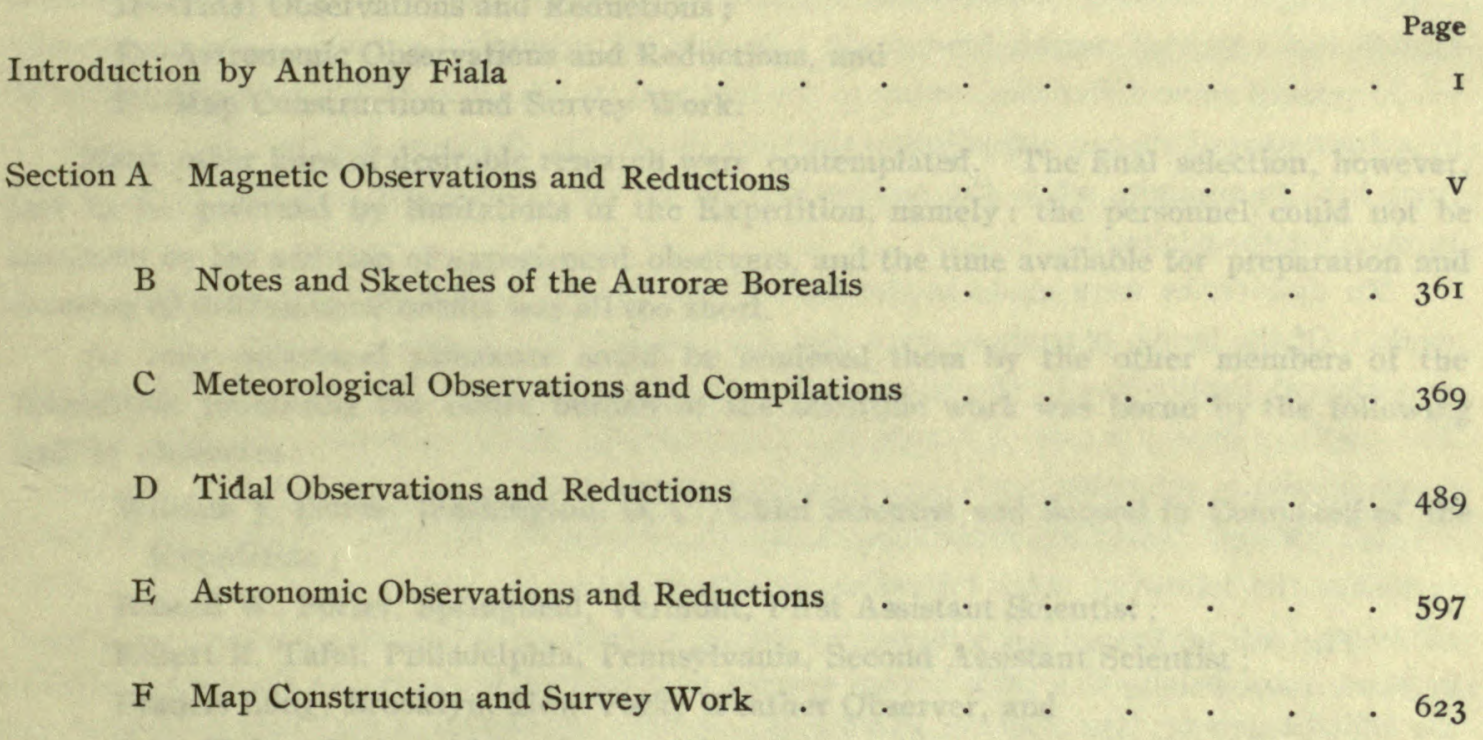




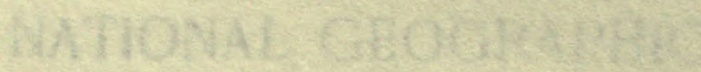

Hersesan.

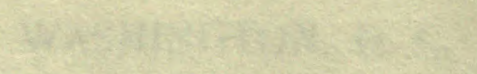

จूละ

I

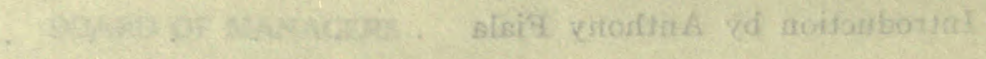

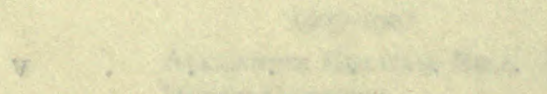

\section{TVMTMOO}

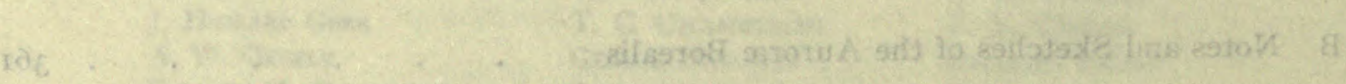

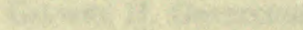

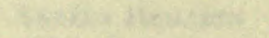

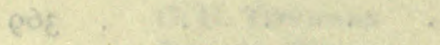

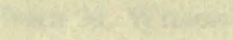

$\cdot 84$

Feद

$\varepsilon s \partial$

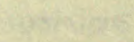

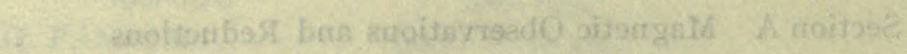

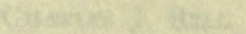

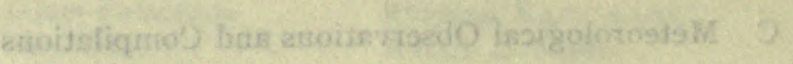

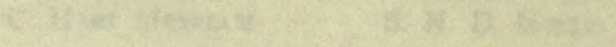

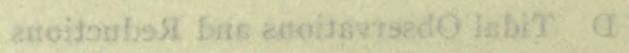

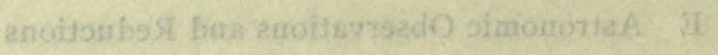

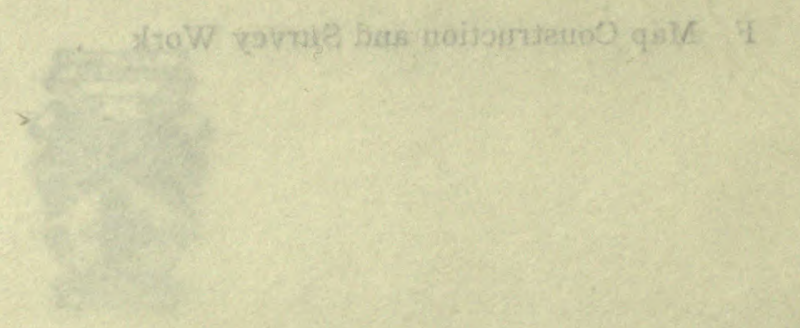




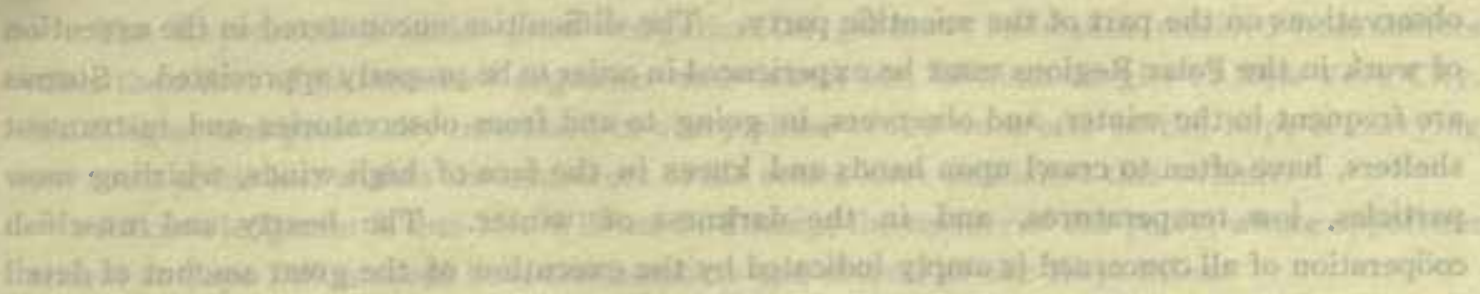

\section{INTRODUCTION}

The scope of the scientific work of the Ziegler Polar Expedition* executed during 1903 to 1905 under the adverse conditions peculiar to the Polar Regions may be summarized by the following section headings of the results reported upon in this volune :

A-Magnetic Observations and Reductions ;

$\mathrm{B}-$ Notes and Sketches of the Auroræ Borealis;

C-Meteorological Observations and Compilations ;

$\mathrm{D}$-Tidal Observations and Reductions;

E-Astronomic Observations and Reductions, and

F-Map Construction and Survey Work.

Many other lines of desirable research were contemplated. The final selection, however, had to be governed by limitations of the Expedition, namely: the personnel could not be increased by the addition of experienced observers, and the time available for preparation and securing of instrumental outfits was all too short.

As only occasional assistance could be rendered them by the other members of the Expedition practically the entire burden of the scientific work was borne by the following staff of observers :

William J. Peters, Washington, D. C., Chief Scientist and Second in Coinmand of the Expedition;

Russell W. Porter, Springfield, Vermont, First Assistant Scientist ;

Robert R. Tafel, Philadelphia, Penusylvania, Second Assistant Scientist:

Francis Long, Brooklyn, New York, Weather Observer, and

Jolnn Vedoe, Boston, Massachusetts. Assistant.

Owing to the loss of the ship, the building of winter quarters, and the almost constant sledging of coal and supplies until far into the winter, there was little time for otlier than the work of providing shelter and food and the preparations for the spring sledge jonrney northward. In addition to the scientific work, observers were called upon for other dnties in these exigencies, besides taking part in the sledge journeys and assisting in the liauling of the instrument from Teplitz Bay to the relief ship. The amount of work accomplished in the consequently scant time available is sufficient evidence of the indefatigable and persistent prosecution of the

* The popular narrative of the Expedition, "Fighting the Polar Ice," by Coumuauder Fiala, has heen published by Messrs. Donbleday, Page \& Co. The volume, now in its second edition, contains considerable information about the handling of Siberian ponies and dogs, the best clothing and equipment for Arctic work, and some useful directions for Polar photography.-ED. 
observations on the part of the scientific party. The difficulties encountered in the execution of work in the Polar Regions must be experienced in order to be properly appreciated. Stornis are frequent in the winter, and observers, in going to and from observatories and instrument shelters, have often to crawl upon hands and knees in the face of high winds, whirling snow particles, low temperatures, and in the darkness of winter. The hearty and unselfish coöperation of all concerned is amply indicated by the execution of the great amount of detail work that is reported upon in this volume.

The natural features and natural history of the Franz Josef Archipelago could lave been studied to advantage but for the lack of trained men, while the impossibility of transporting collections discouraged any systematic attempt to secure specimens. It might, however, be noted that coal was discovered by Mr. Anton Vedoe at Cape Flora in August, I904, and was used during the following winter. The vein is a lignite of poor quality, which, however, burns freely. Brown coal was found by Mr. Russell Porter on Coalmine Island, Booth Channel, at a high elevation. Another vein containing fossils was discovered by Mr. Anton Vedoe at Cape Washington, the eastern extremity of Ziegler Island. Traces of coal were also found at Cape Richthofen, and without doubt other deposits would have been uncovered on the different islands had extended search been made. A detailed geological survey of the Archipelago would present some difficulties, owing to the fact that its islands are for the most part covered by a dome-shaped ice-cap extending to the sea. Strata are, however, exposed on Alger Island from base to summit, while the southern coast of the Arclipelago presents nany opporturities for the geologist.

No discoveries were made in the flora of the Islands during the two brief summers of work. Of the fauna, ptarmigan were seen for the first time in the Archipelago, and several were shot at Teplitz Bay in the summer of 1904, as also on Alger Island and at Rubini Rock. The nesting place of a pair of brants was discovered by Messrs. Stewart and Joln Vedoe at Camp Ziegler in the summer of 1905 , and the eggs secured.

Mr. Miller, Assistant Ornithologist of the American Musenm of Natural History, furnishes the following notes regarding the Ptarmigan:

"The pair of Ptarmigan collected by the Fixpedition on Alger Island, Franz Josef Land, in June, I 904 , belong to a little-known species of considerable rarity in collections. This is the Spitzbergen or Hyperborean Ptarmigan, Lagopus hyperborens, a very near relative of two well-known species, the Alpine Ptarmigan, L. mutus of the mountains of Linrope, and the Rock Ptarmigan, L. mpestris of the Arctic regions of both hemispheres. From both these species it differs in larger size and the presence of a greater amount of white on the tail feathers, though in the latter respect there is considerable individual variation. In habits it does not differ from its near relatives.

"The Spitzbergen Ptarmigan was first described by Sundevall in 1838 , and it is represented by a colored plate in Elliot's Monograph of the Tetraonida. It had not before been recorded outside of Spitzbergen, and from the fact that no Ptarmigan had previously been observed on Franz Josef Land it seems likely that the birds found there in 1904 liad been blown over from Spitzbergen.

" The present pair of birds is an excellent illustration of the fact, already recorded, that the male of this species retains the white winter plumage considerably later in the spring than does the female. The male is wholly pure white, while the female, although taken at the same time, is in the brown plumage of summer." 
The Expedition is under great obligation for generous assistance received from sources other than that of its lamented organizer and donor, Mr. William Ziegler of New York City. Mr. Ziegler was personally interested in every phase of the work and in the hope of carrying out some of his last wishes the executors of his estate have published this volume.

Acknowledgment is due Mr. William S. Champ, the rescuer of the party, whose opportune arrival at Cape Dillon saved both the members and records of the Expedition.

The National Geographic Society, through its former President, Dr. Alexander Graham Bell, and its present President, Dr. Willis I. Moore; its Vice-President, Henry Gannett; its Secretary, O. P. Austin, and its Editor, Gilbert H. Grosvenor, and through its members individually, has given encouragement and assistance in many ways, both in the initiation and completion of the work of the Expedition. It was to this organization that Mr. Ziegler extended the privilege of selecting the scientific leader and it was by the unanimous action of its Board of Managers that Mr. Peters was commissioned in this capacity. The scientific work accomplished conforms, in general, with the suggestions made by the Research Committee of this Society of which Professor G. K. Gilbert was chairman.

Grateful acknowledgnients are due Professor Geelmuyden, Director of the Christiania Observatory, who loaned a Repsold Circle when at the last moment it appeared that one could not be obtained.

Mr. O. H. Tittmann, Superintendent of the United States Coast and Geodetic Survey, on the part of himself and the members of his Bureau, extended every possible lielp in the way of instruction and suggestion. Through his courtesy the Expedition had also the use of the instrumental outfit necessary for the execution of the magnetic work.

Dr. I. A. Bauer, Director of the Department of Terrestrial Magnetism of the Carnegie Institution of Washington, devised the plan of observation best suited to the limited instrumental ontfit and conditions to be encotnitered, which plan experience proved successful. He has further suggested the general scheme of reduction of this portion of the observations.

Professor Willis L. Moore, Chief of the United States Weather Burean, supplied a number of instruments for use in the meteorological observations.

General A. W. Greely, of tlie United States Army, extended assistance by many valuable suggestions as the result of his own wicle experience in Polar work; he also arranged for the loan of some meteorological instruments from the United States Signal Corps.

The task of preparing the scientific results for publication was taken up by Mr. Peters during September, r905, upon the return of the Expedition. He was unable to complete this labor personally owing to his association on January I, 1906, with the Department of Terrestrial Maguetism of the Carnegie Institution of Washington as Commander of the Magnetic Survey Yaclit. In his absence, through the courtesy of Dr. L. A. Bater, Director, the burden of the completion of compilation, computation, editing, and publication of the results has been borne by Mr. J. A. Fleming, of the Department of Terrestrial Magnetism. The priucipal assistance in the great amount of detail work necessary has been rendered by Messrs. E. $H$. Bowen, C. C. Craft, W. B. Corse, and W. N. Ross.

ANThony Fiala

New York City, July' I, I907 


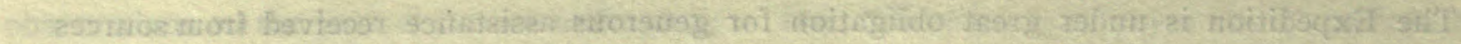

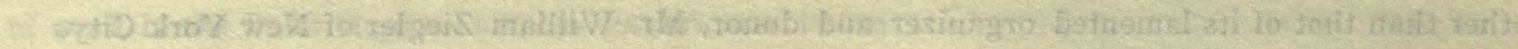

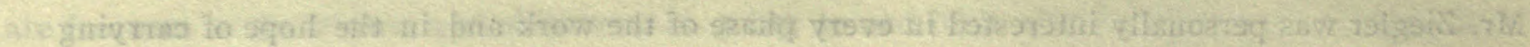

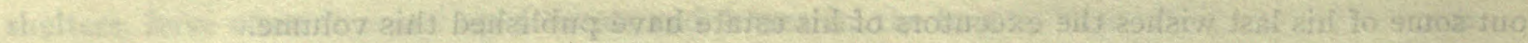

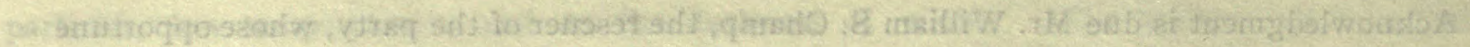

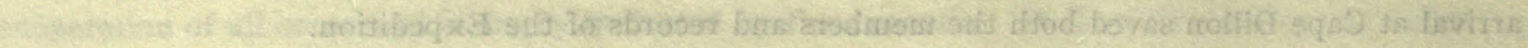

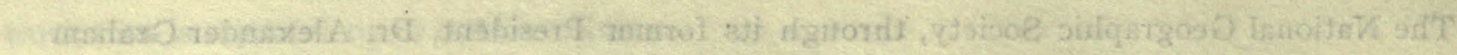

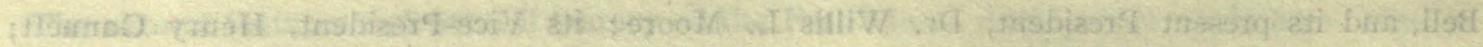

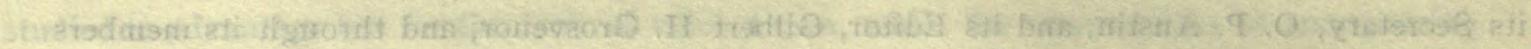

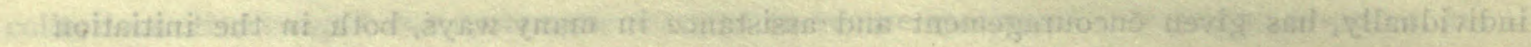

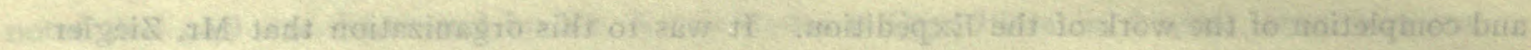

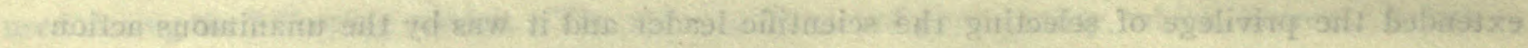

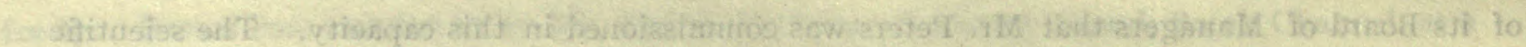

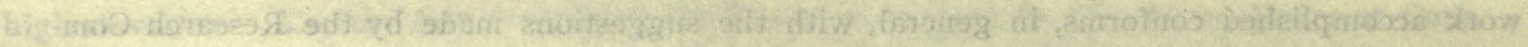

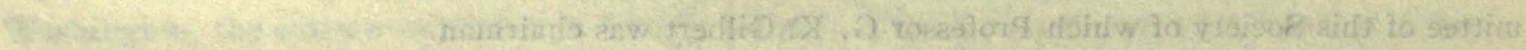

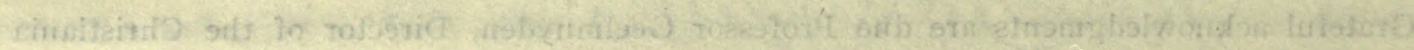

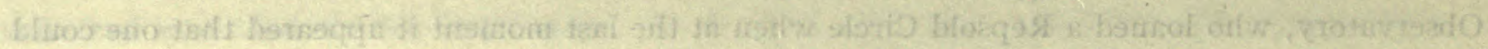

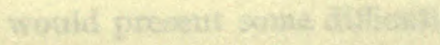

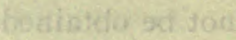

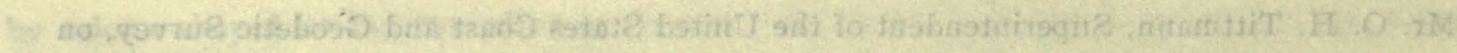

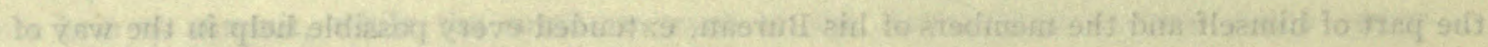

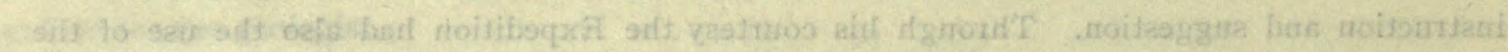

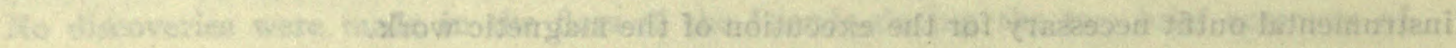

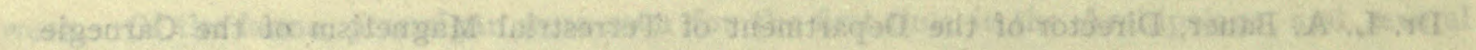

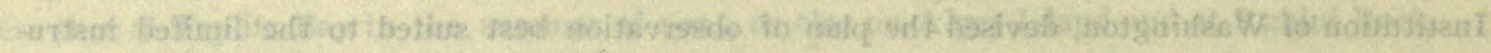

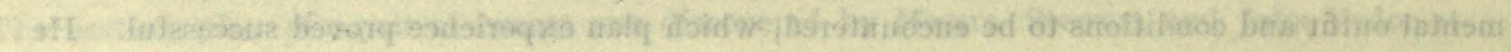

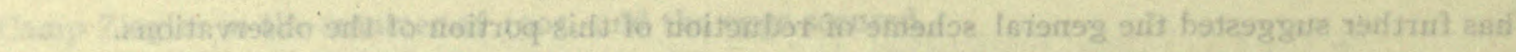

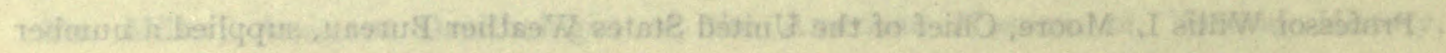

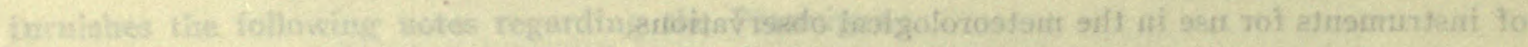

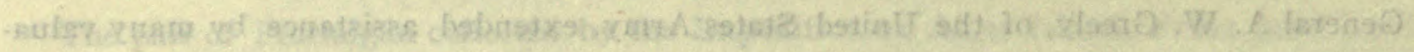

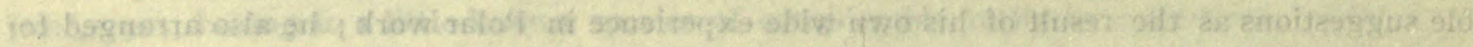

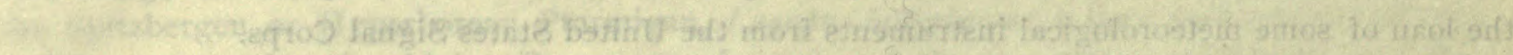

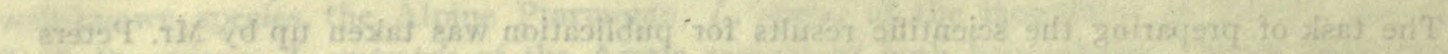
2init of

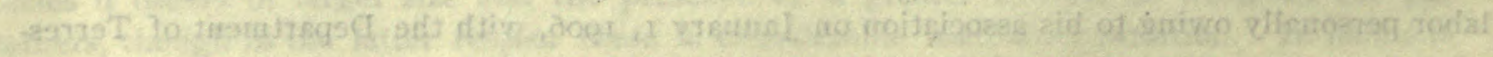

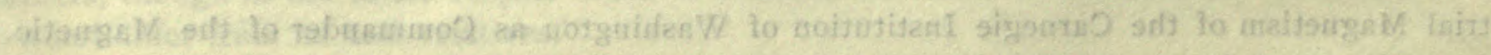

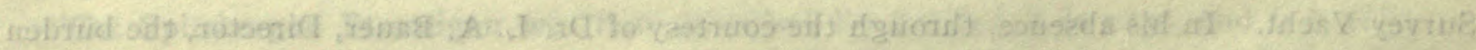

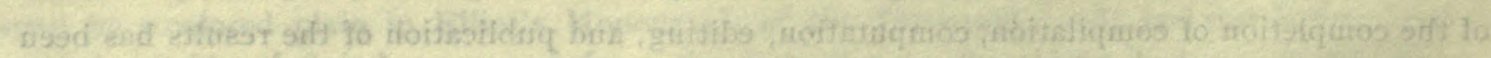

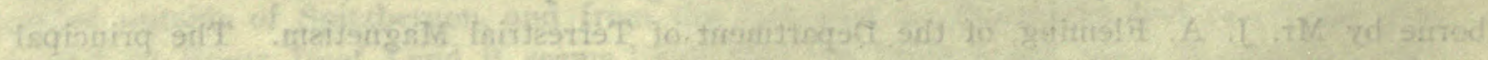

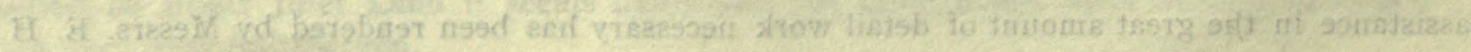

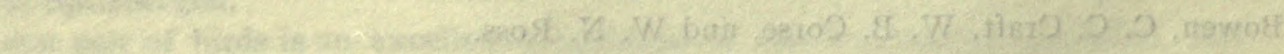

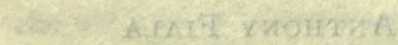

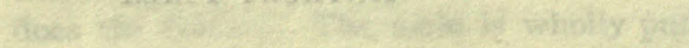

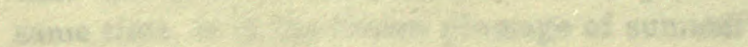

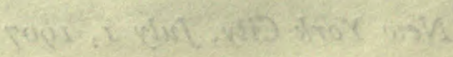


Page iii: Contents, Ist line, for "I" read "v".

Page iii : Contents, and line, for " $v$ " read "I".

Page 8 : I Ith last line, for " $\frac{C}{\sin \mu}$ " read " $\frac{C}{\sin u}$ ".

Page IO: 2nd last line of 2 nd last paragraph, take out comma after " $6765 \gamma$ ".

Page I 3 : last line of 2 nd paragraph, for " $3 \mathrm{~h} 52 \mathrm{~m} 37 \mathrm{~s}\left(5^{\circ} \circ 9^{\prime}\right) \mathrm{E}$ " read " $3 \mathrm{~h} 5 \mathrm{Im} 56 \mathrm{~s}\left(57^{\circ}\right.$ $\left.59^{\prime}\right) \mathrm{E} "$.

Page I16: tabulation of azimuths, for " 108 oo 53 " read " I80 $\infty 53$ ".

Page 297: station number 3 , for " 58 o9" read " 5759 ".

Page 303: 5th line, for "range of 32 , while" read "range of $32^{\prime}$, while".

Page 303 : 6th line, for "viz., 97. I" read "viz., 97.' 1 ".

Page 320: last line, for " 58 o9" read " 57 59".

Page 371 : illustration numbers, for " 4 ", "5", and "6", read " 5 ", "6", and " 4 ".

Page 371 : under illustration list, for " 478 " read " 477 ".

Page 375: longitude east of Greenwich, for " $57^{\circ} 56^{\prime \prime}$ " read " $57^{\circ} 58^{\prime \prime}$ ".

Page 391 : Ist line, for "attz" read "at Teplitz".

Page 392 : Ist line, omit "Tepli".

Page 392 : last line under columns Reading of Fahrenheit Thermometer, for " $-15.5|-15.2|$

$$
-23.8 \text { " read "-17.0|-15.5|-15.2". }
$$

Page 398 : last line under columns Reading of Fahrenheit Thermometer, for "-207.6|-285.6" read " $-276.0 \mid-207.6$ ".

Page 418 : 2nd last line, under column Mean of Extremes, for " +380.0 " read " +380.9 ".

Page 449: longitude east of Greenwich, for " $57^{\circ} 56^{\prime \prime}$ " read " $57^{\circ} 58^{\prime \prime "}$.

Page 477 : formula at head of tabulation, for " $\Delta p=B_{1} \sin \left(\theta-C_{1}\right)-B_{2} \sin \left(2 \theta-C_{2}\right)-B_{3}$ $\sin \left(3 \theta-C_{3}\right)$ " read " $د p=B_{1} \sin \left(\theta+C_{1}\right)+B_{2} \sin \left(2 \theta+C_{2}\right)+B_{3} \sin \left(3 \theta+C_{3}\right) "$.

Page 482 : last line of 2 nd last paragraph, for "figures 4 and 5 " read "figures 5 and 6 ".

Page 495: 6th last line, for " $57^{\circ} 56^{\prime}$ ( 3 b 5 Im $43 \mathrm{~s}$ )" read " $57^{\circ} 58^{\prime}$ ( $3 \mathrm{~h} 5 \mathrm{Im} 53 \mathrm{~s}$ )".

Page 543: longitude east of Greenwich, for " $57^{\circ} 56^{\prime \prime}$ " read " $57^{\circ} 58^{\prime \prime " . ~}$ 


\section{A TA T}

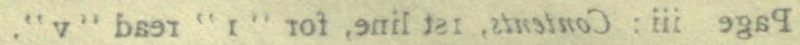

$$
\begin{aligned}
& \text { "I" bBgr " }
\end{aligned}
$$

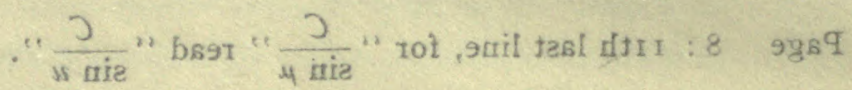

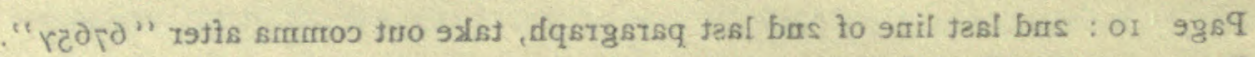

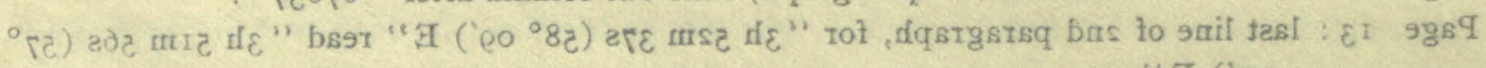

$$
\text { "अ ('e己 }
$$

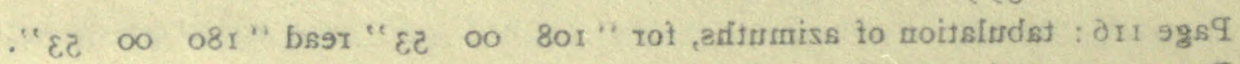

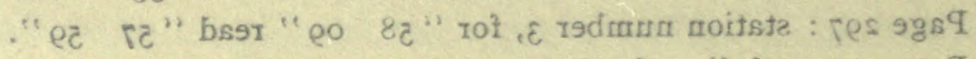

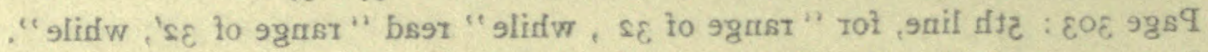

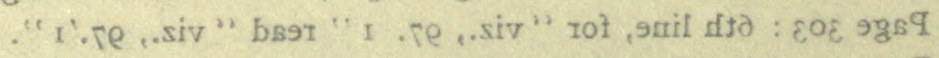

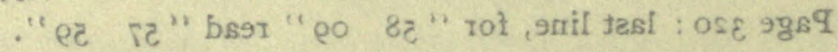

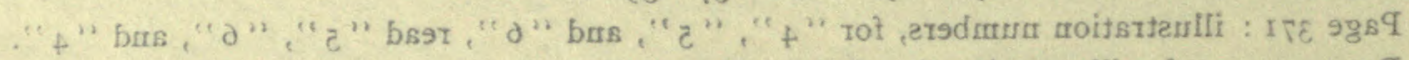

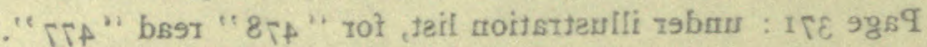

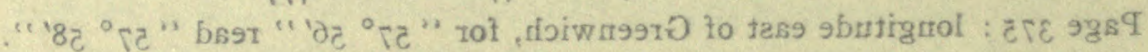
"stilqg'T ts "bs9t "stts" rot, gttil ter : ree 9gs

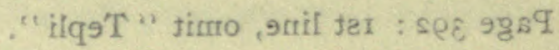

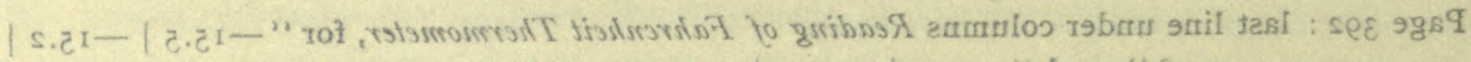

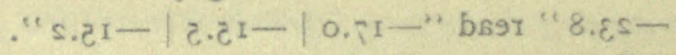

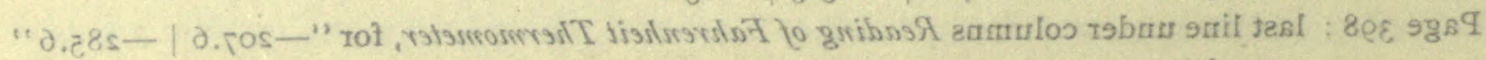

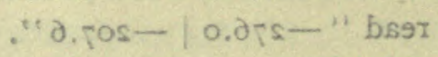

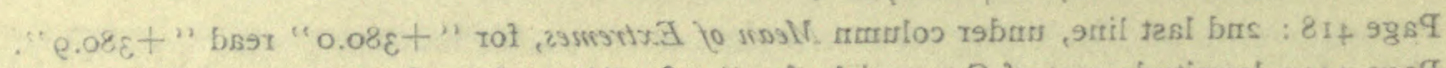
" "8己 "

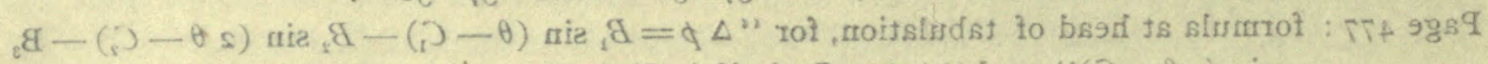

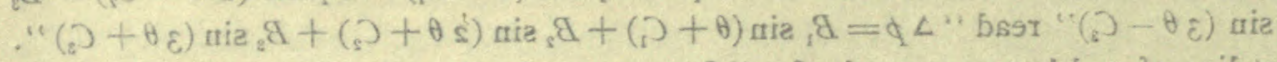

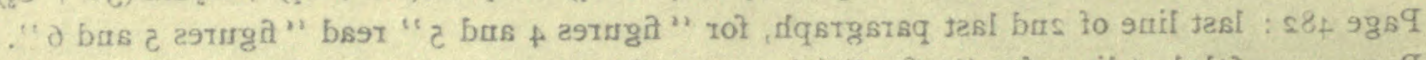

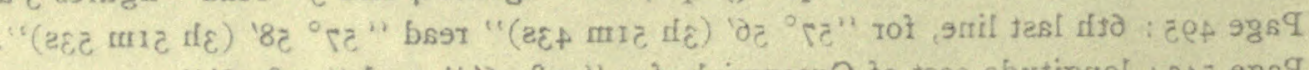

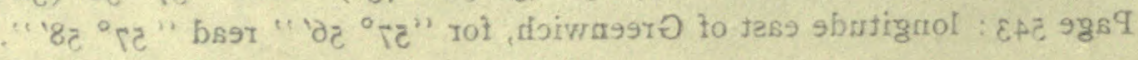




\section{SECTION A}

\section{MAGNETIC OBSERVATIONS \\ AND \\ REDUCTIONS}

BY

W. J. PETERS

In Charge of Scientific Work of the Expedition

AND

J. A. FLEMING

Department Terrestrial Magnetism, Carnegie Institution of Washington 


\title{
A nOITDва
}

\section{2ИOITAVЯAZGO JTIИDAM}

\author{
GWA \\ 2nolTouda9
}

\author{
Y8

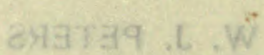 \\ QMA \\ DAmb.JA A. A
}

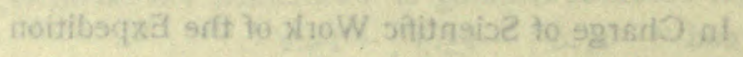

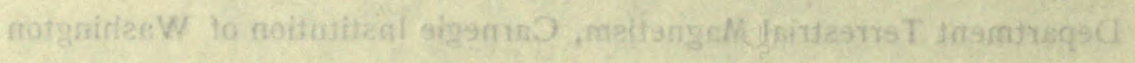




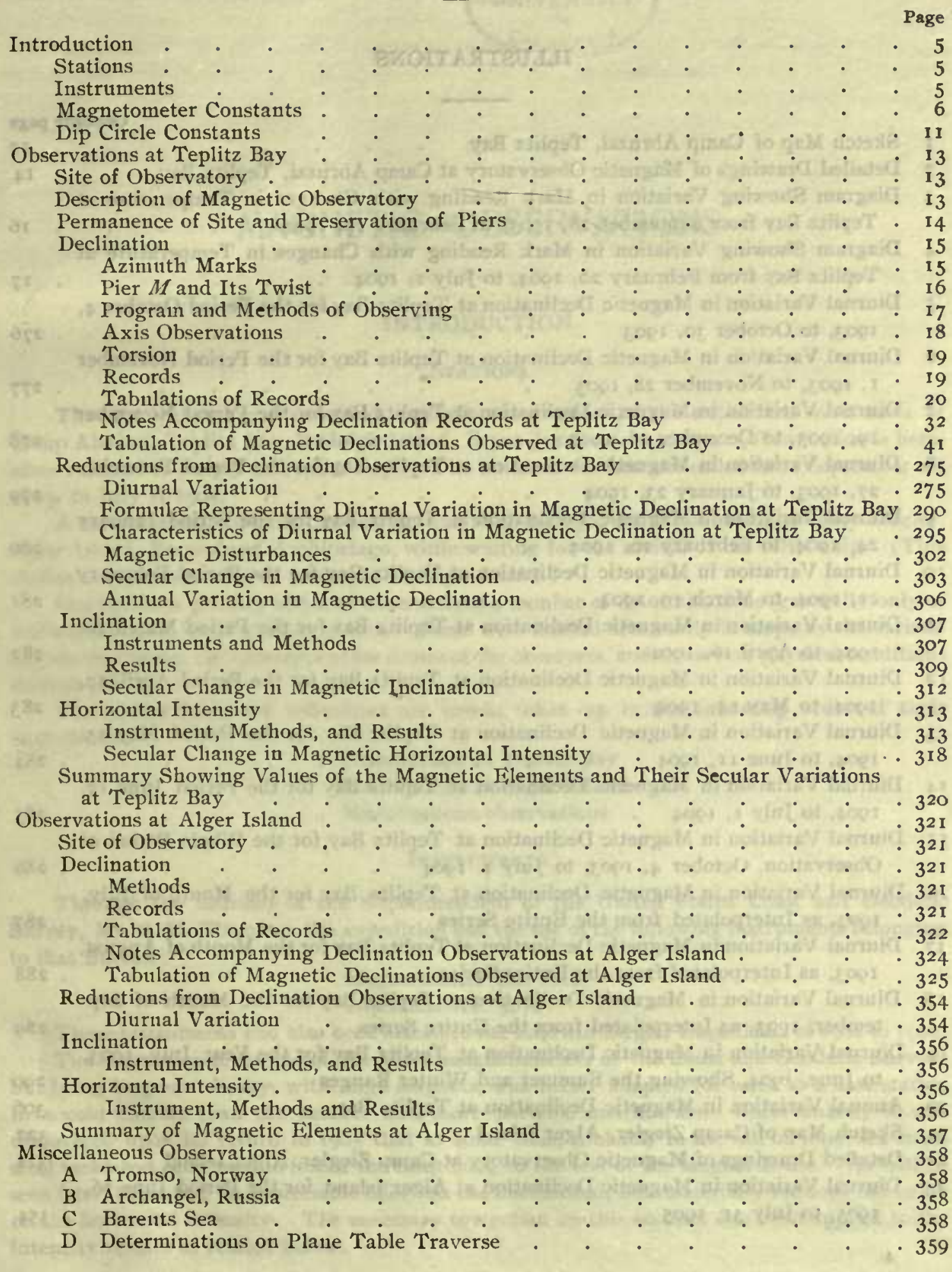




\section{ILLUSTRATIONS}

Sketch Map of Camp Abruzzi, Teplitz Bay

Diagram Showing Variation in Mark Reading with Changes in Temperature at Teplitz Bay from February 20, 1904, to July 1, 1904 . • • • • . 17

Dinrnal Variation in Magnetic Declination at Teplitz Bay for the Period October 4, 1903, to October 30,1903 . . . . . . . . . . 276

Diurnal Variation in Magnetic Declination at Teplitz. Bay for the Period November 1, 1903, to November 28,1903

Diurnal Variation in Magnetic Declination at Teplitz Bay for the Period November 29, 1903, to December 26, 1903 . . . . . . . . . . . 278

Dinrnal Variation in Magnetic Declination at Teplitz Bay for the Period December 27, 1903, to January 23, 1994 . . . . . . . . 279

Diurnal Variation in Magnetic Declination at Teplitz Bay for the Period January 24, I904, to February 20, 1904 . . . . . . . 280

Diurnal Variation in Magnetic Declination at Teplitz Bay for the Period February 21, 1904, to March 19, 1904 . . . . . . 281

Diurnal Variation in Magnetic Declination at Teplitz. Bay for the Period March 20, 1904, to April I6, 1904 . . . . . . . . . . . 282

Diurnal Variation in Magnetic Declination at Teplitz Bay for the Period April i7, I904, to May I4, I904

Dinrnal Variation in Magnetic Declination at Teplitz Bay for the Period May I5, I904, to Julle II, I904

Diurnal Variation in Magnetic Declination at Teplitz Bay for the Period June 12, I904, to July I, I904

Diumal Variation in Magnetic Declination at Teplitz Bay for the Whole Period of Observation, October 4, 1903, to July I, I904 . . . . . . . 286

Diurnal Variation in Magnetic Declination at Teplitz Bay for the Month of July, 1903, as Interpolated from the Entire Series

Diurnal Variation in Magnetic Declination at Teplitz Bay for the Month of August, I 903, as Interpolated from the Entire Series

Diurnal Variation in Magnetic Declination at Teplitz Bay for the Month of September, 1903, as Interpolated from the Entire Series

Diurnal Variation in Magnetic Declination at Teplitz Bay for the Year July, r 903 , to June, 1904, Showing the Summer and Winter Ranges Annual Variation in Magnetic Declination at Teplitz Bay a . . . . . . 306 Sketch Map of Camp Ziegler, Alger Island . . . . . . . . . . . 322 Detailed Drawings of Magnetic Observatory at Camp Ziegler, Alger Island . $\quad 324$ Diurnal Variation in Magnetic Declination at Alger Island for the Period June 26, 1905, to July 31, 1905 . 


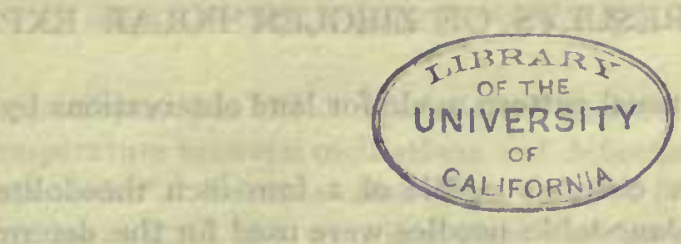

\section{MAGNETIC OBSERVATIONS}

\section{INTRODUCTION}

\section{STATIONS}

The principal and most extended magnetic observations of the expedition were made at Camp Abruzzi, Teplitz Bay, Rudolph Island, Franz Josef Archipelago. (This station is hereafter referred to simply as Teplitz Bay.) The observations here extended from September 28, 1903 , to July I, 1904 .

A second series, covering the period June 26 to July 30, 1905, was made at Camp Ziegler, Alger Island, Franz Josef Archipelago, while waiting for the arrival of the relief ship. (This station is hereafter referred to simply as Alger Island.)

Besides these primary series of observations a number of determinations (generally only of declination) were made at other points. These were limited necessarily in scope by the various exigencies arising, the numerous other duties of the observers, and the severe physical conditions encountered.

The observations and reductions are herein taken up in the following order of their importance :

\section{Observations at Teplitz Bay \\ Observations at Alger Island \\ Miscellaneous observations}

\section{INSTRUMENTS}

Through the courtesy of the Superintendent of the United States Coast and Geodetic Survey, Mr. O. H. Tittmann, the expedition had the use of the following instruments belonging to that Bureau :

\section{Magnetometer No. IIII}

Dip circle No. 5676

These instruments had also been loaned to the Baldwin-Ziegler Expedition of 1901 to 1902.

The magnetometer is one of the older magnetometers of the Coast and Geodetic Survey, but being large and heavy, with large magnets, was better adapted for work at a base station in a cold climate than the smaller and more portable instruments. Unfortunately it was discovered upon its return in 1905 that at some time, probably in the fall of 1899 , six sinall steel tacks had been used to fasten the cloth hood to the end of the magnet-house. As will be seen later, these had no appreciable effect on the declination, but materially reduced the value of the horizontal intensity. The necessary correction on this account has been applied to the intensity results as related below. 
The dip circle is of the usual pattern made for land observations by L. Casella of London, England.

In addition to these, the compass needle of a four-inch theodolite by Berger \& Sons of Boston, U. S. A., and the plane-table needles were used for the determination of declination at several random stations.

The following compilation of constants for the primary magnetic outfit has been prepared by Mr. D. L. Hazard, of the United States Coast and Geodetic Survey, under the direction of the Chief of the Division of Terrestrial Magnetism of that Bureau.

\section{MAgNETONETER CONSTANTS}

A new brass deflection bar was supplied in April, 1903, to take the place of the old one not returned by the first Ziegler Expedition. It is a single straight bar 88 centimeters long. The deflection distances, as determined by the United States Bureau of Standards, are 30.019 centimeters and 40.025 centimeters at $28^{\circ} .75$ Centigrade.

The moment of inertix of intensity magnet No. 4 and stirrup has been determined several times by Mr. W. J. Peters, as follows :

\begin{tabular}{|c|c|c|c|c|}
\hline Place & Date & $\begin{array}{l}\text { No. of } \\
\text { sets }\end{array}$ & $\log K_{30}$ & Weight \\
\hline Washington, D. C. . & May, 1903 & I I & $2.45^{8} 34$ & 2 \\
\hline Cheltenham, Md. . & Sept., I 905 & II & $2.45^{8 I 2}$ & I \\
\hline Cheltenham, Md. . & Oct., I905 & 10 & 2.45919 & 2 \\
\hline Value adopted, welg & hted mean & & $2.45^{864}$ & \\
\hline
\end{tabular}

The temperature coefficient of the intensity magnet has been determined by special obser va tions at various times, as follows:

\begin{tabular}{|c|c|c|c|c|c|}
\hline 6 and 4 & Date & Observer & $\begin{array}{l}\text { Mean temp. } \\
\text { Cent. }\end{array}$ & 9 & $\begin{array}{l}\text { No. of } \\
\text { sets }\end{array}$ \\
\hline $\begin{array}{l}\text { Philadelphia } \\
\text { San Francisco } \\
\text { District of Columbia }\end{array}$ & $\begin{array}{l}\text { I } 848 \text {, Dec. } \\
\text { I } 884 \text {, Oct. } \\
\text { I90o, May }\end{array}$ & $\begin{array}{l}\text { J. S. Ruth . } \\
\text { R. A. Marr : } \\
\text { J. B. Baylor : }\end{array}$ & $\begin{array}{l}20.0 \\
20.1 \\
15.0\end{array}$ & $\begin{array}{r}.000252 \\
230 \\
192\end{array}$ & $\begin{array}{l}4 \\
6 \\
2 \\
7\end{array}$ \\
\hline
\end{tabular}

From regular intensity observations values have been derived as follows :

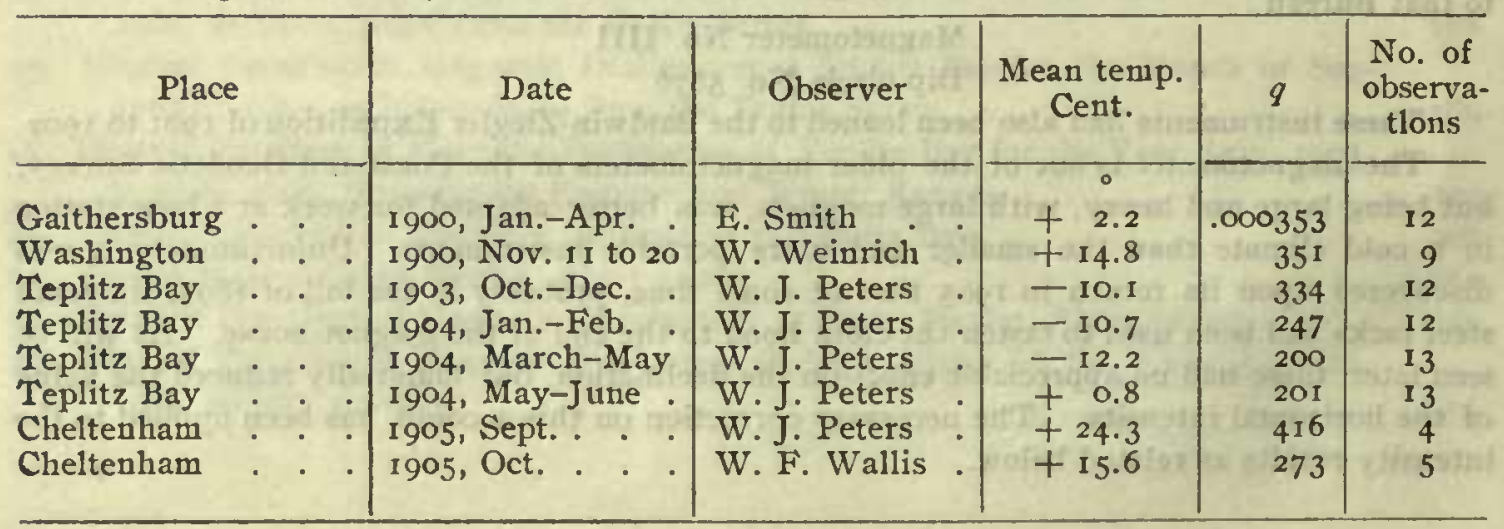


The value $q=0.000192$ was used in the computation of horizontal intensity at Teplitz Bay. Only the difference in temperature between oscillations and deflections is involved in this computation, and it rarely exceeded $2^{\circ}$. For this value of $q$ a value of $\left(t^{\prime}-t\right)$ of $2^{\circ}$ would affect the value of $H$ only I I $/ 4 \gamma$ (.0000I25 C. G. S.), so it is evident that no revision of the computation is required. For the reduction of the values of the magnetic moment to the same temperature in order to obtain an estimate of the accuracy of the observations, a value of $q=0.00030$ was adopted as the best value to be obtained from the above varying values. It is probable that $q$ varies with the temperature, increasing as the temperature increases, but the observations are not sufficiently accurate to determine the relation.

The induction coefficient has been determined as follows:

\begin{tabular}{|c|c|c|c|c|}
\hline Date & Observer & ins of & $\begin{array}{l}\text { No. of } \\
\text { sets }\end{array}$ & $\mu=m h$ \\
\hline \multirow[t]{2}{*}{$\begin{array}{l}\text { April, } \\
\text { Sept., }\end{array}$} & $\begin{array}{l}\text { J. B. Baylor } \\
\text { W. J. Peters }\end{array}$ & & $\begin{array}{l}8 \\
4\end{array}$ & $\begin{array}{l}5 \cdot 39 \\
5.87\end{array}$ \\
\hline & value adopted & $\operatorname{sins} 0$ & .. & 5.63 \\
\hline
\end{tabular}

The first distribution coefficient, $P$, has been determined from deflections at two distances as follows :

\begin{tabular}{|c|c|c|c|c|c|}
\hline Place & Dafe Date & sot- & $\begin{array}{l}\text { No. of } \\
\text { sets }\end{array}$ & $P$ & Weight \\
\hline $\begin{array}{l}\text { Various stations } \\
\text { Teplitz Bay } \\
\text { Cheltenham } \\
\text { Cheltenham }\end{array}$ & $\begin{array}{l}\text { Nov., 1899-Nov., } \\
\text { 1903-1904 } \\
\text { Sept., } 1905: \\
\text { Oct., } 1905\end{array}$ & $\begin{array}{l}1900 \\
\vdots \vdots\end{array}$ & $\begin{array}{r}33 \\
54 \\
6 \\
7\end{array}$ & $\begin{array}{r}-2.30 \\
0.00 \\
-1.49 \\
-2.08\end{array}$ & \multirow{2}{*}{ 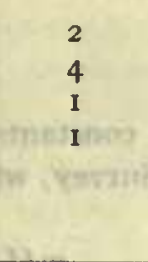 } \\
\hline Mean value & adopted $\cdot \cdot$ & & & -1.0 & \\
\hline
\end{tabular}

The scale value of long magnet has been determined at various times, the recent values being :

\begin{tabular}{|c|c|c|c|}
\hline Place & Date & Observer & Scale value \\
\hline $\begin{array}{l}\text { Gaithersburg, Md. } \\
\text { Washington, D. C. } \\
\text { Teplitz Bay, . } \\
\text { Cheltenham, Md. }\end{array}$ & $\begin{array}{l}\text { 1899, Nov. } 18 \\
\text { 1900, May } 7 \\
1903-1904 . \\
\text { 1905, Sept. } 21\end{array}$ & $\begin{array}{l}\text { E. Smith. } \\
\text { J. B. Baylor : } \\
\text { W. J. Peters : } \\
\text { W. J. Peters. }\end{array}$ & $\begin{array}{l}1 \\
1.56 \\
1.54 \\
1.60 \\
1.56 \\
1.5\end{array}$ \\
\hline \multicolumn{3}{|c|}{ Mean value adopted....} & I.57 \\
\hline
\end{tabular}

The constants adopted for magnet No. 4 of magnetometer No. IIII for the reduction of the Teplitz Bay observations are as follows : 
Corrected distances on new brass deflection bar at $0^{\circ}$ Centigrade :

$\begin{array}{cccc}r & \log r & \log 1 / 2 r^{3} & \log C \\ 30.003 \mathrm{~cm} . & 1.47716 & 4.13045 & \overline{5} .86889 \\ 40.003 \mathrm{~cm} . & 1.60209 & 4.50524 & 5.49441\end{array}$

For an increase in temperature of $1^{\circ}$ Centigrade $\log C$ decreases 0.000025 One division of scale $=\mathrm{I}^{\prime} \cdot 57$

Temperature coefficient : $q=0.00030$ for $1^{\circ}$ Centigrade

Induction coefficient : $\mu=5.63$;

$$
\text { When } \begin{array}{rlrl}
r & =30 \mathrm{~cm} . \quad \log \left(1+\frac{2 \mu}{r^{3}}\right) & =0.00018 \\
& =40 \mathrm{~cm} . & & =0.00008
\end{array}
$$

Distribution coefficient : $P=-1 . \infty$;

$$
\text { When } \begin{array}{rlrl}
r & =30 \mathrm{~cm} . \quad \log \left(1-\frac{P}{r^{2}}\right) & =0.00048 \\
& =40 \mathrm{~cm} . & & =0.00027
\end{array}
$$

Moment of inertia :

$\begin{array}{rr}\text { Temp. Cent. } & \log \pi^{2} K \\ -20^{\circ} & 3.45^{2} 5^{2} \\ -10 & 262 \\ 0 & 273 \\ +10 & 283 \\ +20 & 294\end{array}$

These constants are adapted for the methods of computation in use by the Coast and Geodetic Survey, where the following formulæ are used in computing horizontal intensity :

$$
\begin{aligned}
& m H=\frac{\pi^{2} K}{T^{2}} ; \quad T^{2}=T^{\prime 2}\left[1+\frac{h}{f}\right]\left[\mathrm{I}-\left(t^{\prime}-t\right) q\right]\left[\mathrm{I}+\mu \frac{H}{m}\right] \\
& \frac{H}{m}=\frac{C}{\sin \mu} ; \quad C=\frac{2}{r^{\prime \prime}\left(1-\frac{P}{r^{2}}\right)\left(\mathrm{I}+\frac{2 \mu}{r^{2}}\right)} \quad \text { and } \\
& \log H=1 / 2\left(\log \frac{\dot{H}}{m}+\log m H\right) .
\end{aligned}
$$

In these formulæ,

$H=$ horizontal intensity

$m=$ magnetic moment of magnet

$T^{\prime}=$ observed time of one oscillation corrected for rate of chronometer

$h=$ angle through which magnet is turned by turning the torsion head through an angle $f$

$t^{\prime}=$ temperature of oscillations

$t=$ temperature of deflections

$u=$ deflection angle 
The observations are arranged so that two sets of deflections come between two sets of oscillations, and $t$ and $t^{\prime}$ do not usually differ very much. Consequently an erroneous value of $q$ has little effect on the resulting value of horizontal intensity.

To determine the effect of the stecl tacks on declinations as observed we have the following declination observations at the Cheltenham Magnetic Observatory:

(a) Before removal of tacks, by W. J. Peters :

\begin{tabular}{cc}
$\begin{array}{c}\text { Date } \\
\text { 1905 }\end{array}$ & $\begin{array}{c}\text { Declination } \\
\text { west }\end{array}$ \\
September 19 & \multicolumn{1}{c}{,} \\
September 20 & 518.6 \\
September 20 & 20.0 \\
September 21 & 19.2 \\
September 21 & 18.7 \\
September 22 & 18.9 \\
September 22 & 19.8 \\
Mear & 17.2 \\
\cline { 2 - 2 } & 518.9
\end{tabular}

(b) After removal of tacks, by W. F. Wallis :

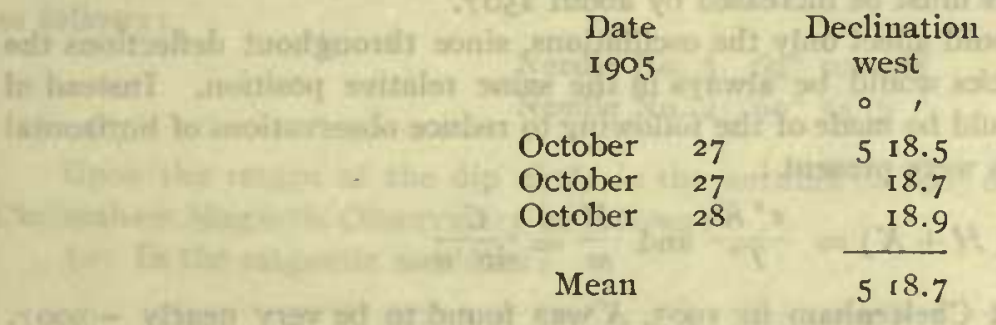

The results by the Observatory magnetometer No. 26 were :

$\begin{array}{cc}\begin{array}{l}\text { Date } \\ 1905\end{array} & \begin{array}{c}\text { Declination } \\ \text { west }\end{array} \\ & 0 \\ \text { September } & 518.7 \\ \text { October } & 518.9\end{array}$

All the above observatious lave been corrected for diurual variation. They show that for declinations determined with Magnetometer No. IIII no correction is required.

To determine the effect of the steel tacks in horizontal intensity observations we have the following results at the Cheltenham Magnetic Observatory:

(a) Before removal of tacks, by W. J. Peters:

$\begin{array}{cc}\text { Date } & \begin{array}{c}\text { Horizontal } \\ \text { I } 905\end{array} \\ \text { intensity }\end{array}$


(b) After removal of tacks, by W. F. Wallis :

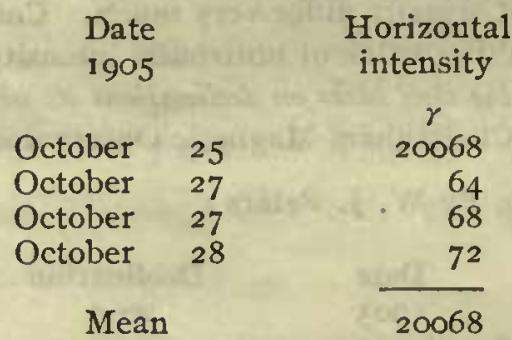

Observations by Magnetometer No. 26 gave the following results :

$\begin{array}{cc}\begin{array}{c}\text { Date } \\ 1905\end{array} & \begin{array}{c}\text { Horizontal } \\ \text { intensity }\end{array} \\ \begin{array}{c}\text { September } \\ \text { October }\end{array} & 20057 \\ \text { Mean } & 65 \\ \end{array}$

Consequently values of horizontal intensity determined with Magnetometer No. IIII before the removal of the tacks must be increased by about $250 \%$.

The presence of tacks would affect only the oscillations, since throughout deflections the suspended magnet and the tacks would be always in the same relative position. Instead of the ordinary formulæ, use shonld be made of the following to reduce observations of horizontal intensity made when the tacks were present :

$$
m(H+X)=\frac{\pi^{2} K}{T^{2}} \text { and } \frac{H}{m}=\frac{C}{\sin u}
$$

From the observations at Cheltenham in $1905, X$ was found to be very nearly $-500 \gamma$. The application of this correction to the Teplitz Bay and Alger Island observations has been made as follows: The combination of the above equations in the usual way to eliminate $m$ gives the value of $V \overline{H(H+X)}$, from which $H$ may be derived when $X$ is known. At Teplitz Bay the average value of $\sqrt{H(H+X)}$ was $6510 \gamma$, from which $H=6765 \gamma$, for $X=-500 \gamma$, or $H=\sqrt{H(H+X)}+255 \gamma$. The same correction applies for Alger Island.

The last column in the following table gives the mean value of $\log m$ reduced to $20^{\circ}$ Centigrade for varions gronps of observations and furnishes the means for comparing the magnetic moment of the intensity magnet at Teplitz Bay and Alger Island and in the United States :

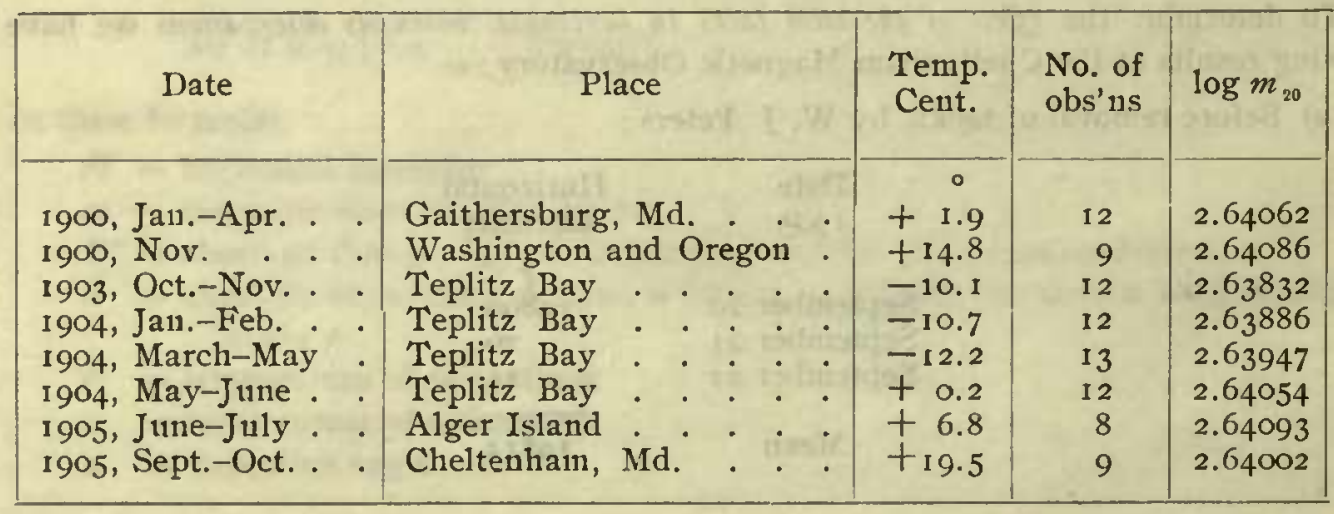


Observations in the United States before and after the instrument was used at Teplitz Bay show very little change in the magnetic moment of the intensity magnet. The observations at Teplitz Bay, however, indicate a gradual increase from beginning to end of the series. The cause of this change is not clear, as magnets are usually found to lose their strength with age. It is clearly not due to an erroneous temperature coefficient, as the mean temperatures of three of the groups are nearly the same. An error of .00087 in $\log m$ corresponds to an error of one part in 500 in $H$, which at Teplitz Bay would be only $13 \gamma$.

\section{Drp CrRcha Constants}

So little time was available between the return of the instrument by the first Ziegler Expeditiou and its reissue to the second, that no extended comparisons could be attempted. Observations were made at the Coast Survey Office in Washington as follows on May $1 \mathrm{r}$, I903:

Needle No. $369^{\circ} 55^{\prime} .8 \mathrm{~N}$

Needle No. $4 \quad 69^{\circ} 56^{\prime} .3$

The normal dip for the station was $69^{\circ} 56^{\prime} .0 \mathrm{~N}$. Observations were also made in two planes making an angle of $60^{\circ}$ with the magnetic meridian, so that the needles rested upon nearly the same parts of the pivots as at Teplitz Bay. These observatious of May 23, 1903, resulted as follows :

Needle No. $369^{\circ} 50^{\prime} .2 \mathrm{~N}$

Needle No. $4 \quad 69^{\circ} 53^{\prime} .6$

Upon the return of the dip circle in the autumu of 1905 observations were made at the Cheltenham Magnetic Observatory as follows :

(a) In the magnetic meridian:

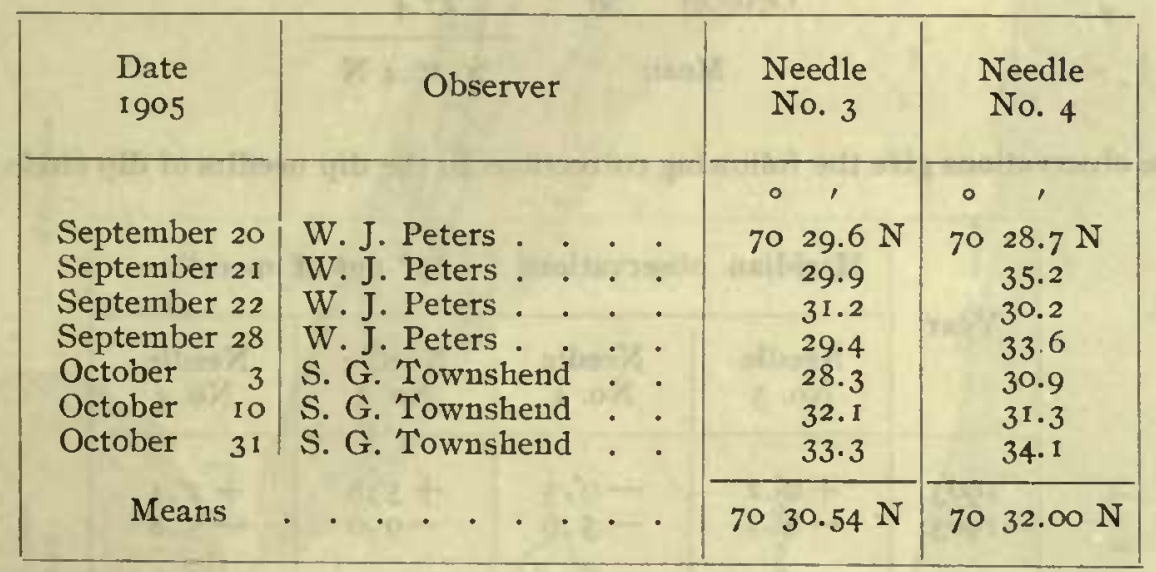


(b) In planes making an angle of $60^{\circ}$ with the magnetic meridian :

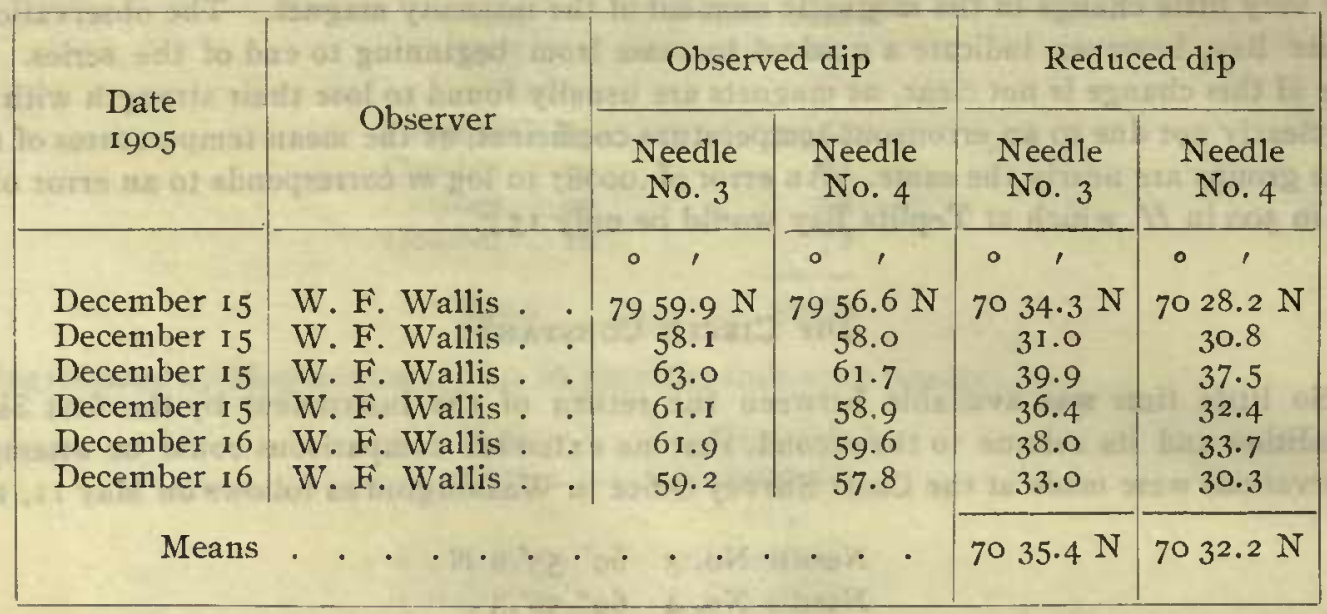

The normal dip for this station is $70^{\circ} 26^{\prime} .4 \mathrm{~N}$, as derived from the following observations with earth inductor:

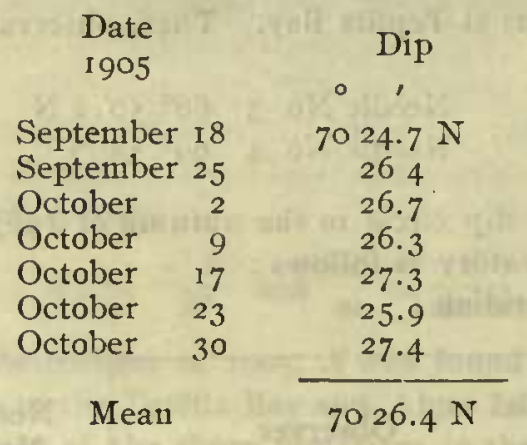

These observations give the following corrections to the dip needles of dip circle No. 5676 :

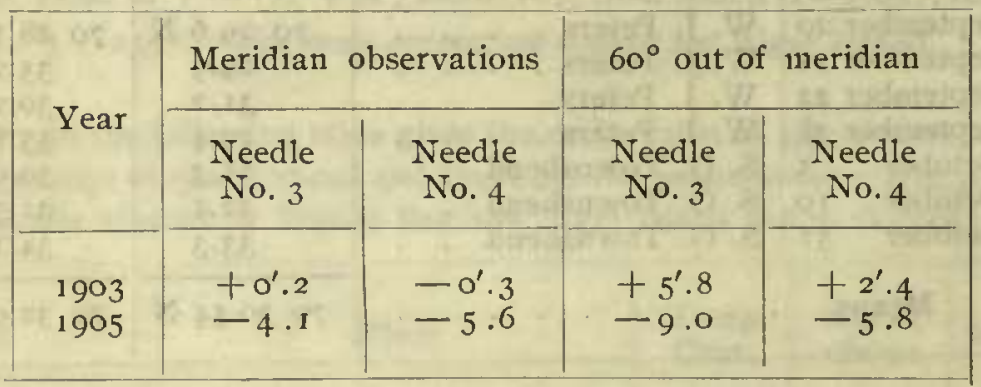

In view of the change indicated between I 903 and 1905 and the probability that the correction required becomes less as the dip increases, it is deened best to apply no correction to the dip observations. 


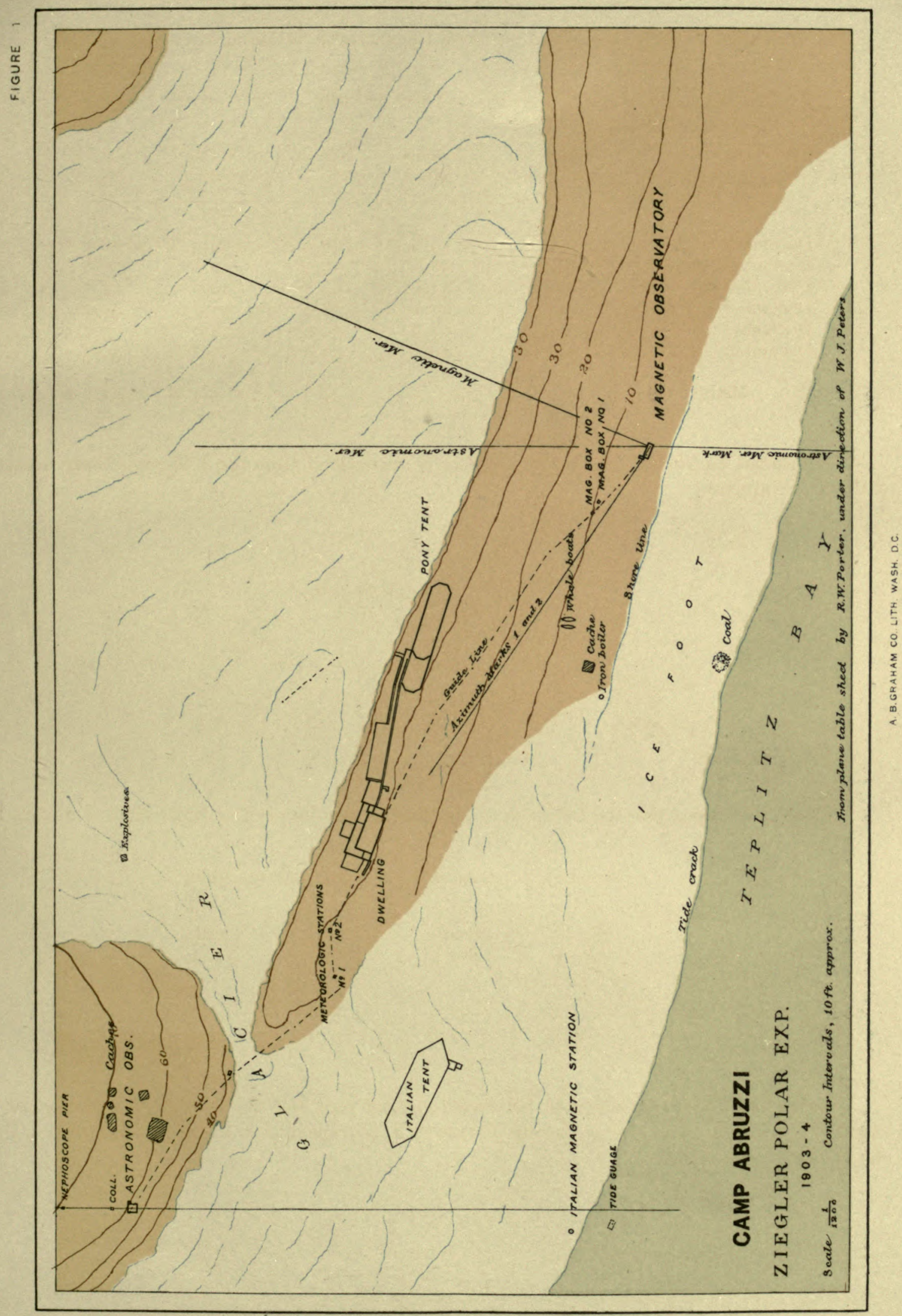





\section{OBSERVATIONS A'T TEPLITZ BAY}

\section{SITE, OF OBSERVATORY}

The magnetic station occupied by the Italian Expedition at Teplitz Bay was examined with a view to reoccupying it, but at the time it was found inpossible to accurately identify the point ; besides it was on the ice-foot, stubject to overflow during summer thaws and to possible movement. Under the circumstances it seemed advisable to select another location which could be more conveniently recovered in the future. It was also intended to make observations in a tent at a point as near to the Italian station as conld be fixed from data appearing in their publications, but owing to the lateness of the season and the hurried debarkation, which required the help of all, this plan could not be carried ont during the period of daylight in 1903 and was, therefore, postponed to the following spring.

Six or seven points were examined. All of these, which were fairly well distributed over the small area free from ice, gave indications of local magnetic attraction, varying from $40^{\prime}$ to $2^{\circ}$. The exposed surface is basalt and contains considerable disseminated magnetite in minute grains. The point finally selected was the one which appeared to be the least affected and at the same time reasonably free from the destructive effect of ice. It is on the shore of Teplitz Bay about 6 feet above sea level and 6 meters from the water edge, in latitude $81^{\circ} 47^{\prime} 30^{\prime \prime} \mathrm{N}$ and longitude $3 \mathrm{~h} 52 \mathrm{~m} 37 \mathrm{~s}\left(58^{\circ} \mathrm{og}\right)$ E.

\section{DESCRIPTION OF MAGNETIC OBSERVATORY}

The observatory is 4.56 meters long, 1.82 meter wide, 1.82 meter to the eaves, and 2.45 meters to the ridge. Its length is parallel to the magnetic prime vertical and allows a distance of 2.1 meters between the magnetometer and dip circle piers.

The structure consists of a framework of wood. The floor, roof, and east and west sides are boarded. The north and south walls are each made of two layers of canvas, the outer layer extending over the board roof. The joints and fastenings are made by dovetailing, nortising, or by large brass screws. The canvas is held by copper tacks and brass nails, or by wooden cleats held in place by brass screws. Snow was banked against the walls up to the eaves and subsequent drifts finally buried the observatory in an even field. A shelf extends north and south across the middle of the room and serves both for a brace and for a table. Two lights were used on this shelf-a brass bull's-eye lantern and a copper kerosene lamp. Their positions are shown in the plan which is drawn to scale. The bull's-eye lantern was used for illuminating the scale and rested on a small, wooden tripod stand with an adjustable wooden footscrew. Two large windows-one in the east wall and one in the west walladmitted light on the return of day. The iron nails nsed in the window frames were all removed.

A copper stove with copper stack was installed with a view to heating the room, if in extreme cold weather it became necessary to put in new fibers or do any other work requiring delicate manipulation. It was used but twice. The lamps and stove were each brought within 30 centimeters of the nagnetometer, revolved and carried around the magnetometer without producing any noticeable effect.

The original carrying magnetometer case was placed under the east window after adjustingpins, screw-drivers, and other magnetic articles and material were removed. Magnet No. 4, used in declination observations, was stored in this case when not in use.

There were two brass hooks on the east wall for garments and two on the south wall to hold the deflection bar in its case. 
The timepiece was a watch* regulated approximately to local time beginning at midnight. It hung on a small brass hook screwed into the middle stud of the south wall.

The piers are of pine, 35 centimeters in diameter. That for the magnetometer is 2.3 meters long and is sunk 0.8 meter in coarse, frozen gravel, leaving 1.25 meter above the floor. The pier for the dip circle is 2.15 meters long, is 0.8 meter under the surface, and 1.10 meter above the floor. These piers are marked $M$ and $D$ respectively on the west side near the top.

There is a vestibule 0.75 meter by 1.3 meter by 1.25 meter, with doors opening into the observatory and ontwards into the open air.

In cold weather considerable trouble was experienced from the collection of ice on the mirror, eyepiece, reading glass, and circle. The roof and walls of the observatory became studded with small ice crystals, the incessant fall of which covered the iustrument and necessitated cleaning every day or two.

During the construction of the observatory a memorandum was kept of every iron tool used and its removal was assured before magnetic work began. This precaution was necessary because of the frequent snowfalls. The building is absolutely non-magnetic and the only iron near by was the small adjusting pin, used to reduce the amplitude of the oscillations after the nagnet was disturbed. This pin was placed vertically on pier $D$ beyond the sphere of influence and has since been brought away.

In the spring of I 904 a bear attempted to break into the observatory while Mr. John Vedoe was observing. He failed to drive it away by noise or voice and was liberated from the embarrassing situation by finally arousing the pack dogs, whose barkings brought aid from the house. As a safeguard against recurrence of such visits a revolver was afterward placed at the far end of the sight shaft 4.5 meters distant from the magnetometer. On March 1, I904, this was taken away and instead a rifle was left outside standing against maguet box No. I.

\section{Permanence of Site and Presegvation of Piers}

Judging from the condition in which the living quarters of the Italian Expedition were found, it is to be concluded that the observatory with its piers may stand for a long time, but snow will collect about the building and hold a small supply of water coming from the summer thaws until it freezes again. Ice, accumulating annually in this manner, may gradually rise in the hut to the top of the piers. The distance and azimuth of the astronomic brick pier will, however, furnish a means of recovering the precise point, should it be buried in a future field of ice. The astronomic observatory is so situated as to be swept clear of snow by the strong winds, unless a decided and permanent change in the prevailing direction should occur.

On the cessation of observations (July I, r904) the observatory was left undisturbed with every article in place excepting the instruments.

*In the tabulations of results the times by watch are listed as "Chronometer time." 


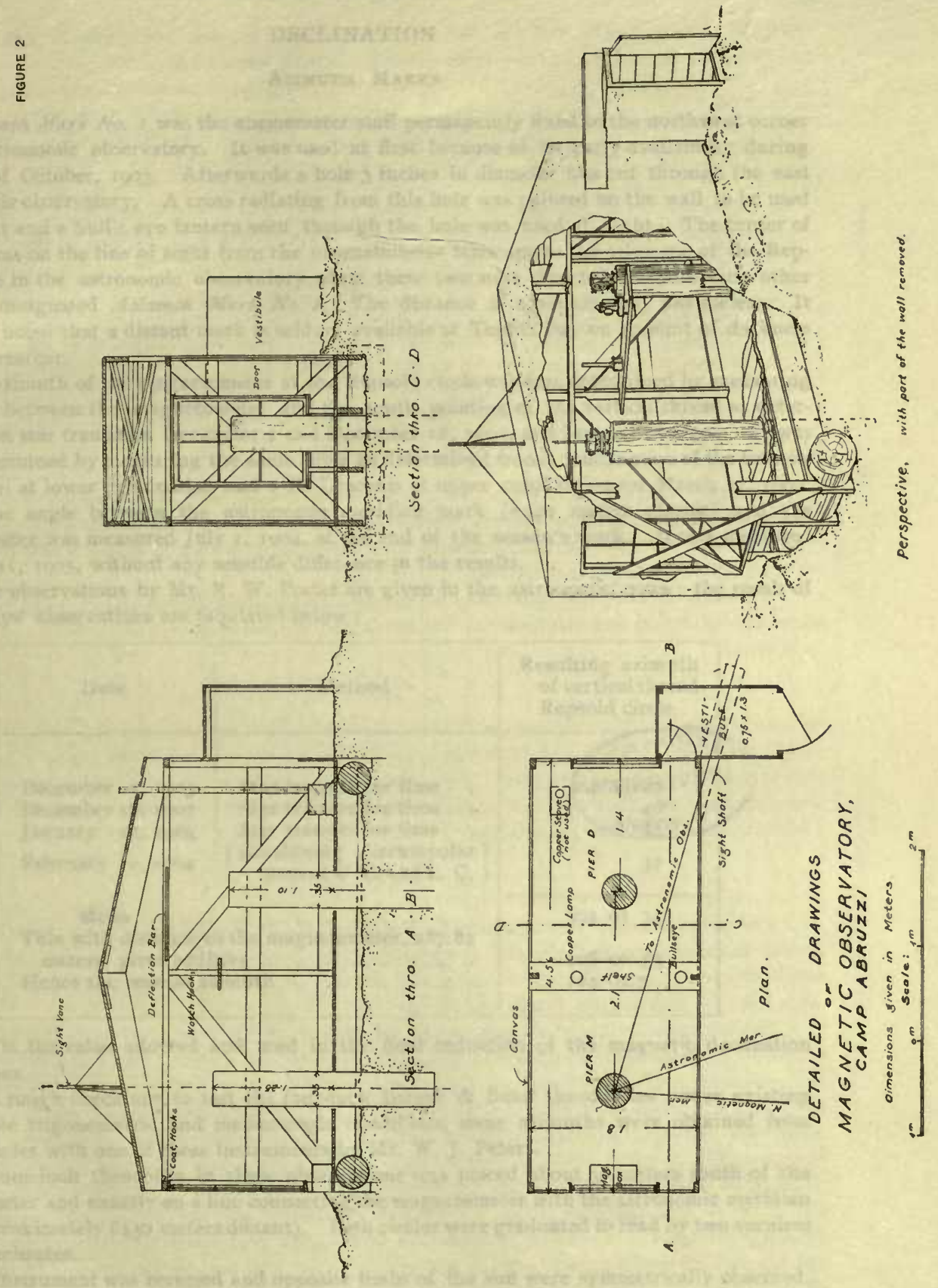




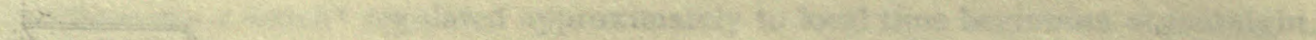

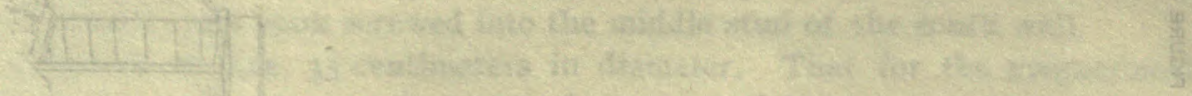
\begin{tabular}{l|l} 
(3) \\
if \\
in
\end{tabular}

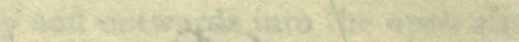

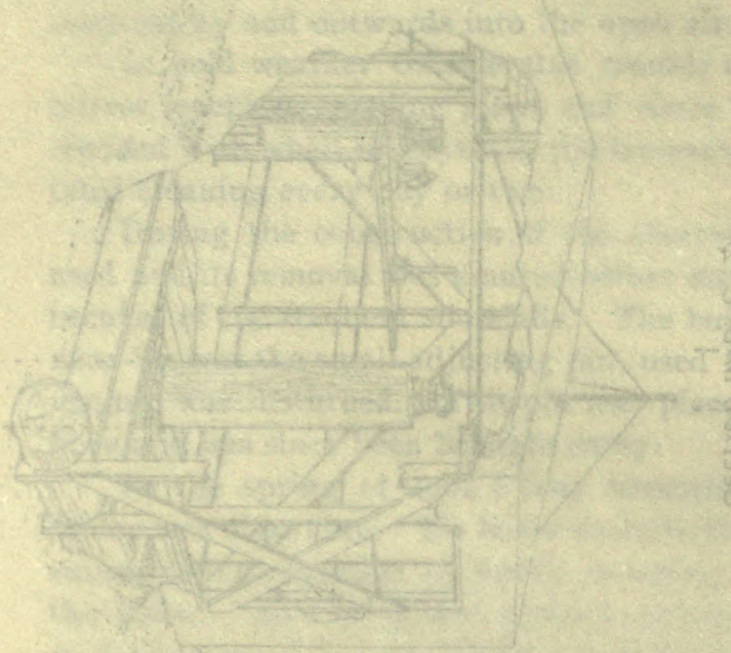

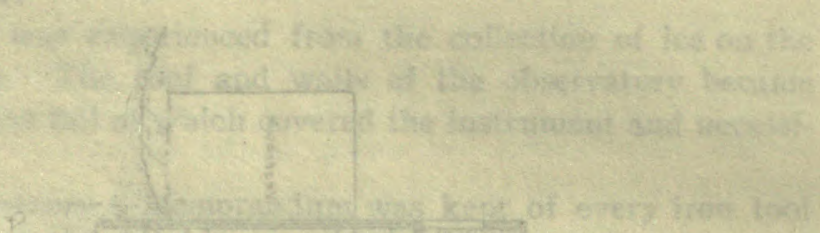
P.
Q
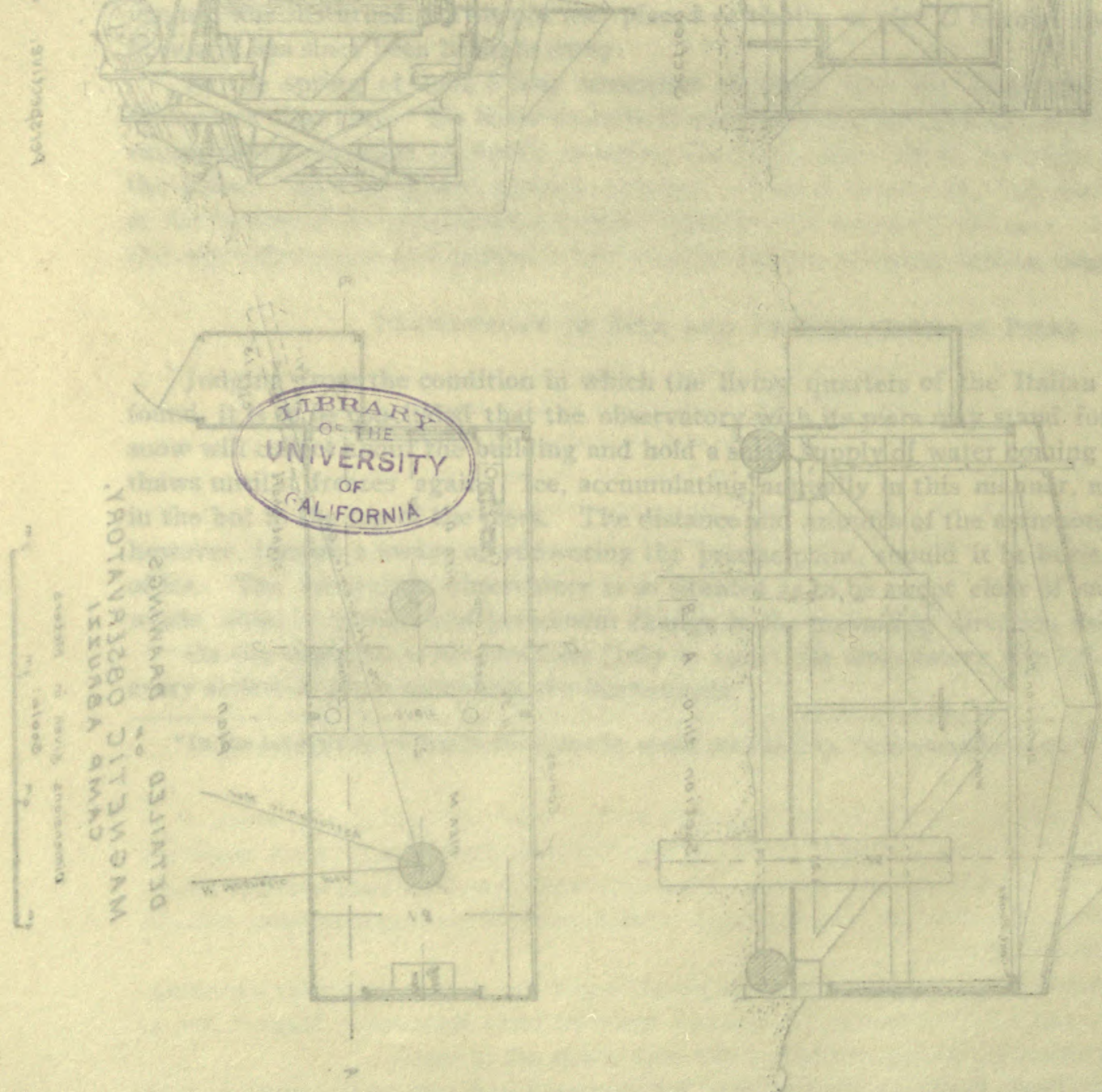

3

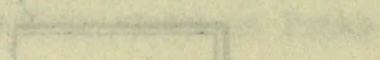

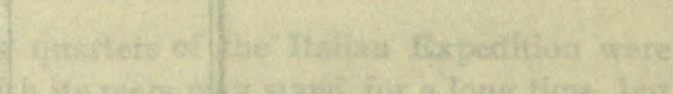

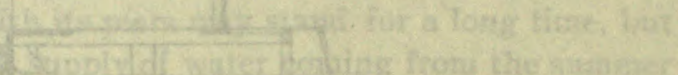

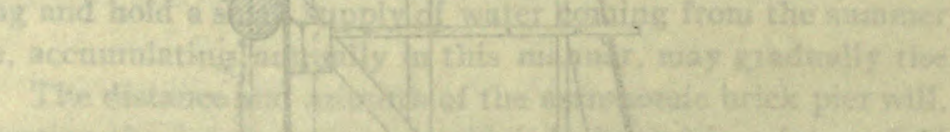

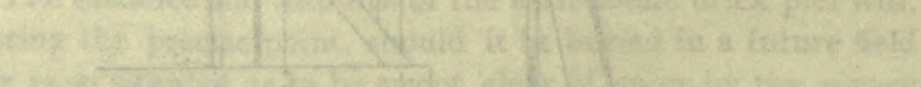

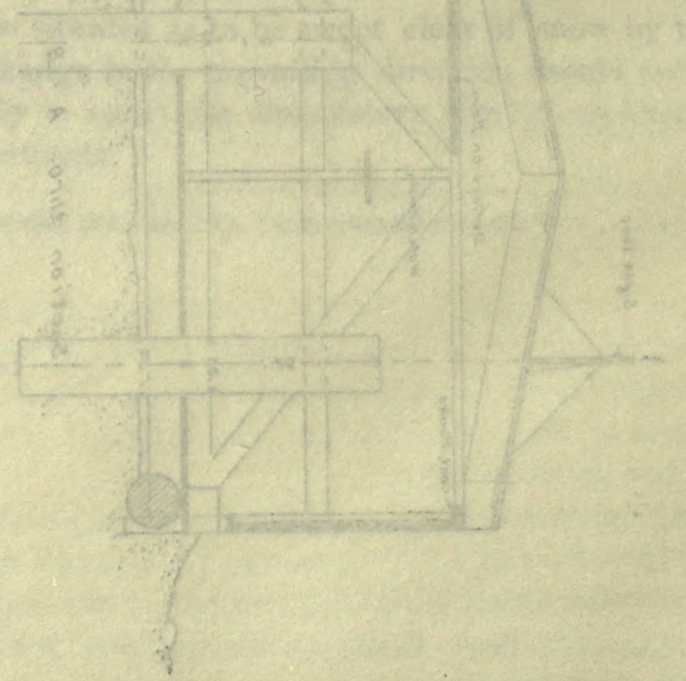




\section{DECLINATION}

\section{AzIMUTII MARKS}

Azimuth Mark No. I was the anemoneter staff permanently fixed to the northwest corner of the astronomic observatory. It was used at first because of its early availability during daylight of October, 1903. Afterwards a hole 3 inches in diameter was cut through the east wall of this observatory. A cross radiating from this hole was painted on the wall to be used in daylight and a bull's eye lantern seen througl the bole was used at night. The center of the liole was on the line of sight from the magnetometer telescope to the telescope of the Repsold circle in the astronomic observatory when these two were directed towards each other and it is designated Azimuth Mark No. 2. The distance is approximately 288 meters. It should be noted that a distant mark is seldom available at Teplitz Bay on account of darkness or thick weather.

The azimuth of the magnetometer at the Repsold circle was first determined by measuring the angle between the magnetometer and the south pointing of the vertical thread as determined from star transit on December 2 and December 18, 1903, and January 27, 1904. It was again determined by measuring the same angle as determined from a combination of the transits of $\eta$ Cephei at lower culmination and I H. Draconis at upper culmination on March I7, 1904 . Finally the angle between the astronomic meridian mark ( 6440 meters distant) and the magnetometer was measured July I, I 904, at the end of the seasou's work. It was measured on April I I, 1905, without any sensible difference in the restilts.

These observatious by Mr. R. W. Porter are given in the astronomic notes; the result of of four days' observations are tabulated below :

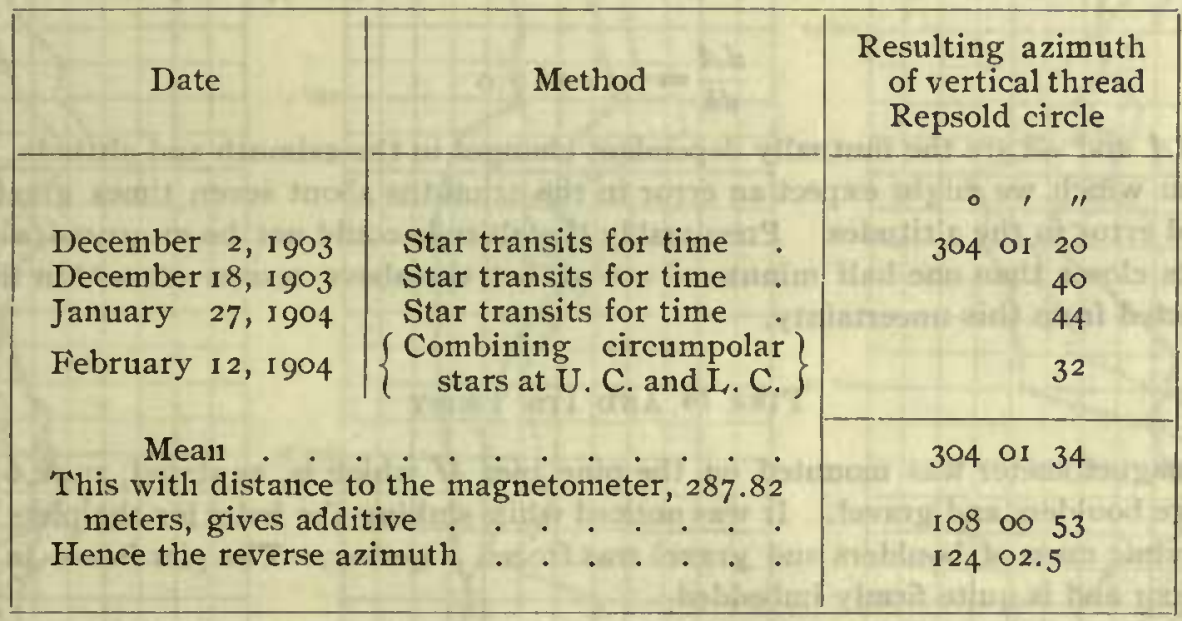

This is the value adopted and used in the final reduction of the magnetic declination observations.

For a rough check and to test the four-inch Berger \& Sons' theodolities under existing unfavorable trigonometric and meteorologic conditions, some azimutls were obtained from solar altitudes with one of these instruments by Mr. W. J. Peters.

The four-inch theodolite in these observatious was placed about 36 meters south of the magnetometer and exactly on a line connecting the magnetometer with the astronomic meridian mark (approximately 6430 meters distant). Both circles were graduated to read by two verniers to single minutes.

The instrument was reversed and opposite limbs of the sun were symmetrically observed. The means of each set of four pointings with the corresponding approximate local civil times, 
reckoned from midnight, and the deduced azimuth of the mark are given in the following tabulation in deriving which the value for latitude used was $81^{\circ} 47 \circ^{\prime} 5 \mathrm{~N}$ :

\begin{tabular}{|c|c|c|c|c|c|}
\hline $\begin{array}{l}\text { Date } \\
1904\end{array}$ & $\begin{array}{l}\text { Local civil } \\
\text { time }\end{array}$ & Altitude & $\begin{array}{l}\text { Angle between } \\
\text { sun and mark }\end{array}$ & Temp. C. & $\begin{array}{l}\text { Resulting } \\
\text { azimuth }\end{array}$ \\
\hline$p 1$ & $h m s$ & o & $0 \quad 1$ & 0 & 0 \\
\hline June 3 & $16 \quad 2507.4$ & 2520.62 & 5759.6 & -3.5 & 214.2 \\
\hline June 3 & $\begin{array}{lll}17 & 18 & 17.8\end{array}$ & 2330.12 & 8121.1 & -3.5 & 14.7 \\
\hline June 6 & $\begin{array}{lll}6 & 18 & 36.4\end{array}$ & 2304.67 & 9051.6 & -2.9 & 13.9 \\
\hline June 6 & $\begin{array}{lll}6 & 27 & 34.7\end{array}$ & 2324.00 & 8836.4 & -2.9 & 12.2 \\
\hline June 6 & 173422.8 & $23 \quad 19.94$ & 8500.1 & -3.8 & 13.0 \\
\hline June 6 & I7 3958.0 & $2307 \cdot 3^{8}$ & $\begin{array}{ll}86 & 36.4\end{array}$ & -3.8 & 17.1 \\
\hline June 7 & $\begin{array}{lll}6 & 32 & 32.2\end{array}$ & $2339 \cdot 50$ & 8732.8 & -0.2 & 14.0 \\
\hline June 7 & $\begin{array}{lll}6 & 40 & 28.2\end{array}$ & 2336.38 & 8533.3 & -0.2 & 12.1 \\
\hline $\tan x$ & an .... & . $\cdot$ & . & . . . & 2 \\
\hline
\end{tabular}

The angle at pier $M$ between this astronomic meridian nark and azimuth mark No. 2, measured with the circle of the magnetometer, was $121^{\circ} 49^{\prime} \cdot 3$; whence the azimuth of mark No. 2 is $124^{\circ}$ o3.'2, a determination with the small instrument which agrees within one minute of the Repsold circle determination.

By differentiating the spherical triangle and substituting values of the latitude, declinations and altitudes, and azimuths for the first observations of June 3 and June $6, A$. M., there will result

$$
\frac{d A}{d h}=7.5 \text { and } 7.0
$$

in which $d A$ and $d h$ are the mutually dependent changes in the azimuth and altitude, respectively; from which we might expect an error in the azimuths about seven times greater than an assumed error in the altitudes. Presumably the altitude could not be measured with these instruments closer than one-half minute of arc so that the above results are within the limits to be expected from this uncertainty.

\section{PIER M AND ITS TWIST}

The magnetometer was mounted on the pine pier $M$ which is, as stated, sunk 0.8 meter among large boulders and gravel. It was noticed while sinking the holes for the piers that the whole morainic mass of boulders and gravel was frozen together. The pier is not in contact with the floor and is quite firmly imbedded.

The horizontal circle of magnetometer No. IIII is rigidly connected with the base which receives the footscrews and, therefore, has no independent motion. As the instrument was left mounted and undisturbed after each day's observations, the various pointings on the azimuth mark taken from time to time should presumably have given the same circle readings. Very soon after the beginning of the winter's work it was noticed that these readings began to vary. The striding level, as well as the stationary level, was carefully examined at each pointing of the mark. When the circle readings were about their maximum and minimum the telescope was reversed in its Y's without disclosing any appreciable collimation error. Readings were taken when the temperature of the hut, practically the same as the outside, had reached its lowest point, to see if any of the effect was due to the passage of the line of sight from the warmer air of the hut to the colder air outside. 

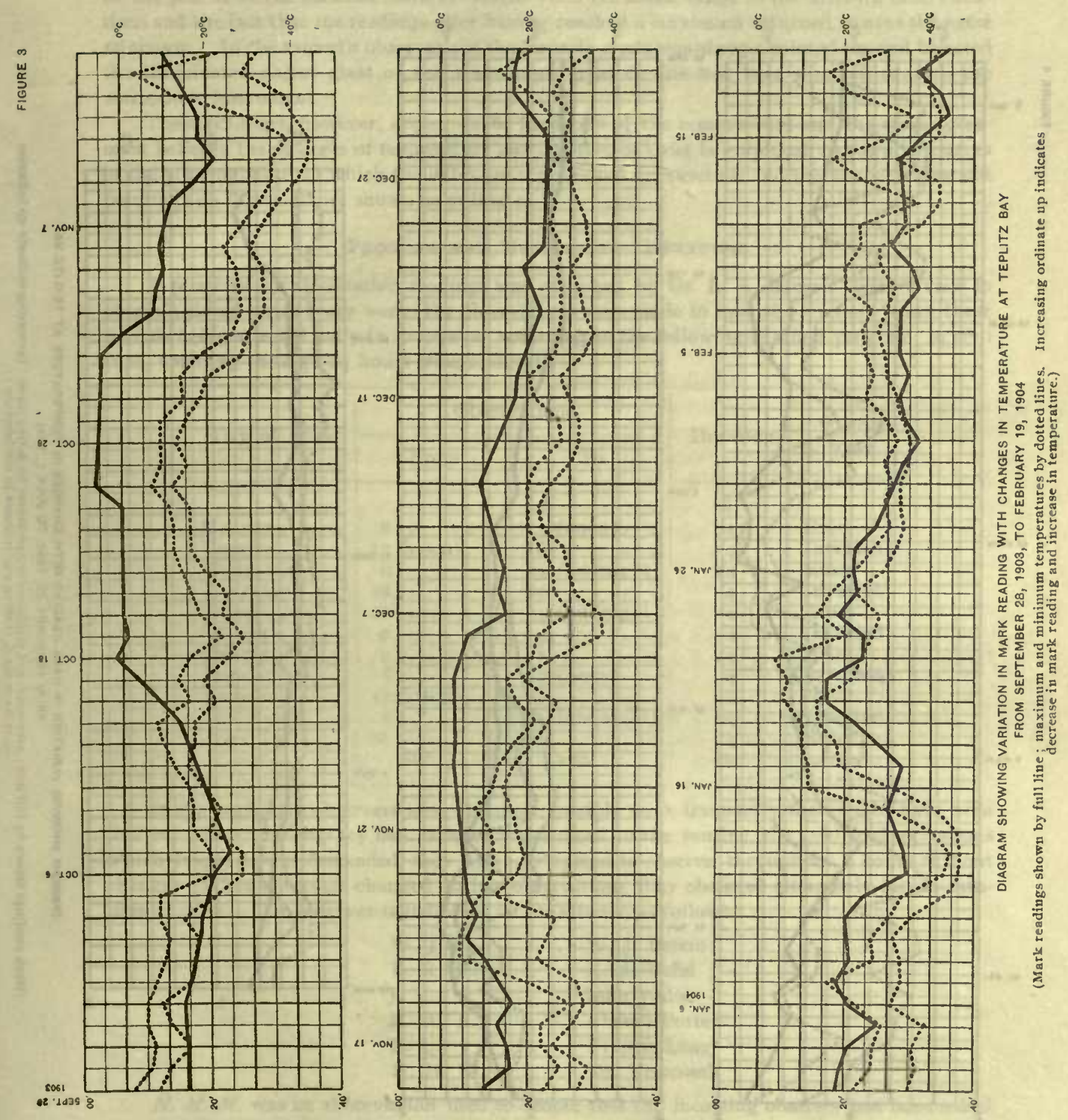


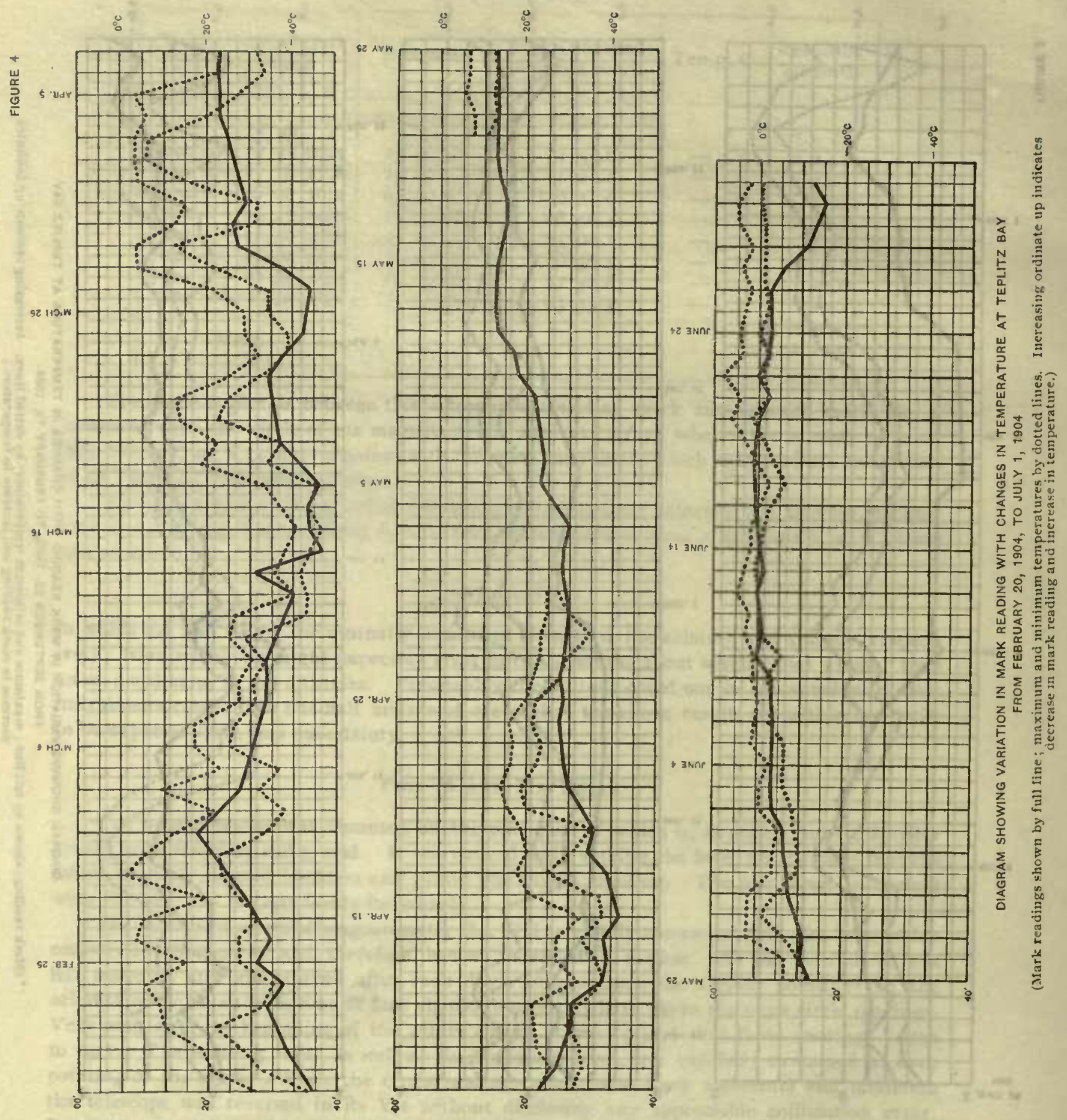


That these changes in the circle reading cannot be ascribed to any motion of translation of the pier $M$ or the azimuth mark is evident from the small range in the azimuth determinations and the fact that the readiugs after having reached a maximum returned to near the same minimum. In the azimuth observations the Repsold circle was always pointed on, and bisected the illuminated object glass of the magnetometer after this had been airected towards the astronomic instrument.

These readings, however, appear to be fuuctions of the temperature and the close agreement between the changes of temperature and changes of twist is graplically shown by curves in the plate opposite on which the dates are represented by abscissæe and the temperatures and pointings on the mark are shown as ordinates.

\section{Program AND METhods OF OBSERVING}

A program for declination readings was arranged by Dr. L. A. Bauer. With a view to obtain a closed series every week, the observations were made to extend over four hotirs, these periods being selected daily in succession according to the following detailed program, in civil time, connting throngh 24 honrs from midnight :

\begin{tabular}{|c|c|c|c|c|}
\hline \multirow{2}{*}{ Day of week } & \multicolumn{2}{|c|}{ Observations } & \multirow{2}{*}{ Duration } & \multirow{2}{*}{$\begin{array}{l}\text { Magnet } \\
\text { scale }\end{array}$} \\
\hline & From & To & & \\
\hline & $h$ & $h$ & $n$ & \\
\hline Monday. & $8 \cdot$. & 12 (noo11). & 4 & Errect \\
\hline Tuesday. . & 12 (noon). & 16. & 4 & Inverted \\
\hline Wednesday. & $0 .$. & 24 (midnight) & 24 & Erect \\
\hline Thursday. & 16 & $20 \cdot \cdot \cdot$ & 4 & Inverted \\
\hline Friday . . & $20 . .5$ & 24 (midnight) & 4 & Erect \\
\hline Snnday . . & $\circ \cdot . \quad \cdot$ & 4. & 4 & Inverted \\
\hline Sunday . . & $4 \cdot \cdot \cdot$. & 8. & 4 & Erect \\
\hline Monday. . . & & 12 (1100n). & 4 & Inverted \\
\hline 'Tresday. & 12 (noon). & & 4 & Erect \\
\hline Wednesday. . & o. . . . & 24 (midnight) & 24 & Inverted \\
\hline $\begin{array}{c}\text { Thursday . } \\
\text { etc. }\end{array}$ & ${ }^{16}$ etc. & ${ }^{20}$ etc. & $\begin{array}{l}4 \\
\text { etc. }\end{array}$ & $\begin{array}{l}\text { Erect } \\
\text { etc. }\end{array}$ \\
\hline
\end{tabular}

In the beginning observers were changed, possibly more frequently than might have been desired. Later, when they had become accustomed to the routine, the cold, and privations which longer hours demanded, they willingly agreed to observe throughout 8 hours withont change. When observers changed during observations they observed alternately for $10 \mathrm{~min}$ utes or more. The observer is indicated by his initials, as follows :

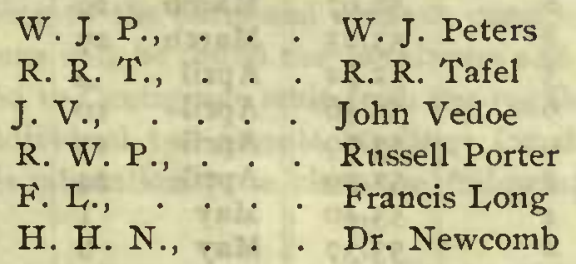

$N$. M. M. was an abbreviation used to denote that the incoming observer had been asked previously to his first observation if he had divested himself of all magnetic materials. It appears at every change of observers in the original record, but is left out of the published notes for lack of space.

Observations were made at every two minutes. Sometines one would be accidentally taken later than planned, in which case the tenths of a minute were noted. 
The time of one oscillation being about ro seconds, the observer commenced at ro seconds of the recorded time, and noted the scale reading at the end of the oscillation then occurring, whether left or right; then the opposite extreme reading of the following oscillation. The recorded time 1s, therefore, within 5 seconds of the time corresponding to the mean reading.

During magnetic disturbances the ten-second oscillation sometimes disappeared and the scale appeared motionless for ten seconds or more. This is indicated in the original by the remark "Quiescent," but in the published record it is shown by identical readings, scale right and left. Again during these disturbances the scale occasionally moved steadily and slowly (recorded "slowly decreasing" or "slowly iucreasing") for 10 or even 60 seconds, and the record then shows the division that transited at the recorded time. Where the note "slowly increasing " appears in record, the reading, as tabulated on pages 41 et seq., is followed by the letter $a$, thus : $25.8 a$; where the note "slowly decreasing" appears in record, the tabulated reading is followed by the letter $b$, thus: $25.8 b$. As the observer had to glance at the watch in this case, the observation may be in error 5 seconds of time.

\section{AXIS OBSERVATIONS}

Observations for axis were made inmediately before and after the declination observation for the day, unless the disturbances were too great. Magnet No. 4, used in declination readings, is cylindrical, requiring some two minutes to place the scale truly horizontal. It was considered advisable to make these axis observations as rapidly as possible, owing to the fact that the changes in declination do not, in general, vary uniformly with the time, even over the short interval (sixteen minutes) usually consumed in making axis observations (United States Coast and Geodetic Survey method). Accordingly in the three positions of the scale $E, I, E$, or $I, E, I$, the ends of consecutive oscillations were read as soon as the magnet was made nearly stationary instead of waiting for consecutive two-minute periods. Even then some very discordant results were obtained, and these have been arbitrarily rejected, and the mean of the remaining results for the week ending Sunday $8 \mathrm{~A}$. M. are the values used in the final reductions. The values adopted at Teplitz Bay are shown in the following tabulation :

\begin{tabular}{|c|c|c|c|c|c|}
\hline \multirow{2}{*}{$\begin{array}{l}\text { Week ending } \\
\text { at } 8 \mathrm{~A} \text {. M. } \\
\text { Stunday. }\end{array}$} & \multirow{2}{*}{$\begin{array}{l}\text { Number of } \\
\text { determina- } \\
\text { tions }\end{array}$} & \multirow[b]{2}{*}{ Axis } & 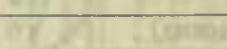 & \multirow{2}{*}{$\begin{array}{l}\text { Number of } \\
\text { determina- } \\
\text { tions }\end{array}$} & \multirow{2}{*}{ Axis } \\
\hline & & & $\begin{array}{c}\text { Week ending } \\
\text { at } 8 \text { A. M. } \\
\text { Sunday }\end{array}$ & & \\
\hline & & $d$ & 1904 & & $d$ \\
\hline October 4 & 6 & 53.12 & February 21 & 4 & $53.3^{6}$ \\
\hline October II & 7 & 52.88 & February 28 & 6 & 53.74 \\
\hline October 18 & 6 & 53.15 & March 6 & 5 & 53.41 \\
\hline October 25 & 5 & $53 \cdot 5^{2}$ & March & 8 & 52.90 \\
\hline November I & 8 & 52.97 & March & 8 & 53.35 \\
\hline November 8 & 8 & 53.25 & March & 5 & $53.4^{2}$ \\
\hline November i5 & 3 & 52.62 & April & 5 & 53.29 \\
\hline November 22 & 6 & $53 \cdot 36$ & A pril & 5 & 53.28 \\
\hline November 29 & 9 & 53.69 & April & 5 & 53.07 \\
\hline December 6 & 7 & 53.39 & April & 8 & 53.20 \\
\hline December ${ }_{3} 3$ & 4 & 53.40 & May & 7 & $53.3^{8}$ \\
\hline December 20 & 8 & 53.37 & May & 10 & 53.27 \\
\hline December 27 & 5 & $53 \cdot 43$ & May & II & 53.65 \\
\hline 1904 & & & May & 9 & 53.69 \\
\hline January & tis 28 & $53 \cdot 3^{2}$ & May & I 1 & $53.7 \mathrm{I}$ \\
\hline January & $\begin{array}{r}5 \\
7\end{array}$ & 53.82 & June & II & $53.5 \circ$ \\
\hline Jalluary 17 & 8 & 53.47 & June & I0 & 53.45 \\
\hline January 24 & 6 & 53.77 & June & $\begin{array}{r}9 \\
9\end{array}$ & 53.44 \\
\hline January 31 & 8 & 53.64 & June & 9 & 5.3 .45 \\
\hline February 7 & 5 & 53.47 & July $\quad I$ & 4 & 53.81 \\
\hline February 14 & 4 & 53.69 & & & \\
\hline
\end{tabular}




\section{TorsIon}

Two fibers were used until Thursday, October I, 1903, when they were found broken. After this four fibers were used. New fibers were inserted March 27, 28, and 29, 1904, the last serving through the remainder of the observatious. Several attempts to use two fibers alone failed, they being only sufficiently strong to stispend the torsion weight for but part of a day.

Observations for torsion were made before and after regular declination readings, excepting when the disturbances interfered. After the day's work the torsion weight was substituted for the magnet, the plane of detorsion was determined and the torsion weight was left suspended until the next observations. When torsion had accunulated during observations and made necessary a shift of torsion head, the effect of $90^{\circ}$ of torsion is noted in the footnotes to tabulations.

\section{RECORDS}

The chronological program arranged by Dr. I. A. Bauer could 11 ot be adhered to as closely as night have been desired during the fall and winter, owing to prevailing strong wiuds, when snow drifted to such an extent as to make travel to and from the observatory botli difficult and dangerous. Under these conditions two men lolding on to a leading line would struggle backwards to tlie observatory and dig out the entrance. This was filled again in a few moments, imprisoning the observer until again liberated by outside aid.

In order to economize space, the original notes have been tabulated as far as possible, with the corresponding resuits. On pages 20 to 26 will be found the readings of the azimuth mark under the headings Azimuth Mark No. 2 and Azimuth Mark No. I. In the column headed Pointing will be found the letters $B, A$, which indicate respectively that the reading of the azimuth mark has been taken before or after the declination observations of the day. Where both letters appear the mean is published; when none is given the azimuth marks were invisible on account of drifting snow or dense fog.

The readings corresponding to the position of the telescope when pointed on the magnet are given under the heading Circle reading of magnet. They are the mean of the readings taken before, after, and sometimes during the period of declination observations. When the telescope with the circle has been shifted during declination observation the values are omitted $i^{n}$ this table, and will be found on pages 26 to 31 , with corresponding time. This last mentioned table therefore shows when disturbances, so great as to require a shifting of the horizontal circle, have occurred. The times of observation first following these shiftings of horizontal sircle in any day's work are also indicated in tabular summary of two-minute declinatious by asterisks (*).

The circle reading of the true south is given for each day on which declination observations have been made. Where there is no corresponding reading on an azimuth mark it has been found by interpolating according to the dates and without considering the temperature effect.

In the temperature columns will be found the maximum and the minimum thermometer readiugs for the day, converted to Centigrade scale from the regular meteorologic record.

The reductions of the individual two-minute readings for declination are tabulated on pages $4 \mathrm{r}$ et seq. To make this tabulation quite clear the following specimen computation for November I, 1903, is given :

The scale readings for 5 h oom are (see page $7 \mathrm{I}$ ) left $59^{d} \cdot 8$, right $65^{d} \cdot 3$, the mean of which is $62^{d} \cdot 5$.

The axis for week ending November I, 1903 (see page 18 ), is $53^{d} .0$, whence the difference, scale minus axis $(\mathrm{S}-\mathrm{A}), 9^{d} \cdot 5$, which converted into arc (see constants, page 8 ) is $+14^{\prime} \cdot 9$. 
The circle reading is found on page 27 , under "Circle reading of magnet," for corresponding time $67^{\circ} \circ 7^{\prime} \cdot 3$, whence magnetic south meridian reads $67^{\circ} 22^{\prime} .2$.

The true south meridian for the corresponding day and time is (see page 2 I) $42^{\circ} 09^{\prime} \cdot 4$.

Therefore the east declination, nncorrected for any change in the plane of detorsion, is $25^{\circ} \mathrm{I} 2^{\prime} .8$.

But the plane of detorsion has shifted $29^{\circ}$ in the direction of increasing azimuths in 9 h $49 \mathrm{~m}$ (see bottom page $7 \mathrm{I}$ ) and the effect of $90^{\circ}$ of torsion as observed is $24^{\prime} \cdot 5$ (see bottom page $7 \mathrm{I}$ ), which gives by interpolation according to time a correction of $-4^{\prime} \cdot 4$, whence the final value of last magnetic declination as observed at 5 h oom on November 1,1903 , is $25^{\circ} 08^{\prime} .4$.

Note that in the tabulation of reductions of declination observations the results are entered to the nearest minute; in those cases where the figure in the tenths of a minnte is 5 the rule is followed to take the nearest even minute as the result to be tabulated.

TABULATIONS OF RECORDS

Circle readings of azimuth marks, magnet, and truc south at Teplitz Bay

\begin{tabular}{|c|c|c|c|c|c|c|c|}
\hline \multirow{2}{*}{$\begin{array}{l}\text { Point- } \\
\text { ing }\end{array}$} & \multirow{2}{*}{ Date } & \multirow{2}{*}{$\begin{array}{l}\text { Azimuth mark } \\
\text { No. } 2 \text {, cross }\end{array}$} & \multirow{2}{*}{$\begin{array}{l}\text { Azimuth mark } \\
\text { No. 1, } \\
\text { anemometer } \\
\text { staff }\end{array}$} & \multirow{2}{*}{$\begin{array}{l}\text { Circle } \\
\text { reading of } \\
\text { magnet }\end{array}$} & \multirow{2}{*}{$\begin{array}{l}\text { Circle } \\
\text { reading } \\
\text { true south }\end{array}$} & \multicolumn{2}{|c|}{$\begin{array}{l}\text { Cent. tempera- } \\
\text { ture }\end{array}$} \\
\hline & & & & & & Max. & Min. \\
\hline & & & & & 0 & & \\
\hline B & & & 16625 & $\circ$ & & & \\
\hline & & & $\begin{array}{l}10625.5 \\
16625.2\end{array}$ & & $\begin{array}{ll}42 & 17.4 \\
42 & 17.2\end{array}$ & $\begin{array}{l}2.1 \\
-\quad 5.6\end{array}$ & - \\
\hline & & 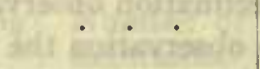 & & . & $4^{2} \quad 17.1$ & -6.7 & -14 \\
\hline 31 & & & W. & 63 & 421 & -5.4 & - IO \\
\hline B & & . & 166 & 64 & $4^{2}$ & -5 & -9 \\
\hline i & & - 1. & 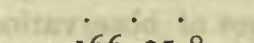 & . & $4^{2}$ & - & 15. \\
\hline $\begin{array}{l}A \\
A\end{array}$ & & $\cdot \cdot$ & 166 & & & $-s$ & \\
\hline B & & & $\begin{array}{l}16620 \\
16626\end{array}$ & $\begin{array}{l}643 \\
65 \text { I }\end{array}$ & $\begin{array}{ll}42 & 1 \\
42 & 1\end{array}$ & $\begin{array}{l}\text { - } 10.0 \\
-\quad 8.2\end{array}$ & - \\
\hline & & &. & & 42 I & $-8 I$ & 20 \\
\hline B & 8 & & the 1 & . & 421 & -20.7 & - \\
\hline B & 9 & & 1662 & 645 & 422 & - & -26 \\
\hline i & & & & 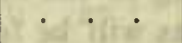 & $422 c$ & - & -20 \\
\hline & & & & & Is & & \\
\hline A & & & I $66 \quad 26.7$ & 6505.0 & I & & 18 \\
\hline B & & & 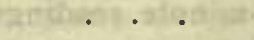 & . $\cdot$ & 42 & $-I$ & -15 \\
\hline & & & 172. & . . . & &.- & \\
\hline A & & A & & 644 & & -8 & \\
\hline . & & & &. & 42 I & $-I$ & -20 \\
\hline & & & . & & & & - 18 \\
\hline B & & & & & 4 & & -24 \\
\hline A & 19 & I & & & $t^{2}$ II I. 8 & -2 & -27 \\
\hline A & 20 & . & & 6441 & 42 I I. 4 & $-\mathrm{r} 8.2$ & -22 \\
\hline B & & & & & & $-I$ & -23 \\
\hline & & & & & 42 III. & - I & \\
\hline
\end{tabular}


Circle readings of azimuth marks, magnet, and true south at Teplitz Bay-Continued

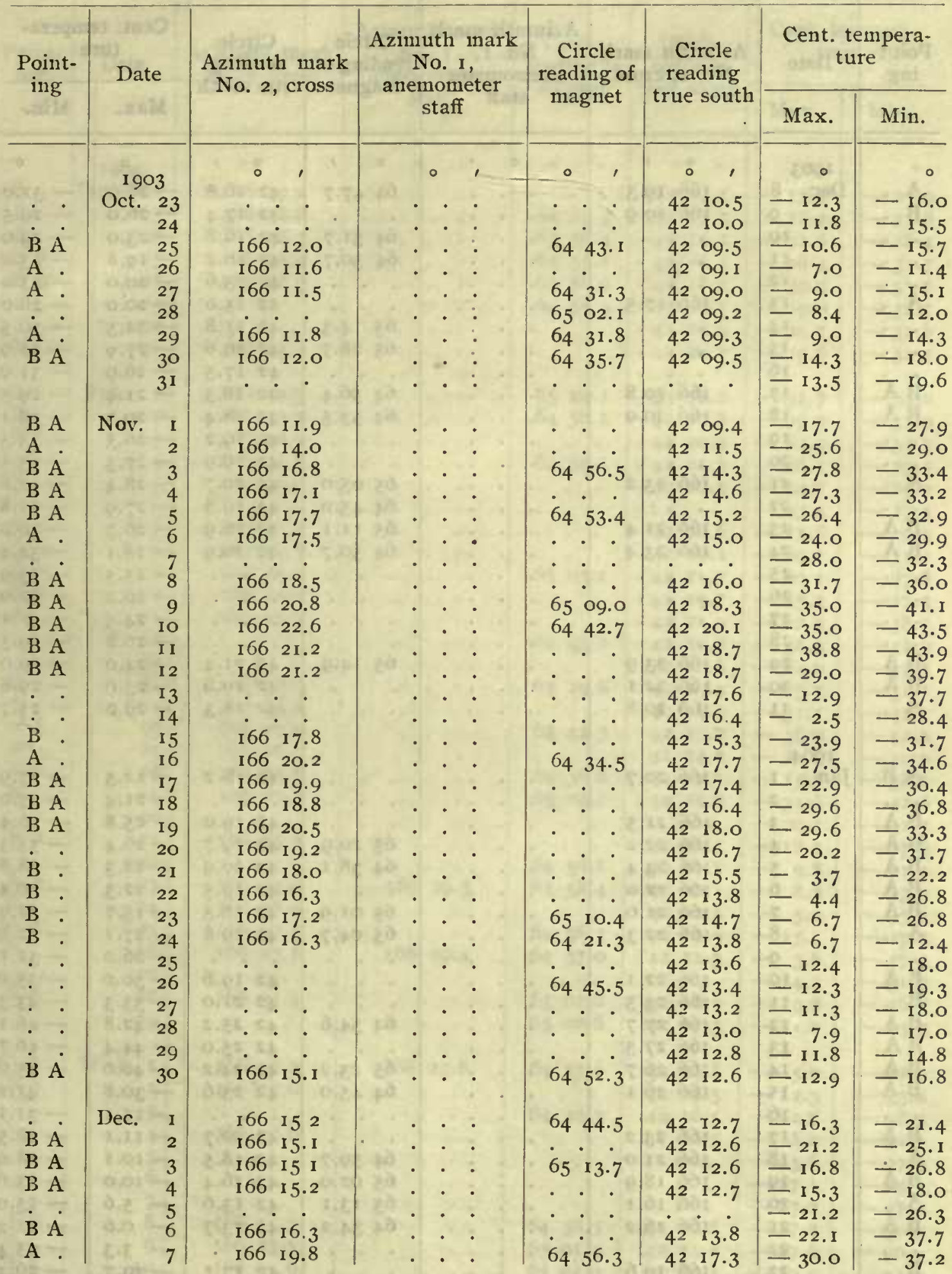


Circle readings of azimuth marks, magnet, and true south at T'eplitz. Bay-Continued

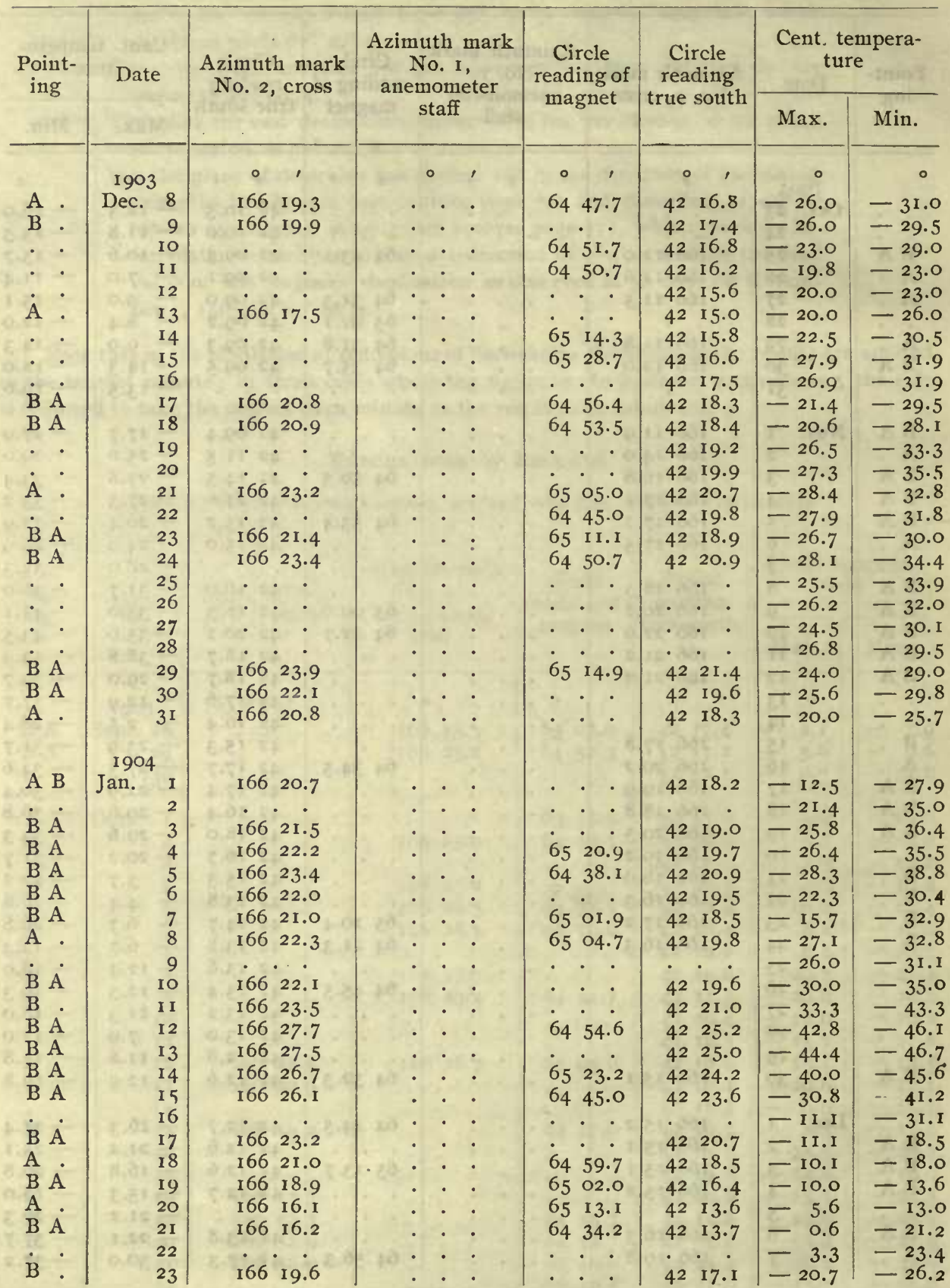


Circle readings of azimuth marks, magnet, and true south at Teplitz Bay-Continued

\begin{tabular}{|c|c|c|c|c|c|c|c|c|c|}
\hline \multirow{2}{*}{$\begin{array}{l}\text { Point- } \\
\text { ing }\end{array}$} & \multicolumn{2}{|c|}{ Date } & \multirow[t]{2}{*}{$\begin{array}{l}\text { Azimuth mark } \\
\text { No. } 2 \text {, cross }\end{array}$} & \multirow{2}{*}{\multicolumn{2}{|c|}{$\begin{array}{l}\text { Azimuth mark } \\
\text { No. I, } \\
\text { anemometer } \\
\text { staff }\end{array}$}} & \multirow{2}{*}{$\begin{array}{l}\text { Circle } \\
\text { reading of } \\
\text { magnet }\end{array}$} & \multirow{2}{*}{$\begin{array}{l}\text { Circle } \\
\text { reading } \\
\text { true south }\end{array}$} & \multicolumn{2}{|c|}{$\begin{array}{l}\text { Cent. tempera- } \\
\text { ture }\end{array}$} \\
\hline & xati & & & & & & & Max. & Min. \\
\hline & I904 & & 01 & & & $\circ$ & $\circ$ & $\circ$ & \\
\hline A & Jan. 2 & & I 66 I 7.2 & . & . & $\cdot \cdot \cdot$ & $42 \quad 14.7$ & -13.4 & -27.9 \\
\hline & 2 & & 166 I 8.8 & & & 6455.3 & 42 I & & \\
\hline & 2013 2 & & $\mathbf{I}$ & 20 & & 6556.4 & 42 I & 一 & \\
\hline B A & 42 & & 9 & 20 & & 645 I.I & $42 I$ & 一 & 一 \\
\hline B A & 2 & & 16620.7 & 24. & . & 6450.9 & 4218 & - & \\
\hline & 512 & & $\cdot \cdot$ & & . & 6458.9 & 4219 & - & 一 \\
\hline$\therefore$ & $2 \quad 3$ & & $\cdot \cdot \cdot$ & & .. & $\cdot \cdot \cdot \cdot$ & $42 \quad 19.9$ & 一 & - \\
\hline B & Hic & & I $66 \quad 23.2$ & 6 & $\cdot \cdot$ & & 42207 & -2 & -34 \\
\hline A & Feb. & & $\begin{array}{ll}166 & 24.7\end{array}$ & . & . . & 6524.1 & $42 \quad 22.2$ & -31 & -35.0 \\
\hline & $1+t$ & & t & 199. &. & $645^{8.1}$ & 422 & - & \\
\hline B A & dian & & I & 68. & . & ; . & $42 \quad 20.4$ & - & \\
\hline A. & 70.25 & 4 & I & . & . & 6459.1 & 4221 & - & \\
\hline A. & $5 \times 2$ & 5 & 23.0 & & . &.$\quad \cdot$ & $42 \quad 20$ & - & - \\
\hline & a:er & 6 &. & that. & . $\cdot$ & . &.$\quad \cdot$ & - & \\
\hline B A & Gens: & & 23.1 & • & .. & . & 4220.6 & - & \\
\hline B A & 2185 & & 624.7 & . & .. & $0^{\circ}$ & 4222 & - & - \\
\hline B A & Eitiz & 9 & 4.2 & . & . . & 65 O3.I & $42 \quad 21.7$ & - & \\
\hline & 2 & & 622.2 & . & . & . $\cdot$ & 42 I & - & \\
\hline A & $315=1$ & & 16623.5 & & . & - . & 4221 & - & \\
\hline . & 2 & & $\cdot \cdot \cdot$ & . & . & 6443.2 & $42 \quad 20$ & -3 & \\
\hline & & I & $\therefore$ & - & - & $\therefore \cdot$ & 4220 & -2 & - \\
\hline A & 94 & & 622.9 & . & . & 6529.8 & 4220 & - & \\
\hline A & 48 & I5 & .0 & & . & $\cdot \cdot \cdot$ & 422 & -3 & - \\
\hline B A & & It & 7 & - & - & $644^{2.5}$ & 422 & - & 一 \\
\hline$B$ : & nis.. & & .7 & . & . & & 422 & 一 & \\
\hline B A & 1 & & I & . & & 6436.6 & 4222 & - & \\
\hline B. & 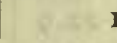 & 19 & 27.7 & - & - · & 6524.1 & $42 \quad 25.2$ & -3 & - \\
\hline & $a^{2}$ & &. & . & . & . . . & . . . & -2 & - \\
\hline B A & 2 & 21 & 16625.2 & & . & & $42 \quad 22.7$ & - I & \\
\hline & 2 & 2 & & & . . & 6456.8 & 4221 & - & $-2 \mathrm{I} .4$ \\
\hline B & & 23 & & I6 & 529.3 & $645^{8.4}$ & 4220 & - & \\
\hline & 2 & 2. & o & . & . $\cdot$ & . . . & 4222 & -5 & - \\
\hline B A & 2 & & & & $\therefore$ & 6458.3 & 42 & - I5 & - \\
\hline B. & 2 & & 16623.7 & 160 & 29.4 & 6437.0 & $4221 \cdot 3$ & -3 & - \\
\hline & 2 & 27 & $\cdot \cdot$ & b & $\cdot \cdot$ & $\cdot \cdot \cdot$ & . . $\cdot$ & -4 & - \\
\hline B A & & & 16621.4 & . & - & 65 or. 6 & $42 \quad 18.9$ & -19.6 & \\
\hline & & & $\cdot \cdot$ & - & . & 6502.8 & $42 \quad 17 \cdot 3$ & -1.0 & $-13 \cdot 3$ \\
\hline B A & ch & & 1 & 166 & 23.8 & 6457.6 & $42 \quad 14.8$ & -5 & -22.9 \\
\hline B A & & & & & & 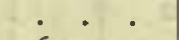 & 4214. & -12 & -33.8 \\
\hline . & & 3 & $\cdot$ & & $\cdot \cdot$ & 6420.3 & 4216 & -2 & - \\
\hline A & & 4 & I66 21.0 & 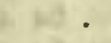 & 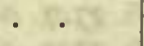 & .. & $4^{2} \quad 18.5$ & -8 & $-3 I$. \\
\hline & & 5 & . & 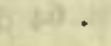 & - . & & . . . & -23.6 & -37.6 \\
\hline & & 6 & & & & & & & $-24 \cdot 3$ \\
\hline & & 7 & & & 0 & & & -1 & - \\
\hline & 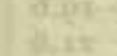 & & I6 & . & .. & $\begin{array}{ll}64 & 58.3 \\
65 & \text { OI. } 4\end{array}$ & $\begin{array}{ll}42 & 20.8 \\
42 & 21.2\end{array}$ & - & - \\
\hline & G I & I0 & 16623.6 & & & 6504.9 & 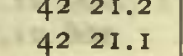 & $\begin{array}{l}-27.8 \\
-30.5\end{array}$ & $\begin{array}{r}3.5 \\
-34.2\end{array}$ \\
\hline
\end{tabular}


Circle readings of azimuth marks, magnet, and true south at Teplitz Bay-Contiuued

\begin{tabular}{|c|c|c|c|c|c|c|c|c|c|}
\hline \multirow{2}{*}{$\begin{array}{l}\text { Point- } \\
\text { ing }\end{array}$} & Date & \multirow[t]{2}{*}{$\begin{array}{l}\text { Azimuth mark } \\
\text { No. 2, cross }\end{array}$} & \multirow{2}{*}{\multicolumn{2}{|c|}{$\begin{array}{c}\text { Azimuth mark } \\
\text { No. I, } \\
\text { anemometer } \\
\text { staff }\end{array}$}} & \multirow{2}{*}{\multicolumn{2}{|c|}{$\begin{array}{l}\text { Circle } \\
\text { reading of } \\
\text { magnet }\end{array}$}} & \multirow{2}{*}{$\begin{array}{l}\text { Circle } \\
\text { reading } \\
\text { true south }\end{array}$} & \multicolumn{2}{|c|}{$\begin{array}{l}\text { Cent. tempera- } \\
\text { ture }\end{array}$} \\
\hline & $\operatorname{sank}$ & & & & & & & Max. & Min. \\
\hline & 1904 & 101 & & & $\circ$ & ' & $\circ \quad$ & x & $\circ$ \\
\hline B A & $M^{\prime} \mathrm{ch}$ I I & $166 \quad 24.4$ & & . $\cdot$ & 64 & 49.2 & $42 \quad 21.9$ & -25.0 & -27.9 \\
\hline$\therefore$ & $\mathrm{r} 2$ & . & 305. & . . & & & .. & -26.2 & -42.8 \\
\hline B A & I 3 & I 66 26. I & 48 & & 65 & 05.1 & $42 \quad 23.6$ & -40.6 & -43.8 \\
\hline A. & I 4 & 16622.3 & bit. & & 65 & 4I. I & $42 \quad r 9.8$ & -36.0 & -42.2 \\
\hline B A & r 5 & 16628.6 & 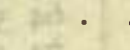 & - & 64 & 42.4 & 4226.1 & $-3^{8.6}$ & -44.4 \\
\hline B A & I6 & $\begin{array}{ll}166 & 27.4\end{array}$ & 150 & · & & 8 & $42 \quad 24.9$ & -40.7 & $\begin{array}{l}-46.3 \\
-43.8\end{array}$ \\
\hline A . & $\begin{array}{r}17 \\
18\end{array}$ & $\begin{array}{r}\text { I } 6627.2 \\
\text { I } 6628.2\end{array}$ & • & . & $\begin{array}{l}65 \\
65\end{array}$ & $\begin{array}{l}00.3 \\
04.8\end{array}$ & $\begin{array}{ll}42 & 24 \cdot 7 \\
42 & 25 \cdot 7\end{array}$ & $\begin{array}{r}-37.5 \\
-33.8\end{array}$ & $\begin{array}{l}-43.8 \\
-45.6\end{array}$ \\
\hline & I9 & . & . & .. & . &. & . . . & -19.0 & $\begin{array}{r}-33.9 \\
-\end{array}$ \\
\hline B A & 20 & 16624.8 & & - & - & & $42 \quad 22.3$ & -22.3 & -37.7 \\
\hline . & $2 \mathrm{I}$ & $\cdot \cdot$ & 180 & . & 64 & 499 & $42 \quad 21.8$ & $-\mathrm{r} 3.3$ & -22.9 \\
\hline$\therefore$ & 22 & $\cdot$ & - & . & 65 & $27 \cdot 7$ & 4221.4 & -12.9 & -25.2 \\
\hline A. & 23 & $166 \quad 23.5$ & f & . & 64 & 52.2 & 4221.0 & -25.2 & -31.7 \\
\hline A & 24 & 16625.2 & . & - & 65 & 06.8 & $\begin{array}{ll}42 & 22.7\end{array}$ & -31 & -38.3 \\
\hline B & & 16627.0 & - & - & 64 & 25.7 & $42 \quad 24 \cdot 5$ & -29.0 & -39.4 \\
\hline$\dot{0}$ & 26 & . & - & . & . & & . $\cdot$. & -29.0 & -34.4 \\
\hline A & 27 & I66 27.7 & - & - & & - $\cdot$ & $42 \quad 25.2$ & -21.2 & -34.9 \\
\hline B. & 28 & $\begin{array}{lll}166 & 24.8\end{array}$ & $7 \mathrm{R}^{2}$ & . & 65 & 00.4 & $42 \quad 22.3$ & -4.0 & -21.2 \\
\hline B A & 29 & 16620.8 & - & - & 64 & 42.2 & $42 \quad 18.3$ & -3.3 & -12.4 \\
\hline B A & 30 & $\begin{array}{lll}166 & 20.2\end{array}$ & . & . & - & & $4^{2}$ I $7 \cdot 7$ & -12.4 & -30.9 \\
\hline B A & $3 r$ & 16621.6 & 164 & · & 64 & 36.3 & 42 I9. I & -15.1 & -31.7 \\
\hline $\mathrm{B}$. & April I & 16621.0 & 21. & - & . & & $42 \quad 18.5$ & -4.0 & $-\mathrm{I} 5 . \mathrm{I}$ \\
\hline$\therefore \cdot$ & 2 & $\cdot \cdot$ & • & - & - & - & •. $\cdot$ & -3.4 & -5.6 \\
\hline A. & 3 & I66 20.0 & 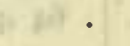 & . & . & . & $42 \quad 17.5$ & -3.3 & -6.2 \\
\hline A : & 4 & $\begin{array}{ll}\text { I66 } & 19.3\end{array}$ & & . & - & & $42 \quad 16.8$ & -5.6 & -19.6 \\
\hline B A & 5 & I 66 I 9.3 & (A) & . & 64 & $57 \cdot 4$ & $42 \quad 16.8$ & - 3.4 & -27.9 \\
\hline B A & 6 & 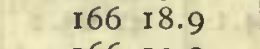 & 240 & . & - & & $42 \quad 16.4$ & -22.9 & $-33 \cdot 3$ \\
\hline B A & .7 & $\begin{array}{ll}166 & 19.2\end{array}$ & - & - & - & - & $42 \quad 16.7$ & -23.4 & -31.4 \\
\hline B A & 8 & 16621.0 & & . & - & & $42 \quad 18.5$ & -25.3 & -33.1 \\
\hline res & 9 & $\cdot \cdot \cdot$ & . & . & . & . & . . . & -23.4 & -32.3 \\
\hline A B & Io & I66 22.2 & 5 & . $\cdot \cdot$ & - & - & $42 \quad 19.7$ & -22.5 & -30.1 \\
\hline A & I I & ז66 22.2 & . & . $\cdot$ & & & $42 \quad 19,7$ & $-21 \cdot 3$ & $-3^{2} \cdot 3$ \\
\hline B A & $\mathrm{I} 2$ & I66 25.2 & 141. & . & 65 & 02.7 & $42 \quad 22.7$ & -32 & -38.3 \\
\hline B A & 13 & ง.66 $25 \cdot 7$ & fas & .. & - & & $4^{2} \quad 23.2$ & -27.6 & -36.5 \\
\hline A. & I4 & I $66 \quad 25 \cdot 3$ & . & . . & 64 & $5 \mathrm{I} .4$ & $4^{2} 22.8$ & -27.1 & -32.9 \\
\hline B A & 15 & I66 26.5 & 6800 & - & 65 & 09.3 & $42 \quad 24.0$ & -32.8 & -37.9 \\
\hline$\dot{\mathrm{p}} \dot{\mathrm{i}}$ & 16 & 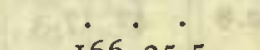 & 1002. & - & $\cdot$ & & $\cdot$. & -20.7 & $-37 \cdot 3$ \\
\hline B A & I7 & I66 $25 \cdot 5$ & . & - & - & - & $42 \quad 23.0$ & -17.4 & $-24 \cdot 5$ \\
\hline A : & 18 18 & $166 \quad 23.7$ & 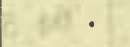 & $\cdot$ & & - & $42 \quad 21.2$ & -20.0 & -30.9 \\
\hline B A & 19 & I66 24.2 & & · & & & $\begin{array}{ll}42 & 21.7\end{array}$ & - Ig.r & $-3^{6.1}$ \\
\hline & 20 & 2.5 & 120 & $\cdot \cdot$ & 64 & 39.1 & $4^{2} \quad 20.5$ & -15.7 & -19.6 \\
\hline A . & $2 \mathrm{I}$ & I66 21.8 & r 66 & 27.7 & 64 & $43 \cdot 7$ & 42 I 9.4 & -13.4 & $-r 8.9$ \\
\hline B A & 22 & 16621.5 & 166 & $27 \cdot 3$ & 64 & 39.6 & 42 I 8.1 & $-\mathrm{I} 6.2$ & -22.6 \\
\hline ;. & 23 & $\cdot 0^{\circ}$ & $\therefore$ & $\therefore$ & - & & - $\cdot$ & -16.8 & -24.0 \\
\hline 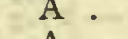 & 2., 24 & I66 2I.I & I66 & 27.1 & 65 & 00.3 & $42 \quad$ I 8.8 & $-\mathrm{r} 5.7$ & $-21 \cdot 3$ \\
\hline A . & 25 & r 6621.4 & 166 & 27.1 & 65 & 08.7 & $42 \quad 19.0$ & -19.6 & -22.2 \\
\hline B A & 26 & 16621.2 & 20. & . $\cdot$ & 64 & 54.0 & $42 \quad 18.7$ & -21.6 & -28.1 \\
\hline B A & 27 & 16621.6 & 202. & . . & . & & 42 I9. I & -22.9 & -29.5 \\
\hline
\end{tabular}


Circle readings of azimuth marks, magnet, and true south at Teplitz Bay-Continued

\begin{tabular}{|c|c|c|c|c|c|c|c|c|}
\hline \multirow{2}{*}{$\begin{array}{l}\text { Point- } \\
\text { ing }\end{array}$} & \multirow[t]{2}{*}{ Date } & \multirow[t]{2}{*}{$\begin{array}{l}\text { Azimuth mark } \\
\text { No. } 2 \text {, cross }\end{array}$} & \multirow{2}{*}{$\begin{array}{l}\text { Azimuth mark } \\
\text { No. I, } \\
\text { anemoineter } \\
\text { staff }\end{array}$} & \multirow{2}{*}{\multicolumn{2}{|c|}{$\begin{array}{l}\text { Circle } \\
\text { reading of } \\
\text { magnet }\end{array}$}} & \multirow{2}{*}{$\begin{array}{l}\text { Circle } \\
\text { reading } \\
\text { true south }\end{array}$} & \multicolumn{2}{|c|}{$\begin{array}{l}\text { Cent. tempera- } \\
\text { ture }\end{array}$} \\
\hline & & & & & & & Max. & Min. \\
\hline & 1904 & 。 & $0 \quad 1$ & - & & $\circ$ & 0 & \\
\hline A : & April 28 & $-10^{\circ}$ & $166 \quad 27.9$ & 64 & 44.9 & 4219.6 & -23.2 & -30.3 \\
\hline B A & & I 6622.0 & I66 27.2 & 64 & 34.8 & 42 I8. I & -24.4 & -29.9 \\
\hline .. & 3 & $6 \times 5$. & . & . & & . . $\cdot$ & -24.7 & $-27 \cdot 3$ \\
\hline B A & May & I $66 \quad 21.3$ & $c^{\circ}-0^{\circ}$ & - & . & $42 \quad$ I 8.8 & . & . . . \\
\hline A. & 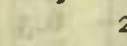 & 16621.5 & $24 \quad$ I $66 \quad 27.4$ & & . $\cdot$ & 42 I 9.1 & . & . \\
\hline B A & 84 & 16621.9 & $\cdot \cdot$ & 64 & 34.8 & $42 \quad 19.4$ & 68 & . . \\
\hline B A & 2. & I66 20.4 & $=0$ & & & $42 \quad 17.9$ & e. & ... \\
\hline B A & $7 \frac{1}{2}$ & I 66 I9. I & I $66 \quad 24.4$ & 65 & 02.8 & 42 I 5.6 & t. & .. \\
\hline B A & 83 & 16619.4 & 16624.6 & & & $42 \mathrm{I}$ & ? & .. \\
\hline B A & (5) & $166 \quad 18.8$ & 16624.0 & & .7. & 42 I & 5 & $\because \cdots$ \\
\hline B & 85 & $166 \quad 18.6$ & . . . & 65 & 09.2 & 42 I & Exe. & . 1. \\
\hline B & $3 .+$ & 166 I 7.I & . & 64 & $5 \mathrm{I} . \mathrm{I}$ & $42 \div 4.6$ & 5 & . . \\
\hline B & 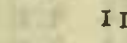 & $166 \quad 16.7$ & H & - & - $\cdot$ & 42 I & 2. & . . \\
\hline B & 84 & I $66 \quad 15.2$ & I $66 \quad 20.7$ & . & . & 42 I & . & .. \\
\hline B A & it & 166 I 5.0 & I $66 \quad 20.5$ & . & - & 42 II. 5 & . & . . . \\
\hline B A & I & 166 I5. I & I $66 \quad 20.7$ & & & 42 I I . 7 & • & . \\
\hline & It & . & $\cdot \quad \cdot$ & 65 & I 2.1 & 4213.0 & $\cdot 1 \cdot 1$ & . . \\
\hline A & 17 & $166 \quad 16.0$ & .. & 65 & I 1.2 & $42 \quad 13.5$ & . & . \\
\hline A. & I 8 & $166 \quad 16.0$ & & & - $\cdot$ & 42 I 3.5 & - & . \\
\hline B A & IS & $166 \quad 15 \cdot 3$ & I $66 \quad 20.2$ & 64 & 26.2 & $42 \quad 12.4$ & . & - \\
\hline B A & $2 c$ & $166 \quad 15.2$ & I $66 \quad 20.5$ & - & & $42 \quad 11.7$ & & \\
\hline & 2 & .0 & $\cdot \cdot \cdot$ & - & - & . . . & -7.3 & -10.5 \\
\hline B A & 2 & $166 \quad 15.3$ & 16620.3 & & & $42 \quad 12.5$ & -7 & -17 \\
\hline A & 23 & $\cdot \cdot \cdot$ & 16621.2 & 65 & $04 \cdot 3$ & 4213.0 & -5.3 & -12.1 \\
\hline A & 24 & 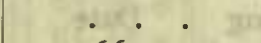 & 16621.5 & - & . & 4213 & $-6 . c$ & -12.3 \\
\hline A. & 2 & $166 \quad I 5.2$ & Fin. & 65 & OI.O & $42 \quad 12.7$ & -6.7 & - II \\
\hline B A & 2 & 166 I 4.2 & 16620.1 & 64 & 07.1 & $42 \quad 10.9$ & -6.9 & - 9.6 \\
\hline B A & 27 & I 66 I $4 \cdot 3$ & I66 I9.3 & 64 & 51.0 & $42 \quad 10.7$ & +2.0 & -6.7 \\
\hline & 68 & 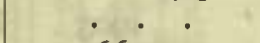 & . & - & - & . . . & + & -0.8 \\
\hline B & 3 & 166 I 3.0 & $\begin{array}{ll}\text { I } 66 & 17.7\end{array}$ & & & 4209.3 & + & - \\
\hline B A & $3 c$ & I 66 I 3.0 & $F \cdot \cdot \cdot \cdot$ & 65 & 07.8 & $42 \quad 10.5$ & -3.9 & - \\
\hline B A & 31 & I 66 I 2.6 & ? & 64 & 48.7 & 42 IO. I & -4.1 & - 9.7 \\
\hline B A & June & $166 \quad 12.5$ & I 66 I 8.2 & & & 42 og. I & - & - \\
\hline B A & $\ln 20$ & I 66 II. 5 & 710. & 64 & 26.6 & 4209.0 & - & - \\
\hline B A & 48 & I 66 I I. 3 & $\begin{array}{lll}166 & \text { I } 6.8\end{array}$ & 64 & 01.7 & 4207 & - & - \\
\hline & ay & $\therefore \cdot$ & $\cdot$ & . & & $. \quad . \quad$. & -2.7 & - \\
\hline & $\varphi^{\circ}$ & I 66 II.5 & I $66 \quad$ I 6.5 & . & . & $42 \quad 07.9$ & + & - \\
\hline & 20 & $166 \quad 11.5$ & L. $\cdot \cdot$ & & - & 4209 & + & - \\
\hline & 19 & 166 I1.2 & & . & & 4208.7 & 0.5 & - \\
\hline & $5 \pi$ & I66 II. 4 & $166 \quad 16.8$ & & & $42 \quad 07.9$ & + & - \\
\hline & 9 & I $66 \quad 09.9$ & $\begin{array}{lll}166 & 16.3\end{array}$ & 64 & 14.2 & 4207 & 0.6 & - \\
\hline & Io & I $66 \quad$ I0. 3 & 16616.1 & 64 & 42.8 & 4206.9 & + & - \\
\hline & I I & $\cdot \cdot$ & 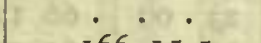 & - & & $1 \cdot \cdot \cdot$ & 1.6 & -0.5 \\
\hline & 12 & 16610.0 & I 66 I 5.5 & & & 4206.5 & + & - \\
\hline A. & I3 & I $66 \quad 10.6$ & 166 I5.8 & 65 & 09.2 & $\begin{array}{ll}42 & 07.9\end{array}$ & + & - \\
\hline & & $\cdot \cdot \cdot 2$ & $150 \cdot \cdot \cdot$ & 64 & 42.6 & $\begin{array}{lll}42 & 07.8\end{array}$ & I. 0 & 0.5 \\
\hline
\end{tabular}


Circle readings of azimuth marks, magnet, and true south at Teplitz Bay-Concluded

\begin{tabular}{|c|c|c|c|c|c|c|c|c|}
\hline \multirow{2}{*}{$\begin{array}{c}\text { Point- } \\
\text { ing }\end{array}$} & Date & \multirow{2}{*}{$\begin{array}{l}\text { Azimuth mark } \\
\text { No. } 2 \text { cross }\end{array}$} & \multirow{2}{*}{$\begin{array}{c}\text { Azimuth mark } \\
\text { No. I, } \\
\text { anemometer } \\
\text { staff }\end{array}$} & \multirow{2}{*}{\multicolumn{2}{|c|}{$\begin{array}{l}\text { Circle } \\
\text { reading of } \\
\text { magnet }\end{array}$}} & \multirow{2}{*}{$\begin{array}{l}\text { Circle } \\
\text { readiug } \\
\text { true south }\end{array}$} & \multicolumn{2}{|c|}{$\begin{array}{l}\text { Cent. tempera- } \\
\text { ture }\end{array}$} \\
\hline & xalla & & & & & & Max. & Min. \\
\hline & 1904 & $\circ \quad$ & $0 \quad 1$ & 。 & ' & - & $\circ$ & 。 \\
\hline A & 15 & 16610.0 & .0. & . . & . & $42 \quad 07.5$ & +1.0 & - \\
\hline & 16 & $66 \quad 09.7$ & $\cdot \cdot \cdot$ & & . . & $42 \quad 07.2$ & $-c$ & $\div$ \\
\hline B & 17 & 16609.6 & I66 I5.6 & 64 & J 4.5 & $\begin{array}{ll}42 & 06.3\end{array}$ & -2. & - \\
\hline & I8 &.$\quad$. & . . & . . & . $\quad$ & . . . & +0. & 一 \\
\hline & I9 & I $66 \quad 09.6$ & . & . . & & 4207.1 & + & - \\
\hline & 2 & 66 10. I & 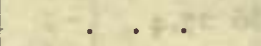 & 65 & 13.2 & 4207.6 & + & + \\
\hline & 21 & $166 \quad 10.9$ & at & .. & .. & $\begin{array}{ll}42 & 08.4\end{array}$ & + & - \\
\hline & 2 & 6 10.I & . & & . $\cdot$ & $42 \quad 07$ & + & - \\
\hline & 23 & $\begin{array}{lll}\mathbf{1} 66 & 10.7\end{array}$ & . & 63 & 32.8 & 4208.2 & + & - \\
\hline B A & 24 & I66 II.2 & . . & 64 & $42 . I$ & $\begin{array}{ll}42 & 08.7\end{array}$ & + & - \\
\hline & 25 &. & . .5 & . . & $\cdot$. & .. & + & - \\
\hline & 26 & 6 II.0 & . . & . & & $\begin{array}{ll}42 & 08.5\end{array}$ & + & - \\
\hline & 27 & $6 \quad 12.2$ & is & & & $\begin{array}{ll}42 & 09.7\end{array}$ & & - \\
\hline B A & 28 & I $66 \quad$ I 4.3 & . & 64 & $5^{1.2}$ & 42 I1.8 & + & - \\
\hline & 29 & I 66 I 5.2 &.$\quad$. & . . & & $42 \quad 12.7$ & + & - \\
\hline & 30 & I66 16.0 & . & 64 & 36.1 & $4^{2} \quad 13.5$ & +4.9 & - 0 . \\
\hline B A & July I & $166 \quad 14.9$ & 2010 & . & & $42 \quad 12.4$ & +1.7 & -0. \\
\hline
\end{tabular}

Circle reading of magnct for days on which the circle was shifted at Teplitz Bay

\begin{tabular}{|c|c|c|c|c|c|c|c|c|c|c|c|c|}
\hline Date & \multicolumn{2}{|c|}{$\begin{array}{l}\text { Chr'r } \\
\text { time }\end{array}$} & $\begin{array}{c}\text { Circle } \\
\text { reading } \\
\text { of magnet }\end{array}$ & Date & \multicolumn{2}{|c|}{$\begin{array}{l}\text { Chr'r } \\
\text { time }\end{array}$} & \multicolumn{2}{|c|}{$\begin{array}{l}\text { Circle } \\
\text { reading } \\
\text { of magnet }\end{array}$} & Date & \multicolumn{2}{|c|}{$\begin{array}{l}\text { Chr'r } \\
\text { time }\end{array}$} & $\begin{array}{l}\text { Circle } \\
\text { reading } \\
\text { of magnet }\end{array}$ \\
\hline & 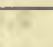 & & $x_{1+1}$ & $2+2$ & & 01 & & & $7+1$ & & & \\
\hline 1903 & h & m & 0, & I 903 & & $\mathrm{~m}$ & - & ' & 1903 & h & $\mathrm{m}$ & 。 \\
\hline Sept. 30 & & $\infty$ & $644^{0.6}$ & Oct. 4 & & 28 & 65 & 19.7 & Oct. 8 & 17 & $5^{2}$ & 6522.0 \\
\hline & o & 48 & 65 I0.0 & & & $\infty$ & 64 & 33.7 & & & 54 & $64 \quad 12.5$ \\
\hline (es) & IO & $5^{8}$ & 6536.0 & 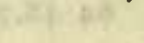 & & 22 & 64 & I0.7 & & & 04 & $653^{2.0}$ \\
\hline & I I & Io & 6455.0 & & & $5^{8}$ & 65 & 07.5 & & & & 6443.0 \\
\hline 40 & 20 & 28 & 66 II.3 & & & 54 & 64 & 14.5 & 11 & 18 & 20 & 6536.2 \\
\hline 4.15 & 20 & 34 & 6704.2 & 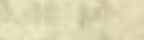 & & 08 & 64 & 59.0 & I3 & 12 & $\infty$ & 6429.4 \\
\hline natro & 20 & 36 & $65 \quad 52.2$ & 40,50 & 9 & 42 & 63 & $5^{2.5}$ & & 13 & $3^{2}$ & 6532.0 \\
\hline 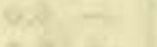 & 20 & 42 & $6455 \cdot 3$ & 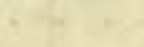 & & 54 & 64 & 17.3 & & I 3 & 48 & 6500.5 \\
\hline 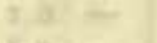 & 21 & 06 & 6610.0 & & 10 & 44 & 63 & 24.5 & & I 4 & $\infty$ & $\begin{array}{ll}64 & 17.5\end{array}$ \\
\hline $6 i 5$ & $2 I$ & 10 & 6502.3 & & IO & $5^{2}$ & 63 & 54.5 & I4 & o & $\infty$ & 6530.7 \\
\hline ?. & 21 & 28 & 6552.2 & & IO & 54 & 64 & I6.0 & & 0 & $\mathrm{O}_{4}$ & $655^{8.7}$ \\
\hline is & 2 I & $3^{8}$ & $66 \quad 52.0$ & & 22 & 36 & 66 & 07.0 & & o & IO & 6535.0 \\
\hline Eह & 21 & 42 & $\begin{array}{lll}65 & 06.7\end{array}$ & Eat $R$ & 22 & 44 & 66 & $3^{8.0}$ & & I & 04 & 6443.3 \\
\hline $\cos (x)$ & 21 & 48 & 6629.0 & 640 & 22 & 46 & 65 & 36.5 & trit & I & 26 & $\begin{array}{ll}65 & 29.7\end{array}$ \\
\hline D) - & 2 I & $5^{2}$ & 6520.1 & & 23 & $\infty$ & 66 & I 2.7 & & 2 & 08 & $66 \quad 19.8$ \\
\hline Oct. 4 & 0 & $\infty$ & 6452.7 & & 23 & 06 & 65 & 30.5 & & 2 & 44 & 6721.0 \\
\hline & 4 & 48 & 6529.7 & & 23 & & 65 & I I. 2 & & 2 & 46 & 6757.3 \\
\hline $\sin 2 \pi$ & 6 & $5 \mathrm{I}$ & $\begin{array}{lll}66 & 05.8\end{array}$ & 8 & 16 & 02 & 64 & 46.5 & . & 2 & $5^{\circ}$ & $66 \quad 39.0$ \\
\hline
\end{tabular}


Circle reading of magnet for days on which the circle was shifted at Teplitz Bay-Continued.

\begin{tabular}{|c|c|c|c|c|c|c|c|c|c|c|c|}
\hline \multirow{2}{*}{$\frac{\text { Date }}{1903}$} & \multicolumn{2}{|c|}{$\begin{array}{l}\text { Chr'r } \\
\text { time }\end{array}$} & $\begin{array}{c}\text { Circle } \\
\text { reading } \\
\text { of magnet }\end{array}$ & \multirow{2}{*}{$\begin{array}{c}\text { Date } \\
1903\end{array}$} & \multicolumn{2}{|c|}{$\begin{array}{l}\text { Chr'r } \\
\text { time }\end{array}$} & $\begin{array}{c}\text { Circle } \\
\text { reading } \\
\text { of magnet }\end{array}$ & \multirow{2}{*}{$\frac{\text { Date }}{1903}$} & \multicolumn{2}{|c|}{$\begin{array}{l}\text { Chr'r } \\
\text { time }\end{array}$} & $\begin{array}{c}\text { Circle } \\
\text { reading } \\
\text { of magnet }\end{array}$ \\
\hline & h & $\mathrm{m}$ & & & h & $\mathrm{m}$ & & & h & & $\therefore \quad 1$ \\
\hline Oct. I 4 & & $5^{6}$ & 68 ог.о & Nov. I & I & $\mathrm{O} 2$ & 6646.8 & Nov. I & 4 & 26 & 7132.6 \\
\hline & & 04 & 6606.0 & 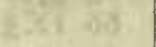 & I & 04 & $\begin{array}{ll}68 & 04.6\end{array}$ & & 4 & 28 & $\begin{array}{ll}71 & 33.3\end{array}$ \\
\hline 5.50 हो) & 3 & I 2 & $67 \times 9.3$ & 0,1520 & I & 08 & 67 I I. 8 & $4 \cot 3 \lambda$ & 4 & 30 & $68 \quad 34.9$ \\
\hline $\tan$ ox & 3 & 18 & $\begin{array}{ll}66 & 31.3\end{array}$ & 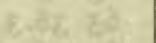 & I & I 4 & $\begin{array}{ll}66 & 19.7\end{array}$ & $6 x+y 0$ & 4 & & \\
\hline$y=28$ & 3 & 20 & 68 00.0 & $\cos y$ & I & 28 & 6457.0 & $(5,14)=3$ & 4 & & 66 I 5.3 \\
\hline हैहि रूे & 3 & 26 & $66 \quad 53.3$ & bxas ed & I & 30 & $65 \quad 17.5$ & $0.04,28$ & 4 & & 6621.8 \\
\hline If id & 3 & 30 & 6754.7 & bital to & I & 3 & $66 \times 3.5$ & 7,109 से & 4 & & 714.3 \\
\hline $40 b^{-1}$ bet & 3 & 36 & $\begin{array}{ll}68 & 49.7\end{array}$ & 5251 हो & I & 36 & 6450.7 & 6.82 . 45 & 4 & & 6645 \\
\hline 4.35 is & 3 & 38 & 67.58 .0 & $4, x_{2}, 13$ & I & 42 & 6507.2 & for 35 & 4 & & 6704 \\
\hline $8.11>8$ & 3 & 44 & 6653.3 & $5+5$ zin & 2 & ro & $6407 \cdot 3$ & S.er ot & 4 & & 6635 \\
\hline 0,83 of & 3 & 5 & $655^{\mathrm{I}}$ & ace +30 & 2 & I4 & $65 \quad 27.2$ & 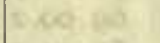 & 4 & & 663 \\
\hline fiats at & 4 & If & 643.5 & 251.28 & 2 & I & $\begin{array}{lll}66 & 31.4\end{array}$ & $2-109 \times 2$ & 4 & & $67 \circ$ \\
\hline $2=8$ is & 4 & I 8 & 73 & 5.06 7nt & 2 & 2 & 6 & F.tes rè & & & 6850.2 \\
\hline weily & 4 & 26 & 6659 & 45428 & 2 & 2 & 683 & $2.7+20$ & & & $7 \mathrm{II}$ \\
\hline H.5x ea & 4 & 28 & 6730 & C. & 2 & 2 & 664 & $1.21 \mathrm{~A}$ & & & 514 \\
\hline $5.7 b \cdot 18$ & 4 & $42-12$ & 661 & wex 28 & 2 & & 67 & $82+13$ & & & 820 \\
\hline 12028 & 8 & 58 & $\begin{array}{ll}65 & 26.8\end{array}$ & Gist in & 2 & 28 & $\begin{array}{ll}68 & 28\end{array}$ & 280 & & & 674 \\
\hline $1,8 x \geqslant 0$ & 18 & 4 & 6438.3 & 1.160 ot & 2 & 30 & 67 10 0 & $1+2+8$ & & & 68 I 7.6 \\
\hline $8+2, t^{2}$ & & 5 & 65 I 2.0 & $2,6+14$ & 2 & 3 & 6545.3 & E.t.2. & & & 6726.9 \\
\hline $2 x+2$ en & & o & $64 \mathrm{r} 2$ & $5.12,98$ & 2 & & I 5 & -170 & & & .5 \\
\hline $2-50 z^{a}$ & & I & 6505.3 & $2+64: 28$ & 2 & 4 & 6535.2 & Wes in & & & 63 \\
\hline 0.01209 & & 26 & $6404 \cdot 3$ & a.je 28 & 3 & & 6622. & $1+127$ & & & $\begin{array}{ll}68 & 09.9\end{array}$ \\
\hline $8 \operatorname{cin} 29$ & 2 & 36 & 6527 & $1.02,10$ & 3 & & 6714. & (2.71) & & & 53 \\
\hline $284+6$ & & 38 & 6502.3 & 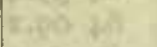 & 3 & I & 6905.6 & 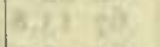 & & & 45 \\
\hline $\sin 3 x$ & & 52 & 6600.2 & 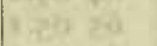 & 3 & & 7022.6 & 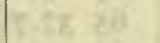 & & & 5 \\
\hline$(x+2)$ & & 56 & 6739 & teos $y=$ & & & 6846 . & .22 in) & & & 5 \\
\hline aktos & & $\mathrm{O} 2$ & 6536.0 & and 34 & 3 & & 6539.8 & 6.61208 & & & $65 c$ \\
\hline 5.7520 & & 30 & $6555 \cdot 3$ & $=.9020$ & 3 & & 6518.5 & $2-25,23$ & & & 62 \\
\hline sit it 33 & & & 6 & $x=2$ हो) & 3 & & $\alpha$ & 56 & & & : \\
\hline 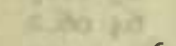 & & 4 & 53 & 8.002 in & 3 & & 3 & 6 axt & & & 64 \\
\hline I 6 & & oc & 49 & ofal th & 3 & & $652 \mathrm{I}$ & 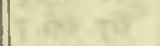 & & & 67 \\
\hline$(3+2)$ & & I0 & 36.8 & te an & 3 & & 6754 & ( & & & 5 \\
\hline 152123 & & 24 & 6422.7 & 2.7120 & 3 & & 5 & $6,4 d y$ & & & 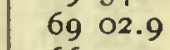 \\
\hline D.es $x \mathrm{a}$ & & 04 & 64 10. & $0 x+26$. & 3 & & 338 & (5.82. 50 & 6 & & 6655 \\
\hline $5, t+28$ & & 06 & 559.3 & $2+3 \times 1$ & 3 & & 72 OI & 1.01 ob & & & 734 \\
\hline rison & & 08 & 65 & $8<8$ & 3 & & 35 & (2) है & & & 1 \\
\hline 0.7z:an & & 10 & 6609.5 & $6 x+25$ & 3 & & 7453 & 2.618 & & & 5 \\
\hline $5.11, y 0$ & & IC & $6453 \cdot 3$ & 2.21 dit & 3 & & 7206 & & & & 6 \\
\hline 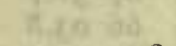 & 23 & 5 & 6457 & 6,01 -8 & 3 & & $702 \mathrm{I}$ & 62 & & & c \\
\hline I 8 & 0 & or & $6424 . \mathrm{I}$ & fol $5=$ & 3 & & $79 \mathrm{Ig}$ & 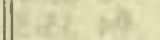 & 6 & & $884^{2}$ \\
\hline $6 x^{2}=2$ & 4 & 08 & 65 I 5.2 & Es & 4 & o & 6855 & .26000 & & & 6625 \\
\hline 26 & 7 & 59 & 66 oI & $2 x^{2}$ & 4 & & 6653 & $(\sin 20$ & & & \\
\hline 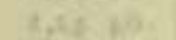 & 9 & 5 & $645^{I}$ & 1.478 & 4 & & 657 & 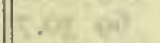 & & & 3 \\
\hline Nov. I & 0 & a & $66 \quad 40.7$ & $176=0$ & 4 & I & 6652 & atic ar & & & 68 Iо. \\
\hline & 0 & of & 6723. & 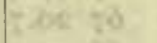 & 4 & & 6930 & o.te 80 & & & 6855. \\
\hline & 0 & I2 & $66 \quad 39.5$ & 6.480 & 4 & & 7108 & Figr. 05 & c & & 6735 \\
\hline & 0 & 14 & 6530.5 & W.25 od & 4 & 18 & $\begin{array}{ll}70 & 32\end{array}$ & $\therefore 22^{2}$ & 6 & & 6736. \\
\hline 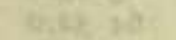 & 0 & 36 & $66 \quad 14.8$ & 6.0820 & 4 & 2 & 6636. & 0.10720 & 6 & & 6719. \\
\hline 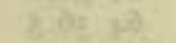 & 0 & 38 & $\begin{array}{ll}67 & 19.7\end{array}$ & E.vig. 82 & 4 & 22 & 6635. & 100 & 7 & & 68 I 5.3 \\
\hline $8.2 x<29$ & 0 & 46 & 6606.5 & $8(0) 20^{\circ}$ & 4 & 24 & 6934.3 & 1.0. & 7 & 24 & 6709.4 \\
\hline
\end{tabular}


Circle reading of magnet for days on which the circle was shifted at Teplitz Bay-Continued

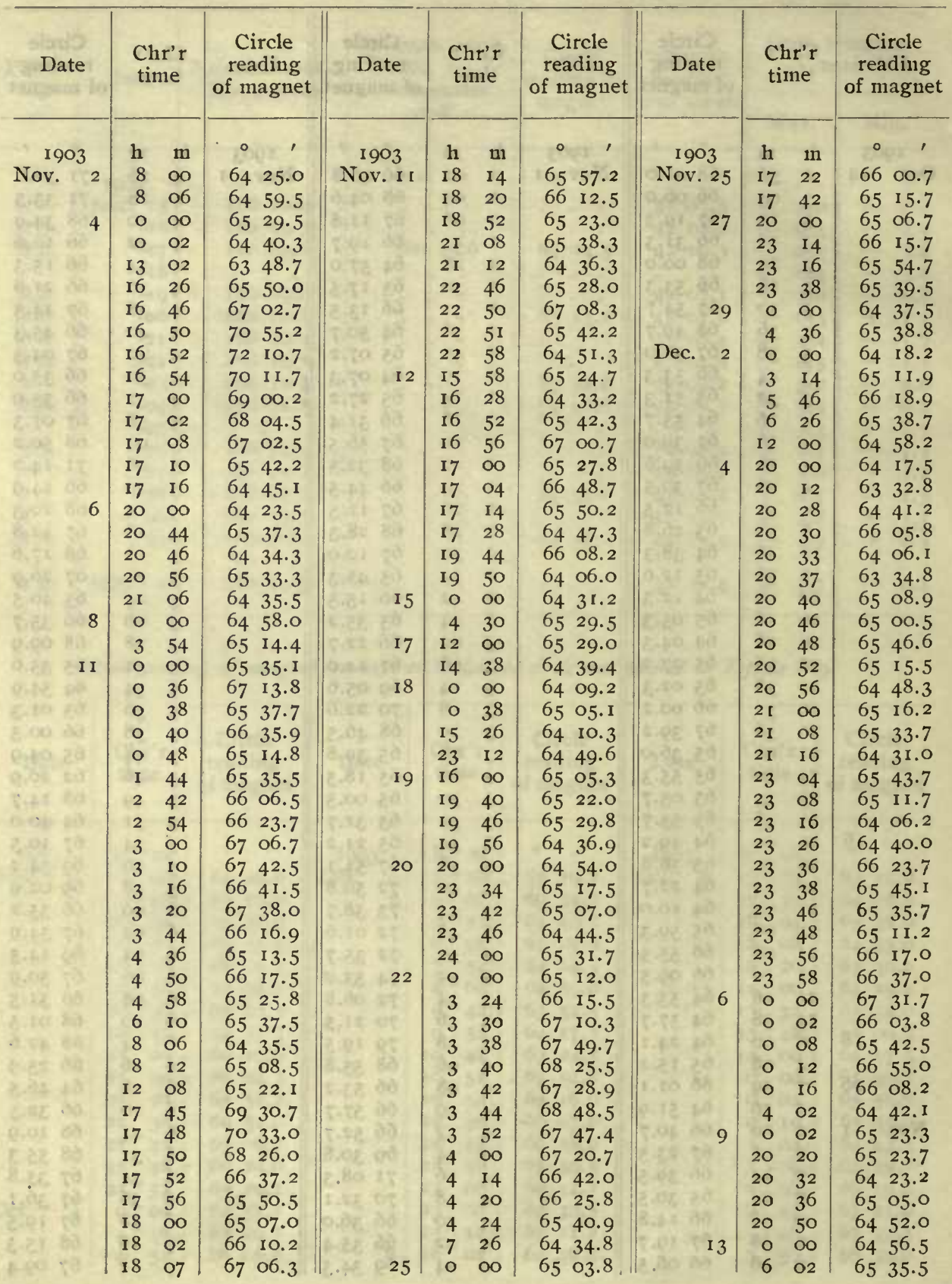


Circle realing of magnet for days on which the circle was shifted at Teplitz Bay-Continued

\begin{tabular}{|c|c|c|c|c|c|c|c|c|c|c|}
\hline Date & $\begin{array}{l}\text { Chr'r } \\
\text { time }\end{array}$ & $\begin{array}{c}\text { Circle } \\
\text { reading } \\
\text { of wagnet }\end{array}$ & I) a & & $\begin{array}{l}\text { Chr'r } \\
\text { time }\end{array}$ & $\begin{array}{c}\text { Circle } \\
\text { reading } \\
\text { of magnet }\end{array}$ & Date & & $\begin{array}{l}\text { Chr'r } \\
\text { time }\end{array}$ & $\begin{array}{c}\text { Circle } \\
\text { reading } \\
\text { of magnet }\end{array}$ \\
\hline 1903 & $\mathrm{~h} \quad \mathrm{~m}$ & 0 , & 1904 & & $m$ & & 1904 & & li & \\
\hline Dec. 16 & O $\quad 02$ & 6445.4 & Jan. & 1 & .52 & $-68-06.2$ & Feb. & 3 & 50 & 6739.5 \\
\hline 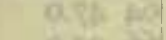 & $20 \quad 5^{8}$ & 6522 & 70 & & 54 & $645^{2.8}$ & 19232 & & $5^{2}$ & $66 \quad 11.5$ \\
\hline 0.50 is & $21 \quad 02$ & $6641 \cdot 3$ & 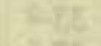 & & I9 56 & 6401.0 & 9085 & & 52 & 6537.5 \\
\hline thts & $21 \quad 08$ & $65 \cup 8.7$ & 25 & & $19 \quad 59$ & 6428.1 & 5.963 & & I9 & $6643 \cdot 3$ \\
\hline 534 & $21 \quad 10$ & 6650.9 & $=61$ & Pat & 2000 & $634^{8 . I}$ & 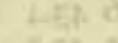 & & $19 \quad 16$ & 6538.5 \\
\hline $6,5 x$ & $2 I$ & $654^{6.7}$ & 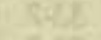 & 3 & $0 \quad \infty$ & $\begin{array}{lll}65 & 03.8\end{array}$ & $50+3$ & & 2I 24 & 6728.3 \\
\hline fere & $2 I$ & 6453 & 720 & & 308 & 6530.2 & 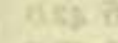 & & $2 \mathrm{I} \quad 26$ & 6628.2 \\
\hline was & $21 \quad 16$ & $\begin{array}{ll}66 \quad 25 \\
6\end{array}$ & 45 & 6 & - 00 & $65 \quad 18.4$ & 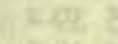 & 20 & $2 \mathrm{I} \quad 30$ & $65 \quad 12.2$ \\
\hline 400 & $21 \quad 20$ & 6544.5 & 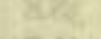 & 20 & $5 \quad 36$ & 6606.6 & Eet + & 70 & 22 & 6622.3 \\
\hline 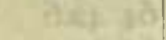 & $21 \bigcirc 26$ & 6450 & 517 & 10) & $6 \quad 29$ & $\begin{array}{ll}65 & 30.4\end{array}$ & $\cos 1 \mathrm{~s}$ & 00 & 22 & 6453.2 \\
\hline$=28$ & $22 \quad 42$ & $652 \mathrm{I}$. & 80 & 20 & I0 08 & 6455.6 & 20 & 5 & 2000 & 6456.2 \\
\hline 21120 & $0 \quad 00$ & 6453. & +34 & 워 & 2208 & 6654.0 & 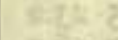 & & $21 \quad 14$ & 6822.0 \\
\hline 70120 & 306 & 6557.7 & $7 \times 3$ & . & $22 \quad 12$ & 66 o3. I & 8201 & & $21 \quad 16$ & 6724.7 \\
\hline 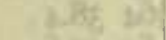 & $3 \quad 32$ & 6640. & $1+2$ & 80 & 22 & $6521 \cdot 3$ & $\sin x$ & 00 & $21 \quad 25$ & 6508.0 \\
\hline oy 80 & $\begin{array}{ll}3 & 44\end{array}$ & 6536 & Pon & 10 & oo & 6443. & $942=$ & 20 & $21 \quad 28$ & $643^{6.3}$ \\
\hline 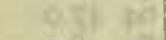 & 716 & 6441 & ATH & 23 & 18 & 6527.6 & 1581 & 20 & 21 & 6520.2 \\
\hline 30 & 00 & 6428. & $8 e^{2}$ & $M 2$ & $3^{2}$ & $\begin{array}{ll}66 & 16.7\end{array}$ & $2, n=$ & & 22 & $\begin{array}{lll}66 & 23.7\end{array}$ \\
\hline $808+10$ & $7 \quad 12$ & 6256. & 232. & 30 & $5^{8}$ & 6520.2 & $\sin \theta$ & 50 & 22 & 6743.0 \\
\hline Q85, हते & I 4 & $652 \mathrm{I}$. & $\cos$ & dy & 14 & $\begin{array}{ll}66 & 10.8\end{array}$ & $2+21$ & 15 & 22 & 6557.2 \\
\hline 0.53 ; 35 & I 8 & 6423 & (2)े & is & 56 & 6737.5 & 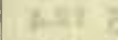 & हd & 22 & 6427.2 \\
\hline pers 30 & 24 & 65 or & attat & 20 & 24 & 6642 & $8 \times 2$ & 19 & 23 & $65 \quad 24.9$ \\
\hline$\$ 10.20$ & 42 & 662 & axt & II & $\infty$ & $6528=$ & $2 x+1$ & 7 & oo & 6501.6 \\
\hline 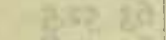 & 48 & 652 & $\pm x$ & 20 & 50 & $\begin{array}{ll}66 & 23.8\end{array}$ & $5.28 \mathrm{c}$ & (w) & Io & 6533.4 \\
\hline $3 x+2 y$ & 34 & 6427 & $=60$ & 20 & I 8 & 6535.1 & 1.3. & 20 & 16 & 6456.2 \\
\hline $87 x+8$ & $5^{2}$ & 6334 & dict & $t^{2}$ & $3^{8}$ & $645^{6.2}$ & 284 & 180 & I8 & $\begin{array}{lll}65 & 48.7\end{array}$ \\
\hline 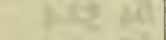 & 10 & 6444 & $R=2$ & 13 & 04 & 6434.0 & $\sec 23$ & 20 & I 02 & $\begin{array}{ll}65 & 13.9\end{array}$ \\
\hline s.y. 30 & II & 6405 & $26 x$ & 3) & 14 & 6447.1 & 2861 & (i) & $4 \quad 54$ & 6634.2 \\
\hline 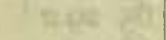 & 12 & 6530 & 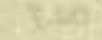 & (d) & 246 & 6528.9 & 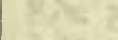 & (2) & 5 & 5548.0 \\
\hline $6=2+5$ & 17 & 6429.3 & $1+20$ & 8 & $10 \quad 06$ & 6455 & ang & (60) & 00 & 6457.7 \\
\hline $3 I$ & $20 \quad 00$ & $655^{8} 7$ & ato & 5) & $23 \quad 14$ & $\begin{array}{lll}66 & 12.3\end{array}$ & c.ent & 8 & oo & 65 II. 5 \\
\hline 52080 & $21 \quad 10$ & $66 \quad 17.8$ & 3245 & 2i) & $23 \quad 30$ & 6606.4 & $8+4=$ & is & 14 & 6424.5 \\
\hline $2=420$ & 21 & 6450.9 & sis is & 10 & 23 & $65 \circ 5$ & 01091 & IO & Oo & 6457.1 \\
\hline 100.80 & $2 \mathrm{I}$ & $66 \quad 14.7$ & Spe & 17 & $0 \quad 00$ & 6505 & 2807 & 20) & 22 & 6620.7 \\
\hline 125.65 & 2 I & 65 II.8 & 409 & 20 & 0 & 6604.0 & tho & 8 & 22 & 6434.7 \\
\hline 804.93 & $2 t$ & 6555 & 882 & ( & 400 & 6459 & 300 & (1) & 22 & 6534.7 \\
\hline Acs $\$$ & $2 I$ & 6512. & $92 x$ & 23 & $20 \quad 00$ & 6515. & owe 7 & ते & 2300 & $663^{1.7}$ \\
\hline $412+8$ & $23 \quad 24$ & 6627 . & Bis & (it) & $22 \quad 12$ & 6507.0 & axe +1 & 18 & 23 & 6449.0 \\
\hline 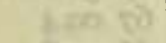 & 23 & 6521. & 6सt & 25 & 22 & 6453 & 543 ? & 20 & 23 & 6534.7 \\
\hline 8.0020 & 23 & 66 I 9.8 & & & 22 & 6544 & $x \cdot \varepsilon^{2}+3$ & 40 & 23 & 6430.3 \\
\hline $\mathrm{c}^{2} \mathrm{z} 2 \mathrm{~s}$ & $23 \quad 53$ & 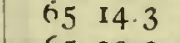 & diz & 24 & 0.02 & 6443 & 200 & II & oo & 6459.7 \\
\hline 20005 & $23 \quad 55$ & 6519 & $=28$ & a) & 406 & 6526 & दरका & & 1936 & 6630.7 \\
\hline$x+2=10$ & 23 & 6648.8 & $x_{20}$ & 3 I & - $\infty$ & $\begin{array}{ll}65 & 04.8\end{array}$ & zon है & 00 & $193^{8}$ & 6742.3 \\
\hline$t=30$ & 23 & 66 I 53 & BE: & 2) & $1 \quad 12$ & $65 \quad 55.9$ & sest $z$ & 2) & $19 \quad 42$ & 6647.2 \\
\hline 028 & 2400 & 6456.7 & $2 ., 10$ & $\theta^{2}$ & 130 & 66.31 .7 & 8.181. & 20 & 19 52 & 6724.7 \\
\hline 1904 & & & extet & खे & 136 & $6655 \cdot$ & 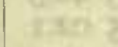 & दृ? & 2000 & 6829.0 \\
\hline Jan. I & 1600 & 6455.8 & the & $\Rightarrow$ & $4 \quad 04$ & 6433. & 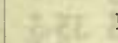 & I 5 & 800 & 6618.0 \\
\hline$x=30$ & I8 50 & 6611.2 & soge & 2it & 08 & 6531.7 & $x^{2}+2$ & & $\begin{array}{ll}9 & 28\end{array}$ & 6525.0 \\
\hline 1003,80 & $\begin{array}{ll}19 & 08\end{array}$ & 6521.7 & & & $\begin{array}{l}7 \\
7\end{array}$ & 6422.9 & *20 & 17 & 000 & 6444.8 \\
\hline $20(2)$ & $\begin{array}{ll}\text { I9 } & 34 \\
\text { I9 } & 48\end{array}$ & $\begin{array}{ll}64 & 34 \cdot 3 \\
66 & 39 \cdot 3\end{array}$ & Feb. & 3 & $\begin{array}{ll}0 & 00 \\
0 & 16\end{array}$ & $\begin{array}{ll}65 & 52.5 \\
66 & 03.5\end{array}$ & +200 & & $\begin{array}{ll}0 & 12 \\
2 & 32\end{array}$ & $\begin{array}{ll}65 & 19.4 \\
66 & 02.2\end{array}$ \\
\hline
\end{tabular}


Circle reading of magnct for days on which the circle avas shifted at Teplitz Bay-Continued

\begin{tabular}{|c|c|c|c|c|c|c|c|c|c|c|c|c|}
\hline \multirow{2}{*}{$\begin{array}{c}\text { Date } \\
1904\end{array}$} & \multicolumn{2}{|c|}{$\begin{array}{l}\text { Chr'r } \\
\text { time }\end{array}$} & \multirow{2}{*}{$\begin{array}{c}\begin{array}{c}\text { Circle } \\
\text { reading } \\
\text { of magnet }\end{array} \\
\circ,\end{array}$} & Date & \multicolumn{2}{|c|}{$\begin{array}{l}\text { Chr'r } \\
\text { time }\end{array}$} & \multirow{2}{*}{$\begin{array}{c}\begin{array}{c}\text { Circle } \\
\text { reading } \\
\text { of magnet }\end{array} \\
\circ, \quad\end{array}$} & \multicolumn{2}{|c|}{ Date } & \multicolumn{2}{|c|}{$\begin{array}{l}\text { Chr'r } \\
\text { time }\end{array}$} & $\begin{array}{c}\text { Circle } \\
\text { reading } \\
\text { of magnet }\end{array}$ \\
\hline & & & & I904 & $\mathrm{h}$ & & & 1904 & & h & & \\
\hline $\mathrm{l}^{1} \mathrm{cb} . \quad 17$ & & 30 & 6627.7 & M'ch 27 & & $5^{2}$ & 6552.0 & April & 3 & I & $3^{8}$ & $653^{2.8}$ \\
\hline $6+1 x^{2}$ & & 06 & & $=$ & & 00 & $65 \quad 16.2$ & in & & I & & 6447.0 \\
\hline 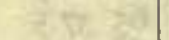 & & IO & 9 & 30 & 0 & $\infty$ & 533 & 4.74 & & & & \\
\hline $4 \times+6$ & & 14 & 664 & 15: 1 & & 46 & 6537 & soret & & & & 6737.7 \\
\hline 35.25 & & 28 & 6643.4 & April I & 20 & $\infty$ & 64 10.2 & Q.02 & & 5 & 48 & $\begin{array}{lll}66 & 25.7\end{array}$ \\
\hline $28=-3$ & 6 & 44 & 6542.2 & 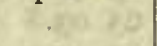 & 20 & I 2 & 6433.7 & $8+4$ & & 6 & 50 & 654 \\
\hline 65.28 & 8 & & 664 & $\operatorname{tag} x$ & 20 & I4 & 50 & $=2$ & 4 & 8 & & (5) \\
\hline E. & 8 & & $65:$ & (x)it zit & & If & 51 & 0.35 & & 8 & 26 & $65 \mathrm{I}$ \\
\hline $45 x+20$ & 12 & & 6449 & $90+2$ तd & 0 & 20 & $33^{8}$ & & & I I & $\infty$ & 3.4 \\
\hline $522+6)$ & & O & 6612 & $y_{0}(0,2)$ & 20 & 23 & 4.41 & & 6 & 0 & $\infty$ & 42.6 \\
\hline (2x) $x$ & & & & $0,82,10$ & & & & $=10$ & & 5 & 20 & \\
\hline $658 \times$ & & & 2 & 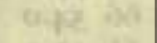 & 20 & 27 & 6 & Exes & & 5 & 54 & I. 3 \\
\hline$x+5=7$ & 21 & 2 & .7 & ifto do & 20 & 29 & 632 & & & 6 & 46 & $\begin{array}{lll}65 & 10.7\end{array}$ \\
\hline ater 20 & & & 612.2 & $2,16 \times 3$ & 0 & 32 & 45 & & & I 2 & & 6438.4 \\
\hline 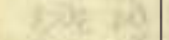 & 21 & 28 & 642 & $8+2+x$ & & 3 & 64 I0.7 & $(1+2)^{2}$ & & 14 & 22 & 5.6 \\
\hline $5:=71$ & 21 & 3 & 632 & cirte 32 & 20 & 3 & 517 & dist & & & & 6447.0 \\
\hline 1.5060 & 2 & 3 & II & F.oy pey & 20 & 4 & 20 & $145 x$ & E. & 23 & & $65 \quad 58.2$ \\
\hline 2.5 ? & & 3 & II & 505,73 & 20 & 4 & 3 & 8,96 & & 23 & 8 & 6459.8 \\
\hline 6.72 is & & & ? & $2.45-40$ & 20 & 4 & $6 \propto$ & E,15 & & 23 & 30 & 2.2 \\
\hline $58-1$. & 22 & $\mathrm{O} 4$ & 1 & $2+15=$ & 20 & 5 & 4 & 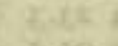 & 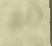 & 23 & & 452.6 \\
\hline$P+5=19$ & 22 & Is & 23 & & $2 \mathrm{I}$ & o & 4 & 6.10 & 7 & I6 & $c$ & 5422.4 \\
\hline 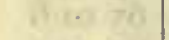 & 2 & & s & & $2 \mathrm{I}$ & & & Q6et & & 19 & $c$ & 01.2 \\
\hline 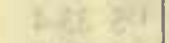 & & & ( & 6,2584 & & & 3 & ale & & I9 & & \\
\hline 460.74 & & & & 1328 & & & 0 & a,se & 8 & 20 & & 6445.8 \\
\hline $2 \mathrm{I}$ & 0 & & ( & $6,10+4$ & 21 & 24 & 3 & QHe & & 20 & 56 & $64 \quad 17.8$ \\
\hline efer 20 & 4 & & & $7+45$ is & 23 & I\& & I & $6=1$ & 10 & 0 & $\infty$ & 5452.4 \\
\hline 24 & 0 & & 3 & $158+14$ & & 2 & 12 & 5200 & & 3 & 44 & 7.2 \\
\hline 2042 & 3 & & 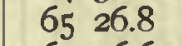 & 82520 & 2 & 28 & 02 & 2.008 & & 4 & & 9.2 \\
\hline & Io & 18 & 36 & (2) 39 & 23 & $3 I$ & 703 & & & 4 & I 4 & 56 I5.3 \\
\hline M'ch 2 & 0 & $\infty$ & & $\cos 20$ & 23 & 3 & 501 & C R 2 & & 4 & $3^{2}$ & 9.2 \\
\hline $21=19$ & 2 & Is & & $4=15$ & 23 & 40 & & 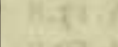 & & 4 & & 5.2 \\
\hline 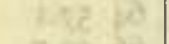 & 20 & It & 03 & the & 23 & 4 & & & & 4 & & 6642.3 \\
\hline$[002 \times$ & 2 & 22 & 08 & 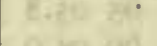 & 23 & 4 & +59 & Fin & & 4 & 4 & 6630.8 \\
\hline (12. & $2 c$ & 2. & & & 23 & 48 & & $8+1$ & & 4 & & $25 \cdot 3$ \\
\hline the & & $2\}$ & & 80 & 23 & 5 & 5 & 923 & & 4 & & 6749.6 \\
\hline-42 & 20 & 32 & & & 23 & 5 & 3 & & & 5 & & 6637.6 \\
\hline 250 & 20 & $3<$ & 57 & & 0 & 0 & 35 & & & 5 & & 6751.2 \\
\hline $1+5,5$ & 20 & & & 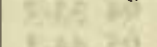 & 0 & I & 15. & ons. & & 5 & & \\
\hline 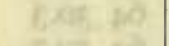 & 21 & & & 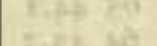 & 0 & & 17 & 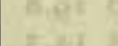 & & 5 & & $\begin{array}{lll}66 & 09.8\end{array}$ \\
\hline 4 & 20 & o & & & 0 & I & 6354 & 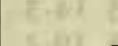 & & 6 & & 65 I 5.2 \\
\hline & 20 & 0 & 27. & & 0 & & 6438.2 & & II & 8 & $\infty$ & $\begin{array}{lll}66 & 09 \cdot 3\end{array}$ \\
\hline 40 & 20 & & & & o & 28 & & & & 8 & & 6436.1 \\
\hline E.5? & 20 & 0 & & & 0 & 30 & & $=$ & & 9 & & 21.2 \\
\hline$y+x=y$ & 20 & IC & & & o & 32 & 67 or. & & 13 & 0 & & \\
\hline 96 16 & 0 & $\alpha$ & 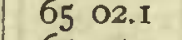 & & 0 & $3^{8}$ & $653^{8.5}$ & & & 4 & & 6555.8 \\
\hline 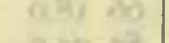 & 20 & 34 & & & 0 & 40 & (1) & 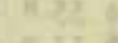 & 0 & 12 & & 6506.6 \\
\hline 20 & 0 & 0 & & & 0 & 43 & 50.2 & 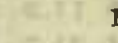 & & 0 & & 6453.4 \\
\hline act & 7 & & & & & $5^{2}$ & 6539 & 40 & & 4 & & 6549.7 \\
\hline 27 & 0 & & 6500.4 & & & IO & 6425.8 & 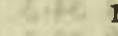 & 18 & 8 & $\infty$ & $65 \quad 19.5$ \\
\hline
\end{tabular}


Circle reading of magnet for days on which the circle was shifted at Teplitz Bay-Concluded

\begin{tabular}{|c|c|c|c|c|c|c|c|c|c|c|c|}
\hline Date & \multicolumn{2}{|c|}{$\begin{array}{l}\text { Chr'r } \\
\text { time }\end{array}$} & $\begin{array}{c}\text { Circle } \\
\text { reading } \\
\text { of magnet }\end{array}$ & Date & \multicolumn{2}{|c|}{$\begin{array}{l}\text { Chr'r } \\
\text { time }\end{array}$} & $\begin{array}{c}\text { Circle } \\
\text { reading } \\
\text { of magnet }\end{array}$ & Date & \multicolumn{2}{|c|}{$\begin{array}{l}\text { Chr'r } \\
\text { time }\end{array}$} & $\begin{array}{c}\text { Circle } \\
\text { reading } \\
\text { of magnet }\end{array}$ \\
\hline & h & 111 & - & 1904 & h & 111 & - & 1904 & h & $\mathrm{m}$ & 0, \\
\hline April 18 & 8 & 32 & $644 I .9$ & May 18 & 1 & 20 & $64 \quad 40.7$ & Junc 8 & 4 & $3^{8}$ & 6529.5 \\
\hline & & 56 & $64 \quad 09.9$ & & 2 & 52 & 6524.4 & & o & $\infty$ & 6426.8 \\
\hline & 9 & 58 & 6436.3 & & 9 & 08 & 6425.7 & & 5 & 14 & $\begin{array}{lll}65 & 05.8\end{array}$ \\
\hline 19 & 12 & oo & 6530.2 & & Io & 20 & 6521.4 & I 5 & 0 & oo & 6449.2 \\
\hline & 15 & 52 & 6440.7 & & IO & 32 & 6419.0 & & 17 & 10 & $\begin{array}{lll}64 & 09.2\end{array}$ \\
\hline 27 & o & oo & $65 \quad 33.3$ & & I8 & 56 & 6321.8 & & 18 & 06 & 6328.8 \\
\hline & 3 & 42 & 6509.0 & & I9 & I 4 & 6422.2 & & 18 & $5^{2}$ & 6247.2 \\
\hline May & 0 & oo & $643^{6.4}$ & 20 & 20 & $\infty$ & 6359.1 & & 20 & 48 & 6207.4 \\
\hline & 3 & 50 & $653^{8.3}$ & & 20 & 22 & 6259.8 & & $2 \mathrm{I}$ & $3^{8}$ & 6345.8 \\
\hline & 4 & 22 & 6637.0 & & 20 & 24 & 6350.3 & & $2 I$ & 46 & 6303.2 \\
\hline & 7 & 56 & 6719.7 & & 20 & 30 & 63 03.1 & 16 & 16 & $\infty$ & 640 \\
\hline & 8 & $\infty$ & 6459.8 & & 21 & $\infty$ & 6346.8 & 4 & I8 & 06 & 6326.7 \\
\hline & IO & 46 & 6448.7 & & 22 & I2 & 6409.3 & 19 & 0 & $\infty$ & 6430.4 \\
\hline & 0 & $\infty$ & 6425.2 & & 22 & I6 & $65 \quad 12.3$ & & 3 & & 4.0 \\
\hline & I & I 8 & 6503.7 & & 22 & 24 & 6423.0 & $2 I$ & 12 & & 6446.6 \\
\hline & I3 & 06 & 6422.2 & 22 & o & oo & 6453.9 & & 15 & 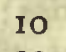 & 64 or.7. \\
\hline & 20 & $\infty$ & 6421.2 & $\therefore$ & 2 & $4^{6}$ & 6530.2 & 22 & 0 & & $\begin{array}{ll}64 & 14.7\end{array}$ \\
\hline & 23 & I 8 & 6522.6 & 24 & 12 & $\infty$ & 6533.2 & & I & $5^{8}$ & 6459.0 \\
\hline & 23 & 22 & 6416.4 & & 13 & 50 & 65 OI.2 & & 5 & 22 & 655 \\
\hline & 0 & $\infty$ & 6442.5 & & I 5 & 06 & 6420 & & II & 3 & 6458.7 \\
\hline & 3 & 42 & 6526.8 & 29 & o & $\infty$ & $64 \quad 13.8$ & 26 & 0 & & 635 \\
\hline & 0 & oo & 6457.5 & & $I$ & I 8 & 6527.2 & & 4 & $\infty$ & 65 \\
\hline & 4 & 52 & 6550.7 & & 4 & OI & 6600.7 & & 5 & 34 & 6555.4 \\
\hline & 12 & 44 & 6500 & June & o & $\infty$ & 6353 & 27 & 8 & o & \\
\hline & 16 & oo & 6305.3 & & o & 40 & 6443.8 & & 8 & 22 & 64 \\
\hline & I8 & 26 & 64 о6. I & & 3 & 48 & 6528.2 & & IO & 04 & 8.8 \\
\hline & 18 & 32 & 63 I0.0 & & 9 & $5 \mathrm{I}$ & 6427.9 & & 10 & 36 & 9.2 \\
\hline & 20 & $\infty$ & 6357.4 & 5 & 0 & $\infty$ & 6442.8 & & I I & 14 & 8.2 \\
\hline & 22 & 58 & 6453.6 & & 1 & I0 & $65 \quad 17.2$ & & 0 & $\infty$ & 6433.8 \\
\hline & 23 & $\infty$ & $65 \quad 41.5$ & 6 & 8 & $\infty$ & 6507.6 & & 5 & 06 & 6543.6 \\
\hline & 23 & 04 & & tant & 8 & 42 & 30 & $y^{5}$ & 7 & & 6456.9 \\
\hline 15 & 0 & oo & 6409.8 & & 8 & 4 & $65 \quad 16.2$ & & I3 & 48 & 58.0 \\
\hline & 2 & 52 & 65 o3.8 & 7 & I2 & $\infty$ & 6441.2 & & 23 & 38 & 6510.3 \\
\hline & 4 & $3^{8}$ & $\begin{array}{lll}66 & 05.5\end{array}$ & & 13 & I8 & $\begin{array}{ll}65 & 48.3\end{array}$ & & 23 & 48 & 64 II. 3 \\
\hline & 5 & Io & 6642.8 & & I4 & I 4 & $\begin{array}{lll}65 & 02.2\end{array}$ & July & 20 & $\infty$ & 6335.5 \\
\hline & 6 & 58 & 6550.0 & 3 & 15 & 26 & 64 17.8 & & 20 & & 624 \\
\hline 8 & 0 & oo & 6405.1 & & 15 & 46 & 6327.5 & 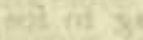 & $2 I$ & 50 & 6322.8 \\
\hline & o & 44 & 6456.9 & 8 & 0 & oo & 6455.1 & sircue & 22 & 04 & 6407.0 \\
\hline & I & & $6547 \cdot 3$ & & & & & & & & 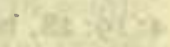 \\
\hline
\end{tabular}




\section{No'tes Accompanying Declination Records at Teplitz Bay}

October, 1903.-4, auroral display about 9:30, fog prevailing since o:00; 4:03:48. daylight begins. $7: 09: 24$, ${ }^{*}$ oscillation checked with steel pin; $7: 09: 46$, scale passed off thread, 4.5 estimated; $7: 10: 36$, oscillation checked with pin; $7: 10: 32$, quiescent for 10 seconds.-12, snow drifting, cloudy, daylight about 6 A. M., bright moon makes it difficult to determine daylight in cloudy weather.-14, slight anrora in east on beginning, sky 50 per cent clouded, windy; $14: 07: 00$, daylight begins; $14: 20: 30$, cloudy and foggy ; 14:24:00, thick, cloudy weather.-15, cloudy and foggy entire period of observation.-16:21:00, aurora appears; $16: 21: 32$, aurora disappears gradually overhead, low fog; $16: 21: 36$, aurora again appears extending from north to southwest, but not very intense ; $16: 22: 34$, wind rising ; I6: $22: 58$, aurora has disappeared. -18 , on beginning light aurora from southeast to sonthwest, clear and still; 18:00:48, aurora has disappeared; $18: 03: 34$, scale went from $40^{d} .8$ to $42^{d} .5$ in 30 seconds. - 19 , foggy; 19:09: 13 , scale traveled from $9: 13$ to $9: 15$ and then stopped. -21 , clear; $21: 04: 12$, clouded over; $21: 07: 00$, daylight begins; $21:$ r6:06, daylight ends; $21: 18: 3^{8}$ clouded entirely; $21: 20: 18, R$. R. T. reports aurora in northwest.-22:16:00, daylight ends, clear; $22: 18: 42$, very high wind in gusts; $22: 19: 32$, faint auroral light in southwest.-23, observations omitted on account of blizzard.-25, foggy, clear overhead, still; 25:03: 10, very slowly increasing; 25:03: 12, very slowly increasing; 25:03:24, wind begins to rise; 25:04:00, partly cloudy; 25:07:00, daylight begins; 25:08:22, starts to decrease; $25: 08: 40$, continues to increase from $22^{d} .2 .-27$, northeasterly storm; $27: 14: 26$, wind subsides; $27: 15: 00$, wind rises. -28 , cloudy and calm; 28:02:06, clouds breaking away overhead, foggy; $28: 03: 14$, took 30 seconds to make oscillation; $28: 03: 50.5$, took one minute to make this oscillation beginning at $3: 50 ; 28: 07: 34$, daylight begins; $28: 11: 20$, cloudy and calm, light snowflakes; 28:17:50, snowing.-29: 18:00, clouded entirely.30: $22: 30$, overcast and cloudy.

November, 1903.-I:00:00, auroral light is waning as observer begins; I:00:22, stopped for an instant and continued to decrease; $1: 00: 28$, clear sky, aurora has disappeared; $1: 00: 50$, aurora appears again, not very brilliant, clear sky; $1: 01: 12$, passed $9^{d} .0$ and came back to recorded values, aurora not very brilliant from magnetic northeast to zenith and magnetic southwest; r:or:22, aurora growing faint in northeast and zenith, southwest remains the same; $1: 01: 26$, jumps off scale from $70^{d} .2 ; x: 01: 38$, brilliant light again northeast, zenith and west; $1: 01: 58$, aurora faituter and in southeast; $1: 02: 10$, aurora mostly in north; $1: 04: 12$, a gradual change from $4: 12$ to $4: 14.5 ; 1: 07: 10$, aurora disappeared; 1:08: I2, daylight begiris.-2:08:15, cloudy, completely overcast. $-3: 12: 32$, steady at $33 \mathrm{~m}$ and begins to decrease, no oscillation.-4:00: I4, cloudy and snowing; 4:00:46, observations suspended to give order relating to ice gain signals; $4: 04: 12$, clear sky; $4: 07: 58$, daylight begins ; 4:17:12, faint aurora stretching in line overhead from magnetic northeast to southwest; 4: 19: 28, bright, moonlight night; $4: 22: 20$, faint aurora from overhead to magnetic southwest; $4: 22: 44$, aurora has disappeared.-5:16:00, thick fog prevails throughout observations. -8:00: 10, clear, light fog on horizon; 8:04: 12, clear, moonlight, still.-9:08: 22, magnet had to be raised; 9:08:50, stationary for to seconds or more; $9: 09: 30$, stationary for 10 seconds or more; 9:09:52, reaches $48^{\mathrm{d}} .0$ at $9: 53 ; 9: 09: 56$, reaches $38^{\mathrm{d}} .7$ at $9: 57.2 .-$ I $1: 00: 34$, reaches $7 \mathrm{Id}$ at $0: 34.5 ; 11: 00: 56$, faint aurora in zenith; $11: 01: 42$, reading

* The flrst figure indicates day of month, the others the hour and minute on chronometer, civil reckoning from o hour through 24 hours, thus : $23: 21: 08$ means 23 d day of month at 21 h osm or 9 h $08 \mathrm{~m}$ P. M. Directions unless _otherwise'specified are magnetic. 
estimated; II:03:30, decreasing, but stopped at this; $11: 03: 32.3$, decreasing, but stopped at this; $11: 03: 42$, scale goes off at $3: 42.5 ; 11: 09: 20$, decreased to $49^{d} .1$ at $9: 20.3 ; 11: 10: 10$, begius to decrease without oscillating; $11: 14: 04$, faint aurora in magnetic north; $11: 16: 00$, aurora in magnetic south; $11: 17: 32$, aurora, east to southeast; $11: 17: 45$, oscillation had to be reduced with the pin as scale swung beyond the thread; II: $17: 50$, aurora, north to sonthest ; 11:19:32, faint aurora, north to sonthwest; $11: 21: 06$, aurora inagnetic east to west throngh south; $11: 21: 26$, aurora has disappeared; $11: 21: 50$, faint auroral light from magnetic south to west; $11: 22: 06$, aurora from west to east through zenith; 11:22: 14 , aurora from sonth through west, faint; $11: 22: 26$, reached $75^{\mathrm{a}}$ and returned to this.12: $16: 00$, faint aurora magnetic north to southwest at begiuning; $12: 16: 50$, aurora from northeast by east to south; $12: 16: 56$, aurora extends in ill-defined bauds from northeast to southwest, a bright band extends through the south, the others between this last and zenith, one through zenith; $12: 17: 10$, aurora, three briglit streams northeast to soutliwest through zenith and throngl southern sky; I 2: 17:30.3, aurora extending $45^{\circ}$ vertically from horizon northeast ; $12: 18: 24$, wo aurora ; $12: 18: 40$, bright a turora northeast to east, $45^{\circ}$ above horizon; 12: 19: 36 , auroral band east to south, $10^{\circ}$ above horizon; 12: 19:50, auroral streak north to west, $10^{\circ}$ above horizon.-I3, omitted on acconnt of blizzard, hint inaccessible.-I5:20:16, partly clondy. - 7 , faint aurora at beginning, single strip northeast to southwest through zenith ; $17: 12: 20$, sky overcast ; $17: 14: 32$, partly cloudy, faint aurora in west, vertical strips from $15^{\circ}$ to zenith; $17: 14: 40$, faint aurora west to east through zenith; $17: 14: 50$, faint al1rora east to west through zenith and very faint patch in north $45^{\circ}$ above horizon; $17: 15: 10$, aurora growing stronger in east, patch to north has disappeared; $17: 15: 22$, scale stopped at $67^{d} .9$ and then increased to $68^{d} .8 ; 17:$ r $5: 46$, aurora has practically disappeared.-1 $8: 07: 58$, aurora in zenith extends to all points of the horizon, strongest in east and southeast; 1 8:08: 18, aurora has grown very dim, especially in the east; $18: 08: 36$, aurora has disappeared; 18:09: 36 , partly cloudy; $18: 10: 34$, overcast ; $18: 11 ; 56$, overcast ; $18: 11: 14$, clear in zenith, very faint aurora from northeast to southwest through zenith; $18: 12: 48$, aurora has disappeared; $18: 13: 04$, faint aurora starts in zenith with increasing streamers extending $30^{\circ}$ to southwest ; $18: 13: 08$, faint aurora, uortheast to southwest, stronger in southwest; $18: 13: 24$, aurora increasing in strength, extending from zenith to horizon from north to east and north to southwest, stronger in east; $18: 13: 30$, aurora stronger in north, has disappeared in east ; 18: 18: 14, aurora through zenith from northeast to southwest; $18: 18: 46$, aurora has almost disappeared; 18: 18:56, faint aurora in broken patches from north to east and half way to zenith ; 18:19:08, aurora has disappeared; $18: 19: 58$, very faint aurora in north, west and south ; 18: 20: 10, aurora has disappeared; $18: 20: 22$, faint aurora in south and southwest ; 18: 20: $3^{2}$, aurora in arch from northeast to northwest, vertex of arch $45^{\circ}$ above horizon, also streamers from zenith to south and west ; $18: 20: 42$, very faint aurora extends in spots from east to west through zenith; $18: 21: 00$, horizontal circle accidentally moved; $18: 21: 06$, a urora has disappeared; $18: 23: 12$, clear sky, no aurora; $18: 24: 55$, telescope reversed on azimuth mark and gave same reading.-19: 6 : oo, faint aurora at beginning from northeast to southwest through zenith, stronger in southwest; 19: $16: 30$, aurora has disappeared; 19:19:36, aurora reappears from northeast to southwest along horizon.-20:20:00, southeast blizzard throughout observations. - 22:01:58, strong gale from south with flying snow; $22: 04: 54$, decreased to $27^{d} \cdot 0 .--23: 08: 30$, increased to $42^{d} .9 ; 23: 08: 36$, decreased to $27^{d} \cdot 7 ; 23:$ I I: 22 , decreased to $25^{\circ} .2 ; 23: \tau 1: 38$, this oscillation in 30 seconds. $-25: 00: 00$, blizzard, 48 to 60 miles pər hour, had to dig ont entrance; $25: 11: 16$, calm; $25: 12: 50$, wind rising ; $25: 18: 54$, aurora northeast to southwest throngh south at $45^{\circ}$ altitude; 25:21:06, cloudy.-26: 16:00, eastsoutheast blizzard.-27:20:00, blizzard continues; $27: 19: 30$, plane of detorsion is $35^{\circ}$, this change since last reading probably due to slackening of fiber before $10: 00$ in an attempt to get intensity observations.-30: $1 \mathrm{r}: 3^{8}$, foggy and snowing. 
December, 1903.-I: 12:22, overcast.-2:01:14, slowly decreasing after this reading; 2:02:05, 80 per cent thin clouds; $2: 03: 40$, clear, caln1, moonlight night; $2: 03: 58$, increased to $54^{d} .2 ; 2: 05: 50$, clear, moonlight, no auroral light ; $2: 05: 54$. I, 30 seconds for this oscillation; $2: 07: 40$, aurora extending from zenith to within $10^{\circ}$ of horizon from north to west through zenith; $2: 07: 58$, aurora from northeast through zenith; $2: 08: 18$, anrora has almost disappeared ; $2: 08: 24$, anrora grows stronger from zenith to nortlieast and southwest ; $2: 08: 46$, aurora very faint ; $2: 09: 02$, aurora has practically disappeared; $2: 09: 10$, aurora grows stronger in southwest, has disappeared in northeast ; $2: 10: 06$, anrora has disappeared; $2: 11$ : 54 , faint aurora northeast to southwest through zenith; $2: 12: 22$, anrora extending from zenith in all directions, chiefly northeast and soluthwest, strongest in southwest ; $2: 12: 34$, aurora in band from northeast to south $15^{\circ}$ above horizon, also faint patches in southwest ; $2: 12: 42$, aurora has almost disappeared in southwest ; $2: 12: 50$, a urora very faint ; $2: 13: 10$, aurora has disappeared; $2: 16: 58,50$ per cent clouded, no aurora; $2: 18: 04,75$ per cent clonded; $2: 21: 58$, aurora in band from east to west througli zenith, stronger in east; $2: 22: 06$, aurora lias disappeared. $-4: 20: 00$, moon visible through clouds during most of observations, very well defined halo.-6:00:44, decreased to $26 \mathrm{~d} ; 6: 01: 38,90$ per cent thin clouds; $6: 03: 12$, sky overcast entirely; 6:05:50, clear sky.-7:08:00 moon has ill-defined halo, no aurora; $7: 11: 24$, very faint aurora in vertical stripes from horizon $11 p$ to $15^{\circ} ; 7: 11: 48$, anrora just starting in northwest and north-northrvest, one vertical stripe in each direction, $15^{\circ}$ long, half way between horizon and zenith. $-8: 14: 22$, aurora in several bands from northeast to southwest through zenith; $8: 14: 34$, aurora growing stronger to eastward; $8: 14: 46$, anrora has almost disappeared ; 8:15:06, aurora grows stronger to the eastward; $8: 15: 18$, aurora grows weaker in the east; $8: 15: 28$, increased to $36^{4} .9 ; 8: 15: 38$, aurora in well-defined arch from northeast to southwest, vertex of arch in southeast $15^{\circ}$ above horizon ; another arch later appeared above the first vertex southwest $50^{\circ}$ above horizo11.- $9: 00: 00$, aurora during axis observations; $9: 02: 16$, wind rises; $9: 03: 32$, high wind; $9: 05: 48$, increasing, but stopped a moment here; $9: 08: 02$, shoveling snow from entrance, no time for observers to alternate; 9: 10: 10, calm; 9:10:48, wind rises; $9: 14: 14$, shoveling snow from entrance, stopped at I $4: 24 ; 9: 18: 22$, shoveling snow from entrance; $9: 20: 48$, had to clieck needle with steel pin. 10: $17: 10$, increased to $56^{\mathrm{d}} .2 ; 10: 17: 14$, decreased to $53^{\mathrm{d}} \cdot 7 .-16: 16: 3^{8}$, shoveling suow from entrance; 16:16:50, no time for more alternation on acconnt of drift against door of hut; 16:2r:30, clecked with steel pin one ininute before observation; $16: 22: 50$, checked with steel pin one minute before observation.-1 7: 16:00, partly clondy.-r 8:22:20, aurora in snake-like clonds and streaks from southeast to southwest $20^{\circ}$ above horizon, constantly changing position and shape; $18: 22: 3^{2}$, aurora forms arch from east to west through zenith, also streak from south to west $15^{\circ}$ above horizon; $18: 22: 58$, aurora growing weak ; 18:23: 12, aurora in streaks southwest to west $15^{\circ}$ above horizon ; 18:23:42, a11rora has disappeared. $-21: 08: 08$, aurora from zenith to nortleast to north; 21:08:42, aurora has disappeared.-22:12:00, had to dig out entrance.-23:03:37, decreased to $33 \mathrm{~d} ; 23: 03: 50.2$, 30 secouds for this oscillation; $23: 07: 32,40$-mile wind and drifting snow, had to dig ont entrance; $23: 12: 52$, aurora from northeast to southwest through zenith; $23: 13: 48$, faint auroral light in uorth; $23: 14: 56$, had to dig out entrance; $23: 15: 04$, ten auroral streams from northeast, not very bright ; $23: 18: 16$, clear; $23: 21: 28$, very faint aurora, streaked northeast to southwest; $23: 21: 34$, aurora growing considerably brighter in east; $23: 21: 40$, aurora has mottled appearance in east, practically gone in west; $23: 21: 56$, aurora has disappeared entirely in west and increased in east, with vertical streamer from horizon to zenith in east and mottled appearance in south ; $23: 22: 02$, anrora has very much diminished to one suall spot in northeast, with mottled appearance in southwest increasing; $23: 22: 08$, cliecked motion with steel pin; $23: 22: 24$, aurora has disappeared; $23: 22: 36$, aurora east to west through zenith, 
partly mottled and partly moving streamers; 23:22:44, aurora growing stronger in west, suake-like streak in soutliwest from zenith to horizon, where it is mottled, very faint in northeast; $23: 22: 52$; snake-like streak northeast to southwest through zenith, also a horizontal streamer $10^{\circ}$ above horizon sontheast to sonthwest; $23: 23: 28$, a urora has disappeared. -25 , observations were not taken on account special request of Mr. Fiala to be present at Christmas dinner.-26, the fibers were found broken, the suspension tube was takeu to the dwellinghouse to attach new fibers, meanwhile a strong blizzard came up preventing return to hut.-29: $14: 46$, clear, moonlight. $-30: 00: 00$, clondy; $30: 06: 14$, increasing to $38 \mathrm{~d}$ in one minute; $30: 06: 22$, stops at $29^{d} .3 ; 30: 06: 46$, stops at $22^{4} .0$ and then contintes to decrease to $6: 48 ; 30: 07: 04$, reaclied $42^{d} \cdot 5$ after this reading; $30: 07: 08$, rapidly decreasing to $9^{d} \cdot 5$; 30:07: 10, increases to $6 \mathrm{I}^{\mathrm{d}} \cdot 9 ; 30: 06: 20$, faint aurora through zetith to southwest, fog all along horizon ; 30:08:00, clouded all over; 30:09:54, increased to $39^{\mathrm{d}} .1 ; 30: 09: 24$, faint aurora through zenith to northeast and southwest; $30: 09: 56$, clear sky, no aurora; $30: 13: 16$, clear and moonlight ; $30: 15: 08$, increased to $38 \mathrm{~d} ; 30: 20: 18$, clear, moonlight, no aurora ; $30: 23: 44$, clouded over.-3 I: 2 I:00, overcast and snowing.

January, I904.-1: I7: 14, snowing.-3:00:00, clear, moonlight niglit, 110 aurora.4:08:00, clear, moonlight, no aurora ; 4:09:58, scale increases rapidly. - 5: I 2: 14, temperature falling rapidly outside; $5: 12: 44$, aurora in streak $I 5^{\circ}$ above horizon from west to north; $5: 12: 50$, faint aurora northeast to southwest through zenith; $5: 12: 56$, aurora growing stronger, streak becoming wavy; 5:13:00, aurora growing stronger in northeast, now two streaks; 5: I 3: I 2, aurora a series of bands close together northeast to southwest through zenith, stronger in northeast ; $5: 13: 24$, aurora growing fainter, particularly in southwest; $5: 13: 32$, aurora stronger in southwest; $5: 13 ; 44$, aurora growing stronger in northeast; $5: 14 ; 02$, aurora very faint; $5: 14: 08$, aurora in broad band from horizon to zenith northeast, extremely faint in southwest from zenith to horizon in band; $5: 14: 16$, aurora in three wavy bands from northeast to southwest through zenith; $5: 14: 54$, aurora has entirely disappeared.-6:04:18, scale decreases unsteadily; 6:10:00, high wind, snowing; $6: 13: 08$, wind moderating; $6: 16: 28$, calm; 6:16:20, overcast ; $6: 16: 36$, snowing ; $6: 18: 50$, calm, overcast, light snow ; $6: 21: 14$, temperature rising very rapidly outside. $-7: 16: 00$, easterly wind, about 35 miles an hour; 7: 19:42, calm. -8:20:00, easterly wind, about 25 miles an hour.-10:00:26, faint a urora, north wind, 20 miles; 10:06:06, after this small oscillation scale continues to increase.11:08:30, aurora northeast to southwest through zenith.-12:12:00, overcast and foggy; 12: $15: 28$, aurora, faint, northeast to southwest, one arch with vertex $60^{\circ}$ above horizon in sontheast, another streak parallel to arch and above it ending in zenith; I2: 15:40, streak and arch have united to form one broad band stronger in northeast. -13:03: 10, magnet vibrating up and down; 13:03: I4, vertical vibrations have ceased; I3:04:16, magnet vibrating up and down; 13:04:20, clear, light fog on horizon; $13: 04: 28$, vertical vibrations have ceased; I3:05: 16, magnet checked with adjusting pin; 13:06:22, clear and calın; 13:07:50, scale continues to decrease after reading of $56^{\mathrm{d}} .5$ taken; I $3: I_{3}: 44$, arc-shaped aurora from northeast to northwest ; I3: $14: 56$, aurora still northeast to northwest and bulk increased by about two ; 13: $15: 40$, aurora has disappeared; I3: 16: I6, faint aurora north to west in arch northwest $10^{\circ}$ above horizon; $13: 16: 3^{2}$, aurora very faint; $13: 17: 40$, aurora has practically disappeared; I 3:20:04, faint aurora in streak, northeast to southwest, stronger in northeast; 13:20:24, aurora has disappeared entirely in southwest; $13: 20: 32$, aurora in two arches northeast to southwest, vertices of both in southeast, the one $20^{\circ}$ and the other $50^{\circ}$ above the horizon ; 13:20:48, aurora in broad irregular band, northeast to southwest through zenith; $13: 21: 28$, aurora has disappeared; $\mathrm{I}_{3}: 22: 26$, aurora reappears in two streaks from northeast to south $10^{\circ}$ above and parallel to horizon, changing rapidly to mottled appearance; $13: 22: 30$, aurora 
from zenith northeast to south, snaky streamers with transverse rays; $13: 22: 38$, aurora extremely faint; $13: 22: 44$, aurora grows stronger; $13: 22: 56$, allora very faint; $13: 23: 16$, $78^{\mathrm{d}} .7$ estimated; $\mathrm{r} 3: 23: 36$, aurora growing faint.-I $5: 20: 00$, clear sky; $15: 2 \mathrm{r}: 32$, hazy along horizon; 15:21:38, overcast.-I 7:00:00, overcast.-i9: 12:00, one fiber of the suspension broken during observations this day, but it apparently does not touch tube.20:00:00, hroken fiber removed before observations, magnet remaining suspended by bit three fibers; 40 mile wind from sontheast, drifting snow; 20:00:26, magnet checked with adjusting pin ; $20: 07: 46$, sloveling snow from entrance; $20: 17: 04$, very faint aurora in spots west to sonth $10^{\circ}$ above horizon; 20: 18: I8, overcast; 20:20:22, calm.-22, observations omitted this day on account of blizzard, wind being 60 to 70 miles per hour.-23:20:00, partly cloudy; $23: 2 \mathrm{I}: 36$, aurora in irregular band fron east to sonth and from horizon to $10^{\circ}$ above horizon ; $23: 2 \mathrm{~s}: 46$, aurora growing stronger and moving considerably ; $23: 22: 06$, strong anrora from zenith to horizon northeast to south, stronger in east and moving rapidly ; 23: 22: 14 . aurora from zenith in all directions, moving rapidly; $23: 22: 24$, aurora fainter, it is now in the northeast and southwest to south ; stronger in southwest to south, where it consists of snaky clonds; $23: 22: 46$, anrora fainter; $23: 22: 58$, anrora in irregnlar moving circular streaks having their centers in zenith ; $23: 23: 18$, anrora in irregular, snaky streak from zeuith to northeast and southwest ; $23: 23: 34$, aurora in irregular streaks and spots in all parts of the sky; 23:23:56, scale decreases, stopped here and then continnes to decrease.-24:02:22, wind rising ; 24:03:46, foggy, except in zenith; 24:05:18, wind light; 24:06:30, calm, cloudy, Snow. - 25:08:00, overcast.-26:12:00, partly cloudy, wind sontheast, velocity 35 miles; 26: 13:20, calm.-27:00:00, clear, moonlight night, light wind, 110 aurora; $27: 10: 36$, hazy $10^{\circ}$ above horizon; $27: 12: 00$, sky hazy; $27: 14: 18$, sky clear, except in the south; 27: 14:56, scale decreases; $27: 16: 14$, calm, clear, moonlight; $27: 19: 30$, thin clouds, stars and moon visible, light wind from east ; $27: 19: 50$, instrument slightly out of level ; it was probahly leveled by striding level which appears level at present but probably has thin suow particles on axis; instrument not disturbed by attempting adjustment since level but slightly ont ; fiber hangs free in center of tube ; 27:21:40, magnet starts from its quiet phases ; calm, moonlight, faint clouds all over sky but stars visible through then ; $27: 22: 30$, scale snddenly increases to this: 27:24:02, striding level taken off, cleaned, reversed and found to be in good adjustment ; the plate level is slightly out of adjustment ; the wves appear clear.-28: r6:00, plate level adjusted before observations began; $28: 16: 12$, very faint anrora northeast from horizon to zenith; $28: 16: 54$, aurora has disappeared; $28: 17: 06$, very faint aurora in southwest, zenith to horizo11; $28: 17: 45$. altrora has disappeared; $28: 18: 56$, aurora in irregnlar horizontal streak south to southwest $10^{\circ}$ above horizon ; 28 : 19:04, 110 aurora ; $28: 19: 26$, after this scale increased to $32^{\mathrm{d}} .8 ; 28: 19: 36$, aurora in irreg 11 lar horizontal streak northeast to east $10^{\circ}$ above horizon; $28: 19: 44$, anrora northeast zenith to horizon; irregular horizontal band southeast to south-southwest $10^{\circ}$ above horizon; $28: 19: 52$, anrora stronger from zenith in all directions northwest to southwest, horizontal streak northeast $10^{\circ}$ above horizo11.- $3 \mathrm{I}$, magnet was dropped on floor in afternoon; 3 I: or:52, wind rises, no anrora.

Febmary, 1904. - 2: 12:20, calm, overcast ; $2: 14: 38$, scale increased to $38^{d} \cdot 9 .-3: 00: 02$, scale decreased to $45^{d} .0$, then increased to $66^{\circ} .0 ; 3: 00: 44$, scale stopped a moment here and then continned to rod; $3: 00: 50$, scale reached 4 od on this swing; $3: 0 \mathrm{r}: 38$, wind has risen ; 3:05: I 2 and $\mathrm{r} 4$, scale increases very slowly; 3:07:30, decreasing slowly and irregularly; $3: 07: 36$, wind in sq11alls, clear in north, moon visible, stars visible in worth; $3: 07: 44$, shoveling snow from entrance; $3: 08: 22$, high wind through $20: 00 ; 3: 14: 46$, cloudy; $3: 15: 50$, shoveling snow from entrance; $3: \mathrm{r} 8: 50$, wind verv strong, at end observations wind light, from northeast.-4: $6: 00$, high wind, clear overhead, cloudy in south; 4: 19:02, almost 
calm, clear overhead, hazy to $30^{\circ}$ above horizon.-5:2r:06, scale stopped here, then decreased; $5: 21: 18$ to 22 , unable to check magnet with pin, had to stop motion with block and then check with pin; 5:22: I0, aurora in northeast and zenith of irregular shape, light wind, clear overhead; $5: 22: 38$, allrora faint in east; $5: 23: 12$, aurora in faint streaks from zenitli to northeast and east. $7: 00: 06$, hazy $20^{\circ}$ above horizon, aurora in south; 7:00:24, faint streaks of aurora in south and worth; 7:01:08, arc-shaped aurora from zenith to northwest; 7:01:24, scale decreasing; 7:01:44, aurora lias disappeared, sky overcast, calm, few stars to be seen; $7: 02: 28$, faint streaks of aurora from northeast to zenith, more stars visible ; $7: 03: 34$, no aurora visible, calın, sky hazy; $7: 04: 34$, scale increasing; $7: 04: 42$, scale increasing.-9: 14:42, daylight ends, about 5 hours' duration; $9: 15: 24$, very faint aurora in northeast.-10:00:00, faint aurora in south at $10^{\circ}$ altitude, bank of clouds below; ro:01:06, scale increasing but does $110 t$ pass $48^{\mathrm{d}} .2$; I0:0 $1: 52$, scale reaches $45^{\mathrm{d}} .5$ at $1: 53 ;$ 10:03:50, scale quiescent at $35^{\mathrm{d}}$. I and then continues decreasing almost imperceptibly; ro:07:28, wind of 10 miles velocity from southwest, cloudy, no aurora; ro: $08: 22$, scale decreased to $42^{\mathrm{d}} .0$; 10:08:34, scale increased about 7 divisions; 10:09:24, scale increasing; I0: 10:50, scale decreasing; I0: $12: 44$, scale increases to $55^{\mathrm{d}} .0$; $10: 16: 38$, overcast ; $10: 22: 12$, scale decreasing rapidly; scale reads $17^{\text {d }} .1$ at $22: 14.4$; I0:22:54, decreasing off scale.- II: 19:34, brilliant aurora from northeast to southeast.-I2:20:00, sky clear, no aurora; I2:22:56, bear trying to break into observatory ; $12: 23: 20$, aurora in southeast; $12: 23: 40$ to 48 , observer investigating outside to see if bear is gone.-I4:00:00, cloudy, light wind; 14:06:18, scale increased to $60^{d} .1 .-17: 01: 08$, scale decreasing to $21^{\mathrm{d}} .2 ; 17: 01: 42$, scale increased to $64^{4} \cdot 5 ;$ I $7: 0 \mathrm{r}: 56$, scale decreasing; $17: 03: 00$, high wind during balance this day's work; I 7:03:40, scale decreasing; $17: 05: 58$, scale decreasing to $4 \mathrm{r}^{\mathrm{d}} .0$ after $5: 00 ; 17: 06: 42$, magnet checked with adjusting pin; $17: 07: 02$, increasing; $17: 07: 42$, shoveling snow from entrance ; 17:08: $3^{8}$, daybreak; $17: 08: 42$, magnet oscillating vertically, hut perfectly steady in high wind; $17: 08: 58$, vertical oscillation has ceased; $17: 12: 08$, shoveling snow from entrance; I 7: $16: 05$, no more alternating on account of snow drifting against entrance to hut.- $18:$ I $7: 48$, faint aurora in northeast, wavy streak from horizon to zenith; $18: 18: 22$, aurora northeast to sontluwest through zenith; $18: 18: 48$, after this oscillation scale decreased to $30^{\text {d }} .0$; $18: 18: 52$, aurora much stronger in northeast where it consists of many irregular bands; I8: I9:48, after this scale increased to $42^{\mathrm{d}} .8 ; 18: 20: 00$, maguet dropped from top of pier to floor.- I9, high wind throughout observations this day.-20:00:56, very faint aurora.-23, duration of daylight, 8 hours. -24 , clear and calm at beginning of observations, wind rising to 60 miles an hour at $7: 10$ and continuing until abont $14: 00$, when it began to slacken, being light at end of day's work; $24: 07: 30, \mathrm{~J}$. V. enters hut, has two small iron rings on person, these are removed; $24: 08: 08$, reading increased on next oscillation to $55^{\mathrm{d}} \cdot 2 ; 24: 10: 32$, magnet checked with adjusting pin ; $24: 16: 00$, temperature rising rapidly outside all day; $24: 16: 58$, daylight ends; $24: 19: 26$, magnet vibrating up and down; $24: 21: 48$, scale increasing to $55^{\mathrm{d}} .8$ at $2 \mathrm{I}: 49.8 ; 24: 2 \mathrm{I}: 52$, very faint aurora east to southeast; $24: 22: 38$, aurora becoming much stronger and extending from east to south. $-28: 00: 00$, sky overcast, revolver placed for first time in far end of sight shaft; $28: 00: 56$, pocket knife found on person and removed; 28:05: 26 and 28, scale increasing.

March, I904.-I:I2:00, partly cloudy and snowing; revolver not taken to hut.-2:00:00, revolver not taken to hut; occasional gusts of wind; $2: 06: 14$, daylight begins ; $2: 12: 26$, wind increasing; $2: 14: 18$, wind diminishing, clear; $2: 16: 14$, light clouds in south, balance sky clear, moderate wind; $2: 17: 22$, daylight ends; $2: 19: 00$, sky clear, wind very light; $2: 20: 00$, moon rises in east with very distinct four-armed cross through center; $2: 20: 16$, scale increased to $66^{d} .0 ; 2: 20: 20$ and 24 , magnet checked with adjusting pin ; $2: 20: 30$, irregular spiral aurora 
in south and zenith; $2: 20: 42$, very light wind, faint aurora in south, cross still in moon; 2: $20: 52$, aurora from zenith to east and west ; $2: 21: 12$, faint aurora in west, dark clouds in east and west ; $2: 21: 38$, aurora has disappeared, partly cloudy in east, wind light. $-3: 16: 00$, revolver not taken to hut; high wind. $-4: 20: 00$, revolver not taken to hit, aurora in north; 4: $20: 46$, passage to vestibule drifted in so much that observer cannot very well observe weather conditions or aurora. -6 , preparations for sledge journey being completed, no time to carry out observations this day. -7 , sledge party left this A. M. $-9: 06: 26$, observations interrupted by failure of light. -1 3:02:46, magnet vibrating up aud down; $13: 04 ; 02$, daylight begins; I $3: 06: 36$, increasing but stopped a moment at this reading. $-16: 03: 42$, daylight begins; 16:08: 18, magnet taken out and replaced (not being level in stirrup either way); 16: 12: 0 , clear overhead, light fog on ice field; $16: 12: 32$, scale increasing almost imperceptibly; $16: 16: 32$, observer can see no cat1se for this jump of needle; $16: 21: 18$, daylight ends; $16: 22: 22$, sky clear; $16: 22: 54$, aurora from northeast to zenith increasing in intensity; $16: 23: 10$, very faint aurora in zenith and southwest. $-17 ; 16: 00$, instrument removed from pier in morning to clean grooves in which foot-screws set.- I $8: 22: 00$, wind velocity about 20 miles an hour, sky hazy. - 20:00:00, instrument cleaned in morning, revolver not taken to hut; 20:03:28, daylight begins.-21:11:36, revolver not taken to hut; owing to 60 to 70 mile wind hut conld not be reached before $11: 30 .-22: 12: 00$, revolver not taken to hut; 22:14:21, checked magnet with adjusting pin.-23:00:00, revolver not taken to hit; 23:00:06, maguet checked with adjusting pin; 23:03:18, daylight begins; 23:05:30, wind ceases; 23:06:46, wind in gusts; 23: 08: 58, light northerly wind, sky cloudy; 23: 15:54, wind from northwest; 23: 16:04, trouble with light cause of delay in observations; $23: 22: 06$, sufficient light to see dwelling distinctly through flying snow; $23: 23: 18$, daylight ends.-24: $16: 00$, revolver not taken to hut. $-25: 24: 10$, fiber broke.-26:11:30, put in four new fibers.-27:02:46, daylight begins; $27: 05: 22$, decreased to this, stopped, and then increased.-28:08:00, fibers found broken; three new fibers put in and observations started as soon as torsion taken out; fibers again broken during intensity observations and replaced by four new fibers at $23: 00$.29: I3:54, scale increased to $57^{\mathrm{d}} \cdot 4$ at $13: 55 \cdot 2$. - 30:00:00, sky partly cloudy, north-110rtheast wind of velocity 48 miles per hour; 30:00: 12, observer found tin box in pocket and put same away where would not affect instruments; $30: 06: 00$, wind decreasing; 30: 12:41.7, scale reached $28^{\mathrm{a}} .7$, decreased steadily, stationary for a moment at $16^{4} .8$, decreased to $10^{4} .0$ after reading at $12: 4^{6}$, then increased to $18^{d} .7$, and then oscillated as shown at $12: 50 ; 30: 15: 04$, scale decreased to $\mathrm{I}^{4} \cdot 6 ; 30: 15: 54$, magnet vibrating up and down; $30: 20: 02$, scale increased to $22^{\mathrm{d}} \cdot 9$, stopped, decreased to $22^{\mathrm{d}} \cdot 1 ; 30: 20: 10.6$, scale increased to $51^{\mathrm{d}} \cdot 7 ; 30: 20: 38$, increased to $49^{\mathrm{a}} .1$, stopped, then increased to $5 \mathrm{I}^{\mathrm{d}} .9$ at $20: 38.9 ; 30: 20: 44$, scale decreased to $38^{\mathrm{a}} \cdot 7$, stopped, then decreased to $34^{4} .9$ at $20: 44.2 ; 30: 20: 46$, then increased to $36^{\mathrm{d}} .2 ; 30: 21: 13$, scale increased to $26^{\mathrm{a}} .2 ; 30: 21: 14$, decreasing to $28^{4} .1 ; 30: 21: 4^{0}$, reading $6^{\mathrm{a}} .4$ estimated; $30: 21: 46$, daylight ends.

April, 1904.-1:20:00, revolver not taken to hut; $1: 20: 06.5$, decreased to $28^{4}$.0.$3: \infty 0: \infty$, revolver not taken to hut; $3: 00: 36.4$, scale increased to $78^{\mathrm{d}} .1 ; 3: 00: 38$, scale decreased until passed out of field of view; $3: 02: 26$, daylight begins. $-4: 08: 00$, revolver 110t taken to hut ; $4: 10: 58$, scale increased to $72^{\mathrm{d}} .9 .-5: 12: 00$, revolver not taken to hut.$6: 02: 00$, sky clear, calm; $6: 16: 14$, instrument slightly out of level. $-7: 16: 00$, sky clear, calm. $-8: 20: 00$, sky clear, light north wind.-10:02:08, scale decreased to $65^{\mathrm{a}} \cdot 2$; 10:03:20, scale increased to $52^{a} .2 ; 10: 05: 44$, reading $78^{1} .7$ estimated.-1 $1: 08: 00$, northeast wind of 40 miles per hour velocity.-12: 12:00, revolver not taken to hut; I2:12:08, magnet oscillating up and dow1.-13:00:00, light southwest wind; $13: 07: 00$, wind south, liazy, light snow; 13:09:12, from quiescence scale decreased; 13:16:59, scale $110 \mathrm{w} 20^{4} .6$.- 
14: 16:00; sky clear, light north. wind.-15:22:20, light east wind, hazy around horizon.I 7:04:10, magnet checked with adjusting pin; 17:07:02, scale increased to $31^{\mathrm{d}} \cdot 3 ; 17: 07: 06$, scale decreased to 6 d $.7 ; 17: 07: 08$, scale increased to $32^{d} .9 ; 17: 07: 12$, scale increased to $46^{d} .8$; 17:07:20, scale decreased to $29^{d} \cdot 3 .-18: 08: 00$, sky hazy, wind variable.-19:12:00, revolver not taken to hut, calm, sky clear; 19: $15: 10$, soutleast wind rising.-20:00:00, revolver not taken to hut, southeast wind of velocity 45 miles an hour, drifting snow ; 20:07:54, wind velocity now 60 miles an hour.-21:16:00, revolver not taken to hut, wind soutlieast and east in gusts; $21: 18: 10$, magnet oscillating" vertically.-22:20:00, revolver not taken to hut, wind south and east-southeast. $-24: 00: 00$,-revolver not taken to hut, wind from southeast. -26:12:00, revolver not taken to hut, wind north and northwest.-27:00:00, revolver not taken to hut, wind northwest; $27: 06: 34.5$, scale now reads $50^{d} .8 ; 27: 11: 18$, no apparent cause for this movement of magnet; sky clear, light nortliwest wind; 27:13:20, caln1.-28:16:00, after this date revolver no longer taken to hut, wind from northeast.29: 20:00, wind light north to calm.

May, I904.-1:00:00, sky clear, wind light north to calm.-2:10:42, reading increased off scale to about $79^{\mathrm{d}}$; north wind. $-3: 12: 18$, scale decreased to $49^{\mathrm{d}} \cdot 3$; wind north-northeast to north; $3: 13: 30$, scale decreased to $30^{\mathrm{d}} .8 ; 3: 13: 34$, scale increased to $39^{\mathrm{d}} \cdot 9 .-4: 00: 00$, calm ; 4:03:50, partly cloudy around horizon; 4:08:02, calm and clear; 4:09:34, clouding up; $4:\ulcorner 2: 48$, scale increasing almost imperceptibly, but returns to reading at $12: 51 ; 4: 12: 56$, scale increasing very slowly to $78^{d} .6$ and returns to reading at $12: 58 ; 4: 14: 50$, sun breaking through clouds, clouds disappearing; $4: 17: 20$, sky again overcast; $4: 22: 00$, scale decreased to $35^{\mathrm{d}} .0 ; 4: 22: 12$, scale decreased to $37^{\mathrm{d}} .1$; wind from northeast. $-5: 16: 00$, wind from north-11ortheast. $-6: 20: 00$, wind from northeast, sky overcast, snowing.- $8: 00: 00$, wind calm to east; $8: 03: 38$, reading $79 .{ }^{\mathrm{d}} \mathrm{O}$ estimated. $-9: 08: 00$, wind from southeast to east.-

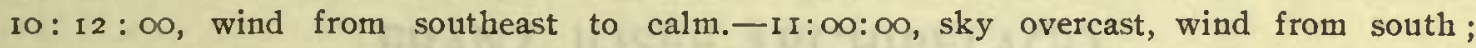
$11: 08: 54$, wind has shifted through east-southeast to east, clouds mostly in horizon; I I:09: 14 , scale increasing from $2 I^{d} .6$ to $22^{d} .8$, then quiescent ; I I : $10: 24$, sky again completely overcast; II: 12: 10, sun breaking through clouds; II: 6 : 10, scattering cirro-cumulus clouds, wind has subsided; $11: 23: 56$, magnet oscillating vertically. - 12 , wind from east.13:20:00, in turning magnet stirrup slipped and made several turus in fiber, it took until 20: oo to recover plane of detorsion; wind calm; I3:20: 18 , magnet checked with adjusting pin ; 13:20:36, observer found a pocket knife on his person; the same was removed outside of hut between $20: 36$ and $20: 38$. - 15 , wind from north; $15: 02: 42$, scale decreasing; 15:06:06, scale increased to $49^{d} .7 ; 15: 07: 36$, reading $70^{\text {do }}$ estimated. - 17 , wind from northnorthwest.- 18 , wind from east, northeast, and north; sky overcast generally throughout observations; 18:06:12, scale increasing; 18:09:54, sun appears for a short time.-19, wind from north-northeast to north.-20, wind north, snow; 20:20: 15.8, scale increased to $67^{d} \cdot 0 .-22$, northeast wind. -23 , northeast wind; $23: 11: 47 \cdot 6$, scale decreased to $22^{d} \cdot 3 .-$ 24 , northeast wind. -25 , sky overcast, light southeast and east wind, snowing; $25:$ I $1: 38$, scale increasing to $42^{\mathrm{d}} .6 ; 25: 13: 46$, scale decreased to $31^{\mathrm{d}} \cdot 6 ; 25: 13: 48$, scale increased to $37^{\mathrm{d}} .0$ and then decreased to quiescence at $13: 50 .-26$, sky overcast, variable wind.-27, east to south wind, snowing at end.-29, sky overcast, light east-southeast wind increasing in strength to east wind and snow at end; 29:0I:26, magnet checked with adjusting pin; 29:07:36, scale increasing. - 30, west-southwest wind; $30: 08: 04.4$, scale decreased to $57^{\mathrm{d}} .9$. -31 , southwest to south-southeast wind at end.

June, 1904.-I, sky overcast, variable winds; $1: 00: 14$, scale decreasing; $1: 05: 48$, scale increasing; 1:06:29.1, scale decreased to $16^{\mathrm{d}} .9$ and then increased steadily to $64^{\mathrm{d}} \cdot 1$ at $6: 32.2$; 
$\mathrm{r}: 06: 41.9$, scale increased steadily to $60^{\mathrm{a}} .9$ at this time; $\mathrm{r}: 10: 38$, fog on horizon; $1: 22: 00$, sçale decreased to $53^{4} \cdot 2 .-2$, cloudy, strong east to north wind.-3, sky generally overcast, southeast wind. -5 , east wind to calm; $5: 04: 59$, scale decreased to $55^{\mathrm{d}} \cdot 5$ at this time; $5: 05: 45 \cdot 2$, scale decreased to $20^{2} .4$ at this time. -6 , east-southeast to south-southwest wind.7 , south-southeast wind; $7: 12: 18.5$, scale increased to $64^{d} .1 ; 7: 14: 12$, reading $79^{d} \cdot 3$ estimated.8 , southeast to southwest wind at end, generally cloudy; $8: 04: 35$, scale increased to $76^{4} \cdot 9$; $8: 05: 58$, scale increasing to $52^{d} .1 ; 8: 0.5: 59.3$, scale increased to $6 \mathrm{r}^{\mathrm{d}} .0 .-9$, southwest wind, suowing, sky overcast, hazy.-12, east to south-southeast wind, sky overcast; 12:04;04, scale increasing; r 2:06: 22, scale decreasing. -13 , east-southeast wind; $13: 08: 40.5$, scale increased to $55^{d} \cdot 9 .-14$, easterly wind of velocity 40 to 50 miles per hour; snow. -15 , sky overcast, east to northeast wind at end; $15: 07: 44$, scale increasing; $15: 10: 06$, scale increases to $55^{d} \cdot 6$, becomes quiescent and then continues to $56^{d} \cdot 3 ; 15: 14: 02$, scale decreases to $59^{d} \cdot 3$; r 5: 15: 30, first rain of the season begins.-16, west-northwest wind; cloudy; drifting snow.I 7, cloudy; west wind.-19, southeast, east, calm to south wiud, partly cloudy; 19:03:40, light fog; 19:05:22, scale increased to $52^{4}$.0, fog gone; 19:05:24, scale increasing; 19:05: 26, scale decreasing; 19:06:58, strong south wind. -20 , calm to northeast wind.-2 1 , eastsoutheast to east wind. -22 , sky clear, calm.-23. light east-southeast wind.-24, calm.26 , east to southeast to south-southeast wind, cloudy; $26: 03: 38$, raining; $26: 06: 22$, fine hail. -27 , southeast wind.-28, partly cloudy, calm.-29:09:00, calm, foggy, cloudy; 29: 1 4: 40 , scale increases to $48^{d} .5$, then decreases to quiescence; $29: 19: 54$, clear overhead, variable wind; 29:21:50, east-southeast wind increasing rapidly. -30 , southeast wind.

July, 1904.-1, sky overcast, snowing, northwest wind; I:21:22.7, scale iucreased to $53^{\mathrm{d}} \cdot \mathrm{O}$. 


\title{
MAGNETIC OBSERVATIONS
}

\section{TABULATION OF MAGNETIC DECLINATIONS}

\author{
OBSERVED AT
}

TEPLITZ BAY STATION, RUDOLPH ISLAND

FRANZ JOSEF ARCHIPELAGO

SEPTEMBER 28, 1903, TO JULY 1, 1904

NORTH LATITUDE : $81^{\circ} 47^{\prime} .4$

LONGITUDE EAST OF GREENWICH: $3^{\mathrm{h}} 52^{\mathrm{m}}$ 


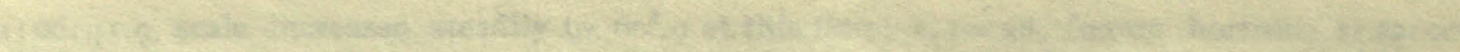

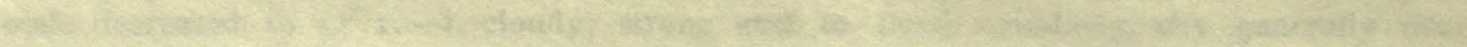

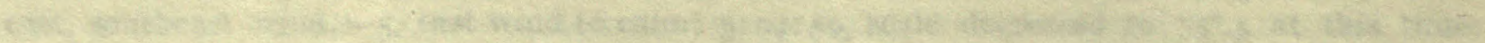

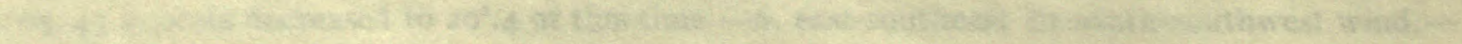

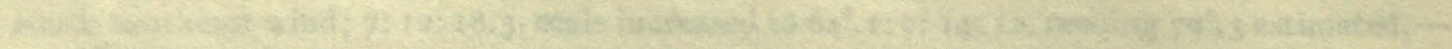

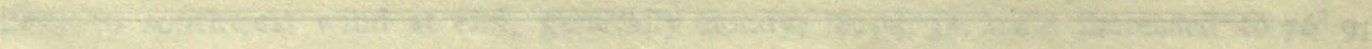
8.

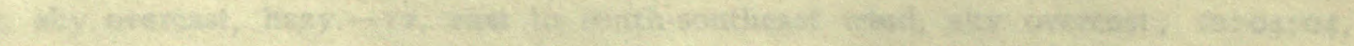

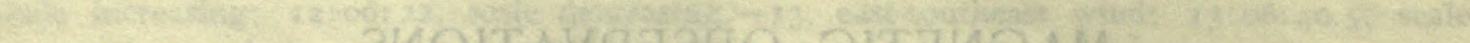

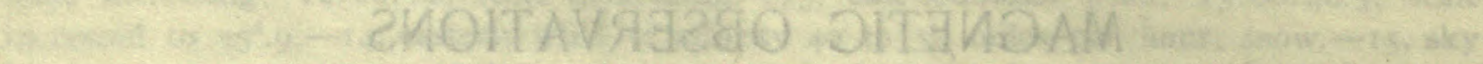

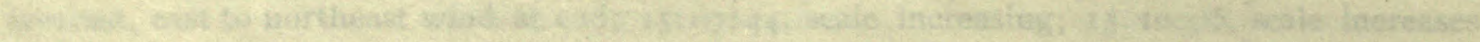

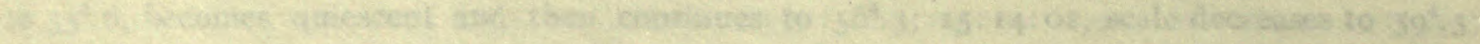

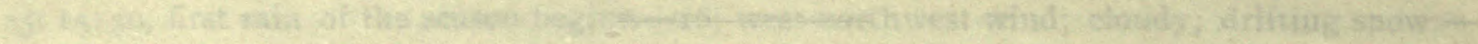

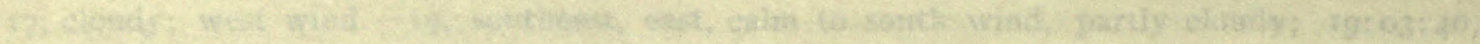

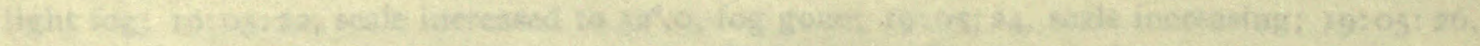

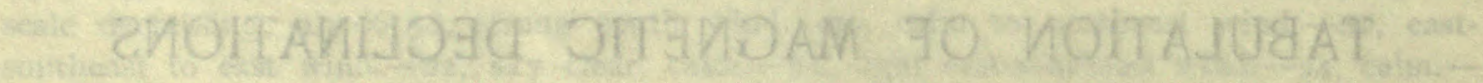

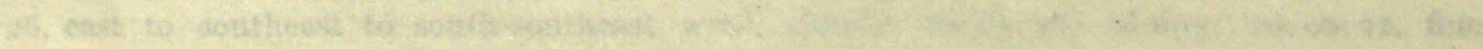

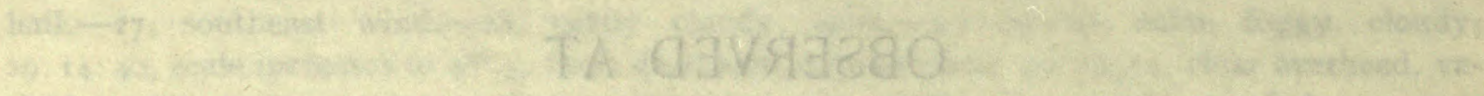

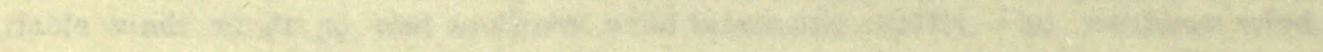

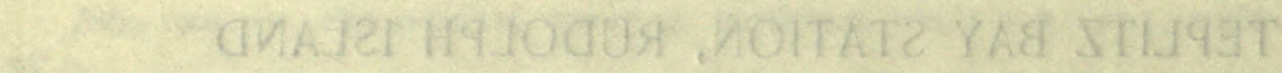

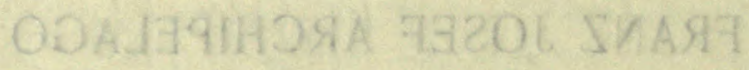

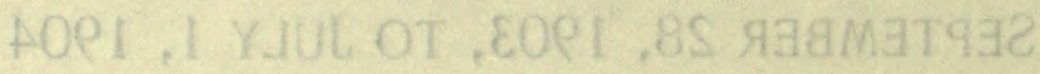

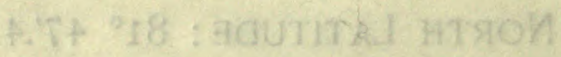

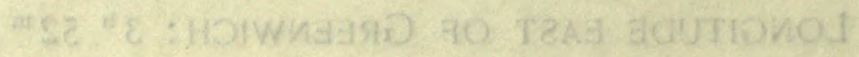


Tabulation of magnetic declinations observed at Teplitz Bay

\begin{tabular}{|c|c|c|c|c|c|c|c|c|c|c|c|c|c|c|c|c|c|c|c|c|}
\hline \multicolumn{5}{|c|}{ Monday, September 28, I903 } & \multicolumn{5}{|c|}{ Magnet scale erect } & \multicolumn{6}{|c|}{ Tuesday, September 29, I903 } & \multicolumn{5}{|c|}{ Magnet scale inverted } \\
\hline \multirow[t]{2}{*}{$\begin{array}{l}\text { Chr'r } \\
\text { time }\end{array}$} & \multicolumn{2}{|c|}{$\begin{array}{l}\text { Scale } \\
\text { readings }\end{array}$} & \multirow{2}{*}{$\begin{array}{l}\text { East } \\
\text { decli- } \\
\text { nation }\end{array}$} & $\begin{array}{l}\text { Tenip. } \\
\text { C. }\end{array}$ & $\begin{array}{l}\text { Chr'r } \\
\text { time }\end{array}$ & $\begin{array}{l}\text { Sec } \\
\text { read }\end{array}$ & $\begin{array}{l}\text { ale } \\
\text { ings }\end{array}$ & East & $\begin{array}{l}\text { Temp. } \\
\text { C. }\end{array}$ & $\begin{array}{l}\text { Chr'r } \\
\text { time }\end{array}$ & $\begin{array}{r}\text { Sc } \\
\text { read }\end{array}$ & $\begin{array}{l}\text { ale } \\
\text { lings }\end{array}$ & Ea & & $\begin{array}{l}\text { Temp. } \\
\text { C. }\end{array}$ & $\begin{array}{l}\text { Chr'r } \\
\text { tinse }\end{array}$ & $\begin{array}{l}\text { Sr } \\
\text { rea }\end{array}$ & $\begin{array}{l}\text { e } \\
\text { igs }\end{array}$ & $\begin{array}{c}\text { East } \\
\text { decli- }\end{array}$ & Temp. \\
\hline & L,eft & Right & & & & L.eft & Right & & & & Left & Right & nati & & & & Left & Right & nation & \\
\hline $\mathrm{h} \mathrm{m}$ & $\mathrm{d}$ & d & - & • & $\mathrm{h} \mathrm{m}$ & d & d & $\circ \quad$ & • & $\mathrm{h} \mathrm{m}$ & d & d & - & & $\circ$ & $\mathrm{h} \mathrm{m}$ & d & d & $\circ \quad$ & $\circ$ \\
\hline 900 & & & & $x^{2}$ & II 00 & 46.5 & 48.0 & 2238 & & 1200 & 46.3 & 44.7 & 224 & & -2.1 & I4 00 & 46.0 & $43 \cdot 5$ & 2248 & -2.0 \\
\hline 02 & & & & wix & 02 & $46 . \overline{8}$ & 48.7 & & (6) & 02 & 49.9 & 49.4 & & I & & 02 & $4^{8} .5$ & 46.3 & 44 & \\
\hline $\begin{array}{l}04 \\
06\end{array}$ & & & & $=0$ & 04 & 46.0 & 47.6 & 38 & & 04 & $5 \mathrm{I} \cdot 3$ & 5 I. 0 & & 8 & ty & 04 & 49.2 & 48.0 & 42 & $t=$ \\
\hline $\begin{array}{l}06 \\
08\end{array}$ & & 619 & & 6 & 06 & 45.7 & 47.0 & 37 & -1.6 & 06 & 48.5 & 46 I & & 4 & & 06 & 46.6 & 44.6 & 47 & We \\
\hline Jo & & & & 9 & $\begin{array}{l}08 \\
10\end{array}$ & $\begin{array}{l}45.2 \\
44.6\end{array}$ & $\begin{array}{l}45 \cdot 3 \\
45 \cdot 3\end{array}$ & $\begin{array}{l}35 \\
35\end{array}$ & $=$ & $\begin{array}{l}08 \\
\text { 10 }\end{array}$ & $\begin{array}{l}40.5 \\
48.5\end{array}$ & $\begin{array}{l}44.3 \\
46.3\end{array}$ & & $\begin{array}{l}7 \\
4\end{array}$ & a & $\begin{array}{l}08 \\
10\end{array}$ & 45.3 & 42.6 & $\begin{array}{l}50 \\
50\end{array}$ & 61 \\
\hline 12 & & & & ar & 12 & 46.0 & 46.3 & 37 & (at) & I2 & 43.4 & 41.9 & & 2 & & 12 & $\begin{array}{l}45 \cdot 4 \\
49 \cdot 3\end{array}$ & $\begin{array}{l}42.4 \\
46.3\end{array}$ & $\begin{array}{l}50 \\
44\end{array}$ & \\
\hline 14 & & & 1 & 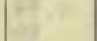 & 14 & 44.8 & 46.0 & 36 & & 14 & 42.2 & 38.5 & & 5 & -2.1 & I4 & 47.6 & 44.6 & 46 & -2.0 \\
\hline 16 & & & & Bi & 16 & $44 \cdot 3$ & 45.3 & 35 & If & I6 & 43.8 & $4 \mathrm{I} \cdot 3$ & 225 & & & I6 & 45.6 & 43. & 48 & \\
\hline 18 & & & 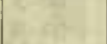 & 58 & I8 & 43.7 & 44.4 & 33 & $=$ & 18 & 37.6 & 35.0 & 230 & & aㅏ & I 8 & $47 \cdot 3$ & 44 & 46 & 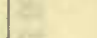 \\
\hline 20 & & 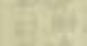 & & $t=$ & 20 & 45.3 & 45.6 & 36 & & 20 & $4 \mathrm{I} .5$ & 39.1 & 225 & & a & 20 & 48.6 & 46 & 44 & cat \\
\hline 22 & & $x,=8$ & & ta & 22 & 44.6 & 45.6 & 35 & $-\mathrm{I} .4$ & 22 & 42.6 & 41.2 & & & 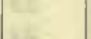 & 22 & 48.4 & 46.6 & 44 & $=$ \\
\hline $\begin{array}{l}24 \\
26\end{array}$ & & 18 & & $x$ & 24 & 45.0 & 45.9 & 36 & $y^{2}$ & 24 & 45.5 & 43.8 & & 8 & 16 & 24 & 46.3 & 44.5 & 47 & 5 \\
\hline $\begin{array}{l}20 \\
28\end{array}$ & & & & Le & $\begin{array}{l}26 \\
28\end{array}$ & $\begin{array}{l}45.3 \\
45.4\end{array}$ & $\begin{array}{l}45.6 \\
46.5\end{array}$ & $\begin{array}{l}36 \\
36\end{array}$ & $t$ & $\begin{array}{l}26 \\
28\end{array}$ & 42.3 & 40.4 & & $\begin{array}{l}4 \\
0\end{array}$ & 2 & 26 & 45.3 & 44. & $\begin{array}{l}48 \\
48\end{array}$ & \\
\hline 30 & 54.5 & 57.8 & 2252 & -2.9 & 30 & 44.9 & $46 . \mathrm{I}$ & 36 & & $\begin{array}{l}28 \\
30\end{array}$ & $\begin{array}{l}51.0 \\
55.6\end{array}$ & $\begin{array}{l}48.3 \\
51.0\end{array}$ & & 5 & -2.0 & $\begin{array}{l}28 \\
30\end{array}$ & $\begin{array}{l}45.4 \\
46.7\end{array}$ & & $\begin{array}{l}48 \\
47\end{array}$ & -1.8 \\
\hline 32 & 53.6 & 55.0 & 50 & & 32 & $44 \cdot 3$ & 45.9 & 35 & 28 & 32 & 57.0 & 52.3 & & 3 & & 32 & 44.0 & $\begin{array}{l}44.7 \\
42.5\end{array}$ & 51 & \\
\hline 34 & 48.6 & 50.4 & 42 & 25 & 34 & 43.3 & 45.0 & 34 & s. & 34 & 67.0 & 57. & & I & 8 & 34 & 44.0 & 42.6 & 51 & $t^{2}$ \\
\hline 36 & $45 \cdot 3$. & 47.0 & 37 & 8 & 36 & 44.0 & 45.3 & 34 & & 36 & 63.4 & 57.7 & & 4 & $a$ & 36 & 46.0 & 44.5 & 48 & $3 x$ \\
\hline 38 & $\mathrm{Mi}$ & ssed & & s & 38 & 45.3 & 47.0 & 37 & $-\mathrm{I} .2$ & 38 & $57 \cdot 3$ & 53.2 & & 2 & ta & 38 & 44.5 & 43.0 & 50 & 3 \\
\hline 40 & 47.8 & 49.2 & 40 & 0 & 40 & 39.9 & 42.8 & 29 & & 40 & 50.4 & 46.4 & 4 & 3 & $y$ & 40 & $46 \cdot 3$ & $45 \cdot 5$ & 46 & +1 \\
\hline $4^{2}$ & 49.8 & 5 ז. 6 & 44 & ( & 42 & 42.5 & 44.4 & 32 & & 42 & 65.9 & 56.2 & & 3 & 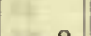 & 42 & 46.4 & $44 \cdot 3$ & 48 & $5=$ \\
\hline 44 & 46.2 & 48.6 & 39 & -2.6 & 44 & 42.3 & 44.6 & 32 & & 44 & 68.0 & 52.3 & 2 & 4 & -I. 8 & 44 & 46.5 & $44 \cdot 3$ & 47 & 19 \\
\hline 46 & $43 \cdot 4$ & $47 \cdot 5$ & 36 & & 46 & 41.0 & 44.5 & 3 I & 111 & 46 & 55.0 & 50.9 & & 6 & 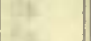 & 46 & $45 \cdot 5$ & 43.6 & 49 & 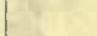 \\
\hline 48 & 43.6 & 47.8 & 36 & as & 48 & 45.2 & 48.0 & 38 & tis & 48 & 60.8 & 50.4 & & I & 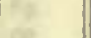 & $4^{8}$ & 44.5 & 43. & 50 & tra \\
\hline 50 & 46.0 & 49.3 & 39 & 1 & 50 & $44 \cdot 3$ & 46.9 & 36 & mat & 50 & 62.4 & 57.9 & & & 5 & 50 & 44.9 & 43 & 49 & 0 \\
\hline 52 & 48.0 & 50.8 & 42 & $=3$ & $5^{2}$ & 40.6 & 43.8 & 30 & -1.0 & 52 & 60.0 & 56. & & 7 & 3 & 52 & 45.6 & 43 & 48 & 2 \\
\hline 54 & 45.6 & 49.0 & 38 & & 54 & 37.0 & 41.3 & 26 & & 54 & 57.7 & 55.0 & & 0 & 36 & 54 & 46.7 & 43. & 48 & \\
\hline 56 & 45.5 & 48.5 & 38 & -2.4 & 56 & 37.2 & 40.6 & 25 & $=$ & 56 & 60.4 & 58 & & & & 56 & 46.5 & 43 & 48 & \\
\hline 58 & 46.4 & 48.7 & 39 & & 58 & 39.4 & 43.0 & 29 & & 58 & 58.0 & 56. & & 9 & & 58 & 45.6 & 43.5 & 48 & \\
\hline 1000 & 46.6 & 49.4 & 40 & & 1200 & 42.3 & 45.6 & 34 & & 1300 & 57.7 & 56.7 & & 9 & -1.6 & I5 00 & 46.9 & 44.5 & 47 & \\
\hline 02 & 47.0 & 49.7 & 40 & $\because z$ & O2 & 45.0 & 46.9 & 36 & ar & 02 & 62.0 & 59.3 & & 3 & & 02 & 47.8 & 45. & 46 & 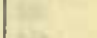 \\
\hline 04 & 47.2 & 50.0 & 4I & te & 04 & 43.9 & 46.3 & 35 & 8 & 04 & 63.1 & 60.6 & & 2 & & 04 & 48.2 & 45. & 45 & \\
\hline 06 & 45.0 & 48.0 & 37 & (2) & 06 & 40.9 & 43.2 & 30 & $=$ & 06 & 63.2 & 60. & 2 & & 10 & 06 & 48.6 & 45. & 45 & \\
\hline 08 & 45.9 & 47.8 & 38 & & 08 & 40.7 & 43.0 & 30 & s. & 08 & 60.9 & $59 . \mathrm{I}$ & & & nt & o8 & 48.2 & 45. & 45 & set \\
\hline IO & 48.0 & 50.4 & 42 & -2.2 & 10 & 41.9 & 44.0 & 32 & & IO & 57.7 & 56.5 & 2 & 9 & & I0 & 48.0 & 45.2 & 45 & 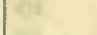 \\
\hline 12 & 47.0 & 50.7 & $4 I$ & & 12 & & & & & I2 & 58.4 & 55.8 & & & 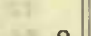 & I2 & 48.8 & & 44 & \\
\hline 14 & 46.9 & 49.8 & 40 & te & I4 & & & & & I4 & 59.0 & 55.6 & & 8 & - I. 8 & I4 & 5I.I & 48. I & 4I & -1.7 \\
\hline 16 & $49 \cdot 5$ & 52.4 & 44 & & I6 & R. R & $\mathrm{T}$ & & 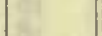 & I6 & 59.7 & 56.2 & & & & I6 & 54.5 & 50 & 36 & \\
\hline I8 & 48.2 & 50.3 & 42 & & I8 & obse & rved & & ate & I8 & 60.2 & 58.2 & & 6 & 9 & I8 & 55.3 & 50. & 35 & \\
\hline 20 & 49.8 & 52.0 & 44 & to & 20 & $\mathrm{I} 2 \mathrm{~h}$ & $02 \mathrm{~m}$ & & (c) & 20 & 61.4 & $59 \cdot 5$ & & 4 & 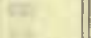 & 20 & 52.5 & 48.5 & 39 & . \\
\hline 22 & 50.6 & 52.5 & 45 & & 22 & to $e$ & end & & 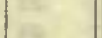 & 22 & 57.0 & 56.2 & & 0 & & 22 & 54.0 & 50.2 & 37 & 4 \\
\hline 24 & 51.0 & 52.4 & 45 & $-2 . I$ & 24 & & & & 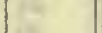 & 24 & 53.5 & 53.3 & & & 1 & 24 & 54.8 & & 36 & E \\
\hline 26 & 58.0 & 6̆. & 58 & & 26 & & & & 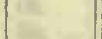 & 26 & 55.9 & & 8 & & 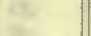 & 26 & 56.5 & 47 & 37 & \\
\hline 28 & 59.2 & $6 \mathrm{I} .5$ & 2259 & & 28 & & 70 & 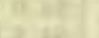 & L & 28 & 54.5 & 52.5 & ? & & & 28 & 54.5 & 46 & 40 & \\
\hline 30 & 62.5 & 64.2 & 2304 & 4 & 30 & & 20 & $2+12$ & 4 & 30 & 52.3 & & & & $-I$. & 30 & $49 . \mathrm{I}$ & & 42 & $-\mathrm{I}$. \\
\hline 32 & 50.3 & 5 I. 8 & 2244 & & 32 & & & & & 32 & 51.6 & 50.3 & & & & 32 & 46.8 & 45 & 46 & \\
\hline 34 & 46.0 & 47.8 & 38 & 8 & 34 & & 210 & & 30 & 34 & 48.9 & $47 \cdot 3$ & 4 & & E & 34 & 46.0 & 44.6 & 47 & 32 \\
\hline 36 & 47.0 & 48. & 39 & & 3 & & 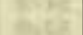 & & -8 & 36 & 46.0 & 45 & & & & 36 & 46.0 & & 47 & \\
\hline $3^{8}$ & 46.8 & 48.3 & 39 & $-2 . I$ & 3 & & & & & 38 & 49.7 & & 4 & & & 38 & 46.6 & & $4^{6}$ & 28 \\
\hline 40 & 45.2 & 47.5 & 37 & & 40 & & 315 & & as & 40 & 50.6 & 49. & 4 & o & & 40 & 43.5 & 43.5 & 50 & 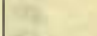 \\
\hline 42 & 46.3 & 48. & 38 & & 42 & r. & 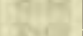 & & a & 42 & 46.7 & 44 & 4 & 7 & 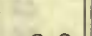 & 42 & 42.0 & 40 & 54 & \\
\hline 44 & 44.4 & 45.2 & 35 & & 44 & & & & & 44 & 48.0 & $45 \cdot 3$ & 4 & & -2. & 44 & 43.0 & $4 \mathrm{I} .5$ & 52 & -1.7 \\
\hline 46 & 41.6 & 43.6 & $3 I$ & & 46 & $x^{2}$ & $7 x=$ & 20 & 50 & 46 & 38.3 & 37.5 & 225 & 9 & 32 & 46 & 43.4 & 38.6 & 2254 & \\
\hline 48 & 44.0 & 44.3 & 34 & & 48 & 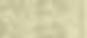 & & & 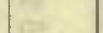 & 48 & 36.8 & 36.6 & 230 & & to & 48 & 36.9 & 33.9 & 2303 & \\
\hline 50 & $45 \cdot 5$ & 46.3 & 36 & & 50 & & tones & & 10 & 50 & 44.4 & 43.6 & 225 & 0 & 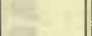 & 50 & 41.6 & 37.0 & $22 \quad 57$ & \\
\hline 52 & 45.3 & 47.0 & 37 & -2.0 & 52 & fint & 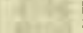 & 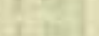 & 6 & 52 & $4 \mathrm{I} \cdot 3$ & 40.5 & 5 & 4 & $a^{2}$ & $5^{2}$ & 36.9 & 35.2 & 2302 & 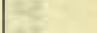 \\
\hline 54 & 48.2 & $49 \cdot 3$ & $4 I$ & & 54 & (1) & & & 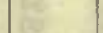 & 54 & 41.8 & $4 \mathrm{I} \cdot 5$ & 2 & & E & 54 & 42.8 & 39.5 & 2254 & 78 \\
\hline 56 & 50.0 & $5 \mathrm{I} .4$ & 44 & & 56 & & & & 8 & 56 & 43.2 & 43.0 & 5 & & & 56 & 44.7 & 42.2 & $5 I$ & \\
\hline 58 & 47.6 & 49.0 & 40 & & 58 & 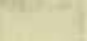 & whe & & & 58 & 44.6 & 43.4 & & & & & 44.4 & 43.0 & 50 & \\
\hline & & & & & & & & & & & & & & & & & 40.1 & 44.7 & 47 & -1.7 \\
\hline
\end{tabular}

Correction to local mean time is $+3 \mathrm{~h} 52 \mathrm{~m} 32 \mathrm{~s}$.

Torsion head at gh $30 \mathrm{~m}$ read $12^{\circ}$ and at end read the same.

Observers-R. R. T. and W. J. P., who alternated from $9 \mathrm{~h} 58 \mathrm{~m}$ to Iob $18 \mathrm{~m}$
Correction to local mean time + Im 40 s.

Torsion head at I Ih $55 \mathrm{~m}$ read $327^{\circ}$ and at end read the same.

Observers-W. J. P. and R. R. T. 
Tabulation of magnetic declinations observed at Teplitz Bay-Continued

\begin{tabular}{|c|c|c|c|c|c|c|c|c|c|c|c|c|c|c|c|c|c|c|c|c|}
\hline \multicolumn{5}{|c|}{ Wednesday, September 30, 1903} & \multicolumn{5}{|c|}{ Magnet scale erect } & \multicolumn{5}{|c|}{ Wednesday, September 30, 1903} & \multicolumn{6}{|c|}{ Magnet scale erect } \\
\hline \multirow{2}{*}{$\begin{array}{l}\text { Chr'r } \\
\text { time }\end{array}$} & \multicolumn{2}{|c|}{$\begin{array}{l}\text { Scale } \\
\text { readings }\end{array}$} & $\begin{array}{c}\text { East } \\
\text { decli- }\end{array}$ & Temp. & Chr'r & $\begin{array}{l}\text { Sct } \\
\text { read }\end{array}$ & $\begin{array}{l}\text { ale } \\
\text { lings }\end{array}$ & $\begin{array}{c}\text { East } \\
\text { decli- }\end{array}$ & Temp. & Chr'r & $\begin{array}{r}\mathrm{Sc} \\
\text { read }\end{array}$ & $\begin{array}{l}\text { ale } \\
\text { lings }\end{array}$ & $\begin{array}{l}\text { East } \\
\text { decli- }\end{array}$ & Temp. & Chr'r & $\begin{array}{l}\text { Sca } \\
\text { readi }\end{array}$ & $\begin{array}{l}\text { ale } \\
\text { ings }\end{array}$ & $\begin{array}{l}\text { Eas } \\
\text { decli }\end{array}$ & & Temp. \\
\hline & Left & Right & & & & I,eft & Right & & & & Left & Right & & & & $L_{0}$ eft & Right & atio & & \\
\hline $\mathrm{h} \mathrm{m}$ & d & d & $\circ \quad$ & & $\mathrm{h} \mathrm{m}$ & d & $\mathrm{d}$ & - , & & $\mathrm{h} \mathrm{m}$ & d & d & $\circ \quad$ & ○ & $\mathrm{h} \mathrm{m}$ & d & & & & - \\
\hline 000 & 47.2 & 49.0 & 2216 & -5.2 & 200 & $5 r .6$ & 52.9 & $225^{2}$ & -3.6 & 400 & 65.8 & 67.7 & $23 I 4$ & $-5 . I$ & 600 & 56.2 & 58.0 & 225 & & $-5 \cdot 3$ \\
\hline 02 & $47 \cdot 3$ & 49.5 & 16 & set & 02 & 5 I. 9 & 53.4 & 52 & $\sqrt{3}$ & 02 & 67.0 & 68.4 & 16 & & 02 & 71 & $3 a$ & 232 & & \\
\hline 04 & 49.8 & 52.0 & 20 & 7 & 04 & 53.3 & 54.7 & 54 & & 04 & 63.1 & 64.4 & 09 & wit & 04 & 59.4 & 60.6 & & 04 & \\
\hline 06 & 49.3 & 51.4 & 19 & tax & 06 & 53.0 & 54.2 & 54 & B. & 06 & 6I.I & 62.5 & 06 & ar & 06 & 59.6 & 62.3 & & 05 & \\
\hline 08 & $5 \mathrm{I} .3$ & 53.0 & 22 & 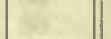 & 08 & 53.6 & 55.0 & 55 & tar & a8 & 60.4 & 61.9 & 05 & $6 x$ & 08 & 63.9 & 64.9 & & II & \\
\hline Io & 56.5 & 59.0 & 31 & 단 & I0 & 54.7 & 56.4 & 57 & at & 10 & 64.9 & 66. & 13 & 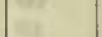 & IO & 64.6 & 65.9 & & I2 & \\
\hline I 2 & 58.0 & 61.0 & 34 & & 12 & 56.0 & 57.1 & 58 & & 12 & 66.5 & 68.5 & I 5 & & 12 & 59.1 & 60.8 & & 04 & \\
\hline 14 & 58.2 & 61.0 & 34 & -5.4 & 14 & 55.8 & 56.8 & 58 & -3.7 & 14 & 71.3 & 72.2 & 22 & -5.2 & 14 & 58.2 & 62.3 & & 04 & -5.5 \\
\hline 16 & 57.6 & 60.4 & 33 & - & 16 & 56.0 & 57.2 & 58 & & 16 & 66.0 & 66.2 & 2313 & & 16 & 56.2 & 58.4 & & $\infty$ & \\
\hline 18 & 58.0 & 60.5 & 33 & 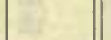 & I 8 & 56.3 & 57.4 & 59 & 4 & 18 & 54.6 & 56.8 & 2257 & is & 18 & 68.7 & 70.5 & & 19 & E. \\
\hline 20 & 59.4 & 62.0 & 35 & ne & 20 & $54 . \mathrm{I}$ & 55.0 & 55 & 15 & 20 & 55.6 & 57.2 & 2258 & 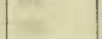 & 20 & 61.9 & 63.5 & & 08 & $a$ \\
\hline 22 & 60.3 & 63.2 & 37 & $k=$ & 22 & 53.7 & 54.7 & 55 & 2 & 22 & 57.8 & 59.4 & 2302 & & 22 & 66 & I $a$ & & 13 & $=$ \\
\hline 24 & 59.8 & 62.3 & 36 & 2. & 24 & 51.7 & 52.6 & 52 & & 24 & $55 . I$ & 57.0 & 2257 & & 24 & 58.9 & 62.4 & & 05 & tw. \\
\hline 26 & 60.8 & 63.2 & 38 & ta & 26 & 52.6 & 53.4 & 53 & te & 26 & 60.9 & 61.4 & 2305 & tes & 26 & 73 & $2 a$ & 232 & 24 & as \\
\hline 28 & 59.9 & 62.3 & 36 & ty & 28 & 53.4 & 54.8 & 54 & & 28 & 64.4 & 64.8 & II & & 28 & 55.7 & 58.0 & 225 & 59 & \\
\hline 30 & 59.6 & 61.8 & 35 & -4.8 & 30 & 51.2 & 52.2 & $5 \mathrm{I}$ & -3.3 & 30 & $6 \mathrm{i} .7$ & 62.2 & 07 & -5.0 & 30 & 71.8 & 72.8 & 232 & 23 & -5.7 \\
\hline 32 & 60.4 & 62.4 & 36 & i & 32 & 48.6 & 50.0 & 47 & $y^{2}$ & 32 & 64.4 & 65.6 & I2 & & 32 & 60.0 & 63.2 & 23 & 06 & \\
\hline 34 & 61.3 & 62.7 & 38 & 3 & 34 & 47.5 & 48.9 & 45 & res & 34 & 62.6 & 64.0 & 09 & & 34 & 53.7 & 56.0 & 225 & 56 & \\
\hline 36 & 59.6 & 60.5 & 34 & $y$ & 36 & 46.5 & 47.9 & 44 & 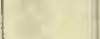 & 36 & 67.7 & 68.3 & 16 & 8 & 36 & $57 \cdot 3$ & 60.4 & 23 & 02 & \\
\hline 38 & 6I.I & 62.4 & 37 & 6 & 38 & 45.5 & 47.0 & 42 & e & 38 & 64.0 & $64 \cdot 3$ & 10 & W. & 38 & 59.6 & 64.8 & & 07 & \\
\hline $4^{\circ}$ & 61.8 & 62.3 & 38 & $y$ & 40 & $45 \cdot 3$ & 46.7 & 42 & nat & 40 & 6 i.9 & 64.0 & 08 & se & 40 & 56.1 & 65.5 & & 05 & \\
\hline 42 & 63.0 & 63.3 & 39 & 8 & 42 & 46.7 & 47.7 & 44 & & 42 & 62.7 & $6_{4} .1$ & 09 & & 42 & 61.7 & 62.0 & & 07 & \\
\hline 44 & 66.4 & 66.8 & 45 & $-4 \cdot 4$ & 44 & 44.4 & $45 \cdot 3$ & 40 & -3.8 & 44 & 62.9 & 64.3 & 09 & -5.1 & 44 & 65.8 & $67 \cdot 3$ & 231 & 14 & -5.8 \\
\hline 46 & 69.0 & 70.0 & 49 & . & 46 & 43.3 & 43.8 & 38 & & 46 & 64.2 & 64.6 & 10 & & 46 & 50.4 & 51.3 & 22 & 49 & \\
\hline $48 *$ & 48.5 & 51.8 & 48 & 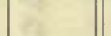 & 48 & $43 \cdot 3$ & 44.2 & 38 & 38 & 48 & 64.9 & 67.0 & 13 & t & 48 & 61.8 & 62.2 & 23 & 07 & \\
\hline 50 & 48.6 & 50.9 & 48 & $w^{2}$ & 50 & 42.3 & 43.0 & 36 & $\theta$ & 50 & 62.3 & 63.8 & 08 & & 50 & 65.2 & 66.2 & & 13 & a \\
\hline 52 & 5 I. 8 & 54.8 & 53 & ce & 52 & 40.8 & 41.3 & 34 & $\mathrm{GE}$ & 52 & 57.4 & 59.0 & or & & 52 & 64.2 & 66.0 & & 12 & 5 \\
\hline 54 & 48.5 & 50.2 & 47 & $1=$ & 54 & 41.5 & 42.0 & 35 & If & 54 & 58.5 & 60.4 & 03 & y & 54 & $5^{8} .3$ & 60.5 & & 03 & 4 \\
\hline 56 & 52.2 & 56.6 & 55 & & 56 & 43.7 & $44 \cdot 3$ & 38 & 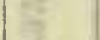 & 56 & 61.7 & 63.9 & 08 & & 56 & 63.0 & 66.1 & & II & \\
\hline 58 & $54 . \mathrm{I}$ & 57.4 & 57 & & 58 & 46.1 & 46.8 & 42 & & 58 & 57.1 & 60.0 & 23 or & & 58 & 61.6 & 63.3 & & 08 & \\
\hline I 00 & 52.8 & 56.9 & 56 & -3.8 & 300 & 48.6 & 49.4 & 46 & $-4 \cdot 2$ & 500 & 53.9 & 56.7 & 2256 & -5.0 & 700 & 59.9 & 62.4 & & 06 & -5.8 \\
\hline 02 & 47.3 & 50.2 & $4^{6}$ & s. & $\mathrm{O} 2$ & 50.2 & 51.2 & 49 & ( & 02 & 54.8 & 57.8 & 58 & . & 02 & 66.2 & 66.8 & & 14 & \\
\hline 04 & 46.5 & 49.2 & 45 & (19) & 04 & 51.9 & 53.3 & 52 & 16 & 04 & 54.7 & 58.1 & 58 & io & 04 & 58.9 & 63.8 & & 06 & \\
\hline 06 & 46.2 & 48.6 & 44 & 5. & 06 & 52.5 & 53.6 & 53 & 8 & 06 & 52.9 & 57.4 & 5 & 8 & 06 & 62.3 & 64.9 & & 09 & \\
\hline 08 & 47.6 & 49.5 & 46 & 6 & 08 & 53.7 & 54.4 & 54 & $6 x$ & 08 & $54 \cdot 3$ & 58. & 58 & 8 & 08 & 55.7 & 59.5 & & 00 & 8 \\
\hline Io & 44.5 & 45.4 & 40 & (4) & 10 & 54.8 & 55.6 & 56 & ot & Io & 51.9 & 55 & 54 & Bet & 10 & 58.5 & 59.7 & & 02 & \\
\hline 12 & 44.3 & 46.0 & 40 & & 12 & 56.5 & 56.5 & 2258 & & 12 & 52.4 & 54 & 5 & & 12 & 70 & $.3 a$ & 232 & 20 & \\
\hline 14 & 50.4 & 51.8 & 50 & -3.6 & I4 & 59.8 & 59.8 & 2303 & $-4 \cdot 3$ & I 4 & 54.9 & 58 & & -5.0 & 14 & 56 & $.8 b$ & 22 & 59 & \\
\hline 16 & 47.6 & 48.9 & 45 & & 16 & 59.1 & 59.8 & 03 & ( & 16 & 55.9 & 58.5 & & ( & 16 & 63.2 & 64.2 & 23 & 10 & -5.9 \\
\hline 18 & 47.9 & 49.1 & 46 & 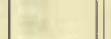 & I 8 & 58.4 & 59.3 & 02 & if & I8 & 54.2 & 58. & & at & 18 & 60.3 & 62.0 & & 06 & \\
\hline 20 & 51.8 & 54.5 & 53 & uet & 20 & 58.6 & 59. & 02 & ete & 20 & 56.2 & 58.0 & 5 & & 20 & 62.4 & 63.2 & & 08 & \\
\hline 22 & 49.7 & 51.5 & 49 & 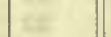 & 22 & 59.0 & 59.9 & 03 & $5=$ & 22 & 52.9 & 55.7 & 55 & & 22 & 62.2 & 63.1 & & 08 & \\
\hline 24 & 50.3 & 52.4 & 50 & III & 24 & 60.8 & 61.5 & 05 & if & 24 & 52.0 & 54. & & & 24 & 64.9 & 68.1 & & 14 & \\
\hline 26 & 47.4 & 48.8 & 45 & ot & 26 & 61.7 & 62.3 & of & e & 26 & 52.8 & 54.8 & & & 26 & 62.2 & 63.2 & & 08 & \\
\hline 28 & 44.4 & 46.2 & $4 I$ & $x^{2}=$ & 28 & 62.4 & 63.2 & 08 & & 28 & 54.0 & 55.8 & & & 28 & 66.5 & 67.4 & & 14 & \\
\hline 30 & 47.4 & 48.8 & 45 & -3.4 & 30 & 63.1 & 63.9 & 09 & -4.5 & 30 & 54.3 & 55.4 & 5 & -5 & 30 & 61.9 & 63.0 & & 08 & -6.0 \\
\hline 32 & 49.1 & 5I.7 & 49 & ( & 32 & 63.9 & 64.9 & I0 & $=$ & 32 & $5 \mathrm{I} .3$ & 55.0 & & & 32 & 66.0 & 66.8 & & 14 & \\
\hline 34 & 47.1 & 49.4 & 45 & it & 34 & 63.3 & 64.2 & 09 & 37 & 34 & 48.3 & 51.8 & & $y$ & 34 & $6 r .9$ & 63.0 & & 08 & \\
\hline 36 & 48.2 & 50.0 & 47 & 15 & 36 & 64.2 & 64. & II & $\omega^{2}$ & 36 & 48.3 & 52.3 & 48 & & 36 & 63.3 & 64.7 & & IO & \\
\hline 38 & 51.7 & 53.6 & 52 & . & 38 & 65.8 & 66. & I4 & r. & 38 & 49.0 & 52.9 & & & 38 & 62.4 & 63.2 & & 08 & \\
\hline 40 & 51.0 & 53.3 & $5 \mathrm{I}$ & at & 40 & 64.8 & 64.8 & II & & 40 & 48.0 & 51.7 & & & 40 & 64.2 & 66.3 & & 12 & \\
\hline 42 & 48.4 & 50.4 & 47 & 2 & 42 & 64.8 & 65.2 & 12 & & 42 & 5 I. 6 & 54.8 & 2253 & & 42 & 64.2 & 65.3 & 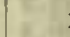 & II & \\
\hline 44 & 48.3 & 50.7 & 47 & -3.5 & 44 & 64.2 & 64.8 & II & -4.8 & 44 & 56.9 & 59.7 & 230 & -4.9 & 44 & 63.3 & 65.3 & & Io & -6.4 \\
\hline 46 & 49.1 & 51.0 & 48 & & 46 & 62.1 & 62.8 & 07 & & 46 & 50.8 & 58.8 & 225 & & 46 & 67.2 & 68.2 & & 16 & \\
\hline 48 & 50.5 & 52.4 & 50 & 12 & 48 & 62.2 & 62.8 & 08 & 8 & 48 & 59.3 & 63.0 & 2306 & & 48 & 66.2 & 69.5 & & 16 & \\
\hline 50 & 49.7 & 50.2 & 48 & 28 & 50 & 61.4 & 62.6 & 07 & $\theta$ & 50 & 53.6 & $55 \cdot 3$ & 225 & & 50 & 68.0 & 69.3 & & 17 & \\
\hline 52 & 49.9 & 51.2 & 49 & (1) & 52 & 61.2 & 62.3 & 06 & $\Rightarrow$ & 52 & 52.3 & 54.1 & 2253 & . & 52 & 66.9 & 70.8 & & 18 & \\
\hline 54 & 51.7 & 53.0 & 52 & 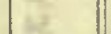 & 54 & 57.5 & 58.0 & 00 & (1) & 54 & 6I.I & 62.0 & 2306 & & 54 & 65.5 & 68.1 & & 14 & \\
\hline 56 & 52.2 & 53.8 & 53 & 25 & 56 & 57.8 & 59.1 & OI & 5 & 56 & 63.2 & 65.4 & 10 & & 56 & 66.2 & 67.8 & & 15 & \\
\hline 58 & 53.0 & 54.7 & 54 & 55 & 58 & 63.5 & 64.3 & 10 & 2 & 58 & 56.9 & 59.4 & OI & & 58 & 64.2 & 64.8 & & II & \\
\hline & & & 7.61 & & & & & & & & & & & & & & & & & \\
\hline
\end{tabular}

Observers-W. J. P. and R. W. P., who alternated from ol $16 \mathrm{~m}$ to Observers-R. W. P. and R. R. T., who alternated 4 h $18 \mathrm{~m}$ to 4 h $28 \mathrm{~m}$. ols $18 \mathrm{~m}$. 
Tabulation of magnetic declinations observed at Teplitz Bay-Continued

Wednesday, September 30, $1903 \quad$ Magnet scale erect Wednesday, September 30, I903 Magnct scale crect

\begin{tabular}{|c|c|c|c|c|c|c|c|c|c|c|c|c|c|c|c|}
\hline \multirow{2}{*}{$\begin{array}{l}\text { Chr'r } \\
\text { time }\end{array}$} & $\begin{array}{l}\text { Scale } \\
\text { readings }\end{array}$ & \multirow{2}{*}{$\begin{array}{l}\text { East } \\
\text { decli- } \\
\text { nation }\end{array}$} & \multirow{2}{*}{$\begin{array}{l}\text { Temp. } \\
\text { C. }\end{array}$} & \multirow{2}{*}{$\begin{array}{l}\text { Chr'r } \\
\text { time }\end{array}$} & $\begin{array}{l}\text { Scale } \\
\text { readings }\end{array}$ & \multirow{2}{*}{$\begin{array}{l}\text { East } \\
\text { decli- } \\
\text { nation }\end{array}$} & \multirow{2}{*}{$\begin{array}{l}\text { Temp. } \\
\text { C. }\end{array}$} & \multirow{2}{*}{$\begin{array}{l}\text { Chr'r } \\
\text { time }\end{array}$} & $\begin{array}{l}\text { Scale } \\
\text { readings }\end{array}$ & \multirow{2}{*}{$\begin{array}{l}\text { East } \\
\text { decli- } \\
\text { nation }\end{array}$} & \multirow{2}{*}{ Temp } & \multirow{2}{*}{$\begin{array}{l}\text { Chr'r } \\
\text { time }\end{array}$} & $\begin{array}{c}\text { Scale } \\
\text { readings }\end{array}$ & \multirow{2}{*}{$\begin{array}{l}\text { East } \\
\text { decli- } \\
\text { nation }\end{array}$} & \multirow{2}{*}{ Temp. } \\
\hline & Left Right & & & & Left Right & & & & Right & & & & Left Right & & \\
\hline
\end{tabular}

81
(04)

(0)

(1)

18

2

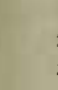

2

3

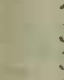

3

38

4

4

4

46
48
5
5
5
5
90

\begin{tabular}{|c|c|c|c|c|c|c|c|c|c|c|c|c|c|c|c|c|c|c|c|c|c|}
\hline d & d & & , & 0 & $\mathrm{~h} \mathrm{~m}$ & d & d & 。 & , & 0 & $\mathrm{~h} \mathrm{~m}$ & $\mathrm{~d}$ & d & 。 & ' & 0 & $\mathrm{~h} \mathrm{~m}$ & d & d & & 。 \\
\hline 64.4 & 67.5 & 23 & I3 & -7.0 & I0 00 & 9.4 & II. 3 & 21 & 46 & & 1200 & 49.5 & 52.1 & 223 & 34 & -6.6 & 1400 & 42.8 & 47.8 & $22 \quad 26$ & -7.1 \\
\hline 71.0 & 71.5 & 23 & $2 \mathrm{I}$ & & 02 & 15.3 & I8. 3 & & 56 & -7.6 & 02 & 50.5 & 53.5 & & 36 & & 02 & $4 \mathrm{I} . \mathrm{I}$ & 46.0 & 23 & \\
\hline 36.3 & $37 \cdot 3$ & 22 & 27 & $p^{2}$ & 04 & 17.5 & 18.8 & & 58 & & 04 & 50.7 & 53.7 & & 36 & & 04 & 37.3 & 42.7 & I7 & (4) \\
\hline 37.6 & 40.0 & & 30 & 80 & 06 & 12.7 & 14.7 & & $5 I$ & . & 06 & 56.2 & 59.3 & & 45 & & 06 & 41.2 & $45 \cdot 3$ & 22 & +0 \\
\hline 35.9 & 37.8 & & 27 & 10 & 08 & 16.5 & 19.1 & & 58 & w & 08 & 51.7 & 53.4 & & 37 & 17 & os & 42.9 & 47.2 & 25 & \\
\hline 32.0 & 35.5 & & 22 & we & 10 & 12.9 & 16.4 & & 52 & t & I0 & 43.0 & 44.9 & & 23 & m & IO & 44.4 & 48.2 & 27 & 01 \\
\hline 35.2 & 38.2 & & 27 & 8 & 12 & 14.6 & 18.3 & & 55 & . & 12 & 38.3 & 40.7 & & I7 & 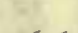 & J2 & 47.0 & 50.6 & $3 I$ & \\
\hline 36.6 & 39.8 & & 30 & -7.0 & 14 & 13.7 & 16.3 & & 53 & -7.8 & 14 & 40.0 & 42.7 & & 20 & -6.6 & I4 & 48.6 & 52.4 & 34 & -7.2 \\
\hline 34.2 & 37.3 & & 26 & & 16 & 13.0 & 16.5 & & 53 & & 16 & 46.2 & 47.7 & & 28 & & 16 & $43 \cdot 3$ & 46.6 & 25 & \\
\hline 40.8 & 45.0 & & 37 & $2 t$ & 18 & 16.0 & $2 \mathrm{I} .2$ & & 59 & & 18 & 48.3 & 50.8 & & 32 & & 18 & 44.2 & $47 . I$ & 26 & \\
\hline 33.8 & 38.3 & & 26 & 68 & 20 & 12.8 & 17.0 & & 53 & 6 & 20 & 45.2 & 46.4 & & 27 & $a$ & 20 & 45.2 & 48.2 & 28 & $\approx$ \\
\hline 38.0 & 40.0 & & $3 I$ & 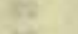 & 22 & 12.5 & 16.4 & & 52 & an. & 22 & 49.3 & 51.8 & & 34 & 드. & 22 & 44.8 & 47.6 & 27 & 67 \\
\hline 35.3 & 36.3 & & 26 & $1=$ & 24 & 14.3 & 18.5 & & 55 & 10 & 24 & 43.9 & 46.4 & & 25 & ye & 24 & 44.2 & 46.9 & 26 & $t$ \\
\hline 29.5 & 32.1 & & I8 & $n=$ & 26 & 8.8 & 26.0 & $2 \mathrm{I}$ & 57 & 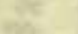 & 26 & 52.3 & 54.9 & & 39 & 8 & 26 & 44.8 & 47.9 & 27 & \\
\hline 32.2 & 33.4 & & 21 & $7 x$ & 28 & 10.4 & 30.6 & 22 & 02 & & 28 & 53.8 & 56.2 & & $4 \mathrm{I}$ & & 28 & 45.7 & 48.2 & 28 & \\
\hline 29.9 & 31.8 & & 18 & -7.0 & 30 & 20.4 & 24.5 & & 05 & 9 & 30 & 50.3 & 51.0 & & .34 & -6.8 & 30 & 47.0 & 49.2 & 30 & -7.6 \\
\hline 28.4 & 29.5 & & 15 & & 32 & 15.0 & 26.0 & & 02 & 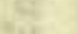 & 32 & 45.7 & 48.2 & & 28 & & 32 & 48.6 & 51.0 & 33 & \\
\hline 29.4 & 31.6 & & 17 & 2 & 34 & 17.0 & 24.3 & & 02 & -7.0 & 34 & $5 \mathrm{I} .2$ & 52.4 & & 36 & at & 34 & 46.1 & 48.2 & 28 & \\
\hline 30.5 & 34.5 & & 21 & 3. & 36 & 15.0 & 25.0 & & OI & & 36 & 48. I & 49. I & & $3 I$ & & 36 & $45 . \mathrm{I}$ & 47.2 & 27 & \\
\hline 30.5 & 36.0 & & 22 & 70 & 38 & 13.5 & 36.3 & & o9 & & 38 & 42.2 & 43.8 & & 22 & 4 & $3^{8}$ & 45.8 & 47.2 & 28 & \\
\hline 30.4 & 37.6 & & 23 & (a) & 40 & 26.0 & 35.0 & & 17 & 6 & 40 & 43.2 & 43.8 & & 23 & (y) & 40 & 47.4 & 48.9 & 30 & 0 \\
\hline 27.2 & 35.8 & & 19 & 24 & 42 & 22.0 & 28.9 & & 09 & $\Rightarrow$ & 42 & 42.8 & 44.2 & & 23 & 4 & 42 & 47.9 & 49.2 & $3 I$ & \\
\hline 42.5 & 44.0 & & 37 & $\rightarrow 7.0$ & 44 & 17.4 & 34.8 & & II & -6.9 & 44 & 40.0 & 40.6 & & 18 & -7.0 & 44 & 49.1 & 50.6 & 33 & \\
\hline 41.7 & 51.3 & & 42 & & 46 & 13.5 & 33.5 & & 06 & 20 & 46 & 45.7 & 46.0 & & 27 & & 46 & 49.4 & 5 I. 4 & 34 & -8.0 \\
\hline 26.3 & 33.4 & & I6 & 60 & 48 & II.O & 32.5 & & 0.4 & & 48 & 43.8 & 44.5 & & 24 & 46 & 48 & 48.8 & 50.4 & 32 & \\
\hline 29.5 & 39.9 & & 24 & 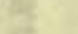 & 50 & 17.5 & 27.2 & 4 & 05 & Fi & 50 & 40.5 & 40.6 & & 18 & 3 & 50 & 49.7 & 51.4 & 34 & . \\
\hline 26.7 & 33.0 & & 16 & 6 & 52 & 9.4 & 29.5 & & $\infty$ & 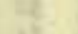 & 52 & 45.6 & 45.8 & & 26 & 2 & 52 & 48.3 & 49.8 & 32 & \\
\hline 23.5 & 32.8 & & 14 & ${ }^{2}$ & 54 & 12.8 & 30.3 & & 03 & 10 & 54 & 47.4 & 47.8 & & 29 & E & 54 & 48.7 & 50.4 & 32 & $\mathrm{k}^{2}$ \\
\hline 24.9 & 32.9 & & 15 & $e^{-2}$ & 56 & 19.0 & 25.6 & & 05 & & 56 & 48.0 & 48.8 & & 30 & & 56 & 45.8 & 46.9 & 27 & \\
\hline 3 I. 4 & 36.0 & & 22 & 17 & $5^{8 *}$ & 19.3 & 25.6 & & 31 & & 58 & 43.0 & 45.0 & & 24 & $1+5$ & $5^{8}$ & 48.2 & 49.6 & $3 \mathrm{I}$ & \\
\hline 24.5 & 31.0 & & 13 & 7 & I I 00 & 15.3 & 29.0 & & 30 & -6.5 & 1300 & 40.0 & 41.8 & & I9 & -7.0 & 1500 & 50.0 & $5 \mathrm{I} \cdot 3$ & 34 & -8.0 \\
\hline 37.2 & 45.8 & & 35 & -7.0 & 02 & 17.8 & 27.0 & & $3 \mathrm{I}$ & & $0 z$ & 41.8 & 43.5 & & 22 & & 02 & 49.0 & 50.0 & 32 & \\
\hline 22.4 & 35.8 & & 15 & & 04 & 24.5 & 25.8 & 3 & 35 & 5 & 0.4 & 47.6 & 48.5 & & 30 & Ses. & 04 & 47.4 & 49.8 & $3 I$ & +5 \\
\hline 29.2 & 35.4 & & 20 & ? & 06 & I I. I & 25.7 & & 24 & & 06 & 49.3 & 49.9 & & 32 & 3 & 06 & 47.1 & 48.3 & 29 & \\
\hline 24.8 & 35.0 & & I6 & 20 & os & 16.0 & $2 \mathrm{I} \cdot 3$ & & 25 & (b) & 08 & 46.6 & 47.9 & & 29 & 150 & 08 & 47.3 & 48.3 & 30 & 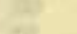 \\
\hline 30.4 & 35.4 & & $2 \mathrm{I}$ & & I0 & 42.2 & 54.6 & & 31 & & Io & 48.9 & 50.3 & & 32 & & 10 & 47.2 & 48. I & 29 & \\
\hline 29.4 & 33.8 & & I9 & 4 & $12^{*}$ & 44.7 & 50.2 & & 29 & $a$ & 12 & 46.4 & 48.8 & & 29 & 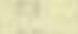 & 12 & 48.2 & 49.2 & $3 \mathrm{I}$ & \\
\hline 26.3 & 31.7 & & 15 & 62 & I4 & 41.8 & 50.8 & & 27 & -6.7 & I4 & 43.6 & 45.5 & & 2.4 & $\rightarrow .0$ & I4 & 48.8 & 49.6 & 32 & -8.1 \\
\hline 24.8 & 29.4 & & 12 & & 16 & 31.8 & 62.3 & & 28 & 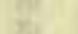 & I6 & 44.7 & 46.3 & & 26 & & 16 & 48.9 & 49.8 & 32 & \\
\hline 30.6 & 32.6 & & 19 & -7.0 & 18 & 43.5 & 58.3 & & 34 & 21 & 18 & 45.4 & 47.6 & & 28 & 81 & 18 & 48.8 & 49.7 & 32 & Hes \\
\hline 23.8 & 25.8 & & 08 & & 20 & 29.8 & 70.4 & & 33 & & 20 & 44.4 & 46.0 & & 26 & & 20 & 49.4 & 50.1 & 33 & \\
\hline 19.9 & 22.4 & 22 & 0.3 & 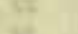 & 22 & 40.2 & 48.5 & & 24 & $\rightarrow$ & 22 & 45.3 & 46.5 & & 27 & 15 & 22 & 48.9 & 49.4 & 32 & 6. \\
\hline 14.0 & 17.0 & $2 I$ & 54 & 14 & 24 & 29.6 & 59.0 & & 24 & 6 & 24 & 44.7 & 46.3 & & 26 & 1 & 24 & 48.2 & 48.7 & 3 I & \\
\hline 17.0 & 20.3 & & 59 & 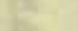 & 26 & 19.5 & 63.8 & & 20 & & 26 & 47.4 & 48.2 & & 30 & 06 & 26 & 48.6 & 48.8 & $3 \mathrm{I}$ & \\
\hline 16.2 & 19.5 & & 58 & -1 & 28 & 25.0 & 57.6 & & I9 & -7.0 & 28 & 44.8 & 45.9 & & 26 & & 28 & 49.0 & 49.3 & 32 & \\
\hline I6. I & 18.8 & & 57 & -7.0 & 30 & 35.5 & 43.1 & & 16 & & 30 & 41.2 & 42.8 & & 20 & -6.9 & 30 & 49.8 & 49.9 & 33 & -8.0 \\
\hline 9.8 & 12.2 & & 47 & & 32 & 27.5 & 50.0 & & 15 & 15 & 32 & 41.3 & 42.7 & & 20 & & 32 & 49.2 & 49.5 & 32 & \\
\hline 12.2 & 14.2 & & 50 & 8 & 34 & 33.5 & 54.0 & & 23 & & 34 & 51.8 & 53.7 & & 37 & & 34 & 48.5 & 48.7 & $3 \mathrm{I}$ & 45 \\
\hline 12.8 & 15.8 & & 52 & a & 36 & 37.4 & 56.7 & & 28 & $=$ & 36 & 59.3 & 60.9 & & 49 & & 36 & 46.2 & 46.8 & 28 & \\
\hline I 4.2 & 16.5 & & 54 & 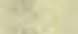 & 38 & 47.6 & 51.2 & * & 32 & & 38 & 46.4 & 47.0 & & 28 & 20 & $3^{8}$ & 45.3 & $46 . I$ & 26 & \\
\hline I3. I & I6. 3 & & 53 & 4 & 40 & 48.4 & 53.0 & & 34 & 39 & 40 & 46.2 & 46.6 & & 27 & 4 & 40 & 45.3 & 46.8 & 27 & 7 \\
\hline 12.3 & 14.6 & & $5 \mathrm{I}$ & 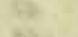 & 42 & 46.7 & 50.7 & & 31 & & 42 & 63.5 & 64.0 & & 54 & & 42 & 44.9 & 46.8 & 26 & -4 \\
\hline 16.4 & 16.6 & & 56 & $\rightarrow . I$ & 44 & 42.8 & 47.8 & & 26 & & 44 & 40.2 & 47.4 & & 23 & -6.9 & 44 & 42.2 & 44.0 & 22 & $\rightarrow .9$ \\
\hline 15.5 & 15.7 & & 54 & & 46 & 44.8 & 47.4 & & 27 & -7.0 & 46 & 51.2 & 55.0 & & $3^{8}$ & & 46 & 42.0 & 44.3 & 22 & \\
\hline 14.4 & 17.2 & & 54 & 145 & 48 & 44.2 & 46.7 & & 26 & & 48 & 40.6 & 43.5 & & 20 & & 48 & 40.8 & 43.6 & $2 \mathrm{I}$ & A \\
\hline 16.0 & 17.5 & & 56 & 88 & 50 & 45.5 & 48. I & te & 28 & 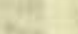 & 50 & 50.2 & 54.2 & & 36 & 9 & 50 & 41.9 & 45.6 & 23 & 8 \\
\hline 16.0 & 17.5 & & 56 & at & 52 & $45 . \mathrm{I}$ & 47.2 & & 27 & 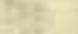 & 52 & 52.0 & 56.9 & & 40 & 3 & 52 & 42.3 & 45.8 & 24 & \\
\hline 15.8 & 16.2 & & 55 & 18 & 54 & 45.0 & 48.0 & & 28 & $F^{2}$ & 54 & 42.7 & 44.7 & & 23 & E. & 54 & 41.3 & 44.7 & 22 & 18 \\
\hline 15.3 & I5.6 & & 54 & 6 & 56 & 49.6 & 52.4 & & 35 & & 56 & 28.2 & 33.0 & & 03 & & 56 & 43.7 & 46.3 & 25 & \\
\hline II .2 & I 4.2 & & 49 & 45 & 58 & 48.2 & $5 \mathrm{I} .2$ & & 33 & 27 & 58 & 37.2 & 42.6 & & 17 & - & 58 & Overl & l'k'd & & \\
\hline
\end{tabular}

Observers-R. R. T. and W. J. P., who alternated from 8 h $02 \mathrm{~m}$ to Observers-R. W. P. and R. R. T., who alternated from I 5 h $40 \mathrm{~m}$ $8 \mathrm{~h} \mathrm{I} 4 \mathrm{~m} ;$ W. J. P. and R. W. P., who alternated fron in $48 \mathrm{~m}$ to to $15 \mathrm{~h} 54 \mathrm{~m}$. (W. J. P. I2h $48 \mathrm{~m}$ to $13 \mathrm{~h} 24 \mathrm{~m}$.) $12 \mathrm{~h} 02 \mathrm{~m}$. 
Tabulation of magnetic declinations observed at Teplitz Bay-Continued

\begin{tabular}{|c|c|c|c|c|c|c|c|c|c|c|c|c|c|c|c|c|c|c|c|c|}
\hline \multicolumn{5}{|c|}{ Wednesday, Scptember 30, 1903} & \multicolumn{5}{|c|}{ Magnet scale erect } & \multicolumn{5}{|c|}{ Wednesday, Scptember 30, I903 } & \multicolumn{6}{|c|}{ Magnet scale crect } \\
\hline \multirow{2}{*}{$\begin{array}{l}\text { Chr'r } \\
\text { time }\end{array}$} & \multicolumn{2}{|c|}{$\begin{array}{l}\text { Scale } \\
\text { readings }\end{array}$} & $\begin{array}{c}\text { East } \\
\text { decli- }\end{array}$ & Temp. & Chr'r & $\begin{array}{r}\text { Sc } \\
\text { read }\end{array}$ & $\begin{array}{l}\text { e } \\
\text { lgs }\end{array}$ & $\begin{array}{c}\text { East } \\
\text { decli- }\end{array}$ & Temp. & Chr'r & $\begin{array}{r}\text { Sc } \\
\text { read }\end{array}$ & gs & $\begin{aligned} \text { East } \\
\text { decli }\end{aligned}$ & Temp. & Chr'r & $\begin{array}{r}\text { Sc } \\
\text { read }\end{array}$ & $\begin{array}{l}\text { e } \\
\text { igs }\end{array}$ & $\begin{array}{r}\text { Eas } \\
\text { decl }\end{array}$ & ast & Temp \\
\hline & L,eft & Right & & & & Lent & Right & & & & I,eft & Right & & & & I,eft & Right & nati & & \\
\hline $\mathrm{h} \mathrm{m}$ & d & d & $\circ \quad$ & • & $\mathrm{h} \mathrm{m}$ & d & $\mathrm{d}$ & & 0 & h m & $\mathrm{d}$ & $\mathrm{d}$ & & - & $\mathrm{h} \mathrm{m}$ & d & & & ' & 。 \\
\hline 600 & 45. I & 48.0 & 2228 & -7.7 & 1800 & 49.2 & $51 \cdot 3$ & 2233 & -7.4 & 2000 & $45 \cdot 3$ & 46.5 & 2227 & & 2200 & 31.8 & 43.8 & 223 & 39 & -6.0 \\
\hline 02 & 46.8 & 48.7 & 29 & ( & 02 & 49.2 & 52.3 & 34 & & 02 & 45.7 & 47.3 & 27 & & & 36.3 & 43.2 & & 42 & \\
\hline 04 & 46.7 & 49.0 & 30 & $y=$ & 04 & 50.7 & 53.2 & 36 & & 04 & $45 \cdot 3$ & 47. & 28 & 3 & 04 & 51.2 & 62 & 3 & 8 & \\
\hline 06 & 47.2 & 49.1 & 30 & 3 & 06 & 50.7 & 52. & 36 & at & 06 & 44.0 & 46 & 26 & ts & 06 & 37.8 & $5 \mathrm{I}$ & & 50 & \\
\hline 08 & 46.6 & $49 \cdot 3$ & 30 & 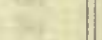 & 08 & 5 I.I & 53.2 & 36 & A & 08 & 44.0 & $4^{6}$ & 26 & a & 08 & 34.0 & 46 & & 43 & \\
\hline 10 & 46.9 & 48.8 & 30 & & 10 & 51.3 & 53. & 36 & & IO & 44.7 & 47 & 27 & $0 x$ & IO & & & & 24 & \\
\hline 12 & 47.8 & 49.0 & 30 & & 12 & 52.1 & 53 & 38 & & 12 & 44.7 & 47. & 27 & $\mathrm{ks}$ & I2 & 20.6 & & & 20 & \\
\hline I4 & 47.2 & 48.8 & 30 & $\rightarrow .6$ & 14 & 50.3 & $5 \mathrm{I}$. & 35 & $\rightarrow 7.4$ & 14 & 44.5 & 46. & 26 & & 14 & 9.8 & & & I9 & -6.0 \\
\hline 16 & 48. I & 49.8 & $3 \mathrm{I}$ & ( & 16 & 48.3 & 50.3 & 32 & a1. & 16 & 44.5 & 46. & 26 & -6.3 & 16 & 25.0 & 28 & & 22 & \\
\hline 18 & 49.2 & $5 \mathrm{I} . \mathrm{I}$ & 33 & 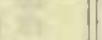 & 18 & 48. I & 51.2 & 32 & in & 18 & 45.0 & 47. & 27 & & I & 26.7 & 30 & & 24 & \\
\hline 20 & 48.6 & 50.3 & 32 & & 20 & 49.0 & 52.0 & 34 & 플 & 20 & 44.6 & 46 & 26 & & 2 & 27.4 & & & 25 & \\
\hline 22 & 49.2 & $5 \mathrm{I} . \mathrm{I}$ & 33 & 2 & 22 & 49.4 & 52. & 34 & $2 x$ & 22 & 43.8 & 45. & 25 & & 22 & 27.6 & 30 & & 26 & \\
\hline 24 & 48.9 & 50.8 & 33 & ${ }^{2}$ & 24 & & 52 & 34 & at & 24 & 37.8 & $4 \mathrm{r}$. & I7 & & 2 & & & & 28 & \\
\hline 26 & 48.9 & 50.8 & 33 & $2=$ & 26 & 0.1 & 52. & 35 & 80 & 26 & 4 I. 6 & 44. & & 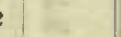 & 2 & 30.6 & & & 30 & \\
\hline 28 & 48.8 & 50.8 & 33 & & 28 & 50.8 & 53. & 36 & & $28^{*}$ & 38.8 & $5 \mathrm{I} \cdot 3$ & 2342 & & 28 & 3I.I & 33 & & 0 & \\
\hline 30 & 48.2 & 50.9 & 32 & $\rightarrow 7.5$ & 30 & 5 I. 3 & 53. & 37 & -7.0 & 30 & Over & l'k'd & & & 30 & 30.5 & 32 & & 29 & \\
\hline 32 & 47.9 & 50.6 & 32 & - & 32 & 50.3 & 52 & 35 & . & 32 & 33.0 & 54.5 & 30 & & 3 & $3 \mathrm{I} .4$ & & & 30 & -6.0 \\
\hline 34 & 48.2 & 51.1 & 32 & 25 & 34 & 49.6 & 56. & 38 & 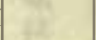 & $34^{*}$ & I 4.5 & 17 & 48 & -6.2 & 3 & $3 \mathrm{I} .4$ & & & 30 & ( \\
\hline 36 & 47.8 & 49.9 & 3 & 8 & 36 & 48.8 & 50 & 33 & & $36^{*}$ & 35. & 38. & 2310 & z & 3 & 3 & & & 29 & \\
\hline 38 & 48.2 & 49.9 & 32 & 8 & $3^{8}$ & 49.0 & 51. & 33 & 한 & 38 & 18.7 & $2 \mathrm{I}$. & 2243 & A & 3 & 4 & & & 9 & \\
\hline 40 & 48.5 & 50.2 & 32 & cet & 40 & 49.5 & 51. & 34 & in & 40 & I 5.2 & I7 & 37 & 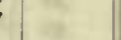 & 40 & 32.3 & 34 & & 32 & \\
\hline 42 & 48.9 & 50.4 & 32 & & 42 & .2 & 52 & 35 & $=$ & $42^{*}$ & 34.1 & $4 I$ & I4 & & 42 & 32.7 & & & 2 & \\
\hline 44 & 49.2 & 50.8 & 33 & -7.5 & 44 & 49.6 & $5 \mathrm{I}$ & 34 & & 44 & 30.5 & 36. & 07 & -6.0 & 44 & 31.6 & 33 & & 31 & -6.0 \\
\hline 46 & 49.9 & 50.3 & 33 & (2) & 46 & 9.7 & $5 \mathrm{I}$ & 34 & A & 46 & 29.7 & 36 & & & 4 & 28.3 & & & 6 & \\
\hline 48 & 49.9 & 50.4 & 33 & Bर & 48 & 49.7 & 50. & 33 & in & 48 & 33.7 & 35 & & & 4 & 28.4 & 29 & & 25 & \\
\hline 50 & 49.9 & 50. & 3 & 2 & 50 & 8.3 & 49. & 32 & & 50 & 37.2 & & 18 & (1) & 5 & 29.3 & & & 7 & \\
\hline 52 & 49.2 & 4 & 3 & $x^{2}$ & 52 & 47.6 & 48.8 & 30 & & 52 & 37.1 & 38 & & s. & 5 & 3I. I & 32 & & 0 & 34 \\
\hline 54 & 48.9 & 49.6 & 3 & & 54 & 46.4 & 48.0 & 29 & & 54 & 46.8 & 56 & & & 5 & 30. & & & 9 & \\
\hline & 49.2 & 50. & 3 & & 56 & 46.6 & 47.5 & 28 & & 56 & 47.4 & 52 & & & 5 & $3 \mathrm{I} .2$ & & & 0 & \\
\hline $5^{8}$ & 50.6 & 51.8 & 3 & & 58 & 46.0 & 47. & 28 & & 58 & 52.7 & 63 & 4 & & 5 & 31.8 & & & 0 & \\
\hline 00 & 51.9 & 53.1 & 3 & -7.6 & 1900 & 45.2 & 46.6 & 27 & -7.7 & 2100 & 53.6 & 60 & 2244 & -6.0 & 2300 & 32.2 & 33.3 & & $3 I$ & -6.0 \\
\hline 02 & $5^{1.9}$ & 52.9 & 3 & 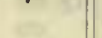 & 02 & & 47.2 & 27 & . & 02 & 64.8 & 72 & 230 & 0 & 02 & $3 \mathrm{I} . \mathrm{O}$ & & & 9 & 0 \\
\hline 04 & 51.0 & 51.9 & 3 & $x_{0}$ & 04 & 46.0 & 47.9 & 28 & 30 & 04 & 54.0 & & 22 & & o & 30. & $3 \mathrm{I}$ & & 9 & \\
\hline$o$ & 51.8 & 5 & 3 & 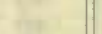 & 06 & & 48. & 28 & & $06^{*}$ & & & 23 & & & 30.5 & 31 & & 8 & we \\
\hline 08 & 50.6 & 50.8 & 3 & s & 08 & 46.2 & 48. & 28 & 8 & 08 & 18.0 & 29. & 23 & ( & or & 29.5 & 30 & & 26 & \\
\hline 10 & 49.2 & 49.7 & 3 & a & I & 47.0 & 48 & 29 & & $10^{*}$ & 46.5 & 49.3 & 22 & 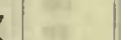 & 10 & 30.0 & 31 & & 28 & at \\
\hline 12 & 49.8 & 50.0 & 3 & & I 2 & 3 & 47. & 27 & & 12 & 40.6 & 43 & 2 & & 1 & & & & 8 & \\
\hline 14 & 9.6 & 5 & 3 & -7.6 & I4 & & 48.5 & 29 & -6.8 & 14 & 42.2 & 43 & & -6.0 & I & 28.3 & 2 & & 25 & \\
\hline 16 & 49.9 & 50.8 & & . & I & 5.6 & 48 & 2 & & 16 & & & & & & 27.7 & & & 4 & -5.9 \\
\hline Iह & & 50. & 3 & tit & I & & 49. & 3 & & I8 & 32. & & & & I & 27.4 & 28 & & 3 & \\
\hline $2 x$ & 49.2 & 4 & 3 & er & 2 & 48.9 & & & in & 20 & & & & e & 2 & & & & 24 & 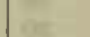 \\
\hline 22 & 48.9 & 5 & 3 & a & 2 & 49.2 & 50 & 3 & $=$ & 22 & & & & & 2 & 27.4 & 28.4 & & 4 & \\
\hline 2. & 9.0 & 5 & 3 & & 2 & 48 & 50 & 3 & t. & 24 & 47 & & & & & & 23 & & I6 & \\
\hline 2 & .9 & 50. & 3 & tet & 2 & 51.2 & 52 & 3 & 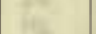 & & & & 22 & & 2 & & 0 & & 24 & \\
\hline 28 & .3 & 50. & 3 & & 28 & 49.2 & 50 & 33 & & $28^{*}$ & & 62 & 23 & & 28 & & 29 & & 26 & \\
\hline 30 & 50.2 & 51.0 & 34 & -7.5 & 30 & 49.4 & 50.2 & 33 & -6.7 & 30 & 47.5 & 53. & 233 & -6. & 30 & 29.6 & 30 & & 27 & -5.8 \\
\hline 32 & 0.2 & 51. & 3 & 80 & 32 & & 50 & 33 & $x^{2}$ & 32 & & & 22 & 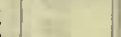 & 32 & 8 & $3 \mathrm{I}$ & & 28 & \\
\hline 34 & 9.9 & 51.0 & 3 & is & 3 & 49.6 & 50 & 33 & u & 34 & 25.5 & 46. & 23 & & 3 & 33. & & & 32 & t \\
\hline 36 & & 5 & 3 & $E^{2}$ & 3 & & $5 I$ & 34 & 8 & 36 & 32.7 & 49 & 23 & & 3 & & 35 & & 35 & \\
\hline 38 & 50.3 & 51.4 & 3 & f & 3 & & 51. & 34 & & $3^{*}$ & & & 24 & & 3 & & & & & \\
\hline 40 & 1.1 & & & 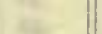 & 4 & 0.6 & $5 \mathrm{I}$. & 35 & e & 40 & 37.0 & 63 & 24 & se & 4 & 32.7 & 33 & & 2 & \\
\hline 42 & $5 \mathrm{I} .4$ & 52.8 & 36 & & 42 & & $5 \mathrm{I}$. & 34 & 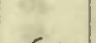 & $42^{*}$ & & & 22 & & 4 & & & & 29 & \\
\hline 44 & 52.1 & 53.0 & 37 & -7.5 & 44 & 48.9 & 50. & 32 & -6.7 & 44 & 64.0 & 66 & 230 & $-6 . c$ & 4 & 30.0 & 30. & & 27 & -5.7 \\
\hline & 51.7 & & 37 & ( & 46 & 47.4 & 48.5 & 30 & & 46 & 18.1 & & 22 & & 4 & & $3 I$ & & 29 & \\
\hline 48 & 50.9 & 52.7 & 36 & E & 48 & 46.8 & 47.8 & 29 & & $48^{*}$ & 68.0 & & 24 & & & & 32 & & & \\
\hline 50 & & 52.0 & 35 & e & 50 & 47.3 & 49. & 30 & $w$ & 50 & II. 6 & 38.0 & 23 & & 50 & 33.8 & 34. & & 3 & \\
\hline 52 & 50.1 & 52.0 & 35 & at & 52 & 45.6 & 47 & 28 & & $52^{*}$ & 16.0 & 34.5 & 22 & & 52 & & 36 & & & \\
\hline 54 & 50.4 & 52.2 & 35 & $t_{12}$ & 54 & 44.9 & 46. & 26 & & 54 & 19.3 & & & & 54 & 40.5 & 44 & & 46 & s. \\
\hline 56 & 50.1 & 52.2 & 35 & 68 & 56 & 45.2 & 46.0 & 26 & & 56 & & & & & & 45.2 & & & 52 & \\
\hline 58 & 49.9 & 51.7 & 34 & $E$ & 58 & $45 \cdot 7$ & 47.0 & 27 & -6.5 & 58 & 40.6 & 51.4 & & & 58 & 47.0 & 48. & 225 & 55 & \\
\hline & & & & & & & & & & & & & & & 60 & 57.4 & 58.1 & & & $-5 \cdot 5$ \\
\hline
\end{tabular}

Observers-R. R. T. and W. J. P., who alternated from Igh $52 \mathrm{~m}$ to 20 h oGm. (W. J. P. altcrnated R. R. T. also from $\mathrm{I} 8 \mathrm{~h} \mathrm{I} 6 \mathrm{~m}$ to $\mathrm{I} 8 \mathrm{~h}$ 22111 and observed readings from $\mathrm{r} 8 \mathrm{~h} 24 \mathrm{~m}$ to $\mathrm{I} 8 \mathrm{~h} 44 \mathrm{~m}$.)
Correction to local mean time +375 .

Torsion head at oh oom read $339^{\circ}$ and at $24 h_{1} 30 \mathrm{~m}$ read the same. Observer-W. J. P. 
Tabulation of magnetic declinations observed at Teplits Bay-Continued

Thursday, October I, 1903

Magnet scale inverted

Friday, October 2, 1903

Magnet scale erect

\begin{tabular}{|c|c|c|c|c|c|c|c|c|c|c|c|c|c|c|c|c|c|c|c|}
\hline \multirow{2}{*}{$\begin{array}{l}\text { Chr'r } \\
\text { time }\end{array}$} & \multicolumn{2}{|c|}{$\begin{array}{l}\text { Scale } \\
\text { readings }\end{array}$} & \multirow{2}{*}{$\begin{array}{l}\text { East } \\
\text { deeli- } \\
\text { nation }\end{array}$} & \multirow{2}{*}{$\begin{array}{l}\text { Temp. } \\
\text { C. }\end{array}$} & \multirow{2}{*}{$\begin{array}{c}\text { Chr'r } \\
\text { time }\end{array}$} & \multicolumn{2}{|c|}{$\begin{array}{l}\text { Seale } \\
\text { readings }\end{array}$} & \multirow{2}{*}{$\begin{array}{c}\text { East } \\
\text { deeli- } \\
\text { nation }\end{array}$} & \multirow{2}{*}{$\begin{array}{l}\text { Temp } \\
\text { C. }\end{array}$} & \multirow{2}{*}{$\begin{array}{l}\text { Chr'r } \\
\text { time }\end{array}$} & \multicolumn{2}{|c|}{$\begin{array}{l}\text { Scale } \\
\text { readings }\end{array}$} & \multirow{2}{*}{$\begin{array}{l}\text { East } \\
\text { decli- } \\
\text { nation }\end{array}$} & \multirow{2}{*}{$\begin{array}{c}\text { Temp. } \\
\text { C. }\end{array}$} & \multirow{2}{*}{$\begin{array}{l}\text { Chr't } \\
\text { time }\end{array}$} & \multicolumn{2}{|c|}{$\begin{array}{l}\text { Seale } \\
\text { readings }\end{array}$} & \multirow{2}{*}{$\begin{array}{l}\text { East } \\
\text { decli- } \\
\text { nation }\end{array}$} & \multirow{2}{*}{ Temp. } \\
\hline & L.eft & Right & & & & r,eft & Right & & & & Left & Right & & & & L,eft & Right & & \\
\hline
\end{tabular}

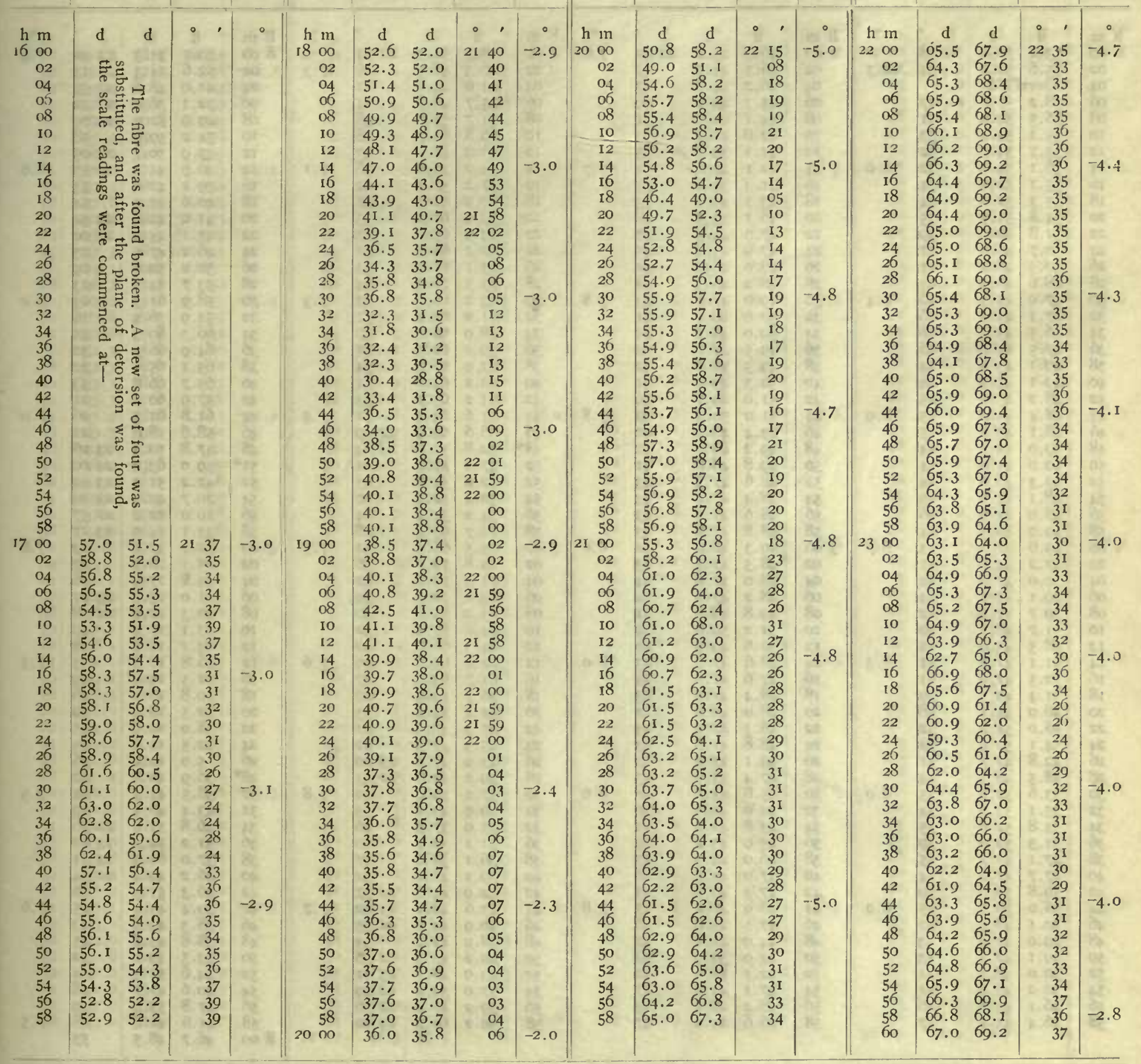

Correction to local mean time - Im 02s. $90^{\circ}$ torsion $=20 .^{\prime} 6$.

Torsion head at $7 \mathrm{~h}$ oom read $72^{\circ}$ and at $201 \mathrm{2} 26 \mathrm{~m}$ read $89^{\circ}$.

Observer-R. R. T.
Correction to local mean time is - $1 \mathrm{~m}$ o6s.

Torsion head at $19 \mathrm{~h} 45 \mathrm{~s}$ rẹad $222^{\circ}$ and at $24 \mathrm{~h}$ zom read the same, Observer-R. R. T: 
Tabulation of magnetic declinations observed at Teplitz Bay-Continued

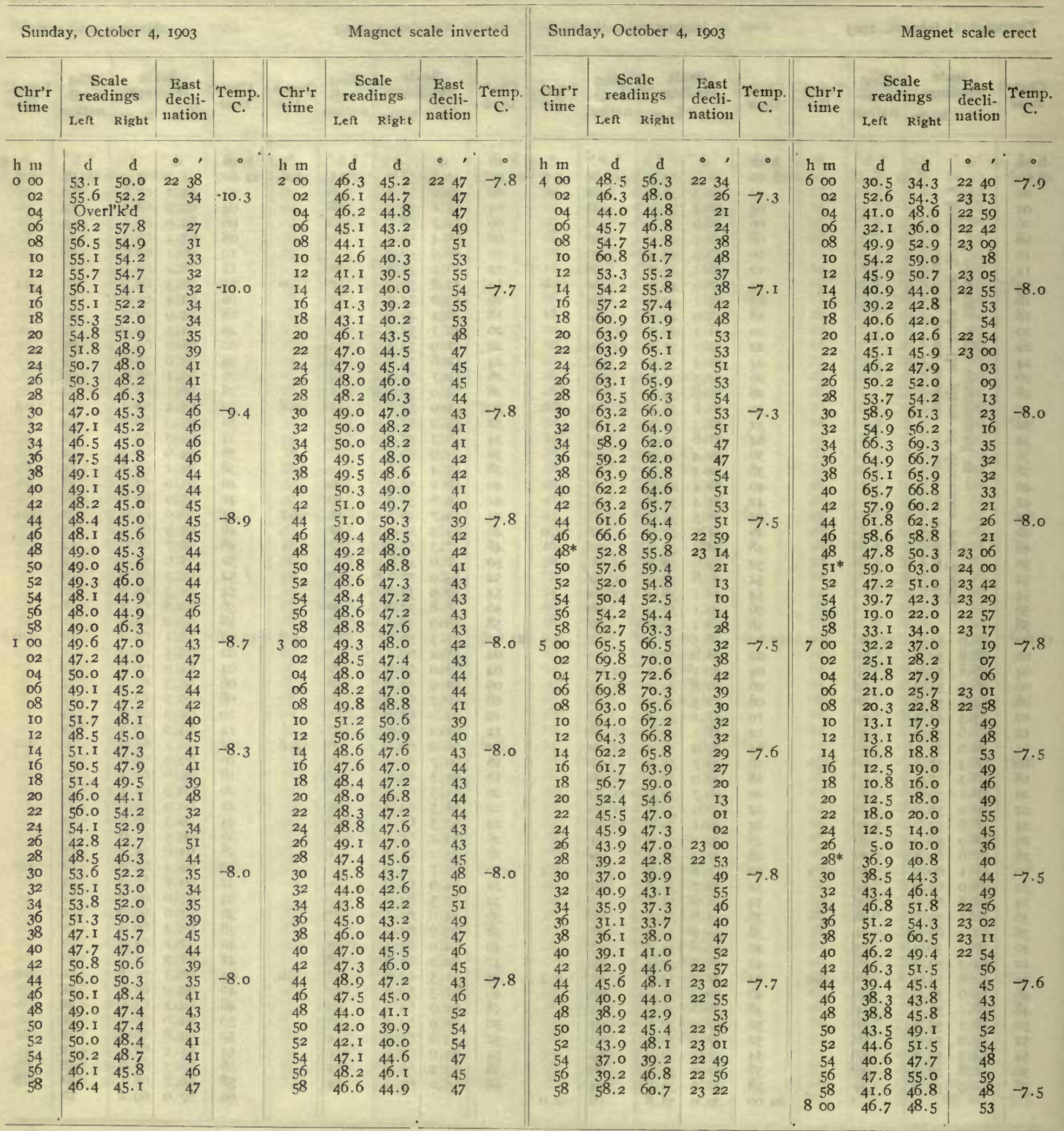

Observers-R. R. T. (W. J. P. $2 \mathrm{~h} 44 \mathrm{~m}$ to 3 h $24 \mathrm{~m}$, alternated R. R. T. to 3 li $46 \mathrm{~m}$.)
Correction to local mean time + Im ogs.

Torsion head at $23 \mathrm{~h}$ oom read $219^{\circ}$ and at $8 \mathrm{~h} 15 \mathrm{~m} \mathrm{read} \mathrm{the} \mathrm{same.}$

Observers-R. R. T. (W. J. P. $7 \mathrm{~h} 14 \mathrm{~m}$ to $8 \mathrm{~h} 20 \mathrm{~m}$, alternated R. R. T. to $8 \mathrm{~h} 32 \mathrm{~m}$.) 
Tabulation of magnetic declinations observed at Teplitz Bay-Continued

Monday, October 5, 1903

Magnet scale inverted

'Tuesday, October 6, 1903

Magnet scale erect

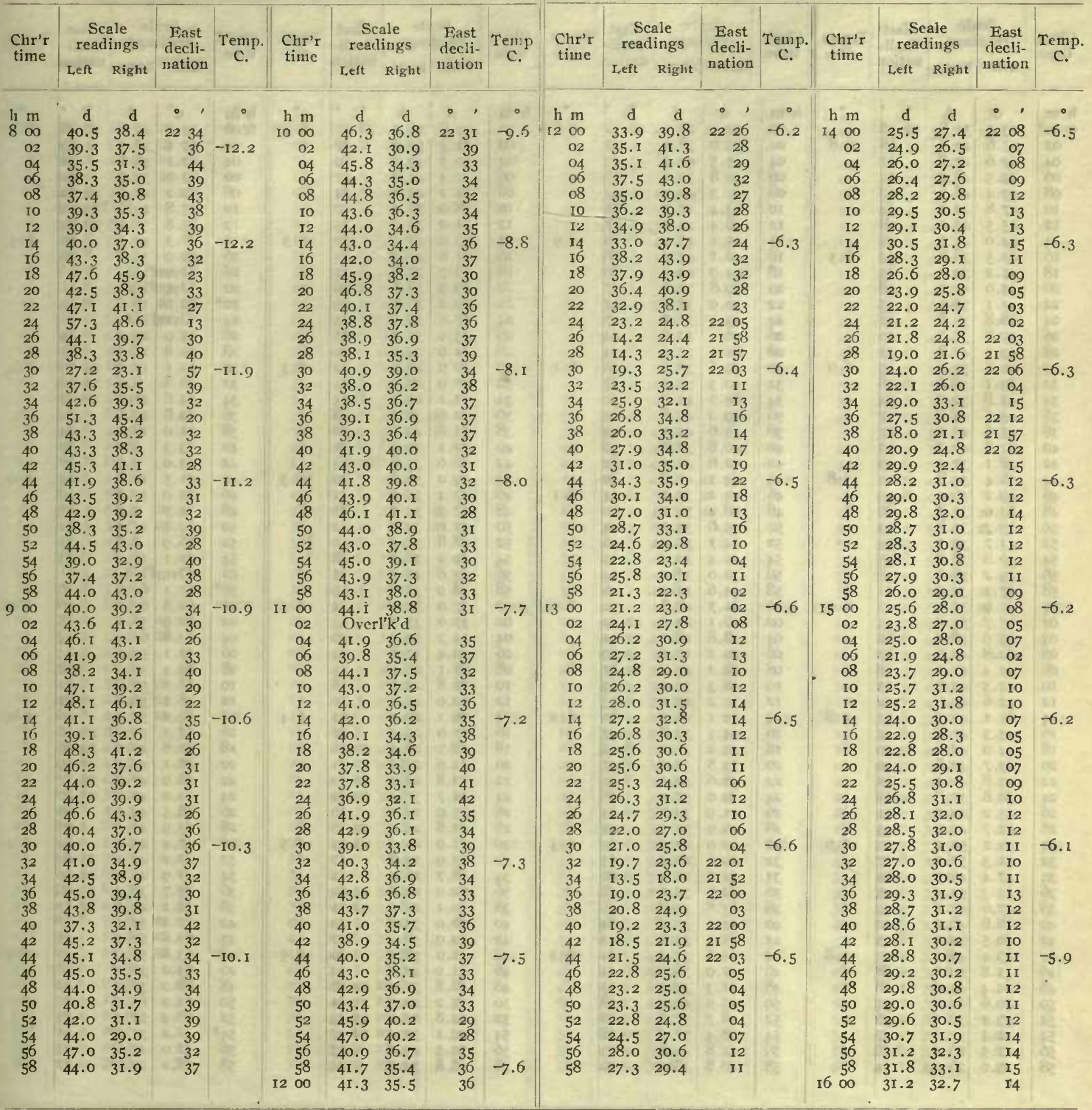

Correction to local mean time is + Im 02s.

Torsion head at $8 \mathrm{~h}$ oom read $2 \mathrm{I}^{\circ}$ and at the end read the same. Observers-W. J. P., R. R. T., and F. L.
Correction to local mean is $+58 \mathrm{~s}$. $90^{\circ}$ torsion $=25 .^{\prime} \mathbf{r}$.

Torsion head at $\mathrm{I} 2 \mathrm{~h}$ oom read $216^{\circ}$ and at $\mathrm{i} 6 \mathrm{~h} 35 \mathrm{~m}$ read $233^{\circ}$.

Observer-R. R. T. (W. J. P. I3h I $2 \mathrm{~m}$ to $14 \mathrm{~h}$ o2m, alternated R. R. T. to $14 \mathrm{~h} I 4 \mathrm{~m}$.) 
Tabulation of magnetic declinations observed at Teplitz Bay-Continued

\begin{tabular}{|c|c|c|c|c|c|c|c|c|c|c|c|c|c|c|c|c|c|c|c|c|}
\hline \multicolumn{6}{|c|}{ Wednesday, October 7, 1903} & \multicolumn{4}{|c|}{ Magnet scale inverted } & \multicolumn{7}{|c|}{ Wednesday, October 7, 1903} & \multicolumn{4}{|c|}{ Magnet scale inverted } \\
\hline \multirow{2}{*}{$\begin{array}{l}\text { Chr'r } \\
\text { time }\end{array}$} & \multicolumn{2}{|c|}{$\begin{array}{c}\text { Scale } \\
\text { readings }\end{array}$} & $\begin{array}{c}\text { East } \\
\text { decli- }\end{array}$ & & Chr'r & $\begin{array}{r}\text { Sc } \\
\text { read }\end{array}$ & $\begin{array}{l}\text { ale } \\
\text { lingss }\end{array}$ & $\begin{array}{l}\text { East } \\
\text { decli- }\end{array}$ & Temp. & & $\begin{array}{r}S c \\
\text { read }\end{array}$ & $\begin{array}{l}\text { ale } \\
\text { lings }\end{array}$ & $\begin{array}{l}\text { Ea } \\
\text { dec }\end{array}$ & & & & $\begin{array}{l}\text { Sc } \\
\text { read }\end{array}$ & $\begin{array}{l}\text { ale } \\
\text { lings }\end{array}$ & $\begin{array}{l}\text { East } \\
\text { decli- }\end{array}$ & \\
\hline & Left & Right & & & & Left & Right & & & & Left & Right & & & & & L,eft & Right & & \\
\hline h $\mathrm{m}$ & d & $d$ & & $\bullet$ & $\mathrm{h} \mathrm{m}$ & d & d & $\circ$, & & $\mathrm{h} \mathrm{m}$ & d & d & & & & h $\mathrm{m}$ & $\mathrm{d}$ & $\mathrm{d}$ & & $\bullet$ \\
\hline $000^{*}$ & 53.0 & 51.8 & 2216 & & 200 & 30.1 & 28.7 & 2252 & -5.8 & 400 & 49.8 & 49.1 & 22 & 21 & -6.3 & 600 & 33.2 & 30.0 & 2248 & -6.5 \\
\hline 02 & 51.6 & 49.8 & 18 & -6.8 & 02 & 39.0 & 38.3 & 37 & & 02 & 5 I. 3 & 50.2 & & I8 & & 02 & 39.4 & 36.0 & 39 & \\
\hline 04 & 49.6 & 47.8 & $2 I$ & & 04 & 45.5 & 42.2 & 29 & & 04 & 50.3 & 49.7 & & I9 & & 04 & $42 . I$ & 36 & 36 & \\
\hline 06 & 49.5 & 48.2 & $2 \mathrm{I}$ & 6 & 06 & 40.8 & 37.9 & 36 & & 06 & 42.4 & & & 32 & & 06 & $37 \cdot 3$ & 3.3 & 42 & \\
\hline 08 & 50.4 & 48.3 & 20 & int & 08 & 42.3 & 40.3 & 33 & 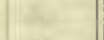 & 08 & $4 \mathrm{I} \cdot 2$ & 40. & & 34 & & as & 32.2 & & 2250 & \\
\hline Io & 47.8 & 45.7 & 24 & 81 & 10 & 43.4 & $4 \mathrm{I} \cdot 5$ & $3 \mathrm{I}$ & $2 t$ & Io & 38.0 & 36.3 & & 40 & & I0 & 25.8 & 20 & 302 & \\
\hline I2 & 45.5 & 43.0 & 28 & 8 & I2 & 46.4 & 44.2 & 27 & 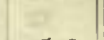 & I2 & $4 \mathrm{I} .2$ & 39.0 & & 35 & & I2 & 45.8 & 36. I & $22 \quad 34$ & \\
\hline 14 & 44.0 & 41.0 & $3 \mathrm{I}$ & & 14 & 45.1 & 43. & 29 & -5.9 & 14 & 43.9 & 42.9 & & 30 & -6.3 & 14 & 48.3 & 40.2 & & -6.5 \\
\hline 16 & 42.5 & 38.8 & 34 & -6.5 & 16 & 46.1 & 43. & 28 & & 16 & $4 \mathrm{I} .8$ & 40 & & 33 & & I6 & 39.9 & & 39 & \\
\hline I8 & 41.2 & 39.2 & 35 & $4 x^{2}$ & 18 & 43.9 & 42.2 & 30 & 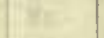 & I8 & 39.7 & 37. & & 37 & & 18 & Over & rl'k'd & & \\
\hline 20 & 45.0 & 44.0 & 28 & 37 & 20 & 39.8 & 37.7 & 37 & $E x$ & 20 & 39. & 38. & & 36 & & 20 & Over & rl'k'd & & 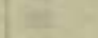 \\
\hline 22 & $4 \mathrm{I} . \mathrm{I}$ & $39 \cdot 3$ & 35 & 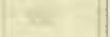 & 22 & 36.9 & 35.4 & $4 \mathrm{I}$ & 20 & 22 & 39.2 & 36 & & 39 & 28 & $22^{*}$ & 22.0 & 16.0 & 45 & 15 \\
\hline 24 & 46.8 & 38.8 & $3 \mathrm{I}$ & 35 & 24 & 33.3 & 31.7 & 47 & 1 & 24 & 37.0 & 35 & & $4 \mathrm{I}$ & & 24 & Over & rl'k'd & & \\
\hline 26 & 37.8 & 35.7 & 40 & 3 & 26 & 36.1 & 32. & 44 & & 26 & 33 & $3 \mathrm{I}$ & & 47 & & 26 & 25.9 & 20 & 39 & \\
\hline 28 & 43.2 & 41.8 & $3 \mathrm{I}$ & & 28 & 42.2 & 39.4 & 34 & & 28 & $3 I$ & 29 & & 50 & & 28 & 20.8 & 15.2 & 47 & \\
\hline 30 & 40.8 & 39.0 & 35 & -6.0 & 30 & 39.6 & 36. & 38 & -6.1 & 30 & 30.9 & 28 & & 52 & -6.3 & 30 & 15.1 & 12 & 2253 & -6.4 \\
\hline 32 & 39.1 & 36.6 & 38 & 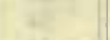 & 32 & 40.3 & 37.7 & 37 & & 32 & 30.9 & 28. & & 5 & & 32 & I0. 8 & 8.4 & 2300 & \\
\hline 34 & 41.8 & 39.0 & 34 & 34 & 34 & 44.7 & $4 \mathrm{I}$. & 30 & 74 & 34 & 31.2 & 29 & & 5 & y & 34 & 24 & 21 & 2238 & \\
\hline 36 & 45.6 & 44.0 & 27 & ne & 36 & 49. & 45 & 2 & 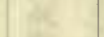 & 36 & & & & 4 & 8 & 3 & 29. & 22 & 34 & \\
\hline 38 & 45.6 & $45 . I$ & 27 & $x^{2}=$ & 38 & 45.6 & 42. & 29 & res & 38 & 31 & 29 & & 5 & (2) & 3 & 21.0 & I8.2 & 44 & 24 \\
\hline 40 & $4 \mathrm{I} .8$ & 41.3 & 33 & bet & 40 & 40.8 & 37.7 & 36 & 60 & 40 & 32 & & & 5 & $a$ & 4 & I3 & 2 & 57 & bes \\
\hline 42 & 38.1 & 37.0 & 39 & & 42 & 42.8 & 40.4 & 32 & t & 42 & 36.0 & 33 & & 43 & & 42 & 13 & 9.9 & 57 & \\
\hline 44 & 36.4 & 34.8 & 42 & -5.8 & 44 & 44. I & $4 \mathrm{I}$ & 30 & & 44 & 39 & 36 & & 3 & -6.3 & 44 & $2 \mathrm{I}$ & 16.8 & 45 & -6.0 \\
\hline 46 & 39.2 & 36.0 & 39 & & 46 & 42.2 & 39.5 & 34 & -6.1 & 46 & 37 & 35 & & $4 I$ & & 4 & 24.0 & 17.9 & 42 & \\
\hline 48 & 33.0 & 32.8 & 46 & Rx & 48 & 38.2 & 36.1 & 40 & $x^{2}$ & 48 & 37 & 34 & & 4 & & 4 & 14. & 9 & 55 & \\
\hline 50 & 39.3 & 35.8 & 39 & 8 & 50 & 35.6 & 33.0 & 44 & $x^{2}$ & 50 & 37 & 34 & & 4 & 24 & 50 & 19.1 & & $5 I$ & \\
\hline 52 & 41.7 & 39.1 & 34 & +5 & 52 & 34. & 32 & 46 & at & 52 & 38 & & & 4 & Be: & 5 & & & 55 & 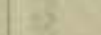 \\
\hline 54 & 40.8 & 38.8 & 35 & 18 & 54 & 35 . & 32.5 & 45 & 25 & 54 & 39 & 36 & & 38 & $k^{2}$ & 54 & 22.1 & 18.9 & 43 & \\
\hline 56 & 39.2 & 36.6 & 38 & 2 & 56 & 36. & 33.2 & 44 & $a^{2}$ & 56 & & $4 \mathrm{I}$ & & 3 & & & Over & rl'k'd & & \\
\hline 58 & 37.4 & 34.9 & $4 \mathrm{I}$ & & 58 & 34.6 & 32. & 46 & & $5^{8}$ & & 35 & & 4 & & $58^{*}$ & 6I.I & 60 & 36 & \\
\hline I 00 & 35.9 & 35.3 & 42 & -6.0 & 300 & $3 \mathrm{I} .7$ & 28.8 & 50 & -6.2 & 500 & 37 & 34 & & 42 & -6.3 & 700 & 61.8 & 60.1 & 36 & -6.0 \\
\hline 02 & 35.7 & 34.0 & 43 & $(106=$ & 02 & $26 . I$ & 23.2 & 2259 & 2aty & 02 & 37 & 33 & & 4 & 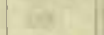 & 02 & 60 & 58 & 38 & 4tit \\
\hline 04 & 39.9 & 38.5 & 36 & 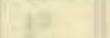 & 04 & I8.8 & I6. I & 23 II & we & 04 & 34 & & & 4 & 10 & 0 & 63.8 & 61.0 & 34 & \\
\hline 06 & 42.8 & 41.6 & 3 & 27 & 06 & 20. & 17. & $23 \circ 9$ & gar & 06 & & & & 4 & & 0 & 57 & & 43 & $x$ \\
\hline 08 & 42.4 & $4 \mathrm{I} . \mathrm{I}$ & 32 & 60 & 08 & 28.2 & 24.2 & 2257 & Bis. & 08 & 28 & 26 & & 5 & 35 & 08 & & & 38 & 35 \\
\hline 10 & 42.3 & 40.7 & 33 & hir & IO & 36.3 & 33.4 & 43 & at & IC & 26 & & & 5 & tir & I & & & $4 \mathrm{I}$ & at \\
\hline 12 & 42.6 & 40.6 & 32 & II & I2 & 30.2 & 27.2 & 53 & & I2 & 32.0 & 29 & & 5 & & 12 & 58 & 56.8 & $4 I$ & \\
\hline 14 & 43.1 & $4 \mathrm{I} .5$ & $3 \mathrm{I}$ & -5.8 & 14 & $32 . I$ & 28.3 & 50 & -6.2 & 14 & 29 & 26 & & 54 & -6.4 & I & 56 & 53.5 & 6 & -6.2 \\
\hline 16 & 43.3 & $4 \mathrm{I}$. & 3 & 24 & 16 & 34 . & 30 & 4 & 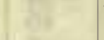 & 16 & 27 & 25 & & & & I & 55 & & 2246 & \\
\hline 18 & 44.0 & 42.6 & 3 & tat & $\mathrm{I} \varepsilon$ & & 31. & 4 & 12 & I & & & 22 & & 3 & I & & 40.5 & 2306 & ki \\
\hline 20 & 43.7 & 42.8 & 30 & tet & 20 & 33. & 3I. I & 47 & ext & 20 & 24.6 & 23 & 23 & or & 8 & 2 & 48 & 44. I & 259 & ec \\
\hline 22 & 42.8 & $41 \cdot 3$ & 32 & $3 x$ & 22 & 36.6 & $34 . I$ & 42 & 2 & 22 & 23 & 22 & 23 & 02 & t & 22 & 48.0 & 47.2 & 2257 & $1=$ \\
\hline 24 & 38.8 & 37. & 38 & 13 & 24 & 39. & 37. & 38 & 15 & 24 & & & 22 & 42 & it & 2 & & 40.7 & 2307 & $x=$ \\
\hline 26 & 43.2 & 42.7 & 31 & 24 & 26 & 41.9 & 39. & 34 & 26 & 26 & 25 & 24 & & 59 & E & 2 & 46.5 & 44. I & oo & \\
\hline 28 & 41.7 & $4 I . C$ & 33 & & 28 & 36.9 & 34. & 42 & & 28 & & 24 & 22 & 58 & & 2 & & & 05 & \\
\hline 30 & 50.2 & 50.1 & 19 & -6.0 & 30 & 32.0 & 29. & 50 & -6.3 & 30 & 21.9 & 20. & 23 & 05 & -6.5 & 3 & 47.4 & 43 & $\infty$ & -6.4 \\
\hline 32 & 51.3 & $5 \mathrm{I} . \mathrm{I}$ & 17 & 5 & 32 & 29.8 & 27. & 53 & 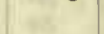 & 32 & & & & & - & 3 & & & 2300 & \\
\hline 34 & 47.4 & 46.7 & 24 & F & 34 & 31.2 & 29.3 & 50 & a & 34 & 18.7 & I6 & & II & wh & 3 & 47 & 45.0 & 2259 & \\
\hline 36 & 53.3 & 52.5 & I & 28 & 36 & & & 48 & & 3 & & & 23 & & & & & & 23 & \\
\hline 38 & 47.6 & 47.4 & 2 & 25 & 38 & 33.8 & 31.8 & 46 & xis & 38 & & 29 & 22 & 5 & 2 & 3 & 50 & 45 & 257 & \\
\hline 40 & 48.6 & 48.2 & 22 & $8 t$ & 40 & 34. & & 44 & wit & 4 & & & & 43 & $=$ & 4 & & & 47 & the \\
\hline 42 & 50.5 & 50.1 & 19 & & 42 & 35. & 33. & 43 & & 42 & 39.9 & 38 & & 37 & & 42 & 63.8 & 58.9 & 35 & \\
\hline 44 & 49.4 & 49.3 & 20 & -5.8 & 44 & 34.8 & 33. & 44 & -6.3 & 44 & 4 I. & 38 & & 36 & -6.5 & 44 & 63.8 & 58.9 & 35 & -6.5 \\
\hline 46 & 51.8 & 50.7 & I8 & 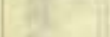 & 46 & 37.0 & $3^{6 .}$ & $4 I$ & & 46 & & 40.8 & & 34 & & 4 & 6.3 .0 & 56.0 & 38 & \\
\hline 48 & 44.4 & 44.0 & 28 & 28. & 48 & $4 I \cdot 3$ & 39.0 & 35 & As & 48 & $3^{8}$ & 37.4 & 22 & 38 & & 48 & 68.0 & 58.7 & 32 & $E^{2}$ \\
\hline 50 & 40.6 & 40.2 & 34 & 60 & 50 & & 44.4 & 28 & (6.). & 50 & & & 23 & OI & 68 & 50 & & 65.0 & 25 & a \\
\hline 52 & 39.2 & 39.1 & 36 & $\sqrt{2}$ & 52 & 38.7 & 37.8 & 38 & $x^{2}$ & 52 & 22.8 & 22.0 & 23 & 03 & $\mathrm{c}^{2}$ & 52 & 68.4 & 67.2 & 25 & \\
\hline 54 & 32.4 & 32.3 & & 12 & 54 & 38.4 & 37.2 & $3^{8}$ & 42 & 54 & 40.1 & 37.4 & 22 & 37 & Na & $54^{*}$ & $4 \mathrm{I} .2$ & 31.7 & $2 \mathrm{I}$ & \\
\hline 56 & 27.5 & 26.2 & 56 & 8 & 56 & 41.8 & 40.8 & 33 & & 56 & & 45.0 & & 23 & 3 & 56 & & 45.6 & 03 & \\
\hline 58 & 29.0 & 26.9 & 54 & $\because$ & 58 & 48.8 & 48.0 & 22 & ter & 58 & 36.8 & $32, \mathrm{I}$ & & 44 & 82 & 58 & 28 . I & 24.6 & 37 & \\
\hline & & & & & & & & & & & & & & & & & & & & \\
\hline
\end{tabular}

Observers-W. J. P. and R.W. P. alternated from oh $14 \mathrm{~m}$ to oh $26 \mathrm{~m}$; Observer-R. R. T.

R. W. P. and R. R. T. alternated from 3 h $52 \mathrm{~m}$ to 4 h $04 \mathrm{~m}$. 
Tabulation of magnetic declinations observed at Teplitz Bay-Continued

\begin{tabular}{|c|c|c|c|c|c|c|c|c|c|c|c|c|c|c|c|c|c|c|c|}
\hline \multicolumn{5}{|c|}{ Wednesday, October 7, 1903} & \multicolumn{5}{|c|}{ Magnet scale inverted } & \multicolumn{5}{|c|}{ Wednesday, October 7, 1903} & \multicolumn{5}{|c|}{ Magnet scale inverted } \\
\hline \multirow{2}{*}{$\begin{array}{l}\text { Chr'r } \\
\text { time }\end{array}$} & \multicolumn{2}{|c|}{$\begin{array}{l}\text { Scale } \\
\text { readings }\end{array}$} & \multirow{2}{*}{$\begin{array}{l}\text { East } \\
\text { decli- } \\
\text { nation }\end{array}$} & Temp. & $\begin{array}{l}\text { Chr'r } \\
\text { time }\end{array}$ & $\begin{array}{l}\text { Sca } \\
\text { readi }\end{array}$ & $\begin{array}{l}\text { ale } \\
\text { lings }\end{array}$ & $\begin{array}{c}\text { East } \\
\text { decli- }\end{array}$ & Temp. & $\begin{array}{l}\text { Chr'r } \\
\text { time }\end{array}$ & $\begin{array}{l}\text { Sc } \\
\text { read }\end{array}$ & $\begin{array}{l}\text { ale } \\
\text { ings }\end{array}$ & $\begin{array}{c}\text { East } \\
\text { decli- }\end{array}$ & Temp. & Clur'r & $\begin{array}{r}S \\
\text { rea }\end{array}$ & $\begin{array}{l}\text { le } \\
\text { ngs }\end{array}$ & $\begin{aligned} \text { East } \\
\text { decli }\end{aligned}$ & Temp. \\
\hline & Left & Right & & & & L,en & Right & nation & & & I.eft & Right & & & & L,eft & Right & natic & \\
\hline $\begin{array}{l}h \mathrm{~m} \\
800\end{array}$ & d & d & $\cdot 1$ & & $\mathrm{~h} \mathrm{~m}$ & d & d & & - & h m & d & d & $\cdot$ & & $\mathrm{h} \mathrm{m}$ & d & d & - & \\
\hline 800 & 26.8 & 18.3 & 2243 & -6.5 & 1000 & 30.6 & 25.8 & 2237 & & 1200 & 31.8 & 25.6 & 2235 & -6.2 & I4 00 & 39.7 & 39.1 & 2218 & -6.8 \\
\hline 02 & 59.4 & 53.3 & 2150 & tring & 02 & 26.8 & 25.6 & 40 & -6.0 & 02 & 31.2 & 23.3 & $\begin{array}{l}37 \\
\end{array}$ & $\theta$ & 02 & $37 \cdot 1$ & 37.1 & 22 & 6 \\
\hline 04 & 62.2 & 43.0 & 2156 & $+4^{7}$ & 04 & 28.5 & 22.5 & $4 \mathrm{I}$ & & 04 & 33.3 & 26.2 & 33 & -16 & 04 & 38.3 & 38.2 & 20 & $p^{n}$ \\
\hline 06 & 46.0 & 17.9 & $22 \quad 29$ & no & 06 & 45.3 & 35.3 & 18 & in & 06 & $35 . \mathrm{I}$ & 27.1 & $3 \mathrm{I}$ & 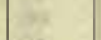 & 06 & 38.7 & 38.3 & 20 & (5) \\
\hline $08^{*}$ & 56.9 & $37 . I$ & 49 & Re & 08 & 52.0 & 42.0 & 08 & Bet & $\infty 8$ & 34.2 & 27.0 & 32 & 28 & 08 & $37 \cdot 3$ & 37.2 & 22 & 10 \\
\hline IO & Over & $r l^{\prime} k^{\prime} d$ & & cat & 10 & 37.0 & 30.3 & 29 & 6 & 10 & 36.4 & 29.9 & 28 & art. & 10 & $4 \mathrm{I} .4$ & 40.5 & 16 & bit \\
\hline I2 & 46.0 & 36.7 & 58 & & I2 & 25.0 & 16.0 & 49 & & 12 & 37.4 & $3 I .4$ & 26 & & 12 & 41.2 & 40.6 & 16 & 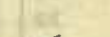 \\
\hline 14 & 68.8 & 55.0 & 26 & -6.5 & 14 & $4 \mathrm{I} \cdot 3$ & $33 \cdot 3$ & 23 & -6.2 & 14 & 38.6 & 32.8 & 24 & -6.1 & I4 & 42.8 & 42.3 & I3 & -6.9 \\
\hline 16 & 69.8 & 62.8 & I9 & 27 & 16 & 27.6 & 23.8 & $4 \mathrm{I}$ & tit & I6 & 38.2 & 33.8 & 24 & & 16 & 45.0 & 44.I & IO & \\
\hline 18 & 68.7 & 65.5 & 2218 & 68 & 18 & 51.8 & 47.2 & 04 & (K) & 18 & 37.6 & 32.9 & 25 & 111 & 18 & 41.8 & $4 \mathrm{I} . \mathrm{I}$ & 15 & ter \\
\hline 20 & 34.8 & 30.9 & 2312 & ant & 20 & 41.8 & $34 . I$ & 22 & cot & 20 & 38.1 & 32.3 & 25 & 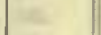 & 20 & 49.2 & 48.1 & 04 & $c^{2}$ \\
\hline 22 & 40.0 & 35.8 & 2304 & $=$ & 22 & 30.3 & 25.6 & 38 & tex & 22 & 38.2 & 33.8 & 24 & 0 & 22 & 45.4 & 42.6 & II & 16 \\
\hline 24 & 67.0 & 62.0 & 2222 & 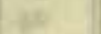 & 24 & 34.3 & 28.8 & 32 & 18 & 24 & $4 \mathrm{I} .0$ & 35.6 & 20 & 16 & 24 & 40.9 & 38.4 & 18 & $t=$ \\
\hline 26 & 45.0 & 43.2 & 54 & 8 & 26 & 35.6 & $35 \cdot 3$ & 26 & $(x$ & 26 & 40.5 & 35.7 & 20 & ac & 26 & 43.1 & 41.6 & I4 & A \\
\hline 28 & 48.5 & 45.8 & 49 & & 28 & 46.0 & 40.3 & 14 & & 28 & 47.8 & 43.7 & 08 & & 28 & 40.8 & 39.6 & I7 & Es \\
\hline 30 & 67.1 & 62.0 & 22 & -6.3 & 30 & 46.3 & 34.3 & 18 & -6.2 & 30 & 49.2 & 44.7 & 07 & -6.0 & 30 & 40.2 & 38.7 & I8 & -7.0 \\
\hline 32 & $6 \mathrm{I} \cdot 3$ & 58.3 & 29 & ate & 32 & 45.6 & 39.2 & 15 & & 32 & 51.1 & 48.9 & 02 & 6 & 32 & 39.8 & 38.8 & I8 & ( \\
\hline 34 & 46.0 & 46.0 & $5 \mathrm{I}$ & in & 34 & 46.8 & 31.0 & 20 & $K^{2}$ & 34 & 47.8 & 44.2 & 08 & 15 & 34 & 37.7 & 36.7 & 22 & 10 \\
\hline 36 & 67.0 & 61.7 & 22 & $x y$ & 36 & 48.0 & 30.0 & 2220 & E & 36 & 46.4 & 42.9 & I0 & & 36 & 37.9 & 36.8 & 22 & W \\
\hline 38 & 47.8 & 43.0 & 52 & $\mathrm{Ag}$ & 38 & 60.0 & 50.0 & 2I 55 & 15 & 38 & 49.0 & 47.2 & 05 & h & 38 & 38.4 & 37.3 & 21 & B \\
\hline 40 & 50.8 & 41.8 & 50 & 78 & 40 & 76.6 & 66.5 & $\begin{array}{r}29 \\
\end{array}$ & as & 40 & $5 \mathrm{I}$. & 48.7 & 0 & 10 & 40 & 40.2 & 38.7 & & 10 \\
\hline 42 & 69.7 & 60.3 & $2 I$ & & 42 & Over & l'k'd & $x^{-2}$ & & 42 & 48.2 & 45.2 & 07 & & 42 & 40.8 & 39.3 & I) & Fin \\
\hline 44 & 54.5 & $45 . I$ & 45 & -6.1 & $44^{*}$ & 42.5 & 31.8 & 30 & -6.2 & 44 & 48.7 & 46.1 & 2206 & -6.2 & 44 & 42.7 & $4 \mathrm{I} . \mathrm{I}$ & I4 & -7.1 \\
\hline 46 & 64.0 & 58.2 & 27 & 9 & 46 & 21.8 & 19.2 & 56 & (n) & 46 & 52.6 & 50.4 & 2159 & at & 46 & 45.8 & 44.7 & & 80 \\
\hline 48 & 60.3 & 57.0 & 2231 & 25 & 48 & 28.6 & 15.0 & 2I 54 & 4 & 48 & 49.4 & 47.3 & 2204 & 6. & 48 & 48.2 & 46 & 22 & 144 \\
\hline 50 & $4 \mathrm{I} \cdot 3$ & 34.3 & 2304 & wet & 50 & 13.2 & 8.1 & 22 I2 & Aff & 50 & 46.3 & $44 \cdot 3$ & 09 & 104 & 50 & 54.1 & 52.1 & 2157 & a \\
\hline $5^{2}$ & 58.0 & 47.2 & $4 \mathrm{I}$ & 39 & $52^{*}$ & 29.0 & 15.5 & 24 & 4 & 52 & 45.8 & 43.2 & 10 & e & 52 & 57.0 & 55.7 & 5 & $x=$ \\
\hline 54 & 58.0 & 48.2 & 40 & is & $54^{*}$ & 26.0 & 17.2 & 46 & at: & 54 & 43.8 & $42 . I$ & I3 & 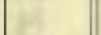 & 54 & 55.8 & 55.3 & & 62 \\
\hline 56 & 42.3 & 25.6 & Io & 34 & 56 & $3 \mathrm{I} .3$ & 23.6 & 37 & an & 56 & 42.7 & 40.8 & I5 & & 56 & 55.0 & 53.9 & & 82 \\
\hline 58 & 59.8 & 52.8 & 35 & & $5^{8}$ & 32.3 & 27.2 & 33 & & 58 & $44 . I$ & 40.8 & I4 & & 58 & $53 \cdot 3$ & 52.7 & 21 & \\
\hline 900 & 66.0 & 65.8 & 20 & -6.0 & II OO & 33.3 & 24.3 & 35 & -6.0 & 1300 & 47.0 & 44.5 & 08 & -6.3 & I5 & 51.2 & 50.1 & 220 & 7.2 \\
\hline 02 & 38.0 & 35.2 & 2306 & wis & 02 & 39.7 & 32.3 & 24 & . & 02 & 46.1 & 43.4 & IO & - & 02 & 47.7 & 46.3 & or & r \\
\hline 04 & 63.2 & 55.2 & 2230 & $\tan$ & 04 & $4 I .5$ & 34.3 & 21 & $a^{\circ}$ & 04 & 43.2 & 40. & I4 & 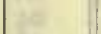 & 04 & 48.6 & 47.7 & in & $1^{7}$ \\
\hline 06 & 57.5 & 47.2 & $4 \mathrm{I}$ & 60 & 06 & 40.5 & 33. & 22 & 4. & 06 & 39.6 & 36.9 & 2 & & 06 & 46.5 & 45.2 & & $m$ \\
\hline 08 & 53.0 & 40.6 & 50 & thet & 08 & 43.3 & 36 & 18 & 65 & 08 & 38. & 35.3 & 23 & 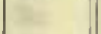 & 08 & 43.3 & 42.1 & th & Ear \\
\hline IO & 49.7 & 37.7 & 2254 & ati & Io & 35.8 & 29.9 & 28 & $\ln x$ & IC & 36.6 & 34.4 & 2 & & IO & 44.3 & 43.9 & 0 & in \\
\hline I2 & & 22.4 & $23 \mathrm{I} 4$ & & I2 & $4 \mathrm{I} .3$ & 35 . & & & 12 & 34. & 32. & 27 & & 12 & $4 \mathrm{I} .7$ & $4 \mathrm{I}$ & & wi \\
\hline 14 & 64.8 & 44.3 & $22 \quad 38$ & -6.1 & 14 & 49.0 & 42.2 & 08 & -6.2 & 14 & 38.0 & 35.2 & 23 & -6.5 & I4 & 39.8 & 39.2 & 8 & -7.3 \\
\hline 16 & 53.0 & 37.0 & 52 & 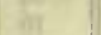 & 16 & 49.8 & 41.5 & 08 & ten & 16 & & 31.3 & 30 & & 16 & 37.8 & 36 & & \\
\hline I8 & 54.4 & 42.2 & 47 & $=$ & I8 & 49.0 & 41.3 & 09 & Ant & 18 & 33. & 32.0 & 29 & 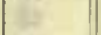 & I8 & $4 \mathrm{I} .9$ & 40.6 & & int \\
\hline 20 & $6 \mathrm{r} .6$ & 44.7 & 40 & $\cos$ & 20 & 48.0 & 40.6 & II & we & 20 & 34.8 & 33.3 & 27 & 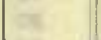 & 20 & 40.2 & 39.1 & & int \\
\hline 22 & 66.5 & 43.0 & 37 & $5=$ & 22 & 43.6 & 36.6 & 17 & $8 x$ & 22 & 34 & 33. & 2 & 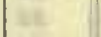 & 22 & 43.2 & 42.2 & 13 & 34 \\
\hline 24 & 60.6 & 35.0 & 48 & 3 & 24 & 42.3 & & 20 & is & 24 & & 33.5 & 2 & 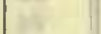 & 24 & 41.9 & 39.7 & & 18 \\
\hline 26 & 46.8 & 46.3 & 50 & A & 26 & 45.7 & 36.8 & 15 & in & 26 & 36. & 36. & 2 & 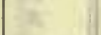 & 26 & 37.2 & 36 & & 16 \\
\hline 28 & 69.5 & 66.5 & I6 & & 28 & 46.8 & 39.8 & 12 & & 28 & 37. & 37.1 & 21 & & 28 & 33.7 & 33.1 & & 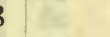 \\
\hline 30 & 63.2 & 51.4 & 33 & -6.0 & 30 & 44.0 & 37.2 & 16 & -6.2 & 30 & 37. & 36.0 & 23 & -6 & 30 & 37.7 & 37 & & -7.4 \\
\hline 32 & 65.7 & 59.7 & 25 & 28 & 32 & 43.3 & 38.4 & 16 & 67 & 32 & 35.7 & 34.5 & 25 & W2 & 32 & 32.8 & 32.8 & 2 & -2 \\
\hline 34 & $64 \cdot 3$ & 60.0 & 25 & Et & 34 & 40.6 & 34.6 & $2 I$ & & 34 & & & 24 & & 34 & 36.0 & 35 & & 14 \\
\hline 36 & 75.0 & 69.3 & Io & Wit & 36 & 40.3 & 34. & 2 & 28 & 30 & $40 . I$ & 39.3 & I & 10 & 3 & 34.5 & 33 & F & \\
\hline 38 & 45.8 & 42.6 & & E & 38 & 40.6 & 33.8 & 22 & $\pi$ & 38 & 41.6 & & I6 & $x$ & 38 & 35.4 & & & 72 \\
\hline 40 & 65.8 & 55.8 & $22 \quad 28$ & in & 40 & 40.5 & .33 .0 & 22 & es. & $4^{\circ}$ & 38.7 & 36.2 & 22 & 0 & 40 & 33.6 & 33.2 & 28 & is \\
\hline $42^{*}$ & $5^{8} .1$ & 55.2 & 2128 & & 42 & 44.6 & 38.2 & I5 & & 42 & 41.8 & 40.2 & I6 & & 42 & 35.8 & & & 58 \\
\hline 44 & 27.3 & 24.3 & 22 I6 & -6.0 & 44 & 42.5 & 35.5 & 19 & -6.3 & 44 & $43 . I$ & 42.1 & 13 & -6.6 & 44 & 35.4 & 34 & & \\
\hline 46 & 10.6 & 4.5 & 45 & 6 & 46 & $34 . I$ & & 33 & ch & 46 & 43.6 & 42.5 & 13 & & 46 & 36.3 & 34.6 & 2 & -7.1 \\
\hline 48 & 27.5 & 16.8 & 22 & 58 & 48 & 29.4 & 20.8 & $4 \mathrm{I}$ & 8 & 48 & 41.8 & 40.5 & 16 & 37 & 48 & 38.4 & 36.3 & 2 & ( \\
\hline & 22.5 & 17.5 & 50 & ate & 50 & 30.7 & 23.3 & 38 & 68 & 50 & 42.7 & 41.4 & I4 & & 50 & 37.2 & & 2 & $a=$ \\
\hline 52 & 18.4 & I6.0 & 30 & $3 i$ & 52 & 33.6 & 26.1 & 33 & sec & 52 & 42.7 & 42.0 & 14 & 16. & 52 & 36.9 & 36.6 & e. & 62 \\
\hline $54^{*}$ & 20.0 & 20.0 & & 35 & 54 & 34.6 & 27.3 & 32 & $2 x$ & 54 & 39.0 & 38.4 & 19 & $y^{2}$ & 54 & 36.3 & 35.7 & 2. & 12 \\
\hline 56 & 51.0 & 48.2 & 03 & 8 & 56 & 33.9 & 27.4 & 32 & 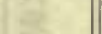 & 56 & 40.3 & 39.7 & I7 & & 56 & 37.6 & 35.5 & fe & $1=$ \\
\hline 58 & 41.2 & 38.8 & I8 & 6 & 58 & 33.9 & 27.4 & 32 & 80 & 58 & 40.9 & 39.7 & I7 & 78 & $5^{8}$ & 40.1 & 35.4 & & 24 \\
\hline & & $8 x$ & & & 100 & & & & & & & & & & & & & & \\
\hline
\end{tabular}

Observers-R. R. T. and W. J. P. alternated from $8 \mathrm{~h} 24 \mathrm{~m}$ to $8 \mathrm{ll} \quad$ Observers-R. W. P. and R. R. T. alternated from $15 \mathrm{~h} 46 \mathrm{~m}$ to I6h 32m. W. J. P. and R. W. P. alternated from $1 \mathrm{Ih} 54 \mathrm{~m}$ to $12 \mathrm{~h} 08 \mathrm{~m}$. $02 \mathrm{~m}$.

(W. J. P. $13 \mathrm{~h} 08 \mathrm{~m}$ to $13 \mathrm{~h} 38 \mathrm{~m}$.) 
Tabulation of magnetic declinations observed at Teplitz Bay-Continted

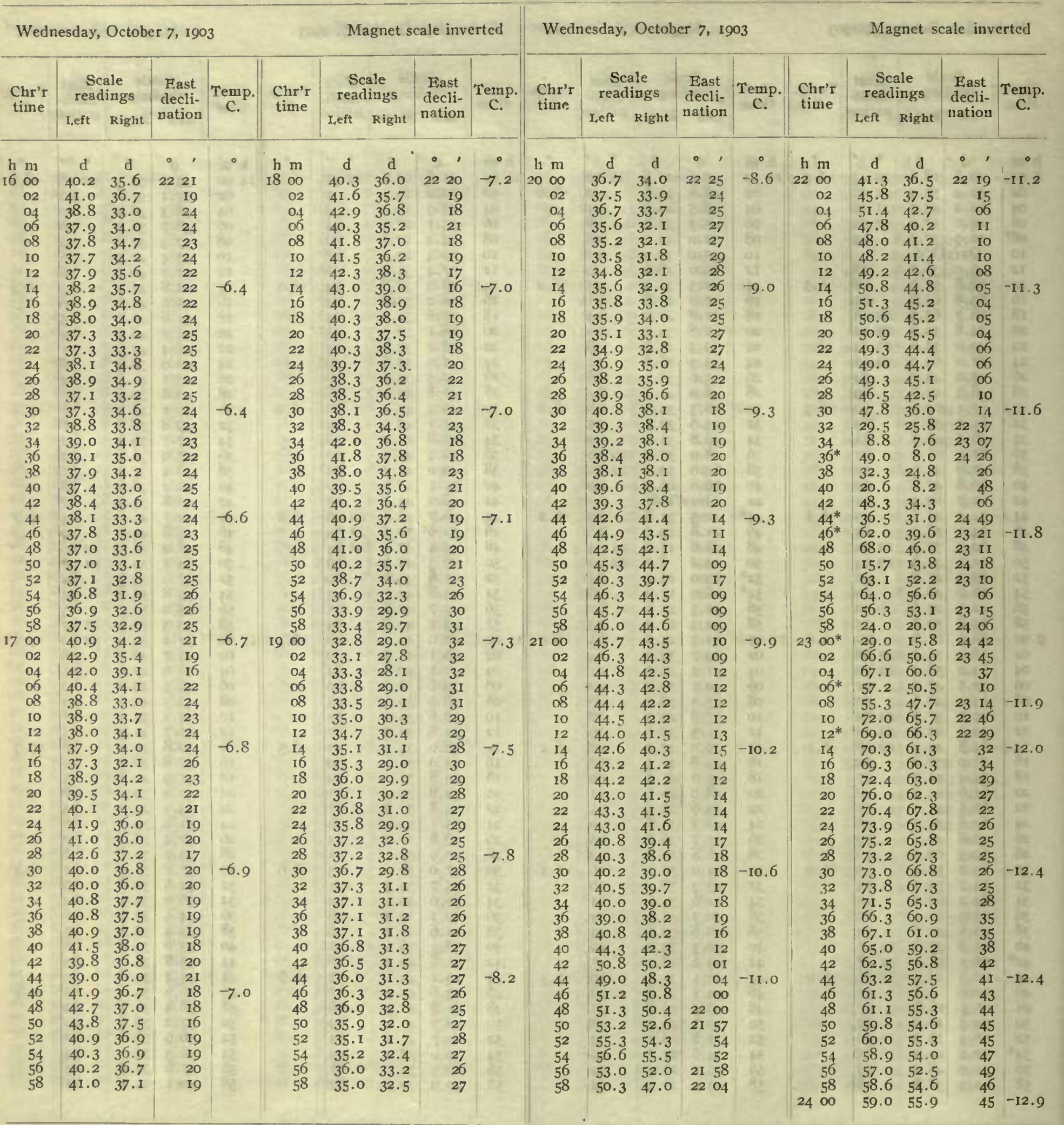

Observers-R. R. T. and W. J. P. alternated from $20 \mathrm{~h} 28 \mathrm{~m}$ to $20 \mathrm{~h}$ $38 \mathrm{~m}$. (W. J. P. I8h $12 \mathrm{~m}$ and $18 \mathrm{~h} 38 \mathrm{~m}$ to $\mathrm{I} 8 \mathrm{~h} 42 \mathrm{~m}$.)
Correction to local mean time is + Im $06 \mathrm{~s}$.

Torsion head at oh oon read $23^{\circ}$ and at $24 \mathrm{~h}$ oom read the same.

Observers-R. R. T. and W. J. P., who alternated from $20 \mathrm{~h} 28 \mathrm{~m}$ to $20 \mathrm{~h} 38 \mathrm{~m}$. 
Tabulation of magnetic declinations observed at Teplitz Bay-Continued

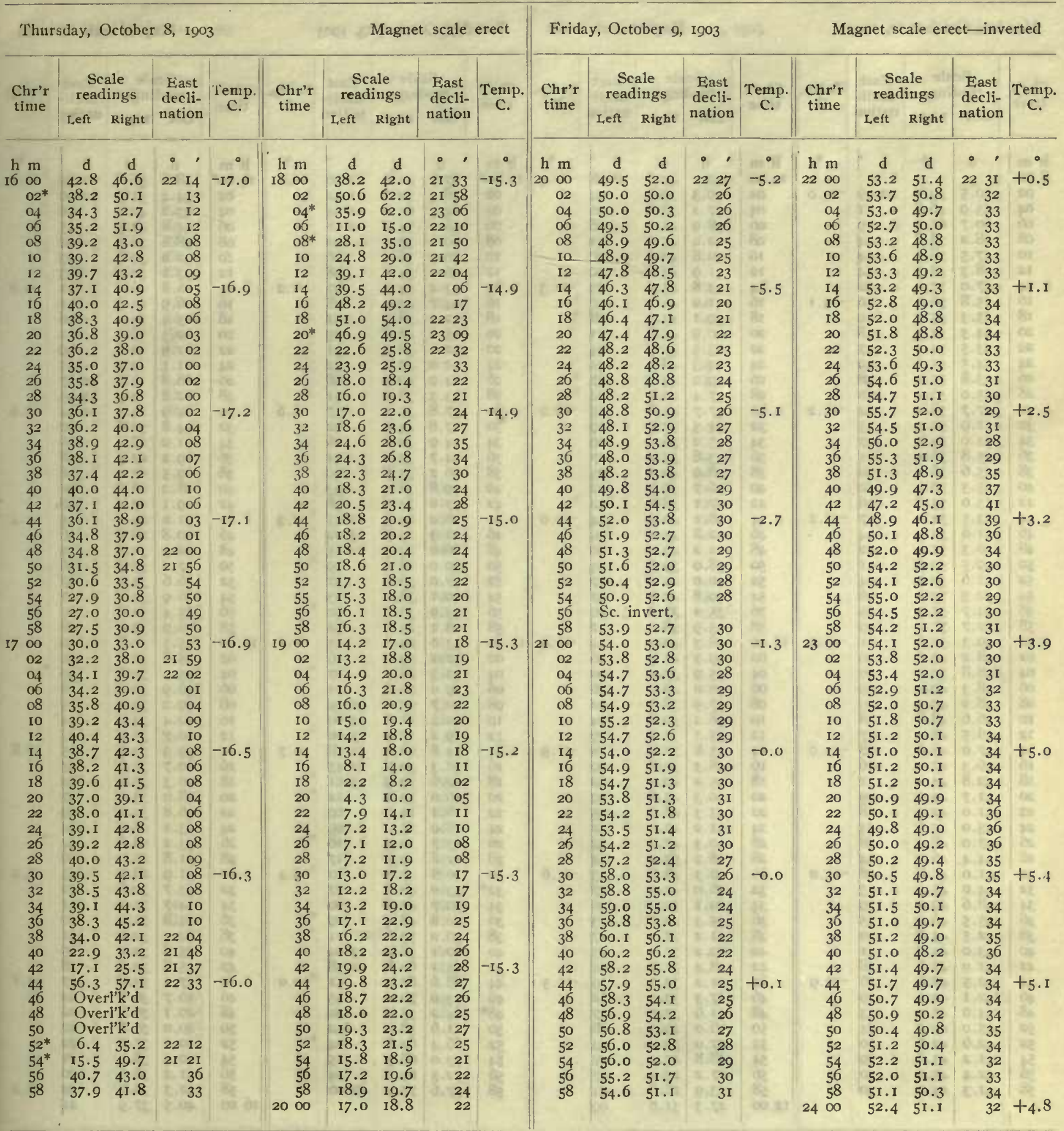

Correction to local mean time is + Im $33 s$.

Torsion head at ol $14 \mathrm{~m}$ read $233^{\circ}$ and at the end read the sane.

Observers-R. R. T. (W. J. P. I8h $30 \mathrm{~m}$ to $181158 \mathrm{~m}$.)
Correction to iocal mean time is $\operatorname{Im} 34 \mathrm{~s}, 90^{\circ}$ torsion $=26 .^{\prime} 06$. Torsion head at $201 \mathrm{com}$ read $270^{\circ}$ and at the end read $264^{\circ}$. Observer-R. R. T. 
Tabulation of magnetic declinations observed at Teplitz Bay-Continued

\begin{tabular}{|c|c|c|c|c|c|c|c|c|c|c|c|c|c|c|c|c|c|c|c|c|}
\hline \multicolumn{6}{|c|}{ Monday, October I2, 1903} & \multicolumn{4}{|c|}{ Magnet scale erect } & \multicolumn{6}{|c|}{ Tuesday, October 13,1903} & \multicolumn{5}{|c|}{ Magnet scale inverted } \\
\hline \multirow{2}{*}{$\begin{array}{l}\text { Chr'r } \\
\text { time }\end{array}$} & \multicolumn{2}{|c|}{$\begin{array}{l}\text { Scale } \\
\text { readings }\end{array}$} & $\begin{array}{c}\text { East } \\
\text { decli- }\end{array}$ & Temp. & & $\begin{array}{l}\text { Sc } \\
\text { read }\end{array}$ & gs & $\begin{array}{c}\text { East } \\
\text { decli- }\end{array}$ & & & rea & $\begin{array}{l}\text { le } \\
\text { ngs }\end{array}$ & $\begin{array}{c}\text { East } \\
\text { decli- }\end{array}$ & & & $\begin{array}{r}\text { Sc } \\
\text { read }\end{array}$ & igs & $\begin{array}{r}\text { Eas } \\
\text { decl }\end{array}$ & $\begin{array}{l}\text { ast } \\
\text { cli- }\end{array}$ & \\
\hline & Left & Right & & & & Left & Right & nation & & & Left & Right & nation & & & Left & Right & & & \\
\hline $\mathrm{h} \mathrm{m}$ & d & d & . ' & - & $\mathrm{h} \mathrm{m}$ & d & & & & & & & & & & & & & & \\
\hline 800 & & & 40 & $\operatorname{lin}=$ & I0 00 & 40.2 & 42.8 & 2228 & -12.4 & $1200^{*}$ & 67.7 & 40.4 & 2210 & -0.6 & I4 $00^{*}$ & 58.6 & 56.8 & $2 I$ & 52 & -0.2 \\
\hline 02 & 50.5 & 59.5 & 2249 & -13.0 & 02 & 39.3 & 39.5 & 24 & 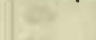 & 02 & 78.3 & 52.1 & 2152 & & 02 & 54.4 & 51.5 & & oo & \\
\hline 04 & 55.9 & 56.8 & $5 \mathrm{I}$ & & 04 & 37.2 & 38.8 & 22 & for & 04 & 64.5 & 63.0 & 2154 & 167 & 04 & 43.2 & 42.2 & & 16 & ant \\
\hline 06 & .7 & $6 \mathrm{r} .5$ & 59 & & 06 & 38.9 & $4 \mathrm{I} .2$ & 26 & 8 & 06 & 56.0 & 54.2 & 2208 & 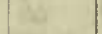 & 06 & & 41.5 & & 15 & 30 \\
\hline 08 & & $5^{8}$ & 54 & 35 & & 2.0 & 45.0 & $3 I$ & 26 & 08 & 60.2 & 57.1 & 02 & and & 08 & 38.6 & & & 24 & tete \\
\hline Io & 57.5 & 58.1 & 54 & 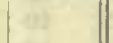 & IO & 40.8 & 43. & 29 & $(1)$ & I0 & 56.3 & 56.1 & 06 & out & 10 & 32.0 & 30.8 & & 33 & eff \\
\hline 12 & & 58 & 54 & 6. & 12 & I. 9 & & 30 & et & I2 & 56.2 & 54.1 & 08 & & 12 & 33.2 & .0 & & 33 & \\
\hline 14 & 5 & 57.0 & 52 & wa & I4 & 3.8 & 45 & 33 & -12.3 & I & 57.4 & 54 & 07 & $-I .2$ & 14 & 26.8 & 26.2 & & $4 I$ & -0.2 \\
\hline 16 & & 56 & 50 & $-13 . I$ & & & & 30 & $1=-2$ & IC & 44.7 & & 28 & & 16 & 26.9 & 25.9 & & $4 \mathrm{I}$ & \\
\hline 18 & 5 & 55.7 & 50 & 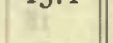 & I8 & 9. I & & 28 & ty & I & 42.2 & 38 & 31 & & 3 & 23.4 & & & 6 & 6 \\
\hline 20 & & 54 & 48 & $=$ & 20 & & & $3 I$ & ser. & 2 & 45.8 & & 25 & bet & 2 & 20.3 & & & 52 & Ex \\
\hline 22 & 5 & 54. & 47 & is & 2 & 3.5 & $47 \cdot 3$ & 34 & $3=$ & 22 & 42.2 & 38 & $3 \mathrm{I}$ & $1=$ & 2 & 21.9 & 21.0 & & 49 & $E$ \\
\hline 24 & & 5 & & $y^{2}$ & 2 & & & 35 & 1. & 2. & & & 32 & & & II.I & I9.4 & & I & 17. \\
\hline 26 & 3.8 & 55. & 48 & 2 & 26 & 9.2 & 43.2 & 27 & $2=$ & 26 & 44.0 & & 2 & $1=$ & 2 & I6.8 & I 5.4 & 22 & 57 & $a$ \\
\hline 28 & 5.0 & 56 & 50 & is & 28 & & 40 & 24 & $x^{2}$ & 28 & & & 3 & F & & 12.8 & 9.3 & 3 & 5 & \\
\hline 30 & & 58.2 & 54 & -13.2 & 30 & 7.7 & $4 \mathrm{I}$ & 25 & -12.2 & 30 & 37.8 & 35 . & 37 & -1.6 & 30 & 24.9 & 24.5 & 22 & 14 & -1.0 \\
\hline 32 & .9 & 52.9 & 45 & 4 & 32 & $4 \cdot 3$ & 37. & I9 & & 3 & 47.8 & 44 & & & & 4.8 & 32.6 & & 30 & \\
\hline 34 & & 4.0 & 47 & is & 34 & & 36 & I9 & 3. & 3 & 44.9 & & 26 & ise & 3 & 31.0 & 28.4 & & 36 & 4 \\
\hline 36 & & 56.4 & 51 & 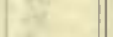 & 36 & 3.8 & 35 & 17 & $x^{2}$ & 3 & 34 & & $4 I$ & $12=$ & & 29.2 & 27 & & 8 & \\
\hline 38 & & 56.1 & 50 & 20 & & & & 25 & $x_{2}$ & & & & & f & 3 & 29.5 & 4 & & 8 & 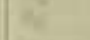 \\
\hline 40 & & 56.0 & 50 & 4 & 40 & 36.7 & 39. & 22 & 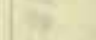 & 4 & 39.8 & 38 & 3 & 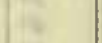 & 4 & 28.2 & 26.6 & & 0 & 46 \\
\hline 42 & & 53.8 & 4 & 20 & 4 & & & 22 & $1=$ & 4 & 37 & & & 19 & 4 & 9. I & 27.2 & & 88 & 4 \\
\hline 44 & & 54.1 & 47 & -13.2 & 44 & 38 & 40. & 24 & $-\mathrm{I} 2.0$ & 4 & 28.0 & 25 & 225 & -1.3 & 44 & 31.2 & 29.1 & & 35 & -1.4 \\
\hline 46 & & 52.4 & 44 & & 4 & 42. & 43 & 30 & 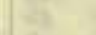 & 4 & 21.3 & & 230 & & & 8 & 28 & & 36 & \\
\hline 48 & 5 & 51.2 & 4 & e & 48 & & & 30 & lot & 4 & 26 & & 225 & 1 & 4 & 32.9 & 31.3 & & 32 & Fe. \\
\hline 50 & & 50.6 & $4 \mathrm{I}$ & aty & 5 & & & 24 & $=$ & 5 & & & & & & & & & & 2 \\
\hline 52 & & 52.5 & 44 & 3it. & 5 & & $4 \mathrm{I}$ & 27 & Q2 & 5 & 37.0 & & 3 & & 5 & & & & 7 & sed \\
\hline 54 & 52.3 & 53.7 & 46 & {$\left[\begin{array}{c}0 \\
0\end{array}\right.$} & 54 & 9.8 & 42.7 & 27 & 24 & 5 & & & & & 5 & 36 & & & 27 & $\mathrm{r}^{2}$ \\
\hline 56 & & 55. & 49 & 62 & 5 & & 37 & 20 & 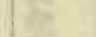 & 5 & & & & & & & 32.8 & & 9 & \\
\hline 58 & & 57.0 & 49 & & 5 & & 38 & $2 I$ & & 5 & 28.2 & & & & & & & & 8 & \\
\hline 900 & .3 & 50.6 & $4 \mathrm{I}$ & -13.2 & II 00 & 3.3 & 40 & 24 & -12.0 & 1300 & 33.0 & & 4 & $-I . I$ & I5 0 & 35.5 & 31.8 & & 0 & -2.2 \\
\hline 02 & & 49.6 & 40 & 2.0 & 02 & & $4 \mathrm{I}$ & 26 & - & 02 & 32.2 & & 4 & & 0 & 5.8 & 31.0 & & 30 & 9 \\
\hline 04 & & 50.2 & 40 & test & 0 & & & 20 & (tas & 0 & & & 4 & & o & & & & & (60) \\
\hline 06 & & 49.4 & 39 & 0 & 0 & & & 26 & (y) & & 9.0 & & & & & & 32 & & & in \\
\hline 08 & & 49.3 & & 3. & o & & & 22 & 2 & 0 & 7 & & 22 & & & 2 & & & 5 & 168 \\
\hline IO & & 48.8 & 38 & wi & Is & & 39 & 24 & 82 & Is & 2.4 & & 230 & & I & 4 & & & 4 & ter \\
\hline 12 & & 48.4 & 37 & wit & & & & I9 & (61) & & & & 230 & & & & & & & \\
\hline 14 & 47.0 & 48.6 & 38 & -13.0 & I 4 & & & 18 & -II.9 & I & 24.8 & & 2258 & -0.7 & I & & 33 & & 5 & -2.8 \\
\hline 16 & & 47.4 & 3 & - & & & & 09 & & & & & 2245 & & & & & & & is \\
\hline 18 & 42.8 & 44.4 & & tet & I & 5.2 & 40 & 22 & $3 i$ & I & 2 & & 230 & & I & & .2 & & 4 & Hin \\
\hline 20 & 1.7 & 43.0 & 26 & tat & $2 c$ & & 44 & 29 & nar & 2 & & & 1 & & & & & & & tes \\
\hline 22 & & 42.3 & & 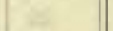 & 2 & & 48 & 3 & $=$ & & 18. & & 0 & & 2 & 6.8 & & & 9 & 112. \\
\hline 24 & 45.0 & 45.6 & 3 & 16 & 2 & .2 & 47. & $3^{2}$ & $y=$ & 2 & 18.0 & & 0 & & & & & & & \\
\hline 26 & & & & F & & & & 32 & & & & & & & & & 28 & & 4 & $\mathrm{~s}$ \\
\hline 28 & 44.4 & 46.2 & 34 & & 28 & 48.2 & 55 & 44 & & 28 & & 9 & I & & 2 & 31.8 & 27.9 & & & \\
\hline 30 & & 41.8 & 8 & $-I 3.0$ & 30 & & 48 & 33 & -II. & & 10.8 & & 2 & -0. & 3 & 31.9 & 28.7 & & 5 & -3.2 \\
\hline 32 & & & 3 & -30 & 3 & & & 27 & . & 3 & 53.0 & & I & & 3 & 7 & 30.8 & & 2 & 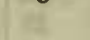 \\
\hline 34 & & & & it & 3 & & & IC & $y$ & & & & & & & & 3I.I & & 3I & ir \\
\hline 36 & & 46. & 3 & $c^{2}$ & 3 & & & 23 & 20 & 3 & 50 & & I & & & & & & 30 & \\
\hline 38 & & 43.8 & & 32 & 3 & & & I7 & $x$ & & & & I & & & & & & 3 I & \\
\hline 40 & 4. & 44.5 & & we & 4 & & 37. & 15 & e. & 4 & 60.1 & & 230 & & & & & & 9 & ie \\
\hline 42 & & & & & 42 & & & I4 & & & & & $22 \quad 58$ & & & & & & 8 & \\
\hline 44 & 40.5 & 40.8 & 26 & -12.9 & 44 & 32.2 & 39. & I9 & -II. & 4 & 68.6 & 67 & 51 & -0.0 & 4 & 37.2 & & & 27 & $-3 . I$ \\
\hline 46 & $42 . I$ & 42.5 & 29 & (2) & 46 & 27.3 & 33. & II & & & 73.0 & & & & & 40 & & & 22 & \\
\hline 48 & 44.0 & 45.0 & & 36 & 48 & & & I7 & 28 & & & & & & 48 & & 36.8 & & 22 & \\
\hline 50 & & 45.5 & 34 & $E$ & 50 & 26.0 & 29. & 06 & 7 & 50 & 6 I. 6 & & 3 & is & & 40.0 & 37.6 & & 22 & \\
\hline 52 & & 42.8 & & $2=$ & 52 & & & 23 & $=$ & 52 & 66.4 & & 2 & E & 52 & & & & 2 & \\
\hline 54 & 41.2 & 43.8 & 29 & $1+$ & 54 & 38.8 & 45.2 & 29 & & 54 & 73.0 & 68. & I & & 54 & 40.4 & 38.2 & & $2 \mathrm{I}$ & \\
\hline jo & 42.4 & $43 . I$ & 30 & & 56 & 27.2 & & 07 & & 5 & 72.5 & 69.2 & I & & 56 & $4 \mathrm{I} .2$ & 38.6 & & 20 & \\
\hline 58 & $4 \mathrm{I} \cdot 3$ & 44.0 & 30 & 8 & 58 & 23.9 & 28.8 & 04 & & 58 & 77.6 & 75.2 & 2206 & & & 42.3 & 38.7 & & I9 & -3.2 \\
\hline & 720. & 47.0 & 4 & 64 & 1200 & 27.3 & 31.8 & 09 & & & & & & $\omega^{2}$ & 1600 & 40.5 & 37.9 & & 21 & \\
\hline
\end{tabular}

Correction to local mean time is +55 s. $90^{\circ}$ torsion $=24^{\prime} 7$.

Torsion head at $8 \mathrm{~h} 02 \mathrm{~m}$ read $246^{\circ}$ and at I2ll oom read $249^{\circ}$.

Observers-W. J. P. and R. R. T., who alternated from $9 \mathrm{~h} 44 \mathrm{~m}$ to roh oom.
Correction to local mean time is +425 .

Torsion head at $12 \mathrm{~h}$ oom read $327^{\circ}$ and at the end read the same.

Observers-W. J. P. and R. R. T., change at $14 \mathrm{~h} 04 \mathrm{~m}$. 
Tabulation of magnetic declinations observed at Teplitz Bay-Continued

\begin{tabular}{|c|c|c|c|c|c|c|c|c|c|c|c|c|c|c|c|c|c|c|c|}
\hline \multicolumn{5}{|c|}{ Wednesday, October I4, 1903} & \multicolumn{5}{|c|}{ 4agnet scale erect } & \multicolumn{5}{|c|}{ Wednesday, October 14, 1903} & \multicolumn{5}{|c|}{ Magnet scale erect } \\
\hline \multirow{2}{*}{$\begin{array}{l}\text { Chr'r } \\
\text { time }\end{array}$} & \multicolumn{2}{|c|}{$\begin{array}{l}\text { Scale } \\
\text { readings }\end{array}$} & \multirow{2}{*}{$\begin{array}{c}\text { East } \\
\text { decli- } \\
\text { nation }\end{array}$} & Temp. & & $\begin{array}{r}\text { Sca } \\
\text { readi }\end{array}$ & $\begin{array}{l}\text { ale } \\
\text { ings }\end{array}$ & $\begin{array}{c}\text { East } \\
\text { decli- }\end{array}$ & Temp. & Chr'r & $\begin{array}{r}\text { Sc } \\
\text { read }\end{array}$ & gs & $\begin{array}{c}\text { East } \\
\text { decli- }\end{array}$ & & & $\begin{array}{r}\text { Se } \\
\text { read }\end{array}$ & igs & $\begin{array}{c}\text { East } \\
\text { decli- }\end{array}$ & \\
\hline & L,eft & Right & & & & Left & Right & nation & & & Left & Right & nation & & & Left & Right & nation & \\
\hline $\mathrm{h} \mathrm{m}$ & d & a & $\cdot$, & $\bullet$ & $\mathrm{h} \mathrm{m}$ & a & d & - , & 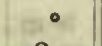 & $\mathrm{h} \mathbf{m}$ & d & d & & 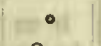 & $\mathrm{h} \mathrm{m}$ & & d & & $\circ$ \\
\hline $00^{*}$ & 53.9 & 57.8 & 2317 & -9.6 & 200 & 54.6 & 58.5 & 2317 & -8.3 & 400 & 47.1 & 54.9 & 2330 & -8.2 & 600 & 37.8 & $39 \cdot 4$ & 2332 & -8.0 \\
\hline 02 & 63.0 & 66.8 & 32 & & 02 & 55.8 & 61.7 & $2 I$ & & 02 & 39.0 & 46.7 & & 64 & 02 & 35.2 & 38.8 & 30 & \\
\hline $04^{*}$ & 22.6 & 36.1 & 2304 & tin & 04 & 61.0 & 66.0 & 28 & 13 & 04 & 37.1 & 45.6 & 15 & 10 & 04 & 33.1 & 37.0 & 26 & 80 \\
\hline 06 & I 4.8 & 29.6 & 2252 & \$9. & 06 & 72.2 & 77.8 & 46 & 10 & 06 & 42.3 & 50.4 & 23 & Q & 06 & 30.6 & 33.8 & 22 & W \\
\hline 08 & 15.4 & 17.8 & 2244 & bi: & 08* & 42.6 & 44.0 & 2347 & $a^{4}$ & 08 & 42.7 & 51.1 & 24 & No & 08 & 29.9 & 33.7 & $2 \mathrm{I}$ & 5 \\
\hline $10^{*}$ & 1.8 & 3.2 & 2158 & (13). & 10 & 50.5 & 54.2 & 24 OI & 6 & Io & 44.9 & 50.1 & 25 & wir & 10 & 29.8 & 33.7 & 21 & ns \\
\hline 12 & 56.0 & 63.2 & 2328 & & 12 & 57.7 & 58.8 & 10 & 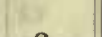 & 12 & 56.0 & 67.8 & 47 & 67 & 12 & 29.0 & & 20 & +7 \\
\hline 14 & 48.0 & 53.4 & 14 & -8.8 & I 4 & 56.2 & 56.9 & 2408 & -8.2 & 14 & 58.9 & 59.7 & 2343 & -8.3 & 14 & 29.0 & 33.1 & 20 & -7.8 \\
\hline 16́ & 40.8 & 45.8 & 2302 & & I6 & 37.6 & 40.1 & 2340 & & $16^{*}$ & 44.5 & 55.9 & $22 \mathrm{I4}$ & & I6 & 27.5 & 32.9 & 19 & \\
\hline 18 & $40 . I$ & 41.8 & $225^{8}$ & Er & 18 & 24.3 & 29.2 & $2 I$ & 4 & $18^{*}$ & 23.1 & 33.2 & 2442 & & 18 & 28.0 & 32.8 & 19 & 5t \\
\hline 20 & 37.4 & 38.8 & 22.54 & we & 20 & 15.2 & 21.9 & 08 & ex & 20 & 20.3 & 35.3 & 42 & 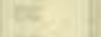 & 20 & 29.2 & 33. & 20 & 08 \\
\hline 22 & $44 . \mathrm{I}$ & 46.5 & 2305 & $=8$ & 22 & 25.2 & 31.4 & 23 & E & 22 & 10.4 & 19.3 & 21 & 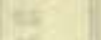 & 22 & 27.1 & 31. & 17 & we \\
\hline 24 & 55.4 & 62.1 & 26 & $t^{2}$ & 24 & 41.2 & 47.5 & 48 & 142 & 24 & 10.2 & 18.9 & 2421 & 16 & 24 & 24.2 & 29. & 13 & Fin \\
\hline 26 & 41.7 & 45.6 & 02 & $3 x$ & 26 & 36.9 & $4 I . I$ & 40 & $=$ & $26^{*}$ & 63.9 & 74.9 & 2508 & 64 & 26 & 22.8 & 26.0 & 10 & 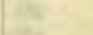 \\
\hline 28 & 44.8 & 49.2 & 2308 & ta & 28 & 48.2 & 51.8 & 57 & & $28^{*}$ & $47 \cdot 4$ & 56.9 & II & 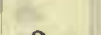 & 28 & 26.1 & & & 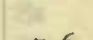 \\
\hline 30 & 37.2 & 41.8 & 2256 & -8.5 & 30 & 46.1 & 53.3 & 57 & -8.4 & 30 & 53.1 & 59.7 & 18 & -8.0 & 30 & 25.1 & 28 & 14 & -7.6 \\
\hline 32 & $3^{2} .5$ & 37.2 & 49 & 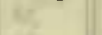 & 32 & 23.3 & 35.6 & 25 & & 32 & & 58. & I4 & 45 & 32 & 23.9 & & 12 & ( \\
\hline 34 & 29.2 & 33.5 & 43 & 14 & 34 & 23.6 & 30.6 & 21 & at & 34 & 63.3 & 64.8 & 30 & 45 & 34 & 25.2 & 28. & 14 & in \\
\hline 36 & 32.5 & 37.4 & 49 & 2 & 36 & 19.3 & 28.1 & 16 & & 36 & 44 & & 2508 & Q & 36 & 28.3 & 30 & 18 & 18 \\
\hline 38 & $36 . \overline{8}$ & 41.2 & 55 & 20 & 38 & 29.8 & $3^{8} .9$ & 33 & Ent & 38 & 13.9 & 19 & 2416 & 80 & 38 & 27.6 & 30. & 17 & ats. \\
\hline 40 & 36.1 & 44.4 & 57 & 48 & 40 & 40.5 & 48.6 & 2349 & (45) & 40 & & & 2408 & 6 & 40 & 26. & & 14 & 4. \\
\hline 42 & 27.2 & $3 \mathrm{I} .4$ & 40 & & 42 & 53.3 & 64.3 & 24 iI & 7 & $42^{*}$ & 38.8 & 48.2 & 2340 & 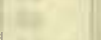 & 42 & 28.1 & 29.5 & 17 & Whit \\
\hline 44 & 16.6 & 22.3 & 24 & -8.6 & $44^{*}$ & 54.4 & 58.0 & 2508 & -8.4 & 44 & 35.8 & 43.6 & 2334 & -7.9 & 44 & 27.1 & 28.2 & 15 & -7.5 \\
\hline 46 & 15.8 & 21.9 & 24 & 4 & $46^{*}$ & 50.2 & 62.8 & 2545 & 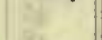 & 46 & 56. & 60.5 & 2403 & & 46 & 26.3 & 27.6 & 14 & \\
\hline 48 & 19.3 & 24.2 & 28 & 89 & 48 & 16.8 & 29.9 & 2453 & 84 & 48 & 60. & 65.8 & II & 80 & 48 & 29.2 & 29.9 & 18 & 8 \\
\hline 50 & 19.5 & 23.9 & 28 & 64 & $50^{*}$ & 52.9 & 60.1 & 27 & at & 50 & 62. & 73. & 18 & ter & 50 & 29.2 & & 18 & 48 \\
\hline 52 & 16.7 & 20.6 & 23 & int & 52 & 34.0 & 45.9 & or & ant & 52 & 55. & & 08 & * & 52 & 27.8 & & 16 & $\sqrt{2}$ \\
\hline 54 & 13.9 & 18.3 & 19 & $\theta$ & 54 & 62.1 & 66.1 & 2429 & 18 & 54 & 60.0 & 76.0 & 24 I8 & 45 & 54 & 27.0 & 28. & I5 & $y^{2}$ \\
\hline 56 & 14.2 & 17.8 & 19 & 18 & $56^{*}$ & 36.8 & 56.2 & 2533 & 92 & 56 & 54.5 & 55.8 & $235^{8}$ & & 5 & 26.1 & 27. & 13 & as \\
\hline 58 & 12.7 & 15.9 & 16 & & $5^{8}$ & 47.3 & 51.9 & 38 & & 58 & & 42.0 & 34 & 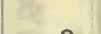 & 58 & 26.9 & 27. & 14 & $x^{2}$ \\
\hline 100 & 12.9 & 15.9 & I6 & -8.5 & 300 & 25.4 & 42.0 & 2513 & -8.4 & 500 & 36.7 & 39.1 & $3 \mathrm{I}$ & -7.8 & 700 & 24.3 & 25.0 & 10 & -7.3 \\
\hline 02 & I2. I & 15.0 & 15 & 201 & 02 & 21.2 & 26.5 & 2457 & 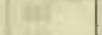 & 02 & 32.8 & 35. & 25 & 3 & 02 & 21.8 & 23. & 07 & \\
\hline $04^{*}$ & 47.1 & 50.3 & 19 & 10 & $04^{*}$ & 46.6 & 60.1 & 2349 & 10 & 04 & 26.8 & 30.9 & 17 & we & 04 & 23.1 & 23. & 08 & 40 \\
\hline 06 & 47.0 & 50.7 & 19 & (4) & 06 & 44.0 & 55.0 & 43 & 9 & 06 & 25.1 & & I4 & ger & & 25.3 & 26 & 12 & t \\
\hline 08 & 46.3 & 49.6 & 18 & 84 & 08 & 43.3 & 61.2 & 2347 & nes & 08 & 24.0 & 26.7 & II & 8 & 08 & 28.7 & 29.2 & 17 & 60 \\
\hline Io & 48.4 & $5 \mathrm{I} . \mathrm{I}$ & 20 & we & 10 & 68.8 & 71.8 & 2415 & 6 & 10 & 21.3 & 23. & 07 & wef & 10 & 31.3 & & 21 & $0 r$ \\
\hline I2 & 52.2 & 55.0 & 26 & & $12^{*}$ & 34.8 & 49.2 & 44 & & 12 & 19.0 & 21.1 & 03 & 24 & 12 & 29.9 & $3 \mathrm{I}$ & I9 & 27 \\
\hline 14 & 55.0 & 58.0 & $3 I$ & -8.4 & 14 & 32.7 & 40.8 & 36 & -8.4 & I4 & 19. & 21 & 04 & -8.0 & I4 & 30.4 & 31.9 & 20 & -7.3 \\
\hline 16 & 58.8 & 61.5 & 37 & & 16 & 28.6 & 30.4 & 25 & & 16 & 20.8 & 22. & 05 & & IC & 30.3 & & 20 & \\
\hline 18 & 62.9 & 66.2 & 44 & 14 & I8* & 55.7 & 57. & 2419 & Et & 18 & & & 06 & we & If & 31.7 & 32.3 & 22 & 6 \\
\hline 20 & 67.1 & 70.0 & 50 & $\infty$ & $20^{*}$ & 30.6 & $4 I .5$ & 2516 & $e$ & 20 & 20.6 & 22.7 & 05 & on & 20 & 32.4 & 34. & 24 & $\omega$ \\
\hline 22 & 66.8 & 69.2 & 49 & 6 & 22 & 36.5 & 40.6 & 2519 & ex & 22 & 20.2 & 22.1 & 05 & 14: & 22 & 33.0 & 34. & 25 & 5 \\
\hline 24 & 70.1 & 74.0 & 55 & 18 & 24 & 23.6 & 24.2 & 2456 & K & 24 & 20.8 & 23.0 & 06 & Mis & & 34.2 & & 26 & 14 \\
\hline $26^{*}$ & 38.9 & 45.2 & 55 & 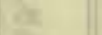 & $26^{*}$ & 22.2 & 34.9 & 2357 & Br & 26 & 21.6 & $24 \cdot 3$ & 07 & as & 26 & 32.0 & 33.8 & 23 & \\
\hline 28 & $36 . \mathrm{I}$ & 42.0 & 50 & & 28 & 56.5 & 66.1 & 2448 & & 28 & 22.7 & & 09 & ( & 28 & 33.5 & & 25 & 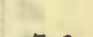 \\
\hline 30 & 38.6 & 43.8 & 53 & -8.4 & $30^{*}$ & 36.6 & 48. I & 2520 & -8.2 & 30 & 23.8 & 25.8 & Io & -8.0 & 30 & 32.8 & & 24 & -7.3 \\
\hline 32 & 40.5 & 46.0 & 57 & 148 & 32 & 46.2 & 55.8 & 2534 & 122 & 32 & 28.0 & & I6 & tar & 32 & 32.8 & & 24 & 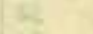 \\
\hline 34 & 40.1 & 44.2 & 2255 & is & 34 & 69.2 & 69.9 & 2603 & 12 & 34 & 27.0 & 28. & 15 & 18 & 34 & 33.6 & 34.8 & 25 & \\
\hline 36 & 42.4 & 47.9 & 2300 & & $36^{*}$ & 27.8 & 38.0 & 2600 & & 36 & 29.0 & 31. & 19 & 06 & 3 & 33.4 & & 25 & (45. \\
\hline 38 & 44.8 & 49.2 & 02 & 35 & 38* & Over & $r l^{\prime} k k^{\prime} d$ & 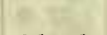 & 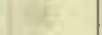 & 38 & 33.2 & 35.2 & 25 & 38 & 38 & 33.7 & & 26 & 103 \\
\hline 40 & 48.2 & 54.1 & 09 & 4 & 40 & 28.4 & 45.7 & $25 \mathrm{I} 5$ & 117 & 40 & & & $2 \overline{8}$ & 04 & 40 & 33.0 & & 25 & 97 \\
\hline 42 & 51.7 & 57.0 & 14 & & 42 & 20.0 & 27.1 & 2454 & & 42 & 39.3 & 41.1 & 35 & 4 & 42 & 32.1 & 34 & 23 & 6 \\
\hline 44 & 54.3 & 59.2 & 18 & -8.3 & $44^{*}$ & 38. I & 45.2 & 18 & -8.1 & 44 & 38.7 & $41 \cdot 3$ & & -8.0 & 44 & 34.0 & & 26 & -7.2 \\
\hline 46 & 48.6 & 50.6 & 07 & & 46 & 31.3 & 44.2 & 24 I2 & $x_{1}$ & 46 & 40.0 & 42.4 & 36 & 40 & 46 & 34.0 & & 26 & \\
\hline 48 & 42.8 & 50.1 & 02 & 24 & 48 & 25.2 & 32.6 & 2358 & 5 & 48 & 44.0 & 45 & 42 & 64 & 48 & 33.9 & & 26 & 18 \\
\hline 50 & 57.8 & 60.2 & 22 & at & 50 & 9.2 & 15.9 & 32 & 08 & 50 & 44.7 & 46.6 & 43 & 68 & 50 & $33 . \bar{I}$ & & 24 & 128 \\
\hline 52 & 60.0 & 64.2 & 26 & R & $52^{*}$ & 43.0 & 52.4 & 25 & at & 52 & 43.0 & 44.9 & 40 & 28 & 52 & 34 . I & & 26 & 42 \\
\hline 54 & 62.2 & 65.8 & 29 & $P 2$ & 54 & 43.8 & 52.0 & .25 & 12 & 54 & 42.0 & 44. & 39 & in? & 54 & 33.8 & 36.1 & 26 & R \\
\hline 56 & 59.5 & 61.7 & 24 & 52 & 56 & 43.3 & 50.9 & 24 & & 56 & 41.2 & 44.0 & 38 & 02 & 56 & 33.0 & 34.9 & 25 & 182 \\
\hline 58 & 59.7 & 62.3 & 24 & $\sqrt{3}$ & 58 & 45.8 & 53.0 & 28 & 25 & 58 & 39.2 & 43.0 & 36 & $\sqrt{2}$ & 58 & 33.3 & 35.2 & 25 & Ee \\
\hline
\end{tabular}


Tabulation of magnetic declinations observed at Teplitz Bay-Continued

\begin{tabular}{|c|c|c|c|c|c|c|c|c|c|c|c|c|c|c|c|c|c|c|c|}
\hline \multicolumn{5}{|c|}{ Wednesday, October I4, 1903} & \multicolumn{5}{|c|}{ Magnet scale erect } & \multicolumn{5}{|c|}{ Wednesday, October I4, 1903} & \multicolumn{5}{|c|}{ Maguxet scale erect } \\
\hline \multirow{2}{*}{$\begin{array}{l}\text { Chr'r } \\
\text { time }\end{array}$} & \multicolumn{2}{|c|}{$\begin{array}{l}\text { Scale } \\
\text { readings }\end{array}$} & \multirow{2}{*}{$\begin{array}{c}\text { East } \\
\text { decli- } \\
\text { nation }\end{array}$} & Temp. & Chr'r & & $\begin{array}{l}\text { ale } \\
\text { lings }\end{array}$ & $\begin{array}{c}\text { East } \\
\text { decli- }\end{array}$ & Temp. & Chr'r & $\begin{array}{r}\text { Sca } \\
\text { readi }\end{array}$ & $\begin{array}{l}\text { ale } \\
\text { lings }\end{array}$ & $\begin{array}{c}\text { East } \\
\text { decli- }\end{array}$ & Temp. & Chr'r & $\begin{array}{l}\text { Sce } \\
\text { read }\end{array}$ & $\begin{array}{l}\text { ale } \\
\text { lings }\end{array}$ & $\begin{array}{c}\text { East } \\
\text { decli- }\end{array}$ & Temp. \\
\hline & Left & Right & & & & I,eft & Right & nation & & & Left & Right & nation & & & Left & Right & natiou & \\
\hline $\mathrm{h} \mathrm{m}$ & d & d & $\circ$, & $\circ$ & $\mathrm{h} \mathrm{m}$ & d & d & $\circ \cdot$ & $11^{\circ}$ & $h \mathrm{~m}$ & d & d & & & $\mathrm{h} \mathrm{m}$ & & a & $\circ$, & • \\
\hline 800 & 33.5 & 35.2 & 2325 & -7.0 & 1000 & 29.8 & 38.4 & 2239 & -6.5 & 1200 & 29.0 & 30.0 & 2232 & -5.6 & I4 00 & 32.8 & 33.8 & 2238 & -5.1 \\
\hline 02 & 35.1 & 36.0 & 27 & . & 02 & 42.7 & 46.1 & 2256 & & 02 & 31.6 & 31.8 & 36 & & 02 & $31 \cdot 3$ & 32.9 & 36 & \\
\hline 04 & 34.9 & 36.3 & 27 & wat & 04 & $47 \cdot 3$ & 49.3 & 2302 & wi & 04 & 35.2 & 36.2 & 42 & we & 04 & 29.0 & 30.5 & 32 & 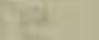 \\
\hline 06 & 34.1 & $36 . I$ & 27 & wit & 06 & 49.2 & 51.4 & 05 & 35 & 06 & 45.0 & $4^{6} .0$ & 57 & tक & 06 & 27.9 & 29.1 & $3 \mathrm{I}$ & \\
\hline 08 & 33.7 & 35.4 & 26 & bet & 08 & 50.6 & 51.6 & 2306 & $\Delta x$ & 08 & 35.4 & 38.2 & 44 & $8=$ & 08 & 26.7 & 27.6 & 28 & \\
\hline Io & 32.0 & 34.2 & 24 & ot & Io & 41.0 & 42.8 & 2252 & int & IO & 27.5 & 30.0 & $3 \mathrm{I}$ & $\ln t$ & IO & 23.3 & 24.7 & 24 & \\
\hline 12 & 30.7 & 33.0 & 21 & Eis & I2 & 46.8 & 48.0 & 2300 & & 12 & 39.4 & 40.9 & 49 & 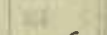 & I2 & 22.8 & 24.0 & 23 & 55 \\
\hline 14 & 27.0 & 29.9 & 16 & -7.0 & I4 & 54.4 & 56.3 & I3 & -6.5 & I4 & 35.6 & 37.6 & 2243 & -5.6 & I4 & 21.6 & 22.8 & 21 & -5.0 \\
\hline 16 & 24.0 & 26.9 & II & & x6 & 51.5 & 53.0 & 2308 & & 16 & 48.1 & $5 \mathrm{I} .2$ & 2304 & & 16 & 21.6 & 22.7 & $2 \mathrm{I}$ & \\
\hline 18 & 24.0 & 26.7 & II & 30 & 18 & 44.5 & 45.0 & 2256 & atit & 18 & 56.5 & 58.7 & 16 & (1) & I8 & 24.1 & $24 \cdot 3$ & 24 & Ais \\
\hline 20 & 22.3 & 25.4 & 09 & $x$ & 20 & 37.2 & 37.7 & 44 & fint & 20 & 56.9 & $57 \cdot 4$ & 15 & net & 20 & 25.8 & 26.9 & 27 & $\cos$ \\
\hline 22 & 20.0 & 23.0 & 05 & we & 22 & 36.8 & 38.2 & & et: & 22 & 49.4 & 50.2 & 04 & 2 & 22 & 25.6 & 26.5 & 27 & $\approx$ \\
\hline 24 & 19.6 & 23.3 & 05 & $3=$ & 24 & 36.8 & 37.8 & 44 & F & 24 & 50.1 & 50.9 & 05 & 15 & 24 & 23.0 & 23.6 & 22 & is \\
\hline 26 & 18.5 & 23.3 & 04 & $x$ & 26 & 37.3 & 38.3 & & lose & 26 & Šlow1 & ly in- & 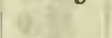 & 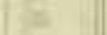 & 26 & 17.2 & 18.2 & 14 & \\
\hline 28 & 19.9 & $24 \cdot 3$ & 06 & 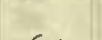 & 28 & 32.2 & 32.3 & 36 & & 28 & creas & sing & & $x=2$ & 28 & I5. 8 & 17.4 & I2 & \\
\hline 30 & 21.5 & 24.5 & 08 & -6.7 & 30 & 35.5 & 37.0 & 43 & -6.5 & 30 & 51.1 & 51.8 & 07 & -5.4 & 30 & 17.8 & 19.3 & 15 & -5.0 \\
\hline 32 & 22.0 & 25.3 & 09 & $x^{\circ}$ & 32 & 34.4 & 36.4 & $4 I$ & 2 & 32 & 49.8 & 50.4 & 05 & 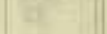 & 32 & 23.0 & $24 \cdot 4$ & 23 & \\
\hline 34 & 22.0 & 24.0 & 08 & 37 & 34 & 30.2 & 30.9 & 34 & is. & 34 & 5 I. 2 & 52.8 & 07 & (20) & 34 & $2 \mathrm{I} .4$ & 22.9 & $2 \mathrm{I}$ & is \\
\hline 36 & 19.7 & 22.0 & 2304 & $x=$ & 36 & 25.5 & 26.8 & 27 & 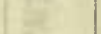 & 36 & 52.4 & 53.9 & 09 & az & 36 & 25.0 & 26.4 & 26 & \\
\hline 38 & І6́.0 & 17.4 & $225^{8}$ & $\pi$ & 38 & 27.1 & 28.9 & 30 & $F^{2}$ & 38 & 57.8 & 59.4 & 18 & 20 & 38 & 25.9 & 27.0 & 27 & a \\
\hline 40 & 16.0 & 17.6 & 58 & 6 & 40 & 32.5 & 32.8 & 37 & a & 40 & 58.3 & 59.7 & 18 & 68 & 40 & 25.2 & 26.4 & 26 & I \\
\hline 42 & 15.6 & 16.2 & 57 & & 42 & 32.5 & 33.8 & 38 & $6 y$ & 42 & 57.2 & 59.2 & 17 & 4 & 42 & 25.3 & 25.6 & 26 & 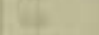 \\
\hline 44 & 15.0 & 16.2 & 56 & -6.7 & 44 & 35.4 & 37.0 & 43 & & 44 & 55.7 & 58.0 & 15 & -5.4 & 44 & 23.9 & 24.4 & 24 & -5.0 \\
\hline 46 & 13.6 & 14.7 & 2254 & & 46 & 29.8 & 31.6 & 34 & -6.6 & 46 & 55.7 & 58.4 & 15 & & 46 & 19.5 & 20.9 & 17 & \\
\hline 48 & 17.8 & 18.6 & 2300 & $n^{2}$ & 48 & 28.7 & 29.7 & 32 & the & 48 & 55.8 & 58.4 & 15 & Xy & 48 & I9.0 & 20.1 & I7 & 4 \\
\hline 50 & 15.0 & 18.4 & 2258 & 5 & 50 & 28.7 & $3 I .5$ & 33 & at & 50 & 55.9 & 58.4 & 15 & tas & 50 & 21.4 & 23.3 & 2I & 68 \\
\hline $5^{2}$ & 23.6 & 25.4 & 23 IO & 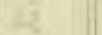 & $5^{2}$ & 3I.I & 32.5 & 36 & $n^{2}$ & 52 & 50.2 & 52.6 & 07 & 12 & 52 & $24 . i$ & 25.0 & 24 & \\
\hline 54 & $23 . I$ & $25 . \dot{I}$ & 2309 & til & 54 & 33.5 & $35 . I$ & 40 & $y i$ & 54 & 52.4 & 54.9 & IO & 12 & 54 & 25.3 & 26.9 & 27 & N2 \\
\hline 56 & 14.7 & 17.6 & 2257 & (1) & 56 & 27.8 & 30.6 & 32 & 3. & 56 & 50.2 & 52.9 & 07 & & 56 & 27.2 & 28.0 & 29 & \\
\hline $58^{*}$ & 17.5 & 36.0 & 28 & & 58 & 23.0 & 25.5 & 24 & & 58 & 50.2 & 53.3 & 07 & $a^{2}$ & 58 & 29.0 & 30.0 & 32 & \\
\hline 900 & 9.1 & 14.5 & 04 & -6.5 & II 00 & 27.4 & 30.7 & 31 & -6.4 & I3 00 & 47.4 & 50.0 & 2302 & -5.4 & I5 00 & 28.0 & 29.2 & 31 & -4.8 \\
\hline 02 & 14.0 & 38.0 & 27 & 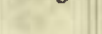 & 02 & 34.8 & 36.2 & 42 & (2) & 02 & $43 . i$ & 45.7 & 2256 & & 02 & $27 \cdot 3$ & 29.0 & 30 & \\
\hline 04 & 29.6 & 34.6 & 36 & firt & 04 & 35.2 & 37.2 & 43 & ait & 04 & 39.0 & 41.9 & 49 & 14 & 04 & 27.1 & 28.9 & 30 & 16 \\
\hline$\infty 6$ & 28.8 & 36.8 & 37 & $x^{2}$ & 06 & 35.0 & 39. & 44 & 18 & 06 & 37.9 & 40.2 & 47 & 30 & 06 & 20.5 & 27.5 & 24 & 16 \\
\hline 08 & 32.6 & 37.2 & 40 & (67) & 08 & 38.3 & $4 I .9$ & 49 & $8 \mathrm{E}$ & 08 & 38.5 & 41.3 & 48 & 29 & 08 & 25.3 & 28.0 & 28 & (6) \\
\hline Io & 33.8 & 37.8 & 42 & (2) & Io & 38.9 & 42.7 & 50 & 01 & 10 & 38.5 & 40.4 & 47 & wir & Io & 27.2 & 30.9 & 31 & tet \\
\hline 12 & 35.0 & 38.3 & 43 & & 12 & 39.6 & 41.6 & 50 & & 12 & 39.5 & 40.9 & 49 & t & 12 & 28.6 & 30.7 & 32 & \\
\hline I 4 & 29.3 & 33.8 & 35 & -6.5 & I4 & 38.7 & 40.8 & 48 & -6.0 & I 4 & 39.7 & 40.5 & 49 & -5.4 & 14 & 27.7 & 30.7 & 32 & -4.8 \\
\hline I6́ & 28.6 & 32.0 & 33 & (5) & I6́ & 43.6 & $46 . c$ & 56 & min & 16 & 37.5 & 39.2 & 46 & & I6́ & 27.6 & 31.2 & 32 & \\
\hline I8 & 33.0 & 35.6 & 40 & $8=$ & 18 & $45: 4$ & 46.0 & 58 & Fit & I8 & 37.3 & 38.8 & 45 & tits & 18 & 28.6 & 31.4 & 33 & iff \\
\hline 20 & 33.5 & 37.0 & $4 \mathrm{I}$ & $=$ & 20 & 43.2 & 44.3 & 54 & (18. & 20 & 38.3 & 38.8 & 46 & 68 & 20 & 28.6 & 30.8 & 32 & 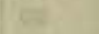 \\
\hline 22 & 32.0 & 35.6 & 39 & 13 & 22 & 33.0 & 36. & 40 & ter & 22 & 35.3 & 35.7 & 42 & $\mathrm{~s}=$ & 22 & 29.0 & 31.6 & 33 & 35 \\
\hline 24 & 41.0 & 44.0 & 52 & 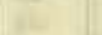 & 24 & 33.2 & 33.9 & 38 & 10 & 24 & 33.6 & $34 \cdot 3$ & 39 & in & 24 & 28.0 & 30.6 & 32 & in \\
\hline 26 & 42.2 & 45.2 & 54 & $=$ & 26 & 28. I & $3 \mathrm{I}$. & 32 & & 26 & 32.0 & 32.0 & 36 & & 26 & 28.0 & 30.6 & 32 & \\
\hline 28 & 41.8 & 44.0 & 53 & & 28 & 23.0 & 25.0 & 24 & & 28 & 36.7 & $37 \cdot 3$ & 44 & & 28 & 28.6 & 3I.I & 33 & \\
\hline 30 & 35.3 & 37.7 & 43 & -6.5 & 30 & 22.5 & 23.0 & 21 & -6.0 & 30 & 37.9 & 38.4 & 46 & $-5 \cdot 4$ & 30 & 30.7 & 33.7 & 36 & -3.9 \\
\hline 32 & 36.3 & 38.8 & 45 & 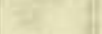 & 32 & 23.5 & 24.5 & 23 & 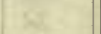 & 32 & 37.9 & 38.9 & 46 & $\theta^{2}$ & 32 & 34.0 & 34.8 & 40 & \\
\hline 34 & 37.2 & 39.2 & 46 & 15 & 34 & 21.8 & 23.5 & $2 I$ & & 34 & 35.9 & 36.6 & 43 & 31 & 34 & 35.0 & 35.9 & $4 \mathrm{I}$ & \\
\hline 36 & 37.1 & 39.3 & 46 & & 36 & 19.6 & 20.8 & I8 & 26 & 36 & 36.4 & 37.4 & & & 36 & 34.9 & 36.5 & 42 & \\
\hline 38 & $4 \mathrm{I} \cdot 3$ & 44.0 & 53 & $w$ & 38 & 20.0 & 20.3 & 17 & & 38 & 27.0 & 28.0 & 29 & We & 38 & $33 . \overline{8}$ & 36.7 & $4 \mathrm{I}$ & 12 \\
\hline 40 & 46.3 & $47 \cdot 3$ & 59 & 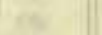 & 40 & 22.0 & 22.4 & 20 & 6 & 40 & 37.2 & 38.2 & 45 & $m$ & 40 & $34 \cdot 3$ & 36.8 & 42 & a \\
\hline 42 & $45 \cdot 3$ & 47.3 & 2258 & 18 & 42 & 21.6 & 22.6 & 20 & & 42 & 38.1 & 38.8 & 46 & & 42 & 36.8 & 39.9 & 46 & \\
\hline 44 & 45.8 & 48.6 & 2300 & & 44 & 19.9 & 20.2 & 17 & -5.5 & 44 & 37.9 & 38.4 & 46 & -5.3 & 44 & 35.9 & 38.4 & 44 & -3.8 \\
\hline 46 & $44 \cdot 5$ & 46.6 & 2257 & -6.5 & 46 & 20.0 & 21.0 & I 8 & & 46 & 37.8 & 38.9 & 46 & & 46 & 35.8 & 37.3 & 43 & \\
\hline 48 & 44.0 & 46.3 & 57 & 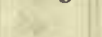 & 48 & 24.0 & 24.1 & 24 & it. & 48 & 37.9 & 38.8 & 46 & 65 & 48 & 37.5 & 41.0 & 47 & \\
\hline 50 & 45.8 & 47.3 & 2259 & $y=$ & 50 & 23.5 & 23.5 & 23 & 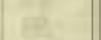 & 50 & 37.9 & 39.3 & 46 & eg & 50 & 38.2 & 42.8 & 49 & $\alpha$ \\
\hline 52 & 47.4 & 49.4 & 2302 & Ext & $5^{2}$ & 24.0 & 25.0 & 24 & 2 & 52 & 37.2 & 38.0 & 45 & 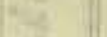 & 52 & 37.3 & $4 \mathrm{I} .7$ & 48 & Q \\
\hline 54 & $47 \cdot 3$ & 48.7 & 23 oI & 18 & 54 & 18.7 & 20.8 & 17 & 182 & 54 & 36.2 & 37.8 & 44 & at & 54 & 38.1 & 41.3 & 48 & 23 \\
\hline 56 & 40.3 & 42.0 & 2250 & 8 & 56 & 12.6 & 13.2 & 06 & Du' & 56 & 36.7 & 37.7 & 44 & $x^{2}$ & 56 & 37.3 & 39.6 & 46 & \\
\hline 58 & 31.0 & 32.3 & 36 & $x=$ & $5^{8}$ & 23.2 & 25.8 & 24 & 3 & 58 & 34.2 & 35.9 & $4^{I}$ & 10 & 58 & 38.6 & 39.8 & 47 & 921 \\
\hline
\end{tabular}

Observers-R. R. T. and W. J. P. alternated from $8 \mathrm{~h} 26 \mathrm{~m}$ to $8 \mathrm{~h} 38 \mathrm{~m}$.

Observers-W. J. P. and R. W. P. altcrnated $12 \mathrm{~h} 04 \mathrm{~m}$ to $12 \mathrm{~h} 18 \mathrm{~m}$. (F. L. made a few alternations with W. J. P. IIh $04 \mathrm{~m}$ to I Ih $\mathrm{I} 4 \mathrm{~m}$.) R. W. P., F. I., and R. R. 'T. alternated I4h $58 \mathrm{in}$ to 16 h o2m. (R. R. T. I3h $20 \mathrm{~m}$ to $\mathrm{r} 3 \mathrm{~h} 56 \mathrm{~m}$.) 
Tabulation of magnetic declinations observed at Teplitz Bay-Continued

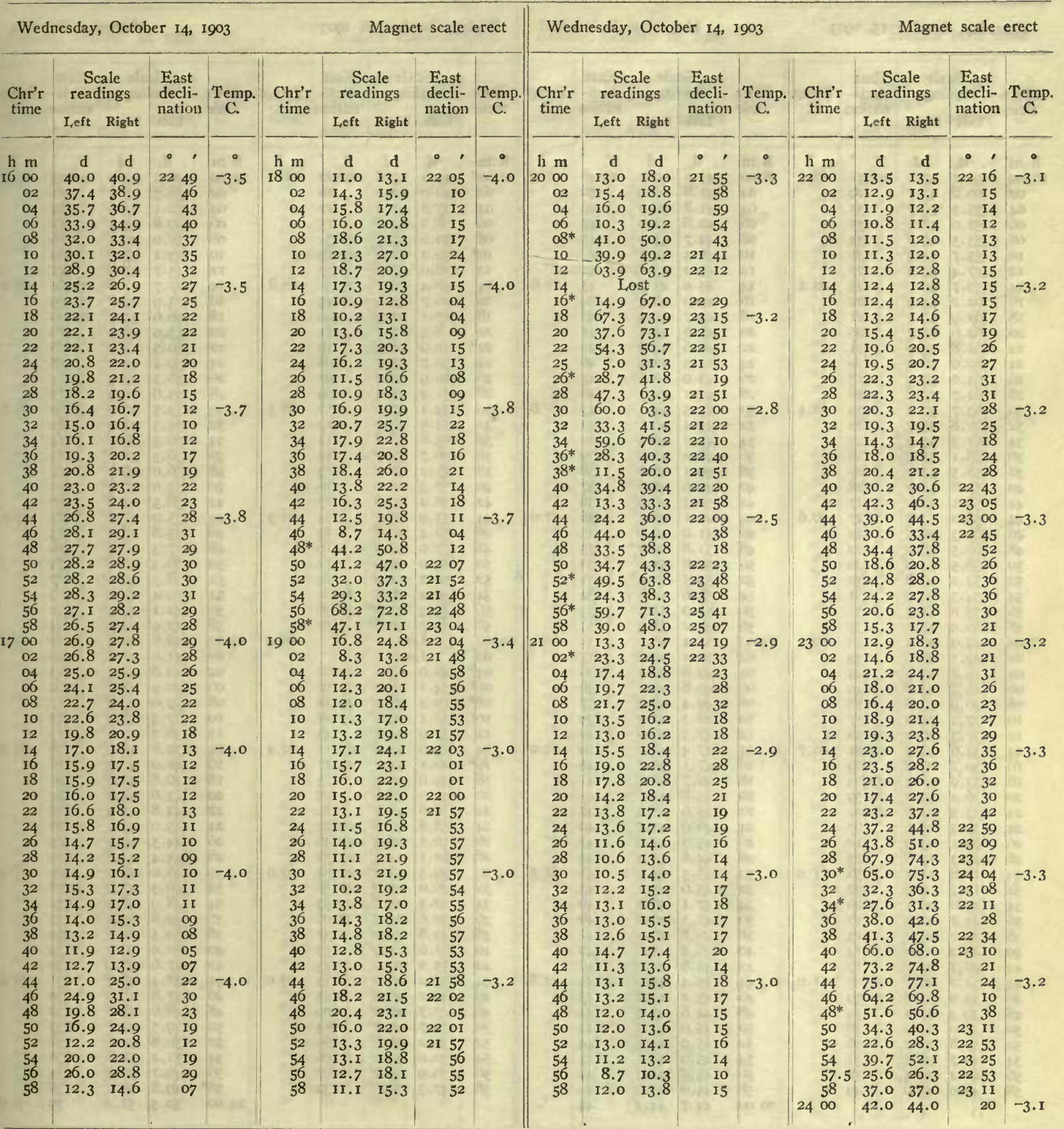

Observers-R. R. T. and W. J. P. alternated from $20 \mathrm{~h} 20 \mathrm{~m}$ to $20 \mathrm{~h}$ 32m. (IV. J. P. I8h $20 \mathrm{~m}$ to I $8 \mathrm{~h} 26 \mathrm{~m}$.)
Correction to local mean time is +30 s. $90^{\circ}$ torsion $=26 . .^{\prime} \mathrm{I}$.

Torsion head at oh oom read $327^{\circ}$ and at $24^{\mathrm{hl}}$ rom read $324^{\circ}$.

Observers-R. R. T. and W. J. P., who alternated from 20 h $20 \mathrm{~m}$ to 2oll $32 \mathrm{~m}$. 
Tabulation of magnetic declinations observed at Teplitz Bay-Continued

\begin{tabular}{|c|c|c|c|c|c|c|c|c|c|c|c|c|c|c|c|c|c|c|c|c|c|c|}
\hline \multicolumn{4}{|c|}{ Thursday, October I 5, } & \multicolumn{2}{|c|}{ I903 } & \multicolumn{5}{|c|}{ Magnet scale inverted } & \multicolumn{6}{|c|}{ Firiday, October I6, I903 } & \multicolumn{6}{|c|}{ Magnet scale erect } \\
\hline Chr's & $\begin{array}{l}\text { Sc } \\
\text { read }\end{array}$ & $\begin{array}{l}\text { ale } \\
\text { ings }\end{array}$ & $\begin{array}{l}\mathrm{Ea} \\
\text { dec }\end{array}$ & $\begin{array}{l}\text { st } \\
\text { eli- } \\
\text { ion }\end{array}$ & Temp. & Chr'r & $\begin{array}{r}\mathrm{Sc} \\
\text { read }\end{array}$ & $\begin{array}{l}\text { ale } \\
\text { ings }\end{array}$ & $\begin{array}{c}\text { East } \\
\text { decli- } \\
\text { nation }\end{array}$ & Temp. & $\begin{array}{l}\text { Chr'r } \\
\text { time }\end{array}$ & $\begin{array}{r}\mathrm{Sc} \\
\text { read }\end{array}$ & $\begin{array}{l}\text { le } \\
\text { ngs }\end{array}$ & $\begin{array}{l}\text { Ea } \\
\text { dec } \\
\text { nat }\end{array}$ & $\begin{array}{l}\text { ast } \\
\text { cli- } \\
\text { tion }\end{array}$ & Temp. & Chr'r & $\begin{array}{r}\mathrm{Sc} \\
\text { reac }\end{array}$ & e & Ea & $\begin{array}{l}\text { ast } \\
\text { cli- } \\
\text { ion }\end{array}$ & Temp. \\
\hline & Left & Right & & & & & Left & Right & & & & Lueft & Right & & & & & $\mathrm{L}_{\mathrm{r}}$ eft & Right & & & \\
\hline I6 00 & 59.1 & 47.0 & 22 & $3 I$ & -0.0 & I8 00 & 43.1 & 40.7 & 2248 & -1.3 & $2000^{*}$ & 45.2 & 45.8 & 22 & 22 & $-I I .2$ & 2200 & 26.2 & 35.0 & 22 & 03 & -9.0 \\
\hline 02 & 60.6 & 47.7 & & 29 & $\sqrt{45}+2$ & 02 & 41.8 & 40.0 & 50 & win & 02 & 40.0 & 42,2 & & 16 & t n & 02 & 38.3 & 47.2 & & 22 & \\
\hline 04 & 55.3 & 50.7 & & $3 I$ & $\theta$ & 04 & 41.8 & 40.2 & 49 & $t^{4}$ & 04 & 37.7 & 40.2 & & 12 & & 04 & 32.1 & 43.6 & & I4 & 1 \\
\hline 06 & 52.0 & 48.6 & & 35 & 19 & 06 & 42.8 & 42.0 & 47 & nit & 06 & $3^{8.2}$ & 42.8 & & I5 & & 06 & 29.8 & $43 . I$ & & 12 & \\
\hline a8 & 53.3 & 49.8 & & 33 & he & 08 & 44.7 & 43.0 & 45 & 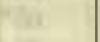 & 08 & 50.6 & 55.5 & 22 & 34 & bis & 08 & 38.0 & 39.3 & & 16 & 5 \\
\hline 10 & 55.7 & 51.5 & & 30 & sis & IO & 46.3 & 44.7 & 42 & tat & Io* & 50.2 & 52.0 & 23 & I9 & 80 & 10 & 33.1 & 35.9 & & 09 & 7 \\
\hline 12 & 57.4 & 53.4 & & 27 & 21 & 12 & 45.2 & 43.8 & 44 & $z^{2}+1$ & 12 & 58.0 & 67.2 & & 37 & 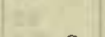 & 12 & 33.7 & 37.0 & & I I & it: \\
\hline 14 & 59.7 & 56.0 & & 23 & -0.2 & I4 & 45.0 & $43 \cdot 3$ & 45 & -1.6 & I4 & 58.4 & 60.1 & 23 & 31 & $-I 0.8$ & 14 & 27.7 & 31.8 & 22 & $\mathrm{O} 2$ & -9.0 \\
\hline 20 & 62.6 & 57.7 & th & 20 & 10 & 20 & 47.0 & 45.2 & $4 I$ & 65 & 20 & 8.5 & II. 7 & G. & I4 & 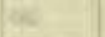 & 20 & 33.9 & 36.0 & & IO & $\alpha$ \\
\hline 22 & 62.8 & 55.6 & & $2 \mathrm{I}$ & na & 22 & 46.6 & 44.9 & 42 & $x^{2}$ & $22^{*}$ & 40.9 & 68.3 & & 10 & 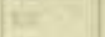 & 22 & 40.3 & 42.2 & & 20 & ac \\
\hline 24 & 62.9 & 56.2 & 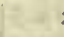 & 20 & $1=$ & 24 & $44 \cdot 3$ & 42.4 & 46 & $2 x$ & 24 & 50.7 & 55.7 & 8 in & 08 & is & 24 & 39.1 & 41.2 & & I 8 & $2+2$ \\
\hline 26 & 64.0 & 57.9 & & I8 & $y=$ & 26 & 43.0 & 41.6 & 47 & his & 26 & 50.9 & 54.7 & $A-1$ & 07 & 14 & 26 & 36.3 & 39.2 & & I4 & \\
\hline 28 & 64.7 & 57.8 & & I 8 & $x^{2}=$ & 28 & $44 . I$ & 42.7 & 46 & 50 & 28 & 51.9 & 55.4 & & 09 & $x_{2}=$ & 28 & 35.6 & 38.3 & & I3 & \\
\hline 30 & 63.7 & 57.2 & & 19 & -0.4 & 30 & 45.5 & $44 \cdot 3$ & 43 & $-\mathrm{I} .8$ & 30 & 54.1 & 57.0 & & 12 & -10.5 & 30 & 33.5 & 36.0 & & 10 & -8.7 \\
\hline 32 & 61.8 & 55.7 & L & 22 & 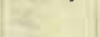 & 32 & 45.4 & 46.6 & 42 & $-2=$ & 32 & 54.9 & 57.0 & & 12 & 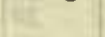 & 32 & 35.3 & 37.4 & & 12 & \\
\hline 34 & $6 \mathrm{I} .3$ & 55.3 & F. & 22 & $1=$ & 34 & 47.7 & 46.6 & 40 & 16 & 34 & 55.8 & 57.8 & & 14 & ar & 34 & 36.5 & 39.2 & & 14 & 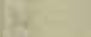 \\
\hline 36 & 58.1 & 51.5 & wir & 28 & cos & 36 & 48.2 & 47.0 & 39 & $\sqrt{6}$ & 36 & 56.7 & 57.7 & & 14 & 4 & 36 & 36.5 & 38.8 & & I4 & 18 \\
\hline $3^{8}$ & 58.5 & 52.0 & & 27 & Eit & 38 & $49 . I$ & 47.9 & 38 & $t^{4}$ & 38 & 57.2 & 57.8 & 4. & I5 & 2 & 38 & 36.0 & 39.1 & & I4 & \\
\hline 40 & 57.8 & 51.2 & 2 & 28 & $4+2$ & 40 & 49.6 & 48.6 & 37 & 14 & 40 & $57 \cdot 3$ & 60.0 & & 16 & 014 & 40 & 33.0 & 36.9 & & IO & 4 \\
\hline 42 & 58.1 & 52.2 & 6 & 27 & (2) & 42 & 50.3 & $49 \cdot 3$ & 36 & 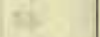 & 42 & 58.2 & 59.8 & & 17 & 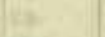 & 42 & $3^{8} .5$ & 41.8 & & I 8 & we \\
\hline 44 & 58.0 & 53.3 & 61 & 27 & $\multimap 0.6$ & 44 & 49.1 & 48.1 & $3^{8}$ & -2.0 & 44 & 53.9 & 59.7 & & 14 & -10.3 & 44 & 35.9 & 37.8 & & I 3 & -9.0 \\
\hline 46 & 60.3 & 56.5 & it) & 22 & ( & 46 & 51.0 & 50.0 & 35 & $84=$ & 46 & 52.4 & 59.8 & & 13 & $(x-2)$ & 46 & 35.8 & 38.4 & & I 3 & \\
\hline 48 & 60.5 & 56.2 & a & 22 & Fin & 48 & 51.8 & 50.7 & 33 & 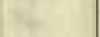 & 48 & 57.0 & $59 \cdot 3$ & $=$ & 16 & 6 & 48 & 37.3 & 40.3 & & 16 & 4 \\
\hline 50 & 58.5 & 54.0 & (10.1) & 26 & ens & 50 & 52.8 & 51.6 & 32 & $w$ & 50 & 55.0 & 59.9 & & 15 & 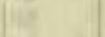 & 50 & 36.3 & 38.8 & & I4 & \\
\hline 52 & 54.2 & 49.8 & ty & 32 & t & 52 & 53.5 & 52.4 & 31 & 2 & 52 & 50.1 & $5 I .2$ & & 04 & $t^{2}$ & 52 & 38.3 & 40.6 & & 17 & \\
\hline 54 & $54 \cdot 3$ & 50.9 & 4 & $3 I$ & (6) & 54 & 52.8 & 52.0 & 32 & 12 & 54 & 49.2 & $49 \cdot 3$ & 22 & 02 & wat & 54 & 39.4 & 4 I. 4 & & I9 & \\
\hline 1700 & 49.9 & 46.3 & & $3^{8}$ & -0.7 & I9 00 & 51.4 & 50.9 & 34 & -2.1 & $2 \mathrm{I} 00$ & I. & ost & & & $-โ 0.0$ & 2300 & 38.6 & 40.3 & & 57 & -9.2 \\
\hline $\mathrm{O} 2$ & $49 . \mathrm{I}$ & 46.6 & mint & 39 & 172 & $\mathrm{O} 2$ & 50.2 & 49.6 & 35 & tat & 02 & & ost & $1=$ & & (x) & 02 & 41.0 & 41.8 & & 20 & 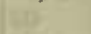 \\
\hline 04 & 45.8 & 42.9 & & 44 & 14 & 04 & 49.8 & 49.1 & 36 & (11) & $04^{*}$ & 48.0 & 67.1 & 22 & 02 & $y$ & 04 & 42.8 & 44.3 & & 23 & re \\
\hline 06 & 49.8 & 43.8 & & 40 & $14 \%$ & 06 & 49.8 & 49.1 & 36 & at: & $06^{*}$ & 34.1 & 36.2 & 23 & 16 & fiat & 06 & 40.9 & 41.8 & th & 20 & 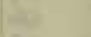 \\
\hline 08 & 36.9 & 33.8 & & 58 & $6 \%$ & 08 & 49.4 & 48.6 & 37 & 16 & $08^{*}$ & 19.2 & 58.0 & 24 & I8 & $16=$ & 08 & 39.0 & 39.5 & & I7 & hat \\
\hline 10 & 37.2 & 35.0 & & 57 & fy & 10 & 48.3 & $47 \cdot 4$ & 39 & 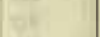 & IO* & 33.1 & 39.2 & 23 & 28 & 6 & IO & $37 \cdot 5$ & 38.3 & & I5 & ir \\
\hline $\mathrm{I} 2$ & $37 \cdot 3$ & 36.2 & 22 & 56 & 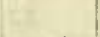 & I2 & 47.3 & 46.8 & 40 & $\sqrt{2}+2$ & 12 & 37.9 & 54.5 & & 44 & $=1 x$ & 12 & 36.2 & 37.4 & & 13 & atis \\
\hline 14 & 34.6 & 32.3 & 23 & OI & -0.7 & I4 & $47 \cdot 4$ & 46.7 & 40 & -2.2 & 14 & 23.6 & 32.0 & 23 & I5 & -9.4 & 14 & 39.8 & 40.6 & & I8 & -9.3 \\
\hline 16 & 30.2 & 26.8 & & 09 & (1) & I6 & $47 . I$ & 46.7 & 40 & the & $16^{*}$ & 20.1 & 54.2 & 22 & 13 & 4 & I6 & 40.0 & 40.9 & & 18 & \\
\hline I8 & 33.9 & 30.3 & 23 & 03 & (1) & I 8 & 46.7 & 46.5 & $4 I$ & E & I 8 & 39.0 & 50.5 & a.s. & 25 & 14. & I8 & 40.8 & 41.8 & & 20 & t \\
\hline 20 & 36.2 & 33.2 & 22 & 59 & $y=$ & 20 & 45.8 & 45.6 & 42 & 2 & 20 & $4 \mathrm{I} .0$ & 48.6 & 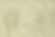 & 25 & f & 20 & 43.6 & 44.2 & & 24 & -4 \\
\hline 22 & 33.9 & 31.8 & 23 & 02 & $1=$ & 22 & 45.6 & $45 \cdot 4$ & 42 & - & 22 & 38.8 & 42.1 & in & 19 & (x) & 22 & 44.8 & 45.4 & & 26 & 24 \\
\hline 24 & 31.3 & 28.6 & & 07 & $x^{2}$ & 24 & 46.0 & 45.7 & 42 & 2 & 24 & 38.8 & 46.8 & 22 & 22 & y & 24 & 45.0 & 45.3 & & 26 & t+ \\
\hline 26 & 29.1 & 23.9 & af & 12 & $4=$ & 26 & $45 \cdot 3$ & 44.9 & 43 & $=$ & 26 & IO. I & 29.3 & 21 & 46 & & 26 & 44.5 & 45.0 & & 25 & \\
\hline 28 & 21.3 & 17.8 & & 23 & 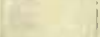 & 28 & 44.8 & $44 \cdot 4$ & 44 & & 28 & 22.1 & 24.4 & $2 \mathrm{I}$ & 52 & (1) & 28 & $44 \cdot 4$ & 44.8 & & 25 & \\
\hline 30 & I1 .8 & I 1.2 & $f(t)=$ & 36 & -1.0 & 30 & $45 \cdot 3$ & 44.9 & 43 & -2.5 & 30 & 29.3 & 33.2 & 22 & 04 & -9.0 & 30 & 46.0 & 46.0 & & 27 & -0.3 \\
\hline 32 & I2. 2 & 9.0 & 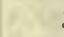 & 37 & (42-2y & 32 & 45.6 & $45 . \mathrm{I}$ & 43 & ar & 32 & 26.0 & 29.0 & $2 I$ & 58 & ( & 32 & 46.5 & 47.0 & & 28 & \\
\hline 34 & 23.0 & 20.1 & 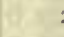 & 20 & W & 34 & 47. I & 46.6 & 40 & at & 34 & 22.3 & 25.8 & $2 I$ & 5.3 & H & 34 & 48.9 & 48.9 & & 32 & \\
\hline 36 & 24.7 & 22.0 & 9 & I7 & & 36 & $47 \cdot 3$ & 46.6 & 40 & $x-$ & 36 & 30.6 & $34 \cdot 3$ & 22 & 06 & 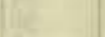 & 36 & 49.8 & 50.1 & & 33 & \\
\hline 38 & 27.2 & 25.2 & $x+1$ & I3 & +4 & $3^{8}$ & 46.8 & 46.5 & $4 I$ & 4 & $3^{8}$ & 49.8 & 54.2 & & 37 & th & 38 & 50.5 & 50.5 & & 34 & \\
\hline 40 & 26.2 & 24.0 & (1) & 14 & (15) & 40 & 46.2 & 45.6 & 42 & 62 & 40 & 61.6 & 65.9 & & 55 & 07 & 40 & 50.0 & 50.0 & & 33 & \\
\hline 42 & 28.1 & 25.2 & . & 12 & 15 & 42 & 45.8 & $45 \cdot 3$ & 42 & & 42 & 55.2 & 59.2 & & 45 & ix & $4^{2}$ & 47.6 & 48.0 & & 30 & \\
\hline 44 & 32.2 & 29.8 & 23 & 05 & $-I .0$ & 44 & 45.4 & 45.0 & 43 & -2.8 & 44 & 33.2 & 40.2 & & I3 & -9.0 & 44 & 48.4 & 48.8 & & $3 I$ & -9.4 \\
\hline 46 & 35.9 & 33.8 & 22 & 59 & 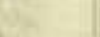 & 46 & 45.2 & 44.8 & 43 & 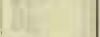 & 46 & 42.2 & 46.8 & & 25 & s. & 46 & 47.8 & 48.0 & & 30 & \\
\hline 48 & 39.3 & 37.1 & & 54 & & $4^{8}$ & 44.8 & $44 \cdot 3$ & 44 & $x^{2}=$ & 48 & 45.7 & 49.2 & & 29 & 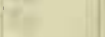 & $4^{8}$ & 48.6 & 48.8 & & 32 & \\
\hline 50 & 37.0 & 34.2 & & 58 & C & 50 & 45.0 & 44.5 & 44 & 0 & 50 & 33.7 & 40.7 & & 14 & 2 & 50 & 49.5 & 49.7 & & 33 & \\
\hline 52 & 37.8 & 36.2 & & 56 & -7 & 52 & 45.0 & $44 \cdot 5$ & 44 & 22 & 52 & 32.2 & 36.8 & & 09 & 2 & 52 & 50.8 & 51.6 & & 36 & \\
\hline 54 & 40.7 & 39.1 & & $5 I$ & $y^{2}$ & 54 & 45.0 & $44 \cdot 5$ & 44 & 18 & 54 & 30.0 & 34.2 & & 06 & 12 & 54 & 5 I. 2 & 51.6 & & 36 & \\
\hline 56 & 41.2 & 39.4 & & $5 I$ & fint & 56 & 45.0 & $44 \cdot 3$ & 44 & $x^{2}$ & 56 & 30.9 & 35.2 & & 07 & & 56 & 49.7 & 50.5 & & 34 & \\
\hline $5^{8}$ & 42.0 & 40.0 & & 49 & $1=$ & 58 & 44.6 & 43.9 & 44 & $y=$ & 58 & 47.3 & 50.6 & & 32 & & $58^{*}$ & 49.8 & 50.2 & & 38 & \\
\hline 1425 & & 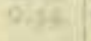 & 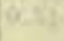 & & (w) is & 2000 & $44 \cdot 7$ & $44 \cdot 3$ & 44 & & & & & & & & 2400 & 50.5 & 51.2 & & 39 & -9.0 \\
\hline
\end{tabular}

Correction to local mean tinc is +30 .

'Torsion head at $15 \mathrm{~h} 40 \mathrm{~m}$ read $327^{\circ}$ and at $20 \mathrm{~h} 24 \mathrm{~m}$ read the same.

Observers $-\mathrm{R}$. R. T. and W. J. P., who alternated from $18 \mathrm{~h} \mathrm{I} 8 \mathrm{~m}$ to I $8 \mathrm{~h}$ 3om.
Correction to local mean tinc is $+44 \mathrm{~s} .90^{\circ}$ torsion $=25 \cdot 3$.

Torsion liead at $20 \mathrm{~h}$ oom read $336^{\circ}$ and at $24 \mathrm{~h} 25 \mathrm{ml}$ read $34 \mathrm{r}^{\circ}$.

Observers-R. R. T. and W. J. P., who alternated from $22 \mathrm{l} 14 \mathrm{~m}$ to $22 \mathrm{~h} 30 \mathrm{~m}$. 
Tabulation of magnetic declinations observed at Teplitz Bay-Continued

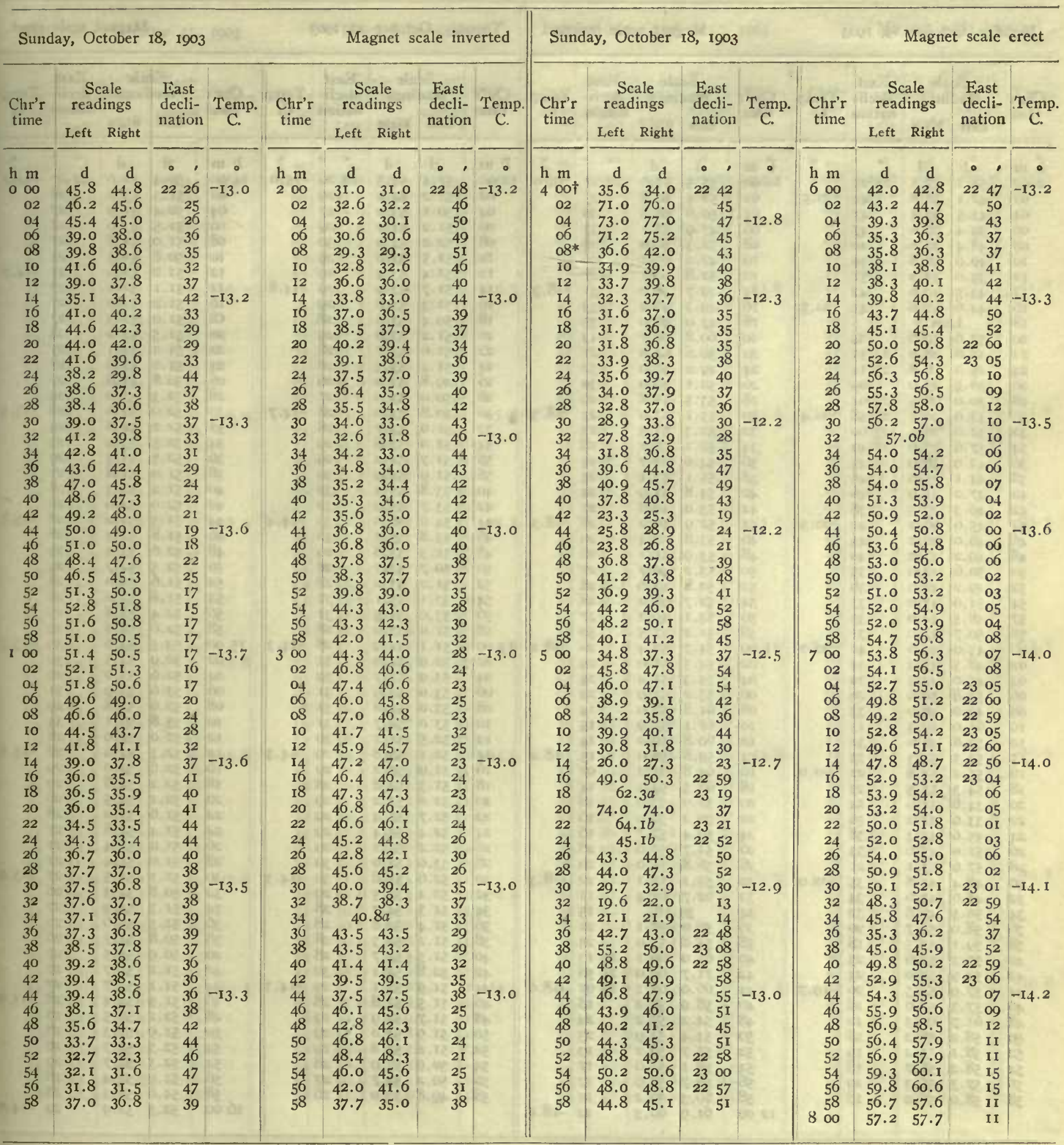

Observer-W. J. P.
Correction to local mean time is +55 .

Torsion head at oh oom read $340^{\circ}$ and at $8 \mathrm{~h} 25 \mathrm{~m}$ read the same.

Observers -W. J. P. and R. R. T., who alternated 3 h $58 \mathrm{~m}$ to $4 \mathrm{~h} 14 \mathrm{~m}$.

$\dagger$ Scale inverted for readings at $4 \mathrm{~h}$ oom. 
Tabulation of magnetic declinations observed at Teplitz Bay-Continued

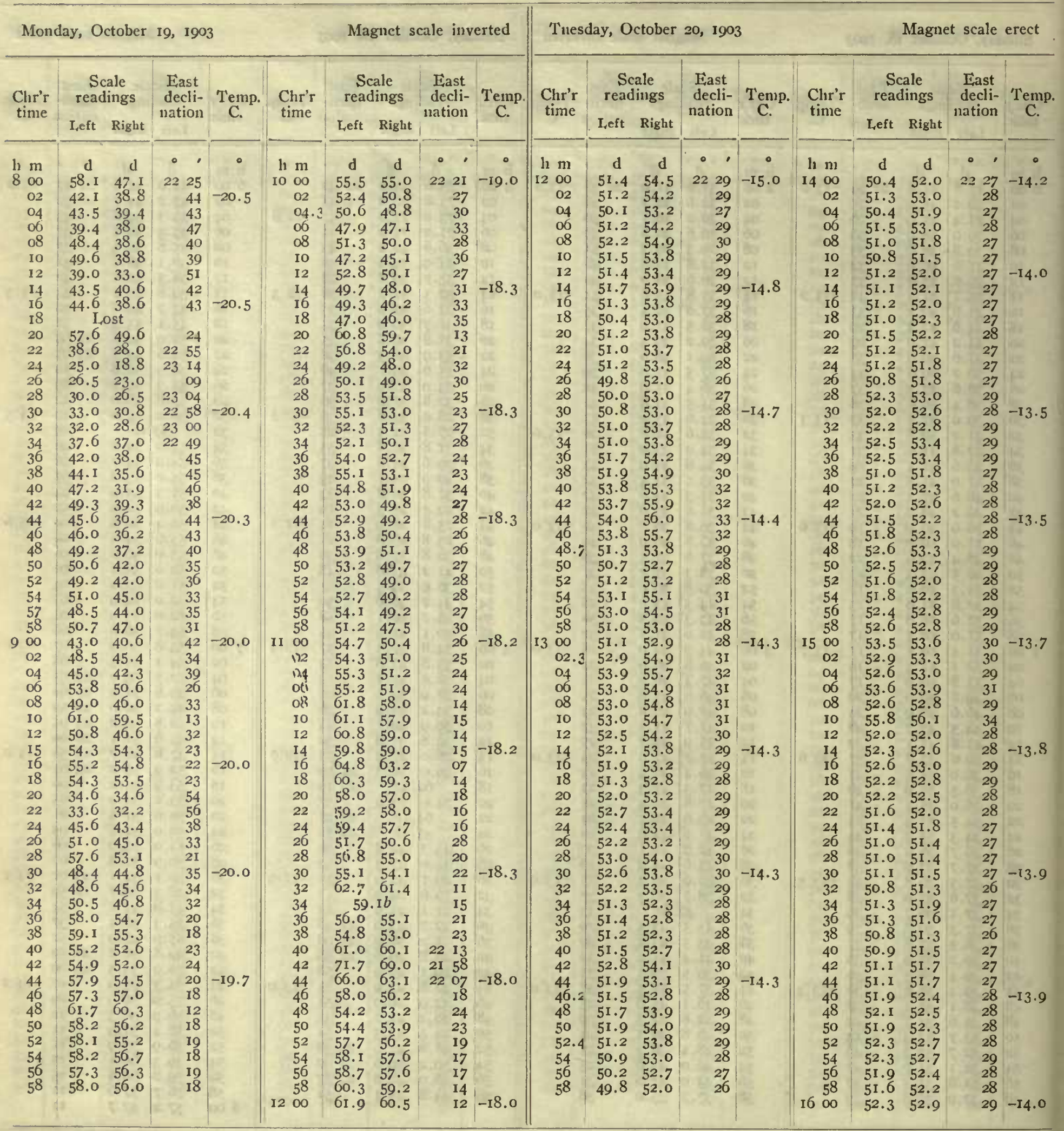

Correction to local mean time is $+\mathrm{Im}$ I 5 s.

Torsion head at $8 \mathrm{~h}$ oom read $339^{\circ}$ and at $\mathrm{I} 2 \mathrm{~h}$ zon read the same.

Observers-W. J. P. and R. R. 'T., who alternated from 9 h $46 \mathrm{~m}$ to roh ooin.
Correction to local mean time is $+\mathrm{Im} 37 \mathrm{~s}$.

Torsion head at $\mathrm{I}$ ih $30 \mathrm{~m}$ read $339^{\circ}$ and at $16 \mathrm{~h} 15 \mathrm{~m}$ read the same.

Observers-R. R. T. and W. J. P., who alternated from I 4 h $10 \mathrm{~m}$ to I4h $20 \mathrm{~m}$. 
Tabulation of magnetic declinations observed at Teplitz Bay-Continued

\begin{tabular}{|c|c|c|c|c|c|c|c|c|c|c|c|c|c|c|c|c|c|c|c|}
\hline \multicolumn{2}{|c|}{ Wednesday } & \multicolumn{2}{|c|}{ October 21, } & \multirow{3}{*}{$\begin{array}{c}1903 \\
\text { Temp. } \\
\text { C. }\end{array}$} & \multicolumn{5}{|c|}{ Magnet scalc inverted } & \multicolumn{5}{|c|}{ Wednesday, October 21, 1903} & \multicolumn{5}{|c|}{ Magnet scale it1verted } \\
\hline \multirow{2}{*}{$\begin{array}{l}\text { Chr'r } \\
\text { time }\end{array}$} & $\begin{array}{r}\mathrm{Sc} \\
\text { read }\end{array}$ & $\begin{array}{l}\text { ale } \\
\text { ings }\end{array}$ & $\begin{array}{l}\text { East } \\
\text { decli- }\end{array}$ & & Chr'r & $\begin{array}{r}\text { Sc } \\
\text { rcac }\end{array}$ & $\begin{array}{l}\text { ale } \\
\text { ings }\end{array}$ & $\begin{array}{l}\text { East } \\
\text { decli- }\end{array}$ & Temp. & Chr'r & $\begin{array}{r}\text { Sc } \\
\text { read }\end{array}$ & ings & $\begin{array}{l}\text { East } \\
\text { decli- }\end{array}$ & & Chr'r & $\begin{array}{r}\text { Sc } \\
\text { read }\end{array}$ & lings & $\begin{array}{l}\text { East } \\
\text { decli- }\end{array}$ & Tenip. \\
\hline & Left & Right & & & & $L_{\text {,eft }}$ & Right & & & & I.eft & Right & & & & Left & Right & & \\
\hline $\mathrm{l}_{1} \mathrm{~m}$ & d & d & $\cdot \quad$ & 0 & $\mathrm{~h} \mathrm{~m}$ & d & d & & $\bullet$ & $h \mathrm{~m}$ & $\mathrm{~d}$ & d & & - & $\mathrm{h} \mathrm{m}$ & $\mathrm{d}$ & d & & 3 \\
\hline 000 & $5 \mathrm{I} .2$ & 48.6 & 2234 & -16.2 & 200 & 48.2 & 48.0 & 2237 & -15.5 & 400 & 52.0 & 50.8 & 2232 & -14.2 & 600 & 49.0 & 48.0 & 2236 & -12.0 \\
\hline 02 & 50.0 & 48.0 & 36 & & 02 & 49.0 & 49.0 & 36 & & 02 & $52 . I$ & 50.2 & 32 & & 02 & 49.0 & 48.0 & 36 & \\
\hline 04 & 50.5 & 48.3 & 35 & & 04 & 48.9 & 48.9 & 36 & & 04 & $5 t .2$ & 50.2 & 33 & & 04 & 48.8 & 47.8 & 37 & \\
\hline 06 & 50.2 & 48.0 & 35 & & 06 & 49.6 & 49.6 & 35 & & 06 & 50.3 & 49.2 & 34 & & of & 48.8 & 47.8 & 37 & 20 \\
\hline 08 & 50.4 & 48.2 & 35 & & 08 & 49.4 & 49.3 & 35 & & 08 & 48.5 & 47.8 & 37 & $y^{4}$ & 08 & 49.1 & 48.2 & 36 & w \\
\hline Io & 49.8 & 47.5 & 36 & at & 10 & 49.0 & 48.8 & 36 & & IO & 47.7 & 47.1 & 38 & 19 & I0 & 49.3 & 48.3 & 36 & 31 \\
\hline 12 & 49.0 & 47.1 & 37 & & 12 & 47.8 & 47.8 & 37 & & 12 & 47.2 & 46.3 & 39 & $1=1$ & 12 & 49.2 & 48.2 & 36 & \\
\hline 14 & $49 \cdot 3$ & $47 \cdot 3$ & 37 & -16.2 & 14 & 48.0 & 48.0 & 37 & -15.3 & 14 & 48.0 & 47.0 & 38 & -13.8 & 14 & 48.8 & 47.5 & 37 & -11.8 \\
\hline 16 & 50.0 & 48.2 & 35 & & 16 & 48.3 & 48.0 & 37 & & 16 & 48.6 & 46.4 & 38 & & 16 & 48.2 & 47.5 & 37 & \\
\hline 18 & 49.5 & 47.8 & 36 & & 18 & 49.2 & 49.0 & 35 & & 18 & 48.8 & 46.8 & 37 & 64 & 18 & 47.3 & 46.8 & 39 & (6in \\
\hline 20.3 & 50.2 & 8.6 & 35 & $1 \mathrm{c}$ & 20 & 47.8 & 47.5 & 38 & 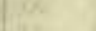 & 20 & 48.8 & 47.0 & 37 & tis & 20 & 46.8 & 46.5 & 39 & N \\
\hline 22 & 50.0 & 48.6 & 35 & xat & 22 & 46.2 & 46.0 & 40 & & 22 & 48.9 & 47.1 & 37 & wis & 22 & 47.8 & 47.2 & 38 & 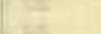 \\
\hline 24 & 50.6 & 49.2 & 34 & 10 & 24 & 45.8 & 45.6 & $4 \mathrm{I}$ & & 24 & 48.2 & 47.2 & 38 & 2t & 24 & 48.0 & $47 \cdot 5$ & 38 & E. \\
\hline 26 & $5 \mathrm{I} .3$ & 49.9 & 33 & $=$ & 26 & 45.6 & 45.4 & $4 \mathrm{I}$ & & 26 & 48.3 & 47.0 & 38 & 5 & 26 & 48.0 & 47.7 & 37 & +2 \\
\hline 28 & 52.6 & 51.3 & $3 \mathrm{I}$ & 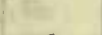 & 28 & 45.6 & 45.6 & $4 \mathrm{I}$ & & 28 & 48.9 & 47.3 & 37 & & 28 & 48.4 & 48.0 & 37 & \\
\hline 30 & 53.1 & 51.8 & 30 & -16.0 & 30 & 46.0 & 45.8 & 4I & -15.2 & 30 & 48.8 & 47.1 & 37 & -13.4 & 30 & 48.8 & 48.0 & 36 & -11.6 \\
\hline 32 & 53.6 & 52.3 & 29 & & 32 & 45.4 & $45 . \mathrm{I}$ & 42 & & 32.3 & 48.7 & 47.0 & 37 & & 32 & 48.7 & 48.2 & 36 & \\
\hline 34 & 53.1 & 52.0 & 30 & & 34 & 46.6 & 46.2 & 40 & & 34 & 48.0 & 46.5 & 38 & & 34 & 48.7 & 48.5 & 36 & \\
\hline 36 & 52.2 & 51.2 & $3 \mathrm{I}$ & & 36 & 46.8 & 46.4 & 39 & $a^{2}$ & 36 & 48.2 & 46.8 & 38 & & 36 & 49.1 & 48.8 & 36 & $x$ \\
\hline 38 & 52.4 & 51.2 & 31 & & 38 & 46.7 & 46.3 & 40 & 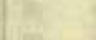 & 38 & 48.2 & 47.0 & 38 & & 38 & 49.1 & 48.8 & 36 & Ne \\
\hline 40 & 53.0 & 52.0 & 30 & & 40 & 47.2 & 47.0 & 39 & & 40 & 48.8 & 47.1 & 37 & 44 & 40 & 48.8 & 48.1 & 36 & 4 \\
\hline 42 & 52.6 & 51.6 & 3I & & 42 & 47.2 & 47.0 & 39 & +3 & 42 & 49.7 & 48.3 & 36 & 138 & 42 & 48.2 & 48.0 & 37 & $\rightarrow$ \\
\hline 44 & 52.6 & 51.8 & $3 \mathrm{I}$ & -16.0 & 44 & 47.3 & 47.2 & 38 & -15.1 & 44 & 51.2 & 49.8 & 33 & -13.0 & 44 & 48.0 & 47.8 & 37 & -11.5 \\
\hline 46 & 52.6 & 51.4 & $3 \mathrm{I}$ & & 46 & 47.6 & 47.2 & 38 & & 46 & 50.8 & 49.7 & 34 & & 46 & 48.2 & 47.7 & 37 & \\
\hline 48 & 52.2 & 51.2 & $3 \mathrm{I}$ & & 48 & 47.5 & $47 \cdot 3$ & 38 & $y$ & 48 & 47.9 & 46.3 & 38 & & 48 & 48.0 & 47.7 & 37 & 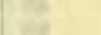 \\
\hline 50 & 52.6 & 51.6 & $3 I$ & & 50 & 47.6 & 47.4 & 38 & $a^{2}$ & 50 & 47.5 & 46.6 & 39 & 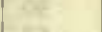 & 50 & 47.8 & 47.2 & 38 & \\
\hline 52 & 52.5 & 48.0 & 34 & 3 & 52 & 48.0 & 47.8 & 37 & 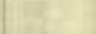 & 52 & 48.6 & 47.6 & 37 & & 52 & 48.1 & 48.0 & 37 & I \\
\hline 54 & 51.2 & 49.3 & 34 & $1=$ & 54 & 52.8 & 52.4 & 30 & W & 54 & 48.9 & 47.9 & 36 & $2_{4}$ & 54 & 49.0 & 48.2 & 36 & M \\
\hline 56 & 51.1 & 9.4 & 34 & & 56 & 47.8 & 47.4 & 38 & & 56 & 48.0 & 46.9 & 38 & & 56 & 48.9 & 47.9 & 36 & \\
\hline 58 & 51.2 & 48.8 & 34 & Ne & 58 & 48.1 & 47.0 & 37 & 180 & 58 & 47.8 & 47.0 & 38 & 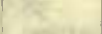 & 58 & 49.8 & 47.8 & 36 & \\
\hline I 00 & 51.2 & 49.6 & 33 & & 300 & 47.4 & 47.2 & 38 & -15.0 & 500 & 48.6 & 47.9 & 37 & -12.9 & 700 & 52.8 & 47.2 & 34 & -11.4 \\
\hline 03 & 50.9 & 49.4 & 34 & -15.8 & 02 & & 46.3 & 40 & & 02 & 48.2 & 48.1 & 37 & & 02 & 51.8 & 48.2 & 34 & \\
\hline 04 & 50.8 & 49.4 & 34 & & 04 & 46.0 & 45.6 & $4 \mathrm{I}$ & & 04 & 47.2 & 47.2 & 38 & & 04 & $5 \mathrm{I} .3$ & 47.9 & 35 & ) \\
\hline 06 & 50.6 & 0.3 & 34 & & 06 & 45.6 & 45.2 & $4 \mathrm{I}$ & ar & 06 & 47.0 & 46.8 & 39 & g & 06 & 51.2 & 48.0 & 35 & 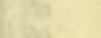 \\
\hline 08 & 50.4 & 9.3 & 34 & $\infty$ & 08 & 46.3 & 45.7 & 40 & 60 & 08 & 47.0 & 47.0 & 39 & E & 08 & $5 \mathrm{I} . \mathrm{I}$ & 49.0 & 34 & \\
\hline 10 & & 0.0 & 35 & 07 & 10 & & 46.8 & 39 & (6) & IO & 48.2 & 48.0 & 37 & 65 & 10 & 51.0 & 48.0 & 35 & 21 \\
\hline 12 & 49.8 & 8.6 & 35 & 61 & 12 & 48.2 & 47.8 & 37 & 71 & 12 & 49.7 & 49.1 & 35 & 14 & 12 & 50.2 & 46.1 & 37 & atr \\
\hline 14 & 49.4 & 48.4 & 36 & -15.6 & 14 & 49.3 & 48.8 & 36 & -14.9 & 14 & 50.0 & 49. I & 35 & -12.8 & 14 & 50.1 & 46.2 & 37 & -10.9 \\
\hline 16 & $49 . \mathrm{I}$ & 48.2 & 36 & & 16 & 50. I & 49.9 & 34 & & I6 & 49.6 & 49.2 & 35 & & 16 & 50.2 & 46.6 & 36 & \\
\hline 18 & 49.3 & 48.4 & 36 & & 18 & 50.6 & 50.4 & 33 & $a^{2}$ & I8 & $49 . I$ & 49.0 & 36 & 71 & 1 & 49.0 & 45.6 & 38 & $2 a$ \\
\hline 20 & 50.0 & $49 . I$ & 35 & & 20 & 51.4 & 51.2 & 32 & 106 & 20 & 49.2 & 48.4 & 36 & F & $2 c$ & 50.1 & 46.0 & 37 & 8 \\
\hline 22 & 50.2 & 49.3 & 35 & E & 22 & 52.3 & $52 . I$ & $3 \mathrm{I}$ & (21) & 2. & 48.8 & 47.8 & 37 & $1=$ & 22 & 50.2 & 46.1 & 37 & 4 \\
\hline 24 & 50.6 & 49.6 & 34 & F & 24 & 52.5 & 52.3 & 30 & 48 & 2 & 49.7 & 48.2 & 36 & 18 & 24 & 49.0 & 45.2 & 38 & 14 \\
\hline 26 & 50.6 & 49.8 & 34 & & 26 & 53.0 & 53. & 29 & ix. & 28 & 49.2 & 47.9 & 36 & $x$ & 2 & 48.8 & 46.2 & 38 & 20 \\
\hline 28 & 51.0 & 50.1 & 33 & $(25)$ & 28 & 52.6 & 52.2 & 30 & 3 & 28 & 49.2 & 48.0 & 36 & & 28 & 49.7 & 47.8 & 36 & in \\
\hline 30 & 51.0 & 50.0 & 33 & -15.6 & 30 & 51.6 & 51.3 & 32 & 26 & 30 & 49.0 & 47.2 & 37 & -12.6 & 30 & 48.8 & 46.5 & 38 & -10.8 \\
\hline 32 & & 50.0 & 34 & & 32 & & 50.4 & 33 & -14.0 & 32 & 49.0 & 48.0 & 36 & & 32 & 48.8 & 47.0 & 37 & 13 \\
\hline 34 & 50.2 & 49.6 & 34 & & 34 & 49.6 & 49.6 & 35 & & 3 & 49.0 & 48.0 & 36 & & 34 & 49.8 & 48.8 & 35 & \\
\hline 36 & & 49.4 & & te & 36 & 49.6 & & 35 & & 36 & 49.0 & 48.0 & 36 & & 36 & 49.1 & 48.0 & 36 & {$[8$} \\
\hline 38 & 50.0 & 49.3 & 35 & 10 & 38 & 50.0 & 49.8 & 34 & 2 & 38 & 48.7 & 47.2 & 37 & & 38 & 49.8 & 47.5 & 36 & 60 \\
\hline 40 & .3 & 49.7 & 34 & 18 & 40 & & 49.4 & 35 & 70 & $4 c$ & 48.3 & 47.0 & 38 & 18 & 40 & 49.3 & 47.3 & 37 & 2 \\
\hline 42 & 50.7 & 50.1 & 33 & 145 & 42 & 48.3 & 48.0 & 37 & 14 & 42 & 48.0 & 46.8 & 38 & 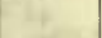 & 42 & 50.0 & $48 . \mathrm{I}$ & 36 & \\
\hline & 50.9 & 50.5 & 33 & $-\mathrm{I} 5.5$ & 44 & 47.1 & 46.8 & 39 & $-I_{4} .5$ & & 48.0 & 46.9 & 38 & -12.2 & 44 & 48.8 & 46.4 & 38 & -10.8 \\
\hline 46 & 50.8 & 50.6 & 33 & & 46 & 47.0 & 46.6 & 39 & & 46 & 47.7 & 46.7 & 38 & & 46 & 49.9 & 47.0 & 36 & \\
\hline 48 & 50.8 & 50.6 & 33 & $\omega$ & 48 & 46.2 & 46.0 & 40 & At & 48 & 47.8 & 46.9 & 38 & B4 & 48 & 49.5 & 46.9 & 37 & 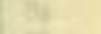 \\
\hline 50 & 50.3 & 50.0 & 34 & 13 & 50 & 47.1 & 46.8 & 39 & Ex & 50 & 48.9 & 48.1 & 36 & & 50 & 50.0 & $47 . I$ & 36 & - \\
\hline 52 & 49.3 & 49.2 & 35 & 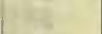 & 52 & 48.0 & & & 78 & 52 & 49.1 & 48.7 & 36 & & 52 & 50.0 & $47 . I$ & 36 & Q \\
\hline 54 & 48.9 & 48.6 & 36 & 27 & 54 & 48.8 & 48.3 & 36 & IE & 54 & 49.9 & 49.2 & 35 & 13. & 54 & 50.1 & 47.0 & 36 & ke \\
\hline 56 & $49 \cdot 3$ & 48.8 & 36 & at & 56 & 50.0 & 49.3 & 35 & (P) & 56 & 49.8 & 49.0 & 35 & (15) & 56 & 49.0 & 46.5 & 38 & \\
\hline 58 & 48.3 & 48. I & 37 & 6 & 58 & $5 \mathrm{I} . \mathrm{I}$ & 50.8 & 33 & F & 58 & 49.2 & 48.2 & 36 & & $5^{8}$ & 49.0 & 46.4 & 38 & \\
\hline
\end{tabular}

Obscrvers-W. J. P. and R. R. T. alternated from $3 \mathrm{~h} 54 \mathrm{~m}$ to 4 h $08 \mathrm{~m}$.

Obscrvers-R. R. T. and R. W. P. alternated from 7 h $52 \mathrm{~m}$ to $8 \mathrm{~h}$ rom. 
Tabulation of magnctic declinations observed at Teplitz Bay-Continued

\begin{tabular}{|c|c|c|c|c|c|c|c|c|c|c|c|c|c|c|c|c|c|c|c|}
\hline \multicolumn{5}{|c|}{ Wednesday, October 2I, 1903} & \multicolumn{5}{|c|}{ Magnet scale inverted } & \multicolumn{5}{|c|}{ Wednesday, October 21, 1903} & cal & \multicolumn{4}{|c|}{ Magnet scale inverted } \\
\hline \multirow{2}{*}{$\begin{array}{l}\text { Chr'r } \\
\text { time }\end{array}$} & \multicolumn{2}{|c|}{$\begin{array}{l}\text { Scale } \\
\text { readings }\end{array}$} & $\begin{array}{l}\text { Tiast } \\
\text { decli- }\end{array}$ & Temin. & Chr'r & $\mathrm{Scac}$ & $\begin{array}{l}\text { ale } \\
\text { lings }\end{array}$ & $\begin{array}{l}\text { East } \\
\text { decli- }\end{array}$ & Temp. & Chr'r & $\begin{array}{l}\text { Sc } \\
\text { read }\end{array}$ & $\begin{array}{l}\text { ale } \\
\text { lings }\end{array}$ & East & Tenı. & Chr'r & $\begin{array}{l}\text { Sce } \\
\text { read }\end{array}$ & $\begin{array}{l}\text { ale } \\
\text { lings }\end{array}$ & $\begin{array}{l}\text { East } \\
\text { decli- }\end{array}$ & Temp. \\
\hline & Left & Right & nation & & & Left & Right & nation & & & Left & Right & nation & & & t.eft & Right & 11 & \\
\hline h $\mathbf{~ m}$ & d & d & $\circ \quad$, & 0 & h m & d & $\mathrm{d}$ & . ' & - & $h \mathrm{~m}$ & d & d & $\cdot \quad$ & 0 & h in & d & d & $\cdot$, & $\circ$ \\
\hline 800 & $49 \cdot 3$ & 46.2 & 2238 & -10.8 & 10 00 & 51.2 & 50.2 & 22.33 & -11.2 & 1200 & 51.6 & 50.7 & 2232 & -11.1 & I4 00 & $53 . \mathrm{I}$ & 51.0 & 2231 & -120 \\
\hline 02 & 49.0 & 45.9 & 38 & - & 02 & 50.1 & 49.8 & 34 & (ntas & 02 & 50.9 & $49 \cdot 5$ & 34 & 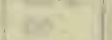 & 02 & 53.6 & 51.4 & 30 & $x^{-1}$ \\
\hline 04 & 50.0 & 47.7 & 36 & in & 04 & 48.8 & 48.0 & 36 & $\sqrt{6}=$ & 04 & $5 \mathrm{I} \cdot 3$ & 50.1 & 33 & Ine & 04 & 53.4 & 51.0 & $3 I$ & Iats \\
\hline 06 & 49.0 & 47.2 & 37 & 65 & 06 & 47.2 & 46.8 & 39 & 68 & 06 & 52.5 & 51.5 & 31 & $m$ & 06 & 53.6 & 51.1 & 30 & . 6 \\
\hline 08 & 48.3 & 47.1 & 38 & 87 & 08 & 47.1 & 46.3 & 39 & 80 & 08 & 51.9 & $5 \mathrm{I} . \mathrm{I}$ & 32 & $x$ & 08 & 55.3 & 53.7 & 27 & As \\
\hline 10 & 48.2 & 46.8 & $3^{8}$ & $x^{2}$ & IO & 48.6 & 47.5 & 37 & 64 & I0 & 50.7 & 50.2 & 33 & as & IO & 55.3 & 52.7 & 28 & vif \\
\hline I2 & 48.2 & 47.2 & 38 & & 12 & 49.6 & 48.5 & 36 & $|3|$ & I 2 & 51.0 & 50.0 & 33 & & 12 & $55 \cdot 3$ & 52.7 & 28 & \\
\hline 14 & 49.3 & 48.2 & 36 & -10.6 & I 4 & 49.9 & 48.8 & 35 & - II.I & I4 & 51.3 & 50.3 & 33 & -11.0 & 14 & 55.7 & 53.0 & 27 & \\
\hline I6 & 48.8 & 47.2 & 37 & & I6 & 50.1 & 49.0 & 35 & tent & I6 & 52.7 & 52.1 & 30 & 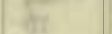 & 16 & 54.9 & 52.8 & 28 & -11.9 \\
\hline 18 & 49.1 & 48.1 & 36 & 35 & I 8 & 51.4 & 50.4 & 33 & sir. & I8 & $53 \cdot 3$ & 52.3 & 30 & fif & I8 & 53.3 & 51.6 & 30 & (x) \\
\hline 20 & 49.2 & 48.4 & 36 & $a$ & 20 & $5 \mathrm{I} . \mathrm{I}$ & 50.0 & 33 & t & 20 & 51.3 & 50.6 & 33 & ar. & 20 & 54.6 & 52.0 & 29 & fin \\
\hline 22 & 48.2 & 47.7 & 37 & act & 22 & 51.7 & 50.1 & 33 & 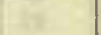 & 22 & $52 . \mathrm{I}$ & 51.2 & 31 & 6 & 22 & 55.9 & 53.5 & 27 & 52 \\
\hline 24 & 48.7 & 48.0 & 37 & 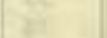 & 24 & 51.3 & $50 . I$ & 33 & n & 24 & 51.6 & 51.2 & 32 & 3 & 24 & 56.0 & 53.7 & 26 & \\
\hline 26 & 48.6 & 48.2 & 36 & & 26 & $5 \mathrm{I} \cdot 3$ & 50.3 & 33 & te & 26 & 51.3 & 50.7 & 32 & E & 26 & 54.9 & 53.3 & 28 & \\
\hline 28 & 48.7 & 48.0 & 37 & & 28 & 50.6 & 49.9 & 34 & & 28 & 51.8 & 51.5 & $3 I$ & 2 & 28 & 54.1 & 51.6 & 30 & \\
\hline 30 & 48.2 & 48.1 & 37 & -10.6 & 30 & 50.7 & 49.6 & 34 & -III. I & 30 & 51.6 & 51.0 & 32 & -11.0 & 30 & 54.7 & 53.0 & 28 & -II. 6 \\
\hline 32 & 49.2 & 48.7 & 36 & ter & 32 & 50.5 & 49.0 & 34 & $x^{2}+2$ & 32 & 52.6 & 51.8 & $3 \mathrm{I}$ & 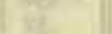 & 32 & 54.8 & 52.1 & 29 & ( \\
\hline 34 & 48.2 & 48.1 & 37 & 37 & 34 & 50.2 & 48.7 & 35 & $y$ & 34 & 53.9 & 53.3 & 28 & $y$ & 34 & 55.0 & 53.0 & 28 & at \\
\hline 36 & 48.8 & 48.6 & 36 & E & 36 & 50.1 & 48.8 & 35 & 5 & 36 & 54.0 & 53.5 & 28 & 6 & 36 & 55.4 & 52.6 & 28 & E. \\
\hline 38 & 49.0 & 48.7 & 36 & 32 & 38 & 50.8 & 48.2 & 35 & $x^{2}$ & 38 & 53.5 & 52.8 & 29 & 8 & 38 & 55.5 & 53.6 & 27 & \\
\hline 40 & 49.7 & 48.7 & 35 & e & 40 & 49.9 & 48.4 & 35 & if & 40 & 52.6 & 52.5 & 30 & 28 & 40 & 55.5 & 53.3 & 27 & \\
\hline 42 & 50.4 & 49.7 & 34 & & 42 & 51.6 & 50.0 & 33 & & 42 & 52.7 & 52.4 & 30 & 14: & 42 & 55.7 & 54.3 & 26 & \\
\hline 44 & 50.0 & 49.7 & 34 & -IO.I & 44 & 51.6 & 50.3 & 33 & $-I 1.0$ & 44 & 52.4 & 51.3 & 31 & -I I. I & 44 & 56.6 & 54.6 & 25 & -II.I \\
\hline 46 & 49.2 & 48. I & 36 & 15 & 46 & 50.1 & 49.2 & 35 & 18 & 46 & 52.2 & 50.9 & 32 & $1=$ & 46 & 55.6 & 55.0 & 26 & \\
\hline 48 & 48.7 & $48 . I$ & 36 & 82 & 48 & 50.7 & 49.1 & 34 & 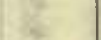 & 48 & 52.6 & 51.2 & 31 & 86 & 48 & 56.1 & 54.7 & 26 & \\
\hline 50 & 49.2 & 48.8 & 36 & 3 & 50 & 50.8 & 49.9 & 34 & 3 & 50 & 52.5 & $5 \mathrm{I} . \mathrm{I}$ & $3 \mathrm{I}$ & 3 & 50 & 55.9 & 54.6 & 26 & $n$ \\
\hline 52 & 49.0 & $48 . I$ & 36 & $\theta$ & 52 & $51 . \mathrm{I}$ & 50.I & 33 & 3 & 52 & 52.7 & 51.5 & $3 \mathrm{I}$ & 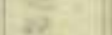 & 52 & 54.0 & 53.0 & 28 & 117 \\
\hline 54 & 48.7 & 47.8 & 37 & 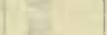 & 54 & 52.0 & 50.9 & 32 & 9 & 54 & 51.4 & 50.3 & 33 & 39 & 54 & 53.3 & 52.7 & 29 & \\
\hline 56 & $49 \cdot 3$ & 48.0 & 36 & ce & 56 & 5 I.I & 49.9 & 33 & 6 & 56 & 51.3 & 50.3 & 33 & 8 & 56 & 53.6 & 53.0 & 20 & \\
\hline 58 & 50.1 & 49.8 & 34 & 8 & $5^{8}$ & 51.6 & 50.4 & 32 & सis & 58 & 52.7 & 51.4 & 31 & FE & 58 & 54.3 & 53.8 & 28 & \\
\hline 900 & 49.2 & 48.7 & 36 & -10.3 & II 00 & 50.9 & 49.9 & 33 & -II.I & 1300 & 53.9 & 52.7 & 29 & -II.5 & 1500 & 54.6 & 54.0 & 27 & -10.8 \\
\hline 02 & 49.6 & 48.2 & 36 & $x^{2}$ & 02 & $5 \mathrm{I} . \mathrm{I}$ & 49.9 & 33 & 100 & 02 & 53.7 & 52.9 & 29 & (c) & 02 & 55.5 & 54.6 & 26 & \\
\hline 04 & 50.0 & 48.0 & 36 & we & 04 & 50.3 & 48.4 & 35 & a & 04 & 53.3 & 51.3 & 30 & in & 04 & 56.0 & 55.3 & 25 & \\
\hline 06 & 51.2 & 49.0 & 34 & art & 06 & 51.2 & 50.3 & 33 & 20 & 06 & 53.4 & 52.7 & 29 & $\operatorname{son}$ & 06.3 & 54.6 & 54.1 & 27 & In \\
\hline 08 & 52.5 & 50.5 & 32 & 27 & 08 & 50.8 & 50.2 & 33 & 65 & 08 & 53.8 & 53.0 & 29 & Wh & 08 & 53.5 & 53.0 & 29 & B. \\
\hline 10 & $5 \mathrm{I} . \mathrm{I}$ & 49.8 & 33 & cat & IO & 51.8 & 50.9 & 32 & nt. & IO & 53.5 & 52.9 & 29 & 6 & 10 & 53.1 & 52.6 & 30 & \\
\hline 12 & 50.0 & 48.2 & 35 & it & 12 & 50.5 & 50.0 & 34 & 91. & 12 & 53.4 & 52.4 & 29 & (1) & 12 & 53.3 & 52.9 & 29 & \\
\hline 14 & 51.0 & 49.7 & 34 & $-\mathrm{II} \cdot 4$ & I4 & 50.7 & 49.8 & 34 & & 14 & 52.3 & 51.4 & $3 \mathrm{I}$ & -I I. 6 & 14 & 53.3 & 52.7 & 29 & -11.0 \\
\hline 16 & 50.2 & 47.6 & 36 & & 16 & 51.0 & 50.8 & 32 & -10.9 & 16 & 51.4 & 50.4 & 32 & 2tit & 16 & 54.0 & 53.4 & 29 & \\
\hline I8 & 50.9 & 48.7 & 34 & 7 & I8 & 52.0 & $5 \mathrm{I} .3$ & 32 & 1. & I 8 & 51.8 & 50.5 & 32 & 82 & I8 & 55.5 & 55.0 & 26 & 12 \\
\hline 20 & 50.9 & 48.9 & 34 & 6 & 20 & 51.6 & $5 \mathrm{I} . \mathrm{I}$ & 32 & in & 20 & 52.4 & 51.7 & $3 \mathrm{I}$ & $\alpha$ & 20 & 55.0 & 54.4 & 27 & is \\
\hline 22 & 48.9 & 47.1 & 37 & $5=$ & 22 & 5 I.I & 50.5 & 33 & in & 22 & 52.4 & 51.4 & 31 & 2 & 22 & 53.5 & 53.0 & 29 & 는 \\
\hline 24 & 48.8 & 47.1 & 37 & 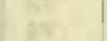 & 24 & 50.5 & 49.7 & 34 & in & 24 & 52.3 & 51.7 & 31 & in & 24 & 53.3 & 52.3 & 30 & \\
\hline 26 & 46.9 & 45.3 & 40 & & 26 & 50.8 & 50.2 & 33 & in & 26 & 53.0 & 52.2 & 30 & 8 & 26 & 53.3 & 53.0 & 29 & \\
\hline 28 & 48.8 & 46.6 & 38 & $y_{1}$ & 28 & 50.8 & 50.1 & 33 & & 28 & 53.2 & 52.3 & 30 & 120 & 28 & 53.3 & 53.0 & 28 & \\
\hline 30 & 48.7 & 46.7 & 38 & $-\mathrm{I} 1 \cdot 3$ & 30 & 51.9 & 50.9 & 32 & -10.9 & 30 & 52.5 & $5 \mathrm{I} .8$ & $3 I$ & -12. & 30 & 55.0 & 54.4 & 27 & -11.0 \\
\hline 32 & 47.5 & 46.6 & 39 & $x^{2}=$ & 32 & 51.0 & 50.1 & 33 & (x) & 32 & 53.6 & 52.4 & 29 & a & 32 & 54.1 & 53.6 & 28 & (20) \\
\hline 34 & 46.2 & 45.8 & 40 & 16 & 34 & 51.4 & 49.9 & 33 & in & 34 & 53.7 & 52.7 & 29 & M. & 34 & 53.6 & 53.0 & 29 & \\
\hline 36 & $46 . I$ & 45.7 & 40 & & 36 & 52.0 & 50.2 & 32 & 60 & 36 & 52.8 & 52.2 & 30 & te. & 36 & 53.4 & 53.0 & 29 & be \\
\hline 38 & 46.6 & 45.7 & 40 & 6 & 38 & 50.5 & 49.1 & 34 & 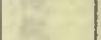 & 38 & $53 \cdot 3$ & 52.7 & 29 & $\theta_{1}$ & 38 & 53.7 & 53.3 & 28 & has \\
\hline 40 & 46.2 & 45.2 & $4 I$ & of & 40 & 51.7 & 49.6 & 33 & 20 & 40 & 52.5 & 52.0 & $3 \mathrm{I}$ & (iii) & 40 & 54.2 & 53.8 & 28 & 04 \\
\hline 42 & 49.8 & 48.5 & 35 & & 42 & 50.7 & 49.0 & 34 & & 42 & 53.5 & 53.3 & 29 & 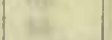 & 42 & 54.2 & 54.0 & 28 & \\
\hline 44 & 50.4 & 49.8 & 34 & $-\mathrm{II} .2$ & 44 & 51.0 & 49.0 & 34 & -10.9 & 44 & 52.5 & $\begin{array}{l}5.3 \\
52.0\end{array}$ & $3 I$ & -12.0 & 44 & 53.9 & 53.5 & 28 & -II.0 \\
\hline 46 & 47.9 & 46.8 & 38 & $\sqrt{6}$ & 46 & 52.0 & 50.3 & 32 & (2) & 46 & 52.3 & 51.9 & $3 I$ & 28 & 46 & 54.4 & 53.9 & 28 & \\
\hline 48 & 48.7 & 48.0 & 37 & Fit & 48 & 51.2 & 49.5 & 34 & 58 & 48 & 52.2 & 51.6 & $3 I$ & its & 48 & 54.3 & 54.1 & 27 & \\
\hline 50 & 50.2 & 49.4 & 34 & 60 & 50 & 50.1 & 48.6 & 35 & 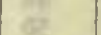 & 50 & 53.5 & 53.0 & 29 & ex & 50 & 54.0 & 53.2 & 28 & \\
\hline 52 & 52.0 & 51.1 & 32 & es & 52 & 50.8 & 49.5 & 34 & th & 52 & 54.8 & 53.0 & 28 & 3 & 52 & 55.2 & 54.3 & 27 & 3 \\
\hline 54 & 52.0 & 51.3 & $3 I$ & $3 x$ & 54 & 52.6 & 50.5 & 32 & $3 x$ & 54 & 54.3 & 53.5 & 28 & 0 & 54 & 54.3 & 53.7 & 28 & \\
\hline 56 & 51.7 & 50.8 & 32 & 82 & 56 & 52.2 & 50.8 & 32 & 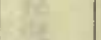 & $\begin{array}{l}54 \\
56\end{array}$ & 53.8 & 52.6 & 29 & & $\begin{array}{l}24 \\
56\end{array}$ & $\begin{array}{l}34.3 \\
54.1\end{array}$ & 52.9 & 28 & \\
\hline 58 & 51.0 & 50.7 & 33 & 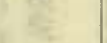 & 58 & 51.5 & 50.5 & 32 & 25 & 58 & 53.4 & 52.0 & 30 & 8 & 58 & 54.1 & 52.9 & 28 & \\
\hline
\end{tabular}

Observer-R. W. P.

Observers-R. W. P. and W. J. P. alternated I2h o2m to $12 \mathrm{~h} 22 \mathrm{~m}$. W. J. P. and R. R. T. alternated ish 5om to 16 h $06 \mathrm{~m}$. 
Tabulation of magnetic declinations observed at Teplitz Bay-Continued

\begin{tabular}{|c|c|c|c|c|c|c|c|c|c|c|c|c|c|c|c|c|c|c|c|}
\hline \multicolumn{6}{|c|}{ Wednesday, October 21, 1903} & \multicolumn{4}{|c|}{ Magnet scale inverted } & \multicolumn{5}{|c|}{ Wednesday, October 21, 1903} & \multicolumn{5}{|c|}{ Magnet seale inverted } \\
\hline \multirow{2}{*}{$\begin{array}{l}\text { Chr'r } \\
\text { time }\end{array}$} & \multicolumn{2}{|c|}{$\begin{array}{l}\text { Scaie } \\
\text { readings }\end{array}$} & \multirow{2}{*}{$\begin{array}{l}\text { East } \\
\text { decli- } \\
\text { nation }\end{array}$} & & Chr'r & $\begin{array}{r}\mathrm{Se} \\
\text { read }\end{array}$ & $\begin{array}{l}\text { alc } \\
\text { lings }\end{array}$ & $\begin{array}{l}\text { East } \\
\text { decli- }\end{array}$ & & & $\begin{array}{r}\text { Sca } \\
\text { rcadi }\end{array}$ & $\begin{array}{l}\text { ale } \\
\text { ings }\end{array}$ & $\begin{array}{l}\text { East } \\
\text { decli }\end{array}$ & & & read & dings & East & \\
\hline & L,eft & Right & & & & Left & Right & & & & $L_{1}$ eft & Right & & & & Left & Right & & \\
\hline $\mathrm{h} \mathrm{m}$ & d & d & - ' & $0^{\circ}$ & $\mathrm{h} \mathrm{m}$ & d & d & & $1^{\circ}$ & $\mathrm{h} \mathrm{m}$ & -00 & d & &,$\quad 0$ & $\mathrm{~h} \mathrm{~m}$ & d & $\mathrm{d}$ & .1 & $\circ$ \\
\hline 1600 & 54.0 & 53.0 & 2228 & $-\mathrm{II} . \mathrm{O}$ & 1800 & 54.1 & 52.1 & 2229 & -3.7 & 2000 & 58.8 & 55.2 & 222 & $23-10.4$ & 2200 & 52.4 & 40.3 & 2240 & -9.9 \\
\hline 02 & 5.3 .2 & 52.3 & 30 & & 02 & 54.1 & 52.0 & 29 & & 02 & 58.3 & 55.0 & & 24 & 02 & 51.0 & 43.2 & & \\
\hline 04 & $53 \cdot 3$ & 52.7 & 29 & 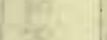 & 04 & 53.8 & 51.9 & 30 & & 04 & 57.8 & 55.0 & & wit. & 04 & 53.0 & 44.9 & & 76 \\
\hline о6 & 53.2 & 52.1 & 30 & 3 & 06 & 53.8 & 52.0 & 29 & & 06 & 58. I & 54.0 & & y & 06 & 55.0 & 45.6 & 34 & an \\
\hline 08 & 53.7 & .52 .2 & 29 & te. & 08 & 53.7 & 52.2 & 29 & & 08 & 57.8 & 53.8 & & tho & 08 & 53.0 & 44.0 & 36 & . \\
\hline 10 & 53.1 & 52.2 & 30 & nd & Io & 54.0 & 52.0 & 29 & 48 & 10 & 56.9 & 53.3 & & 아 & 10 & 53.9 & 40.0 & 39 & $\Rightarrow t$ \\
\hline 12 & 5.3 .5 & 52.5 & 29 & & 12 & 53.8 & 52.2 & 29 & & 12 & $57 \cdot 4$ & $54 \cdot 3$ & & 25 & 12 & 61.0 & 41.2 & 32 & 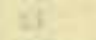 \\
\hline I4 & 53.0 & 52.0 & 30 & -10.8 & 14 & 5.3 .2 & 51.9 & 30 & -9.8 & 14 & 60.9 & 57.9 & & $\begin{array}{ll}19 & -10.4\end{array}$ & 14 & $49 \cdot 3$ & 41.2 & 42 & -9.9 \\
\hline I6 & 52.8 & $5^{2.2}$ & 30 & & I6 & 52.8 & 52.0 & 30 & & 16 & 58.0 & 55.0 & & 24 of & 16 & 71.2 & 51.2 & 16 & \\
\hline 18 & 53.0 & 53.0 & 29 & 34 & 18 & 52.5 & 51.8 & $3 \mathrm{I}$ & & IS & 48.8 & 45.0 & & 39 & 18 & 56.1 & 5.5 .8 & 25 & 61 \\
\hline 20 & 52.7 & $52 . I$ & 30 & $z^{2}$ & 20 & 52.8 & 51.8 & 30 & & 20 & 39.4 & 37.8 & 225 & 16. & 20 & 52.3 & 48.4 & 34 & E4 \\
\hline 22 & 52.8 & 52.1 & 30 & 4 & 22 & 52.8 & 51.8 & 30 & 3 & 22 & 3.5 .0 & 12.0 & 231 & xis & 22 & 50.9 & 47.6 & 35 & 6 \\
\hline 24 & 53.3 & 52.6 & 29 & EE & 24 & 53.2 & $52 . \mathrm{I}$ & 30 & $\mathrm{k}$ & 24 & 38.5 & 36.8 & 225 & $3=$ & 24 & 53.2 & 47.4 & 34 & HE \\
\hline 26 & 53.6 & 52.9 & & $x$ & 26 & 54.0 & 53.0 & 28 & ne & 26 & 50.0 & 48.9 & & tr & 26 & 55.2 & $47 . I$ & 32 & (a) \\
\hline 28 & 53.0 & 52.2 & 30 & -2 & 28 & 54.6 & 53.6 & 28 & $=$ & 28 & 61.0 & 56.8 & & 20 & 28 & 60.3 & 45.9 & 29 & \\
\hline 30 & 54.7 & 53.9 & 27 & -11.0 & 30 & 54.6 & 53.6 & 28 & -9.9 & 30 & 65.1 & 59.8 & & $15-10.6$ & 30 & 63.3 & 45.0 & 28 & -9.8 \\
\hline 32 & 54.9 & $54 . \mathrm{I}$ & 27 & & 32 & 54.4 & 53.4 & 28 & & 32 & 66.5 & 59.9 & & 13 & 32 & $6 \mathrm{I} .5$ & $45 \cdot 5$ & & \\
\hline 34 & $55 . \mathrm{I}$ & $54 \cdot 3$ & 27 & A & 34 & 54.5 & 5.3. & 28 & 11 & 34 & 63.3 & 57.1 & & +2 & 34 & 55.2 & 53.5 & 27 & 18 \\
\hline 36 & 54.8 & 53.8 & 27 & (3) & 36 & 54.4 & 53.3 & 28 & (x) & 36 & 56.6 & 53.0 & & 8 & 36 & 55.2 & 54.0 & 27 & \\
\hline $3^{8}$ & 55.9 & 54.6 & 26 & 3 & $3^{8}$ & 54.5 & 53.6 & 28 & 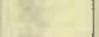 & 38 & 61.3 & 54.0 & & we & 38 & 52.6 & 50.8 & $3 I$ & 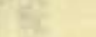 \\
\hline 40 & 55.5 & 54.2 & 26 & 10 & 40 & 54.5 & 53.7 & 28 & 84 & 40 & $6 \mathrm{I} .4$ & 54.2 & & 6 & 40 & 52.1 & 49.2 & 33 & 63 \\
\hline 42 & 56.3 & $54 . I$ & 26 & - & 42 & 54.8 & 54.2 & 27 & frang & 42 & $6 r .0$ & 54.0 & & $22 \quad 4 \quad 6$ & 42 & 52. & 49.7 & 32 & 43 \\
\hline 44 & 57.8 & 55.6 & 24 & -10.8 & 44 & 55.0 & 54.0 & 27 & -10.1 & 44 & 60.4 & 52.6 & 2. & $24-10.6$ & 44 & 52.8 & 49.5 & 32 & -9.5 \\
\hline 46 & 56.9 & 54.1 & 25 & & 46 & 54.7 & 53.8 & 27 & & 46 & 58.4 & 51.7 & & 26 & 46 & 52.6 & 48.9 & 33 & \\
\hline 48 & 56.2 & 54.8 & 25 & 3 & 48 & 54.5 & 53.2 & 28 & 38 & 48 & 58.8 & 52.0 & & 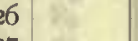 & 48 & $5 \mathrm{I} .2$ & 47.8 & 35 & (14) \\
\hline 50 & 56.0 & 53.2 & 27 & a: & 50 & & 53. & 28 & 2 & 50 & 57.2 & 51.5 & & $A$ & 50 & 51.0 & 48.1 & 35 & nate \\
\hline 52 & $57 \cdot 3$ & $54 \cdot 3$ & 25 & 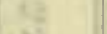 & 52 & 4.5 & 53.5 & 28 & है & 52 & 58.6 & $52 \cdot 3$ & & c5 & 52 & $5 \mathrm{I} . \mathrm{I}$ & 48.6 & 34 & 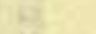 \\
\hline 54 & 56.4 & 54.0 & 26 & N & 54 & 54 & 53. & 28 & 122 & 54 & 58.6 & 50.1 & & 5 & 54 & 51.9 & 49.6 & 33 & 13 \\
\hline 56 & 55.8 & 54.0 & 26 & 73 & 56 & 54.2 & 53.6 & 28 & 2 & 56 & 59.0 & 51.0 & & ge & 56 & 52.9 & 50. & 1 & \\
\hline 58 & 55.3 & 54.0 & 27 & 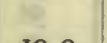 & 58 & & 53.1 & 28 & $\sqrt{25}+2$ & 58 & 56.2 & $54 \cdot 4$ & & 26 & 58 & 54.0 & 51.1 & 30 & \\
\hline 1700 & 55.9 & 54.0 & 26 & -10.0 & 1900 & 54.0 & 5.3 .1 & 28 & -10.2 & 2100 & 57.5 & 55.0 & & $24-10.5$ & 2300 & 56.2 & 53.0 & 27 & -9.6 \\
\hline 02 & 55.0 & 54.5 & 27 & $x^{2}+x^{2}$ & 02 & & 53.0 & 28 & 60 & 02 & 56.8 & $55 . I$ & & 25 & 02 & 53.5 & 50.6 & $3 \mathrm{I}$ & (20) \\
\hline 04 & 55.8 & 53.8 & 26 & wit & 04 & .8 & 53.0 & 29 & Fet & 04 & 56.7 & 54.2 & & 36 & 04 & 53.I & 50.5 & 31 & 36 \\
\hline 06 & 55.9 & $53 \cdot 5$ & 27 & 8 & 06 & & 52. & 29 & 90 & 06 & 56.2 & 54.6 & & in & 06 & 53.2 & 5I & $3 \mathrm{I}$ & $\infty$ \\
\hline 08 & 56.0 & 53.7 & 26 & 60 & 08 & 3.6 & 52.9 & 29 & Wh & 08 & 57.5 & 54.7 & & ibs & 08 & 48.8 & 46.3 & 38 & to \\
\hline Io & 55.6 & 53.1 & 27 & 711 & 10 & 54.0 & 53. & 28 & " & 10 & 56.8 & 54. & & m & 10 & 49.9 & 45 & 38 & ines \\
\hline 12 & $55 \cdot 3$ & 53.1 & 27 & 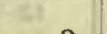 & 12 & .5 & 52.8 & 29 & at & 12 & 53.7 & 52.3 & & 29 & 12 & 50.9 & 47.6 & 35 & \\
\hline 14 & 55.0 & 53.2 & 28 & -9.8 & 14 & 53.9 & 52.9 & 29 & -10.5 & 14 & $55 \cdot 3$ & 52.8 & & $8-10.3$ & 14 & 55.0 & 49.4 & $3 \mathrm{I}$ & -9.7 \\
\hline I6 & 5.1 & 52.0 & 28 & & 16 & .8 & 53.0 & 29 & & 16 & 55.8 & 53.2 & & 27 & 16 & 47.7 & 40.9 & 43 & 31 \\
\hline 18 & 5.3 & 51.9 & 28 & Kis & 18 & 54.1 & 53 & & (6) & 18 & & 52. & & 84 & 18 & & & 224 & this \\
\hline 20 & 5.0 & 52.0 & 28 & 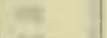 & 20 & & 53. & 28 & TE & 20 & 55.1 & 51.1 & & 06 & 20 & 25.8 & I8.9 & 23 & $\infty$ \\
\hline 22 & 54.9 & 52.1 & 28 & se & 22 & 54.2 & 53.8 & 28 & $1=$ & 22 & 56.0 & $5 \mathrm{I} .7$ & & $x$ & 22 & & 25.8 & 230 & is \\
\hline 24 & & 52.3 & 28 & 14 & 24 & & 53 & 28 & k & 2 & 54.8 & 50 & & 34 & 24 & 45 & 34 & 225 & it \\
\hline 26 & 5.1 & 53.9 & 27 & s5 & 26 & 54.1 & 53 & 28 & tat & 26 & 1 & $5 \mathrm{I}$. & & 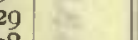 & 26 & 36.1 & 25 & 23 & trt \\
\hline 28 & 1.5 & 5.3 .0 & 28 & ans & 28 & & 53. & 28 & 2. & 28 & 55.2 & 51.8 & & 28 & 28 & 43.9 & 35 & 225 & \\
\hline 30 & 54.4 & 53.1 & 28 & -9.6 & 30 & .7 & 53.7 & 27 & $n^{\prime}$ & 30 & 53.9 & 51.7 & & $30-10.0$ & 30 & 30.8 & 20.2 & 2312 & -9.8 \\
\hline 32 & & $5 \mathrm{I} .5$ & 30 & 125 & 32 & & 53 & & $a_{2}$ & 32 & 55.1 & 52.0 & & 28 & 32 & 37.5 & 28.7 & & \\
\hline .34 & 3.9 & 50.0 & 3 & $k$ & 34 & & 53.1 & 28 & 15: & 34 & 54.7 & 51.7 & & Kin & 34 & 41.0 & 30.6 & 2256 & \\
\hline 36 & & 50.7 & 3 & 내 & 36 & & & & 35 & 36 & & $5 \mathrm{I}$. & & 6x & 3 & 34.4 & $3 I$ & 23 or & $6 x$ \\
\hline 38 & 53.7 & 51.0 & 30 & tes & 38 & & 53.8 & 27 & 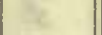 & 38 & 54.2 & 51.8 & & 185 & 38 & 35.1 & 33.1 & 2259 & the \\
\hline 40 & 4.0 & $5 I .5$ & 30 & $\pi 8$ & 40 & & 54. & 27 & in & 40 & $54 \cdot 5$ & $5 \mathrm{I}$. & & 147 & 45 & 36.1 & 33 & 58 & 67 \\
\hline 42 & 53.9 & 51.4 & 30 & 3 & 42 & & 54 & 27 & 20 & 42 & 53.7 & 50.7 & 3 & 30 & 42 & 45.5 & 43 & 42 & \\
\hline & 54.0 & 51.3 & 30 & -9.3 & 44 & & 54. & & -10.6 & & 53.9 & $5 \mathrm{I} \cdot \mathrm{I}$ & & $30-10.1$ & & 51.7 & 49 & 33 & -9.8 \\
\hline 46 & 54.1 & 52.2 & 29 & $x^{2}$ & 46 & 55.8 & 54 . & 26 & $107=$ & 46 & Over & l'k'd & & 10 & 46 & 54.6 & 52.2 & 29 & \\
\hline 48 & & 52.2 & 29 & 4 & 48 & & & 24 & 18 & 48 & 5 I. 0 & 50.0 & & 82 & 48 & 55.8 & $53 \cdot 3$ & 27 & \\
\hline 50 & 53.8 & 52.2 & 29 & 109 & 50 & 5.8 & & 25 & $\sigma$ & 50 & 56.0 & 5.5 .0 & 2 & 60 & 50 & 55.2 & 53.8 & 27 & $1=$ \\
\hline 52 & $54 . \mathrm{I}$ & 52.3 & 29 & 2 & 52 & & 53.2 & 27 & $2 z$ & 52 & $5^{6.0}$ & 46.0 & & wr & 52 & 56.5 & 54 & 25 & \\
\hline 54 & $54 \cdot 5$ & 52.6 & 28 & 18 & 54 & 56.1 & 54.1 & 26 & $\mathrm{R}^{2}$ & 54 & 52.0 & 42.0 & F & 12 & 54 & 57.1 & 52.8 & 26 & 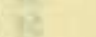 \\
\hline 56 & 54.2 & 52.3 & 29 & 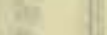 & 56 & 56.7 & 54.8 & 25 & 85 & 56 & 53.0 & 41.0 & & 39 & 56 & 56.4 & 52.0 & 27 & -2 \\
\hline 58 & 53.9 & 52.1 & 29 & 68 & 58 & 57.1 & 55.8 & 24 & $\sqrt{3}$ & 58 & 52.0 & 43.2 & 3 & tes & 58 & 56.0 & 52.0 & 28 & $\rightarrow$ \\
\hline & & & & & & & & & & & & & & $\cos \mathrm{me}$ & 2400 & 55.8 & 52.0 & 28 & -9.1 \\
\hline
\end{tabular}

Observers-R. R. T. and R. W. P. alternated $\mathrm{I} g \mathrm{~h}, 48 \mathrm{~m}$ to $20 \mathrm{oh} 02 \mathrm{~m}$. (W. J. P. $17 \mathrm{~h} 24 \mathrm{~m}$ to $18 \mathrm{~h} 42 \mathrm{~m}$. F. L. $16 \mathrm{~h} 54 \mathrm{~m}$ to $17 \mathrm{~h} 44 \mathrm{~m}$.)
Correction to local mean time is + Im 54 s.

Torsion head at oh oom read $339^{\circ}$ and at the end read the same. Observers-R. W. P. (F. L. 21 h $50 \mathrm{~m}$ to $22 \mathrm{~h} 30 \mathrm{~m}$.) 
Tabulation of magnetic declinations observed at Teplitz Bay-Continued

\begin{tabular}{|c|c|c|c|c|c|c|c|c|c|c|c|c|c|c|c|c|c|c|c|}
\hline \multicolumn{5}{|c|}{ Thursday, October 22, 1903} & \multicolumn{5}{|c|}{ Maginet scale erect } & \multicolumn{5}{|c|}{ Sunday, October 25, 1903} & \multicolumn{5}{|c|}{ Magnet scale ercct } \\
\hline \multirow{2}{*}{$\begin{array}{l}\text { Chr'r } \\
\text { time }\end{array}$} & \multicolumn{2}{|c|}{$\begin{array}{l}\text { Scale } \\
\text { readings }\end{array}$} & \multirow{2}{*}{$\begin{array}{c}\text { Fast } \\
\text { decli- } \\
\text { nation }\end{array}$} & Temp. & Chr'r & $\begin{array}{l}\mathrm{Sc} \\
\text { read }\end{array}$ & $\begin{array}{l}\text { ale } \\
\text { lings }\end{array}$ & East & Temp. & Chr'r & $\begin{array}{r}\text { Sc } \\
\text { read }\end{array}$ & $\begin{array}{l}\text { alc } \\
\text { lings }\end{array}$ & $\begin{array}{l}\text { East } \\
\text { decli- }\end{array}$ & Temp. & Chr'r & $\begin{array}{l}\text { Sce } \\
\text { read }\end{array}$ & $\begin{array}{l}\text { ale } \\
\text { ings }\end{array}$ & East & Temp. \\
\hline & Left & Right & & & & Left & Right & & & & Left & Right & divin & & & Left & Right & & \\
\hline h $\mathrm{m}$ & d & d & $\circ$, & $\circ$ & h $\mathrm{m}$ & d & $\mathrm{d}$ & & $\circ$ & $\mathrm{h} \mathrm{m}$ & d & d & & $\bullet$ & $\mathrm{l} \mathrm{m}$ & d & d & & $\bullet$ \\
\hline 600 & 36.2 & 38.7 & 2340 & +4.7 & 1800 & 32.8 & 33.1 & 2333 & +1.9 & 000 & 52.5 & 52.6 & 2232 & -10.7 & 200 & 47.9 & 48.2 & 2225 & -8.1 \\
\hline 02 & 34.6 & 37.0 & 38 & & 02 & 31.2 & 31.9 & 3I & & 02 & 51.0 & 51.6 & 30 & $\operatorname{lag}^{2}$ & 02 & 47.9 & 48.1 & & \\
\hline 04 & 34.8 & 36.8 & 38 & & 04 & 30. I & $3 \mathrm{I} .0$ & 30 & & 04 & 52.0 & 52.0 & $3 \mathrm{I}$ & $3 x$ & 04 & 47.8 & 48.0 & 25 & \\
\hline 06 & 33.2 & 34.9 & 35 & & of & 26.8 & 28.2 & 25 & is & 06 & 52.6 & 53.1 & 32 & Dis & 06 & 48.6 & 48.7 & 26 & \\
\hline 08 & 32.5 & 34.2 & 34 & 49 & 08 & 26.0 & 27.7 & 24 & tits & 08 & 53.0 & 53.6 & 33 & 65 & 08 & 49.0 & 49.2 & 27 & \\
\hline 10 & 33.2 & 34.4 & 35 & & 10 & 25.2 & 27.2 & 23 & (5) & 10 & 52.6 & 53.1 & 32 & 61 & 10 & 50.3 & 50.5 & 29 & \\
\hline 12 & 35.9 & 37.0 & 39 & & I2.2 & 25.6 & 27.5 & 23 & 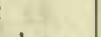 & 12 & 50.9 & 51.3 & 30 & & 12 & 50.7 & 51.0 & 29 & \\
\hline 14 & 34.9 & 36.3 & 38 & +4.4 & 14 & 26.0 & 28.1 & 24 & +1.9 & 14 & 51.8 & 52.2 & $3 I$ & -10.6 & 14 & 50.9 & $5 \mathrm{I} . \mathrm{I}$ & 30 & -8.0 \\
\hline 16 & $33 \cdot 3$ & 34.8 & 35 & & 16 & 24.2 & 26.8 & 22 & ting & 16 & 51.5 & 52.1 & 31 & (1) & 16 & 51.6 & 51.7 & 31 & \\
\hline 18 & 31.0 & 32.5 & 32 & wit & I8 & 22.8 & 24.6 & 19 & 15 & 18 & 52.1 & 52.3 & 32 & A & I8 & 52.9 & 53.1 & 33 & \\
\hline 20 & 38.2 & 39.8 & 43 & & 20 & 22.8 & 24.7 & I9 & ant & 20 & 54.7 & 54.7 & 36 & $m$ & 20 & 49.3 & 49.6 & 27 & \\
\hline 22 & 37.5 & 39.0 & 42 & 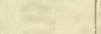 & 22 & 23.2 & 25.0 & 20 & $2=$ & 22 & $56 \cdot 3$ & 56.5 & 38 & c & 22 & 54.8 & 55.3 & 36 & \\
\hline 24 & 31.2 & 32.8 & 32 & 16 & 24 & 23.9 & 25.1 & 20 & $4-$ & 24 & 56.1 & 56.9 & 38 & $w$ & 24 & 55.3 & 55.6 & 36 & \\
\hline 26 & 34.7 & 36.2 & 37 & 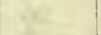 & 26 & 24.3 & 25.8 & 21 & N & 26 & 52.2 & 52.8 & 32 & & 26 & 55.6 & 56.0 & 37 & \\
\hline 28 & 30.2 & 31.8 & 30 & & 28 & 22.8 & 23.9 & 18 & $x^{2}$ & 28 & 52.3 & 53.0 & 32 & & 28 & 56.2 & 56.6 & 38 & \\
\hline 30.3 & 29.2 & 30.2 & 28 & +3.8 & 30 & 21.6 & $23 \cdot 3$ & I7 & +2.3 & 30 & 52.8 & 53.2 & 33 & -10.3 & 30 & 56.4 & 56.8 & 38 & -7.8 \\
\hline 32.6 & 26.2 & 28.0 & 24 & & 32 & 21.6 & 23.4 & I7 & & 32 & 53.3 & 53.8 & 34 & ( & 32 & 56.2 & 56.6 & 38 & \\
\hline 34 & 26.0 & 26.5 & 23 & HE & 34 & 21.6 & 23.2 & 17 & $3 x$ & 34 & 53.4 & 53.9 & 34 & & 34 & 55.2 & 55.6 & 36 & \\
\hline 36.3 & 24.1 & 24.8 & 20 & 8 & 36 & 21.0 & 22.5 & 16 & nt & 36 & 53.6 & 53.9 & 34 & & 36 & 54.6 & 54.8 & 36 & \\
\hline $3^{8}$ & 23.2 & 26.0 & 20 & 7 & 38 & 21.5 & 22.8 & 17 & 12 & 38 & 52.8 & 53.4 & 33 & 팔 & $3^{8}$ & 54.0 & 54.3 & 34 & \\
\hline 40 & 23.8 & 26.0 & $2 \mathrm{I}$ & 44 & 40 & 23.2 & 24.4 & 19 & $6 x$ & 40 & 53.0 & 53.5 & 33 & $6 y$ & 40 & 54.3 & 54.6 & 35 & \\
\hline 42 & 21.1 & 23.2 & 17 & 51 & 42 & 23.3 & 24.5 & 19 & 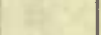 & 42 & 52.5 & 53.0 & 32 & & 42 & 54.6 & 54.8 & 36 & \\
\hline 44 & 20.1 & 23.1 & I6 & +2.0 & 44 & 23.2 & 24.2 & 19 & & 44 & 52.3 & 52.6 & 32 & -10.1 & 44 & 54.8 & 55.2 & 36 & -7.5 \\
\hline 46 & 18.8 & $2 \mathrm{I} .4$ & 13 & & 46 & 23.1 & 24.1 & 19 & +2.9 & 46 & 5 I. 8 & 52.3 & 3I & | & 46 & 55.6 & 55.8 & 37 & \\
\hline 48.2 & I7.4 & 20.5 & II & $\theta$ & 48 & 25.1 & 25.8 & 22 & & 48 & 52.0 & 52.3 & 3I & 7 & 48 & 55.2 & 55.4 & 36 & \\
\hline 50 & 21.0 & 23.8 & 17 & 8 & 50 & 26.2 & 28.0 & 24 & ate & 50 & 52.0 & 52.3 & 31 & 68 & 50 & 55.8 & 56.2 & 38 & \\
\hline 52 & 22.8 & 25.4 & 20 & & 52 & 25.2 & 26.0 & 22 & 0 & 52 & 51.5 & 52.0 & $3 \mathrm{I}$ & $n_{1}^{2}$ & 52 & 56.6 & 57.1 & 39 & \\
\hline 54 & 25.3 & 27.8 & 23 & H & 54 & 24.0 & 24.8 & 20 & te & 54 & 50.9 & 51.2 & 30 & 12. & 54 & 57.2 & 57.6 & $4^{\circ}$ & \\
\hline 56 & 28.2 & 30.8 & 28 & $\alpha$ & 56 & 23.7 & 24.5 & 20 & & 56 & 50.6 & 51.0 & 29 & (1) & 56 & 57.8 & 58.2 & $4 \mathrm{I}$ & \\
\hline 58 & 30.9 & 33.2 & 32 & $x^{2}$ & 58 & 23.7 & 24.3 & 20 & & 58 & 50.0 & 50.7 & 29 & 15 & $5^{8}$ & 58.1 & 58.3 & $4 I$ & \\
\hline$\infty$ & 33.1 & 35.0 & 35 & +3.1 & 1900 & $23 . I$ & $23 \cdot 3$ & I8 & +2.6 & I 00 & 49.6 & 50.0 & 28 & -9.5 & 300 & 56.9 & 57.3 & 39 & -7.2 \\
\hline 02 & 35.0 & 37.0 & 38 & & 02 & 23.7 & 24.0 & 19 & $\cos$ & 02 & 49.8 & 50.2 & 28 & 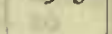 & 02 & 54.6 & 55.0 & 36 & \\
\hline 04 & 35.3 & 37.7 & 39 & 10 & 04 & 23.0 & $23 \cdot 3$ & 18 & $t^{2}$ & 04 & 50.0 & 50.4 & 28 & $16 \%$ & 04 & 51.0 & 51.6 & 30 & \\
\hline 06 & 36.3 & 38.2 & 40 & Wor & 06 & 24.0 & 24.3 & 20 & 84 & 06 & 50.0 & $5 \mathrm{I} .1$ & 29 & 10 & 06 & 48 & $.0 b$ & 25 & \\
\hline 08 & 36.2 & 38.1 & 40 & 87 & 08 & 22.8 & 23.3 & 18 & A) & 08 & 51.3 & 51.8 & 30 & ibs & 08 & 46.5 & 46.5 & 23 & \\
\hline IO & 35.4 & 37.2 & 39 & 47 & 10 & 23.7 & 24.1 & 19 & 61 & IO & 51.4 & $5 \mathrm{I} .9$ & $3 I$ & eir. & IO & 47 & $.5 a$ & 24 & \\
\hline 12 & 35.8 & 37.0 & 39 & then & I2 & 24.4 & 24.6 & 20 & 31 & 12 & 51.6 & 51.8 & 31 & ar & I2 & 50 & $.2 a$ & 28 & \\
\hline 14 & 35.2 & 36.8 & 38 & +2.1 & 14 & 20.6 & 20.9 & 14. & +1.9 & 14 & 51.3 & 51.6 & 30 & -9.1 & 14 & 52.3 & 52.3 & 32 & -7.1 \\
\hline I6 & 34.0 & 35.0 & 36 & & I6 & 21.7 & 21.8 & 16 & & 16 & 51.0 & 51.3 & 30 & 2 & 16 & 54.8 & 54.8 & 36 & \\
\hline I8 & 33.4 & 34.0 & 35 & hats & 18 & 21.0 & 21.2 & 15 & 78 & 18 & 51.0 & 51.3 & 30 & xit & I8 & 54.5 & 54.8 & 35 & \\
\hline 20 & $3 \mathrm{I} .8$ & 32.0 & 32 & c8 & 20 & 21.0 & 21.0 & 15 & $\alpha$ & 20 & 51.3 & 51.8 & $3^{0}$ & ces & 20 & 54.7 & 54.9 & 36 & \\
\hline 22 & 31.8 & 32.7 & 32 & tis & 22 & 21.2 & 21.6 & 15 & $y=$ & 22 & 51.3 & 51.6 & 30 & ter & 22 & 52.5 & 52.7 & 32 & \\
\hline 24 & 32.4 & 32.7 & 33 & is & 24 & 21.3 & 21.8 & 16 & 36 & 24 & 50.8 & $5 \mathrm{I} .3$ & 30 & $t=$ & 24 & 52.3 & 52.5 & 32 & \\
\hline 26 & 33.2 & 33.8 & 34 & 6 & 26 & 23.3 & 23.5 & 19 & 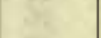 & 26 & 51.0 & $5 \mathrm{I} .5$ & 30 & - & 26 & 52.6 & $53 . \mathrm{I}$ & 32 & \\
\hline 28 & 34.2 & 34.8 & 36 & 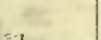 & 28 & 23.6 & 24.0 & 19 & & 28 & 50.8 & $5 \mathrm{I} \cdot 3$ & 30 & & 28 & 53.6 & $54 . I$ & 34 & \\
\hline 30 & 33.8 & 34.2 & 35 & +2.5 & 30 & 22.7 & 23.2 & I8 & +0.6 & 30 & 50.8 & 51.3 & 30 & -8.8 & 30 & 52.6 & 53.0 & 32 & -6.9 \\
\hline 32.5 & 35.0 & 36.0 & 38 & & 32 & 25.0 & 25.6 & 22 & corst & 32 & 50.7 & 51.1 & 30 & & 32 & 51.3 & 51.6 & 30 & \\
\hline 34 & 36.9 & 37.2 & 40 & 46 & 34 & 23.9 & 24.0 & 19 & 45 & 34 & 50.3 & 50.8 & 29 & 18 & 34 & 51.0 & 5 I. 3 & 30 & \\
\hline 36 & .35 .6 & 36.8 & 39 & 15 & 36 & 23.5 & 24.4 & 19 & 2 & 36 & 50.0 & 50.3 & 28 & $w^{2}$ & 36 & 50.8 & 51. & 30 & \\
\hline 38 & 21). 4 & 31.0 & 29 & 24 & 38 & 24.0 & 25.0 & 20 & $\sqrt{n}$ & 38 & 50.6 & 51.0 & 29 & $n^{*}$ & 38 & 50.8 & $5 \mathrm{I} .2$ & 30 & \\
\hline 40 & 30.3 & 31.6 & 30 & 64 & 40 & 22.8 & 23.2 & 18 & at & 40 & 51.0 & 5 I. 3 & 30 & on & 40 & 51.2 & 51.4 & 30 & \\
\hline 42 & 32.8 & $34 . \mathrm{I}$ & 34 & 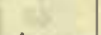 & 42 & 21.3 & 21.9 & I6 & 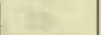 & 42 & 51.2 & 51.5 & 30 & & 42 & $5 \mathrm{I} .5$ & 51.6 & 30 & \\
\hline 44 & 32.3 & 33.2 & 33 & +2.2 & 44 & 20.9 & 21.2 & 15 & -0.0 & 44 & 51.3 & 51.6 & 30 & -8.5 & 44 & 51.5 & 51.9 & $3 \mathrm{I}$ & -6.6 \\
\hline 46 & 33.0 & 34.0 & 34 & (5) & 46 & 22.0 & 22.8 & 17 & - & 46 & 50.6 & 51.0 & 29 & 9.8 & 46 & 52.8 & $53 . \mathrm{I}$ & 33 & \\
\hline 48 & 340 & 35.1 & 36 & 74 & 48 & 21.6 & 22.2 & 16 & 8 & 48 & 50.9 & $5 \mathrm{I} .1$ & 30 & 8. & 48 & 52.0 & 52.3 & $3 \mathrm{I}$ & \\
\hline 50 & 35.7 & 36.0 & 38 & act & 50 & 21.8 & 22.4 & 17 & $x$ & 50 & 50.9 & $5 \mathrm{I} .1$ & 30 & $n^{2}$ & 50 & $5 \mathrm{I} \cdot 3$ & 51.5 & 30 & \\
\hline 52 & 34.9 & 35.2 & 37 & xt & 52 & 20.9 & 21.2 & I5 & $2 c$ & 52 & 50.0 & 50.0 & 28 & at. & 52 & 51.0 & 51.3 & 30 & \\
\hline & 37.2 & 37.7 & 40 & A2 & 54 & 19.8 & 20.0 & 13 & $\sqrt{32}$ & 54 & 50.0 & 50.3 & 28 & 12 & 54 & 52.0 & 52.6 & 32 & \\
\hline 56 & $34 \cdot 3$ & 34.9 & 36 & $x y$ & 56 & 19.2 & 20.0 & I3 & \pm & 56 & 49.3 & 49.6 & 27 & 8 & 56 & 53.0 & 53.5 & 33 & \\
\hline $5^{8}$ & 33.8 & 34.2 & 35 & de & 58 & 20.1 & 20.5 & 14 & & 58 & 48.5 & 48.7 & 26 & yz & $5^{8}$ & 53.5 & 53.9 & 34 & \\
\hline & & & & $\gamma$ & 2000 & 21.2 & 21.9 & 16 & -0.3 & & & & & & & & & & \\
\hline
\end{tabular}

Correction to local mean time is $+2 \mathrm{~m} 25$ s.

Observer-W. J. P

Torsion head at oh oom read $339^{\circ}$ and at ol zom read the same.

Observers- R. R. T. and W. J. P., who alternated $18 \mathrm{~h} I 8 \mathrm{~m}$ to $\mathrm{I} 8 \mathrm{~h}$ 3om. 
Tabulation of magnetic declinations observed at 'Teplitz Bay-Continued

\begin{tabular}{|c|c|c|c|c|c|c|c|c|c|c|c|c|c|c|c|c|c|c|c|}
\hline \multicolumn{5}{|c|}{ Sunday, October 25, Ig03 } & \multicolumn{5}{|c|}{ Magnet scale inverted } & \multicolumn{5}{|c|}{ Monday, October 26, 1903} & \multicolumn{5}{|c|}{ Magnet scale erect } \\
\hline \multirow[t]{2}{*}{$\begin{array}{l}\text { Chr'r } \\
\text { time }\end{array}$} & \multicolumn{2}{|c|}{$\begin{array}{l}\text { Scale } \\
\text { readings }\end{array}$} & \multirow[t]{2}{*}{$\begin{array}{c}\text { East } \\
\text { decli- } \\
\text { nation }\end{array}$} & $\begin{array}{l}\text { Temp. } \\
\text { C. }\end{array}$ & $\begin{array}{l}\text { Chr'r } \\
\text { time }\end{array}$ & $\begin{array}{r}\text { Sc } \\
\text { read }\end{array}$ & $\begin{array}{l}\text { ale } \\
\text { ings }\end{array}$ & $\begin{array}{c}\text { East } \\
\text { decli- } \\
\text { nation }\end{array}$ & Temp. & $\begin{array}{l}\text { Chr'r } \\
\text { time }\end{array}$ & $\begin{array}{l}S c \\
\text { read }\end{array}$ & ale & $\begin{array}{l}\text { Liast } \\
\text { decli- } \\
\text { nation }\end{array}$ & Temp. & $\begin{array}{l}\text { Chr'r } \\
\text { time }\end{array}$ & $\begin{array}{r}\mathrm{Sc} \\
\text { read }\end{array}$ & lings & $\begin{array}{r}\text { East } \\
\text { decli- } \\
\text { nation }\end{array}$ & $\begin{array}{l}\text { Temp. } \\
\text { C. }\end{array}$ \\
\hline & Left & Right & & & & Left & Right & & & & Left & Right & & & & Left & Right & & \\
\hline $\mathrm{h} \mathrm{m}$ & d & d &.$\quad$ & 0 & h m & d & $d$ & $\cdot$, & $\circ$ & $\mathrm{h} \mathrm{m}$ & d & $d$ & $\circ \quad$, & $7^{\circ}$ & $\mathrm{h} \mathrm{m}$ & d & d & & $\bullet$ \\
\hline 4 oI & 54.8 & 53.6 & 2232 & -6.2 & 600 & 56.1 & 55.3 & 2230 & -4.8 & 800 & $34 \cdot 7$ & 39.2 & 2327 & -9.0 & 10 00 & 53.0 & 55.0 & 2244 & -6.8 \\
\hline 02 & 56.0 & 55.0 & 30 & 10 & 02 & $53 . \mathrm{I}$ & 49.7 & 37 & & 02 & $34 \cdot 5$ & 38.7 & 26 & & 02 & 54.8 & 56.0 & 46 & \\
\hline 04 & 55.8 & 55.0 & $3 I$ & 10 & 04 & 50.6 & 48. I & & 10 & 04 & 35.5 & 40.2 & 28 & $f^{2}$ & 04 & 57.2 & 59.0 & 51 & 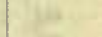 \\
\hline 06 & 56.1 & 55.3 & 30 & 10 & 06 & 51.9 & 49.6 & 38 & 10 & 06 & 28.3 & 34.5 & I8 & o & 06 & 56.0 & 57 & 49 & -8 \\
\hline 08 & 57.3 & 57.0 & 28 & no & 08 & 55.5 & 52.9 & 32 & wh & 08 & 32.8 & 36.5 & 23 & 84 & 08 & 57.2 & 58 & 50 & 20 \\
\hline I0 & 56.5 & 55.9 & 29 & $n t$ & IO & 55.0 & 52.9 & 33 & Fit & Io & 29.5 & 35.1 & 20 & aff & IO & 56.3 & $57 \cdot 3$ & 49 & $\pi 7$ \\
\hline I2 & 54.8 & 54.0 & 32 & 4 & I2 & 54.5 & 51.8 & 34 & 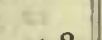 & 12 & 26.7 & 3 I. 2 & I4 & & 12 & 51.9 & 52.9 & 42 & \\
\hline 14 & 55.2 & 51.2 & 34 & -6.0 & I4 & 53.0 & 50.8 & 36 & -4.8 & 14 & 26.6 & $3 \mathrm{I} .2$ & I4 & -8.7 & 14 & 52.9 & 53.0 & 43 & -6.5 \\
\hline 16 & 51.8 & 51.0 & 37 & & 16 & 47.9 & 46.2 & 44 & in & I6 & 23.5 & 27.8 & 09 & & 16 & 54.2 & 54.8 & 45 & \\
\hline 18 & 52.5 & 51.5 & 36 & 31 & I8 & 51.0 & 49.2 & 39 & Wi & 18 & 24.3 & 32.3 & I3 & 44 & 18 & 54.0 & 55.2 & 45 & 64 \\
\hline 20 & 55.8 & 53.8 & 32 & 65 & 20 & 55.9 & 53.0 & 32 & 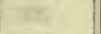 & 20 & 25.9 & 27.0 & Io & 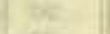 & 20 & 56.2 & 57.3 & 49 & ete \\
\hline 22 & 55.9 & 54.8 & 31 & 6 & 22 & 51.4 & 49.2 & 39 & 25 & 22 & 29 & $\cdot 3^{b}$ & I5 & et & 22 & 57.9 & 59.0 & $5 \mathrm{I}$ & 65 \\
\hline 24 & 54.1 & 52.8 & 34 & 75 & 24 & 50.0 & 46.8 & 42 & is & 24 & 22.5 & 23.4 & 05 & 3 & 24 & 60.0 & 6I.I & 55 & $1=$ \\
\hline 26 & 54.0 & 52.0 & 34 & $=$ & 26 & 50.0 & 47.2 & $4 I$ & $\theta=$ & 26 & 22.4 & 23.8 & 05 & 8 & 26 & 58. I & 59.1 & 52 & \\
\hline 28 & 51.7 & 50.1 & 38 & 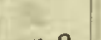 & 28 & 51.0 & 48.3 & 40 & 60 & 28 & 22.0 & 23.3 & 04 & & 28 & 57.2 & 58.8 & $5 \mathrm{I}$ & \\
\hline 30 & 50.9 & 49.6 & 39 & -5.8 & 30 & $55 . \mathrm{I}$ & 51.7 & 34 & -4.8 & 30 & 19.2 & 20.8 & 2300 & -8.5 & 30 & $56 . \mathrm{I}$ & 57.2 & 48 & -6.6 \\
\hline 32 & 48.8 & 47.7 & 42 & & 32 & 56.0 & 55.0 & 30 & 70 & 32 & 17.6 & 19.2 & 2258 & 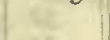 & 32 & 55.0 & 57.0 & 48 & \\
\hline 34 & 47.1 & 45.8 & 45 & 18 & 34 & 54.7 & 53.0 & 33 & He: & 34 & 22.6 & 24.8 & 2306 & 35 & 34 & 55.0 & 57.2 & 48 & 24 \\
\hline 36 & 50.7 & 49.8 & 39 & Ax & 36 & 55.1 & 53.9 & 32 & II & 36 & 21.6 & 23.2 & 04 & a & 36 & 56.0 & 57.7 & 49 & \\
\hline 38 & 50.9 & 49.7 & 39 & 3 & 38 & 53.8 & 52.8 & 34 & ${ }^{2} \mathrm{~s}$ & 38 & 21.5 & 23.2 & 04 & 15 & 38 & 58.6 & 59.9 & 52 & 3 \\
\hline 40 & 48.8 & 48.2 & $4 \mathrm{I}$ & 75. & 40 & 55.0 & 54.0 & 32 & 69 & 40 & 20.8 & 22.2 & 03 & 08 & 40 & $57 \cdot 3$ & 58.9 & $5 \mathrm{I}$ & $\theta$ \\
\hline 42 & 49.2 & 48.2 & $4 \mathrm{I}$ & 4 & 42 & 55.7 & 54.5 & $3 \mathrm{I}$ & 4 & 42 & 25.0 & 25.5 & 08 & 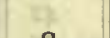 & 42 & 58.7 & 59.5 & 52 & \\
\hline 44 & 49.2 & 48.0 & $4 \mathrm{I}$ & -5.5 & 44 & 56.8 & 55.2 & 30 & -4.8 & 44 & 21.0 & 22.9 & 03 & -8.3 & 44 & 57.1 & 58.5 & 50 & -6.6 \\
\hline 46 & 47.5 & 45.7 & 44 & & 46 & 55.0 & 53.2 & 33 & & 46 & 22.0 & 23.8 & 05 & 10 & 46 & 57.2 & 57.9 & 50 & ( \\
\hline 48 & 47.8 & 46.0 & 44 & 4 & 48 & 51.0 & 50.0 & 38 & at & 48 & 20.2 & 20.8 & OI & 4 & 48. & 55.7 & 57.4 & 48 & 8 \\
\hline 50 & 49.0 & 47.9 & 42 & 아 & 50 & 54.1 & 53.4 & 33 & $c$ & 50 & 21.0 & 22.2 & 0 & & 50 & 45 & $8 b$ & 32 & $n$ \\
\hline 52 & 49.2 & 47.6 & 42 & $=$ & 52 & 53.5 & 52.1 & 35 & 28 & 52 & 10. 8 & 31.8 & 02 & 2 & 52 & 43. & $8 a$ & 28 & 8 \\
\hline 54 & 52.0 & 49.2 & 38 & 122 & 54 & 55.1 & $54 . I$ & 32 & 12 & 54 & 22.2 & 26.2 & 2307 & 12 & 54 & 53 & $8 a$ & 44 & II. \\
\hline 56 & $54 \cdot 3$ & 5I.I & 35 & 28 & 56 & 52.8 & 52.0 & 35 & & 56 & 16.9 & 20.0 & 2258 & 28 & 56 & 58.8 & 59.2 & 52 & Int \\
\hline 58 & 54.7 & 51.9 & 34 & 5 & $5^{8}$ & 54.9 & 54.0 & 32 & & $5^{8}$ & 18.9 & 22.0 & 23 OI & 3 & 58 & 60.3 & 60 & 55 & \\
\hline$\infty$ & 54.5 & 51.8 & 34 & $-5 \cdot 3$ & 700 & 55.8 & $54 . I$ & 31 & -4.8 & 900 & 17.4 & 20.6 & 2259 & -7.8 & II OO & 61.0 & 61.0 & 55 & -6.6 \\
\hline 02 & 55.0 & 51.9 & 34 & 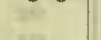 & 02 & 57.2 & 55.8 & 29 & & 02 & 18.8 & 20.9 & 2300 & & 02 & 59.8 & 60.7 & 54 & (n) \\
\hline 04 & 55.1 & 52.6 & 33 & 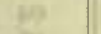 & D4 & 57.0 & 55.8 & 29 & 60 & 04 & 19.2 & 20.8 & $\infty$ & $F^{2}$ & 04 & 59.2 & 61. & 54 & in \\
\hline 06 & 55.1 & 52.2 & 3.3 & $\infty$ & 06 & 55.7 & 55.3 & 30 & 6 & 06 & 19.6 & 20.4 & $\infty$ & se & 06 & 60.2 & $6 \mathrm{I} .4$ & 55 & 63 \\
\hline os & 53.9 & 51.2 & 35 & (9) & as & 50.2 & 49.2 & 40 & 85 & 08 & 20.2 & 20.6 & 23 OI & 20 & 08 & 52.5 & 53.8 & 43 & W \\
\hline Io & 52.3 & 50.1 & 37 & 61 & I0 & 53.5 & 52.2 & 35 & (11) & IO & I5. 4 & 16.9 & 2254 & $\theta$ & IO & 42.2 & 44.0 & 27 & 6 \\
\hline I2 & 5I.I & 49.2 & 39 & t) & 12 & 55.0 & 54.0 & 32 & & I2 & 15.8 & I8. 4 & 56 & 24 & I2 & 46.3 & 47.7 & 33 & \\
\hline 14 & 52.2 & 50.1 & 37 & -5.0 & 14 & 57.2 & 56.0 & 29 & -4.8 & 14 & 18.9 & 19.4 & 59 & -7.5 & 14 & 49.8 & 51.6 & 39 & -6.6 \\
\hline I6 & $5 \mathrm{I} . \mathrm{I}$ & 49.2 & 39 & & 16 & 56.6 & 55.5 & 30 & & 16 & 18.9 & 19.9 & 59 & & 16 & 54.1 & 57.1 & 47 & \\
\hline 18 & 49.7 & 48.4 & $4 I$ & $2 t$ & I8 & 58. I & 57.0 & 2 & $4 y$ & I8 & I0.9 & II. & 47 & U. & I8 & 56.2 & 59 & 50 & te \\
\hline 20 & 49.0 & 47.2 & 42 & 10 & 20.3 & 56.6 & 55.9 & 29 & 84 & 20 & 10.0 & II.O & 45 & 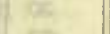 & 20 & 52.4 & 55.0 & 44 & 20 \\
\hline 22 & 48.0 & 46.0 & 44 & 50 & 22 & 56.9 & 54.8 & 30 & $6=$ & 22 & 10.2 & 12.0 & 46 & 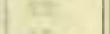 & 22 & 50.9 & 54.4 & 42 & 78 \\
\hline 24 & $7 . I$ & 45.0 & 45 & 18 & 2 & 57.6 & 55.9 & 29 & 14 & 24 & IO. I & 12.2 & 46 & 45 & 24 & 51.8 & 54.8 & 43 & 42 \\
\hline 26 & 48.6 & 47.0 & 42 & $y$ & & 57.2 & 54.9 & 3 & \pm & 26 & 10. 8 & 12.9 & 47 & & 26 & 49.7 & $5^{2.2}$ & 40 & 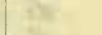 \\
\hline 28 & 48.8 & 47.3 & 42 & $\sqrt{6}$ & 28 & 55.3 & 53.8 & 32 & $=$ & 28 & 9.6 & II. 3 & 45 & $=$ & 28 & $5 \mathrm{I} .2$ & 54.0 & 42 & \\
\hline .30 & 50.0 & 49.3 & 40 & -4.9 & 30 & & 51.2 & 37 & -5.0 & 30 & 7.7 & 8.8 & 42 & -7 & 30 & & 53 & 42 & -6.5 \\
\hline 32 & 9.8 & 48.0 & $4 \mathrm{I}$ & & 32 & 58. I & 56.1 & 28 & 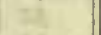 & 32 & 9.5 & II .2 & 45 & 2 & 32 & 50.6 & 52.4 & 40 & 42 \\
\hline 34 & 47.8 & 46.1 & 44 & ter & 34 & 55.8 & 53.7 & 32 & 34 & 34 & 7.4 & 8.5 & $4 \mathrm{I}$ & 12 & 34 & 53.5 & & 45 & 21 \\
\hline 36 & 50.7 & 49.5 & 39 & 12. & 36 & 57.8 & 55.5 & 29 & 4 & 36 & 9.0 & 9.2 & 43 & as & 36 & 53 & 55.2 & 45 & as \\
\hline 38 & 52.5 & 50.7 & 36 & 32 & 38 & 54.8 & 52.4 & 33 & ts & 38 & 10.6 & II.I & 46 & ALL. & 38 & 52.8 & 54.1 & 43 & $x^{2}$ \\
\hline 40 & 51.9 & 50.1 & 38 & ap. & 40 & 58.0 & 55.4 & 2 & 68 & 40 & 9.3 & 9.8 & 44 & 78 & 40 & 51.2 & 52.4 & $4 \mathrm{I}$ & +6 \\
\hline 42 & $49 . I$ & 47.2 & 42 & & 42 & 56.1 & 54.2 & $3 I$ & 4 & 42 & 9.2 & 9.8 & 44 & 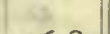 & 42 & 53.0 & 54.0 & 44 & 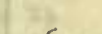 \\
\hline 44 & 48.2 & 46.2 & 44 & -4.8 & 44 & 53.0 & 51.6 & 36 & -5.0 & 44 & 8.1 & 8.8 & 42 & -6.8 & 44 & 54.3 & 55.3 & 46 & -6.5 \\
\hline 46 & 52.2 & 50.8 & 37 & & 46 & 58.8 & 57.3 & 26 & & 46 & 8.2 & 10.3 & 43 & 45 & 46 & 53.8 & 55.4 & 45 & 78 \\
\hline 48 & 53.8 & $5 \mathrm{I} .3$ & 35 & 34 & 48 & 52.8 & 51.8 & 36 & 278 & 48 & 8.2 & 10.0 & 43 & His & 48 & 57.8 & 59.9 & 52 & 84 \\
\hline 50 & 51.9 & 49.3 & 38 & WE & 50 & 55.9 & 53.8 & 32 & nis & 50 & 9.0 & 10.5 & 44 & (u) & 50 & 55.8 & 57.9 & 49 & - \\
\hline 52 & 52.0 & 49.8 & 38 & 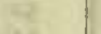 & 52 & 54.2 & 53.7 & 33 & 14 & 52 & 8.1 & 10.5 & 43 & 4 & 52 & 57.5 & 59.1 & $5 I$ & 마일 \\
\hline 54 & 51.6 & 49.1 & 39 & te & 54 & 57.0 & 56.8 & 28 & $\sqrt{2}$ & 54 & 8.9 & 9.9 & 44 & +2 & 54 & $59 . \mathrm{I}$ & 61.3 & 54 & 2 \\
\hline 56 & 51.8 & 49.8 & 38 & & 56 & 58.9 & 58.7 & 25 & 25 & $56^{*}$ & 47.2 & 55.3 & 40 & 3 & 56 & 58.9 & & 54 & \\
\hline 58 & 55.1 & 52.6 & 33 & 2 & 58 & 58.8 & 58.2 & 26 & -24 & 58 & 47.2 & 55.2 & 40 & 8 & 58 & 56.3 & 59.8 & 17.0 & -6 \\
\hline & & & & & 800 & 51.8 & 50.8 & 37 & -5.1 & & & & & & 1200 & 55.0 & 57.8 & 48 & \\
\hline
\end{tabular}

Correction to local mean time is $+2 \mathrm{~m} 255$.

Torsion head at oh oom read $339^{\circ}$ and at the end rcad the sanc.

Observers-W. J. P. and R. R. 'T., who alternated 4 h $04 \mathrm{~m}$ to 4 h I6 1 .
Correction to local mean time is $\operatorname{Im} 51 \mathrm{~s} .90^{\circ}$ torsion $=25^{\prime} \mathrm{I}$.

Torsion heacl at $8 \mathrm{~h} \mathrm{oom} \mathrm{read} 336^{\circ}$ and at $\mathrm{I} 2 \mathrm{~h} 53 \mathrm{~m}$ read $339^{\circ}$.

Obscrvers-W. J. P. and R. R. T., who alternated $8 \mathrm{~h} 40 \mathrm{~m}$ to $8 \mathrm{~h} 52 \mathrm{~m}$. 
Tabulation of magnetic declinations observed at Teplitz Bay-Continued

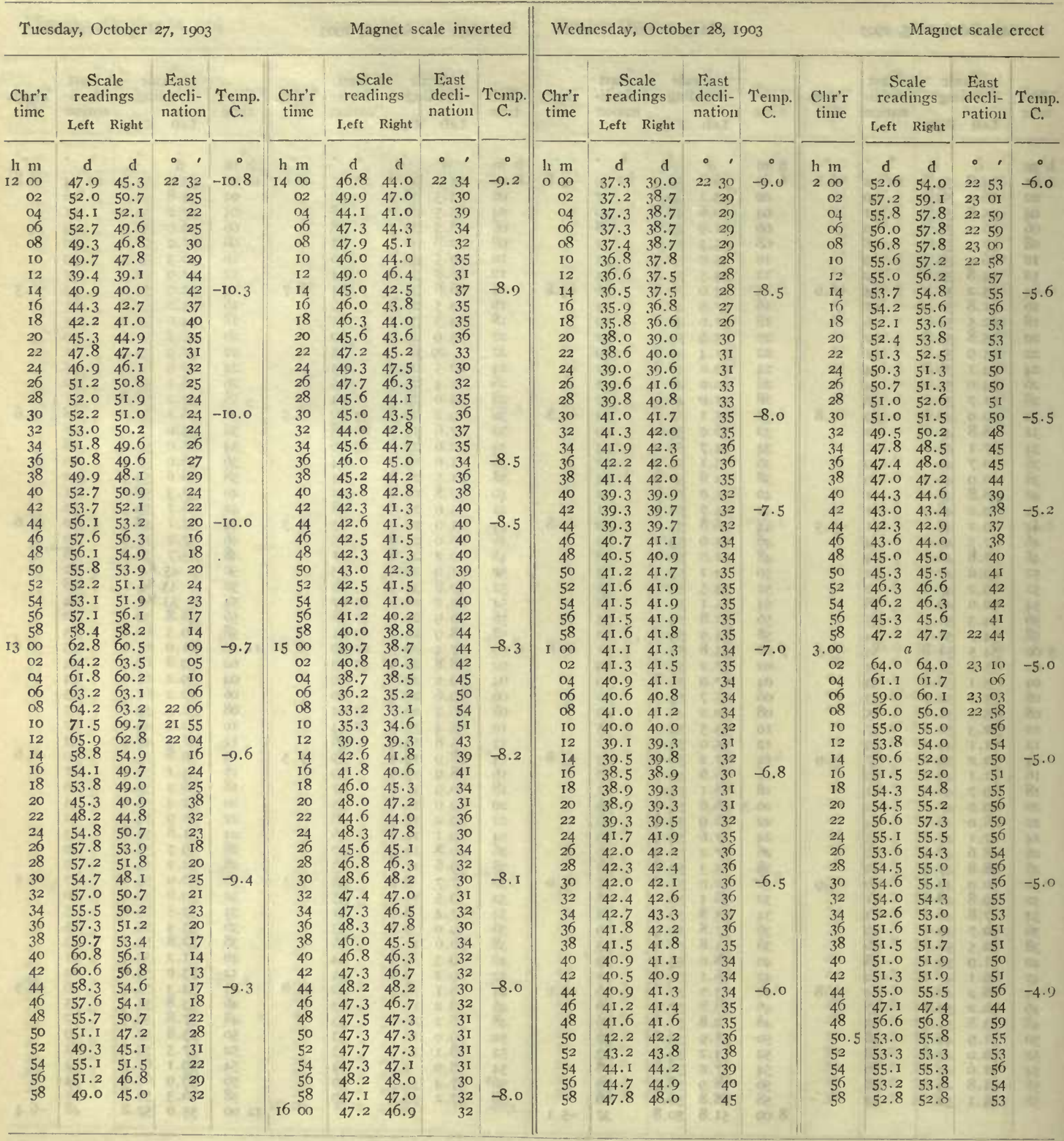


Tabulation of magnetic declinations observed at Teplitz Bay-Continued

\begin{tabular}{|c|c|c|c|c|c|c|c|c|c|c|c|c|c|c|c|c|c|c|c|}
\hline \multicolumn{5}{|c|}{ Wednesday, October 28, 1903} & \multicolumn{5}{|c|}{ Magnet scale erect } & \multicolumn{5}{|c|}{ Wednesday, October 28, I903 } & \multicolumn{5}{|c|}{ Magnet seale erect } \\
\hline \multirow{2}{*}{$\begin{array}{l}\text { Chr'r } \\
\text { time }\end{array}$} & \multicolumn{2}{|c|}{$\begin{array}{l}\text { Scalc } \\
\text { readings }\end{array}$} & \multirow{2}{*}{$\begin{array}{c}\text { East } \\
\text { decli- } \\
\text { ration }\end{array}$} & Temp. & Chr'r & $\begin{array}{r}\text { Sc } \\
\text { read }\end{array}$ & $\begin{array}{l}\text { ale } \\
\text { ings }\end{array}$ & Eas & Temp. & Chr'r & $\begin{array}{r}\mathrm{Sc} \\
\text { read }\end{array}$ & $\begin{array}{l}\text { ale } \\
\text { ings }\end{array}$ & Fiast & Temp. & Chr'r & $\begin{array}{r}\mathrm{Sc} \\
\text { read }\end{array}$ & $\begin{array}{l}\text { ale } \\
\text { lings }\end{array}$ & $\begin{array}{l}\text { East } \\
\text { decli- }\end{array}$ & Temp. \\
\hline & Left & Right & & & & Left & Right & & & & Left & Right & & & & I,eft & Right & & \\
\hline $\mathrm{h} \mathrm{m}$ & d & d & & 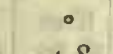 & h $\mathrm{m}$ & d & d & $\circ$ & $\circ$ & h $\mathrm{m}$ & d & d & $\circ$, & 10 & h $\mathrm{m}$ & d & d & $\circ$, & 0 \\
\hline 400 & 53.2 & $53 \cdot 3$ & 225 & -4.8 & 600 & 65.9 & 66.2 & 231 & -4.0 & 8 on & $47 . I$ & 48.1 & 2244 & $-3 \cdot 3$ & 1000 & 37.8 & 38.0 & 2229 & -2.5 \\
\hline 02 & 54.8 & 55.2 & & & 02 & 57.0 & 57.4 & 230 & & 02 & 45.2 & 46.4 & 42 & 5 & 02 & 38.9 & 39.1 & 31 & \\
\hline 04 & 56.6 & 57.1 & & an & 04 & 50.9 & 51.8 & 225 & 14 & 0.4 & 48.2 & 49.2 & 46 & 49 & 04 & 39.0 & 39.2 & $3 I$ & 317 \\
\hline 06 & 56.1 & 56.6 & & $f_{10}$ & 06 & 55.8 & 56.2 & 225 & 34 & 06 & 44.2 & 45.8 & 40 & 90 & 06 & 40.0 & 40.7 & 33 & $\infty$ \\
\hline 08 & 54.2 & 54.8 & & 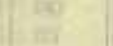 & 08 & 60.6 & 62.4 & 230 & 10 & as & 4.3 .9 & 45.1 & 40 & 20 & os & 38.1 & 38.2 & 30 & 8 \\
\hline 10 & 54.2 & 54.6 & & 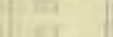 & 10 & 64.8 & 65.4 & & $n t$ & $10-$ & -46.5 & 47.5 & 44 & pr & 10 & 36.3 & 36.9 & & or \\
\hline 12 & 54.8 & 55.3 & & 41 & 12 & 65.7 & 68.7 & & 25 & 12 & 44.6 & 45.7 & 40 & ats & 12 & 38.0 & 38.5 & 30 & 47 \\
\hline 14 & 54.2 & 55.0 & & -4.5 & 14 & 67.1 & 70.0 & & -3.9 & 14 & 44.4 & 44.9 & 40 & -3.0 & 14 & 38.4 & 39.0 & 30 & -2.3 \\
\hline I6 & 5I.I & 52.0 & & & 16 & 68.2 & 69.4 & & & 16 & 45.0 & 45.9 & 41 & & I6 & 38.0 & 38.7 & 30 & \\
\hline 18 & $47 \cdot 3$ & 48.2 & & 3 & I8 & 66.2 & 67.6 & & 2 & I8 & 43.2 & 43.4 & 38 & 18 & 18 & 37.2 & 38.2 & 29 & 24 \\
\hline 20 & 44.6 & 45.2 & & 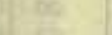 & 20 & 67.8 & 69.1 & ? & os & 20 & 42.0 & 4.3 .0 & 36 & 65 & 20 & 36.9 & 37.3 & 28 & 185 \\
\hline 22 & $43 . I$ & 43.8 & & $\mathrm{c}$ & 22 & 63.7 & 66.2 & I & is: & 22 & $44 . I$ & 45.3 & 40 & 64 & 22 & 37.2 & 37.8 & 29 & $=$ \\
\hline 24 & 42.7 & $43 . I$ & & 6 & 24 & 62.7 & 65.9 & I & $H^{2}$ & 24 & 4.3 .7 & 45.8 & 40 & 75: & 24 & 37.4 & 38.2 & 29 & in \\
\hline 26 & 43.2 & 44.1 & & 15 & 26 & 58.9 & 61.9 & 0 & 8 & 26 & 38.6 & 39.9 & 3I & nes & 26 & 37.0 & 38.0 & 29 & 18 \\
\hline 28 & 44.2 & 44.8 & & 10 & 28 & 60.2 & 62.2 & 0 & 85 & 28 & 38.4 & 39.8 & $3 I$ & 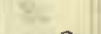 & 28 & 36.4 & 37.6 & 28 & Fis \\
\hline 30 & 45.0 & 46.1 & & -4.2 & 30 & 62.0 & 65.0 & 0 & -3.9 & 30 & 40.2 & 41.7 & 34 & -2.8 & 30 & 35.8 & 36.9 & 27 & -2.2 \\
\hline 32 & 45.2 & 46.0 & & & 32 & 61.9 & 62.3 & 230 & 5 & .32 & 37.3 & 38.5 & 29 & 45 & 32 & $35 \cdot 3$ & 36.6 & & 4 \\
\hline 34 & 45.2 & 46.8 & & 0 & 34 & 55.5 & 57.9 & 225 & 18. & 34 & 37.8 & 39.0 & 30 & 16 & 34 & 35.2 & 36.4 & 26 & IL \\
\hline 36 & 45.7 & 46.8 & & c5 & 36 & 55.1 & 56.9 & & 38 & 36 & 43.0 & 43.9 & 38 & 8. & 36 & 35.9 & 36.9 & 27 & 3. \\
\hline $3^{8}$ & 44.9 & 46.0 & & 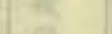 & 38 & 53.0 & 55.4 & 5 & 吸 & 38 & 43.2 & 43.6 & $3^{8}$ & as & 38 & 35.5 & 37.2 & & 8 \\
\hline 40 & 45.5 & 46.6 & & 8 & 40 & 54.2 & 56.6 & $=$ & 와 & 40 & 39 & $2 b$ & (1) $3 \mathrm{I}$ & 0 & 40 & 35.9 & 37.1 & 27 & et \\
\hline 42 & 46.8 & 47.8 & & v & 42 & 55.2 & 57.6 & 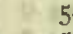 & $4+2$ & 42 & 38.8 & 39.0 & $3 I$ & 41 & 42 & 35.0 & 36.2 & & $x^{2}$ \\
\hline 44 & 45.1 & 46.2 & 4 & -4.1 & 44 & 55.2 & 57.9 & 5 & -3.7 & 44 & 39.0 & 39.8 & 32 & -2.8 & 44 & 36.3 & 37.7 & 28 & -2.2 \\
\hline 46 & 47.2 & $48 . I$ & & & 46 & 55.0 & 57.2 & 225 & 3 & 46 & 39.8 & 41.2 & 33 & $x=$ & 46 & 36.2 & 37.8 & 28 & 4 \\
\hline 48 & 46.8 & 48.0 & & ra & 48 & 56.1 & 59.0 & 230 & H6 & 48 & 38.6 & 40.7 & 32 & Cy & 48 & 36.3 & 37.5 & 28 & 46 \\
\hline 50 & 47.6 & 49.2 & to & $=$ & 50 & 54.3 & 57.8 & 225 & 60 & 50 & 37.9 & 38.2 & 29 & $\alpha$ & 50 & 37.1 & 38.4 & & $Q$ \\
\hline 52 & 47.1 & 48.0 & & 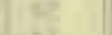 & 52 & 55.2 & 58.2 & 5 & 78 & 52 & 35.9 & 36.6 & 26 & -2 & 52 & 37.6 & 38.5 & 29 & 5 \\
\hline 54 & 49.2 & 50.0 & & 12 & 54 & 54.4 & 58.0 & & 14 & 54 & 33.5 & $35 \cdot 3$ & 24 & 18 & 54 & 37.5 & 38.2 & 29 & 18 \\
\hline 56 & 49.7 & 50.8 & & & 56 & $5 \mathrm{I} .2$ & 55.6 & 225 & 18 & 56 & 39.2 & 40.9 & 32 & fe & 56 & 37.8 & 38.5 & & 8 \\
\hline 58 & 47.8 & 49.2 & at & & 58 & 58.8 & 63.8 & 230 & 2 & 58 & 40.6 & 41.6 & 34 & 82. & 58 & 38.7 & 39.4 & $3 I$ & $8 t$ \\
\hline 00 & 47.8 & 48.8 & $\pi$ & -4.0 & 700 & 50.2 & 56.2 & 225 & -3.6 & 900 & 36.0 & $37 \cdot 3$ & 27 & -2.7 & II $\infty$ & 37.9 & 38.4 & & -2.1 \\
\hline 02 & 51.9 & 52.3 & t & & 02 & 54.0 & 58.0 & 5 & 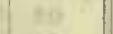 & $\mathrm{O} 2$ & 36.2 & 37.0 & 27 & $620^{\circ}$ & 02 & 36.6 & 37.5 & 28 & $\cos$ \\
\hline 04 & $53 . \bar{T}$ & 53.9 & 5 & $Y^{\prime} ?$ & 04 & 52.8 & 58.4 & es, & 46 & 04 & 37.9 & 39.1 & 30 & 40 & 04 & 35.9 & 36.6 & & 3ip \\
\hline 06 & 56.2 & $57 \cdot 4$ & 225 & 20 & 06 & 52.9 & 57.7 & 4 & 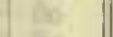 & 06 & 37.0 & 38.0 & 29 & $\infty$ & 06 & 35.6 & 36.1 & 26 & M \\
\hline 08 & 58.7 & 59.6 & 230 & hrt & 08 & 51.3 & 56.0 & 5 & 108 & 08 & 36.0 & 37.3 & 27 & 80 & 08 & 35.0 & 36.0 & 25 & $6 n$ \\
\hline 10 & 57.0 & $57 \cdot 3$ & 225 & Q2 & 10 & 50.6 & 53.9 & 225 & 다 & I0 & 37.6 & 38.7 & 30 & 64 & 10 & 36.1 & 37.1 & 27 & Q4 \\
\hline 12 & 56.2 & 57.1 & 5 & 42 & 12 & 55.8 & 58.8 & 230 & 6 & I2 & 34.6 & 35.2 & 24 & 3. & 12 & 36.0 & 37.0 & 27 & bi \\
\hline I4 & 54.8 & 55.2 & & $-4 \cdot 0$ & 14 & 52.0 & 55.7 & 225 & -3.6 & 14 & 39.5 & 40.6 & 32 & 64 & 14 & 36.0 & 36.9 & & -2.2 \\
\hline 16 & 51.9 & 52.3 & & & I6 & 54.2 & 57.2 & 5 & 4 & I6 & 38.5 & 39.0 & 30 & -2.7 & I6 & 37.1 & 37 & 28 & \\
\hline I8 & 50.7 & 51.4 & & (1) & I8 & 56.0 & 58.2 & 5 & 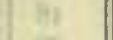 & 18 & 36.9 & 37.5 & 28 & $81^{\circ}$ & I8 & 37.5 & 38. I & & at \\
\hline 20 & 50.9 & $52 . I$ & & tan & 20 & 54.0 & 56.1 & 3 & 15 & 20 & $37 \cdot 3$ & 37.6 & 28 & 6 & 20 & 37.7 & 38.2 & 29 & as \\
\hline 22 & 50.9 & 52.1 & & 11 & 22 & 54.2 & 55.0 & 8 & at & 22 & 40.6 & 40.6 & 34 & $=$ & 22 & 38.8 & 39.0 & 0. $3 \mathrm{I}$ & ats \\
\hline 24 & 48.8 & 49. I & & 18 & 24 & 53.2 & 54.0 & 5 & 36 & 24 & 42.3 & 42.9 & 37 & 18 & 24 & 39.3 & 39 & 32 & 14 \\
\hline 26 & 49. I & 49.8 & & 15 & 26 & 48.9 & 49.9 & 4 & 9 & 26 & 38.5 & 39.2 & $3 \mathrm{I}$ & $=$ & 26 & 39.6 & 39 & 32 & 8 \\
\hline 28 & 49.7 & 50.0 & 4 & $\sqrt{3}$ & 28 & 47.7 & 49.0 & 4 & Bs: & 28 & 39.7 & 39.9 & 32 & R2 & 28 & 38.6 & 38.9 & 30 & fis \\
\hline 30 & 51.2 & 52.6 & 225 & -4.0 & 30 & 49.3 & 51.8 & 4 & -3.4 & 30 & $4 \mathrm{I} .4$ & 42.2 & 35 & -2.5 & 30 & 37.6 & & & -2.3 \\
\hline 32 & 57.2 & 58.0 & 230 & & 32 & $47 . \mathrm{I}$ & 48.9 & 20. 4 & 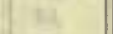 & 32 & 40.0 & 40.9 & 33 & tis & 32 & 37.8 & 38.2 & & at) \\
\hline 34 & 51.0 & 51.9 & 225 & 22 & 34 & 48.6 & 50.0 & 4 & R & 34 & 38.8 & $40 . \bar{I}$ & 32 & A & 34 & 37.3 & 37.7 & & H \\
\hline 36 & 54.2 & 55.8 & 225 & 28 & 36 & 49.0 & 50.0 & 4 & tat & 36 & 38.2 & 39.1 & 30 & 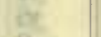 & 36 & 36.9 & 37. & & ex \\
\hline $3^{8}$ & 57.8 & 58.2 & 230 & 88 & 38 & 50.8 & 51.6 & 5 & 82 & 38 & 40.8 & $4 \mathrm{I} .7$ & 34 & 92 & 38 & 36.7 & & 28 & $\gamma_{8}$ \\
\hline 40 & 63.9 & 65.2 & I & 8 & 40 & 49.8 & 51.0 & 4 & 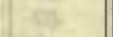 & 40 & $40 . I$ & 40.2 & 33 & (4) & 40 & 36.1 & 36.4 & 26 & 68 \\
\hline 42 & 61.7 & 63.2 & 0 & 3. & 42 & 50.3 & 50.7 & 4 & 35 & 42 & 36.4 & 36.4 & 127 & 6 & 42 & 35.7 & 35 & 26 & 45 \\
\hline 44 & 61.3 & 63.3 & 0 & -4.0 & 44 & 51.2 & 52.3 & 75 & -3.4 & 44 & 33.2 & 33.8 & 22 & -2.6 & 44 & 35.7 & 35.9 & & -2.2 \\
\hline 46 & 63.7 & 64.2 & I & 8 & 46 & 47.9 & 48.0 & 4 & 14 & 46 & 40.0 & 40.0 & 32 & 5 & 46 & 36.2 & 36 & 27 & 9 \\
\hline 48 & 62.6 & 63.8 & if & 16 & 48 & 50.1 & 51.2 & 4 & 86 & 48 & 41.8 & 42.3 & 36 & 16. & 48 & 35.8 & 36.2 & & Fis \\
\hline 50 & 63.0 & 64.9 & 2 & 69 & 50 & 46.7 & 48.2 & 4 & $\mathrm{e}$ & 50 & 38.0 & 39.2 & 30 & 65 & 50 & 36.5 & 36.7 & 27 & vet \\
\hline 52 & 61.1 & $63 . \mathrm{I}$ & 0 & 92 & 52 & 46.7 & 49.0 & 2. 4 & $\mathrm{R}$ & 52 & 36.1 & 36.3 & 26 & 18 & 52 & $37 \cdot 5$ & 37.6 & 8.29 & $\div$ \\
\hline 54 & 62.2 & 64.6 & 6 & 83 & 54 & 47.8 & 50.1 & 4 & 13 & 54 & 35.8 & 36.5 & 26 & 12 & 54 & 37.8 & 38.0 & 29 & 3 \\
\hline 56 & 63.2 & 63.9 & 0 & as & 56 & 47.3 & 48.8 & 4 & 8 & 56 & 36.0 & 36.4 & 26 & 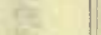 & 56 & 38.0 & 38.2 & 30 & 8 \\
\hline 58 & 55.5 & 59.7 & 0 & 8 & 58 & $48 . I$ & 50.0 & 4 & (A3) & 58 & 37.0 & 37.2 & 28 & Wat & 58 & 38.2 & 38.4 & 30 & $t^{2}$ \\
\hline
\end{tabular}

Observers-W. J. P. and R. R. T. alternated from 4 h $18 \mathrm{~m}$ to $4^{\text {h }} 34 \mathrm{tn}$. R. R. T. and R. W. P. alternated from 7 h $52 \mathrm{~m}$ to $8 \mathrm{~h} 08 \mathrm{~m}$.
Observers-R. W. P. (R. R. T. observed readings from $8 \mathrm{~h} 32 \mathrm{~m}$ to gh $06 \mathrm{~m}$.) 
Tabulation of magnetic declinations observed at Teplitz Bav-Continued

\begin{tabular}{|c|c|c|c|c|c|c|c|c|c|c|c|c|c|c|c|c|c|c|c|}
\hline \multicolumn{5}{|c|}{ Weduesday, October 28, 1903} & \multicolumn{5}{|c|}{ Magnet scale erect } & \multicolumn{5}{|c|}{ Wednesday, October 2S, I903 } & \multicolumn{5}{|c|}{ Magnet scale ercet } \\
\hline \multirow{2}{*}{$\begin{array}{l}\text { Chr't } \\
\text { time }\end{array}$} & \multicolumn{2}{|c|}{$\begin{array}{l}\text { Scale } \\
\text { readings }\end{array}$} & \multirow{2}{*}{$\begin{array}{l}\text { East } \\
\text { decli- } \\
\text { nation }\end{array}$} & Temp. & Chr'r & $\begin{array}{r}\text { So } \\
\text { read }\end{array}$ & $\begin{array}{l}\text { ale } \\
\text { lings }\end{array}$ & East & 'Temp. & Chr'r & $\begin{array}{r}\text { Se } \\
\text { reac }\end{array}$ & igs & $\begin{array}{l}\text { East } \\
\text { decli- }\end{array}$ & Temp. & Chr'r & $\begin{array}{r}\text { So } \\
\text { reac }\end{array}$ & le & $\begin{array}{l}\text { East } \\
\text { decli- }\end{array}$ & Temp. \\
\hline & Ieft & Right & & & & I,eft & Right & & & & Left & Right & & & & Left & Right & nation & \\
\hline $\mathrm{h} \mathrm{m}$ & d & d & ० ' & $\bullet$ & h m & d & $\mathrm{d}$ & & • & h $\mathrm{m}$ & $\mathrm{d}$ & d & & • & h m & $d$ & d & & • \\
\hline 1200 & 37.1 & 37.2 & 2228 & -2.2 & 1400 & $34 \cdot 3$ & 35.7 & 222 & -1.5 & 1600 & 31.4 & 32.4 & 2220 & -1.7 & I8 00 & 31.0 & 31.7 & 2219 & -1.2 \\
\hline 02 & 36.2 & 36.8 & 27 & & 02 & 34.8 & 35.8 & & & 02 & 31.8 & 32.9 & 20 & & 02 & 30.7 & $3 I \cdot 3$ & 18 & \\
\hline 04 & 35.9 & 36.9 & 27 & (12) & 04 & 33.8 & 35.1 & 2 & & 04 & 32.9 & 33.8 & 22 & 34 & 04 & 29.6 & 30.2 & 17 & \\
\hline 06 & 36.6 & 37.0 & 28 & 2 & 06 & 33.6 & 34.9 & 2 & & 06 & 33.6 & 34.4 & 23 & 8 & 06 & 28.8 & 29.4 & I 5 & \\
\hline 08 & 38.0 & 38.7 & 30 & at: & 08 & $33 \cdot 5$ & 34.7 & 2 & nt & 08 & 35.0 & 35.9 & 25 & the & 08 & 26.5 & 27.0 & 12 & \\
\hline 10 & 36.0 & 36.9 & 27 & in & I0 & 33.0 & $34 \cdot 3$ & 22 & wis & IO & 34.5 & 35.9 & 25 & 21 & 10 & 23.8 & 24.2 & 07 & \\
\hline 12 & 35.5 & 36.8 & 26 & $=$ & 12 & 34.6 & 35.0 & 2 & & I2 & 34.9 & 36.0 & 25 & $x^{2}$ & 12 & 22.3 & 24.2 & 06 & \\
\hline 14 & 36.8 & 37.8 & 28 & -2.2 & 14 & 34.5 & 36.7 & 26 & -1.6 & 14 & 34.8 & 36.8 & 26 & $-1 \cdot 3$ & 14 & 23.2 & 25.0 & 08 & -1.2 \\
\hline I6́ & 38.0 & 39.2 & 30 & & 16 & 32.6 & 33.6 & 22 & & 16 & 35.9 & 37.4 & 27 & & 16 & 23.9 & 25.3 & 08 & \\
\hline 18 & 36.4 & 36.9 & 27 & Elif & 18 & 32.4 & 33.0 & 2 & 3 & I8 & 36.0 & 37.8 & 28 & h. & 18 & 23.8 & 25.4 & 08 & \\
\hline 20 & 36.8 & 38.0 & 28 & 65 & 20 & 31.5 & 32.2 & 20 & $=$ & 20 & 36.0 & 38.0 & 28 & 106 & 20 & 25.4 & 27.2 & II & \\
\hline 22 & 46.3 & 47.2 & 43 & 4 & 22 & 32.5 & 33.0 & 21 & 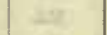 & 22 & 35.5 & 37.4 & 27 & & 22 & 26.4 & 29.1 & 13 & \\
\hline 24 & 38.5 & 38.9 & 30 & In & 24 & $3 \mathrm{r} .8$ & 32.4 & 20 & 14 & 24 & 35.5 & 37.2 & 27 & & 24 & 27.8 & 30.8 & 16 & \\
\hline 26 & 30.5 & 30.9 & & & 26 & 32.6 & 33.6 & 22 & isc & 26 & 34.7 & 36. & 25 & & 26 & 29.0 & 32.0 & 18 & \\
\hline 28 & 24.5 & 25.0 & 08 & 2a & 28 & 32.5 & 33.3 & 21 & 3yin & 28 & 33.9 & 36.0 & 24 & & 28 & 32.0 & 34.9 & 22 & \\
\hline 30 & 23.8 & 24.6 & 08 & -1.5 & 30 & 32.3 & 33.0 & 2. & -1.6 & 30 & 34.8 & 36.3 & 25 & -1.2 & 30 & 33.0 & 35.3 & 23 & -1.3 \\
\hline 32 & 27.1 & 28.3 & 13 & $24 y^{2}$ & 32 & 32.3 & 33.4 & 21 & 12 & 32 & 35.5 & 36.8 & 26 & & 32 & 34.3 & 37.0 & 26 & \\
\hline 34 & 29.3 & 30.7 & 17 & wis & 34 & 33.5 & 34.1 & 2 & $y$ & 34 & 36.1 & 36.9 & 27 & ta & 34 & 34.2 & 36.9 & 25 & \\
\hline 36 & 29.6 & 31.3 & 17 & $t^{t}$ & 36.2 & 33.5 & 34.2 & 2 & 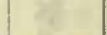 & 36 & 36.0 & 36.9 & 27 & 8 & 36 & 35.9 & 38.2 & 28 & \\
\hline $3^{8}$ & 3I. 8 & 32.8 & 20 & 8. & 38 & 32.6 & 33.2 & 2 & 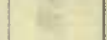 & 38 & 35.5 & 36.3 & 26 & se & 38 & 36.1 & 37.9 & 28 & \\
\hline 40 & 32.5 & 33.8 & 22 & ar & 40 & 32.9 & 33.4 & 2 & 4 & 40 & 35.2 & 36.3 & 26 & fit & 40 & 36.2 & 37 & 28 & \\
\hline 42 & 37.8 & 39.2 & 30 & in & 42 & 33.2 & 33.8 & 2 & & 42 & 34.9 & 35.7 & 25 & & 42 & 37.0 & 38.3 & 29 & \\
\hline 44 & 31.0 & 32.6 & 20 & -1.5 & 44 & 33.8 & 34.3 & 23 & -1.8 & 44 & 34.7 & 35.7 & 25 & -1.2 & 44 & 37.2 & 38.7 & 29 & -1.2 \\
\hline 46 & 32.2 & 33.5 & $2 \mathrm{I}$ & & 46 & 33.8 & 34.4 & 2 & 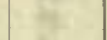 & 46 & 35.0 & 35.7 & 25 & & 46 & 40.2 & $41 \cdot 3$ & 34 & \\
\hline 48 & 31.9 & 33.4 & 2 I & 4 & 48 & 33.2 & 33.6 & 2 & 4 & 48 & $34 \cdot 3$ & 35 . I & 24 & & 48 & $4 \mathrm{I} . \mathrm{I}$ & 42.7 & 5 & \\
\hline 50 & 33.0 & 33.9 & 22 & W & 50 & 32.6 & 33.3 & 21 & ftil & 50 & 34.0 & 35.2 & 24 & (6) & 50 & 40.8 & 42.1 & & \\
\hline 52 & 32.9 & 33.8 & 22 & 21 & 52 & 31.6 & 32.2 & 2 & $\frac{1}{8}$ & 52 & 34.0 & 35.2 & 24 & a & 52 & 39.2 & 40.8 & 32 & \\
\hline 54 & 32.1 & 33.8 & $2 \mathrm{I}$ & 17 & 54 & 30.4 & 31.0 & I & $x$ & 54 & 34.2 & 35.2 & 24 & t & 54 & $4 \mathrm{I} .0$ & 42.2 & 5 & \\
\hline 56 & 31.9 & 34.3 & 22 & 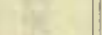 & 56 & $3 \mathrm{I} .0$ & 31.6 & I & 2 & 56 & 34.0 & 34.7 & 24 & te & 56 & 40.3 & $4 \mathrm{~T}$. & 34 & \\
\hline 58 & $3 \mathrm{I} .3$ & 33.5 & 20 & the & 58 & 32.8 & 33.1 & 2 & $x^{2}=$ & 58 & 3.3 .8 & 34.2 & 23 & $=$ & 58 & 36.2 & 38 & 8 & \\
\hline 1300 & $3 \mathrm{I} .4$ & 34.3 & 21 & -1.5 & 1500 & 31.8 & 32.4 & 2 & -2.0 & 1700 & 33.1 & 3.8 & 22 & $-\mathrm{I} .2$ & I9 00 & 34.2 & 35.2 & 24 & -1.2 \\
\hline 02 & 33.5 & 35.0 & 23 & 16. & 02 & $3 \mathrm{I} .4$ & 31.9 & I & $w^{2}$ & 02 & 33.2 & 33.8 & 22 & 39 & 02 & 34.1 & 34 & 24 & \\
\hline 04 & 33.5 & 35.2 & 24 & 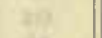 & 04 & 30.7 & 31.2 & I & at? & 04 & 33.0 & 33.8 & 22 & 10. & 04 & 34.2 & 35.8 & 25 & 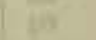 \\
\hline 06 & 32.8 & $34 \cdot 3$ & 22 & ins & 06 & 30.6 & 31.3 & I & 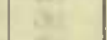 & 06 & 33.2 & 34.3 & 23 & tw & 06 & 34.1 & 35.7 & 24 & \\
\hline 08 & 33.0 & 34.0 & 22 & 50 & 08 & 29.8 & 30.4 & I & $8 y$ & 08 & 33.9 & 35.0 & 24 & l. & 08 & 32.9 & 33.1 & & \\
\hline I0 & 33.2 & 34.3 & 23 & 801 & 10 & 30.6 & 31.2 & I) & t & IO & 34 . I & $35 \cdot 3$ & 24 & 8 & I0 & 30.1 & 32.1 & 9 & \\
\hline I2 & 34.8 & 35.5 & 25 & 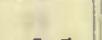 & 12 & 30.3 & 31.0 & I & 145 & I & & 35.9 & 25 & $1=$ & 12 & 30.2 & & & \\
\hline 14 & 34.3 & 34.9 & 24 & -1.5 & 14 & 30. I & 30.9 & I & -1.9 & 14 & 34.2 & 35.8 & 25 & $-1 \cdot 3$ & 14 & 3I.I & 32.5 & 20 & -1.2 \\
\hline I6 & 33.5 & $34 \cdot 3$ & 23 & & I6 & $3 \mathrm{I} .5$ & 31.9 & I & 10 & It & & & & & 16 & $3 \mathrm{I} .2$ & 32 & 19 & \\
\hline 18 & 32.1 & 32.6 & 20 & it & 18 & 31.6 & 32.0 & 2 & a & I8 & 36.8 & 37.2 & 28 & हो। & 18 & 29.0 & 31 & 17 & \\
\hline 20 & 33.6 & 33.8 & 23 & unt & 20 & 29.5 & 30.3 & I & 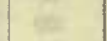 & 20 & 35.6 & 36.8 & 26 & $=$ & 20 & 29.0 & 30.4 & 16 & \\
\hline 22 & 34.0 & 34.5 & 23 & $=8$ & 22 & 29.7 & 30.3 & I & 10 & 22 & 35.0 & 35.9 & 25 & 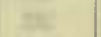 & 22 & 29.3 & 3 I. .2 & I7 & \\
\hline 24 & 34.9 & 34.9 & 24 & $y^{2}$ & 24 & 30.3 & 30. & I & 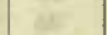 & 24 & 33.8 & 34.8 & 24 & $x$ & 24 & 30.7 & 32.1 & I0 & \\
\hline 26 & 34.3 & $34 \cdot 3$ & 24 & 16 & 26 & 29.3 & 30.0 & I & in & 26 & 32.3 & 33.6 & $2 \mathrm{I}$ & $1=$ & 26 & 30.9 & 32.1 & 19 & \\
\hline 28 & 33.8 & 34.2 & 23 & 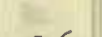 & 28 & 30.2 & $3 \mathrm{I}$. I & I & & 28 & 31.8 & 31.9 & 20 & $-1 \cdot 3$ & 28 & 30.1 & 32. I & IO & \\
\hline 30 & 35.2 & 35.6 & 25 & -1.6 & 30 & 30.5 & $3 \mathrm{I} .2$ & I & -1.9 & 30 & 30.9 & 32.1 & I9 & & 30 & 28.9 & 30.2 & 16 & -1.3 \\
\hline 32 & 36.0 & 36.5 & 26 & 64 & 32 & 30.8 & 31.6 & I & $\cdots$ & 32 & 31.0 & 32.1 & I9 & 2 & 32 & 32.5 & 32.8 & $2 \mathrm{I}$ & \\
\hline 34 & 36.8 & 36.8 & 28 & ke & 34 & 30.5 & $3 \mathrm{I} .2$ & I & H. & 34 & 30.8 & 32.0 & 19 & & & 31.8 & 32.6 & 20 & \\
\hline 36 & 35.0 & 35.6 & 25 & & 36 & 31.6 & 32.5 & 2 & es: & 3 & 31.0 & 32.2 & 19 & & 3 & 32.0 & 32.8 & 20 & 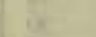 \\
\hline 38 & 34.3 & 34.6 & 24 & $x^{2}$ & 38 & 31.0 & 31.8 & I & f. & 38 & 31.7 & 32.3 & 20 & 8 & 38 & 30.8 & 31.8 & I9 & 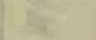 \\
\hline 40 & 34.3 & 34.5 & 24 & 64 & 40 & $3 \mathrm{I} .2$ & 31.5 & I & $\Leftrightarrow$ & 40 & 31.8 & 32.9 & 20 & 10 & 40 & 28.7 & 29.7 & 16 & \\
\hline 42 & $33 \cdot 3$ & 33.8 & 22 & & 42 & 32.3 & 32.8 & 2 & & 42 & $3 I . I$ & 32.7 & 20 & $x$ & 42 & 28. I & 29.2 & 15 & \\
\hline 44 & 32.3 & 32.8 & 21 & -1.6 & 44 & 32.3 & 32.8 & 2 & -1.9 & 44 & 30.4 & 31.9 & 19 & $-1 \cdot 3$ & & 27.2 & $28 . \mathrm{I}$ & 13 & -1.4 \\
\hline 46 & $31 \cdot 3$ & 31.6 & I9 & & 46 & $3 \mathrm{I} .8$ & 32.2 & 2 & & 46 & 30.7 & 31.9 & 19 & & 46 & 26.5 & 27.8 & 12 & \\
\hline 48 & 30.2 & 30.6 & 17 & 2 & 48 & 30.8 & 31.2 & I & at & 48 & 30.4 & 32.0 & 19 & 8 & 48 & 25.8 & 27.0 & II & \\
\hline 50 & 32.8 & 33.6 & 22 & 2 & 50 & $3 \mathrm{I} .3$ & 31.9 & I & as & 50 & 30.2 & 31.0 & I8 & mi & 50 & 26.2 & 27.4 & 12 & \\
\hline 52 & 31.8 & 32.6 & 20 & a & 52 & 31.8 & 32.0 & 2 & 25 & 52 & 30.5 & 31.2 & I8 & 단 & 52 & 26.9 & 28.5 & 13 & \\
\hline 54 & 31.8 & 33.2 & $2 I$ & 12 & 54 & $3 \mathrm{I} \cdot 5$ & 32.1 & 2 & 32 & 54 & 30.9 & 31.6 & I9 & 42 & 54 & 27.4 & 29.2 & I4 & \\
\hline 56 & 31.9 & 33.2 & 2I & 28 & 56 & 31.9 & 32.2 & 2 & के & 56 & 30.6 & $3 \mathrm{I} .2$ & 18 & ist & 56 & 27.9 & 29.1 & 14 & \\
\hline 58 & 33.8 & 35.4 & 24 & the & 58 & $3 \mathrm{I} .5$ & 31.9 & I & & 58 & 31.2 & 31.8 & I9 & 4 & 58 & 27.7 & $29 . I$ & 14 & hit \\
\hline
\end{tabular}

Observers-R. W. P. and W. J. P., who alternated from I2lı $04 \mathrm{n}$ Observers - R. R. T. and R. W. P., who alternated from Igh 48 m

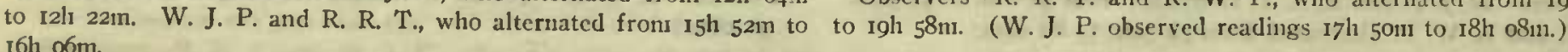
$16 \mathrm{~h} 06 \mathrm{~m}$. 
Tabulation of magnetic declinations observed at Teplitz Bay-Continued

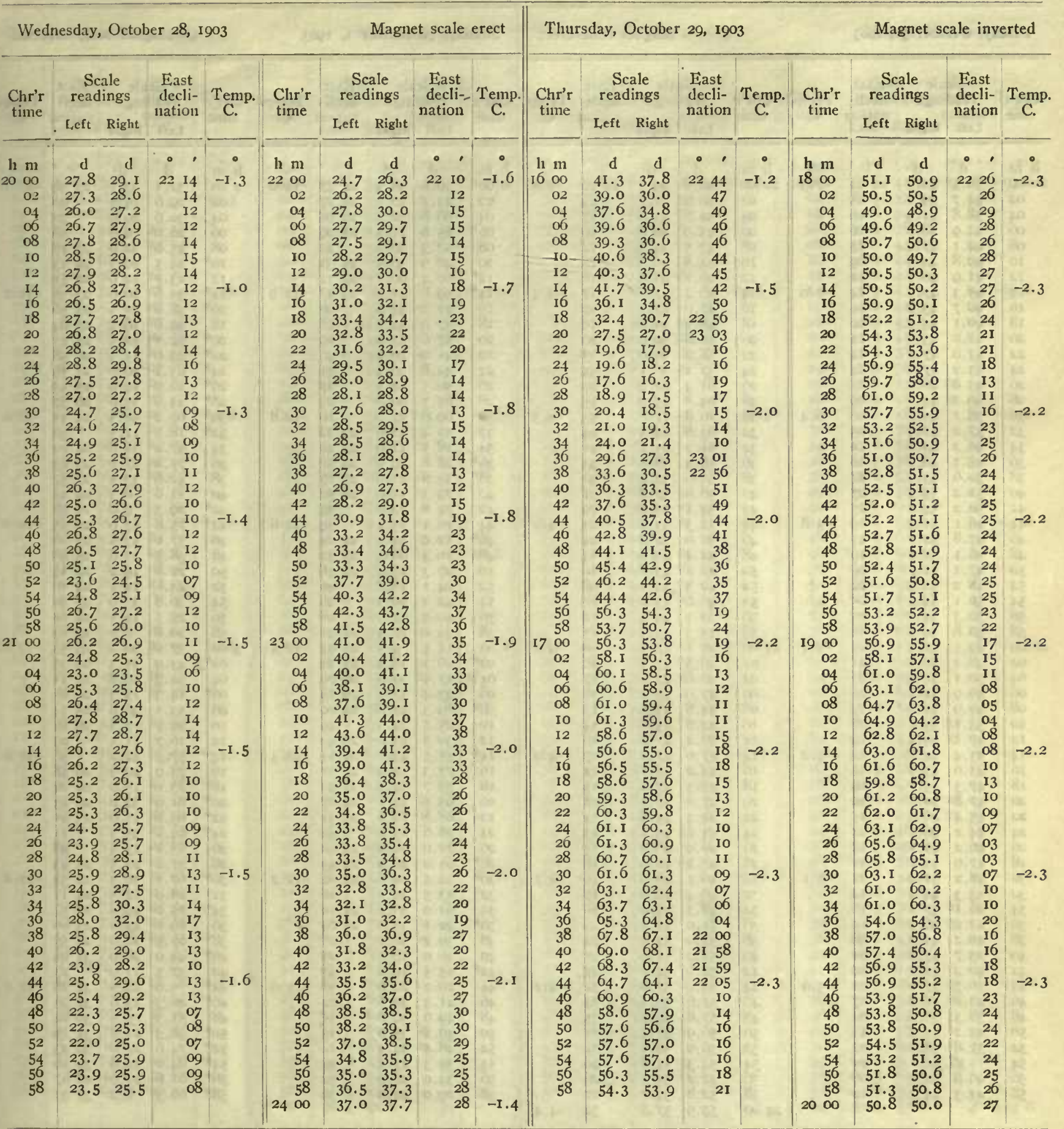

Correction to local mean time is $+\operatorname{Im} 58 \mathrm{~s}$.

Torsion head at oh oom read $339^{\circ}$ and at the end read the same. Observer-R. W. P.
Correction to local mean time is $+2 \mathrm{~m}$ 11s. $90^{\circ}$ torsion $=22 .^{\prime} 4$.

Torsion head at oh $22 \mathrm{~m}$ read $339^{\circ}$ and at $20 \mathrm{~h} 47 \mathrm{~m}$ read $34^{\circ}$.

Observers-W. J. P. and R. R. T., who alternated from $18 \mathrm{~h} 16 \mathrm{~m}$ to I8h 30m. 
Tabulation of magnetic declinations observed at Teplitz Bay-Continued

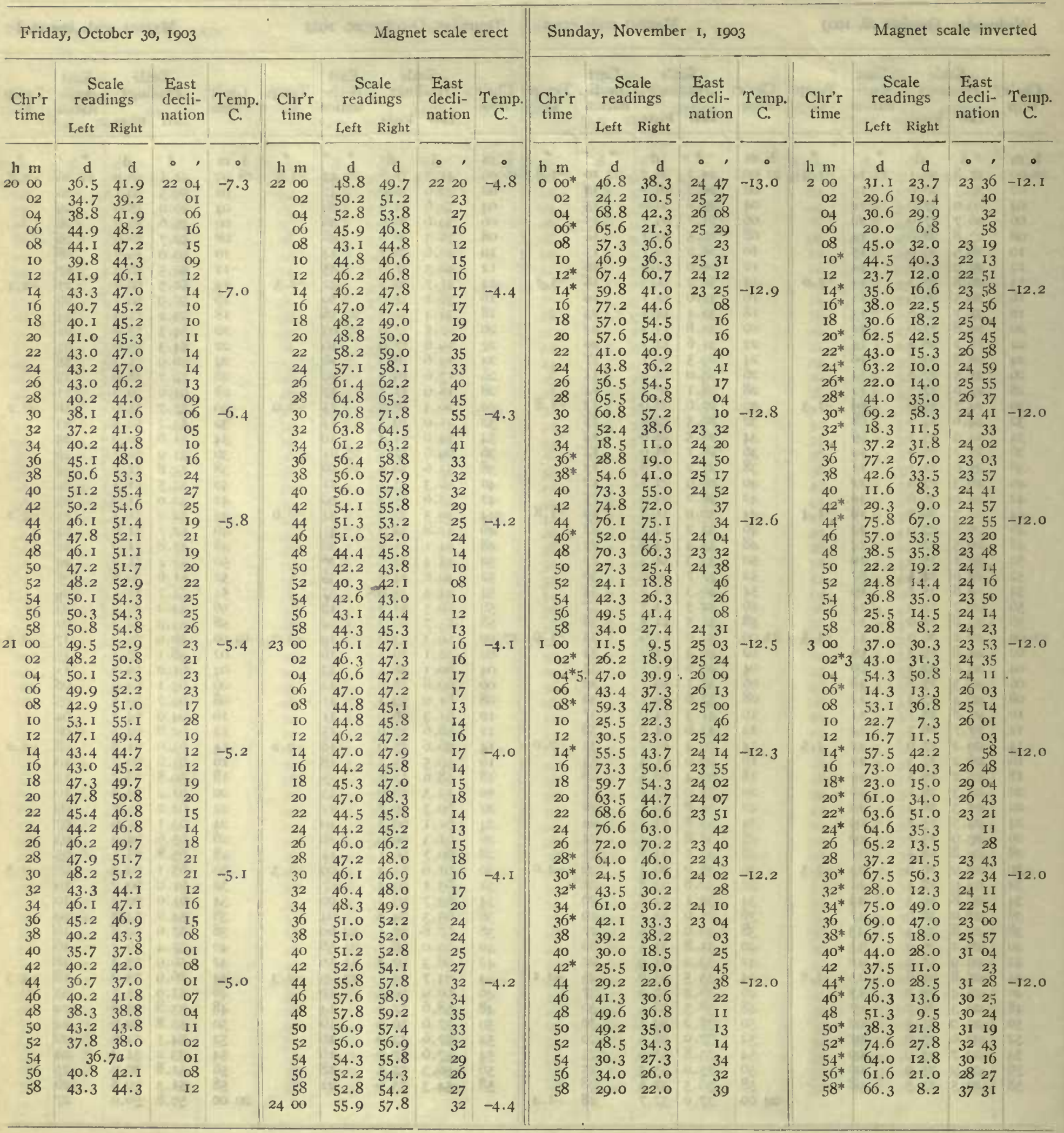


Tabulation of magnetic declinations observed at Teplitz Bay-Continued

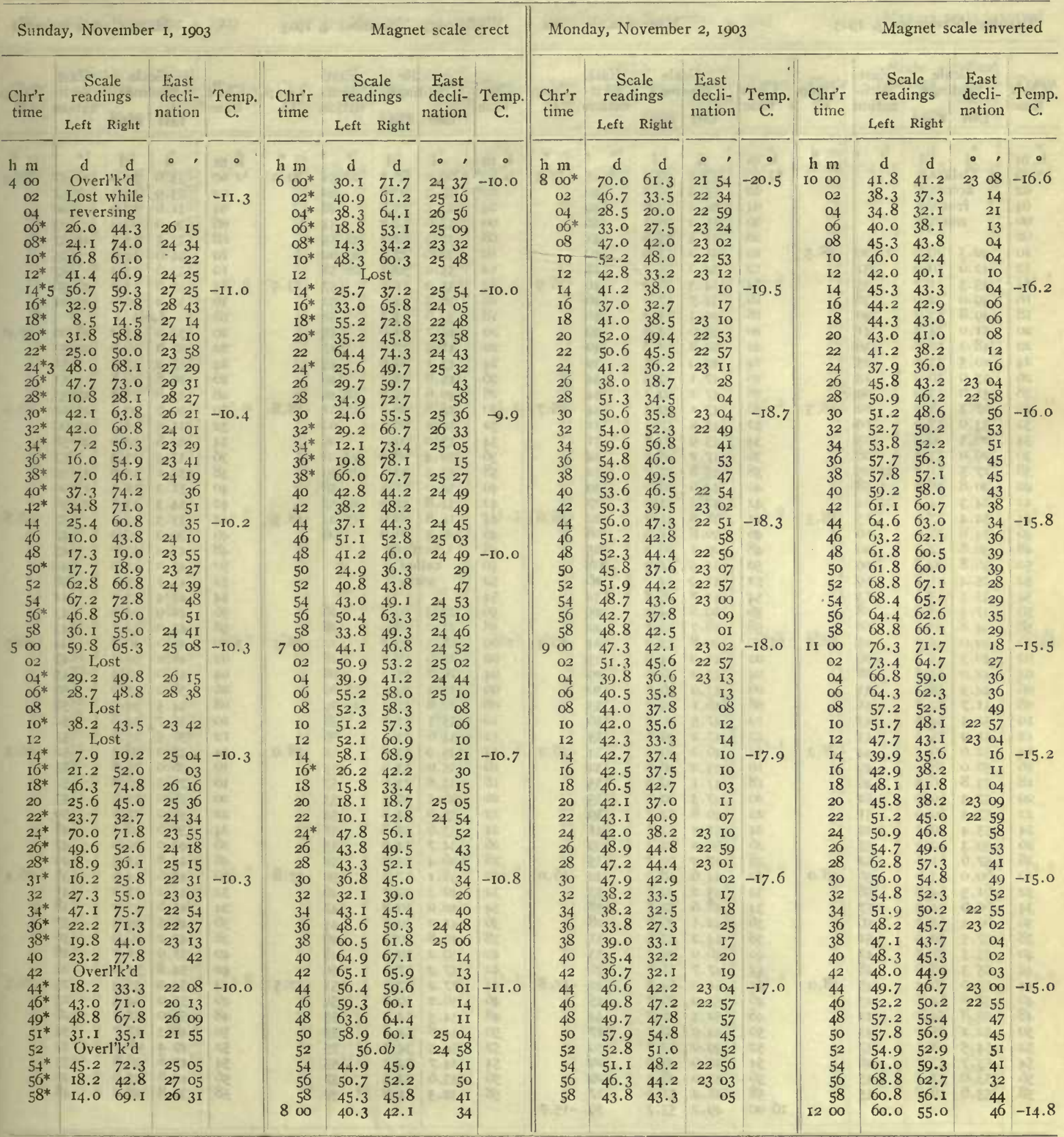

Correction to local mean time is $+2 \mathrm{~m} 36 \mathrm{~s} .90^{\circ}$ torsion $=24 .^{\prime} 5$.

Torsion head at $23 \mathrm{~h} 3 \mathrm{om}$, Octoher $3 \mathrm{I}$, rcad $34^{\circ}$ and at $91 \mathrm{l} 19 \mathrm{~m}$, November I, read $\mathrm{I}^{\circ}$.

Observers-W. J. P. and R. R. T., who alternated from 3 h $58 \mathrm{in}$ to $4 \mathrm{~h} 18 \mathrm{~m}$.
Correction to local mean time is $+2 \mathrm{~m} 38 \mathrm{~s}$. $90^{\circ}$ torsion $=24 .^{\prime} \mathrm{o}$.

Torsion head at $7 \mathrm{~h} 401 \mathrm{n}$ read $339^{\circ}$ and at $12 \mathrm{l} 29 \mathrm{~m}$ read $321^{\circ}$.

Observers-W. J. P. and R. R. T., who alternated from gh $22 \mathrm{~m}$ to glı $36 \mathrm{ni}$. 


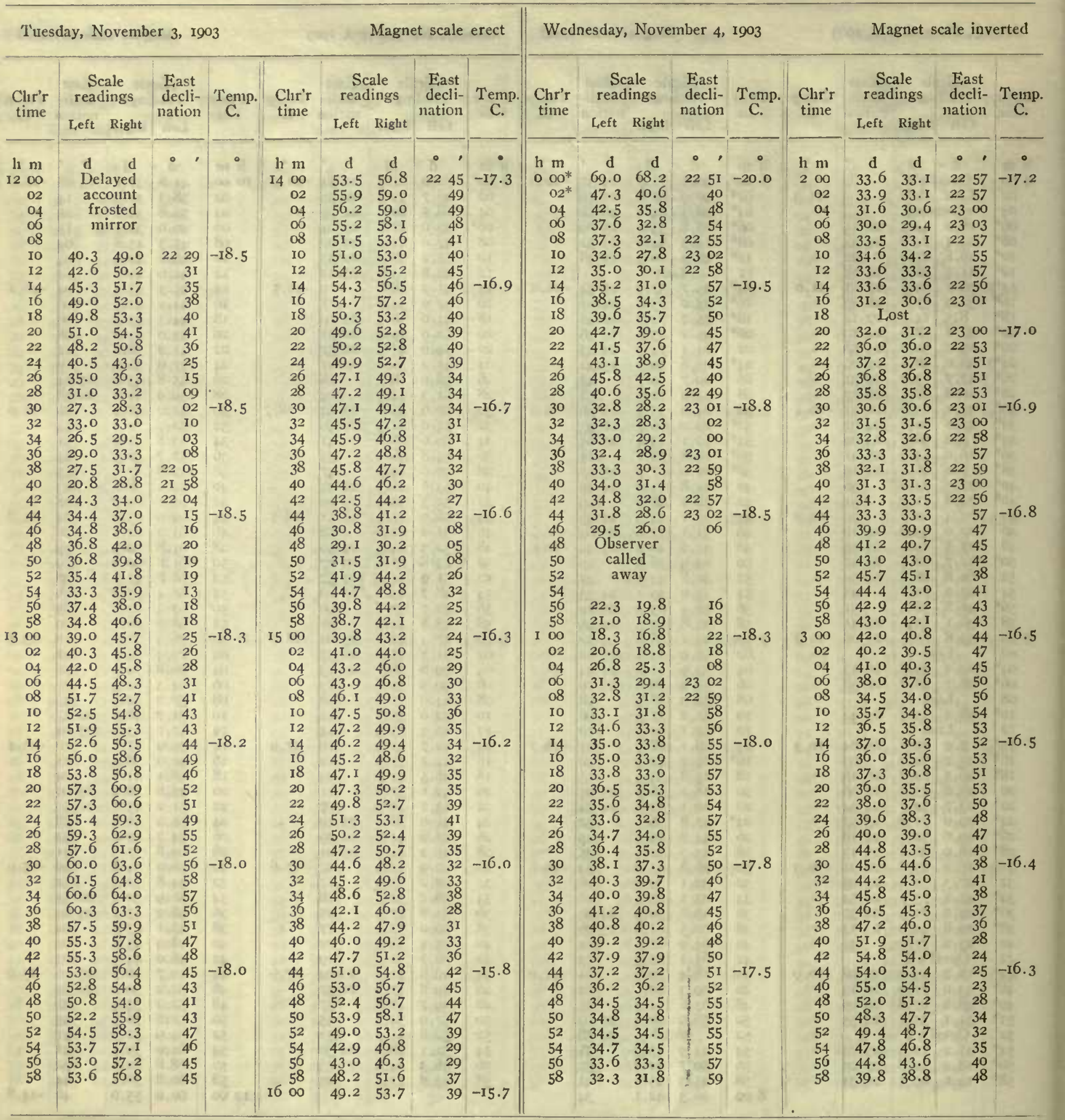

Correction to local mean time is $+2 \mathrm{~m} 37 \mathrm{~s}$.

Torsion head at II $44 \mathrm{~m}$ read $315^{\circ}$ and at $16 \mathrm{~h}$ 3om read the same.

Observers-W. J. P. and R. R. T., who alternated from 13 h $46 \mathrm{~m}$ to
Observers-W. J. P. and R. R. 'T., who alternated from 3 h $56 \mathrm{~m}$ to 4h rom.

$14 \mathrm{~h}$ oom. 
Tabulation of magnetic declinations observed at Teplitz Bay-Continued

\begin{tabular}{|c|c|c|c|c|c|c|c|c|c|c|c|c|c|c|c|c|c|c|c|c|c|}
\hline \multicolumn{5}{|c|}{ Wednesday, November 4, I903 } & \multicolumn{6}{|c|}{ Magnet scale inverted } & \multicolumn{5}{|c|}{ Wednesday, November 4, 1903} & \multicolumn{6}{|c|}{ Magnet scale inverted } \\
\hline \multirow{2}{*}{$\begin{array}{l}\text { Chr'r } \\
\text { time }\end{array}$} & \multicolumn{2}{|c|}{$\begin{array}{l}\text { Scale } \\
\text { readings }\end{array}$} & East & Temp. & Chr'r & $\begin{array}{r}\mathrm{Sc} \\
\text { read }\end{array}$ & $\begin{array}{l}\text { ale } \\
\text { lings }\end{array}$ & Eas & $\begin{array}{l}\text { ast } \\
\text { cli- }\end{array}$ & Temp. & Chr'r & $\begin{array}{l}\text { Sc } \\
\text { readi }\end{array}$ & $\begin{array}{l}\text { ale } \\
\text { ings }\end{array}$ & $\begin{array}{l}\text { East } \\
\text { decli- }\end{array}$ & Temp. & Chr'r & $\begin{array}{r}\mathrm{Sc} \\
\mathrm{read}\end{array}$ & 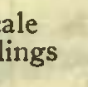 & $\begin{array}{l}\text { Eas } \\
\text { decl } \\
\text { nati }\end{array}$ & & Temp. \\
\hline & L,eft & Right & & & & Left & Right & & & & & Left & Right & latron & & & L,eft & Right & & & \\
\hline$h \mathrm{~m}$ & d & d & $\circ \quad$ & $\circ$ & h m & d & d & $\bullet$ & , & $\circ$ & $\mathrm{h} \mathrm{m}$ & $\mathrm{d}$ & d & $\circ$, & $\bullet$ & $\mathrm{h} \mathrm{m}$ & $\mathrm{d}$ & d & $\cdot$ & , & 0 \\
\hline 400 & 37.1 & 35.9 & 2252 & $-16 . I$ & 600 & 22.6 & 22.3 & 231 & 14 & -15.4 & 800 & 44.0 & 39.6 & 2244 & II $5 . I$ & 1000 & 41.0 & 37.8 & 224 & 47 & -15.2 \\
\hline 02 & 39.8 & 34.8 & $5 \mathrm{I}$ & $1=y^{2}$ & 02 & 22.8 & 22.0 & & 14 & & 02 & 42.8 & 38.8 & 45 & $x^{-2}$ & 02 & 48.6 & 43.8 & & 37 & s \\
\hline 04 & 39.2 & 33.9 & 52 & 79 & 04 & 26.0 & 25.0 & & 09 & 10 & 04 & 43.8 & 40.0 & 44 & 1 & 04 & 42.0 & 36.2 & & 48 & 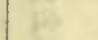 \\
\hline 06 & 37.2 & 32.2 & 55 & $\varphi^{\circ}$ & 06 & 27.8 & 27.0 & 23 & 06 & (t) & 06 & 43.8 & $4 \mathrm{I} .2$ & 43 & +4 & 06 & $40 . I$ & 33.8 & & 51 & \\
\hline as & 34.8 & 30.3 & 58 & set & 08 & 33.2 & 32.2 & 22 & 58 & 3 & 08 & $4 \mathrm{I} .2$ & 38.8 & 46 & 6 & 08 & 35.9 & 30.4 & & 57 & . \\
\hline 10 & $35 . I$ & 31.0 & 57 & int & 10 & 31.0 & 29.0 & 23 & 02 & Ent & Io & 43.4 & 40.8 & 43 & wit & I0 & 37.4 & 33.5 & & 54 & ax \\
\hline 12 & 37.9 & 34.2 & 53 & 1 & 12 & 30.6 & 29.0 & & 02 & 34 & I2 & 42.2 & 40.0 & 45 & tes & 12 & 40.5 & 35.6 & & 50 & \\
\hline 14 & 42.8 & 38.1 & 46 & -16.0 & 14 & 3I.0 & 26.7 & & 04 & -15.4 & I4 & $44 \cdot 3$ & $4 \mathrm{I} .8$ & 42 & -15.2 & 14 & 39.7 & 35.1 & & $5 \mathrm{I}$ & -I $5 . I$ \\
\hline 16 & $44 \cdot 3$ & 39.5 & 44 & $y$ & 16 & 26.2 & 24.0 & & I0 & & 16 & 43.0 & $4 \mathrm{I} . \mathrm{I}$ & 43 & & I6 & 43.8 & 39.0 & & 44 & \\
\hline 18 & $42 . \overline{8}$ & 37.7 & 46 & Her & 18 & 30.8 & 29.0 & $c$ & 02 & 24 & I8 & 45.6 & 43.2 & 40 & (4) & 18 & 48.6 & 45.5 & & 35 & Wr \\
\hline 20 & 43.7 & 39. I & 44 & Ex & 20 & 32.0 & 30.8 & $23 c$ & 00 & 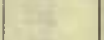 & 20 & 48. I & 46.7 & 35 & is & 20 & 50.8 & 46.0 & & 33 & 46 \\
\hline 22 & 45.9 & 41.0 & $4 \mathrm{I}$ & 10 & 22 & 36.2 & 35.9 & 225 & 53 & $6=$ & 22 & 41.2 & 40.2 & 45 & 68 & 22 & 45.0 & 40.3 & & $4^{2}$ & at \\
\hline 24 & 46.8 & 42.3 & 39 & $P R$ & 24 & 38.2 & 37.8 & & 50 & 65 & 24 & 34.8 & 33.2 & 56 & 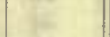 & 24 & 37.0 & 31.6 & & 55 & H \\
\hline 26 & 45.8 & 41.2 & $4 \mathrm{I}$ & $a^{2}$ & 26 & 40.2 & 39.2 & & 47 & (3) & 26 & 35.8 & 34.2 & 54 & 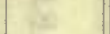 & 26 & 35.0 & 31.9 & & 57 & the \\
\hline 28 & 46. I & 42.2 & 40 & 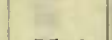 & 28 & 39.2 & 37.3 & & 49 & 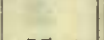 & 28 & 36. I & 33.0 & 55 & -2 & 28 & 38.7 & $35 \cdot 3$ & 225 & $5 \mathrm{I}$ & \\
\hline 30 & $45 \cdot 5$ & 4I. I & $4 I$ & -15.9 & 30 & 33.6 & 32.9 & 225 & 57 & -15.4 & 30 & 36.3 & 35.2 & 53 & -15.2 & 30 & 32.5 & 28.8 & 230 & OI & -15.0 \\
\hline 32 & 43.2 & 39.8 & 44 & & 32.1 & 31.8 & 29.0 & 23 & 02 & & 32 & 40.4 & 34.8 & 50 & 8 & 32 & 32.5 & 29.5 & & OI & \\
\hline 34 & 43.2 & 39.2 & 45 & E & 34 & 34.8 & 32.9 & 225 & 56 & a & 34 & 39.8 & 31.2 & 54 & 41 & 34 & 33.4 & 29.5 & 230 & $\infty$ & W \\
\hline 36 & 44.4 & 40.0 & 43 & 8 & 36 & 42.0 & 40.0 & 4 & 45 & a. & 36 & 42.8 & 37.0 & 47 & as & 36 & 35.3 & $3 \mathrm{I} .2$ & 225 & 57 & \\
\hline 38 & 44.8 & 42.1 & $4 \mathrm{I}$ & iz & 38 & 4 1. 8 & 40.0 & & 45 & 4 & 38 & 34.9 & 3I. I & $22 \quad 57$ & 0 & 38 & 3 I. 6 & 29.0 & 230 & 02 & Itis. \\
\hline 40 & $48 . I$ & 45.3 & 36 & (17) & 40 & 45.8 & 43.2 & & 39 & 217 & 40 & 34.1 & 28.2 & 2300 & 48 & 40 & 33.6 & 29.2 & & $\infty$ & $\omega \theta$ \\
\hline 42 & $47 \cdot I$ & 44.8 & 37 & 10 & 42 & 40.5 & 39.0 & & 47 & 30 & 42 & 38.3 & 32.3 & 2254 & $1=8$ & 42 & 31.0 & 28.6 & & 02 & 6 \\
\hline 44 & 38.8 & 37.2 & 50 & -15.8 & 44 & 36.0 & 34.2 & tet & 54. & $-15 \cdot 3$ & 44 & 42.2 & 37.3 & 47 & -I 4.9 & 44 & 22.0 & 19.4 & & 17 & -14.7 \\
\hline 46 & 36.5 & 34.0 & 54 & & 46 & 39.0 & 37.6 & & 49 & & 46 & 47.9 & 43.8 & 37 & 26 & 46 & 20.7 & I8. I & & I9 & $=0$ \\
\hline 48 & 35.2 & 33.2 & 56 & 10 & 48 & $45 . \mathrm{I}$ & 42.2 & & $4 \mathrm{I}$ & 20 & 48 & 47.4 & 40.8 & 40 & 8 & 48 & 25.3 & 22.8 & & 12 & 16 \\
\hline 50 & 37.0 & 35.8 & $5^{2}$ & 5 & 50 & 47.8 & 46.2 & & 35 & 7 & 50 & 40.0 & 33.9 & $5 \mathrm{I}$ & in & 50 & 32.5 & 29.5 & & OI & 2 \\
\hline 52 & 35.8 & 34.0 & 55 & है & 52 & 40.2 & 38.0 & & 48 & $=$ & $5^{2}$ & 38.4 & 32.7 & 54 & 30 & $5^{2}$ & 31.2 & 28.3 & & 03 & 4 \\
\hline 54 & 33.2 & 31.9 & 2258 & $w$ & 54 & 40.2 & 39.1 & 22 & 47 & "2. & 54 & 42.8 & 38.8 & 45 & WI & 54 & 28.0 & 24.7 & & 08 & We \\
\hline 56 & 29.9 & 29.0 & 2303 & & 56 & 30.8 & 29.0 & 23 & 02 & & 56 & 39.5 & 36.8 & 49 & & 56 & 26.5 & 24.0 & & I0 & \\
\hline 58 & 24.8 & 23.8 & II & & 58 & 35.5 & 32.3 & 22 & 56 & & 58 & 38.6 & 36.2 & $5 \mathrm{I}$ & & 58 & 26.0 & 23.5 & & Io & \\
\hline $5 \infty$ & 21.9 & 21.0 & 2316 & -15.7 & 700 & 33.4 & 28.8 & 23 & $\infty$ & $-15 \cdot 3$ & 900 & 42.8 & 40.3 & 44 & -I5.I & II 0 & 23.0 & 19.3 & & 16 & -14.6 \\
\hline 02 & 34.2 & 33.2 & 2256 & 10 & 02 & 39.0 & 34.0 & 22 & 52 & & 02 & 43.4 & 41.8 & 42 & & 02 & 26.0 & 22.0 & & 12 & (2) \\
\hline 04 & 30.7 & 29.7 & 2302 & An & 04 & 32.8 & 28.0 & 23 & 02 & (11I) & 04 & 44.0 & 41.2 & 42 & 10 & 04 & 29.0 & 25.0 & & 07 & if \\
\hline 06 & $37 . I$ & 35.9 & 2252 & we & 06 & 37.9 & 36.0 & 22 & 5 I & the & 06 & 43.9 & $4 I \cdot 5$ & 42 & the & 06 & 30.1 & 25.0 & 0 & 06 & 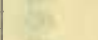 \\
\hline 08 & 38.2 & 37.8 & 50 & 60 & 08 & 4 I. 5 & 37.0 & 22 & 48 & that & 08 & 42.5 & 39.0 & 45 & 158 & 08 & 24.8 & 20.0 & & I 4 & 68 \\
\hline IO & 38.3 & 37.3 & 50 & 34 & Io & 30.2 & 26.0 & 23 & 05 & 54 & 10 & 40.9 & 36.5 & 49 & 3 & 10 & 27.2 & 23.8 & & 09 & or \\
\hline 12 & 36.8 & 36.3 & 52 & eff. & 12 & 37.7 & $3 I .1$ & 22 & 55 & & 12 & 45.9 & 43.5 & 39 & & 12 & 23.9 & 20.4 & 1 & I 5 & 64 \\
\hline 14 & 35.0 & 34.2 & 2255 & $H$ & I4 & 44.6 & 41.2 & & 42 & -15.3 & I4 & 49.0 & $44 . \overline{8}$ & 36 & $-15 . I$ & I4 & 19.3 & 17.0 & & 21 & \\
\hline 16 & 28.0 & 27.1 & 2306 & 22 & I6 & 42.0 & 35.8 & & 48 & & It & 46.2 & 43.8 & 39 & & 16 & 22.9 & 19.6 & & I6 & -14.7 \\
\hline 18 & 30.9 & 30.0 & 2302 & ith & I8 & 39. I & 35.3 & & 5 I & 41 & I 8 & 41.8 & 38.8 & 46 & 2 & I8 & 28.0 & 23.2 & & 09 & in \\
\hline 20 & $33 . \mathrm{I}$ & 32.9 & 2257 & $\cos$ & 20 & 43.2 & $40 . I$ & & 44 & 67 & 20 & 44.8 & 43.5 & 40 & $v e$ & 20 & 25.6 & $2 I . I$ & & 13 & in \\
\hline 22 & 31.8 & 30.7 & 2300 & war & 22 & 35.0 & 31.3 & & 57 & 3t & 22 & 45.7 & 44.7 & 38 & ne & 22 & 18.9 & I 4.9 & & 23 & $y^{2}$ \\
\hline 24 & 36.5 & 35.0 & 2253 & 10 & 24 & 36.0 & 30.8 & & 57 & in & 24 & 51.3 & 49.5 & 30 & $y$ & 24 & 23.9 & 15.8 & & 18 & 4 \\
\hline 26 & 36.5 & 36.0 & 52 & $x$ & 26 & 48.2 & 45.0 & & 36 & $x=$ & 26 & 51.4 & 50.4 & 29 & & 26 & 20.3 & I 3.5 & & 23 & \\
\hline 28 & 35.7 & 34.8 & 54 & & 28 & 40.3 & 32.8 & & 52 & & 28 & $49 . \mathrm{I}$ & $47 . I$ & 34 & & 28 & 17.9 & 10.8 & & 27 & \\
\hline 30 & 34.0 & 33.2 & 2256 & -15.7 & 30 & 34.8 & 29.3 & & 59 & -I 5.2 & 30 & 50.2 & 49.0 & $3 \mathrm{I}$ & -15.0 & 30 & 21.0 & I5.2 & & $2 \mathrm{I}$ & -14.5 \\
\hline 32 & 31.4 & 29.0 & 2302 & 10 & 32 & 55.8 & 52.5 & & 24 & & 32 & 53.5 & 48.0 & 30 & ( & 32 & 24.4 & 19.1 & & 15 & 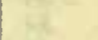 \\
\hline 34 & $30 . I$ & 28.0 & & $\mathrm{k}^{2}$ & 34 & 45.2 & 39.8 & & 42 & & 34 & 53.8 & 52.5 & 26 & II & 34 & 17.0 & 12.0 & & 26 & 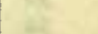 \\
\hline 36 & 33.8 & $3 \mathrm{I} .0$ & $225^{8}$ & $n$ & 36 & 38.9 & 34.0 & & 52 & & 36 & 52.6 & $48 . I$ & 30 & & 36 & 19.0 & I3.1 & & 24 & \\
\hline 38 & 35.4 & $35 . I$ & 54 & Q & 38 & 56.1 & 51.0 & & 25 & & 38 & 48.8 & 44.7 & 36 & = & 38 & 25.1 & 20.9 & & 13 & \\
\hline 40 & 45.0 & 42.3 & $4 \mathrm{I}$ & 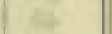 & 40 & 38.9 & 35.2 & & 5I & 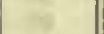 & 40 & 44.5 & 39.2 & 44 & $y$ & 40 & 28.5 & 24.3 & & 08 & 64 \\
\hline 42 & 33.8 & 31.2 & 58 & & 42 & 40.5 & 36.1 & & 49 & & 42 & 45.5 & 40.5 & 42 & & 42 & 29.3 & 23. & & 08 & \\
\hline 44 & 61.0 & 60.8 & I3 & -15.6 & 44 & 45.4 & 41.8 & & $4 \mathrm{I}$ & -15.1 & 44 & 45.6 & 41.0 & $4 \mathrm{I}$ & -15.2 & 44 & 34.0 & 29.0 & & 00. & -14.2 \\
\hline 46 & 63.0 & 61.3 & I2 & & 46 & 44.8 & 41.7 & & $4 \mathrm{I}$ & & 46 & 50.6 & 48.3 & 32 & & 46 & 32.9 & 30. I & 230 & Do & \\
\hline 48 & 44.8 & 44.2 & 2239 & 16. & 48 & 39.2 & 36.3 & & 50 & a & 48 & 48.2 & 38.0 & 42 & 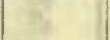 & 48 & $33 \cdot 3$ & 30.3 & 225 & 59 & \\
\hline 50 & 27.0 & $25 \cdot 3$ & 2308 & w & 50 & 36.9 & 33.8 & & 54 & gE & 50 & 43.2 & 38.8 & 45 & ix & 50 & 33.0 & 29.3 & $23 \mathrm{C}$ & 00 & ? \\
\hline 52 & 19.0 & 18. I & 20 & the & 52 & 44.8 & 42.2 & & 4I & 2 & 52 & 43.4 & 40.4 & 44 & $\sqrt{3}$ & 52 & 30.8 & 27.0 & & 04 & \\
\hline 54 & 22.2 & 21.9 & I5 & +2 & 54 & 42.8 & 39.4 & & 45 & 42 & 54 & $43 \cdot 3$ & 41.2 & 43 & $t^{2}$ & 54 & $3 \mathrm{I} \cdot 3$ & 27.8 & 230 & 03 & Bet \\
\hline 56 & 15.2 & 13.6 & 27 & 2 & 56 & 39.8 & 36.8 & & 49 & 18 & 56 & 40.7 & 39.6 & 46 & 12 & 56 & 33.9 & 30.8 & 225 & 59 & \\
\hline 58 & 19.0 & 18.0 & 20 & $(86)$ & $5^{8}$ & $41 \cdot 3$ & 38.0 & & 47 & & 58 & 46.6 & 39.8 & $4 I$ & 8 & 58 & 36.6 & 33.9 & & 54 & \\
\hline & & & & & 64 & bit: & & & & & & & & & & & & & & & \\
\hline
\end{tabular}

Observer-R. R. T.
Observers-R. R. T. and R. W. P., who alternated from $8 \mathrm{~h} 24 \mathrm{~m}$ to 8 h $38 \mathrm{~m}$; R. W. P. and W. J. P., who alternated from Ioh $32 \mathrm{~m}$ to Ioll $42 \mathrm{~m}$. F. I. observed readings from $\mathrm{Ith} 06 \mathrm{~m}$ to $12 \mathrm{~h} 58 \mathrm{~m}$. 
Tabulation of magnetic declinations observed at Teplitz Bay-Continued

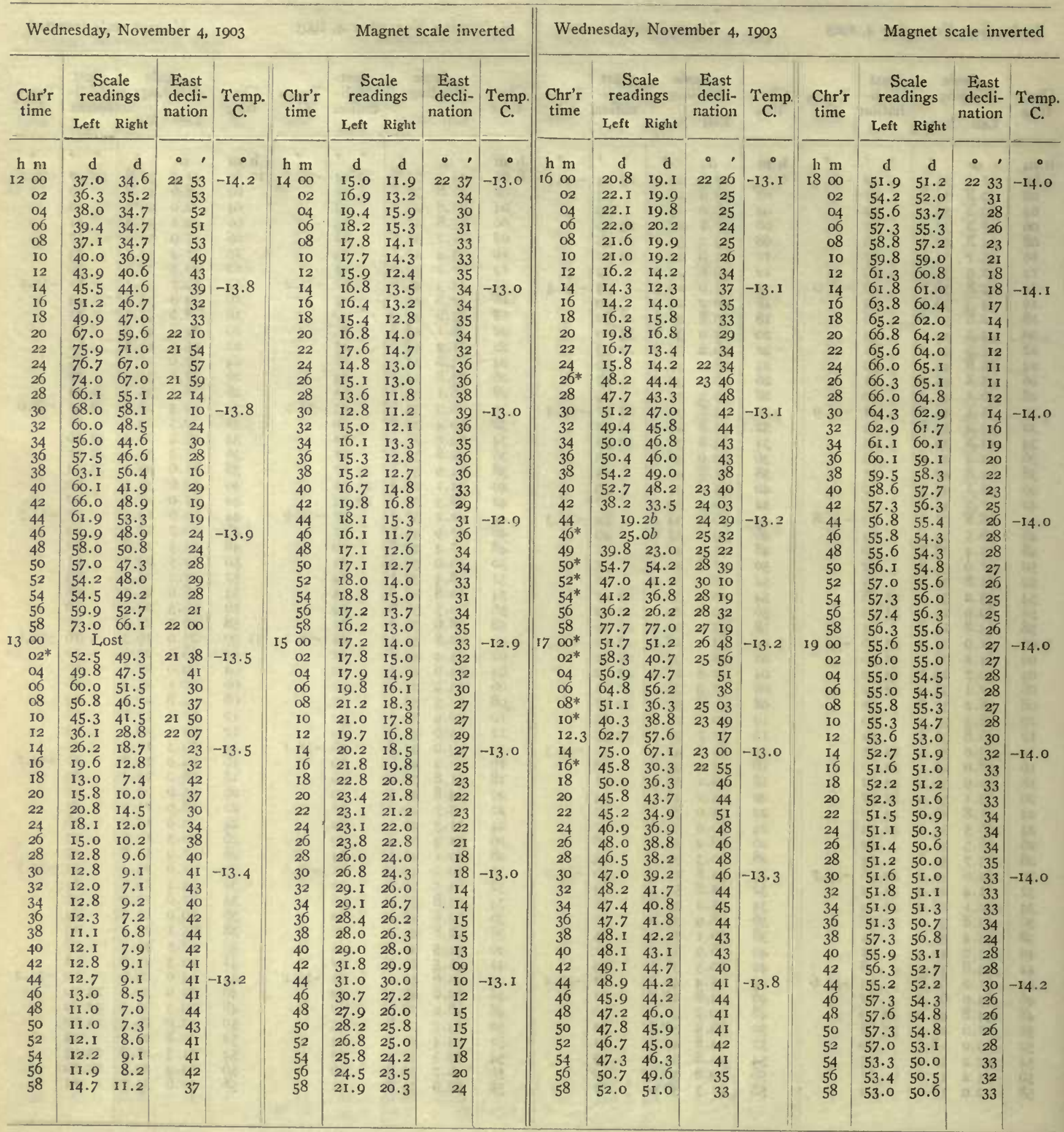


Tabulation of magnetic declinations observed at Teplitz Bay-Continued

\begin{tabular}{|c|c|c|c|c|c|c|c|c|c|c|c|c|c|c|c|c|c|c|c|}
\hline \multicolumn{5}{|c|}{ Wednesday, November 4, 1903} & \multicolumn{5}{|c|}{ Magnet scale inverted } & \multicolumn{6}{|c|}{ Thursday, November 5,1903} & \multicolumn{4}{|c|}{ Magnet scale erect } \\
\hline \multirow{2}{*}{$\begin{array}{l}\text { Chr'r } \\
\text { time }\end{array}$} & \multicolumn{2}{|c|}{$\begin{array}{l}\text { Scale } \\
\text { readings }\end{array}$} & \multirow{2}{*}{$\begin{array}{c}\text { East } \\
\text { decli- } \\
\text { nation }\end{array}$} & Temp. & Chr'r & $\begin{array}{l}\mathrm{Sc} \\
\text { read }\end{array}$ & $\begin{array}{l}\text { ale } \\
\text { lings }\end{array}$ & $\begin{array}{l}\text { East } \\
\text { decli- }\end{array}$ & Temp. & Chr'r & $\begin{array}{r}\mathrm{Sc} \\
\text { read }\end{array}$ & le & $\begin{array}{l}\text { I'ast } \\
\text { decli- }\end{array}$ & & Chr'r & $\begin{array}{r}\mathrm{Sc} \\
\text { read }\end{array}$ & $\begin{array}{l}\text { cale } \\
\text { dings }\end{array}$ & East & Temp. \\
\hline & Left & Right & & & & Left & Right & & & & Left & Right & & & & L,eft & Right & & \\
\hline $\mathrm{h} \mathrm{m}$ & d & $\mathrm{d}$ & $\cdot$, & $\bullet$ & $\mathrm{h} \mathrm{m}$ & a & d & $\cdot 1$ & - & $\mathrm{h} \mathrm{m}$ & d & d & & 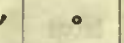 & $\mathrm{h} \mathrm{m}$ & d & d & $\cdot 1$ & 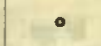 \\
\hline 2000 & 54.8 & 52.4 & 2230 & -14.4 & 2200 & 60.1 & 59.0 & 2221 & $-15 \cdot 0$ & 1600 & 43.2 & 56.3 & 2233 & $3-18.0$ & 1800 & 52.9 & 53.4 & 2238 & -15.0 \\
\hline 02 & 55.3 & 53.0 & 29 & & 02 & 59.8 & 58.2 & 21 & & 02 & 43.8 & 56.1 & 33 & 3 & 02 & 51.8 & 52.7 & 37 & \\
\hline 04 & $55 \cdot 3$ & 53.0 & 29 & dite & 04 & 60.5 & 59.0 & 20 & & 04 & 47.8 & 49.8 & 31 & $3=$ & 04 & 51.9 & 52.7 & 37 & Fo \\
\hline 06 & 54.8 & 52.8 & 29 & 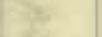 & 06 & 60.3 & 58.9 & 20 & 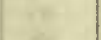 & 06 & 47.8 & 49.7 & 31 & te & 06 & 52.9 & 53.3 & 38 & $\infty$ \\
\hline 08 & 54.7 & 52.3 & 30 & het & 08 & 60.3 & 58.6 & 21 & 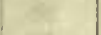 & 08 & 49.2 & 50.8 & 33 & $\tan$ & 08 & 49.0 & & 33 & 80 \\
\hline 10 & 53.2 & 51.2 & 32 & as & 10 & 60,8 & 59.2 & 20 & t & 10 & $-47 \cdot 4$ & 49.8 & 31 & 46 & Io & 48.0 & 48.5 & 30 & of \\
\hline 12 & 55.6 & 52.6 & 29 & 的 & I2 & 59.5 & 57.2 & 23 & $=$ & 12 & 49.0 & 50.7 & 33 & 3 & I2 & 48.2 & 49.0 & 3I & $-1+1$ \\
\hline 14 & 52.6 & 50.3 & 33 & $-\mathrm{I} 4.4$ & 14 & 56.5 & 54.2 & 27 & -15.0 & 14 & 44.9 & 46.8 & 27 & -18.0 & I4 & 50.8 & 51.2 & 35 & -14.8 \\
\hline 16 & 53.0 & 51.0 & 32 & & 16 & 52.0 & $5 \mathrm{I} . \mathrm{I}$ & 33 & & 16.1 & 46.0 & $47 \cdot 4$ & 28 & 120 & 16 & 52.4 & 52.8 & 37 & \\
\hline 18 & 52.0 & 50.2 & 34 & 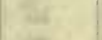 & I8 & 54.8 & 50.6 & $3 I$ & (1) & 18 & 46.9 & 48.5 & 30 & 86 & 18 & 48.2 & 49.1 & 31 & cit. \\
\hline 20 & 52.2 & 50.9 & 33 & $z=$ & 20 & 57.9 & 49.2 & 30 & a & 20 & 49.2 & 50.7 & 33 & 45 & 20 & 47.7 & 48.0 & 30 & etes \\
\hline 22 & 53.6 & 51.6 & 31 & 6. & 22 & 66.3 & 58. I & 16 & 46 & 22 & 51.4 & 53.3 & 37 & in: & 22 & 45.0 & 46.0 & 26 & is \\
\hline 24 & 55.2 & 53.4 & 29 & {[} & 24 & 59.8 & 51.3 & 27 & if & 24 & 52.8 & 54.0 & 38 & 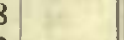 & 24 & 43.8 & 45.2 & 25 & 38 \\
\hline 26 & 51.6 & 50.0 & 34 & $=$ & 26 & 62.9 & 54.9 & 22 & ne & 26 & 53.8 & 55.0 & 40 & 6 & 26 & 39.5 & 39.7 & 17 & ats \\
\hline 28 & 57.6 & 56.3 & 25 & 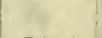 & 28 & 64.2 & 57. & 19 & 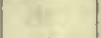 & 28 & $5 \mathrm{I} .7$ & 52 . & 37 & & 28 & 33.0 & 33.2 & 07 & \\
\hline 30 & 58.6 & 57.3 & 23 & -14.5 & 30 & 64.8 & 58.0 & 18 & -15.0 & 30 & 48.2 & 50.1 & 32 & $2-17.4$ & 30 & 38.0 & 39.2 & 15 & $-\mathrm{I} 4.3$ \\
\hline 32 & 59.1 & 57.4 & 23 & & 32 & 65.8 & 59.0 & I6 & & 32.5 & 50.6 & 53 & 30 & 6 & 32 & 37.2 & 37.8 & 14 & ( \\
\hline 34 & 58.1 & 56.8 & 24 & HE & 34 & 66.8 & 60.6 & 14 & $\mathrm{x}^{2}$ & 34 & 49.8 & 52. & 35 & ty & 34.2 & 35.6 & & II & 11 \\
\hline 36 & 57.3 & 55.6 & 25 & is & 36.1 & 66.1 & 60. & 15 & 12 & 36 & 50.7 & 53.0 & 36 & Ny & 36 & 39.3 & 40.1 & 17 & it \\
\hline 38 & 56.9 & 55.6 & 26 & $3 x^{2}$ & $3^{8}$ & 66.3 & 60.8 & 14 & 37 & 38 & 50.6 & 52 & 36 & Fit & 38 & 38.6 & 39.7 & 16 & \\
\hline 40 & 55.2 & 54.0 & 28 & 18 & 40 & 67.0 & 61.3 & 13 & 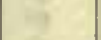 & 40 & 48.8 & 50.2 & 32 & cis & 40 & 39.9 & 40.6 & 18 & 8 \\
\hline 42 & 54.8 & 53.4 & 29 & 2 & 42 & 67.6 & $6 \mathrm{I}$. & 13 & 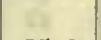 & 42 & 49.3 & $5 I$ & 34 & 4 & 42 & $4 \mathrm{~T} .8$ & 42 & 21 & \\
\hline 44 & 53.3 & 52.3 & $3 \mathrm{I}$ & -14.6 & 44 & 66.8 & 6I.I & I4 & -15.0 & 44 & 51.0 & 52.9 & 36 & $5-17.0$ & 44 & 41.5 & 43 & 21 & -14.1 \\
\hline 46 & 53.0 & 52.0 & 32 & & 46 & 65.9 & 60.3 & 15 & & 46 & 51.9 & 54 . & 38 & 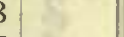 & 46 & 42.5 & 43 & 22 & 0 \\
\hline 48 & 55.8 & 54.6 & 27 & & 48 & 65.1 & 60.0 & 16 & 84 & 48 & $5 \mathrm{I} .2$ & 53.8 & 37 & 8 & 48 & 42.5 & 43 & 22 & ent \\
\hline 50 & 54.3 & 53.3 & 30 & $k=$ & 50 & 62.0 & 56.7 & $2 \mathrm{I}$ & 20 & 50 & 51.9 & 53 & 38 & 18 & 50 & 43.0 & & 23 & ine \\
\hline 52 & 53.9 & 53.0 & 30 & 28 & 52 & 59.8 & 54.8 & 24 & a & 52 & 53.0 & 55 & 39 & $x$ & 5 & 42.6 & 43 & 22 & 18 \\
\hline 54 & 52.5 & $5 \mathrm{I} .3$ & 33 & 14 & 54 & 7.8 & 52.3 & 28 & [i & 54 & 50.2 & 52 & 35 & 15 & 5 & 40.8 & 42 & 20 & 62 \\
\hline 56 & 51.7 & 50.8 & 34 & 29 & 56 & $55 . \mathrm{J}$ & 51.2 & $3 I$ & & 56 & 49.7 & 5 I. & 34 & 4 & 5 & $4 I .4$ & 43 & $2 \mathrm{I}$ & 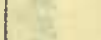 \\
\hline 58 & 50.2 & 49.5 & 36 & & 58 & .7 & 50.0 & 32 & 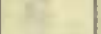 & 58 & 48.7 & 49 & $3 \mathrm{I}$ & & 58 & 43.2 & & 24 & \\
\hline 2100 & 49.4 & 48.7 & 37 & 2 & 2300 & 50.8 & 45.7 & 38 & -15.0 & 1700 & 46.3 & 47.8 & 28 & $3-16.5$ & 1900 & 44.0 & 46.0 & 25 & -14.0 \\
\hline 02 & 50.3 & 49.9 & 35 & 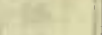 & 02 & 46.8 & 42.8 & 44 & 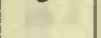 & 02 & 48.2 & 49.2 & $3 \mathrm{I}$ & 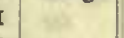 & 02 & $45 . I$ & $47 \cdot 3$ & 27 & - \\
\hline 04 & 51.6 & 50.8 & 34 & 19 & 04 & 41.8 & 36.1 & 53 & E & 04 & 48.4 & 49 & $3 \mathrm{I}$ & B. & 0. & 47.0 & 48 & 29 & 60 \\
\hline$\infty$ & 52.6 & 52.0 & & 20 & 06 & .0 & & 57 & 80 & 06 & 49.2 & & 32 & 150 & 0 & 47.6 & & $3 \mathrm{I}$ & 68 \\
\hline 08 & 52.1 & 51.3 & 33 & 24 & 08 & .8 & 32. & 59 & 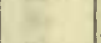 & 08 & 50.8 & 51.8 & 35 & 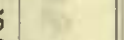 & 08 & 52.2 & & 39 & kit \\
\hline IO & 52.3 & 5 & 3 & $x$ & IO & .8 & & 59 & $17 t$ & Io & 49.3 & & 33 & 61 & 10 & 49.4 & & 35 & 64 \\
\hline 12 & 52.9 & 52.3 & 31 & -15 & I & .5 & 3 & 56 & & I & 49.2 & 49. & 32 & & 12 & $47 \cdot 5$ & 49 & $3 \mathrm{I}$ & \\
\hline 14 & 52.1 & & 3 & -14.8 & 14 & 41.9 & & 52 & -15.0 & I. & 52.2 & 52.8 & 37 & $7-16.0$ & 14 & 45.1 & 43.2 & 24 & $-\mathrm{I} 4.0$ \\
\hline 16 & 52.2 & 51.6 & 3 & & I6 & 44.8 & 40.9 & 47 & & I & 50.1 & 52 & 35 & 5 & I & $43 . I$ & & 25 & $i^{\circ}$ \\
\hline 18 & 52.2 & 5 & 3 & 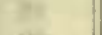 & 18 & 48.3 & & 42 & & I & 48.2 & 50.2 & 32 & 35 & I8 & $43 . I$ & 46.1 & 25 & 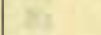 \\
\hline 20 & 52.2 & 5 I. & & per & 20 & 47.2 & 44. & 42 & ne & 20 & 47.1 & & 3 & 18: & & 42.4 & 45 & 23 & 68 \\
\hline 22 & 52.8 & 52.2 & 3 & 3x- & 22 & 50.3 & 49.3 & 36 & 65 & 22 & 48.9 & 50. & 33 & it & 22 & 40.8 & 44.0 & $2 \mathrm{I}$ & \pm 1 \\
\hline 24 & & 5 I. & & $y=$ & & .4 & & 25 & 18 & & & & 3 & 16 & & 37.2 & & I6 & is \\
\hline 26 & 51.0 & 5 & 3 & its & 2 & .1 & & 24 & & & 48.8 & 50 & 32 & ( & 2 & $4 \mathrm{I} . \mathrm{I}$ & 42 & 21 & 30 \\
\hline 28 & & 50.1 & & $x^{2}$ & 28 & & 56.8 & 22 & & 28 & 47.2 & 48 & 30 & & 2 & & & 13 & \\
\hline 30 & 50.6 & 49.9 & 35 & $-I 5.0$ & 30 & 63.9 & 60.2 & 17 & -14.9 & 30 & 48.7 & 49.7 & 32 & -15.1 & 30 & 42.0 & 43 & 22 & -14.0 \\
\hline 32 & & 5 & & & 32 & 2.9 & & 33 & & & 9.6 & & 34 & & & 43.4 & 44 & 24 & $0^{\circ}$ \\
\hline 34 & .0 & 5 & 2 & 18 & 3 & 61.1 & & 22 & & 3 & I & & 3 & & 3 & & & 24 & $3=$ \\
\hline 36 & & & & & & .6 & & & 6 & & 50.8 & & 3 & & & 2 & & 24 & 5. \\
\hline 38 & 53.0 & 51.4 & 3 & fts & 38 & 1.5 & 71.3 & 00 & $k=$ & 38 & 51.9 & 53 & 38 & $x^{2}$ & 38 & 3 & & 27 & $=$ \\
\hline 40 & & & & 요 & 40 & & & 06 & & $4 c$ & 52.1 & & 38 & & & 6.0 & & 28 & \\
\hline 42 & .3 & 52.2 & 3 & & 42 & .3 & 67.8 & 05 & & 42 & 52.1 & & 38 & & 42 & & 46.6 & 27 & 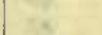 \\
\hline & 56.0 & 54.9 & 27 & -15.0 & & 72.0 & 69.0 & 03 & -15.0 & & 52.3 & & 39 & -15.5 & 44 & 45.6 & 47.0 & 27 & \\
\hline 46 & 56.5 & 55.6 & 26 & & 46 & 68.1 & & ro & & 46 & 54.1 & & 40 & . & 46 & 44.9 & 45.6 & 26 & -13.8 \\
\hline 48 & 56.9 & 56.0 & 25 & it & 48 & 67.2 & 65. & I0 & 5 & 48 & 54.6 & 55 & $4 \mathrm{I}$ & 24 & 48 & 42.0 & 42.9 & 21 & - \\
\hline 50 & 57.2 & 56.3 & 25 & we & 50 & 68.7 & 67. & 07 & 68 & 50 & 54.3 & & $4 \mathrm{I}$ & $1=$ & 50 & 43.0 & 44 & 23 & \\
\hline 52 & 58.4 & 57.5 & 23 & 13 & 52 & 68.0 & 66.4 & 09 & 6 & 52 & 53.9 & 54. & 40 & 3 & 52 & 42.9 & 44. & 23 & 12 \\
\hline 54 & 58.8 & 58.0 & 22 & 32 & 54 & 66.2 & 65.7 & II & 18 & 54 & 53.2 & 54.0 & 3 & $3=$ & 54 & 41.4 & 42 & 20 & \\
\hline 6 & 58.5 & 58.0 & 23 & 6 & 56 & 64.5 & 62.9 & I4 & & 56 & 53.2 & & 39 & & 56 & 41.8 & 43.2 & $2 \mathrm{I}$ & \\
\hline 58 & 60.0 & 58.3 & $2 I$ & 3 & $\begin{array}{r}58 \\
2400\end{array}$ & $\begin{array}{l}64.9 \\
65.0\end{array}$ & $\begin{array}{l}63.8 \\
63.3\end{array}$ & $\begin{array}{l}13 \\
13\end{array}$ & -15.0 & 58 & 53.0 & 53.4 & 3 & a & 2000 & $\begin{array}{l}41.2 \\
39.5\end{array}$ & $\begin{array}{l}42.9 \\
40.8\end{array}$ & $\begin{array}{l}21 \\
18\end{array}$ & -13.3 \\
\hline & & & & & & 05.0 & 03.3 & 13 & -15.0 & & & & & & 2000 & 39.5 & & 10 & \\
\hline
\end{tabular}

Correction to local mean time is $+2 \mathrm{~m}$ 50s. $90^{\circ}$ torsion $=27.9$.

Torsion head at oh oom read $315^{\circ}$ and at $24 \mathrm{~h} 2 \mathrm{Im}$ read $310^{\circ}$.

Observers - W. J. P. and R. R. T., who alternated from $22 \mathrm{~h} 06 \mathrm{~m}$ to $22 \mathrm{~h} 16 \mathrm{~m}$.
Correction to local mean time is $+5 \mathrm{~m}$ IIs. $90^{\circ}$ torsion $=27 .^{\prime} 9$.

Torsion head at $15 \mathrm{~h} 20 \mathrm{~m}$ read $355^{\circ}$ and at $20 \mathrm{~h} \mathrm{I} 6 \mathrm{~m}$ read $350^{\circ}$.

Observers-R. R. 'T. and W. J. P., who alternated from $18 \mathrm{~h} 22 \mathrm{~m}$ to $18 \mathrm{~h} 36 \mathrm{~m}$. 
Tabulation of magnetic declinations observed at Teplitz Bay-Continued

\begin{tabular}{|c|c|c|c|c|c|c|c|c|c|c|c|c|c|c|c|c|c|c|c|}
\hline \multicolumn{5}{|c|}{ Friday, November 6,1903} & \multicolumn{5}{|c|}{ Magnet scale inverted. } & \multicolumn{5}{|c|}{ Sunday, November 8, 1903} & \multicolumn{5}{|c|}{ Magnet scale erect } \\
\hline \multirow{2}{*}{$\begin{array}{l}\text { Chr'r } \\
\text { time }\end{array}$} & \multicolumn{2}{|c|}{$\begin{array}{c}\text { Scale } \\
\text { readings }\end{array}$} & \multirow{2}{*}{$\begin{array}{l}\text { East } \\
\text { decli- } \\
\text { nation }\end{array}$} & Temp. & & $\begin{array}{r}\mathrm{S} \\
\text { rea }\end{array}$ & $\begin{array}{l}\text { cale } \\
\text { dings }\end{array}$ & $\begin{array}{l}\text { East } \\
\text { decli- }\end{array}$ & Temp. & Chr'r & $\begin{array}{r}\mathrm{S} \\
\mathrm{rea}\end{array}$ & $\begin{array}{l}\text { cale } \\
\text { clings }\end{array}$ & $\begin{array}{l}\text { East } \\
\text { decli- }\end{array}$ & Temp. & Chr'r & $\begin{aligned} \text { Sc } \\
\text { reac }\end{aligned}$ & $\begin{array}{l}\text { le } \\
\text { ngs }\end{array}$ & $\begin{array}{l}\text { East } \\
\text { decli- }\end{array}$ & Temp \\
\hline & $I_{e}$ ft & Right & & & & Left & Right & & & & L,eft & Right & & & & L,eft & Right & & \\
\hline $\mathrm{h} \mathrm{m}$ & d & d & & . & $\mathrm{h} \mathrm{m}$ & d & $\mathrm{d}$ & $\cdot \cdot$ & 20 & $\mathrm{~h} \mathrm{~m}$ & d & $d$ & & $0^{\circ}$ & & d & $\mathrm{d}$ & & \\
\hline 2000 & 45.2 & 44.1 & 2222 & $-17 \cdot 3$ & 2200 & 47.2 & 46.8 & $2230-$ & -15.5 & $000^{*}$ & 41.4 & 47.8 & 2228 & -19.8 & 200 & 49.2 & 49.8 & 2236 & -17.6 \\
\hline 02 & 43.0 & 41.6 & 26 & & 02 & 46.9 & 44.9 & 32 & & 02 & 41.8 & 47.6 & 29 & & 02 & 49.4 & 50.1 & 36 & \\
\hline 04 & 38.2 & 36.7 & 33 & to & 04 & 47.2 & 45.2 & 32 & E & 04 & 40.0 & 45.8 & 26 & 12 & 04 & 50.8 & $5 \mathrm{I} .5$ & 39 & \\
\hline 06 & 42.8 & 40.0 & 7 & E. & 06 & 48.8 & 46.8 & 29 & se & 06 & 43.5 & 49. & $3 \mathrm{I}$ & 16 & 06 & 50.3 & 51.0 & 38 & \\
\hline 08 & 44.0 & 42.0 & 25 & E. & 08 & 49.8 & 47.9 & 27 & 78. & 08 & 47.8 & 52.8 & 37 & W & 08 & 51.3 & 52.0 & 39 & is \\
\hline 10 & 39.7 & 36.8 & 32 & Fi & 10 & 9.1 & 47.6 & 28 & का7 & 10 & 48.0 & 53.3 & 38 & 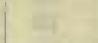 & 10 & 52.2 & 52.6 & $4 \pi$ & Air \\
\hline 12 & & 37.7 & 32 & 15 & 12 & 49.7 & 48.0 & 27 & $6 r$ & 12 & 47.3 & 52.0 & 36 & -19.4 & 12 & 51.0 & 51.6 & 39 & \\
\hline 14 & 37 & 35.0 & 35 & -17.0 & I4 & 47.8 & 46.1 & $30-$ & -15.3 & I4 & 50.2 & 54.0 & 40 & & I4 & 50.3 & 50.9 & 38 & -17.3 \\
\hline I6 & 35.8 & 34.9 & 37 & & 16 & 46.8 & 43.7 & 33 & -3 & 16 & 50.5 & & $4 \mathrm{I}$ & 01 & 16 & 49.8 & 50.2 & 37 & \\
\hline 18 & & $3 b$ & 40 & $\mathrm{Ri}$ & 18 & 45.1 & 42.6 & 35 & $80 x$ & I8 & 53.8 & 57.8 & 46 & ? & 18 & 49.0 & 49.8 & 36 & 54 \\
\hline 20 & 34.8 & 34.1 & $\begin{array}{l}40 \\
38\end{array}$ & $\approx$ & 20 & 45.2 & 43.7 & $\begin{array}{l}35 \\
34\end{array}$ & $=$ & 20 & 59.0 & 62.0 & $\begin{array}{l}40 \\
5.3\end{array}$ & na & 20 & 49.8 & & 37 & \\
\hline 22 & & & 44 & Et & 22 & & & 35 & at & 22 & & & 48 & $\Rightarrow$ & 22 & 52.0 & & $4 \mathrm{I}$ & \\
\hline 2 & & & 55 & 78 & 2. & & & 5 & Fi & 2. & & & 45 & rs & & & & $4 \mathrm{I}$ & \\
\hline & & 28. & & ta & 26 & & & & 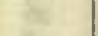 & 2 & $5 \mathrm{I}$ & & & E & & & & $4 \mathrm{I}$ & \\
\hline 28 & 32.8 & 29 & 43 & $1=$ & 28 & 45.3 & 43. & 35 & $=$ & 28 & 49 & & 39 & & 28 & 4.7 & & 45 & \\
\hline 30 & 33.0 & 28.9 & 43 & -17.0 & 30 & 44.2 & 42. & 36 & -15.0 & 3 & $47 \cdot 3$ & 50 & 35 & -19.0 & 30 & 4.3 & 55. & 44 & -17.1 \\
\hline 32 & 37.8 & 30.0 & 39 & & 32 & & 40.5 & 38 & & 32 & 47.1 & 49.0 & 34 & 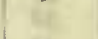 & 32 & 52.5 & & 42 & \\
\hline 34 & 36.7 & 34.2 & 37 & 1 & 34 & & 38.6 & $4 \mathrm{I}$ & & 34 & $5 \mathrm{I} . \mathrm{I}$ & & $4 \mathrm{I}$ & B & 34 & $5 \mathrm{I} .2$ & & 39 & \\
\hline 36 & 35 & 33.0 & 39 & . & 36 & 42.2 & 39. & 40 & 8 & 36 & 49.3 & & 38 & ac & 36 & 52.3 & & $4 \mathrm{I}$ & \\
\hline 38 & 34 & 33.8 & 38 & 4 & 38 & 45.I & 42. & 35 & fit & $3^{8}$ & 51.7 & 53.3 & $4 \mathrm{I}$ & ifc & 38 & 53.8 & 54.3 & 43 & \\
\hline $4^{0}$ & 27 & 20.6 & 54 & -4 & 40 & 4.9 & 42.2 & 36 & int & 40 & 52.4 & 54. & 42 & 14 & 40 & 54.3 & 5 & 44 & \\
\hline 42 & & 18.0 & 2256 & & 42 & 4.2 & 41.0 & 37 & 64 & 42 & & & $4 \mathrm{I}$ & & 42 & & & 45 & \\
\hline $44^{*}$ & 56.1 & 49.8 & 2323 & -56.8 & 44 & 40.9 & 38.1 & 42 & -14.8 & 44 & 52.3 & 54 & 42 & -18.6 & 44 & 57.0 & & 49 & -17.0 \\
\hline $46^{*}$ & 41.8 & 25.0 & 2250 & & 46 & $4 \mathrm{I} .2$ & 37.4 & 42 & & 46 & & & 39 & 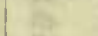 & 46 & 58.1 & & $5 \mathrm{I}$ & \\
\hline 48 & $3 r$ & 16.3 & 2306 & & $4^{8}$ & .2 & 37. & $4 \mathrm{I}$ & 4 & $4^{8}$ & & & 39 & 180 & 48 & & & 52 & \\
\hline 50 & & 17.2 & 4 & & $\begin{array}{l}40 \\
50\end{array}$ & & 40 & $\begin{array}{l}41 \\
37\end{array}$ & 45 & 5 & & & 37 & as & 50 & & & 55 & \\
\hline 5 & & & $c$ & E & 52 & & & 37 & 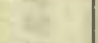 & 5 & & & 45 & $a$ & 52 & & & $\begin{array}{ll}32 & 58\end{array}$ & \\
\hline 5 & & 15 & 06 & & 54 & & $\begin{array}{l}40 \\
40\end{array}$ & 38 & 62 & 5 & & 54 & $\begin{array}{l}43 \\
42\end{array}$ & 32 & 54 & & & 2302 & \\
\hline & 57.8 & $4 \mathrm{~T}$ & 4 & & 56 & & & 39 & & 5 & & & 43 & $x^{2}$ & 56 & & & & \\
\hline & 70.6 & 57. & $c$ & & 58 & & & 39 & & 58 & & & 42 & & 58 & & & & \\
\hline & 78.2 & 65.3 & 49 & -16.3 & 2300 & & & 40 & -14.7 & I 00 & & & 43 & -18.2 & 300 & & & 06 & -16.9 \\
\hline 0 & & 52.7 & & 100 & 02 & & 38 & 40 & & 02 & & & 50 & - & 02 & & & 08 & \\
\hline & & 56.0 & & in & 04 & & & 40 & & a. & & & 47 & 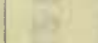 & o. & & & II & \\
\hline & & 33.3 & 2245 & 8 & 06 & 41.8 & 38 & $4 \mathrm{I}$ & 4 & o & & 61 & 54 & as & a & & & 13 & \\
\hline of & 44 & 36. & 4 & 8 & 08 & & & 42 & 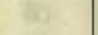 & 0 & & 63 & 57 & B. & 0 & & & I4 & \\
\hline IC & $5 I$ & $4 \mathrm{I}$ & 3 & 6 & I & & & 42 & 24 & I & & & 5I & กิ & II & & & 15 & \\
\hline I 2 & & & I & & I & & & 40 & & 1 & & & 49 & & & & & 17 & \\
\hline I4 & 48 & 37 & 37 & -16.0 & 14 & & & $4 \mathrm{I}$ & -14.7 & I & & & 49 & -18.2 & I. & & & 17 & -16.6 \\
\hline I & & 26 & 57 & & I & & & 38 & & I & & & 57 & tets & I & & & 16 & \\
\hline I\& & & 30 & 4 & 84 & 18 & & & 38 & 15 & I8 & & & & (1) & I8 & & & 14 & in \\
\hline $2 C$ & & 28 & 5 & $\Theta$ & 2 & & & 38 & 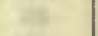 & 2 & & & & $=$ & 20 & & & I8 & 16 \\
\hline 22 & & 36 & 3 & $=5$ & 22 & & & 35 & e & 22 & & & 04 & $\omega$ & 22 & & & II & $E$ \\
\hline 24 & & 43 & 2 & f & 24 & & 4I. & 38 & if & 24 & & & & 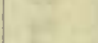 & 24 & & & 09 & 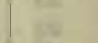 \\
\hline 26 & 49 . & 40 & 34 & E & 26 & 4 & 39 . & 40 & . & 26 & & $6 \mathrm{I} .3$ & & & 26 & & & 04 & 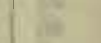 \\
\hline 28 & 48.7 & $4 I$ & 33 & & 28 & & & 42 & & 28 & & & 56 & & 28 & & & 56 & \\
\hline 30 & 5 & 50 & $\begin{array}{l}50 \\
20\end{array}$ & -15.8 & 30 & 40.8 & 38 & 42 & $-I_{4} .6$ & 3 & 58.6 & 58. & $5 \mathrm{I}$ & -18.0 & 30 & 60.5 & 61.2 & 54 & -16.5 \\
\hline 32 & & 45 & 27 & & 32 & & & 42 & & 3 & & & 50 & & & & & 49 & \\
\hline 3 & 4 & 36 & & 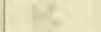 & 3 & & & 42 & & 3 & & & & & & & & & \\
\hline 31 & & 29. & 2 & & 36 & & & $4 \mathrm{I}$ & & 3 & & & & & & & & & \\
\hline 38 & 26. & 24 & 2 & & 3 & & 37 & 4 & & 3 & 48 & & 36 & & 3 & & & & \\
\hline $4 c$ & & 24 & $c$ & & 4 & & & & & 4 & & & 43 & & & & & & \\
\hline 4 & 18 & 13. & 1 & & 42 & & & 38 & & 4 & & & 45 & & 4 & & & 2 & \\
\hline & I & 9 & 27 & -15.6 & 44 & & & 37 & -14.6 & 4 & & & $\begin{array}{l}4 J \\
43\end{array}$ & -17.0 & & & & 55 & -16.5 \\
\hline & 14.6 & 9 & 25 & & 46 & & & 38 & & 4 & 8 & & 42 & & 46 & & & 59 & \\
\hline $4^{8}$ & 23 & 20 & I & 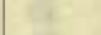 & 48 & & & 9 & 4 & 48 & & .0 & 40 & Mi & 48 & & & 59 & \\
\hline 50 & & 20 & 1 & & 5 & & & 9 & 9 & 5 & & $.0 t$ & 37 & ats & $5 c$ & & & $5^{8}$ & \\
\hline 52 & 17. & 15 & 2319 & 5 & 52 & & & 40 & 4 & 52 & & 50.2 & 37 & 82 & 52 & 6ז.6 & & 56 & \\
\hline 54 & 32.2 & 29. & 2256 & E & 54 & & & 42 & 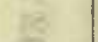 & 5 & 54 & 54 & 44 & te & $54^{*}$ & $51 \cdot 3$ & 52.6 & 57 & \\
\hline & $4^{2}$ & $4 \mathrm{I} .0$ & 39 & & & $4 \mathrm{I} .8$ & & 40 & & 56 & 56.3 & 56.9 & 47 & 10 & 56 & 54.3 & 56.3 & 2302 & \\
\hline & 45.8 & 45.0 & 33 & 8 & 58 & 42 & 40.8 & 39 & 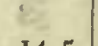 & 58 & $5 \mathrm{I} . \mathrm{I}$ & 52.3 & 40 & E & 58 & 52.9 & 55.0 & oo & 3 \\
\hline & & & & & & 41.0 & $40 . \mathrm{I}$ & $4^{\circ}-$ & $-14 \cdot 5$ & & & & & & & & & & \\
\hline
\end{tabular}

Correction to local mean time is $+5 \mathrm{~m} 36 \mathrm{~s}$.

Torsion head at $19 \mathrm{hl} 4 \mathrm{Om}$ read $350^{\circ}$ and at $24 \mathrm{~h} 20 \mathrm{~m}$ read the same.

Olsserver-W. J. P.

Observer-R. R. T. 
Tabulation of magnetic declinations abserved at $T c p l i t z B a y-$ Continued

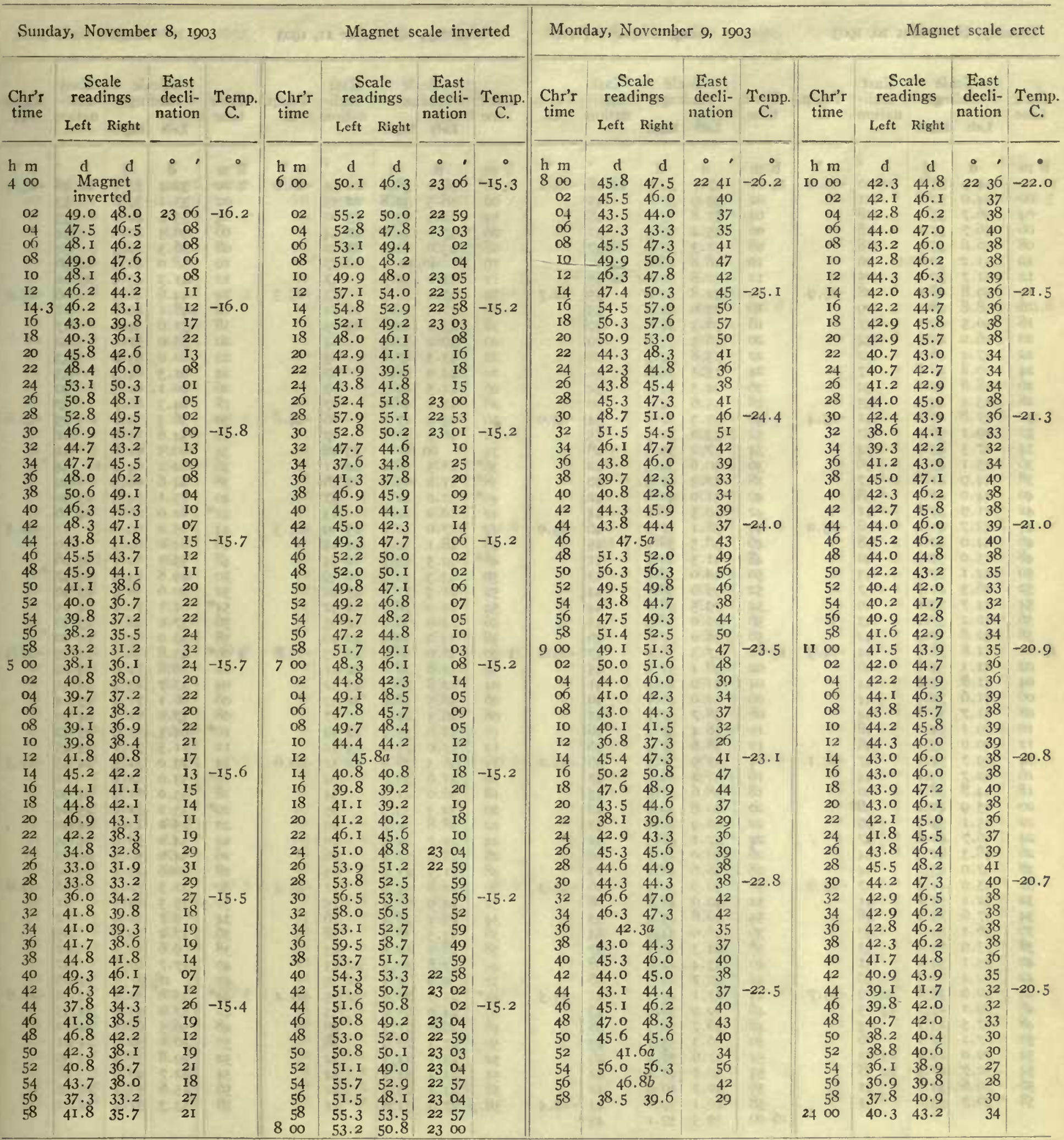

Correction to local mean time is $+7 \mathrm{~m}$ I2s. $90^{\circ}$ torsion $=27.6$.

Torsion head at oh oom read $351^{\circ}$ and at gh $\mathrm{I} 5 \mathrm{~m}$ read $354^{\circ}$.

Observers-W. J. P. and R. R. T., who alternated from th o6m to 4 li $16 \mathrm{~m}$.
Correction to local mean tinc is $+2 \mathrm{~m} 3.45$.

Torsion head at $7 \mathrm{~h} 20 \mathrm{~m}$ read $356^{\circ}$ and at $\mathrm{I} 2 \mathrm{~h} 30 \mathrm{~m}$ rcad the same.

Observers-W. J. P. and R. R. T., who alternated gh $56 \mathrm{~mm}$ to roli $04 \mathrm{~m}$. 
Tabulation of magnetic declinations observed at Teplitz Bay-Continued

\begin{tabular}{|c|c|c|c|c|c|c|c|c|c|c|c|c|c|c|c|c|c|c|c|c|c|c|}
\hline \multicolumn{5}{|c|}{ T'uesday, November 10, 1903} & \multicolumn{6}{|c|}{ Magnet scale inverted } & \multicolumn{6}{|c|}{ Wednesday, November I1, Ig03 } & \multicolumn{6}{|c|}{ Magnet scale ereet } \\
\hline \multirow{2}{*}{$\begin{array}{l}\text { Chr'r } \\
\text { time }\end{array}$} & $\begin{array}{r}\mathrm{S} \\
\text { reac }\end{array}$ & $\begin{array}{l}\text { ale } \\
\text { ings }\end{array}$ & $\begin{array}{l}\text { East } \\
\text { decl }\end{array}$ & Temp. & Chr'r & $\begin{array}{r}\mathrm{Sc} \\
\mathrm{read}\end{array}$ & & $\begin{array}{r}\mathrm{Ea} \\
\mathrm{dec}\end{array}$ & $\begin{array}{l}\text { ast } \\
\text { cli- }\end{array}$ & Temp & Chr'r & $\begin{array}{l}\text { Sca } \\
\text { readi }\end{array}$ & & $\begin{array}{l}\text { Ea } \\
\text { dec }\end{array}$ & & & & $\begin{array}{r}\mathrm{Sc} \\
\text { read }\end{array}$ & $\begin{array}{l}\text { le } \\
\text { ngs }\end{array}$ & & & Temp. \\
\hline & Left & Right & & & & Left & Right & & & & & Left & Right & & & & & Left & Right & & & \\
\hline $\mathrm{h} \mathrm{m}$ & $\mathrm{d}$ & d & $\cdot$ & • & $\mathrm{h} \mathrm{m}$ & $\mathrm{d}$ & $\mathrm{d}$ & 0 & & $\bullet$ & h $\mathrm{m}$ & d & & & & & $\mathrm{h} \mathrm{m}$ & $d$ & d & & & • \\
\hline 1200 & 54.9 & 53.9 & 222 & -26.0 & 1400 & 37.7 & 34.7 & 22 & 48 & -23.1 & $0.00^{*}$ & 38.0 & 38.8 & & & -26.0 & 200 & 32.4 & 35.6 & & 48 & -23.0 \\
\hline 02 & 54.0 & 52.8 & & $1-000$ & 02 & 39.9 & 37.2 & & 45 & & 02 & 38.5 & 39.8 & & & & 02 & 35.1 & 39.6 & & 53 & \\
\hline 04 & 56.7 & 55.1 & & (a) & 04 & 37.8 & 35.0 & & 48 & $t^{3}$ & 04 & $4 \mathrm{I} \cdot 5$ & 43.1 & 23 & 00 & & 04 & 35.3 & 40.1 & & 53 & \\
\hline 06 & 56.7 & 55.2 & & W & 06 & 35.4 & 33.3 & & 51 & 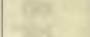 & 06 & 37.0 & 39.2 & 22 & & & 06 & 36.3 & 40.1 & & 54 & \\
\hline 08 & 56.6 & 55.1 & & C & 08 & $31 \cdot 3$ & 30.0 & & 57 & w & 08 & 43.0 & $44 \cdot 3$ & 23 & 02 & & 08 & 36.3 & 39.3 & & 54 & \\
\hline 10 & 51.2 & 50.2 & & nit & 10 & 28.8 & 26.8 & 23 & 02 & ar & 10 & 40. & $4 a$ & 22. & .57 & ta & 10 & 38.0 & & 22 & 56 & \\
\hline I2 & 51.7 & 50.1 & & $1=15$ & 12 & 32.8 & 30.6 & 22 & 55 & & I2 & 34.0 & 34.0 & & 47 & 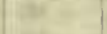 & 12 & $4 \mathrm{I} \cdot 3$ & 43.9 & 23 & or & \\
\hline$I_{4}$ & 9.8 & 48.0 & & -25.9 & 14 & 32.8 & 31.2 & & 55 & -22.7 & I4 & 37.0 & 38.3 & & 53 & $-25 \cdot 5$ & 14 & 44.6 & 46.5 & & 06 & -23.0 \\
\hline 16 & 48.8 & $48 . I$ & & & 16 & 32.0 & $3 \mathrm{I} .2$ & & 55 & & 16 & $37 \cdot 3$ & 38.7 & 22 & 54 & & 16 & 48. I & 49.8 & & II & \\
\hline 18 & 50.5 & $49 . \mathrm{I}$ & & Wh & I8 & 36.3 & 34.8 & & 49 & & 18 & 43.0 & 44.8 & 23 & 03 & at & I8 & 51.6 & 54.0 & & 17 & \\
\hline 20 & 6.0 & $44 \cdot 3$ & & 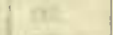 & 20 & 36.3 & 35.0 & & 49 & as & 20 & 50.2 & 52.5 & & 14 & & 20 & 55.0 & & & 22 & as \\
\hline 22 & 47.4 & 46.2 & & 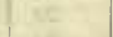 & 22 & 35.8 & 34.5 & & 50 & $=$ & 22 & $43 \cdot 3$ & 47.6 & & 05 & the & 22 & 2.0 & 63.8 & & 33 & ae \\
\hline 24 & 5 & 48.8 & & 2 & 24 & 5.3 & 33. & & 51 & W & 24 & & 48.8 & & 07 & 38 & 24 & I. 5 & & & 33 & 40 \\
\hline 26 & 48.8 & 47.6 & & 10. & 26 & 34.5 & 33.2 & & 52 & 8 & 26 & 44.5 & $49 \cdot 3$ & & 07 & 40 & 26 & 60.1 & & & 30 & 15 \\
\hline 28 & $5 \cdot 4$ & $44 \cdot 3$ & & I & 28 & 35.6 & $34 \cdot 3$ & & 50 & 2 & 28 & 44.9 & 48.0 & & 07 & trat & 28 & $4 \cdot 3$ & & & 37 & \\
\hline 30 & 7.8 & 47.0 & & -25.4 & 30 & 33.3 & 32.0 & & 54 & -23.0 & 30 & 44.9 & 47.8 & & 06 & -24.8 & 30 & 6.5 & 59.2 & & 25 & -22.6 \\
\hline 32 & 49.1 & 46.4 & & & 32 & 3.0 & 31.5 & & 55 & & 32 & 46.0 & 47.3 & & 07 & & 32 & 54.6 & 55 & & $2 \mathrm{I}$ & \\
\hline 34 & $47 \cdot 3$ & 43.9 & & Ex & 34 & 37.2 & 35 & & 48 & 政 & 34 & 60 & $0 a$ & 23 & 28 & 5 & 34 & 60.5 & 62 & & 31 & 2 \\
\hline 36 & 45.1 & 4 & & 2 & 36 & 40.6 & 39 & & 42 & $=$ & $36^{*}$ & 21.5 & 28.0 & 24 & & ME & 36 & 62.2 & & & 34 & \\
\hline 38 & 44.9 & 41.8 & & 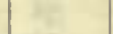 & 38 & 42.3 & $4 \mathrm{~T} .3$ & & 40 & 2 & $38^{*}$ & 61.0 & 65.5 & 23 & 36 & ne & 38 & 64.6 & & & 39 & \\
\hline 40 & 44.0 & 41.2 & & 4 & 40 & 41.0 & 40.0 & & 42 & 9 & $40^{*}$ & 37.0 & 42.3 & & 57 & 46 & 40 & 73.1 & 74.3 & 23 & & \\
\hline $4^{2}$ & 41.9 & 39.8 & & 7 & 42 & 39.5 & 38.2 & & 44 & $\Rightarrow$ & 42 & 10.2 & 12. 6 & & 13 & -2 & $42 *$ & 62.0 & & 24 & 06 & \\
\hline 44 & 43.7 & 41.4 & & -25.0 & 44 & 38.1 & $37 \cdot 3$ & & 46 & -23.0 & 44 & 37.0 & 45.0 & 23 & 59 & $-24 \cdot 3$ & 44 & 65.0 & 69.8 & & II & -22.5 \\
\hline $4^{6}$ & 4.0 & 42.0 & & & 46 & 38.5 & 38.0 & & 45 & & & & 54.7 & 24 & & & & 64.0 & 69.3 & & Io & \\
\hline $4^{8}$ & 43.0 & 39.9 & & 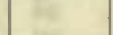 & 48 & 1.3 & 40.7 & & $4 \mathrm{I}$ & 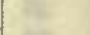 & $48^{*}$ & 49.5 & 53.7 & 22 & 54 & (a) & 48 & 59.3 & 69 & 24 & 06 & \\
\hline 50 & .I & 37. & & 5 & 50 & & & & 43 & 6 & 50 & & 53.3 & 22 & 49 & 6 & 50 & & & & & \\
\hline 52 & 46.3 & 40.7 & & $y=$ & 52 & 39.0 & 38.6 & & 44 & 3 & 52 & 61.5 & 77. & 23 & 22 & $\theta$ & 52 & 50.6 & & & & \\
\hline 54 & 4 & 3 & & fa & 54 & 39.5 & 38.6 & & 44 & E & 54 & 73.0 & 78.0 & 23 & 32 & & $54^{*}$ & 6̆. & 70 & 24 & 2 & \\
\hline 56 & 42.2 & 37. & & a & 56 & 9.0 & 38.3 & & 45 & & 56 & I 4.0 & 26.6 & 22 & 05 & se & 56 & .9 & & & & \\
\hline 58 & 2.8 & 3 & & & 58 & 38.0 & 37.6 & & 46 & & 58 & 34.2 & 50.9 & 22 & 40 & & 58 & 59.0 & 65 & 24 & 20 & \\
\hline oo & 41.8 & 36.3 & & -24.5 & 1500 & $37 \cdot 3$ & 36.6 & & 47 & -23.2 & 100 & 56.0 & 58.3 & 23 & 03 & -23.8 & $300^{*}$ & 43.8 & 58.3 & 24 & 45 & -22.3 \\
\hline 02 & $41 . I$ & 34. & & & 02 & 38.4 & 37.9 & & 45 & & 02 & 34.6 & 38.6 & 22 & $3 \mathrm{I}$ & & 02 & 59.8 & 72.0 & 25 & 09 & \\
\hline 04 & I. 8 & 35.6 & & & 04 & 41.0 & & & $4 \mathrm{I}$ & for & 04 & & 78.5 & 23 & & e & 04 & & & & & 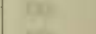 \\
\hline 06 & 1,6 & 35.9 & & & 06 & 42.0 & 40.8 & & 40 & w & 06 & 5.5 & 38.8 & 22 & 33 & & of & 66.1 & & 25 & I4 & 10 \\
\hline 08 & 1.7 & 36. & & & 08 & 38.7 & 37. & & 45 & 72 & 08 & 26.5 & 29.3 & & 17 & & or & & & & & $w$ \\
\hline I0 &.$I$ & 34.2 & & the & 10 & 38.4 & 37.4 & & 46 & ai & 10 & 53.0 & 55.5 & 22 & 59 & & $10^{*}$ & 64.3 & & & 49 & \\
\hline I2 & 4 & 35 . & & 7 & 12 & 38.6 & 37. & & 46 & & 12 & .0 & 75. & 23 & 31 & & 12 & $6 \mathrm{I} .5$ & 71 & 25 & 45 & \\
\hline 14 & 39.7 & 34.9 & & $-24 . I$ & 14 & 37.9 & 37. & & 46 & -23.2 & 14 & & 36.8 & 22 & & -23.6 & I4. & 23.0 & & 24 & 45 & -22.3 \\
\hline I6 & & & & & I6 & & 36 & & 47 & & 16 & 31.6 & 33.0 & & 24 & & $16^{*}$ & 33.0 & 44. & & OI & \\
\hline 18 & 4.8 & 38. & & 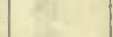 & I8 & 38.6 & 37 & & 45 & 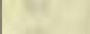 & I8 & & $4 \mathrm{I}$ & & 34 & & I8 & & & & & \\
\hline 20 & 45.9 & 4 & & 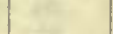 & 20 & 40.8 & 39.8 & & 42 & 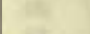 & 20 & & 33.3 & & 2 & & $20 *$ & 3.2 & 52 & 25 & 04 & \\
\hline 22 & 5. I & & & xa & 22 & 46.9 & & & 32 & 5 & 22 & & 30 & & $2 c$ & 마요 & 2 & & & & & 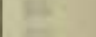 \\
\hline 24 & 5.7 & 40.6 & & 14 & 24 & 47.1 & 46.5 & & 32 & 12 & 2 & .7 & $24 \cdot 3$ & & 10 & & 2. & 34.0 & 38 & 24 & 54 & \\
\hline 26 & 48.3 & 42.3 & & & 26 & .0 & & & & & 26 & 8 & 22.2 & & 06 & & 2 & & & 25 & 2 & \\
\hline 28 & 47.0 & 43.5 & & 30 & 28 & 48.6 & 48. I & & 29 & & 28 & & & & 09 & & 28 & & & 25 & 04 & \\
\hline 30 & 5.9 & $4 I$. & & -23.9 & 30 & 50.9 & & & 26 & -23.3 & 30 & 22.8 & 24.6 & & II & $-23 \cdot 4$ & 30 & 32.5 & 32.5 & 24 & 48 & -22.0 \\
\hline 32 & 42. I & 39.8 & & & 32 & 49.9 & 48.8 & & 28 & & 32 & 24.9 & 28. & & 15 & & 32.3 & 15.5 & & & $2 \mathrm{I}$ & \\
\hline 34 & & 35 & & & 34 & 46.8 & 45.4 & & 33 & ge & 34 & .4 & 28.4 & & 16 & - & 34 & 9.7 & Io & & I2 & \\
\hline 36 & .1 & 34. & & & 36 & 46.0 & 44.9 & & 34 & & 36 & & & & 28 & & 36 & 17.2 & & & 25 & \\
\hline 38 & 33 & & & & 38 & 44.3 & 43 & & 37 & & 38 & .2 & 19.8 & & 03 & & 38 & 9.6 & IO & & 12 & \\
\hline 40 & & & & rest & 40 & 43.4 & & & & 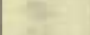 & 40 & 17.8 & 20.2 & 22 & 03 & & 40 & 17.7 & 20.6 & & 27 & \\
\hline 42 & 32. I & & & $x^{2}$ & 42 & 42.7 & 42.3 & & 38 & & 32 & 0.0 & 2.3 & 21 & 35 & 14 & 42 & I8 & $5^{b}$ & 24 & 26 & \\
\hline 44 & 30.0 & 28.6 & & -23.7 & 44 & 41.1 & & & $4 \mathrm{I}$ & $-23 \cdot 5$ & $44^{*}$ & 25.6 & 26.6 & 22 & 35 & -23.2 & $44^{*}$ & 45.0 & 52.6 & 23 & 52 & -22.0 \\
\hline 46 & 29. I & 25.2 & 230 & & 46 & 39.9 & 38 & & 43 & & 46 & 24.0 & 25.6 & & 33 & & 46 & 47.0 & & 23 & 55 & \\
\hline 48 & 27.5 & & & 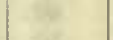 & 48 & 36.7 & 36. & & 48 & 8 & 48 & 26.0 & 27.3 & & 3 & 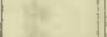 & 48 & 56 & & 24 & I & \\
\hline 50 & $27 . I$ & 22.6 & & & 50 & 36.3 & & & 49 & tre & 50 & & 28.9 & & 38 & 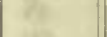 & 50 & 50.0 & 58.6 & 24 & OI & \\
\hline 52 & 29.0 & 25.0 & & & 52 & 37.6 & 38. & & 46 & & 52 & 23.7 & $24 \cdot 3$ & & 32 & & 52 & 49.8 & 56.3 & 23 & 5 & \\
\hline 54 & 32.7 & 27.9 & 225 & & 54 & 40.7 & 40.4 & & 42 & & 54 & 23.3 & 24.3 & & 32 & & 54 & 42.7 & & & 48 & \\
\hline 56 & 36.6 & 32.6 & & & 56 & 40.7 & 40. & & 42 & & 56 & 23.4 & $25 \cdot 3$ & & 32 & & 56 & 45.6 & 55.2 & 23 & 55 & \\
\hline $5^{8}$ & 36.3 & 33.0 & & & 58 & 40. I & 39.8 & & 43 & -23.4 & 58.3 & 27.6 & 31.6 & & $4 I$ & $-x$ & 58 & $53 \cdot 3$ & 57.3 & 24 & & \\
\hline & & & & & & 39.5 & 39.1 & & 43 & & & & & & & & & & & & & \\
\hline
\end{tabular}

Correction to local mean time is $+3 \mathrm{~m}$ 06s.

Torsion head at $\mathrm{inh}$ oom read $356^{\circ}$ and at $\mathrm{i} 6 \mathrm{~h}$ zom read the same.

Ohserver-W. J. P.

Observers - R. R. T. and W. J. P., who alternated $13 \mathrm{~h} 56$ in to 14 l $\infty \mathrm{m}$. 
Tabulation of magnetic declinations observed at Teplitz Bay-Continued

\begin{tabular}{|c|c|c|c|c|c|c|c|c|c|c|c|c|c|c|c|c|c|c|c|}
\hline \multicolumn{5}{|c|}{ IVednesday, November II, 1903} & \multicolumn{5}{|c|}{ Magnet scale erect } & \multicolumn{5}{|c|}{ Wednesday, November II, I903 } & & & $\mathrm{Ma}$ & t scale & erect \\
\hline \multirow{2}{*}{$\begin{array}{l}\text { Chr'r } \\
\text { time }\end{array}$} & \multicolumn{2}{|c|}{$\begin{array}{l}\text { Scale } \\
\text { readings }\end{array}$} & \multirow{2}{*}{$\begin{array}{l}\text { East } \\
\text { decli- } \\
\text { nation }\end{array}$} & \multirow{2}{*}{ Temp. } & Chr'r & $\begin{array}{r}\mathrm{Sc} \\
\text { read }\end{array}$ & $\begin{array}{l}\text { ale } \\
\text { lings }\end{array}$ & $\begin{array}{l}\text { East } \\
\text { decli- }\end{array}$ & Temp. & Clır'r & $\begin{array}{r}\mathrm{Sc} \\
\mathrm{read}\end{array}$ & $\begin{array}{l}\text { le } \\
\text { lgs }\end{array}$ & $\begin{array}{l}\text { East } \\
\text { decli- }\end{array}$ & & & $\begin{array}{r}\mathrm{Sc} \\
\text { read }\end{array}$ & $\begin{array}{l}\text { ale } \\
\text { lings }\end{array}$ & $\begin{array}{l}\text { East } \\
\text { decli- }\end{array}$ & \\
\hline & Left & Right & & & & Left & Right & & & & Left & Right & & & & Left & Right & & \\
\hline li $\mathrm{m}$ & d & $a$ & &,$\quad 0^{\circ}$ & h m & d & d & & & $\mathrm{h} \mathrm{m}$ & d & d & & & h m & & & $\circ$, & \\
\hline 400 & 63.0 & 68.6 & 24 & $9-22.0$ & 600 & 34.2 & 35.9 & 2240 & -20.1 & 800 & 20.7 & 22.1 & 2230 & -19.0 & Io 00 & 46.8 & 48.0 & 2242 & \\
\hline 02 & 63.7 & 68.7 & r & 9 & 02 & $45 . \mathrm{I}$ & 47.5 & 2257 & ng & 02 & 20.8 & 23.2 & 3I & 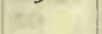 & 02 & 46.8 & 48.0 & & -19.5 \\
\hline 04 & 55.0 & 58.3 & 24 & 4 & 04 & 54.2 & 55.8 & 23 II & 10 & 04 & 13.2 & 17.1 & 20 & (46. & O4 & 47.6 & 48.3 & 43 & \\
\hline 06 & 39.1 & 44.2 & 23 & $7=$ & 06 & 53.2 & 55.7 & Io & & $06^{*}$ & $42 . I$ & 50.9 & 07 & 29 & 06 & 50.6 & 51.3 & 47 & 25 \\
\hline 08 & $4 \mathrm{I} \cdot 3$ & 43.2 & & ter & 08 & 49.8 & 53.7 & 2306 & (0) & 08 & 48.9 & 58.9 & 19 & bef & 08 & 50.1 & 50.5 & & 10 \\
\hline 10 & 36.8 & 37.2 & t. & $4 i$ & 10* & 27.3 & 46.2 & 2254 & Wr & Lo. & 63.7 & $73 \cdot 3$ & 42 & bir & IO & 49. & $.0 b$ & 44 & $\pi t$ \\
\hline 12 & 45.6 & 46.1 & & 8 & 12 & 21.2 & 39.9 & 2244 & -1 & $12^{*}$ & 51.7 & 57.7 & 53 & at: & 12 & 44.0 & 46.8 & & $\pm x$ \\
\hline 14 & 44.8 & 47.7 & & \begin{tabular}{l|l}
8 & -21.9
\end{tabular} & 14 & 34.6 & 51.1 & 2303 & -20.0 & I4 & 50.2 & $55 \cdot 3$ & 50 & & 14 & 42.6 & 43.6 & 35 & \\
\hline 16 & 42.4 & 48.3 & & 7 & I6 & 38.2 & 58.2 & 12 & & 16 & 47.2 & $54 \cdot 3$ & 2247 & -18.8 & 16 & $4 \mathrm{I} \cdot 3$ & 42.0 & 33 & -19.6 \\
\hline 18 & 39.2 & 44.7 & & 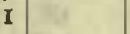 & 18 & 34.0 & 50.7 & 230.3 & & 18 & 59.1 & 64.2 & 2304 & & 18 & 36.0 & 39.6 & 27 & 10 \\
\hline 20 & 45.9 & 54.2 & & sat & 20 & 30.2 & 45.8 & 2256 & Q. & 20 & 52.6 & 57.4 & 2254 & ase & 20 & 39.8 & 44.2 & 33 & $=8$ \\
\hline 22 & 49.2 & 54.5 & & at & 22 & 25.9 & 41.8 & 49 & 82 & 22 & 56.1 & 60.2 & 2259 & 62 & 22 & $4 \mathrm{I} .0$ & $45 \cdot 3$ & 35 & es \\
\hline 24 & 30.2 & 34.8 & & 38 & 24 & 32.2 & 45.0 & 57 & 4 & 24 & 60.9 & 65.8 & 2307 & 2 & 24 & 37.0 & 41.0 & 29 & is \\
\hline 26 & $2 \mathrm{I} . \mathrm{I}$ & 25.3 & & a & 26 & 32.6 & 44.3 & 57 & 2 & 26 & 66.5 & 70.2 & 14 & ar & 26 & 40.6 & 42.8 & & Wos \\
\hline 28 & I5. I & 21.7 & & 29 & 28 & 30.8 & 43.5 & 55 & 20 & 28 & 63.7 & 67.2 & Io & & 28 & 48.1 & 50.5 & 45 & E. \\
\hline 30 & 24.4 & 27.2 & 1 & \begin{tabular}{l|l}
6 & -21.4
\end{tabular} & 30 & I 4.2 & 25.2 & 27 & 172- & 30 & 58.8 & $6 I .5$ & 02 & -18.7 & 30 & 46.3 & 48.3 & 42 & -19.7 \\
\hline 32 & 37.2 & 42.7 & 613 & 8 & 32 & 14.2 & 24.7 & 27 & -19.8 & 32 & 57.7 & 60.9 & 2300 & $1=$ & 32 & 40.0 & 41 & $3 I$ & \\
\hline 34 & 30.3 & 36.8 & 232 & tht & 34 & 26.9 & 36.8 & 46 & & 34 & 52.1 & 55.8 & 2252 & & 34 & 45.6 & 47.8 & $4 \mathrm{I}$ & 12 \\
\hline $36^{*}$ & 44 & I $b$ & 22 & & 36 & 31.6 & 44.6 & 2256 & cete. & 36 & 48.2 & 51.2 & 45 & $1=$ & 36 & 45.0 & 45.8 & 39 & as \\
\hline 38 & 24.9 & 25.2 & & $2=$ & 38 & 39.9 & 49.4 & 2306 & 38 & 38 & $5 \mathrm{I} .3$ & 54.9 & 2251 & A4 & 38 & 46.3 & 48.2 & $4 \mathrm{I}$ & Pi \\
\hline 40 & 20.2 & 20.9 & & $k^{2}=$ & 40 & 36.4 & $47 \cdot 3$ & 02 & fif & 40 & 57.2 & 60.9 & 2300 & bi & 40 & $48 . I$ & 49.6 & 44 & 6 \\
\hline 42 & 27.2 & 29.2 & & a) & 42 & $45 \cdot 3$ & 55.3 & 2315 & $x^{2}=2$ & 42 & 55.0 & 58.8 & 2256 & 25. & 42 & 46.0 & $47 \cdot 3$ & 40 & 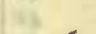 \\
\hline 44 & 45 & $5 a$ & & B. & 44 & 33.2 & 39.7 & 2253 & -19.6 & 44 & 55.8 & 60.2 & 58 & -18.9 & 44 & 44.6 & 45.0 & 38 & -19.6 \\
\hline 46 & 53.6 & 56.1 & 22 & is & 46 & II. 3 & 20.6 & $2 \mathrm{I}$ & & 46 & 55.5 & 58.8 & 57 & & 46 & 47.5 & 48.6 & 43 & 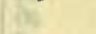 \\
\hline $4^{8}$ & 63 & $0 a$ & 23 & that & 48 & 27.8 & $34 \cdot 3$ & 45 & 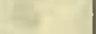 & 48 & 51.6 & 55.5 & 51 & his & 48 & 51.5 & 52.6 & & As \\
\hline $50^{*}$ & 39.3 & 45.2 & & at. & 50 & 30.9 & 38.5 & $5 I$ & ant & 50 & 53.0 & 57.1 & 2254 & int & 50 & 51.5 & 52.5 & & wit \\
\hline 52 & 35.8 & 38.7 & & 6 & 52 & 30.3 & 38.9 & $5 \mathrm{I}$ & (6it & 52 & 59.0 & 63.2 & 2303 & $r=$ & 52 & 49.4 & 51. & 46 & {$[B$} \\
\hline 54 & II. 7 & I 8.2 & $23 c$ & 17 & 54 & 24.2 & 31.9 & 40 & ar & 54 & 59.2 & 6 ז.9 & 2302 & Es & 54 & 52.0 & 52 & & int \\
\hline 56 & 9.8 & I5.9 & 22 & 68 & 56 & 29.9 & 38.8 & 2250 & tat & 56 & 55.5 & 58.6 & 2257 & nte & 56 & 51.5 & 53.2 & 49 & tho \\
\hline $58^{*}$ & 22.7 & 39.3 & & 3 & 58 & 36. I & 44.4 & 2300 & tei & 58 & 60.0 & 62.0 & 2303 & & 58 & 49.2 & 49. & & \\
\hline$\infty$ & 26.9 & 45.5 & 24 & I -20.7 & 700 & 37.8 & 48.2 & 2304 & -19.3 & 900 & 56.8 & 59.0 & 2258 & -18.5 & II 00 & 48.6 & 51.5 & 46 & -19.6 \\
\hline 02 & $34 \cdot 3$ & 51.3 & 22 & 的 & 02 & 30.3 & 37.5 & 2249 & - & 02 & 54.5 & 56.3 & 54 & 20. & 02 & 50.6 & 55.6 & $5 \mathrm{I}$ & - \\
\hline 04 & 46.1 & 63.9 & 23 & in & 04 & 28.2 & 34.3 & 2245 & 10 & 04 & 52.5 & 54.9 & 2252 & tho & 04 & 54.9 & 59. & 57 & 10 \\
\hline 06 & 51.6 & 66.0 & & 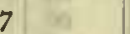 & 06 & 45.2 & 53.9 & 23 I4 & $\delta$ & 06 & 60. & 62.2 & 2304 & 60 & 06 & & & 53 & in \\
\hline 08 & 50.1 & 63.2 & 23 & 100 & 08 & $55 . \mathrm{I}$ & 60.3 & 2327 & $m$ & 08 & 68.3 & 70.4 & 16 & 36 & 08 & 48.6 & 52 & 47 & wo \\
\hline I0 & 40.2 & 51.2 & 225 & 34 & IO & 32.8 & 40.2 & 2254 & $\infty$ & IO & 66.0 & 68.5 & 13 & wit & IO & $44 \cdot 5$ & 46.3 & 39 & oit \\
\hline 12 & $34 . I$ & $45 \cdot 3$ & & 73 & I2 & $55 . \mathrm{I}$ & $6 \mathrm{I} . \mathrm{I}$ & 2327 & 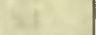 & 12 & 65.0 & 67.0 & I I & ar: & 12 & 47.8 & 50 & 45 & $=1$ \\
\hline I4 & 26.8 & 36.4 & & $4-20.3$ & I4 & 46.3 & 52.0 & 13 & -19.3 & 14 & 62.0 & 64.2 & 2306 & $-\mathrm{I} 9.0$ & 14 & 42.5 & 46.2 & 37 & -19.8 \\
\hline 16 & 23.2 & $3 \mathrm{I} .5$ & & 7 & 16 & 58.8 & 62.2 & $3 I$ & & 16 & 54.6 & 55.6 & 2254 & $x^{2}$ & 16 & 39.0 & 43 & 32 & $x^{2}$ \\
\hline 18 & 29.8 & 38.2 & & Wi & I8 & 55.7 & $6 \mathrm{I} . \mathrm{I}$ & 28 & int & I8 & 54.0 & 55.4 & 53 & $3 n$ & 18 & 47.5 & 51.5 & 45 & 31 \\
\hline 20 & $4 \mathrm{I} \cdot 3$ & 50.3 & & net & 20 & $52 . \mathrm{I}$ & 56.1 & $2 I$ & es & 20 & 53 & $.0 b$ & 50 & 200 & 20.2 & 45.3 & & $4 \mathrm{I}$ & $1+x$ \\
\hline 22 & 40.3 & 49.1 & 22 & kats & 22 & 55.9 & 60.1 & 27 & ate & 22 & 47.0 & 50.6 & 44 & 58 & 22 & 44.6 & 46.9 & 39 & sis \\
\hline 24 & 45.2 & 5 I. 3 & 23 & is & 24 & 54.4 & 60. & 26 & $a t$ & 24 & 50.6 & $54 \cdot 3$ & 49 & is & 24 & & & 45 & is \\
\hline 26 & 45.1 & 5 I.I & $23 c$ & E & 26 & 49.1 & 56.0 & 18 & Ba & 26 & 53.1 & 57.6 & 54 & 6 & 26 & 48.3 & 50.6 & 45 & lata \\
\hline 28 & 35.8 & 42.2 & 22 & $7=$ & 28 & 42.2 & 47. & 07 & & 28 & 46.7 & 52.3 & 45 & me & 28 & 46.7 & & 43 & in \\
\hline 30 & 26.2 & 31.9 & & \begin{tabular}{l|l}
0 & -20.3
\end{tabular} & 30 & 40.8 & 46 & 2304 & -19.2 & 30 & 41.6 & 44. & 34 & -19.2 & 30 & 44.2 & 48. & 40 & -20.0 \\
\hline 32 & 20.9 & 24.9 & & 0 & 32 & 35.8 & 39. & 2256 & & 32 & & & 52 & ( & 32 & 44 . & & 39 & 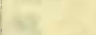 \\
\hline 34 & 22.4 & $3 \mathrm{i} \cdot 3$ & $22=$ & t. & 34 & 45.0 & 48.1 & 23 o9 & & 34 & 56. & & 57 & ax & 34 & 44. & 46 & 38 & lace \\
\hline 36 & 47.8 & 52.2 & $23 c$ & 8 & 36 & 41.0 & 41.8 & or & & 36 & 53.2 & 56 & 53 & aes & 3 & 43. I & 45 & 37 & 3 \\
\hline $3^{8}$ & 45.0 & 48.8 & 225 & $t^{2}$ & 38 & 41.9 & 42.2 & 2302 & the & 38 & 53.0 & 56 & 53 & we & 38 & 44.2 & 47 & 39 & 68 \\
\hline 40 & 33.7 & 36.5 & & 10 & 40 & 37.7 & 39.9 & 2257 & 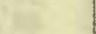 & 40 & 48.2 & $53.0^{\circ}$ & 47 & t & 40 & 48.0 & & 45 & 10 \\
\hline 42 & 34.2 & 38.1 & & 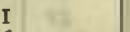 & 42 & 45.6 & 46.9 & 2309 & & 42 & 3 I. 2 & 37.0 & $2 \mathrm{I}$ & & 42 & 49.3 & 5 I. & 47 & \\
\hline 44 & 42.8 & 48.8 & 225 & $6-202$ & 44 & 41.9 & 45.0 & 04 & -19.0 & 44 & 37.0 & 42.0 & 29 & -19.6 & 44 & & & 46 & -20.0 \\
\hline 46 & 53.3 & 58.3 & 231 & 2 & 46 & $42 . I$ & 46.0 & 2305 & - & 46 & 43.6 & 47.0 & 38 & & 46 & 46.8 & 48.8 & 42 & 10 \\
\hline 48 & 47.8 & $52 . \mathrm{I}$ & 230 & is & 48 & 38.5 & 41.8 & 2259 & $t_{4}$ & 48 & 43.5 & 45.9 & 37 & $y_{4}$ & $4^{8}$ & 46.3 & 48.7 & 42 & the \\
\hline 50 & 32.3 & 36.6 & 223 & ate & 50 & $34 . I$ & 38.8 & 53 & & 50 & 52.9 & 55.5 & 52 & oe & 50 & 45.0 & 46.7 & 39 & $\sqrt{102}$ \\
\hline 52 & 29.2 & 35.1 & 3 & $3 t$ & 52 & $37 \cdot 4$ & 40.3 & 57 & teis & 52 & 56.3 & 59.3 & $5^{8}$ & $w$ & 52 & 40.8 & 42.8 & 33 & 18 \\
\hline & $1 \overline{6} .3$ & $2 \mathrm{I} .5$ & & at? & 54 & 24.9 & 28.9 & $3^{8}$ & it. & 54 & 53.6 & 56.5 & 54 & 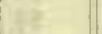 & 54 & $4 \mathrm{I} . \mathrm{I}$ & 43.2 & 33 & at \\
\hline 56 & 28.2 & $30 . I$ & & 4 & 56 & 35.5 & 37.8 & 54 & 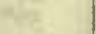 & 56 & 46.3 & 49.3 & 42 & & 56 & 39.5 & $4 \mathrm{I} .3$ & $3 \mathbf{I}$ & 68 \\
\hline 58 & 45,8 & 48.6 & & k & 58 & 28,2 & 3 I. 6 & 43 & & 58 & 44.0 & 45.0 & 37 & 3 & 58 & 38.3 & 39.8 & 29 & 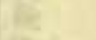 \\
\hline
\end{tabular}

Observers-W. J. P. and R. R. T., who alternated from $4^{\text {h }} 04 \mathrm{~m}$ to 4 h $12 \mathrm{~m}$.
Observers-R. R. T. and W. J. P., who alternated from $8 \mathrm{~h} 44 \mathrm{~m}$ to $8 \mathrm{~h} 54 \mathrm{~m}$. 
Tabulation of magnetic declinations observed at Teplitz Bay-Continued

\begin{tabular}{|c|c|c|c|c|c|c|c|c|c|c|c|c|c|c|c|c|c|c|c|c|c|c|}
\hline \multicolumn{5}{|c|}{ Wednesday, November II, 1903} & \multicolumn{6}{|c|}{ 2.24agnet scale erect } & \multicolumn{12}{|c|}{ Wednesday, November II, 1903} \\
\hline \multirow{2}{*}{$\begin{array}{l}\text { Chr'r } \\
\text { time }\end{array}$} & \multicolumn{2}{|c|}{$\begin{array}{l}\text { Scale } \\
\text { readings }\end{array}$} & \multirow{2}{*}{$\begin{array}{l}\text { East } \\
\text { decli- } \\
\text { nation }\end{array}$} & 'Temp. & Chr'r & $\begin{array}{r}\mathrm{Sc} \\
\text { read }\end{array}$ & $\begin{array}{l}\text { ale } \\
\text { lings }\end{array}$ & $\begin{array}{l}\text { Ea } \\
\text { dec }\end{array}$ & $\begin{array}{l}\text { ast } \\
\text { cli- }\end{array}$ & Temp. & Chr'r & $\begin{array}{r}\text { Sc } \\
\text { read }\end{array}$ & $\begin{array}{l}\text { ale } \\
\text { lings }\end{array}$ & $\begin{array}{l}\text { Eas } \\
\text { decl }\end{array}$ & & & & Sc & $\begin{array}{l}\text { ale } \\
\text { lings }\end{array}$ & $\begin{array}{l}\text { Eas } \\
\text { decl }\end{array}$ & & Temp. \\
\hline & L,eft & Right & & & & Left & Right & & & & & Left & Right & & & & & Left & Right & & & \\
\hline $1 \mathrm{~m}$ & d & d & $\cdot 1$ & ant & $\mathrm{h} \mathrm{m}$ & d & d & - & & 0 & $h \mathrm{~m}$ & d & d & & & & $\mathrm{h} \mathrm{m}$ & $d$ & d & & , & \\
\hline 1200 & 36.8 & 37.8 & 2226 & -20.0 & I4 00 & 38.4 & 39.8 & 22 & 42 & -20.6 & 1600 & 40.0 & $4 I \cdot 3$ & & & -18.8 & $1800^{*}$ & 37.1 & 75.0 & & 54 & -18.3 \\
\hline 02 & 34.8 & 35.1 & 22 & & 02 & 39.4 & 40.6 & & 44 & & 02 & 39.3 & 40.8 & & 43 & & $02^{*}$ & 38.0 & 53.8 & & $4 \mathrm{I}$ & \\
\hline 04 & 36.2 & 37.2 & 25 & wa & 04 & 40.0 & 41.0 & & 45 & & 04 & 39.2 & 40.8 & & 43 & 24 & 04 & 31.7 & 51.9 & 233 & 35 & (11) \\
\hline 06 & 37.2 & 37.7 & 26 & $(x)$ & 06 & 36.7 & 37.7 & & 39 & & 06 & 39.6 & $4 I .2$ & & 44 & 49 & $07^{*}$ & 5.8 & 37.8 & 240 & 00 & ins \\
\hline $08^{*}$ & 38.3 & 38.9 & 42 & 120 & 08 & 36.2 & $37 \cdot 3$ & & 39 & & 08 & 37.4 & 38.8 & & $4 \mathrm{I}$ & Es. & 08 & 6.0 & 34.0 & 235 & 56 & is \\
\hline IO & 35.0 & 35.8 & 37 & 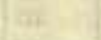 & I0 & 33.8 & 35.8 & & 36 & & 10 & 36.4 & 37.3 & & 38 & 45 & 10 & 13.1 & 33.3 & 240 & OI & 65 \\
\hline 12 & 32.3 & 33.0 & 32 & $2-1$ & 12 & 34.0 & 36.5 & & 36 & thent & I2 & 34.4 & 35 . & & 35 & & 12 & 9.3 & 35.8 & 240 & 00 & \\
\hline 14 & 34.7 & 35.0 & 36 & -19.8 & 14 & Lo & st & & & $-\mathrm{I} 9.4$ & 14 & 33.2 & 33.2 & & 33 & -19.0 & $14^{*}$ & 30.1 & 60.8 & 232 & 27 & -18.0 \\
\hline 16 & 30.0 & 31.2 & 29 & & 16 & 30.7 & 41.7 & & 38 & & 16 & 33.9 & 34.2 & & 34 & & 16 & 55.2 & 74.8 & & 58 & \\
\hline 18 & 32.9 & 33.0 & 33 & if & 18 & 28.7 & 38.7 & & 34 & & 18 & 34.6 & 35.3 & & 35 & Bit & I8 & L. & ost & & & (c) \\
\hline 20 & 33.0 & 34.6 & 34 & (10 & 20 & 29.2 & 38.4 & & 34 & 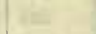 & 20 & 33.9 & 34. & & 34 & $=6$ & $20^{*}$ & $27 . \overline{8}$ & 58.0 & & 38 & $\alpha$ \\
\hline 22 & 34.9 & 36.8 & 37 & 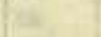 & 22 & 30.2 & 38.2 & & 34 & $=$ & 22 & 32.7 & 33.7 & & 33 & is & 22 & 22.5 & 49.6 & & 28 & 푼 \\
\hline 24 & 40.4 & 42.2 & 46 & $=$ & 24 & 29.3 & 36.6 & & 32 & & 24 & 35.4 & 36.9 & & 37 & $y^{4}$ & 24 & 23.2 & 47.3 & & 26 & 12 \\
\hline 26 & 44.9 & 46.8 & 53 & $=$ & 26 & 29.1 & 35.9 & & 32 & & 26 & 35.2 & 37. & & 37 & as & 26 & 28.3 & 50.8 & & 33 & E \\
\hline 28 & 48.0 & 50.0 & 2258 & & 28 & 30.6 & 36.5 & & 33 & & 28 & 38.0 & 39.9 & & 42 & E. & 28 & 19.7 & 40.8 & & 19 & \\
\hline 30 & 51.3 & 54.0 & 2304 & - I9.9 & 30 & 31.5 & 35.9 & & 34 & -I8.8 & 30 & 42.2 & 42.6 & & 47 & -19.4 & 30 & 21.1 & 40.5 & & 20 & -18.0 \\
\hline 32 & 54.2 & 56.0 & 07 & & 32 & 34.2 & 37.8 & & 37 & & 32 & 42.3 & 43.2 & & 48 & -2.7 & 32 & 22.9 & 42.7 & & 23 & ( \\
\hline 34 & 56.6 & 58.2 & II & E & 34 & 36.2 & 39.4 & & 40 & 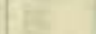 & 34 & 44.3 & 44. & & 51 & 12 & 34 & 17.2 & 35 & & I3 & II \\
\hline 36 & 55.4 & 57.8 & 10 & 8 & 36 & 39.2 & 42.7 & & 45 & 2 & 36 & 46.0 & 47.9 & & 54 & (5) & 36 & 12.5 & 29.2 & & 04 & \\
\hline 38 & 56.0 & 57.8 & IO & b & 38 & 39.2 & 42.8 & & 45 & tit & 38 & 45.0 & 47. & & 53 & $\beta$ & 38 & 10.7 & 26.4 & 230 & & \\
\hline 40 & 58.0 & 58.8 & 13 & wet & 40 & 38.2 & $4 I .4$ & & 43 & as & 40 & 45.3 & 47 & & 53 & 114 & 40 & 9.0 & 23.9 & 225 & 57 & $=$ \\
\hline 42 & 63.0 & 64.0 & $2 I$ & ha & 42 & 37.9 & 41.6 & & 43 & & 42 & 46.0 & 46.8 & & 54 & 78 & 42 & 13.0 & 16.2 & & 54 & \\
\hline 44 & 62.1 & 63.0 & 19 & -19.7 & 44 & 38.2 & 41.7 & & 43 & -18.6 & 44 & 46.0 & 47. & & 54 & -10.3 & 44 & 8.5 & 9.9 & & 46 & -18.0 \\
\hline 46 & 60.1 & 62.0 & 17 & & 46 & 40.9 & 44.2 & & 47 & & 46 & 44.0 & 44.8 & & 50 & & 46 & 7.6 & 10.7 & & 46 & . \\
\hline 48 & 54.7 & 55.0 & 07 & 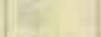 & 48 & 39.9 & 42.9 & & 46 & 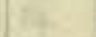 & 48 & 42.0 & $42 \cdot 3$ & & 47 & a & 48 & 7.0 & 9.5 & & 44 & he \\
\hline 50 & 52.2 & 53.7 & 04 & 6 & 50 & 38.2 & 44.1 & & 45 & & 50 & 40.9 & 41.9 & & 46 & 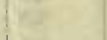 & 50 & 6.5 & 9.7 & & 44 & \\
\hline 52 & 51.2 & 53.4 & 03 & 13 & 52 & 38.1 & 40.8 & & 43 & & 52 & 38.0 & 38 & & $4 I$ & 8 & $52^{*}$ & 39.0 & $4 \mathrm{I} .0$ & & 44 & \\
\hline 54 & 51.0 & 52.9 & 02 & 15 & 54 & 36.2 & 38.2 & & 39 & & 54 & 36.6 & 38. & & 40 & & 54 & 38.5 & 40.4 & & 44 & 4) \\
\hline 56 & 51.4 & 52.0 & 02 & $c$ & 56 & 37.0 & 39.1 & & 40 & 5 & 56 & 37.0 & 38 & & 40 & & 56 & 38.2 & 40.5 & & 44 & \\
\hline 58 & 50.3 & 51.8 & OI & 25 & 58 & 37.8 & 39.8 & & 42 & & 58 & 36.2 & 38.5 & & 39 & & 58 & 38.5 & 40.6 & & $\begin{array}{l}47 \\
44\end{array}$ & \\
\hline 1300 & 53.9 & 54.6 & 06 & -19.8 & I5 00 & 39.5 & $4 \mathrm{I} .3$ & & 44 & -18.5 & 1700 & 34.7 & 37.6 & & 37 & -18.5 & I9 00 & 39.6 & 41.6 & & 45 & -18.0 \\
\hline 02 & 54.0 & 55.0 & 07 & 100 & 02 & 38.8 & 40.8 & & 43 & 120.0 & 02 & 33.6 & 35.2 & & 35 & 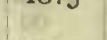 & 02 & 39.6 & 41.6 & & 45 & \\
\hline 04 & 49.3 & 51.3 & $\infty$ & 10 & 04 & 37.6 & 39.3 & & 41 & +1 & 04 & 33.7 & 34.0 & & 34 & 10 & 04 & 38.0 & 40.0 & & 4.3 & \\
\hline 06 & 52.5 & 53.4 & 04 & $6 x$ & 06 & 37.7 & 39. & & $4 \mathrm{I}$ & $n$ & 06 & 32.8 & 35 & & 34 & $\infty$ & 06 & .36 .6 & 38.5 & & $4 \mathrm{I}$ & he \\
\hline 08 & 54.0 & 55.9 & 07 & 6. & 08 & 38.2 & 40.2 & & 42 & (3) & 08 & 34.2 & 36.6 & & 36 & kno & 08 & 35.4 & 37.0 & & 38 & Ay \\
\hline 10 & 49.9 & 51.0 & oo & 71 & 10 & 40.2 & 42.2 & & 45 & at & IO & & 35 . & & 35 & at & Io & 34.5 & 35.8 & & 37 & $5 r$ \\
\hline 12 & 51.7 & 53.2 & 03 & 14 & 12 & 41.9 & 43.9 & & 48 & & 12 & 35.9 & 37.6 & & 38 & & 12 & 33.5 & 35.0 & & 35 & \\
\hline 14 & 51.9 & 52.6 & 03 & -I9. I & 14 & 43.2 & 45. & & 50 & -18.5 & I4 & 5.3 & 38. & & 39. & -18.3 & I4 & 34.5 & 36.5 & & 38 & -I8. \\
\hline 16 & $53 . \mathrm{I}$ & 54.3 & 05 & & 16 & 43.9 & 45.8 & & $5 I$ & & 16 & 33.9 & 35.7 & & 35 & & 16 & 39.6 & 42.8 & & 46 & \\
\hline 18 & 54.0 & 56.3 & 07 & 61 & 18 & 46.1 & 47. & & 55 & $4 t$ & I8 & 32.1 & 33 & & 32 & F & I8 & 44.5 & 47.0 & & 53 & \\
\hline 20 & 52.4 & 54.3 & 05 & re & 20 & $47 \cdot 5$ & 50.0 & 22 & 57 & is & 20 & 31.0 & 32.9 & & $3 \mathrm{I}$ & $=$ & 20 & 47.0 & 49.0 & 225 & 57 & $1 \pm$ \\
\hline 22 & 51.9 & 53.3 & 0.4 & cE & 22 & 49.8 & 50. & & 00 & $\omega$ & 2 & 31.8 & 33.4 & & 32 & as & 22 & 49.5 & 51.3 & 230 & OI & 14 \\
\hline 24 & 50.6 & 51.7 & 23 ol & E & 24 & 47.8 & 50.3 & & 58 & $t$ & 2 & 31.8 & $\begin{array}{l}53.4 \\
33.4\end{array}$ & & 32 & is & 24 & 46.5 & 49.5 & 225 & 57 & \\
\hline 26 & 48.7 & 49.3 & 258 & es & 26 & 47.1 & & 22 & 57 & 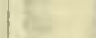 & 26 & 3 I. 2 & $\begin{array}{l}53.4 \\
33.2\end{array}$ & & 32 & s. & 26 & 45.5 & 46.6 & & 54 & the \\
\hline 28 & 45.0 & 45.6 & 52 & 62. & 28 & 48.9 & 51.8 & 23 & oo & & 28 & & 27.1 & & 22 & 6 & 28 & $\begin{array}{l}45.5 \\
39.4\end{array}$ & 42.0 & & 46 & \\
\hline 30 & 44.2 & 45.8 & 52 & -19.0 & 30 & 50.0 & 52.4 & & OI & -18.5 & 30 & 25.2 & 22.2 & & I5 & -18.5 & 30 & 42.5 & 44.6 & & 50 & $-\mathrm{I} 8.6$ \\
\hline 32 & 44.0 & 46.8 & 52 & & 32 & 52.8 & 54. & & 05 & 1 & 3 & & 30.5 & & 27 & $1-1$ & 32 & 42.3 & 44.6 & & 50 & \\
\hline 34 & 45.0 & 47.6 & 54 & is & 34 & 55.5 & 57.3 & & 09 & 12 & 3 & 56.1 & 66.0 & 23 & 17 & 10 & 34 & 44.9 & 46.2 & & 53 & \\
\hline 36 & 40.8 & 43.0 & 47 & & 36 & 56.9 & & & II & & 36 & 37.8 & 44.7 & & 45 & te & 36 & 46.0 & 47.4 & & 55 & $6 z$ \\
\hline 38 & 44.8 & 46.4 & $\begin{array}{l}47 \\
52\end{array}$ & 145 & 38 & 54.7 & 55.8 & & 07 & 21 & 38 & 29.5 & $\begin{array}{l}44 \\
35\end{array}$ & & $\begin{array}{l}45 \\
32\end{array}$ & 2 & 38 & 39.0 & $4 \mathrm{I} .0$ & & 44 & \\
\hline 40 & 44.4 & 46.6 & 52 & 17 & 40 & 54.0 & 55. & & 06 & - & 40 & 32.5 & 36.8 & 22 & 35 & $e^{2}$ & 40 & 41.0 & 43.2 & & 48 & \\
\hline 42 & 43.6 & 45.4 & $5 I$ & 6 & 42 & 53.1 & 54.4 & & 05 & & 42 & 72.2 & 73.8 & 23 & 35 & is & 42 & Lo & & & & \\
\hline & 42.8 & 44.3 & & -I9.0 & & 50.4 & 52.1 & 23 & OI & -18.6 & $44 * 6$ & $4 \mathrm{I} .5$ & 52.5 & 27 & 03 & 48. & 44 & 41.9 & & & 50 & -18.5 \\
\hline 46 & 41.0 & 42.0 & 46 & - & 46 & 48.9 & 48.9 & 22 & $5^{8}$ & -10.0 & $\begin{array}{l}44 \\
46\end{array}$ & & ost & & & & $\begin{array}{l}44 \\
46\end{array}$ & 43.6 & 45.6 & 225 & 52 & \\
\hline 48 & 42.0 & 43.5 & 48 & 0 & 48 & 46.7 & 47.0 & & 54 & & $48 *_{2}$ & 19.7 & 20.2 & 272 & 23 & 14 & 48 & 50.0 & 52.3 & 230 & 02 & \\
\hline 50 & 42.1 & 43.6 & 48 & 8 & 50 & 46.0 & 46.3 & & $\begin{array}{l}54 \\
53\end{array}$ & $=2$ & $50^{*}$ & 39.2 & 62.0 & 260 & & te & 50 & 56.9 & 59.3 & & 13 & ee \\
\hline 52 & 40.8 & $4 I .4$ & 45 & ter & 52 & 44.9 & 45.3 & & 52 & cas & $52^{*} 2$ & 34.1 & 57.7 & 24 & 08 & $=$ & 52 & 63.2 & 66.8 & & 24 & \\
\hline 54 & 40.8 & 40.9 & 45 & 18 & 54 & 43.2 & 44.0 & & 49 & & 54 & 7.8 & 40.9 & 233 & & & 54 & 62.9 & 65.7 & & 23 & $\mathrm{~B}$ \\
\hline 56 & 38.0 & 38.0 & $4 \mathrm{I}$ & & 56 & 41.9 & 42.5 & & 47 & & $\begin{array}{l}54 \\
56^{*}\end{array}$ & 19.2 & 50.5 & & 04 & & 56 & 59.7 & 64.8 & & I9 & \\
\hline 58 & 31.8 & 32.8 & $3^{2}$ & & 58 & 40.0 & 4I. I & & 44 & & 58 & 39.9 & 64.9 & & $3 \mathrm{I}$ & & 58 & 57.6 & 61.6 & & 15 & 82 \\
\hline
\end{tabular}

Ohservers-W. J. P. and F. L., who alternated from I2h I I m to I2h $24 \mathrm{~m} ; \mathrm{I}$. L. and W. J. P., who alternated from I2lı $58 \mathrm{~m}$ to I3l I2m; W. J. P. and R. R. T., who alternated from 14 h $08 \mathrm{~m}$ to $14 \mathrm{hl} 14 \mathrm{~m}$.
Observers-R. R. 'I. and W. J. P., who alteruated from I8h $40 \mathrm{~m}$ to I8h 50in; W. J. P. and R. R. T., who alternated from Igh $34 \mathrm{~m}$ to 19l1 $44 \mathrm{~m}$. 
Tabulation of magnetic declinations observed at Teplitz Bay-Continued

\begin{tabular}{|c|c|c|c|c|c|c|c|c|c|c|c|c|c|c|c|c|c|c|c|c|}
\hline \multicolumn{5}{|c|}{ Werlnesday, November II, I903 } & \multicolumn{6}{|c|}{ Magnet scale erect } & \multicolumn{5}{|c|}{ Thursday, November 12, 1903} & \multicolumn{5}{|c|}{ Magnet scale erect-inverted } \\
\hline \multirow{2}{*}{$\begin{array}{l}\text { Chr'r } \\
\text { time }\end{array}$} & \multicolumn{2}{|c|}{$\begin{array}{l}\text { Scale } \\
\text { readings }\end{array}$} & \multirow{2}{*}{$\begin{array}{l}\text { East } \\
\text { decli- } \\
\text { nation }\end{array}$} & Temp. & & $\begin{array}{l}\text { Se: } \\
\text { read }\end{array}$ & $\begin{array}{l}\text { ale } \\
\text { lings }\end{array}$ & Eas & & Temp. & Chr'r & $\begin{array}{l}\text { Sc: } \\
\text { read }\end{array}$ & $\begin{array}{l}\text { ale } \\
\text { lings }\end{array}$ & East & & & $\begin{array}{l}\text { Sc: } \\
\text { read }\end{array}$ & ngs & East & \\
\hline & Left & Right & & & & Left & Right & & & & & Left & Right & & & & Left & Right & & \\
\hline $\begin{array}{r}\mathrm{h} \mathrm{m} \\
20 \mathrm{OO}\end{array}$ & $\underset{5 I . I}{d}$ & $\begin{array}{c}d \\
57.8\end{array}$ & $\begin{array}{cc}0 & \\
23 & 07\end{array}$ & ${ }_{-18.3}^{\circ}$ & h m & $\sin ^{d}$ & $d_{-5}^{d}$ & $\stackrel{0}{22}$ & & . & & $d$ & d & 1 ' & 10 & $\mathrm{~h} \mathrm{~m}$ & $\mathrm{~d}^{\mathrm{d}}$ & $\mathrm{d}^{\mathrm{d}}$ & $\circ \quad$ & $\begin{array}{c}\circ \\
-18.8\end{array}$ \\
\hline 02 & 43.3 & $\begin{array}{l}57.0 \\
47.9\end{array}$ & 2253 & $\left.\right|^{-10.3}$ & $\begin{array}{r}22 \\
00 \\
02\end{array}$ & $\begin{array}{l}54.0 \\
53.1\end{array}$ & $\begin{array}{l}50.5 \\
57.8\end{array}$ & 2 & 2 & $-17 \cdot 7$ & $\begin{array}{l}00 \\
02\end{array}$ & $\begin{array}{l}37 \cdot 3 \\
37 \cdot 3\end{array}$ & $\begin{array}{l}37.9 \\
38.1\end{array}$ & $\begin{array}{r}22 \\
43 \\
44\end{array}$ & -10.0 & $\begin{array}{r}1800 \\
02\end{array}$ & $\begin{array}{l}40.2 \\
47 \cdot 3\end{array}$ & $\begin{array}{l}43.7 \\
44.7\end{array}$ & $\begin{array}{r}2244 \\
42\end{array}$ & -10.0 \\
\hline 04 & 39.2 & 44.1 & & k: & 04 & 58.8 & 62.6 & & 0 & 18 & 04 & 37.0 & 37.4 & 44 & 110 & 04 & 50.4 & 47.7 & & flar \\
\hline 06 & 38.8 & 42.4 & 45 & 6 & 06 & 43.0 & 56.5 & 221 & & 27 & 06 & 37.6 & 37.9 & 44 & 100 & 06 & 50.8 & 48.6 & 36 & 98 \\
\hline 08 & 36.0 & 39.0 & $4 \mathrm{I}$ & 6 & 08 & 33.6 & 35.6 & 214 & & & 08 & 36.6 & 36.8 & 43 & 68 & 08 & 5 I. 8 & 49.3 & 35 & 100 \\
\hline 10 & 37.3 & 41.8 & 44 & 101 & 10 & 36.3 & 43.4 & & 8 & $=1$ & $10-$ & 36.5 & 37.3 & 43 & ater & IO & 52.4 & 49.9 & 34 & 37 \\
\hline 12 & 33.6 & $37 . \mathrm{I}$ & 37 & 2 & 12 & 33.1 & 44.6 & & 6 & Har & 12 & 37.1 & 37.8 & 43 & 13 & 12 & 54.8 & 52.4 & 30 & $1^{171}$ \\
\hline 14 & $36 . I$ & 39.8 & $224 \mathrm{I}$ & -18.4 & 14 & 35.7 & 40.3 & & 5 & & 14 & $37 \cdot 3$ & 38.2 & 44 & -19.0 & I4 & 56.0 & 53.6 & 28 & -18.5 \\
\hline 16 & $47 \cdot 3$ & 52.1 & 2300 & & 16 & 29.7 & 33.0 & & 4 & -17.4 & 16 & 36.0 & 37.0 & 42 & & 16 & 55.2 & 53.0 & 29 & \\
\hline 18 & $4 \mathrm{I} . \mathrm{I}$ & 47.2 & 2251 & $\mathrm{FH}$ & 18 & 25.6 & 31.6 & 214 & & & I8 & 35.7 & 36.1 & $4 \mathrm{I}$ & 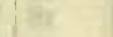 & 18 & 52.5 & 54.5 & 30 & Bi \\
\hline 20 & 42.1 & 47.2 & 52 & $\mathrm{~Pa}$ & 20 & 52.0 & 56.6 & 222 & & - & 20 & 34.0 & 34.9 & 39 & 0 & 20 & 55.2 & 53 & 29 & at: \\
\hline 22 & 42.0 & 51.5 & 55 & Ex & 22 & 70.1 & 70.3 & & 5 & $z^{2}$ & 22 & 32.8 & 34.8 & 38 & 10 & 22 & 8.0 & 46 & 40 & $1 \leq$ \\
\hline 24 & 40.2 & 45.7 & 49 & 하 & 24 & 60 &.$o b$ & & 29 & 74 & 24 & $\mathrm{Sc}$ & alc & & $y^{2}$ & 24 & 48.0 & 46.3 & 40 & 23 \\
\hline 26 & 31.0 & 37.2 & 35 & 6 & 26 & $6 \mathrm{I} \cdot 3$ & $61 \cdot 3$ & & II & 120 & 26 & inve & rted & as & 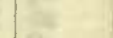 & 26 & $45 \cdot 3$ & 43. & & a \\
\hline 28 & 28.5 & 37.9 & 34 & & 28 & 60.0 & 62.1 & & I & & $28^{*}$ & 46.6 & 36.1 & 34 & 28 & 28 & 45.3 & & 44 & \\
\hline 30 & 28.6 & 33.0 & 30 & -18.4 & 30 & 3.6 & 55. & & I & -17.5 & 30 & 46.3 & 36.3 & 34 & $-\mathrm{I} 9.0$ & 30 & 47.8 & 46 & 40 & -18.5 \\
\hline 32 & 31.5 & 35.4 & 34 & & 32 & 53.8 & 56.3 & & 1 & & 32 & 42.7 & 42.2 & 32 & - & 32 & 48.1 & 46.0 & 41 & \\
\hline 34 & 37.7 & 46.4 & 48 & Fin & 34 & 6.8 & 48.9 & & 0 & He & 34 & $45 \cdot 3$ & 45.3 & 27 & $k$ & 34 & 48.4 & & 39 & 14 \\
\hline 36 & 33.0 & $4 I .3$ & 40 & 40 & 36 & I.I & 44.6 & & 2 & & 36 & 4I. & 39.8 & 35 & N & 36 & 48.9 & 47.8 & 39 & $a^{2}$ \\
\hline $3^{8}$ & 43.6 & 47.2 & 2253 & 18 & 38 & 2.5 & 44. & & 4 & 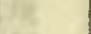 & 38 & 35.8 & 34.9 & 43 & 2e & 38 & 43.2 & $4 \mathrm{I}$ & 49 & 87 \\
\hline 40 & 55.9 & 74.1 & 2324 & 197 & 40 & 52.0 & 52.6 & & 7 & 54 & 40 & 27.9 & 30.3 & 53 & os & 40 & 37.7 & 36 & 57 & ne. \\
\hline 42 & 18.3 & 49.2 & 2235 & 10 & 42 & 62.3 & 65.0 & & 5 & 14 & 42 & 7 & 32.6 & 46 & 24 & 42 & 6.5 & 35.3 & 58 & \\
\hline 44 & 26. I & 34.5 & 29 & -18.5 & 44 & 61.0 & $7^{2.3}$ & 224 & 0 & -17.5 & 44 & 36.7 & 35.1 & 42 & Er & 44 & 38.3 & 37.6 & 2255 & -18.6 \\
\hline 46 & 21.6 & 27.6 & 20 & & $46^{*} 3$ & $5 \mathrm{I} .2$ & 65.5 & $23 \mathrm{I}$ & 8 & & 46 & 36.8 & 33.8 & 43 & -19.0 & 46 & 34.8 & 33.6 & 23 OI & 194 \\
\hline $4^{8}$ & 17.9 & 25.8 & 16 & (1). & 48 & Los & & & & (6) & 48 & 30.6 & 28.1 & 225.3 & 2 & 48 & 33.6 & 32.3 & 2303 & 16 \\
\hline 50 & 19.6 & 26.8 & 18 & ine & $50^{*}$ & 6.0 & 25.5 & 235 & 2 & & 50 & 19.8 & I8.8 & 2308 & 10 & 50 & 40 & 38 & 2253 & 160 \\
\hline 52 & 20.7 & 27.4 & 19 & dis & $51^{*}$ & 32.3 & 37.0 & 225 & 5 & 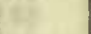 & $52^{*}$ & $5 \mathrm{I} .0$ & 40.3 & 2336 & $\mathrm{cos}$ & 52 & 45.5 & 44.0 & 44 & 82 \\
\hline 54 & 1.7 & & 25 & $\mathrm{~F}^{2}$ & 54 & 12.3 & 21.0 & & 7 & 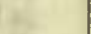 & 54 & 33.0 & & & 62 & 54 & & & 47 & 13 \\
\hline 56 & 26.9 & 33.2 & 29 & 8 & 56 & 6.0 & 14.3 & & 7 & if & $56^{*}$ & 50.3 & 38.8 & 57 & $\omega$ & $5^{6}$ & 42.7 & 41.7 & 2248 & dx \\
\hline 58 & 26.9 & $35 \cdot 3$ & 31 & & $58^{*}$ & 37.0 & 53.8 & & II & 2 & 58 & 74.3 & 64.3 & 24 I8 & $5=$ & 58 & 32.4 & & 2304 & $1+2$ \\
\hline 00 & 27.9 & 34.7 & 31 & -18.1 & 2300 & 42.6 & 60.0 & & I & $-17 \cdot 3$ & I7 $00^{*} \mathrm{I}$ & 38.9 & 23.1 & 2345 & -19.0 & 00 & 27.8 & 26.8 & 12 & -18.3 \\
\hline 02 & 24.0 & 30.2 & 24 & ta & 02 & 52.8 & 56.3 & & 6 & & 02 & 22.9 & 6.0 & 2410 & - & 02 & 17.8 & 15.4 & 29 & 10 \\
\hline 04 & 4.5 & 33.6 & 27 & $j^{n}$ & 04 & 53.5 & 57.3 & & 77 & [8? & $04^{*}$ & 51.9 & $39 . \mathrm{I}$ & 43 & 16 & 04 & 26.9 & 24 & & 10 \\
\hline 06 & .4 & 65.1 & 2256 & 16 & $\infty$ & 59.3 & 62. & & 6 & 64 & 06 & 73.3 & 57.6 & I2 & 8 i & $\alpha$ & 7.4 & & 2258 & \\
\hline $08^{*}$ & 26.7 & 65.6 & 2309 & ser & 08 & 56.2 & 59.2 & & $I$ & $x$ & 08 & 70.7 & 60.2 & I2 & 30 & 08 & 46.9 & 44.6 & & 6 \\
\hline IO & Lo & st & & Dit & IO & 48.6 & 51.6 & & 9 & 61 & 10 & 75.1 & 65.9 & 04 & nit & 10 & 50.5 & 48.1 & 38 & bat \\
\hline $12^{*}$ & 14.9 & 24.8 & 2126 & 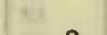 & 12 & $55 . \mathrm{I}$ & 58.5 & & 9 & $x=$ & 12 & & 67.2 & 2450 & & 2 & 5.8 & & 30 & \\
\hline 14 & 54.6 & 55.6 & 2222 & -17.8 & 14 & 49.6 & 52.3 & & 0. & -17.3 & $14^{*}$ & 56.3 & 48.7 & 2334 & -18.8 & 14 & 57.4 & 54.8 & 27 & -18. I \\
\hline 16 & I. 6 & 44.4 & 02 & & 16 & 57.4 & 60.0 & & 2 & & 16 & & & 48 & 101 & & 57.4 & 52.7 & & to \\
\hline I8 & 1.3 & 45.8 & 06 & (ii) & I8 & 54.0 & 57.8 & & 8 & W. & I8 & 52.7 & 46.1 & 38 & wat & 18 & 55 & 50.7 & 32 & 84 \\
\hline 20 & .8 & 47.2 & 06 & ex & 2 & & 56. & & 6 & we & 20 & 5.2 & & 17 & 18 & 20 & 58.8 & 49 & 30 & os \\
\hline 22 & 3.2 & 48.1 & 07 & 4 & 2 & .8 & 50.0 & & 7 & 20 & 22 & 4.0 & 68 & 04 & $\sqrt{4}$ & 22 & 54 & 50 & 32 & 158 \\
\hline 24 & & & 05 & it & 24 & .8 & 53 & & 4 & 13 & 24 & & 69 & 04 & $y=$ & 2 & & & $3 I$ & if \\
\hline 26 & 58.8 & 60 & 29 & A & 26 & 59.3 & 60 & & 14 & & 26 & & & 2300 & 86 & 2 & & & 30 & $\mathrm{X}$ \\
\hline 28 & 6 & 68. & 40 & 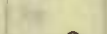 & 28 & & 60 & & 14 & & $28 * 2$ & 41.2 & 32.2 & 2256 & & 28 & & 52 & 29 & 2 \\
\hline 30 & 62.2 & 65.2 & 35 & -17.8 & 30 & .3 & 63. & & 8 & & 30.3 & 3.8 & & 53 & $-\mathrm{I} 8.8$ & 30 & 56.8 & & 29 & -18. \\
\hline 32 & 53.9 & 57.2 & 22 & ( & 32 & & 65. & & 0 & & 32 & 46.5 & 40.2 & 46 & -1 & 32 & 52.9 & $5 \mathrm{I}$ & 33 & \\
\hline 34 & 53.2 & 55. & 20 & 18 & 34 & 64.2 & 70 & & 55 & -17.3 & 34 & I & & $4 I$ & 2 & 3 & 7.2 & 55 & 28 & 14 \\
\hline $3^{6}$ & 52.8 & 55. & 20 & & 36 & & 69. & & 6 & & 36 & & & 37 & & & 67.7 & & 13 & \\
\hline 38 & 52.0 & 52. & 17 & es & 38 & 68.0 & 71. & & 0 & 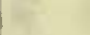 & 38 & 52. & 47 & 36 & & 38 & 69.1 & 66. & Io & 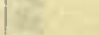 \\
\hline 40 & 54.2 & 55.2 & $2 I$ & & 40 & 60.1 & 65. & & 8 & & 40 & 52.9 & 48.2 & 34 & we & 40 & 63.3 & 53.2 & 24 & 18 \\
\hline 42 & & 42.2 & 27 & $x^{2}=$ & $4^{2}$ & 59.4 & & & 6 & & 42 & & 47.7 & 36 & & 42 & $49 \cdot 3$ & 45.1 & & \\
\hline 44 & 58.0 & 60.4 & 28 & -17.7 & 44 & 59.3 & 62.8 & & 6 & -17.3 & 44 & 50.8 & 47.3 & 37 & -18.8 & $44^{*}$ & 68.3 & 66.1 & $233 I$ & $-18,0$ \\
\hline & 52.8 & 55.9 & 20 & & 46 & 61.6 & 66.5 & & 0 & & 46 & 51.0 & 47. & 36 & & 46 & $58 . \mathrm{I}$ & 55.7 & 2347 & 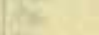 \\
\hline $4^{8}$ & 48.2 & 52. & 14 & a & 48 & 60.8 & 66.2 & & 0 & te & $4^{8}$ & 50.9 & 47. & 36 & (2) & 48 & Over & l'k'd & & ha \\
\hline 50 & $5 \mathrm{I} .2$ & & 17 & ing & 50 & 61.8 & 66.4 & & I & ite & 50 & 50.1 & 47.1 & 37 & ut & $50^{*}$ & 44.5 & 14.7 & $22 \quad 28$ & ay \\
\hline 52 & 46.5 & 48.8 & Io & 8 & 52 & 62.5 & 67.5 & & 2 & it & 52 & 50.2 & 47.3 & 37 & ne & 52 & 45 & & I5 & 2. \\
\hline & 46.9 & 48.8 & 10 & & 54 & 63.1 & 67.5 & & 3 & 42 & 54 & $4^{8.9}$ & 45 & 39 & +2 & 54 & 51.3 & 33.9 & 07 & \\
\hline 56 & 53.0 & 55.6 & 20 & & 56 & 59.5 & $64 \cdot 3$ & & 7 & & 56 & 46.9 & 44.1 & 42 & E & 56 & 41.8 & 34.2 & 22 I5 & \\
\hline 58 & 55.9 & 59.4 & 25 & $\sqrt{z}$ & 58 & 54.5 & 58.3 & 3 & & & 58 & 46.8 & 44.2 & 43 & 2 & 58 & 72.4 & $6 \mathrm{i} .2$ & 2129 & \\
\hline & & & & 60 & 2400 & 61.2 & 64.6 & & 19. & -17.2 & & & & & & 2000 & 51.7 & 40.8 & 2202 & -17.5 \\
\hline
\end{tabular}

Correction to local mean time is $+3 \mathrm{~m} 545.90^{\circ}$ torsion $=28 . ' 7$.

Torsion head at ol oom read $356^{\circ}$ and at 24 h $15 \mathrm{~m}$ read $35 \mathrm{I}^{\circ}$.

Observers-R. R. T. and W. J. P., who alteruated from 21 h $52 \mathrm{~m}$ to $22 \mathrm{~h} 02 \mathrm{~m}$.
Correction to local mean time is $+4 \mathrm{~m} 54 \mathrm{~s} .90^{\circ}$ torsion $=29^{\circ}$.

Torsion head at $15 \mathrm{~h}$ oom read $344^{\circ}$ and at $20 \mathrm{~h}$ rom read $329^{\circ}$.

Observers-R. R. T. (W. J. P. I7h $14 \mathrm{~m}$ to $18 \mathrm{~h} 40 \mathrm{~m}$, alternated to I8h 52m.) 
Tabulation of magnetic declinations observed at 'Teplitz Bay-Continued

\begin{tabular}{|c|c|c|c|c|c|c|c|c|c|c|c|c|c|c|c|c|c|c|c|}
\hline \multicolumn{5}{|c|}{ Sunday, November 15, 1903} & \multicolumn{5}{|c|}{ Magnct scale inverted } & \multicolumn{5}{|c|}{ Sunday, November 15,1903} & \multicolumn{5}{|c|}{ Magnet scale erect } \\
\hline \multirow{2}{*}{$\begin{array}{l}\text { Chr'r } \\
\text { time }\end{array}$} & \multicolumn{2}{|c|}{$\begin{array}{c}\text { Scale } \\
\text { readings }\end{array}$} & \multirow{2}{*}{$\begin{array}{l}\text { East } \\
\text { decli- } \\
\text { nation }\end{array}$} & Temp. & & $\begin{array}{l}\text { Sez } \\
\text { read }\end{array}$ & ale & East & Temp. & Chr'r & $\begin{array}{l}\text { Sca } \\
\text { read }\end{array}$ & $\begin{array}{l}\text { ale } \\
\text { lings }\end{array}$ & $\begin{array}{l}\text { East } \\
\text { decli- }\end{array}$ & Temp. & & $\begin{array}{r}\mathrm{Se} \\
\text { read }\end{array}$ & igs & East & Temp \\
\hline & Left & Right & & & & Left & Right & & & & L,eft & Right & & & & $\mathrm{I}, \mathrm{eft}$ & Right & & \\
\hline h m & $\mathrm{d}$ & d & $\circ$ & 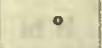 & $\mathrm{h} \mathrm{m}$ & d & d & & & $12 \mathrm{~m}$ & u & a & $\cdot \quad$ & $\circ$ & $\mathrm{h} \mathrm{m}$ & a & & $\circ$ & \\
\hline 0 oo* & 46.3 & 40.3 & 2230 & -15.0 & 200 & 32.8 & 29.1 & 2250 & $-14 \cdot I$ & 400 & Scale & made & & & 600 & 36.0 & 36.8 & 2249 & -11.7 \\
\hline 02 & 46.3 & 41.0 & & & 02 & 31.7 & 29.2 & 51 & & 02 & ere & ect & & $12 \ln 3$ & 02 & 33.7 & $34 \cdot 5$ & & 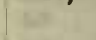 \\
\hline 04 & 44.9 & 39.6 & 32 & wa & 04 & 30.9 & 28.8 & 52 & 10 & 04 & 73.1 & 75.3 & 2250 & $-\mathrm{I} 2.8$ & 04 & 33.4 & & 45 & \\
\hline of & 44.9 & 39.8 & 32 & 20 & 06 & 28.9 & 27.1 & 54 & 6 & 06 & 73.1 & 76.2 & 50 & Ao: & o & 34.2 & & 46 & \\
\hline 08 & $44 \cdot 3$ & 39.4 & 33 & w & os & 29. I & 26. & 54 & F & 08 & 73.8 & 77.1 & 52 & Nar & 08 & $33 \cdot 3$ & & 45 & \\
\hline I0 & 47.8 & 43.5 & 27 & na & I0 & 30.6 & 28.6 & 52 & 106 & I0 & 73.6 & 76.4 & $5 \mathrm{I}$ & bif & 10 & & & 48 & in \\
\hline 12 & 46.0 & 42.0 & 29 & & 12 & 35.0 & 33.3 & 45 & 64 & 12 & 73.1 & & 50 & the & 12 & $34 . \mathrm{I}$ & & 46 & Bs \\
\hline I4 & 45.8 & 42.2 & 29 & -14.6 & 14 & 35.7 & 34. & 44 & -14.0 & 14 & 73.7 & 75.8 & $5 \mathrm{I}$ & -12.7 & I4 & 33.8 & & 45 & $-\mathrm{II} .7$ \\
\hline 16 & 44.6 & 40.9 & 32 & & 16 & 35.7 & 31 & 46 & & 16 & 73.2 & & $5 \mathrm{I}$ & (9) & 16 & 33.7 & & 47 & \\
\hline I8 & 46.9 & 44 & 26 & BST & I8 & 35.3 & & 46 & Bet & 18 & 74.7 & & 52 & $\nexists i$ & Is & & & & ilir \\
\hline 20 & 43.3 & 40.0 & 33 & fo & 20 & 35.2 & 32.1 & 46 & os & 20 & 74.8 & 77.4 & 53 & ex & 20 & 30.6 & 6 & 42 & $=$ \\
\hline 22 & 41.8 & $3^{8}$. & 35 & 2 & 22 & 35.2 & 32.2 & 46 & Ele & 2 & 75.1 & & 53 & 12 & & & & 44 & hat \\
\hline 24 & 6.6 & 38.5 & 36 & $3 x$ & 24 & 4.0 & 30.9 & 48 & is: & 24 & 74.9 & 77.2 & 53 & $x$ & 24 & 2.5 & & 45 & 15 \\
\hline 2 & & 36. & 38 & & 26 & 31.8 & 28 & $5 I$ & & 26 & & & 53 & 185 & & & & & \\
\hline 28 & & 37.1 & 38 & $2=$ & 28 & 30.0 & 27.7 & 53 & $t=5$ & 28 & 76.8 & 78 & 55 & $y_{2}$ & 28 & 33.3 & & 45 & \\
\hline 30 & $4 \mathrm{I} . \mathrm{I}$ & 38.9 & 36 & -14.7 & 30 & 32.1 & 29.5 & 50 & -14.0 & $30^{*}$ & 36.8 & 42 & 54 & -12.4 & 30 & 31.8 & 33.1 & 42 & -11.3 \\
\hline 32 & 41.7 & 38.1 & 36 & & 32 & 37.2 & 33 & 43 & 10 & 32 & 37.1 & & 54 & $x^{2}$ & 3 & $3^{\text {I. } 6}$ & & 42 & \\
\hline 34 & 42.9 & 39.3 & 34 & ith & 34 & 38. I & 34 & 4 & 115 & 34 & & & 55 & $k$ & 3 & . I & & 45 & \\
\hline 36 & 42.3 & 39 & 34 & 32 & 36 & 37.0 & 33 & 43 & 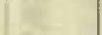 & 36 & & & 54 & ar & 3 & & & & \\
\hline 38 & 39.8 & 36. & 38 & FP & 38 & 33.6 & 29. & 49 & 28 & 38 & 38.7 & 41.7 & 55 & $\mathbb{N}$ & 38 & 31.5 & & 42 & \\
\hline 40 & 39.8 & 37. & 38 & 08 & 40 & $33 . I$ & 28 & 50 & $=$ & 4 & & & 57 & n) & 4 & & & 39 & es \\
\hline 42 & 40.8 & 39. & 36 & $=8$ & 42 & 33.8 & 29 & 48 & 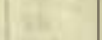 & 42 & 37 & 39 & 53 & 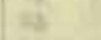 & 42 & 32.8 & & 44 & \\
\hline 44 & 41.0 & 38 & 36 & -14.6 & 44 & 33.2 & 29.2 & 50 & -13.9 & 44 & 34. & 36.8 & 48 & $-12 . I$ & 44 & 34.2 & & 46 & $\mid-11.2$ \\
\hline 4 & 41.6 & 37 & 36 & $x_{7}$ & 46 & 34.0 & 27. & 50 & & 46 & 33 & 35.7 & 46 & , & 4 & & & 44 & \\
\hline 48 & 40.7 & 37. & 37 & 12 & 48 & $33 . I$ & 27. & $5 I$ & $=$ & 48 & & 38 & 50 & 74 & 4 & .8 & & & \\
\hline 50 & 40.9 & 38. & 36 & 28 & 50 & 3.2 & 27. & $5 \mathrm{I}$ & $E$ & 50 & 37 & 39.8 & 52 & hera & 5 & I & & & loe \\
\hline 52 & $42 . \overline{7}$ & 40. & 34 & 78 & 52 & 34.8 & 29.4 & 48 & $1=2$ & 52 & & & 48 & fie & 5 & & & $4 \mathrm{I}$ & 17 \\
\hline 54 & 42.5 & 3 & 34 & 12 & 54 & & 27 & $5 I$ & te & 54 & & & 49 & 18 & 5 & & & 42 & \\
\hline 56 & 42.0 & 3 & 35 & 80 & 56 & 32.3 & 27 & $5 \mathrm{I}$ & & 56 & 38.2 & & 52 & 82 & 5 & $3 \mathrm{I}$ & & 43 & \\
\hline 58 & 41.2 & 39. & 35 & ted & 58 & 2.4 & 28.2 & $5 \mathrm{I}$ & & 58 & 35.3 & 37 & 48 & 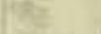 & 58 & 31.1 & & 2 & \\
\hline 100 & 4I. 8 & 39.8 & 34 & -14.7 & 300 & 32.6 & 29.0 & 50 & -13.8 & 500 & 33.0 & 34 & 44 & -12.0 & 700 & 31.9 & & 43 & -I I.I \\
\hline 02 & 41.3 & 38.3 & 36 & ( & 02 & & & 49 & 50 & 0 & & & 45 & $\operatorname{ann}$ & 0 & & & 42 & \\
\hline 04 & 39.8 & 37.1 & 38 & 10 & 0 & 2.8 & 30. & 4 & fys. & 0 & & & 49 & (x) & 0 & 30.3 & & 42 & \\
\hline 06 & 40.0 & 3 & 38 & $x^{2}$ & 0 & & & 48 & Sy & 0 & & & 47 & 27 & & & & & Ser \\
\hline 08 & 40.9 & 37.3 & 37 & Whe & 08 & 32.3 & 30.2 & 50 & 86 & 08 & 32 & 34 & 44 & by & 08 & $3 \mathrm{I} . \mathrm{I}$ & & 42 & \\
\hline I0 & 40.3 & 37.2 & 38 & $6 x$ & 10 & 31.8 & 29.3 & $5 \mathrm{I}$ & 6: & 10 & & & $4 \mathrm{I}$ & eir & I & & & 42 & \\
\hline 12 & 40.5 & 3 & 37 & $1+5$ & I2 & & 30. & 50 & 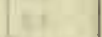 & I & 35 & & 49 & ist & I & 1.4 & & 42 & \\
\hline 14 & 39.8 & 36.8 & 38 & -14.6 & I4 & 32.2 & 30.0 & 50 & 15 & 14 & & & 49 & -12.0 & I4 & 30.3 & II. I & 40 & -11.0 \\
\hline 16 & 39.2 & 36.3 & 39 & & I6́ & 3.6 & 30. & 48 & tet & I6 & & 33 & 42 & Far & I & 29.9 & & 42 & \\
\hline I8 & 39.0 & $36 . I$ & 40 & 178 & I8 & 3.1 & 29.7 & 49 & Xr. & I & 2.5 & & 45 & 81 & I & & & 44 & Int \\
\hline 20 & 39.1 & 36. & 39 & 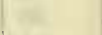 & 2 & & & & r & 2 & & & 47 & $\pi 8$ & 2 & & & jo & $=$ \\
\hline 22 & 37.7 & 36.8 & 40 & 5 & 22 & 31.8 & 29.3 & 5 & Lis & 22 & $3 \mathrm{I} .4$ & 34 & 43 & st & 22 & 30.3 & & 42 & ets \\
\hline & 38.8 & 36.3 & 40 & 2E & 24 & & & 4 & 1 & 2 & & & & is & & & & jo & \\
\hline 26 & 37.8 & 35 & $4 \mathrm{I}$ & $\Delta$ & 2 & 34.8 & 32.8 & 45 & s. & 26 & 35 & & 49 & " & 2 & 29.6 & & $4 I$ & \\
\hline 28 & & & 39 & $x^{2}=$ & 28 & & 33.4 & 45 & & 28 & & & 47 & 18= & 2 & 3 I. 9 & & 45 & \\
\hline 30 & 38. I & 35.9 & 40 & -14. & $3 c$ & 34.8 & 33.1 & 45 & $-I_{3}$ & 30 & & & 42 & -12. & 3 & 32.0 & & 44 & -10.8 \\
\hline 32 & 37.3 & & 43 & 12 & 32 & 34.0 & 33 & 46 & $1-0$ & 32 & & & $4 I$ & (at & 3 & & & 40 & \\
\hline 34 & 35.8 & 32. & & at & 34 & & 33. & 45 & 44 & 3 & & & 42 & A & 3 & 29 & & 40 & \\
\hline 3 & 36.6 & 33.2 & 44 & -1 & 36 & 34.8 & 34.6 & 44 & 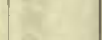 & 3 & & & 4 & te & & & & 37 & \\
\hline 38 & 36.4 & 33. & 44 & 4. & 38.3 & 33.9 & 33.2 & 46 & 8 & 38 & 31 . & 33. & 43 & 14 & 3 & 28.8 & & 38 & \\
\hline 40 & 35.8 & 33.2 & 44 & (1) & 40 & $34 \cdot 3$ & 33 & 45 & 5 & 40 & & 33 & 43 & 8\% & $4 c$ & 36.2 & 39 & $5 \mathrm{I}$ & \\
\hline 42 & 34.9 & 32.1 & 46 & 14 & 42 & & & 44 & 4 & 42 & & & 44 & 10 & 4 & 35.9 & & 49 & \\
\hline 44 & 34.3 & $31 \cdot 3$ & 47 & -14.2 & 44 & 34.8 & 34.2 & 44 & $-\mathrm{I} 3 \cdot 3$ & 44 & $35 . I$ & & 47 & - II.9 & 4 & 30.3 & 31 & 40 & -10.7 \\
\hline 46 & 33.8 & 29.8 & 48 & $x^{2}$ & 46 & 34.6 & 32.0 & 46 & $1-2$ & 46. & $35 . \mathrm{I}$ & & 47 & & 4 & 31.2 & & $4 \mathrm{I}$ & \\
\hline 48 & 35.0 & 30.9 & 47 & 74 & 48 & & 31.8 & 47 & in & 48 & 33.2 & & 44 & 10 & 4 & 33.2 & & 45 & \\
\hline & 34.9 & 30.8 & & of & 50 & & 32.6 & 46 & 10 & 50 & 33.4 & 34.8 & 45 & $n=$ & 5 & 31.0 & 32.8 & 42 & \\
\hline 52 & 34.3 & 29.9 & 48 & $=2$ & 52 & 34.6 & 32.3 & 46 & 17 & 52 & 34.2 & & 46 & 125 & 52 & 32.5 & & 43 & 10 \\
\hline & 33.7 & 29.6 & 49 & 12 & 54 & 33.8 & 31.7 & 47 & I2 & 54 & 36.1 & 37 & 50 & it & 54 & & & 45 & \\
\hline 56 & 33.4 & 29.4 & 49 & ic & 56 & 34.4 & 32.4 & 46 & (2) & 56 & 33.4 & 34.4 & 45 & 80 & 56 & 31.8 & 32.9 & 42 & \\
\hline 58 & 33.0 & 28.9 & 50 & 18 & 58 & $35 \cdot 3$ & 34.9 & 43 & 16 & 58 & 34.6 & 36.0 & 47 & $18 x$ & 58 & 32.7 & 34.2 & 44 & 8 \\
\hline & 2018 & 1258 & $7+32$ & & & & & & & & & ant & 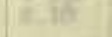 & 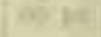 & 800 & 31.9 & 33.2 & 43 & \\
\hline
\end{tabular}

Observer-R. R. T.
Correction to local mean time is $+5 \mathrm{~m} 58 \mathrm{~s}$.

Torsion head at oh oom read $324^{\circ}$ and at the end read the same.

Observer-R. R. T. 
Tabulation of magnetic declinations observed at Teplitz Bay-Continued

\begin{tabular}{|c|c|c|c|c|c|c|c|c|c|c|c|c|c|c|c|c|c|c|c|}
\hline \multicolumn{5}{|c|}{ Monday, November 16,1903} & \multicolumn{5}{|c|}{ Magnet scale inverted } & \multicolumn{10}{|c|}{ Tuesday, November 17, I903 } \\
\hline \multirow{2}{*}{$\begin{array}{l}\text { Chr'r } \\
\text { time }\end{array}$} & \multicolumn{2}{|c|}{$\begin{array}{l}\text { Scale } \\
\text { readings }\end{array}$} & \multirow{2}{*}{$\begin{array}{l}\text { East } \\
\text { decli- } \\
\text { nation }\end{array}$} & \multirow{2}{*}{$\begin{array}{l}\text { Temp. } \\
\text { C. }\end{array}$} & Chr'r & $\begin{array}{r}\text { S } \\
\text { rea }\end{array}$ & $\begin{array}{l}\text { ale } \\
\text { lings }\end{array}$ & $\begin{array}{l}\text { East } \\
\text { decli- }\end{array}$ & Temp. & Chr'r & $\begin{array}{l}\mathrm{Sc} \\
\text { reac }\end{array}$ & ngs & $\begin{array}{l}\text { East } \\
\text { decli- }\end{array}$ & & & $\begin{array}{r}\text { Sc } \\
\text { read }\end{array}$ & $\begin{array}{l}\text { le } \\
\text { ings }\end{array}$ & $\begin{array}{l}\text { East } \\
\text { decli- }\end{array}$ & \\
\hline & Left & Right & & & time & $L_{r}$ eft & Right & & & & Left & Right & & & & Left & Right & & \\
\hline $\mathrm{h} \mathrm{m}$ & d & d & $\therefore \quad$ &. & $\mathrm{h} \mathrm{m}$ & d & d & 0, & & $\mathrm{~h} \mathrm{~m}$ & d & $\mathrm{d}$ & & & $\mathrm{h} \mathrm{m}$ & d & $d$ & & $\bullet$ \\
\hline 800 & $4 \mathrm{I} .8$ & 35.7 & 2240 & -22.0 & I0 00 & 39.3 & 37.9 & 2240 & -18.2 & $1200^{*}$ & 40.2 & $44 \cdot 4$ & 2254 & $-14 \cdot 7$ & I4 00 & 14.7 & 15.9 & 2212 & -13.8 \\
\hline 02 & 39.8 & 36.8 & 40 & 20.0 & 02 & & & 40 & & 02 & 38.8 & 44.2 & 2253 & $(19$ & 02 & 12.2 & I3. I & & \\
\hline 04 & $4 \mathrm{I} . \mathrm{I}$ & 37.8 & 39 & 16. & 04 & 39.8 & 37.3 & 40 & 46 & 04 & $4^{8.5}$ & 51.6 & 2306 & wiv & 04 & II. I & 12.0 & 06 & 10 \\
\hline 06 & 40.8 & 37.8 & 39 & 10 & 06 & 39.4 & 37.4 & 40 & r & 06 & $45 . \mathrm{I}$ & 48.8 & 23 OI & 10 & 06 & 13.2 & 14.8 & IO & 10 \\
\hline os & 43.8 & 39.6 & 35 & 68 & 08 & 39.5 & 37.3 & $4^{\circ}$ & 60 & 08 & 40.6 & 43.3 & 2254 & 186 & 08 & 15.8 & 17.9 & I4 & 85 \\
\hline IO & 44.8 & 39.2 & 35 & $\ln 8$ & IO & 39.6 & 38. I & 40 & 아 & I0 & 39.1 & 40.3 & 50 & 하 & IO & 18.2 & 20.2 & I8 & bi \\
\hline I2 & 43.8 & 38.9 & 36 & $61=$ & 12 & 39.9 & 39.1 & 39 & 41 & 12 & 39.9 & 42.7 & 53 & 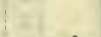 & I2 & I 4.3 & I6. I & I2 & 87 \\
\hline I4 & 42.2 & 37.5 & 38 & -21.5 & I4 & 39.3 & 37.7 & 40 & -17.8 & 14 & 44.0 & 46.3 & 2259 & -14.6 & 14 & 14.0 & 14.8 & IO & -13.6 \\
\hline I6 & 42.3 & 35.2 & 40 & (11) & 16 & 38.9 & 37.0 & $4 I$ & -80 & I6́ & 45.9 & 48.3 & 2302 & -7 & 16 & 8.9 & II. 7 & 04 & \\
\hline I8 & 42.0 & 35.1 & 40 & Wi & I8 & 39.3 & 36.3 & $4 I$ & bit & 18 & 43.8 & 46.0 & 2258 & Sit & 18 & 8.2 & 10.8 & 03 & ait \\
\hline 20 & 42.9 & 35.9 & 39 & is & 20 & 39.8 & 37.0 & 40 & $m$ & 20 & 40.5 & 42.2 & 53 & 106 & 20 & II. 7 & I4. I & 08 & es \\
\hline 22 & 42.8 & 36.4 & 38 & Wx & 22 & 40.0 & 37.8 & 40 & 3 & 22 & 37.7 & 40.0 & 49 & 66 & 22 & 15.8 & 8.6 & 15 & 15 \\
\hline 24 & 4.2 & 38.2 & 36 & EF & 24 & 39.8 & 37.7 & 40 & x & 24 & 33.8 & 35.8 & 42 & ye & 24 & 19.4 & 21.7 & & 18 \\
\hline 26 & 2.8 & 37.0 & 38 & E & 26 & 39.6 & 37 & 40 & tar & 26 & 31.0 & 32.9 & 38 & the & 26 & I7. 4 & $2 \mathrm{I} .5$ & I8 & 18 \\
\hline 28 & 2.2 & 36.8 & 39 & ter & 28 & 40.1 & 38 & 39 & (3). & 28 & $27 . I$ & 29.8 & 32 & ithe & 28 & 16.8 & 17.8 & I5 & Pat \\
\hline 30 & 43.0 & 37.9 & 37 & -21.0 & 30 & 39.8 & 38.6 & 39 & -17.7 & 30 & 25.2 & 27.9 & 29 & -14.5 & 30 & 17.3 & 18.1 & I6 & -13.4 \\
\hline 32 & 2.7 & 35.7 & 39 & 148 & 32 & 40.5 & 37 & 39 & 4 & 32 & 22.2 & & 25 & (2) & 32 & 9.3 & II .2 & 04 & (2) \\
\hline 34 & 40.6 & 38.8 & 38 & & 34 & 39.8 & 37 & 40 & ME & 34 & 24.7 & $28 . I$ & 29 & H & 34 & IO. I & II.9 & 05 & H. \\
\hline 36 & 9.8 & 38.0 & 40 & 24 & 36 & 39.5 & & $4 \mathrm{I}$ & 6 & 36 & 26. I & 29.8 & 32 & in & 36 & 6.8 & 8.2 & 00 & (x) \\
\hline 38 & 38.9 & 37.4 & 4I & Re & 38 & 39. & 37.3 & 40 & $E_{2}$ & 38 & 25.8 & 29.1 & $3 \mathrm{I}$ & A & $38^{*}$ & 37.8 & 43.3 & 02 & $2 A$ \\
\hline 40 & 39.7 & 38. I & 40 & 8 & 40 & 39.8 & & 40 & es: & 40 & 24.9 & & 29 & ct: & 40 & 37.0 & 43 & OI & 64 \\
\hline 42 & 9.4 & 38.4 & 40 & & 42 & 40.9 & & 38 & 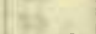 & 42 & 24.3 & 28. & 29 & tis & 42 & 40.7 & 45 & 06 & $5 i$ \\
\hline 44 & 39.2 & 38.0 & 40 & -20.9 & 44 & 42.0 & 40.5 & 36 & $-\mathrm{I} 7.8$ & 44 & 22.9 & 25 & 26 & -14.4 & 44 & 38.0 & $42 . I$ & OI & -13.2 \\
\hline 46 & 39.9 & 36.2 & $4 \mathrm{I}$ & 10. & 46 & $4 \mathrm{I} .5$ & 40. & 37 & -8 & 46 & 23.3 & 26.2 & 26 & (17) & 46 & 41.9 & 45.9 & 07 & \\
\hline $4^{8}$ & 0.3 & 37.2 & 40 & 10 & 48 & & & 38 & 8 & 48 & 23.0 & 25.9 & 26 & 8 & 48 & 40.2 & & 05 & 8 \\
\hline 50 & 0.8 & 37.9 & 39 & at: & 50 & 7 & 39 & 38 & $\infty$ & 50 & 23.8 & 25 & 27 & nat & 5 & 37.9 & $4 \mathrm{I}$ & OI & $n^{n}$ \\
\hline 52 & 0.9 & 37.8 & 39 & 65 & 52 & 40. & 39 & 38 & 6 & 52 & 23.4 & & 26 & 13 & 52 & 38.2 & $4 \mathrm{I}$ & OI & 31 \\
\hline 54 & 9.2 & 37.0 & $4 \mathrm{I}$ & 12 & 54 & 40.1 & & 38 & 8 & 54 & 22.0 & 23. & 24 & . & 54.2 & 38.3 & $4 \mathrm{I}$ & $\infty$ & 48 \\
\hline 56 & 1.0 & & 39 & & 56 & & & 38 & & 56 & 23.9 & & 26 & in & 56 & 38.2 & & 2200 & 10 \\
\hline 58 & 3.0 & 40.3 & 35 & & 58 & 39.8 & 39. & 38 & $-\mathrm{I} 8.0$ & 58 & 26.0 & & 30 & 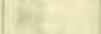 & 58 & 36.4 & 39.8 & 2I 58 & Ws \\
\hline 900 & 2.8 & 40.3 & 36 & -20.4 & II 0 & 39.5 & 39.2 & 39 & 1000 & I3 00 & 24.8 & 26.0 & 28 & -14.2 & I5 00 & $4 \mathrm{I} . \mathrm{I}$ & 44.8 & 2206 & $-13 . I$ \\
\hline 02 & 0.8 & 38.6 & $3^{8}$ & $x^{2}$ & 02 & 39.3 & & 39 & we & 02 & 24.2 & $25 . I$ & 26 & an & 02 & 44.5 & 46.2 & 09 & 60 \\
\hline 04 & 9.3 & $37 \cdot 3$ & 40 & $x=$ & 0 & 9.6 & & 39 & in & 04 & 20.6 & $2 \mathrm{I}$. & $2 I$ & 180 & 0 & .6 & 50 & I5 & 18 \\
\hline 06 &.$I$ & 37. & $4 \mathrm{I}$ & 16 & & & & 39 & W & 06 & 21.8 & & 23 & 10 & & & & & 0 \\
\hline 08 & 39.1 & 38. I & 40 & 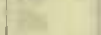 & 08 & 39.6 & 39. & 39 & N. & 08 & 19.8 & 20. & 20 & A. & 08 & 49.4 & 52 & 18 & the \\
\hline IO & 39.1 & 37.9 & 40 & of & & & & 40 & 61 & I0 & I8 & & I7 & wit & & & & I 8 & ar \\
\hline I2 & 8.2 & 37.2 & 42 & & I & . I & 39. & 39 & & 12 & 20.0 & & 20 & (14) & I 2 & 48.8 & 50.9 & I6 & ai \\
\hline I4 & 38.4 & $37 \cdot 3$ & 4I & -19.8 & I4 & & & 39 & $-\mathrm{I} 8.0$ & I4 & & & 24 & $-\mathrm{I} 4.2$ & I 4.5 & $56 . I$ & & & $-\mathrm{I} 3 . \mathrm{I}$ \\
\hline I6 & 0.0 & 36.7 & $4 \mathrm{I}$ & the & It & .0 & & 38 & & 16 & $26 . \mathrm{I}$ & & 30 & & I6 & 60 & & 33 & \\
\hline 18 & & & $4 \mathrm{I}$ & fint & I & & & 3 & $t$ & I 8 & & & 26 & i & I & & & 39 & ith \\
\hline 20 & 9.8 & 37 & 40 & $\log$ & 2 & .1 & & 3 & 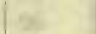 & 20 & 24. & & 27 & ex & 20 & 63 & & 39 & 100 \\
\hline 2 & 6 & & 40 & s. & 2 & & & 3 & 10 & 2 & & & 2 & at & 22 & & & 44 & 14 \\
\hline 2 & 9.8 & 38 & 40 & is & 2 & & & 3 & 45 & 2 & 2I. & & 2 & is & 24 & 68.8 & & 47 & is \\
\hline 26 & .6 & 38.2 & 40 & R & 26 & 40.6 & & 37 & & 26 & & $2 \mathrm{I}$. & 21 & is: & 26 & 7I & $7 \mathrm{I}$ & $5 \mathrm{I}$ & 8 \\
\hline 28 & 9.2 & 38. I & 40 & $1=$ & 28 & 39.3 & 38 & 39 & & 28 & 22.6 & 23. & 24 & & 28 & 2.8 & 73 & 53 & ?he \\
\hline 30 & 9.0 & 38.2 & 40 & -19.0 & 30 & 40.0 & 39. & 38 & -18.0 & 30 & 21.4 & $2 \mathrm{I}$. & 22 & $-14 . I$ & 30 & 71.9 & 72.3 & $5 \mathrm{I}$ & -13.2 \\
\hline 32 & & & 40 & 120 & 32 & & & 38 & $1=x^{2}$ & 32 & & & 22 & 28 & 32 & & & 52 & $10=$ \\
\hline 34 & 9.8 & 37.8 & 40 & in & 34 & & 40 & 3 & is & 34 & $2 \mathrm{I}$ & 22 & 22 & & 3 & 72.8 & 73 & 53 & A6 \\
\hline 36 & 9.9 & 38.0 & 40 & ax & 36 & & & & 2 & 36 & 21.8 & & 22 & 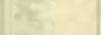 & 36 & I & & 53 & $\alpha$ \\
\hline 38 & 39.4 & 37. & 40 & ac & 38 & 41.8 & 40 & 3 & anc & 38 & 23.2 & 23. & 25 & E. & 38 & & & 54 & 28 \\
\hline 40 & $39 . \mathrm{I}$ & & $4 \mathrm{I}$ & 10 & 40 & & & & 10 & 40 & & & 23 & $=$ & 40 & & 74 & 55 & of \\
\hline 42 & 37.8 & 36. & 42 & & 42 & & 40 & 36 & & 42 & 22. I & 22. & 23 & 5 & 42 & & 77 & 58 & ixs \\
\hline & 38. I & 36. & 42 & -18.0 & & 42.8 & 42.2 & 34 & $-\mathrm{I} 8.2$ & 44 & 22.3 & 23.6 & 24 & -14. & & 73.8 & 74 & 55 & -13.2 \\
\hline 46 & 38.2 & 36.8 & 42 & 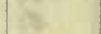 & 46 & & 42.9 & 32 & the & 46 & 21.9 & 22.7 & 23 & the & 46 & 72.8 & 74 & 53 & ton \\
\hline 48 & 39.7 & 38.3 & 39 & 86 & 48 & 4 & & 2 & 15 & $4^{8}$ & 20.4 & $2 \mathrm{I}$. & $2 \mathrm{I}$ & FE. & 48 & 72.1 & & 52 & 26 \\
\hline 50 & .3 & 38 & 40 & (ex & 50 & & & 4 & $\operatorname{los}$ & 50 & 22. & & 24 & 100 & 50 & & & 5I & 100 \\
\hline 52 & & & 39 & Es & 52 & & $4 \mathrm{I}$ & 3 & (at & 52 & 23.2 & 23 & 25 & haf: & 52 & 69.6 & 71.2 & 49 & \\
\hline 54 & 40.2 & 38.7 & 39 & 34 & 54 & $43 . I$ & 42. & 34 & 12 & 54 & 21.8 & 22 & 22 & 12 & 54 & 67.9 & 69.8 & 46 & ar \\
\hline 56 & & 37. & 40 & 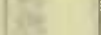 & 56 & 42.8 & 42.2 & 34 & & 56 & 20.2 & 20.9 & 20 & 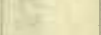 & 56 & 66.8 & 68.8 & & 12 \\
\hline 58 & 39.8 & 38.0 & 40 & 88 & $5^{8}$ & 42.7 & 41.9 & 34 & -I8. I & 58 & 18.2 & 19.5 & I7 & hic & 58 & 66.3 & 67.8 & 43 & \\
\hline & & & & & 1200 & 42.7 & 39.2 & 36 & & & & & & & I6 00 & 64.9 & 66.1 & $4 \mathrm{I}$ & -13.2 \\
\hline
\end{tabular}

Correction to local mean time is $+6 \mathrm{~m}$ ors. $90^{\circ}$ torsion $=25^{\prime} \cdot 6$. Torsion head at 7 h $30 \mathrm{~m}$ read $324^{\circ}$ and at $12 \mathrm{~h}$ 3om read $321^{\circ}$. Observer-R. R. T.
Correction to local mean time is $+6 \mathrm{~m}$ I5s.

Torsion head at IIh oom read $318^{\circ}$ and at the end read the same. Observer-R. R. T. 
Tabulation of magnetic declinations observed at Teplitz Bay-Continued

\begin{tabular}{|c|c|c|c|c|c|c|c|c|c|c|c|c|c|c|c|c|c|c|c|}
\hline \multicolumn{5}{|c|}{ Wednesday, November 18, 1903} & \multicolumn{5}{|c|}{ Magnet scale inverted } & \multicolumn{5}{|c|}{ Wednesday, November 18, 1903} & \multicolumn{5}{|c|}{ Magnet scale inverted } \\
\hline \multirow{2}{*}{$\begin{array}{l}\text { Chr'r } \\
\text { time }\end{array}$} & \multicolumn{2}{|c|}{$\begin{array}{l}\text { Scale } \\
\text { readings }\end{array}$} & \multirow{2}{*}{$\begin{array}{c}\text { East } \\
\text { decli- } \\
\text { nation }\end{array}$} & Ternp. & Chr'r & $\begin{array}{l}\mathrm{Se} \\
\text { read }\end{array}$ & $\begin{array}{l}\text { ale } \\
\text { dings }\end{array}$ & $\begin{array}{l}\text { East } \\
\text { decli- }\end{array}$ & Temp. & Chr'r & $\begin{array}{l}\text { Sca } \\
\text { read }\end{array}$ & $\begin{array}{l}\text { ale } \\
\text { lings }\end{array}$ & $\begin{array}{l}\text { East } \\
\text { decli- }\end{array}$ & Temp. & Chr'r & $\begin{array}{r}\text { Sca } \\
\text { readi }\end{array}$ & $\begin{array}{l}\text { ale } \\
\text { lings }\end{array}$ & $\begin{array}{l}\text { East } \\
\text { decli- }\end{array}$ & Tenıp. \\
\hline & Left & Right & & & & Left & Right & & & & I.eft & Right & & & & L,eft & Right & & \\
\hline $\mathrm{h} \mathrm{m}$ & d & d & $\cdot ;$ & $\circ$ & $\mathrm{h} \mathrm{m}$ & d & d & $\cdot \quad \cdot$ & - & $\mathrm{h} \mathrm{m}$ & & d & & $=0$ & $\mathrm{~h} \mathrm{~m}$ & d & d & $\circ$, & $\bullet$ \\
\hline $000^{*}$ & 39.0 & 38.0 & 2216 & -17.7 & 200 & $47 \cdot 5$ & 45.7 & 2259 & -15.8 & 400 & 36.8 & 35.0 & & -15.8 & 600 & 44.6 & 44.6 & & -16.0 \\
\hline 02 & 39.5 & 38.4 & I6 & 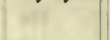 & 02 & 48.5 & 46.5 & 58 & & 02 & 36.0 & 34.4 & 17 & & 02 & 46.0 & 45.6 & OI & \\
\hline 04 & 39.6 & 38.3 & I6 & wa & 04. & 49.4 & 47.8 & 56 & $4 n$ & 04 & 35.4 & 34.0 & 18 & 46 & 04 & 44.6 & 43.9 & 03 & Fa \\
\hline 06 & 39.9 & 38.6 & 15 & $a r$ & 06 & 50.8 & 49.2 & 54 & mo & 06 & 34.4 & 33.0 & 20 & 32 & 06 & 41.8 & $41 \cdot 3$ & 07 & 64 \\
\hline 08 & 39.0 & 37.8 & 16 & 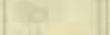 & 08 & 51.2 & 49.2 & 54 & 200 & 08 & 35.4 & 33.8 & I8 & $A$ & 08 & 42.8 & 42.3 & 06 & \\
\hline 10 & 37.2 & $3^{6.3}$ & 19 & 81 & I0 & 52.1 & 50.3 & 52 & 01 & Io & 37.8 & 36.4 & 14 & 24 & I0 & 3.3 .0 & 32.5 & $2 I$ & \\
\hline 12 & 35.3 & 34.8 & 22 & $y^{2}$ & 12 & 50.5 & 48.6 & 55 & Fits & 12 & $4 \mathrm{I} \cdot 4$ & 40.8 & 08 & 28 & 12 & 28.4 & 27.4 & 29 & \\
\hline I4 & 33.5 & 33.3 & 24 & -17.3 & 14 & 48.6 & 46.6 & 2258 & -15.7 & 14 & 45.4 & 45.0 & 02 & -16.0 & 14 & 33.8 & 32.2 & $2 \mathrm{I}$ & -15.9 \\
\hline I6 & 30. I & 29.5 & 30 & & 16 & 47.2 & 45.2 & 2300 & & 16 & 46.2 & $45 . I$ & 2301 & & 16 & 40.0 & 38.2 & II & \\
\hline I8 & 30.6 & 29.4 & 30 & 6 & I8 & 47.5 & 45.5 & 2300 & 81 & I8 & 50.5 & 49.4 & 2254 & Bt & 18 & 41.8 & 40.6 & 08 & 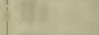 \\
\hline 20 & 28.0 & 26.6 & 34 & nes & 20 & 49.3 & $47 \cdot 3$ & 2257 & 28 & 20 & 54.4 & 53.5 & 48 & es & 20 & 43.0 & 41.0 & 07 & Es \\
\hline 22 & 23.2 & 21.7 & 42 & $3=$ & 22 & 51.8 & 49.8 & 53 & s5 & 22 & 56.4 & $55 \cdot 3$ & 45 & $=$ & 22 & 45.0 & 43.3 & 03 & $4=$ \\
\hline 24 & 22.3 & 21.0 & 43 & $t_{-}$ & 24 & 53.0 & $5 \mathrm{I} .3$ & $5 I$ & tt & 24 & 56.0 & 55.0 & 45 & bx & 24 & 39.0 & 37.5 & I3 & \\
\hline 26 & 22.0 & 20. I & 44 & te & 26 & 50. I & 49.0 & 55 & -1 & 26 & 53.1 & 51.9 & 50 & tis & 26 & $35 \cdot 3$ & 33.8 & I8 & \\
\hline 28 & 20.5 & 18.5 & 46 & $x$ & 28 & 48.8 & 47.6 & 57 & & 28 & 52.6 & 51.6 & 51 & ( & 28 & 33.4 & 32.3 & $2 \mathrm{I}$ & \\
\hline 30 & 17.3 & 15.3 & 51 & -17.0 & 30 & 49.9 & 48.3 & 55 & -15.7 & 30 & 60.3 & 59.1 & 39 & -I6.II & 30 & 34.5 & 3.3 .4 & I9 & -15.8 \\
\hline 32 & 17.3 & 15.7 & 5 I & & 32 & 51.6 & 50.5 & 52 & & 32 & 60.8 & 59.9 & 38 & W. & 32 & 29.0 & 26.8 & 29 & \\
\hline 34 & 14.3 & II. 4 & 2256 & & 34 & 54.2 & 53.0 & 48 & E & 34 & 64.9 & 63.3 & 32 & 2 & 34 & 29.7 & 28.3 & 27 & tot \\
\hline 36 & 9.3 & 7.0 & 2304 & & 36 & 55.9 & 54.4 & 46 & ter & 36 & 66.2 & 64.5 & 30 & ets & 36 & 28.6 & 27.0 & 29 & \\
\hline $38^{*}$ & 45.3 & 38.7 & of & ne & 38 & 54.2 & 52.6 & 49 & 12 & 38 & 65.0 & 64.3 & 31 & 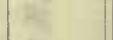 & 38 & 25.2 & 24.0 & 34 & 4 \\
\hline 40 & 40.3 & 39.1 & 10 & its. & 40 & 52.3 & 51.0 & 52 & (17) & 40 & 64.3 & 64.0 & 32 & 64 & 40 & 24.2 & 23.6 & 35 & $\omega$ \\
\hline 42 & 40.3 & 38.8 & 10 & & 42 & 52.5 & 51.5 & $5 \mathrm{I}$ & $x=8$ & 42 & 65.3 & 64.4 & $3 \mathrm{I}$ & 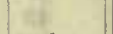 & 42 & 27.2 & 26.2 & $3 \mathrm{I}$ & 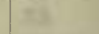 \\
\hline 44 & 38.8 & 37.7 & 13 & -16.6 & 44 & 52.8 & 51.6 & 51 & -15.6 & 44 & 68.0 & 66.5 & 27 & $-16 . I$ & 44 & 29. & $5^{b}$ & 26 & -15.8 \\
\hline 46 & 38.3 & $37 \cdot 3$ & 13 & & 46 & $55 . I$ & $54 . I$ & 47 & - & 46 & 65.6 & 64.5 & 30 & & 46 & 22.9 & 22.6 & 37 & \\
\hline 48 & 38.0 & $3^{6.6}$ & 14 & 64 & 48 & 58.0 & 57.0 & 42 & 1 & 48 & 62.7 & 62.3 & 34 & = & 48 & 22.0 & 21.2 & 39 & \\
\hline 50 & 39.6 & 38.0 & 12 & 20 & 50 & 58.3 & 57.1 & 42 & ace & 50 & 65 & $.3 a$ & 30 & at & 50 & 24.0 & 23.3 & 36 & $\alpha$ \\
\hline 52 & 40.0 & 38.0 & II & $\theta$ & 52 & 58.8 & 57.6 & $4 \mathrm{I}$ & wa & 52 & 64.6 & 64.6 & $3 \mathrm{I}$ & 15 & 52 & 23.6 & 23.4 & 36 & 24 \\
\hline 54 & 42.8 & 41.2 & 07 & 34 & 54 & 61.3 & 60.0 & 37 & 14 & 54 & 62.2 & 62.2 & 35 & $1+2$ & 54 & 30.1 & 29.3 & 26 & +2 \\
\hline 56 & 42.6 & 39.4 & 08 & & 56 & 62.3 & 61.4 & 36 & 10. & 56 & 61.4 & 61.2 & 36 & & 56 & 26.2 & 25.8 & .32 & \\
\hline 58 & 42.0 & 40.0 & 08 & & 58 & 60.9 & 60.4 & 37 & $1=$ & 58 & 58.4 & 58.4 & $4 \mathrm{I}$ & & 58 & 19.8 & 18.6 & 42 & \\
\hline 100 & 43.8 & 42.3 & 05 & -16.3 & 300 & 60.6 & 60.6 & 37 & -15.6 & 500 & 55.8 & 55.3 & 45 & $-\mathrm{I} 6.1$ & 700 & 18.5 & 17.6 & 44 & -15.8 \\
\hline 02 & 47.0 & 45.6 & 2300 & & 02 & 59.2 & 58.8 & 40 & & 02 & 53 & $.5 b$ & 48 & 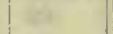 & 02 & 23.5 & 22.6 & 36 & \\
\hline 04 & 50.1 & 49.3 & 2254 & $y e$ & 04 & 57.6 & 56.9 & 43 & $n$ & 04 & 52.3 & 52.0 & $5 \mathrm{I}$ & 6 & 04 & 20.2 & 19.5 & $4 I$ & \\
\hline 06 & $5 \mathrm{I}$ & $.0 b$ & 52 & Mr & 06.3 & 53.8 & 53.0 & 49 & $3 \%$ & 06 & 52.6 & 52.2 & 50 & 40 & 06 & $25 \cdot 3$ & 25.3 & 33 & w \\
\hline 08 & 50.6 & 50.0 & 54 & 16 & 08 & 56.2 & 55.5 & 45 & 1he & 08 & 54.3 & 54.0 & 48 & 85. & 08 & 31.3 & 30.9 & 24 & ans \\
\hline 10 & 50.0 & 49.8 & 54 & $\ln$ & Io & 57.2 & 56.2 & 44 & nt & Io & 54.5 & 54.2 & 47 & 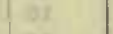 & IO & 29.0 & 28.3 & 28 & 61. \\
\hline 12 & 51.0 & 50.8 & 53 & & 12 & 56.2 & 54.6 & 46 & 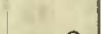 & 12 & 55.0 & 54.3 & 47 & 18 & 12 & 30.3 & 30.3 & 25 & $=$ \\
\hline 14 & 58.0 & 56.5 & 43 & -16.2 & I4 & 51.9 & 50.3 & 52 & -15.8 & I4 & 58.1 & 57.1 & 42 & $-I 6 . I$ & 14 & 29.3 & 28.7 & 27 & -15.8 \\
\hline 16 & 58.0 & 55.6 & 43 & & 16 & 48.0 & 46.2 & 2258 & & I6 & 56.7 & 55.9 & 44 & 5 & 16 & 29.0 & 27.8 & 28 & \\
\hline 18 & 60.0 & 58.3 & 40 & 32 & 18 & 43.1 & 42.0 & 2306 & 87 & I8 & 57.9 & 57.0 & 42 & 24 & 18 & 26.5 & 25.5 & 32 & Min \\
\hline 20 & 55.9 & 54.9 & 46 & 5 & 20 & 42.8 & $4 r \cdot 3$ & 07 & $=5$ & 20 & 56.6 & 55.0 & 45 & $\approx$ & 20 & 25.3 & 22.7 & 35 & $\omega$ \\
\hline 22 & 58.0 & 56.8 & 42 & 35 & 22 & 37.8 & 36.8 & 14 & 77 & 22 & 57.2 & 56.8 & 43 & 178 & 22 & 28.3 & 24.9 & 31 & att. \\
\hline 24 & 55.5 & 54.6 & 46 & rest & 24 & 38.0 & 36.0 & I4 & 14 & 24 & 58.9 & 58.0 & $4 \mathrm{I}$ & +5 & 24 & 29.4 & 25.6 & 29 & 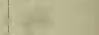 \\
\hline 26 & 49.4 & 47.8 & $5^{6}$ & E. & 26 & 35.4 & 33.8 & I8 & & 26 & 56.8 & 56.0 & 44 & $\approx$ & 26 & 32.0 & 28.8 & 25 & \\
\hline 28 & 51.8 & 51.0 & 52 & & 28 & 36.0 & 33.8 & I8 & & 28 & 55.4 & 55.0 & 46 & & 28 & 27.5 & 24.3 & 32 & \\
\hline 30 & 55.3 & 54.3 & 46 & -16.0 & 30 & 34.6 & 32.0 & 20 & -15.8 & 30 & 57.8 & 57.0 & 42 & -16.1 & 30 & 28.6 & 25.6 & 30 & -16.3 \\
\hline 32 & 54.5 & 54.2 & 47 & & 32 & 30.8 & 28.2 & 26 & : & 32 & 61.7 & 61.7 & 36 & 18 & 32 & 31.0 & 27.3 & 27 & \\
\hline & 56.6 & 55.1 & 45 & HF & 34 & 33.3 & 31.0 & 22 & 36 & 34 & 67.0 & 66.3 & 28 & 18 & 34 & 30.2 & 26.0 & 28 & \\
\hline 36 & 56.6 & 54.6 & 45 & at & $3^{6}$ & 30.6 & 28.4 & 26 & & 36 & $5^{8}$ & $.0 b$ & 41 & & 36 & 27.5 & 24.6 & 32 & \\
\hline 38 & 57.1 & 55.6 & 44 & 48 & 38 & 29.9 & $28 . I$ & 27 & at & 38 & 55.0 & 53.9 & 47 & W & 38 & 29.4 & 25.8 & 29 & \\
\hline 40 & 56.0 & 54.8 & 46 & 47 & 40 & $3 \mathrm{I} .5$ & 30.4 & 24 & 04 & 40 & 54.6 & 54.0 & 47 & 27 & 40 & 29.9 & 27.3 & 28 & \\
\hline 42 & 55.3 & 53.7 & 47 & & 42 & 32.6 & 31.4 & 22 & - & 42 & 57.0 & 56.3 & 44 & & 42 & 27.8 & 26.3 & 30 & \\
\hline 44 & 52.6 & $5 \mathrm{I} .2$ & 51 & -16.0 & 44 & 33.6 & 31.9 & $2 \mathbf{I}$ & -15.8 & 44 & 56.9 & 56.3 & 44 & -16.0 & 44 & 28.3 & 26.3 & 30 & -16.0 \\
\hline 46 & 51.7 & 50.3 & 52 & & 46 & 34.4 & 31.6 & $2 I$ & & 46.2 & 56.0 & 55.5 & 45 & 8. & 46 & 21.8 & 18.6 & $4 I$ & \\
\hline 48 & 51.5 & 50.2 & 53 & Bes & 48 & 34.5 & 32.0 & 20 & 4 & 48 & 55.8 & 55.0 & 46 & Ka & 48 & $25 \cdot 3$ & 23.7 & 34 & \\
\hline 50 & 50.8 & 49.5 & 54 & $\mathrm{R}^{2}$ & 50 & 34.6 & 32.1 & 20 & 18 & 50 & 53.2 & 52.8 & 49 & E2 & 50 & 29.0 & 27.3 & 28 & $=$ \\
\hline 52 & 50.7 & 49.3 & 54 & tes & 52 & 37.3 & 35.3 & I 5 & $1=$ & 52 & 50.2 & 49.8 & 2254 & E & $5^{2}$ & 3 I.o & 29.0 & 25 & \\
\hline 54 & 51.0 & 49.4 & 54 & 12 & 54 & 35.9 & 33.4 & I8 & $1^{2}$ & 54 & 45.6 & 45.2 & 23 oI & k8 & 54 & 33.6 & 28.8 & 24 & 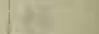 \\
\hline 56 & 50.5 & 48.8 & 55 & 02 & 56 & 38.2 & 36.3 & 14 & 32 & 56 & 46.6 & 45.3 & 00 & 42 & 56 & 36.0 & 34.6 & 17 & \\
\hline 58 & 48.5 & 47.0 & $5^{8}$ & $y^{7}$ & 58 & 37.6 & 35.2 & 15 & $1=$ & 58 & 44.5 & 43.5 & $\mathrm{O}_{4}$ & 24 & 58 & 36.0 & $35 \cdot 3$ & 17 & 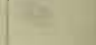 \\
\hline & $7 x$ & & 0.14 & $(x)$ A & & & & & & & & & & & & & & & \\
\hline
\end{tabular}


Tabulation of magnetic declinations observed at Teplitz Bay-Continued

\begin{tabular}{|c|c|c|c|c|c|c|c|c|c|c|c|c|c|c|c|c|c|c|c|c|}
\hline \multicolumn{6}{|c|}{ Wednesday, November 18, 1903} & \multicolumn{5}{|c|}{ Magnet scale inverted } & \multicolumn{5}{|c|}{ Wednesday, November 18,1903} & \multicolumn{5}{|c|}{ Magnet scale inverted } \\
\hline \multirow{2}{*}{$\begin{array}{l}\text { Chr'r } \\
\text { time }\end{array}$} & \multicolumn{2}{|c|}{$\begin{array}{l}\text { Scale } \\
\text { readings }\end{array}$} & $\begin{array}{l}\text { Eas } \\
\text { dec } \\
\text { nati }\end{array}$ & $\begin{array}{l}\mathrm{t} \\
\mathrm{li}- \\
\text { on }\end{array}$ & $\begin{array}{l}\text { Temp. } \\
\text { C. }\end{array}$ & $\begin{array}{l}\text { Chr'r } \\
\text { time }\end{array}$ & $\begin{array}{r}\text { Sc } \\
\text { read }\end{array}$ & $\begin{array}{l}\text { ale } \\
\text { lings }\end{array}$ & $\begin{array}{l}\text { East } \\
\text { decli- } \\
\text { nation }\end{array}$ & Temp. & $\begin{array}{l}\text { Chr'r } \\
\text { time }\end{array}$ & $\begin{array}{l}\mathrm{Sc} \\
\text { read }\end{array}$ & $\begin{array}{l}\text { ale } \\
\text { lings }\end{array}$ & $\begin{array}{c}\text { East } \\
\text { decli- } \\
\text { 11ation }\end{array}$ & $\begin{array}{c}\text { Temp. } \\
\text { C. }\end{array}$ & $\begin{array}{l}\text { Chr't } \\
\text { time }\end{array}$ & $\begin{array}{l}\text { Sc: } \\
\text { read }\end{array}$ & $\begin{array}{l}\text { ale } \\
\text { lings }\end{array}$ & $\begin{array}{c}\text { East } \\
\text { decli- } \\
\text { nation }\end{array}$ & Temp. \\
\hline & Left & Right & . & & & & L,eft & Right & & & & Left & Right & 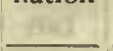 & & & I,eft & Right & & \\
\hline $\mathrm{h} \mathrm{m}$ & d & d & $\circ$ & ' & & h m & d & d & & 0 & li $\mathrm{m}$ & a & d & $\because ;$ & & h m & a & d & $\cdot 1$ & $0^{\circ}$ \\
\hline 800 & 39.4 & 38.9 & 231 & II & $-I_{5} .8$ & 1000 & 52.2 & 51.4 & 2251 & -15.0 & 1200 & 62.8 & 59.6 & 2236 & -13.8 & 1400 & 65.6 & 64.9 & 2230 & $-13 \cdot 5$ \\
\hline 02 & 38.0 & $37 \cdot 3$ & & 4 & in & 02 & 49.4 & 49.2 & 55 & is & 02 & 56.2 & $54 \cdot 3$ & 46 & 6 & 02 & 63.8 & 63.0 & 33 & a \\
\hline 04 & 38.2 & 36.9 & & 4 & 46 & 04 & 48.8 & 47.6 & 57 & 13 & 04 & 53.0 & 49.7 & 52 & - & 04 & 65.0 & 63.8 & 32 & 10 \\
\hline 06 & 38.6 & 40.3 & & II & 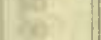 & 06 & 53.0 & 50.2 & 2252 & 80 & 06 & 49.0 & 46.4 & 2258 & 5 & 06 & 66.4 & 65.7 & 29 & (N) \\
\hline 08 & 40.8 & 39.3 & & IO & te & 08 & 39.0 & 38.3 & $23 \mathrm{I} 2$ & 15 & 08 & 45.7 & 41.9 & 2304 & 8 & 08 & 66.1 & 64.9 & 30 & 80 \\
\hline IO & 45.6 & 45.2 & & I & tot & Io & $3^{6.5}$ & 34.4 & I7 & int & 10 & 48.4 & 45.2 & 2259 & $6:$ & I0 & 65.5 & $64 . \bar{I}$ & 3I & $w$ \\
\hline 12 & 42.0 & 40.4 & & 88 & Pet & 12 & 35.9 & $35 . \mathrm{I}$ & I7 & 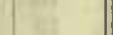 & 12 & 50.0 & 48.9 & 55 & & 12 & 65.6 & 64.6 & 30 & $1=5$ \\
\hline$I_{4}$ & 45.7 & 43.9 & & 02 & -I5.6 & 14 & 34.2 & 32.9 & 20 & & I4 & 48.7 & 45.2 & 2259 & -13.8 & 14 & 67.0 & $66 . I$ & 28 & -13.6 \\
\hline 16 & $45 . \mathrm{I}$ & 42.8 & & 94 & 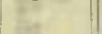 & 16 & 38.9 & 38.5 & 12 & -15.0 & 16 & 47.4 & 41.7 & 2303 & 10 & 16 & 68.8 & 67.7 & 26 & \\
\hline 18 & 35.2 & 34.6 & & 18 & 4 & I8 & 45.9 & 43.9 & 02 & (a) & 18 & 45.0 & 39.9 & 06 & 56 & 18 & 68.2 & 66.8 & 27 & 34 \\
\hline 20 & 40.2 & 37.8 & & I I & 58 & 20 & $45 \cdot 3$ & 42.3 & 2304 & 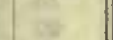 & 20 & 47.6 & 41.8 & 02 & as & 20 & 70.8 & 69.7 & 22 & int \\
\hline 22 & 39.9 & 36.2 & & 3 & ex & 22 & 48.2 & 46.8 & 2258 & 6 & 22 & 47.9 & $4 \mathrm{I} .2$ & 2303 & 65 & 22 & 70.0 & 68.2 & 24 & $6=$ \\
\hline 24 & 37.8 & 34.8 & & 16 & 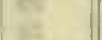 & 24 & 48.8 & 46.4 & 58 & & 24 & 49.5 & 43.7 & 2259 & 1 & 24 & 66.2 & 65.0 & 30 & in \\
\hline 26 & 39.8 & 36.1 & & 13 & E & 26 & 48.0 & $46 . \mathrm{I}$ & 2259 & & 26 & 53.4 & 49.0 & 52 & 8 & 26 & 63.3 & 62.2 & 34 & $=$ \\
\hline 28 & 36.3 & 33.7 & & 18 & & 28 & 44.5 & 43.0 & 2304 & & 28 & $5 \mathrm{I} \cdot 9$ & 47.7 & 54 & & 28 & 65.5 & 64.2 & $3 \mathrm{I}$ & 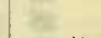 \\
\hline 30 & 37.6 & 35.6 & & 15 & -15.4 & 30 & 48.1 & 45.6 & 2259 & -14.8 & 30 & 48.9 & 44.9 & 59 & -13.7 & 30 & 64.5 & 63.4 & 32 & -13.7 \\
\hline 32 & 40.9 & $3^{8} .9$ & & IO & 10 & 32 & 47.2 & $46 . I$ & 2259 & $1=$ & 32 & 57.1 & 52.8 & 46 & 138 & 32 & 63.0 & 62.1 & 34 & 56 \\
\hline 34 & 45.5 & 44.0 & & 02 & $y$ & 34 & 46.2 & $45 . I$ & 23 oI & & 34 & 60.0 & 56.2 & $4 I$ & is & 34 & 62.6 & 61.8 & 35 & 38 \\
\hline 36 & $4 \mathrm{I} . \mathrm{I}$ & 40.0 & & 09 & 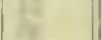 & 36 & 57.0 & 53.5 & 2246 & 3 & 36 & 55.2 & 50.8 & 49 & & 36 & 62.0 & 61.5 & 36 & EE \\
\hline 38 & 47.4 & 45.7 & & 00 & 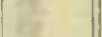 & 38 & 62.1 & 59.6 & 37 & a & 38 & $58 . I$ & 53.3 & 45 & es & 38 & 60.8 & 60.0 & 38 & W \\
\hline 40 & $42 . I$ & 40.0 & & 08 & 3 & 40 & 54.9 & 51.7 & 2249 & $m$ & 40 & 60.2 & 54.7 & 42 & & 40 & 61.5 & 60.9 & 36 & 6 \\
\hline 42 & 47.4 & 45.4 & & 00 & 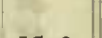 & 42 & 44.9 & 43.0 & 2304 & & 42 & 59.6 & 53.4 & 44 & & 42 & 64.0 & 63.5 & 33 & \\
\hline 44 & 37.7 & 36.7 & & 14 & -15.3 & 44 & $44 . I$ & 41.8 & 05 & -14.6 & 44 & 57.7 & 52.1 & 46 & -13.9 & 44 & 65.4 & 64.4 & $3 \mathbf{I}$ & -14.0 \\
\hline 46 & 30.1 & 26.3 & & 28 & & 46 & 42.1 & $4 \mathrm{I} .2$ & 07 & & 46 & 60.8 & 52.7 & 44 & $x^{2}$ & 46 & 69.0 & 67.0 & 26 & $x=$ \\
\hline 48 & 35.9 & 32.7 & & 19 & H. & 48 & 39.3 & 38.3 & 12 & & 48 & 61.2 & 54.9 & 42 & हbe & 48 & 66.2 & 63.9 & 30 & $x_{4}$ \\
\hline 50 & 39.7 & 35.7 & 231 & 13 & tat & 50 & 41.2 & 39.6 & 09 & (2) & 50 & 58.7 & 51.6 & 46 & ce & 50 & 64.8 & 62.8 & 32 & 78 \\
\hline 52 & 51.7 & 47.9 & 22 & 54 & 28 & 52 & 44.8 & 43.3 & 2304 & W & 52 & 53.0 & $48 . I$ & 53 & is & 52 & 66.0 & 63.3 & $3 \mathrm{I}$ & 28 \\
\hline 54 & 52.3 & 48.2 & & 54 & 2 & 54 & 49.5 & 48.2 & 2256 & & 54 & 56.0 & 51.2 & 48 & & 54 & 65.5 & 63.2 & 32 & \\
\hline 56 & 55.2 & 52.0 & & 48 & & 56 & 50.3 & 49.2 & 2254 & . & 56 & 58.6 & 53.8 & 44 & & 5 & 74.0 & 71.3 & I8 & \\
\hline 58 & 52.6 & 49.2 & & 53 & & 58 & 44.8 & 44.2 & 2303 & & 58 & 60.0 & 56.0 & 42 & & 58 & 74.6 & 72.2 & 17 & \\
\hline 900 & 57.8 & 54.7 & & 44 & -15.2 & II 00 & $40 . I$ & 40.1 & IO & -14.4 & I3 00 & 63.1 & 59.3 & 36 & -13.9 & I5 00 & 72.8 & 70.8 & 20 & -14.0 \\
\hline 02 & 55.8 & 52.8 & & 47 & 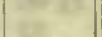 & 02 & 37.5 & 36.2 & I5 & & 02 & 63.5 & 60.1 & 36 & ( & 02 & $74 \cdot 3$ & 71.7 & I8 & 1 \\
\hline 04 & 52.4 & 50.2 & & 52 & 6 & 04 & 46.9 & 45.2 & 00 & & 04 & 63.0 & $60 . I$ & 36 & te & 04 & 73.8 & 71.6 & 18 & 10 \\
\hline 06 & 52.7 & 52.2 & & 50 & 6 & 06 & 42.2 & 40.9 & 2307 & $y$ & $\infty$ & 68.8 & 64.8 & 28 & tes & 06 & $73 \cdot 3$ & 71.5 & I9 & (x) \\
\hline 08 & 58.8 & 56.2 & & 42 & sis & 08 & 47.2 & 48. I & 2259 & 6 & 08 & 68.9 & 64.6 & 28 & 6 & 08 & 74.4 & 72.1 & I8 & 65 \\
\hline Io & 58.3 & 55.0 & & 44 & is & I0 & 50.I & 48.1 & 55 & & I0 & 66.4 & 63.3 & $3 \mathrm{I}$ & 8 & I0 & 70.2 & 68 & 24 & hat \\
\hline 12 & 51.2 & 48.1 & 22 & 55 & & 12 & 56.6 & 52.8 & 47 & & 12 & 64.8 & $6 r .6$ & 33 & & 12 & 70.9 & 69.0 & 23 & \\
\hline 14 & 45.9 & 41.8 & 23 & 04 & $-I 5.2$ & I4 & 61.0 & 59.0 & 38 & $-14 . I$ & I4 & 60.9 & 58.3 & 39 & $-\mathrm{I} 4.0$ & I 4 & $74 . I$ & 72.2 & I8 & -14.0 \\
\hline 16 & $40 . I$ & 36.4 & & I3 & (n) & I6́ & 49.4 & 48.7 & 56 & & 16 & 61.2 & 59.2 & $3^{8}$ & ati & 16 & 72.2 & 70.3 & $2 \mathrm{I}$ & tev \\
\hline I8 & 41.8 & $37 \cdot 3$ & & I0 & in & I8 & 47.3 & 46.2 & 59 & & 18 & 59.6 & 58.2 & 40 & yt & 18 & 73.5 & 72.0 & I8 & in \\
\hline 20 & 40.0 & 37.2 & & I2 & at & 20 & 50.7 & 49.2 & 54 & 6. & 20 & $54 . \mathrm{I}$ & $53 \cdot 3$ & $4^{8}$ & $6=$ & 20 & $75 . I$ & 72.7 & 17 & as \\
\hline 22 & 43.2 & 39.9 & 23 & 07 & ats & 22 & 50.3 & 49.8 & 54 & $=$ & 22 & 52.4 & 50.3 & 52 & I & 22 & 74.9 & 71.6 & I8 & ex \\
\hline 24 & 52.8 & 49.3 & 22 & 52 & 17 & 24 & 49.8 & 49.2 & 2255 & tat & 24 & 53.7 & 52.2 & 50 & $=$ & & 77.0 & 74.2 & 14 & je \\
\hline 26 & 43.6 & 42.2 & 23 & 05 & 8 & 26 & 44.7 & 44. & 2303 & & 26 & 56.8 & 54.1 & 46 & & $26^{*}$ & 43.8 & 38.2 & I4 & th \\
\hline 28 & 45.7 & 43.8 & & 02 & & 28 & 53. I & 52.9 & 2249 & & 28 & 63.0 & 6I.I & 35 & & 28 & 42.2 & 36.9 & I6 & \\
\hline 30 & 47.0 & 45.2 & $23 c$ & $\infty 0$ & $-15 . I$ & 30 & 58.9 & 58.6 & 40 & $-I 4 . I$ & 30 & 64.7 & 6 т.8 & 33 & $-14 . C$ & 30 & $44 . I$ & 37.1 & 14 & $-I_{4} .0$ \\
\hline 32 & 52.2 & 50.6 & 22 & $5^{2}$ & 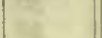 & 32 & 62.6 & 61.2 & 35 & 5 & 32 & 64.1 & 63.2 & 33 & 10 & 32 & 46.9 & 36.8 & 12 & 12 \\
\hline 34 & 51.8 & 48.9 & 22 & 54 & & 34 & 56.9 & 55.9 & 44 & & 34 & 66.1 & $64 \cdot 3$ & 30 & te & 3 & 43.2 & 38.0 & 14 & $M$ \\
\hline 36 & $46 . I$ & 45.6 & 23 & OI & S & 36 & 56.4 & 55. & 45 & 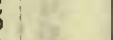 & 36 & 65.8 & $64 \cdot 3$ & 30 & 3 & 36 & 42.9 & 38.5 & 14 & $3 x$ \\
\hline 38 & 39.9 & 37.1 & & 12 & & 38 & 59.4 & 58.0 & 40 & 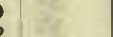 & 38 & 65.7 & 64.0 & $3 I$ & & 38 & 41.8 & 37.2 & 16 & \\
\hline 40 & $42 . \overline{8}$ & 39.9 & 23 & 08 & 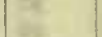 & $4^{0}$ & 54.6 & 53.2 & 48 & ten & 40 & 65.7 & 63.4 & 3I & ver & 40 & 40.9 & 36.9 & 17 & 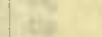 \\
\hline 42 & 48.8 & 47.9 & 22 & 57 & & 42 & 56.0 & 54.5 & 46 & & 42 & 62.9 & 62.2 & 34 & & 42 & 39.9 & 36.4 & 18 & \\
\hline 44 & $45 . I$ & 43.2 & 23 & 03 & -15.0 & 44 & 51.9 & $49 \cdot 3$ & s. & $3-I 4.0$ & 44 & 65.1 & 62.6 & 32 & -13.9 & 44 & $43 \cdot 3$ & 39.6 & I3 & $-\mathrm{I} 4.0$ \\
\hline 46 & 48.2 & 46.9 & 22 & $5^{8}$ & & 46 & 49.7 & $48 . I$ & 3 & & 46 & 68.0 & 67.8 & 26 & & 46 & 39.9 & 36.7 & 18 & \\
\hline $4^{8}$ & 54.9 & 52.8 & & 48 & 8 & 48 & 51.8 & 49.7 & 3 & & 48 & 68.8 & $67 \cdot 5$ & 26 & E & 48 & 39.9 & 37.8 & 17 & 5 \\
\hline 50 & 52.5 & 50.1 & & 52 & in & 50 & 56.6 & 54.1 & 46 & 28 & 50 & 69.8 & 69.1 & 24 & e & 50 & 28.5 & 25.2 & 36 & $a^{2}$ \\
\hline 52 & 49.0 & 47.9 & & 57 & 12 & $5^{2}$ & 53.4 & 51.9 & 50 & 3 & 52 & 67.1 & 67.0 & 27 & 8 & 52 & 26.5 & 24.6 & 38 & at \\
\hline 54 & 53.8 & 51.8 & & 50 & 28 & 54 & 50.9 & 50.2 & 53 & 58 & 54 & 65.9 & 65.4 & 30 & & 54 & 33.0 & 30.0 & 28 & \\
\hline 56 & 53.9 & 52.6 & 3 & 49 & 3 & 56 & 53.7 & $5 \mathrm{I} .3$ & r & $x^{2}$ & 56 & 63.1 & 61.9 & 34 & ia & 56 & 40.8 & 38.2 & 16 & (1) \\
\hline 58 & 58.5 & 56.1 & & 43 & 8 & 58 & 62.8 & 61.7 & 35 & 30 & 58 & 63.7 & 62.3 & 34 & 13 & 58 & 48.0 & 47.0 & 03 & 28 \\
\hline & & 6,25 & 6.8 & & & & & & & & & & & & & & & & & \\
\hline
\end{tabular}

Observers-W. J. P. and R. R. T., who alternated from $8 \mathrm{~h}$ oom to 8 h Ionl.
Observers-R. R. T. and R. W. P., who alternated from 13 h $36 \mathrm{~m}$ to I4h $46 \mathrm{~m}$. 
Tabulation of magnetic declinations observed at Teplitz Bay-Continued

\begin{tabular}{|c|c|c|c|c|c|c|c|c|c|c|c|c|c|c|c|c|c|c|c|c|}
\hline \multicolumn{5}{|c|}{ Wednesday, November I8, I903 } & \multicolumn{5}{|c|}{ Magnet scale inverted } & \multicolumn{5}{|c|}{ Wednesday, November I8, 1903} & \multicolumn{6}{|c|}{ Magnet scale inverted } \\
\hline \multirow{2}{*}{$\begin{array}{l}\text { Chr'r } \\
\text { time }\end{array}$} & \multicolumn{2}{|c|}{$\begin{array}{l}\text { Scale } \\
\text { readings }\end{array}$} & \multirow{2}{*}{$\begin{array}{l}\text { East } \\
\text { decli- } \\
\text { nation }\end{array}$} & & Chr'r & $\mathrm{Sc}$ & $\begin{array}{l}\text { ale } \\
\text { ings }\end{array}$ & $\begin{array}{l}\text { East } \\
\text { decli- }\end{array}$ & & Chr'r & $\begin{array}{l}\text { Sca } \\
\text { readi }\end{array}$ & $\begin{array}{l}\text { ale } \\
\text { ings }\end{array}$ & $\begin{array}{l}\text { Fast } \\
\text { decli- }\end{array}$ & & & $\begin{array}{l}\text { Sca } \\
\text { readi }\end{array}$ & & Eas & ist & Temp. \\
\hline & Left & Right & & & & Left & Right & & & & Left & Right & & & & Left & Right & & & \\
\hline$m$ & d & d & $\circ \cdot$ & c & $\mathrm{h} \mathrm{m}$ & d & $\mathrm{d}$ & $\cdot$, & 0 & $\mathrm{~h} \mathrm{~m}$ & d & d & & & $\mathrm{h} \mathrm{m}$ & $\mathrm{d}$ & d & - & & \\
\hline$\infty$ & 44. & $8 b$ & 2208. & -14.2 & 1800 & 30.0 & 29.0 & 2232 & -14.8 & 2000 & 46.5 & $43 . I$ & 2208 & $-I 4.4$ & 2200 & 28.9 & 27.4 & 223 & 33 & $-14 \cdot 6$ \\
\hline 02 & 43. I & 42.1 & II & & 02 & 28.6 & 28.0 & 34 & & 02 & 48.7 & 43.8 & 06 & & 02 & 26.2 & 25.6 & & 37 & \\
\hline 04 & 45.1 & $44 \cdot 3$ & 08 & & 04 & 26.9 & 26.1 & 36 & & 04 & 45.8 & $41 \cdot 3$ & 10 & & 04 & 24.7 & 24.2 & & 39 & \\
\hline 06 & 46.6 & 44 & 06 & s & 06 & 26.8 & 25.9 & 37 & 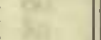 & 06 & 41.8 & 37.9 & 16 & & 06 & 23.8 & 23.2 & & 40 & \\
\hline a8 & 1.9 & 40.3 & I3 & & 08 & 27.9 & 27.2 & 35 & & 08 & 39.6 & 35 . & I9 & & 08 & 24.5 & 23.5 & & 40 & \\
\hline I0 & 42.0 & 40.0 & I3 & & IO & 26.2 & 25.8 & 37 & & I0 & 38.2 & 34 & 21 & & IO & 23.7 & 23.3 & & 40 & \\
\hline I2 & 38.0 & 36.6 & I9 & & I2 & 4.0 & 23.9 & $4 \mathrm{I}$ & & I2 & 38.0 & 34 & 21 & & I. & 22.0 & 21.7 & & 43 & \\
\hline I4 & 40.1 & 39.9 & I5 & -14.2 & I4 & 23.8 & 23.2 & $4 \mathrm{I}$ & $-I_{4} .8$ & I4 & 39.1 & 35.8 & 20 & $-\mathrm{I}_{4} .6$ & 14 & 20.0 & I9. 3 & & 47 & -14.7 \\
\hline 16 & 42.6 & $4 I .5$ & 12 & & 16 & 21.7 & 20.1 & 45 & & 16 & 39.7 & 34.9 & 20 & 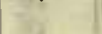 & I & 19.0 & 17.9 & & 49 & ( \\
\hline 18 & 44.8 & 43.0 & 09 & & 18 & 13.9 & I 4.4 & 56 & , & I8 & 41.7 & 37 & 16 & 11 & 18 & 18.6 & 17.8 & & 49 & re \\
\hline 20 & 41.8 & $4 I .1$ & I & $y$ & 20 & 15.7 & 14.9 & 54 & & 20 & 42.8 & 38 & I4 & & 20 & 18.3 & 17.9 & & 49 & 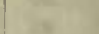 \\
\hline 22 & I. I & 41.0 & I4 & & 22 & .5 & 19.6 & 47 & $=$ & 22 & 44.5 & $4 I$ & II & & 2 & 19.0 & 18.3 & & 48 & 8 \\
\hline 24 & 37.0 & 36.0 & 21 & $=$ & 24 & 27.7 & 25. & 36 & $x^{2}$ & 24 & 46.5 & 43.2 & 08 & & 2 & 20.3 & 19.9 & & 46 & \\
\hline 2 & .9 & 37.6 & IS & & 26 & .9 & 32. & 27 & & 26 & 47.8 & & 06 & & 26 & 23.3 & 23.7 & & $4 \mathrm{I}$ & \\
\hline 28 & $4 \mathrm{I} .2$ & 40.8 & 13 & & 28 & 38.8 & 38.8 & I7 & & 28 & 48.7 & 45 & 04 & & 28 & 25.6 & 25.3 & & 38 & \\
\hline 30 & 44.9 & 43.4 & 09 & -14.4 & 30 & .8 & 40.1 & 15 & -14.6 & 30 & 46.3 & 44 & 07 & -14. & & 25.6 & 25.3 & & 38 & $-\mathrm{I} 4.8$ \\
\hline 32 & 43.2 & 42.0 & II & & 32 & 45.8 & 42.8 & 09 & & 32 & 43.2 & 40. & I3 & & 3 & 24.0 & 23.5 & & 40 & \\
\hline 34 & I. I & 39. & I & 5 & 34 & & 44. & 07 & & 34 & & & I5 & s. & & & 2.6 & & $4^{2}$ & \\
\hline 3 & 39.0 & 37. & Is & 10 & 36 & 50.1 & 47. & 2202 & 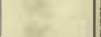 & 36 & $0 . I$ & 37 & I & ta & 3 & & 25.6 & & 37 & Fi \\
\hline 3 & 37.2 & 36.7 & 2 & 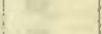 & 38 & & 49. & 59 & & 38 & 37.8 & 35 & I & 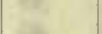 & & 28.4 & 28.0 & & 33 & \\
\hline 40 & 37.8 & 37.2 & I & $x$ & 40 & 48.7 & 47. & 03 & & 40 & 36.9 & & 2 & ta & 4 & 29.0 & 28.6 & & 32 & sin \\
\hline 42 & 37.8 & 37.5 & I9 & -3 & 42 & 43.8 & 42.2 & II & & 42 & 9.7 & 34 & 20 & & 4 & 30.3 & 29.8 & & 30 & \\
\hline 44 & 36.2 & 35.3 & 22 & -14.4 & 44 & 44.2 & 42.5 & I0 & -14.3 & 44 & 36.0 & 33 & 24 & $-I_{4} .6$ & 4 & 31 & $3 I .0$ & & 29 & \\
\hline 46 & & 35.8 & 20 & & 46 & 41.2 & 40. & I4 & L & 46 & 33.5 & & 26 & & 4 & 32.6 & 32.3 & & 27 & $-T_{4} .8$ \\
\hline 48 &. $\mathrm{I}$ & 28.8 & 32 & a & 48 & 41.0 & 39. & I5 & & 48 & 31.8 & & & & 4 & 30 & 30. I & & 30 & \\
\hline 50 & 33.4 & $3 \mathrm{I} .4$ & 2 & 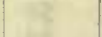 & 50 & 1.2 & 39 & I5 & & 50 & 30.9 & 30 & & & 5 & 3 & 26.3 & & 35 & 5. \\
\hline 52 & & 32.1 & 2 & & 52 & & 37. & 18 & & 52 & 0.9 & & 3 & & 52 & 5 & 23.3 & & 40 & \\
\hline 54 & 37.4 & 36.2 & 2 & & 54 & $38 . \bar{I}$ & 36. & 20 & 5 & 54 & 31.3 & & & & 5 & 0 & 20. & & 44 & \\
\hline 56 & 40.9 & 40.5 & 14 & & 56 & .6 & 34. & 24 & & 56 & 0.5 & & 3 & & & 20. & 17.2 & & 48 & \\
\hline 58 & 39. & & I6 & & 58 & 36.6 & $35 \cdot 3$ & 22 & & 58 & 30.9 & 29.8 & $3 \mathrm{I}$ & & 5 & 18.4 & 16.0 & & 50 & \\
\hline Do & 37.9 & 37.8 & 19 & $-\mathrm{I} 4.5$ & 1900 & 34.9 & 33. & 24 & -14.3 & 2100 & Lo & st & & -14. & 2300 & I8. I & 16.3 & & 50 & -I5.0 \\
\hline 02 & 36. & $6 b$ & 20 & & 02 & 35.9 & 35.2 & 22 & & $02 *$ & 32.2 & 26.8 & $3 I$ & & 02 & 18.0 & 15.1 & & 52 & \\
\hline 04 & 35.5 & $35 \cdot 3$ & 22 & w & 04 & 37.8 & 37.0 & 20 & & 04 & 32.1 & 27.3 & $3 I$ & 8 & 0 & 16.6 & 14.3 & & 53 & \\
\hline 06 & & 35.0 & 23 & 64 & 06 & .5 & 35 . & 22 & & 06 & 32.8 & $27 . \mathrm{I}$ & 3 & & o & I3. I & II. 3 & 22 & 58 & 6 \\
\hline 08 & & .2 & 2 & 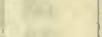 & 08 & & 33 & 25 & 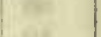 & 08 & 34.9 & 29.3 & 27 & & a & II. 3 & 10.0 & & ol & \\
\hline I0 & 33.3 & 32.3 & 27 & in & I0 & .4 & 32 . & 27 & & 10 & 36.1 & & 2 & & I & 8.7 & 7. & & 05 & \\
\hline 12 & & 3 & 30 & 64 & I2 & & & 30 & & 12.2 & 35.5 & 30. & 25 & & $12^{*}$ & 30 & 29 & & 10 & \\
\hline I4 & 31.9 & $3 \mathrm{I} .3$ & 28 & -14.6 & I4 & .8 & 30. & 30 & -14.4 & I4 & 35.0 & 30.2 & 26 & $-\mathrm{I}_{4}$. & I4 & 34.3 & 29.8 & & 07 & -14.9 \\
\hline 16́ & 32.1 & 1.8 & 28 & . & 16 & 1.8 & 29. & 30 & & 16 & 35.3 & & 25 & -4. & & & 31.6 & & 04 & - \\
\hline 18 & 7 & 31.2 & & 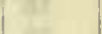 & IE & & 29 & 30 & & I\& & 33.2 & 29.8 & & & I & 2 & 32.2 & $23 c$ & 03 & \\
\hline 20 & .7 & 35.5 & 2 & test & 20 & & 27. & 33 & & $2 c$ & 32.9 & 29. & 2 & & 2 & & 36.8 & 22 & 57 & 8 \\
\hline 22 & & 4 & I3 & 는 & 22 & 28.2 & 25. & & 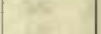 & 2 & 34.2 & & & & & 42.8 & 40.0 & & 52 & \\
\hline 2 & 42.1 & 41.8 & 12 & te & 24 & 7.9 & 25 . & 36 & & 2 & 34.9 & 31 . & 25 & & 24 & $44 \cdot 3$ & 42.0 & & 49 & \\
\hline 2 & & I. I & & & & & & 34 & & & 35 & & & & & & 41.7 & & 50 & \\
\hline 28 & & 5.9 & $2 I$ & & 28 & 1.8 & 29.4 & 30 & & 28 & $34 . I$ & $3 \mathrm{I}$ & 26 & & 28 & 3 & 40.8 & & 51 & \\
\hline 30 & 37.2 & 36.8 & 20 & -14.7 & 30 & 3. I & 31 & 28 & -14.4 & 30 & 33.8 & 33 & 25 & $-I 4.6$ & 30 & 43.5 & $4 \mathrm{I} .2$ & & 50 & -15.0 \\
\hline 32 & & 33.8 & 24 & & 32 & & 32. & 25 & & 32 & 35.9 & & 23 & & 32 & 44.3 & 41.8 & & 50 & \\
\hline 34 & & 38.1 & 18 & 4 & 34 & & 33. & 24 & & 31 & 34.3 & 30. & & & 34 & & 43.4 & & 47 & \\
\hline & & 36.2 & & & & & 34. & 23 & & 36 & & & & & & $4^{6}$ & 43 & & $4^{6}$ & \\
\hline 38 & 31.9 & 31.7 & 2 & & 38 & 7.1 & 34 . & 22 & & 38 & & 32. & 2 & & 3 & & 43 & & 47 & \\
\hline 40 & & 26.2 & & ta & 40 & & & $2 I$ & & $4 C$ & 37.8 & 34. & 21 & & 40 & 47 & 45. & & 44 & \\
\hline 42 & 25.1 & $24 \cdot 5$ & 39 & & 42 & & 35. & 20 & & 42 & 37.7 & 33.8 & 21 & & 42 & & 42.6 & & 49 & \\
\hline 44 & 26.8 & 26.1 & 37 & $-I_{4} .7$ & 44 & & 36. & 19 & -14.5 & & & $33 . I$ & 23 & $-I_{4}$ & 44 & 45.6 & 44.3 & & 47 & -15.0 \\
\hline 46 & 26.8 & 25.8 & 37 & & 46 & .9 & 35. & 20 & & 4 & 36.8 & 35 . & $2 \mathrm{I}$ & & 46 & 45.4 & 43.8 & & 47 & \\
\hline 48 & & 25.0 & & & 48 & & 36. & 20 & & 48 & 36.3 & 36.0 & 21 & & 48 & & 45.3 & & & \\
\hline 50 & 28.9 & 28. I & 33 & 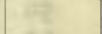 & 50 &.$I$ & 37. & 18 & & 50 & 35.8 & 35.2 & 22 & & 50 & & 45 & & 45 & \\
\hline 52 & 30.8 & 30.1 & 30 & 34 & 52 & 42.2 & 39. & I4 & as & 52 & 36.1 & 35.7 & $2 I$ & 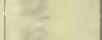 & 52 & 43.6 & 41.6 & & 0 & \\
\hline 54 & 29.2 & 28.8 & 33 & 1 & 54 & 43.3 & 40. & 12 & 10 & 54 & $35 . I$ & 34.2 & 23 & & 54 & 42.3 & 40.3 & & 52 & \\
\hline 56 & & 29.9 & 30 & $\frac{4}{4}$ & 56 & 44.3 & 40.6 & 12 & & 56 & $33 \cdot 3$ & 32.2 & 26 & & 56 & 39.8 & 37.6 & & 56 & \\
\hline 58 & 33.8 & 32.8 & 26 & & 58 & 44.9 & $4 \mathrm{I} .4$ & IO & & $5^{8}$ & $3 I .3$ & 29.8 & 30 & & $\begin{aligned} 58 \\
2400\end{aligned}$ & $\begin{array}{l}38.3 \\
38.0\end{array}$ & $\begin{array}{l}36.9 \\
36.6\end{array}$ & & $\begin{array}{l}58 \\
58\end{array}$ & -15.0 \\
\hline
\end{tabular}

Observers-R. W. P. and R. R. 'T, who alternated from $\mathrm{I} 8 \mathrm{~h} \mathrm{I} 6 \mathrm{~m}$ to I8h zom.
Correction to local mean time is $+6 \mathrm{~m} 28 \mathrm{~s} .90^{\circ}$ torsion $=24^{\prime} 6$. Torsion head at oh oom read $318^{\circ}$ and at $24 \mathrm{~h} 30 \mathrm{~m}$ read $308^{\circ}$. Observers-R. R. T. and W. J. P., who alternated from 20 h Iom to $22 \mathrm{~h}$ 20m. 
Tabulation of magnctic declinations observed at Teplitz Bay-Continued

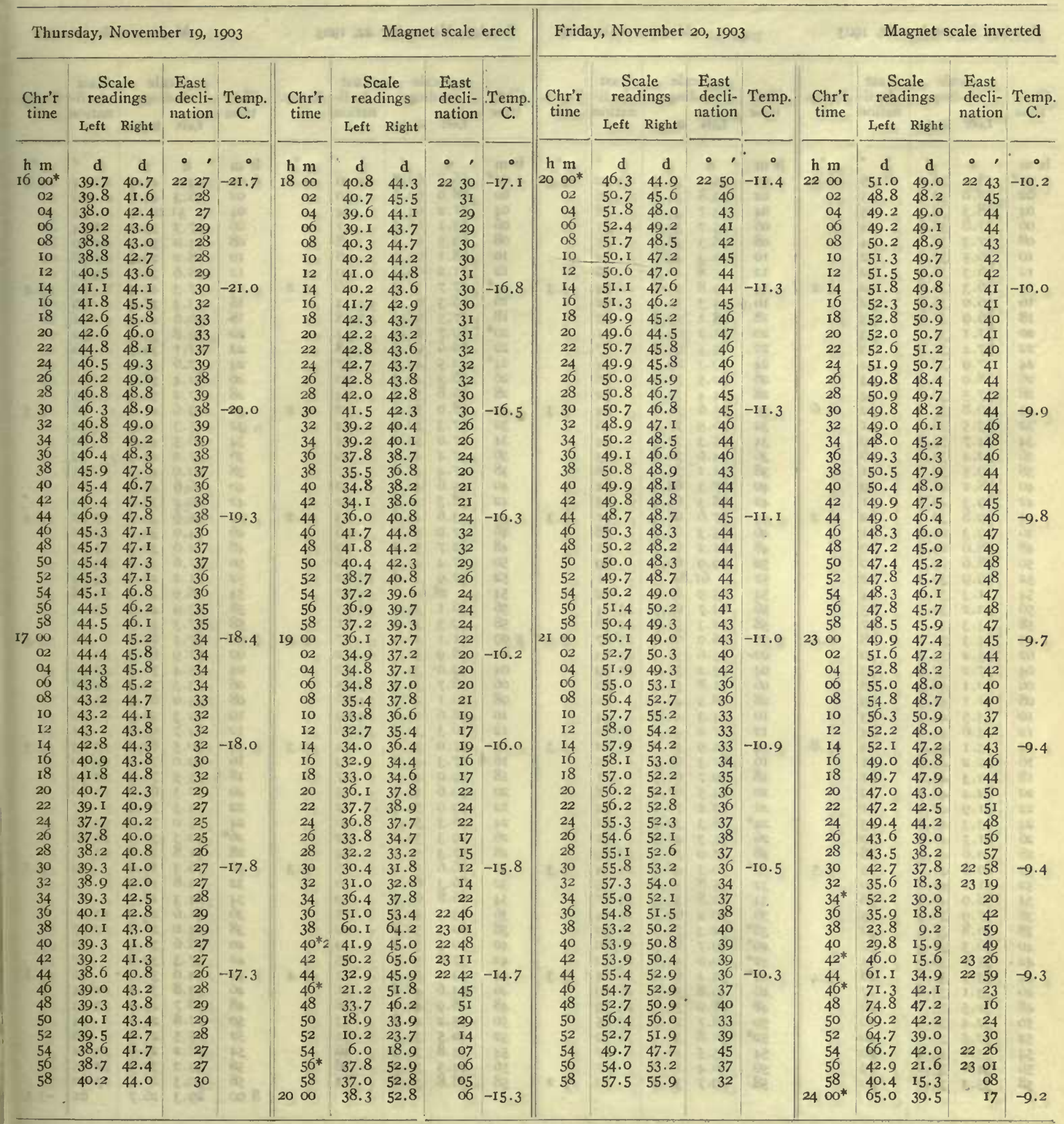

Correction to local mean time is $+8 m 49 s$.

Torsion head at $14 \mathrm{~h} 25 \mathrm{~m}$ read $308^{\circ}$ and at the end read the same. Observer-R. R. T.
Correction to local mean time is $+9 \mathrm{~m}$ o3s.

Torsion head at 19 h $30 \mathrm{~m}$ read $318^{\circ}$ and at the end read the same. Observer-R. R. T. 
Tabulation of magnetic declinations observed at Teplitz Bay-Continued

\begin{tabular}{|c|c|c|c|c|c|c|c|c|c|c|c|c|c|c|c|c|c|c|c|c|c|}
\hline \multicolumn{6}{|c|}{ Sunday, November 22, 1903} & \multicolumn{5}{|c|}{ Magnet scale erect } & \multicolumn{5}{|c|}{ Sunday, November 22, 1903} & \multicolumn{6}{|c|}{ Magnet scale inverted } \\
\hline \multirow{2}{*}{$\begin{array}{l}\text { Chr'r } \\
\text { time }\end{array}$} & \multicolumn{2}{|c|}{$\begin{array}{l}\text { Scale } \\
\text { readings }\end{array}$} & East & Temp. & Chr'r & $\begin{array}{r}\mathrm{Sc} \\
\text { read }\end{array}$ & $\begin{array}{l}\text { ale } \\
\text { dings }\end{array}$ & $\begin{array}{l}\text { Eas } \\
\text { dec }\end{array}$ & & Temp. & Chr'r & $\begin{array}{l}\text { Sc: } \\
\text { read }\end{array}$ & $\begin{array}{l}\text { ale } \\
\text { lings }\end{array}$ & East & Temp. & Chr't & $\begin{array}{r}\text { Sca } \\
\text { readi }\end{array}$ & $\begin{array}{l}\text { ale } \\
\text { lings }\end{array}$ & $\begin{array}{l}\text { Eas } \\
\text { decl }\end{array}$ & st & Teinp. \\
\hline & L,eft & Right & & & & Left & Right & & & & & Left & Right & & & & Left & Right & & & \\
\hline h $\mathrm{m}$ & d & d & $\cdot 1$ & . & $\mathrm{h} \mathrm{m}$ & $\mathrm{d}$ & $\mathrm{d}$ & & , & $\bullet$ & $\mathrm{h} \mathrm{m}$ & & & $\bullet \quad$, & $\bullet$ & h $\mathrm{m}$ & d & $d$ & & $\cdot$ & - \\
\hline $000^{*}$ & 38.0 & 39.0 & 2235 & -6.0 & 200 & 35.1 & $35 \cdot 3$ & & & $-3 \cdot 3$ & $400^{*}$ & 66.8 & 62.8 & 2449 & $-3 \cdot 1$ & 600 & 44.0 & 41.9 & 234 & & -3.4 \\
\hline 02 & 38.0 & 39.3 & 35 & & 02 & 33.8 & $34 \cdot 3$ & & 28 & & 02 & 68.0 & 60.6 & 50 & & 02 & 46.3 & 44.8 & & 39 & \\
\hline 04 & 38.3 & 39.3 & 35 & Y I & 04 & 33.7 & 34.2 & & 28 & 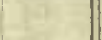 & 04 & 71.2 & 64.6 & 44 & 25 & 04 & 51 & $.0 b$ & & & \\
\hline 06 & 37.8 & 38.6 & 34 & ter & 06 & 35.7 & 36.0 & & $3 I$ & & 06 & 73.1 & 66.3 & $4 \mathrm{I}$ & 34 & 06 & 37.3 & 35.1 & 235 & & \\
\hline 08 & 37.3 & 38.1 & 34 & 84 & 08 & 38.3 & 38.7 & & 35 & Gert & 08 & 69.5 & 61.3 & 48 & 69 & 08 & 34.0 & 30.2 & 240 & & \\
\hline 10 & 36.5 & 37.3 & 32 & tris & IO & $42 \cdot 3$ & 43.9 & & 42 & 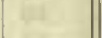 & 10 & 68.1 & 63.0 & 48 & 01 & 10 & 28.2 & 26.3 & 240 & 08 & \\
\hline 12 & 36.3 & 36.9 & 32 & . & 12 & 45.8 & 46.0 & & 46 & y & 12 & 71.2 & 69.3 & 40 & -5 & 12 & 38.6 & 36.3 & 235 & & 1 \\
\hline 14 & 36.3 & 36.8 & 32 & -5.2 & I4 & 49.8 & 51.4 & 22 & 54 & $-3 \cdot 3$ & $14^{*}$ & 57.7 & 51.7 & 26 & -3.2 & 14 & $47 \cdot 3$ & 43.4 & & 40 & -3.4 \\
\hline 16́ & 36.0 & 36.8 & 32 & & 16.9 & 55.3 & 56.3 & 23 & & . & 16 & 66.3 & $6 \mathrm{I} \cdot 3$ & 2412 & & 16 & 43.3 & 38.9 & & 46 & \\
\hline 18 & 36.9 & 37.4 & 33 & Hi & 18 & 58.1 & 59.8 & & 07 & 41 & 18 & 77.5 & 68.0 & $235^{8}$ & 34t & I8 & 46.6 & 42.2 & & $4 I$ & \\
\hline 20 & 37.1 & 37.7 & 33 & $x$ & 20 & 61.2 & 62.1 & & II & $2 x$ & $20^{*}$ & 69.7 & 61.I & 53 & 46 & 20 & 39.8 & 35.4 & & $5^{2}$ & \\
\hline 22 & 37.4 & 37.9 & 33 & $x^{2}$ & 22 & 65.5 & 65.7 & & 17 & sa & 22 & 69.6 & 67.8 & 48 & $+x$ & 22 & 41.1 & 36.3 & & 50 & \\
\hline 24 & 37.9 & 38.5 & 34 & 15 & 24 & 69.3 & 69.9 & & 24 & 10 & $24^{*}$ & 52.5 & 42.2 & 37 & ie & 24 & 57.6 & 52.1 & & 25 & \\
\hline 26 & 38.0 & 38.3 & 34 & & 26 & 72.5 & 72.6 & & 28 & a & 26 & 56.3 & 46.3 & 30 & yc & 26 & 62.4 & 58.4 & & 16 & \\
\hline 28 & 37.5 & 38.0 & 34 & & 28 & 74.4 & 74.8 & & 32 & (n) & 28 & 56.8 & 53.8 & 24 & 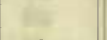 & 28 & 57.2 & 52.3 & & 25 & \\
\hline 30 & 36.8 & 37.3 & 32 & -4.6 & 30 & 75.6 & 76.8 & & 34 & $-3 \cdot 3$ & 30 & 58.0 & 54.6 & 22 & -3.2 & 30 & 49.6 & 45.6 & & 36 & -3.5 \\
\hline 32 & 35.5 & 36.1 & $3 \mathrm{I}$ & & 32 & 76.9 & 77.7 & & 36 & & 32 & 57.3 & 52.9 & 24 & & 32 & $45 \cdot 3$ & 42.3 & & 42 & \\
\hline 34 & 34.7 & 35.3 & 29 & 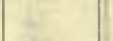 & 34 & $75 \cdot 3$ & 77.5 & & 34 & a & 34 & 57.0 & 53.2 & 24 & E & 34 & 46.5 & 43.3 & & 40 & se \\
\hline 36 & 32.4 & 33.1 & 26 & ne & 36 & 74.6 & 76.0 & & 33 & $x^{2}$ & 36 & 47.4 & 44.4 & 39 & $y$ & 36 & 47.2 & 44.5 & & 39 & \\
\hline 38 & 29.5 & 30.0 & 21 & 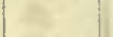 & 38 & 71.1 & 73.1 & & 28 & W & 38 & 47.4 & 47.0 & 37 & es & $3^{8}$ & 50.0 & 47.2 & & 35 & \\
\hline 40 & 28.2 & 28.6 & 19 & (4) & 40 & 67.3 & 68.9 & & 21 & $\sqrt{45}$ & 40 & 45.6 & 42.7 & 42 & 7 & 40 & 46.6 & 43.3 & & 40 & 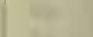 \\
\hline 42 & 29.3 & 29.7 & 21 & 145 & 42 & 68.3 & 69.9 & & 23 & the & 42 & 38.3 & 36.9 & 52 & t & 42 & 40.2 & 38.0 & & 50 & \\
\hline 44 & 29.8 & 30.3 & 22 & -4.2 & 44 & 67.6 & 68.3 & & 21 & -3.3 & 44 & 43.6 & 43.1 & 43 & -3.2 & 44 & 35.6 & 33.6 & 23 & 57 & -3.4 \\
\hline 46 & 29.3 & 29.9 & 21 & & 46 & 65.3 & 66.7 & & 18 & & 46 & 47.9 & 46.9 & 36 & & 46 & 34.0 & 30.1 & & $\rho I$ & \\
\hline 48 & 28.6 & 29.2 & 20 & & 48 & 61.2 & 61.7 & & II & 2 & 48 & 52.7 & 51.9 & 29 & 68 & 48 & 32.6 & 30.3 & 24 & 02 & \\
\hline 50 & 28.6 & 29.1 & 20 & 60 & 50 & 59.9 & 60.6 & & 09 & 5 & 50 & 47.4 & 47.4 & 36 & at & 50 & 37.6 & 35.8 & 235 & 53 & \\
\hline 52 & 29.9 & 30.3 & 22 & 2 & 52 & 61 & $.8 a$ & & II & 27 & 52 & 37.9 & 37.1 & 2352 & $=$ & $5^{2}$ & 31.2 & 28.8 & 240 & 04 & \\
\hline 54 & $3 \mathrm{I} \cdot 3$ & 31.6 & 24 & 1 & 54 & 65.9 & 67.0 & & 19 & 10 & 54 & 28 & $.0 b$ & 2407 & 48 & 54 & 28.9 & 26.4 & $24 c$ & 08 & \\
\hline 56 & 32.2 & 32.5 & 25 & & 56 & 62.7 & 63.7 & 23 & 14 & 3 & 56 & 36.1 & $35 \cdot 5$ & 2355 & & 56 & 43.9 & 41.3 & 23 & 44 & \\
\hline $5^{8}$ & 33.3 & 33.5 & 27 & 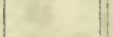 & 58 & 52.3 & 53.7 & 22 & 58 & 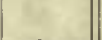 & $5^{8}$ & 39.3 & 37.6 & $5 I$ & 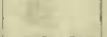 & $5^{8}$ & 57.3 & 51.9 & & 25 & \\
\hline I 00 & 34.3 & 34.5 & 28 & -4.0 & 300 & 58.0 & 60.0 & 23 & 07 & $-3 \cdot 3$ & 500 & $41 . I$ & 40.3 & 47 & -3.2 & 700 & 67.5 & 64.1 & & 08 & -3.5 \\
\hline 02 & 35.2 & 35.4 & 30 & & 02 & 55.6 & 57.1 & 23 & 03 & & 02 & 38.9 & 37.0 & 52 & & 02 & 71.9 & 67.3 & $23 c$ & 02 & \\
\hline 04 & 37.2 & 37.6 & 33 & 60 & 04 & 53.2 & $54 \cdot 3$ & 22 & 59 & pat & 04 & 39.0 & 35.6 & 52 & 47 & 04 & 75.4 & 71.2 & 22 & 56 & \\
\hline 06 & 40.1 & 40.3 & 38 & w. & 06 & 51.8 & 54.2 & & 58 & (3) & 06 & 36.7 & 34.3 & 2355 & tat & 06 & 73.3 & 70.3 & 22 & $5^{8}$ & \\
\hline 08 & $4 \mathrm{I} .1$ & 41.7 & 39 & tet & 08 & 48.9 & 49.1 & & 51 & Ay & os & 30.1 & 27.6 & 2406 & 14 & 08 & 65.0 & 61.9 & 23 & II & \\
\hline 10 & 43.5 & 44.1 & 43 & of & I0 & $44 \cdot 3$ & 44.9 & & 44 & & 10 & 28.6 & 25.1 & 2409 & ent & 10 & 59.3 & 56.5 & & 20 & 45 \\
\hline 12 & 43.6 & 44.6 & 44 & (2) & 12 & 47.0 & 49.0 & 22 & 50 & 12 & 12 & 36.3 & 33.5 & 2356 & - 45 & 12 & 64.7 & 63.0 & & II & \\
\hline I4 & 43.1 & 44.0 & 43 & -3.8 & 14 & 54.9 & $57 \cdot 3$ & 23 & 02 & $-3 \cdot 3$ & I4 & 37.0 & 35.2 & 54 & -3.3 & 14 & 70.5 & 67.5 & 23 & 03 & -3.5 \\
\hline 16 & 42.2 & 43.1 & $4 \mathrm{I}$ & & 16 & 62.3 & 65.5 & & I5 & & I6. 6 & 47.3 & 44.3 & 39 & & 16 & 79.0 & 74.7 & 22 & 50 & \\
\hline 18 & 42.3 & 43.2 & 41 & 47 & 18 & 59.7 & 62.9 & & II & 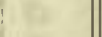 & I8 & 49.0 & 45.0 & 37 & 7. & 18.5 & 75.5 & 72.6 & 22 & 55 & \\
\hline 20 & 42.4 & 43.2 & 42 & 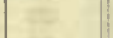 & 20 & 64.5 & 67.0 & & 18 & - & 20 & 59.6 & 49.7 & 25 & 12 & 20 & 72.3 & 69.8 & 23 & $\infty$ & \\
\hline 22 & 41.9 & 42.9 & $4^{I}$ & 20 & 22 & 73.2 & 76.1 & & 32 & & 22 & 59.0 & 54.3 & 22 & as & 22 & 74.3 & 71.3 & & 57 & \\
\hline 24 & $43 \cdot 3$ & 43.7 & 43 & Fr & $24^{*}$ & 28.3 & 39.7 & & 31 & & 24 & 56.3 & $5 \mathrm{i} .7$ & 26 & $F^{2}$ & 24. & 75.0 & 72.0 & 23 & 56 & \\
\hline 26 & 41.5 & 41.7 & 40 & $y=$ & 26 & 35.3 & 44.9 & & $4 \mathrm{I}$ & & 26 & 59.3 & 54.7 & 22 & 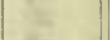 & $26 *$ & 40.3 & 32.0 & 22 & 48 & \\
\hline 28 & 40.6 & 41.0 & 38 & 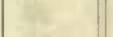 & 28 & 47.0 & 56.3 & 23 & 59 & & 28 & 57.3 & 53.1 & 24 & 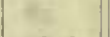 & 28 & 31.8 & 23.8 & $23 c$ & OI & \\
\hline 30 & 40.8 & 41.6 & 39 & -3.5 & $30^{*}$ & 30.3 & 48.3 & 24 & 34 & -3.3 & 30 & 66.6 & 56.2 & 15 & -3.3 & 30 & 26.3 & 19.6 & & $\infty 9$ & -3.5 \\
\hline 32 & $4 \mathrm{I} \cdot 2$ & 41.8 & 40 & & 32 & 35.8 & 55.8 & & 45 & & 32 & 66.3 & 62.1 & 10 & & 32 & 24.8 & 18.0 & & II & \\
\hline 34 & 38.9 & 40.0 & 36 & $y z=$ & 34 & 52.8 & 55.3 & 24 & 57 & 186 & 34 & 66.8 & 63.3 & 09 & est & 34 & $24 \cdot 3$ & I8.0 & & 12 & \\
\hline 36 & 37.9 & 38.9 & 35 & or & 36 & 60.2 & 69.3 & 25 & 14 & & 36 & 63.3 & 59.6 & 15 & est & 36 & 31.6 & 24.0 & 23 & or & \\
\hline 38 & 36.8 & $37 \cdot 3$ & 32 & ta & $38^{*}$ & 52.3 & 73.2 & 25 & 50 & wh & 38 & 60.5 & 56.8 & 19 & 26 & $3^{8}$ & 34.0 & 26.6 & 22 & 57 & \\
\hline 40 & 36.3 & 36.9 & 32 & 18 & $40^{*}$ & 49.7 & 68.3 & 26 & 20 & by & 40 & 60.5 & 56.8 & 19 & 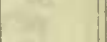 & 40 & 38.6 & 31.9 & & 50 & 0 \\
\hline 42 & 35.9 & 36.6 & 31 & $7-4$. & $42^{*}$ & 20.0 & 44.8 & 24 & 42 & 71. & 42 & 57.8 & 52.0 & 25 & $y+2$ & 42 & $47 \cdot 3$ & 39.6 & & 37 & \\
\hline & 33.9 & 34.6 & 28 & -3.4 & $44^{*}$ & 33.2 & 60.0 & 26 & 24 & $-3 \cdot 3$ & 44 & 59.0 & 53.0 & 23 & -3.4 & 44 & 54.6 & 52.2 & & $2 \mathrm{I}$ & -3.5 \\
\hline 46 & 33.1 & 33.6 & 27 & & 46 & 49.8 & 50.3 & 26 & 29 & & 46 & 53.6 & 48.6 & $3 \pi$ & & 46 & 33.8 & 31.2 & 22 & 54 & \\
\hline 48 & 32.5 & 33.1 & 26 & 26 & 48 & 28.0 & 33.5 & 25 & 59 & & 48 & 48.9 & 44.5 & $3^{8}$ & fise & 48 & 28.7 & 25.2 & 23 & 03 & \\
\hline 50 & 32.4 & 32.9 & 26 & Ift & 50 & 18.7 & 25.3 & 25 & 45 & (1) & 50 & 45.2 & 40.8 & 43 & - & 50 & 27.8 & 24.1 & & 04 & \\
\hline 52 & 32.5 & 33.0 & 26 & ate & $52^{*}$ & 30.8 & 31.6 & 24 & 59 & & 52 & 40.5 & 35.7 & $5 \mathrm{I}$ & If & 52 & 25.5 & $2 \mathrm{r} .4$ & & 08 & \\
\hline 54 & 33.0 & 33.4 & 26 & 12 & 54 & 15.5 & 18.7 & & 37 & $t^{2}=$ & 54 & 43.8 & 39.3 & 46 & 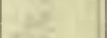 & 54 & 28.2 & 25.8 & $23 c$ & 02 & \\
\hline 56 & 34.6 & 35.0 & 29 & 20 & 56 & 10.4 & 12.0 & & 27 & & 56 & 44.5 & 36.3 & 48 & 3 & 56 & 33.2 & 30.9 & 22 & 55 & \\
\hline 58 & 35.3 & 35.6 & 30 & $t^{2}$ & 58 & 29.7 & 33.3 & 21 & 59 & -3.2 & 58 & 38.8 & 35.8 & 52 & 68 & $5^{8}$ & 30.3 & 27.1 & 23 & $\infty$ & \\
\hline & & & & & & & & & & & & & & & & 800 & 29.3 & 26.7 & & or & -3.5 \\
\hline
\end{tabular}

Observer-W. J. P.

Correction to local inean time is $+9 \mathrm{~m} 32 \mathrm{~s}$.

Torsion head at oh oom read $314^{\circ}$ and at $8 \mathrm{~h} \mathrm{I} 7 \mathrm{~m}$ read the samc.

Observer-W. J. P. 
Tabulation of magnetic declinations observed at Teplitz Bay-Continued

\begin{tabular}{|c|c|c|c|c|c|c|c|c|c|c|c|c|c|c|c|c|c|c|c|}
\hline \multicolumn{5}{|c|}{ Monday, November 23, 1903} & \multicolumn{5}{|c|}{ Magnet scale erect } & \multicolumn{5}{|c|}{ Tuesday, November 24, 1903} & \multicolumn{5}{|c|}{ Magnet scale inverted } \\
\hline \multirow{2}{*}{$\begin{array}{l}\text { Chr'r } \\
\text { time }\end{array}$} & \multicolumn{2}{|c|}{$\begin{array}{l}\text { Scale } \\
\text { readings }\end{array}$} & \multirow{2}{*}{$\begin{array}{c}\text { East } \\
\text { decli- } \\
\text { nation }\end{array}$} & Temp. & Chr'r & $\begin{array}{r}\mathrm{Sc} \\
\text { read }\end{array}$ & $\begin{array}{l}\text { lings } \\
\text { lings }\end{array}$ & $\begin{array}{l}\text { East } \\
\text { decli- }\end{array}$ & Temp. & Chr'r & $\begin{array}{l}\mathrm{Sc} \\
\text { read }\end{array}$ & $\begin{array}{l}\text { ale } \\
\text { lings }\end{array}$ & $\begin{array}{l}\text { East } \\
\text { decli- }\end{array}$ & Temp. & Chr'r & $\begin{array}{l}\text { Se } \\
\text { read }\end{array}$ & lings & $\begin{array}{l}\text { East } \\
\text { decli- }\end{array}$ & Temp. \\
\hline & Left & Right & & & & Left & Right & & & time & Left & Right & & & tim & Left & Right & nation & \\
\hline $\mathrm{h} \mathbf{m}$ & $\mathrm{d}$ & d & - ' & 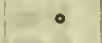 & $\mathrm{h} \mathrm{m}$ & d & a & . ' & • & $h \mathrm{~m}$ & d & d & $\circ \quad$ & & $\mathrm{h} \mathrm{m}$ & d & $d$ & - & \\
\hline 800 & 37.8 & $4^{2} .9$ & 2235 & -10.0 & IO 00 & $37 \cdot 3$ & 38.6 & 2231 & -6.0 & 1200 & 43.7 & 42.3 & 2224 & -6.5 & 1400 & 32.0 & 29.9 & 2243 & $-4 \cdot 7$ \\
\hline 02 & 39.2 & 43.8 & 37 & & 02 & 37.2 & 39.0 & $3 I$ & & 03 & 42.6 & 40.9 & & & 02 & 32.6 & 30.8 & 42 & 7. \\
\hline 04 & $4 \mathrm{I} \cdot 5$ & 42.9 & 38 & E & 04 & 29.8 & 32.8 & $2 \mathrm{I}$ & 5 & 04 & 42.8 & $4 \mathrm{I} .4$ & 26 & 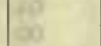 & 04 & 32.4 & 30.3 & 43 & os \\
\hline 06 & 38.1 & 40.2 & 33 & & 06 & 29.7 & 31.2 & 19 & & 06 & 45.7 & 43.8 & 22 & & 06 & 31.9 & 30.3 & 43 & 8 \\
\hline 08 & $37 \cdot 3$ & 40.2 & 32 & 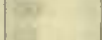 & 08 & 28.8 & 29.8 & 17 & N & 08 & 48.8 & 45.3 & 18 & & 08 & 32.6 & 30.9 & 42 & $m$ \\
\hline Io & 38.8 & 42.4 & 35 & an & IO & 33.3 & 34.3 & 24 & tet & I0 & 49.8 & 46.9 & I6 & & Io & $32 . I$ & 30.6 & 43 & $\mathrm{at}$ \\
\hline I2 & 34.6 & 37.9 & 28 & & 12 & 32.8 & 35.0 & 25 & & 12 & 53.1 & $49 . I$ & 12 & & 12 & 32.6 & 30.9 & 42 & \\
\hline I4 & 35.8 & 39. I & 30 & -9.5 & I4 & 29. I & $3 \mathrm{I} .2$ & 19 & -6.2 & I4 & $5 \mathrm{I} .7$ & 48.9 & 13 & -6.2 & 14 & 32.3 & 31.0 & 42 & -4.6 \\
\hline I6 & 37.4 & 39.9 & 32 & & 16 & 34.0 & 36.8 & 27 & & 16 & 48.2 & 45.2 & 19 & & 16 & $32 . I$ & 30.4 & 43 & . \\
\hline 18 & 37.4 & 39.1 & $3 I$ & & 18 & 35.0 & 38.4 & 29 & & 18 & 46.1 & 42.2 & 23 & & 18 & 31.9 & 30.1 & 43 & of \\
\hline 20 & 32.3 & 33.5 & 23 & 20. & 20 & 35.9 & 38.8 & 30 & & 20 & 43.1 & 38.1 & 28 & 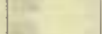 & 20 & $30 . \overline{6}$ & 29.0 & 45 & 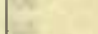 \\
\hline 22 & 35.0 & 36.3 & 27 & 10 & 22 & 34.2 & 37.3 & 27 & E & 22 & 44.7 & 40.2 & 25 & 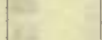 & 22 & 30.6 & 29.2 & 45 & E. \\
\hline 24 & 38.2 & 39.3 & 32 & 20 & 24 & 39.1 & 41.2 & 34 & & 24 & 42.9 & 37.8 & 29 & & 24 & 30.9 & 29.5 & 44 & 5 \\
\hline 26 & 38.1 & 38.5 & 32 & 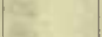 & 26 & $4 I . I$ & 42.2 & 37 & & 26 & 43.8 & 38.6 & 27 & . & 26 & 30.4 & 28.8 & 45 & E \\
\hline 28 & 29.7 & 30.2 & 18 & & 28 & 41.2 & 42.1 & 37 & & 28 & 44.3 & 39.2 & 26 & & 28 & 29.8 & 28.2 & 46 & \\
\hline 30 & 32 & $.0 a$ & 22 & & 30 & 39.1 & 39.9 & 33 & -5.9 & 30 & 41.9 & $37 \cdot 3$ & 30 & -6.1 & 30 & 29.0 & 27.8 & 47 & $-4 \cdot 3$ \\
\hline 32 & 39.9 & 42.1 & 36 & -9.0 & 32 & 35.3 & 35.8 & 27 & & 32 & 42.9 & 39.7 & 27 & & 32 & 30.0 & 28.3 & 46 & \\
\hline 34 & 33.8 & 34.4 & 25 & & 34 & 34.8 & 35.3 & 26 & & 34 & 44.2 & 40.4 & 25 & & 34 & 30.0 & 28.6 & 46 & \\
\hline 36 & 33 & $3^{b}$ & 24 & & 36 & 32.0 & 33.7 & 23 & E. & 36 & 44.4 & 41.3 & 25 & & 36 & 30.0 & 28.9 & 46 & E. \\
\hline 38 & 28.5 & 29.3 & 17 & & 38 & $33 . I$ & 35.0 & 25 & & 38 & 45.0 & 42.3 & 23 & & 38 & 30.4 & 29.3 & 45 & 6 \\
\hline 40 & 39.6 & 40.0 & 2234 & & 40 & 32.7 & 34.1 & 24 & . & 40 & 47.2 & 44.8 & 20 & & 40 & 30.7 & $29 . \overline{8}$ & 44 & . \\
\hline 42 & 60.1 & 60.3 & 2306 & & 42 & 32.3 & $34 . \mathrm{I}$ & 24 & & 42 & 45.8 & 43.1 & 22 & & 42 & 29.2 & 28.3 & 47 & 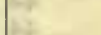 \\
\hline 44 & 47.2 & 50.9 & 2248 & -8.4 & 44 & 32.8 & 34.7 & 24 & -5.6 & 44 & $44 \cdot 3$ & 41.7 & 24 & -6.0 & 44 & 29.5 & 27.9 & 47 & -4.1 \\
\hline 46 & 37.0 & 39.2 & 3I & & 46 & 36.0 & 37.6 & 29 & & 46 & 42.5 & 40.1 & 27 & & 46 & 29.8 & 28.6 & 46 & \\
\hline 48 & 40.7 & 43.9 & 38 & & 48. & 37.0 & 39.0 & $3 \mathrm{I}$ & & 48 & 42.0 & 39.3 & 28 & & 48 & 30.2 & 29.1 & 45 & 60 \\
\hline 50 & 35.8 & 39.1 & 30 & & 50 & 35.5 & 36.9 & 28 & & 50 & 40.9 & 38.2 & 30 & & 50 & 32.0 & 31.0 & 42 & \\
\hline 52 & 33.9 & 37.3 & 27 & & 52 & $34 . \mathrm{I}$ & 35.7 & 26 & & 52 & 39.1 & 37.3 & 32 & & 52 & 32.0 & 30,6 & 43 & 3 \\
\hline 54 & 35.0 & 38.2 & 29 & & 54 & 36.0 & 36.9 & 28 & & 54 & 39.1 & 38.0 & $3 \mathrm{I}$ & & 5 & 29.9 & 28.2 & 46 & III \\
\hline 56 & $35 . \mathrm{I}$ & 37.8 & 28 & & 56 & 36.1 & 36.8 & 28 & & 56 & 37.7 & 36.2 & 34 & & 56 & 30.0 & 28.7 & 46 & \\
\hline 58 & 34.3 & 37.9 & 28 & & 58 & 36.7 & 37.3 & 30 & & 58 & 39.0 & 37.2 & 32 & & 58 & 30.7 & 29.1 & 45 & \\
\hline$\infty$ & 35.0 & 36.9 & 28 & -8.0 & II $\infty$ & 35.9 & 36.3 & 28 & $-5 \cdot 4$ & 1300 & $39 \cdot 3$ & 37.1 & 32 & -5.8 & I5 00 & 30.8 & 29.8 & 44 & -4.0 \\
\hline 02 & 37.1 & 38.2 & 30 & & 02 & 35.8 & $36 . I$ & 28 & & 02 & 38.7 & $37 \cdot 3$ & 32 & & 02 & 31.0 & 30.2 & 44 & \\
\hline 04 & 39.0 & 40.2 & 34 & s. & 04 & 39.7 & 40.4 & 34 & va & 04 & 37.0 & 35.9 & 35 & 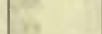 & 04 & $3 \mathrm{I} .3$ & 30.5 & 43 & 5 \\
\hline 06 & 38.0 & 39.1 & 32 & = & $\infty 6$ & 38.4 & $40 . I$ & 33 & 6 & 06 & 36.1 & 35.0 & 36 & s. & 06 & 31.9 & 31.0 & 43 & . \\
\hline 08 & 35.6 & 36.8 & 28 & & 08 & 36.2 & 38.0 & 30 & & 08 & 35.9 & 35.0 & 36 & & 08 & 30.9 & 30.0 & 44 & III \\
\hline 10 & 36.7 & 37.9 & 30 & 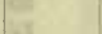 & 10 & 33.2 & 34.3 & 24 & cos & Io & 37.2 & 36.7 & 34 & & I0 & 30.9 & 30.1 & 44 & . \\
\hline 12 & 37.0 & 38.4 & $3 \mathrm{I}$ & & I2 & 34.2 & 35.3 & 26 & & 12 & $35 . \mathrm{I}$ & 34.9 & 37 & & I2 & 30.6 & 30.0 & 44 & \\
\hline 14 & 33.2 & 35.8 & 26 & -7.6 & 14 & 36.6 & 37.8 & 30 & -5.2 & 14 & 35.2 & $34 . \overline{8}$ & 37 & -5.4 & I4 & 29.9 & 29.3 & 45 & -3.8 \\
\hline 16 & 34.1 & 37.1 & 27 & & 16 & 37.2 & 38.9 & $3 I$ & & 16 & 36.0 & 34.6 & 36 & & 16 & 29.9 & 28.9 & 46 & \\
\hline 18 & 36.8 & 40.3 & 32 & to & 18 & $3 \mathrm{I} . \mathrm{I}$ & $32 . \mathrm{I}$ & $2 \mathrm{I}$ & 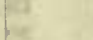 & 18 & 36.3 & 35.2 & 36 & & 18 & 29.1 & 28.7 & 47 & te \\
\hline 20 & 3I.I & 34.5 & 23 & & 20 & 29.5 & 30.6 & 18 & & 20 & 36.6 & 35.5 & 35 & & 20 & 31.6 & 30.1 & 43 & \\
\hline 22 & 31.8 & 35.6 & 24 & . & 22 & 27 & $.8 b$ & 15 & & 22 & 35.6 & 34.6 & 37 & & 22 & 32.2 & $3 \mathrm{I} .4$ & 42 & \\
\hline 24 & 32.1 & 34.8 & 24 & & 24 & 26.6 & 28. I & 14 & & 24 & 34.6 & 33.2 & 39 & & 24 & 32.0 & 3I. I & 42 & $=$ \\
\hline 26 & 29.2 & 31.9 & I9 & 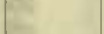 & 26 & 30.1 & 30. I & 19 & & 26 & 34.7 & 33.7 & 38 & & 26 & 31.6 & 30.6 & 43 & 2 \\
\hline 28 & 34.8 & 36.3 & 27 & & 28 & 27.3 & 27.8 & 15 & & 28 & 32.9 & 31.9 & $4 \mathrm{I}$ & & 28 & 30.3 & 29.5 & 45 & 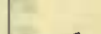 \\
\hline 30 & 34.5 & $35 . \mathrm{I}$ & 26 & -7.2 & 30 & 24.8 & 25.7 & II & -5.1 & 30 & 31.0 & 30.6 & 43 & -5.2 & 30 & 28.9 & 28.0 & 47 & -3.6 \\
\hline 32 & 37.8 & 38.3 & $3 \mathrm{I}$ & & 32 & 22.0 & 22.8 & 07 & & 32 & 30.2 & 29.9 & 45 & & 32 & 28.0 & 26.8 & 49 & \\
\hline & 39.2 & 40.7 & 34 & & 34 & 25.5 & 25.8 & 12 & & 34 & 30.4 & 29.8 & 45 & & 34 & 28.3 & 27.1 & 48 & 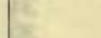 \\
\hline 36 & 36.6 & 37.2 & 29 & & 36 & 26.8 & 27.2 & 14 & & 36 & 32.2 & 32.0 & $4 I$ & & 3 & 27.5 & 26.4 & 50 & 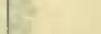 \\
\hline 38 & 36.3 & 36.7 & 29 & & 38 & 29.7 & 32.4 & 20 & & 38 & 33.3 & 32.9 & 40 & & 38 & 28.0 & 26.9 & 49 & \\
\hline 40 & 34.3 & 35.0 & 26 & 8 & 40.3 & 32.3 & $35 . \mathrm{I}$ & 24 & & 40 & 32.2 & 32.2 & $4 \mathrm{I}$ & & 40 & 28.7 & 27.9 & 47 & \\
\hline & $33 \cdot 3$ & $34 \cdot 3$ & 24 & & 42 & 34.5 & 36.8 & 27 & & 42 & 32.8 & 32.1 & $4 \mathrm{I}$ & & 42 & 29.2 & 28.6 & 47 & \\
\hline 44 & 29.2 & 30.3 & 18 & -6.8 & 44 & 34.9 & 37.2 & 28 & -5.0 & 44 & 33.8 & 32.9 & 40 & -4.9 & 44 & 29.7 & 28.9 & 46 & -3.4 \\
\hline 46 & 28.0 & 28.3 & I6 & & 46 & 35.0 & 36.9 & 28 & & 46 & 32.7 & 32.3 & $4 \mathrm{I}$ & & 46 & 30.6 & 29.7 & 45 & \\
\hline 48 & 28. I & 31.0 & I8 & $=$ & 48 & 35.1 & 39.0 & 30 & 8 & 48 & 30.9 & 28.9 & 45 & & 48 & 31.8 & 31.0 & 43 & E \\
\hline 50 & 35.6 & 35.8 & 27 & $=3$ & 50 & 33.4 & 37.7 & 27 & & 50 & 28.8 & 27.3 & 48 & & 50 & 32.8 & 31.6 & 41 & \\
\hline 52 & 36.8 & 37.9 & 30 & 8 & 52 & 30.8 & 34.9 & 23 & se & 52 & 29.2 & 27.2 & 48 & & 52 & 30.6 & 29.0 & 45 & 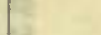 \\
\hline 54 & 35.5 & 37.7 & 29 & & 54 & 33.2 & $36 . \overline{8}$ & 26 & & 54 & 30.8 & 29.2 & 45 & & 54 & 29.8 & 28.2 & 46 & es \\
\hline 56 & 36.2 & 37.3 & 29 & & 56 & 35.9 & 39.1 & 30 & & 56 & 32.2 & 30.1 & $\begin{array}{l}45 \\
43\end{array}$ & & 56 & 29.1 & 28.1 & 47 & \\
\hline 58 & 37.1 & 40.7 & 32 & & 58 & 35.7 & 38.5 & 30 & & 58 & 32.4 & 30.3 & 43 & & & 28.9 & 27.8 & 47 & \\
\hline & & & & & 1200 & 36.0 & 39.7 & $3 \mathrm{I}$ & & & & & & & I6 00 & 28.9 & 27.9 & 47 & -3.2 \\
\hline
\end{tabular}

Correction to local mean time is $+28 \mathrm{~s}$.

Torsion head at $7 \mathrm{~h} 25 \mathrm{~m}$ read $310^{\circ}$ and at $12 \mathrm{~h} 15 \mathrm{~m}$ read the same.

Observer-R. R. T.
Correction to local mean time is - 22 .

Torsion head at inh $52 \mathrm{~m}$ read $307^{\circ}$ and at $16 \mathrm{~h} 25 \mathrm{~m}$ read the same. Observer-R. R. T. 
Tabulation of magnetic declinations observed at Teplitz Bay-Continued

\begin{tabular}{|c|c|c|c|c|c|c|c|c|c|c|c|c|c|c|c|c|c|c|c|}
\hline \multicolumn{5}{|c|}{ Wednesday, November 25, 1903} & \multicolumn{5}{|c|}{ Magnet scale erect } & \multicolumn{5}{|c|}{ Wednesday, November 25, 1903} & \multicolumn{5}{|c|}{ Magnet scale erect } \\
\hline \multirow{2}{*}{$\begin{array}{l}\text { Chr'r } \\
\text { time }\end{array}$} & \multicolumn{2}{|c|}{$\begin{array}{c}\text { Scale } \\
\text { readings }\end{array}$} & \multirow{2}{*}{$\begin{array}{c}\text { East } \\
\text { decli- } \\
\text { nation }\end{array}$} & Temp. & Chr'r & $\begin{array}{r}\mathrm{Sc} \\
\text { read }\end{array}$ & $\begin{array}{l}\text { ale } \\
\text { lings }\end{array}$ & $\begin{array}{l}\text { East } \\
\text { decli- }\end{array}$ & Temp. & Chr'r & $\begin{array}{l}\mathrm{Sc} \\
\text { read }\end{array}$ & $\begin{array}{l}\text { ale } \\
\text { dings }\end{array}$ & $\begin{array}{l}\text { East } \\
\text { decli- }\end{array}$ & Temp. & Chr'r & $\begin{array}{r}\text { Sc } \\
\text { read }\end{array}$ & $\begin{array}{l}\text { ale } \\
\text { lings }\end{array}$ & $\begin{array}{l}\text { East } \\
\text { decli- }\end{array}$ & Temp \\
\hline & Left & Right & & & time & Left & Right & nation & C. & time & $L_{\text {,eft }}$ & Right & nation & & time & Left & Right & nation & \\
\hline h. $\mathrm{m}$ & d & $\mathrm{d}$ & $\therefore \quad$ & $\because$ & $\mathrm{h} \mathrm{m}$ & d & d & $\circ \quad$ & $\because$ & h m & d & d & $\circ \quad$ & & $\mathrm{h} \mathrm{m}$ & d & d & . & $\bullet$ \\
\hline $000^{*}$ & 39.7 & 41.7 & 2230 & -9.8 & 200 & 46.3 & 46.6 & 2239 & -6.3 & 400 & 46.2 & 46.5 & 2239 & -5.2 & 600 & 47.5 & 48.3 & 2241 & -4.7 \\
\hline 02 & 40.9 & 42.6 & $3 I$ & 6 & 02 & $45 . \overline{8}$ & 46.3 & 38 & 60 & 02 & 46.8 & 47.0 & 40 & 2 & 02 & 50.6 & 51.0 & 46 & \\
\hline 04 & 42.3 & 43.8 & 33 & yit & 04 & 45.8 & 46.2 & 38 & 00 & 04 & 47.0 & 47.4 & 40 & $j e$ & 04 & 51.6 & 52.3 & 47 & \\
\hline 06 & 43.0 & $44 \cdot 3$ & 34 & 81 & 06 & 46.3 & 46.8 & 39 & 16 & 06 & 46.9 & $47 \cdot 3$ & 40 & 9 & $\infty$ & 50.3 & 50.9 & 45 & \\
\hline 08 & 43.0 & $44 \cdot 5$ & 34 & ex & 08 & 46.3 & 46.8 & 39 & in & 08 & 47.2 & 47.6 & 40 & 1 & 08 & 46.6 & 47.8 & 40 & 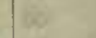 \\
\hline 10 & 43.2 & 44.4 & 35 & $m$ & IO & 46.3 & 46.8 & 39 & ait & 10 & 47.4 & 47.7 & 40 & $=$ & 10 & 43.5 & 44.5 & 35 & at \\
\hline 12 & 43.2 & 44.3 & 34 & & 12 & 46.6 & 47.1 & 39 & & 12 & 47.7 & 47.8 & $4 I$ & $a$ & 12 & 46.1 & 46.4 & 38 & \\
\hline 14 & 43.0 & $44 \cdot 3$ & 34 & -9.2 & 14 & 46.6 & 47.0 & 39 & -6.1 & 14 & 48.4 & 48.6 & 42 & & 14 & 45.8 & 46.0 & 38 & -4.6 \\
\hline 16 & $43 \cdot 3$ & 44.6 & 35 & $x^{2}$ & 16 & 46.8 & 47.0 & 39 & & 16 & $49 \cdot 3$ & 49.5 & 43 & -5.1 & 16 & 41.9 & 42.6 & 32 & \\
\hline 18 & 43.0 & $44 \cdot 3$ & 34 & $a^{2}$ & 18 & 48.2 & 48.4 & 42 & at & 18 & 48.8 & 49.3 & 43 & (1) & 18 & 44.6 & 45.6 & 37 & \\
\hline 20 & 43.6 & 44.6 & 35 & ne & 20 & 47.8 & 48.2 & $4 I$ & $H$ & 20 & 49.3 & 49.5 & 43 & est & 20 & 50.6 & 5 I. 7 & 46 & $E$ \\
\hline 22 & 43.6 & 44.6 & 35 & 12 & 22 & 47.5 & 47.8 & $4 I$ & L & 22 & 48.1 & 48.5 & 42 & 18 & 22 & 49.3 & 50.3 & 44 & Et \\
\hline 24 & $43 \cdot 5$ & 44.6 & 35 & 15: & 24 & $47 \cdot 3$ & 47.7 & 40 & $y$ & 24 & 47.7 & 48.0 & $4 \mathrm{I}$ & in & 24 & 47.9 & 48.6 & 42 & is \\
\hline 26 & 44.2 & 44.9 & 36 & ty & 26 & 48.2 & 48.4 & 42 & 58 & 26 & 47.0 & $47 \cdot 3$ & 40 & 8 & 26 & 47.0 & 47.6 & 40 & Ein \\
\hline 28 & 44.2 & 45.8 & 37 & & 28 & 48.6 & 48.8 & 42 & & 28 & 46.6 & 47.0 & 39 & & 28 & 48.3 & 48.9 & 42 & \\
\hline 30 & 43.8 & 44.6 & 35 & -8.7 & 30 & 48.8 & 49.2 & 43 & -6.0 & 30 & 46.0 & 46.6 & 39 & -5.0 & 30 & 48.3 & 48.9 & 42 & \\
\hline 32 & 43.6 & 44.0 & 35 & - & 32 & 49.3 & 49.4 & 43 & & 32 & 47.2 & 47.5 & 40 & ( & 32 & 46.6 & 47.6 & 40 & -4.6 \\
\hline 34 & 43.6 & 44.2 & 35 & & 34 & 47.6 & 47.8 & $4 \mathrm{I}$ & 3 & 34 & 47.6 & 47.8 & $4 \mathrm{I}$ & 32 & 34 & 42.6 & 43.2 & 33 & \\
\hline 36 & 43.0 & 43.6 & 34 & (1) & 36 & 46.0 & 46.2 & 38 & 78 & 36 & 47.7 & 47.9 & $4 \mathrm{I}$ & 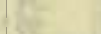 & 36 & 40.5 & 41.9 & $3 \mathrm{I}$ & \\
\hline 38 & 43.5 & $44 \cdot 3$ & 35 & ty & 38 & 45.7 & 46.0 & 38 & 8 & 38 & 48.0 & 48.2 & $4 \mathrm{I}$ & & 38 & 46.8 & 48.0 & 40 & \\
\hline 40 & 44.0 & 44.9 & 36 & 20 & 40 & 46.3 & 46.6 & 39 & 13 & 40 & 47.8 & 48.3 & 4I & . & 40 & 41.6 & 42.8 & 32 & \\
\hline 42 & $44 \cdot 3$ & 45.0 & 36 & & 42 & 46.6 & $47 \cdot 3$ & 39 & & 42 & 47.9 & 48.2 & $4 \mathrm{I}$ & & 42 & 39.7 & $4 \mathrm{I} \cdot 3$ & 30 & \\
\hline 44 & 44.6 & $45 \cdot 5$ & 37 & -8.2 & 44 & $47 \cdot 3$ & 47.9 & $4 I$ & -6.0 & 44 & $47 \cdot 3$ & 47.6 & 40 & -5.0 & 44 & $45 \cdot 3$ & 46.7 & 38 & -4.6 \\
\hline 46 & 44.8 & 45.5 & 37 & & 46 & 47.6 & 48.3 & $4 \mathrm{I}$ & & 46 & 47.0 & $47 \cdot 3$ & 40 & & 46 & 49.7 & 50.7 & 45 & \\
\hline 48 & 45.3 & 45.6 & 37 & 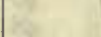 & 48 & 48.0 & 48.6 & 42 & 9 & 48 & 46.0 & 46.0 & 38 & & 48 & 42.6 & 43.6 & 34 & \\
\hline 50 & 46.4 & 47.0 & 39 & 38 & 50 & 48.3 & 48.9 & 42 & $=$ & 50 & 44.3 & 44.9 & 36 & 15 & 50 & 40.8 & 41.6 & $3 \mathrm{I}$ & \\
\hline 52 & 46.0 & 46.6 & 39 & is & 52 & 48.6 & 49.2 & 43 & $E$ & 52 & 43.8 & 44.0 & 35 & 3 & 52 & 43.0 & 43.6 & 34 & \\
\hline 54 & 45.6 & 46.0 & 38 & 3 & 54 & 48.1 & 48.6 & 42 & a & 54 & 42.6 & 43.6 & 33 & & 54 & 43.6 & 44.4 & 35 & \\
\hline 56 & 47.8 & 48.5 & $4 I$ & 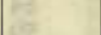 & 56 & 47.3 & 47.9 & 41 & & 56 & $43 \cdot 3$ & 43.8 & 34 & & 56 & 42.3 & 43.6 & 33 & \\
\hline 58 & 49.3 & 49.6 & 43 & & 58 & 47.2 & 47.8 & 40 & & $5^{8}$ & 43.1 & 43.5 & 34 & -5.0 & $5^{8}$ & 41.2 & 42.6 & 32 & \\
\hline 100 & 48.3 & 48.8 & 42 & -7.8 & 300 & 47.0 & 47.6 & 40 & -5.8 & 500 & 43.4 & 43.6 & 34 & & 700 & 42.3 & 43.7 & 33 & -4.6 \\
\hline 02 & 48.5 & 48.7 & 42 & & 02 & 46.7 & $47 \cdot 3$ & 40 & & 02 & 43.3 & 43.7 & 34 & $=$ & 02 & 43.0 & 44.3 & 34 & \\
\hline 04 & 48.3 & 49.6 & 43 & 3 & 04 & 46.9 & $47 \cdot 3$ & 40 & in & 04 & 44.2 & 44.4 & 35 & in & 04 & 44.6 & 45.6 & 37 & 10 \\
\hline 06 & 48.0 & 48.4 & 42 & S. & 06 & 47.2 & 47.7 & 40 & 6 & 06 & 44.3 & 44.6 & 36 & $\infty$ & 06 & 45.0 & 46.6 & 38 & \\
\hline 08 & 47.9 & 48.5 & 42 & w. & 08 & 46.7 & 47.3 & 40 & 8 & os & 43.6 & 43.8 & 34 & 18 & 08 & 44.7 & 46.3 & 37 & \\
\hline 10 & $47 \cdot 3$ & 47.7 & 40 & get & 10 & $47 . I$ & 47.5 & 40 & it & 10 & $43 . I$ & 43.3 & 34 & & 10 & 42.8 & 44.5 & 34 & \\
\hline 12 & 47.6 & 48.0 & 41 & & 12 & 47.8 & 48.3 & 41 & $\leq 4$ & 12 & 42.8 & 42.9 & 33 & & 12 & 42.6 & 43.5 & 33 & \\
\hline 14 & 48.1 & 48.5 & 42 & $-7 \cdot 3$ & 14 & 47.9 & 48.6 & 42 & & 14 & 43.9 & 44.0 & 35 & -5.0 & 14 & 41.7 & 42.7 & 32 & -4.6 \\
\hline 16 & 46.6 & $47 \cdot 3$ & 40 & & 16 & 48.3 & 48.8 & 42 & -5.6 & 16 & 45.3 & 45.6 & 37 & & 16 & 41.3 & 41.6 & $3 \mathrm{I}$ & \\
\hline 18 & 47.6 & 48.3 & $4 \mathrm{I}$ & a. & 18.2 & 48.8 & 49.5 & 43 & - & 18 & 46.5 & 46.8 & 39 & 8 & 18 & 42.3 & 42.7 & 33 & \\
\hline 20 & 48.6 & 49.0 & 42 & . & 20 & 49.8 & 49.2 & 44 & 8 & 20 & 48.3 & 48.6 & 42 & 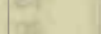 & 20 & 42.3 & 43.1 & 33 & \\
\hline 22 & 48.3 & 48.6 & 42 & E. & 22 & 48.7 & 49.3 & 43 & 5 & 22 & 50.6 & 50.6 & 45 & 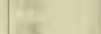 & 22 & 41.3 & 42.3 & 32 & \\
\hline 24 & 47.6 & 48.3 & 41 & & 24 & 47.9 & 48.6 & 42 & & 24 & 51.6 & 51.8 & 47 & sis & 24 & 40.9 & 41.8 & 31 & \\
\hline 26 & 48.0 & 48.4 & 42 & E & 26 & 47.6 & 48.3 & $4 \mathrm{I}$ & & 26 & 54.7 & 54.9 & 52 & 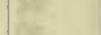 & 26 & 42.2 & 42.6 & 32 & \\
\hline 28 & 48.6 & $49 \cdot 3$ & 43 & & 28 & 47.6 & 48.0 & $4 \mathrm{I}$ & & 28 & 56.1 & 56.3 & 54 & & 28 & 41.6 & 41.6 & 31 & \\
\hline 30 & $47 \cdot 3$ & 47.9 & $4 I$ & -7.0 & 30 & 48.1 & 48.4 & 42 & -5.4 & 30 & 51.9 & 52.4 & 48 & -4.9 & 30 & 43.3 & 44.1 & 34 & $-4 \cdot 5$ \\
\hline 32 & 47.5 & 48.2 & 41 & & 32 & 47.9 & 48.2 & $4^{I}$ & & 32 & 47.4 & 47.7 & 40 & & 32 & 42.7 & 43.7 & 34 & \\
\hline 34 & 47.8 & 48.2 & 41 & tet & 34 & $47 \cdot 3$ & 47.7 & 40 & & 34 & 45.8 & 46.0 & 38 & & 34 & 40.3 & 40.8 & 30 & \\
\hline 36 & 46.3 & 46.6 & 39 & 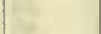 & 36 & 47.3 & 47.6 & 40 & & 36 & 47.0 & 47.3 & 40 & & 36 & 39.3 & 40.7 & 29 & \\
\hline 38 & 45.8 & 46.3 & 38 & & 38 & 46.8 & 47.2 & 40 & & 38 & 48.2 & 48.6 & 42 & & 38 & 38.5 & 41.3 & 29 & \\
\hline 40 & 45.6 & 46.1 & 38 & 3 & 40 & 46.8 & 47.2 & 40 & & 40 & 46.8 & $47 . I$ & 40 & & 40 & 43.2 & 44.9 & 35 & \\
\hline 42 & 46.5 & 46.8 & 39 & & 42 & $47 \cdot 3$ & 47.6 & 40 & & 42 & 45.3 & $45 \cdot 3$ & 37 & & 42 & 47.5 & 49.3 & 42 & \\
\hline 44 & 46.1 & 46.3 & 38 & -6.8 & 44 & 46.5 & 46.8 & 39 & $-5 \cdot 3$ & 44 & 45.0 & 45.2 & 37 & -4.8 & 44 & 43.8 & 45.9 & 36 & $-4 \cdot 5$ \\
\hline 46 & 46.4 & 46.8 & 39 & & 46 & 46.5 & 46.9 & 39 & & 46 & 42.7 & 43.0 & 33 & & 46 & $4 \mathrm{I} .2$ & $43 \cdot I$ & 32 & \\
\hline 48 & 47.2 & 47.6 & 40 & a & 48 & 46.7 & 47.3 & 40 & 5 & 48 & 43.6 & 43.6 & 34 & F & 48 & $47 \cdot 3$ & 49.1 & 42 & \\
\hline 50 & 46.9 & 47.3 & 40 & & 50 & 46.9 & $47 \cdot 3$ & 40 & & 50 & 54 & 1.5a & 52 & & 50 & 42.4 & $44 \cdot 3$ & 34 & \\
\hline 52 & 46.3 & 46.9 & 39 & 3 & 52 & 47.3 & 47.7 & 40 & 6 & 52 & $57 \cdot 3$ & 57.9 & 56 & 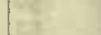 & 52 & 42.2 & 43.7 & 33 & \\
\hline 54 & 46.1 & 46.6 & 39 & 2 & 54 & 47.6 & 47.8 & $4 I$ & & 54 & 55.0 & 55.6 & 53 & & 54 & $44 \cdot 3$ & 46.2 & 37 & \\
\hline 56 & 46.4 & 46.9 & 39 & 65 & 56 & 47.2 & 47.6 & 40 & & 56 & 54.0 & 54.9 & $5 \mathrm{I}$ & & 56 & 43.2 & 45.8 & 36 & \\
\hline 58 & 46.6 & 47.1 & 39 & & 58 & 46.6 & 47.0 & 39 & & 58 & $5 \mathrm{I} .5$ & 52.4 & 47 & & 58 & $4 \mathrm{I} .9$ & $44 \cdot 4$ & 34 & \\
\hline & & 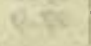 & dis & 6 & & & & & & & & 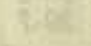 & ate & 72 & & & & & \\
\hline
\end{tabular}


Tabulation of magnetic declinations observed at Teplitz Bay-Continued

\begin{tabular}{|c|c|c|c|c|c|c|c|c|c|c|c|c|c|c|c|c|c|c|c|}
\hline \multicolumn{5}{|c|}{ Wednesday, November 25,1903} & \multicolumn{5}{|c|}{ Magnet scale erect } & \multicolumn{5}{|c|}{ Wednesday, November 25, 1903} & \multicolumn{5}{|c|}{ 1. Magnet scale erect } \\
\hline \multirow{2}{*}{$\begin{array}{l}\text { Chr'r } \\
\text { time }\end{array}$} & \multicolumn{2}{|c|}{$\begin{array}{l}\text { Scale } \\
\text { readings }\end{array}$} & \multirow{2}{*}{$\begin{array}{c}\text { East } \\
\text { decli- } \\
\text { nation }\end{array}$} & 'Temp. & Chr'r & $\begin{array}{r}\mathrm{Sc} \\
\text { read }\end{array}$ & $\begin{array}{l}\text { ale } \\
\text { lings }\end{array}$ & $\begin{array}{l}\text { Eas } \\
\text { decl }\end{array}$ & Temp. & Chr'r & $\underset{\text { rea }}{S c}$ & $\begin{array}{l}\text { e } \\
\text { ngs }\end{array}$ & $\begin{array}{l}\text { East } \\
\text { decli- }\end{array}$ & Temp. & Chr'r & S & ngs & $\begin{array}{l}\text { East } \\
\text { decli- }\end{array}$ & Temp. \\
\hline & Left & Right & & & & Left & Right & & & & Left & Right & & & & Left & Right & & \\
\hline $\mathrm{h} \mathrm{m}$ & d & d & $\cdot \quad$ & $0^{\bullet}$ & h $\mathrm{m}$ & d & d & & $7^{\circ}$ & $\mathrm{h} \mathrm{m}$ & $\mathrm{d}$ & d & $\circ$, & 0 & $\mathrm{~h} \mathrm{~m}$ & d & d & $\circ \quad$ & • \\
\hline 800 & $4 \mathrm{I} .7$ & 43. I & 2233 & -4.2 & 1000 & 32.3 & 40.7 & & -5.0 & 1200 & 41.2 & 43.1 & 2232 & -4.6 & 1400 & 39.2 & 4I. 8 & $22 \quad 29$ & -5.0 \\
\hline 02 & 42.2 & $44 . I$ & 34 & $x^{2}$ & 02 & $35 . I$ & 44.2 & 2 & & 02 & 42.1 & 44.1 & 34 & & 02 & 39.5 & 42.0 & 30 & \\
\hline 04 & 43.0 & 44.8 & 35 & 19e & 04 & 35.2 & $44 . I$ & 2 & 61 & 04 & 41.8 & 43.5 & 33 & Fia & 04 & 39.4 & 41.8 & 30 & 19 \\
\hline 06 & $4 \mathrm{I} . \mathrm{I}$ & 42.4 & 3I & 6 & 06 & 36.3 & 44.3 & 2 & & 06 & 40. I & $4 \mathrm{I} \cdot 3$ & 30 & $t^{2}$ & 06 & 39.3 & 41.5 & 29 & 20 \\
\hline 08 & 38.3 & 40.2 & 27 & 혼. & 08 & 34.6 & 41.8 & 2 & t & 08 & 39.2 & 40.8 & 29 & 86 & 08 & 40.1 & 42.2 & 30 & 6 \\
\hline I0 & 37.2 & 39. I & 26 & 4 & Io & 38.6 & 45.8 & 3 & urt & Io. & -40.3 & $42 . I$ & $3 I$ & 64 & Io & $4 I .3$ & 43.2 & 32 & at \\
\hline 12 & 38.8 & 40.7 & 28 & wit & I2 & 37.2 & 44.7 & 3 & $\theta$ & 12 & 40.8 & 42.0 & $3 \mathrm{I}$ & as & I2 & 39.2 & $4 \mathrm{I} .0$ & 29 & att \\
\hline 14 & 38.3 & 40.9 & 28 & -4.0 & 14 & 36.5 & 44.8 & 3 & $-5 . I$ & 14 & 40.0 & $4 \mathrm{I} .2$ & 30 & -4.4 & I4 & 39.0 & $4 \mathrm{I} .0$ & 29 & -5.0 \\
\hline 16 & 37.3 & 39.7 & 26 & & 16 & 34.0 & 41.2 & 2 & & I6́ & 39.4 & 40.8 & 29 & & 16 & 39.4 & $4 \mathrm{I} .2$ & 29 & \\
\hline 18 & 37.7 & 39.2 & 26 & Rir & 18 & 35.3 & $42 . I$ & 2 & & I8 & 40.1 & 41.7 & 30 & 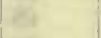 & I8 & 38.8 & 40.1 & 28 & Wir \\
\hline 20 & 39.0 & 41.0 & 29 & $\theta$ & 20 & 36.0 & 42.1 & 2 & a. & 20 & 41.0 & 42.2 & $3 I$ & (4) & 20 & 38.4 & 40. I & 27 & $\omega$ \\
\hline 22 & 38.6 & 40.0 & 28 & tex & 22 & 38.0 & 44.0 & 3 & 6 & 22 & 41.8 & 43.0 & 32 & 62 & 22 & 39.8 & 4I. 4 & 30 & 6 \\
\hline 24 & 40.0 & $42 . I$ & 30 & iz & 24 & 38.3 & 44.2 & 3 & 16 & 24 & 42.0 & 43.0 & 33 & in & 24 & 40.5 & 42.0 & 31 & 14 \\
\hline 26 & 43.7 & $46 . I$ & $3^{6}$ & 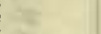 & 26 & 39.3 & 44.2 & 3 & $\%$ & 26 & 42.2 & 43.6 & 33 & 18 & 26 & 40.5 & 42.0 & 3I & is \\
\hline 28 & 45.2 & $47 . I$ & 38 & $3 x$ & 28 & 35.8 & 40.6 & 2 & 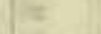 & 28 & 43.0 & 44.0 & 34 & 12 & 28 & $40 . \overline{8}$ & 42.2 & $3 I$ & 15 \\
\hline 30 & 44.9 & 48.3 & 39 & $-4 . I$ & 30 & 36.2 & 40.8 & 2 & -5.0 & 30 & 43.7 & 44.7 & 35 & $-4 \cdot 3$ & 30 & 40.4 & 42.2 & $3 I$ & -5.0 \\
\hline 32 & 47.6 & $49 . I$ & 42 & 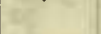 & 32 & 37.1 & 42.3 & 2 & & 32 & 42.6 & 44.0 & 34 & & 32 & 41.0 & 42.4 & $3 \mathrm{I}$ & \\
\hline 34 & 46.3 & 49.2 & $4 \mathrm{I}$ & 12 & 34 & 38.7 & $43 . I$ & 3 & 16 & 34 & 42.2 & 43.3 & 33 & 35 & 34 & 40.2 & 42.0 & 30 & te \\
\hline 36 & 39.3 & 43.5 & 3 I & 뭉 & 36 & 38.9 & $43 . I$ & 3 & 8 & $3^{6}$ & 41.6 & 42.8 & 32 & ist & 36 & 40 & 42.3 & $3 I$ & is \\
\hline 38 & 37.7 & 39.7 & 27 & 18 & 38 & 38.5 & 42.8 & 3 & & 38 & 40.1 & $4 \mathrm{I} \cdot 3$ & 30 & IE & 38 & 39.9 & $4 \mathrm{I} . \mathrm{I}$ & 29 & E \\
\hline 40 & 38.8 & $4 I .9$ & 29 & $\sigma^{2}$ & 40 & 39. I & 42.9 & 3 & 4 & 40 & 39.3 & 40.3 & 28 & 18 & 40 & 39 & 40.3 & 28 & 0 \\
\hline 42 & 32.6 & 34.9 & 19 & in & 42 & 40.2 & 43.7 & 3 & 18 & 42 & 40.7 & $41 . \mathrm{I}$ & 30 & 8 & 42 & 39 . I & 40.2 & 28 & \\
\hline 44 & 36.2 & 38.1 & 24 & $-4 \cdot 3$ & 44 & 38.1 & 41.9 & 2 & -4.9 & 44 & 42.4 & 42.8 & 33 & -4.2 & 44 & 39.9 & 40.9 & 29 & -5.0 \\
\hline 46 & $43 . I$ & 45.0 & 35 & & 46 & 39.0 & 42.0 & 2 & & 46 & 41.9 & 42.5 & 32 & 87 & 46 & 39.0 & 40.0 & 28 & \\
\hline 48 & $43 \cdot 3$ & 45.5 & 36 & 10 & 48 & 39.7 & 42.9 & 3 & te & 48 & 42.3 & 42.7 & 33 & 84 & 48 & 39. I & 40. I & 28 & 6 \\
\hline 50 & 43.8 & 44.8 & 35 & $=$ & 50 & 37.4 & 40.6 & 2 & & 50 & 42.7 & 43.0 & 33 & ot: & 50 & 39.2 & 40. I & 28 & 07 \\
\hline 52 & $4 \mathrm{I} .4$ & 42.8 & 32 & $=2$ & 52 & 37.6 & 40.7 & 2 & & 52 & 42.2 & 43. I & 33 & 6 & 52 & 39.8 & 40.7 & 29 & 2 \\
\hline 54 & 44.3 & 46.0 & 37 & 14 & 54 & 36.2 & 38.6 & 2 & & 54 & 42.2 & 42.8 & 33 & the & 54 & 39 & 40.0 & 28 & \\
\hline 56 & 44.4 & 45.2 & 36 & & 56 & 38.2 & 39.6 & 2 & & 56 & 42.8 & 43.3 & 33 & 25 & 56 & 37.8 & 38.8 & 26 & \\
\hline 58 & 40.3 & 40.7 & 30 & in & 58 & 41.0 & 42.7 & 3 & & 58 & 43.2 & 43.9 & 34 & & 58 & 38.0 & 38.8 & 26 & \\
\hline 900 & 36.8 & 38.2 & 25 & $-4 \cdot 4$ & II 00 & 41.6 & 43.2 & 3 & -4.8 & I3 00 & 43.3 & 43.9 & 34 & -4 & I5 00 & 38.6 & 39.3 & 27 & -5.2 \\
\hline 02 & 35.8 & 38.0 & 24 & & 02 & 38.9 & 40.8 & 2 & & 02 & 43.9 & 44.9 & 35 & & 02 & 38.8 & 39.7 & 27 & \\
\hline 04 & 38.6 & 42.5 & 30 & 14 & 04 & 40.8 & 41.8 & 3 & 10 & 04 & 43.8 & 44.8 & 35 & if & 04 & 38 & 38.8 & 26 & es \\
\hline 06 & 38.9 & 42.9 & 30 & in & 06 & 40.5 & 41.8 & 3 & & 06 & 43.8 & 44.6 & 35 & Dit & $\infty 6$ & 38.1 & $3^{8} .9$ & 26 & Es \\
\hline 08 & 42.6 & 46.2 & 36 & we & 08 & 40.2 & 41.6 & 3 & if & 08 & 43.0 & 44.7 & 35 & Ex & 08 & 37.8 & 38.2 & 26 & se \\
\hline IO & 38.3 & 41.7 & 29 & in & IO & 39.3 & 40.9 & 2 & $\pi$ & Io & 42.1 & 43.7 & 33 & b) & 10 & 37.9 & 38.4 & 26 & $3 f$ \\
\hline I 2 & 37.7 & 41.4 & 28 & $x^{2}+2$ & I2 & 39.3 & 40.8 & 2 & (1) & I2 & 41.7 & 42.8 & 32 & bix & 12 & & 38.0 & 25 & $y^{2}$ \\
\hline 14 & 40.7 & 43.2 & 32 & $-4 \cdot 5$ & 14 & $40 . I$ & 41.6 & 3 & -4.8 & 14 & 41.0 & 42.0 & 31 & -4.7 & 14 & 37.7 & 38.0 & 25 & -5.2 \\
\hline 16 & 42.6 & 44.7 & 34 & & 16 & 41.6 & 43.0 & 3 & & 16́ & 39.6 & $4 \mathrm{I} .2$ & 29 & & 16 & & 38 & 26 & \\
\hline 18 & 33.3 & 37.6 & 22 & 10 & I8 & 41.9 & 43.0 & 3 & & 18 & 39.8 & 41.2 & 29 & Bs & 18 & 38.8 & 39.2 & 27 & it \\
\hline 20 & 37.0 & 43.0 & 29 & L & 20 & 41.9 & $43 . I$ & 3 & & 20 & & 40.0 & & act & 20 & 39.3 & 39.7 & 28 & onf \\
\hline 22 & 43.8 & 48.7 & 38 & tis & 22 & 40.9 & $4 \mathrm{I} .8$ & 3 & 4 & 22 & 38.0 & 40.8 & 28 & nat & 22 & 39.9 & 40.2 & 29 & $=$ \\
\hline 24 & $43 . I$ & 47.9 & 37 & $y=$ & 24 & $41 \cdot 3$ & 42.8 & 3 & 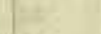 & 24 & 38.3 & 40.9 & 28 & 25 & 24 & 40.2 & 40.5 & 29 & is \\
\hline 26 & 39.8 & 43.8 & 32 & es & 26 & 39.9 & 41.5 & 3 & 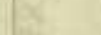 & 26 & 39.2 & 42.7 & 30 & 6 & 26 & $40 . \mathrm{I}$ & 40.2 & 29 & $=$ \\
\hline 28 & 38.0 & 40.9 & 28 & t & 28 & 39.9 & 41.2 & 2 & & 28 & 38.3 & 39.2 & 27 & & 28 & 40.1 & 40.3 & 29 & 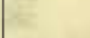 \\
\hline 30 & 39.5 & 43.0 & $3 I$ & -4.6 & 30 & 41.0 & 42.9 & 3 & -4 & 30 & 37.7 & 40.5 & 27 & -5 & 30 & $40 . I$ & 40 & 29 & -5.2 \\
\hline 32 & 36.9 & 40.8 & 27 & ${ }^{\circ}$ & 32 & 40.7 & $43 . I$ & 3 & & 32 & 37.4 & 40.2 & 27 & & 32 & 40.2 & 40.7 & 29 & \\
\hline & 32.1 & 33.3 & 17 & & 34 & 39.8 & 42.2 & 3 & & 34 & 38.0 & 40.0 & 27 & 18 & & 39.9 & 40.5 & 29 & \\
\hline 36 & 34.1 & 38.0 & 22 & is & 36 & 38.7 & 40.5 & 2 & & 36 & 38.2 & 39.9 & 27 & & 36 & 41.0 & 41.8 & $3 \mathrm{I}$ & \\
\hline 38 & 32.2 & 36.2 & 20 & $1=$ & 38 & 37.7 & 39.7 & 2 & 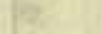 & 38 & 38.3 & 42.1 & 29 & & 38 & 42.1 & 42.5 & 32 & \\
\hline 40 & 34.0 & 39.7 & 24 & ef & 40 & 39.2 & $4 \mathrm{I} .4$ & 2 & B & 40 & 38.0 & 42.2 & 29 & & 40 & 41.9 & 42.3 & 32 & $\theta$ \\
\hline 42 & 34.8 & 43.7 & 27 & $2+5$ & 42 & 39.0 & 40.6 & 2 & & 42 & 38.9 & 42.6 & 30 & & 42 & 42.2 & 42.8 & 33 & \\
\hline 44 & 34.1 & 41.6 & 25 & -4.8 & 44 & 39.8 & 41.2 & 2 & -4.7 & 44 & 39.5 & 44.0 & 31 & -5.0 & 44 & 43.8 & 44.2 & 35 & -5.2 \\
\hline 46 & 36. I & 42.9 & 28 & & 46 & 40.9 & 42.7 & 3 & & 46 & 40.0 & 43.1 & 31 & 18 & 46 & 43.7 & 44.0 & 35 & \\
\hline 48 & 35.4 & 43.2 & 28 & e & 48 & 41.5 & 43.2 & 3 & te & 48 & 38.8 & $4 \mathrm{I} .7$ & 29 & E & 48 & $43 \cdot 3$ & 43.6 & 34 & 14 \\
\hline 50 & 35.3 & 42.2 & 27 & at & 50 & 41.4 & 43.0 & 3 & & 50 & 37.8 & 4I.I & 28 & & 50 & 43.2 & & 34 & 2 \\
\hline $5^{2}$ & 36.1 & 43.8 & 28 & Q & 52 & 43.2 & 44.7 & 3 & 2 & 52 & 39.2 & 42.7 & 30 & $E$ & 52 & 43.5 & 43.8 & 34 & \\
\hline 54 & 37.2 & 43.9 & 29 & iz & 54 & 42.8 & $44 \cdot 3$ & 3 & & 54 & 39.5 & 42.7 & 30 & 2 & 54 & 43.7 & 43.9 & 35 & \\
\hline 56 & 32.5 & 41.0 & 23 & & 56 & 41.6 & 43.0 & 3 & & 56 & 39.8 & 42.7 & 31 & & 56 & 43.4 & 43.8 & 34 & \\
\hline 58 & $34 . I$ & 42.2 & 26 & & 58 & 41.8 & 43.7 & 3 & 3 & 58 & 39.8 & 42.2 & 30 & & 58 & 44.1 & 44.3 & 35 & 6 \\
\hline & & & & & & & & & & & & & & & & & & & \\
\hline
\end{tabular}


Tabulation of magnetic declinations observed at T'eplitz Bay-Continued

\begin{tabular}{|c|c|c|c|c|c|c|c|c|c|c|c|c|c|c|c|c|c|c|c|c|}
\hline \multicolumn{6}{|c|}{ Wednesday, November 25, 1903} & \multicolumn{5}{|c|}{ Magnet scale erect } & \multicolumn{5}{|c|}{ Wednesday, November 25, 1903} & \multicolumn{5}{|c|}{ Magnet scale erect } \\
\hline \multirow{2}{*}{$\begin{array}{l}\text { Chr'r } \\
\text { time }\end{array}$} & \multicolumn{2}{|c|}{$\begin{array}{l}\text { Scale } \\
\text { readings }\end{array}$} & \multirow{2}{*}{\multicolumn{2}{|c|}{$\begin{array}{l}\text { Fast } \\
\text { decli- } \\
\text { nation }\end{array}$}} & & Chr'r & $\begin{array}{l}\text { Sca } \\
\text { read }\end{array}$ & $\begin{array}{l}\text { ale } \\
\text { ings }\end{array}$ & $\begin{array}{l}\text { East } \\
\text { decli- }\end{array}$ & Temp. & Chr'r & $\begin{array}{l}\text { Sce } \\
\text { read }\end{array}$ & $\begin{array}{l}\text { ale } \\
\text { ings }\end{array}$ & $\begin{array}{l}\text { East } \\
\text { decli- }\end{array}$ & & Chr'r & $\begin{array}{l}\text { Sc } \\
\text { read }\end{array}$ & lings & $\begin{array}{l}\text { East } \\
\text { decli- }\end{array}$ & \\
\hline & Left & Right & & & & & Left & Right & & & & Left & Right & & & & Left & Right & & \\
\hline h $\mathbf{m}$ & d & d & & & - & $\mathrm{h} \mathrm{m}$ & d & d & $\cdot$, & $\bullet$ & $\mathrm{h} \mathrm{m}$ & d & u & $\cdot \quad$ & & h m & & & - & $\bullet$ \\
\hline 1600 & $44 \cdot 3$ & 44.7 & 223 & & $-5 \cdot 3$ & I8 00 & 42.1 & 43.8 & 2245 & $-5 \cdot 4$ & 2000 & 27.6 & 28.0 & 2221 & -5.8 & 2200 & 25.6 & 28.9 & 2220 & -5.0 \\
\hline 02 & 44.7 & 44.8 & & & & 02 & 41.8 & 43.8 & 45 & & 02 & 27.0 & $27 \cdot 3$ & 20 & & 02 & 28.9 & 32.6 & 26 & \\
\hline 04 & $44 . I$ & $44 \cdot 3$ & & & 19 & 04 & 43.0 & 44.9 & 47 & 20 & 04 & 27.8 & 28.1 & 22 & We & 04.2 & $33 \cdot 3$ & $35 \cdot 3$ & & \\
\hline 06 & 44.7 & 44.8 & & & 6 & 06 & 41.0 & 43.0 & $\begin{array}{r}44 \\
42\end{array}$ & 85 & $\begin{array}{l}06 \\
08\end{array}$ & $\begin{array}{l}29.0 \\
27.0\end{array}$ & $\begin{array}{l}29.5 \\
27.3\end{array}$ & $\begin{array}{l}24 \\
20\end{array}$ & 8 & $\begin{array}{l}06 \\
08\end{array}$ & 33.5 & 37.2 & 33 & \\
\hline $\begin{array}{l}08 \\
\text { I0 }\end{array}$ & $\begin{array}{l}44.4 \\
44.0\end{array}$ & $\begin{array}{l}44.6 \\
44.2\end{array}$ & $\begin{array}{l}3 \\
3\end{array}$ & & in & $\begin{array}{l}08 \\
\text { I0 }\end{array}$ & & $\begin{array}{l}41.4 \\
40.1\end{array}$ & & 80 & $\begin{array}{l}08 \\
10\end{array}$ & 25.3 & $\begin{array}{l}27.3 \\
26.0\end{array}$ & $\begin{array}{l}20 \\
18\end{array}$ & $6 x$ & $\begin{array}{l}08 \\
\text { I0 }\end{array}$ & $\begin{array}{l}31.3 \\
32.3\end{array}$ & $\begin{array}{l}34.7 \\
35.2\end{array}$ & 30 & \\
\hline $\begin{array}{l}10 \\
12\end{array}$ & 43.9 & $\begin{array}{l}44.2 \\
44.1\end{array}$ & & & 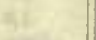 & 12 & $\begin{array}{l}38.3 \\
37.4\end{array}$ & $\begin{array}{l}40.1 \\
38.7\end{array}$ & 37 & 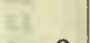 & 12 & 26.6 & 27.0 & 20 & ti & 12 & $\begin{array}{l}31.7 \\
31.5\end{array}$ & $\begin{array}{l}35.2 \\
34.3\end{array}$ & $\begin{array}{l}31 \\
30\end{array}$ & ty \\
\hline 14 & 43.9 & 44.1 & & & $-5 \cdot 3$ & I4 & 37.5 & 38.9 & 38 & -5.8 & 14 & 28.0 & 29.0 & 23 & -6.0 & I4 & $3 \mathrm{I} . \mathrm{I}$ & 33. & 28 & -4.9 \\
\hline 16 & $43 . I$ & $43 \cdot 3$ & & & & 16 & 40.0 & 40.6 & $4 \mathrm{I}$ & & 16 & 25.8 & 28.2 & 20 & as & I6 & 33.6 & 36. & 33 & \\
\hline 18 & 43.3 & 43.7 & & & $n$ & 18 & 40.8 & 41.9 & 43 & 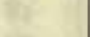 & 18 & 26.3 & 28.7 & $2 \mathrm{I}$ & (is & 18 & 27.0 & 30.3 & 23 & \\
\hline 20 & 43.4 & 43.6 & & & 27 & 20 & 44.2 & 44.3 & 47 & $=$ & 20 & 26.3 & 27.0 & 20 & $a$ & 20 & 29.3 & 32 & 26 & $3=$ \\
\hline 22 & 42.9 & $43 \cdot 3$ & & & 32 & 22 & 43.0 & 44.0 & 46 & 5 & 22 & 26.3 & 28.3 & $2 \mathrm{I}$ & a & 22 & 29.2 & & 26 & Lis \\
\hline 24 & 42.8 & 43.2 & & & {$[2$} & 24 & 43.2 & 44.2 & 46 & is & 24 & 28.7 & 30.5 & 24 & 14 & 24 & 30.6 & 32.6 & 27 & (5) \\
\hline 26 & 42.6 & 43.0 & & & $=$ & 26 & 39.3 & 41.6 & $4 \mathrm{I}$ & si & 26 & 31.6 & 32.7 & 28 & 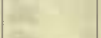 & 26 & 31.8 & 33.2 & 29 & \\
\hline 28 & 42.5 & 42.9 & & & & 28 & 46.9 & $47 \cdot 3$ & 52 & & 28 & 26.3 & 29.7 & 22 & 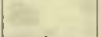 & 28 & $3 \mathrm{I} .3$ & 33.0 & 28 & \\
\hline 30 & 43.0 & 43.2 & & & -5.2 & 30 & 46.7 & 47.4 & 52 & -5.9 & 30 & 25.6 & 26.6 & 19 & -5.9 & 30 & $33 \cdot 3$ & 33.9 & $3 \mathrm{I}$ & -4.9 \\
\hline 32 & 44.0 & 44.2 & & & 8 & 32 & 40.8 & 42.3 & 43 & & 32 & 35.2 & 36.6 & 34 & & 32 & 32.6 & 33.6 & 30 & \\
\hline 34 & 44.9 & 45.1 & & & 5 & 34 & 38.5 & 38.9 & 38 & 18 & 34 & 31.8 & 32.3 & 28 & N. & 34 & 30.3 & $3 \mathrm{I}$ & 26 & \\
\hline 36 & $45 \cdot 4$ & 45.8 & & & (2) & 36 & 37.2 & 38.2 & 37 & $x$ & 36 & 32.3 & 33.3 & 29 & 28 & 36 & 29.5 & 30 & 25 & \\
\hline 38 & 44.8 & 45.2 & & & 2 & 38 & 38.2 & 38.4 & $3^{8}$ & tes & 38 & 34.4 & 36.4 & 33 & 24 & 38 & 32.5 & 34.7 & $3 \mathrm{I}$ & \\
\hline 40 & 43.7 & 44.0 & & & 6 & 40 & 36.6 & 37.0 & 36 & 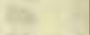 & 40 & 43.8 & 46.8 & 49 & 69 & 40 & 33.5 & 34 & 31 & \\
\hline 42 & 42.9 & 43.2 & & & $4:=5$ & 42 & 33.2 & 33.7 & 30 & & 42 & 42.0 & 46.3 & 47 & & 42 & 34.9 & 36.3 & 34 & \\
\hline 44 & 42.8 & 43.0 & & & -5.2 & 44 & 34.0 & 34.2 & $3 I$ & -5.8 & 44 & 36.3 & 39.0 & 37 & -5 & 44 & 31.8 & 34.2 & 30 & -4.8 \\
\hline 46 & 42.5 & 42.7 & & & & 46 & 38. & $3 a$ & 38 & & 46 & 35.2 & 38.0 & 35 & & 46 & 33.6 & 36.0 & 32 & \\
\hline 48 & 42.6 & 42.9 & & & 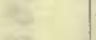 & 48 & $45 \cdot 3$ & 45.4 & 49 & 3. & 48 & 33.0 & 34.6 & $3 \mathrm{I}$ & 64 & 48 & 33.2 & 35.0 & I & \\
\hline 50 & 42.1 & 42.3 & & & a & 50 & 46.6 & 47.4 & $5^{2}$ & $\theta \pi$ & 50 & 37.1 & 38.1 & 37 & 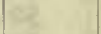 & 50 & 32.1 & 33.8 & 29 & \\
\hline 52 & 41.6 & 41.9 & & & 2 & 52 & 37.6 & 40.9 & 39 & 142 & 52 & 40.3 & $43 \cdot 3$ & 43 & 4 & 52 & 32.9 & 33.6 & 30 & \\
\hline 54 & 40.2 & 40.5 & & & 2 & 54 & $34 . I$ & 37.8 & 34 & 14 & 54 & 35.6 & 37.6 & 2235 & K. & 54 & 31.8 & 33.3 & 29 & \\
\hline 56 & 39.7 & 40.0 & & & 28 & 56 & 33.0 & 36.4 & 32 & & 56 & 46.8 & 58.8 & 23 OI & & 56 & 33.0 & 34 & $3 \mathrm{I}$ & \\
\hline 58 & 39.2 & 39.3 & & & $z^{2}=1$ & 58 & 3I.I & 33.8 & 29 & & 58 & 46.6 & 62.6 & 04 & & 58 & 32.5 & 34.1 & 30 & \\
\hline 1700 & 38.9 & 39.3 & & & 79 & I9 00 & 23.5 & 25.3 & 16 & -5.4 & 2100 & 48.2 & 68.2 & 09 & $-5 \cdot 3$ & 2300 & 30.4 & 32.3 & 27 & -4.8 \\
\hline 02 & 38.8 & 39.0 & & & $-5 \cdot 3$ & 02 & 19.8 & 23.2 & 12 & 20 & 02 & 66.8 & 79.0 & 2332 & 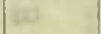 & 02 & 33.0 & 34.5 & $3 \mathrm{I}$ & \\
\hline 04 & 37.8 & 38.2 & & & & $04 \cdot 3$ & 24.2 & 28.2 & I9 & 19 & 04 & Lo & st & & in & 04 & 3 I. 6 & 33.3 & 29 & \\
\hline 06 & 37.8 & 38.0 & & & 6 & 06 & 27.2 & 30.6 & 23 & 60 & 06 & 27.7 & 36.3 & $22 \quad 28$ & 60 & 06 & 33.3 & 35.5 & 32 & v) \\
\hline 08 & 37.6 & 37.8 & & & 67 & 08 & 27.1 & 30.5 & 23 & (Bes & 08 & 29.0 & 37.0 & 30 & Wo & 08 & 33.8 & 36.2 & 33 & \\
\hline Io & 34.8 & 35.0 & & & $\theta$ & IO & 30.2 & 32.6 & 27 & or: & 10 & 27.2 & 34.8 & 26 & ate & I0 & 34.6 & 37.0 & 34 & f \\
\hline I2 & 34.2 & 35.8 & & & & I2 & 31.9 & 34.0 & 29 & 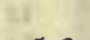 & 12 & 30.3 & 37.5 & 3I & 45 & I2 & 35.2 & 37.7 & 35 & \\
\hline I4 & 34.1 & 35.0 & & & -5.2 & I4 & $3 \mathrm{I} .5$ & $34 . I$ & 29 & -5.2 & 14 & 26.3 & $3.3 \cdot 3$ & 25 & -5 & I4 & 34.5 & 37.2 & 34 & -4.9 \\
\hline 16 & 40.6 & 42.2 & & & & 16 & 29.3 & $3 \mathrm{I} \cdot 3$ & 25 & & 16.2 & $24 \cdot 3$ & 28.2 & 19 & $y^{\circ}$ & 16 & 34.4 & 36.8 & 34 & \\
\hline I8 & 49.0 & 50.2 & 4 & & 815 & I8 & 25.6 & 28.3 & 20 & 4 & I8 & 23.6 & 28.4 & I9 & fit & 18 & 35.6 & 37.8 & 35 & 14 \\
\hline 20 & 57.4 & 58.0 & 225 & & 15 & 20 & $2 \overline{8} .7$ & 3I.I & 25 & $a$ & 20 & 22. I & 28.7 & I8 & as & 20 & 33.3 & 35.0 & $3 \mathrm{I}$ & \\
\hline $22^{*}$ & 32.1 & 38.0 & 23 & & $4 x$ & 22 & 31.2 & 32.6 & 28 & $=$ & 22 & 28.2 & 31.6 & 25 & his & 22 & 31.0 & 32 & 27 & \\
\hline 24 & 17.8 & 24.2 & 225 & & 18 & 24 & 32.3 & 33.3 & 29 & 16 & 24 & 29.8 & 31.3 & 26 & 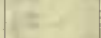 & 24 & 33.8 & 37.2 & 34 & $\mathrm{t}$ \\
\hline 26 & 19.0 & 24.9 & 5 & & 6 & 26 & 31.5 & 32.6 & 28 & & 26 & $2 \mathrm{~T} .4$ & 22.8 & I3 & 든 & 26 & 32.5 & 34.3 & 30 & \\
\hline 28 & 21.8 & 22.8 & $y$ & & The & 28 & 32.0 & 32.8 & 29 & 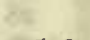 & 28 & 26.7 & 29.2 & 22 & . & 28 & 32.5 & 34.0 & 30 & \\
\hline 30 & I9. I & 21.2 & 3 & & -5.2 & 30 & 30.4 & 31.2 & 26 & -5.2 & 30 & 26.7 & 33.1 & 25 & -5.0 & 30 & 34.3 & 35.3 & 32 & -5.0 \\
\hline 32 & 18.9 & 19.3 & 5 & & 2 & 32 & 29.9 & 30.9 & 25 & & 32 & 40.0 & 45.6 & 45 & $E^{2}$ & 32 & $35 \cdot 3$ & 36.3 & 34 & \\
\hline & 22.0 & $23 \cdot 3$ & 225 & & 14 & 34 & 30.4 & 31.3 & 26 & Et & 34 & 36.5 & $4 \mathrm{I} .7$ & 39 & 4 & 34 & 34.6 & 36.6 & 34 & \\
\hline 36 & 23.3 & 26.0 & 230 & & & 36 & 30.4 & 31.2 & 26 & 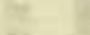 & 36 & 26.8 & 34. & 26 & 7 & 36 & 32.7 & $34 \cdot 3$ & 30 & \\
\hline 38 & 13.5 & 17.0 & 224 & & 26 & 38 & 30.6 & 31.4 & 26 & $\mathrm{z}$ & 38 & 29.6 & 37. & $3 \mathrm{I}$ & 4 & 38 & 32.8 & 34.2 & 30 & \\
\hline 40 & 10.5 & 14.2 & & & $4 h$ & 40 & 30.6 & 31.2 & 26 & 3 & 40 & 26.3 & 38.6 & 29 & 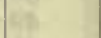 & 40 & 34.5 & 36.1 & 33 & \\
\hline $42^{*}$ & 43.9 & 46.0 & & 8 & & 42 & 29.9 & 30.7 & 25 & 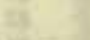 & 42 & 28.6 & 36.8 & 29 & t & 42 & 36.5 & 37.8 & 36 & \\
\hline 44 & 43.9 & 46.6 & te & & $-5 \cdot 3$ & 44 & 30.7 & 31.3 & 26 & $-5 \cdot 5$ & 44 & 32.8 & 37.2 & 33 & -5.0 & 44 & 35.8 & 36.9 & 35 & -5.0 \\
\hline 46 & 42.3 & 45.6 & & & & 46 & 30.7 & 31.3 & 26 & & 46 & 32.8 & 36.2 & 32 & & 46 & 36.2 & 36.5 & 35 & \\
\hline 48 & 40.0 & 42.6 & 4 & & 8 & 48 & 29.7 & 30.3 & 25 & 64 & 48 & 36.3 & 39.6 & 37 & 4 & 48 & 34.4 & 35.5 & 33 & \\
\hline 50 & 41.8 & $44 \cdot 3$ & & & e. & 50 & 29.0 & 29.6 & 24 & 92 & 50 & 34.0 & 38.6 & 35 & 8 & 50 & 36.4 & $37 \cdot 3$ & 36 & \\
\hline 52 & 43.7 & 45.4 & 4 & & 22 & 52 & 29.9 & 30.2 & 25 & EI & 52 & 27.2 & 31.2 & 24 & 25 & 52 & 36.9 & 37.4 & 36 & \\
\hline 54 & 42.4 & $44 \cdot 3$ & & 6 & 3 & 54 & 30.4 & 30.8 & 26 & 18 & 54.2 & 25.4 & 27.8 & 20 & 4 & 54 & 37.3 & 37.8 & 37 & \\
\hline 56 & $41 \cdot 5$ & 44.0 & & & $=$ & 56 & 29.7 & 29.9 & 25 & $Q$ & 56 & 28.4 & 31.7 & 25 & & 56 & 33.8 & 34.6 & $3 I$ & \\
\hline 58 & 42.3 & 43.8 & 4 & & ta: & 58 & $24 . I$ & 24.3 & 16 & QR & 58 & 26.8 & 29.2 & 22 & 4 & $5^{8}$ & 34.2 & 34.9 & 32 & \\
\hline & & & & & & & & & & & & & & & & & 36.3 & 37.6 & 36 & \\
\hline
\end{tabular}

Observers-R. W. P. and W. J. P., who alternated from Igh oom to Igh $14 \mathrm{~m}$.
Correction to local mean time is - 05 s.

Torsion head at on oom read $307^{\circ}$ and at $24 \mathrm{~h} 25 \mathrm{~m}$ read the same. Observer-W. J. P. 
Tabulation of magnetic declinations observed at Teplitz Bay-Continued

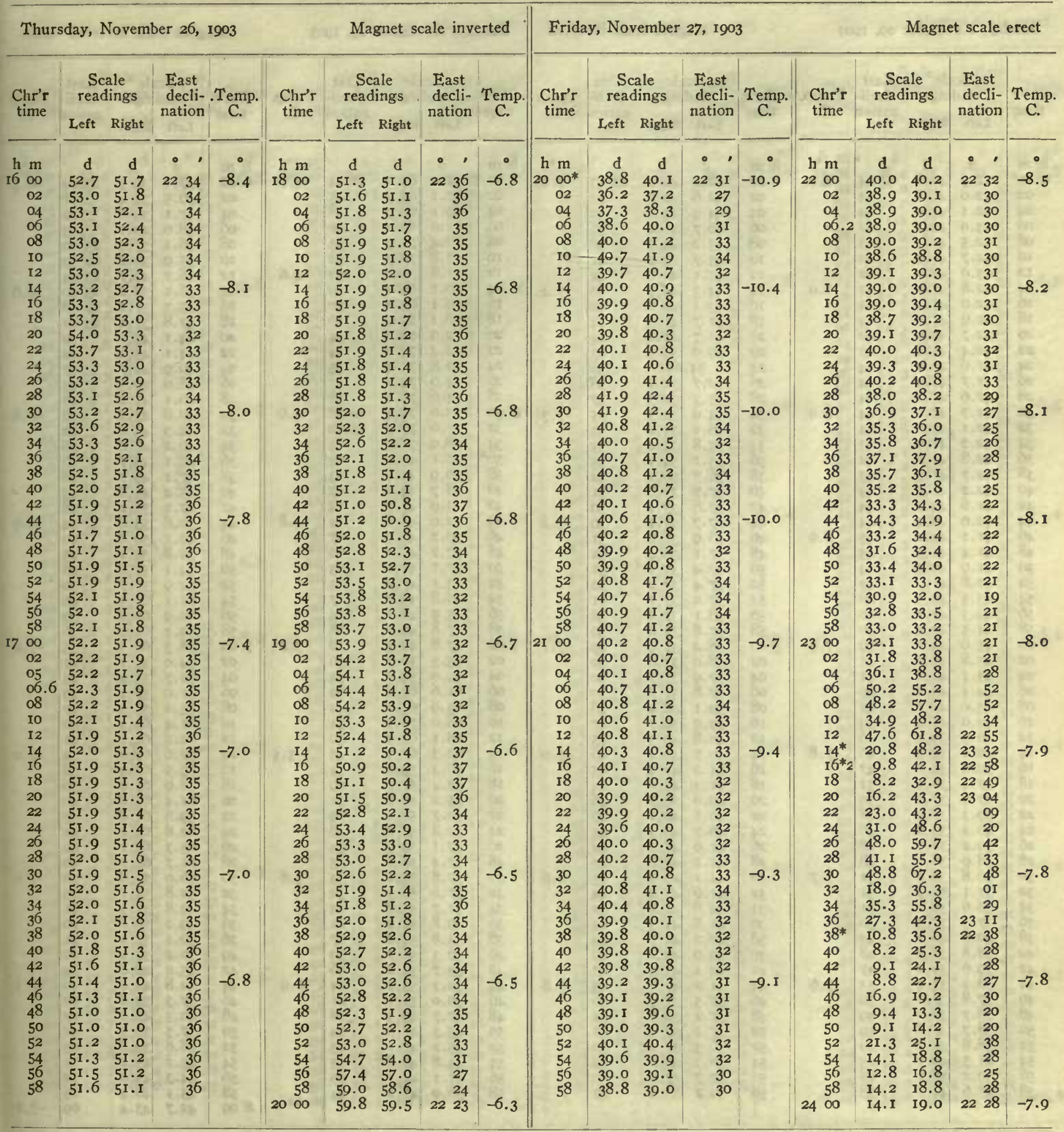

Correction to local mean time is - o2s.

Torsion head at $15 \mathrm{~h} 4 \mathrm{Im}$ read $307^{\circ}$ and at $20 \mathrm{~h}$ 3om read the same. Observer-R. R. T.
Correction to local mean time is $-35 \mathrm{~s}$

Torsion head at Igh $30 \mathrm{~m}$ read $352^{\circ}$ and at $24 \mathrm{~h}$ oom read the same. Observer-R. R. T. 
Tabulation of magnetic declinations observed at Teplitz Bay-Continued

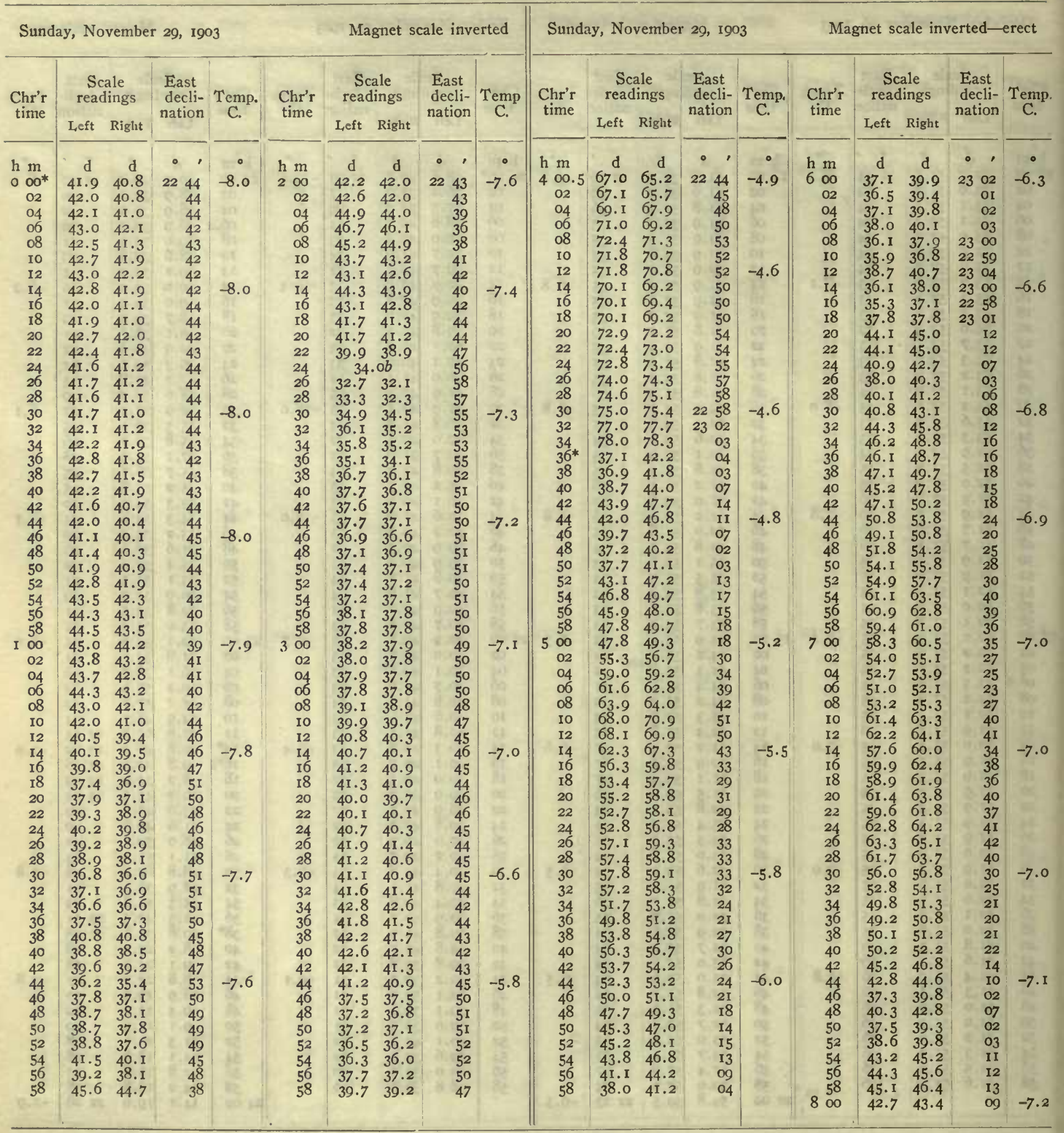


Tabulation of magnetic declinations observed at Teplitz Bay-Continued

\begin{tabular}{|c|c|c|c|c|c|c|c|c|c|c|c|c|c|c|c|c|c|c|c|}
\hline \multicolumn{5}{|c|}{ Monday, November 30 , I903 } & \multicolumn{5}{|c|}{ Magnet scale inverted } & \multicolumn{5}{|c|}{ Tuesday, December I, 1903} & \multicolumn{5}{|c|}{ Magnet scale erect } \\
\hline \multirow{2}{*}{$\begin{array}{l}\text { Chr'r } \\
\text { time }\end{array}$} & \multicolumn{2}{|c|}{$\begin{array}{l}\text { Scale } \\
\text { readings }\end{array}$} & \multirow{2}{*}{$\begin{array}{l}\text { East } \\
\text { decli- } \\
\text { nation }\end{array}$} & & Chr'r & & $\begin{array}{l}\text { ale } \\
\text { lings }\end{array}$ & $\begin{array}{l}\text { East } \\
\text { decli- }\end{array}$ & & Chr'r & $\begin{array}{r}\mathrm{Sc} \\
\mathrm{read}\end{array}$ & $\begin{array}{l}\text { ale } \\
\text { lings }\end{array}$ & $\begin{array}{l}\text { East } \\
\text { decli- }\end{array}$ & & & $\begin{array}{r}\mathrm{Sc} \\
\text { read }\end{array}$ & lings & $\begin{array}{l}\text { East } \\
\text { decli- }\end{array}$ & \\
\hline & Left & Right & & & time & Left & Right & nation & & tim & Left & Right & & & $\operatorname{tin}$ & Left & Right & nation & \\
\hline $\mathrm{h} \mathrm{m}$ & $d$ & d & & & $\mathrm{h} \mathrm{m}$ & d & $\mathrm{d}$ & & & $\mathrm{h} \mathrm{m}$ & & d & & & h m & & & & \\
\hline 800 & 61.0 & 59.2 & 2229 & -10.3 & 10 00 & 61.0 & $59 . \mathrm{I}$ & 2229 & -6.8 & 1200 & 46.1 & 47.1 & 2221 & -8.0 & I4 00 & 48.4 & 48.6 & 2224 & -6.8 \\
\hline 02.3 & 60.2 & 58.1 & 3 I & & 02 & $6 \mathrm{r} .3$ & 59.1 & 29 & & 02 & 55.2 & 56.1 & 35 & & 02 & 49.0 & 49.1 & 25 & \\
\hline 04 & 57.9 & 56.1 & 34 & & 04 & $6 \mathrm{r} .0$ & 58.8 & 29 & & 04 & $54 \cdot 5$ & 54.9 & 34 & & 04 & $49 . \mathrm{I}$ & 49 & 25 & \\
\hline$\infty 6$ & 57.7 & 56.3 & 34 & & 06 & 58.9 & 57.6 & 32 & & 06 & 48.9 & 49.9 & 26 & & 06 & 5I.I & & 28 & 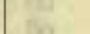 \\
\hline 08 & 55.8 & 54.5 & 37 & & 08 & 59.2 & 57.9 & 32 & & 08 & 49.1 & 50 & 26 & & 08 & 50.9 & 51 & 28 & 0 \\
\hline I0 & $54 . I$ & 53.2 & 39 & 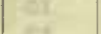 & 10 & 59.1 & 58. & 32 & & Io & -48.3 & 49 & 25 & & Io & 52.9 & & 31 & \\
\hline 12 & 53.9 & 52.1 & 40 & fer & 12 & 58.8 & 57.9 & 32 & & 12 & 50.1 & 51.2 & 27 & & 12 & 4.1 & & 33 & \\
\hline I4 & 51.9 & 50.5 & 43 & -9.8 & I4 & 58.3 & 57.3 & 33 & -6.4 & I4 & 49.2 & 50.2 & 26 & -7.8 & 14 & 53.9 & 54.9 & 33 & -6.7 \\
\hline I6́ & 51.4 & 50.2 & 44 & & 16 & 59.1 & 58.7 & $3 \mathrm{I}$ & & I6 & 50.9 & 51.7 & 29 & & 16 & 55.3 & & 35 & \\
\hline I8 & 53.2 & 52.0 & $4 I$ & & 18 & 60.7 & 59.3 & 29 & & I8 & 49.8 & 50. & 27 & E & 18 & 54.8 & 54 & 34 & 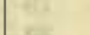 \\
\hline 20 & 52.6 & 52.0 & $4 \mathrm{I}$ & 146 & 20 & 60.2 & 58. & 30 & & 20 & 53.1 & 53 & 32 & & 20 & 54.3 & & 34 & $\mathrm{x}$ \\
\hline 22 & 54.8 & 52.2 & 40 & 촌 & 22 & 58.9 & 58.6 & $3 I$ & & 22 & 50.2 & $5 \mathrm{I}$. & 28 & & 22 & 53.8 & 54 & 33 & E \\
\hline 24 & 57.9 & 55.9 & 34 & 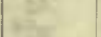 & 24 & 58.5 & 57. & 33 & & 24 & 51.7 & 52 & 29 & a & 24 & 5.0 & & & 5 \\
\hline 26 & 57.7 & 55.3 & 35 & & 26 & 60.7 & 57 & $3 \mathrm{r}$ & & 26 & $57 \cdot 3$ & 58. & 39 & & 2 & 3.9 & & 33 & 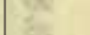 \\
\hline 28 & 57.2 & 55.2 & 35 & & 28 & 60.0 & & $3 \mathrm{I}$ & & 28 & 55.4 & 57 & 3 & & 28 & 4.0 & & & \\
\hline 30 & 58.1 & 57.0 & 33 & -9.3 & 30 & 62.1 & 59. & 28 & -6.2 & 30 & 46.8 & 48. & 23 & -7.6 & 3 & 3.9 & & 33 & -6.5 \\
\hline 32.3 & $63 \cdot 3$ & 62.1 & 25 & & 32 & $6 \mathrm{I} . \mathrm{I}$ & 59 & 29 & & 32 & 45.9 & 47 & 2 & & 3 & & & & \\
\hline 34 & 57.5 & 56.1 & 34 & & 34 & 61.0 & 58. & 30 & & 34 & 48.3 & 49 & 2 & & 3 & 4.8 & & & 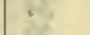 \\
\hline 36 & 45.3 & 44.8 & 2253 & & 36 & 60.9 & 58 & 3 & & 36 & 49.7 & 50 & 2 & & & $5 . \mathrm{I}$ & & & 5 \\
\hline 38 & 39. I & 36.6 & 2304 & & 38 & 59.3 & 57. & 32 & & 38 & 55.1 & 55 & 3 & & 38 & 55.2 & & 35 & \\
\hline 40 & 45.7 & 42.3 & 2254 & 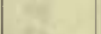 & 40 & & 57 & 3 & ser & $4 c$ & $53 \cdot 3$ & 55 & 3 & 6 & 4 & & & & 2 \\
\hline 42 & 56.3 & 56.1 & 35 & 9 & 42 & 60.1 & 58. & $3 \mathrm{I}$ & 24 & 42 & 52.2 & 53 & 3 & 6 & 4 & 5.6 & & & \\
\hline 44 & 56.6 & 56.1 & 35 & 14 & 44 & 60.5 & 58 & 30 & -6.1 & 44 & 45.3 & 46.9 & 20 & -7.4 & 4 & 55.8 & 56 & & -6.4 \\
\hline 46 & 48.1 & 47.7 & 48 & is & 46 & 60.9 & 58. & 30 & & 46 & 47.0 & 49. & 2 & & 4 & 56.0 & & & \\
\hline 48 & 50.0 & 48.6 & 46 & 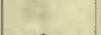 & 48 & 60.8 & 58. & 30 & & 48 & 48.1 & 49. & 2 & 8 & 4 & 56.3 & & & 5 \\
\hline 50 & 50.0 & 48.9 & 46 & -8.7 & 50 & 60.6 & 58. & 30 & & 50 & 49.4 & $5 \mathrm{I}$. & 2 & 4 & 5 & 56.3 & & & 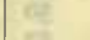 \\
\hline 52 & 56.1 & 54.9 & 36 & & 52 & 61.1 & 59. & 29 & & 52 & 47.8 & 49.2 & 2. & 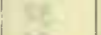 & 52 & 54.9 & 55 & 3 & 5 \\
\hline 54 & 55.7 & 54.2 & 37 & & 54 & 61.3 & 59 & 28 & 14 & 54 & 46.6 & 48 & 2 & 1 & 5 & & & & 8 \\
\hline $5^{6}$ & 56.0 & 55.0 & 36 & & 56 & 61.2 & 59. & 29 & & 50 & 45.4 & 47.6 & 2 & & 5 & 54.3 & & & \\
\hline 58 & $57 . \mathrm{I}$ & 55.4 & 35 & & 58 & 61.2 & 59. & 29 & & 58 & 45.0 & 46 & 2 & & 5 & & & & \\
\hline 900 & 58.1 & 56.2 & 34 & -8.2 & II 00 & 58.9 & 57. & 33 & -6.1 & I3 00 & 45.3 & 46. & $2 c$ & -7.3 & 1500 & 54.8 & & 34 & -6.2 \\
\hline 02 & 60.2 & 56.8 & 32 & & 02 & 57.9 & 56 & 34 & & 02 & 43.9 & 45. & I & & 02 & & & & \\
\hline 04 & 60.1 & 57.4 & $3 \mathrm{I}$ & ) & 04 & 57.8 & 56. & 3 & & o & 39.1 & 40 & I & & o & .0 & & & c \\
\hline 06 & 58.4 & 56.3 & 34 & [a & $\infty 6$ & 57.5 & & 3 & & o & 43.8 & & I & ty & $\infty$ & & & & 6 \\
\hline 08 & 57.5 & 54.3 & 36 & 190 & 08 & 59.7 & 58 & 3 & & 08 & 44.0 & 45 & I & & 08.3 & 57.3 & & & \\
\hline Io & 58.1 & 55.0 & 35 & 60 & IO & 63.1 & & 2( & 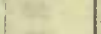 & I & & & I & 5 & I0 & 58.2 & & & 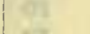 \\
\hline 12 & 60.7 & 57.7 & 3 I & I) & I2 & 61.4 & 60.8 & 28 & 287 & I & 9.3 & 39.8 & I & & 12 & 59.1 & & $4 \mathrm{I}$ & \\
\hline I4 & 61.8 & 59.3 & 29 & -7.7 & I4 & 59.5 & 59.0 & $3 \mathrm{I}$ & -6.0 & I4 & 40.7 & $4 \mathrm{I} .7$ & 13 & -7.1 & 14 & 59.2 & & $4 \mathrm{I}$ & -6.2 \\
\hline I6 & 59.4 & 57.7 & 32 & & 16 & 57.4 & 57. & 34 & & IC & 42.4 & 42. & I & & I & 60.0 & & & \\
\hline I 8 & 60.3 & 57.9 & 3 & 24 & I8 & 58.1 & 56. & 34 & & I 8 & 44.0 & 44.2 & I & & 18 & 60.3 & & & \\
\hline 20 & $6 \mathrm{I} .2$ & 59. & 2 & 18 & 20 & 59.4 & 57. & 3 & & 2 & 43.9 & 44. & I & & 20 & 61.3 & & & \\
\hline 22 & 60.6 & 59. & 3 & axi & 22 & $59 . \mathrm{I}$ & 57. & 3 & $=$ & 22 & 45.1 & 45 & I & & 22 & 61.9 & & & 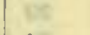 \\
\hline 24 & 60.3 & 59. & 3 & 3 & 2 & & & 3 & Ba & & & 42 & I & & 2 & 61 & & & 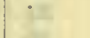 \\
\hline 26 & 60 & 59.2 & 29 & gs & 26 & & 57. & 31 & & 2 & 43.8 & 44 & I & & 26 & $6 r$ & & & E \\
\hline 28 & 60.3 & 59. & 30 & 15 & 28 & 58.6 & & 3 & 115 & 2 & & & I & Wint & 2 & $6 \mathrm{r} .2$ & & & \\
\hline 30 & 59.7 & 58.2 & 3I & $-7 \cdot 3$ & 30 & 7.9 & 55. & 34 & -5.9 & 30 & 42.7 & 42.8 & I & -7.0 & 30 & 61.8 & 62 & 45 & -6.2 \\
\hline 32 & 60. & 5 & & & 32 & & & 3 & & & & & I & & 3 & 62.8 & & & \\
\hline 34 & 60.7 & 59.9 & 2 & & 34 & 59.2 & 57 & 3 & & 3 & 46.3 & 47 & 2 & & 3 & 61.5 & & 5 & \\
\hline 36 & 60.3 & & & & 36 & & & 3 & & & 46.2 & 46 & 2 & & & & & & \\
\hline 38 & 60.3 & 60.0 & 29 & 10 & 38 & 59.4 & 57.8 & 32 & tat & 38 & 42.8 & 43 & I & & 38 & 60.3 & & 3 & \\
\hline 40 & 58.9 & 57.9 & 32 & 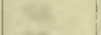 & 40 & & & 2 & $\theta$ & & & & I & $\omega$ & & 60.1 & & &. \\
\hline 42 & 58. I & 57.2 & 33 & 14 & 42 & 6 1.8 & 60.6 & 28 & $y_{4}$ & 42 & & & I & 4 & 42 & 60.4 & & 3 & \\
\hline & 57.2 & 55.4 & 35 & -7.0 & & 60.3 & 59.7 & 29 & -5.7 & 44 & 46.0 & 47.0 & 2 & -7 & 44 & 60.2 & 60 & 3 & -6.1 \\
\hline 46 & 55.7 & 54.6 & 37 & & 46 & 59.8 & 58. & 30 & & 4 & 46.7 & 47.4 & 2 & & 46 & 59.6 & & & \\
\hline 48 & 56.2 & 55.1 & 36 & 4 & 48 & 60.3 & 59. & 3 & 4 & 4 & & & 2 & 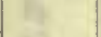 & 4 & 58.8 & & & $x^{2}$ \\
\hline 50 & 58.7 & 57.9 & 32 & 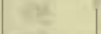 & 50 & 6r.I & 60. & 28 & $x^{2}$ & 50 & 47.8 & 48.1 & 2 & fa & 50 & 57.7 & & 39 & 2 \\
\hline 52 & 59.3 & 58.4 & & & 52 & 60.3 & & 29 & er & & 47.8 & & 2 & & & 56.8 & & & 6 \\
\hline 54 & 60.0 & 59.2 & 30 & & 54 & 60.3 & 59.3 & 30 & ex & 5 & 48.7 & 48 & 2 & 12 & 54 & 56.2 & & 36 & $\because$ \\
\hline 56 & 61.7 & 60.4 & 28 & oc & 56 & 59.3 & 58.3 & $3 \mathrm{I}$ & & 5 & 48.1 & 48.3 & 2 & & 56 & 56.3 & & & \\
\hline 58 & 60.9 & 60.0 & 29 & 18 & 58 & 58.3 & 57.7 & 33 & & 58 & 47.7 & 47.9 & 23 & & 58 & 56.2 & & 36 & \\
\hline & & & & & & 60.1 & 60.0 & 29 & & & & & & & I6 & 55.8 & 56.0 & & \\
\hline
\end{tabular}

Correction to local mean time is - Im 30 .

Torsion head at $7 \mathrm{~h} 36 \mathrm{~m}$ read $339^{\circ}$ and at $\mathrm{I} 2 \mathrm{~h}$ 20m read the same.

Observer-R. R. T.
Correction to local mean time is +25 s. $90^{\circ}$ torsion $=22 .^{\prime} 6$.

Torsion head at roh $10 \mathrm{~m}$ read $336^{\circ}$ and at $16 \mathrm{~h} 25 \mathrm{~m}$ read $34 \mathrm{I}^{\circ}$. Ohserver-R. R. T. 
Tabulation of magnetic declinations observed at Teplitz Bay-Continued

\begin{tabular}{|c|c|c|c|c|c|c|c|c|c|c|c|c|c|c|c|c|c|c|c|}
\hline \multicolumn{5}{|c|}{ Wednesday, December 2, 1903} & \multicolumn{5}{|c|}{ Magnet scale inverted } & \multicolumn{5}{|c|}{ Wednesday, December 2, 1903} & \multicolumn{5}{|c|}{ Magnet scale inverted } \\
\hline \multirow{2}{*}{$\begin{array}{l}\text { Chr'r } \\
\text { time }\end{array}$} & \multicolumn{2}{|c|}{$\begin{array}{l}\text { Scale } \\
\text { readings }\end{array}$} & \multirow{2}{*}{$\begin{array}{c}\text { East } \\
\text { decli- } \\
\text { nation }\end{array}$} & $\begin{array}{l}\text { Temp. } \\
\text { C. }\end{array}$ & $\begin{array}{l}\text { Chr'r } \\
\text { time }\end{array}$ & $\begin{array}{l}\text { Sca } \\
\text { readi }\end{array}$ & $\begin{array}{l}\text { ale } \\
\text { lings }\end{array}$ & $\begin{array}{l}\text { East } \\
\text { decli }\end{array}$ & & Chr'r & $\begin{array}{r}\mathrm{Sc} \\
\text { read }\end{array}$ & le ngs & Ea & & & $\begin{aligned} \text { Se } \\
\text { read }\end{aligned}$ & $\begin{array}{l}\text { le } \\
\text { ings }\end{array}$ & $\begin{array}{l}\text { Eas } \\
\text { dec }\end{array}$ & Temp \\
\hline & I,eft & Right & & & & Left & Right & & & & Left & Right & & & & L,eft & Right & & \\
\hline$h \mathrm{~m}$ & $d$ & d & $\cdot 1$ & $\circ$ & $\mathrm{h} \mathrm{m}$ & d & d & - ' & $\cdot$ & h m & d & d & & • & $h \mathrm{~m}$ & d & $\mathrm{d}$ & - & $\bullet$ \\
\hline $000^{*}$ & 32.3 & $31 \cdot 3$ & & $-13 \cdot 3$ & 200 & 40.8 & 40.6 & 2226 & -9.9 & 400 & 47.8 & 46.2 & 230 & -9.3 & 600 & 56.0 & 55.0 & 2403 & -9.1 \\
\hline 02 & 31.9 & 31.6 & 40 & $x$ & 02 & 41.4 & $4 \mathrm{I} \cdot 3$ & & & 02 & $45 \cdot 3$ & $45 \cdot 3$ & & 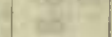 & 02 & 64.6 & 62.1 & 2351 & \\
\hline 04 & $3 I \cdot 5$ & 30.3 & $4 \mathrm{I}$ & 80 & 04 & 40.9 & 40.5 & 26 & 20 & 04 & 37 & $3 b$ & & +5 & 04 & 69.8 & 68.6 & 41 & \\
\hline 06 & 31.2 & 30.4 & $4 \mathrm{I}$ & 290 & 06 & 37.6 & 37.6 & 30 & (3) & 06 & 40.0 & $3^{8.3}$ & & ar & 06 & 69.4 & 67.5 & & \\
\hline 08 & $33 \cdot 3$ & 33.0 & 37 & 20 & 08 & 37.4 & 37.2 & 31 & 86 & 08 & 37.0 & 35.8 & & 60 & 08 & 70.1 & 68 & & \\
\hline I0 & $34 \cdot 4$ & $34 \cdot 3$ & 35 & of & 10 & 36.8 & 36.5 & 32 & tro & 10 & 39.5 & 38.6 & 2 & (1) & 10 & 71.0 & 69.2 & 4 & \\
\hline 12 & 36.5 & 36.0 & 33 & & 12 & $34 \cdot 3$ & 33.7 & 36 & & 12 & 39.6 & $39 \cdot 3$ & 2 & & I2 & 70.2 & & 41 & \\
\hline 14 & 38.6 & 38. I & 29 & -12.6 & 14 & 33.3 & 33.0 & 37 & -9.8 & 14 & 36.6 & 36.0 & 2 & -9.3 & 14 & 63.8 & 62 & 51 & -9.0 \\
\hline 16 & 39.3 & 39.0 & 28 & 98 & 16 & 31.6 & 31.3 & 40 & 21 & 16 & 37.9 & $37 \cdot 3$ & 2 & $x^{2}$ & I6 & 63.9 & 62.6 & & \\
\hline I8 & 37.0 & 36.2 & 32 & 64 & I8 & 31.3 & 30. & $4 I$ & 4 & 18 & 32.6 & 32.3 & & Wt & 18 & 69.3 & 67.5 & & \\
\hline 20 & 35.0 & 34.0 & 35 & 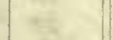 & 20 & 30. I & 29.6 & 43 & 15 & 20 & 30.6 & 29.6 & 3 & $\pi$ & 20 & 70.1 & 68 & 4 & \\
\hline 22 & $33 \cdot 3$ & 32.7 & 38 & 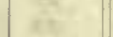 & 22 & 27.8 & 27.3 & 46 & (5) & 22 & 32.9 & 31.3 & 3 & & 22 & 75.2 & 72 & & \\
\hline 24 & 35.0 & 34.5 & 35 & is & 24 & 26.7 & 26.0 & 48 & in & 24 & 40.6 & 38.6 & 2 & & 24. & 79.0 & 77.5 & & \\
\hline 26 & 37.6 & 36.3 & $3 \mathrm{I}$ & in & 26 & 25.5 & 25.3 & 50 & Ex & 26 & 47.5 & 46.7 & 0 & & $26^{*}$ & 56.7 & & & \\
\hline 28 & 37.5 & 37.2 & 31 & & 28 & 24.6 & $24 . I$ & 51 & & 28 & 47.5 & 47.1 & 0 & & 28 & 6. .3 & 55 & & \\
\hline 30 & 37.8 & 37.0 & 31 & -12.0 & 30 & $24 \cdot I$ & 23.6 & 52 & -9.6 & 30 & 47.8 & 46.8 & 0 & -9.3 & 30 & 57.2 & & 2 & -9.0 \\
\hline 32 & $37 \cdot 3$ & 36.3 & 32 & a & 32 & 23.5 & 22.7 & 53 & & 32 & 43.1 & 42.0 & I & tye & 32 & 59.3 & 57 & I & \\
\hline 34 & 34.9 & $34 \cdot 3$ & 35 & N & 34 & 21.4 & 21.3 & 56 & & 34 & 40.8 & 39.9 & & & 34 & 61 & & & \\
\hline 36 & 33.1 & 32.5 & 38 & 3 & 36 & 19.3 & 19.0 & 2259 & & 36 & 36.9 & 36 & & 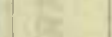 & 3 & 60.2 & & & \\
\hline 38 & 34.6 & 34.0 & 35 & De. & 38 & 18.4 & 18.3 & 23 OI & $a^{2}$ & 38 & 35.3 & 32.7 & 3 & 6 & 38 & 59.8 & 58 & & \\
\hline 40 & 33.3 & 32.8 & 37 & $a$ & 40 & 18.8 & 18.3 & $\infty$ & nt & 40 & 31.6 & 31 & 3 & oit & 40 & 58.8 & 58 & & \\
\hline 42 & $3 \mathrm{I} .3$ & 31.0 & 40 & & 42 & 18.0 & 17.6 & OI & & 42 & 34.8 & 34.2 & 2 & & 42 & 62.7 & $6 \mathrm{I}$ & I2 & \\
\hline 44 & $34 \cdot 3$ & 33.7 & 36 & $-I I \cdot 3$ & 44 & 17.8 & 17.6 & 02 & -9.6 & 44 & 30.0 & 29.5 & 3 & -9.1 & 44 & 59.3 & 58 & $\mathrm{I} 7$ & -9.0 \\
\hline 46 & 28.0 & 27.6 & 46 & & 46 & 18.3 & 18.3 & OI & & 46.4 & 30.0 & 29.3 & & 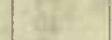 & 46 & 62.5 & $6 I$ & & \\
\hline 48 & 28.6 & 28.5 & 45 & 45 & 48 & 17.0 & 17.0 & 03 & 28 & 48 & 34.6 & 33 & & at & 48 & 67 & & & \\
\hline 50 & 29.5 & 29.3 & 43 & $\Leftrightarrow$ & 50 & 17.2 & 17.0 & 02 & of & 50 & 34.4 & 33 & & 2 & 5 & $6 I$ & 60. & & \\
\hline 52 & 32.3 & 32.3 & 39 & $\sqrt{3}$ & 52 & 16.0 & I5.8 & 04 & $3 x$ & 52 & 41.6 & 39 & I & 52 & 52 & 56 & 55 & & \\
\hline 54 & $3 \mathrm{I} .0$ & 30.6 & 41 & in & 54 & 15.8 & 15.4 & 05 & $y^{2}$ & 54 & 43.6 & 41.0 & I & BS & 54 & 56.3 & 55 & & \\
\hline 56 & 28.8 & 28.8 & 44 & a & 56 & 14.6 & 14.6 & 06 & tos & 56 & 50.3 & 47.5 & 0 & & 56 & 56.3 & & & \\
\hline 58 & 27.9 & 27.1 & 46 & & 58 & 14.3 & 13.7 & 07 & & 58 & 53.6 & $5 I$ & 230 & & 58 & 55 & & & \\
\hline 100 & 23.8 & 22.5 & 53 & -II.0 & 300 & 13.5 & 13.3 & 08 & -9.5 & 500 & 56.1 & 52.6 & 225 & -9.1 & 700 & 58.9 & 58 & I & -9.0 \\
\hline 02 & 23.0 & $22 . I$ & 54 & in & 02 & 14.3 & 13.8 & 07 & 25 & 02 & 59.3 & 56.8 & 5 & & 02 & 55.0 & 53. & & \\
\hline $\begin{array}{l}04 \\
06\end{array}$ & $\begin{array}{l}24.6 \\
24.6\end{array}$ & $\begin{array}{r}23.8 \\
24.3\end{array}$ & 5I & te & 04 & 14.6 & I 4.3 & 07 & in: & 04 & 59.8 & 57.2 & 5 & & 04 & 53 & 52. & & \\
\hline $\begin{array}{l}06 \\
08\end{array}$ & 26.3 & $\begin{array}{l}24.3 \\
26.0\end{array}$ & $\begin{array}{l}5 I \\
48\end{array}$ & 82 & o6 & 13.7 & I3.7 & 08 & 68 & 06 & 60.5 & 58. & & 政 & 06 & 56.1 & & & \\
\hline I0 & 27.3 & 26.8 & $\begin{array}{l}48 \\
47\end{array}$ & 27 & 08 & 13.0 & 13.0 & 09 & $3 y$ & 08 & 60.8 & 57.9 & 5 & & 08 & 44.7 & 44 & & \\
\hline 12 & 27.6 & 27.5 & $\begin{array}{l}47 \\
46\end{array}$ & 30 & I0 & 10.6 & I0. 4 & I & 60 & 10 & 60.1 & & 5 & 6 & 10 & 46.0 & & & \\
\hline 14 & 25.6 & 25.3 & 49 & -10.5 & 12 & 8.0 & $7 \cdot 3$ & I8 & & I2 & 62.6 & 60 & 4 & & 12 & 36.8 & 35 & 5 & \\
\hline 16 & 21.6 & 21.6 & & & $\begin{array}{l}14^{*} \\
16\end{array}$ & 44.8 & 40.0 & I7 & -9.4 & I4 & 63.7 & 6I. & 4 & -9.1 & 14 & 44.6 & 44.0 & 40 & -9.0 \\
\hline 18 & 21.8 & 21.2 & 56 & Ar. & $\begin{array}{l}10 \\
18\end{array}$ & $\begin{array}{l}39.3 \\
38.8\end{array}$ & 38.7 & 22 & $\sqrt{2}-2$ & I6 & 6r.I & 59.8 & & & I6 & 44.5 & & & \\
\hline 20 & & I & 58 & ${ }^{2}$ & 20 & $\begin{array}{l}30.0 \\
38.3\end{array}$ & $\begin{array}{l}30.4 \\
37.9\end{array}$ & $\begin{array}{l}23 \\
23\end{array}$ & 4 & $\begin{array}{l}18 \\
20\end{array}$ & 58.3 & 57.0 & 5 & 81 & 18 & 42.3 & & & \\
\hline 22 & .8 & 21.8 & 54 & m & 22 & $\begin{array}{l}50.3 \\
36.3\end{array}$ & 36.2 & $\begin{array}{l}23 \\
26\end{array}$ & $\theta$ & $\begin{array}{l}20 \\
22\end{array}$ & 55.8 & 54.3 & 225 & te & 20 & $4 \mathrm{I} . \mathrm{I}$ & 38.4 & 48 & \\
\hline 24 & 26.6 & 25.8 & 48 & 15. & 24 & 36.1 & 35.9 & 27 & X & 24 & $\begin{array}{l}53.3 \\
53.0\end{array}$ & $\begin{array}{l}5 \mathrm{I} . \\
5 \mathrm{I} .\end{array}$ & 230 & ant & $\begin{array}{l}2 \\
2\end{array}$ & $\begin{array}{r}45 \\
52.6\end{array}$ & $.0 a$ & $\begin{array}{l}39 \\
20\end{array}$ & \\
\hline 26 & 25.6 & 24.7 & 50 & & 26 & 36.0 & 34. & 2 & 32 & 26 & 50.0 & 49.0 & 0 & & 2 & 49.3 & 48 & & \\
\hline 28 & 25.6 & 25.0 & 50 & & 28 & 35.8 & 35.0 & 28 & & 28 & & & & & 2 & 52.3 & 49 & 30 & \\
\hline 30 & & 24.7 & 50 & -10.2 & 30 & 36.6 & 36.3 & 26 & -9.2 & 30 & 45.1 & 43 & I. & $-9 . c$ & 30 & 44.0 & 4I. & 43 & $-9 . I$ \\
\hline 32 & 26.0 & 25.3 & 49 & $x^{2}$ & 32 & 32.6 & 31.8 & 33 & & 32 & 43.2 & & I & 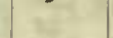 & 32 & 38.2 & 35 & & \\
\hline 34 & 25.1 & 24.5 & 50 & at & 34 & $35 \cdot 5$ & 34.5 & 28 & & 34 & 39.4 & 38 & & & 3 & 42.6 & & & \\
\hline 36 & 25.0 & 2 & & 2 & & $37 \cdot 5$ & 37.3 & 24 & 3 & 36 & 40. & 39.1 & 2 & & 3 & 49.2 & 45 & & \\
\hline $\begin{array}{l}38 \\
40\end{array}$ & 23.2 & 2 & 53 & 82 & 38 & 43.8 & 42.8 & & 15 & 38 & 36.6 & & & 36 & 3 & 49.2 & & & \\
\hline $\begin{array}{l}40 \\
42\end{array}$ & & & 53 & 28 & 40 & 48.8 & 47.6 & 2308 & 6 & 40 & 27.0 & 23 & 4 & $\mathrm{si}$ & 4 & 45.0 & 41.8 & & \\
\hline 42 & 21.8 & & 55 & & 42 & 57.6 & 56.3 & & & 42 & 20.3 & I8.8 & 235 & & 4 & 44.6 & 40. & & \\
\hline 44 & 29.2 & 28.8 & 44 & -10.0 & 44 & 56.0 & 55.4 & 2256 & -9.2 & 44 & 8.5 & 6.8 & $24 \mathrm{I}$ & -9.0 & 4 & 36.9 & & 23 & -9.0 \\
\hline 46 & 30. I & 29.7 & 42 & a & 46 & 52.0 & 51.0 & 2302 & & $46^{*}$ & 56.0 & 48.0 & & & 4 & 32.3 & & 24 & \\
\hline 48 & $3 I .3$ & 30.9 & $4 \mathrm{I}$ & fis & 48 & 55.0 & 54.5 & 2257 & Me & 48 & 48.8 & 45.6 & I & 36 & 48 & $23 . I$ & & & \\
\hline 50 & 31.6 & & & 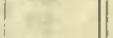 & 50 & 60.4 & 58.6 & 50 & & 50 & 46.2 & 42.6 & 2 & s. & 50 & 21.3 & 16.7 & 20 & \\
\hline 52 & $33 \cdot 3$ & 32.8 & 38 & z & 52 & 63.2 & 61.8 & 45 & & 52 & 46.9 & 44.0 & I & is & 52 & $2 \mathrm{I} .4$ & 17.6 & $24 \mathrm{I}$ & \\
\hline 54 & $34 \cdot 3$ & 34.0 & 36 & $x^{2}$ & 54 & 60.6 & 59.5 & & & 54.1 & 58.0 & 52.8 & 0 & 18 & 54.2 & 34.6 & 30.3 & & \\
\hline $\begin{array}{l}56 \\
58\end{array}$ & $37 \cdot 3$ & 37.3 & 31 & & 56 & 54.8 & 54.3 & 2258 & 38 & 56 & 57.6 & 57.2 & 240 & & & 35.9 & 31.2 & & \\
\hline 50 & 39.4 & 39.3 & . & & 58 & 52.8 & & 2300 & & 58 & 59.3 & 57.6 & & 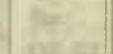 & 58 & $33 \cdot 3$ & 29.9 & 2400 & \\
\hline & & & & & & & & & & & & & & & & & & & \\
\hline
\end{tabular}

Observer-W. J. P.

nbservers - W. J. P. and R. R. T., who alternated from $7 \mathrm{~h} 48 \mathrm{~m}$ to $7 \mathrm{~h} 58 \mathrm{~m}$. 
Tabulation of magnetic declinations observed at Teplitz Bay-Continued

\begin{tabular}{|c|c|c|c|c|c|c|c|c|c|c|c|c|c|c|c|c|c|c|c|c|}
\hline \multicolumn{5}{|c|}{ Wednesday, December 2, 1903} & \multicolumn{6}{|c|}{ Magnet scale inverted } & \multicolumn{5}{|c|}{ Wednesday, December 2, 1903} & \multicolumn{5}{|c|}{ 100 $=$ Magnet scale inverted } \\
\hline \multirow{2}{*}{$\begin{array}{l}\text { Chr'r } \\
\text { time }\end{array}$} & \multicolumn{2}{|c|}{$\begin{array}{l}\text { Scale } \\
\text { readings }\end{array}$} & $\begin{array}{l}\text { East } \\
\text { decli- }\end{array}$ & Temp. & Chr'r & & cale & $\begin{array}{l}\text { Ea } \\
\text { dec }\end{array}$ & $\begin{array}{l}\text { ast } \\
\text { cli- }\end{array}$ & Temp. & Chr'r & $\begin{array}{r}S \\
\text { rea }\end{array}$ & $\begin{array}{l}\text { ale } \\
\text { dings }\end{array}$ & $\begin{array}{l}\text { East } \\
\text { decli- }\end{array}$ & Temp. & & & ile & $\begin{array}{l}\text { East } \\
\text { decli- }\end{array}$ & Temp. \\
\hline & Left & Right & $\begin{array}{c}\text { nation } \\
\end{array}$ & & & Left & Right & & & & & Left & Right & nation & & & L,eft & Right & & \\
\hline h $\mathrm{m}$ & & d & $\circ$, & & h m & & 0 & & & & h m & $d$ & & & & h m & $\mathrm{d}$ & $\mathrm{d}$ & $\circ \quad$ & $\circ$ \\
\hline 800 & 36.8 & 33.4 & 2355 & -8.8 & 10 00 & 64.8 & 62.1 & & & -8.6 & I $200^{*} 3$ & 56.1 & 50.8 & 2246 & -8.7 & 1400 & 51.2 & 49.2 & 2251 & -8.5 \\
\hline 02 & 37.8 & 35.1 & 53 & - & 02 & 66.3 & 64.0 & & 08 & & 02 & 57.1 & 52.9 & 43 & & 02 & $47 \cdot 9$ & 46.7 & 55 & -3 \\
\hline 04 & 45.9 & $4 \mathrm{I} .2$ & 42 & & 04 & 65.9 & 64.0 & & 08 & & 04 & 59.8 & 55.8 & 39 & 3 & 04 & 46.9 & 45.5 & 2257 & 30 \\
\hline 06 & 50.9 & 48.3 & 32 & 3 & 06 & 63.8 & 61.9 & & 12 & & 06 & 59.1 & $55 \cdot 3$ & 40 & 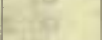 & 06 & 42.2 & 4I. & 2304 & Do \\
\hline 08 & 55.1 & 49.9 & 28 & 8 & 08 & 63.2 & 63.2 & & II & & 08 & 60.0 & 56.1 & 39 & & 08 & 42.3 & $4 \mathrm{~T} . \mathrm{I}$ & 04 & 20 \\
\hline IO & 44.2 & 38.2 & 45 & a & I0 & 62.2 & 6 1.8 & & I3 & & 10 & 59.2 & 55.7 & 40 & & IO & 41.0 & 39.5 & 06 & er \\
\hline 12 & 45.5 & 38.2 & 44 & & I2 & 62.0 & 61.5 & & I3 & & 12 & 58.7 & 54.7 & 4I & & $\mathrm{I} 2$ & 41.7 & 40.3 & 05 & \\
\hline I4 & 38.3 & 33.7 & 54 & -8.7 & I4 & 58.8 & 58.5 & & 18 & -8.6 & 14 & 59.9 & 56.3 & 38 & -8.6 & I4 & 43.8 & 42.5 & 02 & -8.5 \\
\hline I6 & 35.2 & 3 I. 0 & 58 & & I6 & 57.8 & 57.4 & & 20 & & I6 & 59.8 & 56.4 & 38 & 68 & I6 & 44.5 & 43.5 & OI & रे \\
\hline I8 & 38.6 & 32.7 & 54 & tor & I8 & 57.1 & 56.6 & & 21 & & 18 & 57.7 & 54.0 & 42 & & I8 & 42.2 & 4I.I & 04 & \\
\hline 20 & 36.8 & $30 . I$ & 2358 & the & 20 & 56.0 & 55.0 & & 23 & se & 20 & 59.9 & 57.7 & 37 & as & 20 & 40.3 & 39.5 & 07 & ex \\
\hline 22 & 28.0 & 22 . I & 24 II & 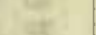 & 22 & 55.0 & 54.5 & & 24 & & 22 & 65.8 & 62.1 & 29 & & 22 & 39. I & 38.3 & 09 & 68 \\
\hline 24 & 20.3 & I7.8 & 20 & int & 24 & 57.7 & 56.1 & & 21 & & 24 & 58.8 & 54.0 & $4 \mathrm{I}$ & & 24 & 40.0 & 39.5 & 07 & in \\
\hline 26 & $24 \cdot 3$ & 20.8 & 2415 & & 26 & 56.0 & 54.8 & & 23 & & 26 & 58.9 & 56.1 & 39 & & 26 & 41.6 & 40 & 05 & \\
\hline 28 & 34.0 & 3I.I & 2359 & & 28 & 56.2 & 55.9 & & 22 & & 28 & 55.2 & 52.4 & 45 & & 28 & 4I. I & 40.4 & 06 & \\
\hline 30 & 20.4 & I5. 3 & 2422 & -8.7 & 30 & 60.9 & 60.2 & & 15 & -8.7 & 30 & 54.1 & $50 . i$ & 48 & -8.7 & 30 & 42.5 & 42.1 & 03 & -8.5 \\
\hline 32 & 29.7 & 25.9 & 2406 & $=$ & 32 & 60.9 & 60.5 & & 15 & & 32 & 51.9 & 47.5 & 52 & 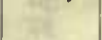 & 32 & 41.0 & 39 & 06 & ( \\
\hline 34 & 37.3 & 34.6 & 2354 & in & 34 & $6 \pi \cdot 3$ & 60.1 & & 15 & & 34 & 50.1 & 46.7 & 54 & & 34 & 37.8 & 37.2 & I I & 4 \\
\hline 36 & 43.7 & 39.1 & 45 & 2 & 36 & 6I.I & 60.2 & & 15 & & 36 & 55.3 & 50.7 & 46 & & $3^{6}$ & 36.8 & 35 & I3 & F \\
\hline 38 & 39.8 & 34.0 & 52 & 8 & $3^{8}$ & 62.1 & 61.7 & & 13 & & 38 & $57 . \mathrm{I}$ & 52.3 & 44 & int & 38 & 36.6 & 35.3 & I3 & 6 \\
\hline 40 & 43.7 & 36.9 & 47 & & 40 & 63.1 & 62.0 & & 12 & & 40 & 54.0 & 49.5 & 48 & Fet & 40 & 34.9 & 33 & 16 & 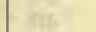 \\
\hline 42 & 45.0 & 38.7 & 44 & & 42 & 63.9 & 63.1 & & II & & 42 & 48.1 & 44.8 & 2257 & & 42 & 34.5 & 33 & 16 & \\
\hline 44 & 47.4 & 40.8 & $234 \mathrm{I}$ & -8.7 & 44 & 63.6 & 62.8 & & II & -8.7 & 44 & 46.2 & 42.7 & 2300 & -8.8 & 44 & 35.3 & 34 & 15 & -8.5 \\
\hline 46 & 33.0 & 29.0 & 2402 & - & 46 & 62.7 & 62.0 & & 12 & & 46 & 44.6 & 40.9 & 2302 & & 46 & 33.3 & 32 & I8 & (15) \\
\hline 48 & 42.9 & 37.8 & 2347 & & 48 & 61.9 & 60.9 & & 14 & & 48 & 47.5 & 43.8 & 2258 & 78 & 48 & 33.0 & 32.3 & I8 & \\
\hline 50 & 49.8 & $44 \cdot 3$ & 36 & a & 50 & 61.8 & 60.3 & & 14 & & 50 & 49.3 & $45 \cdot 3$ & 55 & ne & 50 & 32.4 & 31.6 & 19 & 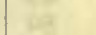 \\
\hline 52 & 55.7 & 48.1 & 29 & & 52 & 62.0 & 60.8 & & 14 & & 52 & 56.1 & 51.7 & 45 & 6 & 52 & 34.8 & 33 & & 31 \\
\hline 54 & 49.9 & 44.0 & 37 & & 54 & 63.1 & 62.1 & & 12 & & 54 & 59.2 & 55.8 & 39 & . & 54 & 34.6 & 34. & I6 & \\
\hline 56 & 52.8 & 46.1 & 33 & & 56 & 66.2 & 65.0 & & 07 & & 56 & 60.7 & 57.2 & 37 & 8 & 5 & & & I5 & \\
\hline 58 & 47.6 & 43.0 & 39 & & 58 & 68.8 & 68.0 & & 03 & & 58 & 58.8 & 56.0 & 40 & & 58 & 37.8 & 36 & 12 & \\
\hline 900 & 49.2 & 44 . I & 37 & -8.7 & II 00 & 67.9 & 67.1 & & 04 & -8.7 & I3 00 & 55.7 & 54.2 & 44 & & 1500 & 38.3 & 37 & 10 & -8.5 \\
\hline 02 & 52.2 & 47.7 & 32 & & 02 & 67.7 & 66.9 & & 04 & . & 02 & 50.7 & 47.8 & 52 & -8.9 & 02 & 38.0 & 37.1 & II & - \\
\hline 04 & 57.9 & 52.8 & 23 & & 04 & 62.8 & 62.6 & & 12 & & 04 & 46.7 & 44.8 & 58 & 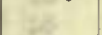 & 04 & 39.9 & 38.7 & 08 & er \\
\hline$\infty 6$ & 59.1 & 54.8 & $2 I$ & & $\infty$ & 64.7 & 64.7 & 23 & 09 & & 06 & 48.3 & 46.2 & 55 & B & $\infty$ & $4 \mathrm{I} \cdot 3$ & 40 & 05 & 6 \\
\hline 08 & 60.7 & 56.9 & I8 & (y) & 08 & 74.6 & 74.1 & 22 & 54 & & 08 & 48.8 & 47.0 & 54 & $\mathrm{f}$ & 08 & $44 \cdot 3$ & 42 & oI & Fet \\
\hline I0 & 58.7 & 56.1 & 20 & & Io & 73.4 & 72.7 & & 56 & & I0 & 53.0 & 50.5 & 48 & ix & To & 45.6 & 43.6 & 2300 & ai \\
\hline 12 & 60.2 & $56 . I$ & I9 & & 12 & 71.0 & 70.8 & 22 & 59 & & 12 & $53 . \mathrm{I}$ & 51.4 & 48 & & 12 & 47.1 & 45.5 & 2257 & \\
\hline I4 & 57.8 & 54.2 & 22 & -8.7 & 14 & 68.6 & 66.0 & 23 & 05 & -8.6 & 14 & 51.7 & & 50 & -9. & I4 & 48 & 46 & & -8.5 \\
\hline I6 & 60.1 & 56.2 & I9 & (1) & I6. 2 & 70.9 & 68.3 & & OI & & 16 & $50 \cdot 3$ & 48.6 & 52 & in & I & 48.0 & 46.1 & 56 & \\
\hline I8 & 61.8 & 57.3 & 17 & & 18 & 66.3 & 63 & & 08 & & 18 & 48.9 & 47.2 & 54 & Fit & I8 & 47 & 45 & 57 & Fit \\
\hline 20 & 61.6 & 56.7 & 17 & a & 20 & 63.3 & 60.4 & se & 13 & & 20 & 45.4 & 44.7 & 59 & cas & 20 & 46.6 & 45.4 & 57 & cos \\
\hline 22 & 63.2 & 58.9 & I4 & & 22 & 64.2 & 62.1 & & II & & 22 & 48.7 & 48. I & 54 & & 2 & 45.6 & 44 & 2259 & \\
\hline 24 & 63.7 & 58.8 & 14 & a & 24 & $64 \cdot 3$ & 62.6 & & II & & 24 & 51.6 & 49.8 & 50 & a & 24 & 45.0 & 44.1 & 2300 & \\
\hline 26 & 67.6 & 62.0 & 08 & & 26 & 65.1 & 63.1 & & 10 & & 26 & 59.6 & 56.1 & 39 & 8 & 2 & 45 & 44. & & \\
\hline 28 & 65.8 & 61.0 & I I & & 28 & 65.7 & 63.9 & & 08 & & 28 & 58.9 & 58.4 & 38 & & 28 & 46.0 & 45 & $5^{8}$ & \\
\hline 30 & 64.7 & 60.1 & 12 & -8.7 & 30 & 66,9 & 65.3 & it & 06 & -8.6 & 30 & 61.1 & 60. & 34 & -9.0 & 30 & 46.5 & & 57 & -8.6 \\
\hline 32 & 64.2 & 60. & I3 & & 32 & 66.3 & 64.9 & & 07 & & 32 & $5^{8} .4$ & 57. & 39 & 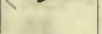 & 3 & 47.2 & 46 & 56 & . \\
\hline 34 & 62.7 & 58.8 & 15 & is & 34 & 71.2 & 69.2 & a & $\infty$ & & 34 & 58.3 & 57 & 3 & & 3 & & 47.1 & 55 & \\
\hline 36 & 63.8 & 60.0 & I3 & & 36 & 63.1 & 62.1 & & 12 & & 36 & 58.7 & 56 & 4 & 8 & 3 & 48 & 47 & 54 & \\
\hline 38 & 63.4 & 60.2 & I3 & 5 & 38 & 62.2 & 60.0 & & 14 & a & 38 & 60.8 & 58.6 & 36 & $x$ & 38 & 49 & 48 & 53 & \\
\hline 40 & 65.3 & 61.4 & II & & 40 & 63.8 & 61.7 & & 12 & & 40 & 61.9 & 60.2 & 34 & in & 40 & 50 & 49 & 52 & \\
\hline 42 & 65.1 & 62.1 & Io & & 42 & 64.7 & 62.3 & & II & & 42 & $65 . I$ & & 29 & & 42 & 53 & & 48 & \\
\hline 44 & 65.1 & 61.0 & II & -8.7 & 44 & 65.1 & 62.3 & & 10 & -8. & 44 & 64.2 & 62.8 & 30 & -8.7 & 44 & 54 & & 46 & -8.7 \\
\hline 46 & 62.8 & 59.7 & 14 & . & 46 & 59.7 & 58.6 & & 18 & $x^{2}$ & 46 & 64.7 & 63.3 & 29 & & 46 & 55.3 & 53.8 & 44 & 1. \\
\hline 48 & 62.7 & 58.8 & 15 & & 48 & 64.1 & 62.9 & & II & & 48 & 62.0 & 60. & 34 & & 48 & 56.3 & 54. & 43 & \\
\hline 50 & 63.9 & 59.2 & I4 & $c^{2}$ & 50 & 68.2 & 66.2 & & 05 & & 50 & 59.3 & 58.2 & 38 & +5 & 50 & 55.7 & 54.3 & 43 & 16 \\
\hline 52 & 64.3 & 61.9 & II & & 52 & 68.8 & 67.0 & & 04 & & 52 & 55.5 & 54.0 & 44 & & 52 & 55.0 & & 45 & \\
\hline 54 & 64.1 & $61 . I$ & 12 & & 54 & 68.2 & 66.9 & 23 & 04 & . & 54 & 52.8 & 51.0 & 48 & ax & 54 & 56.0 & 55 & 43 & \\
\hline 56 & 63.5 & 62.1 & 12 & & 56 & 73.3 & 71.5 & & 57 & & 56 & $5^{1} \cdot 3$ & 49.4 & $5 I$ & tro & 56 & & 54.6 & 43 & \\
\hline 58 & 65.8 & 63.5 & 09 & & 58 & 74.7 & 73.1 & & 54 & 85 & 58 & 51.9 & 49.8 & 50 & 128 & 58 & 56.6 & 55.4 & 42 & E \\
\hline & & 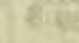 & $1, \phi^{2}$ & & 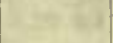 & & & & & & & & & & & & & & & \\
\hline
\end{tabular}


Tabulation of magnetic declinations observed at Teplitz Bay-Continued

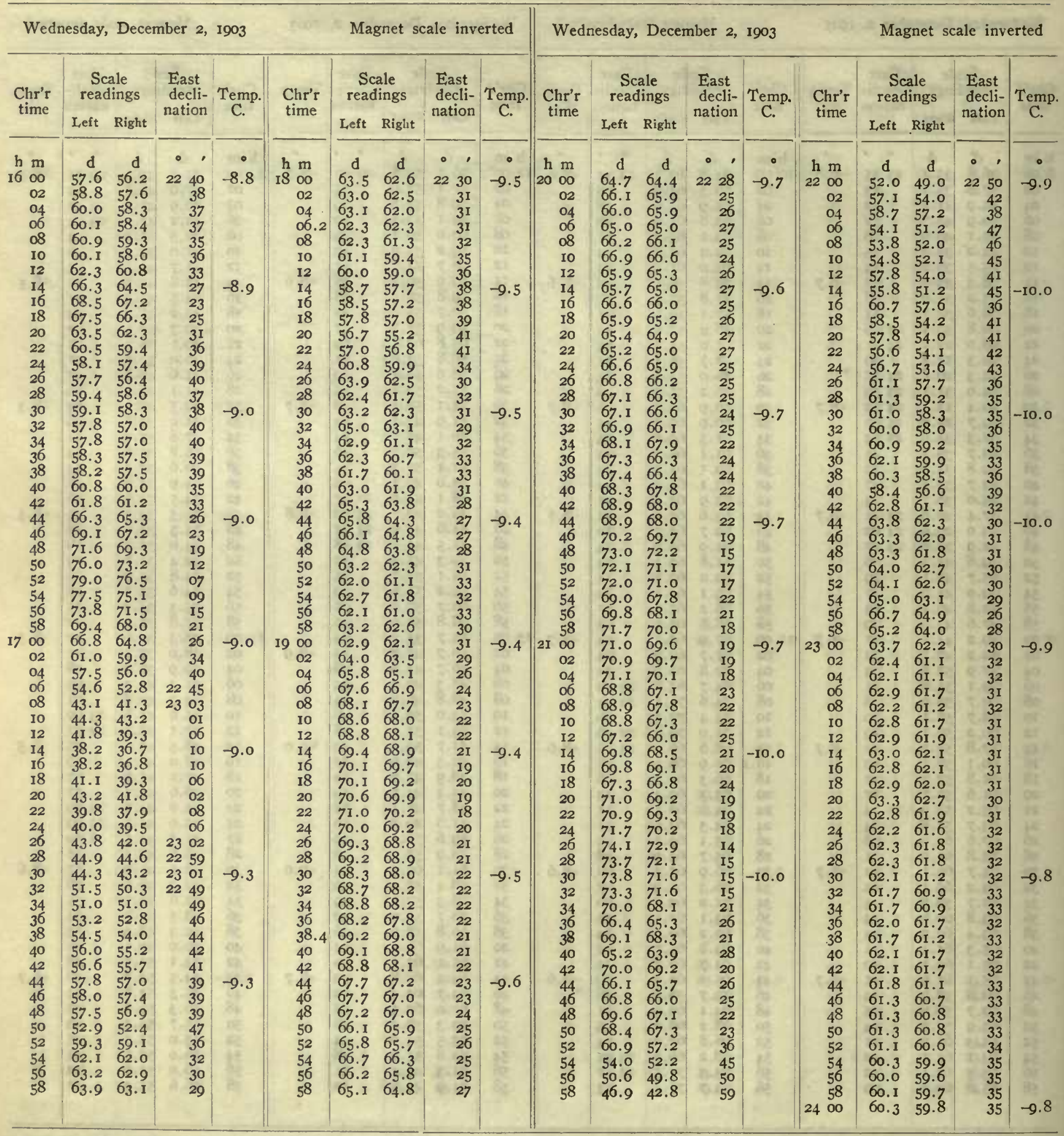

Observers-W. J. P. and R. R. T., who alternated from 18 h $20 \mathrm{~m}$ to I8h $30 \mathrm{~m}$.
Correction to local mean time is - $3 \mathrm{~m}$ 02s.

Torsion head at oh oom read $34 \mathrm{I}^{\circ}$ and at $24 \mathrm{~h}$ 20m read the same. Observer-R. R. T. 
Tabulation of magnetic declinations observed at Teplitz Bay-Continued

\begin{tabular}{|c|c|c|c|c|c|c|c|c|c|c|c|c|c|c|c|c|c|c|c|c|}
\hline \multicolumn{6}{|c|}{ Thursday, Decemer 3, 1903} & \multicolumn{5}{|c|}{ Magnet scale erect } & \multicolumn{6}{|c|}{ Friday, December 4, 1903} & \multicolumn{4}{|c|}{ Magnet scale inverted } \\
\hline \multirow{2}{*}{$\begin{array}{l}\text { Chr'r } \\
\text { time }\end{array}$} & \multicolumn{2}{|c|}{$\begin{array}{l}\text { Scale } \\
\text { readings }\end{array}$} & East & Temp. & Chr'r & $\begin{array}{r}\text { So } \\
\text { reac }\end{array}$ & lings & F & & & Chr'r & $\begin{array}{r}\mathrm{Sc} \\
\text { read }\end{array}$ & $\begin{array}{l}\text { cale } \\
\text { dings }\end{array}$ & East & & Chr'r & $\begin{array}{l}\text { Sc: } \\
\text { read }\end{array}$ & $\begin{array}{l}\text { ale } \\
\text { lings }\end{array}$ & East & Temp. \\
\hline & Left & Right & & & & Left & Right & & & & & Left & Right & & & & Left & Right & & \\
\hline n $\mathrm{m}$ & $\mathrm{d}$ & d & & 。 & $\mathrm{h} \mathrm{m}$ & d & d & • & & - & $\mathrm{h} \mathrm{m}$ & d & d & . ' & . & $\mathrm{h} \mathrm{m}$ & d & d & & $\bullet$ \\
\hline 1600 & $40 . I$ & 42.7 & 2242 & $-5 \cdot 5$ & I8 00 & $34 . I$ & 34.4 & 223 & 31 & -6.6 & 2000 & 53.9 & 52.8 & 2205 & & 2200 & 39.9 & 36.2 & & -9.3 \\
\hline 02 & 39.3 & 41.8 & 41 & & 02 & $35 . \mathrm{I}$ & 35.5 & & & & O2* & 54.7 & 53.9 & 2203 & -10.0 & 02 & 38.0 & $34 \cdot 3$ & & 5 \\
\hline 04 & $39 . \mathrm{I}$ & 41.2 & 4 & & 04 & 36.2 & 36.7 & & 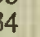 & & 04 & $57 \cdot 3$ & 56.8 & 2159 & & 04 & 43.7 & 38.8 & 3 & ys \\
\hline 06 & 38.1 & 39.9 & & $\mathrm{~N}$ & 06 & 36.8 & 37.0 & & & 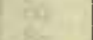 & 06 & 58.1 & 57.0 & 58 & 45 & 06 & 56.3 & 52.3 & I & 7 \\
\hline 08 & 36.8 & 39.8 & 3 & 26 & 08 & 36.5 & 36. & & 35 & $b^{2}$ & 08 & 59.0 & 58.2 & 56 & 10 & 08 & 60.9 & 58.3 & & 80 \\
\hline Io & 37.1 & 39.9 & 38 & es. & IO & 36.2 & 36.2 & & 34 & $(10)$ & I0 & 70.2 & 69.2 & 39 & a․ & IO & 65.2 & 62.5 & & bl \\
\hline 12 & 37.1 & 39.8 & 37 & & 12 & 35.7 & 35.9 & & 33 & 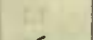 & $12^{*}$ & 49.5 & 45.2 & 30 & 51 & I2 & 63.2 & 62.7 & 0 & $2 \pi$ \\
\hline 14 & 36.0 & 38.2 & 36 & -5.8 & I4 & 34.9 & 35. & & 32 & -6.5 & 14 & 54.8 & $50 . I$ & 22 & -9.7 & I4 & 66.9 & 63.0 & & -9.3 \\
\hline I6́ & 35.8 & 37.9 & 35 & & 16 & $35 \cdot 3$ & 35 & & & & 16 & 58.8 & 57.8 & $21 \quad 12$ & & 16 & 64.2 & 60.9 & 0 & -8 \\
\hline 18 & 36.8 & 38.9 & 37 & 4 & I8 & 35.1 & 35 & & & 41 & I8 & 69.6 & 64.2 & 2059 & Ba & I8 & 66.0 & 62.3 & 220 & 4 \\
\hline 20 & 36.3 & 38.3 & 36 & 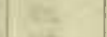 & 20 & 35.7 & 35 & & & as & 20 & 71.0 & 65.9 & 57 & 06 & 20 & 67.2 & $64 . I$ & 215 & 62 \\
\hline 22 & 35.3 & 37.6 & 34 & 23 & 22 & 36.3 & 36. & & 44 & 54 & 22 & 73.4 & 65.0 & 2055 & $\Leftrightarrow$ & 22 & 66.8 & 64.3 & 215 & nat \\
\hline 24 & $35 . I$ & 37.0 & 34 & $1=$ & 24 & 36.8 & 36 & & & 5 & 24 & 65.4 & 55.8 & 2109 & 15 & 24 & 64.0 & 61.9 & 220 & 14 \\
\hline 26 & 34.9 & 36.6 & I & 2 & 26 & 35.8 & 35. & & & 38 & 26 & $37 . \mathrm{I}$ & 30.2 & 2151 & 15 & 26 & 67.2 & 64.6 & 215 & $\therefore$ \\
\hline 28 & 35.3 & 36.9 & 34 & & 28 & 35.1 & 35 . & & 2 & & $28^{*}$ & 75.8 & 43.5 & 22 I9 & as & 28 & 68.8 & 66.3 & 5 & tes \\
\hline 30 & 34.6 & 36.1 & 33 & -6.0 & 30 & 34.9 & 34.9 & & 2 & -6.4 & $30^{*}$ & 64.9 & I4. I & 2415 & -9.5 & 30 & 69.5 & 67.8 & 5 & -9.3 \\
\hline 32 & 35.1 & 36.7 & 34 & & 32 & 34.7 & 34 & & 2 & & $33^{*}$ & 51.2 & $44 \cdot 3$ & 2202 & 20 & 32 & 68.0 & 66.2 & 5 & 7 \\
\hline 34 & 35.6 & 36.9 & 34 & art & 34 & 33.8 & 33 & & 0 & 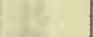 & 34. & 42.2 & 26.7 & $22 \quad 23$ & tes & 34 & 68.4 & 66.5 & 5 & 16 \\
\hline 36 & 35.9 & 37.1 & 35 & & 36 & $33 \cdot 3$ & 33. & & 10 & & $37^{*}$ & 59.5 & I9.8 & 2144 & $x$ & 36 & 69.2 & 66.2 & s & nes \\
\hline 38 & 34.8 & 36.1 & 33 & & 38 & 34.2 & 34 & & & & 38.2 & 78.2 & 40.7 & 2113 & net & 38 & 68.1 & 64.0 & $x$ & the \\
\hline 40 & $35 \cdot 3$ & $36 \cdot 3$ & 33 & t & 40 & $34 . I$ & $34 . I$ & & I & & $40^{*}$ & 34.8 & 14.2 & 2342 & wat & 40 & 70.9 & 64.0 & 215 & 18 \\
\hline 42 & 35.6 & 36.8 & 34 & & 42 & 33.3 & 33 & & 0 & 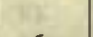 & 42 & 60.3 & 30.1 & 09 & 6 & 42 & 57.1 & 5 I.9 & 221 & 58 \\
\hline 44 & 35.8 & 36.7 & 34 & -6.2 & 44 & 33.8 & 33 & & 0 & -6.4 & 44 & 63.2 & 36.8 & 02 & -9.2 & 44 & 49.9 & 43.8 & 2 & -9.3 \\
\hline 46 & 35.8 & 36.8 & 34 & $3^{5}$ & 46 & 33.8 & 33 & & & & $46^{*}$ & 63.2 & 23.4 & 04 & & 46 & 56.8 & 50.5 & I & 20 \\
\hline 48 & 35.2 & 36.1 & 33 & 하잔 & 48 & 32.4 & 33 & & & 6 & $48 * 5$ & 55.4 & 20.5 & 58 & 14 & 48 & 56.3 & 51.3 & I & (8) \\
\hline 50 & 5.9 & 36.8 & 34 & 8 & 50 & 2.3 & & & 8 & 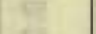 & 50 & 78.3 & $4 \mathrm{I} \cdot 3$ & 24 & 98 & 50 & 57.8 & 51 & I & 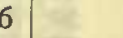 \\
\hline 52 & 6.2 & $37 . I$ & 35 & (x) & 52 & 32.9 & 33 & 2 & 9 & 4 & $52^{*}$ & 67.8 & 16.3 & $2 \mathrm{I}$ & 6 & 52 & 55.0 & & & 8 \\
\hline 54 & 36.8 & 37.6 & 36 & $x^{2}$ & 54 & 33.8 & 33 & & & $P^{2}$ & 54 & 50.8 & I8. I & 2333 & 15: & 54 & 48.6 & 48.0 & 2 & $t$ \\
\hline 56 & 35.9 & 36.5 & 34 & & 56 & 32.1 & 32.8 & & 8 & 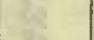 & $56^{*}$ & 76.8 & $33 . I$ & 2233 & 18? & 56 & 40.0 & 37.8 & 4 & I \\
\hline 58 & 34.6 & 35.2 & 32 & & 58 & I.I & $3 \mathrm{I} .2$ & & 6 & 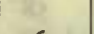 & $\quad 58.2$ & 66.3 & 31.2 & 2243 & 的 & $5^{8}$ & 28.8 & 27.8 & 225 & 10 \\
\hline I7 00 & 34.2 & 35.0 & 32 & -6.3 & I9 00 & 31.2 & $3 \mathrm{I} .2$ & & 6 & -6.5 & $2100^{*}$ & 46.3 & 16.1 & 2338 & -9.0 & 2300 & 21.0 & 12.1 & $23 I$ & -9.3 \\
\hline 02 & 4.6 & 35.2 & 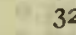 & & 02 & 31.2 & 3I. & & 6 & & 02 & 54.8 & 15.9 & 32 & & 02 & 12.5 & 10.8 & 2 & \\
\hline 04 & 34.2 & 34.9 & 31 & Zn & 04 & $3 \mathrm{I} .9$ & $3 \mathrm{I} .9$ & & 27 & [? & 04 & 66.4 & 34.7 & 08 & 16 & $04^{*}$ & 59.7 & 47.7 & 3 & 16 \\
\hline$o$ & 4.2 & 34.9 & 3 & 90 & 06 & 1.9 & & & & as & 06 & 45 & 13.8 & $234 \mathrm{I}$ & 20 & 06 & 71.2 & & $23 \mathrm{I}$ & 82 \\
\hline 08 & 34.1 & 34.8 & 3. & foy & 08 & 32.3 & 32.5 & & 8 & ne. & $08 *$ & 35.8 & 9.3 & 24 I0 & (a) & $08^{*}$ & 65.8 & 53.7 & 224 & (6) \\
\hline 10 & 2.4 & 33.2 & & (7) & & 33. & $9 a$ & 3 & 0 & Br & 10 & 38.0 & 15.6 & 2403 & ats & Io & 57.6 & & 230 & 매 \\
\hline 12 & 2.4 & 33. I & 20 & & I2 & 34.2 & $34 \cdot 4$ & 3 & $\mathbf{I}$ & & 12 & 70.3 & 51.0 & 23 IO & at & 12 & 48.5 & 38.2 & $23 \mathrm{I}$ & -9.2 \\
\hline I 4 & 3.2 & 33.8 & 3 & -6.4 & I4 & 34.7 & 34.9 & 3 & 2 & -6.7 & 14 & 70. & 50.1 & 2310 & -9.0 & I4 & 70.1 & & 224 & \\
\hline 16 & 3.9 & $34 \cdot 3$ & 3 & & 16 & $3 \cdot 3$ & 33.4 & 3 & & & $16^{*}$ & 41.9 & 19.5 & 2254 & & $16^{*}$ & 46.2 & 29.6 & I & at \\
\hline 18 & 4.6 & 35 . & 3 & 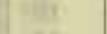 & 18 & 2.1 & 32 & 2 & & & 18 & $43 . \bar{I}$ & 22.1 & & e & I8 & & 23.6 & 2 & (bit \\
\hline 20 & H.I & 34.8 & 31 & nt & 20 & 1.5 & 31.9 & 2 & & 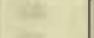 & 20 & 43. & 23.5 & 50 & 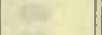 & 20 & 38.0 & 25.2 & 2 & 15 \\
\hline 22 & 3.8 & 34.2 & 31 & 27 & 22 & 1. 6 & 31.9 & 2 & & 3 & 22 & & 29.6 & 40 & c5: & 22 & I & 19.2 & 3 & tiz \\
\hline 24 & 3.7 & $34 . \mathrm{I}$ & 3 & 13 & & 2.2 & 32. & 2 & 8 & te & 2 & 53. & 34.6 & 33 & $y=$ & 24 & 20.8 & 9. & & $1=$ \\
\hline 26 & 3.8 & 34.2 & 31 & 18 & 26 & $3 \mathrm{I} .2$ & $32 . I$ & 2 & & & $2 t$ & 52.3 & 33.8 & 35 & 4 & $26^{*}$ & 38.5 & 29. & 5 & $x$ \\
\hline 28 & f.I & 34.7 & & & 2 & 1.7 & 32. & 2 & & & 28 & 55 & & 28 & $=$ & 28 & 54.0 & 48.0 & (1) & 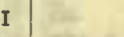 \\
\hline 30 & 3.3 & 33.9 & 30 & -6.5 & 30 & 31.9 & 32.0 & 2 & & -6.7 & 30 & 49.3 & 46.9 & 27 & -9.1 & 30 & 50.9 & 46.5 & 223 & -9.3 \\
\hline 32 & 1.8 & 35.1 & & & 32 & II & & 2 & & & 3 & & & 26 & 2 & 32 & 20.3 & 14.6 & & \\
\hline 34 & 3.7 & 34.2 & 3 & 12 & 34 &.$I$ & 29 & 2 & & 18 & 3 & 64.2 & & 2204 & & 34. & $4 \mathrm{I} \cdot 3$ & 29.0 & 22 & 16 \\
\hline 36 & & & & \& & & 3.9 & & 2 & & & 36 & & 68.8 & & & & & & 24 & \\
\hline 38 & 2.6 & 33.9 & 3 & 2 & 38 & 29.0 & 29.2 & 2 & & & 38 & 68.2 & $64 . I$ & 2158 & A & $38^{*}$ & 61.1 & 52.3 & 232 & nc \\
\hline 40 & 3.9 & & 3 & os & 4 & 9.9 & 30.1 & 2 & & 94 & 40 & 51.9 & 47.6 & 2224 & 34 & 40 & 68.9 & 59.7 & 1 & 션 \\
\hline 42 & .3 & 34.8 & 3 & & 42 & 29.9 & 30.0 & 2 & & & 42 & 47.3 & 45.2 & 30 & $x^{2}$ & 42 & 45.9 & 35.8 & (A) & w \\
\hline 44 & $34 . I$ & 34.6 & 31 & -6.6 & 44 & 30.0 & 30.2 & 2 & 5 & -66 & 44 & 43.2 & 40.4 & 37 & -9.2 & 44 & 71.1 & 55.0 & I & -9.3 \\
\hline 46 & & 34.4 & 3 & & 46 & 29.2 & 29.7 & 2 & & & 46 & 37.1 & 34.7 & 46 & & $46^{*}$ & 41.0 & 20.6 & & \\
\hline 48 & & 35 & 3 & 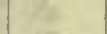 & 48 & 29.0 & 29. & 2 & & & 48 & & 33.3 & 49 & 37 & $48 * 3$ & 39.4 & 21.0 & & 4 \\
\hline 50 & & 35.7 & 3 & 108 & 50 & 9.0 & 29.2 & 2 & & 18 & 50 & & 37.9 & $4 \mathrm{I}$ & n: & 50.2 & $3 \mathrm{I} \cdot 5$ & 12.3 & & 9 \\
\hline 52 & & 34.4 & 3 & 28 & 52 & 29.2 & 29.5 & 2 & & 말 & 52 & 38.2 & $37 . \mathrm{I}$ & 43 & 32 & 52 & 36.8 & 21.2 & & $6 t$ \\
\hline 54 & 33.7 & 33 & 3 & 82 & 54 & 29.8 & 30.2 & & & 13 & 54 & 39.8 & 35.6 & 43 & $t^{3}$ & 54. & 37.1 & 19.8 & 23 & ks \\
\hline & 33.0 & & 29 & $=$ & 56 & 30.7 & 30.9 & 2 & 6 & & 56 & 35.0 & 3I.I & 50 & $6=$ & $56^{*}$ & 60.3 & 39.0 & & \\
\hline 58 & $33 \cdot 5$ & 33.8 & 30 & . & 58 & 32.1 & 32.3 & & & 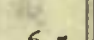 & 58 & 39.8 & 35.7 & 43 & \& & $58^{*} 2$ & 42.8 & 21.7 & & t] \\
\hline & & & & & 2000 & 31.8 & $32 . \mathrm{I}$ & & & & & & & & & & 31.2 & 9.3 & & -9.3 \\
\hline
\end{tabular}

Correction to local mean time is - IOS.

Torsion head at $15 \mathrm{~h} 15 \mathrm{~m}$ read $344^{\circ}$ and at $20 \mathrm{~h} 241 \mathrm{n}$ read the same. Obscrver-R. R. T.
Correction to local mean time is $-22 \mathrm{~s} .90^{\circ}$ torsion $=23^{\prime} 3$.

Torsion head at $19 \mathrm{~h} 15 \mathrm{~m}$ read $324^{\circ}$ and at $24 \mathrm{~h} 15 \mathrm{~m}$ read $333^{\circ}$.

Observer-R. R. T. 
Tabulation of magnetic declinations observed at Teplitz Bay-Continued

\begin{tabular}{|c|c|c|c|c|c|c|c|c|c|c|c|c|c|c|c|c|c|c|c|}
\hline \multicolumn{6}{|c|}{ Sunday, December 6, 1903} & \multicolumn{4}{|c|}{ Magnet scale erect } & \multicolumn{5}{|c|}{ Sunday, December 6, 1903} & \multicolumn{5}{|c|}{ Magnet scale inverted } \\
\hline \multirow{2}{*}{$\begin{array}{l}\text { Chr'r } \\
\text { time }\end{array}$} & \multicolumn{2}{|c|}{$\begin{array}{l}\text { Scale } \\
\text { readings }\end{array}$} & \multirow{2}{*}{$\begin{array}{c}\text { East } \\
\text { decli- } \\
\text { nation }\end{array}$} & Temp. & Clir'r & $\begin{array}{l}\text { Sca } \\
\text { read }\end{array}$ & $\begin{array}{l}\text { ale } \\
\text { lings }\end{array}$ & $\begin{array}{l}\text { East } \\
\text { decli- }\end{array}$ & Temp. & Chr'r & $\begin{array}{l}\mathrm{Sc} \\
\text { read }\end{array}$ & $\begin{array}{l}\text { ale } \\
\text { dings }\end{array}$ & East & Temp. & Clir'r & $\begin{array}{l}\text { Sca } \\
\text { read }\end{array}$ & $\begin{array}{l}\text { ale } \\
\text { lings }\end{array}$ & $\begin{array}{l}\text { East } \\
\text { decli- }\end{array}$ & Temp. \\
\hline & L,eft & Right & & C. & time & L,eft & Right & & C. & time & Left & Right & natior & & time & Left & Right & nation & \\
\hline $\mathrm{h} \mathrm{m}$ & d & d & - & $\bullet$ & $h \mathrm{~m}$ & d & d & $\circ$, & 。 & $\mathrm{h} \mathrm{m}$ & d & $\mathrm{d}$ & & - & $\mathrm{h} \mathrm{m}$ & d & $\mathrm{d}$ & & $\circ$ \\
\hline $000^{*}$ & 27.0 & 42.6 & 2449 & -14.6 & 200 & 18.5 & 18.9 & 2300 & -11.0 & 400 & Lo & & & & 600 & 43.6 & 40.3 & 2247 & -10.0 \\
\hline & 21.0 & 32.6 & 2308 & 7. & 02 & 18.8 & 19.3 & 2300 & & $\mathrm{O} 2^{*}$ & 38.5 & 38.0 & 2252 & -9.5 & 02 & 46.0 & $43 \cdot 3$ & 42 & \\
\hline 04 & $13 \cdot 3$ & 26.3 & 2257 & jar & 04 & .16 .8 & 17.0 & 2257 & 10 & 04 & 38.6 & 38.1 & & & 04 & 43.0 & 41.0 & 46 & 36 \\
\hline 06 & 24.3 & 35.0 & 2313 & 60 & 06 & 13.0 & I3. 3 & 51 & $x^{2}$ & 06 & 36.8 & 36.0 & 2255 & 30 & 06 & $44 \cdot 3$ & 42.3 & 44 & \\
\hline $08^{*}$ & $4 \mathrm{I} .0$ & 61.8 & 26 & 20 & 08 & 10.4 & 10.8 & 47 & 40 & 08 & 32.3 & 32.0 & 2302 & & 08 & 44.0 & $42 . I$ & 45 & W. \\
\hline 10 & 54.0 & $61 \cdot 3$ & 2335 & ate & 10 & 9.8 & 10.1 & 46 & 24 & I0 & 27.3 & 27.3 & 09 & 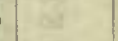 & 10 & 43.2 & 42.0 & 45 & $=1$ t \\
\hline $12^{*}$ & 21.4 & 38.3 & 2404 & (2) & 12 & 8.6 & 9.0 & 44 & $7=$ & 12 & 24.3 & 23.3 & 15 & ty & 12 & 44.1 & 41.0 & 45 & \\
\hline 14 & 7.0 & 20.6 & 2339 & -13.8 & 14 & I0.0 & 10.3 & 46 & -10.6 & I4 & 22.5 & 22.2 & 17 & -9.4 & 14 & 40.3 & 37.7 & 51 & -10.0 \\
\hline $16^{*}$ & 30.0 & 47.4 & 31 & & 16 & 12.0 & 12.3 & 49 & & 16 & 23.5 & 23.0 & 16 & & 16 & 42.0 & 39.8 & 48 & \\
\hline 18 & 36.2 & 55.0 & 42 & 74 & 18 & 12.7 & I3.0 & $5 I$ & bit & I8 & 29.3 & 29.1 & 2306 & 6 & 18 & 43.1 & $4 \mathrm{I} .5$ & 46 & $8 \mathrm{t}$ \\
\hline 20 & 36.5 & 38.5 & 29 & $x=$ & 20 & 13.7 & I3.9 & 52 & is & 20 & 35.0 & $34 . I$ & 2258 & 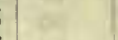 & 20 & 45.8 & 45.0 & $4 I$ & 05 \\
\hline 22 & 38.0 & 40.2 & $3^{2}$ & 28 & 22 & $15 \cdot 3$ & 15.5 & 55 & $3=$ & 22 & 35.3 & 34.3 & 58 & $\mathrm{ev}$ & 22 & 46.9 & 46.1 & 39 & ss \\
\hline 24 & 37.8 & 40.0 & 31 & - & 24 & 16.3 & 17.0 & 57 & th & 24 & 36.3 & 35.7 & 56 & $\mathrm{k}$ & 24 & 49.6 & 48.9 & 35 & is \\
\hline 26 & 40.5 & 42.6 & 36 & & 26 & 17.5 & 18.0 & 58 & 15 & 26 & 36.7 & 35.9 & 55 & 86 & 26 & 47.5 & 46.1 & 39 & \\
\hline 28 & 47.6 & 48.5 & 46 & & 28 & 15.4 & 15.6 & 55 & & 28 & $3^{8} .3$ & 37.0 & 53 & 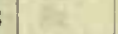 & 28 & 46.1 & 45.0 & $4 \mathrm{I}$ & 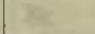 \\
\hline 30 & 53.9 & 57.5 & 58 & -13.0 & 30 & 16.7 & 16.8 & 57 & -10.3 & 30 & 37.8 & 37.0 & 53 & -9.5 & 30 & 46.3 & 44.7 & 41 & -10.2 \\
\hline 32 & 53.8 & 56.7 & 57 & ra & $3^{2}$ & 16.4 & I6. 8 & 57 & ( & 32 & 39.5 & 38.3 & 51 & 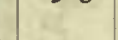 & 32 & 40.7 & 40.3 & 49 & 121 \\
\hline 34 & 48.4 & 48.6 & 47 & k & 34 & I8.0 & 18.3 & 2259 & 38 & 34 & 39.5 & 37.6 & 52 & 12 & 34 & $4 I .3$ & 40.5 & 48 & \\
\hline 36 & 46.2 & 47.7 & 44 & ac & 36 & 18.4 & 18.6 & 2300 & twa & 36 & 40.3 & 38.7 & 50 & & 36 & 39.8 & 38.8 & 51 & \\
\hline $3^{8}$ & $43 \cdot 3$ & 46.2 & 41 & 3 & 38 & I7.9 & I8. I & 2259 & ine & 38 & 42.3 & 40.9 & 47 & 6 & 38 & 35.6 & 35.3 & 57 & \\
\hline 40 & 35.9 & 36.5 & 27 & 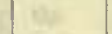 & 40 & 20,0 & 20.7 & 2302 & $\sqrt{3}$ & 40 & 44.8 & 43.5 & 43 & E & 40 & 37.9 & 37.0 & 54 & 74 \\
\hline 42 & 45. & oa & 41 & & 42 & 20.5 & 20.9 & 03 & $16=$ & 42 & 46.8 & 45.6 & 40 & L4 & 42 & 42.6 & $4^{1} \cdot 5$ & 46 & \\
\hline 44 & 28 &.$o b$ & I4 & -12.5 & 44 & 19.6 & 20.2 & 02 & -10.2 & 44 & 45.3 & 44.6 & 42 & -9.5 & 44 & 39.9 & 37.8 & 51 & -10.3 \\
\hline 46 & 22.5 & 23.6 & 07 & & 46 & 20.9 & 21.3 & 04 & & 46 & 46.2 & $45 \cdot 5$ & 40 & & 46 & 38.0 & 35.8 & 54 & \\
\hline 48 & 27.3 & 28.0 & Is & 5 & 48 & 21.0 & 21.3 & 04 & 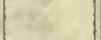 & 48 & 42.5 & 41.3 & 46 & 8 & 48 & 39.8 & 38.2 & $5 I$ & 48 \\
\hline 50 & 29.6 & 30.6 & 18 & 02 & 50 & 21.7 & 21.8 & 05 & & 50 & 40.2 & 38.3 & 51 & (19) & 50 & 44.5 & 41.6 & 45 & \\
\hline 52 & 33.7 & 34.3 & 24 & 28 & 52 & 21.3 & 21.3 & 04 & 46 & 52 & 37.0 & 34.5 & 5 & ${ }^{2}$ & 52 & 42.6 & 39.5 & 48 & 28 \\
\hline 54 & 39.1 & 40.6 & 33 & $\mathrm{~K}$ & 54 & 19.7 & 20.0 & 2302 & WI & 54 & 36.5 & 33.9 & 225 & $x^{2}$ & 54.3 & 42.5 & $4 I \cdot 3$ & 47 & \\
\hline 56 & 35.3 & 37.2 & 27 & & 56 & 17. & $6 b$ & 2258 & & 56 & $34 \cdot 3$ & 32.6 & 23 oc & $d z$ & 56 & 43.3 & 42.3 & 45 & \\
\hline 58 & 34.9 & 37.1 & 27 & 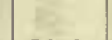 & 58 & 14.6 & 14.6 & 54 & & 58 & 28.6 & 26.0 & o & & 58 & 44.1 & 42.6 & 44 & \\
\hline I 00 & 29.0 & 31.0 & 18 & -12.0 & 300 & 14.0 & I $4 . I$ & 53 & -I0.0 & 500 & 25.9 & 23.9 & I & -9.6 & 700 & $45 . I$ & 43.9 & 42 & -10.6 \\
\hline 02 & 20.4 & 24.0 & 05 & 32 & 02 & 15.2 & 15.5 & 55 & $8=$ & 02 & 24.6 & $23 \cdot 3$ & $I$ & 20 & 02 & 45.0 & $43 \cdot 5$ & 43 & 60 \\
\hline 04 & 25.7 & 29.2 & 13 & 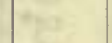 & 04 & 15.5 & I 5.5 & 55 & 72 & 04 & 24.8 & 22.2 & $I$ & $3 x$ & 04 & $47 \cdot 3$ & 44.5 & 40 & 3it \\
\hline 06 & 30.2 & $3 I .6$ & Ig & $8 \%$ & 06 & 15.0 & 15.0 & 54 & 10 & 06 & 21.1 & 19.7 & 20 & 21 & 06 & 47.0 & 44.8 & 40 & \\
\hline 08 & 25.0 & 26.3 & II & $y=$ & 08 & 14.7 & 15.0 & 54 & $n$ & 08 & 27.3 & 25.1 & I & 80 & 08.3 & 48.6 & 47.2 & 37 & 65. \\
\hline I0 & 26.3 & 27.7 & I3 & $\tan$ & I0 & 13.7 & 13.9 & 52 & $3 x$ & I0 & 29.8 & 29.1 & $23 \circ$ & ef & I0 & 52.3 & 50.7 & 31 & as \\
\hline 12 & 26.2 & 28.2 & 13 & & 12 & 15.0 & 15.5 & 54 & & 12 & 35 & $.0 a$ & 225 & & I2 & 50.9 & $49 \cdot 3$ & 34 & \\
\hline 14 & 30.3 & 32.7 & 20 & -II.9 & I4 & 18.0 & 18.5 & 59 & -10.0 & $I_{4}$ & 44.0 & 43.2 & 224 & -9.8 & 14 & 51.7 & 50.7 & 32 & -11.0 \\
\hline I6 & 35.1 & 36.7 & 27 & $x^{2}$ & 16 & 16.3 & 16.7 & 56 & & I6. 2 & 33.0 & 31.0 & 230 & (2) & I6 & 46.8 & 45.4 & 40 & \\
\hline 18 & 29.5 & 30.1 & Ij & |ff & 18 & 14.0 & 14.3 & 53 & it & 18 & 27.9 & 25.9 & IS & 84 & I8 & 48.8 & 48.1 & 36 & \\
\hline 20 & 26.7 & 27.3 & $I_{3}$ & 36 & 20 & 14.3 & 15.0 & & +16 & 20 & 21.2 & 22.6 & I & in & 20 & 49.5 & 47.2 & 36 & is \\
\hline 22 & 30.6 & 30.9 & Ig & $E$ & 22 & 17.3 & 17.7 & 2258 & te & 22 & 23.4 & 22.6 & I & at & 22 & 42.6 & 40.7 & 47 & 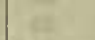 \\
\hline 24 & 29.5 & 30.2 & I7 & ate & 24 & 18.6 & 19.4 & 2300 & 64 & 24 & 22.3 & 21.8 & IS & E & 24 & 50.3 & 48. I & 35 & \\
\hline 26 & 30.3 & 31.5 & 23 IS & Ex & 26 & 20.0 & 20.4 & 2302 & $x^{2}$ & 26 & 31.8 & 30.6 & 0 & $\theta$ & 26 & 43.9 & 40.5 & 46 & \\
\hline 28 & 18 & $5^{b}$ & 225 & 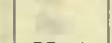 & 28 & 17.0 & 17.7 & $225^{8}$ & & 28 & 32.5 & 31.5 & 230 & $b^{2}$ & 28 & 44.6 & 42.0 & 44 & \\
\hline 30 & I3. I & 13.3 & 225 & -II. 4 & 30 & 16.6 & 17.0 & 57 & -9.9 & 30 & 39.5 & 39.0 & 225 & -9.9 & 30 & 44.9 & 43.1 & 43 & -II.O \\
\hline 32 & 19. & $5 a$ & 230 & 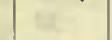 & 32 & 15.2 & 15.7 & 55 & - & 32.3 & 42.3 & 40.8 & 4 & $x^{2}$ & 32 & $44 \cdot 3$ & 40.9 & 45 & (n) \\
\hline 34 & 22.7 & 23.3 & of & we & 34 & 15.0 & $15 \cdot 3$ & 54 & ine & 34 & 42.8 & 40.4 & 4 & k. & 34 & 46.0 & 41.8 & 43 & \\
\hline 36 & 18.7 & 20.3 & 2301 & Fin & 36 & 14.0 & 14.0 & 52 & 2 & 36 & 38.8 & 38.5 & 5 & & 36 & 48.6 & 45.9 & 38 & \\
\hline 38 & $17 \cdot 3$ & 18.0 & $225 \delta$ & 176 & 38 & I3.6 & I3.9 & 52 & 8. & 38 & $39 . \mathrm{I}$ & 38.3 & 225 & 2 & 38 & 41.3 & 38.3 & 50 & E \\
\hline 40 & 17.6 & 18.6 & 5 & ras & 40 & 13.0 & 13.1 & 51 & (1) & 40 & 30.6 & 30.2 & 230 & e & 40 & 41.2 & 39.5 & 49 & \\
\hline 42 & I6. 4 & I7. 3 & 57 & & 42 & 13.0 & 13.0 & $5 I$ & & 42 & 28 & $.3 a$ & $23 a$ & & 42 & 41.5 & 40.3 & 48 & \\
\hline 44 & I5.3 & 16.3 & 5 & -II.I I & 44 & 13.2 & 13.7 & 51 & -9.8 & 44 & 35.2 & 33.8 & 225 & $3-10.0$ & 44 & $42 . \overline{8}$ & 40.5 & 47 & $-I I .0$ \\
\hline 46 & I7. 4 & 18.4 & 58 & 10 & 46 & 15.0 & 15.5 & 54 & & 46 & 44.6 & 44.3 & 4 & & 46 & 45.3 & 42.8 & 43 & \\
\hline 48 & I 4.4 & 15.3 & 5 & 28 & 48 & 15.3 & 15.6 & 55 & 8 & 48 & $42 . I$ & 41.3 & 4 & 28 & 48 & 48.2 & 47.0 & 38 & \\
\hline 50 & I5.8 & 17.0 & 5 & 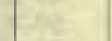 & 50 & I3. 3 & I3.6 & 51 & 52 & 50 & 4 I. 6 & 40. I & 4 & iat & 50 & 45 & $6 b$ & $4 I$ & Q \\
\hline 52 & 17.6 & I8.6 & 5 & $2=$ & 52 & II. 4 & 12.0 & 49 & 34 & 52 & 38.6 & 36.3 & 5 & ser & 52 & 46.7 & 46.3 & 39 & +2 \\
\hline 54 & 18.0 & 18.8 & 2255 & is & 54 & 13.0 & 13.4 & 51 & tat & 54 & 40.0 & 38.0 & 5 & 1) & 54 & 50.3 & 49.8 & 34 & \\
\hline 56 & Ig. 2 & 20.2 & 23 o. & 0 & 56 & 13.4 & 14.0 & 52 & 18 & 56 & 40.6 & 37.8 & 5 & & 56 & 44.3 & 42.2 & 44 & \\
\hline 58 & I6́.6 & 17.3 & 225 & tos? & 58 & I3.8 & 14.0 & 52 & 93 & 58 & $4 I .5$ & 37.8 & 5 & $z=$ & 58 & $45 . I$ & 44.0 & 42 & \\
\hline 6.67 & orise & S. & Lit & On $x$ & & & & & & & 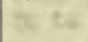 & . & & wits & 800 & 44.0 & 40.0 & 46 & -II.0 \\
\hline
\end{tabular}


Tabulation of magnetic declinations observed at Teplitz Bay-Continued

\begin{tabular}{|c|c|c|c|c|c|c|c|c|c|c|c|c|c|c|c|c|c|c|c|c|}
\hline \multicolumn{5}{|c|}{ Monday, December 7, 1903} & \multicolumn{5}{|c|}{ Magnet scale erect } & \multicolumn{5}{|c|}{ Tuesday, December 8, 1903} & \multicolumn{6}{|c|}{ Magnet scale inverted } \\
\hline \multirow{2}{*}{$\begin{array}{c}\text { Chr'r } \\
\text { time }\end{array}$} & \multicolumn{2}{|c|}{$\begin{array}{l}\text { Scale } \\
\text { readings }\end{array}$} & $\begin{array}{l}\text { East } \\
\text { decli- }\end{array}$ & Temp. & Chr'r & $\begin{array}{r}\text { Sca } \\
\text { readi }\end{array}$ & $\begin{array}{l}\text { ale } \\
\text { ings }\end{array}$ & $\begin{array}{l}\text { East } \\
\text { decli- }\end{array}$ & Temp. & Chr'r & $\begin{array}{l}\text { Sca } \\
\text { read }\end{array}$ & $\begin{array}{l}\text { ale } \\
\text { lings }\end{array}$ & $\begin{array}{l}\text { East } \\
\text { decli- }\end{array}$ & Temp & Chr'r & $\begin{array}{l}\mathrm{Sc} \\
\text { read }\end{array}$ & $\begin{array}{l}\text { ale } \\
\text { lings }\end{array}$ & $\begin{array}{l}\mathrm{Ea} \\
\mathrm{dec}\end{array}$ & ast & Tèmp \\
\hline & Left & Right & & & & Left & Right & & & & Left & Right & 11antion & & & Left & Right & Hati & tion & \\
\hline h $\mathrm{m}$ & d & d & $\cdot \cdot$ & $\bullet$ & h $\mathrm{m}$ & d & d & $\cdot 1$ & 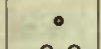 & $\mathrm{h} \mathrm{m}$ & $\mathrm{d}$ & u & $\cdot \cdot$ & & h m & $\mathrm{d}$ & d & - & - & . \\
\hline 800 & 53.0 & 54.7 & 2240 & -22.7 & 100 & 52.1 & 58.1 & 2242 & -18.8 & 1200 & 49.9 & 48.7 & & -18.7 & 1400 & 47.5 & 46.6 & 22 & $4 I$ & $-15 . I$ \\
\hline 02 & 52.7 & 53.9 & 39 & 1 & 02 & 49.7 & 55.0 & 37 & & 02 & 48.8 & 47.0 & 39 & ( & 02 & 49.2 & 47.9 & & 39 & \\
\hline 04 & 54.7 & 56.9 & 43 & 60 & 04 & 48.2 & 54.3 & 36 & & 04 & 52.3 & 51.0 & 34 & 6 & 04 & 51.6 & 50.1 & & 35 & 6 \\
\hline 06 & $5 \mathrm{I} . \mathrm{I}$ & 53.5 & 37 & 4 & 06 & 48.6 & 52.3 & 34 & & 06 & 53.8 & 52.3 & $3 \mathrm{I}$ & $x$ & 06 & 56.8 & 55.1 & & 27 & w \\
\hline 08 & 54.9 & $55 . \mathrm{I}$ & 42 & 4 & 08 & $4 \mathrm{I} .8$ & 47.2 & 25 & Ab & 08 & 54.2 & 52.7 & $3 I$ & 6 & 08 & 56.4 & 55.2 & & 27 & 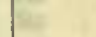 \\
\hline 10 & 52.5 & 53.9 & 39 & $x$ & IO & 36.7 & 40.7 & 16 & 1 & 10 & 54.1 & 53.2 & $3 \mathrm{I}$ & in & Io & 57.6 & 56.8 & & 25 & 0 \\
\hline 12 & 50.4 & $54 \cdot 3$ & 37 & 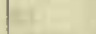 & 12 & 42.8 & 46.2 & 25 & & 12 & 53.1 & 52.2 & 32 & & 12 & 54.5 & 53.6 & & 30 & \\
\hline 14 & 51.2 & 54.2 & 38 & -22.0 & I4 & 39.2 & 43.3 & 20 & -18.7 & 14 & 50.0 & 49.7 & 36 & -18.2 & 14 & 54.1 & 53.1 & & $3 \mathrm{I}$ & -15.0 \\
\hline 16 & 52.3 & 55.0 & 39 & 60 & 16 & 43.2 & 46.1 & 25 & & 16 & 50.7 & 49.4 & 36 & & 16 & 54.9 & 53.7 & & 30 & \\
\hline I8 & 45.5 & 48.4 & 29 & Fit & 18 & 38.4 & 43.2 & 19 & & 18 & 50.5 & 50.0 & 36 & 13 & I8 & 56.4 & 55.6 & & 27 & \\
\hline 20 & 47.0 & $49 . I$ & 31 & $\mathrm{se}$ & 20 & $4 \mathrm{I} \cdot 5$ & 45.8 & 24 & Et & 20 & 47.9 & 47.3 & 40 & $1=$ & 20 & 57.9 & 55.9 & & 25 & in \\
\hline 22 & 49.1 & 52.8 & 35 & Es & 22 & 38.3 & $44 \cdot 3$ & 20 & & 22 & 48.4 & 46.7 & 40 & $E$ & 22 & 54.5 & 51.2 & & 32 & $=$ \\
\hline 24 & 45.6 & 49.0 & 30 & in & 24 & 43.0 & 47.2 & 26 & & 24 & 49.2 & 47.7 & 39 & t & 24 & 55.2 & $5 I \cdot 3$ & & 32 & H \\
\hline 26 & 42.9 & 45.2 & 24 & E & 26 & 43.8 & 48.1 & 27 & & 26 & 50.9 & 48.6 & 37 & a & 26 & 51.5 & 48.2 & & 37 & . \\
\hline 28 & 46.2 & 48.0 & 29 & & 28 & 42.8 & 46.5 & 25 & & 28 & 50.2 & 48.8 & 37 & & 28 & $49 . I$ & 46.6 & & 40 & \\
\hline 30 & 45.9 & 48.1 & 29 & -21.3 & 30 & 42.3 & 47.1 & 25 & -18.4 & 30 & 51.1 & 48.8 & 36 & -17.8 & 30 & 51.7 & 48.8 & & 36 & -14.7 \\
\hline 32 & 38.9 & 41.4 & 18 & (2n & 32 & 38.0 & 43.1 & 19 & 1 & 32 & 53.0 & 51.3 & 33 & (2) & 32 & 49.2 & 46.8 & & 39 & \\
\hline 34 & 36.1 & $37 . \mathrm{I}$ & 13 & 26. & 34 & 37.0 & 43.7 & 19 & tete & 34 & 54.4 & 5 I. 8 & 3 I & & 34 & 45.2 & 42.7 & & 46 & it \\
\hline 36 & 46.2 & $47 . I$ & 28 & 6 & 36 & 39.3 & $44 \cdot 3$ & 21 & E & 36 & 47.1 & 43.8 & 43 & 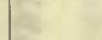 & 36 & 42.7 & 39.2 & & 50 & ( \\
\hline 38 & 43.7 & 44.7 & 25 & ic & 38 & 36.6 & 39.0 & 15 & te & 38 & 48.7 & 46.2 & 40 & E. & 38 & 39.2 & 37.2 & & 55 & t \\
\hline 40 & 40.1 & 40.7 & 19 & 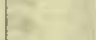 & 40 & 46.2 & 47.8 & 29 & 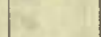 & 40 & 50.9 & 48.9 & 36 & 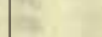 & 40 & 38.0 & 35.7 & & 57 & 8 \\
\hline 42 & 40.3 & 42.1 & 20 & & 42 & 44.3 & 46.8 & 27 & & 42 & 53.7 & 50.9 & 33 & & 42 & 37.8 & 34.9 & 22 & 58 & \\
\hline 44 & 40.2 & 41.7 & 19 & -20.7 & 44 & 44.3 & 46.8 & 27 & -18.2 & 44 & 52.9 & 50.2 & 34 & -17.3 & 44 & 34.8 & 32.0 & 23 & 02 & \\
\hline 46 & 45.6 & 47.7 & 28 & $x^{2}$ & 46 & 44.8 & 47.2 & 27 & & 46 & 51.1 & 48.8 & 36 & (3) & 46 & 35.0 & 32.6 & & 02 & -14.8 \\
\hline 48 & 40.2 & 4I.I & 19 & Let & 48 & 43.9 & 45.9 & 26 & 17 & 48 & 47.7 & 45.7 & 41 & I & 48 & 35.7 & 33.2 & & OI & ( \\
\hline 50 & 38.9 & 40.2 & 17 & $t$ & 50 & 42.9 & 44.9 & 24 & & 50 & 46.6 & 44.8 & 43 & 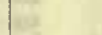 & 50 & 36.3 & 34.2 & 23 & $\infty$ & Do \\
\hline 52 & 45.2 & 45.9 & 27 & 2 & 52 & 41.2 & $4 \mathrm{I} .9$ & 20 & & 52 & 48.6 & 47.0 & 40 & 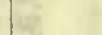 & 52 & 37.7 & 35.7 & 22 & 57 & D. \\
\hline 54 & 41.8 & 42.4 & 21 & 2 & 54 & 42.1 & 43.8 & 23 & & 54 & 52.8 & 51.1 & 33 & & 54 & 37.4 & 35.9 & 22 & 57 & \\
\hline 56 & 39.9 & 40.9 & 19 & 2 & 56 & $4 \mathrm{I} . \mathrm{I}$ & 42.4 & $2 \mathrm{I}$ & & 56 & 53.3 & 52.2 & 32 & & 56 & 35.3 & 33.9 & 23 & $\infty$ & \\
\hline $5^{8}$ & 41.0 & 43.8 & 22 & & 58 & 42.8 & 43.8 & 23 & & 58 & 48.3 & 47.8 & 39 & & 58 & 38.8 & 37.3 & 22 & 55 & \\
\hline 900 & 45.8 & 49.4 & 30 & -20.1 & II 00 & $4 \mathrm{I} . \mathrm{I}$ & 42.0 & 20 & -17.9 & 1300 & 53.9 & 51.8 & 32 & -16.8 & 1500 & 38.0 & 36.9 & & 56 & -14.7 \\
\hline 02 & 35.2 & 37.4 & 12 & the & 02 & 41.0 & 42.7 & $2 I$ & - & 02 & $6 \mathrm{I} .2$ & 60.9 & I9 & or & 02 & 37.7 & 36.6 & & 56 & 07 \\
\hline 04 & $37 \cdot 3$ & 38.8 & 15 & 5 & 04 & 40.9 & 42.7 & 2 I & E & 04 & 62.8 & 61.9 & I7 & tive & 04 & 37.0 & 35.8 & & 58 & DE \\
\hline 06 & 39.0 & 40.6 & 18 & 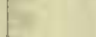 & 06 & $44 . I$ & $45 . I$ & 25 & $n$ & 06 & $6 \mathrm{I} .1$ & 60.4 & 19 & 8 & 06 & 35.7 & 35.0 & 22 & 59 & 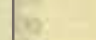 \\
\hline 08 & 46 & $.3^{a}$ & 28 & De & 08 & 38.2 & 40.8 & 17 & I & 08 & 54.4 & 53.2 & 30 & $6 x=$ & 08 & 35.6 & 34.3 & 23 & 00 & 5 \\
\hline I0 & 39.0 & 39.8 & 17 & 10 & 10 & 45.2 & 46.0 & 27 & IIF & 10 & 58.8 & 57.8 & 23 & it & I0 & 33.0 & $3 \mathrm{i} . \overline{8}$ & & 04 & tor \\
\hline 12 & 36.3 & 40.2 & I5 & & 12 & 42.8 & 43.9 & 23 & & I2 & 62.0 & 60.4 & 19 & & 12 & 30.2 & 29.1 & & 08 & \\
\hline 14 & 41.8 & 45.2 & 24 & -19.3 & 14 & 38.9 & 39.9 & I7 & -17.7 & 14 & 61.0 & 60.2 & 20 & -16.3 & I4 & 29.0 & 27.8 & & I0 & -14.6 \\
\hline I6 & 36.8 & 39.3 & I5 & It & 16 & 40.6 & 43.3 & $2 \mathrm{I}$ & & 16 & 59.3 & 57.3 & 23 & $=$ & I6 & 28.6 & 27.2 & & II & \\
\hline 18 & 35.2 & 39.0 & 14 & i & 18 & 42.8 & 44.3 & 24 & 12 & 18 & 55.0 & 52.7 & 30 & (17) & I8 & 28.8 & 27.9 & & 10 & I \\
\hline 20 & 38.2 & 40.6 & I7 & E & 20 & 37.9 & 40.2 & 16 & A & 20 & 49.0 & 47. I & 39 & e & 20 & 26.9 & $25 . \overline{8}$ & & 13 & 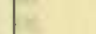 \\
\hline 22 & 40.8 & 42.1 & 20 & $E$ & 22 & 40.1 & 42.2 & 20 & 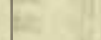 & 22 & 48.2 & 46.8 & 40 & $E$ & 22 & 30.7 & 29.9 & & 07 & $=$ \\
\hline 24 & 42.0 & 44. I & 23 & it & 24 & $4 \mathrm{I} .8$ & 44.2 & 23 & ic & 24 & 49.1 & 47.8 & 39 & 12 & 24 & 31.8 & $3 I .2$ & & 05 & 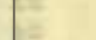 \\
\hline 26 & 37.7 & 39.7 & 16 & $E$ & 26 & 41.8 & 44.0 & 23 & $E$ & 26 & 50.9 & 49.6 & 36 & Las & 26 & 33.1 & 32.9 & 23 & 03 & \\
\hline 28 & 41.8 & 41.8 & $2 \mathrm{I}$ & & 28 & 42.7 & 44.9 & 24 & 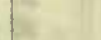 & 28 & 52.8 & 51.3 & 33 & & 28 & 36 & $1 a$ & 22 & 58 & \\
\hline 30 & 44.6 & 44.8 & & -19.2 & 30 & 44.9 & 47.0 & 27 & in & 30 & 51.1 & 50.0 & 35 & -16.0 & 30 & 37.6 & 36.9 & & 56 & -14.4 \\
\hline 32 & 36.0 & 43.8 & 18 & - & 32 & 45.8 & 47.2 & 28 & Fit & 32 & 50.9 & 49.7 & 36 & & 32 & 38.8 & 38.0 & & 55 & \\
\hline 34 & 36.5 & 41.6 & I6 & 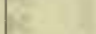 & 34 & 43.8 & 45.9 & 26 & 1 & 34 & 54.2 & 52.9 & 31 & & 34 & 38. I & $37 \cdot I$ & 22 & 56 & \\
\hline 36 & 32.8 & 39.9 & I2 & 4 & 36 & 40.2 & $4 \mathrm{I} .9$ & 20 & t & 36 & 56.0 & 55.2 & 27 & 8 & 36 & 35.6 & 34.5 & 23 & 0 & 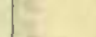 \\
\hline 38 & 35.0 & 41.2 & I5 & F. & 38 & 38.8 & 40.8 & 18 & $=$ & 38 & 55.9 & 55.1 & 28 & 4 & 38 & & 34.2 & & $\infty$ & \\
\hline 41 & 28.6 & 34.8 & 05 & 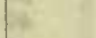 & 40 & 43.9 & 46.2 & 26 & 8 & 40 & 56.1 & $55 \cdot 3$ & 27 & & 40 & 32.8 & 31.4 & & 04 & \\
\hline 42 & 34.3 & $4 \mathrm{I} .4$ & 15 & & 42 & $45 . \overline{8}$ & 47.3 & 28 & & 42 & 54.0 & 53.0 & $3 I$ & & 42 & 32.8 & 31.3 & & 05 & \\
\hline 44 & 3 1. 8 & $41 . \dot{I}$ & 12 & -19.0 & 44 & 47.0 & 48.7 & 30 & -17. & 44 & 51.0 & 49.3 & 36 & -15.6 & 44 & 33.9 & 32.3 & & 03 & -14.1 \\
\hline 46 & 34.7 & $44 . I$ & I7 & $x^{-}$ & 46 & 46.3 & 48.3 & 30 & ( & 46 & 50.3 & 49.6 & 36 & 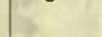 & 46 & 34.8 & 33.2 & 23 & OI & \\
\hline 48 & $4 \mathrm{I} . \mathrm{I}$ & 49.8 & 26 & $=$ & 48 & 44.8 & 46.3 & 27 & 1 & 48 & 49.1 & 48.9 & 38 & s. & 48 & 36.9 & 35.9 & 22 & 58 & \\
\hline 50 & 43.3 & 50.9 & 29 & 2 & 50 & 42.6 & 43.9 & 23 & 1 & 50 & $4^{8} .3$ & $47 \cdot 3$ & 40 & 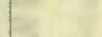 & 50 & 36.4 & 35.5 & & 58 & \\
\hline 52 & 37.8 & 45.2 & 20 & 12 & 52 & 50.1 & 50.8 & 34 & $1=$ & 52 & 47.7 & 47.0 & 40 & E & 52 & 36.0 & 35.0 & 22 & 59 & \\
\hline 54 & 35.0 & 40.3 & I4 & 2 & 54 & 46.1 & 47.3 & 29 & 6 & 54 & 47.0 & 46.2 & $4 \mathrm{I}$ & 2 & 54 & 35.0 & 33.8 & 23 & OI & \\
\hline 56 & 38.8 & $45 \cdot 3$ & $2 \mathrm{I}$ & E & 56.2 & 43.8 & 45.7 & 25 & 5 & 56 & 47.1 & 46.5 & 4I & = & 56 & 33.7 & 33.0 & & 02 & \\
\hline 58 & 38.8 & 44.8 & $2 I$ & z & 58 & 42.8 & $8 b$ & 22 & $=$ & 58 & 48.5 & 47.6 & 39 & $=$ & & 32.3 & 3I.I & & 05 & \\
\hline & & & & & 1200 & 38. & & 15 & & & & & & & 1600 & 30.9 & 30.0 & & 07 & -13.9 \\
\hline
\end{tabular}

Correction to local mean time is - 13s.

Torsion head at $7 \mathrm{~h} 3 \mathrm{~m}$ read $336^{\circ}$ and at $12 \mathrm{~h}$ 3om read the same. Observer-R. R. T.
Correction to local mean time is - 07s.

Torsion head at in $30 \mathrm{~m}$ read $347^{\circ}$ and at $16 \mathrm{~h}$ 20m read the same. Observer-R. R. T. 
Tabulation of magnetic declinations observed at Teplitz Bay-Continued

\begin{tabular}{|c|c|c|c|c|c|c|c|c|c|c|c|c|c|c|c|c|c|c|c|}
\hline \multicolumn{5}{|c|}{ Wednesday, December 9, 1903} & \multicolumn{5}{|c|}{ Magnet scale erect } & \multicolumn{10}{|c|}{ Wednesday, December 9, 1903} \\
\hline \multirow{2}{*}{$\begin{array}{l}\text { Chr'r } \\
\text { time }\end{array}$} & \multicolumn{2}{|c|}{$\begin{array}{l}\text { Scale } \\
\text { readings }\end{array}$} & \multirow{2}{*}{$\begin{array}{l}\text { East } \\
\text { decli- } \\
\text { nation }\end{array}$} & \multirow{2}{*}{ Temp. } & Chr'r & $\begin{aligned} \text { Sc } \\
\text { reac }\end{aligned}$ & $\begin{array}{l}\text { ale } \\
\text { lings }\end{array}$ & $\begin{array}{l}\text { East } \\
\text { decli- }\end{array}$ & Temp. & Chr'r & $\begin{array}{r}\text { Sc } \\
\text { read }\end{array}$ & & $\begin{array}{l}\text { East } \\
\text { decli- }\end{array}$ & Temp. & Chr'r & $\begin{array}{l}\text { Sc } \\
\text { read }\end{array}$ & $\begin{array}{l}\text { cale } \\
\text { dings }\end{array}$ & $\begin{array}{l}\text { East } \\
\text { decli- }\end{array}$ & Temp. \\
\hline & Left & Right & & & & Left & Right & & & & Left & Right & & & & Left & Right & & \\
\hline h $\mathrm{m}$ & d & $d$ & $\therefore$ & $\int_{0}^{-12}$ & $\mathrm{~h} \mathrm{~m}$ & $d$ & & - í & $\therefore$ & h m & $d$ & d & $\therefore$ & $\therefore$ & $\mathrm{h} \mathbf{m}$ & d & d & & $\therefore$ \\
\hline $\begin{array}{ll}0 \\
02 *\end{array}$ & 36.1 & $\begin{array}{l}0 \text { st } \\
37.4\end{array}$ & 2240 & -17.0 & $\begin{array}{r}200 \\
02\end{array}$ & $\begin{array}{l}4 \mathrm{I} .6 \\
4 \mathrm{I} .8\end{array}$ & $\begin{array}{l}41.6 \\
42.0\end{array}$ & $\begin{array}{r}2248 \\
48\end{array}$ & $-14 \cdot 5$ & $\begin{array}{r}400 \\
02\end{array}$ & $\begin{array}{l}53.5 \\
5 \mathrm{I} . \mathrm{I}\end{array}$ & $\begin{array}{l}54 . I \\
5 I .9\end{array}$ & $\begin{array}{rl}23 & 08 \\
04\end{array}$ & $-14 \cdot 5$ & $\begin{array}{r}600 \\
02\end{array}$ & $\begin{array}{l}45.7 \\
53.0\end{array}$ & $\begin{array}{l}47.2 \\
54.8\end{array}$ & $\begin{array}{l}2257 \\
2308\end{array}$ & -15.0 \\
\hline 04 & 38.0 & 38.1 & 42 & 180 & 04 & $4 \mathrm{I} .0$ & $4 \mathrm{I} .3$ & 47 & & 04 & $52 . \mathrm{I}$ & 52.5 & 05 & 1 & 04 & 52.5 & 55.3 & 08 & ถู \\
\hline $\begin{array}{l}06 \\
8\end{array}$ & 39.0 & 40.1 & & 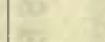 & 06 & 40.3 & 40.6 & 46 & . & 06 & 53.5 & 54.3 & 08 & & 06 & 58.3 & 60.3 & 2317 & \\
\hline of & 39.3 & 39.9 & 44 & $E$ & 08 & 40.5 & 40.7 & 46 & t & $\begin{array}{l}08 \\
\text { to }\end{array}$ & $54 \cdot 3$ & $\begin{array}{l}55.0 \\
56.8\end{array}$ & $\begin{array}{l}09 \\
09\end{array}$ & III & 08 & 44 & .06 & 2253 & \\
\hline IO & 38.5 & 39.5 & 43 & G & Io & 41.2 & $4 \mathrm{I} \cdot 4$ & 47 & 1 & $\begin{array}{l}\text { I0 } \\
\text { I2 }\end{array}$ & 50.0 & $\begin{array}{l}50.8 \\
57.9\end{array}$ & $\begin{array}{l}12 \\
13\end{array}$ & II & I0 & 34.8 & 36.0 & 39 & \\
\hline 12 & 38.3 & 39.3 & 43 & & I2 & 40.8 & 4I.I & 47 & & & 58.0 & 58.9 & 13 & & 12 & 42.3 & 44.0 & $225 \mathrm{I}$ & \\
\hline & 37.3 & 37.8 & $4 \mathrm{I}$ & -16.5 & 14 & 40.6 & 40.6 & 46 & -14.4 & I4 & 50.0 & 58 & 15 & -14.5 & 14 & 47.8 & 50.8 & 23 OI & -15.0 \\
\hline & 38.4 & 39.0 & 43 & & 16 & 40.0 & 40.2 & 46 & & $\begin{array}{l}16 \\
-8\end{array}$ & 69.0 & 59 & 16 & & 16 & 41.0 & 43.2 & 2250 & \\
\hline & 39.3 & 39.3 & 44 & E & I8 & 40.5 & 40.8 & 46 & 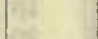 & 18 & & 60.8 & I8 & 4 & I8 & 38.1 & $4 \mathrm{I} .5$ & 46 & \\
\hline 2 & 40.6 & 40.3 & 45 & $=$ & 20 & 46.0 & 46.3 & 55 & $x 6$ & 20 & 63.0 & 63.8 & 23 & $E$ & 20 & 39.4 & $4 \mathrm{I} .9$ & 47 & \\
\hline 2 & $39 . I$ & 39.6 & 44 & $=$ & 22 & $4 \mathrm{I} .5$ & 41.8 & 48 & $=$ & 22 & 65.5 & 66.8 & 27 & $1=$ & 22 & $4 \mathrm{I} .7$ & 43.3 & 50 & \\
\hline 2. & & 38.3 & 42 & $t$ & 24 & 42.3 & 42.6 & 49 & 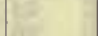 & 24 & 67.8 & 68.8 & 30 & 5 & 24 & 38.2 & 40.2 & 45 & \\
\hline & 38.0 & 38.3 & 42 & $\mathrm{E}$ & 26 & 42.3 & 42.6 & 49 & 14 & 26 & 67.3 & $68 . \mathrm{I}$ & 29 & 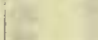 & 26 & 35.4 & 37.5 & $4 \mathrm{I}$ & \\
\hline & 38.6 & 38.8 & 43 & $=$ & 28 & 42.2 & 42.3 & 49 & & 28 & 67.5 & 68.5 & 30 & $=$ & 28 & 35.6 & 37.7 & 4I & \\
\hline $3 c$ & 38.6 & 39.0 & 43 & & 30 & & 42 & 49 & $-\mathrm{I} 4.4$ & 30 & 67.0 & 67.6 & 29 & -14.7 & 30 & 40.3 & 43.2 & 49 & -15.2 \\
\hline 32 & & 39.0 & 43 & & 32 & 42.0 & 42 & 49 & & 32 & 66.3 & 66.5 & 27 & & 32 & $4 \mathrm{I} .7$ & & 5I & \\
\hline 3. & & & $5 \mathrm{I}$ & -16.1 & 34 & $4 \mathrm{I} .4$ & $4 \mathrm{I}$ & 47 & 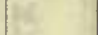 & 34 & 63.9 & $6_{4 . \mathrm{I}}$ & 23 & & 34 & 36.9 & 39 & 43 & \\
\hline & & 46 & 54 & & 3 & 40.6 & $4 \mathrm{I}$. & 46 & & 36 & 6 ז. 6 & 62 & 20 & & 36 & 37.6 & & 43 & \\
\hline 38 & & $47 \cdot 3$ & 56 & 8 & 3 & 41.2 & 41.6 & 47 & & 38 & 63.0 & 63 & 22 & & 38 & 44.4 & 45.7 & 54 & \\
\hline 40 & & 47.3 & 56 & 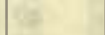 & $4 c$ & 42.4 & 43. & 49 & & 40 & $6 \mathrm{I} .6$ & $6 \mathrm{I}$ & 20 & & 40 & 46.7 & 48 & 58 & \\
\hline 42 & & 47.8 & 57 & & 42 & 42.4 & 42. & 49 & & 42 & 62.5 & 63 & 22 & & 42 & 3 & & 47 & \\
\hline 44 & 44.3 & 44.8 & 2252 & -15.6 & 44 & 43.9 & 44.6 & 52 & -14.3 & 44 & 63.9 & 65 & 24 & -14.7 & 44 & 37.7 & 40 & 45 & -15.2 \\
\hline 46 & 50.4 & 51.2 & 2302 & & 46 & 47.5 & 47.5 & 57 & & 46 & 66.6 & 67 & 28 & & 46 & 38.7 & 41.7 & 47 & \\
\hline 48 & 50.6 & 5I.I & 02 & $t$ & 48 & 48.1 & 48.6 & 58 & & 48 & $66 . \mathrm{I}$ & 66 & 27 & 1 & 48 & 42.3 & & 52 & \\
\hline 50 & $5^{1 .} .6$ & 52.5 & 04 & & 50 & $46 \cdot 3$ & 46.7 & 56 & & 50 & 67.8 & & 30 & & 50 & 44.3 & & 55 & \\
\hline 52 & 51.9 & 52.5 & 04 & 1 & 52 & 45.8 & 46.0 & 34 & & 52 & 68.5 & 69 & 31 & & 52 & & & 45 & \\
\hline 54 & 51.0 & 51.9 & 03 & & 54 & 48.0 & 48.2 & 22 & & 54 & 67.1 & & 29 & & 54 & 29.4 & & 3I & \\
\hline 56 & 50.6 & 51.6 & 02 & & 56 & & 49.6 & 23 & & 56 & 65.7 & 66 & 26 & & 56 & 0.3 & & 34 & \\
\hline 58 & 49.8 & 50.7 & 02 & & 58 & & 50 & 02 & & 58 & 67.5 & 67.8 & 29 & & $5^{8}$ & 5.3 & & $4 \mathrm{I}$ & \\
\hline 100 & 48.7 & 49.3 & 00 & -15.2 & 300 & 50.0 & 50 & 23 or & -14.3 & 500 & 69.3 & 69.3 & 32 & -14.8 & 700 & & & 33 & -15.2 \\
\hline 02 & 49.8 & 50 & OI & & 02 & & & & & 02 & 68.3 & & 32 & & 02 & 28.6 & & 30 & \\
\hline 04 & 48.6 & 49.0 & 00 & 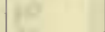 & 04 & & 47 & 56 & b & 04 & 60.1 & & I9 & a) & 04 & 35.4 & & 40 & \\
\hline & 48. & 49.0 & 300 & 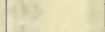 & & & & 53 & 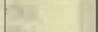 & of & & & II & 8 & & & & 47 & \\
\hline 0 & 48.6 & 48 & 9 & is & & & & & 64 & o & & & 18 & & 08 & & & 42 & \\
\hline & 48. & 48 & 9 & 4 & I & & 43 & 52 & ff: & 1 & & $5^{6}$ & & & & & & 39 & \\
\hline $\mathbf{I} 2$ & 48 & 48 & 59 & 1. & $\mathrm{I}$ & & 42 & 50 & & 1 & & & 257 & & I & & & 36 & \\
\hline I. & 48.5 & 48.7 & 59 & -15.1 & I. & 41.0 & 4I. & 48 & -14. & 14 & 36.2 & 37 & $4 \mathrm{I}$ & -14 & 14 & 6 & & 50 & -15.2 \\
\hline IC & 47.7 & 48.3 & 58 & & I & & & 48 & & 16. & & & 36 & & & & & 58 & \\
\hline 18 & 46.6 & 46.9 & 56 & $t$ & IE & $4 \mathrm{I}$ & 41 & 49 & t5 & I & & & 39 & & & & & 48 & \\
\hline 20 & $45 \cdot 3$ & 45.6 & 54 & & 2 & & 42 & 49 & te & 2 & 42.8 & & $5 \mathrm{I}$ & & 2 & & & 43 & \\
\hline 22 & & 44 & 53 & 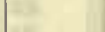 & 2. & & & 50 & & 2. & & & 48 & 8 & 2. & & & 54 & \\
\hline 24 & 43 & 43 & $5 I$ & 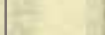 & 2. & & & $5 \mathrm{I}$ & 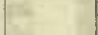 & 24 & & & 36 & Fe & 24 & & & 55 & \\
\hline 26 & 43 & 43 & $5 I$ & 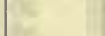 & 26 & & & 52 & 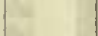 & 26 & & & 29 & $=$ & 26 & 3.3 & & 54 & \\
\hline 28 & 43 & 43 & 51 & 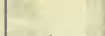 & 28 & & & 54 & & 28 & & & 25 & & 28 & & & 49 & \\
\hline 30 & 42 & 42.6 & 50 & -15.1 & 30 & & & 55 & $-\mathrm{I} 4.4$ & 30 & 26 & & 27 & -15. & & 44.6 & 46.5 & 55 & -15.2 \\
\hline 32 & 42. & 43 & 50 & & 32 & & & 55 & & 32 & & $3 \mathrm{I}$ & $3^{2}$ & & $3^{2}$ & 44.4 & & 55 & \\
\hline 34 & 42 & 42 & 50 & & 34 & & 46 & 56 & 8. & 34 & 31.6 & & 35 & & 3 & & 46.0 & & \\
\hline 36 & 42 & 42 & 50 & & & & 47 & & & 3 & & & 43 & & & & 53.4 & & \\
\hline 3 & 43. & 43 & $5 \mathrm{I}$ & & & & $4^{8}$ & 22 & & 3 & & & 43 & & & 48.3 & & & \\
\hline 40 & 43 & 44 & 5I & & 4 & & 50.0 & 2302 & & 4 & $4 \mathrm{I}$. & 42 & 50 & & & & & 53 & \\
\hline 42 & 44 & 44 & 53 & & 42 & $5 \mathrm{I}$ & $5 \mathrm{I}$ & 04 & & 42 & 42.7 & 43 & $5 I$ & & & & & 49 & \\
\hline 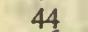 & 44. & 44 & 52 & -14.8 & 4 & 52 & 53 & 06 & & 44 & 44.6 & 45.0 & 54 & & & & 48.5 & 58 & -15.2 \\
\hline & 43. & 43 & 52 & & 46 & 55. & & II & -14.5 & 46 & 45 & $0 a$ & 54 & & & 6 & & 59 & \\
\hline 48 & 43. I & 43.1 & 50 & 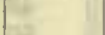 & 48 & 62.3 & 63. & 22 & & 48 & & $3 a$ & 2309 & & 4) & 47. & & 8 & \\
\hline 50 & 43.3 & $43 \cdot 5$ & 51 & & 50 & 59. & $5^{b}$ & 17 & & 50 & 48.3 & 50.0 & 02 & & 50 & 43.9 & 45.6 & 55 & \\
\hline 52 & 43.9 & 44.0 & 5I & & 52 & 51. & $5^{b}$ & 04 & & 52 & 48.0 & 49 & $\infty$ & & 52 & 44.1 & 47.5 & 2256 & \\
\hline 54 & 43.7 & 43.8 & $5 \mathrm{I}$ & & 54 & 50.6 & 50.8 & 03 & & 54 & 48.6 & $49 . I$ & 00 & & 54 & 47.8 & 51.8 & 2302 & \\
\hline 56 & $43 . I$ & 43.3 & 50 & & 56 & & $5 b$ & 13 & & 56 & 49.6 & 50.4 & 2302 & & 56 & 41.8 & 46.6 & 2254 & \\
\hline 58 & 42.3 & 42.5 & 49 & & 58 & 56.8 & 58.1 & I3 & & 58 & 45.2 & 45.4 & 2255 & & 58 & 36.8 & 39.6 & 44 & \\
\hline & & & & & & & & & & & & & & & & & & & \\
\hline
\end{tabular}


Tabulation of magnetic declinations observed at Teplitz Bay-Continued

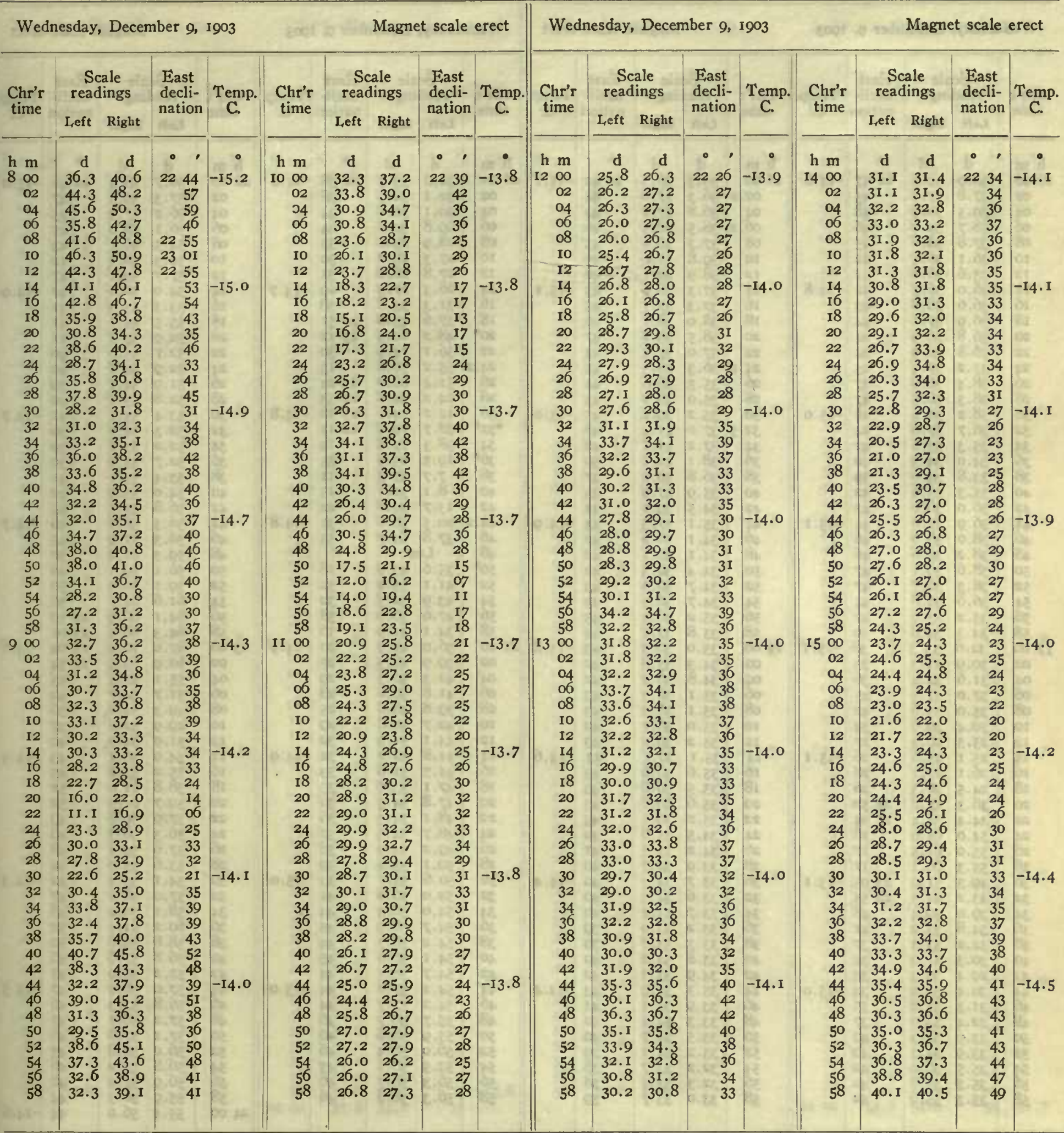

Observers-W. J. P. and R. R. T. changed at $8 \mathrm{~h}$ Iom; no time for alternating observations account snow drifts.
Observers-R. R. T. and W. J. P., who alternated from $14 \mathrm{~h} 26 \mathrm{~m}$ to I4h $36 \mathrm{~m}$. 
Tabulation of magnetic declinations observed at Teplitz Bay-Continued

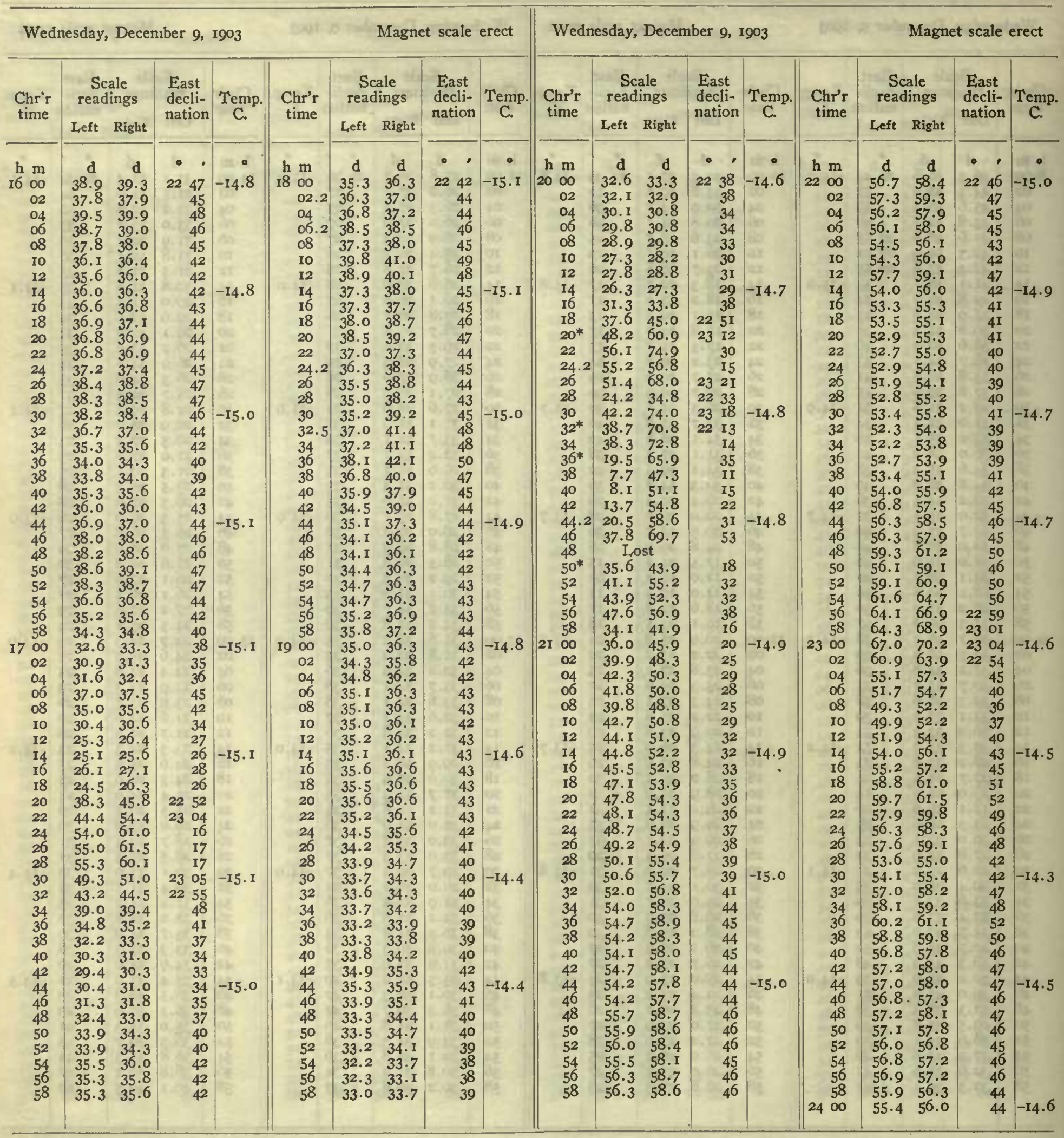

Observers-W. J. P. and R. R. T., who alternated from I8h $30 \mathrm{~m}$ to I8h $36 \mathrm{~m}$.
Correction to local mean time is $+36 \mathrm{~s} .90^{\circ}$ torsion $=25^{\circ} \mathrm{o}$. Torsion liead at oh com read $5^{\circ}$ and $24 \mathrm{~h} 15 \mathrm{~m}$ read $345^{\circ}$. Observer-R. R. T. 
Tabulation of magnetic declinations observed at Teplitz Bay-Continued

\begin{tabular}{|c|c|c|c|c|c|c|c|c|c|c|c|c|c|c|c|c|c|c|c|c|}
\hline \multicolumn{5}{|c|}{ Thursday, December Io, 1903} & \multicolumn{6}{|c|}{ Magnet scale inverted } & \multicolumn{5}{|c|}{ Friday, December Ir, 1903} & \multicolumn{5}{|c|}{ Magnet scale erect. } \\
\hline \multirow{2}{*}{$\begin{array}{l}\text { Chr'r } \\
\text { time }\end{array}$} & \multicolumn{2}{|c|}{$\begin{array}{l}\text { Scale } \\
\text { readings }\end{array}$} & East & Temp. & Chr'r & $\begin{array}{r}\mathrm{Sc} \\
\text { read }\end{array}$ & $\begin{array}{l}\text { ale } \\
\text { ings }\end{array}$ & $\begin{array}{l}\mathrm{Ea} \\
\mathrm{de}\end{array}$ & $\begin{array}{l}\text { st } \\
\text { cli- }\end{array}$ & Temp. & Chr'r & $\begin{array}{r}\mathrm{Sc} \\
\text { reac }\end{array}$ & $\begin{array}{l}\text { ale } \\
\text { lings }\end{array}$ & $\begin{array}{l}\text { East } \\
\text { decli- }\end{array}$ & Temp. & Chr'r & $\begin{array}{l}\mathrm{Sc} \\
\text { read }\end{array}$ & ngs & $\begin{array}{l}\text { East } \\
\text { decli- }\end{array}$ & \\
\hline & Left & Right & & & time & Left & Right & & & $\mathrm{C}$ & time & Left & Right & & & time & Left & Right & nation & \\
\hline h m & d & d & - & 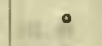 & $\mathrm{h} \mathrm{m}$ & d & d & - & ' & - & $\mathrm{h} \mathrm{m}$ & d & d & - & 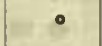 & h m & d & d & - & $\bullet$ \\
\hline 1600 & 57.2 & 53.6 & 2232 & -17.4 & 1800 & 54.6 & 54.2 & 22 & 33 & $-14 \cdot 7$ & 2000 & 5 I.I & 51.9 & 2232 & -15.0 & 2200 & 54.2 & 54.6 & 2236 & -12.0 \\
\hline 02 & 56.3 & 52.9 & 33 & 10 & 02 & 55.7 & 55.3 & & 32 & & 02 & 51.8 & 52.6 & 33 & & 02 & 54.2 & 54.4 & 36 & \\
\hline 04 & 56.1 & 53.0 & 33 & ver & 04 & 56.1 & 55.9 & & $3 \mathrm{I}$ & & 04 & 52.7 & 53.2 & 34 & & 04 & 53.3 & 53.8 & 35 & in \\
\hline 06 & 55.8 & 52.8 & 34 & 8. & 06 & $55 . \mathrm{I}$ & 55.1 & & 32 & $=$ & 06 & $53 . I$ & 53.9 & 35 & at & 06 & $54 . \mathrm{I}$ & 54.2 & 36 & 10 \\
\hline 08 & $55 \cdot 5$ & 53.0 & 34 & 16 & 08 & 55.0 & 54.8 & & 33 & Wo. & 08 & 53 & $.2 b$ & 34 & & 08 & 55.7 & 56.0 & 38 & Es \\
\hline IO & 55.8 & 53.2 & 33 & Fit & IO & 54.6 & 54.3 & & 33 & $a$ & I0 & 53.8 & 53.8 & 35 & 4 & IO & 53.8 & $54 . \mathrm{I}$ & 35 & 1 \\
\hline 12 & 55.7 & $53 \cdot 3$ & 33 & 5 & 12 & 55.9 & 55.8 & & 31 & 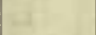 & 12 & 53.1 & 53.2 & 34 & & 12 & 52.5 & 52.8 & 33 & 21 \\
\hline 14 & $55 . \mathrm{I}$ & 53.0 & 34 & -17.0 & I4 & 53.9 & 53.9 & & 34 & -14.6 & I4 & 53. I & $53 . I$ & 34 & $-I 4 \cdot 3$ & 14 & 52.7 & 52.9 & 34 & -12.0 \\
\hline 16 & $55 . \mathrm{I}$ & 53.2 & 34 & & 16 & 54.6 & 54.2 & & 33 & & 16 & 53.2 & 53.2 & 34 & & 16 & 53.2 & 53.8 & 35 & \\
\hline I8 & 54.6 & 52.9 & 34 & ar & 18 & 55.7 & 55.5 & & 31 & 64 & 18 & 53.0 & $53 . I$ & 34 & & 18 & 53.1 & 53.3 & 34 & 11 \\
\hline 20 & 54.1 & 52.4 & 35 & s & 20 & 55.8 & 55.8 & & $3 \mathbf{I}$ & 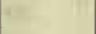 & 20 & 52.7 & 52.8 & 33 & & 20 & 52.9 & 53.2 & 34 & 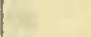 \\
\hline 22 & 54.2 & 52.8 & 35 & 65 & 22 & 55.2 & 55.2 & & 32 & $=$ & 22 & 52.2 & 52.3 & 33 & 6 & 22 & 53.6 & 54.2 & 35 & $1=$ \\
\hline 24 & 54.7 & 53.3 & 34 & $=$ & 24 & 55.1 & 55.1 & & 32 & $=$ & 24 & 52.2 & 52.2 & 33 & 1 & 24 & 50.7 & 51.3 & $3 \mathrm{I}$ & $1-$ \\
\hline 26 & 54.7 & 53.2 & 34 & - & 26 & $55 \cdot 5$ & 55.3 & & 32 & - & 26 & 52.9 & 52.9 & 34 & & 26 & 50.8 & $52 . \mathrm{I}$ & $3 I$ & 1 \\
\hline 28 & $54 \cdot 3$ & 53.2 & 34 & & 28 & $55 . \mathrm{I}$ & 55.0 & & 32 & & 28 & 53.1 & 53.1 & 34 & 1 & 28 & 51.2 & 52.2 & 32 & 1 \\
\hline 30 & $55 . \mathrm{I}$ & 54.0 & 33 & -I6.6 & 30 & 55.4 & 55.2 & & 32 & -14.2 & 30 & 53.4 & 53.7 & 35 & -13.7 & 30 & 50.2 & 51.2 & 30 & -12.0 \\
\hline 32 & 56.2 & 55.3 & $3 I$ & 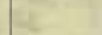 & 32 & $55 . \mathrm{I}$ & 54.9 & & 32 & & 32 & 53.2 & 53.2 & 34 & & 32 & 50.2 & 51.3 & 30 & \\
\hline 34 & 52.8 & 52.2 & 36 & E. & 34 & 54.4 & 54. I & & 34 & & 34 & 53 & $.0 b$ & 34 & & 34 & 49.4 & 50.2 & 29 & \\
\hline 36 & 54.2 & 53.0 & 35 & & 36 & 53.9 & 53.6 & & 34 & & 36 & 51.8 & 51.8 & 32 & & 36 & 5I.I & 51.9 & 32 & \\
\hline 38 & 56.4 & 55.7 & $3 I$ & 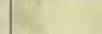 & 38 & 53.4 & 53.1 & & 35 & & 38 & $5^{1} .9$ & 51.9 & 32 & 5 & 38 & 52.9 & 53.4 & 34 & \\
\hline 40 & 55.9 & 55.2 & 32 & 6 & 40 & 53.6 & 53.2 & & 35 & & 40 & 51.7 & 51.8 & 32 & . & 40 & 50.1 & 50.6 & 30 & 18 \\
\hline 42 & 55.9 & 55.3 & $3 I$ & & 42 & 53.9 & 53.7 & & 34 & & 42 & 50.9 & 51.2 & 31 & & 42 & 50.1 & 50.9 & 30 & \\
\hline 44 & 55.8 & 55.2 & 32 & -I6.2 & 44 & 53.9 & 53.6 & & 34 & -14.0 & 44 & $5 \mathrm{I} .2$ & 51.8 & 32 & -13.3 & 44 & 52.9 & 53.1 & 34 & -II.9 \\
\hline 46 & 55.7 & 55.0 & 32 & $t$ & 46 & 53.0 & 52.8 & & 36 & -7 & 46 & $5 I .9$ & 52.4 & 33 & & 46 & 52.2 & 52.9 & 33 & \\
\hline 48 & 56.8 & 56.2 & 30 & 6 & 48 & 53.2 & 53.1 & & 35 & 8 & 48 & 52.1 & 52.4 & 33 & 3 & 48 & 52.3 & 52.9 & 33 & 12 \\
\hline 50 & 58.1 & 57.7 & 28 & if & 50 & 52.9 & 52.7 & & 36 & & 50 & 52.3 & 52.7 & 33 & $=$ & 50 & 53.2 & 53.9 & 35 & t. \\
\hline 52 & 55.7 & 55.2 & 32 & & 52 & 52.7 & 52.2 & & 37 & & 52 & 52.8 & 53.1 & 34 & 12 & 52 & 52.8 & 53.2 & 34 & 4 \\
\hline 54 & 55.7 & 55.2 & 32 & & 54 & 52.4 & 52.2 & & 37 & & 54 & 52.3 & 52.8 & 33 & 8 & 54 & 52.0 & 52.4 & 33 & \\
\hline 56 & 55.7 & 55.2 & 32 & & 56 & 53.7 & 53.3 & & 35 & & 56 & 52.9 & 53.1 & 34 & & 56 & 51.9 & 52.8 & 33 & \\
\hline 58 & 56.0 & 56.0 & $3 I$ & & 58 & 52.9 & 52.7 & & 36 & & 58 & 53.2 & 53.4 & 34 & & $5^{8}$ & 52.8 & 53.2 & 34 & \\
\hline 1700 & 56.8 & 56.3 & 30 & $-\mathrm{I} 5.8$ & I9 00 & 52.8 & 52.6 & & 36 & -13.9 & 2100 & 53.1 & 53.1 & 34 & $-\mathrm{I} 2.9$ & 2300 & 5 I. 8 & 52.3 & 32 & -II.8 \\
\hline 02 & 57.0 & 56.8 & 30 & & 02 & 53.2 & 53.1 & & 35 & & 02 & 53.1 & 53.1 & 34 & & 02 & 53.8 & 54.2 & 36 & \\
\hline 04 & 56.5 & 56.1 & 30 & & 04 & 53.0 & 52.7 & & 36 & & 04 & 52.9 & 53.0 & 34 & $=$ & 04 & 53.9 & 54.9 & 36 & n \\
\hline 06 & 55.2 & 55.1 & 32 & ar & 06 & 53.0 & 52.8 & & 36 & $\theta$ & 06 & 53.1 & 53.1 & 34 & 53 & 06 & 55.0 & 55. & 38 & $\theta$ \\
\hline 08 & 54.7 & $54 \cdot 3$ & 33 & 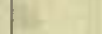 & 08 & 53.0 & 52.9 & & 36 & $1+$ & 08 & 52.9 & 52.9 & 34 & 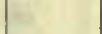 & 08 & 53.8 & $54 . I$ & 35 & W \\
\hline I0 & 55 & $8 a$ & 31 & & 10 & 53.2 & 53.0 & & 35 & & I0 & 52.2 & 52.2 & 33 & 1 & IO & 53.6 & 53.9 & 35 & 1 \\
\hline 12 & 56.9 & 56.9 & 30 & & 12 & 53.8 & 53.3 & & 35 & & 12 & 52.4 & 52.6 & 33 & & 12 & $54 . \mathrm{I}$ & 54.8 & 36 & $y$ \\
\hline 14 & 54 & 66 & 33 & -15.5 & 14 & 53.1 & 53.0 & & 35 & -13.9 & I4 & 52.7 & 52.9 & 34 & -12.7 & I4 & 55.2 & 55.9 & 38 & -II.7 \\
\hline I6 & 54.7 & 54.7 & 33 & (1) & 16 & 53.7 & 53.2 & & 35 & & I6 & 53.4 & 53.7 & 35 & & 16 & 54.7 & 55.3 & 37 & \\
\hline 18 & 57.0 & 57.0 & 29 & 1 & 18 & 53.9 & 53.6 & & 34 & & 18 & 54.0 & 54 & 36 & 4 & 18 & & & 38 & b \\
\hline 20 & 55.5 & $55 \cdot 3$ & 32 & tit. & 20 & $54 . \mathrm{I}$ & 53.9 & & 34 & 6 & 20 & 54.5 & 54.8 & 36 & 8 & 20 & 55.8 & 56.2 & 39 & 1 \\
\hline 22 & 54.7 & 54.2 & 33 & 4 & 22 & 54.2 & 54.0 & & 34 & 1. & 22 & 54.2 & 54.2 & 36 & E & 22 & 56.2 & 56.9 & 39 & 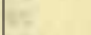 \\
\hline 24 & 55.4 & 55.2 & 32 & $\theta$ & 24 & 54.1 & 54.0 & & 34 & & 24 & 54.2 & 54. & 36 & 5 & 24 & 55.3 & & 38 & 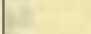 \\
\hline 26 & 55.3 & 55.1 & 32 & t. & 26 & 54.2 & 54.1 & & 34 & & 26 & 54.1 & 54.2 & 36 & e & 26 & 57.2 & & $4 I$ & 8 \\
\hline 28 & 54.8 & 54.2 & 33 & & 28 & 54.0 & 53.9 & & 34 & & 28 & 54.2 & 54.3 & 36 & & 28 & 57.9 & 58.8 & 42 & 1. \\
\hline 30 & 54.8 & 54.2 & 33 & -15.0 & 30 & 53.8 & 53.3 & & 35 & -13.9 & 30 & 54.1 & 54.1 & $3^{6}$ & -12.3 & 30 & 56.8 & 57.0 & 40 & $-\mathrm{II} .7$ \\
\hline 32 & 54.2 & 53.9 & 34 & & 32 & & & & 35 & & 32 & 53.9 & & 35 & & 32 & 56.3 & 56.9 & 40 & \\
\hline 34 & $55 . \mathrm{I}$ & $55 . \mathrm{I}$ & 32 & & 34 & 53.8 & 53.6 & & 34 & & 34 & 53.3 & 53.8 & 35 & & 34 & 56 & Ia & 39 & \\
\hline 36 & 57.3 & 57.1 & 29 & & 36 & 53.5 & 53.2 & & 35 & & 36 & 53.8 & 53.9 & 35 & $t$ & 36 & 54.8 & $55 . I$ & 37 & \\
\hline 38 & $56 . I$ & 55.7 & $3 I$ & te & 38 & 53.8 & 53.4 & & 35 & & 38 & 53.8 & 53.9 & 35 & & 38 & 54.8 & $55 . \mathrm{I}$ & 37 & \\
\hline 40 & 55.0 & 55.0 & 32 & $E$ & 40 & & 53.5 & & 35 & 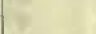 & 40 & $53 . \mathrm{I}$ & 53.1 & 34 & 15 & 40 & 53.8 & 54.0 & 35 & 1 \\
\hline 42 & 56.1 & 56.1 & $3 I$ & $1=$ & 42 & 53.8 & 53.7 & & 34 & 16 & 42 & 52.8 & 52.9 & 34 & & 42 & 52.8 & 53.0 & 34 & \\
\hline 44 & 56.0 & 55.9 & 3I & -14.9 & 44 & 53.8 & 53.4 & & 35 & -13.9 & 44 & 52.4 & 52.8 & 33 & -12.0 & 44 & 54.9 & 55.0 & 37 & $-\mathrm{II} .7$ \\
\hline 46 & 54.2 & 54.2 & 34 & & 46 & 53.9 & 53.8 & & 34 & & 46 & 53.0 & 53.1 & 34 & & 46 & 53.4 & 53.8 & 35 & \\
\hline 48 & 54.8 & 54.8 & 33 & & 48 & 54.0 & 54.0 & & 34 & & $4^{8}$ & 53.0 & & 34 & & 48 & 53.2 & 53.9 & 35 & \\
\hline 50 & 57.1 & 57.0 & 29 & & 50 & 53.9 & 53.8 & & 34 & & 50 & 53.2 & 53.8 & 35 & & 50 & 56.1 & & 39 & \\
\hline 52 & $56 . I$ & 56.1 & $3 I$ & & 52 & 54.7 & $54 \cdot 3$ & & 33 & & 52 & 53.8 & 54.0 & 35 & & 52 & 56.0 & 56.2 & 39 & \\
\hline 54 & 54.7 & 54.2 & 33 & & 54 & 54.1 & 54.0 & & 34 & & 54 & 54.2 & 54.6 & 36 & & 54 & $54 . I$ & 54.6 & 36 & \\
\hline 56 & 53.2 & 53.2 & 35 & & 56 & 54.1 & 53.8 & & 34 & & 56 & 54.7 & 54.9 & 37 & & 56 & 54.4 & 54.7 & 36 & \\
\hline 58 & 53.8 & 53.7 & 34 & & 58 & 53.8 & $53 \cdot 3$ & & 35 & $-\mathrm{I}_{3} .8$ & 58 & 54.8 & 54.9 & 37 & & & 54.2 & 54.5 & 36 & \\
\hline & & & & 58 & 2000 & 53.9 & 53.2 & & 35 & & & & & & & 2400 & 54.0 & 54.8 & 36 & -11.7 \\
\hline
\end{tabular}

Correction to local mean time is + Im 49 s. $90^{\circ}$ torsion $=24 .{ }^{\prime} 6$. Torsion head at $15 \mathrm{~h} 40 \mathrm{~m}$ read $347^{\circ}$ and at $20 \mathrm{~h} 20 \mathrm{~m}$ read $34 \mathrm{I}^{\circ}$. Observer-W. J. P.
Correction to local mean time is - 20 s.

Torsion head at $\mathrm{I} g \mathrm{~h} 45 \mathrm{~m}$ read $344^{\circ}$ and at $24 \mathrm{~h}$ I $5 \mathrm{~m}$ read the same. Observer-R. R. T. 
Tabulation of magnetic declinations observed at Teplitz Bay-Continued

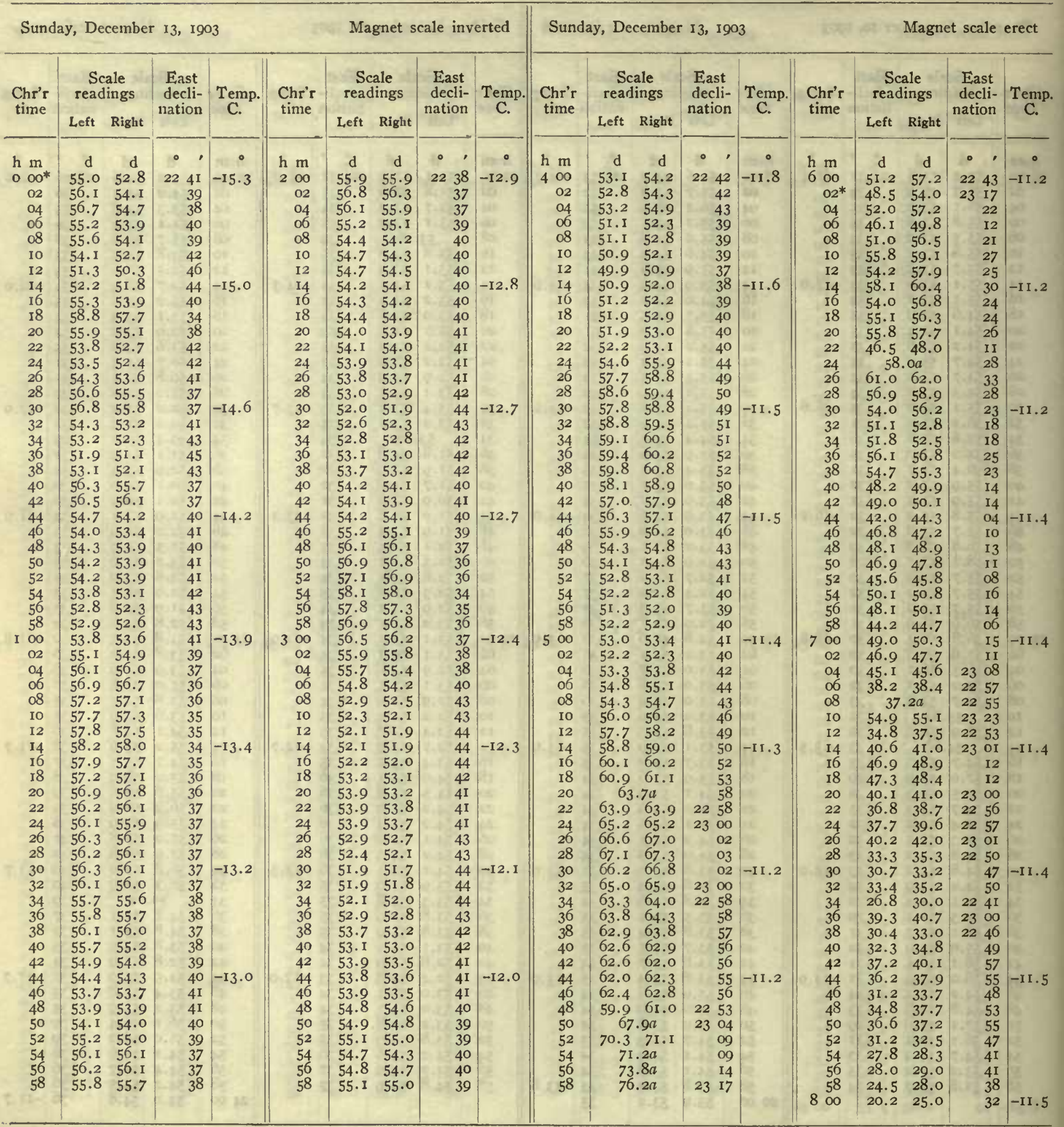

Observer-W. J. P.

Correction to local mean time is -45 s.

Torsion liead at oh oom read $350^{\circ}$ and at gh $30 \mathrm{~m}$ read the same.

Observer-W. J. P. 
Tabulation of magnetic declinations observed at Teplitz Bay-Continued

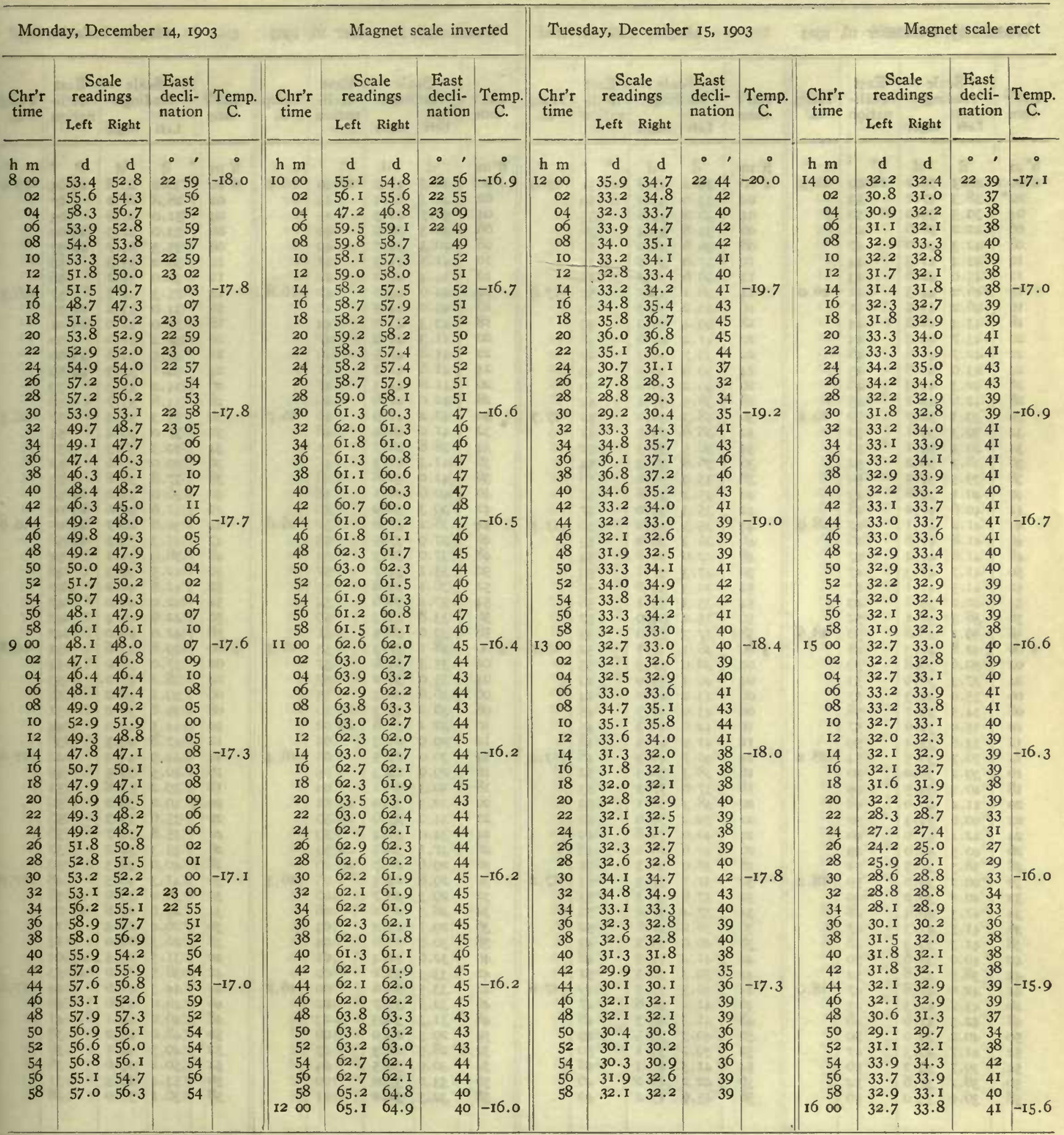

Correction to local mean time is -035 . $90^{\circ}$ torsion $=24 .^{\prime} \mathrm{x}$. Torsion head at $7 \mathrm{~h} 30 \mathrm{~m}$ read $350^{\circ}$ and at $12 \mathrm{~h} 20 \mathrm{~m}$ read $356^{\circ}$. Observer-R. R. T.
Correction to local mean time is $-18 \mathrm{~s} .90^{\circ}$ torsion $=24 .^{\prime} 9$. Torsion head at $12 \mathrm{~h}$ oom read $35 \mathrm{I}^{\circ}$ and at $\mathrm{I} 6 \mathrm{~h}$ oom read $34 \mathrm{I}^{\circ}$. Observer-R. R. T. 
Tabulation of magnetic declinations observed at Teplitz Bay-Continued

\begin{tabular}{|c|c|c|c|c|c|c|c|c|c|c|c|c|c|c|c|c|c|c|c|c|}
\hline \multicolumn{5}{|c|}{ Wednesday, December I6, 1903} & \multicolumn{5}{|c|}{ Magnet scale inverted } & \multicolumn{5}{|c|}{ Wednesday, December 16, 1903} & \multicolumn{6}{|c|}{ Magnet scale inverted } \\
\hline \multirow{2}{*}{$\begin{array}{l}\text { Chr'r } \\
\text { time }\end{array}$} & \multicolumn{2}{|c|}{$\begin{array}{l}\text { Scale } \\
\text { readings }\end{array}$} & \multirow{2}{*}{$\begin{array}{c}\text { East } \\
\text { decli- } \\
\text { nation }\end{array}$} & Temp. & Chr'r & $\begin{array}{l}\text { Sca } \\
\text { readi }\end{array}$ & $\begin{array}{l}\text { ale } \\
\text { ings }\end{array}$ & $\begin{array}{c}\text { East } \\
\text { decli- }\end{array}$ & Temp. & Chr'r & $\begin{array}{l}\text { Sci } \\
\text { read }\end{array}$ & $\begin{array}{l}\text { ale } \\
\text { ings }\end{array}$ & $\begin{array}{l}\text { East } \\
\text { decli- }\end{array}$ & Temp. & Chr'r & $\begin{array}{l}\text { Sc } \\
\text { read }\end{array}$ & $\begin{array}{l}\text { ale } \\
\text { ings }\end{array}$ & $\begin{array}{l}\text { East } \\
\text { decli }\end{array}$ & & \\
\hline & Left & Right & & C. & & Left & Right & & & & Left & Right & & & & Left & Right & natior & & \\
\hline $\mathrm{h} \mathrm{m}$ & d & d & - ' & 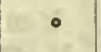 & $\mathrm{h} \mathrm{m}$ & d & d & $\cdot 1$ & $\circ$ & h $\mathrm{m}$ & d & d & & & h $\mathrm{m}$ & d & d & - & ' & - \\
\hline 0.0 .2 & $\begin{array}{l}40.3 \\
38.3\end{array}$ & $\begin{array}{l}39.1 \\
37 . I\end{array}$ & 2249 & -20.5 & 200 & 39.2 & 37.8 & 2251 & -17.9 & 400 & 46.3 & $45 \cdot 3$ & 2240 & -16.9 & 600 & 30.2 & 29.8 & 230 & 5. & -16.2 \\
\hline $\begin{array}{l}02^{+} \\
04\end{array}$ & $\begin{array}{l}38.3 \\
39.5\end{array}$ & $\begin{array}{l}37.1 \\
39.0\end{array}$ & $\begin{array}{l}53 \\
50\end{array}$ & 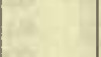 & $\begin{array}{l}02 \\
04\end{array}$ & $\begin{array}{l}33.6 \\
36.6\end{array}$ & $\begin{array}{l}33.0 \\
35.8\end{array}$ & $\begin{array}{l}59 \\
55\end{array}$ & Q & $\begin{array}{l}02 \\
04\end{array}$ & $\begin{array}{l}44 \cdot 3 \\
42.7\end{array}$ & $\begin{array}{l}43.4 \\
42.3\end{array}$ & $\begin{array}{r}43 \\
45\end{array}$ & & $\begin{array}{l}02 \\
04\end{array}$ & $\begin{array}{l}28.8 \\
31.0\end{array}$ & $\begin{array}{l}28.2 \\
30.6\end{array}$ & $\begin{array}{l}0 \\
0\end{array}$ & 3 & \\
\hline 06 & 42.2 & 38.6 & 48 & 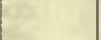 & 06 & 35.8 & 35.4 & 56 & & 06 & 40.3 & 39.9 & 49 & & 06 & 33.0 & 32.3 & 230 & 0 & \\
\hline 08 & 42.3 & 40.0 & 47 & & 08 & 36.6 & 36.0 & 55 & 10 & 08 & 40.3 & 39.8 & 49 & & 08 & $35 \cdot 3$ & 34.7 & 225 & 7 & \\
\hline I0 & 44.4 & $4 \mathrm{I} .3$ & 44 & & I0 & 37.0 & 36.8 & 54 & 97 & Io & 41.5 & 4 I. 3 & 47 & 21 & Io & 36.0 & 35.3 & & 6 & \\
\hline 12 & 45.0 & 42.6 & 43 & & 12 & 38.6 & 38.2 & 52 & & I2 & 44.4 & 44.0 & 42 & & 12 & 36.0 & $35 . \mathrm{I}$ & & 6 & \\
\hline I4 & 46.2 & $44 \cdot 3$ & $4 \mathrm{I}$ & -20.0 & 14 & 38.1 & 37.5 & 52 & -17.8 & I 4.1 & 44.6 & 44.0 & 42 & -16.8 & 14 & 35.3 & $34 \cdot 3$ & & 57 & -16.5 \\
\hline 16 & 46.2 & 43.5 & $4 I$ & & 16 & 40.2 & 39.9 & 49 & & I6 & 45.0 & $44 \cdot 5$ & 42 & & I6 & 34.0 & 33.2 & 225 & 99 & \\
\hline I8 & 43.4 & 42.2 & 44 & 4 & 18 & 40.4 & 40.0 & 49 & $t$ & I8 & $43 \cdot 3$ & 43.0 & 44 & & 18 & 33.0 & 32.0 & 230 & I & \\
\hline 20 & 43.7 & 42.3 & 44 & & 20 & $4 \mathrm{I} .2$ & 40.6 & 48 & 12 & 20 & $4 \mathrm{I} \cdot 3$ & 40.9 & 47 & ce & 20 & 32.2 & 31.0 & & 22 & \\
\hline 22 & 42.7 & 41.6 & 46 & 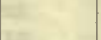 & 22 & 39.7 & 39.3 & 50 & $E$ & 22 & 41.8 & 41.5 & 46 & 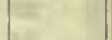 & 22 & 33.5 & 32.6 & 230 & so & \\
\hline 24 & 43.0 & 41.8 & 45 & $F$ & 24 & 39.9 & 39.5 & 49 & 16 & 24 & 41.6 & 41.3 & 47 & trat & 24 & 34.1 & 33.3 & 225 & 59 & \\
\hline 26 & 42.3 & $4 \mathrm{I} \cdot 3$ & 46 & E. & 26 & 39.2 & 38.8 & 50 & ch & 26 & 41.6 & 41.3 & 47 & c & 26 & 34.2 & $33 \cdot 3$ & & 59 & \\
\hline 28 & 42.6 & $42 . \mathrm{I}$ & 45 & & 28 & 38.5 & 38.1 & 52 & $=$ & 28 & 42.0 & 41.6 & 46 & 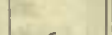 & 28 & 34.6 & 33.7 & & 58 & \\
\hline 30 & 39.3 & 38.7 & 50 & -19.5 & 30 & 38.3 & 38.0 & 52 & -17.7 & 30 & 42.2 & 41.9 & 46 & -16.7 & 30 & 35.3 & $34 \cdot 3$ & & 57 & -16.5 \\
\hline 32 & 39.3 & 39.1 & 50 & & 32 & 38.6 & 38.5 & $5 I$ & & 32 & 41.0 & 40.4 & 48 & & 32 & 37.0 & 36.4 & & 54 & \\
\hline 34 & 39.4 & 39.1 & 50 & 5 & 34 & 38.5 & 38.3 & $5^{2}$ & & 34 & 38.8 & 38.7 & $5 I$ & & 34 & 39.3 & 38.3 & & 51 & \\
\hline 36 & 38.3 & 38.2 & 52 & 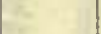 & 36 & 36.8 & 36.3 & 54 & & 36 & $41 \cdot 3$ & 41.3 & 47 & 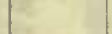 & 36 & 39.0 & 37.6 & & 52 & \\
\hline $3^{8}$ & 39.5 & 39.5 & 50 & 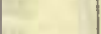 & $3^{8}$ & 35.9 & 35.6 & 56 & & 38 & 41.0 & 40.8 & 48 & & $3^{8}$ & 37.8 & 36.8 & & 53 & \\
\hline 40 & $40 . I$ & 39.8 & 49 & 1 & 40 & 35.2 & 34.8 & 57 & $y$ & 40 & 40.6 & 40. I & 48 & ( & 40 & 36.0 & 35.2 & 225 & 56 & $y$ \\
\hline 42 & 40.6 & 40.2 & 48 & 1 & 42 & 35.3 & 35.0 & 57 & $H$ & 42 & 39.3 & $3^{8} .9$ & 50 & 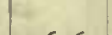 & 42 & 31.6 & 30.6 & 230 & 3 & \\
\hline 44 & $4 \mathrm{I} .2$ & $4 \mathrm{I}$. I & 47 & -18.9 & 44 & 35.5 & 33.0 & 58 & $-17 \cdot 5$ & 44 & 38.2 & 37.8 & 52 & -16.6 & 44 & 30.6 & 28.7 & 230 & 05. & -16.6 \\
\hline 46 & 41.8 & 41.6 & 46 & & 46 & 36.3 & 36.0 & 55 & & 46 & 37.9 & 37.5 & 53 & & 47 & 35.3 & $33 \cdot 3$ & 225 & 58 & \\
\hline 48 & 4I. 6 & $4 \mathrm{I} .4$ & 47 & t & 48 & 37.0 & 36.6 & 54 & 4 & 48 & 37.0 & 36.3 & 54 & 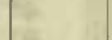 & 48 & 38.9 & 35.1 & & 54 & \\
\hline 50 & 41.7 & $4 \mathrm{I} \cdot 3$ & 47 & $=$ & 50 & 37.3 & 37.1 & 53 & z & 50 & 35.1 & 34.7 & 57 & & 50 & 42.5 & 39.3 & & 48 & \\
\hline 52 & 41.5 & $4 \mathrm{I} . \mathrm{I}$ & 47 & 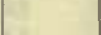 & 52 & $35 \cdot 3$ & 34.6 & 57 & 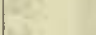 & 52 & 36.1 & 35.8 & 55 & & $5^{2}$ & 42.8 & 40.2 & & 47 & \\
\hline 54 & 40.2 & 39.8 & 49 & 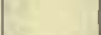 & 54 & $35 \cdot 3$ & 35.1 & 56 & 28 & 54 & 37.1 & 36.6 & 54 & 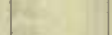 & 54 & 42.0 & 40.0 & & 47 & \\
\hline 56 & 38.3 & 38.1 & 52 & & 56 & 35.2 & 34.9 & 57 & & 56 & 33.8 & 33.3 & 59 & & 56 & 42.6 & 40.4 & & 47 & \\
\hline $5^{8}$ & 39.2 & 38.8 & 50 & -18.6 & 58 & 37.3 & 37.0 & 53 & & 58 & 39.0 & $3^{8} .5$ & 51 & & $5^{8}$ & 42.0 & 40.0 & & 47 & \\
\hline 100 & 40.8 & 40.3 & 48 & & 300 & 38. I & 37.7 & 52 & -17.5 & 500 & 38.1 & 37.3 & 53 & -16.5 & 700 & 39.6 & 37.6 & & $5 \mathrm{I}$ & -16.8 \\
\hline 02 & 39.6 & 38.8 & 50 & 0 & 02 & 38.0 & 37.5 & 53 & & 02 & $35 \cdot 3$ & 34.6 & 57 & & 02 & 37.0 & 36.0 & & 54 & \\
\hline 04 & 37.3 & 36.9 & 53 & 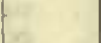 & 04 & 40.3 & 39.9 & 49 & 60 & 04 & 35.0 & 34.2 & 57 & $E$ & $0_{4}$ & 38.2 & 37.2 & & 53 & 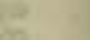 \\
\hline 06 & 35.0 & 34.3 & 57 & $=$ & 06 & 43.2 & 43.0 & 44 & 60 & 06 & 36.6 & 36.3 & 55 & ta & 06 & 39. I & 38.2 & & $5 \mathrm{I}$ & \\
\hline 08 & 34.5 & $34 . \mathrm{I}$ & 58 & 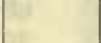 & 08 & 43.0 & 42.5 & 45 & th & 08 & 36.2 & $35 \cdot 3$ & 2256 & 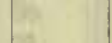 & 08 & 39.6 & 38.0 & & $5 \mathrm{I}$ & 5 \\
\hline 10 & 35.0 & 34.6 & 57 & 1 & To & 42.6 & 42.3 & 45 & 4 & Io & 32.1 & 31.8 & 2302 & 4 & I0 & 44. I & 42.0 & & 44 & \\
\hline 12 & 33.8 & 33.4 & 59 & & I2 & $4 I .9$ & $4 \mathrm{I} .5$ & 46 & -1 & 12 & 29.9 & 29.3 & os & 1 & I2 & 47.8 & 46.3 & & 38 & \\
\hline 14 & 36.7 & 36.5 & 54 & -18.2 & I4 & 40.7 & 40.3 & $4^{8}$ & $-\mathrm{I} 7.4$ & 14 & 30.0 & 29.5 & 05 & -16.4 & I4 & 44.9 & 44.3 & & 42 & -170 \\
\hline 16 & 39.2 & 39.0 & 50 & & 16.2 & 38.3 & $3^{8} .3$ & 52 & & 16 & 29.9 & 29.3 & 05 & & I6 & 40.5 & 39.5 & & 49 & \\
\hline 18 & 40.0 & 39.5 & 49 & 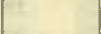 & 18 & 37.6 & $37 \cdot 3$ & 53 & 1 & 18 & 30.3 & 30.1 & 0 & 4 & I8 & 39.2 & 37.8 & & $5 \mathrm{I}$ & 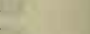 \\
\hline 20 & $4 \mathrm{I} .2$ & 40.0 & 48 & 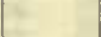 & 20 & $3^{8} .3$ & 38.1 & 52 & 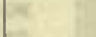 & 20 & 32.3 & 32.0 & 2301 & $=$ & 20.3 & 40.0 & 39.0 & & 50 & \\
\hline 22 & 40.0 & 39.3 & 50 & 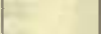 & 22 & 39.2 & 39.0 & 50 & 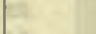 & 22 & 34.0 & 33.6 & 2259 & $t^{2}$ & 22 & 35.6 & 35.3 & & 56 & 5 \\
\hline 24 & $42 . I$ & $4 \mathrm{I} . \mathrm{O}$ & 46 & $=$ & 24 & 40.0 & 39.8 & 49 & $=$ & 24 & 34.0 & 33.4 & 59 & 4 & 24 & 35.8 & 34.7 & & 56 & \\
\hline 26 & 40.5 & 40.0 & 49 & 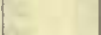 & 26 & 40.6 & 40.2 & 48 & 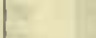 & 26 & 37.1 & 36.5 & 225 & 8 & 26 & 35.3 & 34.2 & & 57 & \\
\hline 28 & 38.3 & 37.5 & 52 & (1) & 28 & 38.9 & 38.3 & $5 I$ & $1=$ & 28 & 29.6 & 28.7 & $23 \circ$ & & 28.2 & 40.0 & 39.3 & & 50 & \\
\hline 30 & 40.2 & 39.0 & 50 & -18.2 & 30 & 37.7 & $37 \cdot 3$ & 53 & -17.2 & 30 & 29.8 & 29.3 & 0 & -16.3 & 30 & $41 \cdot 3$ & 40.5 & & 48 & -17.0 \\
\hline 32 & 40.2 & 39.2 & 49 & & 32 & 37.8 & $37 \cdot 3$ & 5.3 & & $3^{2}$ & 29.3 & 28.7 & o & & 32 & 36.0 & 33.0 & 225 & 58 & \\
\hline 34 & 4 I. 3 & 40.4 & 48 & 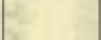 & 34 & 37.4 & 37.4 & 53 & tc. & 34 & 28.3 & $28 . \mathrm{I}$ & os & C. & 34 & 29.0 & 25.8 & 230 & 09 & \\
\hline 36 & 41.5 & 41.3 & 47 & & 36 & $3^{8} .2$ & 37.6 & 52 & 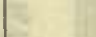 & 36 & 29.7 & 29.3 & 05 & & 36 & 26.4 & 22.4 & & 14 & \\
\hline 38 & 41.3 & 39.9 & 48 & 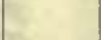 & 38 & 40.0 & 39.5 & 49 & 1 & 38 & 30.9 & 30.6 & 04 & & 38 & 26.3 & 22.0 & & 14 & \\
\hline 40 & 40.0 & 38.6 & 50 & 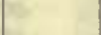 & 40 & 41.2 & 40.5 & 48 & 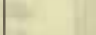 & 40 & 32.6 & 32.4 & 01 & te & 40 & $3 \mathrm{I} .5$ & 29.5 & 230 & 04 & \\
\hline 42 & 38.9 & 37.8 & 52 & & 42 & 39.3 & 38.5 & 5I & & 42 & 33.3 & 33.1 & oc & & 42 & 38.5 & 36.0 & 225 & 53 & \\
\hline 44 & 42.0 & 40.8 & 47 & -18.0 & 44 & 40.3 & 39.5 & 49 & -17.0 & 44 & 33.5 & 32.6 & or & -16.2 & 44 & $44 \cdot 3$ & 41.6 & & 44 & -17.0 \\
\hline 46 & 43.2 & 42.3 & 45 & & 46 & 41.2 & 40.8 & 47 & . & 46 & 32.3 & 31.7 & 02 & & 46 & 46.0 & 45.0 & & 40 & \\
\hline 48 & 42.3 & 41.6 & 46 & 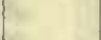 & 48 & 41.3 & 40.3 & 48 & it: & 48 & 31.3 & 30.3 & 0 & 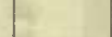 & 48 & 46.0 & $44 . I$ & & 4I & \\
\hline 50 & 40.2 & 39.0 & 50 & & 50 & 40.3 & 39.5 & 49 & & 50 & 29.3 & 29.0 & oc & & 50 & 48.0 & 45.2 & & 38 & \\
\hline 52 & 41.7 & 40.3 & 47 & 1 & 52 & 39.7 & $39 . I$ & 50 & & 52.2 & 28.9 & 28.3 & of & 5 & 52 & 49.3 & $44 . I$ & & 38 & \\
\hline 54 & 39.7 & 39.2 & 50 & & 54 & 41.3 & 40.3 & 48 & & 54 & 30.9 & 30.5 & o. & 2 & 54 & 50.2 & 45.6 & & 36 & \\
\hline 56 & 38.2 & 37.6 & 52 & $=$ & 56 & 42.2 & 41.0 & 46 & & 56 & 33.0 & 33.0 & oc & & 56 & 53.4 & 49.3 & & $3 \mathrm{I}$ & \\
\hline 58 & 36.0 & 35.0 & 56 & & 58 & 45.9 & 45.2 & 40 & 2 & 58 & 32.3 & 32.0 & o. & & 58 & 56.1 & 51.0 & & 28 & \\
\hline
\end{tabular}


Tabulation of magnetic declinations observed at Teplitz Bay-Continued

\begin{tabular}{|c|c|c|c|c|c|c|c|c|c|c|c|c|c|c|c|c|c|c|c|c|}
\hline \multicolumn{5}{|c|}{ Wednesday, December I6, 1903} & \multicolumn{5}{|c|}{ Magnet scale inverted } & \multicolumn{5}{|c|}{ Wednesday, December I6, 1903} & \multicolumn{6}{|c|}{ Magnet scale inverted } \\
\hline \multirow{2}{*}{$\begin{array}{l}\text { Chr'r } \\
\text { time }\end{array}$} & \multicolumn{2}{|c|}{$\begin{array}{l}\text { Scale } \\
\text { readings }\end{array}$} & $\begin{array}{l}\text { East } \\
\text { decli- }\end{array}$ & Temp. & Chr'r & $\begin{array}{r}\mathrm{Sc} \\
\text { read }\end{array}$ & $\begin{array}{l}\text { ale } \\
\text { lings }\end{array}$ & $\begin{array}{l}\text { East } \\
\text { decli- }\end{array}$ & & Chr's & $\begin{array}{l}\mathrm{Sc} \\
\text { read }\end{array}$ & $\begin{array}{l}\text { dings } \\
\text { ding }\end{array}$ & $\begin{array}{l}\text { East } \\
\text { decli- }\end{array}$ & & Chr'r & $\begin{array}{r}\text { Sc } \\
\text { read }\end{array}$ & $\begin{array}{l}\text { ale } \\
\text { lings }\end{array}$ & $\begin{array}{l}\text { Eas } \\
\text { dec }\end{array}$ & $\begin{array}{l}\text { st } \\
\text { cli- }\end{array}$ & \\
\hline & Left & Right & & & & L.eft & Right & & & & Left & Right & & & & Left & Right & & & \\
\hline $\mathrm{h} \mathrm{m}$ & d & d & $\circ \quad$ & $\circ$ & $h \mathrm{~m}$ & d & d & & $\circ$ & $\mathrm{h} \mathrm{m}$ & $d$ & d & & & $\mathrm{h} \mathrm{m}$ & $d$ & d & & & ${ }^{\circ}$ \\
\hline 800 & 54.0 & 51.7 & 2229 & -17.0 & 1000 & 50.7 & 48.4 & 2234 & -16.4 & 1200 & 47.7 & $47 \cdot 3$ & 2237 & -16.2 & I4 00 & $49 . I$ & 48.9 & & 35 & -16.2 \\
\hline 03 & 53.2 & 48. I & 32 & & 02 & 44.1 & $42 . I$ & 44 & & 02 & 47.7 & 47.3 & 37 & $=0$ & 02 & $50 . I$ & 50.0 & & 33 & $=$ \\
\hline 04 & 49.3 & $45 . I$ & 38 & 20 & 04 & 45.9 & $45 . \mathrm{I}$ & 40 & 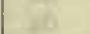 & 04 & 47.7 & 47.6 & 37 & 20 & 04 & 49.8 & 49.3 & & 34 & 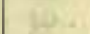 \\
\hline 06 & 47.2 & 42.5 & $4 \mathrm{I}$ & A & 06 & $5 \mathrm{I} . \mathrm{I}$ & 50.2 & 32 & & 06 & 48.7 & 48. & 36 & n & 06 & 49.2 & 49.2 & & 34 & 80 \\
\hline 08 & 48.2 & 44.2 & 39 & E & 08 & 51.9 & 50.9 & $3 I$ & 60 & 08 & 48. I & 48.1 & 36 & wa & 08 & 51 & $.8 b$ & & 30 & ial \\
\hline 10 & 51.7 & 47.2 & 34 & nt & I0 & 50.2 & 48.9 & 34 & $2 t$ & 10 & 48.0 & 47.9 & 36 & - & 10 & 53.8 & 53.3 & & 28 & at \\
\hline 12 & 51.8 & 47.8 & 34 & & 12 & 47.9 & 45.9 & 38 & & I2 & 47.8 & 47.6 & 37 & & 12 & 53.2 & 53.0 & & 28 & \\
\hline 14 & 41.9 & 38.7 & 48 & -17.0 & 14 & 46.8 & 44.3 & 40 & -16.4 & I4 & 47.8 & 47.5 & 37 & -16.2 & 14 & 53.0 & 52.9 & & 29 & -16.3 \\
\hline 17 & $4 \mathrm{I} . \mathrm{I}$ & 37.2 & 50 & & I6 & 48.2 & 46.3 & 38 & & I6 & 47.7 & 47.2 & 37 & की & I6 & 52.7 & $52 . \bar{I}$ & & 30 & tat \\
\hline 18 & 44.9 & 40.9 & 44 & 16 & 18 & $47 \cdot 5$ & 45.7 & 38 & int & 18 & 47.6 & 47.2 & 37 & 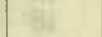 & 18 & 53.4 & 53.0 & & 28 & Bar \\
\hline 20 & $47 . I$ & $44 \cdot 3$ & 40 & $\infty$ & 20 & 48.9 & 46.9 & 36 & re & 20 & 47.9 & 47.7 & 37 & 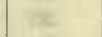 & 20 & 34.1 & & & 27 & 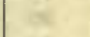 \\
\hline 22 & 49.7 & $41 . I$ & 40 & 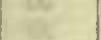 & 22 & 47.0 & 45.9 & 39 & tian & 22 & 48.0 & 47.8 & 36 & 3 & 22 & $5 \mathrm{I} .5$ & 50.8 & & 32 & st: \\
\hline 24 & 42.8 & 40.0 & 47 & $=$ & 24 & 42.9 & 42.9 & 44 & & 24 & 47.7 & 47.1 & 37 & & 24 & 51.2 & 50.7 & & 32 & 14 \\
\hline 26 & 44.8 & 42.2 & 43 & & 26 & 44.8 & 44.2 & 42 & & 26 & 48.2 & 47.8 & 36 & & 26 & 50.4 & 50.2 & & 33 & 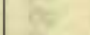 \\
\hline 28 & 44.9 & 41.8 & 44 & & 28 & 47.0 & 46.2 & 38 & & 28 & 47.2 & 47.0 & 38 & & 28 & 50.8 & $50 . I$ & & 33 & 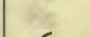 \\
\hline 30 & 40.0 & 37.0 & $5 \mathrm{I}$ & -16.9 & 30 & 50.0 & 49.7 & 34 & -16.3 & 30 & 47.8 & $47 . I$ & 37 & -16.3 & 30 & 50.3 & 49.7 & & 33 & -16.3 \\
\hline 32 & $4 I \cdot 3$ & $37 \cdot 5$ & 50 & & 32 & 50.2 & 49.2 & 34 & 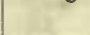 & 32 & 48.1 & 47.6 & 37 & $1=x$ & 32 & 50.3 & & & 33 & er \\
\hline 34 & 45.4 & 42.2 & 43 & 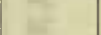 & 34 & 46.0 & 45.9 & 40 & 68 & 34 & 49.0 & 48.6 & 35 & $x_{2}$ & 34 & 50.8 & 50 & & 32 & IY \\
\hline 36 & 46.8 & 43.6 & $4 \mathrm{I}$ & 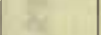 & 36 & 46.2 & 45 & 40 & - & 36 & 48.3 & 47.8 & 36 & it & 36 & 50.0 & 49 & & 34 & स \\
\hline 38 & 39. I & 36.9 & 52 & it & 38 & 46.0 & $45 . I$ & 40 & $\mathrm{E}$ & 38 & 49.0 & 48.6 & 35 & 26 & 38 & 50.0 & 49.7 & & 34 & 残 \\
\hline 40 & 36.0 & 33.7 & 57 & $\infty$ & 40 & 47.0 & 46.0 & 39 & & 40 & 48.8 & 48.1 & 36 & $n$ & 40 & 49.2 & 49.0 & & 35 & 8 \\
\hline 42 & 42.6 & 42.4 & 45 & & 42 & 47.7 & 46.7 & 38 & & 42 & 49.0 & 48.8 & 35 & & 42 & 49.0 & 48.6 & b & 35 & \\
\hline 44 & 45.9 & 45.2 & 40 & -16.9 & 44 & 50.6 & 49.9 & 33 & -16.3 & 44 & 49.9 & 49.2 & 34 & -16.2 & 44 & 49.0 & 48.7 & e & 35 & -16.3 \\
\hline 46 & 45.9 & 45.0 & 40 & 5 & 46 & 48.9 & 48.0 & 36 & & 46 & 49.0 & 48.8 & 35 & 18 & 46 & $49 . I$ & 48.8 & 1 & 35 & 34 \\
\hline 48 & 45.9 & 45.0 & 40 & 4. & 48 & 49.1 & 47.7 & 36 & A & 48 & 47.9 & 47.3 & 37 & 26 & 48 & 48.4 & 48.1 & & 36 & 16 \\
\hline 50 & 42.7 & 41.2 & 46 & we & 50 & 46.9 & 45.9 & 39 & nes & 50 & 47.7 & 47. & 37 & 07 & 5 & 48.2 & 47 & & 36 & 109 \\
\hline 52 & 43.2 & 42.0 & 45 & $e^{2}$ & 52 & 47.8 & 46.8 & 37 & 13 & 52 & 47.8 & 47.8 & 37 & 67 & $5^{2}$ & 48.2 & $47 \cdot 7$ & & 36 & 62 \\
\hline 54 & 46.2 & $45 . I$ & 40 & in & 54 & 48.9 & 48.0 & 36 & ret & 54 & 47.3 & 47.0 & 38 & $y$ & 54 & 48.0 & 47.7 & & 37 & HY \\
\hline 56 & 47.9 & 46.7 & 37 & & 56 & 48.9 & 48.0 & 36 & & 56 & 47.8 & $47 \cdot 3$ & 37 & & 5 & 47.9 & 47 & & 37 & \\
\hline 58 & 46.3 & $45 . I$ & 40 & & 58 & 49.3 & 48.9 & 35 & & $5^{8}$ & 49.2 & 48.9 & 35 & & $5^{8}$ & 47.9 & 47.5 & & 37 & \\
\hline 90 & 44.9 & 44. I & 42 & -16.8 & II 00 & 49.9 & 48.9 & 34 & -16.3 & 1300 & 49.4 & 48.9 & 35 & -16.1 & 1500 & 47.9 & 47.4 & t & 37 & -16.3 \\
\hline 02 & 45.7 & 45.0 & $4 \mathrm{I}$ & & 02 & 49.8 & 48.8 & 34 & $x^{2}$ & 02 & 48.7 & 47.9 & 36 & $x=$ & 02 & $47 \cdot 3$ & 47.1 & & $3^{8}$ & 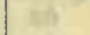 \\
\hline 04 & $40 . I$ & 38.2 & 50 & it & 04 & 49.8 & 48.8 & 34 & & 04 & 48.8 & 48.1 & 36 & in & 04 & 46.3 & 46.1 & & 39 & Wp \\
\hline 06 & 39.8 & 38.3 & 50 & 86 & 06 & 50.7 & $49 . I$ & 33 & & 06 & 48.9 & 48.2 & 36 & & 06 & 45.7 & & & 40 & 60 \\
\hline 08 & 40.9 & 40.I & $4^{8}$ & 6. & 08 & 51.9 & 50.7 & 31 & $5 \%$ & 08 & 49.7 & 49.0 & 34 & 3 & 08 & 45.2 & 45.0 & & $4 \mathrm{I}$ & 6 \\
\hline I0 & 44.0 & 42.2 & 44 & & Io & 52.5 & 50.1 & $3 I$ & +5 & 10 & 49.2 & 48.6 & 35 & $\theta$ & I & & 44 & & $4 \mathrm{I}$ & cr \\
\hline 12 & 45.7 & 43.2 & 42 & & I2 & 53.6 & 52.0 & 29 & & I2 & 49.7 & $49 . I$ & 34 & & I2 & 44.6 & 44.2 & & 42 & \\
\hline I4 & 47.6 & 46.0 & 38 & -16.7 & I4 & 54.8 & 53.8 & 26 & -16.2 & 14 & 50.1 & 49.8 & 33 & -16.1 & I4 & 44. I & 43.7 & & 43 & -16.4 \\
\hline I6 & 50.3 & 48.4 & 34 & 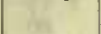 & 16 & 57.2 & 55.9 & 23 & 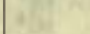 & 16 & 51.2 & 51.0 & 32 & 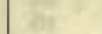 & 16 & 45.2 & 45.0 & & $4 \mathrm{I}$ & 20 \\
\hline 18 & 50.9 & 49.2 & 33 & ent & I8 & 59.0 & 57. & 20 & 85 & Iह & 50.0 & 49 & 33 & 34 & I & 45.8 & & & 40 & at \\
\hline 20 & 55.6 & 53.8 & 26 & 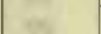 & 20 & 54.9 & 52.0 & 28 & 34 & 20 & 49.9 & 49.2 & 34 & of & 20 & 45.3 & 45 & & $4 \mathrm{I}$ & Et \\
\hline 22 & 54.0 & 52.0 & 28 & $=$ & 22 & 54.7 & 53.2 & 27 & in & 22 & 51.8 & $5 I .3$ & $3 \mathrm{I}$ & 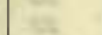 & 2 & & 45.6 & & 40 & $x$ \\
\hline 24 & 52.8 & 51.4 & 30 & & 24 & 52.2 & 50.4 & $3 I$ & & 2. & $48 . I$ & 47 & 3 & 38 & 2 & 46.1 & 9 & & 40 & is \\
\hline 26 & 53.2 & 52.1 & 29 & A & 26 & 55.7 & 54.8 & 25 & & 2 & 47.9 & 47.8 & 3 & & 2 & 45.8 & 45 & & 40 & is \\
\hline 28 & $54 \cdot 3$ & 53.7 & 27 & & 28 & 51.7 & 50.1 & 32 & & 28 & $47 \cdot 3$ & 47.1 & 38 & & 28 & 45.9 & & & 40 & \\
\hline 30 & $52 . I$ & 50.2 & 32 & -16.6 & 30 & 49.8 & 49.2 & 34 & -16.2 & 30 & 46.2 & 45.9 & 40 & -16.0 & 30 & 45.9 & 45.7 & & 40 & -16.3 \\
\hline 32 & 47.0 & 46.3 & 38 & & 32 & 48.1 & 47.0 & 37 & & 32 & 47.2 & 46.9 & 38 & $1=x$ & 32 & $45 . \mathrm{I}$ & 45.0 & & $4 \mathrm{I}$ & $1=0$ \\
\hline 34 & 52.0 & 51.I & $3 \mathrm{I}$ & if & 34 & 48.7 & 47.6 & 36 & 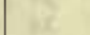 & 34 & 48.1 & 47.9 & 36 & & 34 & 44.4 & 44.2 & & 42 & $x$ \\
\hline 36 & 50. I & 50.1 & 33 & $3^{2}$ & 36 & 48. I & 46.9 & 37 & & 3 & 48.0 & 47.8 & 36 & & 3 & $45 \cdot 3$ & & & $4 I$ & 10 \\
\hline 38 & 50.1 & 49.2 & 34 & $\alpha$ & 38 & 48.0 & 47.0 & 37 & ch & 38 & 47.9 & 47.6 & 37 & 2x & 38 & 44.8 & 44.8 & & 4I & ist \\
\hline 40 & 47.9 & 47.0 & 37 & b8: & 40 & 47.7 & 46.7 & $3^{8}$ & & 40 & 47.7 & 47.2 & 37 & & 40 & 44.7 & 44.4 & & 42 & in \\
\hline 42 & 44.3 & 42.6 & 44 & & 42 & 48.2 & 47.3 & 37 & & 42 & $47 \cdot 3$ & 47.0 & 38 & & 42 & 44.5 & 44.2 & & 42 & \\
\hline & 49.1 & 47.9 & 36 & -16.4 & 44 & 48.5 & $47 . \overline{6}$ & 36 & -16.2 & 44 & 47.2 & $47 . I$ & 38 & -I6.I & 44 & 44.4 & 44.2 & & 42 & -16.3 \\
\hline 46 & 50.7 & 49.6 & 33 & $x^{2}$ & 46 & 47.8 & 46.9 & 37 & $y_{2}$ & 46 & 48.2 & 48.0 & 36 & 18 & 46 & 44.0 & 43.8 & & 43 & 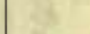 \\
\hline 48 & 50.8 & 49.7 & 33 & iv. & 48 & 47.2 & 46.7 & 38 & $x$ & 48 & 48.8 & 48.6 & 35 & 58 & 48 & 44.3 & 44.2 & & 42 & Et \\
\hline 50 & 48.9 & 47.7 & 36 & (iv & 50 & 47.2 & 47.2 & 38 & 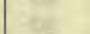 & 50 & 48.8 & 48.0 & 36 & 18 & 50 & $45 . I$ & 45 & & $4 I$ & in \\
\hline & 47.0 & 46.1 & & & 52 & 48.7 & 48.4 & 36 & Ea & 52 & 46.9 & 46.8 & 38 & (kis & 52 & 44.8 & 44.8 & & $4 I$ & E. \\
\hline 54 & 47.7 & 46.4 & 38 & 10 & 54 & 48.6 & 48.4 & 36 & b) & 54 & 47. I & 46.9 & 38 & $x_{1}$ & 54 & 44.8 & 44.8 & & 4I & $k^{2}$ \\
\hline 56 & 48.8 & 47.8 & 36 & $\$$ & 56 & 48.0 & 48.0 & 36 & ne & 56 & 47.0 & 47.0 & 38 & cit & 56 & 45.0 & 45.0 & & $4 I$ & E. \\
\hline 58 & 48.3 & $47 \cdot 3$ & 37 & se & 58 & 47.7 & 47.3 & 37 & 84 & 58 & 47.0 & 47.0 & 38 & 28 & 58 & 45.2 & 45.1 & & $4 I$ & 9 \\
\hline & & & & & & & & & & & & & & & & & & & & \\
\hline
\end{tabular}

Observer-R. R. T.

Observer-R. R. T. 
Tabulation of magnetic declinations observed at Teplitz Bay-Continued

\begin{tabular}{|c|c|c|c|c|c|c|c|c|c|c|c|c|c|c|c|c|c|c|c|c|}
\hline \multicolumn{5}{|c|}{ Wednesday, December 16, 1903} & \multicolumn{5}{|c|}{ Magnet scale inverted } & \multicolumn{5}{|c|}{ Wednesday, December 16,1903} & \multicolumn{6}{|c|}{ Magnet scale inverted } \\
\hline \multirow{2}{*}{$\begin{array}{l}\text { Chr'r } \\
\text { time }\end{array}$} & \multicolumn{2}{|c|}{$\begin{array}{l}\text { Scale } \\
\text { readings }\end{array}$} & $\begin{array}{l}\text { East } \\
\text { decli- }\end{array}$ & Temp. & Chr'r & $\begin{array}{l}\text { Scal } \\
\text { readir }\end{array}$ & $\begin{array}{l}\text { ale } \\
\text { ings }\end{array}$ & East & Temp. & Chr'r & $\begin{array}{l}\mathrm{Sc} \\
\text { read }\end{array}$ & $\begin{array}{l}\text { le } \\
\text { ngs }\end{array}$ & $\begin{array}{l}\text { East } \\
\text { decl }\end{array}$ & & & & $\begin{array}{l}\text { le } \\
\text { hgs }\end{array}$ & $\begin{array}{l}\text { Eas } \\
\text { dec }\end{array}$ & $\begin{array}{l}\text { sst } \\
\text { cli- }\end{array}$ & \\
\hline & Left & Right & & & & Left & Right & & & & Left & Right & & & & Left & Right & & & \\
\hline h n1 & d & d & 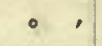 & 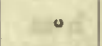 & $\mathrm{h} \mathrm{m}$ & d & d & . 1 & & $\mathrm{~h} \mathrm{~m}$ & d & d & & & $h \mathrm{~m}$ & d & d & & ' & \\
\hline 1600 & $45 \cdot 5$ & 45.2 & $224 \mathrm{I}$ & -16.3 & 1800 & 46.2 & 45.2 & 2240 & -16.0 & 2000 & $49 \cdot 3$ & 49.0 & 223 & $5-16.0$ & 2200 & 54.0 & 53.0 & 22 & 33 & -15.8 \\
\hline 02 & 45.7 & 45.5 & 40 & & 02 & 46.3 & 45.2 & 40 & & 02 & 50.5 & 49.8 & & & 02 & 53.9 & 53.0 & & 33 & \\
\hline 04 & 46.3 & 46. I & 39 & & o & .5 & 45. & 40 & & 04 & 51.2 & 50.6 & 3 & & 04 & 5.2 & 54 & & 31 & \\
\hline 06 & & 6.7 & 38 & & & & & 40 & & 06 & & 50.8 & & & 06 & & & & 29 & \\
\hline 08 & 47.0 & 7.0 & 38 & 10 & 08 & .5 & $45^{\circ}$. & 39 & 3 & 08 & 3.4 & 52.3 & & & 08 & 6 & & & 30 & \\
\hline IO & 47.3 & 47.2 & 38 & & IO & 46.6 & 46.0 & 39 & 01 & 10 & & 53.2 & & 68 & IO & I & & & 32 & \\
\hline 12 & & 47.2 & 38 & otrat & 12 & 7.0 & 46.3 & 38 & 4 & 12 & 50.3 & 49.6 & 3 & 3 & I. & 0 & & & 31 & \\
\hline I4 & 47.1 & 47.0 & 38 & -16.3 & 14 & $47 \cdot 3$ & 47. & 38 . & -16.0 & 14 & 54.0 & 53.3 & 2 & $8-16.0$ & 14 & 4.0 & 53 & & 33 & -16.0 \\
\hline I6 & .2 & 7.1 & 38 & & 16 & $47 \cdot 3$ & & 38 & & 16 & & 53.3 & & & 16 & 5.4 & & & 30 & \\
\hline I8 & 47.1 & 46.9 & $3^{8}$ & 41 & I8 & 46.5 & 46.3 & 39 & & 18 & & 53. & & & 18 & $5^{8}$ & $0 a$ & & 26 & \\
\hline 20 & 47.0 & 6.9 & 38 & $6^{2}$ & 20 & 6.3 & & 39 & 5 & 20 & & 53. & & a & 20 & 58.7 & 58.5 & & 25 & \\
\hline 22 & 46.7 & 46.2 & 39 & 5 & 2 & 5.0 & & 40 & $\mathrm{cs}$ & 22 & & 3 & & & 22 & 3.2 & 58 & & 26 & \\
\hline 24 & & 4 & 39 & fo & & & & 40 & 12 & 24 & & & & & 24 & & & & 8 & \\
\hline 26 & & 46.2 & 39 & $\leqslant$ & 2 & & $46 . I$ & 39 & 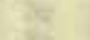 & 26 & & & & & 26 & 7.0 & 57 & & 27 & \\
\hline 28 & & 6.6 & 39 & 25 & 2 & & & 40 & $8=$ & 28 & & & 3 & & 28 & & & & 30 & \\
\hline 30 & $45 \cdot 3$ & $45 . I$ & $4 \mathrm{I}$ & -16.3 & 30 & & 46 & 39. & -16.0 & 30 & & 50.0 & 3 & $3-16.0$ & 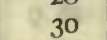 & & & & 30 & -16.0 \\
\hline 32 & & 44.7 & $4 I$ & 100 & 32 & & 46.5 & 38 & & 32 & 50.0 & 49.3 & 3 & & 32 & & & & 35 & \\
\hline 34 & & 44.8 & 4I & 12 & 3 & & & 39 & t? & 34 & & & & & 34 & & & & 22 & \\
\hline 3 & & 45.8 & 40 & 28 & 36 & 46.4 & 46.2 & 39 & 2 & 36 & 50.2 & 49. & & & 36 & 2.5 & 50 & & 6 & \\
\hline 32 & & 4 & $4 \mathrm{I}$ & F & 38 & & 46. & 38 & 48 & 38 & & 49. & & & 3 & & & 2 & 6 & \\
\hline 40 & & 46.8 & 37 & 17 & 40 & 46.7 & 46. & 38 & 10 & 40 & & 48. & & 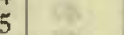 & & & 6 & 3 & 6 & \\
\hline 42 & .0 & 5.1 & 39 & 6 & 4 & 46. & 46.6 & 38 & 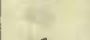 & 42 & & 47. & & aㅐ & & & $4 \mathrm{I} . \mathrm{O}$ & & 8 & \\
\hline 44 & 48.0 & 45.0 & 39 & -16.3 & 44 & 47.5 & 47.2 & 37 & -16.0 & 44 & & 48 & 3 & $5-16.0$ & 44 & 4.8 & & 22 & 53 & -16.0 \\
\hline 46 & 47. & 44.6 & 39 & & & & & 36 & & 46 & & & & & & & & & I0 & \\
\hline 48 & 47.5 & 4.3 & 40 & 4 & 48 & 48.0 & 48.0 & 36 & 4 & 48 & & $\begin{array}{l}40 \\
48\end{array}$ & & & 4 & & & 22 & 6 & \\
\hline 50 & & & 40 & 4 & & & 48.3 & 36 & or: & 50 & 48.4 & 47.4 & & $\operatorname{cog}$ & 5 & 3 & & & 8 & \\
\hline 52 & 47.2 & 4.8 & 40 & $\mathrm{~B}^{2}$ & 52 & & 47.2 & 37 & 4 & 52 & & 52.2 & & 8 & 52 & & & & 6 & \\
\hline 54 & & 5.0 & 39 & B & 54 & .6 & $47 \cdot 3$ & 37 & 6 & 54 & & 50.0 & & & 54 & & 65. & & 4 & \\
\hline 56 & & 5.6 & 38 & & 56 & & & 38 & & 56 & & & & & & & & & 6 & \\
\hline 58 & 48.2 & 46.0 & 38 & & 58 & 46. & $46 . I$ & 39 & & $58^{*}$ & & 28.0 & 233 & & 58 & 3.4 & 61. & & 50 & \\
\hline$\infty 0$ & 48.3 & 46.3 & 37 & -16.2 & 1900 & 46.8 & 46.2 & 39. & -15.8 & 2100 & & 24.0 & I & \begin{tabular}{l|l}
9 & -16.0
\end{tabular} & 2300 & & & & 39 & -16.0 \\
\hline 02 & 48.3 & 46.5 & 37 & & 02 & 46.9 & 46.6 & 38 & & $02^{*}$ & & 36.3 & 243 & & 02 & & 60 & & 51 & \\
\hline 04 & 48.3 & 46.5 & 37 & $\omega^{3}$ & 0 & & 47.0 & 38 & 10 & 04 & & 7.0 & 250 & E & & & & & 54 & \\
\hline 06 & 48.6 & 47.0 & 37 & $\theta^{2}$ & 06 & 47. & 47.0 & 38 & $\alpha$ & 06 & & 65.3 & 240 & ge & a & & & & $\begin{array}{l}34 \\
48\end{array}$ & \\
\hline 08 & 48.1 & 4 & & F & os & & & 37 & G & as & & 30.0 & 231 & 6 & o & & & & 46 & \\
\hline 10 & .6 & 6.3 & 38 & at & IC & 48. & 48. & 3 & of & I0 & & & 245 & it & I & & & & 46 & \\
\hline $\mathrm{I} 2$ & & 4 & 38 & 69 & I? & 48.3 & 48.0 & 36 & & $13^{*}$ & 76.0 & 52.0 & $23 \mathrm{I}$ & & 12 & 9.0 & 67. & & 40 & \\
\hline I4 & 47.8 & 46.0 & 38 & -16.0 & I4 & & 47.1 & 37 & -16.0 & $14^{*}$ & & 34.0 & 224 & $\begin{array}{ll}5 & -15.8\end{array}$ & I & & & & 34 & -16.1 \\
\hline 16 & 47.6 & 46.3 & 38 & & 16 & 46.8 & 46.4 & 38 & & $16^{*}$ & 47 & 34.3 & 242 & & I6 & & & & $4 \mathrm{I}$ & \\
\hline 18 & & 5.4 & 38 & r & I\& & & & 4 & 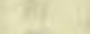 & & & & & & I & & & & & \\
\hline 20 & 48. I & 46.8 & 37 & at & $2 c$ & & $45 . \mathrm{I}$ & $4 \mathrm{I}$ & $a$ & $20^{*}$ & 66.8 & 45.0 & 232 & & 20 & 5.8 & 66 & & 43 & \\
\hline 22 & 47.9 & 46.7 & 37 & es & 22 & & & 4 & $\mathrm{se}$ & 22 & & & & 4 & & & & & & \\
\hline 24 & 48.0 & 46.9 & 37 & $\mathrm{P}^{2}$ & 24 & 45.6 & 45.5 & 40 & is & 24 & 72 & 53.2 & $23 I$ & & 2 & & & & 50 & \\
\hline 26 & 47 & 46.8 & & 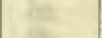 & 26 & & 45. & 40 & $x^{2}$ & 26 & & 3.3 .0 & 223 & & 2 & & & & 49 & \\
\hline 28 & & 46.5 & 38 & & 28 & 4 & & 40 & 84 & 28 & & & 2 & 8 & 28 & & & & 39 & \\
\hline 30 & $47 \cdot 3$ & 46.1 & 38 & -16.0 & 30 & & 45 & 40 & -15.9 & 30 & 67.0 & 65.3 & I & $\begin{array}{ll}3 & -15.5 \\
3\end{array}$ & 30 & 72.3 & 70. & & 35 & -16.0 \\
\hline 32 & & $45 \cdot 3$ & 40 & ton & 32 & & 45.8 & 40 & & 32 & & & & & & & & & 34 & \\
\hline 34 & & 4 & 40 & 48 & 34 & 46 & 45.8 & 40 & & 34 & & 65. & & & 3 & & & & 7 & \\
\hline 36 & & 4 & 41 & 25 & & & & 40 & & 36 & & & & & 3 & & & & 42 & \\
\hline 38 & & 4 & $4 I$ & a & 38 & 48.0 & 47. & 36 & & 38 & & 63 & & & 38 & 1.0 & 70 & & 37 & \\
\hline 40 & 46. & 44 & $4 \mathrm{I}$ & 5 & 40 & & & 37 & 30 & 40 & & & & & & & & & & \\
\hline 42 & & 4 & 40 & 3at & 42 & & 46.3 & 38 & 25 & 42 & 62.3 & 61.3 & 2 & & 42 & 68.3 & & & $4 \mathrm{I}$ & \\
\hline 44 & 46 & 45. & 40 & -16.0 & 44 & 46.8 & 46. & 39 & -16.0 & 44 & 60.8 & & 2 & -15.6 & 44 & & & & 38 & -16.3 \\
\hline & & 45.5 & 39 & & 46 & 46.5 & 46. & 39 & & 46 & 60.0 & 58.8 & & & 46 & & & & 38 & \\
\hline 48 & & & & 82 & 48 & & & 38 & Ae & 48 & & & & & 48 & & & & 40 & \\
\hline 50 & & 46.0 & 39 & 07 & 50 & 47 & 47. & 38 & 44 & 50 & & & & & 50 & 1.0 & & & 40 & \\
\hline 52 & & 45.6 & & 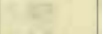 & 52 & & 46. & 39 & $=2$ & 52 & & & 3 & 12 & 52 & & & & 42 & \\
\hline 54 & 46.6 & 45.6 & 39 & 4: & 54 & 45.7 & 45.7 & $\begin{array}{l}39 \\
40\end{array}$ & H5 & 54 & & 53.6 & 3 & - & 5 & 68.5 & 67.8 & & $4 \mathrm{I}$ & \\
\hline 56 & 46.3 & 45.3 & 40 & & 56 & 46.3 & 46. I & 39 & 25 & 56 & & & 3 & $\infty$ & 56 & & & & 40 & \\
\hline 58 & 46.3 & $45 \cdot 3$ & 40 & a & 58 & 47.2 & 46.7 & 38 & 102 & 58 & 54.0 & 53.0 & & (3) & 58 & & 68.1 & & 40 & \\
\hline & & & & & & & & & & & & & & & 2400 & 68.3 & 67.1 & & $4 \mathrm{I}$ & -16.3 \\
\hline
\end{tabular}

Observers-R. R. T. and W. J. P., who alternated from $16 h 44 \mathrm{~m}$ to $16 \mathrm{~h} 46 \mathrm{~m}$.
Correction to local mean time is $+32 \mathrm{~s} .90^{\circ}$ torsion $=25^{\prime} \mathrm{I}$.

Torsion head at $16 \mathrm{~h} 30 \mathrm{~m}$, December I5, read $34 \mathrm{I}^{\circ}$, and at oh $25 \mathrm{~m}$, December 17 , read $346^{\circ}$.

Observer-W. J. P. 
Tabulation of magnetic declinations observed at Teplitz Bay-Continued

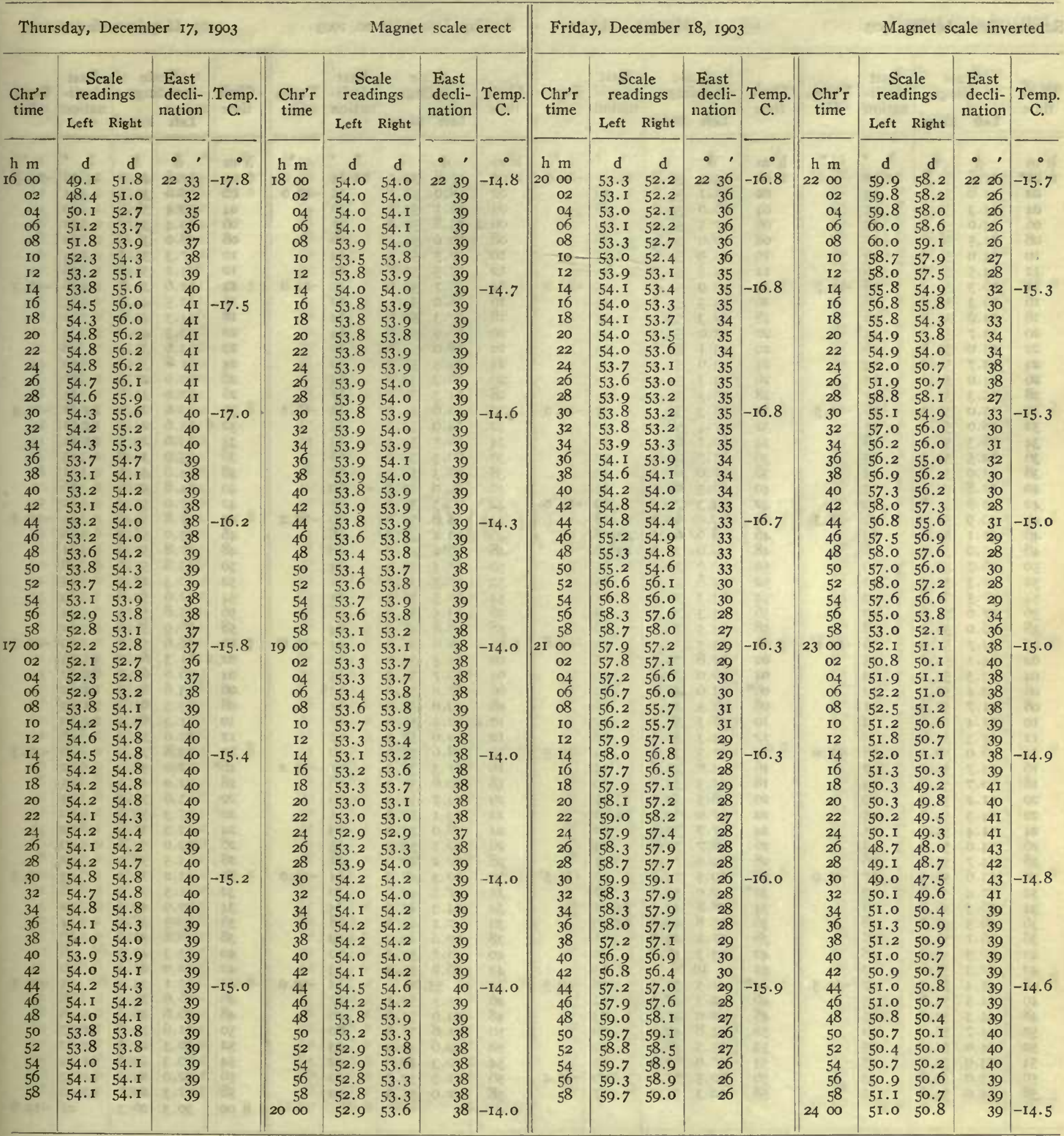

Correction to local mean time is $+45 \mathrm{~s}$.

Torsion head at $15 \mathrm{~h} 10 \mathrm{~m}$ read $344^{\circ}$ and at the end read the same. Observer-R. R. T.
Correction to local mean time is +43 s.

Torsion head at $19 \mathrm{~h} 15 \mathrm{~m}$ read $346^{\circ}$ and at the end read the same. Observer-R. R. T, 
Tabulation of magnetic declinations observed at Teplitz Bay-Continued

\begin{tabular}{|c|c|c|c|c|c|c|c|c|c|c|c|c|c|c|c|c|c|c|c|}
\hline \multicolumn{10}{|c|}{ Sunday, December 20,1903} & \multicolumn{5}{|c|}{ Sunday, Decenber 20, 1903} & \multicolumn{5}{|c|}{ Magnet scale inverted } \\
\hline \multirow{2}{*}{$\underset{\text { tine }}{\text { Chr'r }}$} & \multicolumn{2}{|c|}{$\begin{array}{l}\text { Scale } \\
\text { rcadings }\end{array}$} & \multirow{2}{*}{$\begin{array}{l}\text { Enst } \\
\text { decli- } \\
\text { ration }\end{array}$} & \multirow{2}{*}{$\begin{array}{l}\text { Temp. } \\
\text { C. }\end{array}$} & Chr'r & $\begin{array}{l}\text { Sca } \\
\text { read }\end{array}$ & $\begin{array}{l}\text { ale } \\
\text { lings }\end{array}$ & $\begin{array}{l}\text { East } \\
\text { decli- }\end{array}$ & Temp. & Chr'r & $\begin{array}{l}\text { Sca } \\
\text { readi }\end{array}$ & $\begin{array}{l}\text { ale } \\
\text { ings }\end{array}$ & $\begin{array}{l}\text { East } \\
\text { decli- }\end{array}$ & Temp. & & $\begin{array}{r}\text { Sca } \\
\text { readi }\end{array}$ & $\begin{array}{l}\text { ale } \\
\text { ings }\end{array}$ & $\begin{array}{c}\text { East } \\
\text { decli- }\end{array}$ & Temp. \\
\hline & Left & Right & & & time & L,eft & Right & & & tin & Left & Right & & & & Left & Right & nation & \\
\hline h m & $d$ & d & 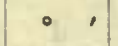 & - & $\mathrm{h} \mathrm{m}$ & d & $\mathrm{d}$ & & 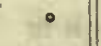 & $\mathrm{h} \mathrm{m}$ & d & d & & - & $\mathrm{h} \mathrm{m}$ & d & d & & - \\
\hline $00^{*}$ & 51.6 & 53.8 & 2232 & 女et & 200 & 55.4 & 55.8 & 2237 & -20.0 & 400 & 57.8 & 52.3 & 2314 & & 600 & 42.6 & 40.0 & 2336 & $-19 \cdot 3$ \\
\hline 02 & 52.0 & 54.6 & 33 & 30 & 02 & 55.1 & 55.6 & 36 & sar & 02 & 58.0 & 53.0 & 13 & -19.0 & 02 & 38.6 & $37 \cdot 5$ & & \\
\hline 04 & 53.3 & 55.6 & 35 & 15 & 04 & 56.0 & 56.8 & 38 & wa & 04 & 56.1 & 51.2 & 16 & 1 & 04 & 39.4 & 38.1 & 40 & \\
\hline 06 & 54.0 & 55.4 & 35 & (nit) & 06 & 57.5 & 58.0 & 40 & $3 y$ & 06.2 & 55.8 & 50.8 & I7 & 16 & 06 & 39.4 & 35.2 & 42 & \\
\hline 08 & 54.5 & 56.6 & 37 & Ant & 08 & 56.0 & 56.6 & 38 & fes & 08 & 56.0 & 51.4 & I6 & hes & 08 & 38.0 & 36.8 & 42 & \\
\hline 10 & 54.0 & 56.0 & 36 & wr & I0 & 53.9 & 54.6 & 35 & $y^{2}$ & 10 & 57.3 & 53.6 & I4 & & 10 & $37 \cdot 3$ & $35 \cdot 3$ & 44 & ay \\
\hline 12 & 54.5 & 56.0 & 36 & xar & 12 & 54.5 & 54.7 & 35 & 5 & 12 & 57.5 & 53.8 & 13 & & 12 & 43.1 & 39.6 & 36 & \\
\hline 14 & 56.3 & 56.3 & 38 & -21.7 & 14 & 53.9 & 54.3 & $35-$ & -20.0 & 14 & 55.7 & 53.0 & 15 & -19.0 & I4 & 42.6 & 39.6 & 36 & -19.3 \\
\hline 16 & 56.3 & 56.9 & 38 & (2) & 16 & 53.5 & 53.8 & 34 & & 16 & 58.0 & 54.6 & 12 & it & 16́ & 42.5 & 40.8 & 35 & \\
\hline 18 & 54.9 & 55.6 & 36 & . & 18 & $53 \cdot 3$ & $53 \cdot 3$ & 33 & 61 & 18 & 57.3 & 53.6 & I4 & is & 18 & $44 \cdot 3$ & 42.6 & 32 & fis \\
\hline 20 & 53.7 & $54 \cdot 3$ & 34 & 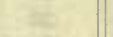 & 20 & 53.0 & $53 \cdot 3$ & 33 & $a=$ & 20 & 56.9 & 53.6 & I4 & W & 20 & 43.3 & 42.3 & 33 & 5 \\
\hline 22 & 55.0 & 55.6 & 36 & $E$ & 22 & 53.1 & 53.6 & 33 & 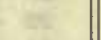 & 22 & 58.0 & 54.0 & I2 & is & 22 & 33.6 & 33.1 & 48 & 34 \\
\hline 24 & 53.7 & $54 \cdot 3$ & 34 & 14 & 24 & 53.6 & 53.9 & 34 & $1^{2}$ & 24 & 59.5 & 56.3 & IO & is & 24 & 31.8 & 30.3 & 52 & \\
\hline 26 & 54.0 & $54 \cdot 3$ & 35 & 22 & 26 & 54.3 & 54.9 & 35 & - & 26 & 56.3 & 54.0 & I4 & & 26 & 30.0 & 27.0 & 56 & \\
\hline 28 & $55 \cdot 3$ & 55.7 & 37 & $y^{2}$ & 28 & 55.3 & 56.0 & 37 & 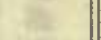 & 28 & 54.3 & 52.4 & I7 & & 28 & 37.0 & 33.6 & 45 & \\
\hline 30 & 55.0 & 55.3 & 36 & -21.5 & 30 & $55 \cdot 3$ & 56.1 & $37-$ & -20.0 & 30 & 56.6 & 54.9 & 13 & -19.1 & 30 & 33.6 & 29.6 & $235 \mathrm{I}$ & -19.3 \\
\hline 32 & 58.0 & 58.3 & $4 \mathrm{I}$ & & 32 & 57.4 & 58.0 & 40 & $x^{2}$ & 32 & 54.9 & 53.5 & 15 & the & 32.6 & 25.6 & 24.4 & 24 OI & 0 \\
\hline 34 & $5^{6.3}$ & 56.5 & 38 & H5 & 34 & 60.1 & 61.3 & 45 & k5: & 34 & 56.5 & $54 \cdot 3$ & 13 & 78 & 34 & 33.6 & 29.5 & $235 \mathrm{I}$ & \\
\hline 36 & 52.5 & 53.5 & 33 & 3 & 36 & 63.7 & 64.0 & 50 & 82 & 36 & 60.3 & 58.2 & 08 & & 36 & 35.9 & 34.6 & 45 & \\
\hline 38 & 53.0 & 54.2 & 34 & E" & 38 & 67.0 & 67.3 & 55 & 4 & 38 & 60.3 & 58.3 & 07 & be & 38 & 44.6 & 44.3 & $3 \mathrm{I}$ & \\
\hline 40 & 52.8 & 53.9 & 33 & tat & 40 & 66.5 & 66.5 & 54 & $0=$ & 40 & 59.0 & 57.3 & $\infty$ & 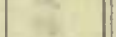 & 40 & 54.8 & 52.6 & 16 & \\
\hline 42 & 50.6 & 51.6 & 30 & & 42 & 69.3 & 70.0 & $59-$ & -20.0 & 42 & 60.3 & 59.2 & 07 & & 42 & 57.0 & 55.2 & 12 & \\
\hline 44 & 49.9 & 51.0 & 29. & -21.3 & 44 & 69.0 & 69.6 & 58 & 12 & 44 & 58.8 & 58.2 & og. & -19.2 & 44 & 57.8 & 56.0 & II & -19.3 \\
\hline 46 & 5I.I & 51.6 & 30 & ( & 46 & 68.6 & 69.1 & 2258 & 28 & 46 & 60.2 & 58.0 & 08 & ( & 46 & 59.0 & 58.3 & 08 & \\
\hline 48 & 55.0 & 55.5 & 36 & 24. & 48 & 70.0 & 70.6 & 2300 & 4 & 48 & 60.2 & 57.7 & 08 & 2 & 48 & 57.8 & 56.0 & II & \\
\hline 50 & 56.7 & 57.3 & 39 & c: & 50 & 70.6 & 71.1 & OI & 6 & 50 & 55.5 & 54.6 & I4 & yis & 50 & 53.8 & 51.5 & 18 & 8 \\
\hline 52 & 57.3 & 57.9 & 40 & al & $5^{2}$ & 72.8 & 73.8 & 05 & $\mathrm{e}=$ & 52 & 57.3 & 56.7 & II & 6 & 52 & 50.3 & 48.9 & 23 & $=1$ \\
\hline 54 & 57.5 & 57.8 & 40 & $R^{2}$ & 54 & 73.3 & 74.1 & 05 & 2: & 54 & 57.5 & 56.3 & II & Not & 54 & 52.6 & 50.8 & 19 & 12 \\
\hline 56 & 57.6 & 57.6 & 40 & (15) & 56 & 73.3 & 74.0 & 05 & $4:$ & 56 & 53.5 & 53.5 & 16 & & 56 & 55.8 & 54.3 & 14 & \\
\hline 58 & 56.0 & 56.3 & 38 & & 58 & 73.5 & 78.3 & 06 & & 58 & 48.8 & 48.3 & 24 & & 58 & 56.9 & 56.0 & 12 & \\
\hline 100 & 54.7 & 54.9 & 36 & -21.0 & 300 & 73.3 & 74.0 & 05 & -19.8 & 500 & 48.6 & 48.2 & 24 & 0 & 700 & 58.6 & 58.0 & 09 & -19.3 \\
\hline 02 & 54. I & $54 \cdot 3$ & 35 & 60 & 02 & 72.8 & 73.7 & 05 & 5 & 02 & $55 \cdot 3$ & 53.0 & 15 & t & 02.2 & $6 \mathrm{r} .3$ & 61.0 & 2305 & \\
\hline 04 & 53.3 & 53.5 & 33 & (64) & 04 & 76.0 & 76.5 & $\infty$ & wa & 04 & 56.7 & $54 \cdot 3$ & 13 & the & 04 & 66.6 & 65.5 & & \\
\hline 06 & 52.4 & 52.7 & 32 & $\sqrt{6}$ & o6* & 33.6 & 40.0 & 12 & bit & 06 & 57.0 & 54.2 & 13 & 60 & 06 & 63.6 & 62.0 & 2302 & \\
\hline 08 & 51.7 & 52.0 & 31 & fis & 08 & 38.5 & 40.1 & I6 & 80 & 08 & 59.0 & 54.8 & II & 160 & 08 & 63.5 & 61.0 & 2303 & 65 \\
\hline 10 & 51.4 & 51.6 & 30 & wh & 10 & 36.3 & 38.1 & I2 & at & 10.2 & 60.2 & 59.0 & 07 & $m$ & 10 & 71.3 & 69.3 & 2250 & Cr \\
\hline 12 & $5 \mathrm{I} .3$ & 51.6 & 30 & 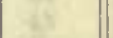 & 12 & 34.3 & 35.1 & 08 & 81 & 12 & 60.6 & 58.3 & 07 & 11 & 12 & 72.0 & 71.0 & 48 & \\
\hline 14 & 56.0 & 56.3 & 38 & -21.0 & 14 & 37.5 & 39.2 & 14 & -19.5 & 14 & $5^{8} .6$ & 56.0 & 10 & & 14 & 73.8 & 72.0 & 46 & -19.3 \\
\hline 16 & 55.7 & 55.7 & 37 & (x) & 16 & 43.6 & 45.8 & 24 & 2.0 & 16 & 61.3 & 58.3 & 07. & -19.2 & $16^{*}$ & 42.0 & 38.9 & 42 & \\
\hline 18 & 50.7 & 50.8 & 29 & atit & 18 & 53.3 & 54.6 & 39 & $5 x$ & I8 & 60.6 & 57.2 & 08 & $x^{2}$ & 18 & 42.0 & 35.3 & 45 & \\
\hline 20 & 51.2 & 51.3 & 30 & est. & 20 & 58.5 & 59.5 & 2347 & ne & 20 & 59.7 & 56.3 & 09 & 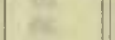 & 20 & 43.6 & 35.3 & 44 & $=$ \\
\hline 22 & $54 \cdot 3$ & 54.7 & 35 & ex & 22 & 67.0 & 69.0 & 24 OI & $\mathrm{az}$ & 22 & 59.1 & 55.3 & II & a & 22 & $45 \cdot 3$ & 38.6 & 40 & es: \\
\hline 24 & 54.4 & 54.6 & 35 & at & 24 & 67.7 & 70.3 & 02 & 12. & 24 & 58.0 & 53.6 & 13 & $1+$ & 24 & $43 \cdot 3$ & 37.8 & 42 & \\
\hline 26 & 54.0 & 54.6 & 35 & & 26 & 68.7 & 71.8 & 04 & te & 26 & 56.5 & 52.5 & 15 & & 26 & 39.0 & 32.8 & 49 & \\
\hline 28 & 54.7 & 55.2 & 36 & r $=$ & 28 & 69.9 & 74.2 & 07 & & 28 & 52.2 & 49.0 & 21 & & 28 & 43.5 & 38.0 & 42 & \\
\hline 30 & 55.6 & 56.5 & 37 & -21.0 & 30 & 69.5 & 74.9 & 07 & -19.5 & 30 & 50.3 & $47 \cdot 3$ & 24 & -19.0 & 30 & 43.2 & 36.4 & 43 & -19.3 \\
\hline 32 & 57.4 & 57.6 & 40 & 0 & $32^{*}$ & 49.0 & 53.0 & I7 & - & 32 & $47 \cdot 3$ & 42.5 & 30 & 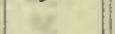 & 32 & 41.5 & 35.0 & 46 & \\
\hline 34 & 56.3 & 56.8 & 38 & az & 34 & 38.8 & 43.0 & 24 OI & tac & 34 & 44.3 & 41.0 & 34 & 2 & 34 & 40.9 & 40.2 & 42 & \\
\hline 36 & 56.5 & 56.7 & 38 & 32 & 36 & 25.8 & 28.8 & 2340 & t5 & 36 & 47.2 & 44.8 & 28 & 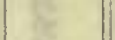 & 36 & 39.3 & 38.5 & 44 & \\
\hline 38 & 56.1 & 56.3 & 38 & ter & 38 & 27.2 & 29.0 & $4 I$ & 6 & 38 & 50.6 & 49.0 & 22 & E. & 38 & 38.8 & 37.5 & 46 & \\
\hline 40 & 56.3 & 56.3 & 38 & 07 & 40 & I3.3 & I6. 3 & 20 & the & 40 & 58.7 & 57.3 & 10 & 7. & 40 & 43.3 & 43.0 & 38 & \\
\hline 42 & 57.3 & 57.6 & 40 & 58 & 42 & IO. & $.0 b$ & I3 & & 42 & 56.6 & 55.3 & 13 & & 42 & 42.0 & $4 \mathrm{I} \cdot 3$ & 40 & \\
\hline & 55.4 & 55.7 & 37 & -20.5 & $44^{*}$ & 44.5 & 51.5 & 08 & -19.5 & 44 & 55. & oa & 14. & -19.0 & 44 & 35.1 & 34.3 & & -19.5 \\
\hline 46 & 54.5 & 54.6 & 35 & ( & 46 & 42.3 & 48.9 & 04 & $x$ & 46 & 55.5 & 51.1 & 17 & - & 46 & 36.6 & 36.3 & 48 & \\
\hline 48 & $54 \cdot 3$ & 54.7 & 35 & the & 48 & 42.0 & 47.1 & 03 & 2 & 48 & 53.6 & 51.6 & I8 & x & 48 & 36.6 & 36.6 & 48 & \\
\hline 50 & 54.7 & 55.3 & 36 & 8 & 50 & 44.8 & 49.0 & 06 & er & 50 & 51.6 & 51.0 & 20 & ef: & 50 & 31.6 & 30.8 & 2257 & \\
\hline 52 & 54.0 & 54.6 & 35 & $2 x$ & 52 & 55.5 & 58.6 & 22 & 0 & 52 & 49.6 & 49.0 & 23 & 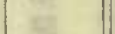 & 52 & 30.3 & 27.9 & & \\
\hline 54 & 53.9 & 54.5 & 35 & 32 & 54 & 57.0 & 60.6 & 25 & & 54 & 50.3 & 49.6 & 22 & if & 54 & 30.3 & 29.3 & 2259 & \\
\hline 56 & 53.0 & 53.6 & 33 & 욜 & 56 & 60.5 & 61.6 & 29 & -19.4 & 56 & 51.3 & 50.7 & 20 & - & 56 & 32.0 & 29.5 & & \\
\hline 58 & 54.0 & 54.6 & 35 & Bit & 58 & 52.3 & 55.3 & 17 & 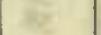 & 58 & 52.0 & 51.0 & 20 & 36 & 58 & 27.3 & 23.7 & 2306 & \\
\hline $\cos$ & r & 30 & $6 x^{\circ}$ & 60 & & & & & & $\mathrm{kr}=$ & & 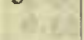 & 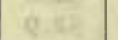 & 0 & 800 & 30.3 & 26.3 & OI & -19.6 \\
\hline
\end{tabular}


Tabulation of magnetic declinations abserved at Teplitz Bay-Continued

\begin{tabular}{|c|c|c|c|c|c|c|c|c|c|c|c|c|c|c|c|c|c|c|c|c|}
\hline \multicolumn{5}{|c|}{ Monday, December 21, 1903} & \multicolumn{6}{|c|}{ 1. Magnet scale erect } & \multicolumn{5}{|c|}{ Tuesday, December 22, 1903} & \multicolumn{5}{|c|}{ Magnet scale inverted } \\
\hline \multirow{2}{*}{$\begin{array}{l}\text { Chr'r } \\
\text { time }\end{array}$} & \multicolumn{2}{|c|}{$\begin{array}{l}\text { Scale } \\
\text { readings }\end{array}$} & East & Temp & Chr'r & $\begin{aligned} \mathrm{Sc} \\
\text { read }\end{aligned}$ & $\begin{array}{l}\text { ale } \\
\text { ings }\end{array}$ & $\begin{array}{l}\text { Ea } \\
\text { dec }\end{array}$ & & Temp. & Chr'r & $\begin{array}{l}\mathrm{Sc} \\
\text { readi }\end{array}$ & $\begin{array}{l}\text { cale } \\
\text { lings }\end{array}$ & $\begin{array}{l}\text { East } \\
\text { decli- }\end{array}$ & Temp. & Chr'r & $\begin{array}{r}\mathrm{Sc} \\
\mathrm{read}\end{array}$ & $\begin{array}{l}\text { ale } \\
\text { ings }\end{array}$ & East & Temp. \\
\hline & Left & Righı & & & & L,eft & Right & & & & & L,eft & Right & & & & Left & Right & nation & \\
\hline $\mathrm{h} \mathrm{m}$ & d & d & $\circ$, & $\circ$ & $\mathrm{h} \mathrm{m}$ & d & d & $\cdot$ & ' & & $h \mathrm{~m}$ & & d & - ' & farts & $\mathrm{h} \mathrm{m}$ & & & & $\circ$ \\
\hline 800 & 58.2 & $59 \cdot 3$ & 2253 & & I0 00 & 70.2 & 70.8 & 23 & II & -20.0 & 1200 & 46.0 & $45 \cdot 4$ & 2237 & -22.2 & 1400 & 48.7 & 47.6 & 2234 & -19.1 \\
\hline 02 & 55.1 & 66.3 & 2256 & -25.0 & 02 & 69.1 & 69.8 & & 09 & tan & 02 & 45.1 & 44.8 & 39 & and & 02 & 47.0 & 46.2 & 36 & - \\
\hline 04 & 62.1 & 66.9 & 2302 & (6) & 04 & 67.7 & 68.1 & & 07 & It & 04 & 45.0 & 44 & 39 & pa & 04 & 46.2 & 45 & 37 & is \\
\hline 06 & 63.9 & 68.0 & 04 & 35 & 06 & 64.7 & 65.1 & & 02 & $x_{0}$ & 06 & 46.4 & 45.6 & 37 & $5=$ & 06 & 46.6 & 46.1 & 36 & 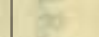 \\
\hline 08 & 67.9 & 69.9 & og & 164 & 08 & 65.2 & 67.0 & & 04 & 32 & 08 & 47.9 & 47.1 & 35 & ho & 08 & 43.9 & 43.0 & $4 \mathrm{I}$ & 25 \\
\hline 10 & 69.9 & 72.1 & 12 & in & 10 & 67.1 & 67.8 & & 06 & 20 & 10 & 46.5 & 46.0 & 36 & $6 r$ & 10 & $43 \cdot 3$ & 42 & 42 & $a$ \\
\hline 12 & 67.1 & 69.7 & 08 & & I2 & 66.9 & 67.1 & & 06 & & 12 & 45.3 & 43. & 39 & er & 12 & 44.7 & & 40 & \\
\hline I4 & 69.3 & 71.3 & II & -24.5 & 14 & 66.8 & 67.2 & & 06 & -19.7 & 14 & 46.1 & 44.8 & 38 & -21.7 & 14 & 46.1 & 45.8 & 37 & -18.9 \\
\hline 16 & 69.1 & 71.3 & II & & I6 & 67.9 & 68.2 & & 07 & & 16 & 47.1 & 45.9 & 36 & $10=$ & 16́ & $47 \cdot 3$ & 47.0 & 35 & \\
\hline I8 & 66.0 & 68.5 & 06 & inf & 18 & 67.8 & 68.0 & & 07 & tht & 18 & 47.1 & 45.8 & 36 & Hat & I8 & 46.1 & 45 & 37 & int \\
\hline 20 & 67.9 & 69.7 & 08 & 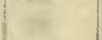 & 20 & 68.7 & 68.9 & & 08 & $n$ & 20 & 46.8 & 44.9 & 37 & of & 20.4 & 46.8 & & 36 & e \\
\hline 22 & 67.9 & 69. & 08 & $\sin$ & 22 & 68. I & 68.7 & & 08 & 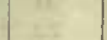 & 22 & 46.6 & 45 & 37 & $y=$ & $22^{4}$ & 47.2 & 46.7 & 36 & 57 \\
\hline 24 & 68.2 & 69.8 & 09 & is & 24 & 68.0 & 68.9 & & 08 & 4 & 24 & 46.8 & 45 & 36 & 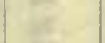 & 24 & 45.1 & & 39 & \\
\hline 26 & 66.3 & 67.9 & 06 & 18 & 26 & 67.2 & 68.2 & & 07 & & 26 & 48.8 & 48.1 & 33 & m & 26 & 46.2 & 45 & 37 & \\
\hline 28 & 65.0 & 66.2 & 2304 & & 28 & 67.9 & 68.7 & & 08 & & 28 & 49.1 & 48 & 33 & 36 & 28 & 47.2 & & 35 & \\
\hline 30 & 62.5 & 63.5 & 2259 & -23.8 & 30 & 64.2 & 65.5 & 23 & 02 & -19.4 & 30 & 47.6 & 46.7 & 35 & -21.0 & 30 & 45.5 & 44.9 & 38 & -18.7 \\
\hline 32 & 61.1 & 61.9 & 57 & ( & 32 & 61.2 & 61.9 & 22 & 57 & 1 & 32 & 47.7 & 46 & 35 & 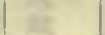 & 32 & $44 . I$ & 43 & 40 & 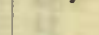 \\
\hline 34 & 61.9 & 63.5 & 2259 & 16 & 34 & 61.2 & 61.5 & & 57 & tet & 34 & 49.7 & 49 & 32 & 11 & 34 & 44.7 & & 40 & \\
\hline 36 & 63.2 & 64.1 & 2300 & (5) & 36 & 59.7 & 60.2 & & 55 & $3 x$ & 36 & 49.3 & 48 & 32 & & 36 & $43 \cdot 3$ & 42.9 & $4 \mathrm{I}$ & \\
\hline 38 & 63.9 & 65.1 & & 35 & 38 & 59.7 & 59.8 & & 54 & 67 & 38 & 49.9 & 49 & 31 & $z_{2}$ & $3^{8}$ & 43.0 & 42 & 42 & 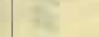 \\
\hline 40 & 65.2 & 66.9 & 04 & $x_{15}$ & 40 & 6т.6 & 62.2 & & 58 & is & 40 & 51.2 & $5 \mathrm{I}$ & 29 & thit & 40 & 43.1 & 43 & 42 & \\
\hline 42 & 62.9 & 63.9 & $\infty$ & & 42 & 62.0 & 62.9 & 22 & 58 & & 42 & 50.6 & 50 & 30 & & 42 & 47.4 & & 36 & \\
\hline 44 & 63.3 & 64.8 & OI & -22.8 & 44 & 62.7 & 63.7 & 23 & 00 & -I9. I & 44 & 49.4 & 49. & 32 & -20.3 & 44 & 47.5 & 47 & 35 & -18.3 \\
\hline 46 & 62.6 & 63.8 & $\infty$ & 76 & 46 & 63.1 & 63.3 & & $\infty$ & 10 & 46 & 48.9 & 48. & 33 & & 46 & 47.9 & 47 & 34 & \\
\hline 48 & 64.3 & 65.9 & 03 & 38 & 48 & 64.2 & $65 . I$ & & 02 & h) & 48 & 48.9 & 48.3 & 33 & & 48 & 49.2 & 48 & 32 & te \\
\hline 50 & 66.8 & 68.0 & 06 & ati & 50 & 4.7 & 65.0 & $23 c$ & 02 & 65 & 50 & 48.2 & 47.4 & 34 & 63 & 50 & 46.9 & 46 & 36 & \\
\hline 52 & 70.6 & 71.3 & I2 & $\omega$ & 52 & 62.0 & 62.8 & 225 & 58 & 3 & 52 & 48.3 & 47.5 & 34 & 3 & 52 & 46.8 & 45.7 & 36 & 3 \\
\hline 54 & 70.7 & 71.8 & 12 & a & 54 & 60.9 & 61.9 & & 57 & & 54 & 49.7 & 48.8 & 32 & $\mathrm{BS}^{-1}$ & 54 & 48.2 & 47 & 34 & \\
\hline 56 & 9.7 & 70.8 & I I & in: & 56 & 57.0 & $57 . \overline{8}$ & & 50 & & 5 & 48.8 & 48.0 & 33 & & 5 & 48.1 & 47 & 34 & \\
\hline 58 & 69.5 & 70.8 & II & $12 x^{2}$ & 58 & 56.3 & 56.8 & & 49 & & 58 & 48.6 & 48.0 & 33 & & 58 & 48.8 & 48 & 33 & \\
\hline 900 & 70.2 & 70.9 & II & -22.0 & II 00 & 45.8 & 46.8 & & 33 & -18.9 & 1300 & 48.9 & 48.1 & 33 & -20.0 & 1500 & 49.9 & 49.0 & 32 & -18.2 \\
\hline 02 & 70.2 & 70.9 & II & 60 & 02 & 44.0 & 44.7 & & 30 & - & 02 & 49.8 & 48.9 & 32 & $\omega$ & 02 & 50.7 & 49 & $3 I$ & $1=$ \\
\hline 04 & 68.8 & 69.7 & og & in & 04 & 43.9 & 44.6 & & 30 & nat & 04 & 49.3 & 48 & 32 & 70 & 04 & 49.2 & 48 & 33 & II \\
\hline 06 & 68.7 & 68.9 & 08 & we. & 06.2 & 43.0 & & & 29 & 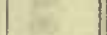 & 06 & & 48 & 32 & 5 & 06 & 48.5 & & 34 & t \\
\hline 08 & 66.3 & 66.9 & 05 & B. & 08 & 40.0 & $40: 5$ & & 24 & A & 08 & 48.2 & 47.6 & 34 & ne. & 08 & 49.6 & 48 & 32 & Wo \\
\hline IO & $7 \cdot 3$ & 67.8 & об & int & IO & $43 \cdot 3$ & & & 29 & 2 & IO & 47 & 47. & 34 & the & I0 & 48.5 & & 34 & of \\
\hline 12 & 68.2 & 68.5 & 08 & & I2 & $45 \cdot 3$ & 45.7 & & 32 & (1) & 12 & 47.2 & 46.5 & 36 & 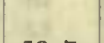 & 12 & $47 . \mathrm{I}$ & 46 & 36 & \\
\hline I4 & 65.1 & 66.0 & 2303 & -21.8 & 14 & 47.5 & 48.0 & & 35 & -18.7 & I4 & & 47 & 34 & -19.7 & 14 & 46.2 & & 37 & -I8.0 \\
\hline 16 & 59.9 & 61.4 & 2256 & dilis & I6 & $49 . I$ & 50.0 & & 38 & $t^{2}$ & I6 & $48 . I$ & 47.8 & 34 & $x^{2}$ & 16 & 46.5 & & 37 & \\
\hline 18 & 56.8 & 57.1 & 50 & $=$ & I8 & 48.3 & 48.8 & & 37 & tat & I8 & & 48 & 32 & in & 18 & 48.7 & & 33 & है \\
\hline 20 & 61.8 & 62.0 & 58 & at & 20 & 9.7 & $5 \mathrm{I} . \mathrm{I}$ & & 40 & ne & 20 & 50.5 & 49.6 & 30 & 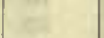 & 20 & 47.8 & 47 & 35 & is \\
\hline 22 & 59.2 & 60.2 & 54 & ats & 22 & 5.8 & 57.7 & & 49 & 6 & 22.4 & 49 & & 32 & 38 & 22 & 47.9 & & 34 & 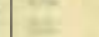 \\
\hline 24 & 58. I & 58.9 & 52 & ie & 24 & .1 & 57. & & $5 \mathrm{I}$ & $n t$ & 24 & 49.9 & 49 & 31 & $2=$ & 24 & 46.8 & & 36 & \\
\hline 26 & 55.9 & & 49 & E & 26 & & 57.9 & & $5 I$ & 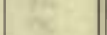 & 26 & 49 & 49 & 32 & & 26 & 47.2 & & 36 & es \\
\hline 28 & 57.2 & 58.0 & $5 I$ & 2 & 28 & 8.2 & 58.9 & & 52 & & 28 & 49. & 49 & 32 & & 28 & 47.8 & 46 & 35 & \\
\hline 30 & & 58.2 & $5 \mathrm{I}$ & -21.2 & 30 & 56.9 & 57.6 & & 50 & -18.3 & 30 & & 49 & 32 & -19.6 & 30 & 47 & 45 & 36 & -17.9 \\
\hline 32 & 3.3 & 58.9 & 52 & 4 & 32 & & & & $5 \mathrm{I}$ & 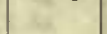 & $3^{2}$ & 50 & & 30 & 1 & 32 & & & 35 & ( \\
\hline 34 & 59.9 & 60.1 & 55 & is & 34 & .2 & 56. & & 48 & & 34 & 49.1 & 48.8 & 32 & it & 34 & 48.8 & 46. & 34 & at \\
\hline 36 & 62.0 & 62.3 & 2258 & B & 36 & 55.5 & 56 & & 48 & F & 36 & & & 34 & & 36 & 48.2 & 46. & 35 & \\
\hline 38 & 64.0 & $64 \cdot 3$ & 23 OI & is & 38 & 54.7 & $55 . \mathrm{I}$ & & 47 & 46 & 38 & 47.4 & 47 & 35 & E & 38 & 49. I & 47.6 & 33 & 8 \\
\hline 40 & 66 & $7 b$ & & $\alpha$ & 40 & 55.0 & 55.9 & & 47 & teit & 40 & & & 37 & & 40 & & $4^{8}$ & 33 & \\
\hline 42 & 66.6 & 66.8 & 05 & & 42 & 53.0 & 53. & & 44 & & 42 & 46.8 & 45.9 & 36 & & 42 & 48.3 & 47. & 34 & \\
\hline 44 & 68. & & 08 & -20.8 & 44 & 57.2 & 57.7 & & 50 & -18.0 & 44 & $47 \cdot 3$ & 46.7 & 35 & -19.2 & 44 & 49. I & 48. I & 33 & -17.8 \\
\hline 46 & 71.2 & 71.3 & 12 & 6 & 46 & 56.3 & 56.8 & & 49 & - & 46 & 48.0 & 46.9 & 35 & ( & 46 & 49.1 & 48. I & 33 & \\
\hline 48 & 69.9 & 69.9 & 10 & Bs & 48 & 57.2 & 57.3 & & 50 & & 48 & 47.6 & 46.8 & & 18 & $4^{8}$ & 48.8 & 47.7 & 33 & \\
\hline 50 & 70.1 & 70.4 & II & the & 50 & 58.1 & 58 & & 52 & 78 & 50 & 45.8 & 44.9 & 38 & $*$ & 50 & 48.6 & 47. & 34 & b \\
\hline 52 & 69.7 & 70.2 & IO & 42 & 52 & 57.2 & 57.8 & & 51 & 62 & 52 & & 43 & $4 \mathrm{I}$ & & 52 & 48.0 & 47.1 & 34 & a \\
\hline 54 & 70.7 & $7 I . I$ & 12 & Hit & 54 & 59.3 & 60.1 & & 54 & 8 & 54 & 46.0 & 44 & 38 & (1) & 54 & 47.8 & 46.9 & 35 & $x$ \\
\hline 56 & 70.6 & 71.2 & 12 & 2 & 56 & 56.9 & $57 \cdot 3$ & & 50 & & 56 & 44.2 & 43.6 & 40 & Wiv & 56 & 48.8 & 47.8 & 33 & $=5$ \\
\hline 58 & 70.1 & 70.9 & II & 2 & 58 & 57.2 & 57.8 & & 51 & & 58 & 47.2 & 46.1 & 36 & 75 & 58 & 49.1 & 48.2 & 33 & \\
\hline & & & & & 1200 & 58.1 & 58.2 & & 52 & -17.9 & & & & & & 16 on & 49.0 & 48.0 & 33 & -17.7 \\
\hline
\end{tabular}

Correction to local mean time is $+37 \mathrm{~s} .90^{\circ}$ torsion $=25 .^{\prime} 4$.

Torsion head at $8 \mathrm{~h} \mathrm{IOm}$, Deccmber 20 , read $348^{\circ}$, and at $\mathrm{r} 2 \mathrm{~h} 30 \mathrm{~m}$, December 21 , read $15^{\circ}$.

Observer-R. R. T.
Correction to local mean time is +30 .

Torsion head at inh $40 \mathrm{~m}$ read $345^{\circ}$ and at the end read the same. Observer-Not noted. 
Tabulation of magnetic declinations observed at Teplitz Bay-Continued

\begin{tabular}{|c|c|c|c|c|c|c|c|c|c|c|c|c|c|c|c|c|c|c|c|}
\hline \multicolumn{5}{|c|}{ Wednesday, December 23, 1903} & \multicolumn{5}{|c|}{ Magnet scale erect } & \multicolumn{10}{|c|}{ Wednesday, December 23, 1903} \\
\hline \multirow{2}{*}{$\begin{array}{l}\text { Chr'r } \\
\text { time }\end{array}$} & \multicolumn{2}{|c|}{$\begin{array}{l}\text { Scale } \\
\text { readings }\end{array}$} & \multirow{2}{*}{$\begin{array}{l}\text { East } \\
\text { decli- } \\
\text { nation }\end{array}$} & \multirow{2}{*}{$\begin{array}{c}\text { Temp. } \\
\text { C. }\end{array}$} & Chr's & $\begin{array}{r}\text { Sca } \\
\text { readi }\end{array}$ & $\begin{array}{l}\text { ale } \\
\text { lings }\end{array}$ & $\begin{array}{l}\text { East } \\
\text { decli- }\end{array}$ & Temp. & Chr's & $\begin{array}{l}\text { Scal } \\
\text { readir }\end{array}$ & $\begin{array}{l}\text { ale } \\
\text { ings }\end{array}$ & $\begin{array}{l}\text { East } \\
\text { decli- }\end{array}$ & Temp. & & $\begin{array}{l}\text { Sca } \\
\text { read }\end{array}$ & le & $\begin{array}{c}\text { East } \\
\text { decli- }\end{array}$ & Temp. \\
\hline & Left & Right & & & & Left & Right & & & & Left & Right & & & & Left & Right & & \\
\hline $\mathrm{h} \mathrm{m}$ & d & d & $\circ$, & $\circ$ & $h \mathrm{~m}$ & d & d & - ' & $\circ$ & $\mathrm{h} \mathrm{m}$ & d & d & - & 8 & $\mathrm{~h} \mathrm{~m}$ & d & & - & 0 \\
\hline 000 & 52.6 & 55.6 & 2253 & -18.0 & 200 & $53 \cdot 4$ & $54 \cdot 3$ & 2253 & -17.4 & 400 & 66.3 & 66.3 & 2312 & -16.7 & $6 \mathrm{~m}$ & 51.3 & 51.6 & 2249 & -16.9 \\
\hline 02 & 60.3 & 64.6 & 2306 & & 02 & $54 \cdot 3$ & 55.4 & 54 & & 02 & 68.3 & $3^{b}$ & I6 & & 02 & 52.6 & 52.9 & $5 \mathrm{I}$ & \\
\hline 04 & 68.8 & 72.8 & 2320 & & 04 & 52.3 & 53.3 & $5 \mathrm{I}$ & & 04 & 61.2 & $2 b$ & 2304 & & & 5 I. 4 & & & \\
\hline 06 & 50 & $o b$ & 2247 & & 06 & 55.0 & $56 . \mathrm{I}$ & 56 & 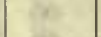 & 06 & 54.5 & $5^{b}$ & 2254 & & 06 & 50.2 & 50 & & \\
\hline 08 & 51.3 & 52.3 & 50 & & 08 & 55.6 & 56.6 & 56 & (1) & 08 & 48.8 & $8 b$ & 45 & ter & 08 & & & & \\
\hline IO & 49.8 & 51.6 & 48 & tut & 10 & 55.8 & 56.6 & 57 & -2 & 10 & 46.6 & $6 b$ & 42 & 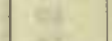 & IC & I. 4 & 52 & 50 & $\alpha \varepsilon$ \\
\hline I2 & 48.0 & 49.3 & 45 & & 12 & 54.5 & 55.2 & 54 & $1=$ & 12 & 45.3 & 45.6 & 40 & 271 & 12 & 53.4 & 54 & 53 & 18 \\
\hline 14 & 49.8 & 50.1 & 47 & -18.0 & I4 & 54.1 & 54.9 & 54 & -17.3 & 14 & 44.0 & 44.2 & 38 & -16.7 & I4 & 52.5 & 53 & $5 \mathrm{I}$ & -17.0 \\
\hline 16 & 49.0 & 51.0 & 47 & & 16 & 54.7 & 55.3 & 55 & & 16 & 44.2 & 44. & 38 & & If & 50.4 & $5 I$ & 48 & \\
\hline I8 & 53.3 & 54.9 & 53 & & I8 & 56.9 & 57. & 58 & & I\& & 46.6 & 46. & 42 & & I\& & 53.4 & & 53 & \\
\hline 20 & 45.3 & 48.5 & 42 & ax & 20 & 56.8 & 57.6 & 58 & 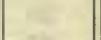 & 20 & 47. & $6 a$ & 43 & & 20 & 56.6 & & 58 & 15 \\
\hline 22 & .3 & 45.6 & 39 & 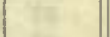 & 22 & 54.7 & 55.3 & 55 & the & 22 & $49.8^{\circ}$ & 49. & 47 & 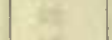 & & & & 56 & as \\
\hline 24 & 3.3 & 43.9 & 37 & 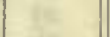 & 24 & 51.7 & 53.5 & 51 & 80 & 24 & .0 & 49. & 45 & & 2 & & 51 & 48 & 15 \\
\hline 26 & 7.3 & 49.3 & 44 & 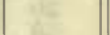 & 26 & 50.6 & 51. & 4 & & 26 & & 48. & 45 & & & & & 50 & \\
\hline 28 & 4.4 & 47.5 & 40 & $x^{-2}$ & 28 & 52.3 & 53.1 & $5 \mathrm{I}$ & 1 & 28 & .7 & 49.0 & 45 & & 28 & & & 48 & \\
\hline 30 & .6 & 47.8 & 42 & -17.8 & 30 & 55.8 & 56. & 56 & -17.1 & 30 & 8 & 48. & 44 & -16.7 & 3 & 50 & 51 & 48 & -16.9 \\
\hline 32 & 5.0 & 48.0 & 42 & & 32 & 54.9 & 55.3 & 55 & & 3 & .8 & 47. & 42 & -2 & 3 & & 54 & 53 & \\
\hline 34 & 44.6 & 46.7 & 40 & & 34 & 52.7 & 53. & 5 & 45 & 34 & 47.6 & 48.0 & 43 & & 34 & 52 & & $5 \mathrm{I}$ & \\
\hline 36 & 4.3 & 46.1 & 39 & & 36 & 50.6 & 51.0 & 48 & (15) & 36 & & 45.5 & & & 3 & & & 48 & \\
\hline 38 & 46.3 & 48.5 & 43 & 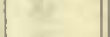 & 38 & 50.8 & 51.2 & 48 & 40 & 3 & 45.4 & 45. & 40 & & 3 & 49.0 & 49 & 45 & \\
\hline 40 & 44.3 & 45.8 & 39 & 9 & 40 & 51.9 & 52. & 50 & AN & 4 & 48.0 & 48. & & f & 4 & & & 3 & - \\
\hline 42 & $4 \mathrm{I} .3$ & 43.5 & 35 & 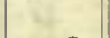 & 42 & 53.0 & 53.6 & 52 & 24 & 42 & 53. & $1 a$ & 52 & & 42 & 5.6 & 45 & 40 & \\
\hline 44 & 47.6 & 48.6 & 44 & -17.8 & 44 & 53.1 & 53. & 5 & ty & 44 & 56.2 & 56.6 & 57 & -16 & 4 & 49 & & 47 & -17.0 \\
\hline 46 & 55.8 & 56.0 & 56 & & 46 & 51.7 & 52. & 50 & (25. & 4 & 56.2 & 56. & 57 & & 4 & 50 & & 47 & \\
\hline 48 & 47.7 & 48.7 & 44 & ter & 48 & 50.6 & 50. & 4 & 20 & 4 & & 58 & & & 4 & & & 50 & \\
\hline 50 & 45.2 & 46.8 & $4 \mathrm{I}$ & te & 50 & 49.6 & 50. & 4 & (61) & 5 & .8 & 58. & 5 & & 5 & 49.8 & 50 & 47 & \\
\hline 52 & 39.7 & 45.6 & 35 & the & 52 & 49.6 & 49 & 4 & 23 & 5 & 4.3 & 54. & & & 5 & Los & st & & 1 \\
\hline 54 & 43.4 & 48.7 & $4 \mathrm{I}$ & & 54 & 50.0 & 50. & 4 & $t^{2}$ & 54 & & 50. & 4 & & 5 & 50.3 & 50 & 47 & \\
\hline 56 & 47.0 & 53.0 & 47 & & 56 & 50.5 & 50 & 4 & & 5 & 49.5 & 50. & 46 & & 5 & 52.4 & & 51 & \\
\hline 58 & 48.4 & 53.0 & 48 & & 58 & 51.3 & 51. & 49 & & 58 & 50.0 & 50. & 47 & & 5 & 55 & & 55 & \\
\hline I 00 & 47.6 & 52.8 & 47 & -17.6 & 300 & 51.8 & 52. & 50 & -17.0 & 500 & 51.6 & 52.6 & 50 & -16.7 & 70 & & & 58 & -16.9 \\
\hline 02 & 52.5 & 58.5 & 56 & & 02 & $5 \mathrm{I} . \mathrm{I}$ & 51. & 49 & & 0 & 52.6 & 53.4 & 52 & & 02 & 57.3 & & 59 & \\
\hline 04 & 53.5 & 56.6 & 55 & e & 04 & 50.3 & 50. & 48 & W & 0 & 54 & 55.0 & 22 & & 0 & & & 57 & a \\
\hline 0 & 52.6 & 55.6 & 53 & $\ln$ & 06 & 49.1 & 49. & 46 & (5) & o & 58. & 59. & 30 & & o & & & 54 & W. \\
\hline 08 & 3.4 & 57. & 56 & 64 & 08 & 48.3 & 48. & 44 & 5 & o & 58. & 59.8 & 0 & 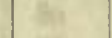 & 0 & & & 8 & int \\
\hline 10 & 55.6 & 58.6 & 58 & 9 & 10 & 48.5 & 48.5 & 4 & 하 & I & & 59. & & te & I & & & & uit \\
\hline 12 & 55.9 & 58.9 & 2258 & 164 & 12 & 48.6 & 48.8 & 45 & 31 & $\mathrm{I}$ & 5 & 59. & 2302 & 6 & I. & & & $\begin{array}{ll}23 & 03\end{array}$ & \\
\hline$I_{4}$ & 58.0 & 59.8 & 23 or & -17.7 & 14 & 49.3 & 49. & 46 & -17.0 & I & 56. & 58. & 2258 & -16.8 & I. & & & 03 & -16. \\
\hline 16 & 5 I. 3 & 54.2 & 2251 & & I & 50.5 & 50.9 & 48 & & I & 5 & 56. & 56 & & I & 59.9 & 60.0 & 02 & \\
\hline 18 & 53.0 & 55.9 & 54 & 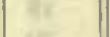 & 18 & 50.3 & 50 & 4 & Wt & I & 54. & 54. & & 2 & I & 62 & $.3 a$ & & \\
\hline 20 & 50.8 & 52.4 & 49 & tw & 20 & 48.8 & 49. & 4 & 25 & 2 & 53. & 54 & & es & $2 c$ & 62.9 & 63 & 08 & E \\
\hline 2 & 7.9 & 49. & 45 & a & 22 & & & 42 & 14 & 2 & $5 \mathrm{I}$ & 51. & 4 & If & 22 & 62.3 & & 07 & \\
\hline 2. & 49.8 & 50.8 & 47 & H & 24 & 46.6 & 46. & 4 & E & 2 & & $5 \mathrm{I}$. & 4 & at & 2 & 59.9 & & 03 & \\
\hline 26 & 50.1 & 51.7 & 48 & $a$ & 26 & 46.6 & 46. & 42 & 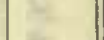 & 2 & 49 & 49. & 46 & is & 2 & 60.0 & & 04 & \\
\hline 28 & 47.2 & 48. & 44 & $=2$ & 28 & 46.6 & 46.7 & 42 & & 28 & & 50 & 47 & & 2 & & & I2 & \\
\hline 30 & 47.7 & 49. I & 44 & -17.6 & 30 & 45.4 & 45.8 & 40 & -16.9 & 30 & 51.6 & $5 \mathrm{I}$ & 50 & -16.9 & 3 & & & I6 & -16.8 \\
\hline 32 & 48.2 & 49. & 45 & & 32 & 43. & $.0 b$ & 36 & & 32.2 & & 54 & 53 & & 3 & & & 15 & \\
\hline 34 & 48.6 & 49. & 45 & & 34 & & $.0 b$ & 3 & & 34. & 54 & 54 & 5 & & 3 & 64.5 & & I2 & \\
\hline & 9.3 & 50.7 & 47 & & 36 & & $.0 b$ & 2 & ir & & & & & & & & & I2 & \\
\hline 38 & 50.7 & 52.1 & 49 & & 38 & 33. & $.7 a$ & 2 & & 3 & 53 & 54 & 5 & 7 & 3 & 63.3 & & IO & \\
\hline 40 & 51.3 & 52.6 & 50 & 10 & 40 & 41. & $.0 a$ & 33 & zat & 40 & 52.3 & 54. & & es & 4 & & & 07 & \\
\hline 42 & 50.3 & $5 \mathrm{I} .5$ & 48 & & 42 & 50.0 & 50.6 & 47 & & 42 & $55 . I$ & 56.3 & 56 & ex & 42 & 60.8 & & 05 & \\
\hline & 51.8 & 53.2 & $5 \mathrm{I}$ & $-17 \cdot 5$ & 44 & 54.7 & 55.4 & 55 & -I6.8 & 44 & 53.6 & 54.6 & 53 & -17.0 & 44 & 59.0 & & 02 & -16.8 \\
\hline 46 & 52.8 & 54.2 & 52 & & 46 & 53. & $.0 b$ & 52 & & 46 & 53.2 & $54 \cdot 3$ & & & 4 & 57.2 & & $\infty$ & \\
\hline 48 & 51.5 & 53.0 & 50 & . & 48 & 46.0 & 46.0 & $4 \mathrm{I}$ & $\sqrt{2}$ & 48 & & 53 & 5 & 20 & 48 & 56.6 & & 02 & \\
\hline 50 & 50.5 & 5 I. 8 & 48 & 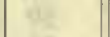 & 50.2 & $42 . \mathrm{I}$ & 42.8 & 35 & at & 50 & 52.6 & & 15 & $\mathrm{e}$ & 5 & 60.0 & & 06 & \\
\hline 52 & 52.3 & 53.5 & $5 \mathrm{I}$ & we & 52 & $40 . \mathrm{I}$ & 40.3 & 32 & $\mathrm{n}^{2}$ & 52 & 52.6 & 53.0 & 5 & th & 52 & 58.3 & & 02 & \\
\hline 54 & 51.9 & 53.0 & $5 \mathrm{I}$ & 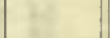 & 54 & 45 & $.0 a$ & 39 & $x^{2}$ & 54 & 50.1 & 50.3 & & ks & 5 & 57.1 & & OI & \\
\hline & $53 \cdot 3$ & 54.3 & 53 & & 56 & 52. & $.5 a$ & 2251 & & 56 & 49 & $3 a$ & 46 & & 5 & 59.1 & 62.1 & 04 & \\
\hline 58 & 53.5 & 54.4 & 53 & & 58 & 59. & & 2302 & & 58 & 51.7 & $5 I .7$ & 50 & 8 & 58 & 60.9 & 63.5 & & \\
\hline
\end{tabular}


Tabulation of magnetic declinations observed at Teplitz Bay-Continued

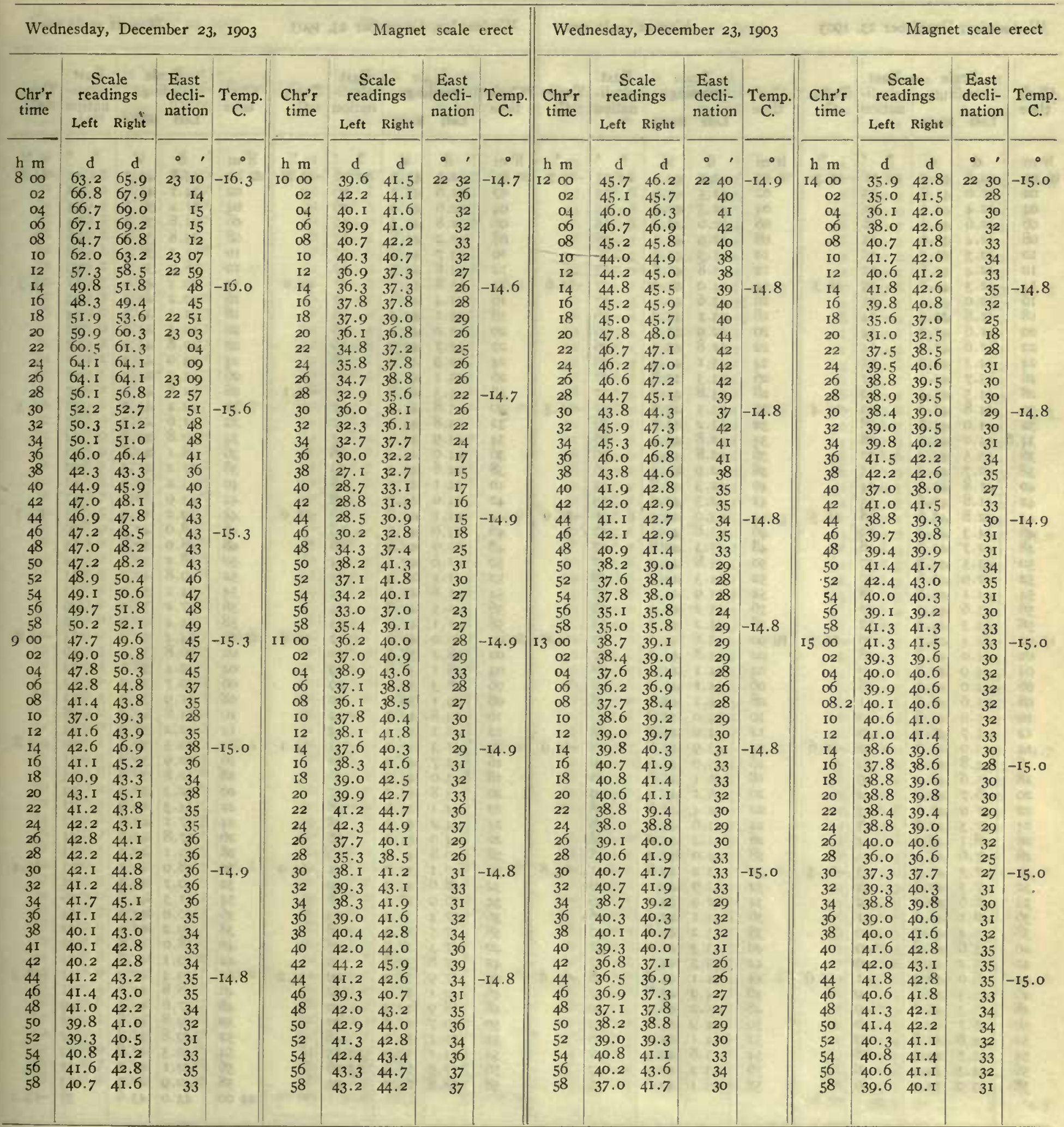


Tabulation of magnetic declinations observed at Teplitz Bay-Continued

\begin{tabular}{|c|c|c|c|c|c|c|c|c|c|c|c|c|c|c|c|c|c|c|c|}
\hline \multicolumn{5}{|c|}{ Wednesday, December 23, I903 } & \multicolumn{5}{|c|}{ Magnet scale erect } & \multicolumn{5}{|c|}{ Wednesday, December 23, 1903} & \multicolumn{5}{|c|}{ Magnet scale erect } \\
\hline \multirow{2}{*}{$\begin{array}{l}\text { Chr'r } \\
\text { time }\end{array}$} & \multicolumn{2}{|c|}{$\begin{array}{l}\text { Scale } \\
\text { readings }\end{array}$} & \multirow{2}{*}{$\begin{array}{c}\text { East } \\
\text { decli- } \\
\text { nation }\end{array}$} & Temp. & Chr'r & $\begin{array}{l}\mathrm{Sc} \\
\text { read }\end{array}$ & $\begin{array}{l}\text { ale } \\
\text { ings }\end{array}$ & $\begin{array}{l}\text { East } \\
\text { decli- }\end{array}$ & Temp. & Chr'r & $\begin{array}{r}\text { Sca } \\
\text { read }\end{array}$ & $\begin{array}{l}\text { ale } \\
\text { lings }\end{array}$ & $\begin{array}{l}\text { East } \\
\text { decli- }\end{array}$ & Temp. & Chr's & $\begin{array}{l}\mathrm{Sc} \\
\text { read }\end{array}$ & $\begin{array}{l}\text { ale } \\
\text { lings }\end{array}$ & $\begin{array}{l}\text { East } \\
\text { decli- }\end{array}$ & Temp. \\
\hline & Left & Right & & & & & Right & nation & & time & Left & Right & & & & Left & Right & & \\
\hline $\mathrm{h} \mathrm{m}$ & d & $\dot{\mathrm{q}}$ & $\circ$, & 0 & $\mathrm{~h} \mathrm{~m}$ & d & d & $\circ \quad$, & $\circ$ & $\mathrm{h} \mathrm{m}$ & d & d & & $\circ$ & $\mathrm{h} \mathrm{m}$ & d & d & $\cdot 1$ & 0 \\
\hline 1600 & 39.8 & 40.2 & 2231 & $-15 \cdot 3$ & 1800 & 42.7 & 42.7 & $22 \quad 35$ & -15.7 & 2000 & 42.9 & 43.3 & 2236 & -14.4 & 2200 & 43.8 & 49.1 & $234 \mathrm{I}$ & -14.2 \\
\hline 02 & $4 I .0$ & 41.8 & 33 & - & 02 & 42.5 & 42.6 & 35 & & 02 & 42.6 & $43 . I$ & 36 & & 02 & 34.7 & 41.6 & & \\
\hline 04 & 42.4 & 43.0 & & 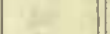 & 04 & 42.3 & 42.5 & 35 & 80 & 04 & 42.6 & 43.0 & 36 & 20 & 04 & $3 \mathrm{I} .2$ & 38.3 & 23 & 3 \\
\hline 06 & 42.6 & 43.0 & 36 & 6 & 06 & 42.6 & 42.8 & 35 & B & $\infty 6$ & 42.8 & $43 \cdot 3$ & 36 & 3 & 06 & 31.8 & 38.7 & 24 & \\
\hline 08 & 42.4 & 43.0 & 35 & We & 08 & 42.9 & 43.1 & 36 & 20 & $\$ 8$ & 43.2 & 43.8 & 37 & $8 s$ & 08 & 35.8 & 37.2 & 26 & 68 \\
\hline Io & 42.3 & 42.7 & 35 & 07 & 10 & $43 \cdot 3$ & 43.3 & 36 & net & IO & 43.7 & 44.1 & 37 & on & Io & 37.1 & 38.3 & 28 & ni \\
\hline 12 & 42.4 & 42.6 & 35 & & 12 & 43.8 & 43.8 & 37 & & I2 & 43.8 & $43 \cdot 3$ & 37 & +1 & 12 & 38.0 & 39.8 & 29 & \\
\hline 14 & 42.3 & 42.7 & 35 & -15.3 & 14 & 44.0 & 44.1 & $37-$ & -15.6 & I4 & 43.8 & 44.0 & 37 & -14.2 & 14 & 39.2 & 41.0 & $3 \mathbf{I}-$ & $-14 \cdot 3$ \\
\hline 16 & 42.0 & 42.4 & 35 & & I6.3 & 43.6 & 43.6 & 37 & $x^{2}$ & I6 & 43.4 & 43.8 & 37 & . & I6 & 40.2 & 42.1 & 33 & \\
\hline 18 & 41.8 & 42.3 & 34 & 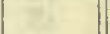 & 18.3 & 43.2 & 43.2 & 36 & $x^{2}$ & I8 & 43.1 & 43.2 & 36 & Ds & 18 & $4 I . I$ & 42.9 & 34 & 상 \\
\hline 20 & 41.6 & 42.2 & 34 & 86 & 20 & 42.9 & 43.0 & 36 & $\alpha^{2}=$ & 20 & 43.0 & 43.2 & 36 & $=$ & 20 & 42.1 & 43.8 & 36 & be \\
\hline 22 & 41.6 & 42.0 & 34 & 2 & 22 & 42.8 & 43.0 & 36 & wa & 22 & 42.7 & 42.9 & 36 & tis & 22 & 42.8 & 44.2 & 37 & 6 \\
\hline 24 & 42.3 & 42.9 & 35 & trat & 24 & 43.1 & $43 \cdot 3$ & 36 & $=$ & 24 & 42.9 & 43.0 & 36 & is & 24 & 43.0 & $44 \cdot 3$ & 37 & \\
\hline 26 & 43.7 & $44 \cdot 3$ & 37 & ( & 26 & $43 \cdot 3$ & 43.8 & 37 & ne & 26 & 43.1 & $43 \cdot 3$ & 36 & $8=$ & 26 & 43.0 & 44.3 & 37 & \\
\hline 28 & $44 \cdot 5$ & 45.1 & 39 & & 28 & 43.8 & 44.0 & 37 & & 28 & 43.3 & 43.8 & 37 & $-14 . I$ & 28 & 42.8 & 44.2 & 37 & \\
\hline 30 & 44.8 & 45.3 & 39 & -15.4 & 30 & 43.5 & 43.8 & 37 & $-15 \cdot 3$ & 30 & 43.8 & 44.0 & 37 & $10=$ & 30 & 42.2 & 43.7 & 36 & -14.3 \\
\hline 32 & 44.0 & 44.7 & 38 & -28 & 32 & 43.2 & 43.7 & 36 & -2. & 32 & 43.8 & 43.9 & 37 & 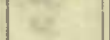 & 32 & 41.6 & $43 \cdot 3$ & 35 & (1) \\
\hline 34.6 & 44.0 & $44 \cdot 5$ & 38 & $y^{2}$ & 34 & 42.8 & 43.0 & 36 & wat & 34 & 43.9 & 44.0 & 37 & H & 34 & 40.8 & 42.8 & 34 & \\
\hline 36 & 43.5 & 44.0 & 37 & $y=$ & 36 & 42.8 & 43.1 & 36 & at & 36 & 44.0 & 44.4 & 38 & is & 3 & 38.1 & 43.8 & 33 & \\
\hline 38 & 42.7 & 42.4 & 35 & $x_{2}$ & 38 & 42.9 & $43 . I$ & 36 & $x^{2}$ & 38 & 44.6 & 45.0 & 39 & 8 & 38 & 37.2 & $4^{\mathrm{I}} \cdot 5$ & 30 & \\
\hline 40 & $4 \mathrm{I} .8$ & 42.3 & 34 & 6 & 40 & 42.3 & 42.8 & 35 & 8 & 40 & 44.1 & 44.7 & 38 & 64 & 40 & 43.2 & 50.3 & 42 & 16 \\
\hline 42 & 41.8 & 42.6 & 35 & & 42 & 42.3 & 42.9 & 35 & & 42 & 44.0 & 44.7 & 38 & $1=5$ & 42 & 43.9 & 56.5 & 47 & \\
\hline 44 & 42.8 & 43.6 & 36 & -15.5 & 44 & 43.0 & 43.2 & 36 & -15.0 & 44 & $44 . I$ & 44.8 & 38 & $-I_{4} .0$ & 44 & 39.2 & 45.9 & 35 & $-14 \cdot I$ \\
\hline 46 & $44 \cdot 3$ & $45 \cdot 3$ & 39 & ( & 46 & 43.8 & $44 . I$ & 37 & - & 46 & 43.8 & 44.7 & 38 & ( & 46 & 38.0 & 40.1 & 30 & a \\
\hline 48 & 42.7 & 43.6 & 36 & -4 & 48 & 43.3 & 43.8 & 37 & 5 & 48 & 42.9 & 43.6 & 36 & 2 & 48 & 28.9 & 30.8 & 15 & 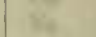 \\
\hline 50 & 43.5 & 44.0 & 37 & (1) & 50 & 43.8 & 44.0 & 37 & 6 & 50 & 41.6 & $42 . I$ & 34 & ne. & 50 & 28.2 & $30 . I$ & 14 & a. \\
\hline 52 & 42.9 & 43.6 & 36 & et: & $5^{2}$ & $44 \cdot 3$ & 44.7 & 38 & a & 52 & 4I.I & 41.9 & 34 & 28 & $5^{2}$ & 39.8 & 40.2 & $3 \mathrm{I}$ & $x^{2}$ \\
\hline 54 & $44 . I$ & 44.8 & 38 & 4 & 54 & 43.9 & $44 . I$ & 37 & $x^{2}$ & 54 & $4 \mathrm{I} .8$ & 42.1 & 34 & Q & 54 & 34.2 & 35.3 & 23 & 17 \\
\hline 56 & 43.6 & 44.5 & 37 & $=$ & 56 & 43.2 & 43.5 & 36 & $2=$ & 56 & 4 I. 5 & 41.8 & 34 & II & 56 & 47.2 & 56.1 & 49 & \\
\hline 58 & $43 \cdot 3$ & 44.3 & 37 & & 58 & 44.2 & 44.2 & 38 & & 58 & 4I. I & 41.5 & 33 & 87 & 58 & 34.4 & 40.9 & 27 & \\
\hline 1700 & 44.4 & 45.3 & 39 & -15.5 & 1900 & 43.8 & 44.1 & 37 & -14.9 & 2100 & 40.0 & 40.6 & 32 & -14.0 & 2300 & 24.4 & 33.2 & 14 & $-I 4.0$ \\
\hline 02 & 44.0 & 44.8 & $3^{8}$ & 60 & 02 & 43.8 & 44.0 & 37 & - & 02 & 38.2 & 38.7 & 29 & i & 02 & 26.8 & 34.4 & 16 & - \\
\hline 04 & 44.9 & 45.7 & 40 & 148 & 04 & 44.1 & 44.4 & 38 & 68 & 04 & 38.1 & 38.8 & 29 & 32 & 04 & 30.4 & 38.5 & 22 & (3) \\
\hline 06 & 44.0 & 44.7 & 38 & whe & 06 & $44 . I$ & 44.4 & 38 & 19 & 06 & 38.3 & 39.2 & 29 & 80 & 06 & 30.7 & 37.2 & 22 & 8 \\
\hline 08 & 45.6 & 46.6 & $4 \mathrm{I}$ & EN & 08 & $44 . I$ & 44.4 & 38 & 16 & 08 & 39. I & 39.9 & 30 & sh & of & 31.9 & 37.1 & 22 & $8 x$ \\
\hline I0 & 45.0 & 45.6 & 40 & 67 & IO & 44.0 & 44.8 & 38 & xyt & Io & 39.8 & 40.7 & 32 & ess & 10 & 34.8 & 41.9 & 28 & 3. \\
\hline 12 & 45.7 & 46.2 & 40 & & 12 & 44.0 & 44.8 & 38 & 50 & 12 & 40.1 & $4 I . I$ & 32 & it & 12 & 32.2 & 38.9 & 24 & \\
\hline 14 & 45.0 & 45.7 & 40 & -15.6 & I4 & 43.9 & 44.8 & 38 & -14.9 & I4 & 41.3 & 42.0 & 34 & -I 4.1 & 14 & 29.5 & 36.1 & 20 & -13.8 \\
\hline 16 & 44.8 & $45 \cdot 4$ & 39 & (2. & 16 & 45.0 & 45.6 & 40 & - & I6 & 42.7 & 43.2 & 36 & 24.0 & 16 & 30.9 & 36.7 & 21 & \\
\hline I8 & 45.6 & 46.2 & 40 & Whe & 18 & 45.1 & 45.6 & 40 & Bet & 18 & 43.0 & 43. & 36 & 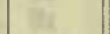 & I & 32.8 & 38.8 & 25 & 郚 \\
\hline 20 & 46.7 & 47.2 & 42 & in & 20 & 45.1 & 45.4 & 39 & 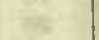 & 20 & 43.8 & 44.0 & 37 & $=$ & 20 & 32.3 & 38.3 & 24 & set \\
\hline 22 & 47.0 & 47.6 & 43 & 5 & 22 & 44.4 & 44.8 & 38 & $s=$ & 22 & 44.1 & 44.5 & 38 & tif & 22 & 34.3 & 40.9 & 27 & $=$ \\
\hline 24 & $47 \cdot 3$ & 47.6 & 43 & is & 24 & 44.6 & 44.9 & 38 & 12 & 24 & 44.2 & 44.7 & 38 & A3 & 24 & 37.1 & 43.6 & 32 & 36 \\
\hline 26 & 48. I & 48.5 & 44 & $E$ & 26 & 44.4 & 44.8 & 38 & is & 26 & 44.0 & 44. & 38 & 2 & 26 & 37.8 & 42.7 & 31 & \\
\hline 28 & 47.6 & 48.0 & 43 & 15 & 28 & 44.0 & 44.2 & 38 & 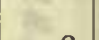 & 28 & 40.8 & $4 \mathrm{I} .7$ & 33 & 1 & 28 & 39.1 & $43 \cdot 3$ & 33 & \\
\hline 30 & $47 \cdot 3$ & 47.5 & 43 & $-15 \cdot 5$ & 30 & 43.2 & 43.8 & 37 & -14.8 & 30 & 40.1 & 39.7 & $3 \mathrm{I}$ & -14.0 & 30 & 38.0 & 42.1 & $3 \mathrm{I}$ & -13.4 \\
\hline 32 & $47 \cdot 3$ & 47.5 & 43 & $=2$ & 32 & 42.9 & 43.1 & 36 & $x^{2}$ & 32 & 38.6 & 40 & 30 & 1 & 32 & 40.7 & 44.3 & 35 & $1=2$ \\
\hline 34 & 46.6 & 47.0 & 42 & & 34 & 42.9 & 43.1 & 36 & 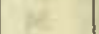 & 34 & 36.9 & 38.6 & 28 & & 34 & $4 \mathrm{I} \cdot 3$ & 44.8 & 36 & \\
\hline 36 & 46.1 & 46.6 & $4 I$ & $y$ & 36 & 43.1 & 43.6 & 36 & Wh: & 36 & 37.4 & 39.4 & 29 & & 36 & 40.0 & 43.2 & 34 & \\
\hline 38 & 45.4 & 45.7 & 40 & $x^{2}$ & 38 & 43.2 & 43.8 & 37 & ate & 38 & 37.5 & 38.9 & 28 & $x$ & 38 & 39.2 & $42 . I$ & 32 & $x^{2}$ \\
\hline 40 & 45.6 & 45.8 & 40 & $\approx$ & 40 & 43.3 & 44.0 & 37 & et & 40 & 37.3 & 38.9 & 28 & 6 & 40 & 38.0 & 40.8 & 30 & 8 \\
\hline 42 & 45.2 & 45.5 & 40 & $3=$ & 42 & 43.3 & 44.0 & 37 & & 42 & 37.8 & 39.6 & 29 & & 42 & 39.1 & 41.8 & 32 & \\
\hline 44 & 46.0 & 46.3 & 4I & -15.6 & 44 & 44.1 & 44.8 & 38 & $-\mathrm{I}_{4} .6$ & 44 & 37.9 & 39.2 & 29 & -14.2 & 44 & 39.4 & 42.0 & 32 & $-13 \cdot 3$ \\
\hline 46 & $45 \cdot 3$ & 45.6 & 40 & a & 46 & 44.9 & 45.8 & 40 & .4 .0 & 46 & $40 . \mathrm{I}$ & $4 \mathrm{I} \cdot 3$ & 32 & 1.7 .6 & 46 & 38.8 & 41.1 & $3 \mathrm{I}$ & \\
\hline 48 & 45.3 & 45.6 & 40 & 20 & 48 & 45.2 & 46.0 & 40 & 78 & 48 & 38.9 & 40.2 & 30 & 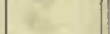 & 48 & 37.6 & 40.1 & 29 & \\
\hline 50 & 45.7 & 45.7 & 40 & $x=$ & 50 & 44.9 & 45.5 & 39 & 81 & 50 & 39.7 & 41.8 & 32 & 68 & 50 & 39.8 & 41.8 & 32 & W \\
\hline 52 & 44.8 & 44.8 & 39 & Ee & 52 & $44 . I$ & 44.9 & 38 & 47 & 52 & 50.7 & 51.0 & 48 & 37 & 52 & 39.8 & 4I. 8 & 32 & \\
\hline 54 & 44.5 & 44.5 & 38 & is & 54 & 43.8 & 44.3 & 37 & ite & 54 & 51.1 & 59.3 & 2255 & Hit & 54 & 40.3 & 41.8 & 33 & $4 t$ \\
\hline 56 & 44.3 & 44.5 & 38 & 20 & 56 & 43.2 & 44.0 & 37 & $6=$ & 56 & 57.9 & 66.9 & 2306 & 36 & 56 & $40 . I$ & $4 \mathrm{I} .2$ & 32 & \\
\hline 58 & 43.0 & 43.0 & 36 & $\sqrt{4}$ & 58 & 43.1 & 43.8 & 36 & 48 & 58 & 58.8 & 64.7 & 05 & 8 & 58 & 40.9 & 41.9 & 33 & \\
\hline & & & & & & & & & & & & & & & & 43.0 & 43.6 & 36 & $-13 \cdot 3$ \\
\hline
\end{tabular}

Observers-W. J. P. and R. R. T., who alternated from $\mathrm{I} 8 \mathrm{~h}$ 20m to I8h $30 \mathrm{~m}$.
Correction to local mean time is $+32 \mathrm{~s}$.

Torsion head at oh oom read $345^{\circ}$ and at $24 \mathrm{~h}$ oom read the same. Observer-R. R. T. 
Tabulation of magnetic declinations observed at Teplitz Bay-Continued

\begin{tabular}{|c|c|c|c|c|c|c|c|c|c|c|c|c|c|c|c|c|c|c|c|}
\hline \multicolumn{5}{|c|}{ Thursday, December 24, 1903} & \multicolumn{5}{|c|}{ Magnet scale inverted } & \multicolumn{10}{|c|}{ Tuesday, December 29, 1903} \\
\hline \multirow{2}{*}{$\begin{array}{l}\text { Chr'r } \\
\text { time }\end{array}$} & \multicolumn{2}{|c|}{$\begin{array}{l}\text { Scale } \\
\text { readings }\end{array}$} & \multirow{2}{*}{$\begin{array}{c}\text { East } \\
\text { decli- } \\
\text { nation }\end{array}$} & \multirow{2}{*}{$\begin{array}{c}\text { Temp. } \\
\text { C. }\end{array}$} & Chr'r & $\begin{array}{l}\text { Sc } \\
\text { read }\end{array}$ & $\begin{array}{l}\text { ale } \\
\text { ings }\end{array}$ & $\begin{array}{l}\text { East } \\
\text { decli- }\end{array}$ & Temp. & Chr'r & $\begin{array}{l}\text { Sc: } \\
\text { read }\end{array}$ & $\begin{array}{l}\text { ale } \\
\text { lingss }\end{array}$ & $\begin{array}{l}\text { East } \\
\text { decli- }\end{array}$ & Temp. & Chr'r & $\begin{array}{l}\text { Sc: } \\
\text { read }\end{array}$ & lings & $\begin{array}{l}\text { East } \\
\text { decli- }\end{array}$ & \\
\hline & Left & Right & & & & Left & Right & & & tin & Left & Right & & & & Left & Right & & \\
\hline $\ln \mathrm{m}$ & d & d & $\circ$, & $\bullet$ & $\mathrm{h} \mathrm{m}$ & d & d & $\cdot 1$ & $0^{\circ}$ & $\mathrm{h} \mathrm{m}$ & d & d & $\cdot \quad$ & 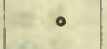 & $\mathrm{h} \mathrm{m}$ & d & d & $\cdot$ & $\bullet$ \\
\hline 600 & 49.7 & $49 \cdot 3$ & 2236 & $-2 \mathbf{I} \cdot 3$ & I8 00 & 48.1 & 46.3 & 2239 & -18.9 & 1200 & 38.8 & 40.9 & 2232 & -20.0 & I4 00 & 39.4 & 40.2 & 2232 & -16.3 \\
\hline 02 & 49.4 & 48.2 & 37 & (2) & 02 & 48.7 & 47.0 & 39 & & 02 & 38.8 & 40.9 & 32 & & 02 & 39.9 & 40.4 & 33 & \\
\hline 04 & 49.4 & 47.9 & 37 & $E$ & 04 & 48.5 & 46.9 & 39 & 8. & 04 & 39.8 & 40.9 & 33 & & 04 & 39.9 & 40.4 & 33 & ex \\
\hline$\infty$ & 49.5 & 47.9 & 37 & D. & 06 & 49.0 & 47.7 & 38 & 8 & $\infty 6$ & 39.8 & $4 \mathrm{I} . \mathrm{I}$ & 33 & & $\infty 6$ & 39.7 & 40.0 & 32 & 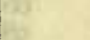 \\
\hline 08 & 9.6 & 48.0 & 37 & . & 08 & 49.4 & $48 . I$ & 37 & 4 & 08 & $39 \cdot 3$ & 40.6 & 32 & & 08 & 39.2 & 39.8 & 32 & 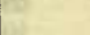 \\
\hline I0 & $49 . I$ & 47.8 & 38 & 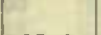 & I0 & 49.6 & 48.3 & 37 & 8 & Io & -38.9 & 40.2 & 32 & & Io & 39.2 & 39.8 & 32 & 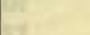 \\
\hline I2 & 49.2 & 48.0 & 37 & -21.0 & 12 & $49 \cdot 3$ & 48.3 & 37 & & 12 & 39.0 & 40.4 & 32 & & 12 & 39. I & 39.7 & 32 & \\
\hline I4 & 49.7 & 48.5 & 36 & 1.5 & I4 & 49.3 & 48.3 & 37 & -18.9 & I4 & 39.2 & 40.8 & 33 & -19.3 & 14 & 39.8 & 40.2 & 33 & -15.9 \\
\hline I6 & 49.3 & 48.6 & 37 & & I6 & 49.7 & 48.9 & 36 & & I6 & 39.3 & 40.9 & 33 & & 16 & 39.7 & 39.9 & 32 & \\
\hline I8 & 49.0 & 48.2 & 37 & E & 18 & 50.2 & $49 \cdot 3$ & 36 & 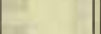 & I8 & 39.3 & 40.8 & 33 & & 18 & 39.2 & 39.8 & 32 & 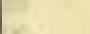 \\
\hline 20 & 49.0 & 48.2 & 37 & 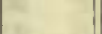 & 20 & 50.4 & 49.4 & 35 & 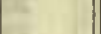 & 20 & 38.4 & 39.8 & $3 \mathrm{I}$ & & 20 & 39. I & 39.3 & $3 \mathrm{I}$ & x. \\
\hline 22 & 49.2 & 48.5 & 37 & 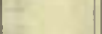 & 22 & 49.9 & 48.9 & 36 & 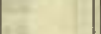 & 22 & $38 . i$ & 39.2 & 30 & & 22 & 39.0 & 39.2 & $3 I$ & 5 \\
\hline 24 & 49. I & $48 . I$ & 37 & 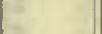 & 24 & 49. I & $48 . I$ & 37 & 1 & 24 & 38.3 & 39.2 & 30 & te & 24 & 39.6 & 39.8 & 32 & 56 \\
\hline 26 & 9. I & 48.2 & 37 & 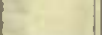 & 26 & 48.3 & 47.6 & 38 & 2 & 26 & 38.8 & 39.7 & 31 & + & 26 & 40.1 & 40.2 & 33 & . \\
\hline 28 & 49.2 & 48.3 & 37 & & 28 & 48.5 & 47.8 & 38 & & 28 & 38.5 & 39.3 & $3 \mathrm{I}$ & & 28 & 40.2 & 40. & 33 & \\
\hline 30 & 49. I & $48 . I$ & 37 & -20.7 & 30 & 48.7 & 47.7 & 38 & -19.0 & 30 & 38.9 & 39.8 & $3 \mathrm{I}$ & -18.8 & 30 & 39.8 & 40.1 & 32 & -15.5 \\
\hline 32 & 48.9 & $48 . I$ & 38 & & 32.3 & 48.7 & 47.4 & 38 & & 32 & 39.1 & 39.8 & 32 & & 32 & 38.7 & 38.9 & $3 \mathrm{I}$ & \\
\hline 34 & 48.9 & 48.0 & $3^{8}$ & C & 34 & 48.2 & $47 . I$ & 39 & 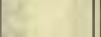 & 34 & 38.8 & 39.3 & $3 \mathrm{I}$ & & 34 & 37.9 & 38. I & 30 & 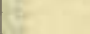 \\
\hline 36 & 48.9 & 48.0 & 38 & 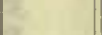 & 36 & 48.2 & $47 . I$ & 39 & L & 36 & 39.0 & 39.4 & $3 \mathrm{I}$ & & 3 & 38.6 & 38.8 & 31 & 2 \\
\hline 38 & 48.7 & 47.9 & 38 & e & 38 & 48.7 & 47.4 & 38 & 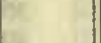 & 38 & 39.2 & 39.8 & 32 & & 38 & 40.0 & 40.3 & 33 & 5 \\
\hline 40 & 48.8 & 48.0 & $3^{8}$ & 6 & 40 & 48.7 & 47.6 & 38 & 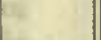 & 40 & 39.2 & 39.9 & 32 & & 40 & 40.1 & 40.6 & 33 & a \\
\hline 42 & 48.7 & 47.8 & 38 & & 42 & 48.2 & $47 \cdot 3$ & 39 & & 42 & 38.7 & 39. I & $3 I$ & & 42 & 39.7 & 40. I & 32 & 4 \\
\hline 44 & 48.5 & 47.7 & 38 & & 44 & 48.9 & $47 \cdot 9$ & 38 & -19.0 & 44 & 38.8 & 39.2 & $3 \mathrm{I}$ & -18.3 & 44 & 39. I & 39.9 & 32 & \\
\hline 46 & 48.9 & 47.9 & 38 & L & 46 & 49.2 & 47.9 & 37 & & 46 & 39. I & 39.9 & 32 & & 46.2 & 39.2 & & 32 & -15.0 \\
\hline 48 & 49.0 & 48.0 & $3^{8}$ & 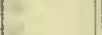 & 48 & 49.1 & 48.0 & 37 & t & 48 & 39.2 & 39.9 & 32 & & 48.2 & 38.9 & 39. & $3 \pi$ & \\
\hline 50 & 49.0 & 48.0 & 38 & & 50 & 49.0 & 47.9 & 38 & & 50 & $3^{88.8}$ & 39.2 & $3 I$ & & 50 & 38.3 & 38. & 30 & c. \\
\hline$j^{2}$ & 48.9 & 48.1 & $3^{8}$ & & 52 & 49.0 & 47.9 & 38 & C. & 52 & 39.0 & 39.2 & $3 I$ & & 52 & 38.3 & 39.3 & $3 \mathbf{I}$ & E \\
\hline 54 & 48.9 & 48.3 & 37 & & 54 & 48.9 & 47.9 & $3^{8}$ & 3 & 54 & 38.8 & 39.2 & $3 \mathrm{I}$ & & 5 & 38.3 & & $3 \mathrm{I}$ & 2 \\
\hline 56 & 49.0 & 48.7 & 37 & & 56 & 49.0 & 48.0 & 37 & & 56 & 39. I & 39.3 & $3 I$ & & 5 & 38.2 & 39.3 & $3 \mathbf{I}$ & 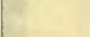 \\
\hline 58 & 49.0 & 48.8 & 37 & & 58 & 49.0 & 47.7 & 38 & - & 58 & 39.9 & $40 . I$ & 33 & & 58 & 38.5 & & 31 & \\
\hline I7 00 & 49.0 & 48.9 & 37 & $-\mathrm{I} 9.8$ & 1900 & $49 . \mathrm{I}$ & 48.0 & 37 & -19.2 & I3 00 & 39.3 & 39.8 & 32 & -17.8 & I5 00 & 38.3 & 39.2 & $3 \mathrm{I}$ & -14.7 \\
\hline 02 & 49.3 & 48.8 & 37 & & 02 & 48.9 & $47 \cdot 7$ & 38 & 1 & 02 & 39.2 & 39.5 & 32 & & 02 & $39 . I$ & & 32 & \\
\hline 04 & 49.4 & 48.8 & 36 & E & 04 & 49.0 & 47.8 & 38 & 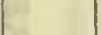 & 04 & 39.0 & 39. & $3 \mathrm{I}$ & $\theta$ & 04 & $39 . I$ & 39 & 32 & 0 \\
\hline об & 49.6 & 49.0 & 36 & 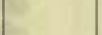 & 06 & 49.2 & 48.1 & 37 & P & 06 & 39.8 & 40 & 32 & & 06 & 39.0 & 39.6 & 32 & 8 \\
\hline 08 & 49.4 & 48.9 & 36 & & 08 & 49.0 & 48.0 & 37 & C & 08 & 39.8 & 40.2 & 33 & & 88 & 39. I & 39. & 32 & \\
\hline IO & 49.0 & 48.7 & 37 & 3 & I0 & 49.0 & $48 . I$ & 37 & 1 & Io & 39.6 & 40 & 32 & 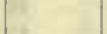 & IO & 39.2 & & 32 & 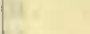 \\
\hline 12 & 49.0 & 48.3 & 37 & res & I2 & 49.2 & 48.0 & 37 & & I2 & 39.7 & 40.2 & 32 & & I2 & 38.1 & 38.9 & 30 & (2. \\
\hline I4 & 49.1 & 48.2 & 37 & -I9.4 & I4 & $49 \cdot I$ & 47.9 & 37 & -19.4 & 14 & 39.3 & 39.9 & 32 & $-17 \cdot 3$ & 14 & 38.9 & 39.8 & 32 & -14.7 \\
\hline I6 & 49.4 & $48 . I$ & 37 & & I6 & 48.3 & 47.6 & 38 & & I6 & 38.9 & 39. & 32 & & I6 & 40.0 & 40 & 33 & \\
\hline I8 & 49.8 & 48.6 & 36 & 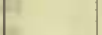 & I8 & 48.9 & 48.0 & $3^{8}$ & 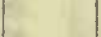 & I8 & 39.1 & 39.8 & 32 & & 18 & 39.2 & & 32 & 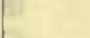 \\
\hline 20 & 50.2 & 49. I & 36 & - & 20 & 49.0 & 47.9 & 38 & 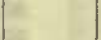 & 20 & 39.1 & 40.0 & 32 & & 2 & 38.1 & 38. & 30 & 1 \\
\hline 22 & 50.9 & 49.4 & 35 & 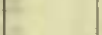 & 22 & 48.9 & 47.6 & 38 & $=$ & 22 & 39.1 & 39.8 & 32 & & 22 & 38.8 & 39.3 & $3 \mathrm{I}$ & 1 \\
\hline 24 & 50.8 & 49.5 & 35 & 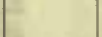 & 24 & 48.8 & 47.9 & $3^{8}$ & 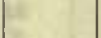 & 24 & 38.9 & 39.4 & $3 I$ & H & 24 & 39.1 & & 32 & 1 \\
\hline 26 & 50.7 & 49.4 & 35 & 2 & 26 & 48.7 & & 38 & 1 & 26 & 39.8 & 40. & 32 & 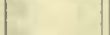 & 2 & 38.8 & 39.3 & $3 I$ & \\
\hline 28 & 50.5 & 49.5 & 35 & & 28.5 & 48. I & 47.6 & 39 & 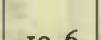 & 28 & 40.2 & 40.8 & 33 & & 28 & 38.9 & & $3 \mathrm{I}$ & 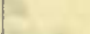 \\
\hline 30 & 50.5 & 49.7 & 35 & -19.1 & 30 & 48.6 & $47 \cdot 7$ & 38 & -19.6 & 30 & 40.1 & 40.7 & 33 & -17.0 & 30 & 39.0 & 39.5 & $3 \mathrm{I}$ & -14.7 \\
\hline 32 & 49.9 & 49.2 & 36 & & 32 & 48.7 & 47.1 & 38 & & 32 & 39.2 & 39.9 & 32 & & 32 & & & $3 I$ & \\
\hline 34 & 49.0 & $48 \cdot 3$ & 37 & 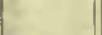 & 34 & 48.9 & $47 \cdot 3$ & 38 & O & 34 & 38.7 & 39.0 & $3 I$ & & 34 & 38.9 & 39.3 & $3 \mathrm{I}$ & \\
\hline 36 & 48.9 & 48.3 & 37 & & 36 & 48.7 & 47.2 & 38 & 2 & 36 & 40.1 & 40.3 & 33 & & 36 & 38.7 & & $3 \mathrm{I}$ & 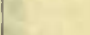 \\
\hline 38 & 49.0 & 48.5 & 37 & 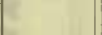 & $3^{8}$ & 49.0 & 47.9 & 38 & 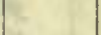 & 38 & 40.0 & 40.2 & 33 & & 38 & 38.3 & 38 & 30 & $=$ \\
\hline 40 & 48.8 & $48 . I$ & 38 & & 40 & 48.6 & 47.7 & 38 & 1 & 40 & 39.2 & 39.9 & 32 & & 40 & & 38 & 30 & 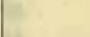 \\
\hline 42 & 48.8 & 47.9 & 38 & & 42 & 48.8 & 48.0 & 38 & 100 & 42 & $39 . \mathrm{I}$ & 39.7 & 32 & & 42 & 38.8 & 39 & $3 I$ & 1 \\
\hline 44 & 48.9 & 48.2 & 37 & -19.0 & 44 & 49.0 & 48.5 & 37 & -19.8 & 44 & 39.6 & $40 . I$ & 32 & -I6.8 & 44 & 38.5 & & $3 \mathrm{I}$ & $-I_{4} .8$ \\
\hline 46 & 49.4 & 48.1 & 37 & & 46 & 49.0 & 48.8 & 37 & & 46 & 39. I & 40.0 & 32 & & 46 & 38.3 & 38.9 & 30 & \\
\hline 48 & 49.6 & $48 . I$ & 37 & & 48 & 49.7 & 49.2 & 36 & 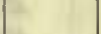 & 48 & 39.2 & 40.1 & 32 & & 48 & 38.9 & & $3 I$ & 2 \\
\hline 50 & 49. I & 47.7 & 38 & & 50 & 50.3 & 49. I & 36 & 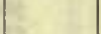 & 50 & 39.2 & 40.1 & 32 & & 50 & 38.3 & 40 & 32 & 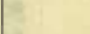 \\
\hline 52 & $49 . I$ & 47.9 & 37 & & 52 & 50.7 & 48.6 & $3^{6}$ & & $5^{2}$ & 39.3 & 40.2 & 32 & & 52 & 38.9 & 40.6 & 32 & 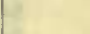 \\
\hline 54 & 48.9 & 47.6 & 38 & & 54 & 49.9 & 47.7 & 37 & & 54 & 39.2 & 40.2 & 32 & & 54 & $39 . \mathrm{I}$ & 40.7 & 32 & $=$ \\
\hline 56 & 48.7 & 47.1 & 38 & & 56 & 49.8 & 47.7 & 37 & & 56 & 39.3 & 40.2 & 32 & & 56 & $3^{88.8}$ & 40.2 & 32 & \\
\hline 58 & 48.2 & 46.7 & 39 & & 58 & 48.9 & 47.8 & 38 & & $5^{8}$ & 39.1 & 39.9 & 32 & & 58 & 39.3 & 40.4 & 32 & \\
\hline & & & & & 2000 & 50.8 & $49 \cdot 3$ & 35 & -19.7 & & & & & & I6 00 & 39.1 & 40.3 & 32 & $-I 4.7$ \\
\hline
\end{tabular}

Correction to local mean time is +2 Is.

Torsion head at $15 \mathrm{~h} 40 \mathrm{~m}$ read $345^{\circ}$ and at $20 \mathrm{~h}$ I $5 \mathrm{~m}$ read the same. Observer-R. R. T.
Correction to local mean time is - Im a8s. $90^{\circ}$ torsion $=24 .{ }^{\prime} 8$.

Torsion head at $7 \mathrm{~h} 55 \mathrm{~m}$ read $201^{\circ}$ and at $16 \mathrm{~h}$ 40m read $210^{\circ}$.

Observer-W. J. P. 
Tabulation of magnetic declinations observed at Teplitz Bay-Continued

\begin{tabular}{|c|c|c|c|c|c|c|c|c|c|c|c|c|c|c|c|c|c|c|c|c|}
\hline \multicolumn{5}{|c|}{ Wednesday, December 30, I903 } & \multicolumn{5}{|c|}{ Magnet scale inverted } & \multicolumn{5}{|c|}{ Wednes day, December 30, 1903} & \multicolumn{6}{|c|}{ Magnet scale inverted } \\
\hline \multirow{2}{*}{$\begin{array}{l}\text { Chr'r } \\
\text { time }\end{array}$} & \multicolumn{2}{|c|}{$\begin{array}{l}\text { Scale } \\
\text { readings }\end{array}$} & $\begin{array}{l}\text { East } \\
\text { decli- }\end{array}$ & Temp. & Chr'r & $\begin{array}{r}\mathrm{Sc} \\
\text { read }\end{array}$ & $\begin{array}{l}\text { ale } \\
\text { ings }\end{array}$ & $\begin{array}{l}\text { East } \\
\text { decli- }\end{array}$ & Temp. & Chr'r & $\begin{array}{r}\text { Sc } \\
\text { read }\end{array}$ & $\begin{array}{l}\text { ale } \\
\text { ings }\end{array}$ & East & Temp. & Chr'r & $\begin{array}{r}\mathrm{Sc} \\
\mathrm{read}\end{array}$ & lings & $\begin{array}{l}\text { Eas } \\
\text { decl }\end{array}$ & st & Temp. \\
\hline & Left & Right & nation & & & Left & Right & nation & & & Left & Right & Tativin & & & Left & Right & & & \\
\hline $\mathrm{h} \mathrm{m}$ & d & d & $\cdot \cdot$ & 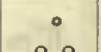 & $h \mathrm{~m}$ & d & d & $\cdot 1$ & & $\mathrm{~h} \mathrm{~m}$ & d & d & $\circ \quad \cdot$ & $\circ$ & $\mathrm{h} \mathrm{m}$ & d & $d$ & - & - & \\
\hline $00^{*}$ & 38.8 & 32.2 & 2237 & -18.8 & 200 & 30.4 & 29.9 & 2245 & -17.4 & 400 & $37 \cdot 3$ & 37.0 & 2234 & -17.0 & 600 & $34 \cdot 3$ & 33.7 & & 39 & -16.5 \\
\hline 02 & 36.7 & $35 \cdot 3$ & 36 & & 02 & 33.2 & 32.3 & $4 \mathrm{I}$ & 20 & 02 & 37.8 & 37.5 & 34 & & 02 & 32.2 & 32.0 & & 42 & \\
\hline $\mathrm{O}_{4}$ & $37 . I$ & $35 \cdot 3$ & 36 & 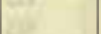 & 04 & 31.9 & $31 \cdot 3$ & 43 & 6 & $0_{4}$ & 39.3 & 39.0 & $3 \mathrm{I}$ & in. & 04 & 33.5 & 33.3 & & 40 & \\
\hline 06 & 37.2 & 35.2 & 36 & & 06 & 32.3 & 32.0 & 42 & & 06 & 40.5 & $40 . I$ & 29 & c. & 06 & $33 . I$ & $33 . \mathrm{I}$ & & $4 \mathrm{I}$ & \\
\hline 08 & Lo & st & & E. & 08 & 32.3 & 32.0 & 42 & 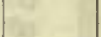 & 08 & 41.3 & 40.9 & 28 & ter & 08 & 3 I. 8 & 31.8 & & 43 & \\
\hline I0 & 37.6 & 35.6 & 35 & trat & 10 & $3 \mathrm{I} .3$ & 3I. I & 44 & $=$ & 10 & 42.3 & 42.1 & 26 & - & I0 & 30.0 & 30.0 & & 46 & \\
\hline I2 & 37.8 & 35.8 & 35 & & I2 & 33.8 & 33.5 & 40 & & 12 & 42.0 & $4 \mathrm{I} .8$ & 27 & & I2 & 30.6 & 30.3 & & 45 & \\
\hline 14 & 37.4 & 35.6 & 36 & -18.3 & I4 & 32.8 & 32.3 & 42 & -17.3 & 14 & $4 \mathrm{I} \cdot 3$ & 40.7 & 28 & -16.9 & I4 & 36 & $.0 a$ & & 36 & -16.4 \\
\hline I6 & 35.9 & $34 . I$ & 38 & Hin & I6 & 32.7 & $32 \cdot 3$ & 42 & $1+x^{2}$ & 16 & 38.8 & 38.2 & 32 & & I6 & & $.0 a$ & & 30 & \\
\hline 18 & 36.6 & 35.1 & 37 & $\%$ & I8 & 33.6 & $33 . I$ & 40 & Cir & 18 & 37.2 & 36.8 & 35 & 4 & I8 & & $.4^{b}$ & & 3I & \\
\hline 20 & 36.6 & 35.2 & 36 & 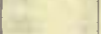 & 20 & $3 \mathrm{I} \cdot 3$ & 30.3 & 44 & 20 & 20 & 35.6 & 35.3 & 37 & & 20 & & $.5^{b}$ & & 39 & \\
\hline 22 & 35.6 & 34.1 & 38 & 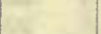 & 22 & 28.3 & 28.0 & $4^{8}$ & Es & 22 & 34.6 & 34.2 & 39 & 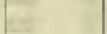 & 22 & & $3^{b}$ & & 45 & \\
\hline 24 & 34.9 & 33.7 & 39 & & 24 & 30.9 & 30.5 & 44 & $=$ & 24 & 34.1 & 33.8 & 39 & & 24 & 30.2 & $30 . I$ & & 45 & \\
\hline 26 & 35.3 & 33.7 & 39 & 4 & 26 & 30.9 & 30.5 & 44 & te & 26 & 32.5 & 32.5 & 42 & & 26 & 37 & $.2 a$ & & 34 & \\
\hline 28 & $35 \cdot 3$ & 33.5 & 39 & & 28 & 29. I & 28.8 & 47 & & 28 & 31.6 & $3 \mathrm{I} .3$ & 43 & & 28 & 37.6 & 37.4 & & 34 & \\
\hline 30 & $34 \cdot I$ & 32.3 & $4 \mathrm{I}$ & $-18 . I$ & 30 & 29.7 & 29.3 & 46 & $-17 \cdot 3$ & 30 & $3 \mathrm{I} .7$ & 31.5 & 43 & -16.9 & 30 & 41.3 & 41.0 & & 28 & -16.3 \\
\hline 32 & $33 \cdot 3$ & $3 \mathrm{I} .3$ & 42 & E. & 32 & 31.0 & 30.8 & 44 & 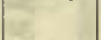 & 32 & $3 \mathrm{I} .8$ & 31.6 & 43 & (19. & 32 & 39.4 & 34.6 & & 35 & \\
\hline 34 & 33.7 & 30.4 & 43 & $u^{\circ}$ & 34 & 35.0 & 34.4 & 38 & Ex & 34 & 31.0 & 30.8 & 44 & & 34 & 37.4 & 37.2 & & 34 & \\
\hline 36 & 3I.I & 29.0 & 46 & 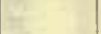 & 36 & 35.6 & $35 \cdot 3$ & 37 & 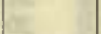 & 36 & 29.6 & 29.1 & 47 & t & 36 & 37.6 & 37.4 & & 34 & \\
\hline 38 & 30.3 & 27.3 & 48 & & $3^{8}$ & 35.4 & 34.2 & 38 & 2 & $3^{8}$ & 29.3 & 29. I & 47 & $y$ & 38 & 35.6 & 35.6 & & 37 & \\
\hline 40 & 30.6 & 29.3 & 46 & $=$ & 40 & 30.0 & 29.0 & 46 & the & 40 & 29.3 & 29.1 & 47 & 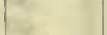 & 40 & 40.9 & 40.6 & & 29 & \\
\hline 42 & 32.6 & 31.3 & 43 & & 42 & 27.0 & 26.7 & $5 \mathrm{I}$ & & 42 & 33.5 & 32.9 & $4 \mathrm{I}$ & & 42 & 43. I & 43.0 & & 25 & \\
\hline 44 & 33.8 & 32.2 & $4 \mathrm{I}$ & -17.9 & 44 & 29.4 & 29.0 & 47 & -17.2 & 44 & 36.8 & 36.8 & 35 & $-\mathrm{I} 6.8$ & 44 & 25 & $.4 b$ & & 53 & -16.4 \\
\hline 46 & 33.2 & 32.5 & 41 & & 46 & 27.8 & 27.4 & 49 & & 46 & 40.0 & 40.0 & 30 & & 46 & 24 & $.5^{b}$ & & 54 & \\
\hline 48 & 32.6 & 31.6 & 42 & 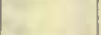 & 48 & 29.2 & 28.2 & 48 & 2 & 48 & $4 \mathrm{I} .3$ & $4 \mathrm{I} . \mathrm{I}$ & 28 & 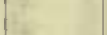 & 48 & 21.8 & $2 \mathrm{I} . \mathrm{I}$ & 225 & 59 & \\
\hline 50 & $33 \cdot 3$ & 32.7 & $4 \mathrm{I}$ & et & 50 & 30.8 & 30.2 & 45 & 5 & 50 & $4 \mathrm{I} .5$ & $4 \mathrm{I} .3$ & 28 & 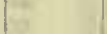 & 50 & 21.0 & 20.2 & 230 & $\infty$ & \\
\hline 52 & 35.3 & $34 \cdot 3$ & 38 & $E$ & 52 & 29.3 & 28.3 & 47 & 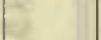 & 52 & 42.0 & 41.9 & 27 & 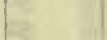 & 52 & 23. I & 22.8 & 225 & 57 & \\
\hline 54 & 34.6 & 33.7 & 39 & 2 & 54 & 27.8 & $27 \cdot I$ & 50 & 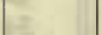 & 54 & $4 \mathrm{I} \cdot 3$ & $4 I .3$ & 28 & 8 & 54 & 26.6 & 26.2 & & $5 I$ & \\
\hline 56 & 35.8 & 35.0 & 37 & & 56 & 29.3 & 28.7 & 47 & & 56 & 40.3 & 40.3 & 29 & 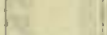 & 56 & 33.7 & 32.7 & & $4 \mathrm{I}$ & \\
\hline $5^{8}$ & 37.1 & 36.0 & 36 & & $5^{8}$ & 28.6 & 28.2 & 48 & & 58 & 40.5 & 40.5 & 29 & & $5^{8}$ & 26 & $.5^{b}$ & & 5I & \\
\hline 100 & 37.6 & 37.0 & 34 & -17.8 & 300 & 28.0 & 27.4 & 49 & -17.2 & 500 & 40.1 & 40.1 & 30 & -16.7 & 700 & 22.0 & 22.0 & & $5^{8}$ & -16.3 \\
\hline 02 & 38.3 & $37 \cdot 3$ & 33 & $2=$ & 02 & 27.5 & $27 . I$ & 50 & $\operatorname{lag}^{2}$ & 02 & $4 \mathrm{I} .3$ & 41.0 & 28 & & 02 & 28 & $.0 a$ & & 49 & \\
\hline 04 & 38.7 & 38.3 & 32 & ca. & 04 & 24.4 & 24.0 & 55 & a & O4 & 41.6 & 41.4 & 27 & & 04.5 & 34.6 & 34.3 & & 39 & \\
\hline 06 & 38.4 & 37.8 & 33 & 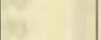 & 06 & 25.3 & 24.8 & 53 & 85 & 06 & 42 & $3^{b}$ & 26 & & 06 & 35 & $.0 b$ & 223 & 38 & \\
\hline 08 & 39.3 & $3^{8} 8.7$ & 32 & 6 & 08 & 26.1 & 25.8 & 52 & te. & 08 & 40 & $3^{b}$ & 29 & & 08 & & $.0 a$ & 230 & 09 & \\
\hline 10 & 36.3 & $35 \cdot 3$ & 37 & 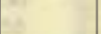 & 10 & 30. I & 27.3 & 48 & Fit & IO & 39 & $3^{b}$ & $3 I$ & & IO & 59.0 & 59.0 & 220 & 00 & \\
\hline 12 & 36.0 & 35.5 & 37 & & 12 & 28.9 & 28.5 & $4^{8}$ & & 12 & 39.2 & 39.2 & 3I & & I $2^{*}$ & 35.3 & 27.5 & 211 & II & \\
\hline I4 & $3^{88.0}$ & 37.4 & 34 & -I7.8 & 14 & 30.9 & 30.5 & 44 & -17.2 & 14 & 36.3 & 35.9 & 36 & -16.5 & I4* & 25.6 & 25.6 & 234 & 45 & -16.2 \\
\hline I6 & 37.0 & 36. I & 36 & 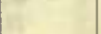 & I6 & 29.9 & 29.5 & 46 & ist & 16 & 35.0 & 34.9 & 38 & & 16 & 50 & $.0 a$ & 230 & 07 & \\
\hline 18 & 36.8 & 36.0 & 36 & & I8 & 27.3 & 26.7 & 50 & 5 & 18 & 34.5 & $34 \cdot 3$ & 39 & 4 & I8* & $47 \cdot 3$ & 37.9 & 222 & $2 \mathrm{I}$ & \\
\hline 20 & 35.5 & 35.3 & 37 & 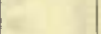 & 20 & 24.8 & 24.8 & 54 & c & 20 & 34.9 & 34.7 & 38 & E & 20 & 22.3 & 14.7 & & $5^{8}$ & \\
\hline 22 & 35.7 & 34.7 & $3^{8}$ & 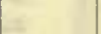 & 22 & 23.0 & 23.0 & 57 & E & 22 & 33 & $.7 b$ & 40 & & 22 & 22.2 & 19.5 & 225 & 55 & \\
\hline 24 & 34.1 & $33 \cdot 3$ & 40 & $=$ & 24 & 22 & $3^{b}$ & 58 & $E$ & 24 & 31.2 & 30.6 & 44 & e & $24^{*} 3$ & 28.0 & 24.5 & 232 & 24 & \\
\hline 26 & 33.0 & 32.3 & 42 & tr. & 26 & 22.2 & 22.0 & 58 & $=$ & 26 & 29.0 & 28.7 & 47 & & 26.2 & 39.6 & 36.8 & & 05 & \\
\hline 28 & 33.3 & 32.4 & $4 \mathrm{I}$ & & 28 & 22.2 & 22.0 & 58 & & 28 & 30.9 & 30.8 & 44 & & 28 & 41.0 & 38.0 & & 03 & \\
\hline 30 & 30.6 & 29.3 & 46 & -17.7 & 30 & $23 \cdot 3$ & 23.3 & 56 & -17.0 & 30 & 33.4 & 33.4 & 40 & -16.4 & 30 & 38.8 & 34.0 & & 08 & \\
\hline 32 & 30.0 & 28.8 & 47 & ( & 32 & 26.7 & 26.0 & $5 \mathrm{I}$ & ( & 32 & 34.2 & 34.0 & 39 & & 32 & 28.3 & 21.8 & & 26 & -16.0 \\
\hline 34 & 30.5 & 28.8 & 46 & 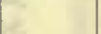 & 34 & 30.3 & 30. I & 45 & 2 & 35 & 34.0 & 33.6 & 40 & & 34 & 38.3 & 29.8 & & 12 & \\
\hline 36 & 32.3 & 30.4 & 44 & & 36 & 33.9 & 33. I & 40 & & 36 & 34.5 & $34 \cdot 3$ & 39 & & 36 & 37.7 & 28.3 & & I4 & \\
\hline $3^{8}$ & $33 \cdot 3$ & 32.0 & $4 \mathrm{I}$ & & 38 & $35 \cdot 3$ & 34.4 & $3^{8}$ & t & 38 & 35.9 & 35.5 & 37 & & 38 & 25.0 & 17.2 & & 32 & \\
\hline 40 & 33.5 & 31.7 & $4 I$ & & 40 & 35.7 & 34.9 & 37 & te & 40 & 34.9 & 34.9 & 38 & & 40 & I6. 5 & 9.0 & & 45 & \\
\hline 42 & $3 \mathrm{I} .0$ & 29.3 & 45 & & 42 & 35.2 & 34.6 & 38 & & 42 & 35.8 & 35.8 & 36 & & $42^{*}$ & 65.5 & 50.0 & & 56 & \\
\hline & 3 I. 3 & 30.3 & & -17.5 & & 36.4 & 35.6 & 36 & -17.0 & & 35.4 & 35.4 & 37 & -16.5 & 44 & 71.5 & 58.3 & & 45 & -16.0 \\
\hline 46 & 3I.I & 30.0 & 45 & ( & 46 & 40 & $0 a$ & 30 & & 46 & 33.8 & 33.8 & 40 & & 46 & 69.9 & 58.7 & & 46 & \\
\hline 48 & 32.1 & 31.0 & 43 & & 48 & 42.5 & 42.3 & 26 & . & 48 & 34.5 & $34 \cdot 3$ & 39 & & $48^{*}$ & 43.0 & 30.0 & & 33 & \\
\hline 50 & 30.9 & 30.1 & 45 & $\alpha$ & 50 & 40.6 & 40.0 & 29 & 5 & 50 & 35.9 & 35.9 & 36 & & 50 & 45.0 & 30.5 & & 31 & \\
\hline 52 & 30.6 & 30.0 & 45 & 5 & 52 & 38.0 & 37.6 & 33 & 2 & 52 & 35.9 & 35.9 & 36 & & $5^{2}$ & 44.0 & 33.2 & & 29 & \\
\hline 54 & 30.0 & 29.3 & 46 & & 54 & 38.3 & 37.8 & 33 & 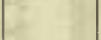 & 54 & 36.3 & 36.3 & 36 & & 54 & 45.8 & 44.2 & & I9 & \\
\hline 56 & $3 \mathrm{I} .5$ & 30.6 & 44 & & 56 & 39.5 & 39.0 & $3 \mathrm{I}$ & & 56 & 36.3 & 36.3 & 36 & & 56 & 49.7 & 48. I & & 13 & \\
\hline 58 & 32.2 & 3I I I & 43 & & 58 & 37.8 & 37.5 & 34 & $E$ & 58 & 36.7 & 36.3 & 35 & & 58 & 53.9 & $53 . \mathrm{I}$ & & 06 & \\
\hline & & $\operatorname{sen}$ & +96 & for & & & & & & & & 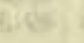 & & 8. & & & & & & \\
\hline
\end{tabular}


Tabulation of magnetic declinations observed at Teplitz Bay-Continued

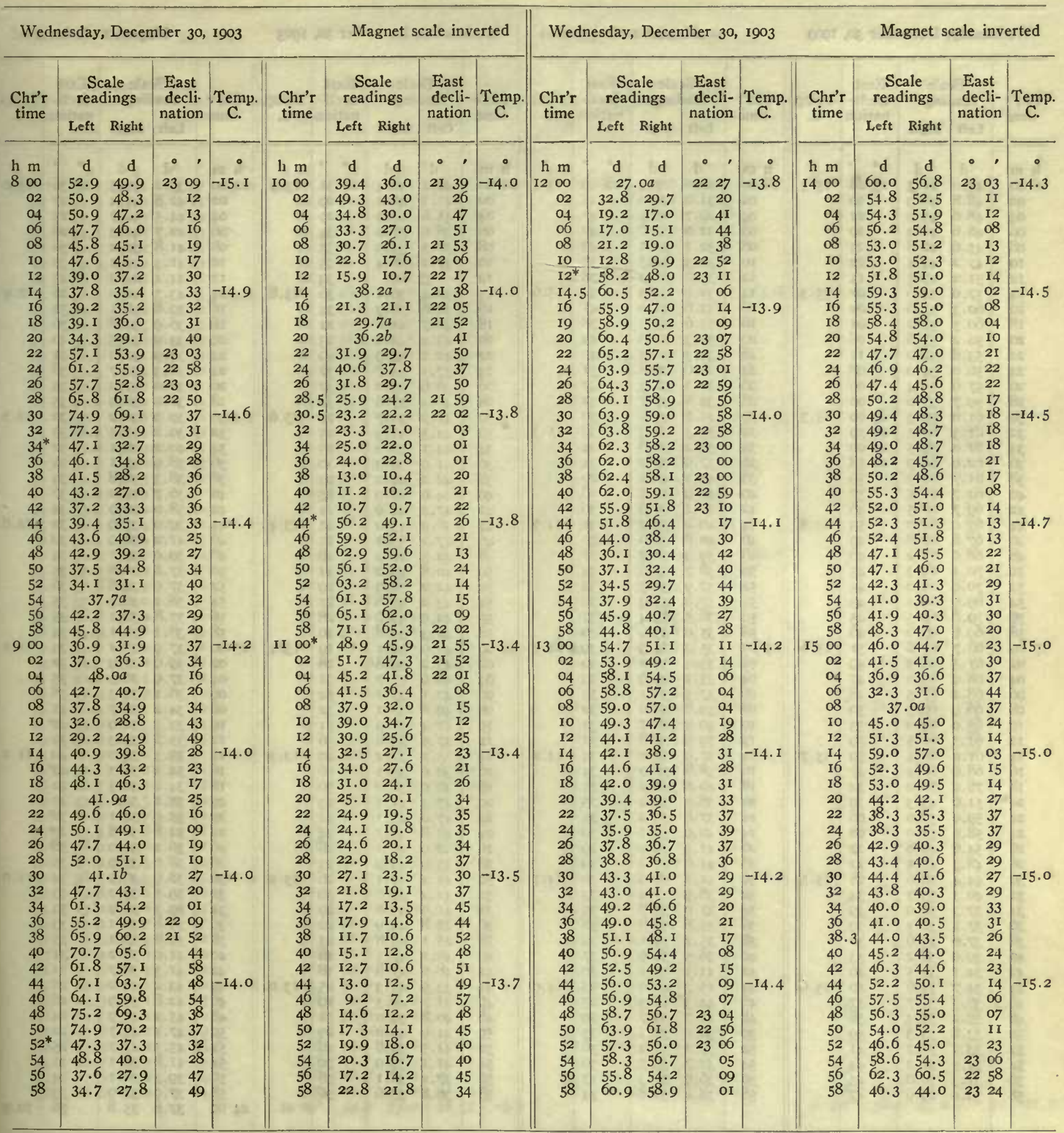

Observer-R. R. T.

Observers-R. R. T. and W. J. P., who alternated from $\mathrm{I}_{4} \mathrm{~h}$ oom to $14 \mathrm{~h} 08 \mathrm{~m}$. 
Tabulation of magnetic declinations observed at Teplitz Bay-Continued

\begin{tabular}{|c|c|c|c|c|c|c|c|c|c|c|c|c|c|c|c|c|c|c|c|c|c|}
\hline \multicolumn{5}{|c|}{ Wednesday, December 30, 1903 } & \multicolumn{6}{|c|}{ Magnet scale inverted } & \multicolumn{6}{|c|}{ Wednesday, December 30, rgo3 } & \multicolumn{5}{|c|}{ Magnet scale inverted } \\
\hline \multirow{2}{*}{$\begin{array}{l}\text { Chr'r } \\
\text { time }\end{array}$} & \multicolumn{2}{|c|}{$\begin{array}{l}\text { Scale } \\
\text { readings }\end{array}$} & $\begin{array}{l}\text { East } \\
\text { decli- }\end{array}$ & Temp. & Chr'r & $\begin{array}{r}\text { So } \\
\text { reac }\end{array}$ & $\begin{array}{l}\text { ale } \\
\text { ings }\end{array}$ & $\begin{array}{r}\text { Ea } \\
\text { dec }\end{array}$ & $\begin{array}{l}\text { ast } \\
\text { cli- }\end{array}$ & Temp. & Chr'r & $\begin{array}{l}\text { Sc } \\
\text { read }\end{array}$ & $\begin{array}{l}\text { ale } \\
\text { lings }\end{array}$ & $\begin{array}{l}\text { Eas } \\
\text { decl }\end{array}$ & & Temp. & Chr'r & $\begin{array}{r}\text { Sc } \\
\text { read }\end{array}$ & $\begin{array}{l}\text { ale } \\
\text { lings }\end{array}$ & $\begin{array}{l}\text { East } \\
\text { decli- }\end{array}$ & Temp. \\
\hline & Left & Right & & & time & Left & Right & & & & time & Left & Right & natic & & C. & time & Left & Right & nation & \\
\hline $\mathrm{h} \mathrm{m}$ & d & d & $\cdot$, & - & $\mathrm{h} \mathrm{m}$ & d & d & & , & $\circ$ & $\mathrm{h} \mathrm{m}$ & d & d & & ' & $=0$ & $\mathrm{~h} \mathrm{~m}$ & d & $\mathrm{d}$ & $\cdot$, & $\bullet$ \\
\hline 1600 & 44.0 & $4 \mathrm{I} \cdot 3$ & 2328 & $-15 \cdot 3$ & 1800 & 28.0 & 27.9 & 22 & 49 & $-15 \cdot 5$ & 2000 & 40.2 & 38.4 & 223 & $3 \mathrm{I}-$ & -13.9 & 2200 & 37.2 & 36.3 & 2236 & -13.0 \\
\hline 02 & 48.8 & 46.3 & 20 & & 02 & 31.0 & 31.0 & & 45 & & 02 & $4 \mathrm{I} .2$ & $40 . \mathrm{I}$ & & 29 & & 02 & 42.8 & 41.4 & 27 & \\
\hline 04 & 48.6 & 45.3 & $2 I$ & 2 & 04 & 29.7 & 29.3 & & 47 & 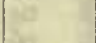 & 04 & 41.6 & 40.6 & & 29 & 60 & 04 & 44.5 & 42.7 & 25 & \\
\hline 06 & 48.6 & 46.3 & 20 & W & 06 & 32.6 & 32.6 & & 42 & 9 & 06 & 38.0 & 37.0 & & 34 & 2 & 06 & 42.7 & 40.3 & 28 & \\
\hline 08 & 45.6 & 42.8 & 25 & 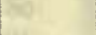 & 08 & 32. I & 32. I & & 43 & 7) & 08 & 38.3 & 37.9 & & 33 & as & 08 & 41.8 & 39.2 & 30 & \\
\hline 10 & 47.2 & 44.5 & 22 & 111 & IO & 32.3 & 32.3 & & 42 & $3 n$ & IO & $37 . \mathrm{I}$ & 36.4 & & 35 & W & I0 & $4 \mathrm{I} \cdot 3$ & $39 . \mathrm{I}$ & 30 & \\
\hline 12 & 51.2 & 49.5 & 15 & & I2 & 35.0 & 35.0 & & $3^{8}$ & & I2 & 37 & $.8 a$ & & 34 & & 12 & 44.4 & 42.3 & 25 & \\
\hline I4 & 57.0 & 54.6 & 07 & $-15 \cdot 3$ & I4 & 32.6 & 32.4 & & 42 & $-I 5.6$ & I4 & 36.4 & 36.1 & & 36 - & -13.8 & I4 & $43 \cdot 3$ & 42.2 & 26 & $-\mathrm{I} 2.9$ \\
\hline I6 & 60.3 & 57.6 & 02 & & 16 & 33.6 & 33.0 & & 4I & & I6 & 38.8 & 36.9 & & 34 & & 16 & 39.8 & 38. I & 32 & \\
\hline I8 & 60.5 & 58.2 & or & 24 & 18 & 35.6 & $35 \cdot 3$ & & $3^{8}$ & 61 & I8 & 46.7 & 44.8 & & $2 \mathrm{I}$ & at & 18 & 36.9 & 35.8 & 36 & \\
\hline 20 & 56.2 & 53.6 & 08 & $=$ & 20 & 32.3 & 31.6 & & 43 & 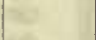 & 20 & 45.4 & 43.9 & & 23 & et & 20 & 34.9 & 33.5 & 40 & \\
\hline 22 & 53.8 & 52.0 & I I & $=$ & 22 & 31.3 & 30.3 & & 45 & $\omega$ & 22 & 42.2 & 39.9 & & 29 & an & 22 & 33.9 & 32.2 & $4 I$ & \\
\hline 24 & 56.0 & 54.0 & 08 & W & 24 & 27.6 & 26.8 & & $5 \mathrm{I}$ & 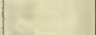 & 24 & 37.1 & 34.7 & & 37 & . & 24 & 36.3 & 34.7 & $3^{8}$ & \\
\hline 26 & 57.6 & 55.4 & 06 & $=$ & 26 & 32.5 & 32.3 & & 42 & E- & 26 & 36.6 & 35.2 & & 37 & E. & 26 & 38.0 & 37.2 & 34 & \\
\hline 28 & 59.7 & 58.0 & 02 & $1=$ & 28 & 39.8 & 37.7 & & 32 & 5 & 28 & 40.2 & 37.8 & & 32 & & 28 & 38.0 & 36.9 & 35 & \\
\hline 30 & $6 \mathrm{I} .0$ & 59.3 & 2300 & -15.5 & 30 & 40.6 & 39.5 & & 30 & & 30 & 42.2 & 39.7 & & 29. & -13.6 & 30 & 39.9 & 38.2 & 32 & -12.3 \\
\hline 32 & 61.3 & 60.5 & $\begin{array}{ll}22 & 59\end{array}$ & & 32 & 39.5 & 38.3 & & 32 & $-\mathrm{I} 5 \cdot 3$ & 32 & 44.3 & $4 \mathrm{I} .9$ & & 25 & & 32 & 39.2 & 37.5 & 33 & \\
\hline 34 & 61.0 & 59.5 & 2300 & $E$ & 34 & 38.2 & 36.8 & & 34 & & 34 & 44. I & 42.2 & & 25 & 4 & 34 & 40.2 & 38.1 & 32 & \\
\hline 36 & 62.0 & 60.9 & $\begin{array}{ll}22 & 58\end{array}$ & $E$ & 36 & 37.9 & 37.2 & & 34 & Et & 36 & 41.9 & 39.3 & & 30 & 16 & 36 & 38.9 & $37 . I$ & 34 & \\
\hline 38 & 61.7 & 60.5 & 59 & 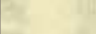 & 38 & 43.2 & $42 . I$ & & 26 & C & 38 & 42.2 & 40.2 & & 29 & 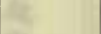 & 38 & 38.3 & 36.8 & 34 & \\
\hline 40 & 62.7 & $6 \mathrm{I} .5$ & 57 & 4. & 40 & 41.9 & $4 \mathrm{I} . \mathrm{I}$ & & 28 & 5 & 40 & 44.0 & 42.9 & & 25 & in & 40 & 36.7 & 35.0 & 37 & \\
\hline 42 & 69.7 & 68.5 & 46 & & 42 & 40. I & 39.6 & & 30 & 10 & 42 & 47.2 & 44.9 & & $2 \mathrm{I}$ & & 42 & 38.0 & 36.9 & 34 & \\
\hline 44 & 65.0 & 64.0 & 53 & $-15 \cdot 5$ & 44 & 40.0 & 39.2 & & $3 I$ & -14.9 & 44 & 46.9 & 45.0 & & $2 \mathrm{I}$ & -13.4 & 44 & 39.9 & 37.9 & 32 & -12.0 \\
\hline 46 & 67.8 & 66.8 & 49 & & 46 & 40.8 & 39.6 & & 30 & & 46 & 47.2 & $45 . \mathrm{I}$ & & $2 \mathrm{I}$ & & 46 & 41.2 & 38.9 & 30 & \\
\hline 48 & 68.0 & 67.2 & 48 & 1 & 48 & 39.9 & 38.7 & & $3 I$ & E & 48 & 42.3 & 40.2 & & 28 & 14 & 48 & 39.0 & 37.2 & 34 & \\
\hline 50.3 & 70.1 & 69.3 & 45 & e & 50 & 38.7 & $37 \cdot 3$ & & 34 & $x$ & 50 & 40.8 & 38.2 & & $3 \mathrm{I}$ & 62 & 50 & 37.8 & 36.8 & 35 & \\
\hline 52 & 70.0 & 69.0 & 45 & $1=$ & 52 & 37.9 & 36.7 & & 35 & $1=$ & 52 & 42.2 & 39.9 & & 29 & 20 & 52 & 37.0 & 35.8 & 36 & \\
\hline 54 & 69.9 & 69.1 & 45 & 2 & 54 & 37.0 & 35.9 & & 36 & $=$ & 54 & 43.2 & 40.8 & & 27 & & 54 & 38.2 & 36.3 & 35 & \\
\hline 56 & 70.6 & 69.5 & 45 & F & 56 & 36.5 & 35.4 & & 37 & & 56 & 42.9 & 40.6 & & 28 & & 56 & 37.8 & 36.1 & 35 & \\
\hline 58 & 69.6 & 68.6 & 46 & 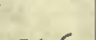 & 58 & 35.0 & 33.9 & & 39 & & 58 & 42.3 & 39.8 & & 29 & & $5^{8}$ & 38.1 & $37 . I$ & 34 & \\
\hline $17 \infty$ & 66.7 & 65.6 & $5 I-$ & -15.6 & 1900 & 35.2 & $33 \cdot 3$ & & 39 & -14.3 & $2 \mathrm{I} 00$ & 42.0 & 39.1 & & 29. & -13.4 & 2300 & 37.9 & 37.1 & 34 & -11.5 \\
\hline 02 & 67.5 & 66.3 & 49 & & 02 & 35.4 & 34.2 & & 39 & tes & 02 & 43.2 & 39.8 & & 28 & & 02 & 39.9 & 37.9 & 32 & \\
\hline 04 & 68.9 & 68.0 & 47 & 0 & 04 & $35 . \mathrm{I}$ & 33.9 & & 39 & do & 04 & 40.9 & 37.7 & & 31 & a & 04 & 37.9 & 36.3 & 35 & \\
\hline 06 & 68.2 & 67.5 & 48 & 0 & 06 & 37.5 & 36. I & & 35 & $=$ & 06 & 40.1 & 37.1 & & 32 & 3 & 06 & 36.1 & 34.3 & 38 & \\
\hline 08 & 66.9 & 66.3 & 50 & - & 08 & 39.8 & 38.6 & & 32 & at & 08 & 40.8 & 37.1 & & 32 & L & 08 & 34.2 & 32.7 & $4 \mathrm{I}$ & \\
\hline 10 & 65.0 & 64.0 & 53 & to & 10 & 40.0 & 38.7 & & 3I & 1 & I0 & $4 \mathrm{I} .4$ & 37.9 & & $3 \mathrm{I}$ & 21 & IO & $33 \cdot 3$ & 31.9 & 42 & \\
\hline 12 & $6 \mathrm{I} \cdot 3$ & 60.5 & 59 & $162=$ & 12 & 39.9 & 38.8 & & $3 \mathrm{I}$ & & 12 & 37.9 & 34.8 & & 36 & (1) & I2 & 35.2 & 33.3 & 39 & \\
\hline 14 & 61.0 & 60.4 & 59 & -15.7 & 14 & 37.9 & 37.0 & & 34 & -14.2 & 14 & 35.2 & 32.2 & & 40 & -13.3 & $\mathrm{I}_{4}$ & $35 \cdot 3$ & 33.0 & 40 & -11.2 \\
\hline I6́ & 68.6 & 68.4 & 47 & & 16 & 38.8 & 37.0 & & 34 & (1) & 16 & 38.0 & 35.1 & & 36 & & 16 & $34 \cdot 3$ & $3 \mathrm{I} \cdot 3$ & 42 & \\
\hline 18 & 78.0 & 78.0 & 32 & 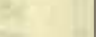 & 18 & 40.8 & 38.2 & & 31 & 4 & 18 & 42.0 & 38.0 & & 30 & 3 & 18 & 34.2 & 3 I. 3 & 42 & \\
\hline $20^{*}$ & 51.6 & 43.3 & 19 & $=$ & 20 & 38.0 & 36.2 & & 35 & III & 20 & $45 \cdot 3$ & $4 \mathrm{I} \cdot 3$ & & 25 & 14 & 20 & 35.2 & $32 . \mathrm{I}$ & 40 & $<$ \\
\hline 22 & 59.0 & 52.0 & 06 & E & 22 & 37.8 & 36.2 & & 35 & $=$ & 22 & 47.0 & 43. I & & 22 & $1=$ & 22 & 35.9 & 34.1 & 38 & \\
\hline 24 & 58.0 & 51.0 & 08 & 2 & 24 & 40.9 & 39.1 & & 30 & g & 24 & 45.8 & 42.7 & & 24 & 13 & 24 & 34.8 & 32.8 & 40 & \\
\hline 26 & 54.6 & 48.8 & 12 & $\mathrm{~F}$ & 26 & 41.9 & 40.2 & & 29 & $\pi$ & 26 & 42.9 & 40.1 & & 28 & 2 & 26 & 36.2 & 34.0 & 38 & \\
\hline 28 & 48.3 & 42.5 & 22 & 5 & 28 & 41.6 & 40.2 & & 29 & 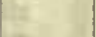 & 28 & 45.9 & 43.9 & & 23 & 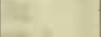 & 28 & 38.0 & 35.9 & 35 & \\
\hline 30 & 44.4 & 38.6 & 28 & -15.5 & 30 & 4I. I & 39.9 & & 30 & -14.1 & 30 & 44.2 & 42. I & & 26 & -13.2 & 30 & 38.9 & 36.9 & 34 & -11.0 \\
\hline 32 & 40.3 & $35 \cdot 3$ & 34 & & 32 & 38.9 & 37.4 & & 33 & $x^{2}$ & 32 & 41.2 & 39.6 & & 30 & & 32 & 39.5 & 37.2 & 33 & \\
\hline 34 & 37.3 & 32.0 & 39 & 64 & 34 & 37.0 & 36.8 & & 35 & 16 & 34 & 41.9 & 39.8 & & 29 & & 34 & 39.3 & 36.9 & 34 & \\
\hline 36 & 37.2 & 32.3 & 39 & & 36 & 35.8 & 34.8 & & 38 & 6 & 36 & 45.8 & 44.9 & & 22 & & 36 & 39.8 & 37.1 & 33 & \\
\hline $3^{38}$ & 37.0 & 35.6 & 36 & C. & 38 & 35.2 & 34.1 & & 39 & ce & 38 & $45 . I$ & 43.8 & & 23 & ace & 38 & 39.8 & 37.2 & 33 & \\
\hline 40 & 39.0 & 38.2 & 33 & t & 40 & 36.5 & 34.9 & & 37 & 14 & 40 & $48 . I$ & 47.5 & & 18 & 14 & 40 & $4 \mathrm{I} . \mathrm{O}$ & 38.2 & 31 & 7 \\
\hline 42 & 34 . I & 33.5 & 40 & 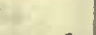 & 42 & $4 \mathrm{I} . \mathrm{I}$ & $39 . \mathrm{I}$ & & 30 & 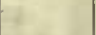 & 42 & 48.6 & 47.8 & & I8 & 4 & 42 & $4 \mathrm{I} .3$ & 38.7 & $3 I$ & \\
\hline 44 & 34.7 & 33.6 & 40 & -15.6 & 44 & 45.7 & 43.9 & & 23 & -14.0 & 44 & 43.9 & $43 \cdot 3$ & & 25 & -13.0 & 44 & 43.2 & 40.2 & 28 & \\
\hline 46.2 & 36.3 & 35.7 & 37 & & 46 & $44 \cdot 3$ & 42.3 & & 25 & & 46 & 41.7 & 40.3 & & 29 & & 46 & 42.8 & 39.9 & 28 & (2) \\
\hline 48 & 35.2 & 34.4 & 38 & . & 48 & $4 \mathrm{I} .7$ & 39.7 & & 29 & 4. & 48 & 40.8 & 39.0 & & $3 \mathrm{I}$ & 4 & 48 & 45.2 & 42.9 & 24 & 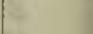 \\
\hline 50 & 33.8 & $33 \cdot 3$ & 40 & e & 50 & 38.0 & 36.1 & & 35 & 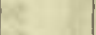 & 50 & 41.9 & 40.2 & & 29 & & 50 & 47.1 & 43.7 & 22 & \\
\hline 52 & 33.2 & 32.7 & $4 I$ & 15 & 52 & 37.1 & 35.2 & & 36 & +2 & 52 & 41.5 & 39.8 & & 29 & $E=$ & 52.3 & $45 . I$ & $4 I .7$ & 25 & \\
\hline & 31.5 & 31.2 & 44 & 2 & 54 & 37.7 & 35.7 & & 36 & 2 & 54 & 41.1 & 39.9 & & 30 . & 2 & 54 & $4 \pi .8$ & 38.1 & $3 \mathrm{I}$ & \\
\hline 56 & 31.3 & 30.8 & 45 & 5 & 56 & 36.9 & $35 \cdot 3$ & & 37 & 2 & 56 & 37.2 & 36.1 & & 36 & & 56 & 39.3 & 36.1 & 34 & \\
\hline 58 & 30.0 & 29.6 & 47 & $=$ & 58 & 38.0 & 36.8 & & 34 & 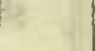 & $5^{8}$ & 36.8 & 35.6 & & 36 & & 58 & 37.9 & 35.9 & $\begin{array}{l}35 \\
36\end{array}$ & . I0. \\
\hline & & & & & & & & & & & & & & & & & & 37.8 & 35.0 & 30 & \\
\hline
\end{tabular}

Observers-W. J. P. and R. R. T., who alternated from $18 \mathrm{~h} 24 \mathrm{~m}$ to $18 \mathrm{~h} 34 \mathrm{~m}$.
Correction to local mean time is - Im I5s.

Torsion head at oh oom read $225^{\circ}$ and at $24 \mathrm{~h}$ zom read the same. Observer-R. R. T. 
Tabulation of magnetic declinations observed at Teplitz Bay-Continued

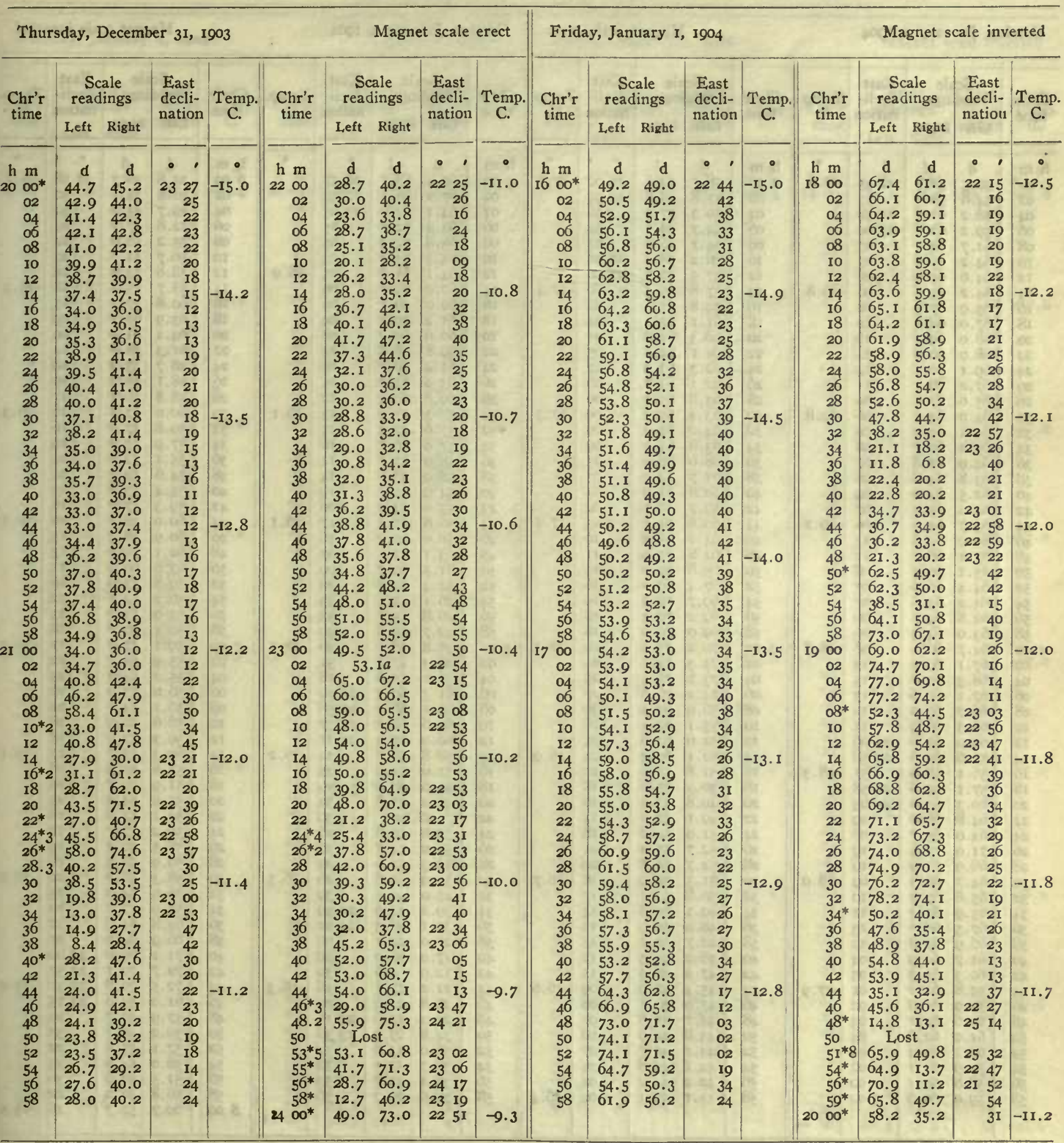

Correction to local mean time is - Im 33 s.

Torsion head at $19 \mathrm{~h} 25 \mathrm{~m}$ read $225^{\circ}$ and at $24 \mathrm{~h}$ rom read the same.

Observers-J. S. V. (R. R. T. observed readings $23 \mathrm{~h} 53.5 \mathrm{~m}$ to 24h com.)
Correction to local mean time is $-\operatorname{Im} 47 \mathrm{~s} .90^{\circ}$ torsion $=222^{\prime} \mathrm{I}$. Torsion head at $15 \mathrm{~h} 30 \mathrm{~m}$ read $228^{\circ}$ and at $20 \mathrm{~h}$ lom read $268^{\circ}$. Observer-R. R. T. 
Tabulation of magnetic declinations observed at Teplitz Bay-Continued

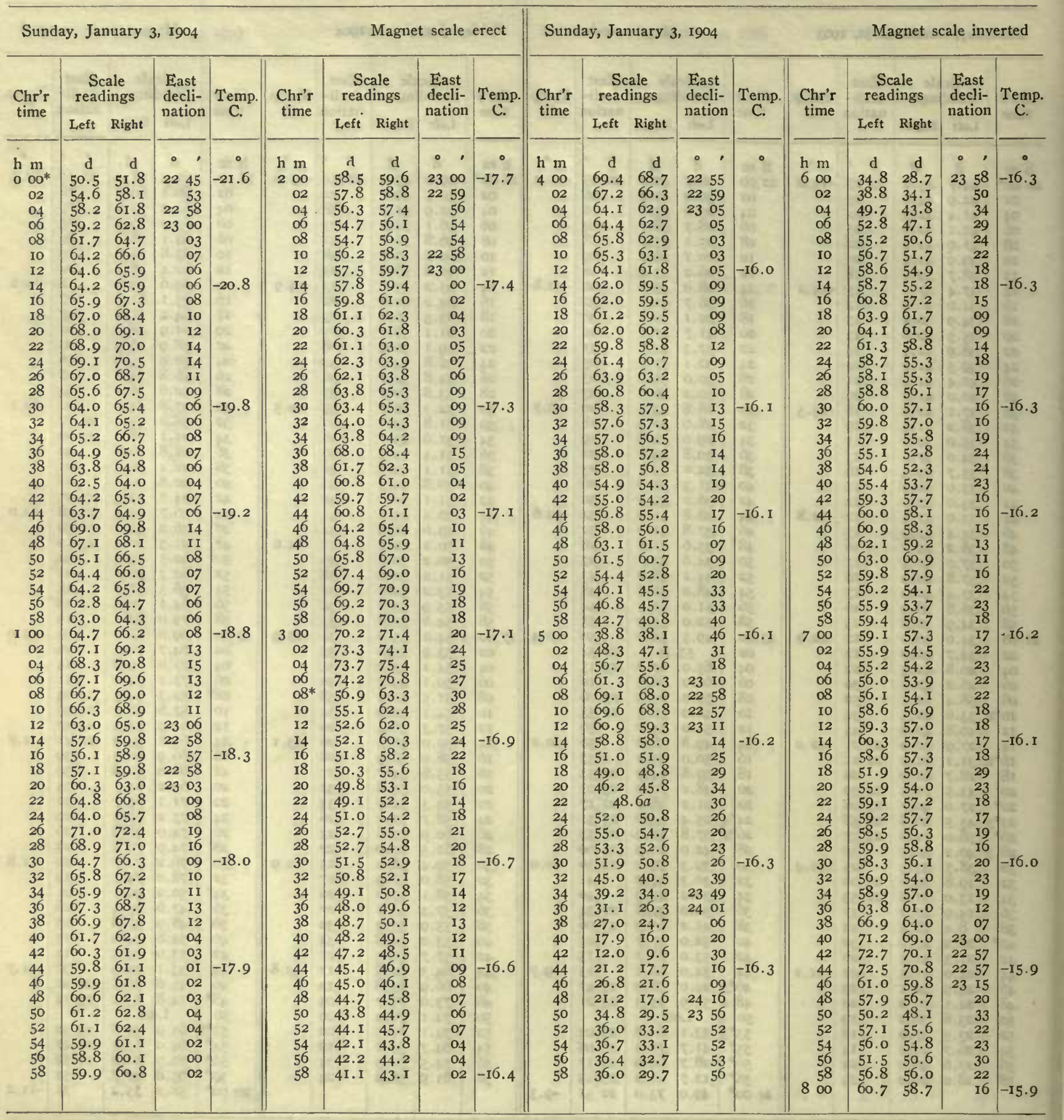


Tabulation of magnetic declinations observed at Teplitz Bay-Continued

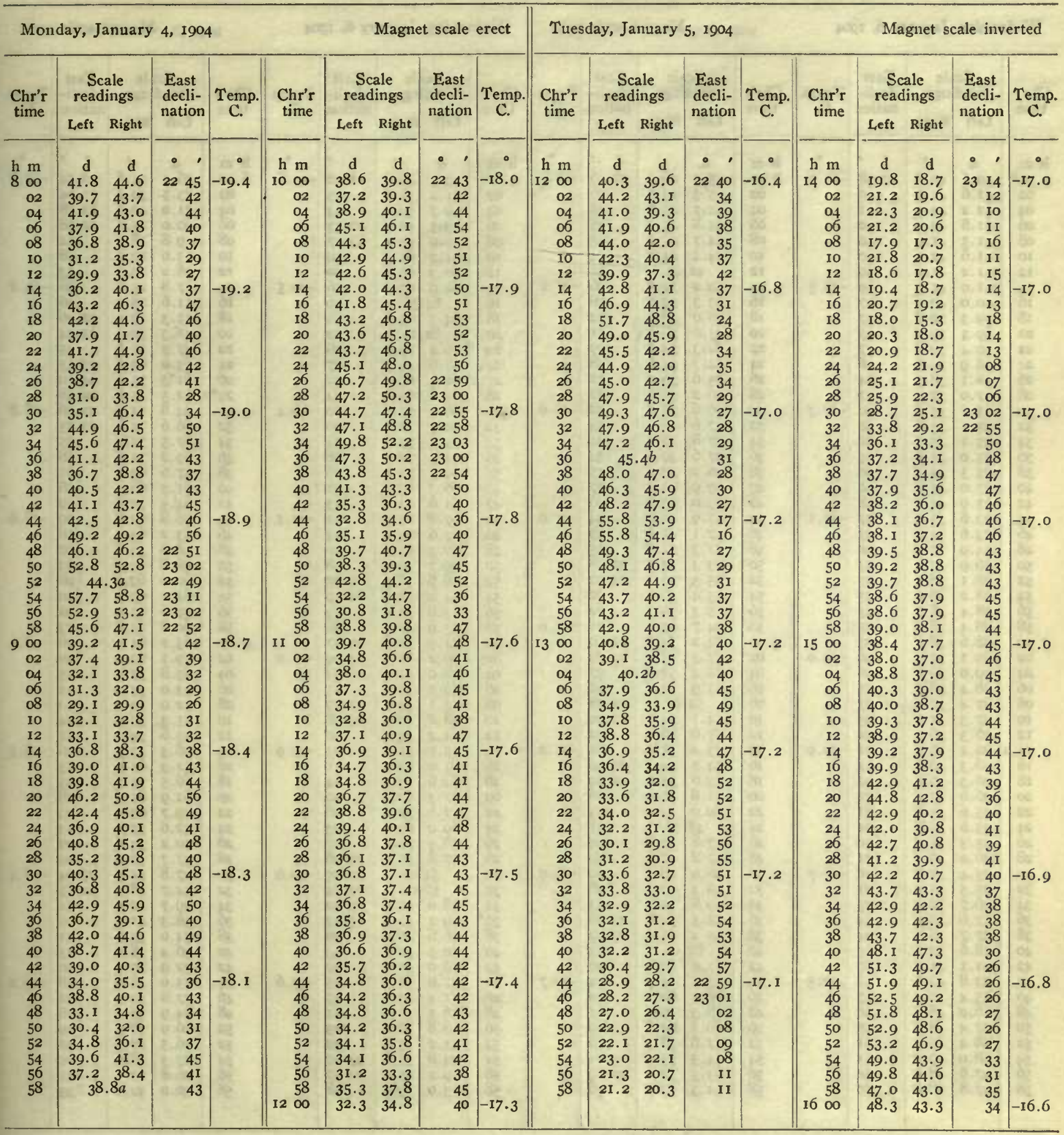

Correction to local mean time is $-2 \mathrm{~m} 32 \mathrm{~s} .90^{\circ}$ torsion $=23 .^{\prime} 6$. Torsion head at $8 \mathrm{~h}$ oom read $175^{\circ}$ and at $12 \mathrm{~h} 30 \mathrm{~m}$ read $130^{\circ}$. Observer-W. J. P.
Correction to local mean time is $-2 \mathrm{~m} 55 \mathrm{~s}, 90^{\circ}$ torsion $=20 .{ }^{\prime} 8$. Torsion head at $1 \mathrm{Ih} 20 \mathrm{~m}$ read $135^{\circ}$ and at $16 \mathrm{~h} 25 \mathrm{~m}$ read $117^{\circ}$ Observer-R. R. T. 
Tabulation of magnetic declinations observed at Teplitz Bay-Continued

\begin{tabular}{|c|c|c|c|c|c|c|c|c|c|c|c|c|c|c|c|c|c|c|c|c|}
\hline \multicolumn{6}{|c|}{ Wednesday, January 6 , rgo4 } & \multicolumn{4}{|c|}{ Magnet scale erect } & \multicolumn{6}{|c|}{ Wednesday, January 6, I904 } & \multicolumn{5}{|c|}{ Magnet scale erect } \\
\hline \multirow{2}{*}{$\begin{array}{l}\text { Chr'r } \\
\text { time }\end{array}$} & \multicolumn{2}{|c|}{$\begin{array}{l}\text { Scale } \\
\text { readings }\end{array}$} & \multirow{2}{*}{$\begin{array}{l}\text { East } \\
\text { decli- } \\
\text { nation }\end{array}$} & Temp. & Chr'r & $\begin{array}{l}\text { Sca } \\
\text { readi }\end{array}$ & $\begin{array}{l}\text { ale } \\
\text { ings }\end{array}$ & $\begin{array}{l}\text { East } \\
\text { decli- } \\
\text { natior }\end{array}$ & Temp. & $\begin{array}{l}\text { Chr'r } \\
\text { time }\end{array}$ & $\begin{array}{r}\text { Sca } \\
\text { readi }\end{array}$ & $\begin{array}{l}\text { ale } \\
\text { ings }\end{array}$ & East & Temp. & Chr'r & $\begin{array}{r}\text { Sca } \\
\text { readi }\end{array}$ & $\begin{array}{l}\text { ale } \\
\text { lings }\end{array}$ & $\begin{array}{l}\text { East } \\
\text { decli }\end{array}$ & & Temp. \\
\hline & Left & Right & & & & Left & Right & & & & Left & Right & & & & Left & Right & & & \\
\hline $\mathrm{h} \mathrm{m}$ & $d$ & d & $\cdot$, & $\bullet$ & $\mathrm{h} m$ & $\mathrm{~d}$ & $\mathrm{~d}$ & $\circ$, & $\bullet$ & $\mathrm{h} \mathrm{m}$ & d & d & . ' & ○ & $\mathrm{h} \mathrm{m}$ & 0 & $\mathrm{~d}$ & & ' & $\circ$ \\
\hline $000^{*}$ & 40.6 & 40.8 & $223^{8}$ & -23.0 & 200 & $45 \cdot 3$ & 46.3 & 2246 & -21.4 & 400 & $49 \cdot 3$ & 49.6 & 2252 & -20.6 & 600 & 16.1 & 24.8 & & 54 & -19.4 \\
\hline 02 & 41.6 & 41.9 & 40 & -3 & 02 & $45 \cdot 3$ & $45 \cdot 5$ & & tat & 02 & 51.2 & 51.6 & 55 & $\Delta$ & 02 & 23.5 & 30.0 & 230 & & - \\
\hline 04 & 42.2 & 42.3 & $4 \mathrm{I}$ & 17 & 04 & $44 \cdot 7$ & 45.7 & 45 & $t^{2}$ & 04 & 49.7 & 50. I & 53 & 20 & 04 & 31.0 & 40.0 & $23 \mathrm{I}$ & 18 & as \\
\hline 06 & 41.8 & 42.0 & 40 & 80 & 06 & 44.8 & 45.4 & 45 & da & 06 & 49.5 & 50.3 & 2253 & as & 06 & 20.0 & 25.5 & 225 & & 6 \\
\hline 08 & $4 \mathrm{I} .5$ & 4 I. 9 & 40 & ans & 08 & 44.7 & 45.2 & 45 & $8 y$ & 08 & 59.0 & 59.0 & 2307 & 64. & 08 & 9.8 & 18.0 & & 44 & wi \\
\hline 10 & 41.6 & 42.0 & 40 & 64 & I0 & 44.4 & 44.6 & 44 & 독 & 10 & 61.0 & $61 \cdot 3$ & 10 & nt: & ro & 13.0 & 20.0 & & 48 & a \\
\hline I2 & 41.2 & 41.2 & 39 & 61 & 12 & 42 & $2 b$ & 4I & 81 & 12 & 60.3 & 60.9 & IO & It & I2 & 16.2 & 23.3 & & 53 & \\
\hline 14 & $4 \mathrm{I} .0$ & $4 \mathrm{I} \cdot 3$ & 39 & -22.5 & 14 & 39.0 & 39.3 & 36 & $-2 \mathrm{r} .2$ & 14 & 64.0 & 65.2 & 16 & -20.5 & I4 & 17.8 & 25.6 & & 56 & -19.2 \\
\hline 16 & 40.0 & 40.3 & 37 & 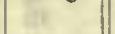 & 16 & 39.3 & 39.6 & 36 & 19 & 16 & 64. & $.5 b$ & 16 & aty & 16 & II. 6 & 18.2 & & 46 & \\
\hline 18 & 39.3 & 39.8 & 37 & 46 & 18 & 38.0 & 38.0 & 34 & 84 & 18 & $64 \cdot 3$ & 64.6 & 16 & dit & 18 & II. 3 & Ig. I & 224 & & Eis \\
\hline 20 & 39.3 & 39.7 & 37 & $a$ & 20 & 35.6 & 36.0 & 31 & g. & 20 & 62 . & $\mathrm{I} b$ & 12 & oE & 20 & 41.3 & 50.3 & 233 & & os \\
\hline 22 & 39.4 & 39.8 & 37 & te & 22 & 34.3 & 34.7 & 29 & $=$ & 22 & 59.4 & 59.9 & 08 & as & 22 & 47.3 & 57.6 & & 45 & $=$ \\
\hline 24 & 38.9 & 39.3 & 36 & is & 24 & $34 \cdot 3$ & 34.6 & 28 & 15 & 24 & 58.6 & 59.3 & 07 & If & 24 & 55.0 & 63.0 & 235 & 55 & 14 \\
\hline 26 & 38.1 & 38.3 & 34 & 8 & 26 & 35.6 & 36.0 & $3 \mathrm{I}$ & $d t$ & 27.2 & 61.3 & 62.3 & 12 & E & 26 & 61.3 & 75.0 & 24 I & 10 & \\
\hline 28 & 37.0 & $37 \cdot 3$ & 33 & thant & 28 & 36.2 & 36.6 & 32 & 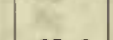 & 28 & 62.6 & 64.2 & 14 & 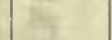 & $29 *_{2}$ & 32.5 & 45.6 & 224 & 48 & \\
\hline 30 & 35.5 & 35.8 & 30 & $-22 \cdot 3$ & 30 & 36.6 & 36.8 & 32 & -21.0 & 30 & 66.9 & 67.6 & 20 & -20.4 & 30 & 34.8 & $54 \cdot 3$ & 225 & 56 & -18.8 \\
\hline 32 & 35.5 & 35.6 & 30 & (2). & 32 & 37.8 & 38.4 & 34 & 0 & 32 & 70.7 & $7 \mathrm{I} .1$ & 26 & 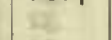 & 32 & 66.6 & 71.6 & 233 & 35 & at. \\
\hline 34 & 35.1 & $35 . \mathrm{I}$ & 30 & 16 & 34 & 37.8 & 38.3 & 34 & 18 & 34 & 75.5 & 77.2 & 34 & 18 & 34 & 67.5 & 71.0 & & 35 & is \\
\hline 36 & 35.0 & 35.0 & 29 & Wit & 36 & 37.6 & 38.0 & 34 & e & 36 & 71.3 & 73.0 & 28 & 8 & 36 & 69.2 & 72.8 & & 38 & \\
\hline 38 & 35.3 & 35.5 & 30 & $\pi$ & 38 & 38.2 & 38.4 & 35 & he & 38 & 69.7 & 72.2 & 26 & 18 & 38 & 57.6 & 63.0 & & 21 & \\
\hline 40 & 35.0 & 35.2 & 30 & 0 & 40 & 39.9 & 40.0 & 37 & 14: & 40 & 65.3 & 69.2 & $2 \mathrm{I}$ & at & 40 & 61.3 & 68.3 & & 28 & ent \\
\hline 42 & 33.9 & 34 . I & 28 & 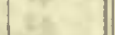 & 42 & 39.0 & 39.3 & 36 & $\Rightarrow$ & 42 & 61.6 & $64 . I$ & I3 & thit & 42 & 64.5 & 70.6 & 3 & 32 & \\
\hline 44 & 33.6 & 33.9 & 27 & -22.1 & 44 & 43.9 & 44.6 & 44 & $-2 \mathrm{I} .0$ & 44 & 67.8 & 69.0 & 22 & -20 . I & 44 & 56.8 & 62.8 & 2 & 20 & -18.6 \\
\hline 46 & 33.6 & $34 . I$ & 27 & tar & 46 & $47 . \mathrm{I}$ & 47.6 & 49 & $1=$ & 46 & 62.6 & 64.0 & I4 & $x^{2}-2$ & 46 & 53.2 & 63. I & & 18 & \\
\hline 48 & 34.9 & 35.5 & 30 & 4 & 48 & 51.3 & 51.8 & 55 & s & 48 & 60.3 & 61.9 & I0 & ay & 48 & 48.8 & 56.8 & & 09 & 6 \\
\hline 50 & 36.9 & 37.4 & 33 & $m$ & 50 & 53.3 & 53.7 & 2258 & at? & 50 & 59.7 & 59.7 & 08 & 56 & 50 & 58.8 & 64.5 & & 23 & $\sqrt{4}$ \\
\hline 52 & 38.1 & 38.5 & 35 & 34 & 52 & 55.5 & 56.1 & 2302 & $\mathrm{R}^{2}$ & 52 & 56.6 & 58.3 & 2305 & te? & 52 & 60.0 & 69.3 & & 28 & 23 \\
\hline 54 & 38.2 & 38.6 & 35 & ta & 54 & 55.4 & 56.0 & 02 & +2 & 54 & 54.0 & 54.5 & 2259 & $k^{2}$ & 54 & 62.2 & 69.5 & & 30 & \\
\hline 56 & 38.4 & 38.7 & 35 & 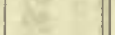 & 56 & 55.3 & 55.7 & 02 & & 56 & 53.0 & 54.9 & 59 & 달 & 56 & 59.6 & 62.0 & & 22 & \\
\hline 58 & 38.4 & 38.8 & 35 & his & 58 & 54.7 & $55 \cdot 3$ & or & -21.0 & 58 & 45.9 & 47.7 & 48 & 8 & 58 & 60.8 & 61.8 & & 23 & \\
\hline 100 & 38.6 & 38.8 & 35 & 60 & 300 & 54.2 & 54.9 & 2300 & 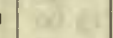 & 500 & 51.9 & 52.8 & 2257 & $60 \times 1$ & 700 & 63.2 & 64.0 & & 26 & -18.5 \\
\hline 02 & 39.1 & 39.3 & 36 & -22.0 & 02 & 53.3 & 53.9 & 2259 & 10 & 02 & 59.2 & 61.8 & 2309 & -20.0 & 02 & 59.1 & 60.0 & & 20 & ( \\
\hline 04 & 39.0 & 39.0 & 36 & 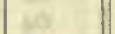 & 04 & 52.2 & 52.8 & 57 & 11t & 04 & 48.0 & 51.3 & 2252 & 10 & 04 & 55.8 & 57.3 & & 15 & 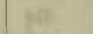 \\
\hline 06 & 38.4 & 38.7 & 35 & 10 & 06 & 51.9 & 52.2 & 56 & 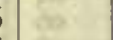 & 06 & 49.0 & 51.6 & 53 & 60 & 06 & 53.0 & 54.4 & & II & \\
\hline 08 & 37.4 & 37.6 & 33 & Aa & 08 & 53. & $.0 a$ & 2258 & 82 & 08 & 53. I & 54.7 & 2259 & 29 & 08 & 52.0 & 54.0 & & () & Ret \\
\hline Io & 37.3 & 37.5 & 33 & ot & IO & $54 . \mathrm{I}$ & 54.7 & 2300 & as & IO & 58.8 & 60.0 & 2308 & ef & 10 & 55.3 & 56.5 & & 14 & \\
\hline 12 & 36.3 & 36.7 & 32 & 10 & 12 & 55.6 & 56.0 & 02 & $57 x$ & I2 & 54.2 & 54.4 & 00 & $y=$ & 12 & 53.6 & $54 . \mathrm{I}$ & & II & \\
\hline 14.2 & 36.4 & 36.8 & 32 & -22.0 & I4 & 55.0 & 55.0 & or & $-2 \mathrm{I} \cdot 2$ & I4 & 55.7 & 57.0 & 03 & -19.9 & 14 & 56.3 & 57.7 & & I6 & -18.5 \\
\hline 16 & 36.3 & 36.6 & 32 & - & 16 & 54.5 & 54.5 & 2300 & ten & I6 & 61.0 & 62.2 & II & 0.8 & 16 & 52.3 & 61.0 & & $I_{5}$ & \\
\hline I8 & 36.3 & 36.6 & 32 & ati & I8 & $53 . \overline{6}$ & 53.6 & 2259 & it & 18 & 62.6 & 63.9 & 14 & Alit: & 18 & 66.5 & 71.3 & & 35 & ins \\
\hline 20 & 36.0 & 37.2 & 32 & 60 & 20 & 51.8 & 52.0 & 56 & ets & 20 & 67.3 & 67.3 & 20 & ac & 20 & 62.9 & 67.0 & & 29 & Fe \\
\hline 22 & 35.6 & 35.8 & $3 I$ & 60 & 22 & 50.0 & 50.0 & 0 & nes & 22 & 72.0 & 72.7 & 28 & $=18$ & 22 & 59.7 & 63.4 & & 23 & es \\
\hline 24 & 35.6 & 35.8 & 31 & if & 24 & 48. & .56 & 51 & 18 & 24 & 69.0 & 69.0 & 23 & 18 & 24 & 52.6 & 57.6 & & I. 3 & \\
\hline 26 & 35.3 & 35.5 & 30 & 35 & 26 & 47.8 & 47.8 & 50 & 22 & 26 & 58.5 & 60.0 & 07 & so & 2 & 50.2 & 54.6 & & Og & \\
\hline 28 & 36.0 & 36.1 & 31 & & 28 & 47.8 & 47.8 & 50 & 82 & 28 & 57.8 & 59.3 & 2306 & Bse & 28 & 47.7 & 52.0 & 3 & $\sigma_{i}$ & \\
\hline 30 & 35.4 & 35.7 & 30 & -21.7 & 30 & 48.0 & 48.3 & 50 & -20.9 & 30 & 54 & $.0 b$ & 2259 & -20.0 & 30 & 47.8 & 51.5 & $23 r$ & $2 \ddot{4}$ & -18.4 \\
\hline 32 & 35.5 & 35.9 & 31 & - & 32 & 46.3 & 46.7 & 48 & - & 32 & 57. & $.0 a$ & 2304 & tat & 32 & 44.7 & 48.0 & 22 & 59 & \\
\hline 34 & 36.7 & $37 \cdot 3$ & 33 & $y^{2}$ & 34 & 49.8 & 50. I & 53 & 46 & 34 & 48.0 & 50.6 & 2252 & B. & 34 & 39.7 & 42.5 & & 51 & \\
\hline 36 & 37.6 & 38.0 & 34 & x & 36 & 51.5 & 52.6 & 2256 & wit & $36^{*}$ & 49.2 & 51.8 & 2342 & $x$ & 3 & 38.3 & $4 \mathrm{I} .5$ & & 49 & \\
\hline 38 & 37.7 & 38. I & 34 & $a^{2}$ & 38 & 55.2 & 55.8 & 2302 & 66 & 38 & 46.6 & 46.6 & 36 & 8 & 38 & 34.0 & 35.6 & & $4 \mathrm{I}$ & \\
\hline 40 & 38.6 & 39.0 & 35 & $\approx$ & 40 & 62 & & I2 & of & 40 & 50.5 & 51.8 & 43 & 64 & 40 & 36.4 & 38.2 & & 45 & wit \\
\hline 42 & 4I. I & $4 \mathrm{I} \cdot 3$ & 39 & & 42 & 67.0 & 68.0 & 20 & 0 & 42 & 52.0 & 55.6 & 47 & $2 x$ & 42 & 32.9 & $35 \cdot 3$ & & 40 & \\
\hline 44 & 43.8 & 44.6 & 44 & -21.5 & 44 & 63.1 & $64 \cdot 3$ & 14 & -20.7 & 44 & 57.0 & 61.3 & 55 & -19.6 & 44 & 45.0 & 45.6 & & $5 \%$ & -18.2 \\
\hline 46 & 45.4 & 47.0 & 47 & the & 46 & 60.5 & 60.7 & IO & (a) & 46 & 51.4 & 53.6 & 45 & & 46 & 27.8 & 30.5 & & 32 & \\
\hline 48 & 46.0 & 48. I & 48 & . & 48 & 58.3 & 59.3 & 07 & 84 & 48 & 56.5 & 57.0 & 5I & 26 & 48 & 24.9 & 28.0 & e & 28 & \\
\hline 50 & 47.4 & 48.4 & 50 & of & 50 & 60.1 & 60.8 & 09 & $n$ & 50 & 48.8 & 56.0 & 45 & of & 50 & 26.0 & 28.3 & i & 29 & \\
\hline 52 & 48.4 & 49.3 & $5 I$ & 31 & 52 & 57.6 & 58.5 & 06 & $=7$ & 52 & 43.0 & 55.6 & 40 & a & 52 & 26.5 & 28.8 & & 30 & \\
\hline 54 & 48.0 & 49.0 & $5 \mathrm{I}$ & 12 & 54 & 54.5 & 55.5 & 23 OI & is & 54 & 24.6 & 31.8 & 2307 & 13 & 54 & 25.3 & 28.6 & es & 29 & A \\
\hline 56 & 47.6 & 48.6 & 50 & & 56 & 53.5 & 54.7 & 2259 & 8 & 56 & 19.2 & 25.4 & 2257 & 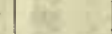 & 56 & 26.8 & 20.9 & IF & $3 \mathrm{I}$ & \\
\hline 58 & 47.8 & 48.8 & 50 & 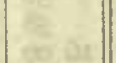 & 58 & 51.3 & 52.3 & 56 & 25 & 58 & 13.0 & I 9.3 & 48 & $\frac{8}{2} 3$ & 58 & 30.0 & 32.0 & & 35 & \\
\hline
\end{tabular}


Tabulation of magnetic declinations observed at Teplitz Bay-Continued

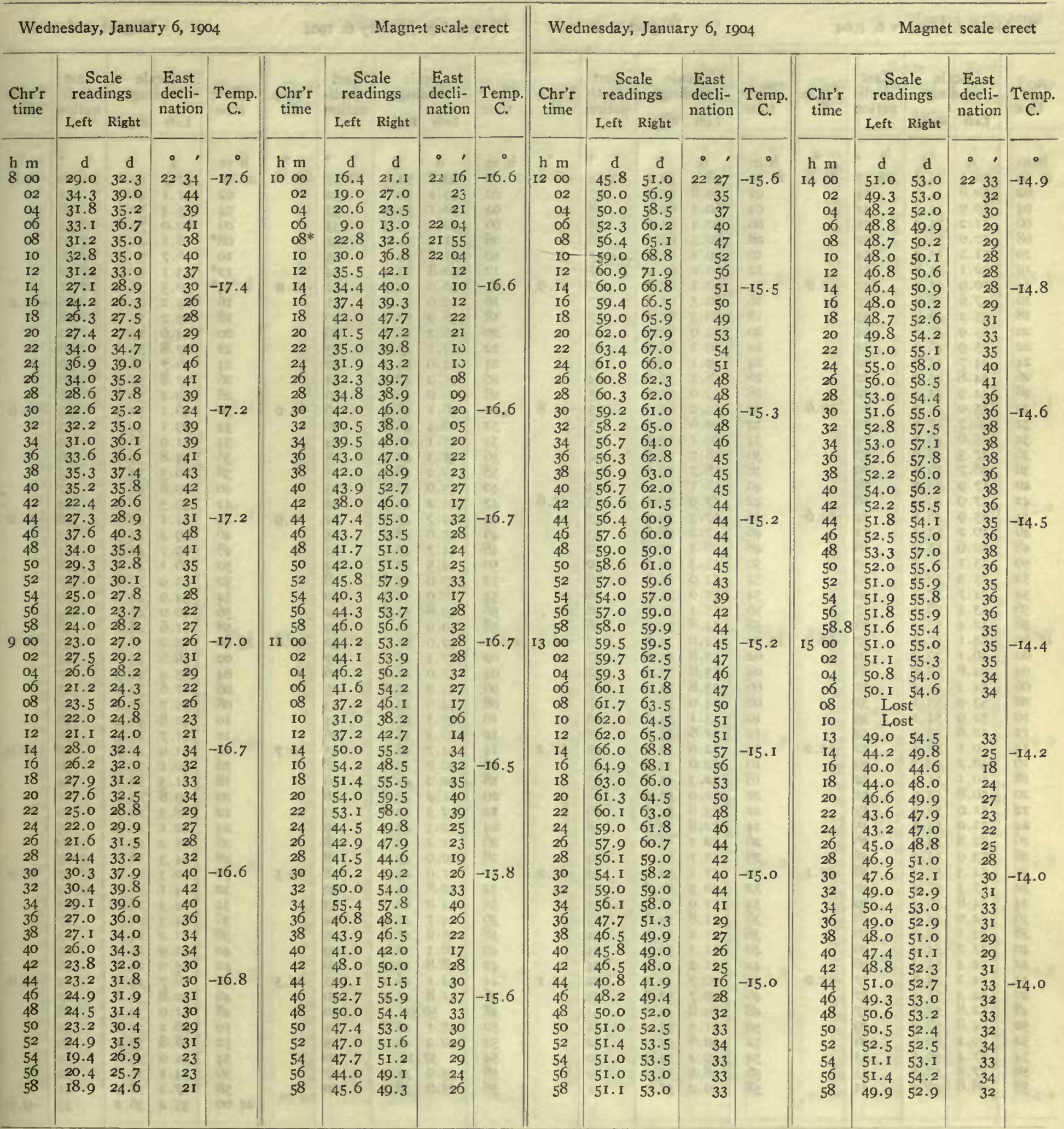


Tabulation of magnetic declinations observed at Teplits Bay-Continued

\begin{tabular}{|c|c|c|c|c|c|c|c|c|c|c|c|c|c|c|c|c|c|c|c|}
\hline \multicolumn{5}{|c|}{ Wednesday, Januarv 6, I904 } & \multicolumn{5}{|c|}{ Magnet scale erect } & \multicolumn{5}{|c|}{ Wednesday, January 6,1904} & \multicolumn{5}{|c|}{ Magnet scale erect } \\
\hline \multirow{2}{*}{$\begin{array}{l}\text { Chr'r } \\
\text { time }\end{array}$} & \multicolumn{2}{|c|}{$\begin{array}{l}\text { Scale } \\
\text { readings }\end{array}$} & \multirow{2}{*}{$\begin{array}{c}\text { East } \\
\text { decli- } \\
\text { nation }\end{array}$} & Temp. & Chr'r & $\begin{array}{l}\text { Sce } \\
\text { read }\end{array}$ & $\begin{array}{l}\text { ale } \\
\text { ings }\end{array}$ & East & Temp & Chr't & $\begin{array}{l}\text { Sca } \\
\text { read }\end{array}$ & $\begin{array}{l}\text { ale } \\
\text { lings }\end{array}$ & East & Temp. & Chr'r & $\begin{array}{l}\text { Se } \\
\text { read }\end{array}$ & $\begin{array}{l}\text { ale } \\
\text { lings }\end{array}$ & $\begin{array}{l}\text { East } \\
\text { decli- }\end{array}$ & Temp. \\
\hline & L,eft & Right & & C. & time & I,eft & Right & nation & & time & Left & Right & nation & C. & & Left & Right & & \\
\hline $\mathrm{h} \mathrm{m}$ & d & d & $\circ \quad$ & - & $\mathrm{h} \mathrm{m}$ & $d$ & d & $\cdot 1$ & - & $\mathrm{h} \mathbf{m}$ & d & d & & & $\mathrm{h} \mathrm{m}$ & d & d & $\cdot$, & 0 \\
\hline 1600 & 50.5 & 53.2 & 2233 & -13.8 & 1800 & 58.3 & 59.4 & 2244 & -12.0 & 2000 & 49.8 & 52.1 & 2232 & -11.0 & 2200 & 37.7 & 49.1 & 2220 & -10.4 \\
\hline 02 & 50.8 & 54.9 & 34 & -2.0 & 02 & 59.8 & 61.3 & 47 & (2) & 02 & 49.2 & 52.8 & 32 & & 02 & 4 I. I & 48.3 & & \\
\hline 0.4 & 50.9 & 54.1 & & wh & 04 & 60.3 & 62.3 & 48 & 15 & 04 & 50.1 & 53.1 & 33 & 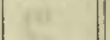 & 04 & 45.4 & 52.2 & 28 & \\
\hline 06 & 51.2 & $55 \cdot 3$ & 35 & (a) & 06 & 60.4 & 62.6 & 48 & 8 & 06 & 50.1 & 53.3 & 33 & 20 & 06 & 60.0 & 70.8 & 2254 & \\
\hline 08 & 50.0 & 53.2 & 32 & $8 x$ & 08 & 60.3 & 62.3 & 48 & 16 & 08 & 5 I.I & 54.3 & 34 & 80 & $08 *$ & $18 . \mathrm{I}$ & 44.3 & 2359 & 47 \\
\hline I0 & 51.2 & 53.9 & 34 & 47 & 10 & 60.1 & 61.8 & 47 & 34 & To & 51.8 & 54.8 & 35 & & 10 & 26.7 & 57.2 & 2416 & 8 \\
\hline 12 & 52.1 & 54.8 & 35 & 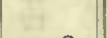 & 12 & 60.0 & 61.9 & 47 & Diva & 12 & 50.8 & 53.4 & 34 & $=1$ & I2* & 33. I & 47.2 & 2322 & 74 \\
\hline 14 & 50.9 & 51.9 & 32 & -12.8 & 14 & 60.1 & 61.8 & 47 & -II.9 & 14 & 50.0 & 52.5 & $\begin{array}{l}3+ \\
32\end{array}$ & -11.0 & $14^{*}$ & 30.5 & 38.0 & $223 I$ & \\
\hline 16 & 47.6 & 48.3 & 27 & trat & 16 & 60.5 & $6 \mathrm{I} .1$ & 47 & & 16 & 49.0 & 51.7 & $3 I$ & & I6 & 25.2 & 29.9 & 20 & -10.2 \\
\hline I8 & 45.2 & $46 . I$ & 23 & Eit & 18 & 60.3 & 61.1 & 47 & Af & I8 & 48.9 & 50.4 & 30 & & 18 & 29.2 & 34.9 & 28 & \\
\hline 20 & 45.9 & 46.8 & 24 & $e^{2}$ & 20 & 59.9 & 60.5 & 46 & 12 & 20 & 46.2 & 48.8 & 26 & 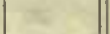 & 20 & 24.6 & 29.9 & 20 & $\sqrt{5}$ \\
\hline 22 & 45.9 & 46.1 & 24 & 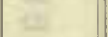 & 22 & 59.1 & 60.3 & 45 & $t$ & 22 & $44 . I$ & 47.2 & 23 & - & 22 & 37.8 & $39 . \mathrm{I}$ & 38 & $a$ \\
\hline 24 & 44.7 & 45.8 & 22 & & 24 & 59.1 & 60.2 & 45 & in & 24 & 44.0 & 46.1 & 22 & $y^{2}$ & 24 & 36.7 & 38.8 & 36 & \\
\hline 26 & 44.8 & 45.3 & 22 & Ee & 26 & 58.7 & 59.8 & 44 & 62 & 26 & 45.0 & 47.1 & 24 & $=$ & 26 & 47.8 & 55.4 & 58 & \\
\hline 28 & 44.9 & 45.4 & 22 & & 28 & 59.0 & 60.0 & 45 & & 28 & 46.0 & 48.1 & 26 & $x^{2}$ & 28 & 49.2 & 53.8 & 58 & \\
\hline 30 & 45.0 & 45.3 & 22 & -12.6 & 30 & 59.2 & 60.3 & 45 . & -11.8 & 30 & 46.1 & 48.7 & 26 & -II.O & 30 & 46.1 & 52.4 & 55 & -10.0 \\
\hline 32 & 46.7 & 47.6 & 25 & $x=$ & 32 & 59.6 & 59.8 & 45 & ( & 32 & 46.4 & 48.8 & 26 & & 32 & 39.1 & 44.2 & 43 & \\
\hline 34 & 45.6 & 46.8 & 24 & $i^{2}$ & 34 & 59.1 & 59.8 & 45 & 18 & 34 & 45.1 & 47.1 & 24 & 16 & 34 & 31.0 & 36.7 & 30 & \\
\hline 36 & 47.3 & 48.9 & 27 & 28 & 36 & 58.7 & 59.6 & 44 & B. & 36 & $45 \cdot 3$ & 47.9 & 25 & iㅛ & 36 & 34.8 & 39.6 & 36 & . \\
\hline 38 & 48.7 & 50.8 & 29 & $4=$ & 38 & 58.7 & 59.7 & 44 & E & 38 & 45.2 & 48.1 & 25 & E & 38 & 26.3 & 33.9 & 25 & 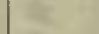 \\
\hline 40 & 47.9 & 50.0 & 28 & 28 & 40 & 58.1 & 59.2 & 43 & 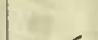 & 40 & 45.0 & 47.9 & 25 & 5 & 40 & 39.8 & 48.7 & 47 & tat \\
\hline 42 & 47.9 & 49.2 & 28 & $7 x^{2}$ & 42 & 57.9 & 59.2 & 43 & -11.6 & 42 & 44.2 & 47.7 & 24 & 185 & 42 & 39.3 & 45.9 & 44 & \\
\hline 44 & 45.9 & 46.8 & 24 & -12.6 & 44 & $57 \cdot 3$ & 59.3 & 43 & the & 44 & 44.7 & 47.7 & 24 & -10.9 & 44 & 4I. I & 48.9 & 48 & -10.0 \\
\hline 46 & 50.2 & 52.4 & 32 & & 46 & 57.2 & 58.8 & 43 & 8 & 46 & 45.0 & 48.3 & 25 & & 46 & 39.4 & 44.3 & 43 & \\
\hline 48 & 52.6 & 55.3 & 36 & (8). & 48 & 56.1 & 57.4 & 40 & (4) & 48 & 44.7 & 47.9 & 24 & & 48 & 39.7 & 44.9 & 44 & \\
\hline 50 & $55 . \mathrm{I}$ & 57.9 & 40 & 36 & 50 & 55.3 & 56.6 & 39 & 41 & 50 & 44.5 & 47.0 & 24 & 68 & 50 & 36.1 & 41.7 & 38 & 60 \\
\hline 52 & $55 \cdot 3$ & 58.1 & 40 & 62 & 52 & 54.9 & 55.7 & 38 & $c=$ & 52 & 47.6 & 49.8 & 28 & 42 & 52 & 35.9 & 39.8 & 37 & 4 \\
\hline 54 & 54.7 & 57.7 & 40 & 15 & 54 & 55.0 & 55.9 & 38 & a & 54 & 48.9 & $5 \mathrm{I} . \mathrm{I}$ & 30 & +4 & 54 & 34.9 & 44.2 & 39 & 82 \\
\hline 56 & 53.4 & 56.1 & 37 & 8 & 56 & $55 . \mathrm{I}$ & 55.8 & 38 & $c$ & 56 & 49.8 & 51.8 & 32 & 20 & 56 & 40.0 & 45.7 & 44 & \\
\hline 58 & 52.4 & 55.3 & 36 & 86 & 58 & 54.8 & 55.4 & 38 & & 58 & 49.9 & 52.1 & 32 & $x=$ & 58 & 41.6 & 48.8 & 48 & \\
\hline 1700 & 54.1 & 55.1 & 37 & -12.3 & 1900 & 54.0 & 54.8 & 37 & $-I I .4$ & 2100 & 49.0 & 51.0 & 30 & -10.8 & 2300 & 33.2 & 38.1 & 33 & -IO. I \\
\hline 02 & 56.1 & 56.9 & 40 & - & 02 & 53.8 & 54.8 & 37 & $-\infty$ & 02 & 47.3 & 48.6 & 27 & 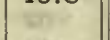 & 02 & 38.6 & 45.8 & 44 & \\
\hline 04 & 54.8 & 55.9 & 38 & 108 & 04 & 53.8 & 55.0 & 37 & 36 & 04 & 47.0 & 48.2 & 26 & the & 04 & 35.4 & 40.3 & 37 & \\
\hline 06 & 54.3 & 55.2 & 37 & $x$ & 06 & 53.8 & 54.8 & 37 & sor & 06 & 45.9 & 47.8 & 25 & Sh. & 06 & 27.6 & 33.3 & 25 & \\
\hline 08 & 53.9 & $55 . I$ & 37 & det & 08 & 54.0 & 55.3 & 37 & Bes & 08 & 44.6 & 45.8 & 23 & $82=$ & 08 & 39.7 & $47 . \mathrm{I}$ & 45 & 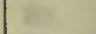 \\
\hline I0 & 52.9 & $54 . \mathrm{I}$ & 35 & of & IO & 53.1 & 54.2 & 36 & ar & 10 & 45.3 & $47 . I$ & 24 & 64 & Io & 42.8 & 46.9 & 48 & 64 \\
\hline 12 & 51.6 & 53.1 & 34 & & 12 & 52.6 & 53.7 & 35 & 34 & 12 & 46.3 & 48.7 & 26 & 27 & 12 & 41.7 & 45.9 & 46 & \\
\hline I4 & 50.1 & 52.8 & 32 & -12.2 & 14 & 52.6 & 53.2 & 35 & -11.2 & I4 & 44.7 & 46.4 & 23 & -10.7 & 14 & 41.9 & 47.8 & 48 & -10.0 \\
\hline I6 & 48.7 & 50.3 & 29 & tar & 16 & 52.6 & 53.8 & 35 & - & $\begin{array}{l}14 \\
16\end{array}$ & $\begin{array}{l}44.7 \\
40.7\end{array}$ & 43.1 & 18 & 10.8 & 16 & 30.1 & 37.4 & 30 & \\
\hline 18 & 50.1 & 51.9 & 32 & 4 & 18 & 52.3 & 53.7 & 35 & 211 & I8 & 40.2 & 42.1 & I6 & 81 & I8 & 21.3 & 26.1 & 14 & \\
\hline 20 & 50.8 & 52.5 & 32 & rester & 20 & 52.9 & 53.9 & 36 & 93 & 20 & 41.2 & 43.8 & I8 & 8 & 20 & 19.8 & 23.2 & II & \\
\hline 22 & 51.0 & 52.4 & 33 & wat & 22 & 53.6 & 54.5 & 36 & 3 & 22 & 42.3 & 44.8 & 20 & $=$ & 22 & 18.3 & 22.7 & 10 & \\
\hline 24 & 52.0 & 53.8 & 35 & $x^{2}$ & 24 & 52.8 & 53.9 & 35 & in & 24 & 42.3 & $44 . I$ & I9 & at & 24 & 22.1 & 26.4 & 15 & \\
\hline 26 & 52.8 & 54.7 & 36 & 2 & 26 & 52.2 & 53.2 & 34 & 28 & 26 & $4 \mathrm{I} .2$ & 43. I & 18 & 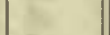 & 26 & 25.2 & 29.3 & 20 & \\
\hline 28 & 53.9 & 55.7 & 38 & $x=$ & 28 & 52.1 & 53.1 & 34 & 2 & 28 & 40.9 & 43.0 & 18 & D. & 28 & 14.3 & I8. I & 2203 & \\
\hline 30 & $54 \cdot 3$ & 56.2 & 38 & -12.1 & 30 & $5 \mathrm{I} .1$ & 52.0 & 33 & -11.2 & 30 & 41.0 & 43.0 & 18 & -10.5 & 30 & 10.3 & 14.4 & 2157 & -10.0 \\
\hline 32 & 55.3 & 57.2 & 40 & (2) & 32 & 50.5 & 52.8 & 33 & on & 32 & 42.8 & 44.9 & 20 & - & 32 & 14.1 & 18.1 & $\begin{array}{ll}22 & 03\end{array}$ & \\
\hline 34 & 54.8 & 56.3 & 39 & 36 & 34 & 51.0 & 53.9 & 34 & $x^{2}$ & 34 & 44.0 & 46.2 & 23 & 35 & 34 & 14.3 & 18.1 & 03 & \\
\hline 36 & 54.0 & 55.8 & 38 & & 36 & 51.8 & 54.6 & 35 & & 36 & 44.7 & 46.9 & 24 & $\sqrt{2}$ & 36 & 24.3 & 26.1 & 17 & \\
\hline 38 & 53.1 & 54.7 & 36 & & 38 & 51.2 & 53.9 & 34 & (ate & 38 & 45.2 & 47.6 & 25 & 86 & 38 & 21.2 & 24.0 & 13 & \\
\hline 40 & 53.7 & 54.2 & 36 & $t_{1}=$ & 40 & $5 \mathrm{I} . \mathrm{I}$ & 53.9 & 34 & $t^{2}$ & 40 & $48 . I$ & 50.4 & 29 & of & 40 & 22.9 & 24.9 & 15 & \\
\hline 42 & 54.4 & $55 \cdot 3$ & 38 & & 42 & 51.2 & 53.2 & 34 & & 42 & 48.8 & 50.3 & 30 & & 42 & 25.0 & 26.8 & 18 & \\
\hline 44 & 54.1 & 54.8 & 37 & -12.0 & 44 & 50.7 & 52.1 & 32 & -II.I & 44 & $44 . I$ & 46.1 & 23 & -10.5 & 44 & 26.7 & 29.6 & 22 & -10.0 \\
\hline 46 & 54.3 & 55.1 & 37 & the & 46 & 50.7 & 52.8 & 33 & & 46 & 41.8 & 45.2 & 20 & & 46 & 30.8 & 32.8 & 27 & \\
\hline 48 & 52.8 & 55.8 & 37 & 20. & 48 & 50.8 & 52.8 & 33 & $F_{2}$ & 48 & 50. & $.0 a$ & 30 & 78 & 48 & 31.9 & 33.4 & 28 & \\
\hline 50 & 54.8 & 56.9 & 39 & 35 & 50 & 51.1 & 53.1 & 34 & $\Rightarrow$ & 50 & 45.1 & 46.8 & 24 & at & 50 & 36.2 & 37.2 & 35 & $=$ \\
\hline 52 & 59.1 & 60.8 & 46 & $=$ & 52 & 50.6 & 52.3 & 32 & 3 & 52 & 40.2 & 40.3 & 15 & the & 52 & 38.8 & 39.7 & 39 & \\
\hline 54 & 57.4 & 59.1 & 43 & 3 & 54 & 49.2 & 51.3 & 30 & $\mathrm{H}$ & 54 & 37. &.$I b$ & 10 & $1=$ & 54 & 34.0 & 35.6 & 32 & \\
\hline 56 & 57.5 & 58.5 & 43 & 3 & 56 & 48.8 & $5 \mathrm{I} .1$ & 30 & 5 & 56 & 35.2 & 35.5 & 07 & ES & 56 & 34.1 & 35.8 & 32 & \\
\hline 58 & 58.6 & 59.9 & 44 & 62 & 58 & 49.2 & 51.9 & 31 & $\sqrt{2}$ & 58 & 35.6 & 38.3 & 10 & tht & 58 & 27.2 & 28.9 & 21 & \\
\hline & & & & & & & & & & & & & & & 2400 & 35.2 & 36.2 & 33 & -9.9 \\
\hline
\end{tabular}


Tabulation of magnetic declinations observed at Teplitz Bay-Continued

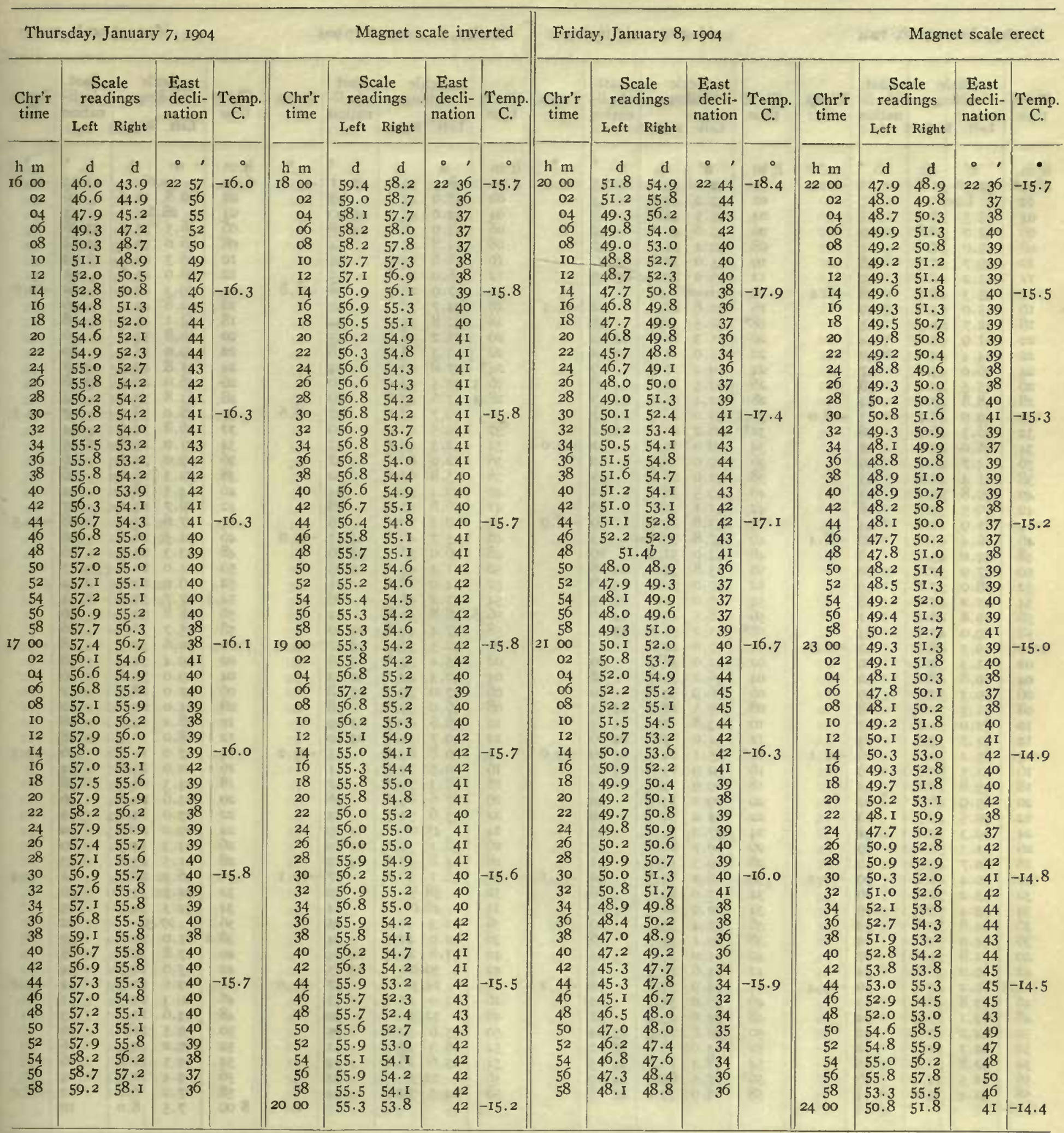

Correction to local mean time is $-46 \mathrm{~s}$.

The torsion head at $15 \mathrm{~h} 25 \mathrm{~m}$ read $102^{\circ}$ and at $20 \mathrm{~h} 25 \mathrm{~m}$ read the same. Observer-J. V.
Correction to local mean time is $-38 \mathrm{~s} .90^{\circ}$ torsion $=17 .^{\prime} 5$.

Torsion head at $20 \mathrm{~h} 25 \mathrm{~m}$, January 7 th, read $102^{\circ}$, and $24 \mathrm{~h}$ 2om read $98^{\circ}$.

Observer-R. R. T. 
Tabulation of magnetic declinations observed at Teplitz Bay-Continued

\begin{tabular}{|c|c|c|c|c|c|c|c|c|c|c|c|c|c|c|c|c|c|c|c|}
\hline \multicolumn{6}{|c|}{ Sunday, January ro, 1904} & \multicolumn{4}{|c|}{ Magnet scale inverted } & \multicolumn{6}{|c|}{ Sunday, January so, 1904} & \multicolumn{4}{|c|}{ Magnet scale erect } \\
\hline \multirow{2}{*}{$\begin{array}{c}\text { Chr'r } \\
\text { time }\end{array}$} & \multicolumn{2}{|c|}{$\begin{array}{l}\text { Scale } \\
\text { readings }\end{array}$} & \multirow{2}{*}{$\begin{array}{l}\text { East } \\
\text { decli- } \\
\text { nation }\end{array}$} & Temp. & Chr'r & $\begin{array}{r}\mathrm{Sc} \\
\mathrm{reac}\end{array}$ & & $\begin{array}{l}\text { East } \\
\text { decli- }\end{array}$ & Temp & Chr'r & $\begin{array}{l}\mathrm{Sc} \\
\text { read }\end{array}$ & ale & $\begin{array}{l}\text { East } \\
\text { decli- }\end{array}$ & Temp. & Chr'r & $\underset{r e a}{S}$ & ale & $\begin{array}{l}\text { East } \\
\text { decli- }\end{array}$ & \\
\hline & Left & Right & & & & Left & Right & & & & Left & Right & tion & & & Left & Right & attont & \\
\hline & d & d & & • & $\mathrm{h} \mathrm{m}$ & d & d & & $\bullet$ & $\mathrm{h} \mathrm{m}$ & d & d & & & $\mathrm{h} \mathrm{m}$ & $d$ & d & & \\
\hline 0 oo* & 37.5 & 37.0 & 2250 & -20.0 & 200 & 42.0 & 39.0 & 2245 & -19.5 & 400 & 48.5 & 50.2 & 25 II & -19.6 & 600 & a & h & 2333 & -19.0 \\
\hline 02 & $\mathrm{LCC}_{5}$ & & & 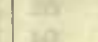 & 02 & 43.2 & 40.9 & $\begin{array}{r}43 \\
30\end{array}$ & & 02 & 41.0 & 47.6 & 2503 & & 02 & 10.8 & $11 \cdot 3$ & 16 & \\
\hline $\begin{array}{l}04 \\
06\end{array}$ & $\begin{array}{l}35.5 \\
32.0\end{array}$ & $\begin{array}{l}33.0 \\
30.8\end{array}$ & $\begin{array}{l}55 \\
59\end{array}$ & 26 & $\begin{array}{l}04 \\
06\end{array}$ & $\begin{array}{r}45.5 \\
43.0\end{array}$ & $\begin{array}{l}43.1 \\
40.1\end{array}$ & $\begin{array}{l}39 \\
44\end{array}$ & 8 & $\begin{array}{l}04 \\
06\end{array}$ & $\begin{array}{l}42.0 \\
33.0\end{array}$ & $\begin{array}{l}43.1 \\
36.0\end{array}$ & $\begin{array}{l}2500 \\
2448\end{array}$ & 6 & $\begin{array}{l}04 \\
06\end{array}$ & 36.5 & 37.0 & 2356 & 8 \\
\hline 08 & 35.0 & 34.0 & 54 & क) & 08 & 40.4 & 38.7 & & 85 & 08 & 24.8 & 27.0 & 34 & 80 & 08 & 46.0 & 50.0 & $24 \mathrm{I} 4$ & 8 \\
\hline Io & 39.7 & 37.9 & 48 & 19 & I0 & 35.0 & 32.8 & 2256 & & Io & 12.6 & 13.9 & $I_{4}$ & at & ro & 40.3 & 42.0 & & 62. \\
\hline I2 & 44.2 & 44.0 & 39 & taㅏ & I2 & 29.0 & 29.0 & 2303 & 4 & I2 & 26.0 & 26.8 & 35 & & 12 & 38.0 & 41.6 & oI & \\
\hline 14 & $45 \cdot 3$ & 44.8 & $3^{8}$ & -18.4 & 14 & Óver & $l^{\prime} k^{\prime} d$ & & & 14 & 27.9 & 28.7 & 38 & -18.8 & 14 & $39 \cdot 3$ & 42.3 & 02 & -19.0 \\
\hline 16 & 45.0 & 43.6 & 39 & & 15 & 30.8 & 28.1 & 02 & -19.5 & 16 & 34.7 & 35.6 & 49 & & 16 & 42.8 & 44.0 & 06 & \\
\hline I8 & 42.7 & 41.3 & 43 & 8 & $18^{*}$ & 30.5 & 30.2 & 45 & & I8 & 30.8 & 33.2 & 44 & 81. & I8 & 44. I & $45 \cdot 3$ & 08 & \\
\hline 20 & $4 \mathrm{I} .9$ & 40.0 & 44 & 50 & 20 & 21.3 & 21.0 & 2359 & $\sigma c$ & 20 & 25.2 & 28.0 & 35 & $y=$ & 20 & 0.8 & $53 \cdot 3$ & 20 & 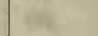 \\
\hline 22 & 44.4 & 42.8 & 40 & es & 22 & 15.0 & II .0 & 2412 & ats & 22 & 13.3 & 18.0 & 18 & ist & 22 & 49.0 & 55.0 & 20 & $\therefore$ \\
\hline 24 & $\begin{array}{r}4.4 \\
46.0\end{array}$ & 45.6 & 37 & is & 24 & 10.2 & 7.0 & 19 & is & $24^{*}$ & 37.8 & 46.3 & 04 & 15 & 24 & $4 \mathrm{I} .8$ & $45 \cdot 3$ & 2407 & its \\
\hline 26 & 45.5 & 44.4 & 38 & ds & 26 & 20.5 & 14.0 & 2406 & & & 42.5 & $49 \cdot 3$ & I0 & $x^{2}$ & 26 & 36.0 & 40.5 & 2358 & 8 \\
\hline 28 & 48.8 & 47.0 & 34 & mas & 28 & 31.4 & 25.6 & 2348 & is & 28.3 & 47.6 & 55.8 & 20 & & 28 & 33.8 & 37.0 & 54 & \\
\hline 30 & 49.9 & 48.7 & $3 \mathrm{I}$ & -18.0 & 30.5 & 30.0 & 28.5 & 2347 & & 30 & 39.7 & 47.5 & 07 & -18.6 & 30 & 36.0 & 38.5 & 57 & -19.3 \\
\hline 32 & 46.5 & 46.5 & 36 & 0 & $32^{*}$ & 26.2 & 21.4 & 2444 & & 32 & 39.5 & 47.0 & 06 & 20 & 32 & 26.8 & 31.0 & 44 & \\
\hline 34 & 46.0 & 45.2 & 37 & IE & 34 & 19.0 & I9.0 & 52 & -19.0 & 34 & 3 & & II & 12 & 34 & 21.0 & 25 & $\begin{array}{l}44 \\
35\end{array}$ & He \\
\hline 36 & 44.7 & 43.7 & 39 & 8 & 36 & I9.0 & 18.9 & 52 & 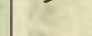 & 3 & & & 27 & \& & 36 & 29.0 & & & \\
\hline 38 & $4 \mathrm{I} \cdot 4$ & 40.0 & 45 & $\dot{ }$ & $3^{8}$ & 31.0 & $28 . \overline{8}$ & 35 & 13 & 38 & 5.8 & 63.8 & 32 & $8 \pi$ & 38 & 2.3 & & $\begin{array}{l}36 \\
36\end{array}$ & A \\
\hline 40 & 39.8 & 37.0 & 48 & 09 & 40 & 39.5 & 36.9 & 22 & 4 & 40 & 50.4 & 59.3 & 24 & 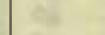 & 40 & 14.5 & 18 & 24 & 5 \\
\hline 42 & 40.5 & 39 & 46 & 48 & 42 & $4 \mathrm{I} . \mathrm{I}$ & 38.0 & 20 & & 42 & 56.8 & & 28 & 4 & 42 & I6.7 & 20. & 27 & 4 \\
\hline 44 & $4 \mathrm{I} .9$ & $4 \mathrm{I} .0$ & 44 & & 44 & 53.8 & 49.8 & 00 & -I8.9 & 44 & $49 \cdot 3$ & 50.1 & I6 & & 44 & 17.6 & 20.2 & 28 & -19.5 \\
\hline 46 & 46.4 & 45.7 & 36 & -18.4 & 46 & 37.8 & 36.8 & 23 & $1 .=$ & 46 & 45.7 & 46.7 & 24 II & -19.0 & 46 & 24.3 & 26.2 & 38 & \\
\hline 48 & 44.0 & 43.7 & 40 & 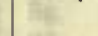 & 48 & 53.0 & 51.0 & 100 & nest & 48 & 36.0 & 37.3 & 2356 & 80 & 48 & 23.3 & 25 & 36 & 6 \\
\hline 50 & 37.9 & 37.6 & 50 & 14 & 50 & 35.8 & 33.0 & 2428 & $-x$ & 50 & $4 \mathrm{I} .4$ & & 2405 & ces & 50 & 21.0 & & 33 & Q \\
\hline 52 & 38.0 & 37.6 & 49 & Cl & 52 & 57.0 & 50.0 & 2358 & 12 & 52 & 44.0 & 48.2 & II & 12 & 52 & 26.9 & $29 . I$ & 42 & 5 \\
\hline 54 & 45.2 & 44 & 38 & ter & 54 & 6.0 & 2.0 & 2515 & $R^{2}$ & 54 & $4 \mathrm{I} .2$ & 45 & of & 4 & 54.5 & 27.7 & 30 & 44 & E. \\
\hline 56 & 49.8 & 48.9 & $3^{\mathrm{T}}$ & 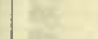 & 56 & 48.5 & 43.0 & 2410 & a & 56. & 38.8 & & 02 & & 56 & 28.8 & & 46 & \\
\hline 58 & 49.0 & 47.7 & 33 & 15 & $58 *$ & 41.2 & 36.0 & 2324 & & 58 & 37.4 & 40.8 & 2400 & & 58 & 55.7 & & 56 & 8 \\
\hline I 00 & 49.3 & 48.7 & 32 & -18.9 & 300 & 47.0 & 42.0 & & -18.9 & 500 & 32.3 & 36.3 & 2352 & -19.1 & 700 & 30. & 32.5 & 48 & $-\mathrm{rg} .5$ \\
\hline 02 & 51.3 & 50 & 29 & & 02.5 & & 38.9 & 20 & & 02 & & & 356 & & 02 & 30 . & 32.4 & 48 & \\
\hline 04 & 49.8 & 48.0 & 32 & 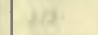 & 04 & 46.2 & 39 & I8 & 14 & 0 & & 42 & 2403 & 16 & 04 & 3 & & 46 & 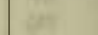 \\
\hline$\infty$ & $\begin{array}{l}49 \\
50\end{array}$ & 50 & & 20 & 06 & & & 30 & 89 & 06 & & & $\begin{array}{lll}3 & 58 \\
4\end{array}$ & 25 & of & 2 & $\begin{array}{l}31 \\
28\end{array}$ & $\begin{array}{l}40 \\
42\end{array}$ & \\
\hline a & $5 \mathrm{I}$. & 51 & 28 & 6 & 08 & & 18.9 & 2354 & (6) & 08 & & & 58 & 82 & 08 & 2 & 22 & 32 & $\sin$ \\
\hline I & 52. & $5 \mathrm{I}$ & 27 & $\mathrm{mi}$ & IO & 13.8 & I I & 2405 & 08 & IC & & 40 & 2359 & or & I0 & I8. & & 28 & tit \\
\hline I2 & 54.7 & 53 & 24 & 44 & I 2 & & 9 & 2406 & nit & $\mathrm{I} 2$ & & & 420 & ist & 12 & 8.0 & & 28 & $\mathrm{~m}$ \\
\hline I4 & 53.2 & & 26 & -19.3 & $14^{*}$ & $35 . \mathrm{I}$ & 24.0 & $23 \quad 39$ & -19.0 & 14 & 59.8 & 63 & 35 & -19.2 & I. & 17.0 & I 8.5 & 26 & - \\
\hline If & $5 \mathrm{I}$ & & 29 & & 16 & & 19.8 & 36 & & IC & & 72. & $5 \mathrm{I}$ & 1-1. & & 22.0 & 23 & 34 & \\
\hline I\& & 49 & & 3 & 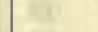 & I\& & & 9. & & 82 & I & & & 47 & $M_{t}$ & I & & & 38 & 4 \\
\hline 20 & 49.9 & 48.6 & 3 & as & 20 & & 14 & 42 & 와 & 2 & 67.6 & 69.6 & 46 & es & 2 & & & 36 & 0. \\
\hline 22 & 50 & 49 & 3 & te & 22 & & & 19 & 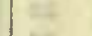 & 22 & 53.6 & 54.3 & 23 & 8 & 22 & & & 36 & E \\
\hline 24 & 50 & & & $t^{-1}$ & 24 & & & 23 & 1 & 24 & 45 & $.0 b$ & 2409 & t & 24 & I & & 30 & \\
\hline 26 & $5 \mathrm{I}$ & & & Zut & 26 & & & 37 & I & 26 & $38 . I$ & 39.1 & 359 & & 26 & 1 & & 20 & \\
\hline 28 & 50 & & 0 & $1=$ & 28 & & 12 & $5 \mathrm{I}$ & & 28 & 45. & & 412 & ine & 28 & I. 5 & & 33 & \\
\hline 30 & 50 & & & -19.2 & 30 & & & 40 & -19.1 & 30 & 56.1 & 56.6 & 27 & -19.I & 30 & 26.2 & 27 . & $4 \mathrm{I}$ & -19.4 \\
\hline 32 & 53 & 52 & 26 & & 32 & & & 28 & & 32 & $5^{8}$ & $.5 b$ & 30 & & 32 & 32.5 & 33. & 50 & \\
\hline 34 & 53.4 & 53 & & FE & 34 & 26.9 & 22.2 & 37 & te & 34 & 46 & $.0 b$ & II & 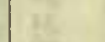 & 34 & 32.3 & 32. & 49 & \\
\hline & 54.8 & 53 & 23 & & $3^{6}$ & 37.4 & & 20 & 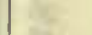 & 36 & 42.3 & 42.3 & 05 & & 36 & 27 & $5^{b}$ & 42 & \\
\hline & 54. & 53 & & 8 & 38 & & & I6 & $x^{2}$ & 38 & & & 04 & f & 38. & & 21.6 & 2 & \\
\hline & $5 \mathrm{I}$. & $5 \mathrm{I}$ & 27 & 6 & 40 & $4 \mathrm{I}$ & 36 & I5 & 04 & 40 & $4 \mathrm{r} .8$ & 42.3 & 2404 & Th & 40 & 7.8 & & 28 & \\
\hline 42 & 49.0 & 46.8 & 34 & 4) & 42 & & 29 & 25 & et & 42 & 30 & $.0 b$ & 2345 & 34 & 42 & 15. & & 24 & \\
\hline & 45 & & 39 & -19. & & 38 & 34 & 18 & -I9. & 44 & 24 & $5^{b}$ & 37 & -19.0 & & 19. & & 31 & -19.4 \\
\hline & 43.8 & 40 & 43 & & 46 & 38 & 32 & 20 & & 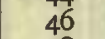 & 21.0 & 21. & $3 \mathrm{I}$ & & & & & $3 I$ & \\
\hline & 43 & & 44 & 4 & 48 & & 25 & 3I & 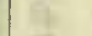 & $4 \varepsilon$ & 32 & $3^{a}$ & 49 & 6 & 4 & 14 & & 3 & \\
\hline 5 & & 40 & 42 & $Q_{2}$ & $5 C$ & 30.8 & 22 & & & 5 & 35.6 & & 55 & 6 & 5 & & & 6 & \\
\hline 5 & & & 42 & at & 5 & & 6 & & & 52 & & & $4 \mathrm{I}$ & 12 & 52 & 0.2 & & 16 & \\
\hline & $4 \mathrm{I}$ & 39 & 45 & $P$ & 54 & 18.3 & II.O & 2453 & & 54 & 25.6 & 26.2 & 39 & 13 & 54 & I0.5 & & I6 & \\
\hline & & 39 & 46 & & $56^{*}$ & 67.5 & 64.0 & 2459 & & 56 & 22 & $3 b$ & 33 & & 56 & 9.4 & 10.6 & I4 & \\
\hline 58 & 42.4 & 39.8 & 44 & & 58 & 60.9 & 59.2 & 2508 & & 58 & 22.3 & 22.7 & 34 & & $\begin{array}{r}58 \\
\end{array}$ & 7.2 & 8.2 & Io & -19.0 \\
\hline & & & & & & & & & & & & & & & 800 & 7.5 & 8.0 & IO & \\
\hline
\end{tabular}

Observers-J. V. and W. J. P., who alternated from $3^{\text {h }} 50 \mathrm{~m}$ to 4h $04 \mathrm{~m}$.
Correction to local mean time is $-225.90^{\circ}$ torsion $=17 .^{\prime} 5$.

Torsion head at oh oom read $93^{\circ}$ and at 9 h $30 \mathrm{~m}$ read $96^{\circ}$.

Observer-W. J. P. 


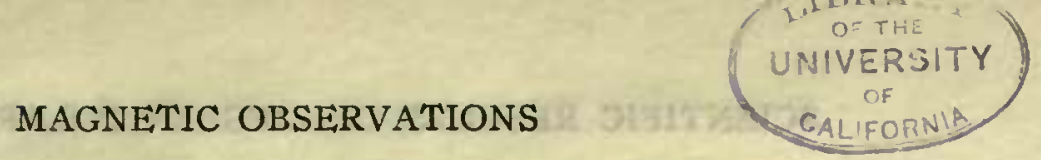

Tabulation of magnetic declinations observed at Teplitz Bay-Continued

\begin{tabular}{|c|c|c|c|c|c|c|c|c|c|c|c|c|c|c|c|c|c|c|c|}
\hline \multicolumn{5}{|c|}{ Monday, January II, I904 } & \multicolumn{5}{|c|}{ Magnet scale inverted } & \multicolumn{5}{|c|}{ Tuesday, January 12, 1904} & \multicolumn{5}{|c|}{ Ifat 2 . Magnet scale erect } \\
\hline \multirow{2}{*}{$\begin{array}{c}\text { Clir'r } \\
\text { time }\end{array}$} & \multicolumn{2}{|c|}{$\begin{array}{l}\text { Scale } \\
\text { readings }\end{array}$} & \multirow{2}{*}{$\begin{array}{c}\text { East } \\
\text { decli- } \\
\text { nation }\end{array}$} & Temp. & Chr'r & $\begin{array}{r}\mathrm{Sc} \\
\text { read }\end{array}$ & $\begin{array}{l}\text { ale } \\
\text { ings }\end{array}$ & $\begin{array}{l}\text { East } \\
\text { decli- }\end{array}$ & Temp. & Chr'r & $\begin{array}{r}\mathrm{Sc} \\
\mathrm{read}\end{array}$ & $\begin{array}{l}\text { ale } \\
\text { dings }\end{array}$ & East & Temp. & Chr'r & $\begin{array}{r}\mathrm{Sc} \\
\text { read }\end{array}$ & $\begin{array}{l}\text { ale } \\
\text { lings }\end{array}$ & $\begin{array}{l}\text { East } \\
\text { decli- }\end{array}$ & Temp. \\
\hline & L,eft & Right & & & & Left & Right & & & & Left & Right & & & & Left & Right & nation & \\
\hline $\mathrm{h} \mathrm{m}$ & d & d & $\circ \cdot$ & {$[0$} & l) $\mathrm{m}$ & d & d & 0 , & 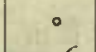 & h m & d & $d$ & & $t$ & $\mathrm{~h} \mathrm{~m}$ & d & d & & 。 \\
\hline $800^{*}$ & 38.0 & 35.8 & 2333 & -23.0 & Io 00 & 52.9 & 50.4 & 2238 & -20.6 & 1200 & 59.9 & 60.3 & 2240 & -29.0 & 1400 & 56.1 & 58.1 & 2235 & -25.8 \\
\hline 02 & 40.8 & 38.7 & 29 & 6 & 02 & 52.0 & 50.3 & 39 & 37 & 02 & 57.8 & 58.9 & 37 & 58 & 02 & 56.7 & 59.2 & 36 & 10 \\
\hline 04 & 37.8 & 36.8 & 33 & ile. & 04 & 50.7 & 48.9 & $4 \mathrm{I}$ & is & 04 & 56.9 & 58.8 & 36 & pa & 04 & 55.2 & 57.8 & 34 & $e^{2}$ \\
\hline$\infty 6$ & 37.8 & 36.4 & 33 & in & 06 & 49.9 & 48.2 & 42 & 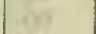 & 06 & 57.0 & 57.7 & 35 & in & 06 & 54.0 & 56.8 & & 65 \\
\hline 08 & 38.9 & 37.2 & 32 & Ba & $\infty 8$ & 51.8 & 49.2 & 40 & 160 & 08 & 54.1 & 55.2 & $3 \mathrm{I}$ & 25 & 08 & 54.3 & 56.7 & 32 & A45 \\
\hline I0 & 42.8 & $4 \mathrm{I} .2$ & 25 & 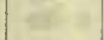 & IO & 48.8 & 46.5 & 44 & 51 & 10 & 55 & $.4 a$ & 32 & 2415 & Io & 54.8 & 56.7 & 33 & at \\
\hline 12 & $4 \mathrm{I} .4$ & $4 \mathrm{I} .0$ & 26 & 51 & I2 & 43.0 & 41.2 & 53 & 年 & 12 & $5 I .2$ & 52.0 & 26 & (1) & 12 & 55.1 & 56.8 & 33 & 00 \\
\hline 14 & 38.2 & 36.2 & 33 & -22.9 & I4 & 45.3 & 43.5 & 50 & -20.6 & 14 & 5 I. 2 & 52.9 & 27 & -28.4 & I4 & 55.0 & 56.8 & 33 & -25.4 \\
\hline I6 & 34.0 & 32.0 & 39 & tai & 16 & 48.2 & 47.2 & 44 & & 16 & 50.1 & 51.6 & 25 & - $=2$ & 16 & 55.9 & 57.5 & 34 & 10 \\
\hline I8 & $3 \mathrm{I} .2$ & 29.3 & 44 & 8 & 18 & 50.2 & 48.9 & 42 & A & 18 & 47.1 & 49.2 & 21 & $76=$ & I8 & 55.1 & 56.4 & 33 & ing \\
\hline 20 & 33.1 & 31.9 & 40 & $\approx$ & 20 & 49.9 & 48.3 & 42 & $x$ & 20 & 46.5 & 47.2 & 19 & $x$ & 20 & 54.7 & 56.4 & & tes \\
\hline 22 & $3 \mathrm{I} .7$ & 29.2 & 44 & $y=$ & 22 & 49.6 & 48.7 & 42 & 18 & 22 & 45.3 & 47.1 & I8 & 28 & 22 & 55.8 & 57.3 & 34 & ef \\
\hline 24 & 32.3 & 30.7 & 42 & if & 24 & 49.0 & 48.0 & 43 & $4=$ & 24 & 46.6 & 49.4 & 21 & 14 & 24 & 55.1 & 56.3 & 33 & 15 \\
\hline 26 & 30.7 & 28.6 & 45 & $A$ & 26 & 48.0 & 47.2 & 44 & 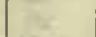 & 26 & 45.0 & 48.3 & 19 & 16 & 26 & 54.2 & 56.0 & 32 & 60 \\
\hline 28 & 28.2 & 25.7 & 49 & ber & 28 & 48.5 & 46.8 & 44 & $x$ & 28 & 52.7 & 53.8 & 29 & 10 & 28 & 53.4 & 55.2 & $3 I$ & 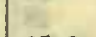 \\
\hline 30 & 25.2 & 22.3 & 54 & -22.3 & 30 & 49. I & 47.8 & 43 & -20.7 & 30 & 54.7 & 60.1 & $3^{6}$ & -28.0 & 30 & 54.0 & $55 \cdot 3$ & $3 I$ & -25.2 \\
\hline 32 & 25.9 & 24.9 & $5 \mathrm{I}$ & 13 & 32 & 50.0 & 49. I & 42 & $x^{2}=$ & 32 & 54.3 & 58.8 & 34 & $1=0$ & 32 & 51.1 & 53 & 28 & at \\
\hline 34 & 24.8 & 24.2 & 53 & 18 & 34 & 48.9 & 47.5 & 44 & it: & 34 & 49.9 & 54.9 & 28 & $\because 8$ & 34 & 5I.I & 52.3 & 26 & 48 \\
\hline 36 & 26.9 & 26.3 & 49 & $\alpha$ & 36 & 49.2 & $48 . \mathrm{I}$ & 43 & & 36 & 45.1 & 49.8 & 20 & 파요. & 36 & $5 \mathrm{I} \cdot 3$ & 53 & 27 & 8 \\
\hline 38 & 28.3 & 25.2 & 49 & the & 38 & 49.8 & 48.9 & 42 & 53 & 38 & 53.2 & 59.1 & 34 & 78 & 38 & 54.3 & 55.7 & 32 & 等. \\
\hline 40 & 27.2 & 26.7 & 49 & 6 & 40 & 49.9 & 48. I & 42 & of & 40 & 52.2 & 57.8 & 32 & of & 40 & 50.5 & 53 & 27 & $\alpha$ \\
\hline 42 & 22.2 & 20.4 & 58 & $=0$ & 42 & 48.0 & 46.5 & 45 & tas & 42 & $52 . I$ & 57.1 & 31 & & 42 & 49.9 & 52 & 26 & \\
\hline 44 & 26.6 & 23.5 & 2352 & -22.0 & 44 & 47.7 & 46.7 & 45 & -20.5 & 44 & 52.6 & 56.5 & $3 \mathrm{I}$ & -27.8 & 44 & 49.8 & 52.2 & 26 & -25.0 \\
\hline 46 & 18.3 & 16.2 & 240.4 & 10 & 46 & 45.4 & 44.0 & 49 & ( & 46 & 53.9 & 56.3 & 32 & & 46 & 52.5 & 55.7 & 30 & 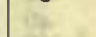 \\
\hline 48 & 18.2 & 17.2 & 03 & 18 & 48 & 46.1 & 44.8 & 48 & 20 & 48 & $55 \cdot 3$ & 57.8 & 34 & 8 & 48 & 55.7 & 58. & 35 & 86. 1 \\
\hline $50^{*}$ & 48. & $7 a$ & Io & $+\frac{\pi}{2}$ & 50 & 47.2 & 45.7 & 46 & Es: & 50 & 55.5 & 57.6 & 34 & $n e$ & 50 & 53.0 & 55 & $3 \mathrm{I}$ & be \\
\hline 52.4 & 55.6 & 54.0 & 24 or & $2 t$ & 52 & 48.2 & 46.8 & 45 & 22 & 52 & 57.1 & 59.0 & 36 & 12 & 52 & 55.2 & 58 & 34 & 24 \\
\hline 54 & 63.8 & 60.9 & 2349 & HY & 54 & 48.4 & $47 \cdot 3$ & 44 & & 54 & 56.4 & 58.2 & 35 & 4 & 54 & $54 \cdot 3$ & 56.1 & 32 & 8 \\
\hline 56 & $69 \cdot 3$ & 66.6 & 40 & & 56 & 50.8 & 48.7 & $4 \mathrm{I}$ & & 56 & $55 . \mathrm{I}$ & 59.1 & 35 & 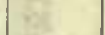 & 56 & 55.2 & 58 & 34 & a \\
\hline 58 & 72.4 & 67.7 & 37 & 10 & 58 & 50.3 & 48.2 & 42 & & 58 & $53 \cdot 3$ & 58.0 & 33 & 28 & 58 & 56.9 & 59.3 & 37 & 165 \\
\hline 90 & $6 \mathrm{r} .2$ & 57.6 & 54 & -21.6 & II 00 & 50.2 & 48.2 & 42 & -20.6 & 1300 & 52.2 & 57.2 & 31 & -27.2 & I5 OO & 53.3 & 55 & $3 \mathbf{I}$ & -25.0 \\
\hline 02 & 69.3 & $65 \cdot 3$ & $4 \mathrm{I}$ & 100 & 02 & 51.8 & 49.9 & 39 & wit & 02 & 55.3 & 59.2 & 35 & 6 & 02 & $54 . I$ & 57.9 & 33 & 20 \\
\hline 04 & 69.3 & 67.1 & 40 & in & 04 & 50.2 & 48.1 & 42 & 16 & 04 & 58.2 & 61.2 & 39 & in & 04 & 55.1 & 57.7 & 34 & 10 \\
\hline 06 & 65.6 & 64.7 & 45 & to & 06 & 51.3 & 48.3 & $4 \mathrm{I}$ & (a) & 06 & 57.7 & 60.1 & 38 & is & 06 & 56.3 & 58.7 & 36 & We \\
\hline 08 & 64.0 & 62.5 & 48 & $\omega$ & 08 & 49.8 & 48.2 & 42 & 14 & 08 & 57.5 & 59.9 & 38 & As & 08 & 58.0 & 59 & 37 & 75 \\
\hline I0 & 71.0 & 69.2 & 37 & of & I0 & 48.0 & 45.7 & 46 & 79 & I0 & 55.1 & 57.7 & 34 & of & I0 & 57.1 & 58.0 & 36 & ciu \\
\hline 12 & 70.0 & 69.2 & 38 & tat & I2 & 54.2 & 52.9 & 35 & 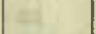 & 12 & 55.2 & 57.8 & 34 & & 12 & 58.2 & 59.9 & 38 & 47 \\
\hline I4 & 69.7 & 68.9 & 38 & -21.2 & I4 & 55.0 & 53.1 & 34 & -20.5 & 14 & 58.2 & 60.2 & 38 & -26.9 & I4 & 58.4 & 59 & 38 & -25.0 \\
\hline 16 & 77.8 & 76.2 & 26 & 24 & I6 & 51.3 & 48.3 & $4 \mathrm{I}$ & & 16 & 59.6 & 61.3 & 40 & 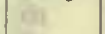 & 16 & 61.0 & 62.7 & 42 & 10 \\
\hline I8* & 49.8 & 44.3 & 24 & 20 & I8 & 50.9 & 48.3 & $4 \mathrm{I}$ & 는 & 18 & 57.2 & & 37 & 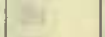 & I8 & 66.3 & 67.8 & 51 & 62 \\
\hline 20 & 49.1 & $42 . I$ & 26 & $\pi$ & 20 & 54.8 & 51.2 & 36 & [7t. & 20 & 57.0 & 58.8 & 36 & at & 20 & 69.8 & 71.7 & 56 & $\infty$ \\
\hline 22 & $5 \mathrm{I} \cdot 3$ & 45.7 & 22 & $-=$ & 22 & 50.9 & 47.9 & 42 & $\theta$ & 22 & 57.1 & 58.8 & 36 & $=4$ & 22 & 66.7 & 68. & 52 & wer \\
\hline 24 & 47.8 & $4 \mathrm{I} .8$ & 28 & 26 & 24 & 53.9 & 50.9 & 37 & 15 & 24 & 55.9 & 57.4 & 34 & 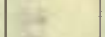 & 24 & 65.2 & 67.2 & 49 & 70 \\
\hline 26 & 48.9 & 42.0 & 27 & is & 26 & 53.4 & 49.7 & 38 & $\alpha$ & 26 & 55.1 & 56.8 & 33 & 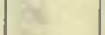 & 26 & 65.0 & & 49 & es \\
\hline 28 & 57.6 & 50.3 & I4 & 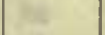 & 28 & 58.5 & 55.4 & 30 & $y=$ & 28 & 54.0 & 55.8 & 32 & 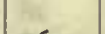 & 28 & 64.2 & 66 & 48 & 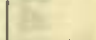 \\
\hline 30 & 62.4 & 55.8 & & -21.0 & 30 & 61.8 & 57.6 & 26 & -20.7 & 30 & 50.3 & 52.0 & 26 & -26.4 & 30 & 62.3 & 64.6 & 45 & $-24 \cdot 7$ \\
\hline 32 & 67.8 & $60 \cdot 3$ & 2258 & $a^{2}$ & 32 & 63.9 & 62.5 & 20 & 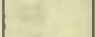 & 32 & 52.0 & 53.0 & 28 & 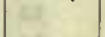 & 32 & 61.1 & 63.1 & 43 & 25 \\
\hline 34 & 77.8 & 66.9 & 45 & 1 & 34 & 61.7 & 59.2 & 24 & $x^{5}$ & 34 & 53.2 & 54.1 & 30 & 216 & 34 & 62.0 & 63.8 & 44 & $x$ \\
\hline 36 & 76.3 & 70.2 & 43 & As & 36 & 58.4 & 56.0 & 29 & & 36 & 52.9 & & 29 & $\theta$ & 36 & 63.5 & 65.0 & 46 & $x^{2}$ \\
\hline $3^{8 *}$ & $53 . \mathrm{I}$ & 47.2 & 40 & Xe & 38 & 55.3 & 53.8 & 34 & 14. & 38 & 50.7 & 52.7 & 26 & a & 38 & 66.1 & 67.7 & 50 & 18. \\
\hline 40 & 51.9 & 46.8 & 42 & 87 & 40 & 53.8 & 52.2 & 36 & 68 & 40 & 50.0 & & 25 & 10 & 40 & 66.0 & & 49 & n) \\
\hline 42 & 53.2 & 48.9 & 39 & 4 & 42 & 52.5 & $49 \cdot 3$ & 39 & $1=$ & 42 & 52.0 & 52 & 28 & & 42 & 63.8 & 64.8 & 46 & 24 \\
\hline 44 & 52.6 & 48.0 & 40 & -20.7 & 44 & $57 . \mathrm{I}$ & 53.0 & 33 & -20.7 & 44 & $53 \cdot 3$ & 54.8 & 30 & -26.0 & 44 & 65.0 & 65.9 & 48 & -24.7 \\
\hline 46 & 53.9 & 48.9 & 38 & $1+5=$ & 46 & 57.8 & 54.3 & $3 \mathrm{I}$ & 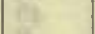 & 46 & 54.2 & 56.9 & 32 & $1+2$ & 46 & 63.8 & 64.8 & 47 & \\
\hline 48 & 55.3 & 51.4 & 35 & 5 & 48 & 54.2 & & 36 & 8 & 48 & 52.2 & 55. & 30 & -2 & 48 & 64.0 & 64.6 & 46 & 1 \\
\hline 50 & $55 \cdot 3$ & 51.8 & 35 & act & 50 & 50.8 & 48.3 & 42 & E & 50 & 51.3 & & 28 & 62 & 50 & 63.2 & 64.0 & 45 & 100 \\
\hline 52 & 54.0 & 50.9 & 37 & 18 & 52 & 51.2 & 48.8 & $4 I$ & 63 & 52 & 52.9 & 55 & 30 & & 52 & 64.6 & & 47 & 80 \\
\hline & 56.1 & 53.2 & 33 & 48 & 54 & 51.9 & 49.2 & 40 & 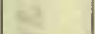 & 54 & 51.9 & & 29 & $z^{2}$ & 54 & 64.9 & 65.1 & 47 & 12 \\
\hline 56 & 56.2 & $53 \cdot 7$ & 33 & as & 56 & 52.0 & 49.4 & 40 & & 56 & 52.5 & & 30 & & 56 & 65.1 & 65.3 & 48 & \\
\hline 58 & $54 . I$ & 51.5 & 36 & $\mathbb{R}$ & 58 & 52.9 & 50.8 & 38 & $1=2$ & 58 & 55.6 & 58.0 & 35 & 8 & & 61.9 & 62.3 & 43 & 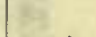 \\
\hline & & & & & 1200 & 52.1 & 49.4 & 40 & -20.6 & & & & & & I6 00 & 69.9 & 70.1 & 55 & $-24 \cdot 7$ \\
\hline
\end{tabular}

Correction to local mean time is - 40 s.

Torsion head at 7 li $00 \mathrm{~m}$ read $96^{\circ}$ and at the end read the same. Observer-R. R. T.
Correction to local mean time is - 49 s.

Torsion head at $1 \mathrm{Ih} 15 \mathrm{~m}$ read $87^{\circ}$ and at the end read the same. Observer-W. J. P. 
Tabulation of magnetic declinations observed at Teplitz Bay-Continued

\begin{tabular}{|c|c|c|c|c|c|c|c|c|c|c|c|c|c|c|c|c|c|c|c|}
\hline \multicolumn{6}{|c|}{ Wednesday, January I3, I904 } & \multicolumn{4}{|c|}{ Magnet scale inverted } & \multicolumn{5}{|c|}{ Wednesday, January I3, I904 } & \multicolumn{5}{|c|}{ Magnet scale inverted } \\
\hline \multirow[t]{2}{*}{$\begin{array}{l}\text { Chr'r } \\
\text { time }\end{array}$} & \multicolumn{2}{|c|}{$\begin{array}{l}\text { Scale } \\
\text { readings }\end{array}$} & \multirow[t]{2}{*}{$\begin{array}{c}\text { East } \\
\text { decli- } \\
\text { nation }\end{array}$} & Temp. & $\begin{array}{l}\text { Chr'r } \\
\text { time }\end{array}$ & $\begin{array}{l}\text { Sca } \\
\text { readi }\end{array}$ & $\begin{array}{l}\text { ale } \\
\text { ings }\end{array}$ & $\begin{array}{l}\text { East } \\
\text { decli- } \\
\text { nation }\end{array}$ & $\begin{array}{c}\text { Temp. } \\
\text { C. }\end{array}$ & $\begin{array}{l}\text { Chr'r } \\
\text { time }\end{array}$ & $\begin{array}{l}\text { Sca } \\
\text { read }\end{array}$ & $\begin{array}{l}\text { ale } \\
\text { ings }\end{array}$ & $\begin{array}{c}\text { East } \\
\text { decli- } \\
\text { nation }\end{array}$ & Temp. & Chr't & $\begin{array}{r}\text { Sca } \\
\text { readi }\end{array}$ & $\begin{array}{l}\text { ale } \\
\text { lings }\end{array}$ & $\begin{array}{l}\text { East } \\
\text { decli- } \\
\text { nation }\end{array}$ & Temp. \\
\hline & Left & Right & & & & L,eft & Right & & & & Left & Right & & & & Left & Right & & \\
\hline h m & $d$ & d & $\circ$, & & $\mathrm{h} \mathrm{m}$ & d & d & & $\circ$ & $\mathrm{h} \mathrm{m}$ & d & d & $\circ$, & & $\mathrm{h} \mathrm{m}$ & d & d & $\circ \quad$ & • \\
\hline 0 & & & & 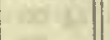 & 200 & 22.0 & 21.2 & & -28.5 & 400 & 54.0 & 53.6 & 2303 & -27.9 & 600 & 57.6 & 57.0 & 2258 & -27.8 \\
\hline 02 & & 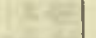 & 5 & $7=$ & 02 & 20.3 & 18.3 & 2303 & & 02 & $55 \cdot 3$ & 55.0 & or & & 02 & 56.8 & 55.6 & 2300 & \\
\hline $04 * 5$ & 26.0 & $25 \cdot 3$ & $2253-$ & -29.2 & 04 & I8. 5 & 17.8 & 04 & $b^{2}$ & 04 & 50.9 & 49.0 & Io & (t) & 0.4 & 50. & $o b$ & 09 & \\
\hline 06.2 & 26.0 & 23.8 & 2254 & & 06 & 10.2 & 9.0 & & we & 06 & 46.2 & 44.2 & I7 & $x$ & 06 & 45.7 & $44 \cdot 3$ & 17 & \\
\hline 08 & 20.3 & 20.1 & 23 oI & $x^{2}$ & 08 & 14.2 & 13.6 & II & $3 x$ & 08 & 47.6 & 45.2 & I5 & 2 & 08 & 47.0 & 46.8 & I4 & $w$ \\
\hline 10 & 27.3 & 22.5 & 2254 & 20 & 10 & 17.6 & 16.6 & 06 & 61 & I0 & $4 \mathrm{I} .6$ & 39.7 & 24 & wit & IO & $45 \cdot 3$ & 41.0 & 20 & \\
\hline 12 & 30.8 & 28.6 & 46 & 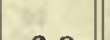 & 12 & 8.6 & 6.4 & $2 I$ & 4 & 12 & 46.0 & 45.0 & 16 & & 12 & 45.3 & 41.0 & 20 & \\
\hline I4 & 28.3 & 26.0 & 2250 & -28.8 & $14^{*}$ & 21.6 & I9.8 & I4 & -28.4 & I4 & 48.6 & 47.2 & I3 & 27.8 & 14 & $44 \cdot 3$ & 40.6 & $2 \mathrm{I}$ & -27.8 \\
\hline I6 & 20.5 & $\mathrm{I} 8.6$ & 2302 & & 16 & $24 \cdot 3$ & 23.6 & 09 & & 16 & 49.5 & 48.0 & 12 & & 16 & 43.0 & 39.4 & 23 & \\
\hline I8 & I 4.0 & 12.0 & 13 & atit & 18 & 28.0 & 26.5 & 2303 & 81 & 18 & 48.4 & 47.0 & 13 & 4 & 18 & 51.6 & 44.0 & I3 & \\
\hline 20 & 14.0 & 12.7 & 23 I2 & 4 & 20 & 34 & $\alpha a$ & 2253 & ne & 20.2 & 45.0 & 44.5 & 18 & 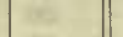 & 20 & 50.2 & 47.8 & II & \\
\hline 22 & 32.0 & 31.2 & 2243 & 62 & 22 & 36.0 & 35.6 & 50 & 178 & 22 & 43.3 & 42.3 & $2 I$ & $=$ & 22 & 45.6 & 39.3 & 2 I & $=$ \\
\hline 24 & 32.6 & $31 \cdot 3$ & 43 & E & 24 & 37.8 & 37.0 & 47 & 45 & 24 & 42.0 & 41.0 & 23 & Es & 24 & 45.0 & 37.5 & 23 & \\
\hline 26 & 38.2 & 38.0 & 33 & cas & 26 & 40.0 & 39.3 & 44 & ne & 26 & 43.5 & $4 I .9$ & 2 I & $E$ & 26 & 45.7 & 43.6 & 18 & \\
\hline 28 & 42.0 & 38.6 & 30 & 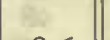 & 28 & 38.2 & 37.6 & 47 & & 28 & 47.3 & 46.3 & 14 & E & 28 & 51.0 & 50.4 & 08 & \\
\hline 30 & 31.6 & 30.3 & 45 & -28.6 & 30 & 38.5 & 38.0 & 46 & -28.3 & 30 & 49.0 & 48.2 & 12 & -27.9 & 30 & 52.3 & 49.4 & 08 & -27.7 \\
\hline 32 & 35.5 & 35.0 & 38 & $1=0$ & 32 & 37.5 & 37.3 & 47 & $-2=$ & 32 & 47.7 & 47.0 & I4 & -2 & 32 & 56.6 & 52.2 & 2302 & \\
\hline 34 & $44 \cdot 3$ & 42.3 & 25 & 46 & 34 & 38.3 & 38.1 & 46 & 42 & 34 & 47.8 & 47.0 & I4 & t5 & 34 & 61.6 & 59.5 & 2253 & \\
\hline 36 & 47.6 & 46.3 & I9 & 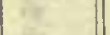 & 36 & 37.2 & 36.4 & 48 & 25 & 36 & 46.6 & 46.0 & I5 & ntes & 36 & 68.7 & 66.5 & 42 & \\
\hline 38 & 44.0 & 43.5 & 24 & $E$ & 38 & 38.3 & 37.6 & 47 & If & 38 & 42.6 & 42.3 & $2 I$ & 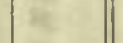 & 38 & 72.0 & 69.3 & 37 & ale \\
\hline 40 & 35.0 & 34.0 & 39 & 95 & 40 & 36.6 & 36.3 & 49 & 6 & 40 & 45.4 & 45.0 & I7 & 0 & 40 & 70.3 & 68.8 & 39 & \\
\hline 42 & 37.7 & 36.6 & 35 & - & 42 & 36.3 & 35.3 & 50 & & 42 & 47.3 & 46.5 & I4 & an & 42 & 62.0 & 60.0 & 52 & \\
\hline 44 & $3 \mathrm{I} \cdot 3$ & 29.5 & $46-$ & -28.7 & 44. & 32.4 & 32.2 & 2255 & -28.2 & 44 & 48.9 & 48.3 & 12 & -27.9 & 44 & 60.3 & 58.6 & 55 & -27.6 \\
\hline 46 & 35.9 & $34 \cdot 3$ & 38 & & $46^{*}$ & 27.0 & 24.0 & 2348 & (n) & 46 & 52.3 & $5 \mathrm{I} .3$ & 07 & & 46 & 63.0 & 61.0 & 51 & \\
\hline 48 & 35.5 & 34.2 & 38 & $=$ & 48 & 42.0 & 39.0 & 24 & 15 & 48 & 55.6 & 55.1 & 23 oI & 20 & 48 & 60.5 & 59.0 & 54 & \\
\hline 50 & 33.0 & 32.6 & 2242 & 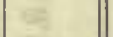 & 50 & 43.6 & 38.0 & 24 & 8 & 50 & 58.6 & 58.3 & 2256 & $\%$ & 50 & 60.6 & 59.2 & 54 & \\
\hline 52 & 20.3 & 19.5 & 2302 & 5 & 52 & 49.0 & 43.0 & 16 & 48 & 52 & 57.2 & 56.0 & 2259 & 43 & 52.2 & 59.9 & 58.4 & 55 & \\
\hline 54 & 21.0 & 20.3 & oI & 22 & 54 & 39.8 & 35.0 & 29 & 13 & 54 & 53.8 & 51.8 & 2305 & 2 & $54 \cdot 3$ & 60.0 & 58.5 & 55 & \\
\hline 56 & I4. & $5^{b}$ & 10 & & 56 & 48.0 & $43 \cdot 3$ & 16 & & 56 & 54.2 & 52.6 & 04 & & 56 & 60.9 & 59.5 & 53 & \\
\hline 58 & II. 5 & II.O & 15 & & 58 & 48.2 & 44.0 & I6 & & 58 & 51.7 & 51.3 & 07 & & 58 & 63.0 & 62.3 & 50 & \\
\hline I 00 & 8.6 & 7.5 & 20 & -28.8 & 300 & 46.8 & 43.3 & 17 & -28.2 & 500 & 53.2 & 51.6 & 06 & -27.8 & 700 & 61.0 & 59.6 & 53 & -27.6 \\
\hline 02 & I5.I & 13.5 & $23 \mathrm{II}$ & tat & 02 & 46.0 & 45.0 & I6 & wit & 02 & 54.1 & 53.0 & 2304 & (2) & 02 & 60.1 & 59.0 & 54 & \\
\hline 04 & 23.3 & 23.3 & 2256 & 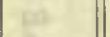 & 04 & 47.5 & 42.8 & I7 & 10 & 04 & 57.2 & 56.5 & 2259 & a & 04 & 60.3 & 58.9 & 54 & \\
\hline 06 & 30.6 & 30.3 & 2245 & (4) & 06 & 56.3 & $53 \cdot 3$ & 02 & in & 06 & 58.1 & 57.3 & 57 & is & 06 & 60.0 & 58.2 & 2255 & \\
\hline 08 & 19.8 & I8. 8 & 2303 & 30 & 08 & 54.3 & $5 \mathrm{I} .7$ & 05 & 60 & 08 & 57.8 & 57.5 & 57 & e & 08 & $57 \cdot 3$ & 55.3 & 2300 & $=$ \\
\hline Io & 26.8 & 24.6 & 2253 & Qi & IO & 49.6 & 48.6 & II & 41 & 10 & 60.0 & 59.7 & 54 & $0 t$ & 10 & 56.7 & 54.6 & 23 OI & 41 \\
\hline 12 & $25 \cdot 3$ & 25.0 & 54 & & I2 & 45.6 & 41.8 & I9 & 44 & 12 & 58. & $.8 b$ & 2256. & 5 & I2 & 58.2 & 56.5 & $22 \quad 58$ & \\
\hline 14 & 26.0 & 24.7 & 53 & -28.8 & 14 & 53.3 & 51.3 & 06 & $x$ & I4 & 57.8 & 53.2 & 23 oI & 145 & I4 & 60.7 & 59.0 & 2254 & $-27 \cdot 5$ \\
\hline 16 & 28.0 & 26.8 & 50 & at & I6 & 56.7 & $54 \cdot 3$ & OI & Bi & 16 & 55.0 & 55.0 & 02 & -27.8 & 16 & $55 \cdot 5$ & 47.8 & 2307 & \\
\hline 18 & 30.2 & 29.2 & 46 & bet & I8 & 51.5 & 50.0 & 08 & $x$ & 18 & 56.3 & 55.6 & 2300 & a & I8 & 53.4 & 51.9 & 05 & \\
\hline 20 & 31.2 & 30.0 & 45 & ets & 20 & 48.2 & 46.8 & I3 & es & 20 & 56.9 & 56.7 & 2259 & $\alpha$ & 20 & 51.6 & 50.8 & 08 & rat \\
\hline 22 & 33.5 & 33.0 & $4 \mathrm{I}$ & $\theta$ & 22 & 49.0 & $47 \cdot 3$ & 12 & Ex & 22 & 58.3 & 58.3 & 56 & se & 22 & 55.5 & 54.0 & 02 & vir \\
\hline 24 & $34 \cdot 4$ & 34.0 & 39 & if & 24 & 51.2 & 49.0 & 09 & 32 & 24 & 60.8 & 60.6 & 53 & is & 24 & 56.7 & 54.7 & 2300 & $F^{2}$ \\
\hline 26 & 36.0 & 35.8 & 37 & 8 & 26 & 57.0 & 55.0 & 00 & $3 \sqrt{1}$ & 26 & 62.5 & 62.3 & 50 & 4 & 26 & 59.5 & 58.0 & 2256 & \\
\hline 28 & 34.6 & $34 \cdot 3$ & 39 & & 28 & 56.0 & 55.2 & 23 oI & & 28 & 63.4 & 63.3 & 48 & & 28 & 59.6 & 59.3 & 55 & \\
\hline 30 & 32.3 & 32.1 & $4^{2}$ & -28.8 & 30 & 59.1 & 58.3 & 2256 & -27.9 & 30 & 63.4 & 63.2 & 48 & -27.7 & 30 & 59.0 & 57.4 & 2256 & -27.7 \\
\hline 32 & 35 & $.4 b$ & 37 & 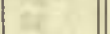 & 32 & 58.0 & 57.3 & 2257 & 0 & 32 & $64 \cdot 3$ & $64 \cdot 3$ & 47 & $0^{2}$ & 32 & 5 I. 4 & 49.0 & 2309 & \\
\hline 34 & 26.6 & 26.3 & 52 & He & 34 & $5 I .3$ & 50.0 & 2308 & int & 34 & 62.9 & 62.4 & 50 & ic & 34 & 48.0 & $45 \cdot 3$ & 15 & \\
\hline 36 & 27.3 & 27.0 & $5 I$ & 15 & 36 & 48.3 & 47.5 & 13 & ta & 36 & 59.5 & 58.9 & 2255 & $x_{2}$ & 36 & 49.0 & 45.2 & I4 & \\
\hline 38 & 24.8 & 23.6 & 55 & 8 & 38 & 47.3 & 46.0 & I5 & nats & 38 & 56.6 & 56.0 & 2300 & 8 & 38 & 52.0 & 48.0 & 09 & \\
\hline 40 & $27 \cdot 3$ & 26.3 & $5 \mathrm{I}$ & E3 & 40 & 54.0 & 52.3 & 0.4 & ofter & 40 & 56.3 & 55.7 & 2300 & Ex & 40 & 51.6 & 46.3 & II & \\
\hline 42 & 30.7 & 29.3 & 46 & & 42 & 54.5 & 53.3 & 03 & & $4^{2}$ & 59. & $.0 a$ & 2255 & & 42 & 58.9 & 52.4 & or & \\
\hline 44 & 32.0 & 30.4 & 44 & -28.5 & 44 & 50.3 & 49.7 & 09 & -27.9 & 44 & 64 & $.0 a$ & 47 & -27.7 & 44 & 56.0 & 49.3 & 05 & -27.8 \\
\hline 46 & 31.0 & 29.5 & 46 & ( & 46 & 50.3 & 49.9 & 09 & ( & 46 & 69.5 & 69.5 & 39 & ( & 46 & $6 \mathrm{I} .3$ & $5 \mathrm{I} .0$ & 2300 & \\
\hline 48 & 29.3 & 26.8 & 49 & 67 & 48 & 50.3 & 49.6 & IO & E. & 48 & 70.5 & 70.3 & 37 & $8 x$ & 48 & 64.4 & $55 \cdot 3$ & 2254 & \\
\hline 50 & 24.7 & 23.5 & 55 & $\theta$ & 50 & 51.6 & 50.0 & 08 & wat & 50 & 68.6 & 68.0 & 4I & Fe & 50 & 59.2 & 56.5 & 57 & \\
\hline 52 & 23.2 & 21.7 & 58 & 랄 & 52 & 56.6 & 53.8 & 23 OI & 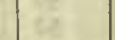 & 52 & 66.0 & 65.2 & 45 & 12 & 52 & 59.5 & 58.0 & 56 & \\
\hline 54 & 25.0 & 23.4 & 55 & 32 & 54 & 59.9 & 57.5 & 2256 & 13. & 54 & 59.6 & 59.0 & 2255 & 42 & 54 & 59.0 & 56.6 & 57 & \\
\hline 56 & 23.8 & $2 \mathrm{I} .6$ & 57 & $\sqrt{7}$ & 56 & 54.0 & 50.3 & 2306 & & 56 & 56.0 & 56.0 & 2300 & & 56 & 6I.0 & 58.6 & 54 & \\
\hline 58 & 26.0 & $24 \cdot 3$ & 54 & ㅊ. & 58 & $54 \cdot 5$ & 51.5 & 05 & get & 58 & 63.3 & 56.6 & 2254 & 72 & 58 & 59.6 & 58.4 & 55 & \\
\hline
\end{tabular}


Tabulation of magnetic declinations observed at Teplitz Bay-Continued

\begin{tabular}{|c|c|c|c|c|c|c|c|c|c|c|c|c|c|c|c|c|c|c|c|}
\hline \multicolumn{5}{|c|}{ Wednesday, January I3, I904 } & \multicolumn{5}{|c|}{ Magnet scale inverted } & \multicolumn{10}{|c|}{ Wednesday, January 13, 1904} \\
\hline \multirow{2}{*}{$\begin{array}{l}\text { Chr's } \\
\text { time }\end{array}$} & \multicolumn{2}{|c|}{$\begin{array}{l}\text { Scale } \\
\text { readings }\end{array}$} & \multirow{2}{*}{$\begin{array}{c}\text { East } \\
\text { decli- } \\
\text { nation }\end{array}$} & \multirow{2}{*}{$\begin{array}{c}\text { Temp. } \\
\text { C. }\end{array}$} & Chr'r & $\begin{array}{r}\mathrm{Sc} \\
\text { read }\end{array}$ & $\begin{array}{l}\text { ale } \\
\text { lings }\end{array}$ & $\begin{array}{l}\text { East } \\
\text { decli- }\end{array}$ & Temp. & Chr'r & $\begin{array}{l}\mathrm{Sc} \\
\text { reac }\end{array}$ & $\begin{array}{l}\text { le } \\
\text { ngs }\end{array}$ & $\begin{array}{l}\text { East } \\
\text { decli- }\end{array}$ & Temp. & & $\begin{array}{r}\mathrm{Sc} \\
\text { reac }\end{array}$ & $\begin{array}{l}\text { ale } \\
\text { ings }\end{array}$ & $\begin{array}{l}\text { East } \\
\text { decli- }\end{array}$ & Temp. \\
\hline & Left & Right & & & & Left & Right & & & & Left & Right & & & & Left & Right & & \\
\hline $\mathrm{h} \mathrm{m}$ & d & & $\cdot$, & $0^{\circ}$ & $\mathrm{h} \mathrm{m}$ & d & & $\circ$, & & $\mathrm{h} \mathrm{m}$ & d & d & $\circ \quad$ & $\stackrel{\circ}{\circ}$ & h $\mathrm{m}$ & d & d & - ' & \\
\hline 800 & 59.9 & 58.0 & 2255 & -26.2 & 1000 & 73.0 & 69.8 & & -26.1 & 1200 & 58.0 & 56.0 & 2225 & -25.8 & 1400 & 55 & $o b$ & 2228 & -25.0 \\
\hline 02 & 62.0 & 60.0 & 52 & & 02 & 75.2 & 73.8 & $3 I$ & & 02 & 58.0 & 57.1 & 24 & & 02 & 53.5 & 52.6 & $3 I$ & 0 \\
\hline 04 & 59.0 & 57.7 & 2256 & (18) & 04 & 76.6 & 74.0 & 30 & 144 & 04 & 56.5 & 56.5 & 26 & 40 & 04 & 53.8 & 53.0 & 30 & 10 \\
\hline 06 & 57.0 & 53.8 & 23 OI & & $06^{*}$ & 57.8 & 56.5 & 24 & 36 & 06 & 55.5 & $55 \cdot 5$ & 27 & 8 & 06 & 56.0 & 55.0 & 27 & 39 \\
\hline 08 & 55.0 & 50.3 & 05 & 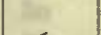 & 08.5 & 66.5 & 58.0 & 17 & 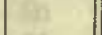 & 08 & $53 \cdot 3$ & 52.3 & $3 I$ & 60 & 08 & 57.0 & 57.0 & 25 & 76 \\
\hline IO & 56.5 & 5 I. 4 & 03 & -26.2 & Io & 56.8 & 55.0 & 26 & 85 & IO & 54.0 & 53.0 & 30 & at & I0 & 55.5 & 55.5 & 27 & 08 \\
\hline I2 & 48.2 & 42.5 & 17 & 和 & 12 & 57.6 & 55.6 & 25 & & 12 & 58.2 & 57.7 & 23 & in & 12 & 54.5 & 54.1 & 29 & 105 \\
\hline 14 & 48.8 & 40.0 & 18 & 34 & I4 & 58.4 & 57.2 & 23 & -26.0 & 14 & 57.3 & 55.9 & 25 & -25.7 & I4 & 55.0 & 55.0 & 28 & -25.0 \\
\hline 16 & 52.5 & 43.8 & I2 & 5 & 16 & 54.5 & 53.8 & 29 & & 16 & 53.0 & 5I.I & 33 & & 16 & 55.1 & 54.0 & 29 & 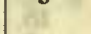 \\
\hline I8 & 48.3 & 34.8 & 23 & Ei & I8 & 57.7 & 54.7 & 26 & 36 & 18 & 53.0 & 52.0 & 32 & War & I8 & 55. I & 54.9 & 28 & $8 r$ \\
\hline 20 & 54.0 & 44.6 & IO & a & 20 & 59.0 & 56.0 & 24 & 68 & 20 & 52.0 & 5I. I & 33 & at & 20 & 58.0 & 56.8 & 24 & 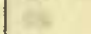 \\
\hline 22 & 52.9 & 42.7 & 13 & $=$ & 22 & 60.0 & $57 . I$ & 22 & 5 & 22 & 54.6 & 53.0 & 30 & ist & 22 & 57.2 & 55.0 & 26 & $\cos$ \\
\hline 24 & 53.6 & 34.2 & 19 & 15: & 24 & 56.0 & 55.0 & 27 & $1=$ & 24 & 58.5 & 51.2 & 28 & 14 & 24 & 58.2 & 57.0 & 24 & 15 \\
\hline 26 & 57.8 & 39.0 & 12 & $x^{2}$ & 26 & 60.0 & 56.0 & 23 & it & 26 & 52.2 & 49.9 & 34 & (e) & 26 & 60.0 & 57.9 & 22 & 36 \\
\hline 28 & 55.0 & 40.5 & I3 & -26.2 & 28 & 59.7 & 54.9 & 24 & $a$ & 28 & 50.9 & 50.0 & 35 & - & 28 & $6 \mathrm{r} .0$ & 58.2 & $2 \mathrm{I}$ & $f=$ \\
\hline 30 & 60.0 & 47.0 & 04 & - & 30 & 56.2 & 50.0 & $3 I$ & -25.8 & 30 & 50.0 & $49 . I$ & 36 & -25.6 & 30 & 60.2 & 57.5 & 22 & -25.0 \\
\hline 32 & 55.0 & 42.5 & I2 & ect & 32 & 53.1 & 48.5 & 34 & & 32 & 50.3 & 50.0 & 36 & ( & 32 & 60.2 & 57.9 & 22 & \\
\hline 34 & 55.0 & 38.6 & I4 & 45 & 34 & $5 \mathrm{I} \cdot 5$ & $48 . I$ & 36 & HE & 34 & 53. I & 53.0 & $3 I$ & 24 & 34 & 58.0 & 55.8 & 25 & $x$ \\
\hline 36 & 53.0 & 34.0 & 20 & 58 & 36 & 48.4 & 45.5 & $4 \mathrm{I}$ & 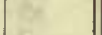 & 36 & 53.2 & 52.5 & 3I & $2 x$ & 36 & 57.3 & 55.0 & 26 & If \\
\hline 38 & $57 \cdot 3$ & 32.8 & $23 \mathrm{I} 7$ & et & 38 & 51.8 & 49.3 & 35 & $8=$ & $3^{8}$ & 55.0 & 55.0 & 28 & ne & $3^{8}$ & 59.8 & 57.5 & 22 & le. \\
\hline 40 & 70.5 & 49.4 & 2254 & $\exists$ & 40 & $5 \mathrm{I} .1$ & 48.6 & 36 & 20 & 40 & 55.8 & 54.9 & 27 & 0 & 40 & $5^{8} .3$ & 56.2 & 24 & 60 \\
\hline 42 & 51.6 & 35.0 & 2320 & 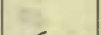 & 42 & 54.5 & 46.1 & 35 & Fit & 42 & 54.2 & 54.0 & 29 & $x^{2}$ & 42 & 58.8 & 56.5 & 24 & 5 \\
\hline 44 & 67.8 & 48.8 & 2256 & -26.5 & 44 & 49.0 & 44.7 & $4 \mathrm{I}$ & & 44 & 56.2 & 55.0 & 27 & -25.5 & 44 & 57.0 & 54.2 & 27 & ats \\
\hline 46 & 69.8 & 44.4 & 58 & 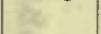 & 46 & 46.0 & 42.8 & 44 & -25.8 & 46 & 55.7 & 54.0 & 28 & & 46 & 55.1 & 52.8 & 30 & \\
\hline 48 & 66.4 & 48.9 & 57 & 8 & 48 & 47.0 & 44.0 & 43 & & $4^{8}$ & 55.3 & 54.8 & 28 & 82 & 48 & 56.3 & 54.0 & 28 & -24.8 \\
\hline 50 & 66.5 & 46.5 & 59 & as & 50 & 53.0 & 50.2 & 33 & ne & 50 & $54 . \mathrm{I}$ & 53.1 & 30 & $=$ & 50 & 55.3 & 53.1 & 29 & $0^{\circ}$ \\
\hline 52 & 72.4 & 50.5 & 52 & ci & 52 & 54.0 & $5 \mathrm{I} .4$ & $3 \mathrm{I}$ & $E$ & 52 & 54.7 & 53.9 & 29 & 6 & 52 & 54.7 & 53.0 & 30 & 12. \\
\hline 54 & 67.0 & 49.0 & 57 & 12 & 54 & 54.5 & 51.8 & $3 \mathrm{I}$ & 5 & 54 & $54 \cdot 5$ & 53.5 & 29 & 12 & 54 & 53.8 & 53.0 & 30 & 32 \\
\hline 56 & 73.0 & 55.8 & 47 & 88 & 56 & 53.8 & 51.2 & 32 & in & 56 & 55.3 & 55.0 & 28 & 82 & 56 & 54.5 & 52.8 & 30 & 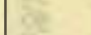 \\
\hline 58 & 73.2 & 58.2 & 45 & $2 y^{2}$ & 58 & 54.6 & 51.0 & $3 \mathrm{I}$ & & 58 & 56.0 & 54.5 & 28 & Ex & 58 & 55.7 & 54.8 & 28 & $x_{2}$ \\
\hline 900 & 72.3 & 60.0 & 44 & -26.8 & II 00 & 57.8 & 55.0 & 25 & -25.9 & 1300 & 56.0 & 53.9 & 28 & wer & 1500 & 56.3 & 55.6 & 26 & -24.8 \\
\hline 02 & 73.2 & 60.7 & 43 & 90 & 02 & 55.6 & 52.4 & 29 & - & 02 & 57.2 & 54.9 & 26 & why & 02 & 55.0 & 54.3 & 28 & $\frac{7}{2}$ \\
\hline 04 & 64.1 & 54.5 & 55 & 30 & 04 & 53.8 & 50.8 & 32 & 8 & 04 & 55.5 & 53.2 & 29 & -25.2 & 04 & 56 & $2 a$ & 26 & is \\
\hline 06 & 72.8 & 62.8 & $4 \mathrm{I}$ & $\sin$ & 06 & 53.0 & 50.9 & 33 & $m$ & 06 & 55.0 & 53.5 & 29 & $x^{2}$ & 06 & 59.0 & 59.0 & 22 & $\omega_{0}$ \\
\hline 08 & 72.2 & 60.5 & 44 & Bo & 08 & 52.0 & 50.0 & 34 & 80 & 08 & 55.2 & 53.0 & 29 & Bs & 08 & 58.0 & 57.2 & 24 & 82 \\
\hline IO & 69.0 & 58.4 & 48 & Ca & I0 & 51.7 & 48.9 & 35 & nis & IO & $54 . \mathrm{I}$ & $5 \mathrm{I} .5$ & 31 & er & I0 & 56.8 & 56.0 & 26 & mit \\
\hline 12 & 66.5 & 54.5 & 53 & & 12 & $5 \mathrm{I} .2$ & 48.2 & 36 & & I2 & 54.9 & 52.7 & 30 & $=1$ & 12 & 58.3 & 57.2 & 24 & it \\
\hline 14 & 74.5 & 64.5 & 39 & -26.7 & I4 & 51.0 & 48.0 & 36 & -26.0 & 14 & 56.0 & 53.5 & 28 & -25.1 & I 4 & 56.4 & 55.1 & 27 & -24.7 \\
\hline 16 & 74.8 & $66 . \bar{I}$ & 37 & [ & 16 & 52.9 & 49.8 & 34 & rat & I6 & 54.8 & 51.0 & $3 I$ & 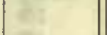 & 16 & 51.7 & 50.4 & 34 & 1. \\
\hline 18 & 73.4 & 66.8 & 38 & Eir & 18 & 53.2 & 50.0 & 33 & $\mathrm{E}_{1}$ & I8 & 54.9 & 51.0 & $3 \mathrm{I}$ & 21 & 18 & 52.0 & 50.9 & 34 & (4) \\
\hline 20 & 68.9 & 62.8 & 45 & 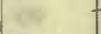 & 20 & 54.2 & 50.7 & 32 & es & 20 & 56.0 & 52.0 & 29 & as & 20 & 52.3 & 51.8 & 33 & ot \\
\hline 22 & 69.5 & 62.2 & 45 & $E$ & 22 & 53.3 & 50.0 & 33 & 14 & 22 & 55.4 & 51.8 & 30 & 6 & 22 & 52.1 & 51.3 & 33 & 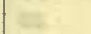 \\
\hline 24 & 72.0 & 67.2 & 39 & it & 24 & 55.0 & 51.8 & 30 & 15 & 24 & & 52.0 & 29 & is & 24 & 53.2 & 52.8 & $3 \mathrm{I}$ & at: \\
\hline 26 & 68.6 & $65 . I$ & 43 & a & 26 & 60.0 & 57.2 & 22 & 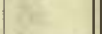 & 26 & 56.5 & 52.1 & 29 & 6 & 26 & 53.8 & 52.8 & 30 & 5 \\
\hline 28 & $6 \mathrm{I} .2$ & 57.5 & 55 & 8 & 28 & 56.8 & 55. & 26 & & 28 & 56.2 & 53.2 & 28 & $\theta$ & 28 & 55.9 & 54.8 & 27 & ( \\
\hline 30 & 68.7 & 65.0 & 43 & 00 & 30 & 58.7 & 57.8 & 23 & -26.0 & 30 & 56.2 & 53.2 & 28 & $-25 . x$ & 30 & 59.8 & 57.8 & 22 & -24.8 \\
\hline 32 & 62.2 & 60.4 & 52 & 22 & 32 & 59.0 & 57.8 & 22 & th & 32 & & 52.9 & 30 & in & 32 & 60.3 & 59.6 & 20 & r \\
\hline 34 & 65.7 & 63.0 & 47 & & 34 & 60.2 & 58.4 & $2 \mathrm{I}$ & 45 & 34 & 54.9 & 53.8 & 29 & II & 34 & 58.7 & 56.6 & 24 & 42 \\
\hline 36 & 73.0 & 70.9 & 35 & -26.2 & 36 & 54.4 & 53.2 & 30 & 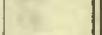 & 36 & 55.0 & 54.0 & 29 & $A^{2}$ & 36 & 56.4 & 54.2 & & $\mathrm{CS}^{2}$ \\
\hline 38 & 71.0 & 70.0 & 37 & $x_{1}$ & 38 & 54.0 & 51.3 & 32 & wr & 38 & 53.6 & 53.0 & 30 & $\mathrm{ks}$ & 38 & 55.8 & 53.7 & 28 & $\mathrm{ki}^{2}$ \\
\hline 40 & 68.1 & 67.0 & 42 & (a) & 40 & 49.2 & 47.2 & 38 & & 40 & 52 & $8 b$ & $3 I$ & bi & 40 & 56.2 & 54.9 & 27 & \\
\hline 42 & 65.5 & 65.0 & 46 & $6 x$ & 42 & 47.9 & 45.7 & $4 I$ & & 42 & 52.0 & 52.0 & 33 & 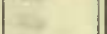 & 42 & 55.3 & 53.8 & 29 & 34 \\
\hline 44 & 64.6 & 62.0 & 48 & & 44 & & 46.7 & 39 & -26.0 & 44 & 52.0 & 51.0 & 33 & & & 52.8 & 50.0 & & -24.5 \\
\hline 46 & 61.8 & 61.0 & 52 & $-26 . I$ & 46 & 48.9 & 47.8 & 38 & (2) & 46 & 53.0 & 52.0 & 32 & 281 & 46 & 54.0 & 52.0 & 31 & ( \\
\hline 48 & 64.6 & 62.9 & 48 & 82 & 48 & 48.9 & 46.6 & 39 & 10 & 48 & 5 I. 3 & 50.3 & 34 & & 48 & 54.0 & 52.5 & $3 I$ & 8. \\
\hline 50 & 65.6 & 65.2 & 45 & $\theta$ & 50 & 49.0 & 47.6 & 38 & nat & 50 & $52 . \mathrm{I}$ & 51.1 & 33 & -25.1 & 50 & 53.9 & 51.9 & $3 \mathrm{~T}$ & $c=$ \\
\hline 52 & 66.7 & 63.8 & 46 & $\mathbf{L}$ & 52 & & 50.5 & 33 & 2 & 52 & 54.8 & 52.1 & 30 & 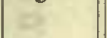 & 52 & 56.0 & 54.0 & 28 & E. \\
\hline 54 & 73.9 & 71.0 & 34 & 22 & 54 & 58.3 & 51.9 & 28 & $y^{2}$ & 54 & 56.0 & 54.4 & 28 & 32 & 54 & 56.2 & 55.2 & 27 & $x^{2}$ \\
\hline 56 & 69.0 & 64.5 & 43 & $a t$ & 56 & 55.0 & 52.5 & 30 & in & 56 & 54.0 & 51.5 & 32 & 68 & 56 & 54.8 & 54.3 & 29 & E \\
\hline 58 & 74.2 & 69.2 & 35 & & 58 & 55.3 & $54 . I$ & 28 & 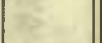 & 58 & 54.8 & 52.5 & & W & 58 & 53.8 & 53.0 & 30 & 3 \\
\hline & & & & $100 y=$ & & & & & & & & & & & & & & & \\
\hline
\end{tabular}


Tabulation of magnetic declinations observed at Teplitz Bay-Continued

\begin{tabular}{|c|c|c|c|c|c|c|c|c|c|c|c|c|c|c|c|c|c|c|c|}
\hline \multicolumn{5}{|c|}{ Wednesday, January I3, 1904} & \multicolumn{5}{|c|}{ Magnet scale inverted } & \multicolumn{5}{|c|}{ Wednesday, January 13, I904 } & \multicolumn{5}{|c|}{ Magnet scale inverted } \\
\hline \multirow{2}{*}{$\begin{array}{l}\text { Chr'r } \\
\text { time }\end{array}$} & \multicolumn{2}{|c|}{$\begin{array}{l}\text { Scale } \\
\text { readings }\end{array}$} & \multirow{2}{*}{$\begin{array}{l}\text { East } \\
\text { decli- } \\
\text { nation }\end{array}$} & Temp. & Chr'r & $\begin{array}{r}\text { Sc } \\
\text { read }\end{array}$ & $\begin{array}{l}\text { ale } \\
\text { ings }\end{array}$ & $\begin{array}{l}\text { East } \\
\text { decli }\end{array}$ & & Chr'r & $\begin{array}{r}\mathrm{Sc} \\
\text { read }\end{array}$ & $\begin{array}{l}\text { ale } \\
\text { ings }\end{array}$ & $\begin{array}{l}\text { East } \\
\text { decli- }\end{array}$ & & & $\begin{array}{r}\mathrm{Sc} \\
\mathrm{rcad}\end{array}$ & $\begin{array}{l}\text { le } \\
\text { ngs }\end{array}$ & $\begin{array}{l}\text { East } \\
\text { decli- }\end{array}$ & \\
\hline & Left & Right & & & & Left & Right & & & & Left & Right & 1 & & & Left & Right & & \\
\hline $\mathrm{h} \mathrm{m}$ & d & d & $\circ \quad$ & $\bullet$ & $\mathrm{h} \mathrm{m}$ & d & d & & 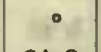 & $\mathrm{h} \mathrm{m}$ & $d$ & $\mathrm{~d}$ & $\cdot:$ & 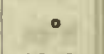 & $\mathrm{h} \mathrm{m}$ & d & (1) & & • \\
\hline 1600 & 52.9 & 52.0 & 2232 & -24.0 & 1800 & 55.3 & 51.0 & 223 & -24.0 & 2000 & $44 \cdot 3$ & 43.0 & 2246 & $-23 \cdot 3$ & 2200 & 49.4 & 48.2 & $223^{8}$ & $-23 \cdot 4$ \\
\hline 02 & 53.0 & 51.5 & 32 & $x^{2}$ & 02 & 54.1 & 51.8 & 31 & Str & 02 & 41.9 & $4 \mathrm{I} . \mathrm{I}$ & 49 & thent & 02 & 49.2 & 47.2 & & 60 \\
\hline 04 & 51.8 & 50. I & 34 & 18 & 04. & 52.9 & 50.8 & 33 & 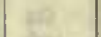 & 04 & 40.2 & 38.4 & 52 & 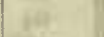 & 04 & 5 I. 3 & 49.2 & 35 & -0 \\
\hline 06 & 51.8 & 49.9 & 34 & ar & 06 & 52.2 & 50.4 & 3 & 60 & 06 & 38.0 & 37.2 & 55 & 208 & 06 & 52.2 & 49.4 & 34 & an \\
\hline 08 & 51.0 & 50.3 & 35 & net & 08 & 52.8 & 50.1 & 3 & $x$ & 08 & 38.9 & $37 \cdot 3$ & 54 & ingr & 08 & 52.9 & 50.6 & 33 & 4 \\
\hline 10 & 51.2 & 50.5 & 34 & oit & I0 & 53.0 & 50.1 & 33 & bif & 10 & 37.6 & 36.9 & 2256 & Wr: & 10 & 53.7 & 5I.I & 32 & int \\
\hline 12 & 52.9 & 50.5 & 33 & 78 & 12 & 52.8 & 50.7 & 6 & 44 & 12 & 34.0 & 33.0 & 2302 & 61 & 12 & 53.2 & 51.0 & 32 & 4 \\
\hline 14 & 54.9 & 53.0 & 30 & -23.8 & 14 & 51.9 & 50.3 & t. & -24.0 & 14 & 34.8 & 33.1 & or & $-23 \cdot 3$ & 14 & 53.2 & 50.9 & 33 & $-23 \cdot 4$ \\
\hline 16 & 54.8 & 52.0 & 30 & & I6 & 51.6 & 50.3 & 3 & $15=$ & 16 & 34.2 & 32.8 & 02 & 60 & 16 & 54.1 & 52.4 & $3^{I}$ & \\
\hline 18 & 53.5 & 51.0 & 32 & क) & 18 & 50.2 & 49.2 & 38 & 7 & 18 & 32.8 & 31.0 & 04 & 81. & 18 & 54.8 & 52.8 & 30 & bs \\
\hline 20 & 56.2 & 54.2 & 28 & $=$ & 20 & $49 . I$ & $48 . I$ & 38 & E & 20 & 35.2 & 32.7 & 23 oI & 6 & 20 & 54.0 & 52.8 & 30 & e. \\
\hline 22 & 56.7 & 54.3 & 27 & 6 & 22 & 49.3 & 48.2 & 38 & 14 & 22 & 37.2 & 35.5 & 2257 & RE & 22 & 52.7 & 50.2 & 34 & $\approx$ \\
\hline 24 & 55.2 & 53.3 & 29 & 12 & 24 & 50.1 & 48.9 & 37 & if & 24 & 39.1 & 37.1 & 54 & is & 24 & $46 . \mathrm{I}$ & 44.9 & 43 & it \\
\hline 26 & 55.3 & 53.8 & 29 & 20 & 26 & 51.0 & 49.7 & 3 & 2 & 26 & 42.0 & 40.0 & 50 & \& & 26 & 38.0 & 36.9 & & 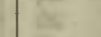 \\
\hline 28 & 56.0 & 53.7 & 28 & knt & 28 & 50.8 & 49.2 & 30 & E & 28 & 43.1 & 40.2 & 49 & $2 \pi$ & 28 & 42.6 & 40.7 & $22 \quad 49$ & \\
\hline 30 & 56.3 & 54.6 & 27 & -23.9 & 30 & 50.8 & 49.3 & 3 & tas & 30 & 44.4 & 41.8 & 46 & -23.6 & 30 & 32.8 & 28.9 & 2306 & -23.4 \\
\hline 32 & 56.3 & 54.6 & 27 & -2 & 32 & 50.7 & 49.7 & 3 & -24.0 & 32 & 45.8 & 42.8 & 45 & - & 32 & 37.9 & 35.1 & 2257 & 20.4 \\
\hline 34 & 56.8 & 54.7 & 27 & $3 E$ & 34 & 51.8 & 50.3 & 3 & $11^{\circ}$ & 34 & 46.2 & 42.9 & 44 & th. & 34 & 16.9 & 14.9 & 2329 & 16 \\
\hline 36 & 56.3 & 54.2 & 28 & 2 & 36 & 52.0 & 50.9 & 3 & 2 & 36 & 46.0 & 43.4 & 44 & (e) & 36 & $34 . I$ & $27 . I$ & 2306 & \\
\hline 38 & 55.7 & 53.8 & 28 & He & 38 & 52.5 & 51.2 & 3. & 34 & 38 & 47.0 & 44.0 & 43 & 2 & 38 & 38.2 & 32.5 & 2259 & 8 \\
\hline 40 & 54.9 & 53.4 & 29 & 6 & 40 & 53.0 & 52.0 & 3 & 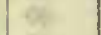 & 40 & 47.7 & 45.0 & 42 & 80 & 40 & 39.2 & 34.2 & 57 & 6 \\
\hline 42 & 56.7 & 54.2 & 27 & $7+$ & 42 & 53.5 & 52.9 & 3 & 62 & 42 & 47.2 & 44.8 & 42 & 6 & 42 & $44 \cdot 3$ & 39.2 & 49 & 94 \\
\hline 44 & 59.8 & 57.0 & 22 & -24.0 & 44 & $53 . \mathrm{I}$ & 52.3 & 3 & -24.0 & 44 & 47.6 & $45 . I$ & 42 & -23.8 & 44 & 45.4 & 41.7 & 46 & -23.6 \\
\hline 46 & 57.5 & 55.1 & 26 & & 46 & 53.1 & 52.0 & 3 & $y^{2}$ & 46 & 47.8 & 44.6 & 42 & - & 46 & 42.9 & 38.9 & 50 & \\
\hline 48 & 57.1 & 54.1 & 27 & 8 & 48 & 52.3 & 51.2 & 3 & 84 & 48 & 47.2 & 44.2 & 42 & tes & 48 & 46.9 & 43.0 & 44 & 4 \\
\hline 50 & 58.1 & 56.2 & 24 & 12 & 50 & 51.3 & 50.3 & 3 & we & 50 & 45.7 & 43.7 & 44 & Q & 50 & 49.9 & 44.2 & 40 & 18 \\
\hline 52 & 57.9 & 55.1 & 26 & 22 & 52 & 50.5 & 49.7 & 3 & 82 & 52 & 46.9 & $45 . \mathrm{I}$ & 42 & Est & 52 & 49.7 & 45.3 & 40 & 2 \\
\hline 54 & 57.4 & $55 . \mathrm{I}$ & 26 & $k^{2}$ & 54 & 50.1 & $49 . I$ & 3 & $k^{2}$ & 54 & $48 . I$ & 46.0 & 40 & $k^{2}$ & 54 & 52.9 & 48.5 & 35 & kt \\
\hline 56 & 57.1 & 54.9 & 26 & 25 & 56 & 50.0 & 49.2 & 3 & 柴 & 56 & 46.9 & 45.2 & 42 & 8 & 56 & 54.3 & 49.5 & 33 & \\
\hline 58 & 57.3 & 55.2 & 26 & 62. & $5^{8}$ & 50.0 & 48.9 & 3 & $x_{2}$ & 58 & 46.0 & 45.2 & 43 & 92 & 58 & $54 . I$ & 50.0 & 33 & \\
\hline I7 00 & 57.3 & 55.2 & 26 & -24.0 & I9 00 & 51.2 & 49.5 & 16 & -24.0 & 2100 & 46.0 & 44.8 & 43 & -23.9 & 2300 & 57.0 & 52.2 & 28 & -23.7 \\
\hline 02 & 54.9 & 53.3 & 29 & 10 & 02 & 50.4 & 48.4 & 3 & $x^{2}$ & 02 & 45.0 & 44.2 & 44 & 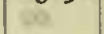 & 02 & 56.0 & 51.1 & 30 & \\
\hline 04 & 54.9 & 53.3 & 29 & +9 & 04 & 50.9 & 48.9 & 3 & 16 & 04 & 45.0 & 43. I & 45 & 10 & 04 & 55.0 & 50.3 & 32 & 0 \\
\hline 06 & 56.0 & 63.9 & 28 & a) & 06 & $50 . I$ & 47.9 & 3 & $\infty 0$ & 06 & 44.2 & 43.0 & 46 & io & 06 & 53.8 & 51.2 & 32 & 8 \\
\hline 08 & 55.4 & 53.0 & 29 & $8 x$ & 08 & 49.3 & 47.3 & c & 8 & 08 & 43.2 & 42.1 & 47 & bi & 08 & 49.9 & 47.8 & 38 & Nat \\
\hline Io & 53.9 & 51.9 & $3 I$ & of & 10 & 48.8 & 47.1 & $\mathrm{x}$. & 97 & IO & 49.3 & 47.0 & 39 & of & I0 & 46.9 & 44.8 & 2242 & $\omega$ \\
\hline$I 2$ & 53.9 & 51.3 & 32 & *1 & 12 & 47.7 & 46.7 & 4 & -1 & 12 & 51.3 & 49.2 & 35 & 21 & 12 & 29 & $.4 b$ & 23 of & 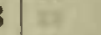 \\
\hline I 4.3 & 53.9 & 52.5 & $3 I$ & 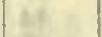 & I4 & 47.2 & 45.8 & 8 & -23.9 & I4 & 50.2 & 49.2 & 36 & -23.8 & $14^{*}$ & 59.7 & 41.0 & 52 & -23.4 \\
\hline I6 & 54.3 & 52.0 & $3 I$ & -24.0 & I6 & 46.8 & 45.7 & k. & & 16 & 50.8 & 48.6 & 36 & & 16 & 78.7 & 65.5 & 2318 & \\
\hline 18 & 54.3 & 52.2 & $3 I$ & 1 & I8 & 46.0 & 45.2 & e & 4 & 18 & 50.2 & 48.2 & 37 & at & 18 & 36.2 & $25 . I$ & 2423 & 51 \\
\hline 20 & 52.9 & $5 \mathrm{I} . \mathrm{I}$ & 33 & 05 & 20 & 46.8 & 44.9 & 4 & W & 20 & 49.9 & 48.1 & 37 & 6 & 20 & 36.3 & $22 . I$ & 2426 & $\approx C$ \\
\hline 22 & 52.7 & 51.2 & 33 & e & 22 & 46.0 & 44.3 & 1 & at & 22 & 51.8 & 49.2 & 35 & ext & 22 & 70.9 & 65.5 & 232.4 & is \\
\hline 24 & 54.2 & 52.3 & 31 & 15 & 24 & 46.1 & 44.3 & 4. & is & 24 & 51.1 & 49.I & 36 & $y=$ & 24 & 78.3 & 69.5 & $23 \quad 15$ & is \\
\hline 26 & 54.8 & 52.3 & 30 & $x$ & 26 & 46.8 & 45.0 & 4 & 8 & 26 & 50.4 & 48 & 37 & $x$ & 26 & 30.0 & 29.1 & 2425 & Sa \\
\hline 28 & 53.8 & 51.3 & 32 & $x<$ & 28 & 44.6 & 42.3 & 4 & 20 & 28 & 50.4 & 48.3 & 37 & $x=$ & 28 & 73.9 & 69.3 & 2319 & \\
\hline 30 & 53.8 & 51.9 & $3 I$ & -24.0 & 30 & 44.9 & $42 . I$ & 4 & -23.8 & 30 & 49.8 & $48 . \mathrm{I}$ & 37 & -23.8 & $30^{*}$ & 45.2 & 30.5 & 2406 & \\
\hline 32 & 53.8 & 51.9 & 31 & 6 & 32 & 43.9 & 42.8 & 4 & $1=$ & 32 & 49.5 & 48.8 & 37 & & 32 & 58.3 & 44.9 & 2344 & $-23 \cdot 5$ \\
\hline 34 & 52.0 & 51.1 & 33 & 45 & 34 & 44.9 & 43.7 & 4 & 16 & 34 & 49.0 & 47.7 & 38 & K & 34 & 66.8 & 52.5 & 32 & \\
\hline 36 & 52.8 & 52.0 & 32 & 80 & 36 & $43 \cdot 3$ & 42.1 & 4 & ax & 36 & 48.7 & $47 . I$ & 39 & ac & 36 & 62.2 & 51.7 & 36 & \\
\hline 38 & 53.0 & 52.3 & 32 & 36 & 38 & 45.6 & $43 \cdot 3$ & 4 & te & 38 & 48.9 & 47.2 & 39 & 2. & 38 & 63.6 & 51.8 & 35 & \\
\hline 40 & 53.9 & 52.2 & 31 & 105 & 40 & 46.4 & $43 \cdot 3$ & 4 & tat & 40 & 48.7 & 46.9 & 39 & os & 40 & 65.4 & 55.4 & 31 & $\theta$ \\
\hline 42 & 53.9 & 52.2 & 31 & 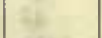 & 42 & 46.0 & 43.2 & 4 & 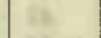 & 42 & 48.2 & 46.9 & 40 & से & 42 & 62.2 & 54.1 & 34 & \\
\hline 44 & 52.3 & 51.2 & 33 & -24.0 & 44 & 46.2 & 43.3 & 10 & $-23 \cdot 5$ & 44 & 47.6 & 46.0 & 41 & -23.7 & 44 & 60.1 & 52.5 & 37 & -23.4 \\
\hline 46 & 54.1 & 52.2 & 31 & 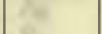 & 46 & 45.8 & 43.2 & $y$ & & 46 & 47.0 & 46.1 & $4 I$ & & 46 & 58.2 & 50.9 & 40 & \\
\hline 48 & 54.2 & 52.4 & 30 & 8 & 48 & 43.9 & $4 I .6$ & 4 & 8 & 48 & 47.2 & 46.7 & 41 & 4 & 48 & 46.1 & 38.6 & 2359 & 9 \\
\hline 50 & 54.0 & 51.3 & 32 & 82 & 50 & 43.8 & 42.0 & 4 & $n$ & 50 & 47.2 & 46.7 & $4 I$ & ez & 50 & 39.2 & 26.8 & 24 I4 & \\
\hline 52 & 54.0 & 50.9 & 32 & $=3$ & 52 & 43.2 & 42.2 & 4 & 4 & 52 & 49.2 & 48.9 & 37 & $x 2$ & 52 & 48.9 & 33.7 & 2400 & 5 \\
\hline & 54.1 & 5 I. I & 32 & 12 & 54 & 44.6 & 43.0 & 0 & 13 & 54 & 50.0 & 49.1 & 36 & 12 & 54 & 72.9 & 60.3 & 2321 & \\
\hline 56 & 54.8 & 50.8 & $3 I$ & 98 & 56 & 44.3 & 43.2 & 4 & a. & 56 & 49.2 & 48.2 & 38 & 8 & 56 & 80.0 & 64.5 & I2 & 6 \\
\hline 58 & 54.8 & 51.2 & $3 I$ & 80 & 58 & 45. I & 43.9 & & 8 & 58 & 49.3 & 48.0 & 38 & 80 & $58 * 2$ & 38.2 & 21.0 & 23 I8 & \\
\hline & & & & & & & & & & & & & & & 2400 & 60.8 & 51.0 & 2237 & $\begin{array}{l}7-23 \cdot 3 \\
\end{array}$ \\
\hline
\end{tabular}

Observer-R. R. T. 
Tabulation of magnetic declinations observed at Teplitz Bay-Continued

\begin{tabular}{|c|c|c|c|c|c|c|c|c|c|c|c|c|c|c|c|c|c|c|c|}
\hline \multicolumn{6}{|c|}{ Thursday, January I4, Ig04 } & \multicolumn{4}{|c|}{ Magnet scale erect } & \multicolumn{6}{|c|}{ Friday, January 15,1904} & \multicolumn{4}{|c|}{ Magnet scale inverted } \\
\hline \multirow{2}{*}{$\begin{array}{l}\text { Chr'r } \\
\text { time }\end{array}$} & \multicolumn{2}{|c|}{$\begin{array}{l}\text { Scale } \\
\text { readings }\end{array}$} & \multirow{2}{*}{$\begin{array}{l}\text { East } \\
\text { decli- } \\
\text { nation }\end{array}$} & Temp. & Chr'r & $\begin{array}{r}\mathrm{Sc} \\
\text { read }\end{array}$ & $\begin{array}{l}\text { ale } \\
\text { ings }\end{array}$ & $\begin{array}{l}\text { East } \\
\text { decli- }\end{array}$ & Temp. & Chr'r & $\begin{array}{l}\mathrm{Sc} \\
\text { read }\end{array}$ & $\begin{array}{l}\text { ale } \\
\text { ings }\end{array}$ & $\begin{array}{l}\text { East } \\
\text { decli- }\end{array}$ & Temp. & Chr'r & $\begin{array}{l}\text { Sca } \\
\text { read }\end{array}$ & $\begin{array}{l}\text { ale } \\
\text { ings }\end{array}$ & $\begin{array}{l}\text { East } \\
\text { decli- }\end{array}$ & \\
\hline & L,eft & Right & & & & Left & Right & & & tin & Left & Right & & & & Left & Right & nation & \\
\hline $\mathrm{h} \mathrm{m}$ & d & d & $\cdot$, & $\circ$ & $\mathrm{h} \mathrm{m}$ & $\mathrm{d}$ & d & $\cdot$, & $\bullet$ & $\mathrm{h} \mathrm{m}$ & d & d & & $\bullet$ & $\mathrm{h} \mathrm{m}$ & d & d & $\circ \quad$ & $\bullet$ \\
\hline 600 & 40.5 & 41.0 & 2239 & 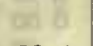 & 1800 & 38.1 & 39.8 & 2236 & -20.0 & 2000 & $44 . I$ & 40.7 & 2239 & $-21 \cdot 4$ & 2200 & 43.9 & 42.1 & 2238 & -17.9 \\
\hline 02 & 40.8 & 41.0 & 39 & -19.4 & 02 & 38.3 & 40.0 & 36 & 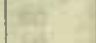 & 02 & 47.6 & 43.9 & 34 & & 02 & 44.8 & 43.6 & 36 & \\
\hline 04 & 40.1 & 40.4 & 38 & 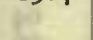 & 04 & 36.8 & 39.8 & 35 & $\sqrt{16}$ & 04 & $47 \cdot 4$ & 43.2 & 34 & 18 & 04 & 45.3 & 44.2 & 35 & 10 \\
\hline 06 & 40.0 & 40.7 & 38 & 20 & 06 & 36.9 & 39.7 & 35 & 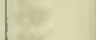 & 06 & 46.6 & 44.0 & 34 & 16 & 06 & 44.8 & 43.7 & 36 & Sy \\
\hline 08 & 39.3 & 40.8 & 38 & 60 & 08 & 36.8 & 39.5 & 35 & W & 08 & 45.6 & 43.0 & 36 & $\theta$ & 08 & 42.1 & $4 \mathrm{I} .2$ & 40 & \\
\hline I0 & 38.8 & 40.3 & 37 & Os: & Io & 36.8 & $39 \cdot 3$ & 34 & $a r$ & -10 & 44.8 & 41.8 & 37 & Ba & IO & 43.3 & 43.1 & 38 & $=$ \\
\hline 12 & 38.7 & 40.3 & 37 & (4) & 12 & 36.6 & 38.9 & 34 & ar & 12 & 43.8 & 42.4 & 38 & $1=$ & 12 & 42.2 & $4 I .9$ & 39 & \\
\hline 14 & 37.9 & 39.8 & 36 & -I9.6 & 14 & 36.8 & 38.9 & 34 & -20.0 & 14 & 43.7 & 42.0 & 38 & -20.9 & I4 & 42.2 & 42.2 & 39 & -17.8 \\
\hline I6 & 37.6 & 39.7 & 36 & & 16 & 36.5 & 39.0 & 34 & & 16 & 44.9 & 42.7 & 37 & & 16 & 42.7 & 4I.I & 40 & \\
\hline 18 & 38. I & 38.8 & 35 & ith & I8 & 36.7 & 38.8 & 34 & ail & 18 & 46.9 & 44.2 & 34 & $8 x$ & 18 & 43.2 & 42.2 & 38 & 63 \\
\hline 20 & 38.7 & 39.5 & 36 & $n$ & 20 & $37 . I$ & 38.9 & 35 & 2 & 20 & 49.2 & 46.8 & 30 & os & 20 & 43.7 & $42 . I$ & 38 & $\theta=$ \\
\hline 22 & 39.1 & 40.8 & 38 & is & 22 & 37.4 & 39.2 & 35 & 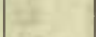 & 22 & 48.2 & 46.2 & 31 & is & 22 & 44.3 & $42 . I$ & 38 & $=$ \\
\hline 24 & 38.7 & 40.2 & 37 & is & 24 & 37.7 & 39.3 & 35 & 12 & 24 & 47.2 & 45.4 & 33 & & 24 & $44 \cdot 3$ & 42.0 & 38 & fif \\
\hline 26 & 38.9 & 40.2 & 37 & is & 26 & 38.0 & 40.0 & 36 & $E^{2}$ & 26 & 46.9 & 45.4 & 33 & $\varepsilon$ & 26 & 44.3 & $42 . \mathrm{I}$ & 38 & Fr \\
\hline 28 & 38.7 & 41.8 & 38 & 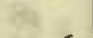 & 28 & 38.7 & 40.8 & 37 & $=2$ & 28 & 47.2 & 44.8 & 33 & & 28 & 43.9 & $42 . I$ & 38 & - \\
\hline 30 & 39.2 & 42.5 & 39 & -19.6 & 30 & 39.2 & 41.0 & 38 & -20.0 & 30 & 47.9 & 45.9 & 32 & -20.0 & 30 & 42.8 & $4 \mathrm{I} . \mathrm{I}$ & 40 & -17.6 \\
\hline 32 & 40.9 & 42.7 & 40 & - & 32 & 39.0 & 41.9 & 38 & $1=$ & 32 & 48.6 & 45.1 & 32 & & 32 & 43.3 & $4 \mathrm{I} . \mathrm{I}$ & 39 & \\
\hline 34 & 40.8 & 42.2 & 40 & the & 34 & 39.2 & 41.6 & 38 & -16 & 34 & 50.5 & $47 \cdot 7$ & 28 & 12 & 34 & $43 . I$ & 41.8 & 39 & FE. \\
\hline 36 & 39.3 & 41.0 & 38 & $1=$ & 36 & 39.0 & 42.0 & 38 & 10 & 36 & 50.3 & 48.3 & 28 & Af & 36 & 43.2 & 41.6 & 39 & 2 \\
\hline 38 & 39.0 & 40.3 & 37 & 86 & 38 & 39.6 & $4 \mathrm{I} .7$ & 39 & Le & 38 & 50.2 & 47.2 & 29 & we & 38 & 42.7 & 40.9 & 40 & 88 \\
\hline 40 & 38.8 & 40.0 & 36 & 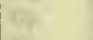 & 40 & 39.9 & 41.8 & 39 & 5 & 40 & 51.9 & 48.9 & 26 & 아 & 40 & 40.3 & 39.0 & 43 & 0 \\
\hline 42 & 39.2 & 40.2 & 37 & 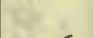 & 42 & 39.7 & $4 \mathrm{I} .3$ & 39 & 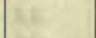 & 42 & 51.9 & 49.9 & 26 & $4+4$ & 42 & 40.0 & 39.0 & 43 & 14 \\
\hline 44 & $39 \cdot 3$ & 40.3 & 37 & -19.6 & 44 & 39.7 & $4 \mathrm{I} . \mathrm{I}$ & 38 & -20.0 & 44 & $52 . \overline{8}$ & 51.0 & 24 & -19.2 & 44 & 40.2 & 39.8 & 43 & -17.3 \\
\hline 46 & 38.8 & 40.2 & 37 & $x^{2}$ & 46 & 39.7 & 41.7 & 39 & & 46 & 53.2 & 50.7 & 24 & & 46 & 41.0 & 39.8 & 42 & \\
\hline 48 & 39.2 & 40.0 & 37 & 89 & 48 & 39.9 & 42.0 & 39 & $\infty$ & 48 & 52.1 & 50.1 & 25 & 40 & 48 & 39.2 & 38.2 & 45 & 10 \\
\hline 50 & 38.8 & 40.3 & 37 & 10 & 50 & 40.5 & 42.4 & 40 & 2 & 50 & 52.1 & 49.9 & 25 & 4 & 50 & 39.3 & 38.2 & 45 & 00 \\
\hline 52 & 38.3 & 40.3 & 36 & 늑 & 52 & 40.3 & 42.1 & 40 & $6 ?$ & 52 & 53.9 & 51.7 & 22 & 5 & 52 & 41.0 & 40.1 & 42 & $=$ \\
\hline 54 & 38.8 & 40.8 & 37 & 185 & 54 & 40.1 & 41.9 & 39 & +2 & 54 & 56.9 & 54.9 & 18 & 20 & 54 & 41.2 & 40.2 & 42 & 12 \\
\hline 56 & 38.9 & 40.8 & 37 & 19 & 56 & 39.1 & 41.0 & $3^{8}$ & VI & 56 & 55.1 & 53.2 & 20 & $x^{4}$ & 5 & 43.1 & 42.1 & 38 & 9 \\
\hline 58 & 38.4 & 40.8 & 37 & $82=$ & 58 & 39.0 & 41.8 & 38 & & 58 & 53.7 & 51.7 & 23 & & 58 & 46.3 & 45.0 & 34 & 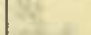 \\
\hline 700 & 38.1 & 40.3 & 36 & -20.0 & 1900 & 39.0 & 41.2 & 38 & $y$ & 2100 & 53.0 & 51.0 & 24 & -18.3 & 2300 & 47.7 & 46.7 & $3 \mathrm{I}$ & -I7.I \\
\hline 02 & 37.9 & 39.8 & 36 & & 02 & 38.5 & 41.8 & 38 & 19 & 02 & 53.8 & 49.7 & 24 & & 02 & 47.8 & 46.1 & 32 & \\
\hline 04 & 37.6 & 39.9 & 36 & 10 & 04 & 38.1 & 42.0 & 38 & 16 & 04 & 54.0 & 49.4 & 24 & 10 & 04 & 50.8 & 48.7 & 27 & \\
\hline 06 & 37.7 & 39.7 & 36 & $\infty$ & 06 & 38.4 & 42.2 & 38 & 3 & 06 & 53.9 & 49.9 & 24 & nis & 06 & 52.4 & 50.2 & 25 & 8 \\
\hline 08 & 37.8 & 40.0 & 36 & War & 08 & 38.2 & 42.2 & 38 & 25 & 08 & 54.2 & 50.5 & 23 & ka & 08 & 54.0 & 52.3 & 22 & 60 \\
\hline I0 & 38.0 & 40.3 & 36 & 6 & IO & 38.3 & $42 . I$ & 38 & tor & I0 & 55.3 & 51.9 & $2 \mathrm{I}$ & 0 & IO & 57.2 & 55.2 & 17 & (111) \\
\hline 12 & 38.5 & 40.3 & 37 & 41 & 12 & 38.7 & 42.0 & 38 & in & 12 & 55.9 & 52.7 & 20 & & 12 & 60.9 & 58.7 & II & 10 \\
\hline 14 & 38.7 & 40.2 & 37 & -20.0 & 14 & 38.8 & 42.0 & 38 & -20.0 & 14 & 56.8 & 54.0 & 18 & $-I 8.0$ & 14 & 59.4 & 57.0 & 14 & -17.0 \\
\hline 16 & 39.2 & 40.0 & 37 & & 16 & 38.4 & $4 I .5$ & 37 & & 16 & 57.0 & 55.8 & 17 & & 16 & 55.7 & 52.8 & 20 & \\
\hline 18 & 39.3 & 40.2 & 37 & 111 & 18 & 38.2 & 41.0 & 37 & hin & 18 & 58.5 & & 15 & Wit. & 18 & 54.0 & 5 I. 7 & 22 & 10 \\
\hline 20 & $39 . \mathrm{I}$ & 40.3 & 37 & 10 & 20 & 38.2 & 40.3 & 3 & 85 & 20 & 53.8 & 52.8 & 22 & $=$ & 20 & $5 \mathrm{I} . \mathrm{I}$ & $49 . I$ & 27 & 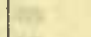 \\
\hline 22 & 39.6 & 40.0 & 37 & $6 x$ & 22 & 38.2 & 40.3 & 36 & fa & 22 & 56.9 & 56.2 & 17 & IE. & 22 & 49.6 & 48.1 & 29 & 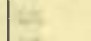 \\
\hline 24 & 39.1 & 40.0 & 37 & +2 & 24 & 38.6 & 40.6 & 37 & t5 & 24 & $56 . I$ & 55.4 & 18 & 12 & 24 & 49.3 & 48.3 & 29 & 24 \\
\hline 26 & 39.7 & 40.8 & 38 & $\infty$ & 26 & 38.7 & 40.8 & 37 & e & 26 & 56.1 & 55.2 & 18 & 16 & 2 & 49.5 & 48.8 & 28 & 69 \\
\hline 28 & 39.8 & $4 \mathrm{I}$. I & 38 & $1=8$ & 28 & 38.3 & 40.4 & 36 & 1 & 28 & 55.8 & 54.9 & 19 & & 28 & 48.7 & 47.8 & 30 & \\
\hline 30 & 39.7 & 40.8 & 38 & -20.0 & 30 & 38.4 & 40.3 & 36 & -20.0 & 30 & 55.1 & 53.9 & 20 & -18.0 & 30 & $47 \cdot 3$ & 46.9 & 31 & -16.9 \\
\hline 32 & 38.9 & $4 \mathrm{I}$. I & 37 & at & 32 & 37.7 & 40.4 & 36 & & 32 & 52.8 & 52.6 & 23 & $\pi$ & $3^{2}$ & 45.7 & 44.2 & 35 & \\
\hline 34 & 39.0 & 41.8 & 38 & 15 & 34 & 37.3 & 39.8 & 35 & & 34 & 48.7 & 48.4 & 29 & & 3 & 45.6 & 44.0 & 35 & 1 \\
\hline 36 & 39.3 & 41.8 & 39 & $1=$ & 36 & 36.8 & 39. & 34 & the & 30 & 48.8 & 48.2 & 29 & & 3 & 45.0 & 43.7 & 36 & \\
\hline 38 & 39. I & 41.0 & 38 & 1 & 38 & 36.7 & $3^{8} .9$ & 34 & 14. & 38 & 44.8 & 44.4 & 35 & i. & 38 & 44.6 & 43.0 & 37 & 2 \\
\hline 40 & 39.0 & 40.0 & 37 & 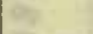 & 40 & 36.7 & 38.3 & 34 & 96 & 40 & 45.9 & 45.2 & 34 & 0 & 40 & 43.9 & 42.8 & 37 & 6 \\
\hline 42 & 38.4 & 40.0 & 36 & 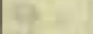 & 42 & 36.4 & 38.0 & 33 & $4 x$ & 42 & 50.3 & 48.8 & 28 & 10.2. & 42 & 44.0 & 42.1 & 37 & \\
\hline 44 & 38.7 & 39.8 & 36 & -20.0 & & 36.3 & 38.0 & 33 & -20.0 & 44 & $5 \mathrm{I} .2$ & 49.9 & 26 & -I7.9 & 44 & 43.7 & 42.0 & 38 & -16.8 \\
\hline 46 & 38.7 & 40.0 & 37 & & 46 & 36.9 & 38.1 & 34 & & 46 & 48.9 & 47.7 & 30 & & 46 & 44.2 & 42.4 & 37 & \\
\hline 48 & 38.6 & 40.0 & 36 & 10 & 48 & 35.6 & 40.7 & 34 & 18 & 48 & 45.1 & 43.9 & 36 & 10 & 48 & 45.9 & 40.9 & 37 & \\
\hline 50 & 38.2 & 39.8 & 36 & 108 & 50 & 36.0 & 40.2 & 35 & oe & 50 & 40.4 & 39.2 & 43 & & 50 & 45.3 & 40.3 & 38 & \\
\hline 52 & 38.0 & 39.7 & 36 & a & 52 & 35.8 & 40.3 & 35 & 42 & 52 & 40.2 & 38.8 & 43 & 2 & 52 & 44.6 & 39.8 & 39 & 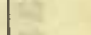 \\
\hline 54 & 38.0 & 39.8 & 36 & 18 & 54 & 35.8 & 40.0 & 34 & is & 54 & 41.4 & 39.3 & 42 & 18 & 54 & 43.0 & 38.3 & 42 & E \\
\hline 56 & 38.3 & 39.8 & 36 & & 56 & 36.0 & 39.9 & 34 & & 56 & 41.8 & 39.9 & 41 & 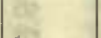 & 56 & 42.9 & 38.4 & 42 & \\
\hline 58 & 38.2 & 39.8 & 36 & 3 & 58 & 36.0 & 39.8 & 34 & & 58 & $4 \mathrm{I} .3$ & 38.9 & 42 & $\cdots$ & $\begin{array}{r}58 \\
-\quad 5\end{array}$ & 42.9 & 38.6 & 41 & \\
\hline & & & & & 2000 & 36.0 & 39.2 & 34 & -20.0 & & & & & & 2400 & 41.3 & 39.0 & 42 & -16.4 \\
\hline
\end{tabular}

Correction to local mean time is $-56 \mathrm{~s}$.

Torsion head at $15 \mathrm{~h} 20 \mathrm{~m}$ read $84^{\circ}$ and at the end read the same. Observer-W. J. P.
Correction to local mean time is - 32 .

Torsion head at $19 \mathrm{~h} 25 \mathrm{~m}$ read $84^{\circ}$ and at the end read the same. Observer-R. R. T. 
Tabulation of magnetic declinations observed at Teplitz Bay-Continued

\begin{tabular}{|c|c|c|c|c|c|c|c|c|c|c|c|c|c|c|c|c|c|c|c|}
\hline \multicolumn{6}{|c|}{ Sunday, January 17,1904} & \multicolumn{4}{|c|}{ Magnet scale erect } & \multicolumn{6}{|c|}{ Sunday, January $x 7,1904$} & \multicolumn{4}{|c|}{ Magnet scale inverted } \\
\hline \multirow{2}{*}{$\begin{array}{l}\text { Chr'r } \\
\text { time }\end{array}$} & \multicolumn{2}{|c|}{$\begin{array}{l}\text { Scale } \\
\text { readings }\end{array}$} & \multirow{2}{*}{$\begin{array}{c}\text { East } \\
\text { decli- } \\
\text { nation }\end{array}$} & Temp. & Chr'r & $\underset{\text { rea }}{S}$ & $\begin{array}{l}\text { ale } \\
\text { lings }\end{array}$ & $\begin{array}{l}\text { East } \\
\text { decli- }\end{array}$ & Temp. & Chr'r & $\begin{array}{r}\mathrm{Sc} \\
\text { reac }\end{array}$ & dings & $\begin{array}{l}\text { East } \\
\text { decli- }\end{array}$ & Temp. & Chr'r & $\begin{aligned} \text { read } \\
\text { se }\end{aligned}$ & & $\begin{array}{l}\text { East } \\
\text { decli- }\end{array}$ & Temp. \\
\hline & Left & Right & & & time & Left & Right & & & time & Left & Right & nation & & tim & Left & Right & nation & \\
\hline & d & d & - & $0^{\circ}$ & $\mathrm{h} \mathrm{m}$ & $\mathrm{d}$ & $\mathrm{d}$ & & & $\mathrm{h} \mathrm{m}$ & d & & & $0^{\circ}$ & $h \mathbf{m}$ & d & d & $\circ$. & - \\
\hline 0 o* & 39.2 & 40.9 & 2224 & -9.0 & 200 & 26.7 & 29.3 & 2304 & -7.3 & $400^{*} 2$ & 51.7 & 51.0 & 2244 & -6.4 & 600 & 45.2 & 41.0 & 2258 & \\
\hline 02 & 38.0 & 39.3 & 22 & 68 & 02 & 24.7 & 28.2 & 02 & & 02 & 52.8 & 49.2 & 44 & & 02 & 51.8 & 49.6 & & -6.6 \\
\hline 04 & 38.6 & $4 \mathrm{I} .7$ & 24 & 30 & 04 & 26.3 & 30.8 & $\begin{aligned} 05 \\
23\end{aligned}$ & ar & 04 & 53.0 & 49.9 & 44 & (16) & 04 & 49.7 & 46.3 & 50 & \\
\hline of & 38.8 & 39.6 & & & & 24.1 & 27.2 & 23 or & 86 & 06 & 54.5 & 54.2 & 39 & 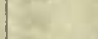 & 06 & 45.1 & 42.2 & 57 & \\
\hline 08 & 40.9 & 43.4 & 28 & min & 08 & 23.0 & 26.9 & 2259 & $\ln 5$ & o8 & 54.6 & 54.1 & 39 & 85 & 08 & 44.1 & 40.9 & 59 & \\
\hline 10 & 47.0 & 48.2 & 36 & uit & 10 & 24.9 & 28.2 & 2302 & oit & I0 & $5 \mathrm{I} . \mathrm{I}$ & 50.7 & 45 & in & 10 & 43.7 & 40.9 & 59 & \\
\hline 12 & 47.5 & 50.1 & 38 & 0 & 12 & 28.1 & 31.0 & 07 & $15-2$ & I2 & 49.0 & 49.0 & $\begin{array}{l}48 \\
48\end{array}$ & 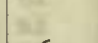 & 12 & 45.6 & $4 \mathrm{I} .5$ & 57 & \\
\hline 14 & 47.8 & 50.0 & 38 & -8.9 & 14 & 24.0 & 26.0 & oo & -7.1 & I4 & 49.2 & 48.6 & $\begin{array}{l}48 \\
48\end{array}$ & -6.5 & 14 & 50.0 & 47.9 & 2249 & -6.6 \\
\hline $\begin{array}{l}16 \\
16\end{array}$ & 46.3 & 49.9 & 37 & 0.9 & $\begin{array}{l}16 \\
16\end{array}$ & 25.7 & 26.2 & or & 18.0 & $\begin{array}{l}14 \\
16\end{array}$ & 48.9 & 47.7 & 49 & -0.5 & 16 & 43.2 & 40.3 & 2300 & \\
\hline 18 & 49.9 & 53.6 & 2243 & 24 & 18 & 26.9 & 27.3 & $\begin{array}{lll}23 & 03\end{array}$ & tis & $\begin{array}{l}10 \\
18\end{array}$ & $\begin{array}{l}40.9 \\
49.1\end{array}$ & 46.3 & $\begin{array}{l}49 \\
50\end{array}$ & ta & 18 & $\begin{array}{l}43.2 \\
44.9\end{array}$ & 42.4 & 2357 & \\
\hline 20 & $\begin{array}{l}49.9 \\
62.8\end{array}$ & 64.0 & 23 OI & $=$ & 20 & 21.6 & 21.8 & 2254 & 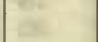 & 20 & $\begin{array}{l}48.1 \\
48.3\end{array}$ & 45. & $5 \mathrm{I}$ & $s$ & 20 & $\begin{array}{l}1.9 \\
.0\end{array}$ & 48.1 & 2249 & \\
\hline 22 & 49.8 & 55.7 & 2244 & tas & 22 & 20.3 & $2 \mathrm{I} .7$ & $\begin{array}{ll}22 & 53\end{array}$ & eet & 22 & $\begin{array}{l}48.3 \\
48.0\end{array}$ & $\begin{array}{l}45 \\
45\end{array}$ & $\begin{array}{l}51 \\
5 \mathrm{I}\end{array}$ & II & 22 & 5.3 & 43.7 & $\begin{array}{r}49 \\
56\end{array}$ & \\
\hline 24 & $\begin{array}{l}49.0 \\
64.1\end{array}$ & 66.7 & 2304 & $y=$ & 24 & 24.8 & 25.2 & 2300 & Liv & 24 & $\begin{array}{l}40.0 \\
47.2\end{array}$ & $\begin{array}{l}45 \\
44 \\
\end{array}$ & $\begin{array}{l}51 \\
53\end{array}$ & is & 24 & $\begin{array}{l}5.3 \\
4.1\end{array}$ & $\begin{array}{l}44.1 \\
44.1\end{array}$ & 56 & \\
\hline 26 & 57.8 & 61.9 & 55 & 8 & 26 & 32.0 & 33.2 & II & $1=$ & 26 & & 44 & 53 & 5 & 26 & .1 & 42.4 & 58 & \\
\hline 28 & $\begin{array}{l}56.9 \\
56.9\end{array}$ & 62.2 & 55 & & 28 & 30.0 & $3 \mathrm{I} .9$ & 09 & 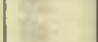 & $\begin{array}{l}20 \\
28\end{array}$ & & & $\begin{array}{l}33 \\
54\end{array}$ & $1 \mathrm{~s}$ & 28 & $\begin{array}{l}44.1 \\
50.4\end{array}$ & $\begin{array}{l}47.4 \\
47.2\end{array}$ & $\begin{array}{l}50 \\
49\end{array}$ & \\
\hline 30 & 57.3 & 64.1 & 57 & -9.0 & 30 & 29.1 & $3 \mathrm{I} .8$ & o8 & -7.1 & $\begin{array}{l}20 \\
30\end{array}$ & & & $\begin{array}{l}54 \\
52\end{array}$ & -6.5 & 30 & $\begin{array}{l}5.4 \\
52.3\end{array}$ & 48.8 & $\begin{array}{l}49 \\
46\end{array}$ & -6.7 \\
\hline 32 & 47.6 & 59.3 & 45 & 8.0 & 32 & 25.0 & 27.8 & 2302 & & $\begin{array}{l}30 \\
32\end{array}$ & 49.2 & $\begin{array}{l}46.3 \\
46.9\end{array}$ & 50 & -0.5 & 32 & 46.1 & 43.2 & 2255 & -0.8 \\
\hline 34 & 49.9 & 58.7 & 47 & 32 & 34 & 22.7 & 25.2 & 2258 & $y$ & 34 & 49.8 & 47.0 & 49 & 37 & 34 & 43.9 & 39.1 & 2300 & \\
\hline 36 & 44.7 & 54.9 & 40 & 15 & 36 & 22.6 & 24.1 & 57 & E. & 36 & 49.5 & 47.7 & 49 & tis & 36 & 45.4 & 42.3 & 2257 & \\
\hline 38 & 41.9 & 51.9 & 35 & 8 & 38 & 23.3 & 25.0 & & in & 38 & 50.7 & 48.4 & 47 & 16 & 38 & 50.2 & 47.0 & 49 & \\
\hline 40 & 52.7 & 57.3 & 48 & 16 & 40 & 22.9 & 24.8 & 2258 & $\Rightarrow$ & 40 & 49.3 & 47.7 & 49 & a & 40 & 5 ז 6 & 48.2 & 47 & \\
\hline 42 & 57.0 & 63.8 & 2256 & & 42 & 25.0 & 26.3 & 2300 & $=$ & 42 & 49.2 & 47.3 & 49 & & 42 & 51.8 & 49. I & 46 & \\
\hline 44 & & 73.1 & 23 II & -8.9 & 44 & 24.9 & 26.7 & 23 or & & 44 & 49.7 & 47.1 & 49 & -6.6 & 44 & 48.1 & & 52 & -6.5 \\
\hline 46 & 62.8 & 76.2 & II & (2) & 46 & 23.9 & 25.8 & 2259 & -7.1 & 46 & 50.3 & 48.2 & 48 & & 46 & 50.9 & 47.7 & 48 & \\
\hline 48 & 6.5 & 74.3 & 12 & 2 & 48 & 23.0 & & 58 & ( & 48 & 49.4 & 47.7 & 49 & 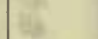 & 48 & 53.3 & 49.7 & 45 & \\
\hline $50^{*}$ & & & 25 & 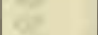 & 50 & & 24.8 & 57 & $=$ & 50 & & & 49 & 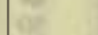 & 50 & & & 49 & \\
\hline 52 & 0.1 & 42.2 & 17 & (⿻) & 52 & 21.6 & 23.1 & 55 & 5 & 52 & 48.7 & 46.6 & $\begin{array}{l}49 \\
50\end{array}$ & 5 & 52 & 48.9 & 45 & $5 \mathrm{I}$ & \\
\hline 54 & 24.9 & 35.4 & 07 & & 54 & 20.2 & 21.7 & 53 & 2 & 54 & $47 \cdot 3$ & 45.2 & 53 & $\Rightarrow$ & 54 & $5 \mathrm{~T} .3$ & & 47 & \\
\hline 56 & & 37.8 & II & 8 & 56 & 18.4 & 20.7 & $5 I$ & 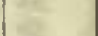 & 56 & 46.2 & 45.0 & 54 & $1=$ & 56 & 49.9 & & 49 & \\
\hline 58 & 28. I & 36.9 & II & E. & 58 & 18.2 & 20.5 & $5 I$ & & 58 & & & 52 & & 58 & 47.4 & 44 & 54 & \\
\hline 100 & 23.6 & 32.2 & 03 & & 300 & 18.9 & 20 & $5 \mathrm{I}$ & -7.1 & 500 & & $\begin{array}{l}430^{\circ} \\
47 .\end{array}$ & 48 & -6.6 & 700 & $\begin{array}{l}44.4 \\
44.9\end{array}$ & & $\begin{array}{l}54 \\
57\end{array}$ & -6.5 \\
\hline 02 & & 34 & 08 & -8.7 & 02 & & $2 \mathrm{I}$ & 53 & 8.1 & 02 & & 50. & 44 & -0.0 & 02 & 44.8 & 44.6 & 55 & 2.5 \\
\hline 04 & & $\begin{array}{l}34 \\
37\end{array}$ & 13 & -0.1 & 04 & 20.1 & 22 & 54 & in. & 04 & & 51. & $\begin{array}{l}4 \\
4 \\
\end{array}$ & 8 & 04 & $\begin{array}{l}44.0 \\
51.3\end{array}$ & & 7 & \\
\hline 06 & 33.8 & 40 & 18 & 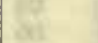 & $\begin{array}{l}06 \\
06\end{array}$ & 19.7 & 22.4 & $\begin{array}{l}4 \\
4\end{array}$ & 8 & of & & $5 \mathrm{II}$ & $\begin{array}{l}4 \\
4 \\
\end{array}$ & , & of & 56.8 & 55.8 & 37 & \\
\hline 08 & & 43 & 2I & $x^{2}$ & os & & & 52 & 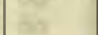 & 08 & & 48. & & As & 08 & 44.0 & & & \\
\hline To & $\begin{array}{l}35 . \\
39 .\end{array}$ & $\begin{array}{l}43 \\
46\end{array}$ & 2 & 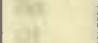 & I0 & I8.9 & 20 & 52 & 8 & I0 & & $\begin{array}{l}40 . \\
45 .\end{array}$ & $\begin{array}{l}47 \\
52\end{array}$ & 30 & $\begin{array}{l}00 \\
10\end{array}$ & $\begin{array}{l}4.0 \\
36.3\end{array}$ & & 23 og & \\
\hline I2 & 38. & 45.9 & 26 & 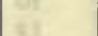 & 12 & I9. I & & 52 & & I & 48.2 & & 52 & & I & & 42.9 & 2257 & \\
\hline I & 25.0 & & 04 & & 14 & 18.9 & 20 & 52 & -7.0 & I & & 47 & 49 & -6 & & 49.8 & 49.2 & 48 & -6.4 \\
\hline 16 & & 38. & 17 & -8.4 & 16 & 19.2 & 21 & 52 & 8 & It & 53 & 49. & $\begin{array}{l}49 \\
44\end{array}$ & $-u$. & I & $\begin{array}{l}49.0 \\
55.2\end{array}$ & $\begin{array}{l}49.2 \\
53.3\end{array}$ & $\begin{array}{l}40 \\
40\end{array}$ & -0.4 \\
\hline I8 & & 46. & 31 & & Is & I8.7 & & 52 & to & $\begin{array}{l}\text { It } \\
\text { I }\end{array}$ & & 51.1 & 42 & & I & 51.9 & 50.1 & 46 & \\
\hline 2 & & & & m. & 2 & & 21 & 5 & 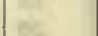 & & & & 4 & & & & & $\begin{array}{l}40 \\
5 \mathrm{I}\end{array}$ & \\
\hline 22 & & 36.8 & I & zes & 2 & & & & ent & 2 & 47.8 & & 53 & 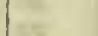 & & 44.8 & 43.6 & $\begin{array}{l}51 \\
57\end{array}$ & \\
\hline 2 & & & 2255 & a & 2 & I8. 6 & & $\begin{array}{l}54 \\
52\end{array}$ & ate & 24 & & & 57 & 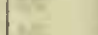 & 2 & & & $\begin{array}{l}57 \\
50\end{array}$ & \\
\hline & & 30 & 2 & 5 & 2 & 7.0 & & 50 & 5 & 26 & & & 57 & 8 & 26 & & & $\begin{array}{l}50 \\
37\end{array}$ & \\
\hline 28 & & 37. & I & & 28 & & 19 & 48 & & 28 & & & 58 & the & 28 & & & & \\
\hline 30 & 2 & & 08 & -8.1 & 30 & & & 45 & -7.0 & 30 & & & 53 & w & 30 & & & $\begin{array}{l}40 \\
58\end{array}$ & \\
\hline 32 & & & 16 & & 32 & & & 45 & & 32 & & & & 2 & 32 & 48.3 & & 52 & -6.4 \\
\hline 34 & & & & & 34 & & & 45 & 4 & 34 & & 44.8 & & 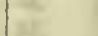 & 34 & & & 46 & \\
\hline 36 & & & & & 36 & & & 46 & & 36 & & 42.5 & 2256 & 8 & 36 & & & I & \\
\hline 38 & & & & the & 38 & & & 47 & re & 38 & & 40.0 & 2300 & & 38 & & $4 \mathrm{I} .2$ & 2259 & \\
\hline 40 & & & & & 40 & & & 49 & & 40 & & 41.8 & 2258 & & 40 & 39.6 & & 2306 & \\
\hline 42 & & & & & 42 & & & 49 & & 42 & & $\begin{array}{l}41.0 \\
45.9\end{array}$ & $5 \mathrm{I}$ & & 42 & 41.0 & 37.8 & 2304 & \\
\hline 44 & & 33. & 10 & & 44 & 16.8 & 19.8 & $\begin{array}{l}49 \\
50\end{array}$ & -7.0 & 44 & & & $\begin{array}{l}51 \\
53\end{array}$ & -6.6 & 44 & 53.8 & 50.9 & $22 \quad 44$ & \\
\hline 46 & 28 & 32 & 2309 & & 46 & 17.8 & 19.9 & $5 \mathrm{I}$ & 7.0 & $\begin{array}{l}44 \\
46\end{array}$ & $\begin{array}{l}41.0 \\
51.0\end{array}$ & $\begin{array}{l}44.3 \\
48.5\end{array}$ & $\begin{array}{l}53 \\
47\end{array}$ & -0. & $\begin{array}{l}44 \\
46\end{array}$ & $\begin{array}{l}59.2 \\
59.2\end{array}$ & 57.2 & $\begin{array}{r}44 \\
35\end{array}$ & \\
\hline 48 & & 26.8 & 2259 & & 48 & 17.1 & 19.2 & 49 & E & 48 & 46 & & & 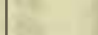 & 48 & & & 38 & \\
\hline 50 & 26.5 & 30.3 & 2305 & & 50 & 14.7 & 17.5 & 46 & 3 & 50 & 48.9 & $\begin{array}{l}43.1 \\
42.0\end{array}$ & $\begin{array}{ll}22 & 54 \\
24\end{array}$ & a & 50 & 48.3 & $\begin{array}{l}46.7 \\
46.7\end{array}$ & 52 & \\
\hline 52 & 25.2 & 29. & 03 & & 52 & I3. I & 16 & 44 & 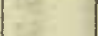 & 52 & 40.8 & 37 & $\begin{array}{lll}23 & 03\end{array}$ & 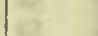 & 52 & & & 53 & \\
\hline 54 & 26.9 & 30. I & 05 & & 54 & I5. I & 18.8 & 47 & 5 & 54 & 44 & 42.0 & 2258 & & 54 & 50.9 & 50. & 47 & \\
\hline & 23.7 & 27.3 & 2300 & & 56 & I 5.2 & I8.2 & 47 & 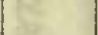 & 56 & $49 . \mathrm{I}$ & 46.0 & $5 \mathrm{I}$ & & 56 & 56.2 & & 39 & \\
\hline 50 & 22.0 & 25 . I & 2257 & & 58 & $\mathrm{I} 4 . \mathrm{I}$ & 16.8 & 45 & ter & 58 & 45.0 & 40.6 & 58 & & 58 & 52.8 & 50.8 & 45 & \\
\hline & & 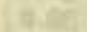 & in & of hat & & & & & & & & & & & 00 & 55.9 & 51.1 & 42 & -6.5 \\
\hline
\end{tabular}

Observer-R. R. T.

Correction to local mean time is $+4 \mathrm{~h}$ ors. $90^{\circ}$ torsion $=\mathrm{r} 3 . .^{\prime} 8$. Torsion head at oh oom read $87^{\circ}$ and at roh oom read $63^{\circ}$.

Observer-R. R. T. 
Tabulation of magnetic declinations observed at Teplitz Bay-Continued

\begin{tabular}{|c|c|c|c|c|c|c|c|c|c|c|c|c|c|c|c|c|c|c|c|}
\hline \multicolumn{4}{|c|}{ Monday, January 18, 1904} & \multicolumn{6}{|c|}{ Magnet scale erect } & \multicolumn{5}{|c|}{ Tuesday, January I9, 1904} & \multicolumn{5}{|c|}{ Magnet scale inverted } \\
\hline \multirow{2}{*}{$\begin{array}{l}\text { Chr'r } \\
\text { time }\end{array}$} & \multicolumn{2}{|c|}{$\begin{array}{l}\text { Scale } \\
\text { readings }\end{array}$} & \multirow{2}{*}{$\begin{array}{c}\text { East } \\
\text { decli- } \\
\text { nation }\end{array}$} & Temp. & Chr'r & $\begin{array}{l}\text { Sc: } \\
\text { read }\end{array}$ & $\begin{array}{l}\text { ale } \\
\text { fings }\end{array}$ & East & Temp. & Chr'r & $\begin{array}{l}\mathrm{Sc} \\
\text { read }\end{array}$ & ngs & $\begin{array}{l}\text { East } \\
\text { decli- }\end{array}$ & Temp. & & $\begin{array}{r}\mathrm{Sc} \\
\text { read }\end{array}$ & $\begin{array}{l}\text { ale } \\
\text { lings }\end{array}$ & $\begin{array}{l}\text { East } \\
\text { decli- }\end{array}$ & Temp. \\
\hline & Left & Right & & & & Left & Right & & & & Left & Right & nation & & & Left & Right & ition & \\
\hline $\mathrm{h} \mathrm{m}$ & d & d & $\cdot \cdot$ & $\bullet$ & h $\mathrm{m}$ & d & $\mathrm{d}$ & $\circ \quad$ & & $\mathrm{h} \mathrm{m}$ & d & d & $\cdot$, & & $\mathrm{h} \mathrm{m}$ & d & d & $\circ$, & $\bullet$ \\
\hline 800 & 51.9 & 53.8 & 2240 & -10.0 & so 00 & 51.7 & 53.0 & 2239 & -6.8 & 1200 & 51.9 & 50.7 & 2249 & -1.8 & 1400 & $54 \cdot 5$ & 52.9 & 2246 & -2.2 \\
\hline 02 & 50.1 & 51.8 & 37 & & 02 & 51.2 & 53. I & 39 & a & 02 & 52.1 & 5I.I & 49 & 35 & 02 & 54.4 & 53. I & & seit \\
\hline 04 & 55.4 & 57.7 & 45 & 8 & 04 & 50.1 & 52.1 & 37 & 3 & 04 & 52.8 & $5 I .3$ & 48 & $\ln$ & 04 & 54.7 & 53.2 & 45 & 48 \\
\hline 06 & 50.7 & 53.9 & 39 & 1 & 06 & 48.9 & 51.0 & 35 & ta & 06 & 53.1 & 50.7 & 49 & & 06 & 54.9 & 53.4 & 45 & 6 \\
\hline 08 & 49.9 & 53.3 & 38 & 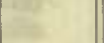 & 08 & $47 \cdot 3$ & 48.3 & 32 & 2 & 08 & 52.7 & 51.2 & 49 & & 08 & 54.9 & 53.6 & 45 & 6 \\
\hline 10 & 50.5 & 53.0 & 38 & . & 10 & 49.9 & 52.4 & 37 & & Io & 52.4 & 50.7 & 49 & tit & 10 & 54.7 & 53.2 & 45 & \\
\hline 12 & 49.2 & 52.3 & 36 & & 12 & 52.7 & $54 \cdot 3$ & $4 I$ & & I2- & 53.3 & 50.5 & 49 & & 12 & 54.6 & 53.1 & 46 & \\
\hline 14 & 53.8 & 57.3 & 44 & -9.1 & 14 & 50.1 & 52.3 & 37 & -6.7 & 14 & 53.7 & $5 \mathrm{I} . \mathrm{I}$ & 48 & -1.8 & 14 & 54.3 & 53.2 & 46 & -2.2 \\
\hline 16 & 52.8 & $54 . \mathrm{I}$ & $4 \mathrm{I}$ & ser & 16 & 48.9 & 49.1 & 34 & & 16 & 53.2 & $52 . I$ & 47 & & 16 & 54.8 & 52.4 & 46 & 54 \\
\hline 18 & 50.9 & 54.1 & 39 & $y$ & 18 & 5 I. 8 & 52.1 & 38 & 67 & 18 & 52. I & 50.9 & 49 & At & 18 & 54.8 & 52.2 & 46 & 6 \\
\hline 20 & 52.0 & 53.8 & 40 & $\alpha$ & 20 & 51.2 & 51.3 & 37 & II & 20 & 52.2 & 50.9 & 49 & is & 20 & 54.7 & 52.2 & 46 & 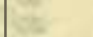 \\
\hline 22 & 48.3 & 51.2 & 35 & an & 22 & 50.7 & 51.0 & 36 & & 22 & 52.9 & 51.9 & 48 & 3 & 22 & 54.9 & 53.0 & 45 & 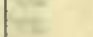 \\
\hline 24 & 53.3 & 55.7 & 42 & ite & 24 & 54.2 & 54.8 & 42 & $z$ & 24 & 53.2 & 52.1 & 47 & t8 & 24 & 54.9 & 53.0 & 45 & $=$ \\
\hline 26 & $5 \mathrm{I} .0$ & 52.3 & 38 & tra & 26 & 49.2 & 50.4 & 35 & 2 & 26 & 52.9 & 51.9 & 48 & & 26 & 54.8 & 53.0 & 45 & 5 \\
\hline 28 & 48.3 & 50.3 & 34 & & 28 & 51.3 & 52.8 & 38 & & 28 & 52.2 & 51.5 & 49 & & 28 & 54.9 & 53.0 & 45 & $1-$ \\
\hline 30 & 53.1 & 54.9 & 42 & -8.9 & 30 & 51.9 & 52.6 & 39 & -6.3 & 30 & 52.8 & 51.6 & 48 & -2.0 & 30 & $54 . \overline{8}$ & 53.7 & 45 & -2.2 \\
\hline 32 & 48.6 & 50.9 & 35 & & 32 & 49.9 & 50.9 & 36 & & 32 & 53.0 & 51.8 & 48 & & 32 & 54.2 & 53.8 & 45 & 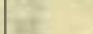 \\
\hline 34 & 49.2 & 51.1 & 35 & 4 & 34 & 50.9 & 51.6 & 37 & & 34 & 53.9 & 51.2 & 48 & 3 & 34 & 54.1 & 53.4 & 46 & 72 \\
\hline 36 & 51.5 & 52.9 & 39 & $\theta$ & 36 & 50.9 & 52.1 & 38 & & 36 & 53.9 & 50.9 & 48 & $x^{2}$ & 36 & 54.2 & 53.2 & 46 & 8 \\
\hline 38 & 56.0 & 57.6 & 46 & & 38 & 51.2 & 53.8 & 39 & & 38 & 53.8 & 50.3 & 48 & & 38 & 54.4 & 53.3 & 46 & $y$ \\
\hline 40 & 48.2 & 50.1 & 34 & 4 & 40 & 53.1 & 55.5 & 42 & & 40 & 53.2 & 50.5 & 49 & & 40 & 54.8 & $53 \cdot 3$ & 45 & 2 \\
\hline 42 & 47.9 & 50.8 & 34 & & 42 & 53.2 & 54.8 & 42 & & 42 & 53.1 & 50.9 & 48 & & 42 & $55 . I$ & 53.3 & 45 & \\
\hline 44 & 51.2 & 54.1 & 39 & -8.3 & 44 & 56.6 & 57.9 & 46 & -6.2 & 44 & 53.8 & 51.2 & 48 & -2.2 & 44 & 55.2 & 53.1 & 45 & -2.2 \\
\hline 46 & 51.9 & 53.1 & 39 & & 46 & 55.1 & 56.4 & 44 & 5 & 46 & 54.1 & 50.9 & 48 & - & 46 & & 52.9 & 45 & $S_{2}$ \\
\hline 48 & 52.9 & 54.3 & 41 & 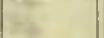 & 48 & 52.2 & 54.7 & 4I & & 48 & 53.9 & 51.2 & 48 & 5 & 48 & 55.6 & 53.1 & 45 & 5 \\
\hline 50 & 51.0 & 52.0 & 38 & $y^{2}$ & 50 & $52 . I$ & 54.5 & 40 & & 50 & 53.1 & 50.9 & 48 & F & 50 & 55.9 & & 45 & in \\
\hline $5^{2}$ & $5^{2.0}$ & 52.5 & 38 & 0. & 52 & 51.0 & 53.9 & 39 & & 52 & 53.1 & $5 \mathrm{I} . \mathrm{I}$ & 48 & - & 52 & 55.7 & 52.8 & 45 & ci. \\
\hline 54 & 51.9 & 52.9 & 39 & 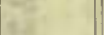 & 54 & 50.9 & 53.9 & 39 & & 54 & $53 \cdot 3$ & $5 \mathrm{I} .7$ & 48 & int & 54 & 55.2 & 52.9 & 45 & 15 \\
\hline 56 & $5 \mathrm{I} . \mathrm{I}$ & 52.3 & 38 & & 56 & 51.9 & 52.5 & 39 & & 56 & $53 \cdot 3$ & 51.8 & 48 & & 56 & 55 & 52.9 & 45 & 58 \\
\hline 58 & 50.7 & 51.9 & 37 & & 58 & 52.2 & 52.9 & 39 & & 58 & 53.2 & $5 \mathrm{I} .5$ & 48 & & 58 & & 53 & 45 & \\
\hline 900 & $5 \mathrm{I} .4$ & $53 . \mathrm{I}$ & 39 & -8.0 & II 00 & 52.0 & 52.9 & 39 & -6.0 & 1300 & 53.2 & 51.2 & 48 & -2.2 & 1500 & 55.3 & 53.3 & 45 & -2.2 \\
\hline 02 & $52 . I$ & 52.9 & 39 & 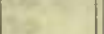 & 02 & 5 I. 4 & 53.2 & 39 & $12 x=$ & 02 & 53.9 & $5 \mathrm{I} . \mathrm{I}$ & 48 & $y^{2}$ & 02 & 55.2 & $53 . I$ & 45 & $y^{2}$ \\
\hline 04 & 52.7 & $54 . \mathrm{I}$ & $4 \mathrm{I}$ & E. & 04 & 53.1 & 53.9 & 40 & & 04 & 54.0 & $5 \mathrm{I} . \mathrm{I}$ & 48 & 8 & 04 & 9 & 52.8 & 45 & 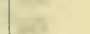 \\
\hline 06 & 53.1 & 55.0 & 42 & 5 & 06 & 52.6 & 54.2 & $4^{I}$ & the & 06 & $54 \cdot 3$ & 51.4 & 47 & 6 & 06.4 & & 52.9 & 45 & 68 \\
\hline 08 & 51.0 & 53.1 & $3^{8}$ & & 08 & 52.8 & 54.3 & $4^{I}$ & & 08 & 54.1 & 51.8 & 47 & 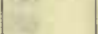 & 08 & 55.8 & 52.8 & 45 & 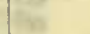 \\
\hline Io & 51.3 & $54 \cdot 3$ & 40 & 34 & 10 & 52.3 & 54.7 & $4 \mathrm{I}$ & 13 & I0 & 54.3 & 42.0 & 47 & (iii & 10 & 55.7 & 52.9 & 45 & 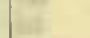 \\
\hline 12 & 50.7 & 53.3 & 38 & & I2 & 52.2 & 54.3 & $4^{0}$ & & I2 & 54.3 & 52.2 & 47 & & 12 & & 52. & 45 & \\
\hline I4 & 50.8 & 54.0 & 39 & -7.7 & I4 & 52.0 & 54.7 & 40 & -5.9 & 14 & 53.9 & 52.2 & 47 & -2.2 & I4 & 55.2 & 53.2 & 45 & -2.3 \\
\hline 16 & $5 \mathrm{I} .1$ & 53.5 & 39 & & I6 & 51.9 & 56.1 & 42 & & 16 & 53.3 & 51.6 & 48 & 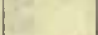 & 16 & 55.2 & 53.1 & 45 & the \\
\hline I8 & 51.1 & 53.4 & 39 & & 18 & 51.6 & $55 . \mathrm{I}$ & 40 & ts & 18 & 54.0 & 52.0 & 47 & 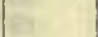 & I8 & 55.1 & $53 \cdot 3$ & 45 & 08 \\
\hline 20 & 51.7 & 53.3 & 39 & aif & 20 & 5 I.9 & 55. & 41 & 8 & 20 & 54.1 & 52.1 & 47 & $=$ & 20 & & 53.8 & 45 & 0 \\
\hline 22 & 50.9 & 52.1 & 38 & 2 & 22 & 51.3 & 55.0 & 40 & 8 & 22 & 54 . I & 51.9 & 47 & 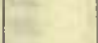 & 22 & 54.9 & 53.9 & 45 & 28: \\
\hline 24 & 52.0 & 53.0 & 39 & & 24 & 51.5 & 55. & 40 & $=$ & 24 & 54.1 & 51. & 47 & wa & 24 & & & 45 & as \\
\hline 26 & 51.9 & 53.0 & 39 & & 26 & 51. I & 53.9 & 39 & E & 26 & 54.0 & 51.7 & 47 & & 26 & 55 & 53.9 & 45 & 68 \\
\hline 28 & 51.6 & 52.2 & 38 & & 28 & 50.8 & 53 & 39 & & 28 & 54.2 & 52 & 47 & 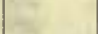 & 28 & & 53 & 45 & \\
\hline 30 & 52.3 & 54.1 & 40 & -7.2 & 30 & 50.1 & 53.2 & 38 & -5.8 & 30 & 54.2 & 52.2 & 47 & -2 & 30 & 55.4 & 53.2 & 45 & -2.3 \\
\hline 32 & $5 \mathrm{I} .2$ & $5 \mathrm{I} .9$ & 38 & & 32 & 50.2 & 53.8 & 38 & & 32 & 54.7 & 52. & 46 & 2 & 32 & & 52 & 45 & 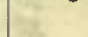 \\
\hline 34 & 52.7 & 53.3 & 40 & & 34 & 50.2 & 53.7 & 38 & th & 34 & 55.0 & 52 & 46 & 38 & 34 & 56.1 & 52 & 45 & 12 \\
\hline 36 & 52.3 & 52.3 & 39 & & 36 & 50.6 & 54.0 & 39 & & 36 & 54.8 & 52.8 & 46 & & 36 & & 52 & 45 & $y$ \\
\hline 38 & 50.3 & 51.2 & 36 & & 38 & 50.3 & 54.1 & 39 & 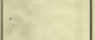 & 38 & $54 \cdot 3$ & 52.9 & 46 & 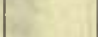 & 38 & 56.2 & 52 & 45 & 0 \\
\hline 40 & 48.7 & 49.8 & 33 & & 40 & 50.7 & 54.2 & 39 & 6 & 40 & 54.2 & 52.8 & 46 & & 40 & 56.2 & 52.7 & 45 & b. \\
\hline 42 & 52.6 & 53.8 & 40 & & 42 & 51.7 & 55.0 & 40 & -6 & 42 & 54.0 & 52.7 & 46 & & 42 & 56.1 & 52.9 & 45 & \\
\hline 44 & 48.9 & 49.7 & 34 & -7.0 & 44 & $52 . I$ & 55.2 & $4 I$ & -5.6 & 44 & 54.2 & 52.9 & 46 & -2.2 & 44 & 56.0 & 53.0 & 45 & -2.3 \\
\hline 46 & 51.0 & 52.0 & 38 & & 46 & 51.8 & 54.8 & 40 & & 46 & 54.7 & 53.2 & 45 & & 46 & 56.2 & 52.9 & 45 & \\
\hline 48 & 52.2 & 53.9 & 40 & & 48 & 52.4 & 55.0 & $4 \mathrm{I}$ & & 48 & 54.9 & 53. & 45 & $E$ & 48 & 56.1 & 52 & 45 & $\mathrm{~s}$ \\
\hline 50 & 48.2 & 50.1 & 34 & & 50 & 51.8 & 54.1 & 40 & & 50 & 54.2 & 52.7 & 46 & $\theta$ & 50 & 55.9 & 53.5 & 44 & gat \\
\hline 52 & 50.1 & 51.7 & 37 & & 52 & 52.2 & 54.5 & 40 & & 52 & $54 . \mathrm{I}$ & 52.1 & 47 & 5 & 52 & 55.8 & & 44 & 3 \\
\hline 54 & 53.2 & $54 . I$ & 4I & & 54 & 52.7 & 54.8 & $4 I$ & & 54 & 54.5 & 52.7 & 46 & & 54 & 55.8 & & 44 & \\
\hline 56 & 51.7 & 52.2 & 38 & & 56 & 52.3 & $56 . I$ & 42 & & 56 & 54.9 & 53.2 & 45 & 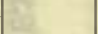 & 56 & 55.9 & 53.8 & 44 & \\
\hline 58 & $49 \cdot 3$ & 50. I & 35 & & 58 & 52.2 & 56.1 & 42 & & 58 & 54.7 & 52.9 & 46 & a & 58 & 55.7 & 53.7 & 44 & \\
\hline & & & & & 1200 & 51.1 & 53.1 & 39 & $-5 \cdot 3$ & & & & & & 500 & $55 . I$ & 53.2 & 45 & -2 . \\
\hline
\end{tabular}

Correction to local mean time is $+3 \mathrm{~m}$ ios. $90^{\circ}$ torsion $=12 . ' 8$.

Torsion head at Ioh com, January 17 , read $63^{\circ}$ and at 13 h Iom, January 18 , read $60^{\circ}$.

Observer-R. R. T.
Correction to local mean time is $+2 \mathrm{~m} 35 \mathrm{~s}$.

Torsion head at IIh $45 \mathrm{~m}$ read $60^{\circ}$ and at the end read the same. Observer-R. R. T. 
Tabulation of magnetic declinations observed at Teplitz Bay-Continued

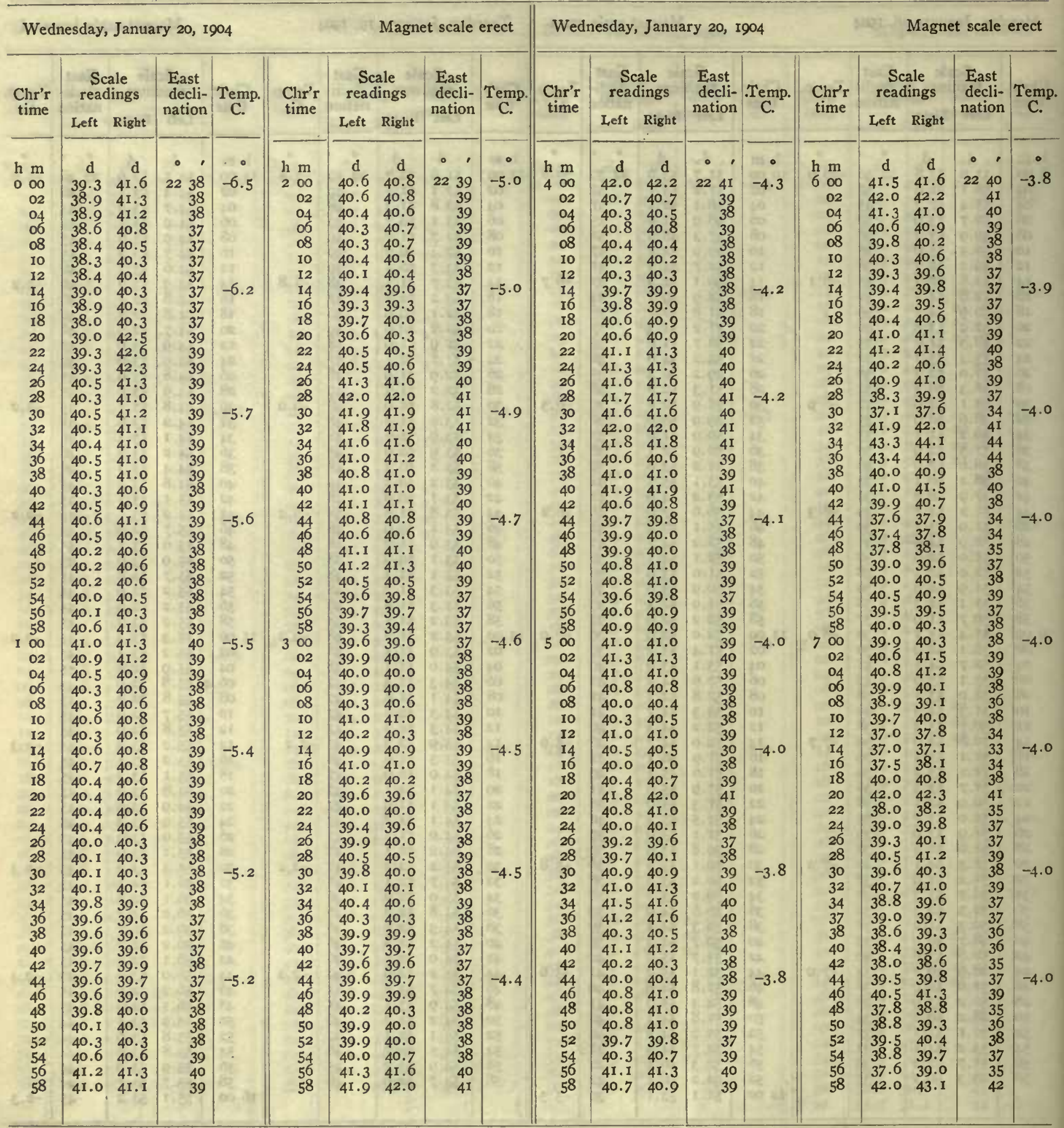

Observer-W. J. P.

Observers-W. J. P. and J. V., who alternated from 7 h $50 \mathrm{~m}$ to 7 h $56 \mathrm{~m}$. 
Tabulation of magnetic declinations observed at Teplitz Bay-Continued

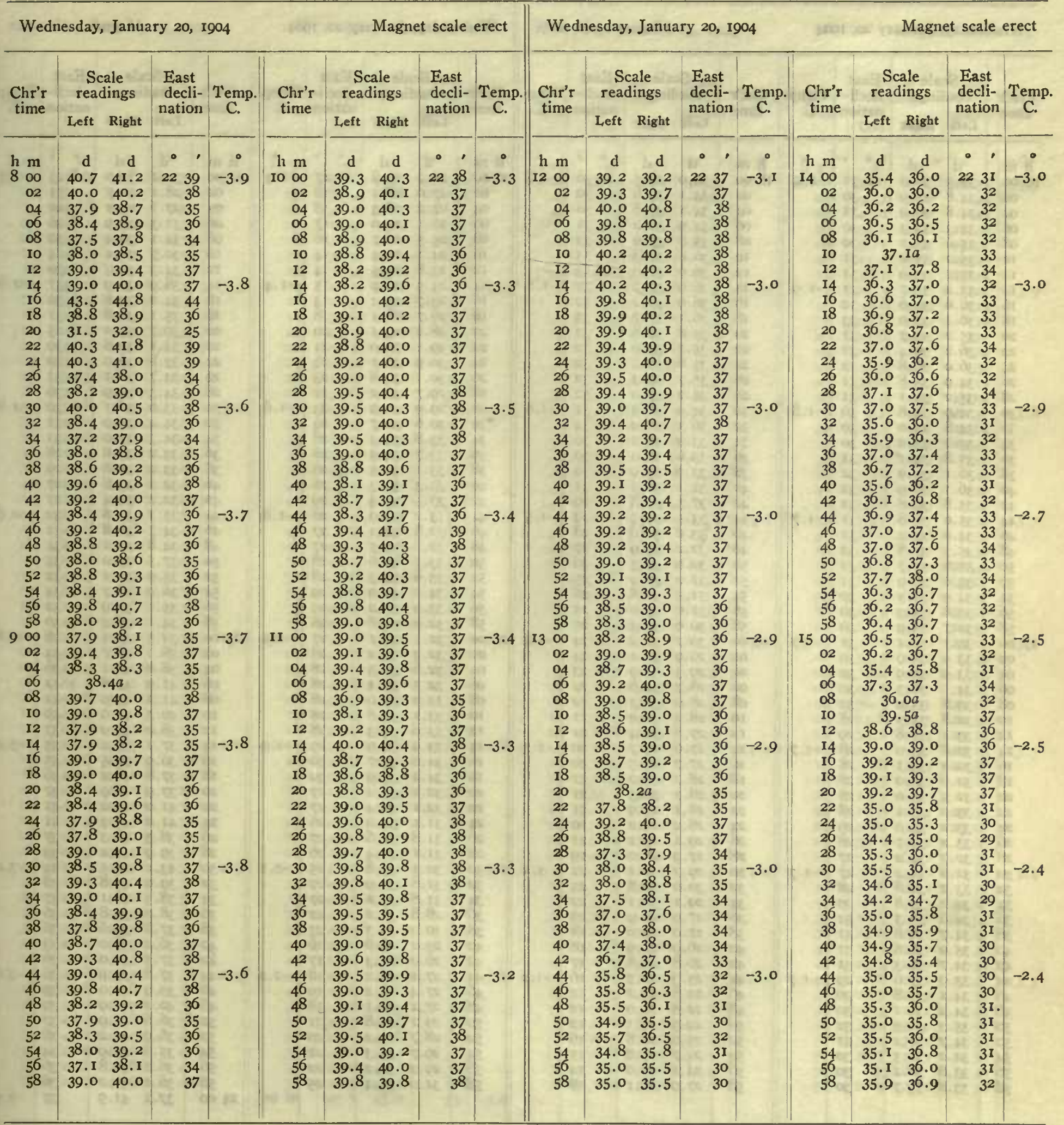

Observer-J. V.
Observers-J. V. and R. R. T., who alternated from $15 \mathrm{~h} 52 \mathrm{~m}$ to $16 \mathrm{~h}$ $04 \mathrm{~m}$. 
Tabulation of magnetic declinations observed at Teplitz Bay-Continued

\begin{tabular}{|c|c|c|c|c|c|c|c|c|c|c|c|c|c|c|c|c|c|c|c|c|c|c|}
\hline \multicolumn{6}{|c|}{ Wednesday, January 20, 1904} & \multicolumn{6}{|c|}{ Magnet scale erect } & \multicolumn{6}{|c|}{ Wednesday, Janttary 20, 1904} & \multicolumn{5}{|c|}{ Magnet scale erect } \\
\hline \multirow{2}{*}{$\begin{array}{l}\text { Chr'r } \\
\text { time }\end{array}$} & $\begin{array}{r}\mathrm{Sc} \\
\text { read }\end{array}$ & $\begin{array}{l}\text { ale } \\
\text { lings }\end{array}$ & $\begin{array}{l}\text { Ea } \\
\text { dec }\end{array}$ & st & Temp. & Chr'r & $\begin{array}{r}\text { Sc } \\
\text { reac }\end{array}$ & $\begin{array}{l}\text { ale } \\
\text { lings }\end{array}$ & $\begin{array}{l}\text { Ea } \\
\text { dec }\end{array}$ & & Temp. & Chr'r & $\begin{array}{r}\mathrm{Sc} \\
\text { reac }\end{array}$ & $\begin{array}{l}\text { ale } \\
\text { lings }\end{array}$ & $\begin{array}{l}\text { Eas } \\
\text { dec }\end{array}$ & $\begin{array}{l}\text { st } \\
\text { cli- }\end{array}$ & Temp. & Chr'r & $\begin{array}{r}\mathrm{Sc} \\
\mathrm{read}\end{array}$ & $\begin{array}{l}\text { ale } \\
\text { lings }\end{array}$ & $\begin{array}{l}\text { East } \\
\text { decli- }\end{array}$ & Teinp. \\
\hline & L,eft & Right & & & & & Leît & Right & & & & & Left & Right & & & & & Left & Right & & \\
\hline h in & d & d & $\circ$ & , & $\circ$ & $\mathrm{h} \mathrm{m}$ & d & $\mathrm{d}$ & 10 & & $=0$ & $\mathrm{~h} \mathrm{~m}$ & d & $d$ & 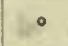 & - & 170 & $\mathrm{~h} \mathrm{~m}$ & $\mathrm{~d}$ & d & 0 & $\bullet$ \\
\hline 1600 & $35 \cdot 5$ & 36.0 & 22 & 31 & -2.2 & 1800 & 34.0 & 35.5 & 22 & & -1.6 & 2000 & 26.2 & 27.8 & 22 & 17 & -1.3 & 2200 & $30 . I$ & 31.0 & 2223 & -2.3 \\
\hline 02 & 34.8 & 35.9 & & 30 & & 02 & $34 . I$ & 35.6 & & 30 & 0 & 02 & 27.0 & 28.1 & & 18 & $10+3$ & 02 & 28.1 & 30.7 & $3 \mathrm{I}$ & \\
\hline 04 & 34.8 & 36.0 & & 3I & thes & 04 & 34 . I & 35.8 & & 30 & 0 & 04 & 27.2 & 28.3 & & 18 & 1 & 04 & 27.1 & 29.7 & 20 & \\
\hline 06 & 34.3 & 35.3 & & 30 & 4 & 06 & 34.9 & 36.2 & & $3 I$ & 1 & 06 & 26.1 & 27.0 & & 17 & Al & 06 & 3 I. 3 & 33.9 & 26 & \\
\hline 08 & 35.0 & 35.2 & & 30 & & 08 & 35.8 & 37.0 & & 32 & 0 & 08 & 25.5 & 26.7 & & 16 & ar & 08 & 34. & 36.4 & 30 & \\
\hline 10 & 35.2 & 35.8 & & $3 I$ & $t$ & I0 & 36.8 & $37 \cdot 3$ & & 33 & 15 & 10 & 24.1 & 25.4 & & I4 & 17 & 10 & 30.7 & 32. I & 24 & \\
\hline I2 & 35.4 & 35.9 & & $3 I$ & & 12 & 36.6 & 37.0 & & 33 & $15=$ & 12 & 23.8 & 25.5 & & I4 & 6 & I2 & 34.3 & 36.3 & 30 & \\
\hline I4 & 35.2 & 35.7 & & $3 \mathrm{I}$ & -1.9 & I4 & 36.9 & 37.2 & & 33 & -1.6 & 14 & 24.5 & 26.2 & & I5 & -1.4 & 14 & 38.9 & 39.9 & 37 & -2.6 \\
\hline 16 & 36.0 & 36.6 & & 32 & & 16 & 36.0 & 37.2 & & 32 & $15=$ & 16 & 23.7 & 25.2 & & I3 & & 16 & 34.2 & 35.8 & 30 & \\
\hline 18 & 36.4 & 37.0 & & 33 & & 18 & 36.1 & 37.7 & & 33 & tr. & 18 & 26.7 & 28.1 & & 18 & 6 & 18 & $35 . \mathrm{I}$ & 36.8 & $3 I$ & \\
\hline 20 & 35.2 & 35.7 & & 31 & 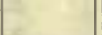 & 20 & 35.8 & 36.8 & & 32 & E & 20 & 28.2 & 29.1 & & 20 & 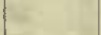 & 20 & 30.7 & 33.2 & 25 & \\
\hline 22 & 36.2 & 36.4 & & 32 & te & 22 & 35.2 & 37.0 & & 32 & $=$ & 22 & 29. I & 30.0 & & 21 & 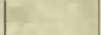 & 22 & 34 . I & 35.8 & 30 & \\
\hline 24 & 36.0 & 36.8 & & 32 & $t$ & 24 & 35.8 & 37.5 & & 32 & 6 & 24 & 30.9 & 32.1 & & 24 & 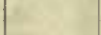 & 24 & 33.8 & 36.0 & 30 & \\
\hline 26 & 34.9 & 35.8 & & 3I & & 26 & 35.7 & $37 \cdot 3$ & & 32 & a & 26 & 30.1 & 30.8 & & 23 & - & 26 & 34.7 & 36.1 & $3 I$ & \\
\hline 28 & 35.2 & 36.1 & & 31 & & 28 & 35.2 & 37.0 & & 32 & -2 & 28 & 29.7 & 30.2 & & 22 & 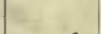 & 28 & 34.3 & 35.9 & 30 & \\
\hline 30 & 34.9 & 35.8 & & 30 & -1.8 & 30 & 34.9 & 36.9 & & $3 I$ & -1.6 & 30 & 29.9 & 30.9 & & 23 & -1.6 & 30 & 33.6 & 36.1 & 30 & -2.8 \\
\hline 32 & 34.8 & 35.0 & & 30 & & 32 & 36.3 & 38.9 & & 34 & & 32 & 30.6 & 31.7 & & 24 & & 32 & 34.4 & 37.0 & $3 I$ & \\
\hline 34 & 35.2 & 35.2 & & 30 & 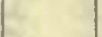 & 34 & 33.9 & 36.2 & & 30 & $\theta$ & 34 & 33.8 & 34.3 & & 28 & 1 & 34 & 34.3 & 37.0 & $3 \mathrm{I}$ & \\
\hline 36 & 35.8 & 36.1 & & $3 \mathrm{I}$ & $y$ & 36 & 33.6 & 36.0 & & 30 & 6 & 36 & 33.7 & 34.3 & & 28 & T & 36 & 33.2 & 33.8 & 28 & \\
\hline 38 & 36.1 & 36.9 & & 32 & & 38 & 33.9 & 35.9 & & 30 & 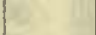 & 38 & 33. I & 34.5 & & 28 & E. & 38 & 33.2 & 35.8 & 29 & \\
\hline 40 & 36. I & 36.9 & & 32 & 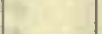 & 40 & 34.1 & 36.9 & & 3 I & 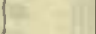 & 40 & 33.3 & 34.3 & & 28 & 4 & 40 & 32.2 & 33.8 & 27 & \\
\hline 42 & 35.8 & 36.8 & & 32 & & 42 & 37.5 & 39.8 & & 36 & & 42 & 33.5 & 35.5 & & 29 & thent & 42 & 32.2 & 34.4 & 27 & \\
\hline 44 & 35.0 & 36.8 & & $3 I$ & -1.7 & 44 & 33.5 & 35.5 & & 29 & -1.5 & 44 & 33.2 & 33.9 & & 28 & -1.8 & 44 & 35.1 & 37.8 & 32 & -2.8 \\
\hline 46 & 34.9 & 37.2 & & $3 \mathbf{I}$ & & 46 & 33.0 & 34.6 & & 28 & & 46 & 32.8 & 34.1 & & 27 & 45 & 46 & 34.7 & 36.8 & 31 & \\
\hline 48 & 35.3 & 37.8 & & 32 & & 48 & 32.2 & 33.9 & & 27 & e & 48 & 32.1 & 33.7 & & 27 & 1 & 48 & 34.8 & 37.1 & $3 \mathrm{I}$ & \\
\hline 50 & 35.6 & 37.7 & & 32 & & 50 & 32.0 & $33 \cdot 3$ & & 26 & w & 50 & 33.1 & 34.6 & & 28 & I & 50 & 37.5 & 39.0 & 35 & \\
\hline 52 & 35.0 & 36.9 & & 31 & & 52 & 31.2 & 32.9 & & 25 & 8 & 52 & 33.6 & 34.3 & Ex & 28 & 1 & 52 & 35.9 & 37.2 & 32 & \\
\hline 54 & 34.9 & $35 \cdot 3$ & & 30 & & 54 & 31.5 & 32.8 & & 26 & & 54 & 34.1 & 34.9 & & 29 & & 54 & 36.9 & 38.7 & 34 & \\
\hline 56 & 34.8 & 35.9 & & 30 & & 56 & 31.0 & 33.0 & & 25 & & 56 & 33.9 & 35.2 & e & 29 & & 56 & $35 \cdot \mathrm{I}$ & 37.9 & 32 & \\
\hline 58 & 34.7 & 35.9 & & 30 & & 58 & 30.9 & 32.3 & & 25 & & 58 & 32.9 & 34.8 & & 28 & & 58 & $34 . I$ & 36.5 & 30 & \\
\hline I7 00 & 34.7 & 36.1 & & $3 \mathrm{I}$ & -1.7 & 1900 & 30.7 & 32.2 & & 24 & -1.4 & 2100 & $32 . I$ & 34.7 & & 27 & -1.9 & 2300 & 32.5 & 35.4 & 28 & -2.8 \\
\hline 02 & 33.9 & 35.9 & & 30 & & 02 & 29.9 & 32.1 & & 24 & 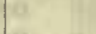 & 02 & 32.2 & 35.2 & & 28 & $1=$ & 02 & 36.1 & 38.7 & 34 & \\
\hline 04 & 33.9 & 35.9 & & 30 & . & 04 & 29.3 & 31.9 & & 23 & e & 04 & 32.6 & 35.3 & & 28 & 19 & 04 & 38.0 & 40.1 & 36 & \\
\hline 06 & 34.1 & 36.3 & & 30 & & 06 & 28.7 & 31.6 & & 22 & b & 06 & 32.3 & 35.4 & & 28 & 3 & 06 & 41.9 & 43.8 & 42 & \\
\hline 08 & 33.8 & 35.9 & & 30 & ? & 08 & 28.0 & 31.3 & & 22 & 9 & 08 & 33.0 & 35.0 & & 28 & p & 08 & 41.2 & 43.9 & 42 & \\
\hline 10 & 32.8 & 35.3 & & 28 & 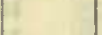 & IO & 28.0 & 31.0 & & 21 & 1 & 10 & 33.1 & 34.8 & 8 & 28 & If & IO & 40.3 & 42.9 & 40 & \\
\hline 12 & 33.1 & 35.8 & & 29 & & I2 & 27.8 & 30.4 & & $2 I$ & & 12 & 33.0 & 34.3 & & 28 & & I2 & 38.0 & 40.9 & 37 & \\
\hline 14 & 32.8 & 35.0 & & 28 & -1.7 & 14 & 27.6 & 29.7 & & 20 & $-1 \cdot 3$ & 14 & 33.9 & 34.9 & c8 & 29 & -2.0 & I4 & 37.1 & 39.2 & 35 & -3.0 \\
\hline 16 & 32.2 & 35.1 & & 28 & & 16 & 27.7 & 29.5 & & 20 & & 16 & 34.0 & 35.7 & e & 30 & & I6 & 36.9 & 39.5 & 35 & \\
\hline 18 & 32.6 & 35.0 & & 28 & t & I8 & 28.7 & 30.2 & & $2 \mathrm{I}$ & r & 18 & 34.2 & 35.9 & & 30 & if & 18 & 37.8 & 40.2 & 36 & \\
\hline 20 & 32.5 & 35.0 & & 28 & & 20 & 28.7 & 30.3 & & $2 I$ & & 20 & 32. I & 34.2 & & 27 & 8 & 20 & 37.9 & 40.9 & 37 & \\
\hline 22 & 33.0 & 35.1 & & 28 & $=$ & 22 & 28. I & 29.9 & & $2 I$ & $=$ & 22 & 32.1 & 34.4 & e & 27 & 5 & 22 & $4 \mathrm{I} .0$ & 43.2 & $4 \mathrm{I}$ & \\
\hline 24 & 33.2 & 35.0 & & 29 & & 24 & 28.2 & 29.2 & & 20 & & 24 & 31.1 & 34.0 & & 26 & & 24 & $4 \mathrm{I} .2$ & 43.3 & $4 \mathrm{I}$ & \\
\hline 26 & 33.2 & 34.7 & & 28 & & 26 & 29.1 & 29.1 & & $2 I$ & & 26 & 31.8 & 34.6 & o & 27 & & 26 & 38.3 & 40.3 & 37 & \\
\hline 28 & 32.7 & 34.2 & & 27 & & 28 & 27.9 & 28.0 & & I9 & & 28 & 3I.I & 34.4 & & 26 & & 28 & 39.9 & 41.2 & 39 & \\
\hline 30 & 32.6 & 34.2 & & 27 & -1.7 & 30 & 29.7 & 30.1 & & 22 & -1.2 & 30 & 30.1 & 33.8 & & 25 & $-2 . I$ & 30 & 43.1 & 44.5 & 44 & -3.0 \\
\hline 32 & 33.2 & 34.8 & & 28 & & 32 & 30.0 & 30.8 & & 23 & & 32 & $3 \mathrm{I} .8$ & 34.9 & & 27 & & 32 & 39.0 & 40.8 & 38 & \\
\hline 34 & 34.2 & 35.8 & & 30 & & 34 & 29.1 & 30. I & & 22 & & 34 & 31.8 & 33.8 & 8 & 27 & & 34 & 38.5 & 40.1 & 37 & \\
\hline 36 & 34.6 & 36.3 & & $3 I$ & & 36 & 27.9 & 28.9 & & 20 & & 36 & 28.8 & 31.2 & & 22 & & 36 & 38.0 & 39.8 & 36 & \\
\hline 38 & 34.7 & 36.6 & & 31 & & 38 & 27.0 & 28.0 & 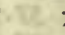 & I8 & & 38 & 30.0 & $3 \mathrm{I} .3$ & (e) & 23 & & 38 & 37.8 & 39.6 & 36 & \\
\hline 40 & 35.0 & 36.8 & & 31 & & 40 & 26.9 & 27.9 & & I8 & & 40 & 25.2 & 26.8 & & 16 & & 40 & 36.2 & 38.0 & 33 & \\
\hline 42 & 34.7 & 36.3 & & $3 \mathrm{I}$ & & 42 & 26.8 & 26.8 & Ac & 17 & & 42 & 26.0 & 27.3 & 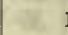 & 17 & & 42 & 37.5 & 40.0 & 36 & \\
\hline 44 & 33.9 & 35.6 & & 29 & -1.7 & 44 & 25.9 & 26.5 & & 16 & -1.2 & 44 & 27.1 & 28.2 & (1) & 18 & -2.2 & 44 & 37.8 & 39.9 & 36 & -3.0 \\
\hline 46 & 34 . I & 35.8 & & 30 & & 46 & 22.8 & 24.2 & & 12 & & 46 & 27.4 & 28.2 & 10 & 19 & & 46 & 39.9 & 42.0 & 39 & \\
\hline 48 & 34.2 & 36.1 & & 30 & & 48 & 22.8 & 24.0 & & 12 & & 48 & 27.1 & 28. I & & 18 & & 48 & 40.2 & 41.9 & 39 & \\
\hline 50 & 35.3 & 36.9 & & 32 & & 50 & 22.9 & 24.1 & & I2 & & 50 & 29.1 & 29.8 & 욜 & 21 & & 50 & 36.6 & 41.9 & 37 & \\
\hline 52 & 34.7 & 36.2 & & 31 & & $5^{2}$ & 25.0 & 26.8 & या & 16 & & 52 & 28.7 & 29.2 & Q & 20 & & 52 & 36.9 & 40.2 & 36 & \\
\hline 54 & 34.2 & 36.5 & & 30 & & 54 & 24.7 & 25.8 & 15 & I5 & & 54 & 29.7 & 30.3 & & 22 & & 54 & 35.8 & 39.9 & & \\
\hline 56 & 33.8 & 36.1 & & 30 & & 56 & $24 . I$ & 26.0 & & I4 & & 56 & 29.2 & 30.2 & ex & 22 & & 56 & 36.2 & 40.8 & 36 & \\
\hline 58 & 33.8 & 35.7 & & 30 & & 58 & 27.0 & 28. I & 26.1 & 18 & & 58 & 31.0 & 32.4 & $=$ & 25 & & 58 & 36.1 & 41.2 & 36 & \\
\hline & & & & & & & & & & & & & & & & & & 2400 & 37.1 & 41.9 & 37 & -2.9 \\
\hline
\end{tabular}

Observer-R. R. T.
Correction to local mean time is - 2 s.

Torsion head at ol oom read $40^{\circ}$ and at the end read the same. Observer-R. R. 'T. 
Tabulation of magnetic declinations observed at Teplitz Bay-Continued

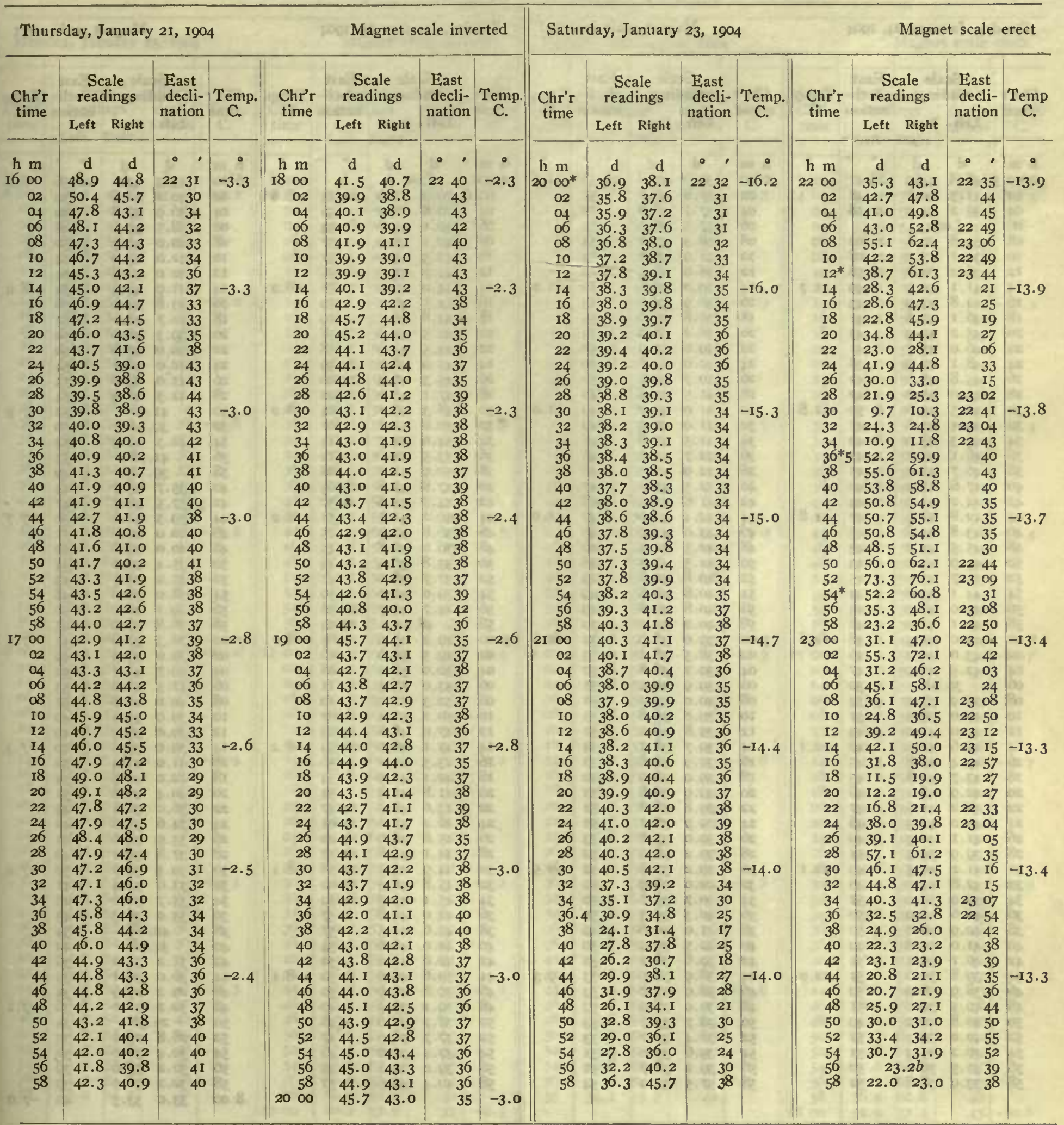

Correction to local mean time is -445 .

Torsion head at $15^{h}$ Iom read $34^{\circ}$ and at the end read the same.

Observer-R. R. T.
Correction to local mean time is $-27 \mathrm{~s}$. $90^{\circ}$ torsion $=14^{\prime}$.

Torsion head at $19 \mathrm{~h} 20 \mathrm{~m}$ read $34^{\circ}$ and at $9 \mathrm{~h} 4 \mathrm{om}$ on the $24^{\text {th }}$ read $28^{\circ}$.

Observers-R. R. T. and J. V., who alternated from $23 \mathrm{~h} 5 \mathrm{~mm}$ to $24 \mathrm{~h}$ com. 
Tabulation of magnetic declinations observed at Teplitz Bay-Continued

\begin{tabular}{|c|c|c|c|c|c|c|c|c|c|c|c|c|c|c|c|c|c|c|c|}
\hline \multicolumn{5}{|c|}{ Sunday, January 24,1904} & \multicolumn{5}{|c|}{ Magnet scale inverted } & \multicolumn{5}{|c|}{ Sunday, January 24,1904} & \multicolumn{5}{|c|}{ 174 Magnet scale erect } \\
\hline \multirow{2}{*}{$\begin{array}{c}\text { Chr'r } \\
\text { time }\end{array}$} & \multicolumn{2}{|c|}{$\begin{array}{l}\text { Scale } \\
\text { readings }\end{array}$} & \multirow{2}{*}{$\begin{array}{l}\text { East } \\
\text { decli- } \\
\text { nation }\end{array}$} & Temp. & Chr'r & $\begin{array}{r}\mathrm{Sc} \\
\text { read }\end{array}$ & $\begin{array}{l}\text { ale } \\
\text { lings }\end{array}$ & $\begin{array}{l}\text { East } \\
\text { decli- }\end{array}$ & Temp. & Chr'r & $\begin{array}{l}\text { Sc: } \\
\text { read }\end{array}$ & lings & $\begin{array}{l}\text { East } \\
\text { decli- }\end{array}$ & & Chr't & $\begin{array}{l}\text { Sc } \\
\text { reac }\end{array}$ & h & $\begin{array}{l}\text { East } \\
\text { decli- }\end{array}$ & \\
\hline & Left & Right & & & time & Left & Right & nation & & & L,eft & Right & & & & Left & Riglit & & \\
\hline h m & $\mathrm{d}$ & d & $\circ \quad$ & 0 & $\mathrm{~h} \mathrm{~m}$ & d & d & $\circ \cdot$ & & $\mathrm{h} \mathrm{m}$ & $\mathrm{d}$ & $d$ & $\circ$, & 0 & $\mathrm{~h} \mathrm{ml}$ & d & d & $\circ$, & $\circ$ \\
\hline 000.5 & 46.8 & 44.9 & $224 I$ & $\cos x$ & 200 & 39.0 & 37.8 & 2253 & -11.6 & 400 & Lo & ast & 814 & 25 & 600 & $43 \cdot 3$ & 43.6 & 2256 & -8.0 \\
\hline $02^{*}$ & 41.5 & 40.7 & 48 & $\operatorname{sen}$ & 02 & 37.8 & 36.7 & 55 & ar & 02.6 & 70.0 & 71.0 & 2255 & -8.6 & 02 & 40.2 & 41.8 & 52 & \\
\hline 04 & 35.2 & 35.0 & $225^{8}$ & 10 & 04 & 37.7 & 36.8 & 55 & 18 & 04 & 70.8 & 71.2 & 56 & 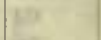 & 04 & 36.9 & 37.8 & 46 & \\
\hline 06 & Lo & st & - & 0 & 06 & 37.5 & 36.3 & 55 & 6 & $06^{*}$ & 40.1 & 44.0 & 54 & $\omega$ & 06 & 38.5 & 39.0 & 48 & 9 \\
\hline 08 & 34.8 & 30.6 & 2302 & 60 & 08 & 38.3 & 37.2 & 54 & $\infty$ & 08 & 42. I & 46.0 & 57 & Gr & 08 & 40.0 & 40.3 & 50 & 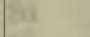 \\
\hline Io & $30 . I$ & 27.0 & 2308 & etit & IO & 38.9 & 38.0 & 53 & it & IO & 42.5 & 45.9 & 57 & or & Io & 42.0 & 43.0 & 54 & \\
\hline I2 & 36.2 & 34.2 & 2258 & tii & I2 & 38.9 & 38.3 & 52 & 65 & 12 & 42.7 & 45.0 & 56 & 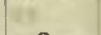 & 12 & 42.0 & 42.6 & 54 & \\
\hline I4 & $41 . I$ & 40.9 & 49 & $-\mathrm{II.7}$ & 14 & 39.0 & 38.6 & 52 & -11.2 & 14 & 44.0 & 46.0 & 58 & -8.2 & 14 & $4 \mathrm{I} .9$ & 42.3 & 54 & -8.0 \\
\hline 16 & 44.8 & 43.9 & 43 & 6y & 16 & 38.9 & 38.0 & 53 & 511 & 16 & 44.5 & $46 . I$ & 59 & 11 & I6 & 43.8 & 44.0 & 56 & \\
\hline 18 & 46.1 & 45.6 & $4 \mathrm{I}$ & Ah & 18 & 36.8 & 36.6 & 55 & 7 & 18 & 44.8 & 46. I & 59 & $=i$ & 18 & 40.7 & $4 \mathrm{I} .0$ & 52 & \\
\hline 20 & 45.2 & 44.3 & 43 & 10 & 20 & 38.2 & 38.0 & 53 & 0 & 20 & 44.5 & 45.0 & 58 & 102 & 20 & 39.3 & 40.0 & 50 & $=$ \\
\hline 22 & 46.0 & 45.7 & $4 \mathrm{I}$ & 6 & 22 & 39.0 & 39.0 & 52 & Es & 22 & 44.5 & 45.9 & 58 & 25 & 22 & 40.0 & 40.0 & 50 & a. \\
\hline 24 & 48.1 & 47.9 & 38 & 16 & 24 & 40.0 & 39.8 & 50 & 1 & 24 & 45.0 & 46.2 & 2259 & is & 24 & 42.0 & $42 . I$ & 54 & is \\
\hline 26 & 45.8 & 45.0 & 42 & is & 26 & 40.7 & 40.2 & 50 & $e$ & 26 & 45.3 & 46.4 & 2300 & $\infty$ & 26 & 43.0 & 43.0 & 55 & \\
\hline 28 & 45.0 & 45.0 & 42 & & 28 & $4 \mathrm{I} .5$ & 40.8 & 48 & E & 28 & $47 . \mathrm{I}$ & 48.0 & 02 & 1 & 28 & 42.3 & 42.3 & 54 & \\
\hline 30 & 40.5 & 40.0 & 50 & -II.8 & 30 & $4 \mathrm{I} . \mathrm{O}$ & 40.2 & 49 & -11.0 & 30 & 48.3 & 49. I & 04 & -8.2 & 6. 30 & $4 I$ & $.0 b$ & 52 & -7.9 \\
\hline 32 & 42.4 & 41.8 & 47 & & 32 & $4 \mathrm{I} .0$ & 40.5 & 49 & & 32 & 50.0 & 5I.I & 07 & 0.2 & 32 & 39 & $.5 a$ & $\begin{array}{l}52 \\
50\end{array}$ & -1.9 \\
\hline 34 & 45.3 & 45.0 & 42 & 10 & 34 & 41.5 & 40.8 & 48 & E & 34 & 54.0 & 54.7 & 13 & 16 & 34 & 42.3 & 42.3 & 54 & \\
\hline 36 & 46.1 & $45 . \mathrm{I}$ & 41 & 10 & 36 & 42.7 & 42.0 & 46 & 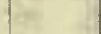 & 36 & 53.5 & 54.0 & 12 & x. & 36 & $4 \mathrm{I}$ & $.7 b$ & 53 & \\
\hline 38 & 46.7 & 45.3 & $4 I$ & 15: & 38 & 42.9 & $4^{2} .3$ & 46 & ter & 38 & 50.9 & 51.2 & 08 & 8 & 38 & 39 & $\mathrm{I} b$ & 49 & \\
\hline 40 & 47.6 & 45.2 & 40 & at & 40 & 42.5 & 42.0 & 47 & 58 & 40 & 47.8 & 47.8 & 2303 & 1 & 40 & 35.7 & 35.7 & 44 & \\
\hline 42 & 44.2 & 44.0 & 44 & & 42 & 41.8 & $4 \mathrm{I} .4$ & 48 & 12 & 42 & 44.0 & 44.8 & 2257 & 65 & 42.5 & 37.5 & 38.5 & 47 & \\
\hline 44 & 42.8 & 41.6 & 47 & -11.8 & 44 & 42.4 & 42.0 & 47 & -11.0 & 44 & 40.2 & $4 I .3$ & $5 I$ & -8.2 & 10 44 & 39.3 & 40.1 & 50 & -7.7 \\
\hline 46 & 43.8 & 42.8 & 45 & t & 46 & 43.0 & 43.0 & 46 & ton & 46 & 36.8 & 37.0 & 45 & 10 & 46 & 40.0 & $4 \mathrm{I} .0$ & $5 \mathrm{I}$ & \\
\hline 48 & 45.5 & 44.2 & 43 & 28 & 48 & 42.5 & 42.5 & 46 & 1 & 48 & 36.0 & 36.8 & 45 & 4 & 48 & 40.0 & 39.0 & 50 & \\
\hline 50 & 44.9 & 44.0 & 43 & m & 50 & 41.5 & 41.5 & 48 & $p$ & 50 & 35.8 & 36.4 & 44 & $E$ & 50 & 35.4 & 36.6 & 44 & \\
\hline 52 & 43.7 & 43.0 & 45 & Ea & 52 & 40.8 & 40.8 & 49 & 12 & 52 & 37.7 & 38.2 & 47 & 3 & 52 & 35.2 & 35.7 & 43 & \\
\hline 54 & 44.1 & 43.8 & 44 & & 54 & 40.8 & 40.8 & 49 & $2 \pi$ & 54 & 39.0 & 40.0 & 1) 50 & & 54 & 38.0 & 39.0 & 48 & \\
\hline 56 & 44.0 & 42.9 & 45 & 5 & 56 & 40.8 & 40.8 & 49 & $=$ & 56 & 44.7 & 44.7 & 2258 & 1 & 56 & 39.1 & 40.0 & 50 & \\
\hline 58 & $42 . \mathrm{I}$ & 41.0 & 48 & & 58 & 40.2 & 40.0 & 50 & & 58 & 48. & $3 a$ & 2304 & & 58 & 36.8 & 37.0 & 45 & \\
\hline 100 & 42.0 & $4 I .3$ & 48 & -12.6 & 300 & 39.0 & 38.9 & 52 & -10.6 & 500 & 49.2 & 49.9 & 05 & -8.2 & 700 & 36.3 & 37.0 & 45 & $-7 \cdot 5$ \\
\hline 02 & 43.0 & 42.2 & 46 & 60 & 02 & 38.5 & 38.2 & 53 & $t^{\prime}$ & 02 & 49.5 & 50.0 & 06 & $\infty$ & 02 & 39.0 & 39.2 & 49 & \\
\hline 04 & 42.5 & 41.9 & 47 & 10 & 04 & 39.2 & 38.8 & 52 & to & 04 & 50.0 & 52.2 & 08 & 10 & 04 & 40.7 & $4 \mathrm{I} . \mathrm{I}$ & 52 & 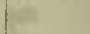 \\
\hline 06 & 39. & .06 & 52 & in & 06 & 39.9 & 39.2 & $5 \mathrm{I}$ & 6 & 06 & 53.0 & 53.5 & II & 0 & 06 & $4 \mathrm{I} .5$ & 42.0 & 53 & \\
\hline 08 & 37.9 & 37.5 & 54 & ino & 08 & 40.0 & 39.0 & $5 \mathrm{I}$ & in & 08 & 52.3 & 52.9 & Io & . & 08 & 40.0 & 40.8 & $5 \mathrm{I}$ & \\
\hline 10 & 40.0 & 39.5 & $5 \mathbf{I}$ & 164 & IO & 39.8 & 36.8 & $5 \mathrm{I}$ & hir & 10 & 50.2 & 51.1 & 07 & Bs & IO & 39.0 & 39.4 & 49 & \\
\hline 12 & 41.0 & 41.0 & 49 & si & I2 & 39.8 & 39.0 & $5 \mathrm{I}$ & is & I2 & 47.9 & 48.8 & 2303 & Ha & I2 & 39.0 & 39 & 49 & \\
\hline 14 & 42.6 & 42.0 & 46 & -12.1 & I4 & 39.7 & 38.7 & $5 \mathrm{I}$ & bi= & 14 & $45 . \mathrm{I}$ & 46.0 & 2259 & -8.2 & 14 & 39.0 & 39.5 & 49 & $-7 \cdot 3$ \\
\hline 16 & 43.4 & 42.8 & 45 & pres & I6 & 39.9 & 38.8 & 5 I & Er & 16 & 43.5 & 44.2 & $\begin{array}{r}56 \\
6\end{array}$ & & 16 & 41.0 & $4 \mathrm{I} .4$ & 52 & \\
\hline 18 & 43.7 & 42.8 & 45 & At & 18 & 39.0 & 38.9 & 52 & It & 18 & $42 . I$ & 42.9 & 54 & Sia & I8 & 42.0 & 42.9 & 54 & HI \\
\hline 20 & 42.9 & 42.1 & $\begin{array}{r}16 \\
1\end{array}$ & $=$ & 20 & 39.9 & 38.8 & $5 \mathrm{I}$ & E & 20 & $4 \mathrm{I} .8$ & 42.2 & 54 & $=$ & 20 & 40.4 & 40.8 & $5 \mathrm{I}$ & 15 \\
\hline 22 & 42.2 & 41.6 & 47 & ine & 22 & 39.8 & 38.8 & $5 I$ & Etr & 22 & 43.6 & 44.5 & 2257 & is & 22 & 36.0 & 36.1 & 44 & \\
\hline 24 & 43.0 & 42.3 & 46 & $1=$ & 24 & 39.9 & 38.7 & $5 I$ & $=$ & 24 & 46.2 & 47.1 & 23 OI & 18 & 24 & 34.6 & 35.0 & 42 & \\
\hline 26 & 4I. I & 40.9 & 49 & $1=$ & 26 & 39.0 & 37.8 & 53 & Ie & 26 & 45.8 & 46.0 & 2300 & 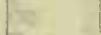 & 26 & 33.9 & 34 & $4 \mathrm{I}$ & \\
\hline 28 & 39.9 & 39.5 & $5 I$ & 2 & 28 & 39.2 & 38.2 & 52 & 8 & 28 & 44. I & 44.8 & 2257 & & 28 & 37.8 & 39.1 & 48 & \\
\hline 30 & $4 \mathrm{I} .2$ & 40.5 & 49 & -12.1 & 30 & 39.2 & 37.8 & 52 & -9.5 & 30 & 44.9 & 45.2 & 2258 & $-8 . I$ & 30 & 39.5 & 39 & 50 & -7.2 \\
\hline 32 & 42.8 & 42.0 & 46 & | & 32 & 40.0 & 38.9 & $5 \mathrm{I}$ & 12 & 32 & 45.8 & 46.3 & 2300 & Is & 32 & 33.6 & 34.4 & $4 \mathrm{I}$ & \\
\hline 34 & 39.9 & 39.0 & $5 I$ & Wit & 34 & 40.7 & 39.7 & 50 & $1=$ & 34 & 45.1 & 45.5 & 2259 & 1 & 34 & 35.7 & 36.0 & 44 & \\
\hline 36 & 40.6 & 40.0 & 50 & & 36 & 40.3 & 39.3 & 50 & 1 & 36 & 44.0 & 44.8 & 57 & iv. & 36 & 35.0 & 36.3 & 43 & \\
\hline 38 & 40.9 & 40.0 & 50 & 1 & 38 & 41.0 & 40.0 & 49 & , & 38 & 43.6 & 44.5 & 57 & e & 38 & 42 & $.0 b$ & 54 & \\
\hline 40 & $4 \mathrm{I} .0$ & 40.0 & 49 & Ei & 40 & 40.5 & 39.5 & 50 & 14 & 40 & 42.0 & 42.6 & 54 & $E$ & 40 & & $.5^{b}$ & 42 & \\
\hline 42 & 40.1 & 39.1 & $5 I$ & 28 & 42 & 39.6 & 38.7 & 52 & 10 & 42 & 40.6 & 41.2 & 52 & & 42 & 31.2 & $3 \mathrm{I} .2$ & 36 & \\
\hline & 39.8 & 39.0 & $5 I$ & -12.0 & 44 & 38.9 & 38.0 & 53 & -9.3 & 44 & 39.6 & 40.0 & 50 & -8. I & 44 & 30.0 & 30. I & 35 & \\
\hline 46 & 38.9 & 38.9 & 52 & & 46 & 38.9 & 37.9 & 53 & & 46 & 39.8 & 40.0 & 50 & t. & 46 & 29.2 & 29.9 & 34 & \\
\hline 48 & 40.3 & 39.2 & $5 I$ & 2 & 48 & 39.0 & 37.9 & 53 & I & 48 & $39 . \mathrm{I}$ & 39.5 & & Bs & 48 & 30.2 & 31.0 & 36 & -7.1 \\
\hline 50 & 40.8 & 40.2 & 49 & is & 50 & 38.7 & 37.7 & 53 & 6 & 50 & 38.7 & 39.0 & 48 & 5 & 50 & 29 & $3 b$ & 34 & \\
\hline 52 & 40.0 & 39.3 & $5 I$ & & 52 & 38.4 & 37.7 & 53 & 2 & 52 & 37.7 & 38.0 & 47 & $E$ & 52 & 28.8 & 29.2 & 33 & \\
\hline 54 & 40.2 & 39.3 & $5 \mathrm{I}$ & 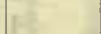 & 54 & 39.6 & 39.0 & $5 \mathrm{I}$ & 12 & 54 & 37.4 & 37.0 & 196 & 2 & 54 & 30.4 & 31.0 & 36 & \\
\hline 56 & 40.5 & 39.6 & 50 & 81 & 56 & 39.2 & 38.7 & 52 & y. & 56 & 39.0 & 39.1 & 49 & 0 & 56 & 32.2 & 33.0 & 39 & \\
\hline 58 & 39.9 & 38.8 & $5 I$ & (2) & 58 & 37.5 & 37.2 & 54 & i & 58 & 40.4 & 40.6 & $5 I$ & 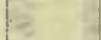 & 58 & 32.2 & 32.9 & 39 & \\
\hline & & & & & & & & & & & & 9ica & E $70=$ & $p 00$ & 800 & 33.0 & 33.5 & 40 & -7.0 \\
\hline
\end{tabular}

Observer-J. V.

Correction to local mean time is $-335.90^{\circ}$ torsion $=14^{\prime}$.

Torsion head at $19 \mathrm{~h} 25 \mathrm{~m}$ on $23 \mathrm{~d}$ read $34^{\circ}$ and at $9 \mathrm{~h} 40 \mathrm{~m}$ read $28^{\circ}$.

Observers-J. V. (W. J. P. axis observations). 
Tabulation of magnetic declinations observed at Teplitz Bay-Continued

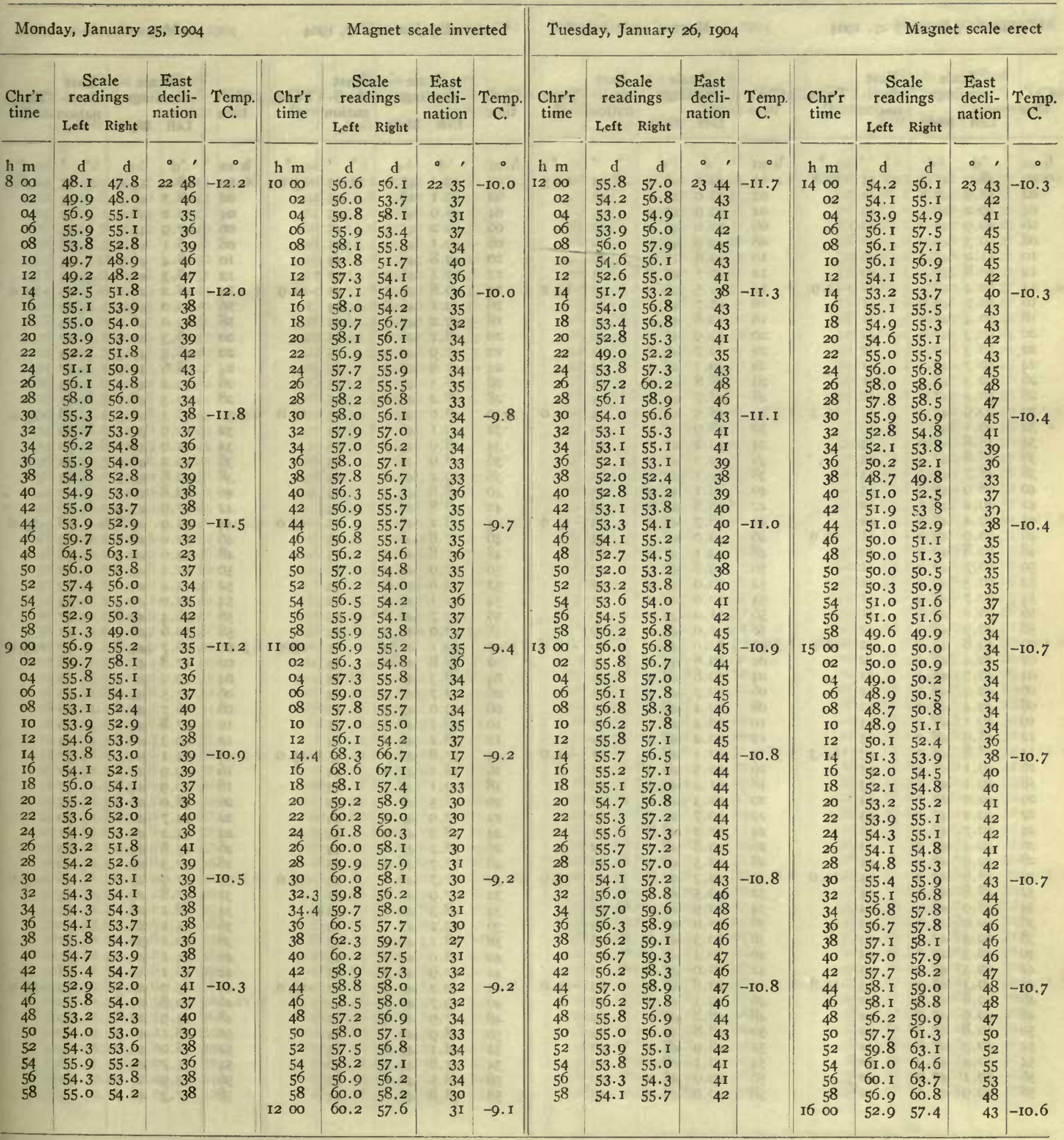

Correction to local mean time is - Im IIs.

Torsion head read $28^{\circ}$ at beginning and ending.

Observer-R. R. T.
Correction to local mean time is - Im 25s.

Torsion head at IIh $40 \mathrm{~m}$ read $35^{\circ}$ and at $\mathrm{I} 6 \mathrm{~h}$ zom read the same. Observer-R. R. T. 
Tabulation of magnetic declinations observed at Teplitz Bay-Continued

\begin{tabular}{|c|c|c|c|c|c|c|c|c|c|c|c|c|c|c|c|c|c|c|c|}
\hline \multicolumn{6}{|c|}{ Wednesday, January 27, 1904} & \multicolumn{4}{|c|}{ Magnet scale inverted } & \multicolumn{6}{|c|}{ Wednesday January 27, 1904} & \multicolumn{4}{|c|}{ Magnet scale inverted } \\
\hline \multirow{2}{*}{$\begin{array}{l}\text { Chr'r } \\
\text { time }\end{array}$} & \multicolumn{2}{|c|}{$\begin{array}{l}\text { Scale } \\
\text { readings }\end{array}$} & \multirow{2}{*}{$\begin{array}{l}\text { East } \\
\text { decli- } \\
\text { nation }\end{array}$} & Temp. & Chr'r & $\begin{array}{l}\text { Sca } \\
\text { readi }\end{array}$ & $\begin{array}{l}\text { le } \\
\text { ings }\end{array}$ & East & Temp. & Chr'r & $\begin{array}{l}\text { Sca } \\
\text { readi }\end{array}$ & $\begin{array}{l}\text { ale } \\
\text { ings }\end{array}$ & $\begin{array}{l}\text { East } \\
\text { decli- }\end{array}$ & Temp. & Chr'r & $\begin{array}{l}\text { Sc } \\
\text { read }\end{array}$ & $\begin{array}{l}\text { ale } \\
\text { ings }\end{array}$ & East & Temp. \\
\hline & Left & Righ! & & & & Left & Right & & & & L,eft & Right & Hatun & & & Left & Right & Mation & \\
\hline $\mathrm{h} \mathrm{m}$ & $d$ & d & $\circ$, & $\circ$ & $\mathrm{h} \mathrm{m}$ & d & d & & $\circ$ & $\mathrm{l} \mathrm{m}$ & d & d & $\circ$, & 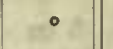 & h $\mathrm{m}$ & $\mathrm{d}$ & d & & $\cdot$ \\
\hline 000 & 48.9 & 47.9 & 2243 & -16.3 & 200 & 49.8 & $47 \cdot 4$ & 2243 & -13.5 & 400 & $49 \cdot 3$ & 47.9 & 2243 & -13.0 & 600 & 48.2 & 45.9 & 2245 & -13.0 \\
\hline 02 & 48.9 & 48.8 & & $e^{2}$ & 02 & 50.0 & $47 \cdot 3$ & 43 & $x^{2}$ & 02 & $49 . I$ & 48. I & 43 & 20 & 02 & 48.3 & 46.7 & 44 & \\
\hline 04 & $49: 2$ & 48.1 & 43 & int & 04 & 49.9 & 47.2 & 43 & $3 \pi$ & 04 & 48.9 & 48.2 & 43 & in & 04 & 50.2 & 48.2 & 42 & +0 \\
\hline 06 & 49.9 & 48.2 & 42 & 20 & 06 & 50.0 & 47.5 & 42 & 50 & 06 & 49.2 & $45 \cdot 3$ & 45 & 66 & 06 & 47.9 & 45.7 & 45 & \\
\hline 08 & 50.3 & 49.2 & $4 \mathrm{I}$ & tos & 08 & 49.9 & 47.5 & 42 & 8. & 08 & 49. I & 45.4 & 45 & 80 & 08 & 46.1 & 44.2 & 48 & tis: \\
\hline IO & 51.0 & 49.3 & 40 & 67 & Io & $49 . \overline{8}$ & 47.6 & 42 & et & I0 & 49.8 & 46.2 & 44 & et & I0 & 48.4 & 46.9 & 44 & 배 \\
\hline I2 & 50.8 & 49.3 & 40 & & 12 & 49.7 & 47.2 & 43 & 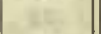 & I2 & 50.1 & 46.9 & 43 & at & 12 & 48.3 & 46.6 & 44 & \\
\hline I4 & 50.4 & $49 . I$ & 4I & -15.9 & I 4 & 49.5 & 47.2 & 43 & -13.2 & 14 & 49.8 & 46.7 & 43 & -12.9 & 14 & 48.2 & 46.2 & 45 & $-\mathrm{I} 3.0$ \\
\hline I6́ & 50.7 & 49.0 & 4I & - 41 & I6 & 49.7 & 47.1 & 43 & 181 & I6 & 49.1 & 46.8 & 44 & & 16 & 50.0 & 48.2 & 42 & \\
\hline I8 & 50.4 & 48.7 & $4^{I}$ & 21 & I8 & 49.8 & 47.1 & 43 & F & 18 & 49.2 & 47.0 & 43 & if & 18 & 49.0 & 48.0 & 43 & 20 \\
\hline 20 & 50.3 & 47.8 & 42 & es & 20 & 49.9 & 47.0 & 43 & es & 20 & $48 . I$ & 46.6 & 45 & in & 20 & 46.3 & 44.9 & 47 & tec \\
\hline 22 & 50.3 & 48.0 & 42 & in & 22 & 49.7 & 46.8 & 43 & $\pm s$ & 22 & 47.9 & 45.9 & 45 & $3=$ & 22 & 48.5 & 47.3 & 44 & $=$ \\
\hline 24 & 50.7 & $48 . I$ & $4 I$ & 12 & 24 & 49.1 & 46.3 & 44 & 16 & 24 & 47.9 & 45.9 & 45 & $f^{2}$ & 24 & 48.6 & 42.5 & 47 & \\
\hline 26 & 50.3 & 48.9 & $4 \mathrm{I}$ & 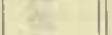 & 26 & 48.8 & 46.7 & 44 & $2 e$ & 26 & 48.3 & 46.3 & 45 & -5 & 26 & 49.2 & 47.7 & 43 & \\
\hline 28 & 50.2 & 47.8 & 42 & 20 & 28 & 48.7 & 47.1 & 44 & 10 & 28 & 48.2 & 46.2 & 45 & 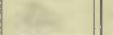 & 28 & 50.3 & 48.9 & $4 \mathrm{I}$ & \\
\hline 30 & 49.9 & 47.3 & 43 & -15.3 & 30 & 48.5 & 47.5 & 44 & -13.0 & 30 & 48.0 & 46.2 & 45 & -13.0 & 30 & 46.3 & $44 \cdot 3$ & 48 & -13.0 \\
\hline 32 & 49.1 & 47.7 & 43 & -0 & 32 & 48.9 & 47.5 & 43 & 4 & 32 & 49.1 & 46.1 & 44 & - & 32 & 40.9 & 39.7 & 56 & \\
\hline 34 & 48.8 & 47.6 & 43 & 36. & 34 & 49.2 & 47.2 & 43 & 27 & 34 & 48.9 & 45.5 & 45 & 15 & 34 & 44.0 & 43.7 & 50 & MA \\
\hline 36 & 48.8 & $47 \cdot 3$ & 44 & des & 36 & 49.2 & 47.6 & 43 & 2 & 36 & 48.9 & 46.1 & 45 & $e^{2}$ & 36 & 49.2 & 48.9 & 42 & \\
\hline $3^{8}$ & 48.7 & 47.9 & 43 & $E_{2}$ & 38 & 49. I & 47.7 & 43 & int & 38 & 49.2 & 46.5 & 44 & $\sqrt{4}$ & 38 & 49.8 & $47 \cdot 3$ & 43 & \\
\hline 40 & 48.9 & 48.2 & 43 & ce. & 40 & 49.1 & 47.9 & 43 & 09 & 40 & 48.8 & 46.2 & 44 & 64 & 40 & 49.8 & 47.9 & 42 & 4 \\
\hline 42 & 49.2 & 48.5 & 42 & 35 & 42 & 48.8 & 47.6 & 43 & Enit & 42 & 48.8 & 46.7 & 44 & 7 & 42 & 47.9 & 45.0 & 46 & 125 \\
\hline 44 & 49.4 & 48.9 & 42 & & 44 & 48.7 & 47.5 & 43 & -13.0 & 44 & 48.2 & 46.7 & 45 & -13.0 & 44 & 45.9 & 43.8 & 48 & $-\mathbf{I} 3.0$ \\
\hline 46 & 50.0 & 49.2 & $4 \mathrm{I}$ & -15.0 & 46 & 49.0 & 47.1 & 44 & 的 & 46 & 47.8 & 46.3 & 45 & & 46 & 52.0 & 49.9 & 39 & \\
\hline 48 & 50.8 & 49.0 & 40 & 的 & $4^{8}$ & 49.1 & 47.2 & 43 & 85 & 48 & 47.8 & 46.3 & 45 & 8 & 48 & 53.0 & 49.9 & 38 & 24 \\
\hline 50 & 50.7 & 49.0 & 4I & cht & 50 & 49.8 & 47.5 & 43 & 20 & 50 & 47.9 & 45.9 & 45 & 8 & 50 & 52.2 & 49.0 & 40 & \\
\hline 52 & 50.3 & 49.0 & $4 \mathrm{I}$ & $=$ & 52 & 50.0 & 47.9 & 42 & 4 & $5^{2}$ & 49.0 & 46.8 & 44 & He & 52 & 45.4 & 44.0 & 49 & \\
\hline 54 & 50.7 & 48.9 & $4 \mathrm{I}$ & & 54 & 50.9 & 48.3 & $4 I$ & 12 & 54 & 50.1 & 47.8 & 42 & 16 & 54 & 42.0 & $4 \mathrm{I} .3$ & 54 & \\
\hline 56 & 50.2 & 48.7 & $4 \mathrm{I}$ & ar & 56 & 51.0 & 49.0 & 40 & ac & 56 & 49.7 & 46.8 & 43 & Bs & 56 & 46.4 & 44.8 & 47 & \\
\hline 58 & 49.9 & 48.0 & 42 & & $5^{8}$ & 51.0 & 49.2 & 40 & 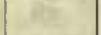 & $5^{8}$ & 48.9 & 46.3 & 44 & & 58 & 50.6 & 48.9 & $4 I$ & \\
\hline 10 & 49.8 & 47.9 & 42 & -14.7 & 300 & 51.0 & 49.2 & 40 & -13.0 & 500 & $49 . \mathrm{I}$ & 46.9 & 44 & $-\mathrm{I} 3.0$ & 700 & 46.7 & 44.8 & 47 & $-\mathrm{I} 3.0$ \\
\hline 02 & 49.4 & 48.4 & 42 & 60 & 02 & 50.8 & 48.9 & $4 I$ & 10 & 02 & 48.8 & 47.2 & 44 & & 02 & 47.8 & 45.2 & 46 & \\
\hline 04 & 49.5 & 48.4 & 42 & 35 & 04 & 50.8 & 48.9 & $4 I$ & (at & 04 & 48.3 & 47.5 & 44 & 16 & 04 & 49.9 & 47.9 & 42 & \\
\hline 06 & 49.3 & 48.3 & 42 & in & 06 & 50.7 & 48.5 & $4 I$ & 32 & 06 & 48.7 & 47.3 & 44 & Ne & 06 & 47.5 & 45.0 & 46 & \\
\hline 08 & 49.4 & 48.2 & 42 & 16 & 08 & 50.4 & 48.3 & $4 \mathrm{I}$ & 110 & 08 & 49.0 & 47.2 & 43 & 16 & 08 & 46.7 & 43.6 & 48 & Ant \\
\hline Io & 49.1 & 48.2 & 43 & nit & IO & 50.4 & 48.4 & $4 I$ & pet & Io & 48.1 & 46.6 & 45 & $6 r$ & 10 & 47.8 & 45.2 & 46 & 4 \\
\hline I2 & 48.9 & 48.2 & 43 & & 12 & 50.2 & 48.3 & 42 & - 28 & I2 & 48.2 & 46.9 & 44 & & 12 & 49.3 & 46.8 & 44 & $\mathrm{sth}$ \\
\hline 14 & 48.8 & 48.0 & 43 & -14.3 & 14 & 50.1 & 48.5 & $4 I$ & -13.0 & 14 & 47.8 & 46.7 & 45 & -13.0 & I4 & $53 . I$ & 50.7 & 37 & 18 \\
\hline 16 & $49 . I$ & 47.3 & 43 & ( & 16 & 50.5 & 48.9 & 41 & ( & 16 & $48 . I$ & 47.2 & 44 & & 16 & 49.9 & 47.8 & 42 & \\
\hline I8 & 49. I & 47.8 & 43 & 24 & I8 & 51.0 & 49.2 & 40 & 2 & I8 & 49.1 & 48.1 & 43 & Ant & I8 & 46.9 & 44.8 & 47 & (1) \\
\hline 20 & 49.2 & $47 \cdot 3$ & 43 & $x$ & 20 & 50.3 & 49.1 & $4^{\mathrm{I}}$ & $9=$ & 20 & 48.5 & 47.8 & 43 & $\alpha$ & 20 & 48.0 & 46.6 & 45 & 8 \\
\hline 22 & 49.2 & 47.2 & 43 & cet & 22 & 50.7 & 49.1 & 40 & 54 & 22 & 47.9 & 47.0 & 44 & inf & 22 & 44.7 & 42.8 & 50 & the \\
\hline 24 & 49.7 & $47 \cdot 4$ & 43 & $1=$ & 24 & 50.2 & 48.8 & $4 \mathrm{I}$ & be & 24 & 48.2 & 47.2 & 44 & is & 24 & 44.2 & 42.2 & 51 & \\
\hline 26 & 49.4 & 47.2 & 43 & 3 & 26 & 49.8 & 48.2 & 42 & 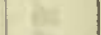 & 26 & 48.2 & 47.2 & 44 & 들 & 26 & 45.8 & 43.0 & 49 & \\
\hline 28 & 49.0 & 46.9 & 44 & & 28 & $49 \cdot 3$ & 48.0 & 43 & & 28 & 47.7 & 46.6 & 45 & 10 & 28 & $47 \cdot 3$ & 45.1 & 46 & \\
\hline 30 & 48.8 & 46.9 & 44 & -14.0 & 30 & 49.3 & 47.8 & 43 & -13.0 & 30 & 47.7 & 46.0 & 45 & -13.0 & 30 & 47.9 & 45.6 & 46 & -13.0 \\
\hline 32 & 48.8 & 46.9 & 44 & 8 & 32 & 49.5 & 47.2 & 43 & $x^{2}$ & 32 & 47.9 & 45.8 & 45 & & 32 & 47.8 & 46.0 & 45 & \\
\hline 34 & 48.9 & 46.9 & 44 & HE. & 34 & 49.9 & 47.2 & 43 & ats & 34 & 48.3 & 46.7 & 44 & (4) & 34 & 48.1 & 46.1 & 45 & \\
\hline 36 & 49.0 & 47.2 & 43 & E: & 36 & 50.0 & 47.1 & 43 & c & 36 & 49.2 & $47 . I$ & 43 & at & 36 & 45.1 & 42.2 & 50 & \\
\hline 38 & 49.3 & 47.3 & 43 & $c^{2}$ & $3^{8}$ & 50.3 & 47.0 & 43 & 7 & 38 & 48. I & 45.9 & 45 & fire & 38 & 40.1 & 38.2 & 57 & \\
\hline 40 & 49.3 & 47.7 & 43 & 62 & 40 & 50.4 & $47 \cdot 3$ & 42 & it5 & 40 & 48.0 & 45.9 & 45 & 48 & 40 & $44 . I$ & 42.2 & $5 I$ & \\
\hline 42 & 49.3 & 47.9 & 43 & & 42 & 50.0 & 47.0 & 43 & & 42 & 47.9 & 46.2 & 45 & 12 & 42 & 46.1 & 43.9 & 48 & \\
\hline 44 & $49 \cdot 3$ & 48.0 & 43 & -13.8 & 44 & 49.8 & 46.9 & 43 & -13.0 & 44 & 49.2 & 47.9 & 43 & -13.0 & 44 & 45.0 & 43.9 & 49 & -12.9 \\
\hline 46 & 49.8 & 47.8 & 42 & $A_{4}$ & 46 & $49 . I$ & $47 \cdot 3$ & 43 & 140 & 46 & 49.7 & 47.7 & 42 & & 46 & 50.8 & 49.8 & 40 & \\
\hline 48 & 49.9 & 47.8 & $=42$ & its & 48 & 48.8 & 47.2 & 44 & 64 & 48 & 47.9 & 46.4 & 45 & Fet. & 48 & 44.9 & 43.8 & 49 & \\
\hline 50 & 50.8 & 47.2 & 42 & 65 & 50 & 48.8 & 47.9 & 43 & $r^{2}$ & 50 & 48.9 & 46.5 & 44 & ce & 50 & 48.1 & 40.9 & 49 & \\
\hline 52 & 50.3 & 47.8 & 42 & $\mathrm{e}^{2}$ & 52 & 48.9 & 48.0 & 43 & 4 & 52 & 48.1 & 45.8 & 45 & $3=$ & 52 & 48.0 & 46.0 & 45 & \\
\hline 54 & 50.4 & 47.9 & 42 & $\mathrm{~F}$ & 54 & $49 . I$ & 48.2 & 43 & 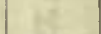 & 54 & 47.8 & 45.2 & 46 & $R^{2}$ & 54 & 45.7 & 44.0 & 48 & \\
\hline 56 & 50.1 & 47.6 & 42 & 62 & 56 & 49.0 & 48.0 & 43 & 25 & 56 & 48.5 & $46 . I$ & 45 & 9 & 56 & 43.9 & 42.3 & $5 I$ & \\
\hline 58 & 49.9 & 47.5 & 42 & $E^{3}$ & 58 & 49.0 & 48.0 & 43 & 8 & 58 & 48.8 & $46 . I$ & 44 & 2 & 58 & $47 \cdot 3$ & 46.9 & 45 & \\
\hline & $f^{4}$ & & & & & & & & & & & & & & & & & & \\
\hline
\end{tabular}


Tabulation of magnetie declinations obscrved at Teplitz Bay-Continued

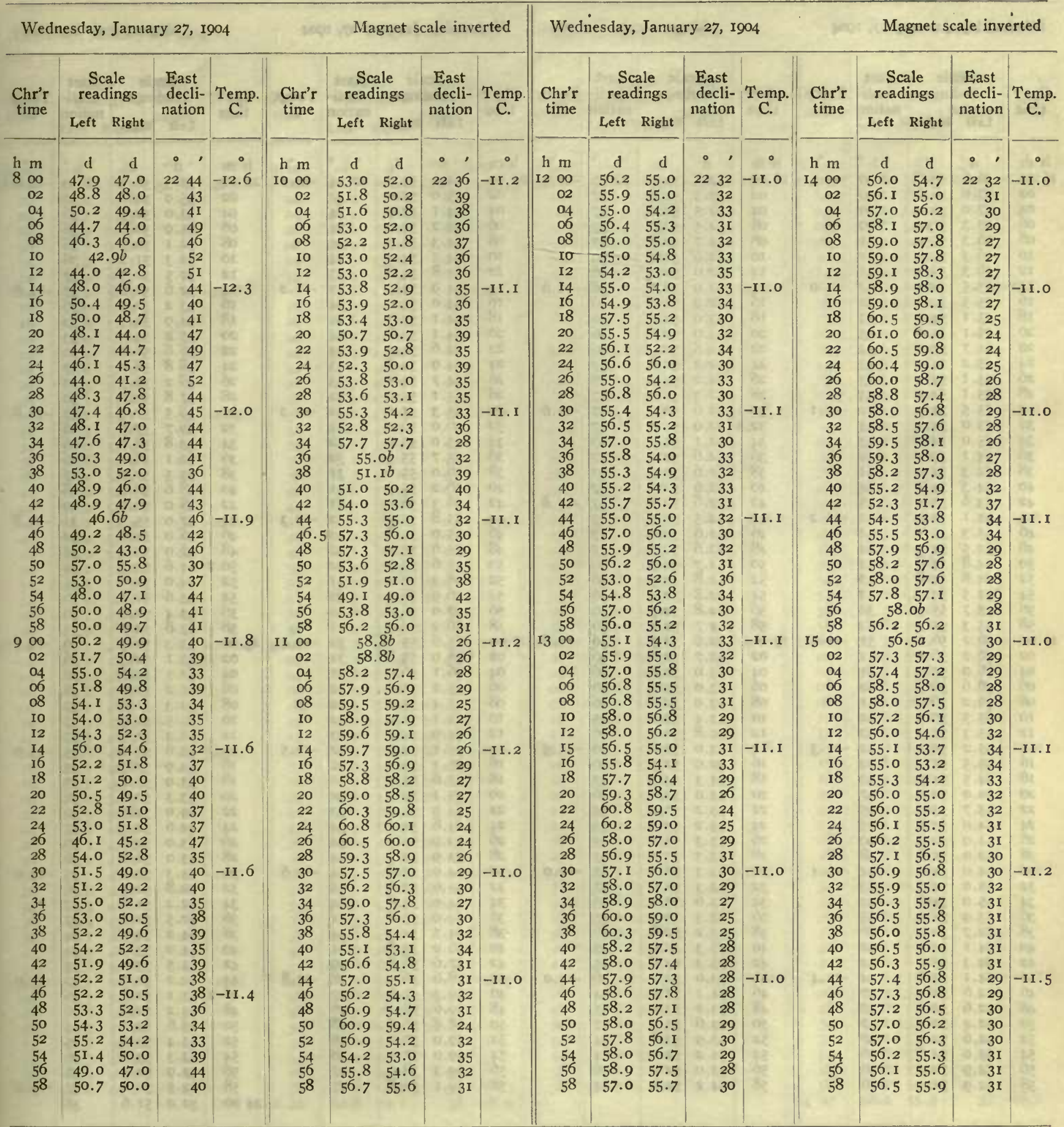


Tabulation of magnetic declinations observed at Teplitz Bay-Continued

\begin{tabular}{|c|c|c|c|c|c|c|c|c|c|c|c|c|c|c|c|c|c|c|c|c|c|}
\hline \multicolumn{5}{|c|}{ Wednesday, Jantsary 27,1904} & \multicolumn{5}{|c|}{ Magnet scale inverted } & \multicolumn{6}{|c|}{ Wednesday, January 27, 1904} & \multicolumn{6}{|c|}{ Magnet scale inverted } \\
\hline \multirow{2}{*}{$\begin{array}{l}\text { Chr'r } \\
\text { time }\end{array}$} & \multicolumn{2}{|c|}{$\begin{array}{l}\text { Scale } \\
\text { readings }\end{array}$} & East & Temp. & Chr'r & $\begin{array}{r}\mathrm{Sc} \\
\text { read }\end{array}$ & $\begin{array}{l}\text { ale } \\
\text { lings }\end{array}$ & East & Temp. & Chr'r & $\begin{array}{r}\mathrm{Sc} \\
\text { reac }\end{array}$ & $\begin{array}{l}\text { ale } \\
\text { lings }\end{array}$ & $\begin{array}{l}\text { East } \\
\text { decl }\end{array}$ & & Temp. & & $\begin{array}{r}\text { So } \\
\text { reac }\end{array}$ & $\begin{array}{l}\text { ale } \\
\text { ings }\end{array}$ & $\begin{array}{ll}\mathrm{E} a \\
\mathrm{dec}\end{array}$ & & Temp. \\
\hline & Left & Right & & & & L,eft & Right & & & & Left & Right & & & & & Left & Right & & & \\
\hline $\mathrm{h} \mathrm{m}$ & d & d & $\circ$, & 0 & $\mathrm{~h} \mathrm{~m}$ & d & d & $\cdot \quad \cdot$ & & h $\mathrm{m}$ & d & d & $\bullet$ & & 0 & $\mathrm{~h} \mathrm{~m}$ & d & d & - & , & ○ \\
\hline I6 00 & 56.6 & 56.6 & & $-\mathrm{II} \cdot 5$ & 1800 & 52.5 & 51.9 & 2237 & $-\mathrm{II} \cdot 5$ & 2000 & 54.0 & 53.9 & 223 & $34-$ & -12.1 & 2200 & 57.3 & 56.3 & 22 & 30 & -12.8 \\
\hline 02 & 57.0 & 56.6 & 30 & 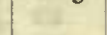 & 02 & 52.3 & 51.8 & 37 & & 02 & 55.0 & 55.0 & & 32 & & 02 & 55.9 & 54.8 & & 32 & \\
\hline 04 & 57.0 & 56.4 & 30 & win & 04 & 52.5 & 52.0 & 37 & 10 & 04 & $54 . \tau$ & 53.9 & & 34 & & 04 & 53.0 & 52.5 & & 36 & \\
\hline 06 & $57 \cdot 3$ & 57.1 & 29 & 80 & of & 52.5 & 52.0 & 37 & do & 06 & 54.2 & 54.0 & & 34 & & 06 & 51.9 & 50.6 & & 38 & \\
\hline 08 & 56.3 & 55.9 & $3 \mathrm{I}$ & 20 & 08 & 52.7 & $52 . \mathrm{I}$ & 37 & 20 & 08 & 54.3 & $54 \cdot 3$ & & 34 & & 08 & $52 . \mathrm{I}$ & 50.9 & & 38 & $t^{2}$ \\
\hline I0 & 56.3 & 55.9 & $3 I$ & ar & 10 & 52.6 & 52,4 & 36 & of & 10 & 54.5 & 54.4 & & 34 & at & IO & 52.3 & 51.2 & & 38 & 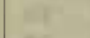 \\
\hline I2 & 56.3 & 55.3 & $3 \mathrm{I}$ & in & 12 & 52.6 & 52.4 & 36 & 17 & I 2 & 54.8 & 54.8 & & 33 & tar & 12 & 52.5 & 51.3 & & 37 & \\
\hline 14 & 55.5 & 54.5 & 32 & $-\mathbf{I} \mathbf{1} .0$ & I4 & 52.9 & 52.6 & 36 & -II.6 & 14 & 54.9 & 54.9 & & 33 & -12.2 & I4 & 52.6 & 5 I. 6 & & 37 & -12.8 \\
\hline 16 & 55.5 & 54.5 & 32 & & 16 & 52.9 & 52.6 & 36 & & I6 & 54.0 & 54.0 & & 34 & & 16 & 53.2 & 52.3 & & 36 & \\
\hline 18 & 55.1 & 54.1 & 33 & ari & I8 & 53.0 & 52.6 & 36 & $\mathrm{St}$ & I8 & $54 . \mathrm{I}$ & 53.7 & & 34 & & 18 & 54.9 & 54.3 & & 33 & \\
\hline 20 & $55 . \mathrm{I}$ & 54.0 & (43 & ece & 20 & 53.0 & 52.6 & 36 & 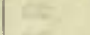 & 20 & $54 . \mathrm{r}$ & 53.8 & & 34 & $\alpha$ & 20 & 55.2 & 54.5 & & 33 & at \\
\hline 22 & 54.7 & 53.9 & 34 & tan & 22 & 52.8 & 52.3 & 36 & 35 & 22 & 54.6 & 54.3 & & 34 & 12 & 22 & 56.6 & 56.0 & & 30 & the \\
\hline 24 & 55.2 & 54.4 & 33 & $y=$ & 24 & 52.3 & 51.8 & 37 & 15 & 24 & 54.9 & 54.6 & & 33 & & 24 & 58.8 & 58.0 & & 27 & \\
\hline 26 & 55.1 & $54 . \mathrm{I}$ & 33 & $\sqrt{5}$ & 26 & 52.2 & 51.9 & 37 & & 26 & 54.7 & 54.5 & & 33 & ter & 26 & 58.2 & 56.3 & & 29 & \\
\hline 28 & 55.1 & $54 . \mathrm{I}$ & 33 & ve & 28 & 52.3 & 51.9 & 37 & $x=$ & 28 & 55.1 & 55.0 & & 32 & 36 & 28 & 57.8 & 56.0 & & 30 & \\
\hline 30 & 55.5 & 54.5 & 32 & -II.I & 30 & 52.6 & 52.0 & 37 & -II.6 & 30 & 55.3 & 55.0 & & 32 & -12.2 & 30 & 59.5 & 57.3 & & 27 & -12.8 \\
\hline 32 & 55.0 & 54.3 & 33 & 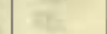 & 32.3 & 52.0 & 51.8 & 37 & & 32 & 56.0 & 56.0 & & $3 I$ & & 32 & 59.7 & 57.9 & & 26 & \\
\hline 34 & 55.0 & 54.0 & 33 & wh. & 34 & $52 . I$ & 51.7 & 37 & 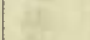 & 34 & 55.0 & 55.0 & & 32 & H. & 34 & 59.8 & 58.0 & & 26 & 18 \\
\hline 36 & 54.1 & $53 \cdot 3$ & 34 & & 36 & 52.6 & 52.2 & 37 & & 36 & 55.7 & 55.5 & & 32 & $\sqrt{2}$ & 36 & 60.1 & 58.2 & & 26 & \\
\hline 38 & $54 \cdot 3$ & 54.0 & 34 & 2 & 38 & 52.6 & 52.3 & 37 & 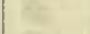 & 38 & 56.0 & 55.6 & & $3 \mathrm{I}$ & 30 & 38 & 59.5 & 57.6 & & 27 & 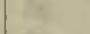 \\
\hline 40 & 54.3 & 54.1 & 34 & aet & 40 & 52.6 & 52.2 & 37 & 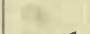 & 40 & 56.2 & 55.9 & & 31 & 88 & 40 & 58.6 & 56.9 & & 28 & \\
\hline 42 & 53.6 & 53.4 & 35 & 16 & 42 & 52.6 & 52.1 & 37 & -11.6 & 42 & 56.3 & 56.0 & & 31 & 하 & 42 & 58.0 & 56.2 & & 29 & 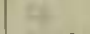 \\
\hline 44 & 54.1 & 53.9 & 34 & -11.2 & 44 & 52.5 & 52.3 & 37 & & 44 & 55.8 & 55.2 & & 32 & -12.3 & 44 & 57.0 & 55.3 & & $3 \mathrm{I}$ & -12.8 \\
\hline 46 & 55.3 & $55 . \mathrm{I}$ & 32 & 128 & 46 & 52.6 & 52.0 & 37 & & 46 & 55.0 & 54.8 & & 33 & & 46 & 56.0 & 54.6 & & 32 & \\
\hline 48 & 54.5 & 54.3 & 34 & 28 & 48 & 52.6 & 52.3 & 37 & 10 & 48 & 55.6 & 55.2 & & 32 & 164 & 48 & 57.0 & 55.3 & & $3 \mathrm{I}$ & \\
\hline 50 & 54.8 & 54.8 & 33 & $\omega$ & 50 & 52.7 & 52.3 & 36 & at & 50 & 55.6 & 55.1 & & 32 & os & 50.2 & 54.6 & 53.3 & & 34 & \\
\hline 52 & 54.8 & 54.8 & 33 & 152 & 52 & 52.8 & 52.5 & 36 & 2 & 52 & 56.3 & 56.1 & & 31 & 5 & 52 & 54.0 & 52.6 & & 35 & \\
\hline 54 & 54.5 & 54.5 & 33 & 82 & 54 & 52.8 & 52.4 & 36 & $H^{2}$ & 54 & 56.9 & 56.4 & & 30 & 12 & 54 & 53.6 & 52.3 & & 36 & \\
\hline 56 & 55.7 & 55.5 & 32 & $w^{2}$ & 56 & 52.8 & 52.5 & 36 & 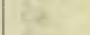 & 56 & 57.0 & 56.6 & & 30 & & 56 & 53.4 & 52.0 & & 36 & \\
\hline 58 & 55.3 & 55.3 & 32 & 1 $13=$ & 58 & 52.7 & 52.5 & 36 & 52 & 58 & 56.2 & 55.9 & & $3 I$ & $12=$ & 58 & 53.8 & 52.6 & & 35 & \\
\hline 1700 & 56.2 & 56.2 & $3 I$ & $-\mathrm{II} .2$ & 1900 & 52.6 & 52.5 & 36 & -11.8 & 2100 & 55.6 & 55.6 & 10 & 32 & -12.4 & 2300 & 53.3 & 52.3 & & 36 & -12.9 \\
\hline 02 & 56.2 & 56.1 & 31 & $1 \cos$ & 02 & 52.7 & 52.6 & 36 & $1=$ & 02 & 56.0 & 55.8 & 13 & $3 I$ & $(159)^{\circ}$ & 02 & 53.1 & 52.3 & & 36 & \\
\hline 04 & 56.0 & 55.9 & $3 \mathrm{I}$ & 16 & 04 & 53.0 & 52.8 & 36 & 10 & 04 & 56.1 & 56.0 & & 31 & 19 & 04 & 52.5 & 51.6 & & 37 & \\
\hline 06 & 56.6 & 56.5 & 30 & oy & 06 & 53.0 & 53. & 36 & at & 06 & 55.2 & 55 & & 32 & ra & 06 & 52.2 & 51.2 & & 38 & \\
\hline 08 & 56.2 & 56.2 & 31 & 81 & 08 & 53.0 & 52.6 & 36 & 80 & 08 & 55.4 & 55.2 & & 32 & $8 x$ & 08 & 52.3 & 51.6 & & 37 & \\
\hline I0 & 55.5 & 55.3 & 32 & $w^{*}$ & 10 & 53.4 & 53.2 & 35 & et & I0 & 55.1 & 54.9 & & 32 & $=1$ & I0 & 52.5 & 51.9 & & 37 & Fis \\
\hline 12 & 55.3 & 55.3 & 32 & 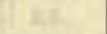 & 12 & 52.8 & 52.5 & 36 & 61 & 12 & 54.9 & 54.7 & & 33 & 58 & 12 & 52.3 & 51.9 & & 37 & 41 \\
\hline I4 & 55.2 & 55.1 & 32 & -II.3 & 14 & 53.0 & 53.0 & 36 & -11.9 & 14 & $55 . \mathrm{I}$ & 54. & & 32 & -12.5 & I4. 3 & 53.6 & 53.0 & & 35 & -13.0 \\
\hline I6 & 55.5 & 55.3 & 32 & 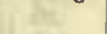 & 16 & 52.9 & 52.7 & 36 & & 16 & 55.3 & 55.1 & & 32 & & 16 & 53.9 & 53.3 & & 35 & \\
\hline 18 & 54.5 & 54.5 & 33 & Wir & 18 & 53.3 & 53 & 35 & 81 & I8 & 55.6 & 55 & & 32 & Mr. & I8 & 53. & & & 35 & \\
\hline 20 & 52.0 & 52.0 & 37 & 6 & 20 & 53.0 & 53.0 & 36 & 62 & 20 & $55 . \mathrm{I}$ & 54. & & 32 & 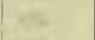 & 20 & 53 & 52.6 & & 36 & E. \\
\hline 22 & 53.7 & 53.3 & 35 & $x:$ & 22 & 53.2 & 53.0 & 35 & 6 & 22 & 54.9 & 54.7 & & 33 & $c=$ & 22 & 53.0 & 52.5 & & 36 & \\
\hline 24 & 54.0 & 53.5 & 35 & fa & 24 & 53.2 & 53.0 & 35 & 18 & 24 & 54.6 & 54 & & 34 & 18 & 24 & 52. & 52.3 & & 37 & \\
\hline 26 & 51.0 & 50.3 & 40 & 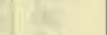 & 26 & 53.3 & 53.1 & 35 & & 26 & 53.9 & 53.5 & & 34 & ats & 26 & 53.0 & 52.6 & & 36 & \\
\hline 28 & 52.0 & 51.6 & 38 & $12 x$ & 28 & $53 \cdot 3$ & 53.1 & 35 & 50 & 28 & 53.9 & 53.5 & & 34 & $x$ & 28 & 53 & 52.6 & & 36 & \\
\hline 30 & 54.0 & 54.0 & 34 & -II.4 & 30 & 53.3 & 53.2 & 35 & -12.0 & 30 & 54.0 & 53.8 & & 34 & -12.6 & 30 & 53.3 & 53.0 & & 35 & -13.0 \\
\hline & 55.0 & 54.7 & 33 & $x^{2}$ & 32 & $53 \cdot 3$ & 53. & 35 & 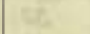 & 32 & 53.6 & 53.6 & & 35 & $76 x=$ & 32 & 52 & 52.4 & & 36 & \\
\hline 34 & 55.0 & 54.6 & 33 & 18 & 34 & 53.6 & 53.3 & 35 & N5 & 34 & 54.0 & 53 & & 34 & & 34 & 52.2 & 51.6 & & 37 & \\
\hline 36 & 53.9 & $53 \cdot 3$ & & & 36 & 53.3 & 53. & 35 & 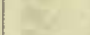 & 36 & 54.0 & 53.8 & & 34 & X & 36 & 52.5 & 52.2 & & 37 & \\
\hline 38 & 52.7 & 52.3 & 36 & 2 & 38 & 53.2 & 52.8 & 36 & $\sqrt{2}$ & 38 & 54.5 & 54.3 & & 34 & Ne & 38 & 54.2 & 54.0 & & 34 & E. \\
\hline 40 & 54.1 & 53.5 & 34 & ar & 40 & $53 \cdot 3$ & 52.9 & 35 & 87 & 40 & 56.0 & 55.8 & & $3 \mathrm{I}$ & 60 & 40 & 54.0 & 53.9 & & 34 & F \\
\hline 42 & 54.3 & 53.9 & 34 & 13 & 42 & $53 \cdot 3$ & 53.0 & 35 & 168 & 42 & 57.6 & 56.8 & & 29 & $=2$ & 42 & 53.5 & 53.0 & & 35 & \\
\hline 44 & $54 . \mathrm{I}$ & 53.6 & 34 & $-\mathrm{II} \cdot 4$ & 44 & 53.0 & 52.7 & 36 & -12.0 & 44 & 58.6 & 56.9 & & 28 & $-\mathrm{I} 2.8$ & 44 & 52.2 & 52.0 & & 37 & $-\mathrm{I} 3.0$ \\
\hline 46 & 52.6 & 52.3 & 37 & t & 46 & 54.1 & 53.9 & 34 & - & 46 & 59.3 & 57.6 & & 27 & - & 46.2 & 53. I & 52.8 & & 36 & \\
\hline 48 & 52.6 & $52 . \mathrm{I}$ & 37 & 18 & 48 & 53.2 & 53 & 35 & $n^{2}$ & 48 & 59.6 & 58.1 & & 26 & 8 & 48 & 53.2 & 52.6 & & 36 & $\omega$ \\
\hline 50 & 52.4 & 52.2 & 37 & a) & 50 & 53.6 & $53 \cdot 3$ & 35 & $e$ & 50 & 58.8 & 56.8 & & 28 & ate & 50 & 53.6 & 53.1 & & 35 & \\
\hline 52 & 52.6 & 52.3 & 37 & 3 & 52 & 53.9 & 53.6 & 34 & cet & 52 & 59.0 & 57.2 & & 28 & 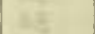 & 52 & 53.4 & 53.0 & & 35 & RE \\
\hline 54 & 53.0 & 52.6 & 36 & W & 54 & 54.2 & 54.0 & 34 & 72 & 54 & 59.0 & 57.5 & & 28 & $F^{2}$ & 54 & 53.3 & 53.0 & & 36 & \\
\hline 56 & 52.9 & 52.4 & 36 & $8 y$ & 56 & 53.0 & 52.9 & 36 & 2 & 56 & 59.3 & 58.0 & & 27 & 10 & 56 & 52.9 & 52.5 & & 36 & \\
\hline 58 & 52.9 & 52.3 & 36 & $8=$ & 58 & 54.2 & 54.2 & 34 & $n=$ & 58 & 59. I & 57.8 & & & $k^{2}$ & 58 & 52.5 & 52.1 & & 37 & -13.0 \\
\hline & & & & & & & & & & & & & & & & 2400 & 52.0 & 51.6 & & 38 & \\
\hline
\end{tabular}

Observer-W. J. P.

Correction to local mean time is - Im I6s.

Torsion head at oh oon read $34^{\circ}$ and at the end read the samc.

Observer-W. J. P. 
Tabulation of magnetic declinations observed at Teplitz Bay-Continued

\begin{tabular}{|c|c|c|c|c|c|c|c|c|c|c|c|c|c|c|c|c|c|c|c|c|c|c|c|}
\hline \multicolumn{6}{|c|}{ Thursday, January 28, I904 } & \multicolumn{6}{|c|}{ Magnet scale erect } & \multicolumn{6}{|c|}{ Friday, January 29, 1904} & \multicolumn{6}{|c|}{ Magnet scale inverted } \\
\hline \multirow{2}{*}{$\begin{array}{l}\text { Chr'r } \\
\text { time }\end{array}$} & $\begin{array}{l}\text { Sct } \\
\text { read }\end{array}$ & $\begin{array}{l}\text { le } \\
\text { igs }\end{array}$ & $\begin{array}{l}\text { Ea } \\
\text { dec }\end{array}$ & $\begin{array}{l}\text { est } \\
\text { cli- } \\
\text { ion }\end{array}$ & Temp. & Chr'r & $\begin{array}{r}\text { Sc } \\
\text { reac }\end{array}$ & $\begin{array}{l}\text { ale } \\
\text { lings }\end{array}$ & $\begin{array}{l}\text { Ea } \\
\text { dec }\end{array}$ & st & Temp. & $\begin{array}{l}\text { Chr'r } \\
\text { time }\end{array}$ & $\begin{array}{l}\text { Sca } \\
\text { readi }\end{array}$ & $\begin{array}{l}\text { le } \\
\text { igs }\end{array}$ & $\begin{array}{l}\text { Eas } \\
\text { dec }\end{array}$ & $\begin{array}{l}\text { st } \\
\text { cli- } \\
\text { ion }\end{array}$ & Temp. & $\begin{array}{c}\text { Chr'r } \\
\text { time }\end{array}$ & $\begin{array}{l}\mathrm{Sc} \\
\text { read }\end{array}$ & $\begin{array}{l}\text { le } \\
\text { ngs }\end{array}$ & $\begin{array}{l}\text { Eas } \\
\text { dec }\end{array}$ & ist & Temp. \\
\hline & Left & Right & & & & & Left & Right & & & & & Left & Right & & & & & L,eft & Right & & & \\
\hline $\mathrm{h} \mathrm{m}$ & d & d & & & ○ & $\mathrm{h} \mathrm{m}$ & $\mathrm{d}$ & d & - & & ${ }^{\circ}$ & $\mathrm{h} \mathrm{m}$ & $\mathrm{d}$ & d & $\circ$ & ' & & $h \mathrm{~m}$ & $\mathrm{~d}$ & d & - & & \\
\hline 1600 & 57.8 & 58.3 & 22 & 39 & -20.8 & 1800 & 47.3 & 50.2 & 22 & 24 & -19.4 & 2000 & 52.9 & 52.2 & 22 & 42 & -19.4 & 2200 & 53.2 & 51.2 & 22 & 44 & -17.2 \\
\hline 02 & 60.0 & 60.8 & & 43 & & 02 & 49.2 & 52.1 & & 27 & & 02 & 53.0 & 52.2 & & $4 \mathrm{I}$ & & 02 & 53.2 & 51.9 & & 43 & \\
\hline 04 & 59.7 & 62.3 & & 44 & & 04 & 48.2 & $5 \mathrm{I} . \mathrm{I}$ & & 26 & & 04 & 53.0 & 51.8 & & 42 & & 04 & 53.2 & 52.0 & & 43 & th \\
\hline 06 & 59.0 & 62.7 & & 43 & & 06 & 48.0 & 50.1 & & 24 & & 06 & 52.7 & 51.5 & & 42 & & 06 & 52.7 & 51.4 & & 44 & 8 \\
\hline o8 & 56.7 & 60.2 & & 40 & $n s$ & 08 & 47.9 & 49.4 & & 24 & & 08 & 52.3 & 5I.I & & 43 & 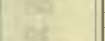 & 08 & 52.3 & 51.3 & & 44 & nt: \\
\hline 10 & 55.0 & 59.1 & & 37 & & IO & $45 \cdot 3$ & 47.1 & & 20 & & IO & 52.1 & 50.9 & & 43 & & IO & 52.2 & 51.2 & & 44 & \\
\hline 12 & 53.9 & 58.7 & & 36 & & 12 & 4 I. I & 42.9 & & 13 & & 12 & 54.2 & 50.0 & & 42 & & 12 & 51.9 & 50.9 & & 45 & \\
\hline 14 & 54.2 & 58.9 & & 37 & -20.8 & 14 & 39.8 & 41.1 & & 11 & -19.2 & 14 & 54.2 & 49.6 & & 42 & -19.0 & 14 & 51.9 & 50.7 & & 45 & -17.0 \\
\hline 16 & 53.0 & 57.4 & & 35 & & 16 & $37 \cdot 5$ & 39.7 & & 08 & & 16 & 54.7 & 50.0 & & 42 & 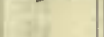 & 16 & 52.9 & 50.1 & & 45 & \\
\hline 18 & 53.8 & 57.9 & & 36 & & 18 & 38.6 & 40.7 & & 09 & & I8 & 54.8 & 50.2 & & $4 I$ & & I8 & 53.2 & 50.9 & & 44 & \\
\hline 20 & 4.9 & 58.2 & & 37 & & 20 & 40.1 & 42.2 & & 12 & & 20 & 54.6 & 50.2 & & 42 & & 20 & 53.9 & 51.1 & & 43 & \\
\hline 22 & 55.2 & 58.5 & & 37 & 10 & 22 & 42.0 & 44.2 & & I5 & & 22 & 54.8 & 50.3 & & $4 I$ & E & 22 & 53.9 & $5 \mathrm{I} .7$ & & 42 & \\
\hline 24 & 53.8 & 57.2 & & 35 & - & 24 & 44.0 & 46.7 & & 18 & & 24 & 54.2 & 50.2 & & 42 & E & 24 & 53.8 & 51.8 & & 42 & \\
\hline 26 & 53.5 & 56.0 & & 34 & 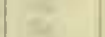 & 26 & $45 \cdot 7$ & 48.3 & & $2 I$ & & 26 & 55.6 & 51.4 & & 40 & & 26 & 53.5 & 51.8 & & 43 & 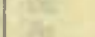 \\
\hline 28 & 53.1 & 55.8 & & 33 & & 28 & 45.0 & 47.1 & & 19 & & 28 & 57.1 & 52.7 & & 38 & & 28 & 53.5 & 52.1 & & 42 & \\
\hline 30 & 52.7 & 55.1 & & 33 & -20.4 & 30 & 43.0 & 45.1 & & I6 & -I9. I & 30 & 56.0 & 53.0 & & 39 & -18.7 & 30 & 53.1 & 52.2 & & 43 & -17.0 \\
\hline 32 & 51.3 & 54.8 & & 31 & & 32 & $4 \mathrm{I} .0$ & 42.3 & & I2 & & 32 & $55 \cdot 3$ & 52.1 & & 40 & & 32 & 54.0 & 53.3 & & $4 I$ & \\
\hline 34 & .0 & $53 \cdot 9$ & & 30 & $y$ & 34 & 38.8 & 39.9 & & 09 & & 34 & 54.2 & 51.0 & & 42 & & 34 & 57.7 & 56.6 & & 36 & \\
\hline 36 & 6 & 52. & & 29 & & 36 & 37.1 & 38,2 & & 06 & & 36 & 52.7 & 49.8 & & 44 & & 36 & $6 \mathrm{I} . \mathrm{I}$ & 60.2 & & 30 & \\
\hline 38 & I. 2 & $54 . I$ & & $3 I$ & & 38 & 36.3 & 37.2 & & 05 & & 38 & 53.1 & 50.1 & & 43 & & 38 & 61.3 & 59.7 & & $3 I$ & \\
\hline 40 & 51.8 & 54.8 & & 32 & 14 & 40 & 35.6 & 35.9 & & 03 & 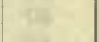 & 40 & 53.9 & 51.1 & & 42 & 9 & 40 & 66.5 & 65.0 & & 23 & 10 \\
\hline 42 & 52.2 & 55.0 & & 32 & $x_{1}$ & 42 & 37.7 & 38.0 & & 06 & $=$ & 42 & 53.9 & 51.0 & & 42 & & 42 & 65.8 & 63.1 & & 25 & 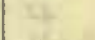 \\
\hline 44 & 52.2 & $54 \cdot 4$ & & 32 & -20.0 & 44 & 37.6 & 38.2 & & 07 & -19.0 & 44 & 53.0 & 50.2 & & 43 & -18.2 & 44 & 64.8 & 64.0 & & 25 & -17.0 \\
\hline 46 & $5^{2.2}$ & 53.8 & & 31 & & 46 & 37.2 & 38.8 & & 07 & & 46 & 53.2 & 49.8 & & 44 & & 46 & 65.3 & $64 \cdot 3$ & & 24 & \\
\hline 48 & 50.9 & 52.8 & & 29 & & 48 & 38.1 & 39.6 & & 08 & & 48 & 53.7 & 50.2 & & 43 & & 48 & 66.0 & 64.6 & & 23 & \\
\hline 50 & 51.2 & 52.9 & & 30 & 6 & 50 & 36.9 & 38.3 & & 06 & & 50 & 53.3 & 50.2 & & 4.3 & & 50 & 64.9 & 63.8 & & 25 & 10 \\
\hline 52 & 9.5 & 5 & & 27 & te & 52 & .9 & 34.8 & 22 & OI & & 52 & 53.8 & 50.3 & & 3 & & 52 & 64.2 & 63.1 & & 26 & 2 \\
\hline 54 & 8.9 & 50.1 & & 26 & & 54 & 30.0 & 31.9 & 21 & 55 & & 54 & 53.9 & 51.2 & & 42 & & 54 & 63.2 & 62.8 & & 27 & the \\
\hline 56 & $48 . I$ & 9.0 & & 24 & $x^{2}$ & 56 & .8 & 31.9 & & 56 & & 56 & 54.2 & 51.9 & & $4 I$ & & 56 & 60.7 & 60.0 & & $3 I$ & a \\
\hline 58 & 7.9 & 48.3 & & 23 & & 58 & 3I. I & 31.9 & & 57 & & 58 & 54.4 & 52.0 & & 41 & & $5^{8}$ & 58.8 & $58 . \mathrm{I}$ & & 34 & \\
\hline 1700 & 48.1 & 48.5 & [a & 23 & -20.0 & 1900 & 24.9 & 26.2 & & 47 & -19.0 & 2100 & 54.7 & 52.3 & & $4 I$ & -18.0 & 2300 & $57 \cdot 3$ & 56.3 & & 37 & -17.0 \\
\hline 02 & 48.1 & 48.8 & & 23 & & $\mathrm{O} 2$ & 21.2 & 22.8 & & 42 & & 02 & 55.8 & 53.5 & & 39 & & 02 & 55.9 & 55.6 & 2 & 38 & \\
\hline 04 & 48.2 & 48.8 & & 24 & & 04 & 24.8 & 25.8 & & 47 & & 04 & 56.1 & 54.2 & & 38 & ES & 04 & 56.3 & 55.9 & & 38 & 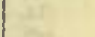 \\
\hline 06 & 48.8 & 48.8 & D & 24 & & 06 & $25 \cdot 9$ & 26.2 & & 48 & & 06 & 56.8 & 54.1 & & 38 & & 06 & 56.7 & 56.1 & & 37 & \\
\hline 08 & 48.1 & 48.7 & & 23 & rat & 08 & 26.5 & 26.9 & $2 I$ & 49 & & 08 & 55.6 & 52.8 & & 40 & & 08 & 57.1 & 56.8 & & 36 & 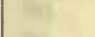 \\
\hline 10 & 47.2 & 7.9 & & 22 & at & IO & 33.6 & $34 \cdot 4$ & 22 & OI & & IO & 54.8 & 52.1 & & $4 I$ & 11 & I0 & 56.0 & 55.2 & & 38 & wit \\
\hline 12 & 7.5 & 48.3 & & 23 & 145 & I 2 & 30.7 & 3I. I & 21 & 56 & & I2 & 55.5 & 53.1 & & 40 & $\sqrt{1}$ & 12 & 54.5 & 53.9 & & $4 I$ & $=12$ \\
\hline 14 & 47.8 & 48.3 & & 23 & -19.9 & 14 & 34.0 & 34.8 & 22 & OI & -19.0 & 14 & 55.5 & 53.5 & & 39 & -17.8 & 14 & 53.9 & 53.1 & & 42 & -17.0 \\
\hline I6 & 48.1 & 48.8 & & 23 & & 16 & 38.0 & 39.2 & & 08 & & 16 & 55.7 & 53.7 & & 39 & & 16 & 53.8 & 53.1 & & 42 & \\
\hline I8 & 48.7 & 49.6 & & 25 & & I8 & 35.2 & 37.0 & 22 & 04 & & I8 & 55.2 & 53.9 & & 39 & & 18 & 54.5 & 54.0 & & $4 I$ & 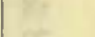 \\
\hline 20 & 49.0 & 50.3 & & 25 & 0 & 20 & 32.1 & 33.8 & $2 \mathrm{I}$ & 59 & & 20 & 54.9 & 53.9 & & 39 & 50 & 20 & 54.7 & 54.1 & & 40 & $t a$ \\
\hline 22 & 49.5 & 50.8 & & 26 & 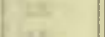 & 22 & 28.7 & 30.4 & & 53 & 14 & 22 & 54.8 & 53.7 & & 40 & - & 22 & 54.2 & 54.0 & & $4 I$ & tar \\
\hline 24 & 48.8 & 49.9 & & 25 & 13 & 24 & 30.9 & $3 I .9$ & & 56 & $1=$ & 24 & 55.2 & 52.8 & & 40 & 217 & 24 & 54.1 & 53.7 & & 42 & y: \\
\hline 26 & 46.1 & 47.9 & 1 & 21 & w & 26 & 29.2 & 30.8 & 21 & 53 & & 26 & 55.1 & 51.9 & & 41 & bin & 26 & 54.9 & $54 \cdot 4$ & & $4 I$ & \\
\hline 28 & 46.2 & 47.0 & t. & $2 I$ & 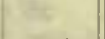 & 28 & 35.1 & 36.3 & 22 & 02 & & 28 & 54.8 & 51.2 & & 42 & 7 & 28 & 54.9 & $54 \cdot 3$ & If & $4 I$ & \\
\hline 30 & 45.7 & 46.1 & & 20 & -19.7 & 30 & 34.1 & 36.8 & 22 & 02 & -18.9 & 30 & 54.5 & 51.8 & & 42 & -17.4 & 30 & $\begin{array}{l}54 \cdot 9 \\
55.2\end{array}$ & $54 \cdot 5$ & & 41 & $-I 6.9$ \\
\hline 32 & 45.8 & 46.1 & & 20 & & 32 & 29.7 & 33.0 & 21 & 56 & & 32 & $54 \cdot 3$ & 51.8 & & 42 & & 32 & 55.0 & 54.2 & A & $4 I$ & (2) \\
\hline 34 & $45 \cdot 3$ & 46.0 & & 19 & Ka & 34 & 28.7 & 33.4 & 21 & 55 & & 34 & 54.8 & 52.2 & & $4 I$ & & 34 & 54.8 & 54.0 & & $4 I$ & 14 \\
\hline 36 & 45.4 & 45.7 & & 19 & ter & 36 & $34 \cdot 5$ & 38.0 & 22 & 03 & & 36 & 55.2 & 52.7 & & 40 & & 36 & 55.0 & 53.6 & & $4 I$ & \\
\hline 38 & 48.1 & 48.3 & & 23 & 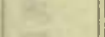 & 38 & 44.1 & 48.0 & 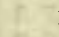 & 19 & & 38 & 54.9 & 52.8 & & 40 & & 38 & & 55.0 & & 39 & \\
\hline 40 & 48.5 & 48.9 & & 24 & 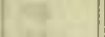 & 40 & 46.0 & 48.9 & & 21 & 14 & 40 & 55.0 & 52.6 & & 40 & 40 & 40 & 62.1 & & & 30 & 71 \\
\hline 42 & 50.2 & 50.8 & & 27 & 1 & 42 & 46.8 & 48.4 & & $2 I$ & 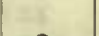 & 42 & 54.9 & 52.7 & & 40 & $x^{2}+4$ & 42 & 62.6 & 61.6 & & 29 & \\
\hline 44 & 51.7 & 52.0 & & 29 & -19.6 & 44 & 64.9 & 67.8 & 17 & $5 I$ & -18.9 & 44 & 54.9 & 52.5 & & 40 & -17.2 & 44 & 61.5 & 59.3 & & 32 & -16.5 \\
\hline 46 & $5 \mathrm{I} .1$ & 52.0 & & 28 & - & 46 & 67.8 & 69.1 & 22 & 54 & & 46 & & 52.8 & & $4 I$ & & 46 & 60.3 & 58.6 & & 33 & \\
\hline 48 & 51.8 & 52.8 & & 30 & & 48 & 75.7 & 78.4 & 23 & 07 & & 48 & 53.8 & 52.2 & & 2 & 5 & 48 & 60.0 & 58.4 & & 33 & 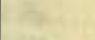 \\
\hline 50 & 50.1 & 51.7 & 2 & 28 & 65 & 50 & 59.2 & 61.9 & 22 & $4 I$ & $c$ & 50 & 53.8 & 51.9 & & 42 & & 50 & 61.1 & 58.2 & & 33 & ate \\
\hline 52 & 48.8 & 50.1 & & 25 & $2+$ & 52 & 43.2 & $52 . I$ & & $2 I$ & X & 52 & 53.2 & 51.3 & & 44 & & 52 & 60.1 & 57.4 & & 34 & $t_{2}$ \\
\hline 54 & 46.7 & 48.2 & & 22 & 3 & 54 & 37.2 & 50.1 & & I5 & 20 & 54 & 53.2 & 51.1 & & 44 & 6 & 54 & 59.9 & 56.7 & & 35 & 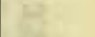 \\
\hline 56 & 46.2 & 48.2 & & 22 & & 56 & 48.4 & 63.8 & & 35 & & 56 & 53.1 & 51.0 & & 44 & & 56 & 58.9 & 55.1 & & 37 & \\
\hline 58 & 46.6 & 49.6 & & 23 & $8=$ & 58 & 48.3 & 62.0 & & 33 & 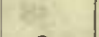 & 58 & 53.3 & $5 I . I$ & & 44 & & 50 & 58.7 & 55.9 & & 36 & \\
\hline & & & & & & 2000 & 62.8 & 79.1 & & 58 & -18.9 & & & & & & & 2400 & 58.1 & 55.3 & & 37 & -16.2 \\
\hline
\end{tabular}

Correction to local mean time is - I2s. $90^{\circ}$ torsion $=12 .{ }^{\prime} 8$. Torsion hcad at 1 hh Iom read $23^{\circ}$ and at 2011 som read $38^{\circ}$. Observer-J. V.
Correction to local mean time is $-37 \mathrm{~s}$. $90^{\circ}$ torsion $=12 .{ }^{\prime} 8$. Torsion head at $20 \mathrm{~h}$ oom read $49^{\circ}$ and at $24 \mathrm{~h} 40 \mathrm{~m}$ read $27^{\circ}$. Observer-R. R. T. 
Tabulation of magnetic declinations observed at Teplitz Bay-Continued

\begin{tabular}{|c|c|c|c|c|c|c|c|c|c|c|c|c|c|c|c|c|c|c|c|}
\hline \multicolumn{6}{|c|}{ Sunday, January 31, Ig04 } & \multicolumn{4}{|c|}{ Magnet scale erect } & \multicolumn{5}{|c|}{ Sunday, January 31, I904 } & \multicolumn{5}{|c|}{ Magnet scale inverted } \\
\hline \multirow{2}{*}{$\begin{array}{l}\text { Chr'r } \\
\text { time }\end{array}$} & \multicolumn{2}{|c|}{$\begin{array}{l}\text { Scale } \\
\text { readings }\end{array}$} & \multirow{2}{*}{$\begin{array}{c}\text { East } \\
\text { decli- } \\
\text { nation }\end{array}$} & Temp. & Chr'r & $\begin{array}{l}\text { Sc: } \\
\text { read }\end{array}$ & $\begin{array}{l}\text { ale } \\
\text { lings }\end{array}$ & East & Temp. & Chr'r & $\begin{array}{l}\text { Sca } \\
\text { read }\end{array}$ & $\begin{array}{l}\text { ale } \\
\text { lings }\end{array}$ & $\begin{array}{l}\text { East } \\
\text { decli- }\end{array}$ & Temp. & Chr'r & $\begin{array}{l}\text { Sci } \\
\text { read }\end{array}$ & $\begin{array}{l}\text { ale } \\
\text { lings }\end{array}$ & East & Temp. \\
\hline & Left & Right & & . & time & Left & Right & nation & C. & time & Left & Right & nation & C. & tim & Left & Right & nation & \\
\hline $\mathrm{h} \mathrm{m}$ & d & d & $\circ \quad \cdot$ & 10 & $h$ in & d & d & & $\circ$ & $\mathrm{h} \mathrm{m}$ & d & d & $\circ \quad$ & $\circ$ & hi $\mathrm{m}$ & & & $\circ$, & 0 \\
\hline $00^{*}$ & 51.3 & 52.0 & 2241 & -18.9 & 200 & 8.0 & 11.0 & 2325 & $-17 \cdot 1$ & 400 & 61.2 & 53.2 & 2429 & & 600 & 60.0 & 58.0 & 2302 & -17.0 \\
\hline 02 & 51.0 & 51.9 & $4 \mathrm{~T}$ & (n) & 02 & 19.8 & 24.0 & 45 & $\theta^{2}$ & 02 & 77.2 & 71.8 & 2402 & & 02 & 57.9 & 57.0 & 05 & \\
\hline 04 & 51.3 & 52.2 & $4 \mathrm{I}$ & $2=$ & 04. & 12.0 & 17.7 & 34 & (45) & $04^{*}$ & 36.2 & 22.1 & $225 \mathrm{I}$ & & 04 & 57.0 & 54.0 & 08 & 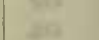 \\
\hline 06 & 51.0 & 51.8 & $4 I$ & 86 & 06 & 14.2 & I8.0 & 36 & 8 & 06 & 28.9 & I 4.0 & 2303 & & 06 & 51.0 & 49.9 & 16 & 6 \\
\hline 08 & 51.0 & 52.3 & $4 \mathrm{I}$ & 20 & 08 & I9. 4 & 23.9 & 45 & 86 & $08 *$ & 54.9 & 36.9 & 23 & & 08 & $45 . I$ & 44.9 & 24 & \\
\hline 10 & 50.0 & 50.3 & 39 & ar & 10 & 10.5 & 12.8 & 2329 & $a$ & 10 & 51.0 & 33.9 & 29 & ne & 10 & 41.5 & 39.5 & $3^{2}$ & \\
\hline I2 & $48 . \mathrm{I}$ & 49.0 & 36 & 0 & 12 & 37.4 & $4 \mathrm{I} . \mathrm{I}$ & 2412 & 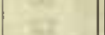 & 12 & 47.5 & 32.0 & 33 & & 12 & 47.3 & 42.5 & 25 & \\
\hline I4 & 47.0 & 47.6 & 34 & -18.5 & 14 & 40.2 & 43.0 & 16 & -17.2 & 14 & 53.1 & 38.8 & 23 & -16.6 & 14 & 49.0 & 46.9 & 20. & -17.2 \\
\hline 16 & 46.7 & $47 \cdot 3$ & 34 & (1) & 16 & 42.4 & 44.2 & 19 & & I6 & 57.1 & 43.2 & 16 & 1 & 16 & 39.1 & 36.0 & 36 & \\
\hline I8 & 46.8 & 41. I & 34 & 89 & 18 & 40.8 & 42.1 & I6 & 3. & I8 & 61. 3 & 48.1 & 09 & 31 & IS & 42.2 & 39.8 & 31 & \\
\hline 20 & 48.2 & 48.5 & 36 & 6 & 20 & 37.7 & 39.0 & 24 II & ace & 20 & 55.0 & 41.0 & 20 & $y$ & 20 & 50.7 & 47.4 & 18 & \\
\hline 22 & 49.8 & 50.7 & 39 & 2 & 22 & 28.6 & 30.9 & 2357 & 25 & 22 & 57.2 & 43.2 & 23 I6 & 2 & 22 & 56.0 & 53.0 & รо & \\
\hline 24 & 52.0 & 52.3 & 42 & R & 24 & 25.0 & 26.1 & 51 & & 24 & 69.8 & 59.7 & 2254 & $=$ & 24 & 59.6 & 57.7 & 03 & \\
\hline 26 & 51.9 & 52.6 & 42 & 3 & 26 & 26.8 & 27.2 & 2353 & & 26 & 67.1 & 56.3 & 58 & & 26 & 56.9 & 54.3 & 2308 & \\
\hline 28 & 51.0 & 51.9 & $4 I$ & & 28 & 34.0 & 34.7 & 2405 & & 28 & 67.0 & 59.0 & 56 & & 28 & 61.9 & 60.8 & 2259 & \\
\hline 30 & 51.4 & 51.8 & $4 I$ & -18.3 & 30 & 37.3 & 38.1 & 10 & -17.2 & 30 & 72.1 & 64.1 & 48 & -16.9 & 30 & 61.I & 60.1 & 2300 & $-17 \cdot 3$ \\
\hline 32 & 53.2 & 54.0 & 44 & ty. & 32 & 37.1 & 38. I & Io & 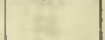 & 32 & 67.0 & 58.0 & 57 & tax & 32 & 59.8 & 58.5 & 02 & \\
\hline 34 & 53.0 & 54.0 & 44 & it. & 34 & 33.0 & 34.0 & 04 & E: & 34 & 68.0 & 60.4 & 54 & $H$ & 34 & 59.6 & 57.9 & 03 & \\
\hline 36 & 51.2 & 52.0 & $4 \mathrm{I}$ & 6 & 36 & 34.6 & 35.8 & 06 & 18 & 36 & 70.5 & 62.1 & 51 & 28 & 36 & 57.0 & $55 \cdot 5$ & 07 & \\
\hline 38 & 49.8 & 50.8 & 39 & 25 & $3^{8}$ & $34 . I$ & 35.2 & 05 & the & 38 & 75.0 & 68.0 & 43 & f & $3^{8}$ & 58.1 & 57.2 & 2305 & \\
\hline 40 & 49.8 & 50.5 & 39 & 6 & 40 & 35.6 & 36.9 & 08 & 다 & 40 & 73.1 & 65.5 & 46 & & 40 & 65.3 & 65.3 & 2253 & \\
\hline 42 & 49.3 & 50.2 & 38 & We & 42 & 35.4 & 36.9 & 08. & +2 & 42 & 70.0 & 61.0 & 2252 & & 42 & 65.8 & 64.0 & 53 & \\
\hline 44 & 49.2 & 50.0 & 38 & -18.0 & 44 & $34 . I$ & 36.3 & 06. & -17.2 & 44 & 58.8 & 50.5 & 2309 & -17.0 & 44 & $64 \cdot 3$ & 62.7 & 55 & -17.2 \\
\hline 46 & 50.1 & 50.8 & 39 & $x^{2}$ & 46 & 36.0 & 37.5 & os & ( & 46 & 59.7 & 55.0 & 2305 & $i$ & 46 & 66.9 & 65.0 & 52 & \\
\hline 48 & 50.2 & 51.0 & 39 & $x^{2}$ & 48 & 33.0 & 34.4 & 04 & th. & 48 & 69.0 & 62.5 & 2252 & n & 48 & 64.1 & 63.6 & 55 & \\
\hline 50 & 49.9 & 50.7 & 39 & 6 & 50 & 34.0 & 37.2 & 07 & ne & 50 & 69.8 & 63.9 & 50 & wit & 50 & 67.2 & 64.0 & 52 & ne \\
\hline 52 & 49.8 & 50.1 & 38 & $E^{2}$ & 52 & 35.2 & 37.1 & 08 & 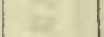 & 52 & 70.0 & 63.5 & 50 & ta & 52 & 67.9 & 65.2 & $5 \mathrm{I}$ & at \\
\hline 54 & 49.2 & 50.1 & 38 & $M$ & 54 & 34.3 & 36.2 & 06 & yz & 54 & 68.5 & 62.0 & 53 & 12 & 54 & 65.8 & 64.0 & 53 & \\
\hline 56 & 46.7 & 48.7 & 35 & $E$ & 56 & 40.7 & 42.0 & 16 & 5 & 56 & 66.2 & 60.5 & 56 & 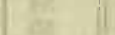 & 56 & 70.0 & 69.8 & 45 & \\
\hline 58 & $4^{48.8}$ & 50.0 & 38 & & $5^{8}$ & 42.0 & 43.7 & 18 & & 58 & 64.9 & 59.0 & 58 & & 58 & 63.3 & 63.0 & 56 & \\
\hline 100 & 50.6 & 52.0 & 40 & -18.0 & 300 & 35.0 & 36.4 & 07 & -17.2 & 500 & 64.2 & 59.1 & 2258 & -17.0 & 700 & 65.9 & 63.1 & 54 & -17.3 \\
\hline 02 & 56.5 & 56.9 & 2249 & tat & 02 & 33.2 & 34.2 & 04 & 50 & 02 & 61.0 & 56.2 & 2303 & $x^{2}$ & 02 & 66.9 & 66.0 & $5 \mathrm{I}$ & \\
\hline 04 & 62.3 & 67.0 & 23 or & 60 & 04 & 33.0 & 33.5 & 03 & 10 & 04 & 63.0 & 58.0 & 2300 & ant & $04^{*}$ & 41.5 & 35.2 & 26 & \\
\hline 06 & 67.0 & 69.6 & 07 & As. & 06 & 31.3 & 32.0 & 2400 & has & 06 & 69.0 & 65.1 & 2250 & S & 06 & 41.0 & 29.0 & 2231 & \\
\hline 08 & 68.3 & 70.0 & 08 & 60 & 08 & 28.3 & 29.7 & 2356 & 80 & 08 & 64.6 & 59.5 & 58 & 85 & 08 & I5.3 & II. 3 & 2305 & 10 \\
\hline 10 & 69.3 & 70.7 & I0 & $3 i$ & 10 & 20.5 & 22.7 & 45 & ai & IO & 65.9 & 62.0 & 55 & & 10.5 & 14.0 & 7.0 & 2310 & \\
\hline $12^{*}$ & 40.2 & 47.8 & 20 & 0 & 12 & 24.2 & 26.5 & $5 \mathrm{I}$ & & 12 & 67.6 & 62.9 & 53 & & 12 & 25.5 & 18.2 & 2252 & \\
\hline 14 & 36.8 & 43.0 & I4 & -18.0 & I4 & 26.8 & 29.2 & 2355 & -17.2 & I4 & 64.0 & 59. I & 2259 & -17.0 & 14 & 36.2 & 31.9 & 223.3 & -17.2 \\
\hline 16 & 53.2 & 69.3 & 47 & $w$ & I6 & 32.9 & 35.1 & 2404 & 100 & I6 & 61.0 & 56.8 & 2303 & $1+x$ & I6 & 13.0 & 10.9 & 23 of & \\
\hline I8 & 27.4 & 68.9 & 27 & 6 & 18 & 37.0 & 38.2 & 10 & $2 \pi$ & 18 & 66.2 & 62.8 & 2254 & ifi & 18 & 14.0 & II.O & 2307 & \\
\hline 20 & $35 \cdot \mathrm{I}$ & 43.8 & I3 & $x=$ & 20 & 33.9 & 35.5 & 05 & 35 & 20 & 58.6 & 54.9 & 2306 & a & 20 & 32.1 & 29.0 & $22 \quad 38$ & \\
\hline 22 & 20.5 & 52.1 & 08 & 68 & 22 & 36.0 & 39.0 & IO & 26 & 22 & 49.9 & 46.9 & 19 & 18 & 22 & 30.1 & 26.4 & $4^{2}$ & $=0$ \\
\hline 24 & 45.0 & 65.8 & $3^{8}$ & $x$ & 24 & 34.2 & 38.1 & 08 & 12 & 24 & 60.0 & 56.7 & 04 & is: & 24 & 26.5 & 21.6 & 49 & \\
\hline 26 & 27.6 & $54 \cdot 3$ & 15 & E & 26 & 36.0 & 39.0 & I0 & ct & 26 & 59.9 & $57 \cdot 3$ & 03 & $\mathrm{H}$ & 26 & 21.3 & I6. 5 & 57 & \\
\hline 28 & 32.9 & 54.0 & 23 I9 & a & 28 & $45 . \mathrm{I}$ & 48.0 & 24 & $18=$ & 28 & 59.9 & 57.2 & 03 & & 28 & 26.3 & 24.0 & 47 & \\
\hline $30^{*}$ & 42.2 & 60.1 & 2407 & $=$ & 30 & 49.4 & 52.1 & $30-$ & -17.2 & 30 & 57.9 & 54.3 & 07 & -17.2 & 30 & $3 \mathrm{r} .4$ & 29.0 & 39 & -17.2 \\
\hline 32 & 36.0 & 54.8 & 2358 & cer & 32 & 50.0 & 51.9 & 3I & $0^{2}$ & 32 & 60.0 & 58.2 & 02 & $x^{2}$ & 32 & 24.9 & 25.5 & 47 & ( \\
\hline 34 & Los & & & 18 & 34 & 41.0 & 45.0 & $24 \quad 18$ & $k$ & 34 & 55.5 & $52 . I$ & II & 28 & 34 & 20.3 & 18.1 & 56 & \\
\hline $36^{*}$ & 33.1 & 42.1 & 2410 & a & 36 & 30.5 & 31.0 & 2359 & & 36 & 54.2 & 51.9 & 12 & & 36 & 26.8 & 23.1 & 47 & \\
\hline 38 & 22.0 & 42.0 & 01 & 82 & 38 & 31.4 & 33.2 & 2402 & be & $3^{8}$ & 57.3 & 55.0 & 2307 & cif. & 38 & $25 \cdot 3$ & 24.0 & 48 & $E$ \\
\hline 40 & 38.0 & 52.3 & 22 & net & 40 & 37.5 & 40.2 & 12 & 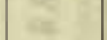 & 40 & 65.0 & 62.0 & 2255 & mi & 40 & 25.2 & 23.0 & 48 & \\
\hline 42 & 38.0 & 53.0 & 22 & & 42 & 35.0 & 36.0 & 2407 & & 42 & 61.3 & 59.9 & 2300 & & 42 & 24.8 & 23.6 & 48 & \\
\hline 44 & 63.0 & 71.1 & 56 & -17.2 & 44 & 22.0 & 24.9 & 2348 & -17.5 & 44 & 56.0 & 53.2 & 08 & -17.2 & 44 & 22.2 & 20.9 & 53 & -17.2 \\
\hline 46 & 40.2 & 49.8 & 22 & 8 & 46 & 17.2 & 20.2 & 40 & r & 46 & 57.0 & 54.5 & 08 & & 46 & 30.9 & 28.6 & 40 & \\
\hline 48 & 40.4 & 52.8 & 24 & 65 & 48 & 12.2 & 14.5 & 32 & $x_{4}$ & 48 & 58.1 & 56.7 & 2305 & & 48 & 26.5 & 24.9 & 46 & \\
\hline 50 & 46.5 & 52.4 & 28 & 60 & 50 & 14.8 & 17.0 & 36 & 10 & 50 & 63.8 & 62.3 & 2256 & at & 50 & 24.2 & 23.2 & 49 & \\
\hline 52 & 57.0 & 64.0 & 46 & ㄷ. & 52 & 21.8 & 23.2 & 46 & 23. & 52 & 55.1 & 52.0 & 23 II & ai & 52 & 32.8 & 30.0 & 37 & \\
\hline & 46.3 & 51.0 & 2427 & 3 & 54 & 25.8 & 27.9 & 53 & $2=$ & 54 & 57.3 & 55.9 & 06 & 2 & 54 & 26. I & 25.0 & 46 & \\
\hline 56 & I9.9 & 22.8 & 2344 & 68 & 56 & 29.2 & 32.3 & 2359 & 20 & 56 & 56.8 & 55.5 & 07 & 85 & 56 & 25.6 & 24.0 & 47 & \\
\hline 58 & 8.2 & 13.2 & 28 & Fi & $5^{8}$ & 42.1 & 46.0 & 2420 & $x^{2}$ & 58 & 59.9 & 58.2 & 02 & 83 & 58 & 25.2 & $24 \cdot 3$ & 48 & \\
\hline 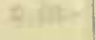 & 2 & 60 & ine & 601 & & & & & & Q. Alth & & $7 \times$ & & $00 \mathrm{c}$ & 800 & 27.7 & 26.2 & 44 & -17.2 \\
\hline
\end{tabular}


Tabulation of magnetic declinations observed at Teplitz Bay-Continued

\begin{tabular}{|c|c|c|c|c|c|c|c|c|c|c|c|c|c|c|c|c|c|c|c|c|c|c|c|}
\hline \multicolumn{6}{|c|}{ Monday, Febrtary 1, I904 } & \multicolumn{6}{|c|}{ Magnet scale erect } & \multicolumn{6}{|c|}{ Tuesday, February 2, 1904} & \multicolumn{6}{|c|}{ Magnet scale inverted } \\
\hline \multirow{2}{*}{$\begin{array}{c}\text { Chr'r } \\
\text { time }\end{array}$} & $\begin{array}{l}\text { Sc } \\
\text { read }\end{array}$ & $\begin{array}{l}\text { ale } \\
\text { lings }\end{array}$ & $\begin{array}{r}\mathrm{E} \\
\mathrm{de}\end{array}$ & $\begin{array}{l}\text { cli- } \\
\text { cli- }\end{array}$ & T'emp. & Chr'r & Sc & $\begin{array}{l}\text { ale } \\
\text { lings }\end{array}$ & Ea & $\begin{array}{l}\text { sst } \\
\text { cli- }\end{array}$ & Temp. & $\begin{array}{l}\text { Chr'r } \\
\text { time }\end{array}$ & $\begin{array}{l}\text { Sc } \\
\text { read }\end{array}$ & $\begin{array}{l}\text { le } \\
\text { ngs }\end{array}$ & Ea & $\begin{array}{l}\text { ist } \\
\text { cli- }\end{array}$ & Temp. & Clir'r & $\begin{array}{l}\text { Sca } \\
\text { read }\end{array}$ & $\begin{array}{l}\text { ale } \\
\text { lings }\end{array}$ & $\begin{array}{l}\text { Eas } \\
\text { decl }\end{array}$ & $\begin{array}{l}\text { ust } \\
\text { cli- }\end{array}$ & Temp. \\
\hline & Left & Right & & & & & Left & Right & & & & & Left & Right & & & & & Left & Right & & & \\
\hline $\mathrm{h} \mathrm{m}$ & d & d & - & , & 0 & $\mathrm{~h} \mathrm{~m}$ & d & $d$ & • & & $\circ$ & $\mathrm{h} \mathrm{m}$ & $d$ & d & & & & $\mathrm{h} \mathrm{m}$ & d & d & & ' & 0 \\
\hline 800 & Lo & & & & -24.0 & 1000 & 36.8 & 49.2 & 22 & 45 & -19.2 & 1200 & 47.6 & 46.7 & & 47 & -14.7 & I4 00 & 36.2 & 35.2 & 23 & 05 & -14.9 \\
\hline 02 & Lo & & & & (t) & 02 & 32.0 & 44.3 & & & ton & 02 & $45 \cdot 3$ & 44.1 & & $5 I$ & (7. & 02 & 35.6 & 35.1 & c & 06 & 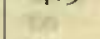 \\
\hline 04 & 42.0 & 45.0 & 22 & 46 & 147 & 04 & 20.2 & 34.2 & & $2 I$ & 34 & 04 & 44.8 & 43.7 & & 52 & 36 & 04 & 38.2 & 38.2 & c & OI & 14 \\
\hline 06 & $43 . I$ & 44.0 & & 46 & $6 y$ & 06 & 14.6 & 30.2 & & 13 & 34 & 06 & 44.9 & 44.0 & & 52 & 95 & 06 & 38.0 & 38.0 & 23 & 02 & $n$ \\
\hline 08 & 46.1 & 48.2 & & 52 & in & 08 & 23.1 & 26.2 & & 16 & 58 & 08 & 47.2 & 45.9 & & 48 & 65 & 08 & 40.1 & 39.7 & 22 & 59 & 86 \\
\hline 10 & 39.6 & $4 I .9$ & & 42 & us: & IO & 24.0 & 32.4 & & 22 & $7^{4}$ & Io. & 45.0 & 42.9 & & 52 & (at) & IO & 41.0 & 40.1 & 5 & 58 & $5 t$ \\
\hline 12 & 42.0 & 43.8 & & 45 & 다 & 12 & 21.9 & 37.8 & & 25 & 37 & 12 & 44.7 & 43.6 & & 52 & 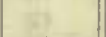 & I2 & 39.8 & 39.2 & 22 & 59 & (t) \\
\hline 14 & 4 I. 2 & 42. I & & 43 & -23.4 & 14 & 28.0 & 39.9 & & $3 I$ & -19.0 & 14 & 46.2 & 44.7 & & 50 & -15.0 & I4 & 39.2 & 38.3 & 23 & 00 & -14.9 \\
\hline I6 & 47.6 & 48.0 & & 53 & & I6 & 32.8 & 42.0 & & 37 & 19.0 & 16 & 45.4 & 44.0 & & 51 & ( & 16 & 37.8 & 37.1 & 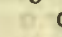 & 03 & 17. \\
\hline 18 & Lo & & & & fet & I 8 & 32.3 & 42.2 & & 36 & Kr & 18 & 45.2 & 43.3 & & 52 & 21 & 18 & 39.9 & 38.3 & 6 & 00 & $2+1$ \\
\hline 20 & 42.0 & 42.0 & & 44 & 16 & 20 & 35.2 & 44.6 & & 40 & $x=4$ & 20 & $49 . \mathrm{I}$ & 44.8 & & 48 & 8 & 20 & 39.3 & 38.3 & & 00 & ch \\
\hline 22 & 39.5 & 42.0 & & 42 & 45 & 22 & 29.7 & 39.3 & & 32 & t5: & 22 & 49.1 & 46.4 & & 46 & a & 22 & 39.3 & 38.1 & 3 & 00 & $\mathrm{c}$ \\
\hline 24 & 43.0 & 45.2 & & 47 & ik & 24 & 32.0 & 40.0 & & 34 & is & 24 & 47.1 & 43.3 & & 50 & 4 & 24 & 39.9 & 39.0 & 23 & 00 & de \\
\hline 26 & 41.0 & 44.2 & & 45 & $m$ & 26 & 34.0 & 39.8 & & 36 & $\frac{5}{2}$ & 26 & 48.5 & 44.2 & & 49 & xe & 26 & .2 & 39.0 & 22 & 59 & $x_{5}$ \\
\hline 28 & 37.0 & 38.8 & & 37 & 86 & 28 & 24.8 & 32.9 & & 23 & $\sqrt{2}$ & 28 & 50.5 & 47.8 & te & 44 & 15 & 28 & 40.8 & 39.4 & 22 & 58 & 22 \\
\hline 30 & 37.2 & 39.8 & 8 & 38 & -23.4 & 30 & I5. I & 23.5 & & 08 & -18.8 & 30 & 47.9 & $44 \cdot 3$ & & 49 & -15.2 & 30 & 39.3 & 38.0 & 23 & OI & $-\mathrm{I} 4.9$ \\
\hline 32 & 38.0 & 40.5 & & 39 & -2.7 & 32 & I6. 2 & 30. I & & 14 & to. & 32 & $45 . \mathrm{I}$ & 41.8 & & 53 & $t^{2}$ & 32 & 38.3 & 38.0 & ( & OI & ( \\
\hline 34 & 43.4 & 46.2 & & 48 & 48 & 34 & I8. 8 & 39.3 & & 23 & in & 34 & 46.9 & 43.6 & set & 50 & 12 & 34 & 37.4 & 37.1 & & 03 & $y=$ \\
\hline 36 & 44.0 & 46.8 & & 49 & ax & 36 & 23.6 & 41.3 & & 29 & & 36 & 45.9 & 41.8 & f & 52 & 4 & 36 & 38.0 & 38.0 & 5 & 02 & 8 \\
\hline 38 & 33.6 & 37.0 & Q & .33 & 18 & 38 & 15.5 & 31.0 & 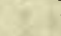 & I4 & & 38 & 45.0 & 4 I. 2 & 6) & 54 & 1 & 38 & 38. & $2 a$ & 23 & OI & dit. \\
\hline 40 & 27.0 & 29.8 & & 22 & $y^{2}$ & 40 & 20.8 & 28.8 & 3 & 17 & $n_{1}$ & 40 & 42.7 & 38.8 & 22 & 57 & 6 & 40 & $4 I . I$ & 40.0 & 22 & 58 & 8 \\
\hline 42 & 29.2 & 30.8 & & 25 & 67 & 42 & $35 \cdot 3$ & 43. I & $n$ & 39 & (1) & 42 & 4I. I & 37.6 & 23 & oo & in & 42 & 41.1 & 40.2 & & 58 & 20 \\
\hline 44 & 27.2 & 29.4 & & 22 & -22.0 & 44 & 27.5 & 41.8 & & 32 & $-\mathrm{I} 8.2$ & 44 & 42.9 & 39.9 & 22 & 56 & -15.3 & 44 & 42.2 & 4 I. I & 22 & 56 & -14.9 \\
\hline 46 & 33.2 & 34.4 & & $3 I$ & - & 46 & 20.7 & 3 I. I & & I9 & & 46 & 41.2 & 38.2 & 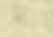 & 59 & - & 46 & 39.7 & $39 . I$ & 23 & oo & 4. \\
\hline 48 & Lo & & & & 14 & 48 & 22.1 & 31.3 & & 20 & & 48 & 42.3 & 39.2 & and & 57 & $1-$ & 48 & 38.6 & 38.2 & c & OI & N \\
\hline 50 & 37.0 & 40.2 & & $3^{8}$ & 34 & 50 & 27.2 & 38.9 & $r$ & 30 & 62 & 50 & 43.7 & 4 I. I & 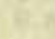 & 55 & 42 & 50 & 40.0 & 38.7 & 23 & 00 & it \\
\hline 52 & 36.4 & 4 I. 0 & & 39 & CE & 52 & 29.6 & 41.3 & & 34 & 4 & 52 & 43.6 & $4 \mathrm{I} .2$ & & 55 & 16 & 52 & 41.0 & 40.2 & 22 & 58 & 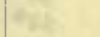 \\
\hline 54 & 26.0 & 32.3 & & 24 & kt & 54 & 29.7 & 38.3 & & $3 \mathrm{I}$ & 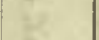 & 54 & 41.5 & 40.1 & $=$ & 57 & $y^{2}$ & 54 & 42.1 & $41 \cdot 3$ & . & 56 & $4 x$ \\
\hline 56 & 31.8 & 34.8 & E & 30 & 17 & 56 & $3 I .5$ & 39.9 & & 34 & -6 & 56 & 40.7 & 39.2 & 9 & 59 & 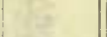 & 56 & 42.8 & 4 I. 7 & 6 & 55 & \\
\hline 58 & 28.0 & 30.2 & b & 24 & $\sqrt{24}=$ & $5^{8}$ & 25.1 & 34.0 & & 24 & & $5^{8}$ & 41.2 & 41.0 & 22 & 57 & & $5^{8}$ & $45 \cdot 3$ & 44.1 & if & $5 I$ & \\
\hline 900 & 25.0 & 27.4 & & 19 & -21.0 & II 00 & 32.3 & 40.4 & & 35 & -18.0 & 1300 & 39.7 & 39.2 & 23 & 00 & $-\mathrm{I} 5.2$ & I5 00 & 44.7 & 44.1 & 1 & 52 & -15.0 \\
\hline 02 & 29.2 & 3.3 .8 & & 27 & (2) & 02 & 34.6 & 42.0 & & 38 & (20.0 & 02 & 41.6 & 39.6 & 22 & 58 & 10 & 02 & 41.6 & $4 \mathrm{I} . \mathrm{I}$ & e & 56 & - \\
\hline 04 & 29.0 & 30.4 & & 24 & Hit & 04 & 26.0 & 33.7 & & 25 & nr & 04 & $4 \mathrm{I} .0$ & 39.5 & t & 58 & $x_{1}$ & 04 & 42.8 & $4 I \cdot 3$ & r & 55 & fig \\
\hline 06 & 33.2 & 36.0 & & 32 & $n$ & 06 & 28.7 & 34.6 & & 28 & (16) & 06 & 42.3 & 40.9 & 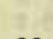 & 56 & av & 06 & 44.0 & 43.2 & a) & 53 & $6 x$ \\
\hline 08 & 30.5 & 37.8 & & 32 & 10 & 08 & 29.1 & 36.1 & & 29 & 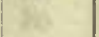 & 08 & 40.7 & 39.0 & 22 & 59 & $x^{2}$ & 08 & $43 \cdot 3$ & 42.9 & & 54 & art \\
\hline IO & 25.8 & 29.5 & & 21 & $m$ & 10 & 29.2 & 34.8 & & 28 & 14t & 10 & 40.4 & 38.3 & 23 & 00 & $y$ & 10 & 43.0 & 42.2 & e & 54 & nar \\
\hline 12 & 32,2 & 37.8 & 22 & 33 & $34=$ & 12 & 31.0 & 35.6 & 14 & 30 & $3 x$ & I2 & 37.1 & 34.8 & 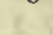 & 05 & & 12 & $44 \cdot 9$ & 43.9 & 6 & 52 & 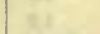 \\
\hline I4 & 57.4 & 62.5 & 23 & 12 & -20.5 & 14 & 30.4 & 35.0 & 18 & 29 & -18.0 & I4 & 34.8 & 33.0 & ta & 08 & -15.0 & 14 & $47 \cdot 3$ & 46.2 & i. & 48 & $-\mathrm{I} 5.0$ \\
\hline 16 & 50.6 & 57.2 & & 02 & (n) & I6 & 32.4 & 36.9 & & 32 & & 16 & 34.0 & 32.7 & & 09 & (5) & 16 & 48.2 & $47 \cdot 3$ & 6 & 46 & r \\
\hline I8 & 58.0 & 66.1 & & 15 & (b) & 18 & 39.3 & 44.0 & & 43 & 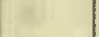 & 18 & 36.9 & 34.7 & & 05 & 11 & I8 & 49.2 & 47.9 & r. & 45 & 7 \\
\hline 20 & 60.0 & 64.2 & 23 & 15 & mer & 20 & 36.6 & 4 I. I & & 39 & 05 & 20 & 37.2 & 35.5 & & 04 & ma & 20 & 50.0 & 48.6 & z & 44 & $\mathrm{nt}^{2}$ \\
\hline 22 & 26.2 & 30.2 & 22 & 22 & $t^{2}$ & 22 & 39.3 & $44 \cdot 5$ & & 44 & 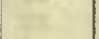 & 22 & 37.3 & 34.8 & 23 & 05 & 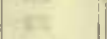 & 22 & 49.7 & 47.8 & K & 45 & 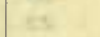 \\
\hline 24 & 23.4 & 27.6 & & I8 & $x^{2}$ & 24 & 34.0 & 36.9 & & 34 & w & 24 & 44.2 & $4 \mathrm{I} .8$ & 22 & 54 & 64 & 24 & 48.9 & 47.4 & e & 46 & it \\
\hline 26 & $33 . I$ & 38.2 & 3 & 34 & 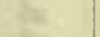 & 26 & 32.3 & 36.0 & & 32 & 10 & 26 & 42.8 & 40.5 & & 56 & 26 & 26 & 48.9 & 47.2 & 0 & 46 & $n$ \\
\hline 28 & 42.0 & 48.9 & & 49 & +2 & 28 & 30.1 & 34.9 & & 29 & & 28 & 41.6 & 39.6 & 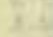 & 58 & & 28 & 49.8 & 48.9 & $y$ & 44 & \\
\hline 30 & 38.0 & 5 I. I & 15 & 48 & -20.0 & 30 & 33.0 & 37.8 & & 34 & -18.0 & 30 & 41.8 & 39.8 & 1 & 57 & -15.0 & 30 & 49.4 & 48.6 & 8 & 44 & -I 5.0 \\
\hline 32 & 21.8 & 32.2 & (1). & 20 & 12 & 32 & 36.7 & 41.6 & & 39 & ( & 32 & 41.8 & 40.4 & & 57 & ( & 32 & 48.9 & 48.2 & 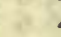 & 45 & - 0 \\
\hline 34 & 15.2 & 23.4 & 1 & 08 & 14 & 34 & 38.6 & 42.9 & & 42 & ant & 34 & 41.9 & 40.7 & & 56 & ze & 34 & 49.1 & 48.3 & & 45 & is \\
\hline 36 & 30.2 & 39.9 & & 33 & $\theta$ & 36 & 3 I. 5 & 34.6 & & 30 & & 36 & 40.6 & 39.9 & 22 & 58 & 4) & 36 & 48.8 & 48.2 & & 45 & \\
\hline 38 & $24 \cdot 3$ & 35.0 & & 24 & $N_{5}$ & 38 & 32.6 & 36.1 & & 32 & r & 38 & 39.6 & 39.1 & 23 & 00 & $x$ & 38 & 47.8 & 46.8 & & 47 & 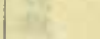 \\
\hline 40 & 32.1 & 41.8 & & 36 & it: & 40 & 25.7 & 30.3 & nt & 22 & 6 & 40 & 39. I & 39.1 & & $\infty$ & $0 y$ & 40 & $47 \cdot 9$ & 47.1 & & 47 & 24 \\
\hline 42 & 32.0 & 39.9 & a & 34 & +5 & 42 & 23.2 & 28.0 & ait & I 8 & 4 & 42 & 37.2 & 36.7 & 1 & 03 & 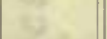 & 42 & 48.0 & 46.3 & & 47 & \\
\hline 44 & 29.0 & 37.4 & 7 & 30 & -19.5 & 44 & 24.9 & $30 . \mathrm{I}$ & & $2 I$ & -17.9 & 44 & 37.2 & 36.3 & $n$ & 04 & -14.9 & 44 & 47.4 & 46.0 & 5 & 48 & -15.0 \\
\hline 46 & 36.9 & 47.2 & 4 & 44 & - & 46 & 26.1 & 30.4 & & 22 & (2.3 & 46 & 37.9 & 36.8 & $=$ & 03 & (1) & 46 & 47.3 & 45.8 & & 48 & (n) \\
\hline 48 & 26.2 & 37.0 & & 28 & 18 & 48 & 28.9 & 32.3 & & 26 & t & 48 & 35.2 & 34.2 & H & 07 & 64 & 48 & 49.1 & 48.7 & & 44 & 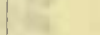 \\
\hline 50 & 29.8 & 41.9 & & 34 & the & 50 & 26.3 & 31.1 & & 23 & ar: & 50 & 35.1 & $34 \cdot 9$ & & 06 & $\mathrm{et}$ & 50 & 53.2 & 50.3 & & 40 & ceste \\
\hline 52 & $3 I . I$ & 42.4 & ar & 36 & s: & 52 & 23.9 & 27.2 & & I8 & ex & 52 & 32.9 & 32.9 & 6 & 10 & as & 52 & 53.9 & 51.2 & & 39 & 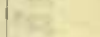 \\
\hline 54 & 32.0 & 44.5 & cy & 38 & 27 & 54 & 23.7 & 26.9 & & I8 & 28 & 54 & 30.9 & 29.8 & $n$ & 14 & 12 & 54 & $53 \cdot 3$ & 51.2 & & 39 & $\mathrm{~B}^{2}$ \\
\hline 56 & 27.1 & 37.8 & 4 & 29 & $c$ & 56 & 28.3 & 31.2 & & 24 & is: & 56 & 32.1 & 31.8 & 1 & II & & 56 & 53.1 & 51.1 & & 40 & c5 \\
\hline 58 & 29.0 & 41.6 & & 33 & 35 & 58 & 32.1 & 34.7 & & 30 & 25= & 58 & $34 \cdot 3$ & 33.1 & 78 & 08 & 38 & 58 & 53.9 & 52.2 & & 38 & $=$ \\
\hline & & & & & & I2 00 & 25.8 & 29.6 & & 2 I & -17.7 & & & & & & & 1600 & 55.0 & 53.3 & & 36 & -14.8 \\
\hline
\end{tabular}

Correction to local mean time is $+\operatorname{Im} 58 \mathrm{~s}$.

Torsion head at $7 \mathrm{~h} 35 \mathrm{~m}$ read $3 \mathrm{I}^{\circ}$ and at the end read the same. Observer-H. H. N.
Correction to local mean time is $-17 \mathrm{~s} .90^{\circ}$ torsion $=13^{\prime} 7$. Torsion head at $\mathrm{IIh} 25 \mathrm{~m}$ read $18^{\circ}$ and at $16 \mathrm{~h} 35 \mathrm{~m}$ read $29^{\circ}$. Observer-R. R. T. 
Tabulation of magnetic declinations observed at Teplitz Bay-Continued

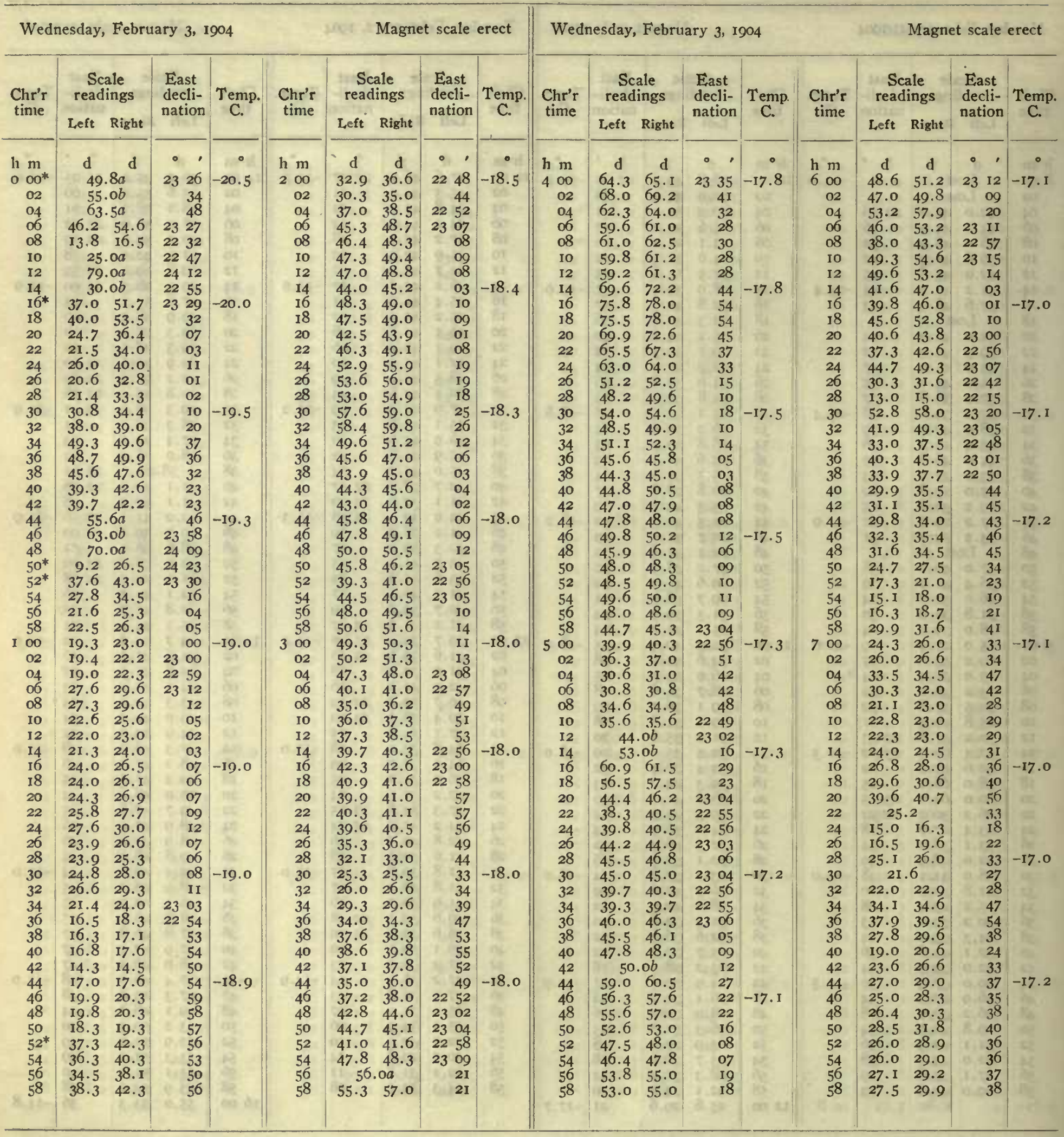


Tabulation of magnetic declinations observed at Teplitz Bay-Continued

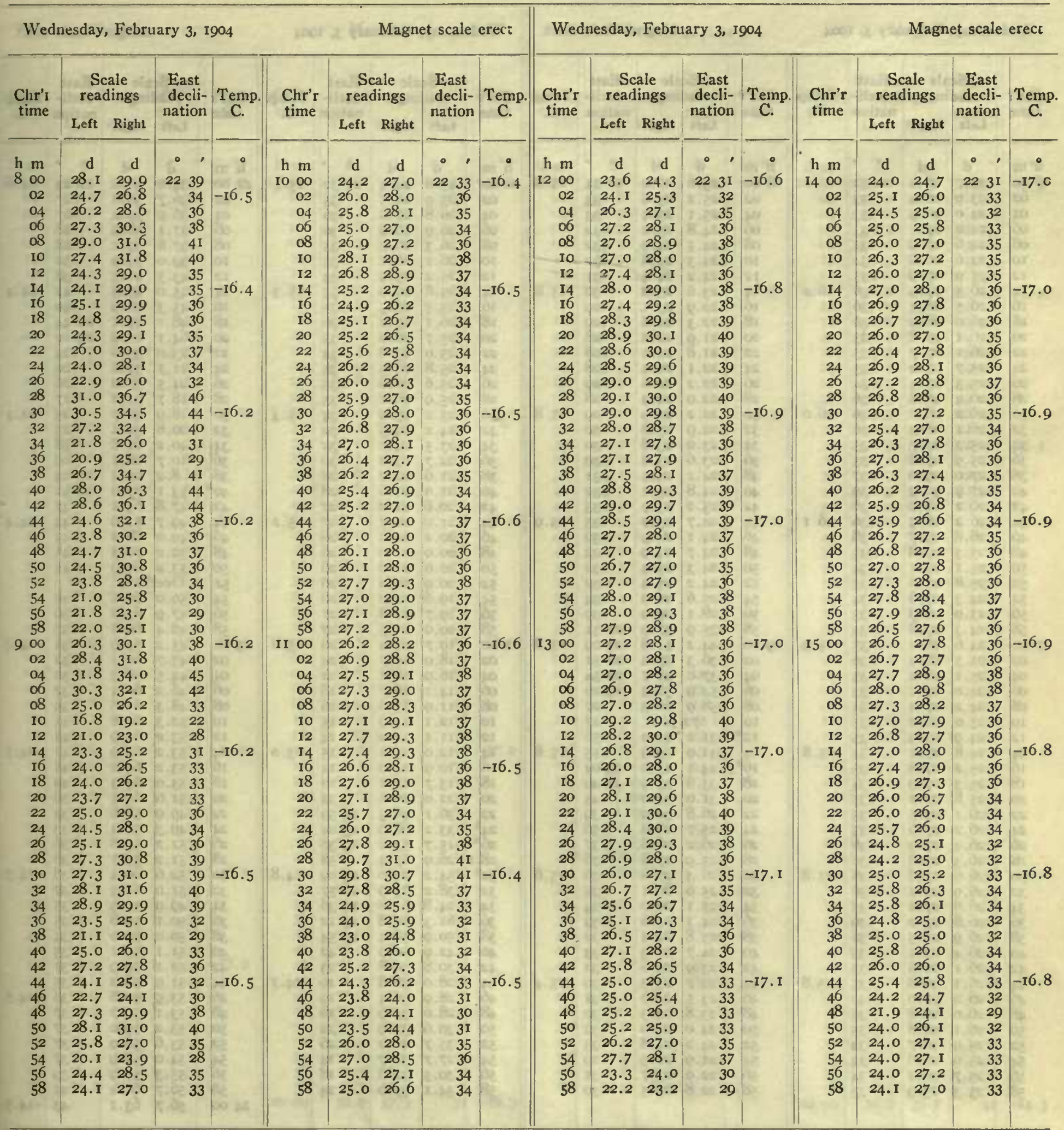


Tabulation of magnetic declinations observed at Teplitz Bay-Continued

\begin{tabular}{|c|c|c|c|c|c|c|c|c|c|c|c|c|c|c|c|c|c|c|c|c|c|c|}
\hline \multicolumn{5}{|c|}{ Wednesday, February 3, 1904} & \multicolumn{6}{|c|}{ Magnet scale erect } & \multicolumn{6}{|c|}{ Wednesday, February 3, 1904} & \multicolumn{6}{|c|}{ Magnet scale erect } \\
\hline \multirow{2}{*}{$\begin{array}{l}\text { Chr'r } \\
\text { time }\end{array}$} & $\begin{array}{r}\mathrm{Sc} \\
\text { read }\end{array}$ & $\begin{array}{l}\text { ale } \\
\text { lings }\end{array}$ & $\begin{array}{c}\text { East } \\
\text { decli- } \\
\text { nation }\end{array}$ & Temp. & $\begin{array}{l}\text { Chr'r } \\
\text { time }\end{array}$ & So & $\begin{array}{l}\text { ale } \\
\text { lings }\end{array}$ & $\begin{array}{r}\text { Eas } \\
\text { dec } \\
\text { natic }\end{array}$ & & Temp. & $\begin{array}{l}\text { Chr'r } \\
\text { time }\end{array}$ & So & $\begin{array}{l}\text { cale } \\
\text { lings }\end{array}$ & $\begin{array}{l}\text { Eas } \\
\text { decl } \\
\text { nati }\end{array}$ & & Temp. & $\begin{array}{l}\text { Chr'r } \\
\text { time }\end{array}$ & $\begin{array}{l}\mathrm{Sc} \\
\text { read }\end{array}$ & $\begin{array}{l}\text { cale } \\
\text { dings }\end{array}$ & Eas & $\begin{array}{l}\text { ast } \\
\text { cli- } \\
\text { ion }\end{array}$ & Temp. \\
\hline & Left & Right & & & & Left & Right & & & & & Left & Right & & & & & Left & Right & & & \\
\hline $\mathrm{h} \mathrm{m}$ & d & $\mathrm{d}$ & - ' & vitio & $\mathrm{h} \mathrm{m}$ & d & d & & ' & 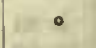 & $h \mathrm{~m}$ & d & d & 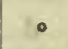 & , & 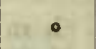 & $h \mathrm{~m}$ & $d$ & d & 0 & , & . \\
\hline 1600 & 23.7 & 26.1 & 2232 & (os $7 x$ & I8 00 & 25.6 & 26.0 & 22 & & -15.9 & 2000 & 23.8 & 24.4 & 223 & & -15.4 & 2200 & 34.0 & 36.0 & & 23 & -14.6 \\
\hline 02 & 23.9 & 26.4 & 33 & tat & 02 & 25.2 & 25.8 & & 3 & & 02 & 25.9 & 26.5 & & 35 & & 02 & 36.7 & 33.8 & & 27 & \\
\hline 04 & 24.0 & 26.5 & 33 & 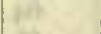 & 04 & 25.1 & 25.4 & & 3 & $t^{n}$ & 04 & 26.8 & 26.8 & & 36 & 판 & 04 & 36.9 & 39.2 & & 27 & \\
\hline 06 & 24.2 & $27 . I$ & 33 & 10 & 06 & 24.8 & 25.2 & & 2 & 90 & 06 & 26.9 & 26.9 & & 36 & & 06 & $37 . I$ & 38.9 & & 28 & \\
\hline 08 & 24.6 & 27.9 & 34 & 6it & os & 24.5 & 24.9 & & 2 & 03 & 08 & 26.3 & 26.3 & & 35 & 6) & 08 & 37.8 & 39.1 & & 28 & \\
\hline ro & 24.6 & 26.8 & 34 & a & Io & 24.3 & 24.8 & & 2 & 208 & IO & 26. I & 26 . I & & 35 & & ro & 39.0 & 39.9 & & 30 & \\
\hline 12 & 25.9 & 27.9 & 35 & $a x$ & 12 & 24.2 & 24.4 & & I & tet & I2 & 25.2 & 25.9 & & 34 & is & 12 & 43.3 & 46.3 & & 38 & \\
\hline 14 & 26.5 & 27.9 & 36 & -16.2 & I4 & 23.6 & 23.8 & & 0 & -15.8 & I4 & 25.8 & 26.0 & & 35 & $-\mathrm{I} 5.3$ & I4 & 45.0 & 49.3 & & 42 & -14.4 \\
\hline 16 & 26.5 & 28 . I & 36 & & 16 & 22.9 & 23.1 & & 9 & & I6 & 25.5 & 26.3 & & 35 & & 16 & 45.3 & 46.9 & & 40 & \\
\hline I8 & 26.0 & 28.0 & 36 & $6 i$ & 18 & 22.3 & 22.8 & & 9 & : & I8 & 26.1 & 27.2 & & 36 & 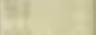 & 18 & 48. I & 49. I & 22 & 44 & \\
\hline 20 & $26 . I$ & 27.8 & 35 & 100 & 20 & 21.9 & 22.2 & & 8 & 100 & 20 & 25.7 & 26.0 & & 35 & & 20 & 64.1 & 65.9 & 23 & Io & \\
\hline 22 & 26.1 & 27.7 & 35 & ar & 22 & $21 . \overline{8}$ & $22 . I$ & & 8 & in & 22 & 25.0 & 25.8 & & 34 & 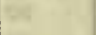 & $22^{*}$ & 23.0 & 35.6 & & 24 & \\
\hline 24 & 25.7 & 27.2 & 35 & 14 & 24 & 22.1 & 22.4 & & 8 & 16 & 24 & 25.8 & 26.5 & & 35 & $1=$ & 24 & 2I. I & 32.8 & & 20 & \\
\hline 26 & 25.5 & 27 . I & 34 & on & 26 & 22.2 & 22.8 & & 9 & 124 & 26 & 25.6 & 26.0 & & 35 & 16 & 26 & 24.5 & 37.0 & & 26 & \\
\hline 28 & 26.4 & 28.8 & 36 & & 28 & 22.0 & 22.3 & & 8 & $x=$ & 28 & 25.9 & 26. I & & 35 & & 28 & 18.7 & 31.2 & & I7 & \\
\hline 30 & 26.2 & 28.8 & 36 & -Í. 0 & 30 & 22.2 & 22.9 & & 29 & -15.8 & 30 & 25.2 & 25.9 & & 34 & -15.3 & 30 & 18.9 & 40.7 & & 25 & -14.4 \\
\hline 32 & 26 . I & 28.3 & 36 & & 32 & 22.9 & 23.8 & & 3o & & 32 & 24.2 & 24.5 & & 32 & & 32 & 20.8 & $39 . I$ & & 25 & \\
\hline 34 & 25.3 & 27.7 & 35 & 14 & 34 & 23.0 & 23.8 & & 30 & 18 & 34 & 23.5 & 24 . I & & 32 & E. & 34 & 40.2 & 61.8 & & $5^{8}$ & \\
\hline 36 & 25.9 & 27.8 & 35 & 127 & 36 & 23.6 & 23.8 & & so & Pro & 36 & 22.7 & 23.2 & & 30 & Is & 36 & 10.7 & 28.7 & & 09 & \\
\hline 38 & 27.0 & 29.0 & 37 & a. & 38 & 24.2 & 24.5 & & I & 17 & 38 & 21.6 & 22.2 & & 28 & E & 38 & 8.2 & 25.4 & 23 & 04 & \\
\hline 40 & 27.9 & 30.0 & 39 & 1. & 40 & 24.8 & 25 . I & & 2 & $\operatorname{lap}$ & 40 & 21.0 & 21.6 & & 28 & $E$ & $40^{*}$ & 53.1 & 77.0 & 22 & $5 \mathrm{I}$ & \\
\hline 42 & 26.2 & 28. I & 36 & 14 & 42 & 25.2 & 25.7 & & 33 & $14=$ & 42 & 20.8 & 21.2 & & 27 & 32 & 42 & 47.8 & 65.1 & & 37 & \\
\hline 44 & 26.2 & 28.9 & 36 & -16. I & 44 & 24.7 & 25.7 & & 33 & -15.9 & 44 & 21.0 & 21.5 & & 27 & -15.2 & 44 & 49.7 & 71.0 & & 44 & $-\mathrm{I} 4.4$ \\
\hline 46 & 26.8 & 29.7 & 38 & & 46 & $24 . I$ & 24.9 & & 2 & & 46 & 20.2 & 21.0 & & 26 & & 46 & 49.0 & 68.2 & & $4 \mathrm{I}$ & \\
\hline 48 & 25.3 & 27.8 & 35 & 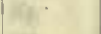 & 48 & 24.7 & 24.9 & & 32 & 8 & 48 & 20.0 & 21.0 & & 26 & $=$ & 48 & 52.2 & 70.3 & & 45 & \\
\hline 50 & 25.1 & $27 . I$ & 34 & at & 50 & 24.3 & 25.3 & & 22 & log & 50 & 20.0 & 21.0 & & 26 & e & 50 & 53.2 & 69.9 & & 45 & \\
\hline 52 & 25.8 & 27.8 & 35 & 12 & 52 & 24.2 & 25.4 & & 2 & Q & 52 & 20.0 & 21.2 & & 26 & $z$ & 52 & Over & rl'k'd & & & \\
\hline 54 & 24.3 & 27.6 & 34 & +4 & 54 & 25.6 & 26.9 & & 34 & 18 & 54 & 19.6 & 21.2 & & 26 & 12 & 54 & 50.0 & 66.6 & & 40 & \\
\hline 56 & 23.9 & 26.9 & 33 & $0=$ & 56 & 28 . I & 29.9 & & 39 & 10 & 56 & I9.7 & 21.8 & & 27 & & 56 & 47.9 & 63.0 & & 36 & \\
\hline 58 & 24.3 & 27.8 & 34 & 16 & 58 & 35.9 & 37.9 & 22 & I & & 58 & 20.0 & 21.5 & & 27 & & $5^{8}$ & 46.4 & 60.8 & & 33 & \\
\hline 1700 & 24.6 & 28.2 & 35 & -15.9 & 1900 & 44.2 & 51.8 & 23 & 9 & -15.8 & 2100 & 19.7 & 21.2 & & 26 & -15.0 & 2300 & 45.2 & 59.9 & & $3 \mathrm{I}$ & -14.4 \\
\hline 02 & 25.5 & 28.9 & 36 & wo & 02 & 36.3 & 37.0 & 22 & SI & & 02 & 18.0 & 19.7 & & 24 & & 02 & 45.7 & 60.3 & & 32 & \\
\hline 04 & 25.1 & 28.2 & 35 & 10 & 04 & 37.0 & 40.7 & 22 & 4 & $10^{\circ}$ & 04 & 16.9 & I8́. I & & 22 & $a$ & 04 & 44.5 & 58.3 & & 30 & \\
\hline 06 & 24.0 & $27 . I$ & 33 & 10 & 06 & 54.9 & 58.3 & 23 & 22 & 00 & 06 & $14 . I$ & I6.7 & & I8 & 10 & 06 & 46.2 & 60.4 & & 32 & \\
\hline 08 & 24.2 & 27.4 & 34 & (a) & 08 & 58.9 & 60.3 & & 27 & bo & 08 & II.9 & I5. 6 & & I6 & $n$ & 08 & 48.3 & 6 r.8 & & 35 & 6 \\
\hline I0 & 25.0 & 28.0 & 35 & ar & I0 & 72 & .76 & & 17 & as & I0 & I2. I & 14.9 & & 15 & $3 i$ & Io & 52.3 & 64.8 & & $4 \mathrm{I}$ & \\
\hline 12 & 24.9 & 27.9 & 35 & $=1$ & I $2^{*}$ & 31.2 & 42.0 & & 6 & Et & 12 & II. I & I4. 2 & & I4 & ir & I 2 & 53.2 & 64.9 & & $4 I$ & \\
\hline I4 & 24.8 & $27 . I$ & 34 & -15.8 & I4 & 31.9 & 39.9 & & 55 & -15.8 & 14 & I2.0 & I5. 2 & & 15 & -15.0 & I4 & 53.2 & 63.9 & & $4 \mathrm{I}$ & $-\mathrm{I} 4.5$ \\
\hline 16 & 25.2 & 27.8 & 35 & & $16^{*} 3$ & 44. I & 45.0 & 23 & 4 & & 16 & 10.9 & I 4.0 & & 14 & & 16 & 53.2 & 64.2 & & $4 \mathrm{I}$ & \\
\hline 18 & 25.3 & 27.3 & 34 & 61 & 18 & 33.6 & 35.9 & 22 & 19 & Se & I8 & 13.7 & I7.I & & 18 & $5 i$ & I8 & 53.0 & 63.3 & & 40 & \\
\hline 20 & 25.8 & 27.2 & 35 & los & 20 & 31,0 & 34.0 & & 15 & $n=$ & 20 & 22.0 & 26.5 & & 32 & $y=$ & 20 & 51.6 & $6 \mathrm{r} .5$ & & 38 & \\
\hline 22 & 26 . 1 & 27.5 & 35 & ex & 22 & 26.8 & 30.0 & & 39 & $=$ & 22 & 36.1 & 46.5 & 22 & 59 & $x$ & 22 & 49.9 & 58.8 & & 34 & \\
\hline 24 & 26.2 & 27.6 & 35 & fis & 24 & 25.7 & 28.0 & & 36 & $=$ & $24^{*}$ & 33.2 & 68.9 & 25 & 04 & $1=$ & 24 & 49.3 & 58.4 & & 33 & 4 \\
\hline 20 & 26.4 & 27.9 & 36 & $w=$ & 26 & 26.2 & 28.8 & & 37 & $i=$ & $26^{*}$ & 15.7 & 53.2 & 23 & 38 & 8 & 26 & 51.2 & 59.8 & & 36 & \\
\hline 28 & 26.3 & 27.8 & 36 & & 28 & 25.9 & 27.8 & & 36 & is & 28.2 & 26.9 & 67.4 & & 58 & $E$ & 28 & 51.2 & 59.7 & & 36 & \\
\hline 30 & 26.6 & 27.6 & 36 & -15.8 & 30 & 26.3 & 27.8 & a. & 36 & -15.6 & $30^{*}$ & 54.2 & 74.0 & 23 & 09 & $-\mathrm{I} 4.8$ & 30 & 52.6 & 60.4 & & 38 & $-\mathrm{I} 4.5$ \\
\hline 32 & 26.9 & 27.7 & 36 & & 32 & 25.9 & $26 . I$ & & 35 & & 32 & 38.2 & 75.9 & 22 & 57 & ( & 32 & 51.0 & 61.3 & & 37 & \\
\hline 34 & 26.6 & 27.1 & 35 & ine & 34 & $25 . I$ & 26.0 & & 34 & 18 & 34 & 24.8 & 61.8 & 22 & 36 & 1 & 34 & 49.9 & 59.9 & & 35 & \\
\hline 36 & 26.8 & 27.2 & 36 & : & 36 & 24.3 & 25.0 & & 33 & & 36 & 61.3 & 70.8 & 23 & I2 & e & 36 & 50.1 & 59.8 & & 35 & \\
\hline 38 & 26.9 & 27.5 & 36 & 12 & 38 & 23.8 & 24.4 & & 32 & 8 & 38 & 50.9 & $5^{8} .4$ & 22 & 54 & lo & 38 & 50.0 & 59.0 & & 34 & \\
\hline 40 & 26.4 & 27.1 & 35 & Af & 40 & 24.7 & 25.3 & & 33 & 48 & 40 & 42.9 & 50.9 & & 42 & 18 & 40 & $5 \mathrm{I} . \mathrm{I}$ & 59.1 & & 35 & W \\
\hline 42 & 26.1 & 26.8 & 35 & 4. & 42 & 23.9 & 24.6 & & 32 & 4 & 42 & 30.8 & 38.8 & & 23 & & 42 & 52.1 & 59.9 & & 37 & \\
\hline 44 & 26 . I & 26.8 & 35 & -I 5.8 & 44 & 22.7 & 23.3 & & 30 & -15.4 & 44 & 28.0 & 36.8 & & 19 & $-\mathrm{I} 4.7$ & 44 & 51.9 & 58.2 & & 35 & \\
\hline 46 & 26.2 & 27.0 & 35 & & 46 & 23.5 & 24.0 & & I & & 46 & 28.9 & 36.4 & & I9 & & 46 & 53.9 & 59.8 & & 38 & $-I 4.5$ \\
\hline 48 & 26.0 & 26.8 & 35 & 10 & 48 & 23.8 & 24.3 & & 32 & A & 48 & 28.6 & 35.9 & & 18 & a & 48 & 53.1 & 59.7 & & 37 & \\
\hline 50 & 26.1 & 26.8 & 35 & es & 50 & 24.0 & 24.0 & & 32 & Det & 50 & 30.6 & 37.9 & & 22 & es & 50 & 53.2 & 59.0 & & 37 & \\
\hline 52 & 25.9 & 26.7 & 34 & iL & 52 & 24.2 & 24.8 & & 33 & E & 52 & 33.7 & 40.2 & & 26 & $=$ & 52 & 53.6 & 58.9 & & 37 & \\
\hline & 25.9 & 26.6 & 34 & 182 & 54 & 21.9 & 22.9 & & 29 & 5 & 54 & 27.7 & 31.7 & & I4 & & 54 & 54.0 & 58.9 & & 37 & \\
\hline 56 & 25.7 & 26.1 & 34 & $d e$ & 56 & 22.9 & 23.7 & & II & Ee & 56 & 30.5 & 32.8 & & 18 & 2 & 56 & 55.3 & 59.9 & & 39 & \\
\hline 58 & 25.3 & 25.9 & 1. 33 & 28 & 58 & 22.8 & 23.5 & & 0 & 20 & 58 & 34.1 & 37.0 & & 24 & & 58 & 56.7 & $6 \mathrm{I} .4$ & & $4 \mathrm{I}$ & \\
\hline & & & & & & & & & & & & & & & & & 2400 & 56.7 & 63.1 & & 43 & -14.5 \\
\hline
\end{tabular}

Observer-R. R. T.

Correction to local mean time is - Im 59s. $90^{\circ}$ torsion $=14^{\prime} 9$. Torsion head at ols oon read $26^{\circ}$ and at 24 h $26 \mathrm{~m}$ read $28^{\circ}$.

Observer-R. R. T. 
Tabulation of magnetic declinations observed at Teplitz Bay-Continued

\begin{tabular}{|c|c|c|c|c|c|c|c|c|c|c|c|c|c|c|c|c|c|c|c|c|}
\hline \multicolumn{6}{|c|}{ 'Thursday, February 4, 1904} & \multicolumn{4}{|c|}{ Magnet scale inverted } & \multicolumn{7}{|c|}{ Friday, February 5, igo4 } & \multicolumn{4}{|c|}{ Magnet scale erect } \\
\hline \multirow{2}{*}{$\begin{array}{l}\text { Chr'r } \\
\text { time }\end{array}$} & \multicolumn{2}{|c|}{$\begin{array}{l}\text { Scale } \\
\text { readings }\end{array}$} & $\begin{array}{l}\text { East } \\
\text { decli- }\end{array}$ & & Chr'r & $\begin{array}{l}\text { Sca } \\
\text { read }\end{array}$ & & East & Temp & Chr'r & $\begin{array}{l}\mathrm{Sc} \\
\text { read }\end{array}$ & $\begin{array}{l}\text { ale } \\
\text { lings }\end{array}$ & East & & & & $\begin{array}{r}\text { Sc } \\
\text { read }\end{array}$ & $\begin{array}{l}\text { le } \\
\text { igs }\end{array}$ & East & Temp. \\
\hline & Left & Right & & & & Left & Right & & & & Left & Right & & & & & Left & Right & & \\
\hline $\mathrm{h} \mathrm{m}$ & $d$ & d & $\cdot$ & 0 & $\mathrm{~h} \mathrm{~m}$ & $d$ & $\mathrm{~d}$ & $\cdot$, & $0^{\circ}$ & h m & d & $d$ & $\cdot$ & , & $\bullet$ & $\mathrm{h} \mathrm{m}$ & $d$ & d & • & \\
\hline 1600 & $54 \cdot 5$ & $54 \cdot 5$ & 2236 & -22.0 & I8 00 & 56.9 & 56.1 & 2233 & -17.9 & $2000^{*}$ & $4 \mathrm{I} .2$ & $4 \mathrm{~T} .9$ & 221 & 17 & -19.5 & 2200 & 35.0 & 42.9 & 2237 & -15.9 \\
\hline 02 & 55.0 & 53.9 & 36 & & 02 & 57.1 & 56.9 & 32 & & 02 & 40.8 & 4I. I & & 6 & & 02 & 35.0 & 42.2 & & \\
\hline 04 & 54.7 & 53.5 & 37 & Hat & 04 & 57.1 & 56.9 & 32 & $y^{3}$ & 04 & 40.9 & $4 \mathrm{I} .2$ & & 6 & & 04 & 37.6 & 46.9 & 42 & \\
\hline 06 & 54.0 & 53.0 & 38 & $=$ & 06 & 56.8 & 56.0 & 33 & we & 06 & 39.9 & 40.5 & & 15 & & 06 & 46.3 & 54.5 & 2255 & \\
\hline 08 & 54.4 & 53.2 & 37 & 10 & 08 & 56.5 & 55.8 & 34 & $\infty$ & 08 & 39.2 & 40.2 & & 14 & & 08 & 61.0 & 68.9 & 2318 & \\
\hline Io & 55.2 & 53.4 & 36 & 15 & 10 & 56.0 & 55.2 & 35 & tif & 10 & 38.9 & 39.4 & & 3 & & Io & 60.3 & 66.1 & 15 & \\
\hline 12 & .2 & 55.2 & 34 & 41 & 12 & 55.9 & 55.2 & 35 & tit & 12 & 37.5 & 38.8 & & 12 & & 12 & 79.0 & 80.0 & 40 & \\
\hline 14 & 56.8 & 55.0 & 34 & -2 I. I & 14 & 55.9 & 55.2 & 35 & -17.7 & 14 & 38.0 & 39.0 & & 12 & -18.8 & $14^{*}$ & 36.0 & 46.8 & 44 & -15.9 \\
\hline 16 & 57.0 & $55 . \mathrm{I}$ & 34 & & 16 & 55.9 & 55.2 & 35 & & 16 & 37.3 & $3^{8} .3$ & & I I & & 16 & 17.0 & 33.0 & 18 & \\
\hline I8 & 58.8 & 56.0 & 32 & 3 & 18 & 56.0 & 55.8 & 34 & 4 & 18 & 37.8 & 38.3 & & II & 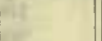 & I8 & 41.8 & 49.8 & 2351 & \\
\hline 20 & $57 \cdot 3$ & 55.4 & 33 & $c$ & 20 & 56.2 & 56.2 & 34 & 5 & 20 & 37.9 & 38.9 & & I2 & & 20 & 63.0 & 77.5 & & \\
\hline 22 & 57.7 & 55.9 & 33 & s & 22 & 56.3 & 56.1 & 34 & tr & 22 & 39.4 & 40.0 & & $I_{4}$ & & $22^{*}$ & 53.3 & 55 & 2524 & \\
\hline 24 & 57.8 & 55.6 & 33 & 4 & 24 & 55.9 & 55.8 & 34 & $=$ & 24 & 39.5 & 40.0 & & I4 & & 24 & 31.1 & 42 & 2456 & \\
\hline 26 & 57.1 & 55.8 & 33 & A & 26 & 55.8 & 55.2 & 35 & 4 & 26 & 40.5 & 41.0 & & 16 & & 26 & 40.5 & 49.0 & 2509 & \\
\hline 28 & & 55.2 & 34 & & 28 & 56.0 & 55.8 & 34 & - & 28 & 1.3 & 42.0 & & I7 & & $28^{*}$ & 38.5 & 48.9 & 2321 & \\
\hline 30 & 57.2 & 56.0 & 33 & -20.0 & 30 & 56.0 & 55.8 & 34 & -17.4 & 30 & $4 \mathrm{I} . \mathrm{I}$ & 41.9 & & I7 & -18.3 & 30 & I 3.5 & 27.7 & 2245 & \\
\hline 32 & 56.9 & 55.8 & 33 & & 32 & 56.2 & 55.8 & 34 & $x^{2}=$ & 32 & 41.9 & 43.0 & & I8 & & $32^{*}$ & 40.0 & 49.0 & 2153 & \\
\hline 34 & 56.0 & 55.0 & 35 & 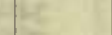 & 34 & 57.0 & 56.1 & 33 & 5 & 34 & 42.2 & 43.9 & & 19 & & 34 & 41.9 & 58.0 & $22 \mathrm{OI}$ & -15.3 \\
\hline 36 & 55.6 & 54.5 & 36 & & 36 & $57 . I$ & 56.6 & 33 & & 36 & 3.0 & 44.2 & & 20 & & 3 & 5 I. 2 & 62.0 & 12 & \\
\hline 38 & 56.5 & 55.7 & 34 & & $3^{8}$ & 56.8 & 56.0 & 33 & $=$ & 38 & 43. I & 44.0 & & 20 & c & 3 & 50.9 & 60.0 & 22 IO & \\
\hline 40 & 56.4 & 55.7 & 34 & 4 & 40 & 56.6 & 56.0 & 33 & * & 40 & 3.5 & $44 . I$ & & 0 & & 40 & 42.5 & 50 & 2156 & \\
\hline 42 & 56.3 & 55.7 & 34 & $1=$ & 42 & 56.4 & 56.0 & 34 & 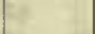 & 42 & $43 \cdot 3$ & 44.2 & & 20 & & 42 & 61.0 & 64.8 & 2221 & \\
\hline 44 & 56.2 & 55.2 & 34 & -19.9 & 44 & 57.1 & 56.6 & 33 & -17.2 & 44 & 42.9 & $43 . I$ & & 19 & -18.0 & 44 & 67.0 & 72.2 & 32 & -15.2 \\
\hline 46 & 55.1 & 54.3 & 36 & & 46 & 58.0 & 57.3 & $3 I$ & $x^{2}$ & 46 & 42.0 & 43.0 & & 18 & & 46 & 61.4 & 66.5 & 23 & \\
\hline $4^{8}$ & 54.9 & 54.2 & 36 & 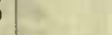 & $4^{8}$ & 58.1 & 57.7 & $3 \mathrm{I}$ & 3 & 48 & 40.8 & $4 \mathrm{I} .2$ & & 16 & 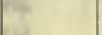 & 48 & 63.2 & 69.0 & 26 & \\
\hline 50 & 56.0 & 55.6 & 34 & & 50 & 58.0 & 57.5 & $3 I$ & & 50 & 39.7 & 40. & & 14 & & 50 & 67.0 & 72 & 32 & \\
\hline 52 & 56.7 & 56.0 & 33 & 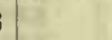 & 52 & 58.2 & 57.9 & $3 I$ & + & 52 & 40.1 & 41.2 & & 15 & & 52 & 65.8 & 70.4 & 30 & \\
\hline 54 & 57.0 & 56.5 & 33 & 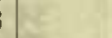 & 54 & 58.8 & 58. & 30 & 2 & 54 & 40.4 & 41.2 & & 16 & & 54 & 60.2 & 64.0 & 20 & \\
\hline 56 & 57.1 & 56.7 & 32 & 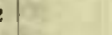 & 56 & 59.0 & 58.5 & 30 & $y$ & 56 & 9.0 & 40.2 & & 14 & & 56 & 62.1 & 67.0 & 24 & \\
\hline 58 & 57.0 & 56.5 & 33 & & 58 & 59.5 & 59.0 & 29 & & 58 & 31.3 & 33.5 & & 02 & & 58 & 65.0 & 69.0 & 28 & \\
\hline I7 00 & 57.9 & 57.5 & $3 \mathrm{I}$ & -I 19.4 & I9 00 & 59.5 & 58.9 & 29 & -17.0 & 2100 & 31.5 & 33.3 & & 02 & -17. & 2300 & 68.8 & 72.0 & 33 & -15.1 \\
\hline 02 & 57.5 & $57 \cdot 3$ & 32 & & 02 & 59.0 & 58.7 & 30 & & 02 & 30.9 & 32.6 & & 02 & & 02 & 69.5 & 71.8 & 34 & \\
\hline 04 & 59.0 & 58.2 & 30 & 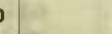 & 04 & 58.8 & 58.2 & 30 & 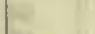 & 0 & & 32. I & 220 & OI & & 04 & 68.2 & 70.6 & 32 & \\
\hline 06 & 57.7 & 57.0 & 32 & 8 & 06 & 58.7 & 58.2 & 30 & $\theta$ & 06 & 25.8 & 29.0 & 2I 5 & 55 & 8 & 06 & 71.0 & 73.0 & 36 & \\
\hline 08 & .7 & 57.2 & 32 & w & 08 & 59.0 & 58.7 & 30 & & of & 21.8 & 26.1 & 214 & & & 08 & 73.0 & & 39 & \\
\hline 10 & 58. I & 57.9 & $3 \mathrm{I}$ & & 10 & 59.0 & 58.5 & 30 & & IO & 26.3 & 35.2 & 220 & 0 & & 10 & 75.6 & 77.0 & 42 & \\
\hline 12 & 9.0 & 58.6 & 30 & & 12 & 57 & $9 a$ & 31 & & 12 & 45.8 & 54.5 & 223 & 30 & te & 12 & 75.0 & 76. & 42 & \\
\hline 14 & 58.6 & 58.2 & 30 & -19.0 & I4 & 57.7 & 57.2 & 32 & -17.0 & $14^{*}$ & 33.0 & 52.2 & 254 & 44 & & I4 & 76.3 & 78.1 & 44 & -15.1 \\
\hline 16 & 57.9 & 57.4 & 31 & & 16 & 57.5 & 57.2 & 32 & & $16^{*}$ & 15.0 & 59.0 & 243 & 38 & & $16^{*}$ & 38.5 & 46.5 & 47 & \\
\hline 18 & 57.2 & 56.9 & 32 & & I8 & 57.8 & 57.5 & 3 & 7 & 18 & Lo & st & & & & 18 & 40.1 & 48.0 & 50 & \\
\hline 20 & 57.0 & 56.7 & 33 & & 20 & 58.6 & 58.1 & 3 & $E$ & 20 & Lo & & the & & $=$ & 20 & & 45.9 & 47 & \\
\hline 22 & 7.0 & 56.5 & 33 & & 22 & 58.3 & 57.9 & 31 & 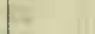 & 22 & Lo & & & & & 22 & 36.7 & 43.0 & 43 & \\
\hline 24 & 6.8 & 56.2 & 33 & & 24 & 58. & 57.8 & 31 & 1 & $25^{*}$ & 21.8 & 29.4 & 220 & 04 & & 24 & 35.0 & $4 \mathrm{I}$. & 40 & \\
\hline 26 & 6.0 & 55.5 & 3 & & 26 & 58.2 & 57.7 & 31 & 8 & 26 & I3.0 & 19.0 & 214 & 49 & & 2 & 34.8 & 40.2 & 39 & \\
\hline 28 & 55.0 & 54.7 & 36 & & 28 & 58.2 & 57.7 & 31 & & $28^{*}$ & & 32.9 & & 39 & & 28 & 34.0 & 39.2 & 38 & \\
\hline 30 & 5.0 & 54.8 & 36 & $5-18.5$ & 30 & 58.3 & 58.0 & $3 \mathrm{I}$ & -16.6 & 30 & 22.8 & 28.0 & & 32 & $-I 6$ & 30 & 34.0 & 39. I & 38 & -15.0 \\
\hline 32 & 55.5 & 55.0 & 35 & & 32 & 58.2 & 58.0 & 3I & & 32 & $2 \mathrm{~T} .2$ & 26.8 & & 30 & & 32 & 33.3 & 38.3 & 37 & \\
\hline 34 & 55.0 & 54.4 & 36 & & 34 & 57.9 & 57.4 & 3 I & & 34 & 23.8 & 29.0 & & & & 34 & & $39 . \mathrm{I}$ & $3^{8}$ & \\
\hline 30 & 54.8 & $54 . \mathrm{I}$ & 36 & & 36 & & 57.8 & 3I & & 36 & & 28.2 & & 3 & & 3 & 36.0 & 40.5 & 40 & 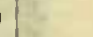 \\
\hline 38 & 55.0 & 54.7 & 36 & & 38 & 58.8 & 58.2 & 30 & 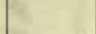 & 38 & 27.8 & 33.6 & & 40 & & 38 & 38.5 & 43. & 44 & \\
\hline 40 & 55.5 & 55.2 & 35 & & 40 & 58.9 & 58.4 & 30 & 1 & 40 & 32.9 & 37.1 & & 47 & & 40 & 39.5 & 44.5 & 46 & \\
\hline 42 & 56.0 & 55.7 & 34 & $\begin{array}{ll}4 & -18.2\end{array}$ & 42 & 58.2 & $58 . \mathrm{I}$ & 3I & 1 & 42 & 37.6 & 42.2 & 215 & 54 & & 42 & 38.0 & $42 . I$ & 43 & \\
\hline 44 & 56.0 & 55.9 & 3 & & 44 & 58.2 & 57.9 & $3 I$ & -16.4 & 44 & 44.6 & 47.9 & $22 \mathrm{C}$ & 04 & -16.0 & 44 & 37.0 & 40.9 & 42 & -15.0 \\
\hline 46 & 55.9 & 55.4 & 35 & & 46 & 57.9 & 57.8 & $3 \mathrm{I}$ & & 46 & 55.4 & 58.0 & & $2 \mathrm{I}$ & & 46 & 38. I & 42.0 & 43 & \\
\hline 48 & 55.9 & 55.2 & 3 & & 48 & 57.5 & 57.5 & 32 & 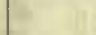 & 48 & 57.8 & 60.0 & & 24 & & 48 & 38. I & 40.8 & 42 & \\
\hline 50 & 56.1 & 55.6 & 3 & & 50 & 57.5 & 57.0 & 32 & & 50 & 67.2 & 71.0 & & 40 & & 50 & 37.9 & 41.0 & 42 & \\
\hline 52 & 56.4 & 55.9 & 3 & & 52 & 58.2 & 57.6 & 3I & & 52 & 71.4 & 75.4 & & 47 & & 52 & 37.3 & 40.3 & $4 \mathrm{I}$ & \\
\hline 54 & 56.2 & 55.8 & 3 & & 54 & 58.6 & 58.0 & 30 & & $54^{*}$ & 47.2 & 57.3 & & 58 & & 54 & 36.1 & 39.0 & 39 & \\
\hline 56 & 56.2 & 55.8 & 3 & & 56 & 57.5 & 56.9 & 32 & & 56 & 46.8 & 57.0 & & 57 & & 56 & 34.9 & 36.9 & 37 & \\
\hline 58 & 56.2 & 55.9 & 3 & & 58 & 58.3 & 57.7 & $3 \mathrm{I}$ & & 58 & 40.2 & 48.0 & & 45 & & & 33.7 & 35.8 & 35 & \\
\hline & & & & & 2000 & 58.0 & $57 \cdot 3$ & $3 I$ & & & & & & & & 2400 & 38.0 & 39.7 & $4 \mathrm{I}$ & -14 \\
\hline
\end{tabular}

Correction to local mean time is +9 s.

Torsion head at $15 \mathrm{~h}$ fom read $28^{\circ}$ and at the end read the same.

Observer-J. V.
Correction to local mean time is $-5 \mathrm{~s} .90^{\circ}$ torsion $=13 .{ }^{\prime} 3$. Torsion head at $19 \mathrm{~h} 30 \mathrm{~m}$ read $28^{\circ}$ and at $24 \mathrm{~h}$ 20m read $32^{\circ}$. Observer-J. V. 
Tabulation of magnetic declinations observed at Teplitz Bay-Continued

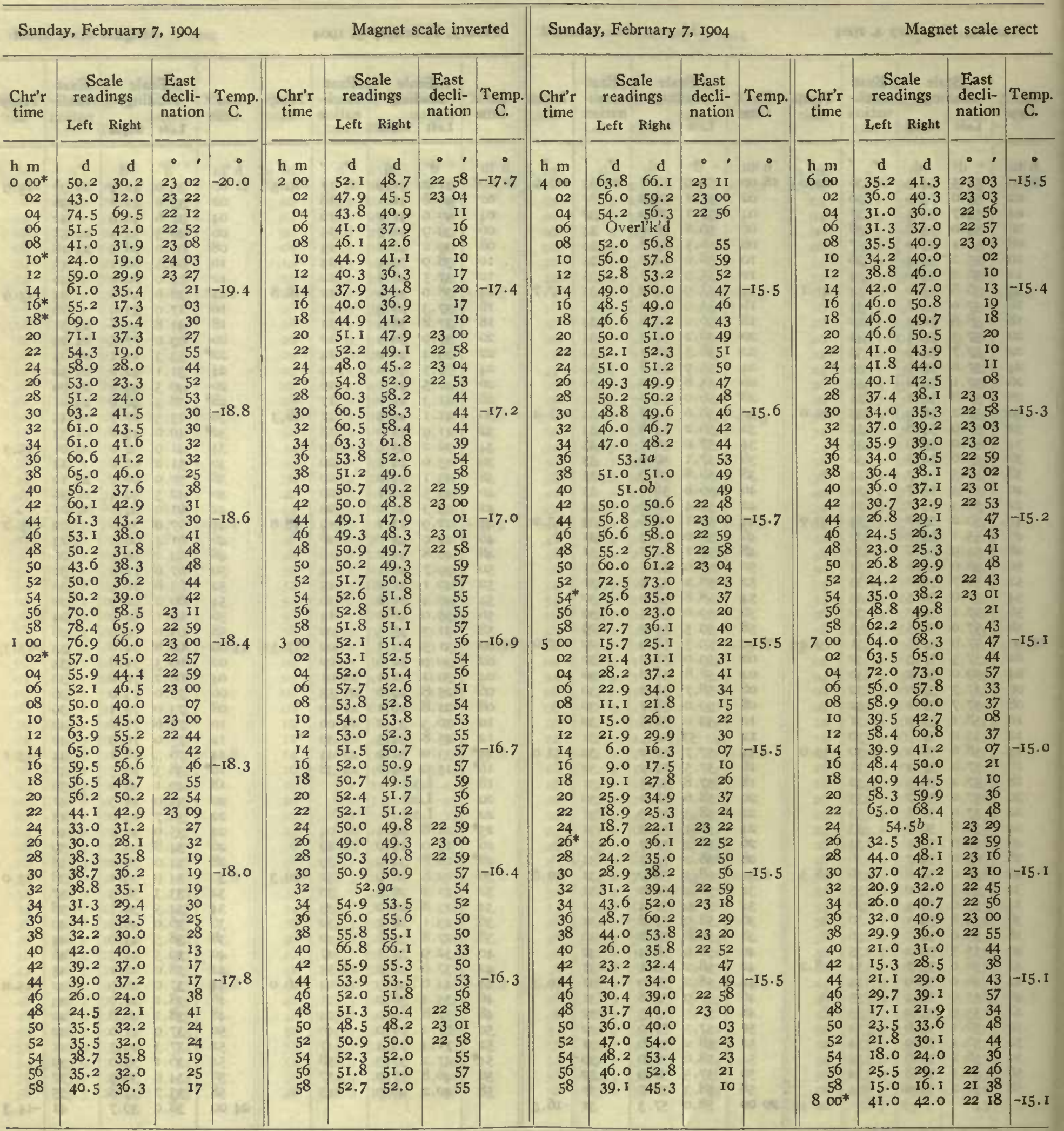


Tabulation of magnetic declinations observed at Teplitz Bay-Continued

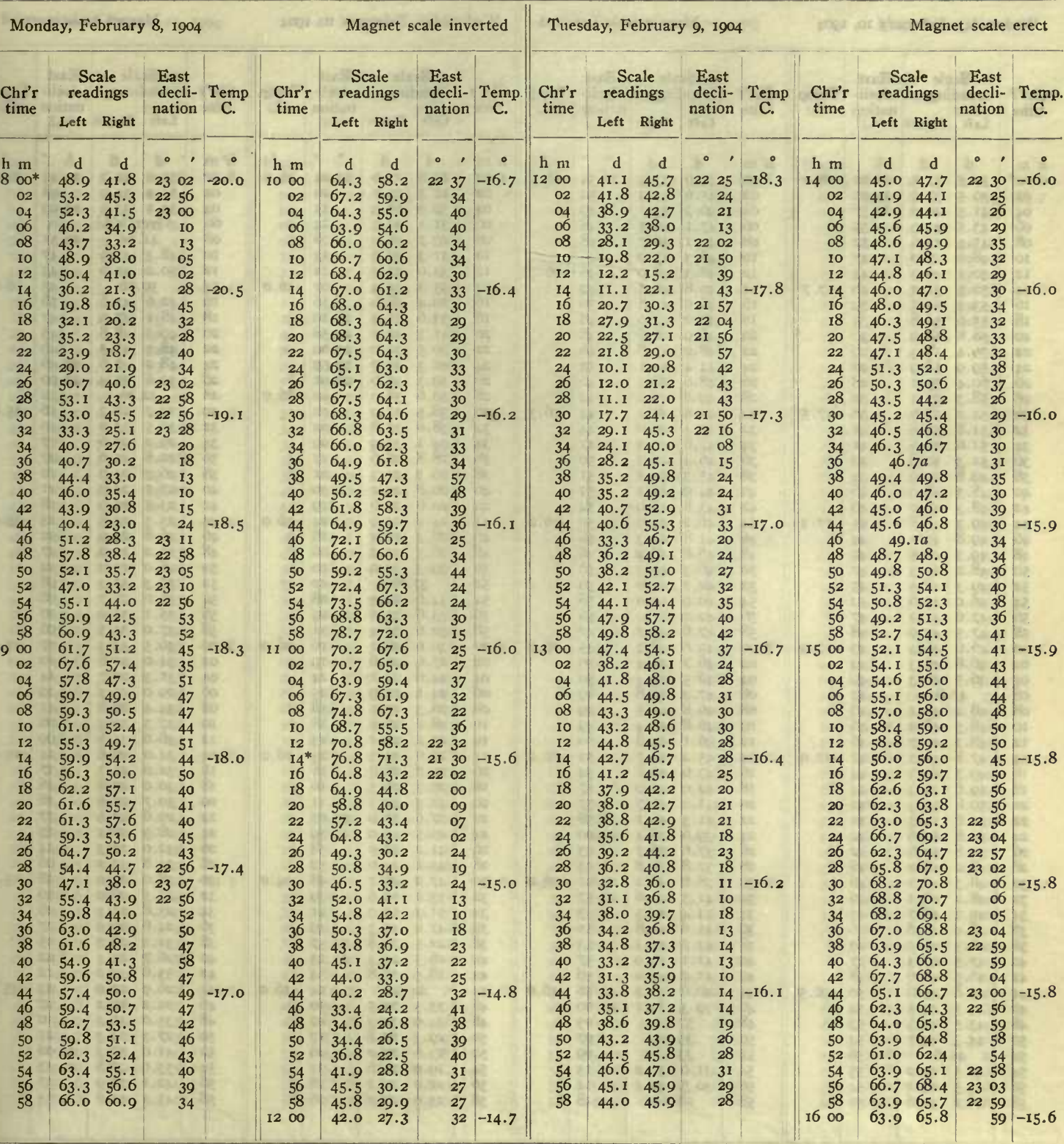

Correction to local mean time is $+4 \mathrm{~m} 23 \mathrm{~s}$.

Torsion head at $7 \mathrm{~h} 25 \mathrm{~m}$ read $29^{\circ}$ and at the end read the same. Observer-H. H. N.
Correction to local mean time is $+535.90^{\circ}$ torsion $=13.8$. Torsion head at $\mathrm{IIh} 25 \mathrm{~m}$ read $25^{\circ}$ and at $16 \mathrm{~h} 42 \mathrm{~m}$ read $38^{\circ}$. Observer-R. R. T. 
Tabulation of magnetic declinations observed at Teplitz Bay-Continued

\begin{tabular}{|c|c|c|c|c|c|c|c|c|c|c|c|c|c|c|c|c|c|c|c|c|c|}
\hline \multicolumn{5}{|c|}{ Wednesday, February 10, 1904} & \multicolumn{5}{|c|}{ Magnet scale inverted } & \multicolumn{6}{|c|}{ Wednesday, February 10, 1904} & \multicolumn{6}{|c|}{ Magnet scale inverted } \\
\hline \multirow{2}{*}{$\begin{array}{l}\text { Chr'r } \\
\text { time }\end{array}$} & \multicolumn{2}{|c|}{$\begin{array}{l}\text { Scale } \\
\text { readings }\end{array}$} & $\begin{array}{l}\text { East } \\
\text { decli- }\end{array}$ & Temp. & Chr'r & $\begin{array}{l}\text { Sca } \\
\text { read }\end{array}$ & $\begin{array}{l}\text { ale } \\
\text { ings }\end{array}$ & $\begin{array}{l}\text { East } \\
\text { decli- }\end{array}$ & Temp & Chr'r & $\begin{array}{r}\mathrm{Sc} \\
\text { reac }\end{array}$ & 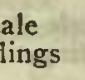 & $\begin{array}{l}\text { Eas } \\
\text { decl }\end{array}$ & & & Chr'r & $\begin{array}{l}\text { Sc } \\
\text { read }\end{array}$ & $\begin{array}{l}\text { ale } \\
\text { ings }\end{array}$ & $\begin{array}{l}\text { Eas } \\
\text { dec }\end{array}$ & cli- & Temp. \\
\hline & Left & Right & & & time & Left & Right & & & & Left & Right & atic & & & & Left & Right & $\operatorname{atc}$ & 10 & \\
\hline $\mathrm{h} \mathrm{m}$ & d & d & - & 0 & $\mathrm{~h} \mathrm{~m}$ & d & d & . & $\bullet$ & $\mathrm{h} \mathrm{m}$ & $d$ & d & & & & $h \mathrm{~m}$ & d & $d$ & - & . & $\bullet$ \\
\hline 0 oo* & 39.5 & 37.1 & 2302 & -18.0 & 200 & 43.6 & $43 \cdot 3$ & 2254 & -16.5 & 400 & 24.0 & 23.6 & 232 & & -16.0 & 600 & 31.0 & 30.3 & & 14 & -16.0 \\
\hline 02 & 39.5 & 37.5 & 23 oI & $\exists$ & 02 & 45.6 & $45 \cdot 3$ & 50 & & 02 & 27.3 & 25.7 & & 20 & and & 02 & $34 \cdot 3$ & 34.0 & & 08 & \\
\hline 04 & 41.5 & 40.0 & 2257 & o & 04 & 46.3 & 45.7 & 49 & 10 & 04 & 30.3 & 29.3 & & I 5 & 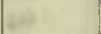 & 04 & 33.5 & 33.5 & & 09 & 10 \\
\hline 06 & 43.5 & 41.9 & 55 & I & 06 & $47 . I$ & 46.5 & 48 & 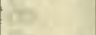 & 06 & 3I.I & 30.3 & & 14 & 1 & 06 & Ove & $l^{\prime \prime} k^{\prime} d$ & & & \\
\hline 08 & 45.3 & 43.6 & 52 & 10 & 08 & 47.1 & 46.7 & 48 & 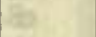 & 08 & 29.7 & 28.3 & & 16 & ta & 08 & $4 \mathrm{I}$ & $o a$ & & 57 & \\
\hline IO & 48.0 & 47.0 & 47 & 10: & 10 & 48.3 & 47.9 & 46 & Wis & I0 & 3 I. 3 & 30.3 & & 13 & & IO & 48.3 & $47 \cdot 3$ & & 47 & \\
\hline 12 & 50.1 & 49.3 & 44 & $1+4=$ & 12 & 48.3 & 48.0 & 46 & -16.4 & 12 & 33.0 & 31.3 & & II & & 12 & 50.2 & 50.0 & & 43 & \\
\hline 14 & 52.5 & 51.2 & 40 & -17.7 & 14 & 46.8 & 46.4 & 49 & (15 & 14 & $35 \cdot 3$ & 34.3 & & 07 & -16.0 & & 50 & $o b$ & & 43 & -16.0 \\
\hline 16 & 53.3 & 52.5 & 39 & & I6 & 46.0 & 45.8 & 50 & $10 x$ & 16 & 29.4 & 28.6 & & 16 & & I6 & 42.6 & 41.6 & & 56 & \\
\hline 18 & 55.6 & 54.8 & 35 & H. & 18 & 47.2 & 47.0 & 48 & ii & 18 & 33.6 & 32.3 & $23 I$ & & Et & I8 & $48 . I$ & 47.0 & & 47 & \\
\hline 20 & 58.8 & 58.0 & 30 & E & 20 & 46.9 & 46.6 & 48 & $=$ & 20 & 41.3 & 40.1 & 225 & 58 & I & 20 & 49.3 & 48.8 & & 45 & \\
\hline 22 & 59.4 & 58.3 & 29 & 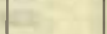 & 22 & 48.2 & 47.8 & 46 & $=$ & 22 & 39.5 & 39.0 & & 00 & $E$ & 22 & 48.3 & 47.5 & & 46 & \\
\hline 24 & 59.9 & 58.9 & 28 & 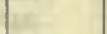 & 24 & 44.4 & 44.1 & 52 & $E$ & 24 & 40.3 & 39.7 & 225 & 59 & Q & 24 & 48.2 & 47.6 & 22 & 46 & \\
\hline 26 & 60.4 & 59.1 & 28 & $E$ & 26 & 48.6 & 48.3 & 46 & Ait & 26 & 39.3 & 38.6 & 230 & OI & & 26 & 39.6 & 39.4 & 23 & DO & \\
\hline 28 & 58.3 & 57.1 & $3 I$ & $=$ & 28 & 49.8 & 49.2 & 44 & & 28 & 38.6 & 38.0 & & 02 & -16.0 & 28 & 32 & $o b$ & & 12 & \\
\hline 30 & 54.2 & 52.6 & 58 & -17.5 & 30 & 49.8 & 49.2 & 44 & -16.3 & 30 & 38.5 & 37.9 & & 02 & - & 30 & 29.7 & 28.7 & & 16 & -I5.8 \\
\hline 32 & 51.8 & 50.8 & $4 \mathrm{I}$ & $10+2$ & 32 & 49.4 & 49.0 & 44 & 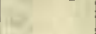 & 32 & 38.0 & 37.8 & & 02 & & 32.2 & 33.5 & 33.0 & & 10 & \\
\hline 34 & 51.6 & 50.8 & $4 I$ & 15 & 34 & 47.6 & $47 \cdot 3$ & 47 & 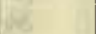 & 34 & 34 . I & 33.3 & & 09 & & 34 & 29.2 & 27.6 & & 17 & \\
\hline 36 & 51.3 & 50.8 & & 8 & 36 & 46.4 & 45.3 & 50 & fir & 36 & 28.8 & 28.6 & & I7 & $y$ & 36 & 22.8 & 21.6 & & 27 & \\
\hline 38 & 49.8 & 49.0 & 44 & 1 & 38 & 45.3 & 44.3 & 51 & tet & 38 & 26.1 & 25.5 & & $2 \mathrm{I}$ & & 38 & 26.3 & 24.9 & & 22 & \\
\hline 40 & 48.3 & 48.0 & 46 & 0 & 40 & 44.9 & 44.3 & 52 & $E$ & 40 & 20.8 & 20.1 & & 30 & E & 40 & 27.5 & 24.3 & & 21 & \\
\hline 42 & 48.3 & 48.0 & 46 & $16=$ & 42 & 43.8 & 43.0 & 54 & & 42 & 20.6 & 20.6 & & 29 & & 42 & 23.5 & 19.9 & & 28 & \\
\hline 44 & 49.3 & 48.8 & 45 & -17.3 & 44 & 46.2 & 44.6 & 50 & -16.2 & 44 & 24.2 & 23.9 & & 24 & -16.0 & 44 & 25.5 & 23.5 & & 23 & -15.6 \\
\hline 46 & 49.8 & 49.2 & & & 46 & 41.8 & 41.3 & 56 & & 46 & 28.3 & 28.1 & & 18 & O & 46 & 26.8 & 24.0 & & 22 & \\
\hline 48 & 49.3 & 48.5 & 45 & 4 & 48 & 48.8 & 48.2 & 46 & 4 & 48 & 36.3 & $35 \cdot 3$ & & 06 & & 48 & 23.2 & 21.2 & & 27 & \\
\hline 50 & 48.2 & 47.3 & 47 & 13 & 50 & 46.3 & 45.3 & 50 & 68 & 50 & 39.3 & 39.0 & & OO & 5 & 50 & 28.0 & 23.2 & & 22 & \\
\hline 52 & 49.3 & 48.5 & 45 & 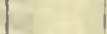 & 52 & 42.7 & 41.6 & 56 & $=$ & 52 & 37 & $.8 b$ & & 02 & & 52 & 35.3 & 33.3 & & 08 & \\
\hline 54 & 46.0 & 45.6 & 50 & 1 & 54 & 40.9 & 40.0 & 58 & & 54 & 34.6 & $34 \cdot 3$ & & 08 & & 54 & 36.0 & 32.7 & & 08 & \\
\hline 56 & 47.6 & 47.2 & 47 & 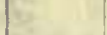 & 56 & 42.2 & 41.0 & 56 & & 56 & 33.6 & 33.3 & & 09 & & 56 & 35.1 & 31.0 & & I0 & \\
\hline 58 & 47.5 & 47.0 & 48 & & 58 & 47.6 & 47.2 & 47 & & 58 & 32.6 & 32.4 & & II & & 58 & 37.0 & 34.5 & & 06 & \\
\hline 100 & $46 . \overline{6}$ & 46.3 & 49 & -17.0 & 300 & 49.8 & 48.2 & 45 & -16.2 & 500 & 30.0 & 28.2 & & 16 & -16.0 & 700 & 36.5 & 33.3 & & 07 & -15.7 \\
\hline 02 & 46.8 & 46.3 & 49 & 1 & 02 & 46.7 & 45.4 & 50 & en & 02 & 27.6 & 26.6 & & 19 & 60 & 02 & 34.0 & 31.8 & & Io & \\
\hline 04 & 46.5 & 46.3 & 49 & 6 & 04 & 46.5 & 45.4 & 50 & 16 & 04 & 24.6 & 24.1 & & 24 & F & 04 & 31.3 & 29.7 & & 14 & \\
\hline 06 & 47 & $8 a$ & 47 & $\pi$ & 06 & 47.2 & 45.8 & 49 & 8 & 06 & 24.1 & 22.9 & & 25 & $n$ & 06 & 29.0 & 25.5 & & 19 & \\
\hline 08 & 48.2 & 48. I & 46 & 6 & 08 & 46.8 & 45.5 & 49 & 6 & 08 & 28.3 & 27.9 & & 18 & $E$ & 08 & 34.4 & 29.8 & & II & \\
\hline 10 & 48.5 & 48.2 & 46 & if & $\infty 9$ & 46.8 & 45.4 & 49 & & IO & 28.2 & 26.8 & & I8 & E & IO & 37.3 & $33 \cdot 3$ & & 06 & \\
\hline 12 & 48.1 & 47.7 & 46 & 11 & I2 & 46.1 & 44.8 & 50 & & I2 & 25.2 & 24 . I & & 23 & & 12 & 36.0 & 31.6 & & $\infty 9$ & \\
\hline 14 & 47.8 & $47 \cdot 3$ & 47 & -17.0 & 14 & 45.2 & 43.7 & 52 & -16.0 & 14 & 25.0 & 24.3 & & 23 & -16.0 & 14 & 34.0 & 26.8 & & 14 & -15.6 \\
\hline 16 & 49.3 & 49.3 & 44 & tro & 16 & 43.6 & 42.6 & 54 & & 16 & 29.7 & 28.8 & & I6 & - & 16 & 32.0 & 24.7 & & 17 & \\
\hline 18 & 49.6 & 49.3 & 44 & is & I8 & 42.0 & 41.0 & 57 & & 18 & 29.3 & 28.5 & & 16 & & 18 & 30.0 & 27.8 & & 16 & \\
\hline 20 & 50.8 & 50.3 & 42 & 12 & 20 & 41.2 & 40.7 & 57 & 6 & 20 & 32.5 & 32.3 & & II & 10 & 20 & 30.7 & 27.6 & & 16 & \\
\hline 22 & 51.6 & 51.3 & 41 & $=$ & 22 & $41: 3$ & 40.1 & 2258 & at & 22 & 37.6 & 37.0 & $23 c$ & 03 & a & 22 & 29.6 & 27.6 & & 17 & \\
\hline 24 & 50.8 & 50.6 & 42 & 15 & 24 & 39.6 & 38.4 & 2300 & 24 & 24 & 40.5 & 40.0 & 225 & 59 & If & 24 & 28.9 & 26.2 & & I9 & \\
\hline 26 & 50.8 & 50.6 & 42 & $1=$ & 26 & 37.6 & 36.7 & 03 & & 26 & 41.2 & 40.6 & & 58 & 2 & 26 & 32.0 & 29.3 & & 14 & \\
\hline 28 & 48.2 & 48.2 & 46 & is & 28 & 37.8 & 36.8 & 03 & & 28 & 45.2 & 44.0 & & 52 & & 28 & 34.8 & 33.0 & & 08 & \\
\hline 30 & 48.3 & 48.1 & 46 & I & 30 & 38.8 & 38.1 & 23 OI & -16.0 & 30 & 47.2 & 46.7 & & 48 & -14.0 & 30 & 28.0 & 27.0 & & 18 & -15.5 \\
\hline 32 & 48.2 & 48.1 & 46 & 1 & 32 & 41.5 & 40.6 & 2257 & & 32 & 53.1 & 52.6 & & 39 & & 32 & 27.5 & 26.0 & & 20 & \\
\hline 34 & 49.2 & 48.7 & & 1 & 34 & 44.4 & 43.3 & & & 34 & 51.3 & 51.0 & & $4 \mathrm{I}$ & & 34 & 23.8 & 21.5 & & 26 & \\
\hline 36 & 48.0 & 47.8 & 46 & K & 36 & 45.6 & 44.8 & $5 I$ & & 36 & 47.0 & 45.5 & & 49 & 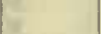 & 36 & 24.6 & 20.0 & & 27 & \\
\hline 38 & 46.5 & 46.5 & 49 & 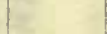 & 38 & 43.7 & 43.3 & 53 & & 38 & 42.5 & 39.8 & 225 & 57 & & 38 & $3 \mathrm{I} . \mathrm{I}$ & 28.3 & & I5 & \\
\hline 40 & 47.5 & 47.3 & 47 & $E$ & 40 & $4 \mathrm{I} . \mathrm{I}$ & 40.9 & 57 & & 40 & 26.5 & $23 \cdot 3$ & 232 & 23 & 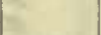 & 40 & 26.7 & 23.4 & & 22 & \\
\hline 42 & 46.9 & 46.6 & 48 & & 42 & 41.9 & 41.2 & 56 & & 42 & I8. 5 & 16.8 & & 34 & & 42 & 33.6 & 30.0 & & 12 & \\
\hline 44 & 45.8 & 45.6 & 50 & -16.5 & 44 & 40.6 & 40.3 & 2258 & -16.0 & 44 & 24 & $.3 a$ & & 24 & -16.0 & 44 & 26.9 & 22.0 & & 23 & -15.4 \\
\hline 46 & 44.5 & 44.5 & 52 & 1 & 46 & 39.3 & 39.1 & 2300 & & 46 & 33.3 & 32.5 & & 10 & & 46 & 24.2 & 20.2 & & 27 & \\
\hline 48 & 43.5 & 43.5 & 53 & 1 & 48 & 37 & $5^{b}$ & 03 & & 48 & 34.9 & 34.3 & & 07 & & 48 & 35.0 & 31.3 & & 10 & \\
\hline 50 & 44.3 & 43.7 & 53 & 2 & 50 & 35. & $o b$ & 07 & - & 50 & 29.3 & 27.8 & & I7 & & 50 & 27.3 & 24.3 & & 21 & \\
\hline 52 & 41 & $8 a$ & 56 & $=$ & 52 & 33. & $5 b$ & 09 & & 52 & 23.6 & 22.0 & & 26 & & 52 & 41.0 & 37.3 & 23 & $\infty 0$ & \\
\hline 54 & 42.5 & 42.2 & 55 & 2 & 54 & 31.0 & 31.0 & I3 & & 54 & 19.9 & 19.6 & & $3 I$ & & 54 & 53.0 & 47.8 & 22 & 43 & \\
\hline 56 & 43.5 & 43.2 & 54 & 7 & 56 & 30.5 & 30.5 & 14 & & 56 & 22.0 & 21.2 & & 28 & & 56 & 42.1 & 39.9 & & 57 & \\
\hline 58 & 46.2 & 45.6 & 50 & aty & 58 & & $1 b$ & 18 & & 58 & 23.0 & 22.2 & & 26 & & 58 & $45 \cdot 3$ & 41.8 & & 53 & \\
\hline
\end{tabular}


Tabulation of magnetic declinations observed at Teplitz Bay-Continued

\begin{tabular}{|c|c|c|c|c|c|c|c|c|c|c|c|c|c|c|c|c|c|c|c|}
\hline \multicolumn{5}{|c|}{ Wednesday, February Io, 1904} & \multicolumn{5}{|c|}{ Magnet scale inverted } & \multicolumn{5}{|c|}{ Wednesday, February 10, I904 } & \multicolumn{5}{|c|}{ Magnet scale inverted } \\
\hline \multirow{2}{*}{$\begin{array}{l}\text { Chr'r } \\
\text { time }\end{array}$} & \multicolumn{2}{|c|}{$\begin{array}{l}\text { Scale } \\
\text { readings }\end{array}$} & \multirow{2}{*}{$\begin{array}{c}\text { East } \\
\text { decli- } \\
\text { nation }\end{array}$} & Temp & Chr'r & $\begin{array}{l}\text { Sea } \\
\text { read }\end{array}$ & $\begin{array}{l}\text { ale } \\
\text { ings }\end{array}$ & $\begin{array}{l}\text { East } \\
\text { decli- }\end{array}$ & Temp. & Chr'r & $\begin{array}{l}\text { Sca } \\
\text { readi }\end{array}$ & $\begin{array}{l}\text { ale } \\
\text { lings }\end{array}$ & East & & & $\begin{array}{l}\text { Sct } \\
\text { read }\end{array}$ & $\begin{array}{l}\text { ale } \\
\text { ings }\end{array}$ & East & Temp. \\
\hline & Left & Right & & & & Left & Right & & & & Left & Right & & & & Left & Right & nation & \\
\hline h $\mathrm{m}$ & d & d & 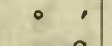 & $\circ$ & $\mathrm{h} \mathrm{m}$ & d & $\mathrm{d}$ & $\cdot \quad \cdot$ & - & h $\mathrm{m}$ & d & d & & & $\mathrm{h} \mathrm{m}$ & 0 & a & & $\cdot$ \\
\hline 800 & 43.1 & 38.0 & 2258 & -15.0 & I0 00 & 55.2 & 53.0 & 2237 & -14.2 & 1200 & 57.2 & 56.1 & 2233 & -14.0 & 1400.5 & 48.0 & 47.5 & 2247 & \\
\hline 02 & 40.0 & 34.9 & 23 o3 & $\theta$ & 02 & 50.0 & 47.9 & 45 & 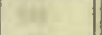 & 02 & 56.9 & 56.1 & 33 & $\theta^{2}$ & 02 & 45.1 & $4 I .5$ & 54 & -13.7 \\
\hline 04 & 37.9 & 35.4 & 2304 & 4 & 04 & 53.4 & 50.4 & 40 & $m$ & 04 & $54 \cdot 3$ & 53.9 & 37 & ja & 04 & 45.8 & $43 . I$ & 52 & $=0$ \\
\hline 06 & 62.5 & 60.8 & 2225 & Ar & 06 & 50. I & 46.9 & 46 & te & 06 & 56.0 & 55.0 & 34 & 168 & 06 & 46.0 & 42.4 & 52 & 168 \\
\hline 08 & 51.2 & 50.4 & 42 & $E$ & 08 & 56.3 & 53.8 & 35 & 6 & 08 & 55.0 & 53.7 & 36 & 20 & 08 & 41.6 & 40.0 & $225^{8}$ & Be \\
\hline 10 & 44.0 & 38.9 & 57 & 97 & IO & 57.2 & 54.9 & 34 & 18. & 10 & 56.3 & 55.7 & 34 & at & Io & 40.0 & 37.8 & $23 \mathrm{OI}$ & of: \\
\hline 12 & 42.2 & 38.9 & 58 & 62 & 12 & 53.1 & 52.8 & 39 & $a^{2} x^{2}$ & 12 & 56.1 & $55 \cdot 3$ & 34 & $x=$ & I2 & 39.8 & 37.2 & 23 oI & \\
\hline I4 & 54.0 & 52.2 & 38 & -15.0 & 14 & 55.9 & 54.5 & 35 & -14.2 & 14 & 58.2 & 58.9 & 30 & -14.0 & I4 & 41.7 & 39.5 & 2258 & -13.8 \\
\hline 16 & 43.5 & 42.2 & 2254 & & 16 & 57.5 & 56.9 & 32 & $x^{2}$ & 16 & 57.9 & 57.2 & $3 \mathbf{I}$ & ( & 16 & 38.0 & 37.0 & 2303 & \\
\hline 18 & 29.3 & 24.8 & 2319 & Eari & I8 & 61.7 & 59.6 & 27 & 24. & 18 & 55.2 & 55.0 & 35 & $3 y$ & I8 & 40.5 & 39.0 & 2259 & (ath \\
\hline 20 & 56.5 & 46.0 & $224 I$ & tat & 20 & 60.5 & 59.2 & 28 & $\sigma$ & 20 & 53.0 & $52 . I$ & 39 & 3 & 20 & 42.0 & 40.3 & 57 & $\cos$ \\
\hline 22 & 58.2 & 54.7 & 2233 & a & 22 & 60.4 & 58.9 & 28 & 46 & 22 & 56.0 & $54 \cdot 3$ & 35 & 35 & 22 & 43.2 & $4 \mathrm{I} .1$ & 56 & $=5$ \\
\hline 24 & 34.2 & 33.2 & 2309 & $E$ & 24 & 61.9 & 59.8 & 26 & $y=$ & 24 & 54.8 & 54.1 & 36 & at & 24 & 43.8 & 42.0 & 54 & $t=$ \\
\hline 26 & 42.0 & 40.8 & 2257 & 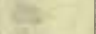 & 26 & 57.0 & $55 \cdot 3$ & 34 & $\approx 7$ & 26 & 58.0 & 56.7 & 32 & 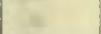 & 26 & $45 \cdot 3$ & 43.5 & 52 & $\sqrt{2}$ \\
\hline 28 & 49.1 & 46.0 & 47 & $t^{2}$ & 28 & 59.2 & 55.1 & 32 & & 28 & 58.3 & 57.5 & $3 \mathrm{I}$ & - & 28 & 49.0 & 48.6 & 45 & \\
\hline 30 & 43.0 & 40.5 & 2256 & -14.9 & 30 & 55.9 & 52.0 & 37 & -14.3 & 30 & $57 . \mathrm{I}$ & 55.8 & 33 & -13.9 & 30 & 47.5 & 46.8 & 48 & -13.7 \\
\hline 32 & 40.2 & 36.9 & 23 or & th & 32 & 55.2 & 52.2 & 37 & - & 32 & 56.2 & 55.1 & 34 & . & 32 & 48.0 & 46.4 & 48 & $\theta^{2}$ \\
\hline 34 & 77.0 & 73.5 & 2204 & 14 & 34 & 54.5 & 51.0 & 39 & 26 & 34 & 58.0 & 57.7 & $3 \mathrm{I}$ & 18 & 34 & 50.4 & $49.5^{\circ}$ & 43 & ac \\
\hline 36 & 60.9 & 59.0 & 2228 & de & 36 & 57.1 & 53.2 & 35 & N & 36 & 55.8 & 55.6 & 34 & 2 & 36 & 50.5 & 49.9 & 43 & ats \\
\hline 38 & I3. 5 & 7.5 & 2345 & $\theta$ & 38 & 60.0 & 57.0 & 30 & fet & 38 & 54.0 & 54.0 & 37 & 2 & 38 & 50.2 & 49.2 & 44 & Fe \\
\hline 40 & 27.3 & 26.1 & 2320 & $\%$ & 40 & 58.0 & 56.0 & 32 & int: & 40 & $55 \cdot 3$ & 54.8 & 35 & 14 & 40 & 51.9 & 51.6 & 40 & by \\
\hline 42 & 52.5 & 49.5 & 2242 & 4 & 42 & 58.5 & 57.6 & 31 & 0 & 42 & & 5.0 & 35 & $1-1$ & 42 & 53.9 & 53.1 & 38 & \\
\hline 44 & 53.0 & 49.0 & 42 & -14.9 & 44 & 57.0 & 55.1 & 34 & $-14 \cdot 3$ & 44 & 48.6 & 43.0 & 50 & & 44 & 55.0 & 53.9 & 36 & -13.5 \\
\hline 46 & 46.0 & 42.6 & 52 & & 46 & 61.1 & 58.9 & 28 & $x$ & 46 & 45.0 & 44.8 & $5 \mathrm{I}$ & -13.9 & 46 & 55.4 & 56.2 & 34 & \\
\hline 48 & 49.3 & $45 \cdot 3$ & 47 & $E$ & 48 & 64.0 & 61.5 & 23 & 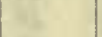 & 48 & 56.1 & 55.3 & 34 & & 48 & 57.0 & 56.0 & 33 & 6 \\
\hline 50 & 49.2 & 47.9 & 46 & ag & 50 & $5^{8} .2$ & 57.0 & $3 \mathrm{I}$ & 18 & 50 & 57.9 & 57.0 & 32 & $1=$ & 50 & 54.8 & 53.8 & 36 & the \\
\hline 52 & 41.9 & 39.0 & 58 & Fiz & $5^{2}$ & 62.7 & 59.9 & 25 & 28 & 52 & 59.8 & 58.9 & 28 & $1=$ & 52 & 55.0 & 54.0 & 36 & $E$ \\
\hline 54 & 44.2 & 37.8 & 57 & 6 & 54 & 61.1 & 59.8 & 27 & Hr & 54 & 56.7 & 55.7 & 34 & & 54 & 55.4 & 53.4 & 36 & 22 \\
\hline 56 & 45.2 & 42.5 & 53 & & 56 & 61.0 & 60.9 & 26 & & 56 & 55.0 & $53 \cdot 3$ & 37 & & 56 & 55.2 & & 36 & 65 \\
\hline 58 & 48.1 & 41.0 & 52 & & 58 & 58. I & 56.0 & 32 & & 58 & 56.8 & 55.3 & 34 & & 58 & 56.0 & & 34 & \\
\hline 900 & 52.3 & 42.2 & 48. & -14.8 & II $\infty$ & 58.2 & 57.2 & $3 I$ & $-I 4 \cdot I$ & 1300 & 59.2 & 57.7 & 30 & -13.8 & I5 OO & 57.5 & 56.5 & 32 & -13.5 \\
\hline 02 & 46.6 & 38.0 & 55 & & 02 & 61.8 & 61.0 & 25 & & 02 & 59.0 & 57.9 & 30 & - & 02 & $58 . \mathrm{I}$ & 57.3 & $3 I$ & \\
\hline 04 & 43.7 & 38.5 & 57 & 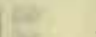 & 04 & 56. & $5^{b}$ & 33 & 17 & 04 & 60.7 & 59.3 & 28 & Te & 04 & 59.2 & & 29 & 19 \\
\hline 06 & 49.8 & 43.2 & 49 & 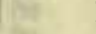 & 06 & 58.0 & 58.0 & $3 I$ & wit & 06 & 63.2 & 61.8 & 24 & 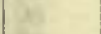 & 06 & 60.9 & 60.0 & 27 & 100 \\
\hline 08 & 43.1 & 38.8 & 58 & (20. & 08 & 58.4 & 56.2 & 32 & has & 08 & 62.4 & 61.0 & 25 & 10 & 08 & & 59.9 & 7 & 8 \\
\hline Io & 50.0 & 47.0 & 46 & (ar & I0 & 53.0 & 50.2 & $4 \mathrm{I}$ & EF & 10 & 61.8 & 60.0 & 26 & 61 & I0 & 61.8 & 60.5 & 26 & in \\
\hline 12 & 48.0 & 44.5 & 49 & 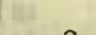 & I2 & 61.1 & 60.0 & 27 & tit & I2 & 57.0 & 55.8 & 33 & 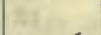 & 12 & & & 28 & \\
\hline 14 & 46.8 & 42.0 & 52 & -14.8 & I4 & 60.4 & 60.0 & 27 & & I4 & 55.4 & 54.7 & 35 & -13.6 & 14 & 62.8 & 61.5 & 25 & -13.3 \\
\hline 16 & $45 . I$ & $4^{1} .9$ & 5.3 & & I6 & 56.5 & 55.0 & 34 & -14.0 & 16 & & 53.0 & 38 & & 16 & 62.5 & 61.8 & 24 & \\
\hline 18 & 49.9 & 45.9 & 46 & & I8 & 57.5 & 55.9 & 33 & I & 18 & 59.8 & 57.5 & 30 & at & I8 & 63.0 & 62.0 & 23 & a \\
\hline 20 & 45.0 & 42.3 & 53 & 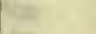 & $20 .:$ & 58.5 & 57.0 & $3 \mathrm{I}$ & $1=$ & 20 & & 58.0 & 30 & tee & 20 & 62.0 & & 5 & $=$ \\
\hline 22 & 45.0 & 41.8 & 54 & $E=$ & 22 & 59.5 & 58.1 & 29 & in & 22 & 58.2 & 57.3 & $3 I$ & $=6$ & 22 & 64.0 & 62.8 & 22 & $E$ \\
\hline 24 & 49.2 & 48.9 & 45 & 18 & 24 & 62.5 & 60.3 & 25 & $1 t^{2}$ & 24 & & 58.0 & 29 & 18 & 24 & 64.9 & 63.8 & 21 & $1 z$ \\
\hline 26 & $49 . I$ & 47.5 & 46 & 16 & 26 & 59. I & 57.7 & 30 & 8 & 26 & 58.1 & 55.9 & 32 & & 26 & 64.5 & 62.9 & 22 & \\
\hline 28 & 53.5 & 50.0 & 40 & Q & 28 & 56.9 & 55.2 & 34 & & 28 & 59.0 & & $3 I$ & 10 & 28 & 63.6 & 62.4 & 23 & \\
\hline 30 & 56.9 & 53.9 & 35 & $-14 \cdot 3$ & 30 & 56.5 & 55.6 & 34 & -14.0 & 30 & 60. & 58.8 & 28 & -13.6 & 30 & 63.9 & 62.0 & 23 & -13.2 \\
\hline 32 & 56.6 & 48.2 & 40 & & 32 & 56.0 & 53.9 & 36 & $x^{2}$ & 32 & 60.8 & 59.0 & 28 & & 32 & 65.0 & 63.0 & $2 \mathrm{I}$ & \\
\hline 34 & 53. I & 51.2 & 40 & 15 & 34 & 55.2 & 54.0 & 36 & Es & 34 & 58.4 & 57.0 & $3 \mathrm{I}$ & 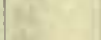 & 34 & 64.0 & 62.9 & 22 & (16 \\
\hline 36 & 56.0 & 47.0 & 41 & 5 & 36 & 53.9 & 52.1 & 38 & & 36 & 60.7 & 59.2 & 28 & & 36 & 64.3 & 63.1 & 22 & \\
\hline 38 & 59.0 & 50.0 & 36 & 2 & 38 & 57.0 & 55.5 & 34 & 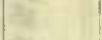 & 38 & 64.7 & 62.3 & 22 & F & 38 & 62.9 & 61.0 & 24 & e. \\
\hline 40 & 58.0 & 5 I. 8 & 36 & 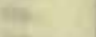 & 40 & & $53 . \mathrm{I}$ & 36 & 0 & 40 & & 64.1 & 20 & $7 y$ & 40 & 63.1 & 61.8 & 24 & 8 \\
\hline 42 & 49.0 & 43.0 & 50 & & 42 & 60.0 & 58.2 & 29 & 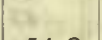 & 42 & 66.2 & 64.0 & 20 & +7 & 42 & 64.0 & 62.0 & 23 & \\
\hline 44 & 54.0 & 52.0 & 38 & $-14 . I$ & 44 & 60.3 & 58.5 & 28 & -14.0 & 44 & 64.2 & 63.6 & $2 I$ & -13.7 & 44 & 64.7 & 61.5 & 23 & -13.2 \\
\hline 46 & 54.6 & 52.8 & 37 & & 46 & 59.6 & 58.2 & 29 & & 46 & 61.9 & 59.8 & 26 & & 46 & 67.2 & 64.4 & 19 & \\
\hline 48 & 55.0 & 54.0 & 36 & 4 & 48 & 6I.0 & 60.0 & 27 & 4 & 48 & 60.7 & 59.0 & 28 & & 48 & 65.0 & 63.0 & 21 & \\
\hline 50 & 55.7 & 54.0 & 36 & & 50 & 57.5 & 55.7 & 33 & 102 & 50 & 58.7 & 57.6 & 30 & & 50 & & 63.8 & $2 I$ & \\
\hline 52 & 53.0 & 50.1 & 4I & & 52 & 59.0 & 57.0 & $3 I$ & 17 & 52 & 55.2 & 55.0 & 35 & Ea & 52 & 63.8 & 62.5 & 23 & \\
\hline 54 & 54.0 & 52.0 & 38 & & 54 & 57.5 & 56.5 & 32 & 13 & 54 & 50.0 & 49.9 & 43 & & 54 & 65.8 & 64.0 & 20 & \\
\hline 56 & 51.6 & 49.0 & 43 & & 56 & 56.9 & 55.3 & 34 & 8 & 56 & 52.0 & 51.1 & $4 \mathrm{I}$ & & 56 & 65.0 & 63.2 & 21 & \\
\hline 58 & 52.2 & 49.9 & 42 & & 58 & 57.2 & 55.9 & 33 & & 58 & 49.7 & 48.8 & 44 & & 58 & 65.5 & 63.7 & 20 & \\
\hline
\end{tabular}


Tabulation of magnetic declinations observed at Teplitz Bay-Continued

\begin{tabular}{|c|c|c|c|c|c|c|c|c|c|c|c|c|c|c|c|c|c|c|c|c|}
\hline \multicolumn{5}{|c|}{ Wednesday, February 10, 1904} & \multicolumn{5}{|c|}{ Magnet scale inverted } & \multicolumn{5}{|c|}{ Wednesday, February Io, I904 } & \multicolumn{6}{|c|}{ Magnet scale inverted } \\
\hline \multirow{2}{*}{$\begin{array}{l}\text { Chr'r } \\
\text { tinie }\end{array}$} & \multicolumn{2}{|c|}{$\begin{array}{l}\text { Scale } \\
\text { readings }\end{array}$} & $\begin{array}{l}\text { East } \\
\text { decli- }\end{array}$ & Temp & Chr't & $\begin{array}{r}\mathrm{Sc} \\
\text { read }\end{array}$ & $\begin{array}{l}\text { ale } \\
\text { lings }\end{array}$ & $\begin{array}{l}\text { East } \\
\text { decli- }\end{array}$ & Tenp. & Chr'r & $\begin{array}{r}\mathrm{Sc} \\
\text { read }\end{array}$ & $\begin{array}{l}\text { ale } \\
\text { lings }\end{array}$ & East & Temp. & Chr'r & $\begin{array}{r}\mathrm{Sc} \\
\text { read }\end{array}$ & $\begin{array}{l}\text { ale } \\
\text { lings }\end{array}$ & Eas & $\begin{array}{l}\text { ast } \\
\text { cli- }\end{array}$ & Temp. \\
\hline & Left & Right & & & & Left & Right & & & & Left & Right & & & & Left & Right & & & \\
\hline h $\mathrm{m}$ & d & d & - ' & 1 & h m & d & d & $\cdot \quad$ & • & h $\mathrm{m}$ & d & $\mathrm{d}$ & & & $\mathrm{h} \mathrm{m}$ & $d$ & $d$ & 0 & ' & . \\
\hline 1600 & 63.0 & 62.1 & 2224 & -13.0 & 1800 & 52.0 & 50.1 & 2242 & -11.8 & 2000 & $55 \cdot 5$ & $54 \cdot 7$ & 2235 & -11.6 & 2200 & 60.9 & 57.9 & & 28 & -11.2 \\
\hline 02 & 63.2 & 62.5 & 23 & & 02 & 51.9 & 48.8 & 43 & $\cos$ & 02 & 58.0 & 56.7 & & & 02 & 63.9 & 61.3 & & 23 & 100 \\
\hline 04 & 65.2 & 64.2 & 20 & ta & 04 & 50.1 & 48.8 & 44 & ta & 04 & 58.1 & 56.6 & 32 & 18 & 04 & 62.9 & 59.2 & & 26 & \\
\hline 06 & 63.1 & 62.9 & 23 & as & 06 & 50.2 & 48.8 & 44 & 8 & 06 & 58.2 & 56.7 & 32 & 180 & 06 & 62.0 & 59.0 & & 27 & S6 \\
\hline 08 & 63.7 & 63.4 & 22 & 36 & 08 & 51.0 & 49.3 & 43 & 65 & 08 & 58.4 & 57.0 & 31 & ent & 08 & 51.0 & 48.5 & & 43 & t5 \\
\hline IO & 62.4 & 62.0 & 24 & 5 & I0 & 50.8 & 48.9 & 44 & 64 & I0 & 60.0 & 58.2 & 29 & ot & I0 & 56.3 & 50.1 & 223 & & 00 \\
\hline 12 & 65.8 & 62.6 & $2 \mathrm{I}$ & . & 12 & 50.7 & 49.2 & 43 & $1-1$ & 12 & 59.4 & 58.3 & 29 & 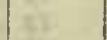 & 12 & 23 & $.0 b$ & 23 & 26 & \\
\hline I4 & 63.5 & 60.8 & 24 & -12.5 & 14 & 51.5 & 50.4 & 42 & -11.8 & 14 & 59.5 & 58.2 & 29 & -11.7 & I4 & 42.2 & 40.3 & 22 & 57 & -11.2 \\
\hline 16 & 63.9 & 61.0 & 24 & (8) & 16 & 51.7 & 50.3 & 42 & & 16 & 58.8 & 57.2 & $3 I$ & & 16 & 32.1 & 31.7 & 23 & 12 & oit \\
\hline 18 & 63.0 & 60.7 & 25 & 182 & 18 & 52.2 & 51.4 & 40 & 14 & 18 & 60.8 & 58.6 & 28 & 28 & $18 * 4$ & $54 . \mathrm{I}$ & 53.6 & 24 & OI & \\
\hline 20 & 62.1 & 59.2 & 27 & as & 20 & 52.4 & $5 \mathrm{I} .4$ & 40 & as: & 20 & 59.9 & 58.2 & 29 & $\infty e$ & $20 * 6$ & 48.8 & 28.9 & 22 : & 38 & \\
\hline 22 & $62 . I$ & 59.0 & 27 & 15 & 22 & 52.3 & 52.0 & 40 & $=$ & 22 & 59.0 & 56.9 & 31 & $x=$ & 22 & 57.9 & 43.2 & & 20 & $=$ \\
\hline 24 & 62.0 & 58.8 & 27 & 127 & 24 & 52.2 & 51.9 & 40 & 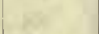 & 24 & 58.5 & 57.0 & $3 \mathbf{I}$ & & 24 & 50.9 & 35.8 & & $3 \mathrm{I}$ & if \\
\hline 26 & 61.7 & 58.8 & 27 & $\operatorname{let}$ & 26 & 50.1 & 50.0 & 43 & 38 & 26 & 58.7 & 57.2 & $3 \mathrm{I}$ & $8 \mathrm{z}$ & 26 & 45.6 & 31.6 & & 39 & \\
\hline 28 & 61.2 & 58.2 & 28 & 2 & 28 & 49.6 & 48.8 & 44 & & 28 & 59.1 & 57.4 & 30. & 16 & 28 & 47.8 & 33.2 & & 36 & \\
\hline 30 & $64 . I$ & 61.3 & 23 & -12.2 & 30 & 49.4 & 49.0 & 44 & -11.8 & 30 & 58.9 & 57.4 & 30 & -II.5 & 30 & .44 .4 & 3 I. 3 & & 40 & - II I. 2 \\
\hline 32 & 66.2 & 64.1 & 19 & Reche & 32 & 49.2 & 48.6 & 45 & 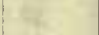 & 32 & 58.8 & 57.6 & 30 & trat & 32 & 38.7 & 29. I & & 46 & \\
\hline 34 & 65.9 & 63.1 & 20 & at & 34 & 49.3 & 48.1 & 45 & 13 & 34 & 58.2 & 57.0 & $3 I$ & Fi & 34 & 50.9 & 42.8 & & 26 & \\
\hline 36 & 63.9 & 60.9 & 24 & $1-2$ & 36 & 50.2 & $4^{8} .9$ & 44 & (6) & 36 & 58.3 & 57.2 & $3 I$ & os & 36 & 58.8 & 50.9 & & 13 & as \\
\hline 38 & 62.8 & 60.0 & 25 & W & 38 & 50.5 & 49.3 & 43 & 6 & 38 & 58.3 & 57.1 & 31 & Ec & 38 & 51.7 & 42.1 & & 26 & 38 \\
\hline 40 & 63.8 & 60.8 & 24 & $y$ & 40 & 50.3 & 49.3 & 44 & uif & 40 & 58.8 & 57.4 & 30 & est & 40 & 49.9 & 41.9 & & 27 & \\
\hline 42 & 62.8 & 60.4 & 25 & 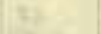 & 42 & 51.7 & 50.3 & 42 & $1=5$ & 42 & 58.4 & 57.2 & $3 \mathrm{I}$ & 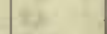 & 42 & 48.6 & 39.3 & & 30 & \\
\hline 44 & 61.7 & 59.6 & 27 & -12.0 & 44 & 52.4 & 51.0 & 40 & -11.8 & 44 & 58.5 & 57.4 & $3 \mathrm{I}$ & -11.4 & 44 & 54.3 & 45.8 & & 2I & -II.I I \\
\hline 46 & 60.4 & 58.5 & 28 & 14 & 46 & 52.2 & 51.0 & $4 I$ & 84 & 46 & 59.3 & 58.8 & 29 & $x$ & 46 & 40.0 & 32.8 & & 42 & \\
\hline 48 & 60.9 & 59.0 & 28 & 13 & 48 & 53.0 & 50.0 & $4 \mathrm{I}$ & $1 R_{0}$ & 48 & 60.2 & 59.2 & 28 & 16 & 48 & 45.4 & 37.8 & & 34 & 6. \\
\hline 50 & 59.7 & 57.6 & 30 & 10 & 50 & 53.1 & 50.0 & $4 \mathrm{I}$ & 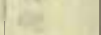 & 50 & 6I.I & 59.6 & 27 & 62 & 50 & 39.7 & 31.0 & & 44 & 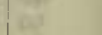 \\
\hline 52 & 59.5 & 57.8 & 30 & 6 & 52 & 53.1 & 48.7 & 42 & $1=$ & 52 & 60.2 & 58.7 & 28 & 28 & 52 & 28.2 & 25 . I & 22 & 58 & 0 \\
\hline 54 & 57.7 & 56.0 & 33 & in & 54 & 52.8 & 48.2 & 42 & 12 & 54 & 60.5 & 58.9 & 28 & 22 & 54 & 15 & $.0 b$ & 23 & 16 & \\
\hline 56 & 56.4 & 55.0 & 34 & 68 & 56 & 52.0 & 48.0 & 43 & 12 & 56 & 60.2 & 58.3 & 29 & & $56^{*}$ & 41.7 & 25.6 & & 47 & \\
\hline 58 & 56.1 & 54.6 & 35 & $18 z$ & 58 & 52.0 & 48.1 & 43 & & 58 & 59.9 & 58.3 & 29 & & 58 & 28.8 & 27.8 & 23 & 55 & \\
\hline I7 00 & 55.3 & 53.9 & 36 & $-12,0$ & 1900 & 51.9 & 48.2 & 43 & -II. 6 & 2100 & 59.7 & 58.1 & 29 & -I I. 4 & $2300^{*}$ & 72.3 & 42.0 & 24 & 07 & -II.O \\
\hline 02 & 56.6 & 55.0 & 34 & $\left(x^{2}+2\right.$ & 02 & $5 \mathrm{I} . \mathrm{I}$ & 48.4 & 44 & in & 02 & 59.0 & 58.1 & 30 & 0 & $02^{*}$ & 69.0 & $23 . I^{-}$ & 22 & $4 \mathrm{I}$ & on \\
\hline 04 & 57.3 & 55.1 & 34 & 10 & 04 & 50.9 & 48.5 & 44 & por & 04 & 59.8 & 58.7 & 29 & 10 & 04 & 70.4 & 24.1 & & 40 & 10 \\
\hline 06 & $57 \cdot 3$ & 55.2 & 34 & ar & 06 & 50.8 & 48.8 & 44 & 10 & 06 & 60.1 & 59.4 & 28 & F & 06 & 72.7 & 38.8 & 22 & 26 & $\infty$ \\
\hline 08 & 57.0 & 54.5 & 34 & Ra & 08 & 5 I. I & 49.2 & 43 & 62 & 08 & 60.6 & 60.1 & 27 & 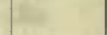 & 08 & 34.0 & 12.7 & 23 & 17 & 100 \\
\hline I0 & 55.8 & 53.3 & 36 & a tes & I0 & 51.4 & 49.8 & 42 & er & 10 & 59.8 & 58.9 & 29 & it & To & $6 \mathrm{i} .0$ & 38.9 & 22 & 35 & \\
\hline I2 & 55.2 & 53.0 & 37 & 14 & I2 & 51.2 & 49.5 & 43 & fx & 12 & 55.0 & 54.1 & 36 & & 12 & 62.8 & 43.0 & & $3 I$ & \\
\hline I4 & 54.0 & 51.6 & 39 & -11.9 & 14 & 51.4 & 49.9 & 42 & -11.7 & 14 & 47.8 & 43.0 & 50 & -11.4 & I4 & 65.1 & 45.0 & & 27 & - II.O \\
\hline 16 & 54.9 & 53.2 & 37 & 1 & 16 & .52 .0 & 50.6 & $4 \mathrm{I}$ & $x^{2}=$ & 16 & 53.0 & 52.3 & 39 & & 16 & 48.8 & 29.1 & 22 & 52 & \\
\hline 18 & 54.1 & 53.0 & 38 & 10 & I8 & $54 \cdot 3$ & 52.5 & 38 & We & 18 & & 62.2 & 15 & 11 & 18 & 37.7 & 19.2 & 23 & 09 & \\
\hline 20 & 55.9 & 54.2 & 35 & $=$ & 20 & 54.2 & 52.4 & 38 & 18 & 20 & 68.8 & 58.1 & 22 & 10 & $20^{*}$ & 36.1 & 34.0 & & 44 & 78 \\
\hline 22 & 56.1 & 55.0 & 34 & $\Leftrightarrow$ & 22 & 54.2 & 52.7 & 38 & $=$ & 22 & 68.9 & 61.8 & 19 & . & 22 & 59.8 & 45.2 & 23 & 17 & \\
\hline 24 & 55.6 & 54.3 & 36 & His & 24 & 53.9 & 52.2 & 38 & 114 & 24 & 69.3 & 61.0 & 20 & lie & 24 & 72.8 & 58.7 & 22 & 56 & \\
\hline 26 & 56.2 & 55.0 & 34 & 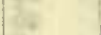 & 26 & 54.2 & 52.6 & 38 & $E$ & 26 & 69.1 & 63.1 & 18 & $=$ & 2 & 66.0 & 52.2 & 23 & 06 & \\
\hline 28 & 56.1 & 55.0 & 34 & E & 28 & 54.0 & 53.0 & 38 & & 28 & 71.2 & 64. & 15 & & 28 & 68.7 & 54.3 & 23 & 03 & \\
\hline 30 & 56.0 & 54.8 & 34 & -II.9 & 30 & $54 . \mathrm{I}$ & 52.2 & 38 & -II.7 & 30 & 69.3 & 63.1 & 18 & -II.4 & 30 & 78.3 & 68.0 & 22 & 44 & $-I I .0$ \\
\hline 32 & 55.2 & 53.8 & 36 & ar & 32 & 53.3 & 52.0 & 39 & I & 32 & 68. I & 62 & 19 & & $32^{*}$ & 44.8 & 28.2 & & 38 & \\
\hline 34 & 53.4 & 51.8 & 39 & +2 & 34 & 52.4 & 51.0 & $4 I$ & $1=$ & 34 & 66.9 & 61.9 & 21 & 2 & 34 & 44.5 & 26.4 & & 39 & \\
\hline & 52.9 & 50.8 & 40 & E. & 36 & 52.4 & 51.2 & 40 & iz & 36 & 67.1 & 62.2 & 20 & If & & 44.0 & 27.8 & & 39 & \\
\hline 38 & 52.2 & 50.1 & $4 \mathrm{I}$ & 12 & 38 & 52.2 & $51 . I$ & $4 \mathrm{I}$ & e & 38 & 66.9 & 62.7 & 20 & 1 & 38 & 44.7 & 27.7 & & 38 & \\
\hline 40 & 52.0 & 49.9 & 42 & 1 & 40 & 52.9 & 51.2 & 40 & tos & 40 & 66.2 & 62.4 & $2 \mathrm{I}$ & $1=$ & 40 & 40.9 & 24.7 & & 43 & \\
\hline 42 & 52.9 & 50.3 & $4 \mathrm{I}$ & (4) & 42 & 53.8 & 51.9 & 39 & & 42 & 67.8 & 62.8 & 19 & 6. & 42 & 39.0 & 24.0 & & 45 & \\
\hline & 52.0 & 49.8 & 42 & -II.9 & 44 & 55.2 & 53.3 & 37 & -II.6 & & 66.1 & 61.8 & 21 & $-11 \cdot 3$ & & $4 \mathrm{I} .4$ & 26.3 & & 42 & - II.O \\
\hline 46 & 51.3 & 49.0 & 43 & 10 & 46 & 55.8 & 53.9 & 36 & os & 46 & 65.8 & 61.4 & 22 & & 46 & 41.8 & 28. I & & 40 & \\
\hline 48 & $5 I .1$ & 48.9 & 43 & $C$ & 48 & 56.0 & 54.8 & 35 & 15 & 48 & 65.9 & 62.2 & 21 & 1 & 48 & 41.0 & 27.2 & & 41 & \\
\hline 50 & 52.1 & 49.9 & 42 & 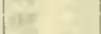 & 50 & 55.3 & 54.5 & 36 & in & 50 & 64.8 & 6I. & 23 & $=$ & 50 & 37.7 & 25.3 & & 45 & \\
\hline 52 & 53.5 & 51.8 & 39 & W & 52 & 54.7 & 53.5 & 37 & $=$ & 52 & 64.8 & 61.8 & 22 & & 52 & 42.2 & 30.2 & & 38 & \\
\hline 54 & 52.1 & 50.6 & $4 I$ & Le & 54 & 54.8 & 53.2 & 37 & 15 & 54 & 64.7 & 61.4 & 23 & 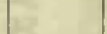 & 54 & $39 \cdot 3$ & 28.2 & & 42 & \\
\hline 56 & 52.1 & 50.3 & 41 & $1=$ & 56 & 55.9 & 54.3 & 35 & ce. & 56 & 63.2 & 60.1 & 25 & $x^{2}$ & 56 & 38.5 & 28.5 & & 42 & \\
\hline 58 & 52.5 & 50.9 & 40 & 23 & 58 & 54.8 & 53.5 & 37 & 5 & 58 & 61.7 & 59.2 & 27 & $=$ & 58 & 39.8 & 29.4 & & $4 \mathrm{I}$ & \\
\hline & & & & & & & & & & & & & & & 2400 & 37.9 & 27.8 & & 43 & - II.O \\
\hline
\end{tabular}


Tabulation of magnetic declinations observed at Teplitz Bay-Continued

\begin{tabular}{|c|c|c|c|c|c|c|c|c|c|c|c|c|c|c|c|c|c|c|c|c|}
\hline \multicolumn{5}{|c|}{ Thursday, February II, 1904} & \multicolumn{6}{|c|}{ Magnet scale erect } & \multicolumn{5}{|c|}{ Friday, February 12, 1904} & \multicolumn{5}{|c|}{ Magnet scale inverted } \\
\hline \multirow{2}{*}{$\begin{array}{l}\text { Chr'r } \\
\text { time }\end{array}$} & \multicolumn{2}{|c|}{$\begin{array}{l}\text { Scale } \\
\text { readings }\end{array}$} & $\begin{array}{l}\text { East } \\
\text { decli- }\end{array}$ & Temp. & Chr'r & $\begin{array}{r}\mathrm{Sc} \\
\text { read }\end{array}$ & $\begin{array}{l}\text { ale } \\
\text { dings }\end{array}$ & $\begin{array}{l}\text { Eas } \\
\text { dec }\end{array}$ & & Temp. & Chr'r & $\begin{array}{l}\mathrm{Sc} \\
\mathrm{reac}\end{array}$ & $\begin{array}{l}\text { ale } \\
\text { lings }\end{array}$ & $\begin{array}{l}\text { East } \\
\text { decli- }\end{array}$ & Temp & & $\begin{array}{l}\text { Sca } \\
\text { readi }\end{array}$ & lings & $\begin{array}{l}\text { East } \\
\text { decli- }\end{array}$ & \\
\hline & Left & Right & & & & Left & Right & & & & & Left & Right & & & & Left & Right & & \\
\hline $\mathrm{h} \mathrm{m}$ & d & d & $\circ \quad$ ' & $0^{\circ}$ & $\mathrm{h} \mathrm{m}$ & d & d & $\bullet$ & & & $\mathrm{h} \mathrm{m}$ & d & d & & & $\mathrm{h} \mathrm{m}$ & u & d & $\cdot \quad$, & \\
\hline $1600^{*}$ & 52.2 & 54.9 & 2238 & -18.7 & 1800 & 50.8 & 52.0 & 223 & & -16.9 & 2000 & 41.8 & $4 \mathrm{I} .2$ & 2242 & -24.9 & 2200 & 40.8 & 39.2 & 2244 & -22.6 \\
\hline 02 & 52.9 & 54.8 & 39 & & 02 & 50.7 & 51.9 & & 35 & & 02 & 41.0 & 40.7 & 43 & & 02 & 41.9 & 39.0 & 43 & \\
\hline 04 & 53.7 & 55.1 & 40 & 6 & 04 & 50.7 & 51.9 & & 35 & 16. & 04 & 41.9 & 40.3 & 42 & 28 & 04 & $4 \mathrm{I} . \mathrm{I}$ & 39.1 & 44 & 10 \\
\hline 06 & 53.8 & 54.9 & 40 & 5: & 06 & 51.0 & 52.1 & & 35 & (2) & 06 & 41.9 & 38.6 & 44 & $x^{2}$ & 06 & 41.2 & 39.0 & & in: \\
\hline 08 & 53.9 & 55.I & 40 & 31 & 08 & 51.8 & 52.9 & & 37 & 50 & 08 & 42.3 & $37 . I$ & 44 & 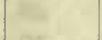 & 08 & 4I. I & 38.9 & 44 & -8 \\
\hline I0 & 52.8 & 54.2 & 38 & (es) & I0 & 52.0 & 53.5 & & 37 & int & I0. & 41,8 & 37.0 & 45 & PI & Io & 40.9 & 38.2 & 45 & \\
\hline 12 & 51.9 & 53.2 & 37 & & I2 & 51.8 & 53.4 & & 37 & & 12 & 41.9 & 37.4 & 45 & 18 & 12 & 40.8 & 38.0 & 45 & =4 \\
\hline 14 & 51.1 & 53.0 & 36 & -18.2 & I4 & 51.7 & 53.8 & & 37 & -16.9 & I4 & 41.8 & 37.2 & 45 & -24.0 & I4 & 40.0 & 38.0 & 46 & -22.5 \\
\hline 16 & 50.8 & 52.3 & 35 & & 16 & 51.7 & 54.1 & & 38 & & I6 & 42.0 & 37.1 & 45 & & 16 & $4 \mathrm{I} . \mathrm{I}$ & 38.0 & 45 & \\
\hline 18 & 49.9 & 52.2 & 34 & firt & I8 & 51.7 & $54 . \mathrm{I}$ & & 38 & int & I8 & 42.4 & 37.7 & 44 & 14 & I8 & 4I. I & 39.0 & 44 & 4 \\
\hline 20 & 49.3 & $5 \mathrm{I} .2$ & 33 & wa & 20 & 51.7 & 54.0 & & 37 & $1=$ & 20 & 42.2 & 37.6 & 44 & ex & 20 & $4 \mathrm{I} .0$ & 39.0 & 44 & 1 \\
\hline 22 & $49 . I$ & 51.3 & 33 & it & 22 & $5 \mathrm{I} . \mathrm{I}$ & 53.2 & & 36 & & 22 & 42.3 & 37.8 & 44 & 45 & 22 & $4^{1} \mathrm{I} .0$ & 39.4 & 44 & $=$ \\
\hline 24 & 49.2 & 51.7 & 34 & $y=$ & 24 & 50.2 & 52.3 & & 35 & $w$ & 24 & 42.9 & 38.2 & 43 & 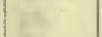 & 24 & 40.9 & 39.5 & 44 & $1=$ \\
\hline 26 & 49.2 & 51.4 & 34 & tets & 26 & 50.2 & $52 . \mathrm{I}$ & & 35 & & 26 & 42.9 & 38.2 & 43 & Ne & 26 & 40.9 & 39.9 & 43 & 0 \\
\hline 28 & 49.6 & 51.6 & 34 & (8) & 28 & 50.1 & 52.0 & & 35 & & 28 & 42.8 & 38.7 & 43 & Fe & 28 & 4I. I & 39.9 & 43 & \\
\hline 30 & 49.3 & 50.8 & 33 & -17.9 & 30 & 50.1 & 51.8 & & 34 & -17.0 & 30 & 42.6 & 39.0 & 43 & -23.9 & 30 & 41.9 & 40.2 & 42 & -22.4 \\
\hline 32 & 49.2 & 51.0 & 33 & ( & 32 & 50.1 & 51.2 & & 34 & & 32 & 42.8 & 39.0 & 42 & & 32 & 43.0 & 40.5 & $4 I$ & \\
\hline 34 & 49.0 & 50.8 & 33 & 18 & 34 & 50.2 & 52. I & & 35 & & 34 & 42.8 & 39.0 & 42 & K & 34 & 43.2 & 40.4 & $4 \mathrm{I}$ & $y$ \\
\hline 36 & 49.2 & 50.8 & 33 & 14 & 36 & 50.7 & 51.9 & & 35 & 6 & 36 & 42.3 & $39 . I$ & 43 & 32 & 36 & 43.8 & 40.9 & 40 & 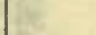 \\
\hline 38 & 49.7 & 50.8 & 33 & 8 & 38 & 50.5 & 51.8 & & 35 & है. & 38 & 41.8 & 38.8 & 43 & Ex & $3^{8}$ & 43.9 & 4I. & 40 & 15 \\
\hline 40 & 50.2 & 51.0 & 34 & xit. & 40 & 50.2 & 5 I. 3 & & 34 & 12 & 40 & $41 . I$ & 39.0 & 44 & 10 & 40 & 44.8 & 41.8 & 39 & 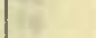 \\
\hline 42 & 50.5 & 51.0 & 34 & & 42 & 49.7 & 50.9 & & 34 & & 42 & 41.0 & 38.7 & 44 & 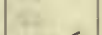 & 42 & 44.8 & $4 \mathrm{I} .2$ & 39 & $1=$ \\
\hline 44 & 50.7 & 51.1 & 34 & -17.7 & 44 & 49.2 & 50.2 & & 32 & -17.0 & 44 & $4 \mathrm{I} . \mathrm{I}$ & 38.6 & 44 & -23.6 & 44 & 45.2 & $4 \mathrm{I} .7$ & 39 & -22.2 \\
\hline 46 & 50.3 & 5 I.I & 34 & & 46 & 49.6 & 51.2 & & 34 & ( & 46 & 41.5 & 37.9 & 44 & & 46 & 46.0 & 41.0 & 38 & $1=$ \\
\hline 48 & 50. I & 50.8 & 34 & $E^{2}$ & 48 & 49.8 & 51.3 & & 34 & B3: & 48 & 42.0 & 38. I & 44 & 5 & 48 & 46.0 & 40.9 & 39 & te \\
\hline 50 & 50.9 & 51.4 & 35. & a & 50 & 50.1 & 51.7 & & 34 & & 50 & 42.4 & 38.8 & 43 & $6=$ & 50 & 46.0 & $4 \mathrm{I} .0$ & 38 & ${ }^{3}$ \\
\hline 52 & 52.1 & 52.3 & 36 & 20 & 52 & 50.2 & 51.9 & & 35 & c & 52 & 42.7 & 38.7 & 43 & 0. & 52 & 45.6 & 42.0 & 38 & 0 \\
\hline 54 & 52.9 & 53.3 & 38 & W. & 54 & 51.3 & 52.6 & & 36 & 3 & 54 & $42 . I$ & 38. I & 44 & 18 & 54 & 42.3 & 42.0 & $4 I$ & $1=$ \\
\hline 56 & $54 \cdot 3$ & 54.8 & 40 & 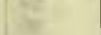 & 56 & 51.8 & 52.9 & & 37 & & 56 & $4 \mathrm{I} .5$ & $38 . I$ & 44 & 58 & 56 & Bea & ar & & 0 \\
\hline 58 & 55.3 & 55.8 & 42 & & 58 & 52.0 & 52.9 & & 37 & & 58 & 41.2 & 38.0 & 44 & 8 & 58 & outsi & ide & & \\
\hline 1700 & 55.2 & 55.8 & 42 & -17.3 & I9 00 & 51.8 & 52.9 & & 37 & -17.0 & 2100 & 40.9 & 37.8 & 45 & -23.4 & 2300 & 45.8 & 42.3 & 38 & $-22 . I$ \\
\hline 02 & $54 . \mathrm{I}$ & 54.9 & 40 & - & 02 & 51.9 & 53.0 & & 37 & ( & 02 & 40.0 & $38 . I$ & 46 & 1 & 02 & 45.6 & 43. I & 37 & in \\
\hline 04 & $54 \cdot 3$ & 55.3 & $4 \mathrm{I}$ & ift & 04 & 52.4 & 53.8 & & 38 & 108 & 0 & 40.7 & 38.4 & 45 & $w$ & 04.5 & $45 \cdot 3$ & 43.2 & 37 & 6 \\
\hline 06 & 54.1 & 55.5 & $4 I$ & $(5$ & 06 & 52.8 & 54 & & 38 & 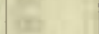 & 06 & 40.9 & 39.0 & 44 & 30 & 06 & 45 & 43. & 37 & W \\
\hline 08 & 54.5 & $55 \cdot 3$ & 4I & a & 08 & 53.0 & 54.7 & & 39 & In & 08 & 41.0 & 39.9 & 43 & E & 08 & 45.0 & 43.0 & 38 & 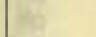 \\
\hline IO & 54.5 & 55.4 & $4 \mathrm{I}$ & se. & 10 & 52.7 & 54 & & 38 & & IO & 41.0 & 40.0 & 43 & in & Io & 44.8 & 43.8 & 37 & ex \\
\hline 12 & 53.8 & 54.7 & 40 & & I2 & 52.6 & 54.0 & & 38 & & I2 & 40.8 & 39.4 & 44 & Lit & I2. 5 & $44 \cdot 3$ & 42.5 & 39 & 78 \\
\hline 14 & 53.9 & 54.7 & 40 & -I7.I & I4 & 52.0 & 52.9 & & 37 & -17.0 & 14 & 40.5 & & 44 & -23.2 & I4 & 44. I & 43.0 & 38 & -22.0 \\
\hline 16́ & 54.0 & 54.6 & 40 & & 16 & 50.8 & 52.0 & & 35 & & 16 & 41.0 & 38.8 & 44 & & I6 & 43.7 & 43.7 & 38 & 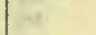 \\
\hline 18 & 55.1 & 56.0 & 42 & $\theta^{4}$ & I8 & 50.8 & 5 I & & 35 & & 18 & 40.7 & 37.4 & 46 & (7i) & 18 & 44.0 & 43.6 & 38 & 1 \\
\hline 20 & 55.2 & 56.0 & 42 & & 20 & 50.1 & 50. & & 34 & & 20 & 40.0 & 37.0 & 46 & 6 & 20 & Over & $r l^{\prime} k{ }^{\prime} d$ & & L \\
\hline 22 & 52.9 & $54 . \mathrm{I}$ & 38 & 4 & 22 & 49.7 & 50.5 & & 33 & & 22 & 40.5 & 37.8 & 45 & E. & 22 & 40.8 & 40.2 & 43 & 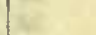 \\
\hline 24 & 52.9 & 53.5 & 38 & & 24 & 49.7 & 50.3 & & 33 & & 24 & 40.8 & 38.0 & 45 & $15^{2}$ & 24 & $4 \mathrm{I} .8$ & 42.3 & $4 \mathrm{I}$ & $1=$ \\
\hline 26 & 54.9 & 55.3 & $4 \mathrm{I}$ & a & 26 & 48. I & & & 30 & 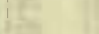 & 26 & 40.2 & 37.8 & 46 & 64 & 26 & 48.5 & 47.8 & 3I & 10 \\
\hline 28 & 54.7 & 55.2 & $4 \mathrm{I}$ & & 28 & 48.0 & 48.3 & & 30 & & 28 & 39.9 & 39.0 & 45 & 6 & 28 & 49.0 & $47 \cdot 4$ & $3 \mathrm{I}$ & 1 \\
\hline 30 & 53.2 & 54.4 & 39 & -17.0 & 30 & 46.0 & 46.0 & & 27 & -17.0 & 30 & 38.9 & 38.4 & 46 & -23.0 & 30 & 49.0 & $47 \cdot 3$ & $3 I$ & -22.2 \\
\hline 32 & $53 \cdot 3$ & 54.5 & 39 & & 32 & 44.0 & 45.0 & & 24 & if & 32 & 38.8 & 37.4 & 47 & 20 & 32 & 49.8 & 48.2 & 30 & Ir \\
\hline 34 & 53.2 & 54.2 & 39 & t & 34 & $42 . I$ & 50.7 & 22 & 27 & & 34 & 38.1 & 37.8 & 47 & & 34 & 49.8 & 48. I & 30 & 7 \\
\hline 36 & 52.2 & 53.2 & 37 & & $36^{*}$ & 49.5 & 59.8 & 24 & I I & & 36 & 38.9 & 38.3 & 46 & & 36 & 50.0 & 48.0 & 30 & \\
\hline $3^{8}$ & 52.2 & 53.2 & 37 & 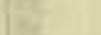 & $38^{*}$ & 27.6 & 48.7 & & 57 & & 38 & 39.0 & 38.2 & 46 & & 38 & 50.0 & 48.2 & 30 & 6 \\
\hline 40 & 52.5 & 53.8 & 38 & & 40 & 6.7 & 32.9 & & 28 & & 40 & 40.3 & 38.2 & 45 & 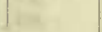 & 40 & Lo & ost & & 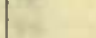 \\
\hline 42 & 52.8 & 53.7 & 38 & & $42^{*}$ & 36.3 & 73.8 & & 28 & & 42 & 39. I & 38.8 & 46 & & 42 & ot & ti & & E. \\
\hline & 52.5 & 53.1 & 37 & -16.9 & 44 & 27.6 & 52.6 & 24 & 05 & -17.0 & 44 & 39.5 & 38.9 & 45 & -22.9 & & acco & ount & & \\
\hline 46 & 52.7 & 53.0 & 37 & & 46 & II. 2 & 34. I & 23 & 37 & & 46 & 39.1 & $3^{8.9}$ & 46 & & 46 & of & & & or \\
\hline 48 & 52.3 & 53.1 & 37 & & 48 & 23.7 & 37.8 & & 50 & & 48 & 39. I & $3^{8} .4$ & 46 & fits. & 48 & be: & ar & & 118 \\
\hline 50 & 51.9 & 52.8 & 37 & 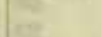 & 50 & 17.5 & 38. I & 23 & 46 & 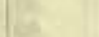 & 50 & 39.8 & 38.4 & 45 & I. & 50 & 46.9 & 45.3 & 34 & $1=$ \\
\hline 52 & 51.2 & 52.0 & 36 & & $52^{*}$ & $4 \mathrm{I} \cdot 4$ & $74 \cdot 3$ & 25 & Io & & 52 & 40.4 & 39.0 & 44 & & 52 & 43.0 & $4 \mathrm{I} .8$ & 40 & \\
\hline 54 & $5 \mathrm{I} . \mathrm{I}$ & 51.9 & 35 & 25 & 54 & 28.7 & 48.5 & 24 & 40 & & 54 & 40.0 & 38.6 & 45 & as & 54 & $4 \mathrm{I} .8$ & 38.7 & 44 & wr \\
\hline 56 & 50.8 & 51.8 & 35 & & 56 & 50.2 & 67.8 & 25 & I2 & & 56 & 40.2 & 39.1 & 45 & & 56 & 37.5 & $33 . I$ & $5 \mathrm{I}$ & $1=$ \\
\hline 58 & 50.7 & 5 I. 8 & 35 & & $5^{8}$ & 71.9 & 76.8 & & $3^{6}$ & & 58 & 40.3 & 39.0 & 45 & & 58 & 39.9 & 32.0 & 50 & \\
\hline & & wack & -2 & 105 & $2000^{*}$ & I3. I & $21 \cdot 3$ & & II & -17.0 & & & & & & 2400 & 40.1 & 31.2 & $5 \mathrm{I}$ & -22.5 \\
\hline
\end{tabular}

Correction to local mean time is $-47 \mathrm{~s}$.

Torsion head at $15 \mathrm{~h} 30 \mathrm{~m}$ read $30^{\circ}$ and at the end read the same.

Observer-R. R. T.
Correction to local mean tine is - Im $12 \mathrm{~s}$.

Torsion head at $19 \mathrm{gh}$ 30m read $28^{\circ}$ and at the end read the same. Observer-J. V. 
Tabulation of magnetic declinations observed at Teplitz Bay-Continued

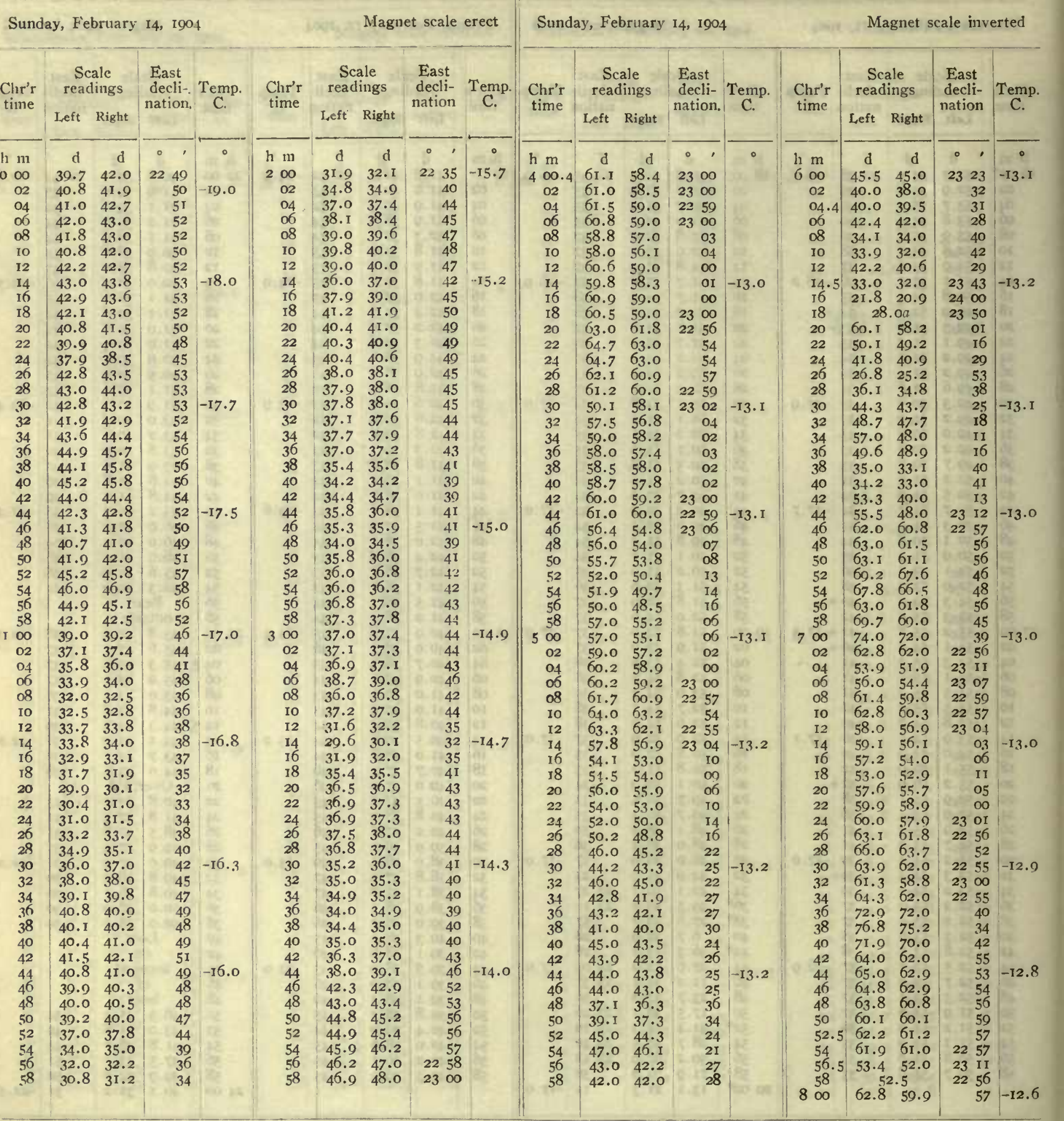


Tabulation of magnetic declinations observed at Teplitz Bay-Continued

\begin{tabular}{|c|c|c|c|c|c|c|c|c|c|c|c|c|c|c|c|c|c|c|c|c|c|c|}
\hline \multicolumn{6}{|c|}{ Monday, February I5, I904 } & \multicolumn{5}{|c|}{ Magnet scale erect } & \multicolumn{6}{|c|}{ Tuesday, February I6, 1904} & \multicolumn{6}{|c|}{ Magnet scale inverted } \\
\hline \multirow{2}{*}{$\begin{array}{l}\text { Chr'r } \\
\text { time }\end{array}$} & Sc & $\begin{array}{l}\text { ale } \\
\text { ings }\end{array}$ & $\begin{array}{l}\mathrm{Ea} \\
\mathrm{dec}\end{array}$ & $\begin{array}{l}\text { st } \\
\text { cli- } \\
\text { ion. }\end{array}$ & Temp. & $\begin{array}{l}\text { Chr'r } \\
\text { time }\end{array}$ & $\begin{array}{r}\text { Sc } \\
\text { read }\end{array}$ & $\begin{array}{l}\text { ale } \\
\text { ings }\end{array}$ & East & Temp. & Chr'r & $\begin{array}{r}\mathrm{Sc} \\
\text { read }\end{array}$ & $\begin{array}{l}\text { ale } \\
\text { lings }\end{array}$ & Ea & cli- & Temp & Chr'r & $\begin{array}{r}\text { Sc } \\
\text { read }\end{array}$ & $\begin{array}{l}\text { le } \\
\text { ngs }\end{array}$ & Eas & ist & Temp \\
\hline & Left & Right & & & & & $L_{s}$ eft & Right & & & & L,eft & Right & & & & & $L_{1} \in f t$ & Right & & & \\
\hline $\mathrm{h} \mathrm{m}$ & d & d & & & $\bullet$ & $\mathrm{h} \mathrm{m}$ & d & d & $\circ \quad$, & & $h \mathrm{~m}$ & d & d & & , & & $\mathrm{h} \mathrm{m}$ & & d & & & 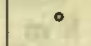 \\
\hline $800^{*}$ & 38.7 & 38.9 & 23 & 33 & -24.0 & 1000 & 31.6 & 35.8 & 2232 & -20.5 & 1200 & 60.6 & 66.8 & $2 I$ & 54 & -25.8 & 1400 & 56.6 & $55 \cdot 5$ & 22 & & $-23 \cdot 4$ \\
\hline 02 & 34.6 & 39.0 & & 30 & ( & 02 & 29.2 & $33 \cdot 3$ & 28 & $-y^{2}$ & 02 & 68.7 & 66.7 & $2 I$ & 55 & -2 & 02 & 56.0 & 54.9 & & I4 & ( \\
\hline 04 & 36.1 & 40.1 & & 32 & (a) & 04 & 29.9 & 32.0 & 27 & 10 & 04 & 57.9 & 54.8 & 22 & I3 & 46 & 04 & 54.2 & 53.0 & & 17 & mats \\
\hline 06 & 35.8 & 39.4 & & $3 I$ & Hry & 06 & 29.6 & 32.0 & 27 & 18 & 06 & 57.1 & 54.8 & & 13 & 10 & 06 & 54.0 & 53.1 & $\theta_{y}$ & I7 & 1) \\
\hline 08 & 25.9 & $29 . I$ & & I5 & 30 & 08 & 30.3 & 31.6 & 27 & 80 & 08 & 58.0 & 56.8 & & II & 15 & 08 & 52.7 & 51.9 & & I9 & 118 \\
\hline IO & 30.8 & 32.5 & & 21 & 64 & Io & $34 . I$ & 35.0 & 33 & $5 t$ & Io & 50.6 & 49.6 & & 22 & at & 10 & 52.8 & 51.9 & Ex & I9 & bif \\
\hline 12 & 27.9 & 30.3 & & 17 & 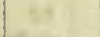 & 12 & 35.9 & 37.2 & 36 & t & I2 & 48.5 & 47.0 & & 26 & ina & 12 & 54.0 & 52.8 & & 17 & 1.t \\
\hline I4 & 28.0 & 30.8 & & I8 & $-23 \cdot 4$ & 14 & 3I. I & $3 I .9$ & 28 & -20.4 & 14 & 50.7 & 49.8 & & 22 & $-25 \cdot 5$ & 14 & 52.6 & 52.6 & & I9 & $-23 \cdot 3$ \\
\hline I6 & 37.7 & 41.9 & & 34 & & 16 & 30.3 & $3 I .5$ & 27 & (n) & I6 & 54.8 & 54.1 & & I6 & ( & I6 & 55.9 & 55.0 & r. & I4 & wis \\
\hline I8 & 38.6 & 40.3 & & 34 & 64 & I8 & 32.1 & $33 \cdot 3$ & 30 & 14 & I8 & 50.0 & 50.0 & & 23 & (16) & I8 & $57 \cdot 5$ & 56.3 & a & 12 & fir \\
\hline 20 & 40.8 & 41.6 & & 36 & 14 & 20 & 34.3 & 35.5 & 33 & wat & 20 & 49.1 & 48.3 & & 25 & $\mathrm{C}^{2}$ & 20 & 60.1 & 59.5 & F & 07 & no \\
\hline 22 & 37.6 & 38.8 & & 32 & 41 & 22 & 34.2 & 35.0 & 33 & $5=$ & 22 & 44.6 & 44.0 & & 32 & 215 & 22 & 61.3 & 60.7 & 8 & 05 & y \\
\hline 24 & 32.3 & $34 \cdot 4$ & & 24 & 14 & 24 & 36.7 & $37 \cdot 4$ & 37 & $1=$ & 24 & 42.4 & 41.5 & & 35 & $x$ & 24 & 61.7 & 60.9 & 22 & 05 & th \\
\hline 26 & 32.2 & 34.4 & & 24 & 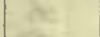 & 26 & 36.5 & 38.0 & 37 & ta & 26 & 42.7 & 42.0 & & 35 & K & 26 & 66.0 & 65.5 & 21 & 58 & 8 \\
\hline 28 & 32.2 & 34.8 & & 24 & $x=2$ & 28 & 26.9 & 38.5 & 30 & $x^{2}$ & 28 & 40.6 & 40.0 & & 38 & 8 & 28 & 56.2 & 56.0 & 22 & 13 & 12. \\
\hline $3^{\circ}$ & 41.8 & 44.1 & & 39 & -22.8 & 30 & 31.9 & 33.2 & 30 & -20.4 & 30 & 39.7 & 38.7 & & 40 & -25.0 & 30 & 61.0 & 60.6 & & 06 & -23.2 \\
\hline 32 & 41.9 & $44 \cdot 9$ & & 40 & 106 & 32 & 34.2 & 34.9 & 33 & 7 & 32 & 40.1 & 38.8 & & 39 & - & 32 & 62.1 & $6 \mathrm{I} .4$ & 6 & 04 & 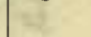 \\
\hline 34 & 45.2 & 48.3 & & 45 & 15 & 34 & 39.5 & 40.1 & 41 & WX. & 34 & 40.7 & 39.0 & & 39 & 14: & 34 & 59.3 & 58.0 & h & $\infty$ & 14 \\
\hline 36 & 40.0 & 44.0 & & 38 & Ir & 36 & 38.7 & 39.9 & 40 & d8 & 36 & 40.6 & 39.0 & & 39 & & 36 & 56.8 & 55.7 & & 13 & 3 \\
\hline 38 & 36.6 & 39.5 & & $3 I$ & Ret & 38 & 35.8 & 36.5 & 35 & Q & 38 & 38.9 & 37.8 & & $4 I$ & 25 & 38 & 56.7 & 55.6 & & I3 & As \\
\hline 40 & 35.2 & 36.3 & & 28 & 64 & 40 & 37.0 & $37 \cdot 3$ & 37 & $7+$ & 40 & 36.8 & 35.7 & & 44 & 64. & 40 & 48.5 & 47.0 & & 26 & 6x \\
\hline 42 & 34.8 & 36.2 & & 27 & 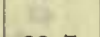 & 42 & $34 \cdot 5$ & 35.1 & 33 & 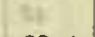 & 42 & 36.9 & 35.6 & & 44 & $2=0$ & 42 & 47.1 & 45.7 & (x) & 28 & $2=$ \\
\hline 44 & 36.2 & 38.1 & & 30 & -22.7 & 44 & $34 \cdot 2$ & $35 \cdot 4$ & 33 & -20.4 & 44 & 39.7 & 38.1 & & 40 & -24.8 & 44 & 43.9 & 42.1 & 2 & 34 & -23.2 \\
\hline 46 & 41.2 & 45.0 & & 39 & $x^{2}$ & 46 & 34.0 & 34.8 & 33 & 187 & 46 & 41.6 & 39.7 & & 37 & ( & 46 & 45.0 & 43.0 & $y$ & 32 & $x^{2}$ \\
\hline 48 & $37 \cdot 9$ & 42.7 & & 35 & 14 & 48 & 34.9 & 35.6 & 34 & 13 & 48 & 37.4 & 36.0 & & 44 & Pa & 48 & 43.6 & 42.0 & & 34 & 28 \\
\hline 50 & 35.3 & 38.8 & & 30 & ces & 50 & 35.4 & 39.2 & 37 & 92 & 50 & 37.2 & 36.7 & & 43 & 60 & 50 & 41.7 & 40.2 & & 37 & nest \\
\hline 52 & $34 . I$ & 34.8 & & 26 & 87 & 52 & 40.0 & 40.4 & 42 & ct & 52 & 36.6 & 36.0 & & 44 & 4 & 52 & 41.6 & 39.6 & & 37 & $x^{2}$ \\
\hline 54 & 36.5 & 39.6 & & 31 & B & 54 & 37.1 & 39.2 & 38 & w & 54 & 33.9 & 33.6 & & 48 & 32 & 54 & 43.0 & 41.8 & & 35 & 14: \\
\hline 56 & 39.2 & 44.0 & & 37 & 8 & 56 & 36.1 & $37 \cdot 3$ & $3^{6}$ & ox & 56 & 35.6 & & & 46 & $6=$ & 56 & 43.1 & $4 I .3$ & & 35 & $=$ \\
\hline 58 & 41.2 & $44 \cdot I$ & & 38 & & 58 & 33.5 & $34 . I$ & 32 & $\sqrt{3}$ & 58 & 37.0 & 36.5 & & 44 & 82 & 58 & 41.9 & 40.8 & & 36 & Pry \\
\hline 900 & $44 \cdot 3$ & 46.5 & & 43 & -22.6 & II 00 & 37.1 & 39.2 & 38 & -20.3 & I3 OO & 40.3 & 38.8 & & 39 & $-24 \cdot 4$ & I5 00 & 43.5 & 42.5 & & 34 & $-23 \cdot I$ \\
\hline 02 & 35.2 & 37.9 & & 29 & tor & 02 & 37.5 & 38.0 & 38 & $10=$ & 02 & $4 I .3$ & 40.1 & & 37 & (1) & 02 & 42.7 & $4 I . I$ & & 35 & (5 \\
\hline O4 & $33 \cdot 4$ & 34.9 & & 25 & thin & 04 & 36.0 & 36.9 & 36 & 40 & 04 & 46.4 & 45.5 & & 29 & 10 & 04 & 43.0 & 41.6 & & 35 & $y^{4}$ \\
\hline 06 & 40.8 & $4 I .5$ & & 36 & $6 n$ & 06 & 36.5 & 36.8 & 36 & We & 06 & 39.7 & 38.9 & & 39 & (6) & 06 & 44.0 & 42.7 & A & 33 & $6 y$ \\
\hline 08 & 40.9 & 41.2 & & 36 & 3 tit & 08 & 35.4 & 36.0 & 35 & 86 & 08 & 36.0 & $35 . I$ & & 45 & 58 & 08 & 43.9 & 42.6 & & 33 & tor \\
\hline 10 & 39.3 & 39.9 & & 34 & $t^{4}$ & IO & 36.3 & $37 \cdot 4$ & 36 & al & IO & 36.1 & 34.7 & & 46 & ot & 10 & 42.6 & 40.9 & a & 36 & wet \\
\hline 12 & 35.2 & 36.5 & & 28 & -1 & I2 & 36.1 & 36.6 & 36 & $1+15$ & I2 & 36.9 & 35.8 & & 44 & 51 & 12 & 41.6 & 40.0 & n & 37 & $\mathrm{dr}$ \\
\hline 14 & 22.8 & 23.9 & & 08 & -22.2 & 14 & 37.8 & 38.5 & $3^{8}$ & $-20 \cdot 3$ & 14 & 38.6 & 37.5 & & 42 & -24.0 & 14 & 39.8 & 38.5 & s & 40 & -23.0 \\
\hline 16 & $22 . I$ & 23.2 & & 07 & cat & 16 & 42.0 & 43.1 & 45 & Int & I6 & 43.1 & 41.0 & & 35 & intat & 16 & 39.1 & 37.9 & E & $4 I$ & $x^{2}+2$ \\
\hline 18 & 22.9 & 25.2 & & 09 & Dis & I8 & 39.2 & 40.5 & $4 I$ & 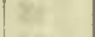 & I8 & 46.6 & 44.9 & & 29 & Bit & I8 & 38.0 & 37.1 & & 42 & At \\
\hline 20 & 17.3 & 19.0 & 23 & $\infty$ & 0 & 20 & 39.0 & 39.9 & 40 & 06 & 20 & 49.6 & 48.0 & & 24 & 0 & 20 & 38.6 & 36.6 & fit & 42 & tas \\
\hline 22 & 15.0 & 16.9 & 22 & 57 & is & 22 & 36.9 & 37.8 & 37 & tet & 22 & 46.1 & 43.9 & & 30 & $6=$ & 22 & 37.2 & 36.6 & f & 43 & $\mathrm{st}$ \\
\hline 24 & Io. I & II. 2 & & 48 & is & 24 & 38.8 & 39.4 & 40 & $1=$ & 24 & 42.1 & .5 & & 36 & 13 & 24 & 37.8 & 36.7 & & 43 & as \\
\hline 26 & 8.9 & IO. I & & 47 & $8=$ & 26 & 39.5 & 39.8 & $4 \mathrm{I}$ & $3 x$ & 26 & 40.0 & 38.0 & & 40 & 80 & 26 & 38.6 & 37.3 & & 42 & A \\
\hline $28^{*}$ & 34.9 & 42.0 & & 39 & $x=0$ & 28 & 36.4 & 37.0 & 36 & $1=$ & 28 & 40.6 & 38.9 & & 39 & 7at & 28 & 38.9 & 37.9 & 8 & 41 & 15 \\
\hline 30 & 33.0 & 38.7 & & 35 & -21.8 & 30 & 36.7 & 37.2 & 37 & -20.3 & 30 & 42.8 & 41.9 & & 35 & -23.9 & 30 & 39.6 & 37.6 & it & 41 & -23.0 \\
\hline 32 & 32.8 & 38.6 & & 35 & (nte & 32 & 31.2 & $33 \cdot 3$ & 29 & (n) & 32 & 44.0 & 42.0 & & 34 & ( & 32 & 38.9 & $37 \cdot 3$ & n & $4 I$ & 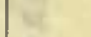 \\
\hline 34 & 30.5 & 36.6 & & 31 & 14. & 34 & 29.4 & 30.1 & 25 & Nit & 34 & 39.6 & 38.0 & & 40 & Ft & 34 & 40.7 & 38.5 & nit & 39 & $\mathrm{~N}$ \\
\hline 36 & 35.2 & 39.9 & & 38 & az & 36 & $29 \cdot 3$ & 30.4 & 26 & int & 36 & 40.6 & 39.2 & & 38 & he & 36 & 40.7 & 38.6 & & 39 & Av \\
\hline 38 & 41.7 & 47.0 & & 48 & Pet & 38 & 40.3 & 42.0 & 43 & Hat & 38 & 42.1 & 41.0 & & 36 & $\varepsilon$ & 38 & 40.1 & 37.2 & & 41 & 6 \\
\hline 40 & 41.2 & 46.8 & & 48 & nes: & 40 & 42.2 & 43.1 & 46 & 60 & 40 & 45.6 & 43.9 & & $3 I$ & 44 & 40 & 39.6 & 37.0 & fis & 41 & 64 \\
\hline 42 & 39.9 & 45.7 & & 46 & $\mathrm{t}=$ & 42 & 36.9 & 38.0 & 37 & 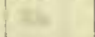 & 42 & $47 \cdot 5$ & 46.2 & & 28 & (2) & 42 & 39.4 & 37.2 & t: & $4 I$ & $71=$ \\
\hline 44 & 40.8 & $45 \cdot 5$ & & 46 & in & 44 & 35.0 & 36.2 & 34 & $-20 \cdot 3$ & 44 & 49.1 & 47.4 & & 26 & -23.8 & 44 & 37.8 & 36.2 & & 43 & -22.9 \\
\hline 46 & 38.8 & 42.9 & & 43 & $\tan$ & 46 & 34.3 & 34.9 & 33 & (n) & 46 & 52.0 & 50.0 & & 21 & (1) & 46 & 40.8 & 38.9 & ef & 39 & -2 \\
\hline 48 & $37 \cdot 3$ & 43.2 & & 42 & -21.0 & 48 & 36.2 & 38.4 & 37 & Mat & 48 & 57.1 & 56.0 & & 12 & $=$ & 48 & $4 I .4$ & 39.6 & & 38 & Nor \\
\hline 50 & 36.8 & 41.3 & & 40 & Q & 50 & 34.6 & 35.8 & 34 & 60 & 50 & 62.1 & 60.0 & 22 & 05 & we & 50 & 41.7 & 39.6 & $a$ & 37 & $\mathrm{c}^{3}$ \\
\hline 52 & 37.2 & 42.0 & & $4 I$ & 12 & 52 & 33.0 & 33.0 & 30 & 12 & 52 & 65.6 & 64.0 & $2 I$ & 59 & 14 & 52 & 40.5 & 38.9 & & 39 & 87 \\
\hline 54 & 36.6 & $4 \mathrm{I} . \mathrm{I}$ & & 40 & 22 & 54 & 33.2 & 33.6 & $3 I$ & $x^{2}$ & 54 & 65.7 & 64.6 & $2 I$ & 59 & $\sqrt{12}$ & 54 & 39.9 & $37 \cdot 4$ & & 41 & N \\
\hline 56 & 36.7 & $4 I .5$ & & 40 & & 56 & 31.8 & $34 \cdot 3$ & 30 & 132 & 56 & 64.0 & 62.8 & 22 & 02 & 16 & 56 & 40.2 & 38.3 & & 40 & Es \\
\hline 58 & $34 \cdot 3$ & 40.0 & & 37 & 82 & 58 & 31.8 & $34 \cdot 5$ & $3 I$ & $a^{2}$ & 58 & 58.1 & 57.1 & a & II & ke & 58 & $4 I . I$ & 39.2 & 9 & 38 & 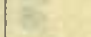 \\
\hline & & & & & & 1200 & 37.0 & $4 \mathrm{I} \cdot 4$ & 40 & -20.1 & & & & & & & I6 00 & 42.3 & 40.9 & & 36 & -22.9 \\
\hline
\end{tabular}

Correction to local mean time is - Im 50 .

Torsion head at $7 \mathrm{~h} 45 \mathrm{~m}$ read $39^{\circ}$ and at the end read the same. Observer-H. H. N.
Correction to local mean time is - Im 51s. $90^{\circ}$ torsion $=15 .^{\prime} 3$. Torsion head at 1 in $25 \mathrm{~m}$ read $36^{\circ}$ and at $16 \mathrm{~h} 26 \mathrm{~m}$ read $46^{\circ}$. Observer-R. R. T. 
Tabulation of magnetic declinations observed at Teplitz Bay-Continued

\begin{tabular}{|c|c|c|c|c|c|c|c|c|c|c|c|c|c|c|c|c|c|c|c|c|}
\hline \multicolumn{5}{|c|}{ Wednesday, February 17,1904} & \multicolumn{5}{|c|}{ Magnet scale erect } & \multicolumn{5}{|c|}{ Wednesday, February I7, 1904 } & \multicolumn{6}{|c|}{ Magnet scale erect } \\
\hline \multirow[t]{2}{*}{$\begin{array}{l}\text { Chr'r } \\
\text { time }\end{array}$} & \multicolumn{2}{|c|}{$\begin{array}{l}\text { Scale } \\
\text { readings }\end{array}$} & \multirow[t]{2}{*}{$\begin{array}{l}\text { East } \\
\text { decli- } \\
\text { nation }\end{array}$} & Temp & $\begin{array}{l}\text { Chr'r } \\
\text { time }\end{array}$ & $\begin{array}{r}\mathrm{Sc} \\
\text { read }\end{array}$ & $\begin{array}{l}\text { ale } \\
\text { lings }\end{array}$ & $\begin{array}{c}\text { East } \\
\text { decli- } \\
\text { nation }\end{array}$ & $\begin{array}{c}\text { Temp. } \\
\text { C. }\end{array}$ & $\begin{array}{l}\text { Chr'r } \\
\text { time }\end{array}$ & $\mathrm{Sc}$ & $\begin{array}{l}\text { ale } \\
\text { dings }\end{array}$ & $\begin{array}{c}\text { East } \\
\text { decli- } \\
\text { nation }\end{array}$ & Temp. & $\begin{array}{l}\text { Chr'r } \\
\text { time }\end{array}$ & $\begin{array}{l}\mathrm{Sc} \\
\text { reac }\end{array}$ & $\begin{array}{l}\text { le } \\
\text { ngs }\end{array}$ & $\begin{array}{l}\text { Eas } \\
\text { decl }\end{array}$ & st & Temp. \\
\hline & Left & Right & & & & L eft . & Right & & & & Left & Right & & & & Left & Right & & & \\
\hline $\begin{array}{l}h \mathrm{~m} \\
0 \infty^{*}\end{array}$ & $\begin{array}{c}d \\
52.3\end{array}$ & 58.0 & $\begin{array}{rr}0 & 1 \\
22 & 23\end{array}$ & $\begin{array}{c}0 \\
-27 . I\end{array}$ & $\begin{array}{l}\mathrm{h} \mathrm{m} \\
2 \infty\end{array}$ & $\begin{array}{c}\text { व } \\
57.8\end{array}$ & $\stackrel{d}{d 8.0}$ & $\begin{array}{l}\circ \\
2302\end{array}$ & $\frac{0}{-22.0}$ & $\begin{array}{l}h \mathrm{~m} \\
4 \infty\end{array}$ & $\begin{array}{l}\mathrm{d} \\
37\end{array}$ & $.2 a^{d}$ & ${ }_{23}^{\circ} 1$ & $\begin{array}{r}0 \\
-10.6\end{array}$ & $\begin{array}{l}h \mathrm{~m} \\
600\end{array}$ & $d_{52}$ & $.0 b^{d}$ & & & ${ }_{-1}^{\circ}$ \\
\hline 02 & 58.3 & 63.0 & & $2 / .1$ & 02 & 57.0 & 57.9 & OI & 20 & 02 & 38.3 & 39.0 & & 19.0 & 02 & 40.0 & 41.5 & & $\begin{array}{l}49 \\
3 \mathrm{I}\end{array}$ & \\
\hline 04 & 65.2 & 73.0 & 45 & 60 & 04 & 59.2 & 60.5 & 05 & Ga & 04 & 40.1 & 40.9 & 18 & 10 & 04 & 40.5 & 40.8 & & $3 I$ & \\
\hline 06 & 62.0 & 60.0 & 40 & $\$$ & 06 & 61.2 & 62. & 08 & 36 & 06 & 45.7 & 46.7 & 27 & $8=$ & 06 & 62.0 & 62.3 & & 04 & \\
\hline 08 & 63.0 & 68.9 & 40 & 5) & 08 & 68.0 & 68.8 & I9 & $2 \mathrm{ne}$ & 08 & 51.5 & 57.9 & 40 & 28 & 08 & 76.5 & 77.0 & 23 & 27 & \\
\hline ro & 69.2 & 73.0 & 2248 & $=$ & IO & 56.5 & 56.9 & $\infty$ & 201 & I0 & 53.0 & 55.0 & 39 & cil & I0* & 36.3 & 46.6 & 24 & 17 & \\
\hline $12^{*}$ & 52.1 & 60.9 & 2300 & 4 & 12 & 58.8 & 59.0 & 04 & $x^{2}$ & I2 & 56.0 & $57 \cdot 3$ & 43 & $=$ & $\mathrm{I} 2$ & 30.4 & 33.5 & & 02 & \\
\hline 14 & 56.1 & 63.0 & 05 & $-25 \cdot 5$ & 14 & 59.1 & 59.3 & 04 & -21.6 & 14 & 57.0 & 58. & 45 & -19.5 & $I 4^{*}$ & 48.0 & 59 & & 25 & \\
\hline 16 & 58.2 & 64.0 & 07 & & 16 & 58.3 & 58.9 & 03 & w5 & 16 & 62.0 & 64.0 & 53 & & 16 & 43.2 & 58 & & $2 I$ & \\
\hline 18 & 6.3 .0 & 68.6 & 15 & $2 \pi$ & 18 & 58.8 & 59.0 & 04 & fir & 18 & 61.5 & 64. & 53 & 60 & 18 & 66.0 & 77 & & 54 & \\
\hline 20 & 68.3 & 76.2 & 25 & $c=$ & 20 & 57.2 & 57.8 & 2302 & $x^{2}$ & 20 & 61.9 & 64.6 & 53 & $=$ & 20 & 65.5 & & & 48 & -18.3 \\
\hline 22 & 68. I & 76.2 & 24 & 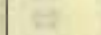 & 22 & 54.0 & 54. & 2257 & ex & 22 & 65.0 & 67. & 58 & ic & 22 & 62.7 & 70. & & 45 & 0.0 \\
\hline 24 & $71 . I$ & 78.0 & 28 & 45 & 24 & 56.2 & 58. & 23 OI & is & 24 & 64.6 & 67.3 & 2358 & 18 & 24 & 64.3 & 70 & 24 & 47 & \\
\hline 26 & 60.6 & 75.0 & 25 & 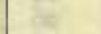 & 26 & 58.1 & 58.7 & 03 & 58 & 26 & 68.6 & 71.5 & 2404 & 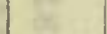 & 26 & 73.1 & 78. & 25 & 0 & \\
\hline 28 & 68.2 & 73.2 & 22 & $1=2$ & 28 & 67.0 & 67.2 & I7 & E & 28 & 74.0 & 76.0 & 12 & & $28^{*}$ & 36.0 & 50 & 24 & 03 & \\
\hline 30 & 61.8 & 68.0 & 13 & -25.0 & 30 & 74.0 & 74.4 & 28 & -20.9 & $30^{*}$ & 58.8 & 60.1 & 2413 & -19.2 & 30 & 36.1 & 49.0 & 24 & 02 & $-18 . I$ \\
\hline 32 & 58.9 & 63.2 & 07 & - & $32^{*}$ & 49.8 & 54.1 & 36 & $x^{2}$ & 32 & 48.5 & 49.1 & 2356 & a & 32 & 26.3 & 44 & 23 & $5 \mathrm{I}$ & \\
\hline 34 & 57.0 & 60.2 & 03 & 14 & .34 & 52.0 & 56 & 39 & 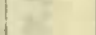 & 34 & 49.0 & 50.3 & 2358 & $x^{2}$ & 34 & 33.0 & 46 & 23 & 77 & \\
\hline 36 & 54.8 & 58.0 & 2300 & 88 & 36 & 49.5 & 52 & 34 & 3 & 36 & 56.6 & 59.5 & $24 \mathrm{II}$ & 18 & 36 & 35.5 & 49 & 24 & & \\
\hline 38 & 49.0 & 53.0 & 2251 & E: & 38 & 46.1 & 49.8 & 29 & 32 & 38 & 58.0 & 60.3 & 12 & Es & 38 & 20.5 & 35 & 23 & 99 & \\
\hline 40 & 46.0 & 48.8 & 46 & (6) & 40 & 47.6 & 50.4 & 31 & set & 40 & 53.2 & 57.9 & 2407 & 68 & 40 & 17.0 & 31 & & 33 & \\
\hline 42 & 4.3 .0 & 45.7 & $4 \mathrm{I}$ & 45 & 42 & 45.3 & 47.9 & 27 & 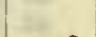 & 42 & 47.9 & 51.0 & 2357 & in & 42 & 9.0 & I7. & & I6 & \\
\hline 44 & 38.2 & 40.9 & 33 & -24.7 & 44 & 40.8 & 42.9 & 20 & -20.8 & 44 & 42.8 & 45.4 & 49 & -19.1 & $44^{*}$ & 42.9 & 45.0 & 23 & 03 & -18.0 \\
\hline 46 & 36.5 & 39.0 & 30 & (2) & 46 & 36.0 & 38.0 & 12 & 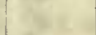 & 46 & $4 \mathrm{I} .0$ & 45. & 47 & & 46 & 32.1 & 35 & 22 & 47 & 13 \\
\hline 48 & 38.8 & 40.2 & 33 & 8 & 48 & 34.1 & 36.9 & 10 & As & 48 & 39.1 & 43.0 & 4 & 2 & 48 & 34.0 & 36 & & 49 & \\
\hline 50.5 & 38.8 & 4I.I & 34 & 68 & 50 & 34.0 & 37. & I0 & $8=$ & 50 & 37.0 & 40 & 4 & 70 & 50 & & & & 8 & $c=$ \\
\hline 52 & $4 \mathrm{I} .0$ & 43.0 & 37 & 8 & 52 & 33.0 & 35.9 & 08 & $w$ & 52 & 34.5 & 37. & 36 & 3 & 52 & 40.8 & 42 & & 59 & \\
\hline 54 & 47.9 & 49.8 & 2248 & 13 & 54 & 29.5 & 32 & 03 & 37 & 54 & 33.0 & 34 & 33 & 3 & 54 & 37.0 & 37 & & 53 & \\
\hline 56 & $\mathrm{I}$ & st & & 2 & 56 & 28.0 & 32.6 & 02 & & 56 & 29.5 & 32.0 & 28 & - & 56 & 30.0 & 30 & & $4 \mathrm{I}$ & \\
\hline 58 & 55.8 & 58.5 & 23 oI & 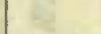 & 58 & 31.6 & 33.8 & 06 & $x=$ & 58 & 27.5 & 30 & 25 & $18=$ & 58 & 28.0 & & & 39 & \\
\hline$\infty$ & 60.0 & 61.0 & 2306 & -24.2 & 300 & 32.1 & 34.8 & 07 & -20.5 & 500 & 23.0 & 26.0 & 18 & - I9.0 & $7 \infty$ & 31.0 & 33 & & 44 & -18.0 \\
\hline 02 & 55.8 & 55.8 & 2259 & 44.0 & 02 & 28.9 & 31.2 & 23 oI & 20.5 & 02 & 15.2 & I6. 5 & 2304 & -300 & 02 & 30.2 & 37 & & 48 & \\
\hline 04 & 45.9 & 46.1 & 44 & 10 & & 21.6 & 28.4 & 2253 & to & 04 & 4.4 & 5. & 2248 & lat & 0 & 37.9 & 38 & & 54 & 10 \\
\hline 06 & 44.0 & 44.8 & $4 \mathrm{I}$ & 40 & 06 & 24.0 & 26.0 & 53 & 100 & $06^{*}$ & 50.0 & 56.1 & 50 & 80 & 06 & 33.5 & 36 & & 49 & \\
\hline 08 & 38 & $o b$ & 3I & is: & 08 & 21.5 & 23.8 & 50 & Ba & 08 & 47.8 & 52.3 & 46 & s. & 08 & 27.0 & 27 & & 37 & 165 \\
\hline 10 & 25.6 & 26.9 & 12 & eft & Io & 21.0 & 23.2 & 1. 49 & ar & IO & 49.7 & 53.0 & 2248 & et & IO & 31.0 & 33.0 & & 44 & \\
\hline 12 & 29.0 & 31.0 & 18 & 24 & 12 & $23 . I$ & 25 & 52 & $=1$ & 12 & 13.1 & 17.0 & 2150 & at & I2 & 5.0 & & & 0 & \\
\hline I4 & 34.7 & 37.2 & 28 & -23.9 & 14 & 25.9 & 28.2 & 2257 & -20.3 & 14 & 40.3 & 53.0 & 2247 & -19.0 & I & 33.7 & 34 & & 47 & -18.0 \\
\hline 16 & 41,2 & 43.5 & 38 & & 16 & 28.7 & 30.8 & 2301 & 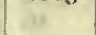 & 16 & & $5 I$ & & & I & & 30 & & 42 & \\
\hline 18 & 43.0 & 44.9 & 40 & bet & 18 & 34.5 & 37.0 & 10 & ar. & 18 & 48.3 & 52.0 & $4 \quad 46$ & Nit & 18 & 35.8 & 37 & & 52 & int \\
\hline 20 & 41.8 & 43.9 & 38 & $=8$ & 20 & 36.6 & 38.5 & 2313 & $x=$ & 20 & 43.5 & 47.0 & 38 & $=$ & 20 & 30.0 & $4 I$. & & 57 & is. \\
\hline 22 & 39.0 & $4 \mathrm{I} .2$ & 34 & $=$ & 22 & 26.1 & 28 . I & 2257 & set & 22 & 36.7 & 41.0 & 28 & $=$ & 22 & 38.0 & 40 & & 5 & $=2$ \\
\hline 24 & 40.3 & 42.8 & 36 & 14 & 24 & 27.0 & 28.9 & 2258 & ab & 24 & 38.1 & 42.5 & 30 & 12 & 24 & 29.8 & 32 & & 43 & \\
\hline 26 & 40.1 & $42 . I$ & 36 & nat & 26 & 29.9 & 32.0 & 2303 & 10 & 26 & 46.0 & 51.2 & 224 & & 26 & 23.0 & 25 & & 32 & \\
\hline 28 & 40.8 & 43.0 & 37 & $y=$ & 28 & 22.8 & 24.4 & 2251 & ite & 28 & 61.9 & 64.0 & 2306 & & 28 & 26.9 & 28 & & 37 & \\
\hline 30 & 48.5 & $5 T .0$ & 2249 & -23.0 & 30 & 20.0 & 21.5 & 47 & -20.1 & 30 & 68.9 & 71.8 & 17 & -18.9 & 30 & 28.2 & 29 & & 40 & -18.0 \\
\hline 32 & 61.0 & 62.9 & 308 & - & 32 & 19.0 & 20.3 & 45 & 30. & 32 & 60.5 & 65.0 & 2305 & ( & 32 & 30.0 & 30 & & $4 \mathrm{I}$ & \\
\hline 34 & 63.0 & 64.0 & & Wit & & 16.0 & & & N & 34 & 55.9 & 57. & & 18 & & 2.5 & & & 46 & \\
\hline 36 & 55.9 & 56.0 & 2250 & $x$ & 36 & 17.0 & 18.4 & 42 & E & 36.5 & 53.0 & 55.0 & 225 & $-y$ & 30 & 33.6 & & & 47 & \\
\hline 38 & 54.9 & 55.0 & 2258 & E & 38 & 22.2 & 22.9 & 50 & $\mathrm{R}^{2}$ & 38.5 & 63.0 & 64.1 & 2307 & kes & 38 & 34.6 & 36 & & 50 & N \\
\hline 40 & 56.3 & 56.8 & 2300 & 27 & 40 & 27.0 & 30.0 & 59 & int & 40 & 71.5 & 73.5 & $2 \mathrm{I}$ & $x^{2}$ & 40 & 27.0 & 27. & & 37 & 16 \\
\hline 42 & & Ia & 07 & 64 & 42 & 18.0 & 20.0 & 44 & $=x$ & 42 & 62.7 & 64.2 & 06 & & 42 & 20.9 & 22.6 & & 28 & 12 \\
\hline 44 & 62.3 & 62.8 & $\infty$ & -22.6 & 44 & 18.8 & 20.0 & 45 & $-20 . I$ & 44 & 64.7 & 66.0 & & -18.7 & 4 & I.3.0 & 17. & & 18 & \\
\hline 46 & 66.8 & & 16 & (2.0 & 46 & 23.2 & 24.0 & 51 & 200 & 46 & 55.2 & 57.2 & 2255 & & 46 & 8.8 & 13 & & II & \\
\hline 48 & 68.0 & 68.8 & I9 & 6 & 48 & 24.3 & 25.2 & 2253 & E. & 48 & $44 \cdot 3$ & 47.0 & 38 & 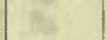 & 48 & I6.0 & 21.9 & & 24 & \\
\hline 50 & 67.0 & 67.2 & 17 & $\psi$ & 50 & 30.2 & $3 \mathrm{I} .7$ & 2303 & 7 & 50 & 50.5 & 51.0 & 47 & est & 50 & 36.6 & 41.6 & 22 & 56 & the \\
\hline 52 & 66.2 & 66.6 & 16 & at & 52 & 30.0 & 30.3 & 02 & sit & 52 & 45 & $.0 b$ & 38 & c5 & 52 & 49.5 & 54.0 & 23 & 15 & \\
\hline 54 & 66.2 & 66.6 & 16 & p & 54 & 30.4 & 30.4 & 02 & if & 54 & 42 & $.0 a$ & 33 & 4 & 54 & 44.5 & 51.9 & 23 & 10 & 3 \\
\hline 56 & 65.2 & 66.0 & 14 & 8 & $\begin{array}{l}34 \\
56\end{array}$ & 32.0 & 32.5 & 05 & E & 56 & 39 & $5 a$ & 29 & 8 & 56 & 23.8 & 29.2 & 22 & 36 & \\
\hline 58 & 60.0 & 60.9 & 06 & $k 2$ & 58 & 33.2 & 34.0 & 07 & be & 58 & 53.2 & 55.9 & 53 & as & 58 & 28.0 & $3 \mathrm{I} .2$ & & $4 \mathrm{I}$ & \\
\hline & & & $(7-4)$ & & & & & & & & & & & & & & & & & \\
\hline
\end{tabular}


Tabulation of magnetic declinations observed at Teplitz Bay-Continued

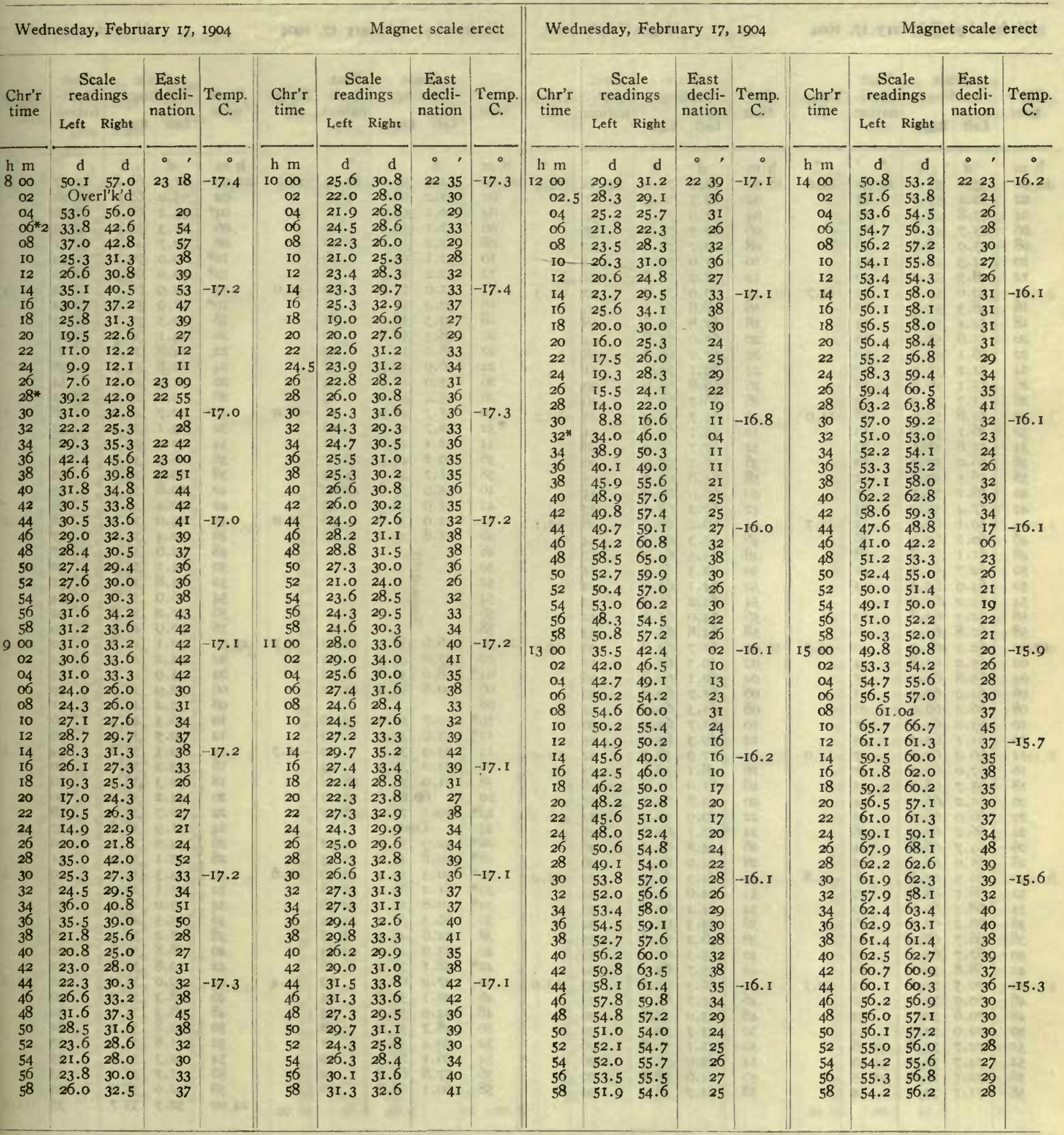


Tabulation of magnetic declinations observed at Teplitz Bay-Continued

\begin{tabular}{|c|c|c|c|c|c|c|c|c|c|c|c|c|c|c|c|c|c|c|c|c|c|}
\hline \multicolumn{4}{|c|}{ Wednesday, February } & 17,1904 & \multicolumn{5}{|c|}{ Magnet scale erect } & \multicolumn{6}{|c|}{ Wednesday, February 17,1904} & \multicolumn{6}{|c|}{ Magnet scale erect } \\
\hline \multirow{2}{*}{$\begin{array}{l}\text { Chr'r } \\
\text { time }\end{array}$} & $\begin{array}{r}\mathrm{Sc} \\
\text { rear }\end{array}$ & $\begin{array}{l}\text { ale } \\
\text { lings }\end{array}$ & East & Temp. & Chr'r & $\begin{array}{l}\text { Sc } \\
\text { read }\end{array}$ & $\begin{array}{l}\text { ale } \\
\text { ings }\end{array}$ & $\begin{array}{l}\text { East } \\
\text { decli- }\end{array}$ & Temp & Chr'r & $\begin{array}{r}\mathrm{Sc} \\
\text { read }\end{array}$ & $\begin{array}{l}\text { ale } \\
\text { lings }\end{array}$ & $\begin{array}{l}\text { East } \\
\text { decl }\end{array}$ & & & Chr'r & $\begin{array}{l}\text { Sca } \\
\text { read }\end{array}$ & lings & Eas! & & Temp. \\
\hline & Left & Right & & & & Left & Right & & & & Left & Right & & & & & Left & Right & & & \\
\hline h m & d & d & & $\bullet$ & $\mathrm{h} \mathrm{m}$ & d & d & $\cdot$, &. & $\mathrm{h} \mathrm{m}$ & $d$ & & & & - & $\mathrm{h} \mathrm{m}$ & a & & - & & $\cdot$ \\
\hline$\infty$ & 55.6 & 57.8 & 2230 & -15.0 & I8 00 & 69.0 & 70.0 & 2250 & -12.4 & 2000 & 53.9 & 58.0 & 222 & & -12.0 & 2200 & 61.0 & 69.8 & 222 & 27 & $-\mathbf{I} .3$ \\
\hline 02 & 52.2 & 54.2 & 25 & & 02 & 69.5 & 70.9 & 51 & & 02 & 52.2 & 56.2 & & & & 02 & 63.0 & 67.0 & & 25 & $x^{2}$ \\
\hline 04 & 45.8 & 48.3 & 15 & kit & 04 & 71.0 & 72.2 & 54 & $t^{2}$ & 04 & $52 . \mathrm{I}$ & 55.9 & & 26 & 18 & $04^{*}$ & 55.2 & 66.4 & & $\infty$ & ate \\
\hline 06 & 45.7 & 48.8 & 15 & 60 & 06 & 72.3 & 73.2 & $\begin{array}{l}34 \\
55\end{array}$ & 50 & 06 & $52 . \mathrm{I}$ & 55.8 & & 26 & 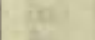 & 06 & 21.3 & 53.0 & 222 & 23 & \\
\hline 08 & 45.9 & 48.8 & 16 & 65 & 08 & 70.2 & 71.0 & 52 & Bes & 08 & 52.2 & 55.9 & & 26 & Bi. & 08 & 10.7 & 48.8 & 221 & II & (1) \\
\hline 10 & $44 \cdot 3$ & 47.7 & 13 & ot & IO & 71.0 & 71.1 & 53 & at & 10 & $52 . I$ & 56.1 & & 26 & (iti & $10^{*}$ & 29.0 & 70.2 & 2I 5 & 54 & as \\
\hline 12 & 48.3 & 51.7 & 20 & $4 x$ & 32 & 71.2 & 71.8 & 54 & 63 & I2 & 52.2 & 56.3 & & 26 & & $\mathrm{I} 2^{*}$ & 30.0 & 69.3 & 222 & 20 & it \\
\hline 14 & 50.0 & 53.1 & 22 & -14.7 & 14 & 70.0 & 71.0 & 52 & -12.3 & 14 & 51.6 & 55.8 & & 26 & -12.0 & I4 & 32.9 & 68.1 & & 22 & -11.3 \\
\hline 16 & 48.3 & 51.2 & 19 & - & I6 & 68.8 & 60.1 & 49 & ar & 16 & 50.3 & 54.9 & & 24 & & $16^{*}$ & 31.9 & 52.8 & 235 & 52 & \\
\hline 18 & 52.4 & 54.8 & 25 & TH & 18 & 67.8 & 67.8 & 48 & then & 18 & 49.3 & 53.0 & & $2 \mathrm{I}$ & 바 & I8 & 50.5 & 74.7 & 242 & 23 & 20 \\
\hline 20 & 55.6 & 57.2 & 30 & 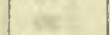 & 20 & 65.0 & 65.8 & 44 & set. & 20 & 48.3 & 51.8 & & 20 & 45 & 20 & 14.1 & $37 \cdot 3$ & 232 & & tat \\
\hline 22 & 56.1 & 57.5 & 30 & $2=$ & 22 & 67.2 & 68.8 & 48 & $2=$ & 22 & 48.0 & 51.1 & & 19 & 67 & 22 & 14.8 & 36.8 & & 26 & in \\
\hline 24 & 54.2 & 55.7 & 27 & 36 & 24 & 60.1 & 70.7 & $5 \mathrm{I}$ & a. & 24 & 46.9 & 49.7 & & 17 & ex & 24 & 22.8 & 43.9 & & 38 & 13 \\
\hline 26 & 55.1 & 56.2 & 28 & 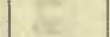 & 26 & 68.8 & 69.9 & 50 & & 26 & 45.0 & 48.1 & & 14 & 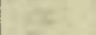 & 26 & 21.9 & 40.9 & & 34 & $1 x$ \\
\hline 28 & 55.7 & 56.9 & 30 & $2=$ & 28 & 66.7 & 67.2 & 46 & & 28 & 45.4 & 48.2 & & 15 & 5 & 28 & 9.1 & 25.2 & 231 & 12 & \\
\hline 30 & 54.8 & 55.9 & 28 & -14.0 & 30 & 64.7 & 65.2 & 43 & -12.2 & 30 & 45.7 & 48.9 & & 16 & -12.0 & $30^{*}$ & 32.7 & 49.6 & 22 & 48 & -II.3 \\
\hline 32 & 56.1 & 57.6 & 30 & 6 & 32 & 65.1 & 65.8 & 44 & - & 32 & 42.7 & 47.0 & & 12 & & 32 & 23.1 & 39.3 & & 32 & \\
\hline 34 & 55.6 & 56.1 & 29 & $x^{2}$ & 34 & 67.7 & 68.2 & 48 & is & 34 & 42.1 & 47.2 & & I I & 18: & 34 & 20.4 & 36.2 & & 28 & it \\
\hline $3^{6}$ & 56.0 & 57.2 & 30 & 10 & 36 & 66.1 & 67.0 & 46 & 8 & 36 & 39.2 & 44.5 & 220 & 07 & ine & 36 & 18.9 & 34.0 & & 24 & 8 \\
\hline 38 & 56.5 & 57.8 & 31 & a & 38 & 65.8 & 66.3 & 45 & $x^{2}$ & 38 & $3 \mathrm{I} .7$ & 36.9 & 215 & 55 & E & 38 & I9. 4 & 33.8 & & 25 & ks \\
\hline 40 & 56.1 & 58.1 & 31 & tre & 40 & 66.0 & 66.8 & 45 & $n^{2}$ & 40 & 35.9 & 39.8 & 220 & 00 & \& & 40 & 19.8 & 33.3 & & 25 & es \\
\hline 42 & 57.4 & 59.4 & 33 & tan & 42 & 67.2 & 68.0 & $\begin{array}{l}40 \\
47\end{array}$ & W & 42 & 34.9 & 38.9 & 215 & 59 & $1=2$ & 42 & 20.4 & 33.8 & & 26 & is \\
\hline 44 & 59.1 & 60.9 & 35 & -13.7 & 44 & 66.2 & 67.1 & 46 & -12.1 & 44 & 32.0 & 37.8 & 5 & 56 & -12.0 & 44 & 23.0 & 34.9 & & 28 & $-\mathrm{II} \cdot 4$ \\
\hline 46 & 60.2 & 61.2 & 36 & - & 46 & 65.3 & 66.3 & 44 & 2.1 & 46 & $3^{1} .8$ & 39.2 & 215 & 57 & $x^{2}=$ & 46 & 22.4 & 34.0 & & 27 & \\
\hline 48 & $6 \mathrm{I} . \mathrm{I}$ & 61.8 & 38 & 6 & 48 & 63.5 & 64.2 & $4 \mathrm{I}$ & 14 & 48 & 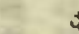 & \pm & & & Lar & 48 & 22.6 & 33.9 & & 27 & \\
\hline 50 & 62.7 & 64.0 & 41 & tat & 50 & 63.3 & 64.2 & 41 & 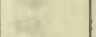 & 50 & & 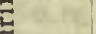 & $F$ & 8 & ne & 50 & 24.1 & $35 \cdot 3$ & & 30 & e \\
\hline 52 & 64.9 & 65.0 & 43 & 5 & 52 & 64.1 & 64.8 & 42 & 28 & 52 & Q & z & r & 8 & 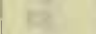 & 52 & 24.8 & 36.1 & & $3 \mathrm{I}$ & 34 \\
\hline 54 & 63.2 & 64.2 & 41 & 17 & 54 & 64.2 & 65.0 & 42 & is & 54 & & 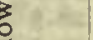 & 0.6 & te & 28 & 54 & 26.9 & 37.8 & & 34 & 18 \\
\hline & 62.3 & 62.8 & 39 & 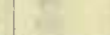 & 56 & 64.0 & 65.0 & 42 & 56 & 56 & & & t. & 8 & de & 56 & 27.2 & 37.8 & & 34 & \\
\hline 58 & 62.8 & 63.3 & 40 & 172 & 58 & 64.8 & 65.3 & 43 & 6 & 58 & & & & & 6 & 58 & 30.9 & 40.6 & & 39 & $\mathbb{2}$ \\
\hline 1700 & 62.7 & 63.0 & 40 & -13.5 & 1900 & $6 \mathrm{r} .7$ & 62.8 & 39 & $-12 . I$ & 2100 & 36.6 & 38. I & 220 & $\infty$ & -11.7 & 2300 & 32.1 & 40.8 & & 40 & -11.4 \\
\hline 02 & 65.2 & 65.8 & 44 & (n) & 02 & 59.3 & 60.0 & 3.5 & 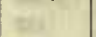 & 02 & 37.6 & 41.9 & & 04 & (5) & 02 & 34.2 & 42.3 & & 43 & \\
\hline 04 & 65.8 & 66.2 & 45 & in & 01 & 60.1 & 60.8 & 36 & 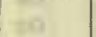 & $04^{*}$ & 8.0 & 36.8 & 225 & 59 & te & 04 & 35.9 & 43.1 & & 45 & +2 \\
\hline 06 & 61.9 & 65.2 & 4.3 & 60 & 06 & $6 \mathrm{r.7}$ & 62.8 & 39 & 86 & 06 & 30.8 & 55.9 & 233 & 32 & 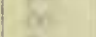 & 06 & 32.9 & 38.7 & & 39 & \\
\hline 08 & 66.2 & 66.9 & 46 & 5 & 08 & $63 \cdot 3$ & 64.0 & 41 & 69 & 08 & & & & & livg & 08 & 33.2 & 40.2 & & $4 I$ & \\
\hline I0 & 63.2 & 64.0 & 41 & $n=$ & ro & 61.7 & 63.0 & 39 & dest & $10^{*}$ & 10.7 & 50.4 & 222 & 29 & $a t$ & IO & 36.3 & $43 \cdot 3$ & & 16 & At \\
\hline 12 & 64.1 & 65.3 & 43 & & 12 & 59.3 & 60.1 & 35 & ar & $12^{*}$ & 33.9 & 35.1 & 2 & 29 & 32 & I2 & 30.5 & 37. & & 36 & \\
\hline 14 & 66.9 & 67.9 & 47 & -13.0 & $\sqrt{4}$ & 57.9 & 58.5 & 32 & -12.0 & 14 & 33.9 & 35.1 & & 29 & $-\mathrm{II} .7$ & I4 & 29.3 & 35.8 & & 34 & -11.4 \\
\hline 16 & 67.0 & 67.9 & 47 & o & I6 & 58.1 & 58.7 & 32 & $\therefore$ & 16 & 33.2 & 33.9 & & 29 & & 16 & 33.2 & 39.1 & & 40 & \\
\hline 18 & 65.7 & 66.7 & 45 & ent & r8 & 61.0 & 61.8 & 38 & W. & I8 & 31.9 & 34.7 & 222 & 28 & 84 & 18 & 32.3 & 38.1 & & 38 & 17 \\
\hline 20 & 65.2 & 66.0 & 44 & $a$ & 20 & 59.8 & 60.1 & 35 & $\alpha$ & 20 & $6 c$ & .00 & 230 & 09 & est & 20 & 34.2 & 39.1 & & 40 & a \\
\hline 22 & 66.0 & 66.4 & 45 & $=$ & 22 & 59.0 & 59.9 & 34 & a & $22^{*}$ & 29.5 & 70.0 & 252 & 27 & 6 & 22 & 32.7 & 37.4 & & 38 & \\
\hline 24 & 66.2 & 67.2 & 4 & $y=$ & 24 & 59.7 & 60.6 & 36 & $y=$ & 24 & $\mathrm{~L}$ & ost & & & $\mathrm{H}$ & 24 & 31.9 & 36.8 & & 37 & \\
\hline 26 & 64.7 & 66.2 & 44 & $E$ & 26 & 54.8 & 66.4 & 36 & net & $26^{*}$ & 42.9 & 56.5 & 234 & 42 & It & 26 & 34.0 & 38.2 & & 40 & \\
\hline 28 & 65.0 & 66.0 & 45 & & 28 & 54.9 & 65.9 & 36 & $x^{2}=$ & $28^{*}$ & 40.8 & 45.8 & 214 & 45 & & 28 & 35.1 & 39.0 & & $4 \mathrm{I}$ & \\
\hline 30 & 66.5 & 67.8 & 47 & -12.8 & 30 & 54.3 & 64.2 & 34 & -12.0 & $30^{*}$ & 20.3 & 55.3 & 204 & 40 & - I I. 6 & 30 & $34 \cdot 3$ & 39.0 & & 40 & -11.4 \\
\hline 32 & 68. I & 68.8 & 49 & $1=$ & 32 & 54.6 & 63.8 & 34 & $x^{2}$ & $32^{*}$ & 61.2 & 69.9 & 230 & 06 & 6 & 32 & 25.1 & 29.9 & & 26 & \\
\hline 34 & 68.3 & 69.8 & 50 & is & 34 & 54.4 & 62.8 & 33 & $y=$ & $34^{*}$ & I0.8 & I9. I & 224 & 46 & 21 & 34 & 23.0 & 26.6 & & 22 & \\
\hline & 66.2 & 67.7 & 46 & es & 36 & 55.3 & 63.1 & & Bes & 36 & 69.3 & 71.7 & $24 \mathrm{I}$ & I4 & $x$ & 36 & 25.5 & 28.8 & & 26 & \\
\hline 38 & 66.7 & 68.2 & 47 & efr. & 38 & 56.2 & 63.6 & $\begin{array}{l}34 \\
35\end{array}$ & An: & 38 & 32.6 & 46.0 & 232 & 25 & A2. & 38 & 27.2 & 30.4 & & 28 & \\
\hline 40 & 67.7 & 69.3 & 49 & 20 & 40 & 55.3 & 62.1 & 33 & $\theta$ & 40 & 15.9 & 21.9 & 225 & 53 & 01 & 40 & 28.8 & 31.6 & & 30 & \\
\hline 42 & 68.8 & 70.2 & 50 & 8 & 42 & 55.7 & 62.1 & 34 & $m$ & $42^{*}$ & 58. I & 71.9 & & 26 & 44 & 42 & 29.8 & 32.7 & & 32 & \\
\hline 44 & 70.5 & 72.2 & 5 & -12.7 & 44 & 56.5 & 62.6 & $\begin{array}{l}34 \\
35\end{array}$ & $-\mathrm{I} 2.0$ & 44 & 47.2 & 60.1 & & 09 & -11.4 & 44 & 29.0 & 31.9 & & $3 \mathrm{I}$ & -11.4 \\
\hline 46 & 69.9 & 71.3 & 52 & 2 & 46 & 55.7 & $6 \mathrm{r.3}$ & 33 & 20 & 46 & 46.1 & 55.8 & & 04 & 34 & 46 & 28.3 & $3 \mathrm{I} .2$ & & 30 & \\
\hline 48 & 70.6 & 72.0 & 53 & Re & 48 & 53.0 & 58.8 & 29 & B. & 48 & 44.9 & 55.1 & & 03 & a. & 48 & 28.3 & $3 \mathrm{I} .4$ & & 30 & \\
\hline 50 & 70.9 & 72.3 & 5. & 6 & 50 & 53.7 & 59.0 & 30 & 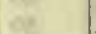 & 50 & 48.7 & 57.8 & & 08 & ats & 50 & 29.8 & 32.7 & & 32 & \\
\hline 52 & 72.7 & 73.8 & 225 & Ez & 52 & 51.1 & 56.8 & 26 & 38 & 52 & 50.4 & 59.9 & & II & L & 52 & 31.8 & 34.1 & & 35 & \\
\hline 54 & 74.7 & 76.1 & $23 \circ$ & A & 54 & 52.0 & 56.7 & 26 & in. & 54 & $56 . \mathrm{I}$ & 64.5 & $y$ & 19 & is & 54 & 32.3 & 34.2 & & 35 & \\
\hline 56 & 72.1 & 73.0 & 225 & Bis & 56 & 54.6 & 58.8 & 30 & (68) & 56 & 57.8 & 65.8 & & 22 & Eis & 56 & 31.7 & 32.3 & E & 33 & \\
\hline 58 & 71.1 & 72.2 & 5 & RE & 58 & 54.7 & 58.9 & 30 & 8 & 58 & 60.1 & 68.8 & 2 & 26 & $\mathrm{E}$ & 58 & 32.0 & 33.5 & & 34 & \\
\hline & & & & & & & & & & & & & & & & 2400 & 32.7 & 33.7 & & 35 & $-I I .4$ \\
\hline
\end{tabular}

Observers-R. W. P. and R. R. T., who alternated from 16 h oom to $16 \mathrm{~h} 02 \mathrm{~m}$.
Correction to local mean time is - Im 555 .

Torsion head at oh oom read $36^{\circ}$ and at the end read the same Observer-R. R. T. 
Tabulation of magnetic declinations observed at Teplitz Bay-Continued

\begin{tabular}{|c|c|c|c|c|c|c|c|c|c|c|c|c|c|c|c|c|c|c|c|}
\hline \multicolumn{5}{|c|}{ Thursday, February I8, 1904} & \multicolumn{5}{|c|}{ Magnet scale inverted } & \multicolumn{5}{|c|}{ Friday, February 19, 1904 } & \multicolumn{5}{|c|}{ Magnet scale erect } \\
\hline \multirow{2}{*}{$\begin{array}{l}\text { Chr'r } \\
\text { time }\end{array}$} & \multicolumn{2}{|c|}{$\begin{array}{l}\text { Scale } \\
\text { readings }\end{array}$} & $\begin{array}{c}\text { East } \\
\text { decli- }\end{array}$ & & Chr'r & $\begin{array}{r}\mathrm{Sc} \\
\text { read }\end{array}$ & $\begin{array}{l}\text { ale } \\
\text { lings }\end{array}$ & $\begin{array}{l}\text { East } \\
\text { decli- }\end{array}$ & Temp. & Chr'r & $\begin{array}{r}\mathrm{Sc} \\
\mathrm{reac}\end{array}$ & $\begin{array}{l}\text { cale } \\
\text { dings }\end{array}$ & $\begin{array}{l}\text { East } \\
\text { decli- }\end{array}$ & & & $\begin{array}{l}\mathrm{Sc} \\
\text { read }\end{array}$ & $\begin{array}{l}\text { le } \\
\text { ngs }\end{array}$ & East & \\
\hline & Left & Right & & & & Left & Right & & & & Left & Right & & & & Left & Right & & \\
\hline $\mathrm{m}$ & d & $\mathrm{d}$ & $\circ \quad$ & $\circ$ & $\mathrm{h} \mathrm{m}$ & d & & & & $h \mathrm{~m}$ & d & $d$ & & & $h \mathrm{~m}$ & d & d & & \\
\hline$\infty$ & 40.2 & 37.0 & 2238 & -22.4 & 1800 & 36.5 & $34 \cdot 7$ & 2242 & -20.7 & 2000 & 37.0 & 37.2 & 2233 & -26.1 & 2200 & 39.1 & $39 \cdot 3$ & 2237 & -23.0 \\
\hline 02 & 39.9 & 36.0 & & 100 & 02 & 38.2 & 36.0 & 40 & $\cos 2$ & 02 & 36.8 & $37 . \mathrm{I}$ & & $\tan$ & 02 & 38.8 & 38.8 & 36 & $y^{2}$ \\
\hline 04 & 40.2 & 36.9 & 38 & 20 & 04 & 40.4 & 38.8 & 36 & 50 & 04 & 37.0 & 37.4 & 33 & 10 & 04 & 39.2 & 39.4 & 37 & jos \\
\hline 06 & 40.8 & 37.2 & 37 & 8 & 06 & 30.9 & 38.7 & 37 & $\infty$ & 06 & 36.9 & 37.3 & 33 & $\mathrm{~m}$ & 06 & 39.4 & 39.8 & 37 & 20 \\
\hline 08 & 40.3 & 37.0 & 38 & BD & 08 & 39.9 & 38.8 & 37 & as & 08 & 36.8 & 37.4 & & 81 & o8 & 39.7 & 40.2 & 38 & 164 \\
\hline IO & $4 \mathrm{I} .2$ & 37.8 & 36 & et & IO & 39.7 & 38.9 & 37 & er & IO & 36.0 & 36.3 & 32 & कi & I0 & 39.9 & 40.5 & 38 & tar \\
\hline 12 & $4 \mathrm{r} .9$ & 38.2 & 36 & Ei & 12 & 39.5 & 38.4 & 37 & 81 & I2 & 37.2 & 37.8 & 8. 34 & 1 at & I2 & 40.0 & 40.9 & 38 & 31 \\
\hline 14 & 40.7 & 37.5 & 37 & -22.2 & 14 & 38.5 & 37.2 & 39 & -20.7 & I4 & 38.0 & 39.0 & 35 & -25.8 & 14 & 41.0 & 41.8 & 40 & -22.9 \\
\hline I6 & 40.8 & 37.8 & 37 & $12 x^{2}$ & I6 & 38. I & 37.0 & 39 & tat & 16 & 40.1 & 10.7 & 38 & 10 & I6 & $4 \mathrm{~T} .0$ & $4 \mathrm{I} .5$ & & 18 \\
\hline 18 & 42.8 & 40.0 & 33 & ta & 18 & 40.0 & 39.0 & 36 & Ent & I8 & 39.7 & 40.0 & 38 & $\mathrm{At}$ & I8 & 40. I & 40.4 & $3^{8}$ & Br \\
\hline 20 & 42.3 & 40.2 & 34 & is & 20 & 40.0 & 38.2 & 37 & $\alpha$ & 20 & 39.0 & 39.5 & 37 & $x$ & 20 & 40.0 & 40.5 & 38 & 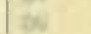 \\
\hline 22 & $41 . \overline{8}$ & 39.7 & 34 & is & 22 & 39.0 & 37.3 & 38 & ex & 22 & $40 . I$ & 40.9 & 39 & ES & 22 & 40.9 & 41.3 & 40 & zat \\
\hline 24 & 42.0 & 40.0 & 34 & $3 z$ & 24 & 37.8 & 36.7 & 40 & Iis & 24 & 30.0 & 39.7 & & & 24 & $4 \mathrm{~T} .0$ & $4 \mathrm{I} .5$ & 40 & \\
\hline 26 & 42.0 & 40.0 & 34 & & 26 & 37.8 & 36.7 & 40 & E & 26 & 38.9 & 39.0 & 36 & 8 & 26 & 40.8 & 41.0 & 39 & \\
\hline 28 & 41.8 & 39.4 & 35 & & 28 & 37.8 & 36.2 & 40 & & 28 & 38.9 & 39.2 & & & 28 & 40.3 & 40 & 38 & \\
\hline 30 & 41.7 & 39.2 & 35 & -22.0 & 30 & 37.8 & 36.1 & 40 & -20.5 & 30 & 39.0 & 39.7 & 37 & -25.4 & 30 & 39.7 & 40.0 & 38 & -22.5 \\
\hline 32 & $4 \mathrm{I} . \mathrm{I}$ & 38.8 & 36 & $1=4$ & 32 & 36.0 & 35.1 & 42 & $1=2$ & 32 & 39.0 & 39.7 & 37 & - & 32 & 39.0 & 30.3 & 36 & \\
\hline 34 & 40.9 & 38.8 & 36 & 말 & 34 & 35.8 & 35.2 & 43 & M. & 34 & 39. I & 39 & 37 & a. & 34 & 38.4 & & 36 & I9: \\
\hline 36 & 41.2 & $39 . \mathrm{I}$ & 35 & 2 & 36 & 34.8 & $34 \cdot 3$ & 44 & E. & 3 & & 39 & 5 & st & 36 & 38.3 & & 36 & 3 \\
\hline 38 & 42.4 & 40.1 & 34 & $\mathrm{ge}$ & 38 & 35.2 & 35.0 & 43 & 98 & 38 & 38.4 & 30. & 36 & & 38 & 39.0 & 39 & 36 & A \\
\hline 40 & 42.2 & 40.1 & 34 & as & 40 & 33.9 & 33.5 & 45 & ta & 40 & 37.9 & 38 & 34 & to & 40 & & & 37 & 6 \\
\hline 42 & 42.3 & 40.7 & 33 & 60 & 42 & 33.2 & $33 . I$ & 46 & 10 & 42 & 40.0 & 40. & 38 & 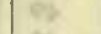 & 42 & 39.8 & 40.0 & 38 & 10 \\
\hline 44 & 42.9 & 41.0 & 33 & -21.9 & 44 & 33.4 & 33.0 & 46 & -20.4 & 44 & $40 . I$ & 40.6 & 38 & -25.0 & 44 & 40.0 & 40.2 & 38 & -22.1 \\
\hline 46 & $43 \cdot \mathrm{I}$ & 42.0 & 32 & & 46 & 34.0 & 33.4 & 45 & 15) & 46 & $40 . I$ & 40.3 & 38 & & 46 & 40.4 & $4 \mathrm{I} .0$ & 39 & $18=$ \\
\hline 48 & 43.8 & 42.4 & $3 I$ & 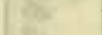 & 48 & 33.8 & 32.2 & 46 & 14 & 48 & 40.3 & 40.9 & 39 & 9 & 48 & 40.5 & $4 \mathrm{I}$ & 39 & 8 \\
\hline 50 & 43.8 & 42.2 & $3 \mathrm{I}$ & 10 & 50 & 30 & $.0 a$ & $5 \mathrm{I}$ & 184 & 50 & 39.3 & 39. & 3 & 86 & 50 & 40.8 & 41.3 & 39 & 16 \\
\hline 52 & 43.6 & $42 . I$ & $3 \mathrm{I}$ & 28 & 52 & 32.8 & 32.8 & 47 & 168 & 52 & 30.0 & 30 & & $\infty$ & 52 & 40.8 & & 39 & Q \\
\hline 54 & 45.2 & 44. I & 28 & 12 & 54 & 33.0 & 32.8 & 47 & $Q$ & 54 & 38.7 & 39 & 36 & 42 & 54 & 40.5 & $4 \mathrm{I} .0$ & 39 & $x$ ? \\
\hline 56 & 44.2 & 43.2 & 30 & 12 & 56 & 34.2 & & 4.5 & 18 & 56 & 30.2 & 39. & 37 & 42 & 56 & & & 38 & 21 \\
\hline 58 & 44.0 & 43.4 & 30 & & 58 & 35.5 & 34.9 & 43 & 12 & 58 & 30.3 & 39 & 37 & 2 & 58 & 40.4 & 40.8 & 39 & 10 \\
\hline 700 & 43.8 & 43.0 & 30 & -2 I. 3 & I9 00 & 35.3 & 34.8 & 43 & -20.2 & $2 \mathrm{I} \infty$ & 40.0 & 40.3 & 38 & -24.8 & $23 \infty 0$ & 40.5 & 40.9 & 39 & -22.0 \\
\hline 02 & 43.2 & 42.5 & $3 \mathbf{I}$ & $\alpha 5$ & 02 & 37.2 & 36.2 & $4 I$ & $10 \%$ & 0 & 40.4 & 40 & 30 & 100 & 02 & 41.6 & 41.9 & 40 & $10 x$ \\
\hline 04 & 43.0 & 42.2 & 32 & $\omega$ & 04 & 37.9 & 36.8 & 40 & 50 & 0 & 40.0 & 40.8 & 38 & $\mathrm{Fu}$ & 04 & 40.8 & $4 \mathrm{~T} . \mathrm{I}$ & 39 & $\operatorname{lin}$ \\
\hline 06 & 42.8 & 42.2 & 32 & is & of & 39.8 & 39.1 & 36 & Wir & of & & & 30 & 10 & 06 & 40.7 & 40.9 & 39 & 19 \\
\hline 08 & $43 . I$ & 42.4 & $3 \mathrm{I}$ & det & 08 & 39.6 & 39.0 & 37 & A6. & 08 & $40 . I$ & 40. & 38 & in & 08 & 40.8 & 41.0 & 39 & Wis \\
\hline IO & 42.9 & 42.2 & 32 & Ro & I0 & 39.8 & 38 & 37 & 180 & Is & & & 38 & $\sqrt{60}$ & I0 & 40.7 & & 30 & 200 \\
\hline 12 & 43.2 & 42.6 & $3 I$ & est & I 2 & 38.9 & 38.0 & 38 & & $\mathrm{I}$ & 30.2 & 30.6 & 37 & 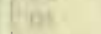 & I 2 & 40.0 & 40.4 & 38 & ait \\
\hline 14 & 43.7 & 42.5 & $3 \mathbf{I}$ & -2 I. I & I4 & 39.4 & 38 & 37 & -20.0 & & & & & $-24 \cdot 3$ & 14 & 40.0 & & 39 & -21.8 \\
\hline 16 & 42.6 & $4 \mathrm{I} \cdot 3$ & 33 & 3 & I6 & 39.7 & 38 & 37 & & I6 & 38.2 & 38.7 & 35 & (3) & I6 & 30.0 & 39.9 & 37 & 1 int \\
\hline 18 & 41.8 & 40.8 & 34 & & I8 & 40. T & 38.8 & 36 & & I8 & 38.9 & 39.0 & & of & I8 & 38.8 & & 36 & She \\
\hline 20 & 40.8 & 30.6 & 35 & 87 & 20 & 40.7 & 39.2 & 36 & 8 & 20 & 39.4 & 39.7 & 37 & 1. & 20 & 38.7 & 39.2 & 36 & Fis \\
\hline 22 & 39.9 & 38.6 & 37 & & 22 & 40.8 & & 35 & & 2 & & & & & 2 & 38.3 & & 36 & \\
\hline 24 & 38.2 & 37.0 & 39 & mer. & 24 & 40.1 & 38 & .36 & We & 2 & 39.6 & 40.0 & 38 & 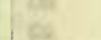 & 24 & 38.5 & 39 & 36 & 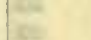 \\
\hline 26 & 38.3 & 37.0 & 39 & . & 26 & 39.0 & 38.0 & 38 & s. & 26 & 39.2 & 39.7 & & 20 & 2 & 39.3 & & 37 & \\
\hline 28 & 35.6 & 35.0 & 43 & $\frac{5}{5}$ & 28 & 39.1 & 38.1 & 38 & & 28 & & 40 & 38 & 1 & 28 & 40.5 & 40 & 39 & \\
\hline 30 & 36.2 & 35. & 42 & -21.0 & 30 & 40.9 & 39.3 & 35 & -20.0 & 30 & 39.8 & 40. & 38 & -24.0 & 30 & 41.4 & 41.5 & 40 & -21.6 \\
\hline 32 & 35.7 & 35.0 & 43 & & 32 & 40.1 & 38.8 & 36 & & 3 & 39.3 & 39 & 37 & & 32 & 41.5 & $4 \mathrm{I} .5$ & 40 & \\
\hline 34 & 35.9 & 35. & 43 & & 34 & 38.0 & & 39 & 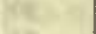 & & & & & & & $4 \mathrm{~T} .6$ & $4 \mathrm{I}$ & 40 & \\
\hline 36 & 36.9 & 36.2 & $4 I$ & 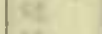 & 3 & 37.1 & 36. I & $4 \mathrm{I}$ & 0. & 3 & 30 & 40 & 38 & & 3 & $4 \mathrm{I} . \mathrm{I}$ & $4 \mathrm{I}$ & 40 & H. \\
\hline 38 & 36.7 & $36 . I$ & $4 \mathrm{I}$ & $k$ & 38 & 37.2 & & $4 \mathrm{I}$ & 4 & 38 & & & & 10 & 38 & $40 . I$ & 40.3 & 38 & 2 \\
\hline 40 & 33.5 & 33.0 & 46 & & 40 & 39.2 & 38.8 & 37 & ate & $4 c$ & 38.7 & & 36 & $\&$ & 40 & 39.3 & 39 & 37 & t \\
\hline 42 & 33.0 & 32.0 & 47 & fic & 42 & $4 \mathrm{~T} .0$ & 40.1 & 35 & Ne & 42 & & 38 & 35 & 162 & 42 & 39. I & & 36 & 8 \\
\hline 44 & 31.8 & 30.2 & 50 & -20.9 & 44 & 42.1 & 41 & 33 & -20. & 44 & 38.8 & 39 & 36 & -23 . & 44 & 39.4 & 39 & 37 & $-2 \mathrm{I} .4$ \\
\hline 46 & 3 ז. 6 & 29.2 & 51 & $1=$ & 46 & 42.9 & 42.2 & 32 & 38 & 46 & 38.7 & 38.9 & 36 & 10 & 46 & 39.4 & 39.8 & 37 & a \\
\hline 48 & 29.8 & 27.2 & 54 & 134 & 48 & 41.9 & $4 \mathrm{I}$. & 33 & 4 & 48 & 39.0 & 39.1 & 36 & (3t) & 48 & 39.5 & 40.0 & 37 & 13 \\
\hline 50 & 27.9 & 26.2 & 56 & 6 & 50 & 40.9 & & $2 \quad 35$ & 28 & 50 & & & 37 & 13 & 50 & 39.8 & 40.1 & 38 & 68 \\
\hline 52 & 29.6 & 27.8 & 53 & 84 & 52 & 38.5 & 38 & 38 & Pas. & 52 & 39.2 & & 38 & 82 & 52 & 40.0 & & 38 & 5 \\
\hline 54 & 32.0 & 30.2 & 49 & $\theta$ & 54 & 40.9 & $\{0.7$ & 17. 34 & ont & 54 & 39.1 & 39. & 37 & 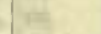 & 54 & 40.4 & 40.7 & 39 & $6 e$ \\
\hline 56 & 33.8 & 32.2 & 47 & az & 56 & 42.9 & 41.2 & 6. 32 & & 56 & 39.0 & 39.2 & 36 & at & 56 & 40.7 & 40.9 & 39 & $=8$ \\
\hline 58 & 35.9 & 34.0 & 10. 44 & ne & 58 & 44.9 & $44 . I$ & 0. 28 & & 58 & 39.1 & 39.3 & 37 & 12 & 58 & 40.2 & 40.6 & 38 & ar. \\
\hline & & & $12 x^{2}$ & $\alpha$ & 2000 & 48.8 & 48.0 & 22 & -20.0 & & & & $3=0$ & & 2400 & 40.7 & 40.8 & 39 & \\
\hline & & & $a^{2}$ & 18 & & & 023 & 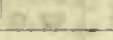 & 25 & & & & & & & & & $.5 \mathrm{xt}$ & \\
\hline
\end{tabular}

Correction to local mean time is $-2 \mathrm{~m}$ oos.

Torsion head at $\mathrm{I} 5 \mathrm{~h} 35 \mathrm{~m}$ read $36^{\circ}$ and at the end read the same. Observer-R. R. T.
Correction to local mean time is - $2 \mathrm{~m}$ i5s.

Torsion head at $\mathrm{Igh} 25 \mathrm{~m}$ read $36^{\circ}$ and at the end read the same. Observer-J. V. 
Tabulation of magnetic declinations observed at Teplitz Bay-Continued

\begin{tabular}{|c|c|c|c|c|c|c|c|c|c|c|c|c|c|c|c|c|c|c|c|}
\hline \multicolumn{5}{|c|}{ Sunday, February 2I, 1904} & \multicolumn{5}{|c|}{ Magnet scale inverted } & \multicolumn{5}{|c|}{ Sunday, February 2I, 1904} & \multicolumn{5}{|c|}{ Hoc: $x$ Magnet scale erect } \\
\hline \multirow[t]{2}{*}{$\begin{array}{l}\text { Chr'r } \\
\text { time }\end{array}$} & \multicolumn{2}{|c|}{$\begin{array}{l}\text { Scale } \\
\text { readings }\end{array}$} & \multirow{2}{*}{$\begin{array}{c}\text { East } \\
\text { decli- } \\
\text { nation }\end{array}$} & Temp. & $\begin{array}{l}\text { Chr'I } \\
\text { time }\end{array}$ & $\begin{array}{l}\text { Sc } \\
\text { reac }\end{array}$ & $\begin{array}{l}\text { ale } \\
\text { lings }\end{array}$ & $\begin{array}{c}\text { East } \\
\text { decli- } \\
\text { nation }\end{array}$ & Temp. & $\begin{array}{l}\text { Chr'r } \\
\text { time }\end{array}$ & $\begin{array}{r}\mathrm{Sc} \\
\text { read }\end{array}$ & $\begin{array}{l}\text { ale } \\
\text { dings }\end{array}$ & East & Temp. & Chr'r & $\begin{array}{r}\text { Sc } \\
\text { reac }\end{array}$ & $\begin{array}{l}\text { le } \\
\text { ngs }\end{array}$ & $\begin{array}{l}\text { East } \\
\text { decli- }\end{array}$ & Temp \\
\hline & Left & Right & & & & Left & Right & tits & & & Left & Right & mation & & & Left & Right & nation & \\
\hline h m & d & d & $\cdot 1$ & & h $\mathbf{m}$ & & d & $\cdot 1$ & & $\mathrm{~h} \mathrm{~m}$ & d & d & $\cdot 1$ & 0 & $\mathrm{~h} \mathrm{~m}$ & $d$ & d & & - \\
\hline $000^{*}$ & 45.9 & 45.2 & 2238 & -18.0 & 200 & 38.9 & $37 \cdot 5$ & 2250 & -16.2 & $400^{*}$ & 50.1 & 52.0 & 2251 & -14.9 & 600 & 52.3 & 55.4 & 2255 & -14.2 \\
\hline 02 & 44.8 & 44.0 & 40 & wat & 02 & 40.3 & 38.7 & 48 & (5) & 02 & 49.9 & 51.8 & 50 & (2) & 02 & 51.1 & 55.4 & 54 & \\
\hline 04 & 43.2 & 41.9 & 43 & $m$ & 04 & 40.2 & 39.0 & 48 & tet & 04 & 49.0 & 51.2 & 49 & (F) & 04 & 50.9 & 55.8 & 54 & tite \\
\hline 06 & 44.2 & 43.1 & 41 & 8 & 06 & 40.8 & 39.9 & 46 & is & 06 & 51.0 & 54.9 & 54 & we & 06 & 51.1 & 56.0 & 55 & wo \\
\hline 08 & $45 . \mathrm{I}$ & 44.2 & 40 & 6. & 08 & 41.2 & 39.3 & 47 & 153 & 08 & 52.2 & 55.8 & 55 & ent & 08 & 51.3 & 56.0 & 55 & lis \\
\hline Io & 44.0 & 43.0 & 41 & or & 10 & 41.8 & 39.9 & 46 & ing & 10 & 53.5 & 56.0 & 56 & 9 & I0 & 51.1 & 55.0 & 54 & ax \\
\hline 12 & 43.8 & 42.8 & 42 & 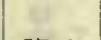 & 12 & 41.8 & 39.9 & 46 & 1 & 12 & 51.9 & 55.0 & 54 & & 12 & 49.9 & 52.0 & $5 \mathrm{I}$ & \\
\hline 14 & 42.6 & $4 \mathrm{I} \cdot 3$ & 44 & $-17 \cdot 3$ & 14 & 42.0 & 40.1 & 45 & $-\mathrm{t} 6 . \mathrm{I}$ & 14 & 49.2 & 52.0 & 50 & $-14 \cdot 3$ & 14 & 49.5 & 52.3 & $5 \mathrm{I}$ & $-I 4 . I$ \\
\hline I6 & 42.8 & 41.2 & 44 & & 16 & 4I. I & 39.2 & 47 & & 16 & 48.9 & 51.I & 49 & & 16 & 51.8 & 53.7 & 53 & $1-7$. \\
\hline 18 & 41.8 & 40.1 & 45 & 21 & 18 & 40.8 & 38.8 & 47 & at & 18 & 48.8 & 51.8 & 50 & 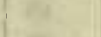 & 18 & 50.8 & 52.5 & 52 & \\
\hline 20 & 40.8 & 39.3 & 47 & at & 20 & 40.2 & 38. I & 48 & $t=$ & 20 & 49. I & 52.0 & 50 & $y$ & 20 & 50.0 & 51.4 & 50 & 15 \\
\hline 22 & 40.1 & 39.1 & 48 & $=$ & 22 & 39.4 & 37.7 & 49 & is & 22 & 49.5 & 51.9 & 50 & tit & 22 & 51.6 & 53.0 & 53 & 대 \\
\hline 24 & 39.4 & 38.9 & 48 & to & 24 & 39.7 & 37.9 & 49 & 145 & 24 & 49.9 & 52.2 & 51 & $\beta^{2}$ & 24 & 51.8 & 54.0 & 54 & \\
\hline 26 & 38.9 & 38.7 & 49 & 8 & 26 & 39.0 & 37.4 & 50 & 64 & 26 & 50.7 & 53.0 & 52 & 2 & 26 & 50.7 & 52.0 & $5 I$ & \\
\hline 28 & 38.3 & 37.9 & 50 & & 28 & 38.8 & $37 \cdot 3$ & 50 & 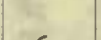 & 28 & 50.0 & 51.8 & 51 & & 28 & 51.0 & 51.9 & 51 & \\
\hline 30 & 39.3 & 38.8 & 48 & -17.1 & 30 & 38.3 & $37 . I$ & $5 \mathrm{I}$ & -16.0 & 30 & 48.5 & 50.2 & 48 & -14.0 & 30 & 49.7 & 51.2 & 50 & .14 .0 \\
\hline 32 & 39.9 & 39.6 & 47 & & 32 & $37 \cdot 3$ & 36.0 & 52 & & 32 & 48.5 & 50.9 & 49 & & 32 & 48.9 & 52.1 & 50 & \\
\hline 34 & 40.1 & 39.3 & 47 & 사 & 34 & 36.3 & 35.2 & 54 & 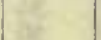 & 34 & 49.0 & 50.0 & 48 & ar & 34 & 49.0 & 52.2 & 50 & \\
\hline 36 & 40.0 & 39.1 & 48 & 30 & 36 & 37.9 & $37 . I$ & 51 & 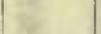 & 36 & 50.2 & $5 I \cdot 3$ & 50 & & 36 & 49.0 & 51.9 & 50 & \\
\hline 38 & 39.6 & 38.8 & 48 & tat & 38 & 37.2 & 36.9 & 52 & 3 & 38 & $5 \mathrm{~T} .1$ & 51.1 & $5 \mathrm{I}$ & es & 38 & 50.0 & 53.2 & 52 & \\
\hline 40 & 37.8 & 37.2 & 51 & th & 40 & 37.8 & $37 \cdot 3$ & $5 I$ & ext & 40 & 5I.I & 51.4 & 51 & it & 40 & 51.8 & 54.7 & 54 & \\
\hline 42 & 37.6 & 37.0 & 51 & & 43 & 37.8 & 37.2 & $5 \mathrm{I}$ & & 42 & 5I.I & 51.8 & $5 \mathrm{I}$ & & 42 & 51.3 & 53.1 & 5.3 & \\
\hline 44 & 37.8 & 36.7 & $5 \mathbf{I}$ & -17.0 & 44 & 37.5 & 37.0 & $5 \mathrm{I}$ & -16.0 & 44 & 52.0 & 52.5 & 53 & -14.0 & 44 & 48.0 & 50.0 & 48 & .14 .0 \\
\hline 46 & 37.1 & 35.7 & 53 & 8 & 46 & 37.2 & 36.6 & 52 & ke & 46 & 51.0 & 52.0 & 51 & i & 46 & 47.1 & 50.0 & 47 & - \\
\hline 48 & 37.8 & 36.8 & $5 I$ & 6 & 48 & 35.8 & 34.8 & 54 & 8 & 48 & 50.5 & 50.8 & 50 & as & 48 & 47.2 & 50.0 & 47 & \\
\hline 50 & 36.6 & 35.9 & 53 & te & 50 & $33 \cdot 3$ & 32.2 & 58 & $1=$ & 50 & 50.5 & 51.0 & 50 & at & 50 & 47.2 & 49.5 & 46 & \\
\hline 52 & 35.9 & 35.1 & 2254 & $\sqrt{2}$ & 52 & 32.2 & 31.7 & 60 & $x$ & 52 & 50.4 & 51.0 & 50 & $N^{3}$ & 52 & 47.0 & 49.5 & 46 & \\
\hline 54 & $31 \cdot 5$ & 30.3 & 2301 & int & 54 & 33.9 & 32.5 & 58 & 3 & 54 & 51.2 & 51.6 & 51 & w & 54 & 47.2 & 50.0 & 47 & \\
\hline 56 & 28.8 & 26.4 & 06 & Bs & 56 & 34.8 & 33.2 & 56 & k & 55.6 & 51.0 & 51.4 & 51 & 8 & 56 & 48.5 & 51.9 & 49 & \\
\hline 58 & 30.8 & 29.1 & $\mathbf{0 3}$ & 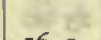 & 58 & 35.6 & $34 \cdot 3$ & 35 & & 58 & 50.8 & 51.0 & 51 & ag vo & 58 & 49.0 & 53.0 & 51 & \\
\hline I $\infty$ & 31.0 & 29.7 & 2302 & -16.9 & 300 & 35.0 & 33.9 & 56 & $-\mathrm{J} 5.8$ & 500 & 50.9 & 51.3 & 51 & -14.0 & 700 & 49.9 & 54.1 & 52 & .14 .0 \\
\hline 02 & 34.0 & 32.8 & 2257 & ta & 02 & 34.6 & 33.7 & 56 & in & 02 & 50.0 & 51.2 & 50 & $x^{2}$ & 02 & 47.1 & 54.1 & 50 & \\
\hline 04 & 35.6 & 34.0 & 55 & 10 & 04 & 33.9 & 33.2 & 57 & $2 n$ & 04 & 50.8 & 52.3 & $5 \mathrm{I}$ & 200 & 04 & 45.8 & 52.1 & 47 & W \\
\hline 06 & 35.8 & 33.8 & 55 & the & 06 & 33.2 & 32.3 & 58 & 16 & 06 & 53.0 & 54.2 & 2255 & 80 & 06 & 45.9 & 51.2 & 47 & \\
\hline 08 & 37.5 & 35.0 & 53 & oft & 08 & $33 \cdot 3$ & 32.7 & 58 & (19t: & 08 & 56.3 & 58.8 & $23 \mathrm{OI}$ & at & 08 & 46.8 & 53.0 & 49 & \\
\hline I0 & 38.2 & 36.2 & 51 & (1) & 10 & 33.7 & 32.9 & 57 & 57 & I0 & 57.7 & 59.5 & 03 & 9 & I0 & 48.9 & 54.2 & $5^{\mathrm{I}}$ & \\
\hline I2 & 38.3 & 36.1 & 51 & 8 & 12 & 33.9 & $33 . \mathrm{I}$ & 57 & & 12 & 56.0 & 58.0 & 2300 & & 12 & 49.9 & 54.9 & 53 & \\
\hline I4 & 38.2 & 36.2 & $5 I$ & -16.8 & 14 & 33.4 & 32.8 & 58 & -15.8 & 14 & 55.0 & 57.0 & 2259 & -14.2 & 14 & 49.4 & 53.0 & $5 \mathrm{I}$ & -14.0 \\
\hline 16 & 38.4 & 36.3 & 51 & & 16 & 33.0 & 32. I & 59 & & I6 & 53.9 & $55 \cdot 3$ & 56 & & I6 & 47.9 & 50.0 & 48 & \\
\hline 18 & 38. I & 36.2 & $5 I$ & $n$ & 18 & 33.6 & 32.2 & 58 & $2=$ & 18 & 50.6 & 52.7 & 52 & & 18 & 47.8 & 50.3 & 48 & \\
\hline 20 & 38. I & 36.2 & 51 & 2 & 20 & $34 \cdot 3$ & 33.0 & 57 & 10 & 20 & 49.9 & 5 I.I & 50 & $=$ & 20 & 48.5 & 50.0 & 48 & \\
\hline 22 & 38.5 & 36.7 & $5 I$ & $x$ & 22 & 36.7 & 34.2 & 54 & 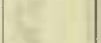 & 22 & 51.7 & 52.3 & 52 & 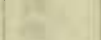 & 22 & 49.5 & 50.0 & 49 & \\
\hline 24 & 39.0 & 37.2 & 50 & se & 24 & 37.8 & 35.2 & 52 & & 24 & 54.5 & 55.0 & 57 & se & 24 & 50.6 & 51.9 & 51 & \\
\hline 26 & 38.8 & 37.0 & 50 & a & 26 & 38.4 & 36.2 & 51 & & 26 & 53.1 & 55.3 & 56 & & 26 & 48.3 & 50.6 & 48 & \\
\hline 28 & 38.8 & 36.9 & 50 & & 28 & 38.6 & 36.8 & 51 & & 28 & 52.2 & 54.6 & 54 & & 28 & $49 . I$ & 51.9 & 50 & \\
\hline 30 & 39.2 & 37.3 & 50 & -16.5 & 30 & 38. I & 36.5 & $5 I$ & -15.7 & 30 & 52.0 & 54.5 & 54 & -14.2 & 30 & 48.8 & 51.5 & 49 & -14.0 \\
\hline 32 & 39.1 & 37.0 & 50 & 130 & 32 & 38.2 & 37.1 & 51 & & 32 & $5 \mathrm{I} . \mathrm{I}$ & 53.0 & 52 & $x^{2}$ & 32 & 49.9 & 52.5 & 51 & \\
\hline 34 & 38.3 & 36.2 & $5 I$ & 8 & 34 & 38.4 & 37.6 & 50 & F & 34 & 51.8 & 53.7 & 53 & 82 & 34 & 51.0 & 54.0 & 53 & \\
\hline 36 & 38.0 & 35.2 & 52 & ne & 36 & 37.3 & 36.8 & 52 & ais & 36 & 50.2 & 53.0 & 51 & 2 & 36 & 50.7 & 52.7 & 51 & \\
\hline 38 & $37 \cdot 3$ & 34.7 & 53 & ans & 38 & 36.8 & 36.0 & 53 & 10 & 38 & 49.0 & 51.0 & 49 & a & 38 & 51.6 & 53.5 & 53 & \\
\hline 40 & 38. I & 34.7 & 53 & in: & 40 & 36.7 & 35.5 & 53 & 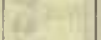 & 40 & 48.9 & 50.1 & 48 & $\theta$ & 40 & 52.5 & 54.5 & 55 & \\
\hline 42 & 36.8 & 34.6 & 54 & & 42 & 36.7 & 35.1 & 53 & & 42 & 47.7 & 49.1 & 47 & 10 & 42 & 51.7 & 53.0 & 53 & \\
\hline 44 & 37.4 & 35.5 & 53 & -16.2 & 44 & 36.8 & $35 . \mathrm{I}$ & 53 & -15.3 & 44 & 47.0 & 48.8 & 46 & $-14 \cdot 3$ & 44 & 51.2 & 52.0 & 52 & -14.0 \\
\hline 46 & 36.8 & 35.1 & 53 & ate & 46 & 36.6 & 35.0 & 53 & wat & 46 & 49.5 & 52.0 & 50 & f & 46 & 51.0 & 53. I & 53 & \\
\hline 48 & 36.1 & $34 \cdot 3$ & 54 & ses & 48 & 36.0 & 34.8 & 54 & $=$ & 48 & $53 \cdot 3$ & 55.6 & 56 & 16 & 48 & 51.5 & 53.0 & 53 & \\
\hline 50 & 37.1 & 35.2 & 53 & k & 50 & 34.7 & 33.9 & 56 & ine & 50 & 55.3 & 58.1 & 60 & $R$ & 50 & 51.8 & 52.1 & 52 & \\
\hline 52 & 37.8 & 36.4 & 5I & 9 & 52 & 34.0 & 33.1 & 6. 57 & w & 52 & 55.0 & 57.0 & 59 & 8 & 52 & 50.0 & 50.3 & 49 & \\
\hline 54 & 38.2 & 36.7 & $5 I$ & Fis & 54 & 35.2 & $34 \cdot 3$ & 55 & E & 54 & 51.0 & & 52 & 18 & 54 & 47.0 & 52.0 & 48 & \\
\hline 56 & 37.7 & 35.9 & 52 & wo $=18$ & 56 & 36.1 & $35 . \mathrm{I}$ & 54 & & 56 & 51.0 & 53.8 & 53 & 100 ts & 56 & 47.1 & 52.0 & 48 & \\
\hline 58 & 37.8 & 36.2 & 52 & & 58 & 35.8 & $35 . I$ & 54 & & 58 & 52.0 & 55.0 & 55 & & 58 & 44.9 & 56.0 & 50 & \\
\hline & & & & & & & & & & & & & & & 800 & 48.0 & 54.0 & $5 I$ & -14.0 \\
\hline
\end{tabular}


Tabulation of magnetic declinations observed at Teplitz Bay-Continued

\begin{tabular}{|c|c|c|c|c|c|c|c|c|c|c|c|c|c|c|c|c|c|c|c|}
\hline \multicolumn{5}{|c|}{ Monday, Fiebruary 22, 1904} & \multicolumn{5}{|c|}{ Magnet scale inverted } & \multicolumn{5}{|c|}{ Tuesday, February 23, 1904} & \multicolumn{5}{|c|}{ Magnet scale erect } \\
\hline \multirow{2}{*}{$\begin{array}{l}\text { Chr'r } \\
\text { time }\end{array}$} & \multicolumn{2}{|c|}{$\begin{array}{l}\text { Scale } \\
\text { readings }\end{array}$} & \multirow[t]{2}{*}{$\begin{array}{l}\text { East } \\
\text { decli- } \\
\text { nation }\end{array}$} & Temp. & $\begin{array}{l}\text { Chr'r } \\
\text { time }\end{array}$ & $\begin{array}{l}\text { Sc: } \\
\text { read }\end{array}$ & $\begin{array}{l}\text { ale } \\
\text { lings }\end{array}$ & $\begin{array}{l}\text { East } \\
\text { decli- }\end{array}$ & Temp. & $\begin{array}{l}\text { Chr'r } \\
\text { time }\end{array}$ & $\begin{array}{l}\text { Sce } \\
\text { read }\end{array}$ & $\begin{array}{l}\text { le } \\
\text { ngs }\end{array}$ & $\begin{array}{l}\text { East } \\
\text { decli- } \\
\text { nation }\end{array}$ & Tems & Chr'r & $\begin{array}{l}\text { Sc: } \\
\text { read }\end{array}$ & $\begin{array}{l}\text { le } \\
\text { ngs }\end{array}$ & $\begin{array}{l}\text { East } \\
\text { decli- }\end{array}$ & Tenıp. \\
\hline & Left & Right & & & & Left & Right & & & & Left & Right & & & & Left & Right & & \\
\hline li $\mathrm{m}$ & d & d & $\circ \quad$ & 0 & $\mathrm{~h} \mathrm{~m}$ & d & d & $\circ 1$ & & $\mathrm{~h} \mathbf{m}$ & d & d & & & $\mathrm{h} \mathrm{m}$ & d & d & $\circ \quad$ & - \\
\hline 800 & 42.2 & 39.8 & 2255 & -16.9 & $10 \infty$ & 50.0 & 45.2 & 2246 & $-13 \cdot 3$ & 1200 & $45 \cdot 9$ & 46.9 & 2227 & -19.4 & 1400 & 42.1 & 43.4 & $222 \mathrm{I}$ & $-19 \cdot 0$ \\
\hline 02 & 43.1 & $4 \mathrm{I} .2$ & 53 & & 02 & 5I.I & 46.4 & 44 & & 02 & 44.6 & $45 \cdot 3$ & 24 & $x^{2}$ & 02 & 44.8 & 45.8 & 25 & -6 \\
\hline 04 & 44.6 & 41.3 & 52 & 10 & 04 & 5 I. I & 47.2 & 44 & is & 04 & 44.8 & 45.8 & 25 & $x 2$ & 04 & 45.7 & 46.3 & 26 & 10 \\
\hline 26 & 46.0 & $43 \cdot 3$ & 50 & 100 & 06 & 52.3 & $47 \cdot 9$ & 42 & $\theta$ & $\infty 6$ & 43.9 & 44.0 & 23 & 2 & $\infty 6$ & 46.7 & $47 \cdot 3$ & 28 & oy \\
\hline os & 46.9 & 44.8 & 48 & 20 & 08 & 51.7 & 48.0 & 43 & 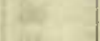 & 08 & 42.8 & 44.2 & 22 & 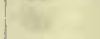 & 08 & 48.2 & 48.7 & 30 & Des \\
\hline IO & 47.6 & $45 \cdot 3$ & 47 & ont & Io & 51.8 & 48.2 & 42 & 4 & Io & $-44 \cdot 8$ & $45 \cdot 3$ & 25 & fin & Io & 48.2 & 48.4 & 30 & ar \\
\hline I 2 & 47.2 & 46.8 & 46 & our & 12 & 50.2 & $47 \cdot 5$ & 44 & wit & 12 & $44 \cdot 5$ & 45.8 & 25 & 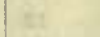 & I2 & 48.5 & 48.9 & 30 & 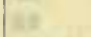 \\
\hline 14 & 49.8 & 48.2 & 43 & $-15 \cdot 5$ & I4 & 49.0 & 46.8 & 46 & -13.2 & I4 & 47.2 & 49.1 & 29 & -20.0 & 14 & 48.0 & 48.4 & 30 & -19.0 \\
\hline 16 & 50.1 & 48.9 & 42 & 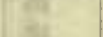 & I6 & $47 \cdot 3$ & 45.8 & 48 & & 16.3 & 47.6 & 49.9 & 30 & his & 16 & $47 \cdot 2$ & 48.2 & 30 & 04 \\
\hline I8 & $49 \cdot 3$ & 48.1 & 43 & wis. & I8 & 47.1 & 45.6 & 48 & (a) & 18 & 48.5 & 50.1 & $3 I$ & 3 & 18 & 46.9 & 48 & 29 & nt \\
\hline 20 & 46.5 & $45 \cdot 3$ & 48 & 18 & 20 & 46.2 & 45.1 & 50 & ats & 20 & 49.2 & 50.1 & 32 & c) & 20 & 47.2 & 48.6 & 29 & 10 \\
\hline 22 & 46.2 & $45 . I$ & 48 & a & 22 & 47.2 & 46.7 & 48 & ats & 22 & 45.9 & $47 \cdot 4$ & 27 & Lat & 22 & 49.2 & 50.1 & 32 & $x$ \\
\hline 24 & 45.9 & $45 \cdot 3$ & 48 & 13 & 24 & 48.2 & 47.6 & 46 & t5 & $24 \cdot 4$ & $45 . I$ & 46.2 & 25 & is & 24 & 49.1 & 49.9 & 32 & is \\
\hline 26 & 46.0 & $45 . I$ & 48 & an & 26 & 49.2 & $47 \cdot 3$ & 46 & 60 & 26 & 49.2 & 50.2 & 32 & 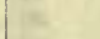 & 26 & 48.9 & 49 & 31 & 4 \\
\hline 28 & 42.8 & 42.0 & 53 & 20 & 28 & 49.8 & 47.9 & 45 & & 28 & 49. I & 50.8 & 32 & $x^{2}$ & 28 & 48.7 & 49 & 31 & $x^{2}$ \\
\hline 36 & 46.3 & 45.0 & 48 & $-14 \cdot 7$ & 30 & 49.6 & $47 \cdot 3$ & 45 & $-I 3.2$ & 30 & 43.8 & 45.2 & 24 & -20.0 & 30 & 48.4 & 48.9 & 30 & -19.0 \\
\hline 32 & 46.6 & 45.3 & 48 & & 32 & 49.9 & 47.6 & 45 & & 32 & 39.8 & 41.0 & 17 & $56=$ & 32 & $47 \cdot 4$ & 48.0 & 29 & $x^{2}$ \\
\hline 34 & $47 \cdot 3$ & 46.2 & 47 & 8r & 34 & 49.5 & $47 \cdot 5$ & 45 & $\sqrt{2}$ & 34 & 38.8 & 39.9 & I6 & 13 & 34 & $47 \cdot 4$ & $47 \cdot 9$ & 29 & $=$ \\
\hline 36 & 48.2 & 47.1 & 45 & set & 36 & 49.6 & $47 \cdot 4$ & 45 & 4 & 36 & 40.2 & $41 \cdot 4$ & I8 & $n$ & 36 & 48. I & 48.6 & 30 & (y. \\
\hline 38 & 50.0 & $47 \cdot 3$ & 44 & 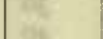 & 38 & 49.9 & 48.1 & 44 & na & 38 & 32.2 & 32.8 & 05 & be & 38 & 49.8 & 50.0 & 32 & 4 \\
\hline 40 & 49.6 & $47 \cdot 5$ & 44 & & 40 & 50.2 & $48 . I$ & 44 & 6 & 40 & 35.8 & 37.8 & 12 & ry & 40 & 50.2 & 50.8 & 33 & 0 \\
\hline 42 & $49 \cdot 3$ & 46.7 & 45 & & 42 & 50.0 & 48.3 & 44 & 4 & 42 & 37.8 & 38.4 & I4 & 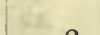 & 42 & 49.8 & 50.0 & 32 & \\
\hline 44 & $49 . I$ & 46.8 & 45 & $-14 \cdot 3$ & 44 & 50.1 & 48.8 & 44 & -13.1 & 44 & 37.2 & 38.1 & 13 & -19.8 & 44 & $49 \cdot 3$ & 50.1 & 32 & -18.9 \\
\hline 46 & 48.3 & 45.8 & 46 & ( & 46 & 49.8 & 47.6 & 45 & & 46 & 39.7 & 40.2 & 17 & $6 y$ & 46 & 49.1 & $49 \cdot 5$ & $3 I$ & $=$ \\
\hline 48 & 46.9 & $44 \cdot 7$ & 48 & min & 48 & 49.6 & 48.7 & 44 & & 48 & 40.3 & $4 I . I$ & I8 & 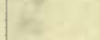 & 48 & 46.9 & $47 \cdot 9$ & 28 & - \\
\hline 50 & $47 \cdot 3$ & 45.6 & 47 & $=1$ & 50 & 49.9 & 48.6 & 44 & 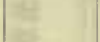 & 50 & 41.2 & $44 . I$ & 21 & in: & 50 & 45.2 & 46.0 & 25 & $y^{2}$ \\
\hline 52 & 47.9 & 45.8 & 47 & e & 52 & 49.4 & 48.0 & 45 & 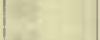 & 52 & 40.8 & 43.8 & 20 & 17 & 52 & 43.0 & 43.2 & 22 & 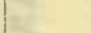 \\
\hline 54 & 9.0 & $47 \cdot 2$ & 45 & & 54 & $49 \cdot 4$ & 48.2 & 45 & 1 & 54 & 41.7 & 45 & 22 & 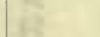 & 54 & 43.0 & 43.6 & 22 & \\
\hline 56 & 50.8 & 48.2 & 42 & & 50 & $49 \cdot 5$ & $47 \cdot 7$ & 45 & 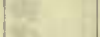 & 56 & 42.8 & $46 . I$ & 24 & & 56 & 44.0 & 44.9 & 24 & \\
\hline 58 & 49.0 & 47.8 & 44 & & 58 & 48.7 & 47.1 & 46 & net & 58 & 42.5 & 46.9 & 24 & & 58 & 45.9 & 46.8 & 27 & \\
\hline 900 & 49.8 & 48.5 & 43 & -14.0 & II 00 & 48.9 & 47.5 & 46 & -13.1 & 1300 & 43.1 & 46.8 & 25 & -19.7 & 1500 & 45.8 & 40.8 & 27 & -18.9 \\
\hline 02 & 48.I & $47 \cdot 3$ & 45 & & 02 & $49 \cdot 3$ & 47.9 & 45 & & 02 & 42.4 & 45.8 & 23 & $x^{2}$ & $\infty 2$ & 45.7 & 46.8 & 26 & 1 \\
\hline 04 & 48.6 & $47 \cdot 3$ & 45 & to & 04 & 49.9 & 47.9 & 45 & 116 & 04 & 44.3 & $47 \cdot 3$ & 26 & th & 04 & 46.0 & $47 \cdot 4$ & 27 & - \\
\hline 05 & 48.9 & 48.0 & 44 & 3 & 06 & 50.2 & 47.7 & 45 & 1 & 06 & 44.6 & $47 \cdot 1$ & 26 & $x$ & $\infty 6$ & 46.8 & 47.9 & 29 & N \\
\hline 08 & 48.8 & 46.1 & 46 & 80 & 08 & 49.8 & 47.3 & 46 & 6 & 08 & 45.1 & 48.8 & 28 & t & 08 & $47 \cdot 3$ & 48.7 & 30 & 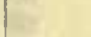 \\
\hline 10 & 47.6 & 46.2 & 47 & 68 & 10 & 50.2 & 47.2 & 45 & in & IO & $43 \cdot 7$ & 46.3 & 25 & W & IO & 49.2 & 50.1 & 32 & H \\
\hline 12 & $47 \cdot 4$ & 46.3 & 47 & (1) & 12 & 49.9 & 47.2 & 46 & L1 & 12 & $47 \cdot 7$ & 50.0 & $3 I$ & & 12 & $49 \cdot 3$ & 50.3 & 32 & \\
\hline 14 & $47 \cdot 6$ & 47.0 & 46 & -13.8 & 14 & 48.9 & 46.2 & 47 & -13.0 & I4 & 46.8 & 49.8 & 30 & -19.7 & I4 & 50.1 & 50.9 & 33 & -18.8 \\
\hline 16 & 48.8 & 47.3 & 45 & & 16 & 51.2 & 48.3 & 44 & 10 & 16 & 45.4 & 48.3 & 27 & ( & 16 & 49.2 & 50.2 & 32 & 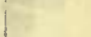 \\
\hline 18 & $47 \cdot 3$ & 46.8 & 47 & Ift & 18 & 51.2 & 49.0 & 43 & in & I8 & 46.7 & $49 \cdot 3$ & 29 & $z_{1}$ & 18 & 49.2 & 50.1 & 32 & 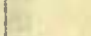 \\
\hline 20 & 48.0 & 46.4 & 46 & ox & 20 & 51.4 & 48.9 & 43 & $1=$ & 20 & 44.2 & 47.0 & 25 & 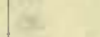 & 20 & 49.8 & 50.4 & 33 & 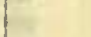 \\
\hline 22 & 47.6 & 45.8 & 47 & 25 & 22 & 51.7 & 48.8 & 43 & 1 & 22 & 43.5 & 45.8 & 24 & 5 & 22 & 49.2 & 50.0 & 32 & 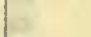 \\
\hline 24 & 47.2 & 45.2 & 48 & 36 & 24 & 51.0 & 48.2 & 44 & 10 & 24 & 43.1 & 45.0 & 23 & nat & 24 & 49.6 & 50.3 & 32 & - \\
\hline 26 & 46.3 & 43.6 & 50 & \% & 26 & 51.1 & 48.7 & 44 & 18 & 26 & $44 \cdot 4$ & $49 \cdot 3$ & 27 & 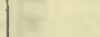 & 26 & 49.8 & 50.9 & 33 & r \\
\hline 28 & 49.9 & $47 \cdot 3$ & 44 & 10 & 28 & 50.7 & 48.8 & 44 & ta & 28 & 44. I & 46.0 & 25 & & 28 & 49.4 & 50.8 & 33 & \\
\hline 30 & 50.4 & 48.3 & 43 & -13.5 & 30 & 52.3 & 47.5 & 44 & -13.0 & 30 & 42.7 & $44 . I$ & 22 & -19.6 & 30 & 48.3 & 49.7 & 31 & -18.8 \\
\hline 32 & $46 . I$ & $44 \cdot 4$ & 50 & 10 & 32 & 51.9 & 47.2 & 44 & $t=$ & 32 & 37.2 & 39.9 & I4 & 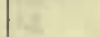 & 32 & 45.8 & 47.0 & 27 & ! \\
\hline 34 & $47 \cdot 2$ & 46.7 & 47 & $x$ & 34 & 51.6 & 46.5 & 45 & $1=$ & 34 & $33 . I$ & 35.5 & 08 & 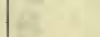 & 34 & $44 \cdot 3$ & $45 \cdot 4$ & 24 & 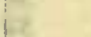 \\
\hline 36 & 48.3 & 47.0 & 46 & 10 & 36 & 51.0 & 46.3 & 46 & 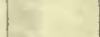 & 36 & 32.1 & 33.9 & 06 & $i^{2}$ & 36 & 43.7 & 44.6 & 23 & 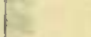 \\
\hline 38 & 48.2 & $47 \cdot 3$ & 46 & (n) & 38 & 50.9 & 46.4 & 46 & Le & 38 & 3 I. 8 & 33.9 & 05 & wit & 38 & 43.2 & 44.2 & 23 & 4 \\
\hline 40 & 49.7 & $47 \cdot 4$ & 44 & 18 & 40 & 51.2 & $47 \cdot 3$ & 45 & 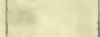 & 40 & 29. I & 31.7 & 02 & & 40 & $4 I .5$ & 42.9 & 20 & 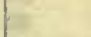 \\
\hline 42 & 48.6 & 47.2 & 45 & 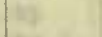 & 42 & 51.6 & 47.8 & 44 & 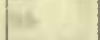 & 42 & 29.8 & 32.2 & 03 & & 42 & 41.2 & 42.1 & I9 & \\
\hline 44 & 49.0 & 46.6 & 45 & $-13 \cdot 5$ & 44 & 51.8 & $48 . I$ & 44 & -12.9 & 44 & $28 . I$ & 30.2 & $\infty$ & -19.3 & 44 & 41.9 & 42.3 & 20 & -18.8 \\
\hline 46 & $49 \cdot 3$ & 47.1 & 45 & (2) & 46 & 52.2 & 48.0 & 44 & 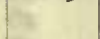 & 46 & 28.8 & $3 I .4$ & OI & $\because$ & 46 & 39.9 & 40.9 & 17 & 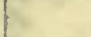 \\
\hline 48 & 48.8 & 48.0 & 45 & 4 & 48 & 52.3 & 48. I & 44 & $1=$ & 48 & 28.5 & 30.3 & $\infty$ & 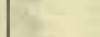 & 48 & 39.1 & 40.0 & 16 & 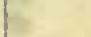 \\
\hline 50 & 49.8 & 46.7 & 45 & $1=$ & 50 & 52.1 & 48.4 & 44 & 1 & 50 & 28. I & 30.3 & $\infty$ & & 50 & 37.8 & 38.0 & I3 & 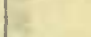 \\
\hline 52 & 49.0 & 45.4 & 47 & 17 & 52 & 52.3 & 49.2 & 43 & $a$ & 52 & $3 I . I$ & 33.2 & 04 & 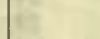 & 52 & $37 \cdot 4$ & 38.1 & I3 & $y$ \\
\hline 54 & 49.2 & $45 . I$ & 47 & 18 & 54 & 52.5 & 48.9 & 43 & 3 & 54 & 33.4 & 35.8 & 08 & $y$ & 54 & 37.9 & 38.4 & 14 & 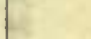 \\
\hline 56 & 49.7 & $45 \cdot 3$ & 46 & 3 & 56 & 52.4 & 48.8 & 43 & 3 & 56 & 36.2 & 38.2 & 12 & & 56 & 36.7 & 37.2 & 12 & 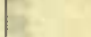 \\
\hline 58 & 50.1 & 44.9 & 46 & 8 & 58 & 53.8 & 48.4 & 42 & & 58 & 38.3 & 40.7 & 16 & $x^{2}$ & 58 & 37.8 & 38.8 & I4 & \\
\hline & & & & & I2 $\infty$ & 51.6 & 49.7 & 43 & -12.5 & & & & & & 1600 & 40.2 & 40.9 & I8 & -18.6 \\
\hline
\end{tabular}

Correction to local mean time is $-2 \mathrm{~m} 39.5 \mathrm{~s} .90^{\circ}$ torsion $=14 .^{\prime} 57$. Torsion head read $36^{\circ}$ and $14^{\circ}$ at beginning and end respectively. Observer-H. H. N.
Correction to local mean time is - Im o6s. $90^{\circ}$ torsion $=16 .^{\prime} 9 \mathrm{r}$. Torsion head read $30^{\circ}$ and $40^{\circ}$ at beginning and end respectively. Observer-R. R. T. 
Tabulation of magnetic declinations observed at Teplitz Bay-Continued

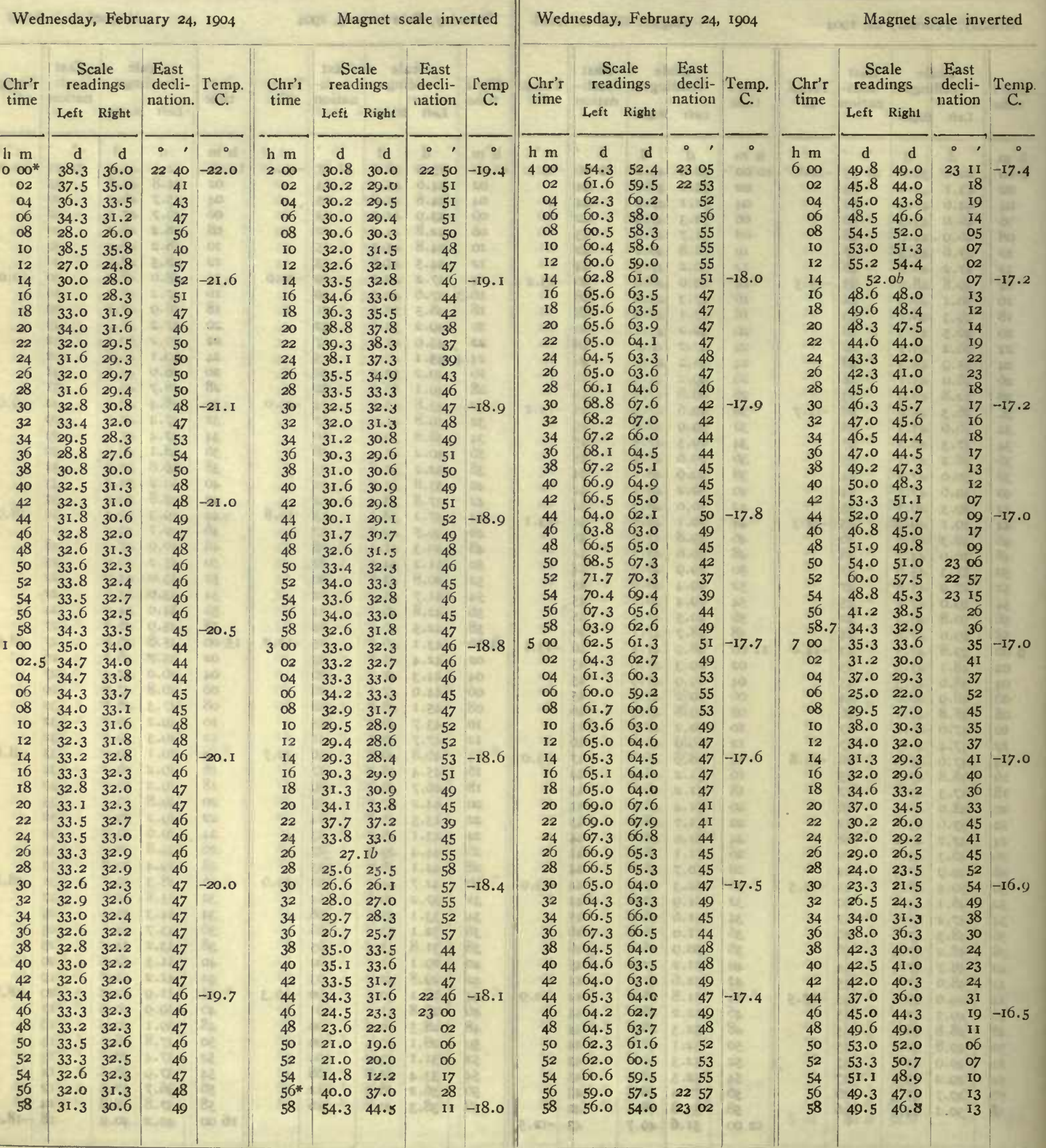

Observer-W. J. P.
Observers-W. J. P. and J. V., who alternated from $7 \mathrm{~h} 46 \mathrm{~m}$ to 7h $52 \mathrm{~m}$. 
Tabulation of magnetic declinations observed at Teplitz Bay-Continued

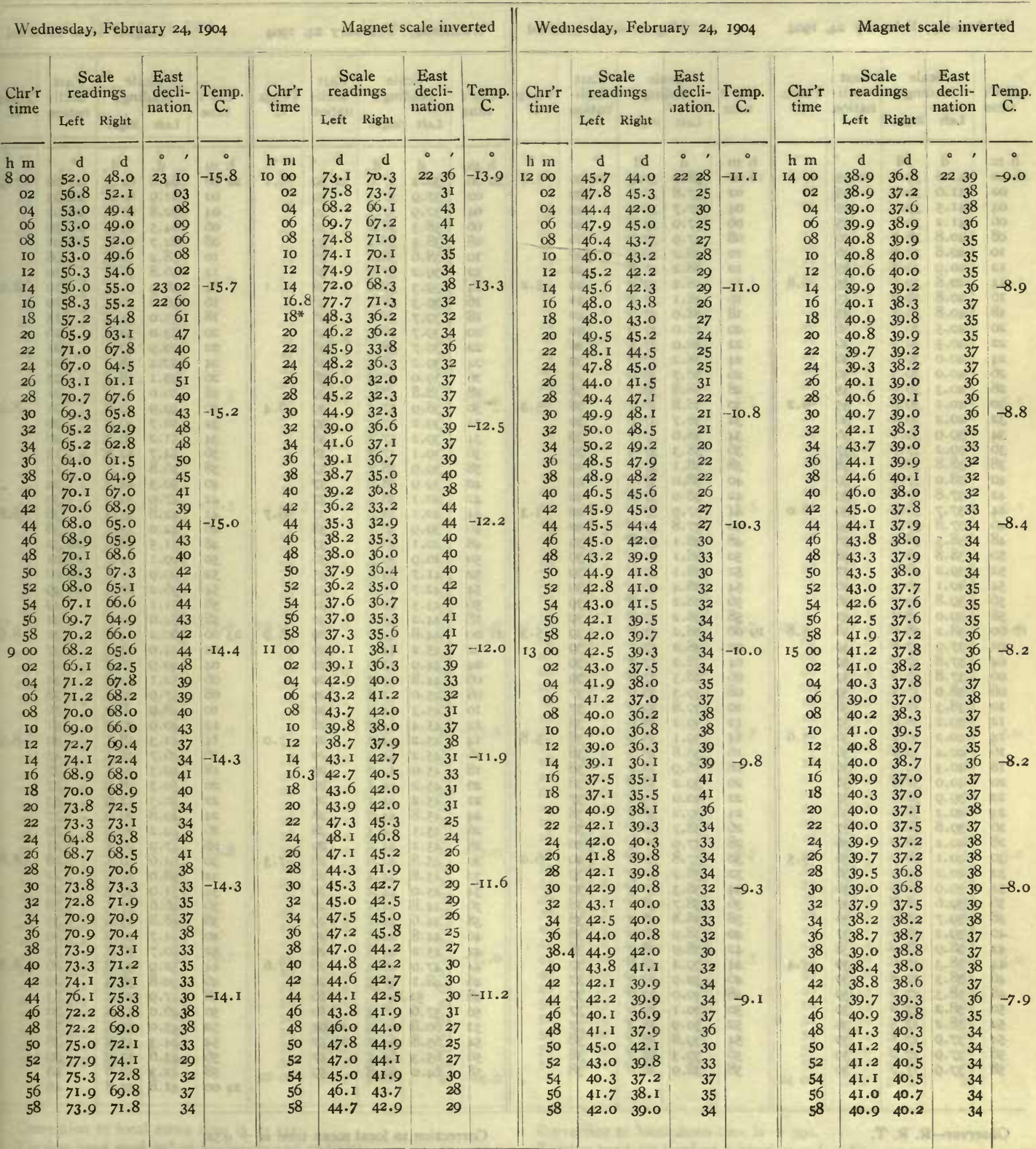

Observer-J. V.

Observers-J. V. and R. R. T., who alternated from $15 \mathrm{~h}$ 50m to I6h oom. 
Tabulation of magnetic declinations observed at Teplitz Bay-Continued

\begin{tabular}{|c|c|c|c|c|c|c|c|c|c|c|c|c|c|c|c|c|c|c|c|c|}
\hline \multicolumn{2}{|c|}{ Wednesday, } & \multicolumn{3}{|c|}{ February 24, 1904} & \multicolumn{5}{|c|}{ Magnet scale inverted } & \multicolumn{5}{|c|}{ Wednesday, February 24, 1904} & \multicolumn{6}{|c|}{ Magnet scale inverted } \\
\hline \multirow{2}{*}{$\begin{array}{l}\text { Chr'r } \\
\text { time }\end{array}$} & $\begin{array}{r}\text { Sca } \\
\text { read }\end{array}$ & $\begin{array}{l}\text { ale } \\
\text { lings }\end{array}$ & $\begin{array}{c}\text { East } \\
\text { decli- }\end{array}$ & Temp. & Chr'r & $\begin{array}{r}\text { Sca } \\
\text { readi }\end{array}$ & $\begin{array}{l}\text { ale } \\
\text { ings }\end{array}$ & $\begin{array}{l}\text { East } \\
\text { decli- }\end{array}$ & Temp. & Chr's & $\begin{array}{l}\text { Sca } \\
\text { read }\end{array}$ & $\begin{array}{l}\text { ale } \\
\text { lings }\end{array}$ & East & Temp. & Chr's & $\begin{array}{r}\text { Sca } \\
\text { readi }\end{array}$ & $\begin{array}{l}\text { ale } \\
\text { ings }\end{array}$ & East & st & Temp. \\
\hline & Left & Right & & C. & time & Left & Right & & C. & time & Left & Right & nation. & C. & time & Left & Right & & & \\
\hline h $\mathbf{m}$ & d & d & $\cdot$, & $\cdot$ & h m & d & d & $\cdot$, & $\cdot$ & $\mathrm{h} \mathrm{m}$ & d & d & $\cdot$, & 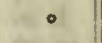 & $\mathrm{h} \mathrm{m}$ & d & d & - & & - \\
\hline 1600 & 41.6 & 39.2 & 2235 & -7.0 & 1800 & 38.0 & $37 \cdot 3$ & 2239 & $-5 \cdot 4$ & 2000 & 38.4 & 37.6 & 2239 & -6.1 & 2200 & 64.0 & 62.3 & 216 & 60 & $-7 \cdot 9$ \\
\hline 02 & 41.6 & 39.2 & 35 & 10 & 02 & 37.8 & 37.2 & 39 & & 02 & 37.9 & 37.7 & 39 & & 02 & 61.9 & 60.2 & 220 & 03 & \\
\hline 04 & 41.5 & 39.2 & 35 & Wes & 04 & 37.7 & 37.0 & 39 & 19 & 04 & 37.9 & 37.7 & 39 & & 04 & 62.8 & 61.0 & 220 & 02 & \\
\hline 06 & 41.2 & 39.1 & 35 & Sor & 06 & 37.9 & 37.0 & 40 & 100 & 06 & 38.3 & 38. & 39 & & 06 & 64.5 & 63.4 & 215 & 59 & \\
\hline 08 & 40.8 & 38.7 & 36 & ins & 08 & 37.9 & 37.7 & 40 & (6) & 08 & 38.5 & 38.1 & 39 & 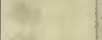 & 08 & 62.7 & 62.0 & 220 & & \\
\hline 10 & 40.8 & 37.8 & 36 & 0 & Io & 38.2 & 36.9 & 40 & 67 & IO & 38.6 & 38.2 & 38 & 01 & I0 & 58.2 & 58.0 & & 08 & \\
\hline 12 & 40.9 & 39.1 & 35 & & 12 & 38.2 & 37.0 & 40 & 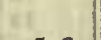 & 12 & 39.2 & 37.9 & 38 & (1) & 12 & 56.8 & 56.8 & & 10 & \\
\hline 14 & 40.8 & 39.0 & 35 & -6.8 & 14 & 38.2 & 37.2 & 40 & -5.2 & 14 & 39.3 & 38.0 & 38 & -6.1 & I4 & 56.9 & 56.1 & & I0 & -8.0 \\
\hline 16 & 40.8 & 39.2 & 35 & chit & 16 & 38.1 & 37.3 & 40 & $x^{2}$ & 16 & 39.3 & 38.1 & 38 & & 16 & 56.8 & 56.1 & & Io & \\
\hline 18 & 40.8 & $39 . I$ & 35 & है. & I8 & 38.0 & 37.2 & 40 & Bi & 18 & 39.7 & 38.2 & 38 & 1 & I8 & 55.0 & 54.2 & & I3 & \\
\hline 20 & 40.5 & 39.1 & 36 & os & 20 & 38.0 & 37.2 & 40 & $\alpha$ & 20 & 39.7 & 38.2 & 38 & $\cos$ & 20 & 53.0 & 52.4 & & 16 & \\
\hline 22 & 40.5 & 39.2 & 36 & $=$ & 22 & 38.0 & $37 \cdot 5$ & 40 & a & 22 & 39.7 & 38.1 & 38 & as & 22 & 49.9 & $49 \cdot 3$ & & $2 \mathrm{I}$ & \\
\hline 24 & 40.0 & 39.1 & 36 & 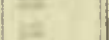 & 24 & 38.2 & 37.8 & 39 & is & 24 & 39.2 & 38.2 & 38 & is & 24 & 49.4 & 48.3 & & 22 & \\
\hline 26 & 40.0 & 39.3 & 36 & 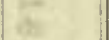 & 26 & 38.7 & 38.0 & 39 & 8 & 26 & 39.0 & 37.9 & 38 & ot & 26 & $49 . I$ & 48.1 & & 23 & \\
\hline 28 & 40.3 & 39.6 & 35 & & 28 & 38.8 & 38.1 & 38 & & 28 & 38.8 & 37.4 & 39 & & 28 & 50.0 & 49.4 & & $2 \mathrm{I}$ & \\
\hline 30 & 40.2 & 39.8 & 35 & -6.2 & 30 & 38.8 & 38.1 & 38 & $-5 \cdot 3$ & 30 & 38.9 & 37.5 & 39 & -6.4 & 30 & 49.9 & 49.6 & & $2 I$ & -8.2 \\
\hline 32 & 40.2 & 39.5 & 36 & & 32 & 38.7 & 37.8 & 39 & 8 & 32 & 39.2 & 37.4 & 39 & $=5$ & 32 & 50.7 & 49.6 & & 20 & \\
\hline 34 & 39.9 & 39.1 & 36 & 6 & 34 & 38.6 & 37.7 & 39 & 92 & 34 & 39.2 & 37.8 & 38 & is. & 34 & 51.2 & 49.2 & & 20 & \\
\hline 36 & 39.2 & 37.7 & 37 & N2 & 36 & 38.3 & 37.4 & 39 & 2 & 36 & 39.1 & 37 & 38 & $g$ & 36 & 54. & 52.8 & & 15 & \\
\hline 38 & 38.8 & 38.1 & 38 & 10 & 38 & 38.1 & 37.1 & 40 & $=$ & 38 & 39.9 & 38. & 37 & & 38 & 56.8 & 5I.I & & 14 & \\
\hline 40 & 38.0 & 37.5 & 39 & E & 40 & 38.0 & 37.1 & 40 & 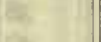 & 40 & 39.9 & 38. & 37 & & 40 & 50.3 & 44.2 & & 24 & \\
\hline 42 & 37.8 & 37.2 & 39 & ait & 42 & 38.0 & 37.2 & 40 & 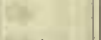 & 42 & $40 . I$ & 38.8 & 37 & & 42 & 47.9 & 39.9 & & 30 & \\
\hline 44 & $37 \cdot 7$ & 36.9 & 39 & -6.0 & 44 & $37 \cdot 9$ & 37.1 & 40 & $-5 \cdot 3$ & 44 & 40.1 & 38. & 37 & -6.7 & 44 & 42.8 & $35 \cdot 3$ & & 38 & -8.8 \\
\hline 46 & 37.8 & 37.2 & 39 & 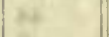 & 46 & 37.8 & 37.2 & 40 & & 46 & 39.9 & 38.1 & 38 & ( & 46 & 42.0 & 35.7 & & 38 & \\
\hline 48 & 38.5 & 38.0 & 38 & 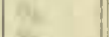 & 48 & 38.1 & 37.8 & 39 & 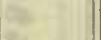 & 48 & $40 . I$ & 38. & 37 & 18 & 48 & $3 \mathrm{I} .5$ & 28.8 & 22 & 52 & \\
\hline 50 & 38.8 & 38.1 & 38 & 8. & 50 & 38. I & 37.9 & 39 & 3 & 50 & 40.9 & 39.3 & 36 & 2 & 50 & $29 . \mathrm{I}$ & 19.7 & 23 & OI & \\
\hline 52 & 39.2 & 38.7 & 37 & lat & 52 & 38.1 & 37.9 & 39 & $E$ & 52 & 41.7 & 39.9 & 35 & a & 52 & 29.9 & 19.7 & $23 c$ & $\infty 0$ & \\
\hline 54 & 40.1 & 39.2 & 36 & 14 & 54 & 38.4 & 38. I & 39 & e & 54 & 42.7 & $4 \mathrm{I} . \mathrm{I}$ & 33 & -2 & 54 & 36.2 & 27.4 & 22 & 49 & \\
\hline 56 & 39.8 & 39.0 & 36 & jy & 56 & 38.2 & 37.8 & 39 & 1.1 & 56 & 43.5 & 42.1 & 32 & 12 & & $5 \mathrm{I} . \mathrm{I}$ & 12.2 & & 49 & \\
\hline 58 & 39.0 & 38.5 & 37 & & 58 & 38.2 & 37.8 & 39 & & 58 & 44.2 & 43.1 & 30 & & 58 & 54.0 & I6.7 & & 44 & \\
\hline 700 & 38.2 & 38.0 & 38 & -5.8 & 1900 & 38.2 & 37.8 & 39 & $-5 \cdot 3$ & 2100 & 44.8 & 44.0 & 29 & -6.9 & 2300 & 60.2 & 24.8 & & 32 & -8.9 \\
\hline 02 & 37.9 & 37.6 & 39 & 6907 & 02 & 38.2 & 37.7 & 39 & $x^{2}$ & 02 & 45.2 & 44.1 & 29 & 95 & 02 & 54.9 & $24 \cdot 3$ & & 37 & \\
\hline 04 & 37.8 & 37.2 & 39 & tas & 04 & 38.2 & 37.7 & 39 & 60 & 04 & 44.9 & 43.8 & 29 & in & 04 & 49.9 & I6.8 & & 47 & \\
\hline$\infty 6$ & 37.8 & 37.2 & 39 & ie & 06 & 38.5 & 37.9 & 39 & 60 & 06 & 44.8 & 43.7 & 29 & id & 06 & $52 . I$ & 22.2 & & $4 \mathrm{I}$ & \\
\hline 08 & 38.0 & 37.5 & 39 & $b s$ & 08 & 39.3 & 38.9 & 37 & $y$ & 08 & 45.0 & 43.8 & 29 & Se. & 08 & 57.9 & 20.2 & & 38 & \\
\hline Io & 38.3 & 37.7 & 38 & 6 & I0 & 39.8 & 39.2 & 37 & 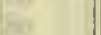 & 10 & 45.3 & 44.0 & 29 & 60 & 10 & 48.9 & 6.0 & & 56 & \\
\hline 12 & 39.2 & 38.8 & 37 & 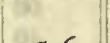 & 12 & 39.2 & 38.9 & 38 & & 12 & 45.4 & 44.2 & 28 & or & 12 & 40.9 & 20.2 & & 51 & \\
\hline 14 & 40.2 & 39.2 & 36 & -5.6 & 14 & 39.1 & 38.9 & 38 & -5.8 & 14 & 45.2 & 44.0 & 29 & -7 & 14 & 42.3 & I8. I & & 52 & -8.9 \\
\hline 16 & 40.4 & 39.2 & 36 & & 16 & 38.8 & 38.3 & 38 & & 16 & 45.2 & 44.0 & 29 & & 16 & 46.7 & $25 . I$ & & 43 & \\
\hline 18 & 40.3 & 39.3 & 36 & ex. & 18 & 38.9 & 38.2 & 38 & & 18 & 45.0 & 44.0 & 29 & ha & 18 & $49 \cdot 3$ & 29.1 & & 37 & \\
\hline 20 & 40.0 & 38.8 & 36 & 20 & 20 & 39.2 & 38.8 & 38 & 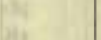 & 20 & 45.2 & $44 . I$ & 29 & 4 & 20 & 50.3 & 28.9 & & 37 & \\
\hline 22 & 39.9 & 38.6 & 37 & or & 22 & 40.0 & 39.2 & 37 & II & 22 & 45.2 & 44.1 & 29 & 6 & 22 & 40.3 & 17.9 & & 53 & \\
\hline 24 & 40.2 & 38.9 & 36 & $\sigma$ & 24 & 39.2 & 38.8 & 38 & 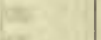 & 24 & 45.8 & 44.8 & 28 & car & 24 & 49.8 & 29.0 & & 37 & \\
\hline 26 & 39.8 & 38.2 & 37 & Es & 26 & 38.7 & 38.1 & 38 & ax & 26 & 45.5 & $44 \cdot 3$ & 28 & 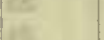 & 26 & 49.2 & 26.7 & & 39 & \\
\hline 28 & 40.2 & 38.9 & 36 & $3=$ & 28 & 37.9 & 37.4 & 40 & & 28 & 45.2 & 44.2 & 29 & & 28 & $47 \cdot 9$ & 26.1 & & $4 \mathrm{I}$ & \\
\hline 30 & 40.1 & 38.9 & 36 & -5.5 & 30 & 37.8 & 37.2 & 40 & -6.0 & 30 & 45.8 & 44.6 & 28 & $-7 \cdot 3$ & 30 & 54.6 & 36.1 & & 28 & .8 .8 \\
\hline 32 & 40.2 & 39.0 & 36 & & 32 & 38.2 & 37.7 & 39 & & 32 & 45.8 & 44.4 & 28 & & 32 & 58.0 & 40.3 & & 2 & \\
\hline 34 & 39.9 & 38.8 & 36 & 2 & 34 & 38.3 & 37.9 & 39 & ty & 34 & 45.0 & 44.2 & 29 & 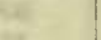 & 34 & 5 I. 0 & 39.3 & & 28 & \\
\hline 36 & 39.6 & 38.8 & 37 & ars. & 36 & 38.7 & 38.2 & 38 & $E$ & 36 & 44.8 & 44.1 & 29 & & 36 & 46.6 & 3I.I & & 38 & \\
\hline 38 & 39.7 & 38.9 & 36 & 14. & 38 & 38.8 & 38.2 & 38 & 08 & 38 & 44.6 & 44.1 & 29 & 18 & 38 & 48.8 & 33.2 & & 35 & \\
\hline 40 & 39.2 & 38.6 & 37 & S. & 40 & 38.9 & 38.2 & 38 & 2 & 40 & 44.8 & 44.1 & 29 & s. & 40 & 44.7 & 30.2 & & 10 & \\
\hline 42 & 38.9 & 38.0 & 38 & 8ic. & 42 & 38.9 & 38.2 & 38 & 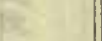 & 42 & 45.2 & 44.6 & 28 & 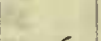 & 42 & 50.7 & 36.7 & & 30 & \\
\hline & 38.3 & 37.6 & 39 & -5.4 & 44 & 39.4 & 38.8 & 37 & 8 & 44 & 47.0 & 46.2 & 26 & -7 & 44 & 52.9 & 39.8 & & 26 & -8.8 \\
\hline 46 & 38.3 & 37.2 & 39 & t & 46 & 39.9 & 39.0 & 37 & a. & 46 & 48.1 & 47.7 & 24 & & 46 & 50.8 & 38.3 & & 29 & \\
\hline 48 & 38.3 & 37.2 & 39 & 3 & 48 & 40.0 & 39.0 & 37 & tat & 48 & 52. & $.8 a$ & 16 & ii & 48 & 47.9 & 36.1 & & 33 & \\
\hline 50 & 38.2 & 37.6 & 39 & in & 50 & 40.1 & 39.0 & 37 & & 50 & 57.6 & 56.2 & Io & 3 & 50 & 46.0 & 33.8 & & 30 & \\
\hline 52 & 38.2 & 37.5 & 39 & 3. & 52 & 39.9 & 38.3 & 37 & 6 & 52 & 60.8 & 60.2 & 2204 & 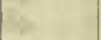 & 52 & 45.0 & $33 \cdot 3$ & & 38 & \\
\hline 54 & 37.9 & 37.1 & 39 & ax & 54 & 39.8 & 38.2 & 38 & 6 & 54 & 65.8 & $64 \cdot 7$ & 2157 & $1=$ & 54 & 44.3 & 33.2 & & 38 & \\
\hline 56 & 37.9 & 37.2 & 39 & . & 56 & 39.3 & 38.2 & 38 & 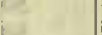 & 56 & 65.3 & 65.1 & 57 & 4 & 56 & 43.6 & 33.2 & & 39 & \\
\hline 58 & 37.9 & 37.2 & 39 & & 58 & 38.9 & 38.0 & 38 & & 58 & 64.0 & 62.8 & 59 & 2 & 58 & 42.3 & 32.6 & & 40 & \\
\hline & & 80 & 10 & $1=$ & & Es & trete & & & & & & & & & 41.8 & 32.8 & & & \\
\hline
\end{tabular}


Tabulation of magnetic declinations observed at T'eplitz Bay-Continued

\begin{tabular}{|c|c|c|c|c|c|c|c|c|c|c|c|c|c|c|c|c|c|c|c|}
\hline \multicolumn{5}{|c|}{ Thursday, February 25, 1904} & \multicolumn{5}{|c|}{ Magnet scale erect } & \multicolumn{6}{|c|}{ Friday, February 26, 1904} & \multicolumn{4}{|c|}{ Magnet scale inverted } \\
\hline \multirow{2}{*}{$\begin{array}{l}\text { Clir'r } \\
\text { time }\end{array}$} & \multicolumn{2}{|c|}{$\begin{array}{l}\text { Scale } \\
\text { readings }\end{array}$} & \multirow{2}{*}{$\begin{array}{c}\text { East } \\
\text { decli- } \\
\text { nation }\end{array}$} & Temp. & Chr'r & $\begin{array}{l}\text { Sc: } \\
\text { read }\end{array}$ & $\begin{array}{l}\text { ale } \\
\text { ings }\end{array}$ & $\begin{array}{l}\text { East } \\
\text { decli- }\end{array}$ & Temp. & Chr'r & $\begin{array}{r}\mathrm{Sc} \\
\text { read }\end{array}$ & $\begin{array}{l}\text { ale } \\
\text { lings }\end{array}$ & $\begin{array}{l}\text { East } \\
\text { decli- }\end{array}$ & Temp. & Chr'r & $\begin{array}{r}\mathrm{Sc} \\
\mathrm{read}\end{array}$ & ngs & East & Tenip. \\
\hline & Left & Right & & & & Left & Rigbt & & & $\ln$ & L,eft & Right & & & & Left & Right & & \\
\hline $\mathrm{h} \mathrm{m}$ & d & d & $\cdot$, & - & $\mathrm{h} \mathrm{m}$ & d & d & - , & & $\mathrm{h} \mathrm{m}$ & d & d & -, & & h $\mathrm{m}$ & d & & & \\
\hline 600 & 52.4 & 53.9 & 2237 & -17.7 & 1800 & 51.7 & 55.0 & 2240 & $-I 6.3$ & 2000 & 39.9 & $34 \cdot 4$ & 2242 & -6.7 & 2200 & 41.2 & 39.8 & 2236 & $-5 \cdot 3$ \\
\hline 02 & 51.7 & 53.8 & 37 & (a) & 02 & $54 \cdot 9$ & 55.2 & 40 & & 02 & 40.1 & 39.0 & 38 & $x^{2}$ & 02 & 40.7 & 39.1 & 37 & 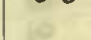 \\
\hline 04 & 52.6 & $54 \cdot 5$ & 38 & wis & 04 & 55.0 & 55.3 & $4 \mathrm{I}$ & 2 & 04 & 40. I & 39. & & 30 & 04 & 41.2 & 39.9 & 36 & (15) \\
\hline 06 & 52.3 & 54.2 & 38 & as & 06 & 54.4 & 54.9 & 40 & & 06 & 40.1 & 39. & 38 & 45 & 06 & 41.2 & 40.3 & 36 & 84 \\
\hline 08 & 52.1 & 54.2 & 37 & ar & 08 & 54.2 & 54.9 & 40 & in & 08 & 40.7 & 39.8 & 37 & (15) & 08 & 42.3 & 41.1 & 34 & $6 y$ \\
\hline 10 & 52.2 & 54.4 & 38 & ca & I0 & 54.2 & 55.0 & 40 & & 10 & 40.8 & 39.9 & 37 & 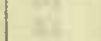 & Io & 41.9 & 40.6 & 35 & $\pi$ \\
\hline 12 & 52.7 & 54.9 & 39 & & 12 & 53.9 & 54.9 & 39 & & 12 & 41.0 & 40. & 36 & & 12 & $40 . \overline{8}$ & 39.7 & 37 & \\
\hline 14 & 52.9 & 54.9 & 39 & -17.9 & 14 & 53.5 & 54.8 & 39 & -16.1 & 14 & 41.0 & 40.3 & 36 & -6.1 & 14 & 41.6 & 40.2 & 36 & -5.2 \\
\hline 16 & 53.1 & 55.0 & 39 & 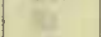 & 16 & 54.0 & 55.0 & 40 & t & 16 & 40.9 & 40. & 36 & if & I6 & 40.9 & 39.8 & 37 & (1) \\
\hline 18 & 54.2 & 55.7 & 40 & int & 18 & $54 \cdot 3$ & $55 \cdot 3$ & 40 & is & 18 & 41.4 & 40.8 & 35 & se & 18 & 40.2 & 39.0 & 38 & at \\
\hline 20 & 53.1 & 54.8 & 39 & 20 & 20 & 54.7 & 55.8 & $4 I$ & tac & 20 & 41.8 & $4 I .3$ & 35 & & 20 & 39.8 & 38 & 39 & try \\
\hline 22 & 53.0 & 54.8 & 39 & itit & 22 & 54.8 & 55.8 & $4 \mathrm{I}$ & ta & 22 & 42.8 & 42.0 & 33 & & 22 & 38.6 & 37.7 & 40 & in \\
\hline 24 & 53.2 & 55.0 & 39 & 20 & 24 & 55.2 & 55.8 & $4 \mathrm{I}$ & & 24 & 42.8 & 42.2 & 33 & & 24 & 38.5 & 37 & 40 & (n) \\
\hline 26 & 53.5 & 55.0 & 39 & E & 26 & 54.9 & 55.2 & 40 & te & 26 & 42.4 & 41.9 & 34 & & 26 & 38.6 & 37 & 40 & tate \\
\hline 28 & $52 . \overline{8}$ & 54.2 & 38 & & 28 & 54.9 & 55.2 & 40 & & 28 & 42.4 & $4 \mathrm{~T} . \overline{8}$ & 34 & & 28 & 37.9 & 37 & $4 I$ & \\
\hline 30 & 52.3 & 54.2 & 38 & -17.8 & 30 & 55.1 & 55.4 & $4 \mathrm{I}$ & -16.0 & 30 & 42.4 & 42.0 & 34 & -5.5 & 30 & 37.4 & 36.8 & 42 & -5.1 \\
\hline 32 & 52.9 & 55.0 & 39 & 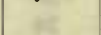 & 32 & 55.1 & 55.7 & $4 I$ & 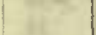 & 32 & 42.8 & 42. & 3 & & 32 & 37.3 & 36 & 42 & (2) \\
\hline 34 & 53.0 & 55.1 & 39 & 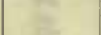 & 34 & 55.0 & 55.7 & $4 \mathrm{I}$ & 28 & 34 & 42.6 & 42.0 & 3 & & 34 & 37.2 & 36.5 & 42 & . \\
\hline 36 & $53 . \mathrm{I}$ & 55.2 & 39 & EF & 36 & 55.2 & 55.7 & $4 \pi$ & zit & 36 & 42.5 & 42.2 & 3 & t & 36 & 37.7 & 37 & $4 \mathrm{I}$ & 政 \\
\hline 38 & 52.8 & 55.0 & 39 & 2 & 38 & 55.4 & 55.9 & $4 \mathrm{I}$ & E. & 38 & $43 . \overline{5}$ & 43.0 & 3 & te. & 38 & 38.0 & 37 & $4 I$ & 3 \\
\hline 40 & 50.7 & 53.0 & 35 & (25. & 40 & 55.5 & 55.9 & 42 & t & 40 & 44.2 & 43. & 3 & & 40 & 38.0 & 37.6 & $4 I$ & . \\
\hline 42 & 49.5 & 51.6 & 33 & & 42 & 55.9 & 56.4 & 42 & av. & 42 & 44.0 & 43.8 & $3 I$ & & 42 & 37.9 & 37 & 41 & \\
\hline 44 & 48.0 & 49.6 & 31 & $-I 7.3$ & 44.3 & 55.8 & 56.7 & 42 & 30 & 44 & 44.2 & 43.9 & 31 & $-5 \cdot 5$ & 44 & 38. I & 37 & $4 \mathrm{I}$ & -5.1 \\
\hline 46 & 46.7 & 48.2 & 28 & t & 46 & 55.6 & 56.4 & 42 & 2 & 46 & $44 \cdot 3$ & $44 . \bar{I}$ & $3 \mathrm{I}$ & - & 46 & 38.2 & 37.6 & 40 & 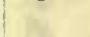 \\
\hline 48 & 46.8 & 48.2 & 29 & 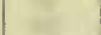 & 48 & 55.7 & 56.3 & 42 & - & 48 & 44.7 & 44. & 3 & 4 & 48 & 38.0 & 37.8 & 40 & $=$ \\
\hline 50 & 47.2 & 48.1 & 29 & & 50 & 55.8 & 56.5 & 42 & 6 & 50 & 44.8 & 44.2 & 3 & 保 & 50 & 38.1 & 37.9 & 40 & 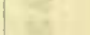 \\
\hline 52 & 47.2 & 47.9 & 28 & 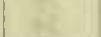 & 52 & 56.0 & 56.9 & 43 & iif & 52 & 45.4 & 45.0 & 2 & & 52 & 37.6 & & $4 I$ & a \\
\hline 54 & 48.4 & 48.8 & 30 & & 54 & 56.2 & 57.0 & 43 & the & 54 & 45.7 & 45 & 2 & & 54 & 37.0 & 36 & 42 & $y^{2}$ \\
\hline 56 & 49.8 & 50.2 & 33 & 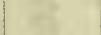 & 56 & 56.1 & 56.9 & 43 & we & 56 & 46.4 & 45 & 2 & & 56 & 36.7 & 36 & 43 & \\
\hline 58 & 50.9 & 51.2 & 34 & & 58 & 55.1 & 57.0 & 42 & & 58 & 45.2 & 44.7 & 29 & & 58 & 36.4 & 36.1 & 43 & \\
\hline 1700 & 51.2 & 51.8 & 35 & -17.0 & 1900 & 55.0 & 56.9 & 42 & -15.6 & 2100 & 44.9 & $44 \cdot 3$ & 30 & -5.7 & 2300 & 37.4 & 37 & 42 & -5.0 \\
\hline 02 & 52.9 & 53.7 & 38 & $x^{2}$ & 02 & 54.7 & 56.4 & $4 \mathrm{I}$ & ( & 02 & 44.9 & $44 \cdot 5$ & 3 & & 02 & 37.8 & 37.5 & 41 & 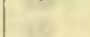 \\
\hline 04 & 54.2 & 55.0 & 40 & & 04 & 54.1 & 56.4 & $4 \mathrm{I}$ & if & 04 & 43.7 & 43.1 & 3 & 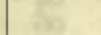 & 04 & 37.5 & 37 & $4 \mathrm{I}$ & $x^{2}$ \\
\hline 06 & 55.6 & 56.1 & 42 & 20 & 06 & 53.9 & 55.9 & 40 & $x^{2}$ & $\infty 6$ & $43 \cdot 3$ & 43 & 3 & we. & $\infty$ & 36.4 & 36 & 43 & e \\
\hline 08 & $57 \cdot 4$ & 58.0 & 45 & & 08 & 54.1 & 55. & 40 & 2 & 08 & 43.0 & 42.6 & 3 & vit & 08 & & 36 & 43 & a \\
\hline I0 & 57.9 & 58.6 & 45 & & IO & 54.2 & 56.0 & $4 \mathrm{I}$ & at & I0 & 41.5 & 41.1 & 35 & & 10 & 36.6 & 36.0 & 43 & \\
\hline 12 & 57.2 & 57.9 & 44 & & I2 & 54.3 & 55. & $4 I$ & & I2 & 40.1 & 39. & & & 12 & 36.5 & 36 & 43 & स \\
\hline 14 & 57.7 & 57.9 & 45 & -17.0 & I4 & 54.1 & 55.8 & 40 & -15.3 & 14 & 39.6 & 39.1 & 38 & -5.7 & 14 & 36.2 & 36.0 & 43 & -5.0 \\
\hline 16 & 57.8 & 58.0 & 45 & $x_{2}$ & 16 & $54 . I$ & 55.9 & 40 & $x^{2}$ & 16 & 39.7 & 39.0 & 3 & 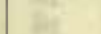 & 16 & 36.1 & 35 & 43 & (n) \\
\hline I8 & 57.4 & 57.8 & 44 & $\theta=$ & IS & 54.8 & 56.2 & $4 \mathrm{I}$ & . & 18 & 39.8 & 39.5 & 3 & & 18 & 36.3 & & 43 & ax \\
\hline 20 & 57.8 & 58.0 & 45 & 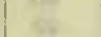 & 20 & 55.1 & 56.8 & 42 & $t^{4}$ & 20 & 42.0 & 41.6 & 34 & trit & 20 & 36.9 & 36 & 43 & 38 \\
\hline 22 & 57.6 & 57.9 & 45 & & 22 & 55.1 & 56.8 & 42 & at & 22 & 42.6 & 42.0 & 3 & & 22 & 36. & 36 & 42 & 3 \\
\hline 24 & $57 \cdot 3$ & 58.0 & 44 & 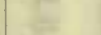 & 24 & 55.3 & 57. & 42 & at & 24 & 44.2 & 44.0 & 3 & 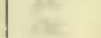 & 24 & 36.8 & 36.4 & 43 & W. \\
\hline 26 & 56.7 & 57.6 & 44 & & 26 & $55 . \mathrm{I}$ & 56. & 42 & & 26 & $47 \cdot 3$ & 46.4 & 26 & & 26 & 36.0 & 35 & 44 & 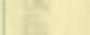 \\
\hline 28 & 56.2 & 57.0 & 43 & & 28 & 55.2 & 56.7 & 42 & & 28 & 48.8 & 47.3 & 25 & & 28 & 35.2 & 35.0 & 45 & \\
\hline 30 & 56.1 & 56.9 & 43 & -16.8 & 30 & 54.2 & 55.8 & $4 \mathrm{I}$ & -15.0 & 30 & 48.6 & 46.3 & 26 & -5.7 & 30 & 36 . & 35 & 44 & -5.0 \\
\hline 32 & 56.1 & 56.9 & 43 & Fiv & 32 & 54.0 & 55.2 & 40 & $x^{2}$ & 32 & 48.0 & 45.4 & 27 & 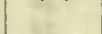 & 32 & 37.8 & 37.0 & $4 \mathrm{I}$ & \\
\hline 34 & 56.0 & 56.9 & 43 & & 34 & 53.8 & 54.9 & 39 & & 34 & 45.2 & $43 \cdot 3$ & $3 I$ & & 34 & 37.9 & 37.1 & $4 I$ & 5 \\
\hline 36 & 55.9 & 56.9 & 43 & t & 36 & 52.0 & 54.0 & 37 & st & 36 & $47 \cdot 3$ & 45.4 & 27 & we & 36 & 37.2 & 36.5 & 42 & the \\
\hline 38 & 55.3 & 57.0 & 42 & co. & 38 & 52.2 & 54.3 & 38 & & 38 & 46.7 & 44.4 & 29 & 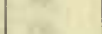 & 38 & 36.5 & 35 & 43 & 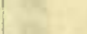 \\
\hline 40 & 55.0 & 56.8 & 42 & $=$ & 40 & 52.8 & 54.4 & 38 & ats. & 40 & 46.5 & 44.6 & 29 & & 40 & 36.1 & 35 & 44 & the \\
\hline 42 & 55.2 & 56.9 & 42 & 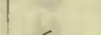 & 42 & 53.1 & 54.7 & 39 & & 42 & 45.8 & 44.0 & 29 & & 42 & 36.4 & & 43 & \\
\hline 44 & 55.3 & 57.0 & 42 & -16.7 & 44 & 53.9 & 55.0 & 39 & -15.0 & 44 & 46.0 & 44.0 & 29 & -5.5 & 44 & 37.0 & 36.8 & 42 & -5.0 \\
\hline 46 & 55.8 & 56.6 & 42 & & 46 & 54.9 & 56.0 & $4 I$ & t & 46 & 44.0 & $4 \mathrm{I} .9$ & 33 & & 46 & 37.8 & $37 \cdot 3$ & $4 \mathrm{I}$ & 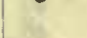 \\
\hline 48 & 55.1 & 56.8 & 42 & 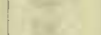 & 48 & 55.4 & 56.4 & 42 & a & 48 & 41.8 & 40.0 & 36 & & 48 & 37.5 & 37.1 & $4 \mathrm{I}$ & 30 \\
\hline 50 & 54.4 & 55.9 & $4 \mathrm{I}$ & & 50 & 55.4 & 56.4 & 42 & 5 & 50 & 42.5 & 40.9 & 34 & & 50 & 37. & & 42 & Es \\
\hline 52 & 54.2 & 55.4 & 40 & 3 & 52 & 55.2 & 56.1 & $4 I$ & H & 52 & $4 \mathrm{I} .4$ & 40.1 & 36 & & 52 & 37.0 & 36.8 & 42 & 48 \\
\hline 54 & $54 \cdot 3$ & 55.8 & 40 & 3. & 54 & 55.2 & 56.2 & $4 \mathrm{I}$ & 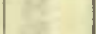 & 54 & 42.0 & 40.3 & 35 & & 54 & 37.4 & 37.1 & 42 & F \\
\hline 56 & 54.4 & 55.4 & 40 & $x^{2}$ & 56 & 55.7 & 56.8 & 42 & 20 & 56 & 41.3 & 40.0 & 36 & & 56 & 38.9 & 37.4 & 40 & 80 \\
\hline 58 & 54.5 & 54.9 & 40 & 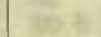 & 58 & 55.9 & 56.7 & 42 & & 58 & 42.1 & 40.8 & 35 & & 58 & 38.7 & 38.2 & 40 & \\
\hline & & & & & 2000 & 55.9 & 56.6 & 42 & -14.9 & & & & & & 2400 & 38.7 & 38.3 & 39 & \\
\hline
\end{tabular}

Correction to local mean time is + 15 s.

Torsion head read $30^{\circ}$ at beginning and ending.

Observer-R. R. T.
Correction to local mean time is +39 s.

Torsion head read $27^{\circ}$ at beginning and ending.

Observer-H. H. N. 
Tabulation of magnetie declinations observed at Teplitz Bay-Continted

\begin{tabular}{|c|c|c|c|c|c|c|c|c|c|c|c|c|c|c|c|c|c|c|c|}
\hline \multicolumn{6}{|c|}{ Sunday, February 28, 1904} & \multicolumn{4}{|c|}{ Magnet scale erect } & \multicolumn{5}{|c|}{ Sunday, February 28, 1904} & \multicolumn{5}{|c|}{ Magnet scale inverted } \\
\hline \multirow{2}{*}{$\begin{array}{l}\text { Chr'r } \\
\text { time }\end{array}$} & \multicolumn{2}{|c|}{$\begin{array}{l}\text { Scale } \\
\text { readings }\end{array}$} & \multirow{2}{*}{$\begin{array}{c}\text { East } \\
\text { decli- } \\
\text { nation. }\end{array}$} & Temp. & Chr't & & $\begin{array}{l}\text { ale } \\
\text { lings }\end{array}$ & $\begin{array}{l}\text { East } \\
\text { decli- }\end{array}$ & Temp. & Chr's & $\begin{aligned} \mathrm{Sc} \\
\mathrm{read}\end{aligned}$ & $\begin{array}{l}\text { le } \\
\text { ngs }\end{array}$ & $\begin{array}{l}\text { East } \\
\text { decli- }\end{array}$ & Hin & Chr'r & $\begin{aligned} \mathrm{Sc} \\
\text { read }\end{aligned}$ & $\begin{array}{l}\text { ale } \\
\text { lings }\end{array}$ & $\begin{array}{l}\text { East } \\
\text { decli- }\end{array}$ & Temp. \\
\hline & Left & Right & & & & Left & Right & & & 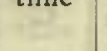 & Left & Right & & & & Left & Right & & \\
\hline $\mathrm{h} \mathrm{m}$ & d & d & & & $\mathrm{h} \mathrm{m}$ & d & d & & 。 & $\mathrm{h} \mathrm{m}$ & d & d & $\circ \quad$, & 0 & $h \mathrm{~m}$ & d & d & $\cdot$ & - \\
\hline 000 & 54.0 & $56 . I$ & 2245 & & 200 & 49.1 & 56.0 & $224 \mathrm{I}$ & $-\mathrm{II} \cdot 9$ & 400.4 & 53.8 & 51.0 & 2245 & $\ln 5$ & 600 & 52.9 & 52.5 & 2244 & -9.5 \\
\hline 02 & 54.0 & 55.8 & & $-14 \cdot 5$ & 02 & 49.3 & 56.2 & $4 \mathrm{I}$ & $\operatorname{sen}$ & 02 & $53 . I$ & 52.0 & 45 & 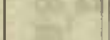 & 02 & 53.9 & 53.5 & 43 & \\
\hline o4 & 53.5 & 56.3 & 45 & 30 & 04 & 50.0 & 56.0 & 42 & $\sqrt{6}$ & 04 & 52.5 & 52.2 & 45 & ate & 04 & 52.6 & 52.3 & 45 & \\
\hline 06 & 53.1 & 57.0 & 45 & 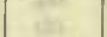 & 06 & 50.0 & 56.3 & 42 & 30 & 06 & 53.0 & 52.8 & 44 & 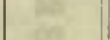 & 06 & $5 \mathrm{I} \cdot 5$ & 51.2 & $4^{6}$ & \\
\hline 08 & 52.2 & 57.0 & 44 & $6 n$ & 08 & 50.8 & 56.8 & 43 & 15 & 08 & 52.0 & 51.8 & 45 & $x^{2}$ & 08 & 51.2 & 51.0 & 47 & \\
\hline Io & 53.0 & 56.0 & 44 & at & 10 & 51.2 & 56.3 & 43 & ese & 10 & 52.0 & 51.9 & 45 & ser & I0 & 51.7 & 51.2 & 46 & \\
\hline I2 & 52.0 & 58.8 & 45 & & 12 & 5I.I & 55.6 & 42 & & I2 & 52.0 & 51.9 & 45 & & I2 & 52.4 & 52.0 & 45 & \\
\hline 14 & 56.2 & 58.2 & 48 & -13.8 & 14 & 50.8 & 54.9 & 4I & -II.4 & 14 & 52.8 & 52.5 & 44 & -I0.0 & I4 & 52.0 & 52.0 & 45 & -9.3 \\
\hline I6 & 50.2 & 58.2 & 43 & 14 & 16 & 51.6 & 53.2 & 4I & & 16 & 53.5 & $53 . \mathrm{I}$ & 43 & 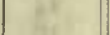 & 16 & 53.0 & 52.3 & 44 & \\
\hline I8 & 50.5 & 58.0 & 43 & ses & 18 & 52.0 & 53.8 & $4 \mathrm{I}$ & II. & 18 & 53.2 & 53.0 & 44 & $\pi$ & 18 & $53 \cdot 3$ & 53.3 & 43 & \\
\hline 20 & 50.5 & 57.2 & 43 & $x^{2}$ & 20 & 52.8 & 54.2 & 42 & 年 & 20 & 52.8 & 52.7 & 44 & - & 20 & 53.0 & 52.7 & 44 & \\
\hline 22 & 50.8 & 57.0 & 43 & $=$ & 22 & 52.9 & 54.9 & 43 & ate & 22 & 52.8 & 52.7 & 44 & 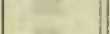 & 22 & 52.3 & 52.3 & 45 & 3 \\
\hline 24 & 51.2 & 56.7 & 43 & 38 & 24 & 52.9 & 54.8 & 43 & B. & 24 & $53 . I$ & 52.9 & 44 & E & 24 & 52.3 & 52.0 & 45 & \\
\hline 26 & 50.0 & 57.2 & 42 & 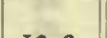 & 26 & 53.0 & 54.9 & 43 & 8 & 26 & 53.4 & $53 . I$ & 43 & E & 26 & 52.3 & 52.2 & 45 & \\
\hline 28 & 50.0 & 57.0 & 42 & $-13 \cdot 3$ & 28 & 53.2 & 55.2 & 43 & & 28 & $53 \cdot 3$ & $53 \cdot 3$ & 43 & -1 & 28 & 52.0 & 52.0 & 45 & \\
\hline 30 & 50.5 & 57.0 & 43 & - & 30 & 53.4 & 55.1 & 43 & $-11 \cdot 3$ & 30 & 53.0 & 53.0 & 44 & -10.0 & 30 & 52.6 & 52.5 & 45 & \\
\hline 32 & 51.6 & 57.8 & 44 & 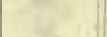 & 32 & 53.7 & 54.5 & 43 & $x_{2}$ & 32 & 53.7 & 53.4 & 43 & $x^{2}$ & 32 & 51.8 & 51.2 & 46 & \\
\hline 34 & 52.7 & 58.0 & 45 & 36 & 34 & 53.5 & 54.0 & 43 & as & 34 & 54.8 & 54.6 & $4 \mathrm{I}$ & $2 y$ & 34 & 49.8 & 49.2 & 49 & \\
\hline 36 & 52.7 & 58.5 & 46 & 3 & 36 & 53.9 & 54.2 & 43 & 38 & 36 & 54.1 & 54.0 & 42 & Bs & 36 & 50.7 & 50.3 & 48 & \\
\hline 38 & 52.0 & 58.0 & 45 & "nt & 38 & 53.7 & $54 . \mathrm{I}$ & 43 & -8 & 38 & $53 . \mathrm{I}$ & 53.0 & 44 & s. & 38 & 52.2 & 52.2 & 45 & \\
\hline 40 & 52.9 & 58.6 & 46 & 20 & 40 & 53.0 & 54.0 & 42 & 8 & 40 & 53.8 & 53.5 & 43 & 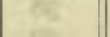 & 40 & 52.7 & 52.3 & 45 & \\
\hline 42 & 53.0 & 58.6 & $4^{6}$ & - & 42 & 53.0 & 54.3 & 42 & on: & 42 & 54.9 & $54 . \overline{8}$ & $4 \mathrm{I}$ & 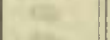 & 42 & 53.0 & 52.9 & 44 & \\
\hline 44 & 52.6 & 58.0 & 45 & -13.0 & 44 & 53.0 & 54.5 & 43 & -II.I & 44 & 54.0 & 54.0 & 42 & -10.0 & 44 & 53.0 & 52.7 & 44 & $-9 \cdot 3$ \\
\hline 46 & 50.8 & 58.2 & 44 & $x=$ & 46 & 52.0 & 55.0 & 42 & & 46 & 53.0 & 52.8 & 44 & & 46 & 52.9 & 52.3 & 44 & \\
\hline 48 & 50.9 & $57 \cdot 7$ & 44 & 27 & 48 & 52.0 & 55.0 & 42 & & 48 & 53.0 & 52.8 & 44 & 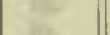 & 48 & 52.2 & 52.2 & 45 & \\
\hline 50 & 51.2 & $57 . \mathrm{I}$ & 43 & a & 50 & 52.0 & 55.0 & 42 & 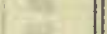 & 50 & 53.8 & 53.6 & 43 & 4h. & 50 & 52.6 & 52. & 45 & \\
\hline 52 & 51.8 & $57 \cdot 4$ & 44 & $x^{2}$ & 52 & 52.5 & 55.5 & 43 & 2 & 52.3 & 52.8 & 52.3 & 45 & 3 & 52 & 52.2 & 51. & 45 & \\
\hline 54 & 51.9 & 57.0 & 44 & a & 54 & 53.1 & 55.7 & 44 & s. & 54 & 52.2 & 52.0 & 45 & 8 & 54 & 52.2 & 52.0 & 45 & \\
\hline 56 & 51.I & 56.7 & 43 & 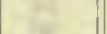 & 56 & 52.8 & 54.9 & 43 & 85 & 56 & 53.0 & 52.8 & 44 & 38 & 56 & $52 . I$ & 51.9 & 45 & \\
\hline 58 & 50.8 & 56.0 & 42 & 10 & 58 & 53.1 & 54.9 & 43 & & 58 & $53 . \mathrm{I}$ & 53.0 & 44 & 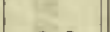 & 58 & 52.2 & 52.0 & 45 & \\
\hline 100 & 50.8 & 56.0 & 42 & -12.9 & 300 & 54.0 & 55.5 & 44 & -II.O & 500 & 52.1 & 52.0 & 45 & -9.9 & 700 & 52.5 & 52.1 & 45 & -9.2 \\
\hline 02 & 52.3 & 56.1 & 43 & - & 02 & 53.2 & 56.0 & 44 & & 02 & 52.0 & 51.5 & 46 & & 02 & 52.5 & 52.0 & 45 & \\
\hline 04 & 54.0 & 57.8 & 46 & a & 04 & 52.0 & 55.2 & 42 & & 04 & 52.9 & 52.8 & 44 & & 04 & 52.5 & 52.0 & 45 & \\
\hline 06 & 54.1 & 57.8 & 46 & and & 06 & $5 \mathrm{I} \cdot 3$ & 55.0 & 42 & 8 & os & 53.8 & 53.5 & 43 & se & 06 & 53.0 & 52.7 & 44 & \\
\hline 08 & 53.7 & 57.0 & 45 & - & 08 & 52.0 & 55.0 & 42 & & 08 & 52.9 & 52. & 45 & & 08 & 52.0 & $5 \mathrm{I}$. & 45 & \\
\hline 10 & $53 \cdot 3$ & 56.0 & 44 & at & IO & 52.0 & 55.2 & 42 & B. & To & $52 . \mathrm{I}$ & $52 . I$ & 45 & 5 & IO & 52.0 & 52.0 & 45 & \\
\hline 12 & 53.9 & 55.7 & 44 & the & 12.4 & 52.1 & 55.7 & 43 & 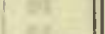 & I2 & 52.8 & 52.5 & 44 & tot & I2 & 52.2 & 52.2 & 45 & \\
\hline 14 & $54 . I$ & 55.2 & 44 & -12.6 & I4 & 51.8 & 55.2 & 42 & -10.9 & I4 & $53 . \mathrm{I}$ & 53.0 & 44 & -9.8 & I4 & 53.0 & 52.0 & 45 & -9.1 \\
\hline 16 & 53.0 & 55.7 & 44 & $1-1$ & 16 & 51.5 & 56.0 & 43 & & 16 & 55.0 & 54.2 & 4I & & 16 & 53.0 & 52.7 & 44 & \\
\hline I8 & 52.0 & 55.0 & 42 & 할. & I8 & 51.9 & 55.5 & 43 & & I8 & 57.0 & 56.0 & 38 & & I8 & 53.2 & 53. & 44 & \\
\hline 20 & 51.8 & 53.7 & $4 \mathrm{I}$ & & 20 & 52.5 & 56.0 & 43 & ate & 20 & 55.0 & 54.9 & $4 \mathrm{I}$ & ant & 20 & 52.2 & 52.1 & 45 & \\
\hline 22 & 51.5 & 52.8 & 40 & $=$ & 22 & 52.7 & 55.9 & 44 & 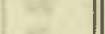 & 22.3 & 54.9 & 54.8 & $4 \mathrm{I}$ & $=$ & 22 & 52.1 & 52.0 & 45 & \\
\hline 24 & 52.3 & 5I. I & 40 & 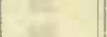 & 24 & 52.6 & 56.0 & 44 & 3 & 24 & 52.5 & 52.0 & 45 & c & 24 & 54.0 & 53.8 & 42 & \\
\hline 26 & 50.4 & 51.9 & 39 & E & 26 & 51.8 & 54.8 & 42 & ta & 26 & 51.3 & 51.0 & 47 & se & 26 & 54.0 & 53.9 & 42 & \\
\hline 28 & 50.2 & $52 . I$ & 39 & & 28 & 52.1 & 54.8 & 42 & & 28 & 53.5 & 53. I & 43 & $=$ & 28 & 53.7 & 53.0 & 43 & \\
\hline 30 & 50.3 & 53.0 & 39 & -12.2 & 30 & 52.9 & 54.9 & 43 & -10.7 & 30 & 54.5 & 54.3 & 42 & -9.8 & 30 & 53.1 & 53.1 & 44 & \\
\hline 32 & 50.0 & 53.2 & 39 & 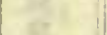 & 32 & 53.0 & 55.2 & 43 & 3 & 32 & 53.1 & 52.3 & 44 & & 32 & 53.5 & 53. & 43 & \\
\hline 34 & $49 . I$ & 53.0 & 38 & $3 x$ & 34 & 53.4 & 55.7 & 44 & Ix- & 34 & 53.2 & 52.2 & 44 & & 34 & 53.0 & 52.9 & 44 & \\
\hline 36 & 48.8 & 52.3 & 38 & 36 & 36 & 52.2 & 54.8 & 42 & 28 & 36 & 53.0 & 52. & 44 & te & 36 & 53.1 & 52. & 44 & \\
\hline 38 & 48.8 & 52.3 & 38 & 3 & 38 & 51.0 & 53.8 & $4 \mathrm{I}$ & $n$ & 38 & 52.2 & 52.2 & 45 & & 38 & 52.9 & 52.9 & 44 & \\
\hline 40 & 49.7 & 53.0 & 39 & 0 & 40 & 52.0 & 54.0 & 42 & 14 & 40 & 53.6 & 53.0 & 43 & & 40 & 52.3 & 52. & 45 & \\
\hline 42 & 50.2 & 53.4 & 40 & aㅏ & 42 & 52.5 & 54.7 & 42 & 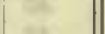 & 42 & 54.2 & 54.0 & 42 & 2 & 42 & 54.2 & 54.0 & 42 & \\
\hline 44 & 50. I & $53 \cdot$ I & 39 & -12.0 & 44 & 53.1 & $55 . \mathrm{I}$ & 43 & -10.5 & 44 & 52.8 & $52 . I$ & 45 & -9.7 & 44 & 54.1 & 53.9 & 42 & -9.0 \\
\hline 46 & 50.4 & 53.9 & 40 & & 46 & 52.7 & 54.8 & 43 & $x^{2}$ & 46 & 51.8 & 51.6 & 46 & & 46 & 53.7 & 53.2 & 43 & \\
\hline $4^{8}$ & 51.8 & 54.2 & 42 & the & 48 & 53.0 & 55.0 & 43 & 8 & 48 & 53.0 & 53.0 & 44 & & 48 & 53.0 & 53.0 & 44 & \\
\hline 50 & 52.8 & 54.9 & 43 & 2 & 50 & 53.2 & 55.0 & 43 & 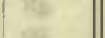 & 50 & 52.0 & 52.0 & 45 & 24 & 50 & 53.3 & 53.2 & 43 & \\
\hline 52 & 52.5 & 54.2 & 42 & $x^{2}$ & 52 & 53.0 & 54.4 & 43 & ac & 52 & 51.2 & 51.0 & 47 & 8 & 52 & 54.0 & 52.1 & 44 & \\
\hline 54 & 50.0 & 56.7 & 42 & an. & 54 & 50.0 & 57.0 & 42 & 135 & 54 & 52.0 & 51.9 & 45 & was & 54 & 54.0 & 53.0 & 43 & \\
\hline 56 & 49.4 & 56.2 & $4 \mathrm{I}$ & 3 & 56 & 50.0 & 56.9 & 42 & $E$ & 56 & 52.2 & 52.0 & 45 & 2 & 56 & 54.0 & 54.0 & 42 & \\
\hline 58 & $49 . I$ & 56.0 & $4 \mathrm{I}$ & 85 & 58 & 49.8 & 52.0 & 38 & 8 & 58 & 51.7 & 51.2 & 46 & 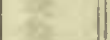 & 58 & 54.3 & 53.9 & 42 & \\
\hline & & C & & 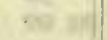 & & & & & & & & & & 160 & $\infty 0$ & 54.0 & 53.9 & & \\
\hline
\end{tabular}

Observer-J. V.

Correction to local mean time is $+17.5 \mathrm{~s}$.

Torsion head at $23 \mathrm{~h} 30 \mathrm{~m}$ read $22^{\circ}$ and at gli $3 \mathrm{Im}$ read the same.

Observer-J. V. 
Tabulation of magnetic declinations observed at Teplitz Bay-Continued

\begin{tabular}{|c|c|c|c|c|c|c|c|c|c|c|c|c|c|c|c|c|c|c|c|}
\hline \multicolumn{6}{|c|}{ Monday, February 29, 1904} & \multicolumn{4}{|c|}{ Magnet scale erect } & \multicolumn{5}{|c|}{ Tuesday, March I, 1904} & \multicolumn{5}{|c|}{ Magnet scale inverted } \\
\hline \multirow{2}{*}{$\begin{array}{l}\text { Chr'r } \\
\text { tine }\end{array}$} & \multicolumn{2}{|c|}{$\begin{array}{l}\text { Scale } \\
\text { readings }\end{array}$} & \multirow{2}{*}{$\begin{array}{c}\text { East } \\
\text { decli- } \\
\text { nation. }\end{array}$} & Temp. & $\begin{array}{l}\text { Clir'r } \\
\text { time }\end{array}$ & $\begin{array}{l}\mathrm{Sc} \\
\text { read }\end{array}$ & $\begin{array}{l}\text { ale } \\
\text { ings }\end{array}$ & $\begin{array}{c}\text { East } \\
\text { decli- } \\
\text { 11ation }\end{array}$ & Temp. & $\begin{array}{l}\text { Chr'r } \\
\text { time }\end{array}$ & $\begin{array}{r}\text { Sc } \\
\text { read }\end{array}$ & $\begin{array}{l}\text { ale } \\
\text { dings }\end{array}$ & $\begin{array}{c}\text { East } \\
\text { decli- } \\
\text { nation }\end{array}$ & Temp. & $\begin{array}{l}\text { Chr'r } \\
\text { time }\end{array}$ & $\begin{array}{r}\mathrm{Sc} \\
\mathrm{read}\end{array}$ & $\begin{array}{l}\text { ale } \\
\text { lings }\end{array}$ & $\begin{array}{l}\text { East } \\
\text { decli- }\end{array}$ & Temp. \\
\hline & Left & Right & & & & Left & Right & & & & Left & Right & & & & Left & Right & & \\
\hline li $\mathrm{m}$ & d & d & $\circ \quad$ & $\circ$ & $\mathrm{h} \mathrm{m}$ & d & d & & - & $\mathrm{h} \mathrm{m}$ & d & $d$ & $\circ \quad$ & & $h \mathrm{ml}$ & $\mathrm{d}$ & d & $\circ \quad$ & • \\
\hline 800 & 53.5 & 55.6 & 2247 & -13.0 & Io $\infty$ & 54.4 & $54 \cdot 7$ & 2247 & -10.2 & 1200 & 52.9 & 51.3 & 2245 & -1.2 & 1400 & 54.9 & 50.3 & 2244 & -2.7 \\
\hline 02 & 51.6 & 53.9 & 45 & & 02 & 54.5 & 54.7 & 47 & & 02 & 53.2 & 51.5 & 44 & & 02 & 55.0 & $49 \cdot 3$ & 45 & \\
\hline 04 & 52.2 & 53.9 & 45 & 10 & 04 & $55 . \mathrm{I}$ & $55 \cdot 3$ & 48 & & 04 & 56.1 & 49.5 & 44 & $1=$ & 04 & 56.5 & 49.I & 44 & Fu \\
\hline 00 & 54.0 & 55.6 & 48 & 8 & 06 & $54 \cdot 3$ & 54.6 & 47 & tir & 06 & 55.8 & 50.2 & 43 & 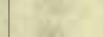 & 06 & 55.8 & 50.0 & 44 & we \\
\hline o8 & $55 \cdot 3$ & 56.8 & 50 & sit & 08 & 53.6 & 54.0 & 46 & 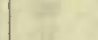 & 08 & 56.0 & 50.2 & 43 & $\infty$ & os & 55.2 & 50.0 & 44 & 20 \\
\hline 10 & 54.6 & 56.4 & 49 & res & 10 & 53. I & 53.4 & 45 & 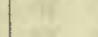 & I0 & 57.0 & 50.7 & 42 & ar & Io & 55.3 & 49.4 & 44 & 92 \\
\hline 12 & $57 \cdot 3$ & 58.2 & 52 & . & 12 & $53 \cdot 7$ & 54.0 & 46 & 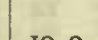 & 12 & $57 \cdot 3$ & 51.8 & 41 & 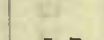 & I2 & 56.0 & 49. I & 44 & -12 \\
\hline 14 & 55.7 & $57 . \mathrm{I}$ & 50 & -12.4 & I4 & $54 . \mathrm{I}$ & 54.7 & 47 & -10.0 & 14 & 57.0 & 52.3 & $4 \mathrm{I}$ & $-\mathrm{I} .7$ & 14 & 56.0 & 49.2 & 44 & -2.9 \\
\hline 16 & 56.0 & $57 \cdot 3$ & 50 & & 16 & 52.6 & 53.0 & 45 & & 16 & 56.9 & 52.7 & 40 & & 16.2 & 56.0 & 48.9 & 44 & \\
\hline I8 & 55.8 & 57.2 & 50 & t & 18 & 52.5 & 52.9 & 44 & a & 18 & 57.4 & 52.2 & 40 & 30 & I8 & 55.4 & 48.3 & 45 & sis \\
\hline 20 & 56.1 & 57.3 & 5 I & 6 & 20 & $54 . I$ & $54 \cdot 3$ & 47 & 16 & 20 & 57.0 & 51.8 & $4 \mathrm{I}$ & $=$ & 20 & 55.7 & 48.4 & 45 & ar \\
\hline 22 & 55.9 & 57.1 & 50 & $a$ & 22 & $54 . \mathrm{I}$ & 54.4 & 47 & 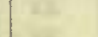 & 22 & 55.8 & 51.1 & 43 & $=$ & 22 & 56.0 & 48.5 & 45 & 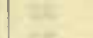 \\
\hline 24 & 56.2 & 57.3 & $5 \mathrm{I}$ & 34 & 24 & 53.6 & 54.0 & 46 & $y=$ & 24 & 55.0 & 51.7 & 43 & 16 & 24 & 55.5 & 47.7 & 46 & 65 \\
\hline 26 & 56.3 & 57.6 & 51 & +8 & 26 & 53.7 & 53.9 & 46 & 난 & 26 & 55.2 & 51.6 & 43 & 8 & 26 & 54.4 & $47 \cdot 4$ & 47 & $\alpha$ \\
\hline 28 & $57 . \mathrm{I}$ & 57.9 & 52 & (2) & 28 & 52.6 & 52.9 & 44 & & 28 & 54.8 & 50.7 & 44 & & 28 & 54.2 & 48.2 & 46 & $n=$ \\
\hline 30 & 56.8 & 57.5 & 51 & -II.8 & 30 & 51.8 & $52 . \mathrm{I}$ & 43 & -10.0 & 30 & 55.0 & 50.4 & 44 & -2.0 & 30 & 53.9 & 49.0 & 46 & -3.0 \\
\hline $3^{2}$ & 56.4 & $57 \cdot 3$ & 51 & $=$ & 32 & 53.5 & $54 . \mathrm{I}$ & 46 & & 32 & 55.0 & 50.3 & 44 & & 32 & 52.2 & 48.5 & 47 & \\
\hline 34 & 56.9 & 57.6 & 51 & 6 & 34 & 53.6 & $54 \cdot 3$ & 46 & 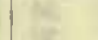 & 34 & $54 \cdot 3$ & 50.3 & 44 & 78 & 34 & 5I.I & 48.8 & 48 & 15 \\
\hline 36 & 55.7 & 56.2 & 49 & 8 & 36 & 53.1 & 53.4 & 45 & 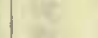 & $3^{6}$ & 54.2 & 49.8 & 45 & 26 & 36 & 50.7 & 46.8 & 50 & 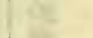 \\
\hline 38 & 55.0 & 55.8 & 49 & 2 & 38 & $53 . \mathrm{I}$ & 53.7 & 45 & 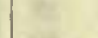 & 38 & 54.8 & 50.8 & 44 & 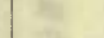 & 38 & 51.8 & 47.0 & 49 & te \\
\hline 40 & 55.1 & 50.0 & 49 & ay & 40 & 53.6 & 53.9 & 46 & $x^{2}$ & 40 & 55.3 & 52.0 & 42 & 4 & 40 & 57.8 & 48.0 & 44 & at \\
\hline 42 & 57.1 & 57.7 & 52 & $1=2$ & 42 & 53.3 & 53.8 & 46 & . & 42 & 56.0 & $52 . \mathrm{I}$ & 42 & $x^{2}$ & 42 & $5 \mathrm{I} .7$ & 46.1 & 50 & $3+4$ \\
\hline 44 & 56.4 & 57.0 & 51 & -II.7 & 44 & 52.8 & $53 . I$ & 45 & -9.8 & 44 & 55.9 & 52.1 & 42 & $-2 . I$ & 44 & 51.9 & $47 \cdot 3$ & 49 & -3.0 \\
\hline 46 & 55.2 & 55.9 & 49 & 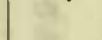 & 46 & 53.7 & 53.9 & 46 & & 46 & 56.2 & 52.0 & 42 & & 46 & 52.0 & 48.7 & 48 & \\
\hline 48 & 54.6 & 55.1 & 48 & $=$ & 48 & 53.1 & 53.6 & 45 & $n$ & 48 & 56.0 & 52.8 & 4I & 12 & 48 & 52.3 & 49.2 & 47 & CE \\
\hline 50 & 55.4 & 55.8 & 49 & zat & 50 & 55.2 & 55.8 & 49 & 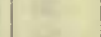 & 50 & 56.0 & 51.8 & 42 & $c$ & 50 & 53.0 & 49.7 & 46 & 02 \\
\hline 52 & 55.7 & 56.2 & 49 & 3 & 52 & $54 \cdot 7$ & 55.0 & 48 & t & 52 & 56. I & 51.6 & 42 & x & 52 & 53.4 & 48.7 & 47 & as \\
\hline 54 & $55 . \mathrm{I}$ & 55.7 & 49 & 8 & 54 & 52.6 & 52.9 & 44 & & 54 & 57.0 & 51.9 & $4 I$ & R8 & 54 & 52.9 & 49.0 & 47 & fe \\
\hline 56 & 55.5 & 56.0 & 49 & tes & 56 & 51.0 & $5 \mathrm{I} .2$ & 42 & & 56 & 55.7 & 51.8 & 42 & & 56 & 52.3 & 49.4 & 47 & $3=$ \\
\hline 58 & $55 . \mathrm{I}$ & 55.6 & 49 & & 58 & 51.9 & 52.2 & 43 & & 58 & 55.7 & 51.8 & 42 & $x=$ & 58 & 53.5 & 50.6 & 45 & . \\
\hline 900 & 54.9 & 55.1 & 48 & $-11 \cdot 3$ & II 00 & 52.8 & 53.0 & 45 & -9.3 & I3 00 & 55.9 & 51.7 & 42 & $-2 . I$ & 1500 & 53.0 & 50.2 & 40 & $-3 . I$ \\
\hline 02 & 54.3 & 54.8 & 47 & & 02 & 52.6 & 52.9 & 44 & & 02 & 54.6 & 52.2 & 43 & & 02 & 51.8 & 50.9 & 46 & \\
\hline 04 & 53.2 & 53.8 & 46 & X. & 04 & 51.9 & 52.2 & 43 & rar & 04 & 54.2 & 52.3 & 43 & tes & 04 & 52.2 & $51 \cdot 3$ & 45 & 25 \\
\hline 06 & 53.0 & 53.4 & 45 & the & 06 & 51.5 & 51.8 & 43 & 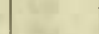 & 06 & 54.0 & 53.0 & 43 & tis & 06 & 53.0 & 51.9 & 44 & 2 \\
\hline 08 & 53.1 & 53.7 & 45 & are & 08 & 5 I.I & $5 \mathrm{I} \cdot 5$ & 42 & t & 08 & 53.8 & 53.0 & 43 & a & 08 & 52.0 & 51.5 & 45 & . \\
\hline 10 & 53.6 & 53.9 & 46 & 0 & IO & 51.6 & 51.9 & 43 & 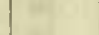 & Io & 54.0 & 52.4 & 43 & t & 10 & 51.7 & 50.6 & 46 & 62 \\
\hline 12 & 53.8 & 54.0 & 46 & 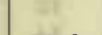 & I2 & 52.0 & 52.4 & 44 & 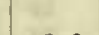 & 12 & 53.2 & 52.4 & 44 & . & I2 & 51.4 & 50.9 & 46 & 列 \\
\hline 14 & $54 . \mathrm{I}$ & 54.2 & 47 & -11.0 & I4 & 52.0 & 52.8 & 44 & -9.3 & I4 & 52.7 & 52.0 & 44 & -2.2 & 14 & $5 I .3$ & 50.8 & 47 & -3.1 \\
\hline 16 & 53.9 & 53.9 & 46 & & I6 & 51.9 & 52.5 & 44 & & I6 & 52.9 & 51.0 & 45 & & 16 & 52.7 & 50.9 & 45 & \\
\hline I8 & 53.6 & 53.9 & 46 & 8 & 18 & $5 \mathrm{I} \cdot 3$ & 51.9 & 43 & 251 & I8 & 53.0 & 50.8 & 45 & $y$ & 18 & 53.9 & 51.8 & 44 & (2) \\
\hline 20 & 54.0 & 54.2 & 47 & $a$ & 20 & 5I.I & $5 \mathrm{I} .4$ & 42 & 2 & 20 & 53. I & 50.2 & 46 & can & 20 & 53.9 & 52.0 & 44 & $3 x$ \\
\hline 22 & 53.8 & 54.0 & 46 & 24 & 22 & 49.5 & 50.9 & 40 & 3 & 22 & 53.9 & 50.5 & 45 & $E$ & 22 & 54.0 & 52.0 & 43 & 178 \\
\hline 24 & 53.9 & 54.2 & 46 & $=$ & 24 & 49.1 & $49 \cdot 5$ & 39 & 18 & 2 & & 51.3 & 42 & t & 24 & 53.3 & 51.7 & 44 & tex \\
\hline 26 & 53.7 & 54.0 & 46 & in & 26 & 49.5 & 49.7 & 39 & $y$ & 26 & 54.6 & $5 \mathrm{I} .2$ & 44 & ate & 26 & 52.9 & 51.2 & 45 & at \\
\hline 28 & 53.9 & 54.1 & 46 & 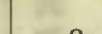 & 28 & 51.4 & 51.7 & 42 & 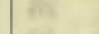 & 28 & 55.0 & 5 โ.2 & 43 & $=$ & 28 & 52.3 & 50.9 & 46 & 26 \\
\hline 30 & 54.1 & 54.2 & 47 & -10.8 & 30 & 53.0 & 53.7 & 45 & -9.0 & 30 & 54.5 & 52.2 & 43 & -2.5 & 30 & 53.0 & 51.9 & 44 & 45 \\
\hline 32 & 54.1 & 54. I & 47 & $1=2$ & 32 & 50.7 & $5 \mathrm{I} .2$ & 42 & & 32 & 54.9 & 52.1 & 43 & & 32 & 52.6 & 52.0 & 44 & $-3 \cdot 3$ \\
\hline 34 & 53.8 & 53.9 & 46 & 28 & 34 & 49.8 & 50.3 & 40 & 18 & 34 & 53.9 & 52.5 & 43 & 15 & 34 & 52.7 & 50.9 & 45 & \\
\hline 36 & 53.7 & 53.9 & 46 & 8 & 36 & 52.2 & 52.7 & 44 & as & 36 & 53.0 & 51.8 & 44 & & $3^{6}$ & 51.0 & 49.4 & 48 & 18 \\
\hline 38 & 53.7 & 53.9 & 46 & ac. & 38 & 52.4 & 52.9 & 44 & 35 & 38 & 51.9 & 50.8 & 46 & & 38 & 51.0 & 48.3 & 49 & a \\
\hline 40 & Ove & rl'k'd & & 04 & 40 & $52 . \mathrm{I}$ & 52.7 & 44 & 10 & 40 & 51.8 & 50.7 & 46 & $0 t$ & 40 & 49.7 & 47.2 & $5 \mathrm{I}$ & 6 \\
\hline 42 & 53.1 & 53.4 & 45 & 24 & 42 & 50.9 & 51.2 & 42 & 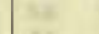 & 42 & 51.2 & 50.0 & 47 & 政 & 42 & 49.7 & 47.0 & 5 I & 54 \\
\hline 44 & 53.1 & 53.3 & 45 & 32 & 44 & 50.2 & 50.8 & $4 \mathrm{I}$ & -9.0 & 44 & 53.2 & 51.4 & 44 & -2.8 & 44 & 47.4 & 44.7 & 54 & -3.2 \\
\hline 46 & 52.9 & 53.0 & 45 & -I0.4 & 46 & 50.6 & 50.7 & $4 I$ & & 46 & 54.2 & 50.2 & 45 & & 46 & 45.6 & 42.9 & 57 & \\
\hline 48 & $53 . \overline{8}$ & 54.0 & 46 & & 48 & 50.7 & 51.2 & 42 & $3 t$ & 48 & 55.0 & 50.9 & 44 & $\pi 7$ & 48 & 44.9 & 43.0 & 58 & 60 \\
\hline 50 & $53 . \mathrm{I}$ & 53.5 & 45 & tip & 50 & 51.8 & 52.0 & 43 & 0 & 50 & 54.2 & 50.8 & 44 & 9 & 50 & 43.2 & 42.0 & 60 & an \\
\hline 52 & 52.6 & 52.9 & 44 & Q & 52 & 51.7 & 51.9 & 43 & 6 & 52 & 53.9 & 48.1 & 47 & a & 52 & 48.3 & 45.6 & 53 & $x=$ \\
\hline 54 & 53. I & 53.4 & 45 & $z$ & 54 & $51 \cdot 3$ & 52.0 & 43 & 2 & 54 & 53.8 & 47.9 & 47 & 15 & 54 & 50.2 & $44 . I$ & 53 & $x^{2}$ \\
\hline 56 & 54. I & 54.2 & 47 & as & 56 & 51.4 & 52.0 & 43 & & 56 & $54 . \mathrm{I}$ & 49.6 & 45 & & 56 & 48.6 & $43 \cdot 3$ & 55 & -2 \\
\hline 58 & 54.2 & 54.4 & 47 & 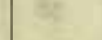 & 58 & 51.9 & 52.3 & 44 & 8 & 58 & 55.0 & 50.6 & 44 & 28. & 58 & 52.0 & 46.3 & 49 & 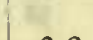 \\
\hline & & & & & 1200 & 51.2 & 51.9 & 42 & -8.5 & & & & & & 1600 & 50.2 & 46.1 & $5 I$ & -3.2 \\
\hline
\end{tabular}

Correction to local mean time is $-28.5 \mathrm{~s}$.

Torsion head at $7 \mathrm{~h} 40 \mathrm{~m}$ read $22^{\circ}$ and at $\mathrm{I} 2 \mathrm{~h}$ zon read $22^{\circ}$. Observer-H. H. N.
Correction to local mean time is -39 s. $90^{\circ}$ torsion $=$ I0.' 63 . Torsion head at $1 \mathrm{ih} 25 \mathrm{~m}$ read $25^{\circ}$ and at $16 \mathrm{~h} 25 \mathrm{~m}$ read $33^{\circ}$. Observer-J. V. 
Tabulation of magnetic declinations observed at Teplitz Bay-Continued

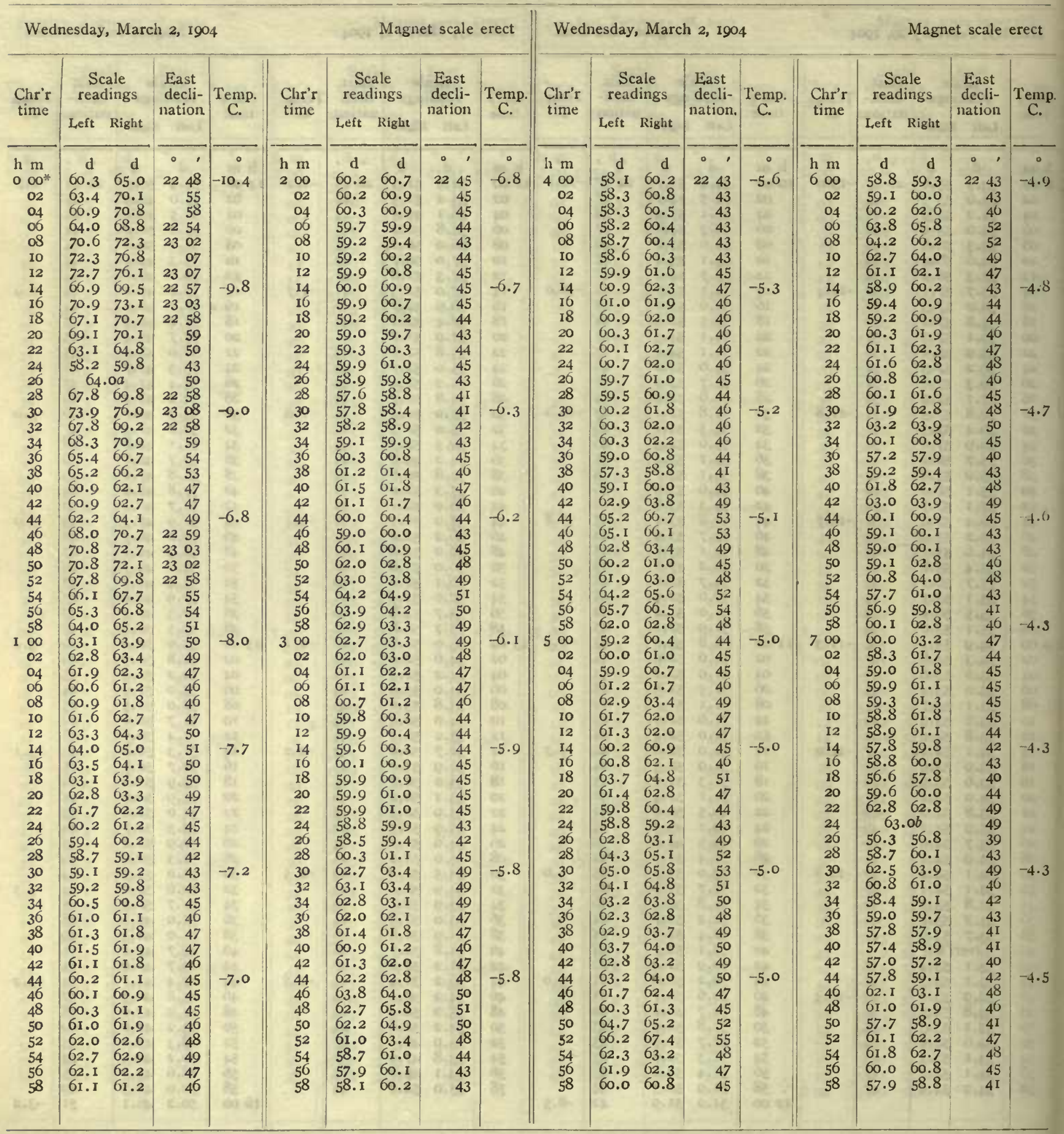


Tabulation of magnetic declinations observed at Teplitz Bay-Continued

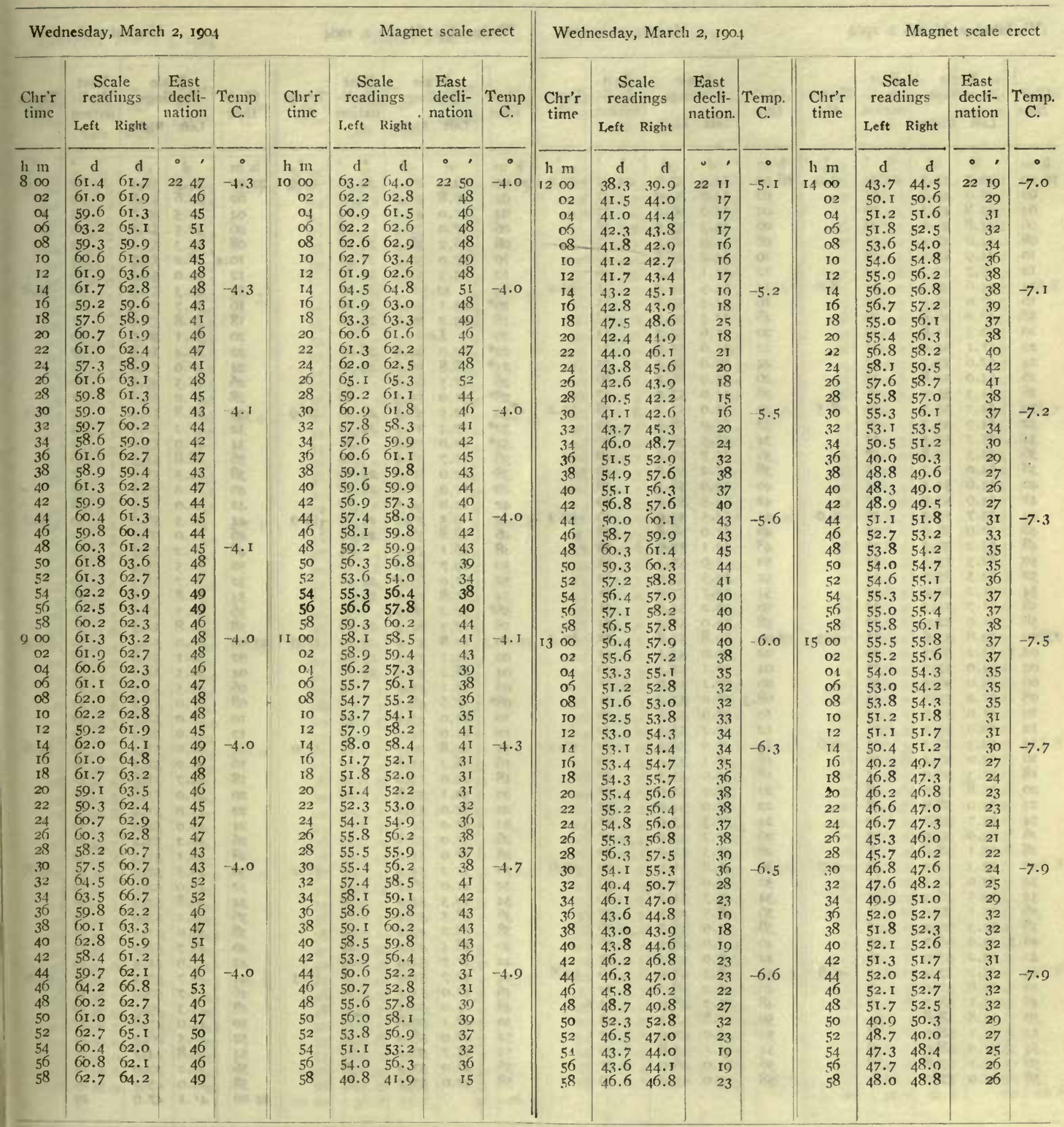

Obscrier-H. H. N.
Ohservers-H. H. N. and J. V., who alternated from $15 \mathrm{~h} 48 \mathrm{~m}$ to I6h oom. 
Tabulation of magnetic declinations observed at Teplitz Bay-Continued

\begin{tabular}{|c|c|c|c|c|c|c|c|c|c|c|c|c|c|c|c|c|c|c|c|c|}
\hline \multicolumn{5}{|c|}{ Wednesilay, March 2, 1904} & \multicolumn{5}{|c|}{ Magnet scale erect } & \multicolumn{7}{|c|}{ Wednesday, March 2, 1904} & \multicolumn{4}{|c|}{ Magnet scale erect } \\
\hline \multirow{2}{*}{$\begin{array}{l}\text { Clir'r } \\
\text { time }\end{array}$} & \multicolumn{2}{|c|}{$\begin{array}{l}\text { Scale } \\
\text { readings }\end{array}$} & $\begin{array}{c}\text { East } \\
\text { decli- } \\
\text { mation. }\end{array}$ & Temp. & Chr'r & $\begin{array}{l}\mathrm{Sc} \\
\text { read }\end{array}$ & $\begin{array}{l}\text { ale } \\
\text { lings }\end{array}$ & $\begin{array}{l}\text { East } \\
\text { decli- } \\
\text { nation }\end{array}$ & Temp. & Chr'r & $\begin{array}{l}\text { Sca } \\
\text { read }\end{array}$ & $\begin{array}{l}\text { ale } \\
\text { lings }\end{array}$ & $\begin{array}{l}\text { Eas } \\
\text { decl }\end{array}$ & & Temp. & Chr'r & $\begin{array}{l}\text { Sca } \\
\text { read }\end{array}$ & $\begin{array}{l}\text { ale } \\
\text { lings }\end{array}$ & East & Temp. \\
\hline & $\mathrm{I}, \mathrm{eft}$ & Right & & & & Left & Right & & & & I.eft & Right & & & & & Left & Right & & \\
\hline h $\mathrm{m}$ & d & d & $\circ \quad$, & $\circ$ & $\mathrm{h} \mathrm{m}$ & d & d & $\circ$, & $\bullet$ & h $\mathrm{m}$ & d & $d$ & - & & $\bullet$ & $\mathrm{h} \mathrm{m}$ & d & त & & - \\
\hline 1600 & 46.7 & 47.7 & 2224 & -7.9 & I8 00 & $54 \cdot 3$ & 57.0 & 2237 & -10.0 & 2000 & 42.5 & 45.2 & 221 & 19 & -10.9 & 2200 & 44.3 & $55 . \mathrm{I}$ & $2=33$ & - II.9 \\
\hline 02 & 45.0 & 45.8 & $2 I$ & - & 02 & 54.6 & 56.0 & 37 & 160 & 02 & 41.8 & 43.2 & & 17 & & 02 & 43.6 & 53.8 & 32 & \\
\hline 04 & 43.8 & 46.1 & 20 & $4^{2}=$ & 04 & 55.2 & 56.2 & 37 & w5 & 04 & 41.0 & $43 . I$ & & 16 & & 04 & 40.5 & 49.0 & 25 & \\
\hline of & 44.8 & 46.6 & 22 & $x$ & 06 & $55 \cdot 3$ & 57.0 & 38 & W & 06 & 37.0 & 37.1 & & 08 & $3 r$ & 06 & 42.5 & 51.0 & 29 & \\
\hline 08 & 44.0 & 47.2 & 21 & ak: & os & 54.9 & 56.2 & 37 & 40 & 08 & 36.2 & 38.5 & 220 & 08 & Bis & 08 & 45.3 & 53.1 & 33 & \\
\hline Io & $44 \cdot 9$ & 47.6 & 22 & $x^{2}$ & 10 & 54.2 & 55.8 & 36 & Hit & 10 & 27.0 & 30.7 & 215 & 55 & 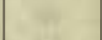 & 10.4 & 48.2 & 53.9 & 35 & 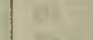 \\
\hline 12 & 45.7 & 47.7 & 23 & & 12 & 54. J & 55.5 & 36 & & 12 & 25.3 & 31.0 & 215 & 54 & & 12 & 49.0 & 54.2 & 36 & \\
\hline I4 & 44.6 & $47 \cdot 3$ & 22 & -8.0 & 14 & 53.5 & 55.2 & 35 & -10.0 & 14 & 42. & $.5 a$ & 22 & 17 & & 14 & 40.3 & 53.9 & 36 & -12.0 \\
\hline 16 & 47.0 & 48.0 & 25 & 3ir & 16 & 52.4 & 55.9 & 35 & 28 & $16^{*}$ & 23.1 & 31.5 & 230 & 08 & & 16 & 51.7 & 56.3 & 40 & \\
\hline I8 & 48.0 & 48.8 & 26 & 27 & 18 & 52.5 & 55.7 & 35 & Af & 18 & 23.2 & 70.0 & 233 & 38 & & 18 & 51.8 & 57.0 & $4 \mathrm{I}$ & \\
\hline 20 & 50.5 & 51.8 & 30 & $e^{2}$ & 20 & 51.6 & 55.2 & 34 & 8. & 20 & $\mathrm{~J}, \mathrm{C}$ & ost & & & T- & 20 & $5 \mathrm{I} . \mathrm{I}$ & 56.0 & 30 & \\
\hline 22 & $5 \mathrm{I} . \mathrm{I}$ & 52.0 & 31 & 32 & 22 & 51.6 & 55.0 & 34 & 18 & $22^{*}$ & 6.0 & 62.0 & 260 & & 6 & 22 & 49.4 & 54.3 & 37 & \\
\hline 24 & 52.6 & 53.7 & 33 & (1) & 24 & 53.0 & 55.4 & 35 & It & $24^{*}$ & 48.8 & 50.0 & 245 & 50 & H & 24 & 47.9 & 51.2 & 33 & \\
\hline 26 & 54.0 & 54.8 & 35 & $8 x^{2}$ & 26 & 53.9 & 56.2 & 36 & & 26 & 47.5 & 60.0 & & 56 & & 26 & 45.5 & 49.9 & 30 & \\
\hline 28 & $54 \cdot 3$ & 55.0 & 36 & & 28 & 54.0 & 57.0 & 37 & & $28^{*}$ & 31.0 & 55.3 & & 60 & & 28 & 46.0 & 50.2 & 31 & \\
\hline 30 & 54.2 & 55.7 & 36 & -8.5 & 30 & 55.0 & 57.2 & 38 & -10.2 & 30 & 12.5 & 48.5 & 24 & 40 & -10.9 & 30 & 46.8 & 51.2 & 32 & -12.1 \\
\hline 32 & 54.9 & 55.2 & 36 & 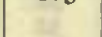 & 32 & 55.2 & 58.5 & 39 & t & $32^{*}$ & 17.4 & $5 I . I$ & 22 & 49 & & 32 & 47.2 & 52.0 & 33 & \\
\hline 34 & 55.2 & 55.5 & 37 & WE. & 34 & 55.5 & 59.0 & 40 & $17 \%$ & $34^{*}$ & 20.0 & 50.0 & & $2 \mathrm{I}$ & 18 & 34 & 47.9 & 53.0 & 34 & \\
\hline 36 & 55.3 & 55.8 & 37 & 28 & 36 & 55.7 & 59.2 & 40 & 27 & 36 & II.7 & 56.1 & & 12 & 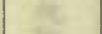 & 36 & 48.8 & 54.7 & 36 & \\
\hline 38 & 54.0 & 54.7 & 35 & $x^{2}$ & 38 & 55.2 & 59.0 & 40 & Ft & 38 & 22.0 & 50.0 & 221 & 16 & dx & 38 & 49.5 & 55.1 & 37 & \\
\hline 40 & 53.6 & 54.1 & 34 & tent & 40 & 54.0 & 58.5 & 38 & hat & 40 & II .O & 38.8 & 21 & 58 & a & 40 & 50.0 & 55.1 & 38 & \\
\hline 42 & 54.9 & 55.9 & 37 & & 42 & 54.5 & 59.0 & 39 & & 42 & 12.0 & 39. T & & 59 & 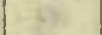 & 42 & 50.0 & 55.6 & 38 & \\
\hline 44 & 53.7 & 55.3 & 36 & -9.0 & 44 & 54.5 & 59.0 & 39 & -10.3 & 44 & 0.5 & 36.9 & 21 & 55 & -10.0 & 44 & 50.3 & 56.0 & 39 & $-\mathrm{T} 2.2$ \\
\hline 46 & 52.9 & 56.0 & 35 & & 46 & 54.0 & 58.8 & 38 & (20 & 46 & 18.8 & 38. 1 & $22{ }^{\circ}$ & 04 & & 46 & 51.0 & 56.0 & 39 & \\
\hline 48 & 53.2 & 56.5 & 36 & 6 & 48 & 52.1 & 57.0 & 36 & ? & 48 & I6. & $4 \mathrm{~T} . \mathrm{I}$ & & 04 & & 48 & $5 \mathrm{I} .2$ & 55.7 & 30 & \\
\hline 50 & 52.5 & 55.3 & 35 & 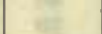 & 50 & 51.0 & 55.4 & 33 & $a$ & 50 & I0.7 & 49.0 & & 13 & 82 & 50 & $5 \mathrm{I} .5$ & 55.4 & 39 & \\
\hline 52 & 52.5 & 55.0 & 34 & 的 & 52 & 50.5 & 53.0 & 32 & 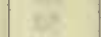 & 52 & 41.0 & 63.0 & 22 & $4 \mathrm{I}$ & (4) & 52 & 51.7 & 55.3 & 30 & \\
\hline 54 & 51.2 & 54.0 & 32 & 9 & 54 & 50.7 & 53.2 & $3 I$ & 27 & 54 & 61.8 & 74.5 & $23 c$ & 06 & 13 & 54 & 50.8 & 54.5 & $3^{8}$ & \\
\hline 56 & 51.7 & 53.2 & 32 & (it) & 56 & 50.2 & 52.9 & $3 I$ & & $56^{*}$ & 32.2 & 78.0 & & 33 & & 56 & 50.5 & 53.5 & 37 & \\
\hline 58 & 51.9 & 52.9 & 32 & & 58 & 53.0 & 55.3 & 35 & & 58 & 17.2 & 62.2 & $23 \mathrm{C}$ & 00 & & 58 & 49.8 & 52.5 & 36. & \\
\hline 1700 & 52.8 & 53.6 & 33 & -0.3 & 1900 & 50.8 & 52.8 & 31 & -10.3 & 2100.3 & 10.8 & 4.3 .0 & 22 & 49 & -II.O & 2300 & 49.0 & 52.4 & 35 & -12.5 \\
\hline 02.3 & 53.2 & 56.1 & 36 & $x^{\circ}$ & 02 & 50.0 & 53.1 & $3 I$ & 100 & 02 & If. I & 57.0 & 230 & 02 & & 02 & 50.1 & 52.8 & 36 & \\
\hline 04 & 54.0 & 57.0 & 37 & the & 04 & 50.1 & 52.3 & 30 & ye & 04 & 16.4 & 46.0 & 225 & & 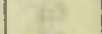 & 04 & 50.5 & 54.1 & 37 & \\
\hline 06 & 53.0 & 56.5 & 36 & 60 & 06 & 50.1 & 52.9 & $3^{T}$ & Wo & 06 & $15 . \mathrm{T}$ & 45.7 & & 54 & & 06 & 49.5 & 5.3 .9 & 36 & \\
\hline 08 & 51.9 & $55 \cdot 3$ & 34 & the & 08.3 & 50.0 & 53.2 & 31 & Co & $08^{*}$ & I7.6 & 48.5 & & 07 & Bis & 08 & 47.0 & $5 \mathrm{~T} .5$ & 33 & \\
\hline Io & 51.0 & 55.0 & 33 & in & Io & 49.0 & 52.5 & 30 & 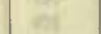 & ro & 21.0 & 53. I & & 13 & 에 & 10 & 47.0 & 52.1 & 33 & \\
\hline 12 & 50.9 & $55 . \mathrm{I}$ & 33 & & 12 & 49.2 & 53.2 & 30 & 4 & 12 & 21.0 & 54.0 & & 17 & +3 & 12 & 47.0 & 52. & 33 & \\
\hline 14 & 52.3 & 56.1 & 35 & -9.6 & 14 & 49.0 & 52.2 & 29 & $-\mathrm{J} 0.4$ & I. & 22.8 & $50 . \mathrm{J}$ & & 12 & -II.O & 14 & 46.0 & $5 T .2$ & 32 & -12.5 \\
\hline 16 & 53.0 & 56.2 & 36 & 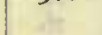 & 16 & 48. I & 53.2 & 29 & (1) & 16 & 26.2 & 51.8 & & 17 & & 16 & 45.0 & 52.1 & $3 I$ & \\
\hline 18 & 51.3 & 56.9 & 35 & $8 t$ & 18 & 49.0 & 53.5 & 30 & hir & 18 & 20.0 & 53.0 & & 20 & 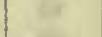 & 18 & 44.7 & 5 r.6 & $3 \mathrm{~T}$ & \\
\hline 20 & 55.8 & 57.0 & 38 & 3 & 20 & 49.9 & 54.0 & 3I & $a s$ & 20 & 30.1 & 52.8 & & 20 & & 20 & 45.0 & $5 T .8$ & $3 \mathrm{I}$ & \\
\hline 22 & 54.0 & 58.9 & $3^{8}$ & $=$ & 22 & 50.0 & 54.0 & 32 & xa & 22 & 32.0 & 53.2 & & 22 & & 22 & 45.0 & 51.6 & $3 T$ & \\
\hline 24 & 54.7 & 59.7 & 40 & as & 24 & 50.3 & 53.0 & $3 \mathbf{I}$ & in & 21 & 35.0 & 54.8 & & 26 & & 24 & 46.0 & 52.0 & 32 & \\
\hline 26 & 54.0 & 59.0 & 39 & Ez & 26 & 50.2 & 53.0 & 31 & est & 26 & 35.0 & 52.8 & & 24 & & 26 & 46.2 & 52.2 & 33 & \\
\hline 28 & 53. I & 58.6 & 38 & & 28 & 49.5 & 52.0 & 30 & & 28 & 36.0 & 53.0 & & 25 & & 28 & 46.1 & $5 \mathrm{~T} .3$ & 32 & \\
\hline 30 & 52.3 & 57.5 & 36 & -9.7 & 30 & 49.8 & 51.9 & 30 & -10.6 & 30 & 37.2 & 54.2 & & 27 & -II.2 & 30 & 46.5 & 52.0 & 33 & -12.8 \\
\hline 32 & 52.0 & 56.3 & 35 & 72 & 32 & 49.0 & 51.5 & 29 & at & 32 & 42.0 & 56.7 & & 33 & & 32 & 47.0 & 52.0 & 34 & \\
\hline & 52.5 & 56.4 & 35 & & 34 & 49.7 & 51.2 & 29 & 20 & 34 & 40.5 & 54.0 & & 20 & & 34 & 48.8 & 52.8 & 35 & \\
\hline 36 & $53 . \mathrm{I}$ & 55.0 & 36 & Q & 36 & 48.5 & $49 \cdot 5$ & 27 & IX & 36 & 38.5 & 50.2 & & 25 & & 36 & $48 . I$ & 52.0 & 34 & \\
\hline 38 & 52.3 & 56.2 & 35 & 8 & 38 & 47.9 & 48.5 & 26 & 8 & 38 & 38.2 & 49.2 & & 24 & & 38 & 48.0 & 5 T. I & 33 & \\
\hline 40.3 & 53.0 & 56.7 & 36 & 8 & 40 & 48.0 & 48.9 & 26 & ns & 40 & 38.4 & 49.7 & & 24 & (x) & 40 & 48.7 & $5 T .2$ & 34 & \\
\hline 42 & 53.2 & 57.0 & 36 & & 42 & 49.0 & 49.3 & 27 & & 42 & 41.7 & 52.0 & & 29 & & 42 & 47.9 & 51.5 & 33 & \\
\hline 44 & 53.2 & 57.4 & 37 & -9.8 & 44 & 48.3 & 48.1 & 26 & -10.8 & 44 & 42.7 & 51.9 & & 30 & $-\operatorname{Tr} .3$ & 44 & 46.7 & 50.4 & 31 & -13.0 \\
\hline 46 & 53.8 & 57.0 & 37 & 3 & 46 & 46.2 & 48.3 & 24 & 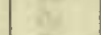 & 46 & 42.0 & 5.3. & & 30 & & 46 & 45.7 & 50.2 & $3 \mathrm{I}$ & \\
\hline $4^{8}$ & $53 . \mathrm{I}$ & 58.7 & 38 & 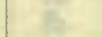 & 48 & $45 \cdot 7$ & 48.0 & 23 & 28 & 48 & 44.0 & 56.1 & & 34 & 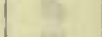 & 48 & 45.3 & 49.3 & 30 & \\
\hline 50 & 54.8 & 58.8 & 39 & $\mathrm{de}$ & 50 & 45.8 & 47.5 & 23 & wat & 50 & 48.4 & 59.8 & & 40 & in & 50 & 43.0 & 47.8 & 27 & \\
\hline 52 & $54 . \tau$ & 58.0 & 38 & 8 & 52 & 45.0 & 47.0 & 22 & ve & 52 & 48.0 & 58.0 & & 39 & a & 52 & $\Delta T .5$ & 46.4 & 24 & \\
\hline 54 & 54.0 & 58.0 & 38 & 12 & 54 & 44.1 & 45.8 & 20 & ait & 5.4 & $\begin{array}{l}40.0 \\
45.7\end{array}$ & 56.2 & & 35 & 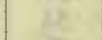 & $\begin{array}{l}52 \\
54\end{array}$ & 38.8 & 44.7 & $2 \mathrm{I}$ & \\
\hline 56 & 54.1 & 57.8 & 38 & 3 & 56 & 44.0 & 46.0 & 21 & 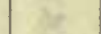 & 56 & 46.0 & 57.3 & & 36 & 2 & 56 & 36.3 & 40.0 & 15 & \\
\hline $5 S$ & 54.0 & 57.0 & 37 & 8 & 58 & 43.0 & 45.5 & 19 & 15 & 58 & 44.0 & $55 . \mathrm{I}$ & & 33 & & 58 & 43.0 & 45.2 & 25 & \\
\hline & & & & & & & & & & & & & & & & 2400 & $4^{i} \cdot \mathrm{I}$ & 47.9 & 25 & \\
\hline
\end{tabular}


Tabulation of magnetic declinations observed at Teplitz Bay-Continued

\begin{tabular}{|c|c|c|c|c|c|c|c|c|c|c|c|c|c|c|c|c|c|c|c|}
\hline \multicolumn{5}{|c|}{ Thursday, March 3, 1904} & \multicolumn{5}{|c|}{ Magnet scale inverted } & \multicolumn{4}{|c|}{ Friday, March 4, Ig04 } & \multicolumn{6}{|c|}{ Magnet scale erect } \\
\hline \multirow{2}{*}{$\begin{array}{l}\text { Chr'r } \\
\text { time }\end{array}$} & \multicolumn{2}{|c|}{$\begin{array}{l}\text { Scale } \\
\text { readings }\end{array}$} & \multirow[t]{2}{*}{$\begin{array}{l}\text { Fast } \\
\text { decli- } \\
\text { nation. }\end{array}$} & Temp. & Chr'r & $\begin{array}{r}\mathrm{Sc} \\
\text { reac }\end{array}$ & lings & East & Temp. & Chr'r & $\begin{array}{r}\mathrm{Sc} \\
\mathrm{read}\end{array}$ & $\begin{array}{l}\text { ale } \\
\text { dings }\end{array}$ & East & Temp. & Chr't & $\begin{array}{l}\mathrm{Sc} \\
\text { read }\end{array}$ & $\begin{array}{l}\text { le } \\
\text { ngs }\end{array}$ & East & Temp. \\
\hline & Left & Riglit & & & & T,eft & Right & & & & Left & Riglit & & & & Left & Right & & \\
\hline & d & 00 & $\circ \quad$ & 0 & $\mathrm{~h} \mathrm{~m}$ & d & d & $\circ$, & & $\mathrm{l}_{1} \mathrm{~m}$ & d & d & & 。 & h $\mathrm{m}$ & d & d & & 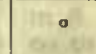 \\
\hline 1600 & 29.6 & 28.8 & 2242 & -18.9 & 1800 & 29.0 & 28.7 & 2242 & -16.3 & $2000^{*}$ & 45.5 & 66.0 & 2246 & $-\mathrm{I} 9.2$ & 2200 & 18.0 & 21.0 & $223 \mathrm{~T}$ & $\operatorname{sen}$ \\
\hline 02 & 29.2 & 28.3 & 43 & $x_{n}$ & 02 & 28.8 & 28.6 & 43 & 10 & $02^{*}$ & 65.3 & 74.7 & 2335 & 1 & 02 & I8.5 & 21.9 & & is \\
\hline 04 & 29.9 & 29.7 & 4I & 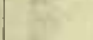 & 04 & 29.1 & 28.8 & 42 & - & $04^{*}$ & $42: 0$ & 50.2 & 49 & 10 & 04 & 17.9 & $21 \cdot 3$ & 31 & $m$ \\
\hline 06 & 29.2 & 28.7 & 42 & if & 06 & 29.2 & 28.9 & 42 & & 06 & 8.7 & 26.1 & 2304 & $t_{0}$ & 06 & I8.5 & 21.8 & & 85 \\
\hline 08 & 30. I & 20.8 & $4 \mathrm{I}$ & 31 & 08 & 20.4 & 29.0 & 42 & & $08^{*}$ & $4 \mathrm{I} \cdot \mathrm{I}$ & 55.9 & 2256 & & 08 & 19.2 & 21.9 & 32 & as \\
\hline Io & 30.9 & 30.6 & 39 & (3) & 10 & 29.7 & 29.2 & $4 \mathrm{I}$ & ga & $10^{*}$ & 52.2 & 69.1 & 2335 & & I0 & 20.2 & 22.8 & & 89 \\
\hline 12 & 30.0 & 28.8 & $4 \mathrm{I}$ & & 12 & 30.3 & 29.8 & 40 & & 12 & 52.0 & 62.2 & 30 & & 12 & 19.8 & 22.2 & 33 & \\
\hline I4 & $3 \mathrm{I} . \mathrm{I}$ & 30.3 & 39 & -18.3 & 14 & 30.8 & 30.2 & 40 & -т6.3 & 14 & 39.1 & 51.0 & II & -19.0 & 14 & 20.0 & 22.9 & 33 & -18.8 \\
\hline 16 & $3 \mathrm{I} \cdot 4$ & 30.5 & 30 & 82 & I6 & $3 I \cdot 3$ & 30.8 & $\quad 39$ & 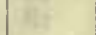 & I6 & 56.0 & 64.0 & 34 & 27 & I6 & I9.3 & 23.0 & 33 & An: \\
\hline 18 & 32.5 & $3 \mathrm{~T} .2$ & 38 & 25 & 18 & 31.0 & 30.6 & 39 & $x^{2}$ & I 8 & 49.8 & 59.8 & 26 & $H$ & 18 & 19.0 & 22.7 & 33 & $8 z$ \\
\hline 20 & 32.0 & 30.8 & $3^{8}$ & $=8$ & 20 & 31.0 & 30.7 & 30 & atr & 20 & 43.0 & 50.8 & 13 & ext & 20 & 18.9 & 22.7 & 33 & 28 \\
\hline 22 & 3 . & 30.8 & $3^{8}$ & is & 22 & 31.3 & 31.1 & 39 & 8. & 22 & 41.0 & 47.7 & on & - & 22 & 20.0 & 23.0 & 34 & ay \\
\hline 24 & 32.3 & $3 \mathrm{~T} . \mathrm{I}$ & 38 & Rs & 24 & 30.0 & 29.5 & $4 I$ & $=$ & 24 & 41.8 & 49.9 & 12 & A & 24 & 22.0 & 24.8 & & the \\
\hline 26 & 32.4 & 31.0 & 38 & Aft & 26 & 30.1 & 20.4 & $4 \mathbf{T}$ & $y^{2}$ & 26 & 38. I & 42.7 & 03 & & 26 & 22.8 & 25.0 & 37 & te \\
\hline 28 & 32.3 & $3^{\mathrm{I}} \cdot 2$ & 38 & & 28 & 30.2 & 29.3 & $4 I$ & & 28 & 35.4 & 40.6 & $\infty$ & & 28 & 22.8 & 25.0 & 37 & \\
\hline 30 & 32.4 & $3 x \cdot 3$ & 38 & -18.0 & 30 & 30.3 & 29.6 & $4 \mathrm{I}$ & -16.3 & 30 & 37.8 & $42 \cdot 3$ & 03 & -19.0 & 30 & 22. I & 24.0 & $3^{6}$ & -18.7 \\
\hline 32 & 32.3 & 31.2 & 38 & ye & 32 & 30.4 & 29.8 & 40 & $y^{2}$ & 32 & 4.3 .5 & 47.9 & 23 I2 & & 32 & $20 . \mathrm{I}$ & 23.0 & 34 & Ix \\
\hline 34 & 3Т.9 & 3 I. . & 38 & $\theta$ & 34 & 29.7 & 29. I & $4 I$ & F & 34 & 37.0 & 38.0 & 2259 & & 34 & 20.3 & 22.7 & 34 & 3. \\
\hline 36 & 3I.I & 30.3 & 30 & tra & 36 & 29.9 & 29.4 & 2. $4 \mathrm{I}$ & 12 & 36 & 31.7 & $35 \cdot 3$ & 52 & $18 x$ & 36 & 20.0 & 22.3 & 33 & 8 \\
\hline 38 & 32.0 & $3 I \cdot 3$ & 38 & $x^{2}$ & 38 & 30.5 & 29.9 & 40 & 6 & 38 & 19.5 & 30.0 & 39 & the & 38 & 20.3 & 22.9 & 34 & 5 \\
\hline 40 & 32.2 & $3 \mathrm{I} .4$ & 38 & tit & 40 & 30.8 & 30.2 & 40 & 32 & 40 & 40.5 & 35.3 & 2259 & & 40 & 20.4 & 22.5 & 33 & m \\
\hline 42 & 32.5 & 31.4 & 38 & -2 & 42 & 30.6 & 30.0 & 40 & $=$ & 42 & 37.2 & 42.0 & 2302 & & 42 & 18.9 & 20.0 & 30 & 4 \\
\hline 44 & 30.2 & 39.6 & $4 \mathrm{I}$ & -17.2 & 44 & 30.5 & 29.9 & 40 & -тб.3 & 44 & 27.0 & 32.0 & 2246 & -19.0 & 44 & 18.7 & 20.1 & 30 & . \\
\hline 46 & 30.8 & 29.7 & 40 & $7=$ & 46 & 30.6 & 30.1 & 40 & 13 & 46 & 25.0 & 30.1 & 43 & 18 & 46 & I8. 6 & 20.0 & 30 & B) \\
\hline 48 & 30.1 & 29.6 & $4 \mathrm{I}$ & 8 & 48 & 30.4 & 30.1 & 40 & a & 48 & 23.8 & 26.8 & 40 & me & 48 & 18.9 & 19.6 & .30 & $\omega$ \\
\hline 50 & 30.0 & 2n. I & $4 I$ & tre & 50 & 30.8 & 30.3 & 40 & w & 50 & 19.0 & 22.0 & $22 \quad 32$ & 4 & 50 & 19.4 & 20.7 & $3 \mathrm{I}$ & is \\
\hline 52 & 30.6 & 29.0 & 40 & (5) & 52 & 31.3 & 31.0 & 30 & it & 52 & 37.5 & $4 \pi .0$ & 23 OI & 25 & 52 & 17.1 & 18.8 & 28 & is \\
\hline 54 & 30.0 & 29.6 & $4 \mathrm{~T}$ & 5 & 51 & .31 .0 & 31.0 & 30 & 3죠 & 54 & 37.0 & 38.9 & 2259 & & 54 & 18.5 & 10.0 & 29 & mo \\
\hline 56 & 30.1 & 29.7 & $4 \mathrm{I}$ & 32 & 56 & 31.5 & $3 \mathrm{I} .3$ & 38 & ins & 56 & 24.5 & 29.0 & 42 & & 56 & 17.0 & 18.0 & 27 & 5. \\
\hline 58 & 20.6 & $20 . \mathrm{T}$ & 42 & & 58 & 32.2 & 31.6 & 38 & & 58 & 22.0 & 28.0 & 39 & & 58 & I6.8 & 17.2 & 27 & \\
\hline 7 on & 29.4 & 28.9 & 42 & -17.3 & in 00 & 32.0 & 31.8 & 38 & -16.2 & 2100 & 30.7 & 36.7 & 53 & -19.2 & 2300 & I6.2 & 16.9 & 26 & -18.8 \\
\hline 02 & 30.2 & 29.9 & 40 & te & 02 & 33.7 & 33.4 & 35 & 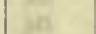 & 02 & 26. ז & 32.0 & 45 & -5 & 02 & 15.0 & 16.2 & 24 & $1-x=$ \\
\hline 04 & 30.5 & 30.2 & 40 & 5 & 04 & 33.7 & 33.1 & 35 & 85 & 04 & $3 I \cdot 3$ & $35 \cdot 3$ & 52 & & 0 & 16.2 & 17.1 & 26 & int \\
\hline of & 30.3 & 29.9 & 40 & Es & o6 & $3.3 \cdot 3$ & 32.9 & 36 & ha & 06 & 28.2 & 34.7 & 49 & h & 06 & 16.2 & 17.0 & 26 & Th. \\
\hline as & 29.8 & 29.2 & $4 \mathrm{I}$ & (6t) & 08 & 33.3 & 33.0 & 36 & $n$ & as & 26.3 & 29.8 & 44 & ter & 08 & I6.0 & 16.8 & 26 & hat \\
\hline I0 & 30.3 & 29.9 & 40 & 67 & to & 32.5 & 32.1 & 37 & (a) & I0 & 23.0 & 26.0 & 38 & 10 & 10 & 15.5 & 16.0 & 25 & ter \\
\hline I2 & 30.4 & $30 . \mathrm{I}$ & 40 & & 12 & 32.3 & 31.7 & 37 & & I2 & 17.0 & 21.3 & 30 & 15 & 12 & 16.3 & 17.3 & 26 & \\
\hline $\mathrm{I} 1$ & 30.0 & 30.4 & 40 & -17.0 & 14 & $3 I . n$ & 31.2 & 38 & -16.1 & 14 & 15.9 & 20.8 & 29 & -19.1 & 14 & 17.3 & 17.9 & 27 & -18.8 \\
\hline I6 & 30.3 & 29.0 & 40 & ( & If & 35.2 & 33.9 & 33 & 1 & 16 & 18.8 & 21.5 & 32 & $1=-2$ & I & 17.8 & 19.4 & 29 & 1: \\
\hline T8 & 20.6 & 29.3 & $4 I$ & os & I8 & 36.8 & 36.2 & 30 & $x$ & I8 & 19. I & 22.0 & .32 & te & 18 & 17.3 & 19.9 & 29 & $\mathrm{~m}$ \\
\hline 20 & 29.8 & 29.2 & $4 \pi$ & $x^{6}$ & 20 & 36.6 & .35 .8 & $3 \mathrm{I}$ & the & 20 & 10.0 & 21.0 & $3 \mathrm{~T}$ & tats & 20 & 15.8 & 18.0 & 26 & on. \\
\hline 22 & 29.8 & 29.3 & 41 & 45 & 22 & $37 \cdot 3$ & 36.8 & 30 & 15 & 22 & 20.0 & 21.8 & 33 & & 22 & 15.7 & I8.0 & 26 & is \\
\hline 24 & 29.9 & 20.3 & $4 I$ & $\mathrm{k}$ & 24 & 36.9 & 35.2 & 31 & is & 24 & 20.7 & 22.0 & 3.3 & 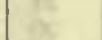 & 24 & 16.2 & 18.2 & 27 & 35 \\
\hline 26 & 20.7 & 20.1 & 41 & E & 26 & 36.0 & 35.3 & 32 & te & 26 & 20.4 & 22.0 & 33 & se & 26 & 17.0 & 19.0 & 28 & ex \\
\hline 28 & 20.8 & & $4 \pi$ & & 28 & 35.0 & 34.9 & 32 & & 28 & 19.7 & 21.8 & 32 & & 28 & 17.0 & 19.3 & 28 & 10 \\
\hline .30 & 20.4 & 28.8 & 14.42 & -16.9 & .30 & 35.4 & 3.4 .7 & 33 & -16.0 & 30 & 19.7 & $2 \mathrm{I} .4$ & 32 & -19.0 & 30 & 19.1 & 21.0 & $3 \mathrm{I}$ & -18.8 \\
\hline .32 & 20.7 & 28.7 & 42 & M & .32 & 35.0 & 35.0 & 32 & (4) & 32 & 20.0 & 21.9 & 33 & 12 & 32 & $2 \mathrm{~T} . \mathrm{O}$ & 21.8 & 33 & $y$ \\
\hline .31 & 20.1 & 28.6 & 42 & 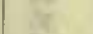 & 31 & 36.4 & 35.5 & $3 I$ & ne & 34.5 & 21.0 & 2.3 .0 & 34 & & 34 & 21.0 & $2 \mathrm{t} .3$ & 33 & 8 \\
\hline 36 & 29.0 & & 42 & 月 & 36 & 37.5 & 36.6 & 30 & 8 & 36.5 & 21.7 & 23.3 & 35 & 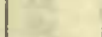 & 3 & 21.0 & 23.7 & 35 & \\
\hline 38 & 20.0 & 28.6 & 42 & $\Leftrightarrow$ & .38 & $.37 . \mathrm{I}$ & 36.7 & 30 & ats & 38 & 21.3 & 23.5 & 34 & & 38 & 21.0 & 23.8 & 35 & tre \\
\hline 40 & 28.2 & 28.0 & 44 & 8 & 40 & 37.0 & 36.7 & 30 & a & 40 & 22.4 & 24.0 & 36 & 10 & 40 & 22.3 & 24.5 & 37 & \% \\
\hline 42 & 27.6 & 27. & 45 & & 42 & 37.2 & 36.4 & 30 & & 42 & 20.7 & 22.0 & 33 & & 42 & 23.5 & 25.9 & 39 & \\
\hline 44 & 28.4 & 28.0 & 43 & -I6.5 & 44 & 37.0 & 36.4 & 30 & -15.9 & 44 & 20.6 & $2 \mathrm{I} \cdot 3$ & 3.3 & -19.0 & 44 & 25.0 & 27.1 & $4 I$ & -18.8 \\
\hline 46 & 28.7 & 28.2 & 4.3 & 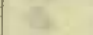 & 45 & 35.7 & 35.3 & 32 & $y^{2}$ & 46 & 20.3 & $2 \pi .3$ & 33 & & 46 & 25.5 & $27 . I$ & $4 \pi$ & \\
\hline 48 & 28.9 & 28.4 & 43 & $\bar{x}$ & 48 & 3.5 .7 & 36.2 & $3 \mathrm{I}$ & 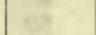 & 48 & 21.0 & $2 \pi .8$ & 33 & & 48 & 25.8 & $27 . I$ & $4 I$ & 6 \\
\hline 50 & 28.7 & 28.3 & 43 & a & 50 & 36.5 & .36 .2 & 31 & W & 50 & I0. 0 & 22.9 & 3.3 & & 50 & 25.0 & 26.7 & $4 I$ & 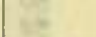 \\
\hline 52 & 29.0 & 28.8 & 42 & 62 & 52 & 36.8 & 35.7 & $3 \mathrm{I}$ & 20 & 52 & I8.6 & $2 \mathrm{I} .2$ & $3 \mathrm{I}$ & 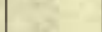 & 52 & 2 โ 8 & 22 & 35 & 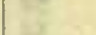 \\
\hline 54 & 20.0 & & 4.3 & & 5.1 & 36.2 & 34.0 & 32 & & 54 & I8.9 & 21.9 & 31 & & 54 & 27.2 & 28.5 & 44 & $\therefore$ \\
\hline $5^{6}$ & 28.6 & 28.6 & 43 & is & 56 & 36.2 & $35 . \mathrm{I}$ & 32 & & 56 & I8. 4 & 21.3 & 31 & & 56 & 29.3 & .33 .0 & 49 & 2 \\
\hline 58 & 28.9 & 28.7 & 42 & & 58 & 35.9 & $34 \cdot 3$ & 32 & & 58 & I8. 5 & 21.0 & $3 I$ & -18.8 & 58 & 30.2 & 33.2 & 50 & \\
\hline & & & & & 2000 & 36.2 & 35.7 & 31 & -т6.0 & & & & & & 2400 & 31.0 & 32.5 & 50 & -18.8 \\
\hline
\end{tabular}

Correction to local mean time is - $1 \mathrm{~m}$ iss.

Torsion head at $15 \mathrm{~h} .35 \mathrm{~m}$ read $18^{\circ}$ and at $20 \mathrm{~h}$ rom read the samc. Observer-H. H. N.
Correction to local mean time is - Im 15.5s.

Torsion head at $10 \mathrm{~h} 25 \mathrm{~m}$ rcaid $16^{\circ}$ and at 24 h $20 \mathrm{~m}$ read the same. Observer-J. V. 
Tabulation of magnetic declinations observed at Teplitz Bay-Continued

\begin{tabular}{|c|c|c|c|c|c|c|c|c|c|c|c|c|c|c|c|c|c|c|c|}
\hline \multicolumn{6}{|c|}{ Tuesday, March 8, I904 } & \multicolumn{4}{|c|}{ Magnet scale erect } & \multicolumn{6}{|c|}{ Wednesday, March 9, 1904} & \multicolumn{4}{|c|}{ Magnet scale inverted } \\
\hline \multirow{2}{*}{$\begin{array}{l}\text { Chr'r } \\
\text { time }\end{array}$} & \multicolumn{2}{|c|}{$\begin{array}{l}\text { Scale } \\
\text { readings }\end{array}$} & \multirow{2}{*}{$\begin{array}{l}\text { East } \\
\text { decli- } \\
\text { nation }\end{array}$} & Temp. & Chr't & $\begin{array}{r}\mathrm{Sc} \\
\mathrm{read}\end{array}$ & $\begin{array}{l}\text { alc } \\
\text { ings }\end{array}$ & East & Temp. & Chr'r & $\begin{array}{l}\text { Sc } \\
\text { reac }\end{array}$ & $\begin{array}{l}\text { ale } \\
\text { lings }\end{array}$ & $\begin{array}{l}\text { East } \\
\text { decli- }\end{array}$ & Temp. & & $\begin{array}{l}\text { Sc } \\
\text { read }\end{array}$ & $\begin{array}{l}\text { le } \\
\text { ings }\end{array}$ & $\begin{array}{l}\text { East } \\
\text { decli- }\end{array}$ & Temp. \\
\hline & Left & Right & & & & Left & Right & & & & Left & Right & & & & Left & Right & & \\
\hline h $\mathrm{m}$ & d & 4 & $\cdot \quad$ & $\bullet$ & $\mathrm{h} \mathrm{m}$ & d & d & ० : & - & $h \mathrm{~m}$ & $\mathrm{~d}$ & d & - & 0 & $\mathrm{~h} \mathrm{~m}$ & d & d & - ' & $\bullet$ \\
\hline 1200 & 53.2 & 54.8 & 2239 & -20.2 & 1400 & 52.0 & 52.5 & 2236 & -19.0 & 000 & 50.3 & 48.9 & 2245 & -20.2 & 200 & 47.9 & 47.6 & 2248 & -19.2 \\
\hline 02 & 52.6 & 54.7 & & $1+11=0$ & 02 & 55.2 & 55.7 & 41 & & 02 & 50.2 & $49 . I$ & 45 & & 02 & 48.3 & 48.0 & 48 & \\
\hline 04 & 51.9 & 52.0 & 36 & 6 & 04. & 48.8 & 49.2 & 31 & & 04 & 50.1 & 49.0 & 45 & & 04 & 47.4 & $47 . I$ & 49 & \\
\hline 06 & 52.3 & 52.5 & 37 & (1) & 06 & 48 & $o b$ & 30 & & 06 & 50.0 & 49. I & 45 & & 06 & 47.8 & 47.7 & 48 & \\
\hline 08 & 51.8 & 53.4 & 37 & 60 & 08 & 46.5 & $47 . I$ & 28 & & 08 & 50.6 & 49.7 & 44 & & 08 & 47.7 & 47.7 & 49 & \\
\hline to & 51.6 & $52 . \mathrm{I}$ & 36 & 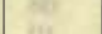 & 10 & 46.3 & 47.5 & 28 & tar & 10 & 50.9 & 49.8 & 44 & & I0 & 47 & $6 b$ & 49 & \\
\hline 12 & 50.8 & 5 I. 3 & 34 & (1) & 12 & 47.6 & 48.0 & 29 & (1) & 12 & 51.0 & 50.2 & 44 & & J2 & 46.9 & 46.7 & 50 & \\
\hline I4 & 54.2 & 54.9 & 40 & -20.0 & 14 & 47.7 & 48.3 & 30 & -19.0 & I4 & 51.7 & 50.8 & 43 & -20.5 & 14 & 47.2 & 46.9 & 50 & -I0.I \\
\hline 16 & $53 . I$ & 53.9 & 38 & & 16 & 47.0 & 48.2 & 29 & & 16 & $5 \mathrm{I} .4$ & 50.7 & 43 & & I6 & 47.8 & 47.5 & 49 & \\
\hline 18 & 52.2 & 52.9 & 37 & zf. & I8 & 46.1 & $47 \cdot 3$ & 28 & Ar. & 18 & 50.9 & 50.3 & 44 & & 18 & 47.8 & 47.6 & 48 & \\
\hline 20 & 52.9 & 53.7 & 38 & & 20 & 45.4 & 46.9 & 27 & & 20 & $5 \mathrm{I} .4$ & 50.8 & 43 & & 20 & 48.6 & 48.4 & 47 & \\
\hline 22 & 52.3 & 53.2 & 37 & $y$ & 22 & 45.9 & 47.3 & 28 & wa & 22 & 51.2 & 50.7 & 43 & & 22 & 48.7 & 48.2 & 47 & \\
\hline 24 & 54.8 & 55.6 & $4 \mathrm{I}$ & 4 & 24 & 44.6 & 45.8 & 25 & 3 & 24 & 50.9 & 50.3 & 44 & 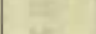 & 24 & 48.3 & 48. I & 48 & \\
\hline 26 & 57.1 & 57.8 & 44 & we & 26 & 44.8 & 46.0 & 26 & $=$ & 26 & 50.6 & 50.1 & 44 & sef & 26 & 48.3 & 48.2 & 48 & \\
\hline 28 & 59.1 & 60.3 & 48 & & 28 & 45.8 & 46.6 & 27 & & 28 & 50.4 & 49.9 & 45 & \pm & 28 & 48.2 & 48. & 48 & \\
\hline 30 & 57.6 & 59.4 & 46 & -19.9 & 30 & 45.2 & 46.5 & 26 & - Г9.0 & 30 & 50.7 & 50.3 & 44 & -20.5 & 30 & 48.2 & 48.0 & 48 & -19.0 \\
\hline 32 & 55.5 & 57.0 & 43 & 0 & 32 & 44.6 & 45.3 & 25 & & 32 & 50.9 & 50.3 & 44 & & 32 & 48.6 & 48.3 & 47 & \\
\hline 34 & $53 . I$ & 54.4 & 39 & & 34 & $43 \cdot 3$ & 44.5 & 23 & 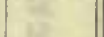 & 34 & 50.8 & 50.3 & 44 & et. & 3 & 48. & 48.5 & 47 & \\
\hline 36 & $54 \cdot 3$ & 55.6 & $4 \mathrm{I}$ & 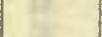 & 36 & $44 . \mathrm{I}$ & 45.0 & 24 & 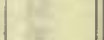 & 36 & 50.2 & 50.0 & 45 & 3 & 36 & 48.8 & 48.6 & 47 & \\
\hline 38 & 55.7 & 56.6 & 43 & 8 & 38 & 44.7 & 45.8 & 25 & & 38 & 50.2 & 49.9 & 45 & & 38 & 48.2 & 47.9 & 48 & \\
\hline 40 & 53.2 & 55.0 & 39 & 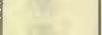 & 40 & 44.7 & 45.4 & 25 & 8 & 40 & 51.0 & 50.8 & 43 & 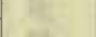 & 40 & 47.8 & 47.4 & 49 & \\
\hline 42 & 5 I. 8 & 53.4 & 37 & (1) & 42 & 47.6 & 48.5 & 30 & (2) & 42 & 51.2 & 50.9 & 43 & 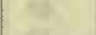 & 42 & 48.7 & 48. & 47 & \\
\hline 44 & 51.7 & 52.6 & 36 & -19.9 & 44 & 48.0 & 40.2 & 31 & -18.9 & 44 & 50.4 & 50.1 & 44 & -20.4 & 44 & 49.0 & 48.8 & 46 & -19.0 \\
\hline 46 & 53.4 & 54.7 & 39 & & 46 & 47.5 & 48.2 & 29 & & 46 & 49.4 & 49.2 & 46 & & 46 & 49.3 & 49.0 & 46 & \\
\hline 48 & 52.9 & 54.9 & 39 & ta & 48 & 47.3 & 48. I & 29 & s. & 48 & 49.8 & 49.3 & 45 & 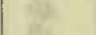 & 48 & 49.2 & 48.8 & 46 & \\
\hline 50 & 54.2 & 56.0 & $4 I$ & (x) & 50 & 49.4 & 49.8 & 32 & 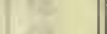 & 50 & 49.2 & 48.8 & 46 & 4 & 50 & 48.9 & 48.5 & 47 & \\
\hline 52 & 57.1 & 58.3 & 45 & 3 & 52 & 49.2 & 49.9 & 32 & 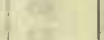 & 52 & 48.7 & 48.4 & 47 & f & 52 & 48.9 & 48. & 47 & \\
\hline 54 & 58.7 & 59.8 & 47 & 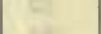 & 54 & 48.5 & 49.3 & $3 \mathrm{I}$ & 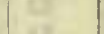 & 54 & 49.4 & $49 . I$ & 46 & 5 & 5 & 40.1 & 48. & 46 & Q \\
\hline 56 & 55.7 & 56.9 & 43 & & 56 & 40.6 & 49.8 & 32 & 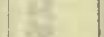 & 56 & 49.8 & 49.3 & 45 & te & 5 & 48.2 & 47. & 48 & \\
\hline 58 & 53.9 & 55.3 & 40 & & 58 & 48.9 & 49.8 & 32 & & 58 & 49.8 & 49.6 & 45 & & 58 & 48.3 & 48. I & 48 & \\
\hline 1300 & 53.8 & 54.9 & 40 & -19.9 & I5 00 & 49.0 & 49.7 & 32 & -18.7 & I 00 & 50.3 & 50. I & 44 & -20.1 & 300 & 48.1 & 47.8 & 48 & -18.7 \\
\hline 02 & 55.4 & 56.5 & 42 & $1=$ & 02 & 50.2 & 50.9 & 34 & - & 02 & 50.9 & 50.6 & 44 & & 02 & 47.9 & 47.7 & 48 & \\
\hline 04 & 56.2 & 56.7 & 43 & a & 04 & 50. I & 50.4 & 33 & 20 & 04 & 51.0 & 50.7 & 43 & 72 & 04 & $47 . I$ & 46. & 50 & \\
\hline 06 & 55.3 & 56.6 & 42 & Ex & 06 & 51.2 & 51.9 & 35 & (6. & 06 & $5 \mathrm{I} . \mathrm{I}$ & 51.0 & 43 & ge & $\alpha$ & 48.3 & 48. & 48 & \\
\hline 08 & 53. I & 54.0 & 38 & $x^{2}$ & 08 & 52.9 & 53.2 & 38 & ta. & 08 & 51.2 & 51.0 & 43 & 9 & 08 & 49.2 & 48.9 & 46 & \\
\hline Io & 54.3 & 55.2 & 40 & (10) & 10 & 53.0 & 53.4 & 38 & 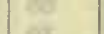 & I0 & 51.9 & 51.6 & 42 & 8 & IC & 50.8 & 50.6 & 44 & \\
\hline I2 & 55.0 & 55.9 & $4 \mathrm{I}$ & $\cos$ & 12 & 51.8 & 52.6 & 36 & 0 & 12 & 53.0 & 52.8 & 40 & 92 & 12 & $5 \mathrm{I} .4$ & 5I.I & 43 & \\
\hline 14 & 53.5 & 54.2 & 39 & -10.6 & 14 & $5 \pi .5$ & 51.8 & 35 & -18.5 & 14 & 54.1 & $54 \cdot 3$ & 38 & -19.9 & I4 & 49.9 & 49.5 & 45 & -18.6 \\
\hline 16 & 53.4 & $54 \cdot 3$ & 39 & s. & 16 & 51.7 & $52 . \mathrm{I}$ & 36 & & 16 & 55.2 & 55.0 & 37 & & I & 49.0 & 48.8 & 46 & \\
\hline I8 & 51.5 & 52.7 & 36 & Ret & 18 & 50.8 & $5 \mathrm{I} .2$ & 34 & & 18 & 55.4 & $55 . \mathrm{I}$ & 37 & & 18 & 47.9 & 47.7 & 48 & \\
\hline 20 & 48.8 & 50.3 & 32 & ar & 20 & 47.6 & 47.9 & 29 & 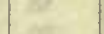 & 20 & 55.1 & 54.8 & 37 & & 20 & 48.4 & 48. & 47 & \\
\hline 22 & 49.8 & 50.7 & 33 & 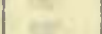 & 22 & 47.4 & 47.8 & 29 & & 22 & 54.4 & $54 . I$ & 38 & 9 & 22 & 48.9 & 48.6 & 47 & \\
\hline 24 & 49.7 & 50.4 & 33 & as & 24 & 47.2 & 47.9 & 29 & ta & 24 & 54.0 & 53.8 & 39 & te & 24 & 49.2 & $40 . I$ & 46 & $\mathrm{x}=\mathrm{s}$ \\
\hline 26 & 50.0 & 50.0 & 33 & & 26 & 48.6 & 48.9 & $3 \mathrm{I}$ & $\theta$ & 26 & 53.2 & 53.0 & 40 & 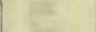 & 26 & 49.2 & 48. & 46 & \\
\hline 28 & 50.8 & 5 I. 8 & 35 & & 28 & 49 & $a d$ & 3I & & 28 & $53 . \mathrm{I}$ & 52.6 & 40 & & 28 & 48.8 & 48.6 & 47 & \\
\hline 30 & 53.3 & $54 . \mathrm{I}$ & 39 & -19.5 & 30 & 49.6 & 49.8 & 32 & -18.4 & 30 & 52.8 & 52.4 & $4 \mathrm{I}$ & -10.9 & 30 & 40.2 & 48.9 & 46 & - 18.2 \\
\hline 32 & 53.9 & 54.4 & 39 & & 32 & 50.2 & 50.7 & 33 & & 32 & 5I.I & 50.8 & 43 & & 32 & 48.1 & 48.0 & 48 & \\
\hline 34 & 55.8 & 57.0 & 43 & 18 & 34 & 50.6 & 51.0 & 34 & 10 & 34 & 49.9 & 49.4 & 45 & 7 & 34 & 48.8 & 48.6 & 47 & \\
\hline 36 & 54.7 & 55.9 & $4 \mathrm{I}$ & 130 & 36 & $5 I \cdot 3$ & 52.0 & 35 & & 36 & 49.5 & 49.2 & 46 & 4 & 36 & 49.1 & 48.8 & 46 & \\
\hline 38 & $53 . \mathrm{I}$ & 53.9 & 38 & & 38 & 48.3 & 49.0 & $3 I$ & & 38 & 48.8 & 48.7 & 47 & & 38 & 48.9 & 47.7 & 47 & \\
\hline 40 & 53.5 & 54.4 & 39 & & 40 & 49.8 & 50.6 & 33 & & 40 & 48.8 & 48.8 & 47 & & 40 & 47.3 & 46. & 50 & \\
\hline 42 & 53.8 & 54.2 & 39 & & 42 & 50.5 & $5 \mathrm{I} .2$ & 34 & & 42 & 49.0 & 48.7 & 47 & & 42 & 46.4 & 46. & $5 \mathrm{I}$ & \\
\hline & 53.5 & 5.3 .9 & 39 & -19.1 & 44 & 50.5 & $5 \mathrm{I} .3$ & 34 & -18.3 & 44 & 48.9 & 48.8 & 47 & -19.6 & 44 & 47.2 & 46.8 & 50 & -18.1 \\
\hline 46 & 53.8 & 54.6 & 39 & $x^{2}$ & 46 & 49.3 & 49.9 & 32 & & 46 & 48.4 & 47.9 & 48 & & 46 & 47.9 & 47.7 & 48 & \\
\hline 48 & 53.6 & 53.8 & 30 & $40 \mathrm{fe}$ & 48 & 50.0 & 50.3 & 33 & & 48 & 46.8 & 46.5 & 50 & 3 & 48 & 47.2 & 46.9 & 50 & \\
\hline 50 & 53.2 & 53.7 & 38 & $x^{2}$ & 50 & 49.8 & $50 . \mathrm{I}$ & 33 & & 50 & 47.0 & 46.8 & 50 & 4 & 50 & 47.3 & 47.0 & 49 & \\
\hline 52 & 54.3 & 54.6 & & at & 52 & 50.3 & 50.4 & 33 & 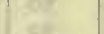 & 52 & 47.3 & 47.2 & 49 & 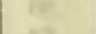 & 52 & 48.8 & 48.3 & 47 & \\
\hline 54 & 52.9 & 53.4 & 38 & 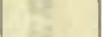 & 54 & 51.8 & 52.1 & 36 & & 54 & 47.2 & 47.1 & 40 & 227 & 54 & 48.5 & 48.2 & 47 & \\
\hline 56 & 53.0 & 53.6 & 38 & Es & 56 & 51.9 & 52.4 & 36 & 12 & 56 & 47.0 & 46.3 & 50 & 38 & 56 & 48.0 & 47.7 & 48 & \\
\hline 58 & 52.9 & 53.8 & 38 & & 58 & 51.0 & 51.7 & 35 & & 58 & 47.3 & 47. I & 49 & & 58 & 47.6 & 47.2 & 49 & \\
\hline 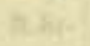 & & $3-5$ & and & $a n$ & I6 00 & 51.2 & 5 I. 4 & 35 & -18.3 & & & & & & & & & & \\
\hline
\end{tabular}


Tabulation of magnetie declinations observed at Teplitz Bay-Continued

\begin{tabular}{|c|c|c|c|c|c|c|c|c|c|c|c|c|c|c|c|c|c|c|c|c|}
\hline \multicolumn{7}{|c|}{ Wcdnesday, March 9, 1904} & \multicolumn{4}{|c|}{ Magnet scale inverted } & \multicolumn{6}{|c|}{ Thursday, March 10, 1904} & \multicolumn{4}{|c|}{ Magnet scale erect } \\
\hline \multirow{2}{*}{$\begin{array}{l}\text { Chr'r } \\
\text { time }\end{array}$} & \multicolumn{2}{|c|}{$\begin{array}{l}\text { Scale } \\
\text { readings }\end{array}$} & $\begin{array}{l}\text { Eas } \\
\text { dec }\end{array}$ & & Temp & Chr'r & $\begin{array}{r}\text { Sc } \\
\text { reac }\end{array}$ & $\begin{array}{l}\text { ale } \\
\text { lings }\end{array}$ & $\begin{array}{l}\text { East } \\
\text { decli- }\end{array}$ & Temp. & Chr'r & $\begin{array}{l}\text { Sc } \\
\text { reac }\end{array}$ & le & $\begin{array}{l}\text { East } \\
\text { decli- }\end{array}$ & Temp. & Chr'r & $\begin{array}{r}\mathrm{Sc} \\
\text { read }\end{array}$ & $\begin{array}{l}\text { ale } \\
\text { lings }\end{array}$ & $\begin{array}{l}\text { East } \\
\text { decli- }\end{array}$ & Temp. \\
\hline & I,eft & Right & natic & & & time & Left & Right & nation & C. & time & Left & Right & nation & c. & time & Left & Right & nation & c. \\
\hline lim & $d$ & d & & , & $\circ$ & $\mathrm{h} \mathrm{m}$ & d & $d$ & $\circ$, & • & $\mathrm{h} \mathrm{m}$ & d & $d$ & $\circ$, & & $\mathrm{h} \mathrm{m}$ & d & d & 0, & $\bullet$ \\
\hline 400 & 48.9 & 48.2 & 224 & & -18.0 & 600 & 43.7 & 43.2 & 2255 & -17.6 & 1600 & 44.9 & 45.8 & 2232 & -23.4 & 1800 & 49.7 & 52.4 & 2241 & -22.9 \\
\hline 02 & 50.0 & 49.7 & & 45 & 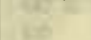 & 02 & 39.8 & 39.1 & 23 OI & & 02 & 43. I & $45 \cdot 3$ & 30 & - & 02 & 50.7 & 52.3 & 42 & (2) \\
\hline 04 & 49.6 & 49.1 & & 46 & 60 & 04 & 44.9 & $44 \cdot 3$ & 2253 & & of & 43.2 & 44.4 & 30 & ta & 04 & 51.8 & 54.8 & 44 & 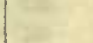 \\
\hline ó & 48.3 & 47.9 & & 8 & 8 & 06 & 48.2 & 47.7 & 48 & 30 & of & 43.5 & 44.6 & 30 & 30 & 06 & 53.9 & 54.5 & 46 & 25 \\
\hline 08 & $47 . \ddot{8}$ & 47.4 & & 49 & me & 08 & 46.7 & 46.1 & 50 & es & 08 & 44.6 & 45.7 & 32 & (1) & 08 & 52.7 & 53.8 & 44 & 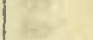 \\
\hline 10 & 47.6 & 47.2 & & 49 & or & 10 & 46.2 & 45.9 & 5 I & we & 10 & -43.8 & 45.9 & $3 \mathrm{I}$ & & 10 & 53.3 & 54.2 & 45 & nat \\
\hline 12 & 48.3 & 47.9 & & 48 & -8 & I2 & 48.7 & 48.1 & 47 & & 12 & 44.4 & 46.2 & 32 & & 12 & 54.0 & $55 \cdot 3$ & 46 & \\
\hline 14 & 47.6 & 47.4 & & 49. & -18.0 & 14 & $5 I .4$ & 51.1 & 43 & -17.5 & I 4 & 44.5 & 46.2 & 32 & -23.0 & 14 & 54.7 & 55.4 & 47 & -22.7 \\
\hline 16 & 47.7 & 47.4 & & 49 & I & 16 & 46.8 & 45.4 & $5 \mathrm{I}$ & (n) & 16 & 45.3 & 46.9 & 33 & $1=$ & 16 & 54.9 & 55.8 & & (2) \\
\hline 18 & 48.4 & 48.1 & & 18 & the & 18 & 41.7 & 40.8 & 59 & (5) & 18 & $47 \cdot 4$ & 48.3 & 36 & W & 18 & 54.7 & 55.3 & 47 & X) \\
\hline 20 & 48.8 & 48.5 & & 47 & & 20 & 41.9 & $41 \cdot 3$ & 58 & & 20 & 48.9 & 50.2 & 38 & we & 20 & 52.8 & 54.1 & 45 & 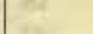 \\
\hline 22 & 49.4 & 49. I & & 66 & te & 22 & 43.3 & 42.9 & 56 & & 22 & 48.3 & 49.5 & 38 & on & 22 & 51.9 & 52.7 & 43 & tat \\
\hline 24 & 49.9 & 49.6 & 4 & 45 & & 24 & 45.8 & 45.2 & 52 & & 24 & 48.5 & 51.2 & 39 & & 24 & $5 \mathrm{I} . \mathrm{I}$ & $5 \mathrm{r} .3$ & $4 \mathrm{I}$ & 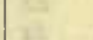 \\
\hline 26 & 50.4 & 49.9 & & 45 & 7a & 26 & 48.7 & 48.3 & 47 & ta & 26 & 48.9 & 50.7 & 39 & 11 & 26 & 49.6 & 51.2 & 40 & 5 \\
\hline 28 & 47.9 & $47 \cdot 4$ & & 19 & & 28 & & ight & & $y=$ & 28 & 50.8 & 54.0 & 43 & & 28 & 48.2 & 49.0 & 37 & \\
\hline 30 & 46.3 & 45.4 & & $\mathbf{I}$ & -18.0 & 30 & & iled & & 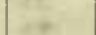 & 30 & 50.7 & 53.8 & 43 & $-23 \cdot 3$ & 30 & 47.7 & 48.2 & $3^{6}$ & -22.7 \\
\hline 32 & 46.9 & 46.1 & & 50 & & 32 & & & & 8 & 32 & 51.2 & 55.0 & 44 & & 32 & 47.2 & 47.9 & 35 & $=x^{2}$ \\
\hline 34 & 48.8 & 48.1 & & 7 & 38 & 34 & & & & gr & 34 & 51.6 & 54.9 & 44 & 10 & 34 & 46.2 & 47.0 & 34 & 3 \\
\hline 36 & 49.3 & 48.7 & & 66 & t & 36 & & & & 3 & 36 & 51.3 & 54.6 & 44 & & 36 & 45.7 & 46.5 & 33 & 20 \\
\hline 38 & 48.5 & 47.7 & 4 & 8 & 8 & 38 & & & & in & 38 & 51.2 & 54.4 & 44 & (3) & 38 & 45.8 & 46.4 & 33 & 3 \\
\hline 40 & 47.1 & 46.2 & 5 & 0 & 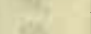 & 40 & & & & $x$ & 40 & $5 \mathrm{I} . \mathrm{I}$ & $54 \cdot 3$ & 44 & & 40 & 45.7 & 46.5 & 32 & (2) \\
\hline 42 & 48.3 & 47.5 & 4 & 8 & 45 & 42 & & & & 1. & 42 & 50.2 & 54.2 & 43 & & 42 & 45.5 & 46.2 & 33 & \\
\hline 44 & 47.1 & 46.3 & 5 & 0 & -18.0 & 44 & & & & 3 & 44 & 51.2 & 53.8 & 43 & $-23 \cdot 3$ & 44 & $45 \cdot 3$ & 46.1 & 32 & -22.4 \\
\hline 46 & 47.2 & 46.9 & & 0 & -5 & 46 & & & 4 & $x^{2}$ & 46 & 51.8 & 54.7 & 44 & & 46 & $45 \cdot 4$ & 45.6 & 32 & \\
\hline 48 & $47 . \mathrm{I}$ & 46.4 & 5 & ;0 & $y^{2}$ & 48 & & & & . & 48 & 52.2 & 55.9 & 45 & & 48 & 45.7 & 46.3 & 33 & $x^{3}$ \\
\hline 50 & 46.0 & 45.8 & 5 & 1 & 5 & 50 & & & & 17 & 50 & 52.3 & 56.4 & 46 & & 50 & 45.6 & 47.8 & 34 & * \\
\hline 52 & 46.5 & 46.1 & 5 & 0 & tar & 52 & $t+$ & 3 & & y. & 52 & 52.2 & 56.5 & 46 & to & 52 & 47.5 & 48.4 & 36 & $2=$ \\
\hline 54 & 48.2 & 47.6 & 4 & 8 & sis & 54 & & & 2 & $\mathrm{y}^{2}$ & 54 & 52.6 & 55.7 & 46 & . & 54 & 47.6 & 48.5 & 36 & Ba \\
\hline 56 & 49. I & 48.6 & & 17 & & 56 & 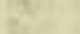 & & tis & tra & 56 & 52.8 & 54.5 & 45 & & 56 & 47.2 & 48.0 & 35 & 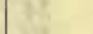 \\
\hline 58 & 48.3 & 47.8 & & 8 & & 58 & & . & & . & 58 & 52.0 & 54.7 & 44 & & 58 & 49.0 & 49.9 & 38 & \\
\hline 500 & 48.2 & 47.7 & & 8 & -18.0 & 700 & 0 & & & & 1700 & $5 \mathrm{I} .4$ & 53.7 & 43 & $-23 \cdot 3$ & 1900 & 50.5 & 51.6 & $4 I$ & -22.3 \\
\hline 02 & 48.2 & 47.9 & & 8 & 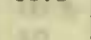 & 02 & 3 & & a & 6. & o2 & 51.2 & 52.9 & 42 & -2.0 & 02 & 53.7 & 54.8 & 46 & . \\
\hline 04 & 47.4 & 47.1 & & 19 & 10 & 04 & & 2 & & and & 04 & 50.1 & 50.9 & 40 & 8 & 04 & 53.1 & $54 \cdot 3$ & 45 & 19 \\
\hline 06 & 47.2 & 46.0 & 5 & 0 & 25 & 06 & & & & Bo & 06 & 49.3 & 49.8 & 38 & ter & 06 & 58.7 & 59.3 & 53 & 12 \\
\hline 08 & $47 \cdot 3$ & 47.1 & 4 & 19 & $b^{3}$ & 08 & & E & & (5) & 08 & 48.2 & 48.6 & 37 & 3 & 08 & 60.2 & 60.9 & 56 & $8^{\circ}$ \\
\hline 10 & 47.0 & 46.7 & 5 & 0 & te & 10 & & & this & 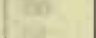 & I0 & 47.5 & 47.8 & 35 & a & IO & 59.6 & 60.3 & 55 & (n) \\
\hline 12 & 48.9 & 48.2 & & 17 & (1) & 12 & 7 & & 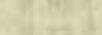 & 20 & 12 & 47.4 & 48.0 & 36 & 80 & 12 & 59.4 & 59.9 & 54 & \\
\hline 14 & 49.9 & 49.7 & & 15 & -17.9 & I4 & 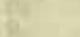 & & & & 14 & 47.6 & 48.9 & 36 & -23.2 & Is & 57.7 & 58.0 & 52 & -22.1 \\
\hline 16 & 50. I & 49.6 & & 15 & & 16 & & & & & 16 & 47.4 & 48.6 & 36 & & 16 & & 58.3 & 52 & \\
\hline 18 & $49 \cdot 3$ & 49.1 & 4 & 6 & inte & 18 & & & & 31 & I8 & 47.3 & 48.8 & 36 & a & 18 & 56.8 & 57.3 & 50 & \\
\hline 20 & 48.8 & 48.6 & 4 & 17 & & 20 & & & & $t_{1}$ & 20 & 47.8 & 49.7 & 37 & & 20 & 55.9 & & 48 & 1 \\
\hline 22 & 47.9 & $47 \cdot 3$ & & 19 & 2 & 22 & & & & . & 22 & 48.1 & 49.7 & 38 & $\pi$ & 22 & 56.1 & 56.3 & 49 & \\
\hline 24 & 47.8 & $47 \cdot 3$ & 4 & 19 & 60 & 24 & & & & 3 & 24 & 48.2 & 50.0 & 37 & 3 & 2 & 57.4 & 58. & 52 & 푼 \\
\hline 26 & 46.5 & 46.1 & 5 & 0 & 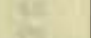 & 26 & & & & $t^{2}$ & 26 & 47.8 & 49.9 & 37 & s & 26 & 58.3 & 58.7 & 53 & $1=$ \\
\hline 28 & 46.4 & 46.3 & & so & & 28 & & & & $x^{2}$ & 28 & 48. I & 49.7 & 38 & & 28 & 62.2 & 62.7 & 59 & \\
\hline 30 & 47.2 & 47.0 & & 19 & -17.9 & 30 & & & & & 30 & 47.7 & 49.8 & 37 & -23.1 & 30 & 61.8 & 62.1 & 58 & -22.1 \\
\hline 32 & 47.3 & 46.9 & & 19 & . & 32 & & . & & 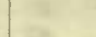 & .32 & 46.6 & 49.1 & 36 & & 32 & $59 \cdot 3$ & 59.5 & 54 & \\
\hline 34 & 47.9 & 47.5 & 4 & 8 & (ats & 34 & & & & $1=$ & 34 & 47.0 & 49.6 & 37 & & 34 & 58.8 & 59.0 & 53 & $4 t$ \\
\hline 36 & 46 & $o b$ & & $I$ & 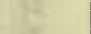 & 36 & & & & 8 & 36 & 48.2 & 49.7 & 38 & & 36 & 59.6 & 60.1 & 55 & res \\
\hline 38 & 50.2 & 50.0 & & 15 & 6 & 38 & & & & & 38 & 46.8 & 48.7 & 36 & & 38 & 59.5 & 59.9 & 54 & \\
\hline 40 & 47.0 & 46.8 & 5 & 0 & 18 & 40 & & & & 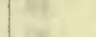 & 40 & 47.0 & 47.5 & 35 & & 40 & 59.4 & 60.0 & 54 & $y$ \\
\hline 42 & 46.7 & 46.6 & & 0 & (1) & 42 & & & & ato & 42 & 47.1 & 47.9 & 35 & & 42 & 58.3 & 58.8 & 53 & \\
\hline 44 & 46.8 & 46.6 & 5 & 0 & -17.7 & 44 & & & & & 44 & 46.6 & 47.0 & 34 & -23.0 & 44 & 57.9 & 58.6 & 53 & -22.0 \\
\hline 46 & 47.3 & 46.8 & & 0 & & 46 & & & & & 46 & 46.5 & 46.8 & 34 & & 46 & 57.6 & 59.7 & 53 & \\
\hline 48 & 47.8 & 47.5 & 4 & 19 & inis & 48 & & . & & ta. & 48 & 47.2 & 47.5 & 35 & se & 48 & 57.8 & 59.5 & 53 & 8 \\
\hline 50 & 46.8 & 46.4 & & 0 & 145 & 50 & & & & tet & 50 & 46.8 & 48.7 & 36 & & 50 & 57.6 & 58.5 & 52 & 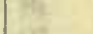 \\
\hline 52 & 46.5 & 46.4 & 5 & 0 & (a) & 52 & & & & 2 & 52 & 48.3 & 50.2 & $3^{8}$ & 5 & 52 & 58.2 & 60.3 & 2254 & \\
\hline 54 & 46.2 & 45.9 & 5 & I & 20 & 54 & & & & $5=$ & 54 & 50.1 & 53.8 & 42 & & 54 & 63.1 & 64.0 & 2300 & \\
\hline 56 & 49.0 & 48.8 & 4 & 6 & 18 & 56 & 18 & & & $\mathrm{~B}_{2}$ & 56 & $51 . I$ & 53.3 & 43 & & 56 & $6 \mathrm{I} .4$ & 61.9 & $225 \%$ & 2 \\
\hline 58 & $48 . \mathrm{I}$ & 47.5 & & 8 & 3 & 58 & $8 x$ & & 685 & . & 58 & 50.6 & 53.4 & 42 & & 58 & 59.8 & 61.0 & $5^{5}$ & \\
\hline & & & & & & & & & & & & & & & & 2000 & 60.9 & 61.7 & 57 & -22.0 \\
\hline
\end{tabular}

Correction to local mean time is $-2 \mathrm{~m} 47 \mathrm{~s}$.

Torsion head at oh oom read $19^{\circ}$ and at 6 h $30 \mathrm{~m}$ read $18^{\circ}$. Observer-H. H. N.
Correction to local mean time is $-3 \mathrm{~m} 33.55 .90^{\circ}$ torsion $=200^{\prime} 4 \mathrm{t}$. Torsion head at 15 h $201 n$ read $15^{\circ}$ and at 20 hom read $13^{\circ}$. Observer-H. H. N. 
Tabulation of magnetic declinations observed at Teplitz Bay-Continued

\begin{tabular}{|c|c|c|c|c|c|c|c|c|c|c|c|c|c|c|c|c|c|c|c|c|c|c|c|}
\hline \multicolumn{6}{|c|}{ Friday, March II, 1904} & \multicolumn{6}{|c|}{ Magnet scalc inverted } & \multicolumn{6}{|c|}{ Sunday, March 13, I904 } & \multicolumn{6}{|c|}{ Magnet scale erect } \\
\hline \multirow{2}{*}{ tille } & $\begin{array}{l}\text { Sc: } \\
\text { Sc: }\end{array}$ & $\begin{array}{l}\text { ale } \\
\text { alc }\end{array}$ & $\begin{array}{l}\text { Eas } \\
\text { Eas } \\
\text { nati }\end{array}$ & & C. & time & & $\begin{array}{l}\text { ale } \\
\text { ale }\end{array}$ & $\begin{array}{l}\text { Eas } \\
\text { Eas } \\
\text { mati }\end{array}$ & & C. & $\begin{array}{l}\text { Chr'r } \\
\text { time }\end{array}$ & $\begin{array}{r}\text { Sc } \\
\text { rea }\end{array}$ & $\begin{array}{l}\text { ale } \\
\text { lings }\end{array}$ & $\begin{array}{l}\text { Fas } \\
\text { dec } \\
\text { natio }\end{array}$ & & Temp. & $\begin{array}{l}\text { Chr'r } \\
\text { time }\end{array}$ & $\begin{array}{r}\mathrm{Sc} \\
\text { read }\end{array}$ & $\begin{array}{l}\text { ale } \\
\text { lings }\end{array}$ & $\begin{array}{l}\text { East } \\
\text { decli } \\
\text { natio }\end{array}$ & & $\begin{array}{l}\text { Temp. } \\
\text { C. }\end{array}$ \\
\hline & L,eft & Right & & & C. & & I.cft & Riglt & & & & & L,eft & Right & & & & & L.cft & Right & & & \\
\hline & d & d & - & , & $\circ$ & l $1 \mathrm{~m}$ & d & $\mathrm{d}$ & & & . & $\mathrm{h} \mathrm{m}$ & 4 & d & & & & $\mathrm{h} \mathrm{m}$ & d & d & - & & 。 \\
\hline 2000 & 58.8 & 57.6 & 22 & I9 & -20.1 & 2200 & $64 \cdot 9$ & 55.8 & 22 & I6 & $-19 \cdot 3$ & $000+$ & 47.8 & 45.8 & 22 & & & 200 & 56.4 & 57.0 & 224 & & -24.0 \\
\hline 02 & 58.5 & $57 \cdot 4$ & & 20 & & 02 & 58.8 & 49.4 & & 25 & & 02.4 & 56.1 & 61.7 & & 51 & -28.7 & 02 & 55.5 & 57.3 & & 48 & \\
\hline 04 & 59.2 & $58 . \mathrm{I}$ & & I8 & & 04 & 59.5 & 51.9 & & 23 & & 04 & 57.7 & 6г.8 & & 52 & & 04 & 55.8 & 56.7 & & 47 & \\
\hline 06 & 60.1 & 59.3 & & 17 & (10 & 06 & 61.8 & 5 I. I & & 22 & & 06 & 58. I & $6 \mathrm{~T} \cdot 9$ & & 53 & 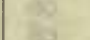 & 06 & 55.9 & 56.7 & & 47 & \\
\hline 08 & 50.9 & 58.7 & & I7 & & 08 & $56 . I$ & 46.9 & 22 & 30 & & 08 & 57.9 & 61.3 & & 52 & 8 & 08 & 56.0 & 56.8 & & 17 & \\
\hline IO & 58.3 & 57.6 & & 20 & 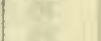 & Io & 32.8 & 21.7 & 23 & 08 & Q & IO & 55.9 & 58.8 & & 48 & 4 & IO & 55.7 & 56.1 & & .15 & \\
\hline I2 & 58.9 & 58.3 & & 18 & & I2 & 49.8 & 43.2 & 22 & 37 & & I2 & 54.9 & 57.1 & & 46 & & 12 & 56.0 & 56.0 & & 45 & \\
\hline 14 & 60.3 & 59.9 & & I6 & -20 . I & I4 & 55.3 & 46.5 & & 30 & -19.0 & 14 & 53.8 & 55.9 & & 44 & -28.0 & 14 & $55 . \mathrm{I}$ & 55.8 & & 45 & -2.3 .8 \\
\hline 16 & 60.4 & 59.7 & & 16 & 200 & I6 & 56.7 & 46.6 & & 29 & & 16 & 51.8 & 53.9 & & $4 \mathrm{I}$ & 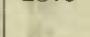 & 16 & 54.7 & 55.0 & & 44 & - \\
\hline 18 & 59.8 & 59.3 & & 17 & tan & I8 & 59.3 & 51.2 & & 24 & a & I8 & 51.0 & $53 \cdot 3$ & & 10 & & 18 & 54.9 & 56.0 & & 45 & \\
\hline 20 & 59.8 & 59.2 & & 17 & ate & 20 & 58.1 & 50.3 & & 25 & ta & 20 & 49.8 & 52.2 & & 38 & & 20 & 59 & $.0 \Omega$ & & $5 \mathrm{I}$ & int \\
\hline 22 & 58.4 & 57.9 & & I9 & & 22 & 54.2 & 47.6 & & 30 & & 22 & 49.9 & 52.1 & & 38 & & 22 & 57.0 & 57.8 & & 88 & \\
\hline 24 & 60.9 & 59.6 & & 16 & 15 & 24 & 54.7 & 47.1 & & 30 & 5 & 24 & 49.0 & 50.9 & & 37 & & 24 & 59.2 & 60.0 & & 52 & th \\
\hline 26 & 60.8 & 59.9 & & 16 & & 26 & 57.8 & 50.9 & & 25 & & 26 & 48. T & 50.1 & & 36 & & 26 & 61.2 & 61.2 & & 54 & \\
\hline 28 & 60.3 & 59.2 & & I7 & $\mathrm{T}$ & 28 & 57.9 & $5 \mathrm{I} .8$ & & 24 & & 28 & 48. I & 50.3 & & 36 & & 28 & 61.0 & $6 \mathrm{I} .2$ & & 54 & \\
\hline 30 & 60.2 & 50.5 & & 16 & -20.2 & 30 & 57.7 & 5 I.7 & & 24 & -18.8 & 30 & 48. I & 49.8 & & 35 & -27.0 & 30 & 60.5 & 6т.J & & 54 & $-23 \cdot 5$ \\
\hline 32 & 61.8 & 60.6 & & I4 & -80 & 32 & 56.9 & 51.2 & & 25 & & 32 & 48.0 & 50.6 & & 36 & & 32 & 60.9 & 63.1 & & 56 & -3.5 \\
\hline 34 & 63.3 & 61.6 & & 12 & 28 & 34 & 58.8 & $54 \cdot 3$ & & 22 & 18 & 34 & 46.3 & 48.8 & & 33 & 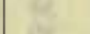 & 34 & $60 . \mathrm{I}$ & C.o & & 54 & \\
\hline 36 & 66.8 & 65.7 & & 06 & 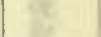 & 36 & 57.8 & 53.6 & & 23 & x & 36 & 46. I & 48.6 & & 33 & 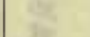 & 36 & 60.7 & 62.8 & & 5.5 & $=$ \\
\hline 38 & 60.6 & 65.3 & & 05 & e & 38 & 46.8 & 43.8 & & 39 & 32 & 38 & $46 . I$ & 48.2 & & 32 & & 38 & 61.0 & 62.8 & & 56 & 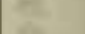 \\
\hline 40 & 71.8 & 69.4 & & 00 & w. & 40 & 55.2 & $5 \mathrm{I} .0$ & & 27 & C. & 40 & 47.2 & $49 . \mathrm{I}$ & & 34 & & 40 & 58. I & 60.1 & & 51 & 10 \\
\hline 42 & 70.9 & 68.6 & & OI & 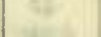 & 42 & 47.9 & 45.4 & & 37 & . & 42 & 47.8 & 49.5 & & 35 & & 42 & 59.9 & 62.0 & & 54 & \\
\hline 44 & 69.5 & 67.2 & & 03 & -20.1 & 44 & 40.0 & 46.0 & & $3^{6}$ & -18.7 & 44 & 48.3 & 50.2 & & 36 & -26.3 & 44 & 61.3 & 63.2 & & 56 & -23.2 \\
\hline $\begin{array}{l}44 \\
46\end{array}$ & 70.0 & 67.3 & & 03 & 20.2 & 46 & 49.9 & 46.2 & & 35 & ( & $\begin{array}{l}44 \\
46\end{array}$ & 49.8 & 50.9 & & 37 & & $\begin{array}{l}44 \\
46\end{array}$ & 60.2 & 62.9 & & 55 & -2.4 \\
\hline 48 & 68.6 & 65.9 & & 05 & 3 & 48 & 49.7 & 47.1 & & 34 & tat & 48 & 50.2 & 51.2 & & 38 & & 48 & 59.2 & 62.2 & & 54 & \\
\hline 50 & 67.4 & 65.2 & & 06 & 9 & 50 & 46. I & 43.4 & & 40 & a & 50 & $5 \mathrm{I} . \mathrm{I}$ & 52.0 & & 39 & & 50 & 60.0 & 63.2 & & 55 & \\
\hline 52 & $67 . I$ & $65 . \overline{6}$ & & 06 & se & 52 & 46.8 & 43.2 & & 40 & wa. & 52 & $53 . \mathrm{I}$ & 53 & & 2 & & 52 & 6 ז. I & 63.8 & & 56 & \\
\hline 54 & 67.0 & 65.5 & & 06 & 2 & 54 & 47.6 & 46.1 & & 37 & 12 & 54 & 55.6 & 56.1 & & 46 & & 54 & 59.7 & 62.2 & & 54 & \\
\hline 56 & 63.5 & 61.6 & & I2 & s. & 56 & 46.2 & 43.7 & & 40 & 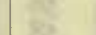 & 56 & 56.8 & 56.8 & & 48 & & 5 & 59.3 & 62.2 & & 54 & \\
\hline 58 & 50.2 & 57.8 & & 18 & 12 & 58 & 42.8 & 39.9 & & 46 & & 58 & 56.1 & 56.8 & & 47 & & 58 & 60.1 & 61.8 & & 54 & \\
\hline 2100 & 56.5 & 54.8 & & 23 & -20.0 & 2300 & 45.5 & $44 \cdot 3$ & & 40 & -18.7 & I DO & 56.2 & 56.5 & & 47 & -25.8 & 300 & 60.4 & 62.0 & & 54 & -23.0 \\
\hline 02 & 58.8 & 57.1 & & 20 & & 02 & 50.2 & 47.4 & & 34 & & 02 & 58.0 & 59.2 & & 6 & & 02 & 61.5 & 63.1 & & 56 & \\
\hline 04 & 51.8 & 50.6 & & 30 & A & 04 & 49.6 & 46.3 & & 35 & 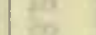 & 04 & 58.9 & 59.8 & & 52 & & 0.4 & 63.6 & 64.0 & $22:$ & 50 & \\
\hline 06 & 50.5 & $49 . I$ & & 32 & ses & 06 & 50.2 & 46.8 & & 31 & 80 & 06 & 57.0 & 57.9 & & 49 & & 06 & 66.1 & 68.0 & 23 & 01 & 8 \\
\hline 08 & 50.9 & 49.2 & & 32 & 2 & 08 & 47.6 & 45.5 & & 37 & 2 & 08 & 57.8 & 58.7 & & 50 & & 08 & 68. I & 60.7 & & 07 & \\
\hline I0 & 52.3 & 50.4 & & 30 & & I0 & 49.8 & $47 . \mathrm{I}$ & & 31 & & I0 & 58.8 & 50. & & $5 \mathrm{I}$ & & I & 67.2 & $6 n .3$ & & 5 & \\
\hline 12 & 50.6 & 49.2 & & 32 & & I2 & $47 \cdot 3$ & 45.5 & & 38 & & 12 & 57.8 & 58. I & & $4 ?$ & & 12 & 64.9 & 66.8 & & 02 & \\
\hline I4 & 5 I. 3 & 49.9 & & $3 \mathrm{I}$ & -19.8 & 14 & 49.6 & 47.7 & & .34 & -18.2 & 14 & 57.0 & 57.4 & & 48 & 25.4 & T4 & 65.2 & 65.8 & & 02 & -23.0 \\
\hline I6 & 50.8 & 49.5 & & 32 & ( & 16 & 47.9 & 45.6 & & 37 & $\sqrt{2}$ & I6 & 57.6 & 58. & & 49 & & 16 & 65.2 & 66.9 & 230 & 02 & \\
\hline I 8 & 51.6 & 5I.I & & 30 & 7) & I8 & 50.8 & 49.1 & & 32 & 0 & 18 & 57.8 & 58 & & 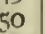 & & I8 & 63.9 & 65.2 & 226 & & \\
\hline 20 & 54.4 & 5.3 .6 & & 26 & 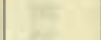 & 20 & 52.9 & 50.7 & & $3)$ & CS: & 20 & 57.9 & 58.8 & & 0 & & 20 & $6 \mathrm{r} .0$ & 63 & & 56 & \\
\hline 22 & 59.9 & 58.0 & & I8 & ㄷ․ & 22 & 52.0 & 50.6 & & 30 & 10 & 22 & & 59 & & 0 & & 2 & 61.8 & 62.8 & & 56 & \\
\hline 24 & 63.5 & 60.7 & & I3 & 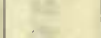 & 24 & 52.2 & 50.9 & & 30 & ta․ & 24 & 58.8 & 59 & & 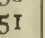 & & 2 & 63.0 & 63.9 & & 58 & \\
\hline 26 & 62.8 & 58.3 & & I 5 & $=$ & 26 & 46.9 & 46.2 & & 37 & a & 26 & 50. & 60 & & 3 & & & 6 & & & 55 & \\
\hline 28 & 45.8 & 40.0 & 22 & 43 & & 28 & 45.2 & 44.0 & & 0 & & 28 & 61.6 & 62. & 22 & 56 & & 2 & 60.8 & 61.8 & & 53 & \\
\hline 30 & 34.6 & I3. 8 & 23 & 12 & $-\mathrm{I} 9.6$ & 30 & 46.3 & 45.4 & & .38 & -18.0 & 30 & 64.4 & 65.0 & 23 & ום & -25.0 & 30 & 50.8 & 6I.I & & 53 & -22.8 \\
\hline 32 & 32.0 & 8.1 & & 19 & & 32 & 45.7 & 44.9 & & 30 & & 32 & $65 . \mathrm{I}$ & 66.9 & & 02 & & & 60.2 & $6 \mathrm{r} . \mathrm{r}$ & & 51 & \\
\hline 34 & 30.2 & IT 8 & 23 & I7 & k & 34 & 46.4 & 45.8 & & 38 & 10 & 34 & 67.2 & 68.0 & & 4 & & 34 & 59.8 & 60.9 & es & 5.3 & \\
\hline & 48.2 & 3I.I & & 48 & 20 & 36 & 46.9 & 46.3 & & 37 & & 36 & 68.9 & 69.0 & & & & & 60.1 & 61.9 & & & \\
\hline 38 & 36.3 & 26.5 & 23 & oI & 2 & 38 & 45.4 & $45 . I$ & & 39 & 78 & 38 & 68.6 & 69.8 & & 07 & & 38 & 61.6 & 62.9 & & 56 & \\
\hline 40 & 60.0 & $5 I .5$ & 22 & 23 & & 40 & 43. I & 42.5 & & 43 & & 40 & 67.1 & 67.8 & & 0.4 & & 40 & 6I.I & 62.1 & & 55 & \\
\hline $\begin{array}{l}40 \\
42\end{array}$ & 58.9 & $\begin{array}{l}51.5 \\
50.3\end{array}$ & & 25 & & 42 & 44.2 & 43.5 & & .12 & & 42 & 64.8 & 65.5 & 23 & OI & & 42 & 59.8 & 60.7 & & 5.3 & \\
\hline 44 & 48.2 & 38.7 & & 42 & -19.5 & 44 & 44.5 & 44.0 & & $4 \mathrm{I}$ & -18.0 & 44 & 63.7 & 64.8 & 22 & 59 & -24.6 & 44 & 57.8 & 50.0 & & 50 & -22.7 \\
\hline 46 & 65.4 & 53.0 & & I7 & & 46 & 44.4 & 43.9 & & 4 I & $y^{2}$ & 46 & 61.7 & 63.0 & & 56 & & 46 & 57.5 & 58.3 & & 40 & \\
\hline 48 & 54.6 & 44.2 & 22 & 33 & 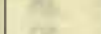 & 48 & 44.3 & 43.8 & & fT & nat & 48 & 62.1 & 63.2 & & & & 48 & 56.2 & 57.1 & & 47 & \\
\hline 50 & 26.8 & 18.3 & 23 & I5 & Q & 50 & 45.6 & 45.0 & & 39 & 0 & 50 & 62.9 & 63. & & 58 & $=$ & 50 & 55.8 & 56.8 & & 47 & \\
\hline 52 & 25.3 & $20 . \mathrm{I}$ & 23 & I5 & 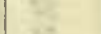 & 52 & 44.7 & 44.5 & & 40 & $2+$ & 52 & 62.7 & 63.8 & & 58 & . & 52 & 53.1 & 53.0 & & 42 & \\
\hline 54 & 5.3 .3 & 40.4 & 22 & 37 & (6) & 54 & 42.6 & 42.3 & & 14 & E & 54 & 60.5 & $6 r .5$ & & 54 & & 54 & 53.9 & 54.2 & & +3 & \\
\hline 56 & 58.7 & 48.8 & & 26 & $2=$ & 56 & 41.8 & 41.2 & & 4.5 & 18 & 56 & 57.9 & 58. & & 50 & The & 56 & 55.4 & 56.4 & & 16 & \\
\hline 58 & 68.2 & 53.7 & & I5 & & 58 & $4 \mathrm{I} .2$ & 40.4 & & 46 & & 58 & 56.9 & 57.6 & & 48 & & 58 & 57.1 & 58.6 & & 10 & \\
\hline & & & & & & 2400 & 40.6 & 39.9 & & 47 & & & & & & & & & & & & & \\
\hline
\end{tabular}

Correction to local mean time is $-3 \mathrm{~m} 47.5 \mathrm{~s}$.

Torsion head at $19 \mathrm{~h} 15 \mathrm{~m}$ rcad $13^{\circ}$ and at oh oom rcad $14^{\circ}$.

Observer-H. H. N.
Observer-R. R. T

$\uparrow$ Scale inverted for this reading. 
Tabulation of magnetic declinations observed at Teplitz Bay-Continued

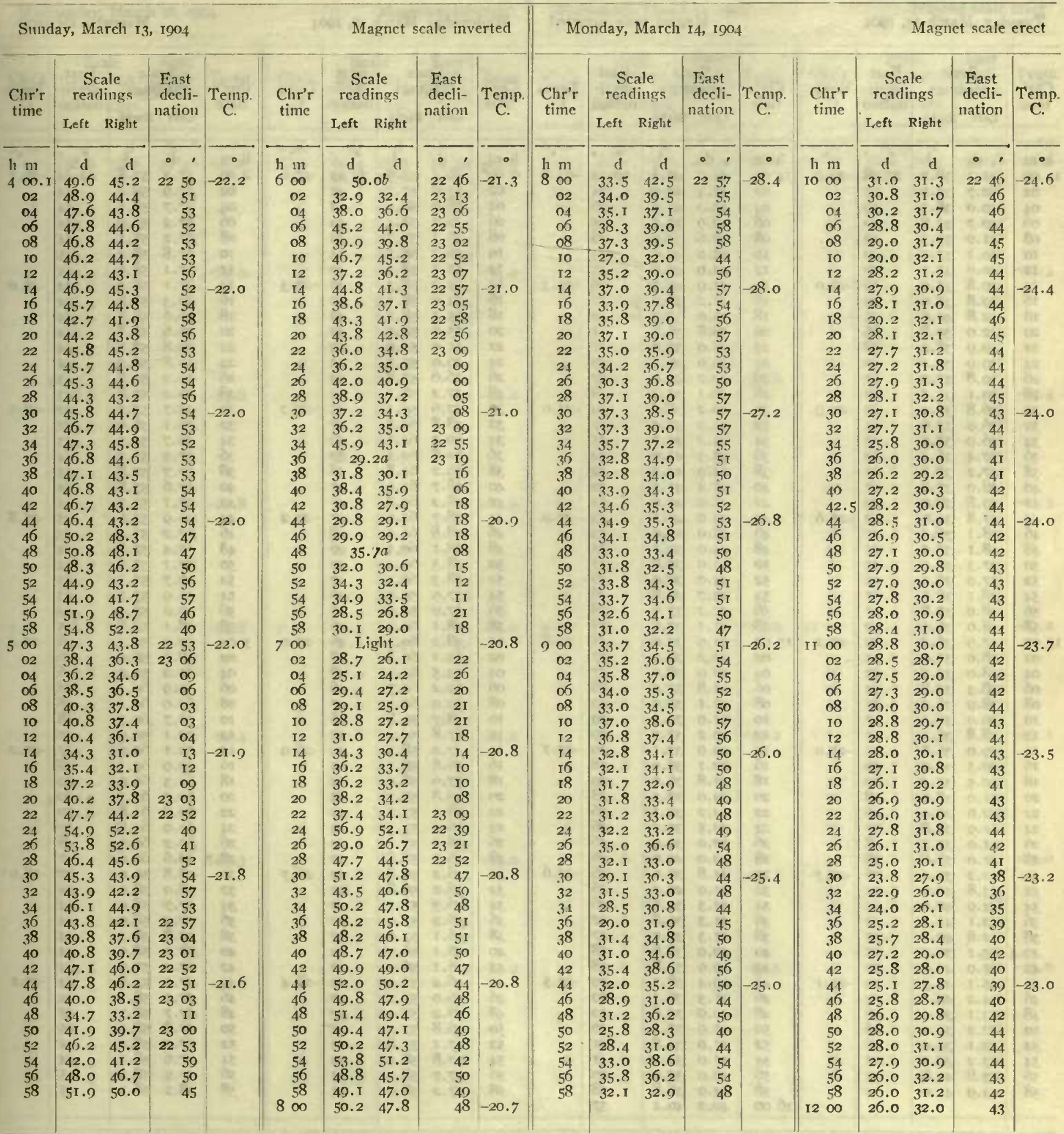

Correction to local mean time is $-24.5 \mathrm{~s}$

Torsion liead at oh oom read $14^{\circ}$ and at $8 \mathrm{~h}$ zom read the same. Observer-R. R. T.
Correction to local mean time is $-59.5 \mathrm{~s}$.

Torsion head at $7 \mathrm{~h} 48 \mathrm{~m}$ read $14^{\circ}$ and at $\mathrm{r} 2 \mathrm{~h} 2 \mathrm{rm}$ read $15^{\circ}$ Observer-J. V. 
Tabulation of magnetic declinations observed at Teplitz Bay-Continued

\begin{tabular}{|c|c|c|c|c|c|c|c|c|c|c|c|c|c|c|c|c|c|c|c|}
\hline \multicolumn{6}{|c|}{ Tnesday, March 15, 1904} & \multicolumn{4}{|c|}{ Magnet scale inverted } & \multicolumn{6}{|c|}{ Wednesday, March 16, 1904} & \multicolumn{4}{|c|}{ Magnet scale erect } \\
\hline \multirow{2}{*}{$\begin{array}{l}\text { Chr'r } \\
\text { time }\end{array}$} & \multicolumn{2}{|c|}{$\begin{array}{l}\text { Scale } \\
\text { readings }\end{array}$} & \multirow{2}{*}{$\begin{array}{c}\text { East } \\
\text { decli- } \\
\text { nation }\end{array}$} & Temp. & Chr'r & $\begin{array}{r}\mathrm{Sc} \\
\mathrm{read}\end{array}$ & $\begin{array}{l}\text { ale } \\
\text { ings }\end{array}$ & East & Temp. & Chr'r & $\begin{array}{r}\mathrm{Sc} \\
\text { read }\end{array}$ & $\begin{array}{l}\text { alc } \\
\text { lings }\end{array}$ & $\begin{array}{l}\text { East } \\
\text { decli- }\end{array}$ & Temp. & Chr'r & $\begin{array}{l}\text { Sca } \\
\text { read }\end{array}$ & $\begin{array}{l}\text { le } \\
\text { ng's }\end{array}$ & $\begin{array}{l}\text { East } \\
\text { decli- }\end{array}$ & Temp. \\
\hline & Left & Right & & & & Left & Right & 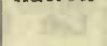 & & & Left & Right & 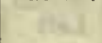 & & & Left & Right & & \\
\hline h $\mathrm{m}$ & $d$ & d & ०, & 0 & in $\mathrm{m}$ & $\mathrm{d}$ & $d$ & $\cdot$. & $\circ$ & h $\mathrm{m}$ & d & d & & & $\mathrm{h} \mathrm{m}$ & d & $d$ & & 0 \\
\hline 1200 & $4 \mathrm{I} . \mathrm{I}$ & 38.0 & 2238 & -26.0 & 1400 & 44.0 & 40.4 & 2234 & -23.8 & $000^{*}$ & 56.1 & 57.9 & 2243 & -27.6 & 200 & 57.2 & 57.8 & 22.44 & -26.1 \\
\hline$\approx$ & 40.7 & 39.7 & 37 & 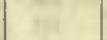 & 02 & 42.4 & 42.0 & 34 & $a^{-a}$ & 02 & 55.9 & $57 \cdot 7$ & 43 & 20 & 02 & 57.9 & 58.2 & 44 & ant \\
\hline 04 & 40.0 & 38.1 & 39 & 401 & 04 & 42.0 & 40.3 & 36 & is & 04 & 56.0 & 58.0 & 43 & (3) & 04 & 57.7 & 58. I & & \\
\hline 06 & 40.7 & 35.8 & 40 & Hit & 06 & 42.9 & 39.9 & 35 & As & 05 & 56.2 & 58.4 & 44 & $\sqrt{65}$ & 06 & 57.2 & 57.9 & 44 & 86 \\
\hline 08 & 41.0 & 35.5 & 40 & 2. & 08 & 42.6 & 39.9 & 36 & 84 & 08 & 56.1 & 57.7 & 43 & 5 & 08 & 57.2 & 58.1 & & is \\
\hline Io & 40.1 & 37.0 & 40 & at & 10 & 43.0 & 40.9 & 34 & ה & 10 & 56.2 & 58.5 & 44 & at & I0 & 57.8 & 58.4 & 45 & \\
\hline 12 & 40.0 & 37.1 & 40 & & 12 & 42.9 & 40.6 & 35 & & 12 & $55 . \mathrm{I}$ & 58.3 & 42 & & 12 & 57.1 & 58.0 & 44 & \\
\hline 14 & 40.8 & 37.0 & 39 & -26.0 & 14 & 41.9 & 40.6 & 36 & $-23 \cdot 3$ & 14 & 55.2 & 58.3 & 42 & -27.3 & 14 & 57.1 & 58.3 & 44 & -26.0 \\
\hline 16 & 39.8 & 38.0 & 39 & (at) & 16 & 41.2 & 38.9 & 37 & 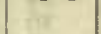 & 16 & 54.9 & $58 . \mathrm{I}$ & 42 & & 16 & 56.3 & 58.8 & 44 & \\
\hline I8 & 42.8 & 40.0 & 35 & Az & 18 & 39.0 & 37.2 & 40 & dis & 18 & $55 . \mathrm{I}$ & $58 . \mathrm{I}$ & 42 & - & 18 & 56.6 & 58.8 & 44 & ite \\
\hline 20 & 42.0 & 39.8 & 36 & $i^{2}$ & 20 & 38.8 & 36.8 & $4 \mathrm{I}$ & NE & 20 & 55.6 & 58.0 & 43 & n & 20 & 56.6 & 58.8 & 44 & sete. \\
\hline 22 & 40.9 & 38.2 & -38 & $1=$ & 22 & 38.0 & 37.0 & (6) 41 & ye & 22 & 55.9 & 57.9 & 43 & is & 22 & 56.8 & 58.7 & 44 & cE: \\
\hline 24 & 39.9 & 37.2 & 40 & 18 & 24 & 39.0 & 38. I & 40 & & 24 & 56.0 & 57.7 & 43 & is & 24 & 56.8 & 58.2 & 44 & \\
\hline 26 & 39.8 & 37.2 & 40 & e & 26 & 40.2 & 40.0 & 37 & 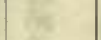 & 26 & 56.6 & 57.8 & 43 & & 26 & 56.2 & 57.8 & 43 & \\
\hline 28 & 39.1 & 37.5 & 40 & & 28 & $40 . \mathrm{I}$ & 39.4 & 38 & & 28 & 57.2 & $58 . \mathrm{T}$ & 14 & & 28 & 56.2 & 57.8 & 43 & \\
\hline 30 & 36.8 & 36.4 & 43 & -25.9 & 30 & 40.2 & 38.2 & 39 & -23.0 & 30 & 57.6 & 59.1 & 45 & -27.2 & 30 & 55.7 & 57.2 & 42 & -25.9 \\
\hline 32 & 36.9 & 35.6 & 43 & $x^{2}$ & 32 & $4 I .2$ & 39.0 & 37 & $x^{2}$ & 32 & 58.8 & 60.7 & 47 & $x^{2}+x^{2}$ & 32 & 56.1 & 56.9 & 42 & \\
\hline 34 & 36.9 & 35.9 & 43 & 32 & 34 & 43.2 & 40.2 & 35 & & 34 & 60.2 & $62 . \pi$ & 50 & tef & 34 & 56.7 & $57 \cdot 3$ & 43 & \\
\hline 36 & 37.4 & 36.4 & 42 & Fe & 36 & $45 \cdot 3$ & 41.9 & 32 & & 36 & 60.8 & 62.5 & 50 & $a^{2}$ & 36 & 57.2 & 57.7 & 44 & \\
\hline 38 & 37.2 & 37.1 & 42 & and & 38 & 44.9 & 42.2 & 32 & h. & 38 & 59.2 & 61.8 & 48 & 85 & 38 & 57.7 & 57.8 & 44 & \\
\hline 40 & 38.0 & 37.2 & $4 I$ & • & 40 & 45.4 & $4 \mathrm{I} \cdot 3$ & 32 & na. & 40 & 58.7 & 61.0 & 47 & 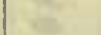 & 40 & 57.6 & 57.8 & 44 & ㅊ. \\
\hline 42 & 39.8 & 38.0 & 39 & & 42 & 45.0 & 40.0 & 33 & & 42 & 58.2 & 60.8 & 47 & & 42 & 57.1 & 57.7 & 44 & \\
\hline 44 & 37.8 & 36.9 & 42 & -25.6 & 44 & 45.0 & 40.1 & 33 & -23.0 & 44 & 58.7 & 61.0 & 47 & -27.2 & 44 & 56.9 & 57.6 & 43 & -25.8 \\
\hline 46 & 38.1 & 36.9 & 41 & Dit & 46 & 45.0 & $4 I .0$ & 33 & 88 & 46 & 58.8 & 61.0 & 48 & $x^{2}$ & 46 & 57.2 & 57.4 & 43 & \\
\hline 48 & 38.1 & 37.0 & $4 \mathrm{I}$ & & 48 & 44.0 & 39.2 & 35 & 2 & 48 & 58.1 & 60.1 & 46 & 5 & 48 & 57.4 & 57.8 & 44 & $+x$ \\
\hline 50 & 37.3 & 36.9 & 42 & t & 50 & 43.0 & 37.8 & 37 & $\theta$ & 50 & 58.3 & 60.4 & 47 & $\infty$ & 50 & 57.1 & 57.6 & 44 & $\theta$ \\
\hline 52 & 37.0 & 36.5 & 42 & 32 & 52 & 43.0 & 38.0 & 36 & ar & 52 & 58.3 & 60.3 & 47 & $\mathrm{~s}^{2}$ & 52 & 56.7 & 57.1 & 43 & is \\
\hline 54 & 38.1 & 36.0 & $4 I$ & in & 54 & 43.7 & 38.8 & 36 & in & 54 & 57.9 & 59.9 & 46 & & 54 & 56.9 & 57.5 & 43 & 87 \\
\hline 56 & 38.0 & 37.8 & $4 \mathrm{I}$ & 15 & 56 & 42.2 & 37.6 & 38 & $A^{2}$ & 56 & 57.0 & 59.9 & 46 & 2 & 56 & 57.0 & 57.4 & 43 & \\
\hline 58 & 38.0 & 36.9 & 42 & Pre & 58 & $4 \mathrm{I} . \mathrm{I}$ & 37.8 & 38 & & 58 & 58.7 & 60.2 & 46 & the & 58 & 58.1 & 58.8 & 45 & \\
\hline 1300 & 37.9 & 36.4 & 42 & -25.2 & 1500 & 41.9 & 37.0 & 38 & -22.8 & I 00 & 58.2 & 60.2 & 46 & -27.0 & 300 & 50.0 & $59 \cdot 3$ & 46 & -25.4 \\
\hline 02 & 38.0 & 35.0 & 43 & o & 02 & 42.2 & 38.2 & 37 & and & 02 & 59.1 & 60.8 & 48 & - & 02 & 58.6 & 50.0 & 46 & \\
\hline 04 & 38.0 & 35.2 & 43 & in & 04 & 42.0 & 38.0 & 37 & the & 04 & 50.5 & $6 \pi . \mathrm{I}$ & 48 & in & 04 & 50.0 & 59.0 & 46 & \\
\hline 06 & 38.3 & 35.8 & 42 & $\sin ^{2}$ & 06 & 41.8 & 37.0 & 38 & 36 & कर & 59.2 & 60.7 & 48 & 68 & 06 & 50.0 & 60.1 & 48 & in \\
\hline 08 & 38.9 & 36.2 & $4 I$ & 84 & 08 & $4 \mathrm{I} . \mathrm{I}$ & 37.0 & 39 & xos & 08 & 50.0 & 60.3 & 47 & his & 08 & 60.0 & 60.2 & 48 & 28 \\
\hline 10 & 39.0 & 36.5 & 4I & ot & Io & $4 \mathrm{~T} .0$ & 37.3 & 39 & et. & To & 58.7 & 59.8 & 46 & at & รо & 59.7 & 60.3 & 48 & $6 t$ \\
\hline 12 & 38.8 & 36.3 & $4 \mathrm{I}$ & tot & 12 & 40.2 & 37.7 & 39 & & 12 & 57.8 & 50.2 & 45 & & 12 & 50.1 & 50.9 & 47 & \\
\hline 14 & 39.0 & 36.8 & $4 I$ & -25.0 & 14 & 40.9 & 37.1 & 39 & -22.7 & I4 & 57.2 & 58.3 & 44 & -26.9 & 14 & 50.1 & 60.0 & & -25.2 \\
\hline 16 & 39.5 & 36.9 & 40 & & I6 & $42 . \mathrm{I}$ & 36.9 & 38 & $x^{2}$ & I6 & 56.8 & 58.1 & 44 & I it & 16 & 59.9 & 60.3 & 48 & \\
\hline 18 & 40.5 & 37.5 & 39 & $x^{2}$ & โ8 & 41.8 & 36.0 & 30 & thy & I8 & 57.0 & 58.0 & 44 & 84 & 18 & 60.3 & 60.9 & 49 & 87 \\
\hline 20 & $4 \mathrm{~T} .0$ & 38.0 & 38 & ant & 20 & 42.0 & $37 \cdot 3$ & 38 & at & 20 & 56.8 & 57.7 & 43 & $\approx$ & 20 & 60.5 & $6 \pi .3$ & 49 & in \\
\hline 22 & 42.0 & 30.0 & 36 & 30 & 22 & 42.2 & 39.1 & 36 & the & 22 & 56.3 & 57.2 & 42 & $E$ & 22 & 60.8 & 61.8 & 50 & $3=$ \\
\hline 24 & $4 \mathrm{I} .9$ & 38.8 & 37 & twat & 24 & 42.5 & 39.2 & 36 & it & 24 & 56.0 & 57.5 & 43 & if & 24 & 60.9 & 61.8 & 50 & \\
\hline 26 & $4 \mathrm{r} .0$ & 37.7 & 38 & wer & 26 & 42.0 & 39.9 & 36 & $(8$ & 26 & 56.0 & 57.1 & 4.3 & 30 & 26 & 60.2 & 60.9 & 48 & \\
\hline 28 & $4 \mathrm{I} .0$ & 37.8 & 38 & 1 & 28 & 42.0 & 40.0 & 36 & & 28 & 56.7 & 55.8 & 42 & & 28.7 & 50.6 & 60.0 & 47 & \\
\hline 30 & 42.2 & 39.4 & 36 & -24.7 & 30 & 41.8 & 39.6 & 36 & -22.6 & 30 & 56.2 & 56.8 & 42 & -26.7 & 30 & 58.8 & 50.0 & 47 & -25.0 \\
\hline 32 & 43.8 & 40.2 & 34 & (20 & 32 & 41.0 & 40.2 & 36 & $\pm r$ & 32 & 56.2 & 56.8 & 42 & 242 & 32 & 58.0 & 58.0 & 44 & \\
\hline 34 & 44.9 & 40.0 & 34 & $H$ & 34 & 41.3 & 39.8 & $3^{6}$ & iy & 34 & 56.3 & 57.1 & 42 & 22 & 34 & 57.7 & 58.0 & 44 & At \\
\hline 36 & 43.1 & $41 . \mathrm{I}$ & 34 & $x$ & 36 & $4 \mathrm{I} \cdot 3$ & 39.8 & 37 & $n$ & 36 & 56.2 & 57.0 & 42 & $x$ & 36 & 57.4 & 58.0 & 44 & \\
\hline 38 & 42.8 & 40.1 & 35 & fir & 38 & $4 \mathrm{~T} .3$ & 39.0 & 37 & 87 & 38 & 56.1 & 57.0 & 42 & 20. & 38 & 57.8 & 58.3 & 44 & II \\
\hline 40.4 & 43.1 & 39.7 & 35 & 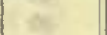 & 40 & 40.9 & 38.6 & $3^{8}$ & 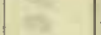 & 40 & 56.1 & 56.9 & 42 & 6 & 40 & 57.3 & 58.0 & 44 & 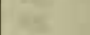 \\
\hline 42 & 45.0 & 40.9 & 33 & 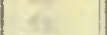 & 42 & 40.9 & 37.8 & 38 & & 42 & 56.1 & 56.9 & 42 & & 42 & 57.2 & 57.9 & 44 & \\
\hline 44 & 45.1 & 41.5 & 32 & -24.1 & 44 & 40.4 & 38.1 & 39 & -22.5 & 44 & 56.2 & 57.2 & 42 & -26.4 & 44 & 57.0 & 57.8 & 44 & 25.0 \\
\hline 46 & 44.2 & $40 . \mathrm{I}$ & 34 & - & 46 & $4 \mathrm{I} \cdot 3$ & 36.3 & 39 & (15 & 46 & 56.0 & 57.2 & 42 & -7 & 46 & 57.1 & 58.1 & & \\
\hline 48 & 43.1 & 39.0 & 36 & R. & 48 & 40.9 & 35.8 & 40 & Fis. & 48 & 56.2 & 57.2 & 42 & $\theta$ & 48 & 57.7 & 58.7 & 45 & \\
\hline 50 & 42.2 & 39.2 & 36 & 20 & 50 & 40.9 & 35.3 & 40 & $\Rightarrow$ & 50 & 56.8 & 57.2 & 43 & $=$ & 50 & 57.8 & 58.7 & 45 & $a$ \\
\hline 52 & 42.1 & 39.9 & 36 & 8 & 52 & 41.0 & 34.8 & $4 I$ & the & 52 & 57.1 & 57.8 & 44 & $a$ & 52 & 57.3 & 58.2 & 44 & 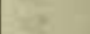 \\
\hline 54 & 43.2 & 39.7 & 35 & iz & 54 & 41.0 & 34.2 & $4 \mathrm{I}$ & 18 & 54 & 57.8 & 58.2 & 44 & 13 & 54 & 57.6 & 58.1 & 44 & (1) \\
\hline 56 & 43.8 & 40.8 & 34 & 25 & 56 & 42.0 & 35.9 & 39 & 4 & 56 & 57.7 & 58.3 & 44 & de & 56 & 57.9 & 58.3 & 45 & \\
\hline 58 & 43.9 & 41.0 & 34 & Bit & 58 & 43.0 & 36.8 & 38 & Ee & 58 & 57.2 & 58.0 & 44 & be & 58 & 58.2 & 58.3 & 45 & no \\
\hline & & 0.8 & ane & $\cos 2=$ & 1600 & 40.2 & 40.1 & 37 & & 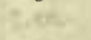 & & 970 & 6 the & 100 $\mathrm{X}$ & & & & & \\
\hline
\end{tabular}

Correction to local mean time is - $9.5 \mathrm{~s}$.

Observer-R. R. T.

Torsion head at $\mathrm{i} 2 \mathrm{~h}$ oom read $14^{\circ}$ and at $16 \mathrm{~h} 35 \mathrm{~m} \mathrm{read} \mathrm{I} 3^{\circ}$.

Observer-J. V. 
Tabulation of magnetic declinations observed at Teplitz Bay-Continued

\begin{tabular}{|c|c|c|c|c|c|c|c|c|c|c|c|c|c|c|c|c|c|c|c|}
\hline \multicolumn{5}{|c|}{ Wednesday, March 16, 1904} & \multicolumn{5}{|c|}{ Magnet scale erect } & \multicolumn{5}{|c|}{ Wednesday, March 16, 1904} & \multicolumn{5}{|c|}{ Magnet scale erect } \\
\hline \multirow{2}{*}{$\begin{array}{l}\text { Chr'r } \\
\text { time }\end{array}$} & \multicolumn{2}{|c|}{$\begin{array}{l}\text { Scale } \\
\text { readings }\end{array}$} & \multirow{2}{*}{$\begin{array}{l}\text { East } \\
\text { decli- } \\
\text { nation }\end{array}$} & Temp. & Chr'r & $\begin{array}{r}\mathrm{Sc} \\
\text { read }\end{array}$ & $\begin{array}{l}\text { ale } \\
\text { lings }\end{array}$ & $\begin{array}{l}\text { East } \\
\text { decli- }\end{array}$ & Temp. & Chr'r & $\begin{array}{l}\text { Sca } \\
\text { read }\end{array}$ & $\begin{array}{l}\text { cale } \\
\text { dings }\end{array}$ & $\begin{array}{l}\text { East } \\
\text { decli- }\end{array}$ & Temp. & Chr'r & $\begin{aligned} \text { Sc: } \\
\text { read }\end{aligned}$ & $\begin{array}{l}\text { ale } \\
\text { iings }\end{array}$ & $\begin{array}{l}\text { East } \\
\text { decli- }\end{array}$ & Temp. \\
\hline & Left & Right & & & time & Left & Right & & C. & time & Left & Right & nation & C. & time & Left & Right & nation & \\
\hline h $\mathrm{ml}$ & d & $d$ & 01 & $\cdot$ & $\mathrm{h} \mathrm{m}$ & $\mathrm{d}$ & d & & & $\mathrm{h} \mathrm{m}$ & d & $\mathrm{d}$ & & & $h$ in & $\mathrm{d}$ & d & $-\dot{ }$ & 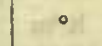 \\
\hline 400 & 58.6 & 58.8 & 2246 & -25.0 & $6 \infty$ & 62.4 & 63.3 & 2252 & -24.0 & 800 & 62.2 & 63.1 & 2252 & -23.9 & $10 \infty$ & 60.2 & 60.3 & 2248 & -23.5 \\
\hline 02 & 57.7 & 58.0 & 44 & & 02 & $6 \mathrm{r} \cdot 3$ & 61.9 & 50 & & 02 & 60.2 & 60.2 & & & 02 & 60.4 & 60.6 & & \\
\hline 04 & 57.2 & $57 \cdot 5$ & 44 & (15) & 04 & 62.1 & 62.7 & 51 & -3 & 04 & 61.2 & 61.8 & 50 & ra & 04 & 59.8 & 60.0 & 48 & 40 \\
\hline 06 & 58.1 & 58.3 & 45 & 50 & 06 & 62.6 & $63 \cdot 3$ & 52 & $x$ & 06 & 61.6 & 62.1 & & 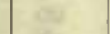 & $\infty 6$ & 58.8 & 59.3 & 46 & 20 \\
\hline 08 & 58.2 & 58.8 & 45 & 59 & 08 & 63.0 & 63.6 & 53 & int & 08 & $6 \mathbf{1} \cdot 3$ & 6 ז.9 & 50 & 86 & 08 & 59.3 & 59.6 & & 65 \\
\hline 10 & 59.0 & 59.2 & 46 & 00 & 10 & 6r.o & 61.7 & 50 & 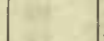 & Io & 61.0 & 61.0 & 49 & 달 & IO & 59.4 & 59.8 & 47 & grt \\
\hline 12 & 59.2 & 59.5 & 47 & 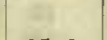 & 12 & 60.9 & 60.9 & 49 & 12x & 12 & 60.4 & $6 \pi .2$ & 49 & 21 & 12 & 59.3 & 59.5 & 47 & $=1$ \\
\hline 14 & 59.2 & 59.8 & 47 & -25.0 & 14 & 62.0 & 62.2 & $5 I$ & -24.0 & 14 & 61.8 & 62.2 & $5 \mathrm{I}$ & 13. & I4 & 58.7 & 59.3 & 46 & -23.5 \\
\hline 16 & 58.8 & 59.7 & 46 & & 16 & 62.7 & 63.2 & 52 & & 16 & 60.2 & 61.0 & 49 & at & 16 & 58.0 & 58.3 & & \\
\hline 18 & 58.3 & 59.1 & 46 & 41 & 18 & 60.9 & $6 \mathrm{I} .0$ & 49 & 2 & 18 & Mag & gnet & & tar & 18 & 57.8 & 58.3 & 44 & Wu \\
\hline 20 & $57 \cdot 9$ & 59.0 & 45 & 10 & 20 & 61.4 & 62.0 & 50 & 5 & 20 & cent & tered & & as & 20.2 & 57.6 & 58.3 & 44 & $x=$ \\
\hline 2.2 & 58.6 & 59.5 & 46 & 25 & 22 & 63.1 & 63.4 & 53 & 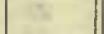 & 22 & & & 4 & 6 & 22 & 57.7 & 58.4 & & 7 \\
\hline 24 & 59.7 & 60.6 & 48 & 25 & 24 & 62.2 & 62.7 & $5 \mathrm{I}$ & L & 24 & $6 \mathrm{II} .5$ & 61.8 & 50 & 15 & 24 & 58.5 & 59.3 & 46 & At \\
\hline 26 & 59.9 & 60.8 & 48 & $a^{2}$ & 26 & 61.6 & 62.0 & 51 & $\leq$ & 26 & 60.1 & 60.3 & 48 & $2=$ & 26 & 59.0 & 60.0 & 47 & in \\
\hline 28.3 & 59.2 & 59.9 & 47 & (12) & 28 & 61.9 & 62.6 & $5 \mathrm{I}$ & tat & 28 & 61.0 & 61.5 & 50 & -23.5 & 28 & 58.5 & 59.3 & 46 & $12=$ \\
\hline 30 & 58.8 & 59.7 & 46 & -24.8 & 30 & 61.0 & 61.7 & 50 & -24.0 & 30 & 61.9 & 62.3 & 5 I & & 30 & 58.3 & 59.3 & 46 & -23.4 \\
\hline 32 & $59 . \mathrm{I}$ & 59.9 & 47 & 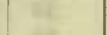 & 32 & 61.0 & 61.3 & 50 & t & 32 & 60.2 & 60.8 & 48 & A. & 32 & 58.2 & 59.0 & 45 & \\
\hline 34 & 59.8 & 60.6 & 48 & 16 & 34 & 62.9 & 63.1 & 52 & 7 & 34 & 58.8 & 60.1 & 47 & ks & 34 & 58.3 & 59.0 & 45 & $y$ \\
\hline 36 & 59.1 & 59.9 & 47 & 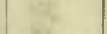 & 36 & 6 1.7 & 62.0 & 51 & & 36 & 59.7 & $6 \pi \cdot 3$ & 48 & 48 & 36 & 58.3 & 58.8 & 45 & g. \\
\hline 38 & 58.9 & 59.1 & 46 & 8 & 38 & 61.2 & 61.7 & 50 & $x^{2}$ & 38 & 60.6 & 62.1 & 50 & 24 & 38 & 58.3 & 58.5 & 45 & 2 \\
\hline 40 & 59.2 & 59.8 & 47. & 5 & 40 & 61.5 & 62.0 & 50 & $y=$ & 40 & 60.0 & 61.6 & 49 & $=$ & 40 & 57.8 & 57.8 & 44 & 178 \\
\hline 42 & 60.1 & 60.4 & 48 & 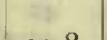 & 42 & 61.9 & 62.1 & 51 & $z_{2}$ & 42 & 60.4 & 61.7 & 49 & 39 & 42 & 57.9 & 57.9 & 44 & 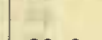 \\
\hline 44 & 60.2 & 60.9 & 48 & -24.8 & 44 & 61.9 & 62.1 & 51 & -24.0 & 44 & 61.3 & $62 . I$ & 50 & 198 & 44 & 57.5 & 57.6 & 44 & $-23 \cdot 3$ \\
\hline 46 & 60.2 & 60.9 & 48 & (2y & 46 & 62.0 & 62.5 & $5 I$ & & 46 & 60.6 & 61.4 & 49 & -22.9 & 46.2 & $57 \cdot 5$ & 57.9 & 44 & 10 \\
\hline 48 & 59.6 & 60.4 & 48 & 6 & 48 & 62.0 & 62.2 & $5 \mathrm{I}$ & $y$ & 48 & 61.0 & 61.7 & 50 & $x^{2}$ & 48 & 57.3 & 57.9 & 44 & 10 \\
\hline 50 & 59.0 & 60.0 & 47 & $\sqrt{8}$ & 50 & 61.3 & 61.8 & 50 & $t^{2}$ & 50 & 60.5 & 61.7 & 50 & $n=$ & 50 & 57.0 & $57 \cdot 3$ & 43 & 100 \\
\hline 52 & 59.8 & 60.8 & 48 & 12 & 52 & 61.0 & 61.4 & 50 & 24 & 52 & 59.9 & 61.0 & 48 & $a^{2}$ & 52 & 56.7 & 57.1 & 43 & $\theta$ \\
\hline 54 & 60.3 & 61.8 & 49 & E & 54 & 6I.1 & 61.8 & 50 & H & 54 & 61.8 & 62.6 & $5 \mathrm{I}$ & 12 & 54 & 56.8 & 57.0 & 43 & 12 \\
\hline 56 & 61.3 & 62.3 & 51 & 5 & 56 & 60.2 & 60.4 & 48 & & 56 & 62.5 & 63.3 & 52 & 78 & 56 & 56.8 & 57.0 & 43 & \\
\hline 58 & 59.3 & 60.2 & 47 & 120 & 58 & 62.0 & 62.7 & $5 I$ & 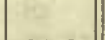 & 58 & 60.5 & $61 \cdot 3$ & 49 & 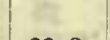 & 58 & $57 \cdot 3$ & 57.9 & 44 & $1=$ \\
\hline$\infty$ & 58.8 & 59.8 & 47 & -24.8 & 700 & 63.3 & 64.0 & 53 & -24.0 & 900 & 58.3 & $59 \cdot 3$ & 46 & -23.0 & 1100 & 57.4 & 57.8 & 44 & -23.2 \\
\hline 02 & 60.1 & $6 \mathrm{r} .5$ & 49 & & 02 & 61.0 & $6 \mathrm{I} \cdot 3$ & 50 & & 02 & 59.4 & 60.2 & 47 & - & 02 & 56.3 & 56.9 & & \\
\hline 04 & 59.7 & 60.6 & 48 & $y^{t}$ & 04 & 61.0 & 6I.I & 49 & 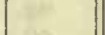 & 04 & 59.3 & 59.7 & 47 & 30 & 04 & 56.0 & 56.3 & 42 & 19 \\
\hline 06 & 58.8 & 59.7 & 46 & 4 & 06 & 62.0 & 62.9 & $5 \mathrm{I}$ & 10 & 05 & 58.6 & 59.6 & 46 & 30 & 06 & 56.3 & 56.7 & 42 & 72 \\
\hline 08 & 58.3 & $59 \cdot 3$ & 46 & ar & 08 & 60.9 & 61.4 & 50 & 15 & 08 & 58.7 & 60.5 & 47 & -3 & 08 & 56.0 & 56.6 & 42 & 3 \\
\hline 10 & 57.0 & 57.8 & 44 & tat & Io & 59.0 & 60.0 & 47 & 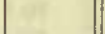 & I0 & 60.6 & 61.6 & 50 & 64 & IO & 56.5 & 56.9 & 42 & (c) \\
\hline 12 & 57.6 & 58.5 & 44 & 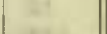 & 12 & 62.5 & 63.3 & 52 & $1=$ & 12 & 60.1 & 61.6 & 48 & 5 & I2 & 56.6 & 57.0 & 43 & 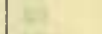 \\
\hline 14 & 62.8 & 63.2 & 52 & $-24 \cdot 4$ & 14 & 61.6 & 62.3 & $5 \mathrm{I}$ & -24.0 & 14 & 61.6 & 62.3 & 5I & -23.2 & I4 & 57.0 & $57 \cdot 3$ & 43 & -23.1 \\
\hline 16 & 63.3 & 63.8 & 53 & ( & 16 & 63.1 & 64.0 & 53 & & 16 & 60.3 & 61.0 & 49 & 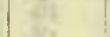 & I6 & 57.3 & 57.4 & 44 & 64 \\
\hline 18 & 61.2 & 6 I.4 & 50 & an & I8 & 60.1 & $6 \mathrm{I} .5$ & 49 & & 18 & 59.8 & 60.8 & 48 & 61 & I8 & 56.5 & 57.0 & 42 & F \\
\hline 20 & 61.0 & 61.7 & 50 & a & 20 & 60.0 & 60.8 & 48 & $y^{2}$ & 20 & 60.3 & 61.0 & 49 & cor & 20 & 56.1 & 56.5 & 42 & 6 \\
\hline 22.3 & 60.9 & 61.8 & 50 & 맘 & 22 & 62.7 & 63.8 & 53 & 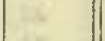 & 22 & 60.3 & 60.6 & 48 & 13 & 22 & 55.1 & 55.5 & 40 & 6 \\
\hline 24 & 60.0 & 61.0 & 48 & 14 & 24 & 60.2 & 61.4 & 49 & 48 & 24 & 60.5 & $6 \mathrm{I} .1$ & 49 & $y=$ & 24 & 54.7 & 54.7 & 39 & n \\
\hline 26 & 63.6 & 64.0 & 55 & c5 & 26 & 61.7 & 63.3 & 52 & the & 26 & 60.3 & 61.0 & 49 & 20 & 26 & 55.0 & $55 \cdot 3$ & 40 & $2=$ \\
\hline 28 & 63.2 & 63.4 & 53 & $y_{2}$ & 28 & 60.3 & 62.1 & 50 & 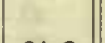 & 28 & $59 . \mathrm{I}$ & 59.6 & 47 & $=$ & 28 & 55.1 & 55.3 & 40 & 1 \\
\hline 30 & 62.1 & 62.1 & 51 & $-24 \cdot 3$ & 30 & 62.7 & 64.8 & 54 & -24.0 & 30 & 59.6 & 60.0 & 47 & 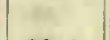 & 30 & 55.6 & $55 . \overline{8}$ & $4 I$ & -23.0 \\
\hline 32 & $6 \mathrm{r} .4$ & $62 . I$ & 50 & & 32 & 62.8 & 64.3 & 53 & & 32 & 60.3 & 60.6 & 48 & $-23 \cdot 3$ & 32 & $55 . \mathrm{I}$ & $55 \cdot 3$ & 40 & \\
\hline 34 & 62. I & 62.7 & 5I & 18 & 34 & 58.6 & 60.8 & 47 & 45 & 34 & 60.3 & 60.6 & 48 & & 34 & 54.3 & 54.7 & 39 & 12 \\
\hline 36 & 62.1 & 62.5 & $5 I$ & 98 & 36 & 60.6 & 62.9 & 50 & 14 & 36 & 60.5 & 61.1 & 49 & 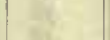 & 36 & 53.8 & 54.2 & 38 & $a$ \\
\hline 38 & 6 б. 6 & 62.0 & $5 \mathrm{I}$ & 20 & 38 & 60.0 & 62.1 & 49 & 8 & 38 & 60.6 & 61.5 & 49 & 25 & 38 & 54.0 & 54.4 & 39 & 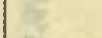 \\
\hline 40 & 61.8 & 62.2 & $5 \mathrm{I}$ & $9=$ & 40 & 59.9 & 62.1 & 49 & 64 & 40 & 60.7 & 61.8 & 50 & 24 & 40 & 54.6 & 55.2 & 40 & ab \\
\hline 42 & 61.2 & 61.8 & 50 & 34 & 42 & $6 \mathrm{I} .1$ & 63.2 & $5 I$ & 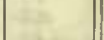 & 42 & 60.6 & 61.5 & 49 & 10 & 42 & 54.6 & 55.0 & 40 & $1=$ \\
\hline 44 & 60.8 & 61.7 & 50 & & 44 & 60.0 & 61.8 & 49 & -24.0 & 44 & 59.8 & 60.6 & 48 & -23.5 & 44 & 54.6 & 55.2 & 40 & -23.0 \\
\hline 46 & 61.3 & 62.0 & 50 & -24.0 & 46 & 60.7 & 62.8 & 50 & & 46 & 59.1 & 60.1 & 47 & & 46 & 54.8 & 55.3 & 40 & \\
\hline 48 & 60.7 & 61.2 & 49 & 5 & 48 & 60.9 & 62.8 & 5 I & 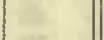 & 48 & 60.3 & 60.6 & 48 & - & 48 & 54.6 & 55.3 & 40 & 15 \\
\hline 50 & 6I.I & $6 \mathbf{1 . 8}$ & 50 & 0 & 50 & 59.8 & 61.8 & 49 & W & 50 & 60.4 & 60.8 & 49 & 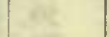 & 50 & 54.1 & 54.5 & 39 & ce \\
\hline 52 & 61.7 & 62.2 & 51 & 달 & 52 & 61.2 & 63.1 & 51 & 28 & 52 & 61.2 & 61.6 & 50 & a & 52 & 54.0 & 54.2 & 38 & 8 \\
\hline 54 & 62.0 & 62.3 & $5 \mathrm{I}$ & ks & 54 & 60.9 & 62.2 & 50 & An & 54 & 61.7 & 62.0 & $5 \mathrm{I}$ & 10 & 54 & 53.1 & 53.3 & 37 & 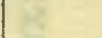 \\
\hline 56 & 60.9 & 61.9 & 50 & 3 & 56 & 59.8 & 60.8 & 48 & (15) & 56 & 61.6 & 62.0 & 51 & 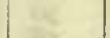 & 56 & 53.6 & 53.7 & 38 & - \\
\hline 58 & 61.3 & 61.9 & 50 & 82 & 58 & 60.9 & $61 \cdot 3$ & 50 & $y=$ & 58 & 60.4 & 60.8 & 49 & 28 & 58 & 53.3 & $53 \cdot 3$ & 37 & 25 \\
\hline
\end{tabular}

Observer-R. R. T.

Observers-R. R. T. and W. J. P., who alternated from 8 h $14 \mathrm{~m}$ to $8 \mathrm{~h} 24 \mathrm{~m}$. 
Tabulation of magnetic declinations observed at Teplitz Bay-Continued

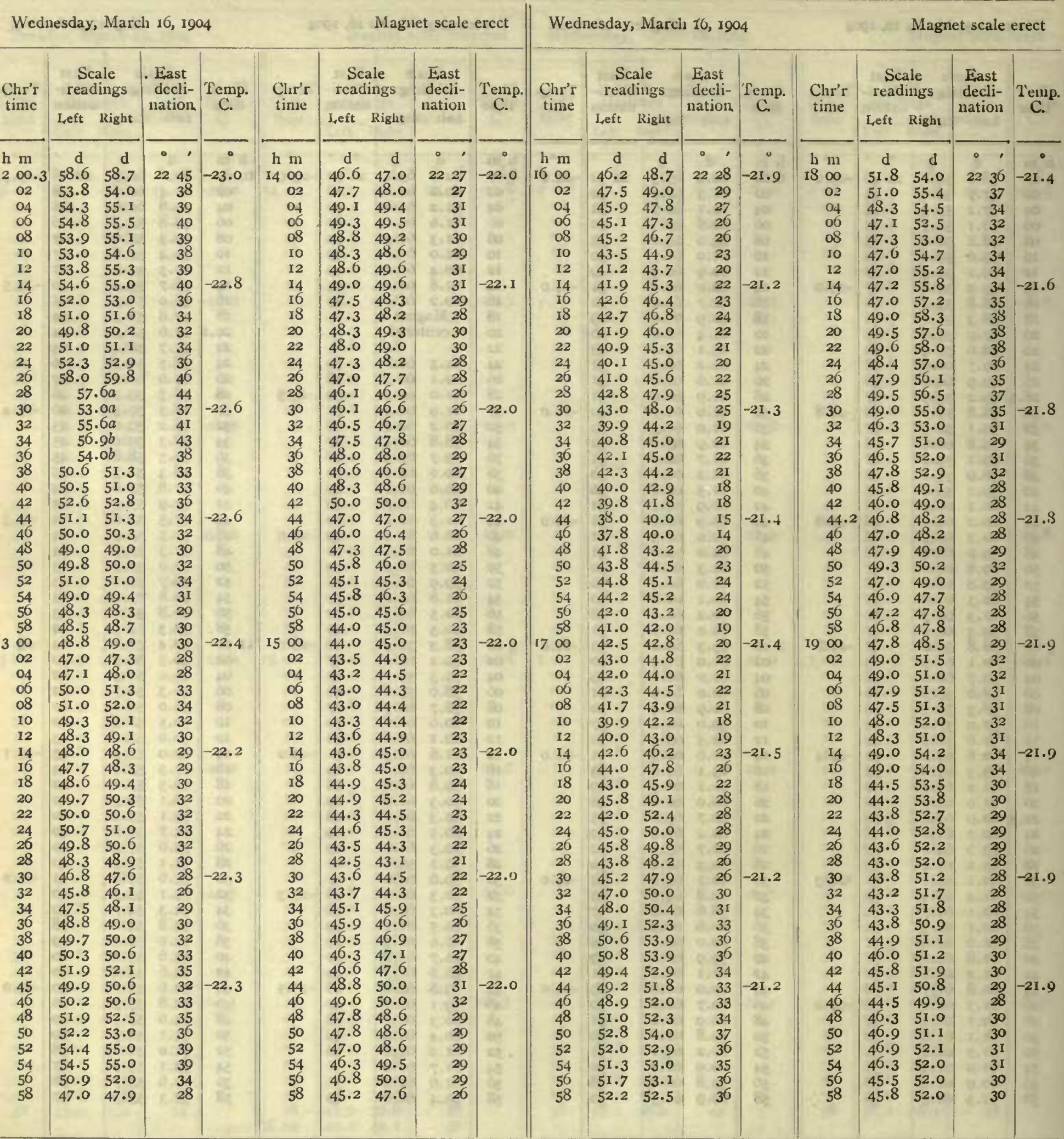

Observers-W. J. P. and J. V., who alternated from rgh $52 \mathrm{~m}$ to Observer-J. V. $\mathrm{I} 6 \mathrm{~h} 02 \mathrm{~m}$. 
Tabulation of magnetic declinations observed at T'eplitz Bay-Continued

\begin{tabular}{|c|c|c|c|c|c|c|c|c|c|c|c|c|c|c|c|c|c|c|c|}
\hline \multicolumn{5}{|c|}{ Wednesday, March I6, Ig04 } & \multicolumn{5}{|c|}{ Magnet scale erect } & \multicolumn{5}{|c|}{ Thursday, March 17, 1904} & \multicolumn{5}{|c|}{ Magnet scale invcrted } \\
\hline \multirow{2}{*}{$\begin{array}{l}\text { Clir'r } \\
\text { time }\end{array}$} & \multicolumn{2}{|c|}{$\begin{array}{l}\text { Scale } \\
\text { readings }\end{array}$} & \multirow{2}{*}{$\begin{array}{l}\text { East } \\
\text { decli- } \\
\text { nation }\end{array}$} & Tenup. & Chr'r & $\begin{array}{l}\text { Sce } \\
\text { read }\end{array}$ & $\begin{array}{l}\text { ale } \\
\text { lings }\end{array}$ & $\begin{array}{l}\text { East } \\
\text { decli- }\end{array}$ & Temp. & Chr'r & $\begin{array}{l}\mathrm{Sc} \\
\text { read }\end{array}$ & $\begin{array}{l}\text { le } \\
\text { ngs }\end{array}$ & $\begin{array}{l}\text { East } \\
\text { decli- }\end{array}$ & I'emp. & Chr'r & $\begin{array}{l}\text { Sca } \\
\text { read }\end{array}$ & $\begin{array}{l}\text { ale } \\
\text { lings }\end{array}$ & East & Temp. \\
\hline & Left & Right & & & & Left & Right & & & & Left & Right & & & & I. eft & Right & & \\
\hline h $\mathrm{m}$ & d & $\mathrm{d}$ & $\circ$, & $\bullet$ & $\mathrm{h} \mathrm{m}$ & d & d & & . & $\mathrm{h} \mathrm{m}$ & d & $\mathrm{d}$ & & & h $\mathrm{m}$ & d & d & $\circ \quad$ & 0 \\
\hline 2000 & 46.2 & 52.1 & 2231 & -22.0 & 2200 & 23.0 & 28.1 & $22 \quad 27$ & -22.4 & I6 00 & 50.8 & 49.8 & 2240 & -26.9 & 1800 & 52.0 & 50.6 & 2239 & -24.2 \\
\hline 02 & 44.8 & 53.0 & 30 & =1t? & 02 & 23.2 & 27.0 & 26 & 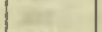 & 02 & 50.8 & 50.0 & 40 & 100 & 02 & 52.1 & 50.6 & 39 & - \\
\hline 04 & $46 . I$ & 53.2 & $3 I$ & $1=$ & 04 & 23.2 & 29.2 & 28 & 12 & 04 & 51.0 & 50.2 & 40 & $=$ & 04 & 51.9 & 50.8 & 39 & ift \\
\hline ó & $45 \cdot 3$ & $52 \cdot 3$ & 30 & war & 06 & 21.9 & 24.8 & 23 & $\infty 0$ & 06 & $5 I \cdot 3$ & 50.3 & 40 & 289 & 06 & 51.8 & 50.9 & 39 & an \\
\hline 08 & 46.0 & 53.0 & $3 \mathrm{I}$ & be & 08 & 20.0 & 24.2 & $2 \mathrm{I}$ & (3y & 08 & 51.7 & 50.2 & 40 & 63 & 08 & 51.8 & $5 I .1$ & 39 & 50 \\
\hline 10 & 49.0 & 55.0 & 35 & tar & 10 & 22.0 & 25.9 & 24 & $6 \mathrm{t}$ & Io & $53 . I$ & 48.7 & 40 & ser & IO & 51.2 & 51.2 & 39 & ox \\
\hline 12 & 55.1 & 60.1 & 44 & thent & 12 & 26.0 & $3 I .2$ & 32 & a & 12 & $53 \cdot 3$ & 49.2 & 39 & & 12 & 51.8 & $5 \mathrm{I} .2$ & 39 & $3+1$ \\
\hline 14 & 57.0 & 63.1 & 48 & -22.0 & I4 & $23 . I$ & 23.8 & 23 & -22.5 & 14 & $54 . I$ & 50.2 & 38 & -26.3 & I4 & 51.9 & $5 I .7$ & 38 & -24.0 \\
\hline 16 & $54 \cdot 5$ & 58.8 & 42 & $\sqrt{1}+2$ & 16 & 27.0 & 31.0 & 32 & 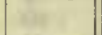 & 16 & 53.2 & 50.4 & 38 & t & 16 & 51.9 & 51.6 & 38 & to \\
\hline 18 & 52.6 & 59.0 & $4 \mathrm{I}$ & $d t$ & 18 & $23 . I$ & 26.0 & 25 & tif & I8 & 52.3 & 50.2 & 39 & 34 & 18 & 52.2 & 51.7 & 38 & it \\
\hline 20 & 56.8 & 57.5 & 43 & E. & 20 & 25.0 & 29.3 & 29 & 20 & 20 & 52.7 & 50.7 & 38 & is & 20 & $52 . I$ & 51.4 & 38 & 6 \\
\hline 22 & 49.0 & 51.5 & 32 & us: & 22 & 27.9 & 31.0 & 33 & and & 22 & 53.8 & 52.0 & 36 & sent & 22 & 52.1 & 51.4 & 38 & sin \\
\hline 24 & 49.2 & $5 \mathrm{~T} .5$ & 32 & in & 24 & 27.2 & 31.1 & 32 & 48 & 24 & 52.8 & 51.2 & 38 & $x^{2}$ & 24 & 52.2 & 51.5 & 38 & 36 \\
\hline 26 & 49.2 & 45.4 & 28 & 65 & 26 & 27.0 & 32.0 & 33 & ax & 26 & 52.9 & 52.1 & 37 & $x$ & 26 & 52.3 & 51.7 & 38 & 68 \\
\hline 28 & 48.5 & $54 \cdot 3$ & 34 & -1 & 28 & 25.0 & 30.0 & 30 & 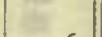 & 28 & 52.8 & 51.9 & 37 & & 28 & 52.2 & $5 I \cdot 3$ & 38 & 6. \\
\hline 30 & 56.2 & 60.6 & 2245 & $-22 . I$ & 30 & 25.8 & 31.0 & $3 I$ & -22.6 & 30 & 52.2 & 51.8 & 38 & -25.9 & 30 & 52.0 & 51.3 & 38 & $-24 \cdot 0$ \\
\hline 32 & 62.1 & 77.8 & 2303 & to & 32 & 25.1 & 32.3 & 32 & 13 & 32 & 52.3 & $5 I .3$ & 38 & ( & 32 & 52.1 & 51.6 & 38 & ( \\
\hline $34^{*}$ & 52.0 & 63.5 & I7 & ic & 34 & 27.0 & 34.0 & 34 & a & 34 & 52.2 & 5 I. I & 38 & $y=$ & 34 & 52.1 & 51.6 & 38 & $y^{2}$ \\
\hline 36 & $57 \cdot 1$ & 60.1 & 19 & at & 36 & 30.0 & 36.8 & 39 & -x & 36 & 51.8 & 51.0 & 39 & 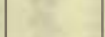 & 36 & 51.9 & $5 \mathrm{I} .2$ & 39 & 48 \\
\hline 38 & 48.0 & 56.9 & $23 \circ 9$ & t & 38 & 28.2 & 35.0 & 36 & 8 & 38 & 5 I. 3 & 50.2 & 40 & $x$ & 38 & 51.7 & 50.7 & 39 & ne. \\
\hline 40 & 36.0 & 48.8 & 2253 & tat & 40 & $27 \cdot 9$ & 31.7 & 34 & $n$ & 40 & 51.0 & 50.0 & 40 & 12 & 40 & 51.1 & 50.7 & 40 & 64 \\
\hline 42 & 27.5 & 40.9 & 40 & co & 42 & 29.9 & 38.8 & 40 & -1 & 42 & $5 \pi .3$ & 50.2 & 40 & & 42 & 50.9 & 50.2 & 40 & \\
\hline 44 & 24.2 & 36. I & 34 & -22.1 & 44 & 28.0 & 36.7 & 37 & -22.7 & 44 & $5 \mathrm{I} .4$ & 50.4 & 40 & -25.4 & 44 & 50.9 & 50.4 & 40 & -23.9 \\
\hline 40 & 2 L. 3 & 34.9 & $3 \mathrm{I}$ & 17 & 46.5 & 32.9 & 36.9 & $4 I$ & (n) & 46 & 52.3 & 50.8 & 39 & (and & 46 & 51.2 & 51.0 & 39 & 0 \\
\hline 48 & 17.1 & 21.8 & I7 & 64 & 48 & 32.0 & 36.0 & 40 & 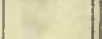 & 48 & 52.9 & $5 \mathrm{I} .2$ & 38 & a & 48 & 51.2 & 51.2 & 39 & 24 \\
\hline 50 & I9.0 & 26.2 & 22 & cent & 50.5 & 33.0 & 36.7 & $4 I$ & rese & 50 & 53.6 & 52.0 & 36 & 10. & 50 & 5 I. I & 50.8 & 40 & $x$ \\
\hline 52 & 17.5 & 29.0 & 23 & tit & 52 & 31.8 & 35.0 & 39 & $z$ & 52 & 54.2 & 52.0 & 36 & $x^{2}$ & 52.3 & 51.1 & 50.8 & 40 & a. \\
\hline 54 & 23.0 & 31.2 & 29 & 36 & 54 & 39.4 & 38.0 & 47 & 3 & 54 & 54.8 & 52.5 & 35 & $y^{2}$ & 54 & 51.0 & 50.8 & 40 & 34 \\
\hline 56 & I9.0 & 31.0 & 26 & ar: & 56 & 37.8 & 38.8 & 47 & . & 56 & 54.8 & 52.7 & 35 & d. & 56 & 51.2 & 50.8 & 39 & \\
\hline 58 & 19.0 & 28.0 & 24 & 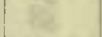 & 58 & 33.0 & 35.2 & 40 & & 58 & 54.7 & 52.7 & 35 & & 58 & 52.2 & 51.5 & 38 & 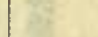 \\
\hline 2100 & I6. 5 & 24.9 & 19 & -22.1 & 2300 & 34.2 & 36.0 & 42 & -22.8 & 1700 & $54 \cdot 3$ & 52.6 & 36 & -25.0 & 1900 & 53.1 & 52.2 & 37 & $-23 \cdot 9$ \\
\hline 02 & 19.9 & 26.0 & 23 & $1-2$ & $\mathrm{O} 2$ & 32.8 & $34 \cdot 3$ & 39 & 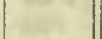 & 02 & 53.9 & 52.8 & 36 & 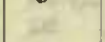 & $\mathrm{O} 2$ & 53.0 & 52.8 & 36 & ( \\
\hline 04 & I8. 0 & 25.1 & 20 & 10 & 04 & $3 I \cdot 3$ & 33.2 & 37 & a & 04 & 53.9 & 53.1 & 35 & 10 & 04 & 53.1 & 52.2 & 37 & $x^{2}$ \\
\hline 06 & I8. 2 & 25.0 & 20 & xy & 06 & 32.9 & 35.0 & 40 & w & 06 & 53.8 & 53.0 & 36 & $4 y$ & 06 & 52.8 & 52.0 & 37 & $x^{2}$ \\
\hline 08 & I8. 2 & 26.0 & $2 I$ & a & 08 & 32.6 & 35.2 & 40 & s. & 08 & 53.8 & 53.1 & 36 & 8 & 08 & 52.2 & 51.9 & 38 & -3 \\
\hline Io & 20.2 & 26.2 & 23 & 08 & IO & 30.0 & 32.7 & 36 & wit & I0 & 53.7 & 53.1 & 36 & 60 & Io & 52.0 & 51.2 & 38 & a \\
\hline I 2 & 23.9 & 28.5 & 28 & 1 & 12 & 31.8 & 33.0 & 38 & & I2 & 53.7 & 53.1 & 36 & 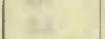 & I2 & 52.1 & 51.3 & 38 & \\
\hline I4 & 24.0 & 32.0 & $3 I$ & -22.2 & I4 & 31.0 & 31.7 & 36 & -22.8 & I4 & 53.4 & 53.0 & 36 & $-24 \cdot 9$ & I4 & 51.8 & 50.9 & 39 & -23.9 \\
\hline 16 & 25.0 & 32.0 & 31 & 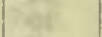 & 16 & 29.9 & 31.7 & 35 & atrat & 16 & $53 \cdot 5$ & 52.8 & 36 & - & 16 & 51.9 & 50.8 & 39 & $-\infty$ \\
\hline 18 & I 4.5 & 26.3 & I9 & $\sqrt{1}$ & I 8 & 29.3 & $31 \cdot 3$ & 34 & 4 & I8 & 53.3 & 52.8 & 36 & $x_{11}$ & I8 & 51.9 & 50.8 & 39 & en: \\
\hline 20 & 12.8 & 23.2 & 15 & $x$ & 20 & 30.0 & 32.0 & 35 & $=$ & 20 & 53.0 & 52.7 & 36 & xin & 20 & 51.2 & 50.3 & 40 & $x=$ \\
\hline 22 & $17 \cdot 2$ & $2 \overline{6} .5$ & $2 \mathrm{I}$ & 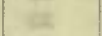 & 22 & $33 \cdot 3$ & 35.0 & 40 & 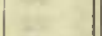 & 22 & 52.9 & 52.7 & 36 & 6 & 22 & 51.6 & 50.0 & 40 & $x^{2}$ \\
\hline 24 & 16.5 & 27.9 & 1.22 & 10 & 24 & 35.2 & 36.1 & 42 & in & 24 & 52.8 & 51.7 & 38 & $x$ & 24 & 51.2 & 49.9 & 40 & \\
\hline 26 & 24.2 & 26.0 & 26 & $\mathrm{WV}$ & 26 & 31.0 & 33.0 & 37 & (1) & 26 & 52.3 & 51.5 & 38 & - & 26 & 50.9 & 49.6 & $4 I$ & \\
\hline 28 & 17.6 & 27.2 & 22 & 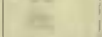 & 28 & 32.0 & $34 \cdot 3$ & 39 & & 28 & 52.2 & $5 I .4$ & 38 & & 28 & 50.3 & 49.2 & $4 I$ & \\
\hline 30 & 20.9 & 32.0 & 28 & -22.2 & 30 & $33 . I$ & 36.0 & $4 I$ & $-22 . ?$ & 30 & 52.1 & 51.0 & 39 & -24.8 & 30 & 50.3 & 49.2 & 41 & -23.8 \\
\hline 32 & 23.0 & 31.9 & 30 & 142 & 32 & 33.2 & 36.5 & 41 & & 32 & 52.2 & 51.2 & 38 & ( & 32 & 50.4 & 49.6 & 41 & ( \\
\hline 34 & 22.9 & 31.2 & 29 & 18 & 34 & 30.9 & $34 . I$ & 38 & $x^{2}$ & 34 & 51.9 & 51.3 & 38 & 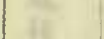 & 34 & 50.3 & $49 \cdot 4$ & $4 I$ & $=$ \\
\hline 36 & 25.0 & 3 I. 3 & $3 I$ & 42 & 36 & 31.0 & 34.2 & 38 & $+3 x^{2}$ & 36 & 52.0 & 51.3 & 38 & 3 & 36 & 50.8 & 49.5 & $4 I$ & 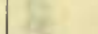 \\
\hline 38 & 24.0 & 29.2 & 28 & a & 38 & 30.8 & 34.1 & 38 & E & 38 & 52.1 & 51.6 & 38 & $=0$ & 38 & 50.5 & 49.3 & $4 I$ & \\
\hline 40 & 22.3 & 25.7 & 24 & 63 & 40 & 31.0 & 34.2 & 38 & 18 & 40 & 52.2 & 51.7 & 38 & 6 & 40 & 50.9 & 50.0 & 40 & \\
\hline 42 & 23.5 & 26.9 & 26 & 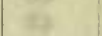 & 42 & 31.0 & 34.6 & 38 & & 42 & 52.2 & 51.9 & 38 & & 42 & 50.8 & 50.0 & 40 & \\
\hline 44 & 19.8 & 22.9 & 20 & -22.3 & 44 & 32.2 & 35.0 & 39 & -22.9 & 44 & 52.0 & 51.8 & 38 & $-24 \cdot 5$ & 44 & 50.8 & $49 \cdot 3$ & $4 I$ & -23.7 \\
\hline 46 & 20.6 & 26.0 & 23 & (n) & 46 & 32.8 & 34.1 & 39 & (1) & 46 & 52.2 & 51.6 & 38 & 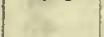 & 46 & 51.3 & 49.7 & 40 & \\
\hline 48 & 21.8 & 24.1 & 23 & 25 & 48 & 32.1 & 33.5 & 38 & 8. & 48 & 52.2 & 51.3 & 38 & & 48 & 50.8 & 49.3 & 4I & \\
\hline 50 & 23.0 & 26.7 & 26 & 6 & 50 & 32.2 & 34.0 & 39 & 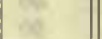 & 50 & 52.1 & 51.2 & 38 & de & 50 & 50.9 & 49.4 & $4 I$ & 35 \\
\hline 52 & 17.2 & 23.2 & 18 & 1. & 52 & 32.1 & 34.0 & 38 & ext & 52 & 52.1 & $5 I . I$ & 38 & $3 x$ & 52 & 50.5 & $49 \cdot 3$ & $4 I$ & - \\
\hline 54 & 22.2 & 29.8 & 28 & 14 & 54 & 31.2 & 36.0 & 39 & 38 & 54 & 52.3 & 51.2 & $3^{8}$ & & 54 & 51.0 & $49 \cdot 3$ & 41 & \\
\hline 56 & 16.5 & 22.2 & I7 & & 56 & 32.0 & 36.9 & 4I & & 56 & 52.1 & 50.8 & 39 & & 56 & 50.9 & 49.3 & $4 I$ & \\
\hline 58 & $22 . I$ & 28.9 & 27 & 13 & 58 & 30.6 & 35.0 & 38 & 3 & 58 & 52.2 & 50.8 & 39 & & 58 & 50.9 & 49.9 & 40 & \\
\hline & & & & & $24 \infty$ & 30.6 & 35.0 & 38 & & & & the & & & 2000 & 50.9 & 49.8 & 40 & -23.7 \\
\hline
\end{tabular}

Correction to local mean time is $+3.5 \mathrm{~s} .90^{\circ}$ torsion $=27^{\prime} 47$. Torsion head at ol oom read $\mathrm{II}^{\circ}$ and at $24 \mathrm{~h} 25 \mathrm{~m}$ read $23^{\circ}$. Observer-J. V.
Correction to local mean time is - $18 \mathrm{~s} .90^{\circ}$ torsion $=26 . ' 53$.

'Torsion head at 15 h $15 \mathrm{~m}$ read $13^{\circ}$ and at 20 h $20 \mathrm{~m}$ read $15^{\circ}$. Observer-R. R. T. 
Tabulation of magnetic declinations observed at Teplitz Bay-Continued

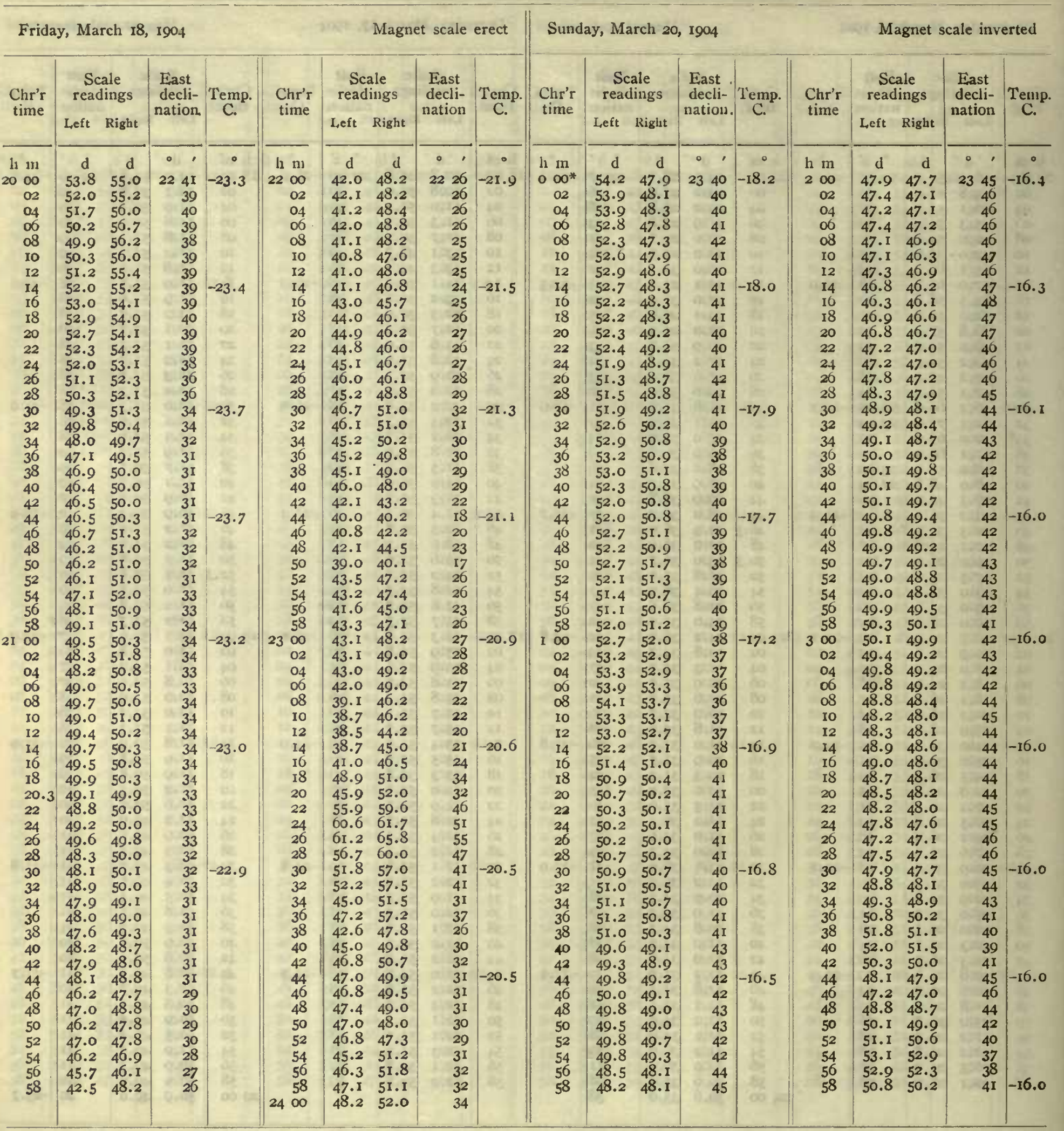

Correction to local mean time is - $1 \mathrm{r} .5 \mathrm{~s}$.

Observer-R. R. T.

Torsion head at $19 \mathrm{~h} 27 \mathrm{~m}$ read $16^{\circ}$ and at $24 \mathrm{~h} 23 \mathrm{~m}$ read the same.

Observer-J. V. 
Tabulation of magnetic declinations observed at Teplitz Bay-Continued

\begin{tabular}{|c|c|c|c|c|c|c|c|c|c|c|c|c|c|c|c|c|c|c|}
\hline \multicolumn{5}{|c|}{ Sunday, March 20, 1904} & \multicolumn{5}{|c|}{ Magnet scale erect } & \multicolumn{5}{|c|}{ Monday, March 21, 1904} & \multicolumn{4}{|c|}{ Magnet scale inverted } \\
\hline \multirow{2}{*}{$\begin{array}{l}\text { Chr'r } \\
\text { time }\end{array}$} & \multicolumn{2}{|c|}{$\begin{array}{c}\text { Scale } \\
\text { readings }\end{array}$} & \multirow{2}{*}{$\begin{array}{c}\text { East } \\
\text { decli- } \\
\text { nation }\end{array}$} & Temp. & Chr'r & $\begin{array}{l}\mathrm{Sc} \\
\text { read }\end{array}$ & $\begin{array}{l}\text { ale } \\
\text { ings }\end{array}$ & $\begin{array}{l}\text { East } \\
\text { decli- }\end{array}$ & Temp. & Chr'r & $\begin{array}{l}\text { Scale } \\
\text { readings }\end{array}$ & $\begin{array}{l}\text { East } \\
\text { decli- }\end{array}$ & Temp. & Chr'r & $\begin{array}{l}\text { Sca } \\
\text { readi }\end{array}$ & $\begin{array}{l}\text { le } \\
\text { ings }\end{array}$ & $\begin{array}{l}\text { East } \\
\text { decli- }\end{array}$ & Temp. \\
\hline & Left & Right & & & & Lefit & Right & & & & Ireft Right & & & & Left 1 & Right & & \\
\hline h $\mathrm{m}$ & d & d & $\circ$, & $\therefore$ & h $\mathrm{nI}$ & d & d & $\circ:$ & $\therefore$ & $\mathrm{h} \mathrm{m}$ & & -, & $\cdot$ & h m & d & d & $\cdot \cdot$ & $\cdot$ \\
\hline 400 & $57 \cdot 3$ & 58.8 & 2344 & -16.0 & 600 & 71.7 & 73.1 & 2406 & -16.9 & 800 & High & 600 & 900 & 1000 & 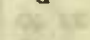 & & & \\
\hline 02 & 58.3 & 59.2 & 45 & 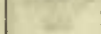 & 02 & $72 . I$ & 73.8 & of & 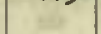 & 02 & winds & 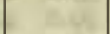 & iw & 02 & $\mathrm{a}$ & & Bat: & 60 \\
\hline 04 & 58.8 & 59.7 & 45 & the & 04 & 70.8 & 71.7 & 04 & wo & of & prevented & 6.1 & 70 & 04 & 35 & & $=0$ & 10 \\
\hline 06 & 59.1 & 59.5 & 46 & 30 & 06 & 70.8 & 72.1 & 05 & 60 & 05 & reaching & e.6. & 10 & 06 & (1) & & $0, x^{2}$ & 100 \\
\hline 08 & 59.0 & 59.2 & 45 & 08 & 08 & 71.3 & 72.1 & 05 & 46 & 08 & observatory & kit & $3 y$ & 08 & 34 & & 3.60 & Ret \\
\hline I0 & 59.2 & 59.4 & 46 & st & ro & 70.2 & 70.9 & 03 & ati & Io & 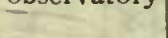 & & at & 10 & 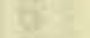 & & 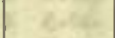 & ait \\
\hline 12 & 59.9 & 60.2 & 47 & & I2 & 69.3 & $69 . \overline{8}$ & 2402 & (68) & 12 & $=1$ & e. & Li: & 12 & $i x$ & 1 & $3 .-67$ & 32 \\
\hline 14 & 60.7 & 61.0 & 48 & -16.0 & 14 & 67.6 & 68.1 & 2359 & -16.9 & I4 & is & 87 & 67 & 14 & 28 & & & Af. \\
\hline 16 & 61.3 & 62.0 & 49 & $+2 x$ & 16 & 67.7 & 68.3 & 2359 & 4 & 16 & e & 3.65 & 36 & 16 & sk & 6 & $7-68$ & int \\
\hline 18 & 61.7 & 62.2 & 50 & 20: & IS & 68.2 & 69.0 & 2400 & $\theta$ & I8 & $3 x$ & +1 & int & 18 & Gto & Het & $3+48$ & ba \\
\hline 20 & 61.6 & 62.1 & 50 & se. & 20 & 69.2 & 70.1 & 02 & Qx & 20 & ac. & 6 & ix & 20 & 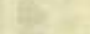 & $c$. & $\cos$ & $\alpha$ \\
\hline 22 & 61.3 & 61.8 & 49 & ax & 22 & 70.6 & 71.4 & 04 & 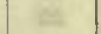 & 22 & $y$ & 85. & $\mathrm{Cs}$ & 22 & 4 & (6) & 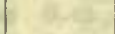 & as \\
\hline 24 & 61.4 & 61.9 & 49 & $y=$ & 24 & 70.0 & 70.8 & 03 & $E$ & 24 & t & a) 5 & 45 & 24 & 25 & (2) & pity & - 85 \\
\hline 26 & 6 ז.9 & 62.1 & 50 & $E$ & 26 & 69.2 & 70.1 & 02 & ne & 26 & 21 & 31 & in & 26 & 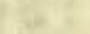 & & 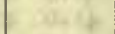 & $\approx$ \\
\hline 28 & 62.2 & 62.4 & 50 & $1=$ & 28 & 69.1 & 70.2 & 02 & & 28 & 56 & $6 t$ & ac & 28 & 7 & & 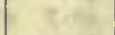 & 2 \\
\hline 30 & 62.8 & 62.9 & $5 I$ & -16.1 & 30 & 69.1 & 69.8 & OI & $-16.1)$ & 30 & 26 & & 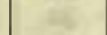 & 30 & 3 & & & 18 \\
\hline 32 & 62.7 & 63.0 & $5 \mathrm{I}$ & $1=$ & 32 & 69.5 & 70.9 & 02 & $x^{2}$ & 32 & ef & 5.2 & in & 32 & 25 & & 54 & +2 \\
\hline 34 & 62.3 & 62.9 & 51 & 12. & 34.5 & 68.7 & 69.7 & OI & in. & 34 & 26 & aft. & 78 & 34 & 36 & & 8.14 & 14 \\
\hline 36 & 61.3 & 61.9 & 49 & $x^{2}$ & 36 & 67.4 & 68.8 & 2400 & ac & 36 & $\alpha$ & w. & $\sqrt{2}$ & 36 & 27 & & $5-6$ & ect \\
\hline 38 & 60.1 & 60.0 & 47 & 2 & 38 & 67.1 & 68.0 & 2358 & ra & 38 & $6 y$ & & $\alpha$ & 38 & af & & ovent & e \\
\hline 40 & 60.1 & 60.4 & 47 & es & 40 & 68.7 & 69.1 & 24 OI & (6) & 40 & es & Wi.e & 4 & 40 & an & it & $\ln 6$ & 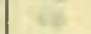 \\
\hline 42 & 60.5 & 6I.0 & 48 & & 42 & 70.0 & 70.9 & 03 & $\log ^{2}$ & 42 & $\Leftrightarrow$ & $7 A$ & 35 & 42 & 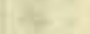 & & 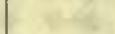 & at: \\
\hline 44 & 60.2 & 60.5 & 47 & -16.3 & 44 & 72.1 & 73.0 & 06 & -17.0 & 44 & 36 & 61 & 12 & 44 & 65 & 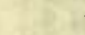 & Hitive & $3=$ \\
\hline 46 & 61.1 & 61.4 & 49 & 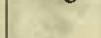 & 46 & 72.9 & 74.0 & 08 & ( & 46 & a & 2 & 6 & 46 & as & & 60 & 6. \\
\hline 48 & $62 \cdot 3$ & 62.9 & $5 I$ & 84 & 48 & 71.7 & 72.2 & 05 & 3 & 48 & xis & 8i: & $2 y$ & 48 & 6 & & 64 & 65 \\
\hline 50 & 62.9 & 63.3 & 52 & is & 50 & $72 . I$ & 72.3 & 06 & 18 & 50 & $x^{2}$ & 8. & $\alpha$ & 50 & act & $=$ & V.ing & 62 \\
\hline 52 & 63.0 & 63.3 & 52 & at. & 52 & 74.9 & 75.8 & II & 5 & 52 & as. & c. ${ }^{2}$ & ac & 52 & 3 & & $3.6 x$ & 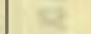 \\
\hline 54 & 62.8 & 63.3 & 5 I & $w^{2}$ & 54 & 73.9 & 74.7 & 09 & 72 & 54 & es & $3 x$ & he & 54 & 64 & & 16.65 & n \\
\hline 56 & $63 . I$ & 64.0 & 52 & of & 56 & 72.0 & 73.3 & 06 & 5 & 56 & 125 & 8 & 85 & 56 & 34 & & 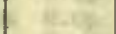 & tis \\
\hline 58 & 63.8 & 64.9 & 54 & & 58 & 71.7 & 72.3 & $\infty$ & & 58 & 78. & 7. & Ies & 58 & (15) & $\mathrm{H}^{2}$ & 68 & 过 \\
\hline 500 & 64.6 & 65.8 & 55 & -16.7 & 700 & 70.2 & 71.4 & 04 & -17.0 & 900 & 6 & EF & 65 & II 00 & H.t. & & Wet & 6 \\
\hline 02 & 64.2 & 65.0 & 54 & 100 & 02 & 72.2 & 72.7 & 2406 & $y=$ & 02 & 65 & 5 & 60 & 02 & 18 & & 7.65 & 60 \\
\hline 04 & 65.5 & 66.1 & 56 & ine & $04 \%$ & 32.9 & 38.8 & 23 I4 & Wir & 04 & 6 & 1. & 14 & 04 & 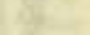 & & 1.25 & inin. \\
\hline 05 & 66.1 & 66.8 & 57 & 60 & 06 & 30.4 & $35 \cdot 3$ & 09 & ine & 06 & af) & 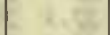 & 30 & 06 & 6 & 7. & s.ret & 6 \\
\hline 08 & $66 . I$ & 66.7 & 57 & 28 & 08 & 30.0 & 34.9 & 2308 & $=$ & 08 & 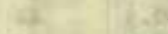 & k. & war & 08 & 60 & & ditit & 65 \\
\hline 10 & 66.9 & 67.2 & 58 & 34 & 10 & 24.0 & 28.2 & 2259 & we & I0 & 34 & a. & on & I0 & wh & & L-36) & wit \\
\hline 12 & 66.2 & 67.1 & 57 & & 12 & $24 \cdot 3$ & 28.8 & 2259 & & 12 & 7 & 85 & $a r$ & 12 & $\theta$ & & 6.58 & ar \\
\hline 14 & 65.9 & 66.9 & 57 & -16.8 & 14 & 28.0 & 31.0 & 2304 & -17.0 & 14 & 12 & 8. & 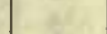 & I4 & 15. & & 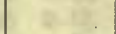 & Mt \\
\hline 16 & 65.0 & 65.8 & 55 & $x^{2}$ & 16 & 29.1 & 32.0 & 06 & t & 16 & t & A. & $(t)$ & 16 & Wh & & 145 & atif \\
\hline 18 & 65.0 & 65.8 & 55 & हi? & 18 & 28.7 & 31.1 & 05 & 84 & 18 & ave & 25 & 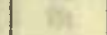 & I8 & ( & $D=$ & 6.62 & the \\
\hline 20 & 65.1 & 66.1 & 55 & is & 20 & 28.0 & 30.9 & 2304 & 2 & 20 & 67 & 78 & is & 20 & 6 & & $1+2=$ & $\alpha$ \\
\hline 22 & 65.0 & 66.3 & 55 & $E$ & 22 & 25.0 & $27 \cdot 3$ & 2259 & 75 & 22 & igit & 2ut & ss & 22 & 26 & & 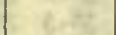 & est \\
\hline 24 & 66.0 & 67.4 & 2357 & 38 & 24 & 24 . I & 26.7 & 58 & $=5$ & 24 & tas. & 54 & F. & 24 & 34 & & $204^{2}$ & 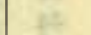 \\
\hline 26 & 67.6 & 68.8 & 2400 & ate & 26 & 23.1 & 25.3 & 56 & 2 & 26 & 78 & & 16 & 26 & & t & we & des \\
\hline 28 & 67.7 & 68.8 & 2400 & & 28 & 23.1 & 25.3 & 56 & & 28 & $z$ & $+a$ & ar & 28 & 28 & & 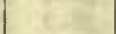 & 185 \\
\hline 30 & $67 . \mathrm{I}$ & 68.3 & 2359 & -16.8 & 30 & 24.5 & 26.0 & 57 & -16.9 & 30 & (ac & nes & $b^{2}$ & 30 & int & & $5=2$ & af \\
\hline 32 & 66.9 & 67.9 & 2358 & $x=$ & 32 & 24.9 & 26.3 & 58 & $x^{2}$ & 32 & se. & $x$ & at & 32 & $\alpha$ & & $\operatorname{mos} 2$ & $\mathrm{E}^{2}$ \\
\hline 34.6 & 67.6 & 69.0 & 2400 & x & 34 & $24 . I$ & 25.4 & 56 & $N_{2}$ & 34 & 20 & & 25 & 34 & & & 600 & \\
\hline 36 & 67.6 & 69.1 & 00 & as & 36 & 22.5 & 24.1 & 54 & its & 36 & $x^{2}$ & the & ac & 36 & 45.3 & 44.0 & 2242 & -16.8 \\
\hline 38 & 67.8 & 69.2 & 00 & 6 & 38 & 21.9 & 23.2 & 53 & $8 c$ & 38 & 3 & es & $8^{2}$ & 38 & 46.1 & & 40 & $1=$ \\
\hline 40 & 68.0 & 69.1 & 2400 & 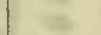 & 40 & 22.3 & 22.9 & 53 & 16 & 40 & a & 6. & 8 & 40 & $4^{6} .2$ & 44.8 & 40 & 68 \\
\hline 42 & 66.9 & 67.9 & 2358 & & 42 & 22.6 & 23.8 & 54 & 73 & 42 & $\mathrm{z}$ & & $\Delta y$ & 42 & 47.0 & 44.7 & 40 & \\
\hline 44 & 66.2 & 67.2 & 57 & -16.8 & 44 & 21.2 & 21.8 & $5 \mathrm{i}$ & -17.0 & 44 & a & $a^{2}$ & 78 & 44 & 48.0 & 44.9 & 39 & -16.8 \\
\hline 46 & 67.2 & 68.2 & 59 & 128 & 46 & 22.7 & 23.8 & 54 & . & 46 & 58 & 0. & 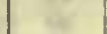 & 46 & 48.1 & 45.1 & 39 & 1 \\
\hline 48 & 67.0 & 67.9 & 58 & he & 48 & 25.0 & 25.4 & 57 & 62 & 48 & $\mathrm{~F}^{2}$ & c. & $*$ & 48 & 48.2 & 44.5 & 39 & 168 \\
\hline 50 & 67.0 & 68.0 & 58 & et & 50 & 19.8 & 19.8 & 49 & a & 50 & 172 & & . & 50 & 46.4 & 45.9 & 40 & 다 \\
\hline 52 & 67.2 & 68.7 & 2359 & xe & 52 & 23.5 & 24.4 & 55 & $\sqrt{2}$ & 52 & $6 x$ & ittes & Wi & 52 & 46.0 & $45 \cdot 5$ & 40 & 67. \\
\hline 54 & 67.7 & 68.8 & 2400 & A2 & 54 & & $.9 a$ & 55 & 42 & 54 & (2) & $x>$ & 12 & 54 & 46.1 & $45 . I$ & 40 & $\because$ \\
\hline 56 & 68. I & 69.3 & 00 & dit & 56 & 21.0 & 21.5 & $5 \mathrm{I}$ & QS & 56 & 124 & 9. & as & 56 & 46.1 & 45.1 & 40 & 80 \\
\hline 58 & 69.7 & 71.1 & 03 & 16 & 58 & 22.8 & 23.2 & 54 & 152 & 58 & wt & (2.) & $\sqrt{15}$ & 58 & 48.3 & $43 . I$ & 40 & \\
\hline & & & & & 800 & $2 \mathrm{I} .2$ & 21.9 & $5 \mathrm{I}$ & -17.0 & & 15 & & $\infty$ & 1200 & 49.0 & 44.0 & 39 & -16.5 \\
\hline
\end{tabular}

Correction to local mean time is - 51s. $90^{\circ}$ torsion $=17 .^{\prime} 58$. Torsion head at oh oon read $27^{\circ}$ and at gh oom read $30^{\circ}$. Observer-R. R. 'T'.
Correction to local mean time is - Is.

Torsion head at $\mathrm{IIh} 05 \mathrm{~m}$ read $28^{\circ}$ and at $\mathrm{I} 2 \mathrm{~h}$ 30m read $27^{\circ}$. Observer-J. V. 
Tabulation of magnetic declinations observed at Teplitz Bay-Continued

\begin{tabular}{|c|c|c|c|c|c|c|c|c|c|c|c|c|c|c|c|c|c|c|c|}
\hline \multicolumn{6}{|c|}{ Tuesday, March 22, Ig04 } & \multicolumn{4}{|c|}{ Magnet scale erect } & \multicolumn{6}{|c|}{ Wednesday, March 23, 1904} & \multicolumn{4}{|c|}{ Magnet scale inverted } \\
\hline \multirow{2}{*}{$\begin{array}{l}\text { Chr'r } \\
\text { time }\end{array}$} & \multicolumn{2}{|c|}{$\begin{array}{l}\text { Scale } \\
\text { readings }\end{array}$} & \multirow{2}{*}{$\begin{array}{l}\text { East } \\
\text { decli- } \\
\text { nation }\end{array}$} & Temp. & Chr'r & $\begin{array}{r}\mathrm{Sc} \\
\text { read }\end{array}$ & $\begin{array}{l}\text { ale } \\
\text { lings }\end{array}$ & $\begin{array}{l}\text { East } \\
\text { decli- }\end{array}$ & Temp. & Chr'r & $\begin{array}{l}\text { Sce } \\
\text { read }\end{array}$ & $\begin{array}{l}\text { ale } \\
\text { lings }\end{array}$ & $\begin{array}{l}\text { East } \\
\text { decli- }\end{array}$ & Temp. & Chr'r & $\begin{array}{r}\mathrm{Sc} \\
\text { read }\end{array}$ & $\begin{array}{l}\text { ale } \\
\text { lings }\end{array}$ & $\begin{array}{l}\text { East } \\
\text { decli- }\end{array}$ & Temp. \\
\hline & Left & Right & & & & L,eft & Right & & & time & Left & Riglit & nation & C. & time & Left & Right & nation & C. \\
\hline $\mathrm{h} \mathrm{m}$ & d & $d$ & $\circ \quad$ & $\therefore$ & $\mathrm{h} \mathrm{m}$ & d & $d$ & $\cdot$, & $\circ$ & $\mathrm{h} \mathrm{m}$ & d & d & 0, & & $\mathrm{~h} \mathrm{~m}$ & d & d & & - \\
\hline 1200 & 39.7 & $4 \mathrm{I} . \mathrm{I}$ & 2246 & -16.2 & 1400 & 39.2 & 40.0 & 2245 & $-14 \cdot 4$ & 000.4 & 50.4 & 44.2 & $224 \mathrm{I}$ & -18.8 & 200 & 47.8 & 46.7 & 2241 & -15.0 \\
\hline 02 & 39.8 & 41.8 & 47 & $1=0$ & 02 & 39.6 & 40.2 & & 60 & 02 & 50.1 & $44 \cdot 3$ & $4 I$ & & 02 & 47.9 & 46.7 & $4 \mathrm{I}$ & \\
\hline 04 & 40.2 & 41.9 & 47 & (19) & 04 & 39.2 & 39.8 & 44 & 10 & 04 & 49.2 & 43.5 & 42 & & 04 & 47.8 & 46.6 & $4 \mathrm{I}$ & 10 \\
\hline 06 & 40.0 & 41.3 & 46 & as & 05 & 26.3 & 45.6 & 39 & $.6 n$ & 06 & 48.0 & 45.8 & $4 I$ & $6=$ & 06 & 47.4 & 46.7 & $4 \mathrm{I}$ & \\
\hline 08 & 39.8 & 41.0 & 46 & Sil & 08 & 27.8 & 44.8 & 40 & 68 & 08 & 48.2 & 45.8 & $4 I$ & 10 & 08 & 47.1 & 46.5 & 42 & \\
\hline 10 & 40.3 & 41.7 & 47 & wiv & 10 & 27.4 & 43.5 & 38 & 31 & IO & $48 . I$ & 45.8 & $4 \mathrm{I}$ & is & 10 & 47.2 & 46.8 & $4 \mathrm{I}$ & \\
\hline 12 & 40.7 & 42.2 & 47 & & I2 & 27.8 & 43.0 & 38 & 35 & 12 & 47.8 & 45.8 & 42 & 7 & 12 & 47.1 & 46.7 & 41 & \\
\hline 14 & 40.7 & 42.3 & 48 & -I 5.8 & I4 & 28.8 & 43. I & 39 & 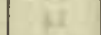 & 14 & 47.9 & $44 \cdot 3$ & 43 & -17.9 & 14 & 47.2 & 46.8 & 41 & -15.0 \\
\hline 16 & 40.7 & 42.1 & 47 & 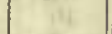 & 16 & 29.3 & 43.0 & 39 & -14.2 & 16 & 48.3 & 44.0 & 43 & & 16 & 47.0 & 46.7 & 42 & \\
\hline 18 & 40.7 & 42.1 & 47 & er & 18 & 29.8 & 42.7 & 39 & 18 & 18 & 47.7 & 43.9 & 43 & wat & 18 & 47.2 & 46.7 & $4 \mathrm{I}$ & at \\
\hline 20 & 40.8 & 42.3 & 48 & $=$ & 20 & 30.1 & 42.1 & 39 & oc & 20 & 48.3 & 44.0 & 43 & $=$ & 20 & 47.9 & 47.2 & 40 & $=$ \\
\hline 22 & 40.8 & 42.3 & 48 & 24 & 22 & 35.8 & 36.8 & 40 & 74 & 22 & 48.2 & 44.5 & 42 & $=5$ & 22 & 48.3 & 47.6 & 40 & 를 \\
\hline 24.5 & 40.9 & 42.3 & 48 & +1 & 24 & 35.6 & 36.7 & 39 & 14 & 24 & 48.6 & 45.1 & 42 & 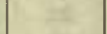 & 24 & 48.7 & 47.9 & 39 & 10 \\
\hline 26 & 41.0 & 41.9 & 47 & 3 & 26 & 35.4 & 36.4 & 39 & & 26 & 48.2 & 45.0 & 42 & 12 & 26 & 49.0 & 48.3 & 39 & \\
\hline 28 & 40.7 & $4 \mathrm{I} .7$ & 47 & 128 & 28 & 35.3 & 36.3 & 39 & 122 & 28 & 47.8 & 44.7 & 42 & 64 & 28 & 49.0 & 48.2 & 39 & \\
\hline 30 & 40.7 & 41.6 & 47 & -15.4 & 30 & 35.6 & 36.8 & 39 & $-\mathrm{I} 4 \cdot \mathrm{I}$ & 30 & 48.1 & 45.8 & $4 I$ & -17.0 & 30 & 48.7 & 47.9 & 39 & -15.0 \\
\hline 32 & 41.3 & 42.2 & 48 & - & 32 & 35.7 & 36.8 & 39 & (- & 32 & 48.0 & 45.8 & $4 \mathrm{I}$ & -80 & 32 & 48.3 & 47.6 & 40 & \\
\hline 34 & 41.9 & 42.9 & 49 & tis & 34 & 35.6 & 36.8 & 39 & $F^{2}$ & 34 & 48.3 & 46.0 & $4 \mathrm{I}$ & $6+46$ & 34 & 48.0 & $47 \cdot 3$ & 40 & 2 \\
\hline 36 & 40.7 & 41.8 & 47 & $c$ & 36 & 35.6 & 36.8 & 39 & 80 & 36 & 48.8 & 46.2 & 40 & 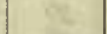 & 36 & 47.8 & 46.9 & $4 \mathrm{I}$ & at \\
\hline 38 & 39.6 & 40.2 & 45 & $=$ & 38 & 35.8 & 37.0 & 40 & 42 & 38 & 48.8 & 46.8 & 40 & tets & 38 & 47.3 & 46.2 & 42 & \& \\
\hline 40 & 40.2 & $4 \mathrm{I} . \mathrm{I}$ & 46 & 16 & 40 & 36.0 & 37.2 & 40 & 6 & 40 & 49.3 & 47.6 & 39 & 6 & 40 & 47.5 & 46.1 & 42 & 5 \\
\hline 42 & 39.9 & 40.7 & 46 & 14 & 42 & 36.1 & 37.1 & 40 & 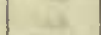 & 42 & 49.6 & 47.8 & 39 & -6 & 42 & 47.8 & 46.2 & $4 \mathrm{I}$ & \\
\hline 44 & 39.0 & 39.9 & 44 & -15.2 & 44 & 35.9 & 37.1 & 40 & -14.0 & 44 & 49.3 & 48.2 & 39 & -16.3 & 44 & 47.6 & 47.1 & 42 & -15.0 \\
\hline 46 & 39.3 & 40.2 & 45 & D & 46 & 35.9 & 36.9 & 40 & ( & 46 & $49 . I$ & 47.8 & 39 & & 46 & 47.9 & 46.3 & $4 \mathrm{I}$ & \\
\hline 48 & 40.3 & 41.2 & 40 & 3 & 48.4 & 35.2 & 36.5 & 39 & 8 & 48 & 48.3 & 47.2 & 40 & fet & 48 & 48.1 & 47.0 & 40 & be. \\
\hline 50 & 39.9 & 40.8 & 46 & $\theta$ & 50 & 35.2 & 36.4 & 39 & (12) & 50 & 47.8 & 46.7 & $4 \mathrm{I}$ & ec & 50 & 48.3 & 47.4 & 40 & 25 \\
\hline 52 & 39.2 & 40.2 & 45 & 2 & 52 & 35.2 & 36.7 & 39 & 0 & 52 & 47.1 & 46.3 & 42 & the & 52 & 48.3 & 47.8 & 40 & \\
\hline 54 & 39.8 & 40.5 & 46 & $\mathrm{n}^{2}$ & 54 & 35.1 & 36.3 & 38 & 17 & 54 & 46.3 & 45.6 & 43 & 12 & 54 & 47.9 & 47.3 & 40 & \\
\hline 56 & 40.0 & 40.8 & 46 & $\theta$ & 56 & 34.8 & 36.0 & 38 & 12 & 56 & 45.7 & 45.0 & 44 & 42 & 56 & 47.6 & 47.2 & $4 \mathrm{I}$ & \\
\hline 58 & 39.8 & 40.6 & 46 & $1=$ & 58 & 35.1 & 36.3 & 38 & 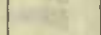 & 58 & 45.7 & 45.1 & 44 & & 58 & 47.2 & 46.9 & $4 \mathrm{I}$ & \\
\hline I3 00 & 39.6 & 40.0 & 45 & -15.0 & 1500 & 35.8 & 36.8 & 40 & -14.0 & I 00 & 46.1 & 45.4 & 43 & -15.7 & 300 & 47.1 & 46.4 & 42 & -15.0 \\
\hline 02 & 39.7 & 40.1 & 45 & ( & 02 & 36.7 & 37.7 & $4 \mathrm{I}$ & 20 & 02 & 46.0 & 45.8 & 43 & & 02 & 46.9 & 46.4 & 42 & \\
\hline 04 & 40.1 & 40.7 & 46 & 200 & 04 & 37.1 & 38.0 & 41 & 16 & 04 & 45.7 & 45.7 & 43 & $716 \%$ & 04 & 46.9 & 46.3 & 42 & in \\
\hline 06 & 40.2 & $41 . I$ & 46 & Ear & 06 & 37.1 & 38.0 & $4 I$ & 16 & 06 & 45.8 & $45 \cdot 3$ & 44 & 00 & 06 & 46.9 & 46.3 & 42 & \\
\hline 08 & 40.1 & 40.7 & 46 & (65) & 08 & 37.4 & 38.3 & 42 & 50 & 08 & 45.8 & 45.2 & 44 & 35 & 08 & 46.8 & $46 . I$ & 42 & $e$ \\
\hline IO & 39.3 & 40.1 & 45 & 171 & 10 & 37.6 & 38.6 & 42 & 61 & 10 & 45.9 & $45 \cdot 3$ & 44 & Ait & 10 & 47.0 & 46.3 & 42 & \\
\hline 12 & 39.2 & 40.0 & 45 & tor & 12 & 37.6 & 38.2 & 42 & tar & 12 & 46.3 & 45.4 & 43 & 124 & 12 & 47.3 & 46.8 & 41 & \\
\hline 14 & 38.9 & 39.7 & 44 & -14.9 & I4 & 37.1 & 37.8 & $4 \mathrm{I}$ & -14.0 & 14 & 46.9 & 45.9 & 42 & -15.2 & 14 & $47 . \overline{8}$ & 47.0 & $4 I$ & -14.9 \\
\hline 16 & 38.1 & 39.2 & 43 & ( & 16 & 36.4 & 37.3 & 40 & i & 16 & 47.3 & 46.3 & 42 & & 16 & 47.8 & 47.1 & $4 I$ & \\
\hline 18 & 38.0 & 39.0 & 43 & 71 & 18 & 36.2 & 37.1 & 40 & bit & 18 & 47.9 & 46.5 & $4 \mathrm{I}$ & t r & 18 & 47.9 & 47.2 & 40 & $=$ \\
\hline 20 & 38. I & 39.0 & 43 & in & 20 & 36.2 & 36.8 & 40 & ved & 20 & 48.2 & $46 . \overline{8}$ & 40 & $0 \leq$ & 20 & 48.0 & 47.0 & 40 & \\
\hline 22 & 38.3 & 39.3 & 43 & 62 & 22 & 36.1 & 36.7 & 40 & is & 22 & 48.8 & 47.1 & 40 & BS & 22 & 48.0 & 47.0 & 40 & 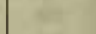 \\
\hline 24 & 38.8 & 39.8 & 44 & ik & 24 & 35.8 & 36.2 & 39 & iz & 24 & 49.1 & 47.2 & 40 & 45 & 24 & 48.3 & 46.3 & $4 \mathrm{I}$ & 34 \\
\hline 26 & 38.4 & 39.3 & 43 & N. & 26 & 35.4 & 36.1 & 38 & 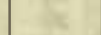 & 26 & 49.2 & 47.4 & 39 & $\alpha$. & 26 & 48.2 & 46.3 & 41 & \\
\hline 28 & 38.2 & 39.0 & 43 & 2 & 28 & 35.5 & 36.0 & 38 & 10 & 28 & 48.8 & 46.9 & 40 & & 28 & 48.0 & 46.3 & $4 \mathrm{I}$ & \\
\hline 30 & 38.8 & 39.3 & 44 & -14.8 & 30 & 35.8 & 36.3 & 39 & -13.0 & 30 & 47.9 & 46.3 & 40 & -15.2 & 30 & 47.8 & 46.3 & $4 I$ & -14.9 \\
\hline 32 & 39.9 & 40.6 & 46 & i & 32 & 35.7 & 36.3 & 39 & - & 32 & 47.4 & 46.0 & 42 & tra & 32 & 47.8 & 46.2 & $4 I$ & \\
\hline 34 & 40.0 & 40.3 & 46 & ic & 34 & $35 \cdot 3$ & 36.1 & 38 & 32 & 34 & 47.1 & 45.9 & 42 & 14 & 34 & 47.4 & 46.0 & 42 & \\
\hline 36 & 39.1 & 39.9 & 44 & 18. & 36 & $35 . \mathrm{I}$ & 35.7 & 38 & 35 & 36 & 47.0 & 45.9 & 42 & & 36 & 47.2 & 46.1 & 42 & \\
\hline 38 & 38.7 & 39.8 & 44 & ent & 38 & 34.8 & 35.4 & 38 & Q & 38 & 47.5 & 46.4 & $4 I$ & in & 38 & 47.1 & 45.9 & 42 & \\
\hline 40 & 39.0 & 40.3 & 45 & $4+$ & 40 & 34.9 & 35.4 & 38 & 64 & 40 & 47.7 & 46.7 & $4 I$ & we & 40 & 47.4 & $45 . \overline{8}$ & 42 & \\
\hline 42 & 39.2 & 40.5 & 45 & at & 42 & 35.3 & 35.8 & 38 & 4 & 42 & 47.8 & 46.8 & $4 I$ & $4+2$ & 42 & 47.3 & 45.8 & 42 & \\
\hline 44 & 39.2 & 40.8 & 45 & -14.7 & 44 & 35.6 & 36.2 & 39 & -13.9 & 44 & 47.8 & 46.8 & $4 \mathrm{I}$ & -15.2 & 44 & 47.1 & 45.6 & 42 & -14.9 \\
\hline 46 & 39.2 & 40.7 & 45 & & 46 & 35.6 & 36.2 & 39 & . & 46 & 48.0 & 47.1 & 40 & rat & 46 & 47.0 & 45.6 & 42 & \\
\hline 48 & 38.5 & 39.9 & 44 & Wh & 48 & 36.0 & 36.7 & 40 & $n$ & 48 & 48.0 & 47.1 & 40 & B & 48 & 47.2 & 45.8 & 42 & \\
\hline 50 & 38.2 & 39.3 & 43 & e? & 50 & 36.6 & 37.2 & 40 & 69 & 50 & 48.1 & $47 \cdot 3$ & 40 & as & 50 & 47.2 & 45.9 & 42 & \\
\hline 52 & 38.6 & 39.5 & 44 & 9 & 52 & 37.8 & 38.2 & 42 & $b^{2}$ & 52 & 47.9 & 47.2 & 40 & at & 52 & 47.2 & 46.0 & 42 & \\
\hline 54 & 38.1 & 38.9 & 43 & 12 & 54 & 38.1 & 38.8 & 43 & $i^{x}$ & 54 & 48.0 & 46.9 & $4 \mathrm{I}$ & R & 54 & 47.2 & 45.8 & 42 & \\
\hline 56 & 38.5 & 39.2 & 43 & the & 56 & $38 . I$ & 38.8 & 43 & at & 56 & 48.0 & 46.8 & $4 \mathrm{I}$ & ie & 56 & 46.8 & 45.4 & 43 & \\
\hline 58 & 39.1 & 39.9 & 44 & Bie & 58 & 37.7 & 38.2 & 42 & 168 & 58 & 47.9 & 46.7 & $4 I$ & bis & 58 & 46.7 & 45.3 & 43 & \\
\hline & & 390.9 & & on & 1600 & 37.1 & 37.7 & $4 I$ & -13.9 & & & & & 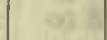 & & & & & \\
\hline
\end{tabular}

Correction to local mean time is $-37 \mathrm{~s} .90^{\circ}$ torsion $=16 .^{\prime} \mathrm{or}$.

Observer-R. R. 'T.

Torsion head at $1 \mathrm{Ih} 30 \mathrm{~m}$ read $24^{\circ}$ and at $16 \mathrm{~h} 20 \mathrm{~m}$ read $22^{\circ}$.

Observer-R. R. T. 
Tabulation of magnetic declinations observed at Teplitz Bay-Continued

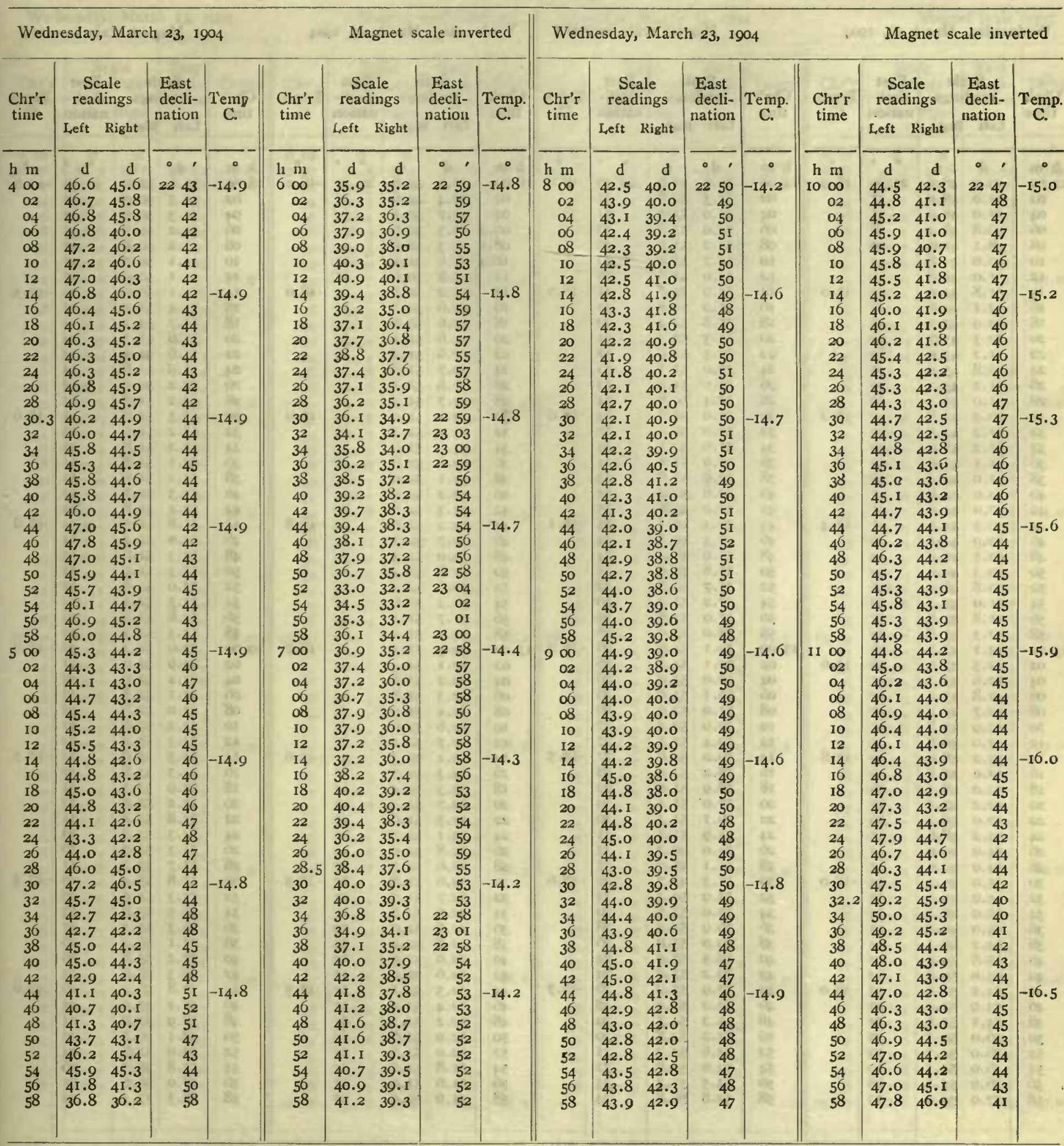

Observers-R. R. T. and J. V., who alternated from 7 h $32 \mathrm{~m}$ to Observer-J. V. 7h $42 \mathrm{~m}$. 
Tabulation of magnetic declinations obscrved at T'eplitz Bay-Continued

\begin{tabular}{|c|c|c|c|c|c|c|c|c|c|c|c|c|c|c|c|c|c|c|c|}
\hline \multicolumn{5}{|c|}{ Wednesday, March 23, I904 } & \multicolumn{5}{|c|}{ Magnet scale inverted } & \multicolumn{5}{|c|}{ Wednesday, March 23, 1904} & \multicolumn{5}{|c|}{ Magnet scale inverted } \\
\hline \multirow{2}{*}{$\begin{array}{l}\text { Chr'r } \\
\text { tine }\end{array}$} & \multicolumn{2}{|c|}{$\begin{array}{l}\text { Scale } \\
\text { readings }\end{array}$} & \multirow{2}{*}{$\begin{array}{c}\text { East } \\
\text { decli- } \\
\text { nation }\end{array}$} & Temp. & Chr'r & $\begin{array}{l}\mathrm{Sc} \\
\text { read }\end{array}$ & $\begin{array}{l}\text { ale } \\
\text { lings }\end{array}$ & $\begin{array}{l}\text { East } \\
\text { decli- }\end{array}$ & Temp. & Chr'r & $\begin{array}{l}\text { Sca } \\
\text { readi }\end{array}$ & $\begin{array}{l}\text { ale } \\
\text { lings }\end{array}$ & $\begin{array}{l}\text { East } \\
\text { decli- }\end{array}$ & Temp. & Chr'r & $\begin{array}{l}\text { Sc } \\
\text { read }\end{array}$ & $\begin{array}{l}\text { ale } \\
\text { lings }\end{array}$ & $\begin{array}{l}\text { East } \\
\text { decli- }\end{array}$ & Temp. \\
\hline & Left & Kight & & & & Left & Right & & & & Left & Right & & & & Left & Right & & \\
\hline li $\mathrm{m}$ & d & $d$ & $\cdot{ }^{\prime}$ & - & $\mathrm{h} \mathrm{m}$ & $d$ & d & $\cdot \cdot$ & $\circ$ & h 111 & d & d & $\cdot$, & o & li $\mathrm{m}$ & d & d & & 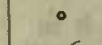 \\
\hline 1200 & 49.2 & 48.1 & 2239 & -16.9 & 1400 & 56.0 & 52.0 & 2230 & -18.0 & 1600 & 52.6 & 49.6 & 2235 & -18.2 & 1800 & 51.6 & 51.0 & 2235 & -17.6 \\
\hline 02 & 51.0 & 48.0 & 37 & 60 & 02 & $54 \cdot 3$ & 52.8 & 3I & 64 & 02 & 52.4 & 49.6 & 35 & & 02 & $5 \mathrm{I} .3$ & 50.9 & 35 & $x^{2}$ \\
\hline 04 & 51.0 & 48.9 & 37 & $\omega$ & 04. & 53.9 & 53.3 & 31 & 10 & 04 & 52.7 & 50.1 & 34 & 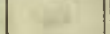 & 04 & $5 \mathrm{I} .3$ & 50.7 & 35 & Fei \\
\hline 06 & 49.8 & 48.2 & 38 & wo & 06 & 54.0 & 53.8 & 30 & 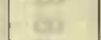 & 06.4 & $53 \cdot 3$ & 50.6 & 34 & ar & 06 & 51.2 & 50.7 & 35 & si \\
\hline 08 & 48.1 & 47.0 & 40 & Eis & 08 & 54.2 & 53.0 & 30 & ito & 08. I & 53.3 & 50.6 & 34 & 6e. & 08 & 51.2 & 50.8 & 35 & 160 \\
\hline Io & 50.0 & 42.8 & 42 & If & 10 & 54.7 & 53.0 & 30 & or & 10.4 & 53.6 & $5 \mathrm{I} . \mathrm{I}$ & 33 & & IO & 51.1 & 50.7 & 35 & 탄 \\
\hline 12 & 51.0 & 42.8 & $4 I$ & & 12 & 55.0 & 52.8 & 30 & & 12 & 53.8 & 50.6 & 33 & & I2 & 51.1 & 50.9 & 35 & \\
\hline 14 & 51.8 & 44.0 & 40 & -17.0 & 14 & 54.9 & 52.7 & 31 & -18.3 & 14 & 53.2 & 50.8 & 33 & -18.0 & I4 & $5 \mathrm{I} . \mathrm{I}$ & 50.9 & 35 & -17.5 \\
\hline 16 & $53 . I$ & 44.6 & 38 & (n) & 16 & 55.0 & 52.8 & 30 & 150 & 16 & 52.6 & 50.7 & 34 & $y_{1}$ & 16 & $5 I .4$ & 51.2 & 34 & \\
\hline 18 & 52.9 & $45 \cdot 3$ & 38 & El & 18 & 55.8 & 52.4 & 30 & 28 & 18 & 52.6 & 50.8 & 34 & bit & 18 & 51.7 & 51.3 & 34 & wis \\
\hline 20 & 52.3 & $45 . I$ & 39 & kn: & 20 & 54.3 & 52.3 & 3 I & we & 20 & 52.6 & 51.1 & 34 & $n=$ & 20 & 51.6 & 51.3 & 34 & $6=$ \\
\hline 22 & 51.2 & 45.0 & 40 & is & 22 & 53.5 & 51.9 & $3^{2}$ & $=$ & 22 & 52.6 & 51.2 & 34 & $E$ & 22 & 51.6 & 51.4 & 34 & in \\
\hline 24 & 50.8 & 45.0 & 40 & 36 & 24 & 53.0 & 51.1 & 33 & $\cos \theta$ & 24 & 52.7 & $5 \mathrm{I} .2$ & 34 & 126 & 24 & 51.9 & 51.8 & 34 & Ms \\
\hline 26 & 51.2 & 45.2 & 39 & 8 & 26 & 53.0 & 51.0 & 33 & & 26 & 52.6 & 5I.I & 34 & 18 & 26 & 52.2 & 51.9 & 33 & \\
\hline 28 & 51.0 & 45.7 & 39 & E & 28 & 52.4 & 50.8 & 34 & & 28 & 52.7 & 51.3 & 33 & tet & 28 & 52.2 & 51.8 & 33 & \\
\hline 30 & 50.3 & 45.8 & 40 & -17.2 & 30 & 52.0 & 50.8 & 34 & -18.0 & 30 & 53.1 & 51.9 & 33 & -17.9 & 30 & 52.3 & 51.8 & 33 & -17.6 \\
\hline 32 & 51.2 & $44 \cdot 3$ & 40 & ( & 32 & 52.0 & 50.0 & 35 & 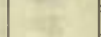 & 32 & 53.1 & 52.3 & 32 & ( & 32 & 52.2 & 51.9 & 33 & 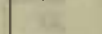 \\
\hline 34 & 51.2 & 45.0 & 40 & x & 34 & 51.9 & 50.5 & 35 & w & 34 & 53.0 & 51.9 & 33 & $\sqrt{25}$ & 34 & 52.2 & 51.8 & 33 & 3 \\
\hline 36 & 51.1 & 44.9 & 40 & 28 & 36 & 52.0 & 51.0 & 34 & (ar. & 36 & 52.6 & 51.6 & 33 & $\varepsilon$ & 36 & 52.1 & 51.8 & 34 & 8 \\
\hline 38 & 51.0 & 44.2 & 40 & the & 38 & 52.9 & 51.6 & 33 & $=$ & 38 & 53.1 & 52.0 & 33 & Ec & 38 & 52.1 & 51.6 & 34 & 28 \\
\hline 40 & 50.0 & 44.0 & $4 \mathrm{I}$ & (1) & 40 & 52.8 & 50.4 & 34 & wat & 40 & 53.2 & 52.3 & 32 & $4^{4}$ & 40 & 52.3 & 51.7 & 33 & $\sqrt{18}$ \\
\hline 42 & 50.0 & 44.0 & $4 I$ & 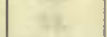 & 42 & 52.0 & 49.8 & 35 & & 42 & 53.3 & 52.8 & 32 & & 42 & 52.4 & 51.8 & 33 & \\
\hline 44 & 50.5 & 44.0 & $4 I$ & $-17 \cdot 4$ & 44 & 52.3 & 49.1 & 35 & -18.1 & 44 & 53.2 & 52.6 & 32 & -17.8 & 44 & 51.8 & 51.5 & 34 & -17.6 \\
\hline 46 & 50.9 & 44.5 & 40 & (2) & 46 & $53 \cdot 3$ & 49.5 & 34 & 28 & 46 & 52.7 & 52.3 & 33 & $x^{2}$ & 46 & 52.0 & 51.4 & 34 & 1 \\
\hline 48 & 50.5 & 45.0 & 40 & 15 & 48 & 55.0 & 48.4 & 34 & 86 & 48 & 53.0 & 52.5 & 32 & 6 & 48 & 52.3 & 51.6 & 34 & \\
\hline 50 & 50.3 & 46.0 & 40 & es & 50 & 55.8 & 49.0 & 33 & 3 & 50 & 52.8 & 52.4 & 32 & $\alpha$ & 50 & 52.3 & 51.9 & 33 & $x=$ \\
\hline 52 & 50.8 & 46.9 & 38 & 2 & 52 & 55.7 & 50.6 & 32 & k2 & 52 & 52.6 & 52.3 & 33 & 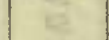 & 52 & 53.0 & 51.8 & 33 & Es \\
\hline 54 & 51.0 & 47.2 & 38 & 11 & 54 & 57.2 & 51.0 & 30 & iz & 54 & 52.2 & 51.6 & 34 & ia & 54 & 53.3 & 52.1 & 32 & $x^{2}$ \\
\hline 56 & 51.8 & 47.8 & 37 & 18 & 56 & 57.0 & 51.8 & 30 & & 56 & 51.7 & 51.1 & 34 & & 56 & 52.8 & 51.3 & 33 & 8 \\
\hline 58 & 52.3 & 47.8 & 36 & $x^{2}$ & 58 & 58.0 & 53.0 & 28 & & 58 & 52.1 & 51.2 & 34 & & 58 & 53.3 & 52.3 & 32 & \\
\hline 1300 & $53 . \mathrm{I}$ & 47.5 & 36 & & 1500 & 59.1 & 54.0 & 26 & -18.2 & 1700 & 52.3 & 51.3 & 34 & -17.7 & 1900 & 53.6 & 52.3 & 32 & -17.6 \\
\hline 02 & 52.5 & 47.6 & $\cdot 36$ & -17.6 & 02 & 60.1 & 54.0 & 26 & in & 02 & 52.1 & 51.4 & 34 & ( & 02 & 53.9 & 53.0 & $3 \mathrm{I}$ & ( \\
\hline 04 & 52.6 & 47.1 & 37 & 10 & 04 & 60.0 & 53.0 & 26 & is & 04 & 52.2 & 51.5 & 34 & wh & $\mathrm{O}_{4}$ & 53.4 & 52.9 & 32 & be \\
\hline 06 & 52.8 & 47.0 & .37 & 50 & 06 & 60.1 & 53.6 & 26 & in & 06 & 52.3 & 51.8 & 33 & $\infty$ & 06 & 53.1 & 52.8 & 32 & 5 \\
\hline 08 & 52.7 & 47.2 & 37 & Bo & 08 & 61.0 & 53.0 & 26 & 62 & 08 & 52.4 & 51.9 & 33 & 62 & 08 & 53.0 & 52.8 & 32 & 23 \\
\hline to & 53.9 & 47.8 & 35 & 61 & I0 & 59.9 & 52.4 & 27 & is. & IO & 52.6 & 52.0 & 33 & 68 & IO & 53.2 & 52.8 & 32 & 21 \\
\hline 12 & 53.0 & 48.8 & 35 & & 12 & 59.4 & 52.5 & 27 & & 12 & 52.7 & 52.1 & 33 & & I2 & 53.3 & 53.2 & 32 & \\
\hline 14 & 53.2 & 48.0 & 36 & -17.8 & 14 & 59.0 & 52.6 & 27 & -18.5 & 14 & 53.1 & 52.3 & 32 & -17.6 & I4 & $53 \cdot 3$ & 53.0 & 32 & -17.5 \\
\hline 16 & 52.0 & 48.8 & 36 & in & 16 & 58.2 & 52.0 & 28 & 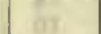 & 16 & 53.3 & 52.7 & 32 & 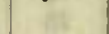 & 16 & 54.3 & 53.8 & 30 & 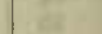 \\
\hline 18 & 52.0 & 48.1 & 36 & 81 & I8 & 58.9 & 51.0 & 29 & 到 & I8 & 53.3 & 52.4 & 32 & ifi & 18 & 53.9 & 53.5 & $3 I$ & \\
\hline 20 & 52.2 & 47.5 & 37 & $2=$ & 20 & 57.0 & 51.1 & 30 & ats & 20 & 52.7 & $5 \mathrm{I} .4$ & 33 & w. & 20 & 53.2 & 52.8 & 32 & 2. \\
\hline 22 & 52.0 & $48 . \mathrm{I}$ & 36 & Fx. & 22 & 56.7 & $5 I .3$ & 30 & 31 & 22 & 52.5 & 51.6 & 33 & 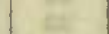 & 22 & 53.8 & 53.4 & $3 \mathrm{I}$ & \\
\hline 24 & 53.2 & 48.6 & 35 & ic & 24 & 55.9 & $5 \mathrm{I} . \mathrm{I}$ & $3 I$ & & 24 & 53.1 & 52.3 & 32 & 34 & 24 & $54 \cdot 3$ & 54.0 & 30 & \\
\hline 26 & 54.0 & 49.0 & 34 & 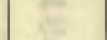 & 26 & 54.0 & 49.2 & 34 & & 26 & 52.8 & 52.0 & 33 & & 26 & $54 \cdot 3$ & 53.9 & 30 & \\
\hline 28 & 54.0 & 49.0 & 34 & & 28 & 5I.I & 48.0 & 37 & & 28 & 52.6 & 51.7 & 33 & & 28 & 53.7 & 53.7 & 31 & \\
\hline 30 & 55.0 & 49.1 & 33 & -18.0 & 30 & $5 \mathrm{I} .3$ & 49.7 & 36 & -18.6 & 30 & 52.6 & $5 \mathrm{I} .8$ & 33 & -17.5 & 30 & 55.8 & 55.4 & 28 & -17.5 \\
\hline 32 & 55.0 & 49.0 & 33 & 1.4 & 32 & 53.9 & 50.4 & 33 & & 32 & 52.7 & 51.9 & 33 & (n) & 32 & 55.3 & 54.9 & 28 & \\
\hline 34 & 55.0 & 48.4 & 34 & $F^{2}$ & 34 & 55.0 & 53.5 & 30 & 3 & 34 & 52.6 & 51.8 & 33 & E & 34 & 56.5 & 55.8 & 27. & \\
\hline & 55.0 & 49.2 & 33 & & 36 & 56.9 & 54.4 & 28 & & 36 & 52.6 & 51.9 & 33 & 25 & 36 & 56.3 & $55 \cdot 3$ & 27 & \\
\hline 38 & 54.2 & $49 . \mathrm{I}$ & 34 & in & 38 & 59.0 & 55.0 & 26 & 14 & 38 & 52.8 & 52.2 & 33 & ai & 38 & 57.1 & 56.2 & 26 & \\
\hline 40 & 55.8 & 49.9 & 32 & $=$ & 40 & 58.5 & 57.0 & 24 & & 40 & 52.6 & 51.9 & 33 & Iff & 40 & 57.2 & 55.8 & 26 & \\
\hline 42 & 55.0 & 50.8 & 32 & & 42 & 59.2 & 57.0 & 24 & & 42 & 52.6 & 52.0 & 33 & & 42 & 58.3 & 56.6 & 25 & \\
\hline 44 & 54.2 & 51.0 & 32 & -18.0 & 44 & 57.9 & 55.2 & 26 & -18.7 & 44 & 52.5 & 52.1 & 33 & -17.5 & 44 & 57.8 & 56.2 & 26 & -17.6 \\
\hline 46 & 53.9 & 51.0 & 33 & 1 = & 46 & 56.2 & 55.0 & 28 & (1) & 46 & 52.4 & 51.9 & 33 & $x^{2}$ & 46 & 58.2 & 57.0 & 25 & \\
\hline 48 & 54.1 & 51.8 & 32 & 6 & 48 & $55 \cdot 3$ & $54 \cdot 3$ & 29 & re & 48 & 52.2 & 51.7 & 34 & 2 & 48 & 58.9 & 57.0 & 24 & \\
\hline 50 & 56.0 & 52.3 & 30 & $e^{2}$ & 50 & 54.2 & 53.8 & 30 & (20) & 50 & 52.0 & 51.6 & 34 & e? & 50 & 58.1 & 56.7 & 25 & \\
\hline 52 & 56.0 & 52.5 & 30 & 35 & 52 & 53.6 & 53.2 & $3 I$ & 28 & 52 & 52.0 & 51.6 & 34 & 2 & 52 & 59.0 & 57.6 & 24 & \\
\hline & 56.0 & 52.5 & & A & 54 & 53.0 & 52.9 & 32 & & 54 & 51.8 & 51.2 & 34 & $\mathrm{n}^{2}$ & 54 & 57.8 & 56.3 & 26 & \\
\hline 56 & 56.9 & $53.5^{\circ}$ & 28 & 8 & 56 & $53 \cdot 5$ & $5 \mathrm{I} .3$ & $\quad 33$ & 6 & 56 & 51.6 & 51.0 & 35 & & 56 & 58.0 & 56.6 & 25 & \\
\hline 58 & 57.0 & 52.9 & 29 & $y$ & 58 & 52.8 & 50.8 & 34 & & 58 & 51.6 & 50.9 & 35 & $B^{2}$ & 58 & 58.5 & $57 \cdot 3$ & 24 & \\
\hline
\end{tabular}

Ohservers-J. V. and W. J. P., who alternated from I5h $46 \mathrm{~m}$ to Observer--IV. J. P. I. 5 h $58 \mathrm{~m}$. 
Tabulation of magnetic declinations observed ut Teplitz Bay-Continued

\begin{tabular}{|c|c|c|c|c|c|c|c|c|c|c|c|c|c|c|c|c|c|c|c|c|c|}
\hline \multicolumn{5}{|c|}{ Wednesday, March 23, I904 } & \multicolumn{7}{|c|}{ Magnet scale inverted } & \multicolumn{5}{|c|}{ Thursday, March 24, I904 } & \multicolumn{5}{|c|}{ Magnet scalc ercet } \\
\hline \multirow{2}{*}{$\begin{array}{l}\text { Chr'r } \\
\text { time }\end{array}$} & \multicolumn{2}{|c|}{$\begin{array}{l}\text { Scale } \\
\text { readings }\end{array}$} & East & Temp & & hr'r & $\begin{array}{l}\text { Sc } \\
\text { read }\end{array}$ & $\begin{array}{l}\text { ale } \\
\text { lings }\end{array}$ & Eas & st & Temp & & $\begin{array}{l}\mathrm{Sc} \\
\text { read }\end{array}$ & $\begin{array}{l}\text { e } \\
\text { igs }\end{array}$ & East & Temp. & Chr'r & $\begin{array}{l}\text { Sc: } \\
\text { read }\end{array}$ & $\begin{array}{l}\text { le } \\
\text { hgs }\end{array}$ & $\begin{array}{l}\text { East } \\
\text { decli- }\end{array}$ & Temp. \\
\hline & Left & Right & & & & & L,eft & Right & & & & & Left & Right & & & & Left & Right & & \\
\hline $\mathrm{h} \mathrm{m}$ & d & d & - ' & $\bullet$ & h & $\mathrm{m}$ & d & d & & & & h $\mathrm{m}$ & d & $d$ & . ' & & $\mathrm{h} \mathrm{m}$ & d & d & - & $\bullet$ \\
\hline 2000 & $59 \cdot 3$ & 58.0 & $22 \quad 23$ & -17.7 & 22 & $\infty$ & 59.3 & 58.0 & 22 & 23 & -18.0 & 1600 & 47.9 & 48.8 & 2236 & 20.7 & 1800 & 47.2 & 47.8 & $22 \quad 35$ & -19.0 \\
\hline 02 & 59.5 & 58.1 & 23 & & & 02 & 60.3 & 58.6 & & 22 & & 02.2 & 47.6 & 48.3 & 36 & & 02 & 48.1 & 48.4 & 36 & \\
\hline 04 & 59.0 & 57.8 & 23 & 10 & & 04 & 60.3 & 58.9 & & 22 & & 04 & $47 \cdot 7$ & 48.3 & 36 & & 04 & 49.1 & 49.2 & 37 & \\
\hline of & 58.5 & $57 \cdot 5$ & 24 & $3 n$ & & 06 & 58.4 & 57.2 & & 24 & & 06 & 48.1 & 48.9 & 36 & & 06 & 49.2 & 49.7 & 38 & \\
\hline os & 58.8 & 57.9 & 24 & 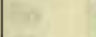 & & 08 & 58.3 & 57.6 & & 24 & & 08 & 47.4 & 48.0 & 35 & & 08 & 40.1 & 40.4 & 38 & 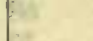 \\
\hline To & 61.0 & 59.4 & 20 & $\mathrm{Cr}$ & & IO & $57 \cdot 5$ & 57.0 & & 25 & & IO & 48.1 & 48.3 & 36 & & 10 & 48.7 & 48.9 & 37 & \\
\hline 12 & $6 \mathrm{6} .5$ & 60.3 & 19 & 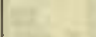 & & 12 & 57.0 & 56.8 & & 26 & & 12 & 48.2 & 48.8 & 36 & & 12 & 47.9 & 48.3 & $3^{6}$ & \\
\hline I4 & 6 т. & 60.3 & 20 & -17.9 & & I4 & 55.7 & 55.5 & & 28 & -18.2 & I4 & 48.2 & 48.8 & 36 & -20.2 & 14 & 47.9 & 48.2 & 36 & -19.0 \\
\hline I6 & 62.3 & $6 I \cdot 3$ & I8 & & & I6 & 55.3 & 55.0 & & 28 & & I6 & 47.5 & 47.8 & 35 & & 16 & $48 . r$ & 48.4 & 36 & \\
\hline I8 & 63.0 & 62.0 & I7 & & & I8 & & 55.3 & & 28 & & I8 & 47.3 & & 35 & & 18 & 48.2 & 48.7 & 36 & \\
\hline 20 & 62.2 & 60.7 & 19 & Hi. & & 20 & 56.1 & 55.7 & & 27 & & 20 & 47.1 & 47.9 & 35 & & 20 & 48.4 & 48.8 & 36 & $=$ \\
\hline 22 & 6 г. 0 & 60.2 & 20 & $t^{2}$ & & 22 & 55.0 & 51.8 & & 20 & & 22 & 46.8 & 47.7 & 34 & & 22 & $47 \cdot 9$ & 48.7 & 36 & 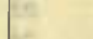 \\
\hline 24 & 58.9 & 58.8 & 2.3 & $t_{0}$ & & 24 & 54.4 & 53.9 & & 30 & & 24 & 45.9 & 47.0 & 33 & & 24 & 48.5 & $48 . n$ & . $\quad 37$ & \\
\hline 26 & 57.2 & 57.1 & 25 & $6 x$ & & 26 & 53.8 & $53 \cdot 3$ & & 31 & & 26 & 46.2 & 47.7 & 34 & & $26^{\circ}$ & 49. I & 49.6 & 38 & \\
\hline 28 & 58.1 & 57.8 & 24 & & & 28 & 54.8 & 54.1 & & . & & 28 & 45.9 & 47.1 & 33 & & 28 & 49.I & 49.7 & 38 & \\
\hline 30 & 57.3 & 56.3 & 26 & -18.0 & & 30 & 55.7 & 54.9 & & 28 & -18.3 & 30 & 45.0 & 46.2 & 32 & -10.7 & 30 & $40 . I$ & 49.4 & 38 & -19.0 \\
\hline 32 & 56.9 & 56.0 & 26 & & & 32 & .8 & 55.0 & & 28 & & 32 & 5.5 & 46.8 & 33 & & & 48.8 & 40.4 & 37 & \\
\hline 34 & 56.3 & 5.5 .9 & 27 & it & & 34 & 56.3 & 55.3 & & 27 & & 31 & 46.2 & 47.8 & 34 & & 3 & 48.2 & 48.9 & 36 & 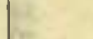 \\
\hline 36 & 57.4 & 56.4 & 26 & & & 36 & 55.6 & 54.5 & & 20 & & 36 & 46.3 & 47.9 & 34 & & 36 & 48.6 & 49.0 & 37 & \\
\hline 38 & $57 \cdot 3$ & 56.3 & 26 & 4 & & 38 & 55.8 & 54.8 & & 28 & senta & 38 & 46.9 & 48.2 & 35 & & 38 & 48.6 & 48.9 & . $\quad 37$ & \\
\hline 40 & 57.0 & 55.6 & 27 & 3 & & 40 & 56. I & $5.5 \cdot 3$ & & 28 & 1 & 40 & 47.2 & 48.4 & 35 & (6) & 40 & 48.7 & 49.0 & 37 & te \\
\hline 42 & 57.2 & 55.8 & 26 & & & 42 & 56.7 & 56.0 & & 27 & 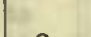 & 42 & 46.9 & 48.2 & 35 & & 42 & 49.6 & 49.9 & 38 & (n) \\
\hline 44 & 58.5 & 57.2 & 2.4 & $-\mathrm{I} 8.0$ & & 44 & 55.8 & 55.3 & & 28 & -18.4 & 44 & 46.9 & 48.0 & 35 & -19.3 & 44 & 49.1 & 49.4 & 38 & -19.0 \\
\hline 46 & 58.3 & 56.9 & 25 & ( & & 46 & 54.0 & 53.5 & & $.3 I$ & & 46 & 46.5 & 47.3 & 34 & & 46 & 49.2 & 49.6 & 38 & \\
\hline 48 & 58.3 & 57.0 & 25 & 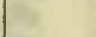 & & 48 & 53.6 & 5.3 .2 & & $3 I$ & 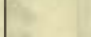 & 48 & 46.2 & $47 . I$ & 33 & & 48 & 49.0 & 49.3 & 37 & \\
\hline 50 & 58.6 & 57.7 & 21 & - & & 50 & 5.3 .0 & 52.4 & . & 32 & 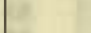 & 50 & 46.0 & $47 \cdot 3$ & 33 & (1) & . 50 & 40.3 & 49.8 & 38 & 2 \\
\hline 52 & 62.3 & 6I. I & I8 & & & 52 & $53 \cdot 3$ & 53.0 & & 32 & 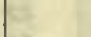 & 52 & 46.2 & 46.9 & 3.3. & 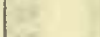 & 52 & 48.9 & 49.3 & 37 & 7 \\
\hline 54 & $6 \mathrm{~s} .5$ & 60.3 & 19 & 18 & & 54 & 54.3 & 53.7 & & 30 & & 54 & 46.7 & 47.0 & .34 & 13 & 54 & 48.3 & 50.1 & 38 & $\therefore$ \\
\hline 56 & $6 \mathrm{I} . \mathrm{I}$ & 59.3 & 20 & & & 56 & 52.8 & 52.3 & & 3.3 & 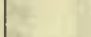 & 56 & 46.6 & 47.1 & 34 & $=$ & 56 & 48.8 & 50.8 & 38 & 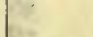 \\
\hline 58 & $6 \mathrm{r} .9$ & 59.3 & 20 & & & 58 & 53.3 & 52.7 & & 32 & & 58 & 46.6 & 46.9 & 34 & 1 & 58 & 48.7 & 50.8 & 38 & \\
\hline 2100 & 62.2 & 60.5 & I9 & -18.0 & 23 & 00 & $52 . \mathrm{I}$ & 51.6 & & 34 & -I8.. & 1700 & 46.7 & A7.0 & 34 & -19.1 & 1900 & 40.2 & 5 & 30 & -19.0 \\
\hline 02 & 62.0 & 60.5 & I9 & (2n & & 02 & 52.1 & 5 ז. & & .33 & & 02 & 46.5 & 46.9 & 34 & ( & 02 & 48.3 & 50.7 & 38 & \\
\hline 04 & 63.4 & $6 \mathrm{r} .8$ & 17 & 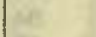 & & 0.1 & 52.6 & 52.3 & & 3.3 & & 01 & 46.7 & 47. I & 34 & & OA & 40.0 & 50.9 & 30 & 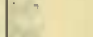 \\
\hline or & 63.7 & 60.5 & I8 & 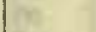 & & on & 52.7 & 52.2 & & 33 & 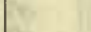 & of & $4^{6.7}$ & 47.0 & 34 & s. & o6 & 48.3 & 50.2 & 38 & 6 \\
\hline 08 & 67.8 & 64.2 & II & ter & & 08 & 52.2 & 51.6 & & 34 & 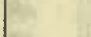 & 08 & 46.0 & 46.2 & 33 & nat & 08 & 47.9 & 49.3 & 36 & 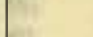 \\
\hline 10 & 66.3 & 62.7 & 14 & 1 & & I0 & $52 . I$ & 51.8 & & .34 & & IO & $47 . \mathrm{I}$ & $47 \cdot 3$ & 34 & 14 & I0 & 48.5 & 49.7 & .37 & 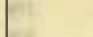 \\
\hline 12 & 60.1 & 58.9 & 22 & 1 & & 12 & $52 . I$ & 52.0 & & 3.3 & & 12 & 6.9 & 47.3 & 34 & 17 & 12 & 48.8 & 40.8 & 38 & \\
\hline 11 & 57.0 & 54.3 & 28 & -I8.0 & & 14 & 52.1 & 51.0 & & 33 & -18.5 & IA & 46.3 & 47.0 & 33 & -I9. I & I4 & 49.7 & 50.5 & 30 & -19.0 \\
\hline If & 65.0 & 6 I. 6 & I6 & ( & & I6 & $52 . I$ & 51.8 & & 34 & & I6 & 45.1 & 4.5 .8 & 32 & - & 16 & 49.3 & 50.3 & 38 & \\
\hline I 8 & 60.7 & 57.3 & 22 & Tas & & I8 & 52.3 & 51.6 & & 34 & 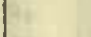 & I8 & 5.7 & 46.0 & 32 & $4 t$ & I8 & 50.0 & 50.8 & 30 & 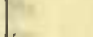 \\
\hline 20 & $6 \mathrm{r} .0$ & 58.3 & 22 & $n$ & & 20 & 52.3 & $5 \pi .4$ & & 3.4 & 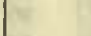 & 20 & 46.0 & 46.2 & 33 & th & 20 & 49.9 & 50.6 & .39 & 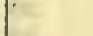 \\
\hline 22 & 59.0 & 57.5 & 23 & an: & & 22 & 52.8 & 51.9 & & 33 & 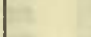 & 22 & 16.0 & 46.6 & 33 & 1 & 22 & 49.9 & 50.5 & 39 & \\
\hline 24 & 55.6 & 52.0 & 31 & 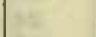 & & 24 & 52.5 & $5 \mathrm{I} .4$ & & 34 & 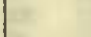 & 21 & 46.2 & 46.7 & 3.3 & $\theta$ & 24 & 40.7 & 50.4 & 39 & \\
\hline 26 & 5 T. 0 & 40.2 & 36 & $y^{2}$ & & 26 & 2.2 & 51.0 & & 34 & & 26 & .8 & 46.4 & 33 & & 26 & 48.7 & 40.3 & $\because .37$ & \\
\hline 28 & 50.3 & 56.6 & 24 & & & 28 & 51.8 & 50.6 & & 3.5 & & 29 &.$I$ & 5.8 & 32 & 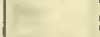 & 28 & 48.0 & 48.8 & - $\quad 36$ & \\
\hline .0 & (n). 8 & 67.2 & 07 & -18.0 & & 30 & 5.3 .8 & 52.8 & n & $3 I$ & -18.5 & 30 & 45.2 & 45.8 & 32 & -19. & 30 & $47 \cdot .3$ & 48. I & 35 & -19.0 \\
\hline 32 & 65.6 & 63.7 & I.3 & (2) & & 32 & 53.3 & 52.3 & & 32 & & 32 & 44.9 & 45.1 & $3 I$ & 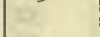 & 3 & 47.2 & 48. I & 35 & \\
\hline 21 & 62.6 & 62.0 & I6 & $y$ & & 31 & 53.5 & 52.4 & & 32 & 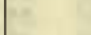 & 31 & $45 . I$ & 45.2 & $3 I$ & 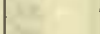 & 3 & 46.9 & 47.7 & 35 & \\
\hline 36 & $6 \pi .8$ & 59.2 & 20 & W & & 36 & 53.6 & 52.4 & & 32 & & 36 & 45.0 & 45.3 & $3 I$ & & 3 & $47 . I$ & 47.8 & 34 & \\
\hline .38 & $54 \cdot 3$ & $5 \mathrm{I} .5$ & 32 & aㅡ. & & 38 & 53.9 & 52.6 & & 32 & & 38 & 44.8 & $45 . I$ & $3 I$ & 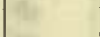 & 3 & 47.0 & $47 \cdot 3$ & 34 & \\
\hline 40 & 54.3 & 52.0 & 32 & t & & 40 & 3.2 & 52.1 & & 32 & & 40 & 5.2 & & $3 I$ & th & 4 & 47.1 & $47 \cdot 4$ & 34 & $=$ \\
\hline$\Delta 2$ & 54.8 & $5 \mathrm{~J} .8$ & $3 I$ & & & 42 & 53.6 & 52.3 & & .32 & & 42 & 5.7 & & 32 & th & 4 & 47.1 & 47.4 & 34 & \\
\hline$A d$ & $6 \mathrm{r} .2$ & 58.8 & $2 \mathrm{I}$ & -18.0 & & 44 & 5.3 .6 & 52.6 & & .32 & -18.5 & $\Delta 4$ & 45.8 & 46.1 & 32 & -10.0 & 44 & 47.3 & 47.8 & $=35$ & -19.0 \\
\hline 46 & 62.0 & 60.3 & 19 & (nt & & 46.4 & 53.0 & 52.2 & & 32 & & 46 & 45.9 & 46.2 & 32 & & 46 & 46.9 & $47 \cdot 3$ & 34 & \\
\hline 48 & 59.5 & 57.6 & 23 & tat & & 48 & 53.0 & 52.1 & & 3.3 & 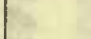 & 48 & 46.9 & 47.0 & 34 & 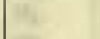 & 48 & 46.4 & $47 . I$ & 34 & 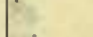 \\
\hline 50 & 50.2 & 57.6 & 2.3 & 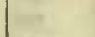 & & 50 & 52.0 & 52.1 & & 33 & 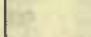 & 50 & 47.0 & 47.3 & 34 & t & 50 & 45.9 & 46.6 & 33 & \\
\hline 52 & 50.3 & 57.6 & 23 & Cry & & 52 & 53.8 & 52.9 & & $3 I$ & 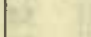 & 52 & 46.8 & 47.1 & 34 & 10 & 52 & 44.9 & 45.7 & $3 I$ & \\
\hline 54 & 58.3 & 56.3 & 25 & 15 & & 54 & $54 . I$ & 53.5 & & .31 & 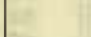 & 51 & $47 \cdot I$ & & 34 & $a^{2}$ & 54 & 43.3 & 44.2 & - 20 & \\
\hline 56 & 57.7 & 56.0 & 26 & 20 & & 56 & $54 \cdot 3$ & 53.5 & & 30 & 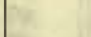 & 56 & 47.2 & 47.6 & 35 & & 56 & 42.8 & 43.9 & $\begin{array}{l}28 \\
\end{array}$ & $\rightarrow$ \\
\hline 58 & 58.3 & 56.7 & 25 & $7 y$ & & 58 & 54.2 & 5.3 .2 & & 31 & & 58 & 47.0 & 47.3 & 34 & 10 & 58 & 42.8 & 43.6 & $\begin{array}{l}\text { - } \quad 28 \\
\end{array}$ & \\
\hline & & & & & & $\infty$ & 54.2 & 53.1 & & $3 I$ & -18.7 & & & & & 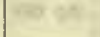 & 2000 & 42.8 & $43 \cdot 3$ & 28 & -19.0 \\
\hline
\end{tabular}

Correction to local mean time is $-24 \mathrm{~s}$.

Torsion head at oh oom read $25^{\circ}$ and at 24 h $20 m$ read the same. Observer-W. J. P.
Correction to local mean time is $+22.5 \mathrm{~s} .90^{\circ}$ torsion $=17 .^{\prime} 59$. Torsion head at $15 \mathrm{~h} 30 \mathrm{~m}$ read $3 \mathrm{I}^{\circ}$ and at $20 \mathrm{~h}$ 20m read $35^{\circ}$. Observer-R. R. T, 
Tabulation of magnetic declinations observed at Teplitz Bay-Continued

\begin{tabular}{|c|c|c|c|c|c|c|c|c|c|c|c|c|c|c|c|c|c|c|c|c|}
\hline \multicolumn{5}{|c|}{ Friday, Marcli 25, I904 } & \multicolumn{5}{|c|}{ Magnet scalc inverted } & \multicolumn{5}{|c|}{ Sunday, March 27, 1904} & \multicolumn{6}{|c|}{ Magnet scale erect } \\
\hline \multirow{2}{*}{$\begin{array}{l}\text { Chr'r } \\
\text { time }\end{array}$} & \multicolumn{2}{|c|}{$\begin{array}{l}\text { Scale } \\
\text { readings }\end{array}$} & $\begin{array}{c}\text { East } \\
\text { decli- } \\
\text { nation. }\end{array}$ & Temp. & $\begin{array}{l}\text { Chr'r } \\
\text { time }\end{array}$ & $\begin{array}{r}\text { Sc } \\
\text { read }\end{array}$ & $\begin{array}{l}\text { ale } \\
\text { lings }\end{array}$ & $\begin{array}{c}\text { East } \\
\text { decli- } \\
\text { nation }\end{array}$ & $\begin{array}{l}\text { Temp. } \\
\text { C. }\end{array}$ & $\begin{array}{l}\text { Chr'r } \\
\text { time }\end{array}$ & $\begin{array}{r}\mathrm{Sc} \\
\text { read }\end{array}$ & $\begin{array}{l}\text { ale } \\
\text { lings }\end{array}$ & $\begin{array}{c}\text { East } \\
\text { decli- } \\
\text { nation. }\end{array}$ & Temp. & $\begin{array}{l}\text { Clir'r } \\
\text { time }\end{array}$ & $\begin{array}{r}\text { Sca } \\
\text { readi }\end{array}$ & $\begin{array}{l}\text { ale } \\
\text { ings }\end{array}$ & $\begin{array}{l}\text { East } \\
\text { decli } \\
\text { natio }\end{array}$ & & Temp. \\
\hline & Left & Right & 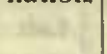 & 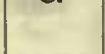 & & Left & Right & atares & & & Left & Right & wan. & & & Left & Right & & & \\
\hline $\mathrm{h} \mathrm{m}$ & d & d & $\therefore \quad$ & ${ }^{\circ}$ & $\mathrm{h} \mathrm{m}$ & d & d & 01 & $\stackrel{0}{\circ}$ & $\mathrm{h} \mathrm{m}$ & d & d & $\circ$, & & $\mathrm{h} \mathrm{m}$ & त & d & & & $\bullet$ \\
\hline 2000 & 39.4 & $39 \cdot 1$ & & -23.0 & 2200 & 42.1 & 40.3 & 2220 & $-2 \mathrm{I} \cdot 2$ & $000^{*}$ & 42.3 & 45.8 & $222 I$ & -25.9 & 200 & 66.2 & 67.0 & & & $-2 \pi \cdot 9$ \\
\hline 02 & 39.2 & 38.8 & $\cdot 24$ & Q & 02 & 42.5 & 40.2 & 20 & 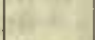 & 02 & 44.0 & 47.0 & 23 & & 02 & 64.1 & 65.0 & & 54 & \\
\hline 04 & 39. I & 38.5 & 24 & 107 & 04 & 42.3 & $4 \mathrm{I} \cdot 5$ & I9 & 2 & 04 & 44.2 & 46.8 & 23 & int & 04 & 65.2 & 66.0 & & 56 & \\
\hline 06 & 39.1 & 38.8 & 24 & (1. & 06. & $43 . I$ & 42.3 & I8 & W & 06 & 46.7 & 49.2 & 27 & 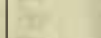 & 06 & 62.3 & 62.8 & & $5 \mathrm{I}$ & \\
\hline 08 & 40.8 & $40 . I$ & 22 & 60 & 08 & 42.1 & 41.0 & 20 & 6 & 08 & 45.9 & 47.9 & 26 & 12 & 08 & 60.6 & $6 r \cdot 3$ & & 48 & \\
\hline Io & $41 \cdot 3$ & 40.2 & $2 \mathrm{I}$ & int & Io & 43.8 & 42.9 & I7 & 67 & Io & 48.2 & 50.9 & 30 & fis & 10 & 59.9 & 60.3 & & 47 & \\
\hline 12 & 42.4 & 40.6 & 20 & & I2 & 41.0 & 40.5 & 21 & & 12 & 53.8 & 54.7 & 37 & & I2 & 50.0 & 59.8 & & 46 & \\
\hline 14 & 42.3 & 40.7 & 20 & -22.7 & . 14 & $4 \mathrm{I} .9$ & $4 \mathrm{I} \cdot 3$ & 20 & -21.0 & I4 & 57.1 & 58.1 & 42 & -25.0 & 14 & 58.0 & 58.7 & & 44 & $-2 \mathrm{I} \cdot 3$ \\
\hline 16 & 43.7 & 41.4 & I8 & 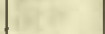 & I6 & 42.3 & 41.8 & I 9 & & 16 & 60.3 & 60.9 & 47 & & 16 & 50.8 & 60.2 & & 47 & \\
\hline 18 & 42.0 & 39.2 & $2 \mathrm{I}$ & a. & 18 & 39.9 & 39.4 & 23 & 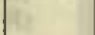 & 18 & 62.1 & 63.9 & $5 \mathrm{I}$ & Wis & 18 & 57.7 & 58.3 & & 44 & \\
\hline 20 & 43.3 & $4 I .0$ & ID & or & 20 & 37.7 & 36.8 & 27 & 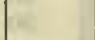 & 20 & 63.2 & 63.8 & 52 & nas. & 20 & 50.3 & 60.1 & & 47 & \\
\hline 22 & $4 \mathrm{I} \cdot 4$ & 40.3 & $2 \pi$ & $c$ & 22 & 38.6 & 38.0 & 25 & $=$ & 22 & 64.7 & 65.3 & 54 & $=$ & 22 & 63.7 & $64 \cdot 3$ & & 53 & \\
\hline 24 & $41 . ?$ & $4 I . I$ & 20 & . & 24 & 44.0 & $42 \cdot 5$ & I7 & 4 & 24 & 63.7 & 64.0 & 52 & $1=$ & 24 & 62.2 & 63.0 & $22 !$ & $5 \mathrm{I}$ & \\
\hline 26 & 42.8 & 40.7 & 20 & $=$ & 26 & 37.6 & $35 \cdot 3$ & 27 & E & 26 & 64.0 & 64.0 & 52 & 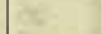 & 26 & 68.2 & 60.8 & 230 & OI & \\
\hline 28 & 42.4 & 40.6 & 20 & & 28 & 37.8 & 36.3 & 27 & & 28 & $6 \pi \cdot 3$ & 6r.9 & 48 & 2 & 28 & 65.1 & $66 . \mathrm{I}$ & 225 & 56 & \\
\hline 30 & $4 \mathrm{I} .2$ & 39.0 & 22 & -22.5 & 30 & 38.4 & 37.5 & 26 & -20.7 & 30 & 61.3 & 62.0 & 49 & -24.0 & 30 & 59.0 & 50.0 & & 46 & -21.0 \\
\hline 32 & 42.9 & 40.2 & 20 & 1 & 32 & 35.8 & 34.9 & 30 & & 32.2 & $6 \pi .2$ & $6 \pi .7$ & $4^{8}$ & & 32 & 55.0 & 57.8 & & $4^{2}$ & \\
\hline 34 & 40.0 & 38.9 & 23 & D. & 34 & 35.9 & 34.7 & 30 & & 34 & 60.2 & 60.8 & 47 & it & 31 & 58.8 & 60.8 & & 47 & \\
\hline 36 & 38.7 & $38 . I$ & 25 & E & 36 & 36.1 & 34.6 & 30 & -5 & 36 & 60.7 & 6 ז..3 & 48 & ive & 36 & $6 \mathrm{x} . \mathrm{I}$ & 63.1 & & 50 & \\
\hline 38 & 40.5 & $37 \cdot 3$ & 24 & 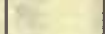 & 38 & 36.3 & 35.4 & 29 & in & 38 & 59.3 & 60.8 & 46 & 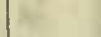 & 38 & 58.7 & 60.8 & & 47 & \\
\hline 40 & 37.8 & 36.6 & 27 & tet. & 40 & 35.9 & 35.5 & 29 & 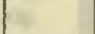 & 40 & 50.7 & 60.6 & 46 & 62 & 40 & 58.2 & 59.9 & & 46 & \\
\hline 42 & 41.7 & 39.5 & $2 I$ & & 42 & 35.0 & 34.3 & $3 I$ & & 42 & 60.2 & 60.8 & 47 & 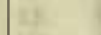 & 42 & 55.0 & 57.0 & & $4 \mathrm{I}$ & \\
\hline 44 & $43.5^{\circ}$ & 41.0 & I9 & -22.3 & 44 & 34.5 & 33.4 & 32 & -20.3 & 44 & $6 r$ & . I $a$ & 48 & -23.7 & 44 & 55.0 & $56 . ?$ & & 40 & -20.0 \\
\hline 46 & $42 . I$ & 40.3 & 20 & & 46 & 33.4 & 32.4 & 34 & 1 & 46 & & $3^{b}$ & 39 & & 46 & 61.7 & 62.8 & 22 ! & 5I & \\
\hline 48 & 43.3 & 42.4 & I8 & Et & 48 & 33.0 & $32 . \mathrm{I}$ & 34 & & 48 & 55.6 & 56.7 & 40 & $8 x$ & 48.5 & 60.1 & 71.2 & 23 & 03 & \\
\hline 50 & 43.5 & 42.7 & 17 & E & 50 & 32.3 & $3 \mathrm{I}: 5$ & 35 & 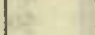 & 50 & 56.3 & 57.5 & $4 I$ & 1 & 50 & 75.1 & 76.0 & & I2 & \\
\hline 52 & 43.2 & 42.6 & I8 & $t^{2}$ & 52 & 32.2 & 30.1 & 36 & 7 & 52 & 58.8 & 50.6 & 45 & $=$ & $52^{*}$ & 39.9 & 46.4 & & I2 & \\
\hline 54 & 42.5 & $42 . \mathrm{T}$ & 19 & . & 54 & 3т.6 & .30 .1 & 36 & $x$ & 54 & 56.3 & 57.4 & $4 \mathrm{I}$ & 17 & 54 & 35.1 & 40.9 & 23 & 04 & \\
\hline 56 & 42.1 & 41.7 & I0 & 28 & 56 & 31.9 & 30.8 & .36 & 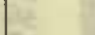 & 56 & 58.0 & 59.1 & 44 & 1 & 55 & 27.0 & 33.5 & 22 & 52 & \\
\hline 58 & 44.3 & 42.9 & I7 & $=$ & 58 & 31.9 & 30.4 & 36 & z & 58 & 58.6 & 50.8 & 45 & K & $5^{8}$ & 25.2 & $3 \mathrm{I} . ?$ & & 50 & \\
\hline 2100 & 43.5 & 42.1 & I8 & -22.0 & 2300 & 32.3 & $3 \mathrm{I} . \mathrm{I}$ & .35 & -20.0 & I $\infty$ & 58.9 & 50.9 & 45 & -23.0 & 300 & 26.3 & 32.8 & & $5 \mathrm{~T}$ & -20.5 \\
\hline 02 & 42.8 & $4 I \cdot .3$ & $\therefore$ in & $17=$ & 02 & 30.8 & 29.9 & .37 & 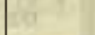 & 02 & 58.0 & 50.0 & 44 & 18 & 02 & 26.4 & 32.8 & & $5 \mathrm{I}$ & \\
\hline 04 & 43.6 & 42.8 & 17 & 16 & 04 & 32.2 & 30.5 & .36 & W & 01 & 60.9 & $6 \pi .8$ & 48 & 280 & 01 & $28 . \mathrm{I}$ & 33.8 & & 53 & \\
\hline of & 42.1 & 40.4 & 20 & 2 & o6 & 31.9 & 30.2 & 36 & in & of & 57.1 & 58.8 & 4.3 & 7 & of & 28.8 & 31.1 & & 54 & \\
\hline 08 & 41.5 & 39.7 & $2 \mathrm{I}$ & 15 & 08 & 32.4 & 30.8 & 36 & n & 08 & 58.7 & 50.1 & 45 & 100 & 08 & 27.1 & 32.0 & & 5I & \\
\hline Io & $4 \mathrm{I} \cdot 3$ & 40.8 & $2 \mathrm{I}$ & ati & To & 33.4 & 30.7 & 35 & 47 & I0 & 62.1 & 63.4 & $5 \mathrm{I}$ & $\theta$ & รo & 25.8 & 31.1 & & 50 & \\
\hline 12 & 41.7 & 30.6 & 2I & ats & 12 & 33.8 & 29.5 & .36 & 1 & I2. & 66. I & 67.1 & 57 & $1=1$ & 12 & 27.0 & 3.3 .2 & & 5.3 & \\
\hline 14 & 41.6 & 39.3 & 22 & -22.0 & 14 & 32.3 & 29.6 & .36 & -20.0 & I $A^{\prime}$ & 6.3 .1 & 6.3 .8 & 52 & -22.8 & I4 & 31. I & 36.2 & 22 & 58 & -20.3 \\
\hline I6. & $4 \mathrm{I} . \mathrm{I}$ & .30 .0 & 22 & 1 & I6 & 31.8 & $2 n .6$ & 37 & & т6 & 57.2 & 57.0 & 43 & & т6 & .36 .3 & $4 \mathrm{~T} .0$ & 23 & of & \\
\hline 18 & 40.3 & 38.5 & 2.3 & Be? & I8 & 3 Tा.8 & 28.7 & .28 & Ba & 18 & $6 \pi .7$ & 62.5 & 50 & Alit & I8 & . $3 \mathrm{~T} .3$ & .35 .0 & 22 & 57 & \\
\hline 20 & 30.7 & 38.6 & 24 & $=$ & 20 & 32.6 & 29.8 & 36 & E & 20 & 50.3 & 60.2 & 46 & 16 & 20 & 28.9 & 33.7 & & 54 & \\
\hline 22 & 40.8 & 30.5 & 22 & in & 22 & 32.8 & 30.1 & 36 & E & 22 & 6т.8 & 62.7 & 50 & $1=$ & 22 & 26.7 & 30.8 & & 50 & \\
\hline 24 & 40.0 & 38.8 & 21 & at & 24 & 31.8 & 29.3 & .37 & 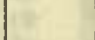 & 24 & 62.8 & 63.8 & 52 & 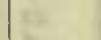 & 24 & 27.4 & $3 \mathrm{I} .2$ & & 5I & \\
\hline 26 & 39.8 & .38 .2 & 24 & $n E$ & 26 & 33.3 & 3 I. 5 & 34 & 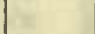 & 26 & 6 Т. & 62.1 & 49 & . & 26 & 21.5 & 25.5 & & 42 & \\
\hline 28 & 40.0 & 38.3 & 24 & 1 & 28 & 33.4 & $3 \mathrm{~T} .2$ & .34 & & 28 & $6 \mathrm{r} .2$ & 62.2 & 40 & 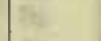 & 28 & 22.0 & 26.7 & & 44 & \\
\hline .30 & 42.0 & 40.2 & 20 & -21.7 & 30 & 33.5 & 30.6 & .35 & -20.0 & .30 & 64.0 & 65.8 & 5.5 & -22.3 & 30 & 24. I & 26.8 & & $\Delta 5$ & -20.0 \\
\hline 32 & 42.2 & 40.7 & 20 & D & 32 & 20.6 & 27.7 & 40 & & .32 & 6.3 .6 & 64.7 & 5.3 & 16. & 32 & 31.5 & 3.3 .2 & 22 & 56 & \\
\hline 34 & 4.3 .4 & 42.8 & 17 & t1 & .31 & 20.5 & 27.8 & 40 & We & 34 & 63.0 & 6.3 .7 & 52 & 4 & 31 & 34.2 & 36.4 & 23 & OI & \\
\hline 36 & 4.3 .6 & 42.3 & I8 & $3 x$ & .36 & 31.8 & 20.3 & 37 & $\mathrm{~F}$ & .36 & 63.4 & 64.0 & 52 & 2 & 36 & 33.1 & 35.7 & 22 & 50 & \\
\hline 38 & $45 \cdot 3$ & 43.2 & . $\quad 16$ & ta & 38 & 30.7 & 28.0 & 30 & 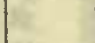 & 38 & 60.8 & 6I. 8 & 40 & he & 38 & 32.1 & 33.0 & 22 & 57 & \\
\hline 40 & 44.1 & 42.7 & 17 & at & 40 & 32.2 & 30.1 & $\begin{array}{r}.36 \\
\end{array}$ & . & 40 & -59.6 & 60.3 & 46 & 7 & 40 & 35.0 & 36.8 & 23 & 02 & \\
\hline 42 & 44.1 & 43.6 & 16 & 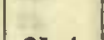 & 42 & 30.8 & 28.2 & 39 & & 42 & 50.8 & 60.7 & 47 & 12 & 42 & 39.0 & $4 \mathrm{~T} \cdot 2$ & & 08 & \\
\hline 44 & 43.3 & $43 . I$ & 17 & -2 I. 4 & 44 & 30.6 & 27.5 & 40 & -19.8 & 44 & 50.2 & $60: 2$ & 46 & -22.0 & 44 & 34.7 & 36.0 & 23 & OI & -20.0 \\
\hline 46 & 43.4 & 42.2 & I8 & & 46 & $3 \mathrm{I} .9$ & 28.2 & 38 & & 46 & 6ז. I & $6 \pi .5$ & 40 & & 46 & 27.0 & 29.3 & 22 & 49 & \\
\hline 48 & 43.2 . & 42.7 & I8 & 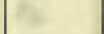 & 48 & $3 I . I$ & 27.6 & 39 & 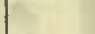 & 48 & 65.1 & 66.6 & 2256 & 1 & 48 & 28.2 & $3 \mathrm{~T} .8$ & 22 & 52 & \\
\hline 50 & 43.0 & 42.2 & I8 & cat & 50 & 30.7 & 27.2 & 40 & Q & 50 & 68. I & 60.2 & 2.300 & tif & 50 & .33 .8 & 36.7 & 23 & OI & \\
\hline 52 & 44.9 & 42.4 & 17 & 122 & 52 & 31.6 & 28.5 & 38 & 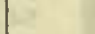 & 52 & 6.3 .4 & 64.4 & $22 \quad 5,3$ & $\mathrm{Et}$ & 52 & $34 . ?$ & 37.6 & & 02 & \\
\hline 54 & 42.7 & $4 \mathrm{I} \cdot 2$ & 19 & 122 & 51 & 32.0 & 28.8 & 37 & 1 & 54 & 67.2 & 68.3 & 59 & 12 & 54 & .34 .8 & 37.2 & 2.3 & 02 & \\
\hline 56 & 4I.? & 40.6 & 20 & 2 & 56 & 31.9 & 29.0 & 37 & & 56 & 67.2 & 68.7 & 50 & ine & 56 & 33.2 & 35.2 & 22 & 59 & \\
\hline 58 & 41.8 & 40.0 & 21 & $18 c$. & 58 & 32.6 & 20.2 & $\begin{array}{r}36 \\
1\end{array}$ & & 58 & 66.7 & 67.9 & 58 & nest & 58 & 29.8 & $3 \mathrm{~T} .8$ & & 54 & \\
\hline & & .0 & $2.67=$ & $60^{\circ}$ & 2400 & 32.3 & 28.3 & 37 & -19.7 & & & & & 091 & & & & & & \\
\hline
\end{tabular}

Correction to local mean time is + I9.5s.

Observer-R. R. T.

Torsion head at igh $30 \mathrm{~m}$ read $35^{\circ}$ and at $24 \mathrm{~h}$ oom read the same.

Observer-H. H. N. 
Tabulation of magnetic declinations observed at Teplitz Bay-Continued

\begin{tabular}{|c|c|c|c|c|c|c|c|c|c|c|c|c|c|c|c|c|c|c|c|}
\hline \multicolumn{5}{|c|}{ Sunday, March 27, I904 } & \multicolumn{5}{|c|}{ Magnet scale inverted } & \multicolumn{6}{|c|}{ Monday, Mareh 28, 1904} & \multicolumn{4}{|c|}{ Magnet scale erect } \\
\hline \multirow[t]{2}{*}{$\begin{array}{l}\text { Chr'r } \\
\text { time }\end{array}$} & \multicolumn{2}{|c|}{$\begin{array}{l}\text { Scale } \\
\text { readings }\end{array}$} & \multirow[t]{2}{*}{$\begin{array}{l}\text { East } \\
\text { decli- } \\
\text { nation }\end{array}$} & Temp. & $\begin{array}{l}\text { Chr'r } \\
\text { time }\end{array}$ & $\begin{array}{l}\text { Sca } \\
\text { readi }\end{array}$ & $\begin{array}{l}\text { ale } \\
\text { ings }\end{array}$ & $\begin{array}{l}\text { East } \\
\text { decli- }\end{array}$ & Temp. & $\begin{array}{l}\text { Chr'r } \\
\text { time }\end{array}$ & $\begin{array}{r}\text { Scal } \\
\text { readir }\end{array}$ & $\begin{array}{l}\text { ale } \\
\text { ings }\end{array}$ & $\begin{array}{c}\text { East } \\
\text { decli- }\end{array}$ & Temp. & Chr'r & $\begin{array}{r}\text { Sca } \\
\text { read }\end{array}$ & $\begin{array}{l}\text { le } \\
\text { ngs }\end{array}$ & East & Temp. \\
\hline & Left & Right & & & & Left & Right & & & & Left $R$ & Right & & & & L,eft & Right & & \\
\hline$h \mathrm{~m}$ & d & d & $\cdot$ & $\circ$ & $\mathrm{h} \mathrm{m}$ & d & $d$ & & & $\mathrm{~h} \mathrm{~m}$ & & d & $\cdot 1$ & & $1 \mathrm{~m}$ & d & d & $\cdot$ & $\bullet$ \\
\hline $400^{*}$ & $49 \cdot 0$ & $47 \cdot 4$ & 230 & -19.8 & 600 & $29 . \mathrm{I}$ & 28.5 & 2333 & -18.9 & 800 & Fibr & re & & Iat 3 & I0 00 & 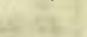 & $x^{2}$ & kiak. & 10 \\
\hline 02 & 48.1 & 47.1 & & rat & 02 & 24.4 & 22.2 & 42 & & 02 & broke & ren; & & 6o & 02 & & 6.98 & 8.12 & (1) \\
\hline 01 & 52.4 & 51.9 & 225 & in & 04 & 40.9 & 40.5 & is & & 04 & had $t$ & to & $13 x$ & 2 & 04 & 15.8 & $3, x=$ & a 7 & b \\
\hline 06 & 50.2 & 48.8 & & 5 & 06 & 34.8 & 32.8 & 25 & & 06 & remo & ove & 3 if & 66 & 06 & 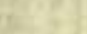 & 1,8 & wate & Wr \\
\hline 08 & 47.5 & 46.2 & 0 & te & 08 & 31.9 & $3 I . I$ & 2329 & & 08 & torsic & ion & & wat & 08 & & 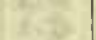 & thitis. & 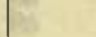 \\
\hline 10 & $45 . \mathrm{I}$ & 44.0 & of & 65 & 10 & 51.9 & 50.9 & 2258 & & 10 & new fi & fibre & $x^{2}$ & $m$ & I0 & & 29 & $6 x^{2}$ & 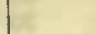 \\
\hline 12.4 & 44.3 & 42.9 & or & & I2 & 42.8 & $42 . I$ & $23 \mathrm{I} 2$ & & 12 & & & & 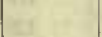 & I2 & & 10 & 3 & 1 \\
\hline 14 & 45.4 & 43.7 & $\alpha$ & -19.7 & 14 & 36.0 & 35.1 & 23 & -18.9 & 14 & & xast & 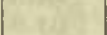 & 81 & 14 & & 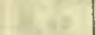 & & \\
\hline 16 & 48.8 & 46.7 & 230 & & 16 & 36.9 & 35.0 & 22 & & 16 & & 3 & s $x^{2}$ & 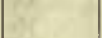 & 16 & & & & tit \\
\hline 18 & 50.8 & 48.9 & 225 & & I8 & 36.2 & 35.1 & 23 & & I8 & & me & $y^{2}$ & Fit & I8 & 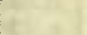 & 32 & $2 \times 28$ & Fit \\
\hline 20 & 49.2 & $47 \cdot 7$ & 2302 & F & 20 & 35.7 & 34.1 & 24 & & 20 & & $y=$ & 50 & $=$ & 20 & & $\mathrm{ft}^{2}$ & $8 x^{2}$ & Es. \\
\hline 22 & 50.0 & 48.3 & oc & & 22 & 34.6 & 33.2 & 25 & & 22 & & & H & $\ldots$ & 22 & & $5=$ & 120 & 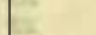 \\
\hline 24 & 50.0 & 48.8 & or & & 24 & 3.3 .7 & 32.4 & 27 & & 24 & & $a^{2}$ & in win & Ex & 24 & & +1.80 & tixte & 18 \\
\hline 26 & 48.0 & $47 . I$ & 0 & & 26 & 38.3 & 36.6 & 20 & & 26 & & $78=5$ & Win & $n$ & 26 & & 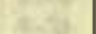 & $125=$ & 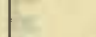 \\
\hline 28 & 48.9 & 48.2 & 230 & & 28 & 37.2 & 35.7 & 22 & & 28 & & 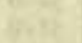 & 10 & $x$ & 28 & & & 58 & $=$ \\
\hline 30 & 51.2 & 50.6 & 225 & -19.4 & 30 & $34 \cdot 3$ & 32.6 & 26 & $-\mathrm{I} 8.8$ & 30 & & e & 10 & H & 30 & & & 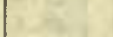 & 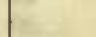 \\
\hline 32 & 48.3 & 47.9 & 230 & . & 32 & 36.2 & 34.8 & 23 & 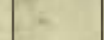 & 32 & & $3 y 0$ & fon & 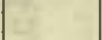 & 32 & 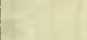 & 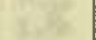 & 1. & L \\
\hline 34 & 47.0 & 46.1 & 0 & & 34 & 35.1 & 33.5 & 25 & & 34 & & 25 & 1.12 & it & 34 & & & & 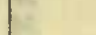 \\
\hline $3^{6}$ & 44.3 & 42.1 & IC & & 36 & 33.5 & 33.1 & 26 & & 36 & & (2) & 60 & 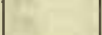 & 36 & 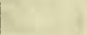 & 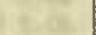 & 0.58 & 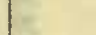 \\
\hline 38 & $4 \mathrm{I} . \mathrm{I}$ & 40.8 & $I_{3}$ & & 38 & 31.0 & 30.2 & 31 & & 38 & & & & 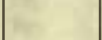 & 38 & E & & 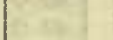 & 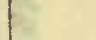 \\
\hline 40 & 42. & I $a$ & I2 & & 40 & 37.8 & 37.2 & 20 & & 40 & & $x^{3}$ & the & D & 40 & & 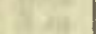 & & 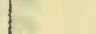 \\
\hline 42 & 47.8 & 47.8 & 2302 & & 43 & 39.2 & 37.8 & I8 & & 42 & & & 9 & 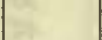 & 42 & & & & . \\
\hline 44 & 52.0 & $5 \mathrm{I} .2$ & 225 & -19.3 & 44 & 41.0 & 38.7 & I6 & & 44 & & 25 & & $t^{2}$ & 44 & & $x^{2}=$ & 20 & E \\
\hline 46 & 48.3 & 47.6 & 2302 & & 46 & 43.3 & 40.8 & I3 & & 46 & & $y=$ & 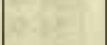 & 15 & 46 & & 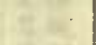 & 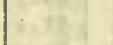 & \\
\hline 48 & 49.8 & 49.1 & or & 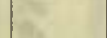 & 48 & 42.9 & 39.1 & 14 & & 48 & & $7=5$ & & 6 & 48 & 56.8 & 50.8 & 2246 & - TO. 3 \\
\hline 50 & 48.1 & 47.2 & 03 & a & 50 & 40.3 & 38.9 & I7 & 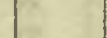 & 50 & & nate & $c x^{2}$ & 6 & 50 & & 50.7 & & -10.3 \\
\hline 52 & 44.8 & 44.8 & 07 & 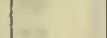 & 52 & 43.7 & 42.2 & I2 & & 52 & & 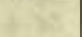 & $1=$ & 1 & 52 & 57.9 & 59 & 43 & 8 \\
\hline 54 & 45 & $\mathrm{I} b$ & $0 ?$ & 3 & 54 & 43.1 & 42.1 & I2 & & 54 & & 11 & 0.7 & y: & 54 & $\begin{array}{l}55 . \\
54 .\end{array}$ & 56 & $\begin{array}{l}43 \\
4 I\end{array}$ & 13 \\
\hline 56 & 46.7 & $45 \cdot 3$ & of & & 56 & 40.9 & 39.8 & I6 & & 56 & & 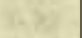 & $6=$ & nat & 56 & 54. & 55 & $\begin{array}{l}41 \\
40\end{array}$ & 5 \\
\hline 58 & $46 . \mathrm{I}$ & 45.6 & of & & 58 & 41.0 & 40.1 & I5 & & 58 & & andis & $1=2$ & $=$ & 58 & & & 40 & -10.7 \\
\hline 500 & 43.7 & 42.9 & IC & $-\mathrm{I} 9.3$ & 700 & 47.4 & 45.8 & 06 & -18.8 & 900 & & & in & 60 & I I 00 & & 55. & 40 & -10.7 \\
\hline 02 & 46.0 & $45 \cdot 3$ & $23 \circ$ & -2.0 & 02 & 37 & $.5 b$ & 20 & & 02 & & 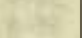 & L2 & 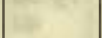 & 02 & 52 & $\begin{array}{l}56 . \\
56 .\end{array}$ & $\begin{array}{l}40 \\
42\end{array}$ & 60 \\
\hline 04 & 51.0 & 50.5 & $225^{8}$ & 2 & 04 & 35.9 & 34.2 & 24 & & 04 & & $\sqrt{25}$ & $6 x$ & $y=$ & 04 & & 56. & 42 & es \\
\hline का & 45.8 & $45 \cdot 3$ & 23 of & $\therefore$ & of & $4 \mathrm{I} .3$ & 39.6 & I6 & tr. & 06 & & 40 & 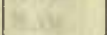 & 105 & 06 & & & 42 & ais \\
\hline os & 46.8 & $45 . \mathrm{I}$ & $23 d$ & & $\infty 8$ & 42.7 & $4 \mathrm{I} .2$ & I3 & 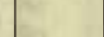 & 08 & & & 130 & No & 08 & 54 & 56 & $4 \mathrm{I}$ & th \\
\hline I0 & 6r. & 57.8 & 2245 & 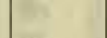 & IO & $44 \cdot 3$ & 42.5 & II & c & I0 & & A & 1028 & 0 & IC & & 58. & 45 & as \\
\hline 12 & 69.4 & 68.9 & 20 & & 12 & 36.2 & $34 . \mathrm{I}$ & 24 & & I2 & & - & $2=1$ & at & 12 & & & 46 & \\
\hline I4 & 53. &.$o b$ & 225 & -19.1 & 14 & 36.9 & 35.7 & 22 & -18.7 & I4 & & ats: & $6 x$ & it & 14 & 58. & 59. & 47 & -10.7 \\
\hline I6 & 27 . & $8 a$ & 233 & & I6 & 40.8 & 39.8 & I6 & & І6 & & ax & the & at & 16 & 58.2 & & 47 & ( \\
\hline 18 & 44. & $2 b$ & & b. & I8 & 42.2 & 39.3 & 15 & & I8 & & 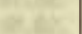 & 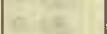 & E. & I8 & & & 44 & te. \\
\hline 20 & 33. & $5^{b}$ & 25 & 6 & 20 & 36.2 & 33.8 & 24 & & 20 & & mis & 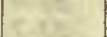 & $x$ & 20 & & & 45 & 8 \\
\hline 22 & 27.3 & 27.3 & 35 & $=$ & 22 & 39.2 & 36.0 & 20 & $=$ & 22 & & to & 5.6 & $=$ & 22 & 57.2 & 58.4 & 45 & t. \\
\hline 24 & 49.0 & 48.2 & 02 & 6 & 24 & 34.1 & 3I. I & 28 & F & 24 & & 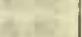 & Ge & it & 24 & & & 44 & \\
\hline 26 & $44 . \mathrm{I}$ & 43.2 & IC & & 26 & 35.0 & 29.9 & 28 & & 26 & & 30 & 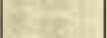 & & 26 & 55 & & 42 & s \\
\hline 28 & 43.1 & 41.6 & $\mathrm{I} 2$ & & 28 & 30.9 & 25.3 & 35 & 5 & 28 & & ain & 2his? & E & 28 & & & $4 \mathrm{I}$ & \\
\hline 30 & 48.9 & 48.2 & 02 & -19.0 & 30 & $42 . I$ & 38.6 & IO & & 30 & & ky & beyt & & 30 & $54 . \mathrm{I}$ & & $4 \mathrm{I}$ & -10.3 \\
\hline 32 & 48.3 & 48.0 & 03 & & 32 & 34.9 & 30.3 & 28 & -18.3 & 32 & & $2 x$ & 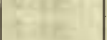 & 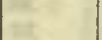 & 32 & & & 43 & $x^{2}$ \\
\hline 34 & $44 . \mathrm{I}$ & 43.1 & & & 34 & 36.3 & 34.6 & 24 & & 34 & & & & if & 34 & 58 & & 47 & $y$ \\
\hline 36 & 36.2 & 35.3 & 22 & E. & 36 & 32.9 & 30.1 & 30 & & 36 & & 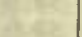 & 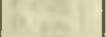 & 语 & 36 & 57.6 & & 46 & tr. \\
\hline 38 & 34.7 & $33 \cdot 3$ & 25 & 10 & 38 & 31.9 & 26.7 & 3.3 & & 38 & & $x=5$ & & & $3^{8}$ & 58.3 & & 47 & 8 \\
\hline 40 & 42.2 & 40.8 & I3 & 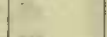 & 40 & 23.9 & I8.8 & 46 & & 40 & & 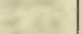 & 3. & is & 40 & 55.0 & & 42 & $\infty$ \\
\hline 42 & 48.0 & 46.7 & 04 & & 42 & 22.8 & 20.1 & 46 & & 42 & & & 60 & tis & 42.2 & 57.0 & 57 & 44 & \\
\hline 44 & 50.8 & 47.2 & or & -18.9 & 44 & I6.6 & 14.2 & 55 & -18.3 & 44 & & dyat & 607 & $x^{2}$ & 44 & 49.1 & $5 \mathrm{I}$ & 34 & -10.2 \\
\hline 46 & 3I.I & 29. I & 31 & & 46 & 20.9 & 16.4 & 50 & & 46 & & 40 & $10 x y$ & 4 & 46 & 49.2 & 50 & 33 & \\
\hline 48 & 47.8 & 46.2 & 04 & 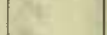 & 48 & $27 . I$ & $26 . I$ & 38 & & 48 & & & t. 15 & 8 & 48 & 50.9 & & 35 & 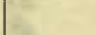 \\
\hline 50 & 46.9 & 46.1 & o 4 & 14 & 50 & 22.9 & 18.8 & 47 & tr. & 50 & & is & $6 y^{2}$ & 18 & 50 & 49.6 & 50 & 33 & \\
\hline 52 & 47.2 & 46.9 & 04 & 20 & 52 & 25.2 & 21.3 & 43 & be & 52 & & $2 \pi$ & i $\quad * 7$ & E & 52 & 51.2 & 53.0 & 36 & D. \\
\hline 54 & 34.4 & 33.6 & 25 & $\omega$ & 54 & 26.8 & 22.3 & $4 \mathrm{I}$ & $1=$ & 54 & & & & & 54 & 56.0 & 57.9 & & \\
\hline 56 & 42. & $2 b$ & 12 & 62 & 56 & 30.2 & 25.6 & 36 & & 56 & & 48 & $\frac{7}{7}$ & 40 & 56 & $57 \cdot 3$ & 58.9 & 46 & 25 \\
\hline 58 & 3I.I & 30.2 & 30 & a & 58 & $3 \mathrm{I} \cdot 3$ & 26.7 & 34 & & 58 & & 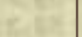 & by & 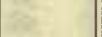 & 58 & 57.7 & 58.5 & 46 & \\
\hline & & 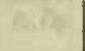 & 0.1. & s. & 800 & 31.7 & 25.8 & & -18.3 & & & $7-2 x$ & 2311 & & & 56.1 & 57.2 & & -9.7 \\
\hline
\end{tabular}

Correction to local mean time is - 9s. $90^{\circ}$ torsion $=12 .{ }^{\prime} 84$. Torsion head at oh oom read $86^{\circ}$ and at Iol rom read $45^{\circ}$. Observer-R. R. T.
Correction to local mean time is $+28 \mathrm{~s}$. $90^{\circ}$ torsion $=9 . .^{\prime} 03$ Torsion head at $10 \mathrm{~h} 45 \mathrm{~m}$ read $294^{\circ}$ and at $12 \mathrm{~h}$ 20m read $297^{\circ}$. Observer-R. R. T. 
Tabulation of magnetic declinations observed at Teplitz Bay-Continued

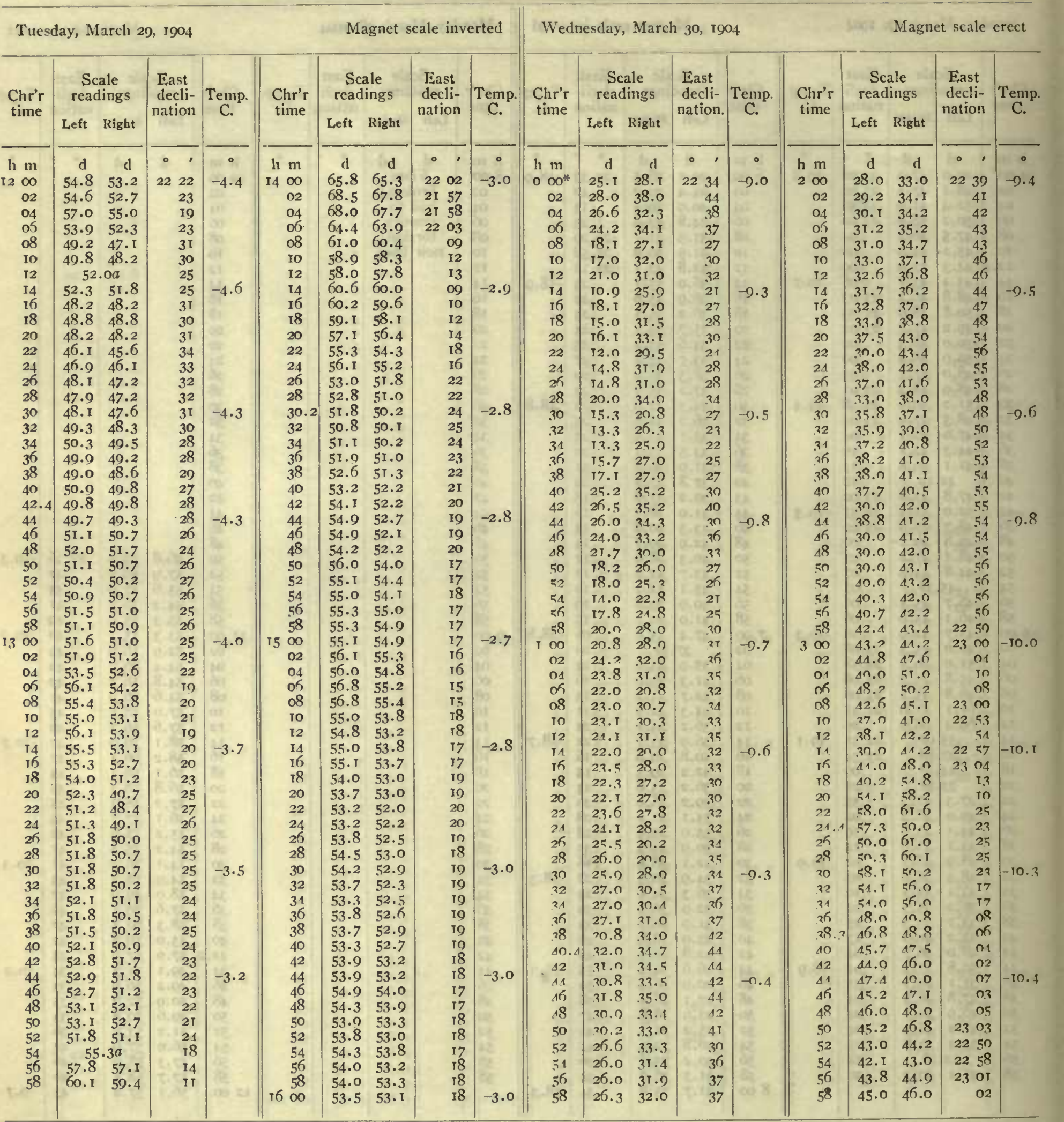

Correction to local mean time is $-7 \mathrm{~s} .90^{\circ}$ torsion $=16 .^{\prime} 48$. Torsion head at $\mathrm{Im} 25 \mathrm{~m}$ read $254^{\circ}$ and at $161 \mathrm{r}$ 20In read $290^{\circ}$.

Observer-J. V. Observer-R. R. T. 
Tabulation of magnetic declinations observed at 'Teplitz Bay-Continued

\begin{tabular}{|c|c|c|c|c|c|c|c|c|c|c|c|c|c|c|c|c|c|c|c|c|c|}
\hline \multicolumn{6}{|c|}{ Wednesday, March 30, 1904} & \multicolumn{6}{|c|}{ Magnet scale erect } & \multicolumn{5}{|c|}{ Wednesday, March 30, 1904} & \multicolumn{5}{|c|}{ Magnet scalc erect } \\
\hline \multirow{2}{*}{$\begin{array}{l}\text { Chr'r } \\
\text { time }\end{array}$} & \multicolumn{2}{|c|}{$\begin{array}{l}\text { Scale } \\
\text { readings }\end{array}$} & $\begin{array}{l}\text { Ea } \\
\text { dec }\end{array}$ & & Temp. & Chr'r & $\begin{array}{r}\mathrm{Sc} \\
\text { read }\end{array}$ & $\begin{array}{l}\text { ale } \\
\text { lings }\end{array}$ & $\begin{array}{l}\text { Eas } \\
\text { decl }\end{array}$ & & Temp. & Chr'r & $\begin{array}{r}\text { Sc } \\
\text { reac }\end{array}$ & $\begin{array}{l}\text { :ale } \\
\text { dings }\end{array}$ & $\begin{array}{l}\text { East } \\
\text { decli- }\end{array}$ & Temp. & Chr'r & $\begin{array}{r}S c \\
\text { read }\end{array}$ & $\begin{array}{l}\text { ale } \\
\text { lings }\end{array}$ & $\begin{array}{l}\text { East } \\
\text { decli- }\end{array}$ & Teinp. \\
\hline & Left & Right & & & & & Left & Right & & & & & Left & Right & & & & $L_{\text {,eft }}$ & Right & & \\
\hline $11 \mathrm{~m}$ & d & $d$ & & & 0 & $\mathrm{~h} \mathrm{~m}$ & d & d & - & & $\bullet$ & $\mathrm{h} \mathrm{m}$ & d & d & & - & $\mathrm{h} \mathrm{m}$ & d & $\mathrm{d}$ & $\circ$ & 。 \\
\hline 400 & 46.8 & $47 \cdot 3$ & 23 & & -10.5 & 600 & 54.0 & 56.3 & 23 & 17 & 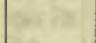 & 800 & 35.5 & 37.2 & $22+7$ & -10.3 & Io $\infty$ & 26.6 & 29.6 & 2234 & -10.0 \\
\hline 02 & 47.8 & 49.0 & & 07 & 10 & 02 & $54 . \mathrm{I}$ & 56.1 & & 17 & $16=$ & 02 & 32.8 & 34.5 & 43 & 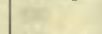 & 02 & 25.6 & 29.1 & 34 & \\
\hline 04 & 48.9 & 50.3 & & 09 & 40 & 04 & 50. I & 52. I & & I I & (1) & 04 & 31.7 & $34 \cdot 3$ & 42 & 160 & 04 & 27.3 & 30.2 & 35 & 15 \\
\hline 06 & 48.0 & 49.0 & & 07 & $2 x$ & 06 & 46.2 & 49.2 & & 06 & (15 & 06 & 30.3 & 31.8 & 39 & 7 & 06 & 26.7 & 29.3 & 34 & (11) \\
\hline 08 & 44.5 & 46.2 & & 02 & Ais & 08 & 43.9 & 47.0 & & 02 & w & 08 & 33.0 & 34.8 & 43 & 67 & 08 & 26.5 & 29.0 & 33 & in \\
\hline 10 & 46.0 & 48.0 & & 05 & Int & IO & 43.0 & 46.0 & 23 & $\infty$ & & 10 & $3 \mathrm{r} \cdot 3$ & 33.0 & $4 \mathrm{I}$ & $a r$ & IO & 27.3 & 29.6 & 34 & as \\
\hline 12 & 44.8 & 46.0 & 23 & 02 & Wi & 12 & 39.0 & 42.3 & 22 & 54 & 10 & 12 & 30.3 & 32.0 & 39 & ar & 12 & 27.5 & 29.7 & 35 & it \\
\hline I4 & 40.8 & 44.0 & 22 & 58 & -10.5 & I4 & 42.5 & 45.5 & 23 & 00 & -10.0 & I4 & 29.2 & 30.7 & 37 & -10.0 & I4 & 27.9 & 30.2 & 35 & -10. I \\
\hline 16 & 38.9 & $4 \mathrm{I} .3$ & & 54 & & I6 & $4 \mathrm{I} .9$ & 44.0 & 22 & 58 & & 16 & 29.5 & $3 \mathrm{I} .3$ & 38 & & 16 & 28.0 & 30.8 & 36 & \\
\hline 18 & 38.0 & 40.8 & & 53 & $F$ & 18 & 40.8 & 42.3 & & 56 & 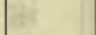 & I8 & 29.3 & 32.3 & 38 & 162 & 18 & 28.3 & 30.7 & 36 & E \\
\hline 20 & 36.9 & 39.2 & & 51 & Fr & 20 & 38.0 & 40.2 & & 52 & $\theta$ & 20 & 3 I. 3 & 32.6 & 40 & $=$ & 20 & 26.3 & 29.7 & 34 & re \\
\hline 22 & 37.0 & 40.0 & & $5 \mathrm{I}$ & $=$ & 22 & 34.2 & 37.0 & 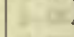 & 46 & $\infty$ & 22 & 34.0 & 35.5 & 45 & $1=$ & 22 & 27.1 & 30.3 & 35 & 2 \\
\hline 24 & 36.5 & 38.6 & & 50 & 18 & 24 & 32.0 & 34.0 & Wis 5 & 42 & $1=$ & 24 & 34.8 & 35.6 & 45 & $1=$ & 24 & 27.0 & 30.3 & 35 & $=$ \\
\hline 26 & 40.2 & 43.0 & & 56 & ic & 26 & 28.9 & 32.0 & $=1.1$ & 38 & 0 & 26 & 31.6 & 32.6 & $4 \mathrm{I}$ & is & 26 & 27.6 & 30.5 & 35 & x \\
\hline 28 & 41.0 & 44.0 & 22 & 58 & os & 28 & 29.0 & 32.3 & 0,1 & 38 & $=$ & 28 & 33.3 & 34.6 & 43 & $=$ & 28 & 27.2 & 29.7 & 34 & 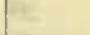 \\
\hline 30 & 43.9 & 46.2 & 23 & 02 & -10.4 & 30 & 34.2 & $37 . \mathrm{I}$ & & 46 & -10.1 & 30 & 31.6 & 32.6 & $4 \mathrm{I}$ & -10.0 & 30 & 26.5 & 29.0 & 33 & -10.2 \\
\hline 32 & 43.2 & 45.0 & 23 & 00 & ets & 32 & 36.8 & 38.9 & $10<5$ & 50 & & 32 & 29.2 & 30.3 & 37 & 142 & 32 & 26.3 & 28.7 & 33 & \\
\hline 34 & 40.0 & 40.8 & 22 & 55 & 18 & 34 & 36.0 & 38.2 & ins & 49 & E & 34 & 29.2 & $3 \mathrm{~T} \cdot 3$ & 38 & 142 & 34 & 26.5 & 29.3 & 34 & th \\
\hline 36 & 38.0 & 39.0 & & 51 & 20 & 36 & 36.0 & 38.2 & t. & 49 & 10 & 36.6 & 32.3 & 32.5 & $4 \mathrm{I}$ & are & 36 & 26.2 & 20. I & 33 & x \\
\hline 38 & 38.5 & 39.0 & & 52 & W & 38 & 36.1 & 38.2 & & 46 & $=$ & 38 & 31.3 & 31.6 & 39 & 1 & 38 & 26.0 & 28.0 & 32 & 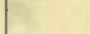 \\
\hline 40 & 30.6 & 40.0 & & 53 & cre & 40 & 32.9 & 38.0 & et & $5 \pi$ & 01. & 40 & 31.7 & 32.0 & 40 & nit & 40 & 25.6 & 27.6 & 31 & $E$ \\
\hline 42 & 38.0 & 38.2 & 8 & $5 \mathrm{I}$ & 12 & 42 & 37.0 & 40.2 & & 46 & $L$ & 42 & 26.8 & 28.0 & 33 & 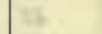 & 42 & 25.6 & 27.3 & 31 & $E x$ \\
\hline 44 & 35.8 & 36.0 & a & 47 & -10.3 & 44 & 34.0 & 37.0 & 12 & 42 & -10.2 & 44 & 30.3 & $3 \mathrm{I} . \mathrm{I}$ & 38 & -10.0 & 44 & 25.6 & 28.3 & 32 & \\
\hline 46 & 34.0 & 35.9 & & 46 & 0.0 & 46 & 3I. I & 34.4 & & 32 & 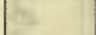 & 46 & 30.3 & $31 \cdot 3$ & 38 & (2) & 46 & 25.6 & 28.6 & 32 & -10.2 \\
\hline 48 & 34.5 & 36.0 & A.t. & 46 & Fit & 48 & 26.0 & 27.0 & 22 & 39 & 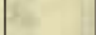 & 48 & 33.8 & 34.0 & 43 & 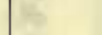 & 48 & 25.0 & 28.3 & $3 \mathrm{I}$ & $x^{2}$ \\
\hline 50 & 33.0 & 34.0 & & 43 & est & 50 & 29.0 & 33.0 & 23 & OI & Q & 50 & 30.6 & 32.2 & 39 & est & 50 & 26.0 & 28.6 & 32 & 98 \\
\hline 52 & 32.0 & 33.0 & & 42 & $=2$ & 52 & 44.8 & 45.1 & 23 & 05 & 2 & 52 & 30.8 & 30.9 & 38 & 0 & 52 & 26.6 & 28.6 & 33 & $t^{2}$ \\
\hline 54 & 33.2 & 34.0 & & 44 & 11 & 54 & 47.0 & 48.0 & 22 & 50 & 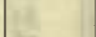 & 54 & 30.8 & 27.6 & 36 & 23 & 54 & 26.2 & 28.0 & 32 & $t^{t}$ \\
\hline 56 & 37.3 & 38.8 & ha & 50 & 09 & 56 & 37.3 & 38.2 & & 48 & 12 & 56 & 29.0 & 30.5 & 37 & Le & 56 & 26.3 & 27.0 & 31 & 20 \\
\hline 58 & $4 \mathrm{I} . \mathrm{I}$ & 41.8 & & $5^{6}$ & 2 & 58 & 36.0 & $37 \cdot 3$ & & 48 & & 58 & 25.2 & 27.2 & $3 \mathrm{r}$ & 2 & 58 & 25.8 & 27.6 & 32 & $=$ \\
\hline 500 & 43. I & 43.9 & 22 & 59 & - T0.3 & 700 & 32.0 & 33.0 & & 42 & -10.3 & 900 & 26.8 & 28.5 & 33 & -10.0 & II 00 & 26.0 & 27.4 & 32 & -10.3 \\
\hline 02 & 45.4 & 46.2 & 23 & 0.3 & 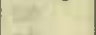 & 02 & $3 I \cdot 3$ & 32.9 & & $4 \pi$ & 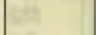 & 02 & 34.6 & 35.6 & 45 & 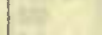 & 02 & 25.0 & 26.4 & 30 & \\
\hline 04 & 48.6 & 49.2 & & 08 & 100 & 04 & 32. I & 32.8 & & $4 \mathrm{I}$ & 0 & 04 & 32.0 & 33.6 & 42 & 160 & 04 & $24 \cdot 3$ & 26.0 & 29 & in \\
\hline 06 & $5 \mathrm{I} .0$ & 51.0 & & 12 & 10 & 05 & 35.0 & 36.0 & & 46 & F & 06 & 27.9 & 30.6 & 36 & No & 06 & 23.7 & 25.2 & 28 & w \\
\hline 08 & 5 I.O & 51.8 & 78 & II & an & 08 & 35.9 & 37.0 & & 48 & $\theta$ & 08 & $3 I .3$ & 32.9 & 40 & 10 & 08 & 24.0 & 25.5 & 28 & trat \\
\hline 10 & 51.8 & 52.0 & $2 x^{2}$ & 12 & (n) & 10 & $34 . \mathrm{I}$ & 38.1 & & 47 & Det & 10 & 29.3 & $3 \mathrm{I} .6$ & $3^{8}$ & $\theta$ & I0 & $23 \cdot 3$ & 24.7 & 27 & 61. \\
\hline I2 & 46.5 & 47.0 & 23 & 04 & int & I2 & 30.0 & 36.0 & & 42 & & I2 & 24.7 & 28.0 & $3 \mathrm{~T}$ & $\mathrm{xt}$ & I2 & 22.7 & 24.7 & 27 & $x^{2}=$ \\
\hline 14 & 42.8 & 44.0 & 22 & 59 & 131 & 14 & 30.8 & 36.2 & & 43 & -10.2 & I4 & 23.8 & 26.5 & 29 & WH & 14 & 24.0 & 25.6 & 20 & -10.3 \\
\hline 16 & 42.0 & 42.6 & & 57 & 20 & เ6́ & 27.2 & 35.0 & & 39 & 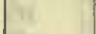 & I6 & 25.8 & 28.3 & 32 & 67 & 16 & 23.0 & 24.3 & 26 & \\
\hline I8 & 40.0 & $4 \mathrm{~T} .0$ & & 54 & 2 & 18 & 27.0 & 35.0 & & 39 & ir & I8 & 27.3 & 30.6 & 35 & -10.0 & 18 & 22.7 & $24 . I$ & 26 & 0 \\
\hline 20 & 37.1 & 38.0 & & 50 & $n$ & 20 & $3 I .3$ & 40.3 & & 47 & $\square$ & 20 & 36.8 & 38.0 & 49 & is & 20 & 23.3 & 25.3 & $\begin{array}{l}48 \\
112\end{array}$ & th \\
\hline 22 & 40.0 & $4 \mathrm{I} .8$ & & 55 & $\pi$ & 22 & 31.0 & 30.0 & & 45 & $=$ & 22 & 27.0 & 29.0 & 34 & is & 22 & 24.1 & $26 . \mathrm{I}$ & 29 & $E$ \\
\hline 24 & 36.0 & 38. I & & 49 & $1 \%$ & 24 & 21.0 & 28.0 & & 29 & 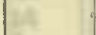 & 24 & 26.9 & 29.8 & 34 & w & 24 & $24 \cdot 3$ & 26.0 & (1) $\quad 29$ & is \\
\hline 26 & 32.3 & 33.9 & & 43 & As & 26 & 24.7 & 27.0 & & $3 \mathrm{I}$ & 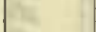 & 26 & 28.5 & $3 I .3$ & 37 & Ie & 26 & 22.3 & 24.0 & 26 & 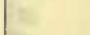 \\
\hline 28 & 32.8 & 37.8 & & 46 & $1=$ & 28 & 30.2 & 30.6 & & 38 & & 28 & 25.8 & 20.1 & 33 & $1=$ & 28 & 18.7 & 22.3 & 22 & 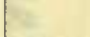 \\
\hline 30 & 37.0 & 38.0 & 7. & 50 & -10.0 & 30 & 32.0 & 37.1 & & 44 & -10.5 & 30 & 24.8 & 28.0 & $3 \mathrm{I}$ & -10.0 & 30 & 18.8 & 23.3 & 22 & -10.4 \\
\hline 32 & 40.0 & 43.0 & 22 & 56 & a & 32 & 31.0 & 35.0 & & 42 & 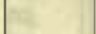 & 32 & $25 . I$ & 28.5 & 32 & ste & 32 & I5.6 & 19.7 & I7 & 1 \\
\hline 34 & 43.6 & 44.8 & 23 & $\infty$ & Iis & 34 & 32.0 & 36.0 & & 44 & 16 & 34 & 27.6 & 30.2 & 35 & 106 & 34 & 15.6 & I9.0 & 17 & 15 \\
\hline 36 & 52.0 & 5.3 .1 & & 1.3 & 2 & 36 & 30.8 & 34.0 & & $4 \mathrm{I}$ & E & 36 & 27.1 & 29.6 & 34 & 12 & 36 & 20.0 & 22.7 & 23 & \\
\hline 38 & 57.1 & 59.0 & & 22 & E. & 38 & 32.1 & 35.0 & & 43 & Eto & 38 & 25.8 & 27.6 & 32 & Iat & 38 & 19.6 & 22.6 & 23 & te \\
\hline 40 & 61.2 & 63.0 & & 28 & 64 & 40 & 38.2 & $4 \mathrm{I} .2$ & & 53 & 10 & 40 & 25.3 & 27.4 & 3I & 10 & 40 & 22.7 & 24.6 & 26 & 51 \\
\hline 42 & 63 & $.9 b$ & & $3 I$ & 6 & 42 & 33.8 & 36.0 & & 45 & 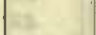 & 42 & 28.3 & 30.5 & 36 & 65 & 42 & 22.5 & $24 \cdot 3$ & 26 & 4 \\
\hline 44 & 62.2 & 64.5 & & 30 & -10.0 & 44 & $3 \mathrm{I} .2$ & 34.1 & & $4 \mathrm{~T}$ & 1 & $\Delta 4$ & 27.3 & 30.6 & 35 & -10.0 & 44 & 22.8 & 25.0 & 27 & $-\mathrm{IO} .5$ \\
\hline 46 & 64.0 & 66.8 & & 33 & 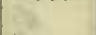 & 46 & 32.5 & 35.2 & & 43 & 0 & 46 & 27.9 & $3 I .3$ & 36 & tis & 46 & 23.3 & 26.5 & 28 & | \\
\hline 48 & 64.1 & 65.1 & & 32 & $\therefore$ & 48 & 37.6 & 39.5 & & $5 \mathrm{I}$ & 11 & 48 & 26.5 & 30.3 & 34 & 80 & 48 & 25.3 & 28.6 & 32 & - \\
\hline 50 & 63.0 & 64.5 & & $3 \mathrm{I}$ & e & 50 & 36.9 & 30.0 & ? & 50 & 皮 & 50 & $24 \cdot 5$ & 28.2 & 31 & vat: & 50 & 25.7 & 28.8 & 32 & 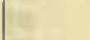 \\
\hline 52 & 61.0 & 62.0 & & 27 & a & 52 & 35.3 & 38.0 & & 48 & 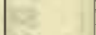 & 52 & 25.1 & 29.1 & 32 & 12 & 52 & 24.3 & 27.1 & 30 & 2 \\
\hline 5.1 & 57.9 & 59.0 & 17 & 22 & 12 & 54 & 31.8 & 33.2 & & $4 \mathrm{r}$ & 2 & 54 & 26.5 & 30.0 & 34 & 10 & 54 & 24.0 & 26.8 & 20 & at \\
\hline 56 & $57 . \mathrm{I}$ & 50.1 & 6 & 22 & 32 & 56 & $3 \mathrm{I} .4$ & 32.6 & 2016 & 40 & 0 & 56 & 28.6 & $3 \mathrm{I} \cdot 3$ & 37 & 2 & 56 & 23.5 & 25.6 & 28 & 파. \\
\hline 58 & 55.6 & 58.2 & 6.: & 20 & a & 58 & 32.5 & 34.0 & 2602 & 42 & 2 & 58 & 27.0 & $3 I .3$ & 37 & 8 & 58 & 22.5 & 25.6 & 27 & $=$ \\
\hline
\end{tabular}

Observers-J. V. and W. J. P., who alternated from 7 h $48 \mathrm{~m}$ to Observer-W. J. P. 7 h $58 \mathrm{~m}$. 
Tabulation of magnetic declinations observed at Teplitz Bay-Continued

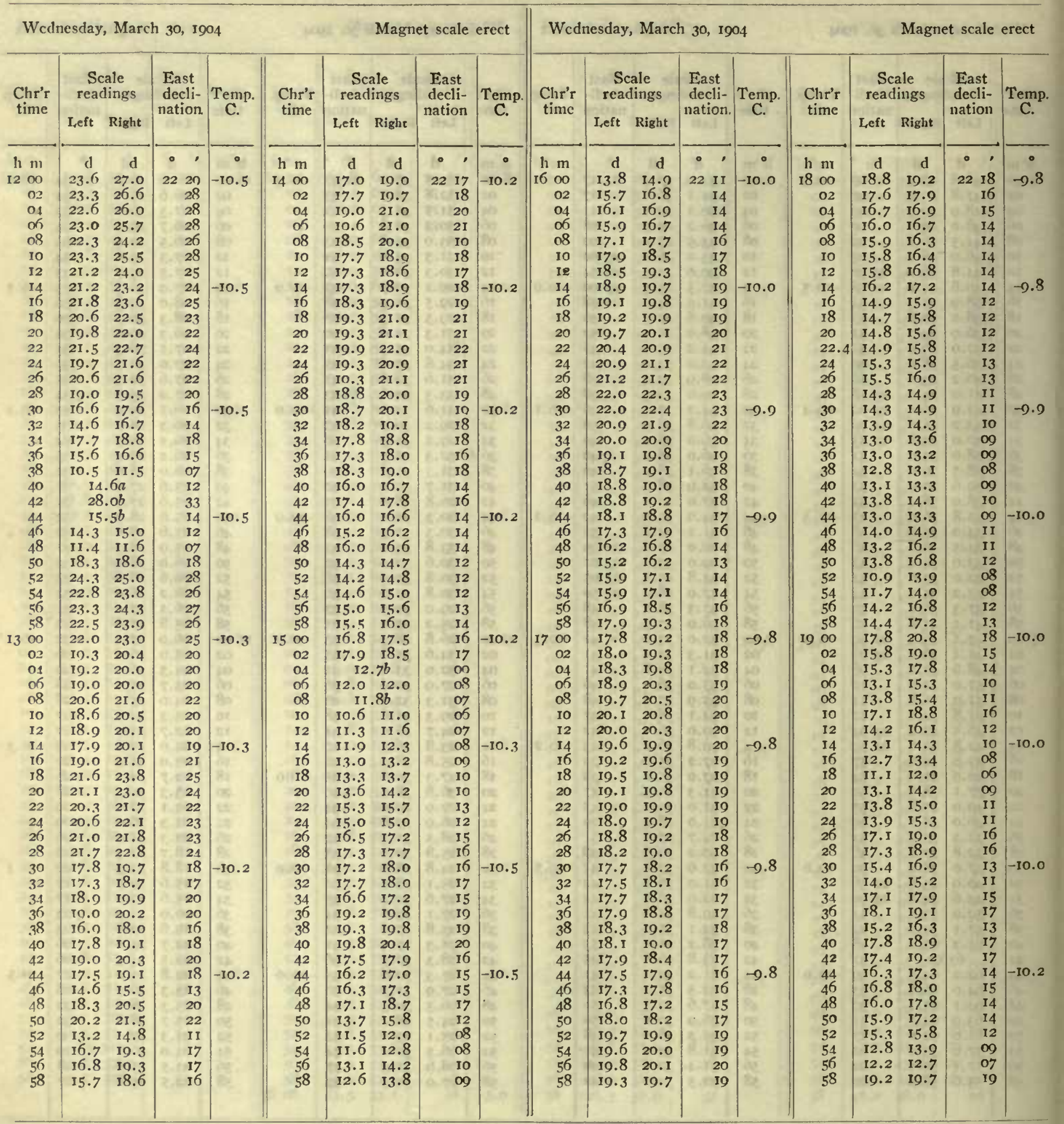

Observers-W. J. P. and R. R. 'T., who alternated from 15 h $36 \mathrm{~m}$ to Observer-R. R. T. I $5 \mathrm{~h} 46 \mathrm{~m}$. 
Tabulation of magnetic declinations observed at Teplitz Bay-Continued

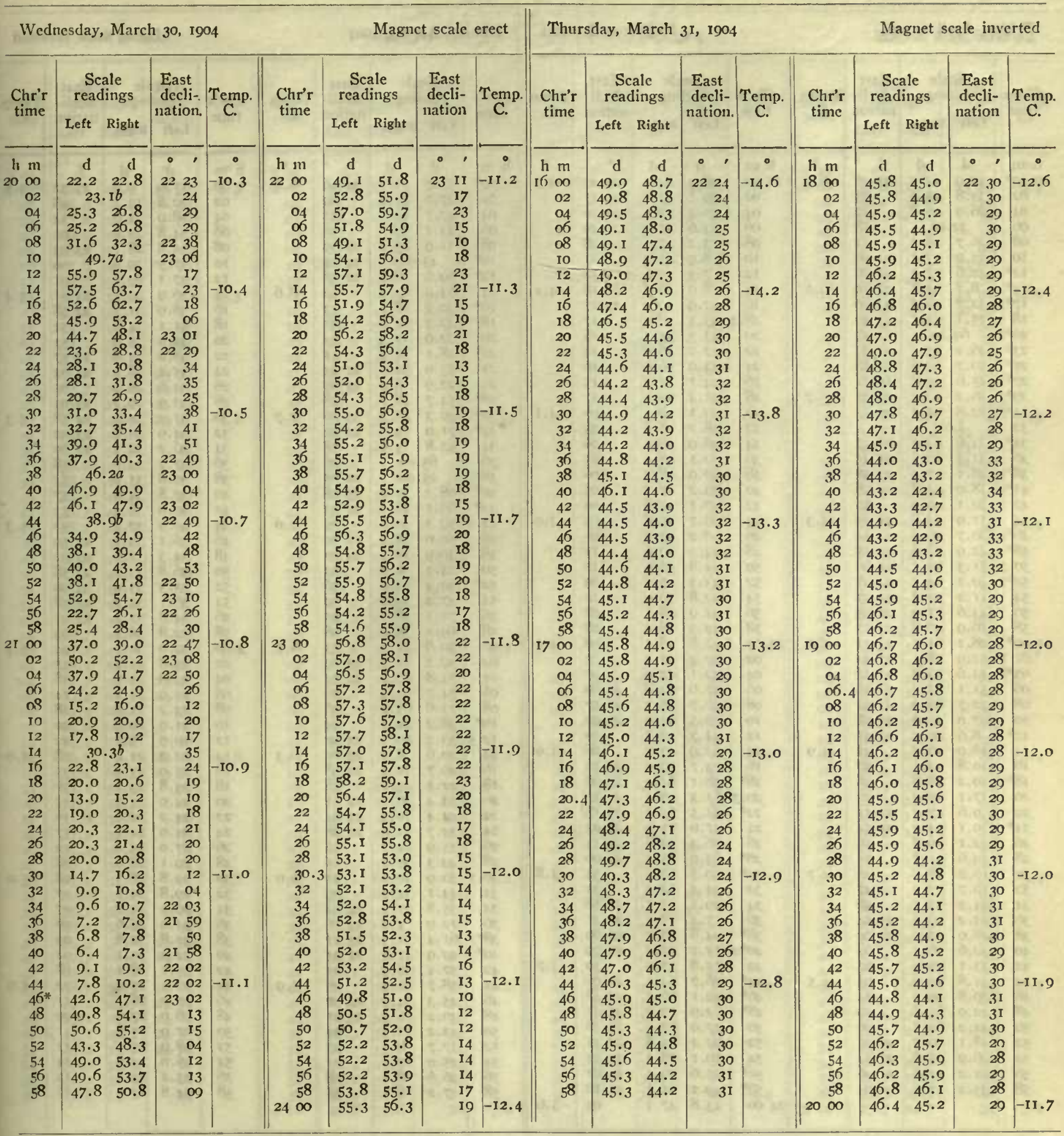

Correction to local mean time is $+\operatorname{IIm} 58.5 \mathrm{~s} .90^{\circ}$ torsion $=$ r6. 68 . Torsion head at oh oom read $278^{\circ}$ and at $24 \mathrm{~h} 20 \mathrm{~m}$ read $304^{\circ}$. Observer-R. R. T.
Correction to local mean time is +43.55 .

Torsion head at $15 \mathrm{~h} 30 \mathrm{~m}$ read $303^{\circ}$ and at $20 \mathrm{~h} \mathrm{r} 5 \mathrm{~m}$ read the same. Observer-R. R. T. 
Tabulation of magnetic declinations observed at Teplitz Bay-Continued

\begin{tabular}{|c|c|c|c|c|c|c|c|c|c|c|c|c|c|c|c|c|c|c|c|c|}
\hline \multicolumn{10}{|c|}{ Friclay, April 1, 1904} & \multicolumn{5}{|c|}{ Sunday, April 3, 1904} & \multicolumn{6}{|c|}{ Magnet scale inverted } \\
\hline \multirow{2}{*}{$\begin{array}{l}\text { Chr'r } \\
\text { time }\end{array}$} & \multicolumn{2}{|c|}{$\begin{array}{l}\text { Scale } \\
\text { readings }\end{array}$} & \multirow{2}{*}{$\begin{array}{c}\text { East } \\
\text { decli- } \\
\text { nation }\end{array}$} & Tenip. & Chr'r & $\begin{array}{r}\text { Sca } \\
\text { readi }\end{array}$ & $\begin{array}{l}\text { ale } \\
\text { lings }\end{array}$ & $\begin{array}{l}\text { East } \\
\text { decli- }\end{array}$ & Ternp. & Clir'r & $\begin{array}{r}\text { Sca } \\
\text { readi }\end{array}$ & $\begin{array}{l}\text { ale } \\
\text { ings }\end{array}$ & $\begin{array}{l}\text { East } \\
\text { decli- }\end{array}$ & & Chr'r & $\begin{array}{r}\text { Sca } \\
\text { readi }\end{array}$ & $\begin{array}{l}\text { ale } \\
\text { ings }\end{array}$ & $\begin{array}{l}\text { Eas! } \\
\text { decli }\end{array}$ & & \\
\hline & Left & Right & & & & I.eft & Right & & & & Left & Right & & & $\mathrm{m}$ & Left & Right & & & \\
\hline$h \mathrm{~m}$ & d & $d$ & $\circ \quad$ & 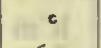 & $\mathrm{h} \mathrm{m}$ & d & & & 0 & h In & d & d & & & $\mathrm{h} \mathrm{m}$ & a & & - & ' & $\circ$ \\
\hline $2000^{*}$ & 42.9 & 43.2 & 2136 & -6.3 & 2200 & 48.3 & $64 \cdot 4$ & 2225 & $-4 \cdot 3$ & $000^{*}$ & 57.8 & 51.7 & 2216 & -8.9 & 200 & 37.9 & 9.8 & 233 & 32 & -5.8 \\
\hline 02 & 34.8 & 35.9 & 24 & the & 02 & 29.1 & 47.2 & $2 \mathrm{I} 57$ & & 02 & 47.9 & 44.8 & 29 & & 02 & 20.8 & 12.1 & & 28 & \\
\hline 04 & 26.9 & 28.8 & & 10 & 04 & 40.4 & 52.9 & 2210 & 10 & 04 & 47.7 & $43 . I$ & $3 I$ & & 04 & 28.9 & 21.0 & & I4 & \\
\hline 06 & 32 & $o b$ & J8 & ty & 06 & 24.6 & $41 \cdot 3$ & 2149 & $x_{1}$ & of & $44 . \mathrm{I}$ & 39.3 & 36 & & 06 & 34.8 & 27.0 & & 05 & \\
\hline 08 & 26.7 & 28.2 & II & 30 & 08 & $\mathrm{I} .3 .2$ & 27.1 & 1. 28 & 6 & o8 & 31.9 & 23.2 & 2259 & 10 & 08 & 35.8 & 28.1 & 230 & 03 & \\
\hline 10 & 33.0 & 33.4 & 2120 & int & I0 & 8.9 & $21 \cdot 3$ & $2 I$ & $\operatorname{cor}$ & $10^{*}$ & 43. & oa & 24 I4 & & 10 & 37.5 & 30.6 & & $\infty$ & \\
\hline $12^{*}$ & 42.6 & 77.9 & $22 \quad 26$ & & 12 & $7 \cdot 3$ & 19.6 & 18 & Wf & $12^{*}$ & 60.6 & 48.8 & 2258 & & 12 & 37.1 & 29.1 & & OI & \\
\hline $14^{*}$ & 8.7 & 52.8 & 2210 & -6.0 & I4 & 8.0 & 19.3 & $\begin{array}{ll}21 & 18\end{array}$ & -4.2 & I 4 & 73.0 & 57.0 & 42 & -8.6 & I4 & 36.9 & 29.7 & 230 & OI & -5.4 \\
\hline $17^{*} 5$ & 32.7 & 56.9 & 2340 & & 16 & 42.9 & 59.0 & $22 \quad 17$ & & $16^{*}$ & 30.7 & 18.2 & 23 & & เ6 & 38.9 & $3 \mathrm{~T} .7$ & 225 & 58 & \\
\hline 18 & 25.1 & $64 \cdot 5$ & 2340 & st: & 18.4 & 43.5 & 58.4 & 17 & 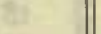 & I8 & 22.2 & II.0 & 35 & & I8 & $4 \mathrm{I} . \mathrm{I}$ & 34.8 & & 54 & \\
\hline $20^{*} 4$ & 69. & Ia & 2145 & Fe & 20 & 43.3 & $57 \cdot 3$ & 16 & at & 20 & 22.9 & II. 3 & 34 & tar & 20 & 40.9 & 35.8 & & 53 & \\
\hline $23^{*}$ & 46.2 & $57 \cdot 3$ & 2221 & $=$ & 22 & 42.1 & 56.0 & 14 & 68 & $22^{*}$ & 48. I & 32.9 & $4 \mathrm{I}$ & & 22 & 38.2 & 32.8 & 225 & 57 & \\
\hline $25^{*}$ & 10.7 & 69.6 & 2258 & fe & 24 & 44.0 & 56.9 & 16 & 15 & 24 & 45.9 & 31.9 & 2243 & & 24 & $.35 . \mathrm{J}$ & 28.3 & 230 & 03 & \\
\hline $27^{*}$ & 15.6 & 49.8 & $195 \mathrm{I}$ & $x^{2}$ & 26 & 44.1 & 55.3 & I5 & $=$ & 25 & 27.9 & 16.9 & 23 on & & 26 & 33.6 & 28.2 & & 05 & \\
\hline $23^{*} 7$ & 16.2 & 22.2 & 2321 & $-5 \cdot 7$ & 28 & $4 \mathrm{~T} .4$ & 52.9 & II & & $28^{*}$ & 54.7 & 48.7 & 48 & & 28 & 3.9 & 29.2 & 230 & 04 & \\
\hline $3 \mathrm{I} * 8$ & I2.8 & 65.0 & 22 I3 & - & 30 & 39.1 & $49 \cdot 4$ & 06 & $-4 \cdot 1$ & $30^{*}$ & $49 \cdot 3$ & 25.3 & 2356 & -7.7 & 30 & 37.2 & 32.4 & 225 & 58 & $-5 \cdot 3$ \\
\hline 32 & Lo & ost & & IIF & 32 & 38.2 & 48.8 & 05 & & $32^{*}$ & 71.0 & 54.7 & 2420 & & 32 & 34.9 & 30.8 & 230 & 02 & \\
\hline $34^{*}$ & 24.6 & 63.2 & 2137 & 12 & 34 & 38.9 & 48.9 & 06 & 15 & 34 & 52.8 & 35.9 & 58 & to & 34 & 36.2 & 32.1 & 226 & 60 & \\
\hline 36 & $3 \mathrm{I} \cdot 3$ & 66.1 & 2145 & X. & 36 & 39.1 & 48.4 & 05 & B. & 36 & 65. & $0 a$ & 26 & & 36 & 37.2 & 32.7 & & 58 & \\
\hline $38 * 2$ & 28. I & 77.3 & 2258 & k & 38 & 40.0 & 48.7 & 06 & s & $3^{8 *}$ & 25. &.$o b$ & 240.5 & 2 & $3^{8}$ & 39.8 & 35.9 & & 54 & \\
\hline $4 r * 6$ & 18.9 & 44.8 & 2138 & 48 & 40 & 39.2 & 47.3 & 05 & $=$ & $40^{*}$ & 68.2 & 31.9 & 25 I8 & 5 & 40 & 37.8 & 34.0 & & 57 & \\
\hline 42 & 30.1 & 49.9 & 50 & $\sqrt{2}$ & 42 & 40.1 & 47.6 & o6 & 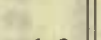 & $43^{*}$ & 60.5 & 46.1 & 2233 & & 42 & 40.0 & 36.4 & & 53 & \\
\hline 44 & II.9 & 32.8 & 2123 & $-5 \cdot 3$ & 44 & 39.7 & 46.8 & 05 & -4.0 & 44.5 & 59.2 & 39.6 & 39 & -7.0 & 44 & 40.9 & 37.2 & & 52 & $-5 \cdot 3$ \\
\hline $46 * 4$ & 20.7 & 56.0 & 2254 & $x^{2}$ & 46 & 40.2 & $47 \cdot 5$ & 06 & & 46 & 50.0 & $35 . \mathrm{I}$ & 2250 & & 46 & 36.1 & 33.1 & 225 & 59 & \\
\hline $48^{*}$ & 42.9 & 72.6 & 2355 & 18 & 48 & 44.3 & 50.9 & 2512 & est & 48 & 30.9 & $15 . \mathrm{J}$ & 2320 & & 48 & 34.3 & 31.6 & 230 & 02 & \\
\hline 50.3 & 51.3 & 70.1 & 2400 & a & 50 & 44.8 & 50.9 & 12 & a & 50 & $24 \cdot 3$ & 6.0 & 3 & & 50 & 36.8 & 33.7 & 225 & 58 & \\
\hline $52^{*} 7$ & 40.1 & 64.5 & 2227 & e & 52 & $43 \cdot 3$ & 49.2 & $\infty$ & ate & $52^{*}$ & 40.4 & 23.0 & 50 & & 52 & 35.3 & 33.0 & 230 & 00 & \\
\hline 54 & 11.2 & 30.2 & 2137 & 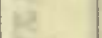 & 54 & 42.8 & 50.0 & то & 12 & 54 & 47.2 & 38.0 & 39 & & 54 & 36.1 & 34.2 & 225 & 58 & \\
\hline 56 & 43.6 & 71.9 & 2235 & $1+2$ & 56 & 41.9 & 48.1 & 08 & 2 & 56 & 70.1 & 58.1 & 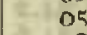 & & 56 & 34.8 & 32.2 & 230 & OI & \\
\hline 58 & I6.9 & 49.8 & 2157 & tat & 58 & 42.3 & 48.2 & 08 & & 58 & 60.1 & 55.0 & 08 & & 58 & 32.8 & 30.2 & 0 & 01 & \\
\hline $2100^{*}$ & 28.1 & $6 \mathrm{I} . \mathrm{J}$ & 23 II & -5.0 & 2300 & 42.8 & 48.5 & 08 & $-4 \cdot 0$ & 100 & 50.8 & $41 \cdot 3$ & 33 & -6.7 & & $32 . n$ & 30.1 & & 04 & -5.1 \\
\hline 02.1 & 52.9 & 78. J & 2344 & $\mathrm{cos}^{2}$ & 02 & $39 \cdot 3$ & 45.2 & 03 & 62 & 02 & 59.2 & $5 \mathrm{r} .7$ & 23 If & & 02 & 25.8 & 23.2 & & 15 & \\
\hline $0.3^{* 8}$ & 44.6 & 73.8 & 2212 & is & 04 & 42.1 & 47.0 & 07 & 10 & 04 & 74.8 & 67.2 & 225 & & 04 & 23.8 & 21.6 & & 18 & \\
\hline 05 & 5 I. I & 73.1 & 2216 & No & 05 & 37.6 & 42.8 & 2200 & 8 & $\sigma_{5}$ & 74.7 & 64.9 & & & 06 & 25.9 & 24.2 & & I 1 & \\
\hline 08 & 32.7 & 60.2 & 2152 & 60 & os & 36.7 & 39.7 & 2157 & 6 & 09 & 71.6 & 65.2 & 55 & & 08 & 23.2 & 21.8 & & 18 & \\
\hline 10 & 27.0 & 49.0 & 21 38 & ar & 10 & 39.5 & 42.9 & 2202 & 61 & $10^{*}$ & 38.2 & 22.7 & 41 & & 10 & 21.9 & 20.8 & & 20 & \\
\hline $12^{*}$ & 8.6 & 40.7 & 2235 & 61 & 12 & 44.7 & 49.1 & 10 & & 12.5 & 33.2 & $20 . \mathrm{I}$ & 2250 & & 12 & 20.9 & 19.2 & & 22 & \\
\hline 14 & 18.0 & 46.7 & 47 & -4.9 & I4 & 55.9 & 57.7 & 26 & -4.0 & 14 & 20.6 & 8.2 & 23 on & -6.3 & 14 & 23.2 & 21.6 & & I8 & -5.0 \\
\hline 16 & 10.8 & 42.1 & 37 & ir & 16 & 73.7 & 76.9 & 2255 & 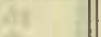 & 16 & 29.7 & 14.2 & 2258 & & 16 & 25 . & 23.5 & & 15 & \\
\hline I8* & 19.2 & 58.8 & 28 & 84 & $18^{*}$ & $58 . \mathrm{I}$ & 61.9 & 24 os & Hit & I8 & 42.5 & 27.0 & 38 & $\mathrm{~s}+\mathrm{s}$ & 18 & 24.8 & 23.2 & & т6 & \\
\hline 20 & 17.1 & 52.9 & 22 & 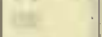 & 20.3 & 61.3 & 70.2 & 2417 & $\pi$ & 20 & 33.4 & 19.0 & $225 \mathrm{I}$ & $x$ & 20 & 24.9 & 23.3 & & 15 & \\
\hline 22 & 9.6 & 47.2 & $22 \mathrm{II}$ & mata & $22^{*}$ & 45.5 & 65.4 & 2258 & Ex & 22 & 24.4 & 10.0 & 2301 & 3 & 22 & 24.2 & 22.8 & & I6 & \\
\hline $24^{*}$ & 19.1 & 54.2 & 2154 & 20 & 24 & 47.0 & 71.0 & 2303 & wh & 24 & 34.7 & 21.6 & 2248 & & 2. & 24.0 & 22.7 & & 17 & \\
\hline 26 & $22 . t$ & 56.8 & 59 & $=$ & 26 & 56.3 & 74.8 & 2314 & ts & 26 & 35.9 & 22.7 & 46 & 7 & 25 & 27.0 & 25.8 & & & \\
\hline 28 & 19.6 & 51.7 & 5.3 & Ex & $28^{*}$ & 63.0 & 75.3 & 2409 & & 28 & 49.3 & 35 . & 2 & & 28 & 29.1 & 28.0 & & 08 & \\
\hline 30 & 16.2 & 48.8 & 48 & $-4 \cdot 7$ & $31^{*}$ & 50.5 & 67.1 & 54 & -3.8 & $30.3^{3}$ & 50.9 & 40.0 & 2 I & -5.0 & 30 & 20.8 & 28.5 & & 08 & -5.0 \\
\hline 32 & 16.7 & 47.4 & 47 & was & 32 & $34 \cdot 3$ & 54.1 & 31 & as. & 32 & $44 . \mathrm{I}$ & 34.6 & 30 & & 32 & 29.8 & 29.2 & & 07 & \\
\hline 34 & 24.2 & 53.3 & 58 & 12 & 34 & 29.1 & $43 \cdot 5$ & .2418 & 15 & 34 & 35.8 & 27.1 & 224 & & 3 & 29.9 & 20.2 & & 07 & \\
\hline 36 & 2.3 .2 & 49.9 & 21 54 & in & 36 & 7.4 & 18.2 & 2341 & & 36 & 12.9 & 7.2 & $23 \mathrm{IC}$ & & 36 & & 28.6 & & 06 & \\
\hline 38. & 28.0 & 52.9 & 2200 & fact & $38^{*}$ & 24.8 & 38.2 & 2309 & $\mathrm{E}$ & $38^{*} ?$ & $6 \mathrm{I} \cdot 4$ & $5 \mathrm{I} .3$ & $23 \mathrm{JI}$ & & 38 & 32.8 & 30.8 & & 03 & \\
\hline 40 & 32.6 & 56.7 & 07 & $\approx$ & $40 * 2$ & 15.2 & 37.5 & 2229 & & $40^{*}$ & 52.9 & 47.8 & 2234 & ite & 40 & 32.0 & $30 . I$ & & & \\
\hline 42 & 33.1 & 55.9 & 07 & 6i: & 42 & 15.8 & 37.9 & 30 & -3.7 & 42 & 57.4 & 46.9 & $3 \mathrm{I}$ & 62 & 42 & 30.1 & 28.1 & & 08 & \\
\hline 44 & 31.8 & 52.0 & 03 & $-4 \cdot 5$ & $44^{*}$ & 46.7 & $64 \cdot 4$ & 22 о6 & 14. & 44 & 58.7 & 49.2 & 29 & -5.9 & 44 & 27.1 & 25.4 . & & 12 & -4.9 \\
\hline 46 & 39.8 & 59. I & 14 & 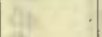 & $46^{*}$ & 49.1 & 66.5 & 2248 & 5 & 46 & 45.3 & 34.9 & 2250 & & 46 & 27.7 & 26.1 & & II & \\
\hline 48 & 42.9 & 61.8 & I9 & tet & $4^{*}$ & 62.9 & 73.8 & 2345 & A4 & 48 & 40.2 & 26.9 & 23 or & 8tis & 48 & 28.9 & 27.0 & & $\infty$ & \\
\hline 50 & 44.2 & $62 . I$ & 20 & क्ष & 50 & 47.9 & 67.1 & 2328 & 2 & 50 & 2.3 .2 & IA. I & 24 & se & 50 & 27.9 & 25.7 & 9 & II & \\
\hline 52 & 48.1 & 66.7 & 2227 & a & 52 & 22.2 & 35.8 & 2244 & $y$ & 52 & 17.7 & 8.5 & 33 & 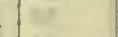 & 52 & 28.0 & 25.9 & & II & \\
\hline & 26.9 & 45.6 & 2I 54 & 10 & 54 & 70.1 & 78.2 & 2354 & 12 & 54 & 25.0 & I5.8 & 21 & 42 & 54 & 26.0 & 23.9 & 마요 & I4 & \\
\hline 56 & 21.2 & 41.8 & 2146 & E: & $56^{*}$ & 25.6 & 39.4 & 2407 & $=$ & 56 & 26.6 & I8. I & 18 & $y=$ & 56 & 25.2 & 23.2 & a & 15 & \\
\hline 58 & 33.2 & 51.7 & 2204 & 2 & $5^{8 *}$ & $\begin{array}{l}49.2 \\
43.8\end{array}$ & $\begin{array}{l}6 \pi \cdot 3 \\
47 \cdot 2\end{array}$ & $\begin{array}{ll}25 & 18 \\
03\end{array}$ & -3.6 & 58 & I7. 2 & 8.1 & 33 & Ale is & 58 & 24.8 & 23.0 & 8. & 16 & \\
\hline & & 9.20 & $17-81$ & $(00) 0$ & 2400 & 43.0 & 47.2 & 03 & & & & tow & & tos & & & & & & \\
\hline
\end{tabular}

Correction to local mean time is - $7 \mathrm{~s}$.

Observer-R. R. T.

Torsion head at $\mathrm{Igh} 30 \mathrm{~m}$ read $332^{\circ}$ and at $24 \mathrm{~h}$ oom read the same.

Observer-R. R. T. 
Tabulation of magnetic declinations observed at Teplitz Bay-Continued

\begin{tabular}{|c|c|c|c|c|c|c|c|c|c|c|c|c|c|c|c|c|c|c|c|}
\hline \multicolumn{6}{|c|}{ Sunday, April 3, 1904} & \multicolumn{4}{|c|}{ Magnet scale erect } & \multicolumn{6}{|c|}{ Monday, April 4, I904 } & \multicolumn{4}{|c|}{ Magnet scale inverted } \\
\hline \multirow{2}{*}{$\begin{array}{l}\text { Chr'r } \\
\text { time }\end{array}$} & \multirow{2}{*}{\multicolumn{2}{|c|}{$\begin{array}{c}\text { Scale } \\
\text { readings } \\
\text { L,eft Right }\end{array}$}} & \multirow{2}{*}{$\begin{array}{l}\text { East } \\
\text { decli- } \\
\text { nation }\end{array}$} & & & & $\begin{array}{l}\text { ale } \\
\text { ings }\end{array}$ & $\begin{array}{l}\text { East } \\
\text { decli- }\end{array}$ & & & $\begin{array}{r}\mathrm{Sc} \\
\text { reac }\end{array}$ & ngs & $\begin{array}{l}\text { East } \\
\text { decli- }\end{array}$ & & & $\begin{array}{r}\mathrm{Sc} \\
\text { read }\end{array}$ & ngs & $\begin{array}{l}\text { East } \\
\text { decli- }\end{array}$ & \\
\hline & & & & & & I,eft & Right & ation & & & Left & Right & & & & I,eft & Right & & \\
\hline & d & d & & $\circ$ & $\mathrm{l} \mathrm{m}$ & d & d & & $\bullet$ & $\mathrm{h} \mathrm{m}$ & d & d & $\circ \quad$ & 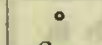 & $\mathrm{h} \mathrm{m}$ & d & d & $\circ$, & $\bullet$ \\
\hline $400 * 6$ & 48.1 & 49.6 & 233 & $-4 \cdot 4$ & 600 & 49.0 & 55.8 & 2407 & $-3 \cdot 3$ & $800^{*}$ & 56.0 & 53.7 & 2343 & -8.7 & 1000 & 35.0 & 34.1 & 2325 & $-4 \cdot 4$ \\
\hline 02 & 48.9 & 50.4 & & T & 02 & 53.2 & 61.2 & 14 & & 02 & 61.2 & 55.3 & 38 & & 02 & 38.2 & 35.8 & $2 \mathrm{I}$ & +8 \\
\hline 04 & 47.3 & 50.8 & 3 & & 04 & 48.5 & 55.9 & 07 & & 04 & 50.2 & 47.1 & 53 & wi & 04 & $45 . I$ & 43.1 & 10 & wial \\
\hline 06 & 49.2 & 51.8 & 4 & & 06 & 60.4 & 67.2 & 25 & & 06 & 52.3 & 48.1 & $5 \mathrm{I}$ & 160 & 06 & $4 \pi \cdot 7$ & 41.0 & 14 & in: \\
\hline 08 & 48.2 & 51.1 & 4 & & 08 & 63.6 & 74.9 & 33 & & 08 & 45.9 & 43.2 & 59 & $(64)$ & 08 & 46.1 & 44.3 & & be \\
\hline то & 47.6 & 50.6 & 3 & & I0 & 62.2 & 69.9 & 28 & 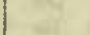 & Io & $54 . \mathrm{I}$ & 50.6 & 47 & & Io & $47 . I$ & 43.9 & 2308 & or \\
\hline 12 & 50.3 & 53.9 & 4 & & 12 & 57.3 & 64.1 & 20 & & 12.4 & 63.8 & 60.3 & 32 & & 12 & 52.8 & 49.9 & 2258 & \\
\hline I4 & 51.8 & 54.9 & 4 & $-4 \cdot 3$ & 14 & 57.8 & 65.9 & 2422 & $-3 \cdot 3$ & I4 & 61.9 & 57.2 & 36 & -8.0 & 14 & 52.0 & 47.8 & 23 or & -4.0 \\
\hline 16 & 50.8 & 54.2 & 4 & & 16 & 37.9 & 45.2 & 2350 & & 16 & 53.2 & 49.9 & 48 & int & 16 & 52.5 & 49.5 & 2259 & 5 \\
\hline 18 & 55.2 & 58.3 & 5 & & 18 & 28. I & 36.0 & 35 & In & 18 & 66.7 & 62.0 & 28 & ins & 18 & 57.4 & 53.0 & 52 & 87 \\
\hline 20 & 56.2 & 59.2 & 5 & & 20 & 22.9 & 30.5 & 26 & & 20 & 63.1 & 59.9 & 33 & $=$ & 20 & $55 . \mathrm{I}$ & 51.7 & 55 & es. \\
\hline 22 & 59.1 & 61.7 & 5 & & 22 & 27.2 & 32.7 & 32 & $=$ & 22 & 65.2 & 59.2 & 32 & 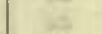 & 22 & 57.2 & 55.0 & $5 I$ & 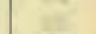 \\
\hline 24 & 61.0 & 62.8 & 235 & & 24 & 37.2 & 41.0 & 46 & & 24 & 68.4 & 61.0 & 28 & Ex & 24 & 56.3 & 53.8 & 53 & \\
\hline 20 & 69.7 & 70.8 & $24 I$ & 3 & 26 & 29.0 & 33.9 & 34 & $x^{2}$ & $26^{*}$ & 53.9 & 46.1 & OI & in & 26 & 57.7 & 55.6 & 50 & ix \\
\hline 28 & 73.6 & 75.2 & 24 I & & 28 & 28.9 & 33.3 & 34 & & 28 & 45.4 & 38.9 & 13 & & 28 & 54.0 & 52.9 & 55 & \\
\hline 30 & 41.8 & 46.9 & 233 & -4.2 & $3 i$ & 33.1 & 38.0 & 40 & $-3 \cdot 3$ & 30 & 44.2 & 36.9 & 16 & -7.2 & 30 & 51.2 & 50.7 & 59 & -3.9 \\
\hline 32 & 49.2 & 54.2 & 4 & & 32 & 27.0 & 30.8 & 30 & & 32 & 43.7 & 37.5 & 15 & (x) & 32 & 51.8 & 50.6 & 2259 & \\
\hline 34 & 47.3 & 52.8 & 4 & & 34 & 20.3 & 24.3 & 20 & & 34 & 51.8 & $45 . \mathrm{I}$ & 03 & ac & 3 & $49 . I$ & 48.3 & 2303 & - \\
\hline 36 & 38.6 & 46.4 & 2 & Fis & 36.7 & 22.0 & 25.9 & 22 & 10 & 36 & 45.9 & 39.3 & I2 & 5 & $3 t$ & 53.8 & 52.8 & 2255 & Fe. \\
\hline 38 & 38.9 & 44.3 & 2 & tr. & 38 & 21.3 & $25 . I$ & 2 I & 2 & 38 & 44.9 & 39.4 & 13 & 2 & 38 & 59.1 & $58 . I$ & 47 & 2 \\
\hline 40 & 38.7 & 44.8 & 2 & & 40 & 27.9 & 33.3 & 33 & & 40 & 36.7 & 33.1 & 24 & (10 & 40 & 63.7 & 62.0 & 40 & a \\
\hline 42 & 38.3 & 46.2 & 2 & & 42 & 35.2 & 37.9 & 42 & & 42 & 42.3 & 37.8 & 16 & & 42 & 67.7 & 66.7 & 34 & \\
\hline 44 & 38.2 & 44.6 & 2 & -4.0 & 44 & 16.3 & 19.6 & I3 & $-3 \cdot 3$ & 44 & 38.3 & 35.6 & $2 \mathrm{I}$ & -6.7 & 44 & 67.3 & 65.2 & 35 & -3.9 \\
\hline 46 & 31.1 & 39.2 & I & & 46 & 22.7 & 23.8 & 2 I & & 46 & 33.2 & 28.3 & $3 \mathrm{I}$ & 18 & 46 & 71.0 & 68.4 & 30 & 10 \\
\hline 48 & 35.9 & 44.8 & 2 & . & 48 & I9. I & 21.8 & 2317 & 12 & 48 & 34.3 & 30.2 & 28 & 8 & 48 & 69.8 & $68 . i$ & 31 & $x^{2}$ \\
\hline 50 & 37.5 & 46.9 & 2 & & $50^{*}$ & 27.7 & 36.2 & 2253 & & 50 & 33.2 & 29.8 & 30 & 02 & 50 & 69.9 & 69.0 & 30 & or \\
\hline 52 & 35.6 & 44.1 & 2 & 3 & 52 & 39. I & 43.8 & 2308 & ate & $5^{2}$ & 31.8 & $27 \cdot 3$ & 33 & 6. & 52 & 71.0 & 69.8 & 29 & at \\
\hline 54 & 36.7 & 42.8 & 2 & & 54 & 47.2 & 53.3 & 2322 & & 54 & 36.3 & $33 \cdot 3$ & 24 & +6 & 54 & 73.0 & 72.3 & 25 & 왕 \\
\hline 56 & 42.8 & 50.5 & 3 & & 56 & 24.2 & 27.8 & 2243 & 6 & 56 & 41.2 & 35.5 & 19 & ia & 56 & 69.8 & 69.3 & 30 & wit \\
\hline $5^{8}$ & 38.7 & 46.8 & 2 & & $5^{8}$ & 17.8 & 21.8 & 2234 & & $5^{8}$ & $34 \cdot 3$ & 31.8 & 27 & & 58 & 70 & $8 a$ & 28 & \\
\hline 500 & 37.2 & 45.7 & 2 & -4.0 & 700 & 45.8 & $47 \cdot 3$ & 2316 & -3.2 & 900 & 48. I & 44.1 & 2307 & -6.0 & II $O O^{*}$ & 40.9 & 3I.I & I4 & -3.7 \\
\hline 02 & 46.2 & 52.9 & 3 & & 02 & 43.3 & 48.0 & 14 & & 02 & 55.8 & 53.1 & 2254 & (63) & 02 & 38.8 & 29.3 & 17 & \\
\hline 04 & 51.7 & 59.8 & 234 & & 04 & 49.0 & 53.0 & 23 & a. & 04 & 62.6 & 59.8 & 43 & at & 04 & $35 \cdot 3$ & 27.9 & $2 \mathrm{I}$ & 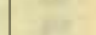 \\
\hline 06 & 59.4 & 67.1 & 240 & & 06 & 55.6 & 57.9 & 32 & & 06 & 52.2 & 50.0 & 59 & 69 & 06 & 36.4 & 28. & 20 & ac \\
\hline 08 & 62.3 & 69.2 & 0 & - & os & 58.7 & $62 . I$ & 37 & & 08 & 56.3 & $53 . \mathrm{I}$ & 53 & 30 & 08 & 36.2 & 28. I & 20 & a \\
\hline I0 & 64.0 & 68.9 & 0 & & IO & 58.2 & 61.0 & 36 & & I0 & 58.8 & 55.3 & 2250 & (a) & IO & 38.8 & 28. I & I8 & \\
\hline I2 & 70.1 & 75.8 & I & & I2 & 58.6 & 60.9 & 36 & & I3 & 49.3 & 48.2 & 2303 & & 12 & 35.1 & 25.4 & 23 & \\
\hline 14 & 66.1 & 72.1 & 24 I & -4.0 & 14 & 53.5 & $57 . \mathrm{I}$ & 30 & -3.2 & I4 & 54.2 & 50.0 & 2257 & -5.8 & 14 & 32.9 & 21.7 & 27 & -3.4 \\
\hline 16 & $53 . \mathrm{I}$ & 60.7 & 235 & ( & 16 & $49 . \overline{8}$ & 52.8 & 23 & & 16 & 62.9 & 60.9 & 42 & (n) & 16 & 29.9 & 20.0 & $3 I$ & ( \\
\hline I8 & 43.1 & 50.9 & 3 & & 18 & 57.6 & 63.9 & 38 & & 18 & 67.0 & 64.3 & 36 & 28 & I8 & 28.4 & 17.7 & 34 & \\
\hline 20 & 37.7 & 44.3 & 2 & s & 20 & 52.8 & 52.8 & 26 & & 20 & 62.3 & 61.9 & 42 & 3 & 20 & 22.0 & 12.8 & 43 & $x^{2}$ \\
\hline 22 & 31.8 & 37.8 & I & wet & 22 & 40.3 & $44 . \mathrm{I}$ & 09 & s. & 22 & 53.3 & 50.2 & 58 & is & 22 & 20.9 & 12.0 & 45 & \\
\hline 24.6 & 27.2 & 32.7 & 0 & & 24 & 54.6 & 59.8 & 32 & & 24.6 & 57.9 & 54.4 & $5 I$ & ia & 24 & 22.2 & I3. I & 43 & aty \\
\hline 26 & 35.4 & 40.8 & 2 & & 26 & 47.1 & 48.9 & I8 & ta & 26 & 53.0 & 49.7 & 2258 & $2 x$ & 2 & 21.7 & 13.8 & 42 & \\
\hline 28 & 30.3 & 36.2 & I & & 28 & 53.9 & 55.2 & 28 & & 28 & 51.8 & 48.7 & 2300 & -1 & 28 & 18.9 & II. 3 & 46 & \\
\hline 30 & 25.1 & 31.8 & 0 & -3.8 & 30 & $64 . I$ & 67.2 & 46 & $-3 . I$ & 30 & 48.3 & 44.9 & 06 & -5.2 & 30 & 23.2 & I5.9 & 40 & -3.4 \\
\hline 32 & 29.0 & 33.2 & I & & 32 & $55 \cdot 3$ & 62.2 & 35 & & 32 & 49.7 & 46.8 & 2303 & s. & 32 & I9.8 & $13 \cdot 3$ & 44 & \\
\hline 34 & $35 . \mathrm{I}$ & 38.4 & I & & 34 & 42.2 & 46.9 & I3 & 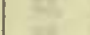 & 34 & 52.2 & 50.0 & 2259 & . & 34 & 16.3 & 12.3 & 48 & 3 \\
\hline 36 & 48. I & 51.2 & 4 & & 36 & 59.3 & 62.4 & $3^{8}$ & & 36 & 49.6 & 46.5 & 2304 & 12 & 36 & 23.4 & 20.0 & 36 & \\
\hline 38 & 54.3 & 58.8 & 235 & & 38 & 62.9 & 67.8 & 45 & & 38 & 43.0 & 40.9 & 13 & (6) & $3^{8}$ & 23.3 & 20.9 & 36 & Fe \\
\hline 40 & 59.7 & 66.0 & 240 & & 40 & 36.0 & 37.2 & 00 & & 40 & 42.1 & 40.2 & 14 & & 40 & 23.4 & $2 \mathrm{I} . \mathrm{I}$ & 35 & \\
\hline 42 & 63.6 & 67.2 & 0 & & 42 & 38. I & 44.5 & 07 & & 42 & 43.4 & 41.0 & 13 & & 42 & 26.9 & 24.6 & 30 & \\
\hline & 68.7 & 72.5 & 24 I & -3.7 & 44 & 44.7 & 50.0 & I7 & -3.0 & 44 & 42.2 & 39.9 & 15 & -5.0 & 44 & $24 . I$ & 22.8 & 34 & $-3 \cdot 3$ \\
\hline $46^{*}$ & 49.2 & 49.7 & $25 \mathrm{I}$ & & 46 & 67.2 & 72.1 & 52 & 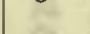 & 46 & 40.9 & 39.4 & 16 & 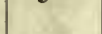 & 46 & 20.4 & 18.9 & 40 & . \\
\hline $48^{*}$ & 23.1 & 24.0 & 232 & & 48 & 58.3 & 59.9 & 35 & the & 48 & 38.9 & 37.3 & 19 & 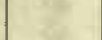 & 48 & 24.9 & $23 \cdot 9$ & 32 & 2 \\
\hline 50.6 & $4 I . I$ & 48. I & 235 & $t_{-1}$ & 50 & 56.3 & 59.8 & 34 & se & 50 & 39.3 & 38.0 & 18 & $\alpha$ & 50 & 26.2 & 25.8 & 30 & tat \\
\hline 53 & 45.4 & 53.2 & 240 & & 52 & 50.2 & 53.7 & 24 & & 32 & 39.9 & 38.0 & 18 & & 52 & 23.8 & 23.6 & 33 & \\
\hline 54 & 50.0 & 55.3 & 0 & 6 & 54 & 48.4 & 51.8 & $2 \mathrm{I}$ & & 54 & 37.6 & 36. I & $2 \mathrm{I}$ & . & 54 & 22.8 & 20.7 & 36 & $=$ \\
\hline 56 & 46.2 & 50.9 & 0 & 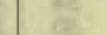 & 56 & 49.2 & 58.2 & 27 & & 56 & 37.6 & 36.1 & 21 & & 56 & 22.1 & 19.3 & 38 & \\
\hline 58 & 47.8 & 54.1 & & 8 & 58 & 47.2 & 52.9 & $2 \mathrm{I}$ & & 58 & 35.0 & 34.3 & 25 & -4.4 & 58 & $24 \cdot 3$ & 19.1 & 36 & tex \\
\hline & & & & & 800 & 49.8 & 55.8 & 26 & -3.0 & 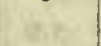 & & & & & 1200 & 25.7 & $2 \mathrm{I} . \mathrm{I}$ & 34 & -3.1 \\
\hline
\end{tabular}

Correction to local mean time is +64 s.

Torsion head at oh oom read $352^{\circ}$ and at gh oom read the same. Observer-R. R. T.
Correction to local mean time is $-\operatorname{Im} 20.5 \mathrm{~s}, 90^{\circ}$ torsion $=\mathrm{I} 6 .^{\prime} \mathrm{oo}$. Torsion head at $8 \mathrm{~h} 35 \mathrm{~m}$ read $355^{\circ}$ and at $12 \mathrm{~h} 25 \mathrm{~m}$ read $360^{\circ}$. Observer-R. R. T. 
Tabulation of magnetic declinations observed at Teplitz Bay-Continued

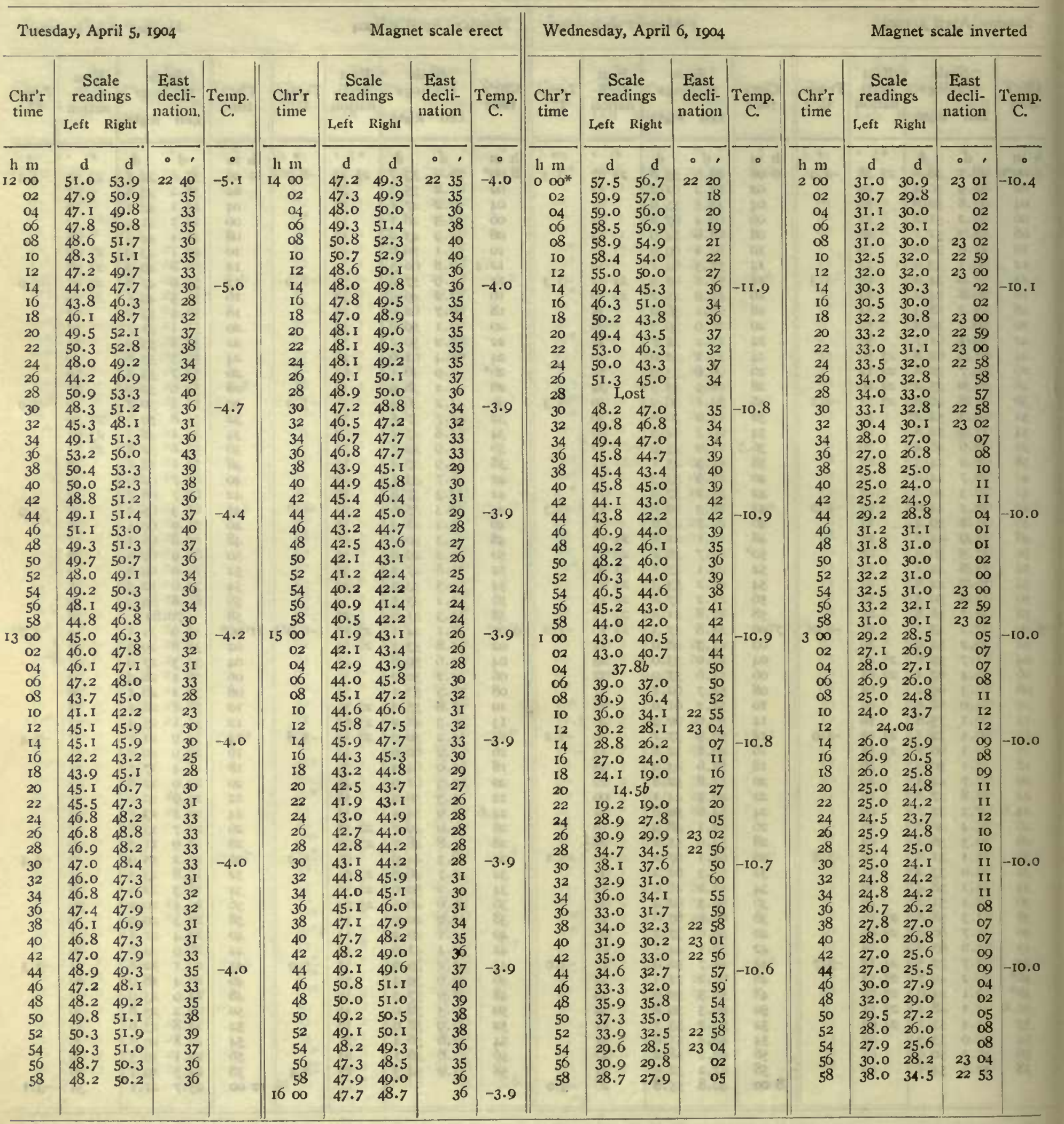

Correction to local mean time is - Im 31.5s. $90^{\circ}$ torsion $=133^{\prime} 78$.

Observer-J. V.

Torsion head at IIh $20 \mathrm{~m}$ read $352^{\circ}$ and at I $6 \mathrm{~h}$ zom read $33 \mathrm{I}^{\circ}$.

Observer-R. R. T. 
Tabulation of magnetic declinations observed at T'eplitz Bay-Continued

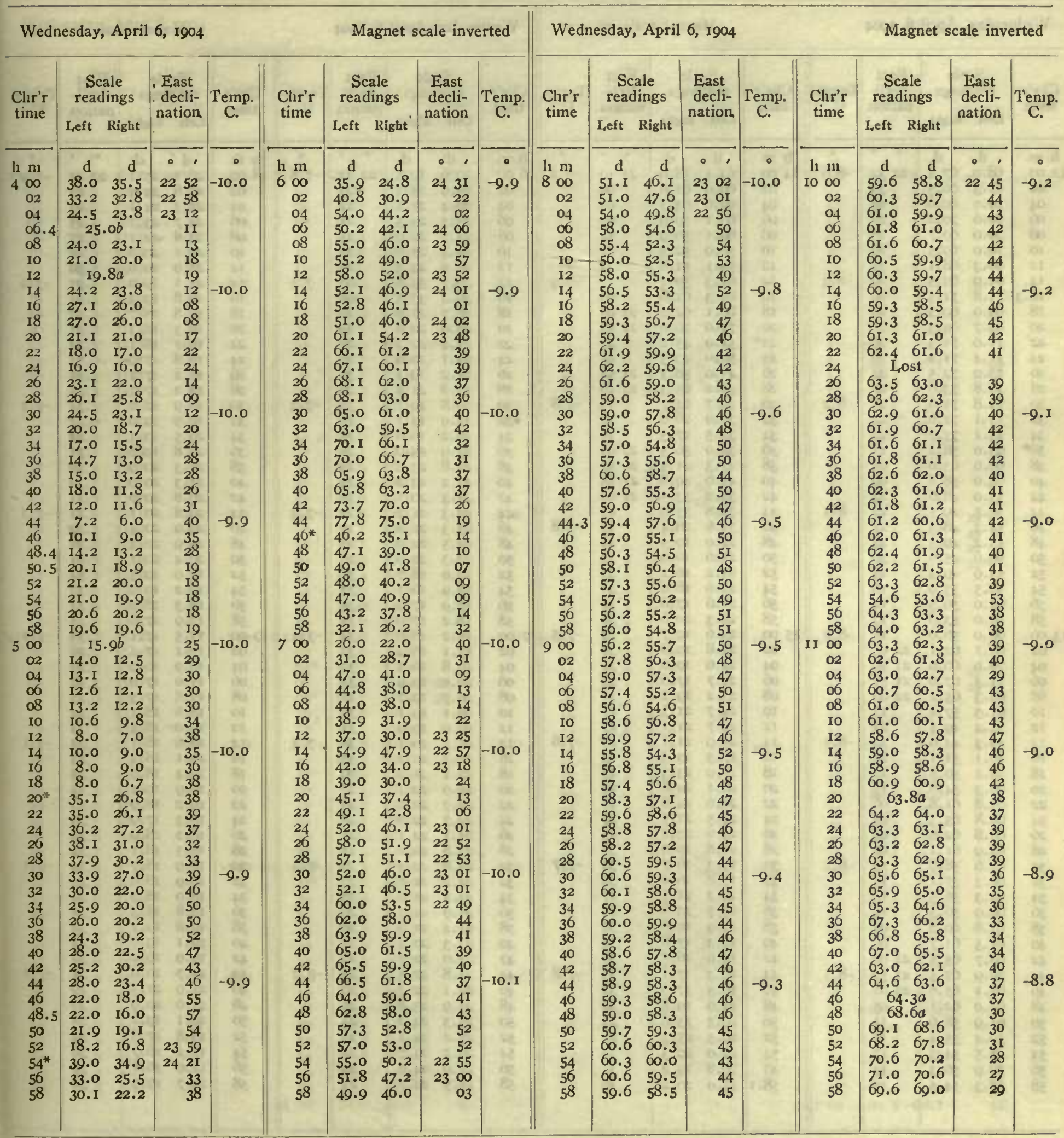

Observers-J. V. and W. J. P., who alternated from 7 h $56 \mathrm{~m}$ to

Observer-W. J. P. 8 horm. 
Tabulation of magnetic declinations observed at Teplitz Bay-Continued

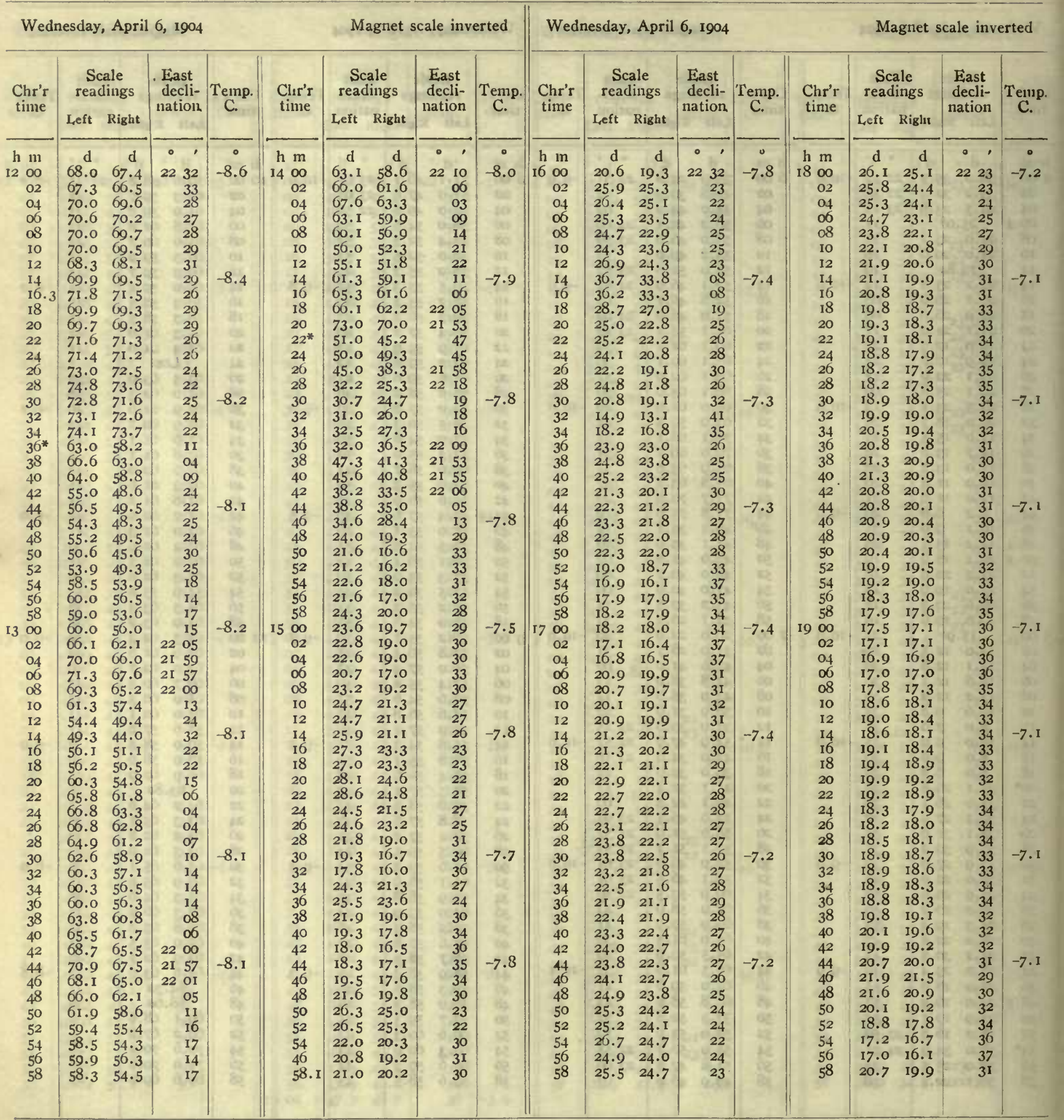

Observers-W. J. P. and R. R. T., who alternated from ish $56 \mathrm{~m}$ to Observer $\rightarrow$ R. R. T. $16 \mathrm{~h} 06 \mathrm{~m}$. 
Tabulation of magnetic declinations observed at Teplitz Bay-Continued

\begin{tabular}{|c|c|c|c|c|c|c|c|c|c|c|c|c|c|c|c|c|c|c|c|}
\hline \multicolumn{6}{|c|}{ Wednesday, April 6, 1904} & \multicolumn{4}{|c|}{ Magnet scale inverted } & \multicolumn{6}{|c|}{ Thursday, April 7, 1904} & \multicolumn{4}{|c|}{ Magnet scale erect } \\
\hline \multirow{2}{*}{$\begin{array}{l}\text { Chr'r } \\
\text { time }\end{array}$} & \multicolumn{2}{|c|}{$\begin{array}{l}\text { Scale } \\
\text { readings }\end{array}$} & \multirow{2}{*}{$\begin{array}{c}\text { East } \\
\text { decli- } \\
\text { nation }\end{array}$} & Temp. & Chr'r & $\begin{array}{r}S c \\
\text { read }\end{array}$ & $\begin{array}{l}\text { fings } \\
\text { lings }\end{array}$ & $\begin{array}{c}\text { East } \\
\text { decli- } \\
\text { nation }\end{array}$ & Temp. & Chr'r & $\begin{array}{l}\text { Sca } \\
\text { read }\end{array}$ & $\begin{array}{l}\text { ale } \\
\text { lings }\end{array}$ & East & Temp. & & $\begin{array}{r}\text { Sca } \\
\text { readi }\end{array}$ & $\begin{array}{l}\text { ale } \\
\text { lings }\end{array}$ & $\begin{array}{l}\text { East } \\
\text { decli- }\end{array}$ & \\
\hline & Left & Right & & & & Left & Right & & & & Left & Right & & & & Left & Pin & & \\
\hline $\begin{array}{r}\text { h m } \\
2000\end{array}$ & d & d & & $\stackrel{7}{\circ}$ & $\mathrm{h} \mathrm{m}$ & d & $\mathrm{d}$ & $\circ \quad$, & 0 & h n & d & d & & & h $\mathbf{m}$ & d & d & $\cdot \cdot$ & \\
\hline $\begin{array}{r}2000 \\
02\end{array}$ & $\begin{array}{l}27.0 \\
20.9\end{array}$ & $\begin{array}{l}26.7 \\
20.2\end{array}$ & 2221 & -7.1 & & 27.1 & 26.8 & 2221 & -7.1 & $16 \mathrm{oO}^{*}$ & 49.9 & 50.5 & & - II.9 & 1800 & 60.4 & 65.9 & $22 \quad 19$ & -10.2 \\
\hline $\begin{array}{l}02 \\
04\end{array}$ & $\begin{array}{l}20.9 \\
19.9\end{array}$ & $\begin{array}{l}20.2 \\
19.1\end{array}$ & $\begin{array}{l}31 \\
32\end{array}$ & tar & 02 & 27.2 & 27.0 & 20 & & 02 & 53.8 & 54.0 & o6 & & 02 & 59.4 & 65.0 & 17 & \\
\hline $\begin{array}{l}04 \\
06\end{array}$ & $\begin{array}{l}19.9 \\
19.2\end{array}$ & $\begin{array}{l}19.1 \\
18.9\end{array}$ & $\begin{array}{l}32 \\
33\end{array}$ & 10 & 04 & 27.1 & 27.1 & 20 & & a4 & 53.0 & 54.0 & & & 04 & 59.0 & 64.8 & 17 & \\
\hline $\begin{array}{l}\infty \\
\infty 8\end{array}$ & $\begin{array}{l}19.2 \\
17.9\end{array}$ & $\begin{array}{l}18.9 \\
17.3\end{array}$ & $\begin{array}{l}33 \\
35\end{array}$ & N & $\infty 6$ & 26.9 & 26.9 & $\begin{array}{l}21 \\
22\end{array}$ & 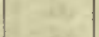 & 06 & 53.8 & 59.8 & II & 6 & 06 & 57.8 & 63.9 & 15 & 60 \\
\hline & 16.9 & $\begin{array}{l}17.3 \\
16.2\end{array}$ & $\begin{array}{l}35 \\
37\end{array}$ & sert & 08 & 26.2 & 26.1 & $\begin{array}{l}22 \\
22\end{array}$ & $=$ & 08 & 55.5 & 60.2 & 12 & 50 & 08 & 55.0 & 60.3 & 10 & is \\
\hline $\begin{array}{l}10 \\
12\end{array}$ & 16.4 & 15.9 & $\begin{array}{l}37 \\
38\end{array}$ & ais & I0 & 25.9 & 25.5 & $\begin{array}{l}22 \\
23\end{array}$ & tint & 10 & 56.0 & 59.8 & 12 & (4) & Io & 56.6 & 61.2 & 12 & if \\
\hline $\begin{array}{l}12 \\
14\end{array}$ & $\begin{array}{l}16.4 \\
\end{array}$ & $\begin{array}{l}13.9 \\
15.9\end{array}$ & $\begin{array}{l}38 \\
38\end{array}$ & 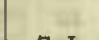 & I2 & 25.6 & 25.0 & $\begin{array}{l}23 \\
24\end{array}$ & & 12 & 52.5 & 60.0 & IO & 10 & 12 & 58.0 & 63.1 & I5 & T. \\
\hline $\begin{array}{l}14 \\
16\end{array}$ & 16.4 & 15.9 & $\begin{array}{l}38 \\
38\end{array}$ & -7.1 & 14 & 25.0 & $24 \cdot 5$ & $\begin{array}{l}24 \\
26\end{array}$ & -7.1 & I4 & 52.0 & $58 \cdot 3$ & 08 & -11.9 & I4 & 57.4 & 62.0 & 13 & -10.1 \\
\hline 16 & 10.3 & 25.9 & 38 & 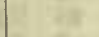 & 16 & 23.9 & $23 \cdot 3$ & 26 & & 16 & 52.0 & 58.9 & 08 & & 16 & 56.3 & 61.0 & 12 & \\
\hline I8 & I6.2 & 15.8 & 38 & sit & 18 & 23.0 & 22.3 & 27 & 14 & 18 & 53.0 & 59 & $\alpha$ & at & I8 & & 58.9 & 08 & $=$ \\
\hline 20 & I6.2 & 15.3 & 38 & in & 20 & 22.5 & 21.9 & 28 & L & 20 & 50.8 & 56.0 & 05 & as & 20 & 53.0 & 58.0 & 07 & 10 \\
\hline 22 & I6. 3 & 15.8 & 38 & $=2$ & 22 & 22.9 & 22.1 & 27 & $=$ & 22 & 53.8 & $59 \cdot 3$ & 22 IO & 15 & & & 59.8 & I0 & 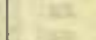 \\
\hline 24 & 17.0 & 16.1 & 37 & (1) & 24 & 23.2 & 23.0 & 26 & 64 & 24 & 47.0 & 49.0 & 2157 & $\pi$ & 24 & .4 & 58.3 & 08 & $E$ \\
\hline 26 & I6. 4 & I5.8 & 38 & 24 & 26 & 24.2 & 23.8 & 25 & $x$ & 26 & 51.0 & 55.0 & 2204 & & & & 59.0 & 08 & $=$ \\
\hline 28 & 15.3 & 14.9 & 39 & $2=$ & 28 & 25.5 & 25.1 & 23 & es & 28 & 55.0 & 59.8 & II & & 28 & & 59.8 & I0 & \\
\hline 30 & 14.3 & I3.9 & $4 \mathrm{I}$ & -7.0 & 30 & 27.1 & 26.3 & 21 & -7.1 & 30 & 55.2 & 59.2 & II & I -11.5 & 30 & & 59.9 & Io & IO. I \\
\hline 32 & 5.0 & I4.1 & 40 & (n) & 32 & 27.1 & 26.1 & 21 & 3 & 32 & 55.2 & 58.2 & IC & & 32 & 55 & 59.9 & Io & 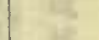 \\
\hline 34 & 16.0 & I5. 4 & 38 & IS. & 34.2 & 28.0 & 27.0 & 20 & 34 & 34 & 54.1 & 57.9 & o. & tix. & 34 & & & 08 & 2 \\
\hline 36 & 5.1 & 14.8 & 39 & e & 36 & 29.1 & 28.1 & 18 & 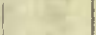 & 36 & 51.7 & 55 & $\infty$ & & 36 & & & $\infty$ & 5 \\
\hline 38 & 14.2 & 13.7 & $4 \mathrm{I}$ & 26 & 38 & 29.0 & 28. I & 18 & int & 38 & 50.7 & $54 . \mathrm{I}$ & 0 & 3 & 38 & & 58.8 & 08 & 2 \\
\hline 40 & I6.7 & I3.I & 39 & 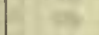 & 40 & 29.2 & 28.7 & 17 & 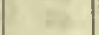 & 40 & 47.0 & 53.0 & $22 \propto$ & 15: & 40 & & 55.5 & 03 & 4 \\
\hline 42 & I 4.2 & 13.7 & $4 I$ & 580 & 42 & 29.9 & 29.1 & 16 & & 42 & 41.5 & 48.3 & 215 & $x^{2}$ & 42 & 50.0 & 53.2 & $\infty$ & 5 \\
\hline 44 & 15.0 & I4. I & 40 & -7.0 & 44 & 29.9 & 29.1 & 16 & -7.0 & 44 & $41 \cdot 3$ & 49.1 & 5 & $-11,1$ & 44 & 49 & 57.2 & 2203 & -10.0 \\
\hline 46 & $15 \cdot 3$ & 14.9 & 39 & -2 & 46 & 30. I & 30.0 & I6 & trat & 46 & 39.9 & 46.0 & 48 & & 46 & & 50.8 & 2156 & \\
\hline 48 & 15.8 & I5.I & 39 & ite & 48 & 30.1 & 30.0 & I6 & 107 & 48 & 37.0 & 45.1 & 4 & & 48 & & 51.0 & 2157 & 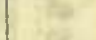 \\
\hline 50 & 16.8 & 15.9 & 37 & $\alpha$ & 50 & 30.9 & 30.9 & 14 & 80 & 50 & 37.8 & 46.0 & 42 & ae & 50.5 & 51.2 & 53.2 & 22 OI & se \\
\hline 52 & 16.2 & 15.7 & 38 & a2 & 52 & 31.4 & 30.6 & I4 & $\theta$ & 53 & 32.0 & 39.0 & 3 & ar & 52 & $57 \cdot 3$ & 59.0 & 10 & t5y \\
\hline 54 & 15.9 & I5. I & 38 & $\because 5$ & 54 & 30.9 & 30.2 & I5 & 35 & 54 & 37.0 & 44.2 & 4 & $\alpha$ & 54 & 73.0 & 75.9 & 36 & Fe \\
\hline 56 & 15.9 & 15.2 & 38 & $z^{2}$ & 56 & 34.9 & $34 \cdot 7$ & 08 & $x^{2}$ & 56 & $39 \cdot 3$ & $45 \cdot 3$ & 4 & & 5 & & 78.0 & 35 & \\
\hline 58 & 14.9 & $14 \cdot 5$ & 40 & 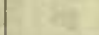 & 58 & 39.9 & 38.7 & oI & & 58 & 39.8 & 45.0 & 4 & 7 & 58 & & 73 & 28 & \\
\hline 2100 & 15.7 & 15.2 & 39 & -7.0 & 2300 & 32.0 & 27.8 & I6 & -7.0 & 1700 & 39.0 & 42.2 & 4 & -11.0 & I9 0 * & 38.0 & 48. I & 25 & -10.0 \\
\hline 02 & 16.9 & 16.0 & 37 & 06 & 02 & I 3.0 & 9.7 & 45 & (3) & 02 & 40.5 & 45.0 & 48 & & 02 & 32.7 & 50.8 & 23 & \\
\hline 04 & 16.9 & 16.0 & 37 & 30 & $04^{*}$ & 42.1 & 36.2 & 53 & WL & 04 & 40.0 & 43.0 & 4 & 6 & 04 & 29.0 & 43.3 & 2214 & $x^{2}$ \\
\hline$\infty 6$ & I6. 3 & 15.7 & 38 & $\mathrm{mp}$ & 06 & 44.3 & $4 \mathrm{I} .2$ & 2247 & & 06 & 44.1 & 47.1 & 5 & 2 & 06 & 9.2 & 13.2 & 2135 & 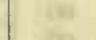 \\
\hline 08 & I5.9 & 15.1 & 38 & 60 & $08^{*}$ & 65.7 & 52.2 & 2333 & (2) & $\infty$ & 44.0 & 47.0 & 5 & 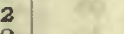 & 08 & 7.0 & II & 32 & 27 \\
\hline Io & I5.I & 14.2 & 40 & 4 & I0 & 57.9 & 46.1 & 44 & ext & Ic & 48.3 & 50 & 5 & 8 & 10 & 14.9 & 21 & 6 & 4 \\
\hline 12 & 15.7 & 14.9 & 39 & & I2 & 59.1 & 50.6 & 39 & & I2 & $49 . \overline{6}$ & 50 & 5 & 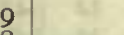 & 12 & 13.5 & I5. & 40 & 34 \\
\hline I4 & I6. I & 15.2 & 38 & -7.0 & I4 & 68.8 & 66.0 & 20 & -7.0 & I4 & 49.0 & 49.8 & 5 & $\begin{array}{ll}8 & -10.9\end{array}$ & 14 & 22.1 & 22.2 & 52 & -9.9 \\
\hline 16 & I5. I & 14.5 & 40 & in & 16 & 66.1 & 61.1 & 26 & & & 45.8 & 48 & 5 & 4 & I & 21 & 30. & 58 & \\
\hline I8 & 15.0 & 14.2 & 40 & (67) & $18^{*}$ & 43.2 & 30.2 & Is & 164 & I & 45.8 & 48. & 5 & 28 & 18 & 14 & 27.0 & 50 & at \\
\hline 20 & 16.4 & I5.6 & 38 & $\mathrm{mx}$ & 20 & 36.1 & 22.0 & 22 & is & 2 & 45.0 & 47 & 5 & ou & 20 & 0 & 25 & 5 & as \\
\hline 22 & 18.0 & I7. 2 & 35 & 16 & 22 & 32.2 & 19.8 & 26 & $\pi$ & 2 & $43 \cdot 3$ & 46. & 5 & 6. & 22 & 17.2 & 27.5 & 53 & 15 \\
\hline 24 & 18.7 & I8. I & 3 & is & 24 & 46.2 & 36.8 & 02 & ts & & 47.0 & & 5 & 7 & 24 & & 22 & & is \\
\hline 26 & 20.0 & 19.6 & 32 & $=$ & 26 & 18 & $.7 b$ & 38 & 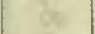 & 26 & 47.2 & 50.1 & 5 & 7 & 2 & 8.5 & 17.8 & 3 & $2 x$ \\
\hline 28 & 21.7 & 2I.I & 29 & 政 & 28 & 26.9 & 24.6 & 27 & & $2 x$ & 49.0 & $5 \mathrm{I}$ & 215 & & 28 & 7.6 & 15.9 & 28 & zat \\
\hline 30 & 22.9 & 21.9 & 28 & -7.0 & $30^{*}$ & 38.3 & 28.0 & 2348 & -7.0 & 30 & 49.8 & 51.2 & 220 & $\begin{array}{ll}0 & -10.8\end{array}$ & $30^{*}$ & 49.8 & 59.8 & 35 & 25 \\
\hline 32 & 23.5 & 22.7 & 26 & ect & $32^{*}$ & 57.0 & 45.2 & 2240 & 59. & 32 & 51.9 & 54.0 & o & & 32 & 48.2 & 56.8 & 3 I & as \\
\hline 34 & 23.9 & 22.9 & 26 & as: & 34 & 53.2 & 43 & 44 & t5 & & $53 \cdot 3$ & & 0 & $t^{2}$ & 3 & 51.9 & 57 & 35 & 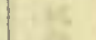 \\
\hline 36 & 24.0 & 23.1 & 26 & & 36 & 63.0 & 52.8 & 29 & & & $53 . \mathrm{I}$ & 54 . & 0 & & 3 & & 60 & 39 & 25 \\
\hline $3^{8}$ & 24.2 & 23.4 & 25 & Es. & 38 & 68.8 & 59.3 & 19 & 2 & 3 & $5^{1.8}$ & 52 & 220 & 6 & 3 & 58.0 & 63 & 44 & 23 \\
\hline 40 & $24 . I$ & $23 \cdot 3$ & 26 & 108 & 40 & 69.9 & 59.1 & 19 & rat & 40 & 49.0 & 50.2 & 215 & $=$ & 40 & 58.9 & 63.2 & 44 & 48 \\
\hline 42 & 24.0 & 23.2 & 26 & $=2$ & 42 & 67.3 & 57.4 & 22 & & 42 & 52.3 & 54.1 & 220 & $1=x^{2}$ & 42 & 61.5 & 66.0 & 48 & 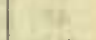 \\
\hline 44 & 24.2 & 23.2 & 26 & -7.0 & 44 & 67.9 & 58.6 & $2 \mathrm{I}$ & -7.0 & 44 & 57.1 & $57 \cdot 5$ & I & $0-10.5$ & 44 & 62.2 & 66.8 & 50 & -10.7 \\
\hline 46 & 24.7 & 23.9 & 25 & 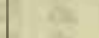 & 46 & $6 \mathrm{I} \cdot 9$ & 53.2 & 30 & 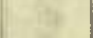 & & 59.0 & 59.5 & I & 3 & 46 & 61.3 & 65.3 & 48 & \\
\hline 48 & 24.8 & 24.1 & 24 & 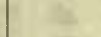 & 48 & 58. I & 50.9 & 34 & 3 & 48 & 55.8 & 63.0 & I & 3 & 48 & 62.9 & 67.8 & $5 I$ & 16 \\
\hline 50 & 25.0 & 24.7 & 24 & $\infty$ & 50 & 49.8 & 42.1 & 48 & 58 & 50 & 56.1 & 62.4 & I & 3 & 50 & 65.0 & 70.7 & 55 & ve \\
\hline 52 & 25.4 & $25 . I$ & 23 & th & 52 & 52.8 & 43.9 & 44 & 32 & 52 & 54.9 & 61.8 & I & 2 & 52 & 65.8 & 70.1 & 55 & 47 \\
\hline 54 & 25.9 & 25.7 & 22 & 12 & 54 & 53.8 & 46.6 & $4 I$ & & 54 & 56.5 & 62.2 & I & 3 & 54 & 64.9 & 69.8 & 54 & Ba \\
\hline 56 & 26.2 & 26.1 & 22 & 3 & 56 & 50.7 & $44 \cdot 3$ & 45 & & 56 & 59.8 & 65.9 & & 8 & 56 & 66.0 & 70.2 & 55 & \\
\hline 58 & 26.8 & 26.5 & $2 I$ & R & 58 & 44.7 & 38.0 & 55 & & 58 & 60.0 & 66.0 & & wat & $\begin{array}{r}58 \\
2000\end{array}$ & $\begin{array}{l}66.0 \\
64.5\end{array}$ & 69.0 & 54 & \\
\hline & & & & & 2400 & 52.6 & 46.0 & 43 & 7.0 & & & & & & 2000 & 04.5 & 08.1 & 52 & \\
\hline
\end{tabular}

Correction to local mean time is - $\operatorname{Im} 46.5 \mathrm{~s} .90^{\circ}$ torsion $=16.764$. Torsion head at oh oom read $336^{\circ}$ and at $24 \mathrm{~h}$ o5m read $344^{\circ}$. Observer-R. R. T.
Correction to local mean time is $-2 \mathrm{~m}$ 08.5s. $90^{\circ}$ torsion $=15 \cdot^{\prime} 78$. Torsion head at 5 h $35 \mathrm{~m}$ read $344^{\circ}$ and at $20 \mathrm{oh} 20 \mathrm{~m}$ read $8^{\circ}$. Observer-J. V. 
Tabulation of magnetic declinations observed at Teplitz Bay-Continued

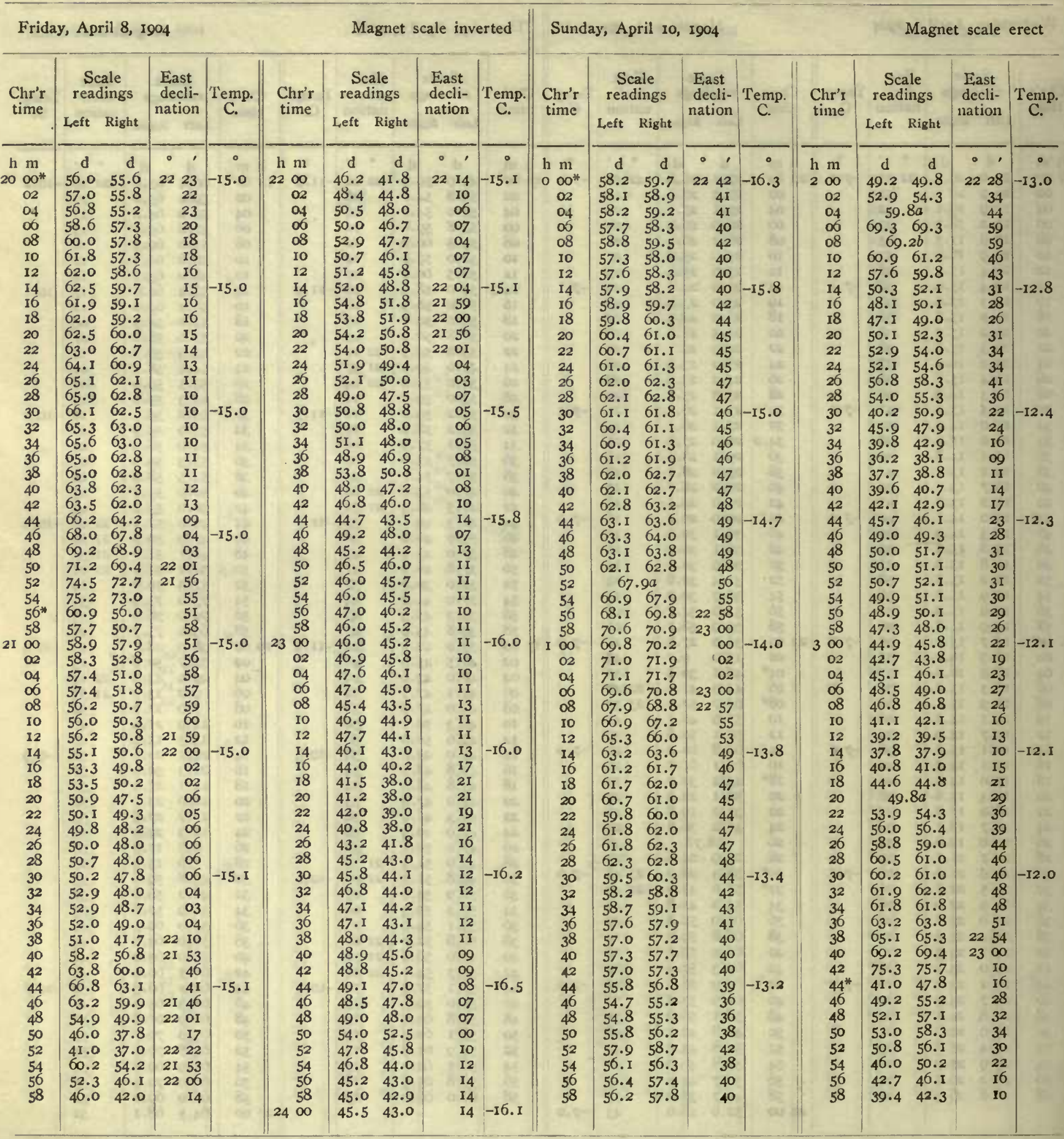


Tabulation of magnetic declinations observed at Teplitz Bay-Continued

\begin{tabular}{|c|c|c|c|c|c|c|c|c|c|c|c|c|c|c|c|c|c|c|c|c|c|}
\hline \multicolumn{6}{|c|}{ Sunday, April 10, 1904} & \multicolumn{5}{|c|}{ Magnet scale inverted } & \multicolumn{7}{|c|}{ Monday, April LI, 1904} & \multicolumn{4}{|c|}{ Magnet scale erect } \\
\hline \multirow{2}{*}{$\begin{array}{l}\text { Chr'r } \\
\text { time }\end{array}$} & \multicolumn{2}{|c|}{$\begin{array}{l}\text { Scale } \\
\text { readings }\end{array}$} & Ea & & & Chr'r & & & $\begin{array}{l}\text { East } \\
\text { decli- }\end{array}$ & Temp. & & $\begin{array}{r}\mathrm{Sc} \\
\text { reac }\end{array}$ & $\begin{array}{l}\text { ale } \\
\text { dings }\end{array}$ & $\begin{array}{l}\text { Eas } \\
\text { dec }\end{array}$ & $\begin{array}{l}\text { ast } \\
\text { cli- }\end{array}$ & & & rea & igs & $\begin{array}{l}\text { East } \\
\text { decli- }\end{array}$ & \\
\hline & Left & Right & & & & & Left & Right & & Z & 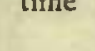 & Left & Right & & & & 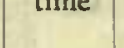 & Left & Right & & \\
\hline $\mathrm{h} \mathrm{m}$ & d & d & $\cdot$ & , & ○ & $h \mathrm{~m}$ & d & d & - & - & $\mathrm{h} \mathrm{m}$ & d & $\mathrm{d}$ & & ' & • & $\mathrm{h} \mathrm{m}$ & a & d & $\circ \quad$ & ○ \\
\hline $400 *$ & 52.0 & 46.1 & 23 & 19 & -11.7 & $600^{*}$ & 47.8 & 39.2 & 23 I5 & -11.0 & $800^{*}$ & 49.1 & 51.8 & 23 & 45 & -14.0 & 1000 & 36.1 & 39.7 & 2238 & -13.8 \\
\hline 02 & 51.9 & 44.7 & & 20 & $x^{2}$ & 02.3 & 51.5 & 46.2 & & 37 & 02 & 54.1 & 54.9 & & 52 & $x_{1}$ & 02 & 36.9 & 39.9 & & (n) \\
\hline 04 & 51.5 & 45.1 & & 20 & 20 & 04 & 48.0 & 43.0 & II & 20 & 04 & 53.0 & 54.2 & & 50 & 69 & 04 & 38.8 & 41.8 & 42 & fis \\
\hline 06 & 48.9 & 42.2 & & 24 & $\omega$ & 06 & 43.9 & 39.0 & 18 & wi & $\alpha \dot{~}$ & 54.6 & 55.9 & & 53 & 16 & 06 & $4 \mathrm{I} \cdot 3$ & 43.9 & 45 & is \\
\hline as & 41.9 & 34.9 & & 35 & 60 & os & 46.3 & 40.9 & 15 & 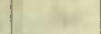 & a8 & 57.2 & $58 . \overline{1}$ & & 57 & 10 & 08 & 42.2 & 44.7 & 46 & $x_{2}$ \\
\hline I0 & 27.9 & 22.6 & & 56 & 81 & 10 & 45.6 & 41.9 & 14 & nis & 10 & 57.2 & 59.7 & & 58 & we & 10 & 42.1 & $44 \cdot 3$ & 46 & 60 \\
\hline 12 & 19.2 & 13.8 & 24 & I0 & $x=7$ & 12 & 41.0 & 37.7 & $2 \mathrm{I}$ & 67 & 12 & 56.3 & $57 \cdot 3$ & & 56 & $a$ & 12 & 41.6 & 43.6 & 45 & In \\
\hline $14^{*}$ & 44.7 & 3 I. 9 & & 22 & -11.7 & 14 & 41.9 & 38.3 & 20 & -10.9 & 14 & 57.2 & 58.4 & & 57 & -13.2 & 14 & 41.4 & $43 . I$ & 44 & -14.0 \\
\hline 16 & 48.2 & 36.9 & & 15 & & 16 & 45.0 & 40.9 & 16 & & 16 & 58.8 & 60.5 & & 60 & & 16 & 40.9 & 42.2 & 44 & (1) \\
\hline 18 & 55.7 & 44.9 & & 03 & F & 18 & 48.8 & 44.7 & 10 & 34 & 18 & 57.1 & 59.0 & & 57 & 37 & I8 & 40.6 & 43.0 & 44 & Wit \\
\hline 20 & 46.9 & 36.3 & & 17 & $=$ & 20 & 50.0 & 45.4 & os & $\alpha$ & 20 & 52.6 & 55.1 & & $5 I$ & 06 & 20 & $41 . I$ & 43.2 & 44 & 56 \\
\hline 22 & 46.5 & $33 . \mathrm{I}$ & & 19 & $x x$ & 22 & 51.0 & 47.1 & 06 & sta & 22 & 48.2 & 52.2 & & 45 & 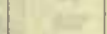 & 22 & 40.0 & 42.3 & 43 & ta \\
\hline 24 & 40.2 & 29.8 & & 27 & 18 & 24 & 52.8 & 48.7 & 2303 & ix & 24 & 35.1 & 38.1 & & 24 & $y=$ & 24 & 39.9 & 41.9 & 42 & His \\
\hline 26 & 44.2 & 32.7 & & 22 & $\alpha$ & 26 & 57.3 & 52.2 & 2257 & 8 & 26 & 30.5 & 33.2 & & 16 & Nx & 26 & 39.2 & $4 \mathrm{I} \cdot \mathrm{I}$ & $4 \mathrm{I}$ & 5 \\
\hline 28 & 34.4 & 27.7 & & 33 & & 28 & 55.8 & 52.1 & 59 & & 28 & 26.0 & 26.7 & & 08 & & 28 & 37.6 & 39.7 & 39 & (kat \\
\hline 30 & 34.0 & 22.7 & 24 & 38 & -11.7 & 30 & 58.8 & 55.2 & 54 & -10.9 & 30 & 15.3 & I8. I & 22 & 52 & -12.8 & 30 & 36.2 & 38.0 & 36 & -14.2 \\
\hline $32^{*}$ & 49.9 & 32.9 & 25 & II & 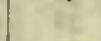 & 32 & 56.9 & 53. I & 57 & 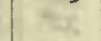 & 32 & 9.9 & 12.9 & & 44 & (n) & 32 & 35.3 & 36.9 & 35 & (2) \\
\hline $34^{*}$ & 54.8 & $28 . \overline{8}$ & 26 & 06 & Es & 34 & 58.9 & 56.3 & 53 & 48 & $35^{*}$ & 52.7 & 61.5 & & 23 & xis & 34 & 35.2 & 36.5 & 34 & ar \\
\hline 36 & 69.0 & 44.8 & 25 & 43 & 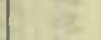 & 36 & 57.8 & $55 . \mathrm{I}$ & 2255 & 8 & 36 & 43.0 & 49.9 & & 06 & 35 & 36 & 36.9 & 37.8 & 37 & 27 \\
\hline $38^{*}$ & $4 \mathrm{I} \cdot \mathrm{I}$ & II. 4 & 25 & 08 & be & 38 & 53.1 & 49.2 & 2303 & & 38 & 49.2 & 54.0 & & 14 & . & 38 & 38.4 & 39.3 & 39 & 8 \\
\hline 40 & 47.7 & 23.0 & 24 & 54 & $\mathrm{a}$ & 40 & 58.9 & 55.2 & 2254 & 5 & 40 & 56.1 & 60.8 & & 25 & 24 & 40 & 39.0 & 40.3 & 40 & 64 \\
\hline 42 & 48.3 & 20.1 & & 55 & 2 & 42.7 & 59.4 & 56.6 & 52 & & 42 & 57.1 & $6 \mathrm{I} .7$ & & 26 & $x^{4}$ & 42 & 38.7 & 39.9 & 40 & 64 \\
\hline $44^{*}$ & 55.9 & 32.1 & & 29 & & 44 & 58.7 & 55.2 & 54 & -10.9 & 44 & 51.9 & 57.2 & & 19 & $-12 \cdot 3$ & 44 & 37.9 & 39.1 & 39 & $-14 \cdot 3$ \\
\hline 46 & 75.7 & 47.5 & & OI & -II.3 & 46 & 59.9 & 57.0 & 52 & - & 46 & 46.1 & 49.8 & & 08 & & 46 & 36.9 & 37.8 & 37 & - \\
\hline 48 & 60.8 & 34.7 & 24 & 23 & . & 48 & 60.8 & 56.7 & $5 \mathrm{I}$ & Win & 48 & 50.1 & 53.1 & & 14 & 28 & 48 & 35.9 & 36.6 & 35 & F. \\
\hline 50 & 27.2 & 6.5 & 25 & II & 68 & 50 & 58.1 & 56.2 & 54 & +2 & 50 & 57.9 & 61.2 & & 26 & 32 & 50 & 35.3 & 36.0 & 34 & $x^{2}$ \\
\hline 52 & 50.8 & $33 \cdot 3$ & 24 & 32 & $=$ & 52 & 65.1 & 62.9 & 43 & 3 & 52 & 59.5 & 63.0 & & 29 & 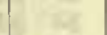 & 52 & 36.2 & 36 & 6 & 4 ate \\
\hline 54 & 3I.I & 7.2 & 25 & 08 & 11 & 54 & 67.1 & 62.6 & 42 & $y^{2}$ & 54 & 58.3 & 6 I.8 & & 27 & ax & 54 & 37.2 & 37.8 & 37 & $y$ \\
\hline $56^{*}$ & $44 \cdot 3$ & 23.7 & 26 & 39 & 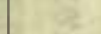 & 56 & 67.7 & 65.9 & 38 & & 56 & 60.0 & 63.7 & & 30 & St. & 56.3 & 37.1 & 37.4 & 37 & $n$ \\
\hline $58^{*}$ & 70.5 & 54.9 & 25 & 18 & 13 & 58 & 62.9 & 61.3 & 46 & & 58 & 60.2 & 64.2 & & 31 & 128 & 58 & 36.1 & 36.8 & 35 & 80 \\
\hline 500 & 44.7 & 26.2 & 26 & or & 83 & 700 & 63.2 & бг.7 & 46 & -10.9 & 900 & 66.2 & 69.2 & & 39 & -12.3 & II 00 & 35.9 & 36.3 & 35 & $-14 \cdot 3$ \\
\hline $02^{*}$ & 32.8 & 14.1 & 25 & 08 & $\mathrm{et}$ & 02 & 55.5 & 51.7 & 59 & -3 & 02 & 63.3 & 65.6 & & 35 & 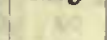 & 02 & 38.8 & 39.3 & 40 & 8 \\
\hline $04^{*} 4$ & 30.9 & 37.0 & 26 & 05 & (a) & 04 & 65.0 & 63.1 & 43 & 8 & 04 & 61.8 & 64.8 & & 32 & 18 & 04 & 39.6 & 40.2 & 41 & 16 \\
\hline 06 & 40.9 & $24 \cdot I$ & 26 & 07 & $x$ & 06 & 58.7 & 58.1 & 52 & in & 06 & 62.2 & 60.1 & & 29 & 10 & 06 & 39.2 & 40.2 & 41 & 50 \\
\hline 08 & $69 . I$ & 53.2 & 25 & 22 & Cov & 08 & 55.0 & 52.7 & 59 & 168 & os & 63.1 & 64.8 & & 33 & $2 \mathrm{ne}$ & 08 & 39.6 & 40.9 & 41 & 18 \\
\hline $10^{*}$ & 63.9 & 47.7 & 24 & 49 & (19) & Io & 66.8 & 66.1 & 39 & ser & Io & 64.2 & 66.9 & & 36 & Xe & Io & 40.0 & $4 \mathrm{I} .3$ & 42 & et \\
\hline 12 & 65.9 & 50.1 & & 45 & & 12 & 59.8 & 58.6 & $5 I$ & 61 & 12 & 66.1 & 68.2 & & 38 & 108 & 12 & 40.0 & 41.7 & 42 & \\
\hline I4 & 67.2 & 58.1 & & 38 & -II.I & I4 & 60 & $.7 a$ & 48 & AN. & 14 & 66.3 & 68.1 & & 38 & -12.4 & 14 & 39.1 & 40.7 & 41 & $-14 \cdot 4$ \\
\hline 16 & 62.3 & 57.6 & & 43 & 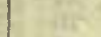 & 16 & 70.1 & 70.1 & 33 & स्ती & 16 & 65.8 & 66.7 & & 37 & & 16 & 37.1 & 38.9 & 38 & -7.7 \\
\hline I8 & 68.7 & 63.2 & & 33 & 64 & 18 & 60.1 & 59.1 & 50 & 14 & 18 & 64.1 & 65.1 & & 34 & fitis & 18 & 36.1 & $37 \cdot 3$ & 36 & at \\
\hline 20 & 69.2 & 66.1 & & 31 & is & 20 & 59.0 & 58.1 & 52 & re & 20 & 65.4 & 65.9 & & 36 & is & 20 & 36.3 & 37.6 & 36 & at \\
\hline $22^{*}$ & $43 . I$ & 34.5 & & 16 & tat: & 22 & 57.0 & 56.0 & 55 & $\mathrm{si}$ & 22 & 67.2 & 67.7 & & 39 & it & 22 & 35.1 & 36.9 & 35 & Net \\
\hline 24 & 49.2 & 37.8 & & 09 & is & 24 & 58.9 & 57.9 & 52 & & 24 & 68.8 & 69.1 & & $4 I$ & ix & 24 & 34.6 & 36.0 & 34 & 18 \\
\hline 26 & 54.9 & 43.2 & & $\infty$ & $E$ & 26 & 64.9 & 63.2 & 43 & 58 & 26 & 70.0 & 70.4 & & 43 & E & 26 & 35.3 & 36.3 & 34 & wat \\
\hline 28 & 52.7 & 44.4 & 24 & oI & & 28 & 64.8 & 64.8 & 42 & & 28 & 71.0 & 71.8 & & 45 & 108 & 28 & 33.0 & 33.8 & $3 \mathrm{I}$ & \\
\hline 30 & 55.7 & 47.6 & 23 & 56 & -11.0 & 30 & 56.9 & 56.1 & 55 & -10.8 & 30 & 73.0 & 73.8 & & 48 & -13.0 & 30 & 32.8 & 33.2 & 30 & -14.7 \\
\hline 32 & 63.3 & 56.1 & & 43 & $12=$ & 32 & 54.6 & 54.2 & 59 & 180 & 32 & 76.2 & 76.9 & & 53 & - & 32 & 32.3 & 32.8 & 29 & -40 \\
\hline 34 & 62.3 & 55.2 & & 45 & 2 & 34 & 56.1 & $55 \cdot 3$ & 56 & 2 & $34^{*}$ & 46.6 & 50.3 & & 54 & Hz & 34 & 31.7 & 32.1 & 28 & \\
\hline 36 & 72.7 & 66.1 & & 28 & 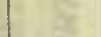 & 36 & 58.1 & 57.9 & 53 & $\sqrt{6}$ & 36 & 46.2 & 51.2 & & 55 & dx & 36 & 33.7 & 34.1 & $3 \mathrm{I}$ & 0 \\
\hline 38 & 73.2 & 65.3 & & 29 & 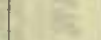 & 38 & 58.8 & $57 \cdot 3$ & 53 & 15 & 38 & 47.4 & 51.8 & & 56 & 21 & 38 & 34.0 & 34.3 & 32 & ies \\
\hline 40 & 68.3 & 60.2 & & 36 & 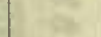 & 40 & 60.1 & 59.0 & $5 I$ & a & 40 & 49.3 & 53.7 & & 59 & $a$ & 40 & 34.0 & 34.6 & 32 & wit \\
\hline 42 & 71.9 & 61.8 & & 32 & 12 & 42 & 58.9 & 57.2 & 53 & 50 & 42 & 49.1 & 53.5 & & 59 & 6 & 42 & 34.2 & 34.8 & 32 & \\
\hline 44 & 78.7 & 68.1 & & 22 & -11.0 & 44 & 56.3 & 54.3 & 57 & -10.7 & 44 & 47.2 & 51.2 & & 55 & $-13 \cdot 3$ & 44 & 34.3 & 35.0 & 33 & -14.9 \\
\hline 46 & 69.4 & $57 . \mathrm{I}$ & & 28 & 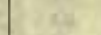 & 46 & 60.1 & 58.6 & $5 \mathrm{I}$ & 108 & 46 & 45.0 & 48.1 & & 51 & & 46 & 34.2 & 34.5 & 32 & 7. \\
\hline 48 & 68.2 & 55.8 & & 40 & 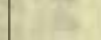 & 48 & 59.0 & 57.1 & 53 & 75 & 48 & 41.7 & 45.2 & & 46 & 6. & 48 & 35.0 & $35 \cdot 3$ & 33 & te \\
\hline 50 & 60.8 & $6 \mathrm{I} . \mathrm{I}$ & & 35 & $n=$ & 50 & 62.0 & 60.0 & 48 & 60 & 50 & 42.1 & 46.1 & & 48 & $n g$ & 50 & 36.1 & 36.1 & 5 & is: \\
\hline 52 & 72.9 & 64.0 & & 30 & Gi & 52 & 64.8 & 63.1 & 44 & - & 52 & 41.7 & 45.4 & & 46 & $x^{2}$ & 52 & 36.3 & 36.8 & 36 & be \\
\hline 54 & 64.8 & 56.8 & & 42 & $B$ & 54 & 63.9 & 61.9 & 45 & 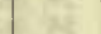 & 54 & 39.7 & 43.2 & & 43 & 12 & 54 & 35.8 & 36.0 & 35 & an \\
\hline 56 & $6 \mathrm{I} .5$ & 57.6 & & 44 & ne & 56 & 64.9 & 62.9 & 44 & Not & 56 & 35.8 & 36.2 & & & 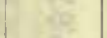 & 56 & 35.3 & 35.9 & & ns \\
\hline 58 & 73.2 & 71.2 & & 24 & 148 & 58 & 65.9 & 63.9 & 42 & & 58 & 35.0 & 38.8 & & 36 & 84 & 58 & 36.6 & 36.8 & 36 & \\
\hline & & & & & & 800 & 68. I & 66.8 & 38 & -10.6 & & - & -5 & & & 60. & 1200 & 36.3 & 36.7 & 36 & -15.0 \\
\hline
\end{tabular}

Correction to local mean time is $-28 \mathrm{~s} .90^{\circ}$ torsion $=14^{\prime} 25$. Torsion head at oh oom read $17^{\circ}$ and at $9 \mathrm{~h} 25 \mathrm{~m}$ read $341^{\circ}$. Observer-R. R. T.
Correction to local mean time is - $1 \mathrm{~m}$ 035. $90^{\circ}$ torsion $=144^{\prime} 29$.

Torsion head at $7 \mathrm{~h} 35 \mathrm{~m}$ read $14^{\circ}$ and at $12 \mathrm{~h} 15 \mathrm{~m}$ read $24^{\circ}$.

Observer-R. R. T. 
Tabulation of magnetic declinations observed at Teplitz Bay-Continued

\begin{tabular}{|c|c|c|c|c|c|c|c|c|c|c|c|c|c|c|c|c|c|c|c|}
\hline \multicolumn{5}{|c|}{ Tuesday, April 12, 1904} & \multicolumn{5}{|c|}{ Magnet scale inverted } & \multicolumn{6}{|c|}{ Wednesday, April 13, 1904} & \multicolumn{4}{|c|}{ Magnet scale erect } \\
\hline \multirow{2}{*}{$\begin{array}{l}\text { Chr'r } \\
\text { time }\end{array}$} & \multicolumn{2}{|c|}{$\begin{array}{l}\text { Scale } \\
\text { readings }\end{array}$} & \multirow{2}{*}{$\begin{array}{c}\text { East } \\
\text { decli- } \\
\text { nation }\end{array}$} & Temp. & Chr'r & $\begin{array}{r}\text { Sc } \\
\text { reac }\end{array}$ & $\begin{array}{l}\text { ale } \\
\text { lings }\end{array}$ & $\begin{array}{l}\text { East } \\
\text { decli- }\end{array}$ & Temp. & Chr'r & $\begin{array}{r}\mathrm{Sc} \\
\text { read }\end{array}$ & $\begin{array}{l}\text { ale } \\
\text { lings }\end{array}$ & $\begin{array}{l}\text { East } \\
\text { decli- }\end{array}$ & Temp. & Chr'r & $\begin{array}{r}\text { Sc } \\
\text { read }\end{array}$ & $\begin{array}{l}\text { dings } \\
\text { dings }\end{array}$ & $\begin{array}{l}\text { East } \\
\text { decli- }\end{array}$ & Temp \\
\hline & Left & Right & & & & Left & Right & & & & Left & Right & & & & Left & Right & & \\
\hline $\mathrm{h} \mathrm{m}$ & d & d & $\cdot \cdot$ & $\circ$ & $\mathrm{h} \mathrm{m}$ & d & d & $\circ \quad$ & $\bullet$ & $\mathrm{h} \mathrm{m}$ & $d$ & d & $\cdot \quad$ & 0 & $\mathrm{~h} \mathrm{~m}$ & $\mathrm{~d}$ & $d$ & $\circ \quad$ & 。 \\
\hline 1200 & 53.9 & 51.7 & $224 \mathrm{I}$ & -19.8 & 1400 & 57.2 & 57.0 & 2234 & -19.8 & $000^{*}$ & 40.2 & 40.9 & 2226 & -22.9 & 200 & 43.8 & 45.2 & & $-23 \cdot 5$ \\
\hline 02 & 52.8 & 50.8 & 42 & & 02 & 57.3 & 57.0 & 34 & & 02 & 38.9 & 39.2 & 24 & & 02 & 38.8 & 40.0 & & \\
\hline 04 & 51.2 & 50.6 & 44 & we & 04 & 57.4 & 57.2 & 34 & 12 & 04 & 37.7 & 38.0 & 22 & & 04 & 37.8 & 38.7 & & \\
\hline 06 & 52.3 & 50.9 & 43 & on & 06 & 57.1 & 57.1 & 34 & 19 & 06 & 36.5 & 38.0 & 21 & & 06 & 38.8 & 40.0 & 24 & \\
\hline 08 & 52.1 & 50.4 & 43 & be & 08 & 57.0 & 56.4 & 34 & 82 & 08 & 37.3 & 39.2 & 23 & 4 & 08 & 40.0 & 42.8 & 28 & \\
\hline Io & 52.2 & 50.9 & 43 & 안 & IO & 56.3 & 55.8 & 36 & 20 & 10 & 37.0 & 38.0 & 22 & & 10 & 43.9 & 44.8 & 32 & \\
\hline 12 & 52.1 & 51.0 & 43 & 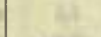 & 12 & 57.0 & 56.0 & 35 & 1 & 12 & 36.8 & 39.2 & 22 & $1=$ & 12 & 42.2 & 43.0 & 30 & \\
\hline 14 & 52.2 & 50.8 & 43 & -20.0 & 14 & 57.2 & 56.3 & 34 & -19.8 & 14 & 37.0 & 39.0 & 22 & -22.9 & 14 & 46.0 & 47.0 & 36 & $-23 \cdot 3$ \\
\hline 16 & 52.2 & 51.3 & 42 & & 16 & 57.8 & 56.1 & 34 & & 16 & 37.2 & 40.3 & 23 & & 16 & 42.2 & 43.8 & 30 & \\
\hline 18 & 53.0 & $52 . I$ & 41 & E & 18 & 57.3 & 55.7 & 35 & 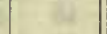 & 18 & 37.7 & 40.5 & 24 & & 18 & 46.2 & & 36 & Alit \\
\hline 20 & 53.8 & 52.6 & 40 & tre & 20 & 57.1 & 55.6 & 35 & as & 20 & 37.6 & 40.4 & 24 & 9 & 20 & 45.0 & 45.3 & 34 & 15 \\
\hline 22 & 53.8 & 52.6 & 40 & $=$ & 22 & 56.8 & $55 . \mathrm{I}$ & 36 & is & 22 & 38.1 & 40.8 & 24 & es & 22 & 45.3 & & 35 & \\
\hline 24 & 53.5 & 52.1 & $4 I$ & Fe & 24 & 56.7 & 55.0 & 36 & it & 24 & 39.2 & 42.0 & 26 & - & 24 & 49.5 & 51.1 & 42 & \\
\hline 26 & 4.0 & 53.0 & 40 & $\mathbb{a}$ & 26 & 56.7 & 55. & 36 & 14 & 26 & 38.8 & 40.3 & 24 & 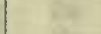 & 26 & 47.0 & 49.4 & 38 & \\
\hline 28 & 55.1 & 54.0 & 38 & $1=$ & 28 & 57.2 & 56.2 & 34 & 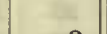 & 28 & 35.0 & 37.0 & 19 & & 28 & 45.0 & 46 & 35 & \\
\hline 30 & 5.1 & 54.2 & 38 & -20.0 & 30 & 57.1 & 56.0 & 35 & -19.8 & 30 & 35.2 & 37 & 20 & -23.0 & 30 & 44.0 & & 32 & -23.0 \\
\hline 32 & 5.2 & 54.9 & 37 & & 32 & 57.8 & 55.2 & 35 & & 32 & 38.5 & 38 & 23 & & 32 & 4.0 & & 32 & \\
\hline 34 & 5.6 & 55.1 & 37 & 15 & 34 & 57.2 & 55 & 35 & & 34 & 40.0 & & 20 & e & 3 & 44.2 & & 34 & \\
\hline 36 & 5.2 & 55.2 & 37 & & 36 & 56.9 & 55.5 & 35 & & 3 & 38.0 & 38.3 & 22 & & 3 & 49.0 & & $4 I$ & \\
\hline 38 & 55.0 & $54 \cdot 3$ & 38 & $5=$ & 38 & 57.0 & 55. & 35 & kn & 38 & 38.9 & 39.7 & 24 & 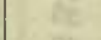 & 38 & 51.0 & 53.8 & 45 & \\
\hline 40 & 4.0 & 53.4 & 39 & 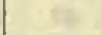 & 40 & 57.8 & 55. & 34 & 44 & 40 & 39.0 & 39 & 24 & 8 & 4 & 52.0 & & 47 & N \\
\hline 42 & 53.3 & 52.3 & $4 I$ & 76 & 42 & 58.0 & 56.0 & 34 & 45 & 42 & $4 \mathrm{I} . \mathrm{I}$ & 42. & 28 & $f=3$ & 4 & 53.2 & 55 & 48 & \\
\hline 44 & 54.0 & 52.9 & 40 & -20.0 & 44 & 57.9 & 55.8 & 34 & -I9.7 & 44 & 42.8 & 43.2 & 30 & -23.2 & 4 & 49.7 & $5 I$ & 42 & -22.8 \\
\hline 46 & 53.2 & 51.9 & 41 & & 46 & 56.2 & 55.0 & 36 & & 46 & 44.2 & 44.7 & 32 & & 4 & 50.1 & 52.2 & 43 & \\
\hline 48 & 53.1 & 51.9 & 4 & 2 & 48 & 55.9 & 54. & 37 & 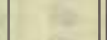 & 4 & 6.0 & 46 & 3 & E & 4 & 45.5 & & 36 & \\
\hline 50 & 2.3 & 51.1 & 4 & 45 & 50 & 55.2 & 54.0 & 38 & 6 & 50 & 42.2 & 43.0 & $3 c$ & a & 5 & 47.2 & & 38 & \\
\hline 52.2 & 3.I & 52. & 4 & 6 & 52 & 5.5 & 54 & 38 & 2 & 5 & & & 2 & 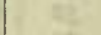 & 5 & 50.7 & & 43 & \\
\hline 54 & 54.1 & 53.1 & 3 & & 54 & 57.6 & 55. & 34 & nit. & 5 & 3.1 & 39. & 2 & the & 5 & 0.9 & 52 & 44 & \\
\hline 56 & 55.9 & 54 & 3 & 2 & 56 & 62.0 & 60 & 28 & 2 & & 8.8 & & 2 & te & & 0.9 & & 44 & \\
\hline 58 & 55.9 & 54.2 & 37 & & 58 & 65.1 & 64.0 & 22 & 18 & 58 & 38.9 & 39.8 & 24 & & 5 & $51 . \overline{8}$ & 52 & 44 & \\
\hline$\infty$ & 56.5 & 54.9 & 36 & -20.0 & $5 \infty$ & 65.1 & 64.2 & 22 & -19.7 & 100 & & & 26 & -23.2 & 30 & 58.8 & & 56 & -22.6 \\
\hline 02 & 57.9 & 56.0 & 34 & 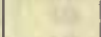 & 02 & 64.0 & 63.3 & 24 & ( & 02 & 37.8 & 45.8 & 28 & & 02 & 53.7 & 55 & 48 & \\
\hline 04 & 58.3 & & 3 & $t^{2}$ & 0 & 62.9 & 62 & 26 & bit & 04 & 37.0 & 45.7 & 28 & k & 04 & 58.0 & 58 & 54 & \\
\hline 06 & 58.2 & 56.1 & 3 & 00 & 0 & 60.8 & 59. & 2 & 80 & & 5.9 & & 2 & 8 & 0 & 57.3 & & 53 & \\
\hline 08 & 58.0 & 56. & 34 & 39 & $\infty$ & 60.4 & 59 & 30 & foe & 08 & 36.2 & 43 & 2 & & a & 57.8 & 58.0 & 54 & \\
\hline 10 & 57.8 & 56.1 & 34 & 96 & I & .0 & 58. & 3 & ar & IO & & & I. & 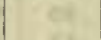 & I & 3.0 & & 47 & \\
\hline I2 & $57 \cdot 3$ & 56.1 & 34 & 97 & I2 & 58.9 & 57.7 & 32 & 10 & 12 & 3 I. 3 & 37.9 & 17 & ty & 12 & 53.7 & 54 & 48 & \\
\hline I4 & 56.0 & 55.4 & 36 & -20.0 & I & 59.8 & 58.5 & $3 I$ & -19.6 & 14 & 34.9 & & 22 & & I & 57.2 & & 53 & -22.6 \\
\hline If & 56.0 & 55.0 & 36 & & If & 58.8 & 56.9 & 33 & -1 & I & 6.9 & 42.1 & 25 & -23.4 & I & 59.0 & 60 & 57 & \\
\hline I8 & 5.8 & 54. & 3 & $4 t$ & I & 58.7 & 57 & 3 & 51 & I & & & 2 & & I & & & & \\
\hline 20 & 57.0 & 55. & 3 & 0 & 2 & 9.0 & 57 & 3 & 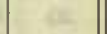 & 2 & 8.0 & & 26 & s. & 2 & 59.8 & $6 I$ & 57 & b \\
\hline 22 & 7.8 & 57. & 3 & $E$ & 2 & 3.3 & 56. & 3 & is & 2 & 9.0 & 43 & 27 & 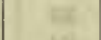 & 2 & 55.8 & & $5 I$ & \\
\hline 2 & 58.0 & 57. & 3 & 12 & 2 & 5.3 & 54. & 3 & 12 & 2 & 3.8 & & 2 & ter & 2 & 55.0 & & 50 & \\
\hline 26 & 58.3 & & 3 & 단 & 2 & & 54. & & $x=$ & 2 & 39.0 & 43 & 2 & & 2 & 56.1 & 56.8 & $5 I$ & \\
\hline 28 & 57.0 & 56.8 & 34 & 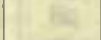 & 28 & 56.8 & 55. & 36 & & 28 & 35.0 & 38 & 20 & & 2 & 57.2 & 57.5 & 53 & \\
\hline 30 & 56.2 & 55.7 & 36 & -19.9 & 30 & 57.7 & 56.2 & 34 & -19.6 & $3 c$ & .0 & 39.9 & 22 & $-23 \cdot 5$ & 3 & 57.0 & 57 & 52 & -22.8 \\
\hline 32 & 5.6 & & & 12 & 3 & & & 3 & $=$ & & & & & & 3 & 56.9 & & & \\
\hline 34 & 57.4 & 56.9 & 3 & 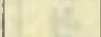 & 3 & 4.0 & 53.8 & 3 & & 3 & 35.6 & 38 & 21 & 18 & 3 & 59.0 & 60 & 2256 & \\
\hline & 58.0 & 57.0 & & 8 & 3 & 4.8 & 54.1 & 38 & c & & 33.5 & & & 18 & & 63.2 & 64 & & \\
\hline 38 & 59.8 & 58.8 & 3 & es. & 38 & 5.3 & 55.1 & 37 & (2) & 38 & & 36. & I & 6 & 3 & 69.8 & 75 & 16 & \\
\hline 40 & 58.3 & 57.1 & 3 & exis & 4 & & & 3 & $x^{2}$ & & .8 & 34. & & c & 4 & 72.8 & & 18 & \\
\hline 42 & 56.4 & 55. & 36 & & 42 & 55.8 & 55.2 & 36 & & 42 & 32.8 & & 16 & 4 & 42 & 74.2 & & 20 & \\
\hline 44 & 57.4 & 56.7 & 34 & -19.8 & 44 & 57.9 & 57.2 & 33 & -19.5 & 44 & 35.4 & 38. & 20 & -23.5 & 44 & 73.4 & 75 & 19 & -22.8 \\
\hline 46 & 57.8 & $57 . I$ & 33 & 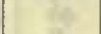 & 46 & 58.3 & 58.2 & 32 & ( & 46 & 35.7 & 38.2 & 21 & & 4 & 71.5 & & 16 & \\
\hline 48 & 57.8 & 57.1 & 3 & Bs & 48 & 58.2 & 58.2 & 32 & 4 & 48 & 34.1 & 37.1 & I\& & An & 48 & $73 . \overline{8}$ & 75 & 20 & \\
\hline 50 & 57.1 & 56.9 & 34 & as & 50 & 59.8 & 59. & 30 & 하자. & & 35.4 & 38 & 2 & 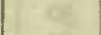 & 5 & 72.0 & & I7 & \\
\hline 52 & 57.4 & 57.0 & 34 & ES & 52 & 59.3 & 59.0 & $3 I$ & 36 & 52 & 36.5 & 39.2 & 22 & $Q$ & 52 & 71.6 & 73 & 16 & $\mathrm{c}$ \\
\hline & 57.8 & 57.0 & 33 & 83 & 54 & 59.8 & 59.3 & 30 & ts & & 39.3 & $4 \mathrm{I} .9$ & 26 & $z^{2}$ & & 73.9 & 76.2 & 20 & \\
\hline 56 & 57.7 & 57.1 & 33 & & 56 & 60.4 & 60.0 & 29 & & 56 & 41.9 & 44.3 & 30 & & 56 & 75.2 & 77.0 & 22 & \\
\hline 30 & 57.7 & 57.2 & 33 & 20 & 58 & 59.6 & 58.9 & 30 & & 58 & $45 \cdot 5$ & 46.3 & 35 & 8 & 58 & 75.8 & 77.0 & 23 & \\
\hline & & & & 100 & I6 00 & 58.9 & 57.9 & 32 & -19.5 & & & & & & & & & & \\
\hline
\end{tabular}

Correction to local mean time is - Im $43 \mathrm{~s} .90^{\circ}$ torsion $=16 .^{\prime} 03$.

Observer-J. V.

Torsion head at $1 \mathrm{Ih} 25 \mathrm{~m}$ read $40^{\circ}$ and at $16 \mathrm{~h} 20 \mathrm{~m}$ read $31^{\circ}$.

Observer-R. R. T. 
Tabulation of magnetic declinations observed at Teplitz Bay-Continued

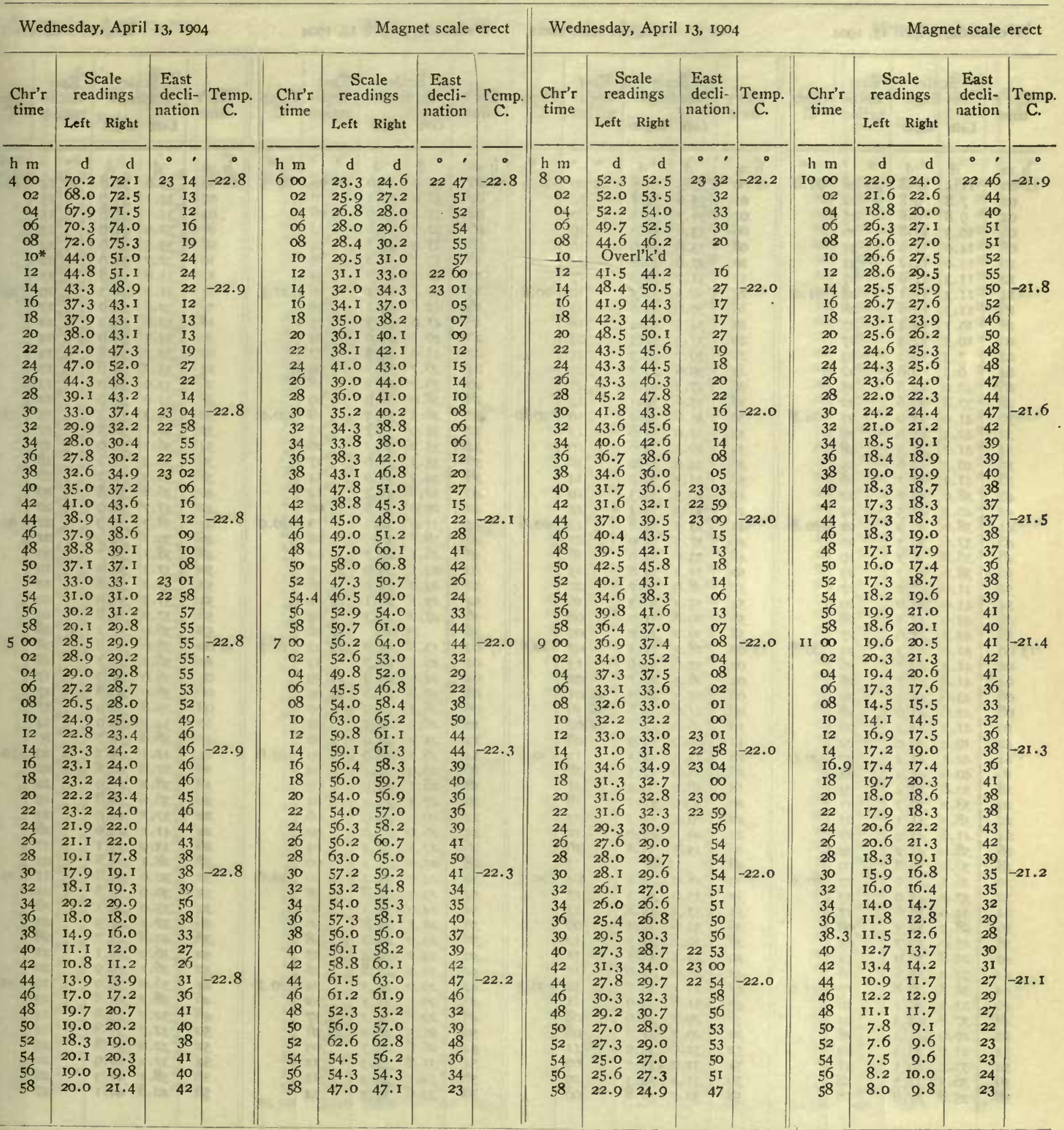

Observers-J. V. and W. J. P., who alternated from $7 \mathrm{~h} 56 \mathrm{~m}$ to Observer-W. J. P. $8 \mathrm{~h}$ o6m. 


\begin{tabular}{|c|c|c|c|c|c|c|c|c|c|c|c|c|c|c|c|c|c|c|c|c|c|c|c|}
\hline \multicolumn{6}{|c|}{ Wednesday, April 13, 1904} & \multicolumn{6}{|c|}{ Magnet scale erect } & \multicolumn{6}{|c|}{ Wednesday, April 13, 1904} & \multicolumn{6}{|c|}{ Magnet scale erect } \\
\hline \multirow[t]{2}{*}{$\begin{array}{l}\text { Chr'r } \\
\text { time }\end{array}$} & $\begin{array}{r}\mathrm{Sc} \\
\text { read }\end{array}$ & $\begin{array}{l}\text { ale } \\
\text { lings }\end{array}$ & $\begin{array}{l}\text { Eas } \\
\text { decl } \\
\text { natio }\end{array}$ & $\begin{array}{l}\text { st } \\
\text { cli- } \\
\text { ion }\end{array}$ & Temp & $\begin{array}{l}\text { Chr'r } \\
\text { time }\end{array}$ & $\begin{array}{r}\mathrm{Sc} \\
\text { read }\end{array}$ & $\begin{array}{l}\text { ale } \\
\text { lings }\end{array}$ & $\begin{array}{l}\text { Eas } \\
\text { dec } \\
\text { nati }\end{array}$ & & Temp & $\begin{array}{l}\text { Chr'r } \\
\text { time }\end{array}$ & $\begin{array}{l}\text { Sca } \\
\text { read }\end{array}$ & $\begin{array}{l}\text { ale } \\
\text { lings }\end{array}$ & $\begin{array}{l}\text { Eas } \\
\text { dec } \\
\text { nati }\end{array}$ & $\begin{array}{l}\text { ist } \\
\text { cli- } \\
\text { inn }\end{array}$ & Temp. & Chr'r & $\begin{array}{l}\mathrm{Sc} \\
\text { read }\end{array}$ & $\begin{array}{l}\text { ale } \\
\text { ings }\end{array}$ & Eas & & Temp. \\
\hline & Left & Right & & & & & Left & Right & & & & & Left & Right & & & & & Left & Right & & & \\
\hline h $\mathrm{m}$ & d & d & $\circ$ & : & $\bullet$ & h $\mathrm{m}$ & d & d & - & $\cdot$ & $\bullet$ & $\mathrm{h} \mathrm{m}$ & d & d & & , & $u^{\circ}$ & h $\mathrm{m}$ & d & d & - & . & $\bullet$ \\
\hline & 11.4 & 12.8 & 222 & 28 & -21.0 & 1400 & 37.3 & 39.8 & 22 & 20 & -21.0 & 1600 & 30.9 & 31.0 & & 08 & -20.5 & 1800 & 33.6 & 34.8 & 22 & I4 & -19.6 \\
\hline 02 & 7.8 & 8.8 & & 22 & & 02 & 35.6 & 30.8 & & 19 & & 02 & 31.9 & 32.1 & & 10 & & 02 & $34 . I$ & 35.9 & & 15 & \\
\hline 04 & 9.8 & 9.8 & & 25 & & 04 & 36.7 & 39.1 & & 20 & & 04 & 31.0 & 31.0 & & 08 & & 04 & 34.1 & 35.8 & & I5 & \\
\hline 06 & 10.3 & ro.8 & & 26 & 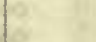 & 06 & 33.6 & 37.0 & & 16 & & 06 & 32.9 & 34.0 & & 12 & & 06 & 32.9 & 34.9 & & 13 & \\
\hline 08 & 14.0 & 14.6 & & 32 & & 08 & 33.8 & 37.6 & & I6 & & 08 & 33.9 & $34 \cdot 3$ & & 14 & & 08 & 33.5 & 35.0 & & 14 & \\
\hline 10 & 14.0 & 14.2 & & 32 & & 10 & 37.6 & 40.8 & & 22 & & 10 & 37.1 & 38.0 & & 19 & & 10 & 32.7 & 34.0 & & 12 & \\
\hline 12 & II. 3 & ri.g & & 27 & & 12 & $3 \mathrm{I} .2$ & 35.2 & & I2 & & I2 & 46.2 & 46.7 & & 3.3 & & 12 & 31.8 & 32.8 & & II & \\
\hline 14 & II. 3 & 13.1 & & 28 & -2 I. 0 & 14 & 30.3 & $33 \cdot 3$ & & I0 & -20.8 & 14 & 42.8 & $44 \cdot 4$ & & 28 & -20.3 & 14 & 31.2 & 32.3 & & 10 & -19.6 \\
\hline 16 & 11.3 & 12.6 & & 28 & & 16 & 30.6 & 35.6 & & 12 & & I6 & 42.8 & 44.1 & & 28 & & 16 & 32.6 & 33.0 & & 12 & \\
\hline 18 & 10.1 & 12.4 & & 27 & & 18 & 34.5 & 39.4 & & I8 & & 18 & $4 I .0$ & 42.3 & & 25 & & 18 & 32.8 & 33.4 & & 12 & \\
\hline 20 & 10.5 & I2. 3 & & 27 & 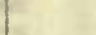 & 20 & 37.3 & 42.5 & & 23 & & 20 & 40.1 & 41.0 & & 24 & & 20 & 34.1 & 35.0 & & 14 & \\
\hline 22 & 7.5 & 10.2 & & 23 & & 22 & 39.7 & 43.3 & & 25 & & 22 & 41.0 & 44.5 & & 27 & & 22 & 33.7 & 34.8 & & 14 & \\
\hline 24 & 6.4 & 9.6 & & 22 & 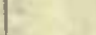 & 24 & 39.6 & $43 \cdot 3$ & & 25 & & 24 & 36.7 & 39.1 & & 20 & & 24 & 33.3 & 34.6 & & 13 & \\
\hline $26 *$ & 40.0 & 42.8 & & 25 & & 26 & 37.3 & 40.5 & & $2 \mathrm{I}$ & & 26 & 36.2 & 37.1 & & 18 & & 26 & 33.0 & 34.0 & & 13 & \\
\hline 28 & 37.8 & 40.6 & & 22 & & 28 & 35.6 & 39.4 & & 19 & & 28 & 38.0 & 38.9 & & 20 & & 28 & 33.0 & 33.9 & & 12 & \\
\hline 30 & 41.0 & 44.0 & & 27 & -20.8 & 30 & 35.6 & 39.2 & & 19 & -20.8 & 30 & 36.5 & $37 \cdot 4$ & & 18 & -20.0 & 30 & $34 \cdot 3$ & 35.5 & & 15 & -19.5 \\
\hline 32 & 42.3 & 45.0 & & 28 & & 32 & 32.0 & 35.6 & & 13 & & 32 & 34.9 & 35.1 & & 15 & & 32 & 36.1 & 36.5 & & 17 & \\
\hline .34 & $4 \mathrm{I} .7$ & 44.3 & & 28 & & 34 & 30.3 & 33.9 & & 10 & & 34.2 & 35.2 & 36.1 & & 16 & & 34 & 36.1 & 36.9 & & 17 & \\
\hline 36 & 40.3 & 42.6 & & 25 & & 36 & 34.0 & 37.0 & & 16 & & 36 & $35 \cdot 3$ & 37.0 & & I7 & & 36 & 36.3 & 37.2 & & 18 & \\
\hline 38 & $4 \mathrm{I} .2$ & 42.7 & & 26 & & 38 & $33 \cdot 3$ & 36.2 & & 14 & & $3^{8}$ & 34.2 & 35.0 & & 14 & & 38 & 36.3 & 37.2 & & 18 & \\
\hline 40 & 38.6 & 40.6 & & 22 & & 40 & 36.5 & 38.3 & & 19 & & 40 & 32.8 & 33.4 & & 12 & & 40 & 36.8 & 37.8 & & 19 & \\
\hline 42 & 40.2 & 41.5 & & 24 & & 42 & 33.3 & 35.4 & & 14 & & 42 & 29.9 & 31.0 & & 08 & & 42 & 37.7 & 38.0 & & 20 & \\
\hline 44 & 38.6 & 40.0 & & 22 & -20.8 & 44 & 33.3 & 34.8 & & 13 & -20.6 & 44 & 31.0 & 32.3 & & I0 & -20.0 & 44 & 38.7 & 39.9 & & 22 & -19.7 \\
\hline 46 & 37.6 & 30.3 & & 20 & & 46 & 37.3 & 39.9 & & 2 I & & 46 & 28.8 & 31.9 & & 08 & & 46 & 30.1 & 39.9 & & 22 & \\
\hline 48 & 36.8 & 38.7 & & 19 & & 48 & 33.6 & 37.0 & & I6 & & 48 & 26.1 & 28.7 & 22 & 03 & & 48 & 38. I & 38.7 & & 20 & \\
\hline 50 & 38.6 & $41 \cdot 3$ & & 23 & & 50 & 33.3 & .4 .8 & & 13 & & 50 & 24.1 & 26.0 & 21 & 50 & & 50 & 37.1 & 38.0 & & 19 & \\
\hline 52 & $4 \mathrm{I} .9$ & 43.2 & & 27 & . & 52 & .38 .9 & 30.7 & & 22 & & 52 & 24.0 & 25.0 & 21 & 58 & & 52 & 37.1 & 37.8 & & 19 & \\
\hline 54 & 43.0 & 44.0 & & 20 & & 54 & 35.3 & 36.3 & & I6 & & 54 & 25.1 & 26.1 & 22 & $\infty$ & & 54 & 37.3 & 38.0 & & 19 & \\
\hline 56 & 42.9 & 43.5 & & 28 & & 56 & 29.8 & $3 \mathrm{Y} \cdot 9$ & & 08 & & 56 & 27.0 & $27 \cdot 3$ & & 03 & & 56 & 37.2 & 37.8 & & 19 & \\
\hline 58 & 42.3 & 42.6 & & 27 & & 58 & 30.2 & 32.2 & & 09 & & 58 & 25 & $.2 b$ & 22 & $\infty$ & & 58 & 36.9 & 37.2 & & 18 & \\
\hline 1300 & 41.0 & 42.3 & & 25 & -20.8 & 1500 & 28.2 & 31.3 & & 07 & -20.6 & 1700 & 13.8 & 14.0 & 21 & 42 & -10.7 & 1900 & 36.6 & 37.8 & & 18 & -19.8 \\
\hline 02 & $4 \mathrm{I} .3$ & 42.7 & & 26 & & $\mathrm{O} 2$ & 32.6 & 31.6 & & I3 & & 02 & 20.1 & 21.7 & & 5.3 & & 02 & 36.1 & 37.7 & & I8 & \\
\hline 04 & 4 T. 8 & 42.7 & & 26 & s. & 04 & 26.4 & 28.7 & & 03 & & 0.4 & 24.0 & 25.0 & 21 & 59 & & 04 & 36.0 & 36.0 & & 17 & \\
\hline 06 & 43.6 & 45.5 & & 30 & & 06 & 25.3 & 26.6 & 22 & OI & & of & 27.8 & 29.8 & 22 & 05 & & 06 & 36.0 & 36.8 & & 17 & \\
\hline 08 & 42.6 & 43.6 & & 28 & & 08 & 21.6 & 23.6 & 2 I & 56 & & 08 & 28.3 & 31.9 & & or & & 08 & 35.8 & 36.9 & & 17 & \\
\hline 10 & $4 \mathrm{I} .3$ & 42.4 & & 26 & & Io & 26.0 & 27.2 & 22 & 02 & & 10 & 26.5 & 29.5 & & 04 & & 10 & 34.2 & 35.0 & & 14 & \\
\hline 12 & 39.4 & 40.0 & & 22 & & 12 & 20.9 & 31.0 & & 08 & & 12 & 25.2 & 27.9 & & 02 & & 12 & 36.8 & 38.7 & & IS & \\
\hline I4 & 42.1 & 43.2 & & 27 & -20.9 & I4 & 33.3 & 34.5 & & 1.2 & -20.5 & I4 & 26.3 & 28.9 & & 03 & -19.6 & 14 & $42 . I$ & 45.3 & & 29 & -19.8 \\
\hline 16 & 40.6 & $4 \mathrm{I} \cdot 4$ & & 24 & & т6 & .36 .6 & 37.5 & & 18 & & т6 & 25.9 & 20.1 & & 03 & & 16 & .37 .6 & 40.8 & & 22 & \\
\hline 18 & 30.8 & 40.4 & & 23 & & 18 & 38.1 & 39.2 & & 21 & & I8 & 26.1 & 28.9 & & 0.3 & & 18 & 38.2 & 41.0 & & 22 & \\
\hline 20 & 38.2 & 39.7 & & 21 & & 20 & 36.6 & 37.2 & & 18 & & 20 & 27.4 & 29.2 & & 04 & & 20 & 36.6 & 38.8 & & 19 & \\
\hline 22 & 38.3 & $4 \mathrm{I}, \mathrm{I}$ & & 22 & & 22 & 40.5 & 4 T. 3 & & 24 & & 22 & 29.0 & 29.9 & & 06 & & 22 & 37.7 & 42.0 & & 23 & \\
\hline 24 & 41.6 & 43.9 & & 27 & & 24 & 40.5 & 42.0 & & 25 & & 24 & 26.0 & 27.0 & & $\alpha$ & & 24 & $4 \mathrm{r} . \mathrm{I}$ & 46.0 & & 28 & \\
\hline 26 & 38.0 & 41.2 & & 22 & & 26 & 35.5 & 35.8 & & 16 & & 26 & $27 \cdot 3$ & 28.3 & & 04 & & 26 & 33.9 & 39.1 & & 17 & \\
\hline 28 & 36.2 & 39.8 & & 20 & & 28 & 33 & $.8 b$ & & $T_{3}$ & & 28 & 25.0 & 25.3 & & 00 & & 28 & 28.4 & 28.8 & & 05 & \\
\hline 30 & 36.5 & 42.0 & & 22 & 2 2т.0 & 30 & 28.0 & 28.2 & & 04 & -20.5 & 30 & 24.9 & 25.4 & 22 & $\infty$ & -19.6 & 30 & 30.0 & 34.8 & & II & -19.9 \\
\hline 32 & 37.3 & 43.0 & & 23 & & 32 & 33.0 & 34.3 & & 13 & & 32 & 23.0 & 24.8 & 21 & 58 & & 32 & 30.9 & 35.8 & & 12 & \\
\hline 34 & 34,0 & 38.6 & & 17 & & 34 & 33.3 & 34.8 & & 13 & & 34 & 24.0 & 25.3 & & 59 & & 34 & 35.0 & 39.1 & & 18 & \\
\hline 36 & 28.3 & 32.5 & & 08 & & 36 & 30.6 & 32.3 & & 09 & & 36 & 22.1 & 23.8 & & 56 & & 36 & 37.4 & 42.9 & & 23 & \\
\hline 38 & 25.0 & 32.7 & & 05 & & 38 & 30.7 & 32.5 & & 10 & & 38 & 23.9 & 24.9 & & 58 & & 38 & 40.8 & 45.7 & & 28 & \\
\hline 40 & 29.8 & 35.0 & & II & & 40 & 31.5 & 33.5 & & II & & 40 & 23.2 & 24.4 & & 57 & & 40 & 36.9 & $4 \mathrm{r} . \mathrm{I}$ & & 21 & \\
\hline 42 & 33.2 & 38.6 & & 16 & & 42 & 31.0 & 33.0 & & 10 & & 42 & 22.1 & 23.8 & & 56 & & 42 & 36.1 & 40.9 & & 20 & \\
\hline 44 & 32.8 & 39.8 & & I7 & -21.0 & 44 & $31 . \mathrm{I}$ & 33.0 & & 10 & -20.5 & 44 & 20.9 & 22.3 & & 57 & -19.7 & 44 & 36.0 & 38.0 & & I9 & -20.0 \\
\hline 46 & 33.3 & 40.7 & & 18 & & 46 & 31.8 & 33.0 & & II & & 46 & 20.2 & 21.1 & & 52 & & 46 & 38.7 & 41.8 & & 23 & \\
\hline 48 & 37.8 & 43.3 & & 24 & 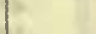 & 48 & 29.7 & 31.0 & & 08 & & 48 & 22.8 & 24.3 & $2 \mathrm{I}$ & 57 & & 48 & 32.7 & 35.8 & & 14 & \\
\hline 50 & 40.9 & 45.3 & & 28 & & 50 & 29.7 & 31.2 & & 08 & & 50 & 25.5 & 26.9 & 22 & or & & 50 & 32.7 & 35.8 & & 14 & \\
\hline 52 & 41.9 & 46.3 & & 29 & & 52 & 31.6 & 33.0 & & II & & 52 & 26.6 & 28.7 & & 03 & & 52 & 37.9 & 41.8 & & 23 & \\
\hline 54 & $37 \cdot 3$ & 40. I & & 21 & & 54 & 31.0 & 31.5 & & $\infty 9$ & & 54 & 31.8 & 33.3 & & II & & 54 & 37.2 & 40.9 & & 21 & \\
\hline 56 & 36.3 & 37.3 & & 18 & & 56 & 31.3 & 32.3 & & 10 & & 56 & 32.1 & $33 \cdot 3$ & & II & & 56 & 38. I & 41.3 & & 22 & \\
\hline 58 & 35.8 & 37.6 & & 18 & & 58 & 30.0 & 30.8 & & 08 & & 58 & 33.6 & $35 \cdot 3$ & & 14 & & 58 & 40.8 & 44.1 & & 27 & \\
\hline
\end{tabular}

Observers-W. J. P and R. R. T., who alternated from 15 h $58 \mathrm{~m}$ to Observer-R. R. T. $16 \mathrm{~h} 08 \mathrm{~m}$. 
Tabulation of magnetic declinations observed at Teplitz Bay-Continued

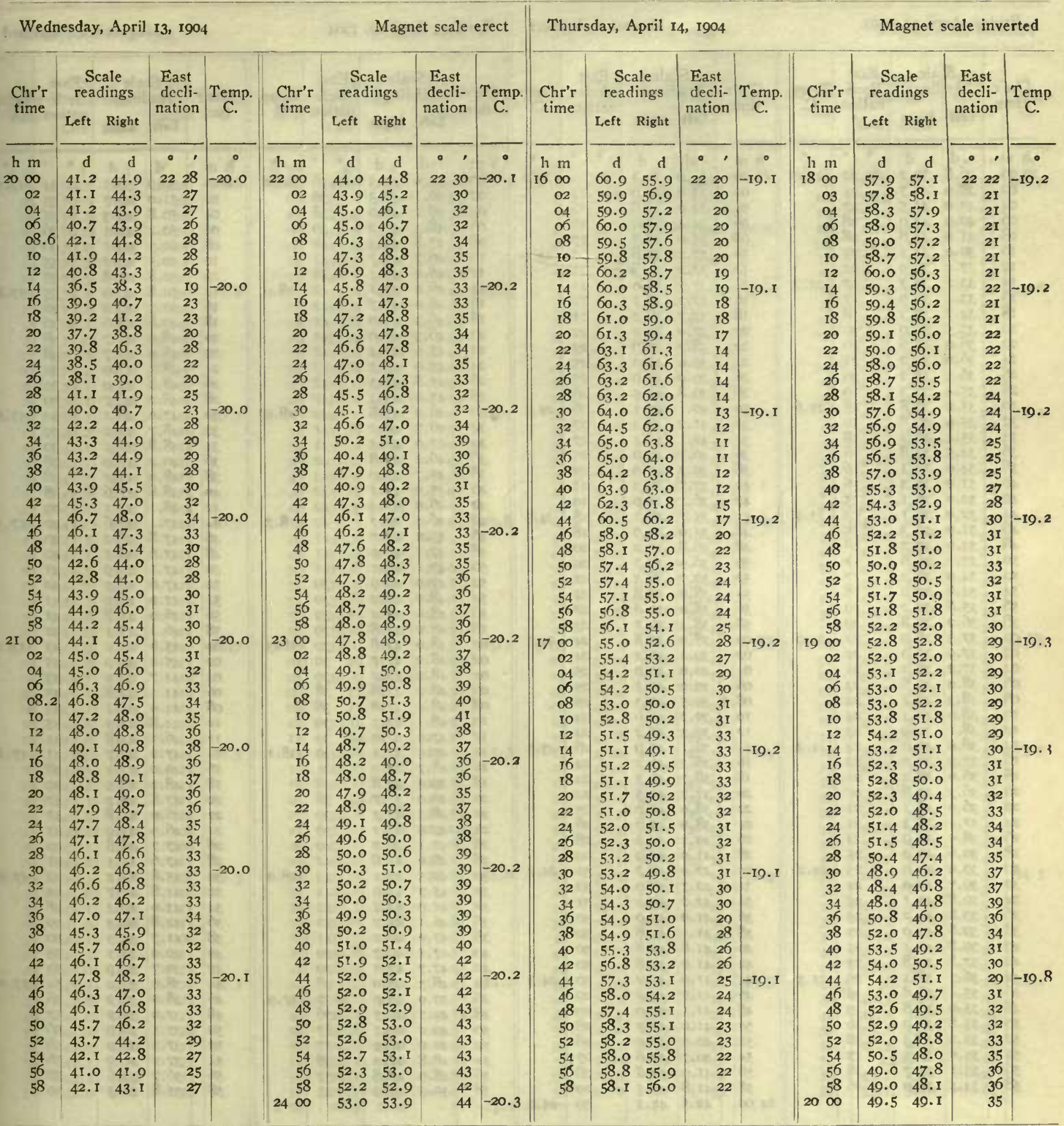

Correction to local mean time is - Im I6s.

Torsion head at oh oom read $35^{\circ}$ and at $24 h_{1}$ rom read the same. Observer-R. R. T.
Correction to local mean time is - 55 s.

Torsion head at $15 \mathrm{~h} 30 \mathrm{~m}$ read $33^{\circ}$ and at $20 \mathrm{~h}$ Igm read the same. Observer-J. V. 
Tabulation of magnetic declinations observed at Teplitz Bay-Continued

\begin{tabular}{|c|c|c|c|c|c|c|c|c|c|c|c|c|c|c|c|c|c|c|c|c|c|c|}
\hline \multicolumn{6}{|c|}{ Friday, April 15, 1904} & \multicolumn{6}{|c|}{ Magnet scale erect } & \multicolumn{5}{|c|}{ Sunday, April 17, 1904} & \multicolumn{6}{|c|}{ Magnet scale inverted } \\
\hline \multirow{2}{*}{$\begin{array}{l}\text { Chr'r } \\
\text { time }\end{array}$} & $\begin{array}{r}\mathrm{Sc} \\
\mathrm{read}\end{array}$ & $\begin{array}{l}\text { ale } \\
\text { lings }\end{array}$ & $\begin{array}{l}\text { East } \\
\text { decli }\end{array}$ & & Temp. & $\begin{array}{l}\text { Chr'r } \\
\text { time }\end{array}$ & $\begin{array}{l}\text { Sca } \\
\text { read }\end{array}$ & $\begin{array}{l}\text { ale } \\
\text { lings }\end{array}$ & $\begin{array}{l}\text { Eas } \\
\text { decl } \\
\text { natio }\end{array}$ & & Temp. & Chr'r & $\begin{array}{l}\mathrm{Sc} \\
\text { read }\end{array}$ & $\begin{array}{l}\text { ale } \\
\text { lings }\end{array}$ & $\begin{array}{l}\text { East } \\
\text { decli- }\end{array}$ & & Chr'r & $\begin{array}{l}\text { Sca } \\
\text { readi }\end{array}$ & $\begin{array}{l}\text { ale } \\
\text { lings }\end{array}$ & East & & Temp. \\
\hline & Left & Right & & & & & Left & Right & & & & & Left & Right & & & & Left & Right & & & \\
\hline$h \mathrm{~m}$ & $d$ & d & $\cdot$ & ' & $\bullet$ & $h \mathrm{~m}$ & $d$ & d & $\cdot$ & , & $\bullet$ & $\mathrm{h} \mathrm{m}$ & d & $\mathrm{d}$ & $\cdot$, & & $\mathrm{h} \mathrm{m}$ & d & d & - & , & $\cdot$ \\
\hline 000 & 36.8 & 39.2 & 222 & & -23.0 & 2200 & 34.0 & 34.4 & 22 & 16. & -24.2 & $00^{*}$ & 50.0 & $49 \cdot 3$ & 2236 & -19.2 & 200 & 50.9 & 50.1 & 22 & 34 & -17.9 \\
\hline 02 & 38.9 & $41 \cdot 7$ & & 25 & - & 02 & 33.0 & $34 \cdot 0$ & & 14 & & 02 & 50.1 & 49.5 & 36 & & 02 & 51.0 & 49.7 & & 35 & \\
\hline 04 & 40.0 & 42.5 & & 27 & ta & 04 & 34.0 & 34.8 & & 16 & & 04 & 49.2 & 47.9 & 38 & & 04 & 50.8 & $49 \cdot 3$ & & 35 & \\
\hline 06 & 40.9 & 43.0 & & 28 & 5 & 06 & 34.4 & 35.0 & & เ6 & & $\infty 6$ & 48.3 & 47.2 & 39 & & 06 & 50.4 & $49 . \mathrm{I}$ & & 36 & \\
\hline a8 & 41.0 & 43.8 & & 28 & ex & 08 & 35.0 & 35.5 & & 17 & & 08 & 47.8 & 46.3 & 40 & & 08 & 50.3 & 49.1 & & 36 & \\
\hline Io & 39.0 & $4 I, I$ & & 25 & 1 & 10 & 37.2 & 37.8 & & 21 & -24.4 & 10 & 46.7 & 45.7 & $4 \mathrm{I}$ & & 10 & 50.5 & 49.2 & & 35 & \\
\hline 12 & 37.2 & 39.9 & & 22 & & 12 & 37.2 & 37.8 & & $2 \mathrm{I}$ & & 12 & 46.0 & $45 . \mathrm{I}$ & 42 & & 12 & 50.8 & 49.8 & & 35 & \\
\hline 14 & 33.2 & 36.7 & & 17 & -23.2 & 14 & 37.7 & 38.0 & & 21 & & 14 & 46.0 & $45 . I$ & 42 & -18.9 & 14 & 50.8 & 49.9 & & 35 & -17.8 \\
\hline I6 & 33.4 & 36.9 & & 17 & & 16 & 37.2 & 38. 1 & & 21 & & 16 & 46.0 & 45.7 & 42 & & I6 & 50.3 & 40.2 & & 36 & \\
\hline 18 & 34.7 & $37 \cdot 3$ & & 18 & t & 18 & 37.7 & 38.2 & & 21 & & 18 & 46.3 & 46.0 & $4 \mathrm{~T}$ & & 18 & 40.8 & 48.6 & & 36 & \\
\hline 20 & 35.5 & 38.9 & & 20 & 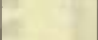 & 20 & 37.0 & 39.0 & & 22 & & 20 & 46.3 & 46.0 & $4 \mathrm{I}$ & & 20 & 48.9 & 47.7 & & 38 & \\
\hline 22 & 36.0 & 38.6 & & 20 & $E$ & 22 & 38.2 & 40.0 & & 23 & & 22 & 45.9 & 45.4 & 12 & & 22 & 48.9 & 47.7 & & 38 & \\
\hline 24 & 36.0 & 30.0 & & 21 & t & 24 & 39.0 & 41.0 & & 25 & & 24 & 45.0 & 45.3 & 42 & & 24 & 48.4 & 47.0 & & 39 & \\
\hline 26 & 36.3 & 38.2 & & 20 & & 26 & 39.0 & 41.0 & & 25 & & 26 & 45.8 & 45.3 & 42 & & 26 & 48.6 & 47.0 & & 39 & \\
\hline 28 & 35.4 & 37.3 & & 10 & & 28 & 39.8 & 41.5 & & 26 & & 28 & 45.9 & 45.4 & 42 & & 28 & 48.2 & 46.7 & & 39 & \\
\hline 30 & 35.2 & 36.8 & & 18 & -23.6 & 30 & 40.1 & 42.1 & & 26 & $-24 \cdot 4$ & 30 & 46.2 & 45.8 & 42 & -I8.9 & 30 & 47.9 & $46 . \mathrm{T}$ & & 40 & -17.6 \\
\hline 32 & 35.9 & 36.8 & & 10 & & 32 & 41.2 & 42.3 & & 27 & & 32 & 46.7 & 46.2 & 41 & & 32 & 48.7 & 47.2 & & 39 & \\
\hline 34 & 35.4 & 36.2 & & 18 & & 34 & $42 . I$ & 42.4 & & 27 & & 34 & 47.0 & 46.3 & $4 I$ & & 34 & 48.8 & 47.1 & & 39 & \\
\hline 36 & 34.5 & 34.8 & & 16 & & 36 & 40.1 & $4 \mathrm{I} .0$ & & 25 & & 36 & 46.2 & 45.8 & 42 & & 36 & 40.2 & 47.9 & & 38 & \\
\hline 38 & 34.0 & 35.1 & & 16 & & 38 & $39 \cdot 3$ & 40.9 & & 25 & & 38 & 45.7 & 45.0 & 43 & & 38 & $48 . n$ & 47.7 & & 38 & \\
\hline 40 & 31.9 & 36.3 & & т6 & & 40 & 37.7 & 38.2 & & 2I & & 40 & 45.9 & 45.1 & 42 & & 40 & 48.8 & 47.6 & & 38 & \\
\hline 42 & 32.0 & 36.1 & & 15 & & 42 & 36.1 & 37.0 & & IO & & 42 & 46.0 & 45.4 & 42 & & 42 & $48 . I$ & 47.1 & & 39 & \\
\hline 44 & 32.0 & 36.1 & & 15 & -23.9 & 44 & 35.7 & 36.1 & & I8 & $-24 \cdot 3$ & 44 & 46.4 & 45.7 & 42 & -18.7 & 44 & 47.4 & 45.6 & & 40 & -17.3 \\
\hline 46 & 31.9 & 35.3 & & 15 & & 46 & 35.0 & 35.9 & & I8 & & 46 & 47.2 & 45.9 & $4 \mathrm{I}$ & & 46 & 47.8 & 46.2 & & 40 & \\
\hline 48 & 34.0 & 37.2 & & I8 & & 48 & 36.8 & 37.2 & & 20 & & 48 & 47.1 & 46.0 & $4 \mathrm{I}$ & & 48 & 47.6 & $46 . I$ & & 40 & \\
\hline 50 & 33.2 & 36.6 & & I7 & & 50 & 36.9 & 37.7 & & 20 & & 50 & $47 . I$ & 46.1 & $4 \mathrm{I}$ & & 50 & $47 . I$ & 45.9 & & $4 \mathrm{I}$ & \\
\hline 52 & 34.0 & 36.2 & & I7 & & 52 & 37.7 & 38.3 & & 22 & & 52 & 47.9 & 46.9 & 39 & & 52 & 47.6 & 46.3 & & 40 & \\
\hline 54 & 34.0 & 37.0 & & I8 & & 54 & 39.0 & 38.8 & & 22 & & 54 & 48.0 & 47.2 & 39 & & 54 & 47.0 & 46.0 & & $4 \mathrm{I}$ & \\
\hline 56 & 35.8 & 37.2 & & 19 & & 56 & 38.0 & 39.0 & & 22 & & 56 & 48.0 & 47.4 & 39 & & 56 & 46.9 & 45.8 & & $4 \mathrm{~T}$ & \\
\hline 58 & 36.2 & 37.8 & & 20 & & 58 & 38.4 & 39. I & & 23 & & 58 & 48.0 & 47.8 & 39 & & 58 & 46.2 & 45.1 & & 42 & \\
\hline 100 & 37.3 & 38.9 & & 22 & -24.0 & 2300 & 38.9 & 39.8 & & 24 & $-24 \cdot 3$ & I 00 & 48.0 & 47.8 & 39 & -18.3 & 300 & 45.6 & 44.2 & & 43 & $-\mathrm{I} 7 \cdot 2$ \\
\hline$\infty$ & 37.9 & 30.0 & & 22 & 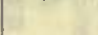 & 02 & 39.4 & 40.2 & & 24 & & 02 & 48.2 & 47.7 & 39 & & 02 & 45.0 & 43.0 & & 45 & \\
\hline 04 & 37.2 & 38.2 & 2 & $2 \mathrm{I}$ & te & 04 & 40.0 & 40.9 & & 25 & & OA & 48.2 & 47.7 & 30 & 8 & 04 & 44.7 & 42.0 & & 45 & \\
\hline 06 & 38.0 & 39.6 & & 23 & (1) & 06 & 39.9 & 40.5 & & 25 & & 06 & 48.2 & 47.8 & 38 & e & 06 & $44 \cdot 5$ & 42.8 & & 45 & \\
\hline 08 & 38.3 & $-40 . I$ & & 24 & 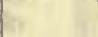 & 08.5 & 39.5 & 40.3 & & 25 & 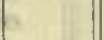 & 08 & 48.4 & 47.8 & 38 & & 08 & 44.0 & $43 . I$ & & 45 & \\
\hline I0 & 38.0 & 40.3 & & 24 & 1 & 10 & 39.2 & 40.0 & 5 & 24 & & 10 & 48.1 & 47.3 & 39 & $y$ & 10 & 44.8 & 43.3 & & 45 & \\
\hline 12 & 38.1 & 39.9 & 2 & 23 & & 12 & $39 \cdot 3$ & 40.2 & & 24 & & 12 & 47.9 & 47.0 & 39 & & 12 & 46.1 & 44.8 & & 4.3 & \\
\hline 14 & .38 .9 & 40.0 & & 24 & -24.2 & I4 & 30.1 & 41.9 & t & 26 & -23.3 & I 4 & 47.9 & 47.1 & 39 & $-I 8 . I$ & I4 & 47.2 & 45.7 & & $4 \mathrm{I}$ & -17.2 \\
\hline 16 & $37 \cdot 3$ & 30.0 & & 22 & $x=12$ & I6 & 38.3 & 40.2 & . & 24 & & I6 & 48.7 & 47.2 & 30 & & I6 & 40.0 & 47.2 & & 38 & \\
\hline 18 & 36.9 & 38.2 & & 21 & 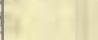 & I8 & 38.7 & 40.3 & an & 24 & & 18 & 49.0 & 47.8 & 38 & E. & I8 & 48.2 & 46. & & 39 & \\
\hline 20 & 36.3 & 37.8 & 1 & 20 & E & 20 & 37.2 & 41.5 & & 24 & & 20 & 40.0 & 47.7 & 38 & & 20 & 47.8 & 46.2 & & 40 & \\
\hline 22 & 36.9 & 38. I & $x$ & 21 & 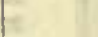 & 22 & 38.0 & 42.0 & & 25 & & 22 & 48.3 & 47.3 & 39 & & 22 & 46.9 & 45.8 & & $4 \mathrm{I}$ & \\
\hline 2.1 & 37.4 & 38.3 & & $2 I$ & 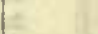 & 24 & 38.0 & 4.3 .0 & & 26 & & 24 & 47.9 & 47.1 & .30 & & 21 & 46.9 & 45.0 & & $4 \mathrm{I}$ & \\
\hline 26 & 38.0 & 39.9 & & 24 & E & 26 & 40.2 & 44.0 & & 28 & & 26 & 47.1 & $4^{6.7}$ & 40 & & 26 & 47.7 & 46.8 & & 40 & \\
\hline 28 & 39.6 & 40.9 & & 25 & & 28 & $4 \mathrm{I} .0$ & 44.9 & th & 29 & & 28 & 46.0 & 45.8 & 42 & & 28 & 47.6 & 45.1 & & 41 & \\
\hline 30 & 39.9 & 40.9 & & 25 & -24.2 & 30 & 42.0 & 45.7 & is & $3 \mathrm{~T}$ & -24.2 & 30 & 45.5 & $45 . \mathrm{I}$ & 43 & -18.0 & 30 & 45.8 & 43.3 & & 44 & -17.0 \\
\hline 32 & 39.7 & 40.0 & te & 24 & 1 & 32 & 42.7 & 45.9 & & 32 & & 32 & 45.7 & 45.0 & 4.3 & 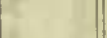 & 32 & 44.8 & 42.7 & & 45 & \\
\hline 34 & 39.8 & 40.1 & & 25 & 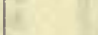 & 34 & 42.2 & 45.2 & $x^{2}$ & $3 I$ & & 31 & 4.5 .9 & $45 . \mathrm{I}$ & 42 & & 31 & 43.0 & 40.3 & & 48 & \\
\hline 36 & 39.8 & 40.2 & & 25 & 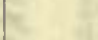 & 36 & 41.9 & 45.0 & 71 & 30 & & 36 & 46.1 & 45.7 & 42 & & 36 & 42.8 & 40.3 & & 49 & \\
\hline 38 & 39.8 & 40.0 & & 25 & te & 38 & 41.5 & 44.8 & & 30 & 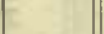 & 38 & 47.0 & 46. & $4 \mathrm{I}$ & & 38 & $4 \mathrm{I} .7$ & 30.3 & & 50 & \\
\hline 40 & 30.8 & 40.1 & 2 & 25 & 5 & 40 & 41.8 & 44.8 & 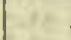 & 30 & & 40 & 47.1 & 46.7 & 40 & & 40 & 40.0 & 38.1 & & 53 & \\
\hline 42 & 37.8 & 39.8 & 2 & 23 & 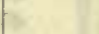 & 42 & 41.9 & 44.8 & & 30 & & 42 & 47.7 & 47.1 & 30 & $y$ & 42 & 30.7 & 37.9 & & 53 & \\
\hline 44 & 36.3 & 38.1 & 2 & 20 & $-24 \cdot 3$ & 44 & 41.9 & 44.7 & & 30 & $-24 \cdot 3$ & 44 & 48.3 & 48.0 & 38 & & 44 & 38.9 & 37.2 & & 54 & -17.0 \\
\hline 46 & 35.4 & 36.8 & I 1 & 19 & - & 46 & 41.2 & 44.2 & & 29 & & 46 & 49.0 & 48.9 & 37 & -17.9 & 46 & 38.0 & 36.2 & & 56 & \\
\hline 48 & 35.9 & 36.8 & 251 & I9 & 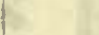 & 48 & 4 I. I & 44.0 & & 29 & & 48.4 & $49 \cdot 7$ & 49.7 & 36 & & 48 & 37.0 & 35.2 & & 57 & \\
\hline 50 & 35.8 & 36.9 & 35 & I9 & 18 & 50 & 41.0 & 43.7 & & 28 & & 50 & 49.2 & $40 . I$ & 37 & & 50 & 37.5 & 36.0 & & 56 & \\
\hline 52 & 35.2 & 36.3 & 1 & 18 & 2 & 52 & 40.9 & 43.0 & & 28 & & 52 & 40.0 & 48.0 & 37 & & 52 & 37.3 & 36.0 & & 56 & \\
\hline 54 & 35.0 & 35.5 & 1 & 17 & 2 & 54 & 40.7 & 42.8 & & 27 & & 54 & 48.8 & 48.3 & 38 & & 54 & 37.2 & 36.0 & & 56 & \\
\hline 56 & $35 . I$ & 35.7 & 15 & I8 & I & 56 & 41.0 & 42.8 & & 28 & & 56 & 48.9 & 48.8 & 37 & & 56 & 36.7 & 35.6 & & 57 & \\
\hline 58 & 34.5 & 35.0 & 1 & 16 & 50 & 58 & 4T. 8 & 43.5 & tit & $\begin{array}{l}29 \\
30\end{array}$ & -24.0 & 58 & 50.3 & 49.9 & 35 & & 58 & 35.8 & $34 \cdot 3$ & & 59 & \\
\hline & & & 1an & & por & 2400 & 42.5 & $44 \cdot \mathrm{I}$ & & 30 & & & & & & & & & & & & \\
\hline
\end{tabular}

Correction to local mean tine is - Im 06.5s.

Observer-R, R. T.

Torsion head at Igll $20 m$ read $34^{\circ}$ and at $24 \mathrm{~h}$ I $7 \mathrm{~m}$ read the same.

Observer-J. V. 
Tabulation of magnetic declinations observed at Teplitz Bay-Continued

\begin{tabular}{|c|c|c|c|c|c|c|c|c|c|c|c|c|c|c|c|c|c|c|c|c|c|c|}
\hline \multicolumn{5}{|c|}{ Sunday, April 17, 1904} & \multicolumn{6}{|c|}{ Magnet scale erect } & \multicolumn{7}{|c|}{ Monday, April 18, 1904} & \multicolumn{5}{|c|}{ Magnet scale inverted } \\
\hline \multirow{2}{*}{$\begin{array}{l}\text { Chr'r } \\
\text { time }\end{array}$} & \multicolumn{2}{|c|}{$\begin{array}{l}\text { Scale } \\
\text { readings }\end{array}$} & East & & Chr'r & $\begin{array}{l}\text { Sca } \\
\text { readi }\end{array}$ & $\begin{array}{l}\text { ale } \\
\text { ings }\end{array}$ & $\begin{array}{l}\mathrm{Ea} \\
\mathrm{dec}\end{array}$ & & Temp. & Chr's & $\begin{array}{l}\text { Sc: } \\
\text { read }\end{array}$ & $\begin{array}{l}\text { le } \\
\text { ngs }\end{array}$ & Eas & $\begin{array}{l}\text { st } \\
\text { cli- }\end{array}$ & Temp. & Chr'r & $\begin{array}{r}\text { Sc } \\
\text { read }\end{array}$ & igs & Ea & cli- & Temp \\
\hline & Left & Right & & & & Left & Right & & & & & Left & Right & & & & & Left & Right & & & \\
\hline $\mathrm{m}$ & $d$ & d & $\cdot$, & 10 & $\mathrm{~h} \mathrm{~m}$ & $d$ & d & $\cdot$ & $\cdot$ & & $\mathrm{h} \mathrm{m}$ & d & d & & , & & $h \mathrm{~m}$ & U & d & - & , & \\
\hline 00 & 69.7 & 74.3 & 2300 & -16.8 & 600 & .43 .0 & 41.0 & 23 & 12 . & -16.0 & $800^{*}$ & 42.9 & 40.2 & 23 & I6 & & 1000 & 27.8 & 22.2 & 22 & 58 & -16.1 \\
\hline 02 & 70.0 & 74.8 & oI & & 02 & 43.1 & 44.2 & & 12 & & 02 & 41.8 & 38.8 & & 18 & B & 02 & 33.0 & 31.0 & & 46 & \\
\hline 04 & 70.2 & 75.0 & oI & r & 04 & 45.0 & 46.0 & & 15 & & 04 & 42.3 & 40.4 & & 16 & 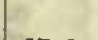 & 04 & 37.0 & 35.9 & & 39 & \\
\hline 06 & 70.6 & 75.0 & oI & 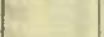 & 06 & 44.2 & 45.2 & & 14 & 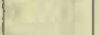 & 06 & 45.2 & 43.8 & & II & -17.0 & о6 & 35.0 & 33.0 & & 43 & \\
\hline 08 & 70.0 & $74 \cdot 3$ & $\infty 0$ & 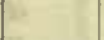 & 08 & 41.6 & 42.7 & & 10 & ty & 08 & 45.4 & 44.1 & & II & & 08 & 37.1 & 35.2 & & 40 & \\
\hline 10 & 71.7 & 72.1 & $\infty$ & 1 & 10 & 40.3 & 42.0 & & 08 & & 10 & -48.0 & 45.5 & & 08 & & 10 & 36.0 & 36.0 & & 40 & \\
\hline 12 & 72.5 & 73.1 & OI & & 12 & 40.1 & 41.9 & & 08 & & 12 & 43.8 & 43.3 & & 13 & & 12 & 37.0 & 36.0 & & 39 & \\
\hline 14 & $73 . I$ & 73.7 & 02 & -16.8 & 14 & 41.0 & 42.3 & & $\infty 9$ & -15.9 & 14 & 44.1 & 42.1 & & 14 & -17.0 & 14 & 29.2 & 28.0 & & 52 & -15.6 \\
\hline 16 & 73.8 & 74.9 & 04 & & 16 & 39.0 & 40.7 & & 07 & & 16 & 44.2 & 40.9 & & 15 & & 16 & 30.4 & 28.0 & & 51 & \\
\hline 18 & 74.1 & 75.1 & 04 & 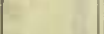 & 18 & 38.8 & 30.7 & & 05 & & 18 & 46.2 & 41.1 & 23 & 13 & 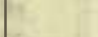 & J 8 & 35.1 & 34.0 & & 42 & \\
\hline 20 & 75.0 & 76.0 & 06 & c & 20 & 37.9 & 38.9 & & 04 & & 20 & 55.6 & 52.0 & 22 & 57 & 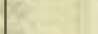 & 20 & 40.2 & 38.8 & & 35 & \\
\hline 22 & 76.2 & 77.1 & 07 & $=$ & 22 & 40.0 & 40.6 & & of & & 22 & 5.3 .1 & 5T.I & & 59 & 1 & 22 & 47.0 & 45.8 & & 24 & \\
\hline 24 & 77.2 & 78.0 & $\infty$ & $E$ & 24 & 39.8 & 40.3 & & $\infty$ & & 24 & 60.2 & 56. & & 50 & 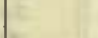 & 24 & 49.9 & 47.6 & & 20 & \\
\hline $26^{*}$ & 38. I & $43 . I$ & 07 & $=$ & 26 & 39.1 & 39.9 & & 05 & & 26 & 61.7 & 58.2 & & 47 & 1 & 26 & 47.6 & 46.9 & & 22 & \\
\hline 28 & 39.1 & 44.8 & 09 & & 28 & 42.2 & 43.2 & & 10 & & 28 & 65.0 & 62.8 & & 40 & 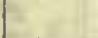 & 28 & $44 \cdot 3$ & 43.2 & & 28 & \\
\hline 30 & 39.3 & 45.0 & 10 & -16.7 & 30 & 40.1 & 41.0 & & 07 & -15.9 & 30 & 72.3 & 69.8 & & 29 & $-17 \cdot I$ & 30 & 48.3 & 47.1 & & 22 & -15.2 \\
\hline 32 & 40.2 & $45 \cdot 3$ & I0 & . & 32 & 37.8 & 38.1 & & 03 & & $32^{*}$ & 54.0 & 52.1 & & 20 & & 32 & 44.3 & 42 & & 28 & \\
\hline 34 & 44.1 & 45.3 & 14 & F & 34 & 36.9 & 37.4 & & 02 & & 34 & 52.1 & 51 & & 22 & 1 & 34 & 44.0 & $4 \mathrm{I}$. & & 19 & \\
\hline 36 & 40.1 & 44.9 & I0 & & 36 & 37.1 & $37 \cdot 7$ & & 02 & & 36 & 54.9 & 52. & & 19 & & 36 & 48.2 & 46 & & 22 & \\
\hline 38 & $4 \mathrm{I} \cdot 7$ & 46.0 & 12 & te & 38 & 36.9 & 37.4 & & 02 & & 38 & 52.9 & 50.6 & & 22 & te & 38 & 51.1 & 50.0 & & 17 & \\
\hline 40 & 42.8 & 47.2 & 14 & 4 & 40 & 37.6 & 38.0 & & 03 & & 40 & 54.7 & 53.7 & & 18 & 1 & $4 c$ & 51.5 & & & 16 & \\
\hline 42 & 43.1 & $47 \cdot 3$ & 14 & & 42 & 38.3 & 38.8 & & 04 & & 42 & 5.3 .9 & 51.5 & & 20 & $x_{2}$ & 42 & 48.1 & 46.5 & & 22 & \\
\hline 44 & 43.0 & 46.9 & 14 & -16.7 & 44 & $36 \cdot 3$ & 37.0 & & OI & -15.8 & 44 & 48.0 & 46.5 & & 29 & -17.0 & 44 & 43.2 & 42.5 & & 20 & -15.0 \\
\hline 46 & 43.5 & 47.5 & 15 & & 46 & 36.3 & 37.6 & 23 & OI & & 46 & 40.3 & 39.2 & & $4 \mathrm{I}$ & & $4 t$ & 44.0 & 42.8 & & 28 & \\
\hline 48 & 45.1 & 48.9 & 17 & & 48 & 35.0 & 36.0 & 22 & 59 & & 48 & 35.0 & 34. & & 49 & 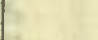 & 48 & 41.9 & 40.2 & & 32 & \\
\hline 50 & 5.3 & 48.9 & 17 & at & 50 & 35.8 & 36.8 & 23 & $\infty 0$ & & 50 & 38.3 & 35.8 & & 45 & 1 & 50 & 41.0 & 39. & & 33 & \\
\hline 52 & 1.7 & 48.1 & 16 & $z$ & 52 & 32.1 & 33.1 & 22 & 54 & & 52 & 37.0 & 35.2 & & 46 & 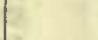 & 5 & 44.1 & & & 28 & \\
\hline 54 & 4.2 & 47.1 & 15 & t & 54 & 35.8 & 37.0 & 23 & $\infty$ & & $54 \cdot 3$ & 65 &.$o b$ & 22 & OI & $E$ & 54 & 47.5 & 46. & & 23 & \\
\hline 56 & 4.8 & 47.2 & 16 & $E$ & 56 & 34.6 & 35.4 & 22 & 58 & & $56^{*}$ & 5.5 .8 & 51.8 & 21 & 47 & & 5 & 49.8 & 48.8 & & 19 & \\
\hline 58 & 44.0 & 46.0 & 14 & & 58 & 35.0 & 36.0 & 107 & 59 & & 58 & 38.3 & 35.0 & 22 & 14 & & 58 & 52.0 & 50.5 & & 16 & \\
\hline 00 & 42.9 & 44.7 & 12 & -16.7 & 700 & 34.1 & 36.1 & 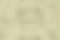 & 58 & -15.7 & 900 & 40.3 & 37.7 & & II & -17.0 & II 00 & 44.0 & 42.7 & & 28 & -14.8 \\
\hline 02 & 42.9 & 45.9 & 13 & & 02 & 29. & $.0 a$ & & 49 & & 02 & 40.0 & 37.0 & & II & & 02 & 45.9 & 48. & & 23 & \\
\hline 04 & 4.7 & 47. & 16 & & 04 & 34.1 & 34.4 & & 57 & & 04 & 38.5 & 36.0 & & 13 & 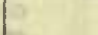 & o. & 48.0 & 46.0 & & 2 & \\
\hline 06 & $45 . \mathrm{I}$ & 47.9 & 16 & & 06 & 17. & $o b$ & & 30 & & 06 & 38.4 & 35.1 & & I 4 & tis & of & 49.7 & 49. & & 18 & \\
\hline 08 & $44 . I$ & 46.0 & 14 & en & 08 & 15 & $7 a$ & CF & 28 & & 08 & 42.0 & 40 & & o7 & (2) & 08 & 51.0 & 50.5 & & 16 & \\
\hline Io & 4.8 & 47.4 & 16 & & 10 & 33.6 & 34.8 & & 57 & & 10 & 46.0 & 44.8 & & $\infty$ & ( & IC & 47.1 & 47. & & 22 & \\
\hline 12 & .8 & 45. & 13 & & 12 & 34. & $3 a$ & 22 & 57 & & I2 & 42.8 & 42.0 & 22 & 04 & & 12 & 42. & $.8 b$ & & 29 & \\
\hline 14 & 43.6 & 46.8 & 14 & -16.5 & 14 & 44.8 & 45.6 & 23 & 14 & $-15 \cdot 3$ & 14 & 47.0 & 46.1 & 21 & 58 & -16.9 & I4 & 39.2 & 38.2 & & 35 & -14.7 \\
\hline 16 & $44 \cdot 3$ & 46.9 & 15 & 8 & & 47.7 & 48.8 & $z^{2}$ & IO & & If & 47.0 & 46.0 & & 58 & & If & 40.2 & 40.0 & & 33 & \\
\hline 18 & 43.2 & 45.7 & $I_{3}$ & t. & 18 & 46.1 & 46.3 & 23 & 16 & & $\mathbf{I}$ & 49.0 & 47.0 & 21 & 55 & $t$ & 18 & 39. & $.9 a$ & & 33 & \\
\hline 20 & $42 . I$ & 44.7 & II & 1 & 20 & 32. & $6 b$ & 22 & 54 & & $2 x$ & 31.0 & 40.0 & 22 & of & 5 & 20 & 43.0 & 42.0 & & 29 & \\
\hline 22 & 0.9 & 43.8 & 10 & & & 32.2 & 32.8 & 22 & 54 & & 2 & 42.2 & & 22 & 06 & 5 & 22 & 40.0 & 39.5 & & 34 & \\
\hline 24 & 40.2 & 42.7 & 08 & 2 & 2 & 37.1 & 37.8 & 23 & 02 & & 2 & 50.4 & 48.0 & 21 & 53 & $\mathrm{~A}$ & 24 & 34.8 & 34 & & 42 & \\
\hline 26 & . I & 42.9 & 09 & & 26 & 4I.I & 41.0 & a & 08 & & 26 & 3 & & 21 & & & 26 & 31.0 & 30.3 & & 48 & \\
\hline 28 & 42.2 & 44.1 & II & & 28 & 46.2 & 47.8 & 23 & 17 & & 28 & $34 . I$ & 29.8 & 22 & 21 & & 28 & 33.0 & 32.5 & & 44 & \\
\hline 30 & D.I & 41.8 & 08 & -16.3 & 30 & 31.3 & 32.7 & 22 & 54 & -15.0 & 30 & 36.9 & 33.0 & & 15 & -16.9 & 30 & 40.1 & 39.6 & & 33 & -14.5 \\
\hline 32.2 & 45.0 & 47. & I6 & 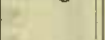 & 32 & 29.2 & 30.7 & 31. & 50 & & 32 & $45 \cdot 5$ & $4 \mathrm{I}$. & & 02 & & 32 & 37 & $6 b$ & & 37 & \\
\hline 34 & 45.1 & 46.1 & & 1 & & .3 & 30. & 76 & 51 & & 34 & 32.2 & 28.9 & & 23 & & 3 & 41.5 & 41.0 & & 31 & \\
\hline 36 & 41.9 & 44.8 & II & & & 32.0 & 32.8 & ter & 54 & & 36 & 29.0 & 22.0 & & 31 & & 36 & 40.3 & & & 33 & \\
\hline 38 & 40.1 & $43 \cdot 3$ & & te & & & 32.0 & 74 & 53 & & 38 & 22.1 & 17.0 & & 40 & & 38 & 50.0 & 48.0 & & 19 & \\
\hline 40 & $4 \mathrm{I} .5$ & 44. & II & 1 & $4 c$ & 30.8 & 31.5 & 32 & 52 & & 40 & 18.8 & 12.0 & & 47 & & 40 & 47.2 & $47 . I$ & & 22 & \\
\hline 42 & .0 & 45.2 & 12 & & 42 & 28.9 & $20 \cdot 3$ & & 49 & & 42 & 23.0 & 19.4 & & 37 & & 42 & 44.0 & 43.9 & & 27 & \\
\hline 44 & 41.7 & 45.7 & 12 & -16.1 & 44 & 27.9 & 28.9 & B & 48 & -14.7 & 44 & 34.9 & 30.0 & & 20 & -16.8 & $44 \cdot 3$ & 38 & $.2 b$ & 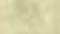 & 36 & -14.2 \\
\hline 46 & 42.8 & 45.9 & 13 & & 46 & 29.1 & 30.7 & $\ln _{5}$ & 50 & & 46 & 34.9 & 29.2 & & 20 & & 46 & 40.3 & 39.9 & & 33 & \\
\hline 48 & 41.0 & 44.6 & II & 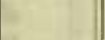 & 48 & & 31.0 & & 51 & & 48 & 38.2 & 24.8 & & 21 & & 48 & 42.9 & 42.0 & & 29 & \\
\hline 50 & 41.2 & $44 . \mathrm{I}$ & 10 & & 50 & 29.8 & 32.2 & 62 & 52 & & 50 & 22.8 & 17.9 & & 39 & & 5o & $45 \cdot 3$ & 45.1 & 8 & 24 & \\
\hline 52 & 41.9 & 44.0 & II & 8 & 52 & 29.8 & 32.0 & 17 & 52 & & 52 & 24.2 & 21.0 & & 35 & & 52 & 47.5 & 48.7 & & & \\
\hline 54 & 42.1 & $44 . I$ & II & te & 54 & 29.1 & 31.0 & 72 & 50 & & 54 & 20.0 & 16.9 & & 42 & 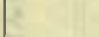 & 54 & 43 & $.0 b$ & & 28 & \\
\hline 56 & 43.5 & 44.9 & 13 & ? & 56 & 29.1 & 30.7 & 125 & 50 & & 56 & 13.9 & 9.1 & & 52 & & 56 & 43.7 & 43.1 & & 27 & \\
\hline 58 & 42.0 & 43.1 & IO & E & $8 \stackrel{58}{00}$ & $\begin{array}{l}27.7 \\
28.9\end{array}$ & $\begin{array}{l}28.9 \\
30.0\end{array}$ & 3. & $\begin{array}{l}48 \\
50\end{array}$ & $-I_{4} \cdot 1$ & $58 *$ & 27.2 & 21.1 & & 59 & S.4 & 58 & 53.1 & 50.0 & 19. & I4 & \\
\hline
\end{tabular}

Correction to local mean time is - Im $26 \mathrm{~s}$.

Torsion head at ol oom read $36^{\circ}$ and at 81 oom rcad the same. Observer-R. R. T.
Correction to local mean time is $-\operatorname{Im} 57.5 \mathrm{~s} .90^{\circ}$ torsion $=16 .^{\prime} 13$ Torsion head at $7 \mathrm{~h} 25 \mathrm{~m}$ read $33^{\circ}$ and at $12 \mathrm{~h} 26 \mathrm{~m}$ read $53^{\circ}$. Observer-J. V. 
Tabulation of magnetic declinations observed at Teplitz Bay-Continued

\begin{tabular}{|c|c|c|c|c|c|c|c|c|c|c|c|c|c|c|c|c|c|c|c|c|c|c|}
\hline \multicolumn{7}{|c|}{ Tuesday, April 19, 1904} & \multicolumn{5}{|c|}{ Magnet scale erect } & \multicolumn{6}{|c|}{ Wednesday, April 20, 1904} & \multicolumn{5}{|c|}{ Magnet scale inverted } \\
\hline \multirow{2}{*}{$\begin{array}{l}\text { Chr'r } \\
\text { time }\end{array}$} & $\begin{array}{r}\text { Sc } \\
\text { read }\end{array}$ & $\begin{array}{l}\text { ale } \\
\text { lings }\end{array}$ & Eas & & Temp. & Chr'r & $\begin{array}{l}\mathrm{Sc} \\
\text { read }\end{array}$ & $\begin{array}{l}\text { ale } \\
\text { lings }\end{array}$ & East & & Temp & Chr'r & $\begin{array}{l}\text { Sca } \\
\text { read }\end{array}$ & $\begin{array}{l}\text { ale } \\
\text { lings }\end{array}$ & $\begin{array}{c}\text { East } \\
\text { decli- }\end{array}$ & Temp. & Chr'r & $\begin{array}{l}\text { Sca } \\
\text { read }\end{array}$ & $\begin{array}{l}\text { ale } \\
\text { lings }\end{array}$ & $\begin{array}{l}\text { East } \\
\text { decli }\end{array}$ & & Temp. \\
\hline & Left & Right & natic & & & & Left & Right & matio & & & & Left & Right & natson & $\therefore$ & time & Left & Right & & & \\
\hline h in & d & $d$ & $\cdot$ & ' & $\therefore$ & $\mathrm{h} \mathrm{m}$ & d & 4 & $\cdot$ & $\therefore$ & & $\mathrm{h} \mathrm{m}$ & 4 & 0 & & & $h \mathrm{~m}$ & d & $d$ & $\cdot$ & & $\circ$ \\
\hline $1200^{*}$ & 49.8 & 51.2 & 230 & 04. & -16.3 & 1400 & 58.9 & 60.1 & $23 I$ & 18 & -16.0 & 000 & 55.7 & 52.1 & $22 \mathrm{I} 8$ & -17.7 & 200 & 32.0 & 30.3 & 225 & 53 & -16.7 \\
\hline 02 & 50.3 & 52.0 & & 05 & & 02 & 50.0 & 60.5 & & & & $\infty$ & 56.3 & 52.1 & 17 & & 02 & 33.6 & 31.1 & & 51 & \\
\hline 04 & 52.0 & 53.8 & & 08 & 6. & 04 & 58.0 & 60.0 & & I8 & & 04 & 56.6 & 53.0 & I6 & if & 04 & 31.7 & $29 . I$ & & 54 & \\
\hline 06 & 53.5 & 54.0 & & 10 & 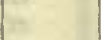 & 06 & 56.9 & 57.8 & & I5 & & 06 & 57.0 & 53.0 & 16 & 6 & 06 & 32.9 & 30.7 & & 52 & \\
\hline 08 & 54.6 & 55.8 & & 12 & 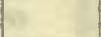 & 08 & 55.9 & 57.1 & & 14 & & 08 & 56.9 & 52.4 & I6 & 0 & 08 & 33.2 & 30.7 & & 52 & \\
\hline 10 & 55.0 & 5.8 & & 12 & V & 10 & 50.3 & 52.0 & & 05 & & ro & 56.9 & 52.3 & I6 & (1) & I0 & 32.9 & 30.2 & & 53 & \\
\hline 12 & 55.2 & 56.5 & & 13 & & 12 & 54.2 & 56.0 & I & 12 & & 12 & 56.1 & 52.3 & 17 & & 12 & 3 I. 0 & 29.2 & & 54 & \\
\hline 14 & 56.0 & 57.0 & & 14 & -16.3 & I4 & 54.8 & 56.2 & & I2 & -15.9 & $\mathrm{I} 4.2$ & 55.2 & 51.4 & T. 8 & -17.3 & $\mathrm{IA}$ & 32.8 & 30.0 & & 5.3 & -16.4 \\
\hline 16 & $55 \cdot 3$ & 56.0 & & 12 & r & 16 & $5 \mathrm{~T} .9$ & 54.0 & $4 y$ & 08 & & I6 & 54.3 & 50.8 & 20 & & 16 & 20.9 & 27.2 & 225 & 57 & \\
\hline 18 & 55.9 & 56.7 & & 13 & 1 & I8 & 50.1 & 51.2 & & 04 & & i8 & 54.0 & 50.7 & 20 & & 18 & 27.9 & 25.8 & 230 & $\infty$ & \\
\hline 20 & 56.1 & 57.0 & & 14 & $=$ & 20 & 47.9 & 49.0 & & or & & 20 & 54.1 & 51.1 & 20 & 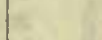 & 20 & 25.0 & 22.9 & & 05 & \\
\hline 22 & 55.4 & 56.2 & & 13 & $E$ & 22 & 46.0 & 48.1 & 230 & $\infty$ & & 22 & 56.4 & 53.5 & 16 & H & 22 & 23.6 & 21 & & 07 & \\
\hline 24 & 55.8 & 56.9 & o & 13 & $=$ & 24 & 46.8 & 48.1 & 225 & 50 & & 24. & 56.9 & 54.1 & 15 & $=$ & 24 & 23.2 & 20.6 & & 08 & \\
\hline 26 & 55.0 & 56.1 & & 12 & $=$ & 26 & 45.0 & 47.0 & & 58 & & 26 & 56.3 & 54.0 & 16 & \& & 26 & 25.3 & 22.7 & & 04 & \\
\hline 28 & 57.2 & 58.7 & 11 & 16 & & 28 & 46.8 & 47.0 & 225 & 59 & & 28 & 56.8 & 54.1 & 15 & & 28 & 24.4 & 22.0 & & क । & \\
\hline 30 & 57.2 & 58.3 & 1 & ז6 - & -16.2 & 30 & 17.2 & 17.8 & 230 & on & & 30 & 57.0 & 54.0 & 14 & -17.1 & 30 & 23.9 & $2 I$ & & 07 & -16.0 \\
\hline 32 & 57.7 & 50.0 & & 17 & 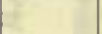 & 32 & 48.3 & 40.5 & & 02 & & 32 & 58.8 & 56.8 & & $C^{2}$ & 32 & $2 \mathrm{r}: 3$ & 18.9 & & II & \\
\hline 34 & 57.0 & 58.1 & & 15 & 8 & 34 & 47.5 & 48.5 & 230 & $\infty 0$ & & 34 & $60 . r$ & 58.4 & $\infty$ & & 31 & I8. & 15.7 & & I6 & \\
\hline 36 & 56.5 & 57.9 & & 15 & 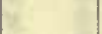 & 36 & 45.5 & 46.2 & 225 & 57 & & 36 & 62.2 & 60.6 & क6 & 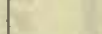 & 35 & I8. I & 15.9 & & 15 & \\
\hline 38 & 56.1 & $57 \cdot 3$ & r & 14 & 1 & 38 & 43.9 & 45.1 & & 55 & & 38 & 62.8 & 61.0 & 05 & & 38 & 18.7 & 17.2 & & 14 & \\
\hline 40 & 56.9 & 57.5 & & 15 & 1 & 40 & 44.9 & 45.0 & 5 & 56 & & 40 & 63.2 & $6 \mathrm{r} .8$ & 04 & & 40 & 21.5 & 20.0 & & 10 & \\
\hline 42 & 56.6 & 57.0 & 8 & 14 & & 42 & 43.2 & 44.1 & 5 & 53 & & 42 & 63.9 & 62.0 & 03 & & 42 & 25.0 & 23.9 & & 04 & \\
\hline 44 & 56.9 & 57.4 & 1 & $15-$ & -16.2 & 44 & 43.2 & 44. I & 5 & 53 & -16.0 & 44 & 63.2 & $6 \mathrm{r} .5$ & 04 & -17.0 & 44 & 26.0 & 24.9 & & 02 & \\
\hline 46 & 55.8 & 56.0 & 1 & 13 & 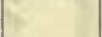 & 46 & 44.0 & 45.0 & $6 i$ & 55 & & 46 & 62.9 & $6 \mathrm{r} . \mathrm{I}$ & 05 & & 46 & 27.1 & 26.0 & & $\infty$ & -15.9 \\
\hline 48 & 56.0 & 56.5 & 151 & 13 & is & 48 & 43.2 & 44.0 & 25 & 5.3 & & 48 & 63.5 & $6 \mathrm{~T} .5$ & 04 & 1 & 48 & 24.7 & 24.0 & & 04 & \\
\hline 50 & 57.9 & 58.1 & 1 & 16 & 2 & 50 & $41 \cdot 3$ & 42.1 & & 50 & & 50 & 61.1 & 50.3 & 08 & 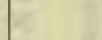 & 50 & 2.3 .1 & 22.7 & & 06 & \\
\hline 52 & 50.0 & 59.3 & 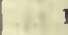 & 18 & $=$ & 52 & 36.7 & 37.0 & & 43 & & 52 & 50.6 & 57.8 & IO & E & 52 & 23.1 & 22.2 & & 07 & \\
\hline 54 & 58.7 & 59.1 & 1 & 18 & 1 & 54 & $35 \cdot 3$ & 36.1 & & $4^{\mathrm{I}}$ & & 54 & 58.9 & 57.0 & II & in & 54 & 25.1 & $21 . \mathrm{I}$ & 230 & 0.1 & \\
\hline 56 & 57.5 & 57.9 & & 16 & & 56 & 36.0 & 37.0 & & 42 & & 56 & 55.4 & 53.7 & 16 & 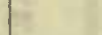 & 56 & 20.8 & 28.9 & 225 & 55 & \\
\hline 58 & 57.6 & 57.9 & 1 & 16 & & 58 & 34.3 & 35.8 & & 40 & & 58 & 54.2 & 52.5 & 18 & & 58 & 30.4 & 30.0 & & 55 & \\
\hline 1300 & 57.2 & 57.5 & 141 & 15 & -16.1 & 1500 & 32.8 & .34 .0 & & 37 & -16.0 & 100 & 52.4 & 50.6 & $2 \mathrm{I}$ & -17.0 & 300 & $2 n . ?$ & 20.0 & & 5 & \\
\hline 02 & 57.1 & 57.9 & 1 & 15 & 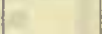 & 02 & 31.3 & 32.2 & & 35 & 8 & 02 & 51.8 & $\Delta 0.3$ & 23 & -5 & $\infty$ & 28.8 & 28. I & 225 & 58 & -15.9 \\
\hline 04 & 58.1 & 58.9 & 1 & 17 & s. & 01 & 29.9 & 30.5 & 3 & 32 & 2. & 0.1 & 50.2 & 48.2 & 25 & 17 & 01 & 27.1 & 25.8 & 230 & $\infty$ & \\
\hline 06 & 58.0 & 58.7 & 1 & 17 & 6 & 06 & 27.5 & 28. I & te. & 29 & 3 & of & 50.3 & $48 . I$ & 25 & 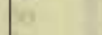 & of & 28.9 & 28.2 & 225 & 57 & \\
\hline 08 & 58.0 & 58.9 & 78 & 17 & 0 & 08 & 26.1 & 27.0 & & 27 & 6 & 08 & 49.0 & 47.8 & 26 & D. & os & 20.7 & $29 . \mathrm{I}$ & & 56 & \\
\hline Io & 60.0 & 61.0 & & 20 & 12 & 10 & 25.5 & 26.2 & A2t & 26 & & Io & 49.7 & 47.7 & 26 & ir & 10 & 20.8 & 29.2 & & 56 & \\
\hline 12 & 59.5 & 60.5 & & 19 & & 12 & 22.3 & 23.0 & $\pi$ & 20 & & 12 & 51.1 & 48.8 & 24 & & 12 & 31.2 & 30.0 & & 53 & \\
\hline & 60.7 & 61.0 & & 20 & -16.1 & 14 & $23 . I$ & 24.0 & o & 22 & - 16.0 & 14 & 51.2 & 49.0 & 23 & -16.9 & 14 & 33.2 & 32.8 & & 50 & -15.0 \\
\hline 16 & 62.6 & 63.0 & & 24 & 1 & 16 & 26.2 & 27.8 & $1=$ & 27 & & 16 & 52.0 & 50.0 & 22 & 1 & I6 & 34.6 & .34 .2 & & 48 & \\
\hline 18 & 64.0 & 64.8 & & 26 & I & 18 & $27 \cdot 3$ & 29.0 & tie & 29 & & 18 & $53 \cdot 3$ & 51.0 & 20 & I & 18 & 34.9 & 34.4 & & $\triangle 8$ & \\
\hline 20 & 64.8 & 65.8 & ts & 28 & E & 20 & 30.1 & 31.2 & 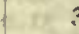 & 33 & & 20 & 54.2 & 51.8 & IO & 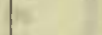 & 20 & 35.3 & .34 .8 & & 47 & \\
\hline 22 & 63.3 & 64.2 & a & 25 & $=$ & 22 & 29.9 & 30.8 & 413 & 33 & $=$ & 22 & 52.4 & 49.7 & 22 & 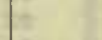 & 22 & 36.0 & 35.5 & & 16 & \\
\hline 24 & 63.1 & 64.2 & & 25 & $=$ & 24 & 26.1 & 27.9 & nt & 27 & & 24 & 51.8 & 40.3 & 2,3 & tr & 21 & .34 .5 & .34 .0 & & 18 & \\
\hline 26 & 64.5 & 65.2 & & 27 & 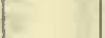 & 26 & 26.1 & 28.0 & & 27 & & 25 & 50.0 & 47.8 & 25 & 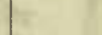 & 26 & 33.0 & 32.3 & & 51 & \\
\hline 28 & 63.2 & 64.9 & 102 & 26 & & 28 & 23.0 & 24.5 & & 22 & & 28 & 49.5 & 47.0 & 26 & & 28 & .32 .6 & 32.0 & & 51 & \\
\hline 30 & 62.1 & 64.5 & 2 & 24 & -16.0 & 30 & 23.0 & 25.0 & & 23 & -16.0 & 30 & 47.7 & $45 . \mathrm{I}$ & 20 & -16.8 & 30 & 33.0 & .32 .7 & & 51 & -15.8 \\
\hline 32 & 62.0 & 63.0 & & 23 & 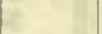 & 32 & 22.9 & 24.7 & 78 & 22 & & 32 & 45.8 & 43.0 & 32 & 1 & 32 & 35.4 & 35.0 & & 47 & \\
\hline 34 & 61.0 & 62.0 & & 22 & & 34 & 23.7 & 25.2 & tis & 23 & & 31 & 45.1 & 42.8 & 32 & 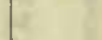 & 34 & 36.1 & 35.7 & & 46 & \\
\hline 36 & 60.3 & 6 ז.8 & ces & 21 & & 36 & 22.7 & 24.3 & & 22 & & .26 & 43.8 & $4 \mathrm{I} . \mathrm{r}$ & 36 & 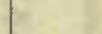 & .65 & .35 .0 & .34 .2 & & 48 & \\
\hline 38 & 61.2 & 62.5 & & 22 & 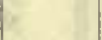 & 38 & 20.0 & 21.5 & & 18 & & 38 & 43.0 & A2. I & 35 & 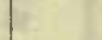 & 38 & 33.9 & 33.2 & & 50 & \\
\hline 40 & 60.9 & 6r.8 & 5 & $2 \mathrm{I}$ & 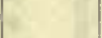 & 40 & I7. I & 19.1 & ลิำ & 13 & & 40 & 40.7 & 38.6 & 40 & & 40 & 32.9 & 32.2 & & $5 \mathrm{I}$ & \\
\hline 42 & 59.2 & 59.8 & & I8 & 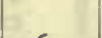 & 42 & 13.7 & 15.2 & it & 08 & & 42 & 30.9 & 37.2 & 42 & & 42 & 33.6 & $33 . I$ & & 50 & \\
\hline 44 & 58.2 & 59.0 & & 17 & -16.0 & 44 & 13.7 & 15.4 & ie & 08 & -16.0 & 44 & .38 .3 & 35.7 & 44 & -16.8 & 44 & 34.7 & $34 . I$ & & 48 & \\
\hline 46 & 59.1 & 59.7 & 150 & 18 & 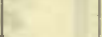 & 46 & 13.9 & 15.5 & & 08 & & 46 & 36.0 & 33.1 & 48 & I & 46 & 37.8 & 37.0 & & 43 & -15.7 \\
\hline 48 & 59.9 & 60.1 & 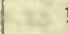 & I9 & 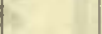 & 48 & 8.0 & ro. 9 & 22 & $\infty$ & & 48 & .34 .4 & 32.1 & 50 & 1. & 48 & $35 . \mathrm{I}$ & 34.6 & & 47 & \\
\hline 50 & 59.9 & 60.2 & 28 & I9 & 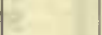 & 50 & 6.5 & 8.4 & $2 \mathrm{I}$ & 57 & & 50 & 33.8 & 31.8 & $5 I$ & 1 & 50 & 40.8 & 40.8 & & 38 & \\
\hline 52 & 59.9 & 60.0 & & 19 & 2 & $52^{*}$ & 35.0 & 40.0 & 5 & 54 & & 52 & 33.4 & $3 \mathrm{~T} .2$ & 5 I & 1 & 52 & 38.9 & 38. I & & 42 & \\
\hline 54 & 57.8 & 58.1 & & 16 & E & 54 & 32.8 & 38.0 & 5 & 51 & & 54 & 33.8 & 31.4 & 51 & $1=$ & 54 & 37.0 & 36.8 & & 44 & \\
\hline 56 & 58.2 & 58.9 & & 17 & $z$ & 56 & 29.9 & 33.7 & $x i$ & 46 & & 56 & 34.0 & 32.0 & 50 & & 56 & 37.0 & 36.7 & 4 & 44 & \\
\hline 58 & 59.0 & 59.6 & 71 & 18 & & 58 & $\begin{array}{l}29.9 \\
29.0\end{array}$ & 34.0 & 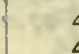 & $\begin{array}{l}46 \\
44\end{array}$ & -16.0 & 58 & .32 .8 & 30.0 & 5.3 & 18 & 58 & $3^{6.4}$ & 36.0 & 4 & 45 & \\
\hline & & & & & & & 29.0 & 32.9 & & 49 & 1 & & & & & & & & & & & \\
\hline
\end{tabular}


Tabulation of magnetic declinations observed at Teplitz Bay-Continued

Wednesday, April 20, 1904
Wednesday, April 20, rgo4

Magnet scale inverted

\begin{tabular}{|c|c|c|c|c|c|c|c|c|c|c|c|c|c|c|c|c|c|c|c|c|c|c|c|}
\hline \multirow[t]{2}{*}{$\begin{array}{l}\text { Chr'r } \\
\text { time }\end{array}$} & \multicolumn{2}{|c|}{$\begin{array}{l}\text { Scale } \\
\text { readings }\end{array}$} & \multirow{2}{*}{\multicolumn{2}{|c|}{$\begin{array}{l}\text { East } \\
\text { decli- } \\
\text { nation }\end{array}$}} & \multirow[t]{2}{*}{$\begin{array}{c}\text { Temp. } \\
\text { C. }\end{array}$} & \multirow{2}{*}{$\begin{array}{l}\text { Chr'r } \\
\text { time }\end{array}$} & \multicolumn{2}{|c|}{$\begin{array}{l}\text { Scale } \\
\text { readings }\end{array}$} & $\begin{array}{l}\text { Fas } \\
\text { decl }\end{array}$ & st & Temp. & Chr'r & Sc & $\begin{array}{l}\text { ale } \\
\text { lings }\end{array}$ & Eas & cli- & Temp. & Chr't & $\begin{array}{l}\text { Sec } \\
\text { read }\end{array}$ & $\begin{array}{l}\text { le } \\
\text { ngs }\end{array}$ & $\begin{array}{l}\text { Eas } \\
\text { decl }\end{array}$ & & Temp. \\
\hline & Left & Right & & & & & Left & Right & & & & & Left & Right & & & & & Left & Right & & & \\
\hline $\mathrm{h} \mathrm{m}$ & d & d & $\bullet$ & ' & $\cdot$ & $\mathrm{h} m$ & $d$ & $d$ & - & ' & • & $\mathrm{h} \mathrm{m}$ & d & d & - & ' & 0 & $\mathrm{~h} \mathrm{~m}$ & d & d & 0 & ' & $\bullet$ \\
\hline 400 & 36.3 & 35.9 & 22 & 45 & -15.5 & 60 & 34. I & 33.0 & 22 & 50 & -15.0 & 800 & 30.4 & 29.1 & 22 & 56 & -15.0 & 1000 & 30.1 & 28.7 & 22 & 56 & $-I 4.6$ \\
\hline 02 & 36.3 & 36.1 & & 45 & & 02 & 29.0 & 28.0 & & 57 & & 02 & 30.8 & 28.8 & & 55 & 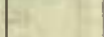 & 02 & 29.4 & 28.0 & 11 & 57 & \\
\hline 04 & 36.0 & 35.9 & & 46 & 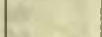 & 04 & 30.8 & $29 \cdot 3$ & & 55 & & 04 & 30.5 & 28.8 & & 56 & 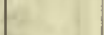 & 04 & 32.0 & 30.0 & cist & 53 & \\
\hline o6 & 36.2 & 36.0 & & 45 & + & of & 30.7 & 30.0 & & 55 & 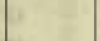 & 06 & 33.0 & 30.9 & & 52 & 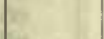 & 06 & 33.5 & 31.8 & & $5 \mathrm{I}$ & 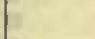 \\
\hline 08 & 36.1 & 36.0 & & 46 & 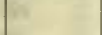 & 08 & 31.0 & 30.0 & & 54 & & as & 34.2 & 32.8 & & 50 & 1 & 08 & 35.0 & 33.3 & 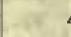 & 48 & 1 \\
\hline IO & 38.0 & 37.7 & & 43 & 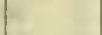 & IO & $34 . I$ & 34.0 & & 49 & & $10-$ & 32.7 & 32.5 & & $5 \mathrm{~T}$ & 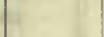 & IO & 37.0 & 35.5 & $\operatorname{tar}$ & 45 & 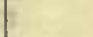 \\
\hline 12 & $37 \cdot 2$ & 36.8 & & 44 & & 12 & 35.8 & 35.0 & & 47 & & 12 & 32.0 & 30.6 & & 53 & 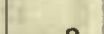 & 12 & 38.5 & 37.0 & 100 & 43 & \\
\hline 14 & 38.8 & $38 . \mathrm{I}$ & & 42 & -15.4 & 14 & $34 \cdot 3$ & 33.1 & & 49 & $-\mathrm{T} 5.0$ & I4 & 34.4 & 33.6 & & 49 & -I 4.8 & 14 & 40.0 & 38.3 & $w^{5}$ & $4 \mathrm{I}$ & -14.5 \\
\hline I6 & 33.1 & 32.8 & & 50 & & I6 & 33.1 & 31.8 & & $5 I$ & & 16 & $35 \cdot 3$ & 33.9 & & 48 & 1 & I6 & 30.6 & 38.0 & $2 x$ & $4^{I}$ & \\
\hline 78 & 36.0 & 35.2 & & 46 & 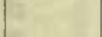 & I8 & 31.0 & 29.2 & & 55 & & I8 & 34.3 & 33.0 & & 49 & i. & I8 & 38.0 & 36.6 & te & 44 & \\
\hline 20 & 36.8 & 36.4 & & 45 & 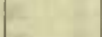 & 20 & 20.3 & 27.9 & & 57 & 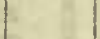 & 20 & 34.6 & 33.3 & & 49 & 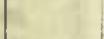 & 20 & 36.0 & $34 \cdot 3$ & re & 47 & 1 \\
\hline 22 & 36.3 & .36 .0 & & 45 & 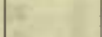 & 22.4 & 28.8 & 27.0 & & 58 & 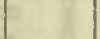 & 22 & 35.3 & 34.0 & & 48 & 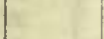 & 22 & 35.8 & 34.2 & in & 47 & \\
\hline 24 & 35.7 & 35.0 & & 47 & - & 24 & 30.1 & 29.0 & & 56 & 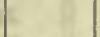 & 24 & 33.8 & 33.0 & & 50 & 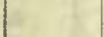 & 24 & 36.3 & 34.0 & Win & 47 & 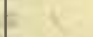 \\
\hline 26 & 33.2 & 32.9 & & 50 & & 26 & 34.1 & 32.7 & & 50 & & 26 & 33.1 & 32.5 & & $5 I$ & 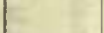 & 26 & 36.6 & $34 \cdot 4$ & 1.5 & 46 & \\
\hline 28 & 33.0 & 32.3 & & $5 I$ & & 28 & 33.9 & 32.7 & & 50 & & 28 & 20.6 & 28.4 & & 57 & & 28 & 37.0 & $34 \cdot 4$ & whe & 46 & \\
\hline 30 & 3 I. I & 30.4 & & 51 & -15.3 & 30 & $33 . \mathrm{I}$ & 32.0 & 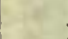 & $5 I$ & $-\mathrm{I} 5.0$ & 30 & 29.5 & 29.0 & & 56 & $-\mathrm{r} 4.8$ & 30 & 36.3 & 34.0 & this & 47 & $-14 \cdot 3$ \\
\hline 32 & 33. I & 32.5 & & $5 I$ & 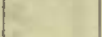 & 32 & 34.0 & 32.0 & & 50 & & 32 & 30.6 & 20.8 & & 55 & 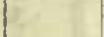 & 32 & $35 \cdot 3$ & 32.6 & & 49 & \\
\hline 34 & 35.1 & $34 \cdot 3$ & & 48 & 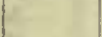 & $34 \cdot 3$ & 33.8 & 32.8 & Et & 50 & & 34 & 29.4 & 28.3 & & 57 & 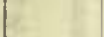 & 34 & 3.5 .3 & 32.8 & evet & 40 & \\
\hline 36 & 36.0 & $35 \cdot 3$ & & 46 & & 36 & $35 \cdot 3$ & 34.6 & 8 & 47 & & 36 & 32.9 & 32.2 & & $5 \mathrm{I}$ & 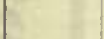 & 36 & 36.3 & 32.4 & is & 48 & \\
\hline 38 & 33.6 & 32.7 & & 50 & 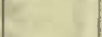 & 38 & $34 \cdot 5$ & 33.9 & 5 & 48 & & 38 & $33 \cdot 3$ & 33.0 & & 50 & 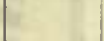 & 38 & 37.2 & 35.3 & $\mathrm{n}$ & 45 & \\
\hline 40 & 39.0 & 38.1 & & 42 & 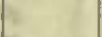 & 40 & 33.1 & 32.7 & ! & 50 & 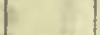 & 40 & 32.5 & 31.8 & & 52 & 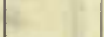 & 40 & $37 \cdot 3$ & 35.3 & 49 & 45 & \\
\hline 42 & 35.8 & 35.2 & & 46 & 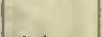 & 42 & 29.8 & 28.8 & 22 & 56 & & 42 & 31.6 & $3 I .3$ & & 53 & 10 & 42 & 35.7 & $34 \cdot 3$ & $t_{1}$ & 47 & \\
\hline 44 & 32.7 & 32. I & & 5I & -15.2 & 44 & 27.0 & 25.7 & 23 & OT & -15.0 & 44 & .30 .8 & 30.3 & & 54 & -14.8 & 44 & 35.4 & 34.0 & & 48 & -14.2 \\
\hline 46 & 34.0 & 34.2 & & 48 & & 46 & 28.4 & 26.8 & 22 & 59 & & 46 & 32.0 & 31.0 & & 53 & 10 & 46.2 & 35.6 & 34.8 & 21 & 47 & \\
\hline 48 & 30.8 & 30.8 & & 54 & 1 & 48 & 29.1 & 28.0 & 22 & 57 & & 48 & 31.8 & 31.0 & & 53 & 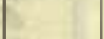 & 48 & 36.0 & $34 \cdot 4$ & it & 47 & \\
\hline 50 & 28.9 & 28.9 & & 57 & $=$ & 50 & 25.8 & 24.2 & 23 & 03 & 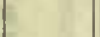 & 50 & 32.6 & 32.0 & & $5 I$ & 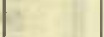 & 50 & 35.2 & $34 \cdot 3$ & & 48 & \\
\hline 52 & 27.9 & 27.9 & & 58 & 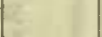 & 52 & 27.2 & 25.8 & 23 & $\infty$ & : & 52 & 34.0 & 33.3 & & 49 & 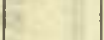 & 52 & 35.6 & 34.6 & & 47 & 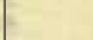 \\
\hline 54 & $27 \cdot 3$ & 27.2 & & 50 & 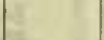 & 54 & 28.9 & 27.8 & 22 & 58 & 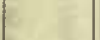 & 54 & 32.8 & 31.8 & & $5 \mathrm{I}$ & 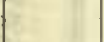 & 54 & $35 \cdot 3$ & 34.6 & bi & 47 & 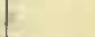 \\
\hline 56 & 28.3 & 27.9 & & 58 & 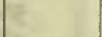 & 56 & $29 \cdot 3$ & 28.2 & & 57 & & 56 & 32.3 & $3 \mathrm{I} \cdot 5$ & & 52 & & 56 & 35.2 & 34.0 & 11 & 48 & \\
\hline 58 & 31.8 & 3 I. 6 & & 52 & & 58 & 27.4 & 26.7 & & 60 & & 58 & 33.6 & 33.0 & & 50 & & 58 & 34.6 & 33.6 & & 48 & \\
\hline 500 & 32.7 & 32.0 & 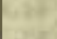 & $5 \mathrm{~T}$ & -15.2 & 700 & 30.8 & 29.2 & & 55 & -15.2 & 900 & 34.4 & 33.6 & & 49 & -14.8 & II $\infty$ & $35 \cdot 3$ & $34 \cdot 5$ & a & 47 & -14.1 \\
\hline 02 & 33.7 & $33 \cdot I$ & & 50 & 1 & 02 & 32.0 & $3 \mathrm{I}$. I & & 53 & 1 & 02 & $34 \cdot 3$ & $33 \cdot 3$ & & 49 & 7 & 02 & 35.9 & 35.3 & 5 & 46 & \\
\hline 04 & 31.7 & 30.4 & & 5.3 & 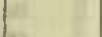 & 04 & 32.5 & $3 I .7$ & & 52 & 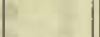 & 01 & 34.0 & $33 \cdot 3$ & & 49 & 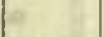 & 04 & 36.4 & 36.1 & A4 & 45 & \\
\hline क & 30.5 & 28.3 & 22 & 56 & 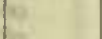 & 06 & 32.8 & 31.8 & 14 & 51 & & 06 & 33.3 & 33.2 & & 50 & 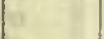 & 06 & 36.6 & 36.5 & & 45 & \\
\hline 08 & 25.4 & $24 . I$ & 23 & 03 & 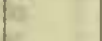 & 08 & 33.0 & 32.0 & He & $5 I$ & 1 & 08 & 33.6 & 32.5 & & 50 & 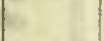 & 08 & 36.0 & 35.6 & tro & 46 & 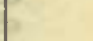 \\
\hline IO & 22.2 & 20.6 & 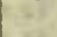 & $\infty$ & 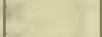 & IO & 31.0 & 29.8 & hr & 54 & & Io & $3 \mathrm{I} \cdot 3$ & 30.3 & & 54 & 1 & Io & $36 . \mathrm{I}$ & 35.9 & ati & 46 & \\
\hline 12 & 25.5 & 23.9 & 23 & 0.3 & 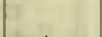 & I2 & 28.0 & 27.0 & 13 & 59 & & 12 & 32.3 & $3 I .0$ & & 52 & 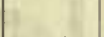 & 12 & 35.5 & $35 \cdot 3$ & tst & 47 & \\
\hline I4 & 30.0 & 28.5 & 22 & 56 & -15.0 & 14 & 30.8 & 29.9 & 145 & 54 & -15.0 & I 4 & $3.5 \cdot 3$ & 34.4 & & 47 & -14.7 & 14 & 35.0 & 34.5 & 11 & 48 & $-14 . I$ \\
\hline т6 & 32.0 & 30.9 & & 53 & & I6 & 33.2 & .32 .1 & 18 & $5 \mathrm{I}$ & & I6 & 36.3 & 34.7 & & 46 & & I6 & $35 \cdot 3$ & 35.3 & & 47 & \\
\hline 18 & 31.0 & 30.0 & & 54 & 1 & I8 & 32.8 & 31.2 & 35 & 52 & & I8 & 33.5 & 31.9 & 4 & $5 I$ & 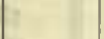 & I8 & $35 \cdot 3$ & $35 \cdot 3$ & tut & 47 & \\
\hline 20 & 28.8 & 27.4 & & 58 & 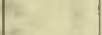 & 20 & 34.1 & 32.9 & 73 & 50 & & 20 & $34 \cdot 3$ & 33.0 & & 49 & 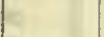 & 20 & 35.4 & 35.2 & 57 & 47 & \\
\hline 22 & 28.3 & 27.1 & & 59 & 2 & 22 & $35 . \mathrm{I}$ & 33.7 & .11 & 48 & 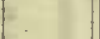 & 22 & 3.3 .8 & 32.3 & & 50 & 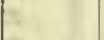 & 22 & $35 . \mathrm{I}$ & 34.6 & 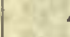 & 47 & \\
\hline 24 & 28.3 & $27 \cdot I$ & & 59 & 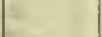 & 24 & 30.8 & 20.9 & 28 & 54 & & 24 & 34.3 & 33.0 & the & 49 & $=$ & 24 & 35.0 & 34.7 & 81 & 47 & \\
\hline 26 & $28 . I$ & 26.9 & & 50 & 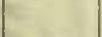 & 26 & 27.9 & 26.4 & (t) & 60 & 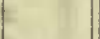 & 26 & 36.6 & 35.0 & & 46 & 1 & 26. & 34.7 & 34.3 & $\sqrt{6} 5$ & 48 & \\
\hline 28 & 30.3 & $20 . \mathrm{I}$ & & 56 & & 28 & $3 I . I$ & 29.9 & 14 & 54 & & 28 & 36.2 & 35.0 & & 46 & & 28 & $35 \cdot 3$ & 35.3 & t1 & 47 & \\
\hline 30 & 32.4 & 31.2 & & 52 & - I5.0 & 30 & 34.9 & 34.0 & & 48 & -15.0 & 30 & 36.7 & $35 . I$ & & 46 & -14.6 & 30 & 36.3 & 36.1 & 18 & 45 & -14.1 \\
\hline 32 & 33.8 & 32.5 & & 50 & 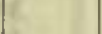 & 32 & 33.7 & .33 .0 & & 50 & & 32 & 35.0 & 34.0 & & 48 & (3. & 32 & 36.8 & 36.3 & in? & 45 & \\
\hline 34 & 33.9 & 32.3 & & 50 & 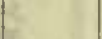 & 34 & 30.0 & $29 \cdot 3$ & & 56 & & 31 & 34.4 & 32.6 & & 50 & 1 & 34 & 37.8 & $37 \cdot 3$ & sos & 43 & \\
\hline 36 & $33 . \mathrm{I}$ & $3 \mathrm{I} .8$ & & 5I & 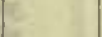 & 36 & 32.0 & 32.0 & & 52 & & .36 & $31 \cdot 3$ & 32.6 & & 50 & & 36 & 39.0 & 37.6 & $x^{3}$ & 42 & \\
\hline 38 & 30.8 & 20.3 & & 55 & 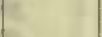 & 38 & 33.9 & 33.2 & & 50 & & .38 & 36.4 & 33.7 & & 47 & 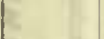 & 38 & .35 .8 & 34.7 & Hat & 47 & \\
\hline 40 & $29 . I$ & 28.1 & & 57 & 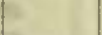 & 40 & $3.3 \cdot 3$ & 32.2 & ty & $5 I$ & & 40 & 34.0 & $3 I .4$ & 4 & $5 I$ & 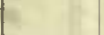 & 40 & 36.4 & $35 \cdot 3$ & +11 & 46 & \\
\hline 42 & 3.3 .2 & 31.8 & & 5 I & . & 42 & 28.9 & 28.2 & 14 & 57 & & 42 & $34 \cdot 4$ & .32 .0 & -28 & 50 & 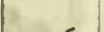 & 42 & 36.6 & 35.7 & 113 & 45 & \\
\hline$\Delta 4$ & 36.0 & 35.0 & & 46 & 2 & 44 & $32 . \mathrm{I}$ & 30.6 & & 53 & -15.0 & 44 & 34.6 & 32.0 & 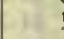 & 50 & -14.6 & 44 & 38.2 & 37.2 & $x^{3}$ & 43 & -14.0 \\
\hline 46 & 34.0 & 33.8 & & 49 & $-\mathrm{I} 5.0$ & 46 & 33.2 & 32.2 & & $5 \mathrm{I}$ & & 46 & 3.3 .6 & $3 I . I$ & +16 & $5 I$ & 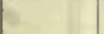 & 46 & 30.7 & 38.6 & is & $4 \mathrm{I}$ & \\
\hline 48 & 27.2 & 26.9 & & 60 & ( & 48 & 33.0 & 31.8 & & 51 & & 48 & $36 . \mathrm{T}$ & 3.3 .8 & $x$ & 47 & 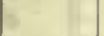 & 48 & 41.3 & 40.0 & in & 38 & \\
\hline 50 & 20.8 & 29.2 & & 56 & t & 50 & 29.7 & 27.2 & 73 & 58 & & 50 & .36 .6 & 35.0 & 3 & 46 & $=$ & 50 & 40.6 & 30.5 & (s) & 39 & \\
\hline 52 & 32.2 & 31.7 & & 52 & L & 52 & 29.7 & 27.9 & 44 & 57 & & 52 & 37.9 & 33.0 & 8 & 49 & 1 & 52 & $39 \cdot 3$ & 38.3 & 104 & $4 I$ & \\
\hline .54 & 34.0 & 33.8 & & 40 & 2 & 54 & 30.2 & $28 . ?$ & & 56 & & 54 & $34 \cdot 3$ & $.33 \cdot 3$ & 32 & 49 & 5 & 54 & 39.6 & 38.8 & $10 x$ & $4 \mathrm{I}$ & \\
\hline 56 & 34.4 & 34.1 & & 48 & 2 & 56 & 31.5 & 30.3 & 43 & 54 & & 56 & 33.4 & .32 .6 & -15 & 50 & 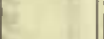 & 56 & 39.4 & $39 \cdot 3$ & $x^{3}$ & $4 I$ & \\
\hline 58 & 37.2 & 37.0 & 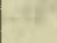 & 44 & $=$ & 58 & 30.6 & 29.0 & & 55 & & 58 & 33.0 & $3 I .5$ & 0 & 52 & 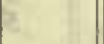 & 58 & 40.5 & 39.5 & ate & 39 & \\
\hline
\end{tabular}

Observers-R. R. T. and W. J. P., who alternated from 7 h $56 \mathrm{~m}$ to

Observer-W. J. P.

$8 \mathrm{~h} 06 \mathrm{~m}$. 
Tabulation of magnetic declinations observed at Teplitz Bay-Continued

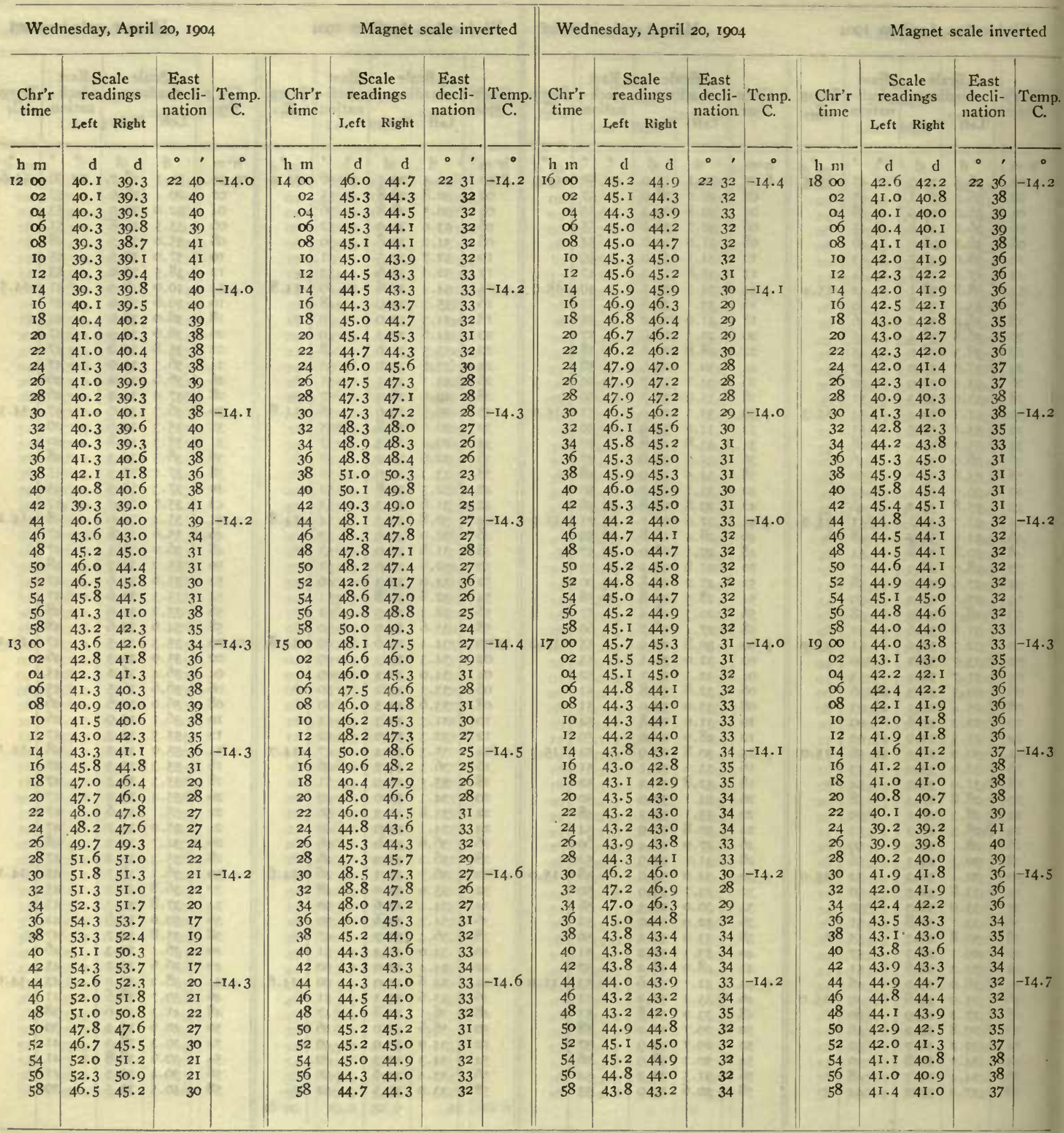

Observers-W. J. P. and J. V., who alternated from $15 \mathrm{~h} 46 \mathrm{~m}$ to Observer-J. V. $16 \mathrm{~h}$ oom. 
Tabulation of magnetic declinations observed at Teplitz Bay-Continued

\begin{tabular}{|c|c|c|c|c|c|c|c|c|c|c|c|c|c|c|c|c|c|c|c|}
\hline \multicolumn{5}{|c|}{ Wednesday, April 20, 1904} & \multicolumn{5}{|c|}{ Magnet scale inverted } & \multicolumn{6}{|c|}{ Thursday, April 2I, 1904} & \multicolumn{4}{|c|}{ Magnet scale erect } \\
\hline \multirow{2}{*}{$\begin{array}{l}\text { Chr'r } \\
\text { time }\end{array}$} & \multicolumn{2}{|c|}{$\begin{array}{l}\text { Scale } \\
\text { readings }\end{array}$} & \multirow{2}{*}{$\begin{array}{l}\text { East } \\
\text { decli- } \\
\text { nation }\end{array}$} & Temp & Chr'r & $\begin{array}{r}\mathrm{Sc} \\
\text { read }\end{array}$ & $\begin{array}{l}\text { ale } \\
\text { lings }\end{array}$ & $\begin{array}{l}\text { East } \\
\text { decli- }\end{array}$ & Temp. & Chr'r & $\begin{array}{l}\text { Sc: } \\
\text { read }\end{array}$ & $\begin{array}{l}\text { ale } \\
\text { ings }\end{array}$ & East & & Chr'r & $\begin{array}{l}\text { Sce } \\
\text { read }\end{array}$ & $\begin{array}{l}\text { ale } \\
\text { ings }\end{array}$ & East & Temp. \\
\hline & Left & Right & & & & Left & Right ${ }^{\circ}$ & & & & Left & Right & & & & Left & Right & & \\
\hline h $\mathrm{m}$ & d & d & $\cdot 1$ & & $\mathrm{~h} \mathrm{~m}$ & d & d & & $\bullet$ & $1 \mathrm{~m}$ & d & d & & & $\mathrm{h} \mathrm{m}$ & d & d & 0, & 1 \\
\hline 200 & $4 \mathrm{I} \cdot 5$ & $4 \mathrm{I} . \mathrm{I}$ & 2237 & -14.8 & 2200 & 46.1 & 46.0 & 2230 & -14.9 & 1600 & 54.2 & 56.2 & 2227 & -11.8 & $18 \infty$ & 55.7 & 55.9 & 2228 & -10.9 \\
\hline 02 & 40.3 & 40.2 & 39 & & 02 & 47.1 & 46.8 & 28 & & 02 & 54.2 & 56.0 & 27 & & 02 & 55.2 & 55.7 & & a \\
\hline 04 & 40.7 & 40.5 & 38 & and & 04 & 48.3 & 48.0 & 27 & 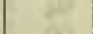 & 04 & 54.2 & 56.0 & 27 & e & 04 & 55.0 & $55 \cdot 3$ & 27 & $\frac{19}{m}$ \\
\hline 06 & $4 \mathrm{I} \cdot 3$ & $4 \mathrm{I} . \mathrm{I}$ & 37 & 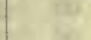 & 06 & 47.0 & 46.8 & 28 & & 06 & 54.8 & 56.1 & 28 & & 06 & $55 \cdot 3$ & 55.9 & 28 & 10 \\
\hline 08 & 41.5 & $4 \mathrm{I} .2$ & 37 & & $\infty 8$ & 46.0 & 45.3 & 31 & & 88 & 55.0 & 56.8 & 28 & 60 & $\infty$ & 55.8 & 56.0 & 28 & 80 \\
\hline IO & 41.8 & $4 \mathrm{I} . \mathrm{I}$ & 37 & & I0 & 46.7 & 46.0 & 29 & & Io & 55.0 & 56.3 & 28 & 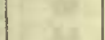 & 10 & 56.0 & 56.4 & 29 & \\
\hline 12 & 42.7 & 42.1 & 36 & & I2 & 46.0 & 45.9 & 30 & & I2 & $54 \cdot 5$ & 56.0 & 27 & & 12 & 56.7 & 56.8 & 30 & \\
\hline I4 & 41.9 & $4 \mathrm{I} .3$ & 37 & -14.8 & 14 & 46.0 & 45.8 & 30 & -14.9 & I4 & 54.2 & 55.8 & 27 & -11.5 & 14 & 56.2 & 56.8 & 30 & -10.9 \\
\hline 16 & $4 I .1$ & 40.9 & 38 & & 16 & 44.9 & 44.4 & 32 & & 16 & 55.1 & 56.9 & 29 & & 16 & 56.2 & 56.2 & 29 & \\
\hline I8 & 42.1 & 41.3 & 37 & & I8 & 44.2 & 44.0 & 33 & Ir & I8 & 55.7 & 57.0 & 29 & & 18 & 56.1 & 56.2 & 29 & ent \\
\hline 20 & 42.8 & $42 . I$ & 36 & & 20 & 43.9 & 43.7 & 33 & 92 & 20 & 54.6 & 56.0 & 28 & se & 20 & 56.2 & 56.8 & 30 & 2 \\
\hline 22 & 43.9 & 42.9 & 34 & $\mathrm{C}^{2}$ & 22 & 43.0 & 42.8 & 35 & 2 & 22 & 53.1 & $54 \cdot 3$ & 25 & . & 22 & 56.7 & 57.0 & 30 & at \\
\hline 24 & 44.0 & 43.0 & 34 & s. & 24 & 42.4 & $42 . I$ & 36 & $y$ & 24 & 52.0 & 53.1 & 23 & & 24 & 56.8 & 57.1 & 30 & $t$ \\
\hline 26 & 45.0 & 44.2 & 32 & & 26 & $43 . I$ & 42.9 & 35 & 13 & 26 & 51.7 & 52.6 & 23 & & 26 & 57.0 & 57.4 & 31 & \\
\hline 28 & 46.0 & 45.5 & 30 & & 28 & $43 \cdot 5$ & 43.1 & 34 & $=$ & 28 & 52.3 & 53.2 & 24 & & 28 & 57.6 & 58.0 & 32 & \\
\hline 30 & 46.9 & $46 . I$ & 29 & -14.8 & 30 & 43.2 & 43.0 & 34 & -14.9 & 30 & 53.9 & 54.9 & 26 & - I I.4 & 30 & 56.2 & 56.8 & 30 & -10.9 \\
\hline 32 & 46.1 & 45.6 & 30 & & 32 & 42.9 & 42.9 & 35 & & 32 & 55.2 & 56.0 & 28 & & 32 & 56.1 & 56.7 & 29 & \\
\hline 34 & 46.0 & 45.2 & $3 \mathrm{I}$ & Le & 34 & 42.7 & 42.2 & 36 & Nat & 34 & 56.0 & 56.9 & 29 & 45 & 34 & 58.1 & 58.5 & 32 & Ac \\
\hline 36 & 48.0 & 47.2 & 27 & 2 & 36 & 42.3 & 42.1 & 36 & 80 & 36 & 56.0 & 56.9 & 29 & 4 & 36 & 57.8 & 58.1 & 32 & $\infty$ \\
\hline 38 & 46.1 & 46.0 & 30 & 68 & 38 & 42.1 & 42.0 & 36 & 3 & 38 & 58.1 & 58.9 & 33 & 0 & 38 & 57.7 & 57.9 & 32 & 2 \\
\hline 40 & 45.2 & 44.2 & 32 & 5 & 40 & 41.5 & 41.2 & 37 & 8 & 40 & 58.8 & 59.5 & 34 & cas & 40 & 57.4 & 57.8 & $3 I$ & कo \\
\hline 42 & 43.8 & 41.8 & 35 & 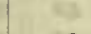 & 42 & 41.2 & 41.1 & 38 & -4 & 42 & 59.0 & 59.9 & 34 & & 42 & 57.3 & 57.8 & $3 I$ & tar \\
\hline 44 & 44.0 & $42 . I$ & 35 & -14.8 & 44 & $4^{\mathrm{I}} .2$ & $4 \mathrm{I} . \mathrm{I}$ & 38 & $-I_{4} . y$ & 44 & 59.2 & 60.1 & 34 & -11.2 & 44 & 57.0 & $57 \cdot 3$ & 30 & -11.0 \\
\hline 46 & 43.8 & 42.5 & 34 & & 46.2 & 40.8 & 40.2 & 38 & & 46 & $59 \cdot 3$ & 60.4 & 35 & $1-.$. & 46 & 57.1 & $57 \cdot 3$ & 31 & \\
\hline 48 & 46.1 & $44 \cdot 4$ & 3I & & 48 & 40.9 & 40.4 & 38 & 48 & 48 & 59.4 & 60.0 & 34 & 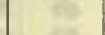 & 48 & 57.3 & 57.8 & $3 I$ & \\
\hline 50 & 46.2 & 43.9 & 32 & & 50 & 40.9 & 40.7 & 38 & 98 & 50 & 59.1 & 59.8 & 34 & 2 & 50 & 57.2 & 57.6 & $3 \mathrm{I}$ & 165 \\
\hline 52 & 45.2 & 42.9 & 33 & & 52 & 41.0 & 40.8 & 38 & 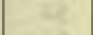 & 52 & 58.8 & 59.1 & 33 & tita & 52 & 57.2 & 57.5 & 31 & 28 \\
\hline 54 & 43.0 & 41.0 & 36 & 2 & 54 & 41.0 & 40.8 & 38 & 28 & 54 & 59.2 & 59.7 & 34 & 12 & 54 & 57.1 & 57.2 & 30 & $\mathrm{E}$ \\
\hline 56 & 44.9 & 42.9 & 33 & & 56 & 41.5 & $4 \mathrm{I} .0$ & 37 & & 56 & 59.1 & 59.7 & 34 & & 56 & 57.0 & 57.0 & 30 & \\
\hline 58 & 43.7 & 42.0 & 35 & & 58 & 42.3 & 42.0 & 36 & & 58 & $59 . \mathrm{I}$ & 60.0 & 34 & & 58 & 56.8 & 57.0 & 30 & \\
\hline$I \infty$ & 45.8 & 43.9 & 32 & $-\mathrm{I} 4.8$ & 2300 & 42.5 & 42.1 & 36 & -14.9 & 1700 & 59.3 & 59.9 & 34 & -II.I & 1900 & 56.8 & 57.0 & 30 & -11.0 \\
\hline 02 & 44.8 & 42.5 & 34 & & 02 & 42.0 & $4 \mathrm{I} .5$ & 37 & & 02 & 59.4 & 59.8 & 34 & & 02 & 56.1 & 56.3 & 29 & \\
\hline 04 & 46.5 & 44.9 & 30 & & 04 & 40.9 & 40.7 & 38 & 19 & 04 & 59.0 & 59.2 & 34 & 3 & 04 & 56.1 & 56.3 & 29 & \\
\hline 06 & $43 \cdot 4$ & $4 \mathrm{I} .2$ & 36 & we & 06 & 40.0 & 39.9 & 40 & (6) & 06 & 58.9 & 59.0 & 33 & 10 & 06 & 56.3 & 57.0 & 30 & - \\
\hline 08 & $45 \cdot 3$ & 43.2 & 33 & & 08 & 39.8 & 39.4 & 40 & 30 & $\infty 8$ & 58.8 & 59.0 & 33 & 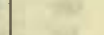 & 08 & 56.9 & 57.2 & 30 & t. \\
\hline IO & $43 . \overline{8}$ & $4 I . I$ & 36 & & 10 & 40.2 & 39.9 & 39 & & IO & 58.9 & 59.0 & 33 & 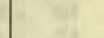 & 10 & 57.0 & 57.7 & 31 & 2 \\
\hline 12 & $43 \cdot I$ & 4 I. I & 36 & $x^{2}$ & 12 & 41.7 & $4 \mathrm{I} .0$ & 37 & 1 & 12 & 58.8 & 59.0 & 33 & $1-1$ & 12 & 57.0 & 57.2 & 30 & Hix \\
\hline 14 & $42 . I$ & 40.5 & 37 & $-\mathrm{I} 4.9$ & 14 & 42.8 & 42.4 & 35 & -14.9 & 14 & $59 . I$ & 59.3 & 34 & -II.O & I4 & 57.0 & 57.0 & 30 & -11.0 \\
\hline 16 & $44 . I$ & 42.9 & 34 & & 16 & $43 . I$ & 42.9 & 35 & & 16 & 59.3 & 60.0 & 34 & & 16 & $57 \cdot 3$ & 58.0 & 31 & \\
\hline 18 & 43.2 & 41.6 & 36 & 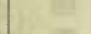 & 18 & 42.8 & 42.1 & 36 & WI & I8 & 60.1 & 60.7 & 36 & & I8 & 57.8 & 58.2 & 32 & 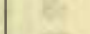 \\
\hline 20 & 43.9 & 42.1 & 35 & 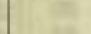 & 20 & 41.0 & 40.7 & 38 & 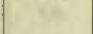 & 20 & 60.1 & 60.7 & 36 & $2 \pi$ & 20 & 58.0 & 58.2 & 32 & 0 \\
\hline 22 & $44 . \mathrm{I}$ & 42.8 & 34 & 난 & 22 & 40.0 & 39.8 & 40 & 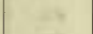 & 22 & 59.0 & 59.7 & 34 & 나 & 22 & 58.0 & 58.1 & 32 & $E$ \\
\hline 24 & 45.2 & 42.0 & 34 & te & 24 & 39.1 & 39.0 & $4 \mathrm{I}$ & ati & 24 & 58.1 & 59.9 & 33 & t & 24 & 58.0 & 58.0 & 32 & 36 \\
\hline 26 & 42.2 & 40.5 & 37 & & 26 & 38.3 & 38.1 & 42 & $e^{2}$ & 26 & 58. I & 58.8 & 32 & & 26 & 58.0 & 58.0 & 32 & 10 \\
\hline 28 & 41.3 & 40.8 & 38 & & 28 & 38.0 & 37.9 & 43 & $1=$ & 28 & 58.4 & 59.0 & 33 & 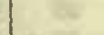 & 28 & 58.0 & 58.1 & 32 & 0 \\
\hline 30 & 42.5 & 42.0 & 36 & -14.9 & 30 & 37.9 & 37.5 & 43 & -14.9 & 30 & 58.6 & 59.1 & 33 & - II .O & 30 & 58.3 & 58.7 & 33 & $-I 1 . I$ \\
\hline 32 & 44.9 & 44.1 & 32 & & 32 & 37.2 & 37.1 & 44 & & 32 & 58.9 & 59.3 & 34 & & 32 & 58.7 & 59.0 & 33 & \\
\hline 34 & 42.9 & 42.3 & 35 & 1e & 34 & 36.9 & 36.8 & 44 & 10 & 34 & 58.7 & 59.0 & 33 & 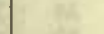 & 34 & 58.9 & 59.3 & 34 & 16 \\
\hline 36 & 43.7 & $43 . I$ & 34 & $z^{2}$ & 36 & 36.5 & 36.3 & 45 & & 36 & 58.3 & 59.0 & 33 & 0 & 36 & 58.9 & 59.3 & 34 & सe \\
\hline 38 & 43.0 & 42.8 & 35 & $\sqrt{6}$ & 38 & 36.2 & 36.0 & 45 & 20 & 38 & 58.3 & 58.9 & 33 & 68 & 38 & 58.4 & 58.9 & 33 & 16 \\
\hline 40 & 43.0 & 42.9 & 35 & Q & 40 & 36.3 & 36.0 & 45 & 26 & 40 & 58.4 & 58.9 & 33 & 27 & 40 & 58.1 & 58.8 & 33 & 45 \\
\hline 42 & $43 . I$ & 43.0 & 35 & 10 & 42 & 36.4 & 36.1 & 45 & & 42 & 58.3 & 58.7 & 33 & $1=$ & 42 & 58.1 & 58.7 & 33 & 25 \\
\hline 44 & 44.0 & 43.9 & 33 & $-\mathrm{I} 4.9$ & 44 & 37.0 & 36.8 & 44 & -14.8 & 44 & 58.7 & 59.0 & 33 & -10.9 & 44 & 58.2 & 58.8 & 33 & he \\
\hline 46 & 45.2 & $45 . \mathrm{I}$ & $3 \mathrm{I}$ & . & 46 & 37.8 & 37.2 & 43 & & 46 & 58.9 & 58.9 & 33 & 0 & 46 & 58.2 & 58.9 & 33 & 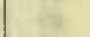 \\
\hline 48 & 44.9 & 44.7 & 32 & 8 & 48 & 38.8 & 38.2 & 42 & 8 & 48 & 58.6 & 58.8 & 33 & 3 & 48 & 58.4 & 59.0 & 33 & ili \\
\hline 50 & 44.0 & 43.9 & 33 & ez & 50 & $40 . I$ & 39.8 & 40 & $\pi$ & 50 & 58.3 & 58.8 & 33 & we & 50 & 58.9 & 59.2 & 33 & $2=$ \\
\hline 52 & 43.0 & 42.4 & 35 & es & 52 & 41.8 & $4 \mathrm{I} . \mathrm{I}$ & 37 & & 52 & 58.2 & 58.2 & 32 & 16 & 52 & 59.1 & 59.8 & 34 & 22 \\
\hline 54 & 42.0 & 41.2 & 37 & $t^{2}$ & 54 & 42.9 & 42.1 & 35 & is & 54 & 57.6 & 57.9 & 32 & $\sqrt{6}$ & 54 & 59.9 & 60.1 & 35 & 34 \\
\hline 56 & 44.1 & 43.7 & 33 & 는 & 56 & 43.0 & 42.5 & 35 & & 56 & 56.9 & 57.2 & 30 & & 56 & 60.0 & 60.4 & 35 & or \\
\hline 58 & 45.2 & 44.8 & 32 & 82 & 58 & 42.3 & 42.0 & 36 & 6 & 58 & 56.0 & 56.2 & 29 & $\sqrt{1}=$ & 58 & 60.1 & 60.4 & 35 & 67 \\
\hline & & & & & 2400 & 42.0 & 41.0 & 37 & & 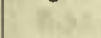 & & & & $m$ & 2000 & 59.8 & 60.7 & 35 & \\
\hline
\end{tabular}

Correction to local mean time is $+\mathrm{Im} .90^{\circ}$ torsion $=16^{\prime} 49$. Torsion head at oh oom read $42^{\circ}$ and at $24 \mathrm{~h} 2 \mathrm{Im}$ read $35^{\circ}$. Observer-J. V.
Correction to local mean time is $+2 \mathrm{~m} 26.5 \mathrm{~s}$.

Torsion head at $15 \mathrm{~h} 35 \mathrm{~m}$ read $38^{\circ}$ and at $20 \mathrm{~h} 12 \mathrm{~m}$ read the same. Observer-R. R. T. 
Tabulation of magnetic declinations abserved at Teplitz Bay-Continued

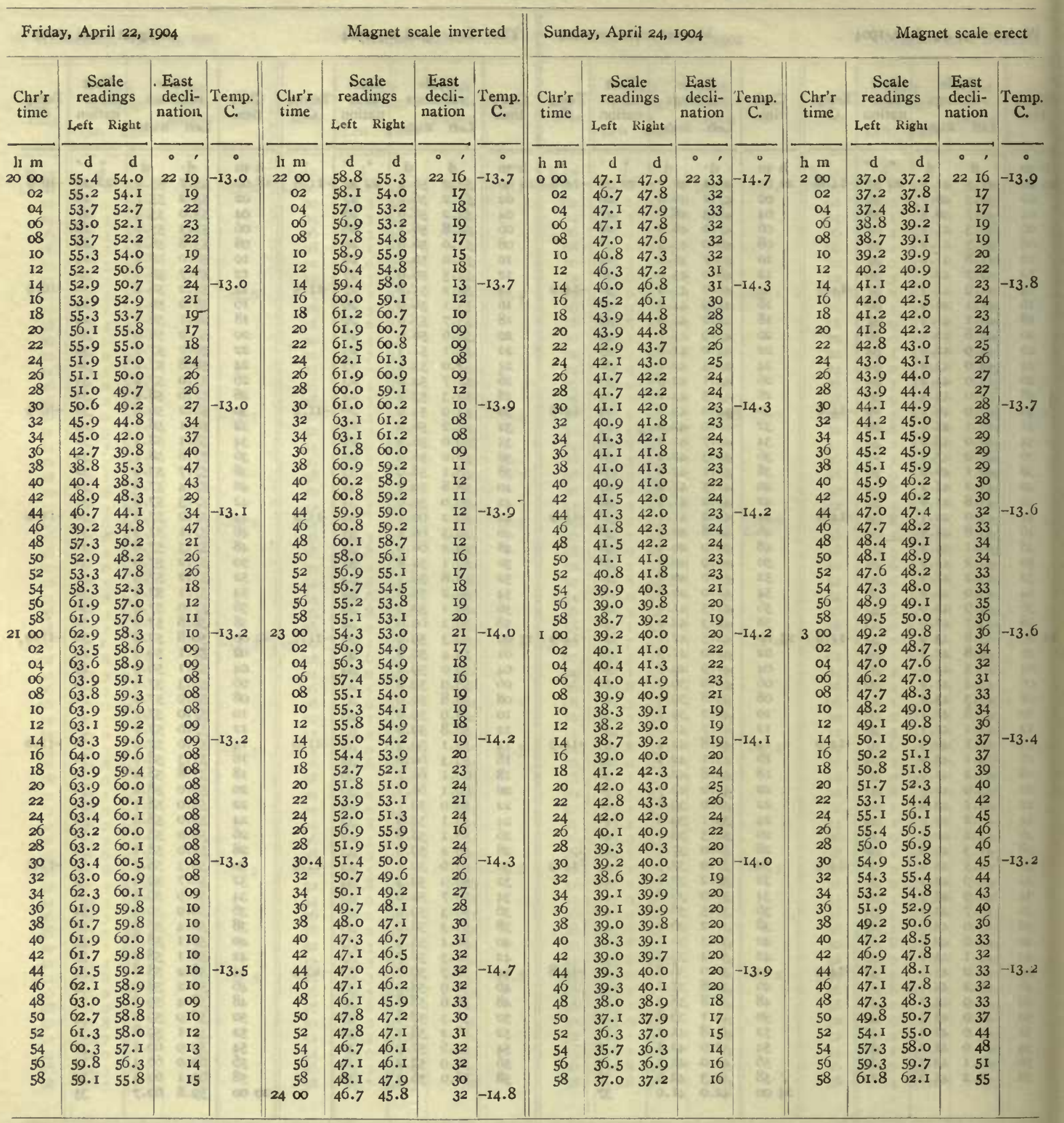

Correction to local mean time is $+2 \mathrm{~m}$ o9s. $90^{\circ}$ torsion $=14^{\prime} 95 . \quad$ Observer-R. R. T.

Torsion head at $19 \mathrm{~h} 30 \mathrm{~m}$ read $38^{\circ}$ and at $24 \mathrm{~h}$ 2om read $42^{\circ}$.

Observer-R. R. T. 
Tabulation of magnetic declinations observed at Teplitz Bay-Continued

\begin{tabular}{|c|c|c|c|c|c|c|c|c|c|c|c|c|c|c|c|c|c|c|c|c|c|}
\hline \multicolumn{6}{|c|}{ Sunday, April 24, 1904} & & \multicolumn{5}{|c|}{ Magnet scale inverted } & \multicolumn{5}{|c|}{ Monday, Apill 25, 1904} & \multicolumn{5}{|c|}{ Magnet scale erect } \\
\hline \multirow[t]{2}{*}{$\begin{array}{l}\text { Chir } \\
\text { time }\end{array}$} & \multicolumn{2}{|c|}{$\begin{array}{l}\text { Scale } \\
\text { readings }\end{array}$} & $\begin{array}{l}\text { Eas } \\
\text { dec } \\
\text { nati }\end{array}$ & $\begin{array}{l}\text { st } \\
\text { cli- } \\
\text { ion }\end{array}$ & $\begin{array}{c}\text { Temp. } \\
\text { C. }\end{array}$ & $\begin{array}{l}\text { Chr'r } \\
\text { time }\end{array}$ & $\begin{array}{l}\text { Sce } \\
\text { read }\end{array}$ & $\begin{array}{l}\text { ale } \\
\text { ings }\end{array}$ & $\begin{array}{l}\text { Eas } \\
\text { decl } \\
\text { natio }\end{array}$ & & Temp. & $\begin{array}{l}\text { Chr'r } \\
\text { time }\end{array}$ & $\begin{array}{l}\text { Sce } \\
\text { read }\end{array}$ & $\begin{array}{l}\text { ale } \\
\text { lings }\end{array}$ & $\begin{array}{c}\text { East } \\
\text { decli- } \\
\text { nation }\end{array}$ & $\begin{array}{c}\text { Temp. } \\
\text { C. }\end{array}$ & $\begin{array}{l}\text { Chr'r } \\
\text { time }\end{array}$ & $\begin{array}{l}\text { Sca } \\
\text { read }\end{array}$ & $\begin{array}{l}\text { ings } \\
\text { ing }\end{array}$ & $\begin{array}{l}\text { East } \\
\text { decli- } \\
\text { nation }\end{array}$ & $\begin{array}{c}\text { Temp. } \\
\text { C. }\end{array}$ \\
\hline & Left & Righı & & & & & Left & Right & & & & & Left & Right & & & & Left & Right & & \\
\hline $\mathrm{n} n$ & d & $\mathrm{d}$ & & & . & $\mathrm{h} \mathrm{m}$ & d & d & & ' & 0 & $\mathrm{~h} \mathrm{~m}$ & 4 & d & & $\bullet$ & $\mathrm{h} \mathrm{m}$ & d & d & $0 \quad$ & - \\
\hline 400 & 41.8 & 37.0 & 23 & 03 & -13.2 & 600 & 36.8 & $35 \cdot 5$ & $23 c$ & 08 & $-I I \cdot 3$ & 800 & 48.8 & 49.8 & 2243 & -14.6 & I0 $\infty$ & 42.1 & 43.0 & 2233 & -13.8 \\
\hline 02 & 40.2 & 35.6 & & 06 & & 02 & 36.2 & $35 \cdot 4$ & & $\log$ & & 02 & 48.0 & 49.0 & 42 & -9 & 02 & $43 \cdot 3$ & 44.2 & 34 & (2tat \\
\hline 04 & 38.3 & 34.2 & & 08 & 45 & 04 & 37.0 & 30.3 & & 08 & wa & 04 & 50.2 & 51.7 & 46 & +6 & 04 & 44.2 & $44 \cdot 9$ & 36 & 40 \\
\hline 06 & 37.9 & $34 . I$ & & $\infty$ & 1 & 06 & 37.7 & 36.4 & & 07 & ra & $\infty 6$ & 52.0 & 54.2 & 49 & 67 & 06 & 42.7 & 43.2 & 33 & $\mathrm{~m}$ \\
\hline 08 & 37.0 & 34.0 & & $\infty$ & at & 08 & 39.8 & 38.5 & & 04 & Wh & 08 & 49.6 & $5 \mathrm{I} \cdot 7$ & 45 & & 08 & 41.8 & 42.1 & 32 & 80 \\
\hline IO & 39.6 & 36.5 & & 05 & 67 & IO & 42.0 & 40.1 & & OI & & IO & 48.8 & 51.0 & 44 & & 10 & 42.3 & 43.0 & 33 & 6 \\
\hline 12 & 40.0 & 37.5 & & 04 & 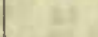 & 12 & $4 I \cdot 5$ & 40.1 & & OI & 1 & I2 & $55 \cdot 3$ & 57.1 & 54 & & 12 & 44.8 & 45.0 & 36 & \\
\hline I4 & 40.3 & 38.0 & & 04 & -12.7 & 14 & 39.9 & 37.8 & & 04 & - I I .O & I4 & 45.4 & 48.3 & 39 & -14.6 & 14 & 44.1 & 44.8 & 36 & -13.7 \\
\hline I6 & $4 \mathrm{I} . \mathrm{I}$ & $39 \cdot 3$ & & 02 & & I6 & 42.2 & 40.7 & $23 c$ & 00 & & 16 & 40.1 & 48.3 & 40 & & 16 & 43.6 & 43.9 & 34 & (n) \\
\hline I8 & $4 I . I$ & 39.9 & & OI & 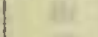 & I8 & 42.9 & 40.9 & 22 & 59 & 20 & 18 & 45.6 & 47.6 & 39 & $4=$ & 18 & 43.0 & 43.4 & 34 & lw \\
\hline 20 & $4 \mathrm{I} . \mathrm{I}$ & 39.9 & & OI & (6) & 20 & $45 \cdot 9$ & 45.0 & 22 & 54 & 0 & 20 & $39 \cdot 3$ & $4 \mathrm{I} \cdot 7$ & 30 & 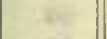 & 20 & 43.7 & 44.0 & 35 & et \\
\hline 22 & 41.8 & 41.0 & & $\infty$ & 6at & 22 & 41.0 & 40.0 & 23 & OI & var & 22 & 44.8 & 48.0 & 39 & $=1$ & 22.4 & $43 . I$ & 44.0 & 34 & $3 t$ \\
\hline 24 & 42.0 & $4 I .2$ & & $\infty$ & 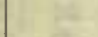 & 24 & 39.0 & 37.9 & & 05 & $y=$ & 24 & 52.7 & 54.0 & 50 & - & 24 & $44 \cdot 2$ & 44.8 & 36 & 16 \\
\hline 26 & 41.9 & $4 I .2$ & & $\infty$ & $a_{2}$ & 26 & 38.1 & 37.0 & & 06 & & 26 & 48.9 & 59.9 & $5 I$ & $=$ & 26 & 45.3 & $45 \cdot 9$ & 37 & $n+$ \\
\hline 28 & $4 I .4$ & $4 I .0$ & & $\infty$ & & 28 & 38.1 & 36.3 & & 07 & & 28 & 48.9 & 50.2 & 44 & $x=$ & 28 & 44.8 & $45 \cdot 4$ & 37 & 12 \\
\hline 30 & 4 I.I & 40.7 & & OI & -12.8 & 30 & 35.8 & 34.0 & & Io & -10.8 & 30 & 48.0 & 48.5 & 42 & -14.6 & 30 & 43.8 & $44 \cdot 3$ & 35 & -13.4 \\
\hline 32 & 40.0 & 39.2 & & 03 & 10 & 32 & 37.1 & 34.2 & & 09 & $1+1$ & 32 & 46.0 & 48.2 & 40 & $x^{2}$ & 32 & 43.1 & $43 \cdot 3$ & 34 & $x^{2}+x^{2}$ \\
\hline 34 & 38.2 & $37 \cdot 3$ & & 06 & 14 & 34 & 40.3 & 38.0 & & 04 & $x^{2}$ & 34 & 50.1 & $5 I .4$ & 46 & $=4$ & 34 & 42.0 & 42.8 & 32 & 35 \\
\hline 36 & 37.0 & $36 . I$ & & 08 & 12. & 36 & 40.8 & 38.1 & & 03 & $n^{2}$ & 36 & 50.1 & 51.9 & 46 & $2=$ & 36 & $43 \cdot 3$ & 43.9 & 34 & $3 x$ \\
\hline 38 & 37.0 & 36.1 & & $\infty 8$ & ces & 38 & 41.2 & 38.9 & 23 & 02 & ent & 38 & $53 \cdot 3$ & 55.2 & $5 I$ & n. & 38 & 45.0 & 45.2 & 37 & 28 \\
\hline 40 & 36.9 & $36 . I$ & & 08 & $1+$ & 40 & 46.9 & 45.0 & 22 & 53 & 14 & 40 & 53.2 & 54.0 & 50 & $7 x^{2}$ & 40 & 44.0 & $44 \cdot 2$ & 35 & 74 \\
\hline 42 & 37.7 & 36.7 & & o7 & 15 & 42 & 46.6 & 45.0 & 22 & 53 & the & 42 & 53.8 & 54.1 & 2250 & 1 & 42 & 46.0 & 46.3 & 38 & $x^{2}$ \\
\hline 44 & 38.5 & 37.6 & & 05 & -12.9 & 44 & 42.5 & 41.0 & 23 & 00 & $-I O . I$ & 44 & 62.2 & 63.0 & 2304 & $-I 4.6$ & 44 & 45.1 & 46.0 & 37 & -13.2 \\
\hline 46 & 39.0 & 37.9 & & 05 & & 46 & 40.9 & 39.0 & 23 & 02 & & 4 & 60.1 & 60.3 & 2300 & (n) & 46 & 42.8 & 43.5 & 34 & 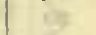 \\
\hline 48 & 37.9 & 37.1 & & 06 & 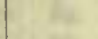 & 48 & 45.0 & 42.0 & 22 & 57 & $y$ & 48 & 56.9 & 57.6 & 2256 & 20 & 48 & 43.3 & 44.7 & 35 & 3 \\
\hline 50 & 38.0 & 36.9 & & 06 & $x=$ & 50 & 47.9 & 45.6 & & 52 & $x^{10}$ & 50 & 55.0 & 55.0 & 2252 & 49 & 50 & $45 \cdot 5$ & 46.7 & 38 & 6 \\
\hline 52 & 37.6 & 36.9 & & 06 & Lt & 52 & 44.9 & 42.9 & & 56 & 2 & 52 & 60.7 & $6 \mathrm{I} .4$ & 23 o2 & $\sqrt{4}$ & 52 & 40.2 & 48.0 & 40 & Er \\
\hline 54 & 39.0 & 38.0 & & 04 & 14 & 54 & 43.9 & $4 I .9$ & & 58 & xe & 54 & 58.7 & 59.7 & 2259 & $3=$ & 54 & 45.0 & 46.8 & 38 & at \\
\hline 56 & 40.2 & 39.5 & & 02 & te & 56 & $43 \cdot 3$ & $4 I .2$ & & 59 & (1) & 56 & 55.2 & 55.9 & 53 & 12. & 56 & 43.1 & 44.9 & 35 & $n$ \\
\hline 58 & 40.3 & 39.9 & 23 & 02 & & 58 & $43 . I$ & $4 I \cdot 9$ & 225 & 58 & $1+x$ & 58 & 52.9 & 54.1 & 50 & 10 & 58 & 42.0 & 44.0 & 33 & 85 \\
\hline 500 & $42 . I$ & 41.6 & 22 & 59 & -12.8 & 700 & 39.9 & 37.9 & 23 & 04 & -10.2 & 900 & 50.9 & 52.7 & 47 & $-14 \cdot 3$ & II 00 & 43.0 & 44.9 & 35 & -13.0 \\
\hline 02 & 42.0 & 40.6 & 23 & 00 & 25 & 02 & 37.5 & 35.9 & & 07 & 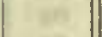 & 02 & $49 . I$ & 50.2 & 44 & 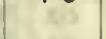 & 02 & 44.1 & 46.2 & 37 & ( \\
\hline 04 & 39.8 & 38.8 & 23 & 03 & 10 & 04 & 33.9 & 32.1 & & I3 & [17 & 04 & 47.8 & 49.1 & 42 & kat & 04 & 42.8 & 45.0 & 35 & 36 \\
\hline 06 & 42.9 & 41.8 & 22 & 59 & 90 & 06 & 33.9 & 32.3 & & I3 & 6 & 06 & 45.6 & 46.9 & 38 & 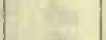 & 06 & 43.3 & 46.1 & 36 & $\mathrm{n}$ \\
\hline 08 & $47 \cdot 2$ & 46.6 & & $5 I$ & tay & 08 & 33.7 & 32.2 & & I3 & $=$ & 08 & 44.0 & 46.2 & 37 & 71 & 08 & 43.2 & 45.9 & 36 & $13 y$ \\
\hline 10 & $49 \cdot 5$ & 48.7 & & 48 & 1 & 10 & 33.0 & 31.9 & & I4 & 315 & IO & 40.9 & 43.8 & 32 & $6 y^{2}$ & Io & 46.8 & 49.1 & $4 I$ & cit \\
\hline 12 & 52.8 & 51.3 & & 43 & 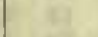 & 12 & $34 \cdot 7$ & $33 \cdot 7$ & & II & $=x$ & 12 & 42.4 & 44.6 & 34 & $1=1$ & 12 & 45.3 & $47 \cdot 3$ & 39 & 7 \\
\hline 14 & 50.4 & 49.9 & 22 & 46 & -12.7 & 14 & 23.7 & 22.1 & & 29 & -10.0 & 14 & $43 \cdot 5$ & 46.0 & 36 & -14.1 & 14 & 41.9 & $44 \cdot 4$ & 34 & -13.0 \\
\hline I6́ & 42.0 & 40.4 & 23 & $\infty$ & & 16 & 29.8 & 28.0 & & 20 & 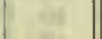 & 16 & 44.9 & 46.9 & 38 & ( & I6. 4 & 45.1 & 46.8 & 38 & $\sqrt{2}$ \\
\hline I8 & 50.3 & 49.0 & 22 & 47 & th & 18 & $37 \cdot 3$ & 35.0 & c & 08 & $x^{4}$ & 18 & 48.9 & 50.5 & 44 & (4) & I8 & 45.2 & 47.7 & 39 & 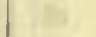 \\
\hline 20 & 47.8 & 47.6 & 22 & 50 & tat & 20 & 40.9 & 39.2 & 23 & 02 & 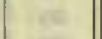 & 20 & 49.0 & 59.8 & 44 & C5 & 20 & $4 \mathrm{I} . \mathrm{I}$ & 43.2 & 32 & 06 \\
\hline 22 & 29.1 & 29.1 & 23 & 19 & 6 & 22 & $45 \cdot 3$ & 43.9 & 22 & 55 & at & 22 & 49.5 & 50.8 & 45 & $4 \pi$ & 22 & 48.2 & 50.1 & 43 & L2 \\
\hline 24 & 25.3 & 24.9 & & 26 & 1 & 24 & 42.9 & 41.8 & 22 & 59 & 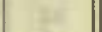 & 24 & $49 \cdot 4$ & 50.8 & 45 & 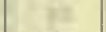 & 24 & 42.6 & 43.6 & 34 & if \\
\hline 26 & $30 . \overline{8}$ & 30.5 & & 17 & 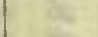 & 26 & 41.9 & 41.0 & 23 & $\infty$ & an & 26 & 47.1 & 48.9 & $4 I$ & in & 26 & 40.0 & 41.0 & 30 & an \\
\hline 28 & 37.7 & 36.9 & & 06 & 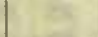 & 28 & 40.1 & 39.1 & & 03 & & 28 & 48.5 & 50.2 & 43 & 1 5 & 28 & 40.5 & 41.9 & 30 & ant \\
\hline 30 & 37.0 & 36.2 & & 08 & -12.4 & 30 & 41.0 & 39.2 & 23 & 02 & -9.6 & 30 & 43.3 & $44 \cdot 7$ & 35 & -14.0 & 30 & 39.2 & 40.8 & 29 & -12.9 \\
\hline 32 & 39.2 & 38.4 & & 04 & $x^{2}$ & 32 & 46.1 & 45.1 & 22 & 54 & $+\sqrt{2}$ & 32 & 46.8 & 48.3 & 40 & ( & 32 & 39.1 & 40.2 & 28 & ( \\
\hline 34 & 39.6 & 38.9 & 23 & $0_{4}$ & 16 & 34 & 46.0 & $45 \cdot 4$ & & 53 & 15 & 34 & 44.9 & 46.1 & 37 & +6 & 34 & 38.9 & 40.0 & 28 & 15 \\
\hline 36 & 43.1 & $4 I .7$ & 22 & 58 & ne & 36 & 49.5 & 49.0 & & 48 & 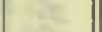 & 36 & 44.2 & $45 \cdot 3$ & 36 & the & 36 & 40.0 & 41.0 & 30 & 6 \\
\hline 38 & 45.2 & 44.2 & & 55 & & 38 & 61 & $.0 a$ & & 29 & 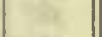 & 38 & 49.0 & 50.0 & 44 & 8 & 38 & 40.0 & $4 I . I$ & 30 & 87 \\
\hline 40 & 43.8 & 42.8 & & 57 & & 40 & 63 & $2 b$ & 22 & 26 & 54 & 40 & 44.1 & 45.8 & 36 & 14 & 40 & $4 \mathrm{I} . \mathrm{I}$ & 42.2 & 31 & 00 \\
\hline 42 & 42.9 & 41.9 & & 58 & & 42 & 38.0 & 36.9 & 23 & 06 & $x^{2}$ & 42 & $43 . I$ & 44.0 & 34 & 1 & 42 & $43 \cdot 3$ & 44.3 & 35 & \\
\hline 44 & 43.9 & 42.8 & & 57 & -12.0 & 44 & 35.8 & 35.4 & & 09 & -9.2 & 44 & 43.0 & $44 . I$ & 34 & -14.0 & 44 & 41.9 & 42.2 & 32 & -12.8 \\
\hline 46 & 42.2 & 41.7 & 22 & 59 & & 46 & 36.9 & 33.2 & 23 & IO & & 46 & 41.2 & 42.3 & $3 I$ & (n) & 46 & 40.1 & 41.7 & 30 & (2) \\
\hline 48 & 39.1 & 38.1 & 23 & 04 & 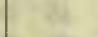 & 48 & 42.1 & 41.6 & 22 & 59 & Fis & 48 & 40.1 & 40.5 & 29 & mat & 48 & 39.1 & 40.3 & 28 & 5 \\
\hline 50.6 & 38.7 & 37.9 & & 05 & 4 & 50 & 39.0 & 37.6 & 23 & 05 & $x$ & 50 & 40.2 & $4 I . I$ & 30 & ec & 50 & 39.1 & 40.0 & 28 & et \\
\hline 52 & 36.2 & 35.2 & & $\infty$ & 6 & 52 & 43.1 & 42.8 & 22 & 58 & 6 & 52 & 41.7 & 42.2 & 32 & -2 & 52 & 39.5 & 40.4 & 28 & 4 \\
\hline 54 & 34.8 & 33.2 & & 12 & 24? & 54 & 45.0 & 44.2 & & 55 & 12 & 54 & 40.1 & 40.9 & 30 & 17 & 54 & 40.1 & 41.2 & 30 & 17 \\
\hline 56 & 31.8 & 31.0 & & 16 & $x^{2}=$ & 56 & 47.8 & $47 . I$ & & 50 & 2 & 56 & 39.0 & 39.9 & 28 & & 56 & 42.0 & 42.6 & 32 & tect \\
\hline 58 & 35.0 & 34.6 & & 10 & 6 & 58 & 49.7 & 49.1 & & 48 & 0 & 58 & 40.9 & 41.9 & $3 I$ & te & 58 & $4 \mathrm{I} \cdot 5$ & 42.9 & 32 & \\
\hline & & & & & & 800 & 46.7 & 45.6 & & 53 & -8.9 & s. & & & & Do & 1200 & 40.7 & 41.9 & $3 I$ & -12.4 \\
\hline
\end{tabular}

Correction to local mean time is $+2 \mathrm{~m}$ 07s. $90^{\circ}$ torsion $=140^{\prime} 54$.

Torsion head at oh oom read $38^{\circ}$ and at 9 h $05 \mathrm{~m}$ read $33^{\circ}$.

Observer-R R. T.
Correction to local mean time is $-5 \mathrm{~s} .90^{\circ}$ torsion $=16^{\prime} 80$.

Torsion head at $7 \mathrm{~h} 35 \mathrm{~m}$ read $24^{\circ}$ and at $12 \mathrm{~h} 15 \mathrm{~m}$ read $26^{\circ}$. Observer-R. R. T. 
Tabulation of magnetic declinations observed at T'eplitz Bay-Continued

\begin{tabular}{|c|c|c|c|c|c|c|c|c|c|c|c|c|c|c|c|c|c|c|c|}
\hline \multicolumn{5}{|c|}{ Tuesday, April 26, I904 } & \multicolumn{5}{|c|}{ Magnet scale inverted } & \multicolumn{5}{|c|}{ Wednesday, April 27, 1904} & \multicolumn{5}{|c|}{ Magnet scale erect } \\
\hline \multirow{2}{*}{$\begin{array}{l}\text { Chr'r } \\
\text { time }\end{array}$} & \multicolumn{2}{|c|}{$\begin{array}{l}\text { Scale } \\
\text { readings }\end{array}$} & \multirow{2}{*}{$\begin{array}{c}\text { East } \\
\text { decli- } \\
\text { nation }\end{array}$} & Temp. & Chr'r & $\begin{array}{r}\text { Sc } \\
\text { read }\end{array}$ & $\begin{array}{l}\text { ale } \\
\text { lings }\end{array}$ & $\begin{array}{l}\text { East } \\
\text { decli- }\end{array}$ & Temp. & Chr'r & $\begin{array}{r}\text { Sct } \\
\text { read }\end{array}$ & $\begin{array}{l}\text { ale } \\
\text { lings }\end{array}$ & East & Temp. & Chr'r & $\begin{array}{l}\text { Sc: } \\
\text { read }\end{array}$ & $\begin{array}{l}\text { ale } \\
\text { lings }\end{array}$ & $\begin{array}{l}\text { East } \\
\text { decli- }\end{array}$ & Temp. \\
\hline & Left & Right & & & & Left & Right & & & & Left & Right & & & time & Left & Right & nation & \\
\hline $\mathrm{h} \mathrm{m}$ & d & d & -, & 0 & $\mathrm{~h} \mathrm{~m}$ & d & d & $\circ$, & 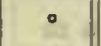 & $h \mathrm{~m}$ & d & d & & 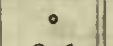 & $h \mathrm{in}$ & d & d & $\circ$, & - \\
\hline 1200 & 46.2 & 44.4 & 2248 & -14.1 & 1400 & 40.0 & 38.8 & 2257 & $-14 \cdot 3$ & $00^{*}$ & 55.5 & 56.7 & 2318 & -18.6 & 200 & 70.2 & 70.3 & $234 \mathrm{I}$ & -19.3 \\
\hline 02 & 49.2 & 48.3 & 43 & . & 02 & 32.0 & 31.8 & 2309 & 20 & 02 & 56.0 & 57.0 & 19 & ( & 02 & 69.8 & 70.1 & 40 & \\
\hline 04 & 46.9 & 45.2 & & 10 & 04 & 35.2 & $34 . I$ & 05 & 10 & 04 & 57.0 & 59.1 & $2 I$ & (is) & 04 & 70.2 & 70.8 & 42 & \\
\hline 06 & $45 . \mathrm{I}$ & 44.1 & 49 & 60 & 06 & 34.0 & 33.2 & 06 & $\infty$ & 06 & 58.8 & 59.9 & 23 & 5 & 06 & 70.1 & 70.6 & $4 \mathrm{I}$ & \\
\hline$\$ 8$ & 48.8 & 46.9 & 44 & 85 & 08 & 35.2 & 34.0 & 05 & 80 & 08 & 58.5 & 59.3 & 23 & 60 & 08 & 70.7 & 71.1 & 42 & \\
\hline 10 & 48.9 & 47.0 & 44 & bis & Jo & 34.2 & 33.2 & 06 & of & I0 & 59.1 & 60.1 & 24 & W & I0 & 71.1 & 71.7 & 43 & vir \\
\hline 12 & 49.0 & 46.9 & 44 & 20 & I2 & 33.8 & 32.0 & 08 & at & I2 & 58.9 & 59.8 & 24 & (t) & I2 & 72.0 & 72.6 & 44 & \\
\hline 14 & 50.6 & 48.3 & 42 & -14.1 & 14 & 34.2 & 32.0 & 07 & $-14 \cdot 3$ & 14 & 58.3 & 59. I & 23 & -18.6 & I4 & 72.1 & 72.7 & 44 & -19.4 \\
\hline 16 & 53.3 & 51.2 & 37 & 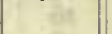 & 16 & 31.8 & 30.7 & IO & (2) & 16 & 57.0 & 57.4 & 20 & tye & 16 & 71.2 & 72.1 & 43 & \\
\hline 18 & 54.9 & 52.2 & 35 & 85 & I8 & 32.3 & $3 \mathrm{I} . \mathrm{I}$ & 09 & (64) & I8 & 57.8 & 58.1 & $2 \mathrm{I}$ & 84 & I8 & 70.3 & 71.0 & 42 & *47 \\
\hline 20 & 54.0 & 50.8 & 37 & 96 & 20 & 28.2 & 26.4 & 16 & 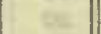 & 20 & 58.2 & 58.9 & 22 & as & 20 & 69.2 & 70.0 & 40 & 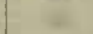 \\
\hline 22 & 53.2 & 51.9 & 37 & 2.58 & 22 & 28.1 & 26.3 & I6 & 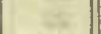 & 22 & 59.8 & 60.1 & 24 & 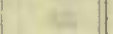 & 22 & 69.5 & 70.1 & 40 & $=E$ \\
\hline 24 & 53.2 & 52.0 & 37 & pit & 24 & 27.8 & 25.6 & 17 & $y=$ & 24 & 60.5 & 60.9 & 26 & $t_{2}=$ & 24 & 69.9 & 70.3 & $4 \mathrm{I}$ & $y^{2}$ \\
\hline 26 & 56.9 & 54.0 & 32 & ca & 26 & 27.8 & 26.0 & I7 & is & 26 & 61.2 & 61.7 & 27 & at & 26 & 70.1 & 70.9 & 42 & \\
\hline 28 & 59.0 & 58.0 & 27 & the & 28 & 27.4 & 26.5 & I7 & & 28 & 61.7 & 62.0 & 27 & 2 & 28 & 71.1 & 71.6 & 43 & \\
\hline 30 & 62.0 & 59.0 & 24 & -14.3 & 30 & 31.0 & 30.0 & II & $-14 \cdot 3$ & 30 & 62.9 & 63.1 & 29 & -18.8 & 30 & 71.2 & 71.8 & 43 & -19.4 \\
\hline 32 & 62.7 & 60.5 & 22 & 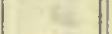 & 32 & 36 & $3 a$ & 2302 & $1=$ & 32 & 63.2 & 63.3 & 30 & 10 & 32 & $71 . I$ & 71.3 & 43 & $x^{2}$ \\
\hline 34 & 6 r.5 & 60.0 & 24 & its & 34 & 43.5 & 43.3 & $225 \mathrm{I}$ & ${ }^{2}$ & 34 & 63.1 & 63.1 & 30 & 14. & 34 & 71.1 & 71.8 & 43 & \\
\hline 36 & 59. I & 58.0 & 27 & 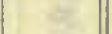 & 36 & 45.2 & 44.4 & 49 & 10: & 36 & 63.7 & 63.9 & 31 & it & 36 & 72.8 & 72.8 & 45 & \\
\hline 38 & 57.7 & 57.0 & 29 & 105 & 38 & 50.8 & 49.8 & 40 & 160 & 38 & 63.8 & 64.0 & $3 \mathrm{I}$ & ine & 38 & 74.0 & $74 . I$ & 47 & \\
\hline 40 & 54.9 & 53.0 & 34 & $=$ & 40 & 54.6 & 53.1 & 35 & 60 & 40 & 64.1 & 64.1 & $3 I$ & 18 & 40 & 74.8 & 74.8 & 48 & \\
\hline 42 & 52.6 & 50.9 & 38 & $1=$ & 42 & 55.1 & 55.0 & 33 & 2 & 42 & 64.3 & 64.7 & 32 & 20 & 42 & 73.8 & 74.0 & 47 & \\
\hline 44 & 56.2 & 55.9 & $3 I$ & $-\mathrm{I} 4.4$ & 44 & 56.8 & 56.3 & 30 & 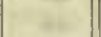 & 44 & 64.2 & $64 \cdot 4$ & 31 & -19.0 & 44 & 73.9 & 73.9 & 47 & -19.5 \\
\hline 46 & 63.2 & 62.1 & $2 \mathrm{I}$ & & 46 & 53.7 & 53.1 & 35 & Di & 46 & 65.2 & 65.7 & 33 & r & 46 & 73.8 & 74.1 & 47 & \\
\hline 48 & 62.9 & 59.2 & 23 & 6 & 48 & 55.8 & 53.7 & 33 & tet & 48 & 65.7 & 66.0 & 34 & 48 & 48 & 74.0 & 74.9 & 48 & \\
\hline 50 & 59.5 & 57.0 & 28 & 8 & 50 & 56.3 & 55.3 & 32 & ex & 50 & 65.9 & 66.0 & 34 & et & 50 & 73.8 & 74.3 & 47 & \\
\hline 52 & 55.2 & 52.9 & 34 & 20 & 52 & 56.0 & 55.3 & 32 & $=1$ & 52 & 64.9 & 65.0 & 32 & 45 & 52 & $74 . I$ & 74.6 & 48 & \\
\hline 54 & 58.3 & 55.1 & 30 & 45. & 54 & 55.2 & 54.7 & 33 & $\mathrm{M}$ & 54 & 64.9 & 65.1 & 32 & is & 54 & $73 \cdot 3$ & 74.1 & 47 & \\
\hline 56 & 57.0 & 55.0 & $3 \mathrm{I}$ & 60 & 56 & 58.0 & 57.0 & 29 & 12. & 56 & 64.9 & 65.1 & 32 & (at & 56 & 74.0 & 75.0 & 48 & \\
\hline 58 & 54.5 & 52.9 & 35 & & 58 & 60.0 & 59.7 & 25 & & 58 & 65.1 & 65.3 & 33 & 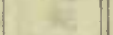 & 58 & 73.9 & 74.8 & 48 & \\
\hline I3 00 & 53.0 & 50.8 & 38 & $-14 \cdot 3$ & I5 00 & 64.0 & 62.1 & 20 & -I $4 . I$ & I 00 & 66.0 & 66.5 & 35 & -19.2 & 300 & 75.0 & 76.1 & 49 & -I9.5 \\
\hline 02 & $5 \mathrm{I} .2$ & 49.9 & 40 & - & 02 & 63.6 & 61.8 & $2 \mathrm{I}$ & 20 & 02 & 66.0 & 67.0 & 35 & 150 & 02 & 74.1 & 75.2 & 48 & \\
\hline 04 & 50.8 & 49.8 & 40 & the & 04 & 61.0 & 59.1 & 25 & sh & 04 & 65.0 & 66.0 & 34 & 10 & 04 & 72.1 & 72.9 & 45 & (z) \\
\hline 06 & 50.9 & 49.3 & 40 & 60 & 06 & 6I.I & 59.8 & 24 & ine & 06 & 64.0 & 64.9 & 32 & iv & 06 & 72.2 & 73.2 & 45 & \\
\hline 08 & 49.0 & 47.8 & 43 & 16 & 08 & 58.9 & 57.1 & 28 & $n$ & os & 65.5 & 66.2 & 34 & 62 & 08 & 73.1 & 74.1 & 46 & \\
\hline Io & 47.7 & 47.0 & 45 & arr & I0 & 58.9 & 56.3 & 29 & $={ }^{2}$ & 10 & 66.8 & 67.2 & 36 & 61 & I0 & 73.1 & 74.0 & 46 & \\
\hline 12 & 45.0 & 43.5 & 50 & 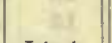 & 12 & 58.0 & 56.0 & 30 & 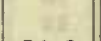 & 12 & 67.8 & 68.1 & 37 & (1) & 12 & 71.9 & 72.8 & 44 & \\
\hline 14 & 42.9 & 42.2 & 52 & -14.4 & 14 & 57.9 & 56.7 & 29 & -14.2 & 14 & 68.6 & 69.0 & 39 & -19.2 & 14 & 72.5 & 73.1 & 45 & -19.4 \\
\hline 16 & 43.9 & 43.8 & 50 & 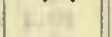 & 16 & 58.0 & 56.8 & 29 & in & 16 & 68.7 & 69.1 & 39 & tate & 16 & $73 . \overline{8}$ & 74.0 & 47 & \\
\hline I8 & 46.1 & 45.0 & 48 & hr & 18 & 54.2 & 52.8 & 35 & 65 & 18 & 69.4 & 69.8 & 40 & Kar & 18 & $74 . I$ & 74.9 & 48 & kx \\
\hline 20 & 50.0 & 49.2 & 41 & 108 & 20 & 54.9 & 50.1 & 37 & $a$ & 20 & 69.2 & 69.2 & 39 & vat & 20 & 76.7 & $77 \cdot 3$ & 52 & 16 \\
\hline 22 & 48.8 & 48.0 & 43 & $k=$ & 22 & 52. I & 51.0 & 38 & wa & 22 & 69.1 & 69.1 & 39 & $\mathrm{E}$ & 22 & 77.1 & 78.1 & 53 & \\
\hline 24 & $5 I .3$ & 50.8 & 39 & 18 & 24 & 52.0 & 50.2 & 39 & 18 & 24 & 68.3 & 68.7 & 38 & itit & 24 & 76.3 & 77.0 & 51 & it \\
\hline 26 & 53.5 & 53.2 & 35 & $\partial$ & 26 & 51.2 & 49.5 & 40 & 8 & 26 & 67.7 & 67.7 & 37 & ate & 26 & 76.1 & 77.0 & $5 \mathrm{I}$ & \\
\hline 28 & 53.2 & 53.0 & 36 & $=$ & 28 & 50.4 & $48 . I$ & 42 & $12 x$ & 28 & 67.8 & 67.8 & 37 & $1=$ & 28 & 76.7 & 77.1 & 52 & \\
\hline 30 & 53.0 & $52 . \mathrm{I}$ & 37 & $-14 \cdot 4$ & 30 & 48.9 & 47.4 & 44 & -14.2 & 30 & 67.9 & 68.0 & 37 & -19.2 & 30 & 76.0 & 77.1 & $5 \mathrm{I}$ & -19.2 \\
\hline 32 & 52.0 & $5 \mathrm{I} .0$ & 38 & $x^{2}$ & 32 & 49.8 & 48.5 & 42 & $1+2=$ & 32 & 69.8 & 69.9 & 40 & $x^{-2}$ & 32 & 74.7 & 75.3 & 49 & \\
\hline 34 & 50.6 & 50.1 & 40 & 12 & 34 & 53.2 & 5I.I & 37 & 41 & 34 & 69.6 & 69.9 & 40 & 15 & 34 & 75.9 & 76.8 & $5 I$ & \\
\hline 36 & 49.7 & $48 \cdot 3$ & 42 & $\mathrm{Si}$ & 36 & $53 . I$ & 51.2 & 37 & (18. & 36 & 69.9 & 70.0 & 40 & 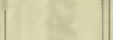 & 36 & 77.3 & 78.0 & 53 & \\
\hline 38 & 48.0 & 46.9 & 45 & ber & 38 & $54 . I$ & 52.8 & 35 & $\theta^{2}$ & 38 & 70.0 & 70.0 & $4 \mathrm{I}$ & $z^{2}$ & 38 & 77.5 & 78.3 & 53 & \\
\hline 40 & 45.9 & 44.9 & 48 & c. & 40 & 52.8 & 51.0 & 38 & os & 40 & 70.3 & 70.8 & $4 \mathrm{I}$ & wate & 40 & 76.0 & 76.3 & 2350 & \\
\hline 42 & $46 . I$ & 45.8 & 47 & 18 & 42 & 53.4 & 50.9 & 37 & - & 42 & 70.9 & 71.0 & 42 & - & $42^{*}$ & 52.7 & 57.1 & 2253 & \\
\hline & 46.2 & 46.0 & 47 & $-14 \cdot 3$ & 44 & 55.1 & 53.7 & 34 & -14.3 & 44 & 70.7 & 70.9 & 42 & -19.2 & 44 & 53.3 & 56.9 & 53 & -19.0 \\
\hline 46 & 48 & $.7 a$ & 43 & . & 46 & 60.0 & $57 . \mathrm{I}$ & 27 & ( & 46 & 70.2 & 70.9 & 42 & $x^{2}$ & 46 & 55.0 & 57.9 & 55 & \\
\hline 48 & $49 . I$ & 48.9 & 42 & ha & 48 & 60.0 & 57.8 & 27 & 34 & 48 & 70.6 & 70.9 & 42 & 28 & 48 & 56.2 & 58.9 & 57 & \\
\hline 50 & 48.3 & 47.8 & 44 & 8 & 50 & 62.0 & 59.8 & 24 & $\frac{75}{6}$ & 50 & 70.1 & 70.2 & 41 & ar & 50 & 57.9 & 60.1 & 2259 & \\
\hline 52 & 47.2 & 47.0 & 45 & $a^{2}$ & 52 & 61.0 & 59.2 & 25 & 만 & 52 & 71.9 & 71.9 & 44 & & 52 & 62.1 & 63.8 & 2305 & \\
\hline 54 & 44.8 & $44 . I$ & 50 & it. & 54 & 64.2 & 62.0 & 20 & A4 & 54 & 72.9 & 73.0 & 45 & 3 & 54 & 62.3 & 64.7 & об & \\
\hline 56 & 45 & $.0 a$ & 48 & $n^{2}$ & 56 & 64.3 & 62.9 & 19 & $n^{2}$ & 56 & 73.1 & 73.1 & 46 & es & 56 & 60.9 & 62.9 & 04 & \\
\hline 58 & 43.3 & 42.7 & 52 & EE & 58 & $6 \mathrm{I} .2$ & 59.9 & 24 & 82 & 58 & 72.0 & 72.2 & 44 & 82 & 58 & 60.9 & 63.0 & 04 & \\
\hline$x^{3}=1$ & 2 & $\theta_{2}$ & 78 & 37 & I6 00 & 58.8 & 57.2 & 28 & -14.4 & $c^{2}$ & $1=$ & & & te- & & & & & \\
\hline
\end{tabular}

Correction to local mean time is $-9.5 \mathrm{~s}$.

Torsion head at IIh $29 \mathrm{~m}$ read $48^{\circ}$ and at $16 \mathrm{~h} 23 \mathrm{~m}$ read the same.

Observer-R. R. T.

Observer-J. V. 
Tabulation of magnetic declinations observed at Teplitz Bay-Continued

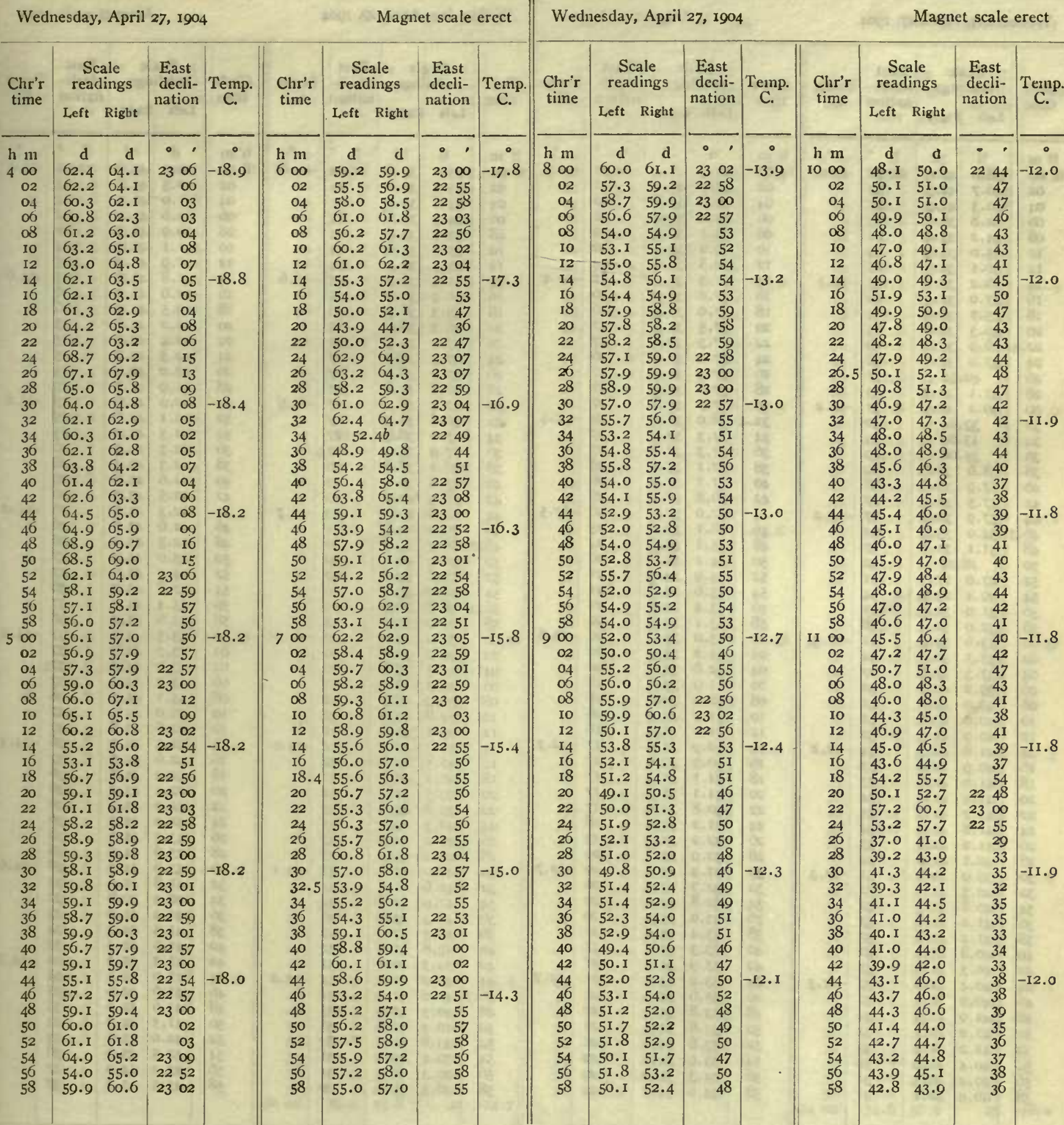

Observers-R. R. T. and J. V., who alternated from $7 \mathrm{~h} 48 \mathrm{~m}$ to $7 \mathrm{~h}$ Observer-J. V. $58 \mathrm{~m}$. 
Tabulation of magnetic declinations observed at Teplitz Bay-Continued

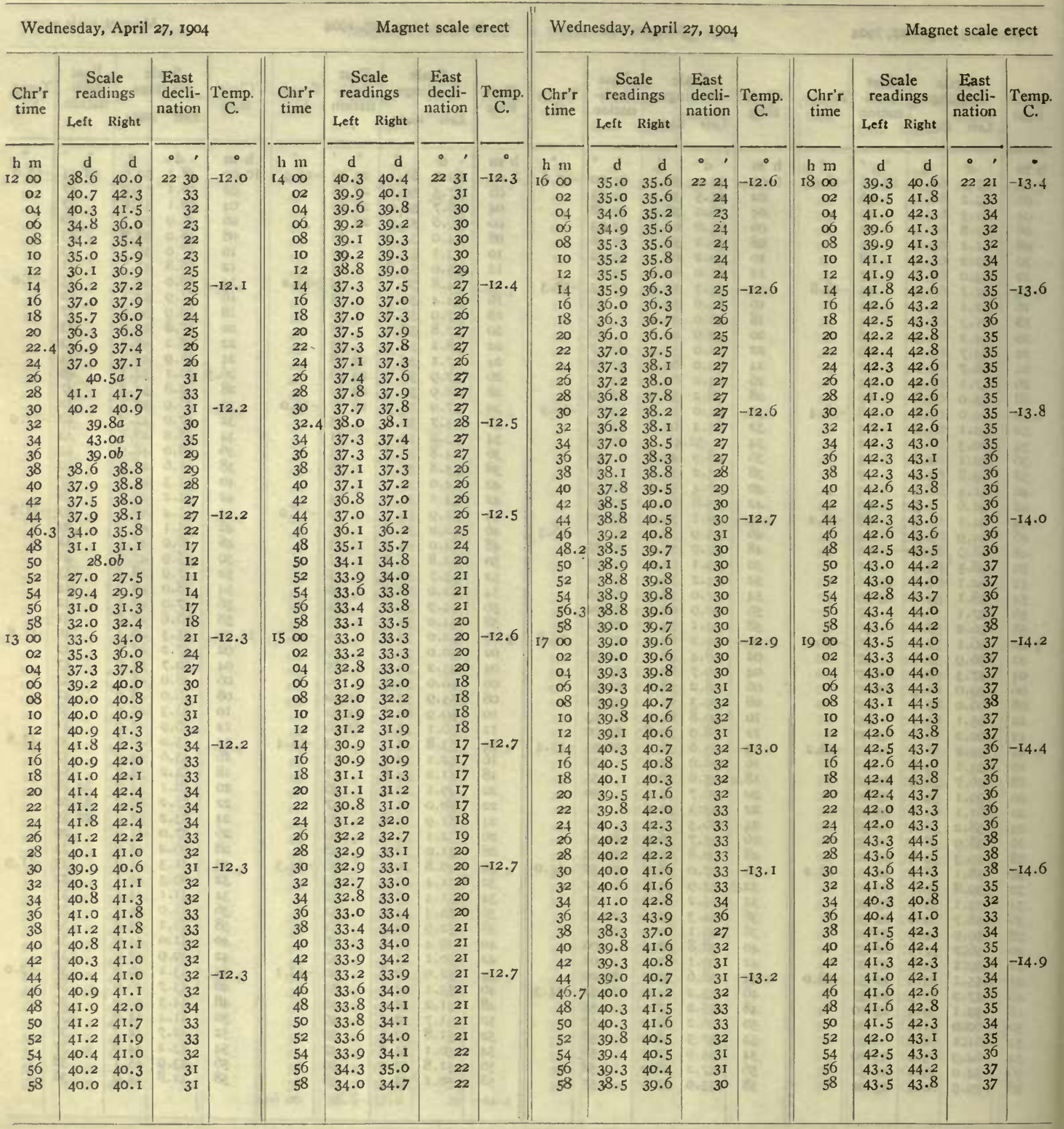

Observers-J. V. and W. J. P., who alternated from I5h $52 \mathrm{~m}$ to

Observer-W. J. P. $16 \mathrm{~h} 02 \mathrm{~m}$. 
Tabulation of magnetic declinations obscrved at Teplitz Bay-Continued

\begin{tabular}{|c|c|c|c|c|c|c|c|c|c|c|c|c|c|c|c|c|c|c|c|}
\hline \multicolumn{5}{|c|}{ Thursday, April 28, 1904} & \multicolumn{5}{|c|}{ Magnet scale inverted } & \multicolumn{5}{|c|}{ Wednesday, April 27, I904 } & \multicolumn{5}{|c|}{ Magnet scale erect } \\
\hline $\begin{array}{l}\text { Chr'r } \\
\text { time }\end{array}$ & \multicolumn{2}{|c|}{$\begin{array}{l}\text { Scale } \\
\text { readings }\end{array}$} & $\begin{array}{l}\text { Fast } \\
\text { decli- } \\
\text { nation }\end{array}$ & $\begin{array}{c}\text { Temp. } \\
\text { C. }\end{array}$ & $\begin{array}{l}\text { Chr'r } \\
\text { time }\end{array}$ & $\begin{array}{r}\text { Sc } \\
\text { read } \\
\text { Left }\end{array}$ & $\begin{array}{l}\text { ale } \\
\text { lings } \\
\text { Right }\end{array}$ & $\begin{array}{l}\text { East } \\
\text { decli- } \\
\text { nation }\end{array}$ & $\begin{array}{c}\text { Temp. } \\
\text { C. }\end{array}$ & $\begin{array}{l}\text { Clir'r } \\
\text { tinse }\end{array}$ & $\begin{array}{r}\text { Sc } \\
\text { reac } \\
\text { Left }\end{array}$ & $\begin{array}{l}\text { cale } \\
\text { dings: } \\
\text { Right }\end{array}$ & $\begin{array}{c}\text { East } \\
\text { decli- } \\
\text { nation. }\end{array}$ & $\underset{\text { C. }}{\text { Temp. }}$ & $\begin{array}{l}\text { Chr'r } \\
\text { time }\end{array}$ & $\begin{array}{r}\text { Sc } \\
\text { read } \\
\text { L,eft }\end{array}$ & $\begin{array}{l}\text { ale } \\
\text { lings } \\
\text { Right }\end{array}$ & $\begin{array}{c}\text { East } \\
\text { decli- } \\
\text { nation }\end{array}$ & $\begin{array}{c}\text { Tenıp. } \\
\text { C. }\end{array}$ \\
\hline $\mathrm{l} \mathrm{m}$ & d & d & $\circ \quad$ & 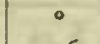 & h m & d & d & $\circ \quad$ & 。 & li $\mathrm{m}$ & d & d & & & $\mathrm{l} \mathrm{m}$ & d & d & . ' & \\
\hline 600 & 53.0 & 51.9 & $22 \quad 27$ & -13.6 & 1800 & 55.2 & 54.7 & $22 \quad 23$ & -13.0 & 2000 & 43.2 & 43.8 & $22 \quad 37$ & -15.0 & 2200 & 43.0 & 43.0 & 2236 & -16.6 \\
\hline 02 & 52.8 & 51.3 & 28 & & 02 & 54.9 & $54 . I$ & 24 & & 02 & 42.6 & 42.9 & 36 & & 02 & 42.8 & $43 \cdot 3$ & 36 & \\
\hline 04 & 52.8 & $5 I . I$ & 28 & & 04 & 54.6 & 53.9 & 24 & & 04 & 42.3 & 42.4 & 35 & & 04 & 42.7 & $43 \cdot 3$ & 36 & 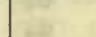 \\
\hline$\alpha$ & $53 \cdot 5$ & 51.9 & 26 & & 06 & 54.8 & 54.0 & 24 & & 06 & 42.3 & 42.6 & 35 & & 06 & 42.6 & 43. & 36 & 12 \\
\hline 08 & 54.7 & $53 \cdot 1$ & 24 & & 08 & 56.0 & 55.2 & 22 & & 28 & 42.3 & 42.4 & 35 & & 08 & $42 \cdot 3$ & 42. & 36 & \\
\hline 10 & 55.5 & 54.1 & 23 & & 10 & 56.1 & 55.8 & $2 I$ & & Io & 42.0 & 42.4 & 35 & & IO & $42 . I$ & 42. & 36 & \\
\hline 12 & 55.7 & 54.7 & 22 & & 12 & 55.0 & $54 \cdot 3$ & 23 & & I2 & -42.0 & 42.6 & 35 & & I2 & $4 I .6$ & 42.4 & 35 & \\
\hline I4 & $55 . \mathrm{I}$ & 54.6 & 23 & $-\mathrm{I} 3.4$ & 14 & 54.0 & 53.4 & 25 & -13.1 & I4 & 41.9 & 42.5 & 35 & -15.2 & I4 & $4 I .5$ & 42. I & 35 & -16.9 \\
\hline I6 & 55.1 & 54.8 & 23 & & I6 & 53.9 & 53.2 & 25 & & 16 & 42.2 & 42.8 & 36 & & 16 & 4I.I & $4 \mathrm{I}$ & 34 & \\
\hline 18 & 54.9 & 54.9 & 23 & & I8 & 54.0 & 53.8 & 24 & & 18 & 42.0 & 42.7 & 35 & 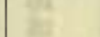 & 18 & 41.6 & 42. & 35 & 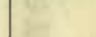 \\
\hline 20 & $54 \cdot 7$ & 54.2 & 24 & & 20 & 54.1 & 53.6 & 25 & & 20 & 42.3 & 42.6 & 35 & $x$ & 20 & $4 I .8$ & 42.6 & 35 & $=$ \\
\hline 22 & 52.7 & 52.7 & 25 & $y^{-2}$ & 22 & 53.0 & 52.1 & 27 & s. & 22 & 42.6 & 43.4 & 36 & 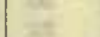 & 22 & $4 I .7$ & 42.3 & 35 & int \\
\hline 24 & 52.0 & 51.7 & 28 & $x^{2}$ & 24 & 52.0 & 51.1 & 28 & 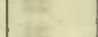 & 24 & 42.8 & $43 \cdot 4$ & 36 & $=$ & 24 & 41.6 & 42.1 & 35 & \\
\hline 26 & 52.4 & 51.9 & 27 & Fin & 26 & 51.9 & 51.0 & 28 & & 26 & 43.0 & 43.6 & 37 & & 26 & $4 I .8$ & 42.2 & 35 & \\
\hline 28 & 52.7 & 52.0 & 27 & 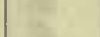 & 28 & 53.1 & 52.2 & 27 & & 28 & 43.3 & 43.8 & 37 & & 28 & 41.6 & 42.0 & 35 & \\
\hline 30 & 52.7 & 52.1 & 27 & $-13 \cdot 3$ & 30 & 55.7 & 54.8 & 22 & $-13 \cdot 3$ & 30 & 43.1 & 43.8 & 37 & -15.5 & 30 & 4 II.8 & 42.2 & 35 & -17.0 \\
\hline 32 & 52.9 & $5 I .9$ & 27 & & 32 & 57.1 & 56.8 & 20 & ( & 32 & $43 \cdot 3$ & 43.8 & 37 & & 32 & 42.1 & 42.2 & 35 & \\
\hline 34 & 53.2 & 52.1 & 27 & 36 & 34 & 58.2 & 58.0 & I8 & & 3 & 43.6 & 43. & 37 & & 34 & 42.3 & 42.3 & 36 & \\
\hline 36 & $54 . I$ & 53.0 & 25 & 10 & 36.4 & 58.0 & 57.7 & I8 & 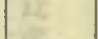 & 36 & 43.8 & 44.0 & 38 & & 36 & 42.0 & 42.2 & 35 & \\
\hline 38 & 55.1 & 54.2 & 23 & & 38 & 57.0 & 56.5 & 20 & & 38 & 43.6 & 43.9 & 37 & $y$ & 38 & 42.0 & 42.2 & 35 & \\
\hline 40 & 55.8 & 55.0 & 22 & 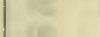 & 40 & 57.0 & 56.2 & 20 & 2 & 40 & 43.7 & 43.7 & 37 & 8 & 40 & 42.5 & 42.7 & 36 & \\
\hline 42 & 56.0 & 55.5 & 22 & we & 42 & 56.9 & 56.1 & 20 & & 42 & 43.6 & 43.8 & 37 & 0 & 42 & 43.0 & $43 \cdot 3$ & 37 & \\
\hline 44 & 55.4 & 55.1 & 22 & $-13 \cdot 3$ & 44 & 56.1 & 56.0 & $2 I$ & & 44 & $43 \cdot 5$ & 43.9 & 38 & & 44 & $43 \cdot 3$ & 43.6 & 37 & -17.2 \\
\hline 46 & 55.9 & 54.9 & 22 & & 46 & 57.1 & 55.7 & $2 I$ & & 46 & 43.4 & 44. & 38 & -15.8 & 46 & 43.3 & 43.7 & 37 & \\
\hline 48 & 57.0 & 56.2 & 20 & & 48 & 57.0 & 56.3 & 20 & & 48 & 43.7 & 44 & $3^{8}$ & & 48 & 43.3 & 43. & 37 & \\
\hline 50 & 56.3 & 55.9 & $2 I$ & - & 50 & 56.8 & 56.1 & $2 I$ & $x^{2}$ & 50 & 45.2 & 45 & 40 & & 50 & $43 \cdot 3$ & 43.9 & 38 & 4 \\
\hline 52 & 54.0 & 53.8 & 24 & & 52 & 56.7 & 56.0 & $2 I$ & 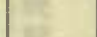 & 52 & 45.6 & 46. & $4 I$ & & 52 & $43 . I$ & $43 \cdot 3$ & 37 & \\
\hline 54 & 52.1 & 51.3 & 28 & 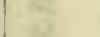 & 54 & 57.0 & 56.3 & 20 & & 54 & 45.6 & 46.0 & $4 I$ & & 54 & 43.0 & 43.6 & 37 & \\
\hline 56 & 52.6 & $5 \mathrm{r} .9$ & 27 & & 56 & $57 \cdot 3$ & 57.0 & Ig & & 56 & $45 \cdot 3$ & 45.8 & 40 & & 56 & 42.6 & 43.1 & 36 & \\
\hline 58 & $54 . I$ & 53.6 & 25 & & 58 & 57.9 & 57.2 & I9 & & 58 & 45.2 & 45. & 40 & & 58 & 42.3 & 43.1 & 36 & \\
\hline$\infty$ & 51.9 & 51.1 & 28 & $-I 3 \cdot 4$ & 1900 & 57.9 & 57.2 & 19 & $-13 \cdot 9$ & 2100 & 44.8 & $45 \cdot 3$ & 40 & -16.0 & 2300 & 42.3 & 42.8 & 36 & -17.4 \\
\hline 02 & 59.1 & 58.8 & 17 & & 02.4 & $58 . I$ & 58.0 & I8 & & 02 & 44.I & 45. & 39 & & 02 & 42.0 & 42.6 & 36 & \\
\hline 04.2 & 58.8 & 58.3 & Is & & 04 & 58.4 & 58.1 & 18 & & 04 & $44 \cdot 5$ & 45 & 39 & & 04 & $4 I . I$ & $4 I .7$ & 34 & \\
\hline 06 & 55.1 & 54.0 & 24 & 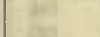 & 06 & 59.1 & 59.0 & 16 & $x^{2}$ & 06 & 44.6 & 44. & 39 & $\pi$ & 06 & 40.8 & 41.0 & 3 & 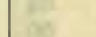 \\
\hline 08 & 51.8 & 50.6 & 29 & ats & 08 & 60.0 & 59.6 & 15 & gar & 08 & $44 \cdot 7$ & 44.7 & 39 & & 08 & 39.3 & 39.9 & $I$ & 3 \\
\hline IO & 49.2 & 48.3 & 3.3 & (1) & Io & 59.7 & 59.1 & 16 & & IO & 44.8 & 44.9 & 39 & & IO & 39.6 & 40.5 & 32 & \\
\hline 12 & 49.9 & 49.1 & $3 I$ & & 12 & 58.2 & 58.0 & I8 & & 12 & 44.8 & 44.9 & 39 & & 12 & 36.8 & 37. & 27 & \\
\hline 14 & 51.8 & 51.0 & 28 & $-13 \cdot 3$ & 14 & 57.9 & 57.9 & I8 & -14.0 & 14 & $44 \cdot 7$ & $45 . I$ & 40 & -16.0 & 14 & 38.3 & 38.6 & 29 & $-17 \cdot 5$ \\
\hline 16 & 53.8 & 52.9 & 25 & & 16 & 58.0 & 58.0 & I8 & & I6 & $44 \cdot 7$ & 45. & 39 & & IC & 36.5 & 37.0 & 27 & \\
\hline 18 & $55 . I$ & 54.2 & 23 & & 18 & 57.9 & 57.5 & 18 & & Is & 44.8 & 45 & 40 & & I & 38.0 & 38. & 29 & \\
\hline 20 & 56.4 & 55.9 & $2 I$ & & 20 & 57.1 & 57.0 & 20 & & 20 & 44.6 & 44.8 & 39 & & 20 & 37.0 & 37.2 & 27 & \\
\hline 22 & 56.8 & 56.1 & $2 I$ & 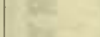 & 22 & 56.8 & 56.3 & 20 & I & 2. & 44.6 & 44.9 & 39 & 4 & 22 & 37.4 & 38.0 & 28 & $t$ \\
\hline 24 & 54.0 & 54.0 & 24 & $\ln$ & 24 & 56.1 & 56.0 & $2 I$ & 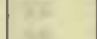 & 24 & 44.9 & 45. & 40 & t & 24 & 36.8 & 37.7 & 28 & F \\
\hline 26 & 53.2 & 52.8 & 26 & & 26 & 55.9 & 55.8 & 22 & & 26 & 44.8 & 45.0 & 40 & & 26 & 37.1 & 38.1 & 28 & \\
\hline 28 & 53.2 & 53.2 & 26 & & 28 & 55.1 & 54.7 & 23 & & 28 & 44.9 & 45.0 & 40 & & 28 & 38.3 & 39.1 & 30 & \\
\hline 30 & 52.6 & 52.2 & 27 & -13.0 & 30 & 56.9 & 56.4 & 20 & $-I 4 . I$ & 30 & 44.6 & $44 \cdot 7$ & 39 & -16.2 & 30 & 37.2 & 38. & 28 & -17.6 \\
\hline 32 & 52.9 & 52.7 & 26 & & 32 & 53.0 & 52.6 & 26 & & 32 & 44.5 & 44.7 & 39 & & 32 & 36.2 & 36.8 & 26 & \\
\hline 34 & 52.9 & 52.5 & 26 & & 34 & 53.9 & 53.7 & 25 & & 34 & $44 \cdot 4$ & 44.8 & 39 & & 34 & 35.3 & 36.2 & 25 & \\
\hline 36 & 53.0 & 53.0 & 26 & & 36 & 3.3 & 52.8 & 26 & & 3 & $4 \cdot 3$ & 44. & 3 & & 3 & & 36.8 & 26 & \\
\hline 38 & 53.9 & 53.7 & 25 & & 38 & 53.1 & 52.9 & 26 & & 38 & 43.6 & 44.0 & 38 & & 38 & & 36. & 25 & \\
\hline 40 & 54.1 & 54.0 & 24 & & 40 & 53.2 & 52.9 & 26 & & 40 & 43.0 & 43.3 & 37 & & 40 & 35.3 & 36.0 & 25 & \\
\hline 42 & 53.9 & 53.9 & 24 & & 42 & $53 \cdot 3$ & 53.1 & 26 & & 42 & $43 \cdot 3$ & 43.8 & 37 & & 42 & 35.6 & 36.0 & 25 & \\
\hline 44 & 52.1 & $5 I .9$ & 28 & -12.9 & 44 & 52.9 & 52.6 & 26 & -14.4 & 44 & 43.7 & $44 \cdot 3$ & 38 & -16.5 & 44 & 35.7 & 36.0 & 25 & -17.8 \\
\hline 46 & 51.4 & 5 I. 2 & 29 & & 46 & 52.4 & 52.2 & 27 & & 46 & 43.8 & 44.6 & 38 & & 46 & 35.5 & 35.8 & 25 & \\
\hline 48 & 52.0 & 51.8 & 28 & & 48 & $53 . \mathrm{I}$ & 53.0 & 26 & & 48 & $44 . I$ & 44.9 & 39 & & 48 & 35.3 & 35.6 & 25 & \\
\hline 50 & 53.8 & 53.0 & 25 & 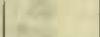 & 50 & 54.8 & 53.9 & 24 & & 50 & 43.8 & & 38 & & 50 & 35.6 & 36.0 & 25 & \\
\hline 52 & 54.8 & 53.9 & 24 & & 52 & 54.8 & 53.9 & 24 & & 52 & 43.6 & 44. & 38 & & 52 & $35 \cdot 3$ & 36.0 & 25 & \\
\hline 54 & 55.2 & $54 . I$ & 23 & $1=$ & 54 & 55.5 & 54.7 & 23 & & 54 & $43 \cdot 3$ & 44. & 37 & & 54 & 35.5 & & 26 & 28 \\
\hline 56 & 55.9 & 54.9 & 22 & & 56 & 56.3 & 55.8 & $2 \mathrm{I}$ & & 56 & 43.1 & 43.6 & 37 & & 56 & 35.0 & 36.0 & 25 & \\
\hline 58 & 55.0 & 54.1 & 24 & $7 x$ & 58 & 56.4 & 55.8 & $2 I$ & & 58 & 43.0 & $43 \cdot 3$ & 37 & & 58 & 35.0 & 36.0 & 25 & \\
\hline & & & & & 2000 & 56.0 & 55.8 & $2 I$ & -14.7 & & & & & & 2400 & 34.8 & 36.0 & 25 & -18.0 \\
\hline
\end{tabular}

Correction to local mean time is -30 s. $90^{\circ}$ torsion $=17 .^{\prime} 58$. Torsion head at $15 \mathrm{~h} 40 \mathrm{~m}$ read $49^{\circ}$ and at $20 \mathrm{~h}$ 20m read $40^{\circ}$. Observer-R. R. T.
Correction to local mean time is $+21.5 \mathrm{~s}$. $90^{\circ}$ torsion $=19$. .' $^{\prime} 19$. Torsion head at ol oom read $57^{\circ}$ and at $24 \mathrm{~h}$ I $5 \mathrm{~m}$ read $42^{\circ}$. Observer-W. J. P. 
Tabulation of magnetic declinations observed at Teplitz Bay-Continued

\begin{tabular}{|c|c|c|c|c|c|c|c|c|c|c|c|c|c|c|c|c|c|c|c|c|}
\hline \multicolumn{5}{|c|}{ Friday, April, 29, 1904} & \multicolumn{5}{|c|}{ Magnet scale erect } & \multicolumn{6}{|c|}{ Sunday, May I, Ig04 } & \multicolumn{5}{|c|}{ Magnet scale inverted } \\
\hline \multirow{2}{*}{$\begin{array}{l}\text { Clir'r } \\
\text { time }\end{array}$} & \multicolumn{2}{|c|}{$\begin{array}{l}\text { Scale } \\
\text { readings }\end{array}$} & $\begin{array}{l}\text { East } \\
\text { decli- } \\
\text { nation }\end{array}$ & Tenp. & Chr'r & $\begin{array}{r}\mathrm{Sc} \\
\text { read }\end{array}$ & $\begin{array}{l}\text { ale } \\
\text { ings }\end{array}$ & $\begin{array}{c}\text { East } \\
\text { decli- } \\
\text { nation }\end{array}$ & Temp. & Clir'r & $\begin{array}{r}\mathrm{Sc} \\
\text { read }\end{array}$ & $\begin{array}{l}\text { ale } \\
\text { lings }\end{array}$ & $\begin{array}{c}\text { East } \\
\text { decli- } \\
\text { nation }\end{array}$ & Temp. & Clir'r & $\begin{array}{r}\mathrm{Sc} \\
\text { read }\end{array}$ & & $\begin{array}{l}\text { East } \\
\text { decli } \\
\text { natio }\end{array}$ & & Temp. \\
\hline & Left & Right & & & & Left & Right & & & & Left & Kiglat & & & & Left & Right & & & \\
\hline h m & d & d & $\circ$ & - & $\mathrm{h} \mathrm{m}$ & d & d & & $\therefore$ & h $\mathrm{m}$ & d & d & & & $\mathrm{l} \mathbf{~ m}$ & $d$ & $\mathrm{~d}$ & $\circ$ & & $\bullet$ \\
\hline 2000 & 33.8 & 36.2 & 2148 & -14.9 & 2200 & 35.7 & 38.2 & $2 I 5 I$ & -16.2 & $00^{*}$ & $47 \cdot I$ & 44.9 & 2229 & -16.8 & 200 & 26.0 & 24.0 & 230 & 02 & -17.5 \\
\hline 02 & $34 \cdot 3$ & 37.1 & & 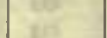 & 02 & 34.0 & 35.1 & 47 & & 02 & 52.0 & 49.0 & 22 & & 02 & 24.2 & 21.0 & & 06 & \\
\hline 04 & 35.0 & 37.2 & 2I 50 & E. & 04 & $37 \cdot 3$ & 39.2 & 53 & ton & 04 & 47.0 & 45.3 & 29 & & 04 & 27.8 & 24.0 & 230 & oI & \\
\hline 06 & 42.0 & 44.8 & 22 oI & & oo & 38.2 & 40.0 & 54 & 28 & 06 & 42.3 & 39.0 & 38 & & 06 & 30.4 & 26.4 & 225 & & \\
\hline 08 & 45.0 & 47.9 & 06 & ate & 08 & 35.7 & 36.1 & 49 & ai & 08 & 45.9 & 44.0 & $3 I$ & 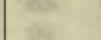 & 08 & 35.1 & 32.5 & & 48 & \\
\hline IO & 51.7 & 56.9 & 18 & at & 10 & 34 & $.8 a$ & 48 & & I0 & 47.2 & 46.9 & 27 & & 10 & 37.1 & $34 . I$ & & 46 & \\
\hline I2 & 52.0 & 56.0 & 18 & $y^{\prime}$ & 12 & & .16 & 50 & & 12 & 47.8 & 46.8 & 27 & & 12 & $37 \cdot 3$ & $34 . I$ & & 45 & \\
\hline 14 & 49.9 & 54.1 & 14 & -15.0 & 14 & 32 & $.9 b$ & 44 & -16.5 & 14 & 44.5 & 43.4 & 32 & -17.0 & 14 & 39.0 & 36.0 & & 42 & -17.5 \\
\hline 16 & $47 \cdot 3$ & 51.3 & I0 & $x^{2}$ & I6 & 32.0 & 33.2 & 44 & $x_{1}$ & 16 & 44.5 & 44.0 & 32 & $0^{\circ}$ & 16 & 36.2 & 33.3 & & 47 & - \\
\hline 18 & $47 . I$ & SI.I & IO & 4 & 18 & 36.1 & $37 \cdot 3$ & 50 & net & 18 & 46.8 & 46.7 & 28 & 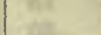 & 18 & 35.0 & 31.9 & & 49 & \\
\hline 20 & 46.4 & 49.8 & 08 & a & 20 & 37.0 & $37 \cdot 3$ & $5 \mathrm{I}$ & We & 20 & 48.4 & 47.9 & 26 & & 20 & 28.3 & 26.1 & & 58 & \\
\hline 22 & 43.9 & 49.9 & $\infty$ & 5in & 22 & 34.0 & 34.9 & 47 & st & 22 & 46.3 & 46.1 & 29 & & 22 & 32.0 & 28.2 & & 54 & \\
\hline 24 & 4I. I & $46 . I$ & 2201 & 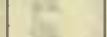 & 24 & 35.2 & 36.1 & 49 & & 24 & 46.9 & 46.6 & 28 & & 24 & 30.1 & 27.9 & & 56 & \\
\hline 26 & 37.2 & 41.0 & 2I 54 & & 26 & 36.0 & 37.0 & 50 & as & 26 & 45.8 & 45.2 & 30 & & 26 & 30. I & 27.9 & & 56 & \\
\hline 28 & 39.0 & 42.1 & 56 & & 28 & 28.0 & 29.8 & 38 & & 28 & 46.8 & 46.0 & 28 & & 28 & 28.3 & 25.7 & 225 & 59 & \\
\hline 30 & 38.0 & $4 I .3$ & 55 & $-15 \cdot 3$ & 30 & 30.0 & 30.7 & 40 & -16.8 & 30 & 48.0 & $47 \cdot 3$ & 26 & -17.1 & 30 & 26.2 & 24.7 & 230 & OI & -17.7 \\
\hline 32 & 37.0 & 40.7 & 54 & & 32 & $35 . I$ & $35 \cdot 3$ & 48 & & 32 & 48.4 & 47.6 & 26 & & 32 & 23.9 & 21.7 & & 05 & \\
\hline 34 & 32.9 & 36.5 & 2I 47 & 13 & 34 & 33.0 & 34.0 & 46 & $=$ & 34 & 48. I & $4 ; .2$ & 26 & & 34 & 22.1 & 20.0 & 230 & 08 & \\
\hline 36 & $40 . \overline{8}$ & $45 . \overline{8}$ & 22 OI & 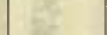 & 36 & 32.2 & 34.2 & 45 & $x^{2}$ & 36 & 49.0 & $48 . I$ & 25 & & 36 & $27 \cdot 3$ & $25 \cdot 3$ & 226 & 60 & \\
\hline 38 & 51.2 & 53.0 & I5 & 20 & 38 & 35.2 & 39.2 & $5 \mathrm{I}$ & 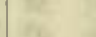 & 38 & 47.2 & 46.1 & $2 S$ & 2 & 38 & 28.9 & 26.9 & & 57 & \\
\hline 40 & 43.2 & 45.9 & 2203 & a & 40 & 35.2 & 38.0 & 50 & . & 40 & 45.6 & 44.9 & 30 & - & 40 & 30.3 & 29.2 & & 55 & \\
\hline 42 & 37.1 & 41.0 & 2154 & 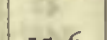 & 42 & 34.8 & 37.8 & 50 & -6 & 42 & 38.9 & $37 \cdot 7$ & $4 \mathrm{I}$ & & 42 & $3 \mathrm{I} .2$ & 29.0 & & 54 & \\
\hline 44 & $33 \cdot 3$ & 35.9 & 47 & -15.6 & 44 & 37.9 & 42.3 & 56 & -16.9 & 44 & 39.0 & 37.0 & 42 & $-I 7.2$ & 44 & 32.0 & 30.2 & & 52 & -17.7 \\
\hline 46 & 28.0 & 34.8 & 42 & & 46 & 40.1 & 44.2 & 59 & & 46 & 38.9 & 37.0 & 42 & $12=$ & 46 & 28.7 & 27.2 & & 57 & \\
\hline 48 & 26.2 & 33.2 & 40 & 5 & 48 & 36.0 & $40 . I$ & 52 & in & 48 & 42.0 & 41.0 & 36 & 6. & 48 & 29.2 & 27.9 & & 56 & \\
\hline 50 & 33.0 & 39.2 & 50 & 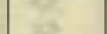 & 50 & 38.8 & 45.0 & 21 59 & $\therefore$ & 50 & 43.0 & 42.2 & 34 & No & 50 & 32.0 & 30.2 & & 52 & \\
\hline 52 & 31.9 & 36.1 & 46 & 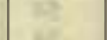 & 52 & 47.0 & 51.2 & 22 I0 & $\frac{2 y}{y}$ & 52 & $44 . I$ & 43.7 & 32 & to & 52 & 33.2 & 31.9 & & 50 & at \\
\hline 54 & 28.7 & 32.3 & $4 \mathrm{I}$ & 3 & 54 & $45 \cdot 4$ & 51.0 & 08 & 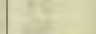 & 54 & 46.1 & 44.7 & 30 & 25 & 54 & 32.5 & 31.0 & & 52 & \\
\hline 56 & 35 . I & 39.1 & 51 & 25 & 56 & 42.8 & 48.6 & 2205 & 35 & 56 & 47.2 & 45.9 & 28 & 85 & 56 & $3 I .0$ & 29.6 & & 54 & \\
\hline 58 & 32.1 & 36.6 & 47 & ene & 58 & 38.9 & 43.1 & 2157 & & 58 & 47.0 & 45. & 28 & & 58 & 28.9 & 27.0 & 225 & 57 & \\
\hline 2100 & 34.0 & 39.1 & 50 & -I5.8 & 2300 & 44.7 & 49.3 & 2207 & -17.0 & 100 & 46.3 & 45.8 & 29 & $-17 \cdot 3$ & 300 & 24.8 & 23.9 & $23 c$ & 03 & -17.8 \\
\hline 02 & 39.2 & 45.3 & 59 & - & 02 & 46.0 & 49.6 & 08 & was & 02 & 45.9 & 45.0 & 30 & 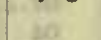 & 02 & $24 . I$ & 22.9 & & 04 & \\
\hline $0_{4}$ & 37.0 & 43.0 & 56 & $m$ & 04 & 46.2 & 49.8 & 08 & ton & 04 & $46 . \overline{8}$ & 45.5 & 29 & 5 & 04 & 25.7 & 24.0 & $23 c$ & 02 & \\
\hline 06 & 38. I & 44.0 & 2157 & 30 & 06 & 55.9 & 58.5 & 23 & $\sin$ & 06 & 46.8 & 45.9 & 28 & 20 & 05 & 29.0 & 27.5 & 225 & 57 & 6 \\
\hline 08 & 41.2 & 46.2 & 2202 & 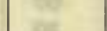 & 08 & Lo & & & $=8$ & os & 46.0 & $45 . I$ & 30 & & 08 & 29.0 & $27 . I$ & & 57 & \\
\hline IO & 40.2 & 45.5 & 2200 & int & I0 & 59.2 & 62.0 & 28 & it & 10 & 46.2 & 45.5 & 29 & tht & IO & 28.0 & 26.3 & & 59 & 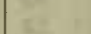 \\
\hline I2 & 37.7 & 42.0 & 2155 & -6 & 12 & 58.2 & 60.9 & 26 & & 12 & 46.7 & 45.8 & 29 & & I2 & 39.0 & 37.9 & & $4 \mathrm{I}$ & \\
\hline 14 & 37.0 & 40.2 & 53 & -16.0 & I4 & 58.3 & 60.2 & 26 & -17.0 & I4 & 46.9 & 46.0 & 28 & -I7.3 & 14 & 30.0 & 29.0 & & 55 & -17.7 \\
\hline 16 & 36.9 & 41.0 & 54 & & 16 & 56.7 & 59.0 & 24 & & 16 & 47.0 & 46.1 & 28 & & 16 & 29.2 & 28.6 & & 56 & \\
\hline I8 & 39.2 & 42.8 & 57 & se. & 18 & $54 . I$ & 55.2 & 19 & 8 & 18 & 47.8 & 46.9 & 27 & $=$ & 18 & 29.0 & 28.8 & 225 & 56 & 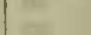 \\
\hline 20 & 40.9 & 43.9 & 59 & $\pi$ & 20 & 58.4 & 60.5 & 26 & yat & 20 & 47.3 & 46.9 & 27 & $=$ & 20 & 24.6 & 24.2 & 23 & 03 & \\
\hline 22 & 41.0 & $44 \cdot I$ & 60 & $=$ & 22 & 60.6 & 62.2 & 29 & ta & 22 & 47.0 & 46.4 & 28 & . & 22 & 23.0 & 23.0 & & 05 & \\
\hline 24 & 39.3 & 42.3 & 57 & $\mathrm{z}$ & 24 & 57.0 & 58.8 & 24 & 3 & 24 & 47.0 & 45.9 & 28 & 5 & 24 & 21 & $.0 b$ & & 08 & \\
\hline 26 & 38.0 & 40.9 & 55 & 0 & 26 & 56.0 & 57.8 & 22 & & 26 & 46.6 & 45.2 & 29 & & 26 & I8.8 & 18.8 & & 12 & \\
\hline 28 & 37.0 & 39.2 & 53 & & 28 & 56.8 & 57.8 & 23 & & 28 & 43.9 & 43.0 & 33 & & 28 & 15.0 & 15.0 & & 18 & \\
\hline 30 & 37.2 & 39.8 & 53 & -16.0 & 30 & 55.6 & 56.0 & 20 & -I7.2 & 30 & 43.4 & $4 \mathrm{I} .9$ & 34 & -17.5 & 30 & 15.7 & 15.4 & & 17 & -17.5 \\
\hline 32 & 37.0 & 38.8 & 52 & - & 32 & 56.9 & 58.1 & 23 & 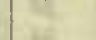 & 32 & 44.2 & $43 . I$ & 33 & -8 & 32 & 15.8 & 15.7 & & I7 & \\
\hline 34 & 38.3 & 40.2 & 54 & 10 & 34 & 53.2 & 55.1 & 18 & 5 & 34 & 45.9 & 44. & 30 & & 34 & I5.0 & I 4.3 & & I8 & \\
\hline 36 & 36.3 & 38.2 & $5 I$ & & 36 & 52.0 & 53.5 & 16 & 2 & 36 & 47.1 & 46.9 & 27 & 25 & 36 & 10 & $.2 b$ & & 25 & \\
\hline 38 & 40.0 & 41.0 & 56 & $a^{2}$ & 38 & 56.2 & 57.7 & 22 & -5 & 38 & 48.0 & 45.2 & 28 & & 38 & 9.5 & 9.2 & & 27 & \\
\hline 40 & $42 . I$ & 43.0 & 60 & $=$ & 40 & 55.8 & 56.2 & $2 I$ & & 40 & 48.6 & 47.0 & 26 & & 40 & 10.2 & 9.8 & & 26 & \\
\hline 42 & 41.9 & 42.9 & 59 & & 42 & 56.8 & 57.5 & 23 & 的 & 42 & 43.0 & 42.0 & 34 & & 42 & 9.7 & 8.9 & & 27 & \\
\hline & 39.2 & 40.3 & 55 & $-16 . I$ & 44 & 55.9 & 56.2 & 21 & $-17 \cdot 3$ & 44 & 35.8 & 34.0 & 46 & -17.6 & 44 & 7.2 & 7.0 & & 30 & -17.3 \\
\hline 46 & 38.1 & 40.2 & 54 & 8 & 46 & $55 \cdot 3$ & 56.1 & 20 & ter & 46 & 35.4 & 33.5 & 47 & (2) & 46 & 9.8 & 9.7 & & 26 & \\
\hline 48 & 39.1 & 42.2 & 57 & $x^{2}$ & 48 & 56.8 & 58.2 & 23 & & 48 & 31.0 & 29.6 & 54 & & 48 & 6.9 & 6.8 & & 30 & \\
\hline 50 & $39 \cdot 3$ & $42 . I$ & 57 & tas & 50 & 58.5 & 58.9 & 25 & ty & 50 & 32.0 & 31.0 & 52 & 3 & $50^{*}$ & $4 I .8$ & $35 . I$ & & 43 & \\
\hline 52 & 39.0 & 42.0 & 56 & 3 & 52 & 53.9 & 54.7 & I8 & & 52 & 34.8 & 33.6 & 48 & 3 & 52 & 38. I & 32.5 & & 48 & \\
\hline 54 & 38.9 & 42.0 & 56 & $e^{2}$ & 54 & 50.9 & 52.0 & I4 & 2 & 54 & 37.5 & 35.0 & 44 & 8 & 54 & 43.0 & 37.2 & & 40 & \\
\hline - & 38.0 & 41.0 & 55 & 然 & 56 & 51.9 & 52.9 & 15 & & 56 & 39.0 & 35.8 & 43 & 8 & 56 & $42 \cdot 3$ & 37.0 & & $4 \mathrm{I}$ & \\
\hline 58 & 34.9 & $37 . I$ & 49 & & 58 & 52.4 & 53.0 & 16 & -17 & 58 & $3 I . I$ & 29.9 & 53 & $\infty$ & 58 & 40.0 & 35.2 & & & \\
\hline & & & & & $\infty$ & 5I.I & 52.0 & 14 & -17.4 & & & & & & & & & & & \\
\hline
\end{tabular}

Correction to local mean time is -56.5 s. $90^{\circ}$ torsion $=18 .^{\prime} 27$.

Observer-J. V.

Torsion head at $19 \mathrm{~h} 29 \mathrm{~m}$ read $40^{\circ}$ and at $201 \mathrm{2} 2 \mathrm{~m}$ read $51^{\circ}$.

Observer-J. V. 
Tabulation of magnetic declinations observed at Teplitz Bay-Continued

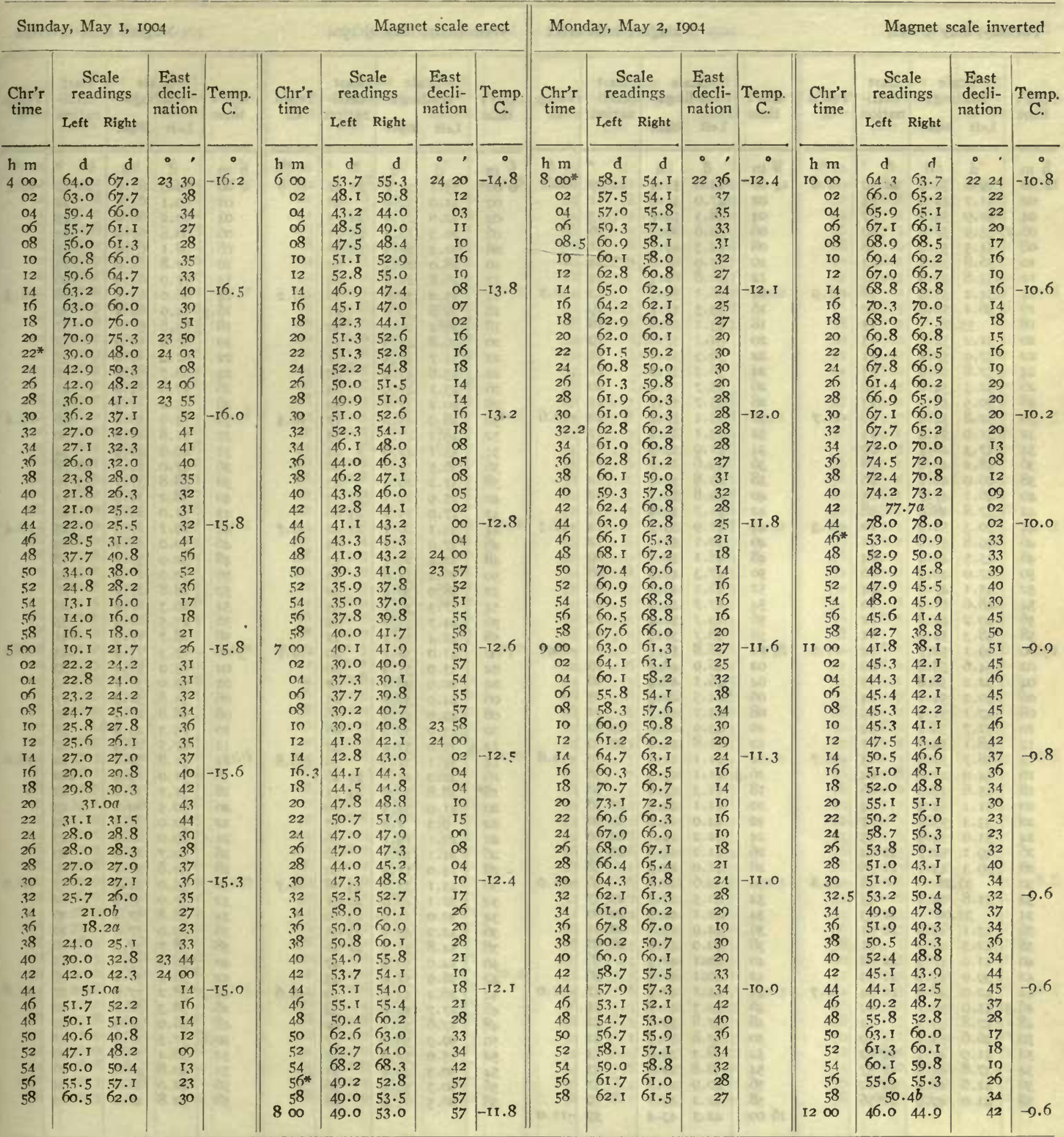

Correction to local mean time is $-8 \mathrm{~s} .90^{\circ}$ torsion = I6.' 35 . Torsion head at oh oom read $51^{\circ}$ and at $8 \mathrm{~h} 45 \mathrm{~m}$ read $49^{\circ}$. Observer-J. V.
Correction to local mean time is $+3 \mathrm{~m} 46 \mathrm{~s} .90^{\circ}$ torsion $=16 .^{\prime} 54$. Torsion head at $7 \mathrm{~h} 35 \mathrm{~m}$ read $58^{\circ}$ and at $12 \mathrm{~h}$ 2om read $45^{\circ}$. Observer-R. R. T. 
Tabulation of magnetic declinations observed at Teplitz Bay-Continued

\begin{tabular}{|c|c|c|c|c|c|c|c|c|c|c|c|c|c|c|c|c|c|c|c|c|c|}
\hline \multicolumn{7}{|c|}{ Tuesday, May 3, 1904} & \multicolumn{5}{|c|}{ Magnet scale erect } & \multicolumn{6}{|c|}{ Wednesday, May 4, I904 } & \multicolumn{4}{|c|}{ Magnet scale inverted } \\
\hline \multirow{2}{*}{$\begin{array}{l}\text { Chr'r } \\
\text { time }\end{array}$} & \multicolumn{2}{|c|}{$\begin{array}{l}\text { Scale } \\
\text { readings }\end{array}$} & Eas & & Temp. & Chr'r & $\begin{array}{r}\text { Sca } \\
\text { read }\end{array}$ & $\begin{array}{l}\text { ale } \\
\text { ings }\end{array}$ & East & & Temp. & Chr'r & $\begin{array}{l}\text { Sca } \\
\text { read }\end{array}$ & $\begin{array}{l}\text { ale } \\
\text { lings }\end{array}$ & East & Temp. & Chr'r & $\begin{array}{l}\text { Sca } \\
\text { read }\end{array}$ & le & $\begin{array}{l}\text { East } \\
\text { dccli- }\end{array}$ & Temp. \\
\hline & Left & Right & & & & & Left & Right & & & & & Left & Right & & & & I,eft & Right & & \\
\hline $\mathbf{h} \mathbf{m}$ & d & d & $\cdot$ & , & $\bullet$ & $\mathrm{h} \mathrm{m}$ & d & d & & & $\bullet$ & $\mathrm{h} \mathrm{m}$ & d & d & & $\bullet$ & $\mathrm{h} \mathrm{m}$ & d & $\mathrm{d}$ & 0, & \\
\hline 1200 & 49.6 & 50.9 & & 10. & -12.0 & 1400 & 44.6 & 51.1 & 220 & & -10.7 & $000 *$ & 33. I & 29.2 & 2242 & -15.9 & 200 & 70.0 & 67.0 & 2222 & -16.4 \\
\hline 02 & 51.1 & 52.9 & & 13 & & 02 & $47 \cdot 0$ & 53.8 & & I & & 02 & 35.0 & 32.0 & 38 & & 02 & 69.1 & 65.9 & 24 & \\
\hline 04 & 52.9 & 55.4 & & 17 & & 04 & 48.3 & 54.7 & & 3 & ats & 04 & 32.0 & 27.0 & 2245 & & 04 & 69.2 & 66.0 & 23 & \\
\hline 06.2 & 55.9 & 57.4 & & 20 & & 06 & 50.6 & 56.3 & & 6 & ten & 06 & 20.3 & 18.1 & 2301 & & 06 & 68.8 & 67.0 & 24 & \\
\hline 08 & $54 \cdot 5$ & 55.9 & & I8 & & 08 & $54 \cdot 3$ & 59.7 & & I & tate & 08 & 22.2 & 20.3 & 2258 & & 08 & 68.I & 65.0 & 25 & \\
\hline 10 & 56.8 & 58.5 & & 22 & test & Io & 55.9 & 60.8 & & 3 & te. & 10 & 28.0 & 22.3 & 52 & & I0 & 67.8 & 64.8 & 25 & \\
\hline I2 & 55.8 & 56.9 & & 20 & & I2 & 56.8 & 60.3 & & 24 & & 12 & 26.8 & 25.2 & 50 & & 12 & 67.0 & 64.5 & 26 & \\
\hline 14 & 54.2 & 55.9 & & 18 & -II.9 & I4 & 56.1 & 59.5 & & 22 & -10.7 & I4 & 35.2 & 32.0 & 38 & -16.2 & 14 & 66.0 & $63 \cdot 3$ & 28 & -16.3 \\
\hline I6 & 56.1 & 56.8 & & 20 & & 16 & 53.5 & 57.7 & & 9 & $7 x$ & 16 & 32.8 & 30.9 & 41 & - & 16.3 & 64.9 & 62.2 & 30 & \\
\hline I 8 & & & & 15 & & I8 & 53.1 & 56.8 & & 8 & se & 18 & 29.2 & 20.5 & 52 & & 18 & 63.6 & 61.8 & $3 \mathrm{I}$ & \\
\hline 20 & 5 I. 2 & 52.8 & & 13 & $y$ & 20 & $54 . I$ & 57.0 & & 19 & tit. & 20 & 46.8 & 45.6 & I9 & a & 20 & 60.0 & 58.0 & 37 & \\
\hline 22 & 57.0 & $57 \cdot 3$ & & $2 \mathrm{I}$ & 6 & 22 & 56.3 & 58.0 & & I & trat & 22 & 51.7 & 49.5 & I2 & 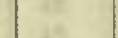 & 22 & 6r.o & 59.0 & 35 & \\
\hline 24 & 57.8 & 58.9 & & 23 & 20 & 24 & 56.3 & 57.9 & & II & 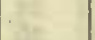 & 24 & 45.6 & 42.9 & 22 & & 24 & 60.7 & 58.9 & 36 & \\
\hline 26 & 52.3 & 54.8 & & 16 & & 26 & $55 \cdot 7$ & 57.4 & & $\infty 0$ & & 26 & 43.1 & 41.9 & 24 & & 26 & 57.8 & 54.9 & $4 I$ & \\
\hline 28 & 50.5 & 53.2 & & 13 & & 28 & 55.7 & 57.7 & & $2 I$ & & 28 & 40.2 & $37 . I$ & 30 & & 28 & 55.2 & 52.3 & 4.5 & \\
\hline 30 & 52.8 & 54.8 & & 16 & -11.7 & 30 & 55.1 & 57.0 & & 20. & -10.6 & 30 & 48.9 & 45.2 & I7 & -16.3 & 30 & 53.0 & 50.6 & 48 & -16.4 \\
\hline 32 & 51.9 & 54.4 & & I5 & & 32 & 53.1 & 55.9 & & 17 & & 32 & 49.1 & $47 . I$ & I5 & & 32 & 51.8 & 49.2 & 50 & \\
\hline 34 & $47 \cdot 3$ & 48.7 & & 07 & 2 & 34 & 51.9 & 53.9 & & 15 & 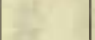 & 34 & 4.3 .1 & 42.0 & 24 & & 34 & 49.9 & 46.0 & 54 & \\
\hline 36 & 48.2 & 49.6 & & 08 & 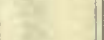 & 36 & 49.6 & 51.7 & & II & $\mathrm{E}^{-}$ & 36 & 36.5 & 34.0 & 3 & & 36 & 47.0 & 44.8 & 2258 & \\
\hline 38 & 46.0 & 48.1 & & 06 & $=$ & 38 & 49.8 & 52.1 & & 2 & 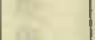 & 38 & 31.2 & 29.4 & 43 & & 38 & 38.8 & 37.7 & 2310 & \\
\hline 40 & 46.1 & 47.7 & & 05 & 7 & 40 & 49.8 & 52.1 & & 12 & 84 & 40 & 25.9 & 22.5 & 2253 & & 40 & 38.2 & 36.9 & II & \\
\hline 42 & 47.8 & 48.9 & & 08 & & 42 & 51.7 & 53.8 & & 14 & -2 & 42 & 17.0 & 15.2 & & & 42 & 31.0 & 28.9 & 23 & \\
\hline 44 & 50.3 & 52.0 & & 12 & -11.3 & 44 & 51.9 & 53.3 & & 4 & -10.5 & 44 & 33.1 & 29.1 & 2242 & -16.7 & 44 & 29.1 & $27 \cdot 3$ & 25 & -16.4 \\
\hline 46 & 49.8 & 5I.I & & II & 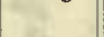 & 46 & 50.1 & 52.0 & & 12 & & 46 & 37.0 & 35.1 & 34 & & 46 & 30.0 & 28.2 & 24 & \\
\hline 48 & 45.5 & 46.4 & & 04 & the & 48 & 48.1 & 50.8 & & 99 & $=$ & 48 & 32.4 & 29.1 & 43 & & 48 & 32.9 & 32.1 & I8 & \\
\hline 50 & 47.0 & 48.3 & & 06 & 6 & 50 & 47.3 & 49.9 & & 88 & 3 & 50 & 36.2 & 34.6 & 3. & 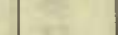 & 50 & 30.0 & 27.9 & 24 & \\
\hline 52 & 45.1 & 47.4 & & 04 & 4 & 52 & 46.1 & 49.0 & & 26 & 10 & 52 & 38.9 & 36.9 & 32 & 3 & 52 & 28.8 & 27.1 & 26 & \\
\hline 54 & 48.6 & 51.9 & & I0 & 37 & 54 & 47.7 & 50.3 & & 99 & 18 & 54 & $37 \cdot 3$ & 36.1 & 33 & & 54 & 29.3 & 27.2 & 25 & \\
\hline 56 & 49.2 & 52.8 & & 12 & Ne & 56 & 49.7 & 52.4 & & 12 & & 56 & 44.7 & 43.6 & 22 & 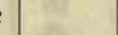 & 56 & 22.5 & 20.3 & 35 & \\
\hline 58 & 48.3 & 52.9 & & II & & 58 & 48.7 & $5^{1} \cdot 3$ & & I0 & & 58 & 42.4 & 39.1 & 27 & & 58 & 18.7 & 17.3 & $4 I$ & \\
\hline 1300 & $49 . I$ & 52.7 & H & 12 & $-I 1.0$ & $15 \infty 0$ & 48.8 & $5 I . I$ & 8 & IO & -10.7 & I 00 & $4 \mathrm{I} .0$ & 38.1 & 29 & -16.7 & 300 & 17.3 & 15.9 & 44 & -16.2 \\
\hline 02 & 54.6 & 58.0 & & 20 & & 02 & 46.9 & 48.8 & & 77 & 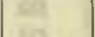 & 02 & 33.9 & $3 \mathrm{I} .9$ & 35 & & 02 & 25.0 & 22.0 & 33 & \\
\hline 04 & 52.2 & 56.0 & 3 & 17 & 19 & 04 & 46.1 & 48.4 & & 66 & 19 & 04 & 30.0 & 26.0 & 47 & & 04 & 33.0 & 31.7 & 19 & \\
\hline 06 & 50.2 & $54 . I$ & & 14 & $x^{3}$ & o6 & 44.5 & 46.3 & & 33 & 60 & 06 & 44.9 & 39.0 & 25 & & $\infty 6$ & $3 . .8$ & 32.9 & 17 & \\
\hline 08 & 47.1 & $5 \mathrm{I} .4$ & & 09 & 20 & o8 & 43.7 & 45.1 & & OI & m & o8 & 49.0 & 47.0 & IC & & os & 28.8 & 27.9 & 25 & \\
\hline 10 & 52.7 & 58.1 & & 19 & 20 & 10 & 43.9 & 46.1 & 22 & 02 & or & IO & 43.0 & 39.8 & 26 & & IO & 25.3 & 23.9 & $3 I$ & \\
\hline 12 & 48.1 & 53.0 & & II & $64 \sqrt{14}$ & 12 & 41.8 & 44.4 & 21 & 59 & 0 & 12.4 & 35.9 & 33.2 & 37 & & 12 & 27.5 & 26.9 & 27 & \\
\hline 14 & 48.7 & 54.8 & & 13 & -10.7 & I4 & 4I.I & 44.1 & & 59 & -10.8 & 14 & 34.1 & 31.8 & 30 & -16.8 & 14 & 31.8 & 30. I & 21 & -16.0 \\
\hline 16 & 44.0 & 47.8 & & 04 & & I6.3 & 4I.I & 43.9 & & 58 & 2 & 16 & 32.2 & 25.5 & 2246 & & 16 & 31.0 & 29.5 & 22 & \\
\hline 18 & 45.6 & 48.6 & & 06 & 24 & I8 & $4 I .3$ & 43.8 & & 58 & 2 & $18^{*}$ & 38.8 & 30.8 & 23 I5 & 1 & I8 & 31.5 & 30.0 & 21 & \\
\hline 20 & 51.1 & 54.2 & 1 & I4 & 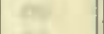 & 20 & $4 I . I$ & 43.9 & 21 & 58 & 18 & 20 & 41.9 & 34.5 & IC & 13 & 20 & 30.5 & 20.1 & 23 & \\
\hline 22 & 53.3 & 55.9 & E & 17 & a & 22 & 42.0 & 44.8 & 22 & $\infty$ & 25 & 22 & 9.3 & 7.0 & 57 & 65 & 22 & 30.0 & 28.0 & 24 & \\
\hline 24 & 51.0 & 54.9 & & 15 & test & 24 & 42.2 & 45.1 & 22 & $\infty$ & ate & 24 & 23.1 & 19.1 & 3 & & 24.5 & 29.0 & 28.0 & 25 & \\
\hline 26 & 49.1 & 52.7 & 22 & 12 & et & 26 & 41.2 & 44.8 & 21 & 59 & & 26 & 24.1 & 19.1 & 36 & & 25 & 30.7 & 20.0 & 23 & \\
\hline 28 & 39.3 & 43.1 & 21 & 56 & - & 28 & 41.0 & 43.3 & & 58 & & 28 & I 4.0 & II. 3 & 50 & & 28 & 32.8 & 31.0 & 19 & \\
\hline 30 & 36. & $.2 b$ & & 48 & -10.8 & 30 & 39.6 & 42.8 & & 56 & -11.0 & 30 & $43 . I$ & 35.8 & 2308 & $\begin{array}{ll}36.7 \\
\end{array}$ & 30 & 35.0 & 34.0 & 15 & -16.0 \\
\hline 32 & 31.8 & 33.1 & & 43 & & 32 & 40.5 & $43 . I$ & 2I & 57 & & 32 & 52.1 & 48.0 & 2251 & & 32 & 37.0 & 35.8 & I2 & \\
\hline 34 & 39. & $.0 a$ & 21 & 53 & 42 & 34 & 42.1 & 44.9 & 22 & 00 & 40 & 34 & 39.0 & 33.9 & $23 \mathrm{I}$ & & 34 & 38.8 & 37.2 & Io & \\
\hline 36 & 43.1 & 44.9 & 22 & OI & 2 & 36 & 42.9 & 45.2 & 22 & OI & $x^{2}$ & 35 & 42.2 & 40.0 & 230 & & 36 & 39.0 & 37.8 & I0 & \\
\hline 38 & 42.3 & 46.3 & & or & ES & 38 & 40.9 & 43.5 & 21 & 58 & (11) & 38 & 54.8 & 51.0 & 2246 & We & 38 & 38.7 & 37.3 & I0 & \\
\hline 40 & 41.9 & $47 \cdot 3$ & 22 & 02 & rat & 40 & $40 . I$ & 42.3 & & 56 & 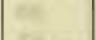 & 40 & 54.0 & 50.9 & 47 & 7 & 40 & 38.0 & 37.0 & II & \\
\hline 42 & 39.9 & 45.9 & $2 \mathrm{I}$ & 59 & 31 & 42 & 40.1 & 41.3 & & 56 & & 42 & 56.9 & 54.0 & 43 & & 42 & 36.2 & 32.1 & 16 & \\
\hline 44 & 42.9 & 48.8 & 22 & 04 & -10.7 & 44 & $41 . I$ & 42.5 & & 57 & $-I I .0$ & 44 & 46.2 & 43.8 & 50 & -16.6 & $44 \cdot 3$ & 38.2 & 37.7 & 10 & -15.8 \\
\hline 46.5 & 45.0 & 52.1 & & 09 & & 46 & 39.3 & 41.0 & & 55 & & 46 & 47.7 & 44.0 & 58 & & 46 & 38.3 & 38.0 & 10 & \\
\hline 48 & 47.8 & 53.3 & & II & tan & 48 & 37.8 & 39.1 & & 52 & (1) & 48 & 57.6 & 54.1 & 42 & $x$ & 48 & 38.0 & 37.5 & 10 & \\
\hline 50 & 46.6 & 53.1 & & Io & at & 50 & 37.8 & 38.8 & & 52 & CE. & 50 & 59.9 & 58.0 & 37 & 69 & 50.6 & 37.5 & 37.0 & II & \\
\hline 52 & 41.9 & 47.1 & 22 & 02 & 81 & 52 & 38.3 & 39.9 & & 53 & 6 & 52 & 55.0 & 52.8 & 45 & R. & 52 & 38.4 & 38.2 & $\infty$ & \\
\hline 54 & 37.8 & 44.8 & 21 & 57 & 27 & 54 & 38.8 & 39.8 & & 53 & 15 & 54 & 50.3 & 49.9 & 51 & 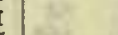 & 54 & 40.8 & 40.0 & 06 & \\
\hline 56 & 41.6 & 48.2 & 22 & 02 & & 56 & 40.9 & 41.8 & & 57 & & 56 & 60.7 & 59.0 & 36 & 54 & 56 & 42.0 & 41.8 & 04 & \\
\hline 58 & 41.0 & 49.9 & 21 & & $\cos 2$ & $\begin{array}{r}58 \\
1600\end{array}$ & $\begin{array}{l}42.2 \\
42.3\end{array}$ & $\begin{array}{l}43.1 \\
43.4\end{array}$ & T & $\begin{array}{l}59 \\
59\end{array}$ & -11.0 & 58 & 66.5 & 65.0 & & $\frac{8}{60}$ & 58 & 42.8 & 42.0 & & \\
\hline & & & & & & & 42.3 & $43 \cdot 4$ & & & -110.0 & & & & & & & & & & \\
\hline
\end{tabular}

Correction to local mean time is $+3 \mathrm{~m} 31.5 \mathrm{~s} .90^{\circ}$ torsion $=16 . .^{\prime} 64$.

Observer-J. V.

Torsion head at $1 \mathrm{Ih} 30 \mathrm{~m}$ read $69^{\circ}$ and at $\mathrm{I} 6 \mathrm{~h} 15 \mathrm{~m}$ read $63^{\circ}$.

Observer-R. R. T. 
Tabulation of magnetic declinations observed at Teplitz Bay-Continued

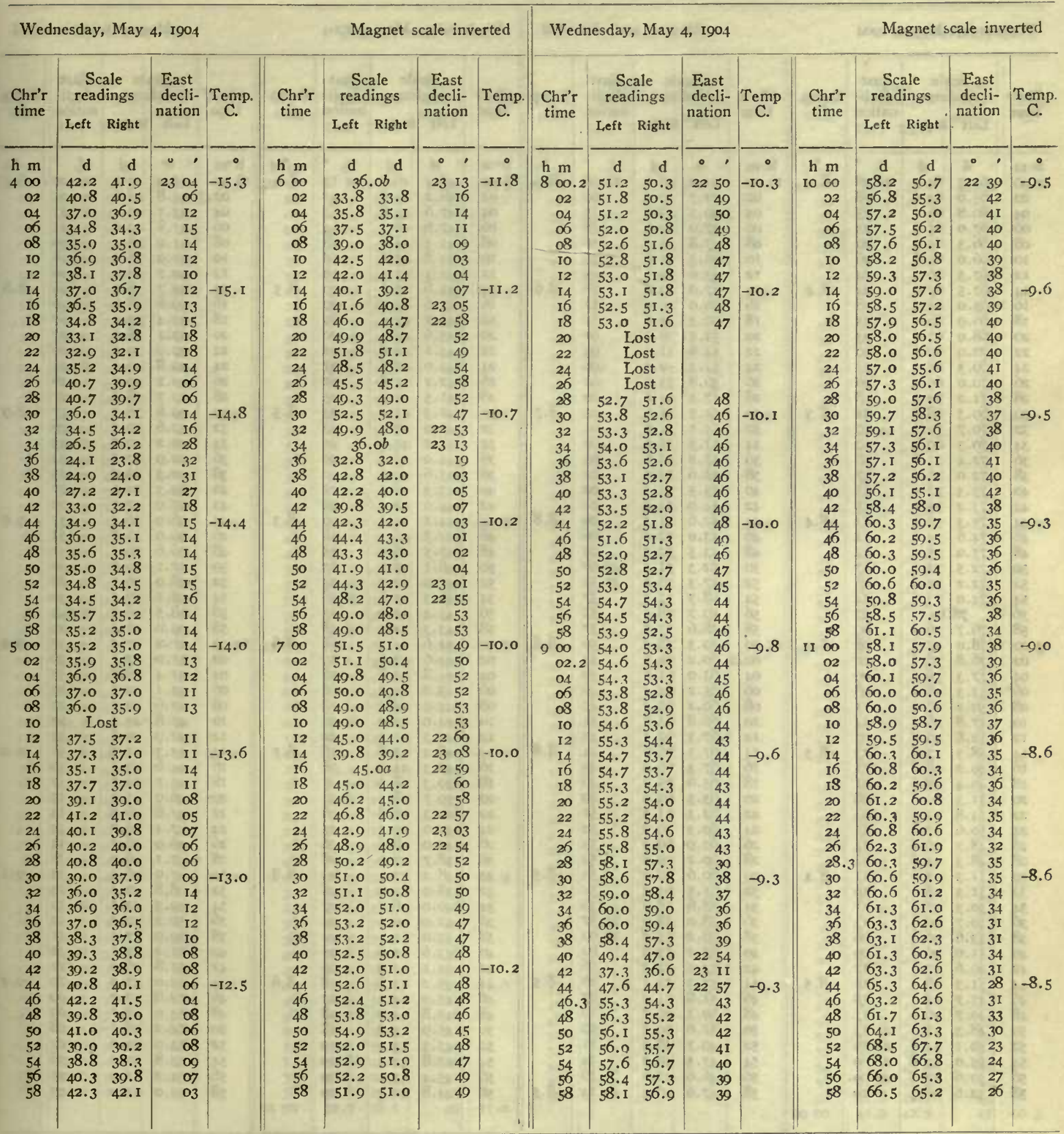

Observers-J. V. and W. J. P., who alternated from 7 h $52 \mathrm{~m}$ to Observer-W. J. P.

$8 \mathrm{~h} \mathrm{orm}$. 
Tabulation of magnetic declinations observed at Teplitz Bay-Continued

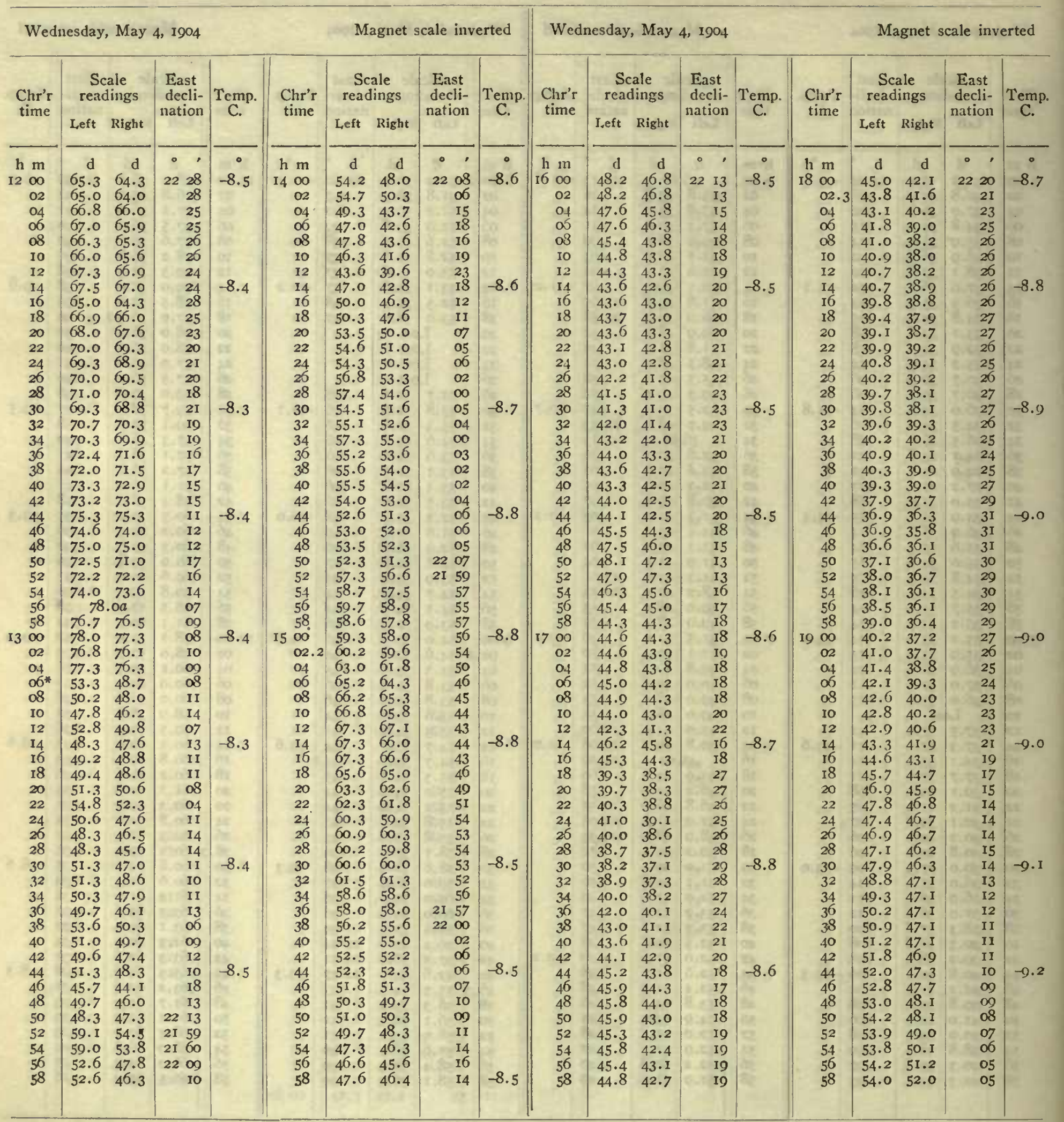


Tabulation of magnetic declinations observed at Teplitz Bay-Continued

\begin{tabular}{|c|c|c|c|c|c|c|c|c|c|c|c|c|c|c|c|c|c|c|c|}
\hline \multicolumn{6}{|c|}{ Wednesday, May 4, 1904} & \multicolumn{4}{|c|}{ Magnet scale inverted } & \multicolumn{6}{|c|}{ Thursday, May 5, 1904} & \multicolumn{4}{|c|}{ Magnet scale erect } \\
\hline \multirow{2}{*}{$\begin{array}{l}\text { Chr'r } \\
\text { time }\end{array}$} & \multicolumn{2}{|c|}{$\begin{array}{l}\text { Scale } \\
\text { readings }\end{array}$} & \multirow{2}{*}{$\begin{array}{c}\text { East } \\
\text { decli- } \\
\text { nation }\end{array}$} & Temp. & Chr'r & $\begin{array}{l}\text { Sca } \\
\text { read }\end{array}$ & $\begin{array}{l}\text { ale } \\
\text { ings }\end{array}$ & East & Temp. & Chr'r & $\begin{array}{l}\text { Sca } \\
\text { read }\end{array}$ & e & East & Temp. & Chr'r & $\begin{array}{l}\text { Sca } \\
\text { readi }\end{array}$ & $\begin{array}{l}\text { ale } \\
\text { lings }\end{array}$ & $\begin{array}{l}\text { East } \\
\text { decli- }\end{array}$ & Temp. \\
\hline & Left & Right & & & & Left & Right & & & & Left & Right & & & & Left & Right & & \\
\hline $\mathrm{h} \mathrm{m}$ & d & d & $\cdot \quad$ & $\bullet$ & h $\mathrm{m}$ & d & d & & $\bullet$ & $h \mathrm{~m}$ & & & $\cdot$, & & $\mathrm{h} \mathrm{m}$ & & A & & \\
\hline $2000 . \mathrm{I}$ & 54.0 & 52.9 & 2204 & -9.3 & 2200 & 39 . & .06 & 2227 & -10.2 & 1600 & 42.6 & 42.6 & 2232 & -9.9 & I8 00 & 40.8 & 41.0 & 2236 & -9.6 \\
\hline 02 & 54.7 & 52.5 & 04 & (2) & 02 & 39.7 & 38.2 & 27 & $1=$ & 02 & 42.1 & 42.4 & 3I & $x^{2}$ & 02 & 41.3 & $4 \mathrm{I} .8$ & & \\
\hline 04 & 53.8 & 52.0 & 05 & 280 & 04 & 47.1 & 44.7 & I6 & at & 01 & 41.0 & 41.0 & $3 \mathrm{I}$ & 610 & 04 & 41.9 & 42.0 & & 20 \\
\hline of & 53.3 & 51.2 & ळ & \% & $\infty$ & 48.7 & 46.3 & 13 & 16 & 06 & 40.8 & 40.8 & 20 & 0 & 06.5 & 42.1 & & 38 & 10 \\
\hline 09 & 53.9 & 51.9 & 05 & th & 08 & 49.7 & 45.9 & 13 & 4 & os. & -39.9 & 40.1 & 28 & ine & 08.2 & 42.3 & 42.8 & 39 & 60 \\
\hline I0 & 53.7 & $52 . I$ & 05 & ant & Io & 50.7 & 48.8 & 10 & 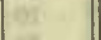 & I0 & 40.1 & 40.3 & 28 & ex: & 10 & $42 . I$ & 42.8 & & W \\
\hline 12 & 53.3 & $52 . \mathrm{I}$ & 05 & t & 12 & 5 T. I & $44 . \mathrm{I}$ & 13 & 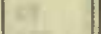 & 12 & 40.0 & 40.8 & 29 & th & 12 & 42.1 & 42.4 & 38 & \\
\hline 14 & 52.9 & 51.9 & 6 & -9.7 & 14 & 45.9 & 41.6 & 19 & -10.3 & 14 & 40.2 & 40.6 & 20 & -9.8 & 14 & 42.0 & 42.3 & & -9.7 \\
\hline 16 & 53.7 & $52 . \mathrm{I}$ & 05 & & 16 & $45 . \mathrm{I}$ & 35.6 & 25 & & 16 & 41.0 & 41.2 & 30 & & 16 & 42.4 & 42.6 & 39 & \\
\hline I8 & 52.2 & 50.9 & o7 & Alt & 18 & 61.3 & 44.5 & 05 & 6. & I8 & $4 \mathrm{~T} .2$ & 41.8 & $3 \mathrm{~T}$ & net & 18 & 42.3 & 42.8 & 39 & nit \\
\hline 20 & 50.9 & 50.2 & $\infty$ & $=$ & 20 & 53.2 & 44.0 & 12 & $6 x$ & 20 & $4 \mathrm{~T} .2$ & 41.4 & $3 \mathrm{I}$ & 18 & 20 & 42.2 & 42.7 & 39 & $e^{2}$ \\
\hline 22 & 50.6 & 49.5 & Io & $\pi$ & 22 & 50.9 & 36.7 & 19 & 10 & 22 & 40.2 & 40.6 & 20 & 18 & 22 & 42.1 & 42.5 & 39 & $t^{2}$ \\
\hline 2.1 & 50.8 & 49.7 & $\infty 9$ & Ex & 24 & 48.9 & 35.8 & 22 & tar & 21 & 38.0 & 38.3 & 26 & iti & 24 & 42.4 & 42.8 & 40 & 15 \\
\hline 26 & $5 \mathrm{I} . \mathrm{I}$ & 49.9 & $\infty$ & $8 \mathrm{~s}$ & 26 & 51.9 & 39.8 & 16 & 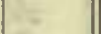 & $2 \pi$ & 48.1 & 48.2 & 32 & 37 & 26 & $42 . I$ & 43.0 & 40 & 6 \\
\hline 28 & 50.2 & 40.2 & Io & 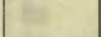 & 28 & 50.9 & 39.7 & 17 & 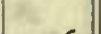 & 28 & Lu & ust & $=$ & 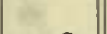 & 28 & 43.5 & 43.8 & $4 \mathrm{I}$ & \\
\hline 30 & 49.7 & 48.1 & II & -9.9 & 30 & 58.1 & 45.2 & 07 & -10.6 & 30 & 38.2 & 30.0 & 27 & -9.8 & 30 & 43.0 & 44.0 & 42 & $\rightarrow .7$ \\
\hline 32 & 49.0 & 48.2 & II & ne & 32 & 49.3 & 33.6 & 23 & $=2$ & 32 & 38.1 & 30.0 & 27 & 到 & 32 & 43.8 & 43.9 & 42 & 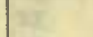 \\
\hline 34 & 49.8 & 48.2 & II & [t & 34 & 51.0 & 34.9 & 21 & 126 & .34 & 38.3 & 38.9 & 27 & tat & 34 & 43.0 & 43.0 & $4 I$ & 28 \\
\hline 35 & 49.9 & 48.2 & II & ac & 36 & 50.7 & 36.7 & 19 & $=$ & 36 & 38.0 & 38.2 & 27 & 86 & 36 & $42 . I$ & 42.4 & 40 & ac \\
\hline $3^{8}$ & 50.7 & 48.9 & I0 & 8 & 38 & 52.3 & 36.9 & 18 & 3 & 38 & 37.7 & 37.8 & 26 & be & .38 & 42.0 & 42.2 & 40 & [6.5 \\
\hline 40 & 51.9 & 50.1 & o8 & $\mathrm{a}$ & 40 & 5I.I & 39.6 & 17 & se & 40 & 37.5 & 37.0 & 25 & 7 & 40 & 42.0 & 42.2 & 40 & $6 y$ \\
\hline 42 & $53 \cdot 3$ & 51.7 & क6 & trat & 42 & 50.1 & 34.8 & 21 & 10 & 42 & 38.2 & 38.8 & 28 & $y=$ & 42.2 & 42.2 & 42.8 & $4 I$ & \\
\hline 44 & 54.3 & 52.0 & 05 & -9.9 & 44 & 46.3 & 34.9 & 24 & -10.8 & 44 & 39.0 & 40.0 & 30 & -9.6 & 44.4 & 42.0 & 42.5 & 40 & -9.8 \\
\hline 45 & 53.7 & $5 \mathrm{I} .2$ & 06 & & 46 & 50.5 & 37.6 & I0 & nat & 46 & 41.0 & $4 \mathrm{I} . \mathrm{I}$ & 32 & 9. & $4^{6}$ & 42.1 & 42.4 & $4 \mathrm{~T}$ & ( \\
\hline 48 & 52.0 & 40.9 & 08 & E & 48 & $48 . I$ & 40.8 & I8 & Bs & 48 & 41.7 & 42.0 & 34 & 1t. & 48 & 42.2 & 42.6 & $4 I$ & te \\
\hline 50 & 50.4 & 48.3 & II & $\theta$ & 50 & 54.5 & 45.5 & Io & 8 & 50 & 41.0 & $4 \mathrm{~T} . \mathrm{I}$ & .32 & a & 50 & 42.1 & 42.8 & $4 \mathrm{I}$ & int \\
\hline 52 & 50.8 & 48.7 & IO & 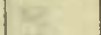 & 52 & 37.8 & 27.1 & 37 & 72 & 52 & 40.0 & 40.2 & $.3 \mathrm{I}$ & $=$ & 52 & 42.0 & 42.2 & $4 I$ & res \\
\hline 54 & 47.0 & 45.8 & 15 & tis & 54 & 23.8 & I4.I & 58 & 12. & 54 & 40.1 & 40.2 & 31 & 32 & 54 & 42.0 & 42.0 & 40 & \\
\hline $5 \%$ & 46.8 & 45.2 & 16 & & 56 & $38 . \mathrm{I}$ & 21.6 & $4 \mathrm{I}$ & 8 & 55 & 40.2 & 40.7 & $3 \mathrm{I}$ & 172 & 56 & 42.0 & 42.2 & $4 \mathrm{I}$ & \\
\hline 58 & 47.7 & 46.1 & 14 & & 58 & 37.8 & 23.7 & 40 & & 58 & 30.9 & 40.1 & $.3 \mathrm{~T}$ & 13 & 58 & 42.0 & 42.3 & $4 \mathrm{I}$ & \\
\hline 2100 & 44.7 & 43.1 & 10 & -9.9 & $23 \infty$ & 27.1 & 12.3 & 57 & -10.8 & ז7 00 & 60.2 & 40.7 & $3 \mathrm{I}$ & -9.3 & I9 on & $4 \mathrm{~T} .0$ & 42.2 & $4 \mathrm{~T}$ & -9.9 \\
\hline 02 & $45 \cdot 5$ & 43.8 & 18 & - & 02 & 48.0 & 32.0 & 25 & 9 & 02 & $4 \mathrm{I} \cdot 3$ & $4 \mathrm{~T} .8$ & .33 & 102 & 02 & $4 \mathrm{I} .7$ & 42.0 & $4 \mathrm{I}$ & $x^{2}$ \\
\hline 04 & 44.2 & 42.1 & 20 & 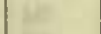 & 04 & 56.9 & 42.6 & 10 & 90 & 01 & 42. I & 42.7 & 35 & if & 0.1 & $4 \mathrm{r} .2$ & 41.8 & 40 & $=$ \\
\hline or & 43.0 & $4 \mathrm{~T} .2$ & 22 & ray & 0 & 55.9 & 42.7 & II & $9 n$ & or & 42.2 & 42.3 & .35 & 80 & or & $4 \mathrm{~T} .2$ & 41.9 & 40 & 17 \\
\hline n? & 44.2 & 43.1 & 20 & 60 & 08 & 52.0 & 39.0 & 16 & 5 & 08 & 41.1 & $4 \mathrm{~T} .2$ & 33 & fins & 08 & $4 \mathrm{I} .5$ & 42.0 & $4 \mathrm{I}$ & 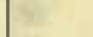 \\
\hline 10 & 41.1 & 4.3. I & 20 & $=$ & 10 & 49.8 & 38.1 & 19 & 2 & 10 & 40.5 & 40.7 & 32 & wit & Io & 41.8 & 42.0 & 41 & 5 \\
\hline 12 & 45.2 & 43.3 & 19 & 52 & 12 & $5 \mathrm{I} .4$ & 40.1 & 16 & 1 & 12.6 & 40.9 & $4 \mathrm{I} .0$ & 3.3 & int & 12 & $4^{4} \cdot n$ & 42.0 & $4 \mathrm{I}$ & \\
\hline 14 & 42.9 & 42.0 & 2 I & -10.0 & 14 & 48.1 & 40.1 & 19 & -10.9 & I4 & 41.0 & $4 \mathrm{I} .2$ & 33 & -0.2 & I1 & $4 \mathrm{~T} .8$ & 42.0 & 42 & -10.0 \\
\hline I6 & 44.1 & 42.4 & 20 & & 16 & 34.4 & 22.0 & 44 & & 15 & 41.5 & $4 \mathrm{I} .0$ & 34 & & T6 & 41.8 & 42.0 & 42 & \\
\hline I8 & 44.1 & $42 . I$ & 20 & 10 & 18 & $3 \mathrm{I} .0$ & 19.3 & 48 & it & I8 & 41.5 & 41.8 & 34 & tate & T8 & $4 \mathrm{I} .9$ & 42.1 & 42 & 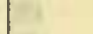 \\
\hline 20 & 4.3 .9 & $4 \mathrm{I} .4$ & $2 \mathrm{I}$ & $\alpha$ & 20 & 39.8 & 25.2 & 37 & str. & 20 & $4 T .5$ & $4 \pi .9$ & 35 & $\cos$ & 20 & $4 \mathrm{~T} \cdot 3$ & 42.0 & 41 & 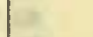 \\
\hline 22 & $43 . \mathrm{I}$ & 40.2 & 23 & $=$ & 22 & 45.0 & 31.6 & 28 & 10 & 22 & 41.9 & 42.0 & & $t=$ & 22 & 42.0 & 42.2 & 42 & 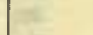 \\
\hline 24 & $4.3 \cdot 3$ & 40.2 & 23 & 10 & 24 & 4I.I & 29.2 & 33 & $n^{2}$ & 24 & 42.1 & 42 & 35 & 14 & 24 & 42.0 & 42.1 & 42 & xe \\
\hline 26 & 4.3 .0 & 40.2 & 23 & $\mathrm{~A}$ & 26 & 35.5 & 24.9 & $4 \mathrm{I}$ & 6 & 26 & 42.7 & 42 & & 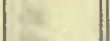 & $2 \pi$ & 41.0 & 42.0 & 42 & $x^{2}$ \\
\hline 28 & 42.4 & 40.2 & 23 & 2 & 28 & 3.3 .9 & 26.2 & $4 I$ & & 28 & $4 \mathrm{I} \cdot \mathrm{I}$ & $4 \mathrm{I} .8$ & 35 & 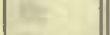 & 28 & $4 \mathrm{I} .8$ & $4 \mathrm{I}$ & 42 & \\
\hline 30 & 42.4 & 40.2 & 23 & -I0.0 & 30 & 36.9 & $22 . I$ & 42 & -10.9 & 30 & 40.0 & & 3.3 & -9.3 & 30 & 41.5 & 41.8 & $4^{2}$ & -IO. I \\
\hline 32 & 42.0 & 39.0 & 24 & $a$ & 32 & 39.0 & 28.0 & 35 & Fi: & 32 & 39.3 & 39.8 & 32 & & 32 & 41.5 & 41.8 & 42 & \\
\hline 34 & 41.8 & 38.7 & 25 & 42 & 34 & 43.9 & 35.0 & 26 & 10 & 34 & 30.8 & 39.9 & 32 & 16 & .3 .1 & 41.8 & 42.0 & 43 & \\
\hline 36 & 40.3 & 38.0 & 27 & $n$ & 36 & 33. I & 25.0 & 42 & & 35 & 40.0 & $40 . I$ & 3.3 & & 36 & $4 \mathrm{~T} .8$ & 42.0 & 43 & $=$ \\
\hline $3^{8}$ & 39.4 & $37 \cdot 3$ & 28 & ne & 38 & 32.5 & 25.2 & 43 & 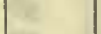 & 38 & 40.4 & 40.7 & 34 & fis & 38.5 & 42.0 & 42.1 & 43 & \\
\hline 40 & 30.8 & 37.2 & 28 & 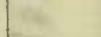 & 40 & 36.8 & 20.0 & 36 & 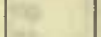 & 40 & 40.2 & 40.5 & 34 & (47. & 40 & 42.0 & 42.3 & 43 & \\
\hline 42 & 38.9 & 36.5 & 29 & at & 42 & 34.1 & 28.1 & 39 & & 42 & 40.1 & $40 . I$ & 3.3 & 67 & 42 & 42.0 & 42.5 & 44 & \\
\hline 4.1 & 39.0 & 36.3 & 20 & -10.1 & 44 & 37.7 & 30.1 & 35 & -11.0 & 44 & 40.0 & $40 . I$ & .33 & $\rightarrow-5$ & 41 & 42.0 & 42.2 & 44 & -10.2 \\
\hline 45 & 39.1 & 37.0 & 28 & 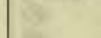 & 46 & $4 \mathrm{I} .2$ & 34.3 & 29 & Y & 46 & 40.0 & 40.1 & 34 & & $4^{6}$ & 41.9 & 42.1 & 44 & 15: \\
\hline 48 & 39.4 & 37.7 & 28 & 28 & 48 & 43.1 & 36.2 & 26 & N & 48 & 40.2 & 40.5 & 34 & 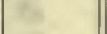 & 48 & 41.9 & 42. & 44 & 4 \\
\hline 50 & 30.1 & 37.7 & 28 & $\mathrm{E}^{2}$ & 50 & 44.4 & 38.3 & 23 & 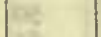 & 50 & 40.9 & $4 \mathrm{r} \cdot \mathrm{T}$ & .35 & -2 & 50 & 41.7 & 42.1 & & 92 \\
\hline 52 & 39.0 & $37 \cdot 3$ & 28 & a & 52 & $35 . I$ & 27.3 & 39 & 22 & 52 & $4 I .2$ & 41.5 & 36 & 16 & 52 & $4 \pi .2$ & 42.0 & 43 & \\
\hline 51 & 42.0 & $40 . \mathrm{I}$ & 24 & 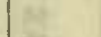 & 51 & 30.8 & 24.1 & 45 & 12 & 54 & $4 \mathrm{~T} .4$ & 41.8 & 15 & 105 & 54 & 41.5 & 42.0 & 43 & \\
\hline $5 \sqrt{5}$ & 40.9 & 39.1 & 25 & 20 & 56 & 26.9 & 20.1 & $5 I$ & 8 & 56 & $4 \mathrm{I} .0$ & 41.9 & 36 & 12 & $5 \%$ & 41.8 & 42.2 & 44 & \\
\hline 58 & 44.0 & 40.7 & 22 & 20 & 58 & 30.8 & 24.5 & 45 & & 58 & 40.8 & 40.9 & 35 & te & 58 & 41.8 & 42.2 & 44 & \\
\hline & & & & & & 32.9 & 28.1 & 40 & $-\mathrm{I} 1.0$ & & & & $x^{2}$ & 0034 & 2000 & 41.9 & 42.7 & 45 & -10.3 \\
\hline
\end{tabular}

Correction to local mean time is $+3 \mathrm{~m} 34 \mathrm{~s} .90^{\circ}$ torsion $=18 .^{\prime} 96$. Torsion head at oh oom read $63^{\circ}$ and at $24 \mathrm{~h}$ o5m read $67^{\circ}$. Observer-R. R. T.
Correction to local mean time is - Im 38.5s. $90^{\circ}$ torsion $=17 .^{\prime} 74$ Torsion head at $15 \mathrm{~h} 40 \mathrm{~m}$ read $67^{\circ}$ and at $20 \mathrm{~h} 2 \mathrm{Im}$ read $346^{\circ}$. Observer-J. V. 
Tabulation of magnetic declinations observed at Teplitz Bay-Continued

\begin{tabular}{|c|c|c|c|c|c|c|c|c|c|c|c|c|c|c|c|c|c|c|c|c|c|}
\hline \multicolumn{6}{|c|}{ Friday, May 6, 1904} & \multicolumn{5}{|c|}{ Magnet scale inverted } & \multicolumn{6}{|c|}{ Sunday, May 8, 1904} & \multicolumn{5}{|c|}{ Magnet scale erect } \\
\hline \multirow{2}{*}{$\begin{array}{l}\text { Chr'r } \\
\text { time }\end{array}$} & \multicolumn{2}{|c|}{$\begin{array}{l}\text { Scale } \\
\text { readings }\end{array}$} & $\begin{array}{l}\text { East } \\
\text { decli- }\end{array}$ & Temp & $\begin{array}{l}\text { Chr'r } \\
\text { time }\end{array}$ & $\begin{array}{l}\mathrm{Sc} \\
\text { read }\end{array}$ & $\begin{array}{l}\text { ale } \\
\text { lings }\end{array}$ & $\begin{array}{r}\text { Eas } \\
\text { dec } \\
\text { nati }\end{array}$ & & $\begin{array}{c}\text { Temp } \\
\text { C. }\end{array}$ & $\begin{array}{l}\text { Chr'r } \\
\text { time }\end{array}$ & $\begin{array}{r}\mathrm{Sc} \\
\text { reac }\end{array}$ & $\begin{array}{l}\text { ale } \\
\text { dings }\end{array}$ & $\begin{array}{c}\text { East } \\
\text { decli- }\end{array}$ & Ternp. & Chr'r & $\begin{array}{l}\text { Sc: } \\
\text { read }\end{array}$ & $\begin{array}{l}\text { le } \\
\text { ngs }\end{array}$ & Eas & st & Temp. \\
\hline & Left & Righ! & & & & Left & Right & that & & & & Left & Right & natron & & & Left & Right & & & \\
\hline h m & d & $d$ & & $\pi$ & $\mathrm{h} \mathrm{m}$ & d & & $\circ$ & & & h $\mathrm{m}$ & d & d & $\circ:$ & & $\mathrm{h} \mathrm{m}$ & d & d & & ' & $\circ$ \\
\hline $2000^{*}$ & 39.2 & 38.0 & & -11.0 & $22 \infty 0$ & $47 \cdot 3$ & 46.1 & 22 & & -II.2 & $000 *$ & 50.6 & 52.1 & 2224 & -11.3 & 200 & 52.8 & 53.5 & & 27 & -10.0 \\
\hline 02 & 39.3 & 37.9 & 28 & 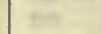 & 02 & 44.5 & 4.3 .7 & & 20 & in & פ2 & 49.1 & 51.6 & 23 & 90 & 02 & 55.2 & 55.9 & & $3 \mathrm{I}$ & \\
\hline 04 & 39.7 & 38.1 & 28 & 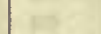 & 04 & 45.5 & 45.0 & & 18 & 0 & 04 & 49.2 & 51.9 & 23 & 39 & 04 & 56.0 & 56.9 & & 32 & \\
\hline 06 & 40.0 & 38.7 & 27 & 30 & $\infty$ & 47.1 & 46.7 & & 16 & an & 06 & 50.3 & 52.1 & 24 & 80 & 06 & 55.0 & 56.8 & & 31 & \\
\hline 08 & 40.2 & 38.9 & 27 & (for & 08 & 44.8 & 44.0 & & 19 & bo & 08 & 50.6 & 52.2 & 24 & the & 08 & 55.3 & 57.0 & & 32 & \\
\hline 10 & 40.0 & 38.5 & & at & 10 & 49.9 & 49.2 & & II & wet & Io & 50.0 & $5 \mathrm{I} .2$ & 23 & bat & 10 & 54.7 & 57.1 & & $3 I$ & \\
\hline 12 & 39.9 & 38.4 & 28 & 18 & 12 & 49.9 & 49.7 & & II & & 12 & 49.2 & 50.8 & 22 & 11 & I2 & 55.1 & 58.1 & & 32 & \\
\hline 14 & 39.2 & 38.0 & 28 & -11.0 & 14 & 49.4 & 48.8 & & I2 & $-\mathrm{II} .2$ & 14 & 49.8 & 5 I. I & 2.3 & -10.9 & I4 & 56.2 & 59.3 & & 34 & -10.0 \\
\hline 16 & 39.1 & 38.0 & 29 & $2 t$ & 16 & 49.1 & 48.6 & & 12 & $t^{1}$ & r & 49.7 & $5 \mathrm{I} .1$ & 23 & 7. & 16 & 57.2 & 60.9 & & 36 & \\
\hline I8 & 39.0 & 37.9 & 29 & Aft & 18 & 49.8 & 49.2 & & II & Ear & 18 & 50. I & 51.9 & 24 & 5 & 18 & 58.8 & 62. I & & 38 & \\
\hline 20 & 38.8 & 37.7 & 29 & $=$ & 20 & 49.7 & 49.0 & & 12 & 68 & 20 & 51.2 & 53.1 & 26 & $E$ & 20 & 57.8 & 61.2 & & 37 & \\
\hline 22 & 38.0 & 37.1 & 30 & itit & 22 & 48.1 & 46.9 & & 14 & m & 22 & 51.5 & 53.1 & 26 & \& & 22 & 58.2 & 61.9 & & 38 & it \\
\hline 24 & 38.2 & 37.5 & 30 & 11 & 24 & 51.0 & 49.9 & & I0 & 12 & 24 & 5I.I & 52.8 & 25 & 17 & 24 & 59.0 & 62.3 & & 39 & $y=$ \\
\hline 26 & 38.3 & 37.5 & 30 & 18 & 26 & 49 & $.7 b$ & & II & Ist & 26 & 51.6 & 52.8 & 26 & is & 26.2 & 58.5 & 61.3 & & 38 & a \\
\hline 28 & .39 .0 & 38.1 & 29 & It & 28 & 48.2 & 48.1 & & 14 & 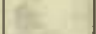 & 28 & $51 . I$ & 52.3 & 25 & it & 28 & 61.8 & 64.1 & & 42 & \\
\hline 30 & 39.5 & 38.8 & 28 & -II.O & 30 & 53.1 & 50.8 & & 08 & -11.3 & 30 & 50.8 & 51.7 & . 24 & -10.8 & 30 & 62.9 & 65.2 & & 44 & -10.0 \\
\hline 32 & 39.5 & 38.9 & 28 & to & 32 & 43.8 & $4 \mathrm{I} . \mathrm{I}$ & & 22 & Ir & 32 & 51.2 & 53.0 & 26 & $x^{2}$ & 32 & 62.0 & 64.2 & & 43 & \\
\hline 34 & $40 . \mathrm{I}$ & 39.5 & 27 & (15) & 34 & 40.2 & 31.0 & & 33 & 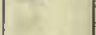 & 34 & 51.6 & 53.3 & 26 & M & 34 & 61.1 & 63.1 & & $4 \mathrm{I}$ & \\
\hline 36 & 39.8 & 39.0 & 27 & ats & 36 & 53.1 & $43 . I$ & & I4 & at & 36 & 52.2 & 54.2 & 27 & 28t & 36 & 61.7 & 63.2 & & 42 & \\
\hline 38 & 39.7 & 39.0 & 27 & 4 & 38 & 51.2 & 41.2 & & 17 & 17 & 38 & 52.3 & 54.5 & 27 & 8 & 38 & 62.8 & 64.0 & & 43 & \\
\hline 40 & 40.1 & 39.6 & 27 & 48 & 40 & 51.9 & 42.1 & & 15 & a & 40 & 50.8 & 53.1 & 25 & es & 40 & 64.1 & 65.2 & & 45 & \\
\hline 42 & 40.6 & 39.9 & 26 & 4 & 42 & 51.5 & 41.0 & & 17 & & 42 & $51 . I$ & 53. I & 26 & & 42 & 64.0 & 65.2 & & 45 & \\
\hline 44 & 39.8 & 39.0 & 27 & -II.I & 44 & 50.2 & 42.3 & & 17 & -11.3 & 44 & 50.2 & 52.7 & 24 & -10.6 & 44 & 62.4 & 63.8 & & 43 & -9.9 \\
\hline 46 & 39.9 & 39.0 & 27 & 1 & 46 & 48.2 & 44.1 & & 17 & (2) & 46 & 50.0 & 52.8 & 24 & 8 & 46 & 60.6 & 62.9 & & 40 & \\
\hline 48 & 40.3 & 39.9 & 26 & R & 48 & $4 \mathrm{I} . \mathrm{I}$ & 34.9 & & 29 & 2. & 48 & 50.1 & 52.8 & 24 & 46 & 48 & 59.7 & 61.3 & & 39 & \\
\hline 50 & $4 \mathrm{I} . \mathrm{I}$ & 40.9 & 25 & at & 50 & 48.9 & 41.0 & & 19 & tor & 50 & 50.0 & 52.3 & -24 & 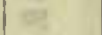 & 50 & 60.0 & 61.4 & & 39 & 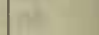 \\
\hline 52 & 41.4 & 41.0 & 24 & tht & 52 & 52.0 & 45.8 & & I2 & ar & 52 & 50.1 & 52.2 & 24 & sit & 52 & 60.5 & 61.3 & & 39 & \\
\hline 54 & 41.9 & $4 \mathrm{I} \cdot 3$ & 24 & is & 54 & 50.8 & 46.0 & & I3 & wit & 54 & 50.1 & 52.3 & 24 & $H$ & 54 & 61.6 & 62.6 & & 41 & 4 \\
\hline 56 & 42.0 & 41.8 & 23 & & 56 & 53.0 & 32.9 & & 22 & 60 & 56 & 49.7 & 51.9 & 23 & 野 & 56 & 63.0 & 64.1 & & 43 & \\
\hline 58 & 43.1 & 42.6 & 22 & 82 & 58 & 53.0 & 41.9 & & 15 & & 58 & 49.9 & 51.0 & 123 & 16 & 58 & 64.1 & 65.3 & & 45 & \\
\hline 2100 & 43.0 & 42.8 & 22 & -II.I & 2300 & 53.0 & 42.2 & $=$ & 14 & -11.4 & 100 & 48.5 & 49.7 & $2 \mathrm{II}$ & -10.4 & 300 & 65.9 & 66.9 & & 48 & -9.9 \\
\hline 02 & 44.0 & $43 . I$ & 21 & 1 & 02 & 55.1 & 44.0 & & II & 6 & 02 & 47.9 & $49 \cdot 3$ & 1. 20 & twithe & 02 & 67.1 & 68.2 & & 50 & \\
\hline 04 & 45.1 & 44.9 & 18 & $2=$ & 04 & 45.8 & 35.3 & & 26 & $=0$ & 04 & 48.3 & 50.2 & $2 \mathrm{I}$ & 19 & $a_{4}$ & 67.1 & 68.9 & & 50 & \\
\hline 06 & 45.3 & 45.3 & 18 & Ant & 06 & $4 I .2$ & 33.3 & & $3 I$ & 6. & 06 & 48.9 & 50.8 & 22 & is & 06 & 64.9 & 66.9 & & 47 & \\
\hline 08 & 47.2 & 46.9 & 15 & Na & 08.3 & 45.9 & 40.6 & & $2 \mathrm{I}$ & 6 & 88 & 49.2 & 50.8 & 22 & $2 x$ & 08 & 65.5 & 68.0 & & 48 & \\
\hline 10 & 47.6 & 47.1 & 15 & oet & 10 & 30.5 & 27.9 & & 43 & at & 10 & 48.8 & 50.0 & $2 \mathrm{I}$ & arr & IO & 66.8 & 69.3 & & 50 & \\
\hline 12 & 46.1 & 45.9 & 17 & inf & I2 & 38.0 & 27.0 & & 38 & & 12 & 49.8 & 51.6 & 23 & $A P$ & 12 & 68.1 & 70.9 & & 53 & \\
\hline I4 & 45.2 & 44.9 & 18 & - II I.2 & 14 & 46.3 & 38.3 & & 23 & -11.5 & 14 & 51.0 & 53.8 & 726 & -10.3 & 14 & 60.2 & 72.0 & & 54 & -9.8 \\
\hline 16 & 45.2 & 45.0 & 18 & & 16 & 45.0 & 35.9 & 22 & 26 & 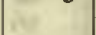 & 16 & 50.9 & 53.1 & 25 & (2) & 16 & 70.0 & 72.9 & & 56 & \\
\hline 18 & 44.8 & 44.3 & 19 & Bit & I8* & 57.3 & 56.0 & 23 & 02 & 24 & I8 & $49 \cdot 3$ & 51.9 & 23 & 42 & I8 & 70.1 & 73.1 & & 56 & \\
\hline 20 & 44.0 & 43.2 & 2 I & $c=$ & 20 & 68.0 & 51.0 & 22 & 57 & es & 20 & 48.6 & 51.0 & 22 & $z=$ & 20 & 70.8 & 73. & & 57 & \\
\hline 22 & 43.2 & 43. I & 2 I & 37t & $22^{*}$ & $49 . I$ & 44.8 & & II & $\Rightarrow$ & 22 & 48.3 & 50.2 & $2 I$ & 15 & 22 & 71.1 & 73.5 & & 57 & \\
\hline 24 & 43.2 & 43.1 & 21 & it & 24 & 47.7 & 37.8 & & 17 & $=$ & 24 & 48.1 & 50.8 & $\quad 2 \mathrm{I}$ & 16 & 24 & 72.0 & 74.0 & & 58 & \\
\hline 26 & 43.9 & $43 \cdot 3$ & 21 & $x=$ & 26 & 25.0 & 18.8 & & 50 & $7=$ & 26 & 47.2 & 50.0 & $\cdots 20$ & 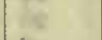 & 26 & $73 . I$ & 75.0 & 220 & 60 & \\
\hline 28 & 44.0 & 43.7 & 20 & 2 & 28 & $22 . \mathrm{I}$ & 14.0 & & 56 & & 28 & 47.6 & 49.5 & 20 & : & 28 & 73.6 & $75 . I$ & 23 & $\infty$ & \\
\hline 30 & 44.0 & 43.9 & 20 & -11.2 & 30 & 41.0 & 31.2 & & 28 & -11.5 & 30 & 47.0 & 49.0 & I9 & -10.2 & $\therefore 30$ & 74.2 & 75.8 & & OI & -9.8 \\
\hline 32 & 44.5 & 44.4 & 19 & $2 x$ & 32 & 47.0 & $4 \mathrm{I} .3$ & & I5 & (n) & 32 & 47.1 & 49.0 & I9 & 2 & 32 & 75.0 & 76.1 & & 02 & \\
\hline 34 & 45.1 & 44.5 & 19 & 17 & 34 & 41.0 & 34.3 & & 25 & in & 34 & 47.2 & 48.3 & I8 & H & 34 & 75.3 & 77.2 & & 03 & \\
\hline 36 & 44.2 & 43.5 & 20 & 48 & 36 & 37.7 & 32.0 & & 30 & 7 & 36 & 47.5 & 48.0 & 18 & $\therefore$ & 36 & 76.1 & 78.2 & & 05 & \\
\hline 38 & 45.0 & 44.2 & 19 & * & 38 & $34 \cdot 3$ & 29.8 & & 34 & 6 & 38 & 49.0 & 49.6 & $2 \mathrm{I}$ & $\ldots$ & 38 & 76.5 & 79.0 & & 06 & \\
\hline 40 & 44.2 & 44.0 & 20 & 28 & 40 & $3 \mathrm{I} .3$ & 26.9 & & 39 & en & 40 & 49.6 & 49.9 & 22 & 6 & 40 & 76.3 & 78.4 & & 05 & \\
\hline 42 & 43.1 & 42.0 & 22 & & 42 & 32.6 & 27.2 & & 37 & & 42 & 50.8 & 50.9 & 23 & 6 & $42^{*}$ & 47.4 & 50.5 & & 05 & \\
\hline & $4 \mathrm{I} .2$ & 40.2 & 25 & -11.2 & 44 & 35.9 & 29.2 & & 33 & -I I 6 & 44 & 50.1 & 50.8 & $\therefore 23$ & -10.1 & 44 & $49 . I$ & 50.8 & & 06 & -9.7 \\
\hline 46 & 41.8 & 40.2 & 25 & (n) & 46 & 37.0 & $3 \mathrm{I} .5$ & & 31 & ntrat & 46 & 48. I & 49.2 & 20 & $x^{2}$ & 46 & 49.1 & 50.3 & & 06 & \\
\hline 48 & 42.9 & 41.1 & 23 & 8 & 48 & 32.0 & 28.1 & & 37 & . & 48 & 47.9 & 49.6 & . 20 & R & 48 & 49.9 & 50.7 & & o7 & \\
\hline 50 & 43.0 & 42.0 & 22 & $\theta^{2}$ & 50 & 37.0 & 33.3 & & 29 & in & 50 & 48. I & 49.4 & 20 & $\propto$ & 50 & 53.9 & 53.9 & & 12 & \\
\hline 52 & 42.2 & 42.0 & 23 & $7^{*}$ & 52 & 26.7 & 26.0 & & 43 & 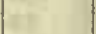 & 52 & 48.3 & 49.2 & $=20$ & 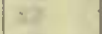 & 52 & 54.1 & 54.9 & & 14 & \\
\hline 54 & 43.1 & 42.2 & 22 & Wr & 54 & 21.7 & 15.1 & & 55 & 0 & 54 & 48.8 & $49 . I$ & $\because \quad 20$ & 12 & 54 & 55.2 & 56.9 & & 16 & \\
\hline 56 & 42.2 & 41.9 & 23 & $7=$ & 56 & 17.9 & 15.3 & & 58 & in & 56 & 49.3 & 49.8 & 21 & 20 & 56 & 54.8 & 56.2 & & 15 & \\
\hline 58 & 45.0 & 44.2 & 19 & 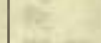 & 58 & 27.1 & 20.2 & & 47 & 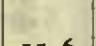 & 58 & 50.9 & 5I.I & 24 & & 58 & $54 \cdot 5$ & 56.1 & & 15 & \\
\hline & & & (x) & $w 5 y=$ & 2400 & 35.0 & 28.0 & & 35 & -11.6 & & & & 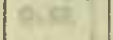 & & & & & & & \\
\hline
\end{tabular}

Correction to local mean time is - Im 02.5s.

Observer-R. R. T.

Torsion head at $19 \mathrm{~h} 33 \mathrm{~m}$ read $66^{\circ}$ and at $24 \mathrm{~h} 23 \mathrm{~m}$ read $67^{\circ}$.

Observer-J. V. 
Tabulation of magnetic declinations observed at Teplitz Bay-Continued

\begin{tabular}{|c|c|c|c|c|c|c|c|c|c|c|c|c|c|c|c|c|c|c|c|c|c|}
\hline \multicolumn{6}{|c|}{ Sunday, May 8, 1904} & \multicolumn{6}{|c|}{ Magnet scale inverted } & \multicolumn{6}{|c|}{ Monday, May 9, I904 } & \multicolumn{4}{|c|}{ Magnet scale erect } \\
\hline \multirow[t]{2}{*}{$\begin{array}{l}\text { Chr'r } \\
\text { time }\end{array}$} & \multicolumn{2}{|c|}{$\begin{array}{l}\text { Scale } \\
\text { readings }\end{array}$} & $\begin{array}{l}\text { Eas } \\
\text { decl } \\
\text { natic }\end{array}$ & & Terng. & $\begin{array}{l}\text { Chr't } \\
\text { time }\end{array}$ & $\begin{array}{r}\text { Sca } \\
\text { readi }\end{array}$ & le & $\begin{array}{l}\text { Eas } \\
\text { decl } \\
\text { natic }\end{array}$ & & $\begin{array}{c}\text { Tenip. } \\
\text { C. }\end{array}$ & $\begin{array}{l}\text { Chr'r } \\
\text { time }\end{array}$ & $\begin{array}{l}\text { Sc } \\
\text { read }\end{array}$ & e & $\begin{array}{l}\text { East } \\
\text { decli- } \\
\text { nation }\end{array}$ & $\begin{array}{l}\text { Temp. } \\
\text { C. }\end{array}$ & $\begin{array}{l}\text { Chr'r } \\
\text { time }\end{array}$ & $\begin{array}{l}\text { Sca } \\
\text { tead }\end{array}$ & $\begin{array}{l}\text { le } \\
\text { ngs }\end{array}$ & $\begin{array}{l}\text { East } \\
\text { decli- } \\
\text { nation }\end{array}$ & Temp. \\
\hline & Left & Right & & & & & L,eft & Right & & & & & Left & Right & & & & Left & Right & & \\
\hline$h \mathrm{~m}$ & d & d & & $\dot{1}$ & $\bullet$ & $\mathrm{h} \mathrm{m}$ & d & d & & ' & & $h \mathrm{~m}$ & d & $\mathrm{d}$ & & & $\mathrm{h}$ nı & d & d & • & $\bullet$ \\
\hline 400 & $5 \mathrm{I} \cdot 7$ & 49.8 & & I6 & $29=$ & 600 & 51.6 & 50.7 & & & $-8 . I$ & 800.4 & 54.9 & 56.2 & 2256 & -6.8 & I0 $\infty$ & 47.0 & 49.4 & 2244 & -5.0 \\
\hline 02 & 50.0 & 48.7 & & I8 & 5 & 02 & 52.1 & 50.2 & & 15 & & 02 & 54.1 & 56.8 & 56 & & 02 & $47 \cdot 3$ & 50.0 & & se \\
\hline 04 & $49 \cdot 4$ & 47.0 & & 20 & $\sqrt{2}$ & 04 & 48.4 & 47.2 & & 20 & & 04 & 52.2 & 56.3 & 54 & & 04 & $47 \cdot 3$ & 49.9 & 45 & ate \\
\hline 06 & 49.7 & $47 \cdot 1$ & & I9 & 25 & 06 & 48.8 & 47.2 & & 20 & $E^{2}$ & 06 & 49.1 & 54.2 & 50 & e. & 06 & 47.1 & 49.7 & & $y$ \\
\hline os & 49.0 & 45.9 & & $\begin{array}{ll}2 \mathrm{I} \\
28\end{array}$ & in & 08 & 48.9 & 48.2 & & 19 & - & os & 48.3 & 53.7 & 49 & are & 08 & 47.9 & 50.3 & 46 & ar \\
\hline 10 & 50.8 & 47.8 & & I8 & at & Io & 50.4 & 49.6 & & 17 & & I0 & 49.0 & 53.3 & 49 & 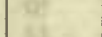 & Io & 47.6 & 50.3 & 46 & ent \\
\hline 12 & 50.5 & 47.8 & & 18 & & I 2 & 49.5 & 49.2 & & 18 & & 12 & 51.7 & 55.8 & 53 & & 12 & 49.0 & $5 \mathrm{I} . \mathrm{I}$ & 47 & 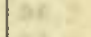 \\
\hline 14 & 47.9 & $46 . I$ & & 22 & $-9 \cdot 3$ & I4 & 48.4 & 47.9 & & 20 & -8.0 & 14 & 52.7 & 56.3 & 54 & -6.7 & 14 & 47.9 & 50.2 & 46 & -5.0 \\
\hline I6 & 45.4 & $43 . \mathrm{I}$ & & 26 & & I6 & 50.1 & 49.4 & & 17 & & I6́ & 53.7 & 56.9 & 56 & & 16 & 46.6 & 48.9 & & \\
\hline I8 & 46.7 & $44 \cdot 7$ & & 24 & est & 18 & 51.0 & 50. I & & 16 & & I8 & 53.2 & 56.1 & 54 & at & 18 & 47.2 & 49.9 & & da \\
\hline 20 & 48.8 & 47.2 & & 20 & 8 & 20 & 50.9 & 49.3 & & 16 & - & 20 & 50.6 & 53.4 & 50 & 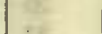 & 20 & 47.9 & 49.8 & 45 & ces \\
\hline 22 & 49.5 & 48.2 & & 19 & & 22 & 50.3 & 40.1 & & 17 & a & 22 & 49.2 & 52.2 & 48 & 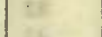 & 22 & $47 \cdot 3$ & 49.2 & 44 & 단 \\
\hline 24 & $42 \cdot .3$ & 47.8 & & I9 & 13 & 24 & $49 \cdot 3$ & 48. I & & I9 & 6 & 24 & 50.2 & 53.1 & 50 & 6 & 24 & 47.1 & 48.9 & 44 & $E$ \\
\hline 25 & 46.8 & $44 \cdot ?$ & & 23 & & 26 & 48.0 & 46.7 & & $2 \mathrm{I}$ & & 26 & 51.6 & 54.1 & 52 & $y^{2}$ & 26 & 47.8 & 49.3 & 45 & 8 \\
\hline 28 & 43.2 & 40.8 & & 29 & & 28 & 48.2 & 47.2 & & 20 & & 28 & 5 I.I & 53.3 & 5 I & & 28 & 47.8 & 40 & 45 & \\
\hline 30 & 40. I & 37.8 & & 34 & $-9 \cdot 3$ & 30 & 45.2 & 43.6 & & 26 & -7.8 & 30 & 51.9 & 53.8 & 52 & -6.4 & 30 & 47.0 & 48.4 & 44 & -4.8 \\
\hline 32 & 37.9 & 35.9 & & 37 & & 32 & 44.5 & 42.4 & & 27 & & 32 & 51.2 & 53.7 & $5 I$ & & 32 & 48.7 & 49.8 & 46 & \\
\hline 34 & 37.0 & 34.8 & & 39 & te & 34 & 44.7 & 43.7 & & 26 & ze & 34 & 52.2 & 54.3 & 52 & 18 & 34 & 47.7 & 48.7 & 44 & 5 \\
\hline 36 & 40.2 & 38.5 & & 34 & & 36 & 45.1 & 43.8 & & 26 & 8 & 36 & 51.7 & 53.7 & 52 & E & 36 & 45.7 & 47 & 42 & \\
\hline 38 & 37.1 & 36.1 & & 38 & 32 & 38 & 44.3 & 43.0 & & 27 & 4 & 38 & 52.9 & 54.6 & 53 & 2 & 38 & 45.0 & 46.1 & 40 & 6 \\
\hline 40 & 38.9 & 37.2 & & 36 & 5 & 40 & 50.3 & 48.9 & & I8 & 25 & 40 & 52.3 & 54.2 & 52 & s. & 4 & 46.6 & 47 & 42 & ta \\
\hline 42 & 37.9 & 37.0 & & 37 & . & 42 & $46 . I$ & 45.0 & & 24 & & 42 & 52.3 & 53.9 & 52 & 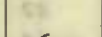 & 42 & 47.0 & 47.7 & 43 & \\
\hline 44 & 38.4 & 37.1 & & 36 & -9.2 & 44 & 47.1 & 46.2 & & 22 & -7.7 & 44 & 50.6 & 51.9 & 49 & -6.1 & 44 & 48.3 & 40.0 & 45 & -4.8 \\
\hline 46 & 36.6 & $35 . I$ & & 39 & & 46 & 52.0 & 51.2 & & I4 & & 46 & 40.8 & 52.0 & 49 & & 46 & 46.0 & 48 & 44 & \\
\hline 48 & 33.5 & 32.2 & & 44 & te & 48 & 54.0 & 53.2 & & II & & 48 & 48.9 & 51.5 & 48 & ine & 48 & 44.8 & 46.1 & 40 & (3) \\
\hline 50 & 34.8 & 33.2 & & 42 & 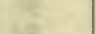 & 50 & 52.3 & 5 I.I & & I4 & ב & 50 & 49.3 & 51.8 & 48 & 0 & 50 & 43.2 & 45.0 & 38 & $\theta$ \\
\hline 52 & 37.8 & 37.2 & & 36 & 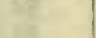 & 52 & 52.1 & 51.3 & & I4 & 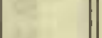 & 52 & 49.8 & $5 I . I$ & 48 & a & 5 & 4.3 .0 & 44.9 & 38 & EE \\
\hline 54 & $4 \mathrm{I} \cdot 2$ & 40.3 & & 3I & 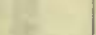 & 54 & 48.9 & 47.8 & & I9 & 3 & 54 & 47.3 & 49.7 & 45 & 6 & 54 & 48.2 & 49. & 46 & 2 \\
\hline 56 & 42.3 & $4 \mathrm{r} \cdot 3$ & & 30 & & 56 & 50.8 & 50.0 & & I6 & & 56 & 44.8 & 46.6 & 40 & c8 & 5 & 44.2 & 45 & 39 & $\cdot$ \\
\hline 58 & 38.7 & $3^{8.2}$ & & 35 & & 58 & 48.9 & 47.9 & & I9 & & 58 & 46.0 & 47.2 & 42 & 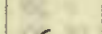 & 5 & 44.0 & 45.7 & 39 & \\
\hline 00 & 35.0 & 35.0 & & 40 & $-9 . I$ & 700 & $50 . \mathrm{I}$ & 49.0 & & I8 & $-7 \cdot 3$ & 900 & 52.1 & 5.3 .1 & $5 \mathrm{I}$ & -6.0 & II $\infty$ & 44.2 & 46.0 & 40 & -4.6 \\
\hline 02 & 32.3 & 32.0 & & 45 & - & 02 & 50.9 & 49.7 & & I6 & & 02 & $55 . \mathrm{I}$ & 57.2 & 57 & & 02 & 44. I & 47.1 & 40 & \\
\hline 04 & 32.0 & $3 \mathrm{I} .5$ & & 46 & & 04 & 47.8 & 47.1 & & 2 I & & 04 & 54.1 & 55.3 & 55 & e & 04 & 44.0 & 47.0 & 40 & 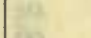 \\
\hline 06 & 34.8 & 34.1 & & $4 \mathrm{I}$ & & 06 & 5I.I & 50. I & & I6 & 2 & 06 & 49.0 & 50.8 & 46 & 58 & o & 44.3 & 47.0 & 40 & (5) \\
\hline os & 37.8 & 37.4 & & 36 & a & 08 & 52.2 & 51.8 & & 14 & - & 08 & $47 . \mathrm{I}$ & 48.8 & 44 & 60 & 08 & 43.9 & & 40 & $\omega$ \\
\hline Io & 38.0 & 37.7 & & 36 & . & I0 & 51.9 & 51.9 & & 14 & & 10 & 50. I & 52.0 & 49 & 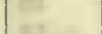 & 10 & 44. & 46 & 40 & 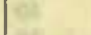 \\
\hline I2 & 37.3 & 37.0 & & 37 & 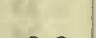 & 12 & 55.3 & 55.1 & & 09 & & 12 & 51.6 & 52.1 & 50 & & 12 & 44.6 & 46 & 40 & \\
\hline I4 & 35.6 & 34.6 & & 40 & -9.0 & 14 & 53.8 & 53.2 & & II & -7.2 & 14 & 51.0 & 52.1 & 50 & -5.9 & I4 & 43.8 & 46.2 & 39 & $-4 \cdot 3$ \\
\hline I6 & 32.4 & $3 \mathrm{I} .3$ & & 45 & & 16 & 56.1 & 55.7 & & 08 & & 16 & 48.3 & 49.3 & 45 & 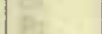 & 16 & 44.2 & 46.7 & 40 & \\
\hline I8 & $3 I .2$ & 30.1 & & 47 & e. & 18 & $53 \cdot 3$ & 52.9 & & I2 & = & 18 & 48.2 & 49. & 45 & 11 & โ8 & 44. & 46.4 & 40 & pis \\
\hline 20 & 28.9 & 27.9 & & $5 \mathrm{I}$ & 5 & 20 & 54.3 & 54.0 & & IO & ar & 20 & 48.6 & 49.4 & 46 & & 20 & 44.2 & 46.3 & 40 & at \\
\hline 22 & 27.2 & 26.6 & & 5.3 & tes & 22 & 58.3 & 57.6 & & 04 & 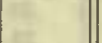 & 22 & 48.3 & 49.2 & 45 & . & 22 & 44. I & & 40 & $=$ \\
\hline 24 & 32.0 & 30.6 & & 46 & 5 & 24 & 57.8 & 57.3 & & 05 & E. & 24 & $49 . \mathrm{T}$ & 50.1 & 47 & C & 24 & 44.0 & 46.2 & 40 & E \\
\hline 26 & $35 \cdot 3$ & 34.6 & & 40 & & 26 & 57.0 & 56.2 & & 06 & & 26 & 49.8 & 50. & 47 & $E$ & & 8 & & 38 & $=$ \\
\hline 28 & 37.0 & 35.7 & & 38 & 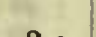 & 28 & 59.1 & 58.7 & & 03 & & 28 & $40 . I$ & 49.9 & 46 & 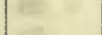 & 28 & 43.2 & 45.6 & 38 & \\
\hline 30 & 37.8 & 37.0 & & 37 & -8.9 & 30 & 60.8 & 59.9 & & or & -7.0 & 30 & 48.8 & 50. I & 46 & -5.7 & 30 & $43 \cdot 3$ & 45.4 & 38 & $-4 \cdot 2$ \\
\hline 32 & 36.3 & 35.3 & & 39 & & 32 & 50.9 & 59.2 & & 02 & & 32 & 49.1 & 50.0 & 46 & & 32 & 43.0 & 45.0 & 38 & 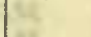 \\
\hline 34 & 38.2 & 37.7 & & 36 & te & 34 & 58.2 & $57 . \mathrm{I}$ & & 05 & 3 & 34 & 40.5 & 50.9 & 48 & (5). & & 43. I & 45 & 38 & 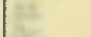 \\
\hline 36 & 40.3 & 39.9 & & 32 & $z$ & 36 & 60.0 & 59.3 & 23 & 02 & & .36 & 48.3 & 50.2 & 46 & 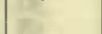 & 36 & 43.2 & 44.0 & 38 & E \\
\hline 38 & 40.2 & 39.9 & & 32 & & 38 & $6 \mathrm{r} \cdot 3$ & 60.9 & 22 & 59 & 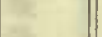 & 38 & 49.0 & 52.0 & 48 & 10 & 38 & 43.2 & 44.8 & 38 & 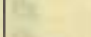 \\
\hline 40 & 42.1 & $4 \mathrm{I} . \mathrm{I}$ & & 30 & E. & 40 & 63.2 & 63.0 & & 56 & 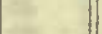 & 40 & 5 I. 0 & 52.8 & 50 & $=$ & 4 & 43.3 & 44.7 & 38 & ct \\
\hline 42 & $37 . I$ & 36.2 & & 38 & & 42 & 64.1 & 63.7 & & 55 & & 42 & 48.9 & 49.8 & 46 & 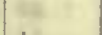 & 42 & 43.0 & 44.0 & 37 & -4 \\
\hline 44 & 34.5 & $34 . I$ & & 4I & -8.6 & 44 & 66.9 & 65.1 & & 52 & -6.8 & 44 & 48.0 & 50.1 & 46 & $-5 \cdot 3$ & 44 & 43.0 & 43.9 & 37 & -4.0 \\
\hline 46 & 37.7 & 37.2 & & 37 & & 46 & 74.1 & 73.2 & & 40 & & 46 & 47.2 & 49.3 & 44 & & $4^{6}$ & 43.1 & 44.1 & 37 & \\
\hline 48 & 43.9 & 42.3 & & 28 & cos & 48 & 77.0 & 76.8 & & 35 & - & 48 & 47.9 & 49.6 & 45 & 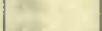 & $4^{8}$ & $43 . I$ & 43.9 & 37 & 3 \\
\hline 50 & $47 \cdot 4$ & 46.6 & & 22 & & 50 & 72. & & 22 & 42 & 5 & 50 & 48.5 & 50.4 & 46 & 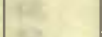 & 50 & 42.7 & 43.9 & 37 & $=$ \\
\hline 52.2 & 40.4 & 39.4 & & 33 & 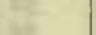 & 52 & 59.8 & $6 b$ & 23 & 02 & & 52 & $48 . I$ & 50.7 & 46 & 12 & 52 & 43.0 & 44.0 & 37 & 2 \\
\hline 54 & 48.0 & 47.8 & & I9 & 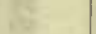 & 54 & 48.8 & $8 b$ & & 19 & $x^{2}$ & 54 & 48.1 & 50.8 & 46 & E. & 54 & 42.7 & $43 . I$ & 36 & \\
\hline 56 & 50.8 & 49.8 & & I6 & & 56 & 56.3 & & & 07 & & 56 & 48. I & 50.7 & 46 & & 56 & 42.1 & 43.I & 36 & 2 \\
\hline 58 & 50.5 & 49. I & & 17 & & 58 & 61.0 & 60.1 & 23 & $\infty$ & & 58 & 47.6 & 49.9 & 45 & & 58 & 42.2 & $43 \cdot 3$ & 36 & \\
\hline & & & & & & $8 \infty$ & 62.9 & 61.7 & 22 & 57 & -6.3 & & & & & & 1200 & 42.3 & 43.2 & 36 & -4.0 \\
\hline
\end{tabular}

Correction to local mean time is $+235.90^{\circ}$ torsion $=17 .^{\prime} 58$. Torsion head at oh oom rcad $67^{\circ}$ and at $8 \mathrm{~h} 15 \mathrm{~m}$ read $62^{\circ}$. Observer-R. R. T.
Correction to local mean time is $+4 s$.

Torsion head at $7 \mathrm{~h} 30 \mathrm{~m}$ read $62^{\circ}$ and at $12 \mathrm{~h} 15 \mathrm{~m}$ read the same. Observer-R. R. T. 
Tabulation of magnetic declinations obscrved at Teplitz Bay-Continued

\begin{tabular}{|c|c|c|c|c|c|c|c|c|c|c|c|c|c|c|c|c|c|c|c|c|}
\hline \multicolumn{6}{|c|}{ Tuesday, May Io, 1904} & \multicolumn{5}{|c|}{ Magnet scale inverted } & \multicolumn{6}{|c|}{ Wednesday, May Ir, 1904} & \multicolumn{4}{|c|}{ Magnet scale erect } \\
\hline \multirow{2}{*}{$\begin{array}{l}\text { Chr'r } \\
\text { time }\end{array}$} & \multicolumn{2}{|c|}{$\begin{array}{l}\text { Scale } \\
\text { readings }\end{array}$} & $\begin{array}{l}\text { East } \\
\text { decli- } \\
\text { nation }\end{array}$ & Ternp. & Chr'r & $\begin{array}{r}\mathrm{Sc} \\
\text { read }\end{array}$ & $\begin{array}{l}\text { ale } \\
\text { lings }\end{array}$ & $\begin{array}{l}\text { Eas } \\
\text { decl }\end{array}$ & & Temp. & Chr'r & $\begin{array}{r}\mathrm{Sc} \\
\text { read }\end{array}$ & $\begin{array}{l}\text { ale } \\
\text { lings }\end{array}$ & East & Temp. & Chr'r & $\begin{array}{r}\mathrm{Sc} \\
\text { read }\end{array}$ & $\begin{array}{l}\text { ale } \\
\text { lings }\end{array}$ & East & Temp. \\
\hline & L,eft & Right & & & & Left & Right & natio & & & & Left & Right & ation & & & Left & Right & & \\
\hline $1 \mathrm{~m}$ & $d$ & d] & $\cdot \quad$ & $\bullet$ & $\mathrm{h} \mathrm{m}$ & d & d & $\cdot$ & & & h $\mathrm{m}$ & d & d & & 3 ing & $\mathrm{h} \mathrm{m}$ & $\alpha$ & (I) & & 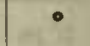 \\
\hline 1200 & 54.7 & 52.7 & 22.36 & -3.9 & 1400 & 67.2 & 66.7 & 22 & 16 & -2.8 & $000 *$ & 50.2 & 51.0 & 2238 & $-4 \cdot 5$ & 200 & 55.1 & 55.8 & 2246 & -5.0 \\
\hline 02 & 55.9 & 55.0 & 34 & (1) & 02 & 67.0 & 66.2 & & 16 & wan & 02 & 50.1 & 50.9 & 38 & 60 & 02 & 56.1 & 56.6 & & \\
\hline 34 & 58.6 & .57 .4 & 30 & $i^{2}$ & 04 & 67.0 & 66.2 & 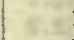 & 15 & a: & 04 & 50.0 & 50.5 & 38 & 30 & 04 & 56.8 & 57.1 & 48 & \\
\hline 06 & 57.9 & 56.2 & $3 \mathrm{I}$ & ta & 06 & 67.8 & 67.1 & & 15 & & 06 & 49.8 & 50.3 & & 86. & 06 & 58.1 & 58.3 & 50 & \\
\hline os & 55.4 & 54.0 & 35 & hat & 08 & 66.7 & 55.9 & & 17 & 2 & 08 & 49.7 & 50.2 & 37 & $x_{n}$ & 08 & 58.7 & 59.0 & $5 I$ & \\
\hline 10 & 56.6 & 55.2 & 33 & tat & 10 & 66.8 & 66.1 & & 17 & $3 x$ & Io & 49.8 & 50.2 & 38 & int & IO & 58.9 & 59.1 & 52 & \\
\hline I2 & 56.8 & $55 . I$ & 33 & & I2 & 66.1 & 65.2 & & 18 & & 12 & 49.9 & 50.3 & 38 & $t 8$ & I2 & 59.9 & 60.1 & 53 & \\
\hline 14 & 55.3 & 53.8 & 35 & -3.9 & 14 & 69.9 & 69.0 & & 12 & -2.6 & 14 & 50.2 & 50.8 & 38 & -4.4 & 14 & 60.2 & 60.5 & 54 & -5.0 \\
\hline I6 & 53.2 & 52.0 & 38 & & 16 & 69.7 & 69.1 & & 12 & 3ren & 16 & 50.5 & 50.8 & 38 & & I6 & 60.0 & 60.3 & 53 & \\
\hline 18 & 54.1 & 53.0 & 37 & 51 & I8 & 70.0 & 69.2 & & 12 & 8 & 18 & 51.0 & 51.2 & 39 & 47 & I8 & 60.5 & 60.8 & 54 & \\
\hline 20 & 55.0 & 53.1 & 36 & $x^{2}$ & 20 & 70.6 & 70.3 & & Io & $\theta^{2}$ & 20 & 51.0 & 51.2 & 39 & ise & 20 & 59.3 & 60.0 & 53 & \\
\hline 22 & 55.0 & 53.1 & 36 & tis & 22 & 72.0 & 72.0 & & 08 & is & 22 & $5 \mathrm{I} . \mathrm{I}$ & $5 \mathrm{~T} .5$ & 40 & s. & 22 & 60.7 & 61.0 & 54 & \\
\hline 24 & 54.9 & 53.0 & 36 & $y$ & 24 & 71.2 & 71.0 & & $\infty 9$ & in & 24 & 50.9 & 51.2 & 39 & it: & 24 & 6I.I & 61.2 & 55 & \\
\hline 26 & 54.7 & $53 . I$ & 36 & 5 & 26 & 70.9 & 70.3 & & 10 & 4 & 26 & 51.6 & 52.0 & 40 & E & 26 & 60.3 & бт.0 & 54 & \\
\hline 28 & 55.6 & 54.0 & 35 & & 28 & 72.0 & 71.0 & & 08 & & 28 & 52.0 & 52.6 & $4 \mathrm{~T}$ & & 28 & 60.0 & 60.2 & 53 & \\
\hline 30 & 56.7 & 55.4 & 33 & -3.8 & 30 & 73.7 & 73.1 & & 06 & -2.3 & 30 & 52.0 & 52.6 & $4 I$ & -4.6 & 30 & 60.2 & $6 \pi .0$ & 54 & $-5 . I$ \\
\hline 32 & 56.4 & 55.2 & 33 & s. & $32 . I$ & 73.7 & 73.1 & & 06 & - & 32 & 52.2 & 52.8 & $4 \mathrm{I}$ & 40 & 32 & 60.8 & 61.2 & 55 & \\
\hline 34 & 56.1 & 55.0 & 34 & th & 34 & 72.9 & 72.5 & & 07 & & .34 & 52.0 & 52.3 & $4 \pi$ & & 34 & 60.7 & 6I.I & 55 & \\
\hline 36 & 56.8 & 55.9 & 32 & m & 36 & 70.7 & 69.9 & & 10 & 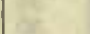 & 36 & 52.8 & 53.1 & 42 & 28 & 36 & $6 r \cdot 4$ & 62.0 & 56 & \\
\hline 38 & 58.0 & 57.5 & 30 & 3 & 38 & 72.0 & 71.0 & & 08 & & 38 & 53.0 & 5.3 .2 & 42 & 30 & 38 & 62.8 & 6.3 .1 & 58 & \\
\hline so & 58.3 & 57.9 & 30 & 28 & 40 & 72.1 & 71.0 & & 08 & $=$ & 40 & 52.9 & 53.4 & 42 & 8 & 40 & 62.3 & 62.9 & 57 & \\
\hline 42 & 58.5 & 58.0 & 29 & & 42 & 73.1 & 72.7 & & 06 & & 42 & 53.0 & 53.7 & 43 & & 42 & 62.4 & 63.0 & 58 & \\
\hline 44 & 59.2 & 58.6 & 28 & -3.8 & 44 & 74.0 & 73.5 & & 04 & $-2 . \mathrm{I}$ & 44 & 52.9 & 53.5 & 42 & -4.8 & 44 & 62.9 & 63.1 & 58 & $-5 . I$ \\
\hline 46 & 59.1 & 58.0 & 29 & . & 46 & 74.8 & 73.3 & & 0.5 & . & 46 & 53.2 & 53.9 & 43 & (7) & 46 & 63.0 & 63.1 & 58 & \\
\hline 48 & 58.7 & 57.7 & 29 & Hita & 48 & 76.2 & 74.8 & & 02 & 상 & 48 & 54.7 & 55.0 & 45 & B. & 48 & 63.1 & $63 \cdot 3$ & 58 & \\
\hline 50 & $59 \cdot 3$ & 58.1 & 20 & W. & 50 & 77.3 & 76.0 & & oI & 8 & 50 & 55.0 & 55.7 & 46 & 9 & 50 & 63.0 & 63.1 & 58 & \\
\hline 52 & 59.4 & 58.3 & 28 & 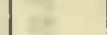 & 52 & 77.9 & 76.4 & & $\infty$ & E. & 52 & 55.0 & 55.7 & 46 & $e^{2}$ & 52 & 63.1 & 63.3 & 58 & \\
\hline 54 & 59.6 & 58.7 & 28 & Wt & 54 & 77.1 & 76.0 & 22 & $\infty$ & is & 54 & 55.1 & 55.3 & 46 & iv & 54 & 63.2 & 63.8 & 59 & \\
\hline 56 & 59.3 & 58.2 & 29 & & 56 & 78.5 & 77.4 & 21 & 58 & $x^{2}$ & 56 & 54.8 & 55.0 & 45 & & 56 & 63.5 & 63.7 & 50 & \\
\hline 58 & 60.2 & 59.3 & 27 & & 58 & 76.8 & 75.9 & 22 & or & & 58 & 54.8 & 55.0 & 45 & & 58 & 63.0 & 63.1 & 58 & \\
\hline 1300 & 60.7 & 60.2 & 26 & -3.5 & 1500 & 74.0 & 73.1 & & 05 & -2.0 & I 00 & 54.0 & 54.2 & 44 & -7.9 & 300 & 62.3 & 62.8 & 57 & $-5 . I$ \\
\hline 02 & 60.1 & 59.2 & 27 & tis & 02 & 74.0 & 73.1 & & 05 & 58 & 02 & 53.9 & 53.9 & 44 & 60 & 02 & 61.7 & $6 \mathrm{r} .8$ & 56 & \\
\hline 04 & 60.9 & 60.0 & 26 & s. & 24 & 75.8 & 73.2 & & 04 & $x^{2}$ & 04 & 53. I & 53.5 & 43 & to & 04 & 61.0 & $6 \mathrm{I} . \mathrm{I}$ & 55 & \\
\hline 06 & $6 \mathrm{r} .7$ & 61.0 & 24 & $x^{2}$ & 06 & 76.3 & 75.0 & & 02 & 85 & 06 & 52.9 & 53.2 & 42 & 18 & 06 & 60.9 & 61.0 & 55 & \\
\hline ns & 63.1 & $62 . I$ & 22 & 18 & 08 & 78.0 & 73.0 & & 02 & 0 & 08 & 52.7 & 53.0 & 42 & As. & 08 & 59.9 & 60.1 & 53 & \\
\hline IO & 61.5 & 60.9 & 25 & wet & Io & 78.1 & 76.4 & & $\infty$ & & Io & 52.5 & 5.3 .0 & 42 & ar & 10 & 59.3 & 50.7 & 52 & \\
\hline 12 & 60.3 & 59.8 & 27 & & I2 & 75.3 & 75.0 & & 03 & & 12 & 52.8 & 53.0 & 42 & & 12 & 58.3 & 58.9 & $5 I$ & \\
\hline I4 & $6.3 . I$ & 62.3 & 22 & -3.2 & I4 & 73.1 & 72.2 & & 07 & -1.9 & I4 & 52.8 & 53.1 & 42 & -5.0 & 14 & 58. I & 58.8 & $5 I$ & -5.1 \\
\hline I6 & 64.0 & $63 . \mathrm{I}$ & 21 & 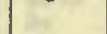 & I6́ & 72.0 & 71.7 & & 08 & 42. & 16 & 52.0 & 53.0 & $4 \mathrm{~T}$ & & 16 & 59.0 & 59.6 & 52 & \\
\hline I8 & 63.6 & 6.3 .1 & 21 & & I8 & 71.2 & 71.0 & & 09 & te & I8 & 51.7 & 52.2 & 40 & 35 & I8 & 59.0 & 59.2 & 52 & \\
\hline 20 & 62.0 & $6 \mathrm{r} . \mathrm{I}$ & 24 & $t=$ & 20 & 70.0 & 70.0 & & II & nets & 20 & 50.0 & 5ा.8 & 10 & $\mathrm{r}$ & 20 & 59.8 & 60.0 & .53 & \\
\hline 22 & 64.6 & 63.8 & 20 & & 22 & 68.8 & 68.3 & & 13 & $=$ & 22 & 50.8 & 51.4 & 39 & se & 22 & 60.4 & 60.7 & 54 & \\
\hline 24 & 65.2 & 64.8 & I9 & we & 24 & 67.8 & 67.8 & & 14 & in & 24 & 5I.I & 51.7 & 40 & 18 & 24 & 60.2 & 60.5 & 54 & \\
\hline 26 & 64.0 & 64.2 & 20 & 20 & 26 & 66.8 & 66.3 & & I6 & & 26 & 52.7 & 53.0 & 42 & s. & 26 & 58.0 & 58.2 & 50 & \\
\hline 28 & 62.8 & 62.1 & 23 & & 28 & 65.9 & 65.3 & & I8 & & 28 & 51.9 & 52.2 & $4 \mathrm{I}$ & & 28 & 57.9 & 58.0 & 50 & \\
\hline 30 & 62.0 & 61.5 & 24 & -3.0 & 30 & 63.3 & 63.2 & & 22 & -1.8 & 30 & 52.2 & 52.8 & 1.1 & -5.0 & 30 & 57.9 & 57.9 & 50 & -5.0 \\
\hline 32.2 & 64.6 & 63.8 & 20 & & 32 & 63.8 & 63.6 & & $2 \mathrm{I}$ & & 32 & 52.8 & 53.0 & 42 & & 32 & 57.9 & 58.3 & 50 & \\
\hline .34 & 64.0 & 63.6 & $2 \mathrm{I}$ & 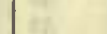 & 34 & 64.6 & 64.2 & & 20 & $y$ & 34 & 52.5 & 52.8 & 42 & & 34 & 58.0 & 58.9 & $5 \mathrm{I}$ & \\
\hline 36 & 64.6 & 64.0 & 20 & & 36 & 64.0 & 64.0 & & 20 & A & 36 & 52.2 & 52.8 & $4 \mathrm{I}$ & & 36 & 58.9 & 59.8 & 52 & \\
\hline 38 & 64.7 & 64.1 & 20 & 5 & 38 & 63.8 & 63.2 & & $2 I$ & ty & 38 & 52.2 & 52.8 & $4 \mathrm{I}$ & Es & 38 & 50.2 & 60.1 & 53 & \\
\hline 40 & 63.0 & 62.1 & 23 & & 40.2 & 63.3 & 63.1 & & 22 & & 40 & 52.2 & 52.8 & $4 \mathrm{I}$ & & 40 & 58.8 & 59.6 & 52 & \\
\hline 42 & 64.3 & 63.5 & $2 \mathrm{I}$ & & 42 & 63.0 & 62.8 & & 22 & 5 & 42 & 52.5 & 52.9 & 42 & & 42 & 58.8 & 59.2 & 52 & \\
\hline 14 & 66.8 & 65.8 & I7 & -29 & 44 & 63.8 & 63.6 & & $2 I$ & $-\tau .7$ & & 52.9 & 52.9 & 42 & -5.0 & 44 & $58 . I$ & 58.8 & $5 \mathrm{I}$ & -4.8 \\
\hline 46 & 64.8 & 63.9 & 20 & & 46 & 63.1 & 62.7 & & 22 & & 46 & 5 T. 9 & $52 . \mathrm{I}$ & $4 \mathrm{I}$ & & 46 & 57.8 & 58.1 & 50 & \\
\hline 48 & 63.0 & 62.0 & 23 & 5 & 48 & 63.3 & 62.8 & & 22 & 5 & 48 & 52.0 & 52.1 & .11 & & 48 & 58.2 & 59.0 & 51 & \\
\hline 50 & 64.8 & 63.5 & 20 & $E$ & 50 & 63.1 & 62.4 & & 22 & 2 & 50 & 52.1 & $5,2.5$ & $4 I$ & & 50 & 59.7 & 60.0 & 53 & \\
\hline 52 & 66.1 & 64.5 & 18 & 5 & 52 & 63.0 & 62.3 & & 22 & 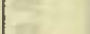 & 52 & 53.0 & 53.2 & 42 & & 52 & 60.5 & 60.8 & 54 & \\
\hline 54 & 64.5 & 63.3 & $2 \mathrm{I}$ & & 54 & 62.2 & 51.9 & & 23 & $y$ & 54 & 53.0 & 53.3 & 42 & 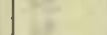 & 54 & 61.1 & 61.6 & 55 & \\
\hline 56 & 65.2 & 64.0 & 19 & & 56 & 62.8 & 62.2 & & 23 & & 56 & 53.8 & 53.9 & 44 & 8 & 56 & 6I.I & $6 \pi .4$ & 55 & \\
\hline 58 & 67.0 & 66.0 & T6́ & & 58 & 62.7 & 62.1 & & 23 & & 58 & 55.1 & 55.2 & 46 & & 58 & 60.8 & 60.9 & & \\
\hline & & is & 1. $2=1$ & or 87 & 1600 & 62.7 & 61.5 & & 23 & $-I .5$ & & 18 & $\cos 18$ & 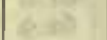 & 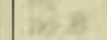 & & & & & \\
\hline
\end{tabular}

Correction to local mean time is -31.5 s. $90^{\circ}$ torsion $=16 . ' 99$.

Torsion head at $\mathrm{Ih} 35 \mathrm{~m}$ read $74^{\circ}$ and at $16 \mathrm{~h} 15 \mathrm{~m}$ read $67^{\circ}$.

Observer-J. V.

Observer-R. R. T. 
Tabulation of magnetic declinations observed at Teplitz Bay-Continued

\begin{tabular}{|c|c|c|c|c|c|c|c|c|c|c|c|c|c|c|c|c|c|c|c|}
\hline \multicolumn{6}{|c|}{ Wednesday, May II, I904 } & \multicolumn{4}{|c|}{ Magnet scale erect } & \multicolumn{6}{|c|}{ Weduesday, May II, 1904} & \multicolumn{4}{|c|}{ Magnet scale erect } \\
\hline \multirow{2}{*}{$\begin{array}{l}\text { Chr'r } \\
\text { time }\end{array}$} & \multicolumn{2}{|c|}{$\begin{array}{l}\text { Scale } \\
\text { readings }\end{array}$} & \multirow{2}{*}{$\begin{array}{c}\text { East } \\
\text { decli- } \\
\text { nation }\end{array}$} & Temp. & Chr'r & $\begin{array}{l}\text { Sci } \\
\text { read }\end{array}$ & $\begin{array}{l}\text { ale } \\
\text { ings }\end{array}$ & $\begin{array}{l}\text { East } \\
\text { decli- }\end{array}$ & Temp. & Chrr'r & $\begin{aligned} \text { Sc } \\
\text { read }\end{aligned}$ & ale & $\begin{array}{l}\text { East } \\
\text { decli- }\end{array}$ & Temp. & Chr'r & $\begin{array}{l}\text { Sce } \\
\text { read }\end{array}$ & $\begin{array}{l}\text { ale } \\
\text { lings }\end{array}$ & $\begin{array}{l}\text { East } \\
\text { decli- }\end{array}$ & Temp. \\
\hline & L,eft & Right & & & time & Left & Right & & C. & time & Left & Right & nation & C. & time & Left & Right & nation & C. \\
\hline $\mathrm{h} \mathrm{m}$ & $d$ & $d$ & - & $\circ$ & $1 \mathrm{~m}$ & d & d & . & • & $\mathrm{l} \mathrm{m}$ & d & d & & - & $\mathrm{h} \mathrm{m}$ & $\mathrm{d}$ & $d$ & $\circ$, & • \\
\hline 400 & 61.1 & $6 \mathrm{I} .2$ & 2255 & $-4 \cdot 4$ & 600 & $32 . \mathrm{I}$ & 33.0 & 2303 & $-4 \cdot 7$ & 800 & 22.7 & 23.5 & 2248 & -2.9 & 1000 & 16.6 & 17.0 & 2239 & -2.5 \\
\hline 02 & 62.2 & 62.3 & 57 & $\operatorname{tas}$ & 02 & 32.5 & 33.6 & 04 & & 02 & 21.5 & 22.2 & 46 & - & 02 & 17.9 & 18.3 & 41 & - \\
\hline 04 & 63.1 & 63.8 & 50 & 5 & 04 & 34.6 & 35.7 & 07 & & 0.4 & 22.0 & 22.3 & 47 & 6 & 04 & 17.4 & 17.6 & 40 & 68 \\
\hline 06 & $63 \cdot 3$ & 63.8 & 2259 & ay & 06 & $33 \cdot 3$ & 34.8 & 06 & & $\infty$ & 22.8 & 23.0 & 48 & $=$ & 06 & 14.4 & 14.6 & 35 & 20 \\
\hline os & 64.9 & $0_{5} .1$ & 2301 & 연 & as & 33.0 & 34.0 & 05 & 17 & 08 & 22.0 & 22.5 & 47 & $m$ & a8 & II.8 & 12.0 & $3 I$ & 68 \\
\hline IO & 65.9 & 66.0 & 0.2 & aㅏ. & I0 & 30.3 & 31.0 & 2300 & 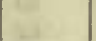 & IO & 22.8 & 22.8 & 48 & Ley & 10 & I3. 3 & 13.4 & 33 & $w$ \\
\hline 12 & 64.2 & 64.8 & 2300 & t5. & 12 & 27.9 & 29.0 & 2257 & & I2 & 22.2 & 22.2 & 47 & & 12 & 16.3 & 16.4 & 38 & \\
\hline 14 & 62.0 & 62.0 & 2256 & -4.2 & 14 & 29.9 & 30.9 & 60 & -4.8 & 14 & 22.8 & 23.0 & 48 & -2.5 & 14 & 15.6 & 16.3 & 37 & -2.7 \\
\hline 16 & 61.9 & 62.0 & 56 & 4 & I6 & 27.8 & 29.0 & 57 & 35 & IO & 23.4 & 23.6 & 49 & $y^{2}$ & 16 & 16.7 & 18.0 & 39 & 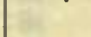 \\
\hline 18 & 63.3 & 64.0 & 59 & ar & I8 & 27.4 & 28.3 & 56 & - & 18 & 23.1 & 23.6 & 49 & ct & 18 & 18.0 & 18.6 & 41 & 6 \\
\hline 20 & 63.3 & 63.9 & 59 & is & 20 & 28.3 & 29.2 & 57 & tat & 20 & 22.0 & 22.2 & 47 & 64 & 20 & 17.6 & 18.8 & $4 \mathrm{I}$ & int \\
\hline 22 & 62.1 & 62.9 & 57 & Is. & 22 & 28.4 & 29.2 & 57 & 난 & 22 & 21.2 & 21.2 & 46 & 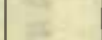 & 22 & 17.3 & 18.7 & 40 & 18 \\
\hline 24 & 61.8 & 62.0 & 56 & $=$ & 24 & 27.3 & 28.5 & 56 & 6 & 24 & 21.8 & 22.2 & 47 & E & 24 & 17.9 & 19.0 & $4^{1} I$ & tat \\
\hline 25 & 63.0 & 63.2 & 2258 & tas & 26 & 26.3 & 27.2 & 54 & 망 & 26 & 22.3 & 22.6 & 47 & $E$ & 26 & 18.5 & 19.6 & 42 & E \\
\hline 28.3 & 67.1 & 67.8 & 2305 & net & 28 & 27.0 & 27.9 & 55 & & 28 & 22.3 & 22.6 & 47 & & 28 & 19.5 & 20.6 & 44 & \\
\hline 30 & 69.8 & 70.0 & 09 & $-4 \cdot I$ & 30 & 28.3 & 29.0 & 57 & -4.8 & 30 & 22.6 & 23.0 & 48 & -2.0 & 30 & I8.8 & I9.8 & 42 & -3.0 \\
\hline 32 & 68.9 & $69 . I$ & 07 & & 32 & 26.3 & 27.1 & 54 & $\%$ & 32 & 23.0 & 23.6 & 49 & & 32 & 18.5 & 19.7 & 42 & . \\
\hline 34 & 69.6 & 69.9 & 08 & W & 34 & $26 . \mathrm{I}$ & 27.0 & 54 & & 34 & 22.3 & $23 \cdot 3$ & 48 & & 34 & 18.6 & 19.6 & 42 & 6 \\
\hline 36 & 64.6 & 64.8 & or & 30 & 36 & 25.5 & 26. I & 53 & a & 35 & 22.3 & 23.5 & 48 & te & 36 & 18.3 & 20.0 & 42 & 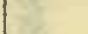 \\
\hline 38 & YI & $.0 a$ & 10 & 28. & 38 & 26.8 & 27.2 & 55 & cis & 38 & 21.8 & 23.6 & 48 & 6 & 38 & I8.5 & 19.6 & 42 & 08 \\
\hline 40 & 76.2 & 76.8 & 19 & 4 & 40 & 28.0 & 28.8 & 57 & 9 & 40 & 21.0 & 22.8 & 47 & 6 & 40 & 19.0 & 20.4 & 43 & 6 \\
\hline 42 & 68.9 & 70.0 & os & 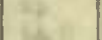 & 42 & 27.8 & 28. I & 56 & & 42 & 20.6 & 22.6 & 46 & & 42 & I8.8 & 20.6 & 43 & \\
\hline 44 & 65.7 & 66.1 & 02 & -4.1 & 44 & 26.8 & 27.0 & 54 & -4.7 & 44 & 22.2 & 22.6 & 47 & & 44 & 18.3 & I9. 4 & 42 & -3.2 \\
\hline 46 & 70.1 & 71.0 & I0 & . & 46 & 20.2 & 26.8 & 54 & (2) & 46 & 20.5 & 21.8 & 45 & -2.0 & 46 & 16.5 & I8. I & 39 & (2) \\
\hline 48 & $7 \mathrm{I} .4$ & 72.1 & 12 & 8 & 48 & 26.0 & 26.3 & 53 & (4) & 48 & 18.8 & 20.4 & 43 & be: & 48 & I5.3 & 17.3 & 38 & t \\
\hline 50. & 74.0 & 75.4 & 16 & wa & 50 & 24.8 & 25.0 & $5 \mathrm{I}$ & & 50 & I9.5 & 20.6 & 44 & $E^{2}$ & 50 & 14.9 & 17.4 & 38 & 2 \\
\hline $52^{*}$ & 43.0 & 48.1 & 24 & 3 & 52 & $24 . I$ & 24.2 & 50 & 3 & .52 & 20.0 & 20.9 & 44 & 2 & 52 & 13.8 & I5.6 & 35 & w. \\
\hline 54 & 47.6 & $5 \mathrm{I} \cdot 3$ & 30 & $a^{2}$ & 54 & 25.0 & $25 \cdot 3$ & 52 & & 54 & I9. 3 & 20.0 & 43 & & 54 & 13.6 & 15.6 & 35 & 20 \\
\hline 56 & $5 \mathrm{I} . \mathrm{O}$ & 56.3 & 36 & $=$ & 56 & 25.8 & 26.3 & 53 & & 56 & 20.3 & 21.0 & 44 & & 56 & 12.8 & 14.5 & 34 & \\
\hline 58 & 48.9 & 52.2 & 32 & 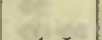 & 58 & 25.3 & 26.0 & 52 & & $5^{8}$ & 23.0 & 23.8 & 49 & & 58 & II.8 & 13.8 & 32 & \\
\hline 500 & 43.8 & 47.6 & 24 & -4.1 & 70 & 24.2 & 25.0 & 51 & -4.2 & 900 & 21.0 & 22.0 & 46 & -2.0 & II 00 & II.0 & 13.0 & $3 \mathrm{I}$ & $-3 \cdot 3$ \\
\hline 02 & 39.8 & 43.4 & 18 & & 02 & 25.6 & 26.0 & 53 & in & 02 & 18.5 & I 8.8 & $4 \mathrm{I}$ & - & 02 & I1.6 & 13.0 & 32 & . \\
\hline of & 35.0 & 38.0 & I0 & tet & 04 & 25.0 & 25.3 & 52 & 15. & 04 & 23 & $3 a$ & 2249 & 25 & $\mathrm{C}_{4}$ & II.7 & 12.3 & $3 \mathrm{r}$ & 28 \\
\hline 05 & 32.0 & 34.0 & 04 & 8 & 05 & 24.9 & 25.1 & 5I & & 05 & 32.0 & 32.2 & 2303 & $\omega_{2}$ & 06 & 11.9 & 12.8 & 32 & 80 \\
\hline os & 29.2 & $3 \mathrm{I} .7$ & 2300 & no & 08 & 25.0 & 26.5 & 53 & & os & 21.9 & 23.3 & 2248 & 2 & 08 & t2. 6 & 13.1 & 32 & 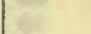 \\
\hline 10 & 28.8 & 30.4 & 2259 & re & I0 & 24.4 & $25 \cdot 3$ & $5 \mathrm{I}$ & & 10 & 14.2 & 1.5 .8 & 36 & 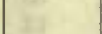 & Io & I3.5 & $\pi 4.5$ & 34 & tra \\
\hline I2 & 30.1 & 32.2 & $23 \mathrm{OI}$ & 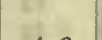 & 12 & 25.0 & 25.7 & 52 & & 12 & 12.8 & I3.0 & 32 & & 12 & I2. 8 & 13.0 & 32 & \\
\hline$I_{4}$ & 30.9 & 32.9 & 02 & -4.2 & 14 & 24.8 & 25.3 & $5 \mathrm{I}$ & -3.8 & I4 & 22.0 & 22.0 & 47 & -2.0 & 14 & 13.3 & 14.0 & 34 & $-3 \cdot 3$ \\
\hline I6 & 33.8 & 35.8 & 07 & t5. & 16 & 24.8 & 25.2 & 51 & - & 16 & 24.9 & 26.1 & 52 & the & 16 & 14.0 & 14.2 & 34 & $6 y^{2}$ \\
\hline I8 & 32.0 & 33.3 & 03 & E. & 18 & 24.9 & 25.5 & 52 & (1) & 18 & 16.7 & 18.0 & 39 & & 18 & 14.0 & 14.3 & 34 & 68 \\
\hline 20 & 31.2 & 33.0 & 03 & is & 20 & 24.8 & 25.1 & 5I & $=$ & 20 & 9.6 & 9.7 & 37 & $1=$ & 20 & I2.I & 12.7 & 32 & $\mathrm{ks}$ \\
\hline 22 & 33.4 & 35.0 & or & te & 22 & 23.9 & 24.5 & 50 & & 22 & 12. I & 13.2 & 32 & & 22 & II.I & 12.1 & 30 & 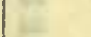 \\
\hline 24 & 30.0 & 31.8 & OI & 5 & 24 & 22.9 & 23.8 & 49 & & 24 & 22.0 & 22.6 & 47 & $x$ & 24 & 13.6 & 13.8 & 34 & 8 \\
\hline 26 & 32.0 & 33.8 & 04 & & 26 & 22.9 & 23.7 & 49 & & 26 & 22.8 & $23 . \mathrm{C}$ & 48 & & 26 & I4.6 & 15.3 & 36 & Ex. \\
\hline 28 & 34.5 & 36.5 & 08 & & 28 & 22.9 & 23.7 & 49 & & 28 & 26.2 & 27.0 & 54 & & 28 & 14.6 & 15.0 & 36 & \\
\hline 30 & 34.8 & 36.6 & as & -4.3 & 30 & 23.6 & 24.0 & 50 & $-3 \cdot 5$ & 30 & 22.8 & 24.4 & 2249 & -2.0 & 30 & 12.3 & 12.4 & 32 & $-3 \cdot 3$ \\
\hline 32 & 40.0 & 42.7 & I7 & & 32 & 24.0 & 25.0 & 51 & & 32 & 33.0 & 33.7 & 2304 & & 32 & II. 9 & I2. I & $3 I$ & \\
\hline 34 & 43.0 & 44.9 & $2 I$ & 8. & 34 & 23.2 & 23.8 & 49 & & 34 & 34.0 & 31.6 & OI & & 34 & 11.6 & 11.9 & $3 \mathrm{I}$ & \\
\hline 36 & 42.9 & 44.9 & $2 I$ & 3 & 36 & 23.2 & 23.9 & 49 & & 36 & 30.3 & 31.7 & 23 oI & 8 & 36 & 10.7 & II.3 & 30 & 35 \\
\hline $3^{8}$ & 42.9 & 45.0 & $2 \mathrm{I}$ & 3 & 38 & 22.8 & 23.2 & 48 & 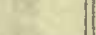 & 38 & 26.1 & $27 \cdot 3$ & 2254 & & 38 & II.7 & 12.3 & $3 \mathrm{I}$ & 5 \\
\hline 40 & 40.0 & 42.0 & I7 & 8 & 40 & 23.4 & 23.8 & 49 & 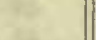 & 40 & 19. I & 19.7 & 43 & & 40 & 13.3 & I 4.0 & 34 & - \\
\hline 42 & 40.2 & 42.2 & I7 & 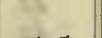 & 42 & 23.2 & 23.8 & 49 & & 42 & 21 & $9 a$ & 47 & & 42 & 13.3 & I4.I & 34 & \\
\hline 44 & 37.6 & 38.9 & I2 & $-4 \cdot 5$ & 44 & 22.9 & 23.4 & 48 & -3.2 & 44 & 26.0 & 25.0 & 53 & ZZ.I & 44 & 12.8 & 13.3 & 33 & $-3 \cdot 3$ \\
\hline 46 & 34.8 & 36.2 & 08 & & 46 & 23.0 & 23.3 & 48 & & 40 & 25.7 & $26 . I$ & 53 & & 46 & 12.3 & 13.0 & 32 & \\
\hline 48 & 34.1 & 36.0 & 07 & 8 & 48 & 22.9 & 23. I & 48 & & 48 & $21 \cdot 3$ & 21.6 & 46 & & 48 & II. 8 & $\mathrm{r} 2.4$ & $3 I$ & 6 \\
\hline 50 & 34.2 & 35.8 & 07 & as & 50 & 23.1 & 23.8 & 49 & & 50 & 18.8 & 19.3 & 42 & & 50 & I0.6 & II .8 & 30 & \\
\hline 52 & $33 . I$ & 34.8 & 05 & Sit & 52 & 22.8 & 23.1 & 48 & & 52 & 19.8 & 20.2 & 44 & & 52 & II.8 & 12.2 & $3 I$ & \\
\hline 54 & 34.8 & 36.0 & 08 & $\sqrt{2}$ & 54 & 23.9 & 24.5 & 50 & 8 & 54 & 19.6 & 20.0 & 43 & & 54 & II. 6 & 12.3 & $3 I$ & ne \\
\hline 56 & $33 \cdot 3$ & 35.1 & 06 & 6 & 56 & $24 \cdot 3$ & 25.0 & $5 I$ & is & 56 & 17.0 & 17.6 & 39 & gi & 56 & II. 3 & I1. .8 & & \\
\hline 58 & 33.0 & 34.2 & 05 & & 58 & 23.0 & $24 . I$ & 49 & & 58 & 16.4 & 16.6 & 38 & & 58 & 11.3 & II 8 & & \\
\hline
\end{tabular}

Observer-J. V.

Oliservers-J. V. and W. J. P., who alteruated from $81100 m$ to 8 h rom. 
Tabulation of magnetic declinations observed at Teplitz Bay-Continued

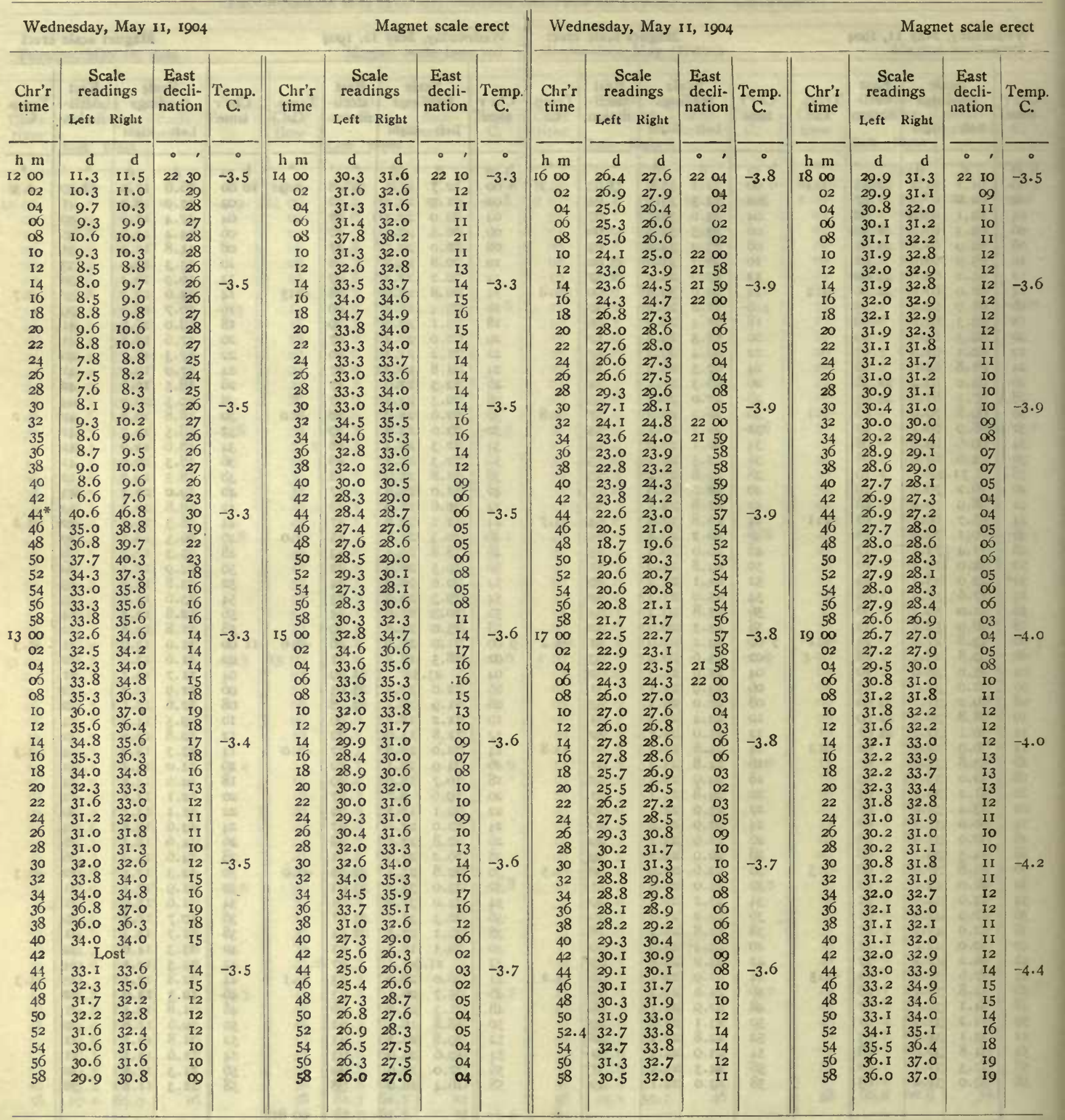


Tabulation of magnetic declinations observed at Teplitz Bay-Continued

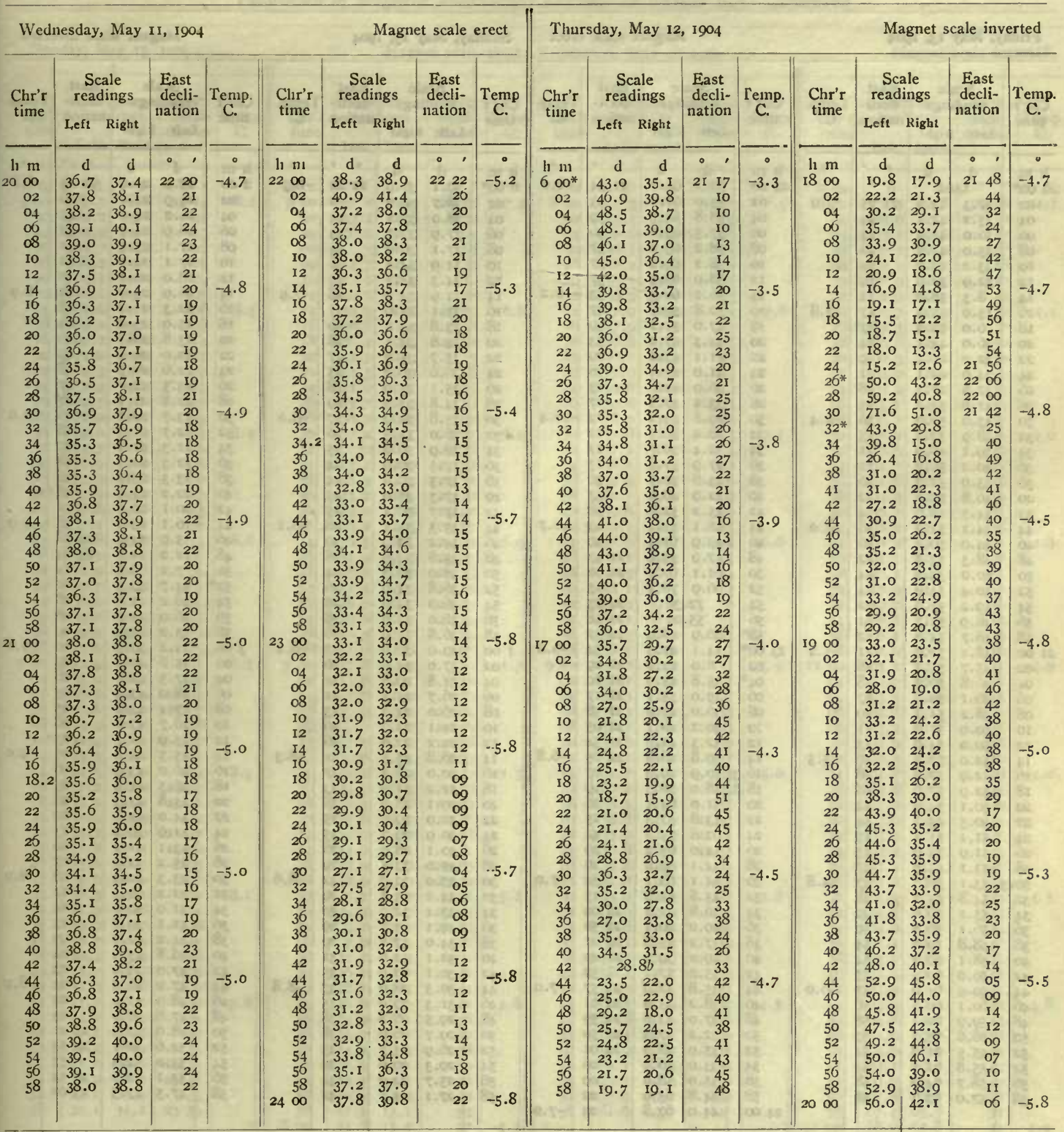

Correction to local mean time is $-25.90^{\circ}$ torsion $=12 . ' 73$. Torsion head at oh oom read $67^{\circ}$ and at $24^{\mathrm{h}} \mathrm{15 \textrm {m }}$ read $63^{\circ}$. Observer-R. R. T.
Correction to local mean time is $-55 \mathrm{~s}$. $90^{\circ}$ torsion $=15$.' $^{\prime} 54$.

Torsion head at $15 \mathrm{~h} 28 \mathrm{~m}$ read $63^{\circ}$ and at $2 \mathrm{Ih}$ I $2 \mathrm{~m}$ read $75^{\circ}$. Observer-J. V. 
Tabulation of magnetic declinations observed at Teplitz Bay-Continued

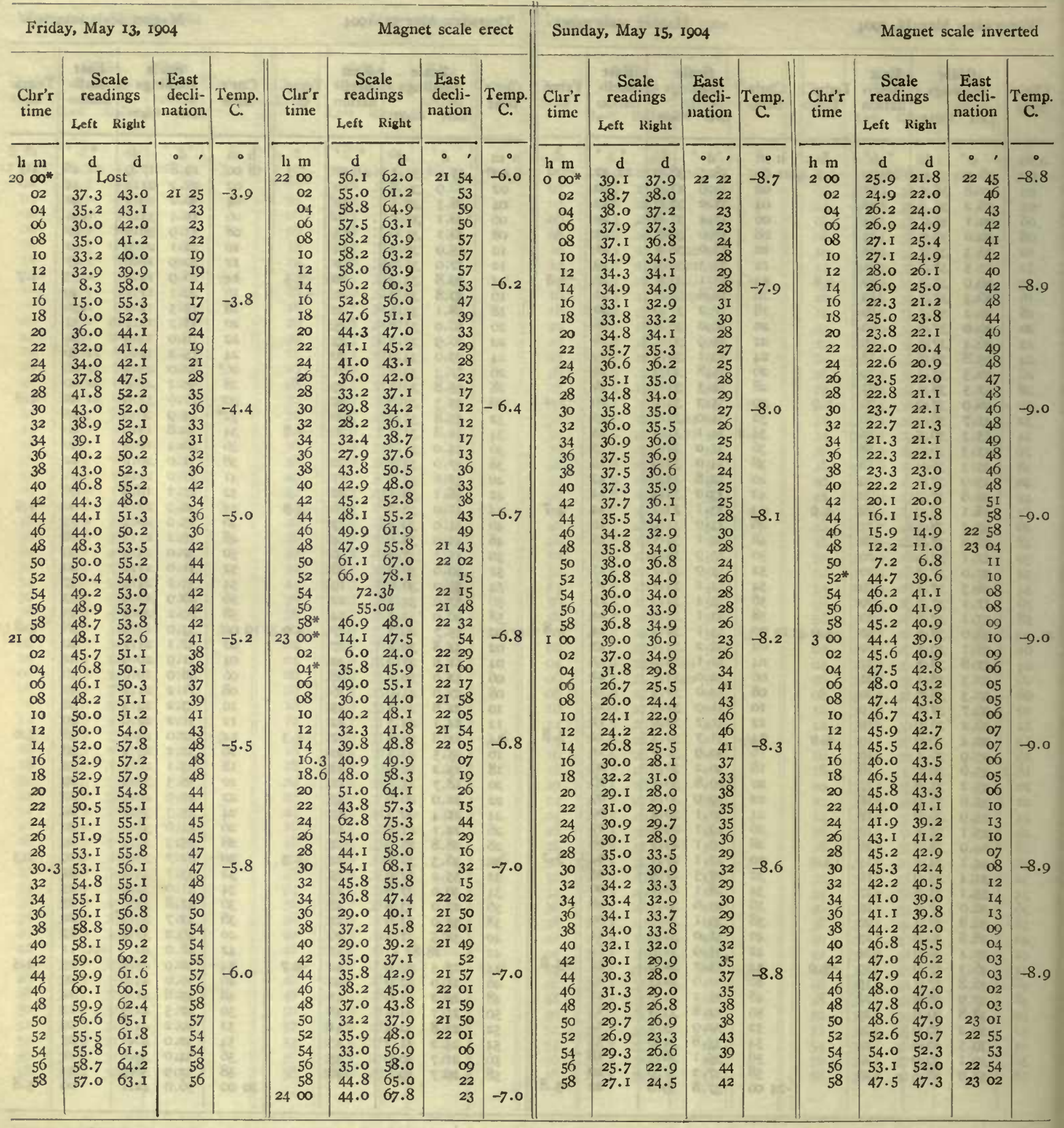

Correction to local mean time is - $1 \mathrm{~m}$ 17.5s.

Observer-J. V.

Torsion head at $19 \mathrm{~h} 58 \mathrm{~m}$ read $81^{\circ}$ and at $24 \mathrm{~h} 27 \mathrm{~m}$ read the same.

Observer-J. V. 
Tabulation of magnetic declinations observed at Teplitz Bay-Continued

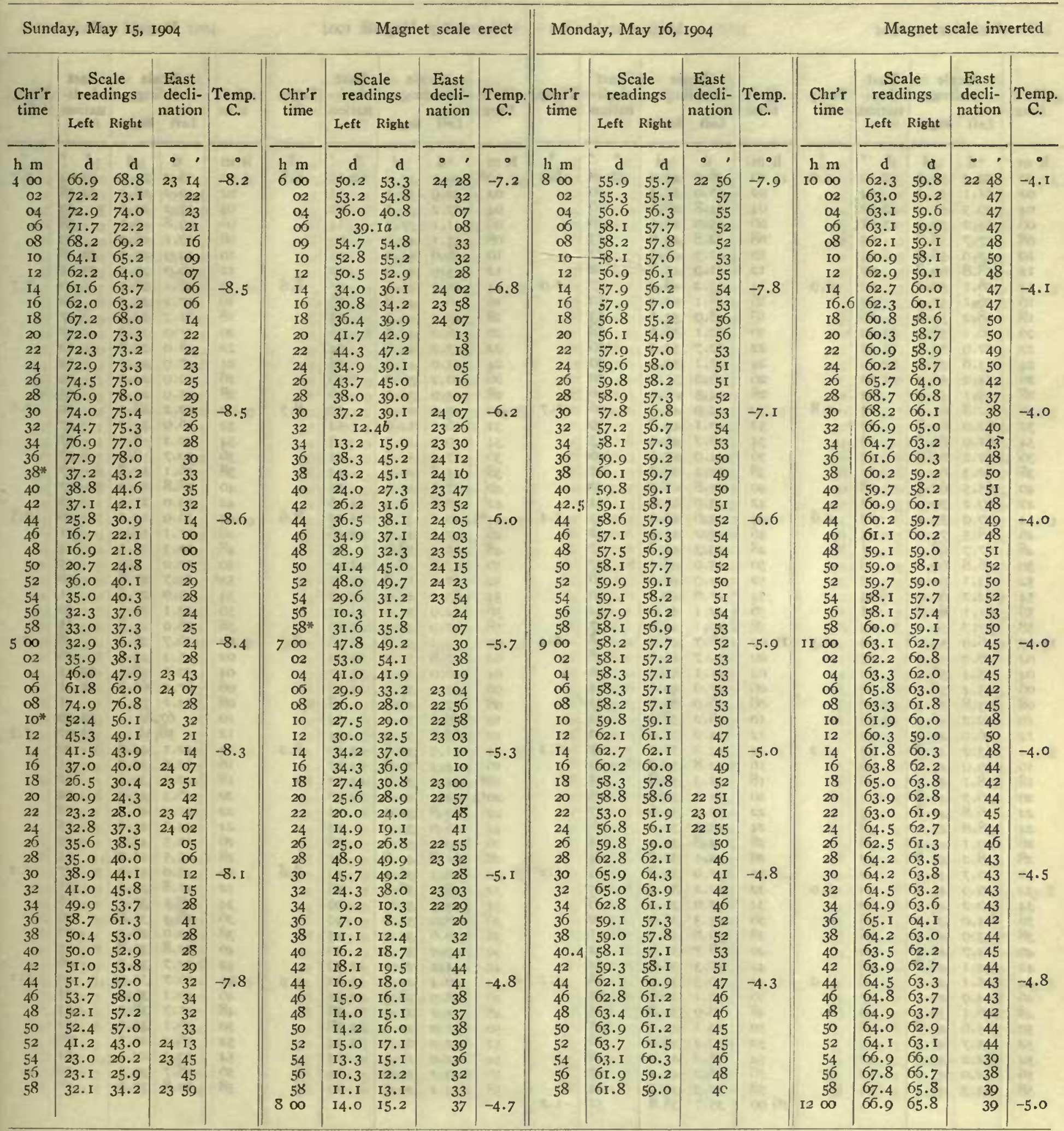

Correction to local mean time is $+6.5 \mathrm{~s}$.

Torsion head at oh oom read $79^{\circ}$ and at $8 \mathrm{~h} 24 \mathrm{~m}$ read the same. Observer-J. V.
Correction to local mean time is $-7 \mathrm{~s} .90^{\circ}$ torsion $=17 .^{\prime} 62$ Torsion head at 7 h $50 \mathrm{~m}$ read $79^{\circ}$ and at $\mathrm{I} 2 \mathrm{~h}$ 20m read $69^{\circ}$. Observer-R. R. T. 
Tabulation of magnetic declinations observed at Teplitz Bay-Continued

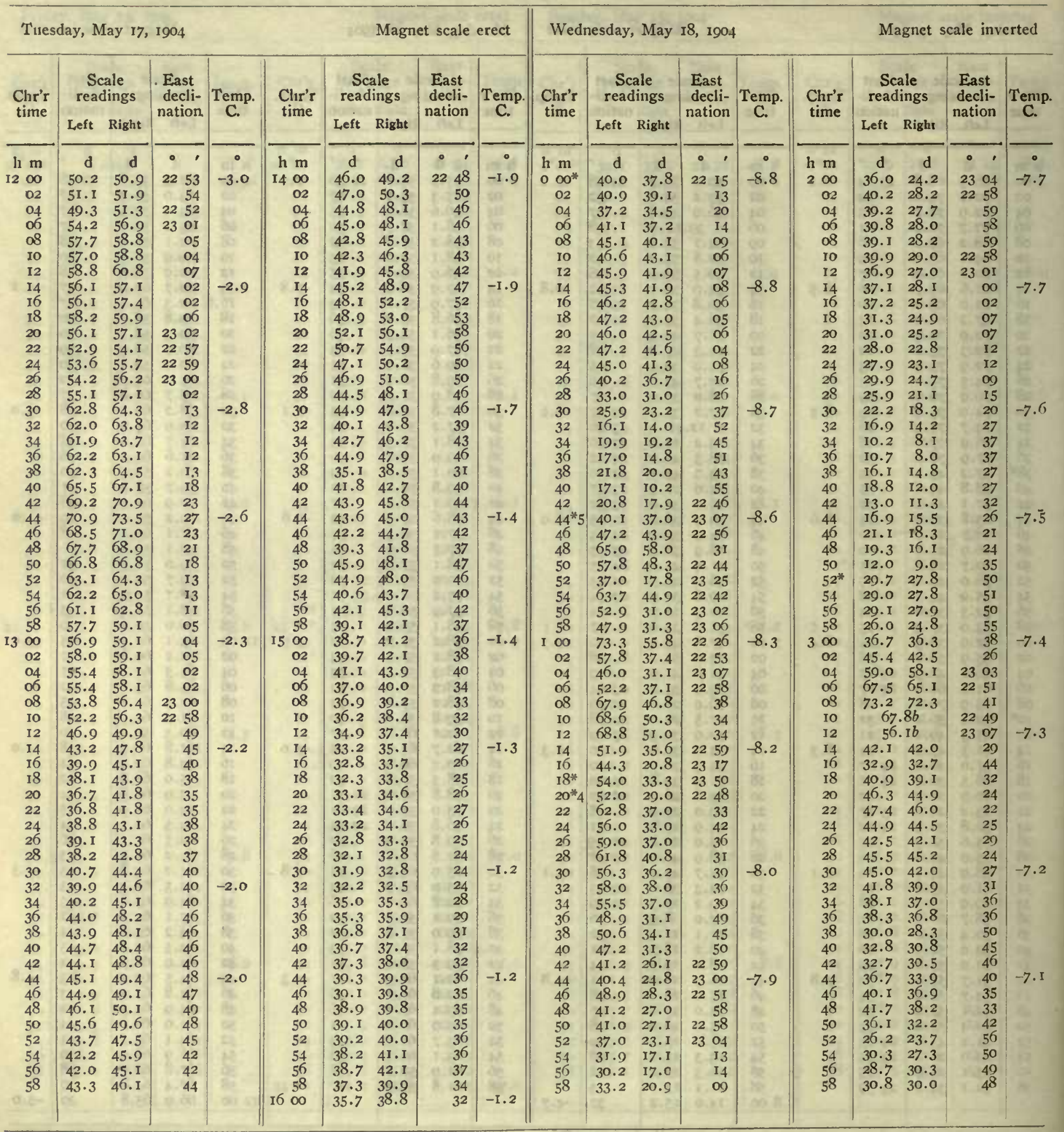

Correction to local mean time is - $17 \mathrm{s.} 90^{\circ}$ torsion $=12 .^{\prime} 17$.

Observer-J. V.

Torsion head at I th $40 \mathrm{~m}$ read $75^{\circ}$ and at $\mathrm{I} 6 \mathrm{~h}$ 20m read $64^{\circ}$.

Observer-R. R. T. 
Tabulation of magnetic declinations observed at Teplitz Bay-Continued

\begin{tabular}{|c|c|c|c|c|c|c|c|c|c|c|c|c|c|c|c|c|c|c|c|c|c|c|c|}
\hline \multicolumn{6}{|c|}{ Wednesday, May 18, I904 } & \multicolumn{6}{|c|}{ Magnet scale inverted } & \multicolumn{6}{|c|}{ Wednesday, May I8, I904 } & \multicolumn{6}{|c|}{ Magnet scale inverted } \\
\hline \multirow{2}{*}{$\begin{array}{l}\text { Chr'r } \\
\text { time }\end{array}$} & read & $\begin{array}{l}\text { dings } \\
\text { ding }\end{array}$ & $\begin{array}{r}\text { Ea } \\
\text { dec }\end{array}$ & $\begin{array}{l}\text { ast } \\
\text { cli- } \\
\text { ion }\end{array}$ & Temp. & Chr'r & $\begin{array}{l}\text { Sca } \\
\text { read }\end{array}$ & $\begin{array}{l}\text { ale } \\
\text { lings }\end{array}$ & Ea & cli- & Temp. & $\begin{array}{l}\text { Chr'r } \\
\text { time }\end{array}$ & Sca & $\begin{array}{l}\text { ale } \\
\text { dings }\end{array}$ & $\begin{array}{l}\text { Eas } \\
\text { decl } \\
\text { natic }\end{array}$ & $\begin{array}{l}\text { st } \\
\text { cli- } \\
\text { ion }\end{array}$ & Iemp. & $\begin{array}{l}\text { Chr'r } \\
\text { time }\end{array}$ & Sca & $\begin{array}{l}\text { ale } \\
\text { lings }\end{array}$ & Eas & & Temp. \\
\hline & Left & Right & & & & & Left & Right & & & & & Left & Right & & & & & Left & Right & & & \\
\hline h $\mathrm{m}$ & d & d & & & $\bullet$ & $\mathrm{h} \mathrm{m}$ & d & d & & & $\bullet$ & $\mathrm{h} \mathrm{m}$ & d & d & & & • & $\mathrm{h} \mathrm{m}$ & d & d & - & & $\bullet$ \\
\hline 400 & 35.0 & 32.8 & 23 & 42 & -7.0 & 600 & 55.1 & $54 \cdot 3$ & 23 & 09 & -5.9 & 800 & 65.8 & 62.0 & 22 & 55 & 20 & 1000 & 25.3 & $24 \cdot 5$ & 22 & 57 & $-4 \cdot 5$ \\
\hline 02 & 32.0 & 30.0 & 23 & 46 & 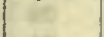 & 02 & $55 . \mathrm{I}$ & 63.9 & & IO & & 02 & 64.0 & $6 \mathrm{I} .2$ & & 57 & w6 & 02 & 36.0 & 33.8 & & 42 & \\
\hline 04 & 24.8 & 20.4 & 24 & $\infty$ & $5=$ & 04 & 56.8 & 55.9 & & 07 & ret & 04 & 69.4 & 67.0 & 22 & 48 & 10 & 04 & 36.0 & 36.0 & & 40 & 20 \\
\hline$\infty 6$ & 21.0 & 17.7 & & 05 & & $\infty$ & 56.9 & 56.1 & & 06 & - & $\infty 6$ & $6 \pi .5$ & 57.1 & 23 & 02 & $6 x$ & 06 & 25.7 & $25 \cdot 5$ & 22 & 56 & 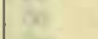 \\
\hline 08 & 24.0 & I9.9 & & OI & hen & as & 60.0 & 59.2 & & 02 & 8 & 08 & 67.3 & 63.3 & 22 & 53 & W & 08 & 23.0 & 22.3 & 23 & OI & wat \\
\hline IO & 22.0 & 19.0 & 24 & 03 & na & IO & 60.9 & 59.8 & 23 & $\infty$ & $f^{4}$ & 10 & 71.7 & 69.3 & & 44 & $n$ & 10 & 28.5 & 27.2 & 22 & 53 & 65: \\
\hline 12 & $25 \cdot 3$ & 20.9 & 23 & 59 & 的 & I2 & 64.3 & 61.8 & 22 & 56 & (n) & I2 & 63.0 & 60.2 & & 58 & txy & 12 & 29.3 & 29.3 & & 50 & 142 \\
\hline I4 & 32.7 & 27.8 & & 48 & -7.0 & I4 & 70.3 & 69.8 & & 45 & -5.8 & I4 & 65.6 & 63.0 & m & 54 & $-5 \cdot 3$ & 14 & $29 \cdot 3$ & 28.3 & & $5 I$ & $-4 \cdot 3$ \\
\hline 16 & 39.8 & 36.1 & & 36 & & 16 & 66.0 & 65.3 & & 52 & & 16 & 64.8 & 63.1 & & 55 & & I6 & $35 \cdot 5$ & $34 \cdot 5$ & 22 & 42 & \\
\hline 18 & 36.9 & 35.2 & & 39 & & I8 & 64.5 & 63.9 & & 54 & W & I8 & 65.5 & 63.5 & & 54 & 41 & 18 & 22.5 & 22.1 & 23 & 02 & 14 \\
\hline 20 & 36.2 & 28.0 & & 45 & 18: & 20 & 65.8 & 64.9 & & 53 & 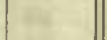 & 20 & 64.6 & 62.6 & & 55 & 64 & $20 *$ & 44.0 & 38.0 & & 28 & 60 \\
\hline 22 & 29.0 & 26.0 & & 52 & 4 & 22 & 66.5 & 64.8 & & 52 & 14 & 22 & 65.2 & 62.7 & & 55 & 4 & 22 & 39.0 & 36.8 & & 33 & 15 \\
\hline 24 & 31.2 & 28.9 & & 48 & 35 & 24 & 67.9 & 65.1 & & $5 \mathrm{I}$ & $1=$ & 24 & 65.6 & 64.2 & 1 & 53 & is & 24 & 50.4 & 48.0 & & 15 & in \\
\hline 26 & 32.2 & 30.3 & & 46 & 10 & 26 & 68.9 & 66.0 & & 49 & ve & 26 & 64.0 & $6 \mathrm{I} .5$ & 22 & 57 & 28 & 26 & 52.0 & 49.2 & 23 & 13 & $x$ \\
\hline 28 & 31.9 & 29.5 & & 47 & & 28 & 62.9 & $6 r .9$ & 22 & 57 & (2) & 28 & $6 \mathrm{r} \cdot 3$ & 59.3 & 23 & $\infty$ & 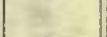 & 28 & 65.3 & 59.7 & 22 & 54 & Ans \\
\hline 30 & 31.2 & 29.0 & & 48 & -6.9 & 30.3 & 49.2 & 47.7 & 2.3 & I9 & -5.8 & 30 & 64.6 & 63.0 & 22 & 55 & -5.0 & 30 & 65.3 & $6 \mathrm{I} \cdot 3$ & 2 & 53 & $-4 \cdot 3$ \\
\hline 32 & $27 \cdot 1$ & 25.0 & & 54 & (n) & 32 & 50.1 & 47.8 & & 18 & & 32 & 64.3 & $62 . I$ & & 56 & ( & $32^{*}$ & $44 \cdot 3$ & 33.6 & 4 & 29 & \\
\hline 34 & 27.1 & 25.2 & & 54 & is & 34 & 55.0 & 51.9 & & I I & 11 & 34 & 62.8 & 60.9 & & 58 & 72 & 34 & 42.2 & 39.8 & E & 25 & 16 \\
\hline 36 & 28.7 & 26.1 & & 52 & a. & 36 & 59.2 & 57.0 & & 04 & Wx & 36 & 69.3 & 65.7 & & 49 & 14 & 36 & 39.0 & 38.2 & E & 29 & 4 \\
\hline 38 & 27.8 & 25.9 & & 53 & 36 & 38 & 60.3 & 58.3 & 23 & 02 & 62 & 38 & $64 \cdot 3$ & 63.2 & 22 & 55 & E & 38 & 40.6 & 39.0 & P & 27 & a \\
\hline 40 & 27.8 & 25.8 & & 53 & at & 40 & 62.2 & 60.0 & 22 & 59 & the & 40 & 59.7 & 56.8 & 23 & 04 & 214 & 40 & 44.0 & 39.2 & & 24 & 15 \\
\hline 42 & 26.1 & 24.8 & & 55 & 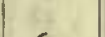 & 42 & $6 \mathrm{r} .2$ & 59.3 & 23 & OI & $3 x$ & 42 & 64.3 & 60.6 & 22 & 57 & $x^{2}$ & 42 & $39 \cdot 3$ & 37.0 & & 30 & $41=$ \\
\hline 44 & 27.8 & 25.7 & & 53 & -6.9 & 44 & 61.8 & 59.7 & & $\infty$ & -5.7 & 44 & $55 \cdot 3$ & 50.5 & 23 & 12 & -5.0 & 44 & 41.6 & 32.8 & & $3 I$ & $-4 \cdot 2$ \\
\hline 46 & 30.9 & 28.2 & & 49 & - & 46 & 58.0 & 56.2 & & 05 & & 46 & 50.8 & 47.0 & 23 & I8 & 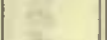 & 46 & 42.3 & 29.3 & & 34 & (7) \\
\hline 48 & 36.0 & 3.6 & & 40 & W & 48 & 60.8 & 58.2 & 8 & 02 & 3 & 48 & 67.2 & 65.4 & 22 & $5 I$ & 3 & 48 & 34.6 & 29.4 & & 40 & 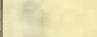 \\
\hline 50 & 31.3 & 28.4 & & 48 & he & 50 & 62.9 & 59.0 & & $\infty$ & (4) & 50 & 73.8 & $7 \pi \cdot 3$ & re & 41 & 14 & 50 & 30.2 & 25.6 & & 46 & 14 \\
\hline 52 & 33.0 & 31.8 & & 44 & 15 & 52 & 58.3 & 55.3 & 7 & $\infty$ & $y$ & 52 & 72.6 & 70.2 & I & 43 & 2 & 52 & $34 \cdot 3$ & 27.4 & & $4 I$ & $t$ \\
\hline 54 & 34.4 & 34.3 & 4 & $4 I$ & & 54 & 58.3 & 55.0 & & 06 & E & 54 & 70.6 & 67.2 & fi & 47 & 12 & 54 & 28.3 & 20.8 & & $5 I$ & 14t \\
\hline 56 & 32.7 & $3 \mathrm{I} \cdot 9$ & 1 & 44 & & 56 & 57.1 & 54.0 & & 08 & 37 & 56 & 70.6 & 67.0 & f & 47 & $y^{1}=$ & 56 & 27.2 & 20.1 & & 53 & \\
\hline 58 & 35.2 & $34 . I$ & th. & $4 I$ & & 58 & 58.0 & 56.0 & 4 & 06 & $4 x$ & 58 & 60.1 & 64.6 & 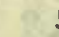 & 50 & & 58 & 26.8 & 22.2 & 7 & $5 I$ & the \\
\hline 500 & 35.0 & 34.I & 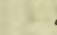 & 40 & -6.7 & 700 & 6 т.o & 58.0 & 23 & 02 & $-5 \cdot 7$ & 900 & 67.6 & 62.5 & & 53 & -4.8 & II 00 & $34 \cdot 2$ & 28.6 & & 40 & -4.0 \\
\hline 02 & 37.8 & 37.0 & & 36 & ( & 03 & 63.0 & 60.0 & 22 & 50 & & 02 & 68.0 & 63.6 & & 52 & ( & 02 & 36.9 & 30.1 & & 37 & r \\
\hline 04 & 40.0 & 38.2 & & 34 & 10 & 04 & 64.0 & 60.5 & & 58 & 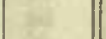 & 04 & 72.2 & 68.6 & & 45 & 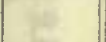 & 04 & 33.6 & 27.8 & & 42 & Hit \\
\hline 06 & 44.2 & 42.9 & & 27 & 10 & 06 & 64.2 & $6 \mathrm{r} .0$ & 22 & 57 & 3 & 06 & 74.6 & 71.3 & & $4 I$ & 4 & 06 & $37 \cdot 7$ & $34 \cdot 5$ & & 33 & ant \\
\hline 08 & 44.7 & 44.2 & & 26 & Wh & 08 & $6 \mathrm{I} .2$ & 58.2 & 23 & OI & = & a8* & $43 \cdot 3$ & 33.8 & & 36 & F & 08 & 37.0 & 35.6 & & 33 & $F_{i}$ \\
\hline 10 & 44.9 & 44.1 & & 25 & 61 & IO & 62.8 & 60.7 & 22 & 58 & $x$ & IO & 36.9 & 36.3 & 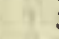 & 39 & wit & 10 & 32.4 & 32.0 & & 39 & 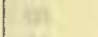 \\
\hline 12 & $43 \cdot 5$ & 43.2 & & 27 & 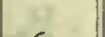 & 12 & 56.6 & 54.0 & 23 & 08 & & I2 & 32.0 & 30.3 & s. & 48 & 19. & 12 & 32.4 & $3 I .0$ & & 40 & 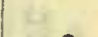 \\
\hline 14 & 47.6 & 46.9 & & 21 & -6.4 & I4 & 54.0 & 51.0 & & 12 & -5.7 & 14 & 27.1 & 26.6 & 1 & 54 & -4.9 & 14 & 41.6 & 37.6 & & 28 & -3.8 \\
\hline I6 & 53.3 & 52.9 & & 12 & & I6 & 60.7 & 57.8 & 23 & 02 & & 16 & 29.8 & 28.9 & & 50 & . & 16 & 41.0 & 37.1 & & 29 & \\
\hline I8 & $57 \cdot 3$ & 55.9 & & 06 & 71 & I8 & 62.0 & 59.8 & 22 & 60 & InIf & I8 & $4 I . I$ & 38.3 & & 34 & 8 & 18 & 42.2 & 38.8 & & 26 & 24 \\
\hline 20 & 56.6 & 55.3 & 1 & 07 & $6=$ & 20 & 62.5 & 60.2 & 22 & 59 & f & 20 & $41 . C$ & 38.3 & 5 & 34 & 15 & 20 & 46.5 & 43.5 & & I9 & $y^{*}$ \\
\hline 22 & 5.5 .1 & $54 \cdot 7$ & & $\infty 9$ & 5 & 22 & 60.8 & 58.3 & 23 & 02 & 257 & 22 & 29.0 & $27 \cdot 7$ & & 52 & $=$ & 22 & 47.0 & $43 \cdot 5$ & & 19 & WE. \\
\hline 24 & 56.3 & 55.8 & & 07 & 11: & 24 & 60.3 & 57.2 & 23 & 0.3 & 41 & 24 & $24 \cdot 3$ & 23.0 & 22 & 60 & 14 & 24 & 45.4 & 41.8 & & 21 & 15 \\
\hline 26 & 56.2 & 56.1 & & 07 & & 26 & 63.2 & $6 \pi .0$ & 22 & 58 & 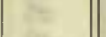 & 26 & 24.0 & 21.7 & 23 & OI & $n$ & 26 & 42.6 & 39.0 & & 26 & 48 \\
\hline 28 & 51 . & $8 b$ & & I4 & & 28 & 64.0 & 61.0 & 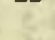 & 57 & & 28 & 29.6 & 28.8 & 22 & 51 & 2 & 28 & 39.0 & 35.8 & & 31 & 14 \\
\hline $3 n$ & 51.8 & 5 I. 2 & 8 & 14 & -6.1 & 30 & 64.0 & $6 \pi .0$ & 22 & 57 & -5.8 & 30 & 32.0 & 29.5 & $=$ & 48 & -4.9 & 30 & 36.3 & 33.0 & & 35 & ( \\
\hline 32 & 54.9 & 54.9 & ( & $\infty$ & (5) & 32 & 61.2 & 59.1 & 23 & OI & (n) & 33 & 33.8 & 31.8 & & 45 & (2) & 32 & 35.8 & 32.6 & & 36 & -3.8 \\
\hline 34 & 60.2 & 59.6 & 23 & OI & $t$ & 34 & 60.0 & 56.9 & 23 & 04 & wi & 34 & 32.3 & $3 \mathrm{I} \cdot 3$ & & 46 & -5 & 34 & 35.6 & 32.6 & & 36 & ( \\
\hline 36 & 67 & oa & 22 & 50 & $z^{2}$ & 36 & 63.0 & 60.2 & 22 & 58 & 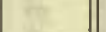 & 36 & $27 \cdot 3$ & 26.0 & 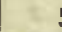 & 55 & 17 & 36 & 36.3 & 33.6 & & 35 & 27 \\
\hline 38 & 67.5 & 67.1 & & 50 & 18. & 38 & 63.9 & 61.9 & 4 & 56 & t & 38 & 26.8 & 25.3 & & 56 & . & 38 & 34.2 & 32.0 & & 38 & 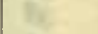 \\
\hline 40 & 63.8 & 63.2 & & 56 & $6=$ & 40 & 62.9 & 6 I. 6 & 22 & 58 & 7 & 40 & 26.8 & 24.3 & . & 56 & $m$ & 40 & 33.0 & 30.8 & & 40 & r*t \\
\hline 42 & 63.0 & 62.9 & & 56 & & 42 & 59.3 & 57.8 & 23 & 03 & 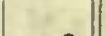 & 42 & 32.8 & $3 I .3$ & $\mathrm{t}$ & 46 & (2) & 42 & 32.6 & 30.6 & & 40 & k \\
\hline 44 & 64 & $8 a$ & & 53 & -6.0 & 44 & 59.0 & $57 \cdot 5$ & & 04 & -5.8 & 44 & $33 . \mathrm{I}$ & 30.8 & 2 & 46 & -4.8 & 44 & 33.8 & 31.8 & & 38 & -3.6 \\
\hline 46 & 60.3 & 68.2 & 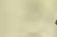 & 47 & $x^{2}$ & 46 & 59.0 & 55.3 & $y$ & 06 & & 46 & 30.9 & 28.5 & 5 & 50 & 的 & 46 & 34.0 & 32.3 & & 38 & \\
\hline 48 & 60.2 & 68.3 & 7 & 47 & kat & 48 & 60.8 & 57.8 & 23 & 02 & ( & 48 & 31.6 & 30.2 & 2 & 48 & 5 & 48 & $34 \cdot 3$ & 32.3 & $n$ & 38 & 14 \\
\hline 50 & 64.8 & 64.2 & 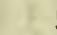 & 54 & $5=$ & 50 & 63.8 & 60.8 & 22 & 57 & we & 50 & 32.3 & 30.3 & 18 & 47 & its & 50 & 34.6 & 33.0 & & 37 & $1=$ \\
\hline 52 & 54.3 & 64.1 & 22 & 54 & 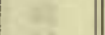 & 52 & 61.2 & 60.8 & 22 & 57 & -5 & 52 & 30.3 & 28.3 & 5 & 50 & 2 & 52 & $34 \cdot 3$ & 32.6 & & 37 & 11 \\
\hline 54 & 59. & $o b$ & 23 & 02 & W & 54 & 58.0 & 55.8 & 23 & 06 & 12 & 54 & 25.6 & $24 \cdot 3$ & 5 & 57 & 10 & 54 & 34.2 & 32.5 & & 38 & 12 \\
\hline 56 & 50.7 & 50.0 & 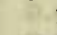 & I6 & & 56 & 63.3 & 60.2 & 22 & 58 & $x^{2}$ & 56 & 24.6 & 24.0 & 22 & 58 & axt & 56 & 35.1 & $33 \cdot 3$ & & 36 & 18 \\
\hline 58 & 52.1 & 50.9 & th & If & 28 & $5^{8}$ & 65.0 & 62.2 & & 55 & $-5 \cdot 7$ & 58 & 23.0 & 22.4 & 23 & & 112 & 58 & 36.3 & $34 \cdot 3$ & & & 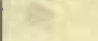 \\
\hline
\end{tabular}

Observers-J. V. and W. J. P., who alternated from $8 \mathrm{~h} 06 \mathrm{~m}$ to $8 \mathrm{~h}$ 12m.

Observer-W. J. P. 
Tabulation of magnetic declinations observed at Teplitz Bay-Continued

\begin{tabular}{|c|c|c|c|c|c|c|c|c|c|c|c|c|c|c|c|c|c|c|c|}
\hline \multicolumn{6}{|c|}{ Wednesday, May 18, 1904} & \multicolumn{4}{|c|}{ Magnet scale inverted } & \multicolumn{6}{|c|}{ Wednesday, May r8, 1904} & \multicolumn{4}{|c|}{ Magnet scale inverted } \\
\hline \multirow{2}{*}{$\begin{array}{l}\text { Chr'r } \\
\text { time }\end{array}$} & \multicolumn{2}{|c|}{$\begin{array}{l}\text { Scale } \\
\text { readings }\end{array}$} & \multirow{2}{*}{$\begin{array}{l}\text { East } \\
\text { decli- } \\
\text { nation }\end{array}$} & \multirow{2}{*}{ Temp. } & Chr'r & $\begin{array}{l}\text { Sé } \\
\text { read }\end{array}$ & $\begin{array}{l}\text { ale } \\
\text { ings }\end{array}$ & $\begin{array}{l}\text { East } \\
\text { decli- }\end{array}$ & Temp. & Chr'r & $\begin{array}{r}\mathrm{Sc} \\
\mathrm{read}\end{array}$ & dings & $\begin{array}{l}\text { East } \\
\text { decli- }\end{array}$ & Temp. & Chr'r & $\begin{array}{r}S c \\
\text { read }\end{array}$ & & $\begin{array}{l}\text { East } \\
\text { decli- }\end{array}$ & Temp. \\
\hline & Left & Right & & & & Left & Right & & & & Left & Right & & & & Left & Right & & \\
\hline $\mathrm{h} \mathrm{m}$ & & & & & & & & & & & $d$ & d & & & & & & & \\
\hline & 36.4 & 34.6 & 2234 & -3.6 & $\infty$ & 38.6 & 37.6 & 2230 & -3.0 & 1600 & 43.9 & $43 \cdot 3$ & $222 I$ & -3.1 & & 37.2 & 36.8 & 2232 & -3.8 \\
\hline $\begin{array}{l}02 \\
04\end{array}$ & $\begin{array}{l}36.3 \\
38.5\end{array}-10$ & & 33 & as & 02 & & $37 \cdot 5$ & $\begin{array}{r}30 \\
28\end{array}$ & $=$ & 02 & $45 \cdot 3$ & 44.7 & 19 & & 02 & 37.9 & 37.1 & 31 & \\
\hline $\begin{array}{l}04 \\
06\end{array}$ & $\begin{array}{l}38.5 \\
30.5\end{array}-1-10$ & $\begin{array}{l}37.1 \\
38.0\end{array}$ & & $x$ & $\begin{array}{l}04 \\
06\end{array}$ & $\begin{array}{r}40.0 \\
4.0\end{array}-120$ & $\begin{array}{l}38.7 \\
4 \mathrm{I} .5\end{array}$ & $\begin{array}{l}28 \\
24\end{array}$ & 50 & 04 & 5.6 & 45 . & I9 & & 04 & 0.1 & & 28 & \\
\hline 08 & $\begin{array}{l}39.5 \\
39.3\end{array}$ & $\begin{array}{l}38.0 \\
37.6\end{array}$ & $\begin{array}{l}29 \\
30\end{array}$ & 20. & $\begin{array}{l}00 \\
08\end{array}$ & $\begin{array}{l}4.9 \\
43.0\end{array}-1-10$ & $\begin{array}{l}41.5 \\
42.7\end{array}$ & $\begin{array}{l}24 \\
23\end{array}$ & 6 & & $\begin{array}{r}46.3 \\
47.6\end{array}$ & 45.7 & 18 & & 06. & I. 8 & $4 \mathrm{I} \cdot \mathrm{I}$ & 25 & \\
\hline IO & 38.9 & 37.3 & 30 & ar. & ro & $\begin{array}{l}43.0 \\
49.0\end{array}-1-10$ & 43.5 & 17 & at & 10 & $\begin{array}{l}47.0 \\
47.8\end{array}$ & $\begin{array}{r}40.8 \\
47.2\end{array}$ & $\begin{array}{l}10 \\
15\end{array}$ & 80 & 08 & 44.7 & 44.0 & 20 & \\
\hline 12 & 38.8 & 37.3 & 30 & st. & 12 & .4 & & 20 & 31 & 12 & 46.3 & 45.3 & 18 & . & $\begin{array}{l}10 \\
12\end{array}$ & $\begin{array}{l}40.8 \\
48.2\end{array}-12-10$ & 40.2 & 17 & \\
\hline I4 & 37.5 & & 32 & -3.6 & 14 & & & 20 & -3.0 & 14 & 45.8 & & 10 & -3.3 & 12 & 48.2 & 47.2 & 15 & \\
\hline 16 & 37.0 & 35.6 & 33 & & 16 & 47.6 & 46.5 & 16 & & 16 & 4.6 & 43 & 20 & 3.8 & It & 9.0 & 48.1 & I4 & -3.5 \\
\hline I8 & $37 \cdot 3$ & & 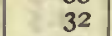 & tax & 18 & & & 21 & 74 & 18 & .0 & 44 & 9 & thi & I8 & .4 & & $\begin{array}{l}14 \\
13\end{array}$ & In \\
\hline 20 & & 36 & 2 & 10 & 20 & & & 09 & कर & 20 & & & 3 & asc & 20 & & & 13 & \\
\hline 2 & & & 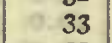 & $=$ & 2 & & 56 & I & $\approx$ & 2 & & & & tz & & & 5 & & $=$ \\
\hline 2 & & & 3 & $y^{2}$ & 2 & 58.6 & 58 & & w & 24 & & 47 & $\mathrm{I}_{4}$ & II & & .I & & & \\
\hline & 37.6 & 36.6 & 3 & 35 & 26 & 60.2 & 59. & 56 & 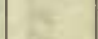 & 26 & 49.3 & 47 & 14 & $x$ & 26 & 9.9 & 48.2 & 13 & \\
\hline 28 & 37.6 & 36.5 & 32 & 20 & 28 & 59.0 & 58 & 58 & 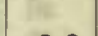 & 28 & 51.6 & & I0 & & 28 & 48.3 & 46.9 & 15 & \\
\hline 30 & $3^{6} .8$ & 35.8 & 33 & -3.5 & 30 & & $59 \cdot 3$ & 56 & -2.9 & 30 & 53.5 & 51 & or & -3.5 & 30 & 46.3 & 45.8 & I8 & $-3 \cdot 3$ \\
\hline 32 & & 37.3 & $3 \mathrm{I}$ & - & 32 & & 61.3 & 53 & a & 32 & & 49 & II & & 32 & 9.1 & 36.6 & 30 & \\
\hline 34 & & 37.3 & 30 & 18 & 34 & & 59 & 56 & te & 34 & .6 & 51 & 08 & is & 34 & & 35.0 & 32 & E: \\
\hline 36 & & & & de & 36 & 60.3 & 59 & 56 & is & 36 & .3 & & 04 & & 3 & .6 & & 17 & \\
\hline 38 & & 36 & 32 & 6 & 38 & & & 51 & Ne & 38 & & & or & Re & 3 & .3 & 48.9 & I2 & \\
\hline 40 & & 36.9 & 31 & 80 & 40 & & 60.8 & 53 & 68 & 40 & & 55 & 02 & of & 40 & & $49 . I$ & 12 & \\
\hline 42 & & & 30 & 45 & 42 & & 66.3 & 45 & & 42 & & & 04 & & 42 & .8 & 47.1 & 12 & \\
\hline 44 & 38.2 & & $3 \mathrm{I}$ & -3.6 & 44 & & 66.1 & 45 & -2.8 & 44 & & & 07 & -3.7 & 4 & 53.9 & & I0 & -3.1 \\
\hline 46 & 37.6 & & $3 \mathrm{I}$ & 3.0 & 46 & 65.8 & 63.9 & 48 & -.0 & 46 & & 52 & of & 3.8 & 4 & 37.9 & 30.1 & 36 & 30 \\
\hline 48 & & & 3 & 4 & 48 & & & 46 & $4 t$ & 48 & & & of & $z_{2}$ & 4 & & 38 & II & \\
\hline 50 & & & 3 & at & 50 & & & & 7 & 50 & & & & es & 5 & & & & c \\
\hline 52 & & & 3 & $=1$ & 52 & & 58 & $2 \mathrm{I}$ & H. & 52 & & & & 22 & 5 & & & & \\
\hline & & & 3 & E & 54 & & 56 & 22 & 16 & 5 & & & & 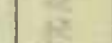 & 5 & & & & \\
\hline & & 38 & 3 & 18 & 56 & 63.0 & 60.3 & & st & 5 & & & & 69 & & 4.1 & 9.0 & & \\
\hline & & & 3 & 32 & 58 & 53.0 & $5 \mathrm{I}$ & & bs & $5 \varepsilon$ & & & & 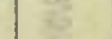 & 5 & & 8.1 & & \\
\hline$\infty$ & $37 \cdot 3$ & 36.5 & 32 & -3.6 & I5 50 & 48.6 & 46.3 & 15 & -2.8 & I7 00 & & 56 & OI & -3.9 & 1900 & & 6.8 & 55 & -3.0 \\
\hline 02 & & & 3I & 20 & 02.4 & $45 \cdot 3$ & 44 & & 10 & 02 & & & $\infty 0$ & - & 02 & & 7.8 & 54 & \\
\hline 04 & 8.8 & 38 & 29 & 10 & 04 & & & 24 & 10 & 0 & & & Do & 18 & 0 & & 8.8 & 54 & \\
\hline of & & & 2 & 10 & or & & & & 34 & ob & & & & Be & 0 & & 10.7 & 53 & \\
\hline o & & & 3 & 80 & 08 & & & & 70 & of & & & I & 60 & o & & 9.7 & 55 & \\
\hline IC & & & 2 & of & IS & & & & Et & I & & & or & or & I & & & 2156 & \\
\hline 12 & & & 2 & 48 & I: & & & & & 12 & & & or & & & & & 201 & \\
\hline 14 & & & 28 & -3.6 & 14 & & $4 \mathrm{I}$ & 3 & -2.8 & 14 & & & $\infty 0$ & -4.0 & $14^{*} 5$ & & 37.9 & I0 & -3.0 \\
\hline 56 & & & 2 & a & 16 & & & & wi & IC & & & or & . & 16 & & & I4 & \\
\hline 18 & & & 2 & Br. & I8 & & & & $8 t$ & I & & & & 72 & I8 & & & 14 & \\
\hline 20 & & & 2 & $a$ & 2 & & & & a & 2 & & & & te & 2 & & & 1 & \\
\hline 22 & & & 36 & 34 & 22 & & & & $=$ & 2 & & & & sa & 2 & & & 16 & $y$ \\
\hline 24 & & & 28 & 15 & 24 & & 43 & & zer & 2 & & & & in & 2. & & 35. & 18 & $=$ \\
\hline & & & 28 & 35 & 26 & & & 25 & 8 & 2 & & & & $E$ & 2 & & 37. & I5 & \\
\hline & & 40 & 26 & 86 & 28 & & 43 & 21 & 15: & 2 & & & & F & 28 & & & 31 & \\
\hline $3 c$ & & & 27 & $-3 \cdot 5$ & 3 & & & & -2.9 & 3 & & & & -4.0 & & & & & -2.9 \\
\hline 32 & & 39 & 27 & . & 32 & & & 3 & res & 3 & & & & 40 & & & & & \\
\hline 34 & 39 & & 28 & 28 & 34 & 45.6 & 45 & 19 & $2 x$ & 3 & & & II & 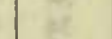 & & & & & \\
\hline 3 & & & & 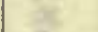 & 36 & & & & QS & 3 & & & & & 3 & & & & \\
\hline 3 & & & 2 & ne & 3 & & & & tie & 3 & & & & E & 3 & & & & \\
\hline 4 & & & & & & & & & . & & & & I I & $\infty$ & & & & & \\
\hline 42 & & & 26 & & 42 & & & & & 4 & & & 99 & & 4 & & & & \\
\hline 44 & & 40. & 27 & -3.3 & 44 & & 49 & & -3.0 & 4 & & & 08 & -4.0 & 4 & & 59.0 & 51 & -2.9 \\
\hline 46 & & & 28 & & 46 & & & & & 4 & & & 09 & & 4 & & & 56 & \\
\hline 48 & & 33 & 3 & 6 & 48 & & & 8. & is & 48 & & & & 6 & 4 & & & $\begin{array}{ll}21 \quad 56\end{array}$ & \\
\hline 50 & & & 28 & $\sigma$ & 50 & & & 17 & or & 50 & 46.3 & 46 & 17 & 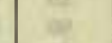 & 5 & & 50.8 & 2204 & \\
\hline 52 & 39 & 39 . & 28 & a & 52 & 48.5 & 48 & 14 & se & 52 & & & 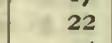 & 8 & 52 & & 51.1 & 05 & \\
\hline 54 & & 38 & 29 & 75 & 54 & 50.3 & 50 & II & k & 54 & 41.6 & 40 & 25 & 3 & 54 & 58.7 & 48.1 & 09 & \\
\hline 56 & & 38 & 30 & \& & 56 & 47.2 & 46 & 16 & be & 56 & 39.7 & 38 & 28 & 28 & 56 & 59.9 & 51.7 & 05 & \\
\hline 50 & 39.5 & 38.2 & 29 & $\pi 3$ & 58 & 44.5 & 43.5 & $2 \mathrm{I}$ & 35 & 58 & $39 \cdot 3$ & 38.2 & 29 & 89 & 58 & 60.9 & 52.7 & 04 & \\
\hline
\end{tabular}


Tabulation of magnetic declinations observed at Teplitz Bay-Continued

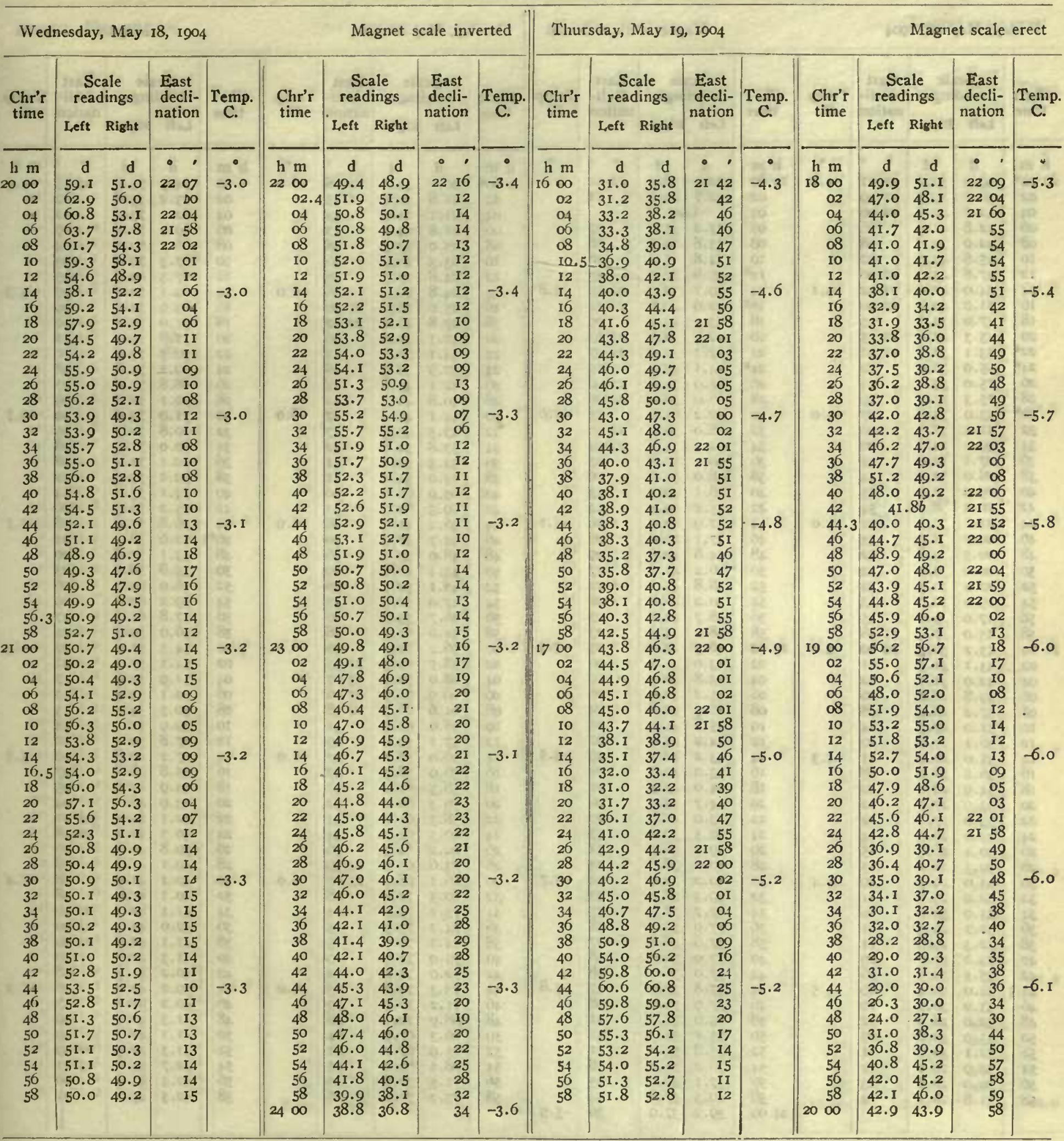

Correction to local mean time is $-1.55 .90^{\circ}$ torsion $=19 .{ }^{\prime} 62$. Torsion head at oh oom read $65^{\circ}$ and at $24 \mathrm{~h}$ in read $67^{\circ}$. Observer-R. R. T.
Correction to local mean time is $+2 \mathrm{~s} .90^{\circ}$ torsion $=16.0^{\prime} 92$. 'Torsion head at $15 \mathrm{~h} 38 \mathrm{~m}$ read $69^{\circ}$ and at $20 \mathrm{~h} 21 \mathrm{~m}$ read $83^{\circ}$. Observer-J. V. 
Tabulation of magnetic declinations observed at Teplitz Bay-Continued

\begin{tabular}{|c|c|c|c|c|c|c|c|c|c|c|c|c|c|c|c|c|c|c|c|c|c|}
\hline \multicolumn{6}{|c|}{ Friday, May 20, 1904} & \multicolumn{5}{|c|}{ Magnet scale inverted } & \multicolumn{6}{|c|}{ Sunday, May 22, 1904} & \multicolumn{5}{|c|}{ Magnet scale erect } \\
\hline \multirow{2}{*}{$\begin{array}{l}\text { Chr's } \\
\text { time }\end{array}$} & \multicolumn{2}{|c|}{$\begin{array}{l}\text { Scale } \\
\text { readings }\end{array}$} & $\begin{array}{l}\text { East } \\
\text { decli } \\
\text { natio }\end{array}$ & - Temp. & $\begin{array}{l}\text { Chr'r } \\
\text { time }\end{array}$ & $\begin{array}{l}\mathrm{Sc} \\
\mathrm{read}\end{array}$ & $\begin{array}{l}\text { ale } \\
\text { lings }\end{array}$ & $\begin{array}{l}\text { Eas } \\
\text { decl }\end{array}$ & & Temp. & Chr'r & $\begin{array}{r}\mathrm{Sc} \\
\text { reac }\end{array}$ & $\begin{array}{l}\text { cale } \\
\text { dings }\end{array}$ & East & & Chr'r & $\begin{array}{r}\mathrm{Sc} \\
\text { read }\end{array}$ & $\begin{array}{l}\text { ale } \\
\text { lings }\end{array}$ & $\begin{array}{l}\text { Eas } \\
\text { decl }\end{array}$ & & Temp. \\
\hline & Left & Right & & & & Left & Riglit & & & & & Left & Right & & & & Left & Right & & & \\
\hline h $\mathrm{m}$ & d & $d$ & $\cdot$ & $\bullet$ & $\mathrm{h} \mathrm{m}$ & d & d & $\cdot$ & & $1^{\circ}$ & $\mathrm{h} \mathrm{m}$ & d & d & & $\bullet$ & $\mathrm{h} \mathrm{m}$ & d & d & $\cdot$ & - & $\bullet$ \\
\hline $2000^{*}$ & 59.0 & 56.2 & 214 & $-4 \cdot 3$ & 2200 & 32.0 & 28.5 & 22 & 12 & $-4 \cdot 6$ & $0 \infty^{*}$ & 30.1 & 34.2 & 220 & -6.0 & 200 & 74.9 & $75 \cdot 3$ & 23 & 15 & -5.9 \\
\hline 02 & 56.1 & 49.3 & & $=0$ & 02 & 32.7 & 29.0 & & & 20 & 02 & 21.8 & 26.9 & 215 & & 02 & 73.2 & 73.6 & & 12 & \\
\hline 04 & 42.1 & 42.0 & 220 & 3 & 04 & L & ost & 2. & & fer & 04 & 21.1 & 24.6 & & 16 & 04 & 72.2 & 73.9 & & 12 & \\
\hline o6 & 42.8 & 41.4 & & 80 & $06^{\circ}$ & 35.2 & 31.0 & & 07 & 5 & of & 21.8 & 24.2 & & Wo & 0 & 69.0 & 71.2 & & 07 & 68 \\
\hline 08 & 42.0 & 39.8 & of & 16 & 08 & 35.8 & 29.9 & & 08 & os & 08 & 25.0 & 27.3 & & 80 & 08 & 65.7 & 69.2 & & 04 & 16 \\
\hline 10 & 45.8 & 42.9 & 0 & or & ro & 24.8 & 15.2 & & 28 & net & 10 & 23.5 & 26.2 & 5 & at & 10 & 67.3 & 70.0 & & 05 & 06 \\
\hline I2 & 47.8 & 43.0 & $22 \propto$ & 14 & $12^{*}$ & 25.8 & 17.0 & & 48 & tet & 12 & 24.8 & 27.3 & 5 & $\mathrm{n}$ & 12 & 67.3 & 60.9 & & 05 & \\
\hline 14 & 54.0 & 47.0 & 2I 5 & -4.1 & 14 & 10.1 & I0.0 & 225 & 59 & -4.8 & 14 & 24.8 & 26.0 & 5 & -6.0 & 14 & 69.1 & 71.1 & & 07 & -5.9 \\
\hline 16 & 51.0 & 40.0 & 220 & 7. & $16^{*}$ & 52.6 & 40.0 & 23 & 12 & th & $\begin{array}{l}4 \\
16\end{array}$ & 25.0 & 26.9 & 215 & & 16 & 69.9 & 72.7 & & $\infty$ & \\
\hline 18 & 47.0 & 14.7 & 2 & $\pi$ & 18 & 53.4 & 43.2 & & 09 & hit & 18 & 27.8 & 29.4 & 220 & an & 18 & $7 \mathrm{I} .0$ & 73.4 & g. & 10 & 43 \\
\hline 20 & $45 \cdot 3$ & 27.0 & $22 \mathrm{I}$ & 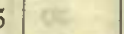 & 20 & 42.3 & 36.7 & $23=$ & 23 & 6. & 20 & 3I.I & 31.8 & 0 & 15 & 20 & 71.8 & $74 . \mathrm{I}$ & & 12 & 85 \\
\hline $22^{*}$ & 53.1 & $37 \cdot 3$ & 210 & is & 22 & $67 . \mathrm{I}$ & 57.9 & 22 & 47 & 4 & 22 & $34 . \mathrm{I}$ & 36.2 & I & 12 & 22 & 70.5 & 73.0 & & 10 & Ex \\
\hline $24^{*}$ & 55.0 & 36.0 & 5 & 156 & $24^{*}$ & 54.1 & $43 \cdot 3$ & & I9 & 10 & 24 & 37.9 & 39.6 & I & ic & 24 & 69.2 & 71.8 & & 08 & it \\
\hline 26 & 75.0 & 55.2 & 2 & 8 & 26 & 61.9 & 52.9 & $22 c$ & & E & 26 & $42 . \mathrm{I}$ & 43.8 & 2 & ine & 26 & 68.8 & 71.0 & & 07 & 64 \\
\hline 28 & $75 \cdot 3$ & 57.2 & I & 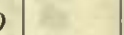 & 28 & 66.0 & 56.8 & 21 & 59 & kE & 28 & 40.8 & 42.1 & 2 & 8 & 28 & 70.1 & 72.4 & & $\infty 9$ & \\
\hline $30 *$ & 53.0 & 34.8 & 0 & -4.0 & 30 & 70.2 & 61.0 & & 53 & -5.0 & 30 & 44.1 & 45.4 & 2 & -6.0 & 30 & 71.0 & 73.0 & & 10 & -5.7 \\
\hline 32 & 51.4 & 37.3 & o & - & 32 & 68.0 & 59.0 & 21 & 56 & (n) & 32 & 43.5 & $\begin{array}{l}45.4 \\
45.2\end{array}$ & 2 & (6. & 32 & 70.8 & 72.3 & & $\infty$ & \\
\hline 34 & 39.0 & 24.1 & 2 & Wh: & 34 & 62.0 & 54.0 & 22 & 04 & [1. & 34 & 53.8 & 54.8 & 4 & ir & 34 & 70.0 & 71.9 & & 08 & x \\
\hline 36 & 27.1 & 12.0 & 4 & $e^{2}$ & 36 & 60.0 & 52.8 & & 07 & de & 36 & 53.3 & $54 . \mathrm{I}$ & 4 & os & 36 & 70.1 & 71.2 & c & 08 & 致 \\
\hline 38 & 28.0 & 12.2 & 4 & an & 38 & 58.0 & 51.0 & & ro & Eit & 38 & 53.0 & 54.1 & $\begin{array}{l}4 \\
4\end{array}$ & F & 38 & 72.2 & 73.9 & & 12 & 85 \\
\hline 40 & 19.9 & 7.0 & 5 & 14 & 40 & 56.1 & 49.5 & & 13 & be & 40 & 65.0 & 66.0 & 6 & 6 & 40 & 74.1 & 75.2 & & 14 & $\sigma_{*}$ \\
\hline 42 & 20.1 & 8.0 & 5 & 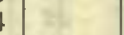 & 42 & 55.2 & 49.4 & & 14 & 4 & 42 & 59.3 & 62.3 & 5 & 62 & 42 & 75.2 & $76: 3$ & & 16 & $\mathrm{x}$ \\
\hline 44 & 2I.I & 9.7 & 5 & -4.0 & 44 & 52.9 & 47.9 & & 16 & $-5 . I$ & 44 & 36.2 & 41.5 & I & -6.0 & 44 & 76.8 & 77.9 & & 18 & -5.7 \\
\hline 46 & 20.7 & 8.7 & 5 & 8 & 46 & 47.1 & 42.0 & & 26 & ( & 46 & 44.7 & 48.0 & 3 & & $46 * 3$ & 52.6 & 58.4 & & 21 & \\
\hline 48 & 20.5 & 9.8 & 5 & 25 & 48 & 44.6 & 40.0 & & 29 & 4 & 48 & 48.0 & $53 . \mathrm{I}$ & 3 & A & 48 & 53.1 & 57.8 & & 20 & ha. \\
\hline 50 & 20.0 & 9.9 & 5 & $w$ & 50 & 46.2 & $4 \mathrm{I} .7$ & & 27 & $\omega$ & 50 & 43.1 & 48.8 & 2 & w. & 50 & 55.8 & 58.9 & & 23 & ele \\
\hline 52 & 20.0 & 10.5 & 5 & \& & 52 & 48.8 & $45 . \mathrm{I}$ & & 22 & Int & 52 & $48 . I$ & $52 . \mathrm{I}$ & 3 & 46 & 52 & 56.9 & 60.7 & & 26 & $x^{2}$ \\
\hline 54 & 19.0 & 10.0 & 5 & 47 & 54 & 50.2 & 46.0 & & 20 & 12 & 54 & 47.0 & 52.9 & 3 & 28 & 54 & 59.2 & 63.0 & & 29 & the \\
\hline 56 & 17.0 & 9.0 & 5 & as & 56 & 48.7 & 44.2 & & 23 & $s=$ & 56 & 46.7 & 53.8 & 3 & & 56 & 57.8 & 61.3 & & 27 & \\
\hline 58 & 15.0 & 7.4 & 2I 5 & b. & 58 & 48.1 & 44.2 & & 23 & & 58 & 54.0 & 61.7 & 4 & 8 & 58 & 56.0 & 59.3 & & 24 & \\
\hline 2100 & 43.2 & 32.5 & $22 \alpha$ & -4.2 & $23 \infty$ & 46.7 & 43.0 & & 25 & $-5 \cdot 3$ & 100 & 56.2 & 62.2 & 5 & -6.0 & $3 \infty$ & $54 \cdot 3$ & 57.9 & & 22 & -5.5 \\
\hline 02 & 43.1 & 31.5 & 0 & & 02 & 43.0 & $4 \mathrm{I} . \mathrm{I}$ & & 30 & 40 & 02 & 58.7 & 63.3 & 225 & & 02 & 54.1 & 57.2 & & $2 \mathrm{I}$ & \\
\hline 04 & 42.0 & $3 \mathrm{I}, \mathrm{I}$ & 0 & te & 04 & 43.8 & 40.9 & & 29 & we & 04 & 68.0 & 72.2 & 230 & 10 & 04 & 51.9 & 55.0 & & 17 & 45 \\
\hline of & 39.8 & 28.8 & 0 & 80 & о6 & 45.2 & 42.9 & & 26 & 90 & 06 & 66.9 & 71.9 & 230 & & $\infty 6$ & $50 . \bar{I}$ & 53.3 & & 15 & \\
\hline od. & 37.2 & 28.2 & $a$ & $8 u$ & 08 & 46.3 & 44.8 & e & 24 & 89 & 08 & 61.6 & 66.7 & 225 & 30 & 08 & 50.3 & 53.4 & 1 & 15 & in \\
\hline ICI & 31.0 & $27 \cdot 3$ & If & 4 & 10 & $45 . \overline{8}$ & 44.2 & & 25 & WiI & Io & 58.0 & 61.5 & 5 & 04 & 10 & 49.9 & $52 . \mathrm{I}$ & & 14 & Tit \\
\hline 12: & 36.1 & 27.0 & IC & ar & 12 & 44.2 & 42.9 & & 27 & 65 & 12 & 57.1 & 59.9 & 4 & 61 & 12 & 47.8 & 50.1 & & 10 & \\
\hline 14 & 35.0 & 27.0 & 5 & $-4 \cdot 3$ & 14 & 43.2 & 42.1 & y & 29 & -5.5 & I4 & 56.0 & 58.1 & 4 & -6.0 & 14 & 47.2 & 49.8 & & 10 & -5.5 \\
\hline 16 & 36.2 & 28.9 & o & & 16 & 43.0 & 42.1 & 9 & 29 & & 16 & 56.0 & 58.7 & 4 & & 16 & 45.8 & 48.1 & & 07 & \\
\hline 18 & 34.9 & 28.0 & I & in & 18 & 44.0 & 43.5 & & 27 & Ef & 18 & 58.5 & 61.4 & $\mathrm{x}$ & 21 & 18 & 44.9 & 47.0 & & 05 & ex \\
\hline 20 & 33.9 & 27.1 & $\%$ & Ar & 20 & 44.7 & 43.7 & & 26 & ot & 20 & 59.3 & 62.0 & 중 & $k=$ & 20 & 43.9 & 46.1 & & 04 & 28 \\
\hline 22 & 33.0 & 27.1 & I. & ex & 22 & 43.0 & $4 \mathrm{I} .9$ & 1. & 29 & tes & 22 & 58.3 & $6 \mathrm{I} .2$ & 8 & $8 \pi$ & 22 & $42 . \mathrm{I}$ & 44.4 & $23 c$ & & net \\
\hline 24 & 32.0 & 26.3 & I. & 20 & 24 & 40.5 & $39 . \mathrm{I}$ & at. & 33 & 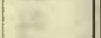 & 24 & 60.3 & 62.7 & 5 & & 24 & 40.3 & 42.4 & 225 & 58 & re \\
\hline 26 & 30.4 & 26.0 & 0 & 80 & 26 & 39.9 & 38. & 6 & 34 & 62 & 26 & 62.2 & $64 \cdot 3$ & 5 & de & 26 & 39.8 & 40.5 & & 56 & \\
\hline 28 & 30.3 & 26.1 & I & $z=$ & 28 & 40.0 & 38.9 & 8 & 34 & $1=$ & 28 & 63.6 & 65.9 & 225 & & 28 & $40 . I$ & 41.7 & is & 58 & \\
\hline 30 & 33.0 & 29.5 & 1 & $-4 \cdot 4$ & 30 & 40.2 & 38.9 & & 34 & -5.7 & 30 & 66.1 & 68. I & 230 & -6.0 & 30 & 40.1 & 41.9 & & 58 & -5.4 \\
\hline 32 & 32.2 & 29.0 & 1. 1 & & 32 & 39.3 & 38.4 & & 35 & the & 32 & 69.5 & 71.8 & a & 4 & 32 & 40.1 & 41.9 & & 58 & \\
\hline 34 & 33.0 & 30.0 & $\mathbf{I}$ & 16 & 34 & 37.7 & 37.0 & & 37 & 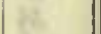 & 34 & 73.2 & 75.0 & I. & & 34 & $4 \mathrm{I} . \mathrm{I}$ & 42.3 & 225 & 59 & \\
\hline 36 & 32.2 & 29.3 & 0. I & dy & 36 & 37.0 & 36.2 & & 38 & ite & 36 & 74.2 & 75.9 & I & & 36 & 42.0 & $43 . I$ & 230 & $\infty$ & \\
\hline 38 & 32.3 & 29.8 & I & 8 & 38 & 37.6 & 36.8 & & 37 & kF & 38 & 74.2 & 76.0 & I. & 26 & 38 & $4 \mathrm{I} . \mathrm{I}$ & 42.6 & 225 & 59 & \\
\hline 40 & 33.2 & 31.0 & 0 & 08 & 40 & 37.0 & 36.6 & & 38 & Des. & 40 & 73.0 & 74.5 & I & is & 40 & 41.3 & 42.7 & & 59 & \\
\hline 42 & 35.5 & 32.9 & 0 & A & 42 & 37.0 & 36.2 & & 38 & & 42 & 72.9 & 74.0 & I & & 42 & 40.2 & $4 \mathrm{I} .5$ & & 58 & \\
\hline 44.5 & 34.0 & 30.2 & 0 & -4.5 & 44 & 37.8 & 37.0 & & 37 & -5.8 & 44 & 73.3 & 74.8 & I. & -6.0 & 44 & 39.2 & 40.6 & & 56 & $-5 \cdot 3$ \\
\hline 46 & 33.0 & 30.0 & I & & 46 & 37.0 & 36.0 & 4 & 38 & & 46 & 71.2 & 72.9 & I & & 46 & 40.2 & $4 \mathrm{I} . \mathrm{I}$ & & 57 & \\
\hline 48 & 32.0 & 28.0 & I & 14 & 48 & 35.0 & 34.0 & & 41 & b & 48 & 68.9 & 70.1 & $\alpha$ & +6 & 48 & $4 \mathrm{I} .3$ & 42.4 & & 59 & \\
\hline & 33.9 & 30.8 & 0 & se & 50 & 35.1 & 34.1 & 6 & 41 & 0 & 50 & 66.3 & 67.8 & 0 & 9 & 50 & $4 \mathrm{I} . \mathrm{I}$ & 42.3 & & 59 & \\
\hline 52 & 32.1 & 30.0 & I & $x$ & 52 & 35.8 & 34.2 & 5. & $4 \mathrm{I}$ & 12 & 52 & 65.9 & 67.0 & 0 & 6 & 52 & $4 \mathrm{I} \cdot 3$ & 42.5 & 225 & 59 & \\
\hline 54 & 30.9 & 29.0 & $I$ & $\mathrm{k}^{2}$ & 54 & 38.2 & 34.9 & 2) 3 & 38 & 18 & 54 & 68.1 & 69.0 & 0 & kz & 54 & 42.7 & 43.6 & 230 & OI & \\
\hline 56 & 30.0 & 26.8 & I & & 56 & 37.1 & 40.0 & & 35 & n & 56 & 71.5 & 72.8 & (1) & & 56 & 42.3 & 43.0 & & $\infty$ & \\
\hline 58 & 31.8 & 28.1 & I & te & 58 & 40.3 & 37.8 & & 34 & & 58 & 75.2 & 76.2 & 1 & ket & 58 & 42.5 & 43.7 & 0 & OI & ty \\
\hline & & & & $\infty 0$ & 2400 & 39.2 & 37.0 & & 36 & -5.5 & & & & & & & & & & & \\
\hline
\end{tabular}

Correction to local mean time is $-4 \mathrm{~s}$. $90^{\circ}$ torsion $=18 .^{\prime} 24$.

Torsion head at $\mathrm{Igh} 35 \mathrm{~m}$ read $83^{\circ}$ and at $24 \mathrm{~h} 2 \mathrm{Im}$ read $89^{\circ}$.

Observer-R. R. T.

Observer-J. V. 
Tabulation of magnetic declinations observed at Teplitz Bay-Continued

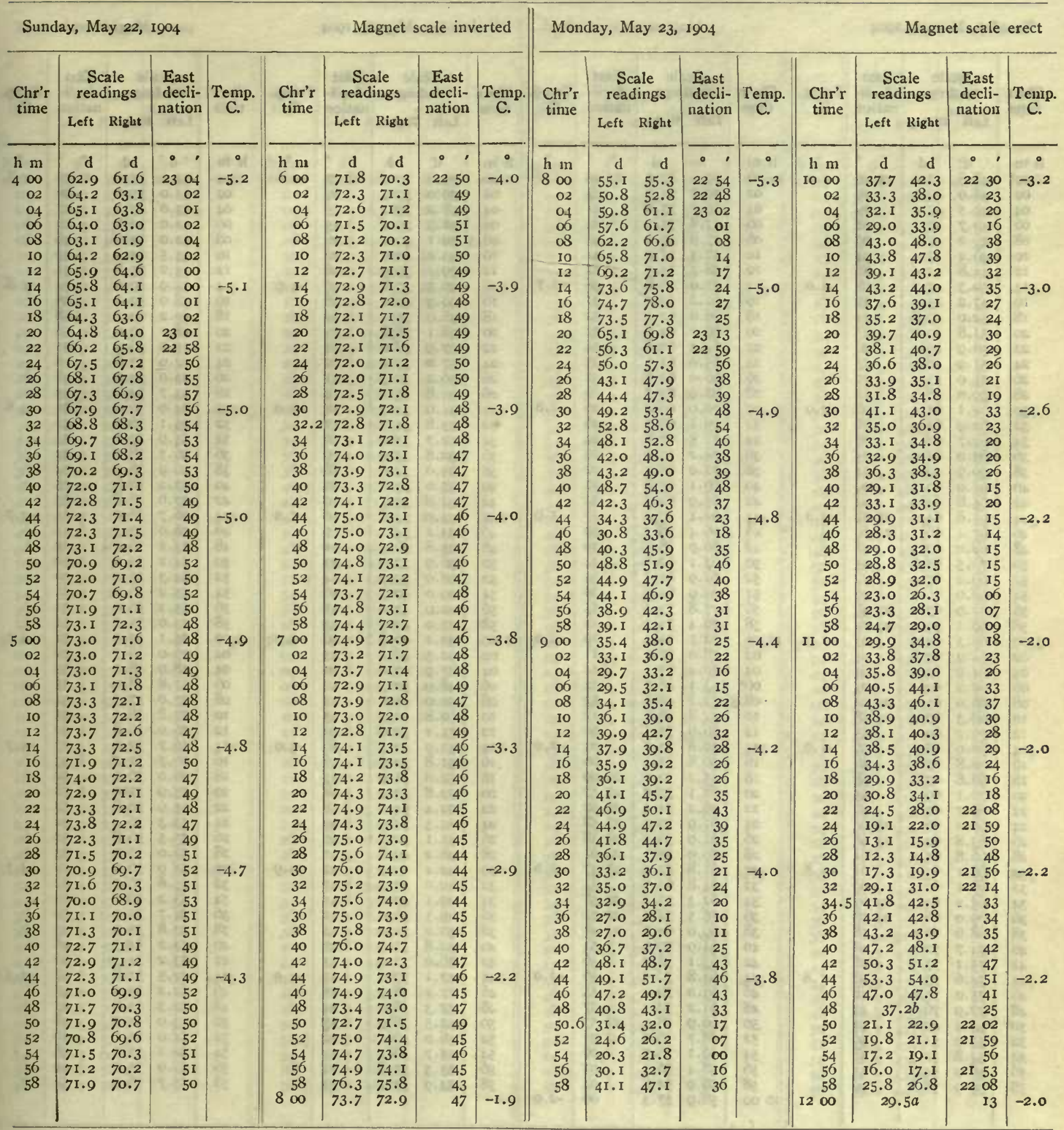

Correction to local mean time is $-22 \mathrm{~s}, 90^{\circ}$ torsion $=18 .^{\prime} 60$. Torsion head at oln oom read $90^{\circ}$ and at $8 \mathrm{~h}$ 2on read $85^{\circ}$. Observer-R. R. T.
Correction to local mean time is $-41.5 \mathrm{~s}$. $90^{\circ}$ torsion $=19 . .^{\prime} 62$. Torsion head at $7 \mathrm{~h} 4 \mathrm{Om}$ read $85^{\circ}$ and at $\mathrm{i} 2 \mathrm{~h}$ 20m read $9 \mathrm{I}^{\circ}$. Observer-R. R. T. 
Tabulation of magnetic declinations observed at Teplitz Bay-Continued

\begin{tabular}{|c|c|c|c|c|c|c|c|c|c|c|c|c|c|c|c|c|c|c|c|}
\hline \multicolumn{6}{|c|}{ 'Tuesday, May 24, 1904} & \multicolumn{4}{|c|}{ Magnet scale inverted } & \multicolumn{6}{|c|}{ Wednesday, May 25, 1904} & \multicolumn{4}{|c|}{ Magnet scale erect } \\
\hline \multirow{2}{*}{$\begin{array}{l}\text { Chr'r } \\
\text { tinze }\end{array}$} & \multicolumn{2}{|c|}{$\begin{array}{c}\text { Scale } \\
\text { readings }\end{array}$} & \multirow{2}{*}{$\begin{array}{c}\text { East } \\
\text { decli- } \\
\text { nation }\end{array}$} & Temp. & Chr'r & $\begin{array}{r}\text { Sc } \\
\text { reac }\end{array}$ & ale & $\begin{array}{l}\text { East } \\
\text { decli- }\end{array}$ & Temp. & Chr'r & $\begin{array}{r}\text { Sc } \\
\text { reac }\end{array}$ & dings & $\begin{array}{l}\text { East } \\
\text { decli- }\end{array}$ & Temp. & Chr'r & $\begin{array}{r}\mathrm{Sc} \\
\mathrm{read}\end{array}$ & $\begin{array}{l}\text { :ale } \\
\text { lings }\end{array}$ & $\begin{array}{l}\text { East } \\
\text { decli- }\end{array}$ & Temp. \\
\hline & Left & Right & & & & Left & Right & & & & Left & Right & & & & Left & Right & & \\
\hline & & $d$ & & 0 & $\mathrm{lim}$ & d & a & & & $h \mathrm{in}$ & & & & & $\mathrm{h} \mathrm{m}$ & $\mathrm{d}$ & d & & \\
\hline $00^{*}$ & 48.8 & 47.9 & 2329 & -2.4 & I4 00 & 59.6 & 57.9 & 2240 & -1.7 & 000 & 39.8 & 40.0 & 2227 & -4.6 & 200 & 54.9 & 55.0 & 2250 & -4.8 \\
\hline $\begin{array}{l}02 \\
04\end{array}$ & 48.9 & & $\begin{array}{l}29 \\
20\end{array}$ & 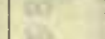 & $\begin{array}{l}02 \\
04\end{array}$ & $\begin{array}{l}50.9 \\
50.7\end{array}$ & $\begin{array}{l}54.7 \\
57.0\end{array}$ & $\begin{array}{r}45 \\
4 \mathrm{~T}\end{array}$ & 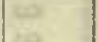 & 02 & 39.0 & 39.9 & 26 & & 02 & 55.0 & 55.0 & 50 & \\
\hline $\begin{array}{l}04 \\
06\end{array}$ & $\begin{array}{l}49.5 \\
49.7\end{array}$ & $\begin{array}{l}47.2 \\
47.1\end{array}$ & $\begin{array}{l}29 \\
28\end{array}$ & 80 & $\begin{array}{l}04 . \\
06 .\end{array}$ & 57.8 & $\begin{array}{l}57.0 \\
55.1\end{array}$ & $\begin{array}{l}41 \\
44\end{array}$ & 25 & $\begin{array}{l}04 \\
05\end{array}$ & $\begin{array}{l}38.2 \\
38.3\end{array}$ & 39.1 & $\begin{array}{l}25 \\
25\end{array}$ & 10 & 04 & 53.0 & 53.3 & 47 & \\
\hline as & 49.2 & 47.2 & & 80 & 08 & 57.9 & 55.4 & 44 & 8 & 08.3 & 39.1 & $\begin{array}{l}39.2 \\
40.0\end{array}$ & $\begin{array}{l}25 \\
26\end{array}$ & 20 & $\begin{array}{l}\infty \\
08\end{array}$ & $\begin{array}{l}2.0 \\
52.0\end{array}$ & 53.0 & $\begin{array}{l}40 \\
46\end{array}$ & \\
\hline Io & 46.4 & 43.5 & 34 & uff & 10 & 57.3 & 55.0 & 44 & a & 10 & $39 . \mathrm{I}$ & 40.0 & 25 & ar & I0 & 53.5 & 54.4 & 49 & \\
\hline 12 & 44.8 & 42.0 & 36 & 81 & 12 & 58.7 & 55.9 & 42 & & 12 & 39.1 & 39.9 & 26 & 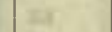 & 12 & 55.2 & 55.8 & 5I & \\
\hline 14 & 50.3 & 47.7 & 28 & -2.0 & 14 & 61.0 & 58.8 & 38 & -1.7 & 14 & 39.5 & 39.8 & 26 & -4.9 & 14 & 55.9 & 59.0 & 54 & -4.8 \\
\hline 16 & 47.2 & & 32 & & 16 & 62.2 & 59.2 & 37 & & 16 & 39.3 & 40.0 & 26 & & I6 & 58.0 & 59.1 & 56 & \\
\hline 18 & 52.0 & 49.8 & 24 & ke & 18 & 66.0 & $62 . I$ & 32 & 8 & 18 & 38.9 & & 25 & in & 18 & 59.2 & 60.2 & 58 & \\
\hline 20 & 51.8 & 49.0 & 25 & 15 & 20 & 65.7 & 62.8 & $3 \mathrm{I}$ & 10 & 20 & .1 & & 24 & es & 20 & $58 . \mathrm{I}$ & 59.0 & 56 & \\
\hline 22 & 50.9 & 49. & 26 & 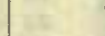 & 22 & 64.3 & 60.8 & 34 & es & 22 & .9 & 38.0 & 24 & as & 22 & 55.0 & & $5 \mathrm{I}$ & \\
\hline 24 & $5 \mathrm{I} .9$ & 48.9 & 25 & 4 & 24 & 65.0 & 61.9 & 33 & 20. & 24 & & & 22 & 14 & 24 & 52.9 & 53 & 48 & \\
\hline 26 & & 53.1 & 20 & 6 & 26 & 66.0 & 62.0 & 32 & e & 26 & 36.1 & & $2 \mathrm{I}$ & 8 & 26 & 53.0 & & 48 & 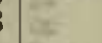 \\
\hline 28 & & 58.2 & 12 & the & 28 & 67.9 & 63.7 & 29 & & 28 & 34.8 & & 19 & 62 & 28 & 53.7 & & 49 & \\
\hline 30 & 62.1 & $6 \mathrm{r} .2$ & 08 & -2.0 & 30 & 70.0 & 66.2 & 26 & -1.8 & 30 & 34.0 & & 18 & -5.0 & 30 & 54.9 & 56.0 & 51 & -4.8 \\
\hline 32 & 62.9 & 62.3 & 06 & 8 & 32 & 70.1 & 66.0 & 26 & 25 & 32 & 33.8 & 34.2 & 17 & & 32 & $55 . \mathrm{I}$ & 56.5 & 52 & \\
\hline 34 & 62.7 & 62.1 & of & $E$ & 34 & 71.5 & 68.0 & 23 & it & 34 & 32.7 & 33 & 16 & 25 & 34 & 55.9 & & 53 & \\
\hline 36 & 63.0 & 62.9 & 05 & \& & 36 & 74.2 & 70.6 & 19 & 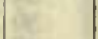 & 36 & 32.8 & & 16 & 18 & 36 & 56.5 & & 54 & \\
\hline 38 & 59.9 & 59.2 & II & AE & 38 & 72.0 & 68.3 & 22 & 20 & 38 & 34.0 & 34.8 & 18 & 6 & 38 & 56.9 & 58.2 & 54 & \\
\hline 40 & 61.0 & 60.5 & $\infty$ & 97 & 40 & 69.1 & 65.8 & 27 & tar. & 40 & 35.0 & 35.8 & 20 & 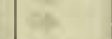 & 40 & 57.2 & & 55 & ; \\
\hline 42 & 65.8 & 64.9 & 02 & & 42 & 65.9 & 62.3 & 32 & & 42 & 34.8 & & 19 & $=8$ & 42 & 57.6 & & 55 & \\
\hline 44 & 65.8 & 64.3 & 2302 & -2.0 & 44 & 62.1 & 59.2 & 37 & -1.9 & 44 & 33.0 & 33 & 16 & -5.0 & 44 & 56.7 & 58.0 & 54 & $-4 \cdot 7$ \\
\hline 46 & 67.4 & $66 . I$ & 2260 & & 46 & 60.4 & 58.1 & 39 & & 46 & 33.2 & & 17 & & 46 & 56.0 & & 53 & \\
\hline 48 & 68.2 & 67.2 & 58 & 8 & 48 & 60.9 & 58.1 & 39 & 5 & 48 & 35.8 & & 21 & 4 & 48 & 55.2 & & & \\
\hline 50 & 70 & 68. & & $\Rightarrow$ & 50 & 63.1 & 62.0 & 34 & e & 50 & & & 23 & of & 50 & 54 & & & \\
\hline 52 & 69 & 67 & 57 & $E$ & 52 & 62.9 & 6r.I & 35 & E & 52 & 0 & & 24 & 2 & 52 & & & & \\
\hline 54 & 68.0 & 66.7 & 59 & & 54 & 65.9 & 64. & 30 & 8 & 54 & .0 & & 26 & F & 54 & 55 & & & \\
\hline 56 & 67. & 67.0 & 2259 & tiv & 56 & 75.8 & 73.3 & I5 & & 56 & & & 31 & & & & & & \\
\hline 58 & 60.1 & 59.5 & 2310 & 6 & 58 & 76.4 & & I4 & & 58 & & & 33 & Bs & 58 & 55.0 & & & \\
\hline 300 & 65.3 & 64.0 & 03 & -1.9 & I5 & & 71.3 & IO & -2.0 & I 00 & & & 36 & -5.0 & 300 & 55.1 & & 51 & -4.7 \\
\hline 02 & 64.9 & 63.8 & 03 & A. & 02 & 78.0 & 77.0 & II & 60 & 02 & 42.3 & 46.2 & 33 & & 02 & 56.0 & & 52 & \\
\hline 04 & 64.9 & 63.5 & 04 & ia & 04 & 76.4 & 75.1 & I4 & 10 & 04 & 47.9 & & $\begin{array}{l}30 \\
40\end{array}$ & 49 & 04 & 58.0 & 58.2 & & \\
\hline 06 & 65.2 & 64.2 & 03 & 6 & $06^{*}$ & 53.1 & 48.7 & 12 & 6 & $\begin{array}{l}0 \\
06\end{array}$ & $\begin{array}{l}48.9 \\
48.9\end{array}$ & $\begin{array}{l}49 \\
50\end{array}$ & 42 & s. & 05 & 57.8 & 58.0 & 55 & \\
\hline os & 66.0 & 65.2 & 2302 & के & 08.5 & 52 & 48.6 & 12 & to & 08 & & & 43 & 6 & o & 57.0 & & & \\
\hline Io & 68.3 & 67. & 2258 & ef & 10 & 52.8 & 48.2 & I2 & ait & IC & 50.8 & & 45 & e & & & & & \\
\hline 12 & 70 & & & & 12 & & 51.5 & 06 & & 12 & & & 46 & & 12 & 56.0 & 56.8 & 5 & \\
\hline 14 & 70.0 & 68. & 56 & -1.9 & $\cdot 14$ & & 53.0 & 03 & -2.0 & 14 & 51.9 & & 46 & -5.0 & 14 & & & 53 & -4.7 \\
\hline 16 & 68.8 & 67. & 58 & & I6 & 56.8 & $5 \mathrm{I}$. & 06 & & I6 & & & 46 & & I6 & & & & \\
\hline 18 & 68.8 & & 58 & के & 18 & & & 2209 & 67 & I8 & & & 45 & 6 & 18 & & & & \\
\hline 20 & 70 & & 5 & $\infty$ & 20 & & & & 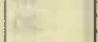 & 20 & & & 48 & 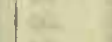 & 0 & & & 5 & \\
\hline 22 & 69 & & 6 & 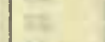 & 22 & & & 02 & ut & 22 & 52.4 & & 48 & is & 22 & & & & \\
\hline 24 & 67 & 66. & 2260 & $y=$ & 24 & & & 58 & $=$ & 24 & 53.3 & & 49 & is & 24 & & & 5 & \\
\hline 26 & 63 & 62 & 2306 & 8 & 26 & & 56 & 2158 & 5 & 26 & & & 46 & 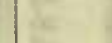 & 26 & & & 2 & \\
\hline 28 & 66. & 65. & or & & 28 & & 55. & 22 or & & 28 & 48.2 & 49.8 & $4 \mathrm{I}$ & his & 28 & & & 5 & \\
\hline 30 & 66. & 65 & OI & -1.8 & 30 & & 57.9 & 2157 & -2.0 & 30 & 49.4 & & 42 & -4 & 30 & 55 & & 52 & -4.7 \\
\hline 32 & 67.1 & 66.2 & 00 & $x^{2}$ & 32 & 64.8 & 60.7 & 53 & rar & 32 & & & $4 \mathrm{II}$ & & 32 & 55.6 & 56 & 5 & \\
\hline & 65.2 & 63.7 & 2303 & ate & 34 & 62.3 & 57.2 & 2158 & (12. & 34 & 49.1 & & $4 \mathrm{I}$ & it & 34 & 55.2 & 56.1 & 51 & \\
\hline & 69 & 66.5 & 2258 & E. & 36 & 59.5 & 54.0 & 2202 & 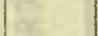 & 36 & & & 42 & 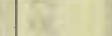 & 36 & & & 50 & \\
\hline 38 & 68.6 & 66. & 58 & 82 & 38 & 69 & 64.7 & 2146 & E. & 38 & & $5 \mathrm{I}$ & 43 & to. & 38 & 53.8 & 55.0 & 49 & \\
\hline $4 c$ & 74.7 & & 48 & 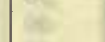 & 40 & & & 53 & & 40 & 52 & & 47 & $\omega$ & & & & & \\
\hline $4^{2}$ & & & 48 & as & 42 & & 58 & 56 & & 42 & 56.0 & & 53 & & & & & 48 & \\
\hline & & & & -1.8 & 44 & & & 56 & -2.0 & & & & 55 & -4.9 & & 52.5 & 53 & & $-4 \cdot 5$ \\
\hline & $\begin{array}{l}74 \\
75\end{array}$ & & & & 46 & & 59 & 55 & & 4 & & & 5 & 7 & & & & & \\
\hline & & & 43 & 80 & 48 & & & & & 4 & & & 5 & 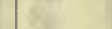 & & & & & \\
\hline & & & 52 & $\omega$ & $5 c$ & & & & & & & & & eve & & & & & \\
\hline & & & & 15 & 52 & & & & & 52 & & & 5 & & & & & & \\
\hline & & & & $y$ & 54 & & & $\infty$ & & 5 & & & & & & & & & \\
\hline & & & & $c$ & 56 & & & OI & & 56 & 55.3 & & 51 & 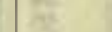 & & & & & \\
\hline 58 & 59.9 & 58.8 & $\begin{array}{l}45 \\
39\end{array}$ & 8 & 58 & 56. & 54.8 & 04 & & 58 & 54.9 & 55.1 & 50 & $\beta$ & 58 & 55.7 & 56.9 & & \\
\hline & & & & $65=$ & 1000 & 58.9 & $57 \cdot 3$ & $\infty$ & -2.0 & & & & & 60) & & & & & \\
\hline
\end{tabular}

Correction to local mean time is $-\mathrm{Im}$ 19.5s. $90^{\circ}$ torsion $=18 . .^{\prime} 60$.

Observer-J. V.

Torsion head at $\mathrm{IIh} 35 \mathrm{~m}$ read $93^{\circ}$ and at $16 \mathrm{~h} 15 \mathrm{~m}$ read $96^{\circ}$.

Observer-R. R. T. 
Tabulation of magnetic declinations obserz'ed at Teplitz Bay-Continued

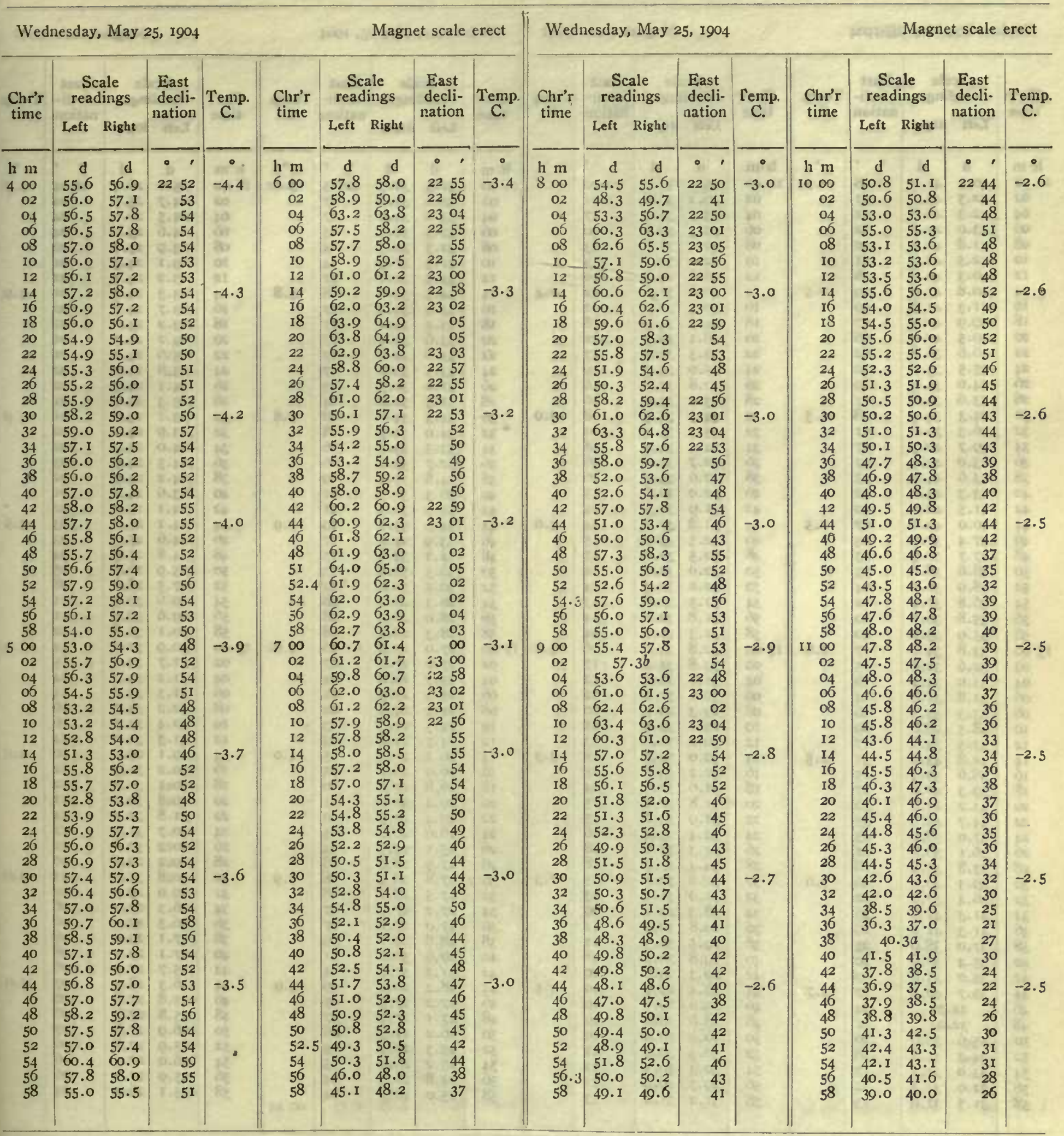

Observers-J. V. and W. J. P., who alternated from $7 \mathrm{l}_{1} 56 \mathrm{~m}$ to Observer-W. J. P. $8 \mathrm{~h} 06 \mathrm{~m}$. 
Tabulation of magnetic declinations observed at Teplitz Bay-Continued

\begin{tabular}{|c|c|c|c|c|c|c|c|c|c|c|c|c|c|c|c|c|c|c|c|}
\hline \multicolumn{5}{|c|}{ Weduesday, May 25, 1904} & \multicolumn{5}{|c|}{ Magnet scale erect } & \multicolumn{6}{|c|}{ Wednesday, May 25, I904 } & \multicolumn{4}{|c|}{ Magnct scale erect } \\
\hline \multirow{2}{*}{$\begin{array}{l}\text { Chr'r } \\
\text { time }\end{array}$} & \multicolumn{2}{|c|}{$\begin{array}{l}\text { Scale } \\
\text { readings }\end{array}$} & \multirow[t]{2}{*}{$\begin{array}{c}\text { East } \\
\text { decli- } \\
\text { nation. }\end{array}$} & Teung. & Clur'r & $\begin{array}{r}\text { Sc } \\
\text { read }\end{array}$ & $\begin{array}{l}\text { ale } \\
\text { ings }\end{array}$ & $\begin{array}{l}\text { East } \\
\text { decli- } \\
\text { nation }\end{array}$ & iemp & Chr'r & $\begin{array}{l}\text { Sc } \\
\text { read }\end{array}$ & $\begin{array}{l}\text { ale } \\
\text { lings }\end{array}$ & East & Teinp. & Chr'r & $\begin{array}{r}\text { Sc } \\
\text { read }\end{array}$ & le & $\begin{array}{l}\text { East } \\
\text { decli- }\end{array}$ & Temp. \\
\hline & Left & Right & & & & L,eft & Right & & & & Left & Right & & & & Left & Right & & \\
\hline li $\mathrm{m}$ & d & d & - ' & 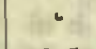 & h m & d & d & & & $\mathrm{h} \mathrm{m}$ & $d$ & $d$ & $\cdot \quad$ & & $\mathrm{h} \mathrm{m}$ & d & d & & $\bullet$ \\
\hline 1200 & 40.5 & 41.0 & 2228 & -2.5 & I4 00 & 35.2 & 36.0 & 2220 & -2.8 & 1600 & 32.3 & 32.5 & 22 I5 & -3.6 & 1800 & 25.0 & 26.4 & 2204 & $-3 \cdot 9$ \\
\hline 02 & 42.5 & $43 . I$ & 3I & 200 & 02 & 36.2 & 36.8 & $2 I$ & va & 02 & 34.0 & 34.0 & 17 & & 02 & 24.7 & 25.9 & & \\
\hline 04 & 42.3 & 42.8 & $3 I$ & 6 & 04 & 33.7 & 34.4 & 17 & 30 & 04 & 33.8 & 34.0 & 17 & xal & 04 & 24.5 & 26.0 & 04 & \\
\hline 06 & 43.0 & 43.6 & 32 & $\mathrm{cos}^{\circ}$ & 06 & 35.3 & $36 . \mathrm{I}$ & 20 & 10 & 06 & 32.3 & 32.5 & 15 & 90 & $\infty 6$ & 24.6 & 25.9 & 04 & \\
\hline 08 & 4I.8 & 42.5 & 30 & (6) & 08 & 30.4 & 31.6 & 13 & $B 2$ & 08 & 32.3 & 32.3 & 15 & 34 & 08 & 23.9 & $25 . \bar{I}$ & 02 & \\
\hline Io & 42.5 & 42.8 & 3I & et & 10 & 32.0 & 32.5 & 14 & 21 & 10 & 31.3 & $3 \mathrm{I} .9$ & 14 & or & Io & 23.1 & $24 \cdot 3$ & or & \\
\hline 12 & 40.3 & 40.8 & 28 & er & 12 & 28.8 & 29.3 & 10 & 312 & 12 & 33.5 & 34.0 & 17 & 67 & 12 & 23.2 & 24.4 & OI & \\
\hline 14 & 40.8 & 41.6 & 29 & -2.5 & I4 & 30.6 & 31.0 & I2 & -2.4 & I4 & 34.3 & 34.6 & 18 & -3.8 & 14 & 23.3 & 24.4 & OI & -3.9 \\
\hline I6 & $41 \cdot 3$ & 41.9 & 29 & (25) & 16 & 30.3 & 31.0 & 12 & ir & 16 & 33.9 & 34.2 & 17 & & I6 & 23.7 & 24.8 & 02 & \\
\hline 18 & 39.9 & 40.5 & 27 & $8 t$ & 18 & 34.5 & 34.9 & 18 & $x$ & 18 & 33.5 & 34.2 & 17 & 81 & 18 & 23.2 & 24.1 & OI & int \\
\hline 20 & 39.5 & 40.0 & 26 & $c$ & 20 & 36.1 & 36.3 & $2 \mathrm{I}$ & $x^{2}$ & 20 & 33.6 & 33.9 & 17 & Ex & 20 & 23.2 & 24.1 & oI & tas \\
\hline 22 & 36.6 & 36.8 & 22 & L6. & 22 & 33.3 & 34.0 & 17 & $=0$ & 22 & 33.6 & 34.0 & 17 & E & 22 & 23.6 & 24.1 & OI & 44 \\
\hline 24 & 35.5 & 35.6 & 20 & 15 & 24 & 35.0 & 35.3 & 19 & 16 & 24 & $33 \cdot 3$ & 33.7 & 17 & 15 & 24 & 23.8 & 24.2 & 02 & 34 \\
\hline 26 & 34.6 & 35.0 & I9 & as & 26 & 32.8 & 33.6 & 16 & - & 26 & 31.0 & 31.5 & 13 & $\notin$ & 26 & 24.1 & 24.5 & 02 & $x^{2}$ \\
\hline 28 & 33.6 & 33.8 & 17 & $=$ & 28 & 33.0 & 33.9 & 16 & 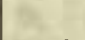 & 28 & $31 \cdot 3$ & 32.0 & 14 & $x^{2}$ & 28 & 23.3 & 23.9 & or & \\
\hline 30 & $34 \cdot 3$ & 34.5 & I8 & -2.5 & 30 & 34.3 & 34.5 & I8 & -3.0 & 30 & 34.3 & 34.6 & 18 & -3.8 & 30 & $23 . I$ & 23.5 & OI & -3.9 \\
\hline 32 & 36.3 & 36.6 & $2 \mathrm{I}$ & 45 & 32 & 35.3 & 35.5 & 20 & the & 32 & 34.0 & 34.3 & 18 & 46 & 32 & 23.9 & $24 . I$ & 02 & \\
\hline 34 & 39.7 & 40.3 & 27 & tes & 34 & 34.4 & 34.4 & 18 & 15 & 34 & $33 \cdot 3$ & 33.5 & 16 & 16 & 34 & 24.0 & $24 \cdot 3$ & 02 & \\
\hline 36 & 43.0 & 43.5 & 32 & alle. & 36 & 32.7 & 33.3 & I6 & a & 36 & 32.7 & 33.0 & 16 & 2 & 36 & 24.5 & $24 \cdot 9$ & 03 & es \\
\hline 38 & 43.6 & 44.0 & 33 & 5 & 38 & 32.6 & 33.0 & 16 & 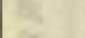 & 38 & 34.8 & 35.5 & 19 & 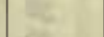 & 38 & 24.2 & 24.9 & 02 & E: \\
\hline 40 & $43 \cdot 3$ & 43.6 & 32 & $\omega$ & 40 & 32.0 & 32.6 & I5 & 6 & 40 & 36.3 & 36.5 & $2 I$ & 50 & 40 & 23.6 & $24 . I$ & OI & tef \\
\hline 42 & $4 \mathrm{I} .5$ & 42.0 & 30 & 64 & 42 & 31.9 & 32.1 & 14 & & 42 & 35.9 & 36.3 & $2 \mathrm{I}$ & 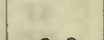 & 42 & $24 . I$ & 25.0 & 02 & \\
\hline 44 & 40.0 & 40.0 & 27 & -2.5 & 44 & 31.8 & 32.2 & 14 & -3.0 & 44 & 34.9 & $35 \cdot 3$ & I9 & -3.9 & 44 & 24.1 & $25 . I$ & 03 & -3.9 \\
\hline 46 & $37 \cdot 4$ & 37.7 & 23 & & 46 & 32.5 & 33.0 & 15 & 7 & 46 & 33.9 & $34 . \mathrm{I}$ & 17 & & 46 & 24.6 & 25.7 & 03 & \\
\hline 48 & 37.7 & 37.9 & 23 & 76 & 48 & 32.5 & 33.0 & 15 & 6 & 48 & 32.2 & 32.6 & 15 & 14 & 48 & 24.8 & 25.8 & 04 & \\
\hline 50 & 38.6 & 38.6 & 25 & tos & 50 & 32.0 & 32.5 & I4 & a & 50 & 32.5 & 32.8 & 15 & 14 & 50 & 25.9 & 26.8 & 05 & es \\
\hline 52 & 38.0 & 38.5 & 24 & 8 & 52 & 32.0 & 32.3 & 14 & 8 & 52 & 30.5 & 30.8 & 12 & 28 & 52 & 27.5 & 28.8 & 08 & 9 \\
\hline 54 & 36.8 & 37.2 & 22 & $B^{2}$ & 54 & $3 \mathrm{~T} \cdot 3$ & 31.6 & 13 & 145 & 54 & 28.3 & 28.6 & 08 & ie & 54 & 29.6 & 30.9 & II & \\
\hline 56 & 35.8 & 36.1 & 20 & $\Leftrightarrow=$ & 56 & $3 \mathrm{I} .8$ & $32 . I$ & 14 & as & 56 & 28.0 & 28.3 & \$8 & 2 & 56 & 29.6 & 30 & I I & \\
\hline 58 & 35.9 & 35.9 & 20 & 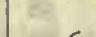 & 58 & 31.3 & 32.1 & I4 & $2 x$ & 58 & 28.3 & 28.5 & 08 & ke & 58 & 30.1 & 31.8 & 12 & \\
\hline 1300 & 33.8 & 34.0 & 17 & -2.6 & 1500 & 32.8 & 33.0 & I6 & -3.2 & 1700 & 27.0 & 27.0 & 06 & -4.0 & I9 00 & 30.9 & 32.1 & I4 & -3.9 \\
\hline 02 & 32.6 & 32.6 & 15 & & 02 & 33.8 & 34.1 & 17 & 68 & 02 & 27.2 & 27.2 & 07 & & 02 & 31.7 & 33.1 & 15 & \\
\hline 04 & 33.0 & 33.2 & I6 & 50 & 04 & 31.8 & 31.8 & 14 & 19 & 04 & 25.7 & 26.0 & 04 & 10 & 04 & 31.2 & $33 \cdot 3$ & 14 & 40 \\
\hline 06 & 31.8 & 31.8 & 14 & so & 06 & 30.6 & 31.0 & 12 & 2 & 06 & $25 \cdot 3$ & 25.3 & 04 & as & 06 & $3 I \cdot 3$ & 33.0 & I4 & 4 \\
\hline 08 & 33.3 & 33.5 & 16 & $y^{0}$ & 08 & 29.3 & 29.7 & 10 & wo & 08 & 24.4 & 24.6 & 02 & 80 & 08 & 32.2 & 33.7 & 16 & in \\
\hline IO & 28.5 & 28.8 & $\infty 9$ & 41 & 10 & 29.0 & 30.5 & II & ou & Io & 24.5 & 24.8 & 03 & et & 10 & 32.4 & 33.7 & I6 & 60 \\
\hline 12 & 33.5 & 33.8 & 17 & 6 & 12 & 30.8 & 31.6 & 13 & 97 & 12 & 24.7 & 24.7 & 03 & 11 & 12 & $3 \mathrm{I} \cdot 3$ & 32.0 & 14 & \\
\hline 14 & 42.8 & 43.0 & $3 I$ & -2.5 & 14 & 32.0 & 32.6 & 15 & $-3 \cdot 3$ & 14 & $26 . \mathrm{I}$ & 26.3 & 05 & -4.0 & 14 & 29.1 & 29.9 & 10 & -4.0 \\
\hline 16 & 38.6 & 39.3 & 25 & & 16 & 32.2 & 33.0 & 15 & & 16 & 26.0 & 26.2 & 05 & & 16 & 30.2 & 31.3 & 12 & \\
\hline I8 & 39.3 & 40.6 & 27 & ent & 18 & 31.6 & 32.6 & 14 & 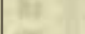 & 18 & 26.8 & 27.2 & 06 & 41 & 18 & 30.1 & 31.0 & 12 & at \\
\hline 20 & 43.2 & 43.8 & 32 & ati & 20 & $3 \mathrm{I} .8$ & 32.8 & 15 & $\approx$ & 20 & 29.4 & 30.0 & II & $\alpha$ & 20 & 29.8 & 30.7 & II & $=$ \\
\hline 22 & $44 \cdot 7$ & 45.6 & 35 & Re & 22 & 31.9 & 33.0 & 15 & 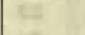 & 22 & 29.4 & 29.9 & IO & $2 x$ & 22 & 31.8 & 32.2 & 14 & $=$ \\
\hline 24 & 37.0 & 38.0 & 23 & 15 & 24 & 32.4 & 33.4 & I6 & 15 & 24 & 26.0 & 26.6 & 05 & $1=$ & 24 & 30.7 & 31.1 & 12 & 14 \\
\hline 26 & 36.1 & 36.8 & $2 \mathrm{I}$ & 32 & 26 & $34 \cdot 3$ & 34.6 & I8 & a & 26 & 25.1 & 25.9 & 04 & 17 & 26 & 30.2 & 30.8 & 12 & \\
\hline 28 & 33.8 & 35.4 & 18 & & 28 & 34.4 & 35.0 & 18 & 5 & 28 & 27.9 & 28.8 & 08 & 20 & 28 & 29.2 & 29.9 & 10 & \\
\hline 30 & 39.3 & 40.0 & 26 & -2.6 & 30 & 32.6 & 32.8 & I5 & $-3 \cdot 3$ & 30.2 & 27.8 & 28.2 & 08 & -3.9 & 30 & 30.8 & 31.3 & I3 & -4.0 \\
\hline 32 & 36.4 & 37.0 & 22 & & 32 & 31.0 & $3 I .4$ & I3 & & 32 & 27.5 & 28. I & 08 & & 32 & 29.0 & 29.9 & 10 & \\
\hline 34 & $4 \mathrm{I} \cdot 3$ & 41.6 & 29 & R & 34 & 28.3 & 29.2 & 09 & ets & 34 & 27.2 & 27.9 & 07 & 16 & 34 & 26.3 & 27.1 & 06 & \\
\hline 36 & 41.3 & 41.6 & 29 & e & 36 & 29.8 & 30.4 & II & Q2 & 36 & & 27.2 & 07 & 17 & 36 & 26.3 & 27.1 & o6 & \\
\hline 38 & 37.0 & 38.0 & 23 & 2 & 38 & 29.5 & 30.3 & II & a & 38 & 28.8 & 29.1 & 09 & 18 & 38 & 27.1 & 29.0 & 08 & \\
\hline 40 & 35.4 & 36.6 & 20 & 9 & 40 & 29.8 & 30.2 & I I & 6. & 40 & 29.6 & 30.0 & II & $\approx$ & 40 & 27.1 & 29. I & 08 & \\
\hline 42 & 35.0 & 35.6 & 19 & 45 & 42 & $29 \cdot 3$ & 29.6 & Io & 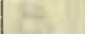 & 42 & $3 I \cdot 3$ & 31.9 & I4 & 67 & 42 & 25.7 & 27.9 & 06 & \\
\hline 44 & 39.7 & 40.8 & 27 & -2.7 & 44 & 29.4 & 29.9 & I0 & -3.5 & 44 & 28.2 & 29.2 & 09 & -3.9 & 44 & 26.3 & 28.2 & 07 & -4.0 \\
\hline 46 & & $3^{b}$ & 34 & & 46 & 29.6 & 29.6 & IO & & 46 & 27.2 & 27.9 & 07 & & 46 & 25.8 & 27.8 & 06 & \\
\hline 48 & 32 & $.0 a$ & 14 & s. & 48 & 28.6 & 28.6 & 09 & 63 & 48 & 27.1 & 27.9 & 07 & 146 & 48 & 25.8 & 27.7 & 06 & \\
\hline 50 & 35.6 & 35.6 & 20 & 8 & 50 & 29.0 & 29.0 & 10 & ae & 50 & 26.2 & $27 \cdot 3$ & 06 & 85 & 50 & 26.9 & 27.8 & 07 & \\
\hline 52 & 34.3 & 34.8 & 18 & 3 & 52 & 30.3 & 30.3 & 12 & 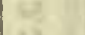 & 52 & $25 \cdot 3$ & 26.9 & 05 & 10 & 52 & 25.9 & 27.8 & 06 & \\
\hline 54 & 33.0 & 34.1 & 17 & 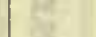 & 54 & 30.3 & 30.3 & 12 & $\beta$ & 54 & 25.2 & 26.9 & 05 & 12 & 54 & 26.0 & 27.7 & 06 & \\
\hline 56 & 32.0 & 32.3 & 14 & 80 & 56 & 29.7 & 29.9 & II & 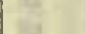 & 56 & 24.8 & 26.3 & 04 & & 56 & 25.9 & 27.7 & 06 & \\
\hline 58 & $3 \mathrm{I} \cdot 3$ & 31.6 & 13 & & 58 & 31.2 & 31.2 & 13 & & 58 & 25.0 & 26.5 & 04 & 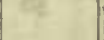 & 58 & $25 . I$ & 20.3 & 04 & \\
\hline
\end{tabular}


Tabulation of magnetic declinations observed at Teplitz Bay-Continued

Wednesday, May 25, $1904 \quad$ Magnet scale erect 1 Thursday, May 26, 1904 Magnet scale inverted

\begin{tabular}{|c|c|c|c|c|c|c|c|c|c|c|c|c|c|c|c|c|c|c|c|}
\hline \multirow{2}{*}{$\begin{array}{l}\text { Chr'r } \\
\text { time }\end{array}$} & \multicolumn{2}{|c|}{$\begin{array}{l}\text { Scale } \\
\text { readings }\end{array}$} & \multirow{2}{*}{$\begin{array}{l}\text { East } \\
\text { decli- } \\
\text { nation }\end{array}$} & \multirow{2}{*}{ Temp. } & \multirow{2}{*}{$\begin{array}{l}\text { Chr'r } \\
\text { time }\end{array}$} & \multicolumn{2}{|c|}{$\begin{array}{l}\text { Scale } \\
\text { readings }\end{array}$} & \multirow{2}{*}{$\begin{array}{c}\text { East } \\
\text { decli- } \\
\text { nation }\end{array}$} & \multirow{2}{*}{ Temp. } & Chr'r & $\begin{array}{r}\mathrm{Sc} \\
\text { rea }\end{array}$ & $\begin{array}{l}\text { cale } \\
\text { dings }\end{array}$ & $\begin{array}{l}\text { East } \\
\text { decli- }\end{array}$ & Temp. & Chr's & $\begin{array}{r}\mathrm{Sc} \\
\text { rea }\end{array}$ & $\begin{array}{l}\text { ale } \\
\text { lings }\end{array}$ & $\begin{array}{l}\text { East } \\
\text { decli- }\end{array}$ & \\
\hline & Left & Right & & & & Left & Right & & & & Left & Right & & & & L,eft & Right & & \\
\hline $\mathrm{h} \mathrm{m}$ & $d$ & d & & $0^{\circ}$ & h m & $d$ & $d$ & & $\bullet$ & $\mathrm{m}$ & d & d & & 。 & $h$ in & & & & \\
\hline $\begin{array}{r}2000 \\
02\end{array}$ & 25.8 & 27.0 & 2205 & -4.1 & 2200 & 18.3 & 19.1 & 21 53 & -4.9 & 1600 & 40.2 & 38.1 & 22 Iو & -3.0 & I8 00 & 40.0 & 37.7 & $22 \quad 20$ & -3.0 \\
\hline 02 & 25.8 & 27.1 & 05 & & 02 & 18.6 & 19.9 & 54 & & 02 & 40.9 & 38.9 & I8 & & 02 & 40.4 & 38.3 & 19 & \\
\hline 04 & 26.3 & 27.9 & 07 & $x^{5}$ & 04 & 2I.I & 23.0 & 58 & 2 & 04 & 4I.I & 39.0 & 18 & 8 & 04 & 40.1 & 38.0 & 19 & (1) \\
\hline $\begin{array}{l}06 \\
08\end{array}$ & 25.3 & 26.4 & 04 & 80 & $\begin{array}{l}05 \\
08\end{array}$ & 20.1 & 22.8 & 58 & 10 & 06 & 41.7 & 39.2 & I7 & 00 & 06 & 40.0 & 37.9 & 19 & 02 \\
\hline IO & $\begin{array}{l}25.0 \\
27.3\end{array}$ & $\begin{array}{l}27.0 \\
29.0\end{array}$ & os & arr & $\begin{array}{l}10 \\
10\end{array}$ & 18.0 & $\begin{array}{r}20.3 \\
10.3\end{array}$ & $\begin{array}{l}54 \\
53\end{array}$ & 10 & 08 & 42.3 & $40 . I$ & I6 & 10 & 08 & $4 \mathrm{I} . \mathrm{I}$ & 38.9 & 18 & 35 \\
\hline 12 & 20.3 & 3. I I & II & if & 12 & 17.3 & 10.3 & 53 & 1 & 10 & 42.0 & 40.0 & I6 & $n$ & IO & 4I.0 & 38.8 & 18 & of \\
\hline 14 & 27.1 & 28.9 & 08 & -4.2 & 14 & 18.2 & 20.1 & 53 & -40 & 12 & 40.7 & $39 . I$ & I8 & & I2 & 40.9 & 38.5 & 18 & \\
\hline $\begin{array}{l}14 \\
16\end{array}$ & 23.0 & 25.8 & 03 & & $\begin{array}{l}14 \\
\mathrm{I} 6\end{array}$ & 17.0 & 20.0 & 54 & -4.9 & 14 & 40.0 & 38.8 & I9 & -3.0 & I4 & 41.9 & 39.2 & 17 & -3.3 \\
\hline 18 & 23.8 & $\begin{array}{l}24.0 \\
24.9\end{array}$ & 02 & 2 & 18 & I9.7 & 22.0 & 58 & 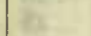 & $\begin{array}{l}16 \\
18\end{array}$ & 40.8 & 39.1 & I8 & 6 & $\begin{array}{l}16 \\
\end{array}$ & $4 \mathrm{I} .5$ & 38.8 & 18 & at \\
\hline 20 & 24.1 & 25.2 & 03 & $\approx$ & 20 & 18.0 & 2I.I & 55 & ix & $\begin{array}{l}10 \\
20\end{array}$ & 40.8 & $\begin{array}{l}40.0 \\
39.9\end{array}$ & 17 & $x=$ & $\begin{array}{l}10 \\
20\end{array}$ & $\begin{array}{l}4 \mathrm{II.0} \\
40.0\end{array}$ & $\begin{array}{l}38.1 \\
37.7\end{array}$ & $\begin{array}{l}18 \\
20\end{array}$ & क्ष \\
\hline 22 & 23.0 & 24.7 & OI & $a$ & 22 & I8.8 & 21.8 & 56 & $E$ & 22 & 40.9 & 40.0 & 17 & 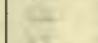 & 22 & 38.8 & 36.5 & 22 & $\because$ \\
\hline 24 & 23.1 & 25.0 & 02 & $\hat{\theta}$ & 24 & 17.3 & 20.5 & 54 & Is & 24 & 40.8 & 39.7 & I7 & 13 & 24 & 9.0 & 36.9 & $2 I$ & is \\
\hline 26 & 22.9 & 24.8 & OI & is & 26 & I9. I & 22.2 & 2156 & 논 & 26 & $4 \mathrm{I} \cdot 3$ & 40.1 & IV & tr & 26 & 8.5 & 36.9 & 21 & ac \\
\hline 28 & 24.7 & 26.6 & 04 & & 28 & 22.3 & 25.0 & 22 oI & & 28 & 41.7 & 40.0 & I6 & 15 & 28 & 8.2 & 36.8 & 22 & \\
\hline 30 & 26.1 & 28.7 & 07 & $-4 \cdot 2$ & 30 & 2.9 & 26.1 & 02 & -5.0 & 30 & 41.9 & 40.2 & 16 & -3.0 & 3 & 8.9 & 37.1 & 21 & -3.0 \\
\hline 32 & 24.6 & 26.7 & 04 & & 32 & 4. I & 27.8 & 05 & & 32 & 42.2 & 40.9 & 15 & & 3 & 0.3 & 38.0 & 20 & \\
\hline 34 & 26.3 & $29 . \mathrm{I}$ & 08 & As & 34 & 5.6 & 29.1 & 07 & EE & 34 & & & 18 & it & & & & 22 & 88 \\
\hline 36 & 31.0 & 33.1 & I4 & 9 & 36 & 6.8 & 30.2 & 09 & 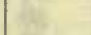 & $\begin{array}{l}34 \\
36\end{array}$ & 39.8 & 38.2 & I9 & 28 & 3 & 0.7 & & 19 & $\alpha$ \\
\hline 38 & 3I.I & 33.0 & I4 & N & 38 & 26.7 & 30.1 & 08 & 8 & 38 & 0 & 39.8 & I7 & 8 & & .1 & & 18 & es \\
\hline 40 & 3.2 & 35.1 & 18 & 을 & 40 & 6.9 & 30.1 & 09 & 막 & 40 & & & I7 & $\omega$ & 4 & & & 17 & 74 \\
\hline 42 & 33.0 & 35.1 & 17 & 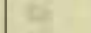 & 42 & 9. I & 32.0 & 12 & & 42 & & & 17 & 4 & & & & I I & is \\
\hline 44 & 33. I & 34.0 & I7 & $-4 \cdot 3$ & 44 & $32 . I$ & 35.2 & I7 & -5.0 & 44 & $4 \mathrm{I} .0$ & 40.0 & 17 & -3.0 & & 3.8 & & 12 & 14 \\
\hline & 3I. I & 33.1 & I4 & & 46 & 5.0 & 37.8 & $2 \mathrm{I}$ & & 46 & 41.9 & 40.5 & 16 & & & 3.3 & 42.8 & 13 & 8 \\
\hline 48 & 30.1 & 32.5 & I 3 & 16 & 48 & 5.4 & 38.0 & 22 & $F_{4}$ & 48 & & & 16 & 24 & & 2.8 & 42.1 & 14 & 5 \\
\hline 50 & 27.3 & 29.9 & 09 & $\mathbb{Q}$ & 50 & & 36. & 20 & vi & 50 & & 40.0 & I7 & at & & & & 15 & 08 \\
\hline 52 & 27.6 & 29.9 & 09 & th & 52 & 3.2 & 35.7 & 18 & 4 & 52 & & & 16 & 25 & & .1 & $4 \mathrm{I}$. & 15 & 8 \\
\hline & 28. I & 30.2 & IO & +2 & 54 & $31 . I$ & 33.7 & 15 & IE & 54 & 42.1 & 40.0 & 16 & $\theta$ & & .0 & $4 \mathrm{I} .7$ & 15 & E \\
\hline & 3.0 & 30. & I0 & & 56 & 3I. I & 33.8 & 15 & & $\begin{array}{l}47 \\
56\end{array}$ & & 42.1 & $\mathrm{I} 3$ & 8 & & .8 & $4 \mathrm{I} .5$ & 15 & a \\
\hline 58 & $29 . I$ & $3 \mathrm{I}$ & II & $\varepsilon$ & & 3I. I & 33.9 & I5 & & 58 & & & 13 & 3 & & 2.1 & & 17 & 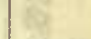 \\
\hline Do & 3I. 6 & 33 & I5 & $-4 \cdot 3$ & 2300 & 29.9 & 32.7 & 13 & -5.0 & $\begin{array}{l}50 \\
700\end{array}$ & $\begin{array}{l}44.1 \\
44.0\end{array}$ & $\begin{array}{l}42.0 \\
42.0\end{array}$ & $\begin{array}{l}13 \\
13\end{array}$ & -3.0 & I9 00 & $\begin{array}{l}2.1 \\
2.1\end{array}$ & 41.9 & 15 & -4.0 \\
\hline 02 & 31.0 & 33 & 14 & & 02 & $33 . I$ & & & & 02 & . I & & 15 & & 02 & 2.2 & 42.1 & 14 & \\
\hline 0 & 29.6 & & I & +1 & o & & 37 & 2 I & Ha & 04 & .7 & 40.6 & 15 & है & 0 & .8 & & 12 & 10 \\
\hline & & 3 I. 9 & I & As & $\alpha$ & & & & & 06 & .0 & & 15 & 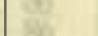 & o & .2 & & 13 & 20 \\
\hline 08 & 28.0 & 30.0 & I & 50 & $\infty 8$ & 4I.I & 43 & 3 & 8 & 08 & 43.2 & & 14 & s & 0 & & 41 & 15 & In \\
\hline Io & 26.1 & 27.3 & 06 & 와 & IO & 43.1 & & 34 & & I0 & 43.2 & & 14 & 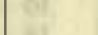 & I & & & 16 & at \\
\hline I2 & 28. I & & 9 & & 12 & 45.3 & 48. & 37 & & I2 & & & 12 & 1 & I & .2 & & 15 & 0 \\
\hline I4 & 25.5 & 26.6 & 05 & -4.4 & 14 & 46.7 & 49. & 39 & -5.1 & I4 & 45.2 & 44.1 & 10 & -3.0 & I & 3. I & 42.7 & 13 & 11 \\
\hline 16 & & 26.6 & & & 16 & 44.8 & 45 & 35 & & 16 & & & 08 & 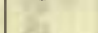 & I & & & 10 & W \\
\hline I8 & & 25 & 2203 & at & If & & 43 & 32 & $n$ & 18 & & & 07 & r & I & & & 09 & 4 \\
\hline 20 & & & 58 & & 2 & & & 3 & 14 & 2 & & & 0 & 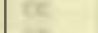 & 2 & & & 08 & Es \\
\hline 22 & & & 57 & Fis & 22 & & & 9 & $F$ & 22 & & & 07 & 1 & 2 & .8 & & 05 & 4 \\
\hline 24 & & & & ex & 24 & & & 27 & $x$ & 24 & & & 10 & 15 & 2. & 50.3 & 49.9 & 02 & 15 \\
\hline 26 & & 2I & 21 58 & & 26 & 43 & 43 & 32 & 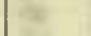 & 26 & & & 10 & $\theta$ & 2 & & & OI & 4 \\
\hline 28 & & & 03 & $v^{2}$ & 28 & & & 38 & & 28 & & & I0 & $\infty$ & 2 & & & 05 & $E$ \\
\hline 30 & & 20.8 & 56 & $-4 \cdot 7$ & 3 & & & 40 & -5.2 & 30 & 45.5 & & 10 & -3 & 3 & & 48.0 & 05 & -4.2 \\
\hline 32 & & & & & 3 & & & 3 & & 3 & & & II & & 3 & & 45.3 & 09 & \\
\hline 34 & & & 56 & Fe & 3 & & & & & 3. & & 42.8 & 12 & 18 & & 45.2 & 45. & I0 & is \\
\hline 36 & & & & & 3 & & 38 & 2 & 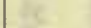 & 3 & & 42. & & 8 & 3 & & 47. & 06 & E. \\
\hline 38 & & & & 18 & 3 & & 39 & 2 & $a$ & 3 & & $4 \mathrm{I} .0$ & 15 & 78 & 3 & & 48.0 & 05 & $E$ \\
\hline 40 & & & & 6 & 4 & & & 2 & 6 & 4 & & & 15 & $\sigma^{\circ}$ & 4 & 46.1 & 45. & 08 & ci \\
\hline $4^{2}$ & & & 55 & 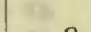 & 42 & & 43 & $3 \mathrm{I}$ & & 42 & & & 15 & 4 & & & 46. & 06 & 48 \\
\hline 44 & I8. I & 20.5 & 54 & -4.8 & 44 & & 44 & $3^{2}$ & -5.3 & 44 & $4 \mathrm{I} .8$ & & 16 & -3.0 & 4 & 48.0 & 47.7 & 05 & -4.2 \\
\hline 46 & & & 50 & & 4 & & 45 & 34 & & 4 & 42.1 & & 16 & & 4 & 49.1 & 48 & 04 & \\
\hline 48 & 17.8 & 18 & 5 & 3 & 48 & & 45 & 34 & 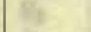 & 4 & & & 15 & 8 & & 50. & & 02 & 5 \\
\hline 50 & I9 & 20. & & 4 & 50 & & 45 & 35 & 6 & 5 & 43.2 & & & & 5 & 50.1 & & 02 & 8 \\
\hline 52 & 20.0 & 20. & 56 & $\mathrm{~s}$ & 52 & & 44 & 32 & 4 & 5 & & & I & & & 50.2 & 50.1 & & 67 \\
\hline 5 & 21.2 & $2 \mathrm{I}$ & 58 & 4 & 54 & & 44 & 33 & & 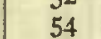 & 40.8 & 38 & 19 & 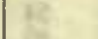 & & 52 & & 9 & \\
\hline & & & $5 \%$ & 48 & & & 45 & 34 & & & & & 19 & & & 52.0 & 50. & 60 & \\
\hline 58 & 20.1 & 20.8 & 56 & 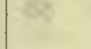 & $\begin{array}{r}50 \\
24 \quad 00\end{array}$ & $\begin{array}{l}44.9 \\
45.2\end{array}$ & $\begin{array}{l}45.9 \\
46.3\end{array}$ & $\begin{array}{l}35 \\
36\end{array}$ & & & 39.9 & 37.2 & 20 & & & 54.1 & 53.I & 56 & 10 \\
\hline & & & & & & & & & & & & & & & & 52.5 & 51.2 & & $-4 \cdot 3$ \\
\hline
\end{tabular}

Correction to local mean time is $+25.55 .90^{\circ}$ torsion $=20 .{ }^{\prime} 87$. Torsion head at oh oom read $100^{\circ}$ and at $24 \mathrm{~h}$ i $3 \mathrm{~m}$ read $102^{\circ}$. Observer-R. R. T.
Correction to local mean time is $-75.90^{\circ}$ torsion $=199^{\prime} 53$.

Torsion head at $15 \mathrm{~h} 29 \mathrm{~m}$ read $102^{\circ}$ and at $201124 \mathrm{~m}$ read $106^{\circ}$. Observer-J. V. 
Tabulation of magnetic declinations observed at Teplitz Bay-Continued

\begin{tabular}{|c|c|c|c|c|c|c|c|c|c|c|c|c|c|c|c|c|c|c|c|}
\hline \multicolumn{6}{|c|}{ Friday, May 27, 1904} & \multicolumn{4}{|c|}{ Magnet scale erect } & \multicolumn{6}{|c|}{ Sunday, May 29, I904 } & \multicolumn{4}{|c|}{ Magnet scale inverted } \\
\hline \multirow{2}{*}{$\begin{array}{l}\text { Chr'r } \\
\text { time }\end{array}$} & \multicolumn{2}{|c|}{$\begin{array}{l}\text { Scale } \\
\text { readings }\end{array}$} & \multirow{2}{*}{$\begin{array}{c}\text { East } \\
\text { decli- } \\
\text { nation }\end{array}$} & Temp & Chr'r & $\begin{array}{r}\text { Sc } \\
\text { read }\end{array}$ & $\begin{array}{l}\text { ale } \\
\text { lings }\end{array}$ & $\begin{array}{l}\text { East } \\
\text { decli- }\end{array}$ & Temp. & Chr'r & $\begin{array}{l}\text { Sca } \\
\text { read }\end{array}$ & $\begin{array}{l}\text { ale } \\
\text { lings }\end{array}$ & $\begin{array}{l}\text { East } \\
\text { decli- }\end{array}$ & Temp. & Chr'r & $\begin{array}{l}\mathrm{Sc} \\
\text { read }\end{array}$ & $\begin{array}{l}\text { ale } \\
\text { lings }\end{array}$ & East & Temp. \\
\hline & Left & Right & & & & Left & Right & 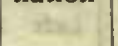 & & & Left & Right & & & & Left & Right & & \\
\hline h m & d & d & $\cdot 1$ & $\bullet$ & h $\mathrm{mI}$ & d & $d$ & & $\cdot$ & $\mathrm{h} \mathrm{m}$ & $\mathrm{d}$ & d & $\cdot 1$ & - & $\mathrm{l}_{1} \mathrm{~m}$ & & d & & • \\
\hline 2000 & 36.3 & 36.9 & 2213 & $-\mathrm{T} .4$ & 2200 & $34 \cdot 9$ & 36.3 & 22 12 & -1.0 & $00^{*}$ & 40.0 & 39.2 & 2227 & +0.2 & 200 & 56.8 & 54.0 & 23 I5 & -0.1 \\
\hline 02 & 36.3 & 37.8 & 14 & 20 & 02 & 28.9 & 30.1 & 02 & $36=$ & 02 & 38.8 & 38.1 & 28 & ten & 02 & 57.8 & $54 \cdot 4$ & 14 & $y^{2}$ \\
\hline 04 & 37.0 & 39.0 & 16 & 30 & 04 & 28.0 & 30.5 & 02 & 10 & 04 & 38.8 & 38.1 & 28 & 15 & 04 & $55 \cdot 3$ & 52.0 & 18 & 10 \\
\hline 06 & 37.1 & 38.9 & 16 & 62 & 06 & 28.0 & 30.5 & 2202 & $\infty 0$ & 06 & 37.9 & $35 \cdot 9$ & $3 I$ & 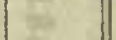 & 06 & 52.8 & 49.7 & 22 & \\
\hline 08 & 36.0 & 37.9 & 14 & aे & 08 & 26.2 & 29.1 & 2 I 59 & tis & 08 & 37.9 & 36.0 & $3 I$ & bi & 08 & 51.9 & 49.0 & 23 & her \\
\hline 10 & 37.0 & 39.5 & I6 & git & 10 & 25.1 & $28 . I$ & 58 & is & 10 & $38 . \overline{8}$ & 37.1 & 29 & $a i$ & I0 & 56.3 & 53.0 & 16 & $a x$ \\
\hline 12 & 38.0 & 40.5 & I8 & & 12 & 2I.I & 24.0 & 5I & & 12 & 40.9 & 38.8 & 26 & 64 & I2 & 58.7 & 56.9 & II & \\
\hline I4 & 36.7 & 39.0 & 15 & $-I .3$ & 14 & 23.8 & 26. I & 55 & -1.0 & 14 & 35.6 & 33.2 & 35 & +o.I & 14 & 53.8 & 51.0 & 20 & 0.1 \\
\hline I6 & $35 \cdot 5$ & 37.8 & I3 & the & 16 & 20.7 & 23.9 & $5 \mathrm{I}$ & dif:- & 16 & 34.0 & 32.2 & 37 & 1 & 16 & 52.2 & 5 I. I & $2 \mathrm{I}$ & \\
\hline I8 & 34.0 & 36.0 & II & xy & I8 & 22.9 & 25.3 & 54 & 2 & 18 & 36.0 & 34.0 & 34 & 84 & 18 & $5 \mathrm{I} . \mathrm{I}$ & 49.6 & 23 & \\
\hline 20 & 35.8 & 37.1 & 13 & a & 20 & 23.7 & 26.2 & 2 I 55 & $2 n$ & 20 & 35.9 & 33.1 & 35 & 06 & 20 & 50.6 & 49.1 & 24 & $=$ \\
\hline 22 & 37.8 & 39.2 & I6 & $=$ & 22 & 26.8 & 29.6 & 2200 & Es & 22 & 34.9 & 32.8 & 36 & 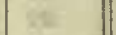 & 22 & 53.9 & 52.1 & I9 & 5 \\
\hline 24 & 38.9 & 40.1 & I8 & ait & 24 & 27.9 & 29.5 & OI & is & 24 & 35.2 & 33.2 & 35 & if & 24 & 56.0 & 54.1 & 16 & \\
\hline 26 & 36.9 & 38.1 & I5 & 8 & 26 & $29 . I$ & 31.0 & 03 & Ex & 26 & 35.3 & 34.0 & 34 & ie & 26 & 52.9 & 51.5 & 20 & \\
\hline 28 & 35.4 & 36.8 & I3 & tar & 28 & 29.0 & 31.0 & 03 & & 28 & 35.2 & 33.3 & 35 & lis & 28 & 53.2 & 51.9 & 20 & \\
\hline 30 & 34.2 & 36.0 & II & -1.3 & 30 & 29.2 & 30.9 & 03 & -1.0 & 30 & 33.2 & 31.8 & 38 & 0.0 & 30 & 53.0 & 51.8 & 20 & $\rightarrow 0 . I$ \\
\hline 32 & 34.0 & 35.0 & IO & 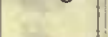 & 32 & 32.9 & 34.2 & 2209 & 8 & 32 & 27.9 & 26.6 & 46 & y. & 32 & 51.1 & 49.7 & 23 & 15e \\
\hline 34 & 35.7 & 36.0 & I2 & if & 34 & 27.0 & 28.3 & 2I 59 & yx & 34 & 27.1 & 25.9 & 47 & it & 34 & 52.7 & 5I.I & $2 \mathrm{I}$ & \\
\hline 36 & 36.2 & 37.0 & I3 & 8. & 36 & 22.9 & $24 . I$ & 53 & 25 & 36 & 27.8 & 26.2 & 46 & of & 36 & 52.9 & $5 \mathrm{~T} .1$ & $2 \mathrm{I}$ & ac \\
\hline 38 & 37.1 & 37.9 & 15 & 2 & 38 & 21.1 & 23.3 & $5 \mathrm{I}$ & 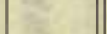 & 38 & 27.3 & 26.2 & 47 & 88 & 38 & 49.1 & 48.4 & 26 & \\
\hline 40 & 37.0 & 37.9 & 15 & E & 40 & 20.0 & 22.0 & 49 & 5 & 40 & 28.0 & 26.6 & 46 & 6 & 40 & $46 . I$ & $45 . I$ & $3 \mathrm{I}$ & 8 \\
\hline 42 & 36.8 & 37.2 & I4 & 14. & 42 & 20.7 & 22.5 & 50 & & 42 & 26.5 & 25.1 & 48 & it. & 42 & 50.0 & 49.2 & 24 & \\
\hline 44 & 34.2 & 35.8 & II & -1.2 & 44 & 20.8 & 22.3 & 50 & -1.0 & 44 & 25.5 & $24 \cdot 3$ & 50 & 0.0 & 44 & 49.9 & 48.0 & 26 & 0.0 \\
\hline 46 & 33.7 & 35.1 & 10 & 3 & 46 & 19.9 & $21 . I$ & 48 & an & 46 & 27.2 & 25.9 & 47 & iy. & 46 & 44.0 & 42.0 & 35 & \\
\hline 48 & 32.8 & 34.2 & $\infty 9$ & 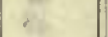 & 48 & 19.8 & 20.9 & 48 & 20 & 48 & 29.9 & 28.0 & 44 & 2 & 48 & 39.0 & 37.9 & 42 & \\
\hline 50 & 32.9 & 35.0 & $\infty$ & 3 & 50 & 19.8 & 20.5 & 48 & $=$ & 50 & 28.9 & 27.8 & 44 & of & 50 & 37.1 & 36.3 & 45 & g \\
\hline 52 & 32.8 & 34.9 & 09 & $\theta$ & 52 & $21 . I$ & 21.7 & 50 & $=$ & 52 & 28.7 & 27.9 & 44 & $\mathrm{gz}$ & 52 & 39.6 & 39.0 & 40 & 3 \\
\hline 54 & $34 . I$ & 36.0 & II & $=2$ & 54 & $2 \mathrm{I} .4$ & 22.0 & 50 & 3 & 54 & 27 & $.1 b$ & 46 & tE & 54 & 44.2 & 43.2 & 34 & 8 \\
\hline 56 & 36.1 & 38.3 & I4 & 2 & 56 & I8. I & I9. I & 45 & 8 & 56 & 26 & $.9 b$ & 47 & $\sqrt{2}$ & 56 & 45.2 & 45.0 & 32 & \\
\hline 58 & 38.6 & 40.9 & I8 & & 58 & 20.9 & 21.2 & 49 & & 58 & 26.0 & 25.8 & 48 & Re & 58 & $46 . I$ & 45.4 & 30 & \\
\hline 2100 & 38.9 & 41.0 & I9 & -1.2 & 2300 & 18.2 & I9. 5 & 46 & -1.0 & I 00 & 24.8 & 24.2 & 50 & -0.1 & 300 & $46 . I$ & 45.8 & 30 & 0.0 \\
\hline 02 & 37.8 & 39.8 & 17 & car & c2 & I6. 3 & 16.5 & 42 & 50 & 02 & 21.2 & 21.0 & 56 & va & 02 & 48.0 & 46.9 & 28 & ren \\
\hline 04 & 38.8 & 39.9 & I8 & 30 & 04 & 18.1 & 18.5 & 45 & $w$ & 04 & 22.6 & 20.9 & 55 & 10 & 04 & 50.9 & 50.0 & 23 & \\
\hline 06 & 38.8 & 40.7 & 18 & Ex & 06 & 18.0 & 18.3 & 44 & (n) & 06 & 24.4 & 23.1 & 52 & 85 & 06 & 49.I & 48.4 & 26 & \\
\hline 08 & 37.2 & 39.1 & 16 & S. & 08 & I8.9 & I9. I & 46 & Ko & 08 & 22.5 & 20.5 & 2255 & 80 & os & 50.0 & $49 \cdot 3$ & & 하 \\
\hline IO & 37.8 & 39.2 & 16 & ait & IO & 15.0 & I5.5 & 40 & tat & I0 & 13.0 & 11.9 & $23 \circ 9$ & tit & 10 & 50.9 & 50.0 & 23 & \\
\hline I2 & 37.5 & 38.9 & 16 & & 12 & 13.0 & I3.I & 36 & 4 & I2 & 14.9 & 14.7 & 06 & 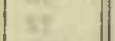 & I2 & 53.1 & 52.0 & 20 & \\
\hline 14 & 37.2 & 38.2 & 15 & $-1 . I$ & 14 & 12.9 & 13.6 & 37 & -1.0 & 14 & Ii.o & 9.2 & 13 & $\multimap 0.1$ & 14 & 49.1 & 48.8 & 26 & $\multimap . I$ \\
\hline 16 & 36.1 & 37.2 & I3 & if & 16 & II. 7 & 12.3 & 35 & tis & I6 & 7.0 & 6.1 & I9 & 政 & 16 & 43.5 & 42.9 & 35 & \\
\hline 18 & 36.3 & $37 . I$ & 14 & ind & 18 & 12.1 & 13.2 & 36 & ilit & $18^{*}$ & 45.8 & 37.9 & 36 & 24 & 18 & $43 . I$ & 42.5 & 35 & \\
\hline 20 & $33 \cdot 3$ & 33.7 & 09 & 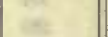 & 20 & II.9 & 12.7 & 35 & $=$ & 20 & 58.0 & 50.9 & I7 & be & 20 & 42.2 & 41.9 & 36 & \\
\hline 22 & 30.8 & 31.2 & 05 & $\cos ^{2}$ & 22 & 10.3 & II.9 & 34 & $=$ & 22 & 68.7 & 60.8 & 23 OI & is & 22 & 44. I & 44.0 & 33 & \\
\hline 24 & 30.9 & 3I.I & 05 & ia & 24 & 10.9 & II.9 & 34 & ats. & 24 & 78.1 & 70.2 & 2246 & $3 t$ & 24 & 50.8 & 50.5 & 23 & \\
\hline 26 & 36.7 & 37.7 & I4 & 5 & 26 & 10.2 & II. 2 & 33 & $E$ & 26 & 69 &.$c b$ & 54 & ic & 26 & 51.3 & 49.9 & 23 & \\
\hline 28 & 30.8 & 31.3 & 05 & & 28 & 10.4 & II. 8 & 34 & & 28 & 70.1 & 70.0 & 52 & ks. & 28 & 46.9 & 44.9 & 30 & \\
\hline 30 & $37 \cdot 3$ & 37.9 & 15 & -I.I & 30 & II .O & 12.0 & 34 & -1.0 & 30 & 69 & $.0 b$ & 2254 & -0.1 & 30 & 39.9 & 38.0 & $4 I$ & $-0 . I$ \\
\hline 32.3 & 40.8 & $42 . I$ & $2 I$ & $x^{2}$ & 32 & II. 2 & I2. 8 & 35 & 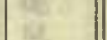 & 32 & 63.5 & 62.9 & 2303 & (c) & 32 & 31.9 & 30.5 & 2353 & \\
\hline 34 & 37 & $.2 b$ & I4 & 7 & 34 & I3. 5 & 14.8 & 38 & is & 34 & 63.0 & 61.8 & 04 & WL & 34 & 22.2 & 21.5 & & \\
\hline 36 & &.$g b$ & 06 & 8 & 36 & 13.7 & 14.8 & 38 & है & 36 & 64.0 & 63.0 & 02 & es & 36 & 21.2 & 20.1 & IO & \\
\hline 38 & 32.6 & 32.9 & 07 & 2 & 38 & 14.2 & 15.0 & 39 & $\frac{c}{2}$ & 38 & 62.9 & 62.1 & 04 & in & 38 & $25 . I$ & 23.0 & 2404 & \\
\hline 40 & 33.7 & 33.9 & 09 & $\theta^{2}$ & 40 & 16.0 & 16.8 & 42 & 2 & 40 & 62.8 & 62.0 & 04 & af & 40 & 31.5 & 30.9 & 2353 & \\
\hline 42 & 35.2 & 36.0 & 12 & 20 & 42 & 17.2 & 17.9 & 44 & 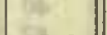 & 42 & 62.8 & 61.9 & 04 & $\frac{75}{4}$ & 42 & 37.0 & 35.8 & 45 & \\
\hline 44 & 36.1 & 36.5 & 13 & -1.1 & 44 & I4 & $.1 b$ & 38 & -1.0 & 44 & 58.0 & $55 . I$ & 2314 & $0 . I$ & 44 & 37.2 & 36.1 & 45 & -0.2 \\
\hline 46 & 36.2 & 37.5 & 14 & 27 & 46 & 13.7 & I4. I & 38 & 4 & 46 & $7 I .3$ & 67.3 & 2253 & Xn & 46 & 37.9 & 36.8 & 44 & \\
\hline 48 & 38.8 & 39.0 & 17 & 8 & 48 & $15 . \mathrm{I}$ & 16.0 & 40 & 8 & 48 & 72.9 & 68.9 & $5 I$ & Fis. & 48 & 37.7 & 36.2 & 44 & \\
\hline 50 & 44.2 & 45.9 & 27 & art & 50 & 16.5 & 17.2 & 42 & 3 & 50 & 72.0 & 67.9 & 2252 & az & 50 & 37.6 & 36.0 & 44 & \\
\hline 52 & $5 I . I$ & 50.3 & 36 & 7 & 52 & 17.1 & 17.9 & 44 & 3 & 52 & 60.8 & 58.1 & 2309 & $=2$ & 52 & 35.9 & 33.8 & 2348 & \\
\hline 54 & 47.9 & $49 . I$ & 32 & & 54 & 18.6 & 10.1 & 46 & 92 & 54 & 58.9 & 55.5 & 12 & E. & 54 & 27.5 & 25.1 & $24 \mathrm{OI}$ & \\
\hline 56 & 49.0 & 49.1 & 33 & 8 & 56 & I8. I & 19.0 & 45 & Bas & 56 & 56. I & 53.6 & I6 & te & 56 & 23.0 & 20.8 & 08 & \\
\hline 58 & 41.9 & 43.8 & 23 & b5 & 58 & 16.4 & 17.9 & 43 & & 58 & 55.2 & 52.6 & I8 & 62 & 58 & 25.0 & 23.0 & 04 & \\
\hline 40 & & & Ease & $\omega 0$ & 2400 & 16.9 & I8. I & 44 & -1.0 & & & & 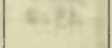 & 0615 & & & & & \\
\hline
\end{tabular}

Correction to local mean time is - 16.5s. $90^{\circ}$ torsion=22.' 23 .

Ohserver-R. R. T.

Torsion head at $19 h \mathrm{I} 5 \mathrm{~m}$ read $102^{\circ}$ and at $24 \mathrm{~h} 34 \mathrm{~m}$ read $101^{\circ}$.

Observer-R. R. T. 
Tabulation of magnetic declinations observed at Teplitz Bay-Continued

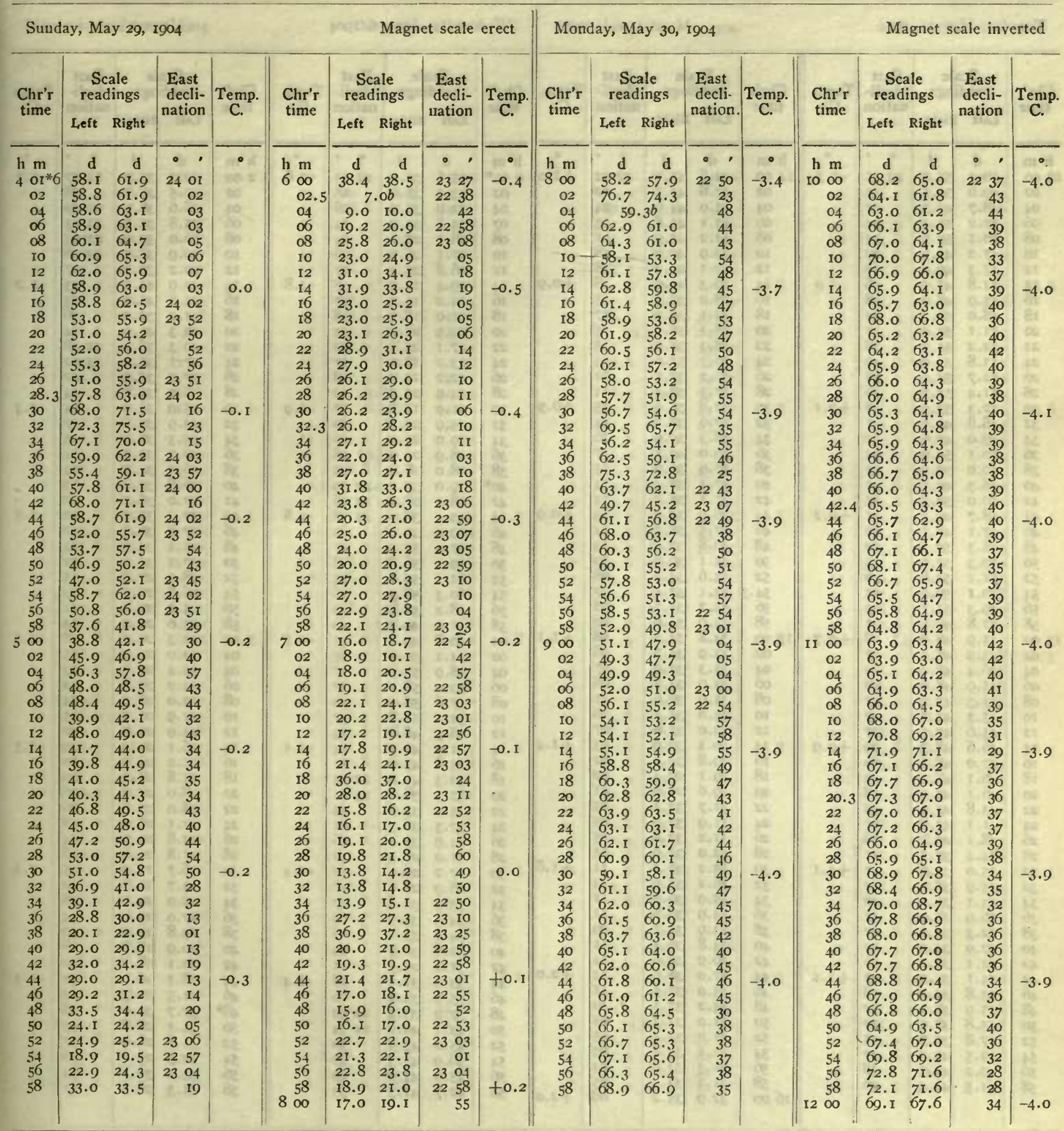

Correction to local mean time is $-54.5 \mathrm{~s}$.

Torsion head at oh oom rcad $102^{\circ}$ and at $8 \mathrm{~h}$ 20m read the same.

Observer-R. R. T.
Correction to local mean time is $+32 \mathrm{~s}$

Torsion head at $7 \mathrm{~h} 25 \mathrm{~m}$ read $104^{\circ}$ and at $12 \mathrm{~h} i 4 \mathrm{~m}$ read the same. Observer-R. R. T. 
Tabulation of magnetic declinations observed at Teplitz Bay-Continued

\begin{tabular}{|c|c|c|c|c|c|c|c|c|c|c|c|c|c|c|c|c|c|c|c|c|}
\hline \multicolumn{5}{|c|}{ Tuesday, May 3I, 1904} & \multicolumn{6}{|c|}{ Magnet scale erect } & \multicolumn{6}{|c|}{ Wednesday, June I, I904 } & \multicolumn{4}{|c|}{ Magnet scale inverted } \\
\hline \multirow{2}{*}{$\begin{array}{l}\text { Chr'r } \\
\text { time }\end{array}$} & \multicolumn{2}{|c|}{$\begin{array}{l}\text { Scale } \\
\text { readings }\end{array}$} & $\begin{array}{l}\text { East } \\
\text { decli- }\end{array}$ & Temp. & Chr'r & $\begin{array}{r}\text { Sc } \\
\text { read }\end{array}$ & $\begin{array}{l}\text { ale } \\
\text { lings }\end{array}$ & Eas & $\begin{array}{l}\text { st } \\
\text { cli- }\end{array}$ & Temp. & Chr'r & $\begin{array}{r}\text { Sc } \\
\text { reac }\end{array}$ & $\begin{array}{l}\text { ale } \\
\text { dings }\end{array}$ & $\begin{array}{l}\text { East } \\
\text { decli- }\end{array}$ & Temp. & Chr'x & $\begin{array}{r}\text { Sc } \\
\text { read }\end{array}$ & $\begin{array}{l}\text { ale } \\
\text { lings }\end{array}$ & $\begin{array}{l}\text { East } \\
\text { decli- }\end{array}$ & Temp. \\
\hline & Left & Right & haterat & & & Left & Right & Hate & nin & & time & Left & Right & & & & Left & Righı & divon & \\
\hline $\mathrm{h} \mathrm{m}$ & d & d & - ' & - & $\mathrm{h} \mathrm{m}$ & d & d & - & & & $\mathrm{h} \mathrm{m}$ & d & $\mathrm{d}$ & & & $\mathrm{h} \mathrm{n}$ & d & d & & - \\
\hline 1200 & 50.9 & 51.7 & 2235 & -0.3 & 1400 & 42.8 & 43.1 & 22 & 22 & +1.0 & $00^{*}$ & 35.6 & $32 . \mathrm{I}$ & 22 I 5 & -2.6 & 200 & 40.2 & 39.2 & 2257 & $-2 . I$ \\
\hline 02 & 49.6 & 50.1 & 33 & $x^{2}$ & 02 & 42.8 & 43.1 & & 22 & $\sin 1$ & 02 & 33.0 & 31.0 & & 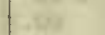 & 02 & 39.5 & 38.3 & 58 & Whe \\
\hline 04 & 48.7 & 49.3 & 32 & Bs & 04 & 43.9 & 44.6 & & 24 & 30 & 04 & 32.9 & 31.0 & I8 & & 04 & 38.6 & 38.0 & & \\
\hline 06 & 50.2 & 51.4 & 34 & 68 & 06 & 43.1 & 43.8 & & 23 & 80 & 07 & 34.8 & 33.9 & I4 & & 06 & 35.8 & 35.3 & 2303 & \\
\hline 08 & 50.1 & 50.7 & 34 & Bit. & 08 & 42.2 & 42.9 & & 21 & 80 & 08 & 28.7 & 28.1 & & 80 & 08 & 36.0 & 35.0 & 2303 & in \\
\hline 10 & 47.2 & 47.6 & 29 & ance & IO & 39.9 & 40.9 & & 18 & or & IO & $27 . I$ & 26.9 & 26 & 48 & 10 & 39.9 & 38.5 & 2258 & \\
\hline 12 & $45 \cdot 3$ & 46.2 & 26 & & I 2 & 40.1 & $4 \mathrm{I} .2$ & & 18 & & 12 & 26.8 & 25.4 & 27 & & 12 & 40.2 & 39.0 & 57 & \\
\hline 14 & 44.8 & 45.5 & 26 & 0.0 & 14 & $39 \cdot 3$ & 40.9 & & 18 & +0.9 & 14 & 19.4 & 19.3 & 38 & -2.8 & 14 & 42.9 & 41.1 & 53 & -2.2 \\
\hline 16 & 45.9 & 48.2 & 28 & wat & 16 & 38.0 & 39.3 & & I 5 & 4 & I6 & 14.2 & I3. 3 & 47 & & 16 & 43.0 & $4 \mathrm{~T} .2$ & 53 & \\
\hline I8 & 48.0 & 50.3 & 32 & Nir & I8 & 39.1 & 39.9 & & 17 & 32 & 18 & 21.0 & I8. 1 & 38 & (6) & 18 & 41.2 & 39.2 & 56 & et \\
\hline 20 & $5 \mathrm{I} . \mathrm{I}$ & 54.0 & 37 & $x^{2}$ & 20 & 38.1 & 38.9 & & 15 & 2 & 20 & 23.9 & $21 . I$ & 33 & $\infty$ & 20 & 42.3 & 40.9 & 54 & $\theta$ \\
\hline 22 & 52.2 & 54.2 & 38 & it & 22 & 38. I & 38.9 & & I5 & 6 & 22 & 27.1 & 22.1 & 30 & Et & 22 & 40.3 & 38.4 & $22 \quad 58$ & $\theta$ \\
\hline 24 & 53.8 & 55.0 & 40 & 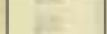 & 24 & 36.7 & 37.6 & & I3 & ant & 24 & 23.2 & 17.1 & 37 & 12 & 24 & $37 . \mathrm{I}$ & $35 \cdot 3$ & 2302 & \\
\hline 26 & 52.7 & $54 . I$ & 38 & S & 26 & 36.2 & 37.1 & & 12 & 2. & 26 & 22.2 & 17.0 & 38 & & 26 & 37.0 & 35.9 & 02 & \\
\hline 28 & 51.4 & 52.3 & 36 & & 28 & 37.7 & 38.1 & & $I_{4}$ & & 28 & 24.9 & 22.0 & 32 & & 28 & 36.0 & 34.7 & 04 & \\
\hline 30 & 52.3 & $53 \cdot 3$ & 38 & to.I & 30 & 37.8 & 38.9 & & I5 & +0.9 & 30 & 21.9 & 17.8 & 37 & -2.9 & 30 & 35.9 & 34.0 & 04 & -2.3 \\
\hline 32 & 48.8 & 49.1 & $3 I$ & trat & 32 & 37.6 & 38.2 & & I4 & 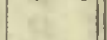 & 32 & 25.9 & 23.1 & 30 & (n) & 32 & 33.9 & 32.8 & 07 & \\
\hline 34 & 49.0 & 49.6 & 32 & 20 & 34 & 38.3 & 39.9 & & I6 & $y_{n}$ & 34 & 29.0 & 25.1 & 26 & a & 34 & $35 \cdot 3$ & 33.9 & 05 & \\
\hline 36 & 50.1 & 50.9 & 34 & 80 & 36 & 39.0 & 40.1 & & I7 & a & 36 & 28.9 & 22.9 & 28 & Af & 36 & 36.2 & 34.9 & 04 & \\
\hline 38 & 50.0 & 50.2 & 33 & 1. & 38 & 37.9 & 39.6 & & 15 & as & 38 & 18.0 & 16.0 & 42 & Iic & 38 & 36.1 & .34 .9 & 04 & \\
\hline 40 & 48.3 & $49 . \mathrm{I}$ & $3 \mathrm{I}$ & a & 40 & $37 . I$ & 38.4 & & I4 & $\infty$ & $40^{*}$ & 39.0 & 37.0 & 2259 & Q37. & 40 & 36.8 & 35.4 & 03 & At \\
\hline 42 & 50.0 & 50.5 & 33 & & $4^{2}$ & 37.1 & 38.3 & & 14 & & 42 & 28.0 & 27.3 & 2316 & & 42 & 37.0 & 36.1 & 02 & \\
\hline 44 & 48.7 & $49 . I$ & $3 \mathrm{I}$ & +0.2 & 44 & 36.9 & 37.9 & & I3 & +0.9 & 44 & 39.2 & 38.9 & 2258 & -2.8 & 44 & 37.9 & 37.2 & 00 & -2.5 \\
\hline 46 & $47 . I$ & $48 . I$ & 29 & 7 & 46 & 37.5 & 38.1 & & 14 & & 46 & 50.9 & 49.9 & 40 & - & 46 & 37.0 & 36.5 & 02 & \\
\hline 48 & 48.3 & 50.I & 32 & Bt & 48 & 36.8 & 37.4 & & I3 & 8 & 48 & 57.0 & 55.3 & $3 I$ & 7 & 48 & $35 . I$ & 34.9 & 04 & \\
\hline 50 & 50.3 & 52.0 & 35 & mat & 50 & $35 . \mathrm{I}$ & 36.0 & & I0 & $a^{2}$ & 50 & 59.1 & 56.8 & 28 & 6 & 50 & 37.1 & 35.9 & 02 & \\
\hline 52 & $5 \mathrm{I} . \mathrm{I}$ & 52.8 & 36 & If & 52 & 33.6 & 34.1 & & 08 & 38 & 52 & 6I.I & 59.0 & 25 & $E^{2}=$ & 52 & 28.2 & 27.8 & 15 & \\
\hline 54 & 50.1 & 52.0 & 35 & $y$ & $54 \cdot 3$ & 33.1 & 33.4 & & 07 & in & 54 & 60.3 & 58.3 & 026 & II & 54 & 26.6 & 25.2 & 19 & \\
\hline 56 & 49.3 & $51 . I$ & 33 & 54 & 56 & 33.7 & $34 . I$ & & 88 & $t=0$ & 56 & 58.2 & 56.9 & 29 & Bit & 56 & 25.2 & 23.7 & 21 & \\
\hline 58 & 47.8 & 49.5 & $3 I$ & 1 & 58 & 33.9 & 34.6 & & 08 & & 58 & 51.0 & 49.4 & 40 & 8 & 58 & 23.0 & 22.2 & 24 & \\
\hline 1300 & 45.9 & 48.0 & 28 & +0.7 & 1500 & 33.8 & 34.2 & & 08 & +I.I & 100 & 50.8 & $49 . \mathrm{I}$ & $4 \mathrm{I}$ & -2.7 & 300 & 20.3 & 20.1 & 28 & -2.5 \\
\hline 02 & 43.9 & $48 . I$ & 27 & $x^{2}$ & 02 & 33.9 & 34.7 & & 08 & +2 & 02 & 54.1 & 52.5 & 35 & 8 & 02 & 22.0 & 21.9 & 25 & -8 \\
\hline 04 & 46.4 & 48.4 & 29 & ia & 04 & $33 \cdot 3$ & 33.9 & & 07 & 35 & 04 & 58.9 & 57.9 & 27 & 18 & 04 & 23.4 & 23.1 & 23 & \\
\hline$\infty 6$ & 45.9 & 48.0 & 28 & $x^{2}$ & 06 & 33.9 & 34.7 & & 08 & 5 & 06 & 59.0 & 58.1 & 27 & 165 & 06 & 24.2 & 23.7 & 22 & \\
\hline 08 & $45 \cdot 3$ & 47.2 & 27 & at & 08 & 35.9 & 36.6 & & I I & 10 & 08 & 57.6 & 56.0 & 30 & 6. & 08 & 23.0 & 22.2 & 24 & \\
\hline 10 & $45 \cdot 3$ & $47 . I$ & 27 & at & IO & 35.0 & $35 \cdot 3$ & & 10 & 6 & IO & 52.2 & 51.8 & 37 & bit. & 10 & 21.9 & 21.5 & 25 & \\
\hline 12 & 45.6 & 47.1 & 27 & & 12 & 33.0 & 33.8 & & 07 & & 12 & 48.5 & 48.0 & 43 & 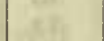 & 12 & 24.9 & 24.7 & 20 & \\
\hline 14 & 46.6 & 48.3 & 29 & +0.9 & 14 & 30.4 & 30.9 & & 03 & $+\mathrm{I} .4$ & 14 & 4I.9 & $4 \mathrm{I} .4$ & & -2.5 & 14 & 26.1 & 25.2 & I9 & -2.5 \\
\hline I6 & 47.0 & 48. I & 29 & 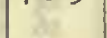 & 16 & 29.8 & 30.1 & & 02 & $=$ & $\begin{array}{l}16 \\
16\end{array}$ & 43.8 & 43.0 & $\begin{array}{l}54 \\
5 I\end{array}$ & & 16 & 25.2 & 24.9 & 20 & \\
\hline 18 & $46 . I$ & 48.2 & 29 & . & I8 & 29.1 & 29.4 & & $\infty$ & 88 & I8 & 42.3 & $4 \mathrm{~T} .0$ & 2254 & 35 . & 18 & 25.8 & 25.1 & 20 & \\
\hline 20 & $46 . I$ & 48.0 & 28 & $a s$ & 20 & 29.6 & 29.9 & & OI & $\alpha$ & 20 & 37.7 & 36.8 & 23 OI & de & 20 & 28.0 & 27.5 & I6 & ath \\
\hline 22 & 43.8 & 45.9 & 25 & 28 & 22 & 31.7 & 32.1 & & 05 & nin & 22 & 35.0 & 33.7 & 05 & ix & 22 & 28.0 & 27.6 & 16 & \\
\hline 24 & $4 \mathrm{I} . \mathrm{I}$ & 43.8 & $2 \mathrm{I}$ & 34 & 24 & 31.9 & 32.9 & & 05 & M & 24 & 37.5 & 36.5 & oI & ter & 24 & $27 . I$ & 26.0 & 18 & \\
\hline 26 & 38.1 & 40.4 & I6 & & 26 & $3 \mathrm{I} .2$ & 32.8 & & 05 & 8 & 26 & 34.9 & 33.1 & 06 & 8 & 26 & 23.8 & 22.0 & 24 & \\
\hline 28 & 34.4 & 37.5 & II & & 28 & 32.0 & 32.9 & & 05 & & 28 & 31.0 & 29.5 & 12 & & 28 & 21.8 & 20.2 & 26 & \\
\hline 30 & 35.0 & 37.0 & II & +I.I & 30 & 35.2 & 36.3 & & I I & +1.8 & 30 & 27.2 & 26.0 & 17 & -2.4 & 30 & 16.7 & 14.2 & 35 & -2.4 \\
\hline 32 & 35.9 & 38.0 & I2 & int & 32 & 36.2 & 37.9 & & I3 & 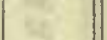 & 32 & 28.9 & 26.6 & 16 & an & 32 & 13.7 & 11.7 & 40 & \\
\hline 34 & 34.1 & 36.2 & IO & at & 34 & 35.0 & 36.8 & & I I & 37 & 34 & 33.2 & 32.8 & 07 & it & 34 & 15.0 & 13.0 & 38 & \\
\hline 36 & 36.9 & 38.9 & I4 & At & 36 & 34.9 & 36.8 & & I I & 6 & 36 & 34.3 & 33.5 & 06 & $\cdot$ & 36 & 17.0 & 15.0 & 34 & \\
\hline 38 & 32.3 & 34.2 & 07 & 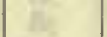 & 38 & 33.9 & 35.7 & & 09 & at & 38 & $\begin{array}{l}34.5 \\
34.2\end{array}$ & 33.1 & 06 & 18 & 38 & I9. 8 & 17.9 & 30 & \\
\hline 40 & 30.9 & 32.0 & 04 & -1 & 40 & 34.8 & 36.5 & & II & 8 & $\begin{array}{l}30 \\
40\end{array}$ & $\begin{array}{l}34.2 \\
32.8\end{array}$ & 30.9 & 09 & 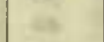 & 40 & 20.9 & 18.8 & 28 & \\
\hline 42 & 32.1 & 33.1 & 06 & & 42 & 36.9 & 38.6 & & 14 & 10 & 42 & $3 \mathrm{I} .2$ & 29.9 & II & 154 & 42 & $20 . I$ & 18.2 & 30 & \\
\hline 44 & $33 . I$ & 34.2 & 07 & +I.I & 44 & 37.0 & 38.6 & & I4 & +2.0 & 44 & 33.0 & 3 I. 9 & 08 & -2.4 & 44 & 18.1 & 16.9 & 32 & -2.3 \\
\hline 46 & 33.8 & $34 \cdot 7$ & 08 & on & 46 & 35.0 & 37.5 & & I I & 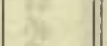 & $\begin{array}{l}44 \\
46\end{array}$ & $\begin{array}{l}33.0 \\
35.0\end{array}$ & $\begin{array}{l}31.9 \\
32.8\end{array}$ & o6 & -2.4 & 46 & II. 5 & 10.3 & 42 & \\
\hline 48 & 35.9 & 37.1 & I2 & $\sqrt{4}$ & 48 & 34.2 & 37.1 & & I0 & 8 & 48 & 37.2 & 35.4 & 2302 & As & $4^{*}$ & 37.7 & 3.3 .0 & 48 & \\
\hline 50 & 35.8 & 37.0 & 12 & $n$ & 50 & $3 \mathrm{I} .0$ & 33.7 & & 05 & 28 & $\begin{array}{l}40 \\
50\end{array}$ & 43.1 & 40.2 & 2254 & R & 50 & 34.1 & 28.8 & 55 & \\
\hline 52 & 37.8 & 39.5 & I5 & 만 & 52 & $3 \mathrm{I} .0$ & 33.4 & & 05 & 3 & $\begin{array}{l}50 \\
52\end{array}$ & 45.0 & 43.0 & 50 & b & 52 & 31.8 & 28.0 & 57 & \\
\hline & 39.5 & 40.5 & 17 & fE & 54 & $3 \mathrm{I} .1$ & 33.8 & & 05 & 17 & $\begin{array}{l}22 \\
54\end{array}$ & $\begin{array}{l}43.0 \\
46.9\end{array}$ & $\begin{array}{l}42.0 \\
45 \cdot 3\end{array}$ & 47 & 47 & 54 & 32.3 & 29.1 & 56 & \\
\hline 56 & 40.2 & $41 . I$ & I8 & of & 56 & 30.3 & 32.8 & & 04 & it & $\begin{array}{l}54 \\
56\end{array}$ & $\begin{array}{l}40.9 \\
45.0\end{array}$ & $\begin{array}{l}45.3 \\
44.0\end{array}$ & 49 & in & $\begin{array}{l}54 \\
56\end{array}$ & 33.2 & 30.1 & 54 & \\
\hline 58 & 41.5 & 42.4 & 20 & 80 & $\begin{array}{r}58 \\
\text { I }\end{array}$ & $\begin{array}{l}31.1 \\
32.9\end{array}$ & $\begin{array}{l}33.4 \\
35.2\end{array}$ & & $\begin{array}{l}05 \\
08\end{array}$ & +2.0 & 58 & 42.7 & $4 \mathrm{I} \cdot 3$ & 53 & $2 y$ & 58 & 31.3 & 28.3 & 57 & \\
\hline & & & 1.05 & & & 30.9 & $35 \cdot 2$ & & & & & & & & & & & & & \\
\hline
\end{tabular}

Correction to local mean time is $+17.5 \mathrm{~s} .90^{\circ}$ torsion $=199^{\prime} 22$.

Observer-J. V.

Torsion head at inh $25 \mathrm{~m}$ read $104^{\circ}$ and at $16 \mathrm{~h} 15 \mathrm{~m}$ read $97^{\circ}$.

Observer-R. R. T. 
Tabulation of magnetic declinations observed at Teplitz Bay-Continued

\begin{tabular}{|c|c|c|c|c|c|c|c|c|c|c|c|c|c|c|c|c|c|c|c|c|c|}
\hline \multicolumn{6}{|c|}{ Wediıcsday, June I, 1904} & \multicolumn{5}{|c|}{ Magnet scale inverted } & \multicolumn{7}{|c|}{ Wednesday, June I, I904 } & \multicolumn{4}{|c|}{ Magnet scale inverted } \\
\hline \multirow{2}{*}{$\begin{array}{l}\text { Chr'r } \\
\text { time }\end{array}$} & \multicolumn{2}{|c|}{$\begin{array}{l}\text { Scale } \\
\text { readings }\end{array}$} & $\begin{array}{c}\text { East } \\
\text { decli- } \\
\text { nation }\end{array}$ & $\begin{array}{c}\text { Temp. } \\
\text { C. }\end{array}$ & $\begin{array}{l}\text { Chr'r } \\
\text { time }\end{array}$ & $\begin{array}{r}\text { Sca } \\
\text { readi }\end{array}$ & $\begin{array}{l}\text { ale } \\
\text { lings }\end{array}$ & $\begin{array}{l}\text { Eas } \\
\text { decl } \\
\text { natio }\end{array}$ & & Temp. & $\begin{array}{l}\text { Chr'r } \\
\text { time }\end{array}$ & $\begin{array}{l}\text { Sca } \\
\text { read }\end{array}$ & $\begin{array}{l}\text { e } \\
\text { igs }\end{array}$ & $\begin{array}{l}\text { East } \\
\text { decli } \\
\text { natio }\end{array}$ & & Temp. & & $\begin{array}{l}\text { Sca } \\
\text { readi }\end{array}$ & le & $\begin{array}{c}\text { East } \\
\text { decli- } \\
\text { nation }\end{array}$ & Tenip. \\
\hline & Left & Right & & & & Left & Right & & & & & Left & Right & & & & & $L_{i}$ eft & Right & & \\
\hline $\mathrm{h} \mathrm{m}$ & d & d & $\cdot$ ' & $\bullet$ & h nı & d & d & & & $\bullet$ & $\mathrm{h} \mathrm{m}$ & 0 & & & & & h m & d & & - , & $\bullet$ \\
\hline 400 & $34 . I$ & 33. I & $235 I$ & -2.2 & 600 & 32.0 & 31.2 & 235 & 55 & -2.2 & 800 & 67.6 & 66.6 & 230 & & -1.0 & 100 & $34 \cdot 3$ & 32.6 & 2253 & -0.8 \\
\hline 02 & 37.9 & 36.0 & 46 & 60 & 02 & 29.1 & 28.3 & & & $7 x$ & 02 & 66.0 & 64.9 & & 02 & 54 & 02 & 34.1 & 33.0 & 52 & \\
\hline 04 & 40.0 & 37.6 & 43 & 72 & 04 & 28.8 & 27.1 & 240 & & 10 & 04 & $67 \cdot 3$ & 65.0 & & OI & 10 & 04 & $3 \mathrm{I} \cdot 5$ & 30.5 & 56 & 10 \\
\hline 06 & 43.7 & 41.5 & 37 & 92 & 06 & 24.2 & 23.1 & & & 6 & 06 & 61.3 & 60.7 & & 09 & 60 & 06 & 33.9 & 32 . & 53 & \\
\hline 08 & 43.0 & 40.9 & 38 & 165 & 08 & 23. I & 22.7 & & 08 & at & o8 & 63.2 & 63.0 & & 06 & 85 & 08 & 34.8 & 34. & $5 \mathrm{I}$ & \\
\hline 10 & 36.9 & 35.5 & 47 & wit & 10 & $28 . I$ & 27.4 & & or & 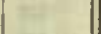 & 10 & 65.9 & 65.2 & & 02 & $=n$ & I0 & 35.6 & 34 & 50 & \\
\hline 12 & 30.9 & 29.2 & 57 & 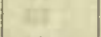 & I 2 & 26.0 & 24.2 & & 05 & 71 & 12 & $6 \mathrm{r} .3$ & $6 \mathrm{r} .0$ & & 09 & & 12 & 33.6 & 32. & 54 & \\
\hline 14 & 35.0 & 3 I. 9 & 52 & -2.2 & 14 & 22.9 & 22.2 & & 09 & -2.0 & 14 & 64.8 & 64.0 & & 04 & -0.8 & 14 & 33.5 & 32.8 & 53 & -0.8 \\
\hline 16 & 36.1 & 32.9 & 50 & & 16 & 26.0 & 25.0 & & 04 & & 16 & 67.0 & 66.8 & & $\infty$ & & 16 & 33.5 & 32.5 & 53 & \\
\hline 18 & 37.0 & 34.0 & 48 & 23 & I8 & 28.6 & 27.1 & 240 & & ta & 18 & 66.3 & 66.0 & 230 & OI & thit & I8 & 32.3 & & 55 & 2 \\
\hline 20 & 36.6 & 33.2 & 49 & 6 & 20 & 32.9 & 30.3 & 235 & & ne. & 20 & 69. & $.5 a$ & 225 & 56 & in & 20 & 34.0 & 32 . & 53 & $6 \pi$ \\
\hline 22 & 35.0 & $32 . I$ & 52 & $=$ & 22 & 38.9 & 38.0 & & 44 & 35 & 22 & 67.1 & 66.6 & 230 & $\infty$ & $c=$ & 22 & 34.6 & 33. & 52 & 2 \\
\hline 24 & 35.3 & 33.8 & 50 & 16 & 24 & 51.7 & 49.8 & & 25 & $y=$ & 24 & 70.6 & 70.3 & 225 & 54 & $F^{5}$ & 24 & 33.8 & 32 & 3 & ic \\
\hline 26 & 35.8 & 33.7 & 50 & $E$ & 26 & 47.0 & $44 . I$ & 233 & 33 & 186 & 26 & 69.3 & 68.5 & 225 & & e & 26 & 33.5 & 32 & 3 & \\
\hline 28 & 30.3 & 28. I & 2358 & $E$ & 28 & 25. & $.8 b$ & 240 & & $6 c$ & 28 & 66.3 & 65.6 & 230 & 02 & 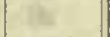 & 28 & 35.5 & 35 & 0 & 20 \\
\hline 30 & 29. I & 27.8 & 2400 & -2.2 & 30 & 28 & $.0 a$ & 240 & or & . & 30 & 67.8 & 67.8 & 225 & 58 & -0.8 & 30 & 35.0 & 34. & $5 \mathrm{I}$ & -0.6 \\
\hline 32 & 25.8 & 24.7 & 04 & 55 & 32.2 & 64.1 & 64.1 & 230 & 04 & 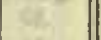 & 32 & $67 \cdot 3$ & 66.8 & 230 & $\infty$ & & 32 & 33.3 & 32 & 54 & 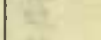 \\
\hline 34 & 20.8 & 18.8 & 13 & 16 & 34 & 60.1 & 59.9 & & 10 & 13 & 34 & 65.6 & 65.0 & & 02 & at & 34 & 33.9 & 32 & 53 & 41 \\
\hline 36 & 23.6 & 21.1 & 09 & 18 & 36 & 66. & $.8 b$ & & $\infty$ & at & 36 & 66.0 & 66.0 & & 02 & $\therefore$ & 36 & 33.5 & 33 & 53 & at: \\
\hline 38 & 27.0 & 25.0 & 2403 & 18 & 38 & 38.8 & 38.8 & & 44 & 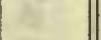 & 38 & 66.1 & 66.0 & 230 & & 25 & $3^{8}$ & 34.5 & 34. & I & 2 \\
\hline 40 & 3 I. 5 & 28.2 & 2357 & ine & 40.5 & 55 & $.0 a$ & & 18 & $r=$ & 40 & 69.2 & 69.0 & 225 & 57 & fas & 40 & & & 50 & 9. \\
\hline 42 & 31.5 & 29.3 & 56 & 0 & 42 & 61.2 & 60.8 & & 19 & -2 & 42 & 69.3 & 68.9 & & 57 & $=$ & 42 & 34.6 & 34 & $5 I$ & 6 \\
\hline 44 & 32.6 & 30.1 & 55 & -2.3 & 44 & $6 \mathrm{I} .6$ & 60.5 & & 09 & -1.7 & 44 & 68.7 & 68.3 & 225 & 58 & -0.8 & 44 & 34.2 & 34. & 52 & -0.5 \\
\hline 46 & 32.1 & 29.0 & 2356 & & 46 & 64.5 & 63.1 & & 04 & 10. & 46 & 63.5 & 63.0 & 230 & 06 & & 46 & 35.2 & 35. & 50 & \\
\hline 48 & 25.8 & 23.1 & 2406 & S8 & 48 & 61.7 & 59.0 & & IO & 80 & 48 & 70.0 & 69.9 & 225 & 55 & 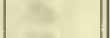 & 48 & 35.5 & & 9 & \\
\hline 50 & 30.7 & 27.0 & 2359 & 92 & 50 & 58.0 & 55.6 & & 16 & Cest & 50 & 70.6 & 70.3 & & 55 & nc & 5 & & & 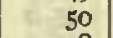 & \\
\hline 52 & 34.4 & 32.0 & 52 & 22 & $5^{2}$ & 56.9 & 56.1 & & I6 & - & 52 & 70.3 & 70.3 & & & 4 & 5 & & & & 2 \\
\hline 54 & 37.0 & 33.2 & 49 & 23 & 54 & 56.1 & 55.0 & I & 18 & & 54 & 0 & 60. & & 5 & If & 5 & 7.1 & 36 & 8 & F \\
\hline 56 & 39.0 & 36.3 & 45 & int & 56 & 58.0 & 58.0 & & 14 & & 56 & & 76. & & 4. & 3 & & & & 9 & \\
\hline 58 & 36.7 & 32.5 & 50 & 2 & 58 & 6 r. 9 & 60.9 & & 08 & & 58 & 76.8 & 76.3 & & 45 & 12 & 58 & 35.3 & 34.8 & 50 & \\
\hline 500 & 36.8 & 33.7 & 49 & -2.3 & 700 & 58.8 & 58.1 & I & 13 & -1.8 & 900 & 71.0 & 70.3 & & 54 & -0.8 & II 00 & Lo & ost & & (6ot Di \\
\hline 02 & 38.9 & 3.3 .7 & 47 & +6 & 02 & 62.2 & 60.0 & & 09 & & 02 & 71.0 & 70.3 & & 54 & $7 x$ & 02 & 37.0 & 36.6 & 48 & -0.3 \\
\hline 04 & 42.0 & 38.2 & $4 \mathrm{I}$ & tat & 0 & 59.3 & 57.7 & & 13 & & 04 & 70.5 & 70.3 & & 55 & wh & 04 & 38.0 & $37 \cdot 3$ & 46 & \\
\hline 06 & 43.0 & 38.8 & 40 & 100 & 06 & 55.3 & 54.9 & & 18 & 64 & $\alpha$ & 71.5 & 71. & & 53 & 00 & & 36.4 & 36 & 8 & 81 \\
\hline 08 & 49.6 & 45.8 & & ter & 08 & & 59. & & IO & & 08 & & 70 & & & 6 & 08 & 38.0 & & 6 & the \\
\hline I0 & 56.0 & 53.0 & 19 & or & I0 & 58.3 & 57.1 & & I4 & 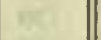 & Ic & 60.8 & 69.3 & & 56 & a & 10 & 30.3 & 38 . & 44 & Hith \\
\hline 12 & 50.5 & 49.1 & 26 & 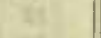 & & & 58.7 & & 12 & 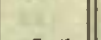 & I & 68.6 & 68.0 & & 58 & 25 & 12 & 38.3 & & 46 & \\
\hline 14 & $45 \cdot 3$ & 44.0 & 34 & -2.3 & 14 & 58. I & 57.2 & & I4 & -1.5 & 14 & 70.6 & 70.3 & & 55 & -0.8 & 14 & 39.6 & 38. & 44 & -0.3 \\
\hline 16 & 42.8 & 41.8 & 3 & & & & 56.2 & & I6 & & 16 & 69.6 & 60.3 & & 56 & & 16.2 & 39.2 & 38. & 4 & \\
\hline 18 & $4 \mathrm{~T} .3$ & 40.8 & 40 & 41 & 18 & 56.5 & 55.2 & & 17 & & 18 & 71.0 & 70. & & 5. & 86 & 18 & 40.3 & & & \\
\hline 20 & 39.9 & 39.6 & 42 & is & $2 C$ & 58.3 & 57.8 & & I4 & & $2 c$ & 72.0 & 71.7 & & 5 & 28 & $2 c$ & 39.6 & & 3 & e \\
\hline 22 & 36.8 & 35.5 & 48 & 13 & 22 & 61.7 & 60.1 & & $\infty$ & & 22 & 71.9 & 70.9 & & & 14 & 22 & & & & wa \\
\hline 24 & 34.9 & 32.6 & $5 I$ & 48 & 2 & 63.2 & 62.9 & & 06 & & 2 & 70.6 & 69.6 & & 5 & $E$ & 24 & 38.0 & 37 & 6 & $y$ \\
\hline 26 & 36.0 & 34.9 & 49 & 24 & 26 & 64.9 & 64. & & 04 & & 26 & 71.0 & 69.9 & & & int & & & & & \\
\hline 28 & 39.0 & 36.9 & 45 & & 28 & 63.1 & $6 r \cdot 5$ & & 07 & & 28 & 71.6 & 71.4 & & 53 & $\sqrt{2}$ & 28 & 38.0 & 37 & 46 & $\sqrt{5}=$ \\
\hline 30 & $4 \mathrm{I} \cdot 3$ & 40.7 & 40 & -2.4 & 30 & 64.2 & 64.1 & & 04 & -1.3 & 30 & 71.2 & 70.0 & & 55 & -0.9 & 30 & 39.0 & & 44 & -0.4 \\
\hline 32 & 38.2 & 38.2 & 44 & (2) & 32 & 62.8 & 61.8 & & 07 & & 32 & 72.5 & 72.0 & & 5 & & 32 & 38.6 & 38.6 & 45 & \\
\hline 34 & 38.2 & 37.9 & 45 & 47. & 34 & 63.8 & 6.3 .5 & 230 & 05 & 14 & 3 & 72.8 & 72.2 & & 5 & 3 & 34 & 39.0 & 39.0 & 44 & 31 \\
\hline 36 & 36.7 & 36.0 & 47 & ty & 36 & & 60.3 & & 55 & & 30 & 72. I & 71.3 & & 53 & & & 40.3 & 40. & 42 & (a) \\
\hline $3^{8}$ & 33.9 & 33.9 & $5 \mathrm{I}$ & सer & 38 & 69.8 & 69.2 & & 56 & & 38 & 70.8 & 70.0 & & & 2 & 38 & 40.6 & 40. & 42 & \\
\hline 40 & 35.7 & 35. & 48 & to & 40 & 70.2 & 60.3 & & 55 & 04 & 40 & 70.0 & 60.8 & & 56 & net & 40 & 40.3 & 39. & 43 & cer \\
\hline 42 & 38.0 & 36.8 & 46 & 12 & 42 & 69.5 & 68.1 & & 57 & & 42 & 72.6 & 72.0 & & 52 & 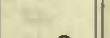 & 42 & $4 \mathrm{I} .2$ & & $4 \mathrm{I}$ & 63 \\
\hline 44 & 38.8 & 37.5 & 44 & -2.4 & 44 & 60.2 & 67.9 & & 57 & -1.1 & 44 & 73.3 & 72.8 & & 51 & -0.8 & & 40.6 & 40.5 & 42 & -0.4 \\
\hline 46 & 40.0 & 39.2 & 42 & & 46 & 0.2 & 69.0 & 5 & 56 & 50 & 46 & 71.0 & 71.0 & & 54 & 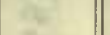 & 46 & 30.2 & 39. & 44 & - \\
\hline 48 & $4 \mathrm{I} .2$ & $4 \mathrm{I} . \mathrm{I}$ & 40 & an & 48 & 70.1 & 69.0 & & 56 & 74 & 48 & 70.1 & 70.0 & & & n & 48 & $3^{88.0}$ & & 46 & 84 \\
\hline 50.3 & $43 \cdot 7$ & 43.0 & 36 & 13 & 50 & 65.2 & 63.1 & 230 & 04 & col & 50 & 73. & $6 a$ & & 50 & wit & 50 & 38.0 & 37.8 & 46 & $n$ \\
\hline 52 & 42.8 & 43.1 & 37 & 6 & 52 & 62.1 & 60.0 & & 9 & है & $51^{*}$ & 42.5 & 37.3 & & & $3=$ & 52 & 39.0 & 38.7 & 44 & 2 \\
\hline 54 & 4.3 .9 & 43.1 & 36 & $f^{2}$ & 54.3 & 68.1 & 64.7 & & OI & 44 & 54 & 32.0 & 30.8 & 225 & 56 & $H 2$ & 54 & 39.6 & 39 & 44 & 34 \\
\hline 56 & 38.2 & 37.8 & 45 & & 56 & 62.2 & 59.9 & W & 99 & 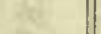 & 56 & 24.5 & $23 \cdot 3$ & 230 & 08 & $t^{2}$ & 56 & 40.1 & & 43 & 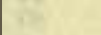 \\
\hline 58 & 36.8 & 36.1 & 47 & & 58 & 64.0 & 62.7 & 0 & & 82 & 58 & 31.0 & 30.3 & 225 & & 28 & 58 & 40.5 & 40.6 & & E. \\
\hline
\end{tabular}

Observer-J. V.

Observers-J. V. and W. J. P., who alternated from $8 \mathrm{~h}$ oom to 8 h rom. 
Tabulation of magnetic declinations observed at Teplitz Bay-Continued

\begin{tabular}{|c|c|c|c|c|c|c|c|c|c|c|c|c|c|c|c|c|c|c|c|}
\hline \multicolumn{6}{|c|}{ Wednesday, June I, 1904} & \multicolumn{4}{|c|}{ Magnet scale inverted } & \multicolumn{6}{|c|}{ Wednesday, June I, 1904} & \multicolumn{4}{|c|}{ Magnet scale inverted } \\
\hline \multirow{2}{*}{$\begin{array}{l}\text { Chr'r } \\
\text { time }\end{array}$} & \multicolumn{2}{|c|}{$\begin{array}{c}\text { Scale } \\
\text { readings }\end{array}$} & \multirow{2}{*}{$\begin{array}{l}\text { East } \\
\text { decli- } \\
\text { nation }\end{array}$} & Temp. & Chr'r & $\begin{array}{l}\text { Sce } \\
\text { read }\end{array}$ & $\begin{array}{l}\text { ale } \\
\text { ings }\end{array}$ & $\begin{array}{l}\text { East } \\
\text { decli- }\end{array}$ & Temp. & Chr'r & $\begin{array}{r}\mathrm{Sc} \\
\mathrm{read}\end{array}$ & $\begin{array}{l}\text { ale } \\
\text { lings }\end{array}$ & $\begin{array}{c}\text { East } \\
\text { decli- }\end{array}$ & Temp. & Chr'r & $\begin{array}{r}\text { Sc } \\
\text { reac }\end{array}$ & $\begin{array}{l}\text { ale } \\
\text { ings }\end{array}$ & East & Temp. \\
\hline & Left & Right & & & & Left & Right & nation & & & Left & Right & & & tin & Left & Right & & \\
\hline h m & $d$ & $d$ & $\circ$, & $\cdot$ & $\mathrm{h} \mathrm{m}$ & $d$ & d & $\cdot \quad$ & $\bullet$ & $\mathrm{h} \mathrm{m}$ & d & $d$ & & • & $\mathrm{h} \mathrm{m}$ & $d$ & d & & - \\
\hline 1200 & 39.9 & 39.6 & 2243 & -0.3 & 1400 & 47.9 & $47 \cdot 7$ & $223 I$ & -0.3 & 1600 & 56.3 & 56.1 & 2218 & -1.2 & 1800 & 47.4 & 46.8 & 2233 & -1.4 \\
\hline 02 & 39.7 & 39.5 & 43 & (2) & 02 & $48 . I$ & 48.0 & 3I & 20 & 02 & 55.8 & 55.6 & & 60 & 02 & $48 . I$ & 46.9 & 32 & \\
\hline 04 & 39.6 & 39.2 & 44 & 10 & 04 & 48.8 & 48.6 & 30 & 10 & 04 & 56.0 & 55.7 & 19 & 19 & 04.1 & 48.0 & 47.0 & 32 & \\
\hline 06 & 39.8 & 39.4 & 43 & 80 & 06 & $49 . I$ & 48.9 & 29 & So & 06 & 56.0 & 56.0 & I8 & 16 & 06 & 48.7 & 47.9 & $3 \mathrm{I}$ & $x^{2}=$ \\
\hline 08 & 40.3 & 39.8 & 43 & (⿻) & 08 & 48.5 & 48.3 & 30 & 40 & 08 & 56.3 & 56.1 & I8 & 5 & 08 & 48.8 & $48 . \mathrm{I}$ & $3 \mathrm{I}$ & \\
\hline 10 & 40.3 & 39.8 & 43 & st & I0 & 48.0 & 47.8 & $3 I$ & ot: & I0 & 56.0 & 56.0 & I8 & 84 & IO & 49.8 & 49.2 & 29 & \\
\hline 12 & 40.5 & 40.3 & 42 & at & 12 & 48.2 & 47.8 & $3 \mathrm{I}$ & 48 & I2 & 54.9 & 54.8 & 20 & 41 & I2 & 50.1 & 49.6 & 29 & -1.0 \\
\hline 14 & 40.3 & 39.6 & 43 & -0.3 & I4 & 48.1 & $47 \cdot 9$ & $3 \mathrm{I}$ & -0.3 & 14 & 54.6 & 54.5 & $2 I$ & 73t & I4 & 50.9 & 50.1 & 28 & \\
\hline I6 & 40.1 & 39.5 & 43 & (x) & 16 & 48.5 & 48.3 & 30 & 4 & I6 & $54 \cdot 3$ & $54 \cdot 3$ & 21 & 308 & I6 & 51.0 & 50.1 & 28 & as \\
\hline I8 & 40.6 & 40.3 & 42 & 81 & 18 & 48.6 & 48.6 & 30 & Whit & 18 & 53.9 & 53.8 & 22 & 8: & 18 & 50. I & 50.1 & 28 & Av \\
\hline 20 & $4 \mathrm{I} .2$ & 40.8 & $4 \mathrm{I}$ & Ne & 20 & 49.6 & 49.3 & 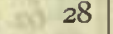 & es & 20 & 53.0 & 53.0 & 23 & we & 20 & $5 \mathrm{I} . \mathrm{I}$ & 50.3 & 27 & 28 \\
\hline 22 & 42.6 & 42.0 & 39 & 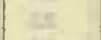 & 22 & $49 \cdot 5$ & 49.2 & 29 & the & 22 & 52.6 & 52.5 & 24 & A. & 22 & 51.4 & 50.5 & 27 & s6 \\
\hline 24 & 43.2 & 42.8 & 38 & is & 24 & 49.6 & 49.5 & 28 & in & 24 & 52.0 & 52.0 & 25 & $x=$ & 24 & 51.9 & 50.8 & 26 & \# \\
\hline 26 & 43.2 & 42.9 & 38 & as & 26 & 50.0 & 49.7 & 28 & $t^{2}$ & 26 & 52.3 & 52.0 & 25 & है & 26 & 52.3 & $5 \mathrm{I} .1$ & 26 & \\
\hline 28 & 43.0 & 42.6 & 38 & Bs & 28 & 49.6 & 49.6 & 28 & $\sqrt{4}$ & 28 & 51.5 & 51.3 & 26 & in & 28 & 53.2 & 52.1 & 24 & \\
\hline 30 & 42.8 & 42.5 & 39 & -0.2 & 30 & 50.2 & 50.0 & 27 & & 30 & 51.3 & 51.0 & 26 & $-\mathrm{I} .5$ & 30 & $54 . I$ & 53.0 & 23 & -0.8 \\
\hline 32 & 43.1 & 42.8 & 38 & 78 & 32 & 51.3 & $5 \mathrm{I} .0$ & 26 & -0.5 & 32 & 51.8 & $5 \mathrm{I} .5$ & 25 & (2) & 32 & 55.4 & 54.1 & $2 \mathrm{I}$ & \\
\hline 34 & 44.3 & 44.2 & 36 & te & 34 & $51 \cdot 3$ & $5 \mathrm{I} .2$ & 26 & 15 & 34 & 52.2 & 52.0 & 25 & kic & 34 & $50 . \mathrm{I}$ & 54.9 & 20 & $y^{2}$ \\
\hline 36 & 45.3 & 45.0 & 35 & 68. & 36 & 50.I & 50.0 & 28 & $a_{2}$ & 36 & 52.5 & 52.1 & 24 & Ats. & 36 & 56.7 & 55.3 & 19 & \\
\hline 38 & 44.6 & 44.0 & 36 & \&. & 38 & 49.0 & 48.8 & 29 & $F^{2}$ & 38 & 52.3 & 52.0 & 25 & 92 & 38 & 56.5 & 55.6 & I9 & te \\
\hline 40 & 44.0 & 43.6 & 37 & E & 40 & $5 \mathrm{I} \cdot 3$ & $5 \mathrm{I} .0$ & 26 & $2 y$ & 40 & 51.7 & $5 \mathrm{I} .3$ & 26 & $\cos$ & 40 & 56.5 & 55.4 & IO & iit \\
\hline 42 & 44.2 & 44.0 & 36 & 45 & 42 & 51.6 & 51.2 & 25 & 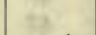 & 42 & $5 \mathrm{I} . \mathrm{I}$ & 50.6 & 27 & -2 & 42 & 56.0 & 55.0 & 20 & \\
\hline 44 & 44.6 & 44.4 & 36 & -0.1 & 44 & 50.6 & 50.5 & 27 & -0.7 & 44 & 50.4 & 50.1 & 28 & -1.8 & 44 & 55.1 & 54.4 & $2 \mathrm{I}$ & -0.9 \\
\hline 46 & 44.8 & 44.6 & 36 & 秘 & 46 & 51.8 & $5 \mathrm{I} .3$ & 25 & (1) & 46 & 49.7 & $49 \cdot 3$ & 29 & . & 46 & 55.1 & 54.2 & $2 \mathrm{I}$ & \\
\hline 48 & 44.9 & 44.6 & 36 & 8 & 48 & 52.2 & 52.0 & 24 & 8 & 48 & 50.0 & 49.7 & 29 & e. & 48 & $55 . \mathrm{I}$ & 54.7 & $2 \mathrm{I}$ & 25. \\
\hline 50 & 45.0 & 44.9 & 35 & wio & 50 & $49 \cdot 3$ & 49.0 & 29 & 10 & 50 & 50.2 & 50.0 & 28 & ap & 50 & 55.4 & 55.0 & 20 & 6 \\
\hline 52 & 45.2 & 45.0 & 35 & at & 52 & 48.0 & 48.0 & $3 \mathrm{I}$ & zhe & 52 & 49.6 & 49.4 & 29 & 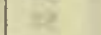 & 52 & 55.9 & 55.2 & 20 & ae \\
\hline 54 & 45.4 & 45.2 & 34 & kx. & 54 & 48.6 & 48.6 & 30 & 14 & 54 & 49.3 & 49.1 & 1829 & 17 & 54 & 55.9 & 55.2 & 20 & \\
\hline 56 & 45.6 & 45.6 & 34 & 38 & 56 & 49.3 & 49.0 & 29 & & 56 & 49.3 & 49.0 & 30 & & 56 & 57.8 & 57.2 & I7 & \\
\hline 58 & 45.8 & 45.6 & 34 & Ey & 58 & 49.3 & $49 \cdot 3$ & 29 & & 58 & $49 \cdot 3$ & 49.1 & 29 & -2.0 & 58 & 58.8 & 58.1 & I6 & \\
\hline 1300 & 46.2 & 46.1 & 33 & -0.1 & I5 00 & 48.8 & 48.7 & 30 & -1.0 & $17 \infty$ & 49.3 & 49.1 & - 29 & 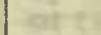 & I9 00 & 59.2 & 58.8 & I4 & -1.0 \\
\hline 02 & 46.4 & 46.4 & 33 & 34 & 02 & 49.0 & 49.0 & 29 & 30 & 02 & 49.0 & 48.8 & 30 & 30 & 02 & 60.1 & 59.0 & I4 & \\
\hline 04 & 47.0 & 46.9 & 32 & 10 & 04 & 49.6 & 49.6 & 28 & ing & 04 & 49.1 & 48.9 & 30 & 19 & 04 & 60.7 & 59.5 & 13 & \\
\hline 06 & 46.3 & 46.0 & 33 & Sus & 06 & 49.6 & 49.5 & 28 & 78 & 06 & 48.5 & 48.2 & $3 I$ & 6 & 06 & $6 \mathrm{I} . \mathrm{I}$ & 60.0 & I2 & \\
\hline 08 & 45.8 & 45.8 & 34 & For & 08 & 50.5 & 50.3 & 27 & sis & 08 & 48. I & 47.9 & $3 \mathrm{I}$ & $8 n$ & 08 & 61.0 & 60.0 & 12 & \\
\hline Io & 46.0 & 45.7 & 34 & bf: & Io & 51.3 & $5 \mathrm{I} \cdot 3$ & 26 & $\theta$ & I0 & 47.9 & 47.7 & 32 & $16 t$ & Io & 6r.I & 60.0 & 12 & \\
\hline 12 & 46.0 & 46.0 & 34 & $y$ & 12 & 51.9 & 51.6 & 25 & & I2 & 48.0 & 47.8 & 32 & $\pm y$ & I2 & $6 \mathrm{I} . \mathrm{I}$ & 60.0 & 12 & \\
\hline 14 & 46.3 & 46.3 & 33 & 0.0 & I4 & 52.7 & 52.3 & 24 & -1.0 & I4 & 48.5 & 48.2 & $3 \mathrm{I}$ & -2.0 & I4 & 60.9 & 60.0 & 12 & -0.9 \\
\hline 16.9 & 47.0 & 46.9 & 32 & the & 16 & 52.5 & 52.3 & 24 & ser & $\begin{array}{l}14 \\
16\end{array}$ & 48.8 & 48.6 & 30 & & 16 & 6 I. 3 & 60.2 & 12 & \\
\hline 18 & 47.2 & 47.2 & 32 & hin & I8 & 52.3 & 52.3 & 24 & 4 & I8 & 48.6 & 48.3 & 31 & ihr & 18 & $6 \mathrm{r} .2$ & 60.5 & 12 & \\
\hline 20 & 47.6 & 47.4 & 3I & os & 20 & 53.0 & 52.6 & 23 & es & 20 & $48 . I$ & 47.6 & 32 & $\alpha$ & 20 & 60.9 & 60.1 & I2 & \\
\hline 22 & 47.7 & 47.5 & 31 & af & 22 & 64.0 & 63.6 & 06 & is & 22 & 48.3 & 48.0 & $3 I$ & tes & 22 & 60.9 & 60.1 & I2 & \\
\hline 24 & 47.6 & 47.5 & $3 \mathbf{I}$ & iz & 24 & 55.1 & 55.0 & 20 & ys & 24 & 48.0 & 47.7 & 32 & iv & 24 & 60.5 & 59.9 & I3 & \\
\hline 26 & 47.9 & 47.9 & 3I & act & 26 & 56.0 & 55.6 & I9 & \& & 26 & 48.5 & 48.2 & & 88 & 26 & 60.5 & 59.8 & I3 & \\
\hline 28 & 48.0 & 47.9 & 3I & At & 28 & 57.3 & 57.1 & 16 & & 28 & 48.7 & 48.3 & 30 & $7 x$ & 28 & 60.9 & 59.8 & 12 & \\
\hline 30 & 48.0 & 47.8 & 3I & $\multimap . \mathrm{I}$ & 30 & 56.6 & 56.5 & I8 & $-I . I$ & 30 & 46.6 & 46.3 & 33 & -2.0 & 30 & 60.9 & 59.9 & I2 & -0.7 \\
\hline 32 & 47.8 & 47.3 & 3I & ace & 32 & 57.0 & 56.6 & I7 & sis & 32 & 46.1 & 45.8 & 35 & as & 32 & 60.9 & 59.9 & 12 & \\
\hline 34 & 47.6 & 47.3 & 32 & $x$ & 34 & 56.6 & 56.5 & $\mathrm{I} 8$ & if & 34 & 46.2 & 45.5 & 35 & iv & 34 & 60.2 & 59.3 & 14 & \\
\hline 36 & 47.0 & 46.9 & 32 & $x^{2}$ & 36 & 57.0 & 57.0 & 17 & 2 & 36 & 45.7 & 45.3 & 35 & & & 59.8 & 59.1 & 14 & \\
\hline 38 & 46.5 & 46.3 & 33 & e. & 38 & 55.8 & 55.6 & 8. 19 & Ne: & 38 & 45.2 & 44.9 & 36 & 42 & 38 & 59.3 & 59.0 & 14 & \\
\hline 40 & 46.3 & 46.0 & 34 & ay & 40 & 55.4 & 55.0 & 20 & 6 & 40 & $44 \cdot 3$ & 44.2 & & 58 & 40 & 59.0 & 58.4 & 15 & \\
\hline 42 & 46.3 & 46.1 & 33 & in: & 42 & 55.3 & 55.0 & 20 & & 42 & 44.3 & 43.9 & 38 & 36 & 42 & 58.0 & 57.0 & 17 & \\
\hline 44 & 47.5 & 47.0 & 32 & -0.2 & 44 & 55.3 & $55 . \mathrm{I}$ & 20 & $-\mathrm{I} .2$ & 44 & 44.4 & 44.0 & 37 & -2.0 & 44 & 56.4 & 56.1 & I9 & -0.4 \\
\hline 46 & 48.2 & 47.8 & 30 & the & 46 & 55.3 & 55.0 & 20 & $E$ & 46 & 44.9 & 44.5 & 37 & 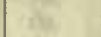 & & 56.3 & 55.7 & I9 & \\
\hline 48 & 48.6 & 48.4 & 30 & 36. & 48 & $55 \cdot 3$ & $55 . \mathrm{I}$ & 20 & 8 & 48 & 45.0 & 44.8 & 36 & ite. & 48 & 57.0 & 56.7 & 18 & \\
\hline 50 & 49.2 & 49.0 & 29 & of & 50 & $55 . \mathrm{I}$ & 54.9 & 20 & net & 50 & 45.8 & 45.6 & 35 & 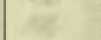 & 50 & 56.1 & 56.0 & 19 & \\
\hline 52 & 49.0 & 48.9 & 29 & 48 & 52 & 55.3 & 55.0 & 20 & $4 x$ & 52 & 46.3 & 45.9 & 34 & at & 52 & 56.7 & 56.1 & I9 & \\
\hline 54 & 48.3 & 48.0 & 30 & it & 54 & 56.3 & 56.3 & I8 & is & 54 & 46.8 & 46.0 & & $y 2$ & 54 & 57.1 & 56.9 & 18 & \\
\hline 56 & 48.1 & 48.0 & 30 & 58 & 56 & 57.1 & 57.0 & 17 & 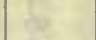 & 56 & 46.6 & 45.9 & 34 & 38 & 56 & 57.2 & 56.9 & 18 & \\
\hline 58 & 48.0 & 47.8 & $3 I$ & $3 y$ & 58 & 56.9 & 56.9 & 17 & $\sqrt{5}$ & 58 & $47 . I$ & 46.0 & 34 & $\pi$ & 58 & 56.8 & 56.1 & 19 & \\
\hline
\end{tabular}


Tabulation of magnetic declinations observed at Teplitz Bay-Continued

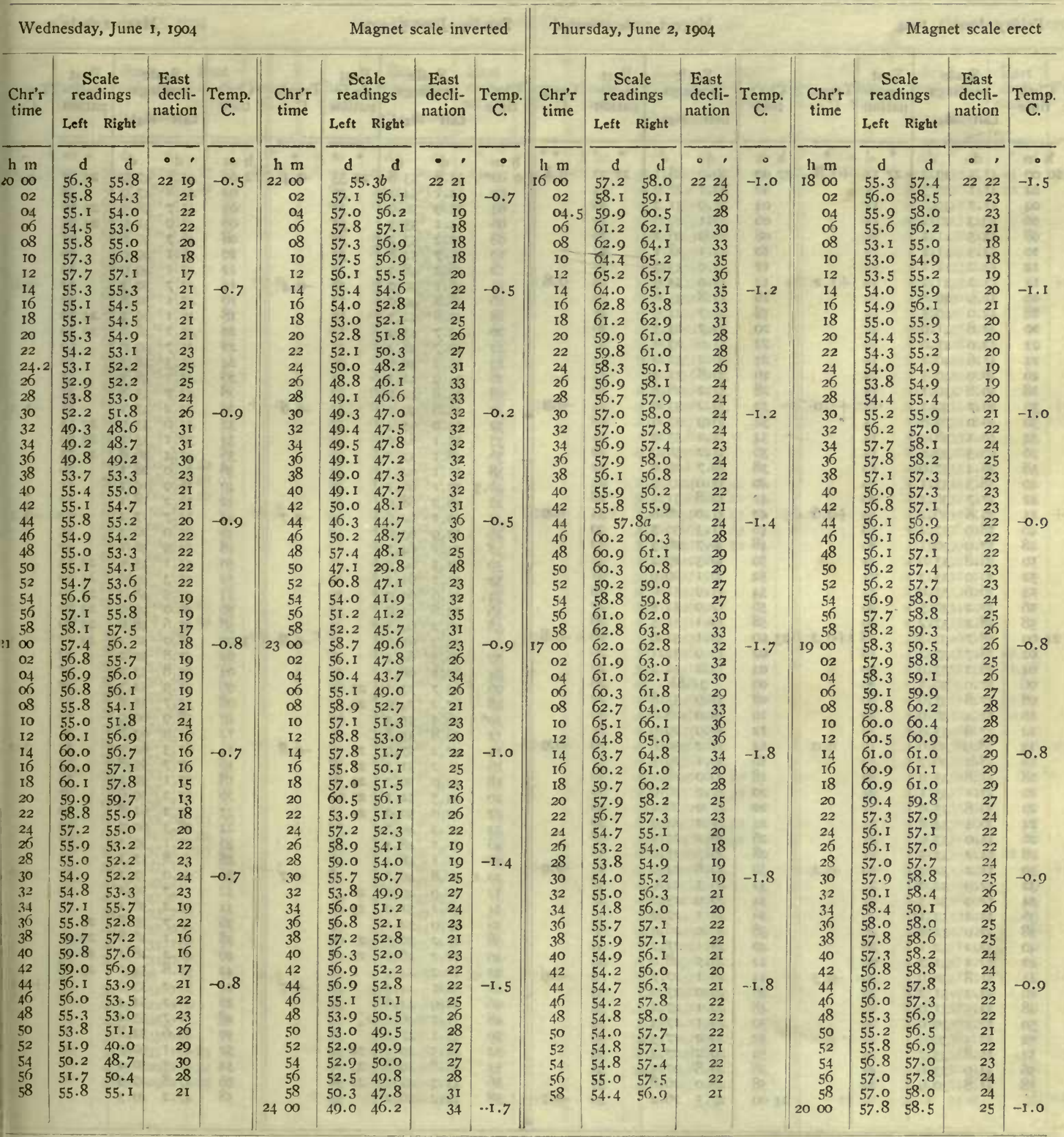

Correction to local mean time is $+39.5 \mathrm{~s} .90^{\circ}$ torsion $=199^{\prime} 53$. Torsion head at oh ooin read $97^{\circ}$ and at 24 h $15 \mathrm{~m}$ read $72^{\circ}$. Observer-R. R. T.
Correction to local mean time is $+\mathrm{Is}$.

Torsion head at $15 \mathrm{hl} 36 \mathrm{~m}$ read $72^{\circ}$ and at $20 \mathrm{~h} \mathrm{IIm}$ read the same. Observer-J. V. 
Tabulation of magnetic declinations observed at Teplitz Bay-Continued

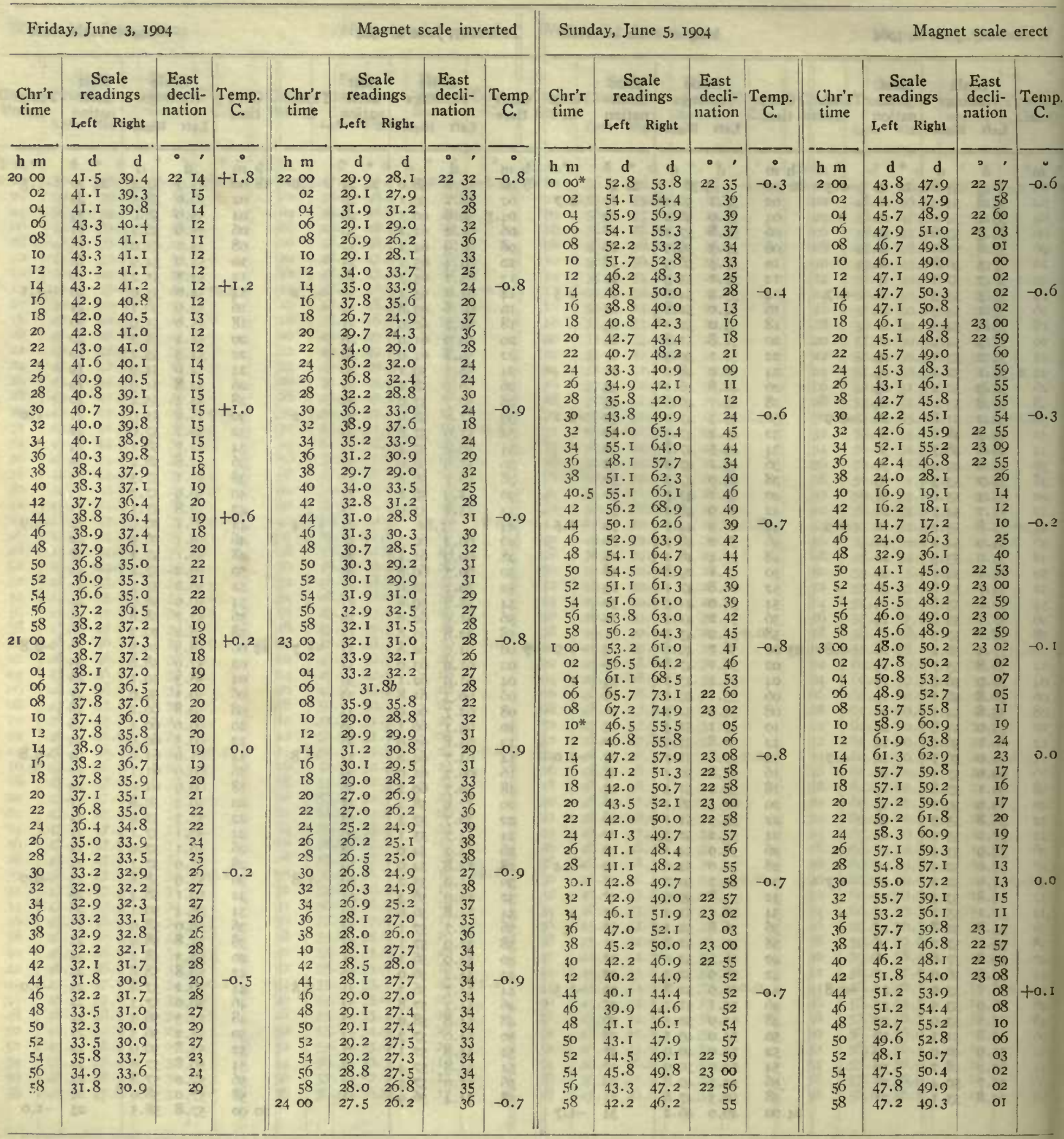

Correction to local mean time is - 2.5 s.

Observer-R. R. T.

Torsion head at $\mathrm{Igh} 42 \mathrm{~m}$ read $72^{\circ}$ and at $24 \mathrm{~h} \mathrm{I} 3 \mathrm{~m}$ read the same.

Observer-J. V. 
Tabulation of magnetic declinations observed at Teplitz Bay-Continued

\begin{tabular}{|c|c|c|c|c|c|c|c|c|c|c|c|c|c|c|c|c|c|c|c|c|}
\hline \multicolumn{6}{|c|}{ Sunday, June 5,1904} & \multicolumn{4}{|c|}{ Magnet scale inverted } & \multicolumn{6}{|c|}{ Monday, June 6, I904 } & \multicolumn{5}{|c|}{ Magnet scale erect } \\
\hline \multirow{2}{*}{$\begin{array}{l}\text { Chr's } \\
\text { time }\end{array}$} & \multicolumn{2}{|c|}{$\begin{array}{l}\text { Scale } \\
\text { readings }\end{array}$} & $\begin{array}{l}\text { East } \\
\text { decli- } \\
\text { nation }\end{array}$ & Teinp. & $\begin{array}{l}\text { Chr'r } \\
\text { time }\end{array}$ & $\begin{array}{l}\text { Sc: } \\
\text { read }\end{array}$ & $\begin{array}{l}\text { ale } \\
\text { ings }\end{array}$ & $\begin{array}{c}\text { East } \\
\text { decli- } \\
\text { nation }\end{array}$ & Temp & $\begin{array}{l}\text { Chr'r } \\
\text { time }\end{array}$ & $\begin{array}{r}\mathrm{Sc} \\
\text { reac }\end{array}$ & $\begin{array}{l}\text { ale } \\
\text { dings }\end{array}$ & $\begin{array}{c}\text { East } \\
\text { decli- } \\
\text { nation }\end{array}$ & Temp & $\begin{array}{l}\text { Chr'I } \\
\text { time }\end{array}$ & $\begin{array}{r}\text { Sc } \\
\text { read }\end{array}$ & $\begin{array}{l}\text { ale } \\
\text { lings }\end{array}$ & $\begin{array}{l}\text { Eas } \\
\text { decl } \\
\text { nati }\end{array}$ & & Temp. \\
\hline & Left & Right & & & & Left & Right & & & & Left & Right & & & & Left & Rigbt & & & \\
\hline $\mathrm{h} \mathrm{m}$ & $d$ & d & $\circ$ & 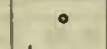 & $\mathrm{h} \mathrm{m}$ & d & d & $\cdot$, & $\bullet$ & $\mathrm{h} \mathrm{m}$ & d & d & 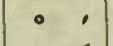 & 0 & $\mathrm{~h} \mathrm{~m}$ & d & d & & & 0 \\
\hline 400 & 58.3 & 57.2 & 2303 & to.3 & 600 & 24.1 & 20.0 & 2359 & 0.0 & $800^{*}$ & 51.1 & 52.8 & & +6.4 & 1000 & 47.7 & 50.2 & & & +7.2 \\
\hline 02 & 60.8 & 59.8 & 2259 & 10 & 02 & 36.9 & 31.0 & 38 & & 02 & 50.8 & 52.7 & & & 02 & $44 . \mathrm{I}$ & 46.6 & 22 & & \\
\hline 04 & 62.8 & $6 \mathrm{I} .2$ & 56 & & 04 & 28.9 & 26.9 & 50 & if & 04 & $49 \cdot 3$ & 52.2 & 54 & in & 04 & 41.8 & $45 . \mathrm{I}$ & & 52 & $p^{2}$ \\
\hline 06 & 62.1 & 61.0 & 57 & $x^{2}$ & 06 & 34 & $.5 b$ & 39 & 25 & 06 & 50.3 & 53.8 & 56 & 81 & 06 & 40.3 & 43.0 & & 49 & $w$ \\
\hline 08 & 63.0 & $62 . \mathrm{I}$ & 55 & 28 & 08 & 39.6 & 37.9 & 32 & Ge & 08 & 50.9 & 53.8 & 57 & 20 & 08 & 34.4 & 37.0 & & 39 & ai \\
\hline 10 & 63.8 & 62.9 & 54 & car & IC & 38.8 & $37 \cdot 5$ & 33 & si & 10.2 & 51.7 & 54.5 & 58 & 6 & 10 & 31.8 & 33.0 & & 34 & wir \\
\hline 12 & 64.1 & $63 \cdot 3$ & 53 & & I2 & $4 \mathrm{I} .9$ & 38.9 & 30 & & 12 & 51.7 & 54.2 & 58 & & 12 & 32.1 & 33.1 & & 34 & E \\
\hline I4 & 63.9 & 63.0 & 54 & +0.9 & I4 & 42.2 & 40.0 & 29 & 0.0 & 14 & 52.8 & 55.0 & 59 & +6.2 & 14 & $3 \mathrm{I} . \mathrm{I}$ & 33.2 & & 34 & +7.2 \\
\hline I6 & 65.0 & 64.2 & 52 & & 16 & 39.8 & 36.1 & 34 & & 16 & 50.9 & 52.8 & 56 & & 16 & 28.8 & 30.8 & & 30 & \\
\hline 18 & 65.8 & 64.9 & $5 I$ & ait & 18 & 42.9 & 39.3 & 29 & If & I8 & $47 . I$ & 49.1 & 50 & & 18 & 35.0 & 35.3 & & 38 & $A 1$ \\
\hline 20 & 64.9 & 64.0 & 52 & . & 20.5 & 38.7 & 34.2 & 36 & & 20 & 48.2 & 49.9 & 52 & 80 & 20 & 35.9 & 37.1 & & $4 \mathrm{I}$ & $\omega^{\circ}$ \\
\hline 22 & 65.2 & 64.0 & 52 & wi & 22 & 56.2 & 50.4 & Io & 18 & 22 & 51.1 & 53.0 & 56 & 4 & 22 & $47 . I$ & 48.9 & & 59 & 6 \\
\hline 24 & 64.6 & 63.1 & 53 & & 24 & 41.0 & 38.5 & $3 \mathrm{I}$ & & 24 & 51.8 & 53.8 & 58 & fle & 24 & 37.7 & 39.9 & & 44 & 315 \\
\hline 26 & 65.2 & 63.8 & $5^{2}$ & & 26 & 58.0 & 53.9 & 06 & & 26 & 53. I & 55.0 & 59 & & 26 & 37.1 & $39 . \mathrm{I}$ & & 43 & $x$ \\
\hline 28 & 65.2 & 63.7 & 52 & & 28 & 45.7 & 41.2 & 25 & & 28 & 49.9 & 52.0 & 55 & & 28 & 36.3 & 37.2 & & $4 \mathrm{I}$ & \\
\hline 30 & 66.1 & 64.1 & 51 & +1.0 & 30 & $45 \cdot 3$ & 39.3 & 27 & 0.0 & 30 & 46.8 & 48.1 & 49 & +6.2 & 30 & 33.9 & 34.9 & & 37 & +7.2 \\
\hline 32 & 66. I & 64.9 & 50 & & 32 & 40.9 & 36.9 & 32 & +2 & 32 & 46. I & $47 \cdot 3$ & 48 & & 32 & 33.1 & 34.2 & & 36 & \\
\hline 34 & 65.9 & 64.8 & 5 I & & 34 & $34 . I$ & 30.2 & 43 & & 34 & 47.9 & 48.9 & $5 I$ & 146 & 34 & 31.4 & 33. I & & 34 & 12 \\
\hline 36 & 62.9 & $6 \mathrm{i} .5$ & 56 & we & 36 & 39.9 & 37.9 & 32 & 38 & 36 & 49.2 & 50.9 & 53 & -8 & 36 & 28.9 & 32.9 & & 32 & $\theta$ \\
\hline 38 & 62.0 & 60.8 & $22 \quad 57$ & res & 38 & 38.9 & 35.8 & 35 & & 39 & 49 & $.0 b$ & 52 & : & 38 & 29.9 & 33.0 & & 33 & E \\
\hline 40 & 59.4 & 58.2 & 23 of & & 40 & 28.9 & 25.2 & $5 \mathrm{I}$ & of & 40 & 18.5 & 24.1 & 22 o8 & 60 & 40 & 32.5 & 34.6 & & 36 & Bf \\
\hline 42 & $6 \mathrm{I} . \mathrm{I}$ & 60.2 & $225^{8}$ & & 42 & 31.9 & 26.6 & $4^{8}$ & & $42^{*}$ & 52.7 & 56.8 & 2129 & & 42 & 29.3 & 32.9 & & 32 & \\
\hline 44 & 60.1 & 59.1 & 60 & to.8 & 44 & 30.3 & 25.7 & 49 & +0.2 & $44^{*}$ & 53.2 & 55.3 & 2308 & +6.2 & 44 & 27.2 & 33.5 & & $3 \mathrm{I}$ & +7.0 \\
\hline 46 & 61.2 & 60.2 & 58 & & 46 & 28.9 & $24 . I$ & 52 & & 46 & 53.0 & 60.9 & $\begin{array}{lll}23 & 13\end{array}$ & & 46 & 32.0 & 36.9 & & 37 & ( \\
\hline 48 & 64.5 & 63.2 & 53 & 8. & 48 & $4 \mathrm{I} .5$ & 37.9 & $3 \mathrm{I}$ & & 48 & 37.7 & 39.9 & 2244 & 2 & 48 & 33.5 & 39.9 & & $4 \mathrm{I}$ & 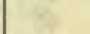 \\
\hline 50 & 63.4 & $62 . I$ & 55 & 3 & 50 & 30.8 & 27. & 47 & ces & 50 & 36.4 & 42.8 & 46 & ivt & 50 & 26.7 & 34.2 & & $3 I$ & 62 \\
\hline 52 & 61.9 & 6I.I & 57 & ce & 52 & 37.1 & 34.8 & 37 & 19. & 52 & 31.9 & 37.5 & 38 & wa & 52 & 27.1 & 32.3 & & 30 & 2 \\
\hline 54 & 60.4 & 59.2 & 59 & & 54 & $42 . I$ & 39. & 30 & $\frac{12}{12}$ & 54 & $37 . \mathrm{I}$ & $44 . I$ & 47 & La & 54 & 27.9 & 33.9 & & 32 & 46 \\
\hline 56 & 60.9 & 60.0 & 2258 & & 56 & 41.9 & 39.8 & 29 & & 56 & 39.6 & 49.9 & 54 & $2=$ & 56 & 27.9 & 34.2 & & 32 & 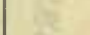 \\
\hline 58 & 59 & $.7 b$ & 2300 & & 58 & 46.8 & 45.4 & $2 \mathrm{I}$ & & 58 & 21.9 & 3 I. 9 & 26 & & 58 & 27.3 & 33.7 & & $3 I$ & \\
\hline 500 & 54.2 & 53.7 & og & +0.3 & 700 & 49.4 & 47.0 & I8 & to.2 & 900 & 35.2 & 43.0 & 45 & +6.3 & II 00 & 35.2 & 40.6 & & 43 & +7.1 \\
\hline 02 & 56.9 & 55.9 & 05 & 100 & 02 & $44 . I$ & 41.0 & 26 & 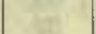 & 02 & 38. I & 46.2 & 50 & & 02 & 41.7 & 47.0 & 22 & 53 & 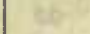 \\
\hline 04 & 54.2 & 53.0 & $\infty$ & & 01 & 36.0 & 33. & 39 & & 04 & 33.7 & $4 \mathrm{I}$. & 42 & 185 & 04 & 50.3 & 57.7 & 23 & 08 & to \\
\hline 06 & 57.1 & 56.2 & 04 & 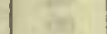 & 06 & 49.7 & $46 . i$ & 18 & X. & 06 & $4 \mathrm{I} . \mathrm{I}$ & 48. I & 53 & wh & 06 & 54.4 & 65.1 & & 17 & \\
\hline 08 & $56 . \mathrm{I}$ & 55.6 & 06 & 8s. & 08 & 47.5 & $44 . \mathrm{I}$ & $2 \mathrm{I}$ & & 08 & 40.0 & 46.8 & 52 & wo. & 08 & $45 . \mathrm{I}$ & 55.5 & 23 & 02 & tes: \\
\hline 10 & 57.9 & 57.0 & 03 & $=$ & Io & 38.1 & 33. & 37 & nots & TO & 39.9 & $47 . I$ & 52 & 20 & 10 & 40.8 & 49.9 & 22 & 54 & Dr \\
\hline 12 & 55.8 & 54.9 & 06 & & I2 & 42.6 & 38. & 30 & & 12 & $4 \mathrm{I} . \mathrm{I}$ & 47.4 & 53 & & 12 & 32. I & $40 . \mathrm{I}$ & & 40 & 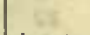 \\
\hline 14 & 54.1 & 52.7 & Io & to.I & I4 & 46.9 & $42 . I$ & 23 & to. I & I4 & 40.9 & 46.8 & 52 & +6.7 & 14 & 32.6 & 38.9 & & 39 & +7.7 \\
\hline I6 & 56.9 & 55.5 & 05 & & I6 & 50.2 & 47. & 17 & & 16 & 45.5 & 50.0 & 58 & & 16 & 29. I & 35.8 & & 34 & \\
\hline 18 & 54.9 & 53. I & 08 & 24 & I8 & 52.2 & 48.3 & 14 & 8 & 18 & 42.9 & 47.8 & 2254 & 84 & 18 & 26.1 & 32.8 & & 30 & pr \\
\hline 20 & 58.8 & $57 . I$ & 02 & & 20 & 51.2 & 47.8 & 16 & & 20 & 51.3 & 57.4 & 2309 & $c=$ & 20 & 24.2 & 3I. I & & 27 & we \\
\hline 22 & 60.9 & 58. I & $\infty$ & N & 22 & 54.2 & 51. & II & 0 & 22 & 46.0 & 54.1 & 02 & 67 & 22 & 26.0 & 33.0 & & 30 & 6 \\
\hline 24 & 60.7 & 58.0 & 2300 & & 24 & 50.8 & 48. & I6 & & 24 & 53.3 & 57.8 & 2310 & $y=$ & 24 & 24.2 & 30.8 & & 27 & it \\
\hline 26 & 64.6 & 60.8 & 2255 & 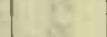 & 26 & 48.8 & 44.6 & 20 & & 26 & 42.2 & $47 \cdot 4$ & 2254 & $6=$ & 26 & 25.9 & 31.2 & & 28 & in \\
\hline 28 & 58.0 & $57 \cdot 3$ & 2303 & & 28 & 5 I.I & 47. & 16 & & 28 & 46.0 & 50.8 & 59 & 85 & 28 & 28.9 & 34.1 & 22 & 33 & \\
\hline 30 & 50.0 & $47 . \mathrm{I}$ & I7 & to. I & 30 & 50.2 & 46.2 & 18 & 0.0 & 30 & 44.9 & 49.7 & 58 & +7.0 & 30 & 38.6 & 43.0 & & 47 & +8.I \\
\hline 32 & 43.9 & 40.2 & 27 & ( & 32 & 50.3 & $47 . I$ & 17 & & 32 & 42.8 & 45.6 & 5.3 & (1) & 32 & 38.2 & 45.3 & & 49 & b \\
\hline 34 & 49.8 & 47.0 & I7 & & 34 & 50.0 & 45. & 18 & & 34 & 40.3 & 41.9 & 48 & 8 & 34 & 34.1 & 41.0 & . & 42 & if \\
\hline 36 & 35.5 & $33 \cdot 7$ & 39 & & 36 & 54.2 & 49. & 12 & & 36 & 39. I & 41.1 & 46 & Ex & 3 & 35.7 & 43.4 & & 45 & mo \\
\hline 38 & 40.0 & 37.9 & 32 & & 38 & 58.9 & 54.8 & 04 & & 38 & $4 \mathrm{I} . \mathrm{I}$ & 43.8 & 50 & Re & 38 & 43.5 & 49.0 & & 56 & We. \\
\hline 40 & 34.0 & 33. I & 41 & at & 40 & 60.0 & 56.0 & 02 & & 40 & 37.3 & 39.2 & 43 & 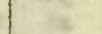 & 40 & 45.2 & $5 I .4$ & & 59 & 28 \\
\hline 42 & 35.0 & 34.9 & 38 & & 42 & 61.2 & 56.0 & 23 or & & 42 & 37.3 & 38.2 & 42 & 63 & 42 & 42.9 & 47.9 & & 55 & \\
\hline 44 & 39 & $.9 b$ & 31 & 0.0 & 44 & 6.3 .6 & 58.1 & 2258 & +0.2 & 44 & 36.3 & 37.1 & 41 & +7.1 & 44 & 43.9 & $47 \cdot 3$ & & 55 & +8.6 \\
\hline 46 & $33 . \mathrm{I}$ & 32.7 & & & 46 & 64.0 & 57.8 & 58 & & 46 & 34.5 & $37 \cdot 3$ & 40 & - & 46 & 38. I & $42 . I$ & & 46 & \\
\hline 48 & 22.9 & 22.2 & 58 & 5 & 48 & 65.0 & 58.8 & 56 & & 48 & 43.0 & 45.6 & 53 & (5) & 48 & 33.8 & 37.4 & & 39 & $18 \mathrm{c}$ \\
\hline 50 & 29.7 & 28.7 & 48 & & 50 & 65.9 & 58.3 & 56 & & 50 & 43.7 & 45.4 & 5.3 & ige & 50 & 34.9 & 39.9 & & 42 & act \\
\hline 52 & $33 \cdot 3$ & 33.0 & $4 I$ & 3 & 52 & 65.3 & 58.1 & 56 & a & 52 & 40.5 & 42.0 & 48 & 31 & 52 & 32.9 & 36.1 & & 38 & we \\
\hline 54 & 35.8 & 35 . I & $23 \quad 38$ & & 54 & 65.3 & 58.3 & 56 & & 54 & 45.2 & 48.8 & 57 & & 54 & 32.2 & 35.9 & & 37 & \\
\hline 50 & 21.3 & 20. I & 2401 & & 56 & 64.9 & 58.2 & 57 & & 56 & 44.8 & 48.4 & 56 & at & 56 & 35.9 & 39.0 & & 42 & 58 \\
\hline 58 & 30.7 & 29.0 & 2346 & & 58 & 66.1 & 60.1 & 54 & & 58 & 41.2 & 45.9 & 52 & 15 & 58 & 3I. I & 34.8 & & 35 & \\
\hline & & & & $w$ & 300 & 67.8 & 01.3 & 52 & to. 8 & & & & & $0 \mathrm{~s}$ & 1200 & 32.2 & 34.0 & & 35 & +8.8 \\
\hline
\end{tabular}

Correction to local mean time is $-38 \mathrm{~s}$.

Torsion head at oh oom read $72^{\circ}$ and at $8 \mathrm{~h} \mathrm{I} 5 \mathrm{~m}$ read the same. Observer-R. R. T.
Correction to local mean time is - Im 04.5s. $90^{\circ}$ torsion $=18 .^{\prime} 90$. Torsion head at $7 \mathrm{~h}$ 3om read $76^{\circ}$ and at $\mathrm{2} 2 \mathrm{~h}$ is $\mathrm{m}$ read $63^{\circ}$. Observer-R. R. T. 
Tabulation of magnetic declinations observed at Teplitz Bay-Continued

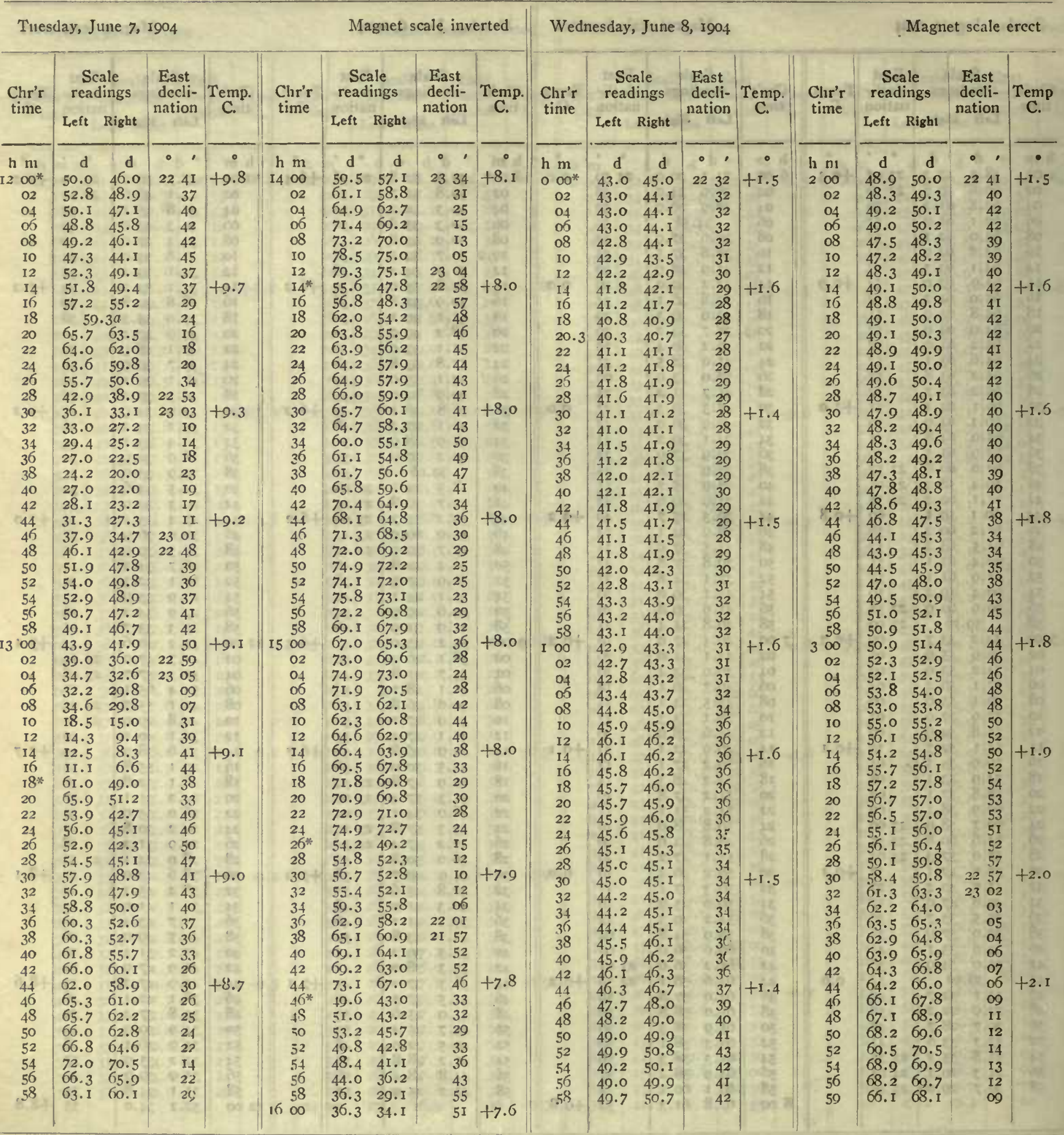


Tabulation of magnetic declinations observed at Teplitz Bay-Continued

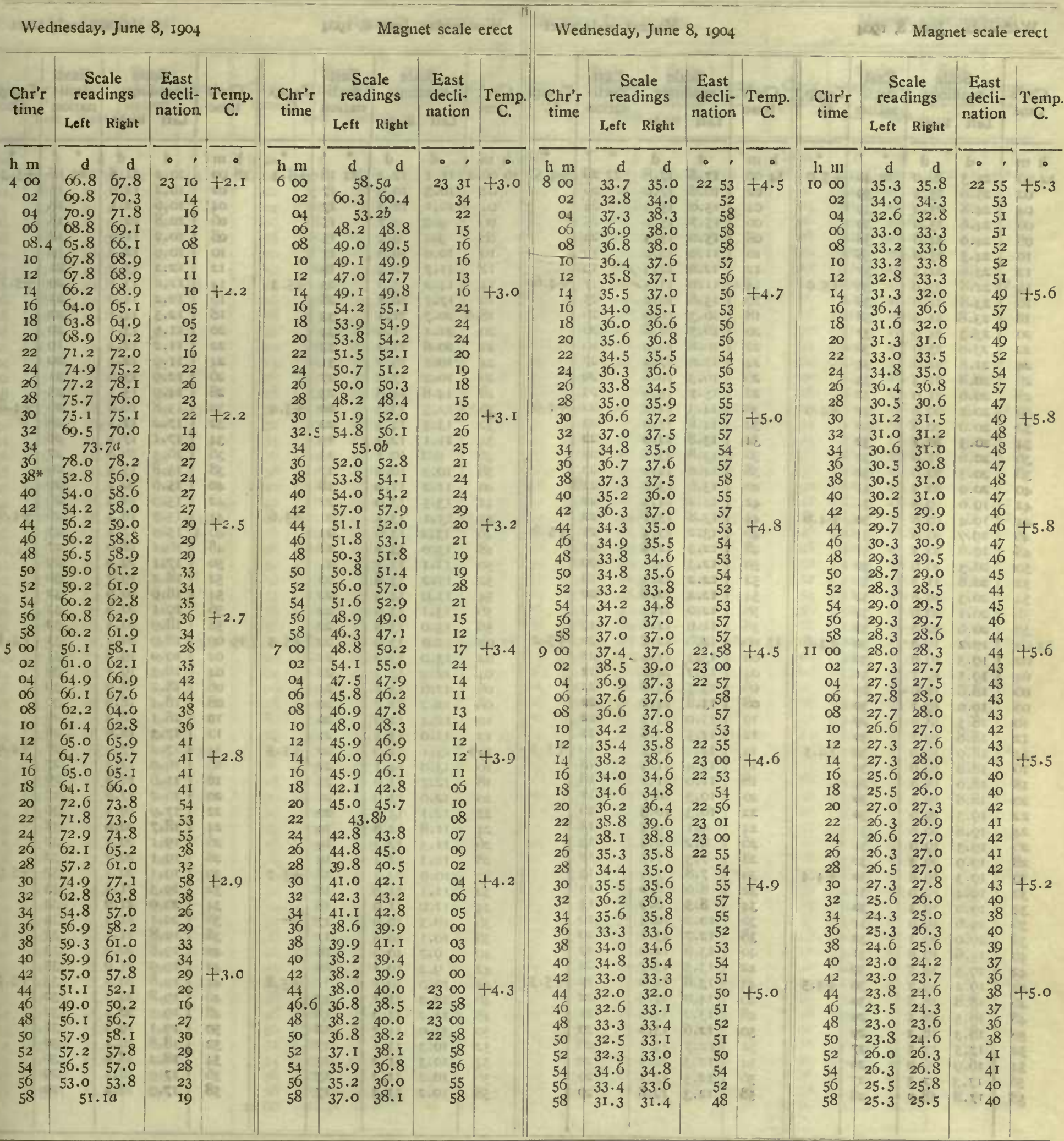

Observer-J. V.

Observers-J. V. and W. J. P., who alternated from $8 \mathrm{~h} .06 \mathrm{~m}$ to $8 \mathrm{~h} 16 \mathrm{~m}$. 
Tabulation of magnetic declinations observed at Teplitz Bay-Continued

\begin{tabular}{|c|c|c|c|c|c|c|c|c|c|c|c|c|c|c|c|c|c|c|c|}
\hline \multicolumn{5}{|c|}{ Wednesday, June 8, I904 } & \multicolumn{5}{|c|}{ Magnet scale crect } & \multicolumn{6}{|c|}{ Wednesday, June 8, Ig04 } & \multicolumn{4}{|c|}{ Magnet scale erect } \\
\hline \multirow{2}{*}{$\begin{array}{l}\text { Chr'r } \\
\text { time }\end{array}$} & \multicolumn{2}{|c|}{$\begin{array}{l}\text { Scale } \\
\text { readings }\end{array}$} & \multirow{2}{*}{$\begin{array}{c}\text { East } \\
\text { Jecli- } \\
\text { lation }\end{array}$} & Temp. & $\begin{array}{l}\text { Chr'r } \\
\text { time }\end{array}$ & $\begin{array}{l}\text { Sca } \\
\text { readi }\end{array}$ & $\begin{array}{l}\text { ale } \\
\text { lings }\end{array}$ & $\begin{array}{l}\text { East } \\
\text { Cecli- } \\
\text { Iation }\end{array}$ & Temp. & Chr'r & $\begin{array}{l}\text { Sec } \\
\text { read }\end{array}$ & $\begin{array}{l}\text { ale } \\
\text { lings }\end{array}$ & - East & Temp. & & $\begin{array}{r}\text { Sca } \\
\text { readi }\end{array}$ & $\begin{array}{l}\text { ale } \\
\text { lings }\end{array}$ & East & Temp \\
\hline & Left & Right & & & & Left & Right & & & & Left & Right & & & & Left & Right & & \\
\hline h m & d & d & $\cdot 1$ & 0 & $\mathrm{~h} \mathrm{~m}$ & d & d & $0 \quad$, & & $h \mathrm{~m}$ & d & d & - ' & & h m & a & a & - , & \\
\hline 1200 & $25 \cdot 3$ & 26.0 & 3240 & +1.5 .0 & 1400 & 17.8 & 17.8 & 2228 & $+4 \cdot 5$ & 1600.2 & II.I & 12.2 & 2218 & $+5 \cdot 3$ & 1800 & 10.2 & 11.8 & 22 I8 & +5.3 \\
\hline 02 & 25.0 & $25 \cdot 5$ & 39 & 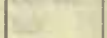 & 02 & 16.8 & 17.0 & 26 & 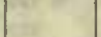 & 02 & 10.3 & 10.9 & 17 & & 02 & 10.2 & 12.0 & I8 & \\
\hline 04 & 24.8 & 25.0 & 39 & To & 04 & 15.0 & $15 \cdot .3$ & 24 & ser & 04 & IO. I & 10.8 & I7 & & 04 & 10.6 & I2. I & 18 & \\
\hline 06 & 4.6 & 25.0 & 39 & - & 06 & 15.7 & I5.7 & 25 & 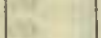 & 06 & $9 \cdot 3$ & 9.7 & I5 & & 06 & 10.8 & 12. I & 18 & \\
\hline 08 & 24.0 & 24.8 & 38 & Fon & 08 & 15.0 & $15 \cdot 3$ & 24 & 6 & 08 & 9.1 & 9.9 & I5 & & 08 & 10.2 & II.9 & & \\
\hline Io & $24 \cdot 3$ & 25.0 & 38 & & I0 & II.9 & 12.0 & I9 & 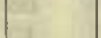 & Io & 10.0 & 10.8 & I7 & & 10 & 10.9 & 12.2 & 19 & \\
\hline 12 & 23.6 & 24.3 & 37 & & 12 & 12.0 & 12.3 & I9 & & 12 & II.7 & 12.4 & I9 & & 12 & 12.2 & 13.9 & $2 \mathrm{I}$ & \\
\hline I4 & 22.8 & 23.6 & 36 & -15.0 & I4 & 11.0 & II. .3 & I7 & +4.6 & 14 & $\mathrm{I}_{4} .2$ & 15.2 & 23 & +5.3 & 14 & 12.0 & 13.1 & 20 & +5.1 \\
\hline 16 & 23.8 & 24.6 & 38 & ba & 16 & 15.8 & 16.2 & 25 & & 16 & I7.I & 18.1 & 28 & & 16 & 12.2 & I 4.0 & 21 & \\
\hline 18 & 23.8 & 24.5 & 38 & 5 & 18 & 16.3 & I6. 6 & 26 & ty & I8. 6 & 20.7 & $21 . I$ & 33 & & 18 & 12.5 & 14.1 & 22 & \\
\hline 20 & $23 \cdot 3$ & 24.0 & 37 & 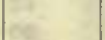 & 20 & 16.5 & 16.8 & 26 & trat & 20 & $21 . \mathrm{I}$ & 21.9 & 34 & 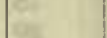 & o & II.9 & I3.I & 20 & \\
\hline 22 & 23.0 & 23.6 & 36 & & 22 & 16.8 & I6.9 & 26 & ta & 22 & 20.8 & 21.2 & 33 & Iis & & II. 2 & 12.4 & 19 & \\
\hline 24 & 21.8 & 22.6 & 34 & & 24 & 17.0 & 17.2 & 27 & 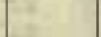 & 24 & I9. I & 19.7 & $3 \mathrm{I}$ & $=$ & 4 & II.9 & 12.7 & 20 & \\
\hline 26 & 20.8 & $2 \mathrm{I} .3$ & 33 & te & 26 & 16.3 & I6.6 & 26 & A & 26 & 16.2 & 17.2 & 27 & & 26 & 12.9 & I3.6 & 2 I & \\
\hline 28 & 20.6 & 21.0 & 32 & & 28 & 15.7 & 15.9 & 25 & & 28 & 14.8 & 14.8 & 23 & & 28 & 14.9 & 15.7 & 25 & \\
\hline 30 & 20.2 & 20.6 & 32 & +4.9 & 30 & 14.3 & 14.3 & 22 & +4.9 & 30 & 12.3 & 13.1 & 20 & +5.4 & 30 & 15.8 & 16.8 & 26 & +4.8 \\
\hline 32 & 20.0 & 20.6 & 32 & 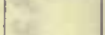 & 32 & 14.8 & 15.0 & 23 & & 32 & 13.5 & I4.4 & 22 & & 3 & 17.2 & 17.2 & 28 & \\
\hline 34 & 20.3 & 20.7 & 32 & 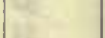 & 34 & 13.8 & 14.0 & 22 & 4 & 31 & II. I & 12.3 & 19 & & 34 & 17.1 & I7. 2 & 28 & \\
\hline 36 & 20.6 & 21.0 & 32 & $1=$ & 36 & 13.8 & I3.8 & 22 & 2 & 36 & 9.2 & I0.I & I5 & & & 16.3 & 16.8 & 27 & \\
\hline 38 & 20.3 & 20.6 & 32 & 5 & 38 & 14.0 & 14.0 & 22 & & 38 & 10.3 & 10.9 & 17 & & 38 & 15.8 & 15.9 & 26 & \\
\hline 40 & 20.0 & 20.3 & $3 \mathrm{I}$ & & 40 & 13.6 & 13.7 & $2 \mathrm{~J}$ & & 40 & II.2 & 11.9 & 18 & & 40 & 15.6 & 16.1 & 26 & \\
\hline 42 & 19.6 & 19.8 & $3 \mathrm{I}$ & 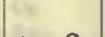 & 42 & I 4.2 & I4. 3 & 22 & 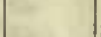 & 42 & I 3.5 & 13.9 & 22 & & 42 & 15.9 & 16.3 & 26 & \\
\hline 44 & 19.4 & 19.8 & 30 & +4.8 & 44 & I 3.8 & 15.7 & 22 & & 44 & $13 . I$ & 13.5 & 21 & +5.6 & 44 & 15.7 & 16.2 & 26 & +4.6 \\
\hline 45 & 19.7 & 20.0 & $3 \mathrm{I}$ & & 46 & 13.2 & I 3.4 & 21 & +5.0 & 46 & 13.2 & I3. 6 & 21 & & 46.4 & 15.9 & I7.2 & 27 & \\
\hline 48 & I9. 8 & 20.2 & $3 I$ & 1 & 48 & 12.3 & 12.8 & $2 \mathrm{I}$ & & 48 & I 3.0 & I 3.2 & 21 & & 48 & 16.1 & 18.0 & 27 & \\
\hline 50 & 20.3 & 20.6 & 32 & 1 & 50 & 12.3 & 12.t. & 20 & & 50 & 13.1 & 13.7 & 2 & & 50 & I7.I & 19.0 & 29 & \\
\hline 52 & 20.8 & $2 \mathrm{I} .2$ & 3.3 & 3 & 52 & 12.2 & 12.5 & 19 & e & 52 & I 3.2 & 13.8 & 22 & at & 5 & I8. 2 & 19.7 & 30 & \\
\hline 54 & 20.3 & 20.8 & 32 & $\ldots$ & 54 & 12.4 & 12.5 & 20 & & 54 & I3.9 & 14.3 & 2 & & 5 & 18.9 & 20.6 & 32 & 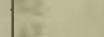 \\
\hline 36 & 20. I & 20.6 & 32 & 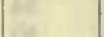 & 56 & 12.0 & 12.3 & 19 & e & 56 & 13.1 & 13.9 & 2 & & & 19.1 & 20.8 & 32 & \\
\hline 58 & 20.6 & 20.8 & 32 & & 58 & II. 9 & 12.1 & 19 & & 58 & 13.0 & 13.8 & 22 & & 58 & 18.9 & 20.4 & 32 & \\
\hline$\infty$ & 19.2 & 19.8 & 30 & +4.6 & I5 00 & T2. 6 & 12.8 & 20 & +5.2 & 1700 & 13.0 & 13.9 & 22 & +5.5 & I 90 & I8.9 & 20.2 & $3 I$ & $+4 . I$ \\
\hline 02 & 19.3 & I9. 6 & 30 & s. & 02 & 13.3 & 13.4 & 21 & & 02 & 13.0 & 13.2 & 21 & & 0 & 19.0 & 19.8 & $3 \mathrm{I}$ & \\
\hline 04 & 19.5 & 19.7 & 3I & to & $0_{4}$ & $I_{4} .2$ & 14.2 & 22 & a) & 04 & I 2.9 & 13.1 & $2 \mathrm{I}$ & & & 18.3 & 19. & 30 & \\
\hline 06 & I8. 8 & 18.9 & 29 & 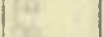 & 06 & 13.5 & 13.8 & 22 & & or & 12.6 & 13.1 & 21 & & & 18.2 & 18 & 0 & \\
\hline 08 & I9.0 & 19. I & 30 & ent & 08 & 13.8 & 14.2 & 22 & & 08 & I 2.2 & 12.7 & 20 & & o & 18.4 & 18.9 & 30 & \\
\hline 10 & 18.5 & 18.0 & 29 & 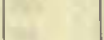 & IO & I3.0 & $13 \cdot 3$ & 22 & & I0 & 12.4 & 12.9 & 20 & & I & I8. I & 18.3 & 9 & \\
\hline 12 & 18.8 & 19. I & 30 & & I2 & I2. 5 & $12 . \overline{8}$ & $2 I$ & & 12 & 13.0 & 13.5 & 2I & & 12 & I7. 3 & I8.0 & 28 & \\
\hline I4 & 18.9 & 19.0 & 30 & $\cdot+4.5$ & 14 & I2. I & I2. 3 & I9 & +5.3 & I4 & 13.1 & 14.0 & 22 & +5.5 & & 17.2 & 17.9 & 28 & +4.0 \\
\hline I6 & 18.8 & 19.0 & 30 & & I6 & II. 4 & II. 4 & 58 & & 16 & 13.0 & $14 . I$ & 22 & & & 16.0 & 17. & 27 & \\
\hline 18 & 18.6 & I8.8 & 29 & test & I8 & II.0 & II. 2 & I8 & trat & I8 & 12.7 & 13.9 & $2 \mathrm{I}$ & & I & 15.6 & 16 & 26 & \\
\hline 20 & 18.8 & I9. 6 & 30 & & 2 & I0. 3 & 10.7 & 17 & & $2 x$ & 12.6 & 13.6 & $2 \mathrm{I}$ & & & I6. 2 & 17.8 & 27 & \\
\hline 22 & I9. 5 & I9. 6 & 30 & 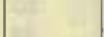 & 22 & 10.3 & I0. 3 & I6 & 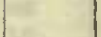 & 2 & I2. I & I3. I & 20 & & 2 & I7.9 & 19.1 & 30 & \\
\hline 24 & 19.8 & 20.0 & $3 \mathrm{I}$ & & 2 & 9. I & 9.4 & I5 & 3 & 2. & II. 8 & 12.8 & 19 & & & I8.7 & 20.0 & $3 \mathrm{I}$ & \\
\hline 26 & 19.3 & I9.9 & $3 \mathrm{I}$ & 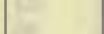 & 26 & 8.2 & 8.4 & I3 & & 2 & II. 3 & 12.4 & I9 & & 2 & I9. I & 20.7 & 32 & \\
\hline 28 & 10.9 & 20.3 & 32 & & 28 & 7.3 & 7.5 & 12 & & 28 & II. I & $12 . I$ & 19 & & 2 & I9.1 & 20 & 32 & \\
\hline 30 & 19.3 & I9. $\overline{8}$ & 30 & $+4 \cdot 4$ & 30 & 7.8 & 7.9 & 13 & & 30 & I0.9 & I2. I & 18 & +5 . & 30 & I9. I & 20.5 & 32 & +3.9 \\
\hline 32 & 19.4 & 19.9 & .31 & & 32 & 8.0 & 8.0 & T3 & t & 3 & II. I & II. 8 & 18 & (1) & 3 & I9. 3 & $2 \mathrm{I}$ & 33 & \\
\hline 34 & 19.0 & I 9.3 & 30 & 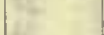 & 34 & 8.5 & 8.9 & I4 & & 3 & II.0 & II.O & I8 & & 3 & 20.0 & $2 \mathrm{I}$ & 34 & \\
\hline 36 & 10.6 & 19.9 & $3 \mathrm{I}$ & C. & 36 & 10.2 & 10.4 & 16 & & 3 & 10.3 & 10.9 & I7 & & & 0.1 & $2 \mathrm{I}$ & 34 & \\
\hline 38 & 20.0 & 20.3 & 32 & I & 38 & 12.7 & $13 . \mathrm{I}$ & 20 & & 3 & I0. I & 10.5 & & & 3 & 19.8 & 2 I & 33 & \\
\hline 40 & 19.6 & I9.8 & $3 \mathrm{I}$ & I & 40 & 13.6 & I 4.0 & 22 & 28 & 4 & 9.7 & I0. 3 & 16 & & 4 & 19.8 & 20 & 33 & \\
\hline 42 & 19.5 & I9. 5 & 30 & T. & 42 & 13.5 & 13.7 & 22 & & 42 & 9.2 & IO. I & 16 & & 4 & 19.9 & 21.0 & 33 & \\
\hline 44 & 19.2 & 19.2 & 30 & +4.5 & 44 & 13.8 & 14.0 & 22 & +5.3 & 44 & 10.0 & 10.7 & I7 & +5.8 & 44 & 19.8 & 20.9 & 33 & +3.7 \\
\hline 46 & I8.8 & I8.8 & 30 & & 46 & I 4.0 & $14 \cdot 3$ & 22 & & 46 & 10.2 & II.I & 17 & & 406 & 19.8 & 20.5 & 32 & \\
\hline 48 & 18.5 & 18.5 & 29 & 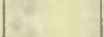 & 48 & 13.2 & 13.3 & $2 \mathrm{I}$ & 1 & 48 & 11.2 & II. 2 & I & 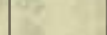 & 48 & 19.5 & 20 & 32 & \\
\hline 50 & I8. 3 & I8. 3 & 29 & 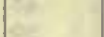 & 50 & 12.6 & I.3.0 & 20 & 3 & 50 & 11.0 & II.7 & 18 & 0 & 5 & I9. I & I9. & 3I & \\
\hline 52 & 17.5 & 17.5 & 27 & 3 & 52 & 12.7 & I3.I & 20 & & 52 & 10.3 & II I I & 17 & & 52 & I8.8 & 19.1 & 30 & \\
\hline 54 & 16.9 & 16.9 & 26 & $\pi$ & 54 & 12.3 & 12.7 & 20 & & 54 & 10.3 & II. 2 & 17 & 37 & 54 & 18.2 & & 30 & \\
\hline 56 & 17.3 & $17 \cdot 3$ & 27 & 10 & 56 & 12.5 & 13.I & 20 & & 56 & 10.2 & II. 2 & 17 & & 56 & 17.9 & I8. 2 & 29 & \\
\hline 58 & 17.8 & 18.0 & 28 & 28 & 58 & 12.3 & 12.8 & 20 & & 58 & 10.2 & II. 3 & I7 & tat & 58 & 17.9 & 18.4 & 29 & \\
\hline
\end{tabular}

Observers-W. J. P. and R. R. T., who alternated from 15 h $52 \mathrm{~m}$ to Observer-R. R. T. I6h $04 \mathrm{~m}$. 
Tabulation of magnetic declinations observed at Teplitz Bay-Continued

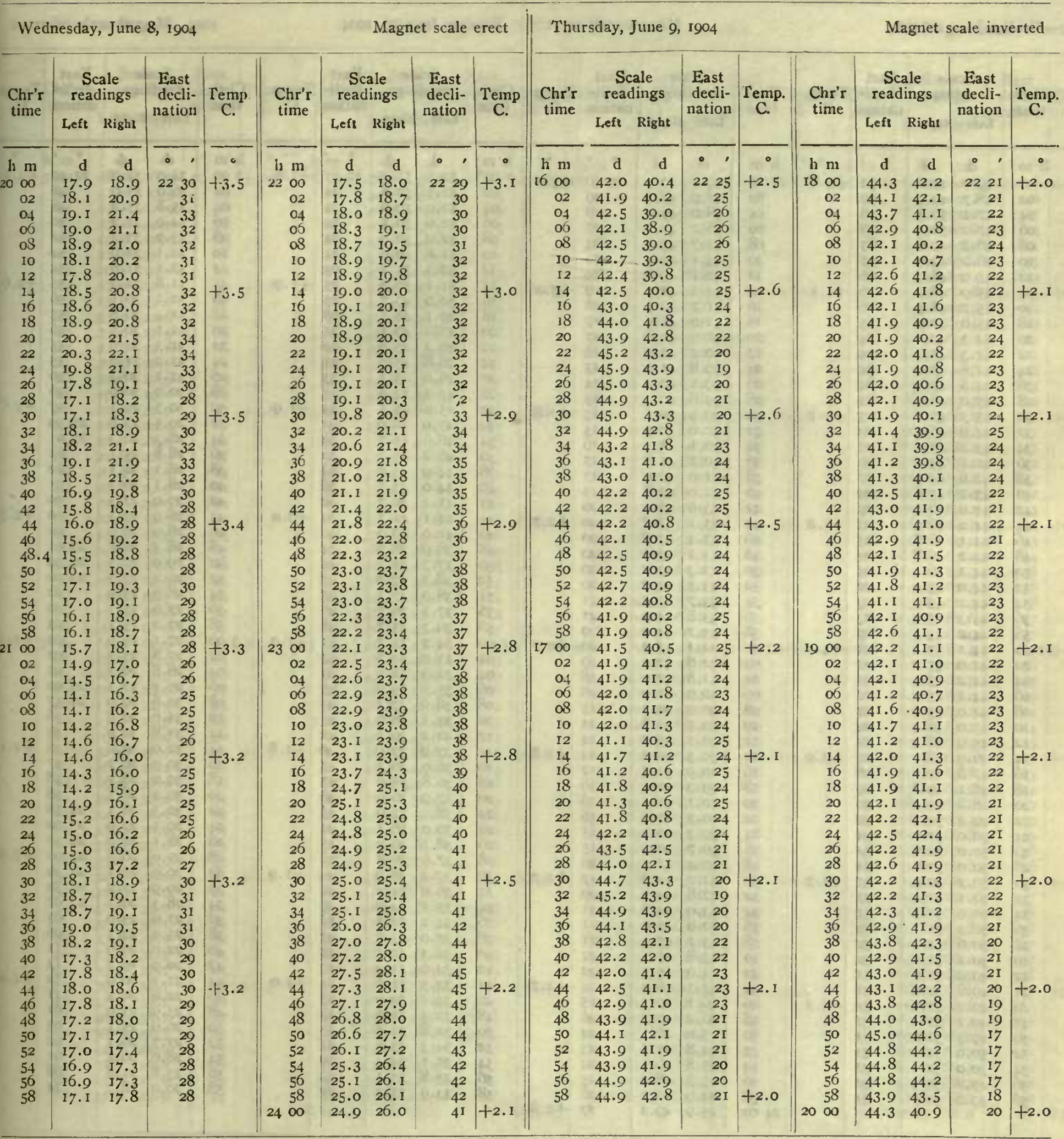

Correction to local mean time is $+07.5 \mathrm{~s} .90^{\circ}$ torsion $=17.94$. Torsion head at oh oom read $60^{\circ}$ and at $24^{\text {h }}$ 2om read $41^{\circ}$. Observer-R. R. T.
Correction to local mean time is $+15 \mathrm{~s} .90^{\circ}$ torsion $=17^{\prime}, 78$. Torsion head at $15 \mathrm{~h} 37 \mathrm{~m}$ read $42^{\circ}$ and at $20 \mathrm{~h} 19 \mathrm{~m}$ read $60^{\circ}$. Observer-J. V. 
Tabulation of magnetic declinations observed at Teplitz Bay-Continued

\begin{tabular}{|c|c|c|c|c|c|c|c|c|c|c|c|c|c|c|c|c|c|c|c|c|}
\hline \multicolumn{5}{|c|}{ Friday, June Io, 1904} & \multicolumn{5}{|c|}{ Magnet scale erect } & \multicolumn{6}{|c|}{ Sunday, June 12, 1904} & \multicolumn{5}{|c|}{ Magnet scale inverted } \\
\hline \multirow[t]{2}{*}{$\begin{array}{l}\text { Chr'r } \\
\text { time }\end{array}$} & \multicolumn{2}{|c|}{$\begin{array}{l}\text { Scale } \\
\text { readings }\end{array}$} & \multirow[t]{2}{*}{$\begin{array}{c}\text { East } \\
\text { decli- } \\
\text { nation }\end{array}$} & Temp. & $\begin{array}{l}\text { Chr'r } \\
\text { time }\end{array}$ & $\begin{array}{l}\text { Sca } \\
\text { read }\end{array}$ & $\begin{array}{l}\text { ale } \\
\text { ings }\end{array}$ & $\begin{array}{l}\text { East } \\
\text { decli- } \\
\text { nation }\end{array}$ & $\begin{array}{l}\text { Temp } \\
\text { C. }\end{array}$ & $\begin{array}{l}\text { Chr'r } \\
\text { time }\end{array}$ & $\begin{aligned} \text { Sc } \\
\text { reac }\end{aligned}$ & $\begin{array}{l}\text { ale } \\
\text { lings }\end{array}$ & $\begin{array}{c}\text { East } \\
\text { decli- } \\
\text { nation }\end{array}$ & $\begin{array}{l}\text { Temp } \\
\text { C. }\end{array}$ & $\begin{array}{l}\text { Chr'r } \\
\text { time }\end{array}$ & $\begin{array}{r}\text { Sc } \\
\text { read }\end{array}$ & $\begin{array}{l}\text { ale } \\
\text { lings }\end{array}$ & $\begin{array}{l}\text { Eas } \\
\text { decli } \\
\text { natio }\end{array}$ & $\begin{array}{l}\text { st } \\
\text { ali- }\end{array}$ & Temp. \\
\hline & Left & Right & & & & Left & Right & & & & Left & Rigbt & 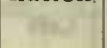 & & & L,eft & Right & & & \\
\hline $11 \mathrm{~m}$ & d & d & $\cdot$ ' & $\bullet$ & h $\mathrm{m}$ & d & d & $\circ \quad$ & $\bullet$ & $h \mathbf{m}$ & d & d & & & h m & d & d & & - & 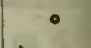 \\
\hline 2000 & 49.0 & 49.6 & 2230 & +4.8 & 2200 & 49.4 & 50.4 & 2230 & +3.0 & $000^{*}$ & 52.5 & 51.2 & 2223 & +2.0 & 200 & 34.9 & 34.0 & & $50-$ & $+2 . I$ \\
\hline 02 & $\begin{array}{l}48.7 \\
47.1\end{array}$ & $\begin{array}{l}49.9 \\
50.3\end{array}$ & $\begin{array}{l}30 \\
28\end{array}$ & 34 & $\begin{array}{l}02 \\
04\end{array}$ & $\begin{array}{l}48.9 \\
48.0\end{array}$ & $\begin{array}{l}50.1 \\
40.0\end{array}$ & & tar & $\begin{array}{l}02 \\
04\end{array}$ & $\begin{array}{l}52.1 \\
52.0\end{array}$ & $\begin{array}{l}50.8 \\
50.9\end{array}$ & $\begin{array}{l}24 \\
24\end{array}$ & $4 y^{2}$ & 02 & 36.0 & 35.0 & & 48 & \\
\hline $\begin{array}{l}04 \\
06\end{array}$ & $\begin{array}{l}47.1 \\
47.0\end{array}$ & $\begin{array}{l}50.3 \\
50.1\end{array}$ & $\begin{array}{l}28 \\
28\end{array}$ & 8 & $\begin{array}{l}04 \\
06\end{array}$ & $\begin{array}{l}48.0 \\
46.2\end{array}$ & $\begin{array}{l}49 \cdot 0 \\
47 \cdot 1\end{array}$ & $\begin{array}{r}28 \\
25\end{array}$ & tart & & $\begin{array}{l}52.0 \\
52.0\end{array}$ & $\begin{array}{l}50.9 \\
50.5\end{array}$ & $\begin{array}{l}24 \\
24\end{array}$ & 80 & $\begin{array}{l}04 \\
06\end{array}$ & $\begin{array}{l}35.8 \\
34.9\end{array}$ & $\begin{array}{l}35.2 \\
34.2\end{array}$ & & $\begin{array}{l}48 \\
50\end{array}$ & \\
\hline 08 & 46.3 & 49.6 & 27 & as & 08 & 44.7 & 45.3 & 23 & 85 & 08 & 52.0 & 50.8 & 24 & 8 & $\begin{array}{l}06 \\
08\end{array}$ & $\begin{array}{l}34.9 \\
33.9\end{array}$ & $\begin{array}{l}34.2 \\
33.2\end{array}$ & & $\begin{array}{l}50 \\
52\end{array}$ & \\
\hline I0 & 46.0 & 49.0 & 27 & 94 & I0 & $43 . I$ & 43.9 & 20 & at & I0 & 51.8 & 49.9 & 24 & es & I0 & 32 . I & $3 \mathrm{I} .6$ & & 54 & \\
\hline 12 & 45.9 & 48.9 & 26 & 4 & I2 & 42.1 & $43 . I$ & 19 & it & 12 & 52.5 & $5 \mathrm{I} . \mathrm{I}$ & 23 & & 12 & 32.1 & $3 \mathrm{I} .8$ & & 54 & \\
\hline 14 & 45.9 & 48.9 & & $+4 \cdot 5$ & 14 & $42 . I$ & 42.9 & Ig & +3.0 & 14 & $51 . \mathrm{I}$ & 50.2 & 25 & +1.9 & 14 & 31.8 & 3I.0 & & 55 & +2.6 \\
\hline 16 & $45 \cdot 7$ & 48.3 & 26 & & 16 & $4 \mathrm{I} \cdot 7$ & 42.7 & 18 & & I6 & 50.9 & $49 \cdot 7$ & 25 & tor & 16 & $3 I . I$ & 30. I & & 56 & \\
\hline 18 & 45.0 & 47.9 & 25 & 64 & 18 & 41.0 & 41.9 & 18. 17 & 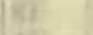 & 18 & $49 . I$ & 48.1 & 28 & its? & I8 & $3 \mathrm{I} .4$ & 30.8 & & 55 & \\
\hline 20 & $44 \cdot 3$ & $47 \cdot \mathrm{I}$ & 24 & $a$ & 20 & 40.9 & 41.6 & 17 & ces & 20 & 47.2 & 46. I & $3 I$ & 2 & 20 & $3 \mathrm{I} . \mathrm{I}$ & 30.5 & & 56 & \\
\hline 22 & $44 \cdot 3$ & 47.0 & 24 & = & 22 & 40.2 & 41.0 & I6 & $E$ & 22 & 46.2 & 45.8 & 32 & 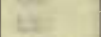 & 22 & $3 \mathrm{I} \cdot 3$ & 30.9 & & 55 & \\
\hline 24 & 44.8 & 47.0 & 24 & is & 24 & 39.8 & 41.0 & 15 & 145 & 24 & 46.8 & 45.9 & $3 I$ & 14 & 24 & $3 \mathrm{~L} .2$ & 31.0 & & 55 & \\
\hline 26 & 44.8 & 47.0 & 24 & $y$ & 26 & 39. I & 40.2 & 14 & 185 & 26 & 46.0 & 45.1 & 33 & & 26 & 3I.I & 30.4 & & 56 & \\
\hline 28 & 45.0 & 46.9 & 24 & $\frac{12}{2}$ & 28 & 39.8 & 40.3 & 15 & 5 & 28 & 45.5 & 44.8 & 33 & & 28 & 30.9 & 30.0 & & 56 & \\
\hline 30 & $45 \cdot 3$ & $47 . I$ & 25 & $+4 . I$ & 30 & 39.9 & 40.6 & 15 & +2.8 & 30 & 45.9 & $45 . I$ & 33 & +1.9 & 30 & 30.5 & 29.1 & & 57 & +2.6 \\
\hline 32 & $44 \cdot 9$ & 46.9 & 24 & 2 & 32 & 40.0 & 40.9 & 15 & it & 32 & 47.0 & 46.0 & $3 \mathrm{I}$ & 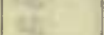 & 32 & 29.5 & 27.8 & 22 & 59 & \\
\hline 34 & 43.2 & 45.2 & $2 \mathrm{I}$ & 12 & 34 & $40 . I$ & 41.1 & 16 & (18. & 34 & 46.6 & 45.7 & 32 & it & 34 & 28.3 & 26.3 & 23 & OI & \\
\hline 36 & $42 . I$ & 44.2 & 20 & 98 & $3^{6}$ & 40.7 & 41.8 & 17 & te & 36 & 46.2 & $45 . I$ & 32 & & 36 & 28.8 & $25 . \mathrm{I}$ & & 02 & \\
\hline 38 & 42.6 & 44.9 & $2 \mathrm{I}$ & 3 & 38 & 41.0 & 42.0 & 17 & is & 38 & 44.9 & 43.9 & 34 & 68 & 38 & 28. I & 26.1 & & 02 & \\
\hline 40 & 43.2 & 45.6 & 22 & 0. & 40 & 42.0 & 42.8 & 19 & $h$ & 40 & 44.0 & $43 . I$ & 36 & & 40 & 28.1 & 26.7 & & or & \\
\hline 42 & 44.0 & 46.1 & 23 & 4 & 42 & 43.0 & 43.2 & 20 & & 42 & $4 \mathrm{I} .2$ & 40.7 & 40 & & 42 & 29.0 & 27.1 & & $\infty$ & \\
\hline 44 & 44.2 & 46.3 & 23 & +4.0 & 44 & $43 . \mathrm{I}$ & 43.5 & 20 & +2.7 & 44 & 42.1 & $4 \mathrm{I} . \mathrm{I}$ & 39 & +2.0 & 44 & 28.9 & 27.1 & & $\infty$ & +2.5 \\
\hline 46 & 44.1 & 46.8 & 23 & & 46 & 43.4 & 43.9 & 20 & $x_{0}$ & 46 & 41.0 & 40.0 & 40 & 7. & 46 & 28.9 & 26.9 & & $\infty$ & \\
\hline 48 & 44.2 & 46.8 & 24 & on & 48 & 43.2 & 43.9 & 20 & as & 48 & 38.9 & 38.2 & 44 & & 48 & 28.5 & 26.8 & 23 & OI & \\
\hline 50 & 44.1 & 46.1 & 23 & $a^{2}$ & 50 & 43.3 & 43.7 & 20 & 10 & 50 & $37 \cdot 7$ & 37.1 & 45 & of & 50 & 29.9 & 27.5 & 22 & 59 & \\
\hline 52 & 44.0 & $45 \cdot 9$ & 22 & at & 52 & 43.5 & 43.7 & 20 & 16z & 52 & 38. I & 37.8 & 45 & a & 52 & 29.2 & 27.5 & 23 & $\infty$ & \\
\hline 54 & $44 . I$ & 45.9 & 23 & 12 & 54 & 43.7 & 43.9 & $2 I$ & & 54 & 38.0 & 37.1 & 45 & & 54 & 28.0 & 25.9 & & 02 & \\
\hline 56 & $44 \cdot 3$ & $45 \cdot 9$ & 23 & cet & 56 & 43.8 & $44 . I$ & 21 & 10 & 56 & 37.1 & 36.7 & 46 & se & 56 & 27.9 & 25.7 & & 02 & \\
\hline 58 & 44.2 & $45 \cdot 3$ & 22 & 102 & 58 & 43. I & 44.0 & 20 & & 58 & 36.3 & 35.7 & 48 & & 58 & 27.2 & $25 . I$ & & 03 & \\
\hline 2100 & $44 . I$ & 45.1 & 22 & +4.0 & 2.300 & 42.4 & 43.9 & 20 & +2.7 & I $\infty$ & 34.2 & 33.3 & 51 & +1.9 & 300 & 27.1 & 25.0 & & 03 & +2.2 \\
\hline 02 & 44.0 & 45.7 & 22 & 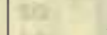 & 02 & 42.6 & 42.9 & I9 & ent & 02 & 33.0 & 32.1 & 53 & 6 & 02 & 27.8 & 25.7 & & 02 & \\
\hline 04 & 44.2 & 45.9 & 23 & 18 & 04 & $4 \mathrm{I} .8$ & 42.0 & I8 & yidi & 04 & 32.9 & 32.0 & 53 & 10 & 04 & 28.8 & 26.7 & & OI & \\
\hline 06 & $44 . I$ & 45.7 & 22 & 9 & 06 & 40.9 & $4 \mathrm{I} .0$ & I6 & Int & 06 & 35.2 & 35.0 & 49 & $\sqrt{y}$ & 06 & 29.2 & 27.1 & 23 & $\infty$ & \\
\hline 08 & 44.0 & 45.9 & 22 & 15 & 08 & 40.0 & 40.1 & I5 & & 08 & 38.0 & 37.1 & 45 & 8. & 08 & 30.6 & 29.0 & 22 & 57 & \\
\hline Io & 45.0 & 46.4 & 24 & $0 \pi$ & IO & 39.5 & 39.8 & 14 & & 10 & 39.1 & 38.9 & 43 & 0 & Io & 31.6 & 30.0 & & 56 & \\
\hline 12 & 45.9 & 46.3 & 24 & 31 & 12 & 39.1 & 39.5 & 14. 14 & & 12 & 40.2 & 39. I & 42 & & 12 & $3^{1.8}$ & 30.0 & & 56 & \\
\hline 14 & 46.9 & 48.2 & 27 & +3.9 & 14 & 39.5 & 40.0 & $\mathrm{I}_{4}$ & +2.8 & I4 & 37.9 & 37.0 & 46 & +1.8 & I4 & 32.0 & 30.0 & & 56 & $+2 . I$ \\
\hline I6 & 47.8 & 49. I & 28 & & 16 & 40.9 & 41.5 & 17 & & I6 & 36.9 & 36.1 & 47 & 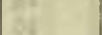 & 16 & 30.9 & 29.2 & & 57 & \\
\hline 18 & 48.1 & 49.8 & 29 & Ex & 18 & 39.9 & 40.9 & 16 & +1 & 18 & 36.8 & 35.9 & 47 & हो & 18 & 30.0 & 28.2 & & 58 & \\
\hline 20 & 48.8 & 49.9 & 30 & $0 t$ & 20 & 40.1 & $4 \mathrm{I} . \mathrm{I}$ & 16 & tos & 20 & 37.9 & 36.9 & $4^{6}$ & es & 20 & 30.2 & 28.5 & & 58 & \\
\hline 22 & 49.3 & 50.5 & 30 & (45) & 22 & 40.3 & 40.7 & I6 & 14 & 22 & 38.0 & 37.0 & 45 & th & 22 & 30.3 & 28.1 & 22 & 58 & \\
\hline 24 & 50.0 & 51.0 & $3 I$ & 14 & 24 & 39.3 & 40.3 & 15 & 15: & 24 & 39.0 & 38. I & 44 & & 24 & 29.1 & 27.0 & 23 & & \\
\hline 26 & 50.0 & 50.8 & $3 \mathrm{I}$ & 56 & 26 & 39.7 & 40.4 & I5 & & 26 & 38. I & 36.9 & 45 & & 26 & 28.1 & 26.0 & 22 & 59 & \\
\hline $2 S$ & 50.0 & 50.6 & $3 I$ & 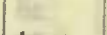 & 28 & 40.4 & $4 \mathrm{I} .2$ & I6 & & 28 & 37.9 & 36.8 & 46 & & 28 & 29.0 & 26.8 & 23 & $\infty$ & +2.0 \\
\hline 30 & 50.2 & 50.9 & 3I & +3.7 & 30 & 41.0 & 42.0 & 17 & +2.7 & 30 & 37.2 & 38.9 & 44 & +1.9 & 30 & 29.9 & 27.1 & 22 & & \\
\hline 32 & 50.1 & 5 I. 0 & 3I & & 32 & 42.8 & 44.2 & 20 & & 32 & 36.0 & 34.2 & 49 & & 32 & 29.9 & 27.2 & 22 & 59 & \\
\hline 34 & 50.0 & 50.9 & 3I & 45 & 34 & 43.0 & 44.7 & 2I & & 34 & 35.5 & 34.9 & 49 & at & 34 & 28.3 & 26. I & 23 & OI & \\
\hline 36 & 50.3 & 5I.I & 32 & 28 & 36 & 42.9 & 45.0 & $2 \mathrm{I}$ & & 36 & 34.2 & 32.8 & 52 & da & 36 & 27.0 & 25.0 & & 03 & \\
\hline 38 & 50.6 & 5 I. 4 & 32 & $x$ & 38 & 43.2 & 45.2 & $2 \mathrm{I}$ & & 38 & 37.0 & 35. & 47 & & 38 & 25.0 & 23.1 & & 06 & \\
\hline 40 & 50.2 & 51.0 & 32 & 4 & 40 & 43.8 & $45 . I$ & 22 & $=5$ & 40 & 32.3 & 3I. I & 54 & a & 40 & 22.0 & 20.9 & & I I & \\
\hline 42 & 49.9 & 51.0 & $3 \mathrm{I}$ & 4 & 42 & 44.0 & 45.2 & 22 & & 42 & 32.9 & 31.9 & 53 & & 42 & 20.9 & 19.2 & & 13 & \\
\hline 44 & 49.8 & 50.8 & 3 I & 15 & 44 & 45.1 & 46.0 & 24 & +2.7 & 44 & 32.3 & 31.6 & 54 & +2.0 & 44 & $2 \mathrm{I} . \mathrm{I}$ & 20.0 & & 12 & +2.0 \\
\hline 46 & 49.2 & 51.2 & $3 I$ & +3.3 & 46 & 46.1 & 47.3 & 25 & $9:=$ & 46 & 32.1 & 30.9 & 55 & & 46 & 22.0 & 20.4 & & II & \\
\hline 48 & 49.1 & 5I.I & $3 I$ & & 48 & 46.2 & $47 \cdot 7$ & 26 & & 48 & 32.3 & 31.0 & 55 & & 48 & 23.9 & 23.0 & & 07 & \\
\hline 50 & 49.3 & 51.2 & 31 & 0 & 50 & 45.0 & 46.1 & 24 & et & 50 & 32.8 & 31.8 & 53 & 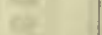 & 50 & 26.9 & 25.1 & & 03 & \\
\hline 52 & 50.0 & 51.0 & 3I & 28 & 52 & 44.8 & 45.5 & 623 & & 52 & 34.3 & 33.8 & $5 I$ & & 52 & 27.7 & 26.1 & & 02 & \\
\hline 54 & 50.0 & 5 I.I & 3I & $\{x$ & 54 & 45.8 & $46 . \bar{I}$ & 24 & & 54 & $35 . I$ & $34 \cdot 3$ & 50 & & 54 & 28.0 & 26.4 & & oI & \\
\hline 56 & 49.8 & 50.9 & $3 I$ & 6 & 56 & 45.5 & 48.0 & & & 56 & 33.8 & $33 . I$ & 52 & & 56 & 23.8 & 21.8 & & 08 & \\
\hline 58 & 49.6 & 50.7 & $3 I$ & 30 & 58 & 44.9 & 49.4 & 26 & & 58 & 33.9 & 33.2 & 52 & 10 & 58 & 20.0 & I8. 2 & & I4 & \\
\hline & & & $(24)$ & 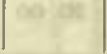 & 2400 & 45.9 & 49.2 & 27 & +2.8 & -5 & & 3,07 & & 609 & & & & & & \\
\hline
\end{tabular}


Tabulation of magnetic declinations observed at Teplitz Bay-Continued

\begin{tabular}{|c|c|c|c|c|c|c|c|c|c|c|c|c|c|c|c|c|c|c|c|c|}
\hline \multicolumn{5}{|c|}{ Sunday, June 12, 1904} & \multicolumn{6}{|c|}{ Magnet scale inverted } & \multicolumn{6}{|c|}{ Monday, June 13,1904} & \multicolumn{4}{|c|}{ Magnet scale inverted } \\
\hline \multirow{2}{*}{$\begin{array}{l}\text { Chr's } \\
\text { time }\end{array}$} & \multicolumn{2}{|c|}{$\begin{array}{l}\text { Scale } \\
\text { readings }\end{array}$} & $\begin{array}{l}\text { East } \\
\text { decli- }\end{array}$ & Temp. & Chr'r & $\begin{array}{l}\mathrm{Sc} \\
\text { read }\end{array}$ & $\begin{array}{l}\text { ale } \\
\text { lings }\end{array}$ & Eas & & Temp. & Chr'r & $\begin{array}{r}\text { Sc } \\
\text { reac }\end{array}$ & $\begin{array}{l}\text { dine } \\
\text { dings }\end{array}$ & $\begin{array}{l}\text { East } \\
\text { decli- }\end{array}$ & & Chr'r & $\begin{array}{r}\text { Sc } \\
\text { read }\end{array}$ & $\begin{array}{l}\text { ale } \\
\text { ings }\end{array}$ & $\begin{array}{l}\text { East } \\
\text { decli- }\end{array}$ & \\
\hline & Left & Right & Hation & & & Left & Right & & & & & Left & Right & & & & Left & Right & & \\
\hline h m & d & d & $\cdot$ & 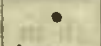 & h m & a & d & & , & & $\mathrm{h} \mathrm{m}$ & $\mathrm{d}$ & & & $\bullet$ & $h$ in & d & & $\cdot \quad$ & 2 \\
\hline 400 & I8.I & 16.9 & 2317 & +2.0 & 600 & 46.8 & $45 \cdot 5$ & 23 & II & +1.8 & 800 & 50.9 & 48.7 & 23 of & +4.8 & I0 $\infty$ & 64.1 & 62.8 & 2246 & +6.7 \\
\hline 02 & 21.0 & 19.3 & .12 & 10 & 02 & 44.8 & 43.9 & & I4 & r & 02 & 50.9 & 49.2 & 07 & 090 & 02 & 63.9 & 62.3 & 46 & \\
\hline 04 & 23.1 & 22.9 & 08 & iit & 04 & $4 \mathrm{~T} .3$ & 40. I & & 19 & $y=$ & 04 & 51.0 & 48.9 & & 5 & 04 & 63.1 & 62.0 & & jas \\
\hline 06 & 23.1 & 22.9 & 08 & 60 & 06 & 44.9 & 43.7 & & 14 & sto & 06 & 51.4 & 49.3 & 06 & 20 & 06 & 64.9 & 63.8 & 44 & 8 \\
\hline 08 & 22.9 & 22.0 & 09 & Whe & 08 & 43.5 & $42 . \mathrm{I}$ & & 16 & 1 & 08 & 5 I. I & 49.2 & 06 & 25 & 08 & 65.1 & 63.9 & & 60 \\
\hline 10 & 21.8 & $2 \mathrm{I} .0$ & II & er & IO & 43.0 & 41.9 & & 17 & 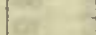 & -10 & 50.9 & 49.2 & 07 & tr. & IO & 64.4 & 63.2 & 45 & \\
\hline 12 & 20.8 & 20.1 & 12 & & I2 & 45.5 & 44.0 & & I3 & & 12 & 48.3 & 46.8 & I0 & (5) & I2 & 65.9 & 64.4 & 43 & \\
\hline I4 & 23.0 & 22.3 & 09 & +1.8 & I4 & 42.9 & 40.9 & & 17 & +1.8 & 14 & 46.2 & 44.9 & I4 & +5.0 & I4 & 64.3 & 62.8 & 45 & +6.1 \\
\hline 16 & 23.9 & 23.0 & 07 & $i^{\circ}$ & 16 & 44.1 & 43.9 & & 14 & 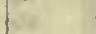 & I6 & 45.9 & 45.1 & 14 & & I6́ & 64.0 & 62.5 & & in \\
\hline 18 & 23.0 & 22.8 & 08 & ini & I8 & 51.0 & 49.9 & & 04 & 1 & I8 & 50.8 & 49.8 & 06 & tar & I8 & 66.0 & 65.1 & 42 & is \\
\hline 20 & 23.0 & 22.9 & 08 & $a$ & 20 & 48.3 & 47.8 & & 08 & Es & 20 & 53.9 & 52.1 & 2302 & $a=$ & 20 & 66.3 & 65.2 & 42 & at \\
\hline 22 & 22.9 & 22.8 & 08 & as & 22 & 43.5 & 43.3 & & 15 & tit. & 22 & 59.9 & 58.6 & 2252 & as & 22 & 65.9 & 65.2 & 42 & Ls \\
\hline 24 & 20.9 & 20.7 & II & is & 24 & 40.8 & 38.3 & & 21 & 18 & 24 & 59.5 & 58.2 & 53 & 26 & 24 & 65.2 & 64. & 43 & 12 \\
\hline 26 & 20.0 & 19.9 & 13 & ax & 26 & 42.8 & 41.9 & & 17 & is & 26 & $6 \pi .3$ & 60.3 & 50 & os & 26 & 65.5 & 65.0 & 43 & 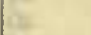 \\
\hline 28 & 19.1 & 19.0 & 14 & & 28 & 50.3 & 49.2 & & 05 & & 28 & $55 . \mathrm{I}$ & 54.8 & 2259 & Ex & 28 & 66.0 & 65 & 42 & \\
\hline 30 & 20.6 & 20.0 & 12 & +1.8 & 30 & 43 & $.9 b$ & & 14 & +1.8 & 30 & 52.5 & 50.9 & 2304 & +5.8 & 30 & 66.7 & 66.1 & & $+6 . I$ \\
\hline 32 & 21.0 & 20.9 & II & 10 & 32 & 43.9 & 42.3 & & 15 & 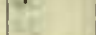 & 32 & 59.8 & 59.1 & 2252 & & 32 & 66.9 & 66.2 & $4 \mathrm{I}$ & \\
\hline 34 & 22.0 & $2 \mathrm{I} .2$ & 10 & it & 34 & 42.5 & 40.9 & & 18 & = & 34 & 54.9 & 54.2 & 60 & tz & 34 & 66.9 & 66.2 & $4 \mathrm{I}$ & 45 \\
\hline 36 & 21.6 & 20.3 & II & $a^{2}$ & 36 & 45.3 & $44 . I$ & & 13 & 10 & 36 & 57.1 & 56.8 & 56 & W & 36 & 66.0 & 65.1 & & 58 \\
\hline 38 & 20.2 & I9. I & 13 & 185 & 38 & $47 . I$ & 45.8 & & 10 & 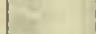 & 38 & 57.9 & 56.9 & 55 & 12. & 38 & 66. I & 65.1 & 42 & (iix \\
\hline 40 & 20.8 & 19.9 & 12 & a & 40 & $47 . \mathrm{I}$ & 45.8 & & IO & os & 40 & 55 & $.0 a$ & 59 & cos & 40 & 66.5 & 65.0 & & nis. \\
\hline 42 & $21 . I$ & 20.0 & 12 & & 42 & $45 \cdot 9$ & 44.2 & & 12 & & 42 & 59.8 & 59.2 & 52 & & 42 & 65.9 & 64.1 & 43 & \\
\hline 44 & 20.6 & 19.7 & 12 & +1.7 & 44 & 48.8 & 46.8 & & 08 & +1.9 & 44 & 58.6 & 57.9 & 54 & +6.1 & 44 & 66.1 & $64 \cdot 3$ & 43 & +6.0 \\
\hline 46 & 19.9 & 17.1 & 15 & 1 & 46 & 47.6 & 45.5 & & 10 & & 46 & 58.1 & 57.1 & $\begin{array}{l}24 \\
55\end{array}$ & 70.2 & 46 & 66.0 & 64.0 & $\begin{array}{l}43 \\
43\end{array}$ & \\
\hline 48 & 19.0 & 16.3 & 17 & 6 & 48 & 48.6 & 46. & & $\infty 9$ & 20 & 48 & 61.9 & 61.1 & 49 & 28 & 48 & 65.2 & 63. & 44 & . \\
\hline 50 & 19.0 & 16.8 & 16 & 8 & 50 & 48.9 & 46.8 & & 08 & 0 & 50 & 62.0 & 6I.I & 49 & 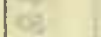 & 50 & 64.1 & 62 & 46 & E \\
\hline 52 & 16.3 & 14.9 & 20 & 8 & 52 & 51.9 & 50.0 & & 03 & 3 & 52 & 59.9 & 59.2 & 52 & 62 & 52 & 64.2 & 62.5 & 46 & $E$ \\
\hline 54 & 15.0 & 12.5 & 23 & 3 & 54 & 44.9 & 43.8 & & 14 & 2. & 54 & 58.0 & 57.2 & 55 & $\mathrm{at}^{2}$ & 54 & 63.1 & & 48 & \\
\hline 56 & 15.0 & 12.2 & 23 & $\sqrt{2}$ & 56 & $46 . I$ & 45.0 & & 12 & & 56 & 64.2 & 62.7 & 45 & we & 56 & 63.3 & 61.6 & 47 & \\
\hline 58 & I6. I & I4. 0 & 21 & & 58 & 50.9 & 49.2 & & 05 & +2.0 & 58 & $6 \mathrm{~T} .8$ & 60.5 & 49 & 2. & 58 & 63.4 & 61.9 & 47 & \\
\hline 500 & 14.9 & II.9 & 23 & +1.6 & $7 \infty$ & 46.0 & 43.3 & & 13 & 6 & 900 & 59.6 & 58.9 & 52 & +6.7 & II 00 & 63.2 & 61.5 & 47 & +5.7 \\
\hline 02 & 13.3 & II.O & 25 & - & 02 & 48.0 & 46.9 & & $\infty 9$ & $=0$ & 02 & 59.5 & 58.3 & 53 & 10 & & 63.9 & 62 & 46 & \\
\hline of & 10.8 & 9.9 & 28 & 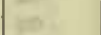 & 04 & 47.2 & 45.9 & & 10 & 18 & 04 & 6r. & 60.7 & 50 & 10 & 04 & 64.6 & 62.7 & 45 & 48 \\
\hline 06 & II. 2 & 10.0 & 28 & 85 & 06 & 48.2 & 47.0 & & 08 & 6 & 06 & $6 \mathrm{r} .2$ & 60.2 & 50 & 60 & & 64.9 & 63. & 45 & 60 \\
\hline 08 & I3.I & I2. I & 24 & 60 & os & 46.0 & 44.9 & & 12 & Bs. & 08 & 60.2 & 59.1 & 52 & 6 & 08 & 65.2 & 63.2 & 44 & 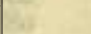 \\
\hline I0 & 13.3 & 12.7 & 24 & or & IO & 45.0 & 44.9 & & 13 & Hit & IO & 60.9 & 60.1 & 50 & oit & IO & & & 44 & cir \\
\hline 12 & 9. I & 8.9 & 30 & & I2 & 46.1 & 45.0 & & 12 & & I2 & $6 \mathrm{r} .2$ & 60.9 & 49 & 4 & 12 & 65.8 & 63.9 & 43 & \\
\hline $14^{\prime \prime} 5$ & 32.9 & 28.0 & 35 & +1.7 & I4 & 49.0 & 48.8 & & 06 & +2.1 & 14 & 62.8 & 62.0 & 47 & +7.0 & I4 & 65.9 & 64.0 & 43 & +5.5 \\
\hline 16 & 30.3 & 27.2 & 38 & 10 & I6 & $45 . \mathrm{I}$ & 44.6 & & 13 & in & I6 & 62.7 & $6 \mathrm{r} .3$ & 48 & & 16 & 66.0 & 63.8 & 43 & \\
\hline I8 & 29.9 & 27.2 & 38 & 89 & I8 & 48 & $.9 a$ & & 06 & 1 & I8 & 62.9 & 61.9 & 47 & $4:$ & I8 & 67.0 & 64.9 & 42 & Wi \\
\hline 20 & 32.6 & 30.2 & 34 & os. & 20 & $5 I .3$ & 51.2 & & 03 & $1=$ & 20 & 65.0 & 64.0 & 44 & os & 20 & 68.0 & 65 & 40 & 06 \\
\hline 22 & 34.0 & 3 I.O & 32 & cas & 22 & 45.9 & 45.8 & & II & 1 & 22 & 64.2 & 63.9 & 45 & 20 & 22 & 68.9 & 66.5 & 39 & $=$ \\
\hline 24 & 33.0 & 30.2 & 34 & is & 24 & 40. I & $40 . I$ & & 20 & Ie & 24 & 63.2 & & 46 & 13 & & & & 39 & 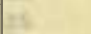 \\
\hline 26 & $3 \mathrm{I} .9$ & 29.0 & 35 & 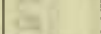 & 26 & 50.1 & $48 . I$ & & 06 & & 26 & 62.6 & 62.1 & 47 & 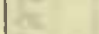 & 26 & 69.0 & 67.1 & 38 & oc \\
\hline 28 & 33.2 & 31.0 & 33 & & 28.4 & 47 & $.0 b$ & & $\infty$ & & 28 & 63.8 & 63.0 & 46 & at & 2 & & 68 & 36 & \\
\hline 30 & 34.8 & 32.1 & $3 \mathrm{I}$ & +1.7 & 30 & 44.9 & 44.5 & & I3 & +2.2 & 30 & 65.2 & 64.7 & 43 & +7.0 & 30 & 70.4 & 68.4 & 36 & +5.3 \\
\hline 32 & $4 \mathrm{I} .5$ & 38.2 & & & 32 & Over & l'k'd & & & 1 & 32 & 64.9 & 64.6 & 44 & & 32 & & 68.2 & 37 & \\
\hline 34 & 41.0 & 39.0 & 20 & is & 34 & 50.3 & $48 . I$ & 23 & 06 & 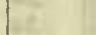 & 34.6 & 63.9 & 63.9 & 45 & Fe & 34 & 68.7 & 67.2 & 38 & vil. \\
\hline 36 & 37.2 & 36.1 & 26 & g & 36 & 55.6 & 54.1 & 22 & 57 & C. & 36 & 64.0 & 63. & 45 & 106 & & 69.1 & & 38 & \\
\hline 38 & 33.5 & $32 . I$ & 32 & 18 & 38 & 53.1 & 52.9 & 23 & $\infty$ & Ac & 38 & 62.8 & 62 & 47 & Z2. & 38 & 69.2 & 68.0 & 37 & \\
\hline 40 & 33.6 & 31.9 & 32 & 9 & 40 & 48.0 & 46.0 & & 09 & 1 & 40 & 62.0 & 62.0 & 48 & 29 & & 69.5 & 68.2 & 37 & \\
\hline 42 & 39.3 & $37 \cdot I$ & 23 & & 42 & 40.9 & 40.6 & & 19 & & 42 & 62.3 & 61.9 & 48 & ax. & 42 & 68.7 & 67.9 & 38 & \\
\hline 44 & 39. I & 38. I & 23 & +1.7 & 44 & 52.9 & 51.9 & & oI & +2.3 & 44 & 62.0 & 61.0 & 49 & +7.0 & 44 & 68.1 & & 39 & $+5 . \mathrm{I}$ \\
\hline 46 & $4 \pi .9$ & 39. I & 20 & & 46 & 48.9 & 48.0 & 23 & 07 & 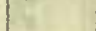 & 46 & 62.7 & 61.7 & 48 & & 46 & 68.0 & 66. & 39 & ( \\
\hline 48 & 40.0 & 38.1 & 22 & s & 48 & 55.0 & 53.6 & 22 & 58 & s. & 48 & 63.1 & 62.1 & 47 & 10 & 48 & 67.6 & 66.2 & 40 & 8 \\
\hline 50 & 39.1 & 38.3 & 22 & Q & 50 & 48.0 & 46.2 & 23 & 09 & 0 & 50 & 62.1 & 61.3 & 48 & 20 & 50 & 67.0 & 66.0 & $4 \mathrm{I}$ & \\
\hline 52 & 44.1 & 42.9 & 15 & 82 & 52 & 52.2 & 51.7 & & 02 & $=$ & 52 & 64.9 & 64.0 & 44 & ats & 52 & 67.3 & 66.0 & $4 \mathrm{I}$ & 18 \\
\hline 54 & 42.1 & 40.8 & I8̆ & 12 & 54 & 53.3 & 51.9 & & OI & Q & 54 & 63.4 & 62.2 & 47 & 18 & 54 & 67.9 & 66.2 & 40 & 4 \\
\hline 56 & 43.1 & 41.9 & 16 & di & 56 & 52.9 & $51 . \mathrm{I}$ & & 02 & 10 & 56 & 63.4 & 62.1 & 47 & & 56 & 67.9 & 66.3 & 40 & \\
\hline 58 & 45.0 & 43.9 & I4 & 8 & 58 & 53.2 & 52.I & & or & & 58 & 63.2 & 62.1 & 47 & & $5^{8}$ & 67.8 & & 40 & \\
\hline & & & & & $8 \infty$ & 52.0 & $5 I .1$ & & 02 & +2.7 & & & & & & 1200 & 66.9 & 66.0 & 4I & +5.0 \\
\hline
\end{tabular}

Correction to local mean time is - Im 22s. $90^{\circ}$ torsion $=19 .^{\prime} 03$. Torsion head at oh oom read $59^{\circ}$ and at $8 \mathrm{~h} 17 \mathrm{~m}$ read $53^{\circ}$. Observer-J. V.
Correction to local mean time is - In 44 s. $90^{\circ}$ torsicn $=18 .^{\prime} 06$.

Torsion head at 7 h $50 \mathrm{~m}$ read $53^{\circ}$ and at $12 \mathrm{~h}$ 20m read $49^{\circ}$. Observer-J. V. 
Tabulation of magnetic declinations observed at Teplitz Bay-Continued

\begin{tabular}{|c|c|c|c|c|c|c|c|c|c|c|c|c|c|c|c|c|c|c|c|c|c|}
\hline \multicolumn{5}{|c|}{ Tuesday, June I4, IgO4 } & \multicolumn{6}{|c|}{ Magnet scale erect } & \multicolumn{6}{|c|}{ Wednesday, June 15,1904} & \multicolumn{5}{|c|}{ Magnet scale inverted } \\
\hline \multirow{2}{*}{$\begin{array}{l}\text { Chr's } \\
\text { time }\end{array}$} & \multicolumn{2}{|c|}{$\begin{array}{l}\text { Scale } \\
\text { readings }\end{array}$} & $\begin{array}{c}\text { East } \\
\text { decli- } \\
\text { nation }\end{array}$ & $\underset{\mathrm{C}}{\text { Iemp. }}$ & $\begin{array}{l}\text { Chr'r } \\
\text { time }\end{array}$ & $\begin{array}{l}\text { Sca } \\
\text { read }\end{array}$ & $\begin{array}{l}\text { ale } \\
\text { lings }\end{array}$ & $\begin{array}{l}\text { East } \\
\text { decli } \\
\text { natio }\end{array}$ & & Temp. & Chr'r & $\begin{array}{l}\text { Sca } \\
\text { read }\end{array}$ & $\begin{array}{l}\text { ale } \\
\text { lings }\end{array}$ & $\begin{array}{l}\text { East } \\
\text { decli }\end{array}$ & remp. & Chr'r & $\begin{array}{l}\text { Sca } \\
\text { read }\end{array}$ & $\begin{array}{l}\text { e } \\
\text { igs }\end{array}$ & East & st & remp. \\
\hline & Left & Right & & & & Left & Right & & & & & Left & Right & & & & Left & Right & & & \\
\hline $\mathrm{h} \mathrm{m}$ & d & d & $\cdot \cdot$ & $\infty$ & $\mathrm{h} \mathrm{m}$ & $d$ & d & $\cdot$ & - & $\bullet$ & $\mathrm{h} \mathrm{m}$ & $d$ & d & - & 0 & h m & d & d & & & $\bullet$ \\
\hline 1200 & 52.6 & 54.2 & 22.37 & +10 & I4 00 & 46.1 & 47.2 & 222 & 24 & to.7 & $000^{*}$ & 54.0 & $53 \cdot 7$ & 224 & +1.3 & 200 & 46.0 & 45.8 & 225 & 54 & +1.0 \\
\hline 02 & 52.2 & 54.6 & 3.5 & 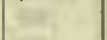 & 02 & 46.7 & $47 \cdot 4$ & & 25 & 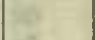 & 02 & 51.0 & 50.9 & 4 & & 02 & $46 \cdot 3$ & 46.0 & & 53 & \\
\hline 04 & 52.3 & 54.5 & 3.5 & int & 04 & $46 \cdot 3$ & $47 \cdot 4$ & & 24 & Q & 04 & 49.8 & $49 \cdot 4$ & 4 & te & 04 & $45 . I$ & 45.0 & & 55 & \\
\hline $0 \hat{s}$ & 52.9 & 54.8 & 36 & 60: & 06 & 46.9 & 47.8 & & 25 & $\$$ & 06 & 47.0 & 46.5 & 5 & & 06 & 42.1 & 42.0 & 22 & 60 & \\
\hline 08 & 52.1 & 54.4 & & $\ln i$ & 08 & 47.0 & 47.8 & & 25 & Fis & os & $45 \cdot 3$ & 45.2 & 5 & & 08 & 41.0 & 40.9 & 23 & OI & \\
\hline 10 & $5 \mathrm{I} . \mathrm{I}$ & 52.0 & 32 & 8 & 10 & 46.9 & 47.6 & & 25 & 04 & 10 & 44.0 & 43.8 & 5 & QII & IO & 37.1 & 36.9 & & 08 & \\
\hline I2 & 50.4 & 52.9 & 32 & is: & 12 & 46.7 & 47.9 & & 25 & & 12 & 42.9 & 42.9 & 5 & a & I2 & $35 . I$ & 35.0 & & II & \\
\hline 14 & 50.8 & 53.1 & 32 & +0.0 & 14 & 46.9 & 48.0 & E & 25 & to.8 & I4 & 43.6 & 43.4 & 225 & $+\mathrm{I} .2$ & 14 & 34.9 & 34.1 & & II & +1.0 \\
\hline 16 & 51.0 & $53 \cdot 3$ & 3.3 & 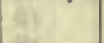 & 16 & 46.3 & $47 \cdot 7$ & & 25 & in & 16 & 42.1 & 41.9 & 230 & & 16 & 34.2 & 33.0 & & I3 & \\
\hline 18 & 50.3 & 52.6 & 32 & fir & 18 & 46.1 & $47 \cdot 4$ & & 24 & (4) & 18 & 41.9 & $4 I . I$ & 230 & & 18 & $34 \cdot 9$ & 34.0 & & I3 & \\
\hline 20 & 49.9 & 52.1 & 31 & $\infty$ & 20 & 42.9 & 44.1 & & 19 & $c 5$ & 20 & 42.9 & 41.8 & 225 & int & 20 & 3i.I & 30.8 & & I7 & \\
\hline 22 & 50.3 & 52.1 & 31 & 14 & 22 & 43.5 & 44.7 & & 20 & $=$ & 22 & 46.0 & 45.4 & 5 & & 22 & 3I. I & 30.9 & & 17 & \\
\hline 24 & 51.1 & 52.9 & 33 & 4t2 & 24 & 44.0 & 44.9 & & $2 \mathrm{I}$ & 14 & 24 & 45.1 & $44 \cdot 4$ & 5 & 12 & 24 & $34 . I$ & 33.8 & & 12 & \\
\hline 26 & 51.7 & 53.4 & 33 & 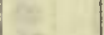 & 26 & 44.7 & 45.6 & & 22 & 8 & 26 & 45.1 & 44.5 & 5 & & 26 & $35 . I$ & 34.0 & & II & \\
\hline 28 & 51.2 & 53.1 & 33 & 5 & 28 & 44.8 & 45.3 & & 22 & 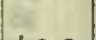 & 28 & 44.2 & 43.9 & 5 & & 28 & 35.0 & 34.1 & & II & \\
\hline 30 & 50.9 & 52.6 & 32 & to.8 & 30 & 45.2 & 46.1 & & 23 & +0.9 & 30 & 43.0 & 42.2 & 5 & +1.2 & 30 & 39.0 & 38.8 & & 04 & +1.0 \\
\hline 32 & 50.1 & 52.1 & 31 & ax & 32 & 45.7 & 46.8 & & 24 & 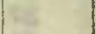 & 32 & 42.8 & 42.7 & 5 & & 32 & 37.0 & 36.3 & & 08 & \\
\hline 34 & 49.2 & 51.7 & 30 & 25 & 34 & 46.2 & 47.1 & & 24 & 12 & 34 & 46.0 & 45.8 & 5 & ts & 34 & 38.9 & 38.1 & & 05 & \\
\hline 36 & $49 . I$ & 51.8 & 30 & $d$ & 36 & 47.1 & 48.0 & & 26 & $1-$ & 36 & 47.1 & 46.8 & & & 36 & 40.2 & 39.6 & & 03 & \\
\hline 38 & 50.0 & 52.9 & 32 & be & 38 & 46.1 & 47.1 & & 24 & $\theta=$ & $3^{8}$ & 47.6 & 47.1 & 5 & 4 & 38 & 39.2 & 38 . I & & 05 & \\
\hline 40 & 50.2 & 53.0 & 32 & 6 & 40 & 46.9 & 47.8 & 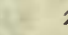 & 25 & E & 40 & 48.1 & 47.1 & 5 & 16 & 40 & 37.0 & 36.0 & & 08 & \\
\hline 42 & 50.2 & 52.7 & 32 & 6 & 42 & 48.7 & 49.2 & & 28 & & 42 & 45.9 & 45.0 & 5 & 5 & 42 & 35.8 & 35.0 & & 10 & \\
\hline 44 & $50 . I$ & 52.0 & $3^{\top}$ & 10.7 & 44 & 48.7 & 49.2 & & 28 & to. 9 & 44 & 46.0 & 45.9 & & +I.I & 44 & 36.9 & 36.2 & & 08 & +1.0 \\
\hline 46 & 50.1 & 53.0 & 32 & 5 & 46 & 49.1 & 49.8 & & 28 & ( & 46 & 42.6 & $4 \mathrm{I} . \mathrm{I}$ & 6 & & 46 & 39.6 & 38.8 & & 04 & \\
\hline 48 & 48.8 & 51.3 & 30 & 164 & 48 & 49.9 & 50.6 & & 30 & 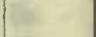 & 48 & 43.6 & 42.2 & & a & 48 & 39.8 & 39.1 & & 04 & \\
\hline 50 & 48.1 & 50.3 & 28 & 2 & 50 & 50.1 & 50.9 & & 30 & E & 50 & 44.0 & $43 . I$ & 5 & est & 50 & 36.3 & 36.1 & & 09 & \\
\hline 52 & 48.7 & 50.8 & 29 & Ait & 52 & 49.9 & 50.3 & & 30 & E & 52 & 45.0 & 44.7 & 5 & 난 & 52 & 33.9 & $33 . I$ & & I3 & \\
\hline 54 & 49.5 & 51.2 & 30 & 22 & 54 & 49.8 & 50.5 & & 30 & 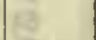 & 54 & 45.8 & 45.0 & 5 & 14 & 54 & 33.4 & 33.0 & & 14 & \\
\hline 55 & 50.3 & 52.1 & 3I & 24 & 56 & 50.1 & 50.8 & (11) & 30 & at & 56 & $45 \cdot 3$ & 44.5 & & 9 & 56 & 34.0 & 33.8 & & I3 & \\
\hline 58 & 50.6 & $52 . \mathrm{I}$ & 32 & ks & 58 & 49.8 & 50.2 & ze & 30 & 7 & 58 & $44 . I$ & 43.9 & & 8. & 58 & 35.8 & 35.3 & & 10 & \\
\hline 1300 & 50.2 & 51.9 & $3 I$ & to.7 & I 500 & 49.1 & 49.9 & & 29 & to. & I $\infty$ & $45 \cdot 3$ & $44 . I$ & 5 & +I. I & 300 & 37.4 & 37.1 & 23 & 07 & +1.0 \\
\hline 02 & 49.2 & $50 . I$ & 29 & 5 & 02 & 50.0 & 50.7 & & 30 & 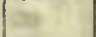 & 02 & 46.0 & 45.8 & & 4 & 02 & 49.0 & 48.8 & 22 & 49 & \\
\hline 04 & 48.9 & 49.6 & 28 & 18 & 04 & 48.4 & 49.1 & & 27 & 0 & 04 & 45.9 & 45.1 & 5 & & 04 & 40.3 & 39.9 & 23 & 03 & \\
\hline 06 & 49.2 & 50.2 & 29 & ㅈt & 06 & $47 \cdot 4$ & 47.9 & & 26 & 6 & 06 & 43.9 & 43.1 & 225 & & 06 & $4 \mathrm{I} .2$ & 40.8 & 23 & OI & \\
\hline 08 & 50.0 & 51.1 & 30 & Bs & 08 & $47 . I$ & 47.7 & & 25 & 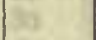 & 08 & 41.8 & $4 \mathrm{I} .0$ & 230 & & 08 & $43 \cdot 3$ & 42.9 & 22 & 58 & \\
\hline I0 & 50.2 & $51 \cdot 3$ & $3 I$ & et & IO & 47.0 & $47 \cdot 4$ & & 25 & ar & IO & 4I. I & 40.1 & 0 & of & IO & 47.0 & 46.1 & & 53 & \\
\hline 12 & 49.8 & 50.8 & 30 & 34) & 12 & 47.7 & 48.0 & & 26 & & 12 & 39.8 & $39 . \mathrm{I}$ & 0 & 41 & I2 & 49.9 & 49.2 & & 48 & \\
\hline I4 & 49.2 & 50.0 & 29 & +0.7 & I4 & 47.2 & 47.9 & & 26 & +0.8 & I4 & 39.8 & 39.6 & & $+\mathrm{I} . \mathrm{I}$ & 14 & 5I.I & 50.9 & & 46 & +1.0 \\
\hline 16 & 48.0 & 49.0 & 27 & 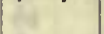 & 16 & 47.7 & 48.1 & & 26 & 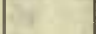 & I6 & $42 . I$ & 4I. 6 & & & I6́ & 50.9 & 50.7 & & 46 & \\
\hline 18 & 47.5 & 48.5 & 26 & Er & 18 & 48.2 & 49.0 & & 27 & 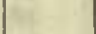 & I8 & 40.1 & 39.3 & 0 & 3 & I8 & 46.8 & 46.5 & 22 & 52 & \\
\hline 20 & 48.7 & 49.7 & 28 & es & 20 & 47.3 & 48.0 & & 26 & 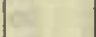 & 20 & 40.0 & 39.7 & & ist & 20 & 41.9 & 40.9 & 23 & or & \\
\hline 22 & 47.9 & 48.9 & 27 & 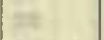 & 22 & 47.9 & 48.8 & & 27 & 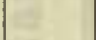 & 22 & 40.0 & 39.2 & o & at & 22 & 32 & $.9 b$ & & I4 & \\
\hline 24 & 49.1 & 50.1 & 29 & 16 & 24 & 47.5 & 48.2 & & 26 & $=$ & 24 & 39.8 & 39.0 & & & 24 & 20 & $.0 b$ & & 34 & \\
\hline 26 & 49.6 & 50.1 & 29 & 5 & 26 & 46.2 & 47.1 & & 24 & 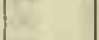 & 26 & 38.2 & 36.8 & 0 & & 26 & 10.8 & 10.5 & & 49 & \\
\hline 28 & 49.5 & 50.0 & 29 & $x^{2}$ & 28 & 47.2 & 48.2 & & 26 & & 28 & 36.9 & 36.8 & 0 & & 28 & 18.5 & I7. I & & 38 & \\
\hline 30 & 50.3 & 50.9 & 30 & to 7 & 30 & 47.8 & 48.8 & & 27 & +0.7 & 30 & 40.7 & 39.9 & 230 & +1.1 & 30 & 26.1 & 24.3 & & 26 & +1.0 \\
\hline 32 & 49.6 & 49.9 & 29 & & 32 & 47.9 & 48.8 & & 27 & 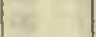 & 32 & 43.8 & $43 \cdot 3$ & 225 & & 32 & 31.0 & 28.8 & & I9 & \\
\hline 34 & 49.8 & $49 . \overline{8}$ & 29 & AE & 34 & 47.9 & 48.9 & & 27 & & 34 & 42.5 & 41.9 & & & 34 & 32.9 & 30.2 & & 16 & \\
\hline 36 & 49.1 & 49.4 & 28 & +2 & 36 & 47.9 & 48.8 & & 27 & 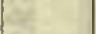 & 36 & 44.0 & 43.9 & & & 36 & $33 . I$ & 31.0 & & 15 & \\
\hline 38 & 49.0 & 49.0 & 28 & ac & 38 & $47 . I$ & 47.9 & & 26 & 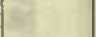 & 38 & 44.2 & $44 . I$ & & & 38 & 34.8 & 32.2 & & I3 & \\
\hline 40 & 48.3 & 48.8 & 27 & 8 & 40 & 46.9 & 47.7 & & 25 & 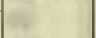 & 40 & 44.2 & $44 . I$ & & 4 & 40 & 35.7 & 33.8 & & II & \\
\hline 42 & 48.2 & 48.6 & 27 & b & 42 & $47 . \bar{I}$ & 48.0 & & 26 & & 42 & 43.8 & 43.6 & & 7 & 42 & 36.2 & 34.2 & & I0 & \\
\hline 44 & 48.2 & 48.9 & 27 & +0.7 & 44 & 49.1 & 49.9 & & 29 & +0.4 & 44 & $44 . I$ & 43.9 & 5 & +1.0 & 44 & 36.3 & 34.8 & & 10 & +I.I \\
\hline 46 & 48.2 & 49.2 & 27 & 17 & 46 & 50.4 & 51.4 & & $3 I$ & 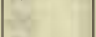 & 46 & 45.7 & 45.2 & & & 46 & 37.9 & 36.0 & & 08 & \\
\hline 48 & 48.1 & 49.1 & 27 & 3 & 48 & 51.9 & 53.0 & & 33 & 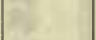 & 48 & 47.0 & 46.9 & 5 & 48 & 48 & 39.0 & 37.1 & & 06 & \\
\hline 50 & 47.9 & 48.9 & 27 & 12 & 50 & 52.6 & 53.9 & & 34 & $=$ & 50 & 47.8 & 47.2 & & A & 50 & 38.8 & 37.0 & & 06 & \\
\hline 52 & 47.2 & 48.2 & 26 & at & 52 & 5 I.I & $52 . I$ & & 32 & D & 52 & 48.2 & $48 . I$ & cit & W & 52 & 34.8 & 32.8 & & I2 & \\
\hline & 46.4 & 47.4 & 25 & it & 54 & 49.2 & 50.1 & & 29 & $E$ & 54 & 48.8 & 48.4 & & & 54 & 30.4 & 29.0 & & I9 & \\
\hline 56 & 46.6 & 47.7 & 25 & 2 & 56 & 46.3 & $47 . I$ & & 24 & 68 & 56 & 47.9 & 47.9 & & & 56 & 27.0 & 25.5 & & 24 & \\
\hline 58 & 46.2 & 47.2 & 24 & 35 & 58 & $43 \cdot 7$ & 44.0 & & 20 & & 58 & 46.9 & 46.8 & & De & 58 & $26 . I$ & 24.7 & & 26 & \\
\hline & & & & $0^{4}$ & 1600 & 40.3 & 4I.I & & 15 & & & & 2,18 & & $\infty$ & & & & & & \\
\hline
\end{tabular}

Correction to local mean time is +19.55 .

Observer-J. V.

Torsion head at $\mathrm{I} 2 \mathrm{~h}$ oom read $5 \mathrm{I}^{\circ}$ and at $\mathrm{I} 6 \mathrm{~h} \mathrm{I} 5 \mathrm{~m}$ read the same.

Observer-J. V. 
Tabulation of magnetic declinations observed at Teplitz Bay-Continued

Wednesday, Junc 15,1904

Magnet scale inverted

Wednesday, June 15,1904

Magnet scale inverted

\begin{tabular}{|c|c|c|c|c|c|c|c|c|c|c|c|c|c|c|c|c|c|c|c|}
\hline \multirow{2}{*}{$\begin{array}{l}\text { Chr'r } \\
\text { time }\end{array}$} & \multicolumn{2}{|c|}{$\begin{array}{l}\text { Scale } \\
\text { readings }\end{array}$} & \multirow{2}{*}{$\begin{array}{c}\text { East } \\
\text { decli- } \\
\text { nation }\end{array}$} & \multirow{2}{*}{ Temp. } & \multirow{2}{*}{$\begin{array}{l}\text { Chr'r } \\
\text { time }\end{array}$} & \multicolumn{2}{|c|}{$\begin{array}{l}\text { Scale } \\
\text { readings }\end{array}$} & \multirow{2}{*}{$\begin{array}{l}\text { East } \\
\text { decli- } \\
\text { nation }\end{array}$} & \multirow{2}{*}{ Temp. } & Chr'r & & $\begin{array}{l}\text { cale } \\
\text { dings }\end{array}$ & $\begin{array}{l}\text { East } \\
\text { decli- }\end{array}$ & Temp & Chr'r & $\begin{array}{r}\text { Sc } \\
\text { reac }\end{array}$ & $\begin{array}{l}\text { ale } \\
\text { lings }\end{array}$ & $\begin{array}{l}\text { East } \\
\text { decli- }\end{array}$ & Temp \\
\hline & Left & Right & & & & Left & Right & & & & Lefft & Right & & & & Left & Right & & \\
\hline $\mathrm{m}$ & d & d & - ' & $0^{\circ}$ & $\mathrm{h} \mathrm{m}$ & $d$ & d & & - & $\mathrm{h}$ in & d & $d$ & & & $\mathrm{~h} \mathrm{n}$ & d & $d$ & - ' & $\bullet$ \\
\hline 02 & 21.8 & 20.0 & & & & 22.1 & 20.2 & 32 & & & 32.3 & $3 \mathrm{I} . \mathrm{I}$ & 23 I6 & +1.9 & 10 & 52.8 & 52.5 & & +2.5 \\
\hline$\infty 0$ & 24.8 & 23.0 & 328 & +I.I & 600 & 29.9 & 26.7 & 2321 & +1.7 & 02 & 32.9 & 32.2 & 15 & & 02 & 52.0 & 5 I. 3 & 45 & \\
\hline 04 & 20.0 & 18.8 & 35 & & $0_{4}$ & 17.3 & 16.2 & 39 & & 04 & 32.1 & 30.9 & 16 & 10 & 04 & 47.6 & 46.9 & 51 & \\
\hline 05 & 18.9 & 17.9 & 37 & 6 & 06 & 18.9 & 18.7 & 36 & ter & 06 & 38.0 & 36.6 & 07 & 10 & 06 & 53 & $.6 a$ & $4 \mathrm{I}$ & \\
\hline 08 & $2 \mathrm{I} . \mathrm{I}$ & 20.9 & 33 & bir & 08 & 22.0 & 21.0 & 32 & 4 & 08 & 36.8 & 35.9 & 08 & 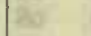 & 08 & 57.0 & 56.7 & 36 & \\
\hline 10 & 26.3 & 25.8 & 25 & or & I0 & 23.8 & 22.8 & 29 & & I0 & 36.4 & 35.5 & 09 & wir & 10 & 56.0 & 56.0 & 38 & \\
\hline 12 & $31 . \mathrm{I}$ & 30.0 & 18 & & I2 & $2 \mathrm{~T} .3$ & 19.6 & 34 & & 12 & 39.2 & 38.0 & 05 & & 12 & 7.0 & 56.5 & 37 & \\
\hline 14 & 30.9 & 29.2 & 18 & +1.2 & 14 & 10.2 & 9.8 & 50 & +1.7 & 14 & 40.5 & 38.6 & 04 & +2.0 & 14 & 8.3 & 58.0 & 34 & +2.6 \\
\hline $\begin{array}{l}16 \\
16\end{array}$ & 26.2 & 25.9 & 25 & 170 & 16 & 13.2 & II. I & 46 & 10. & 16 & 39.0 & 36.9 & 06 & $T 2.0$ & $\begin{array}{l}14 \\
16\end{array}$ & 7.0 & 56.0 & $\begin{array}{l}34 \\
37\end{array}$ & $T 2.0$ \\
\hline 18 & 20.8 & 20.2 & 33 & & I8 & $\begin{array}{r}15.9 \\
6.8\end{array}$ & 14.8 & 42 & & I8 & 36.1 & & IO & $=$ & 18 & .3 & $53 \cdot 3$ & $4 \mathrm{I}$ & \\
\hline 20 & 17.1 & 16.3 & 39 & & 20 & 16.8 & 15.0 & 41 & & 20 & 43.0 & & $\infty$ & & & & & 43 & \\
\hline 22 & 15.0 & 14 & 4. & 5 & 22 & 19.8 & 19.0 & 35 & & 22 & & & 17 & & & & 0.2 & 46 & \\
\hline 27 & & & & & 24 & 20.0 & 19 & 34 & & 24 & 99.0 & 38.5 & 05 & & & 1.8 & 50.3 & 46 & \\
\hline 26 & 15.9 & 15.2 & $4 I$ & & 26 & 17.8 & 17.3 & 38 & & 26 & 34.2 & 34.0 & 12 & $=$ & 26 & 51.8 & 50.6 & 45 & \\
\hline 28 & 13.8 & I3.7 & 44 & & 28 & 17.6 & 17.1 & 38 & & 28 & 29.9 & 28.5 & 20 & & 28 & 3.0 & 51.0 & 44 & \\
\hline 30 & 12.8 & 12.2 & 46 & +1.2 & 30 & 18.3 & 17.9 & 37 & +1.6 & 30 & 35.6 & 34.4 & II & +2.2 & 30 & $4 \cdot 3$ & I. 6 & 42 & +2.5 \\
\hline 32 & 12.0 & II.9 & 47 & & 32 & 20.0 & 19.3 & 35 & & 32 & 27.5 & 26.2 & 23 & & 32 & 4.0 & 51.3 & 43 & \\
\hline 34 & I3. I & 12.5 & 46 & & 34 & & & 36 & & 34 & 34.3 & $33 . I$ & 13 & 14 & 34 & 1. 3 & & 43 & \\
\hline 36 & 14.2 & 13.6 & 4 & & 36 & 20.8 & 19.8 & 34 & 6 & 36 & 34.3 & 33.0 & 13 & & 36 & 3 & 50.2 & 44 & \\
\hline 38 & 16.3 & 15.7 & 4 & & 38 & 20.0 & 18. I & 36 & 4 & 38 & 32.4 & & 16 & W. & 3 & & & 40 & \\
\hline 40 & 18.9 & 18.0 & 37 & & 40 & 19.0 & 17.7 & 37 & 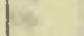 & 40 & 40.3 & 38.6 & 04 & 10 & 40 & .8 & & 34 & \\
\hline 42 & 8.2 & 17.7 & 37 & & 42 & 19.5 & 17.9 & 36 & & 42 & 39.0 & 36.1 & 07 & & 42 & & & 37 & \\
\hline 44 & I8.3 & 18.0 & 37 & +1.4 & 44 & 20.2 & 19.9 & 34 & +1.6 & 44 & 41.3 & 39.8 & 2302 & +2.3 & 44 & & & 49 & +2.6 \\
\hline 46 & 17.3 & 17.1 & 39 & & 46 & 21.2 & 20.4 & 33 & & 46 & 45.8 & $44 \cdot 3$ & $22 \quad 55$ & & 4 & & & 43 & \\
\hline 48 & 14.5 & $14 . \mathrm{I}$ & 43 & & 48 & 23.2 & 22.9 & 29 & & 48 & 2.0 & & 3 OI & $1=$ & 4 & & & $\begin{array}{l}45 \\
47\end{array}$ & \\
\hline 50 & & & 4 & & 50 & 5.0 & $25 . I$ & 26 & 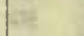 & 50 & 4.5 & & 57 & $\omega_{8}$ & & & & 44 & \\
\hline 52 & 16.9 & 16 & 3 & & 52 & 26.9 & 26.0 & 24 & a & 52 & & & 53 & 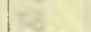 & & & & 42 & \\
\hline 54 & 15.0 & 15 & 4 & & 54 & 32.8 & 31.0 & 16 & y & 5 & .8 & & $\begin{array}{l}53 \\
52\end{array}$ & 18 & $\begin{array}{l}5 \\
5\end{array}$ & & & 46 & \\
\hline & 12.6 & & 46 & & 56 & 33.0 & 31.8 & I5 & & 56 & 46.7 & & 54 & 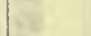 & & 0.6 & & 46 & \\
\hline & IC & $.5^{b}$ & 49 & & $\begin{array}{r}58 \\
-7\end{array}$ & 26.8 & 26.6 & 24 & & 58 & 53.9 & & 43 & & 5 & 52.0 & & 45 & \\
\hline oc & 12.2 & 12.2 & 46 & $+\mathrm{T} .5$ & 700 & 26.8 & 25.4 & 24 & $+\mathrm{t} .6$ & 00 & $55 . \mathrm{I}$ & & 40 & +2.5 & II 00 & & 3.9 & 48 & +2.6 \\
\hline 0 & 8.9 & 18 & 36 & & 02 & & ost & & & 02 & 54.0 & & 42 & & & & & 46 & \\
\hline 0. & 14.1 & 14 & 43 & 14 & & L & ost & & & & 54.0 & 52 & 42 & 10 & & & & 50 & \\
\hline 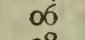 & & & 4 & 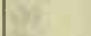 & 06. & 21.3 & 2I.I & 32 & 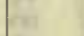 & $\alpha$ & 57.6 & & 36 & 10 & & & & 50 & \\
\hline 08 & 13.2 & & 4 & & 08 & 21.0 & 20 & 33 & & of & 55.6 & 54 & 39 & 100 & of & & & 43 & \\
\hline I0 & 14.9 & & 4 & & & 23.3 & 23.1 & & & I & 1.4 & & $4 \mathrm{I}$ & 10 & & & & 40 & \\
\hline 12 & & & 4 & & I & I8.5 & I8. I & 3 & & & & & 41 & & & & & 44 & \\
\hline Is & I5.9 & & 4 & +1.8 & 14 & 24 & $.5 a$ & 27 & +1.5 & 14 & 55.2 & & 40 & +2.5 & & & & 43 & +2 \\
\hline It & 17.1 & 16. & 4 & & & 27.0 & & 24 & & 16 & 4.5 & & 42 & & & & & 45 & \\
\hline 18 & & & 4 & 61 & 18 & 26.9 & & & & 1 & & & 40 & 6 & & & & 44 & \\
\hline 26 & & & 4 & $a$ & & & & 28 & & 2 & & & 3 & & & & & 48 & \\
\hline 22 & & & 4 & if & 2 & & & & & 22 & & & 4 & 64 & & & & 42 & \\
\hline 24 & & & & 15 & 2 & & & & & 2. & & & $4 \mathrm{I}$ & by & & & & $4 \mathrm{I}$ & \\
\hline 26. & & & 4 & & 2 & & & & & 2 & 50.3 & & 48 & E. & & & & 6 & \\
\hline 28 & & & & & 2 & & & 32 & & 28 & & & 50 & & & & & 32 & \\
\hline 30 & & & 4 & +1.8 & 3 & 25.8 & & 27 & +1.7 & 30 & $5 \mathrm{I} . \mathrm{I}$ & & $4^{6}$ & +2.5 & & & & $\begin{array}{l}32 \\
3 \mathrm{I}\end{array}$ & +2.5 \\
\hline 32 & & & 4 & & 3 & & & 3 & & 3 & 56.0 & 54 & 39 & 172.0 & 3 & 9.8 & & $\begin{array}{l}11 \\
34\end{array}$ & $1+2.5$ \\
\hline 34 & 14.2 & 13 & 4 & & 3 & & 18 & 36 & & 3. & 56.9 & & 37 & & & & & 37 & \\
\hline 36 & & 14 & 4 & $x$ & 36 & & & 34 & & 3 & & & 4 & & & & & 37 & \\
\hline 38 & & & & at. & 38 & & & 3 & 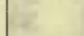 & 3 & & & 48 & W & & & & 37 & \\
\hline 40 & 18.6 & I8. & 3 & $=$ & 40 & & 23 & 28 & 8 & 4 & 51.6 & & 45 & & & 6.6 & & 38 & \\
\hline 42 & & & & & 4 & & 17 & 36 & & 4 & & & 42 & & & 58.3 & & 36 & \\
\hline & & & & & 4 & 22. & & $3 \mathrm{I}$ & +1.8 & 4 & & & 44 & +2.5 & & & & 39 & +2.5 \\
\hline & 2 & 20 & 3 & s & & 24 & & 28 & & & & & 40 & & & & & 37 & \\
\hline & & & 3 & 6 & 4 & 27. & & & & 4 & & & & & & & & 38 & \\
\hline & & & & 8 & 5 & & & & & & & & & & & & & 37 & \\
\hline & I & & & 5 & 5 & & & & & & & & $4 \mathrm{I}$ & & & & & 43 & \\
\hline 5 & & 19 & 3 & 13 & 5 & 28 & 28 & & & 5 & & & 42 & 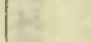 & 5 & 53.1 & $49 \cdot 3$ & 45 & \\
\hline 56 & 28.8 & 26.0 & 23 & & & 30. I & 29 & & $x^{x}$ & 56 & 56.5 & & 38 & & 5 & 54.0 & & 44 & \\
\hline 58 & 32.0 & 29.7 & 17 & 12 & 58 & 29.0 & 28.6 & 20 & & 58 & 47.5 & $47 \cdot 3$ & 51 & $O$ & 58 & 54.0 & 50.8 & 43 & \\
\hline
\end{tabular}

Observer-J. V.

Observers-J. V. and W. J. P., who alternated from $8 \mathrm{~h} 06 \mathrm{~m}$ to $8 \mathrm{~h} \mathrm{I6m.}$ 
Tabulation of magnetic declinations observed at Teplitz Bay-Continued

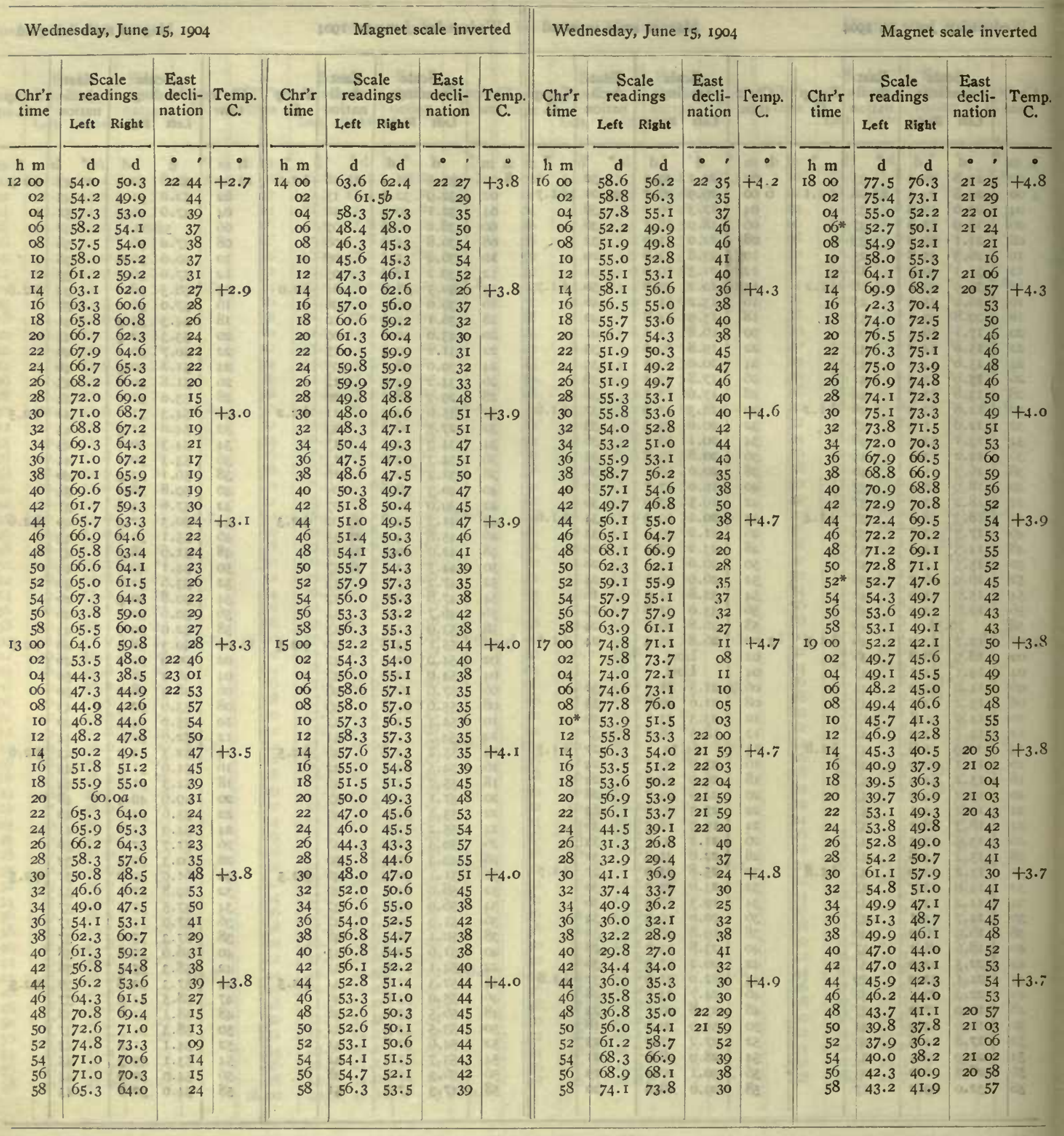

Observers-W. J. P. and R. R. T., who alternated from $15 \mathrm{~h} 38 \mathrm{~m}$ to Observer-R. R. T. Ish 52m. 
Tabulation of magnetic declinations observed at Teplitz Bay-Continued

\begin{tabular}{|c|c|c|c|c|c|c|c|c|c|c|c|c|c|c|c|c|c|c|c|}
\hline \multicolumn{5}{|c|}{ Wednesday, June 15,1904} & \multicolumn{5}{|c|}{ Magnet scale inverted } & \multicolumn{4}{|c|}{ Thursday, June I6, I904 } & \multicolumn{6}{|c|}{ Magnet scale erect } \\
\hline \multirow{2}{*}{$\begin{array}{l}\text { Chr'r } \\
\text { time }\end{array}$} & \multicolumn{2}{|c|}{$\begin{array}{l}\text { Scale } \\
\text { readings }\end{array}$} & \multirow{2}{*}{$\begin{array}{l}\text { Fast } \\
\text { decli- } \\
\text { mation }\end{array}$} & Tennp. & Chr'r & $\begin{array}{l}\text { Sc } \\
\text { read }\end{array}$ & $\begin{array}{l}\text { ale } \\
\text { lings }\end{array}$ & East & Temp. & Chr't & $\begin{array}{l}\text { Sc } \\
\text { read }\end{array}$ & $\begin{array}{l}\text { ale } \\
\text { lings }\end{array}$ & East & Temp. & Chr'r & $\begin{array}{l}\text { Sc: } \\
\text { read }\end{array}$ & $\begin{array}{l}\text { ale } \\
\text { lings }\end{array}$ & $\begin{array}{l}\text { East } \\
\text { decli- }\end{array}$ & Temp. \\
\hline & Left & Right & & & & Left & Right & & & & Left & Right & & & & Left & Right & & \\
\hline h $\mathrm{m}$ & d & d & $\circ$ & 6 & $\mathrm{l} \mathrm{m}$ & d & d & - ' & 0 & $\mathrm{~h} \mathrm{~m}$ & d & d & . ' & & $h \mathrm{~m}$ & d & d & - 1 & - \\
\hline 2000 & 41.1 & 42.9 & $2 c .55$ & +3.7 & 2200 & 44.9 & 32.7 & 2119 & +4.2 & $1600^{*}$ & $32 . I$ & 32.8 & 2125 & +1.0 & I8 00 & 18.9 & $2 I .1$ & 2106 & 0.0 \\
\hline 02 & $43 \cdot 3$ & 42.4 & 56 & & 02 & 48.1 & 36.6 & 13 & & 02 & 32.8 & 34.2 & 27 & & 02 & I6.8 & 18.2 & 2102 & \\
\hline 04 & $44 \cdot 7$ & 43.1 & 55 & 67 & 04 & 42.9 & 30.1 & 22 & & 04 & 29.1 & 3I.I & 22 & 10 & 04 & 14.6 & 17.4 & 2060 & \\
\hline 06 & 45.1 & $44 \cdot 7$ & 53 & & 06 & 41.9 & 30.9 & 22 & ats & 06 & 29.2 & 31.7 & 22 & 30 & $06^{*}$ & 29.1 & 30.4 & 42 & wit \\
\hline 08 & 46.8 & 45.3 & $5 I$ & to & 08 & 44.2 & 33.6 & 18 & $2 x$ & 08 & 31.8 & 33.2 & 26 & 28 & 08 & 28.2 & 34.1 & 44 & $4=$ \\
\hline IO & 43.4 & 42.9 & 56 & et & Io & $4 I .3$ & 31.9 & 22 & $\mathrm{CP}^{5}$ & 10 & 28.2 & 30.1 & 20 & ar: & IO & 30.3 & 35.9 & 48 & 70 \\
\hline 12 & 45.0 & 44.1 & 54 & & 12 & 39.9 & 30.8 & 24 & . & 12 & $26 . \overline{3}$ & 29.6 & I8 & 4 & 12 & 32.8 & 38.1 & 51 & 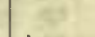 \\
\hline 14 & 42.2 & $4 I .9$ & 58 & +3.8 & 14 & 35.9 & $27 \cdot 3$ & 30 & +4.1 & I4 & 27.4 & 30.8 & 20 & to. 6 & 14 & 32.9 & 38.2 & $5 I$ & to. I \\
\hline 16 & $43 . I$ & 42.6 & 56 & & I6 & 35.7 & 27.8 & 30 & & 16 & 24.0 & $28 . I$ & 15 & & 16 & 33.9 & 35.0 & 50 & \\
\hline I 8 & 44.0 & 43.2 & 55 & Bi & I8 & 33.8 & 27.3 & 32 & N & I8 & I9.0 & $23 . I$ & 07 & hr & I8 & 28.9 & 35.1 & 46 & 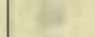 \\
\hline 20 & 44.9 & $44 . I$ & 54 & 16 & 20 & 28. I & 21.9 & 40 & $x^{2}$ & 20 & 25.2 & 32.0 & I9 & 6 & 20 & 28.9 & 34.0 & 45 & $\cos$ \\
\hline 22 & 44.9 & 44.0 & .54 & 48 & 22 & 30.1 & 24.9 & 36 & $=3$ & 22.6 & 30.0 & 32.1 & 23 & t5x: & 22 & 25.7 & 31.0 & 40 & 6. \\
\hline 24 & 47.2 & $46 . I$ & 50 & 10 & 24 & 27.9 & 22.5 & 40 & $x^{2}$ & 24 & 24.8 & 29.0 & 17 & 25 & 24 & 25.1 & 30.2 & 39 & th \\
\hline 26 & 49.9 & 47.9 & 47 & & 26 & 31.8 & 26.0 & 34 & $x$ & 26 & I7. 8 & 20.0 & 2104 & OK & 26 & 25.0 & 30.3 & 39 & 42 \\
\hline 28 & 50.4 & 49.9 & 45 & & 28 & 24.0 & 18.0 & 47 & 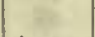 & 28 & 13.0 & 16.7 & 2057 & $\sqrt{1+2}$ & 28 & 25.0 & 29.3 & 38 & \\
\hline 30 & 50.0 & 49.0 & 46 & +3.9 & 30 & I8. I & 13.2 & 55 & +4.0 & 30 & 9.8 & 13.0 & 52 & to. 4 & 30 & 25.2 & 30.7 & 40 & +0.2 \\
\hline 32 & 48.9 & 47.0 & 48 & - & 32 & 17.8 & I3.I & 55 & ( & 32 & I2. 3 & 13.7 & 2055 & t & 32 & $27 \cdot 3$ & 32.0 & 42 & 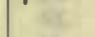 \\
\hline 34 & $55 \cdot 3$ & 53.5 & .35 & the & 34 & 18.3 & 14.3 & 54 & 45 & 34 & 16.3 & 19.9 & 2103 & 12 & 34 & 30.2 & 34.3 & 46 & 12 \\
\hline 36 & 59.2 & 58.4 & 31 & & 36 & 20.3 & 16.2 & $5 I$ & 28 & 36 & 22.0 & 22.9 & 10 & we & 36 & 33.0 & 37.0 & $5 I$ & cate \\
\hline 38 & 55.6 & 54.9 & 37 & 184 & 38 & 17.2 & 12.9 & 56 & $3 /$ & 38 & 25 & $.2 a$ & 14 & 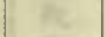 & 38 & 36.8 & 39.3 & 55 & Gr \\
\hline 40 & 53.8 & 53.0 & 40 & 6. & 40 & 14.9 & 11.0 & 59 & 48 & 40 & 28.0 & 28.7 & 19 & 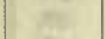 & 40 & 37.1 & 40.1 & 56 & 104 \\
\hline 42 & 63.3 & 61.4 & 26 & (nt) & 42 & 16.9 & 12.8 & 56 & 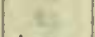 & 42 & 27.0 & 28.1 & 18 & 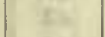 & 42 & 36.5 & 39.2 & 55 & $1+1$ \\
\hline 41 & 66.8 & 63.2 & 22 & $i^{7} .9$ & 44 & I6.9 & 13.8 & 56 & +4.0 & 44 & 41.0 & $4 I .0$ & 39 & +0.3 & 44 & 35.2 & 38.2 & 53 & +0.2 \\
\hline 46 & 72.1 & 69.2 & $20 \quad 13$ & ( & 46 & 17.2 & 14.1 & 55 & tiva & 46 & 45.7 & 46.3 & 47 & & 46 & 34.8 & 38.3 & 53 & \\
\hline $48 *$ & 58.0 & 53.0 & 1957 & & 48 & 14.0 & II. 3 & 60 & he & 48 & 43.0 & 45.8 & 44 & 4 & 48 & 33.9 & 37.0 & $5 I$ & 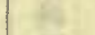 \\
\hline 50 & 49.3 & $4 I$ I I & 2013 & $n$ & 50 & I 4.4 & 12.0 & 59 & ne & 50 & 40.0 & 44.0 & 40 & the & 50 & 37.0 & 40.2 & 2056 & $\cos$ \\
\hline 52 & 53.1 & 52.0 & OI & 48 & 52 & 14.7 & 12.5 & 58 & zis & 52 & $43 \cdot 3$ & $47 \cdot 5$ & 46 & 18 & 52 & 40.0 & 42.8 & $2 \mathrm{I}$ OI & $1 \geq$ \\
\hline 54 & 52.0 & 48.0 & 05 & 93 & 54 & I4.8 & 12.2 & 58 & $\mathrm{H}$ & 54 & $43 \cdot 3$ & 48.1 & 46 & 22 & 54 & 46.0 & 48.9 & IO & 1 \\
\hline 56 & 49.2 & 43.9 & I I & & 56 & 16.0 & 13.9 & 56 & $y=$ & 56 & 43.2 & 46.2 & 45 & it & 56 & 46.8 & 49.2 & II & the \\
\hline 58 & 48.7 & 43.2 & 12 & fis: & 58 & 17.8 & I5.I & 54 & & 58 & 43.1 & 45.3 & 44 & & 58 & 49.8 & 52.5 & I6 & \\
\hline 2100 & 52.2 & 43.4 & 09 & $\therefore 0$ & 2300 & 15.9 & 14.1 & 56 & +3.8 & 1700 & 46.2 & 47.0 & 48 & $\dashv 0.1$ & 1900 & 51.6 & 54.0 & 19 & +0.2 \\
\hline 02 & 43.9 & 36.1 & 21 & 8 & 02 & I3. 9 & 12.0 & 59 & 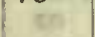 & 02 & 48.1 & 49.8 & $5 \mathrm{I}$ & ( & 02 & 53.7 & 56.0 & 22 & $10=$ \\
\hline 04 & 46.8 & $44 . I$ & I3 & $3 x$ & 04 & 14.6 & 12.4 & 58 & 10 & 04 & 48.1 & 51.9 & 53 & 20 & 04 & 55.0 & 57.8 & 24 & 16 \\
\hline$\infty 6$ & 34.0 & 30.8 & 33 & 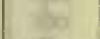 & 06 & 16.7 & 14.5 & 55 & $\ln$ & 06 & 50.8 & 51.9 & 55 & 30 & 06 & 51.3 & 54.1 & I8 & 109 \\
\hline$\infty 8$ & 43.9 & 40.0 & 18 & Her & 08 & 18.9 & I6.8 & 52 & Nat & 08 & 47.8 & 50.3 & 5 I & 80 & 08 & 51.8 & 54.0 & I9 & F \\
\hline 10 & 38.2 & 34.6 & 27 & ent & IO & 17.9 & 15.8 & 53 & hil & Io & 43.9 & 45.0 & 44 & a) & Io & 52.7 & 55.3 & 20 & 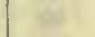 \\
\hline 12 & 38.9 & 32.9 & 27 & & 12 & 15.9 & 13.3 & 57 & 34 & 12 & 46.5 & 48.0 & 49 & sin & 12 & 51.9 & 55.5 & 20 & 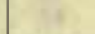 \\
\hline I4 & 35.8 & $3 I \cdot I$ & 31 & $T 4 . I$ & 14 & 17.0 & I 4.8 & 55 & +3.7 & 14 & 46.9 & 49.9 & 50 & 0.0 & 14 & 48.7 & 52.0 & I5 & +0.2 \\
\hline 16 & $34 \cdot 7$ & 31.8 & 32 & |1 & 16 & 20.1 & 18.8 & 49 & ( & I6 & 49.3 & 52.3 & 54 & 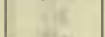 & I6 & 47.4 & 50.9 & I3 & ( \\
\hline 18 & 32.1 & $27 \cdot 9$ & 37 & Alis. & I8 & 20.4 & 19.0 & 49 & 14 & 18 & 46.2 & 51.0 & 5 I & Hit: & I8 & 56.2 & 57.2 & 25 & 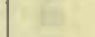 \\
\hline 20 & 31.5 & 28.9 & 36 & $\operatorname{les}$ & 20 & 18.0 & 16.9 & 52 & ns & 20 & 44.9 & 48.3 & $4^{8}$ & 165 & 20 & 63.1 & 68.1 & 39 & p \\
\hline 22 & 37.8 & 30.2 & 30 & 42 & 22 & I6. 2 & 15.7 & 55 & $x$ & 22 & 49.0 & $54 . I$ & 55 & 45 & 22 & 58.9 & 62.8 & $3 I$ & 45 \\
\hline 24 & 22.4 & 15.2 & .54 & 18 & 24 & I8. I & 17.7 & 52 & 14 & 24 & 49.0 & 55.0 & 56 & $3=$ & 24 & 65.0 & 66.0 & 38 & $a_{1}$ \\
\hline 26 & 26.1 & 2 I. 2 & 47 & ns & 26 & 22.1 & 21.8 & 45 & & 26 & 51.8 & 56.1 & 2159 & 15 & 26 & 63.0 & 63.2 & 35 & fart \\
\hline 28 & 25.4 & I9. 5 & 49 & 15 & 28 & 20.8 & $19: 3$ & 48 & $5_{1}$ & 28 & 52.0 & 57.8 & 22 or & 1ke & 28 & 49.0 & 50.7 & I4 & $y=$ \\
\hline 30 & 19.3 & 13.9 & .58 & +4.1 & 30 & I5.8 & 15.1 & 55 & +3.4 & 30 & 50.9 & 53.2 & 2156 & 0.0 & 30 & 46.3 & 47.8 & 10 & to. I \\
\hline 32 & 20. I & 14.2 & 20.57 & $x_{2}$ & 32 & 15.8 & $14 . I$ & 56 & $x^{2}$ & 32 & 55.9 & 59.8 & 2205 & 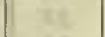 & 32 & 45.9 & 48.0 & $\infty$ & 1 \\
\hline 34 & 16.7 & 10.3 & 2102 & $\mathrm{Ar}^{2}$ & 34 & 17.3 & I6. & 54 & 4 & 34 & 53.1 & 56.8 & 22 OI & +5 & 34 & 45.2 & 47.8 & on & . \\
\hline 36 & 15.2 & 10.5 & 04 & $\sqrt{3 t}$ & 36 & 20.8 & 18.7 & 49 & $3 t$ & 36 & 50.1 & 54.1 & 2156 & $4 x$ & 36 & 40.2 & 42.2 & $\infty$ & 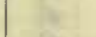 \\
\hline $38 *$ & 66.4 & 51.6 & 30 & 18 & 38 & 23.0 & 2I.I & 45 & b. & 38 & 52.0 & 56.5 & 60 & the & 38 & 45.0 & 46.1 & 07 & 25 \\
\hline 10 & 72.1 & 5.5 .9 & 22 & ifte & 40 & 25.4 & 23.8 & $4 I$ & tar & 40 & 52.5 & 56.1 & 60 & $\mathrm{D}$ & 40 & 40.9 & 41.9 & OI & the \\
\hline 42 & 71.5 & 59.0 & 20 & tax & 42 & 29.2 & 28 . I & 35 & 3 & 42 & 46.9 & 50.8 & $5 I$ & +1 & 42 & 40.8 & 44.6 & 03 & 4 \\
\hline 44 & 74.0 & .58 .2 & $21 \quad 18$ & +4.1 & 44 & 30.1 & 29.0 & 33 & +3.1 & 44 & 51.7 & 54.8 & 58 & 0.0 & 44 & 39.9 & 42.9 & $2 I$ OI & 0.0 \\
\hline $46^{*}$ & 62.7 & 42.0 & 2057 & 1 & 46 & 27.9 & 27.1 & 36 & to & 46 & 42.6 & 45.1 & 43 & s. & 46 & 31.0 & 34.9 & 2047 & (n) \\
\hline 48 & 61.0 & $4 \mathrm{I} .9$ & 59 & A & 48 & 24.8 & 24.1 & $4 I$ & th & 48 & 29.9 & 33.9 & 24 & 25 & 48 & 22.0 & 27.9 & 35 & 2 \\
\hline 50 & 63.9 & 43.1 & 56 & ine & 50 & 25.5 & 24.8 & 40 & ra & 50 & 26.8 & 29.8 & Ig & $\mathrm{nz}$ & 50 & 26.0 & 32.3 & 2041 & PEs \\
\hline 52 & $64 \cdot 3$ & $47 \cdot 3$ & 52 & the & 52 & 25.0 & 24.1 & $4 I$ & 43 & 52 & 29.9 & 31.2 & 22 & $y=$ & 52 & 43.8 & 49.8 & 2109 & 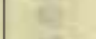 \\
\hline 54 & 63.7 & 47.6 & 20.52 & 73 & 54 & 22.4 & 22.0 & 45 & it & 54 & 31.7 & 34.7 & 27 & 3 & 54 & 43.2 & 50.1 & $\infty$ & $x^{2}$ \\
\hline .56 & 53.8 & 42.3 & $\therefore \begin{array}{lll}1 & 0\end{array}$ & & 56 & 19.9 & 19.1 & 49 & & 56 & 29.9 & .33 .0 & 24 & $n$ & 56 & 51.2 & 56.5 & 20 & 68 \\
\hline $5^{9}$ & 49.3 & 36.7 & 12 & 125 & 58 & I9.0 & 18.9 & 50 & & 58 & 23.8 & 25.8 & I3 & AE & 58 & 49.1 & 53.8 & I6 & $t y$ \\
\hline & & & & & 2400 & 16.9 & 15.3 & 54 & +3.0 & $f^{2}=$ & & [xt & $\mathrm{cr}_{2}$ & 10 & 2000 & 55.0 & 58.0 & 24 & 0.0 \\
\hline
\end{tabular}

Correction to local mean time is -35 s. $90^{\circ}$ torsion $=15^{\prime} 36$. Torsion head at oh oon read $51^{\circ}$ and at $24 \mathrm{~h} 20 \mathrm{~m}$ read $50^{\circ}$. Observer-R. R. ' $\mathrm{l}$ '.
Correction to local mean time is $-0.5 \mathrm{~s} .90^{\circ}$ torsion $=17 .^{\prime} 14$.

Torsion lead at $15 \mathrm{~h} 44 \mathrm{~m}$ read $50^{\circ}$ and at $20 \mathrm{~h} 16 \mathrm{~m}$ read $49^{\circ}$. Observer-J. V. 
Tabulation of magnetic declinations observed at Teplitz Bay-Continued

\begin{tabular}{|c|c|c|c|c|c|c|c|c|c|c|c|c|c|c|c|c|c|c|c|c|}
\hline \multicolumn{6}{|c|}{ Friday, June 17, 1904} & \multicolumn{4}{|c|}{ Magnet scale inverted } & \multicolumn{6}{|c|}{ Sunday, June 19, 1904} & \multicolumn{5}{|c|}{ Magnet scale erect } \\
\hline \multirow{2}{*}{$\begin{array}{l}\text { Chr'r } \\
\text { time }\end{array}$} & \multicolumn{2}{|c|}{$\begin{array}{l}\text { Scale } \\
\text { readings }\end{array}$} & $\begin{array}{c}\text { East } \\
\text { decli- } \\
\text { nation. }\end{array}$ & Temp. & $\begin{array}{l}\text { Chr'r } \\
\text { time }\end{array}$ & $\begin{array}{l}\text { Sca } \\
\text { readi }\end{array}$ & $\begin{array}{l}\text { ale } \\
\text { lings }\end{array}$ & $\begin{array}{c}\text { East } \\
\text { decli- } \\
\text { nation }\end{array}$ & $\begin{array}{l}\text { Temp } \\
\text { C. }\end{array}$ & $\begin{array}{l}\text { Chr'r } \\
\text { time }\end{array}$ & $\begin{array}{l}\text { Sca } \\
\text { read }\end{array}$ & $\begin{array}{l}\text { ale } \\
\text { lings }\end{array}$ & $\begin{array}{c}\text { East } \\
\text { decli- } \\
\text { nation }\end{array}$ & Temp. & Chr'r & $\begin{array}{l}\text { Sca } \\
\text { read }\end{array}$ & $\begin{array}{l}\text { ale } \\
\text { lings }\end{array}$ & Eas & st & Temp. \\
\hline & Left & Right & & & & Left & Right & & & & Left & Right & & & & Left & Right & & & \\
\hline h m & $d$ & d & $\cdot$, & - & $\mathrm{h} \mathrm{m}$ & $d$ & d & $\circ \cdot$ & - & $\mathrm{h} \mathrm{m}$ & d & d & $\cdot \cdot$ & - & $\mathrm{h} \mathrm{m}$ & d & d & & & $\cdot$ \\
\hline 2000 & 46.2 & 45.8 & 2220 & -0.5 & 2200 & 52.0 & 51.3 & 22 II & -1.0 & $000^{*}$ & 59.0 & 59.8 & 2233 & +0.9 & 200 & 52.7 & 53.1 & & 23 & +1.8 \\
\hline 02 & 52.1 & 51.0 & II & $1 \times 0^{\circ}$ & 02 & 52.1 & 51.1 & II & $x^{2}$ & 02 & 59.1 & 60.8 & 34 & 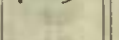 & 02 & 52.9 & 53.7 & & 23 & \\
\hline 04 & 51.8 & 51.0 & II & 20 & 04 & 52.0 & $5 \mathrm{I} . \mathrm{I}$ & II & 160 & 04 & 59.8 & $6 \mathrm{I} .1$ & 34 & 80 & 04 & 49.9 & 50.3 & & 18 & \\
\hline 06 & $45 \cdot 5$ & 44.2 & 22 & 3 & 06 & 51.9 & 50.1 & 12 & 20 & 06 & 60.2 & 61.6 & 35 & 89 & 06 & 51.3 & 52.0 & & 20 & \\
\hline o8 & 49.0 & 47.8 & I6 & 200 & 08 & 51.8 & 50.6 & 12 & 80 & os & 60.4 & 61.8 & 36 & 68 & as & 53.0 & 53.2 & & 23 & \\
\hline Io & 54.2 & 53.0 & 08 & 87 & I0 & 50.8 & 49.9 & 13 & an & I0 & 59.9 & $6 \pi .0$ & 34 & 64 & 10 & 53.0 & 54.0 & & 24 & \\
\hline 12 & 58.3 & 57.2 & OI & $2+1$ & I2 & 50.3 & 49.9 & I3 & 20y & 12 & 59.8 & 60.5 & 34 & 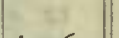 & 12 & 53.9 & 55.1 & & 25 & \\
\hline I4 & 58.9 & $57 \cdot 4$ & 22 or & -0.7 & 14 & 52.1 & 51.3 & I I & -1.0 & 14 & 59.2 & 60.1 & 33 & +0.6 & 14 & 53.1 & 54.1 & & 24 & +2.0 \\
\hline 16 & 59.9 & 58.2 & 2159 & & I6 & 54.8 & 53.9 & 07 & $34^{2}$ & І6์ & 60.6 & $6 \mathrm{I} . \mathrm{I}$ & 35 & & 16 & 53.2 & 54.2 & & 24 & \\
\hline 18 & 59.9 & 58.6 & 2159 & 8 & I8 & 56.7 & 55.5 & 04 & 16 & I8 & 60.8 & 61.3 & 35 & Eit & 18 & 56.0 & $57 \cdot 3$ & & 28 & \\
\hline 20 & 55.9 & 5.40 & 2206 & $\theta$ & 20 & $5 \mathrm{~T} .4$ & 50.8 & 12 & $y=$ & 20 & 60.6 & 61.2 & 35 & $8 x$ & 20 & 59.2 & 60.7 & & 34 & \\
\hline 22 & 52.3 & $5 \mathrm{I} .0$ & II & $=$ & 22 & 55.8 & 55.0 & 05 & 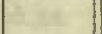 & 22 & 60.9 & 61.3 & 36 & 35 & 22 & 61.0 & 62.0 & & 36 & \\
\hline 24 & 51.7 & 50.2 & 12 & E & 24 & 52.9 & 52.8 & $\infty 9$ & 18 & 24 & 60.7 & $6 \mathrm{r} .9$ & 36 & is & 24 & 60.0 & 60.3 & & 34 & \\
\hline 26 & 51.0 & 49.9 & 13 & 단. & 26 & 52.0 & 51.3 & II & dit & 26 & 61.7 & 62.1 & 37 & ae & 26 & 57.2 & 58.0 & & 30 & \\
\hline 28 & 51.5 & 50.5 & 12 & (5) & 28 & 52.9 & 52.1 & I0 & 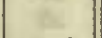 & 28 & 61.3 & 62.0 & 36 & 80 & 28 & 58.0 & 59.1 & & $3 \mathrm{I}$ & \\
\hline 30 & 49.9 & 48.8 & 15 & -0.8 & 30 & 51.0 & 50.0 & 13 & -1.0 & 30 & 60.9 & $6 \mathrm{1} .8$ & 36 & to.s & 30 & 60.0 & 60.3 & & 34 & +2.0 \\
\hline 32 & 48.0 & 46.9 & 18 & (a) & 32 & 47.9 & 46.8 & 18 & 5 & 32 & 60.5 & 61.4 & 35 & 2 & 32 & 60.3 & 61.1 & & 35 & \\
\hline 34 & 47.9 & 46.3 & 18 & in: & 34 & 46.2 & 44.9 & $2 \mathrm{I}$ & Fe. & 34 & 58.9 & 59.4 & 32 & 16 & 34 & 60.1 & 60.9 & & 35 & \\
\hline 36 & 46.9 & 45.8 & 19 & 8 & 36 & 45.0 & 43.7 & 22 & y. & 36 & $55 \cdot 3$ & $56 . \mathrm{I}$ & 27 & 8 & 36 & 59.9 & 60.2 & & 34 & \\
\hline $3^{8}$ & 43.6 & 42.9 & 24 & A & 38 & $44 \cdot 5$ & $42 \cdot 3$ & 24 & 4 & 38 & .58 .9 & 59.9 & 33 & Az & 38 & 60.0 & 60.5 & & 34 & \\
\hline 40 & 44.9 & 43.9 & 22 & 24 & 40 & 45.3 & 43.6 & 22 & os & 40 & 55.0 & 56.1 & 27 & 4 & 40 & 6I.I & 61.9 & & 36 & \\
\hline 42 & 45.8 & 44.6 & 21 & 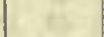 & 42 & $44 . \mathrm{I}$ & 42.9 & 24 & & 42 & 56.9 & 57.7 & 30 & 84 & 42 & 60.9 & 6г.0 & & 35 & \\
\hline 44 & 45.2 & 44.7 & 22 & -1.0 & 44 & 43.9 & 42.1 & 24 & $-1 . I$ & 44 & 58.0 & 59.0 & $3 \mathrm{I}$ & +o.9 & 44 & 61.2 & 61.9 & & 36 & +1.9 \\
\hline 46 & 45.7 & $44 . I$ & 22 & & 46 & 45.0 & 43.7 & 22 & & 46 & 58.0 & 59.1 & 31 & & 46 & 62.3 & 63.1 & & 38 & \\
\hline 48 & 45.9 & 45.2 & 21 & 5 & 48 & 46.2 & 45.9 & 20 & We. & 48 & 57.1 & 58.5 & 30 & 78 & 48 & 62.2 & 63.0 & & 38 & \\
\hline 50 & 45.8 & 45.3 & 21 & 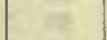 & 50 & 49. I & 48.2 & 16 & E & 50 & 54.9 & 56.1 & 27 & we & 50 & 64.0 & 64.2 & & 40 & \\
\hline 52 & 46.0 & 45.5 & 20 & ets & 52 & 46.9 & 45.7 & I9 & Se & 52 & 53.7 & $54 . \mathrm{I}$ & 24 & है & 52 & 68.2 & 68.8 & & 47 & \\
\hline 54 & 44.9 & 44.9 & 22 & $y$ & 54 & 46.8 & $45 . \mathrm{I}$ & 20 & F & 54 & 52.8 & 54.0 & 23 & 18 & 54 & 66.0 & 66.9 & & 44 & \\
\hline 56 & 42.5 & 42.3 & 26 & 28 & 56 & 43.3 & 41.9 & 25 & 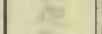 & 56 & 52.2 & 53.8 & 23 & fes & 56 & 64.3 & 65.0 & & $4 I$ & \\
\hline 58 & 41.9 & 4 I. 2 & 27 & de & 58 & 40.7 & 38.9 & 30 & (6) & 58 & 53.1 & 54.2 & 24 & 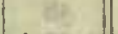 & 58 & 64.4 & 65.8 & & 42 & \\
\hline 2100 & 41.8 & $41 \cdot 3$ & 27 & -1.0 & $23 \infty$ & 38.0 & 36.1 & 34 & $-I . I$ & 100 & $54 . \mathrm{I}$ & 55.4 & 25 & +0.9 & 300 & 67.8 & 68.9 & & 47 & +2.0 \\
\hline 02 & 39.9 & $39 \cdot 3$ & 30 & 6 & 02 & 36.8 & 35.3 & 36 & 301 & 02 & 55.1 & 56.2 & 27 & 8 & 02 & 70.3 & 70.8 & & 50 & \\
\hline 04 & 40.0 & 39.5 & 30 & $\mathrm{M}$ & 04 & $36 . I$ & 35.0 & 36 & 10 & 04 & 55.1 & 56.9 & 27 & $y d$ & 04 & 69.8 & 70.3 & & 49 & \\
\hline 05 & 40.2 & $4 \mathrm{I} . \mathrm{I}$ & 28 & 69 & 06 & 35.6 & 34.3 & 37 & 90 & 06 & 56.6 & 57.8 & 29 & gin & 06 & 65.2 & 67.0 & & 44 & \\
\hline 08 & 39.9 & 39.1 & 30 & 35 & 08 & 36.6 & $35 \cdot 3$ & 36 & 38 & 08 & 56.1 & 57.4 & 29 & Ilo: & 08 & 63.2 & 64.0 & & 39 & \\
\hline 10 & 41.2 & 40.0 & 28 & ar & 10 & 37.0 & 36.0 & 35 & dit & 10 & 56.1 & 57.2 & 28 & 03 & IO & 62.9 & 63.2 & & 38 & \\
\hline I2 & 45.0 & 44.3 & 22 & ix & 12 & 36.8 & 35.9 & 35 & 배 & 12 & 56.2 & 57.0 & 28 & 21 & 12 & 66.7 & 66.9 & & 45 & \\
\hline 14 & 45.3 & 44.9 & $2 I$ & -1.0 & 14 & $38 . \mathrm{I}$ & 36.9 & 33 & $-I . I$ & I4 & 56.0 & 56.8 & 28 & +0.9 & I4 & 64.0 & 64.1 & & 40 & +2.1 \\
\hline 16 & 49.3 & 48.0 & 16 & & 16 & 40.3 & 39.9 & 29 & $14^{2}$ & 16 & 55.5 & 56.2 & 27 & & 16 & 65.0 & 65.6 & & 42 & \\
\hline 18 & 51.0 & 50.8 & 22 I2 & Fit & I8 & 40.2 & 36.0 & 32 & Bi & 18 & 56.1 & 57.0 & 28 & 31 & I8 & 64.2 & 65.2 & & $4 I$ & \\
\hline 20 & 61. . & 59.0 & 2158 & er & 20 & 43.0 & 41.9 & 26 & 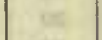 & 20 & 57.1 & 58.1 & 30 & 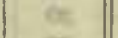 & 20 & 68.8 & 69.1 & & 48 & \\
\hline 22 & 58.3 & 56.9 & 2202 & H & 22 & 45.0 & 43.9 & 22 & $=$ & 22 & 57.1 & 58.1 & 30 & es & 22 & 69.0 & 70.1 & & 49 & \\
\hline 24 & 54.5 & 53.5 & o7 & is & 24 & 46.1 & 45.0 & 21 & H & 24 & 57.8 & 58.2 & $3 \mathrm{I}$ & $\mu$ & 24 & 66.2 & 66.9 & & 44 & \\
\hline 25 & 52.6 & $5 \mathrm{I} . \mathrm{I}$ & II & is & 26 & 45.5 & 44.7 & 21 & 6 & 26 & 57.8 & 58.9 & $3 I$ & 10 & 26 & 63.6 & $64 \cdot 3$ & & 40 & \\
\hline 28 & 52.5 & 51.6 & 10 & tax & 28 & $43 . I$ & 41.2 & 26 & $2 x^{2}$ & 28 & 57.9 & 58.8 & 31 & . & 28 & 63.1 & 63.9 & & 39 & \\
\hline 30 & 52.1 & 50.9 & II & -1.0 & 30 & 42.0 & 40.6 & 27 & -1.2 & 30 & 57.2 & 58.3 & 30 & +1.0 & 30 & 63.1 & 63.2 & & 39 & \\
\hline 32 & 50.6 & 50.1 & I3 & 4 & 32 & 40.8 & $39 \cdot 3$ & 29 & 6 & 32 & 56.0 & 57.1 & 28 & & 32 & $64 \cdot 3$ & 65.0 & & $4 I$ & \\
\hline 34 & 49.1 & 48.3 & I6 & at & 34 & 39.9 & 38.8 & 30 & 18: & 34 & 55.1 & 56.1 & 27 & is & 34 & 65.9 & 66.5 & & 43 & \\
\hline 36 & 50.3 & 49.2 & I4 & x & 36 & 39.8 & 38.8 & 30 & (5) & 36 & $54 . I$ & 55.1 & 25 & 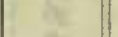 & 36 & 68.7 & 69.7 & & 48 & \\
\hline 38 & 51.8 & 50.3 & 12 & E & $3^{s}$ & 40.3 & 39.2 & 30 & 62 & 38 & 53.2 & 55.2 & 25 & 6 & $3^{8}$ & 71.2 & 72.2 & & 52 & \\
\hline 40 & 50.9 & 50.3 & I3 & 24 & 40 & 41.2 & 40.2 & 28 & of & 40 & 52.7 & $53 \cdot 3$ & 23 & of: & 40 & 72.2 & 72.9 & & 53 & \\
\hline 42 & 51.5 & 51.0 & I2 & & 42 & 42.0 & 40.6 & 27 & & 42 & 51.9 & 52.1 & $2 I$ & 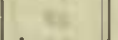 & 42 & $73 \cdot 3$ & 74.2 & & 55 & \\
\hline 44 & 50.4 & 50.2 & I3 & $-\mathrm{I} .0$ & 44 & 46.9 & 45.2 & 20 & -1.2 & 44 & 53.2 & 54.0 & 24 & +1.2 & 44 & 72.5 & 73.5 & & 54 & +2.8 \\
\hline 46 & 50.1 & 49.9 & 14 & pas & 46 & 41.3 & 39.5 & 29 & 182 & 46 & 53.2 & 53.9 & 24 & & 46 & 72.8 & 73.9 & & 55 & \\
\hline 48 & 50.8 & 50.0 & I3 & Bh & 48 & 42.1 & 40.0 & 28 & 84 & 48 & 53.1 & 53.9 & 24 & 4 & 48 & 73.9 & 75.8 & 22 & 57 & \\
\hline 50 & $5 \mathrm{I} \cdot 3$ & 51.1 & 12 & $\approx$ & 50 & 42.7 & 40. I & 27 & 2 & 50 & 52.1 & 52.5 & 22 & os & 50 & 77.1 & 78.1 & 23 & or & \\
\hline 52 & 53.1 & 52.9 & $\infty$ & $\mathrm{s}$ & 52 & 41.0 & 39.2 & 29 & $\theta$ & 52 & 50.3 & 51.0 & 19 & $=5$ & $52^{*}$ & 53.5 & 57.8 & & $\infty$ & \\
\hline 54 & 52.6 & 51.8 & 10 & 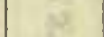 & 54 & 39. I & 37.6 & 32 & P & 54 & $49 \cdot 3$ & 49.8 & 17 & $k$ & 54 & 54.9 & 58.7 & & 02 & \\
\hline 56 & 53.2 & 52.8 & 09 & he & 56 & 37.8 & 36.1 & 34 & 18 & 56 & 48.0 & 49.2 & 16 & 6 & 56 & 54.8 & 57.7 & 23 & or & \\
\hline $5^{8}$ & 52.1 & 51.8 & II & 82 & 58 & 36.7 & 35.5 & 35 & 8 & 58 & 51.0 & 52.0 & 20 & 65 & 58 & 51.9 & 55.0 & 22 & 57 & \\
\hline 6,9 & $x^{-2}$ & & $9=0$ & $9 s$ & 2400 & 35.0 & 34.0 & 38 & -1.2 & & & & & 564 & & & & & & \\
\hline
\end{tabular}

Correction to local mean time is - 55s. $90^{\circ}$ torsion $=17:^{\prime} 30$.

Torsion head at 1 gh $36 \mathrm{~m}$ read $4^{\circ}$ and at $24 \mathrm{~h} 18 \mathrm{~m}$ read $55^{\circ}$.

Observer-J. V.

Observer-J. V. 
Tabulation of magnetic declinations observed at T'eplitz Bay-Continued

\begin{tabular}{|c|c|c|c|c|c|c|c|c|c|c|c|c|c|c|c|c|c|c|c|}
\hline \multicolumn{6}{|c|}{ Sunday, June 19, 1904} & \multicolumn{4}{|c|}{ Magnet scale inverted } & \multicolumn{6}{|c|}{ Monday, June 20, I901 } & \multicolumn{4}{|c|}{ Magnet scale erect } \\
\hline \multirow{2}{*}{$\begin{array}{l}\text { Chr'r } \\
\text { time }\end{array}$} & \multicolumn{2}{|c|}{$\begin{array}{l}\text { Scale } \\
\text { readings }\end{array}$} & \multirow{2}{*}{$\begin{array}{c}\text { East } \\
\text { decli- } \\
\text { nation }\end{array}$} & & Chr'r & $\begin{array}{l}\text { Sca } \\
\text { readi }\end{array}$ & $\begin{array}{l}\text { le } \\
\text { ings }\end{array}$ & $\begin{array}{l}\text { East } \\
\text { decli- }\end{array}$ & & Chr'r & $\begin{array}{l}\mathrm{Sc} \\
\text { read }\end{array}$ & $\begin{array}{l}\text { le } \\
\text { ings }\end{array}$ & $\begin{array}{l}\text { East } \\
\text { decli- }\end{array}$ & & Chr'r & $\begin{array}{l}\text { Sce } \\
\text { read }\end{array}$ & $\begin{array}{l}\text { le } \\
\text { igs }\end{array}$ & $\begin{array}{l}\text { East } \\
\text { decli- }\end{array}$ & \\
\hline & Left & Right & & & & Left & Right & 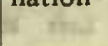 & & & Left & Right & 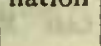 & & & L,eft & Right & 110ivin & \\
\hline li $\mathrm{m}$ & d & d & $\cdot$, & 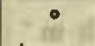 & $\mathrm{h} \mathrm{m}$ & d & d & $\cdot$ ' & $\bullet$ & h $\mathrm{m}$ & & d & $\cdot 1$ & - & h m & d & d & $\cdot$ ' & ${ }^{\circ}$ \\
\hline 400 & 54.0 & 50.0 & 2259 & +3.4 & 600 & 33.0 & 30. I & 2331 & +3.9 & 800 & L & st & & & I0 $\infty$ & 43.9 & 46.8 & 2253 & +11.8 \\
\hline 02 & 54.8 & 50.7 & 58 & - & 02 & 30.9 & 28.8 & 34 & - & 02 & 48.1 & 50.9 & 2260 & +8.1 & 02 & $43 \cdot 5$ & 46.8 & 53 & are \\
\hline 04 & 54.1 & 50.9 & 58 & & 04 & $35 . \mathrm{I}$ & 33.0 & 27 & & 04 & 42.3 & 46.8 & & 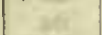 & 04 & 43.6 & 46.9 & 53 & \\
\hline 06 & 53.9 & $5 \mathrm{I} .0$ & 2259 & 6 & 06 & 37.2 & 35.8 & 23 & ag & 06 & 43.2 & 47.2 & 53 & 86 & $\infty 6$ & 43.0 & 46. I & 52 & as \\
\hline 08 & 51.0 & 49.0 & 2302 & $7^{2}$ & 08 & 36.0 & 35.0 & 25 & $5 a$ & 08 & 40.2 & 43.9 & 48 & ma & 08 & $43 . I$ & 46.0 & 2252 & \\
\hline 10 & 50.0 & 48.0 & 04 & 28 & Io & 33.9 & 33.1 & 28 & है। & 1o & 39.3 & 42.7 & 46 & vit & I0 & 48.8 & 50.9 & 2300 & Ex \\
\hline 12 & 49.9 & 47.5 & 04 & & I2 & 30.8 & 29.9 & 33 & & 12 & 37.6 & 41.8 & 44 & & I2 & 46.9 & 48.9 & 2257 & \\
\hline I4 & 49.9 & 47.7 & 04 & +3.2 & If & 32. & $9 b$ & 29 & +3.2 & I4 & 38.1 & 41.4 & 44 & +8.2 & If & 47.2 & 48.4 & 57 & $+12 . I$ \\
\hline 16 & 48.9 & 46.7 & 06 & & I6 & 30.0 & 28.0 & 35 & & I6 & 38.1 & $4 \mathrm{I} . \mathrm{I}$ & 44 & & I6 & 47.3 & 48.9 & 57 & \\
\hline 18 & 49.0 & 47.0 & 05 & 91 & I8 & 31.9 & 31.8 & $3 \mathrm{I}$ & $8:$ & 18 & 38.8 & 41.9 & 45 & & I8 & 46.8 & 47. & 56 & 81 \\
\hline 20 & 47.9 & 46.0 & 07 & 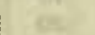 & 20 & 33.2 & 31.8 & 30 & $x=$ & 20 & 37.9 & 40.2 & 4.3 & $o$ & 20 & 47.9 & 48.5 & 57 & 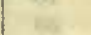 \\
\hline 23 & 47.9 & 46.8 & 06 & iff & 22 & 32.9 & $3 \mathrm{I} \cdot 3$ & 30 & xz & 22 & 37.8 & 40.7 & 4.3 & & 22 & 45.6 & 46.0 & 54 & a \\
\hline 24 & 47.2 & 45.9 & 08 & & 24 & 39.0 & 37.8 & 20 & in. & 24 & 38.8 & 41.9 & 45 & & 24 & 44.2 & 44.9 & 52 & $x^{4}$ \\
\hline 26 & 46.9 & 45.2 & 09 & & 26 & 35.9 & 34.0 & 26 & 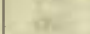 & 26 & 38.0 & 41.0 & 44 & & 26 & 45.7 & 46 & 54 & \\
\hline 28 & $43 \cdot 3$ & $4 \mathrm{I}=5$ & 14 & & 28 & 38.4 & 36.5 & 22 & & 28 & 38.1 & 40.9 & $4 t$ & & 28 & 45.9 & 46.1 & 54 & - \\
\hline 30 & 37.9 & 36.8 & 22 & +3.4 & 30 & 40.8 & 38.3 & I9 & +3.0 & 30 & $3^{8} .9$ & $4 \mathrm{I} \cdot \mathrm{C}$ & 14 & +8.2 & 30 & 44.0 & $45 . I$ & 52 & - \\
\hline 32 & 37.9 & 36.1 & 23 & 8 & 32 & $4 \mathrm{I} .0$ & 39.9 & 17 & y & 32 & 39.1 & 41.2 & 45 & $x^{2}=0$ & 32 & 43. I & 44 & 50 & 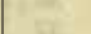 \\
\hline 34 & 40.0 & 38.2 & I9 & & 34 & 38. I & 36.9 & 22 & $y$ & 34 & 40.2 & $4 \% .7$ & 47 & & 34 & $4 \mathrm{I} .4$ & 42.1 & 47 & \\
\hline 36 & 42.0 & 40.4 & 16 & $\operatorname{ke}$ & 36 & 36.7 & $36 . I$ & 24 & $x^{2}$ & 36 & 39.5 & 41.8 & 46 & & 3 & 42.7 & 43 & 50 & \\
\hline 38 & 40.9 & 39.7 & I7 & & 38 & $37 . \mathrm{I}$ & 37.0 & 23 & this & 38 & 41.2 & $43 . I$ & 48 & & 38 & 43.0 & & 50 & \\
\hline 40 & $43 \cdot 3$ & $4 \mathrm{I} \cdot 9$ & 14 & 0 & 40 & $4 \mathrm{r} \cdot 9$ & $4 \mathrm{I} .0$ & I6 & as & 40 & 42.3 & 44.0 & 50 & & 40 & 45.0 & 46.0 & 53 & \\
\hline 42 & 49.0 & $45 \cdot 3$ & 07 & & 42 & 42.8 & $42 . I$ & I4 & & 42 & 42.5 & 43.9 & 50 & & 42 & 46.1 & 47.4 & 55 & +12.7 \\
\hline 44 & 45.0 & 43.9 & I I & +3.8 & 44 & 42. I & 41.0 & 16 & +2.9 & 44 & 43.2 & 44.8 & $5 \mathrm{I}$ & +9.0 & 44 & 46.3 & & 55 & \\
\hline 46 & 45.9 & 42.6 & I I & & 46 & 39.2 & 39.0 & 19 & & 46 & 43.7 & 45.0 & 51 & & 4 & 46.3 & $4^{6 .}$ & 55 & +12.6 \\
\hline 48 & $39 . \mathrm{I}$ & 38.1 & 20 & 25 & $4^{8}$ & 43.5 & 42.2 & 14 & 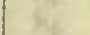 & 48 & 44.0 & 44.9 & $5 \mathrm{I}$ & $\cos$ & 48 & 47.0 & 47.9 & 56 & 72 \\
\hline 50 & $39 \cdot 3$ & 37.7 & 20 & ent & 50 & $44 . \overline{8}$ & 43.8 & II & at & 50 & 48.7 & 49.1 & 59 & 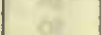 & 50 & 46.1 & 47. & 55 & cos \\
\hline 52 & 36.9 & $35 . \mathrm{I}$ & 24 & et & 52 & 44.9 & 44.0 & II & 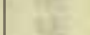 & 52 & $47 \cdot 3$ & 48.2 & 57 & 0 & 52 & 46.1 & & 55 & 2 \\
\hline 54 & 38. I & $37 . I$ & 22 & is & 54 & 42.9 & 42.0 & 14 & 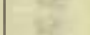 & 54 & 48.9 & 50.0 & 59 & & 54 & 44.7 & 45 & 53 & Ext \\
\hline 56 & 38.8 & 37.9 & $2 \mathrm{I}$ & & 56 & +4.1 & 44.0 & 12 & & 56 & 48.7 & 49.4 & 59 & & & 44.6 & & 53 & E \\
\hline 58 & $4 \mathrm{r} \cdot 9$ & 40.1 & 15 & & 58 & 40.9 & 38.9 & 18 & & 58 & 47.4 & 48.3 & 57 & & 58 & 47.1 & 48.0 & 56 & 5 \\
\hline 500.5 & 40.8 & 39.0 & I8 & $+4 . I$ & 700 & 71.9 & $40 . \overline{6}$ & 16 & +2.7 & 900 & $47 . I$ & 47.8 & 56 & +9.8 & II & 46.8 & 47.2 & 56 & \\
\hline 02 & 4I. I & 39.9 & 17 & . & 02 & $43 . \overline{8}$ & 42.1 & 13 & (1) & 02 & $47 \cdot 3$ & 49.8 & 58 & & 02 & 45.8 & 47. & 55 & + II. \\
\hline 04 & 38.0 & 36.3 & 23 & ia & 04 & 43.0 & 42.0 & I4 & se & 04 & 48.8 & 50.1 & 59 & & 04 & $4 \mathrm{I} .4$ & 43. & 48 & 20 \\
\hline 06 & 36.0 & 35.0 & 25 & 5 & 06 & 39.9 & $39 . I$ & 19 & 81 & 05 & 45.8 & 46.9 & 55 & & 06 & 44.9 & 46 & 53 & \\
\hline 08 & 39.0 & 38.3 & 20 & & 08 & 40.9 & 40.0 & 17 & 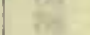 & 08 & 45.0 & 45.8 & 2253 & & 08 & 46.0 & 48. I & 56 & 8 \\
\hline 10 & 25.0 & 24.9 & 42 & e & I0 & 39. I & 39.0 & 20 & & 10 & 50.1 & 50.9 & 23 or & & I0 & 44.8 & 46.9 & 54 & \\
\hline I2 & 23.7 & 22.0 & 45 & & 12 & 40.0 & 38.2 & 19 & & I 2 & 46.3 & 47.0 & 2255 & & 12 & 45.0 & 46.3 & 53 & \\
\hline I4 & 24.0 & 23.2 & 44 & $+4 \cdot 3$ & I4 & 37.7 & 36.9 & 22 & +2.5 & I4 & 44.9 & 46.4 & 53 & +10.1 & 14 & 42.1 & & 49 & + II.0 \\
\hline I6 & 25.5 & $25 . \mathrm{I}$ & $4 \mathrm{I}$ & . & 16 & 43. & $9 a$ & 12 & . & 16 & 46.0 & $47 . I$ & 55 & . & I6 & $4 \mathrm{I} .7$ & 43.8 & 49 & I \\
\hline 18 & 33.0 & 32.8 & 29 & 3. & I8 & 46. I & 44.2 & 10 & he & I8 & 43.0 & 43.9 & 50 & ist & เ8 & 43.0 & 44.9 & $5 I$ & Fet \\
\hline 20 & 39.9 & 38.0 & 20 & $x^{2}$ & 20 & 38.2 & 37.8 & $2 I$ & ist & 20 & 44.0 & 45.0 & 52 & $x$ & 20 & 46.1 & 47 & 56 & at \\
\hline 22 & & $.0 a$ & Io & & 22 & $46 . \mathrm{I}$ & $45 . \mathrm{I}$ & 09 & & 22 & 45.1 & 45. & 53 & & 22 & 46.8 & & 56 & be \\
\hline 24 & 51.0 & 50.0 & OI & 18 & 24 & 40.9 & 40.1 & 17 & , & 24 & 41.9 & $43 . I$ & 49 & & 24.5 & 44.1 & 45.8 & 52 & \\
\hline 26 & 80.9 & 49.9 & 02 & & 26 & $5 \mathrm{I}$. & $0 a$ & or & & 26 & 44.2 & 45.0 & & & 26.3 & 42.0 & 42.8 & 48 & \\
\hline 28 & $4 \mathrm{I}$ & $.0 b$ & 16 & & 28 & 48. & $a b$ & 05 & & 28 & 42.0 & 43.8 & 19 & & 28 & 44.2 & 44.9 & 52 & +10.1 \\
\hline 30 & 36 & $2 b$ & 24 & & 30 & 37.3 & 36.9 & 23 & +2.6 & 30 & 42.0 & 43.9 & 49 & +10.7 & 30 & 44.7 & 45.9 & 53 & ( \\
\hline 32 & $3 \mathrm{I} .2$ & 31.1 & 32 & +4.2 & 32 & 43.0 & 41.0 & 15 & 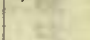 & 32 & $42 . I$ & 44.1 & 50 & & 32 & 39.0 & & 44 & 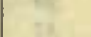 \\
\hline 34 & 29.9 & 28.8 & 35 & & 34 & 40.8 & 40.0 & 18 & the & 34 & 46.9 & & 56 & 4 & 34 & $37 \cdot 3$ & & $4 \mathrm{I}$ & 70 \\
\hline 36 & 28. I & 27.0 & 38 & & 36 & 44.0 & 42.0 & 13 & 18 & 36 & 45.8 & 48.0 & 55 & & 36 & 38.1 & & 42 & \\
\hline 38 & 27.3 & 26. I & 39 & & 38 & 44.8 & 43.9 & II & & 38 & 43.2 & 45.5 & $5 I$ & 8 & 38 & 40.8 & 44.8 & 49 & s. \\
\hline 40 & 27.0 & 25.0 & 40 & & 40 & 46.0 & 45.0 & $\infty$ & & 40 & 45.9 & 48.6 & $5^{5}$ & & 40 & 38.1 & & 42 & \\
\hline 42 & 29.0 & 26. & 37 & & 42 & 46.1 & 44.3 & Io & & 42 & 47.0 & 48.9 & 57 & & 42 & 37.5 & 38.8 & 42 & \\
\hline 44 & $23 . \mathrm{I}$ & 21.0 & 46 & +4.1 & 44 & 45.0 & 44.0 & I I & +2.7 & 44 & 40.5 & 42.0 & 46 & +II.o & 44 & 35.0 & 36.2 & 38 & +0.5 \\
\hline 46 & I9.8 & I6.6 & 52 & $\sqrt{3}$ & 46 & 45.2 & 44.2 & II & & 46 & 41.2 & 43.2 & 48 & 8 & 46 & 35.2 & 36.2 & 38 & \\
\hline 48 & I7.8 & 14.2 & 56 & 8 & 48 & 43.8 & $42 . I$ & 13 & $c_{1}$ & 48 & 42.0 & 44.1 & 49 & 80 & 48 & 36.2 & 37.0 & 39 & \\
\hline 50 & 22.8 & 20.2 & 47 & as & 50 & 46.0 & 44.8 & 10 & Ex & 50 & 38.6 & 4I. 4 & 45 & 8 & 50 & $35 . \mathrm{I}$ & 35 . & 37 & \\
\hline 52 & 20.3 & 18.9 & 50 & as & 52 & 48.9 & 48.0 & 05 & 11 & 52 & 39.1 & 41.9 & 45 & & 52 & 34.0 & & 35 & \\
\hline 54 & 24.2 & 21.5 & 45 & M & 54 & 50.1 & 45.9 & 05 & 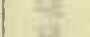 & 54 & 42.2 & 45.4 & $5 \mathrm{l}$ & te & 5 & 36.5 & & 39 & \\
\hline 56 & 25.1 & 22.4 & 44 & & 50 & 42. I & $4 \mathrm{I} \cdot \mathrm{I}$ & 16 & & 56 & 43.9 & 45. & 52 & 34 & 56 & 35.9 & 36.8 & 39 & \\
\hline 58 & 30.0 & 28.8 & 35 & 58 & 58 & 47.8 & 46.0 & 07 & & 58 & 43.7 & 45.2 & $5 \mathrm{I}$ & & 58 & 35.1 & 36. I & 38 & \\
\hline & & & & & 800 & 51.2 & 49.9 & OI & +2.9 & $0 x-4$ & 2) & - & 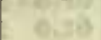 & & 1200 & 36.5 & 37.5 & 40 & +9.0 \\
\hline
\end{tabular}

Correction to local mean time is $-26 \mathrm{~s} .90^{\circ}$ torsion $=18 .^{\prime} 46$. Torsion head at oh oom read $57^{\circ}$ and at 81 i 7 in read $46^{\circ}$. Observer-J. V.
Correction to local mean tinje is $+6 \mathrm{~s}$.

Torsion head at $7 \mathrm{~h} 45 \mathrm{~m}$ read $50^{\circ}$ and at $12 \mathrm{~h}$ 30m read the same. Observer-J. V. 
Tabulation of magnetic declinations observed at Teplitz Bay-Continued

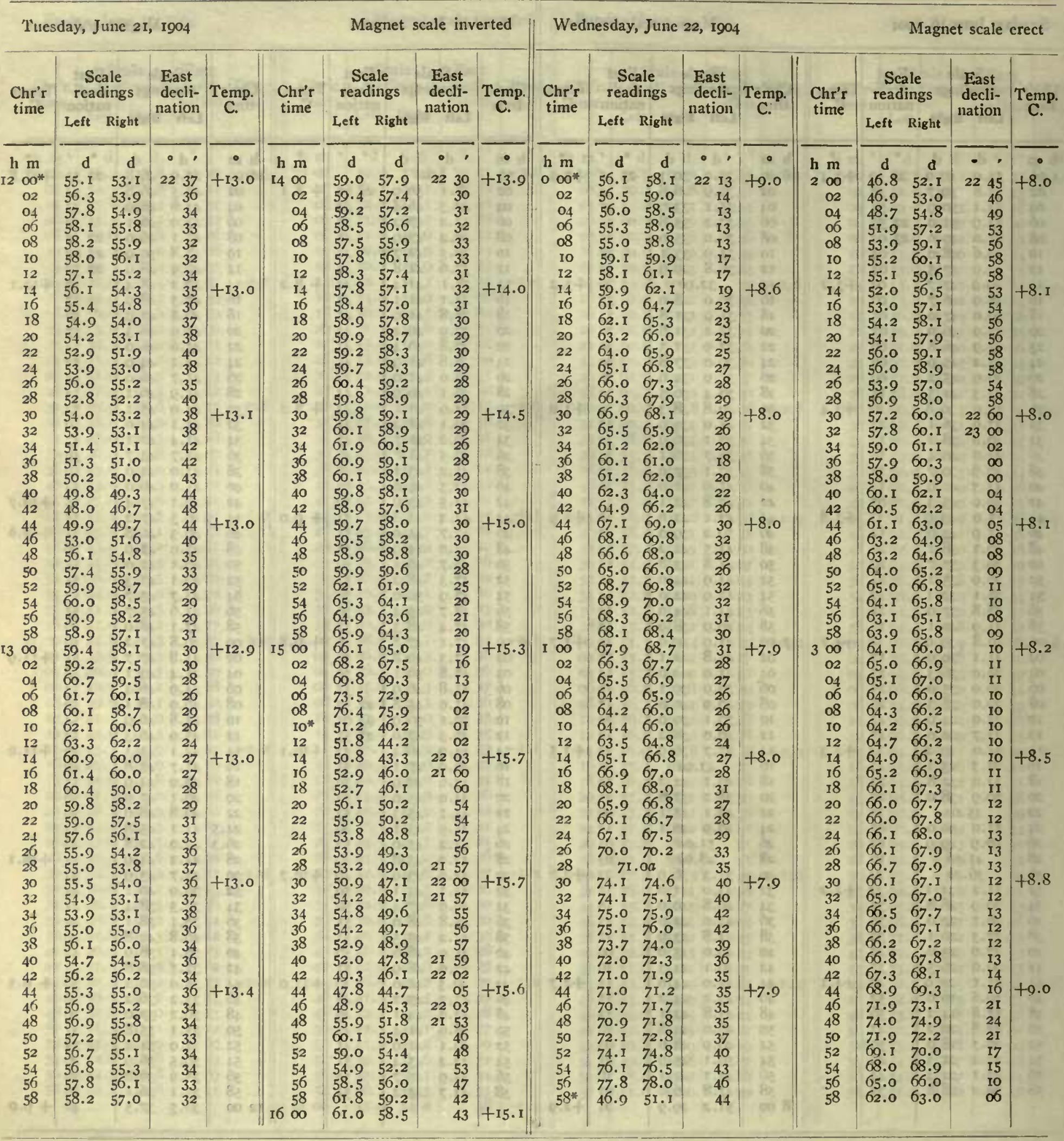

Correction to local mean time is $-16 \mathrm{~s} .90^{\circ}$ torsion $=15^{\prime}{ }^{\prime} 48$.

Observer-J. V.

Torsion hcad at I Ih $30 \mathrm{~m}$ read $53^{\circ}$ and at $16 \mathrm{~h} 25 \mathrm{~m}$ read $46^{\circ}$.

Observer-J. V. 
Tabulation of magnetic declinations observed at Teplitz Bay-Continued

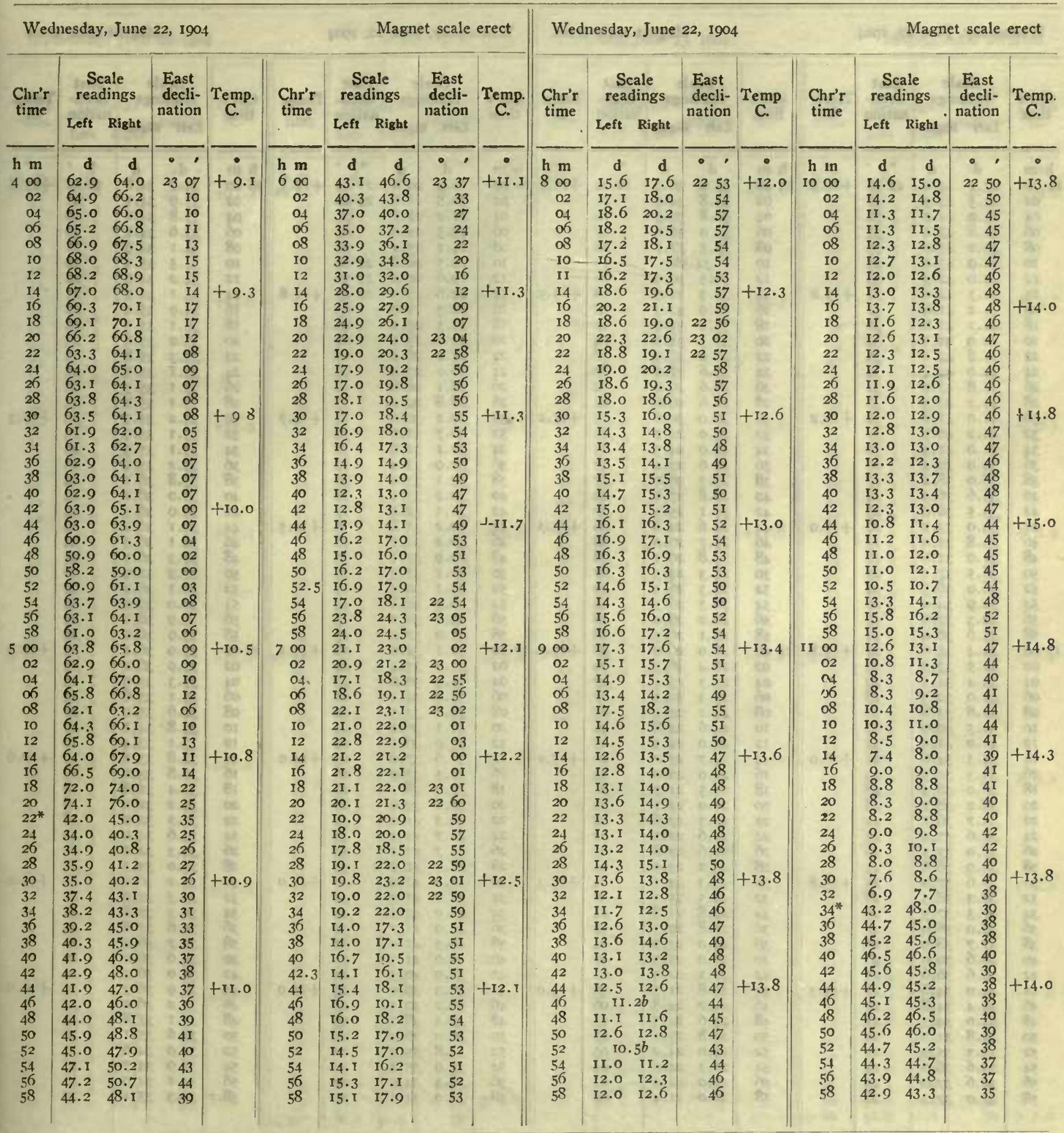

Observers-J. V. and W. J. P., who alternated from 7 h $52 \mathrm{~m}$ to Observer-W. J. P. $8 \mathrm{~h} 02 \mathrm{~m}$. 
Tabulation of magnetic declinations observed at Teplitz Bay-Continued

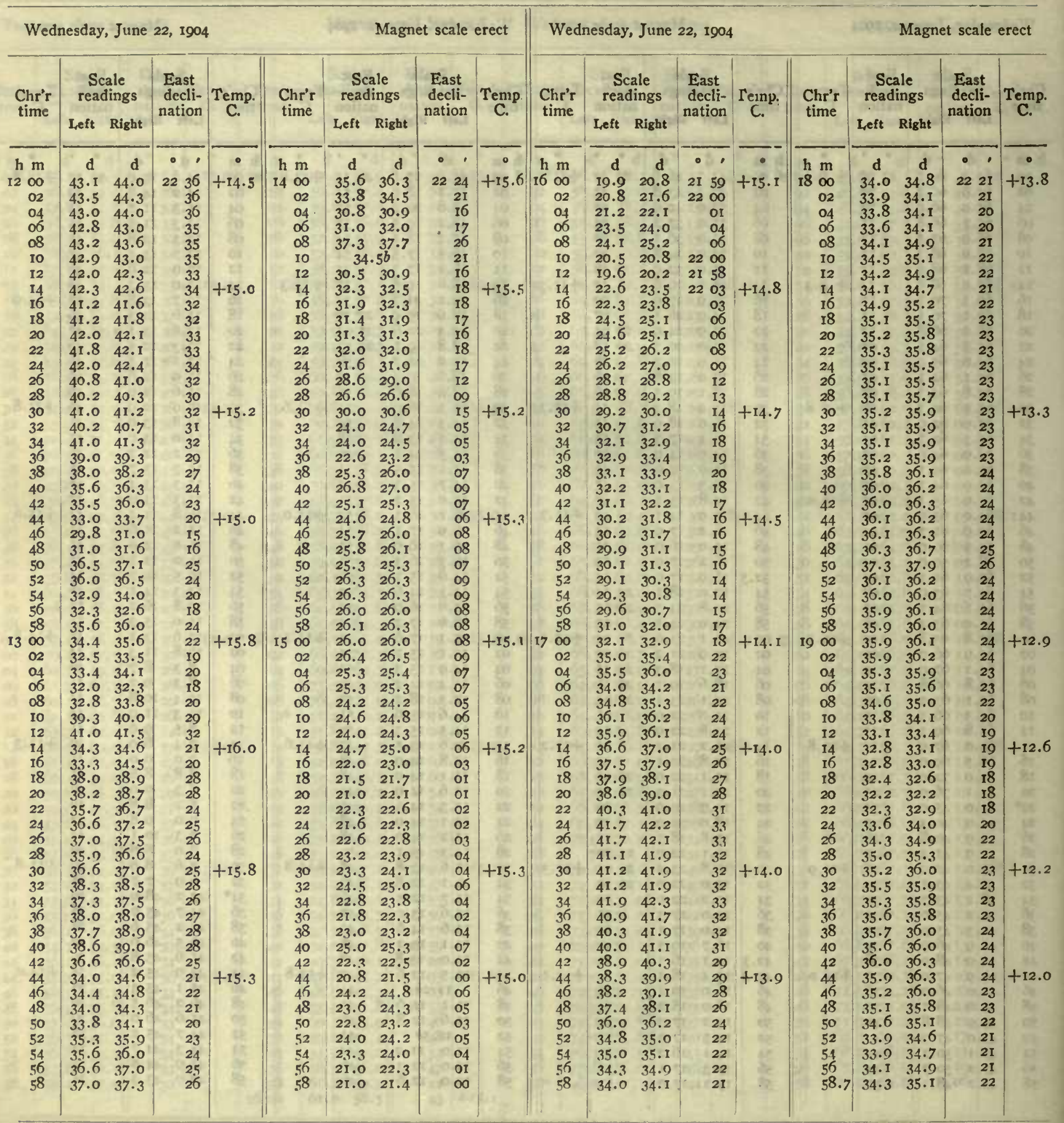


Tabulation of magnetic declinations observed at Teplitz Bay-Continued

\begin{tabular}{|c|c|c|c|c|c|c|c|c|c|c|c|c|c|c|c|c|c|c|c|}
\hline \multicolumn{5}{|c|}{ Wednesday, June 22, 1904} & \multicolumn{5}{|c|}{ Magnet scale erect } & \multicolumn{6}{|c|}{ Thursday, June 23, 1904} & \multicolumn{4}{|c|}{ Magnet scale inverted } \\
\hline \multirow[t]{2}{*}{$\begin{array}{l}\text { Chr'r } \\
\text { time }\end{array}$} & \multicolumn{2}{|c|}{$\begin{array}{l}\text { Scale } \\
\text { readings }\end{array}$} & \multirow[t]{2}{*}{$\begin{array}{c}\text { East } \\
\text { decli- } \\
\text { nation }\end{array}$} & Temp. & Chr'r & $\begin{array}{l}\text { Sci } \\
\text { read }\end{array}$ & lings & $\begin{array}{l}\text { East } \\
\text { decli- }\end{array}$ & Temp. & Chr'r & $\begin{array}{r}\mathrm{Sc} \\
\text { read }\end{array}$ & $\begin{array}{l}\text { ale } \\
\text { dings }\end{array}$ & $\begin{array}{l}\text { East } \\
\text { decli- }\end{array}$ & Temp & Chr'r & $\begin{array}{l}\text { Sca } \\
\text { read }\end{array}$ & lings & East & Temp. \\
\hline & L,eft & Right & & & & Left & Right & & & & Left & Right & & & & Left & Right & & \\
\hline $\mathrm{h} \mathrm{m}$ & $d$ & d & - & 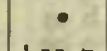 & h m & d & d & $\therefore$, & $\bullet$ & h m & $d$ & d & & & $\mathrm{h} \mathrm{m}$ & d & d & $\cdot$, & $\bullet$ \\
\hline 2000 & 35.0 & 35.6 & 2223 & +11.7 & 2200 & 36.0 & 36.7 & 2224 & +10.4 & 1600 & 48.9 & 44.2 & 2136 & +13.6 & I 800 & 42.0 & 41.0 & 2145 & tro. I \\
\hline 02 & 35.2 & 36.0 & 23 & & 02 & 36.0 & 36.0 & 24 & & 02 & 47.8 & 43.2 & & & 02 & 43.7 & 42.1 & & \\
\hline 04 & 35.1 & 35.8 & 23 & the & 04 & 35.7 & 35.7 & 23 & & 04 & 46.9 & $43 \cdot 3$ & 38 & 10 & 04 & $43 \cdot 3$ & 42.2 & 43 & 78 \\
\hline 06 & 34.7 & $35 \cdot 3$ & 22 & 8 & 06 & 35.0 & 35.1 & 22 & & $\infty 6$ & 46.0 & 42.9 & 39 & 3 & of & 42.9 & 41.9 & & $2 n$ \\
\hline 08 & $35 . \mathrm{I}$ & 35.9 & 23 & 18 & 08 & $34 \cdot 3$ & 34.9 & 22 & & 08 & -45.9 & 42.5 & 39 & 20 & 08 & 41.8 & 40.5 & 45 & ho \\
\hline I0 & 35.2 & 36.0 & 23 & tat & Io & 34.1 & 34.7 & 21 & & ro & $44 \cdot 3$ & 41.9 & 41 & 54 & I0 & 41.9 & 40.2 & & 6 \\
\hline 12 & 35.1 & 35.8 & 23 & & 12 & 34.0 & 34.4 & 2I & & 12 & $44 . I$ & 41.7 & $4 \mathrm{I}$ & Wr & I2 & 41.9 & 40.2 & & at \\
\hline 14 & 35.1 & 35.9 & 23 & tII.6 & I4 & 33.2 & 34.0 & 20 & +10.5 & 14 & $44 . I$ & 41.0 & 42 & +12.7 & 14 & $40 . \mathrm{I}$ & 39.1 & & +9.7 \\
\hline 16 & $35 \cdot 3$ & 35.9 & 23 & & 16 & 33.2 & 34.3 & 20 & & 16 & $43 . I$ & 40.7 & 43 & & 16 & 39.1 & 38.1 & 50 & \\
\hline 18 & 35.5 & 35.9 & 23 & 25 & 18 & 33.8 & 34.4 & $2 \mathrm{I}$ & tik & 18 & 42.1 & $4 \mathrm{I}$ & 44 & 2 & I8 & 38.5 & 37.7 & 50 & सी \\
\hline 20 & 35.0 & 35.2 & 22 & 15 & 20 & 33.7 & 34.9 & 21 & (16 & 20 & 42.8 & 41.8 & 42 & os & 20 & 38.9 & 38.0 & 50 & ns \\
\hline 22 & 35.1 & $35 . I$ & 22 & tis & 22 & 34.0 & 34.9 & 21 & 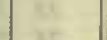 & 22 & 43.1 & 42 & 42 & sit & 22 & 39.1 & 38.9 & $\begin{array}{l}19 \\
\end{array}$ & 36 \\
\hline 24 & 35.2 & 35.7 & 3 & E & 24 & 35.0 & 35.5 & 22 & 85 & 24 & 42.0 & 41. & 43 & Is & 2 & & 39.0 & 49 & is \\
\hline 26 & 35.4 & 35.9 & 23 & 8 & 26 & 35.3 & 36.1 & 23 & & 26 & 4I.I & 4I.I & 44 & 8 & 26 & 39.8 & 38.9 & 49 & 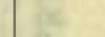 \\
\hline 28 & $35 \cdot 3$ & 35.9 & 23 & 1 & 28 & 35.0 & 35.8 & 23 & & 28 & $4 \mathrm{I} . \mathrm{I}$ & 41.1 & 44 & & 28 & 39.0 & 38.0 & 50 & 6he \\
\hline 30 & 35.1 & 35.6 & 23 & +11.3 & 30 & 34.9 & 35.3 & 22 & +10.I & 30 & 42.0 & 41.9 & 43 & $+12 . I$ & 30 & 39.9 & 39.0 & 48 & +9.1 \\
\hline 32 & 35.2 & 36.2 & 23 & 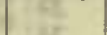 & 32 & 33.1 & $34 . I$ & 20 & & 32 & 42.8 & 42. & 42 & $b^{\circ}$ & 32 & 40.5 & 40.1 & 47 & 10 \\
\hline 34 & 36.0 & 36.9 & 24 & 25 & 34 & 32.1 & 33.2 & 18 & & 34 & 41.9 & 41.2 & 44 & +2 & 34 & $4 \mathrm{I} \cdot 3$ & 40.9 & 46 & k \\
\hline 36 & 36.1 & 37.0 & 2 & 28 & 36 & 32.2 & 33.7 & 19 & & 36 & $4 \mathrm{I} .2$ & $4 \mathrm{I}$. & 44 & $x$ & 36 & $43 . I$ & 42.8 & 43 & E. \\
\hline 38 & 35.5 & 36.3 & 24 & Be & 38 & 32.7 & 33.9 & 20 & & 38 & $4 \mathrm{I} . \mathrm{I}$ & 40.9 & 45 & th & 38 & 44.2 & 43.9 & $4 \mathrm{I}$ & 186 \\
\hline 40 & 35.6 & 36.2 & 24 & 14 & 40 & 33.0 & 34.2 & 20 & & 40 & 4I.I & 40. & 45 & ete & $4 C$ & 43.2 & 43.0 & 43 & $\mathrm{~ns}$ \\
\hline 42 & 35.0 & 35.8 & 23 & & 42 & 33.0 & 34.2 & 20 & & 42 & 41.9 & 4I.I & 44 & & 42 & 42.9 & 42.2 & 44 & \\
\hline 44 & 34.2 & 35.1 & 22 & +II.I & 44 & 32.9 & $34 . I$ & 20 & +10.0 & 44 & 41.2 & 42.3 & 44 & +11.6 & 44 & 42.0 & 41.1 & & +8.9 \\
\hline 46 & 33.1 & 34.0 & 20 & $x^{2}$ & 46 & 32.2 & $34 \cdot 3$ & I9 & & 46 & 42.9 & 41.7 & 43 & & 46 & 42.9 & $4^{\mathrm{T}} \cdot 9$ & 44 & 10 \\
\hline 48 & 32.1 & 32.9 & I & 30 & 48 & 32.1 & 33.0 & I8 & ist & 48 & 42.9 & $4 \mathrm{I}$. & 43 & 84 & 48 & 44.8 & 43.9 & 40 & Fs \\
\hline 50 & 32.2 & 32.7 & 18 & se & 50 & $3 I . I$ & 32.9 & I8 & E & 50 & 42.5 & $4 \mathrm{I}$. & 4 & 02 & 50 & .9 & 45 & 39 & 18 \\
\hline 52 & 2.6 & 33.9 & IS & S & 52 & $3 \mathrm{I} .5$ & 33.0 & I8 & 58 & 52 & 42.2 & $4 \mathrm{I}$. & 43 & s? & 52 & 46.9 & 45 & 38 & 당 \\
\hline 54 & 2.8 & 33.3 & Is & 8 & 54 & 30.2 & $3 \mathrm{I} .9$ & I6 & R & 54 & 41.9 & $4 \mathrm{I}$. & 44 & R & 54 & 47.0 & 45 & 38 & 8 \\
\hline 56 & 33.1 & 33.8 & 2 & de & 56 & 29.9 & 31.2 & I5 & & 56 & $4 \mathrm{I} . \mathrm{I}$ & $4 \mathrm{I}$. & 45 & av & $5 t$ & 46.0 & 45 & 39 & (10) \\
\hline 58 & 3.9 & 34.4 & 21 & & 58 & $29 . I$ & 30.9 & I4 & & 58 & 40.0 & 39. & 46 & & 58 & 45.5 & 44 & 40 & \\
\hline$\infty$ & $34 . I$ & $35 . \mathrm{I}$ & 22 & +II.O & 2300 & 30.0 & $3 \mathrm{T.1}$ & I5 & +9.8 & I7 $\infty$ & 39.9 & 39.2 & 47 & +11.2 & I9 00 & 46.0 & 45.1 & 39 & +8.7 \\
\hline 02 & 3.5 & $34 \cdot 3$ & 2 & 1. & 02 & 29.8 & 3 I. I & 15 & & 02 & 39.9 & 39. & 47 & 10 & 02 & 46.1 & 45.8 & 39 & $\because 2$ \\
\hline 04 & $2 . I$ & 33.1 & Is & 18 & 04 & 30.2 & 31.8 & 16 & 2 & 04 & $40 . I$ & 39 & 47 & & 04 & 45.5 & 45.0 & 40 & 65 \\
\hline 06 & .7 & 32.8 & 18 & 80 & 06 & 30.9 & 32. I & 17 & wa & $\infty 6$ & 40.7 & & 46 & 10 & 0 & 44.9 & & 40 & W. \\
\hline 08 & I. I & 32.2 & 17 & & 08 & 30.6 & 31.9 & 16 & & 08 & 40.3 & 39. & 47 & As & 08 & 44.9 & 44 & 40 & the \\
\hline IO & 1.6 & 33.0 & I\& & & IO & 30.8 & $3 \mathrm{I} .8$ & 16 & a & I0 & 39.9 & 30 & 47 & Git & IO & 44.9 & & 40 & 01 \\
\hline I2 & 3.1 & 34.1 & 20 & & 12 & 31.6 & 32.5 & 18 & & 12 & 30.0 & 38 & 49 & & 12 & 44.9 & 44.8 & 40 & \\
\hline 14 & .8 & 34.7 & $2 \mathrm{I}$ & +11.0 & 14 & 32.9 & 33.5 & 19 & +9.6 & 14 & 38.9 & 38 & 49 & +II. & I4 & 45.9 & 45.9 & 39 & \\
\hline I6 & $3 . I$ & 34.0 & 20 & & I6. 4 & 33.5 & 34.6 & $2 \mathrm{I}$ & & 16 & 38.1 & 38. & 50 & & I6 & 45.9 & 45.6 & 39 & +8.2 \\
\hline 18 & .7 & 33.8 & If & - & 18 & 34.2 & $35 . \mathrm{I}$ & 22 & & I8 & 38.2 & & 50 & Bet & 18 & 46.0 & 45.9 & 39 & \\
\hline 20 & 1.9 & 32.8 & I8 & th & 20 & 34.6 & 35.2 & 22 & 25 & 20 & 38.9 & 38. & 49 & 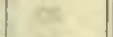 & 20 & 45.5 & 45 & 40 & fe \\
\hline 22 & & $3 \mathrm{r} .7$ & I6 & 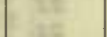 & 22 & 35.0 & 35.7 & 2 & a & 22 & 40.2 & 39. & 47 & $6+$ & 22 & $44 . I$ & 44.0 & 42 & $3=$ \\
\hline 24 & .8 & 30.2 & 14 & & 24 & 35.2 & 36.0 & 23 & 12 & 24 & 41.3 & $4 \mathrm{I}$ & 45 & 45 & 24 & 43.9 & 43 & & 15 \\
\hline 26 & & & I2 & & 26 & 35.9 & 36.3 & 24 & 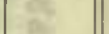 & & 41.6 & $4 \mathrm{~T} . \mathrm{C}$ & & $a^{5}$ & 26 & $43 \cdot 3$ & 43.0 & 43 & $\%$ \\
\hline 28 & 8.0 & 28.8 & 12 & & 28 & 36.0 & 36.3 & 24 & & 28 & $41 . I$ & 40.3 & 46 & $x^{2}$ & 28 & 43.2 & 42. & 44 & \\
\hline 30 & 28.0 & 28.9 & 12 & tir.o & 30 & 36.3 & 36.0 & 25 & +9.7 & 30 & $40 . I$ & 39.5 & 47 & +10.9 & 30 & 43.0 & 42.3 & 44 & +8.0 \\
\hline 32 & 7.9 & 28.0 & 12 & & 32 & 36.2 & 37.6 & 25 & & 32 & 30.2 & & 48 & 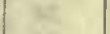 & 32 & 43.0 & 42 & 44 & 121 \\
\hline 34 & .3 & 28.8 & II & & 34 & 36.4 & 37.5 & 2 & & 34 & 38.7 & 38. & 49 & 45 & 3 & 43.1 & 42.3 & 44 & 15 \\
\hline 36 & & 29.1 & I & & 36 & 36.6 & 37.6 & 2 & & 36 & 38.8 & 38. & & 38 & 3 & 44.0 & 43. & 43 & ate \\
\hline 38 & 29.4 & 0.5 & 14 & th & 38 & 36.3 & 37.3 & 25 & & 38 & 39.0 & 38.9 & 9 & es & 38 & 45.0 & 44.2 & $4 I$ & Bi \\
\hline 40 & & 1.0 & I & 2. & 40 & & 37.3 & 2 & 10 & 40 & 39.9 & 39.3 & 48 & 6 & 4 & 45.2 & 44.7 & $4 \mathrm{~T}$ & 60 \\
\hline 42 & & .3 & 16 & 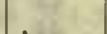 & 42 & 36.6 & 37.3 & 25 & & 42 & 39.5 & 39. & 48 & & 42 & 45.9 & 44.8 & 40 & \\
\hline 41 & 32.2 & 33.0 & 18 & +10.7 & 44 & 36.8 & 37.6 & 26 & +9.7 & 44 & 39.9 & 39. & 48 & +10. & 44 & 45.9 & 44.9 & 40 & +7.8 \\
\hline 46 & .9 & 3.8 & 20 & & 46 & 37.1 & 38.8 & 27 & & $4^{6}$ & 39.0 & 39.8 & 47 & 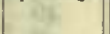 & 4 & 46.0 & & 40 & 20 \\
\hline 48 & & & 2 & & 48 & 37.1 & 38.9 & 27 & es & 48 & 39.8 & 39 & & 4 & 48 & 46.8 & & 38 & \\
\hline 50 & & & 2 & & 50 & 37.5 & $39 . \mathrm{I}$ & 2 & & 50 & 40.0 & 39 & 4 & st & 5 & 47.1 & 46.0 & 38 & $x$ \\
\hline 52 & 4.6 & 35.4 & 22 & & 52 & 37.1 & 39.0 & 2 & 4 & 52 & 40.8 & 40.0 & 46 & 34 & 5 & 47.0 & 45.6 & 38 & te \\
\hline 54 & & 36.1 & 2.3 & $\omega=$ & 51 & 37.3 & 39.1 & 27 & & 54 & $4 \mathrm{I} .0$ & 40 & 46 & 34 & 5 & 48.0 & 47.8 & 36 & AC \\
\hline 56 & & 36.9 & 24 & -1 & 56 & 37.6 & 39.2 & 28 & & 56 & 41.9 & 40.9 & 45 & $=$ & 56 & 49.1 & 48.9 & 34 & \\
\hline 58 & 36.0 & 36.9 & 24 & & 58 & 37.1 & $39 . \mathrm{I}$ & 27 & +9.8 & 58 & 42.1 & $4 \mathrm{I} .0$ & 45 & ne & 58 & 51.0 & 50.4 & 32 & set \\
\hline & & & & & 3400 & 38.0 & 40.0 & 28 & & & & & & & 2000 & 51.9 & $5 \mathrm{~T} . \mathrm{I}$ & 30 & +7.6 \\
\hline
\end{tabular}

Correction to local mean time is - Im o6s. $90^{\circ}$ torsion $=17 .^{\prime} 49$ Torsion head at ol com read $46^{\circ}$ and at $24 \mathrm{~h} 15 \mathrm{~m}$ read $53^{\circ}$. Observer-R. R. T.
Correction to local mean time is -5 s. $90^{\circ}$ torsion $=18 . .^{\prime} 21$.

Torsion head at $15 \mathrm{~h} 42 \mathrm{~m}$ read $54^{\circ}$ and at $20 \mathrm{~h} 20 \mathrm{~m}$ read $39^{\circ}$.

Observer-J. V. 
Tabulation of magnetic declinations observed at Teplitz Bay-Continued

\begin{tabular}{|c|c|c|c|c|c|c|c|c|c|c|c|c|c|c|c|c|c|c|c|}
\hline \multicolumn{6}{|c|}{ Friday, June 24, 1904} & \multicolumn{4}{|c|}{ Magnet scale erect } & \multicolumn{6}{|c|}{ Sunday, June 26,1904} & \multicolumn{4}{|c|}{ Magnet scale inverted } \\
\hline \multirow{2}{*}{$\begin{array}{l}\text { Chr'r } \\
\text { time }\end{array}$} & \multicolumn{2}{|c|}{$\begin{array}{l}\text { Scale } \\
\text { readings }\end{array}$} & \multirow{2}{*}{$\begin{array}{c}\text { East } \\
\text { decli- } \\
\text { nation }\end{array}$} & Temp. & Chr'r & $\begin{array}{l}\text { Sc } \\
\text { read }\end{array}$ & $\begin{array}{l}\text { ale } \\
\text { ings }\end{array}$ & East & Temp & Chr'r & $\begin{array}{l}\text { Sc: } \\
\text { read }\end{array}$ & $\begin{array}{l}\text { ale } \\
\text { lings }\end{array}$ & East & Temp. & Chr'r & $\begin{array}{l}\text { Sc: } \\
\text { read }\end{array}$ & le & $\begin{array}{l}\text { East } \\
\text { decli- }\end{array}$ & Temp. \\
\hline & Left & Right & & & & Left & Right & & & & Left & Right & & & & Left & Right & & \\
\hline $\begin{array}{r}h \mathrm{~m} \\
2000\end{array}$ & $d$ & d & ${ }^{\circ}$, & - & $\begin{array}{r}\text { h m } \\
2200\end{array}$ & & $\stackrel{d}{d 6}$ & $\begin{array}{cc}\cdot & 1 \\
22 & 22\end{array}$ & $\begin{array}{r}\circ \\
+88\end{array}$ & li $\mathrm{m}$ & $d$ & $d$ & $\therefore$ ' & 。 & h $\mathrm{m}$ & d & d & $\therefore:$ & - \\
\hline $\begin{array}{r}2000 \\
02\end{array}$ & $\begin{array}{l}44.0 \\
44.1\end{array}$ & $\begin{array}{l}45 \cdot 3 \\
45.5\end{array}$ & $\begin{array}{r}22 \\
20 \\
20\end{array}$ & $\log 8$ & $\begin{array}{r}2200 \\
02\end{array}$ & $\begin{array}{l}46.0 \\
45.9\end{array}$ & $\begin{array}{l}40.0 \\
46.0\end{array}$ & & +8.8 & $\begin{array}{c}000^{*} \\
02\end{array}$ & $\begin{array}{l}50.1 \\
50.2\end{array}$ & $\begin{array}{l}49.5 \\
49.0\end{array}$ & $\left|\begin{array}{rl}21 & 51 \\
& 5 I\end{array}\right|$ & +5.8 & $\begin{array}{r}200 \\
02\end{array}$ & $\begin{array}{l}3 \mathrm{I} . \mathrm{I} \\
3 \mathrm{I} . \mathrm{I}\end{array}$ & $\begin{array}{l}29.9 \\
29.4\end{array}$ & $\begin{array}{ll}22 & 2 \mathrm{I} \\
& 2 \mathrm{I}\end{array}$ & +5.2 \\
\hline 04 & 44.8 & 45.9 & $2 I$ & ing & 04 & 45.0 & 45.7 & 21 & 10 & 04 & 49.9 & 48.7 & $5 I$ & & 04 & 31.0 & 29.0 & 22 & $=$ \\
\hline 06 & 44.8 & 46. I & $2 \mathrm{I}$ & 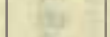 & 06 & $45 . I$ & 45.4 & 20 & 70 & 06 & 48.9 & 47.8 & 53 & & 06 & 30. I & 28.9 & 22 & \\
\hline 08 & 45.0 & $46 . \mathrm{I}$ & $2 I$ & 60 & 08 & 43.7 & 44.1 & 18 & Bo & os & 50.5 & 49.7 & 50 & & 08 & 28.9 & 26.8 & 25 & \\
\hline Io & $45 \cdot 4$ & 46.6 & 22 & 26 & Io & $42 \cdot 3$ & 42.9 & I6 & of & Io & 50.0 & 48.3 & 52 & & Io & 28.0 & 26.1 & 26 & \\
\hline 12 & 45.9 & 46.9 & 22 & +7.6 & 12 & 43.0 & $43 . I$ & 17 & & I2 & 47.7 & 46.8 & 55 & & 12 & 28.0 & 26.8 & 26 & \\
\hline 14 & 46.0 & 47.0 & 23 & ( & 14 & 40.9 & 41.8 & 14 & +8.3 & I4 & 46.0 & 45.2 & 57 & +5.8 & 14 & 28.9 & 27.0 & 25 & +5.2 \\
\hline I6 & $46 . I$ & 46.9 & 23 & 74 & 16 & 43.0 & 43.9 & 18 & $x^{2}$ & 16 & 48.0 & 47.0 & 54 & to & 16 & 29.9 & 28.1 & 23 & \\
\hline 18 & 46.0 & 46.2 & 22 & 84 & 18 & 43.2 & 44.0 & I8 & 80 & I8 & 48.1 & 47.2 & 54 & 8 & 18 & 32.1 & 31.0 & 19 & R. \\
\hline 20 & 45.4 & 46.0 & 2I & 34 & 20 & 42.8 & $43 . I$ & 17 & es & 20 & 48.2 & 47.9 & 53 & ex & 20 & 29.3 & 28 & 23 & $x$ \\
\hline 22 & $45 . I$ & 45.3 & 20 & 는 & 22 & 44.0 & 44.7 & 19 & $\therefore$ & 22 & 48.7 & 47.9 & 53 & is & 22 & 28.1 & 27.8 & 25 & $\sqrt{x}$ \\
\hline 24 & 44.7 & $45 . \mathrm{I}$ & 20 & 18 & 24 & 42.0 & 42.5 & I6 & is & 24 & 49.8 & 48.0 & 52 & 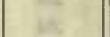 & 24 & 29.2 & 28.2 & 24 & \\
\hline 26 & 44.0 & 44.9 & I9 & As & 26 & 42.9 & 43.4 & 17 & 4 & 26 & 49.7 & 48.1 & 52 & & 26 & 28.9 & 27.9 & 24 & \\
\hline 28 & 44.0 & $44 \cdot 5$ & 19 & 1 & 28 & 43. I & 43.9 & I8 & & 28 & 48.0 & 46.6 & 54 & & 28 & 26.5 & 25.8 & 28 & \\
\hline 30 & 44. I & 44.7 & 19 & +7.9 & 30 & 46.9 & 47.1 & 23 & +8.0 & 30 & 48.3 & 47.4 & 54 & +5.8 & 30 & 27.0 & 25.9 & 27 & +5.1 \\
\hline 32 & 44.9 & $45 . I$ & 20 & ( & 32 & 49.0 & 49.1 & 26 & 8 & 32 & 50.9 & $49 \cdot 3$ & 50 & 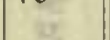 & 32 & 3 I.O & 30.0 & 21 & ( \\
\hline 34 & 44.8 & 45.1 & 20 & He. & 34 & 51.0 & 51.2 & 30 & es & 34 & 50.0 & 48.9 & $5 \mathrm{I}$ & 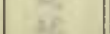 & 3 & 33.9 & 32 & I6 & \\
\hline 36 & $44 . \mathrm{I}$ & 44.9 & 19 & $=$ & 36 & $50 . I$ & 50.1 & 28 & dx & 36 & 48.0 & $47 . I$ & 54 & & 3 & 32.5 & 32.0 & 18 & \\
\hline 38 & 44. I & 44.8 & 19 & les & 38 & 47.2 & 47.8 & 24 & 24 & 38 & 47.9 & 46.9 & 54 & $7 x^{2}$ & 3 & 29.0 & 28.1 & 24 & \\
\hline 40 & 44.4 & 44.8 & 20 & 69 & 40 & 45.0 & $45 . I$ & 20 & ect & 40 & 48. I & 47.0 & 54 & a & 40 & 30.7 & 29.1 & 22 & \\
\hline 42 & $44 \cdot 3$ & 44.8 & I9 & & 42 & 44.2 & 44.9 & 19 & & 42 & 47.9 & 46.9 & 54 & 1 & 42 & 30.0 & 29.2 & 22 & \\
\hline 44 & 44.7 & 44.8 & 20 & +8.0 & 44 & 45.8 & $46 . I$ & 22 & +7.9 & 44 & 47.9 & 46.9 & 54 & +5.9 & 44 & 27.9 & 26.9 & 26 & +5.I \\
\hline 46 & 45.0 & 45.1 & 20 & 84 & 46 & 45.5 & 45.6 & 21 & (n) & 46 & 47.0 & 46.0 & 56 & ( & 46 & 26.1 & $25 . \mathrm{I}$ & 29 & \\
\hline 48 & 44.9 & 45.1 & 20 & 86 & 48 & 44.2 & 44.8 & I9 & 18 & 48 & 48.9 & 48. I & 53 & 15 & 48 & 26.0 & 25.1 & 29 & \\
\hline 50 & 44.2 & 44.9 & I9 & [1] & 50 & 44.3 & 45.0 & 20 & c요 & 50 & $55 . \mathrm{I}$ & 54.7 & 43 & 68 & 50 & 25.9 & 24.9 & 29 & \\
\hline 52 & 44.2 & 44.9 & 19 & $\mathrm{e}^{2}$ & 52 & 45.3 & 45.9 & 2 I & is & 52 & 54.0 & 52.0 & 46 & 8 & 52 & 25.0 & 23.9 & 30 & \\
\hline 54 & 44.2 & 44.6 & 19 & 42 & 54 & 45.0 & 46.8 & 22 & $y=$ & 54 & 56.9 & 56.5 & 40 & 12 & 54 & 24.3 & 23.5 & $3 \mathrm{I}$ & \\
\hline 56 & 44.4 & 44.9 & 20 & 18 & 56 & 44.1 & 45.0 & 19 & 88 & 56 & $55 . \mathrm{I}$ & 54.7 & 43 & & 56 & 23.0 & 22. I & 34 & \\
\hline 58 & 44.2 & 45.0 & 20 & & 58 & 45.8 & 46.1 & 22 & & 58 & 52.0 & $5 \mathrm{I}$. & 48 & & 58 & 22.1 & 21.5 & 35 & \\
\hline 2100 & $44 . I$ & 45.0 & I9 & +8.2 & 2300 & 47.0 & 48.0 & 24 & +7.8 & $I 00$ & $5 \mathrm{I} . \mathrm{I}$ & 50.8 & 49 & +5.9 & 300 & 22.9 & 22.0 & 34 & +5.0 \\
\hline 02 & 44.0 & 45.1 & I9 & 6 & 02 & 46.1 & $47 . I$ & 23 & 15 & 02 & 50.0 & 49.3 & 5I & 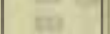 & 02 & 21.8 & 21.0 & 35 & \\
\hline 04 & 43.9 & 45.3 & 20 & tis & 04 & 46.7 & 47.5 & 24 & 10 & 04 & 50.1 & 49.8 & 50 & 10 & 04 & 2I. I & 20.7 & 36 & ent \\
\hline 06 & $44 . I$ & 45.4 & 20 & 85 & 06 & 47.1 & 48.0 & 24 & 18 & 06 & $49 . I$ & 48. I & 53 & 8 & 06 & 21.0 & 20. I & 37 & \\
\hline 08 & 44.9 & 45.8 & $2 \mathrm{I}$ & 69 & 08 & 47.1 & 48.4 & 24 & 8 & 08 & 47.7 & 47.5 & 54 & 8 & $\infty 8$ & 21.9 & $2 \mathrm{I} .7$ & 35 & \\
\hline IO & 44.8 & 45.9 & 2 I & 68 & Io & 47.3 & 48.8 & 25 & ats & 10 & 47.3 & $46 . I$ & 55 & 00 & IO & 21.9 & 2I.I & 35 & \\
\hline I2 & 45.8 & 46.9 & 22 & & I2 & 47.8 & 49.1 & 26 & & 12 & $47 \cdot 5$ & 46.7 & 55 & & 12 & 21.9 & 21.2 & 35 & \\
\hline $\mathrm{I}_{4}$ & 44.9 & 46.8 & 22 & +8.3 & I4 & 48.1 & 49.3 & 26 & +7.8 & 14 & 48.2 & 47.2 & 54 & +5.8 & I4 & 23.2 & 23.0 & 32 & +5.0 \\
\hline 16 & 45.3 & $46 \cdot 3$ & 22 & 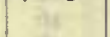 & I6 & 49.0 & 50.0 & 27 & re & 16 & 48.7 & $47 . I$ & 54 & 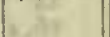 & I6 & 24.8 & 24.0 & $3 \mathrm{I}$ & \\
\hline 18 & 45.8 & 46.9 & 22 & in & I8 & 49.3 & 50.1 & 28 & 21 & I8 & 48.8 & 47.0 & 54 & His & I8 & 26.0 & 25.7 & 28 & \\
\hline 20 & $45 \cdot 7$ & 46.1 & 22 & 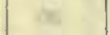 & 20 & 50.3 & 50.4 & 29 & 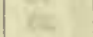 & 20.5 & 49.0 & 46.9 & 54 & os & 20 & 28.8 & 27.9 & 24 & \\
\hline 22 & 45.8 & 45.9 & 22 & $\mathrm{nx}$ & 22 & $5 \mathrm{I} . \mathrm{I}$ & 52.0 & 30 & sx & 22 & 48.5 & 45.9 & 5 & $=$ & 22 & 30. I & 29.2 & 22 & \\
\hline 24 & 44.6 & 44.9 & 20 & $\sqrt{15}$ & 24 & 51.0 & 52.1 & 30 & It & 24 & 49.8 & 45. & 54 & & 2 & 31 . & 30.1 & 2I & \\
\hline 26 & 42.5 & 43.0 & I7 & 85 & 26 & 52.2 & 53.0 & 32 & has & 26 & 48. I & $44 . I$ & 56 & A & 26 & 30.8 & 29.8 & $2 \mathrm{I}$ & \\
\hline 28 & 42.3 & $43 . I$ & I7 & & 28 & 5I.I & 52.0 & 30 & & 28 & 47.9 & 44.0 & 2157 & & 28 & 29.9 & 29.2 & 22 & \\
\hline 30 & 42.9 & $44 . I$ & 18 & +8.7 & 30 & 52.1 & 53.8 & 33 & +7.5 & 30 & 44.9 & $4 \mathrm{I} . \mathrm{I}$ & 22 oิ & +5.6 & 30 & 29.5 & 28.8 & 23 & +5.0 \\
\hline 32 & $43 . I$ & 45.0 & 19 & $x^{2}$ & 32 & 52.8 & 54.9 & 34 & $1+2$ & 32 & 44.0 & 40.9 & 02 & & 32 & 27.9 & 27.1 & 26 & \\
\hline 34 & 42.0 & 43.9 & 17 & te & 34 & 53.0 & 55.0 & 34 & is & 34 & 42.5 & 39. & 05 & a & 34 & 25.9 & 25.0 & 20 & \\
\hline 36 & 42.9 & 44.2 & I8 & 2y & 36 & $53 . \mathrm{I}$ & $55 . \mathrm{I}$ & 35 & te & 36 & 41.8 & 38. & 06 & & & 24.0 & 23.7 & $3 I$ & \\
\hline 38 & 44.1 & 45.4 & 20 & 85 & 38 & 53.7 & 55.3 & 35 & ile & 38 & 40.8 & 37.8 & 07 & $=$ & 38 & 22.0 & 21.0 & 35 & \\
\hline 40 & 45.2 & 46.7 & 22 & is & 40 & $53 . \mathrm{I}$ & 55.1 & 35 & E & 40 & 40.1 & 37.5 & 08 & $F^{2}$ & 40 & 18.1 & I8.0 & $4 \mathrm{I}$ & \\
\hline 42 & 46.0 & 47.0 & 23 & & 42 & 52.4 & $54 . I$ & 33 & & 42 & 39.8 & 37. & 08 & & 42 & 16. I & 15.8 & 44 & \\
\hline 44 & 46.0 & 47.0 & 23 & +8.7 & 44 & 51.8 & 53.3 & 32 & +7.3 & 44 & 30.9 & 37. & 08 & +5.4 & 44 & 14.0 & I 3.3 & 48 & +5.0 \\
\hline 46 & 42.0 & 42.8 & I6 & 184 & 46 & 51.8 & 53.4 & 32 & 28 & 46 & 38.3 & 36.1 & 10 & & 46 & 13.5 & I3. 3 & 48 & \\
\hline 48 & 47.2 & 47.7 & 24 & 9. & 48 & $5 \mathrm{I} . \mathrm{I}$ & 52.8 & $3 \mathrm{I}$ & 16. & 48 & 35.7 & 33.7 & 14 & 8 & 48 & I8.8 & 18.2 & 40 & \\
\hline 50 & 47.7 & 48.0 & 25 & $\theta$ & 50 & 51.0 & 52.3 & 30 & 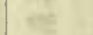 & 50 & 34.0 & 32.0 & I7 & & 50 & I8.2 & 18.0 & 40 & \\
\hline 52 & $47 \cdot 3$ & 47.9 & 24 & 32 & 52 & 50.3 & 51.9 & 30 & 5 & 52 & 33. I & 30.9 & 19 & (2) & 52 & 13.0 & I2.0 & 49 & \\
\hline 54 & $47 . I$ & 47.6 & 24 & H & 54 & 50.2 & 51.3 & 29 & Hit & 54 & 32.0 & $3 \mathrm{I} \cdot 2$ & 19 & & 54 & 12.0 & II. 2 & $5 \mathrm{I}$ & \\
\hline 56 & 46.1 & 47.0 & 23 & E & 56 & 50.0 & $5 \mathrm{I} . \mathrm{I}$ & 29 & 82 & 56 & 30. I & 28. I & 23 & 5 & 56 & II. 8 & II .O & $5 \mathrm{I}$ & \\
\hline 58 & 45.5 & 46.3 & 22 & 18 & 58 & 50.0 & 51.0 & 29 & ins & 58 & 27.7 & 25.8 & 27 & & 58 & II.I & 10.9 & 52 & \\
\hline & & 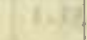 & 5.12 & का $\mathrm{C}$ & 2400 & 49.8 & 50.9 & 29 & & & & ositi & e.ffir & & & & & & \\
\hline
\end{tabular}

Correction to local mean time is $-26 s$.

Observer-J. V.

Torsion head at $16 \mathrm{~h} 52 \mathrm{~m}$ read $40^{\circ}$ and at $24 \mathrm{~h} \mathrm{r} 3 \mathrm{~m}$ read the same.

Observer-J. V. 
Tabulation of magnetic declinations observed at Teplitz Bay-Continued

\begin{tabular}{|c|c|c|c|c|c|c|c|c|c|c|c|c|c|c|c|c|c|c|c|}
\hline \multicolumn{5}{|c|}{ Sunday, June 26, 1904} & \multicolumn{5}{|c|}{ Magnet scale erect } & \multicolumn{6}{|c|}{ Monday, June 27, 1904} & \multicolumn{4}{|c|}{ Magnet scale inverted } \\
\hline \multirow{2}{*}{$\begin{array}{l}\text { Chr'r } \\
\text { time }\end{array}$} & \multicolumn{2}{|c|}{$\begin{array}{l}\text { Scale } \\
\text { readings }\end{array}$} & \multirow{2}{*}{$\begin{array}{l}\text { East } \\
\text { decli- } \\
\text { nation }\end{array}$} & & Chr'r & $\begin{array}{l}\text { Sca } \\
\text { read }\end{array}$ & $\begin{array}{l}\text { ale } \\
\text { lings }\end{array}$ & $\begin{array}{l}\text { East } \\
\text { decli- }\end{array}$ & & & & $\begin{array}{l}\text { ale } \\
\text { lings }\end{array}$ & $\begin{array}{l}\text { Eas } \\
\text { decl }\end{array}$ & & & $\begin{array}{r}\text { Sc } \\
\text { read }\end{array}$ & $\begin{array}{l}\text { ale } \\
\text { lings }\end{array}$ & $\begin{array}{l}\text { East } \\
\text { decli- }\end{array}$ & Temp. \\
\hline & Left & Right & & & & Left & Right & & & & Left & Right & & & & Left & Right & & \\
\hline h $\mathrm{m}$ & d & $d$ & $\cdot$ & 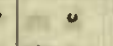 & $\mathrm{h} \mathbf{m}$ & d & d & $\cdot$, & - & $\mathrm{h} \mathrm{m}$ & d & d & & & $\mathrm{h} \mathrm{m}$ & d & & -, & \\
\hline $400^{*}$ & 50.0 & 50.7 & 225 & +5.9 & 600 & 44.0 & 46.2 & 2334 & +4.8 & $800 *$ & 54.7 & $54 \cdot 5$ & & +10.2 & Io $\infty$ & 33.1 & 28.1 & 2307 & +12.2 \\
\hline 02 & 50.8 & 52.1 & 5 & & 02 & 42.0 & 45.1 & & & 02 & 59.3 & 59.1 & & $1=$ & 02 & 18.1 & II. 2 & 32 & \\
\hline 04 & 52.3 & 53.1 & 5 & 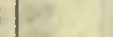 & 04 & 6.9 & 49.0 & 38 & 1 & 04 & 62.0 & 60.0 & & & $04^{*}$ & 63.3 & $49 . I$ & 45 & \\
\hline 06 & 51.9 & 53.2 & 5 & 0 & 06 & 50.0 & $5 \mathrm{I} .9$ & 43 & co & 06 & 60.6 & 56.8 & & $\Delta$ & 06 & 52.4 & 45.5 & 57 & F \\
\hline os & $5 \mathrm{I} .3$ & 53.0 & 5 & 0 & 08 & 52.7 & 54.9 & 48 & 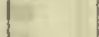 & os & 55.2 & $5 I .3$ & 2 & & 08 & 70.0 & $6 \mathrm{I} .1$ & 31 & \\
\hline 10 & 5I.I & 52.8 & 5 & 5 & 10 & 51.5 & 52.8 & 45 & 1 & 10 & 62.8 & 55.8 & & & 10 & 74.8 & 66.3 & 23 & \\
\hline 12 & $5 \mathrm{I} . \mathrm{I}$ & 52.1 & 5 & & I2 & 49.9 & 50.8 & 42 & 1 & 12 & 72.2 & 66.5 & 230 & & 12 & 74.7 & 67.8 & 22 & \\
\hline 14 & 50.7 & 52.1 & 5 & +5.1 & I4 & 48.1 & 49.0 & 39 & +4.8 & 14 & 74.9 & 69.1 & 225 & +10.I & 14 & 72.9 & 66.1 & 24 & +12.8 \\
\hline 16 & 50.2 & 52.0 & 5 & & 16 & 43.0 & 44.7 & 32 & & I6 & 68.3 & 62.0 & 230 & & 16 & 62.8 & 56.8 & & \\
\hline 18 & 50.0 & 51.0 & 5 & : & 18 & 40.8 & 41.9 & 28 & 1 & 18 & 64.9 & 59.8 & 231 & & I8 & 69.4 & 64.0 & 29 & \\
\hline 20 & 49.0 & 50.2 & 5 & 1 & 20 & 40.9 & 41.9 & 28 & 5 & 20 & 76.3 & 71.8 & 225 & & 20 & 65 & .06 & 32 & \\
\hline 22 & 50.0 & 51.8 & 5 & 16 & 22 & 41.9 & 43.1 & 30 & 5 & $22^{*}$ & 45.9 & $34 . I$ & 5 & & 22 & 63.9 & 63.7 & 33 & \\
\hline 24 & 5I.I & 52.3 & 5 & $=$ & 24 & 39.1 & 41.0 & 26 & 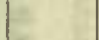 & 24 & & 43.6 & & & 24 & 72.1 & $6 \mathrm{I} .4$ & 29 & \\
\hline 26 & 52.0 & 53.1 & 5 & $1=$ & 26 & $46 . I$ & 48.5 & 37 & $=$ & 26 & 48.0 & 38.4 & & & 26 & 72.7 & 62.0 & 28 & $=$ \\
\hline 28 & 52.3 & 53.7 & 5 & 5 & 28 & $46 . I$ & 48.0 & 37 & 1 & 28 & 50.2 & 42.6 & & & 28 & 69.9 & 58.2 & 33 & \\
\hline 30 & 53.8 & 54.2 & 5 & +5.0 & 30 & 44.7 & 46.1 & 34 & +4.8 & 30 & 48.1 & 40.8 & 4 & +10.9 & 30 & 55.9 & 47.6 & 52 & $+\mathrm{I} 3.0$ \\
\hline 32 & 54.4 & 55.1 & (4) 5 & 10.0 & 32 & 43.0 & 45.0 & 32 & & 32 & 41.0 & 34.8 & 225 & & 32 & 61.5 & 55.9 & 41 & \\
\hline 34 & 55.8 & 56.1 & 226 & 5 & 34 & 43.8 & 44.9 & 33 & & 34 & 28.9 & $2 \mathrm{I} .2$ & 23 & 22 & 34 & 74.7 & 69.8 & 20 & 5 \\
\hline 36 & $57 \cdot 3$ & 59.0 & 230 & 15 & 36 & $4 \mathrm{I} . \mathrm{I}$ & 42.5 & 29 & & 36 & 27.7 & 21.9 & & ax & $36^{*}$ & 53.9 & $51 . \mathrm{I}$ & 2302 & k \\
\hline 38 & 60.8 & 62.0 & a & F. & 38 & 40.0 & 41.0 & 27 & & 38 & 33.4 & 27.0 & 230 & t. & 38 & 59.2 & 56.5 & 2253 & $=$ \\
\hline 40 & 59.9 & 61.0 & o? & 14 & 40 & $40 . I$ & 41.6 & 27 & 1 & 40 & 40 & 35.8 & 225 & 4 & 40 & 63.9 & 58 & 48 & 5 \\
\hline 42 & 59.0 & 59.7 & 0 & $1=$ & 42 & 42.2 & $43 . \mathrm{I}$ & 30 & & 42 & 37.0 & 33. I & 230 & 4 & 42 & 70.2 & 66.2 & 37 & it \\
\hline 44 & 59.1 & 60.9 & $\alpha$ & +4.9 & 44 & 42.1 & 42.5 & 30 & +4.8 & 44 & $3 I .9$ & 27.9 & 0 & +II.2 & 44 & $6 \mathrm{I} . \mathrm{I}$ & 57.3 & 51 & +13 \\
\hline 46 & 59.1 & 60.8 & $\alpha$ & & 46 & $40 . I$ & 40.2 & 26 & & 46 & 24.9 & 27.8 & I & & 46 & 55.4 & 51.8 & & \\
\hline 48 & 58.8 & 59.3 & o. & 4 & 48 & 39.9 & 40.3 & 26 & 5 & 48 & 17.8 & 13.7 & & & 48 & 59.9 & $54 . \mathrm{I}$ & 54 & \\
\hline 50 & 6r.o & $6 \mathrm{r} .9$ & of & 15 & 50 & 36.9 & 38.0 & 22 & E & 50 & & $23 . I$ & I & & 50 & & 60.8 & 47 & \\
\hline 52 & 63.0 & 63.8 & I & $E$ & 52 & 43.1 & 43.7 & $3 I$ & 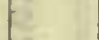 & 52 & 22.2 & I5.8 & 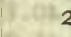 & ? & 52 & 69.5 & 65.9 & 38 & \\
\hline 54 & 63.0 & 64.8 & I & $\mu$ & 54 & 42.7 & 43.6 & 31 & t & 54 & 25.9 & 25 & I & & 54 & 72.3 & $6 \overline{8}$ & 33 & \\
\hline 56 & 64.9 & 67.0 & I & 02 & 56 & 44.1 & 45.0 & 33 & 10 & 56 & 29.1 & 24.0 & & & 5 & 76.3 & $72 . I$ & 8 & \\
\hline 58 & 66.1 & 68.3 & $\mathrm{I}$ & & 58 & $45 . I$ & 45.5 & 34 & & 58 & 24.0 & 18.3 & 232 & & 5 & 76.3 & 71 & 28 & \\
\hline 500 & 67.2 & 69.9 & 2 & +4.8 & $7 \infty$ & 42.5 & $43 . I$ & 30 & +4.7 & 900 & 40.8 & 31.0 & 225 & + II. 8 & II 00 & 72.7 & 69.0 & 33 & +13.3 \\
\hline 02 & 67.1 & 69.1 & Is & & 02 & 40.1 & 41.2 & 27 & & 02 & 39.1 & 30.2 & 230 & & 02 & & 59 & & \\
\hline 04 & 68.3 & 69.1 & 2 & 10 & 04 & 38.6 & & 24 & & 04 & 42.4 & 38.0 & 225 & & 04 & 61.9 & 57 & 50 & \\
\hline 06 & 68.2 & 69.9 & 2 & 10 & 06 & 37.0 & 38.8 & 22 & 5 & 06 & & 30. & 23 & & & & 58 & & 6 \\
\hline$\infty 8$ & 69.0 & 70.3 & 2 & 10 & 08 & 40. & $.9 a$ & 27 & $=$ & 08 & 3I.9 & 26.9 & 23 & & 08 & 3.7 & 60.2 & 47 & \\
\hline Io & 69.9 & 70.9 & 2. & or & Io & $42 . I$ & 42.1 & 29 & & Ic & & 35. & 225 & & I & & 66 & & \\
\hline 12 & 69.7 & 70.4 & 2. & & I2 & 39.2 & 39.8 & 25 & & 12 & I & 37.2 & 225 & & 12 & 78.1 & 74.1 & 24 & \\
\hline 14 & 69.9 & 71.0 & 2. & +4.8 & 14 & 39.9 & 40.1 & 26 & +4.7 & 14 & 28.4 & 24.8 & 23 I & +12.0 & $14^{*}$ & 49.8 & 42 & 40 & +13 \\
\hline I6 & 71.5 & 72.9 & 2. & & 16 & 44.1 & 44.8 & 33 & & 16 & 26.0 & 22.3 & I & & 16 & 45.7 & 38.9 & 46 & \\
\hline I8 & 70.0 & 71.9 & & 4 & 18 & 43.9 & 44.0 & $1 \quad 32$ & 11 & 18 & 36.8 & 32.1 & 230 & 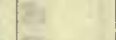 & 18 & 37.1 & $32 . \mathrm{I}$ & 2259 & \\
\hline 20 & 69.1 & 71.0 & 2 & 10 & 20 & 44.3 & 44.9 & 33 & $=$ & 20 & 44.6 & 40.2 & 224 & & 20 & 2I.I & 2 & 2322 & $E$ \\
\hline 22 & 67.4 & 68.3 & I & $1=$ & 2 & 48.1 & 48 & 3 & . & 22 & & & 4 & $\mathrm{E}$ & 2 & 20. & 16 & 24 & 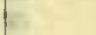 \\
\hline 24 & 60.2 & 70.9 & 2 & $a^{2}$ & 24 & 43.4 & 44.6 & $\begin{array}{l}32 \\
6\end{array}$ & 1 & 24 & $5 \mathrm{I} .8$ & 49 & & $=$ & 24 & 17.8 & 16.2 & 26 & \\
\hline 26 & 69.7 & & & & 26 & 42. & & $\begin{array}{l}29 \\
9\end{array}$ & s & 26 & & & & Xo & & & 19. & 19 & - \\
\hline 28 & 67.0 & 67.0 & I & & 28 & 42.9 & 43. I & $3 \mathrm{I}$ & F & 28 & 52.1 & 48.2 & & $=$ & 28 & 25.8 & 21. I & I6 & \\
\hline 30 & 69.8 & $7 \mathrm{I} .2$ & 2 & +4.7 & 30 & 40.7 & 40.9 & 27 & 5 & 30 & & $42 . I$ & & +12.0 & 30 & 18.9 & 14.5 & 27 & +13 \\
\hline 32 & 70.9 & 71.5 & 2 & & 32 & 43.0 & 43.7 & 8) $3 I$ & 1 & 32 & $48 . I$ & 45.2 & & & 32 & I4. I & 13.1 & 2332 & \\
\hline $34^{*}$ & & 48.2 & 3 & Ex & 34 & 40.2 & 40.8 & 27 & 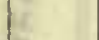 & 34 & 55.0 & 51.6 & & & 34 & 54.5 & 49.1 & 2232 & \\
\hline 36 & 46.3 & $5 \mathrm{I} . \mathrm{I}$ & 4 & in & 36 & & & 25 & 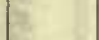 & 36 & 58 & & & 2 & 36 & 56.0 & 53 & 27 & \\
\hline 38 & 44.0 & 48.1 & 3 & W & 38 & 45.0 & 46.6 & 35 & D. & 38 & 61.0 & 56. & & & 38 & 50.1 & $44 \cdot 5$ & 39 & E \\
\hline 40 & 45.0 & 49.0 & & Dit & 40 & 45.0 & 45.6 & 34 & & 40 & & & & (iit & 40 & 53.8 & 44. & 36 & \\
\hline 42 & 46.1 & 50.0 & 3 & & 42 & 45.2 & 46.0 & 35 & & 42 & 57.2 & 49.3 & & & 42 & 61.3 & 50.1 & 26 & \\
\hline 44 & 47.2 & 5I. I & 4 & +4.7 & 44 & 42.0 & 43.3 & 30 & +2. & 44 & & 52.0 & & +12 & 44 & 69.3 & 54.9 & 15 & +14 \\
\hline 46 & 46.8 & 50.3 & 3 & & 46 & 38.9 & 40.0 & 25 & & 46 & 58.1 & 52.2 & & - & 46 & 70.1 & 52.9 & I6̆ & \\
\hline 48 & 45.3 & 49.2 & 3 & 5 & 48 & 36.3 & 37.7 & $2 I$ & 10 & 48 & 67.0 & 61.5 & & & 48 & 68.5 & 52.3 & I8 & \\
\hline 50 & & 50.8 & 4 & Dit & 50 & 38.8 & 39.8 & 25 & E & 50 & 71.9 & 64.1 & L.) & 2 & 50 & 68.4 & 51.2 & I9 & \\
\hline 52 & 46.0 & 49.0 & 3 & 42 & 52 & 37.0 & $37 \cdot 3$ & $2 \mathrm{I}$ & E & 52 & 65.1 & & & a & 52 & 64.8 & 53.3 & 20 & \\
\hline 54 & 45.2 & 48.4 & 3 & 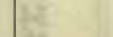 & 54 & 33.6 & 34.5 & 16 & 22 & 54 & 54.9 & 46. & & 3 & 54 & 63.6 & 54.0 & $2 \mathrm{I}$ & 5 \\
\hline 56 & 45.0 & 48.0 & 3 & 10 & 56 & 34.0 & 36.0 & 18 & & 56 & & 39.9 & & & 56 & 56.2 & 45.9 & 33 & \\
\hline 58 & 43.9 & 46.3 & 3 & 12 & 58 & 33.9 & 35.2 & 17 & +4.9 & 58 & 38.9 & 34.0 & 225 & & 58 & 49.9 & 46.6 & $\begin{array}{l}\text { 1. } 37 \\
\end{array}$ & \\
\hline & & & & & & & & & & & & & & & & 48.3 & 41.2 & 43 & +14.0 \\
\hline
\end{tabular}

Correction to local mean time is - Im oos. $90^{\circ}$ torsion $=13^{\prime} 42$. Torsion head at oh oom read $40^{\circ}$ and at $8 \mathrm{~h} 17 \mathrm{~m}$ read $42^{\circ}$. Observer-J. V.
Correction to local mean time is - Im $46 \mathrm{~s}$. No torsion observations. Torsion head at $8 \mathrm{~h}$ com read $43^{\circ}$ and at $12 \mathrm{~h}$ o5m read $33^{\circ}$.

Observer-R. R. T. 
Tabulation of magnetic declinations observed at Teplitz Bay-Continued

\begin{tabular}{|c|c|c|c|c|c|c|c|c|c|c|c|c|c|c|c|c|c|c|c|c|c|}
\hline \multicolumn{6}{|c|}{ Tuesday, June 28,1904} & \multicolumn{5}{|c|}{ Magnet scale erect } & \multicolumn{6}{|c|}{ Wednesday, June 29, 1904} & \multicolumn{5}{|c|}{ Magnet scale inverted } \\
\hline \multirow{2}{*}{$\begin{array}{l}\text { Chr'r } \\
\text { time }\end{array}$} & \multicolumn{2}{|c|}{$\begin{array}{l}\text { Scale } \\
\text { readings }\end{array}$} & $\begin{array}{l}\text { East } \\
\text { decli- }\end{array}$ & Temp. & Chr'r & $\begin{array}{r}\text { Sc } \\
\text { reac }\end{array}$ & $\begin{array}{l}\text { ale } \\
\text { lings }\end{array}$ & Eas & & Temp. & Clir'r & $\begin{array}{r}\text { Sc } \\
\text { reac }\end{array}$ & $\begin{array}{l}\text { ale } \\
\text { dings }\end{array}$ & East & Temp. & Chr'r & $\begin{array}{r}\text { Sci } \\
\text { read }\end{array}$ & $\begin{array}{l}\text { dile } \\
\text { dings }\end{array}$ & East & & Temp. \\
\hline & Left & Right & nation & & & Left & Right & & & & time & Left & Right & nation & C. & time & Left & Right & natio & & \\
\hline $\mathrm{h} \mathrm{m}$ & d & d & $\cdot \cdot$ & $\circ$ & h m & d & d & $\bullet$ & ' & - & h m & d & d & & - & $\mathrm{h} \mathrm{m}$ & d & $d$ & - & ' & • \\
\hline 200 & 46.2 & 47.8 & 2229 & $+2 r .2$ & 1400 & 42.0 & 43.0 & 22 & & +20.0 & $000^{*}$ & 51.8 & 50.9 & 2225 & +5.1 & 200 & 34.0 & 33.2 & 225 & 53 & +4.9 \\
\hline 02 & 44.9 & 45.2 & 26 & in & 02 & 42.2 & 43.7 & & 22 & 8 & 02 & 50.7 & 50.0 & 27 & & 02 & 35.1 & 35.1 & & 50 & \\
\hline $04 \cdot 3$ & 45.3 & 46.4 & 27 & iv & 04. & 42.7 & 43.8 & & 23 & $\theta$ & 04 & 51.1 & 50.2 & 26 & $\rightarrow$ & 04 & 33.3 & 32.8 & & 54 & \\
\hline 06 & $46 . I$ & 46.4 & 28 & 63 & $\infty 6$ & 42.0 & 46.1 & & 24 & 9 & 06 & 51.8 & 50.7 & 25 & 6 & 06 & 34.7 & $34 . I$ & & 52 & \\
\hline os & 46.0 & 46.4 & 28 & 61 & 08 & 43. I & 44. I & & 23 & 0 & 08 & 52.2 & 51.2 & 24 & 20 & 08 & 35.1 & 35.1 & & 50 & \\
\hline 10 & 46.2 & $47 \cdot I$ & 28 & 67 & I0 & 45.3 & 46.0 & & 27 & 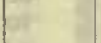 & I0 & 52.4 & 51.7 & 24 & 10 & 10 & 37.8 & 37.2 & & 47 & \\
\hline 12 & 46.8 & 47.0 & 29 & & I2 & 47.0 & 47.6 & & 29 & & 12 & 52.4 & 51.8 & 24 & & 12 & 33.2 & 32.1 & & 54 & \\
\hline 14 & 44.0 & $44 \cdot 4$ & 24 & +20.9 & 14 & 46.3 & 47.0 & & 28 & +20.0 & 14 & 52.0 & $5 \mathrm{I} .2$ & 25 & +5.0 & & 30.9 & 30.1 & 225 & 58 & +4.9 \\
\hline 16 & 43.9 & 44.2 & 24 & 3 & 16 & 43.5 & 44.0 & & 24 & & 16 & 51.3 & 50.9 & 25 & & 16 & 24.1 & 23.9 & $23 c$ & 08 & \\
\hline I8 & 43.5 & 44.4 & 24 & Br & I8 & 44.0 & 45.1 & & 25 & i & 18 & 51.4 & 50.9 & 25 & int & 18 & 21.7 & 20.8 & & 12 & \\
\hline 20 & 45.0 & 45.9 & 26 & oes & 20 & 44.0 & 44.6 & & 25 & $E$ & 20 & 51.8 & $5 \mathrm{I} . \mathrm{I}$ & 25 & 0 & 20 & 24.1 & 22.9 & & 09 & \\
\hline 22 & 45.0 & 46. I & 26 & ctis & 22 & 43.2 & $44 . I$ & & 23 & $E$ & 22 & 52.8 & 52.2 & 23 & $=$ & 22 & 24.4 & $23 . \bar{I}$ & & 08 & \\
\hline 24 & 45.9 & 46. I & 27 & 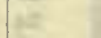 & 24 & 42.0 & 44.0 & & 22 & If & 24 & 52.7 & 52.0 & 23 & it & 24 & 22.2 & 2I.I & & 12 & \\
\hline 26 & 45.2 & 46.2 & 27 & $E$ & 26 & 4I. I & 43.2 & & 2 I & c & 26 & 52.1 & 51.8 & 24 & $E$ & 26 & 19.7 & I8. I & & 16 & \\
\hline 28 & 45.9 & 47.9 & 29 & & 28 & $4 \mathrm{I} .4$ & 42.2 & & $2 \mathrm{I}$ & & 28 & 52.6 & 51.9 & 24 & & 28 & 16.0 & 15.2 & & $2 \mathrm{I}$ & \\
\hline 30 & 45.2 & 47.0 & 27 & +20.4 & 30 & 40.2 & $4 \mathrm{I} . \mathrm{I}$ & & I9 & +19.9 & 30 & 53.1 & 52.6 & 23 & +5.0 & 30 & 17.7 & 16.9 & & 18 & +5.0 \\
\hline 32 & 45.9 & 46.9 & 28 & 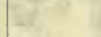 & 32 & 40. I & 42.0 & & 19 & & 32 & 53.0 & $52 . I$ & 23 & & 32 & 17.9 & I7. 8 & & 18 & \\
\hline 34 & 47.2 & 48.9 & 30 & W: & 34 & 40.4 & 42.2 & & 20 & & 34 & 52.7 & 52.1 & 23 & Hz & 34 & 19. I & I8. I & & 16 & \\
\hline 36 & 48.0 & 49.7 & 32 & N & 36 & 39.4 & 40.9 & & 18 & & 36 & 51.0 & 50.3 & 26 & 8 & 36 & 23.9 & 23.0 & & $\infty$ & \\
\hline 38 & 47.7 & 49.1 & 31 & Le & 38 & $4 \mathrm{I} .0$ & 42.0 & & 20 & & 38 & 50.1 & 49.2 & 28 & 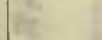 & 38 & 23.9 & $23 . I$ & & $\infty$ & \\
\hline 40 & 47.8 & 48.9 & 31 & E2 & 40 & 4I.I & 42.2 & & 20 & 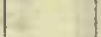 & 40 & 48.8 & 47.8 & 30 & 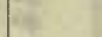 & 40 & 22.9 & 21.9 & & I0 & \\
\hline 42 & 47.1 & 48.3 & 30 & & 42 & 41.9 & $42 . I$ & & $2 I$ & & 42 & 48.2 & 47.4 & 30 & & 42 & 22.9 & 21.7 & & I0 & \\
\hline 44 & 46.7 & 48.0 & 29 & +20.1 & 44 & 42.3 & 42.9 & & 22 & +19.7 & 44 & 48.0 & 47.8 & 30 & +4.9 & 44 & 23.9 & 22.2 & & IO & +5.0 \\
\hline 46 & $4 \mathrm{I} .7$ & $42 \cdot 3$ & $2 I$ & 8 & 47 & 41.8 & 41.7 & & 20 & 0 & 46 & 48.1 & 47.9 & 30 & ( & 46 & 23.5 & 23.0 & & $\infty 9$ & \\
\hline 48 & 46.9 & 48.0 & 29 & 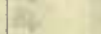 & 48 & 4 I.I & 42.0 & & 20 & 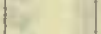 & 48 & 48.8 & 48.3 & 29 & 3. & 48 & 23.8 & 23.1 & & 09 & \\
\hline 50 & 47.2 & 47.8 & 30 & oes & 50 & 40. I & 40.5 & & 18 & 8 & 50 & 48.9 & 48.2 & 29 & E & 50 & $2 \mathrm{I} .9$ & 20.2 & & I3 & \\
\hline 52 & $45 \cdot 3$ & 46.0 & 26 & 52 & 52 & 40. I & 41.0 & & 18 & 1 & 52 & 48.8 & 48.0 & 30 & as & 52 & $20 . \overline{8}$ & I9. I & & 14 & \\
\hline 54 & 45.8 & 46.3 & 27 & 13 & 54 & 41.0 & 41.9 & & 20 & 2 & 54 & 48.0 & 47.2 & $3 \mathrm{I}$ & is & 54 & 18.2 & 17.3 & & 18 & \\
\hline 56 & 47.1 & 48.0 & 30 & ce & 56 & 39.2 & 40.9 & & I8 & Q & 56 & 49.0 & 48.2 & 29 & E & 56 & 18.9 & 18.2 & & I6 & \\
\hline 58 & 46.5 & 47.0 & 28 & & 58 & 40.5 & 40.9 & & I9 & 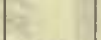 & 58 & 48.0 & 47.5 & $3 \mathrm{I}$ & & 58 & 21.4 & 20.2 & & 13 & \\
\hline 1300 & 46.0 & 46.9 & 28 & +20.0 & I5 00 & 40.2 & 41.0 & & פ' & & 100 & 47.8 & 47.0 & $3 I$ & +4.9 & 300 & 22. I & 2I.I & & I2 & +5.0 \\
\hline 02 & $45 . I$ & 46.2 & 26 & wer & 02 & 40.1 & 4I.I & & I9 & 1 & 02 & 47.9 & 47.1 & $3 I$ & & 02 & 23.8 & $22 . I$ & & ro & \\
\hline 04 & 45.1 & 45.8 & 26 & 20 & 04 & 40.8 & 41.8 & & 20 & 6 & 04 & 47.9 & $47 . I$ & $3 \mathrm{I}$ & yo & 04 & 22.5 & 21.4 & & II & \\
\hline$\infty$ & 45.5 & 46.2 & 27 & 37 & $\infty 6$ & 41.3 & $42 . I$ & & 20 & E & 06 & 47.2 & 46.7 & 32 & 6 & 06 & 20.3 & 19.2 & & 15 & 6 \\
\hline 08 & 46.2 & 47.3 & 28 & in & 08 & 40.8 & 42.0 & & 20 & & 08 & $47 . I$ & 46.4 & 32 & 6 & 08 & 19.9 & 19.0 & & 15 & \\
\hline I0 & 47.1 & 48.1 & 30 & es: & I0 & 40.5 & $4 \mathrm{I} .4$ & & I9 & +19.0 & Io & 46.1 & 45.4 & 34 & it & 10 & 19.6 & 18.9 & & 15 & \\
\hline 12 & 48.0 & 49.0 & $3 I$ & & I2 & 39.3 & 40.8 & & I8 & $7^{-}$ & I2 & 44.9 & 44.3 & 36 & & 12 & 19.0 & 18.2 & & 16 & \\
\hline 14 & 47.6 & 48.0 & 30 & +20.0 & 14 & 39.9 & 41.0 & & 19 & & 14 & 44.7 & 43.9 & 36 & +4.8 & 14 & I6. 9 & 16.2 & & 20 & +5.1 \\
\hline 16 & 46.8 & 47.1 & 29 & & 16 & 40.2 & 41.8 & & I9 & & 16 & 43.8 & 43.2 & 37 & a & 16 & 16.0 & 14.9 & & 21 & \\
\hline I8 & 43.3 & 44.0 & 23 & ist & I8 & 40.0 & 41.9 & & 19 & 10 & I8 & $43 . I$ & 43.1 & 38 & Er & 18 & 16.8 & 15.3 & & 20 & \\
\hline 20 & 42.5 & 42.9 & 22 & es & 20 & 39.0 & 39.0 & & I6 & Es & 20 & 43.3 & 43.1 & 38 & $=$ & 20 & 16.9 & 15.3 & & 20 & \\
\hline 22 & 43.8 & 44.0 & 24 & $5=$ & 22 & 38.1 & 38.2 & & I5 & $=$ & 22 & 43.4 & 43.0 & 38 & 26 & 22 & 15.8 & 14.7 & & 22 & \\
\hline 24 & 44.8 & 45.8 & , 26 & is & 24 & 38.1 & 38.4 & & I5 & Le & 24 & 41.7 & $4 I . I$ & 41 & is & 24 & 15.5 & 14.7 & & 22 & \\
\hline 26 & 46.8 & 47.0 & 29 & 25 & 26 & 37.1 & 37.9 & & 14 & 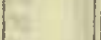 & 26 & 38.9 & 38.3 & 45 & & 26 & 16.0 & 15.7 & & $2 I$ & \\
\hline 28.6 & 48 & $.1 a$ & 30 & & 28 & 38.1 & 38.4 & & I5 & & 28 & 40.2 & 39.8 & 43 & & 28 & 16.7 & 16.1 & & 20 & \\
\hline 30 & 48.9 & 49.1 & 32 & +20.0 & 30 & 37.1 & 37.9 & & 14 & +18.3 & 30 & 39.4 & 38.7 & 44 & +4.8 & 30 & I7. 8 & 17.1 & & 18 & +5.3 \\
\hline 32 & 48.9 & 49.5 & 3I & ic & 32 & 36.9 & 36.9 & & I3 & & 32 & 39.9 & $39 . I$ & 44 & & 32 & 19.0 & 17.4 & & 17 & \\
\hline 34 & $48 . I$ & 48.8 & $3 \mathrm{I}$ & 12 & 34 & 36.2 & 37.5 & & 13 & 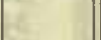 & 34 & 39.9 & 39.8 & 43 & & 34 & I8.6 & 17.5 & & 17 & \\
\hline 36 & 46.0 & 46.2 & 27 & 15 & 36 & 35.9 & 36.9 & & 12 & 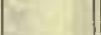 & 36 & 40.7 & 40.2 & 42 & & 36 & 17.3 & 16.4 & & 19 & \\
\hline 38 & 45.8 & 46.0 & 27 & E & 38 & $3^{8.7}$ & 39.0 & & 16 & . & 38 & 40.5 & 40.0 & 42 & he & 38 & 18.0 & I7. I & & 18 & \\
\hline 40 & 49 & $.0 a$ & 32 & re & 40 & 37.7 & 38.0 & & 14 & 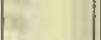 & 40 & 40.8 & 40.1 & 42 & 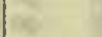 & 40 & 18.0 & 16.9 & & 18 & \\
\hline 42 & 48.0 & 49.0 & $3 \mathrm{I}$ & $x_{2}$ & 42 & 37.7 & 38.2 & & 14 & & 42 & $4 \mathrm{I} .4$ & 40.3 & 42 & & 42 & 17.8 & 16.9 & & 18 & \\
\hline 44 & 45.9 & 46.8 & 28 & +20.0 & 44 & 36.0 & 36.7 & & I2 & +17.8 & 45 & 41.9 & 41.0 & $4 \mathrm{I}$ & +4.9 & 44 & 18.0 & 17.0 & & 18 & +5.8 \\
\hline 46 & 46.1 & 47.0 & 28 & $y^{2}$ & 46 & 36.1 & $37 . I$ & & I2 & 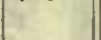 & 46 & 42.0 & 41.0 & 40 & & 46 & 19.3 & I8.0 & & 16 & \\
\hline 48 & 47.0 & 48.4 & 30 & $\theta$ & 48 & 34.5 & 35.8 & & I0 & 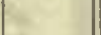 & 48 & 41.9 & 40.7 & $4 I$ & Es & 48 & 18.3 & I7.7 & & 17 & \\
\hline 50 & $47 . I$ & 48. I & 30 & Q & 50 & 34.1 & 35.3 & & $\infty$ & D & 50 & 40.8 & 39.3 & 43 & PS & 50 & 16.9 & 16.2 & & 20 & \\
\hline 52 & 47.2 & 48. I & 30 & $\alpha$ & 52 & 35.1 & 36.3 & & I I & E & 52 & 39.9 & 38.6 & 44 & 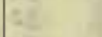 & 52 & 18.0 & 17.2 & & 18 & \\
\hline 54 & 48.0 & 49.0 & $3 \mathrm{I}$ & I & 54 & 37.3 & 38.2 & & 14 & $=$ & 54 & 35.9 & 34.9 & 50 & 2 & 54 & 18.9 & 18.1 & & 16 & \\
\hline 56 & 48.7 & 49.8 & 32 & & 56 & 35.2 & 36.0 & 1.7 & II & & 56 & 34.8 & 33.9 & 52 & be & 56 & I8. I & 17.2 & & 18 & \\
\hline 58 & 46.7 & $47 \cdot 3$ & 29 & & 58 & 36.8 & 36.8 & .2z & I3 & +17.0 & 58 & 32.8 & 31.9 & 55 & $=$ & 58 & 19.5 & I8.9 & $8+1$ & 15 & \\
\hline & & & & & & E= & 2 & & $s$ & & & & & & & & & & & & \\
\hline
\end{tabular}

Correction to local mean time is -36 s.

Observer-R. R. T.

Torsion head at Ith $42 \mathrm{~m}$ read $52^{\circ}$ and at $16 \mathrm{~h} \mathrm{I} 7 \mathrm{~m}$ read the same.

Observer-J. V. 
Tabulation of magnetic declinations observed at Teplitz Bay-Continued

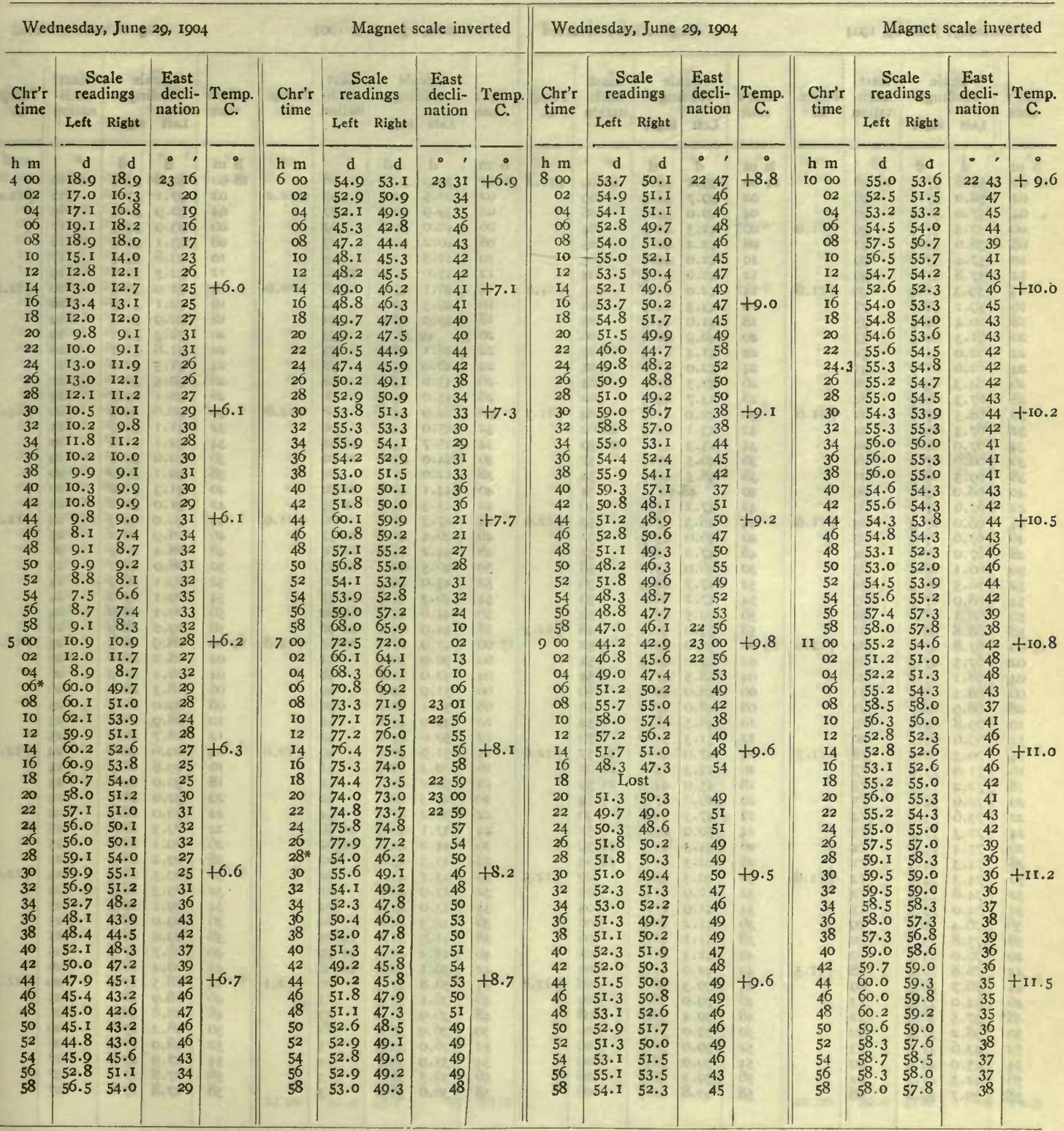

Observer-R. R. T.

Observers-R. R. T. and W. J. P., who alternated from $8 \mathrm{~h} 42 \mathrm{~m}$ to gh oom. 
Tabulation of magnetic declinations observed at Teplitz Bay-Continued

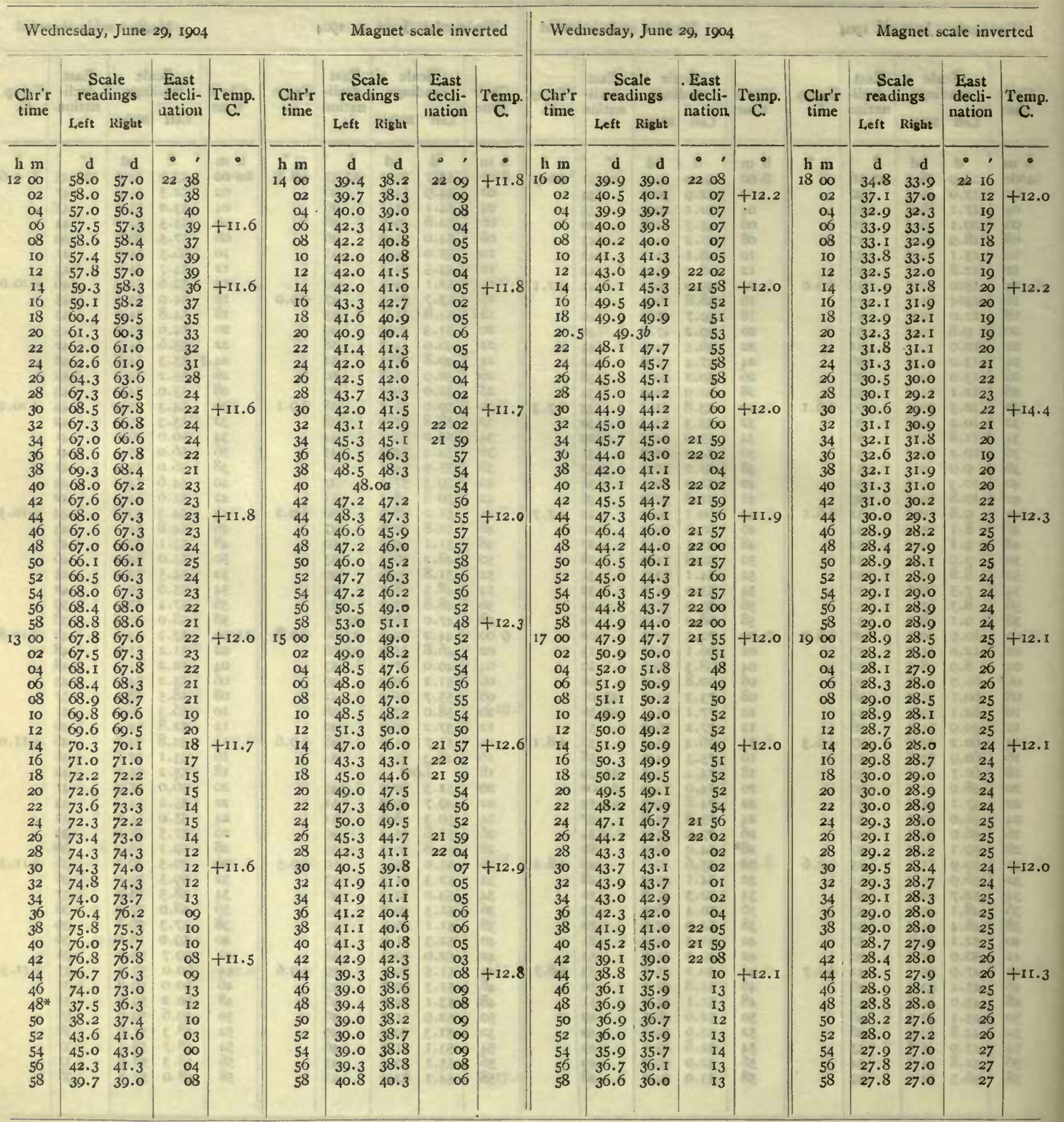


Tabulation of magnetic declinations observed at Teplitz Bay-Continued

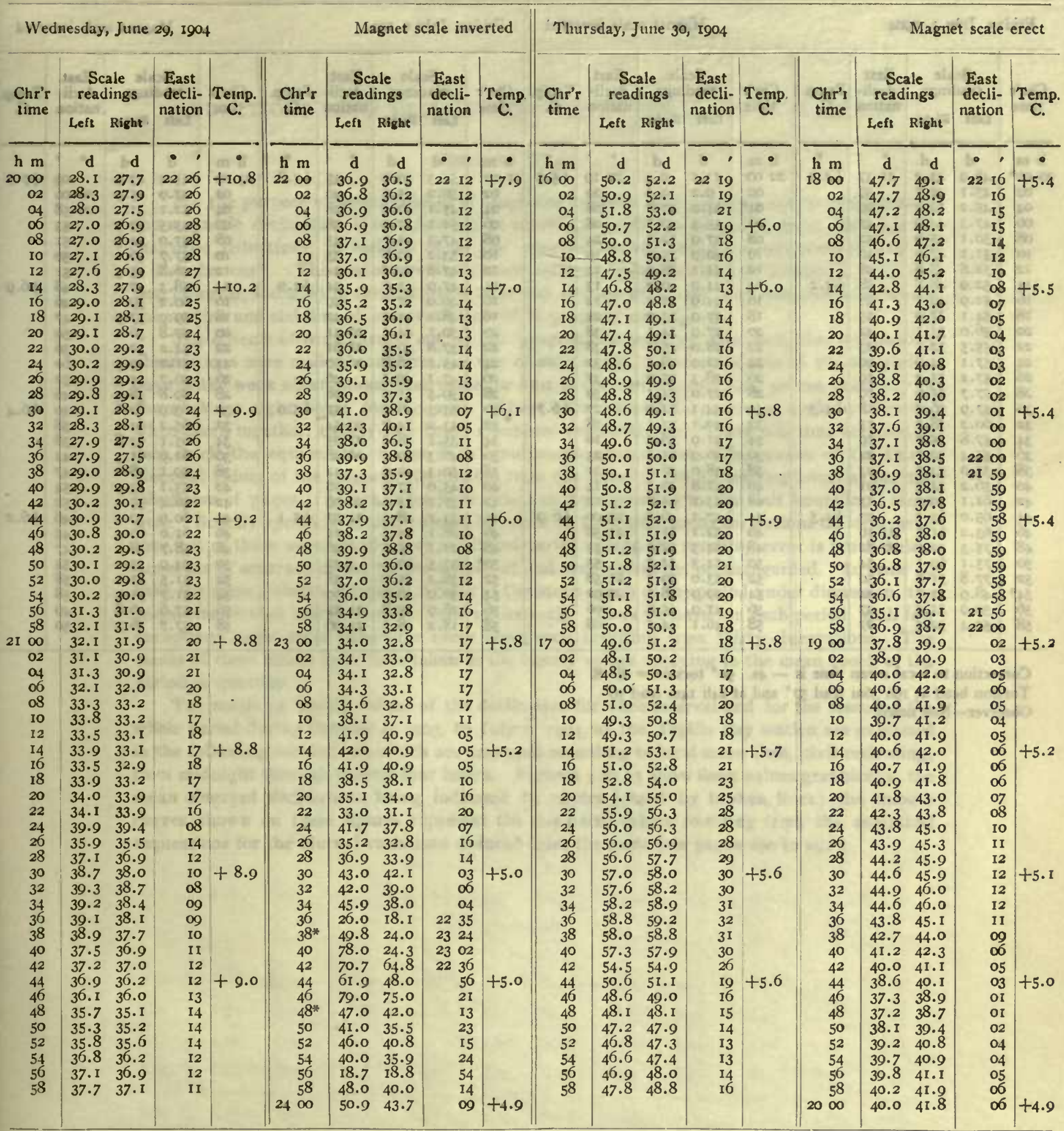

Correction to local mean time is $-58 \mathrm{~s} .90^{\circ}$ torsion $=199^{\prime} 21$. Torsion head at oh oom read $51^{\circ}$ and at 24 h $20 \mathrm{~m}$ read $53^{\circ}$. Observer-J. V.
Correction to local mean time is - Im 26s. $90^{\circ}$ torsion $=17 .^{\prime} 7 \mathrm{C}$ Torsion head at $15 \mathrm{~h} 35 \mathrm{~m}$ read $53^{\circ}$ and at $20 \mathrm{~h} 15 \mathrm{~m}$ read $36^{\circ}$. Observer-Not noted. 
Tabulation of magnetic declinations observed at Teplitz Bay-Continued

\begin{tabular}{|c|c|c|c|c|c|c|c|c|c|c|c|c|c|c|c|c|c|c|c|c|}
\hline \multicolumn{6}{|c|}{ Friday, July I, 1904} & \multicolumn{5}{|c|}{ Magnet scale inverted } & \multicolumn{6}{|c|}{ Friday, July I, I904 } & \multicolumn{4}{|c|}{ Magnet scale inverted } \\
\hline \multirow{2}{*}{$\begin{array}{l}\text { Chr'r } \\
\text { time }\end{array}$} & \multicolumn{2}{|c|}{$\begin{array}{l}\text { Scale } \\
\text { readings }\end{array}$} & \multirow{2}{*}{$\begin{array}{c}\text { East } \\
\text { decli- } \\
\text { nation }\end{array}$} & \multirow{2}{*}{$\begin{array}{c}\text { Temp. } \\
\text { C. }\end{array}$} & \multirow{2}{*}{$\begin{array}{l}\text { Chr'r } \\
\text { time }\end{array}$} & \multicolumn{2}{|c|}{$\begin{array}{l}\text { Scale } \\
\text { readings }\end{array}$} & \multirow{2}{*}{\multicolumn{2}{|c|}{$\begin{array}{c}\text { East } \\
\text { decli- } \\
\text { nation }\end{array}$}} & \multirow{2}{*}{$\begin{array}{l}\text { Temp. } \\
\text { C. }\end{array}$} & \multirow{2}{*}{$\begin{array}{l}\text { Chr'r } \\
\text { time }\end{array}$} & \multicolumn{2}{|c|}{$\begin{array}{l}\text { Scale } \\
\text { readings }\end{array}$} & \multirow{2}{*}{$\begin{array}{c}\text { East } \\
\text { decli- } \\
\text { nation }\end{array}$} & \multirow{2}{*}{ Cemp. } & \multirow{2}{*}{$\begin{array}{l}\text { Chr'r } \\
\text { time }\end{array}$} & \multicolumn{2}{|c|}{$\begin{array}{l}\text { Scale } \\
\text { readings }\end{array}$} & \multirow{2}{*}{$\begin{array}{l}\text { East } \\
\text { decli- } \\
\text { nation }\end{array}$} & \multirow{2}{*}{$\begin{array}{c}\text { Temp } \\
\text { C. }\end{array}$} \\
\hline & Left & Right & & & & Lefit & Right & & & & & Left & Right & & & & Left & Right & & \\
\hline$h \mathrm{~m}$ & d & d & $\cdot \quad$ & $\because$ & $h \mathrm{~m}$ & d & d & & & & h m & d & d & & $m$ & $\mathrm{~h} \mathrm{~m}$ & d & d & • & \\
\hline $2000^{*}$ & 46.9 & 43.2 & 2137 & $+4 . I$ & $22 \infty 0$ & II.I & 9.4 & & I9 & $+5 \cdot 3$ & 2100 & $54 \cdot 3$ & 49.9 & 33 & +4.8 & 2300 & $45 \cdot 4$ & 43.9 & $\infty$ & +7.0 \\
\hline 02 & $45 . I$ & 42.5 & 39 & 0 & 02 & 9.2 & 9.2 & & 20 & & 02 & 50.0 & 45.9 & 40 & & & 47.9 & & 2206 & \\
\hline 04 & 45.2 & 52.2 & 39 & a & $04^{*}$ & 34.2 & 30.8 & & 28 & 0 & 04 & $45 . I$ & 40.3 & 48 & N & 04 & $52 . I$ & 51.1 & 2158 & in \\
\hline$\infty$ & $47 \cdot 3$ & 42.9 & 37 & 60 & 06 & 35.3 & 30. & & 28 & 0 & $\infty$ & 5I.I & 47.0 & 38 & en & $\infty 6$ & 55.5 & 53.9 & 53 & \\
\hline$\infty 8$ & 50. I & 5.9 & 32 & es & 08 & 37.9 & 32.5 & & 24 & 60 & 08 & $47 \cdot 3$ & 44.0 & 43 & 6 & as & 57.0 & $54 \cdot 9$ & $5 \mathrm{I}$ & ? \\
\hline 10 & 5I. I & 7.2 & 30 & et & 10 & 40.9 & & & 19 & en & 10 & & 50.8 & 33 & 61 & IO & & & 50 & \\
\hline 12 & 52.8 & 8.8 & 28 & & 12 & $44 . I$ & 38. & & 14 & ur & 12 & 47.8 & 42.2 & 44 & & 12 & 56.8 & 55 & $5 \mathrm{I}$ & \\
\hline 14 & 52.9 & 48.8 & 28 & +4.2 & 14 & 44.0 & 40. & & 13 & Ir & 14 & 47.9 & 43.0 & 44 & +4.9 & I4 & 58.3 & & 48 & +6.9 \\
\hline 16 & 52.9 & 49.1 & 28 & 18 & 16 & 44.9 & 40.4 & & 12 & ir & 16 & 46.0 & 37.7 & 49 & & 16 & 60.2 & 59 & 46 & \\
\hline I8 & $54 . \mathrm{I}$ & 1.0 & 25 & $8 \mathrm{~s}$ & 18 & 37.0 & 33.9 & & 24 & it & 18 & 48. I & 44.9 & 42 & int & I8 & 59.9 & & 47 & \\
\hline 20 & 56.0 & 53.1 & 22 & ces & 20 & 27.9 & $24 . \mathrm{I}$ & & 38 & $=$ & 20 & 50.1 & 44.1 & $204 I$ & 60 & 20 & 57.8 & 56 & 50 & \\
\hline 22 & 55.5 & 52.1 & 23 & ats. & 22 & 28.0 & 25.1 & & 38 & LE & 22 & 37.8 & 30.9 & $21 \mathrm{OI}$ & 50 & 22 & 54.2 & 52.1 & 56 & \pm \\
\hline 24 & 57.1 & 54.9 & 20 & in & 24 & 32.0 & 28. & & 32 & is & 2 & & 36.8 & 2050 & 14 & 24 & 51.2 & & 60 & \\
\hline 26 & 57.2 & 55.2 & 19 & 26s & 26 & 28.3 & 25.0 & & 37 & E & 26 & 47.1 & 40.1 & 46 & 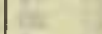 & 26 & 56.7 & 54 & 52 & \\
\hline 28 & 57.7 & 55.6 & 19 & 25 & 28 & 27.0 & 24 & & 39 & & 28 & 59.5 & 51.2 & 28 & $1=$ & 28 & 59.8 & 58 & 46 & \\
\hline 30 & 57.9 & 56.2 & 18 & +4.3 & 30 & 34.1 & 31.9 & & 27 & +6.7 & 30 & 53.3 & 46.3 & 37 & +5.0 & 30 & 63.9 & 62.6 & 40 & +6.4 \\
\hline 32 & 60.2 & 58.0 & 15 & a & 32 & 40.1 & & & 18 & $z^{2}$ & 3 & & 34.7 & 2054 & & 3 & 65.2 & & 38 & \\
\hline 34 & 57.8 & 55.0 & 19 & tis: & 34 & 41.9 & 38.9 & & 16 & I & 34 & 35.1 & 28.2 & 2105 & $y$ & 34 & $65 . I$ & 63.8 & 38 & \\
\hline 36 & 54.4 & 52.1 & 24 & 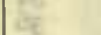 & 36 & & & & 23 & $=$ & 36 & 46.3 & 39.2 & 2048 & $6=$ & 3 & 62.9 & & $4^{2}$ & \\
\hline 38 & 55.0 & 53.1 & 23 & $\varepsilon$ & 38 & 38.0 & 36. & & 21 & 6 & 38 & 50.3 & 42.9 & 42 & Ee & 38 & 59. I & 58 & 47 & \\
\hline 40 & 56.2 & $54 \cdot 3$ & 21 & 20 & 40 & 42.8 & 40 & E & 14 & 8 & 40 & 45.0 & 37.0 & 50 & 6 & 4 & $55 . \mathrm{I}$ & 53 & 5 & \\
\hline 42 & 61.1 & 59.0 & 13 & & 42 & 43.1 & $4 I$ & & 12 & & 42 & 37.1 & 34.0 & 2059 & & 4 & 56.4 & & & \\
\hline 44 & 65.0 & 63.2 & 07 & +4.5 & 44 & 47.1 & 46.0 & 22 & 06 & +6.9 & 44 & 33.1 & 28.2 & 2107 & $+5 . I$ & 4 & 60.0 & 59.3 & 46 & +6.2 \\
\hline 46 & 63.2 & $6 \mathrm{I} .5$ & 2110 & en & 46 & 52.1 & 51 . & $2 I$ & 58 & te & 46 & 28. I & 24.4 & I4 & 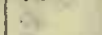 & 46 & 61.0 & 60.3 & 44 & \\
\hline 48 & 75.3 & 72.0 & 2052 & 83 & 48 & 54.5 & 52.9 & $=1$ & 55 & S & 48 & $2 I . I$ & 15.0 & 26 & 8 & 48 & 58.7 & 58.1 & 47 & \\
\hline & 75.1 & 69.1 & 54 & 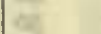 & 50 & 54.3 & 52.3 & & 55 & es: & $50^{*}$ & 37.5 & 30.0 & 42 & 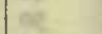 & 5 & 54.9 & & & \\
\hline 52 & 77.8 & 71.8 & 50 & cea & 52 & 53.0 & 50.5 & $2 I$ & 58 & 2 & 52 & 38.2 & 35.0 & 38 & 22 & 52 & 51.5 & $5 \mathrm{I} .1$ & & \\
\hline $54^{*}$ & 48.9 & $4 I .1$ & . 44 & 12 & 54 & 49.9 & $48 . I$ & 22 & 02 & 4 & 54 & 30.9 & 26.9 & 50 & 42 & 54 & 51.7 & & 58 & \\
\hline 56 & 59.9 & 51.9 & 27 & 6 & 56 & 47.0 & 45.8 & & $\infty$ & 5 & 56 & 28.9 & 25.8 & 2152 & 6 & 5 & 57.6 & 56.8 & 49 & \\
\hline 58 & 59.4 & 52.3 & 27 & & 58 & 46.1 & 44.2 & $6 e^{4}$ & 08 & & 58 & 18.2 & I8. I & 2206 & 3 & 58 & 57.7 & 56.2 & 1. & \\
\hline
\end{tabular}

Correction to local mean time is $-45.90^{\circ}$ torsion $=18$.' 18 . Torsion head at $19 \mathrm{hh} 36 \mathrm{~m}$ read $57^{\circ}$ and at $24 \mathrm{~h} 14 \mathrm{~m}$ read $56^{\circ}$.

Observer-Not noted. 


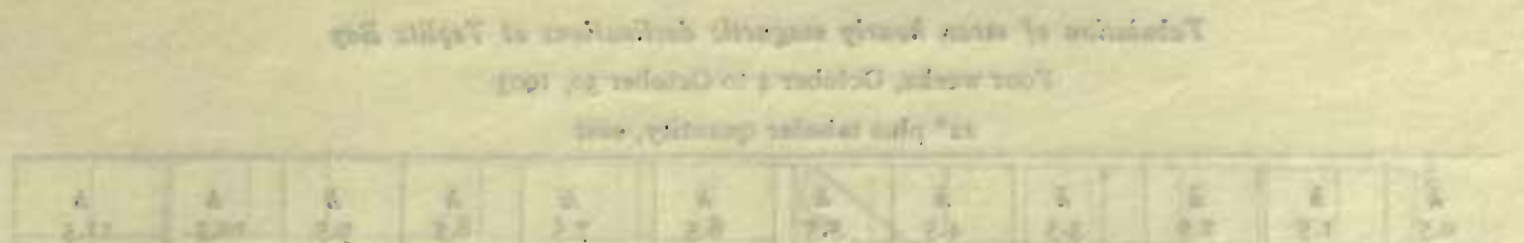

\section{REDUCTIONS FROM DECLINATION OBSERVATIONS AT TEPLITZ BAY}

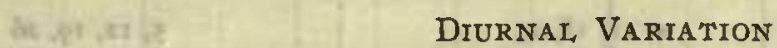

For the purpose of determination of empirical formula to express the diurnal variation in the magnetic declination it was decided, in accordance with the general present tendency in magnetic reductions, to include all of the observed values without elimination of any as "disturbances". The few observations made betweeu September 28 and October 4, 1903, are not, however, included in the discussion as it was deemed that some time was necessary before the routine and stability of observation could be properly established.

The scheme of work carried out, as per program on page 17 , was such that continuous observations were made throughout one day of each week. On each of four of the remaining days of the week observations were made for four hours continuously, and on one other day of the week for eight hours continuously. These latter observations were so made as to cover, when taken together, twenty-four hours numbered consecutively. There was thus obtained in each week the equivalent of two days' continuous observation. The means of the thirty observed values of the magnetic declination from one hour to the succeeding hour, as per the tabulation of pages $4 \mathrm{r}$ to 274 , have been taken as corresponding to the half hours local mean time. Strictly speaking account should be taken of the chronometer corrections on local mean time but as these were usually very small and varied in sign the resulting error is much below the order of accuracy of the results arrived at and may, therefore, be disregarded. In order to have the mean values correspond strictly to the mean epoch of the period under discussion, the series was divided into intervals of four weeks each. Thus we have for each period, with few exceptions, eight mean values for every hour, each resulting from thirty observations. The means of these means have been taken as the hourly values applying to the mean epoch of the period in question.

The resulting hourly values of the declination for each interval and for the mean of the whole period during October 4, 1903 , to July I, I904, at the Teplitz Bay station are exhibited in the following tabulations, which are arranged according to local mean time, civil reckoning, from midnight through twenty-four hours. Figures 5 to 5 show these values graphically, the mean observed declinations being indicated by circles joined by broken lines; the smooth curves shown on these figures represent the computed values resulting from the analytical expressions for the diurnal variations deduced from the same (see pages 290 to $29 \mathrm{l}$ ). 
Tabulation of mean hourly magnetic declinations at Teplitz Bay

Four weeks, October 4 to October 30,1903

$22^{\circ}$ plus tabular quantity, east

\begin{tabular}{|c|c|c|c|c|c|c|c|c|c|c|c|}
\hline $\begin{array}{c}h \\
0.5\end{array}$ & $\begin{array}{c}h \\
1.5\end{array}$ & $\begin{array}{c}h \\
2.5\end{array}$ & $\begin{array}{c}h \\
3.5\end{array}$ & $\begin{array}{c}h \\
4.5\end{array}$ & $\begin{array}{c}h \\
5.5\end{array}$ & $\begin{array}{c}h \\
6.5\end{array}$ & $\begin{array}{c}h \\
7.5\end{array}$ & $\begin{array}{c}h \\
8.5\end{array}$ & $\begin{array}{c}h \\
9.5\end{array}$ & $\begin{array}{c}h \\
10.5\end{array}$ & $\begin{array}{c}h \\
11.5\end{array}$ \\
\hline & \multicolumn{3}{|c|}{ Sunday } & & \multicolumn{3}{|c|}{ Sunday } & $t$ & \multicolumn{2}{|c|}{ Monday } & \\
\hline 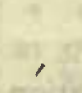 & $4, \mathrm{II}$ & $\begin{array}{r}\text { I8, } 25 \\
,\end{array}$ & $\prime$ & & 4, II , & 8,25 & , & 2 & $\begin{array}{l}5, \mathrm{I} \\
,\end{array}$ & $\begin{array}{c}\text { J9, } 26 \\
,\end{array}$ & , \\
\hline 40.4 & 41.5 & 45.4 & 45.2 & 52.8 & 67.4 & 73.4 & 52.9 & 33.9 & 33.2 & 337 & 35.4 \\
\hline$\cdots$ & $\cdots$ & $\cdots$ & $\cdots$ & $\cdots$ & $\cdots$ & $\cdots \cdot$ & $\ldots$ & 48.7 & 33.7 & 26.1 & 21.1 \\
\hline 28.0 & 36.5 & 40.3 & 28.5 & 39.0 & 50.1 & 58.9 & 63.8 & 44.7 & 27.0 & 27.2 & 17.1 \\
\hline 32. I & 29.1 & 34.0 & 31.4 & 36.4 & 38.4 & 34.9 & 31.0 & 68.8 & 48.1 & 47.9 & 45.7 \\
\hline oract & 2) Ind & 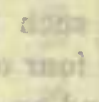 & 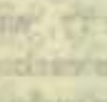 & 48 & $\begin{array}{l}\text { Wed } \\
7,14\end{array}$ & $\begin{array}{l}\text { day } \\
28\end{array}$ & & & & & \\
\hline 32.3 & 32.0 & 36.7 & 44.6 & 39.1 & 50.9 & 45.1 & 44.5 & 44.9 & 33.9 & 20.2 & 22.5 \\
\hline $48 . I$ & 53.0 & 123.0 & 145.9 & 126.2 & 80.7 & 77.5 & 81.9 & 65.9 & $45 \cdot 3$ & 44.0 & 31.7 \\
\hline 33.2 & 34.5 & 37.9 & 35.6 & 36.6 & 36.6 & 36.7 & 36.4 & 36.4 & 35.8 & $34 \cdot 3$ & 33.0 \\
\hline 33.0 & 35.5 & 48.7 & 53.4 & 46.3 & 57.5 & 63.9 & 50.6 & 35.3 & 29.8 & 28.7 & 28.2 \\
\hline 35.2 & 37.4 & 52.3 & 54.9 & 53.8 & 54.5 & 55.8 & 51.6 & $47 \cdot 3$ & 35.8 & 32.8 & $29 \cdot 3$ \\
\hline
\end{tabular}

Tabulation of mean hourly magnetic declinations at Teplitz Bay Four weeks, October 4 to October 30,1903 -Continued

$22^{\circ}$ plus tabular quantity, east

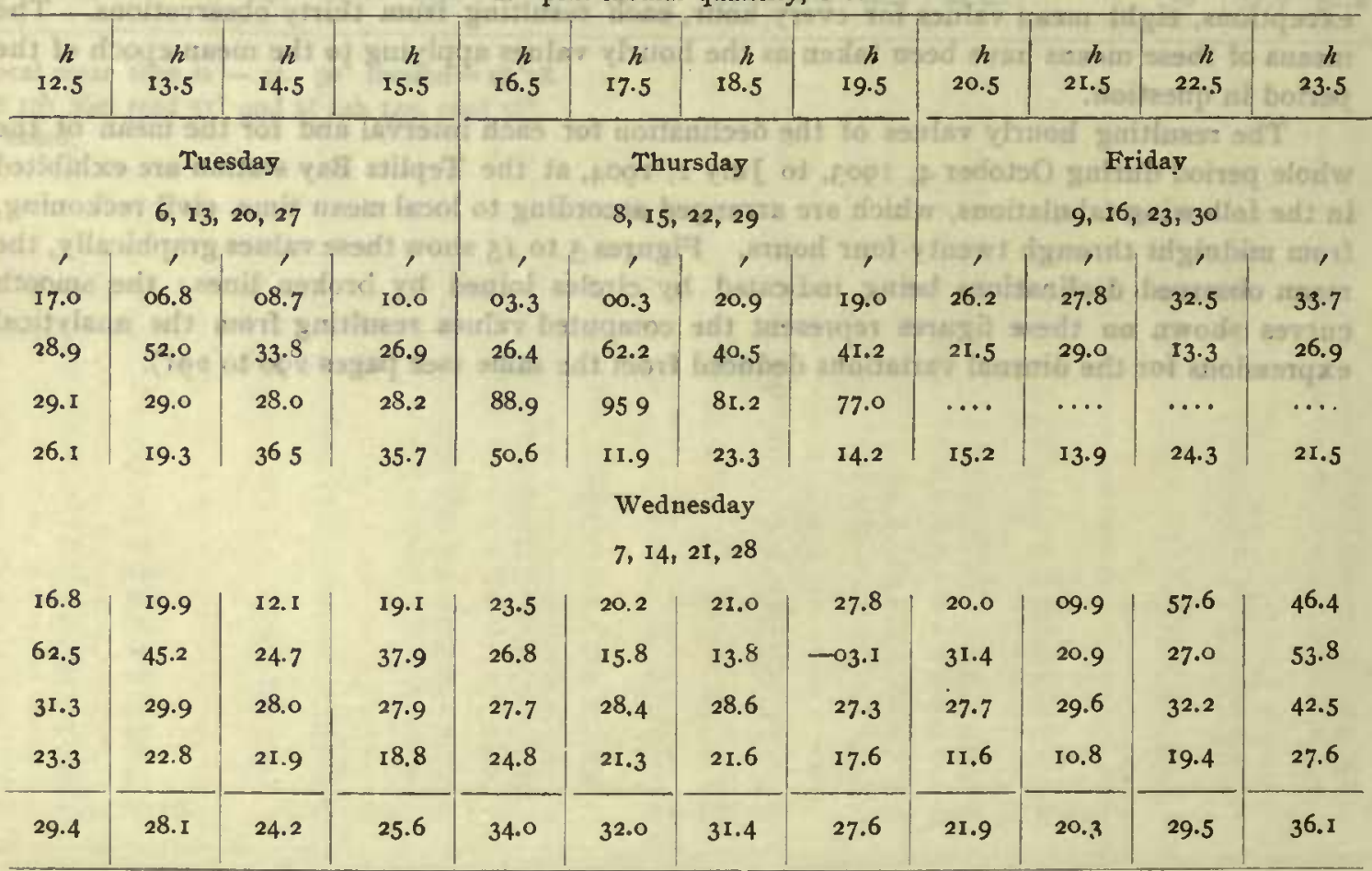

Mean value for the whole period, $22^{\circ} 36^{\prime} 7 \mathrm{E}$. 


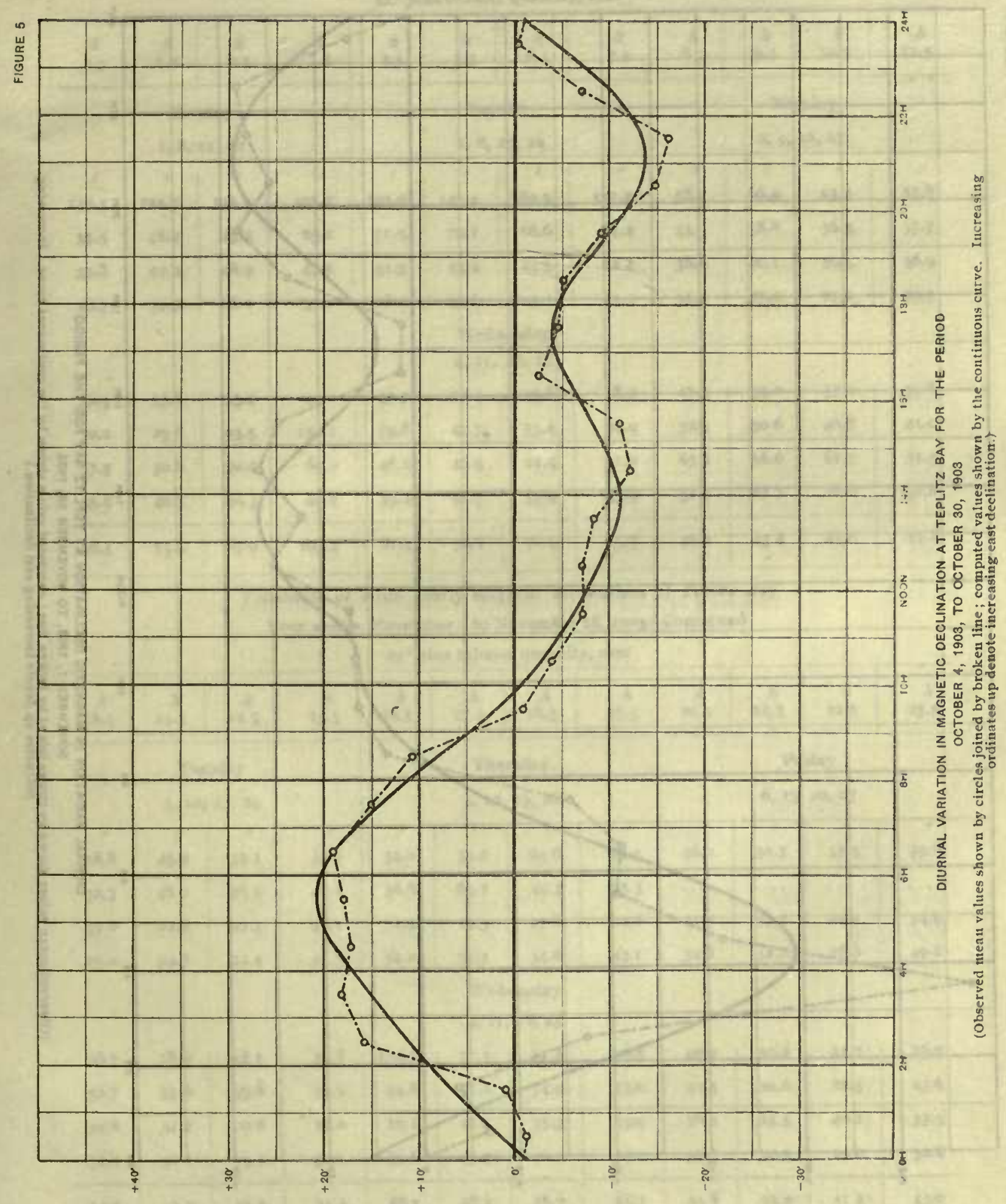




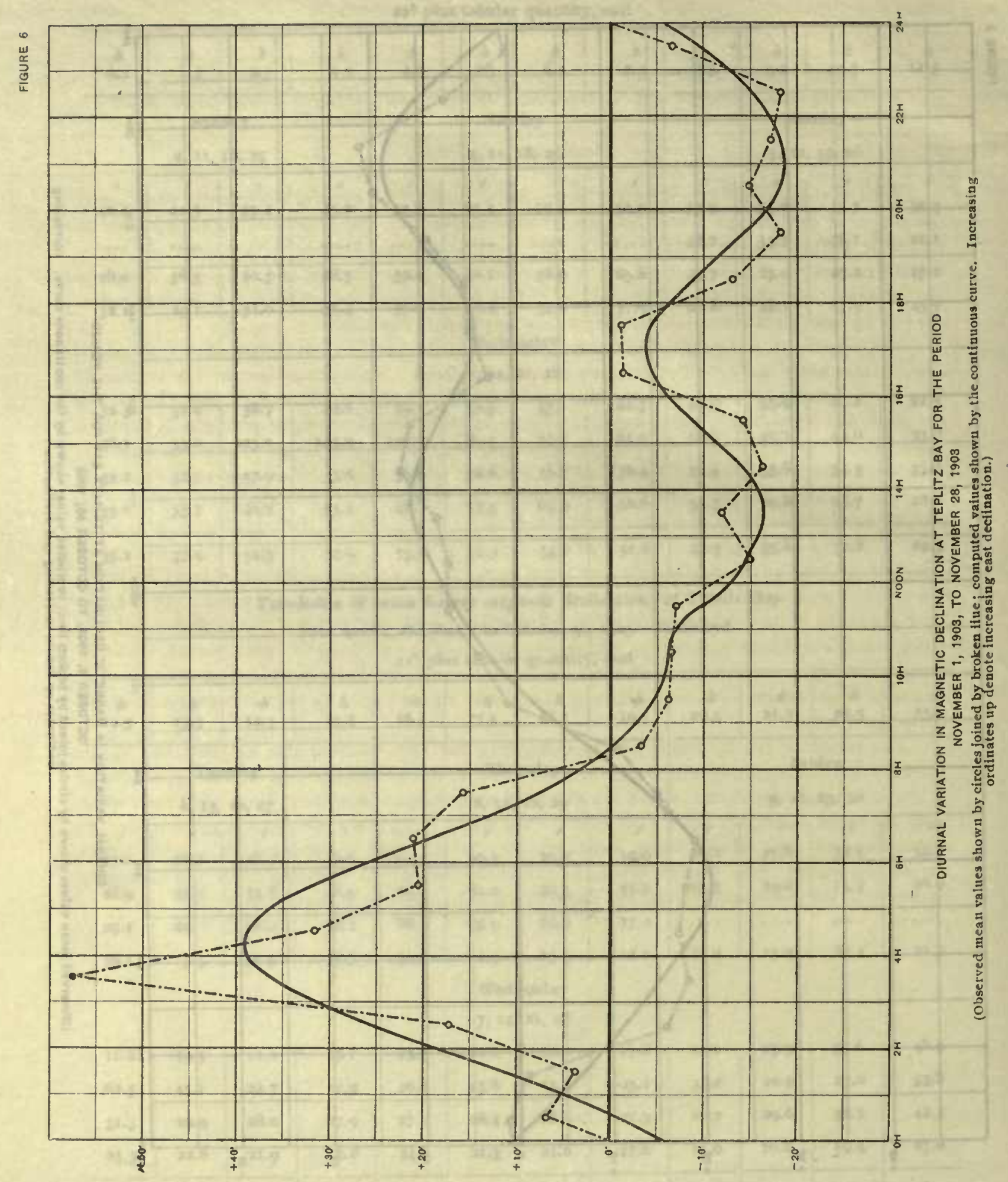


Tabulation of mean haurly magnetic declinatians af Teplifz Bay

Four weeks, November I to November 28 , I 903

$22^{\circ}$ plus tabular quantity, east

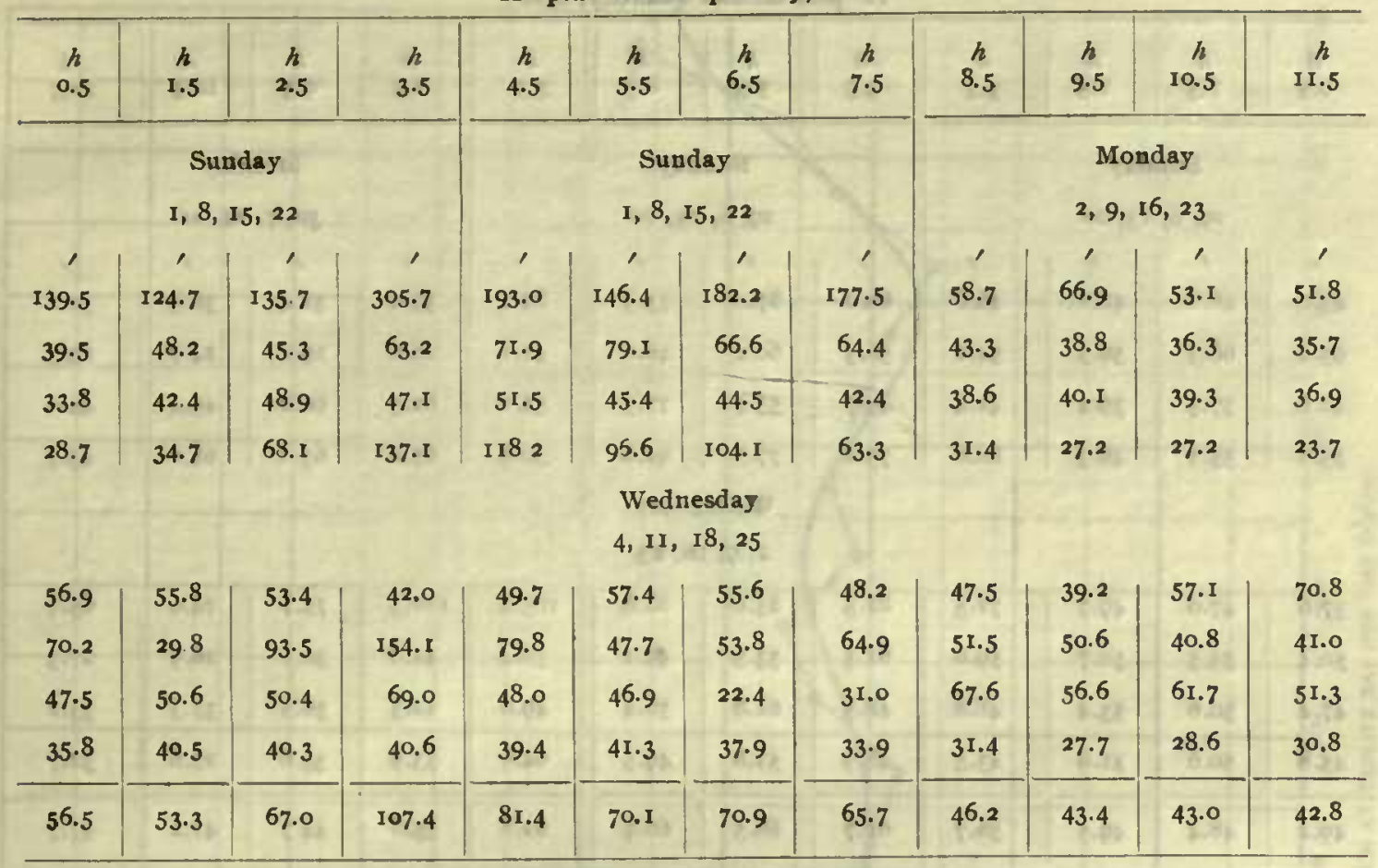

Tabulation of mean hourly magnetic declinations at Teplitz Bay Four weeks, November $\mathrm{I}$ to November 28,1903 -Continued

$22^{\circ}$ plus tabular quantity, east

\begin{tabular}{|c|c|c|c|c|c|c|c|c|c|c|c|}
\hline $\begin{array}{c}h \\
\mathbf{2} 2.5\end{array}$ & $\stackrel{h}{13.5}$ & $\stackrel{h}{145}$ & $\begin{array}{c}h \\
15.5\end{array}$ & I6.5 & $\stackrel{h}{h}$ & I8.5 & $\begin{array}{c}h \\
\text { I9.5 }\end{array}$ & $\begin{array}{c}h \\
20.5\end{array}$ & $\stackrel{h}{21.5}$ & $\begin{array}{c}h \\
22.5\end{array}$ & $\begin{array}{c}h \\
23.5\end{array}$ \\
\hline & \multicolumn{2}{|c|}{ Tuesday } & & \multicolumn{3}{|c|}{ Thursday } & & & \multicolumn{2}{|c|}{ Friday } & \\
\hline & \multicolumn{2}{|c|}{$3,10,17,24$} & & \multicolumn{3}{|c|}{$5,12,19,26$} & & & \multicolumn{2}{|c|}{$6,13,20,27$} & \\
\hline 1 & 1 & 1 & ' & 1 & ' & 1 & 1 & 1 & 1 & 1 & 1 \\
\hline I8.8 & $45 \cdot 4$ & 33.1 & 35.0 & 34.0 & 35.2 & 24.6 & 24.5 & 46,2 & 50.3 & $35 \cdot 3$ & 39.8 \\
\hline 32.3 & 48.0 & 48.5 & 40.1 & 56.8 & 69.7 & $44 \cdot 3$ & $35 \cdot 3$ & $\cdots \cdots$ & $\cdots$ & $\cdots$ & $\cdots$ \\
\hline 39.6 & 22.6 & 07.3 & 40.1 & $34 \cdot 4$ & 29.3 & 27.8 & 24.8 & $44 \cdot 5$ & $37 \cdot 2$ & 44.4 & 54.9 \\
\hline 25.0 & 39.8 & 44.4 & $45 \cdot 3$ & 34.2 & 35.4 & 34.8 & 33.1 & 32.8 & 32.2 & 26.8 & 49.6 \\
\hline & & & & & We & esday & & & & & \\
\hline & & & & & $4, I$ & 8,25 & & & & & \\
\hline 26.7 & 28.0 & 34. I & 20.8 & 134.3 & 77.3 & $2 I .4$ & 30.3 & 29.9 & 30.2 & 21.7 & 26.9 \\
\hline 52.7 & 54.8 & 39.8 & 54.1 & 42.8 & 71.0 & 74.9 & 54.0 & 40.4 & 20.6 & 22.5 & $43 \cdot 6$ \\
\hline 50.8 & 34.2 & 29.6 & 18.0 & 16.1 & 26. I & $25 \cdot 3$ & 23.4 & 18.2 & 25.5 & 40.1 & 53.3 \\
\hline 32.0 & 30.1 & 29. I & 30.1 & 33.6 & 43.5 & 40.2 & 24.4 & $3 I .5$ & 30.5 & 29.0 & 32.8 \\
\hline $34 \cdot 7$ & 37.9 & 33.2 & 35.4 & 48.3 & 48.4 & 36.7 & 31.2 & 34.8 & 32.4 & 314 & 43.0 \\
\hline
\end{tabular}

Mean value for the whole period, $22^{\circ} 49 .^{\prime} 8 \mathrm{H}$. 
Tabulation of mean hourly magnetic declinations at Teplitz Bay

Four weeks, November 29 to December 26, Ig03

$22^{\circ}$ plus tabular quantity, east

\begin{tabular}{|c|c|c|c|c|c|c|c|c|c|c|c|}
\hline $\begin{array}{c}h \\
0.5\end{array}$ & $\begin{array}{c}h \\
I .5\end{array}$ & $\begin{array}{c}h \\
2.5\end{array}$ & $\begin{array}{c}h \\
3.5\end{array}$ & $\begin{array}{c}h \\
4.5\end{array}$ & $\begin{array}{c}h \\
5 \cdot 5\end{array}$ & $\begin{array}{c}h \\
6.5\end{array}$ & $\begin{array}{c}h \\
7 \cdot 5\end{array}$ & $\stackrel{h}{8.5}$ & $\begin{array}{c}h \\
9.5\end{array}$ & $\begin{array}{c}h \\
10.5\end{array}$ & $\underset{11.5}{h}$ \\
\hline & \multicolumn{2}{|c|}{$\begin{array}{c}\text { Sunday } \\
29,6,13,20\end{array}$} & & \multicolumn{4}{|c|}{$\begin{array}{c}\text { Sunday } \\
29,6,13,20\end{array}$} & \multicolumn{3}{|c|}{$\begin{array}{c}\text { Monday } \\
30,7,14,21\end{array}$} & \\
\hline 43.0 & $\begin{array}{c}\prime \\
46.6\end{array}$ & 48. I & $4^{\prime}$ & $\frac{\prime}{60.2}$ & 8 & $\begin{array}{c}\text { ' } \\
73.9\end{array}$ & $\stackrel{\prime}{84 \cdot I}$ & $\begin{array}{c}1 \\
39.0\end{array}$ & $\begin{array}{c}\prime \\
3 I .4\end{array}$ & 30.5 & 31.2 \\
\hline 93.4 & 66.3 & 56.5 & $54 . I$ & 56.5 & 60.0 & 46.0 & 4I.I & 28.6 & 19. 1 & 24.7 & 23.5 \\
\hline 40.6 & 37.5 & $39.4^{i}$ & 41.4 & 43.7 & $55 \cdot 7$ & 77.5 & 55.2 & 62.5 & 60.2 & 49.4 & 44.0 \\
\hline 35.6 & $35 \cdot 1$ & 46.3 & 88.8 & 73.1 & $77 \cdot 3$ & 92.6 & 50.6 & 65.0 & 63.1 & 61.2 & 43.7 \\
\hline & & & & & Wedn & day & & & & & \\
\hline & & & & & 2,9 & 23 & 7 & & & & \\
\hline 37.6 & 47.0 & 49.7 & 71.5 & 81.5 & 83.6 & 88.6 & 105.4 & IIO.3 & 75.7 & 74.0 & 65.7 \\
\hline 50.4 & 53.5 & 50.7 & 59.0 & $8 \mathrm{I} .5$ & 53.5 & 49.I & 52.4 & 44.8 & 36.1 & 26.9 & 27.3 \\
\hline $47 \cdot 4$ & 50.6 & 53.4 & 48.8 & 48.5 & $6 \mathrm{r} .8$ & 56.4 & 49.0 & 42.3 & 36.5 & 37.3 & 33.2 \\
\hline 45.8 & 50.6 & 51.9 & 43.5 & 48.9 & 52.8 & 49.5 & 64.6 & 53.9 & 35.6 & 25.6 & 32.7 \\
\hline 49.2 & 48.4 & 49.5 & 56.7 & 61.7 & $66.5^{\circ}$ & 66.7 & 62.8 & 55.8 & 447 & $4 \mathrm{r} .2$ & 37.7 \\
\hline
\end{tabular}

Tabulation of mean hourly magnetic declinations at Teplitz Bay

Four weeks, November 29 to December 26, 1903-Continued

$22^{\circ}$ plus tabular quantity, east

\begin{tabular}{|c|c|c|c|c|c|c|c|c|c|c|c|}
\hline $\begin{array}{c}h \\
12.5\end{array}$ & $\begin{array}{c}h \\
13 \cdot 5\end{array}$ & $\begin{array}{c}h \\
14.5\end{array}$ & $\stackrel{h}{15.5}$ & $\stackrel{h}{16.5}$ & $\begin{array}{c}h \\
77 \cdot 5\end{array}$ & $\stackrel{h}{18.5}$ & $\begin{array}{c}h \\
19.5\end{array}$ & $\begin{array}{c}h \\
20.5\end{array}$ & $\stackrel{h}{21.5}$ & $\begin{array}{c}h \\
22.5\end{array}$ & $\begin{array}{c}h \\
23.5\end{array}$ \\
\hline & \multicolumn{2}{|c|}{$\begin{array}{l}\text { Tuesday } \\
1,8,15,22\end{array}$} & & & \multicolumn{2}{|c|}{$\begin{array}{l}\text { Thursday } \\
3,10,17,24\end{array}$} & & & \multicolumn{2}{|c|}{$\begin{array}{c}\text { Friday } \\
4,11,18,25\end{array}$} & \\
\hline $\begin{array}{c}\prime \\
27.1\end{array}$ & 18.5 & 32.9 & ${ }^{\prime} 1.0$ & 35.2 & 30.8 & 31.4 & 26.3 & 18.6 & $\begin{array}{c}\prime \\
49.6\end{array}$ & I4. 6 & 80.7 \\
\hline 36.1 & 31.8 & 43.5 & 62.2 & 32.7 & $3^{1.9}$ & 33.5 & 34.6 & 33.4 & 34.9 & 33.2 & 37.1 \\
\hline 41.0 & 39.2 & 39.8 & $37 \cdot 3$ & $3^{8} .5$ & $\cdot 39.1$ & $3^{8.9}$ & 38.4 & 33.7 & 28.2 & 30.6 & 39.6 \\
\hline 34.5 & 34.1 & 36.9 & 34.1 & 37.3 & 36.8 & 37.6 & 37.4 & & & & $\ldots$ \\
\hline & & & & & Wed & sday & & & & & \\
\hline 44.7 & 44.5 & 68.4 & $54 . I$ & 30.2 & $\begin{array}{c}2,9 \\
48.7\end{array}$ & $\begin{array}{l}23 \\
32.5\end{array}$ & 23.0 & 23.3 & 25.2 & 36.7 & 32.3 \\
\hline 30.8 & 35.8 & 30.2 & 33.1 & 43.9 & 43.5 & 44.8 & 41.0 & 37.8 & 38.2 & 45.2 & 46.3 \\
\hline 36.4 & 36.0 & 33.2 & 40.8 & 39.0 & $3^{8.6}$ & $3^{8.5}$ & $3^{8.5}$ & 35.6 & 54.6 & 40.6 & 42.0 \\
\hline 36.9 & 30.1 & 30.8 & $3 \mathrm{I} .4$ & 35.9 & 40.2 & 36.3 & 37.7 & 36.2 & 37.5 & 91.5 & 88.4 \\
\hline 35.9 & 33.8 & 39.5 & 41.8 & 36.6 & $3^{8.7}$ & 36.7 & 34.6 & 31.2 & $3^{8.3}$ & 41.8 & 52.3 \\
\hline
\end{tabular}

Mean value for the whole period, $22^{\circ} 45 \cdot 9 \mathrm{E}$. 


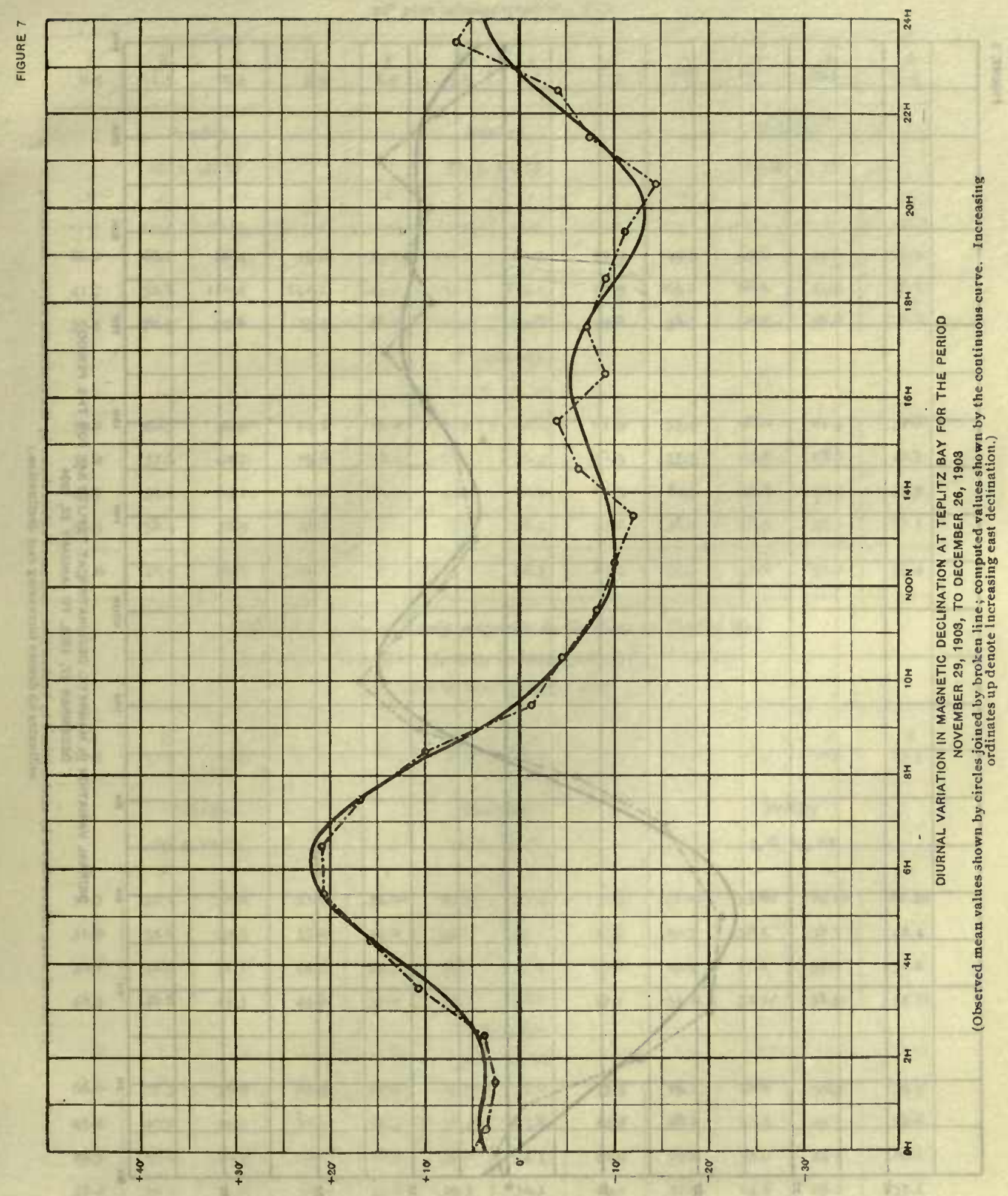




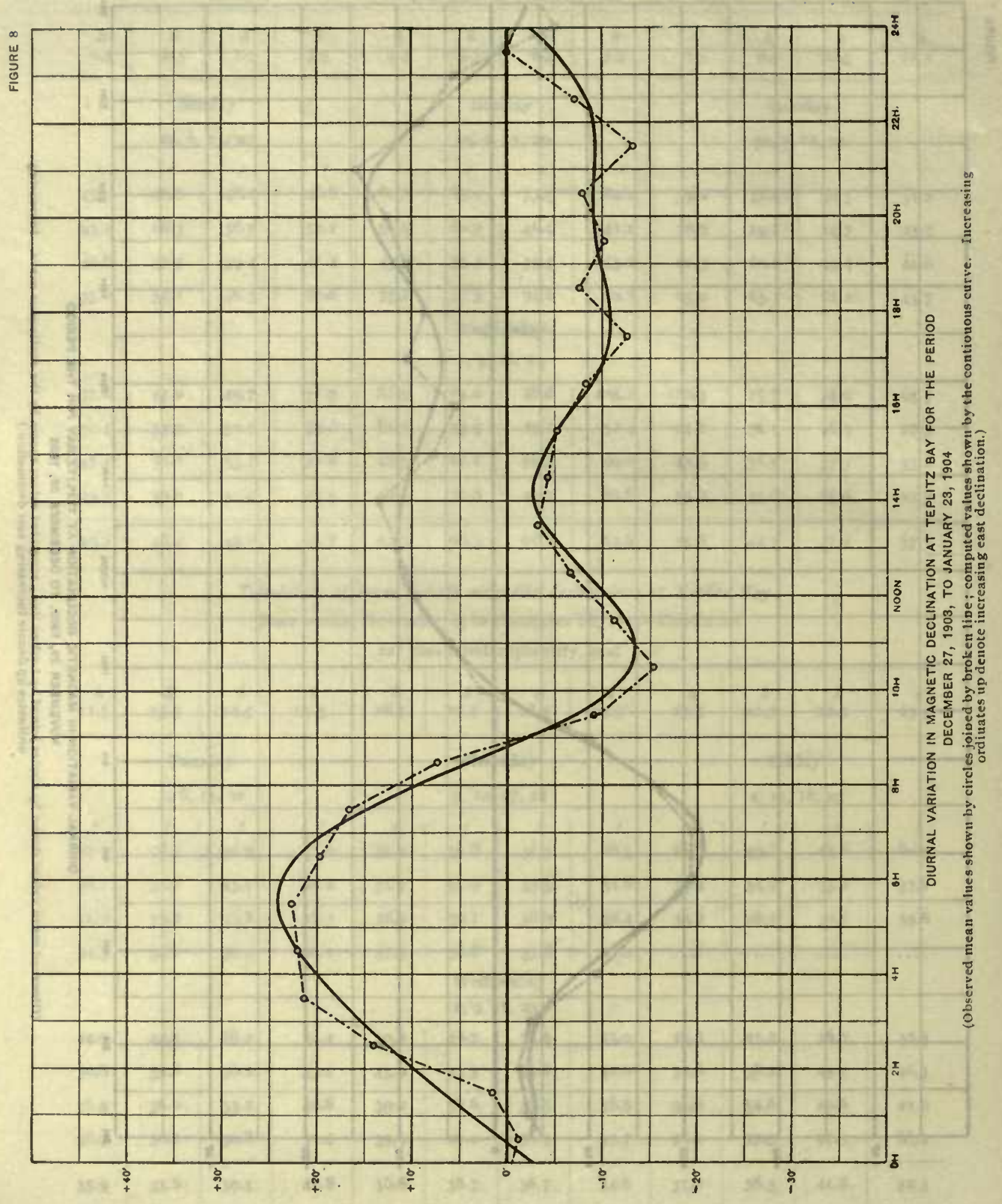


Tabulation of mean hourly magnetic declinations at Teplitz Bay

Four weeks, December 27, I903, to January 23, I904

$22^{\circ}$ plus tabular quantity, east

\begin{tabular}{|c|c|c|c|c|c|c|c|c|c|c|c|}
\hline $\begin{array}{c}h \\
0.5\end{array}$ & $\begin{array}{c}h \\
1.5\end{array}$ & $\begin{array}{c}h \\
2.5\end{array}$ & $\begin{array}{c}h \\
3.5\end{array}$ & $\begin{array}{c}h \\
4.5\end{array}$ & $\begin{array}{c}h \\
5 \cdot 5\end{array}$ & $\begin{array}{c}h \\
6.5\end{array}$ & $\stackrel{h}{h}$ & $\begin{array}{c}h \\
8.5\end{array}$ & $\begin{array}{c}h \\
9.5\end{array}$ & $\begin{array}{c}h \\
10.5\end{array}$ & $\begin{array}{c}h \\
11.5\end{array}$ \\
\hline & \multicolumn{2}{|c|}{$\begin{array}{c}\text { Sunday } \\
27,3,10,17\end{array}$} & & \multicolumn{4}{|c|}{$\begin{array}{c}\text { Sunday } \\
27,3,10,17\end{array}$} & & \multicolumn{2}{|c|}{$\begin{array}{l}\text { Monday } \\
28,4,11,18\end{array}$} & \\
\hline 1 & , & 1 & , & 1 & 1 & 1 & , & ' & , & 1 & , \\
\hline$\cdots$ & $\cdots$ & $\cdots$ & $\cdots$ & $\cdots$ & $\cdots$ & $\cdots$ & $\cdots$ & $\cdots$ & $\cdots$ & $\cdots$ & $\cdots$ \\
\hline 66.2 & 66.6 & 66.4 & 75.2 & 73.5 & IOI.O & 80.7 & -77.9 & 44.7 & 40.6 & 49.6 & 43.2 \\
\hline 41.7 & 32.8 & I 10.4 & 142.1 & I 43.1 & 123.4 & IO9. I & 90.9 & 105.2 & 66.6 & 43.6 & 36.6 \\
\hline 50.5 & 70.9 & 596 & 49.4 & 48.7 & 51.8 & 54.6 & 49.8 & 38.7 & 38.4 & 38.6 & 40.0 \\
\hline & & & & & Wed & esday & & & & & \\
\hline$\partial 8 t^{2}$ & $x-2$ & $2+\pi$ & Fat & 284 & 30,6 & I 3,20 & (6) & $r+1$ & GAts: & 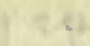 & 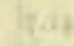 \\
\hline 39.2 & 40.3 & 44.5 & 44.2 & 34.8 & 36.5 & 41.8 & 67.9 & 53.4 & 08.o & or. 3 & $3 \mathrm{I} .8$ \\
\hline 34.4 & 37.6 & 42.7 & 59.6 & 70.0 & 76.0 & 76.9 & 58.3 & 35.3 & 29.6 & 18.0 & 27.7 \\
\hline 48.7 & 5I. I & 64.1 & 67.1 & 71.8 & 55.9 & 62.9 & 60.3 & 64.7 & 43.8 & 30.5 & 31.5 \\
\hline 38.3 & $3^{8.4}$ & $3^{8.9}$ & $3^{8.2}$ & 39.1 & $3^{8.9}$ & $3^{8.3}$ & 37.2 & 36.2 & 36.3 & 37.1 & 37.1 \\
\hline 45.6 & 48.2 & 60.9 & 68.0 & 68.7 & $69 \mathrm{I}$ & 66.3 & 63.2 & 54.0 & 37.6 & 31.2 & 35.4 \\
\hline
\end{tabular}

Tabulation of mean hourly magnetic declinations at Teplitz Bay

Four weeks, December 27, 1903, to January 23, 1904-Continued $22^{\circ}$ plus tabular quantity, east

\begin{tabular}{|c|c|c|c|c|c|c|c|c|c|c|c|}
\hline$h$ & $h$ & $h$ & $h$ & $h$ & $h$ & $h$ & $h$ & $h$ & $h$ & $h$ & $h$ \\
\hline I 2.5 & 13.5 & 14.5 & 15.5 & I6.5 & I 7.5 & I 8.5 & 19.5 & 20.5 & 21.5 & 22.5 & 23.5 \\
\hline & \multirow{2}{*}{\multicolumn{2}{|c|}{$\begin{array}{c}\text { Tuesday } \\
\text { 29, 5, 12, } 19\end{array}$}} & & \multicolumn{4}{|c|}{ Thursday } & & \multicolumn{2}{|c|}{ Friday } & \\
\hline bith & & & 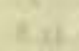 & It: & $3 I, 7$ & $4,2 I$ & 874 & $3 \times 7$ & 1,8 , & 22 & asin \\
\hline ' & 1 & ' & ' & ' & ' & ' & , & ' & 1 & 1 & ' \\
\hline 31.7 & 32.1 & $3 \mathrm{t} .8$ & $3 r \cdot 4$ & $34.9 b$ & $255^{6}$ & $5^{0.5^{b}}$ & $46.9 b$ & $77.0 \Omega$ & $53.6 a$ & 29. I $a$ & $6 \mathrm{r} .4 a$ \\
\hline 31.9 & 54.6 & 59.3 & 37.0 & 44.0 & 39.1 & 40.0 & $41 \cdot 3$ & 39.9 & 38.5 & $3^{8} 7$ & 42.4 \\
\hline 30.1 & 32.5 & 35.7 & 44.5 & $37 \cdot 3$ & 36.7 & 36.9 & 35.8 & 29.9 & 27.2 & $39 \cdot 2$ & 31.2 \\
\hline 48.3 & 46.6 & $45 \cdot 3$ & 44.8 & $37 \cdot 9$ & 34.6 & 38.6 & 37.1 & $34.2 c$ & $32.1 C$ & $58.5 c$ & $55.7 c$ \\
\hline $7 x+4$ & $t=1$ & 74 & 2.7 & $\operatorname{san}$ & $\begin{array}{l}\text { Wed } \\
30,\end{array}$ & $\begin{array}{l}\text { sday } \\
3,20\end{array}$ & $3 \pm 18$ & & 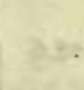 & $\neq=$ & 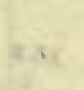 \\
\hline 66.0 & $77 \cdot 3$ & 76.0 & 82.9 & 62.0 & $3^{8.5}$ & 37.6 & $33 \cdot 3$ & 29. I & 28.8 & 33.3 & 34.5 \\
\hline $45 \cdot 4$ & 40.0 & $34 \cdot 3$ & 30. I & 30.3 & 37.4 & 43.8 & 34.2 & 28. I & 21.3 & $45 \cdot 3$ & 24.6 \\
\hline 29.3 & 29.9 & 26.5 & 28.2 & 28.7 & 31.0 & 34.3 & 42.9 & 49.0 & 39.0 & 432 & 86. I \\
\hline 37.2 & 34.2 & 32.6 & 32.0 & 3 r.o & 29.5 & 30. I & I9. I & 22.9 & 24.6 & 29.2 & 37.1 \\
\hline 40.0 & 43.4 & 42.2 & 41.4 & $3^{8.3}$ & 34.0 & 39.0 & 36.3 & $3^{8.8}$ & 33.1 & 39.6 & 46.6 \\
\hline
\end{tabular}


Tabulation of mean hourly magnetic declinations at Teplits Bay

Four weeks, January 24 to February 20, 1904

$22^{\circ}$ plus tabular quantity, east

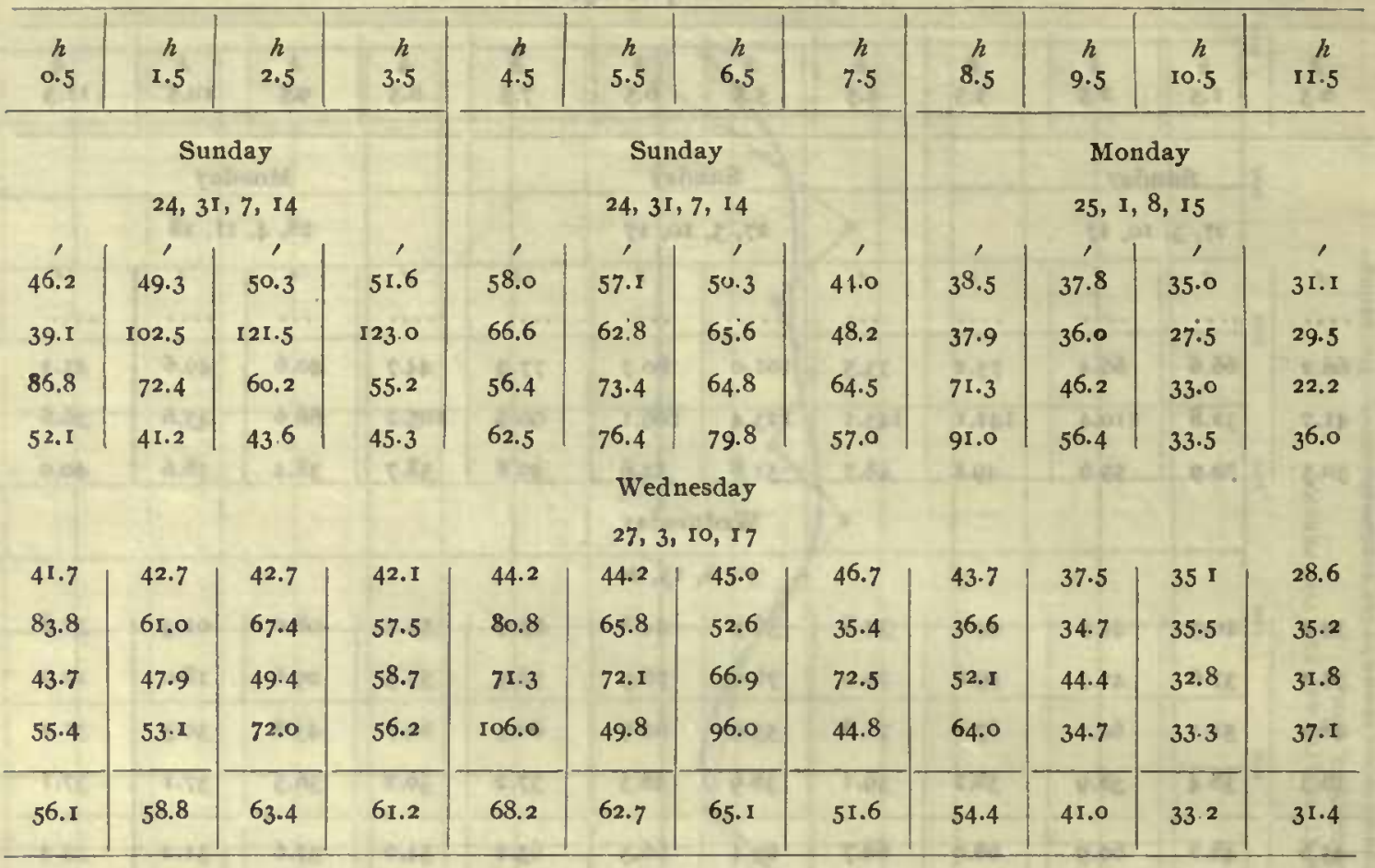

Tabulation of mean hourly magnetic declinations at Teplitz Bay Four weeks, January 24 to February 20, 1904-Continued

$22^{\circ}$ plus tabular quantity, east

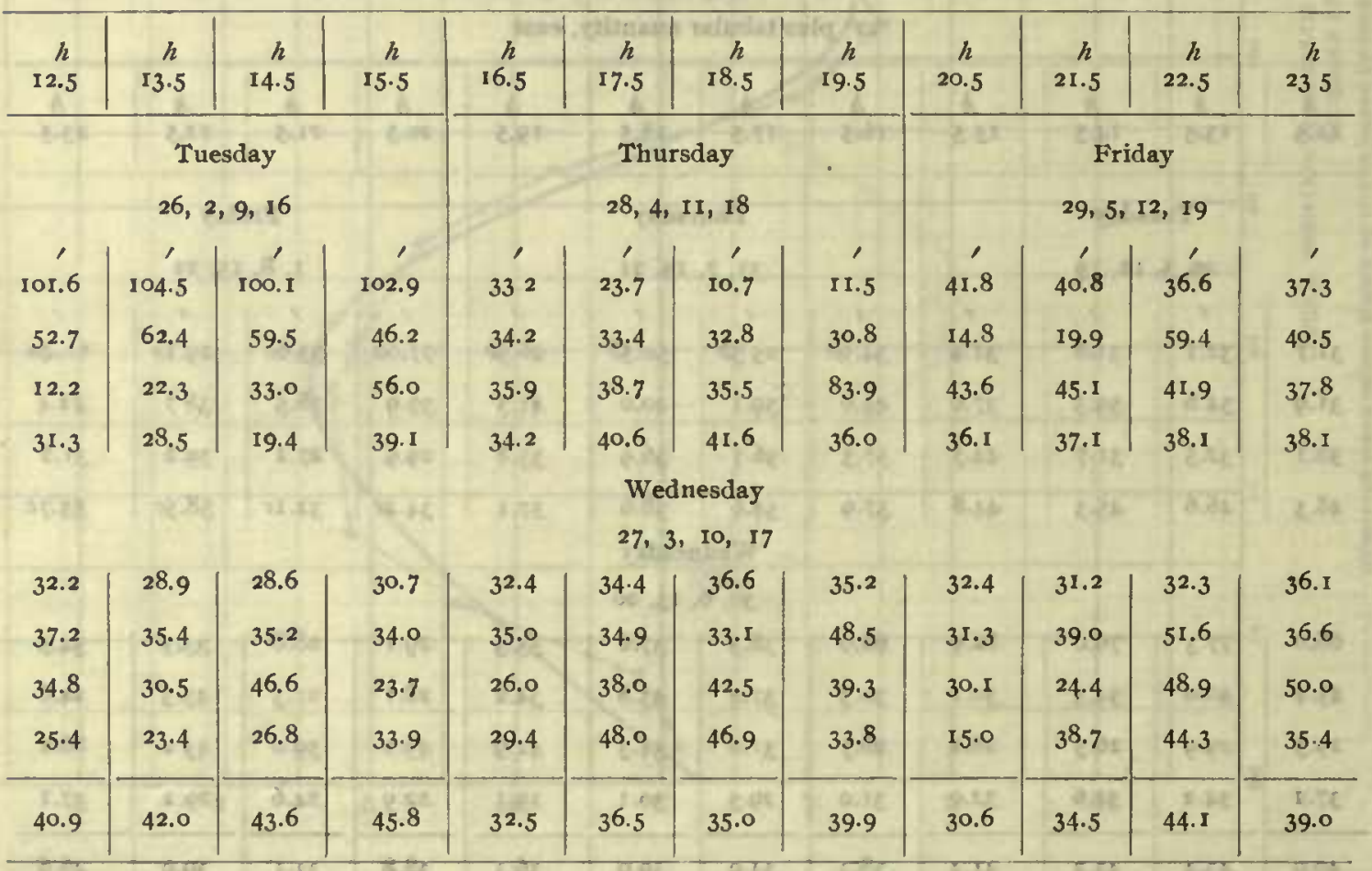

Mean value for the whole period, $22^{\circ} 46^{\prime} 3 \mathrm{E}$. 


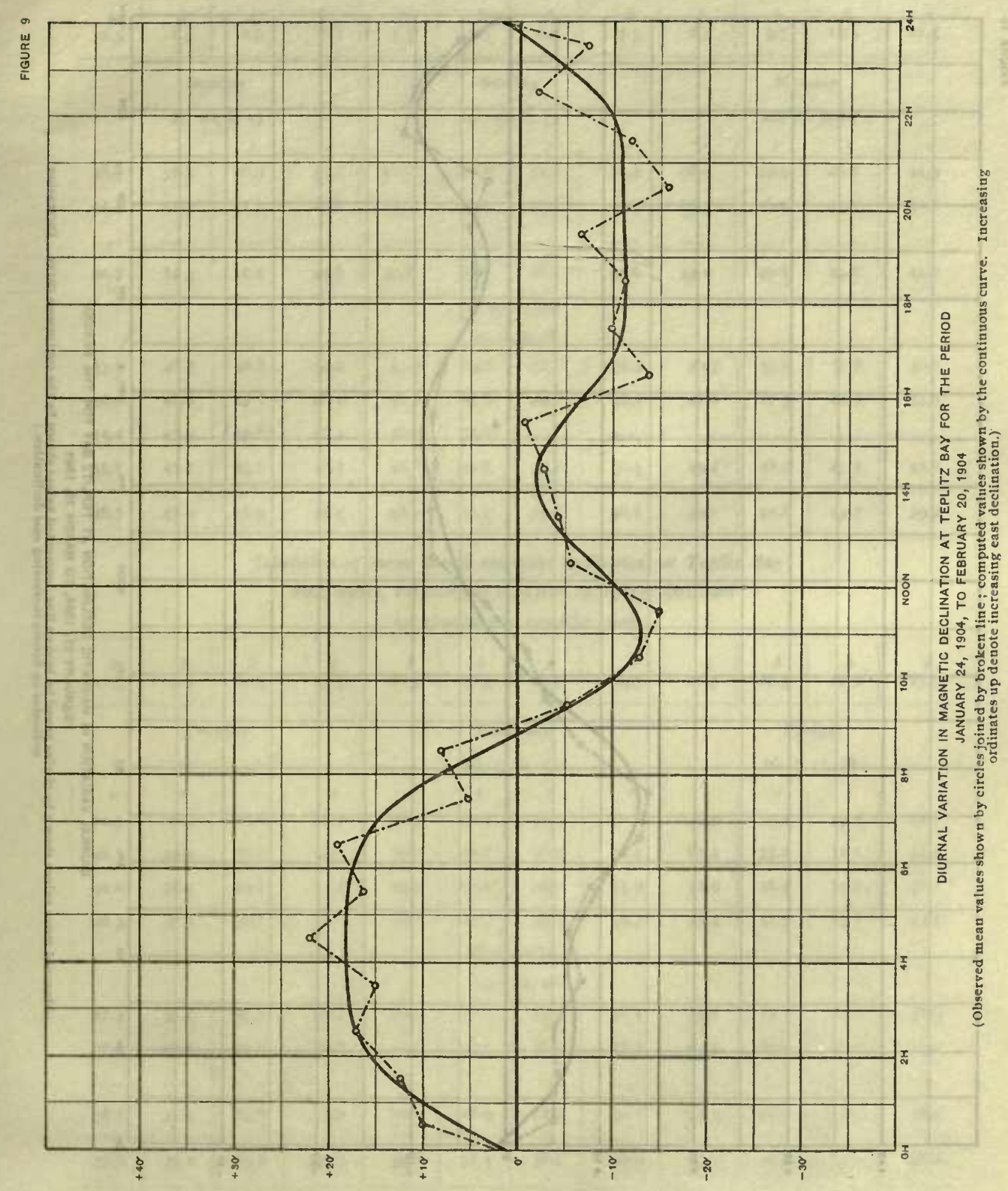




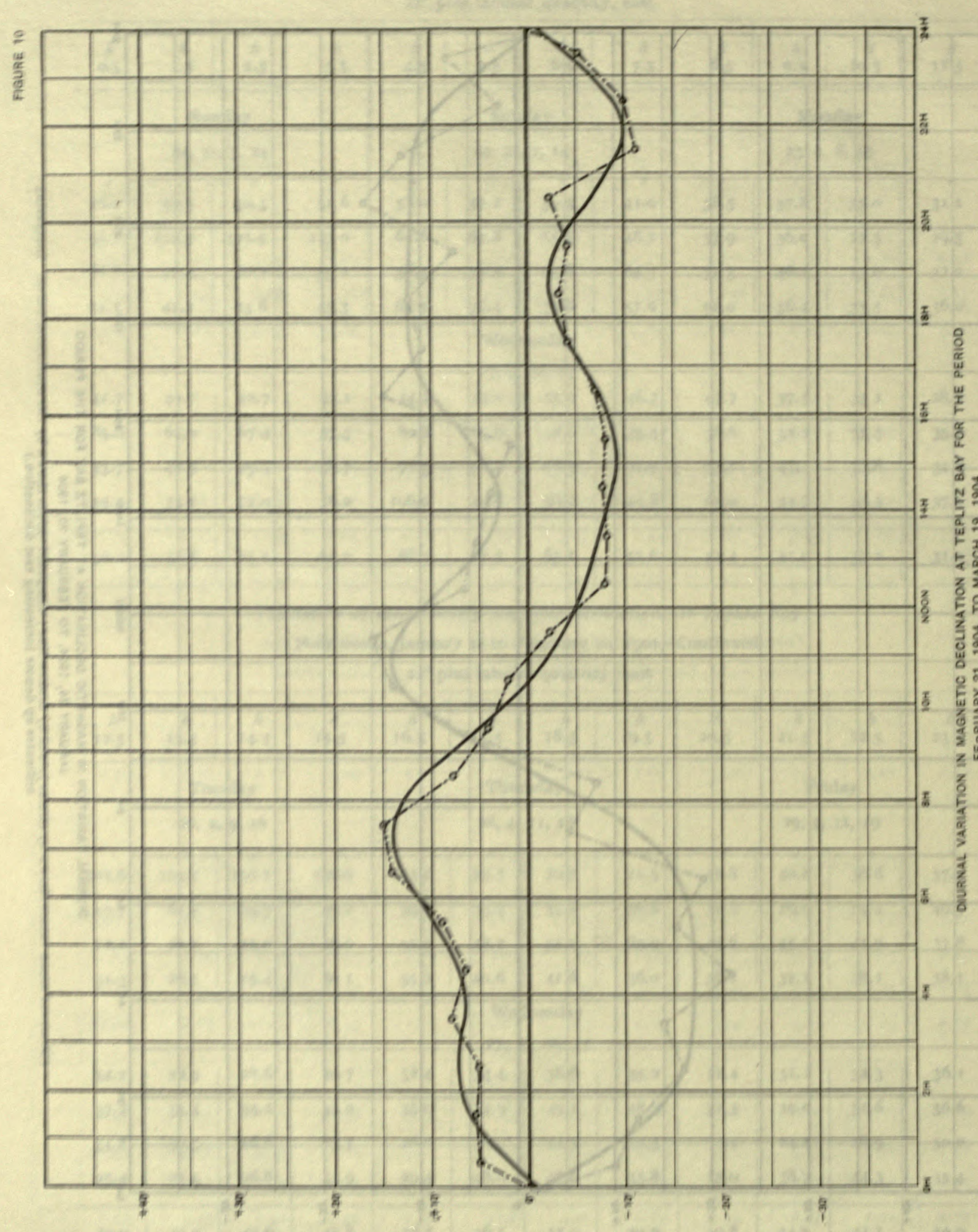


Tabulation of moras howrby magnetic declinations at Teplits Buy

Fovar weeks, Febrwary 21 to Mlarch 19 in 1904

$22^{\circ}$ phes tabelar quantity, east

\begin{tabular}{|c|c|c|c|c|c|c|c|c|c|c|c|}
\hline $\begin{array}{c}b \\
a-5\end{array}$ & $\begin{array}{c}n \\
1.5\end{array}$ & $\begin{array}{c}h \\
2.5\end{array}$ & 35 & $\stackrel{x}{4}$ & $\frac{12}{5-5}$ & $a^{2}$ & $\frac{1}{2.5}$ & $\frac{a}{85}$ & $\frac{1}{95}$ & $\frac{h_{2}}{\mathrm{ma}}$ & 15i. \\
\hline \multicolumn{4}{|c|}{ Surday } & \multicolumn{4}{|c|}{ Surnday } & \multicolumn{4}{|c|}{ Mobdiay } \\
\hline \multicolumn{4}{|c|}{$21,28,6,13$} & \multicolumn{4}{|c|}{$21,28,6,13$} & \multicolumn{4}{|c|}{$22,29,26,14$} \\
\hline 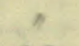 & $?$ & ? & 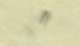 & $n$ & $?$ & $?$ & +4 & $2 n$ & $?$ & $?$ & ? \\
\hline 48.8 & $52 \cdot 3$ & 50.9 & $54+5$ & 50,9 & 53.8 & 54,2 & 50.4 & 46.8 & 45.9 & 45.5 & $4+3$ \\
\hline 44.7 & $4 \pi \cdot 3$ & 42.5 & 42.6 & 43.8 & 44.6 & 45.7 & 43.7 & 49,5 & 46.0 & 45.7 & 42.6 \\
\hline ירירו & $\cdots$ & $\cdots+$ & $\cdots$ & now & $\cdots$ & $m$ & $m$ & $m$ & $m$ & $\sin x$ & 27 \\
\hline $4 a 7$ & 545 & 54.4 & $5+6$ & 52.5 & 58.8 & $67, x$ & 596 & 53,0 & 49.6 & $4,3.8$ & 44.7 \\
\hline \multicolumn{12}{|c|}{$\begin{array}{l}\text { Wedmesday } \\
24,2,94.6\end{array}$} \\
\hline 479 & 46.5. & 46.8. & $54+4$ & 47,9 & 49,6 & 24,6 & 94.5 & 5007 & 372.1 & 37.8 & 3000 \\
\hline 560 & 46.6 & 45.1 & 46.2 & 46.1 & 47,8 & 458 & $4+2$ & 45.6 & 47,5 & 447 & 35.7 \\
\hline 444 & 43.9 & 47.8 & 47.4 & 48.3 & 48.8 & & non & $m$ & $m$ & mon & $m a$ \\
\hline 45.5 & 43.7 & 43.7 & 461 & 46.8 & 49.8 & $5 a .8$ & $50 \cdot 3$ & $49 \cdot 4$ & 485 & 45.2 & 40.6 \\
\hline 467 & 47.0 & 469 & 49.4 & 480 & 50,3 & 558 & 56,6 & 49.2 & 45.8 & $43-27$ & 3992 \\
\hline
\end{tabular}

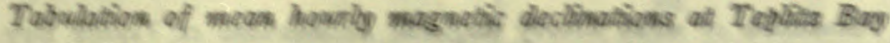

Fower weeks, Febrwary at to Mlarch 29, 1904-Contithwed

$22^{\circ}$ plies tabelar quantitys, east

\begin{tabular}{|c|c|c|c|c|c|c|c|c|c|c|c|}
\hline $\begin{array}{c}4 \\
12.5\end{array}$ & $\frac{\pi}{435}$ & $\frac{1}{4+5}$ & $\frac{14}{25,5}$ & $16 \sqrt{3}$ & $\frac{\pi}{47,5}$ & 18.5 & $\frac{12}{29}$ & $\frac{\pi}{2005}$ & $2 \pi \cdot 5$ & 2025. & 235 \\
\hline & \multicolumn{3}{|c|}{ Thesday } & \multicolumn{4}{|c|}{ Thworsdiay } & & \multicolumn{2}{|c|}{ Fudilay } & \\
\hline - & ? & , & $\rightarrow$ & $n$ & $\rightarrow$ & $\rightarrow$ & $\rightarrow$ & 4 & $n$ & $n$ & $\rightarrow$ \\
\hline 22.5 & 36.6 & 28.6 & 24.7 & 360 & 42.2 & 44.6 & 4007 & 335 & 32.3. & 39.5 & 42.2 \\
\hline 42,5 & $4+2$ & 460 & 48.2 & 397 & 40.7 & 40.2 & 32.5 & 634 & 35.2 & $32 \pi$ & 33.4 \\
\hline tao & $3 \times 4$ & 29,2 & $33-5$ & 395 & 3276 & 390 & 530 & 1129 & 36.4 & 347 & $35=$ \\
\hline 2003 & 37,2 & 36,7 & $3^{50}$ & 38.2 & $372-3$ & 387 & 39.7 & 35.4 & $34-5$ & 288 & 32,4 \\
\hline \multicolumn{12}{|c|}{$24,2,9,26$} \\
\hline $27 \sqrt{3}$ & 35.3 & 35,2 & $36+4$ & 36.4 & 32,6 & 394. & 38.4 & 3720 & 2977 & 23,4 & 38.2 \\
\hline 27,4 & 294 & 34.2 & 28.8 & 30.3 & 36.4 & $36 \pi$ & $297-4$ & 53.4 & 29.8 & 35,5 & 34,6 \\
\hline 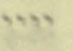 & $+\cdots$ & 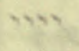 & 320 & $m$ & $\sqrt{2}$ & 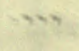 & ment & $m$ & 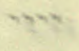 & $m a n$ & $m$ \\
\hline 36.2 & 37.5 & 20,8 & 28.2 & 2220 & 28.9 & 324 & 30,4 & 38.2 & 24.2. & 30.5 & 38.4 \\
\hline 33.7 & 33.2 & 339 & 335 & 34,8 & $327+4$ & $3 \times 8$ & 30,3 & 3907 & 300,3 & $3 \times 4$ & अat: \\
\hline
\end{tabular}




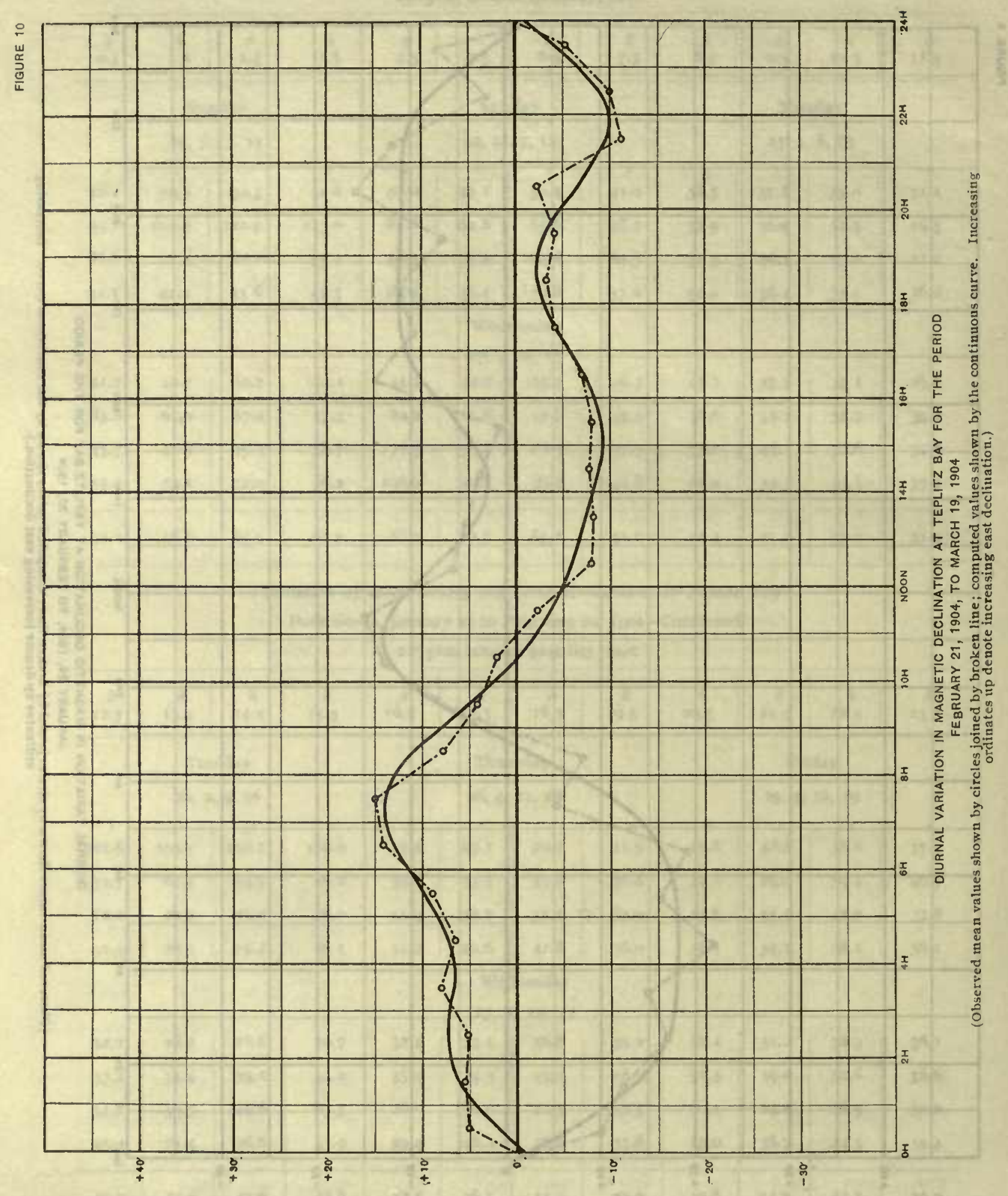


Tabulation of mean hourly magnetic declinations at Teplitz Bay

Four weeks, February 21 to March I9, 1904

$22^{\circ}$ plus tabular quantity, east

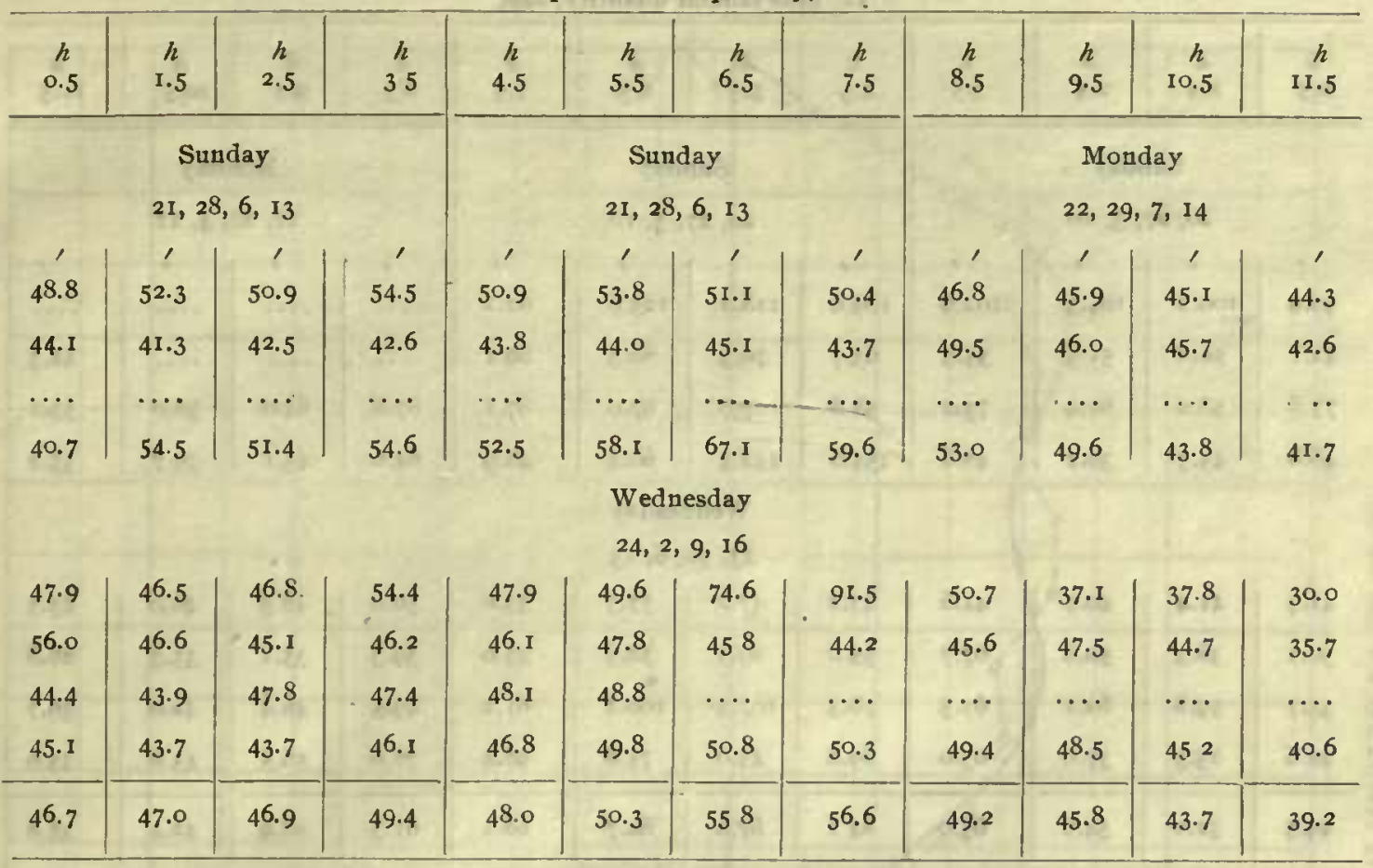

Tabulation of mean hourly maguetic declinations at Teplitz Bay

Four weeks, February 21 to March 19, 1904-Continued

$22^{\circ}$ plus tabular quantity, east

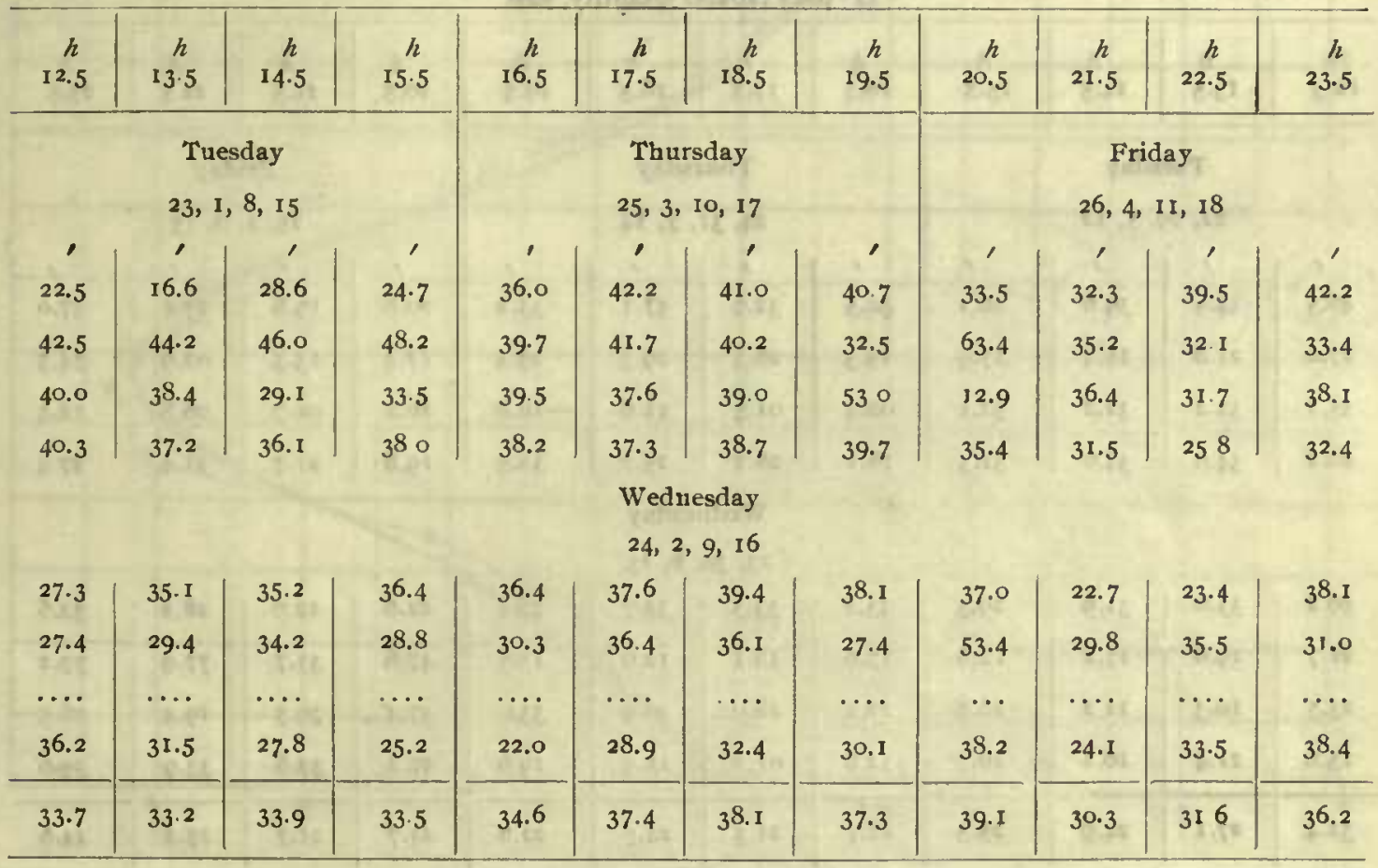

Mean value for the whole period, $22^{\circ} 41 .{ }^{\prime} 6 \mathrm{E}$. 
Tabulation of mean hourly magnetic declinations at Teplitz Bay

Four weeks, March 20 to April 16, 1904

$22^{\circ}$ plus tabular quantity, east

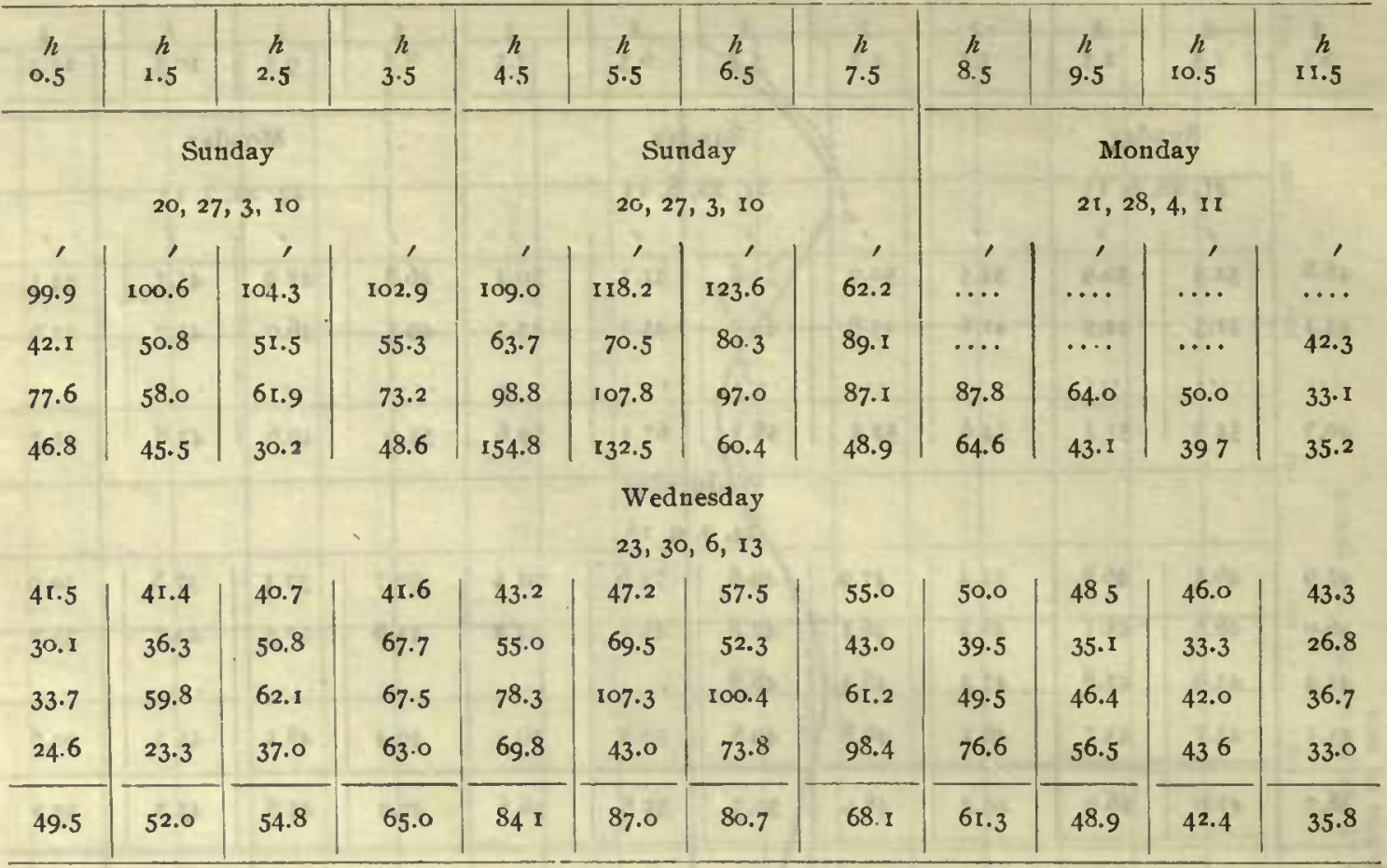

Tabulation of mean hourly magnetic declinations at Teplitz Bay

Four weeks, March 20 to April 16, 1904-Continued

$22^{\circ}$ plus tabular quantity, east

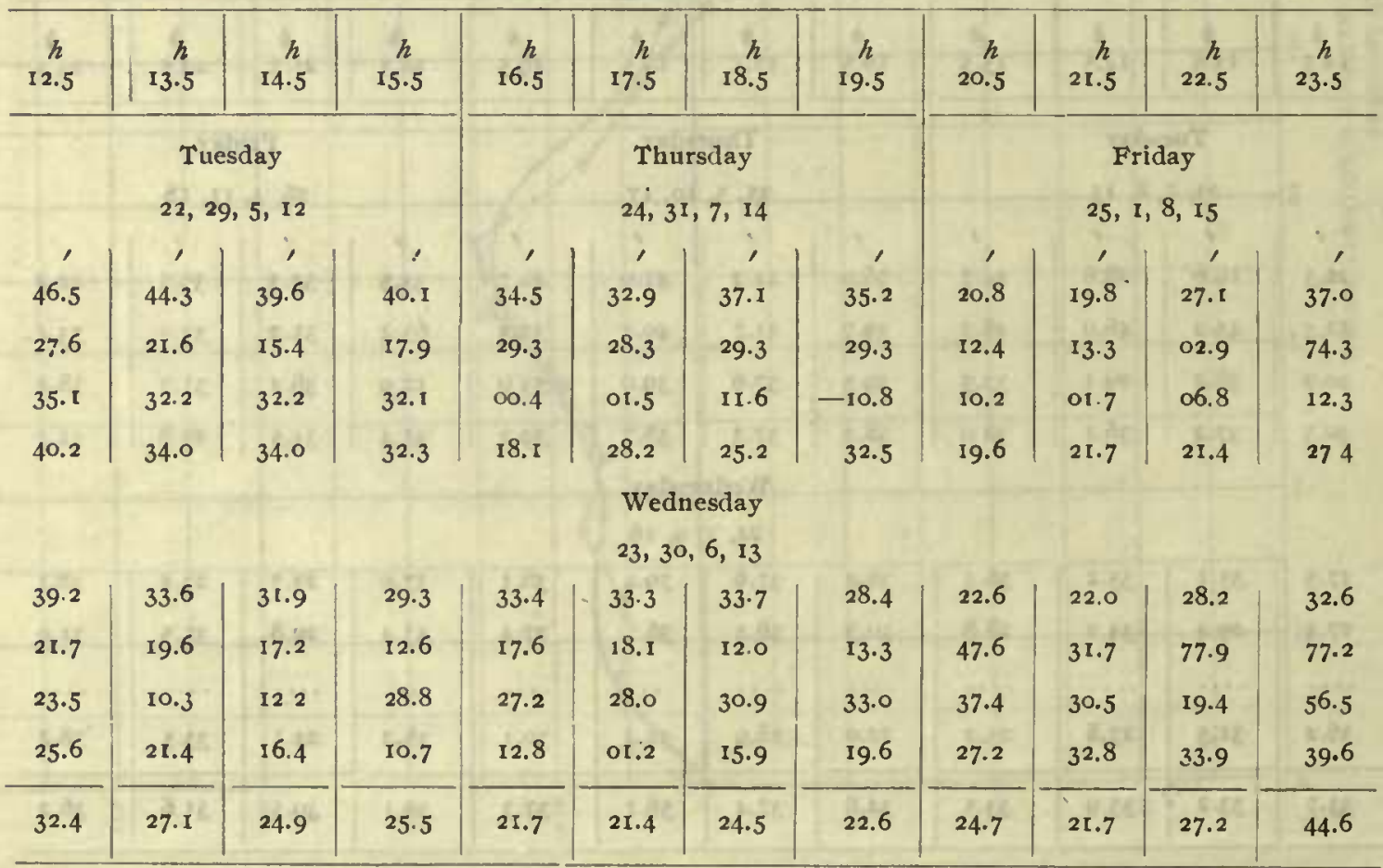

Mean value for the whole period, $22^{\circ} 43^{\prime} 7 \mathrm{E}$. 


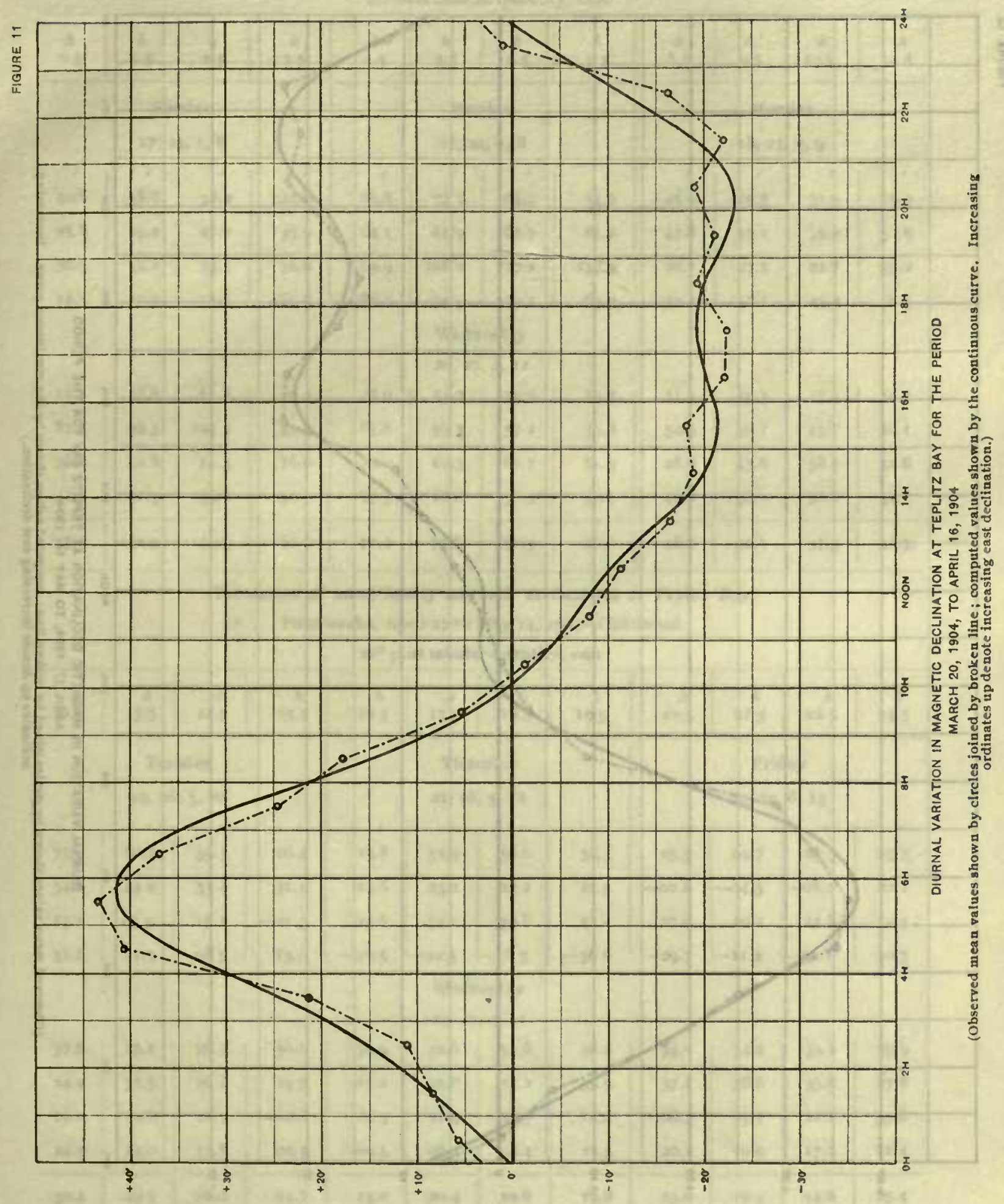




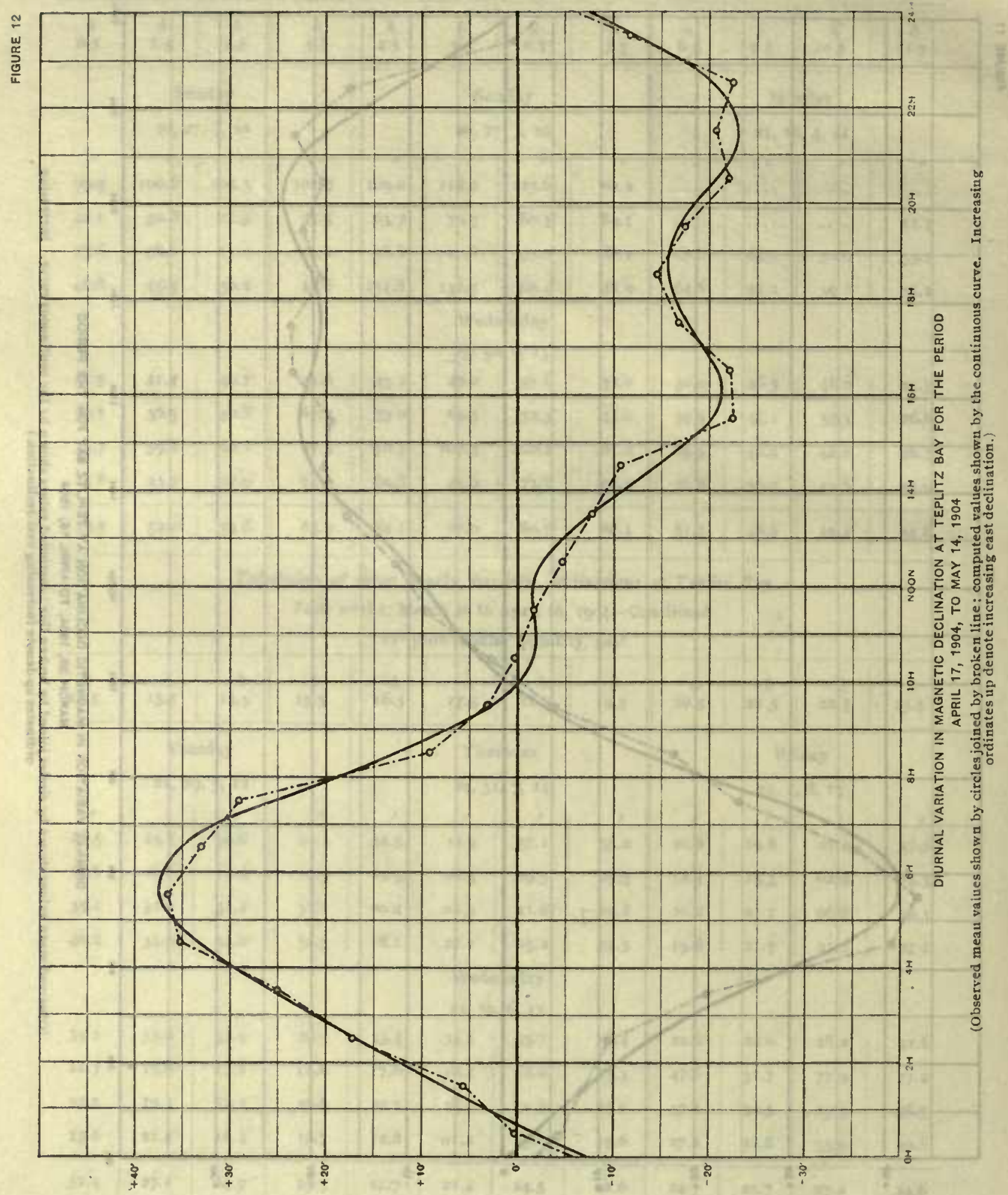


Tabulation of mean hourly magnetic declinations at Teplitz Bay

Four weeks, April I 7 to May I4, 1904

$20^{\circ}$ plus tabular quantity, east

\begin{tabular}{|c|c|c|c|c|c|c|c|c|c|c|c|}
\hline $\begin{array}{l}h \\
05\end{array}$ & $\begin{array}{c}h \\
1.5\end{array}$ & $\begin{array}{c}h \\
2.5\end{array}$ & $\begin{array}{c}h \\
3.5\end{array}$ & $\begin{array}{c}h \\
4.5\end{array}$ & $\begin{array}{c}h \\
5 \cdot 5\end{array}$ & $\begin{array}{c}h \\
6.5\end{array}$ & $\begin{array}{c}h \\
7.5\end{array}$ & $\begin{array}{c}h \\
8.5\end{array}$ & $\begin{array}{c}h \\
9.5\end{array}$ & $\begin{array}{c}h \\
10.5\end{array}$ & $\begin{array}{l}h \\
\text { II } 5\end{array}$ \\
\hline & \multicolumn{2}{|c|}{$\begin{array}{c}\text { Sunday } \\
17,24,1,8\end{array}$} & & \multicolumn{3}{|c|}{$\begin{array}{c}\text { Sunday } \\
I 7,-24, I, 8\end{array}$} & & & \multicolumn{2}{|c|}{$\begin{array}{l}\text { Monday } \\
18,25,2,9\end{array}$} & \\
\hline , & ' & , & 1 & 1 & , & 1 & , & 1) & , & , & 4 \\
\hline 40.6 & $3^{8.8}$ & 38.2 & 47.9 & 68.8 & 72.1 & 64.7 & 54.6 & 43.9 & 19.5 & 31.0 & 29.0 \\
\hline 25.8 & 19.9 & 27.0 & 39.9 & 64.1 & 61.9 & 61.7 & 61.2 & 47.8 & 37.1 & 35.2 & 32.9 \\
\hline $3^{0.3}$ & 35.2 & 55.5 & 76.6 & 99.9 & 108.2 & 127.2 & 137.2 & 26.7 & 27.2 & 22.7 & 35.2 \\
\hline 24.3 & 21.4 & $3^{8.6}$ & 61.2 & 89.0 & 95.9 & 79. I & 63.9 & 50.5 & 47.1 & 43.4 & $3^{8} .4$ \\
\hline & & & & & Wed & sday & & & & & \\
\hline & & & & & 20 & 4, II & & & & & \\
\hline I3. I & 338 & 61.8 & 49.2 & 48.9 & 54.2 & 54.0 & $53 \cdot 3$ & 51.9 & 49.3 & 47.4 & 44.4 \\
\hline 87.5 & 99.3 & I04.3 & 93.3 & 65.8 & 59.7 & 57.2 & 57.8 & 54.9 & $5^{0.1}$ & 43. I & 39. I \\
\hline $3^{6.6}$ & 52.8 & 54.5 & 76.9 & 75.2 & 69.3 & 61.7 & 51.9 & 46.9 & 43.6 & $3^{8} 5$ & 32.6 \\
\hline 41.1 & 41.9 & 54.6 & 52.7 & 67.3 & 68.8 & 56.5 & 50.0 & 46.8 & 46.7 & $3^{8.8}$ & 32.1 \\
\hline 37.4 & 42.9 & 54.3 & 62.2 & 72.4 & 73.8 & 70.3 & 66.2 & 46.2 & 40.1 & 37.5 & $3=5$ \\
\hline
\end{tabular}

Tabulation of mean hourly magnetic declinations at Teplitz Bay

Four weeks, April i7 to May 14, 1904-Continued

$22^{\circ}$ plus tabular quantity, east

\begin{tabular}{|c|c|c|c|c|c|c|c|c|c|c|c|}
\hline $\begin{array}{r}h \\
12.5\end{array}$ & $\begin{array}{c}h \\
13.5\end{array}$ & $\begin{array}{r}h \\
14.5\end{array}$ & $\begin{array}{r}h \\
15.5\end{array}$ & $\begin{array}{r}h \\
16.5\end{array}$ & $\begin{array}{c}h \\
\mathrm{I} 7.5\end{array}$ & $\begin{array}{c}h \\
18.5\end{array}$ & $\begin{array}{c}h \\
195\end{array}$ & $\begin{array}{c}h \\
20.5\end{array}$ & $\begin{array}{c}h \\
21.5\end{array}$ & $\begin{array}{c}h \\
22.5\end{array}$ & $\begin{array}{c}h \\
23.5\end{array}$ \\
\hline & \multicolumn{2}{|c|}{ Tuesday } & & \multicolumn{4}{|c|}{ Thursday } & \multicolumn{4}{|c|}{ Friday } \\
\hline & \multicolumn{2}{|c|}{$19,26,3,10$} & & \multicolumn{3}{|c|}{$2 \mathrm{I}, 28,5,12$} & & \multicolumn{4}{|c|}{$22,29,6$, I3 } \\
\hline , & , & , & , & , & , & , & , & , & , & , & , \\
\hline 73.5 & 80.4 & 59.3 & I6.4 & 29.6 & 32.9 & 30.0 & 32.3 & $25 \cdot 3$ & 097 & I 3.5 & $25 \cdot 3$ \\
\hline 34.6 & 44.0 & 53.4 & 3I. I & 24.8 & 25. I & 22.2 & 21.5 & $\multimap 02.8$ & -04.5 & -08.1 & 20.1 \\
\hline 13. I & 05.9 & I5. I & $-\mathrm{or} \cdot 3$ & 29.6 & 34.1 & 398 & 42.4 & 27.0 & 20.1 & I5 8 & $34 \cdot 3$ \\
\hline 32.1 & 21.5 & 085 & I5.0 & -40.5 & -22.3 & -165 & -36.6 & -29.7 & - II.4 & -14.1 & 10.5 \\
\hline
\end{tabular}

Wednesday

$20,27,4$, I I

\begin{tabular}{r|r|r|r|r|r|r|r|r|r|r|r}
37.6 & 27.1 & 28.9 & 30.0 & 30.9 & 32.8 & 34.6 & 36.2 & 34.0 & 34.2 & 34.2 & 39.9 \\
24.9 & 31.5 & 26.1 & 19.7 & 27.2 & 31.8 & 35.2 & 35.9 & 37.1 & 38.6 & 35.8 & 27.8 \\
19.0 & 09.8 & 06.3 & -02.6 & 18.5 & 21.4 & 26.7 & 14.4 & 08.7 & 23.7 & 22.1 & 33.6 \\
24.5 & 14.0 & 11.6 & 09.5 & -00.3 & 06.5 & 08.4 & 12.5 & 20.1 & 19.9 & 17.2 & 11.4 \\
\hline 32.4 & 29.3 & 26.2 & 14.7 & 15.0 & 20.4 & 22.6 & 19.8 & 15.0 & 16.3 & $14: 6$ & 25.4 \\
\hline
\end{tabular}

Mean value for the whole period, $22^{\circ} 37^{\prime} \mathrm{r} \mathrm{E}$. 
Tobulation of mean hourly magnetic declinations at T'eplitz Bay

Four weeks, May I5 to June II, I904

$22^{\circ}$ plus tabular quantity, east

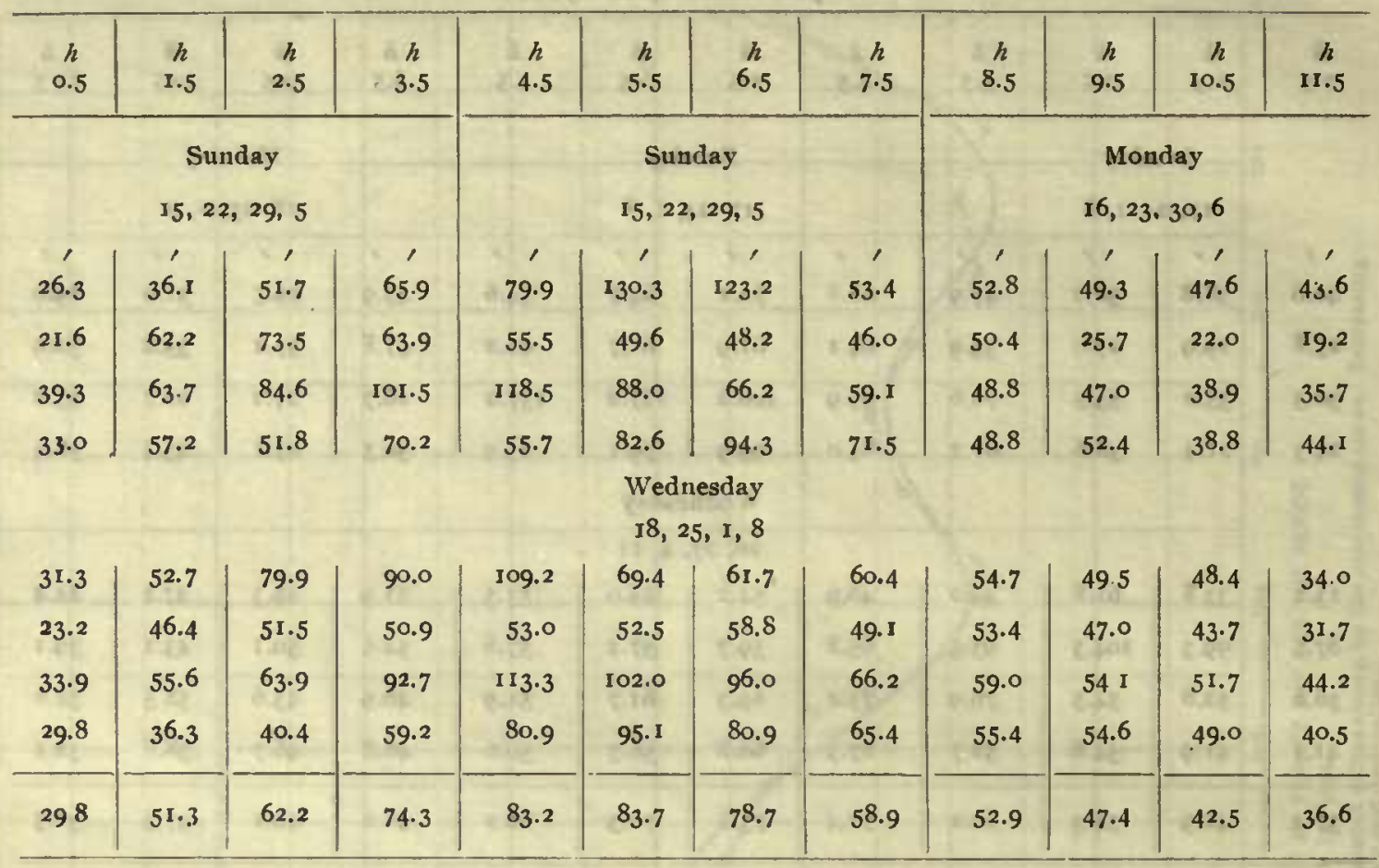

Tabulation of mean hourly magnetic declinations at Teplitz Bay

Four weeks, May I5 to June II, I904-Continued

$22^{\circ}$ plus tabular quantity, east

\begin{tabular}{|c|c|c|c|c|c|c|c|c|c|c|c|}
\hline $\begin{array}{c}h \\
12.5\end{array}$ & $\begin{array}{c}h \\
13.5\end{array}$ & $\begin{array}{c}h \\
14.5\end{array}$ & $\begin{array}{c}h \\
\times 5.5\end{array}$ & $\begin{array}{c}h \\
16.5\end{array}$ & $\begin{array}{c}h \\
17.5\end{array}$ & $\begin{array}{c}h \\
18.5\end{array}$ & $\begin{array}{c}h \\
19.5\end{array}$ & $\begin{array}{c}h \\
20.5\end{array}$ & $\begin{array}{c}h \\
21.5\end{array}$ & $\begin{array}{c}h \\
22.5\end{array}$ & $\begin{array}{c}h \\
23.5\end{array}$ \\
\hline & \multicolumn{2}{|c|}{ Tuesday } & & & \multicolumn{2}{|c|}{ Thursday } & & & \multicolumn{2}{|c|}{ Friday } & \\
\hline & \multicolumn{2}{|c|}{ I $7,24,3 I, 7$} & & & \multicolumn{2}{|c|}{$19,26,2,9$} & & & \multicolumn{2}{|c|}{$20,27,3,10$} & \\
\hline , & , & , & , & , &, & , & , & , & , & , & , \\
\hline 67.9 & 46.5 & 44.8 & $3 I .1$ & -05.9 & 03.5 & $\multimap 02.3$ & -05.1 & -11.6 & 09.9 & 23.9 & 33.0 \\
\hline 74.7 & 55.1 & 32.4 & or. 8 & 16.6 & 13.3 & 17.7 & 06.8 & 13.6 & 15.8 & -04.6 & -20.4 \\
\hline 32.9 & I 8.6 & 14.9 & 07.5 & 27.7 & 24.6 & 21.8 & 25.0 & 15.8 & 24.0 & 28.7 & 33.0 \\
\hline \multirow[t]{2}{*}{47.2} & 89.4 & 48.4 & 09.0 & 23.6 & 22.4 & 22.7 & 20.7 & $24 \cdot 3$ & 28.8 & I9.6 & 19.6 \\
\hline & & & & & \multicolumn{2}{|c|}{$\begin{array}{l}\text { Wednesday } \\
18,25,1,8\end{array}$} & & & & & \\
\hline $3 I \cdot 3$ & 28.9 & 04.7 & 19.7 & IO. I & 09.6 & 15.5 & 06.6 & 09.4 & II. 8 & I 1.8 & 22.8 \\
\hline 25.5 & 21.3 & 15.9 & 13.0 & 15.4 & 06.4 & 03.7 & 09.9 & 08.4 & $\infty .2$ & 04.5 & 30.8 \\
\hline $3^{8.5}$ & $3 \mathrm{I} .7$ & 28.6 & 20.9 & 24.4 & 33.2 & 24.0 & $x 4.6$ & 22.1 & 20.5 & 27.7 & 24.5 \\
\hline 34.4 & 29.7 & 22.5 & 18.8 & 21.8 & 18.9 & 24.2 & 30.6 & 30.5 & 27.6 & 33.7 & 41.0 \\
\hline 44.0 & 40.2 & 26.5 & 15.2 & 16.7 & 16.5 & 15.9 & 13.6 & 14.1 & $17 \cdot 3$ & 18.2 & 23.0 \\
\hline
\end{tabular}

Meau value for the whole period, $22^{\circ} 40^{\prime} 1$ E. 


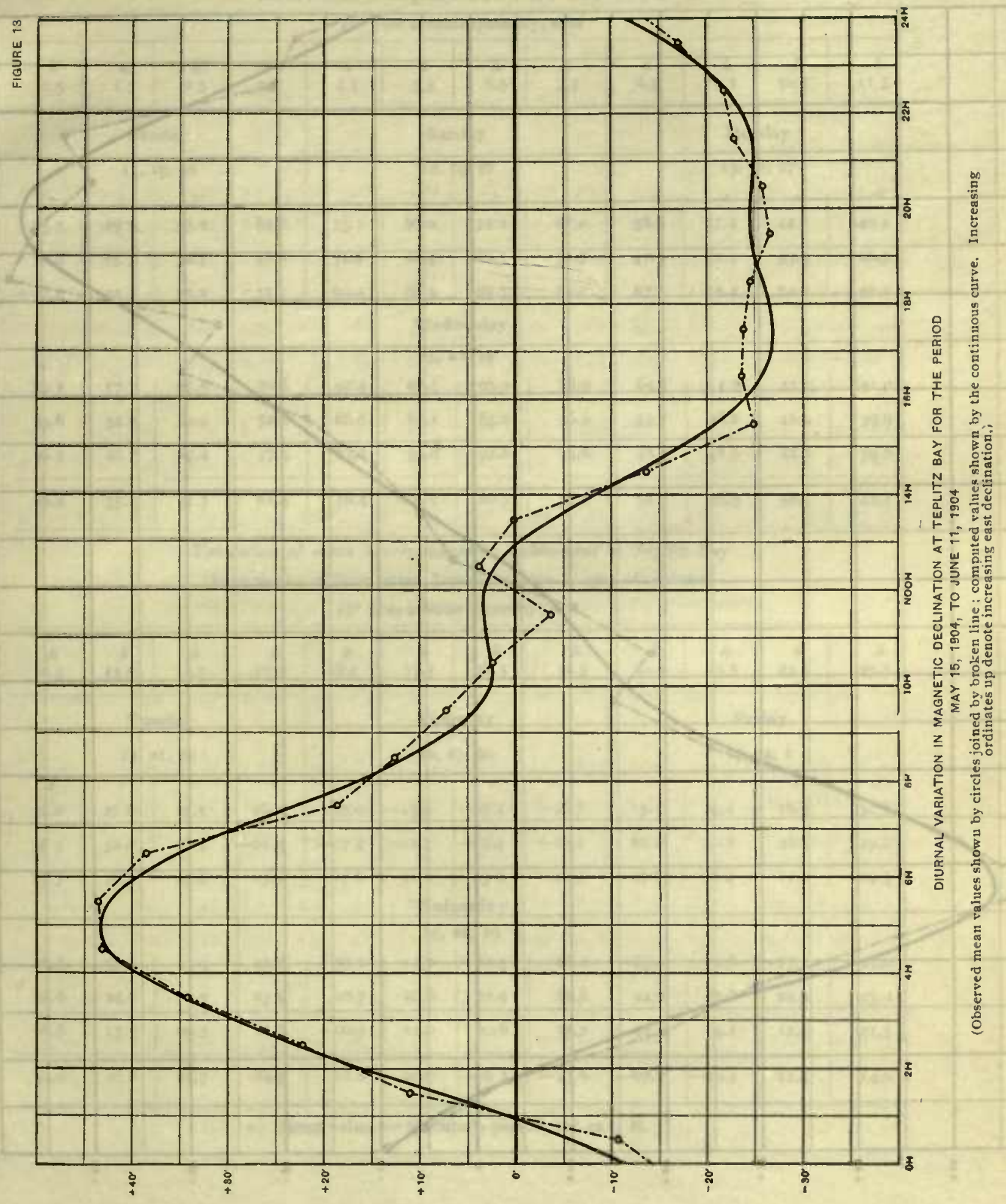




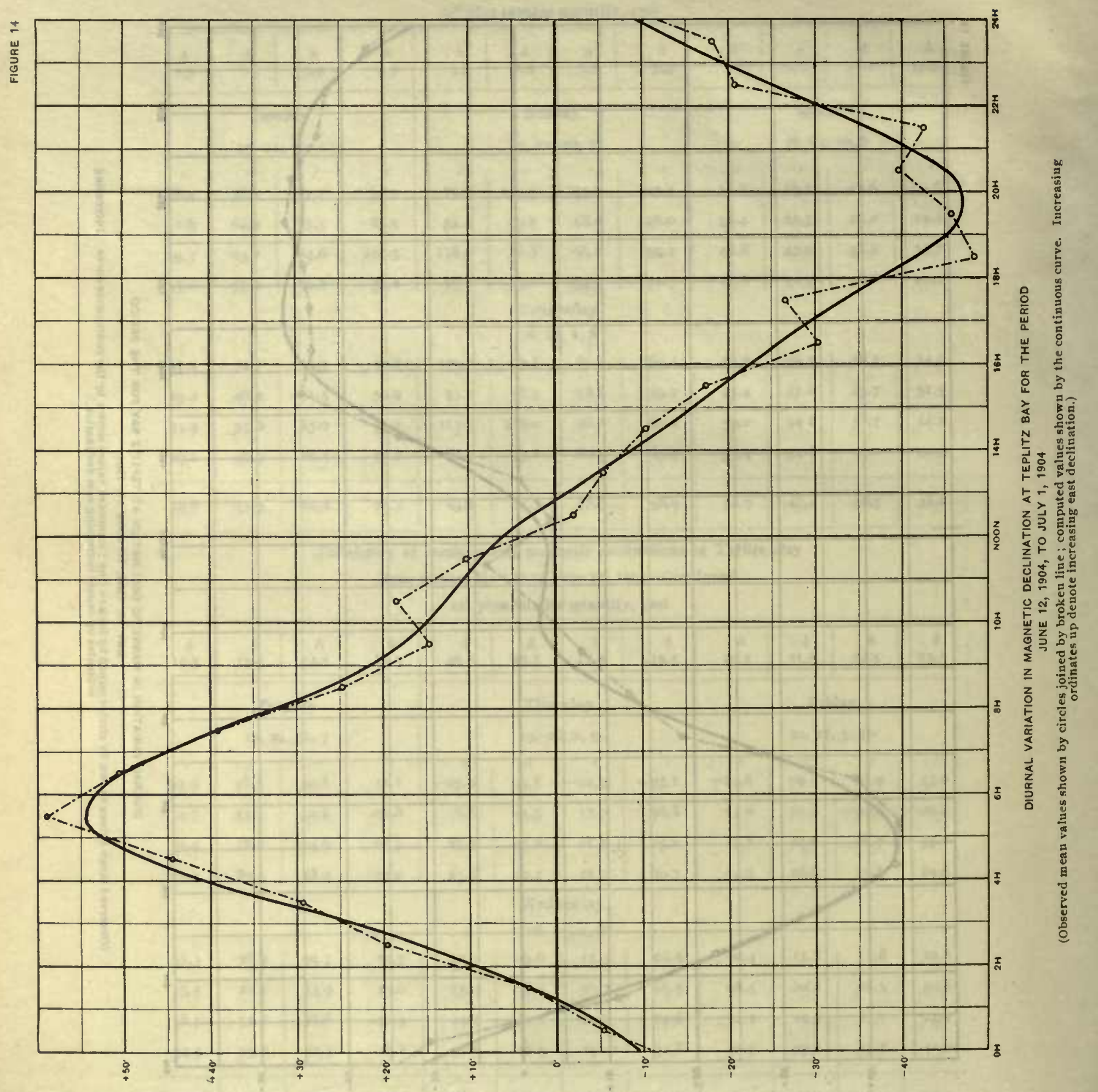


Tabulation of mean hourly magnetic declinations at Teplitz Bay

Three weeks, ending series, Juue 12 to July $\mathrm{r}, 1904$

$22^{\circ}$ plus tabular quantity, east

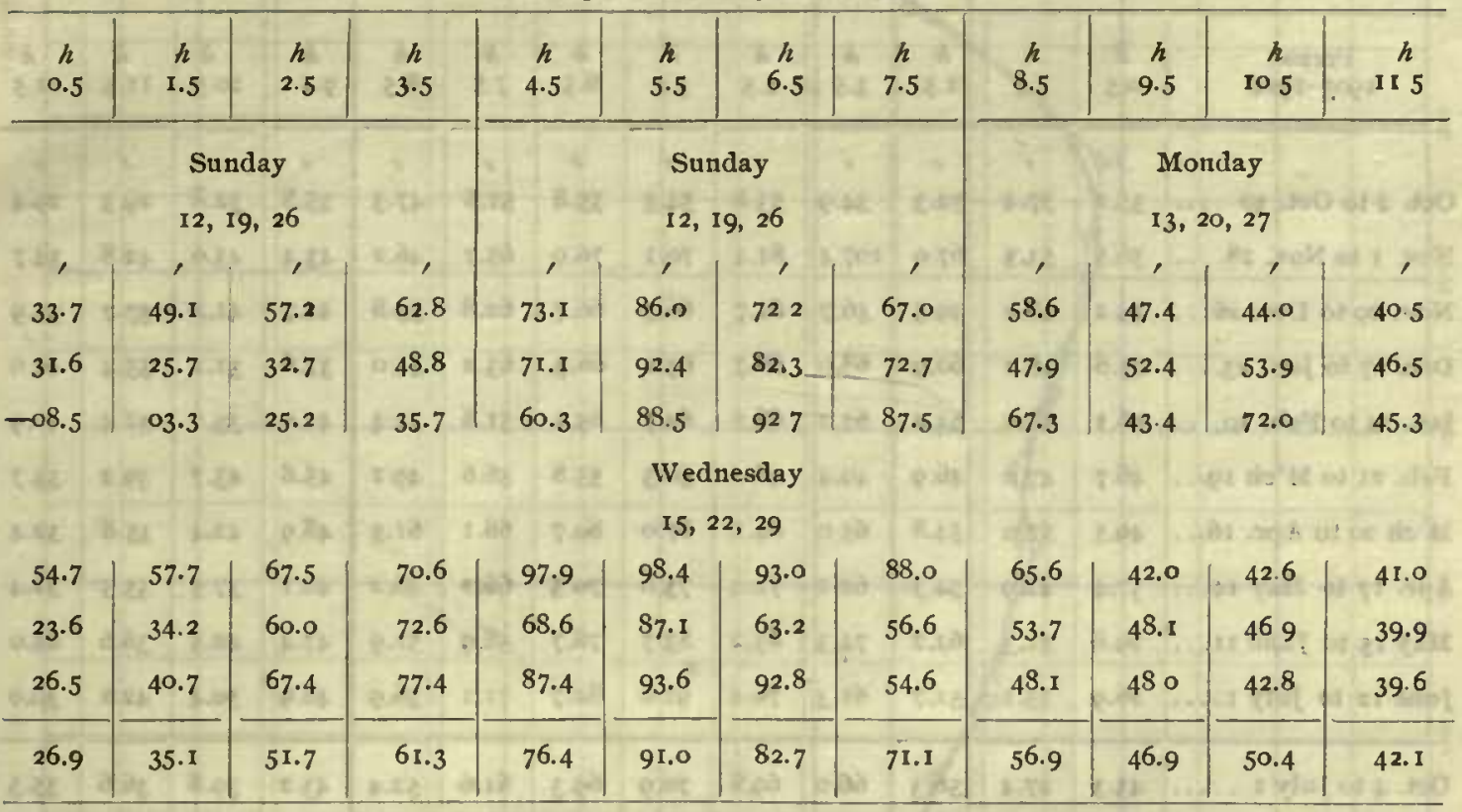

Tabulation of mean hourly magnetic declinations at Teplitz Bay

Three weeks, ending series, June I2 to July I, 1904-Coutiuued

$22^{\circ}$ plus tabular quantity, east

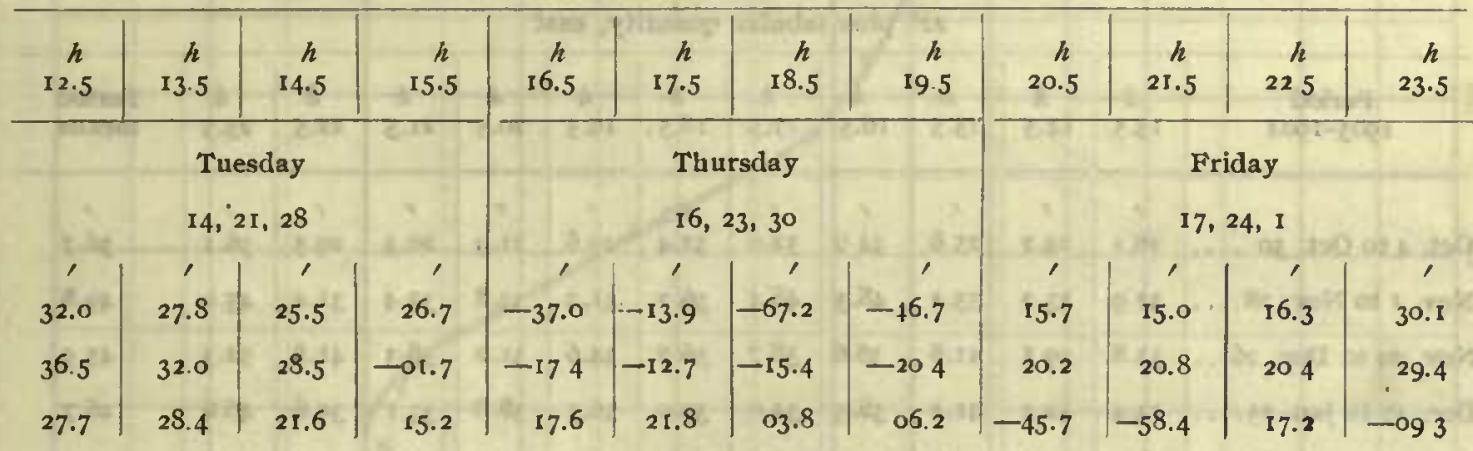

Wednesda y

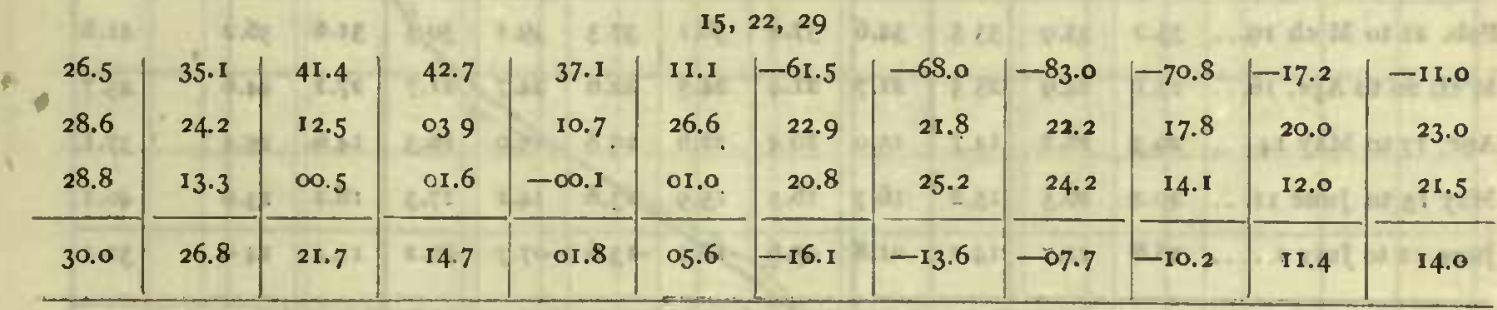

Mean value for the whole period, $22^{\circ} 32 .^{\prime} 1 \mathrm{E}$. 
Summary of mean hourly magnetic declinatians at Teplitz Bay

From observations between October 4, 1903, and July I, 1904

$22^{\circ}$ plus tabular quantity, east

\begin{tabular}{|c|c|c|c|c|c|c|c|c|c|c|c|c|c|}
\hline $\begin{array}{c}\text { Period } \\
1903-1904\end{array}$ & $\begin{array}{c}h \\
0.5\end{array}$ & $\begin{array}{c}h \\
\mathbf{I} .5\end{array}$ & $\begin{array}{c}h \\
2.5\end{array}$ & $\begin{array}{c}h \\
3.5\end{array}$ & $\begin{array}{c}h \\
4.5\end{array}$ & $\begin{array}{c}h \\
5.5\end{array}$ & $\begin{array}{c}h \\
6.5\end{array}$ & $\begin{array}{c}h \\
7.5\end{array}$ & $\stackrel{h}{85}$ & $\begin{array}{r}h \\
9.5\end{array}$ & $\begin{array}{r}h \\
10.5\end{array}$ & $\stackrel{h}{\mathrm{I}}$ & $\begin{array}{c}h \\
12.5\end{array}$ \\
\hline & , & 1 & , & , &, & , & , & ' & ' & , & 1 & , & ' \\
\hline Oct. 4 to Oct. 30. & 35.2 & 37.4 & 52.3 & 54.9 & 5.8 & 54.5 & 55.8 & 51.6 & $47 \cdot 3$ & 35.8 & 32.8 & 29.3 & 29.4 \\
\hline ov. I to Nov. 28. & 56.5 & $53 \cdot 3$ & 67.0 & 107.4 & 81.4 & 70.1 & 70.9 & 65.7 & 46.2 & 43.4 & 43.0 & 42.8 & 34.7 \\
\hline Nov. 29 to Dec. 26 . & 49.2 & 48.4 & 49.5 & 56.7 & 61.7 & 66.5 & 66.7 & 62.8 & 55.8 & 44.7 & 41.2 & 37.7 & $35 \cdot 9$ \\
\hline )ec. 27 to Jan. 23 & 45.6 & 48.2 & 60.9 & 68.0 & 68.7 & 69.1 & 66.3 & 63.2 & 54.0 & 37.6 & 31.2 & 35.4 & 40.0 \\
\hline an. 24 to Feb. 20 & $56 . \mathrm{r}$ & 58.8 & 63.4 & 61.2 & 68.2 & 62.7 & $65 . I$ & 51.6 & 54.4 & 41.0 & 33.2 & 31.4 & 40.9 \\
\hline Feb. 2 I to $M$ 'ch Ig. & 46.7 & 47.0 & 46.9 & 49.4 & 48.0 & 50.3 & 55.8 & 56.6 & 49.2 & 45.8 & 43.7 & 39.2 & $33 \cdot 7$ \\
\hline I'ch 20 to Apr. I6. & 49.5 & 52.0 & 54.8 & 650 & 84.1 & 87.0 & 80.7 & 68. I & $6 \mathrm{r} .3$ & 48.9 & 42.4 & 35.8 & 32.4 \\
\hline Apr. I7 to May $14 \ldots$ & 37.4 & 42.9 & $54 \cdot 3$ & 62.2 & 72.4 & 73.8 & 703 & 66.2 & 46.2 & 40.1 & 37.5 & 355 & 32.4 \\
\hline May Is to June Ir.. & 29.8 & 51.3 & 62.2 & $74 \cdot 3$ & 83.2 & 83.7 & 78.7 & 58.9 & 52.9 & 47.4 & 42.5 & 36.6 & 44.0 \\
\hline June 12 to July I.... & 26.9 & $35 \cdot I$ & $5 \mathrm{r} .7$ & $6 r \cdot 3$ & 76.4 & 91.0 & 82.7 & 71.1 & 56.9 & 46.9 & 50.4 & 42.1 & 30.0 \\
\hline 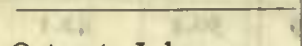 & s. & 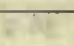 & $t$ & & & 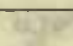 & 17 & & $3 x$ & 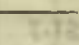 & & & .8 \\
\hline Oct. 4 to July I...... & $43 \cdot 3$ & $47 \cdot 4$ & 56.3 & 66.0 & 69.8 & 70.9 & 69.3 & $6 r .6$ & 52.4 & 43.2 & 39.8 & 36.6 & $35 \cdot 3$ \\
\hline
\end{tabular}

Summary of mean hourly magnetic declinations at Teplitz Bay

From observations between October 4, I903, and July I, 1905-Continued

$22^{\circ}$ plus tabular quantity, east

\begin{tabular}{|c|c|c|c|c|c|c|c|c|c|c|c|c|}
\hline $\begin{array}{l}\text { Period } \\
\text { I903-I } 904\end{array}$ & $\begin{array}{c}h \\
13.5\end{array}$ & $\begin{array}{c}h \\
14.5\end{array}$ & $\begin{array}{c}h \\
15.5\end{array}$ & $\begin{array}{c}h \\
16.5\end{array}$ & $\begin{array}{c}h \\
17.5\end{array}$ & $\begin{array}{c}h \\
\mathrm{x} 8.5\end{array}$ & $\begin{array}{c}h \\
19.5\end{array}$ & $\begin{array}{c}h \\
20.5\end{array}$ & $\begin{array}{c}h \\
21.5\end{array}$ & $\begin{array}{c}h \\
22.5\end{array}$ & $\begin{array}{c}h \\
23 \cdot 5\end{array}$ & $\begin{array}{l}\text { Period } \\
\text { means }\end{array}$ \\
\hline Oct. 4 to Oct. $30 \ldots$ & 2'́s. & $\frac{1}{24.2}$ & '́, & $34^{\circ}$ & 32.0 & 31.4 & ${ }_{27.6}^{\prime}$ & ${ }_{21.9}^{\prime}$ & ${ }_{20.3}^{\prime}$ & $\begin{array}{l}\prime \\
29.5\end{array}$ & 36. & 36.7 \\
\hline Nov. I to Nov. $28 \ldots$ & 37.9 & 33.2 & 35.4 & 48.3 & 48.4 & 36.7 & 31.2 & 34.8 & 32.4 & 31.4 & 43.0 & 49.8 . \\
\hline Nov, 29 to Dec. 26 . & 33.8 & 39.5 & 41.8 & 36.6 & 38.7 & 36.7 & 34.6 & 31.2 & 38.3 & 41.8 & 52.3 & 45.9 \\
\hline Dec, 27 to Jan. 23 . & 43.4 & 42.2 & 41.4 & 38.3 & 34.0 & 39.0 & 36.3 & 38.8 & 33. I & 39.6 & 46.6 & 46.7 \\
\hline Jan. 24 to Feb. $20 \ldots$ & 42.0 & 43.6 & 45.8 & 32.5 & 36.5 & 35.0 & 39.9 & 30.6 & 34.5 & 44. I & 39.0 & 46.3 \\
\hline Feb. $2 \mathrm{I}$ to $\mathrm{M}^{\prime} \mathrm{ch} 19 . .$. & 33.2 & 33.9 & 335 & 34.6 & 37.4 & 38.1 & 37.3 & 39.1 & 303 & 31.6 & 36.2 & 41.6 \\
\hline M'ch 20 to Apr. $16 . .$. & 27.1 & 24.9 & 25.5 & 21.7 & 21.4 & 24.5 & 22.6 & 24.7 & 21.7 & 27.2 & 44.6 & 43.7 \\
\hline Apr. I7 to May 14 & 29.3 & 26.2 & 14.7 & 15.0 & 204 & 22.6 & 19.8 & 15.0 & 16.3 & 14.6 & 25.4 & 37.1 \\
\hline May is to June II... & 40.2 & 26.5 & 15.2 & I6.7 & 16.5 & I5.9 & 13.6 & I4. I & $17 \cdot 3$ & I8.2 & 23.0 & $40.1^{-}$ \\
\hline June 12 to July $I$. & 26.8 & 21.7 & 14.7 & 01.8 & .05 .6 & -16.1 & -13.6 & -07.7 & -10.2 & II. 4 & 140 & 32.1 \\
\hline Oct. 4 to & 34.2 & 31.6 & 29.4 & 27.9 & 29.1 & 26.4 & 24.9 & $24: 2$ & 23.4 & 28.9 & 36.0 & 42.0 \\
\hline
\end{tabular}




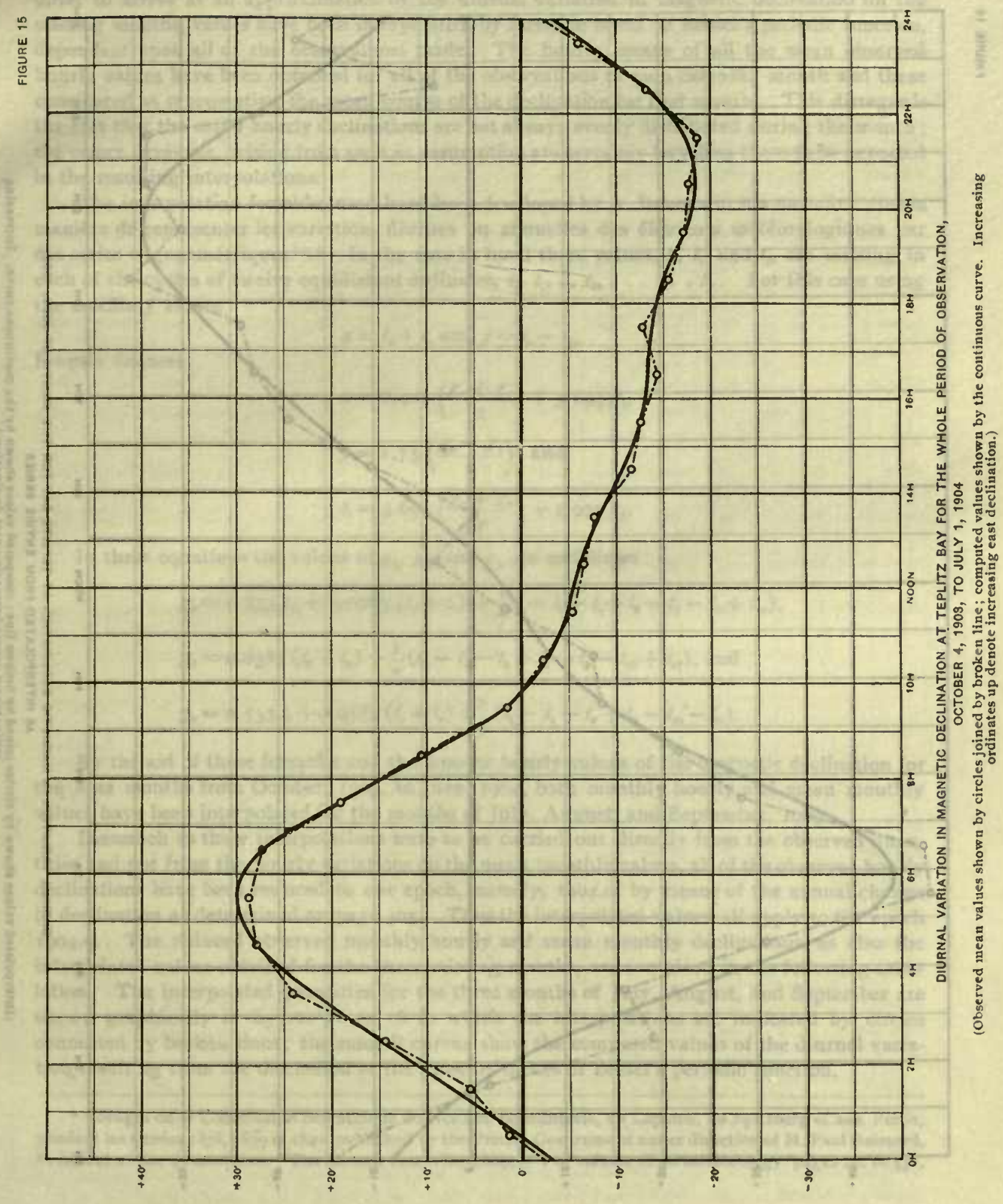




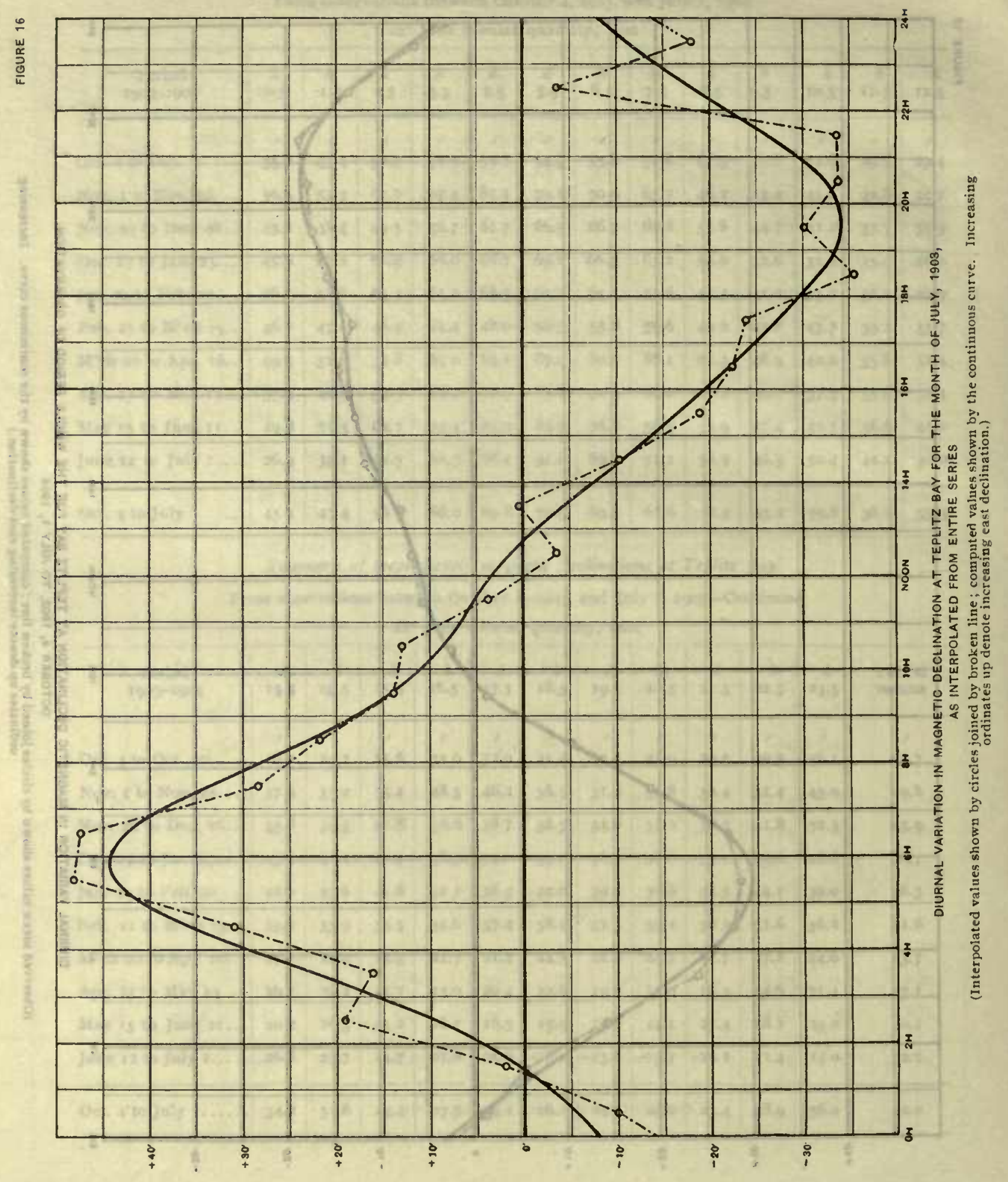


Unfortunately the observations at Teplitz Bay cover only nine months of the year. In order to arrive at an approximation of the diurnal variation in magnetic declination for the missing months, values have been interpolated, by formulæ based on Bessel's periodic function, dependent upon all of the observations made. The hourly means of all the mean observed hourly values have been obtained for all of the observations in each calendar month and these considered as representing the mean course of the declination for that month. This disregards the fact that the mean hourly declinations are not always evenly distributed during the month ; the errors, however, arising from such an assumption are certainly less than those to be expected in the resulting interpolations.

The interpolation formulæ used have been developed by A. Bravais in his memoir "Sur la manière de représenter les variations diurnes ou annuelles des éléments météorologiques par des series trigonométriques" ** In the case in hand three values, $t_{0}, t_{1}$, and $t_{3}$, are missing in each of the cycles of twelve equidistant ordinates, $t_{0}, t_{1}, t_{2}, t_{2}, \ldots \ldots t_{11}$. For this case using the auxiliary values

Bravais deduces

$$
\begin{aligned}
& x=t_{0}+t_{3} \text { and } y=t_{0}-t_{2}, \\
& x=6.929\left(\frac{g_{0}+g_{2}}{2}\right)+3.694 g_{1}, \\
& y=1.75\left(\frac{g_{0}-g_{2}}{2}\right), \text { and } \\
& t_{1}=3.694\left(\frac{g_{0}+g_{2}}{2}\right)+2.970 g_{1} .
\end{aligned}
$$

In these equations the values of $g_{0}, g_{1}$, and $g_{2}$ are as follows :

$$
\begin{aligned}
& g_{0}=0.533 t_{11}+0.0383\left(t_{6}+t_{1}\right)+\frac{1}{7}\left(-t_{3}-t_{4}+t_{6}-t_{8}-t_{8}+t_{10}\right), \\
& g_{1}=0.0383\left(t_{6}+t_{8}\right)+\frac{1}{7}\left(t_{3}-t_{4}-t_{5}+t_{1}-t_{8}-t_{10}+t_{11}\right), \text { and } \\
& g_{2}=0.533 t_{3}+0.0383\left(t_{7}+t_{8}\right)+\frac{1}{7}\left(t_{4}-t_{6}-t_{6}+t_{6}-t_{10}-t_{11}\right) .
\end{aligned}
$$

By the aid of these formula and the known loourly values of the maguetic declination for the nine months from October, 1903, to June, 1904, both monthly hourly and mean monthly values have been interpolated for the mouths of July, August, and September, I903.

Inasmuch as these interpolations were to be carried out directly from the observed quantities and not from the hourly variations on the mean monthly values, all of the observed hourly declinations have been reduced to one epoch, namely, 1904.0, by means of the annual change in declination as determined on page 305. Thus the interpolated values all apply to the epoch 1904.0. The reduced observed monthly hourly and mean monthly declinations, as also the interpolated values obtained for the three missing months, are contained in the following tabulation. The interpolated quantities for the three months of July, Angust, and September are shown graphically in figures 16 to 18 in which the interpolations are indicated by circles connected by broken lines; the smooth curves show the computed values of the diurnal variation resulting from the discussion of the same by means of Bessel's periodic function.

* Voyages de la Commission Scientifique du Nord en Scandinavie, en Laponie, au Spitzberg et aux Feröe, pendent les annees 1838,1839 et 1840 , published by the French Government under direction of M. Paul Gaimard, President of the Commission. The memoir comprises chapter V of volume II on meteorology, pages 29 I to 332. 
Tabulation of monthly mean hourly magnetic declinations at Teplitz Bay

All values reduced to mean epoch $1904{ }^{\circ}$

$22^{\circ} 4 \mathrm{I}^{\prime}$ plus tabular quantity, east

\begin{tabular}{|c|c|c|c|c|c|c|c|c|c|c|c|c|c|}
\hline Savaly Month & $\begin{array}{c}h \\
0.5\end{array}$ & $\begin{array}{c}h \\
1.5 \\
\end{array}$ & $\begin{array}{c}h \\
2.5\end{array}$ & $\begin{array}{c}h \\
3 \cdot 5\end{array}$ & $\begin{array}{c}h \\
4 \cdot 5 \\
\end{array}$ & $\begin{array}{c}h \\
5.5\end{array}$ & $\begin{array}{c}h \\
6.5\end{array}$ & $\begin{array}{c}h \\
7 \cdot 5\end{array}$ & $\begin{array}{c}h \\
8.5 \\
\end{array}$ & $\begin{array}{c}h \\
9.5\end{array}$ & $\begin{array}{c}h \\
10.5\end{array}$ & $\stackrel{h}{11.5}$ & $\begin{array}{c}h \\
\text { I2. } 5\end{array}$ \\
\hline 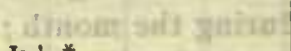 & 11 & & & & 1 & 21 & 1 & 19 & (7) & & & & 16 \\
\hline July$^{*} \ldots \ldots \ldots \ldots \ldots$ & -22.6 & -10.4 & 06.5 & 03.8 & 18.3 & $35 \cdot 7$ & 34.8 & I5.9 & 09.0 & OI.4 & $\infty .4$ & -08.9 & -16.2 \\
\hline August*.......... & -18.5 & - II.9 & 05.6 & -04.0 & 06.0 & 23.4 & 27.4 & Io.o & 07.1 & -00.5 & -0.5 & -10.6 & -20.0 \\
\hline September*........ & $-\infty$ I & -07.8 & 08.6 & 04.9 & $c 6.7$ & I6.6 & 21.5 & 10.1 & 05.8 & $-\mathrm{OI} .8$ & -03.0 & -10.2 & -18.2 \\
\hline October .......... & $-04 \cdot 3$ & $-02 . I$ & 12.8 & 15.4 & $14 \cdot 3$ & I5.I & 16.3 & 12. I & 07.8 & -03.7 & -06.7 & -10.2 & -I0.1 \\
\hline November ......... & I 5.0 & 12.6 & 24.9 & 60.6 & 39.1 & 32.0 & 31.2 & 27.8 & 05.4 & O2.I & 01.6 & OI. 5 & $-05 \cdot 3$ \\
\hline December. ...... & 08.2 & 07.0 & 08.5 & I5.8 & I8.0 & 19.6 & 22. I & 20.2 & 17.0 & OI.2 & -03.0 & -02.9 & -02.1 \\
\hline January......... & 03.9 & 13.4 & 25.0 & 30.7 & 27.0 & 26.6 & 23.5 & I5.7 & 09.6 & $\infty .0$ & -05.4 & -06.9 & 03.2 \\
\hline February & I5.9 & IO. I & I 2.2 & II.I & 23.0 & 18.7 & 24.5 & I6.8 & I3 6 & $\infty .5$ & -05.9 & -07.6 & -11.5 \\
\hline March.. & 075 & 09.7 & II.9 & 15.2 & I5.5 & $2 \mathrm{I} .2$ & 25.7 & I5 I & 05.0 & 03.3 & $\infty .1$ & $-04 \cdot I$ & -06.8 \\
\hline April. & 00.5 & 04.1 & 09.6 & 17.1 & 38.0 & 36.6 & 28. I & 22.1 & 16.4 & 02.6 & -01.7 & -07.8 & -06.3 \\
\hline May.. & -13.4 & 02.0 & 16.7 & $27 \cdot 3$ & $39 \cdot 3$ & 375 & 320 & 19.6 & O4. I & -01.2 & -05.6 & -10.2 & -08.1 \\
\hline June. . & -15.6 & -04.3 & 07.5 & 21.3 & 34.4 & 47.4 & 41.0 & 25.6 & II. 7 & 04.9 & 04.8 & -01.9 & -10.9 \\
\hline October to March... & 077 & 08.4 & 15.9 & 24.8 & 22.8 & 22.2 & 23.9 & 180 & 09.7 & 00.6 & -03.2 & -05.0 & -05.4 \\
\hline April to September. . & -13.1 & -04.7 & 09. I & II.7 & 23.8 & 32.8 & 30.8 & 17.2 & 09.0 & $\infty .9$ & -01.1 & -08.3 & $-13 \cdot 3$ \\
\hline Mean of year. & -02.7 & or.9 & 12.5 & 18.3 & 23.3 & 27.5 & $27 \cdot 3$ & 17.6 & 094 & $\infty .7$ & -02.2 & -06.6 & -09.4 \\
\hline
\end{tabular}

Tabulation of monthly mean hourly magnetic declinations at Teplitz Bay-Continued

All values reduced to mean epoch $\mathrm{rg} 4.0$

$22^{\circ} 4 \mathrm{I}^{\prime}$ plus tabular quantity, east

\begin{tabular}{|c|c|c|c|c|c|c|c|c|c|c|c|c|}
\hline Month & $\begin{array}{c}h \\
\mathbf{1} 3.5\end{array}$ & $\begin{array}{c}h / 2 \\
14.5\end{array}$ & $\begin{array}{c}h \\
15.5\end{array}$ & $\begin{array}{c}h \\
\mathbf{1 6 . 5}\end{array}$ & $\begin{array}{c}h \\
I 7.5\end{array}$ & $\begin{array}{c}h \\
\mathrm{I} 8.5\end{array}$ & $\begin{array}{c}h \\
19.5\end{array}$ & $\begin{array}{c}h \\
20.5\end{array}$ & $\begin{array}{c}h \\
21.5\end{array}$ & $\begin{array}{c}h \\
22.5\end{array}$ & $\begin{array}{c}h \\
23.5\end{array}$ & $\begin{array}{l}\text { Monthly } \\
\text { means }\end{array}$ \\
\hline & & , & 1 & 1 & $\sqrt{1}$ & 1 & , & 1 & 1 & 1 & , & , \\
\hline July*. & -122 & $-23 . I$ & $-3 I .4$ & -35.1 & -36.4 & -48.1 & -42.8 & -46.4 & -46.2 & -16.2 & -30.4 & -12.6 \\
\hline sugust ${ }^{*} . \ldots \ldots$. & -13.8 & -23.9 & -28.5 & -24.5 & -29.9 & -39.6 & -32.7 & -40.2 & -42.6 & -06.4 & -26.4 & -12.4 \\
\hline September ${ }^{*} . \ldots \ldots$. & $-13 \cdot 7$ & $|-21.7|$ & -22.4 & -II.I & -18.7 & -24.4 & $-2 I \cdot 3$ & -27.9 & $-3 I \cdot 3$ & -04.7 & -15.8 & -07.9 \\
\hline October. ........... & $-\mathrm{II} \cdot 4$ & $-15 \cdot 3$ & -13.9 & -05.5 & $-14 \cdot I$ & -11.7 & -14.7 & -18.2 & -18.2 & -09.4 & -04.0 & -03.2 \\
\hline November ..... & $-02 . I$ & -06.8 & -04.6 & 08.3 & 08.4 & -03.3 & -08.8 & -05.2 & -07.6 & -08.6 & 03.0 & 09.2 \\
\hline December.......... & -02.7 & 01.7 & 04.2 & -01.2 & -01.9 & -03.8 & -06.1 & -04.5 & -01.6 & -01.2 & Io.8 & $05 . I$ \\
\hline January....... & 05 I & 03.5 & 02.5 & -06.9 & -08.9 & -05.6 & -07.5 & -06.5 & $-c 9.4$ & $-\infty .9$ & 02.5 & 05.6 \\
\hline $\operatorname{ary} \ldots . . . \cdots \cdot$ & - IO. I & -06.4 & -05.1 & -08.5 & -02.7 & -02.8 & 02,0 & -11.7 & -09.5 & 01.5 & $-02 . I$ & 02.8 \\
\hline March...$\ldots \ldots$ & $-\infty .2$ & $-\mathrm{II} .7$ & -12.1 & -10.9 & -09.8 & -09.3 & -10.4 & -05.7 & -13.7 & -06.0 & -02.5 & $\infty 0.8$ \\
\hline April......... & -08.1 & -10.4 & -18.1 & -21.8 & -20.5 & -17.5 & $-\mathrm{I} 8.2$ & -20.9 & -23.4 & -25.4 & -07.3 & -01.3 \\
\hline May.............. & -19.2 & -26.7 & -33.4 & -38.4 & -34.7 & -32.2 & -37.4 & -38.1 & -32.5 & -34.2 & $-24 \cdot 3$ & -08.8 \\
\hline June..... & -09.7 & -18.8 & -29.1 & -33.5 & -31.0 & -44.7 & -43.4 & -39.7 & -40.4 & -26.5 & $-24 . I$ & -07.4 \\
\hline October to March... & -05.1 & -05.8 & -04.8 & -04.1 & -04.8 & -06.1 & -07.6 & -08.6 & -10.0 & -04.1 & OI. 3 & 03.4 \\
\hline April to September. & -12.8 & -20.8 & -27.2 & -27.4 & -28.7 & -34.4 & -32.6 & -35.5 & -36.1 & -19.0 & -21.4 & -08.4 \\
\hline Mean of year. & -08.9 & -13.3 & -16.0 & -15.8 & -16.7 & -20.2 & $-20 . I$ & -22.1 & -23.0 & -11.5 & -10.0 & -02.5 \\
\hline
\end{tabular}

* These are the mowths for which values are interpolated. 


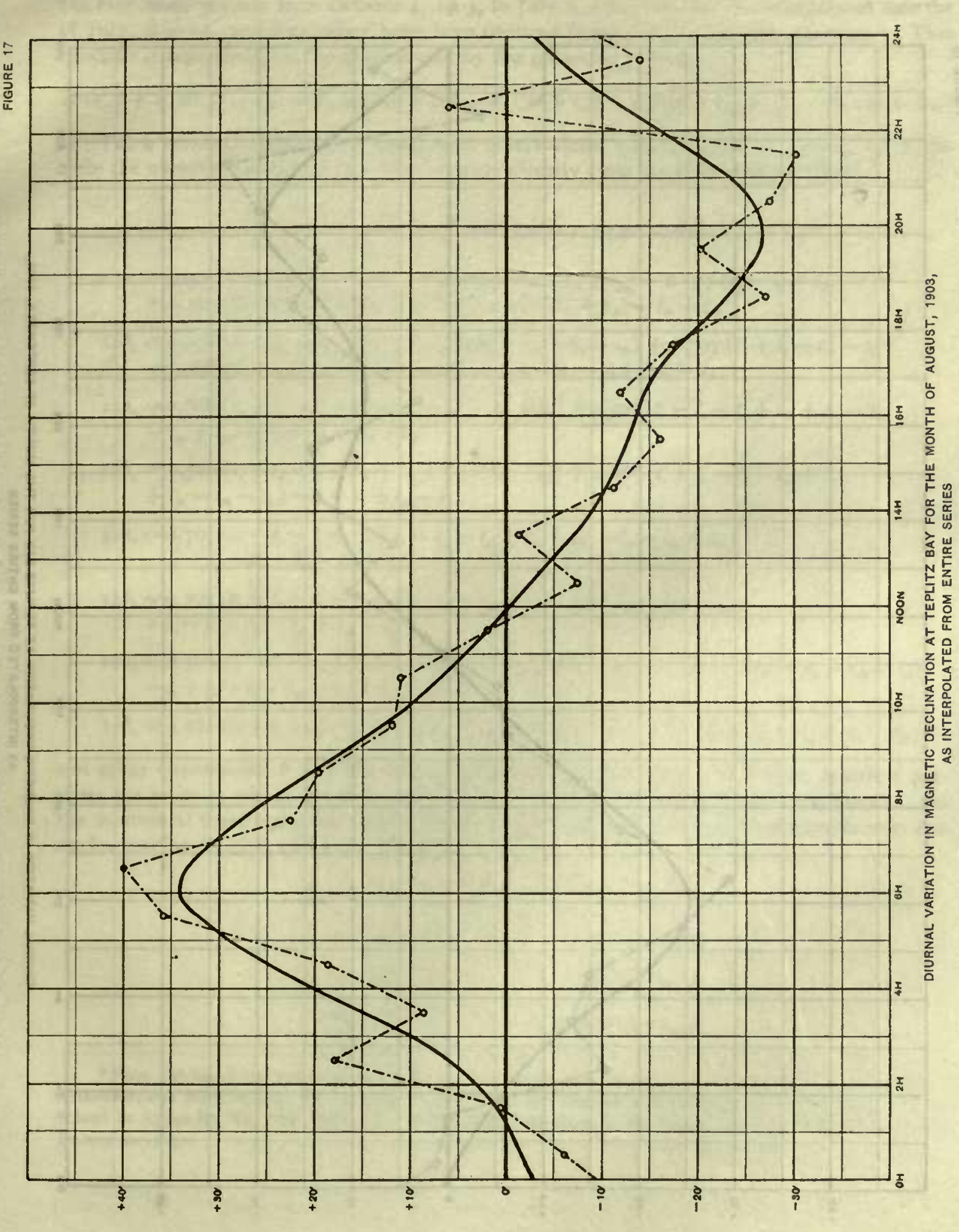




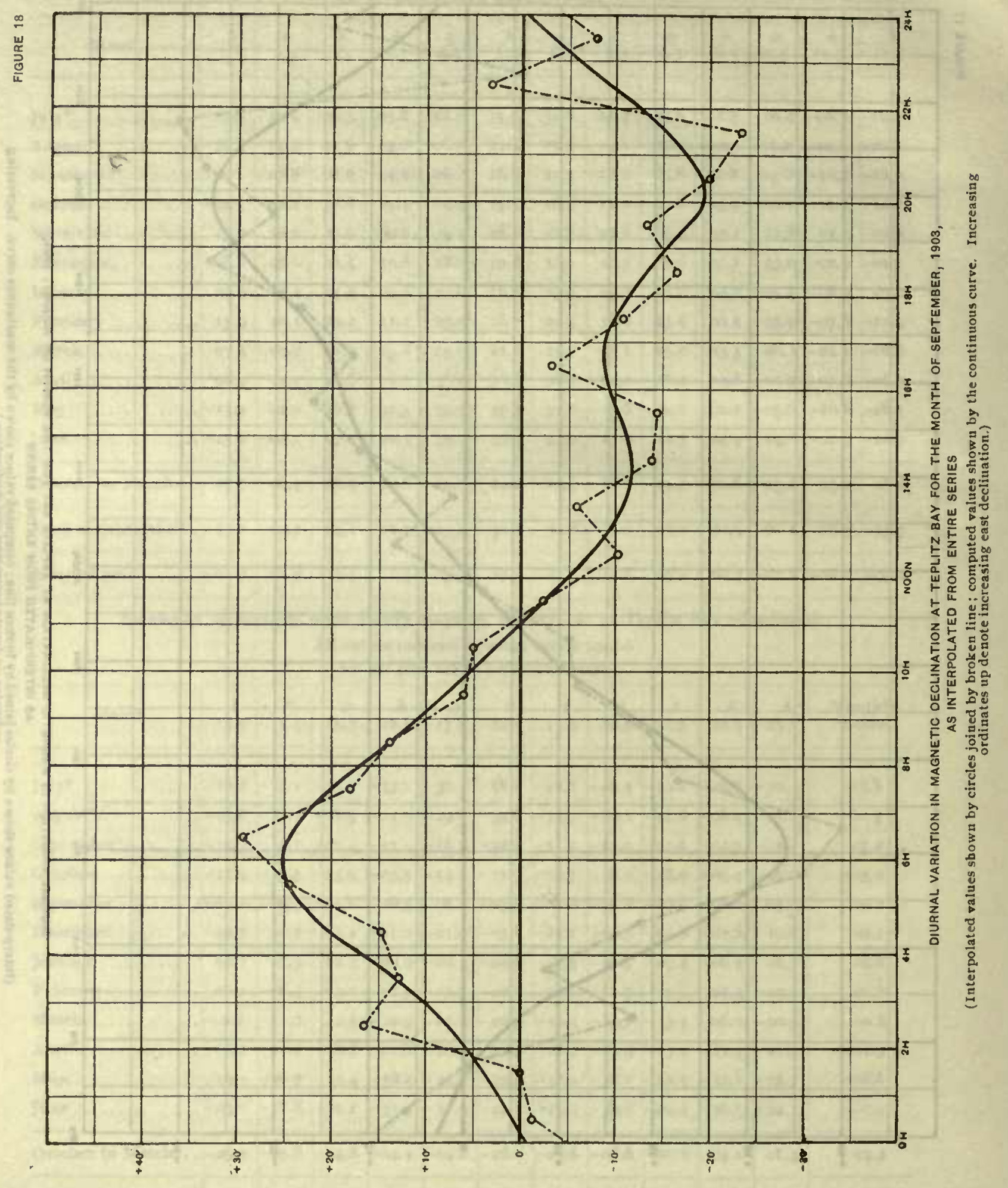


The analytical expressions representing the diurual variation in magnetic declination for the four-week periods from October 4, 1903, to July I, 1904, and for the interpolated months of July, August, and September have been deduced from Bessel's periodic function.* This function is represented in the general case by the following formula :

$D=A+B_{1} \sin \left(\theta+C_{1}\right)+B_{2} \sin \left(2 \theta+C_{2}\right)+B_{3}\left(3^{\theta}+C_{3}\right)+B_{4}\left(4 \theta+C_{4}\right)+\ldots B_{n}\left(n \theta+C_{n}\right)$

For a series of twenty-four equidistant observations, $t_{1}, t_{3}, t_{3}, t_{1}, t_{6}, \ldots \ldots t_{23}, t_{34}$, in the cycle the numerical computations may be made directly from the following equations:

$$
\begin{aligned}
& A=\frac{1}{24}\left(t_{1}+t_{2}+t_{3}+t_{4}+t_{6}+\ldots \ldots t_{4}\right) \\
& \mathrm{I} 2 a_{1}=0.966\left(t_{1}-t_{11}-t_{13}+t_{23}\right)+0.866\left(t_{2}-t_{10}-t_{11}+t_{23}\right)+0.707\left(t_{3}-t_{9}-t_{15}+t_{21}\right) \\
& +0.500\left(t_{1}-t_{8}-t_{18}+t_{30}\right)+0.259\left(t_{5}-t_{7}-t_{17}+t_{19}\right)-t_{12}+t_{24} \\
& 12 b_{1}=0.259\left(t_{1}+t_{11}-t_{13}-t_{23}\right)+0.500\left(t_{2}+t_{10}-t_{14}-t_{28}\right)+0.707\left(t_{3}+t_{9}-t_{15}-t_{21}\right) \\
& +0.866\left(t_{4}+t_{8}-t_{18}-t_{20}\right)+0.966\left(t_{3}+t_{7}-t_{17}-t_{19}\right)+t_{6}-t_{18} \\
& 12 a_{2}=0.866\left(t_{1}-t_{5}-t_{7}+t_{11}+t_{13}-t_{17}-t_{19}+t_{23}\right)+0.500\left(t_{3}-t_{4}-t_{8}+t_{10}+t_{14}-t_{10}\right. \\
& \left.-t_{20}+t_{28}\right)-t_{6}+t_{13}-t_{18}+t_{24} \\
& 12 b_{8}=0.500\left(t_{1}+t_{5}-t_{7}-t_{11}+t_{13}+t_{17}-t_{19}-t_{23}\right)+0.866\left(t_{2}+t_{6}-t_{8}-t_{10}+t_{11}\right. \\
& \left.+t_{18}-t_{20}-t_{32}\right)+t_{3}-t_{3}+t_{15}-t_{31} \\
& 12 a_{3}=0.707\left(t_{1}-t_{3}-t_{5}+t_{7}+t_{9}-t_{11}-t_{13}+t_{15}+t_{17}-t_{19}-t_{31}+t_{33}\right) \\
& -t_{4}+t_{8}-t_{12}+t_{18}-t_{20}+t_{24} \\
& 12 b_{3}=0.707\left(t_{1}+t_{3}-t_{3}-t_{7}+t_{9}+t_{11}-t_{13}-t_{15}+t_{17}+t_{19}-t_{21}-t_{23}\right) \\
& +t_{2}-t_{8}+t_{10}-t_{14}+t_{18}-t_{23} \\
& \mathrm{I} 2 a_{4}=0.500\left(t_{1}-t_{2}-t_{4}+t_{8}+t_{5}-t_{8}-t_{10}+t_{11}+t_{19}-t_{14}-t_{18}+t_{17}+t_{19}-t_{20}-t_{23}+t_{23}\right) \\
& -t_{3}+t_{8}-t_{9}+t_{12}-t_{15}+t_{18}-t_{21}+t_{24} \\
& 12 b_{4}=0.866\left(t_{1}+t_{2}-t_{4}-t_{3}+t_{7}+t_{8}-t_{10}-t_{11}+t_{13}+t_{14}-t_{16}-t_{17}+t_{19}+t_{20}-t_{23}-t_{23}\right)
\end{aligned}
$$

and other expressions of like cluaracter for terms of higher order. For most practical purposes the series is not in general improved by the addition of terms beyond the fourth. On the solution of these equations by substitution of the observed values the term-coefficients and angles may be determined by the following:

$$
\begin{array}{ll}
B_{1}=\sqrt{a_{1}{ }^{2}+b_{1}{ }^{2}} & \tan C_{1}=a_{1} / b_{1} \\
B_{2}=\sqrt{a_{3}{ }^{2}+b_{3}{ }^{2}} & \tan C_{3}=a_{3} / b_{2} \\
B_{2}=\sqrt{a_{3}{ }^{3}+b_{3}{ }^{2}} & \tan C_{3}=a_{3} / b_{3} \\
B_{4}=\sqrt{a_{1}{ }^{2}+b_{1}{ }^{2}} & \tan C_{4}=a_{4} / b_{4}
\end{array}
$$

* First published by Bessel in the Literary Gazette of Jena in 1814 ; also published in his paper in Astrononische Nachrichten, No. 136, May, 1828. See also Bravais' nienoir referred to above and C. A. Schott in Appendix No. $S$ of the Report of the Superintendent of the United States Coast and Geodetic Survey for 1890 . 
In the case of a cycle of twelve equidistant observations :

$$
\begin{aligned}
& A=\frac{1}{12}\left(t_{1}+t_{2}+t_{3}+t_{4}+\ldots \ldots t_{13}\right) \\
& 6 a_{1}=0.866\left(t_{1}-t_{5}-t_{7}+t_{11}\right)+0.500\left(t_{2}-t_{4}-t_{8}+t_{10}\right)-t_{8}+t_{12} \\
& 6 b_{1}=0.500\left(t_{1}+t_{3}-t_{7}-t_{11}\right)+0.866\left(t_{2}+t_{4}-t_{8}-t_{10}\right)+t_{3}-t_{9} \\
& 6 a_{2}=0.500\left(t_{1}-t_{2}-t_{4}+t_{5}+t_{7}-t_{8}-t_{10}+t_{11}\right)-t_{3}+t_{8}-t_{9}+t_{12} \\
& 6 b_{2}=0.866\left(t_{1}+t_{2}-t_{4}-t_{5}+t_{7}+t_{8}-t_{10}-t_{11}\right) \\
& 6 a_{3}=-t_{2}+t_{1}-t_{0}+t_{8}-t_{10}+t_{12} \\
& 6 b_{8}=+t_{1}-t_{3}+t_{5}-t_{7}+t_{9}-t_{11} \\
& 6 a_{4}=0.500\left(-t_{1}-t_{2}-t_{4}-t_{5}-t_{7}-t_{8}-t_{10}-t_{12}\right)+t_{3}+t_{6}+t_{9}+t_{12} \\
& 6 b_{4}=0.866\left(t_{1}-t_{2}+t_{4}-t_{3}+t_{7}-t_{8}+t_{10}-t_{11}\right)
\end{aligned}
$$

On the evaluation of these equations the coefficients $B_{1}, B_{2}, B_{3}, \ldots B_{n}$, and the angles $C_{1}, C_{2}, C_{3}, \ldots C_{n}$ are found as before.*

The results of the discussions for the various periods from the observed and interpolated hourly declinations are as follows, the probable error of a single representation being indicated by the "plus or minus" quantity at the end of each formula :

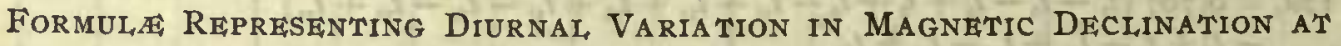 TEPLITZ BAY}

October 4 to October 30,1903 :

$D=22^{\circ} 36 .^{\prime} 7+13 .^{\prime} 67 \sin \left(\theta+2^{\circ} 3 \mathrm{I}^{\prime}\right)+6 .^{\prime} 98 \sin \left(2 \theta+282^{\circ} 25^{\prime}\right)+$

I. $33 \sin \left(3^{\theta}+16^{\circ} 46^{\prime}\right)+1 .^{\prime} 55 \sin \left(4^{\circ}+62^{\circ} 47^{\prime}\right) \pm 0 .^{\prime} 37$

November I to November 28,1903 :

$$
D=22^{\circ} 49 .^{\prime} 8+199^{\prime} 74 \sin \left(\theta+11^{\circ} 44^{\prime}\right)+14^{\prime} 03 \sin \left(2 \theta+303^{\circ} \mathrm{or}^{\prime}\right)+
$$

3. $.^{\prime} 91 \sin \left(3^{\theta}+285^{\circ} 42^{\prime}\right)+3 .^{\prime} 13 \sin \left(4^{\theta}+145^{\circ} 38^{\prime}\right) \pm 0 .^{\prime} 94$

November 29 to December 26,1903 :

$D=22^{\circ} 45^{\prime} 9+13 \cdot^{\prime} 13 \sin \left(\theta+6^{\circ} 09^{\prime}\right)+4 \cdot^{\prime} 97 \sin \left(2 \theta+270^{\circ} 44^{\prime}\right)+$

$4 .^{\prime} 50 \sin \left(3^{\theta}+103^{\circ} 12^{\prime}\right)+2 .^{\prime} 00 \sin \left(4^{\theta}+78^{\circ} 58^{\prime}\right) \pm 0 .^{\prime} 24$

December 27,1903 , to Jantuary 23,1904 :

$D=22^{\circ} 46 .^{\prime} 7+13 .^{\prime} 96 \sin \left(\theta+9^{\circ} 40^{\prime}\right)+8 .^{\prime} 60 \sin \left(2 \theta+295^{\circ} 59^{\prime}\right)+$

3.' $97 \sin \left(3^{\circ}+159^{\circ} 26^{\prime}\right)+2 .^{\prime} 01 \sin \left(4^{\theta}+33^{\circ} \mathrm{oo}^{\prime}\right) \pm 0 .^{\prime} 35$

January 24 to February 20,1904 :

$D=22^{\circ} 46 .^{\prime} 3+13 .^{\prime} 54 \sin \left(\theta+15^{\circ} 04^{\prime}\right)+7 .^{\prime} 72 \sin \left(2 \theta+317^{\circ} 17^{\prime}\right)+$

2.' $19 \sin \left(3^{\circ}+126^{\circ} 14^{\prime}\right)+2 .^{\prime} 79 \sin \left(40+318^{\circ} 22^{\prime}\right) \pm 0 .^{\prime} 48$

February 21 to March 19, 1904 :

$D=22^{\circ} 4 \mathrm{r}^{\prime} 6+9 .^{\prime} 47 \sin \left(\theta+357^{\circ} 48^{\prime}\right)+3 .^{\prime} 65 \sin \left(2 \theta+247^{\circ} 46^{\prime}\right)+$ 2. ${ }^{\prime} 38 \sin \left(3^{\circ}+4^{\circ} 04^{\prime}\right)+2 .^{\prime} 34 \sin \left(4 \theta+34^{\circ} 42^{\prime}\right) \pm 0 .^{\prime} 24$

* These solutions are given by C. A. Schott in Appendix No. 8 of the Report of the Superintendent of the United States Coast and Geodetic Survey for I 890 . 


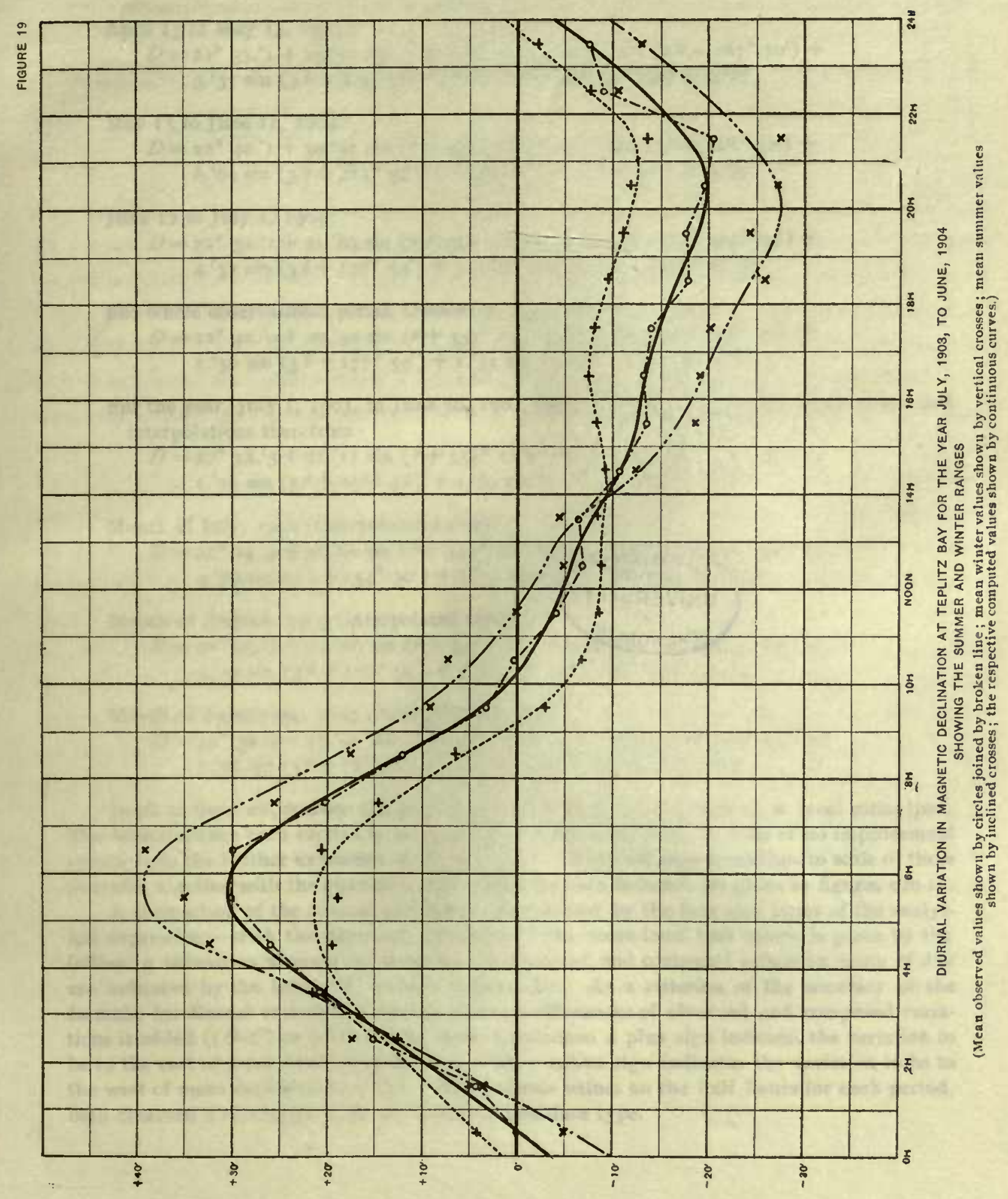



March 20 to April 16, I904:

$$
\begin{aligned}
D= & 22^{\circ} 43 \cdot^{\prime} 7+27 \cdot^{\prime} 93 \sin \left(\theta+0^{\circ} 26^{\prime}\right)+7 \cdot^{\prime} 96 \sin \left(2 \theta+274^{\circ} 24^{\prime}\right)+ \\
& 2 .^{\prime} 67 \sin \left(3 \theta+149^{\circ} 03^{\prime}\right)+3 \cdot^{\prime} 56 \sin \left(40+65^{\circ} 34^{\prime}\right) \pm 0 .^{\prime} 39
\end{aligned}
$$

April 17 to May 14,1904 :

$$
\begin{aligned}
D= & 22^{\circ} 37 \cdot^{\prime} \mathrm{x}+25{\cdot^{\prime}}^{\prime} \mathrm{I} \sin \left(\theta+353^{\circ} 22^{\prime}\right)+8 .^{\prime} 8 \mathrm{I} \sin \left(2 \theta+287^{\circ} \mathrm{I0^{ \prime }}\right)+ \\
& 3 \cdot^{\prime} 37 \sin \left(3^{\theta}+224^{\circ} 52^{\prime}\right)+3 .^{\prime} 8 \mathrm{I} \sin \left(4^{\theta}+24^{\circ} 14^{\prime}\right) \pm 0 .^{\prime} 27
\end{aligned}
$$

May I 5 to June II, 1904 :

$$
\begin{gathered}
D=22^{\circ} 400^{\prime} 1+300^{\prime} 31 \sin \left(\theta+350^{\circ} 15^{\prime}\right)+9 .^{\prime} 14 \sin \left(20+308^{\circ} 5 I^{\prime}\right)+ \\
6 .^{\prime} 64 \sin \left(3^{0}+223^{\circ} 42^{\prime}\right)+1 .^{\prime} 50 \sin \left(4^{0}+31^{\circ} 56^{\prime}\right) \pm 0 .^{\prime} 39
\end{gathered}
$$

June I 2 to July I, I904:

$$
\begin{gathered}
D=22^{\circ} 32 .^{\prime} 1+41^{\prime} 83 \sin \left(\theta+341^{\circ} 19^{\prime}\right)+8 .^{\prime} 65 \sin \left(2 \theta+309^{\circ} 04^{\prime}\right)+ \\
4 .^{\prime} 57 \sin \left(3 \theta+156^{\circ} 34^{\prime}\right)+3 .^{\prime} 88 \sin \left(40+100^{\circ} 30^{\prime}\right) \pm 0 .^{\prime} 53
\end{gathered}
$$

For whole observational series, October $4, x 903$, to July 1, 1904 :

$$
\begin{aligned}
& D=22^{\circ} 42 .^{\prime} 0+20 .^{\prime} 5^{2} \sin \left(\theta+357^{\circ} 14^{\prime}\right)+7 .^{\prime} 69 \sin \left(20+294^{\circ} 16^{\prime}\right)+ \\
& \text { I. }{ }^{\prime} 50 \sin \left(3^{0}+177^{\circ} 59^{\prime}\right)+\text { I. }^{\prime} 5 \mathrm{I} \sin \left(4^{\circ}+47^{\circ} 52^{\prime}\right) \pm 0 .^{\prime} 12
\end{aligned}
$$

For the year, July I, I903, to June 30 , I904, from monthly grouping of observations and interpolations therefrom :

$$
\begin{aligned}
D= & 22^{\circ} 38 .^{\prime} 5+2 \mathrm{r} .^{\prime} \mathrm{II} \sin \left(\theta+354^{\circ} \mathrm{I} 8^{\prime}\right)+6 .^{\prime} 90 \sin \left(2 \theta+29 \mathrm{r}^{\circ} 26^{\prime}\right)+ \\
& \mathrm{r} .^{\prime} 75 \sin \left(3 \theta+156^{\circ} 4^{\prime}\right)+\mathrm{r} .^{\prime} 62 \sin \left(4^{\theta}+58^{\circ} 5^{\prime}\right) \pm 0 .^{\prime} 20
\end{aligned}
$$

Month of July, 1903 (interpolated values) :

$$
\begin{aligned}
D= & 22^{\circ} 24 .^{\prime} 9+32 .^{\prime} 62 \sin \left(0+341^{\circ} 59^{\prime}\right)+5 .^{\prime} 95 \sin \left(2 a+291^{\circ} 36^{\prime}\right)+ \\
& 4^{\prime} 79 \sin \left(30+154^{\circ} 30^{\prime}\right)+2 .^{\prime} 73 \sin \left(4^{\circ}+84^{\circ} 45^{\prime}\right) \pm 0 .^{\prime} 75
\end{aligned}
$$

Month of August, 1903 (interpolated values) :

$$
\begin{aligned}
D= & 22^{\circ} 25^{\prime} 8+24^{\prime} 66 \sin \left(0+343^{\circ} 19^{\prime}\right)+4 .^{\prime} 26 \sin \left(20+273^{\circ} 14^{\prime}\right)+ \\
& 3 .^{\prime} 59 \sin \left(3^{\theta}+116^{\circ} 5 I^{\prime}\right)+2 .^{\prime} 63 \sin \left(40+86^{\circ} 04^{\prime}\right) \pm 0 .^{\prime} 90
\end{aligned}
$$

Month of September, 1903 (interpolated values) :

$$
\begin{aligned}
D= & 22^{\circ} 30 .^{\prime} 9+17 .^{\prime} 95 \sin \left(\theta+35^{\circ} 52^{\prime}\right)+5 .^{\prime} 09 \sin \left(20+272^{\circ} 49^{\prime}\right)+ \\
& 2 .^{\prime} 25 \sin \left(3^{0}+72^{\circ} 56^{\prime}\right)+2 .^{\prime} 28 \sin \left(40+90^{\circ} 30^{\prime}\right) \pm 0 .^{\prime} 66
\end{aligned}
$$

In all of these expressions the angle $\theta$ counts from $15^{\circ}$ as 0.5 hour A. M. local mean time. The formula have been carried to terms of the fourtli order only, as little or no improvement results from the further extension of the function. Graphical representations to scale of these formulæ, together with the quantities from which they are deduced, are given by figures 5 to 19 .

A comparison of the diurnal variations-represented by the four sine terms of the analytical expressions-with the observed quantities at the mean local half hours, is given by the following tabulation wherein the observed, interpolated, and computed values on mean of day are indicated by the letters $O, I$, and $C$ respectively. As a criterion of the accuracy of the formulæ for diurnal variation, a column showing differences of observed and computed variations is added $((O-C)$ or $(I-C))$. In these tabulations a plus sign indicates the variation to be to the east of mean declination for day, while a minus sign indicates the variation to be to the west of mean declination for day. The extreme values on the half hours for each period, both observed and computed, are indicated by bold-face type. 
Tabulation of observed and computed diurnal variation of magnetic declination at Teplitz Bay

\begin{tabular}{|c|c|c|c|c|c|c|c|c|c|c|c|c|c|c|c|}
\hline \multirow{2}{*}{ 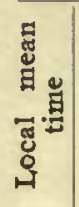 } & \multicolumn{3}{|c|}{ Month of July } & \multicolumn{3}{|c|}{ Month of August } & \multicolumn{3}{|c|}{ Month of September } & \multicolumn{3}{|c|}{$\begin{array}{l}\text { October } 4 \text { to } \\
\text { October } 3^{\circ}\end{array}$} & \multicolumn{3}{|c|}{$\begin{array}{l}\text { November I to } \\
\text { November } 28\end{array}$} \\
\hline & $I$ & $C$ & $I-C$ & $I$ & $C$ & $I-C$ & $I$ & $C_{0}$ & $I-C$ & 0 & $c$ & $O-C$ & 0 & $C$ & $O-C$ \\
\hline$h$ & ' & 1 & 1 & $\cdots$ & 1 & 1 & & & & & , & 1 & & , & , \\
\hline & -10.0 & -5.4 & -4.6 & $-6 . I$ & -1.7 & -4.4 & -1.2 & +1.0 & -2.2 & $-\mathbf{I} .5$ & +1.4 & -2.9 & +6.7 & -0.8 & $+7 \cdot 5$ \\
\hline & +2.2 & +0.4 & +1.8 & +0.5 & +1.0 & -0.5 & +0.1 & +3.8 & -3.7 & +0.7 & +6.4 & -5.7 & +3.5 & + II.8 & $3-8.3$ \\
\hline & +I9.I & +9.8 & $+9 \cdot 3$ & +18.0 & +5.9 & +12.1 & +16.5 & +7.7 & +8.8 & +15.6 & +10.8 & +4.8 & +17.2 & +25.8 & -8.6 \\
\hline & +16.4 & +22.8 & -6.4 & +8.4 & +14.6 & -6.2 & +12.8 & +13.6 & -0.8 & +18.2 & +15.2 & +3.0 & +57.6 & +36.4 & +21.2 \\
\hline & +30.9 & +36.0 & -5.1 & +18.4 & +24.8 & -6.4 & +14.6 & +20.2 & -5.6 & +17.1 & +18.9 & -1.8 & +31.6 & +38.8 & -7.2 \\
\hline & +48.3 & +43.6 & +4.7 & +35.8 & +32.1 & +3.7 & +24.5 & +24.5 & 0.0 & +17.8 & +20.6 & -2.8 & +20.3 & +31.8 & -II.5 \\
\hline & +47.4 & +42.4 & +5.0 & +39.8 & +33.1 & +6.7 & +29.4 & +24.3 & $+5 \cdot x$ & +19.1 & & +0.2 & +21.1 & +19.2 & +1.9 \\
\hline & +28.5 & $+33 \cdot 7$ & -5.2 & +22.4 & +28.0 & -5.6 & +18.0 & +20.0 & -2.0 & +14.9 & +13.9 & +1.0 & $\begin{array}{r}+15.9 \\
\end{array}$ & +7.1 & +8.8 \\
\hline & +21.6 & +22.6 & -1.0 & +19.5 & +20.0 & -0.5 & +13.7 & +13.8 & -0.1 & +10.6 & +7.5 & +3.1 & -3.6 & -0.5 & -3.1 \\
\hline & +14.0 & +13.7 & +0.3 & trr.9 & +12.5 & -0.6 & & +7.9 & -1.8 & -0.9 & & & -6.4 & -3.9 & -2.5 \\
\hline & +13.0 & +8.5 & +4.5 & +10.9 & +6.9 & +4.0 & & +2.6 & +2.3 & & & -0.9 & -6.8 & -6.4 & $-o$ \\
\hline & +3.7 & +5.2 & -1.5 & +1.8 & +2.2 & -0.4 & -2.3 & -2.4 & +0.1 & -7.4 & -6.4 & $-\mathbf{1 . 0}$ & -7.0 & -10.2 & +3 \\
\hline & -3.6 & +1.2 & -4.8 & -7.6 & -2.5 & -5.1 & -10.3 & & -3.0 & & & & -15.1 & -14.7 & -0 \\
\hline & +0.4 & $-4 \cdot 5$ & +4.9 & $-\mathrm{I} .4$ & & & -5.8 & & +5.0 & & -10.8 & & $-\operatorname{Ir} .9$ & -16.6 & +4 \\
\hline & -10.5 & -10.8 & +0.3 & -II.5 & -10.7 & -0.8 & -13.8 & -11.8 & -2.0 & -12.5 & -10.6 & & -16.6 & -14.0 & -2.6 \\
\hline & -18.8 & -16.7 & -2.1 & - 6.1 & -12.9 & -3.2 & -14.5 & -10.3 & -4.2 & $-I I . I$ & -8.3 & & & -8.7 & -5 \\
\hline & -22.5 & -22.0 & -0.5 & -12.1 & & +2.8 & -3.2 & -8.8 & +5.6 & -2.7 & -5.4 & +2.7 & -1.5 & -4.5 & +3.0 \\
\hline & -23.8 & -27.1 & $+3 \cdot 3$ & -17.5 & -18.4 & +0.9 & -10.8 & -9.8 & -1.0 & $-4 \cdot 7$ & -4.2 & -0.5 & -1.4 & -4.7 & $+3 \cdot 3$ \\
\hline & -35.5 & & $-3 \cdot 7$ & -27.2 & & -4.2 & $-\mathrm{r} 6.5$ & -13.5 & -3.0 & $-5 \cdot 3$ & -6.0 & +0.7 & & -9.2 & \\
\hline & -30.2 & -34.2 & +4.0 & -20.3 & -26.4 & +6.1 & -13.4 & -17.6 & +4.2 & -9.1 & -9.9 & +08 & -18.6 & -14.8 & -3.8 \\
\hline & -33.8 & -324 & -1.4 & -27.8 & -25.7 & -2.1 & -20.0 & -18.9 & $-1 . I$ & -14.8 & -13.3 & -1.5 & -15.0 & -18.4 & +3.4 \\
\hline & -33.6 & -26.2 & -7.4 & -30.2 & -20.1 & -10.1 & -23.4 & -15.6 & -7.8 & -16.4 & -13.6 & & & & +1 \\
\hline & -3.6 & -18.0 & +14.4 . & +6.0 & -12.2 & +18.2 & +3.2 & -9.4 & +12.6 & -7.2 & -10.2 & +5.0 & -18.4 & -15.4 & -3.0 \\
\hline & -17.8 & -10.8 & & -14.0 & & & -7.9 & -3.2 & $\mid-4.7$ & -0.6 & -4.5 & +3.9 & -6.8 & -9.8 & +3.0 \\
\hline
\end{tabular}

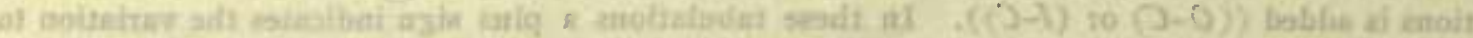

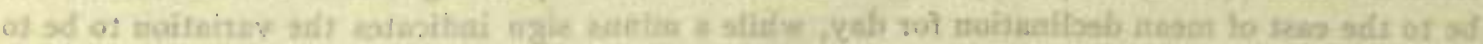

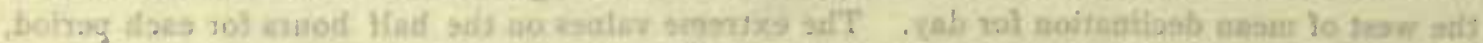


Tabulation of observed and computed diurnal variation of magnetic declination at Tcplitz Bay-Continued

\begin{tabular}{|c|c|c|c|c|c|c|c|c|c|c|c|c|c|c|c|}
\hline \multirow{2}{*}{ 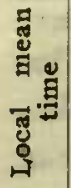 } & \multicolumn{3}{|c|}{$\begin{array}{l}\text { November } 29 \text { to } \\
\text { December } 26\end{array}$} & \multicolumn{3}{|c|}{$\begin{array}{l}\text { December } 27 \text { to } \\
\text { January } 23\end{array}$} & \multicolumn{3}{|c|}{$\begin{array}{l}\text { January } 24 \text { to } \\
\text { February } 20\end{array}$} & \multicolumn{3}{|c|}{$\begin{array}{l}\text { February } 21 \text { to } \\
\text { Mareh } 19\end{array}$} & \multicolumn{3}{|c|}{$\begin{array}{l}\text { March } 20 \text { to } \\
\text { April I6 }\end{array}$} \\
\hline & 0 & $C$ & $O-C$ & & $C$ & $O-C$ & $O$ & $C$ & $O-C$ & $O$ & & $O-C$ & $O$ & $C$ & $O-C$ \\
\hline & & & & & & & & & & & & & & & \\
\hline & & 1 & 0.9 & I. I & +0.4 & -1.5 & +9.8 & +6.3 & 3.5 & +5.1 & +2.0 & $0+3 \cdot I$ & +5 & +3.1 & +2 \\
\hline 5 & +2.5 & +3.6 & $-1 \cdot r$ & +1.5 & +6.6 & $-5 . I$ & +12 & +13.3 & 0.8 & +5 & +6.1 & $1-0.7$ & + & + & +0.2 \\
\hline & +3 & $t$ & -0.9 & +14.2 & +12.6 & +1.6 & +17 & +I7.I & 0.0 & +5 & +2 & $2-1.9$ & $+I$ & & -3.6 \\
\hline & +10.8 & +8 & +1.9 & +21.3 & +17.8 & +3.5 & +14 & +18 & -3.1 & +7.8 & $+c$ & $6+1.2$ & +21.3 & & -3.2 \\
\hline 4.5 & +15.8 & +15 & +0.2 & +22.0 & +22.1 & -0.1 & +21.9 & +17.8 & $+4 . I$ & +6.4 & + & -0.8 & +40.4 & & $\div 5.2$ \\
\hline 5.5 & +20.6 & +21.0 & -0.4 & +22.4 & +24.2 & -1.8 & +16.4 & +17.8 & $-\mathrm{I} .4$ & +8.7 & + & $9-1.2$ & $+4 i$ & & +1.9 \\
\hline 6.5 & +20.8 & +21.6 & -0.8 & +19.6 & +22.1 & -2.5 & +18 & +16 & +2.4 & +14.2 & & $0+1.2$ & +37 & & 2.0 \\
\hline 7.5 & +16.9 & +16.8 & +0.1 & +16.5 & +14.8 & $+\mathbf{I} \cdot 7$ & +5 & +11.8 & -6.5 & +15.0 & & $7+1.3$ & +24 & & 4. I \\
\hline & +9.9 & +8.6 & +1.3 & +7.3 & +3.7 & +3.6 & +8 & & +4.8 & +7.6 & $+\mathrm{I}$ & $7-3 \cdot 1$ & +17.6 & & +2 \\
\hline 9.5 & $-\mathrm{r} .2$ & +0 & -1.6 & -9.1 & -7.0 & -2.1 & & -6.2 & +0.9 & +4.2 & +5 & $2-1.0$ & +5 & & $+I . I$ \\
\hline .5 & -4.7 & $-5 \cdot 5$ & +0.8 & -15.5 & -12.9 & -2.6 & $-13 . I$ & -12.3 & -0.8 & +2.1 & -0.1 & +2.2 & -1 & & +1 \\
\hline & -8.2 & -8.8 & +0.6 & -II.3 & -12.5 & +1.2 & $-\mathbf{I}$ & -12 & -2.5 & -2.4 & $-3 \cdot 7$ & $7+1.3$ & -7 & & -1.6 \\
\hline & & -10.1 & +0.1 & -6.7 & & $1=1$ & & & $+2,5$ & & -5.8 & $8-2.1$ & $-I$ & & -0.8 \\
\hline & -12.1 & -9.8 & -2.3 & -3 & & 170 & -4 & $-3 \cdot 3$ & -1.0 & -8.4 & -7.4 & $4-1.0$ & -16.6 & & -0. \\
\hline & -64 & -8 & +1.9 & -4.5 & -3.0 & -1.5 & -2.7 & $-2 . I$ & -0.6 & -7 & -8.8 & $8+I .1$ & $-\mathbf{I} 8,8$ & & $+1 \mathrm{I}$ \\
\hline .5 & -4.1 & -6.4 & +2.3 & -5.3 & -5 & +0.3 & -0.5 & -4.7 & +4.2 & $-8 . \mathrm{I}$ & -9.0 & $0+0.9$ & -18 & & +3.1 \\
\hline & -9.3 & -5.6 & -3.7 & -8.4 & & +0.6 & -13.8 & -8.6 & -5.2 & & -7.2 & $2+0.2$ & -22 & -20.2 & \\
\hline & -7 & -72 & 0.0 & -12.7 & & -1.9 & -9.8 & & +1.2 & -4.2 & -4.2 & 0.0 & -22 & & \\
\hline 3.5 & & -10 & +1.3 & -7.7 & -10.5 & +2.8 & $-\mathrm{II} \cdot 3$ & -11.3 & 0.0 & -3.5 & -2 & $4-I . I$ & -19.2 & & + \\
\hline & & -13.2 & & -10.4 & & -0.9 & & & +45 & & -3 & $4-0.9$ & -2 & & +1 \\
\hline .5 & -14.7 & -12.6 & $-2 . I$ & -7.9 & -9.2 & $+1 \cdot 3$ & -15 & -10.9 & -4.8 & -2.5 & -6.8 & $8+4 \cdot 3$ & -19.0 & & +3 \\
\hline 5 & -7.6 & -8.1 & +0.5 & $-\mathrm{r} 3.6$ & -9.2 & -4.4 & $-I I$ & -10 & -1.2 & -11 & -9 & -1.7 & -22.0 & & -3 \\
\hline$\cdot$ & -4.1 & $-I$ & -2.2 & $-7 \cdot I$ & -8.3 & & -2.2 & -7.9 & +5 & - Io.0 & -8.8 & $8-\mathrm{r} .2$ & -16.5 & & \\
\hline & +6.4 & +2 & + & -0.1 & & & & & -5.5 & -5.4 & & -1.3 & +0.9 & & \\
\hline
\end{tabular}


Tabulation of abserved and computcd diurnal variation of magnetic declination at Teplitz Bay-Continted

\begin{tabular}{|c|c|c|c|c|c|c|c|c|c|c|c|c|c|c|c|}
\hline \multirow{2}{*}{ 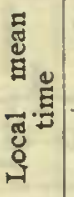 } & \multicolumn{3}{|c|}{ April.17 to May 14} & \multicolumn{3}{|c|}{ May I 5 to June II } & \multicolumn{3}{|c|}{ June 2 to July I } & \multicolumn{3}{|c|}{ October 4 to July I } & \multicolumn{3}{|c|}{ Mean Year } \\
\hline & 0 & C & $O-C$ & $o$ & C & $O-C$ & $O$ & $C$ & $O-C$ & $O$ & C & $O-C$ & $O$ & $C$ & $O-C$ \\
\hline$h$ & & & , & & 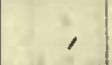 & 1 & & 1 & , & &, & 1 & & & \\
\hline 0.5 & +0.3 & -1.8 & $+2 . I$ & -10.3 & -5.6 & -4.7 & -5.2 & 6.2 & +1.0 & +1.3 & +0.3 & +1.0 & -0.2 & $-0 . \mathrm{I}$ & o. I \\
\hline I. 5 & +5.8 & +8.0 & -2.2 & $+\mathbf{I I . 2}$ & +7.8 & +3.4 & +3.0 & +2.8 & +0.2 & +5.4 & $+7 \cdot 4$ & -2.0 & $+4 \cdot 3$ & $+6 . I$ & -1.8 \\
\hline 2.5 & +17.2 & +16.9 & +0.3 & +22.1 & +22.3 & -0.2 & +19.6 & +15.9 & +3.7 & $+14 \cdot 3$ & $+14: 7$ & -0.4 & +15.0 & +12.9 & +2.1 \\
\hline $3 \cdot 5$ & +25.1 & +25.5 & -0.4 & +34.2 & +349 & -0.7 & +29.2 & +32.6 & -3.4 & +24.0 & +22.0 & $+2: 0$ & +20.7 & +20.4 & +0.3 \\
\hline $4 \cdot 5$ & $+35 \cdot 3$ & +33.4 & +1.9 & +43.1 & +42.5 & +0.6 & $+44 \cdot 3$ & +47.6 & $-3 \cdot 3$ & +27.8 & +27.9 & -0.1 & +25.8 & +27.2 & -14 \\
\hline $5 \cdot 5$ & +36.7 & +37.7 & $-\mathrm{r} .0$ & +43.6 & +42.6 & +1.0 & +58.9 & +54.3 & +4.6 & +28.9 & +30.1 & -1.2 & +30.0 & $+30: 4$ & -0.4 \\
\hline 6.5 & +33.2 & +35.0 & -1.8 & +38.6 & +35.1 & $+3 \cdot 5$ & +50.6 & +50.4 & +0.2 & +27.3 & $+27 . I$ & +0.2 & +29.8 & +28.2 & +1.6 \\
\hline $7 \cdot 5$ & $+29 . \mathrm{I}$ & +25.2 & +3.9 & +18.8 & +23.1 & $-4 \cdot 3$ & +39.0 & +39.0 & 0.0 & +19.6 & +19.4 & +0.2 & +20.1 & $+2 r .0$ & -0.9 \\
\hline 8.5 & $+9 . I$ & +12.5 & -3.4 & +12.8 & +11.6 & +1.2 & +24.8 & +26.5 & -1.7 & +10.4 & +9.9 & +0.5 & +11.9 & +11.8 & +0.1 \\
\hline 9.5 & +3.0 & +2.6 & +0.4 & +7.3 & +4. & +2.8 & $+\mathrm{I} 4.8$ & +17.8 & -3.0 & +1.2 & +1.9 & -0.7 & +3.2 & +3.9 & -0.7 \\
\hline 10.5 & +0.4 & -1.4 & +1.8 & +2.4 & +2.8 & -0.4 & $+\mathrm{IS} .3$ & +12.9 & +5.4 & -2.2 & -2.8 & +0.6 & +0.3 & -1.0 & +1.3 \\
\hline & $-\mathrm{r} .6$ & $-\mathrm{I} .5$ & $-0 . I$ & $-3 \cdot 5$ & +3.4 & -6.9 & +10.0 & +8.7 & +1.3 & -5.4 & -5.0 & -0.4 & -4.2 & -3.6 & -0.6 \\
\hline 2.5 & -4.7 & -2.6 & $-2 . I$ & +3.9 & +2.0 & +1.9 & -2.1 & +2.6 & -4.7 & -6.7 & -6.4 & -0.3 & -6.9 & -5.6 & -1.3 \\
\hline $3 \cdot 5$ & -7.8 & $-7 \cdot 5$ & -0.3 & $+0 . \mathrm{I}$ & -3.6 & +3.7 & $-5 \cdot 3$ & -5.2 & -0.1 & -7.8 & -84 & +0.6 & -6.4 & -8.1 & +1.7 \\
\hline & & & +3. & -13.6 & -12 & $-I .2$ & -10.4 & -12.6 & +2.2 & $-I$ & -10.6 & +0.2 & - Io 8 & -10.6 & - -02 \\
\hline & & -20.2 & -2.2 & -24.9 & -2 & 4.0 & & & +1.6 & - I2.6 & -12.4 & -0.2 & -13.5 & -12.5 & $5-10$ \\
\hline & $-22 . I$ & & -1.4 & -23.4 & -25.8 & +2.4 & $-30 \cdot 3$ & $-25 \cdot 4$ & -4.9 & $-\mathrm{I} 4 . \mathrm{I}$ & -13.2 & -0.9 & $-\mathrm{I}_{3} \cdot 3$ & -13.6 & $5+0.3$ \\
\hline & -16.7 & & $+1 . r$ & -23.6 & & & & $-33 \cdot 3$ & +6.8 & -12.9 & -13.9 & +1.0 & $-\mathrm{I} 4.2$ & -14.8 & +0.6 \\
\hline & -14.5 & & +1.0 & -24.2 & $-25 \cdot 5$ & $+1 \cdot 3$ & -48.2 & $-4 \mathrm{r} .6$ & -6.6 & -15.6 & -15.2 & -0.4 & & -16.7 & -1.1 \\
\hline 19.5 & -173 & -16.8 & -0.5 & -26.5 & -24.6 & $5-1.9$ & $-45 \cdot 7$ & -46.7 & +1.0 & -17.1 & -17.2 & +0.1 & -17.6 & -18.9 & +1.3 \\
\hline .5 & $-22 . \mathrm{I}$ & -20.8 & $-1 \cdot 3$ & -26.0 & -24.6 & $5-1.4$ & -39.8 & $-45 \cdot 1$ & +5.3 & -17.8 & -18.4 & +0.6 & -19.6 & -19.6 & 00 \\
\hline 5 & -20.8 & -23.1 & +2.3 & -22.8 & -24.6 & $5+1.8$ & $-42 \cdot 3$ & -36.6 & $-5 \cdot 7$ & -18.6 & $-\mathrm{r} 7 \cdot 3$ & -1.3 & -205 & -17.5 & 3.0 \\
\hline 22.5 & -22.5 & -20.2 & -2.3 & -21.9 & $-22 \cdot 3$ & $3+0.4$ & -20.7 & -25.0 & +4.3 & -13.1 & -13.1 & 0.0 & -9.0 & -12.7 & +3.7 \\
\hline$\cdot 5$ & $-\mathrm{II.7}$ & -12.2 & +0.5 & -17.1 & -16.0 & $-I . I$ & $-18 . I$ & -14.5 & -3.6 & -6.0 & -6.8 & +0.8 & -7.6 & -6.4 & 1.2 \\
\hline
\end{tabular}


Characteristics of Diurnal, Variation in Magnetic Declination at Teplitz Bay

The characteristic features of the curves, figures 5 to 19 , representing the total diurnal variation in magnetic declination for the various periods discussed are summarized in the tabulation following. In this summary a plus sign denotes a departure to the east of the mean value, while a negative sign denotes a departure to the west of the mean value. The epochs of mean declination are designated I and II, the former being the passage of mean in course of westward movement, and the latter in course of eastward movement of the needle.

\begin{tabular}{|c|c|c|c|c|c|c|c|c|c|c|c|c|}
\hline 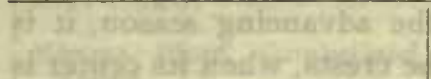 & $10 \mathrm{~s}$ & Pri & & 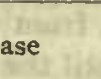 & & 70 & Seconi & dary & e & & & \\
\hline Period & Maxi & munı & Minit & $\mathrm{um}$ & $\begin{array}{l}\infty \\
- \\
-\end{array}$ & Maxis & mum & Minis & $\mathrm{um}$ & 范 & & \\
\hline 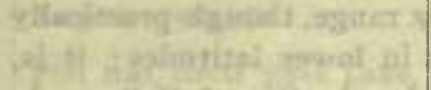 & L.M.T. & Am't & L.M.T. & Am't & 蔦 & L.M.T. & Ain't & L.M.T. & $A m^{\prime} t$ & 宽 & I & II \\
\hline- & & & & & {[} & & 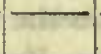 & & & & & $\longrightarrow$ \\
\hline uly (interpolated)...... & 5.8 & $\begin{array}{r}\prime \\
+443 \\
\end{array}$ & $\begin{array}{c}h \\
19.6\end{array}$ & -34.1 & $78 \cdot 4$ & $*^{h}$ & & *h & *' & * & $\begin{array}{c}h \\
12.8\end{array}$ & I.5 \\
\hline ugust & 6.0 & +34.0 & 19.9 & -26.8 & 60.8 & * & * & & * & * & 12.0 & 1.2 \\
\hline pt & $6 \mathrm{I}$ & +25.1 & 20.2 & -19.4 & 44.5 & I6.8 & -8.7 & $14 \cdot 3$ & $-\mathrm{II} .8$ & 3.1 & II.O & 0.2 \\
\hline & 5.6 & +20.8 & & $-\mathbf{r} 3 \cdot 7$ & 34.5 & & $-3 \cdot 9$ & 13.8 & $-I I . I$ & 7.2 & 9.8 & 0.3 \\
\hline $\mathrm{v}$ & 4.2 & +39.2 & $2 \mathrm{I} .0$ & -18.6 & 57.8 & 17.0 & -3.8 & $13 \cdot 5$ & -16.3 & 12.5 & 8.4 & 0.6 \\
\hline ove & 6.2 & +21.9 & 19.8 & $-\mathrm{I} 3 \cdot 3$ & 35.2 & I6.4 & $-5 \cdot 4$ & 12.5 & -10.1 & 4.7 & 9.6 & 22.9 \\
\hline 2.60 & 5.6 & $+24 \cdot 0$ & 8.0 & -10.8 & 34.8 & 14.2 & -2.7 & II.O & $-13 \cdot 3$ & 10.6 & 8.8 & 0.4 \\
\hline nu & 5.2 & & 8.2 & & $29 \cdot 4$ & & -2.0 & & -13.0 & II.O & 8.9 & 23.8 \\
\hline eb & $7 \cdot 3$ & +14.0 & 21.8 & -9.9 & 23.9 & 18 & -.2 .2 & I5. I & $-9: 1$ & 6.9 & 10.5 & 0.1 \\
\hline Marc & 5.6 & $+4 \mathrm{I} \cdot 4$ & 20.2 & -23.0 & 64.4 & & -19.1 & $15 \cdot 5$ & -21.2 & 2.1 & $10 . I$ & 0.0 \\
\hline 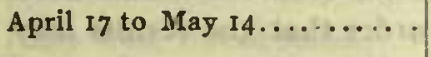 & $5 \cdot 5$ & +37.4 & & -23.1 & 60.5 & 18.6 & $-15 \cdot 7$ & 16.0 & $-2 \mathrm{r} .2$ & $5 \cdot 5$ & 9.9 & 0.7 \\
\hline & 5.0 & $+43 . \mathrm{I}$ & 21.1 & -25.1 & 68.2 & 20.0 & $-24 \cdot 5$ & 17.3 & -26.8 & 2.3 & 12.9 & 0.9 \\
\hline & $5 \cdot 4$ & +54.2 & 198 & $-47 \cdot 1$ & IOI $\cdot 3$ & * & * & * & * & * & 12.8 & 1.2 \\
\hline Summer mean-Oct. to March. & $5 \cdot 7$ & +39.2 & 20.1 & -27.5 & 66.7 & * & * & Afte & * & * & 11.6 & 1.2 \\
\hline & $5 \cdot 4$ & +21.2 & 20.9 & -12.6 & 33.8 & 16.7 & -9.2 & I 4.2 & -7.4 & 1.8 & 9.2 & 23.8 \\
\hline Meat & 5.6 & +30.4 & 20.4 & -19.8 & 50.2 & * & * & * & * & * & 10.2 & 0.5 \\
\hline
\end{tabular}

* For these intervals the secondary phases, while indicated, have their crests no longer definitely marked.

From the above it at once appears that the principal maximum and minimum of east declination occur at earlier and later times respectively than for stations in lower latitudes. The morning extreme is reached almost always between 5 and $6 o^{\prime}$ clock. The afternoon extreme varies between 6 and ro o'clock without any very systematic cycle dependent upon the chronological order of the periods; in the half-yearly means, however, it is earlier for the winter season than for the summer season by about one hour. The increase in diurnal range 
from winter to summer is quite pronounced. In the half-yearly means this difference in range is quite clearly shown by the curves of figure 19, in which the October to March mean is indicated by the dotted line and the April to September mean by the broken line.

In general the curves show also a secondary maximum and minimum between the two principal extremes of day; in several cases the minimum of this second wave exceeds the principal western deviation in amount. As will be noted, this secoudary wave is very prominent in the winter season; with the approach of the summer months it becomes less and less pronounced until in midsummer, though still recognizable, the critical points are no longer definitely marked. In general the time interval between the minimum and maximum of this wave is about two and one-half hours; it is, as a whole, earliest in midwinter, with its center at about one-half hour past noon, from which time, with the advancing season, it is carried forward with diminishing range until the disappearance of the crests, when its center is at about $5 \mathrm{P}$. M. to $6 \mathrm{P}$. M. By reason of this transposition as a whole the effect is practically eliminated in the year's meau and very greatly smoothed out in the winter's mean. These secondary extremes are inuch more pronounced and of greater yearly range, though practically of the same period as for the same feature when noted at stations in lower latitudes; it is, however, superimposed on the mean curve later in the day. Thus for the Girard College observations at Philadelphia, Bache* found the extremes of a similar effect for the winter at 9.7 hours and 13.2 hours, and for the summer at 10.2 hours and 13.2 hours, the seasonal ranges being respectively $0 . ' 7 \mathrm{I}$ and 0. ' 15 .

The change of the epochs of mean declination with the season is very marked and, in general, very regular, being earliest in winter at 9 hours and 23 hours and latest in summer at 13 hours and 1.5 hour local time. In the half-yearly means the epoch $I$ is later in summer than in winter by 2.4 hours, and epoch II by 1.4 hours. On the average for the year epoch I is at 10.2 hours A. M., a time, which as will be seen later, agrees well with that for the same event at stations widely distributed over the Earth.

In order to make a proper comparison of the total solar-diurnal variation in magnetic declination for the mean year at Teplitz Bay with the corresponding phenomenon for stations elsewhere on the Earth, particularly in the Arctic Regions, a compilation of the mean hourly variatious on the mean of year has been prepared for some twenty-four additional locations. In all cases where the published records have been immediately available the observed means have been taken without elimination of the so-called "disturbances" in order that the results may be strictly intercomparable. When, as is the case for many of the stations, the mean observed quantities do not apply to the local mean hours, the values have been plotted to scale and the hourly values taken from the smooth curves drawu through the points plotted. The departures from mean as shown in the tabulation are all referred to the north-seeking end of the needle, a plus sign in licating a movement to the east and a minus sigu a movement to the west of the mean position. The words "maximum" and "minimum" are used in the sense of eastern and western elongations respectively. The stations are ar ranged in the decreasing order of northerly magnetic inclination for the purpose of bringing out clearly the relation between magnetic dip and range of the diurnal variation in declination. The geographical positions, series of observations from which results are taken, the mean dips over the periods of observa-

* Discussion of the magnetic and meteorological observations made at the Girard College Observatory, Philadelphia, in $1840,1841,1842,1843,1844$, and 1845 , by A. D. Bache. Part II. Smithsonian Contributions to Knowledge. Washington, June, 1862. 
tion, as also the approximate corresponding mean sun-spot frequencies accordiug to Dr. Wolf and Professor Wolfer,* arc as follows:

\begin{tabular}{|c|c|c|c|c|c|c|c|}
\hline$\frac{\breve{s}}{\square}$ & Statiın & Latitude & $\begin{array}{c}\text { Longitude } \\
\text { east of } \\
\text { Greenwich }\end{array}$ & $\begin{array}{c}\text { Magnetic } \\
\text { 1uclina- } \\
\text { tion }\end{array}$ & $\begin{array}{l}\text { Magnetic } \\
\text { lalitude }\end{array}$ & Observations & 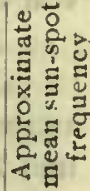 \\
\hline I & Fort Conger, Griunell Land.. & $\stackrel{\circ}{8}$ & $\begin{array}{cc}0 & 1 \\
295 & 16\end{array}$ & ${ }^{\circ}$, & $8006 \mathrm{~N}$ & Aug., 1882 , to July, 1883. & 59 \\
\hline 2 & $\begin{array}{c}\text { Kingua Fjord, Cumberland } \\
\text { Sound. } \ldots \ldots \ldots \ldots \ldots \ldots \ldots\end{array}$ & $6636 \mathrm{~N}$ & 29241 & $8351 \mathrm{~N}$ & $7750 \mathrm{~N}$ & Oct., 1882 , to Sept., $188_{3} \ldots$ & 60 \\
\hline 3 & Teplitz Bay, Rudolph Island. & $8 \mathrm{I} 47 \mathrm{~N}$ & $5^{8} \circ 9$ & $83-12 \mathrm{~N}$ & $7635 \mathrm{~N}$ & Oct., 1903 , to June, $1904 \ldots$ & (8o) \\
\hline 4 & Ssagastyr, Siberia. .......... & $7323 \mathrm{~N}$ & 12636 & $83 \propto 9 \mathrm{~N}$ & $7629 \mathrm{~N}$ & Jan., $188_{3}$, to Dec., $188_{3} \ldots$ & 64 \\
\hline 5 & $\begin{array}{l}\text { Fort Rae, Great Slave Lake, } \\
\text { Canada } . . \ldots \ldots \ldots \ldots \ldots\end{array}$ & $6239 \mathrm{~N}$ & 24446 & $8254 N$ & 76 or $\mathrm{N}$ & Oct., 1882 , to Sept., $1883 \ldots$ & 60 \\
\hline 6 & Ooglaamie, Alaska.......... & $7118 \mathrm{~N}$ & 20320 & $8 \mathrm{I} 24 \mathrm{~N}$ & $7310 \mathrm{~N}$ & Sept., I882, to Aug. I $883 \ldots$ & 60 \\
\hline 7 & Cape Thordsen, Spitzbergen.. & $7828 \mathrm{~N}$ & 1542 & $8027 \mathrm{~N}$ & $7124 \mathrm{~N}$ & Sept., 1882 , to Aug., $1883 \ldots$ & 60 \\
\hline 8 & Jan Mayen Island........... & $710 \mathrm{~N}$ & $35132^{\circ}$ & $7900 \mathrm{~N}$ & $6845 \mathrm{~N}$ & Sept., 1882 , to July, $1883 \ldots$ & 60 \\
\hline 9 & Karmakul Bay, Novaia Zenılia. & $7223 \mathrm{~N}$ & 5242 & $7843 \mathrm{~N}$ & $6815 \mathrm{~N}$ & Oct., 1882 , to Aug., $1883 \ldots$ & 60 \\
\hline 10 & Bossekop, Norway........... & $695^{8 \mathrm{~N}}$ & 2315 & $7626 \mathrm{~N}$ & $6414 N$ & Aug., 1882 , to July, $1883 \ldots$ & .59 \\
\hline II & Sitka, Alaska.............. & $5703 \mathrm{~N}$ & 22440 & $7555 \mathrm{~N}$ & $6321 \mathrm{~N}$ & Irregular series, 1848 to 1862 & $(60)$ \\
\hline 12 & Toronto, Canada.......... & $4339 \mathrm{~N}$ & 28036 & 75 I5 N & $6214 \mathrm{~N}$ & July, I 842 , to June, $1848 \ldots$ & $5 r$ \\
\hline I 3 & Sodanklä, Finland......... & $6724 \mathrm{~N}$ & 2636 & $7448 \mathrm{~N}$ & 6 I $29 \mathrm{~N}$ & Sept., I 882 , to Aug., $1883 \ldots$ & 60 \\
\hline 14 & Ekateriuburg, Siberia ..... & $5649 \mathrm{~N}$ & 6038 & $7039 \mathrm{~N}$ & $5455 \mathrm{~N}$ & Jan., I 893 , to Dec., 1893 . & 83 \\
\hline 15 & De Bilt, Netherlands. ....... & $5206 \mathrm{~N}$ & 511 & $6651 \mathrm{~N}$ & $4928 \mathrm{~N}$ & Jan., I903, to Dec., $1903 \ldots$ & (6o) \\
\hline 16 & Los Angeles, United States.... & $3403 \mathrm{~N}$ & 24145 & $5930 \mathrm{~N}$ & $4020 \mathrm{~N}$ & Oct , 1882 , to Oct., $1889 \ldots$ & 36 \\
\hline 17 & Key West, United State & $2433 \mathrm{~N}$ & $278 \quad 12$ & $5432 \mathrm{~N}$ & $35 \circ 4 \mathrm{~N}$ & March, 1860 , to March, 1866 & 57 \\
\hline 18 & Zi-ka-wei, China......... & $3 \mathrm{I} 12 \mathrm{~N}$ & I21 36 & $4542 \mathrm{~N}$ & $2708 \mathrm{~N}$ & Jan., Ig0I, to Dec., I90I.... & (10) \\
\hline I9 & Colába, India $\ldots \ldots \ldots \ldots \ldots$ & I $854 \mathrm{~N}$ & 7249 & $2130 \mathrm{~N}$ & II $09 \mathrm{~N}$ & Jan., I901, to Dec., I90I.... & (10) \\
\hline 20 & St. Helena Island $\ldots \ldots \ldots \ldots$ & I5 $57 \mathrm{~S}$ & 35420 & $2159 \mathrm{~S}$ & II $25 \mathrm{~S}$ & Sept., I 842 , to Ang., I $847 \ldots$ & 38 \\
\hline 21 & Buitenzorg, Java.. .......... & 6 II S & 10650 & $3033 \mathrm{~S}$ & $1627 \mathrm{~s}$ & Jan., I904, to Dec., I904.... & (8o) \\
\hline 22 & South Georgian Island . .... & $5431 \mathrm{~S}$ & 32400 & $4^{8} 5^{8 \mathrm{~s}}$ & $2953 \mathrm{~S}$ & Sept, 1882 , to Aug., $1883 \ldots$ & 60 \\
\hline 23 & Cape of Good Hope, Africa.... & $3356 \mathrm{~S}$ & IS 29 & $5321 \mathrm{~S}$ & $3354 \mathrm{~S}$ & April, I 841 , to June, I $846 \ldots$ & 29 \\
\hline 24 & Mauritius Island. . . . . . . & $2006 \mathrm{~S}$ & 5733 & $545 \mathrm{I} \mathrm{S}$ & $3523 \mathrm{~S}$ & Jan., I 883 , to Dec., I $883 \ldots$ & 64 \\
\hline 25 & Hobarton, Tasmania........ & $4252 \mathrm{~S}$ & 14728 & $7036 \mathrm{~S}$ & $5451 \mathrm{~S}$ & Jan., $184 \mathrm{I}$, to Sept., I $848 \ldots$ & 49 \\
\hline
\end{tabular}

The references for each of the above series are as follows:

Fort Conger, Grinnell Land-Iuternational Polar Expedition to Lady Franklin Bay, Grinnell Land, by Lieut. A. W. Greely. (Volume 2, reduction of magnetic observations by C. A. Schott.) Washington, $\mathrm{r} 888$.

* As collected and plotted by Mr. W. Ellis in his paper on the relation between diurnal range of magnetic declination and horizontal force and solar spots (Proceedings of the Royal Society. Volume 63, pp. 64-78). The values after I 896 are exterpolations based on Mr. Ellis' curves; these are indicated by enclosure in parentheses.

so 
Kingua Fjord, Cumberland Sound-Die Internationale Polarforschung, 1882-1883; die Beobachtungs-Ergebnisse der Deutschen Stationen, Band I, Kingua Fjord. Herausgegeben von Prof. Dr. G. v. Neumayer und Prof. Dr. C. Börgen. Berlin, I 886.

Teplitz Bay, Rudolph Island-The present reductions.

Ssagastyr, Siberia-Beobachtungen der Russischen Polarstation an der Lenamïndung; astronomische und magnetische Beobachtungen $1882-1884$, bearbeitet von V. Fuss, F. Müller, und N. Jïrgens. Herausgegeben unter Redaction von Dr. A. v. Tillo. 1895 .

Fort Rae, Great Slave Lake, Canada-Report of Superintendent of the United States Coast and Geodetic Survey for 1890 ; Appendix No. 9, by C. A Schott. Washington, 1891.

Ooglaamie, Alaska-Report of Superintendent of the United States Coast and Geodetic Survey; Appendix No. 13, by C. A. Schott. Washington, 1891 .

Cape Thordsen, Spitzbergen-Observations faites au Cap Thordsen, Spitzberg, par l'Expedition Suédoise ; tome I : 4, magnetisme terrestre, par E. Solander. Stockbolm, I888.

Jan Mayen Island-Die Österreichische Polarstation Jan Mayen; Beobachtungs-Ërgebnisse. II Band, II Abtheilung. Magnetische Beobachtnngen auf Jan Mayen 1882-1883, bearbeitet von Linienschiffs-Lieutenant August Gratzl.

Karmakul Bay, Novaia Zemlia-Beobachtıngen der Russischen Polarstation auf Nowaja Semlja; Theil I, magnetische Beobachtungen, bearbeitet von K. Andrejeff. Herausgegeben unter Redaction von R. Lenz. $189 \mathrm{r}$.

Bossekop, Norway-Die Internationale Polarforschung 1882-1883; Beobachtungs-Ergebnisse der Norwegischen Polarstation Bossekop in Alten, herausgegeben von Aksel S. Steen. II Theil. Erdmagnetismus und Nordlicht. Christiania, 1888.

Sitka, Alaska-Same reference as Ooglaamie, Alaska.

Toronto, Canada-Observations made at the magnetical and meteorological observatory at Toronto, in Canada. Volume II. (Abstract of the observations to I 848 by Colonel Edward Sabine.) London, 1853 .

Sodanklä, Finland-Exploration Internationale des régions Polaires, I882-1883 et 18831884 ; Expédition Polaire Finlandaise ; tome II, magnétisme terrestre, par Selim Lemström et Ernest Biese. Helsingfors, 1887 .

Ekaterinburg, Sibcria-Die Beobachtungen des meteorologischen und magnetischen Observatoriums zu Katharinenburg im Jahre 1893, herausgegeben von H. Abels. Ekaterinburg, I894.

De Bilt, Netherlands-Koninklijk Nederlandsch Meteorologisch Institut; Jaarboek, 1903 , B, Aard-magnetisme. Utrecht, 1905 .

Los Angeles, United States-Same reference as Fort Rae, Great Slave Lake, Canada.

Key West, United States-Same reference as Los Angeles, United States.

Zi-ka-wei, Chin $x$-Observatoire magnétique et météorologique de Zi-ka-wei (Chine); bulletin des observations; tome XXVII, année rgor. Shanghai, 1903.

Colaba, India-Magnetical, meteorological, and seismological observations made at the Government Observatory, Bombay, in the years 1900 and 1901, under the direction of N.A. F. Moos, B. Sc., F. R. S. E. Bombay, 1903.

St. Helena Island-Observations made at the magnetical and meteorological observatory at St. Helena. Volume II. (Discussion of observations by Major-General Edward Sabine.) London, 1860 .

Buitenzorg, Java-Observations made at the Royal magnetical and meteorological observatory at Batavia, by Dr. W. van Bemmelen, Acting Director. Batavia, 1906.

South Georgia Island-Die Internationale Polarforschung; Beobacht1ungs-Ergebnisse der Deutschen Stationen, Band II, Süd-Georgien. Herausgegeben von Prof. Dr. G. v. Neumayer und Prof. Dr. C. Börgen. Berlin, 1886. 
Cape of Good Hope, Africa-Observations made at the magnetical and meteorological observatory at the Cape of Good Hope. Volume I. (Abstracts of the observations by Lieut.-Colonel Edward Sabine.) London, 1851.

Mauritius Island-A discussion of the results obtained from the self-recording magnetometers at the Royal Alfred Observatory, Maritius, from 1875 to 1890 , under the direction of C. Meldrum, M. A., L. L. D., F. R. S., edited by T. F. Claxton, F. R. A. S. Mauritius, 1899.

Hobarton, Tasmania - Observations made at the magnetical and meteorological observatory at Hobarton, in Van Diemen Island. Volume I. (Abstracts of the observations by Lieut.-Colone1 Edward Sabine.) London, 1850.

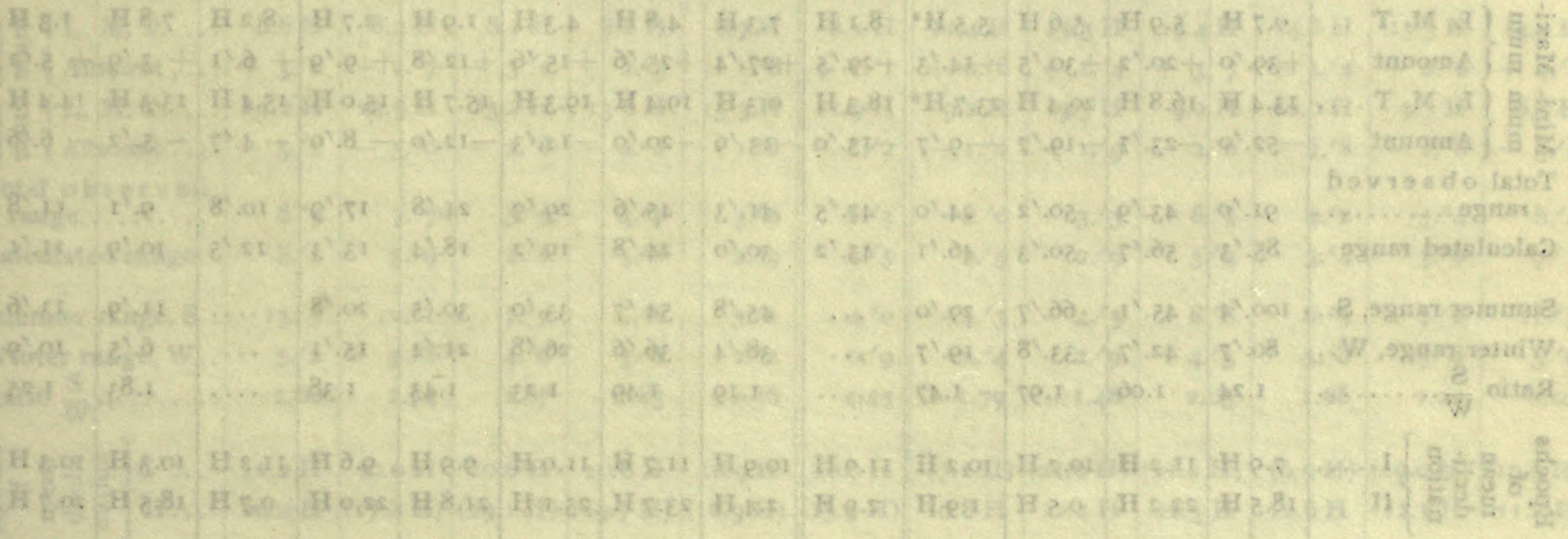


Tabulation of the mean yearly tolal solar-diurnal variation in magnetic declination at various stations [Eastern deflection of north-seeking end from mean is indicated by a plus sign ; western deflection by a minus sign.]

\begin{tabular}{|c|c|c|c|c|c|c|c|c|c|c|c|c|c|}
\hline 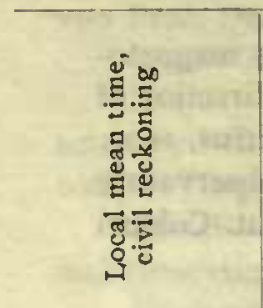 & 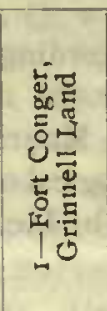 & 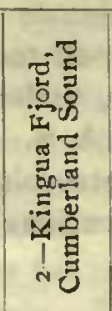 & 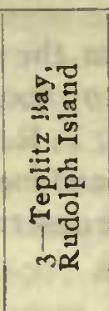 & 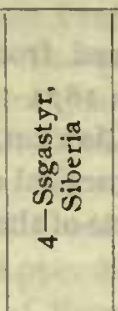 & 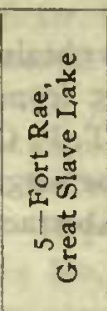 & 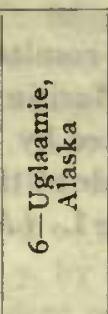 & 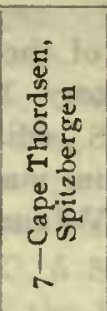 & 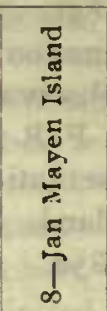 & 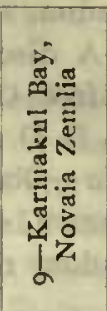 & 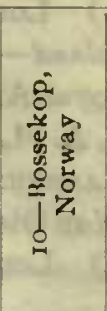 & 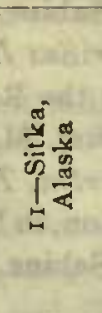 & 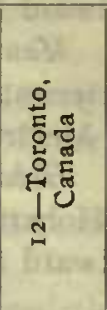 & 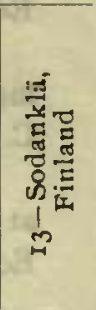 \\
\hline$h$ & & & , & & & 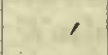 & & 2. & & & & & \\
\hline I & +38.7 & -11.7 & +3.1 & -6.2 & -11.0 & -11.2 & +1.8 & +7.0 & +11.8 & +8.7 & +0.2 & +0.6 & +5 \\
\hline 2 & +36.2 & 15.8 & +9.8 & +0.7 & -6.6 & -3.3 & +6.2 & +10.7 & +12.8 & +9.7 & $+\mathbf{I . 0}$ & +0.5 & +4 \\
\hline 3 & +33.2 & +18.0 & +17.1 & +6.7 & +0.8 & +3 & +10.7 & 13.8 & +11.4 & $\begin{array}{r}+9.9 \\
\end{array}$ & +1.4 & +0.8 & +4 \\
\hline 4 & +29.4 & +19.1 & +23.9 & $+\mathrm{rr.6}$ & +7.4 & +7.9 & +16.2 & +15 & +9.5 & +8.7 & + & +1.2 & +4 \\
\hline 5 & +24.7 & +19.3 & +29.4 & +13.9 & +13.6 & +1 & +2 & $+\mathbf{I}$ & +7.4 & +6.0 & +2.9 & $+\mathrm{r} .8$ & +4 \\
\hline 6 & +18.6 & +20.1 & +30.1 & +14.3 & +2 & +22.1 & +2 & & +5.6 & +4.2 & + & +2 & +3 \\
\hline 7 & +8.6 & +19.9 & +24.9 & +13.2 & +26.2 & +26.9 & +22.3 & +7.8 & +4.9 & $+3 \cdot I$ & +5.3 & +3.5 & +2 \\
\hline 8 & -0.5 & +17.4 & +16.9 & +9.7 & $+2 c$ & +26.3 & $+I$ & +5.7 & +2.5 & +1.8 & .0 & +3 & +2 \\
\hline 9 & -I 2.1 & +10.8 & +7.7 & +4.3 & +2 & $+20 . I$ & $+\mathrm{II} .4$ & +3. & $+\mathbf{I} \cdot 3$ & +0.7 & .3 & +3 & +1 \\
\hline Io & -23.2 & +3.7 & +1.1 & $+\mathrm{I} \cdot 3$ & +16.8 & +7.4 & +6.9 & +2.0 & -0.2 & -0.6 & +3.0 & +0.8 & +0 \\
\hline II & -37.9 & +1.3 & -2.6 & -1.8 & +8.0 & -1.0 & +4.3 & 0.0 & -2.0 & -2.9 & +0.6 & -2.0 & $-\mathbf{I}$ \\
\hline I 2 & $-43 . I$ & -9.0 & -4.9 & $-4 . \mathrm{I}$ & -0.9 & -6.5 & + & -2.3 & -4.3 & -5.2 & -2.1 & -4.2 & -4 \\
\hline 13 & -5 I.I & -15.I & -6.5 & -5.3 & -4.0 & -7.4 & -0.5 & -4.3 & -7.3 & -6.8 & -3.2 & -5.0 & -5 \\
\hline 14 & -50.8 & $-2 \mathrm{I} .2$ & -9.6 & -5.9 & -8.1 & -7.6 & -6.2 & -6.0 & -9.9 & -7.6 & --4.2 & -4.8 & -6 \\
\hline 15 & -46.1 & -20.4 & - II.9 & -5.9 & -10.6 & -7.9 & -9.9 & -7.0 & $-\mathrm{II} .5$ & -7.9 & -4.6 & -3.8 & -6 \\
\hline 16 & $-34 \cdot 2$ & -20.6 & -12.9 & -5.3 & - II.3 & -9.6 & -12.1 & -8.2 & $-\operatorname{rr} .4$ & -7.6 & -4.6 & -2.5 & -5 \\
\hline I7 & -19.9 & -23.6 & -14.0 & -3.7 & -I2.I & -9.9 & -14.3 & - 10.4 & -10.8 & -7.4 & -2.8 & -1.3 & -4 \\
\hline I 8 & $-4 \cdot I$ & -19.4 & -15.7 & -2.4 & -12.9 & -9.7 & -1 & -13.1 & -9.5 & -6.0 & -3.2 & -0.3 & - \\
\hline I9 & +3.1 & -16.1 & -17.8 & -1.9 & -12.5 & -8.0 & -1 & 1.2 & -8.0 & -6.6 & -2.4 & +0.2 & -2 \\
\hline 20 & +13.0 & -15.5 & -19.6 & -2.3 & -II.O & -6.1 & $-I$ & -13.4 & -6.0 & $-5 \cdot 3$ & $-\mathrm{I} .4$ & +0.7 & $-\mathbf{I}$ \\
\hline 21 & +19.0 & -8.8 & -I9. I & -5.0 & -12.0 & -8.7 & $-\mathbf{I}$ & -IO.I & -3.2 & -1.6 & -0.8 & +1.2 & $+\mathrm{s}$ \\
\hline 22 & +27.3 & -0.6 & -15.6 & $-7 \cdot 3$ & $-I I .9$ & $-\mathbf{r o . 5}$ & $-I$ & $-5 \cdot 3$ & +1.0 & $+o . I$ & -0.4 & +1.3 & + \\
\hline 23 & $-35 \cdot 3$ & +3.9 & $-\mathrm{I} 0.2$ & -9.1 & -11.9 & -8.9 & -10.0 & $-\mathrm{I} .5$ & +6.0 & +5.3 & -0.6 & $+\mathrm{I.2}$ & +2 \\
\hline 24 & +35.9 & +9.2 & -3.6 & -9.5 & -12.0 & -13.7 & -3.9 & +4.0 & +9.9 & +7.3 & -0.6 & +0.8 & +4 \\
\hline $2,120.1$ & $0.7 \mathrm{H}$ & $5.9 \mathrm{H}$ & $56 \mathrm{H}$ & $5.5 \mathrm{H}^{*}$ & 8.I H & & & & I.9 H & $2.7 \mathrm{H}$ & $8.2 \mathrm{H}$ & $7.8 \mathrm{H}$ & 1.3 \\
\hline E Amount. & $+39 .^{\prime} 0$ & +20.2 & $+30 . ' 5$ & $+14 \cdot^{\prime} 3$ & $+29 \cdot 5$ & $+27 \cdot 4$ & $+25 .^{\prime} 6$ & $+15 .^{\prime} 6$ & +12.18 & $+9 . ' 9$ & $+6 .^{\prime} 1$ & $+3 \cdot 9$ & $+5 \cdot^{\prime}$ \\
\hline 昌 $\{$. M. T ... & I $3.4 \mathrm{H}$ & $16.8 \mathrm{H}$ & $20.4 \mathrm{H}$ & $23.7 \mathrm{H}^{*}$ & $18.3 \mathrm{H}$ & $0.3 \mathrm{H}$ & $19.4 \mathrm{H}$ & I9.3 H & $15.7 \mathrm{H}$ & $\mathbf{1 5 . 0 ~ H ~}$ & $15.4 \mathrm{H}$ & I $3.3 \mathrm{H}$ & 14.4 \\
\hline Amount & -52.10 & $-23 \cdot 7$ & -59.7 & -9.7 & $-13 . \%$ & $-13 .{ }^{\prime} 9$ & $-20 .{ }^{\prime} 0$ & $-14 \cdot \cdot^{\prime} 3$ & $-12 . ' 0$ & $-8 .^{\prime} \mathrm{o}$ & $-4^{\prime} 7$ & $-5 \cdot^{\prime} 2$ & -6 \\
\hline & & $43 \cdot 9$ & $50 . / 2$ & $24 \cdot{ }^{\prime} 0$ & $42 .^{\prime} 5$ & $41 \cdot 3$ & & 29.9 & $24 \cdot^{\prime} 8$ & 17.9 & $10 . / 8$ & 9.'I & 31 \\
\hline Calculated range. & $85 \cdot^{\prime} 3$ & $56 .^{\prime} 7$ & $50 .^{\prime} 3$ & $46 .^{\prime} I$ & $43 \cdot \cdot^{\prime} 2$ & $30 . ' 0$ & $24 \cdot \cdot^{\prime} 8$ & I9.'2 & I8.' 4 & $13 \cdot^{\prime} 3$ & 12. 5 & 10.'9 & \\
\hline umuer range, S. & I00.' 4 & $45 .^{\prime} \mathrm{I}$ & $66 . ' 7$ & $29 . ' 0$ & & $45^{\prime} 8$ & $54 \cdot 7$ & $33 . \%$ & $30 . ' 5$ & 20.18 & & II.'9 & I3.' \\
\hline Winter range, W.. & $80 . ' 7$ & $42 .^{\prime} 7$ & $33 \cdot \cdot^{\prime} 8$ & $19 \cdot 7$ & & $38 .^{\prime} 4$ & $36 .^{\prime} 6$ & 26.18 & $21.0^{\prime} 4$ & $15 \cdot^{\prime} I$ & & $6 .{ }^{\prime} 5$ & 10. \\
\hline W & I.24 & 1.06 & 1.97 & 1.47 & & 1.19 & J.49 & 1.23 & I.43 & 1. $3^{8}$ & & I. 83 & I \\
\hline 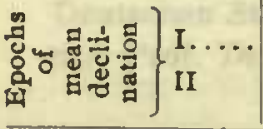 & $\begin{array}{r}7.9 \mathrm{H} \\
\text { I } 8.5 \mathrm{H}\end{array}$ & $\begin{array}{l}\text { II.2 H } \\
22.2 \mathrm{H}\end{array}$ & $\begin{array}{r}10.2 \mathrm{H} \\
0.5 \mathrm{H}\end{array}$ & $\begin{array}{r}10.2 \mathrm{H} \\
1.9 \mathrm{H}\end{array}$ & $\begin{array}{r}\text { I I.9 H } \\
2.9 \mathrm{H}\end{array}$ & $\begin{array}{r}\text { I0.9 H } \\
2.4 \mathrm{H}\end{array}$ & $\begin{array}{l}11.7 \mathrm{H} \\
23.7 \mathrm{H}\end{array}$ & $\begin{array}{l}\text { I I.O H } \\
23.1 \mathrm{H}\end{array}$ & $\begin{array}{r}9.9 \mathrm{H} \\
2 \mathrm{II} .8 \mathrm{H}\end{array}$ & $\begin{array}{r}9.6 \mathrm{H} \\
22.0 \mathrm{H}\end{array}$ & $\begin{array}{r}1 \mathrm{I} .2 \mathrm{H} \\
0.7 \mathrm{H}\end{array}$ & $\begin{array}{l}10.3 \mathrm{H} \\
18.5 \mathrm{H}\end{array}$ & $\begin{array}{l}10.31 \\
20.71\end{array}$ \\
\hline
\end{tabular}

${ }^{*}$ At this station a pronounced second maximum and minimum at $19.3 \mathrm{H}$ and $14.6 \mathrm{H}$ of $-1 . .^{\prime} 8$ and $-6 .^{\prime} \mathrm{o}$ respectively. 
Tabulation of the mean yearly total solar-diurnal variation in magnetic declination at varions stations

[Eastern deflection of north-seeking end fron mean is indicated by a plus sign; western deflection by a minus sign.]

\begin{tabular}{|c|c|c|c|c|c|c|c|c|c|c|c|c|}
\hline 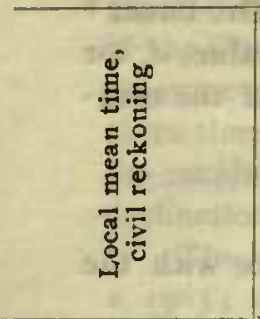 & 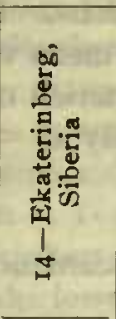 & 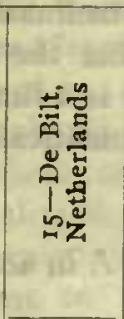 & 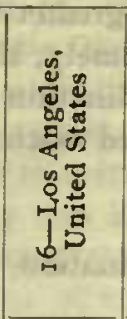 & 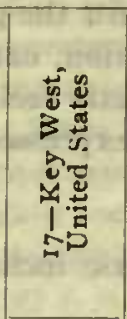 & 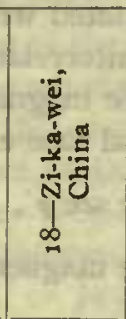 & 兽 & 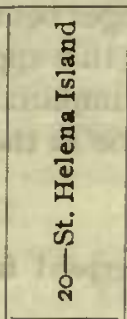 & 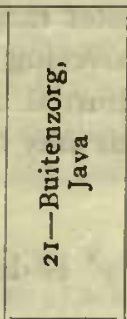 & 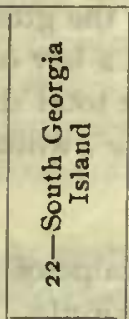 & 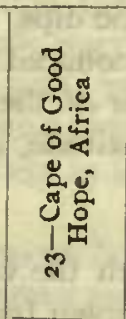 & 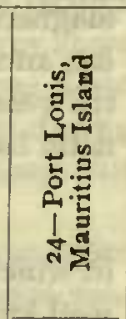 & 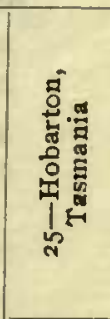 \\
\hline$h$ & , & , & , & 1 & $y=1$ & 1 & & & & & & \\
\hline 1 & +1.0 & +0.9 & 0.0 & 0.0 & $+\mathbf{0 . I}$ & +0.1 & + o. I & 0.0 & -0.8 & +0.5 & 0.0 & $-\mathrm{I}$. \\
\hline 2 & +0.9 & +2.8 & +0.1 & 0.0 & +0.1 & +0.1 & $+\mathbf{0 . 1}$ & $+0 I$ & -10 & +0.5 & & -0.7 \\
\hline 3 & +0.9 & +0.8 & +0.2 & +0.1 & +0.2 & 0.0 & +0.1 & +0.2 & -1.2 & +0.5 & c & -0. \\
\hline 4 & +10 & +1.0 & +0.3 & +0.2 & +0.2 & 0.0 & o o & +0.2 & -14 & +0.4 & - o. I & -0. \\
\hline 5 & +1.3 & +1.5 & $+\circ 6$ & $+0_{4}$ & +0.2 & 0.0 & +0.1 & +0.1 & -1.4 & +0.4 & -0.1 & -0.7 \\
\hline 6 & +1.9 & +2.0 & +1.3 & $+\mathbf{r . 0}$ & +0.5 & +0.4 & +0.4 & +0.1 & -1.5 & +0.3 & -0.2 & $-\mathrm{I} . \mathrm{I}$ \\
\hline 7 & +3.0 & +24 & +2.4 & +2.1 & +1.0 & +0.8 & $+0 . I$ & -0.4 & -1.6 & 0.0 & -0.6 & -2.0 \\
\hline 8 & +3.6 & +2.7 & +3.1 & +25 & +1.7 & $+\mathbf{I . I}$ & -0.8 & -1.3 & -2.3 & -1.0 & -1.6 & -3.0 \\
\hline 9 & +3.7 & +2.0 & +2.6 & +2.2 & +1.8 & +0.7 & $-1 . I$ & $-\mathrm{I} .8$ & -2.6 & -2.2 & -2.6 & -3.5 \\
\hline 10 & +2.6 & 00 & +1.1 & +1.1 & $+\mathrm{I} . \mathrm{I}$ & 0.0 & -0.8 & -1.8 & -2.0 & -2.8 & -2.7 & -2.8 \\
\hline II & +0.5 & -2.6 & -0.8 & -0.2 & -0.1 & -0.8 & +0.2 & $-\mathrm{I} .2$ & -0.5 & --2.2 & -1.9 & -0.9 \\
\hline 12 & -2.0 & $-4 \cdot 5$ & -2.2 & -1.4 & -1.3 & -1.2 & +0.8 & -0.3 & +1.6 & -0.8 & -0.1 & +1.4 \\
\hline I3 & -4.2 & -5.0 & -2.7 & -2.1 & -1.7 & -1.0 & +0.7 & +0.5 & +3.1 & +0.4 & +15 & +3.6 \\
\hline 14 & -5.1 & -4.3 & -2.6 & -2.2 & -1.6 & -0.5 & +0.4 & +1.0 & +3.7 & +1.3 & +2.7 & +4.7 \\
\hline 15 & -4.8 & -2.8 & -2.0 & -1.9 & $-\mathrm{I} . \mathrm{I}$ & -0.1 & 0.0 & +1.4 & +3.5 & $+\mathrm{I} \cdot 3$ & +2.9 & +4.6 \\
\hline 16 & - 3.6 & -1.5 & -1.1 & -1.3 & -0.5 & +0.2 & -0.4 & +1.3 & +2.4 & +1.0 & +2.3 & +3.5 \\
\hline & -2.1 & -0.4 & -0.5 & -0.8 & 0.0 & +0.2 & -0.6 & +0.9 & +15 & +0.4 & +1.1 & +2.2 \\
\hline 18 & -0.9 & 0.0 & -0.2 & -0.4 & 0.0 & 0.0 & -0.4 & +0.5 & +1.0 & +0.2 & +0.2 & +1.2 \\
\hline 19 & -0.3 & +0.3 & 0.0 & -0.2 & -0.1 & -0.1 & -0.1 & +0.4 & +0.5 & +0.2 & 0.0 & +0.5 \\
\hline 20 & 0.0 & +1.0 & 0.0 & +0.1 & -0.1 & -0.1 & +0.1 & +0.2 & +0.4 & +0.2 & -0.1 & -0.2 \\
\hline 21 & +0.3 & +1.2 & +0.1 & +0.2 & $-0 . I$ & -0.1 & +02 & +0.1 & +0.2 & +0.3 & -0.2 & -0.8 \\
\hline 22 & +0.5 & +1.3 & +0.1 & +0.2 & $-0 . I$ & 0.0 & +0.3 & 0.0 & -0.4 & +0.3 & -0.2 & -1.3 \\
\hline 23 & +0.8 & +1.5 & +0.1 & +0.2 & -0.1 & 0.0 & +0.3 & 一 0.1 & -0.5 & +0.4 & -0.2 & -1.5 \\
\hline 24 & +1.0 & +1.3 & 0.0 & + o.I & 0.0 & +0.1 & +0.2 & 0.0 & -0.7 & +0.4 & - & -1.4 \\
\hline 1. $1.1 . .$. & $8.6 \mathrm{H}$ & $8.2 \mathrm{H}$ & 8. I H & $8.2 \mathrm{H}$ & $8.7 \mathrm{H}$ & & $12.4 \mathrm{H}$ & I5. $3 \mathrm{H}$ & $14.4 \mathrm{H}$ & $14.8 \mathrm{H}$ & I5. $3 \mathrm{H}$ & $14.4 \mathrm{H}$ \\
\hline \{ Amount... & $+3 .^{\prime} 8$ & $+2 \cdot 7$ & $+3^{\prime 1}$ & $+2 . ' 5$ & $+1 .{ }^{\prime} 8$ & $+1 .{ }^{\prime} \mathbf{1}$ & +0.18 & $+1 . .^{\prime} 4$ & $+3 \cdot{ }^{\prime} 7$ & $+1 . .^{\prime} 4$ & $+2 .^{\prime} 6$ & +4.18 \\
\hline 目 $\{\mathrm{L}, \mathrm{M}, \mathrm{T}, \ldots$ & $14.2 \mathrm{H}$ & $12.9 \mathrm{H}$ & $13.2 \mathrm{H}$ & $136 \mathrm{H}$ & I3 $3 \mathrm{H}$ & I I.9 H & $9.2 \mathrm{H}$ & $9.5 \mathrm{H}$ & $9.2 \mathrm{H}$ & $10.0 \mathrm{H}$ & $9.7 \mathrm{H}$ & $8.8 \mathrm{H}$ \\
\hline \{ Amount .... & $-5 \cdot^{\prime} I$ & $-5 \cdot 0$ & -2.18 & $-2 .^{\prime} 2$ & -1.18 & $-1 .{ }^{\prime} 2$ & $-1 .{ }^{\prime} 2$ & $-1 . ' 9$ & $-2 . ' 6$ & -2.18 & -2.18 & -3.6 \\
\hline ge......... & $8 . ' 9$ & $7 \cdot 7$ & $5 \cdot 9$ & $4 \cdot{ }^{\prime} 7$ & $3 \cdot 16$ & $2 .^{\prime} 3$ & 2.'o & $3 \cdot{ }^{\prime} 3$ & $6 . ' 3$ & $4 \cdot{ }^{\prime} 2$ & $5 \cdot^{\prime} 4$ & 8.14 \\
\hline Calculated range. . & $8 . ' 2$ & $5 \cdot 9$ & $4 \cdot{ }^{\prime} I$ & $3 . ' 8$ & $2 . ' 9$ & 2.' 5 & $2 .^{\prime} 5$ & $2 . ' 9$ & $3 \cdot 4$ & 3.' 4 & $3 . / 8$ & $7 \cdot^{\prime} 1$ \\
\hline Summer ragge, S.. & $13 \cdot{ }^{\prime} 5$ & IO.'I & $70^{\prime} 7$ & 6.16 & $5 \cdot{ }^{\prime} 2$ & $4 . \%$ & $4 \cdot \cdot^{\prime} 3$ & $4 \cdot{ }^{\prime} 3$ & $8 . ' 8$ & $6 \cdot 3$ & $7 .^{\prime o}$ & II.'3 \\
\hline Winter range, W.. & $5 \cdot 2$ & $5 \cdot 18$ & $4 \cdot 6$ & $3 \cdot 6$ & $2 .^{\prime} 2$ & $0 . ' 9$ & 2.' 4 & 2.'9 & $4 \cdot 3$ & $3 . / 8$ & $5 \cdot{ }^{\prime} 8$ & $5 .^{\prime} 6$ \\
\hline Ratio $\frac{S}{W} \ldots .$. & 2.60 & 1.74 & 1.67 & 1.83 & 2.36 & 4.44 & 1.79 & 1.48 & 2.05 & 1.66 & $\mathrm{I} .2 \mathrm{I}$ & 2.02 \\
\hline 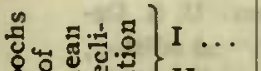 & I I. $2 \mathrm{H}$ & $10.0 \mathrm{H}$ & $10.6 \mathrm{H}$ & $10.8 \mathrm{H}$ & $10.9 \mathrm{H}$ & $10.0 \mathrm{H}$ & $(7 . I \mathrm{H})$ & $(2 \mathrm{I} .6 \mathrm{H})$ & $(2 \mathrm{I} .2 \mathrm{H})$ & $(6.9 \mathrm{H})$ & $(19.0 \mathrm{H})$ & $(19.6 \mathrm{H})$ \\
\hline 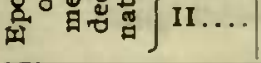 & $20.0 \mathrm{H}$ & $(17.8 \mathrm{H})$ & (I9.I H) & (19.7 H) & $(\mathrm{I} 6.9 \mathrm{H})$ & $(\mathbf{I} 5.3 \mathbf{H})$ & $10.8 \mathrm{H}$ & I $2.4 \mathrm{H}$ & II. $\mathbf{H} \mathbf{H}$ & $12.6 \mathrm{H}$ & I $2.1 \mathrm{H}$ & I $1.4 \mathrm{H}$ \\
\hline
\end{tabular}


An inspection of the mean yearly ranges in diurnal variation for the stations of the above tabulation indicates that these ranges are in some way dependent upon the values of the respective magnetic dips, the greater ranges being associated with the greater inclinations. Dr. Bauer* has announced a law covering this apparent interrelation, namely, that the mean value, $d$, for the year of the total diurnal amplitude of the magnetic declination is a function of the magnetic latitude, $\varphi^{\prime}$, which may be in the general case expressed mathematically as

$$
d=k \sec ^{2} \varphi^{\prime}
$$

in which the value of $\varphi^{\prime}$ is derived from the magnetic iuclination, $I$, in accordance with the well-known formula

$$
\tan \varphi^{\prime}=0.5 \tan Y \text {; }
$$

$k$ is a constaut depending upon the position of the particular series under discussion in the sun-spot cycle. Dr. Bauer's preliminary value of $k$ is 2.'58. Pending greater elaboration by him it has seemed desirable in connection with above compilation to determine values of $k$ by grouping these stations in order of the approximate sun-spot frequencies given on page 297 . Accordingly values have been derived by the method of least squares from groupings of stations as indicated in the following summary of results :

\begin{tabular}{|c|c|c|}
\hline Grouping stations number & $\begin{array}{c}\text { Mean sun-spot } \\
\text { frequency for } \\
\text { grouping }\end{array}$ & $\begin{array}{c}\text { Resulting } \\
\text { value of } k\end{array}$ \\
\hline 3,14, and $21 \ldots \ldots \ldots \ldots \ldots \ldots \ldots$ & 81 & 2.71 \\
$1,2,4$ to $11,13,15,17,22$, and 24... & 60 & 2.52 \\
$12,16,18,19,20,23$, and 25....... & 32 & 2.36 \\
\hline
\end{tabular}

The ranges for the individual stations of the groupings have been calculated using the above values of $k$ and the results placed against the actually observed quantities for each station. The agreement between the observed and computed ranges is, in the main, quite satisfactory.

\section{MAGNETIC DISTURBANCES}

As already stated, those days of observation on which particularly great disturbances in magnetic declination occurred may be readily noted by reference to the tabulation of circle readings of the magnet for the days on which the horizontal circle was shifted (see pages 20 to 26). In view of the fact that no continuous observations were made either for magnetic inclination or intensity in connection with those for declination, it has not been deemed worth while to attempt any general discussion or comparison of these dishurbances. As will be noted from the miscellaneous remarks (pages 32 to 40 ), the magnetic storms were very frequently associated with the aurora borealis. On the other hand, the auroral displays were not always accompanied by magnetic disturbances, and vice versa.

The observations of November I, Ig03, between midnight and 8 A. M., local mean time are of particular interest, as they cover the final portion of the great magnetic storm of October

* A remarkable law, by L. A. Bauer. Journal of Terrestrial Magnetism. Volume II, p. 7o. Cincinnati, 1897. Also United States maguetic declination tables and isogonic charts for Igoz, by L. A. Bauer. U. S. Department of Commerce and Labor, Coast and Geodetic Survey, O. H. Tittmann, Superintendent. 2d edition, p. 51. Wảshington, Ig03. 
3 to November 1, 1903.* The extreme values of declination observed at Teplitz Bay during this period were $37^{\circ} 3 \mathrm{I}^{\prime} \mathrm{E}$ at $4: 0 \mathrm{I} \mathrm{A}$. M. and $20^{\circ} \mathrm{r}^{\prime} \mathrm{E}$ at 5:49 A. M., local mean time, the greatest range thus being $17^{\circ} 18^{\prime}$ in 1.8 hour. During the same absolute period the records of the Cheltenham Magnetic Observatory of the United States Coast and Geodetic Survey show an extreme range of 32 , while the maximum range for the entire storm at this point was about three times as much, viz., 97. I. From this comparison it appears highly probable that dirring the maximum violence of this disturbance on October $3 \mathrm{I}, \mathrm{I} 903$, the extreme range in magnetic declination at Teplitz Bay may have been as much as $52^{\circ}$.

The highest value observed during the entire series was $37^{\circ} 3 \mathrm{r}^{\prime} \mathrm{E}$, as above, on November I, I 903 ; the lowest, $19^{\circ} 5^{I^{\prime}}$ at 8:27 P. M. on June 15,1904 . The absolute observed range was, therefore, $17^{\circ} 40^{\prime}$. The unusual character and magnitude of the disturbance of October $3^{1}$, 1903, is thus at once apparent.

\section{Secular Change in Magnetrc Declination}

The only previous observations of the declination of the magnetic needle at Teplitz Bay available for use in determining the secular change in this element are those made by Commander Cagni, of the Italian Expedition of 1899 and I900. His observations as reported by Professor Palazzo thave been corrected for diurual variation as determined from the preceding discussion. The following table gives a synopsis of these determinations so reduced (the corrections ou accolnt of diurnal variation are the mean values over the respective periods of observation):

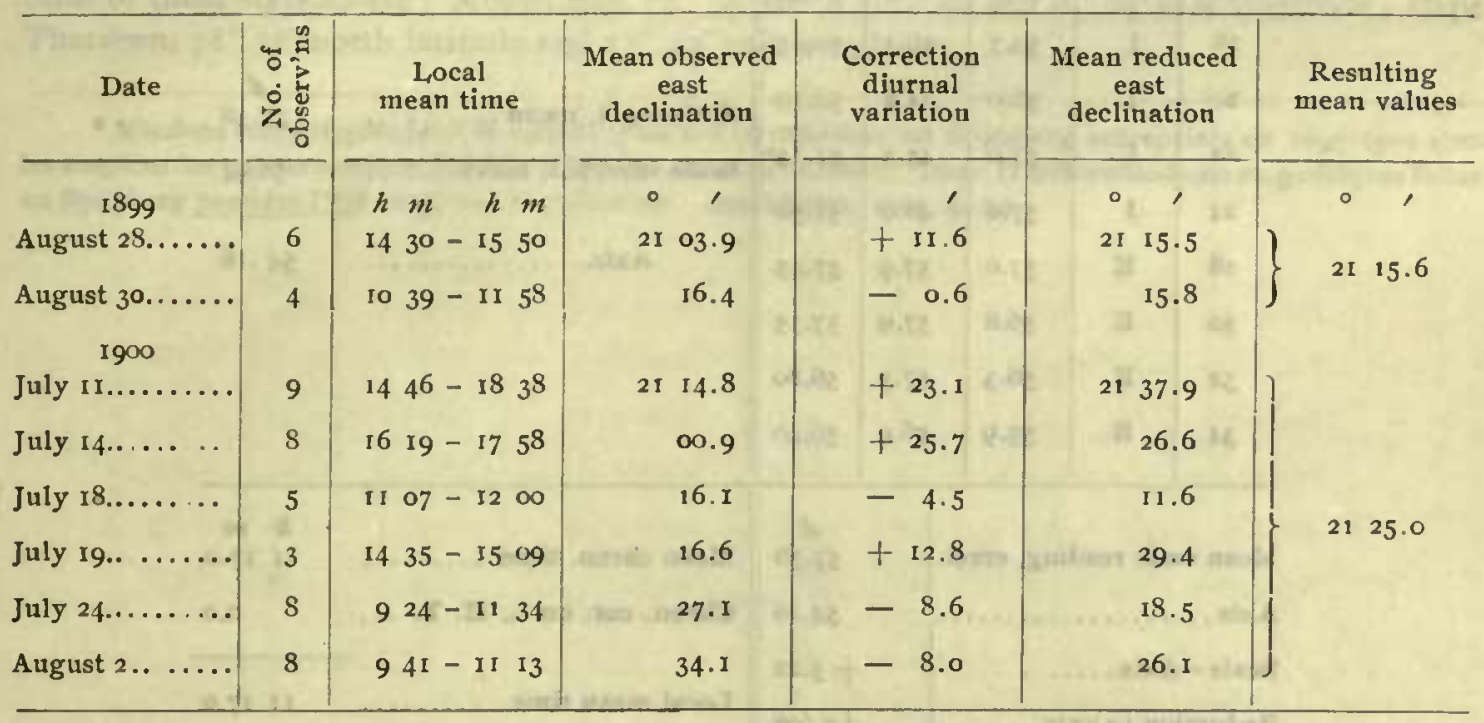

The resulting mean values of $2 I^{\circ} 15 .^{\prime} 6$ and $2 I^{\circ} 25 .^{\prime} 0$ correspond to mean of day for the epochs 1899.66 and 1900.55 respectively.

Unfortunately the multitudinous duties of the various members of the Ziegler Expedition field force prohibited making observations at the Italian station until just shortly prior to the

* Journal of Terrestrial Magnetism and Atmospheric Electricity, volume IX, Ig04, pp. 25-33; also Annalen der Hydrographie und maritimen Meteorologie, I904, Heft III, pp. I12-I27.

† Osservazioni scientifiche esequite durante la spedizione Polare di S. A. R. Luigi Amedeo di Savoia, Duca degli Abruzzi. Milan, r903. Pp. 453-462. (Relazione sulle osservazioni magnetiche fatta dal Professore Luigi Palazzo.) 
final retreat south. As a result but one set of declination observations at Commauder Cagni's station could be made. 'This set is herewith given in its entirety :

\begin{abstract}
Station: Italian station, Teplitz Bay Instrument : Maguetometer IIII Mark : Magnetic observatory
\end{abstract} Magnet : No. 4
Date: June 23, 1904

Observer: W. J. P.

Line of detorsion : $54^{\circ}$

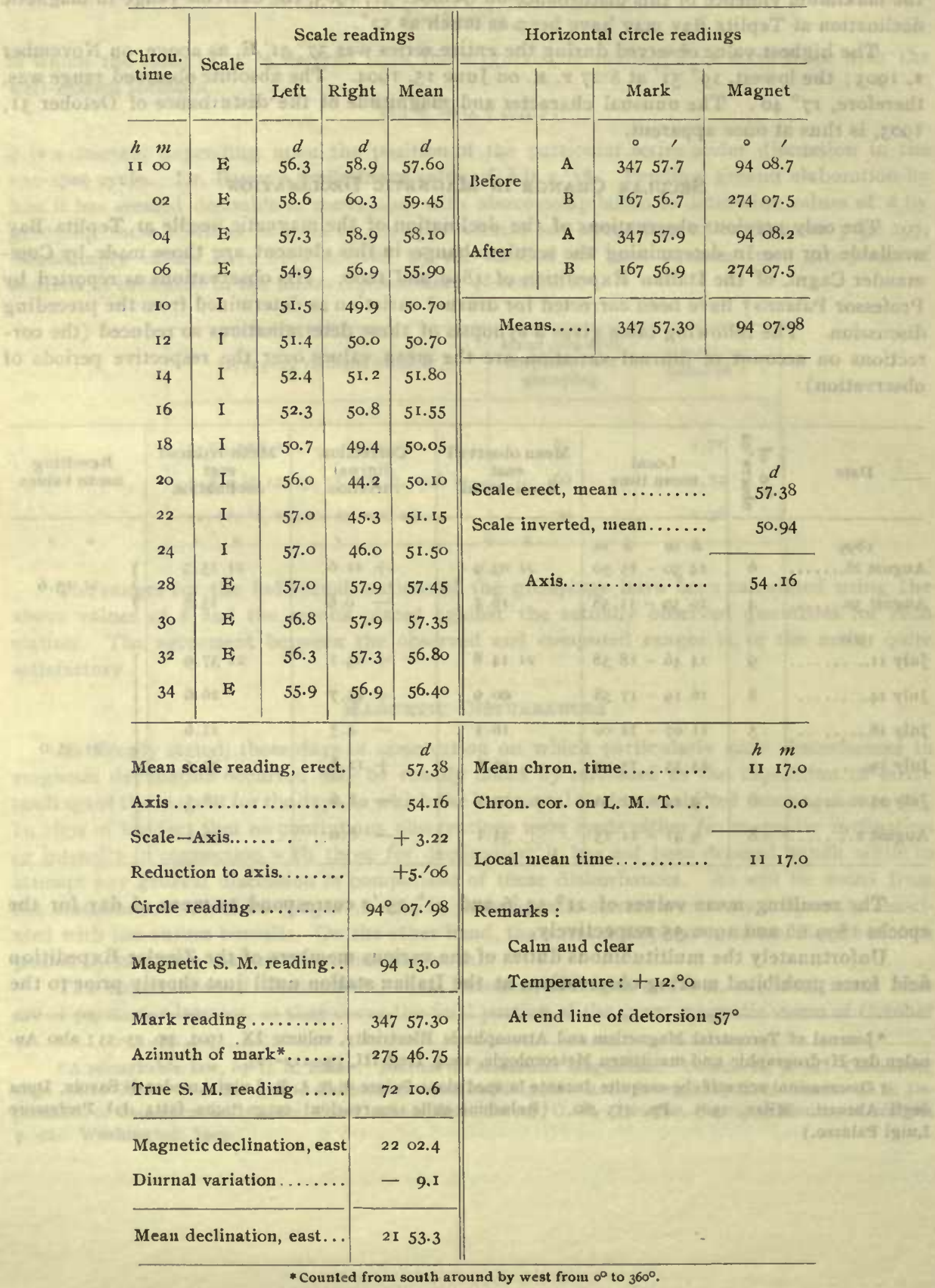


Owing to the fact that no suitable instrument was available, observations at the observatory simultaneous with the above could not be made. The observations nearest in point of time at the but are those of June 22 and June 23. Correcting the two-minute readings of June 22 between 20 hours and 24 hours for diurnal variation a mean value of $22^{\circ} 51^{\prime}$ results. In like manner, the observations on June 23 between 16 hours and 20 hours give a mean value of $22^{\circ} 19^{\prime}$. It thus appears, since the average value during the period June $\mathrm{I} 2$ to July $\mathrm{I}$ is $22^{\circ} 32^{\prime}$, that, while the general magnetic conditions preceding the observations at the Italian station by thirteen to fourteen hours were such as to give declinations high by about I9', the conditions six to seven hours later were such as to give declinations low by about I $^{\prime}$. It therefore seems quite probable that between II and I $2 \mathrm{~A}$. M. the magnetic conditions were about normal, and hence a direct comparison with the preceding may be made.

As already noted, Commander Cagni's results at about the same season of year were $21^{\circ}$ I 5. 6 and $21^{\circ} 25 .^{\prime}$ o for the epochs 1899.66 and 1900.55 , or in the mean $2 I^{\circ} 20 .^{\prime} 3$ for 1900.10 . The result above for epoch 1904.48 is $2 \mathrm{I}^{\circ} 53^{\prime} 3$; hence the apparent effect in the elapsed interval of 4.4 years due to secular variation is an increase of east declination of $33^{\prime}$. Assuming a linear chauge over the interval, the secular change in magnetic declination in the region of Teplitz Bay may be taken as about

$$
\text { + 7.'5 per year. }
$$

It may be noted that this value is of the same magnitude as those deduced by Dr. Solander* for Mossel Bay and Cape Thordsen, Spitzbergen, for the periods 1873.5 to 1899.7 and 1883.2 to 1899.7 , viz., 7.' 3 and $7 .^{\prime} 8$ decreasing west declination respectively. The geographical positions of these stations are : Mossel Bay, $79^{\circ} 53^{\prime}$ north latitude and $16^{\circ}$ o4' east longitude ; Cape Thordsen, $78^{\circ} 28^{\prime}$ north latitude and $15^{\circ} 42^{\prime}$ east longitude.

* Missions scientifiques pour la mesure d'un arc de méridian au Spitzberg entreprises en 1899 -1902 sous les auspices des gouvernements Suédois et Russe-Missiou Suédois. Tome II Déterminations maguétiques faites au Spitzberg pendent l'éte 1899, par E. Solander. Stockholm, 1903, p. 50. 


\section{ANNUAL, VARIATION IN MAgNeTIC Declination}

By means of the preceding value of the annual rate of secular variation in magnetic declination at Teplitz Bay the monthly mean hourly declinations and means were reduced to epoch I904.0 as per tabulation on page 288. By the use of Bessel's periodic function in the particular case of a cycle of twelve equidistant observations the following expression has been obtained, after the methods detailed on page 290 , as representing the annual variation :

$$
\begin{gathered}
D=22^{\circ} 38^{\prime} 5+9 .^{\prime} 27 \sin \left(\theta+244^{\circ} 59^{\prime}\right)+2 .^{\prime} 32 \sin \left(2 \theta+141^{\circ} 07^{\prime}\right) \\
+\mathrm{I} .^{\prime} 4 \mathrm{I} \sin \left(3 \theta+4^{\circ} 45^{\prime}\right) \pm 0 .^{\prime} 33^{*}
\end{gathered}
$$

In this formula the angle $\theta$ counts from the middle of June as $0^{\circ}$, no account being taken of the inequality in length of months. The addition of a fourth term resuits in no improvement, as in this case the probable error is identical with that above. It may be noted that the range of variation on the mean of year is unusually large, the maximum deflection early in December being + 10.' 2 while the opposite extrene about the middle of August is - 10.'o. The computed and observed values are shown graphically to scale in figure 20 . The following summary of variations on the mean yearly value will serve further to indicate the agreement between the observed $(O)$ and computed $(C)$ quautities, easterly deviations being denoted by

\begin{tabular}{|c|c|c|c|c|c|c|c|}
\hline Month & $o$ & C & $O-C$ & Month & $o$ & C & $O-C$ \\
\hline & , & , & , & $2=$ & , & , & ' \\
\hline Juxe..... & -4.8 & -6.8 & +2.0 & December. & +7.6 & +9.7 & -2.1 \\
\hline July. ... & -10.1 & -8.6 & $-\mathrm{x} .5$ & January... & $+8 . x$ & +7.0 & +r.I \\
\hline August & -9.9 & -ro.o & to.I & February. & +5.3 & +5.4 & -0.1 \\
\hline September & -5.4 & -6.8 & $+x .4$ & March .... & +3.3 & +3.9 & -0.6 \\
\hline October.... & -0.7 & +1.8 & -2.5 & April ..... & $+\mathrm{r} .2$ & $\multimap 0.1$ & +1.3 \\
\hline November. & $+\mathrm{Ir} .7$ & +9.0 & +2.7 & May.... & -6.3 & $\cdots 4.4$ & -1.9 \\
\hline
\end{tabular}
a plus sign, and vice versa:

* The probable error of a single representation is $\pm 0 . ' 33$. 


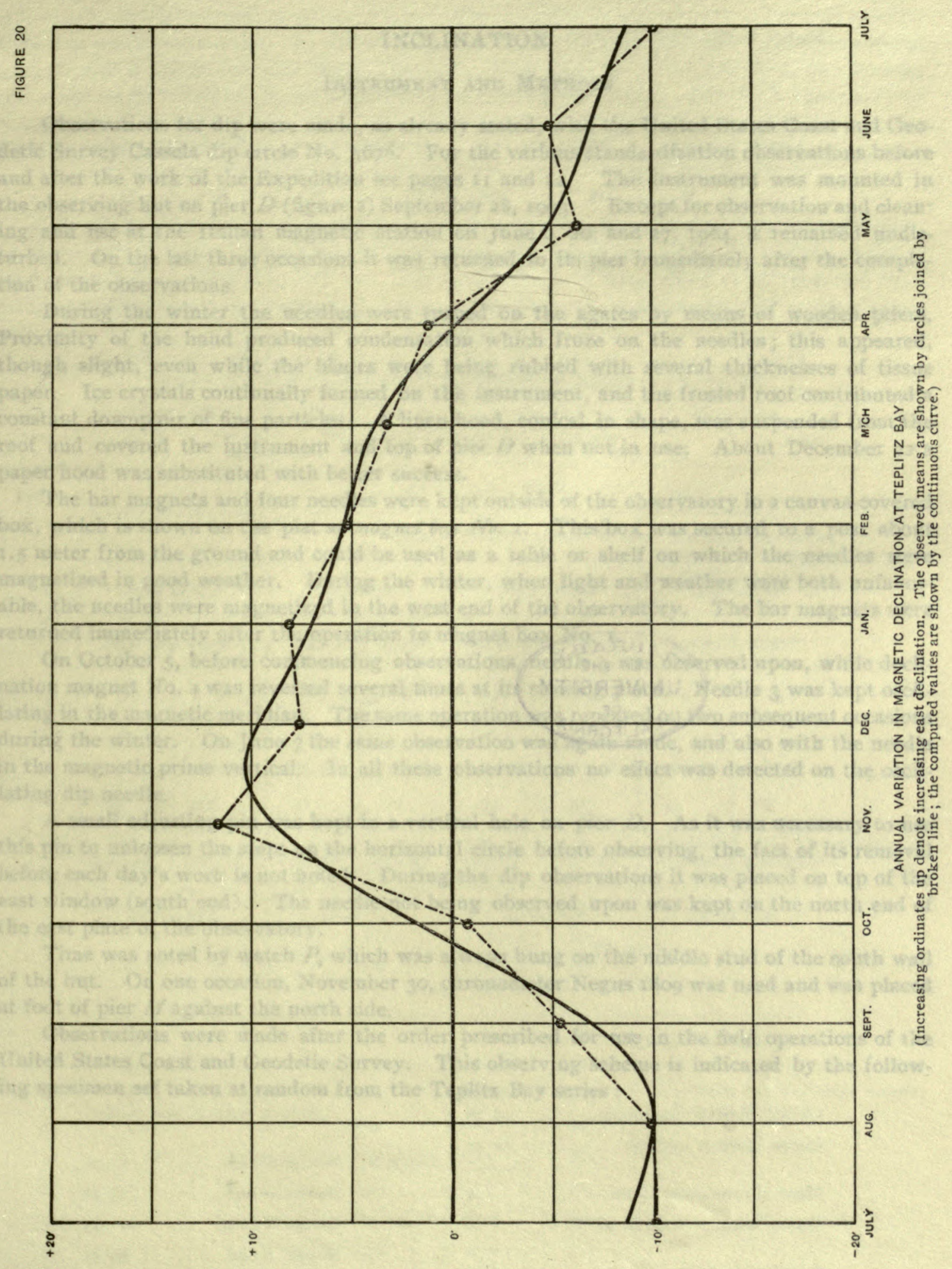




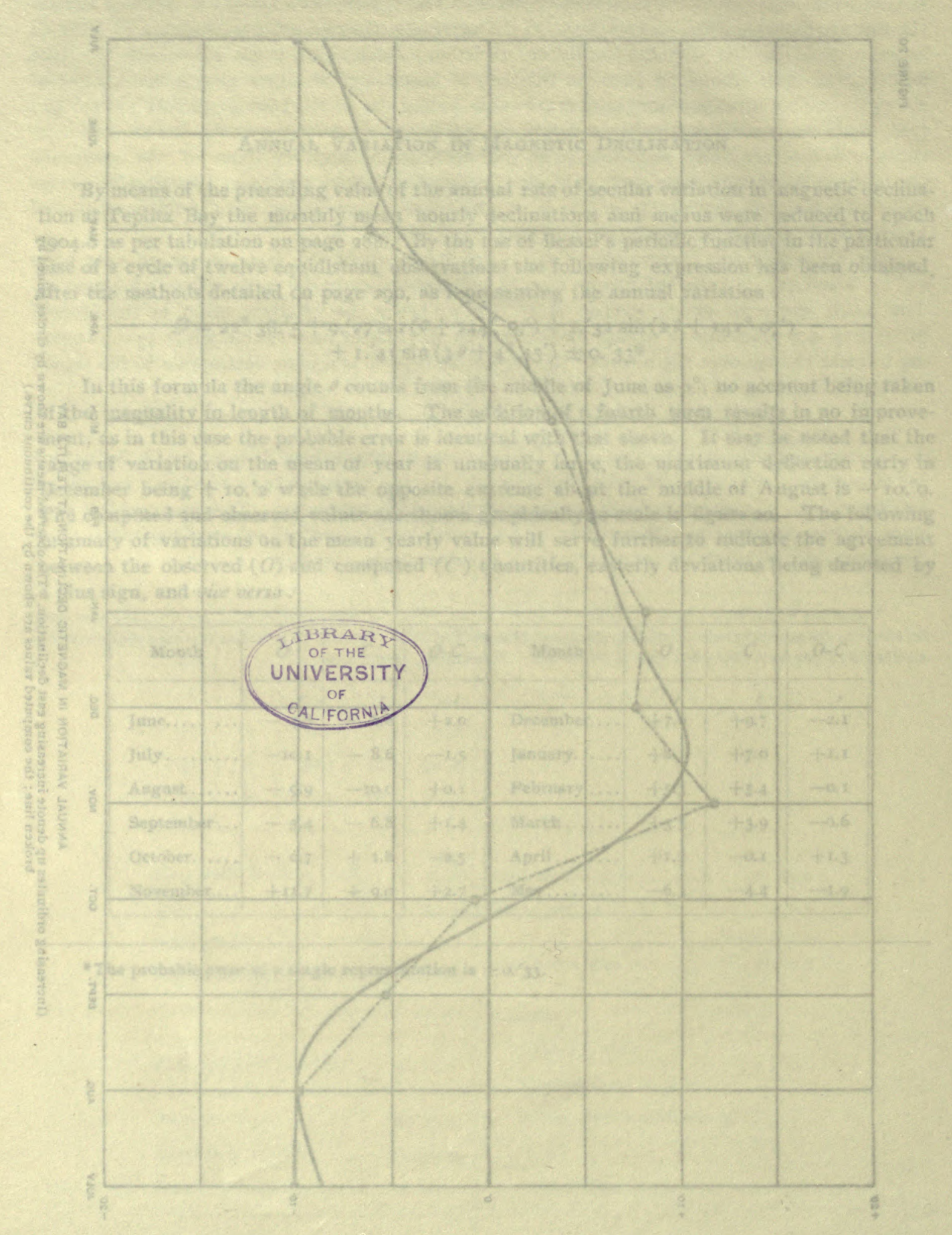




\section{INCLINATION}

\section{INSTRUMENT AND METHODS}

Observations for dip were made, as already stated, with the United States Coast and Geodetic Survey Cassela dip circle No. 5676. For the various standardization observations before and after the work of the Expedition see pages II and 12 . The instrument was mounted in the observing hut on pier $D$ (figure 2) September 28, 1903. Except for observation and cleaning and use at the Italian magnetic station on June 6,20 , and 27,1904 , it remained undisturbed. On the last three occasions it was returned to its pier immediately after the completion of the observations.

During the winter the needles were turned on the agates by means of wooden pliers. Proximity of the hand produced condensation which froze on the needles; this appeared, though slight, even wliile the blades were being rubbed witl several thicknesses of tissue paper. Ice crystals continually formed on the instrument, and the frosted roof contributed a constant downpour of fine particles. A linen hood, conical in shape, was suspended from the roof and covered the instrument and top of pier $D$ when not in use. About December 22 a paper hood was substituted with better success.

The bar magnets and four needles were kept outside of the observatory in a canvas-covered box, which is shown on the plat as magnet box No. $r$. This box was secured to a post about r. 5 meter from the ground and could be used as a table or shelf on which the needles were magnetized in good weather. During the winter, when light and weather were both unfavorable, the needles were magnetized in the west end of the observatory. The bar magnets were returned immediately after the operation to magnet box No. 1 .

On October 5, before commencing observations, needle 3 was observed upon, while declination magnet No. 4 was reversed several times at its stowing place. Needle 3 was kept oscillating in the magnetic meridian. The same operation was repeated on two subsequent occasions during the winter. On June 7 the same observation was again made, and also with the needle in the magnetic prime vertical. In all these observations no effect was detected on the oscillating dip needle.

A small adjusting pin was kept in a vertical hole on pier $D$. As it was necessary to use this pin to unloosen the stops on the horizontal circle before observing, the fact of its removal before each day's work is not noted. During the dip observations it was placed on top of the east window (south end). The needle not being observed upon was kept on the north end of the east plate of the observatory.

Time was noted by watch $P$, which was always hung on the middle stud of the south wall of the hut. On one occasion, November 30 , chronometer Negus I 809 was used and was placed at foot of pier $M$ against the north side.

Observations were made after the order prescribed for use in the field operations of the United States Coast and Geodetic Survey. This observing scheme is indicated by the following specimen set taken at random from the Teplitz Bay series :

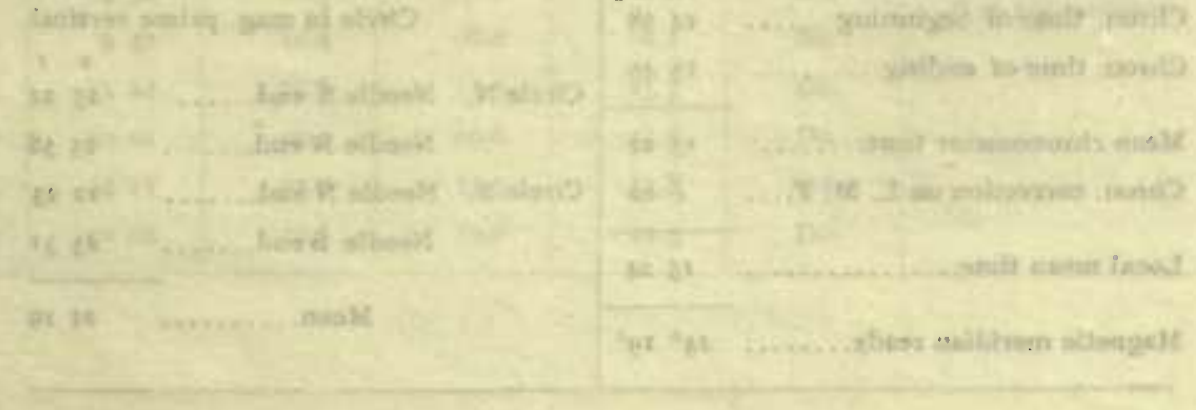



Station : Teplitz Bay
Date: January 18,1904
Observer: W. J. P.
Dip circle No. 5676
Needle No. 3

End of needle marked $A$ down

\begin{tabular}{|c|c|c|c|c|c|c|c|}
\hline \multicolumn{2}{|c|}{ Circle east } & \multicolumn{2}{|c|}{ Circle west } & \multicolumn{2}{|c|}{ Circle west } & \multicolumn{2}{|c|}{ Circle east } \\
\hline \multicolumn{2}{|c|}{ Needle face east } & \multicolumn{2}{|c|}{ Needle face west } & \multicolumn{2}{|c|}{ Needle face cast } & \multicolumn{2}{|c|}{ Needle face west } \\
\hline $\mathrm{S}$ & $\mathrm{N}$ & S & $\mathrm{N}$ & S & s & $\mathbf{s}$ & $\mathrm{N}$ \\
\hline $\begin{array}{cc}0 & 1 \\
82 & 53\end{array}$ & $\begin{array}{c}\circ \\
8255\end{array}$ & $\begin{array}{c}0 \quad \\
8321\end{array}$ & $\begin{array}{l}\circ 1 \\
8310\end{array}$ & $\begin{array}{rr}0 & 1 \\
83 & 27\end{array}$ & $\begin{array}{c}\circ \\
8326\end{array}$ & $\begin{array}{cc}\circ & 1 \\
83 & 13\end{array}$ & $0_{3}^{\circ} 15$ \\
\hline 52 & 55 & I5 & 210 & 23 & 19 & II & 13 \\
\hline minas & की & 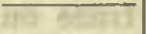 & क्या & 7 & & 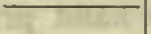 & (nt \\
\hline 8252.5 & 8255.0 & 8318.0 & 8310.0 & 8325.0 & 8322.5 & $83 \quad 12.0$ & 8314.0 \\
\hline $82^{\circ}$ & $3 .^{\prime} 75$ & $83^{\circ}$ & $14 \cdot 10$ & $83^{\circ}$ & $83^{\circ}$ & $83^{\circ}$ & 3.10 \\
\hline
\end{tabular}

Polarities reversed : end of needie marked B down

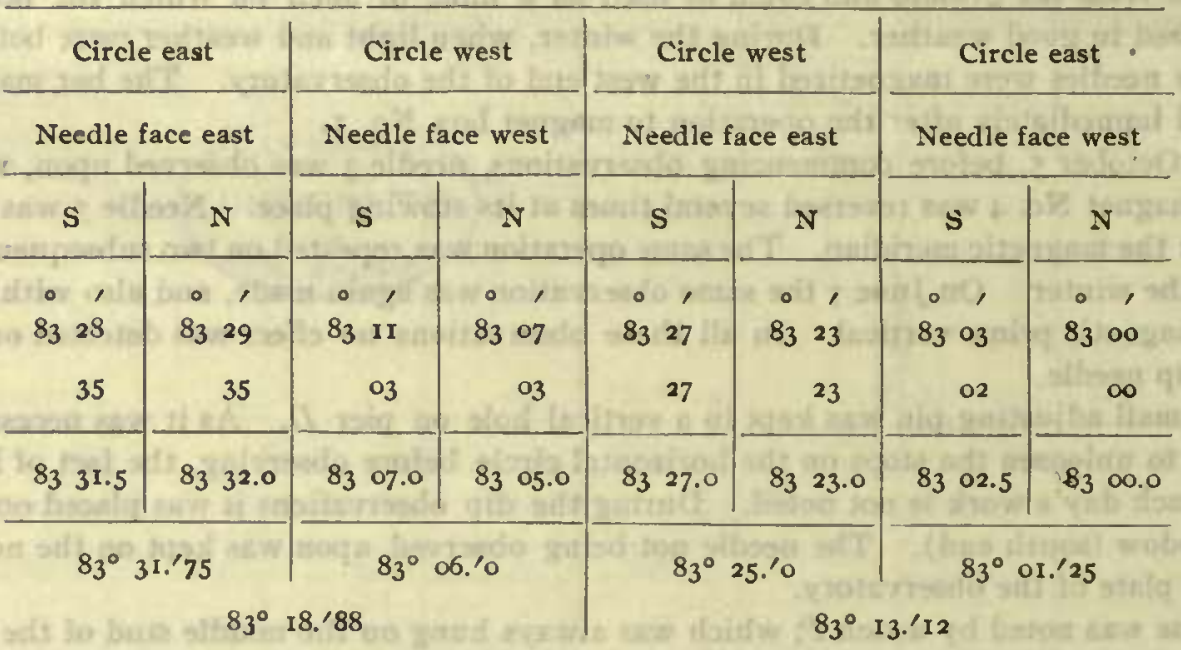

Mean : $83^{\circ} 16 .^{\prime} 0$

Resulting dip : $83^{\circ} 13^{\prime} 6$

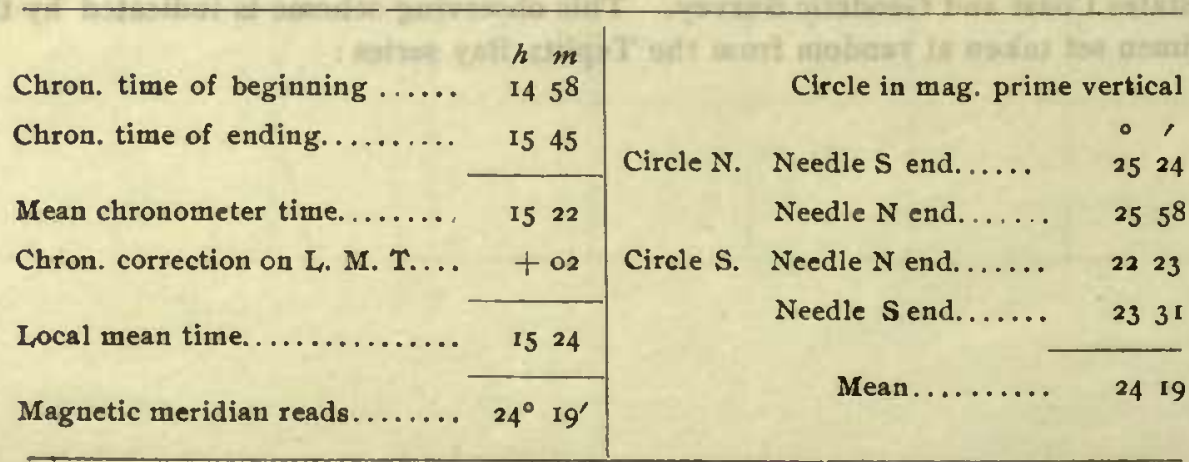


When observing with two needles the observations with first polarity of the first needle were immediately followed by the complete determination with the second needle (polarities of both needles being reversed at the same time), after which observations with the second polarity of the first needle were made. By this arrangement the final means from each needle correspond to practically the same epoch of time. As will be noted from the above specimen, two settings were made on each end of the needle in every position except in those cases where these differed by ten minutes or more of arc, when an additional observation was made and the mean of the three readings taken.

\section{RESULTS}

Only the final results for each needle and their means are shown in the tabulation following, the mean values being adopted without correction (see page 12). The time is the mean of the times noted at beginning and end, and is expressed in local mean time, reckoned from midnight throughout the twenty-four hours. The observer is indicated by the initials (see page 17 ).

Tabular summary of observations of magnetic indination at Teplitz Bay

\begin{tabular}{|c|c|c|c|c|c|c|c|c|c|}
\hline \multicolumn{2}{|c|}{ Date } & L. M. T. & Needle 3 & Needle 4 & Mean & Observer & $\begin{array}{l}6 \pm 7 \\
+4 x \\
\end{array}$ & Renıarks & $\begin{array}{r}15 \\
4 \\
\end{array}$ \\
\hline \multicolumn{2}{|c|}{1903} & $h m$ & 0, & 0, & 0, & & & 18.42 & $\cos ^{2}-x^{2}$ \\
\hline \multirow[t]{5}{*}{ Oct. } & 5 & 1628 & $83 \quad 16.4 \mathrm{~N}$ & $8317.3 \mathrm{~N}$ & $8316.8 \mathrm{~N}$ & W. J. P. & & & \\
\hline & 12 & II 18 & 36.6 & 28.3 & 32.4 & & $\begin{array}{r}\text { Magne } \\
\text { mine }\end{array}$ & $\begin{array}{l}\text { meridian } \\
\text { October } 5\end{array}$ & as deter- \\
\hline & 19 & I5 24 & 08.8 & 10.2 & 09.5 & Do. & 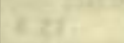 & the $x=$ & 8 \\
\hline & 29 & 1234 & 10.6 & 08.4 & 09.5 & Do. & 5.20 & 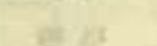 & II \\
\hline & 30 & 1527 & II. I & 12.7 & 11.9 & Do. & $1603=$ & Rete & wi \\
\hline \multirow[t]{2}{*}{ Nov. } & 20 & 1702 & 13.6 & 08.7 & II. 2 & Do. & $(1-2)$ & 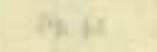 & (n) \\
\hline & 30 & 1952 & 15.9 & 10.8 & 13.4 & No. & $2-19$ & W. & pt \\
\hline \multirow[t]{2}{*}{ Dec. } & 3 & 1506 & 10.7 & I2. 6 & I1. 6 & Do. & $s .11$ & 69 in & it: \\
\hline & 4 & Io Io & 14.4 & 14.4 & 14.4 & Do. & $4 n$ & of p p & 25 \\
\hline \multicolumn{2}{|c|}{7} & 1430 & 09.9 & II. 8 & 10.8 & Do. & \multicolumn{3}{|c|}{$\begin{array}{l}\text { Corrected for observation in } \\
\text { magnetic azimuth } 1^{\circ} \text {. }\end{array}$} \\
\hline \multicolumn{2}{|c|}{19} & 10 $3^{6}$ & 11.5 & 22.6 & 17.0 & Do. & $7 x^{2}$ & fin 8 & 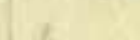 \\
\hline & 21 & 1459 & 16.2 & I8.8 & 17.5 & Do. & was & $58 \times 3$ & e \\
\hline & 25 & 1122 & 09.8 & 13.2 & I 1.5 & Do. & & 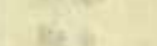 & ant \\
\hline & 26 & 830 & 13.7 & 12.8 & 13.2 & Do. & & & \\
\hline \multicolumn{2}{|c|}{1904} & & & $\left.a^{4}\right)$ & tat & & 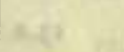 & $\mathrm{mpm}$ & 11 \\
\hline Jan. & 5 & 932 & 11.3 & 13.0 & 12.2 & Do. & & $402 x$ & in \\
\hline & 9 & 950 & 10.5 & 18.2 & 14.4 & Do. & & & \\
\hline & 14 & 1434 & 08.3 & 05.4 & 06.8 & Do. & \multicolumn{3}{|c|}{$\begin{array}{l}\text { Aurora in north of large extent, } \\
\text { but not brilliant. }\end{array}$} \\
\hline & $\begin{array}{l}15 \\
18\end{array}$ & $\begin{array}{r}942 \\
1524\end{array}$ & $\begin{array}{r}12.4 \\
13.6\end{array}$ & $\begin{array}{l}08.0 \\
13.0\end{array}$ & $\begin{array}{l}10.2 \\
13.3\end{array}$ & $\begin{array}{l}\text { Do. } \\
\text { Do. }\end{array}$ & $2+3$ & $x^{2}=$ & 10 \\
\hline & 19 & Io or & 12.4 & 09.6 & II.O & Do. & $2=$ & 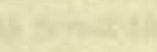 & 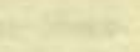 \\
\hline & 25 & I4 II & 12.2 & 13.5 & 12.8 & Do. & 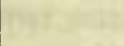 & 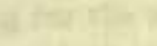 & 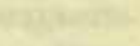 \\
\hline & 26 & 1002 & 12.6 & 10.0 & II.3 & Do. & 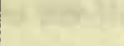 & 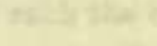 & 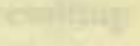 \\
\hline
\end{tabular}


Tabular summary of observations of magnetic inclination at Teplitz Bay-Continued

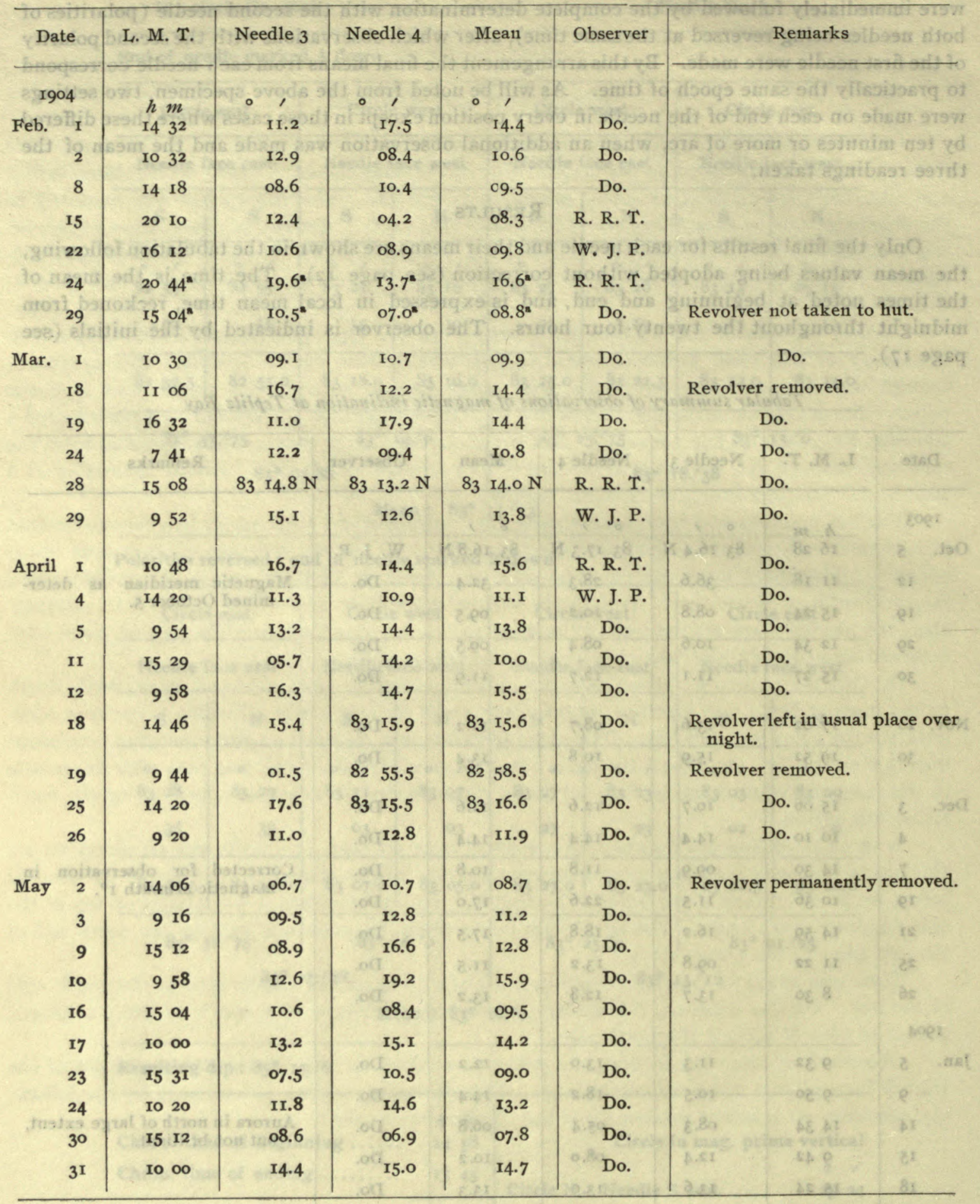

- Needles No. I and No. 2. 
Tabular summary of observations of magnetic indications at Teplitz Bay-Continued

\begin{tabular}{|c|c|c|c|c|c|c|}
\hline Date & L. M. T. & Needle 3 & Needle 4 & Mean & Observer & Remarks \\
\hline 1904 & & 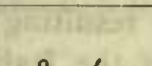 & 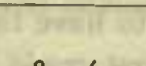 & 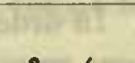 & & 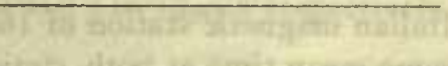 \\
\hline June 6 & $1524^{*}$ & or. $3^{*}$ & $03.5^{*}$ & $02.4^{*}$ & Do. & See foot-note. \\
\hline 6 & 1522 & 17.3 & 12.0 & 14.6 & Do. & 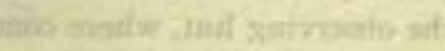 \\
\hline ㄱำ 7 & 1004 & 13.4 & 14.5 & 14.0 & Do. & 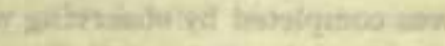 \\
\hline 13 & 1530 & 14.0 & II. 5 & 12.8 & Do. & 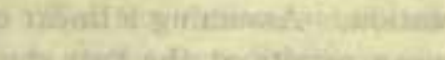 \\
\hline I4 & 946 & 15.2 & 14.8 & 15.0 & Do. & \\
\hline 20 & $1530^{*}$ & or. $9^{*}$ & $04.7^{*}$ & $03 \cdot 3^{*}$ & Do. & $x+2 x^{2}+2$ \\
\hline 20 & 1528 & 09.6 & 12.0 & 10.8 & Do. & \\
\hline $2 \mathrm{I}$ & $1003^{*}$ & $11.2^{*}$ & $08.1^{*}$ & $09.6^{*}$ & Do. & 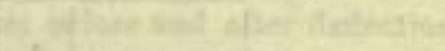 \\
\hline 21 & 953 & 12.0 & I8.o & 15.0 & Do. & 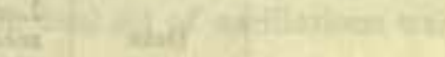 \\
\hline 27 & $1618^{*}$ & $09.8^{*}$ & $19.4^{*}$ & $14.6 *$ & Do. & \\
\hline 27 & 1618 & 18.2 & 20.4 & 19.3 & Do. & \\
\hline
\end{tabular}

* These observations were made at Italian station.

NoTE. - When the observations of June 20 at the Italian station were being made all iron bolt was found at the foot of the pier; needle was not affected wbile it was being removed, but probably the observations of June 6 are vitiated.

The following monthly mean values for magnetic dip result from these observations :

\begin{tabular}{|c|c|c|c|c|c|c|}
\hline \multirow{2}{*}{ Epoch } & \multirow{2}{*}{$\begin{array}{l}\text { No. } \\
\text { obs'ns } \\
\text { each } \\
\text { needle }\end{array}$} & \multicolumn{3}{|c|}{ Dip by } & \multirow{2}{*}{\multicolumn{2}{|c|}{$\begin{array}{l}\text { Resulting } \\
\text { mean } \\
\text { dip }\end{array}$}} \\
\hline & & Needle No. 3 & Need & le No. 4 & & \\
\hline 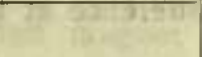 & 20 & 0,1 & 0 & , & 0 & , \\
\hline 1903.80 & 5 & $83 \quad 16.70 \mathrm{~N}$ & 83 & $15.3^{8} \mathrm{~N}$ & 83 & $16.0 \mathrm{~N}$ \\
\hline 1903.80 & $4^{4}$ & $11.72^{\star}$ & & I2. $15^{\circ}$ & & I $1.9^{\circ}$ \\
\hline 1903.90 & 2 & 14.75 & & 09.75 & & I2.3 \\
\hline I903.95 & 7 & I2.3I & & 15.17 & & 13.7 \\
\hline 1904.04 & 8 & 11.66 & & II. 34 & & 11.5 \\
\hline 1904.12 & $7^{b}$ & $12.26^{\circ}$ & 1,2 & $10.02^{b}$ & & II.I" \\
\hline 1904.22 & 6 & I3. 15 & & 12.67 & & I2.9 \\
\hline I904.28 & 9 & 12.08 & & 12.03 & & I2.I \\
\hline I904.37 & 10 & $10.4^{8}$ & & 12.98 & Whet: & II.7 \\
\hline 1904.46 & 7 & 14.24 & & $\mathrm{I} 4.74$ & $8+1$ & 14.5 \\
\hline
\end{tabular}

- Omitting October 12, I903. 'Including two values, needles No. I and No. 2.

In adopting a mean value from this summary it has been deemed best to reject the observations of October 12, 1903, as probably defective, owing to improper setting for the magnetic meridian. From the remaining sixty sets of observations with two needles each the resulting inclination at Teplitz Bay is-

$83^{\circ} 12.4$ for epoch 1904.13. 


\section{Secular Change in Magnetic Inclination}

Observations were made for inclination, as noted in the tabulation of dip results, at the Italian magnetic station of 1899 and 1900 . In order to have the resulting values apply to the same mean time at both stations observations were first made at the Italian station with one polarity of the marked end of each needle; the instrument was then transferred to pier $D$ in the observing hut, where complete sets with each needle were made, and finally the comparison was completed by observing with second polarity of the marked end of each needle at the Italian station. Assuming a linear change in diurnal variation over the period of observation, the mean results at the two stations are directly comparable. The mean resulting difference in magnetic dip between the two locations is 7.' 3 as adopted from the following tabulation of the comparisons :

\begin{tabular}{|c|c|c|c|c|c|c|c|}
\hline Date & $\begin{array}{l}\text { Local } \\
\text { mean } \\
\text { time }\end{array}$ & $\begin{array}{l}\text { Mean dip } \\
\text { observed at } \\
\text { Italian } \\
\text { station }\end{array}$ & $\begin{array}{l}\text { Local } \\
\text { mean } \\
\text { time }\end{array}$ & $\begin{array}{l}\text { Mean dip } \\
\text { observed at } \\
\text { Ziegler } \\
\text { station }\end{array}$ & $\begin{array}{l}\Delta \text { I Zieg- } \\
\text { ler station } \\
\text { to Italian } \\
\text { station }\end{array}$ & Weight & in \\
\hline 1904 & $h m$ & 0, & $h m$ & 0, & & 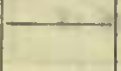 & we \\
\hline June 6 & 1524 & $8302.4 \mathrm{~N}$ & I5 22 & $83 \times 4.6 \mathrm{~N}$ & -12.2 & 1 & 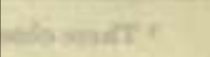 \\
\hline June 20 & 1530 & 03.3 & ${ }_{5} 28$ & 10.8 & -7.5 & 2 & W - sirtovi? \\
\hline June 27 & 1618 & 14.6 & 1618 & 19.3 & -4.7 & 2 & 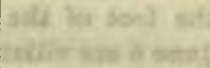 \\
\hline Whe & weight & difference & opted. & $\ldots$ & $-7 \cdot 3$ & Ix 2017 & olfel $56 \%$ \\
\hline
\end{tabular}

The comparison of June 6 is given weight of one against two for each of the other comparisons by reason of the observer's note regarding discovery of iron bolt at foot of pier on June 20. There being no reason to assume any change in local magnetic conditions at so isolated and unfrequented a locality, we may assume the same difference at the time of the Italian Expedition.

Commander Cagni's observations resulted as follows :*

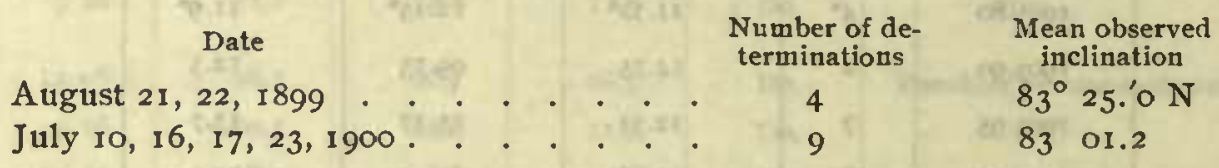

whence the mean value $83^{\circ} 13^{\prime} \mathrm{I} N$ for the epoch 1900.09. Referred to the Ziegler Polar Expedition station, this becomes $83^{\circ} 13^{\prime} I+7 \cdot^{\prime} 3=83^{\circ} 20 .^{\prime} 4$. The value for the epoch I904. I3, as above, is $83^{\circ} \mathrm{r2} .^{\prime} 4 \mathrm{~N}$. Both of these series are uncorrected for diurnal variation. Each, however, consists of observations made at various parts of the day, so that the means may be assumed as applying practically to mean of day at their respective epochs. Thus in four years the northerly magnetic inclination in the region of 'Teplitz Bay has decreased 8'. Hence the rate of change in magnetic inclination due to secular variation, assuming this effect to be a linear function during the elapsed interval, is

$$
-2 \text { per year. }
$$

This value is, while of the same sign, considerably smaller than that taken by Professor Palazzo as a result of his discussion of Commander Cagni's observations, viz., $-24^{\prime}$. He, however, considers this determination as not very reliable, owing to the short period between the inclination observations, and also the great variation between the individual values obtained.

* Osservazioni scientifiche esequite durante la spedizione Polare di S. A. R. Luigi Amedeo di Savoia, Duca degli Abruzzi, 1899-1900. Milan, 1903. Pp. 462-475. (Relazione sulle osservazioni magnetiche fatta dal Professore I,uigi Palazzo.) 
The annual rate above deduced is further confirmed by the fact that it is of practically the same order as values obtained at other Arctic stations. Thus, for example, for Cape Thordsen and Mossel Bay, Spitzbergen, Dr. Solander* has deduced the annual rates of increase in magnetic inclination as $0 .{ }^{\prime} 4$ and $0 .{ }^{\prime} 8$ for the periods 1883.2 to 1899.7 and 1873.5 to 1899.7 respectively.

\section{HORIZONTAL, INTENSITY}

\section{INSTRUMENT, METHODS, AND RESUr,TS}

Observations for liorizontal intensity were made, as already stated, with magnetometer No. IIII loaned by the United States Coast and Geodetic Survey. For the various standardization observations with this instrument before and after the work of the expedition, as also for the constants and methods of reduction used, see pages 6 to Ir. Determinations were made generally on Monday afternoons and Tuesday mornings, but it was not possible before the advent of daylight to adhere strictly to this program. In these observations for intensity the time of a set of fifty oscillations was observed in the usual manner before and after deflection observations. Sometimes these were repeated; at other times the final set of oscillations was lost, owing to the failure to transit of the selected scale division.

The fortietl division, being 11ear the niddle of the șcale, was the one whose transit was chosen. The initial amplitude of the oscillation was usually made about one degree in order to increase the probability of the transit of the fiftieth oscillation. The amplitude of swing was noted at the beginning and end, in scale divisions, but is not published for lack of space. The time was noted by mean time chronometer Negus No. 1809 , which was carried to the observatory for the purpose. It was always placed at the foot and touching the north side of pier $M$ (figure 2). This chronometer was compared daily with the sidereal chronometer Negus No. 1769 , which was 11 sed in the astronomic observations. Determinations of the torsional effect of the four suspension fibers were made before the first and after the last oscillation sets. The temperature was noted at the beginning, middle, and end by a Centigrade thermometer, the bulb of which projected into the magnet-house of the magnetometer. This same thermometer was used in the deflection observations, readings being taken before and after each set.

Magnet No. 2 was suspended during deflection observations. A solid brass bar with a carrier supported magnet No. 4 at thirty and forty centimeters from the center. Eight settings were made and the time and temperature noted as usual. Magnet No. 2 was kept in inagnet box No. 2 outside of the hut (shown on plat) and was brought into the observatory immediately after preceding oscillation observations. From fifteen to thirty minutes elapsed before it was observed upon. After deflection, and before the subsequent oscillation observations, it was returned to inagnet box No. 2.

The observation specimens, pages 314 and 315 , which together yield an absolute value of the horizontal intensity, $H$, will serve to make clear the method of observation and computation. In the tabulation of results given below, each value of $H$ is deduced from two or more such sets of oscillation and one set of deflection observations made in the order stated above.

By the aid of the specimens the accompanying tabulation of the condensed original notes may be readily interpreted. The times given are local mean reckoned from midnight tlirongh tiventy-four hours. The mean deflection angles observed at thirty and forty centimeters are given in the two columns under heading $u$. The columns $t$ and $t^{\prime}$ show the mean temperature readings for the deflection and oscillation observations respectively. The meau time of one oscillation corrected for the rate of chronometer appears under column headed ' $T$ '. The effect of ninety degrees of torsion in the suspension is given in the column $v$. The column headed $H$ gives the finally reduced values for horizontal intensity expressed in gammas, one gamma being 0.0000 I C. G. S. unit. The resulting values of the magnetic noment of maguet No. 4 at $t$ degrees and at twenty degrees Centigrade are shown in the last two columns respectively. Reference to pages 8 and ro will serve to explain the remaining beadings. 
Station : Teplitz Bay

Date: March 19, 1904

Observer: R. R. T.

Instrunient : Magnetometer IIII

Magnet: No. 4, inverted

Clironometer Negus I809, daily rate gaining 2."37 on ulean time

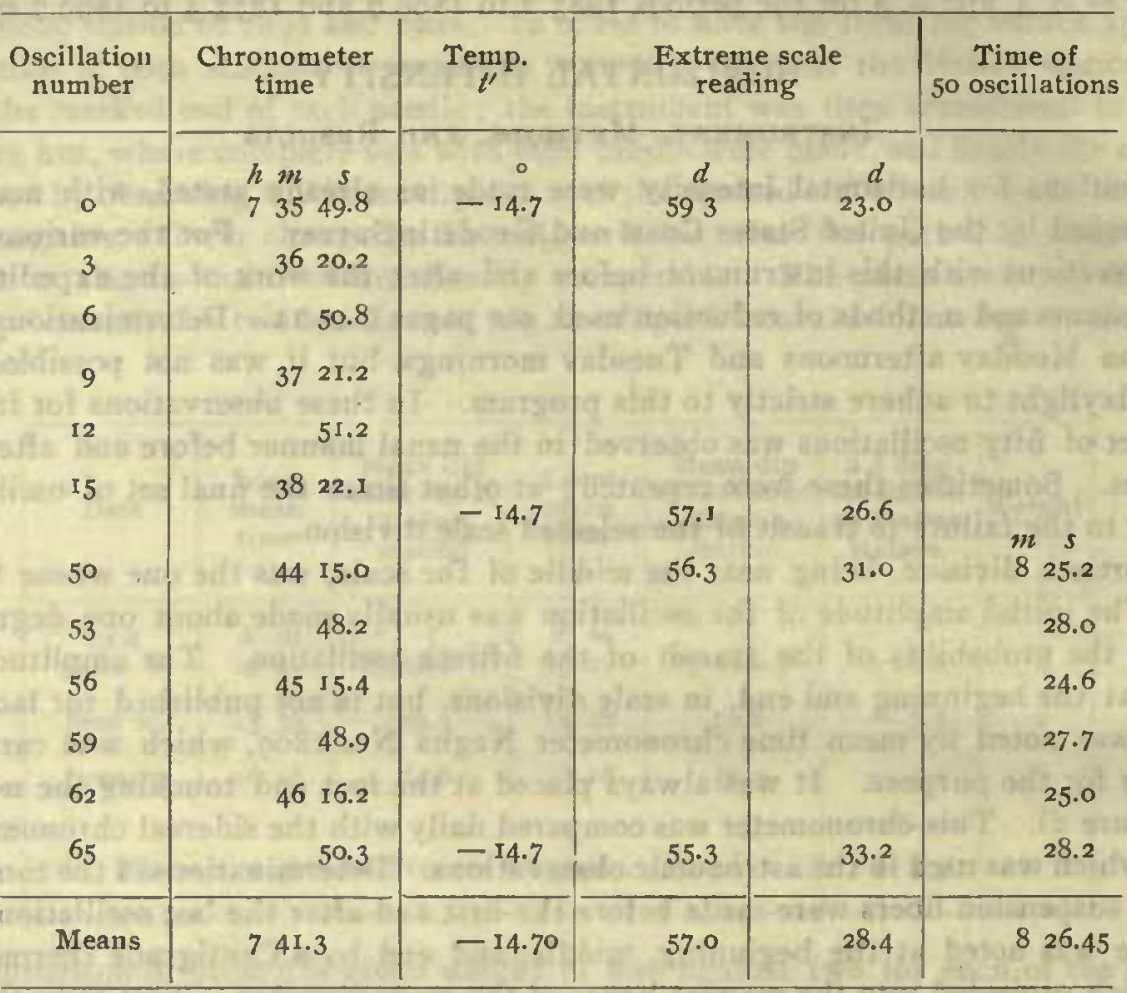

Formulæ: $\mathrm{T}^{2}=\mathrm{T}^{\prime 3}\left(\mathrm{I}+\frac{h}{f}\right)\left(\mathrm{I}-\left(t^{\prime}-t\right) q\right)\left(\mathrm{I}+\mu \frac{\mathrm{H}}{m}\right) ; m(\mathrm{H}+\mathrm{X})^{\dagger}=\frac{\pi^{2} \mathrm{~K}}{\mathrm{~T}^{2}}$

Coefficient of torsion. One div. $=I^{\prime} 57$

\begin{tabular}{|c|c|c|c|c|c|}
\hline $\begin{array}{l}\text { Tors. } \\
\text { circle }\end{array}$ & $\mathrm{Sc}$ & e & Mean. & Diff's & L. M. I. \\
\hline $\begin{array}{l}0 \\
\text { I9 }\end{array}$ & $\begin{array}{c}d \\
40.8\end{array}$ & $\begin{array}{c}d \\
39.8\end{array}$ & $\begin{array}{c}d \\
40.30\end{array}$ & $d$ & $h i n$ \\
\hline Jog & 30.4 & 29.6 & 30.00 & $\begin{array}{r}10.30 \\
10.80\end{array}$ & $\left(t^{\prime}-t\right)=$ \\
\hline 289 & 50.9 & 48.7 & 49.80 & & -0 \\
\hline I9 & 40.6 & 39.2 & 39.90 & & \\
\hline & Mean & $\begin{aligned} & d \\
= & 10.0\end{aligned}$ & $\begin{array}{r}\prime \\
=15.7\end{array}$ & & \\
\hline
\end{tabular}

Remarks :

Chr. to I. M. T. $=+3^{\text {h }} 4^{8 .}{ }^{\mathrm{m}} \mathrm{O}$

Revolver removed to magnet box

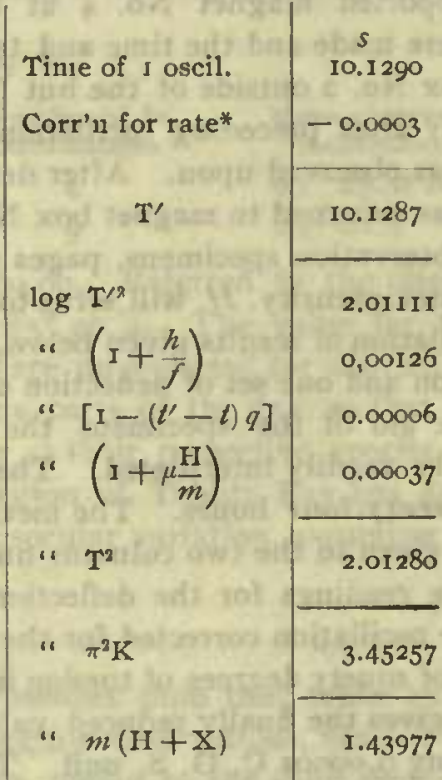

* Plus for losing rate and minus for gaining rate.

† See page 10. 
DHFLECTIONS WITH MAGNETOMETER NO. IIII

Station: Tepiitz Bay

Date: March 19, 1904

Observer: R. R. T.

Magnet No. 4 deflecting at right angles to magnet No. 2 suspended

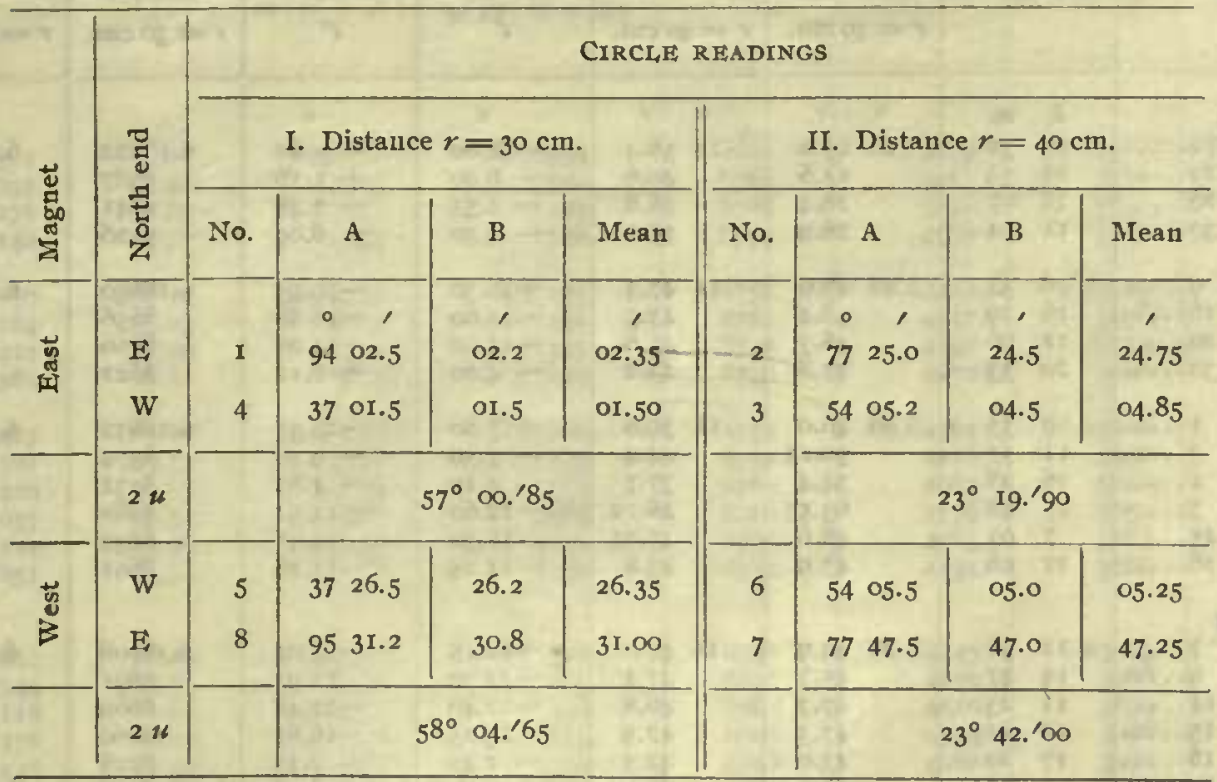

Formule: $\frac{\mathrm{H}}{m}=\left[\frac{2\left(\mathrm{x}+\frac{\mathrm{P}}{r^{3}}+-\right)}{r^{3}\left(\mathrm{r}+\frac{2 \mu}{r^{3}}\right)}\right] \frac{\mathrm{I}}{\operatorname{Sin} u}=\frac{\mathrm{C}}{\operatorname{Sin} u} ; \log \mathrm{H}^{\dagger}=\frac{1}{2}\left(\log \frac{\mathrm{H}}{m}+\log m(\mathrm{H}+\mathrm{X})\right)+255 \gamma$

\begin{tabular}{|c|c|c|}
\hline & I & II \\
\hline & 01 & $\circ \quad$, \\
\hline $2 \|$ (mean) & $57 \quad 32.75$ & 2330.95 \\
\hline$u$ & 2846.4 & II 45.5 \\
\hline
\end{tabular}

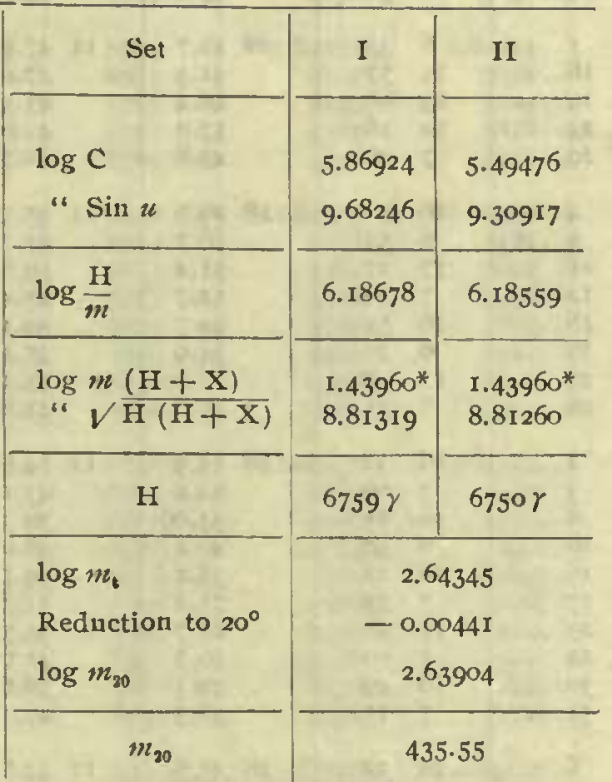

* Mean from four sets oscillations before and after deflections : I.43977, I.43959, 1.43974, and 1.43930 .

† See page ro. 
Tabutar summary of observations of magnetic horizontal intensity at Teptitz Bay

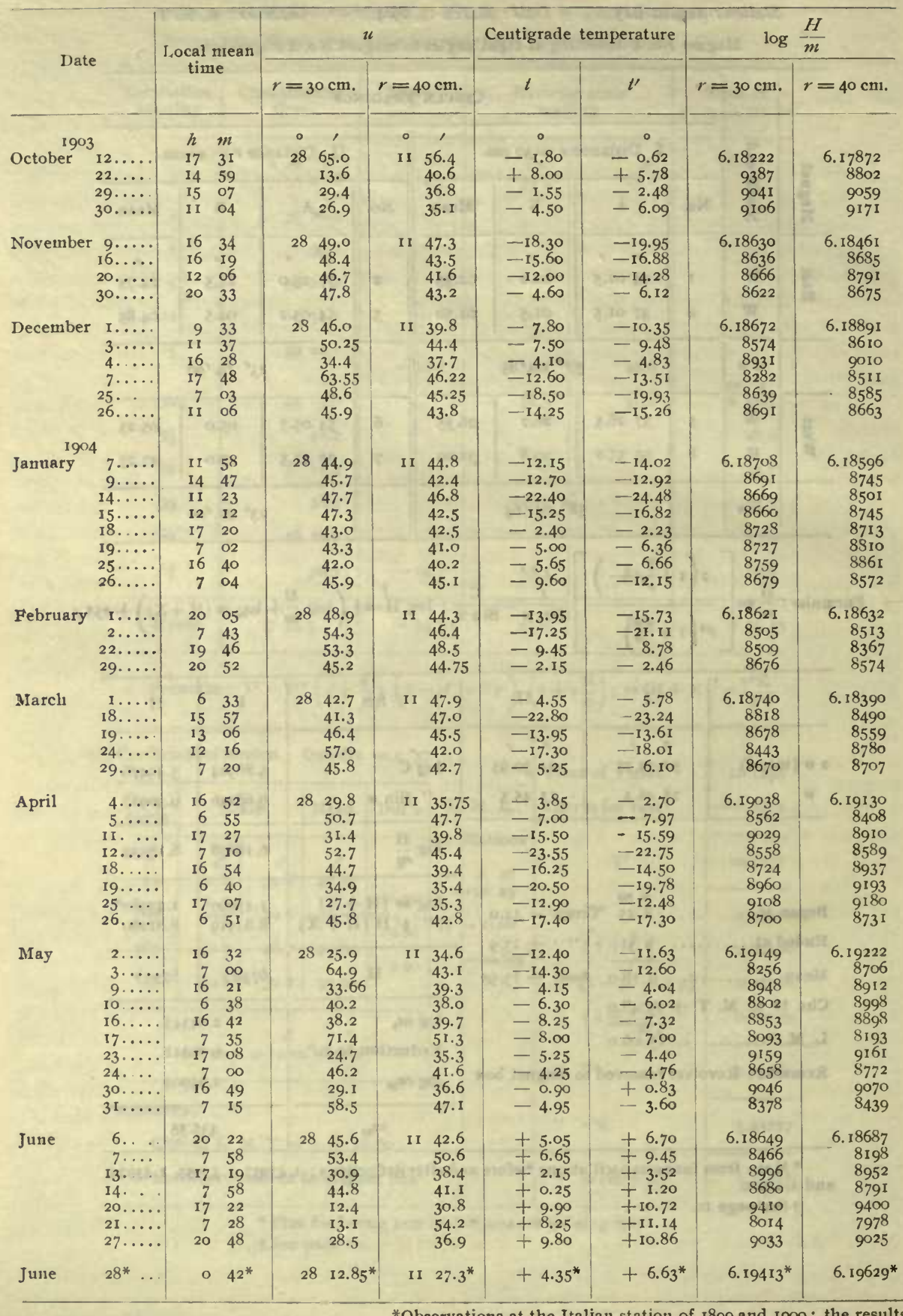


MAGNETIC OBSERVA'TIONS

Tabular summary of observations of magnetic horizontal intensity at Teplitz Bay

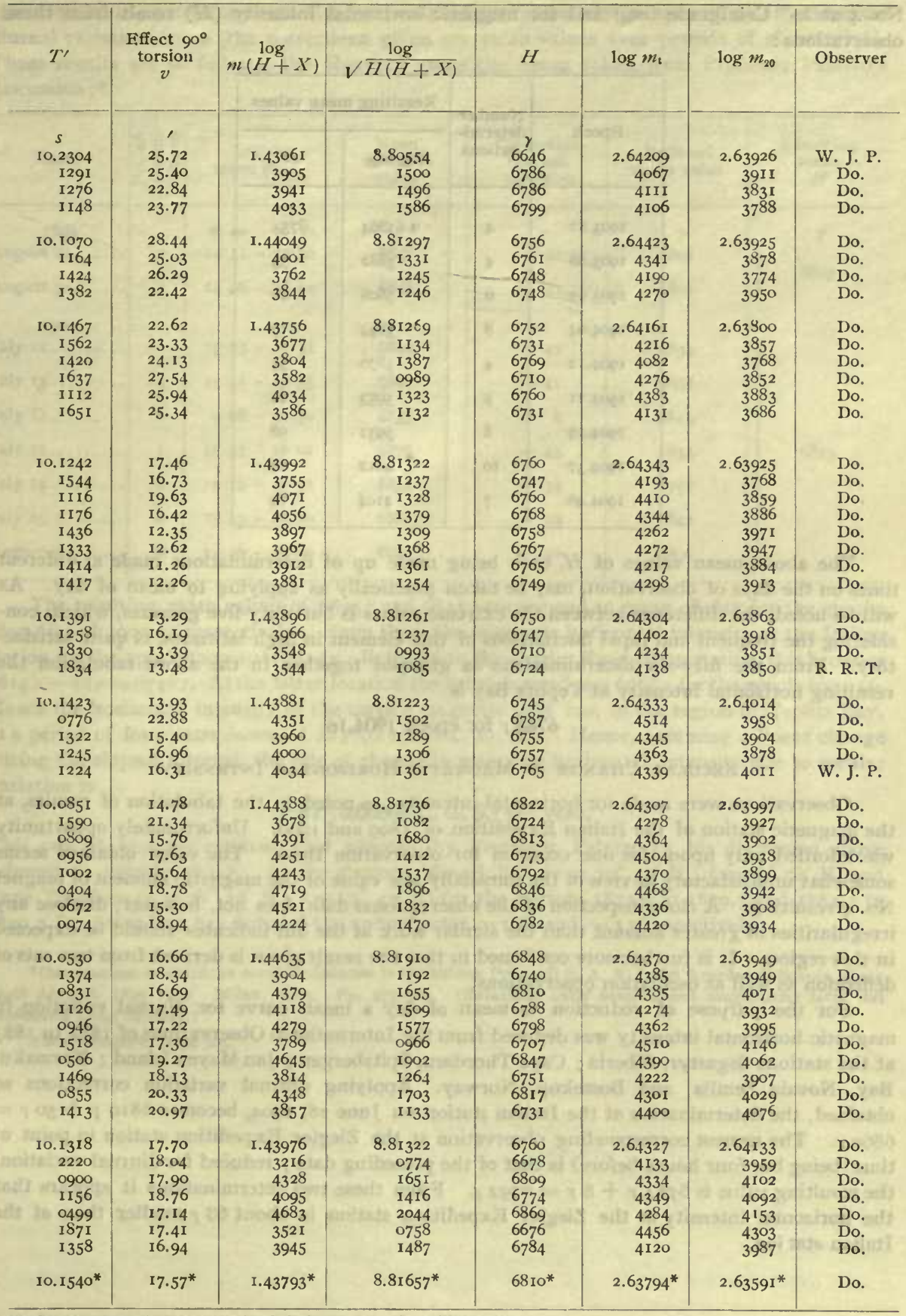

are for two complete sets each of deflections and oscillations. 
The following monthly mean values for the logarithm of the magnetic moment of magnet No. 4 at $20^{\circ}$ Centigrade $\left(m_{20}\right)$ and for magnetic horizontal intensity $(H)$ result from these observations :

\begin{tabular}{|c|c|c|c|}
\hline \multirow{2}{*}{ Epoch } & \multirow{2}{*}{$\begin{array}{c}\text { Number } \\
\text { determi- } \\
\text { nations }\end{array}$} & \multicolumn{2}{|c|}{ Resulting mean values } \\
\hline & & $\log m_{20}$ & $H$ \\
\hline $1903.8 \mathrm{I}$ & 5 & 2.63864 & $\begin{array}{c}\gamma \\
6754\end{array}$ \\
\hline 1903.88 & 4 & $3^{882}$ & 53 \\
\hline I903.95 & 6 & 3808 & 42 \\
\hline 1904.04 & 8 & 3894 & 59 \\
\hline 1904.12 & 4 & 3870 & 33 \\
\hline $1904.2 \mathrm{I}$ & 5 & 3953 & 62 \\
\hline 1904.29 & 8 & 3931 & 98 \\
\hline 1904.37 & 10 & 4012 & 84 \\
\hline 1904.46 & 7 & 4104 & 64 \\
\hline
\end{tabular}

The above mean values of $H$, each being made up of determinations made at different times on the days of observation, may be taken practically as applying to mean of day. As will be noted, the difference between the extreme values is but sixty-five gammas, which, considering the frequent and rapid fluctuations of this element in high latitudes, is quite satisfactory. From the fifty-six determinations as grouped together in the above tabulation the resulting horizontal intensity at Teplitz Bay is

\section{$6768 \gamma$ for epoch 1904.16.}

\section{Secular Change in Magnetic Horizontal Intensity}

Observations were made for horizontal intensity, as noted in the tabulation of results, at the magnetic station of the Italian Expedition of 1899 and 1900. Unfortunately opportunity was afforded only upon this one occasion for observation there. The value obtained seems somewhat unsatisfactory in view of the unusually low value of the magnetic moment of magnet No. 4 resulting. A close inspection of the observational data does not, however, disclose any irregularities of greater amount than the similar work at the hut indicates should be expected in this region. It is furthermore confirmed in that the result given is derived from two sets of deflection as well as oscillation observations.

For the purpose of reduction to mean of day a mean curve for diurnal variation in magnetic horizontal intensity was deduced from the International Observations of 1882 to I883 at the stations Ssgastyr, Siberia ; Cape Thordsen, Spitzbergen ; Jan Mayen Island ; Karmakul Bay, Novaia Zemlia, and Bossekop, Norway. Applying diurnal variation corrections so obtained, the determination at the Italian station on June 28 , 1904, becomes $6810 \gamma+50 \gamma=$ $6860 \mathrm{r}$. The nearest corresponding observation at the Ziegler Expedition station in point of time (being but four hours before) is that of the preceding date ; reduced for diurnal variation, the resulting value is $6784 \gamma+8 \gamma=6792 \gamma$. From these two determinations it appears that the horizontal intensity at the Ziegler Expedition station is about $68 \gamma$ smaller than at the Italian stat ion. 
Inasmuch as Commander Cagni's observations were, with but one exception, made during afternoon hours, his values have also been reduced approximately to mean of day by the same diurnal variation curve (the corrections given are inean values over periods of observation). These results are as follows, the observed quantities being taken from Professor Palazzo's discussion :*

\begin{tabular}{|c|c|c|c|c|c|c|}
\hline Date & 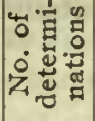 & $\begin{array}{c}\text { Local } \\
\text { mean time }\end{array}$ & $\begin{array}{l}\text { Mean } \\
\text { observed } \\
\text { value }\end{array}$ & $\begin{array}{l}\text { Correction } \\
\text { diurnal } \\
\text { variation }\end{array}$ & $\begin{array}{c}\text { Reduced } \\
\text { mean value }\end{array}$ & $\begin{array}{l}\text { Resulting } \\
\text { mean } \\
H\end{array}$ \\
\hline 1899 & & $h m \quad h m$ & $r$ & $\gamma$ & $\gamma$ & $\gamma$ \\
\hline August $29 . . . .$. & 2 & $1431-1730$ & 6842 & $-4 I$ & 68 or & 60 \\
\hline August $30 . . . .$. & 4 & $1446-1802$ & $684^{9}$ & $-4 \mathrm{I}$ & 6807 & \\
\hline 1900 & & & & & & 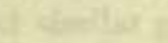 \\
\hline July $x 2 \ldots \ldots \ldots$ & $x$ & $1707-1731$ & 6895 & $-4^{2}$ & 6853 & 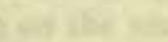 \\
\hline July $13 \ldots .$. & 4 & I5 $41-1835$ & 6892 & $-4 \mathrm{I}$ & $685 I$ & int \\
\hline July $14 . . .$. & 8 & $908-1230$ & 6835 & -6 & 6829 & \\
\hline July $14 \ldots \ldots$ & I & I5 $37-16$ oI & 6876 & -43 & 6833 & 6823 \\
\hline July $24 \ldots \ldots \ldots$ & 8 & 15 10 - 1835 & $683 \mathrm{r}$ & -39 & 6792 & \\
\hline July $25 \ldots . . .$. & 8 & I5 $23-1830$ & $688 \mathrm{I}$ & -39 & $684^{2}$ & \\
\hline August $3 \ldots \ldots$. & 8 & $1440-1809$ & 6849 & -40 & 6809 & \\
\hline
\end{tabular}

The resulting mean values of $6805 \gamma$ and $6823 \gamma$ correspond to mean of day for the epochs 1899.66 and 1900.55 respectively. From these the maguetic horizontal intensity at the Italian station for the epoch 1900. Io is $6814 \gamma$. Referred to the Ziegler Expedition station, this becomes $6814 \gamma-68 \gamma=6746 \gamma$. At the latter location the value derived was $6768 \gamma$ for the epoch 1904.16. Hence the horizontal intensity of the earth's magnetic field has, in the region of Teplitz Bay, in a period of four years increased $22 \gamma(0.00022$ C. G. S.). Hence, assuming a linear change during the elapsed interval, the rate of change in magnetic horizontal intensity due to secular variation is

$$
+6 r(0.00006 \text { C. G. S.) per year. }
$$

This rate of increase confirms that adopted by Professor Palazzo in his discussion above referred to, namely, $+9 \gamma$. At the stations Mossel Bay $\left(\varphi=79^{\circ} 53^{\prime} \mathrm{N} ; \lambda=16^{\circ} \circ 4^{\prime} \mathrm{E}\right)$ and Cape Thordsen $\left(\varphi=78^{\circ} 28^{\prime} \mathrm{N} ; \lambda=15^{\circ} 4^{\prime} \mathrm{E}\right)$ for the periods 1873.5 to 1899.7 and 1883.2 to $1899.7 \mathrm{Dr}$. Solandert has deduced the annual rates $-10 \gamma$ and $-3 \gamma$ respectively.

* Osservazioni scientifiche esequite durante la spedizione Polare di S. A. R. Luigi Amedeo di Savoia, Duca degli Abruzzi, r899-1900. Mitan, 1903. Pp. 475-500. (Relazione sulle osservazioni magnetiche fatta dal Professore Luigi Palazzo.)

† See reference, page 305 . 
Summary Showing Values of the Magnetic El,ements and their Secular VariaTIONS AT TEPLITZ BAY

The following summarizes the mean results obtained by the expedition at the Teplitz Bay station. In the case of the declination the value applies to the mean of both day and year; this may be said to be practically the case likewise for the inclination and intensity.

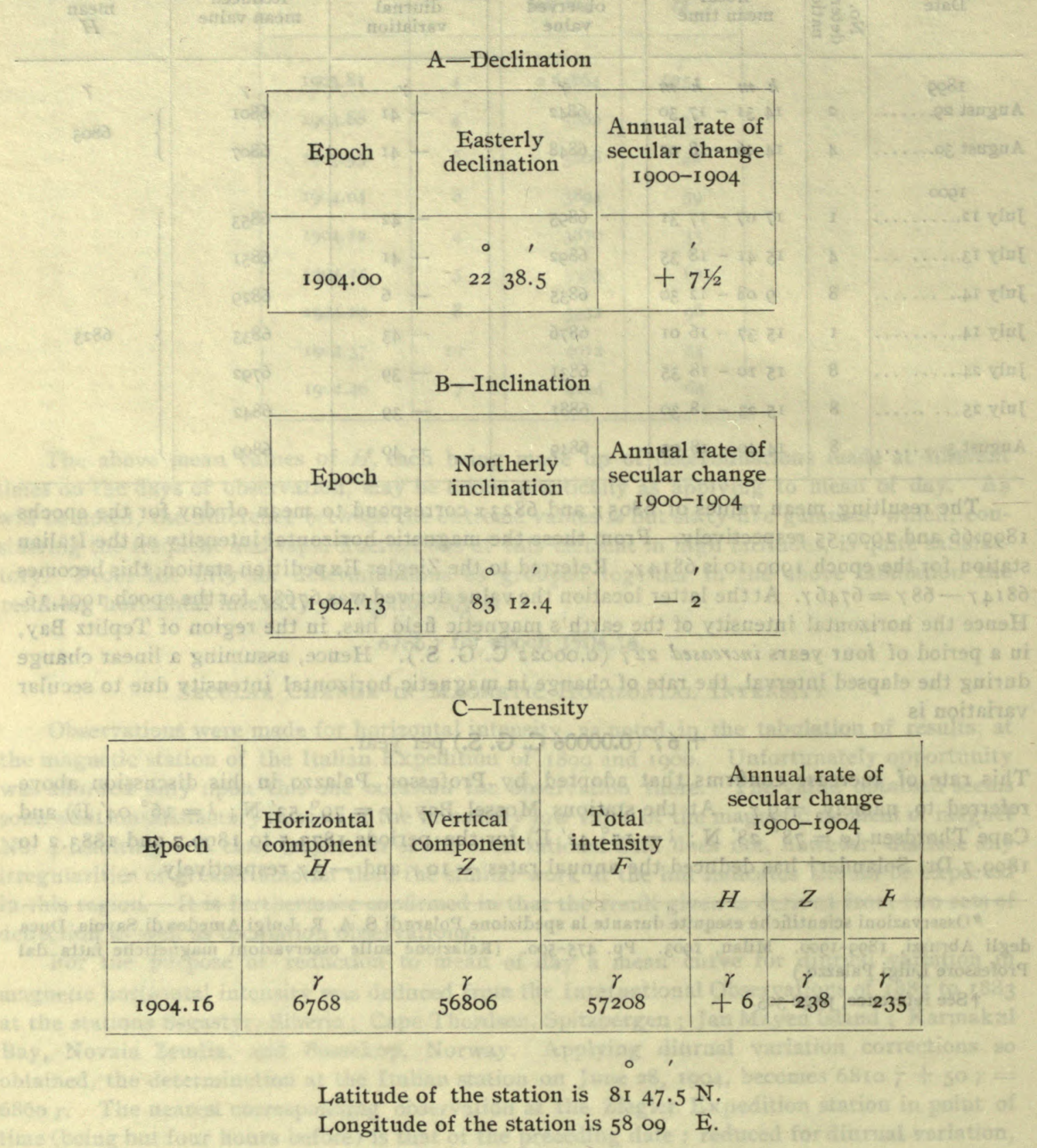




\section{OBSERVATIONS AT ALGER ISLAND}

\section{SITH AND OBSERVATORY}

The stay at Alger Island (Camp Ziegler) being of uncertain duration, dependent upon the arrival of the relief expedition, the observing quarters were of less permanent character than those at Teplitz Bay. The site of the magnetic station is, as shown in figure $2 \mathrm{I}$, some 289.5 meters due north of the astronomic pier. To test for local disturbance observations were made at four points to the magnetic north, east, west, and south, respectively, and each distant about 9r meters from the site proposed. These gave indications of local magnetic attraction, in the maximum, about $30^{\prime}$. Apparently, therefore, the local conditions are more uniform than is the case at the Teplitz Bay site. The construction and dimensions of the observatory are shown in detail by the plan and sections of figure 22. The construction proved very serviceable despite its temporary character. The central pier is of coniferous drift-wood about 20 centimeters in diameter, I.75 meter long, and is sunk some 0.4 meter in frozen gravel, thus leaving a clear height of about I.3 meter. Towards the close of the work a second and similar pier was erected, as shown in the figure, the intention being to mount the dip circle on the same. No use was, however, made of it, the central pier serving for all of the observations. Great care was exercised that no nuagnetic material was used in building the observatory. The pier will undoubtedly remain in good condition for a long time, so that the station may be reoccupied if fiture opportunity offers.

The geographical position of this station is latitude $81^{\circ} 2 I^{\prime} 30^{\prime \prime} \mathrm{N}$ and longitude $3^{\mathrm{b}} 44^{\mathrm{m}} 22^{\mathrm{o}}$ $\left(56^{\circ}\right.$ o5.' 5$) \mathrm{E}$.

\section{DECLINATION}

\section{METHODS}

The methods of observation and record were substantially the same as for the Teplitz Bay series. The regular declination work was begun June 26,1905 , and continued, so far as possible, in accordance with the program of observation outlined on page 17 , until July I, I 905 .

The azimuth mark used was the sonth astronomic meridian mark, distant about 3,9 ro meters. From sun observations with the Repsold circle at the astronomic station on July 16 , $19,20,26$, and 28,1905 , on which days ten determinations were made (see astronomic notes), the azimuth of this mark as referred to the magnetic station pier is $359^{\circ} 59^{\prime}{ }^{\prime} 27$.

So far as this short series of observations goes there is no very decided evidence of pier twist with changes in temperature, as was the case for the Teplitz Bay series.

The observers are indicated by their initials as per list on page 17 .

As for the later observations at Teplitz Bay a suspension of four fibers was used (see page 19).

The following values for axis are used in the final reductions (see page I8):

$\begin{array}{lcc}\begin{array}{c}\text { Week ending at } \\ 8 \text { A. M. Sunday }\end{array} & \begin{array}{c}\text { Number of } \\ \text { determinations }\end{array} & \begin{array}{c}\text { Mean } \\ \text { sxis value } \\ d\end{array} \\ \text { July 2, 1905 } & 9 & 53.54 \\ \text { July 9, I905 } & 7 & 53.62 \\ \text { July I6, I905 } & 8 & 53.10 \\ \text { July 23, 1905 } & 8 & 53.13 \\ \text { July 30, I905 } & 8 & 53.50\end{array}$

\section{RECORDS}

The original notes and results liave been tabulated in the same manner as the Teplitz Bay series (see pages 19 and 20). The readings of azimuth nark will be found on page 322 . The readings corresponding to the position of the telescope appear under the heading, Circle reading of magnet. Where the telescope with circle has been shifted during declination observations this fact has been denoted in the table of resulting declinatious by an asterisk at the time of 
observation first following the change; in these cases the values are omitted in table on page 322 and tabulated separately on page 323 . In the various tabulations the values enclosed in parentheses are interpolated, these being cases where conditions prevented observation of the corresponding quantities.

\section{TABULATIONS OF RECORDS}

Circles readings of azimuth mark, magnet, and true south at Alger Island

\begin{tabular}{|c|c|c|c|c|}
\hline Pointing & Date & Azimuth mark & $\begin{array}{l}\text { Circle reading } \\
\text { of magnet }\end{array}$ & $\begin{array}{l}\text { Circle reading } \\
\text { true south }\end{array}$ \\
\hline B A $\ldots \ldots . .$. & $\begin{array}{c}1905 \\
\text { June } 26\end{array}$ & $\begin{array}{c}0 \\
5938.8\end{array}$ & $\begin{array}{c}\circ \quad, \\
80^{\circ} \text { I } 8.1\end{array}$ & $\begin{array}{c}0, \\
5939 \cdot 5\end{array}$ \\
\hline A & $(100+29$ & 5937.7 & 7955.9 & 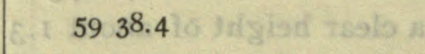 \\
\hline & $\begin{array}{l}1 \text { fHato } \\
28\end{array}$ & 5937.2 & 8027.0 & 5938.0 en batosisy \\
\hline A $\ldots$. & 9. $\quad 29$ & 5936.4 & 79 10.0 & 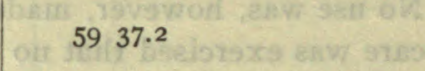 \\
\hline$\ldots \ldots \ldots$ & ifstast 30 & 5935.5 & tot $\ldots \ldots \ldots$ & ais 5936.2 \\
\hline .. & July 2 & 5936.8 & natan & 5937.6 racos stip \\
\hline & 3 & $(5937.0)$ & $80 \times 6.9$ & $(5937 \cdot 7) \quad(2 x)(2 \cdot 80$ \\
\hline & 4 & 5937.2 & $80 \quad 17.6$ & 5937.9 \\
\hline & tivoch & 5937.0 & $\ldots \ldots \ldots$ & 5937.7 \\
\hline & 6 & $5937 \cdot 9$ & n.m. & 5938.6 arti \\
\hline & $\ln 6=7$ & 5936.3 & anc. & 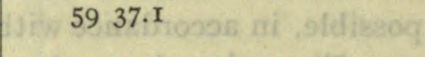 \\
\hline . & 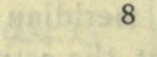 & 5936.5 & ....... & 5937.2 vantixas silfif \\
\hline & & 5935.8 & 8032.9 & 5936.6 \\
\hline & ai $\mathbf{x} \mathbf{I}$ & 5935.2 & 7937.0 & 5935.9 lo dismumisan snat \\
\hline A & 1940412 & 5935.6 & w...... & 5936.4 \\
\hline A... & I4 & 5937.0 & 79 Ir.9 & 5937.7 \\
\hline & 309016 & 5936.2 & w. & 5936.9 arty not 286 \\
\hline A $\ldots$. & $\begin{array}{l}17 \\
0\end{array}$ & 5936.4 & $\cdots \cdots$ & $5937 \cdot 1$ \\
\hline & 18 & 5936.0 & 8000.0 & 5936.7 \\
\hline A... & less & 5936.3 & & 5937.1 \\
\hline & 20 & $5937 \cdot 3$ & 7950.6 & 5938.0 \\
\hline & $2 I$ & $\left(593^{8.1}\right)$ & 7957.0 & (59 $3^{8.8)}$ \\
\hline & 23 & 5938.8 & w..... & $\begin{array}{l}5939.6 \\
\end{array}$ \\
\hline & 24 & 5938.9 & & 5939.6 \\
\hline & 25 & 5938.0 & $\begin{array}{lll}79 & 10.8\end{array}$ & 59.38 .7 \\
\hline & 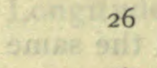 & 5938.8 & A som. & 5939.6 \\
\hline & d Ition 27 & 5938.5 & 7944.6 & 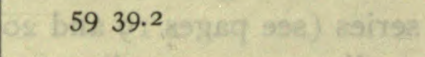 \\
\hline $\begin{array}{l}\ldots \ldots \ldots \ldots \ldots \\
\cdots \cdots \cdots \cdots\end{array}$ & 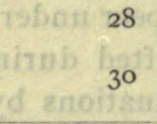 & $\begin{array}{l}5938.8 \\
\left(593^{8.8)}\right.\end{array}$ & $\begin{array}{c}80 \text { o3.8 } \\
\ldots \ldots \ldots \\
\ldots \ldots\end{array}$ & $\begin{array}{l}5939.6 \\
(5939.6)\end{array}$ \\
\hline
\end{tabular}


FIGURE 21

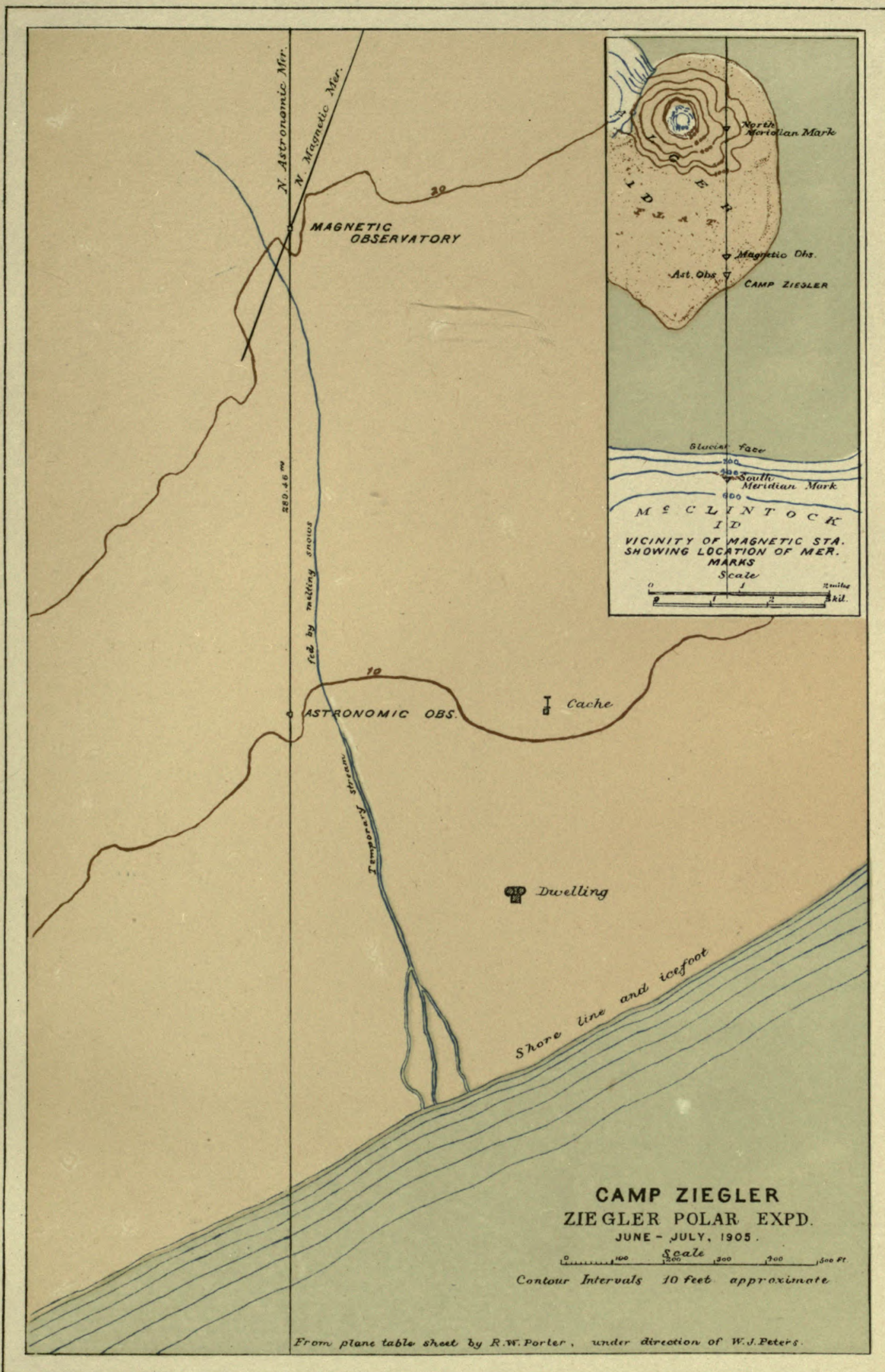


(1) 
Circle readings of magnet for days on which circle was shifted at Alger Island

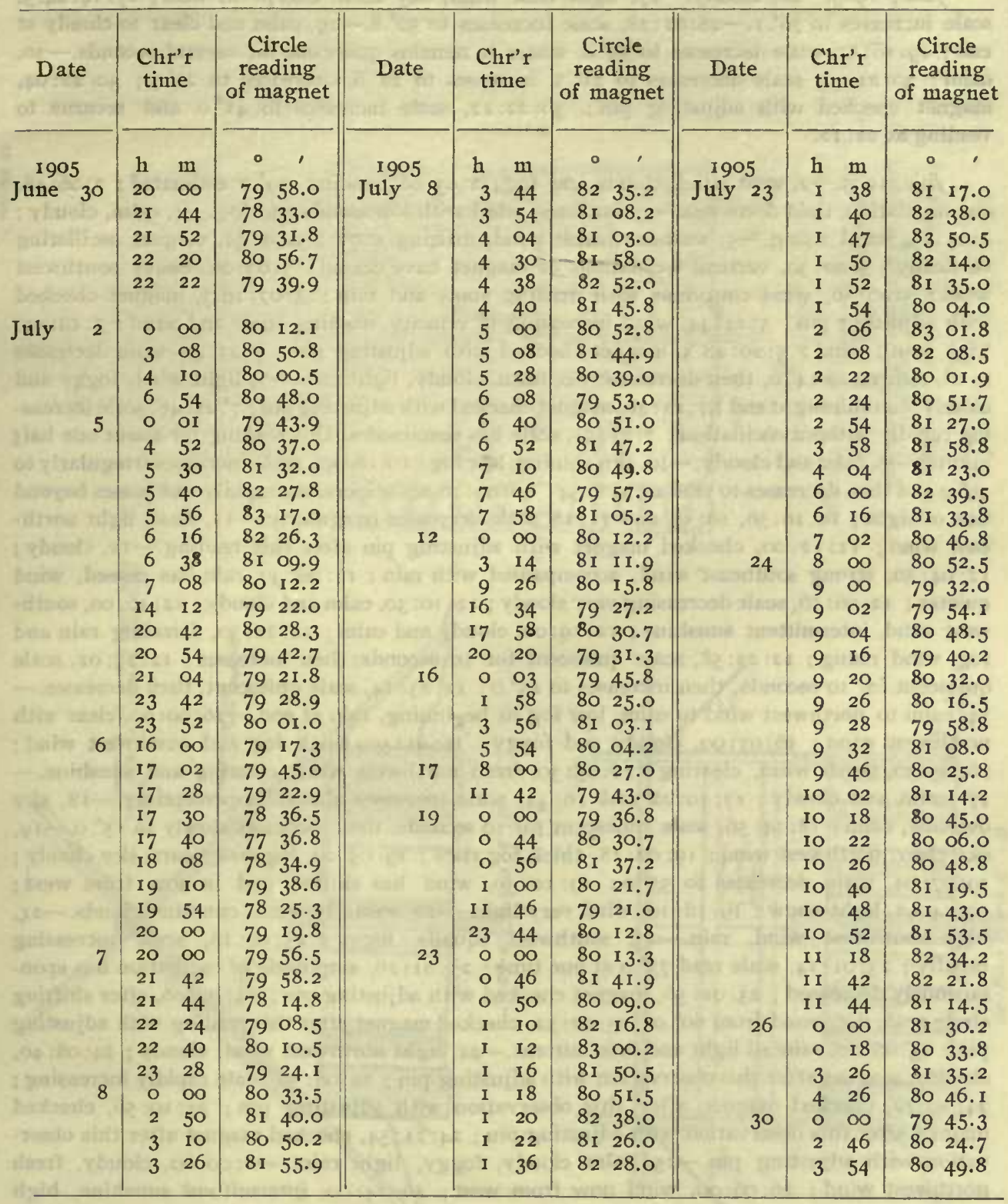




\section{Notes Accompanying Dechination Observations at Alger Island}

June, 1905.-26, cloudy. -27 , light west wind, sky clear except in west; $27: 14: 24.5$, scale increases to $76^{d} .1 .-28: 01: 28$, scale increases to $50^{d} .8 .-29$, calm and clear to cloudy at end; $29: 16: 52$, scale decreases to $35^{\mathrm{d}} .0$, where it remains quiescent for several seconds.-30, calm; 30:21: 12 , scale decreases to $2 \mathrm{I}^{\mathrm{d}} \cdot 3$, increases to $22^{\mathrm{d}} \cdot 6$, decreases to $2 \mathrm{I}^{\mathrm{d}} \cdot 5 ; 30: 22: 04$, magnet checked with adjusting pin; $30: 22: 12$, scale increases to $41^{8} .0$ and returns to reading at $22: 12$.

July, 1905.-2, west wind, clonds and fog; $2: 03: 06$, reading $79^{d} .0$ estimated; $2: 06: 48$, one oscillation, then decreases. -3 , southwest wind with low clouds and fog. -4 , calm, cloudy ; 4:03: 15, wind rising. -5 , west-southwest wind, drifting snow; $5: 01: 54$, magnet oscillating vertically; $5: 02: 30$, vertical oscillations of magnet have ceased; $5: 03: 00$, heavy southivest wind; $5: 06: 56$, wind continues with drifting snow and rain; $5: 07: 10.3$, magnet checked with adjusting pin; 5: 14:44, wind increasing in velocity, drifting snow and sand; 5:19:04, high south wind; $5: 20: 48.3$, magnet checked with adjusting pin; $5: 23: 40$, scale decreases to $o^{d}$, returus to 14.0 , then decreases. -6 , calm, cloudy, light rain. -7, light wind, foggy and cloudy, suu shining at end ; $7: 21: 46$, niagnet checked with adjusting pin ; $7: 21: 48$, scale increasing rapidly without oscillation; $7: 21: 54$, scale has remained at this reading for about one half minute. -8 , calm and clondy. - 10 , snn shining, 10 fog; $10: 08: 5^{2}$, scale increases irregularly to $49^{\circ} .0$ and then decreases to reading at $8: 54$; ro: 09: 16 , scale increases rapidly and passes beyond line of sight ; 10: $10: 56,10: 58$, and Ir: 18 , scale decreases irregularly.-I 1 , clear, light northeast wind; II: $12: 00$, checked magnet with adjusting pin after this reading. -12 , cloudy; I2: $04: 50$, strong southeast wind, accompanied with rain; $12: 06: 31)$, rain has ceased, wind quieter; $12: 06: 56$, scale decreasing very slowly ; $12: 10: 30$, calm and clondy ; $12: 16: 00$, soutl1west wind, intermittent sunshine; 12: 19:00, cloudy and calm; 12:21:30, drizzling rain and fog, wind rising; $12: 22: 5^{8}$, scale quiescent for Io seconds, then increases; I $2: 23: 02$, scale quiescent for Io seconds, then increases to $45^{\mathrm{d}} .0$; $12: 23: 14$, scale quiescent, then decreases.14, calm to northwest wind to calm, low fog in beginning, rain at end._r6:00:00, clear witl 110rthwest wind; 16:01:00, cloudy and foggy; 16:01:50, thick fog and northwest wind; 16: 03: 30, north wind, clearing; 16:05:30, fresh northwest wind, clearing and sunshine.r7, calm and clondy; $17: 10: 22$ and 10: 24 , scale increases almost imperceptibly. - 18 , sky overcast, calm; $18: 14: 56$, scale quiescent for ro seconds, then increases slowly to $15^{\mathrm{d}} \cdot$.o.-19, sky clear, northwest wind; I9: 02: I8, thick fog rises; 19: 05:20, fog disappears, sky cloudy ; 19:07:04, scale decreases to $55^{4} .0 ; 19: 10: 30$, wind has shifted and is now from west; I9: $14: 42$, light snow; 19: $18: 10$, wind very light. -20 , westerly wind, cumnlus clouds. -21 , high southwest wind, rain.-23, southwest squalls, foggy; 23:00:16, scale increasing rapidly; $23: 01$ : 14 , scale read $75^{\mathrm{a}} .0$ at one time; 23 : or: 26 , amplitude of oscillation has spontaneously decreased; $23: 01: 56$, magnet checked with adjusting pin; $33: 02: 06$, after shifting circle scale decreased from $60^{d} .0 ; 23: 02: 32$, checked magnet after this reading with adjusting pin ; 23:06:20, rainfall light and intermittent.—24, light northwest wind, clondy; 24:08:40, checked magnet after this observation with adjusting pin ; $24: 08: 56$, scale rapidly increasing ; 24: 10: 22 , checked magnet after this observation with adjusting pin; $24: 10: 56$, checked magnet after this observation with adjusting pin; $24: 11: 54$, checked magnet after this observatiou with adjusting pin.-25, calm, cloudy, foggy, light rain.-26:00:00, cloudy, fresh northwest wind; 26:06:00, wind 110w from west; 26: 14:00, intermittent sunshine, high cumulo-stratus and cirro-cumulus clouds; $26: 17: 40$, calm, cloudy; 26: 20:50, calm, low, thick fog ; 26:22: 18, fog lifts, sun appears; 26:23: 10, clear sky, a thick fog is slowly coming in from the sea. -27 , calun and cloudy.-28: 20:00, calm, clear; $28: 20: 40$, cloudy; $28: 21: 50$, thick fog; 28: 22:20, scale remains quiescent at the greater reading.-30, thick fog, calm. 


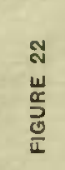
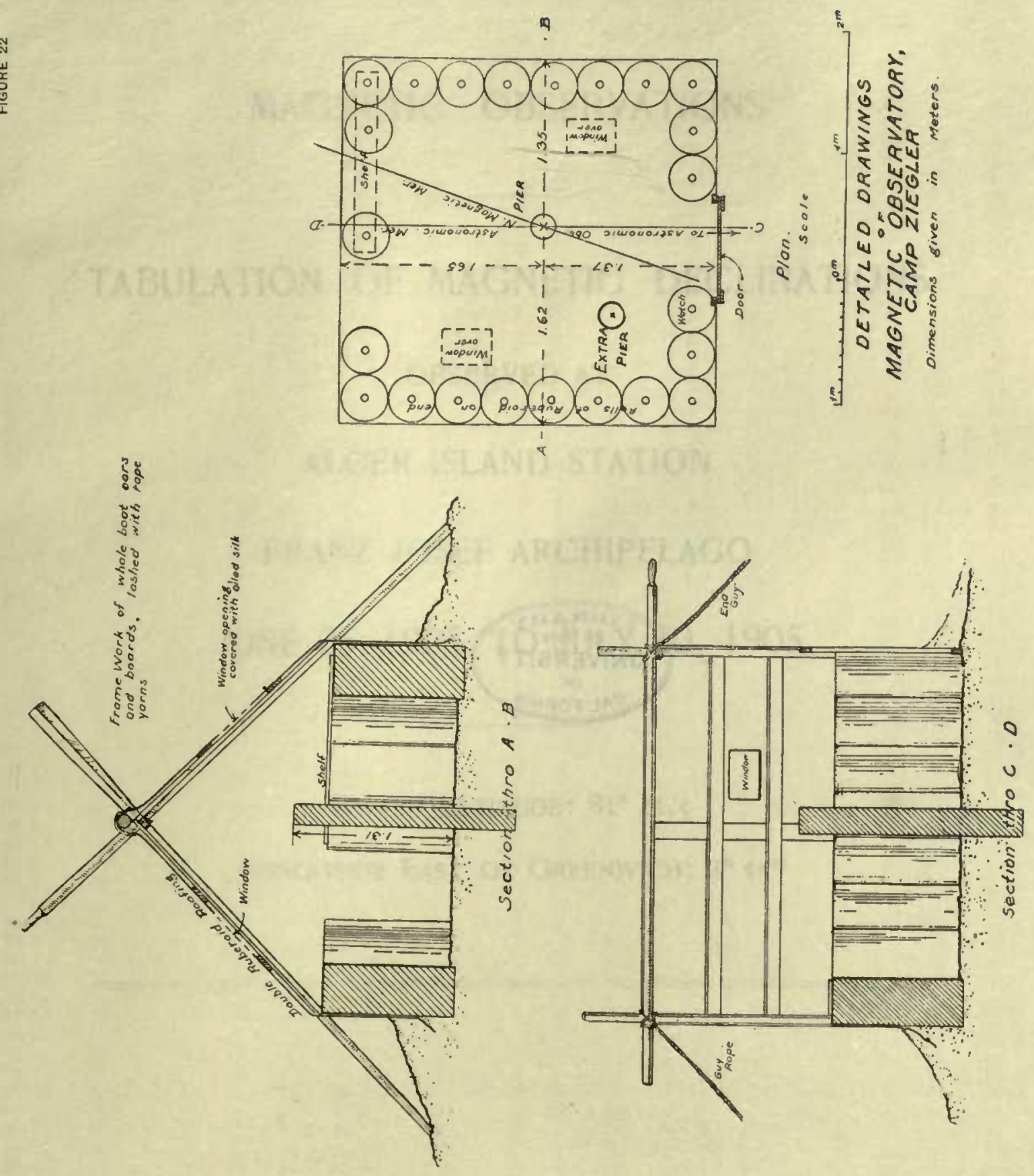



\title{
MAGNETIC OBSERVATIONS
}

TABULATION OF MAGNETIC DECLINATIONS

\author{
OBSERVED AT
}

ALGER ISLAND STATION

FRANZ JOSEF ARCHIPELAGO

JUNE 26, 1905, TO JULY 30, 1905

NORTH LATITUDE: $81^{\circ} 21 .^{\prime} 5$

LONGITUDE EAST OF GREENWICH: $3^{\mathrm{h}} 44^{\mathrm{m}}$ 


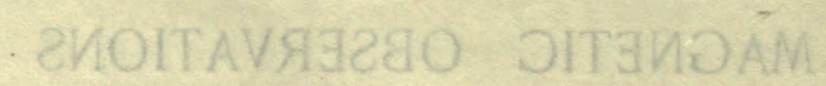

\section{2ИOITAИIJOJO OITHИDAM FO MOITAIUEAT TA Gav93290}

VOITATZ QVIALIZI GJJIA

ODALA9IHOЯA 7320L SИAЯ7

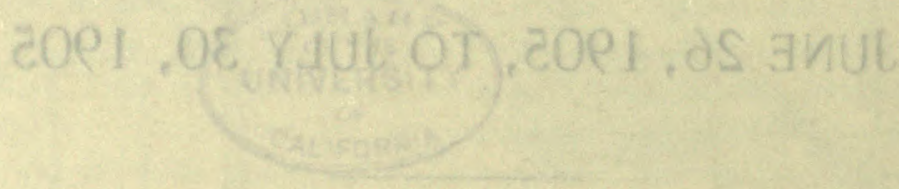

24:5 98 : acuTthad HTson

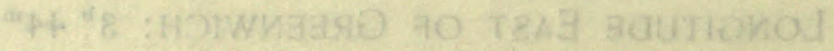

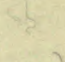


Tabulation of magnctic declinations observed at Alger Island Station

\begin{tabular}{|c|c|c|c|c|c|c|c|c|c|c|c|c|c|c|c|c|c|c|c|c|}
\hline \multicolumn{6}{|c|}{ Monday, June 26,1905} & \multicolumn{4}{|c|}{ Magnet scale erect } & \multicolumn{6}{|c|}{ Tuesday, June 27,1905} & \multicolumn{5}{|c|}{ Magnet scale inverted } \\
\hline \multirow{2}{*}{$\begin{array}{l}\text { Chr'r } \\
\text { time }\end{array}$} & \multicolumn{2}{|c|}{$\begin{array}{l}\text { Scale } \\
\text { readings }\end{array}$} & East & Temp. & Chr'r & $\begin{array}{r}\mathrm{Sc} \\
\text { read }\end{array}$ & $\begin{array}{l}\text { ale } \\
\text { lings }\end{array}$ & East & Temp. & Chr'r & $\begin{array}{r}\mathrm{Sc} \\
\text { read }\end{array}$ & $\begin{array}{l}\text { ale } \\
\text { lings }\end{array}$ & $\begin{array}{l}\text { East } \\
\text { decli- }\end{array}$ & Temp. & & $\begin{array}{r}\mathrm{Sc} \\
\text { read }\end{array}$ & $\begin{array}{l}\text { ale } \\
\text { ings }\end{array}$ & Eas & cli- & Temp \\
\hline & Left & Right & & & & Left & Right & & & & Left & Right & & & & L,eft & Right & & & \\
\hline $\mathrm{h} \mathrm{m}$ & d & d & $\circ$, & 0 & $\mathrm{~h} \mathrm{~m}$ & d & d & . ' & $\bullet$ & $h \mathrm{~m}$ & d & & . & - & $\mathrm{h} \mathrm{m}$ & d & & & & $\bullet$ \\
\hline 800 & & & & in & $10 \infty$ & 47.0 & $47 \cdot 3$ & 2029 & +9.2 & 1200.5 & 59.2 & 58.8 & 2009 & +15.5 & 1400 & 59.3 & 58.9 & & $\infty 9$ & +12.0 \\
\hline 02 & & & & TI & 02 & 47.8 & 48.3 & 30 & & 02 & 58.8 & 58. I & & & 02 & 59.6 & 59.0 & & 08 & \\
\hline 04 & & & 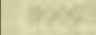 & 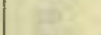 & 04 & 48.7 & 49.1 & $3 \mathrm{I}$ & 45 & 04 & 58.2 & 57.7 & II & & 04 & 61.4 & 61.0 & & 05 & \\
\hline 06 & & se & c net2 & 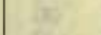 & 06 & 49.0 & 49.4 & 32 & tet & $\infty 6$ & 58.1 & 57.8 & II & & 06 & 61.0 & 60.8 & & $\infty 6$ & te \\
\hline 08 & Ex & Dite & $2=$ & 8 & 08 & 49.3 & 49.7 & 32 & 30 & 08 & 58.9 & 58.2 & I0 & 10 & 08 & 62.I & 61.8 & & 04 & 80 \\
\hline 10 & & & & ent & Io & 49.6 & 50.0 & 33 & 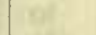 & 10 & 59.1 & 58.8 & 09 & 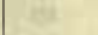 & Io & 62.8 & 62.3 & & 03 & wat \\
\hline I2 & & & 2211 & 47 & I2 & 49.2 & 49.6 & 32 & & 12 & 58.0 & 58.5 & 09 & at & 12 & 64.2 & $64 . I$ & 20 & OI & \\
\hline I4 & & & 5.85 & 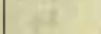 & I4 & 49.2 & 49.6 & 32 & +9.5 & 14 & 58.0 & 57.6 & II & +15.6 & 14 & 67.5 & 66.9 & & 56 & $+I I .4$ \\
\hline I6́ & & 8 & 8.75 & in & 16 & 48.8 & 49.2 & 32 & & 16 & 57.3 & 57.1 & I2 & & 16 & 69.1 & 68.8 & & 53 & \\
\hline I8 & & & 1118 & Ift: & 18 & 48.2 & 48.6 & $3 \mathrm{I}$ & ats. & 18 & 58.7 & 58.7 & $\infty$ & & 18 & 68.1 & 68. I & & 54 & $8 x$ \\
\hline 20 & & 35 & 12.75 & 85 & 20 & 47.0 & $47 \cdot 3$ & 29 & 10 & 20 & 59.1 & 59.1 & 09 & $2 \pi$ & 20 & 70 & $.0 a$ & & 52 & 10 \\
\hline 22 & & 84 & 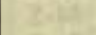 & $c \varepsilon$ & 22 & 47.3 & 47.9 & 29 & $1 \pm$ & 22 & 59. I & 58.9 & 09 & as & 22 & 74 & I $1 a$ & & 45 & Etit \\
\hline 24 & th & 20 & 7.5 & 14 & 24 & 47.9 & 48.2 & 30 & $x^{2}$ & 24 & 50.4 & 59.2 & 08 & Hit. & 24 & 76 & $.0 a$ & & 42 & \\
\hline 26 & Int & 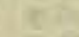 & $11 \times 2$ & Ix & 26 & 48.3 & 48.8 & $3 \mathrm{I}$ & \pm & 26 & 58.3 & 57.9 & 10 & is & 26 & $75 \cdot 3$ & 75.1 & & 43 & \\
\hline 28 & & & & प्रो & 28 & 47.4 & 47.8 & 29 & & 28 & 57.9 & 57.7 & II & the & 28 & $75 . \mathrm{I}$ & 74.8 & & 44 & \\
\hline 30 & 52.5 & 55.6 & 2039 & +7.7 & 30 & 48.0 & 48.6 & 30 & +9.7 & 30 & 57.4 & 57.1 & 12 & +15.6 & 30 & 74.9 & 74.7 & & 44 & fII. \\
\hline 32 & 52.3 & 55.0 & 39 & . & 32 & 48.0 & 48.0 & 30 & & 32 & 57.6 & 57.4 & II I & & 32 & 76.0 & 75 & & 42 & \\
\hline 34 & 52.7 & $55 \cdot 5$ & 40 & 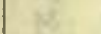 & 34 & 48.2 & 48.4 & 30 & "Et & 34 & 56.8 & 56.2 & 13 & & 34 & 69.8 & 69.2 & I9 & 52 & 47 \\
\hline 36 & 52.0 & $54 \cdot 3$ & 38 & Af & 36 & 48.4 & 48.7 & $3 \mathrm{I}$ & $3 x$ & 36 & 54.0 & 54.5 & 16 & & 36 & 65.2 & 64.9 & 20 & $\infty$ & W \\
\hline 38 & 53.6 & 55.6 & 40 & We & 38 & 48.3 & 48.6 & $3 \mathrm{I}$ & 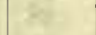 & 38 & 5.5 .8 & 55.0 & 15 & 10 & 38 & 63.2 & 62.8 & & 03 & 2 \\
\hline 40 & 52.8 & 55.6 & 40 & es & 40 & 47.8 & 48.0 & 30 & $a$ & 40 & 56.9 & 56.7 & 12 & 10 & 40 & 6I.I & 60.7 & & 06 & + \\
\hline 42 & 53.2 & 55.1 & 40 & . & 42 & 47.8 & 48.0 & 30 & 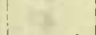 & 42 & 57.2 & 56.9 & 12 & ate & 42 & 59.9 & 59.2 & & 08 & \\
\hline 44 & 53.6 & 56.0 & $4 \mathrm{~T}$ & +8.0 & 44 & 47.7 & 48. I & 30 & +9.6 & 44 & 58.1 & 58.0 & Ie & +15.4 & 44 & 62.9 & 62.1 & & 03 & +10.3 \\
\hline 46 & 55.0 & 56.7 & 42 & 10 & 46 & 48.3 & 48.7 & $3 \mathrm{I}$ & & 46 & 58.8 & 58.2 & 10 & & 46 & 62.9 & 62.8 & 20 & 03 & \\
\hline 48 & 57.6 & 59.8 & 47 & W. & 48 & 48.0 & 48.1 & 30 & 7 & 48 & 58.9 & 58.8 & 09 & 6 & 48 & 67.9 & 67. & to & 56 & 37 \\
\hline 50 & 55.5 & 57.3 & 43 & $a$ & 50 & 46.6 & 46.8 & 28 & kOt & 50 & 60.3 & 50.8 & o7 & $n E$ & 50 & 60.1 & 68.8 & & 53 & 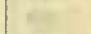 \\
\hline 52 & 55.2 & 57.2 & 43 & (17) & 52 & 46.0 & 46.3 & 27 & 20 & 52 & 59.9 & 58.5 & 09 & 15 & 52 & 66.4 & 66.2 & 19 & 57 & ate \\
\hline 54 & 56.5 & 58.0 & 44 & 32 & 54 & 45.6 & 45.9 & 26 & W & 54 & 59.7 & 58.1 & 09 & 42 & 54 & 63.6 & 63.3 & 20 & 02 & 34 \\
\hline 56 & 54.6 & 56.3 & 42 & I3 & 56 & 44.0 & 44.3 & 24 & & 56 & 59.1 & 58.1 & IO & & 56 & 60.7 & 60.1 & & 07 & 3 \\
\hline 58 & 54.3 & 56.2 & $4 \mathrm{I}$ & & 58 & $44 \cdot 3$ & 44.6 & 24 & & 58 & $6 \mathrm{r} .2$ & 60.4 & 06 & +15.2 & 58 & 59. I & 58.9 & & 09 & \\
\hline 900 & 56.8 & 58.0 & 45 & +8.3 & II 00 & 44.0 & 44.6 & 24 & +9.5 & 1300 & 62.8 & $62 . I$ & 04 & 80 & 1500 & 59.2 & 59.0 & & 09 & + 10.1 \\
\hline 02 & 51.8 & 54.3 & 38 & 16 & $\alpha 2$ & 43.9 & 44.5 & 24 & & 02 & 63.9 & 62.8 & 02 & 40 & 02 & 59.3 & 59.0 & & $\infty$ & \\
\hline 04 & 51.6 & 53.0 & 3 & 18 & 04 & 44.4 & 45.1 & 25 & $=0$ & 04 & 65.1 & 64.4 & 0 & 48 & 04 & 59.9 & 59. & & 08 & 39 \\
\hline 6 & $5.3 \cdot 3$ & 55.2 & 40 & 18 & os & 45.3 & 46.0 & 26 & (2) & 06 & 63.5 & 62.8 & 02 & Wor & 06 & 59.9 & 59.2 & & 08 & $(60$ \\
\hline 08 & 50.7 & 51.6 & 3 & the & os & 46.5 & 47.2 & 28 & he & os & 63.2 & 62.1 & 0 & 80 & 08 & 60.4 & 60 & & 07 & ne \\
\hline IO & 49.6 & 50.5 & 33 & anc & I0 & 47.8 & 48.5 & 30 & 애 & IO & 64.2 & 63.2 & OI & art & IO & 60.1 & 59.9 & te & 07 & $\mathrm{Cr}$ \\
\hline 12 & 51.0 & 51.6 & 3 & & I2 & 46.7 & 48. I & 29 & 27 & I2 & 65.2 & 64.8 & $\infty$ & $=1$ & 12 & 50.8 & 59.3 & & 08 & 제가 \\
\hline I4 & 50.0 & 51.6 & 34 & +8.6 & 14 & 43.8 & 44.3 & 24 & +9.3 & I4 & 63.7 & 63.3 & $\infty$ & +14.9 & 14 & 50.5 & 50.1 & & 09 & +10.0 \\
\hline т6 & 46.0 & 46.4 & 2 & & I6 & 41.6 & 42.6 & $2 \mathrm{I}$ & & 16 & 50.6 & 50.2 & 08 & & 76 & 58.9 & 58. & & $\infty$ & \\
\hline I8 & 51.0 & 51.2 & 35 & 4 & 18 & 42.3 & 43.2 & 22 & wit & I8 & 62.0 & $6 \mathrm{r} .4$ & 05 & 10 & I8 & 59.2 & 58.0 & & on & W. \\
\hline 20 & 53.8 & 54.8 & 4 & $\approx 6$ & 20 & 43.6 & 44.7 & 24 & $\cos$ & 20 & 65.1 & 65.0 & $\infty$ & ${ }^{2}$ & 20 & 62.4 & 61.7 & & 04 & 06 \\
\hline 22 & 53.2 & $54 \cdot 3$ & 3 & the & 2 & 43.3 & 44.6 & 24 & thet & 22 & 65.0 & 64.8 & 0 & 34 & 22 & 63.0 & $6_{3}$ & & OI & 28 \\
\hline 24 & 50.I & 50.5 & 73 & 18 & 24 & & 44.2 & 23 & $t^{2}$ & 24 & 64. & 64.0 & OI & $y=$ & & 64.8 & 64 & 20 & & YA \\
\hline 26 & 49.3 & 49.7 & 3 & $t=$ & 26 & 41.6 & 43. & $2 \mathrm{I}$ & $2 \pi$ & 26 & 63.6 & 63.0 & 2002 & 8 & 26 & 65.9 & 65.8 & 19 & 58 & in \\
\hline 28 & 49.3 & 50.0 & 3 & $y=$ & 28 & $4 \mathrm{I} .3$ & 42.6 & 20 & int & 28 & 66.7 & 65.9 & I0 57 & Tr & 28 & 66.9 & 66.7 & & 57 & $1=$ \\
\hline 30 & 49.0 & 50.4 & 3 & +0.0 & 30 & 41.8 & 43.2 & 2 I & +0.2 & 30 & 62.1 & 61.7 & 2004 & +13. & 30 & 68.0 & 67. & & 55 & tro \\
\hline 32 & 50.2 & 51.0 & 3 & & 32 & 43.3 & 44.6 & 24 & 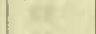 & 32 & 58.2 & 58.1 & To & & 32 & 68.9 & 68.6 & & 54 & \\
\hline 34 & 49.4 & 50.0 & 3 & is & 34 & $43 \cdot 3$ & 44.6 & 24 & 84 & 34 & 54.9 & 54.0 & I6 & 31 & 34 & 70.9 & 70.1 & & $5 \mathrm{I}$ & at \\
\hline 36 & 49.2 & 49.5 & 3 & the & 36 & $4 \pi .2$ & 42.3 & 20 & $2 x$ & 36 & 56.6 & 55.8 & I & be & 36 & 74.0 & 72.8 & & 47 & $\mathrm{~B}^{2}$ \\
\hline 38 & 49.4 & 49.5 & 3 & 26 & 38 & 39.2 & 40.3 & 17 & kt & 38 & 58.0 & 57.9 & & fr & 38 & 73.2 & 72.0 & & 47 & Et \\
\hline 40 & 49.0 & 49.2 & 32 & 64 & 40 & 37.6 & 39.2 & 15 & 208 & 40 & $6 \mathrm{r} .2$ & 6r.o & क & $\sqrt{6}$ & 40 & 73.2 & 72.9 & & 47 & $\mathrm{C}$ \\
\hline 42 & 50.0 & 50.3 & 33 & $\sqrt{4}=$ & 42 & 38.0 & 39.4 & I5 & & 42 & 60.3 & 50.9 & or & 6. & 42 & 74.0 & 73.6 & & 46 & . \\
\hline 44 & 49.6 & 50.0 & 33 & +9.0 & 44 & 35.6 & 36.6 & II & +9.1 & 44 & 58.2 & 58.2 & 10 & & 44 & $7 \mathrm{r} .1$ & 70.8 & & 50 & +10.2 \\
\hline 46 & 49.4 & 50.0 & 33 & & 46 & $3 \mathrm{I} .8$ & 33.0 & 05 & & 46 & 56.0 & 55.9 & I4 & +12.7 & 46 & 70.5 & 70.1 & & $5 \mathrm{~T}$ & \\
\hline 48 & & 50.2 & 33 & in & 48 & 34.5 & 35.3 & 09 & 76 & 48 & 56.4 & 56.2 & I3 & & 48 & 69.0 & 67.9 & I9: & 54 & 16 \\
\hline 50 & 48.8 & 49.4 & 32 & as & 50 & 36.4 & 37.4 & 12 & 여 & 50 & 54.5 & $54 . I$ & 16 & 68 & 50 & 63.7 & 63.0 & 20 & & 2 \\
\hline 52 & 48.0 & 48.3 & $3 I$ & 20 & 52 & 36.6 & 37.6 & I3 & 4 & 52 & 57.1 & 56.5 & I2 & $x^{2}$ & 52 & $6 \mathrm{I} .4$ & 60.8 & & 06 & 2 \\
\hline 54 & 48.3 & 49.2 & $3 I$ & 4 & 54 & 35.2 & 36.3 & II & 14: & 54 & 57.9 & 57.7 & II & $\mathrm{w}$ & 54 & $6 \mathrm{r} .2$ & 60.6 & & 06 & 28 \\
\hline 56 & 48.7 & 49.3 & 32 & 68 & 56 & 34.7 & 36.0 & I0 & & 56 & 55.2 & 55.0 & I5 & 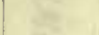 & 56 & $6 \mathrm{I} .9$ & 6r.o & & 05 & 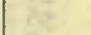 \\
\hline 58 & 46.8 & 47.6 & 20 & 82 & 58 & 35.6 & 36.9 & II & +9.1 & 58 & 59.2 & $59 . \mathrm{I}$ & $\infty$ & the & 58 & 61.8 & 61.2 & $\mathrm{r}$ & 05 & 2 \\
\hline & & & & 00.4 & 1200 & $35 \cdot 3$ & 36.5 & II & & & & & & 801 & 1600 & $6 \mathrm{r} .0$ & 59.0 & & 07 & +10.5 \\
\hline
\end{tabular}

Correction to local mean time is $-21 \mathrm{~s}$.

Torsion head at $8 \mathrm{~h} 30 \mathrm{~m}$ read $246^{\circ}$ and at $13 \mathrm{~h}$ 5om read the same. Obscrver-W. J. P.
Correction to local mean time is - 30 .

Torsion head at $\mathrm{i} 3 \mathrm{~h}$ oom read $258^{\circ}$ and at $16 \mathrm{~h}$ zom rcad the same. Observer-J. V. 
Tabulation of magnetic declinations observed at Alger Island Station-Continued

\begin{tabular}{|c|c|c|c|c|c|c|c|c|c|c|c|c|c|c|c|c|c|c|c|c|}
\hline \multicolumn{6}{|c|}{ Wednesday, June 28, 1905} & \multicolumn{5}{|c|}{ Magnet scale erect } & \multicolumn{6}{|c|}{ Thursday, June 29, 1905} & \multicolumn{4}{|c|}{ Magnet scale inverted } \\
\hline \multirow{2}{*}{$\begin{array}{l}\text { Chr'r } \\
\text { time }\end{array}$} & \multicolumn{2}{|c|}{$\begin{array}{l}\text { Scale } \\
\text { readings }\end{array}$} & $\begin{array}{c}\text { East } \\
\text { decli- } \\
\text { nation }\end{array}$ & $\begin{array}{c}\text { Temp. } \\
\text { C. }\end{array}$ & $\begin{array}{l}\text { Chr'r } \\
\text { time }\end{array}$ & $\begin{array}{r}\mathrm{Sc} \\
\text { read }\end{array}$ & $\begin{array}{l}\text { ale } \\
\text { ings }\end{array}$ & $\begin{array}{r}\text { Ea } \\
\text { dec } \\
\text { nati }\end{array}$ & $\begin{array}{l}\text { ast } \\
\text { cli- } \\
\text { ion }\end{array}$ & & & $\begin{array}{r}\text { S } \\
\text { rea }\end{array}$ & $\begin{array}{l}\text { e } \\
\text { hgs }\end{array}$ & $\begin{array}{l}\text { East } \\
\text { decli- } \\
\text { nation }\end{array}$ & & $\begin{array}{l}\text { Chr'r } \\
\text { time }\end{array}$ & $\begin{array}{l}\text { Sc } \\
\text { reac }\end{array}$ & le & $\begin{array}{c}\text { East } \\
\text { decli- } \\
\text { nation }\end{array}$ & Temp \\
\hline & Left & Right & & & & Left & Right & & & & & Left & Right & & & & Left & Right & & \\
\hline $\mathrm{h} \mathrm{m}$ & $d$ & d & $\cdot$ ' & $0^{\circ}$ & $\mathrm{h} \mathbf{m}$ & d & d & & & 0 & $\mathrm{~h} \mathrm{~m}$ & 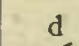 & $\mathrm{d}$ & $\circ \quad$ & $4 \pi$ & $\mathrm{h} \mathrm{m}$ & a & $\mathrm{d}$ & & • \\
\hline 000 & $\cos$ & ost & 10 & $\infty \mathrm{s}$ & 200.5 & 51.0 & 52.9 & & 46 & +5.5 & 1600 & 43.6 & $41 \cdot 3$ & I9 50 & & 1800 & 19.5 & 19.0 & 2029 & +13.0 \\
\hline 02 & $L$ & ost & 0.0 & & 02 & 55.2 & 56.6 & & 53 & 100 & 02 & & 42.7 & & +15.0 & 02 & 19.5 & & 30 & \\
\hline 04 & 37.5 & 37.8 & 2024. & +6.0 & 04 & 55.3 & 56.3 & & 53 & 15 & 04 & 47.5 & 45.7 & & & 04 & 20.0 & 18.8 & 29 & \\
\hline 06 & & 36.4 & 22 & 80 & of & $5 \mathrm{I} \cdot 3$ & 53.0 & & 47 & (89 & 06 & 48.5 & 47.1 & 42 & 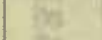 & 0 & 22.0 & 20.6 & 26 & \\
\hline 08 & 39.8 & 40.2 & 28 & 68 & 08 & 48.2 & 50.2 & & 42 & 87 & 08 & 49.3 & 47.7 & 40 & ה4 & 08 & 22.3 & & 25 & \\
\hline I0 & 39.0 & 39.5 & 26 & ait & 10 & 49.3 & 51.5 & & 44 & 04 & Io & 49.0 & 47.7 & $4 \mathrm{I}$ & at & Io & 21.3 & 19 & 27 & \\
\hline 12 & 38.3 & 39.4 & 26 & & 12 & 58.0 & 60.0 & & 58 & 1 & 12 & 50.7 & 48.7 & 39 & $-15=$ & 12 & 21.3 & & 27 & \\
\hline 14 & 36.3 & 37.0 & 22 & +6.0 & 14 & 52.8 & 53.4 & & 48 & +5.2 & 14 & 49.8 & 48.5 & 40 & +15.2 & 14 & 19.7 & I8 & 29 & +13.0 \\
\hline I6 & 31.8 & .3 .2 & 16 & 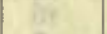 & I6 & 49.0 & 49.5 & & 42 & & & 52.3 & 51.0 & 36 & & 16 & 18.5 & 17 & $3 \mathrm{I}$ & 1 - \\
\hline I8 & 3 I. 2 & 32.0 & 15 & $7 t$ & I8 & 49.5 & 50.5 & & 44 & 8it & I8 & 54.3 & 53.3 & 33 & 37 & If & 21.3 & & 26 & \\
\hline 20 & 31.2 & 33.2 & 16 & 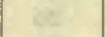 & 20 & 46.5 & 47.6 & & 39 & etr & 20 & 55.0 & 54.1 & 32 & it & 20 & 21.8 & 21 & 26 & \\
\hline 22 & 30.8 & 33.0 & 15 & at & 22 & 46.0 & 46.2 & & 37 & $E$ & 2 & $55 \cdot 3$ & 54.3 & 31 & $1=$ & 22 & 4.3 & & 22 & \\
\hline 24 & 31.1 & 33.1 & 15 & it & 24 & 46.6 & 47.8 & & 39 & 15 & 2 & & 54.7 & 31 & itit & 24 & 4.7 & & 21 & \\
\hline 26 & 32.0 & 33.8 & 17 & 18 & 26 & I & ost & & & & 26 & & 56.0 & 29 & 0 & 26 & 26.8 & & 18 & \\
\hline 28 & 33.3 & 35.7 & 19 & & 28 & 46.5 & 47.8 & & 39 & & 28 & 56.0 & $55 \cdot 3$ & 30 & 22 & 28 & 30.0 & 29 & 13 & \\
\hline 30 & 34.3 & 36.3 & 20 & +6.0 & 30 & 44.6 & 45.5 & & 36 & $+5 . I$ & 30 & 56.3 & 55.8 & 29 & +15.1 & 30 & 33.0 & 32 & 08 & +13. \\
\hline 32 & 35.4 & 36.0 & 21 & 4 & 32 & 44.3 & 45.0 & & 35 & & 32 & 57.8 & $57 \cdot 3$ & 27 & 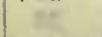 & 32 & $4 \cdot 3$ & & 6 & \\
\hline 34 & $35 \cdot 3$ & 37.1 & 22 & $=2$ & 34 & 46.7 & 47. & & 39 & & 34 & & 57.3 & 27 & 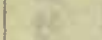 & 3 & 4.5 & & & \\
\hline 36 & 34.0 & 35.4 & 2 & 3 & 36 & 49.4 & 50. & & 43 & & 36 & 57.5 & 56.6 & 28 & 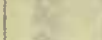 & 3 & 4.1 & & & \\
\hline 38 & 34.2 & 36.3 & 20 & 6. & 38 & 48.4 & 49.5 & & 42 & tat & 38 & 55.0 & 5.3 .8 & 32 & 8 & 38 & 4.1 & & 6 & \\
\hline 40 & 37.0 & 38.4 & 24 & 6 & 40 & 48.4 & 50. & & 42 & 15 & 40 & 50.0 & 48.6 & 40 & 36 & 4 & 4.0 & & 7 & \\
\hline 42 & $39 \cdot 3$ & 40.4 & 28 & 68 & 42 & 50.3 & 52.0 & & 45 & . 20 & 42 & 46.6 & 46.3 & 45 & 24 & 42 & 6 & & 8 & \\
\hline 44 & 38.0 & 40.2 & 26 & +5.9 & 44 & 52.0 & 54.2 & & 48 & +5.0 & 44 & 44.6 & 44.3 & 48 & +14.8 & 4 & $33 \cdot 3$ & & 08 & +13. \\
\hline 46 & 37.8 & 40.4 & 26 & & 46 & 55.1 & 56.8 & & 53 & & 46 & 41.8 & $4 \mathrm{~T} \cdot 4$ & 52 & & 4 & .3 & & II & \\
\hline 48 & $37 \cdot 3$ & 40.3 & 26 & $\theta$ & 48 & 60.3 & 61.6 & $2 I$ & OI & $2 y$ & 48 & 41.0 & 40.6 & 54 & fer & 4 & & & 4 & \\
\hline 50 & 38.5 & 40.5 & 27 & tas & 50 & 62.9 & 64.1 & & 05 & ex & 50 & 40.5 & 40.3 & 1954 & 08 & 5 & 28.0 & 27 & 6 & \\
\hline 52 & $37 \cdot 3$ & 39.7 & 26 & 82 & 52 & 62.0 & 62.9 & $2 \pi$ & 03 & 23 & 5 & 36 & .76 & 2000 & 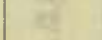 & 5 & 26.5 & & 19 & \\
\hline 54 & 38.5 & 40.5 & 27 & $2^{3}$ & 54 & 59.3 & 61.0 & 20 & 59 & 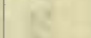 & 54 & 35.5 & 35.5 & 02 & it & 5 & 6.4 & & 19 & \\
\hline 56 & 40.9 & 42.3 & 3 & 89 & 56 & 57.5 & 59. & & 57 & & 56 & 33.0 & 32.3 & 07 & & 5 & 7.6 & & 7 & \\
\hline 58 & 46.0 & 48.1 & 39 & $x^{2}$ & 58 & 57.0 & 59.5 & & 56 & & 58 & 30.6 & 30.0 & 10 & 7ve & 5 & 29.0 & & 6 & \\
\hline$I \infty O$ & 46.1 & 48.4 & 30 & +5.6 & 300 & 57.7 & 59.3 & 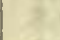 & 57 & +5.0 & 1700 & 3I.I & 30.6 & og & +1.4 .1 & 1000 & 9.3 & & 15 & $+\mathrm{I} 2 . \mathrm{O}$ \\
\hline 02 & 53.0 & $5.5 \cdot 9$ & 50 & $\infty$ & 02 & 57.8 & 59.3 & & 57 & 00 & 02 & 33.0 & 31.0 & 07 & 80 & & & & 18 & \\
\hline 04 & 55.6 & 58.0 & 54 & 10 & 04 & 59.0 & 59.7 & & 58 & in & 0 & 0.0 & 27.6 & 13 & I & 0 & 5.8 & 24 & 20 & \\
\hline$\alpha$ & 58.0 & 59.7 & 57 & ay & & 59.3 & 60.6 & & 59 & Syr & $\alpha$ & & 23.6 & & De & & 4.0 & & 3 & \\
\hline 08 & 53.3 & 55.7 & $5 !$ & (60) & 08 & 58.2 & 59.0 & & 57 & 30 & os & 20.8 & 20.0 & 26 & की & 08 & 1 . 3 & & 27 & \\
\hline 10 & $5 \mathrm{I} .5$ & 54.3 & 48 & $6 r$ & & 59.0 & 59.2 & & 58 & 01 & I & .6 & I9. & 26 & 하. & IO & 19.6 & 19 & & \\
\hline 12 & 50.5 & 52.3 & 46 & & 12 & 59.7 & 60.2 & 20 & 59 & at & I & & IO. I & 27 & is & 12 & 20.0 & 19 & 29 & \\
\hline 14 & 50.7 & 52.0 & 46 & +5.6 & 14 & 60.1 & 61.0 & 21 & Do & +5.0 & I & & 18.3 & 28 & +13. & I & 20.1 & 19 & 29 & $+I_{2}$ \\
\hline I6 & 48.3 & 49.6 & 42 & 68 & It & 61.3 & 62.3 & a & 02 & . & I6 & 16. & 15.7 & 33 & 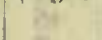 & I & 20.2 & & 20 & \\
\hline 18 & 47.0 & 48.3 & 40 & 87 & Is & 61.6 & $6,2.8$ & 4 & 03 & 루랄 & 18 & 16.7 & 15.3 & 33 & H. & I & 20.8 & & 28 & \\
\hline 20 & 45.8 & $47 \cdot 4$ & 38 & tot & 2 & 60.5 & 61 & $x_{4}$ & OI & $=$ & 2 & & I4.7 & & te & 2 & & & & \\
\hline 22 & 43.7 & 45.2 & 35 & int & 2 & 9.3 & 51.7 & & or & tre & 2 & & 14.1 & 3 & d & 2 & 0.6 & & & \\
\hline & 42.5 & & 3 & 12 & & & & & OI & in & & & & & $1 z$ & & & & & \\
\hline 26 & 45.2 & 45.6 & 36 & 28 & 2 & 50.8 & 62.6 & $2 I$ & OI & xy & 2 & 19.2 & 18.1 & 2 & At & $2 f$ & 23.5 & 22 & 24 & \\
\hline 28 & 50 & & 44 & $7=$ & 28 & 58.0 & 60.5 & 20 & 58 & $x=$ & 28 & .3 & 18.0 & 29 & Var & 28 & 24.2 & & 4 & \\
\hline 30 & 5.5 .7 & 58.0 & 54 & +5.5 & 30 & 56.0 & 58.2 & & 55 & +5.1 & 30 & 17.6 & 17.0 & $3 I$ & +13. & 3 & 3.3 & & 25 & +II. \\
\hline 32 & & 58.0 & 55 & I & & .0 & & & 52 & n & & & I6. & & & 3 & 2.0 & & & \\
\hline 34 & 56.0 & 58.0 & 54 & $k$ & 3 & & 55.8 & & 52 & 30 & 3 & & 17.6 & & 3 & 3 & 20.1 & & & \\
\hline 36 & 48.3 & 49.7 & 4 & $\mathrm{~N}$ & & & 57 & & 53 & ar & 3 & 0.1 & & & As & & & & & \\
\hline 38 & 48.0 & 50.0 & 42 & 8 & 38 & 5.6 & 57 & & 53 & fe. & 38 & & I8. 6 & 28 & Afr & 3 & 1.6 & & 27 & \\
\hline 40 & 49.0 & 49.3 & 42 & ais & $4 c$ & 7.0 & 58.3 & & 55 & $E$ & 4 & 21.7 & 20.3 & 26 & हैं & 40 & 20.9 & 20 & & \\
\hline 42 & 46.7 & 47.5 & 30 & tis & 42 & 6.0 & 58.0 & & 54 & & 42 & & & 27 & & 42 & 21.3 & & 28 & \\
\hline 44 & 52.0 & 5.3 .7 & 48 & +5.5 & 44 & 55.3 & $57 \cdot 3$ & & 5.3 & +5.2 & 44 & 21.0 & 19.6 & 27 & +13.0 & 44 & 21.3 & 20 & 28 & +II. \\
\hline 46 & $54 \cdot 3$ & & 52 & 3 & 46 & & & $\alpha$ & 56 & & 46 & & In. 8 & & & 4 & & & 28 & \\
\hline 48 & 55.0 & 56.3 & 52 & 5 & 48 & 56.6 & 60.0 & 12 & 56 & 81 & 48 & 19.2 & 17.2 & 3 & ? & 48 & 21.8 & 21 & 27 & \\
\hline 50 & 55.8 & 57.2 & 51 & 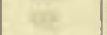 & 50 & 54.8 & 58.0 & & 54 & on & 50 & 23.3 & 22.7 & & 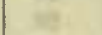 & 50 & 22.0 & & 27 & \\
\hline 52 & 52.0 & 53.6 & 48 & 18 & 52 & 56.6 & 60.0 & 20 & 56 & $E$ & 52 & 24.0 & & 22 & 37 & 52 & 22.3 & & 26 & \\
\hline & 48.5 & 50.3 & 42 & 18 & 54 & 58.3 & 62.3 & & & 4 & 54 & 20.9 & 19.6 & & & & 21.6 & & 28 & \\
\hline 56 & 46.7 & 49.2 & 40 & AS & 56 & 54.0 & 57.2 & 20 & 52 & 8 & $5 \%$ & 20.3 & In. $\mathrm{I}$ & 28 & if & 56 & 21.2 & & 28 & \\
\hline 58 & 47.4 & 49.8 & $4 \mathrm{I}$ & 85 & 58 & 53.0 & 56.7 & & 51 & 87 & 58 & I8.7 & 17.5 & 30 & 8t: & 58 & 22.0 & 20.8 & 27 & +11.2 \\
\hline ant & $\mathrm{TP}$ & & 16: & pos & 400 & 56.6 & 59.8 & & 56 & +5.2 & & & & 7,2 & $x$ & 3000 & 21.8 & 21.0 & 27 & \\
\hline
\end{tabular}

Correction to local mean time is - 3 Is.

No torsion observations made.

Observer-W. J. P.
Correction to local mean time is -20.6 s. $90^{\circ}$ torsion $=22 .^{\prime} 67$. Torsion head at $16 \mathrm{~h}$ oom read $260^{\circ}$ and at $20 \mathrm{~h} 20 \mathrm{~m}$ read $242^{\circ}$. Observer-W. J. P. 
Tabulation of magnetic declinations obscrved at Alger Island Station-Continued

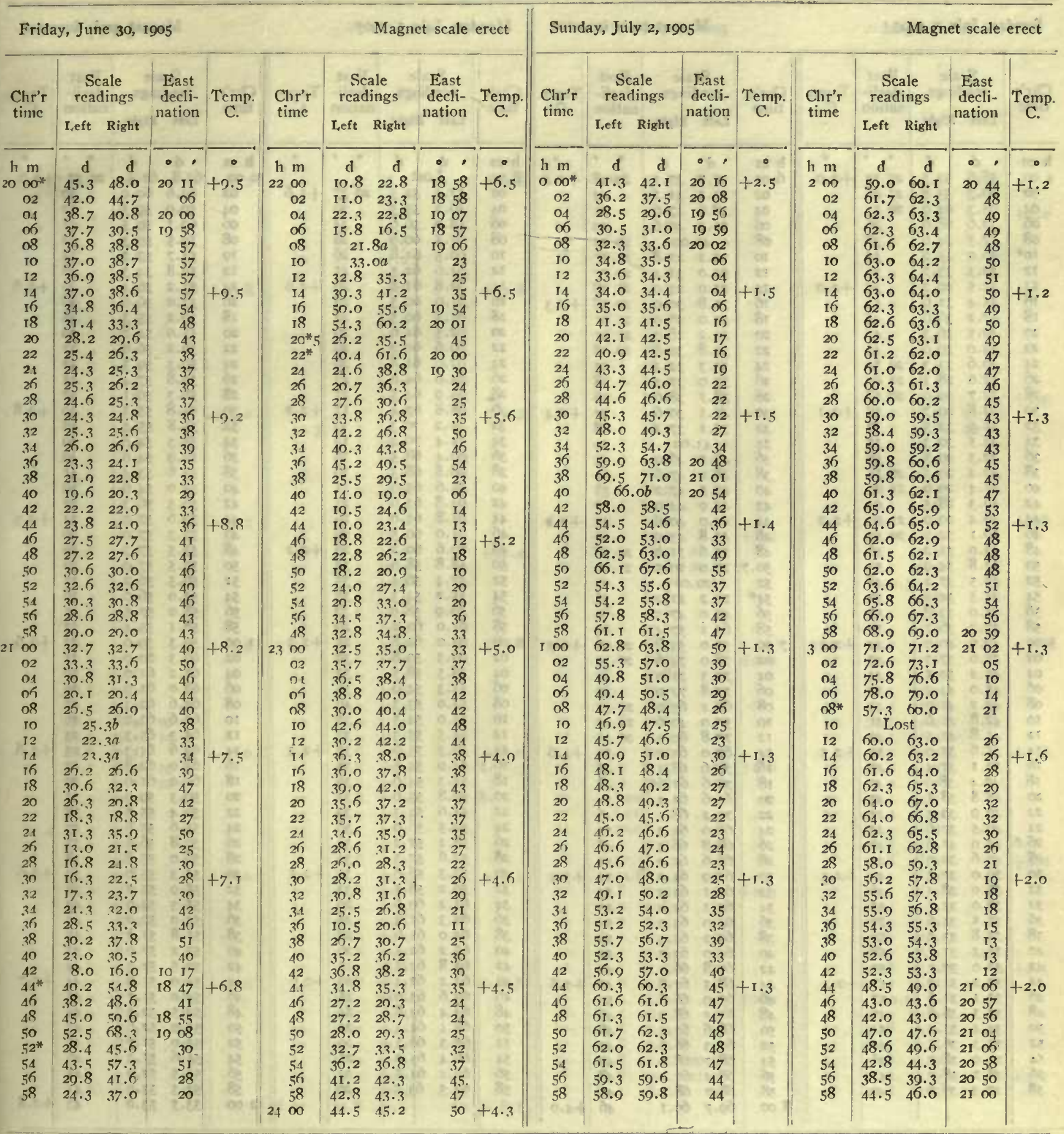

Correction to local mean time is -24 s. $90^{\circ}$ torsion $=18 . ' 59$

Observer-W. J. P.

Torsion head at 2011 oom read $249^{\circ}$ and at $24^{\mathrm{h}}$ oom read $252^{\circ}$.

Observer-W. J. P. 
Tabulation of magnetic declinations observed at Alger Island Station-Continued

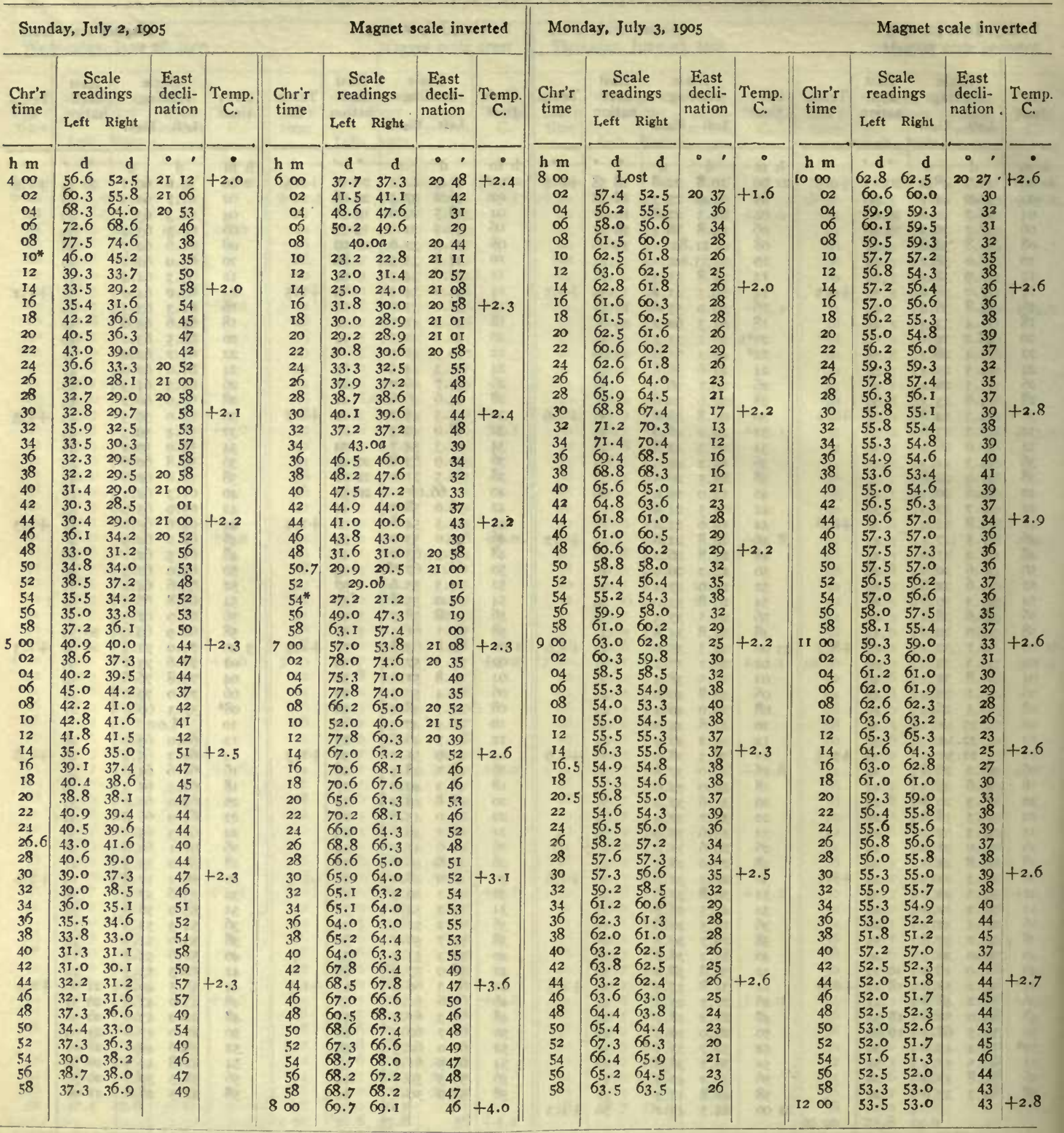

Correction to local mean time is $-29.2 \mathrm{~s} .90^{\circ}$ torsion $=18 .{ }^{\prime} 23$. Torsion head at ol oom read $252^{\circ}$ and at $8 \mathrm{~h}$ lom read $249^{\circ}$. Observer-W. J. P.
Correction to local mean time is $-33 \mathrm{~s}$. $90^{\circ}$ torsion $=20 . ' 27$. Torsion head at $8 \mathrm{~h}$ com read $270^{\circ}$ and at $12 \mathrm{~h} 20 \mathrm{~m}$ read $255^{\circ}$. Observer-W. J. P. 
Tabulation of magnetic declinations observed at Alger Island Station-Continued

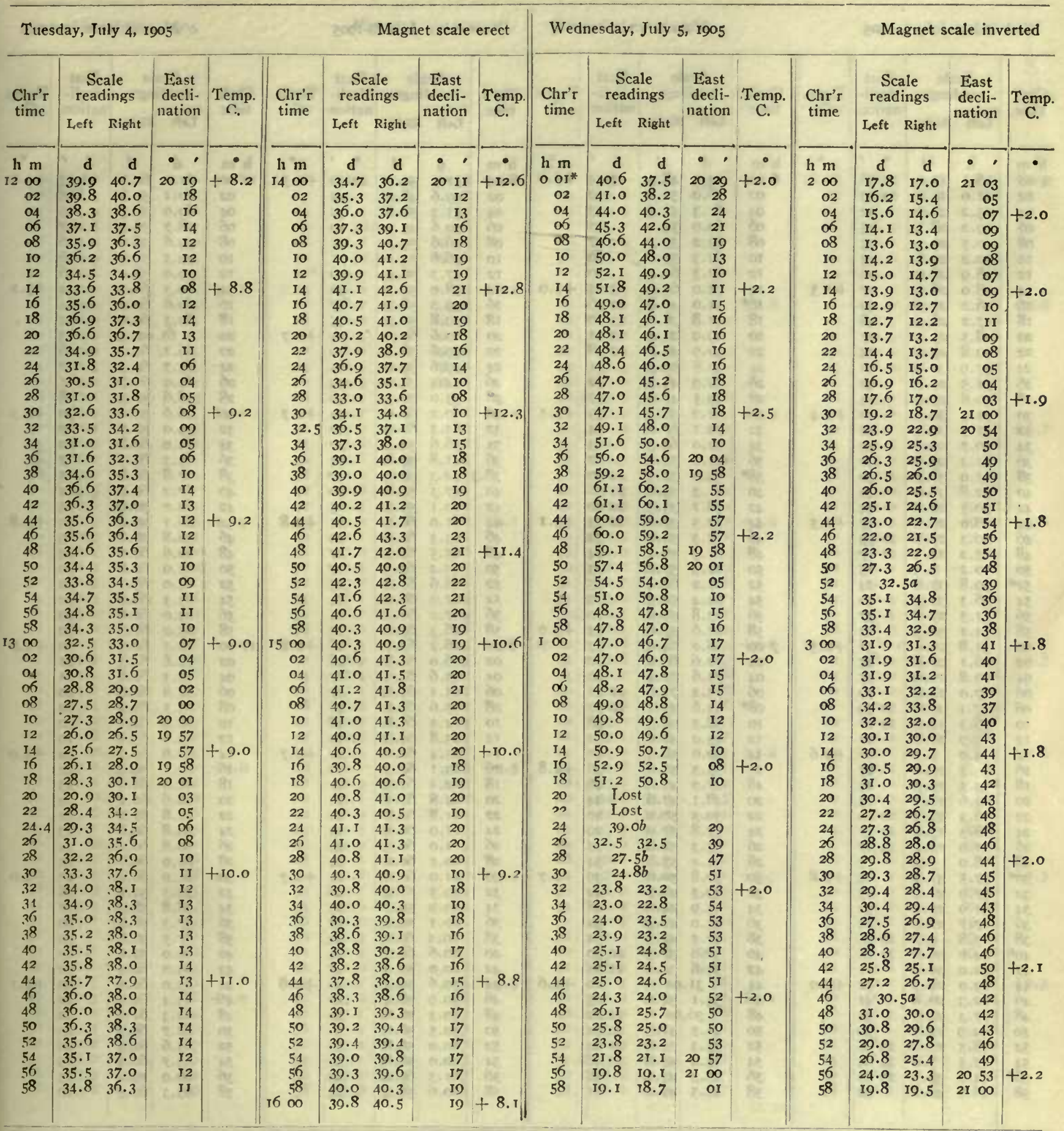

Correction to local mean time is about $-405.90^{\circ}$ torsion $=18 .^{\prime}$ og. Observer-A. F.

Torsion head at $12 \mathrm{~h}$ oom read $28^{\circ}$ and at $\mathrm{I} 6 \mathrm{~h} 15 \mathrm{mr}$ read $253^{\circ}$.

Observer-W. J. P. 
Tabulation of magnetic declinations obserzcd at Alger Island Station-Continued

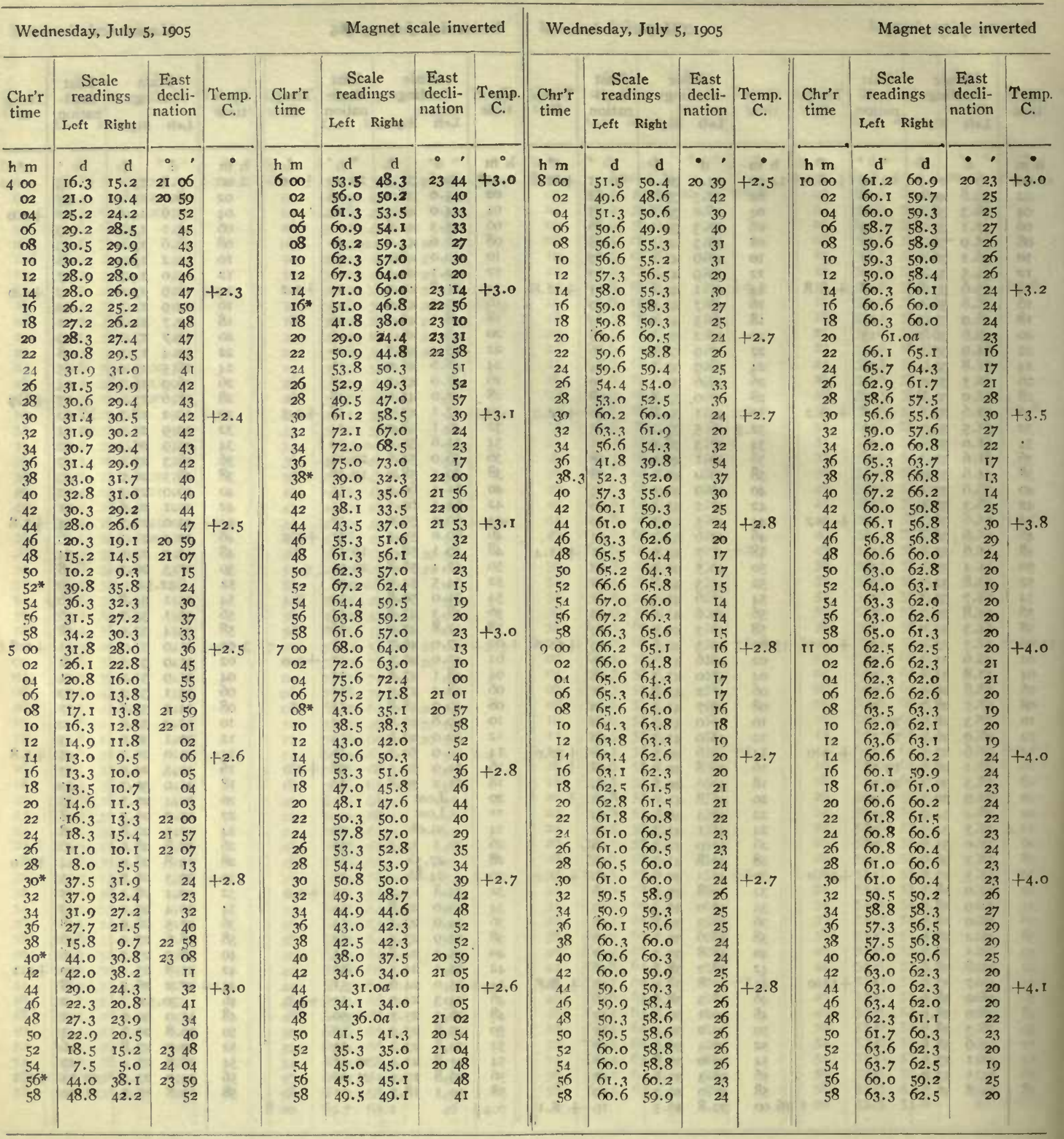

Observers-A. $\vec{F}$. and W. J. P., who alternated from $6 \mathrm{~h} 08 \mathrm{~m}$ to Observer-W. J. P. 6 h $22 \mathrm{~m}$. 
Tabulation of magnetic declinations observed at Alger Island Station-Continued

\begin{tabular}{|c|c|c|c|c|c|c|c|c|c|c|c|c|c|c|c|c|c|c|c|}
\hline \multicolumn{6}{|c|}{ Wednesday, July 5,1905} & \multicolumn{4}{|c|}{ Magnet scale inverted } & \multicolumn{6}{|c|}{ Wednesday, July 5, I905 } & \multicolumn{4}{|c|}{ Magnet scale inverted } \\
\hline \multirow{2}{*}{$\begin{array}{l}\text { Chr'r } \\
\text { time }\end{array}$} & \multicolumn{2}{|c|}{$\begin{array}{l}\text { Scale } \\
\text { readings }\end{array}$} & \multirow{2}{*}{$\begin{array}{c}\text { East } \\
\text { decli- } \\
\text { nation. }\end{array}$} & T'emp. & Chr'r & $\begin{array}{r}\mathrm{Sc} \\
\text { read }\end{array}$ & $\begin{array}{l}\text { ale } \\
\text { ings }\end{array}$ & $\begin{array}{l}\text { Elast } \\
\text { decli- }\end{array}$ & & & $\begin{array}{r}\text { Sc } \\
\text { read }\end{array}$ & $\begin{array}{l}\text { ale } \\
\text { lings }\end{array}$ & $\begin{array}{l}\text { East } \\
\text { decli- }\end{array}$ & & & $\begin{array}{l}\mathrm{Sc} \\
\mathrm{rea}\end{array}$ & $\begin{array}{l}\text { le } \\
\text { igs. }\end{array}$ & $\begin{array}{l}\text { East } \\
\text { decli- }\end{array}$ & \\
\hline & Left & Right & & & & Left & Right? & 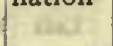 & & & Left & Right & & & & Left & Right & & \\
\hline $\begin{array}{r}h \mathrm{~m} \\
2200\end{array}$ & $\mathrm{~d}$ & $\cos _{d}^{d}$ & & & h m & d & & $\circ \quad$ & & h m & d & d & - & & & & d & : & \\
\hline $\begin{array}{r}1200 \\
02\end{array}$ & $\begin{array}{l}69.0 \\
68.3\end{array}$ & $\begin{array}{l}68.8 \\
68.0\end{array}$ & $\begin{array}{r}2010 \\
12\end{array}$ & +4.0 & $\begin{array}{r}1400 \\
02\end{array}$ & $\begin{array}{l}63.9 \\
65.1\end{array}$ & $\begin{array}{l}63.2 \\
64.8\end{array}$ & $\begin{array}{r}2019 \\
17\end{array}$ & +4.1 & $\begin{array}{l}\infty \\
02\end{array}$ & $\begin{array}{l}33 . I \\
33.8\end{array}$ & $\begin{array}{l}31.8 \\
32.1\end{array}$ & 20.18 & $+3 \cdot 5$ & 02 & $\begin{array}{l}46.7 \\
46.5\end{array}$ & $\begin{array}{l}44.9 \\
45.3\end{array}$ & 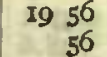 & +3 \\
\hline 04 & $64 \cdot 3$ & 63.8 & 18 & 27 & 04 & 67.4 & 67.1 & 13 & 10 & 04 & 34.1 & $32 \cdot 4$ & 6. 16 & 10 & 04 & 46.6 & 45.5 &, 56 & 25 \\
\hline 06 & 66.8 & 66.2 & 14 & $\omega$ & 06 & 70 & $5 a$ & 08 & 16 & 06 & 33.7 & 32.2 & 17 & 5 & 06 & 46.3 & 45.4 & 56 & . \\
\hline 08 & 63.6 & 62.4 & 20 & 56 & 08 & 75.0 & 74.3 & 2002 & 20 & 08 & 33.6 & 32.1 & 17 & 20 & 08 & 46.0 & 45.0 & 57 & 2. \\
\hline IO & 60.9 & 59.9 & 24 & wi & IO & 78 & $.0 a$ & 1956 & ces & 10 & 33.8 & 32.9 & 16 & 64 & Io & 45.6 & 44.6 & & as \\
\hline 12 & 61.6 & 60.3 & 23 & ist & $12^{*}$ & 49.0 & 44.2 & 55 & $=1$ & 12 & 34.2 & 33.2 & 16 & si. & I2 & 45.2 & 44.2 & 58 & ta: \\
\hline 14 & 60.6 & 59.6 & 24 & +4.1 & 14 & 48.8 & 44.9 & 55 & +4.4 & 14 & $34 \cdot 3$ & 33.1 & 16 & tart & 14 & 44.5 & 43.6 & 59 & \\
\hline 16 & 60.0 & 59.2 & 25 & 0 & 16 & 48.2 & 43.7 & 56 & $\because$ & 16 & 34.0 & 32.7 & 16 & +3.8 & 16 & 44.9 & 44.2 & 58 & +3.0 \\
\hline 18 & 60.0 & 59.3 & 25 & 26 & 18 & 46.2 & 42.2 & 1959 & tii & 18 & 32.5 & 30.9 & 19 & tor & I8 & 45.3 & 44.6 & & $\because$ \\
\hline 20 & 60.2 & 59.3 & 25 & as & 20 & $45 \cdot 3$ & $4 I .2$ & $20 \mathrm{OI}$ & $\infty$ & 20 & $3 \mathrm{I} .2$ & 29.4 & $2 I$ & $\ldots$ & 20 & 45.5 & 44.7 . & & $\therefore$ \\
\hline 22 & 60.0 & 59.1 & 25 & in & 22 & 46.0 & 42.6 & 1959 & . & 22 & 31.2 & 29.8 & 20 & 6 & 22 & 45 & 44.9 & 58 & .5. \\
\hline 24 & 60.3 & 59.5 & 25 & ik & 24 & 46.5 & 42.9 & 1958 & 116 & 24 & 32.4 & 30.3 & 19 & ix. & 24 & 46.0 & $45 \cdot 3$ & & in \\
\hline 26 & 60.6 & 59.8 & 24 & 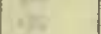 & 26 & $44 \cdot 3$ & 40.8 & 2002 & 10. & 26 & 32.6 & 30.8 & 19 & $g=$ & 26 & 46.1 & 45.4 & 57 & $\therefore$ \\
\hline 28 & 60.1 & 59.1 & 25 & 20 & 28 & 44.4 & $4 \mathrm{I} .0$ & OI & $\because$ & 28.3 & 32.6 & $3 \mathrm{I}$. I & 18 & . & 28 & 45.9 & 45.3 & $\begin{array}{l}56 \\
5\end{array}$ & $\therefore$ \\
\hline 30 & 60.0 & 59.0 & 25 & +4.1 & 30 & 44.7 & 41.7 & OI & +4.4 & 30 & 32.2 & 30.8 & $\therefore 19$ & +3.9 & 30 & 45.6 & 45.0 & & \\
\hline 32 & 60.2 & 59.4 & 25 & 20 & 32 & 44.6 & 42.0 & $\infty$ & E & 32 & 31.9 & 30.7 & 19 & no & 32 & & 45.2 & 57 & +2.7 \\
\hline 34 & 60.0 & 59.2 & 25 & 12 & 34 & 43.8 & $4 I .1$ & 02 & 12 & 34 & 31.6 & 30.4 & 20 & K. & 34 & 45 & 44.9. & 58 & 1.0 \\
\hline 36 & 61.0 & 60.2 & 24 & te & 36 & 42.6 & 40.0 & 04 & 2 & 36 & $3 \mathrm{I} .5$ & 30.5 & 20 & a & 36 & 44.6 & $44 . \bar{r}$ & 59 & 5 \\
\hline 38 & 60.0 & 59.1 & 25 & est & 38 & 42.2 & 39.9 & 04 & $1 / 2$ & 38 & 31.5 & 30.2 & 20 & 82 & 38 & 44.5 & 43.8 & & WA. \\
\hline 40 & 63.5 & 53.3 & 27 & 68 & 40 & 40.8 & 38.9 & 06 & 108 & 40 & 31.3 & 30.1 & 20 & cs & 40 & 45 & 44.2 & 58 & $\therefore$ \\
\hline 42 & 60.8 & 59.8 & 24 & 10 & 42 & 40.5 & 39.0 & $\infty 6$ & s: & 42 & 31.3 & 30.2 & 20 & 4. & 42 & 44.9 & $44 \cdot 3$ & 1958 & 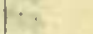 \\
\hline 44 & 60.5 & 59.3 & 25 & +3.9 & 44 & 39.2 & 37.8 & 08 & +4.1 & 44 & 32.0 & 30.9 & I9 & +3.9 & 44 & $44 \cdot 3$ & 43.6 & 2000 & +2.5 \\
\hline 46 & 60.3 & 59.7 & 24 & ( & 46 & 37.1 & 35.9 & II & ro & 46 & 32.5 & 31.4 & 18 & . & 46 & 43.3 & 42.6 & & \\
\hline 48 & 62.1 & 60.9 & 22 & 38 & 48 & 37.6 & 36.5 & IO & 26 & 48 & 32.0 & 31.0 & 19 & 8 & 48 & 42. & 41.9 & & (2) \\
\hline 50 & 62.3 & $6 \mathrm{I} . \mathrm{I}$ & 22 & 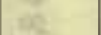 & 50 & 37.5 & 36.4 & Io & 68 & 50 & 32.1 & 31.4 & 19 & ait & 50 & 41.9 & 41.2 & 03 & ge \\
\hline 52 & 62.9 & 61.8 & 21 & 18 & 52 & 37.7 & 36.8 & 10 & es & 52 & 32.7 & 31.8 & 18 & E. & 52 & & 40.3 & 04 & 35 \\
\hline 54 & 62.5 & 61.8 & 2 I & 35 & 54 & 38.1 & 37.2 & 09 & 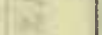 & 54 & 33.0 & 32.1 & 17 & 32 & 5 & 40.3 & 39.6 & 06 & 4 \\
\hline 56 & 63.0 & 62.1 & 20 & Et & 56 & 38.7 & 37.6 & $\infty$ & 08 & 56.3 & 32.5 & 31.7 & 18 & ne & 56 & & & 07 & \\
\hline 58 & 63.6 & 63.1 & 19 & & 58 & 39.0 & 38.0 & 08 & 8 & 58 & $32 . \mathrm{I}$ & 31.2 & 19 & ny & 58 & 38.0 & 37.4 & 09 & \\
\hline 300 & 64.4 & 63.9 & 18 & +4.0 & I5 00 & 38.3 & 37.6 & 09 & +3.8 & 1700 & 31.8 & 30.8 & I9 & +3.6 & 1900 & $\mathrm{Mis}$ & & & \\
\hline 02 & 64.4 & 63.9 & 18 & ( & 02 & 37.9 & $37 \cdot 3$ & 09 & ( & 02 & 31.8 & 30.9 & 19 & 20 & 02 & 38.0 & 37.5 & 09 & +2.5 \\
\hline 04 & 64.2 & 63.7 & 18 & 19 & 04 & 37.4 & 36.7 & IO & 78 & 04 & 32.1 & 31.5 & 18 & 10 & o4 & 39.0 & 38.6 & 08 & $0^{2}$ \\
\hline$\infty 6$ & 64.0 & 63.5 & 19 & 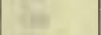 & $\infty 6$ & $37 . I$ & 36.2 & II & $(6)$ & 06 & 32.4 & 31.8 & $=\quad 18$ & 60 & & 40.0 & 39.5 & 06 & , \\
\hline 08 & 63.4 & 63.0 & 19 & . & 08 & 36.8 & 35.7 & 12 & $\omega$ & 08 & 32.8 & 32.0 & 18 & be & 08 & 40.9 & 40.3 & 05 & .: \\
\hline I0 & 61.0 & 60.8 & 23 & Aor: & IO & 35.2 & 34.8 & (1) 14 & 164 & I & & 32.6 & 17 & 64 & I0 & $41 . \overline{6}$ & $4 I . I$ & 04 & os \\
\hline I2 & 61.8 & 61.6 & 22 & ar & 12 & 34.8 & 33.9 & 14 & (3) & 12 & 33.1 & 32.8 & 17 & (c) & 12 & 42.0 & $41 \cdot 3$ & & - \\
\hline I4 & 60 & $.5 b$ & 24 & & 14 & 33.9 & 32. & 16 & (4) & 14 & & 33.7 & I5 & +3.4 & 14 & 42.0 & 41.2 & & +2.4 \\
\hline 16 & 59.0 & 58.8 & 26 & +4.0 & 16 & 32 . I & 31.1 & 19 & +3.5 & 16 & 34.8 & 34.2 & 14 & 4 & 16 & 42.2 & 41.4 & & 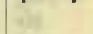 \\
\hline 18 & 60.5 & 60.2 & 24 & ( & 18 & 31.9 & 30.8 & 19 & & 18 & & 34.7 & I4 & 68 & I & 42.6 & 41. & & . \\
\hline 20 & 61 & $.5 a$ & 22 & $\alpha=$ & 20 & $3 \mathrm{I} . \mathrm{I}$ & 30.3 & 20 & 66 & 20 & 34.6 & 33. & 15 & 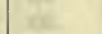 & 20 & 42.9 & 41.8 & & $\therefore$ \\
\hline 22 & 65.8 & 65.3 & 16 & is & 22 & 30.9 & 29.9 & $2 \mathrm{I}$ & ins & 22 & 35.0 & 34.2 & 14 & et. & 22 & 43.6 & 42.3 & & $\therefore$ \\
\hline 24 & 66.1 & 65.8 & 15 & 12 & 24 & 31.3 & 30.2 & 20 & at & 24 & & 34.9 & 13 & ik & 24 & 44.3 & 43. & $20 \infty$ & $\therefore$ \\
\hline 26 & 68 & $.5 a$ & II & $\therefore$ & 26 & 32.0 & 30.8 & 19 & a & 26 & 36.6 & 35.7 & I2 & $i^{2}$ & 20 & 45.1 & 43.9 & I9 58 & $\because$ \\
\hline 28 & 70.4 & 70.0 & 08 & & 28 & $3 \mathrm{I} . \mathrm{I}$ & 30.2 & 20 & $t_{3}$ & 28 & 36.6 & 35.7 & I2 & ins & 28 & 46.0 & 44.6 & & \\
\hline 30 & 70.5 & 70.2 & 08 & +4.0 & 30 & 30.8 & 30.3 & 20 & +3.4 & 30 & 37.0 & 36.0 & II & $+3 \cdot 3$ & 30 & 45.3 & 43.8 & $\begin{array}{l}58 \\
-58\end{array}$ & +2.3 \\
\hline 32 & 72.9 & 72.3 & 05 & (1) & 32 & & 30.0 & 21 & & 32 & 38.0 & 37.0 & (c) 10 & ( & 32 & & 43.8 & 1959 & $: 2$. \\
\hline 34 & 73.3 & 73.0 & 0 & 32 & 34 & 29.8 & 29.0 & 22 & . & 34 & 39.2 & 38.0 & 08 & H & 34 & 44.6 & 43.1 & 20.00 & (2) \\
\hline 36 & 73.7 & 73.1 & 04 & 政 & 36 & 30.2 & 30.0 & 21 & . & 36 & 40.5 & 39.2 & 106 & $\sqrt{5}$ & 3 & 45.2 & & 1959 & $\therefore$ \\
\hline 38 & & .86 & 06 & 2 & 38 & 30.9 & 30.5 & 20 & 120 & 38 & $4 I .9$ & 40.9 & 04 & हe & 38 & 45.3 & 43.3 & I9 59 & $\therefore$ \\
\hline 40 & 69.8 & 69.3 & 09 & 3 & 40 & $3 \mathrm{I} .4$ & 30.8 & 20 & 100 & 40 & 43.5 & $42 . \overline{8}$ & 20 oI & st. & 40 & 44.2 & 42.2 & $20 \mathrm{OI}$ & ej. \\
\hline 42 & 70.1 & 69.5 & 09 & $3 y$ & 42 & 32.0 & 31.9 & 18 & 142 & 42 & 45.0 & 44.5 & 1958 & ... & 42 & 43.8 & 42.0 & oI & \\
\hline & 69.5 & $69 . I$ & 10 & & 44 & 32.7 & 31.8 & 18 & +3.5 & 44 & 45.3 & 44.8 & 58 & 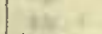 & 44 & 42.2 & 40.2 & & +2.3 \\
\hline 46 & 70.5 & 70.0 & 08 & +4.0 & 46 & 33.0 & 31.9 & 18 & & 46 & 45.0 & $44 \cdot 3$ & 58 & +3.1 & 46 & 41.5 & .40 .0 & 04 & 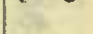 \\
\hline 48 & 67.0 & 66.0 & 14 & ( & 48 & 33.1 & 32.0 & 17 & as & 48 & 45.0 & 44.1 & 58 & 10 & 48 & 41.3 & 40.3 & 04 & . \\
\hline 50 & 69.3 & 68.7 & 10 & 3 & 50 & 33.4 & 32.0 & 17 & . & 50 & 45.1 & 44.2 & 58 & 0 & 50 & 40.1 & 38. & 06 & $:$ \\
\hline 52 & 70.2 & 69.8 & $\infty 9$ & 85 & 52 & & 32. & & 82 & 52 & 44.9 & 43.9 & 59 & se & 52 & 38.4 & 37.6 & & $x=$ \\
\hline 54 & 66.0 & 65.4 & 16 & 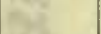 & 54 & 33.1 & 31.9 & 17 & (4) & 54.2 & & 44.3 & 58 & H. & 54 & 37.7 & 37.0 & 10 & i. \\
\hline 56 & 63.3 & 62.7 & 20 & & 56 & 33.1 & 32. & & 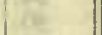 & 56 & 45.8 & 44.9 & & 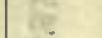 & 56 & 37.6 & 37.0 & 10 & . \\
\hline 58 & 63.8 & 63.1 & 19 & 48 & 58 & 33.0 & 31.8 & 18 & 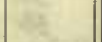 & 58 & 46.6 & 44.9 & 57 & $\therefore$ & 58 & $37 \cdot 3$ & 36.8 & 10 & $\therefore=$ \\
\hline & & & -3 & & & & & & & & & c.06 & 40 & $\therefore$ & & & & & \\
\hline
\end{tabular}

Observers-W. J. P. and A. F., who alternated from $12 \mathrm{~h} 48 \mathrm{~m}$ to I2h $58 \mathrm{~m}$.
Observers-A. F. and W. J. P., who alternated from 17 h $46 \mathrm{~m}$ ito I 8 h oom. 
Tabulation of magnetic declinations observed at Alger Island Station-Continued

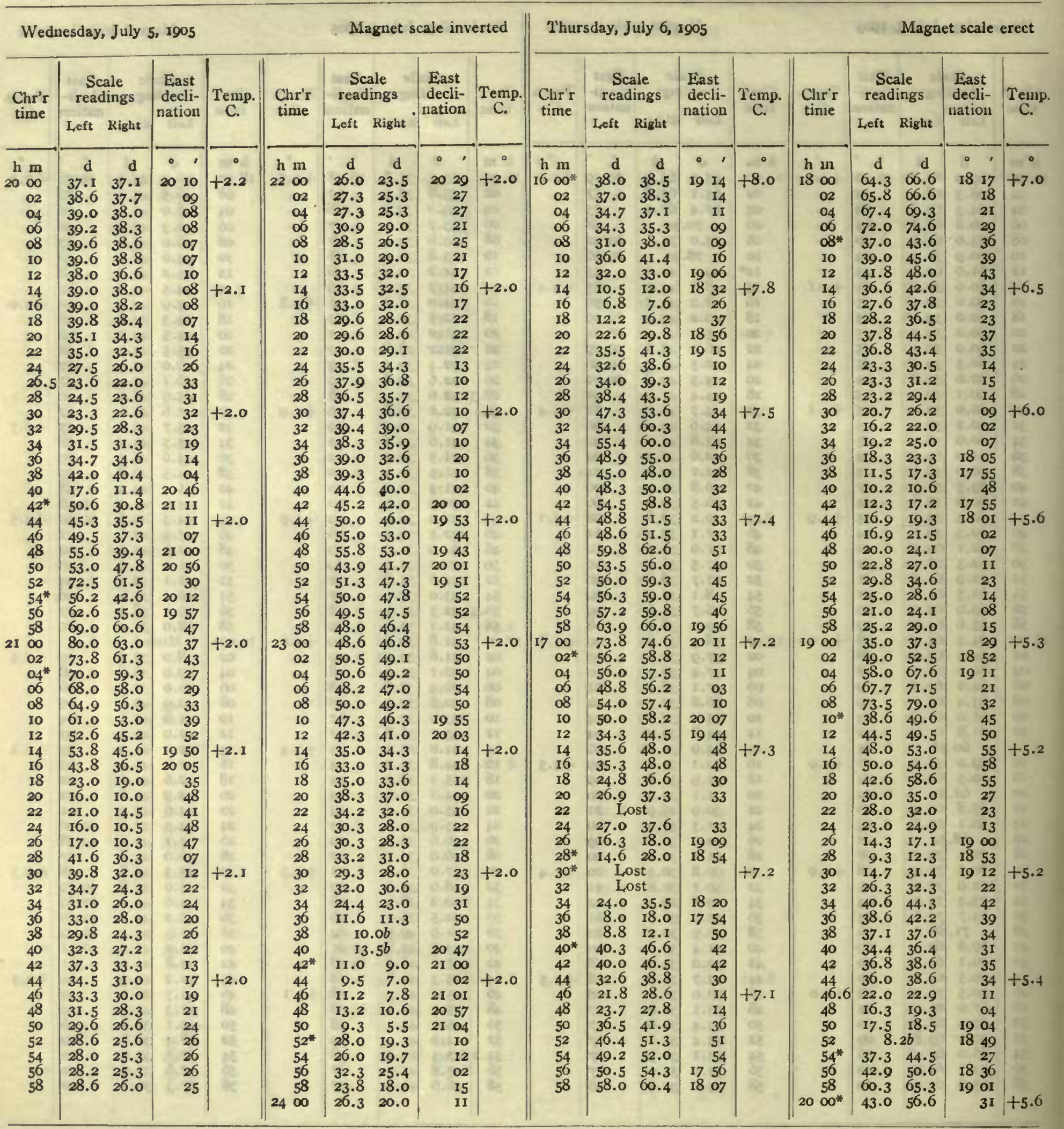

Correction to local mean time is -49 s, $90^{\circ}$ torsion $=177^{\prime} 77$.

Torsion head at oh Iom read $252^{\circ}$ and at $24 \mathrm{~h}$ com read $249^{\circ}$.

Observer-W. J. P.
Correction to local mean time is + Im o2s.

Torsion head at beginning and ending read $252^{\circ}$.

Observer-W. J. P. 
Tabulation of magnetic declinations observed at Alger Island Station-Continued

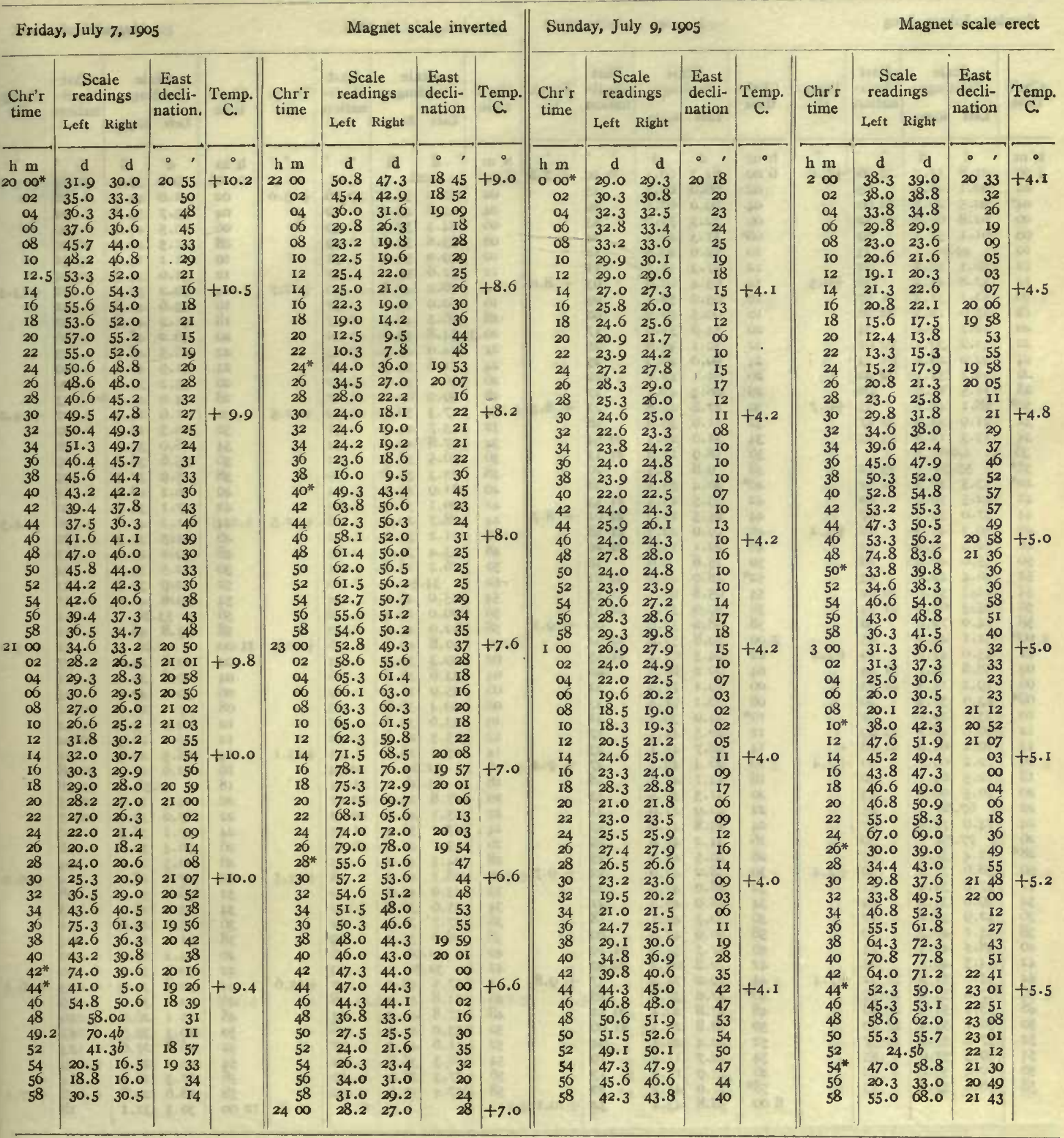

Correction to local mean time is $-0.3 s$.

Observer-W. J. P.

Torsion head at beginning and ending read $252^{\circ}$.

Observer-W. J. P. 
Tabulation of magnetic declinations observed at Alger Island Station-Continued

\begin{tabular}{|c|c|c|c|c|c|c|c|c|c|c|c|c|c|c|c|c|c|c|c|c|c|}
\hline \multicolumn{6}{|c|}{ Sunday, July 9,1905} & \multicolumn{5}{|c|}{ Magnet scale inverted } & \multicolumn{6}{|c|}{ Monday, July 10, 1905} & \multicolumn{5}{|c|}{ Magnet scale erect } \\
\hline \multirow{2}{*}{$\begin{array}{l}\text { Chr'r } \\
\text { time }\end{array}$} & \multicolumn{2}{|c|}{$\begin{array}{l}\text { Scale } \\
\text { readings }\end{array}$} & $\begin{array}{c}\text { Elast } \\
\text { decli } \\
\text { natiol }\end{array}$ & Temp. & $\begin{array}{l}\text { Chr'r } \\
\text { time }\end{array}$ & $\begin{array}{l}\text { Sca } \\
\text { read }\end{array}$ & $\begin{array}{l}\text { ale } \\
\text { lings }\end{array}$ & $\begin{array}{l}\text { East } \\
\text { decil } \\
\text { natio }\end{array}$ & & Temp. & $\begin{array}{l}\text { Chr'r } \\
\text { time }\end{array}$ & $\begin{array}{l}\text { Sca } \\
\text { read }\end{array}$ & $\begin{array}{l}\text { ale } \\
\text { lings }\end{array}$ & $\begin{array}{c}\text { East } \\
\text { decli- } \\
\text { nation }\end{array}$ & Temp. & Chr'r & $\begin{array}{r}\mathrm{Sc} \\
\mathrm{read}\end{array}$ & $\begin{array}{l}\text { ale } \\
\text { lings }\end{array}$ & East & & Temp. \\
\hline & Left & Right & Thation & & & L,eft & Right & Matso & & & & $\mathrm{I}_{\mathrm{e}} \mathrm{eft}$ & Right & & & & Left & Right & & & \\
\hline li $\mathrm{m}$ & d & $d$ & $\circ$ & ${ }^{\circ}$ & h $\mathrm{m}$ & d & $d$ & $\circ$ & $\dot{\prime}$ & $\because 0$ & h $\mathrm{m}$ & $\stackrel{d}{L}$ & $d$ & $\circ$ ' & -0 & h m & d & d & & & 10 \\
\hline $\begin{array}{r}400 \\
02\end{array}$ & $\begin{array}{l}21.8 \\
40.1\end{array}$ & $\begin{array}{l}15.2 \\
20.1\end{array}$ & $\begin{array}{l}2226 \\
03\end{array}$ & +6.0 & $\begin{array}{r}600 \\
02\end{array}$ & $\begin{array}{l}61.7 \\
66.0\end{array}$ & $\begin{array}{l}60.4 \\
62.3\end{array}$ & 205 & $\begin{array}{l}50 \\
45\end{array}$ & +7.8 & $\begin{array}{r}800 \\
02\end{array}$ & $\begin{aligned} \text { Los } & \end{aligned}$ & st 54.0 & 2056 & +13.0 & I0 00 & 39.8 & 39.9 & & & +13.5 \\
\hline $04^{*}$ & $\begin{array}{l}40.1 \\
30.5\end{array}$ & 26.2 & 2200 & $\tan$ & 04 & 69.0 & 67.1 & 3 & $\begin{array}{l}43 \\
39\end{array}$ & $\frac{10}{x a}$ & $\begin{array}{l}02 \\
04\end{array}$ & $\begin{array}{l}52.0 \\
42.8\end{array}$ & $\begin{array}{l}54.0 \\
45.3\end{array}$ & $\begin{array}{r}2050 \\
42\end{array}$ & +13.0 & $\begin{array}{l}02 \\
04\end{array}$ & $\begin{array}{l}41.0 \\
33.7\end{array}$ & $\begin{array}{l}44.0 \\
33.8\end{array}$ & & $\begin{array}{l}38 \\
24\end{array}$ & 36 \\
\hline$\infty$ & 62.8 & 61.8 & $2 \mathrm{II}$ & 30 & $\infty$ & 72.5 & 71.7 & & 33 & 60 & $\infty$ & 54.3 & 56.0 & 59 & 20 & $\infty$ & 42.5 & 44.4 & & 40 & 36 \\
\hline 08 & $6 \pi .1$ & $54 \cdot 3$ & Ig & 60 & $08^{*}$ & $5 \mathrm{I} .3$ & 46.1 & & 24 & 10 & 08 & 53.8 & 55.6 & 59 & 84 & 08 & 41.5 & 46.0 & 204 & 40 & ns \\
\hline 10 & 49 & $.0 a$ & 33 & & I0 & 54.0 & 49.8 & & & wi & IO & 49.0 & 50.5 & 51 & ait & 10 & II. I & 13.6 & I9 5 & $5 \mathrm{I}$ & ot \\
\hline 12 & 56.1 & 55.5 & 22 & & 12 & $57 \cdot 3$ & 55.5 & & 11 & & 12 & 52.6 & 55.2 & 57 & a & 12 & 31.6 & 34.8 & 202 & 24 & 44 \\
\hline 14 & 47.8 & 43.5 & 38 & +6.5 & 14 & 63.4 & 60.0 & & 03 & +8.0 & 14 & 46.2 & 47.8 & 46 & +12.7 & I.4 & 30.2 & 33.0 & & $2 I$ & +13.5 \\
\hline 10 & 36.1 & $3 \mathrm{I} .2$ & 2157 & ( & 16 & 63.0 & 58.5 & & 05 & (a) & 16 & 40.0 & 41.6 & $\begin{array}{r}47 \\
6\end{array}$ & & 16 & 33.6 & 42.8 & & $3 I$ & \\
\hline I8 & 15.0 & 12.5 & 2228 & & I8 & 60.5 & 56.5 & & 08 & in & 18 & 46.5 & 48.5 & 2047 & e & 18 & 41.2 & 42.8 & & 37 & 24 \\
\hline 20 & 18.7 & 14.2 & 24 & . & 20 & $54 . \overline{8}$ & 51.0 & & 17 & 2 & 20.3 & 54.8 & 56.6 & 2100 & a & 20 & 46.2 & 48.4 & & 46 & 다 \\
\hline 22 & 32.2 & 30.1 & 22 o1 & $=$ & 22 & 50.1 & $45.8^{\circ}$ & & 25 & 2 & 22 & 44.0 & $45 \cdot 3$ & 2043 & $\therefore$ & 22 & 46.5 & 48.0 & & 45 & 64 \\
\hline 24 & 50.0 & 48.2 & 2133 & 4 & 24 & 50.0 & 46.0 & & 25 & & 24 & $5 \mathrm{I} .8$ & 54.3 & 56 & 74 & 24 & 41.9 & 46.0 & & 40 & 44 \\
\hline 26 & 32.2 & $3 I . I$ & 2200 & 5 & 26 & 51.1 & 47.7 & & 22 & & 26 & 36.1 & 39.1 & 2032 & 6 & 26 & 35.2 & 37.8 & & 29 & $2=$ \\
\hline 28 & 14.5 & 13.8 & 28 & & 28 & 48.3 & $45 . I$ & & 27 & & 28 & 59.9 & 60.0 & 2107 & $\approx$ & 28 & 44.0 & 47.0 & & 43 & \\
\hline $30^{*}$ & 48.1 & 43.7 & 33 & +6.7 & 30 & 47.8 & 44.8 & & 27 & +8.3 & 30 & 47.3 & 50.3 & 2049 & +12.5 & 30 & 21.6 & 24.2 & & 07 & +14.0 \\
\hline 32 & 41.9 & 32.0 & 2247 & & $3^{2}$ & 41.0 & 38.0 & & 38 & 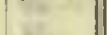 & 32 & 43.7 & $44 . I$ & $4 I$ & . & 32 & 20.3 & 25.3 & & 07 & \\
\hline 34 & 15.3 & 12.8 & 2323 & at & 34 & 31.0 & 27.3 & 205 & 54 & wis & 34 & 37.8 & 40.2 & 34 & 38 & 34 & 28.0 & 31.0 & & 18 & 12 \\
\hline 36 & Lo & ost & & & 36 & 18.9 & 15.8 & $2 \mathrm{II}$ & 13 & 8 & 36 & 36.5 & 38. & 32 & a. & 36 & 35.6 & 38.6 & & 29 & 8 \\
\hline $38 *$ & & $\cdot 5^{a}$ & $23 \mathrm{I} 2$ & 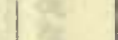 & 38 & $9 . \overline{8}$ & 7.5 & & 26 & 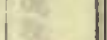 & 38 & 33.3 & 34.8 & c. 26 & E्ट & 38 & 35.9 & 37.3 & & 29 & 28 \\
\hline $40^{*}$ & 53.5 & 49.8 & $22 \mathrm{I} 2$ & 6 & $40^{*}$ & 40.4 & 35.5 & & 38 & & 40 & 31.9 & 33.3 & 24 & $x$ & 40 & 26.2 & 28.0 & & 14 & es \\
\hline 42 & 62.5 & 56.4 & $\infty$ & 28 & 42 & 33.5 & 23.4 & 215 & 53 & & 42 & 30.1 & 31.2 & 20 & 8. & 42 & $37 . I$ & 38.8 & & 30 & $x y$ \\
\hline 44 & 57.0 & 52.3 & o? & 3 & 44 & 25.5 & 21.6 & 220 & oI & +8.7 & 44 & 28.7 & 29.7 & 18 & +12.5 & 44 & $4 \mathrm{I} .2$ & 41.8 & & 36 & +14.5 \\
\hline 46 & 50.3 & 45.0 & I8 & +7.2 & 46 & 29.0 & 22.3 & 215 & 58 & & 46 & $3 \mathrm{I} .2$ & 32.0 & 22 & 10 & 46 & 31.3 & 32.3 & & 21 & \\
\hline 48 & 52.4 & 49.9 & 12 & 1 & 48 & 22.1 & $13 . \overline{8}$ & 22 & 10 & 30 & 48 & 33.4 & 34.0 & 25 & 6 & 48 & 29. I & 29.2 & & 17 & At \\
\hline 50 & 55.0 & 40.0 & 18 & 39 & 50 & 15.8 & 9.0 & & 18 & & 50 & 26.2 & 26.8 & 14 & 20 & 50 & 32.5 & 35.0 & & 24 & 28 \\
\hline 52 & 46.4 & 42.0 & 23 & & $52^{*}$ & 48.0 & 44.8 & 222 & $2 \mathrm{I}$ & & 52 & 34 & $.5 a$ & 26 & 8 & 52 & 32.9 & 33.9 & & 23 & A \\
\hline 54 & 61.1 & 55.0 & 220 & 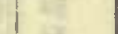 & 54 & 65.8 & 63.5 & 215 & 53 & & 54 & 45.0 & 46.5 & 44 & 12 & 54 & 39.6 & 42.0 & & 35 & 38 \\
\hline 56 & 65.2 & 6I.I & 215 & 3 & 56 & 59.0 & 57.4 & 220 & 03 & & 56 & 29.0 & 29.3 & I8 & 2 & 56 & 53 & $.0 b$ & & 54 & \\
\hline 58 & 74.0 & 72.0 & 3 & 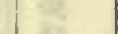 & 58 & 58.2 & 53.6 & & 06 & $\mid+9.1$ & 58 & 42.0 & 43.6 & 40 & & 58 & 43.8 & 46.0 & & $4 \mathrm{I}$ & \\
\hline $500^{*}$ & $5 \mathrm{I} .1$ & 44.0 & 25 & $x^{2}$ & 700 & 59.8 & 57.0 & 220 & 02 & & 900 & 40.8 & 40.8 & 36 & +12.9 & II 00 & 20 & $.0 b$ & & 12 & +15.0 \\
\hline 02 & 55.0 & 47.6 & IS & $1+7.2$ & 02 & 64.5 & $6 \mathrm{I} .3$ & 215 & 55 & 20 & 02 & 39.3 & 39.3 & 34 & 10 & 02 & 22.6 & 24.0 & & 07 & \\
\hline 04 & 38.8 & 32.7 & 214 & & 04 & 69.2 & 66.3 & & 48 & & 04 & 33.6 & 34.8 & 26 & is & 04 & 29.8 & 34.2 & & 21 & in \\
\hline$\infty$ & 19.2 & 14.5 & 22 & & $\infty$ & 79.1 & 77.0 & & 32 & Es & 06 & 35.9 & 36.6 & $\begin{array}{r}29 \\
\end{array}$ & 60 & $\infty$ & 39.2 & 42.5 & & 35 & 80 \\
\hline $08^{*}$ & 43.0 & 37.3 & 29 & & $\infty 8$ & 78.1 & 74.4 & & 35 & & 08 & 30.4 & 30. & 20 & 5. & 08 & 17.2 & 21.6 & $20 \mathrm{C}$ & I & we \\
\hline IO & 33.7 & 30.1 & 4 & 20 & $10^{*}$ & 44.0 & 39.3 & & $3 \mathrm{I}$ & 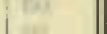 & Io & 33.6 & 33.8 & 25 & ett & Io & 6.9 & 9.9 & I9 4 & 44 & es \\
\hline 12 & 56.8 & 48.7 & 220 & wit & $\mathrm{I} 2$ & 46.5 & 42.1 & & 27 & ast & 12 & 25.5 & 25.6 & 12 & 6 & 12 & 19.0 & 20.3 & $20 \mathrm{c}$ & 02 & ir \\
\hline 14 & 70.2 & 61.8 & 214 & -2 & 14 & 48.7 & 45.1 & & 23 & & 14 & 25.3 & 26.3 & & +13.I & 14 & 18.5 & 20.5 & & OI & M \\
\hline 16 & 72.2 & 69.8 & 40 & +7.4 & 16 & 46.4 & 42.3 & & 27 & +9.4 & 16 & 52 & $.0 a$ & 2054 & & 16 & 22.6 & 25.0 & $20 \mathrm{c}$ & 08 & \\
\hline 18 & 72.5 & 69.7 & $4 c$ & & I8 & $45 . \mathrm{I}$ & $42 . I$ & & 28 & & I8 & 65 & $.0 b$ & 21 & 18: & 18 & I7 & $.3 b$ & 195 & 58 & Br \\
\hline 20 & 70.5 & 68.0 & 4 & & 20 & 41.3 & 39.5 & & 33 & 4 & 20 & $27.8^{\circ}$ & 29.9 & $20 \mathrm{II}$ & ux & 20 & 14.3 & 17.1 & 195 & 55 & \\
\hline 22 & 67 & $.8 b$ & A & & 22 & 42.9 & 41.0 & & $3 \mathrm{I}$ & & 22 & $45 . \mathrm{I}$ & 45.6 & 2043 & $\approx$ & 22 & 27.0 & $32 . I$ & 20 & & is \\
\hline 24 & 74.0 & 72.4 & 3 & & 24 & 45.8 & 42.9 & & 27 & & 24 & 57.2 & 59. & 2I 03 & is & 24 & 37.1 & 38.5 & & 30 & aix \\
\hline 26 & 77 & $.5 a$ & 3 & & 26 & 44.2 & 42.0 & & 29 & & 26 & 43.7 & $45 . \overline{6}$ & 2042 & & 26 & 36.4 & 36.8 & & 28 & \\
\hline $28^{*}$ & 42.4 & 34.8 & 2 & & 28 & 46.5 & 45.0 & & 25 & & 28 & 44.5 & 45. & 43 & ren & 28 & 20.3 & 22.6 & & 04 & \\
\hline 30 & 38.6 & 33.0 & 30 & +7.4 & 30 & 48.2 & 47.0 & & 22 & +9.7 & 30 & 48. I & 49.5 & 48 & +13.5 & 30 & 27.6 & 28.0 & & 14 & +16.0 \\
\hline 32 & 37.8 & 32.6 & 3 & & 32 & 48.0 & 47.5 & & 22 & & 32 & 43.0 & 43. & 39 & 100 & 32 & 36.2 & 37.0 & & 28 & \\
\hline 34 & 39.0 & 33.2 & 2 & 跑 & 34 & 48.9 & 48.3 & & 21 & i & 34 & 35.6 & 36.8 . & 29 & 18 & 34 & 25.7 & 28.9 & & 13 & ite \\
\hline 36 & 42.9 & 38.2 & 22 & 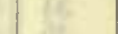 & 36 & 50.7 & 49.1 & & I8 & & 36 & 37.7 & 39.3 & & W & 36 & 25.0 & 26.8 & & II & \\
\hline 38 & $45 . I$ & 40.3 & Is & & 38 & 50.0 & 47.8 & & 20 & & 38 & 28.6 & $3 I .0$ & I9 & 表 & 38 & 21.0 & 22.0 & & 04 & \\
\hline 40 & 48.5 & $43 \cdot 3$ & I 2 & . & 40 & 57.3 & 53.8 & 211 & 10 & & 40 & 40 & $.9 b$ & & & 40 & 24.2 & 25.2 & & 09 & \\
\hline 42 & 54.5 & 49.1 & o & & 42 & 70.8 & 65.2 & 205 & 50 & & 42 & 32. & $.5 a$ & 23 & 26 & 42 & 30.5 & 32.1 & & 20 & \\
\hline 44 & 55.6 & 51.8 & 210 & +7.4 & & 79.0 & 74.3 & & 37 & +9.8 & & & 48.4 & 47 & +13.6 & & 32.8 & 34.0 & & 23 & +16.5 \\
\hline 46 & 57.0 & 53.4 & 205 & & $46^{*}$ & 47.0 & 37.8 & & 38 & & 46 & 34.8 & 37.0 & 28 & $1-80$ & 46 & 30.0 & 31.6 & & 19 & \\
\hline 48 & 58.0 & 54.5 & 205 & (6) & 48 & 37.8 & 29.4 & 205 & 52 & & 48 & 37.8 & 40.2 & $\begin{array}{l}13 \\
\end{array}$ & 2 & 48 & 25 . I & 26 . I & & 10 & \\
\hline 50 & 56.1 & 53.9 & $21 \alpha$ & 4 & 50 & 28.8 & 20.7 & 210 & 06 & & 50 & 43.0 & $44 \cdot 3$ & 40 & a & 50 & 29.3 & 30.3 & & 17 & \\
\hline 52 & 57.8 & 55.0 & 205 & 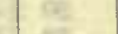 & 52 & 29.4 & 22.1 & & $a_{4}$ & tis & 52 & $4 \mathrm{I} .6$ & & 37 & $=$ & 52 & .26 .8 & 27.6 & & 13 & \\
\hline 54 & 59.5 & 57.0 & 5 & 2 & 54 & 20.7 & 11.8 & & 19 & tas & 54 & 38.3 & 38.8 & & & 54 & 24.4 & 25.4 & & $\infty 9$ & \\
\hline 56 & 60.4 & 58.8 & 5 & 12 & 46 & 23.9 & 17.0 & & 13 & ? & 56 & 37.3 & 38.0 & 30 & & 56 & 27.3 & 28.2 & & 14 & \\
\hline 58 & 58.6 & 57.8 & 5 & & $58 \%$ & 61.0 & 43.2 & & 30 & & 58 & 37.4 & 37.6 & 30 & & 58 & 28.6 & 29.8 & & 16 & +17.1 \\
\hline & & & & & 800 & 58.8 & 43.2 & & 32 & +10.1 & $x^{2}$ & & & 60 & $\infty 61$ & 1200 & 30.3 & 3I. I & & 18 & \\
\hline
\end{tabular}

Correction to local mean time is -3.0 .

Torsion head at beginning and end read $252^{\circ}$.

Observers-W. J. P. and A. F., who alternated from $4^{\mathrm{ll}} \mathrm{o}+\mathrm{m}$ to 4h $14 \mathrm{~m}$.
Correction to local mean time is $-4.6 \mathrm{~s}$. $90^{\circ}$ torsion $=17.87$.

Torsion liead at $8 \mathrm{~h}$ oom read $252^{\circ}$ and at $12 \mathrm{~h} 15 \mathrm{~m}$ read $267^{\circ}$.

Observer-W. J. P. 
Tabulation of magnetic declinations observed at Alger Island Station-Continued

Tuesday, July 11, 1905

- Magnet scale inverted

Wednesday, July 12, 1905

Magnet scale erect

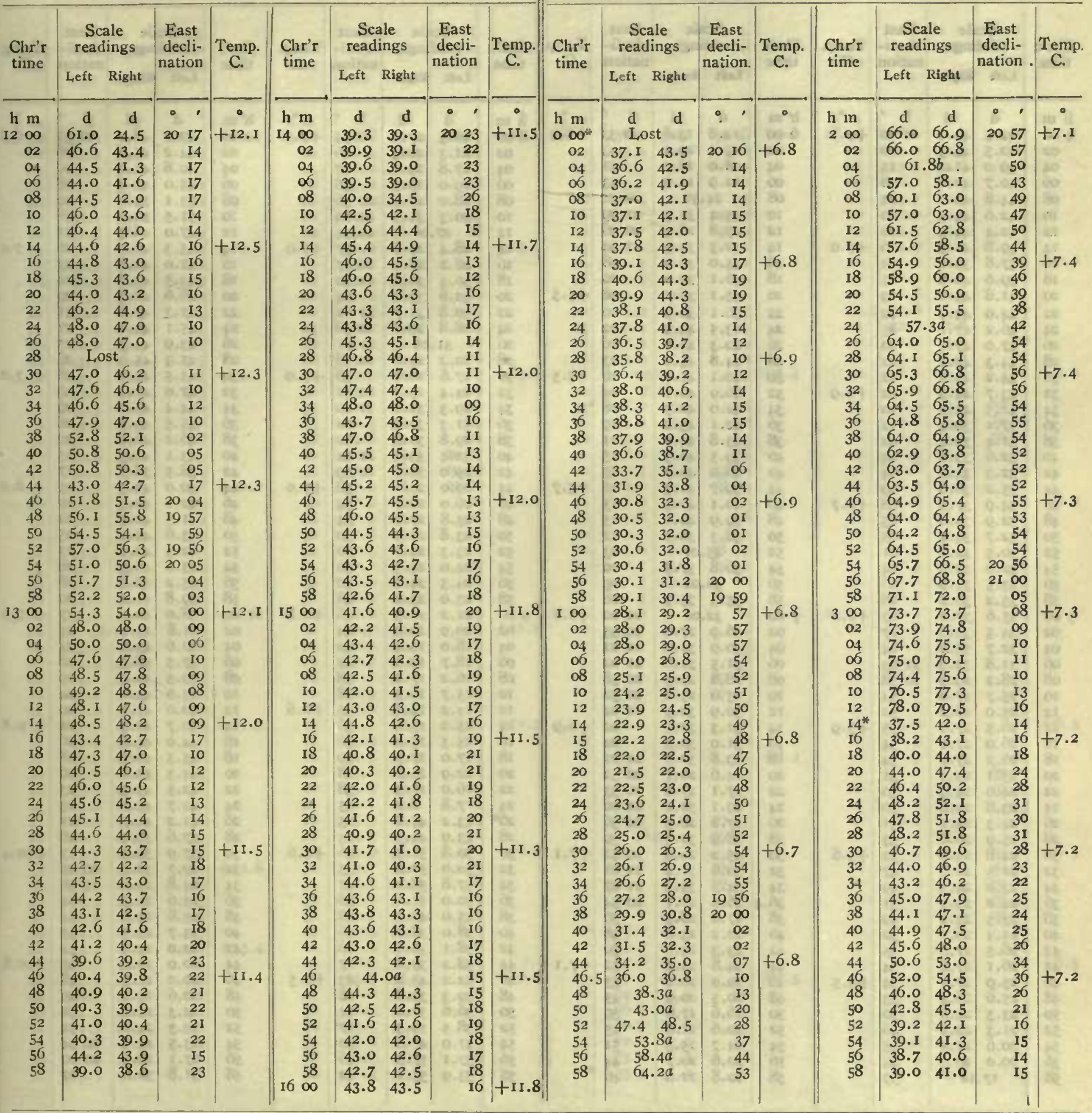

Correction to local mean time is - 10.55 .

Observer-A. F.

Torsion head at $12 \mathrm{~h}$ com read $249^{\circ}$ and at $\mathrm{i} 6 \mathrm{~h}$ oom read the same.

Observer-W. J. P. 
Tabulation of magnetic declinations obscrved at Alger Island Station-Continued

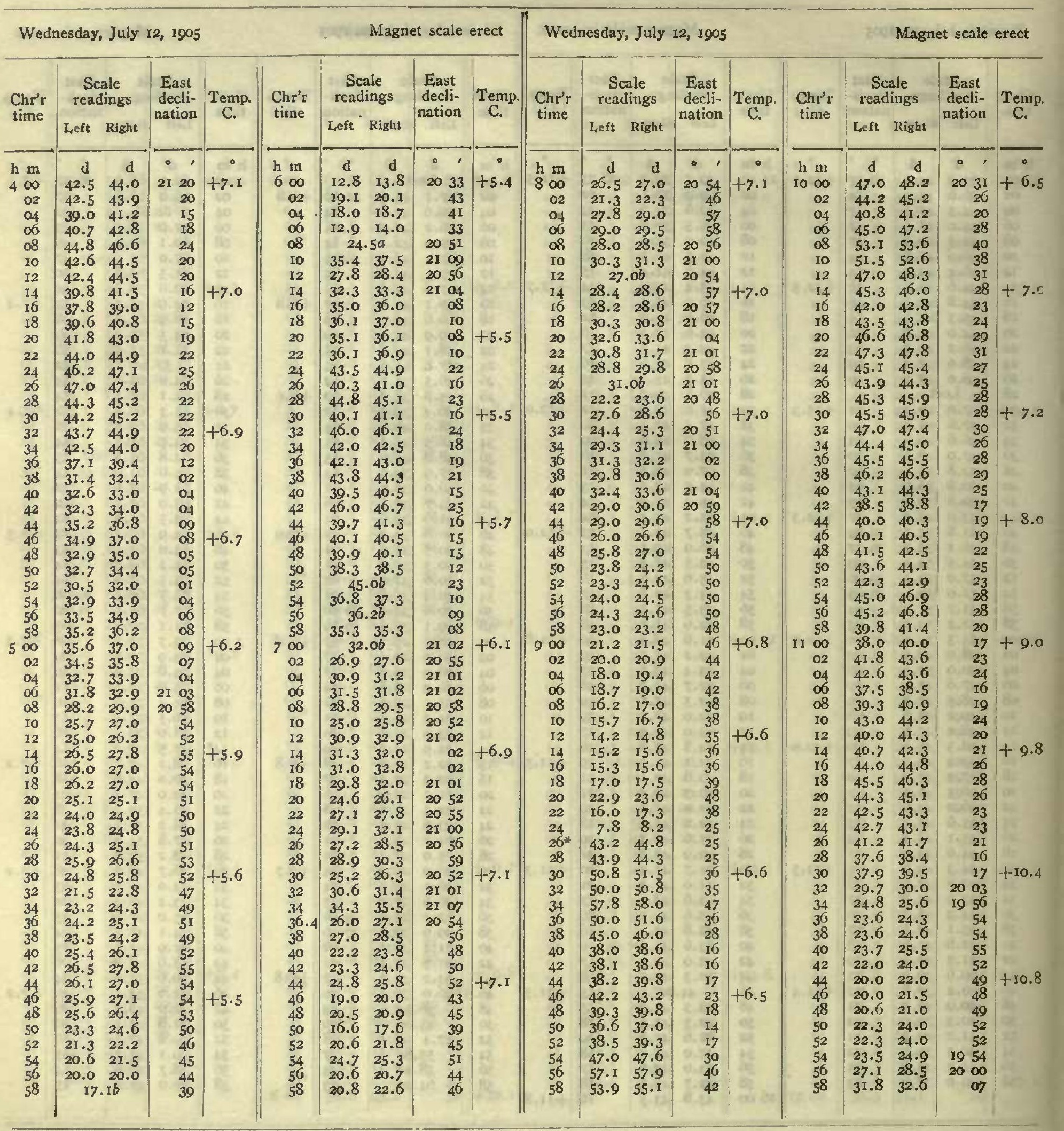

Observers-A. F. and W. J. P., who alternated from $6 \mathrm{~h} 04 \mathrm{~m}$ to Observer-W. J. P. $6 \mathrm{~h} 14 \mathrm{~m}$. 
Tabulation of magnetic declinations observed at Alger Island Station-Continued

Wednesday, July 12, 1905

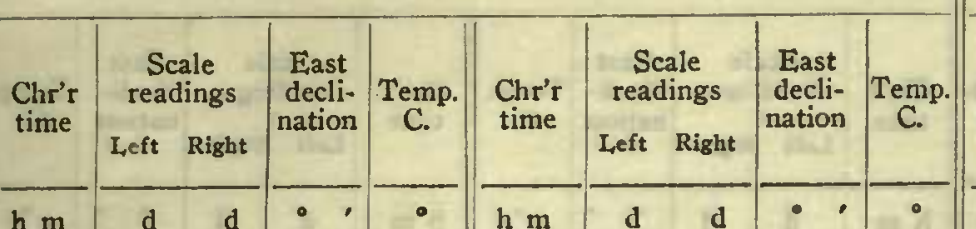

\begin{tabular}{l|rr|r|r}
00 & 32.9 & 34.6 & 2009 \\
02 & 33.0 & 34.0 & 09
\end{tabular} +10.4 14

$04 \quad 33.0 \quad 34.0$

$06 \quad 35.5 \quad 36.1$

$\begin{array}{lll}08 & 37.7 & 38.8\end{array}$

Io 38.139 .1

I2 $37.8 \quad 38.7$

1440.141 .0

$\begin{array}{lll}16 & 40.2 & 41.3\end{array}$

I8 $40.0 \quad 41.3$

$20 \quad 42.0 \quad 43.0$

$22 \quad 43.1 \quad 43.9$

$24 \quad 43.0 \quad 43.4$

$\begin{array}{lll}26 & 42.6 & 43.3\end{array}$

\begin{tabular}{l|ll}
28 & 43.5 & 44.0 \\
30 & 43.5 & 43.8
\end{tabular}

\begin{tabular}{l|ll}
32 & 43.6 & 44.3
\end{tabular}

$\begin{array}{lll}34 & 44.5 & 44.8\end{array}$

$\begin{array}{llll}36 & 44.9 & 45.1\end{array}$

$\begin{array}{lll}38 & 45.3 & 45.9\end{array}$

$40 \quad 42.8 \quad 43.1$

$42 \quad 49.0 \quad 49.6$

$44 \quad 5 I .3 \quad 5 I .5$

$46 \quad 50.0 \quad 50.6$

\begin{tabular}{l|l|l}
48 & 46.4 & 46.4
\end{tabular}

\begin{tabular}{l|ll}
50 & 45.5 & 46.0
\end{tabular}

\begin{tabular}{l|ll}
52 & 43.7 & 44.1
\end{tabular}

\begin{tabular}{l|ll}
54 & 43.2 & 44.0
\end{tabular}

\begin{tabular}{l|ll}
56 & 43.5 & 44.5 \\
58 & 44.0 & 44.8
\end{tabular}

$300 \quad 44.6 \quad 45.1$

$02 \quad 46.1 a$

\begin{tabular}{l|ll}
04 & 45.5 & 45.5
\end{tabular}

of $44.3 \quad 44.8$

$0844.1 \quad 44.6$

I0 45.246 .0

$\begin{array}{lll}12 & 46.2 & 46.7\end{array}$

I4 $47.0 \quad 47.6$

I6 $48.0 \quad 48.2$

$\begin{array}{lll}18 & 46.7 & 47.0\end{array}$

$20 \quad 46.0 \quad 46.5$

$22 \quad 45.5 \quad 45.9$

\begin{tabular}{l|ll}
24 & 44.8 & 45.1
\end{tabular}

\begin{tabular}{l|ll}
26 & 46.0 & 46.1 \\
28 & 46.1 & 47.7
\end{tabular}

\begin{tabular}{l|ll}
28 & 46.1 & 47.7 \\
30 & 45.7 & 46.2
\end{tabular}

$32 \quad 44.6 \quad 45.1$

\begin{tabular}{l|l|l}
34 & 44.2 & 44.8
\end{tabular}

\begin{tabular}{l|ll}
36 & 44.0 & 44.4
\end{tabular}

$\begin{array}{lll}38 & 43.9 & 44 . I\end{array}$

$\begin{array}{lll}40 & 42.5 & 43.0\end{array}$

\begin{tabular}{l|ll}
42 & 43.2 & 43.9
\end{tabular}

$44 \quad \begin{array}{lll}42.8 & 43.3\end{array}$

\begin{tabular}{l|ll}
46 & 40.6 & 41.3 \\
48 & 40.7 & 41.5
\end{tabular}

\begin{tabular}{l|ll}
48 & 40.7 & $4 I .5$ \\
50 & $4 I .4$ & $42 . I$
\end{tabular}

$\begin{array}{llll}52 & 41.5 & 42.1\end{array}$

\begin{tabular}{l|ll}
54 & 41.8 & 42.2
\end{tabular}

$\begin{array}{lll}56 & 40.8 & 41.1\end{array}$

\section{09}

\begin{tabular}{l|l|}
12 \\
16
\end{tabular}

$20+10.2$

20

20

24

24
24
23

23

25

24 + IO. I

25
26

27
28

23

$34+10.2$

35

29

25

24
25

26

$26+$ I I.O 1500

28

28
26

26

29

$30+I I .0$

$3 \mathrm{I}$

30

29
28

26

29

$28+12.8$

26

25

23.

$24+13.4$

24

20

22

22

20
18
$38.3 \quad 39.0$

38.239 .1

$44 \cdot 5 a$

IO $\quad 44.5 \quad 45.8$

I2 $45.0 \quad 46.0$

I4 $40.0 \quad 41.0$

34.936 .1

35.135 .6

37.838 .5

$41.5 a$

42.243 .0

$40.2 \quad 41.3$

39.140 .3

$39.7 \quad 40.4$

39.340 .3

$37.7 \quad 38.3$

$\begin{array}{ll}38.5 & 39.4\end{array}$

$\begin{array}{lll}40 & 32.1 & 32.9\end{array}$

\begin{tabular}{l|ll}
42 & 27.1 & 28.0 \\
44 & 27.2 & 28.4
\end{tabular}

\begin{tabular}{l|ll}
44 & 27.2 & 28.4 \\
46 & 27.6 & 29.1
\end{tabular}

$\begin{array}{lll}48 & 27.5 & 29.0\end{array}$

$\begin{array}{llll}50 & 27.8 & 29.5\end{array}$

\begin{tabular}{l|ll}
52 & 27.3 & 28.9
\end{tabular}

30.231 .0

$56 \quad 31.0 \quad 32.0$

$\begin{array}{lll}58 & 31.0 & 33.3\end{array}$

30.832 .1

30.732 .1

30.932 .1

$30.7 \quad 31.8$

$31.1 \quad 31.9$

$\begin{array}{ll}33.6 & 34.7\end{array}$

35.136 .2

$33.9 \quad 34.9$

32.534 .3

$34.1 \quad 35.7$

$35.0 \quad 36.6$

$32.1 \quad 34.8$

$33.3 \quad 34.5$

$34.3 \quad 35.3$

$\begin{array}{ll}34.9 & 36.2\end{array}$

33.134 .0

$32.8 \quad 33.9$

$33.2 \quad 34.5$

$33.7 \quad 34.9$

$33.4 \quad 35.4$

$31.2 \quad 33.0$

$29.9 \quad 31.3$

$32.5 \quad 33.9$

$32.0 \quad 33.0$

32.934 .0

$33.0 \quad 34.1$

$32.6 \quad 34.2$

$\begin{array}{ll}32.2 & 33.5 \\ 31.4 & 32.8\end{array}$
Wednesday, July I2, 1905

Magnet scale erect

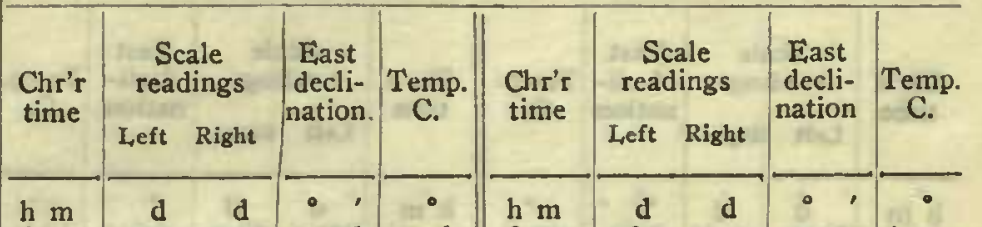

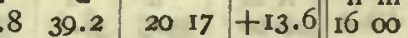

$\begin{array}{r}17 \\ 17\end{array}+13.6$ I6 00

\begin{tabular}{ll|l}
31.0 & 32.0 & $2006+9.6$
\end{tabular}

1800

$36.3 \quad 41.9$

$2032+7.1$

\begin{tabular}{ll|l|llll}
29.0 & 30.3 & 02 & & 02 & 36.0 & 40.8 \\
28.7 & 29.8 & 02 & & 04 & 35.5 & 39.9
\end{tabular}

$06 \quad 36.2 \quad 40.3$

$08 \quad 35.7 \quad 40.0$

$\begin{array}{lll}38 & 34.8 & 35.1\end{array}$

$32.3 \quad 33.4$

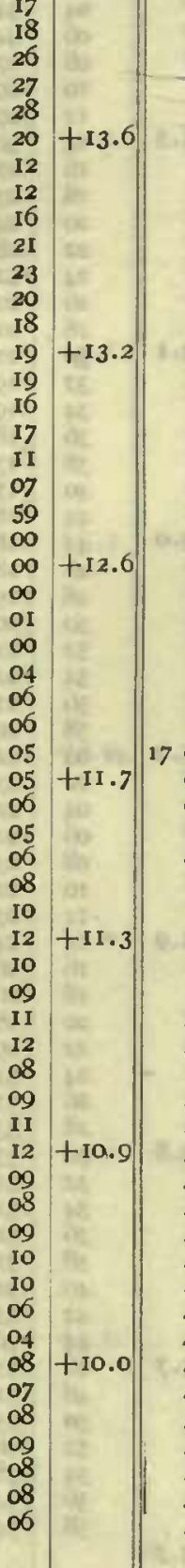

\begin{tabular}{l|ll|l|}
06 & 29.1 & 30.0 & 02 \\
08 & 30.8 & 31.8 & 05
\end{tabular}

10 $\quad 35.0 \quad 39.2$

$\begin{array}{lll}12 & 33.3 & 37.0\end{array}$

$14 \quad 34.9 \quad 36.1$

\begin{tabular}{l|l|l}
16 & 29.2 & 32.2
\end{tabular}

I8 $27.2 \quad 30.6$

$20 \quad 26.8 \quad 29.6$

$\begin{array}{lll}22.9 & 23.6 & 25.6\end{array}$

$\begin{array}{lll}24 & 22.2 & 24.8\end{array}$

$\begin{array}{lll}26 & 21.2 & 23.0\end{array}$

\begin{tabular}{l|ll}
28 & 19.6 & 21.8
\end{tabular}

$\begin{array}{lll}30 & 18.6 & 20.8\end{array}$

\begin{tabular}{l|ll}
32 & 19.0 & 20.9
\end{tabular}

$34 \quad 18.0 \quad 19.5$

$\begin{array}{lll}36 & 16.6 & 18.2\end{array}$

\begin{tabular}{l|ll}
38 & 18.3 & 21.1 \\
40 & 21.0 & 23.1
\end{tabular}

$40 \quad 21.923 .1$

$\begin{array}{lll}42 & 23.8 & 25.0\end{array}$

\begin{tabular}{l|ll}
44 & 25.3 & 26.8
\end{tabular}

\begin{tabular}{l|ll}
46 & 25.8 & 27.5
\end{tabular}

4826.628 .0

\begin{tabular}{l|ll}
50 & 29.1 & 30.9
\end{tabular}

\begin{tabular}{l|ll}
52 & 27.5 & 29.3
\end{tabular}

$\begin{array}{lll}54 & 27.5 & 29.1\end{array}$

$\begin{array}{lll}56 & 28.6 & 30.0\end{array}$

\begin{tabular}{lll|ll}
54 & 14.6 & 18.1 & 18 & 53 \\
\hline & 22.1 & 25.5 & 19 & 05
\end{tabular}

\begin{tabular}{l|ll}
56 & 27.3 & 29.7
\end{tabular}

$34.3 \quad 37.0$

$40.0 \quad 43.1$

12
23
33

\begin{tabular}{l|ll}
02 & 47.3 & 50.0
\end{tabular}

\begin{tabular}{l|ll}
04 & 53.5 & 55.9 \\
06 & 56.3 & 58.9
\end{tabular}

0857.0

$12 \quad 63.5 \quad 65.0 \quad 08$

1464.266 .0

$\begin{array}{lll}6 & 64.2 & 66.5\end{array}$

$\begin{array}{lll}18 & 67.5 & 68.8\end{array}$

67.168 .3

\begin{tabular}{l|ll}
22 & 67.1 & 68.7 \\
24 & 63.0 & 64.8
\end{tabular}

$26 \quad 60.261$.

28

$30 \quad 55.56$

$55.0 \quad 55.5$

$56.6 \quad 57.0$

$3150.0 \quad 59.5$

$36 \quad 59.360 .1$ or

$\begin{array}{lll}38 & 62.1 & 63.0\end{array}$

$\begin{array}{lll}42 & 65.2 & 66.3\end{array}$

$44 \quad 65.6 \quad 66.7$

$46 \quad 65.0 \quad 66.1$

\begin{tabular}{l|ll}
50 & 66.5 & 68.0
\end{tabular}

$\begin{array}{lll}52 & 70.7 & 72.0\end{array}$

$\begin{array}{llll}54 & 72.0 & 73.0\end{array}$

\begin{tabular}{l|ll}
56 & 75.0 & 76.1 \\
$58^{*}$ & 35.3 & 41.0
\end{tabular} \begin{tabular}{l|ll}
58 & 28.3 & 30.2
\end{tabular}

$\begin{array}{lll}900 & 29.1 & 31.3\end{array}$

$02 \quad 29.8 \quad 32.0$

$\begin{array}{lll}04 & 28.6 & 30.2\end{array}$

$06 \quad 29.0 \quad 31.0$

$08 \quad 28.8 \quad 30.0$

Io $31.0 \quad 33.0$

$\begin{array}{lll}12 & 28.8 & 30.0\end{array}$

$\begin{array}{lll}\text { If } & 25.3 & 26.6\end{array}$

$\begin{array}{lll}\text { I6 } & 22.5 & 23.7\end{array}$

I8 20.922 .1

$\begin{array}{lll}20 & 19.2 & 20.3\end{array}$

$24 \quad 15.0 \quad 16.1$

26 I0.5 II.9

2812.0 I 13.

$30 \quad 10.3 \quad 11.6$

$32 \quad 18.4 \quad 10.3$

34 I 4.9 I6. I

\begin{tabular}{l|ll}
36 & 10.6 & 11.6
\end{tabular}

$\begin{array}{lll}38 & \text { I8.2 } & 19.0\end{array}$

\begin{tabular}{l|ll}
40 & 21.1 & 21.8
\end{tabular}

\begin{tabular}{l|ll}
42 & 23.2 & 24.0
\end{tabular}

$\begin{array}{lll}44 & 24.2 & 25.0\end{array}$

$24.5 \quad 25.3$

$25.0 \quad 25.6$

25.826 .4

25.626 .2

$25.6 \quad 25.6$

$\begin{array}{ll}25.3 & 26.0 \\ 24.1 & 24.8\end{array}$

$3 I$
3 I

30

$3 I$

30

26

19 +7.0

I6

I5

I0

06

03

$02+7.0$

2002

I9 58

2002

06

12

I3 +6.9

I4

I6

17

$18+6.5$

I9

17
18 
Tabulation of magnetic declinations observed at Alger Island Station-Continued

\begin{tabular}{|c|c|c|c|c|c|c|c|c|c|c|c|c|c|c|c|c|c|c|c|}
\hline \multicolumn{6}{|c|}{ Wednesday, July 12, 1905} & \multicolumn{4}{|c|}{ Magnet scale erect } & \multicolumn{6}{|c|}{ Friday, July 14, 1905} & \multicolumn{4}{|c|}{ Magnet scale inverted } \\
\hline \multirow{2}{*}{$\begin{array}{l}\text { Chr'r } \\
\text { time }\end{array}$} & \multicolumn{2}{|c|}{$\begin{array}{l}\text { Scale } \\
\text { readings }\end{array}$} & \multirow[t]{2}{*}{$\begin{array}{l}\text { East } \\
\text { decli- } \\
\text { nation }\end{array}$} & Temp. & $\begin{array}{l}\text { Chr'r } \\
\text { time }\end{array}$ & $\begin{array}{l}\text { Sc } \\
\text { read }\end{array}$ & $\begin{array}{l}\text { ale } \\
\text { lings }\end{array}$ & $\begin{array}{c}\text { East } \\
\text { decli- } \\
\text { nation }\end{array}$ & Temp. & Chr'r & $\begin{array}{l}\text { Sc } \\
\text { read }\end{array}$ & $\begin{array}{l}\text { ale } \\
\text { lings }\end{array}$ & $\begin{array}{l}\text { East } \\
\text { decli- } \\
\text { nation }\end{array}$ & Temp. & Chr'r & $\begin{array}{l}\text { Sct } \\
\text { readi }\end{array}$ & $\begin{array}{l}\text { ale } \\
\text { lings }\end{array}$ & East & Temp. \\
\hline & Left & Right & & & & Left & Right & & & & Left & Right & & & & Left & Right & & \\
\hline h in & d & d & $\circ \quad$ & 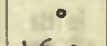 & h $\mathrm{m}$ & -0 & 6 & & & h m & d & & $\cdot ;$ & . & $\mathrm{h} \mathrm{m}$ & d & d & & \\
\hline 2000 & 22.5 & 23.0 & 2007 & +6.3 & $22 \infty$ & 58.9 & 60.9 & 2006 & +4.4 & 2000 & 39.5 & 38.8 & I9 56 & +4.0 & 2200 & 34.5 & $34 \cdot 3$ & 2004 & +4.0 \\
\hline 02 & 21.7 & 22.3 & 06 & 6 & 02 & 65.4 & $67 \cdot 5$ & & $\tan 2$ & 02 & 38.0 & 36.7 & 1959 & 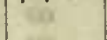 & 02 & 34.2 & 33.9 & & \\
\hline 04 & 21.9 & 22.5 & 06 & tais & 04 & 63.0 & 65.8 & 13 & 10 & 04 & 34.5 & 33.0 & 2005 & In & 04 & $33 \cdot 3$ & 33.0 & 06 & \\
\hline 06 & $21 . \overline{8}$ & 22.2 & 06 & 90 & 06 & 68.3 & 71.0 & & 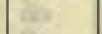 & 06 & 34.0 & 32.1 & 06 & 08 & 06 & 32.7 & 32.3 & 06 & 15 \\
\hline 08 & 20.5 & 20.9 & 03 & sur & 08 & 68.3 & 71.1 & $2 I$ & 60. & 08 & 27.5 & 26.4 & 15 & 20 & o8 & 33.0 & 32.6 & 06 & bs \\
\hline I0 & 18.6 & 18.9 & $20 \infty$ & Oed & 10 & 76.0 & 78.0 & 32 & 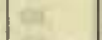 & I0 & 29.0 & 28.0 & 13 & at & 10 & $33 \cdot 3$ & 33.0 & 06 & or \\
\hline 12 & 15.8 & 16.0 & 1956 & 4 & 12 & 73.3 & 76.1 & 29 & & 12 & 29.2 & 27.6 & 13 & $1=$ & I2 & 34.0 & 32.3 & 06 & \\
\hline 14 & II.9 & 12.7 & 50 & +6.1 & 14 & 73.8 & 76.8 & 30 & +4.3 & $I_{4}$ & 30.1 & 29.0 & II & +4.0 & 14 & $33 \cdot 3$ & 32.9 & 06 & +4.0 \\
\hline 16 & $7 \cdot 3$ & 7.8 & 43 & 24 & 16 & 74.0 & 76.6 & 30 & 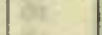 & 16 & 27.9 & 26.1 & 15 & & 16 & 32.3 & 32.3 & 07 & \\
\hline I8 & $7 \cdot 3$ & 7.7 & 43 & At & 18 & 68.0 & 72.0 & 21 & $4 i$ & 18 & 25.3 & 23.8 & 19 & $2 \pi$ & 18 & 32.0 & $3 \mathrm{I} .5$ & 08 & \\
\hline $20 *$ & 43.0 & 48.0 & 43 & cat & 20 & 61.5 & 65.3 & $20 \mathrm{II}$ & set: & 20 & 24.5 & 23.0 & 20 & as & 20 & 31.6 & 30.6 & 09 & int: \\
\hline 22 & 42.5 & 47.0 & 42 & 60 & 22 & 53.3 & 55.7 & 1957 & as & 22 & 28.4 & 26.6 & 14 & as & 22 & 30.0 & 29.2 & II & stiz \\
\hline 24 & 41.3 & 45.2 & $\quad 39$ & 14. & 24 & 50.4 & 52.4 & 52 & in & 24 & 22.0 & 20.5 & 24 & If & 24 & 30.4 & 29.9 & I0 & $y=$ \\
\hline 26 & 40.7 & 44.9 & 39 & 86 & 26 & 50.2 & 52.8 & 52 & Es & 26 & 22.1 & 21.0 & 24 & on & 26 & 3I.I & 30.2 & IO & \\
\hline 28 & 44.6 & 48.2 & 44 & 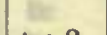 & 28 & $5 \mathrm{I} \cdot 3$ & 53.1 & 1954 & 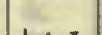 & 28 & 22.3 & 21.4 & 23 & to & 28 & 32.8 & 31.8 & 07 & \\
\hline 30 & 46.4 & 50.0 & 47 & +5.8 & 30 & 56.8 & 59.0 & 2002 & +4.1 & 30 & 21.6 & 20.6 & 24 & +4.0 & 30 & 32.2 & $3 \mathrm{I} .2$ & 08 & +4.0 \\
\hline 32 & 46.2 & 49.6 & 47 & & 32 & 57.0 & 59.1 & 02 & $1=0$ & 32 & 19.5 & 19.0 & 27 & 1 & 32 & 32.3 & 31.7 & 07 & \\
\hline 34 & 48.1 & 51.1 & 49 & 26 & 34 & 65.6 & 67.0 & 16 & 46 & 34 & I9. I & I8. 2 & 28 & is & 34 & 34.5 & 34.0 & 2004 & 12 \\
\hline 36 & 49.9 & 53.1 & 52 & 8 & 36 & 59.5 & 61.5 & 2006 & 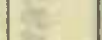 & 36 & 18.3 & I7. 5 & 30 & 8 & 36 & 37.5 & 37.1 & I9 59 & It. \\
\hline 38 & 48.9 & 51.9 & 5I & acc & 38 & 55.5 & 56.0 & 1959 & 8 & 38 & 17.5 & 17.0 & $3 I$ & ats & 38 & 42.0 & 41.8 & 52 & Re \\
\hline 40 & $47 \cdot 3$ & 50.2 & 48 & 요 & 40 & 58.3 & 61.0 & 2005 & $\omega$ & 40 & 17.3 & I6. 2 & $3 \mathrm{I}$ & $\alpha$ & 40 & 40.0 & 39.2 & I9 55 & 4 \\
\hline 42 & $47 \cdot 3$ & 49.9 & 48 & $4 x$ & 42 & 66.8 & 67.1 & 16 & . & 42 & 18.6 & 17.8 & 29 & 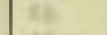 & 42 & 36.0 & 35.8 & $20 \mathrm{OI}$ & 28 \\
\hline 44 & 47.2 & 49.5 & 47 & 15 & 44 & 73.8 & 74.6 & 28 & +4.0 & 44 & 18.8 & 18.1 & 29 & $14=$ & 44 & 37.0 & 36.3 & $\infty$ & \\
\hline 46 & 46.6 & 49.0 & 47 & +5.5 & 46 & 59.0 & 60.6 & 2006 & 8 & 46 & 18.6 & 18.3 & 28 & +4.0 & 46 & 36.2 & 35.6 & OI & +4.0 \\
\hline 48 & 46. I & 48.3 & 46 & क & 48 & 45.6 & 46.6 & 1944 & 86 & 48 & 19.0 & 18.3 & 28 & & 48 & 34.0 & $33 \cdot 3$ & 05 & \\
\hline 50 & $47 . I$ & 49.2 & 47 & ee & 50 & 49.0 & 50.3 & 49 & $e$ & 50 & 20.0 & 18.8 & 27 & $\alpha$ & 50 & 32.2 & $3 \mathrm{I} .0$ & 08 & \% \\
\hline $5^{2}$ & 46.0 & 48.5 & 46 & na & 52 & 44.6 & 45.5 & 42 & at & 52 & 22.0 & 21.6 & 23 & $\mathrm{at}$ & 52 & 33.0 & 32.1 & 07 & 44 \\
\hline 54 & 45.7 & 47.8 & 45 & 12 & 54 & 39.7 & 42.3 & 36 & te & 54 & 22.2 & 21.3 & 23 & E & 54 & 32.9 & 31.9 & 07 & $\mathrm{~N}^{2}$ \\
\hline 56 & 46.0 & 47.9 & 45 & E & 56 & 36.1 & 36.9 & 29 & 08 & 56 & $2 \mathrm{I} .5$ & 21.3 & 24 & 伐 & 56 & 31.2 & 30.4 & 09 & \\
\hline 58 & 45.2 & 46.8 & 44 & 25 & 58 & 36.6 & 36.6 & 29 & 1 & 58 & 20.0 & I9.6 & 26 & 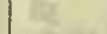 & 58 & 33.0 & 32.2 & 06 & \\
\hline 2100 & 41.2 & 43.3 & 38 & +5.2 & 2300 & 44.0 & 45.0 & $4 I$ & +4.0 & 2100 & 22.8 & 22.1 & . 22 & +4.0 & 2300 & 34.9 & 33.9 & A. 04 & +3.8 \\
\hline 02 & 39.1 & 41.0 & 34 & 80 & 02 & 43.2 & 43.2 & 39 & 26 & 02 & 23.8 & 23.4 & $2 I$ & & 02 & 34.8 & 33.8 & 04 & \\
\hline 04 & 38.5 & 40.1 & 33 & 15 & 04 & $4 \mathrm{I} .9$ & 42.5 & 38 & 10 & 04 & 22. I & 21.8 & 23 & fel & 04 & 34.6 & 33.2 & 04 & 30 \\
\hline 06 & $41 \cdot 3$ & 43.0 & 38 & 80 & 06 & 45.0 & 45.6 & 43 & 3 int & 06 & 22.0 & 21.1 & 24 & (n) & 06 & 36.9 & 35.2 & OI & 90 \\
\hline 08 & 40.8 & 42.6 & 37 & 84 & 08 & $44 \cdot 3$ & $44 \cdot 3$ & 41 & we? & 08 & 23.0 & 23.0 & 22 & 6 & 08 & $36 . I$ & 34.5 & 02 & t \\
\hline I0 & 42.1 & 43.7 & 39 & 91 & 10 & 50 & $.0 a$ & 1950 & 에 & I0 & 22.0 & $2 \mathrm{I} .2$ & 24 & er & 10 & 36.2 & 35.0 & 02 & of \\
\hline 12 & 43.5 & 45.0 & 4I I & 4 & 12 & 59.0 & 59.5 & 2004 & 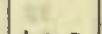 & I2 & 23.8 & 23.3 & $2 I$ & ar: & 12 & 36.0 & 34.6 & 02 & \\
\hline 14 & 46.6 & 47.6 & 46 & +5.0 & 14 & 57.5 & 57.5 & 2002 & +3.9 & 14 & 24.5 & 24.2 & 19 & +4.0 & I4 & 35.4 & 34.0 & 03 & +3.8 \\
\hline 16 & 47.5 & 48.3 & 47 & & 16 & 52.0 & 52.6 & 1954 & & 16 & 26.2 & 26.1 & 16 & & 16 & 35.3 & 33.9 & 03 & \\
\hline I8 & 49.2 & 50.1 & 49 & (4) & I8 & 60.9 & 62.3 & 2008 & atr & 18 & 26.7 & 26.3 & I6 & 81 & 18 & 34.2 & 32.8 & 05 & tin \\
\hline 20 & 48.9 & 49.0 & 48 & od & 20 & 60.5 & $6 \mathrm{I} .1$ & 07 & 06 & 20 & 28.3 & 28.1 & 13 & $\approx$ & 20 & 33.5 & 32.0 & 06 & $\omega$ \\
\hline 22 & 46.0 & 47.1 & 45 & Iat & 22 & 58.0 & 58.5 & 03 & st & 22 & 29.9 & 29.5 & II & +5 & 22 & 32.9 & $3 \mathrm{I} .2$ & 07 & bit \\
\hline 24 & 44.0 & 45.0 & $4 I$ & +4 & 24 & 55.4 & 56.6 & $\infty$ & 18 & 24 & 31.0 & 30.8 & 09 & 146 & 24 & 32.2 & 30.6 & 08 & 8 \\
\hline 26 & 44.6 & 46.0 & 43 & es & 26 & 57.0 & 58.4 & 02 & as & 26 & 31.4 & $3 \mathrm{I} . \mathrm{I}$ & 09 & 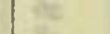 & 26 & 32.3 & 31.0 & 08 & \\
\hline 28 & 43.4 & 44.4 & 40 & $3=$ & 28 & 58.4 & 59.0 & 04 & 0 & 28 & 33.0 & 32.6 & 06 & $1=$ & 28 & 32.0 & 30.6 & 08 & \\
\hline 30 & 44.5 & 45.5 & 42 & +4.8 & 30 & 57.8 & 58.6 & 03 & +3.8 & 30 & 33.0 & 32.8 & 06 & +4.0 & 30 & 30.3 & 29.3 & II & +3.8 \\
\hline 32 & 44.0 & 45.0 & 41 & 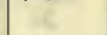 & 32 & 55.8 & 56.8 & $\infty$ & r & 32 & 31.8 & $3 \mathrm{I} .2$ & 08 & ( & 32 & 30.3 & 29.3 & II & \\
\hline 34 & 44.3 & 45.0 & 42 & $2 x$ & 34 & 55.6 & 56.8 & $\infty$ & it & 34 & 3I.I & 31.1 & 09 & 143 & 34 & 31.9 & 31.0 & 08 & 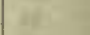 \\
\hline 36 & 43.8 & 44.6 & $4 \mathrm{I}$ & $\theta=$ & 36 & 56.0 & 57.0 & $\infty$ & $x^{2}$ & 36 & 3 I. 9 & 31.8 & 08 & 32 & 36 & 33.2 & 32.6 & 06 & \\
\hline 38 & 46.1 & 47.5 & 45 & Ne. & 38 & 60.8 & $6 \mathrm{I} .1$ & 07 & $2 x$ & 38 & 31.8 & 31.7 & 08 & 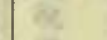 & 38 & $34 \cdot 5$ & 33.6 & 04 & \\
\hline 40 & 47.8 & 48.5 & 47 & it & 40 & 61.0 & 62.0 & 08 & es & 40 & $34 . I$ & 34.1 & 04 & 4 & 40 & 34.0 & 32.4 & 06 & $6=$ \\
\hline 42 & 48.0 & 48.5 & 47 & & 42 & 59.0 & 59.6 & 05 & 8 & 42 & 35.6 & 35.2 & 02 & 25 & 42 & 32.0 & 30.5 & $\infty 9$ & \\
\hline & 49.4 & 50.3 & 50 & +4.6 & 44 & 61.3 & 62.1 & 08 & & 44 & 36.5 & 36.0 & OI & +4.0 & 44 & 32.0 & 30.4 & $\infty 9$ & +3.6 \\
\hline 46 & 50.9 & 52.0 & 52 & $\sigma^{\circ}$ & 46 & 64.5 & 65.3 & 13 & +3.7 & 46 & 35.0 & $34 \cdot 3$ & 03 & r. & 46 & 35.0 & 32.5 & $0 \overline{8}$ & \\
\hline 48 & 52.8 & 54.3 & 56 & Ex & 48 & 71.8 & 72.0 & 24 & (2) & 48 & 32.4 & 32.1 & 07 & 84 & 48 & 33.0 & 31.6 & 07 & \\
\hline 50 & 53.1 & 54.3 & 56 & at & 50 & 70.9 & 71.9 & 24 & fis & 50 & $35 . i$ & 35.0 & 2003 & 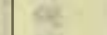 & 50 & 33.1 & 31.9 & 06 & \\
\hline 52 & 54.7 & 55.6 & 1958 & c & 52 & 68.2 & 68.5 & I9 & 82 & 52 & 38.2 & 38.0 & I9 58 & in & 52 & 33.1 & 31.5 & 07 & \\
\hline 54 & 60.1 & 61.5 & 2007 & $y^{3}$ & 54 & 70.4 & 70.6 & 22 & $z^{2}$ & 54 & 38.2 & 37.6 & 1958 & $R$ & 54 & 31.8 & 30.3 & 09 & \\
\hline 56 & 57.6 & 58.0 & 02 & $\alpha$ & 56 & 68.9 & 69.9 & 920 & AE & 56 & 34.0 & 34.0 & 2004 & es & 56 & 29.2 & 27.9 & 13 & \\
\hline 58 & 58.3 & 59.3 & 04 & 82 & 58 & 65.7 & 66.3 & I5 & 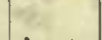 & 58 & 34.1 & 33.7 & 04 & 16 & 58 & 27.0 & 26.0 & & \\
\hline & & & & & 2400 & 63.3 & 63.6 & II & +3.7 & & & & & & $24 \infty 0$ & 25.3 & 24.7 & 18 & +3.5 \\
\hline
\end{tabular}

Correction to local mean time is os.

Torsion head at beginning and ending read $252^{\circ}$.

Observer-W. J. P.
Correction to local mean time is - 19s. $90^{\circ}$ torsion $=18 . ' 05$. Torsion head at $20 \mathrm{~h}$ com read $252^{\circ}$ and at $24 \mathrm{~h} 15 \mathrm{~m}$ read $242^{\circ}$. Observer-W. J. P. 
Tabulation of magnetic declinations observed at Alger Island Station-Continued

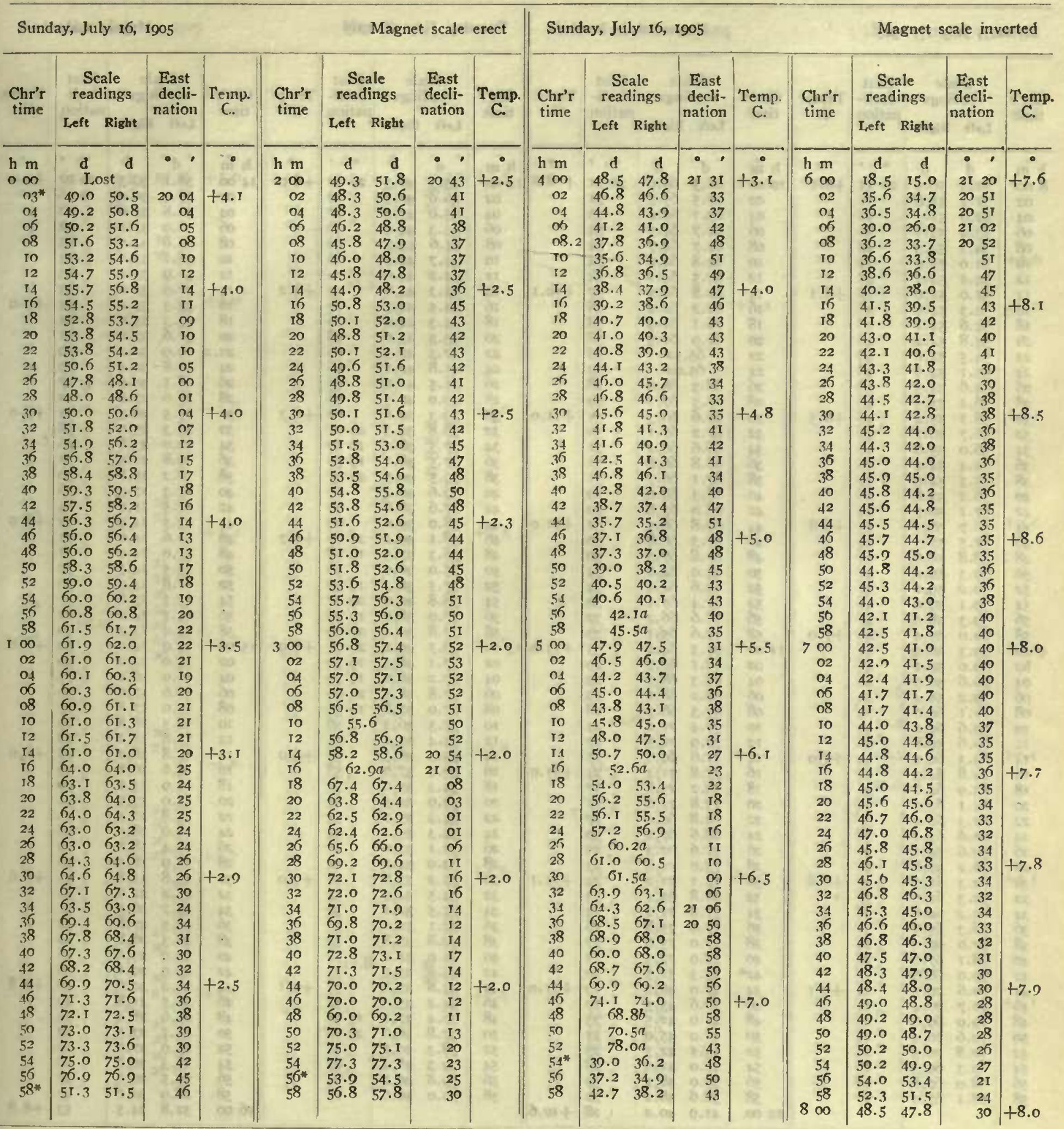

Observer-W. J. P.

Correction to local mean time is $-20 \mathrm{~s} .90^{\circ}$ torsion $=18^{\prime} 70$.

Torsion head at oh oom read $242^{\circ}$ and at 8 h oom read $269^{\circ}$.

Observers-W. J. P. and A. F., who alternated from 4 h $00 \mathrm{~m}$ to 4h rom. 
Tabulation of magnetic declinations observed at Alger Island Station-Continued

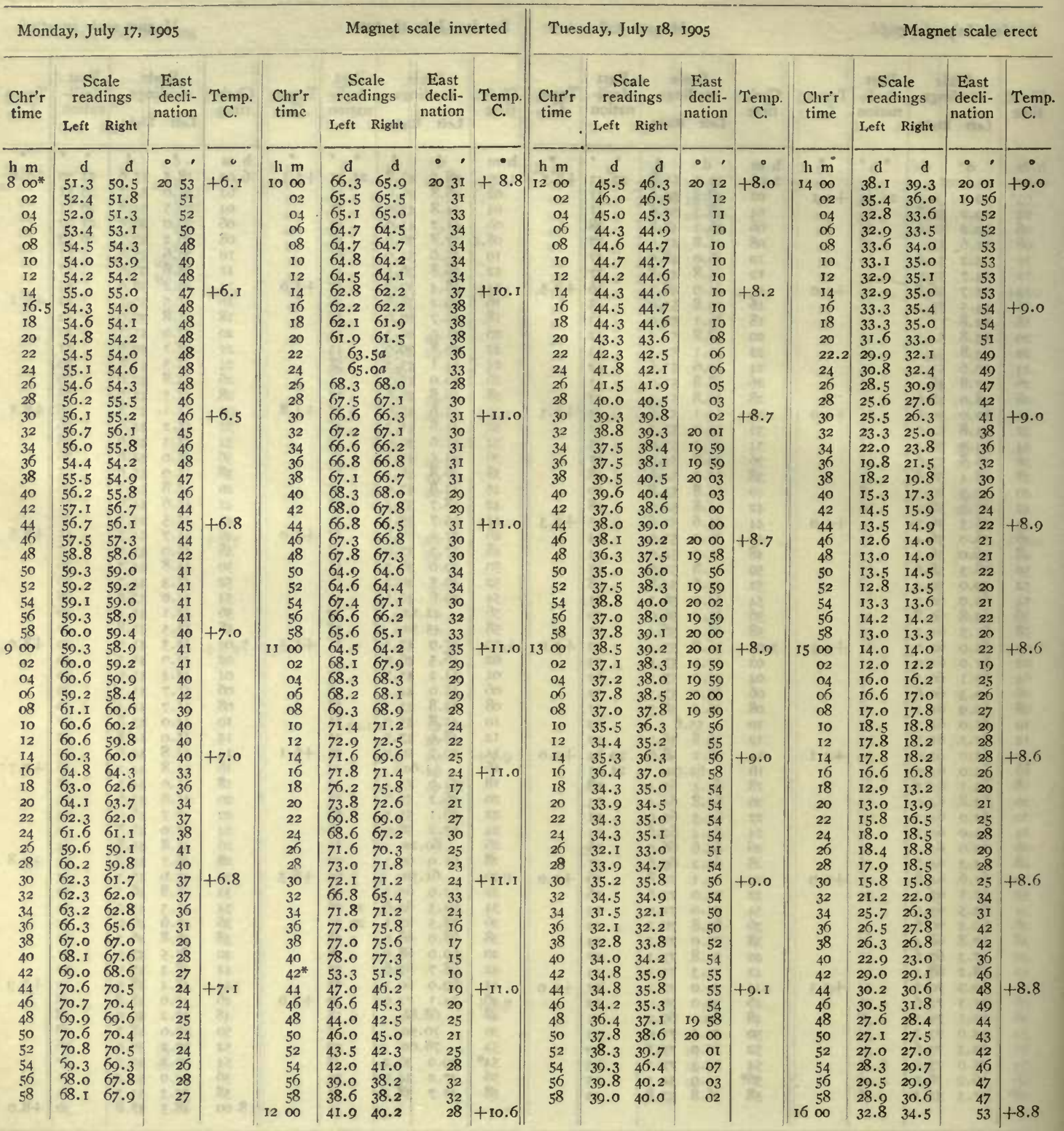

Correction to local mean time is - 22.55. $90^{\circ}$ torsion $=18 . .^{\prime} 02$. Torsion head at $8 \mathrm{~h}$ oom read $272^{\circ}$ and at $\mathrm{I} 2 \mathrm{~h} 59 \mathrm{~m}$ read $250^{\circ}$. Observer-W. J. P.
Correction to local mean timc is about $-155.90^{\circ}$ torsion $=177^{\prime} 40$. Torsion head at $12 \mathrm{~h}$ oom read $246^{\circ}$ and at $16 \mathrm{~h} 25 \mathrm{~m}$ read $255^{\circ}$. Observer-W. J. P. 
Tabulation of magnetic declinations obserzed at Alger Island Station-Continued

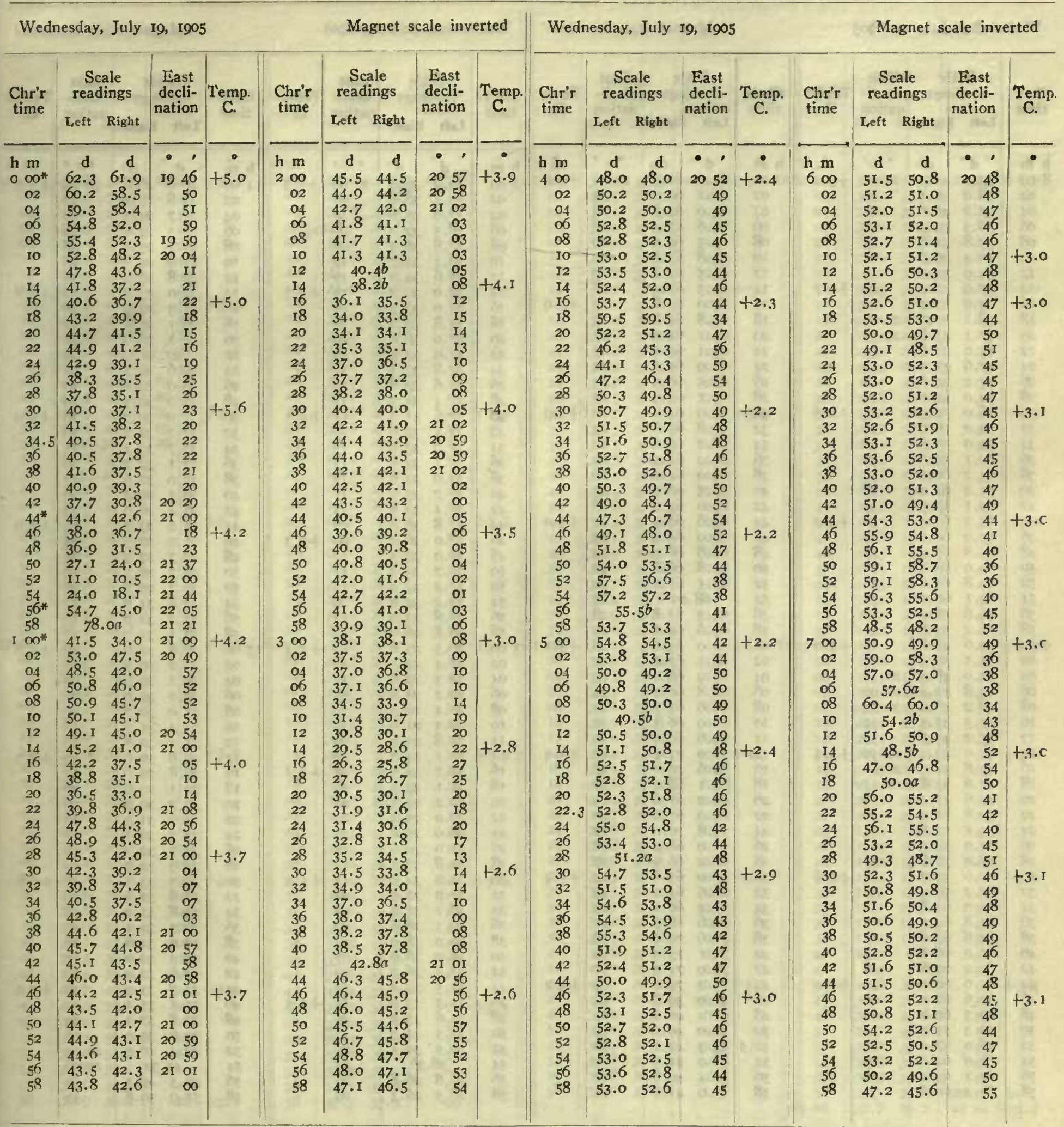


Tabulation of magnetic declinations observed at Alger Island Station-Continued

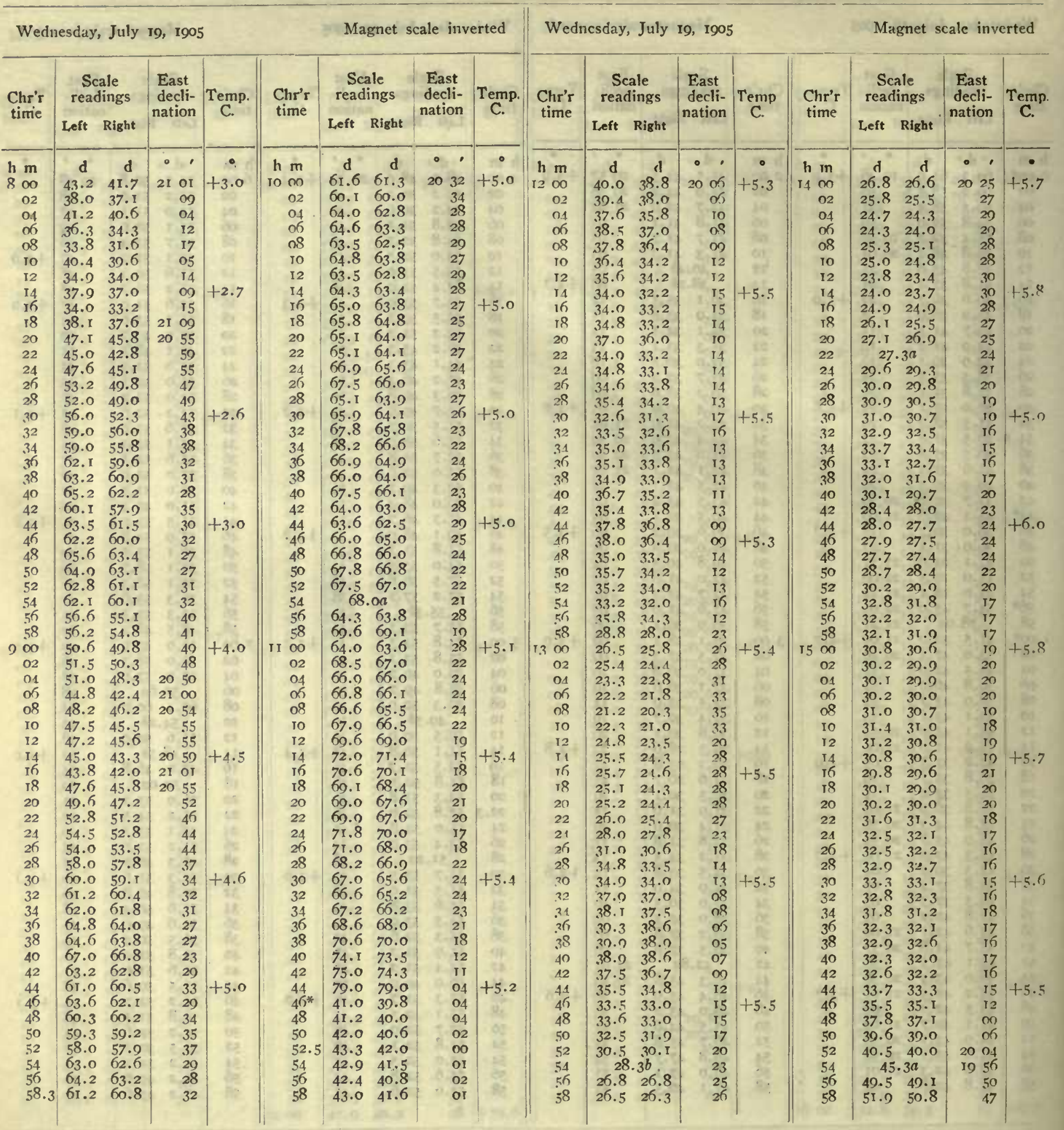


Tabulation of magnetic declinations observed at Alger Island Station-Continued

\begin{tabular}{|c|c|c|c|c|c|c|c|c|c|c|c|c|c|c|c|c|c|c|c|}
\hline \multicolumn{5}{|c|}{ Wednesday, July 19, Igo5 } & \multicolumn{5}{|c|}{ Magnet scale inverted } & \multicolumn{6}{|c|}{ Wednesday, July I9, Ig05 } & \multicolumn{4}{|c|}{ Magnet scale inverted } \\
\hline \multirow{2}{*}{$\begin{array}{l}\text { Chr'r } \\
\text { time }\end{array}$} & \multicolumn{2}{|c|}{$\begin{array}{l}\text { Scale } \\
\text { readings }\end{array}$} & \multirow{2}{*}{$\begin{array}{c}\text { East } \\
\text { decli- } \\
\text { nation }\end{array}$} & $\begin{array}{l}\text { Temp. } \\
\text { C. }\end{array}$ & $\begin{array}{l}\text { Chr'r } \\
\text { time }\end{array}$ & $\begin{array}{l}\text { Sc } \\
\text { read }\end{array}$ & $\begin{array}{l}\text { ale } \\
\text { lings }\end{array}$ & $\begin{array}{c}\text { East } \\
\text { decli- } \\
\text { nation }\end{array}$ & $\underset{\mathrm{C} .}{\text { Temp. }}$ & $\begin{array}{l}\text { Chr'r } \\
\text { time }\end{array}$ & $\begin{array}{l}\text { Sc } \\
\text { read }\end{array}$ & $\begin{array}{l}\text { le } \\
\text { ngs }\end{array}$ & $\begin{array}{c}\text { East } \\
\text { decli- }\end{array}$ & Temp. & Chr'r & $\begin{array}{l}\text { Sc: } \\
\text { read }\end{array}$ & $\begin{array}{l}\text { le } \\
\text { igs }\end{array}$ & $\begin{array}{l}\text { East } \\
\text { decli- }\end{array}$ & $\begin{array}{l}\text { Temp. } \\
\text { C. }\end{array}$ \\
\hline & Left & Right & & & & Left & Right & & & & Left & Right & & & & Left & Right & & \\
\hline $\mathrm{h} \mathrm{m}$ & $\mathrm{d}$ & d & & & $h \mathrm{~m}$ & $d$ & d & & & $\mathrm{h} \mathrm{m}$ & $\mathrm{d}$ & d & $\circ \quad$ & & h $\mathrm{m}$ & d & d & $\cdot 1$ & \\
\hline $6 \infty$ & 51.8 & 51.5 & I9 46 & +5.5 & 1800 & 36.6 & 36.5 & 2010 & & 2000 & $37 \cdot 3$ & $37 \cdot 3$ & $20 \propto 9$ & +5.2 & 2200 & 35.2 & $34 \cdot 5$ & $20 \quad 13$ & +4.0 \\
\hline $\mathrm{O} 2$ & 52.3 & 52.3 & 45 & & 02 & 36.2 & 35.9 & II & & 02 & 37.5 & $37 \cdot 3$ & $\infty$ & 1.0 & 02 & 34.2 & 33.4 & I4 & \\
\hline 04 & 2.6 & 52.4 & 45 & 10 & 04 & 35.8 & 35.2 & 12 & & 04 & 7.5 & $37 \cdot 3$ & $\infty$ & 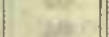 & 04 & 33.2 & 33. & 15 & \\
\hline 06 & 2.5 & 52.1 & 45 & 35 & 06 & 33.9 & 33. I & 15 & & 06 & & $37 \cdot 4$ & 8 & 2 & 06 & 33.0 & 5 & 16 & (1) \\
\hline 08 & 53.4 & 52.9 & 44 & e & 08 & 33.2 & 32.5 & 16 & & $\infty 8$ & & 37.8 & 08 & & $\infty 8$ & 33.2 & 32.8 & I6 & 4 \\
\hline 10 & 54.0 & 53.8 & 43 & & IO & 33.6 & 33.2 & 15 & & IO & .0 & 37.8 & 08 & yer & IO & 32.6 & 32.3 & 16 & t \\
\hline 12 & 53.2 & 53.0 & 44 & & I2 & 35.1 & $34 \cdot 5$ & 13 & & 12 & 37.8 & 37.6 & 08 & & I2 & 33.2 & 32. & 16 & \\
\hline 14 & 52.3 & 52.3 & 45 & & I4 & 35.9 & 35.2 & 12 & +5.8 & 14 & 37.2 & 37.0 & $\infty$ & $+5 . I$ & 14 & $33 \cdot 3$ & 33. & 15 & +4.0 \\
\hline I6 & 48.9 & 48.9 & 50 & +5.4 & 16 & 35.1 & 34.8 & 12 & & 16 & 37.0 & 36.8 & $\infty$ & & I6 & 30.6 & 30.0 & 20 & $x^{-2}$ \\
\hline 18 & 47.6 & $47 \cdot 3$ & 53 & & 18 & 34.6 & 34.0 & I3 & & 18 & 37.2 & 36.6 & $\infty$ & & 18 & 27.2 & 26.5 & 25 & 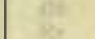 \\
\hline 20 & 47.0 & 46.8 & 54 & & 20 & 34.6 & 33.8 & 14 & & 20 & 37.6 & $37 \cdot 2$ & $\infty$ & & 20 & $24 \cdot 5$ & 24.0 & 29 & \\
\hline 22 & 45.9 & $45 \cdot 5$ & 56 & & 22 & 33.6 & 33.0 & 15 & & 22 & 38.8 & 38.0 & 07 & & 22 & 24.0 & 23.7 & 30 & \\
\hline 24 & 45.1 & 44.8 & 57 & & 24 & 32.2 & 32.0 & 17 & 6. & 24 & 38.0 & 37.5 & 08 & & 24 & $24 \cdot 3$ & 24.1 & 29 & \\
\hline 26 & 44.1 & 43.7 & 1958 & & 26 & 31.0 & 30.3 & 19 & test & 26 & 37.7 & 37.3 & $\infty 8$ & & 26 & 23.7 & 23.5 & 30 & \\
\hline 28 & $43 \cdot 4$ & 43.0 & 2000 & & 28 & 30.8 & 30.1 & 20 & & 28 & $37 \cdot 5$ & $37 \cdot 3$ & $\infty 8$ & & 28 & 22.8 & 22 & $3 I$ & \\
\hline 30 & $44 \cdot 5$ & 44.0 & I9 58 & +5.3 & 30 & 30.5 & 30.0 & 20 & +5.6 & 30 & 37.5 & 37.2 & 09 & +5.0 & 30 & 22.8 & 22.7 & 32 & +4.0 \\
\hline 32 & $4 \cdot 4$ & $44 . I$ & I9 58 & & 32 & 30.5 & 30.0 & 20 & & 32 & $37 \cdot 5$ & 37.2 & $\infty$ & & 32 & 22.6 & 22 & 32 & \\
\hline 34 & 42 & $3^{b}$ & 2001 & & 34 & 30.0 & 29.5 & $2 I$ & & 34 & 38.5 & 37.6 & 08 & & 34 & 21.6 & $21 \cdot 5$ & 34 & \\
\hline 36 & 39.6 & 39.0 & 06 & & 36 & $29 \cdot 4$ & 28.5 & 22 & & 36 & 38.3 & 38.0 & 07 & & 36 & 21.0 & 20 & 35 & \\
\hline 38 & 35 & $4 b$ & 12 & & 38 & 29.1 & 28.0 & 22 & & 38 & 38.2 & 38.0 & 07 & & 3 & 19.8 & 19 & 37 & \\
\hline 40 & 34.2 & 33.9 & I4 & & 40 & 28.8 & 28.3 & 22 & & 40 & 38.4 & 37.7 & 08 & & 40 & 19.5 & & 37 & \\
\hline 42 & 6.0 & 35.8 & II & & 42 & 29.2 & 28.7 & 22 & & 42 & 37.8 & 37.1 & 0 & n & 42 & 19.8 & & 37 & \\
\hline 44 & 34.8 & $34 \cdot 5$ & 13 & +5.1 & 44 & 29.8 & 29.I & $2 I$ & +5.5 & 44 & 37.3 & 37.0 & $\infty$ & 1 & 44 & 16.3 & 16. & 42 & +4.0 \\
\hline 46 & 33.7 & 33.5 & 15 & 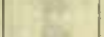 & 46 & 30.5 & 29.8 & 20 & & 46 & 37.1 & 36.7 & $\infty$ & & 46 & 15.8 & I5. & 43 & \\
\hline 48 & $33 \cdot 5$ & 33.2 & I5 & & 48 & 31.0 & 30.5 & 19 & 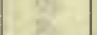 & 48 & 36.9 & 36.5 & 10 & & 48 & 14.5 & I4 & 45 & \\
\hline 50 & $5 \cdot 5$ & 35.2 & 12 & & 50 & $3 I .3$ & 31.0 & I8 & 8 & 50 & 36.6 & 36.0 & IO & 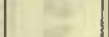 & 50 & 13.3 & I2. & 47 & \\
\hline 52 & $7 \cdot 3$ & 37.0 & $\infty$ & & 52 & $3 I .2$ & 31.0 & I8 & & 52 & 36.1 & 35.8 & II & 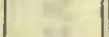 & 52 & 12. & 12 & 48 & \\
\hline 54 & 8.0 & $37 \cdot 7$ & $\infty 8$ & & 54 & 31.6 & $3 I \cdot 3$ & I8 & & 54 & & 35.6 & I & & 5 & 12.2 & & 48 & \\
\hline 56 & 6.5 & 36.1 & IO & & 56 & 31.4 & $3 I \cdot 3$ & 18 & & 56 & & 36 & II & & 5 & 12.5 & I2. & 48 & \\
\hline 58 & 34.5 & 34.1 & 13 & & 58 & $3 I .2$ & 30.8 & 19 & & 58 & & 36.0 & II & & 58 & 13.2 & I3.0 & 47 & \\
\hline 1700 & 33.7 & 33.2 & I5 & +5.0 & 1900 & 31.6 & 31.2 & I8 & +5.7 & II 00 & 36.1 & 35.6 & II & +4.8 & 2300 & 14.5 & I4. & 45 & +3.9 \\
\hline 02 & 34.0 & 33.8 & 14 & & 02 & 32.3 & 32.0 & 17 & & 02 & 35.6 & 35.0 & 12 & & 02 & 15.8 & I5.2 & 43 & \\
\hline 04 & 34.8 & 34.2 & 13 & & 04 & 33.1 & 32.6 & 16 & & 04 & & 35.0 & 12 & & 04 & 17.5 & 17.2 & 40 & te \\
\hline 06 & 34.5 & $34 . I$ & I3 & & 06 & 33.6 & 33.2 & 15 & 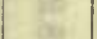 & 06 & & 35.0 & 12 & & 06 & I9. I & 18. & 37 & 8 \\
\hline 08 & 33.5 & 33.0 & 15 & & $\infty 8$ & 33.8 & $33 \cdot 3$ & I5 & Br. & 08 & & 35.2 & 12 & $\ln ^{*}$ & 08 & 21.0 & 20 & 35 & \\
\hline IO & 32.3 & 32.0 & 17 & & 10 & 33.3 & 33.0 & 15 & 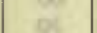 & Io & 36.2 & 35.8 & I I & & IO & 22.0 & 21.6 & 33 & \\
\hline 12 & 32.9 & 32.5 & 16 & & 12 & 32.8 & 32.3 & I6 & 0 & 12 & 37.2 & 36.8 & $\infty$ & & 12 & 22.1 & 21.9 & 33 & \\
\hline I4 & 32.9 & 32.4 & 16 & +5.0 & I4 & 32.3 & 32.0 & I7 & +5.9 & 14 & 38.3 & 37.9 & 07 & +4.3 & I4 & 21.6 & $2 I$ & 34 & +3.8 \\
\hline I6 & 32.3 & 32.1 & 17 & & 16 & 32.2 & 32.0 & 17 & 1.0 & I6 & & 38.6 & $\infty 6$ & & 16 & 21.7 & $2 I .3$ & 34 & 1.8 \\
\hline 18 & 32.1 & 32.1 & I7 & & I8 & 32.2 & 31.9 & I7 & & I8 & 38.6 & 38.2 & or & & I8 & 23.2 & 22.8 & $3 \mathbf{I}$ & $y$ \\
\hline 20 & 33.0 & 33.0 & 16 & & 20 & 32.1 & 31.8 & 17 & o & 20 & 37.4 & 37.2 & $\infty$ & & 20 & 22.5 & 22.2 & 32 & \\
\hline 22 & 35.0 & $34 \cdot 5$ & 12 & & 22 & 32.2 & 31.8 & 17 & to & 22 & 36.7 & 36.4 & 10 & $\mathrm{c}$ & 22 & 21.5 & $21 \cdot 3$ & 34 & \\
\hline 24 & 36.0 & 35.6 & II & & 24 & 32.5 & $3 I .9$ & I7 & va & 24 & 37.6 & $37 \cdot 2$ & $\infty$ & H & 24 & I9. 6 & I8. 8 & 37 & \\
\hline 26 & 37.0 & 36.7 & $\infty$ & & 26 & $33 \cdot 3$ & 32.2 & 16 & sx & 26 & 38.8 & 38.3 & 07 & & 26 & 16.5 & 16.3 & 42 & \\
\hline 28 & 37.2 & 36.9 & 09 & & 28 & 32.8 & 32.4 & I6 & & 28 & 39.0 & 38.6 & $\infty 6$ & & 28 & I5.0 & I3.8 & 45 & \\
\hline 30 & 38.2 & 37.8 & 08 & +5.0 & 30 & 32.9 & 32.5 & I6 & +5.6 & 30 & $39 \cdot 3$ & 38.8 & 06 & $+4 . I$ & 30 & I 3.4 & 12.5 & 47 & +3.6 \\
\hline 32 & 38.8 & 38.2 & 07 & 10 & 32 & 33.2 & 33.0 & I5 & t & 32 & 38 & 38.2 & 07 & & 32 & II. 6 & 12. & 49 & \\
\hline 34 & 38.1 & 37.8 & 08 & 1 & 34 & 34.1 & 33.8 & 14 & ) & 34 & 37.7 & 37.1 & 0 & & 34 & II. I & IO. & 50 & \\
\hline 36 & 38.0 & 37.4 & 08 & (c) & 36 & 34.2 & 33.8 & 14 & $\sqrt{3}$ & 36 & & 36.6 & 0 & . & 36 & I I. 6 & II.O & 50 & \\
\hline 38 & 38. I & 37.6 & 08 & 20 & 38 & 33.8 & 33.5 & I5 & 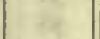 & 38 & & 36.8 & $\infty$ & & 38 & I I. 3 & II. & 50 & \\
\hline 40 & 38.5 & 38.0 & or & as. & 40 & 33.0 & 32.8 & I6 & 6 & 40 & 38.6 & 37.8 & o & & 40 & 10.3 & 9. & 52 & \\
\hline 42 & 39.5 & 39.0 & 06 & is & 42 & 31.9 & 31.9 & I7 & 4 & 42 & 40.0 & 39.2 & 05 & & 42 & 8.2 & 7. & 55 & \\
\hline 44 & 40.7 & 40.0 & 04 & +5.0 & 44 & 31.3 & $3 I .3$ & 18 & 34 & 44 & $4 I .0$ & 40.0 & 04 & +4.0 & $44^{* 2}$ & 40.8 & 38.6 & 57 & +3.4 \\
\hline 46 & 40.3 & 39.5 & 05 & ( & 46 & 32.3 & 32.1 & I7 & +5.3 & & 40.2 & 39.3 & 05 & & 46 & 39.6 & 37. & 58 & \\
\hline 48 & 39.2 & 38.6 & 06 & $\mathrm{ra}$ & 48 & 34.0 & 33.8 & I4 & & 48 & 39.9 & 39.0 & 0 & & 48 & 39.2 & 37. & 59 & \\
\hline 50 & $37 \cdot 3$ & 36.9 & $\infty$ & ne & 50 & $35 \cdot 3$ & 35.0 & I2 & E & 50 & 39.0 & & o & & 50 & 39.1 & 37. & 59 & \\
\hline 52 & 36.0 & 35.3 & II & 18 & 52 & 36.7 & 36.6 & IO & 32 & 52 & & 38.1 & o & & 52 & 39.0 & 37. & 59 & \\
\hline 54 & 35.8 & 35.1 & 12 & 2 & 54 & 37.3 & 37.1 & $\infty$ & HE & 54 & & $37 \cdot 2$ & 08 & & 54 & 39.6 & 38. & 58 & \\
\hline 56 & 36.6 & 35.6 & IO & & 56 & 37.2 & 37.0 & 09 & & 56 & 36.8 & 36.2 & 10 & & 56 & 39.5 & & 59 & \\
\hline 58 & 36.9 & 36.2 & IO & +5.2 & 58 & 37.5 & 37.3 & 09 & & 58 & 35.6 & 35.2 & 12 & & 58 & 39.1 & & 2059 & \\
\hline 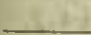 & $x^{2}+2$ & +4 & 2.24 & & & & & & & & & & & & $\infty$ & 37.7 & 35.7 & 2 I OI & +3.4 \\
\hline
\end{tabular}

Observers-A. F. and W. J. P., who alternated from $I 7 \mathrm{~h} 48 \mathrm{~m}$ to I7h $56 \mathrm{~m}$.
Correction to local mean time is - 145 .

Torsion head at ol $00 \mathrm{~m}$ read $255^{\circ}$ and at $24 \mathrm{~h}$ 20m read the same. Observer-W. J. P. 
Tabulation of Magnetic declinations observed at Alger Island Station-Continued

\begin{tabular}{|c|c|c|c|c|c|c|c|c|c|c|c|c|c|c|c|c|c|c|c|}
\hline \multicolumn{6}{|c|}{ Thursday, July 20, 1905} & \multicolumn{4}{|c|}{ Magnet scale erect } & \multicolumn{6}{|c|}{ Friday, July 2I, 1905} & \multicolumn{4}{|c|}{ Magnet scale inverted } \\
\hline \multirow{2}{*}{$\begin{array}{l}\text { Chr'r } \\
\text { time }\end{array}$} & \multicolumn{2}{|c|}{$\begin{array}{l}\text { Scale } \\
\text { readings }\end{array}$} & \multirow[t]{2}{*}{$\begin{array}{l}\text { East } \\
\text { decli- } \\
\text { nation }\end{array}$} & Temp. & $\begin{array}{l}\text { Chr'r } \\
\text { time }\end{array}$ & $\begin{array}{l}\text { Se: } \\
\text { read }\end{array}$ & $\begin{array}{l}\text { ale } \\
\text { ings }\end{array}$ & $\begin{array}{l}\text { East } \\
\text { decli- } \\
\text { nation }\end{array}$ & $\begin{array}{c}\text { Temp. } \\
\text { C. }\end{array}$ & $\begin{array}{l}\text { Chr'r } \\
\text { time }\end{array}$ & $\begin{array}{l}\text { Sc } \\
\text { reac }\end{array}$ & lings & $\begin{array}{c}\text { East } \\
\text { decli- } \\
\text { nation }\end{array}$ & $\begin{array}{c}\text { Temp. } \\
\text { C. }\end{array}$ & $\begin{array}{l}\text { Clir'r } \\
\text { time }\end{array}$ & $\begin{array}{r}\text { Sca } \\
\text { readi }\end{array}$ & $\begin{array}{l}\text { ale } \\
\text { ings }\end{array}$ & $\begin{array}{l}\text { East } \\
\text { decli- } \\
\text { nation }\end{array}$ & Temp. \\
\hline & L,eft & Kight & & & & Left & Righl & & & & Left & Right & & & & Left & Right & & \\
\hline h 111 & d & d & $\cdot 0$ & - & h m & d & d & - i & & $\mathrm{h} \mathrm{m}$ & d & d & - & 0 & li $\mathrm{m}$ & $\mathrm{d}$ & d & . & - \\
\hline 1600 & 48.9 & 49.6 & 2006 & $+7 \cdot 5$ & 1800 & 50.3 & 50.3 & 2008 & +6.4 & 2000 & 53.5 & 50.7 & 2020 & +2.0 & 2200 & 63.3 & 60.8 & 2007 & +1.5 \\
\hline 02 & 49.2 & 50.0 & 07 & . & 02 & 50.0 & 50.0 & 08 & & 02 & 53.2 & 50.3 & 20 & & 02 & 63.1 & $61 \cdot 5$ & 06 & \\
\hline 04 & 50.0 & 50.4 & 08 & (2) & 04 & 49.6 & 49.8 & 07 & no & 04 & 52.9 & 50.5 & 20 & Bit & 04 & 64.8 & 62.6 & 04 & 8 \\
\hline$\infty$ & 50.8 & 51.3 & $\infty$ & is & $\infty$ & $49 . I$ & 49.3 & 06 & is & 06 & 50.6 & 48.3 & 24 & 00 & 06 & 62.3 & 59.8 & $\infty$ & 60 \\
\hline 08 & 50.5 & $51 \cdot 3$ & 09 & oif & 08 & 48.6 & $48 . \overline{8}$ & 06 & or & 08 & 49.2 & 47.0 & 26 & wi & 08 & 59.5 & 57.5 & 12 & 8 \\
\hline $\begin{array}{l}10 \\
12\end{array}$ & $\begin{array}{r}\mathrm{L} \\
50.2\end{array}$ & st & 08 & sir & $\begin{array}{l}\text { I0 } \\
\text { I2 }\end{array}$ & $\begin{array}{l}49.0 \\
49.0\end{array}$ & $\begin{array}{l}49.0 \\
49 . I\end{array}$ & $\begin{array}{l}06 \\
06\end{array}$ & ai & $\begin{array}{l}\text { I0 } \\
\text { I2 }\end{array}$ & $\begin{array}{l}47.0 \\
45.1\end{array}$ & $\begin{array}{l}45.0 \\
43.1\end{array}$ & $\begin{array}{l}29 \\
33\end{array}$ & $\mathrm{xi}$ & $\begin{array}{l}10 \\
12\end{array}$ & $\begin{array}{l}62.3 \\
59.9\end{array}$ & $\begin{array}{l}59.9 \\
58.9\end{array}$ & $\begin{array}{l}08 \\
11\end{array}$ & ar \\
\hline 14 & 49.9 & 50.6 & 08 & & 14 & 49.3 & 49.7 & or & +6.1 & I4 & 46.1 & 44.4 & 31 & $+I .9$ & 14 & 61.0 & 59.5 & 10 & +1.5 \\
\hline 16 & 49.3 & 50.2 & 07 & +7.5 & 16 & $50 . \mathrm{I}$ & 50.7 & 08 & 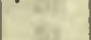 & 16 & 49.0 & 48.0 & 26 & 109 & 16 & 59.1 & 58.0 & 13 & $1-0$ \\
\hline 18 & 48.8 & 49.3 & 06 & 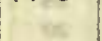 & I8 & $5 \mathrm{I} . \mathrm{I}$ & 51.9 & Io & at & I8 & $55 . \mathrm{I}$ & 54.1 & 16 & 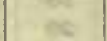 & 18 & 60.0 & $59 \cdot 5$ & II & 25 \\
\hline 20 & 48.9 & 49.2 & 06 & tin & 20 & 52.3 & 53.0 & 12 & ut & 20 & 56.8 & 55.6 & 14 & as & 20 & 58.6 & 58.1 & I3 & 4. \\
\hline 22 & 48.9 & 49.3 & 06 & is & 22 & 52.2 & 53.0 & 12 & a & 22 & 55.6 & $54 \cdot 3$ & 16 & ic & 22 & 58.4 & 57.5 & I4 & ats \\
\hline 24 & 49.7 & 50.1 & 08 & 8 & 24 & 53.0 & 53.6 & I3 & 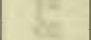 & 24 & 5I.I & 50.3 & 22 & es & 24 & 59.1 & 58.9 & 12 & E \\
\hline 26 & 50.3 & 50.9 & $\infty 9$ & the & 26 & 53.6 & 54.0 & I4 & ac. & 26 & 52.6 & 51.7 & 20 & E. & 26 & 59.3 & 59.1 & 12 & te. \\
\hline 28 & 50.3 & 50.6 & 08 & & 28 & 54.0 & 54.5 & I4 & & 28 & $54 . I$ & 53.0 & 18 & & 28 & 59.1 & 58.9 & 12 & \\
\hline 30 & $50 . \bar{I}$ & 50.5 & 08 & +7.5 & 30 & $54 . I$ & 54.8 & 15 & +5.8 & 30 & 56.6 & 55.6 & I4 & +1.6 & 30 & 57.8 & 57.2 & I5 & $+I .5$ \\
\hline 32 & 50.3 & 50.5 & 08 & & 32 & 53.9 & 54.1 & 14 & & 32 & 56.9 & 56.0 & 14 & & 32 & 54.5 & 53.8 & 20 & \\
\hline 34 & 51.0 & $5 \mathrm{I} . \mathrm{I}$ & $\infty 9$ & the & 34 & $53 \cdot 3$ & 53.7 & 13 & 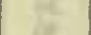 & 34 & 55.8 & 55.0 & 15 & & 34 & 57.2 & 56.6 & 16 & 2 \\
\hline 36 & 54 & $0 a$ & 14 & & 36 & 53.5 & 53.8 & I3 & 80 & 36 & $55 . \mathrm{I}$ & $54 \cdot 3$ & 16 & 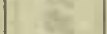 & 36 & 56.2 & 55.6 & I7 & $x$ \\
\hline 38 & $55 . I$ & $55 \cdot 3$ & 16 & 8 & 38 & 53.4 & 53.8 & 13 & nets & 38 & 55.0 & $54 \cdot 3$ & 17 & 6 & 38 & 60.0 & 60.0 & II & 6 \\
\hline 40 & 55.4 & 55.8 & I6 & 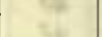 & 40 & 53.0 & 53.3 & 13 & $y$ & 40 & 55.0 & 54.5 & 17 & s. & 40 & 59.1 & 58.8 & 13 & \\
\hline 42 & 54.3 & 54.5 & I5 & & 42 & 52.6 & 53.0 & 12 & & 42 & 57.0 & 55.9 & 14 & & 42 & 61.8 & 6r.o & $\infty 9$ & \\
\hline 44 & 52.5 & 52.8 & I2 & +7.0 & 44 & 52.2 & 52.8 & I2 & +5.5 & 44 & 57.6 & 56.6 & 13 & +1.6 & 44 & 59.1 & 59.0 & I2 & +1.5 \\
\hline 46 & 51.3 & 51.7 & Io & & 46 & 51.5 & 52.0 & Io & . & 46 & 58.5 & 57.1 & 12 & & 46 & 60.3 & 60.3 & IO & \\
\hline 48 & $51 . \mathrm{I}$ & 51.6 & I0 & a. & 48 & 51.7 & 52.1 & II & 5 & 48 & 57.3 & 56.5 & 13 & & 48 & 60.0 & 60.0 & II & 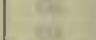 \\
\hline 50 & 51.8 & 52.1 & II & 2. & 50 & 52.2 & 52.8 & I2 & & 50 & 58.7 & 57.3 & 12 & & 50 & $6 \mathrm{I} .2$ & 61.2 & $\infty 9$ & \\
\hline 52 & 51.8 & 52.0 & II & to & 52 & 52.3 & 53.0 & 12 & wa & 52 & 57.8 & 56.6 & 13 & & 52 & $6 \mathrm{r} \cdot 3$ & 6I.I & $\infty$ & \\
\hline 54 & 52.3 & 52.5 & I2 & $x^{2}$ & 54 & 52.5 & 53.0 & I2 & nt) & 54 & 56.8 & 55.2 & 15 & & 54 & 60.0 & 59.3 & 12 & \\
\hline 56 & 52.7 & 52.9 & 12 & 8 & 56 & 52.0 & 52.6 & II & thas & 56 & 56.2 & 54.5 & 16 & 政 & 56 & 60.2 & 59.8 & I I & \\
\hline 58 & 54.0 & $54 . \mathrm{I}$ & I4 & & 58 & 52.0 & 52.8 & I2 & & 58 & 56.0 & 54.6 & 16 & & 58 & 59.2 & 58.8 & 13 & \\
\hline I7 $\infty$ & 55.0 & 55.1 & 16 & +6.8 & 1900 & 52.0 & 52.9 & I2 & $+5 . I$ & 2100 & 55.0 & 52.9 & 18 & & 2300 & 59.1 & 58.9 & I3 & +1.6 \\
\hline 02 & 55.6 & 55.8 & I7 & 10. & 02 & 52.5 & 53.5 & 12 & 120 & 02 & 56.0 & 53.3 & 17 & +1.6 & 02 & 57.8 & 57.6 & 15 & ( \\
\hline 04 & 56.0 & 56.2 & 17 & 80 & 04.2 & 54.2 & 55.0 & I5 & ses & 04 & 54.6 & 51.8 & 20 & 90 & 04 & 56.6 & 56.3 & 17 & 2. \\
\hline 06 & $55 \cdot 4$ & 56.0 & 17 & the & 06 & 54.9 & 56.0 & 16 & No & 06 & 54.8 & 52.3 & 19 & 80 & 06 & 56.7 & 56.3 & 17 & \\
\hline 08 & 54.8 & 55.2 & 16 & gr. & 08 & 55.5 & 56.0 & I7 & gr & 08 & 53. I & $5 \mathrm{I} . \mathrm{I}$ & $2 I$ & & 08 & 58.6 & 58.3 & 14 & net \\
\hline 10 & $54 \cdot 3$ & 54.6 & I5 & 4 & IO & 55.3 & 56.3 & I7 & 4 & I0 & 53.5 & 51.3 & $2 I$ & & 10 & 53.9 & 53.9 & $2 \mathrm{I}$ & \\
\hline 12 & 54.8 & 54.8 & 15 & 15 & I2 & $5 I . I$ & 55.9 & I3 & & 12 & 53.9 & 51.7 & 20 & & I2 & Over] & l'kd & & \\
\hline I4 & 54.3 & 54.6 & 15 & +6.7 & I4 & 54.7 & 55.5 & 16 & $+5 . I$ & 14 & 54.0 & 52.3 & 20 & +1.6 & 14 & 57.2 & 57.1 & I6 & +1.7 \\
\hline I6 & 54.0 & 54.2 & 14 & $x^{2}$ & 16 & 53.1 & 53.7 & 13 & 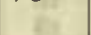 & 16 & 56.1 & 54.3 & 17 & & 16 & 56 & $0 a$ & 18 & - \\
\hline I8 & 53.4 & 54.0 & I4 & & I8 & 53.1 & 53.6 & 13 & & 18 & 57.8 & 55.9 & 14 & & I8 & 58. & $2 a$ & I5 & \\
\hline 20 & 53.2 & 53.8 & 13 & 10 & 20 & 52.0 & 52.4 & I I & 80 & 20 & 55.6 & 53.1 & 18 & & 20 & 56.8 & 56.4 & I7 & \\
\hline 22 & 53.5 & 53.8 & 13 & in & 22 & 53.0 & 53.2 & 13 & 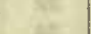 & 22 & 57.0 & 53.7 & 16 & & 22 & 56.6 & 56.6 & 17 & \\
\hline 24 & 53.2 & 53.8 & 13 & 5 & 24 & 52.3 & 52.8 & 12 & in & 24 & 58.0 & 55.0 & 15 & & 24 & 59.6 & 59.3 & 13 & \\
\hline 26 & $53 \cdot 5$ & 54.0 & I3 & & 26 & 52.1 & 52.6 & II & 잘 & 26 & 60.3 & 56.9 & 12 & E. & 26 & 58.4 & 58.4 & I4 & \\
\hline 28 & 53.0 & 53.6 & 13 & & 28 & 50.9 & 5 I. 3 & IO & & 28 & 58.5 & $54 \cdot 5$ & 15 & & 28 & 58.7 & 58.6 & 14 & \\
\hline 30 & 53.3 & 54.1 & 13 & +6.6 & 30 & 50.3 & 50.9 & $\infty$ & +5.0 & 30 & 58.2 & 55.5 & 15 & +1.5 & 30 & 59.9 & 59.5 & 12 & +1.8 \\
\hline 32 & 53.6 & 54.0 & I4 & & 32 & 49.0 & 49.4 & 06 & & 32 & 57.5 & 53.5 & 17 & & 32 & 57.6 & 57.0 & 16 & \\
\hline 34 & $53 \cdot 3$ & 53.9 & I3 & 2 & 34 & 50.0 & 50.3 & 08 & 30 & 34 & 60.0 & 56.5 & I2 & & 34 & 60.0 & 59.8 & I2 & \\
\hline 36 & $53 \cdot 3$ & 53.6 & I3 & 2 & 36 & 49.0 & 49.2 & $\infty 6$ & 92 & 36 & 60.6 & 57.9 & II & & 36 & 67.0 & 66.5 & 02 & \\
\hline 38 & 52.8 & 52.8 & 12 & an & 38 & 49.0 & 49.0 & 06 & (2) & 38 & 58.1 & 54.9 & 15 & E & 38 & 64.8 & 63.8 & 05 & \\
\hline 40 & 52.2 & 52.3 & II & to & 40 & 50.3 & 50.3 & 08 & tat & 40 & 55.8 & 52.2 & 19 & 94 & 40 & 64.8 & 64.0 & 05 & \\
\hline 42 & 52.1 & 52.2 & II & a & 42 & 49.6 & $49 . \overline{8}$ & 07 & & 42 & 57.9 & 55.3 & 15 & 124 & 42 & 58.8 & 58.1 & 15 & \\
\hline 44 & $52 . I$ & 52.5 & II & +6.5 & 44 & 50.5 & 50.5 & 08 & $+5 . I$ & 44 & 58.8 & 56.0 & 14 & +1.5 & 44 & 61.0 & 60.6 & II & +1.8 \\
\hline 46 & 52.8 & 53.0 & 12 & & 46 & 51.7 & 51.9 & I I & & 46 & 59.5 & $57 \cdot 3$ & 12 & 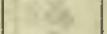 & 46 & 65.3 & $64 \cdot 3$ & 05 & \\
\hline 48 & 53.0 & 53. I & 12 & ce & 48 & 51.8 & 51.8 & II & 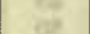 & 48 & 59.5 & 57.3 & 12 & 24 & 48 & 62.5 & $6 \mathrm{i} .3$ & $\infty$ & \\
\hline 50 & 52.2 & 52.5 & I I & & 50 & 52.7 & 53.0 & 12 & & 50 & 60.9 & 58.2 & IO & $Q$ & 50 & 59.7 & 59.3 & 13 & \\
\hline 52 & 52.0 & 52.3 & I I & 20 & 52 & 54.8 & 55.0 & 15 & He & 52 & 62.9 & 60.3 & 07 & CE & 52 & 58.2 & 57.6 & 16 & \\
\hline 54 & 51.6 & 51.9 & IO & be & 54 & 55.6 & 56.0 & 17 & & 54 & 63.2 & 60.8 & 07 & 12 & 54 & 56.9 & 56.3 & 18 & \\
\hline 56 & 51.0 & $5 \mathrm{I} .0$ & $\infty$ & & 56 & 57.5 & 58.0 & 20 & & 56 & 62.5 & 60.3 & 08 & $x=$ & 56 & 56.0 & 55.0 & 20 & \\
\hline 58 & 51.0 & 51.0 & $\infty$ & $=0$ & 58 & 58.9 & 59.7 & 22 & +5.2 & 58 & 64.8 & 62.5 & 04 & 8 & 58 & 52.0 & 51.3 & 26 & \\
\hline & & & & & 2000 & 60.0 & 60.5 & 24 & & & & & & & 2400 & 53.8 & 53.1 & 23 & +1.7 \\
\hline
\end{tabular}

Correction to local mean time is - I3s.

Torsion head at $16 \mathrm{~h}$ oom read $255^{\circ}$ and at $20 \mathrm{~h} 15 \mathrm{~m}$ read the same.

Observer-W. J. P.
Correction to local mean time is $-8 \mathrm{~s}$. $90^{\circ}$ torsion $=16^{\prime} 8 \mathrm{I}$. Torsion head at $20 \mathrm{~h}$ oom read $282^{\circ}$ and at $24 \mathrm{~h} 15 \mathrm{~m}$ read $252^{\circ}$ Observer-W. J. P. 
Tabulation of magnetic declinations observed at Alger Island Station-Continued

Sunday, July 23, I905
Magnet scale erect
Sunday, July 23,1905

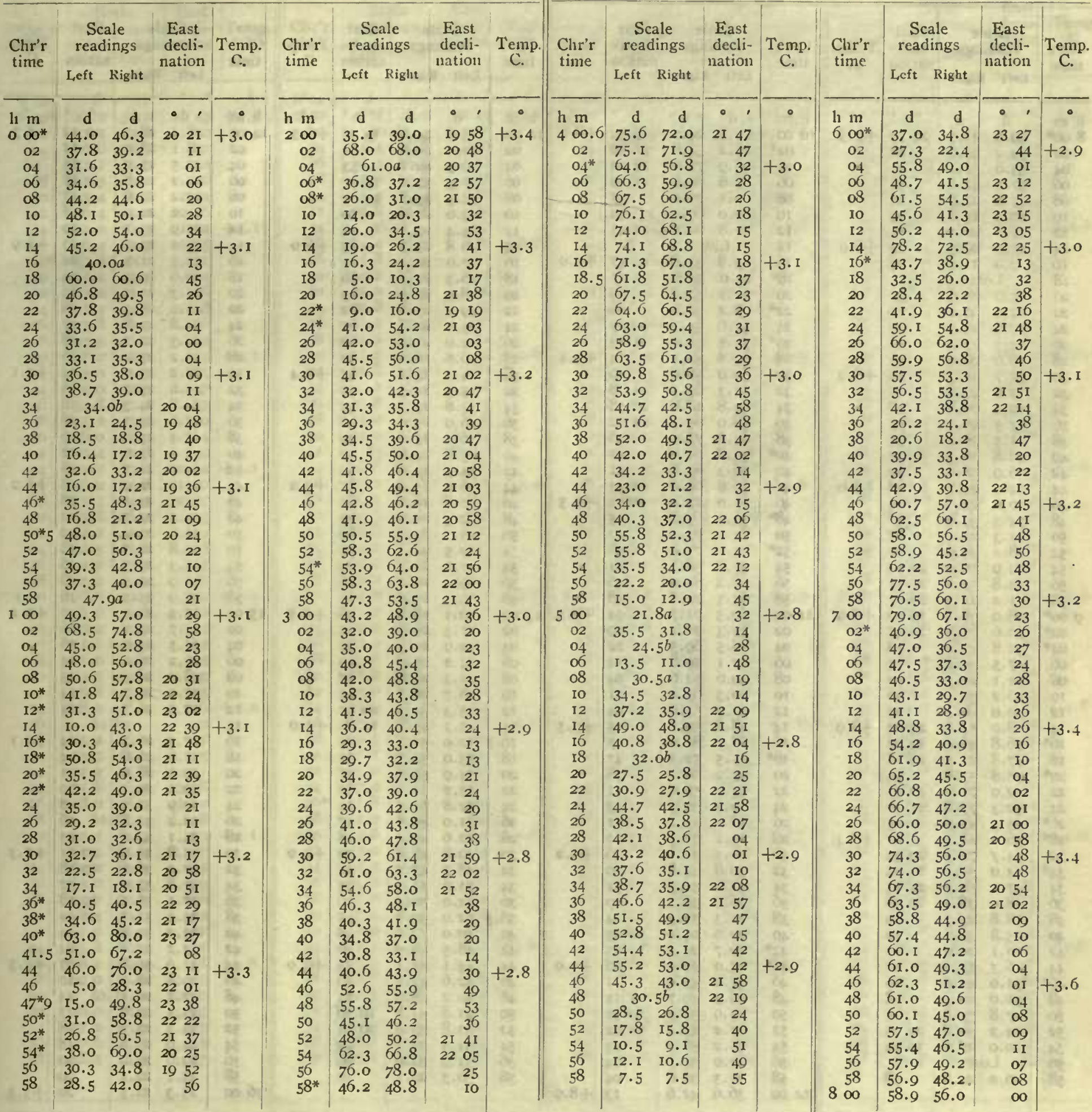

Observers-W. J. P. and A. F., who alternated from 3 h $58 \mathrm{nn}$ to $4 \mathrm{~h} 08 \mathrm{~m}$.
Correction to local mean time is - $6 \mathrm{~s}$

Torsion head at olt oom read $252^{\circ}$ and at gh $25 \mathrm{~m}$ read the same. Observer-A. F. 
Tabulation of magnetic declinations observed at Alger Island Station-Continued

\begin{tabular}{|c|c|c|c|c|c|c|c|c|c|c|c|c|c|c|c|c|c|c|c|}
\hline \multicolumn{6}{|c|}{ Monday, July 24, 1905} & \multicolumn{4}{|c|}{ Magnet scale erect } & \multicolumn{6}{|c|}{ Tuesday, July 25, I905 } & \multicolumn{4}{|c|}{ Magnet scale inverted } \\
\hline \multirow[t]{2}{*}{$\begin{array}{l}\text { Chr'r } \\
\text { time }\end{array}$} & \multicolumn{2}{|c|}{$\begin{array}{l}\text { Scale } \\
\text { readings }\end{array}$} & \multirow[t]{2}{*}{$\begin{array}{c}\text { East } \\
\text { decli- } \\
\text { nation }\end{array}$} & $\begin{array}{c}\text { Temp. } \\
\text { C. }\end{array}$ & $\begin{array}{l}\text { Chr'r } \\
\text { time }\end{array}$ & $\begin{array}{l}\text { Sca } \\
\text { readi }\end{array}$ & $\begin{array}{l}\text { ale } \\
\text { ings }\end{array}$ & $\begin{array}{c}\text { East } \\
\text { decli- } \\
\text { nation }\end{array}$ & Temp. & $\begin{array}{l}\text { Chr'r } \\
\text { time }\end{array}$ & $\begin{array}{l}\text { Sca } \\
\text { read }\end{array}$ & $\begin{array}{l}\text { ale } \\
\text { lings }\end{array}$ & $\begin{array}{c}\text { East } \\
\text { decli- } \\
\text { nation }\end{array}$ & $\begin{array}{l}\text { Temp. } \\
\text { C. }\end{array}$ & $\begin{array}{l}\text { Chr's } \\
\text { time }\end{array}$ & $\begin{array}{l}\text { Sce } \\
\text { read }\end{array}$ & $\begin{array}{l}\text { ale } \\
\text { ings }\end{array}$ & $\begin{array}{c}\text { East } \\
\text { decli- } \\
\text { nation }\end{array}$ & Temp. \\
\hline & Left & Right & & & & Left & Right & & & & L,eft & Right & & & & Left & Right & & \\
\hline $\mathrm{h} \mathbf{m}$ & d & d & $\circ$, & & h m & d & d & & & h m & & & $\circ \quad 1$ & & $\mathrm{~h} \mathrm{~m}$ & & & $\therefore$. & \\
\hline $80^{*}$ & $\begin{array}{l}39.8 \\
30.2\end{array}$ & $\begin{array}{l}41.5 \\
45.6\end{array}$ & $20 \begin{array}{r}53 \\
56\end{array}$ & $+7 \cdot 5$ & $\begin{array}{l}\text { I0 } \infty \\
02^{*}\end{array}$ & $\begin{array}{l}22.8 \\
46.2\end{array}$ & $\begin{array}{l}24.2 \\
54.8\end{array}$ & $\begin{array}{ll}19 & 59 \\
21 & 30\end{array}$ & +8.6 & $12 \underset{00}{02}$ & $\begin{array}{l}45.6 \\
42.2\end{array}$ & $\begin{array}{l}44.6 \\
40.6\end{array}$ & $\begin{array}{ll}19 & 45 \\
51\end{array}$ & +6.5 & $14 \underset{02}{02}$ & $\begin{array}{l}28.4 \\
28.1\end{array}$ & $\begin{array}{l}28.3 \\
28.0\end{array}$ & $20 \quad 12$ & $+4 \cdot 3$ \\
\hline $\begin{array}{l}02 \\
04\end{array}$ & $\begin{array}{l}39.2 \\
33.6\end{array}$ & $\begin{array}{l}45 \cdot 0 \\
37 \cdot 1\end{array}$ & $\begin{array}{l}50 \\
44\end{array}$ & 10 & 04 & 68.9 & 72.6 & 2202 & 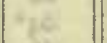 & 04 & 40.5 & 40.1 & 53 & 10 & 04 & 27.5 & 27.0 & 1). 13 & 6 \\
\hline 06 & 37.1 & 41.6 & $5 I$ & $\omega$ & $\infty 6$ & 60.8 & 64.8 & 2149 & 20 & $\infty 6$ & 37.9 & 37.0 & 1957 & His & 06 & 25.2 & 24.8 & I7 & \\
\hline 08 & 17.8 & 22.6 & $2 I$ & 3 & 08 & 60.1 & 76.0 & 57 & 8 & 08 & 34.5 & 33.8 & 2002 & 400 & 08 & 25.2 & 24.6 & I7 & \\
\hline 10 & 10.2 & I5.8 & $\infty$ & +4 & Io & 62.0 & 69.2 & 54 & .1 & I0 & 29.6 & 28.6 & 10 & wir & Io & 24.2 & 24.0 & 18 & \\
\hline 12 & II.I & 20.1 & I3 & 0 & 12 & 52.8 & 62.8 & $2 I 4 I$ & $x 5$ & 12 & $24 \cdot 4$ & 23.8 & 0.18 & tor & I2 & 23.9 & 23.3 & I9 & \\
\hline 14 & 17.7 & 29.3 & 26 & +8.0 & 14 & & st & & 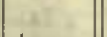 & I4 & 26.8 & 25.5 & 15 & +6.0 & I4 & 23.1 & 22.5 & 20 & +4.9 \\
\hline 16 & 9.0 & II. 9 & 05 & & 16 & 23.8 & 35.8 & 2057 & +9.0 & 16 & 27.0 & 26.2 & 14 & 80 & 16 & 22.3 & 21.7 & 22 & \\
\hline 18 & $\mathrm{LC}$ & ost & & 21 & $18^{*}$ & 20.0 & 38.6 & 27 & & 18 & 26.3 & 24.7 & 16 & 81 & I8 & 20.6 & 20.3 & 24 & 81 \\
\hline 20 & 25.6 & 28.6 & 32 & es & 20 & II. 2 & 32.8 & I6 & de & 20 & 28.0 & 27.5 & 13 & 도 & 20 & $2 \mathrm{I} \cdot 3$ & 2 I. 3 & 23 & oc \\
\hline 22 & 12.0 & 20.2 & 14 & a & $22^{*}$ & 28.2 & 52.8 & 06 & 34 & 22 & 29.1 & 27.8 & 0.12 & tos & 22 & 25.0 & $23 \cdot 3$ & 18 & Ini \\
\hline 24 & 17.8 & 25.0 & 22 & $t=$ & 24 & 52.6 & 55.1 & 2027 & If & 24 & 23.0 & 21.6 & $2 \mathrm{I}$ & tis & 24 & 24.8 & 24.8 & 17 & 10 \\
\hline 26 & 12.5 & 17.5 & 12 & 35 & $26^{*}$ & 53.5 & 58.0 & $2 \mathrm{II}$ & y & 26 & 22.0 & 21.0 & 22 & is & 26 & 24.3 & 24.1 & I8 & \\
\hline 28 & 18.6 & 24.6 & 23 & 8 & 28 & 55.8 & 58.1 & & +9.4 & 28 & 16.8 & I6.0 & 30 & 15 & 28 & 25.2 & 25.2 & I6 & \\
\hline 30 & 32.7 & 35.3 & 42 & +8.0 & 30 & 52.0 & 56.0 & 10 & the & 30 & I4.I & I3. I & 35 & +5.4 & 30 & 27.2 & 27.0 & I4 & +5.1 \\
\hline 32 & 17.8 & 27.8 & 25 & the & 32 & $54 \cdot 3$ & 63.5 & $\begin{array}{ll}21 & 18\end{array}$ & 4. & 32 & I5.3 & 14.5 & 33 & ( & 32 & 25.8 & 25.2 & 16 & \\
\hline 34 & 19.4 & 20.6 & 28 & 35 & 34 & 43.8 & 48.8 & 2058 & 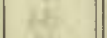 & 34 & 13.8 & 13.3 & 35 & & 34 & 24.8 & 24.5 & I8 & \\
\hline 36 & 33.3 & 48.0 & 53 & 85. & 36 & 58.0 & 65.3 & 2122 & (1) & 36 & 16.0 & I5.0 & 32 & \& & 36 & 25.6 & 25.3 & 16 & \\
\hline 38 & 21.5 & 36.2 & 34 & 8 & 38 & 64.8 & 78.5 & 38 & 82 & 38 & 16.0 & 15.2 & 32 & 26 & 38 & 25.4 & 25.0 & 16 & \\
\hline 40 & 22.8 & 38.5 & 37 & 97 & $40^{*}$ & 54.8 & $6 \mathrm{r} .0$ & 47 & 와 & 40 & 15.8 & I 4.8 & 32 & 6 & 40 & $25 \cdot 3$ & 25.0 & 17 & $\mathrm{e}$ \\
\hline 42 & 26.9 & 28.0 & 32 & & 42 & 56.3 & 64.8 & $5 I$ & 4 & 42 & 14.7 & 14.0 & 34 & 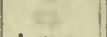 & 42 & 24.5 & 24.1 & 18 & \\
\hline 44 & 24.1 & 25.7 & 28 & +8.0 & 44 & 56.8 & 66.6 & 2153 & (5ne & 44 & 16.2 & 15.6 & $3 \mathrm{I}$ & +5.0 & 44 & 24.2 & 24.0 & 18 & +5.2 \\
\hline 46 & 20.0 & 24.6 & 24 & 15 & 46 & 61.0 & 73.0 & 22 OI & +9.5 & 46 & 20.8 & 19.6 & 24 & 4 & 46 & 24.3 & $24 . I$ & 18 & \\
\hline 48 & 34.3 & 39.0 & 46 & $\sqrt{38}$ & $48 *$ & 56.0 & 65.8 & 15 & $x^{2}$ & 48 & 22.3 & 21.6 & 22 & 14 & 48 & 23.2 & 22.7 & 20 & \\
\hline 50 & $2 \mathrm{I} \cdot 3$ & 24.4 & 25 & at & 50 & 64.0 & 73.1 & 27 & of & 50 & $23 . I$ & 23.0 & 20 & $z$ & 50 & 23.6 & 23.4 & 19 & \\
\hline 52 & 18.2 & 23.0 & 21 & 9 & $52^{*}$ & 63.0 & 71.6 & 36 & Es & 52 & 22.5 & 22.1 & 20 & 5 & 52 & 24.8 & 24.5 & 18 & E \\
\hline 54 & 10.9 & 16.0 & 2010 & 13 & 54 & 57.8 & 58.3 & $2 I$ & 18 & 54 & 21.7 & 21.3 & 22 & thit & 54 & 25.3 & $25 \cdot 3$ & 16 & IfI \\
\hline 56 & 54.8 & 57.8 & $21 \quad 17$ & 145 & 56 & 68.2 & 80.0 & 46 & be & 56 & 19.2 & 19.0 & 26 & & 56 & 25.9 & $25 \cdot 5$ & 16 & \\
\hline 58 & II. 2 & 12.3 & 2007 & 0 & 58 & 64.5 & 72.8 & 38 & . & 58 & 19.3 & 19.0 & 26 & & 58 & 25.9 & 25.5 & I6 & \\
\hline $900^{*}$ & 28.0 & 38. I & 1920 & +8.0 & II $\infty$ & 60.3 & 71.7 & 34 & +9.5 & 1300 & 20.1 & I8.8 & 26 & +4.5 & I5 $\infty$ & 25.3 & 24.9 & 17 & +5.2 \\
\hline $02^{*}$ & 70.6 & 75.8 & 2045 & 20 & 02 & 55.3 & 71.1 & 29 & & $\mathrm{O} 2$ & 21.3 & 20.7 & 23 & 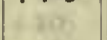 & 02 & 25.7 & $25 . \mathrm{I}$ & 16 & 1 \\
\hline $04^{*}$ & 32.8 & 47.2 & 48 & 10 & 04 & 48.5 & $69 \cdot 5$ & 22 & 80 & 04 & 22.9 & 22.3 & $2 I$ & Q2 & 04 & 25.5 & 25.0 & 16 & ya \\
\hline 06 & 7.5 & 30.2 & I4 & ay & 06 & $47 . I$ & 59.8 & 14 & on & 06 & 24.2 & 23.5 & I9 & & 06 & 26.2 & 26.0 & 15 & \\
\hline 08 & $22 . I$ & 24.7 & 22 & 80 & 08 & 39.6 & 54.6 & 04 & 60 & 08 & 25.3 & 24.6 & 0.17 & fo & 08 & 28.0 & 26.5 & 13 & 20 \\
\hline I0 & 26.2 & 39.4 & 36 & of & 10 & 40.3 & 63.0 & II & 65f & 10 & 28.7 & 28.5 & II & of & 10 & $27 \cdot 3$ & 26.7 & 14 & rest \\
\hline 12 & 20.0 & 28.5 & 23 & 1 & 12 & 37.5 & 57.0 & 04 & 61 & 12 & 35.3 & 34.3 & 20 or & $5 t$ & 12 & 27.1 & 26.5 & 14 & 31 \\
\hline T4 & 8.0 & 15.6 & 2004 & +8.0 & 14 & $48 . \overline{8}$ & 59.7 & 15 & +9.5 & 14 & 43.3 & 43.1 & 1948 & +4.0 & I4 & & & & \\
\hline $16^{*}$ & 36.8 & 46.4 & 1942 & & 16 & 68.6 & 81.0 & 47 & & 16.5 & 52.0 & $5 \mathrm{I} .1$ & 35 & rit & 16 & 27.3 & 26.3 & 14 & +5.1 \\
\hline 18 & 57.8 & 71.5 & 2018 & त61 & $18^{*}$ & 45.5 & 52.3 & 47 & $8 \mathrm{r}$ & 18 & 53.0 & 52.5 & 33 & 61 & 18 & 27.6 & 26.6 & 14 & \\
\hline $20^{*}$ & 38.8 & 57.8 & 44 & 10 & 20 & 35.2 & 50.2 & 38 & $\alpha$ & 20 & 54.9 & 53.9 & $3 I$ & $\mathrm{E}$ & 20 & 29.1 & 28.0 & II & \\
\hline 22 & 42.0 & 71.2 & 2057 & $\mathrm{Ex}$ & 22 & 33.2 & 56.0 & $4 I$ & 55 & 22 & 55.2 & $54 . I$ & 30 & \pm & 22 & 30.1 & 29.1 & 10 & $4 \mathrm{vi}$ \\
\hline 24 & 52.3 & 77.8 & 21 IO & is & 24 & 19.3 & 39.3 & I7 & 12 & 24 & 52.8 & $5 \mathrm{I} .5$ & 34 & is & 24 & 30.9 & 30.2 & 08 & \\
\hline $26^{*}$ & 23.5 & 43.0 & 2005 & & 26 & I6.0 & 35.2 & II I & 0 & 26 & 51.0 & 49.8 & 37 & $\approx$ & 26 & 32.2 & 31.8 & $\infty 6$ & \\
\hline $28^{*}$ & 36.2 & 48.3 & 02 & +8.1 & 28 & 19.2 & 31.4 & Io & $8=$ & 28 & 50.6 & 49.4 & 38 & $8=$ & 28 & 34.1 & 33.2 & $\mathbf{0 3}$ & \\
\hline 30 & 54.7 & 72.3 & 2035 & 60 & 30 & 26.5 & $3 \mathrm{I} . \mathrm{I}$ & 16 & +9.2 & 30 & 49.6 & 48.8 & 39 & +4.0 & 30 & 35.8 & 34.9 & & +5.3 \\
\hline $32^{*}$ & 43.0 & 59.1 & 2124 & 62 & 32 & 30.8 & 43.8 & 29 & & 32 & 48.3 & 47.2 & $4 \mathrm{I}$ & (t) & 32 & 37.2 & 36.6 & 1958 & \\
\hline 34 & 37.2 & 50.2 & $21 \quad 13$ & 14. & 34 & 32.2 & 44.5 & $3 \mathrm{I}$ & 16 & 34 & 51.9 & 51.3 & 35 & $x$ & 34 & 37.8 & 37.0 & 57 & \\
\hline 36 & 28.0 & 38.4 & 2056 & T. & 36 & 26.9 & 36.1 & 20 & 48 & 36 & 52.0 & 50.5 & 36 & & 36 & 37.1 & 36.5 & 1958 & \\
\hline $3^{8}$ & 18.8 & 25.3 & 39 & Re & 38 & 21.3 & 33.3 & I4 & 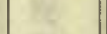 & 38.5 & 52.0 & 50.8 & 35 & b. & 38 & 34.5 & $34 . I$ & 2002 & \\
\hline 40 & 25.8 & 34.2 & 52 & $6=$ & 40 & 20.0 & 24.2 & 2205 & 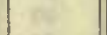 & 40 & 50.0 & 50.0 & 38 & 06 & 40 & 33.2 & 32.8 & 0.04 & \\
\hline 42 & 10.1 & 20.9 & 29 & 64 & $42^{*}$ & 20.7 & 24.2 & 2145 & & 42 & 48.5 & 48.0 & 40 & & 42 & 33.2 & 32.6 & 04 & \\
\hline 44 & 6.3 & 15.2 & $2 \mathrm{I}$ & 48 & $44^{*}$ & 43.0 & 52.0 & 26 & +9.0 & 44 & $45 . I$ & 45.1 & 45 & +4.0 & 44 & 31.6 & 30.8 & 07 & +5.5 \\
\hline $46^{*}$ & 31.0 & 41.8 & 19 & 4 & 46 & 31.0 & 45.0 & II & & 46 & 42.6 & 42.2 & 50 & & 46 & 29.8 & 28.5 & Io & \\
\hline 48 & 31.0 & 40.5 & 18 & 86 & 48 & 36.5 & 39.7 & 21 II & 04 & 48 & 40.2 & 38.6 & 54 & & 48 & 27.1 & 26.1 & I4 & \\
\hline 50 & 27.8 & 37.8 & 14 & SE & 50 & 25.9 & 34.9 & 2059 & $\alpha$ & 50 & $4 \mathrm{~T} .8$ & 41.8 & I9 50 & & 50 & 25.5 & 24.6 & 17 & \\
\hline 52 & 24.2 & 30.8 & 2005 & 82 & 52 & 22.2 & 43.2 & 2102 & Iy & 52 & 33.8 & 33.0 & 2004 & & 52 & $24 \cdot 3$ & 23.7 & I8 & \\
\hline 54 & 10.0 & I7.6 & I9 44 & $P$ & 54 & 35.2 & 56.2 & & 16 & 54 & $34 \cdot 3$ & 34.1 & 02 & & 54 & 24.6 & 23.8 & I8 & \\
\hline 56 & Lc & st & & & 56 & 33.0 & 34.5 & 04 & $2-$ & 56 & $3 \mathrm{I} .9$ & $3 \mathrm{I} .9$ & 06 & I & 56 & 23.6 & 22.0 & 20 & \\
\hline 58 & 20.2 & 24.8 & 58 & 2 & 58 & 31.3 & 36.6 & a4 & & 58 & 30.3 & 29.1 & 10 & & 58 & 23.1 & 22.4 & 20 & \\
\hline & & & & 003 & 1200 & 36.6 & 42.6 & I3 & +8.9 & & & & & & I6 00 & 23.3 & $22 . \dot{5}$ & 20 & +5.3 \\
\hline
\end{tabular}

Correction to local mean time is -5 s.

Torsion head read $252^{\circ}$ at beginning and ending.

Observer-W. J. P.
Correction to local mean time is $+6 \mathrm{~s} .90^{\circ}$ torsion $=17 .^{\prime} 13$. Torsion head at $\mathrm{I} 2 \mathrm{~h}$ oom read $26 \mathrm{I}^{\circ}$ and at $16 \mathrm{~h} 20 \mathrm{~m}$ read $258^{\circ}$. Observer-W. J. P. 
Tabulation of magnetic declinations observed at Alger Island Station-Continued

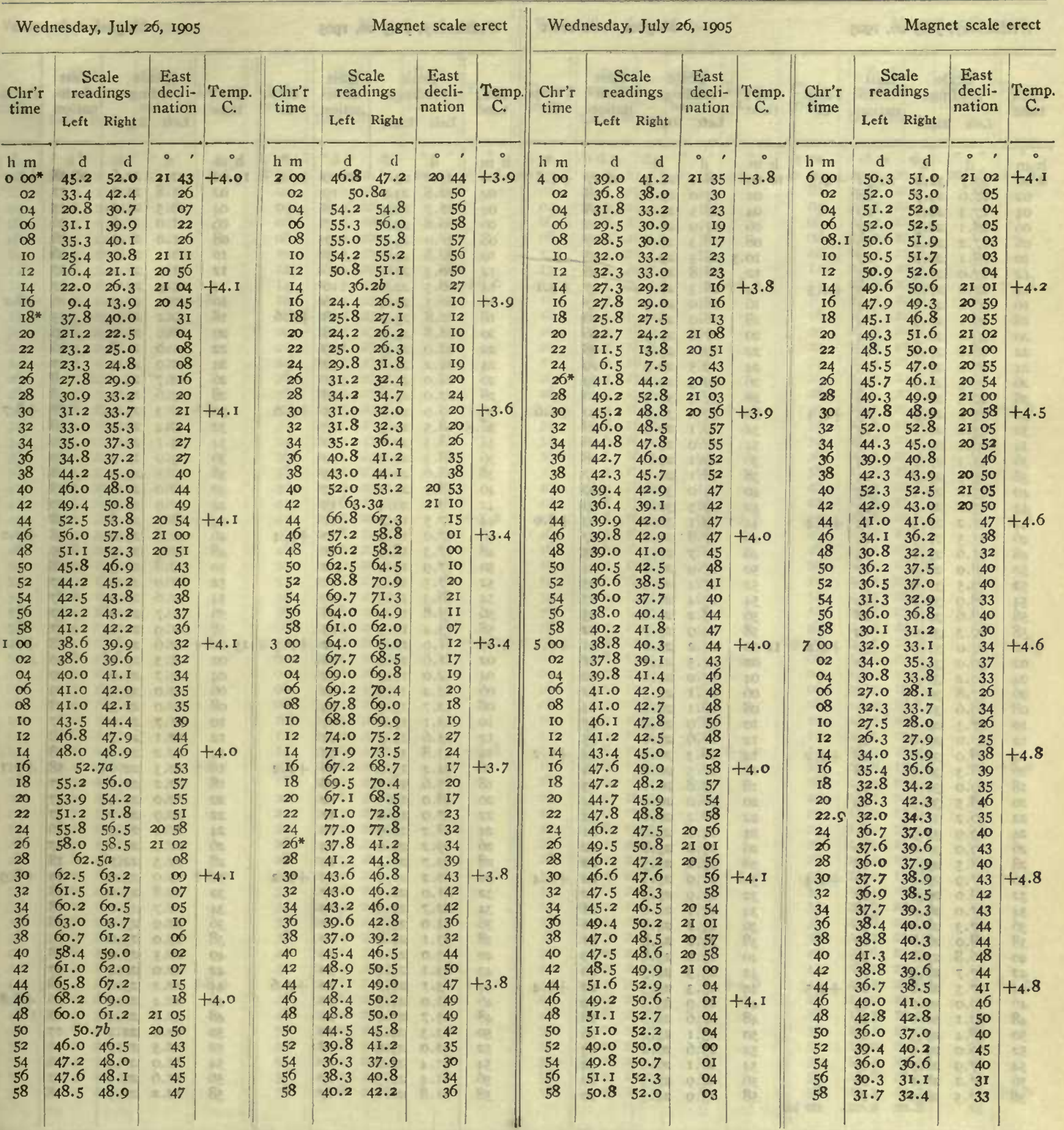

Observer-A. F.

Observers-A. F. and W. J. P., who alternated from $6 \mathrm{~h}$ oam to 6h $12 \mathrm{~m}$. 
Tabulation of magnetic declinations obscrved at Alger Isiand Station-Continued

\begin{tabular}{|c|c|c|c|c|c|c|c|c|c|c|c|c|c|c|c|c|c|c|c|}
\hline \multicolumn{6}{|c|}{ Wednesday, July 26,1905} & \multicolumn{4}{|c|}{ Magnet scale erect } & \multicolumn{6}{|c|}{ Wednesday, July 26,1905} & \multicolumn{4}{|c|}{ Magnet scale erect } \\
\hline \multirow{2}{*}{$\begin{array}{l}\text { Chr'r } \\
\text { time }\end{array}$} & \multicolumn{2}{|c|}{$\begin{array}{l}\text { Scale } \\
\text { readings }\end{array}$} & \multirow{2}{*}{$\begin{array}{l}\text { East } \\
\text { decli- } \\
\text { nation }\end{array}$} & 'Temp. & Chr'r & $\begin{array}{l}\mathrm{Sc} \\
\mathrm{read}\end{array}$ & $\begin{array}{l}\text { ale } \\
\text { lings }\end{array}$ & $\begin{array}{l}\text { East } \\
\text { decli- }\end{array}$ & Temp. & Chr'r & $\begin{array}{r}\text { Sc } \\
\text { reac }\end{array}$ & dings & $\begin{array}{l}\text { East } \\
\text { decli- }\end{array}$ & & Chr'r & $\begin{array}{r}\mathrm{Sc}_{\mathrm{i}} \\
\text { read }\end{array}$ & $\begin{array}{l}\text { ale } \\
\text { lings }\end{array}$ & $\begin{array}{l}\text { East } \\
\text { decli- }\end{array}$ & Temp \\
\hline & Left & Right & & & ne & Left & Right & & & & $L_{\text {reft }}$ & Right & & & & L,eft & Right & & \\
\hline $\mathrm{h} \mathrm{m}$ & d & $d$ & 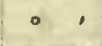 & - & $\mathrm{h} \mathrm{n}$ & $\mathrm{d}$ & d & & $a^{\circ}$ & $\mathrm{h} \mathrm{m}$ & $d$ & d & & & $\mathrm{h} \mathrm{m}$ & d & & $\circ \quad$ & 0 \\
\hline 800 & 30.7 & 32.0 & 2032 & +4.5 & 1000 & 28.1 & 28.5 & 2027 & +4.8 & 1200 & 23.2 & 23.0 & 20 I9 & +6.3 & 1400 & 22.1 & 22.6 & $20 \quad 18$ & +7.9 \\
\hline 02 & 28.6 & 29.7 & 28 & & 02 & 29.0 & 29.0 & 28 & & 02 & 22.8 & 23.6 & I9 & & 02 & 21.8 & 22.3 & I7 & \\
\hline 04 & 27.3 & 28 . I & 26 & & 04 & 28.5 & 29.2 & & Lit & 04 & 21.7 & 22.9 & 18 & $8 a$ & 04 & 20.5 & $2 \mathrm{I} . \mathrm{I}$ & & \\
\hline 06 & 28. I & 28.5 & 27 & 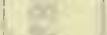 & 06 & 27.9 & 28.2 & 26 & 20 & 05 & 20.8 & 23.2 & I7 & in & $\infty 6$ & 21.0 & 21.6 & & \\
\hline 08 & 27.2 & 28.0 & 26 & 85 & 08 & 28.3 & 28.6 & 27 & Ry & 08 & 21.0 & 23.0 & 17 & 20 & 08 & 21.0 & 21.5 & 16 & \\
\hline I0 & 28 . I & 29.0 & 27 & or & 10.5 & 28.3 & 29. I & 28 & es & 10 & 20.8 & 22.6 & I7 & et & 10 & $2 \mathrm{I} . \mathrm{I}$ & 21.7 & & \\
\hline 12 & 27.0 & $27 \cdot 4$ & 25 & tr & 12 & 28.8 & 29.5 & 28 & 51 & 12 & 20.3 & 22.0 & 16 & 65 & 12 & 21.5 & 22 . I & 17 & \\
\hline 14 & 27.2 & 27.2 & 25 & +4.5 & 14 & 28.3 & 28.7 & 27 & $+5 . I$ & 14 & 20.5 & 22.6 & 16 & \& & 14 & 21.5 & 22.0 & 17 & \\
\hline 16 & 26.6 & 26.8 & 24 & & 16 & 28.0 & 28.5 & 27 & & 16 & 20.5 & 22.4 & 16 & & 16 & 20.9 & $2 \mathrm{I} .2$ & 16 & +8.2 \\
\hline 18 & 26.1 & 26.9 & 24 & C5: & 18 & 28.0 & $28 . \bar{I}$ & 26 & di & I8 & 21.8 & $23 \cdot 3$ & 18 & +6.6 & 18 & 20.2 & 21.0 & 15 & \\
\hline 20 & 26.0 & 26.5 & 24 & 68 & 20 & 27.8 & 27.9 & 26 & 8 & 20 & 21.8 & 23.5 & 18 & 16 & 20 & 20.9 & 21.2 & 16 & e \\
\hline 22 & 26.8 & 27.2 & 25 & 45 & 22 & 29.1 & 29.1 & 28 & $E$ & 22 & 23.0 & 24.5 & 20 & 4 & 22 & 21.0 & 21.8 & 16 & $E$ \\
\hline 24 & 26.6 & 27.6 & 25 & 15 & 24 & 29.5 & 29.5 & 29 & 145 & 24 & 24.2 & 25.8 & 22 & 44 & 24 & 21.9 & 22.4 & 17 & \\
\hline 26 & 25.3 & 26.9 & 23 & & 26 & 29.3 & 29.6 & 29 & 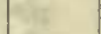 & 25 & 24.0 & 25.3 & 21 & $\therefore$ & 26 & 22.7 & 23.2 & 18 & \\
\hline 28 & 26.3 & 28.7 & 26 & Bs & 28 & 30.3 & 30.3 & 30 & $=$ & 28 & 22.0 & 23.6 & 18 & 85 & 28 & 22.6 & 23.2 & 18 & \\
\hline 30 & 25.9 & 28.5 & 25 & +4.4 & 30 & 30.2 & 30.2 & 30 & +5.6 & 30 & 19.9 & $2 I .3$ & 15 & +7.0 & 30 & 22.3 & 23.0 & 18 & +8.7 \\
\hline 32 & 25.6 & 28.0 & 25 & & 32 & 29.1 & 29.5 & 28 & & 32.5 & 18.5 & 20.5 & I3 & & 32 & 22.4 & 23.2 & 18 & \\
\hline 34 & 26.4 & 29.0 & 26 & $y$ & 34 & 28.0 & 29.3 & 28 & K & 34 & 18.3 & 20.0 & 12 & it & 34 & $23 . I$ & 23.9 & 19 & \\
\hline 36 & $25 \cdot 3$ & 27.7 & 2.4 & ac & 36 & $29 . I$ & 29.6 & 28 & (x) & 36 & 18.3 & 20.0 & 12 & hes & 36 & 23.0 & 23.7 & 19 & \\
\hline 38 & 25.5 & 27.9 & 24 & 2 & 38 & 28 . I & 28.9 & 27 & 8 & 38 & 18.6 & 20.0 & 13 & \#ir & 38 & 22.5 & 23.2 & 18 & \\
\hline 40 & 25.8 & 28.1 & 25 & Q & 40 & 29.0 & 30. I & 29 & 8 & 40 & 18.9 & 20.3 & 13 & os & 40 & 22.0 & 22.6 & 18 & 28 \\
\hline 42 & 25.0 & 26.9 & 23 & 5 & 42 & 29.3 & 30.0 & 29 & 1 & 42 & 19.0 & 20.4 & 13 & 56 & 42 & 22.9 & 23.4 & 19 & 25 \\
\hline 44 & 25.2 & 27.5 & 24 & +4.4 & 44 & $29 . \mathrm{I}$ & 30.0 & 29 & +6.0 & 44 & 17.5 & 19.3 & II & $x^{3}$ & 44 & 22.1 & 22.8 & 18 & \\
\hline 46 & 25.5 & 27.9 & 24 & & 46 & 27.8 & 28.8 & 27 & & 46 & 17.0 & 18.3 & I0 & +7.4 & 46 & 22.6 & 23.0 & 18 & +9.0 \\
\hline 48 & 25.6 & 27.0 & 24 & 8 & 48 & 26.7 & 27.5 & 25 & 6 & 48 & $17 \cdot 3$ & 18.9 & II & & 48 & 22.9 & $23 \cdot 3$ & 19 & \\
\hline 50 & 28.0 & 28.9 & 27 & ov & 50 & 25.9 & 26.8 & 24 & ans & 50 & 17.9 & 19.2 & 12 & Or & 50 & 22.8 & 22.9 & 18 & z \\
\hline 52 & 29.5 & $3 \mathrm{I} .0$ & 30 & 4 & 52 & 25.4 & 26.3 & 23 & 23 & 52 & 17.5 & 18.9 & II & st. & 52 & 21.9 & 22.2 & 17 & ati \\
\hline 54 & 28.9 & 30.2 & 29 & 23 & 54 & 25.4 & 26.3 & 23 & 14 & 54 & 16.3 & I7. 5 & $\infty$ & 4x & 54 & 21.6 & 22.0 & 17 & Rz \\
\hline 56 & 28.6 & 30.1 & 28 & 7 & 56 & 25.2 & 26.0 & 23 & & 56 & I5.8 & 16.9 & 08 & 13 & 56 & 21.7 & 22.0 & 17 & \\
\hline 58 & 29.3 & 30.0 & 29 & 2 & 58 & 24.9 & 25.7 & 22 & 1,8 & 58 & I 3.4 & 14.9 & 05 & 82 & 58 & 21.9 & 22.2 & 17 & \\
\hline$\infty$ & 29.0 & $3 \mathrm{I} .0$ & 30 & +4.2 & II OO & 24.2 & 25.0 & 21 & +6.1 & 1300 & II.9 & 12.9 & 02 & +7.3 & I5 00 & 22.6 & 23.0 & 18 & $+9 . I$ \\
\hline 02 & 30.0 & 31.0 & 30 & & 02 & 24.5 & 24.7 & 21 & 1. & 02 & II. 6 & I2. 4 & OI & & 02 & 23.3 & 23.7 & 19 & \\
\hline 04 & 30.2 & $3 I .6$ & 3I & +6 & 04 & 25.0 & 25.2 & 22 & 20 & 04 & 12.0 & 12.9 & 02 & 10 & 04 & 23.7 & 23.9 & 20 & 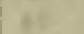 \\
\hline 06 & 27.8 & 30.7 & 28 & 8 & $\infty$ & 25.1 & 25.3 & 22 & जो & of & 12.2 & 12.9 & 02 & 87 & 06 & 23.0 & $23 \cdot 3$ & 19 & \\
\hline 08 & 29.6 & 32.5 & $3 I$ & 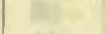 & 08 & 26.1 & 26.9 & 24 & As & 08 & 12.7 & 13.3 & 03 & 86 & 08 & 22.0 & 22.3 & 18 & \\
\hline I0 & $27 \cdot 3$ & 29.8 & 27 & et & IO & 26.9 & 27.0 & 25 & 4 & I0 & 13. I & I 3.8 & 04 & 9t & 10 & 21.7 & 21.9 & 17 & CEt \\
\hline I2 & 27.0 & 29.4 & 27 & th & I2 & 26.6 & 27.0 & 25 & & I2 & 13.6 & I 4.3 & 04 & 4 & 12 & 22. I & 22.5 & 18 & tis \\
\hline I4 & 28.0 & 30.6 & 28 & +4.2 & I4 & 26.3 & 26.9 & 24 & +6.2 & 14 & 13.8 & 14.8 & 05 & 1. & 14 & 22.2 & 22.9 & 18 & \\
\hline I6 & 28.3 & 30.4 & 28 & & 16 & 26.5 & 26.9 & 24 & & 16 & I 4.2 & I 5.2 & 06 & +7.2 & 16 & 22.3 & 22.6 & 18 & +9.1 \\
\hline I8 & 29.0 & 30.8 & 29 & wr & I8 & 25.8 & 26.1 & 23 & 16 & I8 & 15.6 & I6. 5 & 08 & & 18 & 22.2 & 22.5 & 17 & \\
\hline 20 & 28.5 & 30.5 & 29 & x & 20 & 26.0 & 26.2 & 24 & E & 20 & 18.0 & I9. I & 12 & $\alpha$ & 20 & 22.0 & 22.4 & 17 & (2) \\
\hline 22 & 30.2 & 32.0 & $3 \mathrm{I}$ & $2+2$ & 22 & 26.2 & 26.6 & 24 & 15 & 22 & 17.9 & 19.0 & II & 14 & 22 & 21.9 & 22.2 & 17 & \\
\hline 24 & 29.9 & $3 \mathrm{I} .9$ & $3 \mathrm{I}$ & 11 & 24 & 24.6 & 25.0 & 21 & $k$ & 24 & 18.2 & 18.9 & 12 & $y^{2}$ & 24 & 21.4 & 21.9 & 16 & \\
\hline 26 & 29.0 & 30.0 & 29 & $x$ & 26 & 25.0 & 25.8 & 22 & W & 26 & 19.2 & 20.0 & 13 & $\mathrm{~N} 2$ & 26 & 21.9 & 22.2 & 17 & \\
\hline 28 & 28.5 & 29.3 & 28 & 54 & 28 & 25.0 & $25 \cdot 3$ & 22 & 10 & 28 & 19.7 & 20.4 & 14 & 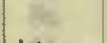 & 28 & 22.3 & 22.7 & 18 & \\
\hline 30 & 27.9 & 29.0 & 27 & +4.4 & 30 & 24.9 & 25.1 & 22 & +6.4 & 30 & 20.2 & 21.2 & 15 & +7.2 & 30 & 23.0 & 23.0 & 19 & +8.9 \\
\hline 32 & 28.0 & 29.0 & 27 & $x^{-2}$ & 32 & 24.9 & 25.3 & 22 & & 32 & 21.0 & 22.5 & 17 & & 32 & 22.8 & 23.0 & 18 & \\
\hline 34 & 27.0 & 28.0 & 26 & $A^{\prime}$ & 34 & $24 . I$ & 24.6 & 2I & & 34 & 22.7 & 24.4 & I9 & 21 & 34 & 22.7 & 23.0 & 18 & \\
\hline 36 & 27.3 & 28.0 & 26 & de & 36 & 24.2 & 24.8 & $2 \mathrm{I}$ & tr: & 36 & 24.0 & 25.3 & $2 \mathrm{I}$ & $x$ & 36 & 22.9 & 23.0 & 18 & \\
\hline 38 & 28.0 & 28.8 & 27 & * & 38 & 24.0 & 24.8 & $2 I$ & 1 & 38 & 23. I & 24.8 & 20 & Re & 38 & 23.0 & 23.0 & 19 & 1 \\
\hline 40 & 26.8 & 27.8 & 25 & of & 40 & 23.0 & 23.8 & 19 & ay & 40 & 23.0 & 24.2 & 20 & 94 & 40 & 22.5 & 23.0 & 18 & \\
\hline 42 & 27.7 & 28.9 & 27 & 4 & 42 & 23.9 & 24.3 & 20 & 14 & 42 & 23. I & 24.4 & 20 & $1=$ & 42 & 22.0 & 22.4 & 17 & 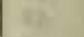 \\
\hline 44 & 27.3 & 28.8 & 26 & +4.5 & 44 & 25.3 & 25.5 & 22 & +6.4 & 44 & 23.1 & 24.7 & 20 & +7.6 & 44 & 22.2 & 23.6 & 18 & \\
\hline 46 & 29.0 & 30.0 & 29 & & 46 & 22.9 & 23.5 & 19 & & 46 & 23.0 & $24 \cdot 3$ & 20 & & 46 & 21.4 & $2 \mathrm{I} .9$ & 16 & +8.8 \\
\hline 48 & 29 . I & 30.0 & 29 & 28 & 48 & 22.6 & 22.9 & 18 & Ba & 48 & 23.7 & 25.0 & $2 \mathrm{I}$ & 84 & 48 & 22.2 & 22.4 & 18 & \\
\hline 50 & 28.4 & 29.5 & 28 & ce & 50 & 23. I & 23.4 & I9 & net & 50 & $23 \cdot 3$ & $24 \cdot 4$ & 20 & of & 50 & 22.0 & 22.2 & 17 & \\
\hline 52 & 29.0 & 29.9 & 29 & Su & 52 & 25.6 & 26.2 & 23 & 18 & 52 & 22.0 & 23.0 & 18 & the & 52 & $2 \pi \cdot 3$ & 21.7 & 16 & \\
\hline 54 & 27.9 & 28.7 & 27 & BI & 54 & 26. I & 26.6 & 24 & $\mathrm{~N} 2$ & 54 & 21.4 & 22.2 & 17 & 18 & 54 & 21.0 & 21.4 & 16 & \\
\hline 56 & 27.2 & 28. I & 26 & ar & 56 & 24.9 & 25.3 & 22 & Q & 56 & $2 I .4$ & 22.3 & 17 & . & 56 & 21.6 & 22 . I & 17 & \\
\hline 58 & 27.5 & 28.0 & 26 & 182 & 58 & 23.9 & $24 \cdot 3$ & 20 & B. & 58 & 21.8 & 22.1 & 17 & Ait & 58 & 22.2 & 22.9 & 18 & \\
\hline & 20 & 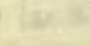 & & & & & & & & & & & & & & & & & \\
\hline
\end{tabular}


Tabulation of magnetic declinations observed at Alger Island Station-Continued

\begin{tabular}{|c|c|c|c|c|c|c|c|c|c|c|c|c|c|c|c|c|c|c|c|c|}
\hline \multicolumn{6}{|c|}{ Wednesday, July 26, 1905} & \multicolumn{5}{|c|}{ Magnet scale erect } & \multicolumn{6}{|c|}{ Wednesday, July 26, 1905} & \multicolumn{4}{|c|}{ Magnet scale erect } \\
\hline \multirow{2}{*}{$\begin{array}{l}\text { Chr'r } \\
\text { time }\end{array}$} & \multicolumn{2}{|c|}{$\begin{array}{l}\text { Scale } \\
\text { readings }\end{array}$} & $\begin{array}{l}\text { East } \\
\text { decli- } \\
\text { nation }\end{array}$ & Temp. & $\begin{array}{l}\text { Chr'r } \\
\text { time }\end{array}$ & $\begin{array}{l}\text { Sca } \\
\text { read }\end{array}$ & $\begin{array}{l}\text { ale } \\
\text { lings }\end{array}$ & $\begin{array}{c}\text { Eas } \\
\text { decli } \\
\text { natio }\end{array}$ & & Temp. & Chr'r & $\begin{array}{r}\text { Sc } \\
\text { read }\end{array}$ & le & $\begin{array}{l}\text { East } \\
\text { decli- }\end{array}$ & Temp. & Chr'r & $\begin{array}{r}\mathrm{Sc} \\
\text { read }\end{array}$ & e & East & Temp. \\
\hline & Left & Right & & & & Left & Right & & & & & Left & Right & & & & L,eft & Right & & \\
\hline h m & d & d & & & h $\mathrm{m}$ & d & d & & & & $\mathrm{ln} \mathrm{m}$ & d & d & & & $\mathrm{h} \mathrm{m}$ & $d$ & d & & \\
\hline 1600 & $\begin{array}{l}22.8 \\
23.0\end{array}$ & $23 \cdot 3$ & 2019 & +8.6 & 1800 & 20.9 & 21.0 & 20 & 5 & +8.0 & 2000 & 22.0 & 22.7 & $20 \mathrm{I8}$ & +6.1 & 2200 & 20.9 & & 2016 & +4.8 \\
\hline 02 & $\begin{array}{l}23.0 \\
22.6\end{array}$ & $\begin{array}{l}23.5 \\
23.0\end{array}$ & 19 & 38 & 02 & 21.0 & $21 \cdot 3$ & & 16 & 180 & 02 & 21.5 & 21.7 & 16 & 50 & 02 & $20 . \overline{8}$ & $21 . \overline{8}$ & I6 & \\
\hline 04 & $\begin{array}{l}22.6 \\
22.4\end{array}$ & 23.0 & 18 & 19 & 04 & 21.2 & 21.5 & & 16 & 38 & O4 & 20.9 & 2I. I & & 30 & 04 & 20.3 & 21.3 & 15 & 60 \\
\hline 06 & & $\begin{array}{l}23.0 \\
22.0\end{array}$ & 18 & to & 06 & 21.6 & 22.0 & & 17 & 60 & of & 20.8 & 2I.I & 15 & & & 21.0 & & т6́ & in \\
\hline 08 & $\begin{array}{l}21.5 \\
21.2\end{array}$ & 22.0 & 17 & Bbs & 08 & 22.0 & 22.3 & & 17 & $=0$ & as & 20.9 & 21.3 & 16 & +5 & 08 & 21.2 & 22. & 16 & \\
\hline 10 & $\begin{array}{l}21.2 \\
21.8\end{array}$ & $\begin{array}{l}21.8 \\
22.2\end{array}$ & 16 & at & Io & 22.3 & 22.8 & & 18 & & 10 & 20.5 & 21.1 & 15 & & I0 & 20.9 & & 16 & \\
\hline 12 & 21.8 & 22.2 & 17 & s) & 12 & 22.9 & 23.0 & & 18 & & 12 & 19.9 & 20.7 & 14 & & 12 & 21.7 & 22 & 17 & \\
\hline 14 & 21.7 & 22.2 & 17 & & I4 & 23.3 & 23.5 & & 19 & +8.0 & 14 & 19.3 & 20.1 & 13 & +6.0 & I4 & 22.0 & 22.9 & 18 & +4.8 \\
\hline 16 & 22.0 & 22.4 & 17 & +8.5 & 16 & 23.4 & 23.6 & & 19 & & 16 & 18.9 & 19.5 & 13 & bint & 16 & 22.0 & 22.9 & 18 & 10 \\
\hline 18 & 23.3 & 24.0 & 20 & $x^{2}$ & I8 & 23.2 & 23.4 & & 19 & ह5. & I8 & 18.5 & 19.0 & 12 & tex & I8 & 20.6 & 21 & 15 & \\
\hline 20 & 23.6 & 24.0 & 20 & कs & 20 & 23.3 & 23.4 & & 19 & $\cos$ & 20 & 18.3 & 18.9 & 12 & Ds & 20 & 21.0 & 22 & 16 & cet \\
\hline 22 & 24.2 & 24.6 & $2 \mathrm{I}$ & E & 22 & 23.4 & 23.4 & & 19 & 20 & 22 & 18.2 & 18.8 & 12 & $E$ & 22 & 20.6 & 21.5 & 16 & \\
\hline 24 & 24.0 & 24.5 & 20 & 35 & 24 & 23.3 & 23.6 & & 19 & I & 24 & 18.0 & 18.5 & 11 & 34 & 2 & 20.3 & 21 . & 15 & \\
\hline 26 & 21 & $.8 a$ & 17 & & 26 & 23.3 & 23.5 & & I9 & (5) & 26 & 17.4 & 17.9 & 10 & 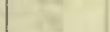 & 2 & 21.0 & 22 & 16 & \\
\hline 28 & 19.2 & 19.8 & 13 & & 28 & 23.3 & 23.5 & & 19 & & 28 & 16.5 & 17.0 & 09 & & 28 & 19.9 & 20. & 14 & \\
\hline 30 & 18.8 & 19.0 & 12 & +8.4 & 30 & 23.3 & $23 . \overline{8}$ & & 19 & +7.6 & 30 & 15.9 & 16.4 & 08 & +5.6 & 30 & 20.1 & 21. & 15 & +5.0 \\
\hline 32 & 19.4 & 19.7 & 13 & (2) & 32 & 23.5 & 23.6 & & 19 & - & 32 & 15.8 & I6. 2 & 08 & (10 & 32 & 20.8 & $2 \mathrm{I}$ & 16 & \\
\hline 34 & 20.5 & 20.5 & I5 & I2. & 34 & 23.3 & 23.7 & & 19 & : & 34 & 15.6 & 16.1 & of & IS & 34 & 21.0 & 22. & 16 & \\
\hline 36 & 20.9 & 21.0 & 15 & $\mathrm{~A}^{2}$ & 36 & 23.1 & 23.1 & & 19 & 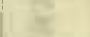 & 36 & 16.1 & I6. 3 & 08 & a & 36 & 20.3 & 21 & & \\
\hline 38 & 21.1 & 21.3 & 16 & th & 38 & 23.2 & 23.2 & & 9 & 4 & 38 & 17.0 & 17.1 & 09 & he & 38 & 21.0 & 21. & & P. \\
\hline 40 & 21.3 & 21.4 & 16 & $=$ & 40 & 23.0 & 23.3 & & 9 & & 40 & 17.9 & 18.0 & II & te & 4 & 21 & 21 . & I6 & \\
\hline 42 & 21.5 & 21.7 & 16 & & 42 & 22.6 & 23.0 & & 8 & & 42 & 17.8 & 17.9 & 10 & 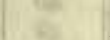 & 42 & 21.8 & 22 . & I7 & \\
\hline 44 & 21.0 & 21.3 & I6 & +8.4 & 44 & 21.9 & 22.3 & & 7 & +7.2 & 44 & 16.8 & 16.9 & $\infty$ & +5.5 & 44 & 22.6 & 23. & 19 & +5.1 \\
\hline 46 & 19.9 & 20.3 & 14 & ( & 46 & $21 . \overline{8}$ & 22.2 & & 17 & & 46 & 15.9 & 16.1 & 08 & 1 & 46 & 22.3 & 23. & & \\
\hline 48 & 19.8 & 20.3 & 14 & $\mathrm{~B}$ & 48 & 21.0 & 21.9 & & 6 & E & 48 & 14.8 & 15.0 & 06 & te & 48 & 22.5 & 23 & & \\
\hline 50 & 19.2 & 19.9 & 13 & ar & 50 & 20.9 & 21.3 & & I6 & 6 & 50 & 14.2 & 14.3 & 05 & wat & $5 c$ & 22.3 & 22 & & w \\
\hline 52 & 19.0 & 19.3 & 13 & 25 & 52 & 21.1 & 21.5 & & 16 & $x^{2}$ & 52 & 14.1 & 14.4 & 0 & $a^{2}$ & 5 & 22.2 & 22 & 8 & 5 \\
\hline 54 & I8. 8 & 19.3 & I & 12 & 54 & 21.6 & 21.9 & & 17 & & 54 & 14.3 & 14.7 & 0 & S & 5 & 22 & 22 & 17 & 3 \\
\hline 56 & 18.4 & 18.9 & 12 & 4 & 56 & 21.8 & $22 . \bar{I}$ & & 17 & & 56 & 14.7 & 14.9 & 0 & & 5 & 22 & 23 & 9 & \\
\hline 58 & 18.4 & 19.0 & 12 & & 58 & $2 I .3$ & 21.9 & & 6 & & || 58 & 14.7 & 15.1 & o6 & 22 & 5 & 21.8 & 22 & 17 & \\
\hline 700 & I8. I & 18.8 & II & +8.3 & 1900 & 20.9 & 21.5 & & 6 & +7.0 & 2100 & 14.8 & 15.1 & 06 & & 2300 & 20.7 & 21.3 & 15 & +5.6 \\
\hline 02.4 & 17.8 & 18.8 & 11 & (n) & 02 & 20.5 & 21.0 & & 5 & nes & 02 & 14.8 & 15.5 & 06 & +5.3 & 02 & 9.8 & & 4 & 120 \\
\hline 04 & 17.7 & 18.4 & II & $=$ & 04 & 20.0 & 20.3 & I & 4 & 4 & 0 & 14.9 & 15.6 & 06 & 00 & o. & 19.0 & I9. & 13 & \\
\hline 06 & 17.3 & 18.0 & I & 86 & 06 & 19.4 & 19.9 & & 3 & 8 & 06 & I5.I & 15.6 & 0 & 68 & & 17.7 & & & 8 \\
\hline 08 & 17.4 & I8.6 & I & 80 & as & 19.2 & 9.8 & & 3 & th & 08 & 15.3 & 15.9 & 0 & Ev & os & 17.0 & 18 & 10 & 8 \\
\hline 10 & 18.0 & 19.0 & I & e & & 19.3 & 0.1 & & 3 & & & & & & 20 & IC & 17.3 & I8 & 0 & 2. \\
\hline 12 & 18.8 & 19.7 & 13 & & I & .0 & 2 & & 4 & & 12 & .3 & 16.8 & 08 & & 12 & 9.5 & I9. & 13 & \\
\hline 14 & 19.1 & 20.0 & I3 & & I & 0.6 & 1.0 & I & 5 & +6.8 & 14 & 16.8 & 17.3 & $\infty 9$ & +5.2 & 14 & & I6 & 08 & \\
\hline 16 & 19.2 & 20.0 & 13 & +8.1 & 16 & 20.6 & I.I & & 5 & . & 16 & 16.8 & 17.2 & 09 & o & I6 & 16.8 & 17 & $\infty 9$ & +6.0 \\
\hline 18 & 19.3 & 20. & 13. & & I & .9 & & & 6 & in & 18 & 15.6 & 16. & & 5 & 18 & 15.3 & I5 & 7 & ( \\
\hline 20 & 19.0 & 19.6 & I3 & & 2 & 20.6 & 21.2 & & 5 & & 20 & 15.5 & 16.5 & & 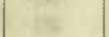 & 20 & & & & 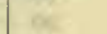 \\
\hline 2 & 18.7 & 19.6 & I & Ex & 2 & 0.3 & 2 & & 5 & tis & 22 & & 17.2 & 09 & as. & 2 & I5.0 & I5. & 06 & AS \\
\hline 2 & 18.2 & 19.5 & I & is & 24 & 0.3 & 2I.I & & & 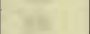 & 24 & 18.8 & 19. & & E & & I5.3 & & 07 & 2 \\
\hline & 18.0 & & I & & & 0.4 & 21.1 & & 5 & & 26 & 19.3 & 20 . & I4 & E & 26 & 14.9 & 15 & $\infty 6$ & 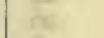 \\
\hline 28 & 17.8 & 18.9 & II & & 28 & 0.5 & 21.3 & & 5 & & 28 & & $2 \mathrm{I}$. & 15 & 86 & 28 & 15.5 & & 7 & \\
\hline 30 & 18.0 & 19.3 & 12 & +8.0 & 30 & 1.0 & & & 6 & +6.6 & 30 & 21.0 & 21.6 & 16 & +5.0 & 30 & 17.4 & 17 & 10 & +6.0 \\
\hline 3 & .0 & & $I$ & & 32 & 1.8 & 21.8 & & 7 & $x^{2}$ & 32 & 1.6 & 22. & & 10. & 3 & 19.1 & & I3 & 10 \\
\hline & & 19. & 12 & & 34 & 21.9 & 22.0 & & & 32 & 34 & 22.2 & 23. & & 18 & 3 & 21.6 & & 17 & \\
\hline 36 & 18.7 & I9.6 & $\mathrm{I} 2$ & 8 & & 1.6 & & & & $\mathrm{Cx}^{2}$ & 36 & 22.5 & & & ne & 3 & 21.8 & 22. & 17 & \\
\hline 38 & & 19.9 & 13 & है & 38 & 21.4 & 21.6 & & & 8 & 38 & 22.3 & 23. & & $\theta$ & 38 & 23.1 & & & c) \\
\hline 40 & 18.8 & 19.6 & 13 & & 40 & 1.1 & 21.3 & & & & $4 c$ & 2.0 & 22. & 18 & 20 & 40 & 20.6 & 21 & 15 & e \\
\hline 42 & 19.0 & 19.4 & 13 & & 42 & 21.1 & 21.3 & & 6 & & 42 & 21.9 & 22.5 & 17 & & 42 & 22.7 & & 19 & \\
\hline 44 & 9.4 & 20.0 & I3 & +8.0 & 44 & 21.1 & 21.5 & 1 & 6 & +6.2 & 44 & 21.4 & 22 . & 17 & +4.8 & 44 & $25 \cdot 3$ & 26.8 & 23 & +5.9 \\
\hline 46 & 20.0 & 20.4 & 14 & ( & 46 & 21.6 & 21.8 & & & & 46 & 21.0 & 21 . & & & & 21.9 & 23.3 & 18 & \\
\hline 48.3 & 20.3 & & IS & 1 & 48 & 22.0 & 22.2 & & 7 & 7 & 48 & 20.8 & 21 & 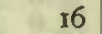 & int & 48 & 18.3 & 19. & 12 & 8 \\
\hline 50 & 20.6 & 21.1 & 15 & 8 & 50 & 21.0 & 22.3 & 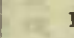 & 7 & से & 50 & 21.0 & 21.8 & & $\alpha^{2}$ & & 16.3 & & & $x^{3}$ \\
\hline 52 & .2 & 21.1 & 15 & $t 5$ & 52 & 21.8 & 22.2 & & 7 & 3 & 52 & 21.0 & 21.6 & 16 & 8 & 52 & 16.0 & I6.6 & 08 & 3 \\
\hline 54 & .4 & 21.3 & 15 & H & 54 & 22.2 & 22.9 & & & y & 54 & 20.1 & 20.9 & 15 & 58 & & 16.4 & & $\infty$ & \\
\hline 56 & 20.3 & $21 . \mathrm{I}$ & 15 & S & 56 & 22.2 & 23.0 & & 8 & $\mathrm{~N}$ & 56 & 19.3 & 20.0 & I3 & or & 56 & 17.8 & 18.6 & II & \\
\hline 58 & 20.1 & 20.7 & 14 & 18 & 58 & 21.8 & 22.6 & & & 8 & 58 & 20.5 & 21.3 & 15 & Bs & 58 & 19.3 & I9.7 & 13 & +6.0 \\
\hline & & & & & & & & & & & & & & & & & 20.2 & & 15 & \\
\hline
\end{tabular}

Observers-A. F. and W. J. P., who alternated from I7h $40 \mathrm{~m}$ to Correction to local mean time is - I2s.

I7h 5om.

Torsion head read $258^{\circ}$ at beginning and ending.

Observer-W. J. P. 
Tabulation of magnetic declinations observed at Alger Island Station-Continued

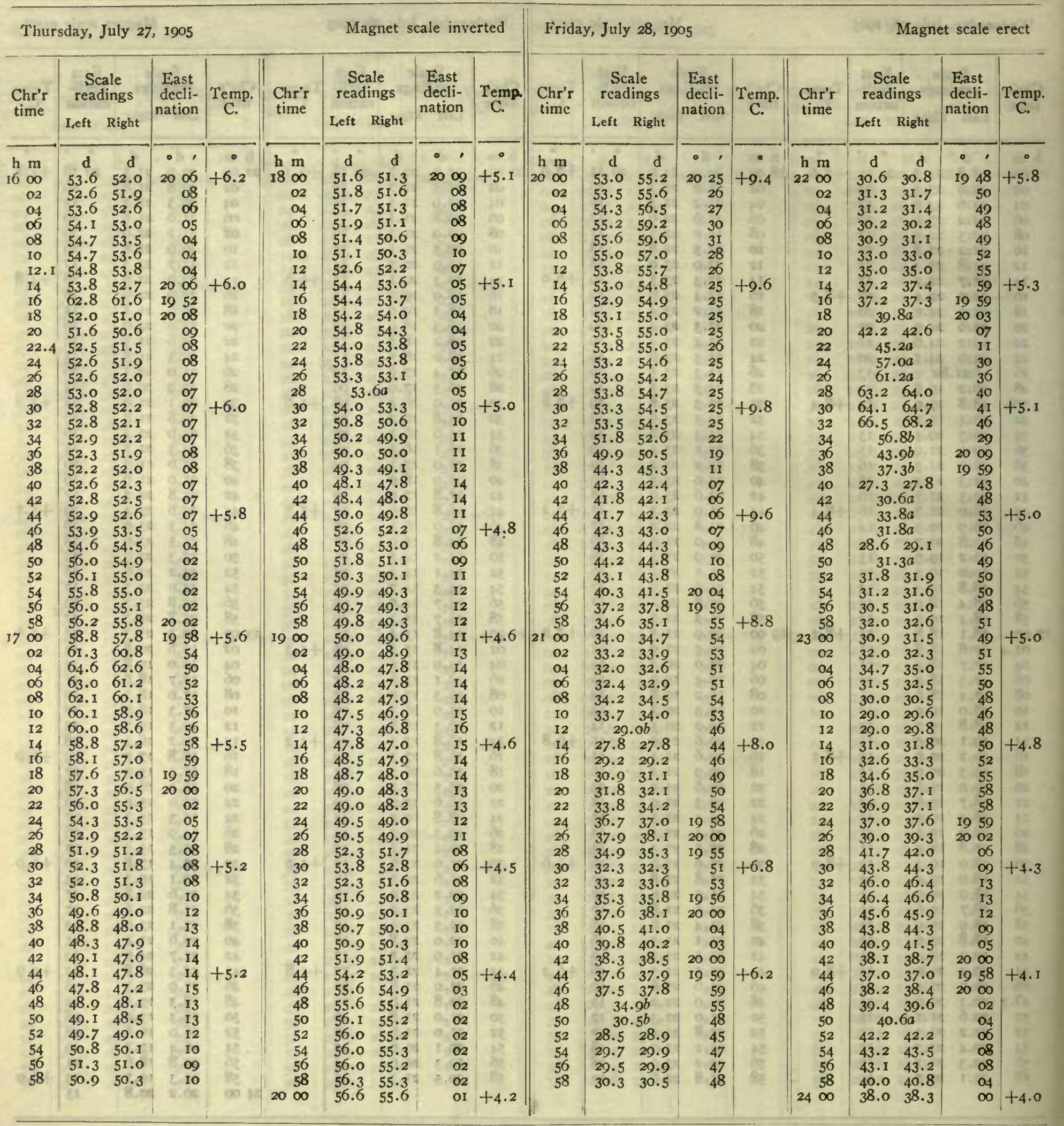

Correction to local mean time is - Ios.

Torsion head read $28^{\circ}$ at beginning and ending. Observer-W. J. P.
Correction to local mean time is - Ios. $90^{\circ}$ torsion $=18 .^{\prime} 33$. Torsion head at $20 \mathrm{~h}$ oom read $25^{\circ}$ and at $24 \mathrm{~h} 20 \mathrm{~m}$ read $252^{\circ}$. Observer-W. J. P. 
Tabulation of magnetic declinations observed at Alger Island Station-Continued

Sunday, July 30, I905 Magnet scale inverted Sunday, July 30, 1905

Magnet scale erect.

\begin{tabular}{|c|c|c|c|c|c|c|c|c|c|c|c|c|c|c|c|c|c|c|c|c|}
\hline \multirow{2}{*}{$\begin{array}{l}\text { Chr's } \\
\text { time }\end{array}$} & \multicolumn{2}{|c|}{$\begin{array}{l}\text { Scale } \\
\text { readings }\end{array}$} & \multirow{2}{*}{$\begin{array}{l}\text { East } \\
\text { decli- } \\
\text { nation }\end{array}$} & \multirow{2}{*}{ Temp. } & \multirow{2}{*}{$\begin{array}{l}\text { Chr'r } \\
\text { time }\end{array}$} & \multicolumn{2}{|c|}{$\begin{array}{l}\text { Scale } \\
\text { readings }\end{array}$} & \multirow[t]{2}{*}{$\begin{array}{c}\text { East } \\
\text { decli- } \\
\text { nation }\end{array}$} & $\begin{array}{c}\text { Temp. } \\
\text { C. }\end{array}$ & $\begin{array}{l}\text { Chr'r } \\
\text { time }\end{array}$ & $\begin{array}{l}\text { Sca } \\
\text { read }\end{array}$ & $\begin{array}{l}\text { e } \\
\text { igs }\end{array}$ & $\begin{array}{l}\text { East } \\
\text { decli- } \\
\text { nation }\end{array}$ & $\begin{array}{l}\text { Temp. } \\
\text { C. }\end{array}$ & Chr'r & $\begin{array}{l}\text { Sc: } \\
\text { read }\end{array}$ & $\begin{array}{l}\text { ale } \\
\text { lings }\end{array}$ & $\begin{array}{c}\text { Ea } \\
\text { dec } \\
\text { nati }\end{array}$ & & Temp \\
\hline & Left & Right & & & & Left & Right & & & & Left & Right & & & & Left & Right & & & \\
\hline h m & d & d & $\cdot 1$ & $\bullet$ & $\mathrm{h} \mathrm{m}$ & d & d & 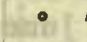 & 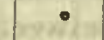 & $\mathrm{h} \mathrm{m}$ & $\mathrm{d}$ & d & - , & & $\mathrm{h} \mathrm{m}$ & d & d & - & - & \\
\hline $0 \infty^{*}$ & 50.6 & 49.0 & 2012 & +4.2 & 200 & $47 \cdot 3$ & 46.3 & $20 \mathrm{IC}$ & +5.0 & 400 & 56.7 & 58.8 & 2I 17 & +5.4 & 600 & $45 \cdot 3$ & $47 \cdot 3$ & 20 & 59 & +5.3 \\
\hline 02 & 53.0 & $5 \mathrm{I} .2$ & $\$ 8$ & & 02 & 45.4 & 44.0 & 2 & & $\mathrm{O} 2$ & 57.0 & 59.0 & & & 02 & 49.7 & 50.1 & 2 I & 04 & \\
\hline 04.5 & 50.2 & 49.3 & I2 & & 04 & $43 \cdot 3$ & 42.7 & 2 & & 04 & 54.5 & 56.8 & 13 & & 04 & $47 \cdot 5$ & 48.5 & & 02 & \\
\hline $06^{\circ}$ & 47.6 & 46.0 & I6 & & 06 & 40.6 & 40.2 & 26 & & 06 & 53.8 & 55.3 & 12 & & 06 & 49.3 & 50.1 & & 04 & \\
\hline 08 & 47.3 & 46.5 & 16 & & 08 & $36 . I$ & 35.9 & 3 & & 08 & 53.0 & 55.0 & II & & 08 & 48.4 & 49.6 & 2 I & 03 & \\
\hline IO & 49.0 & 47.5 & $\mathrm{I}_{4}$ & & 10 & 29.6 & 29.5 & 4. & & 10 & 48.1 & 49.2 & 2102 & & IO & 44.0 & 44.8 & 20 & 56 & \\
\hline 12 & 48.2 & 47.2 & r 5 & & 12 & 26.0 & 24.6 & 50 & & 12 & 41.2 & 42.1 & 2052 & & 12 & 45.2 & 46.0 & & 58 & \\
\hline 14 & 48.2 & 47.1 & I5 & +4.5 & 14 & 19.7 & 18.9 & 205 & +5.0 & 14 & 38.8 & 40.0 & 2048 & +5.6 & 14 & 40.0 & 41.0 & & 50 & \\
\hline 16 & 48. I & 47.3 & r 5 & & 16 & 19.6 & 18.2 & $21 \propto$ & & I6 & 47.2 & 48.0 & 21 oI & & 16 & $4 \mathrm{r}$ & .00 & 20 & $5 I$ & +5.5 \\
\hline 18 & 48.3 & $47 \cdot 3$ & I5 & 15 & 18 & 23.9 & 22.3 & 205 & & I8 & 52.3 & 54.0 & 10 & & 18 & 47.2 & 47.6 & 21 & or & \\
\hline 20 & 49.1 & $48 . I$ & I3 & & 20 & 26.7 & 25.0 & 49 & & 20 & 49.9 & 51.8 & 06 & & 20 & 50.0 & 51.5 & 21 & 06 & \\
\hline 22 & 45.8 & 45.5 & I8 & & 22 & 25.9 & 24.0 & 5 & & 22 & $47 \cdot 3$ & 48.9 & 02 & & 22 & 44.0 & 45.4 & 20 & 56 & \\
\hline 24 & 43.8 & 43.0 & 22 & nitis & 24 & 27.8 & 26.0 & 48 & & 24 & 48.4 & 49.8 & 03 & & 24 & 50.3 & 51.2 & 21 & 06 & \\
\hline 26 & 43.6 & 43.5 & 21 & & 26 & 27.3 & 25.3 & 48 & & 26 & 49.5 & 50.9 & 05 & & 26 & 40.2 & 41.8 & 20 & $5 \mathrm{I}$ & \\
\hline 28 & 39.6 & 39.0 & 28 & & 28 & 30.9 & 28.4 & 43 & & 28 & 50.3 & 51.5 & 06 & & 28 & 45.0 & 45.6 & & 57 & \\
\hline 30 & 40.1 & 40.0 & 27 & +4.5 & 30 & 36.6 & 34.1 & 34 & +5.0 & 30 & 49.2 & 49.9 & 04 & +5.7 & 30 & 39.2 & 40.2 & & 48 & +5.7 \\
\hline 32 & 47.6 & 47.5 & 15 & (and & 32 & 38.0 & 36.2 & 3 & & 32 & 49.1 & 50.2 & 04 & & 32 & 40.8 & 41.2 & & $5 \mathrm{I}$ & \\
\hline 34 & 49.3 & 47.6 & I4 & & 34 & 36.2 & $34 \cdot 3$ & 34 & & 34 & 49.0 & 49.9 & & & 34 & 36.0 & 37.2 & & 44 & \\
\hline 36 & 41.2 & 40.8 & 25 & & 36 & 34.2 & 32.4 & $3 \%$ & & 36 & 50.2 & 50.8 & 06 & & 36 & 34.2 & 35.0 & & 40 & \\
\hline 38 & 44.1 & $43 . I$ & 21 & & 38 & $34 \cdot 3$ & 32.0 & 38 & & 38 & 55.0 & 55.8 & I3 & & 38 & 31.2 & 32.1 & & 36 & \\
\hline 40 & 47.0 & 46.5 & 16 & & 40 & 29.0 & 26.2 & 46 & & 40 & 54.0 & 54.8 & I2 & & 40 & 33.8 & 35.4 & & 40 & \\
\hline 42 & 47.0 & 46.0 & 17 & & 42 & 23.0 & I8. 5 & $205 \%$ & & 42 & 53.3 & 54.2 & IO & & 42 & 38.8 & 40.4 & & 48 & \\
\hline 44 & 44.9 & 44.2 & 20 & & 44 & 14.6 & 9.0 & 211 & & 44 & 56.2 & 57.4 & I 5 & & 44 & 36.0 & 37.2 & & 44 & \\
\hline 46 & 47 & $.8 a$ & I5 & & $46^{*}$ & $35 \cdot 3$ & $30 . I$ & 18 & +5.0 & 46 & 58.5 & 59.5 & & +5.5 & & 37.8 & 39.3 & & 47 & +5.8 \\
\hline 48 & 51.0 & 50. I & I0 & & 48 & 45.5 & 39.9 & 02 & & 48 & 56.2 & 57.6 & $1 \overline{6}$ & & 48 & 36.0 & 37.0 & & 43 & \\
\hline 50 & 51.3 & 50.5 & ro & & 50 & 46.6 & $41 \cdot 3$ & $\infty$ & & 50 & 55.9 & 56.8 & 15 & & 50 & 35.3 & 36.1 & & 42 & \\
\hline 52 & 53.9 & 53.0 & $\infty$ & 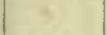 & 52 & 44.1 & 39 . I & o 4 & & 52 & 55.3 & 56.5 & 14 & & 52 & 32.7 & 33.5 & & 38 & \\
\hline 54 & 52.6 & 52.0 & 08 & te & 54 & 36.0 & 31.0 & I6 & $\mathrm{ser}$ & 54 & 56.7 & 57.8 & 16 & & 54 & 33.2 & 34 & & 39 & \\
\hline 56 & 52.7 & 51.9 & 08 & & 56 & 33.5 & 29. I & $2 c$ & & 56 & 56.2 & 57.3 & 15 & & 5 & 35.1 & 36.5 & & 42 & \\
\hline 58 & 51.4 & 50.9 & o) & $x$ & 58 & 37.8 & 33.1 & $I_{4}$ & & 58 & 55.8 & 56 & I5 & +5.3 & 58 & 37.7 & 38.8 & & 46 & \\
\hline 100 & 50.8 & 50.0 & II & +4.9 & 300 & 37.0 & 32.9 & I 4 & +5.0 & 500.5 & 55.8 & 56.9 & 15 & & 700 & 39.1 & 39.5 & & 48 & +6.0 \\
\hline 02 & 51.0 & 50.1 & 10 & & 02 & 38.8 & 34.8 & II & in & 02 & 54.8 & 55.9 & I3 & & 02 & 39.5 & 40 & & 49 & \\
\hline 04 & 50.1 & 49.6 & I2 & & 04 & 40.1 & 36.8 & $\alpha$ & & 04 & 55.0 & 56.2 & I3 & & 04 & 40.2 & 40.8 & & 50 & \\
\hline об & 49.7 & 49.1 & 12 & & 06 & 39.5 & 36.0 & IC & wr & 06 & 55.2 & 56.4 & 14 & & 06 & 43.5 & 44.0 & & 55 & \\
\hline 08 & 49.5 & 49.0 & 12 & & 08 & 36.3 & 33.0 & IS & & 08 & 56.8 & 57.3 & 16 & & 08 & 40.0 & 41.0 & & 50 & \\
\hline 10 & 50.0 & 49.5 & 12 & & I0 & 32.1 & 20.3 & 21 & tent & 10 & 53.0 & 54.2 & 2110 & $=$ & Io & 32.7 & 34.0 & & 38 & \\
\hline 12 & 50. I & 49.9 & II & & 12 & 30.8 & 28.6 & 22 & & 12 & $41 \cdot 5$ & 43.5 & 2053 & & 12 & 33.7 & & & 40 & \\
\hline 14 & 50.2 & $50 . I$ & II & & 14 & 28.0 & 25.9 & 27 & +5.0 & 14 & 35.0 & 36.0 & 42 & +5.2 & 14 & 36.2 & 36.8 & & 44 & +6.2 \\
\hline 16 & 50.3 & 50.0 & II & +5.0 & 16 & 29.0 & 26 & 26 & & 16 & 34.2 & & $4 I$ & & I & 36.2 & 37 & & 44 & \\
\hline 18 & 50.1 & 49.5 & I 2 & & 18 & 3r. I & 28.5 & 22 & & I8 & 39.9 & $4 \mathrm{I}$ & 50 & & 18 & 37.8 & 38. & & 46 & \\
\hline 20 & 50.3 & 49 & II & & 20 & 30. I & 27.7 & 24 & & 20 & 39.2 & 40. & 48 & & 20 & 35.3 & 36.0 & & 42 & \\
\hline 22 & 52.0 & 51.5 & $\infty 8$ & & 22 & 29.0 & 27.0 & 25 & -21 & 22 & 42.3 & 44.5 & 54 & 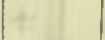 & 22 & 33.8 & 34.2 & & 40 & \\
\hline 24 & $54 \cdot 3$ & 53 & 05 & & 24 & 28.8 & 26.6 & 26 & & 24 & 43.8 & 44. & 56 & & 24 & 32 & $.3 b$ & & 37 & \\
\hline 26 & 51.5 & 50.3 & IO & & 26 & 31.4 & 29.3 & 22 & & 26 & 42.3 & 43. & 2053 & 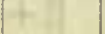 & & 29 & $.2 b$ & & 32 & \\
\hline 28 & 49.5 & 48.4 & 13 & & 28 & 30.9 & 29.1 & 22 & & 28 & 47.0 & 47.9 & 2I oI & & 28 & 27.0 & 27.8 & & 29 & \\
\hline 30 & 48.5 & 47.7 & I 4 & +5.0 & 30 & 29.3 & 27.5 & 24 & +5.0 & 30 & 44.8 & 46.0 & 2058 & +5.1 & 30 & 28.7 & 29.0 & & $3 I$ & +6.6 \\
\hline 32 & 48.7 & 47.6 & 14 & & 32 & 29.0 & 27.2 & 25 & & 32 & 49.5 & 50.6 & 2105 & & 32 & 34.0 & 35.8 & & $4 \mathrm{I}$ & \\
\hline 34 & 52.8 & 5I.I & 08 & & 34 & 30.6 & 29.0 & 22 & & 34 & 47.0 & 48. & or & f & 34 & 38.8 & 40.7 & & 48 & \\
\hline 36 & $55 \cdot 3$ & 54.5 & 04 & & 36 & 32.0 & 30.3 & 20 & & 36 & 50.7 & 52.3 & 07 & & 3 & 38.5 & 39 & & 47 & \\
\hline $3^{8}$ & 53.5 & 52.6 & & & 38 & 33.0 & $3 \mathrm{I} \cdot 3$ & IO & & 38 & 52.5 & 54.0 & IO & $H$ & 38 & 31.6 & 32.8 & & 37 & \\
\hline 40 & 53.7 & 52.8 & 06 & & 40 & 33.5 & 32.0 & 18 & & 40 & 51.0 & 52.4 & 07 & & 40 & 30.7 & $3 I \cdot 5$ & & 35 & \\
\hline 42 & 5 I. 6 & 51.0 & $\infty$ & & 42 & 33.0 & 31.7 & 18 & & 42 & 51.6 & 52.5 & 08 & & 42 & 29.4 & 30 & & 33 & \\
\hline 44 & 50.3 & 49.3 & 12 & +5.0 & 44 & 31.0 & 30.0 & 21 & +5.0 & 44 & 49.0 & 50.0 & 04 & & 44 & $33 \cdot I$ & 34.8 & & 39. & \\
\hline 46 & 48.6 & 47.6 & I4 & & 46 & 30.4 & 29.3 & 22 & & 46 & 47.8 & 49. I & 02 & +5.1 & 46 & 34.8 & 35.8 & & 42 & +6.9 \\
\hline 48 & 45.0 & 44.0 & 20 & & 48 & 32.0 & 31.0 & 20 & & 48 & 48. & $7 b$ & $2 \mathrm{IO}$ & & 48 & 33.6 & 34.6 & & 40 & \\
\hline 50 & 45.2 & $44 . I$ & 20 & & 50 & 33.2 & 32.2 & 18 & & 50 & 44 & $8 a$ & 2057 & & 50 & 31.0 & 32.0 & & 36 & \\
\hline 52 & 45.6 & 44.3 & rg & & 52 & 34.0 & $33 \cdot 3$ & 16 & & 52 & $\because 51:$ & $5 a$ & 2107 & & 52 & 28.8 & 30.0 & & 32 & \\
\hline 54 & 47.4 & 46.4 & 16 & & $54^{*}$ & 52.6 & 51.0 & 13 & & 54 & 51. & $8 b$ & of & & 54 & 30.0 & 31.0 & & 34 & \\
\hline 56 & 47.3 & 46.3 & 16 & & 56 & 52.6 & 51.0 & 13 & & 56 & 47. & & OI & & 56 & $3 \mathrm{I} .2$ & 32.3 & & 36 & \\
\hline 58 & 48.7 & 47.8 & 14 & & 58 & 50.8 & 49.2 & 16 & & 58 & 57. & $4 b$ & I6 & mitra & $\begin{array}{r}58 \\
800\end{array}$ & $\begin{array}{l}36.0 \\
37.5\end{array}$ & $\begin{array}{l}37.0 \\
39.0\end{array}$ & & $\begin{array}{l}44 \\
46\end{array}$ & \\
\hline
\end{tabular}

Observers-W. J. P. and A. F., who alternated from 3 h $58 \mathrm{~m}$ to $4 \mathrm{~h} 08 \mathrm{~m}$.
Correction to local mean time is - Ios.

Torsion head at beginning and ending read $252^{\circ}$.

Observer-A. F. 


\section{REDUCTIONS FROM DECLINATION OBSERVATIONS AT ALGER ISLAND}

\section{DiURNAL VARIATION}

In deriving an expression representing the diurnal variation in magnetic declination over the period of observation at Alger Island the same methods have been used as in the case of the Teplitz Bay series (see pages 275 to 294). A tabulation of the resulting mean magnetic declinations as deduced from the observations between J11ne 26 and July 31,1905 , is given on the following page.

The analytical expression representing the daily variation of the east declination of the needle as deduced from these means is

$$
\begin{aligned}
& D=20^{\circ} 28 .^{\prime} 4+34 .^{\prime} 70 \sin \left(\theta+3^{\circ} 15^{\prime}\right)+110^{\prime} 55 \sin \left(2 \theta+292^{\circ} 31^{\prime}\right) \\
& +7 .^{\prime} 94 \sin \left(30+232^{\circ} 25^{\prime}\right)+1 .^{\prime} 73 \sin \left(4^{\theta}+150^{\circ} 43^{\prime}\right) \pm 0.0^{\prime} 65^{*}
\end{aligned}
$$

the angle $\theta$ counting from $15^{\circ}$ as 0.5 hour A. M. A graplical representation to scale of this formula, together with the mean values from which it is deduced, is given in figure 23. The agreement between the individual observed means and values computed for the corresponding times is given in the following summary, in which the observed and computed values are represented by letters $O$ and $C$ respectively, easterly deviation from the mean value for the whole period being indicated by a plus sign, and vice versa, and extremes being in bold-face type.

Summary of observed and computed diurnal variation of magnetic declination at Alger

\begin{tabular}{|c|c|c|c|c|c|c|c|}
\hline L. M. T. & 0 & C & $O-C$ & L. M. T. & 0 & c & $O-C$ \\
\hline $\begin{array}{c}h \\
0.5\end{array}$ & $\begin{array}{r}\prime \\
-7.4\end{array}$ & $\begin{array}{r}1 \\
-\quad 4.9\end{array}$ & -2.5 & $\begin{array}{r}h \\
12.5\end{array}$ & $\begin{array}{r}\prime \\
-14.4\end{array}$ & $\begin{array}{r}1 \\
-10.9\end{array}$ & $\begin{array}{r}\prime \\
-3.5\end{array}$ \\
\hline I.5 & +10.8 & + II.O & -0.2 & I3. 5 & -18.2 & -17.4 & -0.8 \\
\hline 2.5 & +23.8 & +30.5 & -6.7 & 14.5 & -19.0 & -23.3 & +4.3 \\
\hline 3.5 & +48.7 & +47.3 & +1.4 & I5.5 & -19.7 & -27.2 & +7.5 \\
\hline 4.5 & +48.6 & +55.0 & -6.4 & 16.5 & -32.5 & -28.7 & -3.8 \\
\hline 5.5 & +51.6 & +51.0 & +0.6 & 17.5 & -27.1 & -28.0 & +0.9 \\
\hline 6.5 & +45.4 & +38.1 & $+7 \cdot 3$ & 18.5 & -30.5 & -25.8 & -4.7 \\
\hline 7.5 & +23.2 & +22.5 & +0.7 & 19.5 & -22.7 & -23.0 & +0.3 \\
\hline 8.5 & +8.6 & +10.0 & -1.4 & 20.5 & -16.7 & -20.5 & +3.8 \\
\hline 9.5 & +2.8 & +2.5 & +0.3 & 21.5 & -25.2 & - Ig.I & -6.1 \\
\hline I0. 5 & +7.4 & -1.6 & +9.0 & 22.5 & -23.0 & -17.8 & -5.2 \\
\hline 11.5 & +2.9 & -5.5 & +8.4 & 23.5 & -17.5 & - I4.I & -3.4 \\
\hline
\end{tabular}
Istand for period June 26 to July 3I, 1905

For a value of magnetic declination at Alger Island, practically referring to mean of day, the mean of the tabulated observational means may be taken, viz:

$20^{\circ} 28.4 \mathrm{E}$ for the epoch 1905.53.

* This quantity is the probable error of a single representation. 


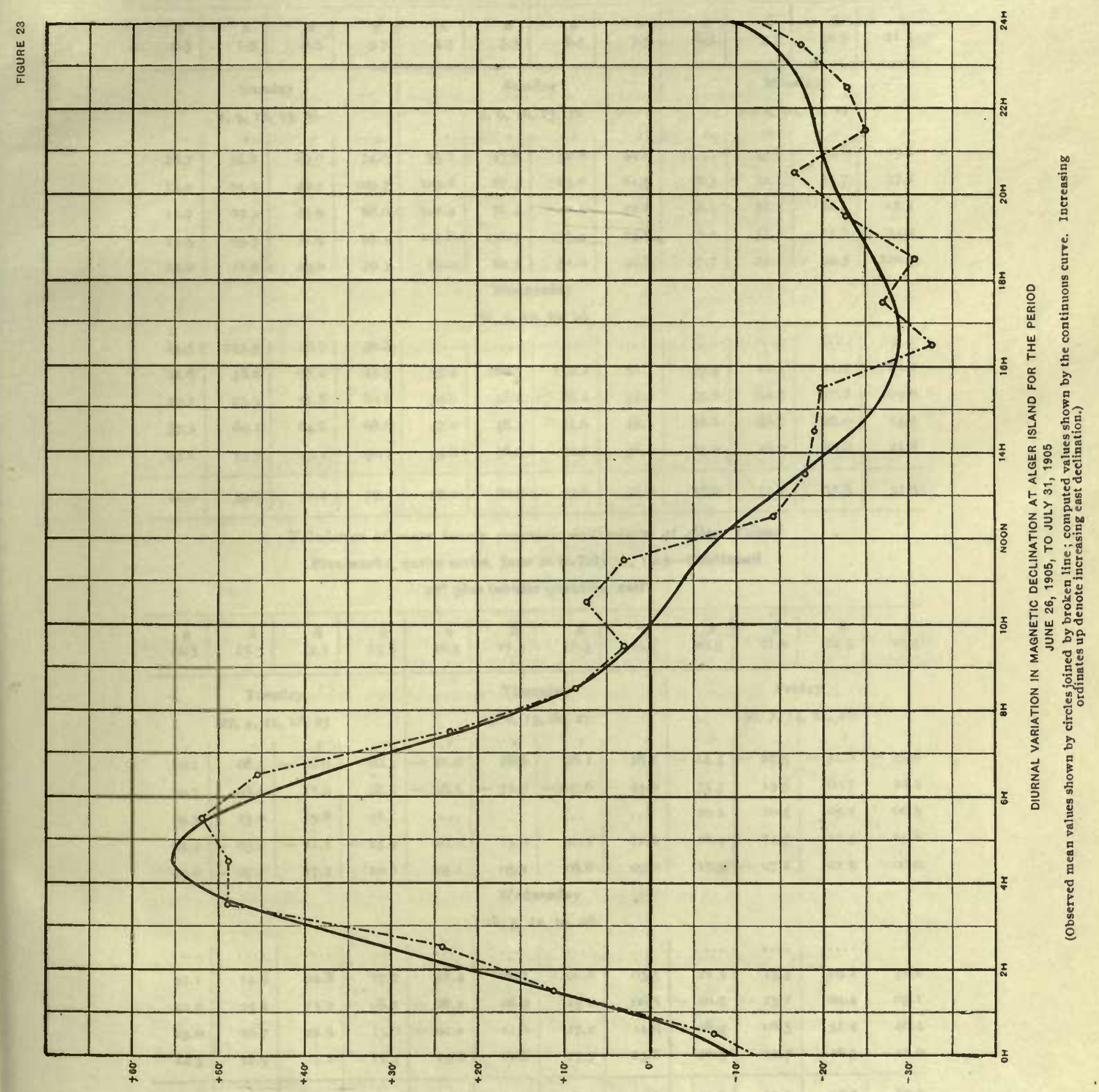




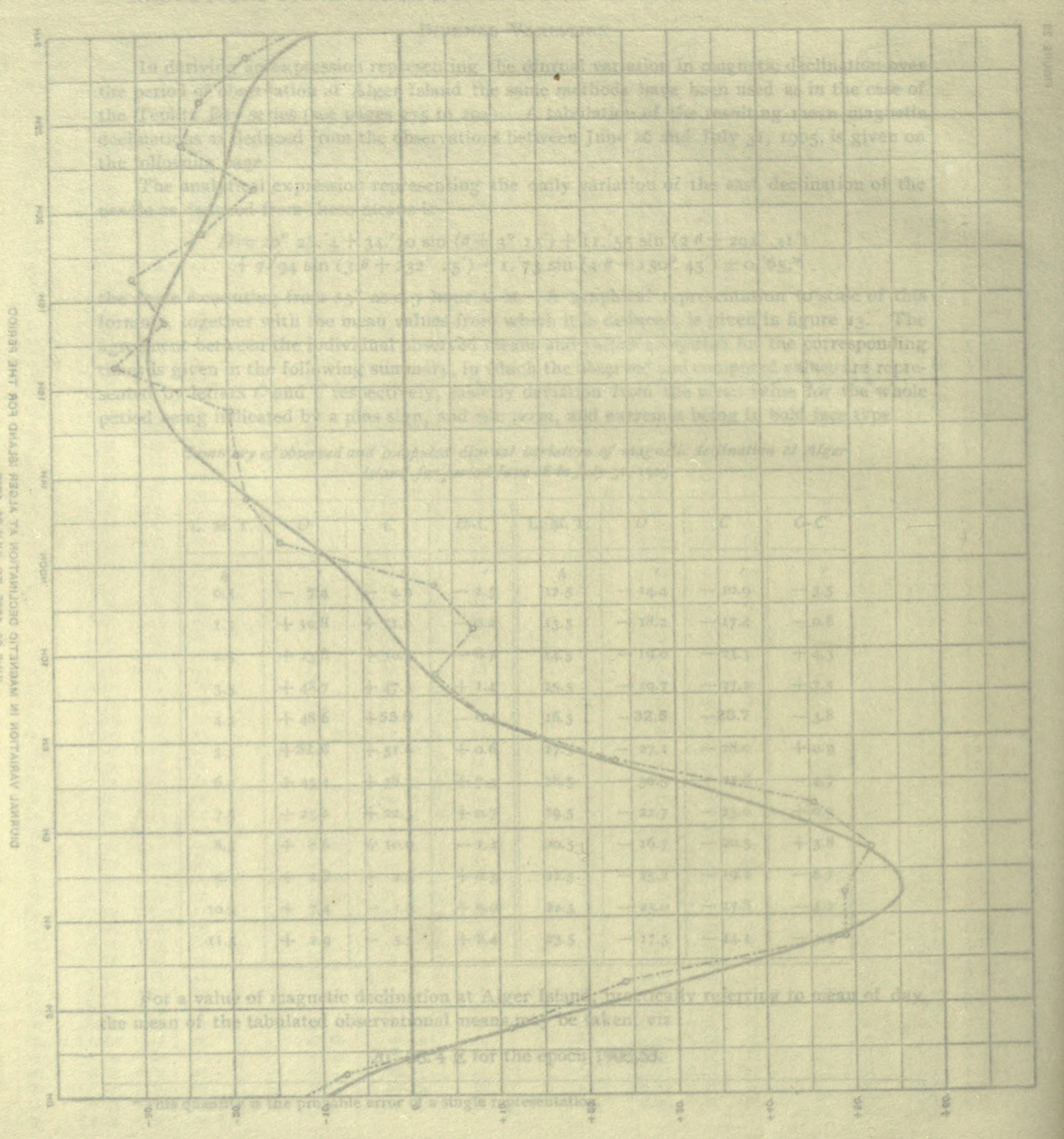


Tabulation of mean hourly magnetic declinations at Alger Island

Five weeks, entire series, June 26 to July 31,1905

$20^{\circ}$ plus tabular quantity, east

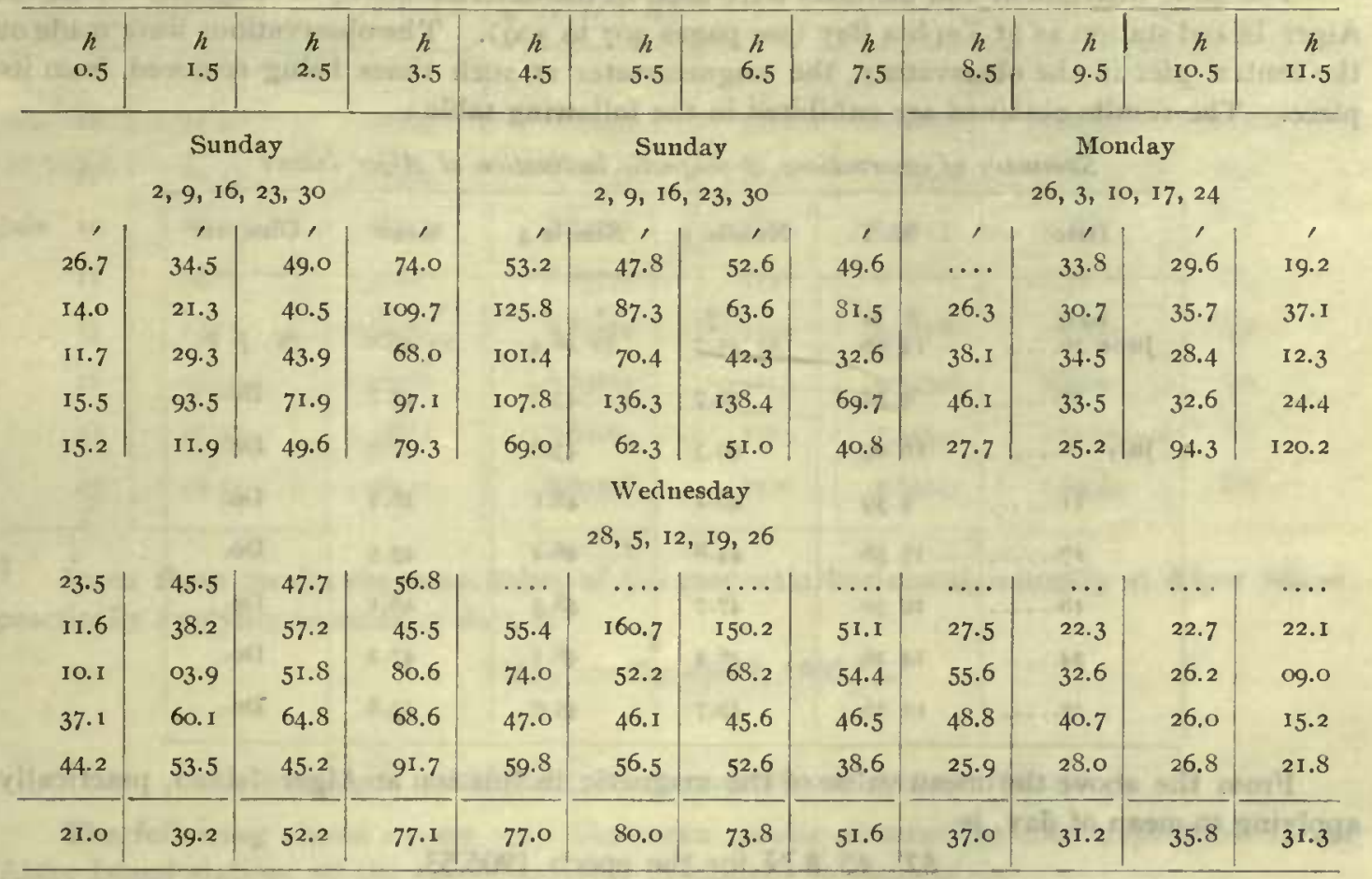

Tabulation of mean hourly magnetic declinations at Alger Island

Five weeks, entire series, June 26 to July 3I, 1905-Continued

$20^{\circ}$ plus tabular quantity, east

\begin{tabular}{|c|c|c|c|c|c|c|c|c|c|c|c|}
\hline $\begin{array}{c}h \\
12.5\end{array}$ & $\begin{array}{c}h \\
\mathrm{I} 3.5\end{array}$ & $\begin{array}{c}h \\
14.5 \\
\end{array}$ & $\begin{array}{c}h \\
\mathrm{I} 5 \cdot 5 \\
\end{array}$ & $\begin{array}{r}h \\
16.5 \\
\end{array}$ & $\stackrel{h}{\text { I } 7.5}$ & $\begin{array}{c}h \\
18.5 \\
\end{array}$ & $\begin{array}{c}h \\
19.5 \\
\end{array}$ & $\begin{array}{c}h \\
20.5 \\
\end{array}$ & $\begin{array}{c}h \\
21.5 \\
\end{array}$ & $\begin{array}{c}h \\
22.5 \\
\end{array}$ & $\begin{array}{c}h \\
23.5 \\
\end{array}$ \\
\hline \multicolumn{4}{|c|}{ Tuesday } & \multicolumn{4}{|c|}{$\begin{array}{l}\text { Thursday } \\
\end{array}$} & \multicolumn{3}{|c|}{$\begin{array}{c}\text { Friday } \\
30,7,14,21,28\end{array}$} & \\
\hline $\begin{array}{c}\prime \\
\text { IO. I }\end{array}$ & 06.5 & $\begin{array}{r}\prime \\
-02.0\end{array}$ & $\infty^{\prime}$ & $\begin{array}{r}\prime \\
-17.0\end{array}$ & $\frac{1}{26.6}$ & I8. & 26.6 & $\begin{array}{r}\prime \\
-\quad 14.4\end{array}$ & $\begin{array}{c}\prime \\
-29.5\end{array}$ & $\begin{array}{r}\prime \\
-31.6\end{array}$ & $\begin{array}{r}\prime \\
-25.6\end{array}$ \\
\hline 10.7 & 08.0 & I 7.2 & I8.5 & -36.5 & -72.9 & -103.6 & -43.0 & 33.5 & 19.5 & 00.7 & 10.2 \\
\hline 09.5 & I5.0 & I 5.8 & I8. I & $\ldots$ & & $\cdots$ & $\ldots$ & 20.2 & I0. 4 & 05.1 & 06.9 \\
\hline 04.4 & -03.4 & -21.4 & -25.9 & 10.0 & 13.0 & I0. 5 & I2. 6 & IS. 4 & 14.5 & I I. 5 & 13.8 \\
\hline 19.0 & -07.7 & 17.4 & I0.6 & 05.I & 05.2 & 08.6 & 09.0 & 17.5 & -07.1 & 01. 9 & $\infty .0$ \\
\hline nnaty & hosir & & $14 x^{2}=$ & whatit & Wedn & nesday & $y=31$ & 120 & & & 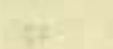 \\
\hline & $m$ & & 7016 & $-(x)$ & $28,5,1$ & $2,19,26$ & & & & & \\
\hline$\cdots \cdot$ & $\cdots$ & $\cdots$ & $\cdots$ & $\cdots$ & $\cdots$ & & $\cdots$ & 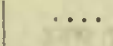 & $\cdots$ & $\cdots$ & \\
\hline 22.1 & 14.6 & 04.8 & 17.2 & 18.4 & OS. I & $-\infty 0.8$ & 03.5 & 21.5 & 13.3 & 09. I & $3 I .1$ \\
\hline 22.9 & $25 \cdot 3$ & 13.0 & 08.2 & -28.1 & 06.9 & 15.2 & 06.5 & -10.5 & -13.1 & 04.4 & 03. I \\
\hline I3.0 & 20.7 & 22.6 & 13.0 & -00.2 & II.O & 17.2 & 14.9 & 08.9 & 08.5 & 31.4 & 46.4 \\
\hline I 4.3 & 12.9 & 17.2 & 17.7 & I 5.8 & I2. 8 & 17.7 & 15.8 & 10.3 & 12.7 & 16.5 & I2.0 \\
\hline I 4.0 & 10.2 & 09.4 & 08.7 & -04.1 & OI.3 & -02.1 & 05.7 & 11.7 & 03.2 & 05.4 & 10.9 \\
\hline
\end{tabular}




\section{INCLINATION}

\section{INSTRUMENT, METHODS, AND RESULTS}

The same instrument and methods were used in the determinations of magnetic dip at the Alger Island station as at Teplitz Bay (see pages 307 to 309). The observations were made on the central pier in the observatory, the magnetometer at such times being removed from its place. The results obtained are exhibited in the following table :

Sunnmary of observations of magnetic inctination at Alger Istand

\begin{tabular}{|c|c|c|c|c|c|c|c|}
\hline Date & L. $\mathrm{M}$ & 1. T. & Needle 3 & Nee & edle 4 & Mean & Observer \\
\hline $\begin{array}{r}1905 \\
\text { June } 26 . \text {. }\end{array}$ & $\begin{array}{r}h \\
14\end{array}$ & $\begin{array}{l}m \\
56\end{array}$ & $\begin{array}{cc}\circ & 1 \\
82 & 45.7\end{array}$ & 82 & ${ }_{46.4}^{\prime}$ & $82 \quad 46.0$ & W. J. P. \\
\hline 27. & 24 & 54 & 46.7 & Ant & 43.6 & 45.2 & Do. \\
\hline July Io. . & 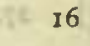 & 09 & 40.3 & ws & 43.6 & 42.0 & Do. \\
\hline II.. & & 59 & 48.2 & 378 & 48.1 & 48.2 & Do. \\
\hline 17. & 13 & 56 & 44.8 & & 46.2 & $45 \cdot 5$ & Do. \\
\hline I8. & 10 & 30 & 47.7 & & 48.5 & 48. I & Do. \\
\hline 24. & 14 & 26 & 46.4 & & 48.1 & 47.2 & Do. \\
\hline 25 & 10 & 16 & 42.7 & Als & 45.0 & 43.8 & Do. \\
\hline
\end{tabular}

From the above the mean value of the magnetic inclination at Alger Island, practically applying to mean of day, is

$82^{\circ} 45 .^{\prime} 8 \mathrm{~N}$ for the epoch 1905.53 .

\section{HORIZONTAL, INTENSITY}

INSTRUMENT, METHODS, AND RESULTS

The instrument and methods used at the Alger Island station were the same as at Teplitz Bay, already described in detail on pages $3^{1} 3^{\text {to }} 3^{15}$. The following tabulation summarizes the work at this station; the various column headings are explained on page $3^{1} 3$.

Summary of observations of magnetic intensity at Alger Island

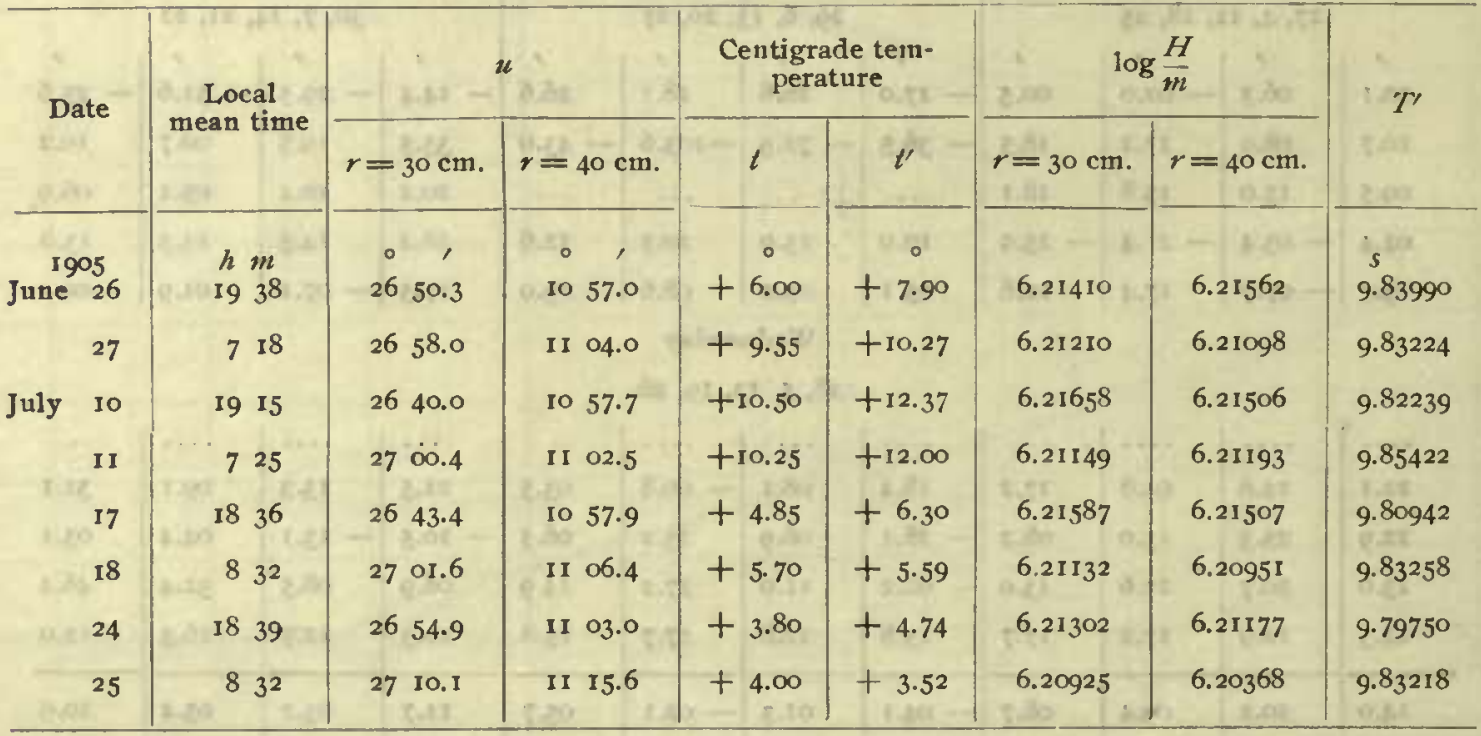


Summary of observations of magnetic intensity at Alger Island-Continued

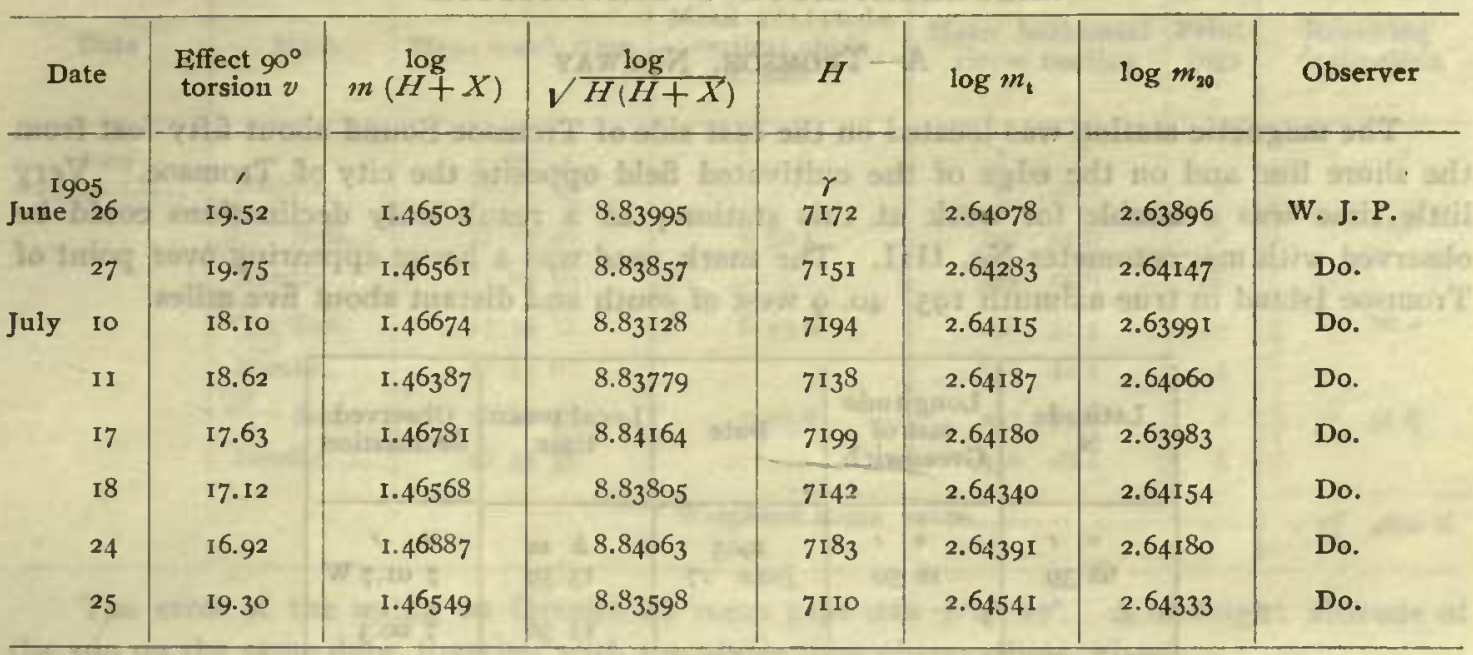

From these results the mean value of the magnetic horizontal intensity at Alger Island, practically applying to mean of day, is

$7161 \gamma$ for the epoch 1905.53.

\section{SUMMARY OF MAGNETIC ELEMENTS AT ALger ISLAND}

The following shows in one view the mean results obtained by the expedition at the Alger Island station, all the values applying practically to mean of day :

\begin{tabular}{|c|c|c|c|c|c|}
\hline Epoch & $\begin{array}{c}\text { East } \\
\text { declination }\end{array}$ & $\begin{array}{c}\text { Northerly } \\
\text { inclination }\end{array}$ & $\begin{array}{c}\text { Horizontal } \\
\text { intensity }\end{array}$ & $\begin{array}{c}\text { Vertical } \\
\text { intensity }\end{array}$ & $\begin{array}{c}\text { Total } \\
\text { intensity }\end{array}$ \\
\hline I905.53 & 0, & 0, & $\gamma$ & $\gamma$ \\
728 & 8246 & 7 I6I & 56395 & 56848 \\
\hline
\end{tabular}

Latitude of station is $8 \mathrm{I} 21.5 \mathrm{~N}$. Longitude of station is 56 05.5 E. 


\section{MISCELLANEOUS OBSERVATIONS}

\section{A-Tromsot, NORWAY}

The magnetic station was located on the east side of Tromsoe Sound about fifty feet from the shore line and on the edge of the cultivated field opposite the city of Tromsoe. Very little time was available for work at this station; as a result only declinations could be observed with magnetometer No. IIII. The mark used was a house appearing over point of Tromsoe Island in true azimuth $195^{\circ} 40 .{ }^{\prime} 9$ west of south and distant about five miles.

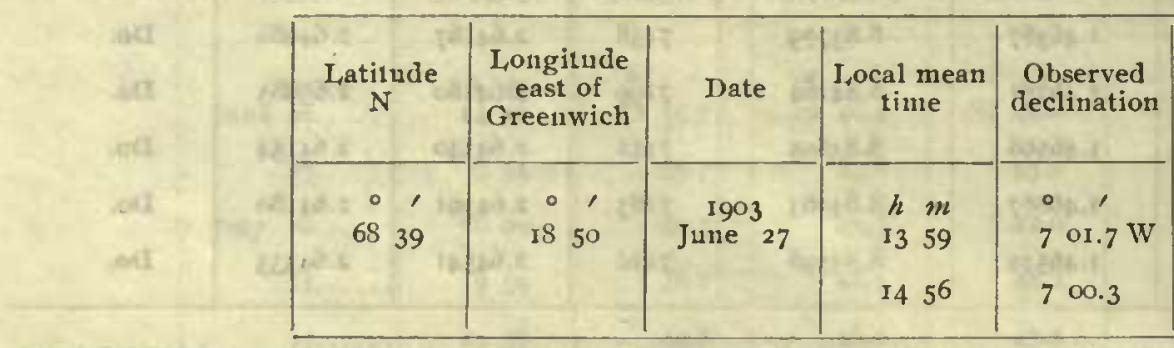

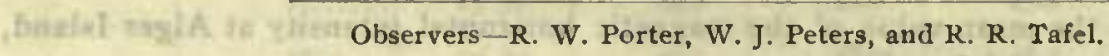

\section{B-ARCHANGEL, RUSSIA}

The magnetic station was located on the south point of a low, flat, sandy island covered with small willows in the Dwina River about west-sonthwest of the old prison in Solomba. The taller and middle spire of the Archangel cathedral is in true azimuth from the magnetic station $344^{\circ} 20 . ' 3$ west of sontli. Complete observations were nuade with magnetometer No. IIII and dip circle No. 5676 . In the following summary of the results at this station all instrumental corrections have been made, but 110 corrections for diurual variations are applied.

\begin{tabular}{|c|c|c|c|c|c|c|c|c|}
\hline $\begin{array}{l}\text { Latitude } \\
\text { N }\end{array}$ & $\begin{array}{l}\text { Longitude } \\
\text { east of } \\
\text { Greenwich }\end{array}$ & Date & $\begin{array}{l}\text { Local meau } \\
\text { time }\end{array}$ & $\begin{array}{c}\text { Observed } \\
\text { declination }\end{array}$ & $\begin{array}{c}\text { Local niean } \\
\text { time }\end{array}$ & $\begin{array}{l}\text { Observed } \\
\text { dip* }^{*}\end{array}$ & $\begin{array}{c}\text { Observed } \\
\text { horizontal } \\
\text { intensity }\end{array}$ & $\begin{array}{c}\text { Observed } \\
\log m_{20}\end{array}$ \\
\hline 01 & 0, & 1903 & $h m$ & 0,1 & $h \quad m$ & 01 & $\gamma$ & \\
\hline 6434 & 4040 & July 3 & 1317 & $722.7 \mathrm{E}$ & $155^{\circ}$ & $7354 . \mathrm{I} \mathrm{N}$ & ... & $\cdots \cdot$ \\
\hline 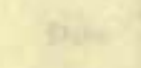 & $x^{2}+x^{2}$ & & 1826 & 728.8 & I5 $4^{8}$ & 7400.6 & & . .... \\
\hline & & July 4 & 1218 & 727.3 & $15 \mathrm{IS}$ & .. & I 4528 & 2.64048 \\
\hline & & & 1332 & 725.0 & I7 42 & $\ldots \ldots$ & $1453^{8}$ & 2.64054 \\
\hline $\tan 10$ & $15 y^{7}$ & & I8 31 & 725.8 & .. & .. & & \\
\hline
\end{tabular}

Observers-W. J. Peters and R. W. Porter. $\quad$ * Needles Nos. 3 and 4 respectively.

\section{C-BARENTS SEA}

Observations were made on the floating ice in Barents Sea. For declination observations a C. I. Berger and Sons' alt-azimuth instrument witl compass needle attached in tube under telescope (see figure 2 of astronomic notes) was used. The methods and results obtained are exhibited in the following summary : 


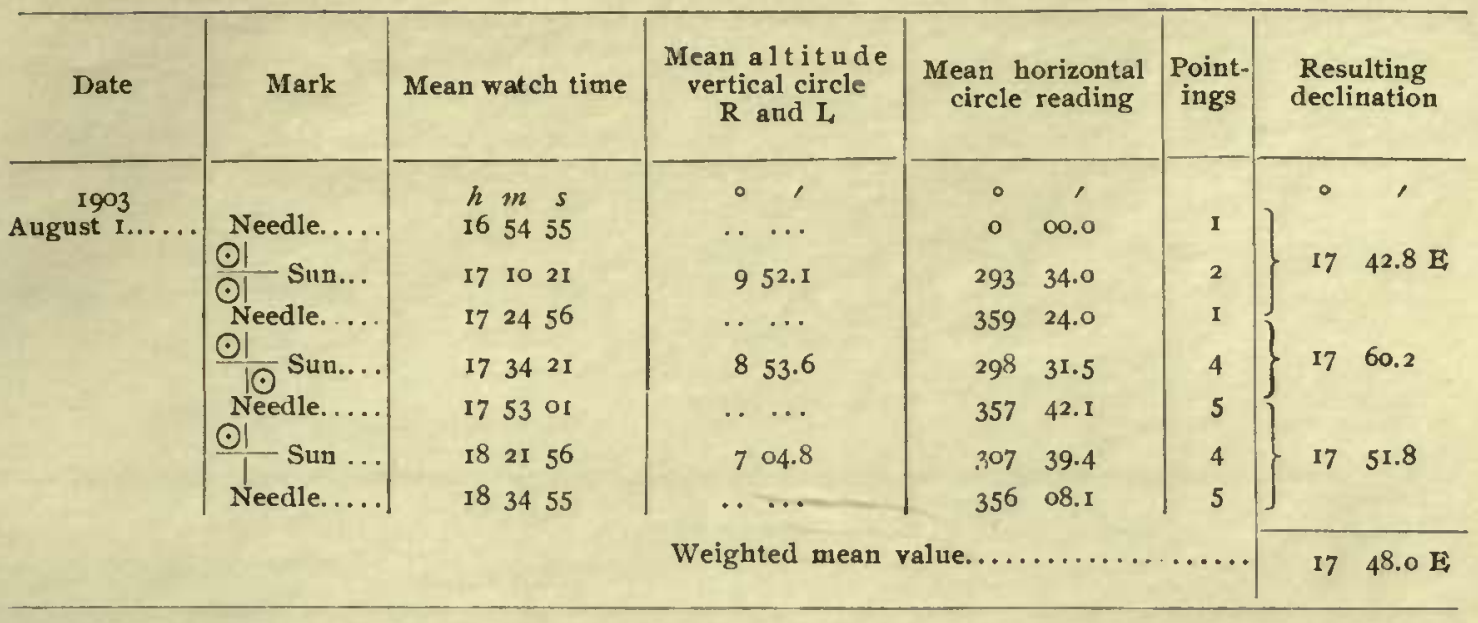

The error of the watch on Greenwich mean time was $+4^{m} 57^{\circ}$. A miduight altitude of the sun on the same date, together with the above time observations, gives :

$$
\begin{aligned}
& \text { Latitude } 77^{\circ} \circ 6^{\prime} \mathrm{N} \text {. } \\
& \text { Longitude } 52^{\circ} \text { 15.' I E of Greenwich. }
\end{aligned}
$$

The magnetic observing tent was set up about six hundred feet distant from the alt-azimuth instrument in a direction $\mathrm{S} 10^{\circ} \mathrm{W}$, and dip observations were made with dip circle

\begin{tabular}{|c|c|c|c|c|}
\hline \multirow{2}{*}{ Date } & \multirow{2}{*}{$\begin{array}{l}\text { Local mean } \\
\text { time }\end{array}$} & \multicolumn{3}{|c|}{ Observed inclination } \\
\hline & & Needle No. 3 & Needle No. 4 & Mean \\
\hline $\begin{array}{c}1903 \\
\text { August I.. }\end{array}$ & $\begin{array}{cc}h & m \\
22 & 3 \mathrm{I}\end{array}$ & $\begin{array}{l}\circ \\
81 \text { oI.7 N }\end{array}$ & $\begin{array}{c}\circ \\
8 \mathrm{I} \\
05.2 \mathrm{~N}\end{array}$ & $\begin{array}{cc}\circ & , \\
81 & 03.4 \mathrm{~N}\end{array}$ \\
\hline
\end{tabular}
No. 5676 in the usual manner, giving the following results :

Observers - W. J. Peters aud R. W. Porter.

\section{D-Determinations on Plane Table Traverse}

In the course of the plane table traverse work from Teplitz Bay to Cape Flora Mr. R. W.

\begin{tabular}{|c|c|c|c|c|c|c|}
\hline Place & $\begin{array}{l}\text { Latitude } \\
\mathrm{N}\end{array}$ & $\begin{array}{l}\text { Longitude } \\
\text { east of } \\
\text { Greenwich }\end{array}$ & $\begin{array}{l}\text { Local mean } \\
\text { date }\end{array}$ & $\begin{array}{c}\text { Azinuth determined } \\
\text { low }\end{array}$ & $\begin{array}{c}\text { No. } \\
\text { needle } \\
\text { pointings }\end{array}$ & $\begin{array}{c}\text { Mean } \\
\text { observed } \\
\text { decliuation }\end{array}$ \\
\hline Cape Norway...... & $\begin{array}{rr}0 \\
81 & 12\end{array}$ & $\begin{array}{cc}\circ & \prime \\
55 & 34\end{array}$ & April ${ }^{1904} \quad h$ & Theorlolite No. II....... & I & 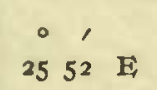 \\
\hline $\left.\begin{array}{r}\text { Hooker Is l and } \\
\text { station XXI.... }\end{array}\right\}$ & $802 I$ & 5312 & June I9, I2.I & $\left\{\begin{array}{r}\text { Theodolite and azimuth } \\
\text { obs. on station XX... }\end{array}\right\}$ & 7 & $1941 \cdot 7$ \\
\hline Rubini Rock....... & 80 I9 & 5248 & June $23,22.0$ & $\left\{\begin{array}{r}\text { Theodolite and azimuth } \\
\text { obs. on station A.... }\end{array}\right\}$ & 5 & 1729.7 \\
\hline Cape Flora.... & 7957 & 4958 & July 9-19..... & $\left\{\begin{array}{c}\text { Plane table needle sta- } \\
\text { tions } \mathrm{B}, \mathrm{D}, \mathrm{E}, \mathrm{L}, \mathrm{K}, \mathrm{M} .\end{array}\right\}$ & 6 & 1457 \\
\hline
\end{tabular}
Porter made the following declination observations : 


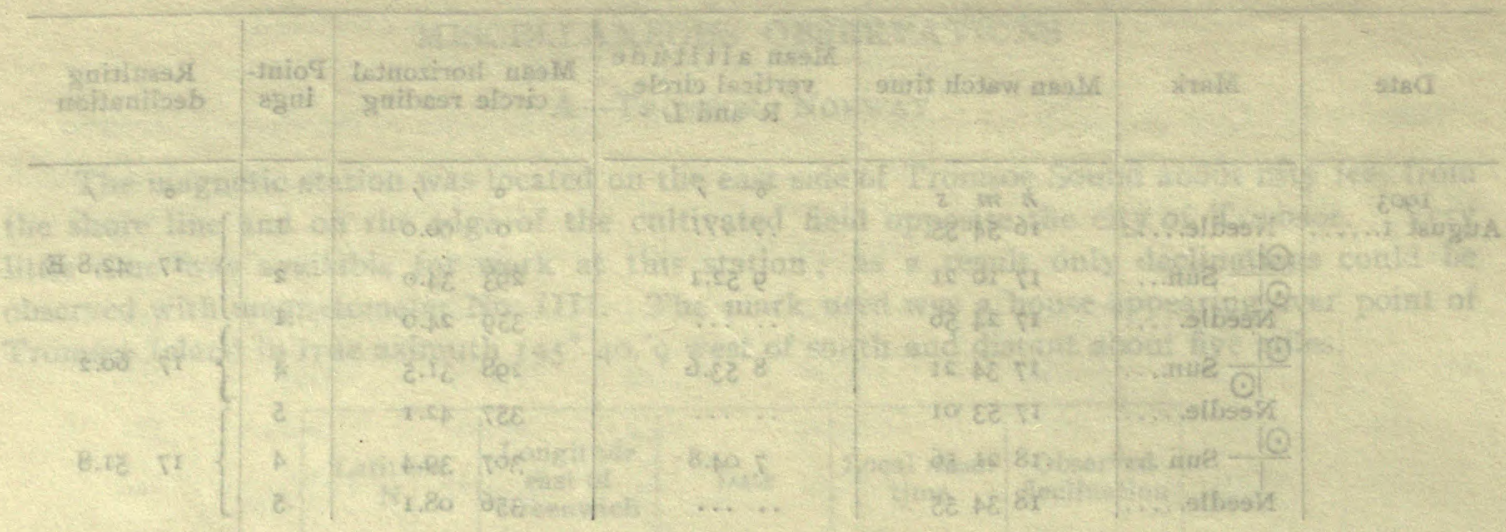

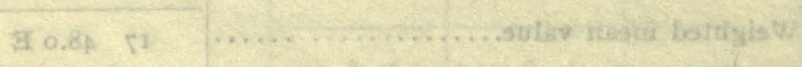

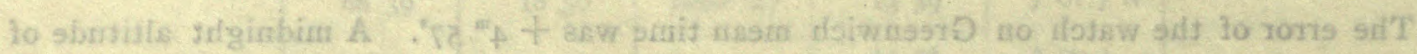

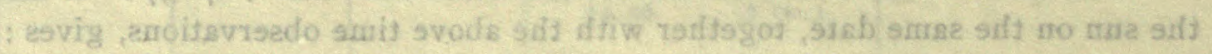

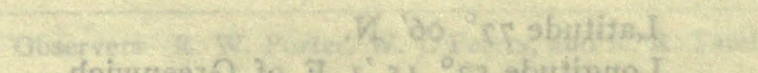

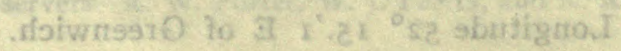

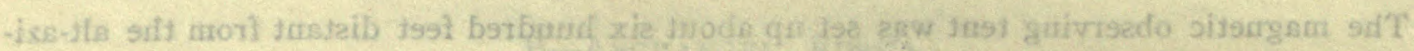

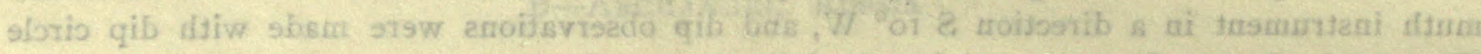

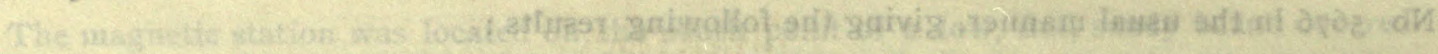

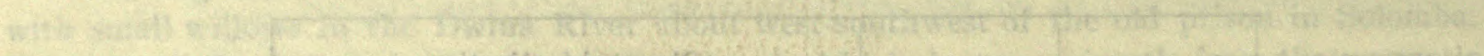

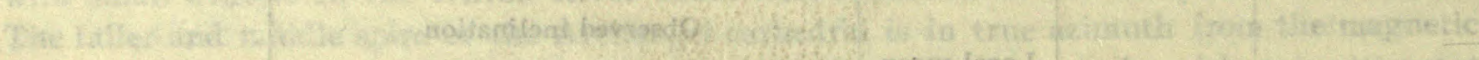

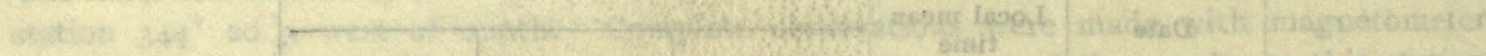

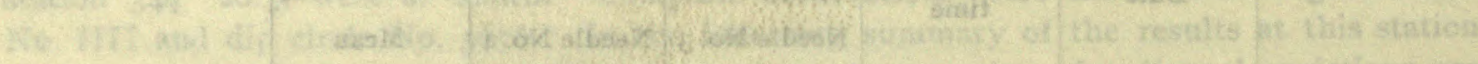

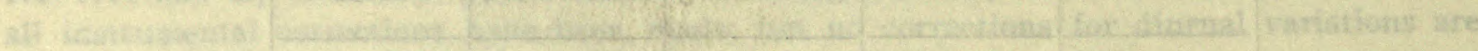
appited

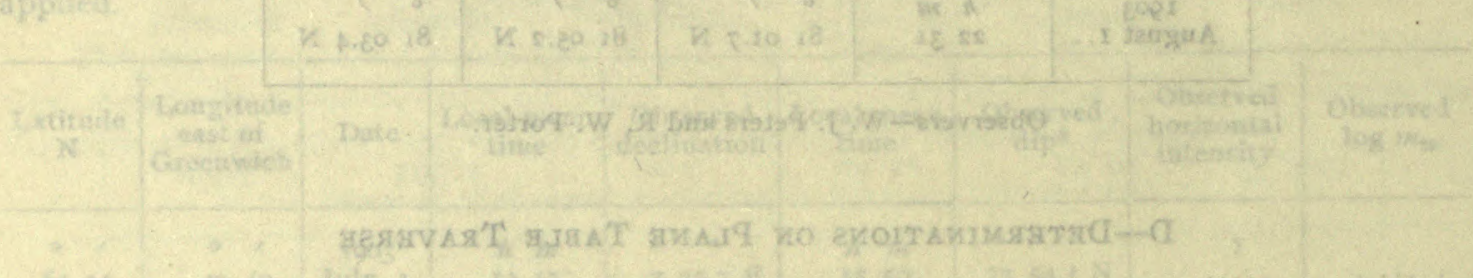

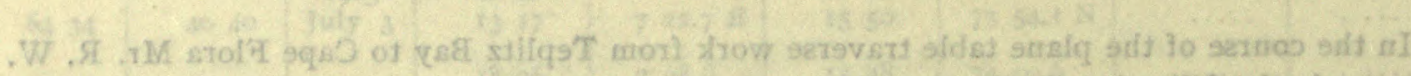

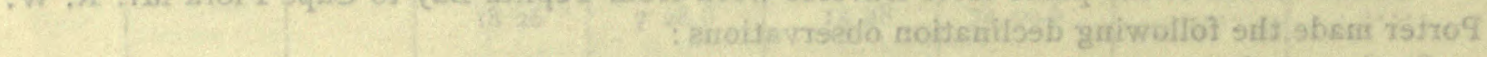

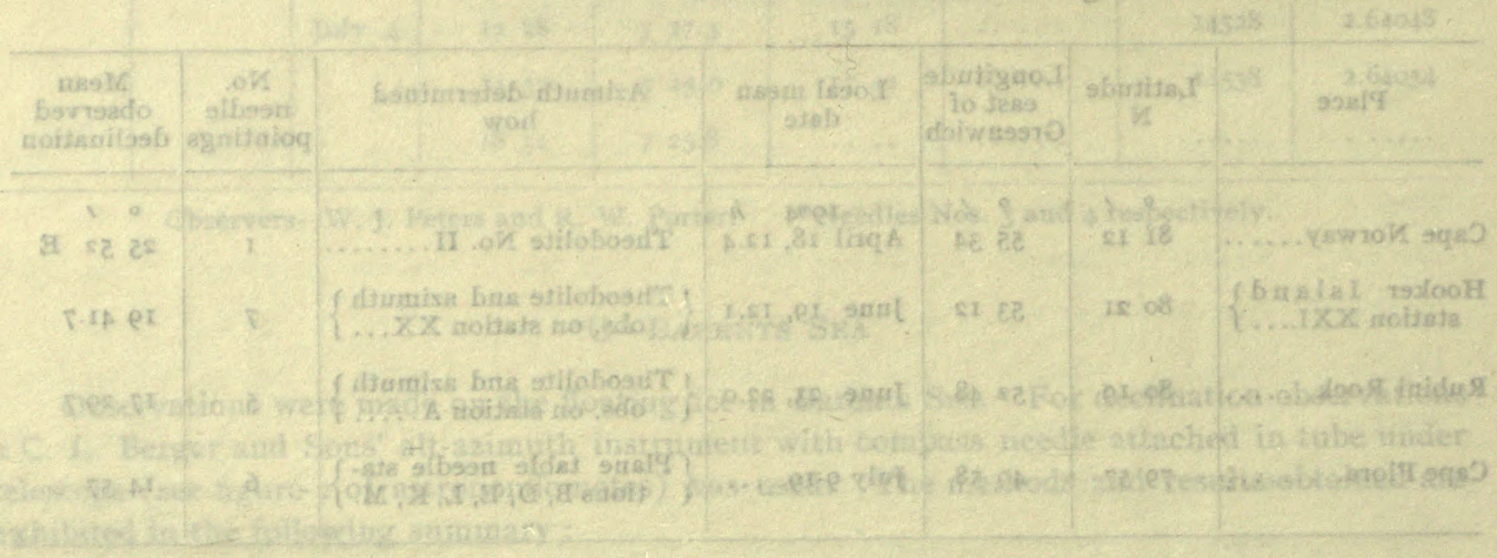




\section{SECTION B}

\section{NOTES AND SKETCHES}

$$
\text { OF THE }
$$

\section{AURORA BOREALIS}

BY

ANTHONY FIALA

Commander of the Expedition 
\& VOIT372.

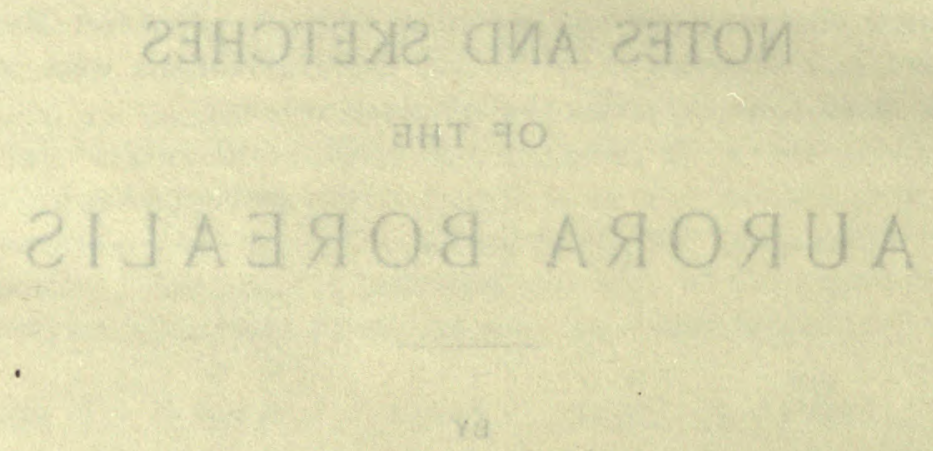

B. IAIA YKOHTKA

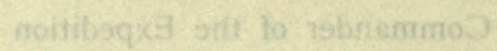

$x^{2}$ 


\section{CONTENTS}

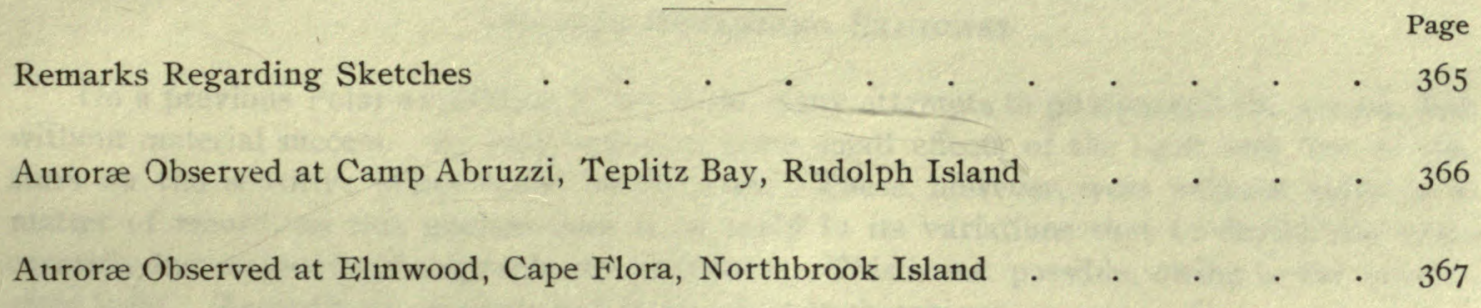

\section{PLATES}

I December 23, I903, 3:30 P. M. II January $23,1904,9: 50$ P. M.

2 December 23, I903, 4:IO P. M. I2 January 23, 1904, 9:56 P. M.

3 December 23, 1903, 4:40 P. M. I3 Jantary 23, 1904, 10:03 P. M.

4 December 23, 1903, 5:15 P. M. I4 January 23, 1904, 10:06 P. M.

5 December 23, 1903, I1:40 P. M. I5 January 23, 1904, 10:09 P. M.

6 December $23,1903,12: 00$ P. M. I6 January $23,1904,10: 13$ P. M.

7 January 2, 1904, 8:00 P. M. 17 January $23,1904,10: 20$ P. M.

8 January 2, 1904, 8:30 P. M. I8 January $23,1904,10: 24$ P. M.

9 January 2, 1904, 9:00 P. M. I9 January 23, 1904, I0:28 P. M.

Io January $23,1904,9: 36$ р. м. 



\section{AURORAL OBSERVATIONS}

\section{REMARKS REgARDING SKETCHES}

On a previous Polar expedition I had made many attempts to photograph the aurora, but without naterial success. By long exposure some small effects of the light with that of the stars on the sensitive plates could be obtained. These, however, were without value as a matter of record, as this phewomenon is so rapid in its variations that to depict the same correctly instantaneous photographs are necessary. This is not possible, owing to the insufficient light. Accordingly recourse had to be taken to sketching.

In connection with the work at the n11agnetic observatory a n11mber of sketclies of the aurora were made, using for the purpose a board with compass attached for orientation. The cardinal points indicated on the plates are accordingly magnetic. The drawings were made upon black sleets of paper, upon eacls of whicln a circle representing the horizon was previonsly drawn in clialk. The sheets were so placed together and pimned at the corners that they conld be torn off as the sketclies were completed. A pin at the center represented the zenith point. Having had some experience in rapid sketcling, it did not take long to place on the paper rongh chalk sketches of the beautiful auroral light, using the board as a plane table, and drawing in the circle of the chalk horizon the display that was taking place in the heavens overheail.

Owing to numerous other duties and on account of the generally prevalent bad weather opportunities for sketching were few. 'The attendaut physical diffictlties for work of this kind in the open air, under the flickering liglit of a sunall lantern, without shelter of any kind, and witl a tenperature of from $30^{\circ}$ to $50^{\circ}$ Fahrenheit below zero, may be readily imagined. In spite of these difficulties some very interesting and, it is believed, representative sketches were secured. The results of this work are shown by plates numbers I to 19, the titles of which give the dates and times of the phenomena so recorded.

In order to make as complete a record as possible of this phenomenon during the time the Expedition was at work, the various auroral notes have been gatlered together from the meteoroloyic records for both the Teplitz. Bay and Cape Flora stations. The notes at Camp Abruzzi between October, 1903, and March, r904, as also for those at Cape Flora, were collected by Sergeant Francis Long, Weather Observer. Those between October, 1904, and February, 1905, were nuade by different nembers of the party, cliiefly by Messrs. Peters, Porter, and Dr. Seitz. The times given are local mean, civil reckoning through twenty-four. hours for the respective statious. The first figure entered indicates the day of the month, those following the hour and unintute, thus: 21:18:10 means the 21st day of the montl at local mean time $18^{\mathrm{h}} 10^{\mathrm{mm}}$ or $6^{\mathrm{h}} 10^{\mathrm{m}} \mathrm{P}$. M. Directions given in these notes are all true, and not magnetic as in the cases of the sketches. The references are to the beginning and ending of the display, the extent of the same and the altitude, thus: $21: 18: 10$ to $20: 20$, $\mathrm{H}$, to $\mathrm{W}$, about $60^{\circ}$, means that an anrora was observed on the 21 st day of the particular month under which the note comes between $18^{\mathrm{h}} 10^{\mathrm{m}}$ and $20^{\mathrm{h}} 20^{\mathrm{m}}$, and that it extended from east to west at an altitude of about $60^{\circ}$. In connection with these notes reference should also be inade to such references of auroræ as are contained in the notes accompanying the declination results at Teplitz Bay on pages 32 to 40 , as these are not included in the following. 


\section{Aurora; Orserved at Camp Abruzzi, Teplitz Bay, Rudolpi Island}

October, 1903-3:21:00 to $22: 00, \mathrm{E}$ to $\mathrm{W}$, about $35^{\circ}$ to $40^{\circ}$. - 21: 19: 1o to $20: 40, \mathrm{E}$ to W, about $25^{\circ}$ to $40^{\circ}$. - 30: $21: 20$ to 22: IO, SE to NW. -3 I: $21: 45$ to 22 : Io from $60^{\circ}$ to $90^{\circ}$.

November, 1903-1:05:00 to 6:15; I: 16: 15 to $18: 00 .-4: 16: 45$ to $17: 30, \mathrm{E}$ to W.I0: I 7: I 5 to I9: IO, E to W.-II: I6: oo to 22:00, E to W, about $35^{\circ}$.- I2: 15:00 to I6: 25, E to W.-I4:20:50 to $2 \mathrm{I}: \mathrm{IO}, \mathrm{W}$ to E. -I $5: 22: 30$ to $23: 10 .-17: 14: 30$ to $22: 10, \mathrm{E}$ to W.18:0 4:00 to $10: 30$; $18: 14: 00$ to $21: 10 .-19: 10: 30$ to $10: 50, E$ to $W$ and $N$; $19: 14: 30$ to

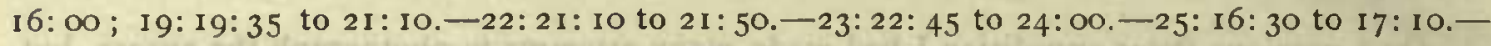
26: $20: 00$ to $21: 00$ from $30^{\circ}$ to $45^{\circ}$.

December, 1903-2:07:00 to 8: 15.-8: 15: 55 to I7:00, E to W; 8: 19: Io to $21: 10 .-$ I $2: 20: 00$ to $21: 30 .-13: 07: 45$ to $18: 30 .-14: 07: 45$ to $9: 10 ; 14: 19: 30$ to $24: 00$. - I $7: 19: 30$ to 24:00.-18: II: 45 to $12: 30 ; 18: 19: 00$ to 23 : I0.-19:23:00 to $24: 00$. - 20: I $9: 30$ to $20: 15$.$21: 07: 30$ to $9: 00 ; 21:$ I $9: 50$ to 2 I: $50 .-22: 19: 50$ to $20: 30 .-23: 07: 45$ to $8:$ I $5 ; 23:$ I I: 45 to $17: 00 ; 23: 22: 40$ to $24: 00$. - $24: 20: 00$ to $22: 30$. - $28: 22: 30$ to $24: 00$.

January, I9O4-2:19:45 to $21: 00$. -4: IO: Io to IO: $20 ; 4: 12: 00$ to $12: 40 .-5: 07: 55$ to

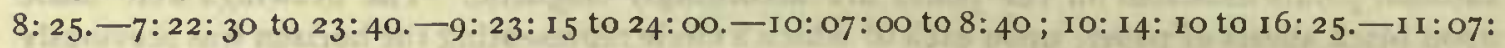
30 to $8: 10$; II: I $5: 25$ to $17: 00$; II: 22: IO to $23: 25$.-I $2:$ I $5: 50$ to I8: IO ; I2: I9: 20 to 22: 25 . I3: II: OO to II: 25 ; I $3: I 2: 00$ to I $2: 40$; I $3: 14: 40$ to $24: 00$-I I 1 II: 5 O to I $2: 40 ;$ I $4: 22: 30$ to $23: 10 .-15: 12: 00$ to $16: 00 ; 15: 16: 30$ to $20: 10 .-20: 12: 10$ to $12: 35$ in S. $-23: 22: 00$ to $24: 00$. $-24: 00: 00$ to $00: 30 .-25:$ I $9: 30$ to 2 I: $10 .-28:$ I $9:$ I 5 to I9: $25 ; 28:$ I9: 40 to $19: 55$. 30: $19: 45$ to $20: 10 ; 30: 20: 30$ to $20: 50 .-31: 20: 15$ to $20: 45$.

February, 1904-1: 15: 10 to $15: 40, \mathrm{E}$ to W.-5: I8: 10 to $18: 40 ; 5: 22: 20$ to $23: 00 .-$ 6: $21: 00$ to $24: 00 .-7: 07: 45$ to $8: 15 ; 7: 19: 15$ to $24: 00 .-8: 15: 30$ to $17: 00 ; 8: 18: 00$ to $24: 00 .-9: 19: 55$ to $20: 25$. - Ir: $19: 00$ to $24: 00$. - I 2: $21: 30$ to $24: 00$. - I6: $19: 15$ to $21: 00$; 16:22:00 to $24: 00$. - I $8: 19: 00$ to $21: 10 ; 18: 22: 00$ to $24: 00 .-23: 20: 25$ to $20: 35 ; 23: 22:$ I 5 to $23: 00$.

March, I904-2: 19:40 to 20:20.-I6:21:35 to 22:00.

October, 1904-19, aurora in E.-28, fine aurora.

November, I904-2, light aurora 3 days.-4, aurora SE to SW.-7, light aurora in SE and W.- $\rightarrow$, light aurora, ESE to SW.-Io, light aurora, E to W.-I2, very light aurora, E to SW.-13, light a urora, SE to middle SW. - 16, light aurora, SSW to SSE.-1 7, brilliant aurora, $E$, to $W$, entire southern hemisphere.-26, light aurora. - 27, aurora $S E$ to $W$. - 29, light aurora, $18: 30$, WNW to E, and $20: 00$, ESE to WSW.

December, 1904-I, aurora NE to WNW; wide auroral bands over S (SSE to SSW).2, slight auroral display in flashes in $\mathrm{N}$ (NNE, to NNW). - 3, straight auroral band over horizol1, SSE to SSW, with light dashes in E, during P. M.- - t, auroral dashes from $12: 45, \mathrm{NW}$ to NE; magnificent aurora from 20: 5 to $21: 30$; bauds SE to WSW changed to waving streams of all colors moving $\mathrm{W}$ to $\mathrm{E}$, fading to a faint ribbon across sky, followed by flashes. - 5, A.M., light aurora, W to NNE, plain band; noon, wide, uncolored arch across zenith, E to W ; P.M., clear aurora NE to E.-6, A.M, aurora, E to W, center zenith.-I2, P.M., light band, E to W.-I 4, I2:00, light dashes in W and "auroral smoke" $N$ to NNE; $18: 00$, dashes in W and band, W to E; 20: 00 , band over $S$ horizon, remained steady ustil $21: 15$, then lengtheued to $\mathrm{W}$ and worked $\mathrm{N}$, expanding, waving, coloring and fading, until at $2 \mathrm{I}: 35$ reaclied zenith; 
corona then formed, remaining overhead until $21: 46$, when corona disappeared and bands gradually faded; this display was brightest and sharpest in E, less distinct and not so liighly colored in W ; gave a very distinct light. $-15, \mathrm{~A}$. M., fine NE to SW arch overhead, faded slowly; $5: 00$, in $\mathrm{S}$ from $\mathrm{E}$ to $\mathrm{W}$, arch and coroua at zenith; $8: 45$, rays all direct from zenith ; 12:00 to 15:00, uncolored arch zenith ESE to WNW, clearer in W; "auroral smoke" in N and $\mathrm{NNE}$. - 16, A. M., rays $\mathrm{N}$ to $\mathrm{W}$ from zenith; $12: 00$, light rays in $\mathrm{NW}$ (N to $\mathrm{W}$ ), "auroral smoke," ENE to NNE.-18, A. M., liglit band NNE to NE; 20:20 to 21:30, band across sky, E to SSW.-20: 20:00, band from W to zenith; ribbon, NE to zenith.—26:12:00, aurora in W to zenith. -28:17:00, flames in E, bands across $S W ; 22: 00$, aurora in S.-29:09:00, wide baud, $\mathrm{SE}$ to $\mathrm{SW}, 45^{\circ}$ either side zenith. - 3o, heavy, dull aurora over $\mathrm{N}$ lıalf of sky, convoluted.

January, 1905-1:12:30, dashes in $\mathrm{W}$, swinging around until $\mathrm{W}$ to $\mathrm{E}$, then changing to brilliant sashes and streamers, working down to W to SIE at $14: 30 ; 1: 20: 00$, light band, SI to SW.-2:20:00, band in W.-3:08:00, very liglit dashes in NE about $45^{\circ}$ toward zenitl ; 3: 20:00, large aurora, E to W, widely spread in E into several moving bands. $-4:$ I 3:00 to 20: 00 , band, $E$ to $W ; 4: 20: 00$, clear auroral display, corona at zenith with waving streamers, $\mathrm{E}$ and W.-8: 12:00, light anrora in N.-I I: 10:00, rays, zenith to W and E; I1: 12:00, light band, SE to SW ; I1: 17:30, band, SE, to SSW; $11: 20: 00$, band, SE to SW.-12:22:00, display in S, convoluted band, ribbon E to W.-13:08:00, dashes and band, E to W; $13: 20: 00$, glow E to SE; 13:21:25, convoluted bands in S zenith.-14:12:00, dashes in NNE; 14:22:30, bright rays and bands over $S$ half sky, E to $W$ and zenith.-17: 10:00, dashes in $W ; 17: 11: 00$, band NE to W; $17: 13: 00$, band E to W. -25:20:00, auroral curtain, E to WSW to SE to SSW to S, movement E. - 26: 20:00, band, E to SW, $20^{\circ}$ altitude.-27:18:00, band, E to SW, $45^{\circ}$ altitude; $27: 19: 30$, aurora in $\mathrm{E}$, convoluted, $248^{\circ}$ to $315^{\circ}$ azimuth, streamers to $68^{\circ}$ azimuth and $50^{\circ}$ altitude.

February, 1905-1:20:00, light band, E to W.-3:20:00, dashes in W and E with bands connecting. -6: 20: 00 , light baud, straight $\mathrm{E}$ to $\mathrm{SW}$, altitude $15^{\circ}-7: 20: 00$, band, E to SSW; convoluted aurora, $\mathrm{E}$ to $\mathrm{S}$ of zenith. $-8: 20: 00$, band, $\mathrm{E}$ to $\mathrm{W} ; 8: 22: 00$, convoluted aurora, E to SW; 8:23:25, glow and "smoke" in SSE.-9:08:00, dashes in NE; 9: 17:00, bands, $\mathrm{E}$ to $\mathrm{W}$, converging in W; $9: 18: 00$, moving bands, E to SW.-I $4: 19: 45$, fine display swinging over entire sky; $14: 20: 00$, orange-colored corona, large cloud-like anrora covering zenith about $15^{\circ}$ on each side, trail to SW tinged with colors. - 21:20:00, band, E to SW, light glow and bands in $\mathrm{E}$.

\section{Aurorai Observed at Elimwood, Cape Flora, Northbrook Island}

Oclober, 1904-18:19:15 to $21: 00 .-19: 19: 00$ to 20:00.-23:21:00 to $21: 40 .-24: 19: 00$, ending during night. $-28: 19: 00$ to $19: 55$.

November, 1904-2:18: 10, ending during night.-4:17:15, ending during niglit.5: 16: 50 to $6: 05: 00, \mathrm{E}$ to W.-6:19:40 to $22: 30 .-7: 18: 30$ to $20: 15 .-9: 17: 50$ to $22: 30 .-$ I2: $20: 50$ to $21: 30 .-18: 12: 40$ to $16: 30$, E to W. -29: I5:00 to $22: 10 .-30: 07: 00$ to $21: 25$.

December, 1904-1:16:00 to $21: 00, \mathrm{E}$ to W.-5:11:20 to $17: 00 .-6: 15: 10$ to $16: 55, \mathrm{E}$ to W. -9: r $3: 30$ to $13: 50$, E to W.-14: or: 00 , ending during night ; $14: 19: 00$ to $19: 55$, Es to W. -15:08:00 to 8:30. - 18: 22: 10 to $23: 20$, E to W. $-26: 14: 00$ to $19: 30, \mathrm{E}$ to W. - 28: 15:10 to $21: 30, \mathrm{E}$ to W.-29: 19:20 to $20: 00$, E to W, $50^{\circ}$. 
January, 1905- $\mathrm{I}: \mathrm{I} 3$ : 10 to $\mathrm{I} 6: 20, \mathrm{E}$ to $\mathrm{W}$, about $65^{\circ}-2: 19: 00$ to $20: 10, \mathrm{E}$ to $\mathrm{W}$, about $55^{\circ}-4: 08: 50$ to $9: 30$, about $35^{\circ} ; 4: 18:$ ro to $23: 50$, about $45^{\circ} .-5: 19: 30$ to $22: 00$, E to W, about $10^{\circ} .-7: 19: 30$ to $21: 00, \mathrm{E}$ to $\mathrm{W}$, about $30^{\circ} .-8$, from $3: 00$ and during early morning, E to W, about $60^{\circ}$. - Ir I: 09:00 to $10: 00, E$ to W, about $60^{\circ}$; Ir: $14: 30$ to $19: 00$, E to W, about $55^{\circ}$.- $\mathrm{r} 2: \mathrm{r} 6:$ o o to $16: 30$, about $55^{\circ}$. - $\mathrm{r} 3: \mathrm{rg}: 30$ to $2 \mathrm{r}: 00$, about $45^{\circ}$. - $\mathrm{r} 4: \mathrm{r} 2: 30$ continued to 19: 50 , between $30^{\circ}$ and $90^{\circ}$; $14: 20: 35$ to $21: 50 .-17: 14: 00$ to $15: 30 .-22: 15: 10$ to $16: 00 .-$ 25: $\mathrm{I6}$ : Io to $24: 00$. between $30^{\circ}$ and $48^{\circ}$. $-26: 14: 00$ to $22: 30,90^{\circ}-27: 14: 00$ to $15: 15$, between $60^{\circ}$ and $70^{\circ}$.

February, $1905-1: 17: 00$ to $17: 40, \mathrm{E}$ to $\mathrm{W}$, about $70^{\circ} .-3: 18: 00$ to $22: 00$, about $70^{\circ}$. 4: $21: 20$ to $22: 30 .-5: 17: 00$ to $19: 30, \mathrm{E}$ to W, about $60^{\circ}$ to $70^{\circ}$. -6: $18: 30$ to $19: 20$, E to W, about $80^{\circ} .-9: 21: 00$ to $24: 00 .-10: 17: 00$ to $23: 00$, about $65^{\circ}$ to $70^{\circ}$. - $12: 18: 00$ to $24: 00, \mathrm{E}$ to W, between $60^{\circ}$ and $80^{\circ}$. $-\mathrm{r} 4: 18: 30$ to $20: 40$. $-\mathrm{r} 9: \mathrm{r} 8: 40$ to $20: 50$. - $21: 19: 00$ to $21: 30$, between $50^{\circ}$ and $55^{\circ}$. $\rightarrow 28:$ ig: 10 to $21: 00$.

March, 1905-I: 19:40 to $21: 30$.

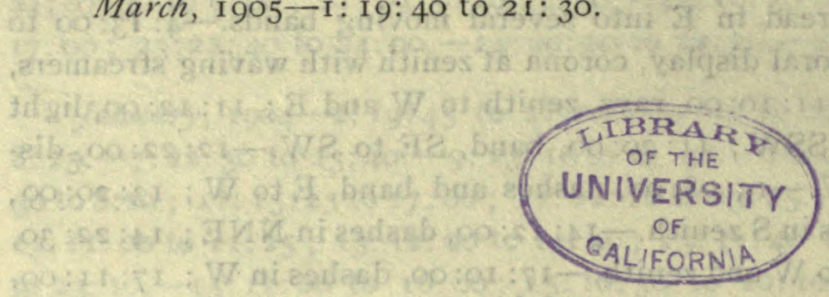

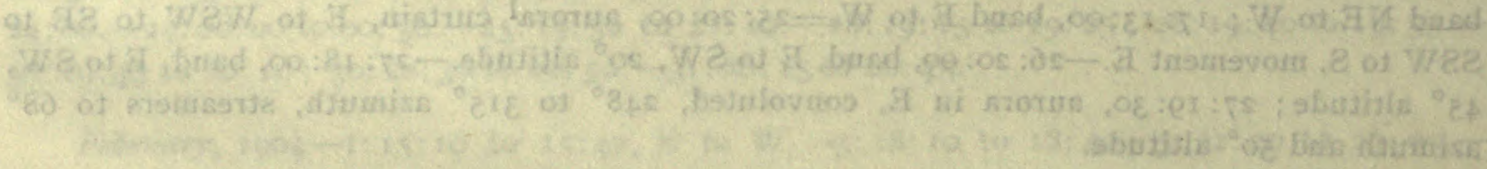

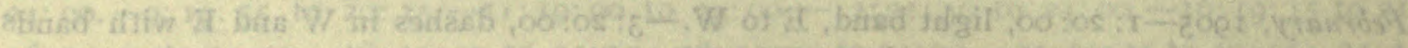

सWदe of

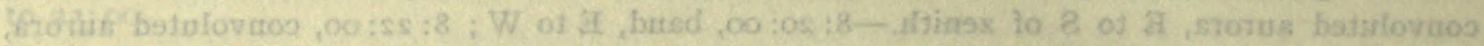

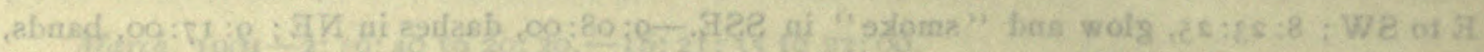

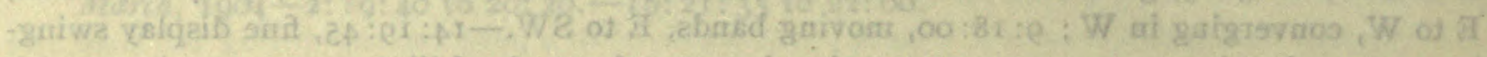

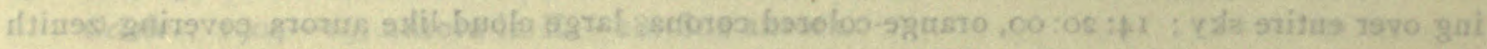

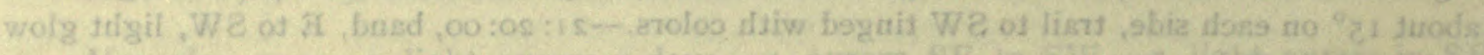




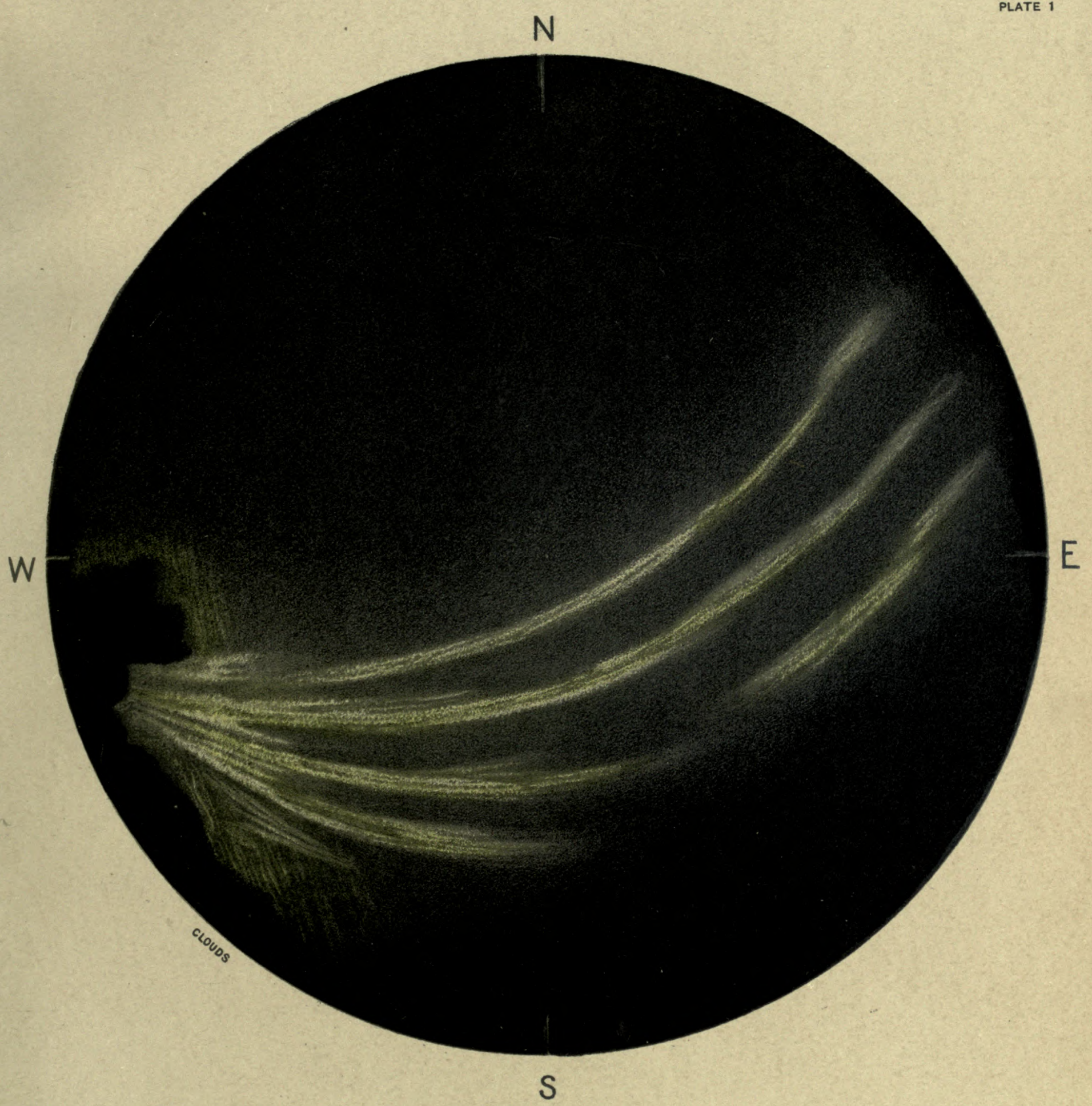

DECEMBER 23, 1903, 15 HR. 30 MIN. 



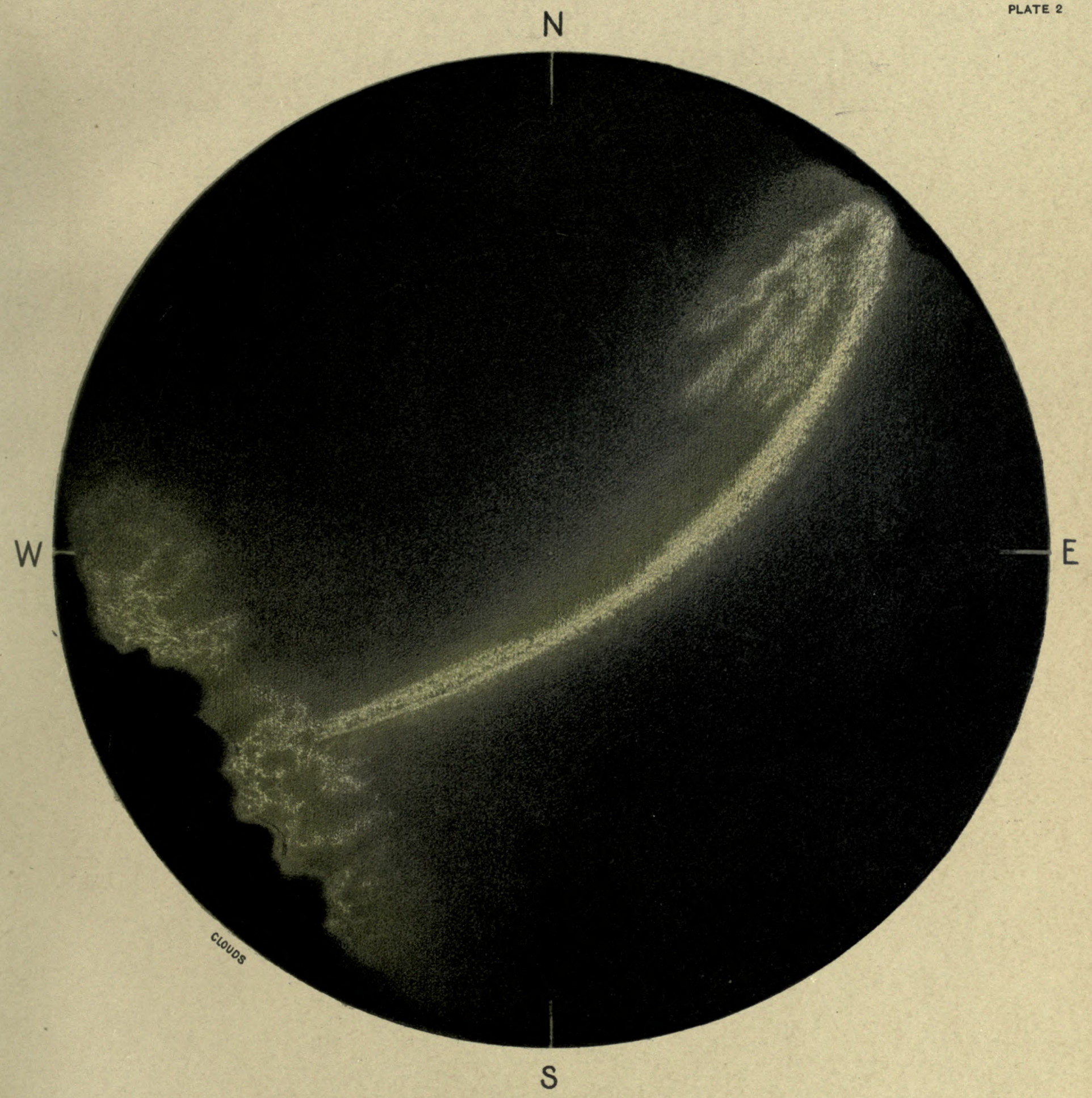

DECEMBER 23, 1903, 16 HR - 10 MIN 



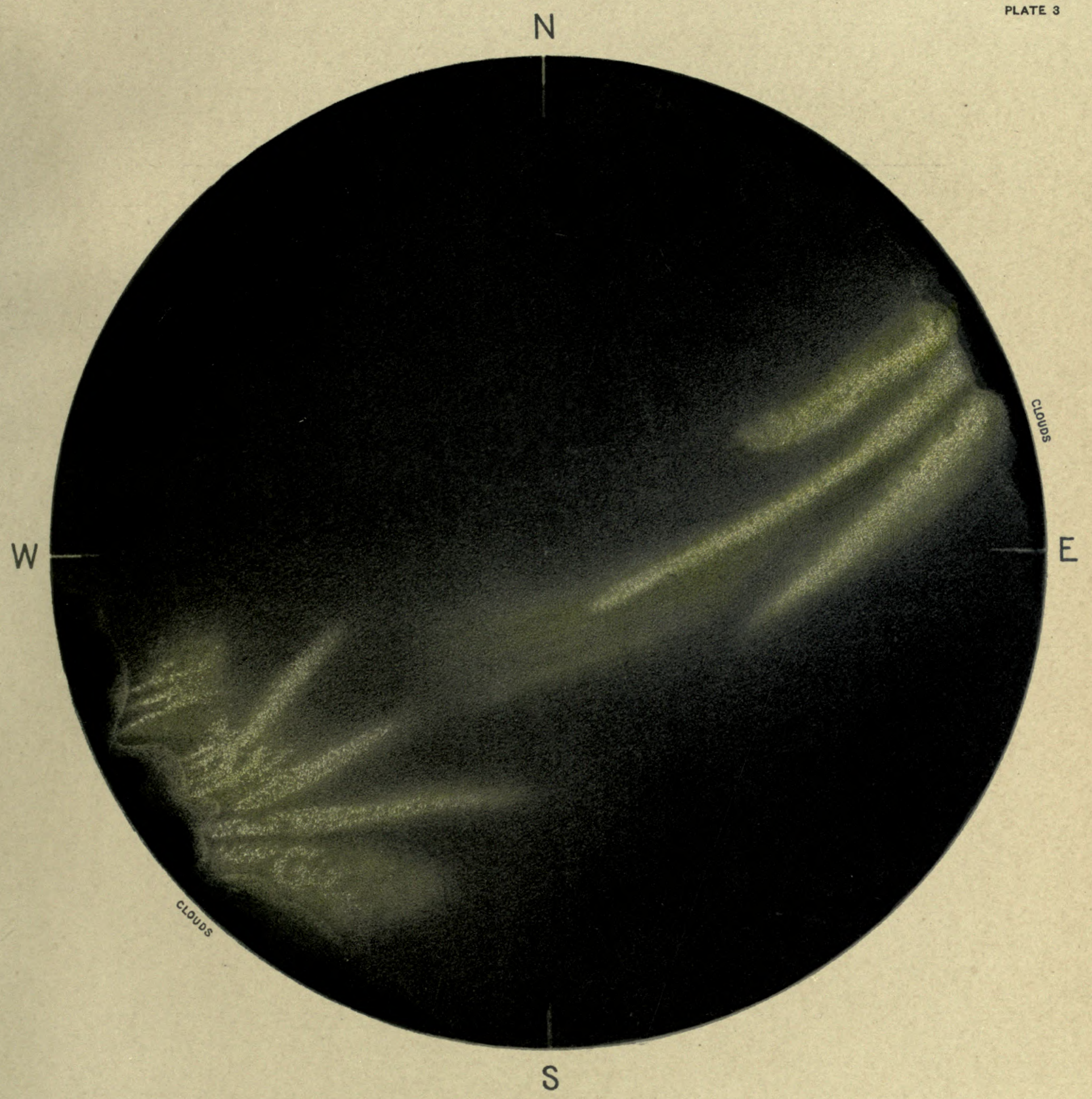

DECEMBER 23, 1903, 16 HR. 40 MIN. 



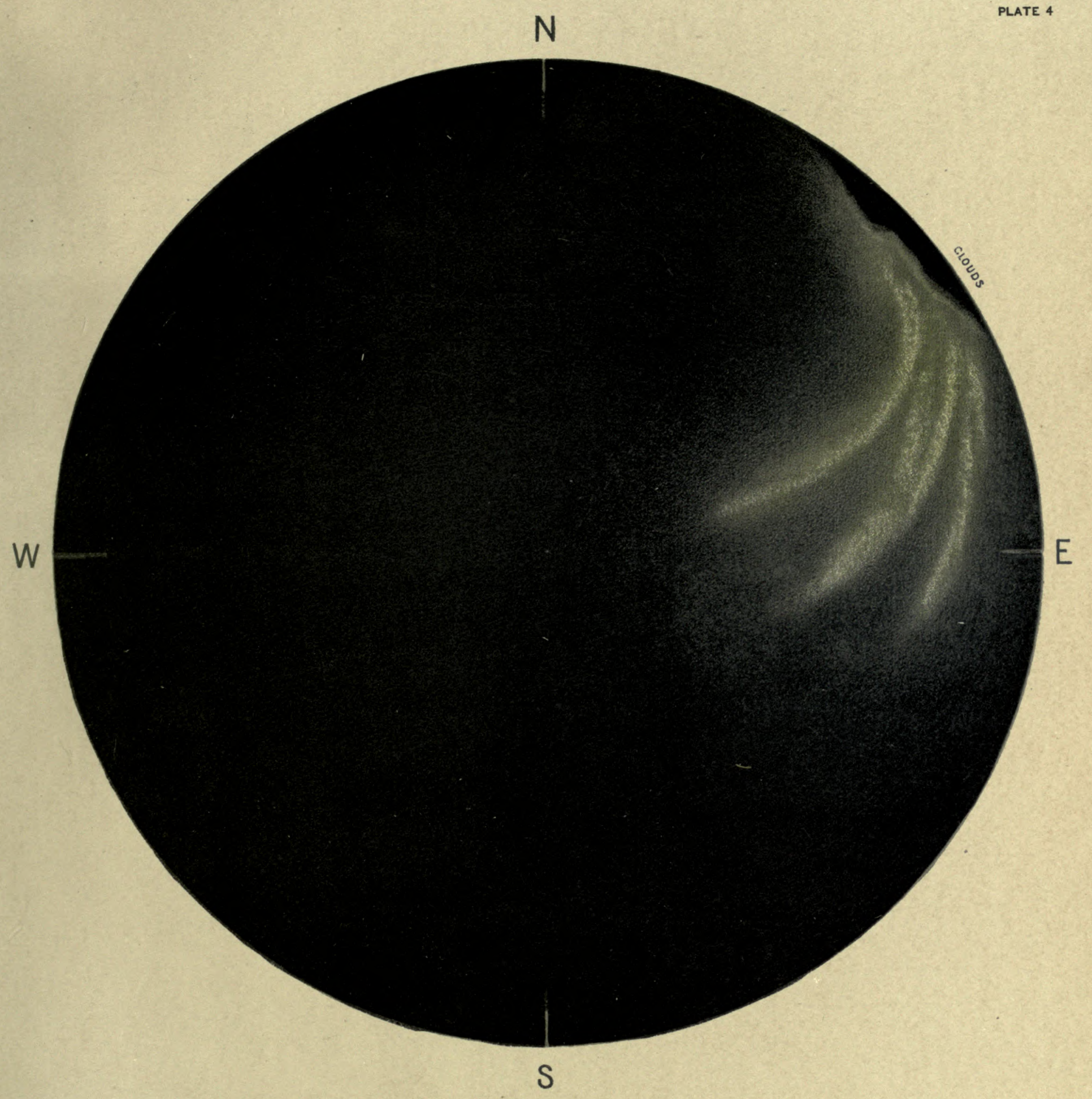

DECEMBER 23, 1903, 17 HR. 15 MIN. 



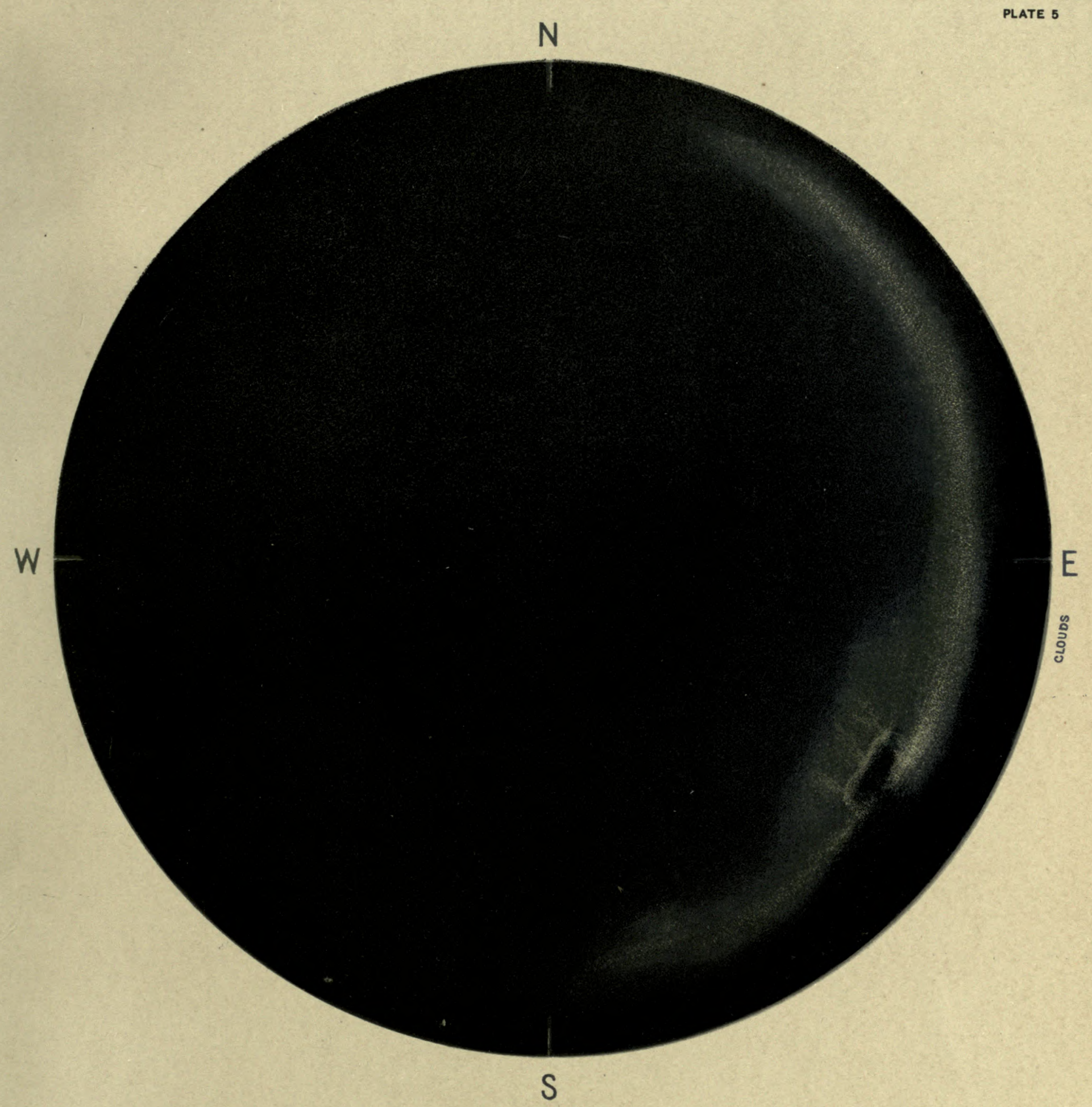

DECEMBER 23, 1903, 23 HR. 40 MIN. 



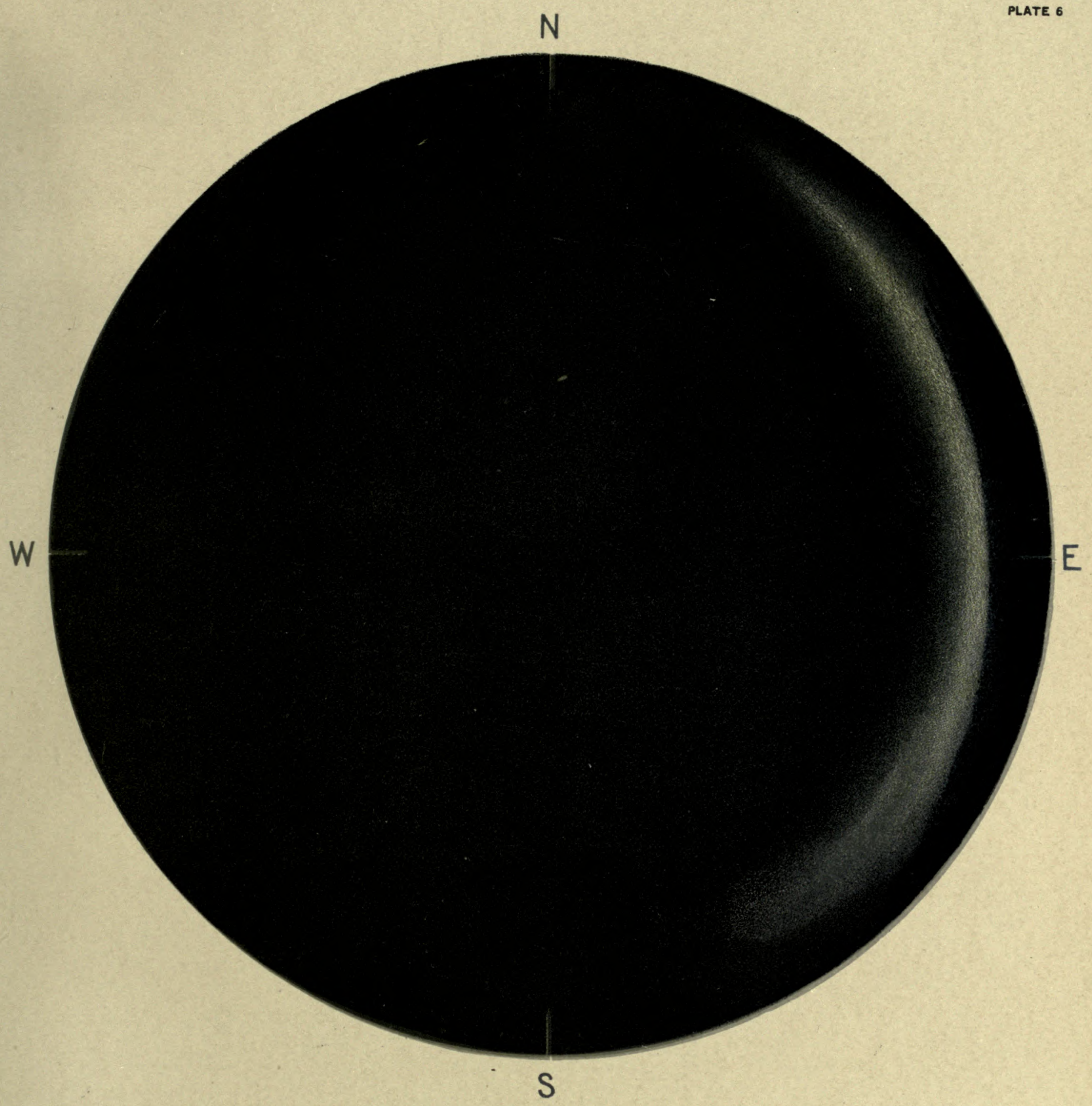

DECEMBER 23, 1903, MIDNIQHT LA8T APPEARANCE 



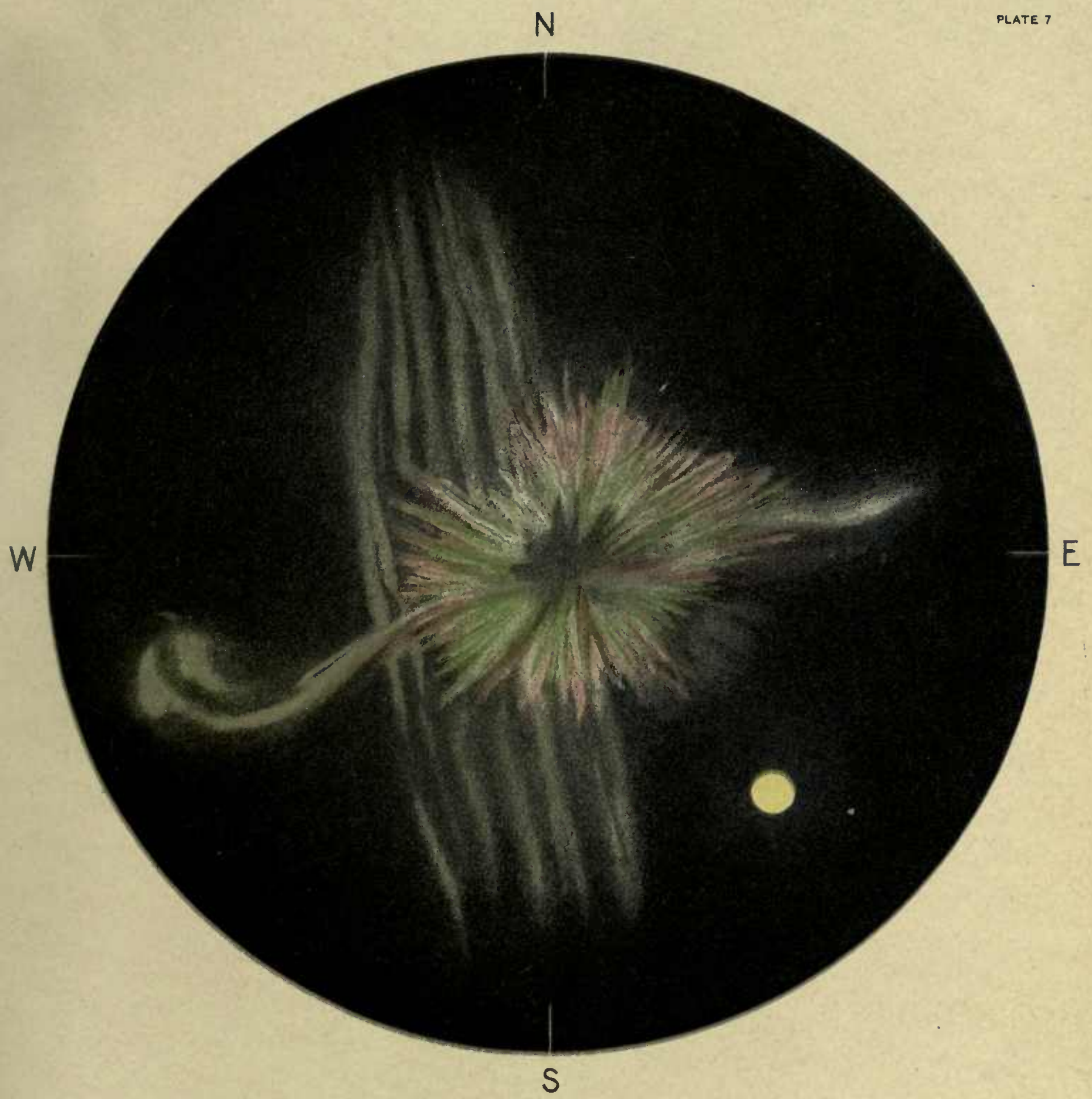

JANUARY 2, 1904, 20 HR. 



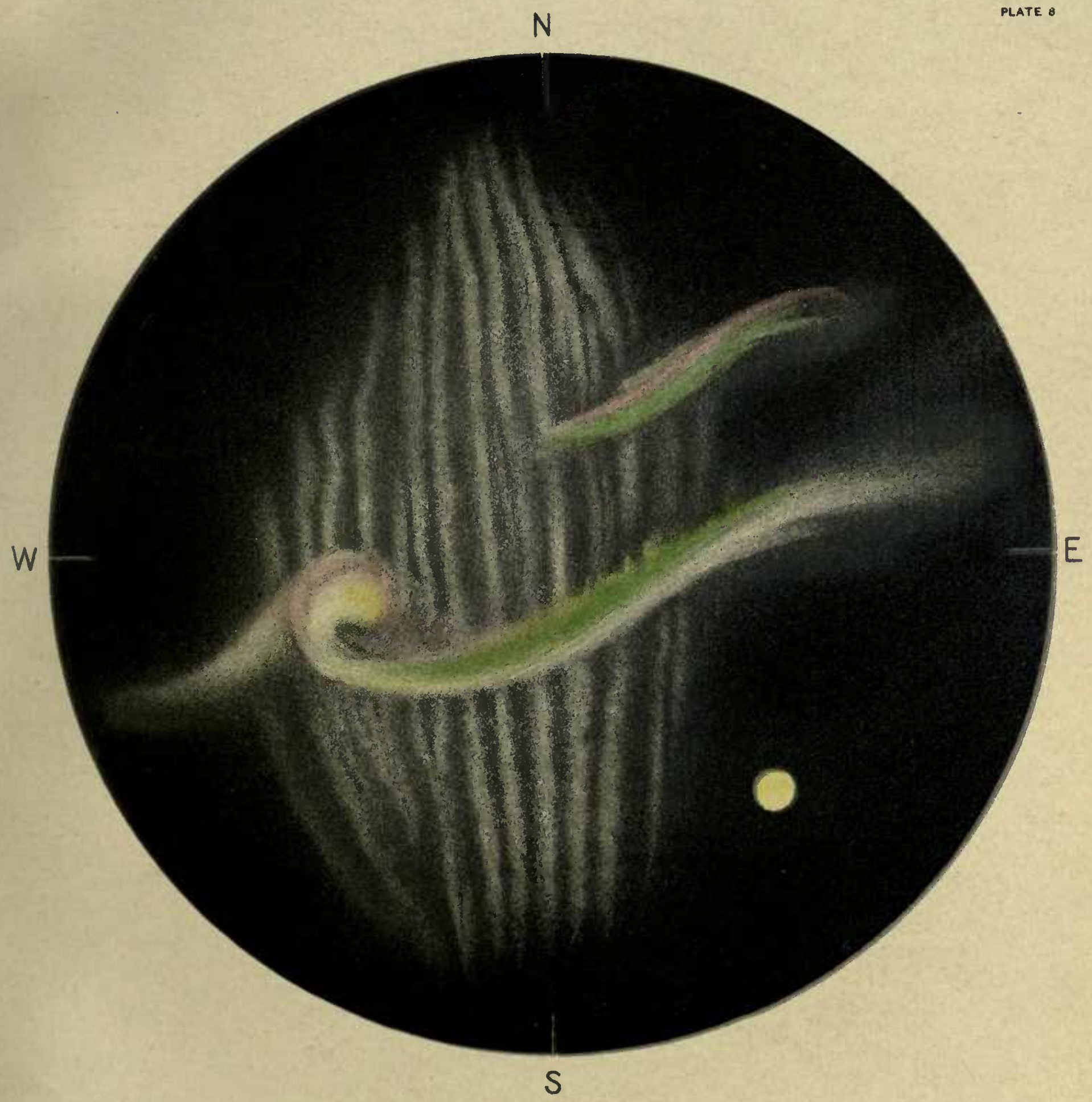

JANUARY 2, 1904, 20 HR. 30 MIN. 



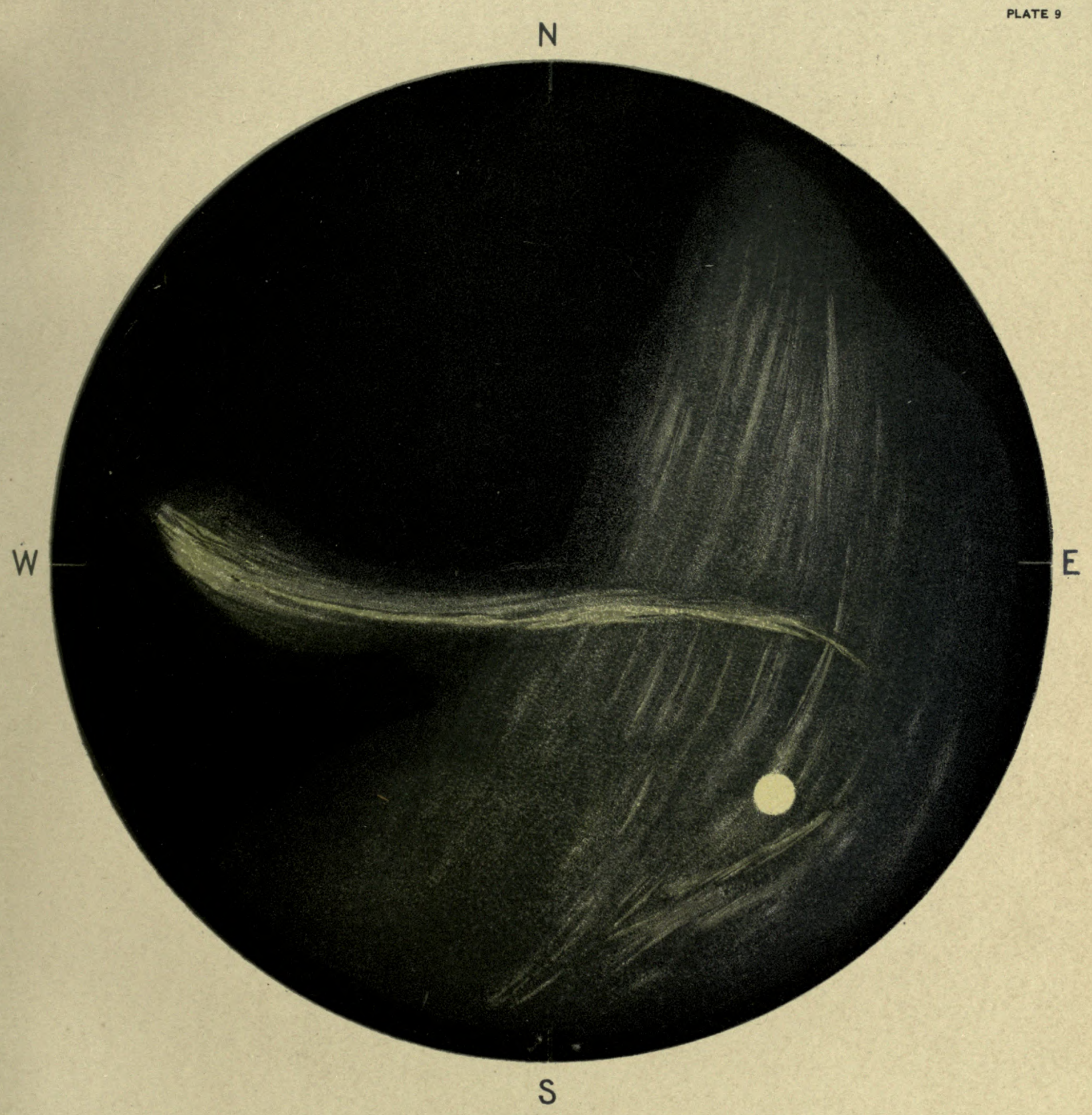

JANUARY 2, 1904, 21 HR. 



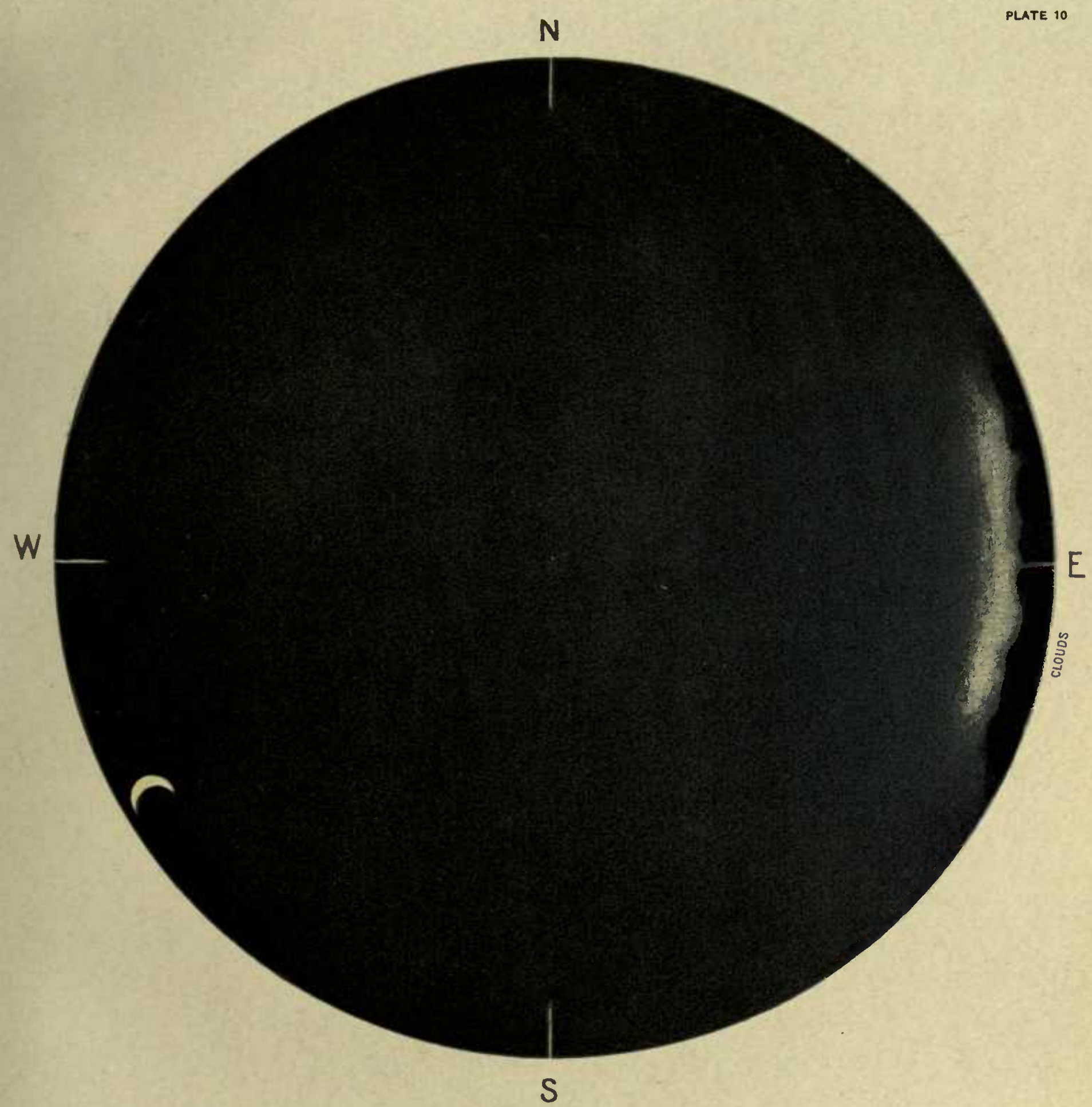

JANUARY 23, 1904, 21 MR. 36 MIN.

FIRST APPEARANCE 



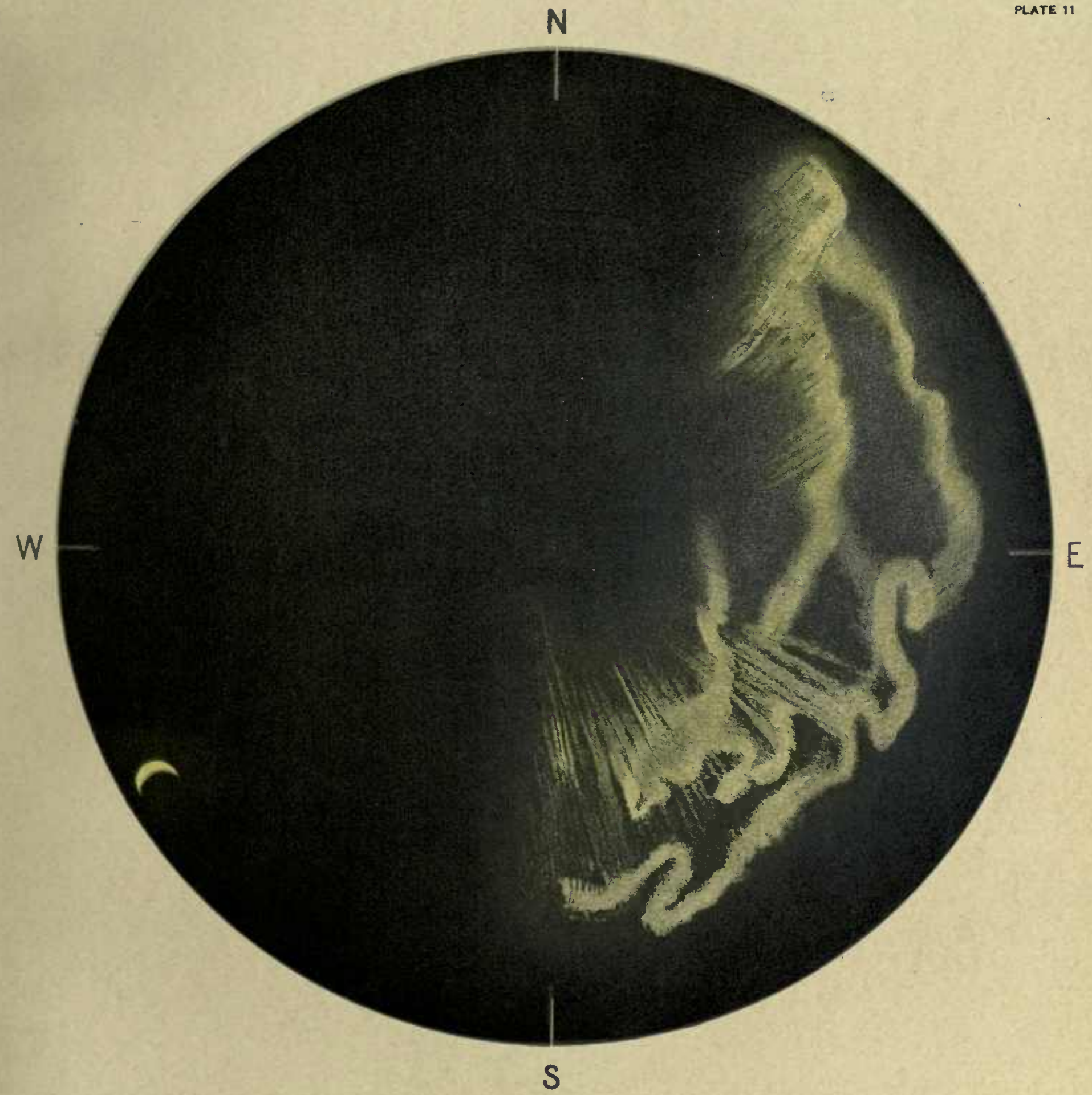

JANUARY 23, 1904, 21 MR. 50 MIN. 


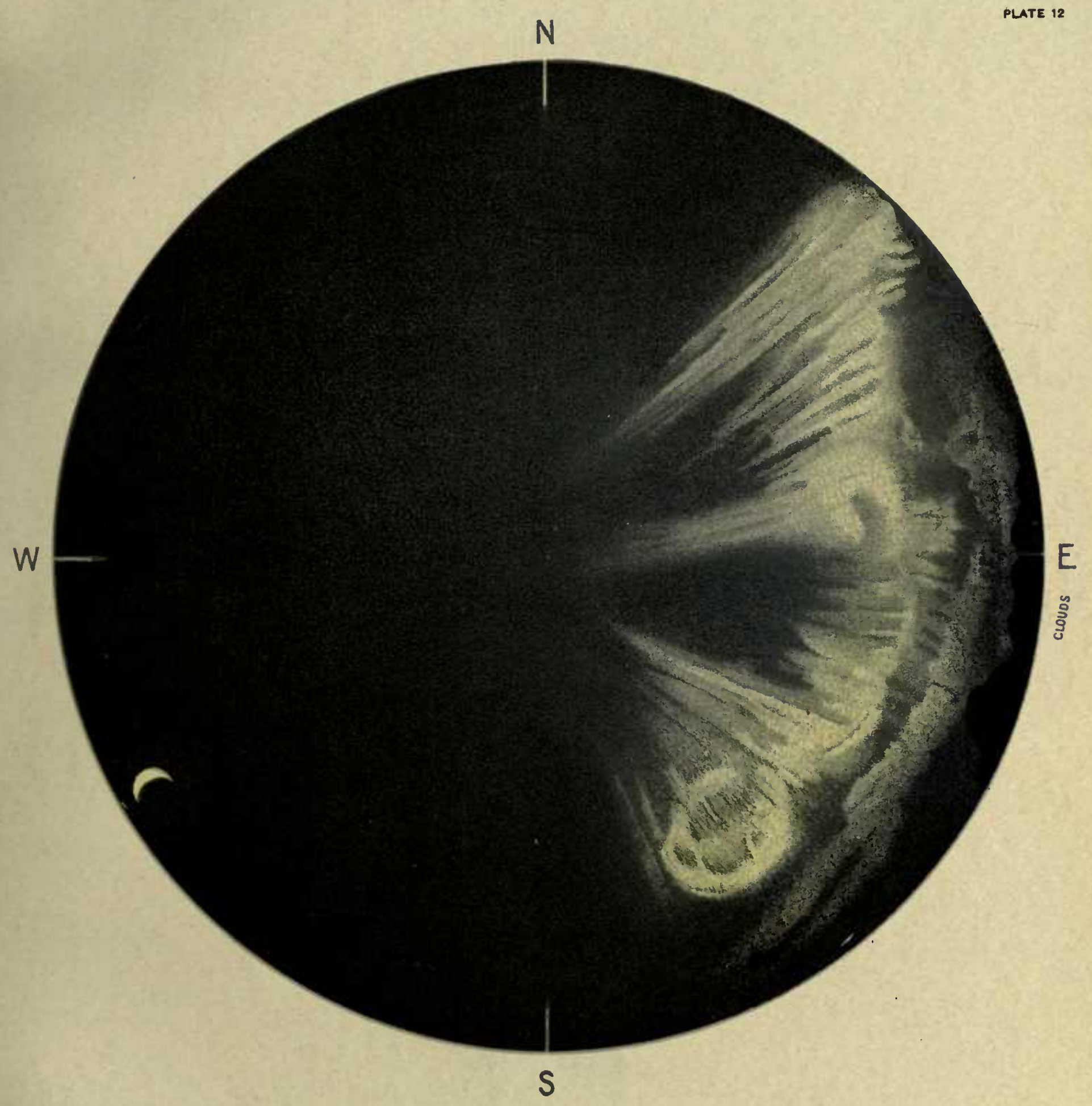

JANUARY 23, 1904, 21 HR. 56 MIN. 



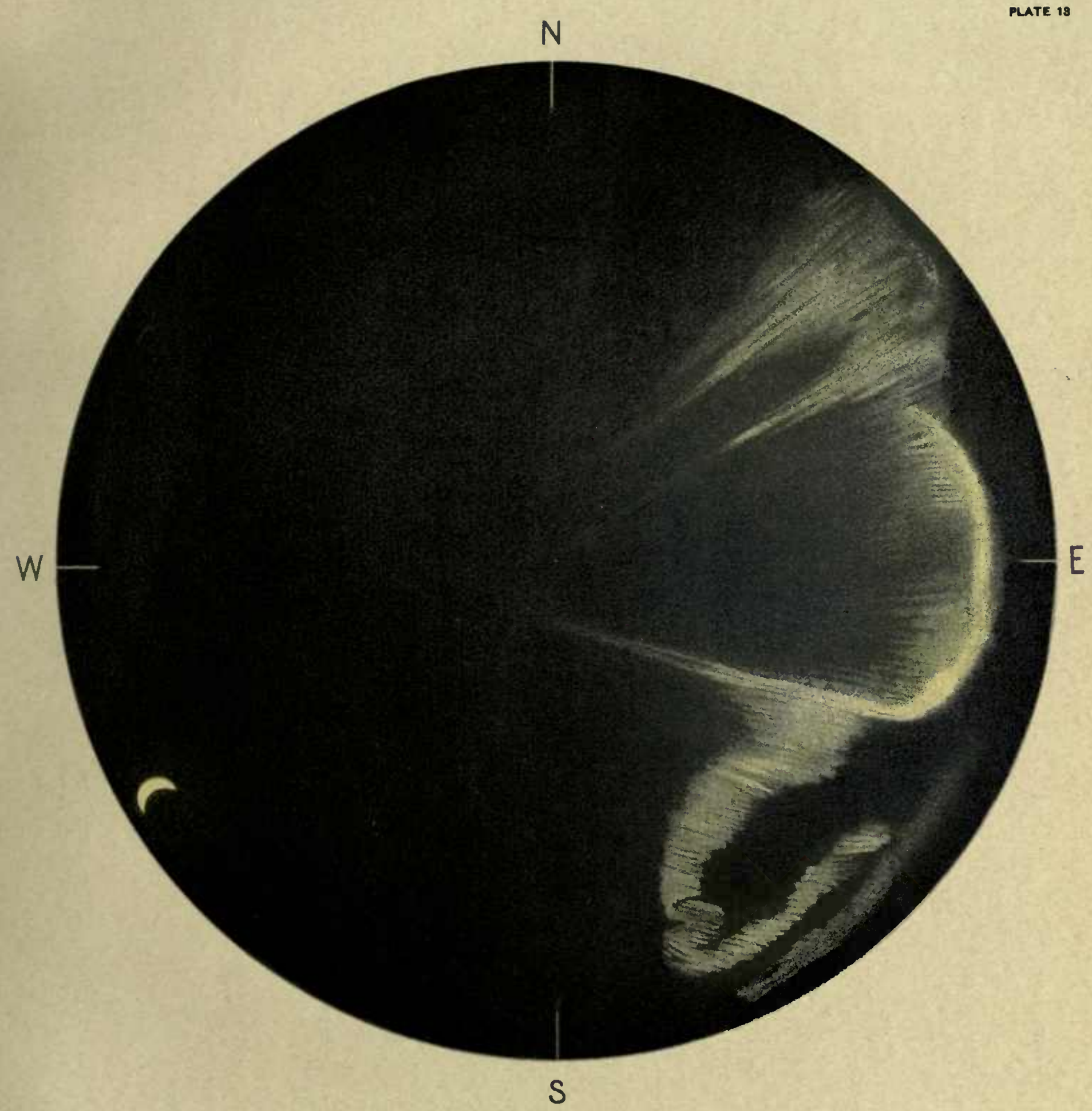

JANUARY 23, 1904, 22 HR. 8 MIN. 



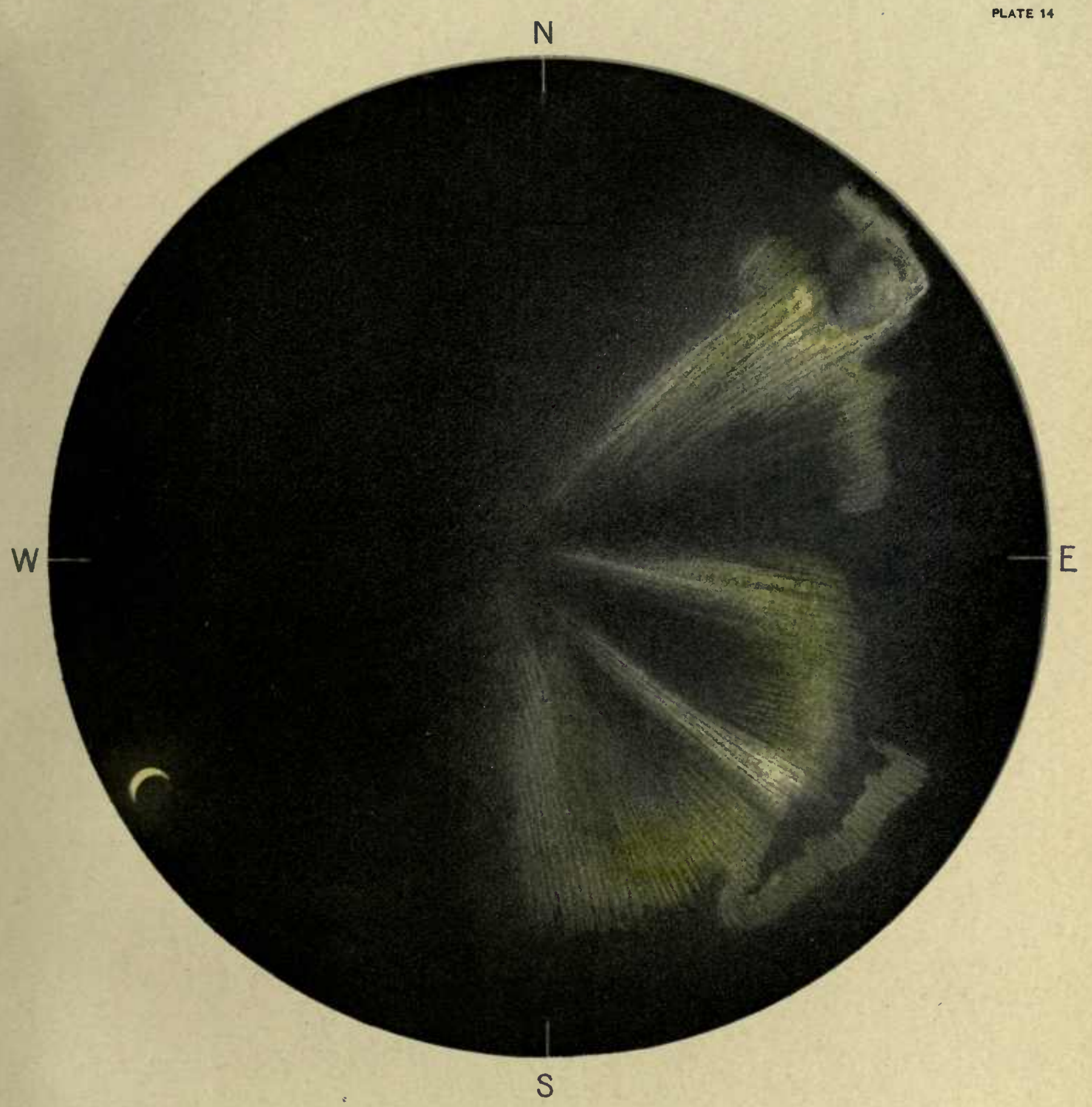

JANUARY 23, 1904, 22 HR. 6 MIN. 



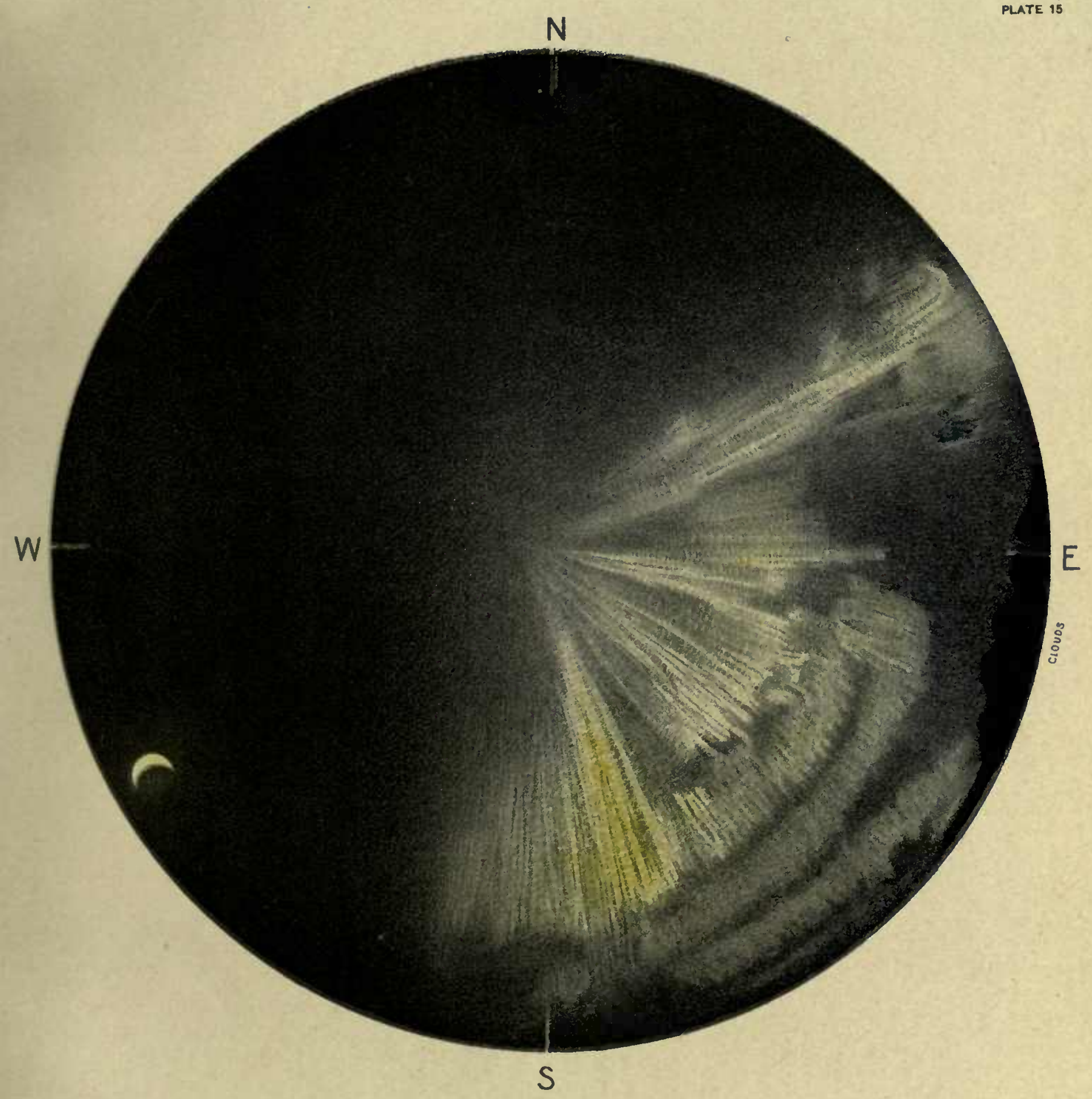

JANUARY 23, 1904, 22 HR. 9 MIN. 



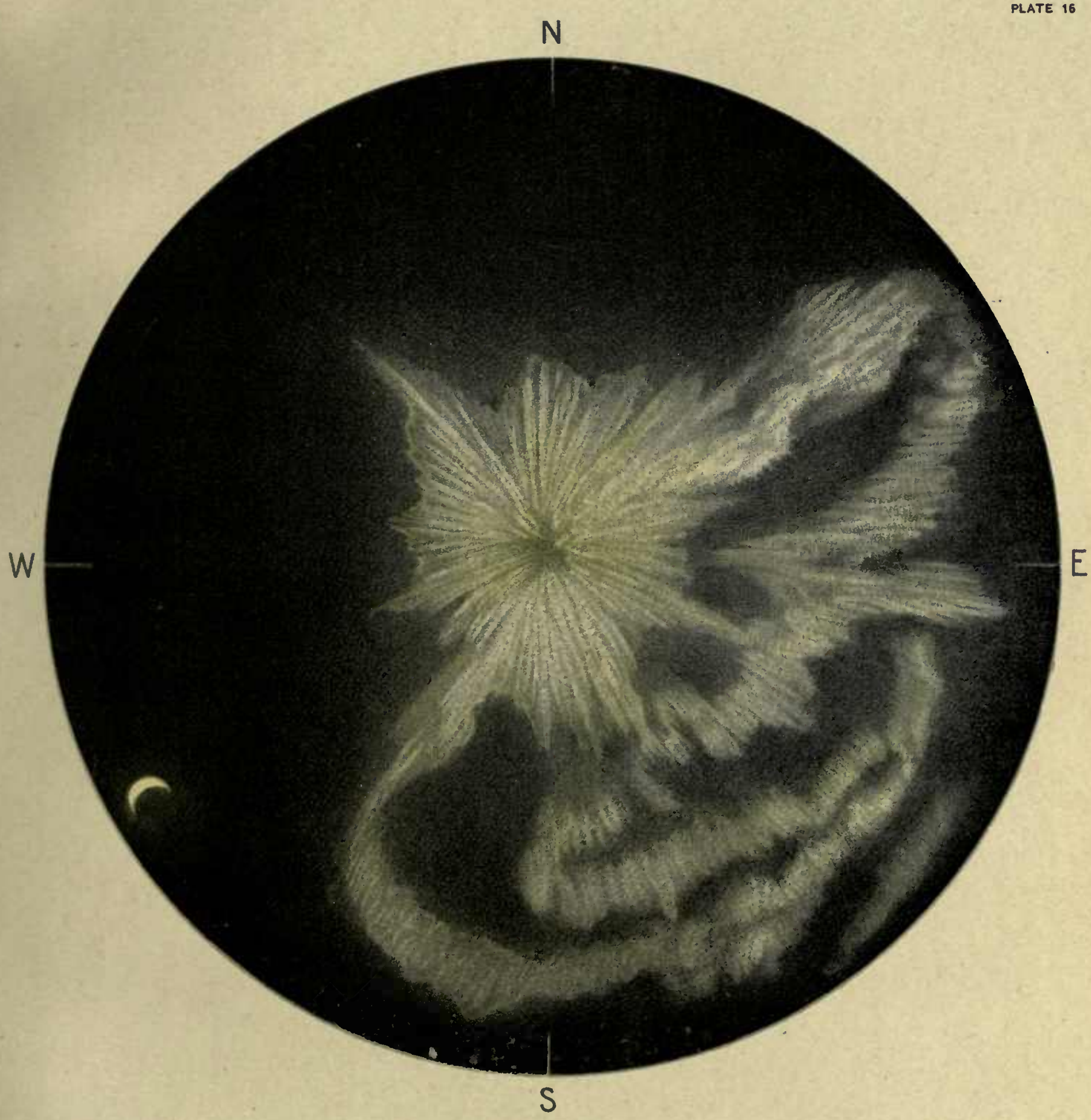

JANUARY 23, 1904, 22 HR. 18 MIN. 



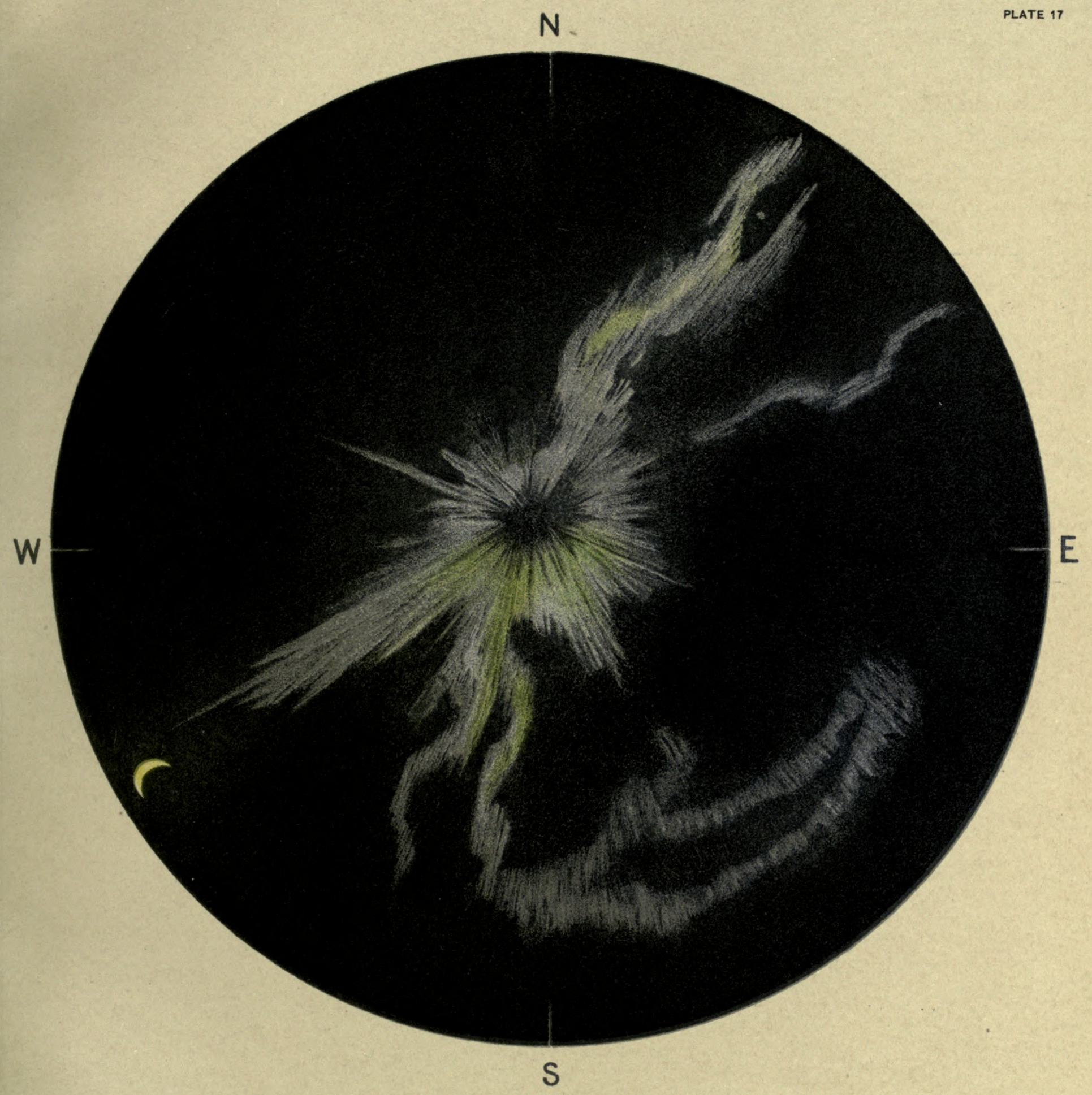

JANUARY 23, 1904, 22 HR. 20 MIN. 


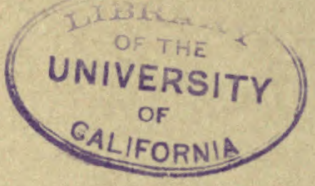




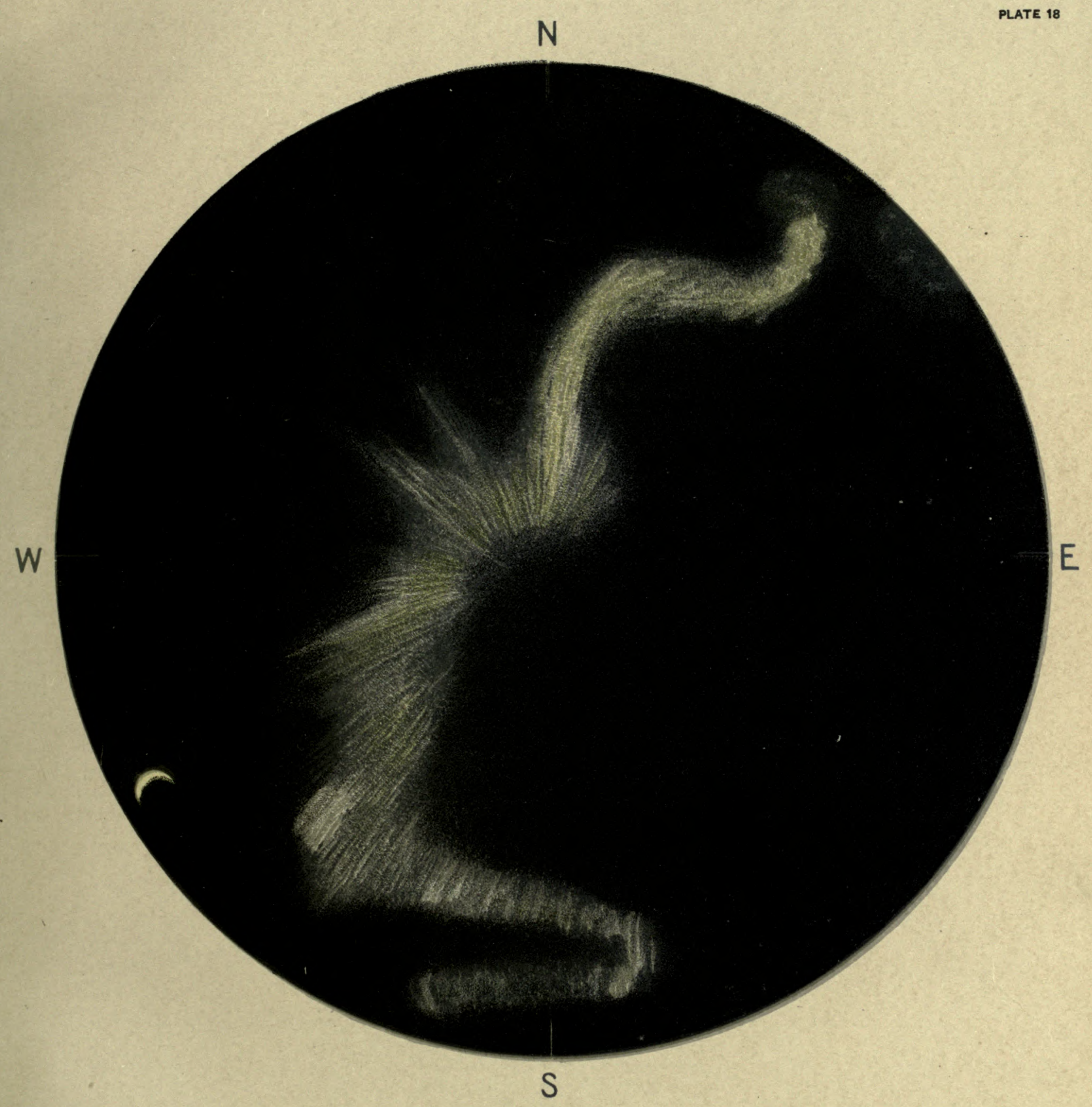

JANUARY 23, 1904, 22 HR. 24 MIN. 



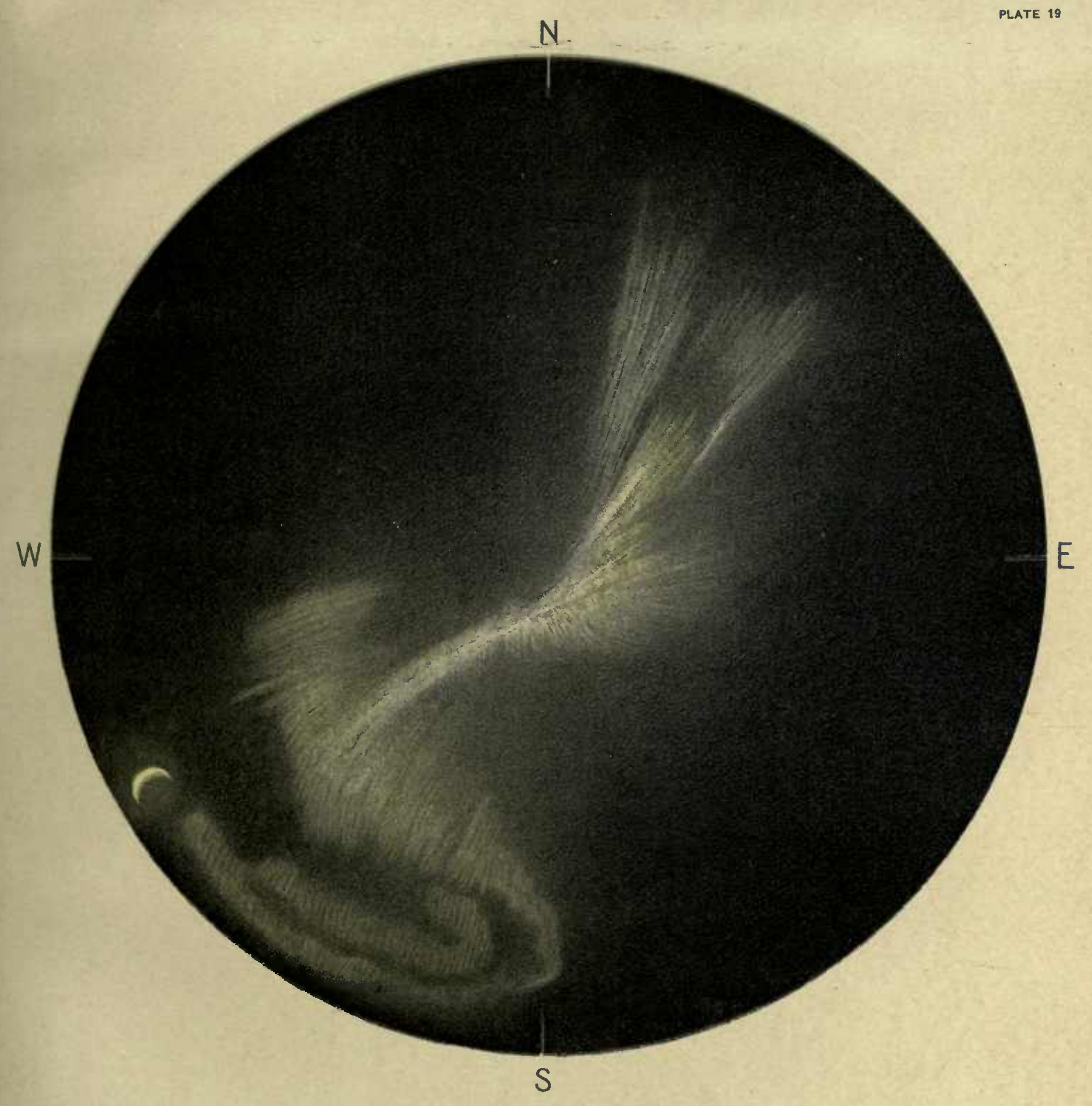

JANUARY 23, 1904, 22 MR. 28 MIN. 



\section{SECTION C}

\section{METEOROLOGICAL OBSERVATIONS \\ AND \\ COMPILATIONS}

BY

W. J. PETERS

In Charge of Scientific Work of the Expedition

AND

J. A. FLEMING

Department Terrestrial Magnetism, Carnegie Institution of Washington 
3 ทоाт

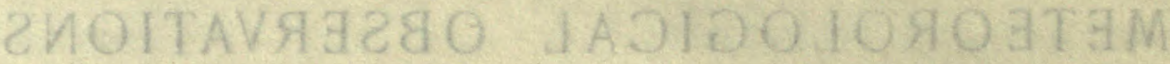

anis

\section{MOITA $119 M 03$}

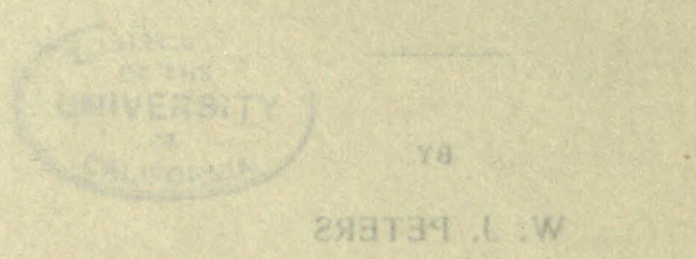

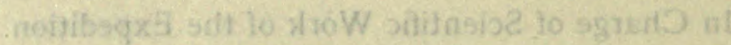

$\mathrm{GMA}$

DYHMA.17 A. A . L

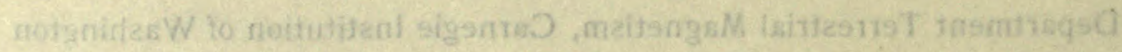




\section{CONTENTS}

Page

Instruments, Stations, and Methods _ . . . . . . . . . . . 373

Records . . . . . . . . . . . . . . . . . . 374

Tabulation of Daily Records at Teplitz Bay . . . . . . . . . . $\quad 375$

Tabulation of Daily Records at Cape Flora _ _ _ . . . . . . . . . 401

Tabulation of Wind Movement at Teplitz Bay . . . . . . . . . 449

Reduction of Observations at Teplitz Bay . . . . . . . . . 472

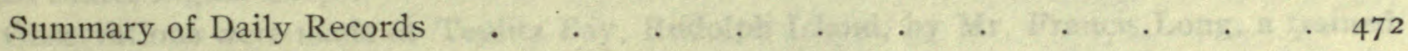

Diurnal Variation in Temperature . . . . . . . . . . . 474

Diurnal Variation in Atmospheric Pressure . . . . . . . . . . . . 476

Diurnal Wind Movement . . . . . . . . . . . . . . . . of . 478

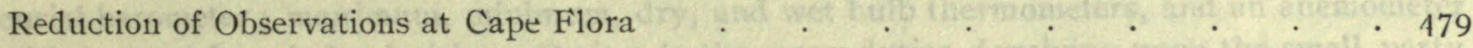

Summary of Daily Records . . . . . . . . . . . 479

Diurnal Variation in Atmospheric Pressure + . . . . . . . . . . . . . . $48 \mathrm{I}$

General Remarks . . . . . . . . . . . . . . . 482

Miscellaneous Observations . . . . . . . . . . . . . . 483

\section{ILLUSTRATIONS}

I Instrument Shelter at Teplitz Bay . . . . . . . . . . 374

2 Diurnal Variation in Temperature at Teplitz Bay . . . . . . . . . . 474

3 Diurnal Variation in Atmospheric Pressure at Teplitz Bay . . . . . 475

4 Barogram and Thermogram for the Period November if to November I7, I903, at Teplitz Bay . . . . . . . . . . . . . . . 476

5 Barogram and Thermogram for the Period January i9 to January 25,1904 , at Teplitz Bay . . . . . . . . . . . . . . . 478

6 Diurnal Variation in Atmospheric Pressure at Cape Flora . . . . . . . 482 


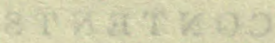

गुका

दारे

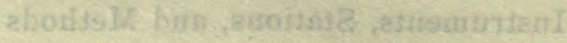

Aff.

टรद

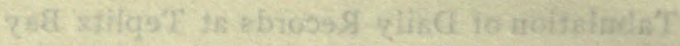

Top

esia

STB

spes:

inte

of?

354

est:

ext

$18 \mathrm{p}$

esp

$28 \&$

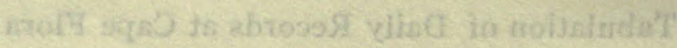

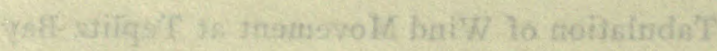

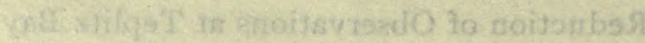

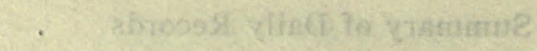

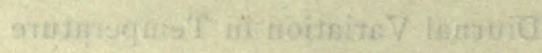

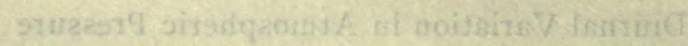

Jusmavolk beriW lsmentid

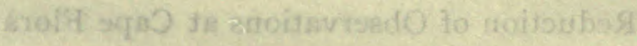
ehomegr velis d to kumentue

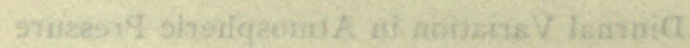

estismgg letacrate

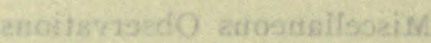

\section{EVOITA STEUIJI}

signe viteorge

718

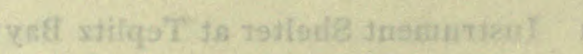

trit

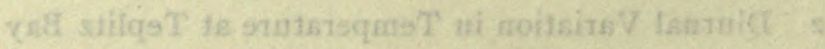

दोiो

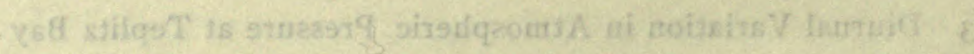

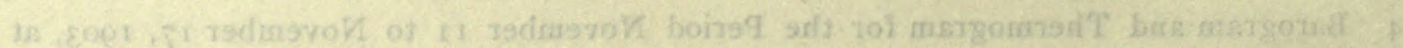
$\partial \mathrm{di}$

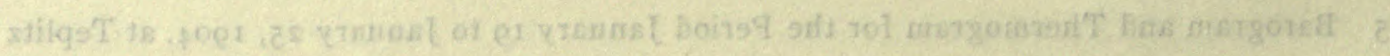
$8 \mathrm{sp}$

ver

$\leq 8$

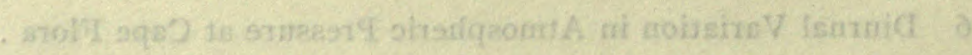




\title{
METEOROLOGICAL OBSERVATIONS
}

\author{
InSTRUMENTS, STATIONS, AND METHODS
}

The instrumettal outfit for the meteorological work consisted of two barometers; two barographs; several aneroids; a nephoscope ; maximu1n, mituinuın, wet, and dry bulb therinometers; thermograpl ; two anemometers, and a single register for recording wind velocities. The greater part of this ontfit was loaned the Expedition through the contesy of Professor Willis Moore, of the United States Weather Burean, and General A. W. Greeley, of the United States Signal Corps.

Observations were nade at Teplitz Bay, Rudolplı Island, by Mr. Francis Long, a trained observer from the United States Weatlier Burean. from September, 1903, * to the end of A pril, 1904, when one division of the Expedition left for Cape Flora; Northbrook Island. At this time the instruments were distributed between the two parties; those left at Teplitz Bay were afterward read or kept in working order by Mr. Spencer W. Stewart and consisted of a mercurial barometer; maximum, minimum, dry, and wet bulb thermometers, and an anemometer with register for wind velocities. Owing to the matny duties devolving upon the small party left at Teplitz Bay, Mr. Stewart could not make observations very regularly or systematically ; in consequence after April 30, 1904, only the resilts for wind movement have been incorporated in this report. Mr. Long personally superintended the transportation of the instruments destined for Cape Flora. These included an aneroid barometer; maximum, minimum, dry, and wet bu1lb thermometers, and an anemometer without register.

On arrival at Teplitz. Bay, in the fall of 1903 , an instrument shelter was set 11 close to the original site used by the Italian Expedition for their meteorological observations (see figure i of "Section A" for a map of the Teplitz Bay station showing respective locations). This shelter was constructed in conformity with the regulations of the United States Weather Bureau, being about 1.5 meter above the surface of the ground, and is very clearly shown in figure $\mathrm{r}$. In it were installed, exposed, wet bulb, maximum and minimum thermometers, and the thermograph. These were all placed at the same level, namely, about to $1 / 2$ meters above the sea. The anemometer was fixed on the astronomical observatory (see figure $I$ as also figure $\mathbf{I}$ of "Section E"), some roo meters from the shelter-house and about 22 meters above sea level. The mercurial barotneter and aneroid, together with the barograpli, were installed in the living-liouse at 9.35 meters above sea level.

Records were made daily at 8 A. M., noon, and 8 P. M., local mean time. The true direction of the wind was determined by eye observations of the weatlier vane. Observations with the nephoscope were foind to be impracticable : during the winter the darkness prohibited any attempt; during the period of daylight the clonds were of such a character and were so low, really not more than fogs, that they presented no well-defined points on which to observe.

At Cape Flora ("Elmwood" of the Jackson-Harmsworth Expeuition of r894 to I897) a valuable series of observations extending from May $2 \mathrm{r}$, 1904, to July 30, 1905, was made by Mr. Long. An improvised instrument shelter, following as closely as possible the regulations of the United States Weather Bureau, was constructed from boxes in which were installed

* Prior to this time and during the voyage of the S. Y. "Anerica" north from Tromso, Mr. Long made regularly daily observations. These covering, as they do, a region already frequently reported upou meteorologically, are not recorded in this volume. 
maximum, minimum, dry, and wet bulb thermometers. An aneroid baroneter was installed in the living-house 15. I meters above sea level. Wind motions were determined by dial readings of the anemometer at $8 \mathrm{~A}$. M., noon, and 8 P. M., local mean time, the true directions being estinuated by eye from weather vane. Temperature and otluer observations were also made at the same times. The elevation of the top of the anemometer staff, which was mounted on the storehouse, was abont 20 meters above sea level. It may be noted that the wind is locally affected at Cape Flora by the proximity of bluffs 350 ineters high. This is especially noticeable when open water affords another means of determining the wind direction.

\section{RECORDS}

The various meteorological instruments were compared with standard instruments before embarking and corrections determined. Unfortunatcly the difficilties and exigencies of the retreat in I 905 were such as to necessitate abandoning practically the entire meteorological outfit. Accordingly only the initial comparisons were available for the correction of the records. This is to be regretted, especially so in the case of the barometers.

The corrected records at the Teplitz Bay and Cape Flora stations are given on pages 375 to 471 ; the final summaries and reductions resulting will be found ou pages 472 to 482 . In view of the fact that all of the records, in conformity with the standards used by the United States Weather Bureau, have been made in the Fahrenheit scale for temperature and in the English measure for wind and atmospheric pressure, these systems have been retained in the various compilations. As will be noted, no records are given for relative humidity, vapor pressure, or dew point; this is owing to the fact that with the insufficient apparatus available no reliable results could be obtained under the condition of prevailing low temperatures.

In addition to the work at these two principal stations numerous irregular observations were made as time permitted at various points. Of these only the records made on the sledge trips are here recorded as being of interest in indicating the conditions of travel in the Archipelago (see pages 483 to 487 ).

Observations preceding 12 hours of September 22, 1903, were made on board the "America" in Teplitz Bay; after that tine they were made at the final station, Camp Abruzzi, Teplitz Bay. The times of observation preceding 12 hours of September 28 are 45 M. M. T. east; on and after that tine local mean time is used; aneroid barometer used through September 21 ; nercurial barometer after that date.

The following abbreviations are used in the tabulations:

$$
\begin{aligned}
& \mathrm{T} \text {-trace of precipitation } \\
& \mathrm{R} \text {-rain } \\
& \mathrm{S}^{\mathrm{d}} \text {-dry snow } \\
& \mathrm{C}-\text { calm }
\end{aligned}
$$

$$
\begin{aligned}
& \mathrm{S}^{\mathrm{m}} \text {-moist snow } \\
& \mathrm{S}^{\mathrm{L}} \text {-sleet } \\
& \mathrm{H} \text {-hail } \\
& \mathrm{DN} \text {-during night }
\end{aligned}
$$

Cloud classifications :

$$
\begin{aligned}
& \text { A-Cu-alto-cumulus } \\
& \text { Ci-cirrus } \\
& \text { Ci-S-cirro-stratus } \\
& \mathrm{Cu}-\mathrm{N} \text {-cumulo-nimbus } \\
& \text { Fr-N-fracto-nimbus } \\
& \mathrm{N}-\text { nimbus } \\
& \text { S-Cu-strato-cumulus } \\
& \text { *-fog } \\
& \mathrm{Ci}-\mathrm{Cu} \text { - cirro-cumulus } \\
& \mathrm{Cu} \text {-cumulus } \\
& \text { Fr-Cu-fracto-cumulus } \\
& \text { Fr-S-fracto-stratus } \\
& \text { S-stratus } \\
& \mathrm{H} \text {-haze } \\
& \text { **-dense fog }
\end{aligned}
$$

Cloud characters appearing in parentheses, thus ( $\mathrm{Ci}-\mathrm{Cu}$ ), refer to upper clouds, all other references being to lower clouds. A totally clouded sky is counted as amonnt of cloudiness ten, a perfectly clear sky being counted zero. Otherwise the tabulations are explained sufficiently by the column headings. 


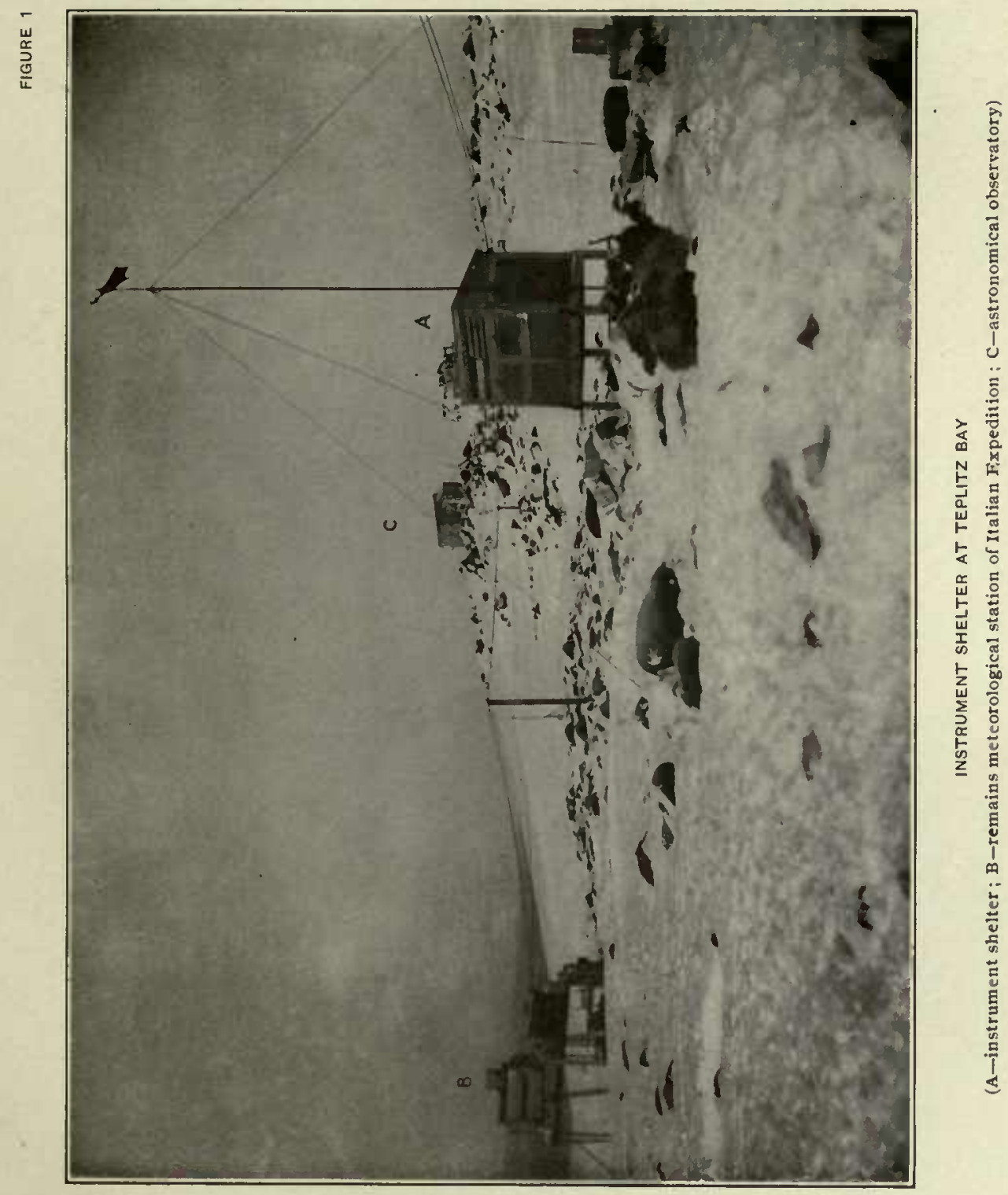





\title{
METEOROLOGICAL OBSERVATIONS
}

\section{TABULATION OF DAILY}

\section{METEOROLOGICAL OBSERVATIONS}

\author{
RECORDED AT
}

TEPLITZ BAY STATION, RUDOLPH ISLAND

FRANZ JOSEF ARCHIPELAGO

SEPTEMBER 1, 1903, TO APRIL 30, 1904

\author{
NORTH LATITUDE: $81^{\circ} 47.5$ \\ LONGitude EAST OF GREENWICH: $57^{\circ} 56^{\prime}$
}




\title{
2ИOITAV93280 IADIDOJOЯOGTAM
}

\author{
YJIAC 70 ИOITAIUSAT \\ 2ИOITAVЯ3280 JADIDOJOЯОЭT3M
}

TA वзवя0039

GИA.J2I H9.JOQUЯ ИOITAT2 YAQ JII.J9GI

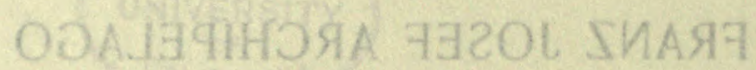

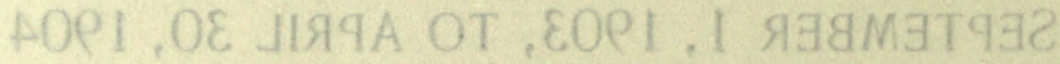

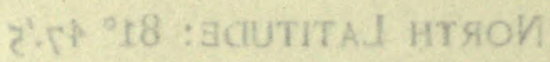

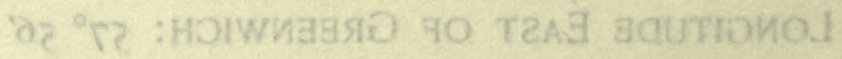


Tabulation of daily meteorological observations at Teplitz Bay during the month of September, 1903 Observer: Francis LoNG

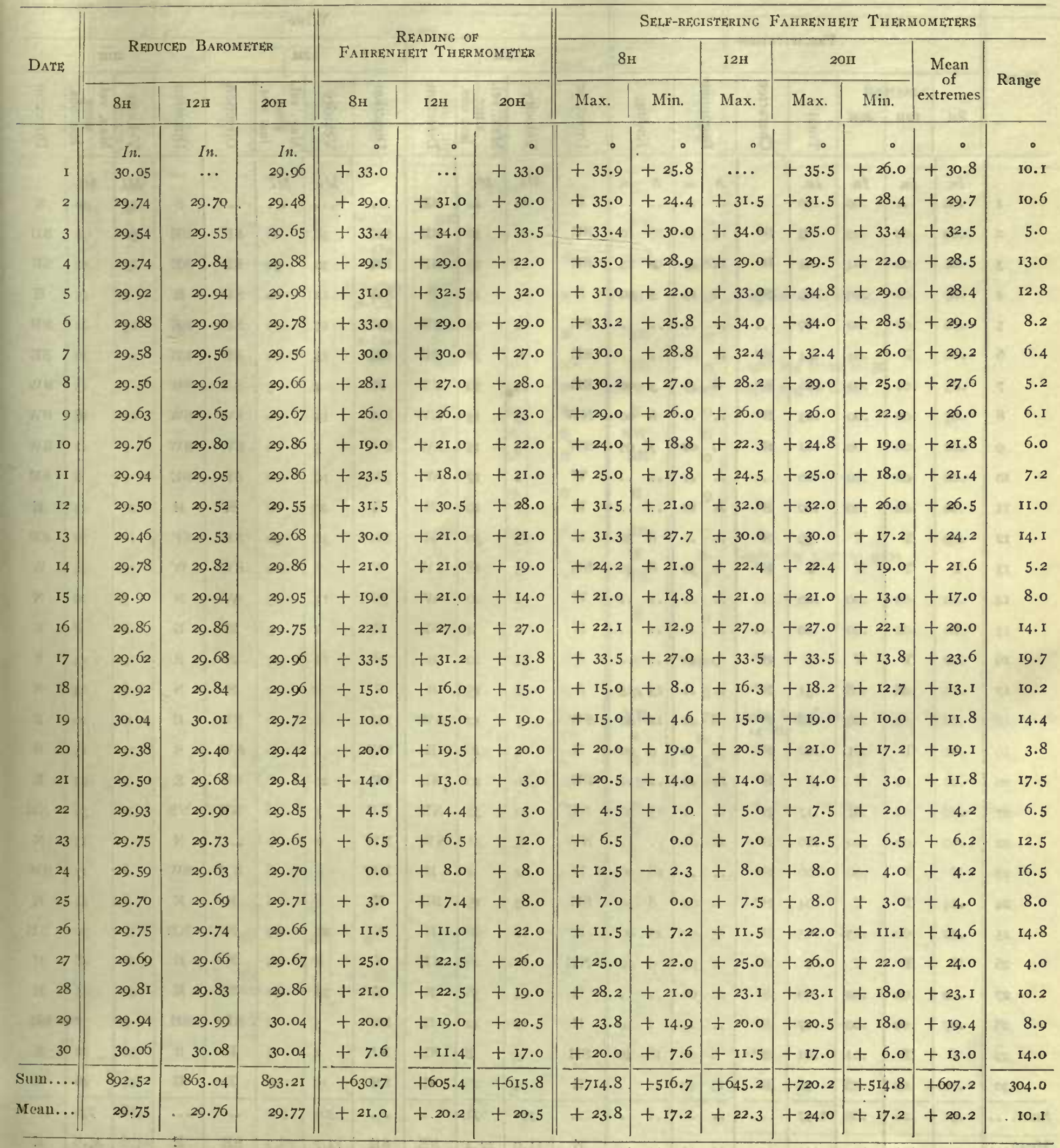


Tabulation of daily metcorological observations at Teplitz Bay during the month of September, 1903-Continued Observer: Francis LoNG

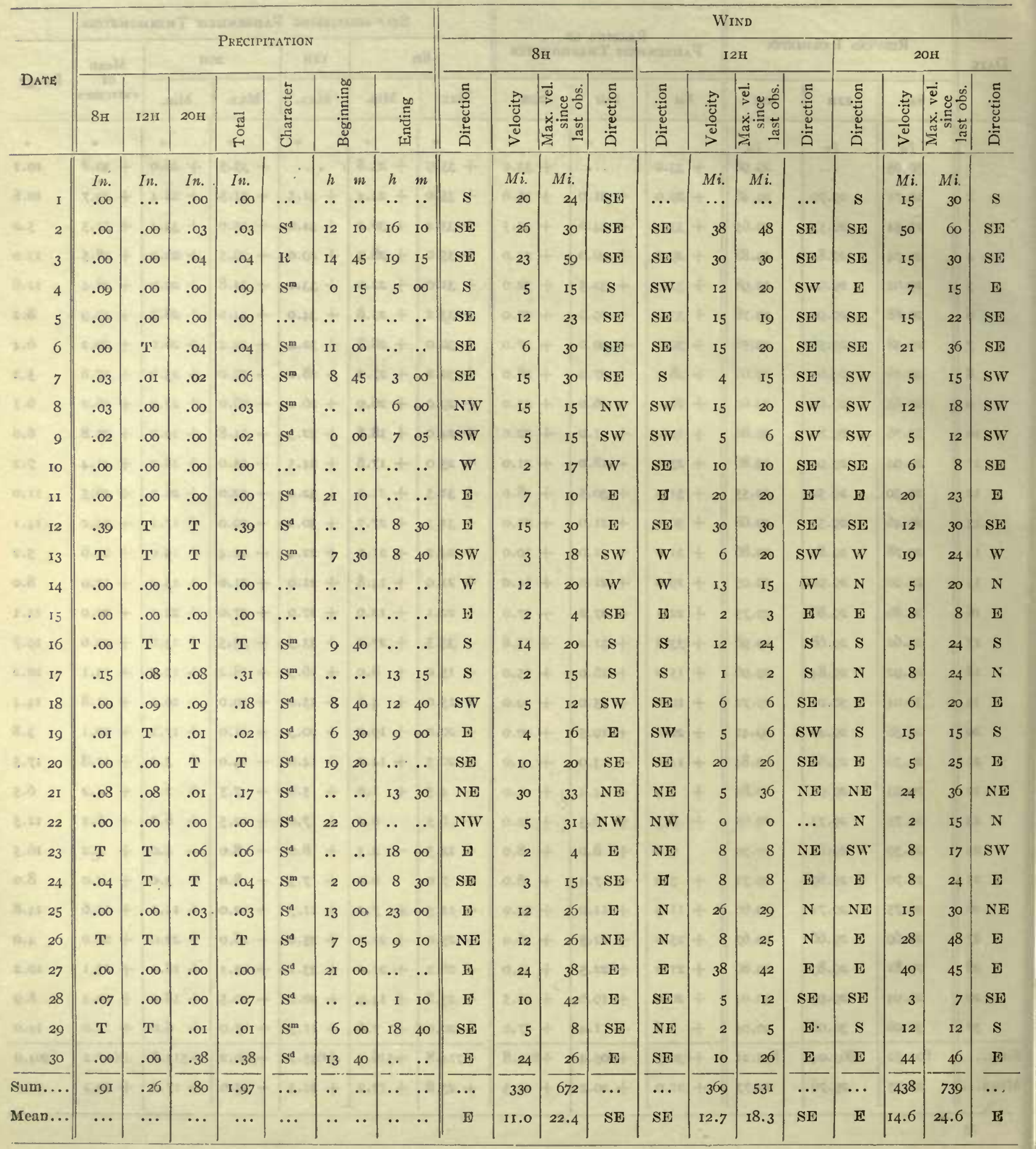


Tabulation of daily metcorological observations at Teplitz Bay during the month of September, 1903-Continued Observer: FRANCIS LONG

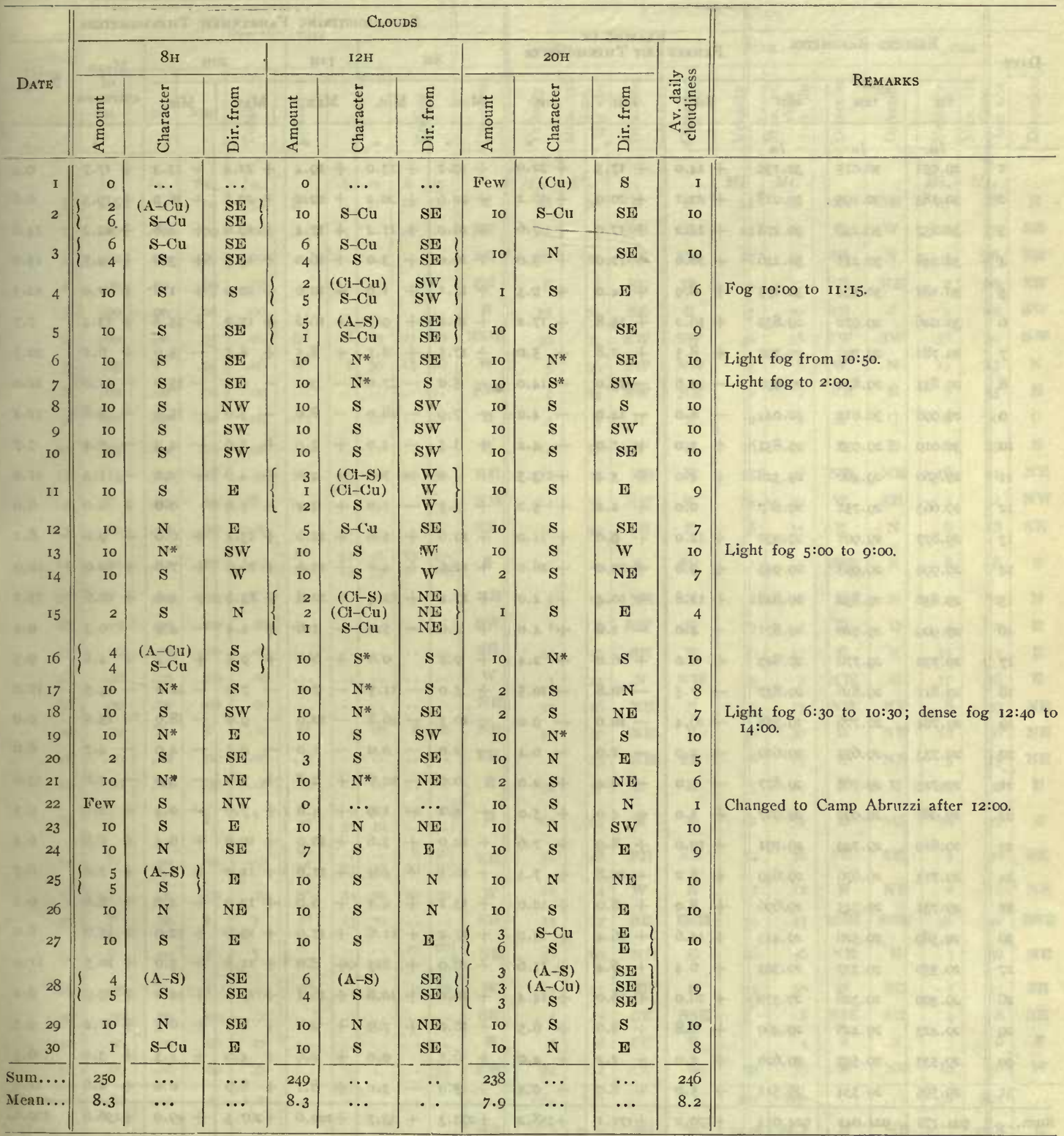


Tabulation of daily meteorological observations at Teplitz Bay during the month of October, 1903 Observer: Francis LoNo

\begin{tabular}{|c|c|c|c|c|c|c|c|c|c|c|c|c|c|c|}
\hline \multirow{3}{*}{ DATE } & \multirow{2}{*}{\multicolumn{3}{|c|}{ REDUCED BAROMETER }} & \multirow{2}{*}{\multicolumn{3}{|c|}{$\begin{array}{c}\text { READING OF } \\
\text { FAIIRENHEIT THERMOMETER }\end{array}$}} & \multicolumn{8}{|c|}{ SELF-REGISTERING FAHRENIIEIT THERMOMETERS } \\
\hline & & & & & & & 81 & & & 12 II & & III & & \\
\hline & $8 \mathrm{H}$ & $\mathrm{I} 2 \mathrm{H}$ & $20 \mathrm{H}$ & $8 \mathrm{H}$ & $\mathrm{I} 2 \mathrm{H}$ & $20 \mathrm{H}$ & Max. & & Min. & Max. & Max. & Min. & & \\
\hline & In. & In. & In. & $\circ$ & 8 & 3. & 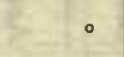 & & $\circ$ & $\circ$ & $\circ$ & $\circ$ & $\circ$ & $\circ$ \\
\hline I & 29.951 & 30.015 & 30.139 & +14.0 & +17.5 & +22.0 & +19.2 & + & I3.0 & +19.4 & +22.4 & +13.3 & +17.7 & 9.4 \\
\hline 2 & 30.183 & 30.195 & 30.218 & +22.1 & +20.0 & +16.2 & +22.6 & + & 20.2 & +22.1 & $+22 . I$ & +16.0 & +19.3 & 6.6 \\
\hline 3 & 30.237 & 30.259 & 30.278 & +14.2 & +17.0 & +7.6 & +16.0 & + & II. 2 & +17.4 & +19.6 & +4.8 & +12.2 & 14.8 \\
\hline 4 & 30.248 & $30.24 I$ & 30.246 & +10.0 & +12.0 & +3.0 & +10.0 & + & 3.0 & +16.0 & +15.9 & +3.0 & +9.5 & 13.0 \\
\hline 5 & 30. 185 & 30. 194 & 30.175 & +8.9 & +4.0 & +9.5 & +13.1 & + & I.O & +10.0 & +10.0 & +1.8 & +7.0 & 12.1 \\
\hline 6 & 30.046 & 29.972 & 29.832 & +16.2 & +15.8 & +17.2 & +16.2 & + & 9.5 & +16.2 & +17.2 & +15.8 & +13.4 & 7.7 \\
\hline 7 & 29.782 & 29.792 & 29.797 & +8.5 & +7.8 & -5.0 & +17.5 & + & 8.5 & +8.5 & +8.5 & -5.0 & +6.2 & 22.5 \\
\hline 8 & 29.833 & 29.870 & 29.930 & -12.6 & $-\mathrm{I} 3.0$ & -14.0 & -5.0 & -- & 17.0 & -9.0 & -9.0 & -15.0 & - II.0 & 12.0 \\
\hline 9 & 29.996 & 30.011 & 30.044 & -8.0 & $-\mathrm{I} 4.0$ & -4.0 & -7.9 & - & I6.0 & -8.0 & -3.6 & -15.6 & -9.8 & I2. 4 \\
\hline I0 & 30.019 & 29.957 & $29.83 \mathrm{I}$ & +3.0 & +1.0 & -4.2 & +3.5 & - & 4.0 & +3.0 & +3.0 & -4.2 & -0.4 & 7.7 \\
\hline II & 29.560 & 29.466 & 29.328 & +4.0 & +3.4 & +3.5 & +4.0 & - & 7.0 & +4.0 & +4.0 & +2.6 & -1.5 & II.O \\
\hline I 2 & 29.663 & & 29.867 & 0.0 & +2.2 & +5.0 & +3.5 & - & I.O & $+\quad 2.2$ & +5.0 & 0.0 & +2.0 & 6.0 \\
\hline 13 & 29.877 & & 29.950 & +12.0 & +9.0 & + II. & +12.0 & + & 5.0 & +12.9 & +13.1 & +6.0 & +9.0 & 8. I \\
\hline I4 & 29.994 & 29.993 & 29.949 & +9.0 & +10.0 & +16.0 & f. 12.6 & + & 4.0 & +10.0 & +16.0 & +7.0 & +10.0 & 12.0 \\
\hline 15 & 29.836 & 29.832 & 29.848 & +12.8 & +10.4 & +4.0 & +17.2 & + & 12.4 & +12.8 & +13.0 & +4.0 & +10.6 & \\
\hline I6 & 29.909 & 29.910 & 29.871 & -4.0 & -1.6 & +4.0 & +4.0 & - & 5.0 & -1.6 & +4.4 & -4.9 & -0.3 & 9.4 \\
\hline I7 & 29.752 & 29.776 & 29.819 & +6.0 & +6.0 & +2.4 & +9.2 & & 0.0 & +6.1 & +9.5 & +2.2 & +4.8 & 9.5 \\
\hline I8 & $29.8 \mathrm{II}$ & 29.816 & 29.837 & -7.5 & -10.8 & - 10.5 & +5.0 & - & II. 5 & -7.0 & -7.0 & -12.0 & -3.5 & 17.0 \\
\hline I9 & 29.784 & 29.776 & 29.758 & -16.4 & - 10.0 & -9.0 & -10.5 & - & I6.8 & -10.0 & -9.0 & -18.0 & -13.5 & 9.0 \\
\hline 20 & 29.733 & 29.671 & 29.610 & -2.0 & -1.0 & -0.4 & -2.0 & - & 9.0 & - I.0 & -0.4 & -4.0 & -4.7 & 8.6 \\
\hline $2 \mathrm{I}$ & 29.723 & 29.768 & 29.877 & -1.0 & -0.4 & +2.0 & 0.0 & - & 10.6 & +2.8 & +3.0 & -3.0 & -3.8 & 126 \\
\hline 22 & 29.987 & 29.997 & 29.927 & +1.0 & +4.0 & +5.0 & +4.2 & - & I. 9 & +4.0 & +7.6 & -0.4 & +2.8 & 9.5 \\
\hline 23 & 29.819 & 29.749 & 29.791 & +10.0 & +8.0 & +7.6 & +10.0 & + & 3.6 & +10.0 & +10.0 & +6.4 & +6.8 & 6.4 \\
\hline 24 & 29.713 & 29.676 & 29.659 & +8.2 & +10.8 & +7.4 & + IO.I & + & $4 \cdot 3$ & +11.0 & +11.0 & +5.0 & +7.6 & 6.7 \\
\hline 25 & 29.731 & 29.743 & 29.696 & +8.0 & +6.0 & +12.0 & +13.1 & + & 4.2 & +8.0 & +12.0 & +4.0 & +8.6 & . I \\
\hline 26 & 29.589 & 29.526 & 29.445 & +15.6 & +16.4 & +13.0 & +17.2 & + & II. 6 & +17.0 & +19.6 & +12.0 & +15.6 & 8.0 \\
\hline 27 & 29.350 & 29.337 & 29.303 & +6.4 & +6.4 & +11.6 & +16.0 & + & 6.3 & +8.0 & +11.8 & +5.0 & +10.5 & II .O \\
\hline 28 & 29.309 & 29.328 & 29.359 & +16.0 & +16.0 & +14.4 & +16.0 & + & 10.8 & +16.1 & +17.0 & +14.2 & +13.9 & 6.2 \\
\hline 29 & 29.423 & 29.428 & 29.469 & +8.8 & +8.0 & +6.5 & +16.0 & + & 7.9 & +9.0 & +8.8 & +6.5 & +11.2 & 9.5 \\
\hline 30 & 29.533 & 29.557 & 29.620 & +2.0 & +2.2 & +4.0 & +6.5 & & 0.0 & +2.6 & +4.0 & $+\quad 1.5$ & +3.2 & 6.5 \\
\hline $3 I$ & 29.595 & 29.534 & 29.541 & +5.0 & +8.0 & +0.4 & +5.0 & - & 3.0 & +8.1 & +8.0 & 0.0 & +2.6 & II. I \\
\hline Sum.... & 924 . I7 I & $924.04 I$ & 924.014 & +170.2 & +I7I.I & +158.2 & +274.3 & + & 33.7 & +220.6 & +267.5 & +49.0 & +156.0 & 319.6 \\
\hline Mean... & 29.812 & 29.808 & 29.807 & +5.5 & +5.5 & +5.1 & +8.8 & + & I.I & +7.1 & +8.6 & +1.6 & +5.0 & 10.3 \\
\hline
\end{tabular}


Tabulation of daily meteorological observations at Teplitz Bay during the month of October, I903-Continued

Observer: FRANCIS LoNC

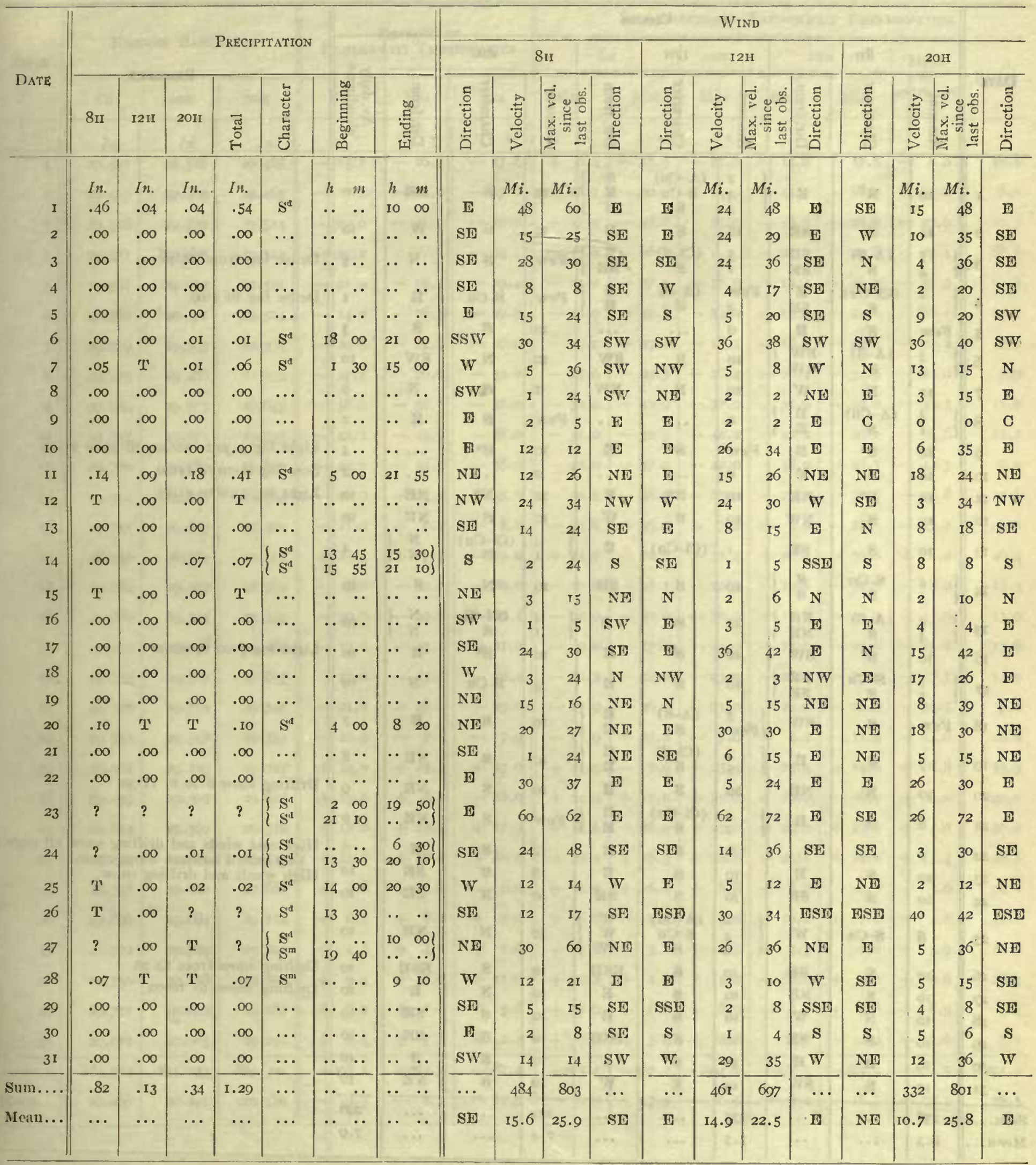


Tabulation of daily metcorological abscrvations at Teplitz Bay during the month of October, Igo3-Continued Observer: Financis Long

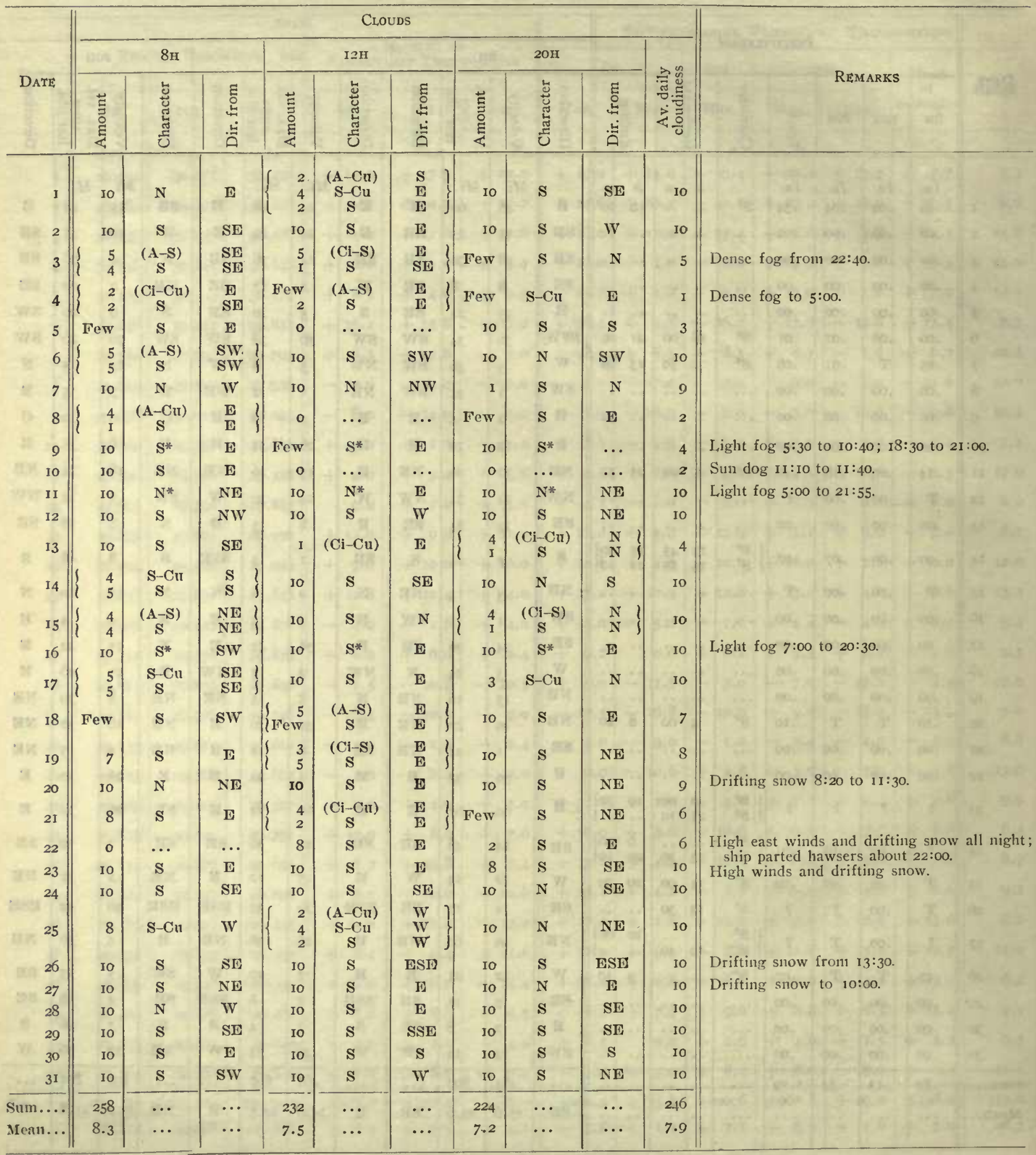


Tabulation of daily metcorological observations at Teplitz Bay during the month of November, 1903 Observer: FRANCIS LONG

\begin{tabular}{|c|c|c|c|c|c|c|c|c|c|c|c|c|c|c|}
\hline \multirow{3}{*}{ DATE } & \multirow{2}{*}{\multicolumn{3}{|c|}{ REDUCÉL BAROMETER }} & \multirow{2}{*}{\multicolumn{4}{|c|}{$\begin{array}{c}\text { READING OF } \\
\text { FAHRENHEIT THERMOMETER }\end{array}$}} & \multicolumn{6}{|c|}{ SELF-REGISTERING Fahrenifeit Therrometers } & \multirow{3}{*}{ Range } \\
\hline & & & & & & & & \multicolumn{2}{|c|}{$8 \mathrm{II}$} & \multirow{2}{*}{$\frac{\text { I2H }}{\text { Max. }}$} & \multicolumn{2}{|c|}{$20 \mathrm{H}$} & \multirow{2}{*}{$\begin{array}{c}\text { Mean } \\
\text { of } \\
\text { extremes }\end{array}$} & \\
\hline & $S_{H}$ & $\mathrm{I} 2 \mathrm{H}$ & $20 I I$ & & 3H & I2II & $2 \mathrm{OH}$ & Max. & Min. & & Max. & Min. & & \\
\hline & In. & In. & In. & & $\cdot$ & & $\circ$ & $=0$ & $\circ$ & - & 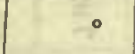 & $\circ$. & $\circ$ & $\circ$ \\
\hline I & $29.57 \mathrm{I}$ & 29.548 & 29.485 & - & 15.0 & -16.6 & -17.8 & +0.4 & $-\mathrm{I} 8.0$ & $-\mathrm{I} 5.0$ & -15.0 & -17.8 & -8.8 & I8.4 \\
\hline 2 & 29.416 & 29.409 & 29.465 & - & 17.2 & - I 4.0 & $-I 7.9$ & $-I 7.1$ & -20.0 & $-\mathrm{I} 4.0$ & -14.0 & -18.2 & -17.0 & 6.0 \\
\hline 3 & 29.528 & 29.530 & 29.514 & -2 & 22.0 & -21.0 & - I9.0 & -17.9 & -28.2 & $-2 \mathrm{I} .0$ & $-\mathrm{x} 8.5$ & -22.0 & -23.0 & = 10.3 \\
\hline 4 & $29.47 \mathrm{I}$ & No obs. & 29.542 & -2 & 23.2 & test & $-24 \cdot 4$ & -17.0 & -26.4 & $\ldots$ & -22.0 & -27.8 & -22.4 & 10.8 \\
\hline 5 & $29.56 \mathrm{r}$ & 29.554 & $29.59 \mathrm{I}$ & -2 & 21.0 & - I8.I & - 19.4 & -21.0 & -27.2 & - I8.0 & -15.4 & -21.0 & $-2 \mathrm{I} \cdot 3$ & II 8 \\
\hline 6 & 29.566 & 29.570 & 29.524 & - & 14.0 & - I6.5 & -20.0 & -10.9 & -10.8 & $-\mathrm{I} 4.0$ & -14.0 & -21.6 & $-\mathrm{I} 6.2$ & 10.7 \\
\hline 7 & 29.329 & 29.303 & 29.334 & -2 & 20.0 & -19.6 & -25.0 & - I9.0 & -23.0 & -18.3 & -18.2 & -26.2 & -22.2 & 8.0 \\
\hline 8 & 29.423 & 29.480 & 29.639 & -2 & $27 \cdot 4$ & -26.6 & -32.0 & -25.0 & -29.3 & -26.4 & -25.4 & -32.9 & -29.0 & 7.9 \\
\hline 9 & 29.680 & 29.673 & 29.645 & -3 & 38.0 & -38.0 & -31.0 & -32.0 & -42.0 & -37.0 & $-3 \mathrm{I} .0$ & -39.0 & -36.5 & II.O \\
\hline Io & 29.657 & 29.674 & 29.733 & - & 42.2 & -43.9 & -46.0 & -31.0 & -42.9 & -42.2 & -42.2 & -46.2 & -38.6 & 15.2 \\
\hline II & 29.780 & 29.789 & 29.820 & - & 42.0 & -44.0 & -38.0 & -42.0 & -47.0 & -42.0 & -38.0 & $-46 . I$ & -42.5 & 9.0 \\
\hline I2 & 29.701 & 29.735 & 29.894 & - & 20.0 & -24.9 & $-35 \cdot 5$ & -20.0 & -38.0 & -20.0 & -20.0 & -39.6 & -29.8 & 19.6 \\
\hline I3 & $29.75 \mathrm{I}$ & 29.663 & 29.309 & - & II. 6 & +7.2 & +8.2 & -10.8 & -36.0 & +7.2 & +9.0 & $-\mathrm{II} .6$ & $-\mathrm{I} 3.5$ & $45 \cdot 0$ \\
\hline I4 & 28.956 & 28.960 & 29.402 & + & 23.5 & +27.4 & - 19.0 & +24.0 & -10.2 & +27.4 & +27.4 & - I9.0 & +4.2 & 46.4 \\
\hline 15 & 29.284 & 29.007 & 28.762 & - & 17.0 & -11.0 & -20.0 & $-\mathrm{x} 6.0$ & -25.1 & - то. 8 & -10.8 & -20.0 & -18.0 & $14 \cdot 3$ \\
\hline I6 & 29.060 & 29.245 & 29.339 & - & 30.0 & -30.0 & -17.4 & -20.0 & -30.0 & -30.0 & -17.4 & -30.4 & -23.9 & 13.0 \\
\hline I7 & 29.216 & 29.198 & 29.280 & - & I3.6 & -13.1 & $-2 I .2$ & -9.0 & -17.4 & -13.0 & -13.0 & -22.6 & -15.8 & I3. 6 \\
\hline I8 & 29.536 & 29.567 & 29.657 & - & 27.0 & -23.0 & -22.4 & -21.2 & -34.2 & -23.0 & -22.4 & -27.0 & -27.7 & 13.0 \\
\hline 19 & 29.726 & 29.750 & 29.745 & - & 21.0 & -28.0 & -25.0 & -21.0 & $-26 . I$ & -21.0 & -21.0 & -28.0 & -24.5 & 7.0 \\
\hline 20 & 29.642 & 29.598 & 29.353 & - & II. 9 & -7.6 & -7.9 & - II.9 & -25.0 & -7.6 & -4.2 & - II.9 & -14.6 & 20.8 \\
\hline 2I & 29.062 & 29.123 & 29. I97 & + & I4.0 & +25.0 & +23.0 & +14.0 & -7.9 & +25.4 & +25.4 & +14.0 & +8.8 & $33 \cdot 3$ \\
\hline 22 & 29.093 & 29.303 & 29.613 & + & 19.0 & +0.5 & -16.0 & +24.0 & +17.0 & +19.0 & +19.0 & -16.0 & +4.0 & 40.0 \\
\hline 23 & $29.6 \mathrm{I}_{4}$ & 29.560 & 29.516 & + & 18.0 & +19.5 & +17.9 & +18.0 & -16.0 & +20.0 & +20.0 & +16.0 & +2.0 & 36.0 \\
\hline 24 & 29.497 & 29.536 & 29.535 & + & 19.5 & +18.0 & +10.4 & $+20 . I$ & +16.5 & +20.8 & +20.8 & +10.0 & +15.4 & Io. 8 \\
\hline 25 & 29.557 & 29.600 & $29.66 \mathrm{I}$ & + & 6.8 & +3.0 & 0.0 & +10.0 & +6.8 & +6.8 & +6.8 & 0.0 & +5.0 & I0.0 \\
\hline 26 & 29.609 & 29.684 & 29.714 & + & 6.5 & +8.2 & +9.0 & +6.8 & -2.5 & +9.0 & + I0. I & +5.0 & +3.8 & 12.6 \\
\hline 27 & 29.772 & 29.709 & 29.702 & + & $4 \cdot 4$ & +4.0 & +1.0 & $+\mathrm{II} .8$ & +3.1 & +4.5 & +4.5 & 0.0 & +5.9 & II. 8 \\
\hline 28 & 29.730 & 29.758 & 29.810 & + & 9.4 & $+\mathrm{I} 5.0$ & + Ir.o & +10.2 & $+\quad 1.6$ & +15.2 & +18.0 & +9.0 & +9.8 & I6. 4 \\
\hline 29 & $29.7 \mathrm{II}$ & 29.642 & 29.282 & + & 6.2 & +7.6 & +5.6 & + II.o & +6.0 & +8.0 & +8.0 & +5.6 & +8.3 & $5 \cdot 4$ \\
\hline 30 & 29.075 & 29.065 & $20.24 \mathrm{I}$ & + & 8.5 & +7.2 & +2.4 & +9.1 & +3.8 & +8.8 & +8.8 & +2.0 & +5.6 & $7 . I$ \\
\hline 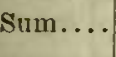 & $884 \cdot 574$ & 855.233 & 885.304 & -2 & 298.3 & $-253 \cdot 3$ & -387.4 & -203.4 & -537.4 & -201.2 & -185.7 & $-483 \cdot 3$ & -372.5 & .495 .2 \\
\hline & 29.486 & 29.491 & 29.510 & - & 9.9 & -8.7 & -12.9 & -6.8 & -17.9 & -6.9 & -6.2 & $-\mathrm{I} 6 . \mathrm{I}$ & -12.4 & 16.5 \\
\hline
\end{tabular}


Tabulation of daily metcorological observations at Teplitz Bay during the month of November, I903-Continued

Observer: FRANCIS LoNG

\begin{tabular}{|c|c|c|c|c|c|c|c|c|c|c|c|c|c|c|c|c|c|c|c|c|c|}
\hline \multirow{3}{*}{ DATE } & \multirow{2}{*}{\multicolumn{9}{|c|}{ PrecritTATION }} & \multicolumn{12}{|c|}{ Wind } \\
\hline & & & & & & & & & & \multirow[b]{2}{*}{ } & \multicolumn{3}{|c|}{$8 \mathrm{H}$} & \multicolumn{4}{|c|}{$12 \mathrm{H}$} & \multirow[b]{2}{*}{ 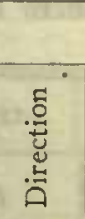 } & \multicolumn{2}{|c|}{$2 \mathrm{OH}$} & \\
\hline & \multirow{2}{*}{$\begin{array}{l}8 \mathrm{II} \\
I n . \\
.00\end{array}$} & \multirow{2}{*}{$\begin{array}{l}\text { I2II } \\
\text { In } \\
.00\end{array}$} & \multirow{2}{*}{\begin{tabular}{|l}
$20 \mathrm{H}$ \\
In. \\
.00
\end{tabular}} & \multirow{2}{*}{$\begin{array}{l}\text { 吾 } \\
\text { Fi } \\
\text { In. } \\
.00\end{array}$} & 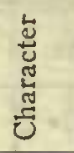 & \multicolumn{2}{|c|}{. } & \multicolumn{2}{|c|}{ 苞 } & & 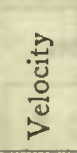 & 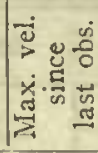 & 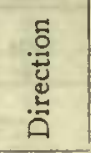 & 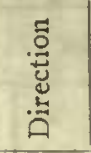 & $\begin{array}{l}\frac{3}{0} \\
\frac{5}{0} \\
3\end{array}$ & 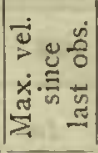 & & & 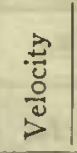 & 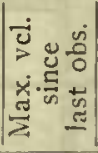 & 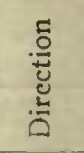 \\
\hline I & & & & & $\ldots$ & $\begin{array}{l}h \\
. .\end{array}$ & $\begin{array}{l}m \\
. .\end{array}$ & $\begin{array}{l}h \\
. .\end{array}$ & $\begin{array}{l}m \\
\ldots\end{array}$ & $N$ & $\begin{array}{r}M i . \\
8\end{array}$ & $\begin{array}{r}M i . \\
\quad 12\end{array}$ & $\mathrm{~N}$ & NW & $\begin{array}{r}M i . \\
5\end{array}$ & $\begin{array}{r}M i . \\
8\end{array}$ & NW & NW & $\begin{array}{r}M i . \\
\text { I2 }\end{array}$ & $\begin{array}{r}M i . \\
\text { I5 }\end{array}$ & NW \\
\hline 2 & .00 & .00 &.$\infty$ & .00 & .. & .. & .. & .. & . & NE & 5 & 15 & NE & NE & 12 & .14 & NE & $\mathrm{NE}$ & IO & 20 & NE \\
\hline 3 & .00 & .00 & .00 &.$\infty$ & ... & .. & .. & .. & .. & NE & 10 & 12 & NE & NE & 8 & 10 & ND & NW & 5 & I5 & $\mathrm{NW}$ \\
\hline 4 & .00 &.$\infty$ & .00 &.$\infty$ & .. & .. & . & .. & . & $N W$ & 5 & I5 & NW & $\ldots$ & ... & $\ldots$ & $\cdots$ & $\mathbf{N}^{\top}$ & 4 & 12 & $N$ \\
\hline 5 & .00 & .00 & .00 & .00 & .. & .. & . & .. & .. & t8 & I & I4 & $N$ & SE & 2 & 3 & E & w & 5 & 12 & W \\
\hline 6 &.$\infty$ & .00 &.$\infty$ & .00 & .. & .. & .. & .. & .. & NW & I & 12 & NW & $\mathrm{NW}$ & 3 & 6 & $\mathrm{NW}$ & $\mathrm{NW}$ & 5 & 6 & NIV \\
\hline 7 & .00 & .00 & .00 & .00 & $\cdots$ & .. & .. & .. & . & ND & 3 & 8 & NE & NE & 5 & 5 & NE & $\mathrm{NW}$ & 2 & 6 & NW \\
\hline 8 &.$\infty 0$ & .00 & .00 &.$\infty$ & $\cdots$ & .. & .. & .. & .. & NE & 2 & 6 & NE & NE & 8 & 10 & $\mathrm{NE}$ & $\mathbf{E}$ & 3 & I0 & E \\
\hline 9 & .00 & .00 & .00 & .00 & $\cdots$ & .. & 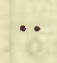 & .. & .. & II & I & 3 & D & NE & I & 2 & $\mathrm{NE}$ & $\mathbf{E}$ & I & 4 & E \\
\hline &.$\infty$ & .00 & .00 & .00 & $\cdots$ & . & 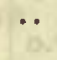 & .. & .. & NE & 5 & 8 & $\mathrm{NE}$ & $\mathrm{E}$ & 6 & 12 & $\mathbf{E}$ & E & 3 & I5 & E \\
\hline II & .00 & .00 & .00 & .00 & $\cdots$ & . & . & . & . & $\mathbf{E}$ & 2 & 4 & $\mathbf{E}$ & NE & 2 & 2 & E & SE & 4 & 4 & $\mathrm{SE}$ \\
\hline I2 & .00 & .00 & .00 & .00 & $\ldots$ & .. & .. & .. & .. & SSW & 12 & I6 & SSW & W & 5 & 12 & SSW & D & 4 & 15 & SSW \\
\hline 13 &.$\infty$ &.$\infty$ & .00 &.$\infty$ & $\ldots$ & $\ddot{0}$ & $\because$ & . & 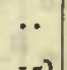 & SE & 32 & 36 & SE & SSE & 30 & 42 & SSD & s & 48 & 52 & $\mathbf{S}$ \\
\hline 14 &.$\infty$ & .01 & $T$ & .OI & $\begin{array}{l}\mathbf{R}^{\mathrm{m}} \\
\mathbf{S}^{\mathrm{d}}\end{array}$ & $\begin{array}{r}8 \\
9 \\
16\end{array}$ & $\begin{array}{l}40 \\
15 \\
00\end{array}$ & $\begin{array}{r}9 \\
12 \\
20\end{array}$ & $\left.\begin{array}{l}15 \\
10 \\
25\end{array}\right\}$ & $\mathrm{s}$ & 10 & 52 & $\mathbf{s}$ & SW & I0 & I2 & s & $W$ & 22 & 26 & W \\
\hline & $T$ & $T$ & .14 & .14 & $\begin{array}{l}\mathbb{S}^{d} \\
\mathbf{S}^{a}\end{array}$ & $\begin{array}{r}7 \\
12\end{array}$ & $\begin{array}{l}\infty \\
30\end{array}$ & $\begin{array}{r}8 \\
17\end{array}$ & $\begin{array}{l}50 l \\
301\end{array}$ & $\mathrm{NE}$ & 2 & 20 & NE & ESE & 29 & $3 I$ & $\mathbf{E}$ & $\mathbf{N}$ & 14 & 32 & $\mathrm{~N}$ \\
\hline 16 & .00 & .00 & .00 &.$\infty 0$ & $\ldots$ & .. & 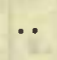 & .. & . & $\mathrm{N}$ & 20 & 27 & $\mathbf{N}$ & W & 15 & 20 & W & SW & 10 & 20 & WV \\
\hline 17 & .00 & $T$ & $T$ & $T$ & $\mathrm{~S}^{4}$ & $I$ & $\infty$ & 4 & 20 & W & 10 & 28 & $\mathrm{~W}$ & W & 10 & I5 & W & $\mathrm{NE}$ & 9 & 12 & $\mathrm{~N}$ \\
\hline I8 & .00 & .00 & .00 & .00 & $\cdots$ & .. & . & .. & . & $\mathrm{NE}$ & 5 & 12 & NW & W & 5 & 8 & $W$ & SE & 4 & 8 & SE \\
\hline 19 & .00 & .00 &.$\infty$ & .00 & ... & .. & .. & .. & . & SW & 5 & Io & STV & SE & 3 & 5 & $\mathbf{s}$ & SE & 8 & 8 & SE \\
\hline &.$\infty$ & .00 & .12 & .12 & $S^{a}$ & 13 & 40 & .. & . & s & 5 & Io & $\mathrm{s}$ & $\mathrm{SE}$ & I5 & I6 & SE & SSID & 36 & 42 & SSE \\
\hline 2I & .15 & $.0 I$ & . 10 & .26 & $\begin{array}{l}\mathbb{S}^{d} \\
\mathbf{S}^{d}\end{array}$ & 10 & $\ddot{\infty}$ & $\begin{array}{l}3 \\
.\end{array}$ & $\begin{array}{c}\text { ol } \\
. .1\end{array}$ & $\mathrm{~s}$ & 24 & 52 & $\mathrm{~s}$ & $\mathrm{~s}$ & 23 & 30 & $s$ & $\mathrm{~s}$ & I5 & 28 & $\mathrm{~S}$ \\
\hline 22 & .16 & .03 & .00 & . I9 & $\mathrm{s}^{\mathrm{m}}$ & .. & . & 10 & 20 & SSE & 20 & 52 & SSE & SW & 20 & 42 & SW & $N$ & 7 & 42 & SWV \\
\hline 23 & .04 & .15 & .02 & $.2 \mathrm{I}$ & $\mathrm{S}^{\mathrm{m}}$ & 3 & 30 & I6 & I 5 & ESE & 28 & 35 & FSE & ESE & 30 & 39 & ESE & 8 & 33 & 48 & SE \\
\hline 24 & .00 & .00 &.$\infty$ & .00 & $\mathrm{~s}^{\mathrm{m}}$ & 20 & 35 & .. & . & ESE & 24 & 48 & ESE & ESE & 24 & 29 & SD & EST & 36 & 46 & ESE \\
\hline & .15 & .00 & .00 & .15 & $\mathrm{~S}^{\mathrm{m}}$ & .. & .. & IO & $\infty$ & ESE & 8 & 60 & ESD & W. & 8 & 38 & SD & $\mathbf{N}$ & 36 & 38 & SE \\
\hline 25 &.$\infty$ &.$\infty$ & .00 &.$\infty 0$ & $\ldots$ & .. & .. & .. & . & E & 35 & 58 & $\mathbf{E}$ & $\mathbf{E}$ & 36 & 48 & E & SE & 40 & 56 & SE \\
\hline & .00 & .00 &.$\infty$ & .00 & $\ldots$ & .. & .. & .. & .. & SE & 15 & 48 & $\mathrm{SE}$ & E & 48 & 49 & D & SD & 27 & 60 & SE \\
\hline 28 & .00 &.$\infty 0$ &.$\infty 0$ &.$\infty$ & $\ldots$ & .. & .. & .. & . & SE & 30 & 48 & SE & $\mathbf{E}$ & 35 & 35 & $\mathbf{E}$ & SE & 36 & 48 & SE \\
\hline 29 &.$\infty$ &.$\infty$ & .04 & .04 & $\mathbf{S}^{\mathrm{m}}$ & 17 & IO & 22 & 30 & ESE & 48 & 56 & ESE & SE & 60 & 60 & SE & ESE & 60 & 66 & ESE \\
\hline 30 & . Io & .03 & .02 & .15 & $S^{t}$ & 3 & 30 & I6 & I0 & SE & 36 & 76 & DSD & $\mathrm{ND}$ & Iо & 15 & ND & W & 23 & 23 & $w$ \\
\hline Sum.... & .60 & .23 & .44 & 1.27 & $\ldots$ & .. & .. & .. & .. & $\ldots$ & $4 \mathrm{I} 3$ & 853 & $\cdots$ & ... & 468 & 618 & $\ldots$ & $\ldots$ & 522 & 789 & $\ldots$ \\
\hline ean.. & $\cdots$ & $\cdots$ & $\cdots$ & $\cdots$ & $\ldots$ & .. & .. & & .. & NE & 13.8 & 28.4 & NE & NE & I6. I & 21.3 & E & S⿴囗十 & 17.4 & 26.3 & SE \\
\hline
\end{tabular}


Tabulation of daily meteorological observations at Teplitz Bay during the month of November, 1903-Continued Observer: FRANCIS LONG

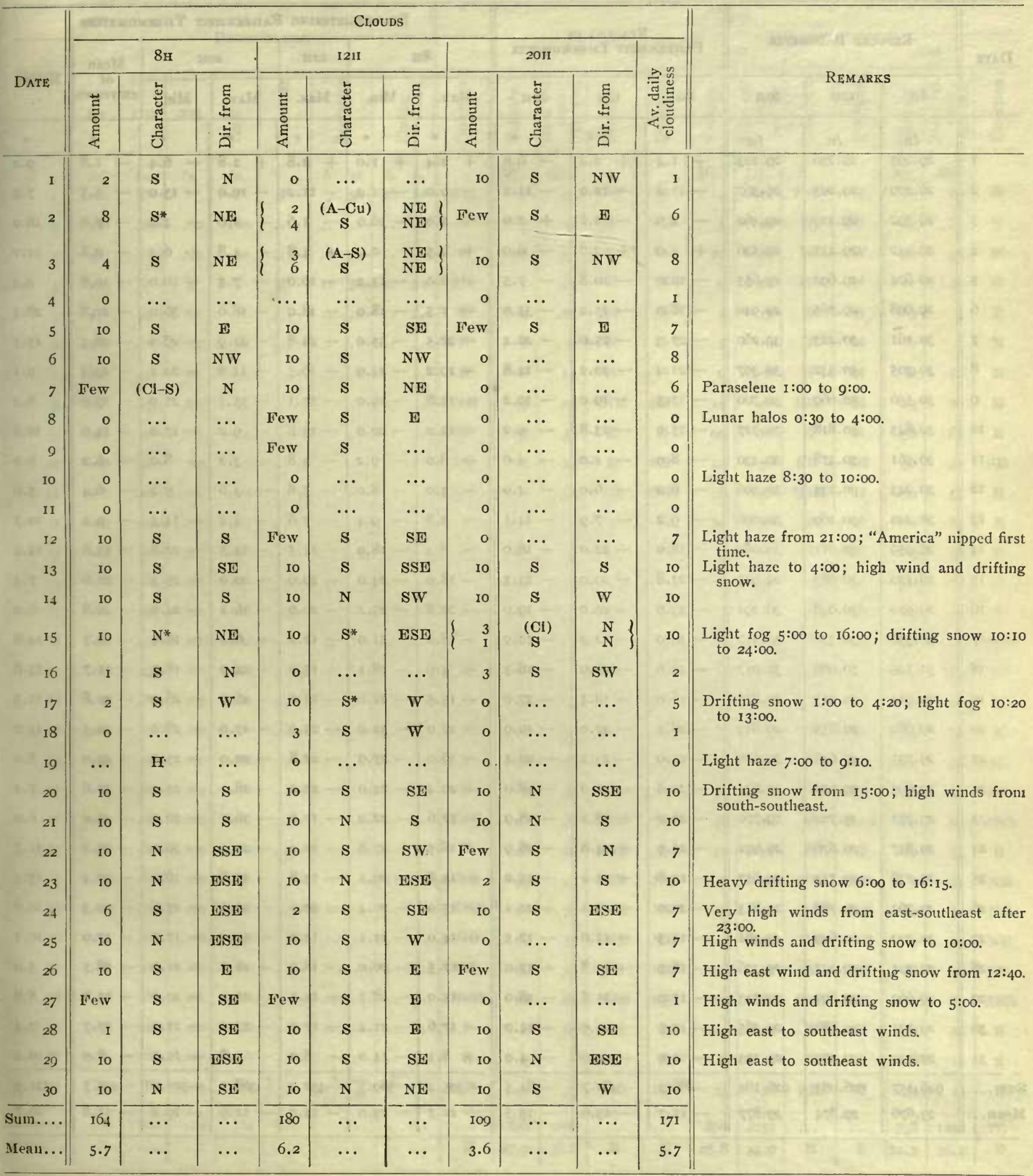


Tabulation of daily meteorological obscrvations at Teplitz Bay during the month of December, 1903 Observer: FRANCIS LONG

\begin{tabular}{|c|c|c|c|c|c|c|c|c|c|c|c|c|c|c|}
\hline \multirow{3}{*}{ DATE } & \multirow{2}{*}{\multicolumn{3}{|c|}{ REDUCED BAROMETER }} & \multirow{2}{*}{\multicolumn{3}{|c|}{$\begin{array}{c}\text { READING OF } \\
\text { FAHRENHEIT THE,RMOMETER }\end{array}$}} & \multicolumn{8}{|c|}{ SELF-REGISTERING FAHRENHEIT THERMIOMETERS } \\
\hline & & & & & & & \multicolumn{3}{|c|}{$8 \mathrm{H}$} & \multirow{2}{*}{$\frac{12 \mathrm{H}}{\operatorname{Max} .}$} & \multicolumn{2}{|c|}{$20 \mathrm{H}$} & \multirow{2}{*}{$\begin{array}{l}\text { Mean } \\
\text { of } \\
\text { extremes }\end{array}$} & \multirow{2}{*}{ Range } \\
\hline & $8 \mathrm{HI}$ & I2II & 2011 & $8 \mathrm{rH}$ & I $2 \mathrm{II}$ & 2011 & $\mathrm{Ma}$ & ax. & Min. & & Max. & Min. & & \\
\hline & In. & $I n$. & In. & $1^{\circ}$ & $n^{\circ}$ & 30 & & $\bullet$ & $\circ$ & - & $\cdot$ & $\circ$ & - & $\circ$ \\
\hline I & 29.295 & 29.236 & 29.223 & +1.4 & +2.2 & -6.0 & + & 2.4 & +1.0 & +2.8 & +2.8 & -6.4 & -1.8 & 9.2 \\
\hline 2 & 29.279 & 29.293 & 29.367 & -11.2 & -12.0 & -11.2 & - & 6.0 & -11.2 & - II.2 & - I0.0 & -13.0 & 9.5 & 7.0 \\
\hline 3 & 29.392 & 29.429 & 29.462 & -2.0 & -2.2 & +2.0 & - & 2.0 & -16.0 & $-\quad 1.6$ & +2.0 & -4.0 & 7.0 & 18.0 \\
\hline 4 & 29.442 & 29.455 & 29.536 & +4.0 & +2.0 & -6.0 & + & $4 \cdot 5$ & 0.0 & +4.8 & +4.8 & -6.4 & -0.8 & II . 2 \\
\hline 5 & 29.674 & 29.692 & 29.655 & - 10.0 & - 10.8 & -7.5 & - & 6.0 & -15.2 & - I0.0 & -7.5 & - 10.0 & -10.6 & 9.2 \\
\hline 6 & 29.698 & 29.765 & 29.940 & -18.0 & -25.2 & $-35 \cdot 0$ & - & 7.5 & -18.0 & -18.0 & -18.0 & -36.0 & -21.8 & 28.5 \\
\hline 7 & $30.16 \mathrm{I}$ & 30.223 & 30.260 & -27.5 & -25.0 & -22.4 & -2 & 27.5 & -35.0 & -24.8 & -21.9 & -29.0 & -28.4 & I3.I \\
\hline 8 & 30.305 & 30.332 & 30.397 & -21.2 & -20.2 & -14.8 & -1 & 19.2 & $-23 \cdot 9$ & -19.5 & -14.8 & -22.1 & -19.4 & 9.1 \\
\hline 9 & 30.556 & 30.669 & 30.700 & -17.5 & -19.0 & -19.2 & -1 & 14.8 & -19.0 & - I5.I & - I5.I & -21.0 & -17.9 & 6.2 \\
\hline 10 & 30.845 & 30.818 & 30.727 & -15.0 & $-\mathrm{I} 3.8$ & -9.2 & -1 & 12.2 & -20.0 & -13.8 & -9.2 & -17.0 & -14.6 & 10.8 \\
\hline II & $30.56 \mathrm{I}$ & 30.478 & 30.430 & -8.0 & -4.0 & -4.0 & - & 8.0 & -9.2 & -3.8 & -3.2 & -8.0 & 6.2 & 6.0 \\
\hline 12 & 30.243 & 30.235 & 30.306 & -6.0 & -6.0 & -4.0 & - & 4.0 & -8.0 & $--\quad 5.8$ & -3.6 & -9.2 & 6.4 & 5.6 \\
\hline 13 & 30.240 & 30.169 & 30.056 & -9.2 & -7.9 & -14.5 & - & 3.8 & -9.4 & -7.6 & -4.2 & -14.5 & 9.2 & 10.7 \\
\hline 14 & 29.959 & 30.011 & 30.089 & $-\mathrm{I} 8.0$ & -22.0 & -18.0 & 一 & 8.4 & $-\mathrm{I} 8.0$ & -14.5 & -14.5 & -22.8 & -15.6 & $\mathrm{I} 4.4$ \\
\hline 15 & 30.130 & 30.083 & 29.945 & -23.8 & -20.0 & -23.5 & -1 & 18.0 & -24.0 & -20.0 & -20.0 & -25.3 & -21.6 & $7 \cdot 3$ \\
\hline 16 & 29.970 & 30.048 & 30.292 & -23.0 & -20.0 & -19.0 & -2 & 22.8 & $-25 \cdot 4$ & -20.0 & -16.2 & -24.0 & -20.8 & 9.2 \\
\hline 17 & 30.330 & 30.356 & 30.317 & -18.0 & -12.2 & -10.9 & -1 & 18.0 & -21.0 & -12.2 & -6.4 & -19.0 & -13.7 & I 4.6 \\
\hline 18 & 30.125 & 30.058 & 30.017 & -16.0 & - 16.0 & -18.5 & - & 4.9 & $-18 . I$ & - I5.9 & -14.9 & -18.5 & 11.7 & I3.6 \\
\hline 19 & 29.914 & 29.911 & 29.875 & -16.0 & -18.4 & -27.0 & -1 & 15.5 & -21.0 & -16.0 & -16.0 & -28.0 & 21.8 & 12.5 \\
\hline 20 & 29.862 & 29.855 & 29.849 & -28.4 & -27.0 & -19.0 & -2 & 27.0 & -32.0 & --23.0 & -17.0 & -28.0 & 24.5 & 15.0 \\
\hline $2 I$ & 29.735 & 29.646 & 29.628 & -24.0 & -23.2 & -20.4 & -1 & 19.0 & $=-27.0$ & -22.8 & -20.0 & -25.0 & 23.0 & 8.0 \\
\hline$z 2$ & 29.695 & 29.670 & 29.644 & -23.8 & -22.0 & -18.0 & -2 & 20.4 & -24.0 & -22.0 & -18.0 & -25.2 & -21.6 & 7.2 \\
\hline 23 & 29.732 & 29.724 & 29.770 & -20.0 & -18.2 & -18.9 & -1 & 17.6 & -22.0 & -17.0 & -16.0 & -22.0 & 19.0 & 6.0 \\
\hline 24 & 29.847 & 29.878 & $29.93 \mathrm{I}$ & --25.0 & -24.0 & -28.9 & $-I$ & 18.5 & -27.8 & -20.4 & -20.4 & -30.0 & -24.2 & II. 5 \\
\hline 25 & 29.738 & 29.713 & 29.657 & -13.8 & -14.0 & -15.0 & $-I$ & I3.8 & -29.1 & -13.8 & -13.8 & -16.0 & $-2 \mathrm{I} .4$ & $\mathrm{I} 5 \cdot 3$ \\
\hline 26 & 29.763 & 29.788 & 29.813 & -20.0 & -23.2 & $-22 . I$ & -1 & 15.0 & -20.4 & -20.0 & -20.0 & -25.6 & -20.3 & 10.6 \\
\hline 27 & $29 \cdot 722$ & 29.649 & 29.461 & -14.5 & - I 5.0 & -17.5 & - I & 14.0 & $-22 . I$ & . 12.0 & -12.0 & -17.5 & -17.0 & IO. I \\
\hline 28 & 29.221 & 29.221 & $29 \cdot 336$ & -18.9 & - Ig.8 & -17.0 & $-I$ & 17.5 & -20.0 & $\ldots$ - 18.9 & -16.0 & $-2 I .0$ & $-\mathrm{I} 8.5$ & 5.0 \\
\hline 29 & 29.663 & 29.692 & 29.743 & -15.0 & - I5.8 & -18.0 & $--I$ & $\mathrm{I} 5.0$ & -18.5 & -14.5 & $-\mathrm{II} .2$ & -20.0 & -15.6 & 8.8 \\
\hline 30 & 29.721 & 29.685 & 29.565 & -17.9 & -20.0 & -14.0 & - I & 17.6 & -21.2 & -17.9 & -14.0 & $-2 \pi .4$ & -17.7 & 7.4 \\
\hline $3 I$ & 29.339 & 29.290 & 29.193 & -10.0 & - 10.0 & -4.0 & - & 8.1 & -14.0 & -8.0 & -3.8 & - 10.0 & -8.9 & 10.2 \\
\hline Sum... & 926.157 & 925.083 & 926.184 & -486.3 & -482.7 & $-48 \mathrm{r} \cdot 5$ & -39 & 95.2 & -589.5 & -432.5 & -368.1 & -595.9 & -490.5 & $33 \mathrm{r} \cdot 3$ \\
\hline Mean... & 29.876 & 29.874 & 29.877 & -15.7 & -15.6 & -15.5 & $-I$ & 12.7 & -19.0 & $-\mathrm{I} 4.0$ & -11.9 & - 19.2 & -15.8 & 10.7 \\
\hline
\end{tabular}


Tabulation of daily metearalogical abservations at Teplitz Bay during the month of December, I903-Continued

Observer: Francis Long

\begin{tabular}{|c|c|c|c|c|c|c|c|c|c|c|c|c|c|c|c|c|c|c|c|}
\hline \multirow[b]{3}{*}{ DATE } & \multirow{2}{*}{\multicolumn{7}{|c|}{ PrECIPITATTON }} & \multicolumn{12}{|c|}{ W WIND } \\
\hline & & & & & & & & \multicolumn{4}{|c|}{$8 \mathrm{H}$} & \multicolumn{4}{|c|}{$121 \mathrm{I}$} & \multicolumn{4}{|c|}{$2 \mathrm{OH}$} \\
\hline & $8 \pi$ & $12 \mathrm{H}$ & $201 \mathrm{I}$ & 胥 & 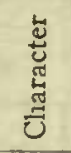 & 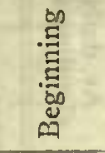 & 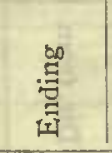 & 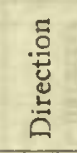 & 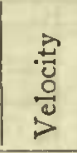 & 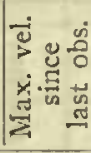 & 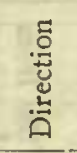 & 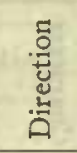 & $\begin{array}{l}\frac{\vec{G}}{0} \\
\frac{0}{0} \\
\end{array}$ & 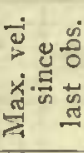 & 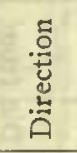 & 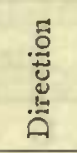 & $\frac{3}{30}$ & 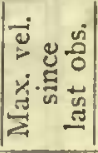 & \\
\hline & In. & In & In. & In. & (a) & $h \quad m$ & $h \quad m$ & & Mi. & Mi. & & & Mi. & Mi. & & & Mi. & Mi. & \\
\hline I & .00 & $\mathrm{~T}$ & 'T & $\mathrm{T}$ & $S^{d}$ & & 1610 & SW & 22 & 30 & SW & W & I7 & 24 & SW & W & 15 & 24 & W \\
\hline 2 &.$\infty$ &.$\infty$ & .00 & .00 & $\cdots$ & .. $\quad$. & .. $\quad$. & W & 5 & 20 & W & NIV & 5 & 10 & w & W & 15 & 15 & W \\
\hline 3 &.$\infty 0$ &.$\infty$ &.$\infty$ &.$\infty$ & $\ldots$ & .. $\quad .$. & & W & 30 & 35 & W & $w$ & 20 & 36 & WV & W & 20 & 36 & W \\
\hline 4 &.$\infty$ & $T$ & $\mathrm{~T}$ & $\mathrm{~T}$ & $S^{\prime 1}$ & II 50 & 1230 & Sw & I4 & 23 & W & W & 8 & 20 & W & $\mathrm{N}$ & II & 20 & w \\
\hline 5 & .00 &.$\infty$ & .00 & .00 & $\ldots$ & . $\quad \cdots$ & .. $\quad .$. & E & 4 & 15 & $\mathrm{~N}$ & D & 2 & 3 & $\mathbf{E}$ & $\mathrm{s}$ & 3 & 5 & $\mathrm{E}$ \\
\hline 6 &.$\infty$ &.$\infty$ &.$\infty 0$ &.$\infty$ & $\ldots$ & .. $\quad .$. & $\begin{array}{ll}. . & . .\end{array}$ & N & 8 & 9 & $\mathbf{N}$ & $\mathbf{N}$ & 5 & 6 & $\mathrm{~N}$ & $\mathbf{N}$ & 6 & 7 & $\mathrm{~N}$ \\
\hline 7 & .00 &.$\infty 0$ & .00 & .00 & $\ldots$ & .. $\quad$. & .. $\quad .$. & N & 6 & 12 & $N$ & C & 0 & 8 & $\mathrm{~N}$ & $\mathrm{~N}$ & 8 & I8 & $\mathrm{N}$ \\
\hline 8 & .00 & .00 & .00 & .00 & $\cdots$ & .. $\quad$. & .. $\quad$. & NE & 5 & 27 & $\mathrm{NL}$ & $\mathrm{SE}$ & 15 & 20 & SE & $\mathbf{E}$ & I5 & 20 & $\mathbf{E}$ \\
\hline 9 & .00 &.$\infty$ & .00 & .00 & ... & .. $\quad .$. & .. $\quad$. & $\mathrm{SE}$ & 38 & 48 & E & E & 60 & 60 & E & E & 58 & 60 & E \\
\hline 10 & .00 &.$\infty 0$ & .00 &.$\infty 0$ & $\ldots$ & .. $\quad$. & .. $\quad .$. & SE & 12 & 53 & $\mathrm{E}$ & SE & 24 & 36 & SE & SE & 36 & 42 & SE \\
\hline II & .00 & .00 & .00 & .00 & $\ldots$ & .... & .. $\quad$. & $\mathrm{SE}$ & 38 & 48 & SE & ESA & 48 & 52 & ESE & $\mathbf{E}$ & 39 & 54 & E \\
\hline 12 & .00 &.$\infty$ & .00 & .00 & $\ldots$ & .. $\quad .$. & .. $\quad .$. & If & 70 & 72 & E & $E$ & 72 & 72 & E & E & 50 & 84 & E \\
\hline 13 & .00 & .00 &.$\infty$ & .00 & $\ldots$ & $\begin{array}{ll}. . & . .\end{array}$ & .. $\quad .$. & I: & 5 & 36 & E & $\mathbf{E}$ & 10 & 15 & $\mathrm{E}$ & E & 24 & 28 & E \\
\hline 14 &.$\infty$ & .00 & .00 & .00 & $\ldots$ & .. $\quad .$. & .. $\quad .$. & $\mathrm{E}$ & 34 & 42 & E & $\mathrm{E}$ & 24 & 45 & $\mathrm{E}$ & $\mathbf{E}$ & 36 & 38 & E \\
\hline 15 & .00 &.$\infty$ &.$\infty$ & .00 & $\ldots$ & $\begin{array}{ll}. . & . .\end{array}$ & .. $\quad$. & 14 & 33 & 36 & $\mathrm{E}$ & $\mathbf{E}$ & 15 & 35 & $\mathrm{E}$ & E & 35 & 42 & $\mathbf{E}$ \\
\hline 16 & .00 &.$\infty 0$ & .00 & .00 & $\ldots$ & $\begin{array}{ll}. . & . \cdot\end{array}$ & .. $\quad .$. & E & 62 & 72 & E & SE & 62 & 72 & $\mathrm{SE}$ & W & I 2 & 72 & ESE \\
\hline 17 & .00 & .00 & .00 & .00 & ... & .. $\quad .$. & .. $\quad .$. & ESE & 60 & 60 & ESE & ESD & 48 & 60 & SE & SE & 20 & 60 & SE \\
\hline 18 &.$\infty$ & .00 & .00 &.$\infty$ & $\ldots$ & .. $\quad .$. & .. $\quad .$. & E & 36 & 36 & E & SW & 23 & 48 & E & s & 4 & 48 & $\mathbf{E}$ \\
\hline 19 & .00 & .00 & .00 & .00 & $\ldots$ & .. $\quad .$. & .. & I & 22 & 24 & E & $\mathrm{s}$ & 14 & 24 & $\mathbf{E}$ & E & 32 & 48 & 团 \\
\hline 20 & .00 &.$\infty$ & .00 & .00 & $\ldots$ & .. $\quad .$. & .. $\quad .$. & $\mathbf{E}$ & 48 & $50^{\circ}$ & $\mathbb{E}$ & E & 48 & 48 & $\mathbf{E}$ & $\mathrm{SE}$ & 25 & 58 & SE \\
\hline $2 \mathrm{I}$ & .00 &.$\infty 0$ &.$\infty$ &.$\infty 0$ & $\ldots$ & $\ldots \quad \cdots$ & $\begin{array}{ll}. . & . .\end{array}$ & II & 8 & 30 & E & NE & 2 & 10 & NE & SE & 15 & 19 & SE \\
\hline 22 & .00 &.$\infty 0$ & .00 & .00 & $\ldots$ & 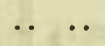 & .. $\quad .$. & ESE & 46 & 46 & ESE & $\mathrm{E}$ & 48 & 50 & E & ESE & 60 & 70 & ESE \\
\hline 23 &.$\infty 0$ &.$\infty$ &.$\infty$ & .00 & $\ldots$ & .. $\quad .$. & .. $\quad .$. & $\mathrm{E}$ & 46 & 66 & ESE & E & 42 & 54 & $\mathbf{E}$ & $\mathrm{E}$ & I2 & 54 & $\mathrm{E}$ \\
\hline 24 & .00 & .00 & .00 & .00 & $\ldots$ & .. $\quad .$. & .. $\quad .$. & $\mathrm{NE}$ & 23 & 25 & $\mathrm{Ne}$ & NE & 5 & 20 & $\mathrm{NE}$ & NE & 5 & 41 & NE \\
\hline 25 & .00 & .00 & .00 & .00 & $\ldots$ & $\begin{array}{ll}. . & . .\end{array}$ & . $\quad .$. & NNE & 17 & 36 & $\mathrm{NE}$ & NE & 28 & 32 & $\mathrm{NE}$ & NE & 30 & 32 & $\mathrm{NE}$ \\
\hline 26 &.$\infty$ &.$\infty$ & .00 &.$\infty$ & ... & .. $\quad .$. & $\begin{array}{ll}. . & . .\end{array}$ & $\mathrm{NE}$ & 14 & 33 & NE & $\mathrm{NE}$ & 4 & 15 & NE & $\mathrm{NE}$ & 5 & I5 & $\mathrm{NE}$ \\
\hline 27 &.$\infty$ &.$\infty$ & .00 &.$\infty$ & $\ldots$ & .. $\quad .$. & .. .. & SE & 23 & 25 & SE & SE & 32 & 35 & SE & $\mathrm{SE}$ & 48 & 48 & SE \\
\hline 28 &.$\infty$ &.$\infty$ & .00 & .00 & $\ldots$ & .... & .. $\quad .$. & SSE & 82 & 84 & SSE & $\mathrm{SE}$ & 72 & 84 & SSE & $S E$ & 72 & 84 & SSE \\
\hline 29 &.$\infty$ &.$\infty$ & .00 & .00 & $\ldots$ & $\begin{array}{ll}. . & . .\end{array}$ & .. $\quad .$. & $\mathrm{s}$ & 8 & 60 & SE & $\mathrm{s}$ & 12 & 20 & $\mathrm{~S}$ & E & 5 & 20 & $s$ \\
\hline 30 & .00 & .00 &.$\infty$ & .00 & $\ldots$ & $\begin{array}{ll}. . & \ldots\end{array}$ & .. $\quad .$. & If & 3 & 6 & E & E & 2 & 5 & E & $\mathrm{s}$ & 8 & II & $\mathbf{S}$ \\
\hline 31 & ' & $\mathrm{T}$ & .04 & .04 & $\begin{array}{l}\mathbf{S}^{d} \\
\mathbf{S}^{d}\end{array}$ & $\begin{array}{rr}6 & 00 \\
13 & 30\end{array}$ & $\left.\begin{array}{cc}8 & 30 \\
. . & . .\end{array}\right\}$ & SE & 36 & 38 & $\mathrm{SE}$ & SE & 33 & 36 & SE & $s$ & 34 & 49 & $S$ \\
\hline m. & .00 & .00 & .04 & .04 & $\ldots$ & .. $\quad .$. & .. $\quad .$. & ... & 858 & 1197 & ... & $\ldots$ & 800 & 1055 & ... & $\ldots$ & 758 & 1222 & $\ldots$ \\
\hline & $\ldots$ & $\ldots$ & $\ldots$ & 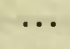 & $\ldots$ & .. $\quad$. & .. $\quad .$. & IV & 27.7 & 38.6 & E & E & 25.8 & 34.0 & E & E & 24.5 & 39.4 & $\mathbf{E}$ \\
\hline
\end{tabular}


Tabulation of daily meteorological observations at Teplitz Bay during the month of December, 1903-Continued Observer: FRANCIS LoNG

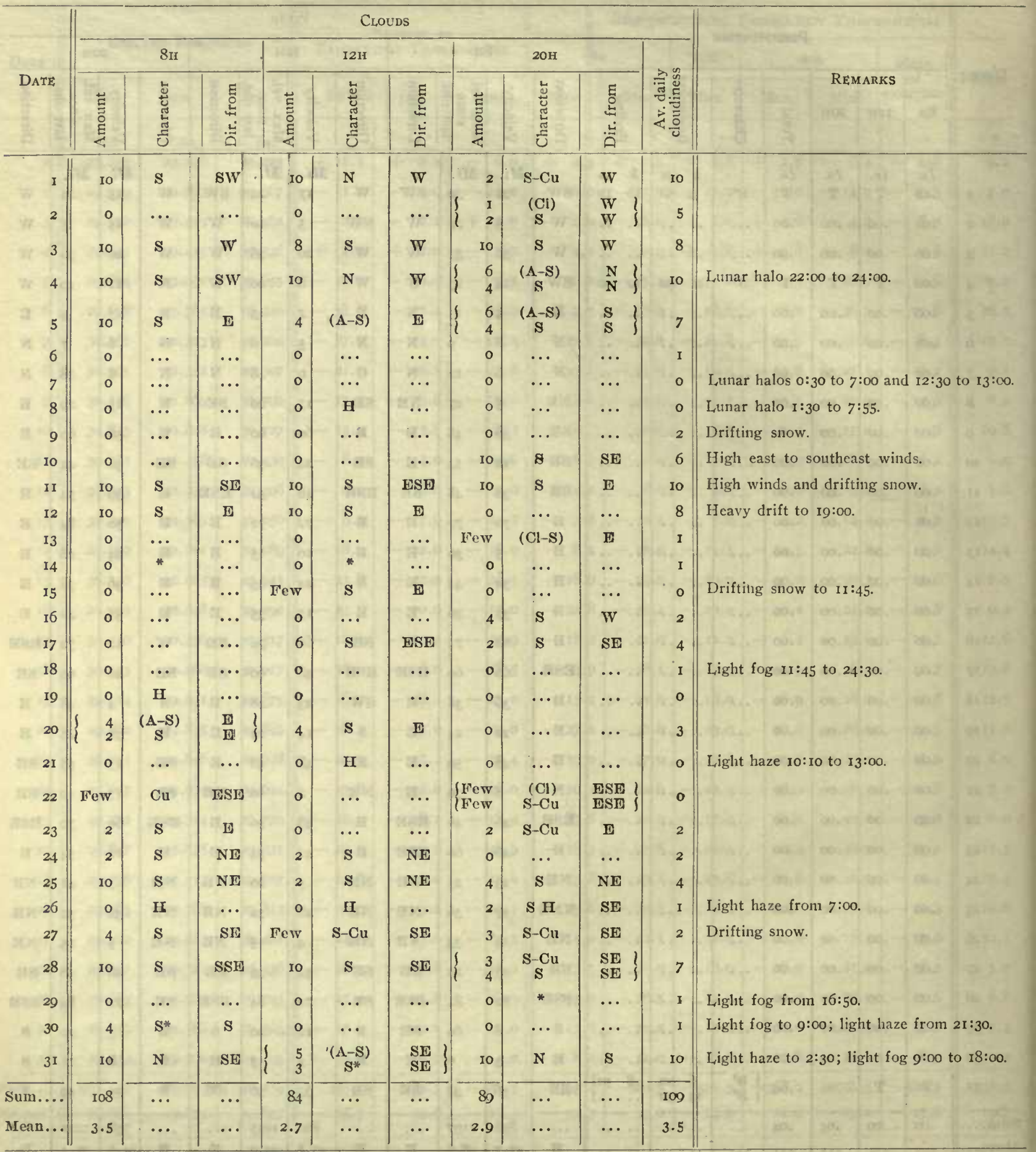


Tabulation of daily meteorological observations at Teplitz Bay during the month of January, 1904

Obscrver: FRANCIS LONG

\begin{tabular}{|c|c|c|c|c|c|c|c|c|c|c|c|c|c|c|c|}
\hline \multirow{3}{*}{\multicolumn{2}{|c|}{ DATE }} & \multirow{2}{*}{\multicolumn{3}{|c|}{ RE,DUCED BAROMETER }} & \multirow{2}{*}{\multicolumn{4}{|c|}{$\begin{array}{c}\text { READING OF } \\
\text { FAHREN HEIT TIIER MOMETER }\end{array}$}} & \multicolumn{6}{|c|}{ SELF-REGISTERING FAIRENHEIT THFRMOMETLRS } & \multirow{3}{*}{ Range } \\
\hline & & & & & & & & & \multicolumn{2}{|c|}{ SII } & \multicolumn{3}{|c|}{ 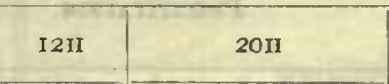 } & \multirow{2}{*}{$\begin{array}{c}\text { Mean } \\
\text { of } \\
\text { extrenses }\end{array}$} & \\
\hline & & $8 I I$ & $12 \mathrm{II}$ & $20 \mathrm{HI}$ & & Вн & $\mathrm{I} 2 \mathrm{H}$ & 2011 & Max. & Min. & Max. & Max. & Min. & & \\
\hline & & In. & In. & $I n$. & & - & 。 & $0^{\prime}$ & - & $\circ$ & 0 & 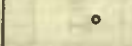 & - & - & - \\
\hline & I & 29.286 & 29.338 & 29.273 & - & 10.0 & -17.5 & $-\quad 9.8$ & $+\quad 3.8$ & -10.0 & - I0.0 & -9.8 & - I8.0 & -7.1 & 21.8 \\
\hline & 2 & 29.186 & 29.304 & 29.564 & - & I I. 0 & -24.0 & -30.0 & -6.4 & - II. 2 & - II.O & $-I I .0$ & -31.0 & $-I 8.7$ & 24.6 \\
\hline & 3 & 29.564 & $29 \cdot 354$ & 29.287 & - & 28.4 & -18.0 & $-I 5.4$ & -28.4 & -33.6 & - I8.0 & -14.2 & -28.4 & -23.9 & 19.4 \\
\hline & 4 & 29.603 & 29.662 & 29.639 & - & $27 \cdot 5$ & -30.0 & -23.1 & $-\mathbf{I 5 . 4}$ & -29.4 & -27.5 & -23.0 & -32.0 & -23.7 & I6. 6 \\
\hline$x$ & 5 & 29.410 & 29.541 & 29.862 & - & 21.0 & -29.0 & -37.0 & -18.8 & -23.1. & -21.0 & -21.0 & -38.0 & -28.4 & 9.2 \\
\hline & 6 & 29.552 & 29.317 & 29.036 & - & 21.0 & $-I 5.0$ & -8.0 & -21.0 & -40.0 & $\rightarrow I 5.0$ & -8.0 & -22.6 & -24.0 & 32.0 \\
\hline & 7 & 29.050 & 29.129 & 29.233 & - & I5.0 & -20.0 & $-24 \cdot 1$ & +4.0 & $-\mathrm{I} 5.0$ & - I 5.0 & -15.0 & -27.2 & - II. 6 & $3 I .2$ \\
\hline & 8 & 29.289 & 29.248 & 29.148 & - & 23.5 & -21.8 & -16.8 & -23.0 & $-27 . I$ & -21.0 & -16.7 & -24.2 & -21.9 & I0. 4 \\
\hline & 9 & 29.173 & 29.162 & 29.151 & - & 17.0 & $-I 6.9$ & -24.0 & -14.6 & -22.0 & $\div \mathrm{I} 6.2$ & -16.2 & -24.3 & - I9.4 & 9.7 \\
\hline & IO & $29 \cdot 366$ & 29.425 & 29.448 & - & 22.0 & -25.0 & -30.1 & -22.0 & -30.0 & -22.0 & -22.0 & -31.0 & -26.5 & 9.0 \\
\hline & II & 29.467 & 29.492 & 29.604 & - & 29.8 & -37.0 & $-45 \cdot 4$ & -28.0 & $-3 I .0$ & -29.8 & -29.8 & -46.0 & -37.0 & 18.0 \\
\hline & 12 & 29.704 & 29.732 & 29.701 & - & 46.0 & $\rightarrow 47.2$ & -50.0 & -45.0 & -48.0 & -46.0 & -46.0 & -50.9 & -48.0 & 5.9 \\
\hline a & I3 & 29.772 & 29.823 & 29.871 & - & 49.9 & -50.2 & -48.0 & -49.9 & -52.0 & -49.8 & -48.0 & -52.0 & -50.0 & $4 \cdot 0$ \\
\hline 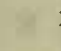 & I4 & 29.954 & 29.977 & 30.032 & - & 47.8 & -47.0. & -40.0 & -47.8 & -50.0 & -46.0 & -40.0 & -50.0 & -45.0 & 10.0 \\
\hline & I5 & 29.921 & 29.902 & 29.891 & - & 26.9 & $-24 \cdot 0$ & -24.0 & -26.9 & -42.2 & -24.0 & -23.4 & -26.9 & -32.8 & I8.8 \\
\hline & 16 & 29.665 & 29.494 & 29.267 & - & I0.0 & -3.0 & † II.O & -10.0 & -24.0 & -3.0 & +12.2 & -10.0 & -5.9 & 36.2 \\
\hline & 17 & 29.404 & 29.485 & 29.476 & + & 4.0 & +3.0 & 0.0 & +12.2 & +3.2 & +4.0 & +4.0 & -1.0 & +5.6 & 13.2 \\
\hline & I8 & 29.381 & $29 \cdot 379$ & 29.410 & + & II.9 & +10.2 & + II.9 & +14.0 & 0.0 & +12.0 & +12.0 & +10.0 & +7.0 & 14.0 \\
\hline 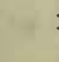 & 19 & 29.468 & 29.455 & 29.369 & + & II. 8 & +14.0 & +10.0 & +12.2 & +8.8 & +14.0 & +14.2 & +7.7 & + II.0 & 6.5 \\
\hline & 20 & 29.155 & 29.156 & 29.136 & + & II.O & +18.0 & +15.0 & $+\mathrm{II} .2$ & +8.9 & +19.0 & +22.0 & +11.0 & +15.4 & I3. I \\
\hline III & $2 I$ & 28.693 & 28.570 & 28.843 & + & I 5.0 & +23.5 & -6.0 & +15.5. & +3.0 & +24.0 & +30.8 & -6.0 & +12.4 & 36.8 \\
\hline It & 22 & 28.250 & 28.243 & 28.370 & + & 26.0 & +25.1 & - 5.0 & +26.0 & - 10.0 & +26.0 & +26.0 & -5.0 & +8.0 & 36.0 \\
\hline a & 23 & 28.724 & 28.823 & 28.997 & $\ldots$ & II.O & $-I 2.0$ & -13.0 & -5.0 & -11.0 & - II.O & -11.0 & -15.0 & -10.0 & I0. 0 \\
\hline & 24 & 29.168 & 29.227 & 29.264 & + & $8: 2$ & +2.0 & 0.0 & +8.2 & -18.0 & +8.0 & +8.2 & 0.0 & -4.9 & 26.2 \\
\hline & 25 & 29.414 & 29.412 & 29.446 & - & 2.5 & -4.0 & 7.0 & +3.0 & -2.5 & $-2 . I$ & -2.1 & -7.0 & -2.0 & I0.0 \\
\hline & 26 & 29.500 & 29.552 & 29.619 & - & $I \cdot 4.0$ & $-I 5.0$ & -16.0 & -7.0 & -15.0 & -14.0 & $-I 4.0$ & - I9.0 & -13.0 & 12.0 \\
\hline & $2 ;$ & $29.65 I$ & 29.673 & 29.749 & - & I8.o & -19.0 & -24.0 & $-\mathrm{I} 5.4$ & -20.0 & $-\mathrm{I} 6.0$ & -16.0 & -25.0 & -20.2 & 9.6 \\
\hline & 28 & 29.904 & 29.952 & 29.993 & -2 & 27.0 & -25.0 & -26.0 & -22.0 & -28.2 & -25.0 & -23.8 & -27.6 & $-25 . I$ & 6.2 \\
\hline & 29 & 29.905 & 29.857 & 29.852 & -2 & 22.2 & -21.6 & -22.4 & +21.6 & -26.9 & -20.2 & -20.2 & -23.2 & -23.6 & 6.7 \\
\hline & 30 & 29.949 & 29.988 & 30.082 & -2 & 23.0 & -23.0 & -23.0 & -22.0 & -25.0 & -22.8 & $-2 I \cdot 4$ & -26.8 & $-24 . I$ & 5.4 \\
\hline & $3 I$ & 30.225 & 30.266 & 30.349 & -2 & 25.0 & -28.0 & -28.0 & +21.2 & $\div 29.6$ & -24.0 & -24.0 & -30.2 & -25.7 & 9.0 \\
\hline $\mathrm{m}$ & & 912.763 & 912.938 & 913.962 & $-4 c$ & $61: 6$ & $-477 \cdot 4$ & -548.2 & -361.3 & -650.9 & $-403 \cdot 4$ & -347.2 & -660.6 & I3. I & 501.5 \\
\hline & & 29.444 & 29.449 & $29 \cdot 483$ & -1 & $14 \cdot 9$ & $-\mathrm{I} 5.4$ & -17.7 & ․ II.7 & $-21 \cdot 0$ & -13.0 & $-I I .2$ & $\div 21.6$ & -16.6 & .2 \\
\hline
\end{tabular}


Tabulation of daily meteorological observations at Teplitz Bay during the month of January, 1904-Continued Observer: FRANCIS LONG

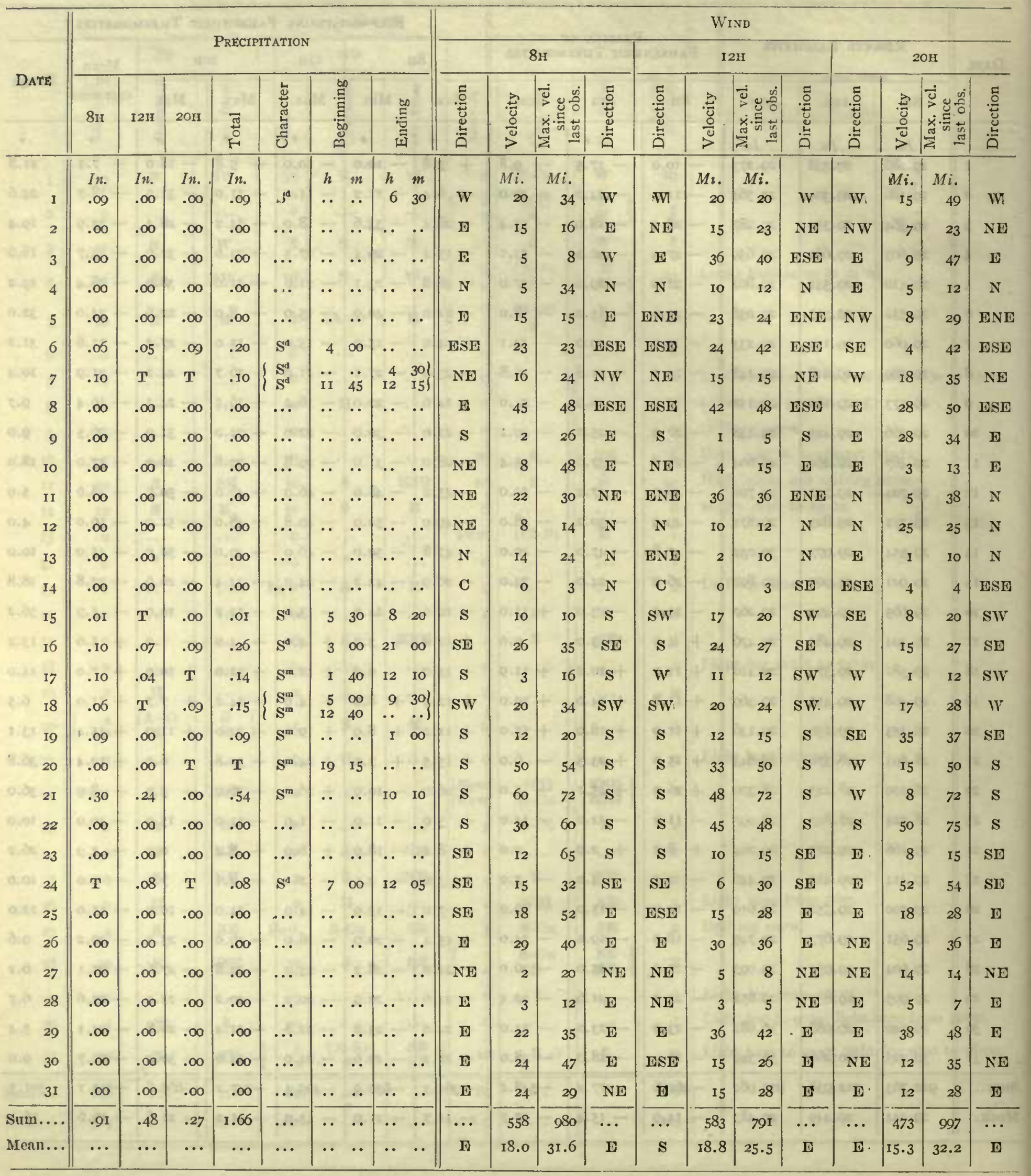


Tabulation of daily meteorological observations attz Bay during the month of January, Igo-4-Continued

Observer: Francis Long

\begin{tabular}{|c|c|c|c|c|c|c|c|c|c|c|c|c|c|}
\hline \multirow[b]{3}{*}{ DATE: } & \multicolumn{10}{|c|}{ Whan } & \multirow{3}{*}{\multicolumn{3}{|c|}{ REMARKS }} \\
\hline & \multirow[b]{2}{*}{$\begin{array}{l}\text { 宸 } \\
\text { 戛 } \\
\end{array}$} & \multicolumn{2}{|l|}{$8 \mathbf{H}$} & \multirow[b]{2}{*}{ 苨 } & \multirow{2}{*}{ 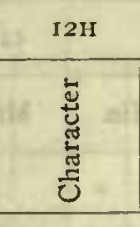 } & \multirow{2}{*}{$\begin{array}{l} \\
E \\
0 \\
\dot{2} \\
\dot{a} \\
\end{array}$} & \multirow[b]{2}{*}{$\begin{array}{l}\text { 苐 } \\
0 \\
\text { E } \\
\end{array}$} & \multicolumn{2}{|c|}{$20 \mathrm{H}$} & \multirow{2}{*}{ 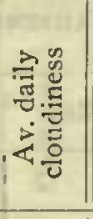 } & & & \\
\hline & & 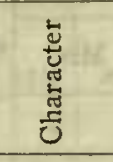 & 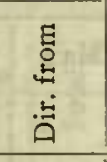 & & & & & 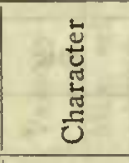 & 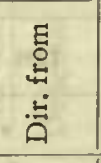 & & & & \\
\hline I & o & $\ldots$ & $\cdots$ & $\begin{array}{l}2 \\
\text { I }\end{array}$ & $\begin{array}{l}(\mathrm{Ci}-\mathrm{S}) \\
\mathrm{S} \mathrm{H}\end{array}$ & $\left.\begin{array}{l}\mathrm{W} \\
\mathrm{W}\end{array}\right\}$ & IO & $\mathrm{S}-\mathrm{Cu}$ & W & 8 & Lumar halo $7: 45$ to $8: 30$ & 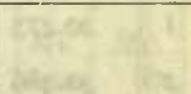 & \\
\hline 2 & 10 & $\mathbf{S}^{*}$ & $\mathbf{E}$ & o & $\cdots$ & $\cdots$ & Few & (CI) $\mathrm{H}$ & SE & I & 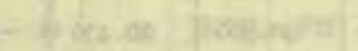 & 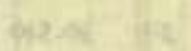 & \\
\hline 3 & $\left\{\begin{array}{l}5 \\
3\end{array}\right.$ & $\begin{array}{l}(\mathrm{A}-\mathrm{S}) \\
\mathrm{S}^{*}\end{array}$ & $\begin{array}{l}\mathbf{E} \\
\mathbf{E}\end{array}$ & $\begin{array}{l}5 \\
3\end{array}$ & $\underset{\mathrm{S}^{*}}{(\mathbf{A}-\mathbf{S})}$ & $\mathbf{E}$ & $\begin{array}{l}4 \\
2\end{array}$ & $(\mathrm{~A}-\mathrm{S})$ & $\begin{array}{ll}\mathbf{E} & 1 \\
\mathbf{E} & 1\end{array}$ & 8 & $\begin{array}{l}\text { Light fog } 7: 00 \text { to } 18: 00 \text {; } \\
\text { to } 14: 35 .\end{array}$ & drifting snow & $10: 45$ \\
\hline 4 & I & * & $\cdots$ & 0 & 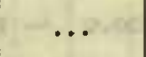 & & o & $\ldots$ & $\cdots$ & I & Lunar halo $23: 10$ to $24: 00$ & o. & \\
\hline 5 & $\left\{\begin{array}{l}2 \\
2\end{array}\right.$ & $\begin{array}{l}(\mathrm{Ci}-\mathrm{S}) \\
\mathrm{S} \mathrm{H}\end{array}$ & $\mathrm{E}\}$ & Few & $\mathbf{S} \mathbf{H}$ & ENE & 2 & (Ci) $\mathbf{H I}$ & $\mathrm{NW}$ & I & Lunar halo to $1: 00$; light 1 & haze $6: 10$ to 2 & $24: 00$. \\
\hline 6 & 10 & $\mathrm{N}^{*}$ & ESE & I0 & $N^{*}$ & ESE & I0 & $\mathrm{N}^{*}$ & SE & IO & Light fog from 6:00. & inctiks & \\
\hline 7 & 10 & $\mathbf{S}^{*}$ & NE & To & $\mathbf{N}^{*}$ & NE & 0 & $\ldots$ & $\cdots$ & 8 & Light fog to $16: \infty$ & wates & \\
\hline 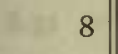 & Irew & $\mathbf{S}$ & $\mathbf{E}$ & Few & $\mathbf{S}$ & ESE & IO & s & $\mathbf{E}$ & 5 & Drifting snow $6: 00$ to 16 & $6: 40$ & r \\
\hline 9 & Io & $\mathbf{S}$ & S & 10 & $\mathbf{s}$ & $\mathbf{S}$ & o & * & $\cdots$ & 8 & Light fog $17: 00$ to $21: 00$. & $\cdot 120=1+x$ & 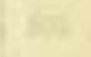 \\
\hline IO & 0 & $\cdots$ & $\cdots$ & 0 & $\ldots$ & $\cdots$ & o & $\mathbf{H}$ & $\cdots$ & 0 & Haze $18: 40$ to $20: 40$ & $600 x+2$ & \\
\hline II & o & $\cdots$ & $\cdots$ & 0 & $\ldots$ & $\cdots$ & 0 & $\ldots$ & $\cdots$ & o & Haze from 22:00. & & \\
\hline 12 & Io & S H & NE & 4 & $S^{*}$ & $\mathbf{N}$ & o & $\cdots$ & $\cdots$ & & Haze to $8: 15$; light fog 8 : & : I5 to $13: 30$ & \\
\hline 13 & o & * & $\cdots$ & Few & $\mathbf{s}$ & SE & $\circ$ & * & $\cdots$ & o & Light fog 6:00 to $9: 10$ anc & d from $18: 40$. & \\
\hline 14 & 0 & $\cdots$ & $\cdots$ & 0 & $\cdots$ & $\cdots$ & 0 & * & $\cdots$ & o & Generally light fog. & 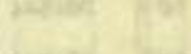 & \\
\hline 15 & Io & $\mathbf{N}^{*}$ & $\mathbf{S}$ & Few & $\mathbf{s}$ & SE & $\circ$ & $\ldots$ & $\cdots$ & 6 & Light fog to $8: 25$ & 10506 & \\
\hline 16 & I0 & $\mathbf{N}^{*}$ & SE & Io & $\mathbf{N}^{*}$ & $\mathbf{S}$ & 10 & $\mathrm{~N}^{*}$ & $\mathbf{S}$ & 10 & Light fog $3: 00$ to $20: 30$. & tactis & \\
\hline 17 & I0 & $\mathrm{N}^{*}$ & $\mathrm{~s}$ & Io & $\mathrm{N}^{*}$ & W & 6 & $S^{*}$ & W & 9 & Light fog $3: 30$ to $22: 30$. & $\sin (x)$ & . \\
\hline 18 & I0 & $\mathbf{N}^{*}$ & Sw & 10 & $\mathbf{S}^{*}$ & sw & 10 & $\mathrm{~N}^{*}$ & W & IO & Generally foggy. & 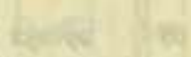 & 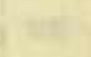 \\
\hline 19 & 10 & $\mathbf{S}^{*}$ & $\mathbf{s}$ & I0 & $\mathbf{S}$ & $\mathbf{S}$ & 10 & $\mathrm{~s}$ & SE & 9 & Light fog to $8: 30$; driftin & ng snow from & $19: 30$ \\
\hline 20 & I0 & $\mathbf{S}$ & $\mathbf{S}$ & $\begin{array}{l}5 \\
4\end{array}$ & $\underset{S}{(A-S)}$ & $\left.\begin{array}{l}\mathrm{s} \\
\mathrm{s}\end{array}\right\}$ & 10 & N & W & 8 & Drifting snow to I I:00. & & \\
\hline $2 \mathrm{I}$ & I0 & $\mathbf{N}$ & $\mathbf{S}$ & 10 & $\mathbf{S}$ & s & Few & $\mathbf{S}$ & $\pi$ & 8 & Litente & wyice: & \\
\hline 22 & 10 & $\mathbf{S}$ & $\mathbf{s}$ & Io & $\mathbf{s}$ & $\mathbf{s}$ & 7 & $\mathbf{S}$ & s & 10 & 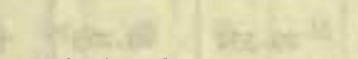 & atate & \\
\hline 23 & Few & $\mathbf{s}$ & $\mathbf{S E}$ & Few & $\mathbf{s}$ & SE & 4 & $\mathbf{S}$ & SE & 2 & Variable winds. & & \\
\hline 2.4 & Io & $\mathbf{N}$ & SE & Io & $\mathrm{N}^{*}$ & SE & & $\cdots$ & $\cdots$ & 7 & & 16zeste & \\
\hline 25 & 7 & $\mathbf{S}$ & SE & Io & S & ESE & & $\begin{array}{c}(\mathrm{Ci}-\mathrm{S}) \\
\mathrm{S}\end{array}$ & $\left.\begin{array}{l}\mathbf{E} \\
\mathbf{G}\end{array}\right\}$ & 6 & $\cos ^{2} \cos x$ & ance & \\
\hline 26 & 0 & $\cdots$ & - & 2 & $\mathbf{S}$ & SE & $\left\{\begin{array}{l}2 \\
2\end{array}\right.$ & $\begin{array}{l}(\mathrm{Ci}-\mathrm{S}) \\
(\mathrm{A}-\mathrm{S})\end{array}$ & $\left.\begin{array}{l}\mathbf{E} \\
\mathbf{E}\end{array}\right\}$ & I & (8): ift & $\sqrt{4}+8 x$ & \\
\hline 27 & 4 & S & E & $\begin{array}{l}2 \\
2\end{array}$ & $\stackrel{(A-S)}{S H}$ & $\begin{array}{ll}\text { SE } \\
\text { SE }\end{array}$ & Few & (Ci) & $\mathbf{E}$ & 3 & Light haze 9:25 to $14: 00$ & thos. & \\
\hline 28 & 0 & $\cdots$ & $\cdots$ & o & $\cdots$ & $\cdots$ & 0 & $\cdots$ & $\cdots$ & o & & $x_{n+3}=$ & \\
\hline 29 & & $\cdots$ & $\cdots$ & o & $\cdots$ & $\cdots$ & & $\cdots$ & $\cdots$ & $\circ$ & Drifting snow. & & \\
\hline 30 & 0 & $\cdots$ & $\cdots$ & o & $\cdots$ & $\cdots$ & 0 & $\cdots$ & $\cdots$ & o & 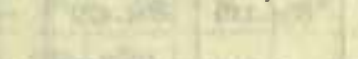 & & \\
\hline $3 I$ & & $\cdots$ & $\cdots$ & o & ... & $\cdots$ & 0 & $\cdots$ & $\cdots$ & 0 & $\tan x$ & & \\
\hline , & 164 & $\cdots$ & $\cdots$ & I.40 & $\cdots$ & $\cdots$ & 103 & $\cdots$ & $\cdots$ & 145 & & & \\
\hline Mean... & $5 \cdot 3$ & $\cdots$ & $\cdots$ & $4 \cdot 5$ & $\cdots$ & $\cdots$ & 3.3 & $\cdots$ & $\cdots$ & 4.7 & & & \\
\hline
\end{tabular}


Tabulation of daily meteorological observations at Tepli Teplitz Bay during the month of February, 1904 Observer: FRANCIS LONG

\begin{tabular}{|c|c|c|c|c|c|c|c|c|c|c|c|c|c|c|}
\hline \multirow{3}{*}{ DATE } & \multirow{2}{*}{\multicolumn{3}{|c|}{ REDUCED BAROMETER }} & \multirow{2}{*}{\multicolumn{3}{|c|}{$\begin{array}{c}\text { READING OF } \\
\text { FAIIRENHEIT THERMOMETER }\end{array}$}} & \multicolumn{8}{|c|}{ SELLF-RFGISTERING FAHRENHEIT THERMOMETERS } \\
\hline & & & & & & & \multicolumn{3}{|c|}{$8 \pi$} & \multirow{2}{*}{$\frac{\text { I2II }}{\text { Max. }}$} & \multicolumn{2}{|c|}{$20 \mathrm{H}$} & \multirow{2}{*}{$\begin{array}{c}\text { Mean } \\
\text { of } \\
\text { extremes }\end{array}$} & \multirow{2}{*}{ Range } \\
\hline & $8 \mathbf{H}$ & I2II & $20 \mathrm{H}$ & $8 \mathrm{H}$ & $12 \mathrm{H}$ & $20 \mathrm{H}$ & Max. & & Min. & & Max. & Min. & & \\
\hline & $I n$. & In. & In. & 15 & 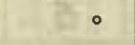 & 0 & - & & $\circ$ & $\circ$ & $\circ$ & $\circ$ & $\cdot$ & $\circ$ \\
\hline $\mathbf{I}$ & 30.473 & 30.509 & 30.575 & -29.0 & -28.2 & -27.0 & -27.6 & - . & $3 \mathrm{I} . \mathrm{I}$ & -26.0 & -25.0 & -30.6 & -28.0 & 6.1 \\
\hline 2 & 30.486 & $30.44 I$ & 30.552 & -23.2 & -21.8 & -23.0 & -23.0 & - & $27 \cdot 4$ & -20.6 & -20.0 & -25.0 & -23.7 & 7.4 \\
\hline 3 & 30.540 & 30.506 & 30.430 & -22.0 & -20.0 & $-\mathrm{I} 6.0$ & - I8.0 & - & 25.0 & $-\mathrm{II} .4$ & - II.9 & -24.0 & -18.2 & I3.6 \\
\hline 4 & 30.424 & 30.376 & 30.376 & - I9.0 & -20.0 & -19.0 & - I4.9 & - & 19.0 & - I9.0 & - 18.9 & $-2 I .0$ & -18.0 & 6.1 \\
\hline 5 & 30.128 & 30.049 & 29.922 & $-I_{5} .0$ & -17.0 & -12.0 & -15.0 & . - & 20.0 & -15.0 & - II. 2 & -17.0 & $-\mathrm{I} 5.6$ & 8.8 \\
\hline 6 & 29.92 I & 29.932 & 30.044 & $-\mathrm{I} 2.0$ & -12.2 & $-\mathrm{I} 7.0$ & $-\mathrm{II} .8$ & - & 14.0 & - 10.0 & - 10.0 & -17.0 & $-\mathrm{I} 3.5$ & 7.0 \\
\hline 7 & 30.001 & 29.978 & 29.982 & - 19.0 & -14.0 & -20.0 & -18.6 & - & 21.0 & -14.0 & $-\mathrm{I} 4.0$ & -20.0 & -17.5 & 7.0 \\
\hline 8 & 30. 149 & 30. 188 & 30.084 & $-I 5.0$ & -21.0 & -4.0 & $-\mathrm{I} 5.0$ & - & 24.1 & -15.0 & -4.0 & -22.0 & $-\mathrm{I} 4.0$ & 20.1 \\
\hline 9 & 30.253 & 30.266 & 30.300 & - II.O & -9.9 & - 16.0 & -4.0 & - & I7. 2 & -7.0 & -7.0 & -17.8 & -10.9 & 13.8 \\
\hline 10 & 30.154 & 30.067 & $29.88 \mathrm{I}$ & -12.0 & - II.O & -10.0 & -12.0 & - & 17.0 & - II.O & - 10.0 & -12.2 & -13.5 & 7.0 \\
\hline II & 29.690 & 29.640 & $29.65 \mathrm{I}$ & - I9.0 & -19.0 & -37.0 & $-9 . I$ & - & I9.0 & -18.5 & $-\mathrm{I} 8.5$ & -37.0 & -23.0 & 27.9 \\
\hline 12 & 29.807 & 29.870 & 29.964 & $-4 \mathrm{I} \cdot 9$ & -43.0 & -42.5 & -37.0 & - & 42.2 & -41.8 & -39.8 & -43.6 & -40.3 & 6.6 \\
\hline 13 & 29.911 & 29.805 & 29.695 & -35.0 & -26.0 & $\div 12.0$ & -35.0 & - & 43.0 & -25.0 & - II.0 & -35.0 & -27.0 & 32.0 \\
\hline 14 & 29.524 & $29 \cdot 479$ & $29.66 \mathrm{I}$ & $-4 \cdot 0$ & +4.0 & -24.0 & -2.9 & - & 6.0 & +4.0 & +4.0 & $-24 \cdot 0$ & - 10.0 & 28.0 \\
\hline 15 & 29.724 & 29.729 & 29.749 & -27.0 & -32.0 & -36.0 & -24.0 & - & 31.8 & -27.0 & -27.0 & -43.0 & -.33 .5 & 19.0 \\
\hline I6 & 29.905 & 29.976 & 29.999 & -41.0 & -43.0 & -40.1 & -35.0 & - & 43.4 & -33.0 & -33.0 & -44.2 & -38.6 & II . 2 \\
\hline 17 & 29.770 & 29.590 & 29.282 & -21.0 & -19.0 & -7.5 & -21.0 & - & 40.0 & $-\mathrm{I} 8.6$ & -5.3 & -21.0 & -22.6 & $34 \cdot 7$ \\
\hline 18 & 29.252 & 29.347 & 29.622 & -22.2 & $-32 . \mathrm{I}$ & -36.9 & +4.0 & - & 22.2 & -22.2 & -22.2 & -36.9 & $-\mathrm{I} 6.4$ & 40.9 \\
\hline 19 & 29.911 & 29.945 & 29.928 & -42.0 & -42.0 & $-24 \cdot 9$ & -42.0 & - & 44.6 & -41.9 & $-24 \cdot 9$ & -44.2 & -34.8 & 19.7 \\
\hline 20 & 29.596 & 29.529 & 29.497 & - 19.0 & -14.0 & -9.9 & -18.0 & - & 24.9 & -13.6 & -4.0 & -19.0 & $-\mathrm{I} 4.4$ & 20.9 \\
\hline 21 & 29.744 & 29.708 & 29.567 & -12.0 & -9.0 & -4.0 & -5.6 & - & I7.4 & -8.3 & -1.0 & -13.0 & -9.2 & 16.4 \\
\hline 22 & 29.438 & 29.354 & 29.283 & +6.2 & +6.0 & +14.0 & +7.0 & - & 6.4 & +6.2 & +14.5 & +2.6 & +4.1 & 21.0 \\
\hline 23 & 29.479 & 29.666 & 29.945 & -15.0 & -25.0 & -27.5 & +16.4 & - & I 5.0 & -15.0 & -15.0 & -27.5 & $-\quad 5.6$ & 43.9 \\
\hline 24 & 29.245 & 28.970 & 29.002 & -5.0 & +20.0 & +5.0 & -5.0 & - & 31.5 & +20.0 & +23.1 & -5.0 & -4.2 & 54.6 \\
\hline 25 & 29.165 & 29.226 & 29.472 & -9.0 & -15.0 & -16.8 & +5.0 & - & 9.0 & -9.0 & -9.0 & -17.4 & -6.2 & 22.4 \\
\hline 26 & 29.419 & 29.316 & 29.185 & +5.0 & +16.5 & +24.1 & +5.2 & - & I7.0 & +16.8 & +25.6 & +5.0 & +4.3 & 42.6 \\
\hline 27 & 29.619 & 29.691 & 29.665 & - I5.0 & -20.0 & -16.0 & $+24 . I$ & - & I5.0 & -15.0 & $-\mathrm{I} 5.0$ & -25.0 & -0.4 & 49. I \\
\hline 28 & 29.676 & 29.731 & 29.840 & -3.5 & -5.2 & -6.9 & -3.5 & - & 16.0 & -3.0 & -3.0 & -7.4 & -9.5 & 13.0 \\
\hline 29 & 29.470 & 29.234 & 29.326 & +4.0 & +23.5 & +22.6 & +4.0 & - & $9.9^{\circ}$ & +24.0 & +30.2 & +4.0 & +10.2 & 40.1 \\
\hline Sum.... & 864.874 & 864 . II 8 & 864.479 & -493.6 & -449.4 & -440.3 & -332.3 & -6 & 570.1 & -369.9 & -263.2 & -638.2 & -450.0 & 640.9 \\
\hline Mean... & 29.823 & 29.797 & 29.810 & $-I 5.5$ & -15.2 & -23.8 & -11.5 & - & 23. I. & -12.8 & - 9.J & -22.0 & $-15 \cdot 5$ & 22.1 \\
\hline
\end{tabular}


Tabulation of daily meteoralogical abservations at Teplitz Bay during the Month of Fcbruary, I904-Continued Observer: FRANCIS LoNG

\begin{tabular}{|c|c|c|c|c|c|c|c|c|c|c|c|c|c|c|c|c|c|c|c|}
\hline \multirow{3}{*}{ DATE } & \multirow{2}{*}{\multicolumn{7}{|c|}{ PRECIPITATION }} & \multicolumn{11}{|c|}{ WIND } & \multirow[b]{3}{*}{ 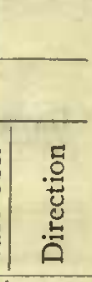 } \\
\hline & & & & & & & & \multicolumn{4}{|c|}{$8 \mathrm{H}$} & \multicolumn{4}{|c|}{$\mathrm{I} 2 \mathrm{H}$} & \multicolumn{3}{|c|}{2011} & \\
\hline & 8 в & I2II & $2 \mathrm{OH}$ & $\begin{array}{l}\bar{\pi} \\
\text { 今 } \\
\text { F }\end{array}$ & 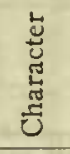 & 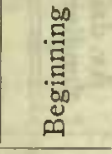 & 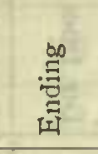 & & 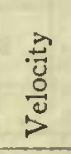 & 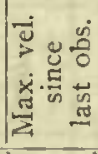 & 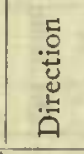 & 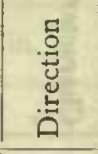 & $\frac{\vec{s}}{0}$ & 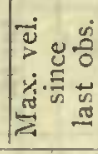 & 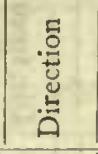 & 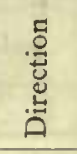 & $\mid \begin{array}{l}\vec{t} \\
0 \\
0 \\
0\end{array}$ & 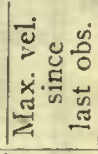 & \\
\hline I & $\begin{array}{l}I n . \\
.00\end{array}$ & $\begin{array}{l}\text { In. } \\
.0 c\end{array}$ & $\begin{array}{l}\text { In. } \\
. \infty\end{array}$ & $\begin{array}{l}\text { In. } \\
. \infty\end{array}$ & ... & $\begin{array}{ll}h & n \\
. . & . .\end{array}$ & $\begin{array}{ll}h & m \\
. . & . .\end{array}$ & E & $\begin{array}{r}M i . \\
4\end{array}$ & $\begin{array}{r}\text { Mi. } \\
24\end{array}$ & $\mathrm{E}$ & $\mathbf{E}$ & $\begin{array}{r}M i . \\
4\end{array}$ & $\begin{array}{r}M i . \\
15\end{array}$ & NE & NE & $\begin{array}{r}M i . \\
\text { I2 }\end{array}$ & $\begin{array}{r}M i . \\
17\end{array}$ & $\mathbf{E}$ \\
\hline 2 & .00 & .00 & .00 & .00 & $\ldots$ & .. $\quad .$. & .. $\quad .$. & D & 8 & 24 & E & ENE & 21 & 30 & ENE & SE & 4 & 30 & ENE \\
\hline 3 & .00 & .00 & .00 & .00 & .. & . $\quad$. & .. $\quad$. & $\mathbf{E}$ & 25 & 35 & $\mathrm{E}$ & I) & 46 & 46 & $\mathbf{E}$ & E & 58 & 60 & E \\
\hline 4 &.$\infty$ & .00 & .00 & .00 & ... & .. $\quad .$. & .. $\quad$. & $\mathbf{E}$ & 44 & 59 & $\mathbf{E}$ & E & 48 & $5 \pi$ & $\mathbf{E}$ & $\mathrm{NE}$ & 5 & 58 & $\mathbf{E}$ \\
\hline 5 & $\mathrm{~T}$ & $\mathrm{~T}$ & .00 & $\mathrm{~T}$ & $S^{4}$ & 730 & $8 \quad 15$ & $\mathrm{NE}$ & 20 & 20 & NE & $\mathrm{NE}$ & 20 & 26 & NE & E & 40 & $4 I$ & $\mathbf{E}$ \\
\hline 6 &.$\infty$ & .00 & .00 &.$\infty$ & .. & .. $\quad .$. & .... & $\mathrm{NE}$ & 12 & 42 & E & $\mathbf{E}$ & 36 & 48 & E & $\mathbf{E}$ & 10 & 48 & E \\
\hline 7 &.$\infty$ & $\mathbf{T}$ & $\mathrm{T}$ & $\mathrm{T}$ & $\mathbf{S}^{d}$ & II $\infty$ & 1230 & $\mathrm{NE}$ & 15 & 20 & E & SE & 5 & 16 & $\mathbf{E}$ & E & I & 16 & $\mathbf{E}$ \\
\hline 8 & .00 &.$\infty$ &.$\infty$ &.$\infty$ & ... & .. $\quad .$. & .. $\quad .$. & s & 4 & 20 & s & $\mathbf{E}$ & 5 & 16 & $\mathbf{E}$ & SE & 30 & 48 & $\mathrm{SE}$ \\
\hline 9 & .00 & .00 &.$\infty 0$ &.$\infty$ & .. & $\begin{array}{ll}. . & . .\end{array}$ & .. $\quad$. & s & 10 & 48 & SSE & SE & 3 & 24 & SE & $\mathrm{NE}$ & 3 & 24 & SE \\
\hline 10 & .00 &.$\infty$ & .02 & .02 & $S^{4}$ & 1540 & .. $\quad$. & w & 15 & 17 & W & w & 17 & 17 & W & sw & 15 & 25 & SW \\
\hline II & .09 & .05 & $\mathbf{T}$ & .14 & $\mathbb{S}^{d}$ & $\quad \cdots \quad$. & I2 45 & $N$ & 8 & 29 & $\mathrm{~N}$ & $\mathrm{NE}$ & Io & 12 & $\mathbf{N}$ & $\mathrm{N}$ & 14 & 30 & $\mathbf{N}$ \\
\hline 12 &.$\infty$ &.$\infty$ & .00 &.$\infty$ & $\cdots$ & $\ldots \quad \ldots$ & .. $\quad .$. & $\mathrm{N}$ & 5 & 17 & $\mathrm{~N}$ & $\mathrm{~N}$ & I & 3 & $N$ & $\mathrm{~N}$ & I & 5 & $\mathbf{N}$ \\
\hline 13 & .00 & .oI & .10 & $.1 \mathrm{I}$ & $\mathrm{S}^{\mathrm{d}}$ & 10 $\infty$ & $20 \quad 45$ & SE & 16 & 16 & SE & s & I0 & 16 & $\mathrm{SE}$ & SW & 23 & 24 & SW \\
\hline 14 & .02 & .05 & .03 & .10 & $S^{d}$ & I $\infty$ & 1645 & SW & 16 & 36 & sw & SIV & 30 & 35 & SW & NE & 10 & 35 & SW \\
\hline 15 &.$\infty$ &.$\infty$ & .00 & .00 & $\ldots$ & .. $\quad$. & .. $\quad .$. & W & 3 & 20 & $\mathbf{N}$ & $\mathrm{C}$ & 0 & IO & W & $\mathbf{E}$ & 6 & I9 & $\mathrm{E}$ \\
\hline I6 & .00 & .00 &.$\infty$ & .00 & $\ldots$ & .. $\quad .$. & .. $\quad .$. & $\mathbf{N}$ & 12 & 24 & $\mathrm{~N}$ & $\mathrm{~N}$ & 3 & 20 & $\mathbf{N}$ & N & I & 20 & $\mathbf{N}$ \\
\hline 17 & .00 & .00 & .00 & .00 & .. & .. $\quad$. & .. $\quad .$. & SE & 36 & 38 & SE & SE & $4^{8}$ & 48 & $\mathrm{SE}$ & $\mathrm{SE}$ & 48 & 54 & $\mathrm{SE}$ \\
\hline 18 & .00 & .00 &.$\infty$ &.$\infty$ & .. & .. $\quad .$. & .. $\quad .$. & W & 20 & 52 & $\mathrm{SE}$ & WV & I6 & 20 & w & W & 8 & 24 & W \\
\hline 19 & .00 & .00 & .00 & .00 & $\cdots$ & .. $\quad .$. & .. $\quad .$. & v & I & 14 & W & C & 0 & 4 & $\mathbf{N}$ & s & 8 & 12 & s \\
\hline 20 &.$\infty$ &.$\infty 0$ &.$\infty 0$ & .00 & $\cdots$ & $\quad \cdots \quad \cdots$ & .. $\quad$. & SE & 48 & $6_{3}$ & $\mathrm{SE}$ & E & 42 & 60 & $\mathrm{SE}$ & s & 12 & 60 & SE \\
\hline $2 \mathrm{I}$ & .00 & .00 &.$\infty 0$ & .00 & $\cdots$ & $\begin{array}{ll}. . & .\end{array}$ & .. $\quad$. & $N$ & 2 & 15 & s & $\mathbf{E}$ & 25 & 28 & E & E & 58 & 58 & D \\
\hline 22 & .00 & .00 & .00 & .00 & $\cdots$ & . $\quad .$. & .. $\quad .$. & $\mathrm{s}$ & 32 & 60 & E & SE & 48 & 50 & SE & $\mathrm{s}$ & 36 & 50 & SE \\
\hline 23 & $T$ & $\mathrm{~T}$ &.$\infty$ & $\mathrm{T}$ & $S^{d}$ & 700 & $9 \infty$ & $\mathrm{NW}$ & 20 & 47 & $\mathrm{~s}$ & NW & $I_{5}$ & 24 & NW & NE & 8 & 24 & NW \\
\hline 24 & .00 & .07 & .01 & .08 & $S^{d}$ & $9 \quad 30$ & 1430 & SSE & 50 & 66 & SSE & SSW, & 36 & 48 & SSE & W & 18 & 48 & SSW \\
\hline 25 & .00 & .00 & .00 & .00 & .. & . $\quad$. & .. $\quad .$. & W & 20 & 29 & W & $w$ & 20 & 24 & WV & NW & Io & 25 & $w$ \\
\hline 26 & .03 & .10 & .12 & .25 & $\mathbf{S}^{m}$ & $5 \infty$ & I8 50 & $s$ & 12 & 13 & W & SE & 12 & I7 & $\mathrm{s}$ & w & 24 & 28 & w \\
\hline 27 & .00 &.$\infty$ &.$\infty 0$ &.$\infty$ & ... & $\begin{array}{lll}\text {.. } & . .\end{array}$ & .. $\quad$. & $\mathrm{NW}$ & 15 & 48 & W & NIV & 12 & 20 & NW & $\mathbf{S}$ & 3 & 20 & $\mathrm{NW}$ \\
\hline 28 & .02 & $T$ & .00 & .02 & $\mathrm{~S}^{\mathrm{m}}$ & 500 & 920 & s & 5 & I6 & $\mathrm{s}$ & W & I0 & 20 & w & SE & I & 20 & W \\
\hline 29 & .06 & .15 & .or & .22 & $\ldots$ & .. $\quad .$. & .. $\quad .$. & B & 52 & 60 & $\mathbf{E}$ & s & 36 & 56 & $\mathbf{E}$ & w & II & 56 & $\mathbf{E}$ \\
\hline Sum.... & .22 & .43 & .29 & .94 & $\cdots$ & .. $\quad .$. & .. $\quad .$. & $\ldots$ & 530 & 972 & $\cdots$ & $\ldots$ & 579 & 804 & $\ldots$ & ... & 478 & 979 & $\ldots$ \\
\hline Mean... & $\ldots$ & $\cdots$ & $\ldots$ & $\ldots$ & $\ldots$ & .. $\quad .$. & .. & E & 18.3 & 33.5 & $\mathbf{E}$ & IV & 20.0 & 27.7 & I & E & 16.5 & 33.8 & $\mathbf{E}$ \\
\hline
\end{tabular}


Tabulation of daily metcorological obscrvations at Teplitz Bay during the month of Ficbruary, 1904-Continued Observer: FRANCIS LONG

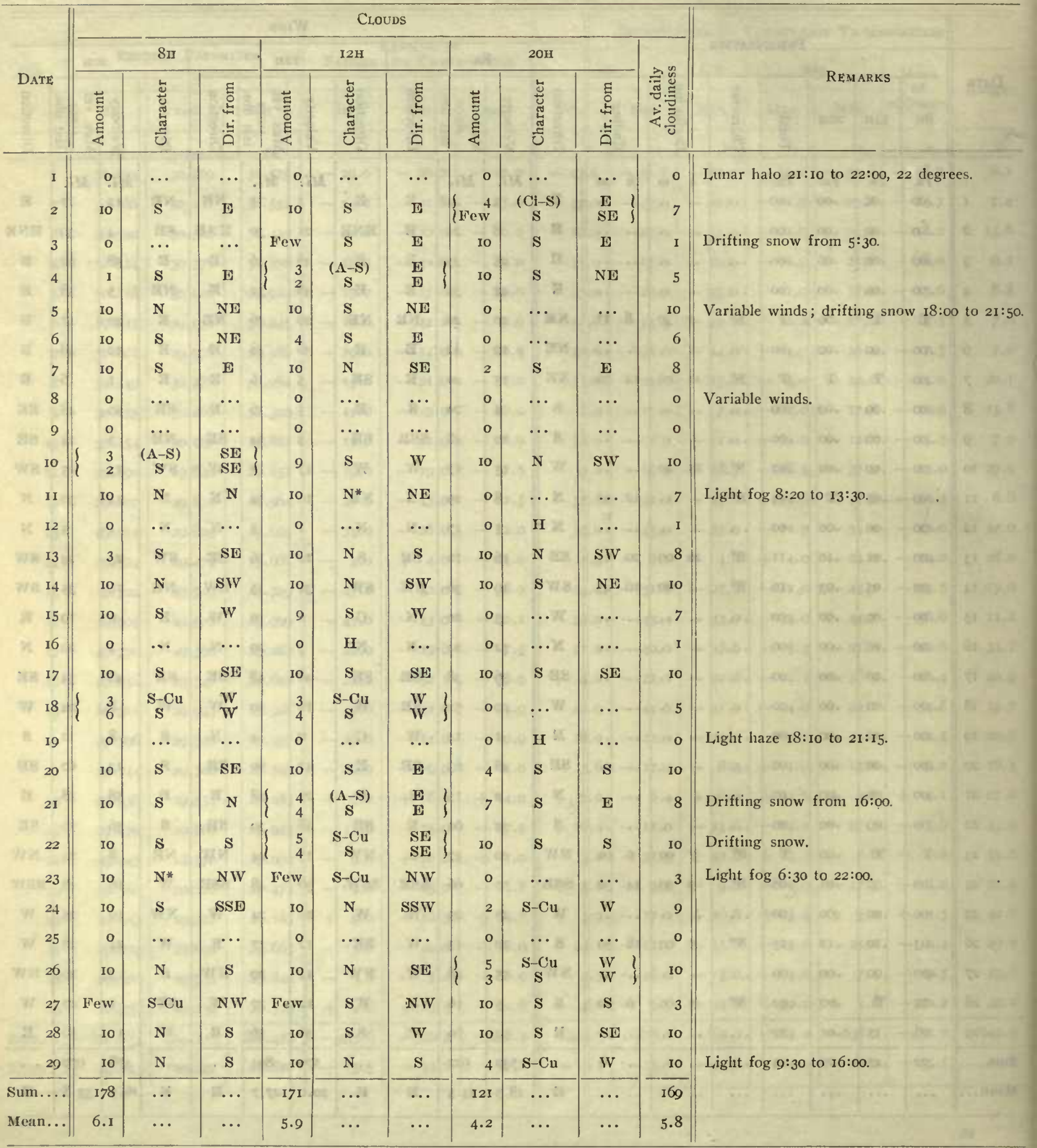


Tabulation of daily meteorological observations at Teplitz Bay during the month of March, 1904

Observer: Francis Lonc

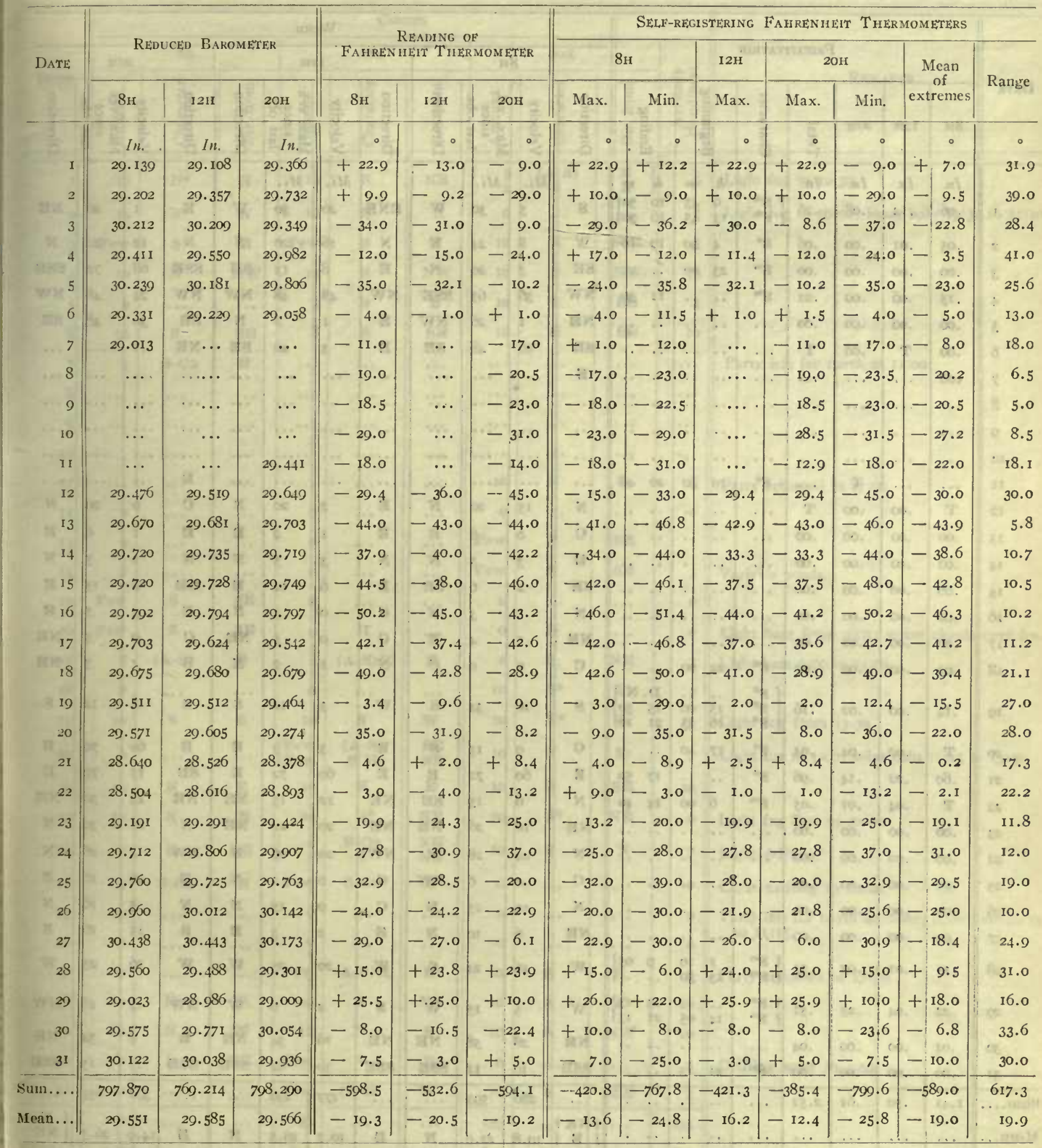

Between Marcli 7 and 11, 1904, observer was with party on trip to Cape Fligely; the thermometer readings during this interval have been taken from the thermograph records. 
Tabulation of daily metcorological observations at Teplitz Bay during the month of March, igo4-Continued Observer: Francis Lonc

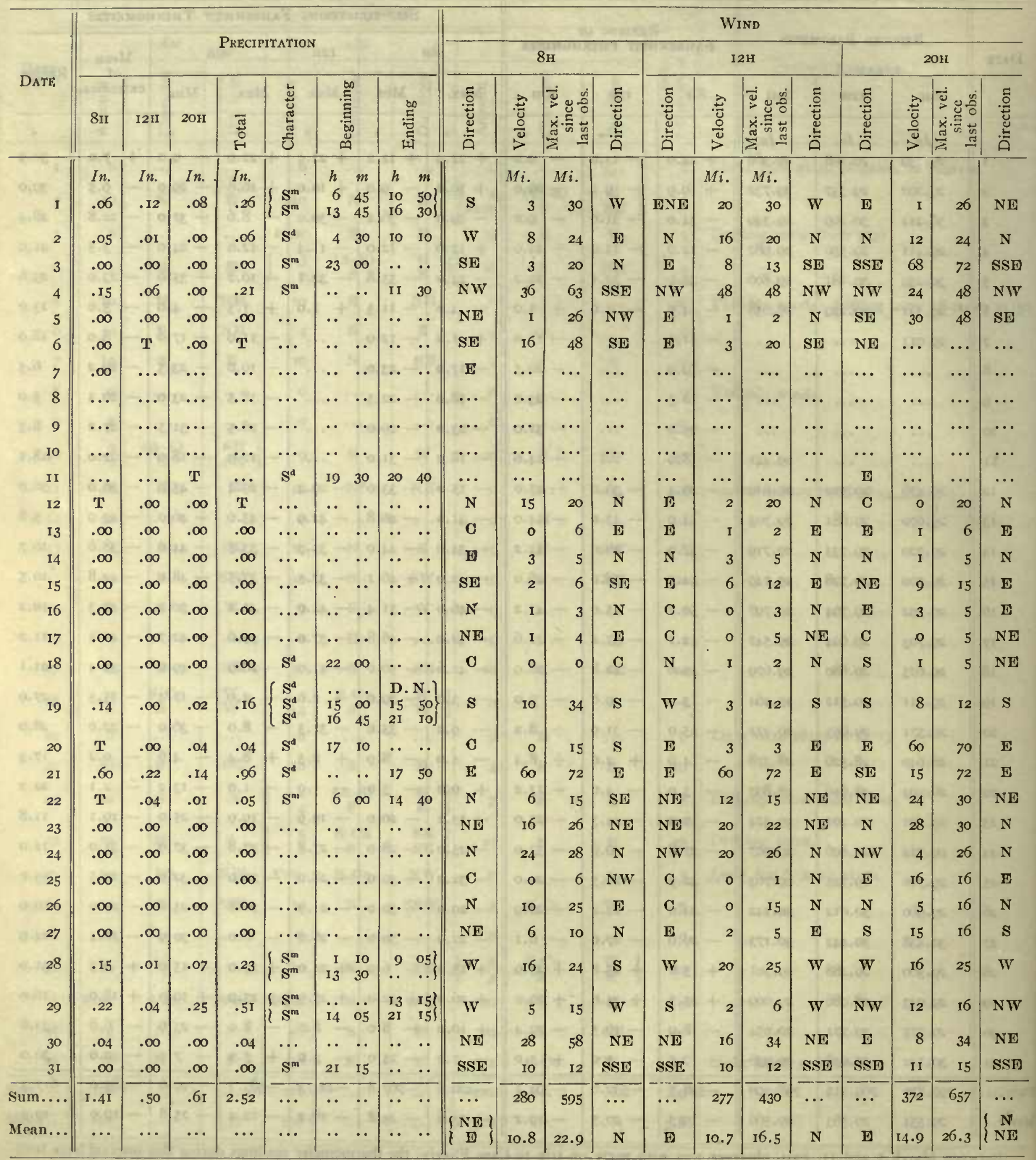


Tabulation of daily metcorological observations at Teplitz Bay during the month of March, 1904-Continued

Observer: Francis LoNG

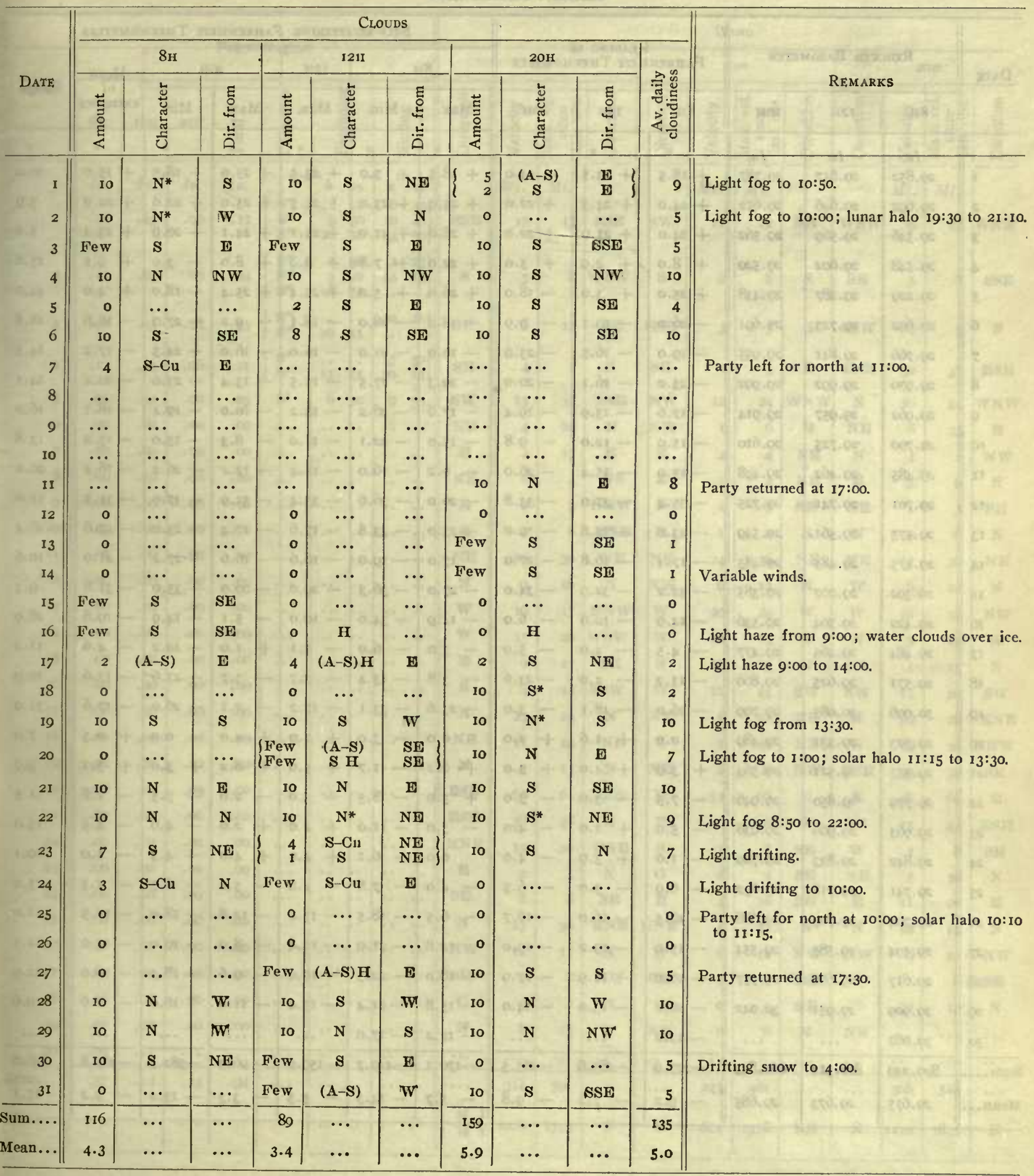


Tabulation of daily meteorological observations at Teplitz Bay during the month of April, 1904 Observer: FrancIS LoNG

\begin{tabular}{|c|c|c|c|c|c|c|c|c|c|c|c|c|c|}
\hline \multirow{3}{*}{ DATE } & \multirow{2}{*}{\multicolumn{3}{|c|}{ REDUCED BAROMETER }} & \multirow{2}{*}{\multicolumn{3}{|c|}{$\begin{array}{c}\text { READING OF } \\
\text { FAHRENIIETT TIIERMOMETER }\end{array}$}} & \multicolumn{6}{|c|}{ SELF-REGISTERING FAIIRENHEIT THERMOMETERS } & \multirow{3}{*}{ Range } \\
\hline & & & & & & & \multicolumn{2}{|c|}{$8 \mathbf{H}$} & \multirow{2}{*}{ Max. } & \multicolumn{2}{|c|}{$2 \mathrm{OH}$} & \multirow{2}{*}{$\begin{array}{l}\text { Mean } \\
\text { of } \\
\text { extremes }\end{array}$} & \\
\hline & $8 \mathrm{II}$ & $\mathrm{I} 2 \mathrm{H}$ & $2 \mathrm{OH}$ & $8 I I$ & I2H & 2011 & Max. & Min. & & Max. & Min. & & \\
\hline & $I_{n}$ & In & In. & & 。 & -0 & 。 & $\ldots$ & 。 & - & - & 。 & - \\
\hline I & 29.852 & 29.817 & 29.787 & +18.5 & +23.5 & +24.0 & +18.5 & +5.0 & +23.5 & +25.0 & +18.5 & +15.0 & 20.0 \\
\hline 2 & 29.692 & 29.646 & 29.612 & +24.0 & +24.3 & +22.0 & +25.9 & +23.0 & +24.5 & +25.0 & +22.0 & +24.0 & 3.9 \\
\hline 3 & 29.546 & 29.569 & 29.562 & +24.0 & +21.9 & +22.0 & +26.0 & +21.0 & +24.1 & $+24 \cdot I$ & +20.9 & +23.4 & 5.1 \\
\hline 4 & 29.548 & 29.602 & 29.520 & +8.0 & +2.9 & +5.0 & +22.0 & +7.8 & +8.0 & 8.0 & 13.0 & +9.5 & 25.0 \\
\hline 5 & 29.229 & 29.287 & 29.438 & $+25 \cdot 0$ & $+3 \cdot 0$ & $-\mathrm{I} 8.0$ & +26.0 & +5.0 & $+25 \cdot 4$ & $+25 \cdot 4$ & -18.0 & +4.0 & 44.0 \\
\hline 6 & 29.692 & 29.723 & $29.69 \mathrm{I}$ & -26.0 & -19.1 & -9.9 & - I8.0 & -28.0 & - I8. I & -9.2 & $\div 27.0$ & -18.6 & 18.8 \\
\hline 7 & 29.766 & 29.841 & 29.955 & - I9.0 & -16.5 & -23.9 & - 10.0 & -21.0 & -16.0 & -16.0 & $\div 24.5$ & -17.2 & $14 \cdot 5$ \\
\hline 8 & 29.970 & 29.992 & 29.992 & -23.0 & -16.1 & -20.0 & -20.3 & -27.5 & -15.5 & -13.4 & -23.0 & -20.4 & I4. I \\
\hline 9 & 29.962 & 29.957 & 29.914 & -17.6 & -13.9 & - I9.4 & - 17.6 & -26.2 & -13.2 & -10.0 & -19.4 & - I8.I & 16.2 \\
\hline 10 & 29.790 & 29.725 & 29.610 & -15.0 & -12.0 & -9.8 & -15.0 & -22.1 & - II.O & -8.3 & -15.0 & -15.2 & 13.8 \\
\hline II & 29.485 & 29.462 & 29.498 & -15.0 & -15.4 & -26.0 & -6.2 & -16.0 & $-15 \cdot 4$ & -12.2 & -26.2 & -16.2 & 20.0 \\
\hline 12 & 29.701 & 29.741 & 29.725 & -35.4 & -37.0 & -33.8 & -26.0 & -36.6 & -35.4 & -31.9 & -37.0 & $-3 \mathrm{I} \cdot 5$ & II.O \\
\hline 13 & 29.575 & $29.56 \mathrm{I}$ & 29.549 & -23.0 & -17.8 & -19.0 & -23.0 & -33.8 & -17.6 & -17.4 & -23.0 & -25.6 & 16.4 \\
\hline 14 & 29.475 & 29.485 & 29.485 & -17.4 & -16.8 & -27.0 & -17.0 & - 19.0 & -16.6 & - 16.6 & -27.2 & -21.9 & 10.6 \\
\hline 15 & 29.302 & 29.297 & $29 \cdot 385$ & -32.2 & -32.9 & -34.0 & -27.0 & $-3^{6.3}$ & -29.0 & -29.0 & -35.0 & -31.6 & 9.3 \\
\hline I6 & 29.422 & 29.504 & 29.536 & -14.9 & -10.0 & -6.0 & -14.9 & -34.0 & -10.0 & $-5 . I$ & -14.9 & -19.6 & 28.9 \\
\hline 17 & 29.484 & $29 \cdot 465$ & 29.477 & -4.5 & -4.0 & -5.0 & - 3.0 & -6.0 & -3.1 & 1.0 & -10.2 & 4.6 & II. 2 \\
\hline 18 & 29.571 & 29.625 & 29.860 & $-\mathrm{I} 3.2$ & -4.0 & -23.6 & -3.8 & -13.4 & -3.7 & $3 \cdot 7$ & -23.6 & -13.6 & 19.9 \\
\hline 19 & 30.096 & 30.085 & 29.799 & -26.0 & $-\mathrm{I} 8 . \mathrm{I}$ & -.3 .0 & -23.6 & -33.1 & -17.2 & $-2 . I$ & -26.0 & -17.6 & 31.0 \\
\hline 20 & 29.323 & 29.331 & 29.489 & 0.0 & +1.6 & +2.0 & 0.0 & -3.0 & +2.0 & 4.0 & 0.0 & +0.5 & 7.0 \\
\hline 21 & 29.557 & 29.521 & 29.563 & $3 \cdot 9$ & +4.0 & +3.0 & +6.2 & $-1.7^{\circ}$ & +5.0 & 8.2 & +3.0 & 3.2 & 9.9 \\
\hline 22 & 29.779 & 29.850 & 29.946 & -7.5 & -3.0 & -3.0 & +3.0 & -8.5 & -3.0 & 2.0 & -7.5 & -2.8 & 11.5 \\
\hline 23 & 29.963 & $29.966^{\circ}$ & 29.938 & -5.0 & $+\quad$ I.0 & -4.0 & -3.0 & - II.0 & +2.0 & 2.0 & -4.6 & 4.5 & 13.0 \\
\hline 24 & 29.842 & 29.833 & 29.789 & - I:0 & +3.9 & -4.0 & $\quad 0.0$ & -6.1 & +4.0 & 4.0 & $-4 \cdot \dot{3}$ & -1.0 & IO. 1 \\
\hline 25 & $29.74 \mathrm{I}$ & 29.718 & 29.692 & -6.0 & -3.0 & -6.5 & -4.0 & -7.8 & -2.8 & -3.0 & $\div 6.5$ & $5 \cdot 3$ & 5.0 \\
\hline 110126 & 29.704 & 29.705 & 29.688 & $-16: 5$ & - 16.0 & -16.7 & -6.5 & - 18.5 & .15 .0 & - 12.8 & -18.0 & -12.5 & 12.0 \\
\hline 27 & 29.594 & 29.585 & 29.554 & - I5:0 & -13.2 & -19.0 & -12.8 & -21.0 & -12.8 & 8.9. & -19.0. & -15.0 & 12.1 \\
\hline 28 & 29.617 & 29.668 & 29.757 & -18.0 & -11.9 & -17.9 & -19.0 & -22.5 & -10.0 & 9.6. & -18.0 & -16.0 & 12.9 \\
\hline 29 & 29.909 & 29.951 & 30.042 & -16.0 & -13.0 & -14.0 & - I5.8 & $-.2 \mathrm{I} .4$ & -13.0 & $-\mathrm{I} 1.8$ & -16.0 & $-\mathrm{I} 6.6$ & 9.6 \\
\hline 30 & 30.062 & $\cdots$ & $\ldots$ & -12.2 & $\because$ & (一 & -12.2 & -17.0 & & $\ldots$ & $\cdots$ & $\cdots$ & $\cdots$ \\
\hline Sum.... & 890.249 & 860.512 & 860.853 & -207.6 & -285.6 & -285.5 & -170.1 & -429.7 & - I 59.9 & -96.3 & -382.5 & -265.8 & 436.8 \\
\hline Mern... & 29.675 & 29.673 & 29.685 & 9.2 & -7.2 & $-\quad 0.8$ & -5.7 & $-\mathrm{I} 4.3$ & -5.5 & $3: 3$ & $-13: 2$ & -9.2 & I5. I \\
\hline
\end{tabular}


Tabulation of daily meteorological observations at Teplitz Bay during the month of April, 1904-Continued

Observer: Francis LoNG

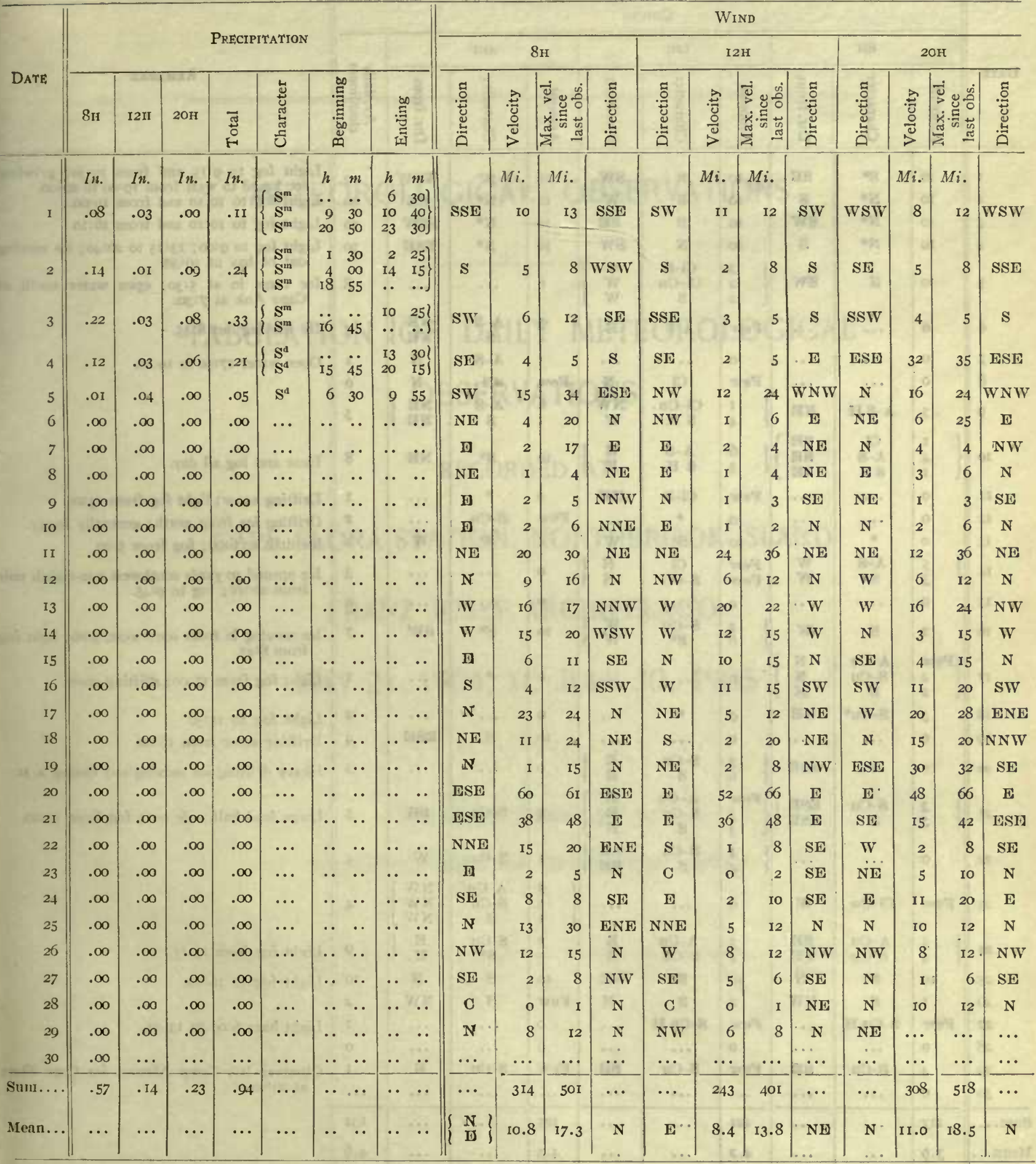


Tabulation of daily meteorological observations at Teplitz Bay during the month of April, Ig04-Continued

Observer: Francis LoNG

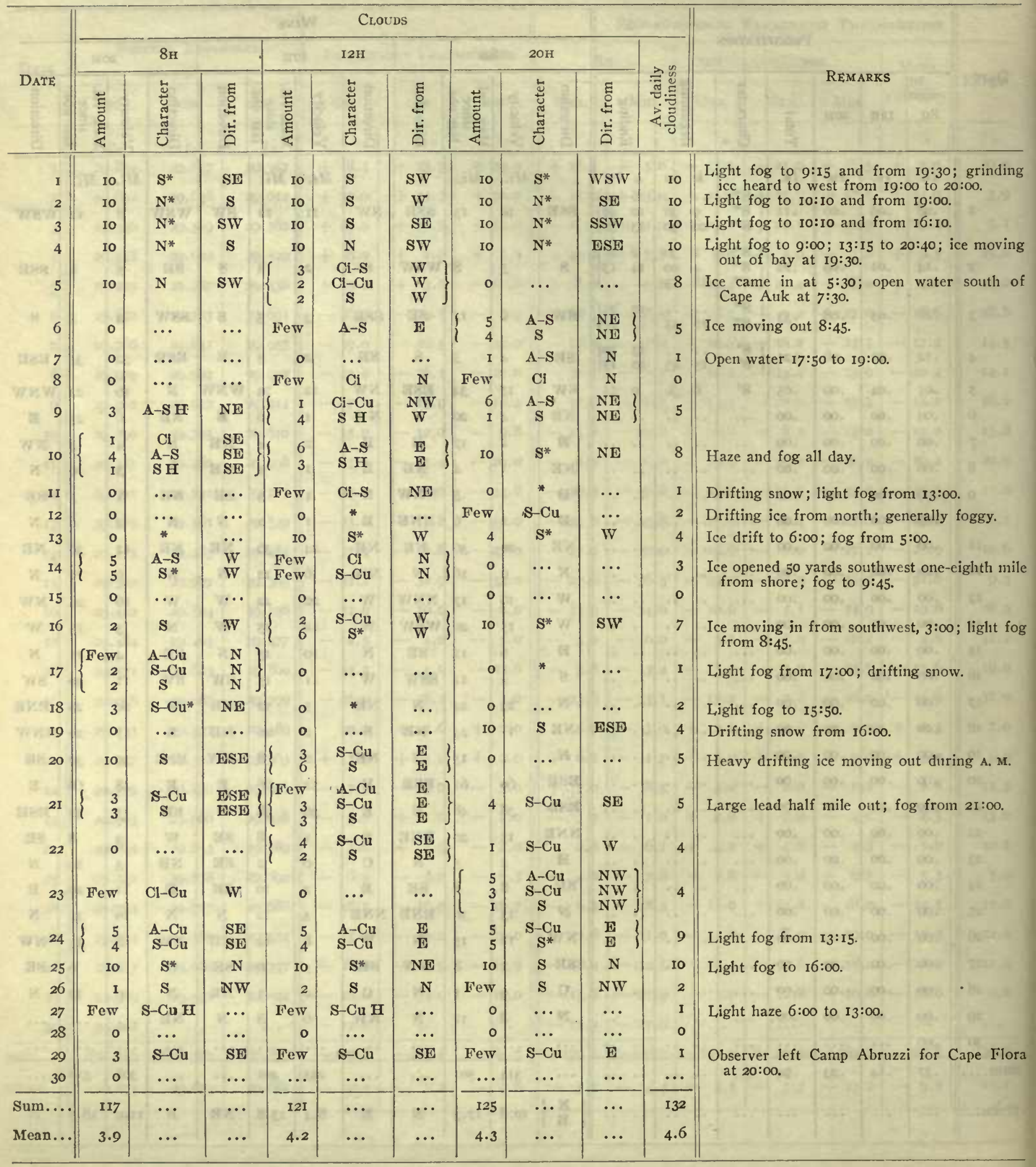




\title{
METEOROLOGICAL OBSERVATIONS
}

\section{TABULATION OF DAILY METEOROLOGICAL} OBSERVATIONS

\author{
RECORDED AT
}

CAPE FLORA STATION, NORTHBROOK ISLAND

FRANZ JOSEF ARCHIPELAGO

MAY 21, 1904, TO JULY 30, 1905

NORTH LATITUDE: $79^{\circ} 57^{\prime}$

LONGITUDE EAST OF GREENWICH: $49^{\circ} 59^{\prime}$ 


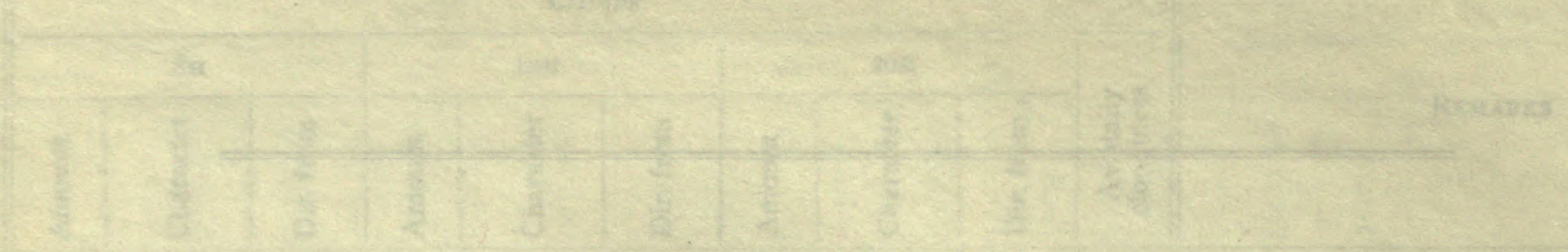

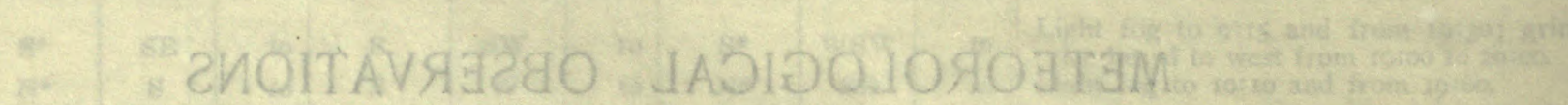
sith

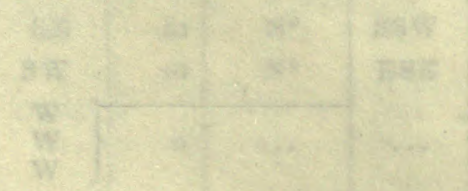

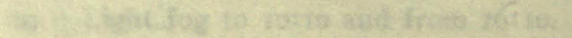

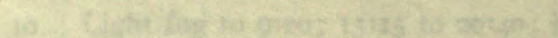

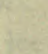

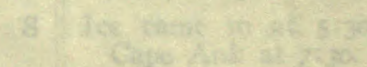

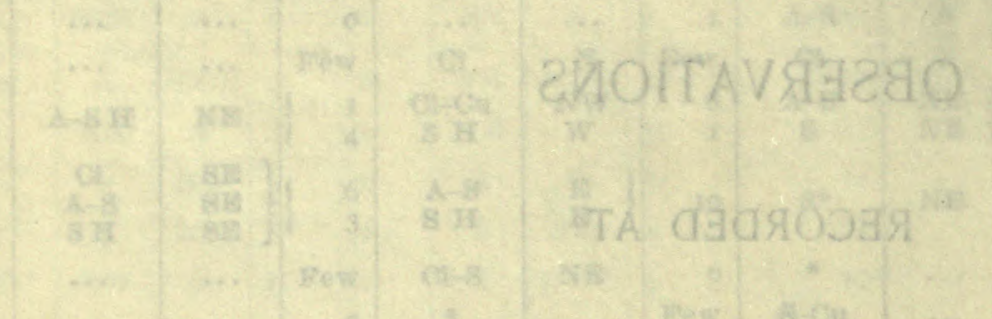

MOITAIUEAT

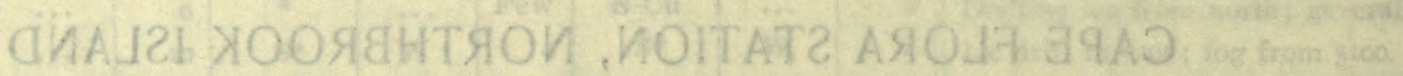

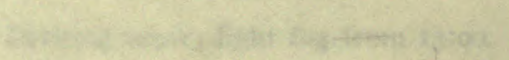

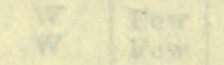

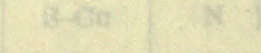

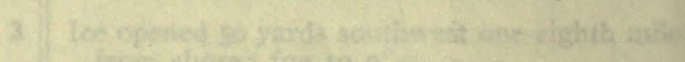
OОА. ЗЯННОЯА 7Э2ОL ГИАЯЯ

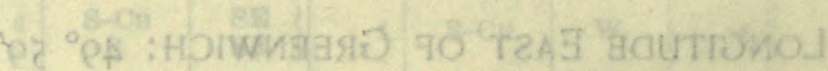

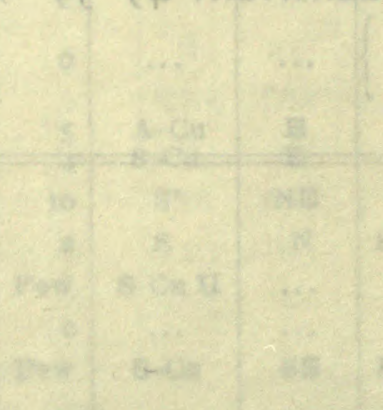


Tabulation of daily meteorological observations at Capc Flora during the month of May, Igo4

Observer: FRANCIS LaNG

\begin{tabular}{|c|c|c|c|c|c|c|c|c|c|c|c|c|c|}
\hline \multirow{3}{*}{ DATE } & \multirow{2}{*}{\multicolumn{3}{|c|}{ ANEROID BAROMETER }} & \multirow{2}{*}{\multicolumn{3}{|c|}{$\begin{array}{c}\text { READING OF } \\
\text { FAHRENHEIT THERMOMETER }\end{array}$}} & \multicolumn{7}{|c|}{ SFLF-REGISTERING FAHRENHEIT THERMOMETERS } \\
\hline & & & & & & & \multicolumn{2}{|c|}{$8 \mathrm{H}$} & \multirow{2}{*}{$\begin{array}{l}\text { I2II } \\
\text { Max. }\end{array}$} & \multicolumn{2}{|c|}{$20 \mathrm{H}$} & \multirow{2}{*}{$\begin{array}{c}\text { Mean } \\
\text { of } \\
\text { extremes }\end{array}$} & \multirow{2}{*}{ Range } \\
\hline & $8 \mathrm{r}$ & $\mathrm{I} 2 \mathrm{H}$ & $20 \mathrm{H}$ & $8 \mathrm{H}$ & $12 \mathrm{H}$ & $20 \mathrm{H}$ & Max. & Min. & & Max. & Min. & & \\
\hline & In. & In. & In. & -5 & 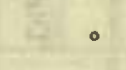 & 3. & . & $\circ$ & 。 & - & - & - & - \\
\hline & $\cdots$ & $\cdots$ & $\cdots$ & an: $\cdots$ & $\cdots$ & $\cdots$ & $\cdots$ & $\cdots$ & $\cdots$ & $\cdots$ & $\ldots$ & $\cdots$ & $\cdots$ \\
\hline 2 & $\cdots$ & & $\cdots$ & $=\cdots$ & $\cdots$ & $\cdots$ & $\cdots$ & $\cdots$ & $\cdots$ & $\cdots$ & $\cdots$ & $\ldots$ & ... \\
\hline 3 & $\cdots$ & & $\cdots$ & $\cdots$ & $\ldots$ & $\ldots$ & 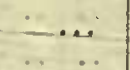 & $\ldots$ & $\cdots$ & $\cdots$ & $\cdots$ & $\ldots$ & .. \\
\hline 4 & $\cdots$ & & $\cdots$ & $\cdots$ & $\ldots$ & $\quad \cdots$ & $\cdots$ & $\cdots$ & $\cdots$ & $\cdots$ & $\ldots$ & $\cdots$ & $\cdots$ \\
\hline 5 & $\cdots$ & $\cdots$ & $\ldots$ & $\cdots$ & $\cdots$ & $\cdots$ & $\cdots \cdots$ & $\cdots$ & $\cdots$ & $\ldots$ & $\ldots$ & $\ldots \cdots$ & ... \\
\hline 6 & $\ldots$ & & $\ldots$ & $\ldots$ & $\ldots$ & $\ldots$ & $\ldots$ & $\cdots$ & $\ldots$ & $\ldots$. & $\ldots$ & $\ldots$ & $\ldots$ \\
\hline 7 & $\cdots$ & & $\cdots$ & $\cdots$ & $\ldots$ & $\cdots$ & $\cdots$ & $\ldots$ & $\cdots$ & $\cdots$ & $\ldots$ & $\cdots$ & $\cdots$ \\
\hline 8 & $\cdots$ & & $\cdots$ & .. & $\ldots$ & $\ldots$ & $\ldots$ & $\ldots$ & $\cdots$ & $\ldots$ & $\ldots$ & $\cdots$ & $\cdots$ \\
\hline 9 & $\cdots$ & $\cdots$ & $\cdots$ & $\ldots$ & $\ldots$ & $\ldots \cdots$ & $\cdots$ & $\cdots$ & $\cdots$ & $\ldots$ & $\ldots$ & $\ldots \cdots$ & $\ldots$ \\
\hline Io & $\ldots$ & $\ldots$ & $\ldots$ & $\ldots$ & $\ldots$ & $\cdots$ & $\ldots$ & $\ldots$ & $\ldots$ & $\ldots$ & $\ldots \ldots$ & $\ldots$ & $\ldots$ \\
\hline II & $\ldots$ & & $\cdots$ & $\ldots$ & $\ldots$ & $\because \quad \therefore$ & $\therefore$ & $\therefore$ & $\cdots$ & $\therefore$ & $\cdots \ldots$ & $\cdots$ & $\ldots$ \\
\hline 12 & $\cdots$ & 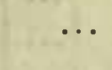 & $\ldots$ & $\ldots$ & ... & $\cdots$ & $\ldots$ & $\because$ & $\cdots$ & $\cdots$ & $\cdots$ & $\cdots$ & $\ldots$ \\
\hline 13 & $\ldots$ & 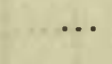 & & ... & $\cdots$ & $\cdots$ & $\cdots \cdots$ & $\because$ & $\cdots$ & $\ldots$ & $\ldots$ & $\ldots \cdots$ & $\cdots$ \\
\hline 14 & $\ldots$ & & $\ldots$ & $\cdots$ & & $\cdots$ & $\ldots$ & $\cdots$ & $\cdots$ & $\cdots$ & $\ldots$ & $\ldots$ & $\cdots$ \\
\hline 15 & $\ldots$ & & $\cdots$ & $\cdots$ & $\ldots$ & $\ldots$ & $\cdots$ & $\therefore$ & $\cdots$ & $\cdots$ & $\cdots$ & $\cdots$ & $\cdots$ \\
\hline 16 & $\cdots$ & $\cdots$ & $\cdots$ & $\cdots$ & & $\cdots$ & $\cdots$ & $\cdots$ & $\cdots$ & $\cdots$ & $\cdots$ & $\cdots$ & $\cdots$ \\
\hline 17 & ... & $\ldots$ & ... & $\cdots$ & $\ldots$ & $\cdots$ & $\cdots$ & $\cdots$ & $\cdots$ & $\ldots$ & $\cdots$ & $\ldots$ & $\cdots$ \\
\hline 18 & ... & $\ldots$ & $\ldots$ & ... & $\ldots$ & $\cdots$ & $\cdots$ & $\cdots$ & $\cdots$ & $\cdots$ & $\cdots$ & $n, \cdots$ & $\cdots$ \\
\hline 19 & $\cdots$ & $\cdots$ & .. & $\cdots$ & $\cdots$ & $\cdots$ & $\cdots$ & $\cdots$ & $\cdots$ & $\cdots$ & $\cdots$ & $\cdots$ & $\cdots$ \\
\hline 20 & . & $\ldots$ & $\ldots$ & $\quad \ldots$ & $\ldots$ & $\ldots$ & $\ldots$ & $\cdots$ & $\ldots$ & $\ldots$ & $\ldots$ & $\ldots$ & $\ldots$ \\
\hline $2 I$ & 29.70 & 29.68 & 29.72 & +16.5 & +16.7 & +18.0 & +17.1 & +13.2 & +17.4 & +19.0 & +15.1 & +16.1 & 5.8 \\
\hline 22 & 29.62 & 29.62 & 29.60 & +15.0 & +17.0 & +19.0 & +19.0 & +11.0 & +18.0 & +19.0 & $+\quad$ I.o & + 10.0 & I8.o \\
\hline 23 & 29.66 & 29.74 & 29.84 & +15.5 & +16.3 & +18.0 & +19.0 & +10.5 & +16.3 & +22.5 & +12.2 & +16.5 & 12.0 \\
\hline 24 & 29.87 & 29.90 & 30.05 & +16.5 & +18.4 & +14.2 & +18.0 & +10.0 & +19.0 & $+2 \mathrm{I} .4$ & +14.2 & +15.7 & II. 4 \\
\hline 25 & 30.20 & 30.20 & 30.20 & +19.5 & +20.0 & +18.0 & +20.0 & $+\mathrm{II} . \mathrm{o}$ & +20.0 & +20.0 & +17.9 & +15.5 & 9.0 \\
\hline 26 & 30.14 & 30.08 & 30.006 & +17.0 & +18.5 & +20.4 & +18.0 & +15.0 & +18.8 & +20.4 & +16.6 & +17.7 & $5 \cdot 4$ \\
\hline 27 & 29.86 & 29.84 & 29.83 & +29.0 & +33.1 & $+34 . I$ & +29.0 & +20.0 & +35.6 & +35.6 & +29.0 & +27.8 & 15.6 \\
\hline 28 & 29.84 & 29.84 & 29.75 & +33.8 & +34.4 & +31.0 & +34.1 & +31.2 & +35.0 & +35.0 & +30.6 & +32.8 & 4.4 \\
\hline 29 & 29.72 & 29.74 & 29.70 & +33.0 & +30.4 & +25.0 & +34.0 & $+3 \mathrm{I} .0$ & +32.3 & +32.3 & +24.0 & +29.0 & 10.o \\
\hline 30 & 29.72 & 29.74 & $29.8 \mathrm{I}$ & +20.1 & +20.5 & +15.8 & +25.0 & +19.0 & +20.5 & +20.5 & +15.0 & +20.0 & 10.0 \\
\hline 31 & 29.87 & 29.89 & 29.93 & +20.5 & +22.0 & +22.5 & +21.0 & +14.7 & +22.2 & +24.5 & +20.0 & +19.6 & 9.8 \\
\hline Sum.... & 328.20 & 328.27 & 328.41 & +236.4 & $+247 \cdot 3$ & +236.0 & +254.2 & +186.6 & +255.1 & +270.2 & +195.6 & +220.7 & ..111.4 \\
\hline Mean... & 29.84 & 29.84 & 29.86 & +21.5 & +22.5 & +21.5 & $+23 . I$ & +17.0 & +.23 .2 & +24.6 & +.17 .8 & +20.1 & $10 . x$ \\
\hline
\end{tabular}


Tabulation of daily meteorological observations at Cape Flora during the month of May, 1904-Continued Observer: Francis LoNG

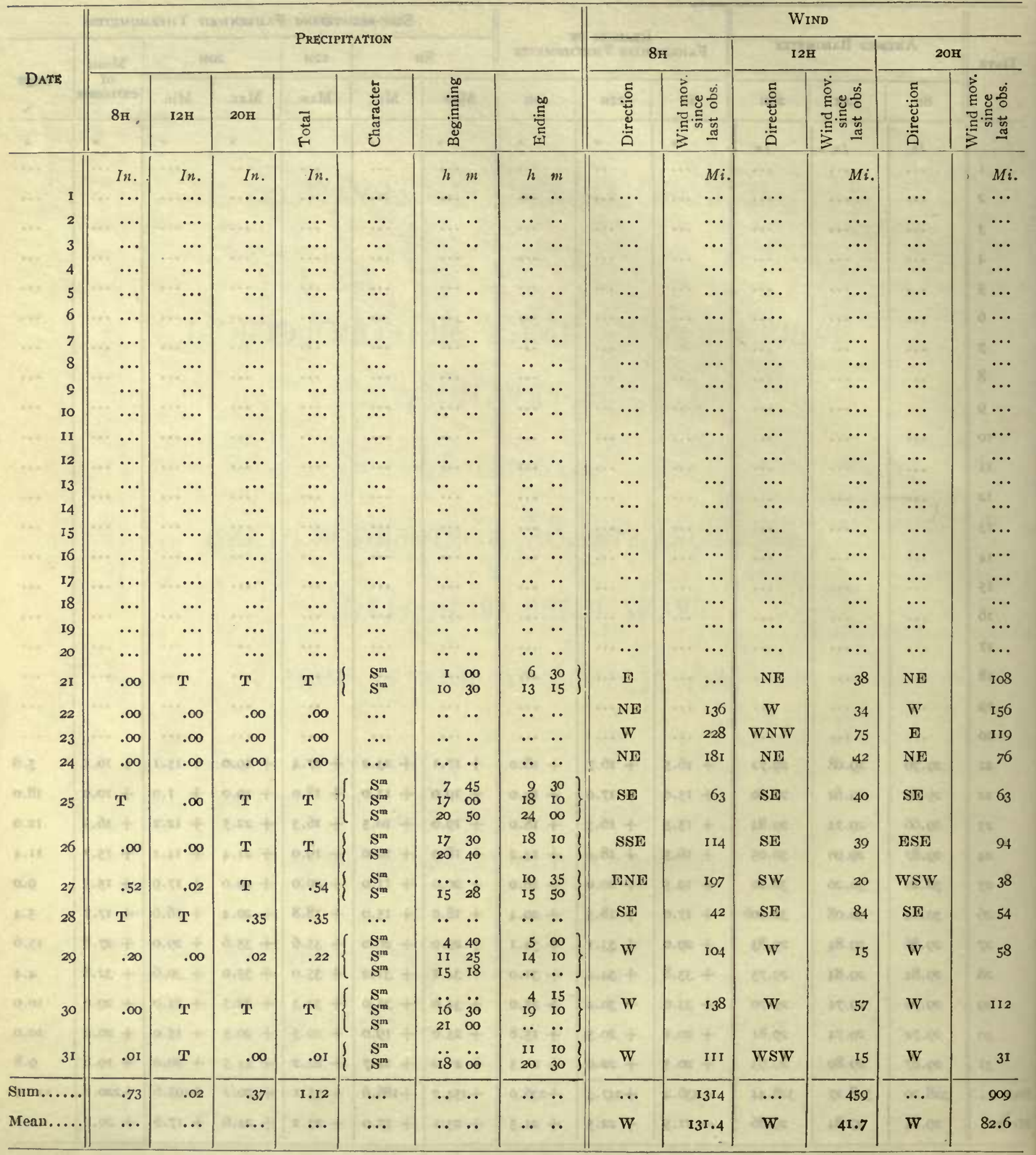


Tabulation of daily meteorological observations at Cape Flora during the month of May, Ig04-Continued Obscrver: FRANCIS LONG

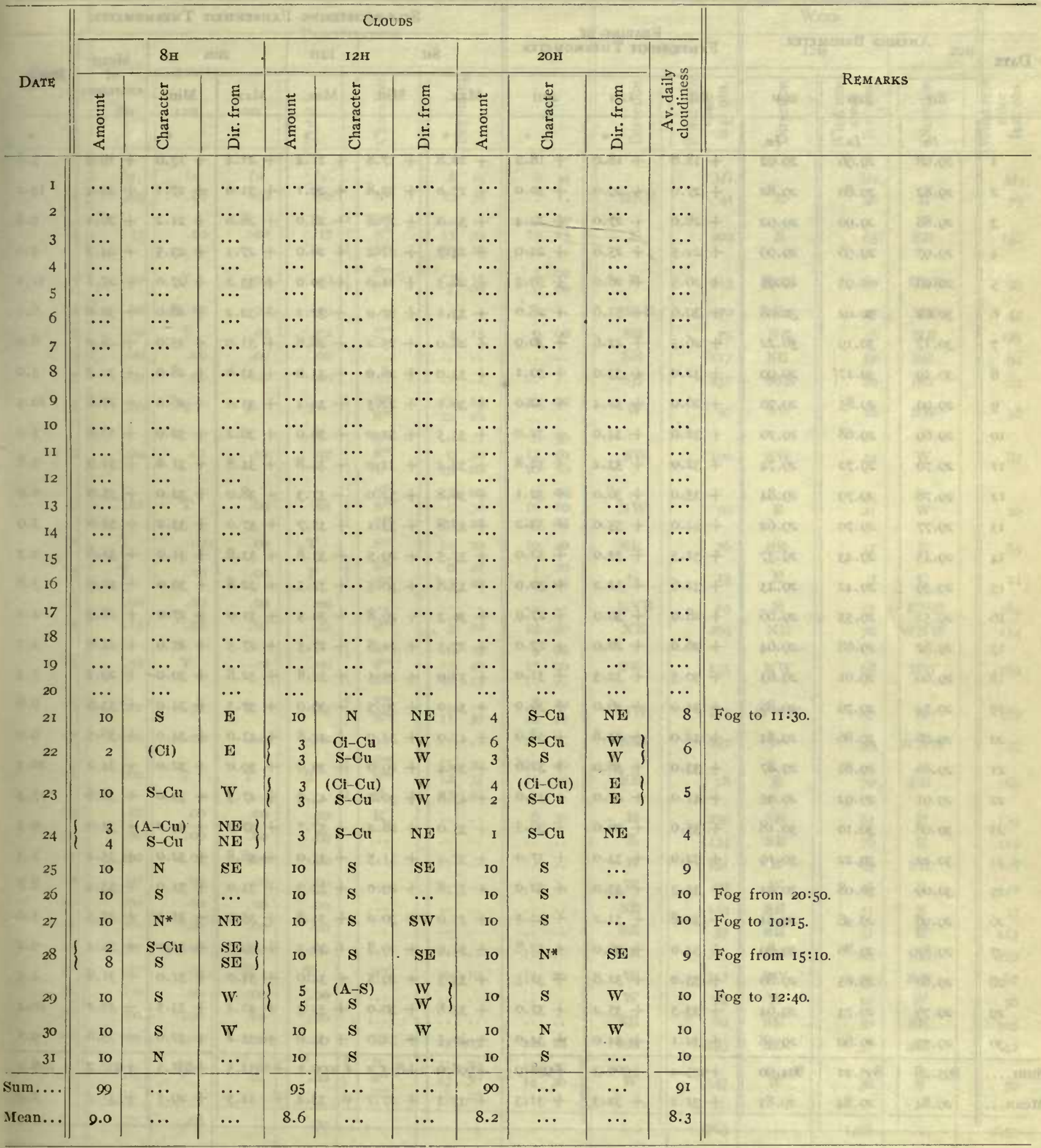


Tabulation of daily metcorological observations at Cape Flora during the month of June, 1904

Observer: Francis Long

\begin{tabular}{|c|c|c|c|c|c|c|c|c|c|c|c|c|c|}
\hline \multirow{3}{*}{ Date } & \multirow{2}{*}{\multicolumn{3}{|c|}{ ANEROID BARONETER }} & \multirow{2}{*}{\multicolumn{3}{|c|}{$\begin{array}{l}\text { READING OF } \\
\text { FAHRENHEIT THERMOMETER }\end{array}$}} & \multicolumn{7}{|c|}{ SELF-REGISTERING FAHRENHEIT THERMOMETERS } \\
\hline & & & & & & & 8 & & $12 \mathrm{H}$ & 2 & 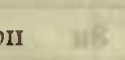 & & \\
\hline & $8 \mathrm{HI}$ & $\mathrm{I} 2 \mathrm{HI}$ & $20 \mathrm{H}$ & $\mathrm{SH}_{\mathrm{H}}$ & I2II & 2011 & Max. & Min. & Max. & Max. & Min. & & fonge \\
\hline 1 & $\begin{array}{c}\text { In. } \\
29.98\end{array}$ & $\begin{array}{l}\ln . \\
29.96\end{array}$ & $\begin{aligned} \ln . \\
29.92\end{aligned}$ & $\begin{array}{r}\circ \\
+\quad 18.0\end{array}$ & $\begin{array}{r}\circ \\
+18.0\end{array}$ & +18.2 & $\begin{array}{r}\circ \\
+22.8\end{array}$ & $\begin{array}{r}0 \\
+\quad 17.8\end{array}$ & $\begin{array}{r}0 \\
+21.4\end{array}$ & $\begin{array}{r}0 \\
+\quad 21.4\end{array}$ & $\begin{array}{r}0 \\
+\quad 17.0\end{array}$ & $\begin{array}{r}0 \\
+\quad 19.9\end{array}$ & $\stackrel{\circ}{5.8}$ \\
\hline 2 & 29.82 & 29.80 & 29.82 & +27.0 & +29.0 & +30.0 & $+27.0^{\circ}$ & +17.8 & $+29 . \mathrm{I}$ & +31.0 & +27.1 & $+24 \cdot 4$ & 13.2 \\
\hline 3 & 29.88 & 29.90 & 29.92 & +28.0 & +27.0 & +22.4 & +31.0 & +27.8 & +28.0 & +28.0 & +21.2 & +26.1 & 9.8 \\
\hline 4 & 29.97 & 29.96 & 29.99 & $+24 \cdot 5$ & +25.0 & +24.0 & +24.7 & +21.2 & +26.0 & +27.1 & +23.5 & $+24 \cdot 2$ & 5.9 \\
\hline 5 & 29.96 & 29.95 & 29.95 & +26.5 & +28.0 & +30.3 & +26.5 & $+2 \mathrm{r} .0$ & +30.0 & +33.2 & +27.0 & +27.1 & 12.2 \\
\hline 6 & 30.02 & 30.02 & 30.08 & +30.6 & +31.6 & +28.0 & +33.1 & +27.0 & +32.2 & +32.2 & +28.0 & +30.0 & 6. I \\
\hline 7 & 30.17 & 30. I9 & 30.22 & +26.5 & +27.6 & +30.9 & +28.0 & +25.2 & +28.6 & $+3 \mathrm{I} .0$ & +25.0 & +28.0 & 6.0 \\
\hline 8 & 30.19 & 30.17 & 29.99 & +32.0 & +33.0 & +30.1 & +33.0 & +28.0 & +33.0 & +33.0 & +28.0 & +30.5 & 5.0 \\
\hline 9 & 29.90 & 29.85 & 29.70 & +26.0 & +32.4 & +32.0 & +30.1 & +22.5 & +32.4 & +33.0 & +26.0 & +27.8 & I0. 5 \\
\hline IO & 29.69 & 29.68 & 29.70 & +32.0 & +34.0 & +32.0 & +33.5 & +31.0 & +36.0 & +36.2 & +32.0 & +33.6 & 5.2 \\
\hline II & 29.70 & 29.72 & 29.74 & +32.0 & +33.4 & +32.8 & +32.4 & +31.0 & +34.8 & +34.8 & +31.0 & +32.9 & 3.8 \\
\hline 12 & 29.78 & 29.79 & 29.84 & +35.0 & +36.0 & +32.1 & +36.8 & +32.0 & +37.3 & +38.0 & +32.0 & +35.0 & 6.0 \\
\hline 13 & 29.77 & 29.70 & 29.62 & +34.0 & +35.0 & +33.2 & +35.8 & +33.1 & +35.7 & +37.0 & +33.2 & +35.0 & 3.9 \\
\hline 14 & 29.45 & 29.43 & $29 \cdot 37$ & +31.5 & +33.0 & +32.0 & +33.5 & +29.5 & +33.8 & +33.8 & +31.0 & +31.6 & 4.3 \\
\hline 15 & 29.39 & 29.42 & 29.43 & +32.6 & +32.2 & +30.0 & +33.8 & +30.5 & +32.5 & +32.8 & +30.0 & +31.9 & 3.8 \\
\hline 16 & 29.52 & 29.55 & 29.66 & +28.0 & +30.0 & +27.0 & +30.3 & +26.8 & +30.5 & +31.0 & +27.0 & +28.9 & 4.2 \\
\hline 17 & 29.67 & 29.68 & 29.64 & +26.0 & +26.0 & +27.0 & +27.5 & $+2 \mathrm{I} .8$ & +27.5 & +27.5 & +25.0 & +24.6 & $5 \cdot 7$ \\
\hline 18 & 29.60 & 29.61 & 29.63 & +30.5 & +32.5 & +31.0 & +31.0 & +25.5 & +32.8 & +32.8 & $+30.0^{\circ}$ & +29.2 & $7 \cdot 3$ \\
\hline 19 & 29.74 & 29.76 & 29.85 & +34.0 & +36.0 & +36.0 & +34.0 & +30.5 & +36.0 & +37.3 & +34.0 & +33.9 & 6.8 \\
\hline 20 & 29.86 & 29.86 & 29.84 & +42.0 & +34.8 & +38.0 & +43.0 & +34.0 & +42.8 & +43.0 & +34.0 & +38.5 & 0.0 \\
\hline $2 \mathrm{I}$ & 29.86 & 29.85 & 29.87 & +33.0 & +38.0 & +32.6 & +39.4 & +29.0 & +39.0 & +39.0 & +32.0 & +34.2 & 10.4 \\
\hline 22 & $29.9 \mathrm{I}$ & 29.92 & 29.95 & $+4 \mathrm{I}: 0$ & +42.0 & +35.0 & +43.8 & +30.0 & +43.0 & +47.3 & +35.0 & +38.6 & $17 \cdot 3$ \\
\hline 23 & 30.08 & 30. 10 & 30.18 & +35.0 & +36.6 & +36.5 & +35.0 & +28.4 & +37.7 & +37.7 & +34.0 & +33.0 & 9.3 \\
\hline 24 & 30.22 & 30.22 & 30. 19 & +32.0 & +33.0 & +37.0 & +37.4 & +31.5 & +33.0 & +38.8 & +32.0 & +35.2 & $7 \cdot 3$ \\
\hline 25 & 30.09 & 30.08 & 30.04 & +32.3 & +33.0 & +32.0 & +37.8 & +29.0 & +33.0 & +33.0 & +31.0 & +33.4 & 8.8 \\
\hline 26 & 29.98 & 29.98 & 29.94 & +32.8 & +34.2 & +32.5 & +35.0 & +30.0 & +34.8 & +35.0 & +31.0 & +32.5 & 5.0 \\
\hline 27 & 29.87 & 29.86 & 29.89 & +34.0 & +36.0 & +32.8 & +34.0 & +29.8 & +36.3 & +39.0 & +32.0 & +34.4 & 9.2 \\
\hline 28 & 29.85 & 29.85 & 29.80 & +33.0 & +32.8 & +32.5 & +33.5 & +29.5 & +34.0 & +34.0 & +31.0 & +3 I. 8 & 4.5 \\
\hline 29 & 29.76 & 29.74 & 29.64 & +33.5 & +35.2 & +37.0 & +33.8 & +30.0 & +35.3 & +40.4 & +33.5 & +35.2 & I0. 4 \\
\hline 30 & 29.59 & 29.60 & 29.48 & +32.1 & +34.0 & +34.0 & +40.5 & +31.0 & +34.0 & +35.2 & +32.0 & +35.8 & 9.5 \\
\hline Sum.... & 895.28 & 895.21 & 894.90 & +935.4 & +969.3 & $+938: 9$ & $+\infty x 8.0$ & +832.2 & +1000.5 & +1033.5 & +885.5 & +937.2 & 216.2 \\
\hline ean... & 29.84 & 29.84 & 29.83 & +31.2 & +32.3 . & +31.3 & +33.3 & +27.7 & +33.4 & +34.5 & $+29.5^{\circ}$ & +31.2 & 7.2 \\
\hline
\end{tabular}


Tabulation of daily metcorulogical abservations at Cape Flora during the month of June, 1904-Continued Observer: FRANCIS LoNG

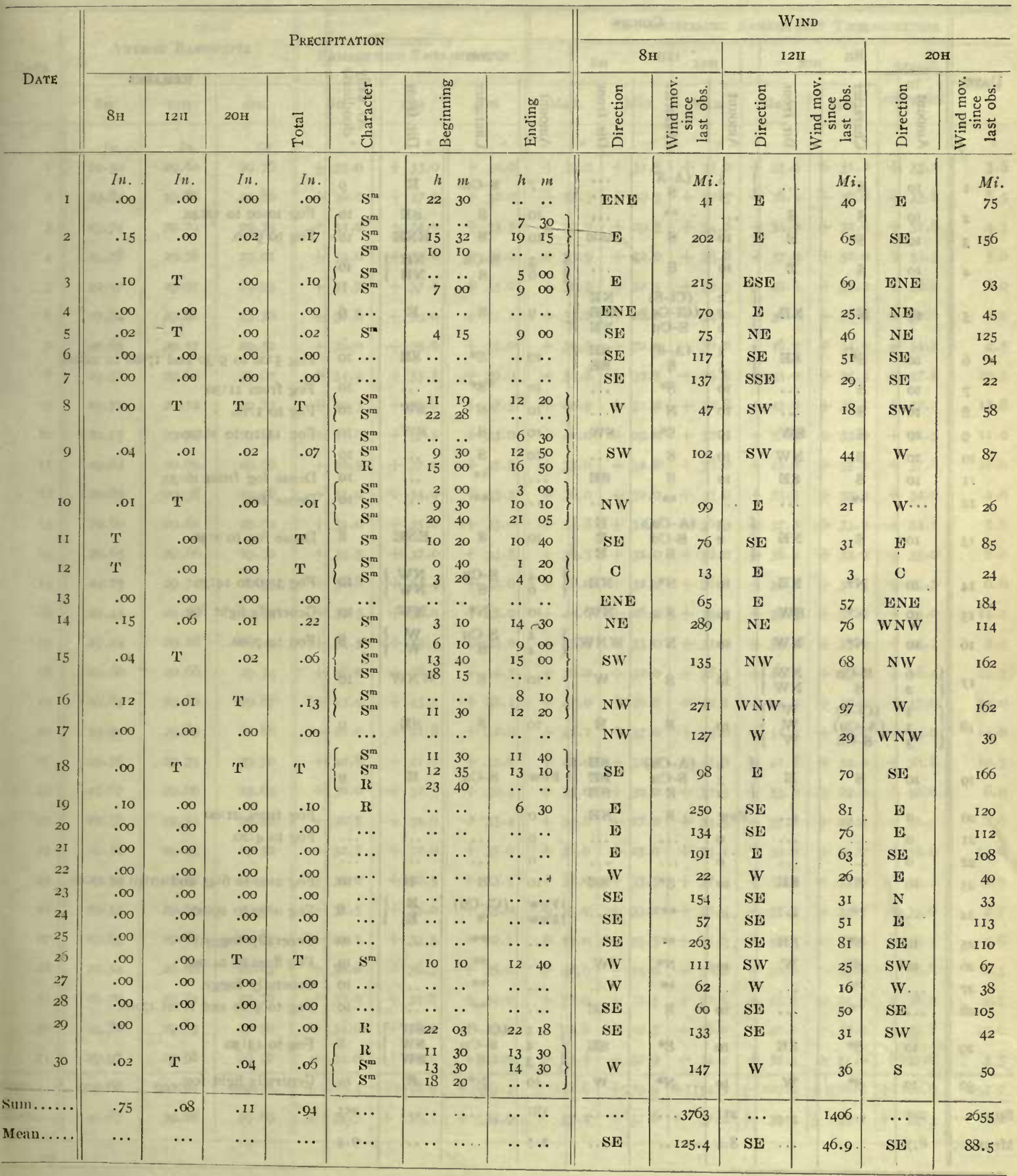


Tabulation of daily meteorological observations at Cape Flora during the month of June, 1904-Continued Observer: Francis LoNG

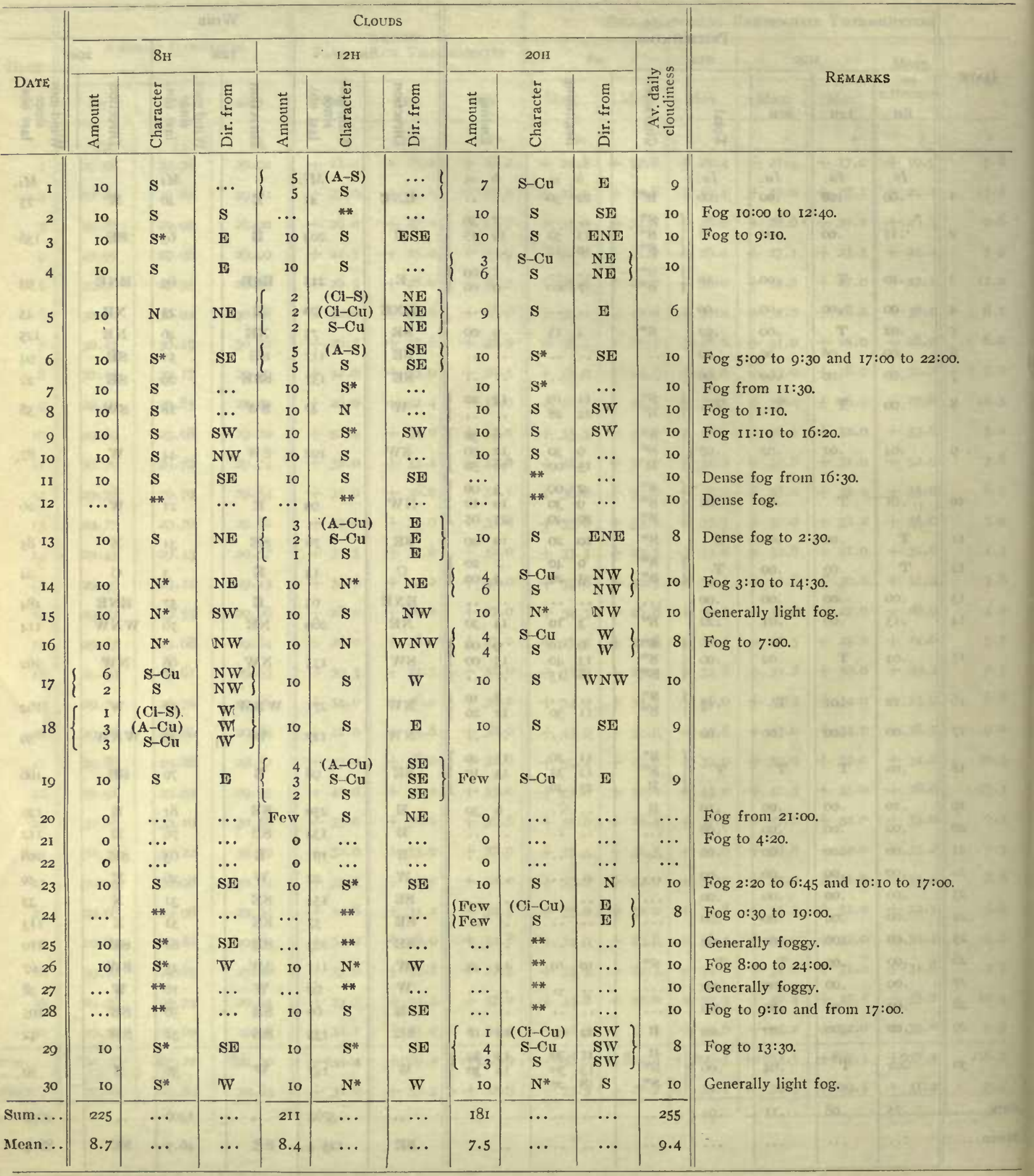


Tabulation of daily meteorological observations at Cape Flora during the month of July, I904

Observer: FRANCIS LONG

\begin{tabular}{|c|c|c|c|c|c|c|c|c|c|c|c|c|c|}
\hline \multirow{3}{*}{ DATE: } & \multirow{2}{*}{\multicolumn{3}{|c|}{ ANEROID BAROMETER }} & \multirow{2}{*}{\multicolumn{3}{|c|}{$\begin{array}{c}\text { READING OF } \\
\text { FAHRENHEIT THERMOMETER }\end{array}$}} & \multicolumn{7}{|c|}{ SELF-REGISTERING FAHRENHEIT THERMOMETERS } \\
\hline & & & & & & & \multicolumn{2}{|c|}{$8 \mathrm{H}$} & \multirow{2}{*}{$\frac{\mathrm{I} 2 \mathrm{H}}{\mathrm{Max}}$} & \multicolumn{2}{|c|}{$2 \mathrm{OH}$} & \multirow{2}{*}{$\begin{array}{c}\text { Mean } \\
\text { of } \\
\text { extremes }\end{array}$} & \multirow{2}{*}{ Range } \\
\hline & $8 \mathrm{H}$ & $\mathrm{I2H}$ & $2 \mathrm{OH}$ & $8 \mathrm{H}$ & $\mathrm{I} 2 \mathrm{H}$ & $2 \mathrm{OH}$ & Max. & Min. & & Max. & Min. & & \\
\hline & In. & In. & In. & & 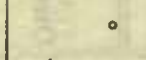 & 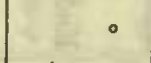 & 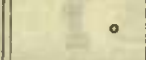 & $\circ$ & $\circ$ & $\circ$ & & ○ & 。 \\
\hline$I$ & 29.50 & 29.60 & 29.76 & +35.0 & +33.0 & +32.0 & +35.0 & +31.5 & +35.0 & +35.0 & +31.2 & +33.1 & 3.8 \\
\hline 2 & 29.82 & 29.86 & 29.86 & +36.0 & +37.5 & +32.0 & $+37 \cdot 3$ & +29.5 & $+37 \cdot 5$ & +38.0 & +30.0 & +33.8 & 8.5 \\
\hline 3 & 29.74 & 29.74 & 29.74 & +33.0 & +34.0 & +33.5 & +33.0 & +29.5 & +34.0 & +34.0 & +32.0 & +31.8 & 4.5 \\
\hline 4 & 29.78 & 29.78 & 29.91 & +33.0 & +33.6 & +36.0 & +33.9 & +32.0 & +33.6 & +37.0 & +32.0 & +34.5 & 5.0 \\
\hline 5 & 29.97 & 29.97 & 29.93 & +35.7 & +37.0 & +33.0 & +36.5 & +32.5 & $+39 \cdot 3$ & +40.0 & +32.0 & +36.0 & 8.0 \\
\hline 6 & 29.84 & $29.8 \mathrm{I}$ & 29.76 & +30.8 & +34.0 & +33.1 & +33.2 & +28.0 & +34.0 & +36.0 & +30.8 & +32.0 & 8.0 \\
\hline 7 & 29.63 & 29.57 & 29.52 & +36.0 & +35.0 & +34.0 & +36.5 & +32.0 & +36.0 & +36.0 & +34.0 & +34.2 & $4 \cdot 5$ \\
\hline 8 & 29.57 & 29.60 & 29.67 & +34.4 & +35.5 & +39.0 & +34.8 & +32.5 & +35.5 & +43.0 & +34.4 & +37.8 & 10.5 \\
\hline 9 & 29.71 & 29.72 & 29.73 & +40.3 & +39.0 & +37.5 & +40.5 & +31.2 & +40.8 & +43.0 & +37.5 & +37.1 & II. 8 \\
\hline I0 & 29.75 & 29.74 & 29.64 & +35.0 & +37.5 & +33.0 & +40.0 & +29.0 & +37.5 & +37.5 & +33.0 & +34.5 & II.O \\
\hline II & 29.63 & 29.69 & 29.69 & +36.5 & +37.0 & +35.5 & +37.0 & +32.8 & +38.0 & +42.8 & +35.0 & +37.8 & 10.0 \\
\hline 12 & 29.63 & 29.58 & 29.58 & +35.0 & +34.0 & +33.3 & +36.0 & +32.0 & +35.0 & +35.5 & +33.0 & +34.0 & 4.0 \\
\hline 13 & 29.60 & $29.6 \mathrm{I}$ & 29.62 & +34.0 & +35.6 & +33.0 & +34.8 & +31.2 & +36.9 & +37.0 & +33.0 & +34.1 & 5.8 \\
\hline I4 & 29.65 & 29.66 & 29.72 & +35.5 & +37.0 & +33.5 & +36.5 & +32.0 & +38.0 & +38.0 & +33.0 & +35.0 & 6.0 \\
\hline 15 & 29.75 & 29.74 & 29.59 & +36.8 & +34.4 & +33.0 & +38.0 & +32.4 & +36.8 & +39.0 & +32.5 & +35.7 & 6.6 \\
\hline I6 & 29.44 & 29.38 & 29.33 & +39.5 & +37.0 & +35.0 & +40.0 & +26.0 & +39.5 & +39.5 & +33.0 & +33.0 & 14.0 \\
\hline I7 & 29.24 & 29.28 & .29 .44 & +34.5 & +33.5 & +33.0 & +35.4 & +33.0 & +35.0 & +35.0 & +33.0 & +34.2 & 2.4 \\
\hline I8 & 29.60 & 29.66 & 29.74 & +36.0 & +38.4 & +43.5 & +36.0 & +32.0 & +38.4 & +44.7 & +35.0 & +38.4 & 12.7 \\
\hline 19 & 29.85 & 29.86 & 29.87 & +43.0 & +45.5 & $+42 . I$ & +47.0 & +40.0 & +47.1 & +54.0 & +40.0 & +47.0 & 14.0 \\
\hline 20 & 29.90 & 29.89 & 29.88 & +40.0 & +41.8 & +42.0 & +42.1 & +37.0 & +43.0 & +51.0 & +39.0 & +44.0 & I4. 0 \\
\hline $2 I$ & 29.78 & 29.75 & 29.70 & +40.8 & +41.0 & +35.0 & +42.0 & +31.5 & +41.8 & +41.8 & +34.0 & +36.8 & 10.5 \\
\hline 22 & 29.67 & 29.66 & 29.65 & +30.0 & +32.5 & +30.5 & +35.0 & +30.0 & +32.5 & +33.0 & +29.0 & +32.0 & 6.0 \\
\hline 23 & 29.56 & 29.54 & 29.49 & +32.5 & +34.0 & +33.4 & +33.0 & +27.2 & +34.5 & +35.8 & +29.4 & +31.5 & 8.6 \\
\hline 24 & 29.34 & 29.43 & 29.52 & +32.5 & +32.0 & +35.0 & +36.0 & +25.0 & +33.1 & +39.0 & +29.0 & +32.0 & $: 4.0$ \\
\hline 25 & 29.54 & 29.58 & 29.54 & +34.0 & +36.5 & +33.5 & +35.0 & +33.5 & +37.9 & +37.9 & $+33.0^{\circ}$ & +35.4 & 4.9 \\
\hline 26 & 29.55 & 29.57 & 29.66 & +34.5 & +35.0 & +35.0 & +35.0 & +33.2 & +35.4 & +35.9 & +33.5 & +34.6 & 2.7 \\
\hline 27 & 29.63 & 29.63 & 29.61 & +40.1 & +34.5 & +39.5 & +43.0 & +33.5 & $+4 \mathrm{I} .0$ & +41.0 & +33.2 & +38.1 & 9.8 \\
\hline 28 & 29.54 & 29.56 & 29.63 & +38.0 & +36.3 & +35.0 & +40.0 & +34.0 & +38.0 & +40.0 & +33.5 & +36.8 & 6.5 \\
\hline 29 & 29.70 & 29.74 & 29.76 & +36.0 & +34.8 & +34.0 & +37.0 & +33.5 & +37.0 & +43.0 & +34.0 & +38.2 & 9.5 \\
\hline 30 & 29.80 & 29.83 & 29.90 & +34.0 & +38.0 & +33.4 & +34.6 & +29.5 & +38.0 & +42.1 & +33.0 & +35.8 & 12.6 \\
\hline 31 & 29.97 & 29.98 & 29.99 & +34.0 & +34.0 & +32.0 & +34.5 & +32.9 & +34.4 & +34.5 & +31.0 & +32.8 & $3 \cdot 5$ \\
\hline Sum.... & 919.68 & 920.01 & 920.43 & +1106.4 & $+\operatorname{III} 8.9$ & +1083.3 & +1148.6 & +979.9 & +1154.5 & +1220.0 & +1026.0 & +1102.0 & 253.7 \\
\hline & 29.67 & 29.68 & 29.69 & +35.7 & +36.1 & +34.9 & $+37 \cdot 1$ & +31.6 & +37.2 & +39.4 & +33.1 & +35.5 & 8.2 \\
\hline
\end{tabular}


Tabulation of daily metcorological observations at Cape Flora during the month of July, 1904-Continued Observer: Francis Lonc

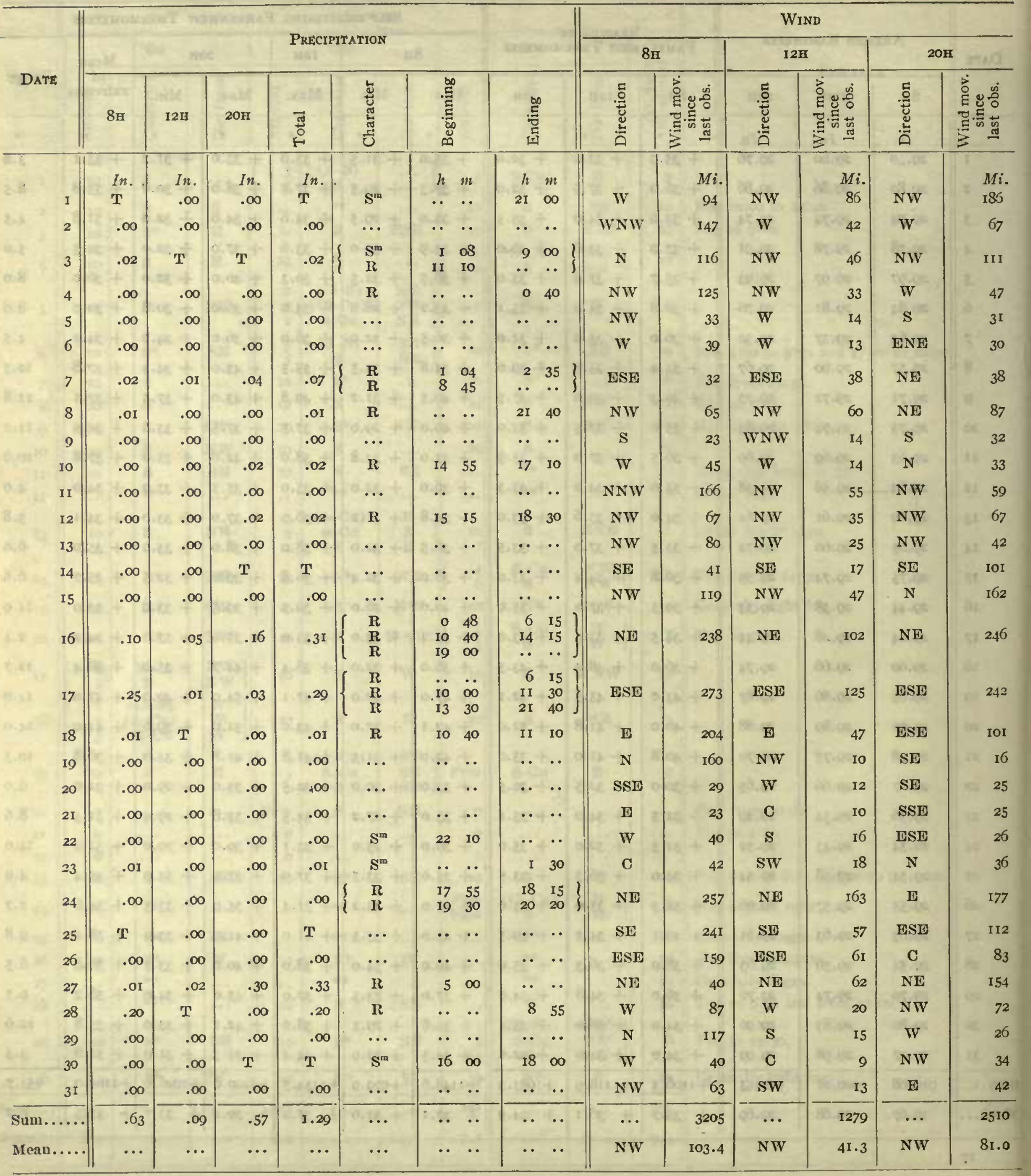


Tabulation of daily meteorological observations at Cape Flora during the month of July, 1904-Continued

Observer: FRANCIS LONG

\begin{tabular}{|c|c|c|c|c|c|c|c|c|c|c|c|c|c|c|}
\hline \multirow{4}{*}{ DATE } & \multicolumn{10}{|c|}{$\begin{array}{ccc}\text { Chouds } \\
\end{array}$} & \multirow{2}{*}{\multicolumn{3}{|c|}{ 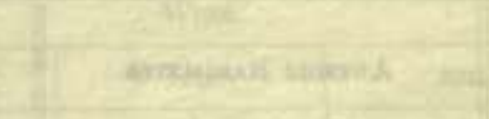 }} & \\
\hline & \multicolumn{3}{|c|}{$8 \mathrm{H}$} & \multicolumn{3}{|c|}{$12 \mathrm{H}$} & \multicolumn{3}{|c|}{$20 \mathrm{H} \quad \mathrm{T}=\mathrm{x}$} & \multirow{3}{*}{ 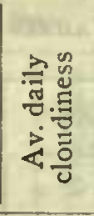 } & & & & \\
\hline & & & & & & & & & & & & REMA & KS & \\
\hline & $\begin{array}{l}\mathrm{E} \\
\overline{5} \\
\frac{0}{4}\end{array}$ & 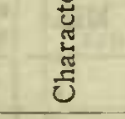 & 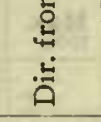 & $\begin{array}{l}\text { हूँ } \\
\text { हू } \\
\text { है }\end{array}$ & 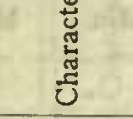 & 离 & 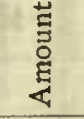 & 苞 & 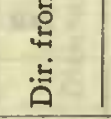 & & माह & & 145 & \\
\hline I & 6 & $\stackrel{\text { S-Cu }}{\text { S }}$ & $\underset{w}{w}$ & $\begin{array}{l}6 \\
2\end{array}$ & $\stackrel{\text { s-Cu }}{\text { S }}$ & $\begin{array}{ll}n W \\
N W\end{array}$ & 4 & S-Cu & $N W$ & 8 & Isine & $\infty 60=$ & wowe & \\
\hline 2 & 5 & s-Cu & NW & 5 & $\stackrel{\text { S-Cu }}{\mathrm{S}}$ & w & 10 & $\mathrm{~S}$ & $\mathrm{~W}$ & 10 & Fog from 20:45 & 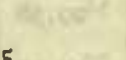 & $20 \alpha$ & \\
\hline 3 & Io & $\mathrm{N}^{* *}$ & $\mathbf{N}$ & 10 & $N^{* *}$ & NW & Io & s & $\mathrm{NW}$ & 10 & Fog to $18: 10$. & 5. & पर्शे & 18 \\
\hline 4 & Io & $\mathbf{S}^{*}$ & NW & Io & $S^{*}$ & NW & $\begin{array}{l}6 \\
4\end{array}$ & $\begin{array}{l}\text { S-Cu } \\
\text { S }\end{array}$ & $\frac{W}{W}$ & I0 & Fog 2:15 to 13 & :20 and $f$ & om 23:00. & \\
\hline 5 & Io & s & $\mathrm{NTW}$ & 0 & * & $\ldots$ & $\begin{array}{l}4 \\
5\end{array}$ & $\mathbb{S}^{*}$ & $\mathbf{s}$ & 7 & Generally light & fog. & & 8 \\
\hline 6 & Io & $\mathbf{S}^{*}$ & W & 10 & $\mathrm{~S}^{*}$ & w & IO & $s$ & $\mathrm{NE}$ & 9 & I'og to $14: 50$ & & glus & 8 \\
\hline 7 & I0 & $\mathbf{s}$ & $\mathrm{E}$ & 10 & $\mathrm{N}$ & E & Io & $\mathbf{N}$ & $\mathrm{NE}$ & Io & & & & \\
\hline 8 & $\begin{array}{l}3 \\
6\end{array}$ & $\underset{S}{S-C u}$ & $\begin{array}{l}\text { NW } \\
\text { NW }\end{array}$ & $\begin{array}{l}5 \\
4\end{array}$ & $\underset{\mathrm{S}}{\mathrm{s}-\mathrm{Cu}}$ & $\left.\begin{array}{l}\text { NWW } \\
\text { NW }\end{array}\right\}$ & $\left\{\begin{array}{r}3 \\
\text { Few } \\
2\end{array}\right.$ & $\begin{array}{l}(\mathrm{Cl}-\mathrm{S}) \\
(\mathrm{A}-\mathrm{Cu}) \\
\mathrm{S}-\mathrm{Cu}\end{array}$ & $\begin{array}{l}\text { NE } \\
\text { NE } \\
\text { NE }\end{array}$ & 5 & $\operatorname{los} \theta=$ & $b y=0$ & While & \\
\hline 9 & 0 & & $\ldots$ & Few & $(\mathrm{A}-\mathrm{Cu})$ & $N W$ & 0 & $\ldots$ & $\ldots$ & 0 & 4he & 78.65 & 99:5 & we \\
\hline Io & Few & $\begin{array}{c}(A-C u) \\
S\end{array}$ & w & 9 & s & $w$ & 10 & $\mathbf{s}$ & $\mathbf{N}$ & 7 & Fiog from $20: 3$ & 30. & $\operatorname{los} \infty$ & 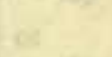 \\
\hline II & $\left\{\begin{array}{l}5 \\
4\end{array}\right.$ & $\underset{S}{S-C u}$ & $\begin{array}{l}\text { NW } \\
\text { NWV }\end{array}$ & $\begin{array}{l}5 \\
4\end{array}$ & $\underset{\mathrm{S}}{\mathrm{S}-\mathrm{Cu}}$ & $\begin{array}{l}N W \\
N W\end{array}$ & Few & $\mathrm{s}-\mathrm{Cu}$ & NW & 5 & Fog to $5: 20$ an & ad from 2 & :oo. & $y$ \\
\hline 12 & Io & $s$ & NW & Io & $\mathbf{S}^{*}$ & $\mathrm{NW}$ & $\ldots$ & ** & & Io & Generally fogg & sy. & & \\
\hline 13 & Io & $\mathbf{S}^{*}$ & $\mathrm{NW}$ & ... & $* *$ & NIV & Io & $\mathbf{S}^{*}$ & $\mathrm{NW}$ & IO & Fog to & & 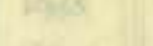 & 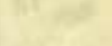 \\
\hline 14 & Io & $\mathrm{S}^{*}$ & SE & Io & $\mathbf{S}^{*}$ & SE & Io & $\mathbf{N}^{*}$ & SE & Io & Fog $1: 30$ to 20 & 50. & 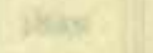 & or $2 x$ \\
\hline 15 & $\begin{array}{l}2 \\
2 \\
4\end{array}$ & $s$ & & $\begin{array}{r}3 \\
2 \\
\text { Few }\end{array}$ & $\begin{array}{l}(\mathrm{Ci}-\mathrm{Cu}) \\
\mathrm{S}-\mathrm{Cu}\end{array}$ & $\underset{\mathbf{N}}{\mathbf{E}}$ & $\begin{array}{l}3 \\
6\end{array}$ & $\underset{\text { S }}{\text { S-Cu }}$ & $\begin{array}{l}\mathrm{N} \\
\mathrm{N}\end{array}$ & 9 & thene & $2 x=5$ & $\cos =x$ & $412=$ \\
\hline 16 & 10 & s & NE & Io & $\mathrm{N}$ & NE & 10 & $\mathbf{N}$ & NE & I0 & High north & $t$ gale fro & $9: 30$. & $m$ \\
\hline I7 & Io & $s$ & ESE & 10 & s & ESE & to & $\mathrm{N}^{*}$ & ESE & 10 & High east-sout & (1) & ; fog from & $12: 30$ \\
\hline 18 & Io & 8 & SE & $\begin{array}{l}3 \\
6\end{array}$ & $\underset{\text { S }}{\text { S-Cu }}$ & $\underset{\mathbf{E}}{\mathbf{E}}$ & $\left\{\begin{array}{l}2 \\
2 \\
2\end{array}\right.$ & $\begin{array}{l}(\mathrm{Ci}-\mathrm{S}) \\
\text { (Ci-Cu) } \\
\text { S-Cu }\end{array}$ & $\begin{array}{l}\text { NE } \\
\text { NE } \\
\text { NE }\end{array}$ & 8 & Fog to $7: 00$ & beat? & extes & 57 \\
\hline I9 & $\left\{\begin{array}{r}\mathrm{I} \\
\text { Few } \\
\mathrm{I}\end{array}\right.$ & & $\begin{array}{l}N \\
N \\
N\end{array}$ & $\left\{\begin{array}{l}\text { Few } \\
\text { Few }\end{array}\right.$ & $\underset{S}{(\mathrm{Ci}-\mathrm{Cu})}$ & $\begin{array}{l}\text { NW } \\
\text { NW }\end{array}$ & $\begin{array}{l}7 \\
2\end{array}$ & $\underset{\text { S }}{\mathrm{S}-\mathrm{Cu}}$ & $\begin{array}{l}\mathrm{N} \\
\mathrm{N}\end{array}$ & 2 & 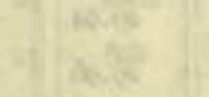 & $\cos \left(\frac{10}{20}\right.$ & 20 & \\
\hline 20 & $\left\{\begin{array}{r}1 \\
2 \\
\text { Few }\end{array}\right.$ & $\begin{array}{l}(\mathrm{Cl}-\mathrm{Cu}) \\
(\mathrm{A}-\mathrm{Cu}) \\
\mathrm{S}-\mathrm{Cu}\end{array}$ & $\begin{array}{l}\mathrm{N} \\
\mathrm{N} \\
\mathrm{N}\end{array}$ & $\begin{array}{l}4 \\
2\end{array}$ & $\underset{\mathrm{S}-\mathrm{Cu}}{(\mathrm{A}-\mathrm{Cu})}$ & W & Few & s & $\mathrm{N}$ & 4 & & W. & 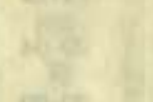 & $=$ \\
\hline $2 \mathrm{I}$ & $\left\{\begin{array}{l}4 \\
2\end{array}\right.$ & $\begin{array}{c}(\mathrm{A}-\mathrm{Cu}) \\
\mathrm{S}-\mathrm{Cu}\end{array}$ & $\left.\begin{array}{l}\mathrm{w} \\
\mathrm{w}\end{array}\right\}$ & $\left\{\begin{array}{r}\text { Few } \\
2 \\
6\end{array}\right.$ & $\begin{array}{l}(\mathrm{Cl}-\mathrm{S}) \\
(\mathrm{A}-\mathrm{S}) \\
\mathbf{S}\end{array}$ & $\underset{W}{W}$ & & ** & ‥ & 6 & Fog from 17:0 & & $\cos x$ & \\
\hline 22 & Io & $\mathbf{S}^{*}$ & W & 10 & $S^{*}$ & s & Io & $\mathbf{s}$ & SE & 10 & Generally fogg & . & $\cos \alpha$ & wa $>60$ \\
\hline 23 & Io & s & $\cdots$ & 10 & $\mathbf{s}$ & $\mathbf{E}$ & Few & $\mathbf{S}$ & SE & 4 & Fog to $4: 30$ & & is & in $=18$ \\
\hline 24 & $\left\{\begin{array}{l}4 \\
4\end{array}\right.$ & $\underset{S}{\mathrm{~S}-\mathrm{Cu}}$ & $\left.\begin{array}{l}\mathrm{NE} \\
\mathrm{NE}\end{array}\right\}$ & $\begin{array}{l}1 \\
4 \\
3\end{array}$ & $\begin{array}{l}\text { (Ci-Cu) } \\
\text { S-Cu } \\
\text { S }\end{array}$ & $\begin{array}{l}\mathrm{NE} \\
\mathrm{NE} \\
\mathrm{NE}\end{array}$ & 10 & $\mathrm{N}^{*}$ & E & 10 & Fog from $16: 3$ & & $\ln 3 x$ & \\
\hline 25 & Io & $\mathbf{S}^{*}$ & SE & $\begin{array}{l}4 \\
6\end{array}$ & $\underset{\mathrm{S}}{\mathrm{S}-\mathrm{Cu}}$ & $\begin{array}{l}\text { SE } \\
\text { SE }\end{array}$ & 10 & $\mathbf{s}$ & ESE & Io & Fog to $9: 30$ & & bise & $\approx$ \\
\hline 26 & Io & s & ESE & 10 & $s$ & ES & Ic & $\mathbf{s}$ & 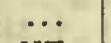 & 10 & & & 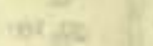 & $\mathrm{x}$ \\
\hline 27 & Io & (9) & $\mathrm{NE}$ & 10 & $\mathrm{~N}^{*}$ & $\mathrm{NE}$ & 10 & $\mathbf{N}$ & NE & 10 & Fog II:00 to & $12: 40$. & & \\
\hline 28 & $\begin{array}{l}5 \\
4\end{array}$ & $\stackrel{(A-S)}{S}$ & $\stackrel{W}{W}$ & 10 & $\mathrm{~S}^{*}$ & W & so & $\mathrm{s}$ & NW & I0 & Fog II:50 to I & I3:00. & 10 & \\
\hline 29 & $\left\{\begin{array}{r}2 \\
\text { Few }\end{array}\right.$ & $\stackrel{(\mathrm{Ci}-\mathrm{S})}{\mathrm{S}}$ & $\begin{array}{l}\mathrm{N} \\
\mathrm{N}\end{array}$ & $\begin{array}{l}2 \\
3 \\
2\end{array}$ & $\begin{array}{l}(\mathrm{Ci}-\mathrm{CH}) \\
(\mathrm{A}-\mathrm{S}) \\
\mathrm{S}\end{array}$ & $\begin{array}{l}\text { W } \\
W \\
\text { w }\end{array}$ & 10 & s & W & 4 & 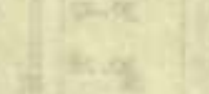 & & 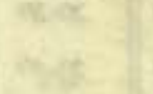 & \\
\hline 30 & $\left\{\begin{array}{l}2 \\
\text { LFew }\end{array}\right.$ & $\begin{array}{c}(\mathrm{Ci}-\mathrm{Cu}) \\
\mathrm{S}-\mathrm{Cu}\end{array}$ & W & $\begin{array}{l}\text { I } \\
4 \\
2\end{array}$ & $\begin{array}{l}(\mathrm{Ci}-\mathrm{S}) \\
(\mathrm{A}-\mathrm{Cu}) \\
\mathrm{S}\end{array}$ & $\left.\begin{array}{l}N W \\
N W \\
N W\end{array}\right\}$ & Io & s & & 4 & & & $\frac{x+5}{20}$ & \\
\hline $3 \mathrm{I}$ & $\cdots$ & ** & $\cdots$ & $\cdots$ & ** & $\cdots$ & $\cdots$ & ** & $\cdots$ & 10 & Dense fog fror & m 7:00. & & \\
\hline & 23 & $\cdots$ & $\ldots$ & 234 & $\ldots$ & $\ldots$ & 222 & ... & $\ldots$ & & & & & \\
\hline & 7.9 & $\cdots$ & $\ldots$ & 8. I & $\ldots$ & $\ldots$ & 7.9 & ... & $\cdots$ & 7.8 & & & & \\
\hline
\end{tabular}


Tabulation of daily meteorological observations at Cape Flora during the month of August, I904 Observer: Francis LoNG

\begin{tabular}{|c|c|c|c|c|c|c|c|c|c|c|c|c|c|c|c|}
\hline \multirow{3}{*}{ DAte } & \multirow{2}{*}{\multicolumn{3}{|c|}{ ANEROID BAROMETER }} & \multirow{2}{*}{\multicolumn{3}{|c|}{$\begin{array}{c}\text { READING OF } \\
\text { FAHRENHEIT THERMOMETER }\end{array}$}} & \multicolumn{9}{|c|}{ SELF-REGISTERING FAHRENHEIT THERMOMETERS } \\
\hline & & & & & & & \multicolumn{4}{|c|}{$8 \pi$} & \multirow{2}{*}{$\frac{\mathrm{I} 2 \mathrm{H}}{\mathrm{Max} .}$} & \multicolumn{2}{|c|}{$20 \mathrm{HI}$} & \multirow{2}{*}{$\begin{array}{c}\text { Mean } \\
\text { of } \\
\text { extremes }\end{array}$} & \multirow{2}{*}{ Range } \\
\hline & $8 \mathrm{H}$ & $12 \mathrm{H}$ & $20 \mathrm{H}$ & $8 \mathrm{H}$ & I2H & $20 \mathrm{H}$ & & Max. & & Min. & & Max. & Min. & & \\
\hline & In. & In. & In. & $\circ$ & -2 & $\circ$ & (4) & $\circ$ & & - & - & $\circ$ & ○ & . & - \\
\hline I & 29.91 & 29.90 & 29.83 & +35.0 & +34.0 & +30.0 & + & 35.0 & + & 30.0 & +36.0 & +36.0 & +30.0 & +33.0 & 6.0 \\
\hline 2 & 29.60 & $29.5^{8}$ & 29.43 & +33.5 & +33.4 & +33.2 & + & 33.5 & + & 30.0 & +33.5 & +34.0 & +32.6 & +32.0 & 4.0 \\
\hline 3 & 29.37 & 29.30 & 29.27 & +34.5 & +34.5 & +33.0 & + & 35.5 & + & 33.0 & +34.5 & +35.0 & +33.0 & +34.2 & 2.5 \\
\hline 4 & 29.29 & 29.36 & 29.50 & +30.5 & +30.4 & +29.0 & + & 33.0 & + & 30.0 & +30.5 & +32.8 & +29.0 & +31.0 & 4.0 \\
\hline 5 & 29.49 & 29.50 & 29.52 & +33.0 & +.34 .0 & +33.6 & + & 33.0 & + & 27.0 & +34.0 & +34.4 & +32.4 & +30.7 & 7.4 \\
\hline 6 & 29.60 & 29.67 & 29.67 & +35.0 & +36.5 & +40.0 & + & 35.0 & + & 33.0 & +36.8 & +41.0 & +35.0 & +37.0 & 8.0 \\
\hline 7 & 29.80 & 29.84 & 29.82 & +36.0 & +39.0 & +36.5 & + & 40.0 & + & 33.6 & +40.0 & +42.3 & +36.0 & $+3^{8.0}$ & 8.7 \\
\hline 8 & $29.7 \mathrm{I}$ & 29.65 & 29.54 & +35.0 & +33.1 & +35.0 & + & 36.5 & + & 34.0 & +35.0 & +35.0 & +33.0 & +34.8 & 3.5 \\
\hline 9 & 29.56 & 29.58 & 29.64 & +34.0 & +36.0 & +35.0 & + & $35 . I$ & + & 33.0 & +36.8 & +38.0 & +34.0 & +35.5 & 5.0 \\
\hline 10 & 29.78 & 29.84 & 29.93 & +34.0 & +37.0 & +37.0 & + & 36.0 & + & 27.5 & +37.4 & $+4 I .4$ & +34.0 & +34.4 & 13.9 \\
\hline II & 29.91 & 29.88 & 29.84 & +40.0 & +40.5 & +38.0 & + & 42.0 & + & 34.0 & +42.1 & +43.2 & +38.0 & +38.6 & 9.2 \\
\hline 12 & 29.84 & 29.83 & $29.8 \mathrm{I}$ & +35.0 & +36.9 & +37.0 & + & 40.0 & + & 33.8 & $+37 . \mathrm{I}$ & $+4 \mathrm{I} . \mathrm{I}$ & +34.0 & +37.4 & $7 \cdot 3$ \\
\hline 13 & 29.84 & 29.90 & 30.04 & +35.0 & +35.0 & +31.0 & + & 37.0 & + & 34.0 & +35.0 & +35.0 & +31.0 & +34.0 & 6.0 \\
\hline 14 & 30.26 & 30.28 & 30.24 & +32.5 & +35.0 & +33.0 & + & 35.0 & + & 28.0 & +36.8 & +38.1 & +32.0 & +33.0 & IO. I \\
\hline 15 & 30.12 & 30.12 & 30. 10 & +37.0 & +43.5 & +39.0 & + & 39.2 & + & 30.0 & +44.0 & +47.8 & +37.0 & +38.9 & 17.8 \\
\hline 16 & 30.06 & 30.04 & 30.00 & +38.0 & +43.0 & +36.0 & + & 40.0 & + & 32.0 & +43.0 & +45.0 & +36.0 & +38.5 & 13.0 \\
\hline I7 & 29.99 & 29.98 & 29.97 & +38.0 & +38.0 & +39.0 & + & -38.6 & + & 35.0 & +39.0 & +46.0 & +38.0 & +40.5 & II.O \\
\hline I8 & 29.95 & 29.95 & 29.94 & +36.5 & +37.4 & +35.0 & + & 40.0 & + & 31.0 & +41.2 & $+4 \mathrm{I} .2$ & +35.0 & +36.1 & 10.2 \\
\hline 19 & 29.95 & 29.96 & 29.96 & +35.0 & +36.9 & +34.0 & + & 36.2 & + & 33.9 & +38.0 & +38.0 & +34.0 & +36.0 & 4. I \\
\hline 20 & 29.94 & 29.90 & 29.95 & +36.0 & +35.1 & +33.5 & + & 36.0 & + & 32.0 & +36.0 & +36.0 & +32.9 & +34.0 & 4.0 \\
\hline $2 \mathrm{I}$ & 29.99 & 30.00 & 30.02 & +30.0 & +31.0 & +30.8 & + & .33 .9 & + & 28.9 & +31.0 & +32.2 & +29.0 & $+3 I .4$ & 5.0 \\
\hline 22 & 30.09 & 30.14 & 30.17 & +33.0 & +33.5 & +34.5 & + & 33.0 & + & 29.0 & +34.0 & +38.9 & +31.2 & +34.0 & 9.9 \\
\hline 23 & 30.22 & 30.25 & 30.26 & +36.0 & +37.0 & +33.8 & + & 40.0 & + & 34.0 & +40.0 & +41.0 & +33.0 & +37.0 & 8.0 \\
\hline 24 & 30.24 & 30.22 & 30.19 & +33.5 & +36.0 & +32.0 & + & 33.8 & + & 27.0 & +37.0 & +45.0 & +32.0 & +36.0 & 18.0 \\
\hline 25 & 30.15 & 30.13 & 30.06 & +30.0 & +36.5 & +33.0 & + & 32.0 & + & 24.1 & +36.5 & +42.8 & +30.0 & +33.4 & 18.7 \\
\hline 26 & 30.04 & 30.00 & 30.04 & +30.0 & +29.0 & +29.5 & + & 33.0 & + & 25.0 & +31.0 & +31.8 & +25.0 & +29.0 & 8.0 \\
\hline 27 & 30.10 & 30.14 & 30.16 & +29.0 & +33.0 & +30.0 & + & 29.5 & + & 28.0 & +33.2 & +34.0 & +29.5 & +31.0 & 6.0 \\
\hline 28 & 30.21 & 30.23 & 30.28 & +31.0 & +29.1 & +26.0 & + & 31.0 & + & 29.0 & +32.1 & +32.1 & +26.0 & +29.0 & 6. I \\
\hline 29 & 30.30 & $30 \cdot 30$ & 30.27 & +24.0 & +24.0 & +21.5 & + & 26.0 & + & 20.5 & +24.0 & +25.0 & +21.5 & +23.2 & 5.5 \\
\hline 30 & 30.26 & 30.26 & 30.28 & +30.0 & +31.0 & +32.0 & + & 30.1 & + & 19.0 & +31.0 & +32.0 & +29.5 & +25.5 & 13.0 \\
\hline 31 & 30.22 & 30.16 & 29.98 & +32.0 & +32.8 & +27.0 & + & 32.0 & + & 30.0 & +32.8 & +32.8 & +27.0 & +29.9 & 5.8 \\
\hline Sum.... & 927.79 & 927.89 & 927.71 & +1042.0 & +1082.1 & +1027.9 & $+\mathrm{I}$ & 1090.9 & & 929.3 & +1109.3 & +1168.9 & +990.6 & +1047.0 & 259.7 \\
\hline Mean.. & 29.93 & 29.93 & 29.93 & +33.6 & +34.9 & +33.2 & + & 35.2 & + & 29.7 & +35.8 & +37.7 & +32.0 & +33.8 & 8.4 \\
\hline
\end{tabular}


Tabulation of daily meteorological observations at Cape Flora during the month of August, 1904-Continued Observer: FRANCIS LONG

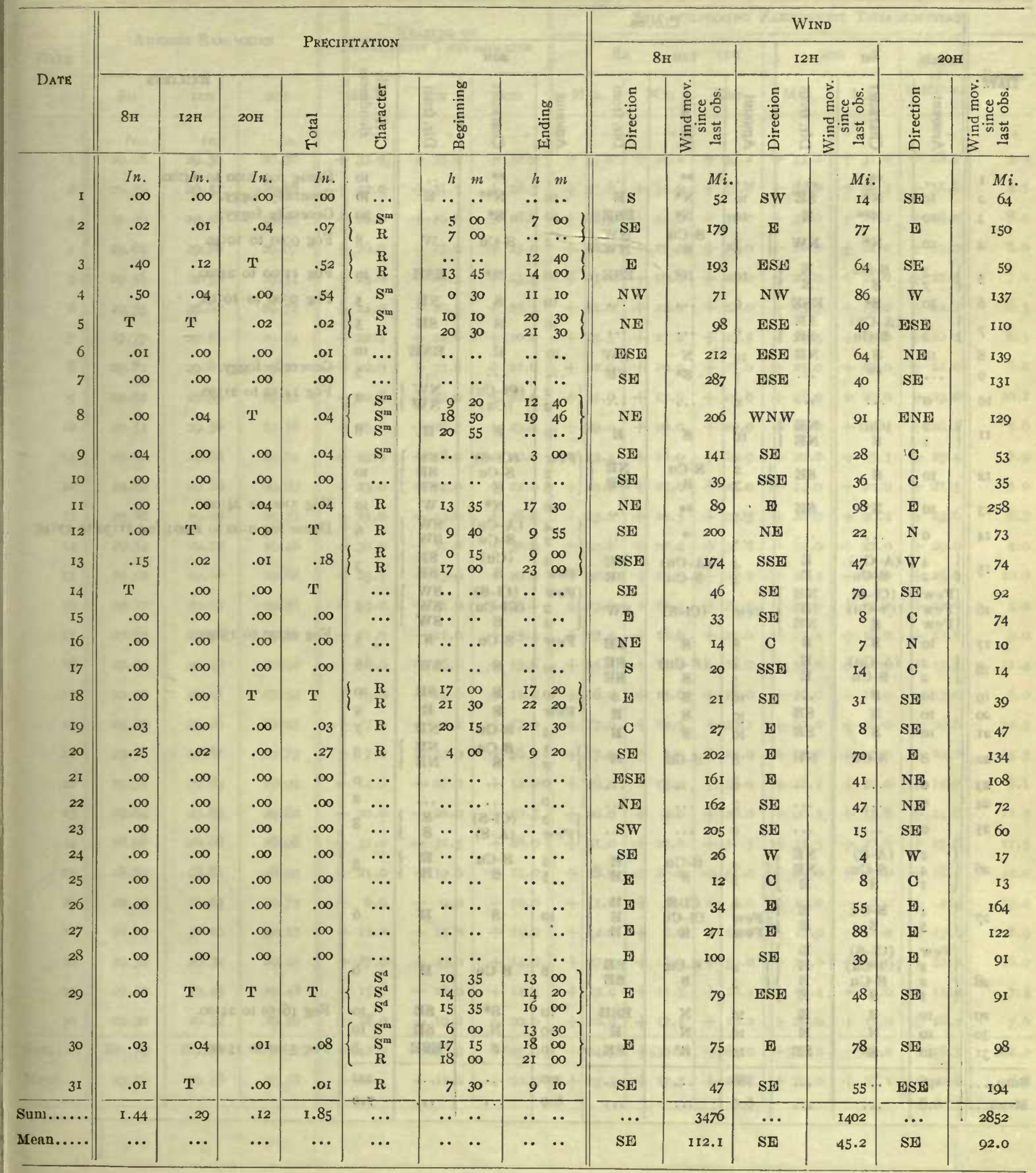


Tabulation of daily meteorological observations at Cape Flora during the month of August, Ig04-Continued Observer: Francis LoNG

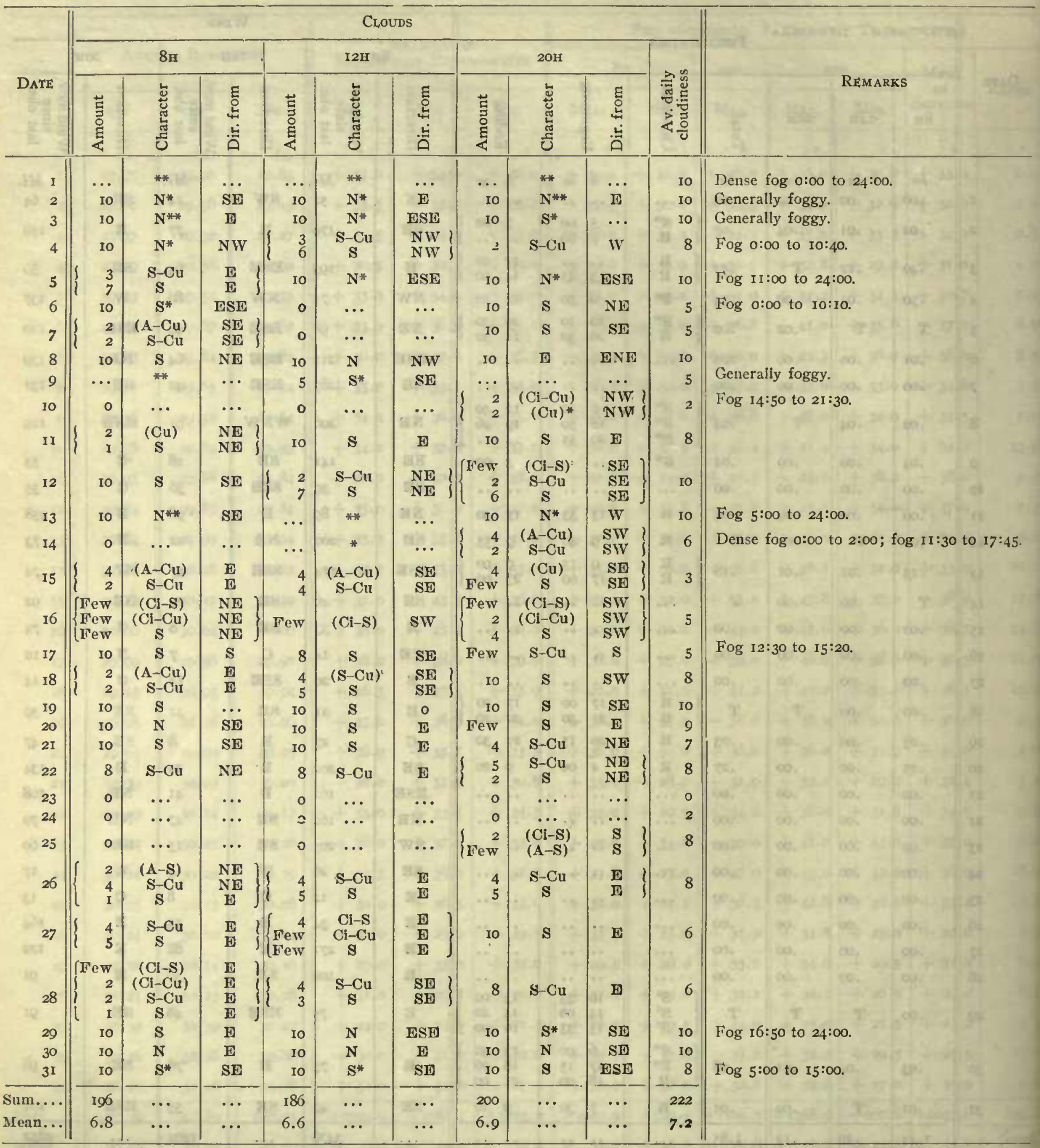


Tabulation of daily meteorological observations at Cape Flora during the month of September, Igo4

Observer: Francis LoNG

\begin{tabular}{|c|c|c|c|c|c|c|c|c|c|c|c|c|c|}
\hline \multirow{3}{*}{ DATE } & \multirow{2}{*}{\multicolumn{3}{|c|}{ ANEROID BAROMETER }} & \multirow{2}{*}{\multicolumn{3}{|c|}{$\begin{array}{l}\text { READING OF } \\
\text { FAITRENHEIT THERMOMETER }\end{array}$}} & \multicolumn{7}{|c|}{ SELF-REGISTERING FAHRENHEIT THERMOMETERS } \\
\hline & & & & & & & 8 & & $\mathrm{I2H}$ & & $\mathrm{OH}$ & & $\operatorname{mon}$ \\
\hline & $8 \mathrm{II}$ & $\mathrm{I} 2 \mathrm{H}$ & $2 \mathrm{OH}$ & $8 \mathrm{H}$ & $\mathrm{I} 2 \mathrm{H}$ & $20 \mathrm{HI}$ & Max. & Min. & Max. & Max. & Min. & & $\operatorname{Nan}$ \\
\hline & & In. & In. & & $0^{\circ}$ & & -0 & 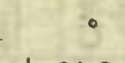 & $\circ$ & $\bullet$ & $\bullet$ & & $\cdot$ \\
\hline I & 29.68 & 29.58 & 29.56 & +33.0 & +33.0 & +32.0 & +33.0 & +24.2 & +33.2 & +33.2 & +31.0 & +28.7 & 9.0 \\
\hline 2 & 29.56 & 29.52 & 29.58 & +32.0 & +31.5 & +28.5 & +33.0 & +31.0 & +32.0 & +32.0 & +28.5 & +30.8 & 4.5 \\
\hline 3 & 29.46 & 29.48 & 29.55 & +28.0 & +30.0 & +28.0 & +28.5 & +24.0 & +30.0 & +32.0 & +28.0 & +28.0 & 8.0 \\
\hline 4 & 29.64 & 29.70 & 29.67 & +26.0 & +25.0 & +23.0 & +28.0 & +25.0 & +26.0 & +26.0 & +22.0 & +25.0 & 6.0 \\
\hline 5 & 29.56 & 29.52 & 29.54 & +23.0 & +25.5 & +23.5 & +23.0 & +19.0 & +26.0 & +26.0 & +21.0 & +22.5 & .0 \\
\hline 6 & 29.55 & 29.62 & 29.72 & +24.0 & +20.5 & +25.5 & +24.0 & +22.0 & +24.0 & +25.5 & +19.0 & +22.2 & 5.5 \\
\hline 7 & 29.77 & $29.8 \mathrm{I}$ & 29.84 & +22.2 & +22.0 & +20.0 & +25.5 & $+2 \mathrm{I} .2$ & +23.5 & +23.5 & +19.0 & +22.2 & 6.5 \\
\hline 8 & 29.82 & $29.8 \mathrm{I}$ & 29.72 & +22.0 & +23.0 & +21.0 & +22.0 & +19.0 & +25.0 & +25.0 & +20.0 & +22.0 & 6.0 \\
\hline 9 & 29.62 & 29.59 & 29.52 & +20.8 & +22.0 & +28.0 & +21.0 & +17.9 & +22.0 & +28.0 & +20.0 & +23.0 & 10. I \\
\hline Io & 29.53 & 29.56 & 29.58 & +29.0 & +26.5 & +23.4 & +29.0 & +26.0 & +29.0 & +29.0 & +23.4 & +26.2 & 5.6 \\
\hline II & 29.60 & 29.66 & 29.74 & +19.0 & +18.0 & +20.0 & +23.4 & +16.0 & +19.9 & +20.0 & +15.5 & +19.4 & 7.9 \\
\hline 12 & 29.82 & 29.88 & 29.89 & +23.0 & +24.0 & +22.0 & +23.2 & +19.0 & +24.0 & +24.0 & +21.0 & +21.5 & 5.0 \\
\hline$I_{3}$ & 29.88 & 29.89 & 29.73 & +21.0 & +23.0 & +28.0 & +23.0 & $+\mathrm{I} 9.3$ & +23.5 & +28.0 & +20.2 & +23.6 & 8.7 \\
\hline I4 & 29.54 & 29.52 & 29.48 & +30.0 & +31.0 & +30.0 & +30.0 & +27.0 & +31.0 & +31.0 & +27.0 & +29.0 & 4.0 \\
\hline 15 & 29.52 & 29.60 & 29.68 & +23.5 & +23.9 & +20.0 & +30.0 & +23.5 & +26.2 & +26.2 & +20.0 & +25.0 & 10.0 \\
\hline I6 & 29.77 & 29.82 & 29.56 & +23.0 & +21.0 & +25.0 & +23.0 & +19.1 & +23.5 & +25.0 & +20.0 & & 5.9 \\
\hline I7 & 29.62 & 29.58 & 29.33 & +21.0 &.+21.0 & +25.4 & +27.0 & +18.0 & +21.0 & +25.4 & +18.0 & & 9.0 \\
\hline 8 & 29.34 & 29.42 & 29.42 & +18.0 & 1.0 .0 & +15.6 & +25.4 & +17.0 & +18.0 & +18.0 & +14.0 & +19.7 & II 4 \\
\hline .19 & $29 \cdot 32$ & $29.4 \mathrm{I}$ & 29.54 & $T 15.0$ & +14.6 & +11.0 & +16.0 & +15.6 & +15.0 & +16.0 & +10.0 & +13.0 & 6.0 \\
\hline 20 & 29.29 & 29.26 & 29.25 & +17.0 & +20.1 & +17.0 & +17.0 & +10.5 & +20.1 & +23.0 & +.16 .0 & +16.8 & 12.5 \\
\hline 2I & 29.58 & 29.67 & 29.73 & +7.0 & +12.0 & +16.0 & +17.0 & +3.5 & +12.0 & +17.0 & +7.0 & +10.2 & 13.5 \\
\hline 22 & 29.74 & 29.84 & 29.93 & +14.0 & +16.0 & +12.0 & +16.0 & +13.0 & +16.0 & +18.1 & +12.0 & +15.0 & 6.1 \\
\hline 23 & 29.84 & 29.83 & 29.62 & +20.0 & +20.0 & +20.5 & +20.0 & +9.0 & +20.0 & +20.5 & +17.8 & +14.8 & II 5 \\
\hline 24 & 28.98 & 28.98 & 28.98 & +31.0 & +31.5 & +28.0 & +31.0 & +20.0 & +31.5 & +31.5 & +27.6 & +25.8 & 11.5 \\
\hline 25 & 28.79 & $28.8 \mathrm{I}$ & 28.93 & +21.0 & +19.0 & +11.0 & +28.0 & +20.0 & +21.0 & +21.0 & +9.0 & +18.5 & 19.0 \\
\hline 26 & 29.44 & 29.53 & 29.73 & +6.0 & +9.0 & +10.0 & +11.0 & +3.5 & +9.0 & +10.0 & -2.0 & +4.5 & 13.0 \\
\hline 27 & 30.04 & 30.10 & 30.12 & +12.0 & +15.0 & +15.0 & +12.0 & +9.0 & +15.0 & +17.9 & $+\mathrm{II} .0$ & +13.4 & 8.9 \\
\hline & 29.50 & 29.32 & 29.44 & +27.0 & +29.0 & +14.0 & +27.5 & +14.0 & +30.0 & +30.0 & +14.0 & +22.0 & 16.0 \\
\hline 29 & 29.45 & 29.45 & 29.48 & +15.8 & +16.0 & +14.5 & +19.0 & + II. 5 & +16.4 & +16.4 & +14.0 & +15.2 & $7 \cdot 5$ \\
\hline 30 & 29.50 & 29.54 & 29.56 & +18.0 & +19.2 & +20.0 & +18.0 & +12.4 & +19.2 & +20.0 & +18.0 & +16.2 & 7.6 \\
\hline & 886.75 & $887 \cdot 30$ & 887.42 & +642.3 & +658.9 & +627.9 & +704.5 & +530.2 & +683.0 & +719.2 & +542.0 & +618.7 & 264.2 \\
\hline & 29.56 & 29.58 & 29.58 & +21.4 & +22.0 & +20.9 & +23.5 & +17.7 & +22.8 & +24.0 & +18.1 & +20.6 & 8.8 \\
\hline
\end{tabular}


Tabulation of daily meteorological observations at Cape Flora during the month of September, 1904-Continued Observer: FRANCIS LONG

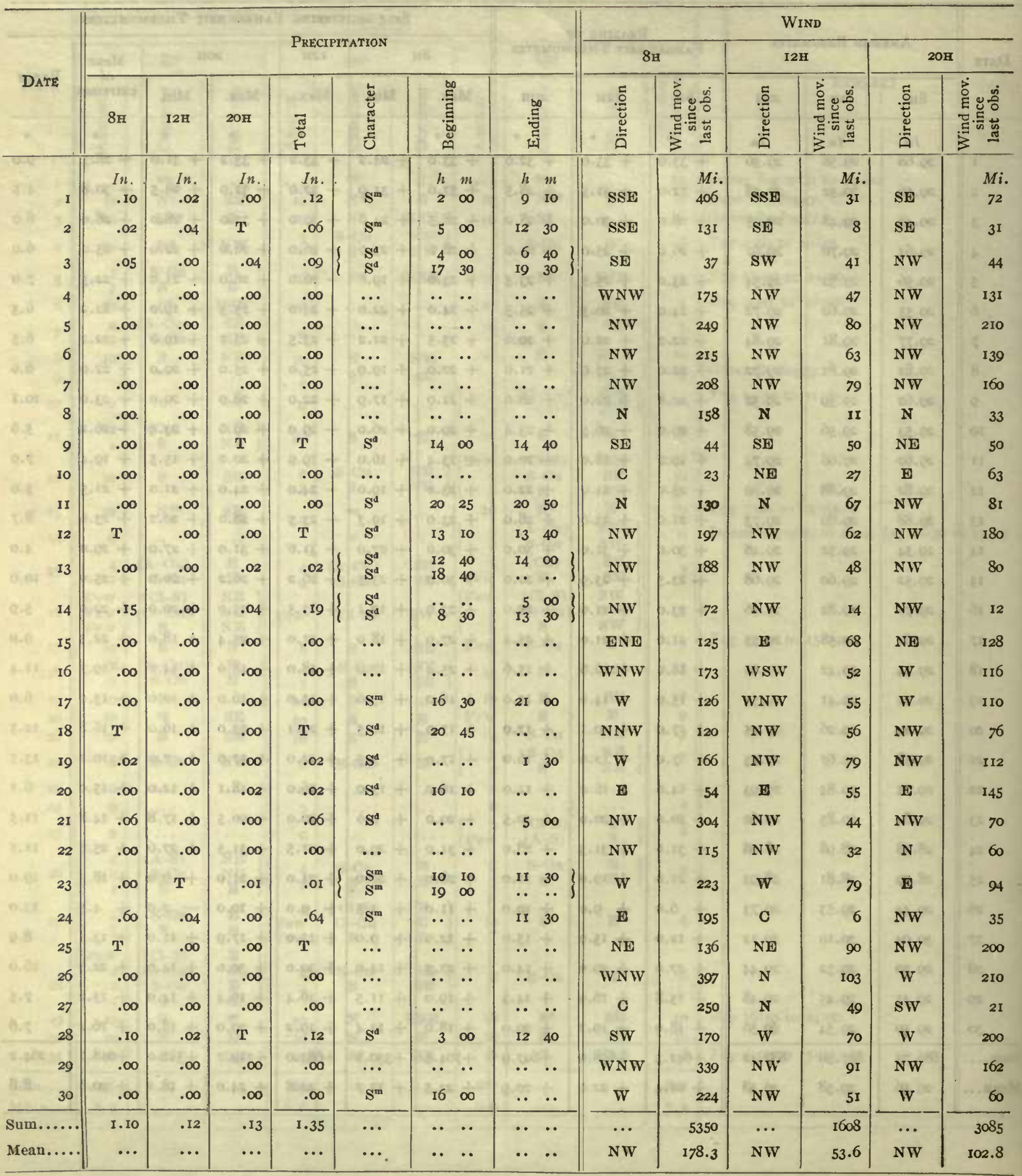


Tabulation of daily meteorological observations at Cape Flora during the month of September, 1904-Continued Observer: Francis LONG

\begin{tabular}{|c|c|c|c|c|c|c|c|c|c|c|c|c|c|c|c|}
\hline \multirow[b]{3}{*}{ DATE } & \multicolumn{10}{|c|}{ 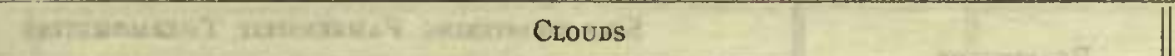 } & \multirow{2}{*}{\multicolumn{4}{|c|}{ REMARKS }} & \multirow{3}{*}{$2+4 \pi$} \\
\hline & $\frac{342}{1}$ & \multicolumn{2}{|l|}{$8 \mathrm{H}$} & \multicolumn{3}{|c|}{$\begin{array}{r}\mathbf{1 2 H} \\
\end{array}$} & \multirow[b]{2}{*}{ 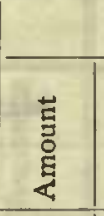 } & \multicolumn{2}{|c|}{2011} & \multirow[b]{2}{*}{ 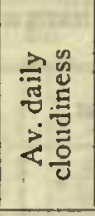 } & & & & & \\
\hline & 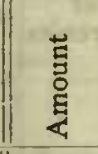 & 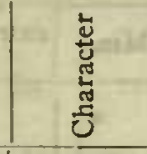 & 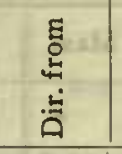 & $\begin{array}{l}\text { 䓂 } \\
\text { 量 }\end{array}$ & 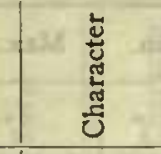 & $\begin{array}{l}\text { E } \\
\text { 岕 } \\
\text { 㐫 } \\
\end{array}$ & & 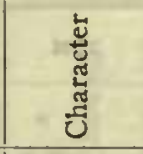 & 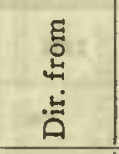 & & & 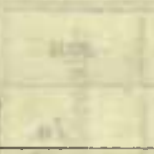 & $\begin{array}{r}\text { REMARKS } \\
\end{array}$ & $\therefore$ & \\
\hline I & 10 & $\mathbf{N}$ & SSE & Io & $\mathrm{s}$ & $\ldots$ & Io & $s$ & SE & 10 & & 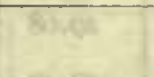 & काल & 8.68 & \\
\hline & 10 & $\mathbf{N}$ & SSE & 10 & $\mathbf{N}$ & SE & Io & $\mathbf{S}^{*}$ & SE & 10 & Fog 1 & $18: 30$ to 24 & $1: 00$ & $\arg 0$ & 36 \\
\hline 3 & $\begin{array}{l}4 \\
4\end{array}$ & $\stackrel{(\mathrm{A}-\mathrm{Cu})}{\mathrm{S}-\mathrm{Cu}}$ & $\left.\begin{array}{l}\text { SE } \\
\text { SE }\end{array}\right\}$ & 10 & $\mathbf{s}$ & sw & 10 & $\mathbf{s}$ & $\mathrm{NW}$ & Io & Fog 0 & $0: 00$ to $4: 3$ & o. & 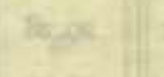 & 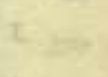 \\
\hline 4 & 10 & $\mathbf{s}$ & NW & $\begin{array}{l}4 \\
5\end{array}$ & $\underset{\mathrm{S}}{(\mathrm{S}-\mathrm{Cu})}$ & $\left.\begin{array}{l}\text { NW } \\
\text { NW }\end{array}\right\}$ & 8 & $\mathrm{~s}-\mathrm{Cu}$ & NW & 8 & & 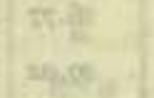 & 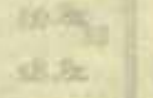 & $\begin{array}{l}0406 \\
58\end{array}$ & 2. \\
\hline 5 & $\begin{array}{l}4 \\
6\end{array}$ & $\begin{array}{l}\text { (S-Cu) } \\
\text { S }\end{array}$ & $\left.\begin{array}{l}\mathrm{NW} \\
\mathrm{NW}\end{array}\right\}$ & 10 & $\mathrm{S}-\mathrm{Cu}$ & NT & Io & s-Cu & $\mathrm{NW}$ & 10 & High & northwest & winds. & Higs & \\
\hline 6 & 10 & s & NW & 7 & $\mathrm{~S}-\mathrm{Cu}$ & $\mathbb{N W}$ & 10 & $\mathbf{s}$ & NW & 9 & ${ }_{1}$ & abse & ceios. & $E=$ & $t$ \\
\hline 7 & 10 & s & NW & $\begin{array}{l}2 \\
4\end{array}$ & $\underset{\mathrm{S}-\mathrm{Cu}}{(\mathrm{Ci}-\mathrm{Cu})}$ & $\left.\begin{array}{l}\mathbb{N} W \\
N W\end{array}\right\}$ & 10 & $s$ & NW & 8 & ${ }^{2}$ & chipe & psios & $\alpha \theta$ & 8 \\
\hline 8 & 10 & s & N & $\begin{array}{l}2 \\
4 \\
2\end{array}$ & $\begin{array}{c}(\mathrm{A}-\mathrm{Cu}) \\
\text { S-Cu } \\
\text { S }\end{array}$ & $\left.\begin{array}{l}\mathbf{N} \\
\mathbf{N} \\
\mathbf{N}\end{array}\right\}$ & Io & $\mathbf{s}$ & $\mathbf{N}$ & 9 & & 46., & 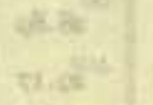 & $\frac{6025}{x+25}$ & $e^{8}+2$ \\
\hline 9 & $\begin{array}{l}4 \\
4\end{array}$ & 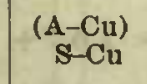 & \begin{tabular}{|} 
SE \\
$\mathbf{S E}$
\end{tabular} & $\begin{array}{l}4 \\
5\end{array}$ & $\mid \begin{array}{c}(\mathrm{A}-\mathrm{Cu}) \\
\text { S-Cu }\end{array}$ & $\left.\begin{array}{l}\text { SE } \\
\text { SE }\end{array}\right\}$ & Io & $S$ & ... & 10 & & 0 ine & so.cent & wion & $x+7=$ \\
\hline Io & $\begin{array}{l}4 \\
4\end{array}$ & $\stackrel{(\mathrm{A}-\mathrm{Cu})}{\mathrm{S}-\mathrm{Cu}}$ & $\begin{array}{l}\mathbf{N} \\
\mathbf{N}\end{array}$ & 10 & s & $\mathrm{NE}$ & Io & $\mathbf{s}$ & E & 9 & & we $8 \mathrm{t}$ & casest & enives & $\mathrm{si}^{2}$ \\
\hline 11 & $\begin{array}{l}2 \\
4\end{array}$ & $\underset{\mathrm{S}-\mathrm{Cu}}{(\mathrm{A}-\mathrm{Cu})}$ & $\stackrel{N}{N}$ & $\begin{array}{l}1 \\
5\end{array}$ & $\underset{\mathrm{S}-\mathrm{Cu}}{(\mathrm{A}-\mathrm{Cu})}$ & $\left.\begin{array}{l}\mathbf{N} \\
\mathbf{N}\end{array}\right\}$ & 10 & s & $N W$ & 7 & & $\mathrm{~m}, \mathrm{i}$ & & $\begin{array}{l}\text { potan } \\
\text { athes }\end{array}$ & $x^{3}$ \\
\hline 12 & 10 & $\mathbf{s}$ & NW & $\begin{array}{l}3 \\
7\end{array}$ & $\underset{\mathrm{S}}{(\mathrm{S}-\mathrm{Cu})}$ & $\begin{array}{l}\text { NW } \\
\text { NW }\end{array}$ & $\begin{array}{r}3 \\
+6\end{array}$ & $\begin{array}{c}(\mathrm{S}-\mathrm{Cu}) \\
\mathrm{S}\end{array}$ & $\left.\begin{array}{l}\mathrm{NW} \\
\mathrm{NW}\end{array}\right\}$ & 8 & & $8,6 x$ & senthet & Nerk & si 4 \\
\hline 13 & $\begin{array}{l}4 \\
3 \\
1\end{array}$ & $\begin{array}{c}(\mathrm{A}-\mathrm{Cu}) \\
\mathrm{S}-\mathrm{Cu} \\
\mathrm{S}\end{array}$ & $\left.\begin{array}{l}\text { NW } \\
\text { NW } \\
\text { NW }\end{array}\right\}$ & Io & $\mathbf{s}$ & NW & Io & & NW & 9 & Fog I & I $5: 40$ to 24 & :oo. & $\theta+4$ & ans \\
\hline 14 & 10 & $\mathrm{~S}^{*}$ & $N w$ & Io & $\mathbf{N}^{*}$ & NW & .. & ** & $\ldots$ & Io & Gener: & rally foggy & & & \\
\hline 15 & 10 & s & ENE & $\begin{array}{l}4 \\
6\end{array}$ & $\begin{array}{c}(\mathrm{S}-\mathrm{Cu}) \\
\mathrm{S}\end{array}$ & $\underset{\mathbf{E}}{\mathbf{E}}$ & $\begin{array}{l}3 \\
5\end{array}$ & $\begin{array}{c}(\mathrm{S}-\mathrm{Cu}) \\
\mathrm{s}\end{array}$ & $\left.\begin{array}{l}\mathrm{NE} \\
\mathrm{NE}\end{array}\right\}$ & 9 & Fog 0 & $0: 00$ to $6: 0$ & o. & terte & 80 \\
\hline 16 & 10 & s & NW & 8 & $s$ & WSW & 8 & $\mathbf{S}$ & W & 8 & & cosese & zitics: & ase & 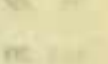 \\
\hline $\begin{array}{l}17 \\
18\end{array}$ & $\begin{array}{r}10 \\
3\end{array}$ & $\begin{array}{c}S \\
(\mathrm{~S}-\mathrm{Cu})\end{array}$ & $\begin{array}{c}\text { W } \\
\text { NNW }\end{array}$ & I0 & s & $\begin{array}{c}n t i \\
m\end{array}$ & I0 & $\mathbf{N}$ & $\begin{array}{l}N W \\
N W\end{array}$ & to & & $2=$ & aes & . & \\
\hline 19 & ${ }_{1}^{3}$ & $\underset{\substack{S \\
s}}{S-C u}$ & $\left.\begin{array}{c}\text { NNW } \\
W \\
\text { W }\end{array}\right\}$ & 3 & $s-C_{11}$ & NW & 3 & s-Cu & $\begin{array}{l}N W \\
N W\end{array}$ & $\begin{array}{l}\text { Io } \\
3\end{array}$ & & $23 \cdot .98$ & nsees & Dever: & $=1+4$ \\
\hline 20 & I0 & $\mathbf{s}$ & E & Io & s & $\mathbf{E}$ & Io & $\mathrm{N}^{*}$ & E & 10 & Fog I & I6:30 to 24 & :00. & tor & 45 \\
\hline $2 \mathrm{I}$ & 3 & $\mathrm{~S}-\mathrm{Cu}$ & $\mathrm{NW}$ & $\begin{array}{l}3 \\
6\end{array}$ & $\begin{array}{l}(\mathrm{S}-\mathrm{Cu}) \\
\mathrm{S}\end{array}$ & $\left.\begin{array}{l}\mathrm{NW} \\
\mathrm{NW}\end{array}\right\}$ & 9 & $\mathbf{s}$ & NW & 7 & & & & 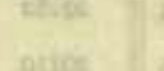 & \\
\hline 22 & $\begin{array}{l}2 \\
2\end{array}$ & $\underset{S-C u}{(\mathrm{Cl}-\mathrm{Cu})}$ & $\begin{array}{l}\mathrm{NW} \\
\mathrm{NW}\end{array}$ & o & & & Few & $s$ & $\mathbf{N}$ & I & & 1925 & $25-c$ & wase & \\
\hline 23 & Io & $\mathrm{s}$ & W & IO & s & w & I0 & N & $\mathbf{E}$ & 10 & & & & & \\
\hline 24 & I0 & $\mathbf{N}^{*}$ & $\mathbf{E}$ & ... & ** & $\ldots$ & 10 & s & NW & 10 & Foggy & sy to $18: 00$. & & & \\
\hline 25 & $\left\{\begin{array}{l}2 \\
3\end{array}\right.$ & $\underset{\mathrm{S}-\mathrm{Cu}}{(\mathrm{Cl}-\mathrm{Cu})}$ & $\begin{array}{l}\mathrm{NE} \\
\mathrm{NE}\end{array}$ & $\begin{array}{l}2 \\
6\end{array}$ & $\underset{\mathrm{S}-\mathrm{Cu}}{(\mathrm{Ci}-\mathrm{Cu})}$ & $\left.\begin{array}{l}\mathrm{NE} \\
\mathrm{NE}\end{array}\right\}$ & 10 & s & $\mathrm{NW}$ & 8 & High & northeast & wind sh & ing to no & iwest at \\
\hline $\begin{array}{l}26 \\
27\end{array}$ & $\begin{array}{r}\text { Few } \\
0\end{array}$ & $\begin{array}{c}(\mathrm{Ci}-\mathrm{Cu}) \\
\ldots\end{array}$ & $\begin{array}{c}\mathrm{NW} \\
\ldots\end{array}$ & $\begin{array}{l}\text { Few } \\
\text { Few }\end{array}$ & $\stackrel{\mathrm{S}}{\mathrm{Cl}-\mathrm{S}}$ & $\underset{N W}{N}$ & $\begin{array}{r}\text { Few } \\
\text { I0 }\end{array}$ & $\begin{array}{c}\mathrm{s}-\mathrm{Cu} \\
\mathrm{s}\end{array}$ & $\begin{array}{l}\text { W } \\
\text { SW }\end{array}$ & $\begin{array}{l}3 \\
3\end{array}$ & $\begin{array}{l}\text { High } \\
\text { Cam }\end{array}$ & $\begin{array}{l}\text { northwest } \\
\text { mp Abruzzi }\end{array}$ & $\begin{array}{l}\text { gale to } 5: 3 \\
\text { at } 11: 30 \text {. }\end{array}$ & :30. Party & left for \\
\hline 28 & Io & $\mathrm{N}^{*}$ & SW & 10 & $\mathrm{~N}^{*}$ & W & Few & s & w & 7 & Fog 6 & $6: 00$ to $15:$ & oo. & कolt: & te $x^{2}$ \\
\hline 29 & 0 & $\cdots$ & $\cdots$ & 0 & $\cdots$ & $\cdots$ & 3 & $\mathbf{s}$ & $N W$ & 1 & & west to no & rthwest wi & ind all nigh & t. \\
\hline 30 & $\begin{array}{l}2 \\
5 \\
\end{array}$ & $\begin{array}{l}(\mathrm{A}-\mathrm{Cu}) \\
\mathrm{S}-\mathrm{Cu}\end{array}$ & $\begin{array}{l}W \\
W\end{array}$ & 10 & $s$ & $\mathrm{NW}$ & 10 & $s$ & $w$ & 8 & & Pextort & $4=08$ & aetik & thes \\
\hline & 230 & $\cdots$ & $\cdots$ & 223 & $\cdots$ & & 238 & & $\cdots$ & 235 & & $n$ & $7 x=$ & 3 & $y^{3}$ \\
\hline ean... & 7.7 & ... & $\cdots$ & 7.7 & $\cdots$ & ... & 8.2 & $\cdots$ & $\cdots$ & 7.8 & & $\pi$ & 648 & 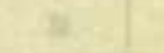 & 2thent \\
\hline
\end{tabular}


Tabulation of daily meteorological observations at Cape Flora during the month of October, 1904 Observer: FRANCIS LONG

\begin{tabular}{|c|c|c|c|c|c|c|c|c|c|c|c|c|c|}
\hline \multirow{3}{*}{ DATE } & \multirow{2}{*}{\multicolumn{3}{|c|}{ ANEROID BAROMETER }} & \multirow{2}{*}{\multicolumn{3}{|c|}{$\begin{array}{c}\text { READING OF } \\
\text { FAHRENHEIT THERMOM ETER }\end{array}$}} & \multicolumn{7}{|c|}{ SELF-REGISTERING FAIIRENHEIT THERMOMETERS } \\
\hline & & & & & & & \multicolumn{2}{|c|}{$8 \mathbf{H}$} & \multirow{2}{*}{$\begin{array}{c}\mathrm{I} 2 \mathrm{H} \\
\mathrm{Max} .\end{array}$} & \multicolumn{2}{|c|}{$20 \mathrm{H}$} & \multirow{2}{*}{$\begin{array}{c}\text { Mean } \\
\text { of } \\
\text { extremes }\end{array}$} & \multirow{2}{*}{ Range } \\
\hline & $8 \mathrm{H}$ & $12 \mathrm{H}$ & 2011 & $8 \mathrm{H}$ & $12 \mathrm{H}$ & $20 \mathrm{H}$ & Max. & Min. & & Max. & Min. & & \\
\hline & In. & In. & In. & $\circ$ & $\bullet$ & 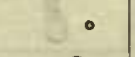 & 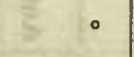 & 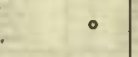 & $\circ$ & $\circ$ & $\therefore$ & $\circ$ & - \\
\hline I & 29.58 & 29.66 & 29.68 & +21.0 & +22.5 & +18.0 & +21.0 & +17.0 & +22.5 & +22.5 & +17.0 & +19.8 & $5 \cdot 5$ \\
\hline 2 & 29.56 & 29.58 & 29.40 & +14.0 & +14.0 & +13.0 & +18.0 & +12.0 & +14.0 & +15.0 & $+\mathbf{1 1 . 0}$ & +14.5 & 7.0 \\
\hline 3 & 29.28 & 29.36 & 29.35 & +25.0 & +28.0 & +23.0 & +25.2 & +13.0 & +28.0 & +28.0 & +23.0 & +20.5 & 15.0 \\
\hline 4 & 29.00 & 28.93 & 28.77 & $+2 \mathrm{I} .0$ & +24.0 & +28.0 & +23.0 & +19.0 & +26.0 & +28.0 & +27.0 & +23.5 & 9.0 \\
\hline 5 & 28.74 & 28.82 & 29.02 & +24.0 & +23.3 & +20.8 & +28.0 & +23.2 & +24.0 & +24.0 & +20.0 & +24.0 & 8.0 \\
\hline 6 & 29.14 & 29.22 & 29.28 & +20.0 & +21.2 & +21.2 & +23.6 & +19.0 & +21.2 & +21.2 & +18.6 & $+2 I . I$ & 5.0 \\
\hline 7 & 29.28 & 29.30 & 29.30 & +24.0 & +25.0 & +23.4 & +24.0 & +20.0 & +25.0 & $+25 \cdot 3$ & +22.2 & +22.6 & $5 \cdot 3$ \\
\hline 8 & 29.20 & 29.20 & 29.23 & +27.0 & +28.0 & +30.0 & +27.3 & +20.1 & +29.0 & +30.0 & +25.2 & +25.0 & 9.9 \\
\hline 9 & 28.98 & 28.89 & 28.84 & +25.0 & +25.0 & +25.6 & +30.0 & +24.0 & +25.0 & +26.2 & +24.0 & +27.0 & 6.0 \\
\hline 10 & 29.07 & 29.17 & 29.43 & +18.6 & +19.0 & +9.0 & +25.6 & +18.0 & + 19.0 & +19.0 & +9.0 & +17.3 & I6. 6 \\
\hline II & 29.60 & 29.67 & 29.70 & +5.5 & +10.0 & +15.0 & +9.0 & +5.0 & +10.0 & +15.0 & +4.0 & +9.5 & II.O \\
\hline 12 & 29.43 & 29.23 & 29.20 & +26.0 & +31.5 & +31.0 & +26.4 & +12.0 & +31.5 & +33.0 & +22.1 & +22.5 & 21.0 \\
\hline 13 & 28.90 & 28.87 & 29.12 & +27.5 & +28.0 & +9.5 & +31.5 & +27.0 & +28.0 & +28.0 & +9.0 & +20.2 & 22.5 \\
\hline 14 & 29.33 & 29.34 & 28.92 & +2.9 & +5.0 & +10.0 & +9.5 & -1.0 & +5.0 & +10.0 & +2.1 & +4.5 & II.O \\
\hline 15 & 28.90 & 28.96 & 29.38 & +12.0 & +10.5 & +10.5 & +12.0 & +8.0 & +17.0 & +17.0 & +7.0 & +12.0 & 10.0 \\
\hline 16 & 29.70 & 29.72 & 29.65 & +9.9 & +13.4 & +24.0 & +10.5 & +2.5 & +13.4 & +24.0 & +8.2 & +13.2 & 21.5 \\
\hline 17 & 29.52 & 29.54 & 29.53 & +33.5 & +32.5 & +32.8 & +34.0 & +24.0 & +33.5 & +33.5 & +31.8 & +29.0 & I0.0 \\
\hline 18 & 29.54 & 29.55 & 29.55 & +27.0 & +24.0 & +9.5 & +32.8 & +27.0 & +27.0 & +27.0 & +7.0 & +19.9 & 25.8 \\
\hline 19 & 29.68 & 29.78 & 29.93 & +7.0 & +3.0 & +5.0 & +9.5 & +4.0 & +8.0 & +8.0 & 0.0 & +4.8 & 9.5 \\
\hline 20 & 29.86 & 29.85 & 29.68 & +4.0 & +6.0 & +3.0 & +8.0 & +2.0 & +7.0 & +9.0 & +2.0 & +5.5 & 7.0 \\
\hline 21 & $29 \cdot 3^{8}$ & $29 \cdot 36$ & 29.36 & +17.4 & +21.0 & +9.5 & +18.0 & 2.0 & +21.0 & +21.0 & +9.0 & +11.5 & 19.0 \\
\hline 22 & 29.48 & 29.45 & 29.34 & +4.0 & $+\quad 5.5$ & +2.0 & +9.5 & 2.0 & +5.5 & +10.2 & +2.0 & +6.1 & 8.2 \\
\hline 23 & 29.62 & 29.76 & 29.88 & -2.0 & -6.0 & 0.0 & +4.0 & 4.0 & -2.0 & 0.0 & -7.0 & -1.5 & II.O \\
\hline 24 & 29.82 & 29.82 & 29.64 & $-\quad 1.0$ & 0.0 & +3.0 & 0.0 & 4.6 & 0.0 & +3.0 & -3.0 & -0.8 & 7.6 \\
\hline 25 & 29.10 & 29.09 & 29.30 & +12.0 & +10.0 & +3.0 & +12.0 & 3.0 & +12.0 & +12.0 & +3.0 & +7.5 & 9.0 \\
\hline 26 & 29.60 & 29.71 & 29.71 & -5.0 & $-\quad 5.8$ & -7.0 & +4.0 & - & -4.0 & -4.0 & -7.0 & -1.5 & II.0 \\
\hline 27 & 29.67 & 29.70 & 29.72 & -3.0 & $-2 . I$ & -1.0 & 0.0 & 1.2 & +0.2 & +0.2 & -5.0 & $\rightarrow 2.4$ & 5.2 \\
\hline 28 & 29.71 & 29.72 & 29.76 & +2.0 & +2.5 & -4.0 & +2.0 & 3.0 & +3.0 & +3.0 & -4.4 & -0.7 & $7 \cdot 4$ \\
\hline 29 & 29.70 & 29.74 & 29.75 & -6.8 & -5.5 & -4.1 & -3.2 & -9.2 & -4.8 & -3.2 & -8.8 & -6.2 & 6.0 \\
\hline 30 & 29.52 & 29.45 & 28.90 & +5.0 & +6.5 & +11.0 & +6.0 & -4.0 & +6.9 & +11.0 & +5.0 & +3.5 & 15.0 \\
\hline $3 I$ & 28.40 & 28.48 & 28.60 & +10.0 & +7.1 & -2.0 & +20.0 & +9.5 & +10.5 & +10.5 & -2.0 & $\begin{array}{r}+9.0 \\
\end{array}$ & 22.0 \\
\hline Sum. & 910.29 & 911.92 & 910.92 & +407.5 & +427.1 & +372.7 & +501.2 & $+28 \mathrm{r} \cdot 3$ & +462.4 & +507.4 & +272.0 & +380.0 & 358.0 \\
\hline Mean... & 29.36 & 29.42 & 29.38 & +13.1 & +13.8 & +12.0 & +16.2 & +9.1 & +14.9 & +16.4 & +8.8 & +12.3 & 11.5 \\
\hline
\end{tabular}


Tabulation of daily meteorological observations at Cape Flora during the month of October, 1904-Continued Observer: Francis Long

\begin{tabular}{|c|c|c|c|c|c|c|c|c|c|c|c|c|c|c|c|}
\hline \multirow[b]{3}{*}{ DATE } & \multirow{2}{*}{\multicolumn{8}{|c|}{ Precipitation }} & \multicolumn{7}{|c|}{ Wind } \\
\hline & & & & & & & & & \multicolumn{2}{|c|}{$8 \mathrm{H}$} & \multicolumn{3}{|c|}{ I2H } & \multicolumn{2}{|c|}{$20 \mathrm{HI}$} \\
\hline & $8 \mathrm{H}$ & $\mathrm{I} 2 \mathrm{H}$ & 2011 & $\begin{array}{l}\vec{\pi} \\
\stackrel{5}{0} \\
\xi\end{array}$ & 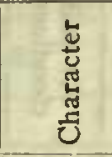 & \multicolumn{2}{|c|}{ 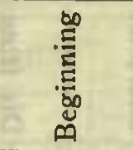 } & 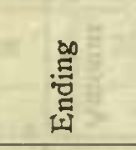 & 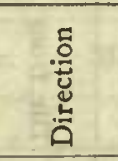 & 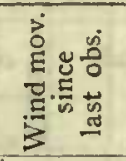 & 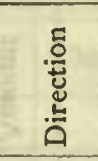 & & 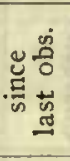 & 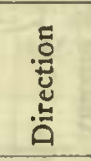 & 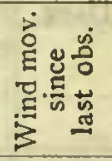 \\
\hline I & $\begin{array}{l}\text { In. } \\
.03\end{array}$ & $\begin{array}{l}I n . \\
T\end{array}$ & $\begin{array}{l}\text { In. } \\
.00\end{array}$ & & $\mathbf{S}^{\mathbf{m}}$ & $\begin{array}{l}h \\
. .\end{array}$ & $\begin{array}{l}m \\
. .\end{array}$ & $\begin{array}{ll}h & m \\
9 & \text { I0 }\end{array}$ & $\mathbf{E}$ & $\begin{array}{r}M i \\
86\end{array}$ & ENE & & $\begin{array}{r}M i \\
51\end{array}$ & $\mathbf{E}$ & $\begin{array}{l}M i \\
\mathrm{I}_{4} 9\end{array}$ \\
\hline 2 & .00 & .00 &.$\infty 0$ & .00 & $\ldots$ & 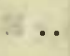 & .. & $\therefore$ & $\mathbf{E}$ & 548 & $\mathbf{E}$ & & 179 & E & 370 \\
\hline 3 & .00 & $\mathrm{~T}$ &.$\infty$ & $\mathrm{T}$ & $\mathrm{S}^{\mathrm{m}}$ & 9 & $\infty$ & 10 30 & E & 452 & E & & 55 & E & $\ldots$ \\
\hline 4 & .00 & .00 & $T$ & T & $\mathrm{S}^{\mathrm{m}}$ & II & $\infty$ & 1240 & ENE & ... & ENE & & $\ldots$ & NE & 1406 \\
\hline 5 & .00 & .00 &.$\infty$ & .00 & ... & .. & .. & .... & $\mathbf{N}$ & 282 & $N$ & & 143 & NE & 144 \\
\hline 6 & .20 & .00 & .01 & .21 & $\left\{\begin{array}{l}\mathbb{S}^{\mathrm{d}} \\
\mathbb{S}^{\mathrm{m}}\end{array}\right.$ & $\begin{array}{l}\text { D } \\
19\end{array}$ & $\begin{array}{l}N . \\
\infty\end{array}$ & $\left.\begin{array}{cc}7 & 30 \\
21 & 00\end{array}\right\}$ & NNE & 174 & c & & 7 & D & 33 \\
\hline 7 & $T$ & .00 & .00 & $\mathrm{~T}$ & ... & . & .. & .. $\quad$. & $E$ & 106 & SE & & 66 & E & 138 \\
\hline 8 & .00 & .04 & .02 & .06 & $\left\{\begin{array}{l}S^{\mathbf{m}^{\mathrm{m}}} \\
\mathbf{R}\end{array}\right.$ & $\begin{array}{r}9 \\
15\end{array}$ & $\begin{array}{l}40 \\
00\end{array}$ & 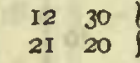 & E & 228 & E & & 104 & SE & 168 \\
\hline 9 & .15 & .20 & .40 & .75 & $\mathrm{~S}^{\mathrm{m}}$ & D & N. & 2110 & ESE & 262 & SE & & 73 & $w$ & II2 \\
\hline Io & .02 & .00 & .00 & .02 & $\cdots$ & *. & $\cdots$ & ... & WNW & 326 & W & & 80 & w & 168 \\
\hline II & .00 & .00 & $.0 \mathrm{r}$ & .01 & $\mathrm{~S}^{\mathrm{m}}$ & I3 & 30 & $16 \quad 15$ & $\mathrm{NW}$ & I74 & $\mathrm{NW}$ & & 46 & D) & 68 \\
\hline I2 & .00 & .04 & $T$ & .04 & $\mathrm{~S}^{\mathrm{m}}$ & ro & $\infty$ & 1300 & SE & 329 & SE & & 125 & sw & 135 \\
\hline I3 &.$\infty$ & .00 & .00 & .00 & $\cdots$ & .. & .. & .. $\quad$. & s & I03 & $\mathbf{E}$ & & 63 & $N W$ & 64 \\
\hline 14 & .00 & .00 & .06 & .06 & $S^{d}$ & 18 & 15 & D.N. & W & 243 & W & & 42 & E & 120 \\
\hline 15 & .04 & .00 & .02 & .06 & $S^{d}$ & 12 & 50 & 15 00 & $\mathbf{c}$ & 271 & E & & 32 & NE & 115 \\
\hline I6 & .00 &.$\infty$ &.$\infty$ & .00 & $\ldots$ & .. & .. & .... & $\mathbf{E}$ & 8 & SSW & & Io & ESE & 83 \\
\hline 17 & .00 & $\mathbf{T}$ & .or & $.0 I$ & $\left\{\begin{array}{l}\mathrm{R} \\
\mathrm{R}\end{array}\right.$ & $\begin{array}{l}\text { II } \\
\text { I2 }\end{array}$ & $\begin{array}{l}40 \\
08\end{array}$ & $\begin{array}{ll}\text { I1 } & 46 \\
\text { I2 } & 25\end{array}$ & ESE & IIO & E & & $2 \mathrm{I}$ & ENE & 33 \\
\hline I8 & .00 & .00 & .00 & .00 & ... & .. & .. & .. $\quad$. &.$N$ & 95 & $\mathrm{~N}$ & & $\ldots$ & $\mathrm{NE}$ & $\mathrm{II}_{4}$ \\
\hline I9 & .00 &.$\infty 0$ & .00 & .00 & & .. & .. & .... & N & 270 & W & & 42 & $\mathbf{w}$ & 37 \\
\hline 20 & .00 &.$\infty 0$ &.$\infty$ & .00 & ... & .. & .. & .. $\quad$. & E & 94 & ID & & 74 & E & 163 \\
\hline $2 \mathrm{I}$ & .00 & .02 & .09 & . II & $\mathrm{S}^{\mathrm{d}}$ & 9 & 15 & 1630 & SE & $\mathrm{A}$ r & $\mathrm{s}$ & & 19 & NE & 56 \\
\hline 22 & .00 & .00 & .00 & .00 & ... & .. & .. & .. $\quad$. & $\mathrm{NE}$ & 179 & $w$ & & 12 & NE & 175 \\
\hline 23 & .00 & .00 & .00 & .00 & $\ldots$ & .. & .. & .. $\quad$. & NW & 293 & $\mathrm{~N}$ & & 60 & $\mathrm{~N}$ & II6 \\
\hline 24 & .00 & .00 &.$\infty 0$ &.$\infty$ & $\ldots$ & .. & .. & .. $\quad .$. & W & 139 & NW & & 29 & $\mathbb{E}$ & 59 \\
\hline 25 & .00 & .02 & $T$ & .02 & $S^{d}$ & 9 & 40 & 1230 & . D & 389 & $\mathbf{E}$ & & 159 & NW & II 5 \\
\hline 25 & .00 & .00 & .00 & .00 & $\ldots$ & .. & .. & .. $\quad .$. & $\mathrm{NW}$ & 286 & NE & & 88 & N & 92 \\
\hline 27 & .00 & .00 & .00 & .00 & $\ldots$ & .. & .. & .. .. & NE & 109 & NE & & 62 & NE & 97 \\
\hline 28 & .00 & .00 & .00 & .00 & $\ldots$ & .. & .. & .. $\quad .$. & NW & 162 & NW & & 57 & $\mathrm{~N}$ & 69 \\
\hline 29 & .00 & .00 & $T$ & $\mathrm{~T}$ & $\mathbf{S}^{\mathrm{d}}$ & 18 & 30 & D.N. & $\mathrm{NE}$ & 68 & NE & & 16 & $\mathbf{E}$ & 60 \\
\hline 30 & .05 & .00 & .40 & .45 & $\mathbf{S}^{\mathrm{d}}$ & 16 & $\infty$ & .. & $\mathbf{E}$ & 199 & E & & $4 I$ & D & 236 \\
\hline $3 \mathrm{I}$ & .50 & .15 & .02 & .67 & $\mathbf{S}^{\mathrm{d}}$ & .. & .. & I3 00 & WNW & 334 & NW & & III & $\mathrm{NW}$ & I 84 \\
\hline Sum.. & .99 & .47 & I.04 & 2.50 & ... & .. & .. & .. & $\ldots$ & 6402 & ... & & 1867 & ... & 4879 \\
\hline & $\ldots$ & ... & ... & ... & ... & .. & .. & .. $\quad$.. & B & 213.4 & E & & 64.4 & DD & 162.6 \\
\hline
\end{tabular}


Tabulation of daily meteorological observations at Cape Flora during the month of October, 1904-Continued Observer: Francis LoNG

\begin{tabular}{|c|c|c|c|c|c|c|c|c|c|c|c|}
\hline \multirow[b]{3}{*}{ DATE } & \multicolumn{4}{|c|}{$4 \pi+2)$} & \multicolumn{2}{|c|}{ Clouds } & & & & \multirow[b]{3}{*}{ 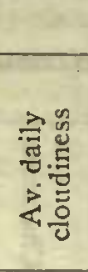 } & \multirow[b]{3}{*}{ REMARKS } \\
\hline & ats & $8 \mathrm{H}$ & intat & \multicolumn{3}{|c|}{$12 \mathrm{H}$} & \multicolumn{3}{|c|}{ 20н } & & \\
\hline & $\begin{array}{l}\stackrel{\rightleftarrows}{E} \\
\text { ¿ } \\
\text { E }\end{array}$ & 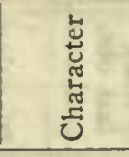 & 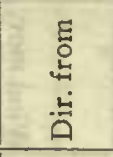 & 节 & 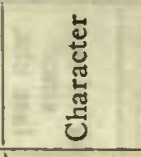 & 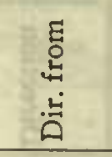 & 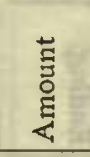 & 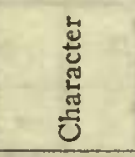 & 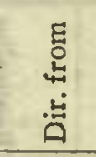 & & \\
\hline I & 10 & & $\mathbf{E}$ & $\left\{\begin{array}{l}4 \\
3\end{array}\right.$ & $\begin{array}{l}(\mathrm{A}-\mathrm{S}) \\
\mathrm{S}-\mathrm{Cu}\end{array}$ & W $\}$ & Io & s & $\mathbf{D}$ & & Drifting snow from $16: 00$. \\
\hline $0 \leq 2$ & $\begin{array}{l}2 \\
2 \\
2\end{array}$ & $\begin{array}{c}(\mathrm{Cl}-\mathrm{Cu}) \\
(\mathrm{A}-\mathrm{Cu}) \\
\mathrm{S}\end{array}$ & $\begin{array}{l}\mathbf{E} \\
\mathbf{E} \\
\mathbf{E}\end{array}$ & $\begin{array}{l}2 \\
2 \\
3\end{array}$ & $\begin{array}{l}(\mathrm{Cu}) \\
\text { S-Cu } \\
\text { S }\end{array}$ & $\left.\begin{array}{l}\mathbf{E} \\
\mathbf{E} \\
\mathbf{E}\end{array}\right\}$ & Io & $\mathbf{S}$ & E & 8 & Drifting snow and heavy east gale. \\
\hline & $10^{\circ}$ & $\mathbf{S}^{*}$ & E & - 10 & $\mathbf{s}$ & $\mathbf{E}$ & Io & s & E & Io & East gale to $5: 00 ;$ fog $5: 30$ to $10: 40$ \\
\hline 4 & Io & $\mathbf{S}^{*}$ & ENE & I0 & $\mathbf{N}^{*}$ & ENE & Io & $\mathbf{S}^{*}$ & NE & 10 & High winds; drifting snow $11: 00$ to $12: 40$; \\
\hline 5 & 10 & $\mathbf{S}$ & $\mathbf{N}$ & IO & $\mathrm{S}$ & $\mathrm{N}$ & Io & S & NE & & Drifting snow. \\
\hline 6 & $\begin{array}{l}2 \\
2 \\
4\end{array}$ & $\begin{array}{l}(\mathrm{A}-\mathrm{Cu}) \\
\mathrm{S}-\mathrm{Cu} \\
\mathrm{S}\end{array}$ & $\begin{array}{l}\mathrm{NE} \\
\mathrm{NE} \\
\mathrm{NE}\end{array}$ & $\begin{array}{l}2 \\
3 \\
3\end{array}$ & $\begin{array}{c}(\mathrm{A}-\mathrm{Cu}) \\
\mathrm{S}-\mathrm{Cu} \\
\mathrm{S}\end{array}$ & $\underset{N}{N}$ & Io & $\mathbf{N}$ & $\mathbf{E}$ & 10 & 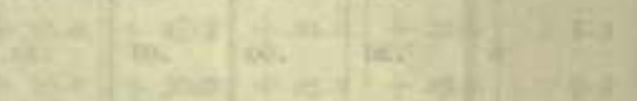 \\
\hline$=7$ & I0 & S & $\mathbf{E}$ & Io & $\mathbf{S}$ & SE & 10 & S . & $\mathbf{E}$ & 10 & Light drifting snow from $16: 00$. \\
\hline 8 & $\begin{array}{l}2 \\
3 \\
3\end{array}$ & $\begin{array}{c}(\mathrm{A}-\mathrm{Cu}) \\
(\mathrm{A}-\mathrm{S}) \\
\mathrm{S}\end{array}$ & $\begin{array}{l}\mathbf{E} \\
\mathbf{E}\end{array}$ & Io & $\mathbf{N}$ & $\mathbf{E}$ & Io & $\mathbf{N}$ & SE & Io & High east wind and drifting snow to $13: 00$. \\
\hline 9 & 10 & $\mathbf{N}$ & ESE & Io & $\mathbf{N}$ & SE & I0 & $\mathbf{N}$ & W & Io & Drifting snow $5: 00$ to $9: 30$. \\
\hline Io & $\begin{array}{l}2 \\
8\end{array}$ & $\underset{\mathrm{S}}{\mathrm{S}-\mathrm{Cu}}$ & $\left.\begin{array}{c}\text { WNWV } \\
\text { NW }\end{array}\right\}$ & 8 & 24 & W & Few & $\mathbf{S}-\mathbf{C u}$ & W & 6 & Generally west gale. \\
\hline II & 3 & $\mathrm{~S}-\mathrm{Cu}$ & NW & 10 & $\mathbf{S}$ & $N W$ & Io & s & $\mathbf{E}$ & & 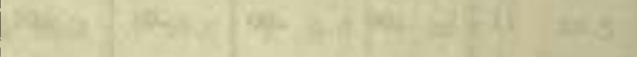 \\
\hline 12 & I0 & $\mathbf{S}$ & SE & Io & $\mathbf{N}$ & SE & Io & S a & SW & Io & High southeast wind and drift to $9: 15$. \\
\hline 13 & $\cdots$ & ** & $\cdots$ & Io & $\mathbf{S}$ & $\mathbf{E}$ & 9 & $\mathbf{S}$ & $N W$ & & Dense fog to $9: 30$ \\
\hline I4 & 4 & $\mathbf{s}$ & $\mathbf{W}$ & $\left\{\begin{array}{l}4 \\
3\end{array}\right.$ & $(\mathbf{A}-\mathbf{S})$ & $\mathbf{W}$ & 10 & $\mathbf{N}$ & $\mathbf{E}$ & 6 & 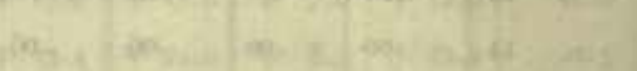 \\
\hline 15 & $\begin{array}{l}4 \\
5\end{array}$ & $\underset{S}{(A-S)}$ & $\mathbf{E}$ & Io & $s$ & $\mathbf{E}$ & o & $\cdots$ & $\cdots$ & 7 & 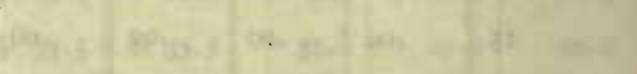 \\
\hline 16 & Io & $\mathbf{S}^{*}$ & $\mathbf{E}$ & 10 & $\mathbf{S}^{*}$ & SSW & IO & $S^{*}$ & ESE & 10 & Light fogs $6: 00$ to $13: 30$ and $17: 00$ to $24: 00$. \\
\hline I7 & $\cdots$ & ** & $\cdots$ & ... & ** & . & ... & ** & ... & I0 & Generally foggy. \\
\hline I8 & 10 & s & $\mathbf{E}$ & .. & ** & & I 0 & $\cdots$ & ... & 7 & Fog early A. M. and Io:00 to I5:00. \\
\hline (19 19 & Few & $\mathbf{S}$ & $\mathbf{N}$ & 0 & $\ldots$ & 14. & $\ldots 0$ & .... & ... & 0 & Solar halo II:50 to $12: 20$ \\
\hline 20 & Few. & $(\mathbf{A}-\mathbf{S})$ & $\mathbf{E}$ & & $\cdots$ & $\cdots$ & $\ldots$ & $\cdots$ & $\cdots$ & 2 & Fog from I8: $3 \circ$ \\
\hline 21 & IO & $\mathbf{S}$ & SE & 10 & $\mathbf{N}^{*}$ & $\mathbf{s}$ & Io & $(\mathrm{S})$ & $\mathrm{NE}$ & & Fog $9:$ Is to I $7: 00$ \\
\hline 22 & $\begin{array}{l}3 \\
3\end{array}$ & $\underset{S}{(A-S)}$ & $\begin{array}{l}\mathrm{NE} \\
\mathrm{NE}\end{array}$ & $\begin{array}{l}3 \\
6\end{array}$ & $\frac{(\mathrm{A}-\mathrm{Cu})}{\mathrm{S}}$ & W & Io & $\mathbf{S}$ & NE & 8 & Sun disappears for winter. \\
\hline 23 & Few & $\mathbf{S}$ & $\mathbb{N W}$ & Few & $\mathbf{s}$ & $\mathbf{N}$ & $\begin{array}{r}3 \\
5\end{array}$ & $\begin{array}{l}(\mathrm{A}-\mathrm{Cu}) \\
\mathbf{S}\end{array}$ & $\begin{array}{l}\mathrm{N} \\
\mathrm{N}\end{array}$ & $\mathbf{I}$ & Very clear and cold. \\
\hline 24 & $\left\{\begin{array}{l}5 \\
3\end{array}\right.$ & $\underset{\mathbf{S}}{(\mathrm{A}-\mathrm{S})}$ & $\underset{W}{W}$ & & $\underset{\mathrm{S}}{(\mathrm{A}-\mathrm{Cu})}$ & $\left.\begin{array}{l}\mathrm{W} \\
\mathrm{NW}\end{array}\right\}$ & 2 & $(\mathrm{Ci})$ & $\mathbf{E}$ & & 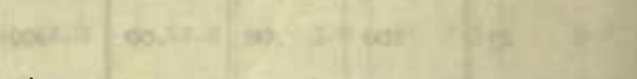 \\
\hline $\begin{array}{l}25 \\
26\end{array}$ & $\begin{array}{l}\text { Io } \\
\text { Few } \\
\text { Few }\end{array}$ & $\begin{array}{c}S \\
(C i-S) \\
(A-S)\end{array}$ & 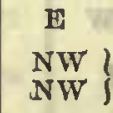 & 10 & $\mathbf{N}^{*}$ & $\ldots$ & $\begin{array}{l}\text { 10 } \\
0\end{array}$ & $\cdots$ & NW & Io & $\begin{array}{l}\text { East gale to II:00; fog } 10: 00 \text { to } 13: 00 \text { and } \\
\text { 17:00 to 21:00. } \\
\text { High drifting wind to } 9: 00 \text {. }\end{array}$ \\
\hline 27 & 9 & S & NE & 10 & $\mathbf{s}$ & NE & 10 & $\mathbf{S}$ & NE & I0 & $\begin{array}{ll}n \\
n\end{array}$ \\
\hline 28 & 10 & $\mathbf{S}$ & NW & 10 & $\mathbf{S}$ & NW & Few & Ci-s & $\mathbf{N}$ & 7 & $\cos .3 \times 130$ \\
\hline 29 & I & $\mathbf{s}$ & E & $\left\{\begin{array}{l}4 \\
4\end{array}\right.$ & $\begin{array}{c}(\mathrm{A}-\mathrm{Cu}) \\
\mathrm{S}\end{array}$ & $\left.\begin{array}{l}\mathbf{E} \\
\mathbf{E}\end{array}\right\}$ & Io & $\mathbf{N}$ & $\mathbf{E}$ & & 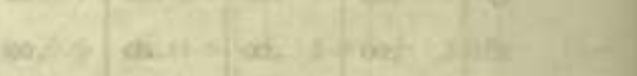 \\
\hline 30 & Io & S & E & 10 & S & $\mathbf{E}$ & 10 & $\mathbf{N}$ & E & 10 & Drifting from $16: \infty$ \\
\hline $3 I$ & 10 & $\mathrm{N}$ & WNW & I0 & $\mathbf{N}$ & $\mathrm{NW}$ & Io & $\mathbf{S}$ & NW & & \\
\hline Sum.... & 212 & $\cdots$ & & 230 & - & & 219 & $\cdots$ & & 239 & \\
\hline Mean... & 7.3 & .. & -.. & 7.9 & $\ldots$ & •. & 7.1 & & & 7.7 & 2. \\
\hline
\end{tabular}


Tabulation of daily meteorological observations at Cape Flore during the month of November, 1904

Observer: Francis LoNG

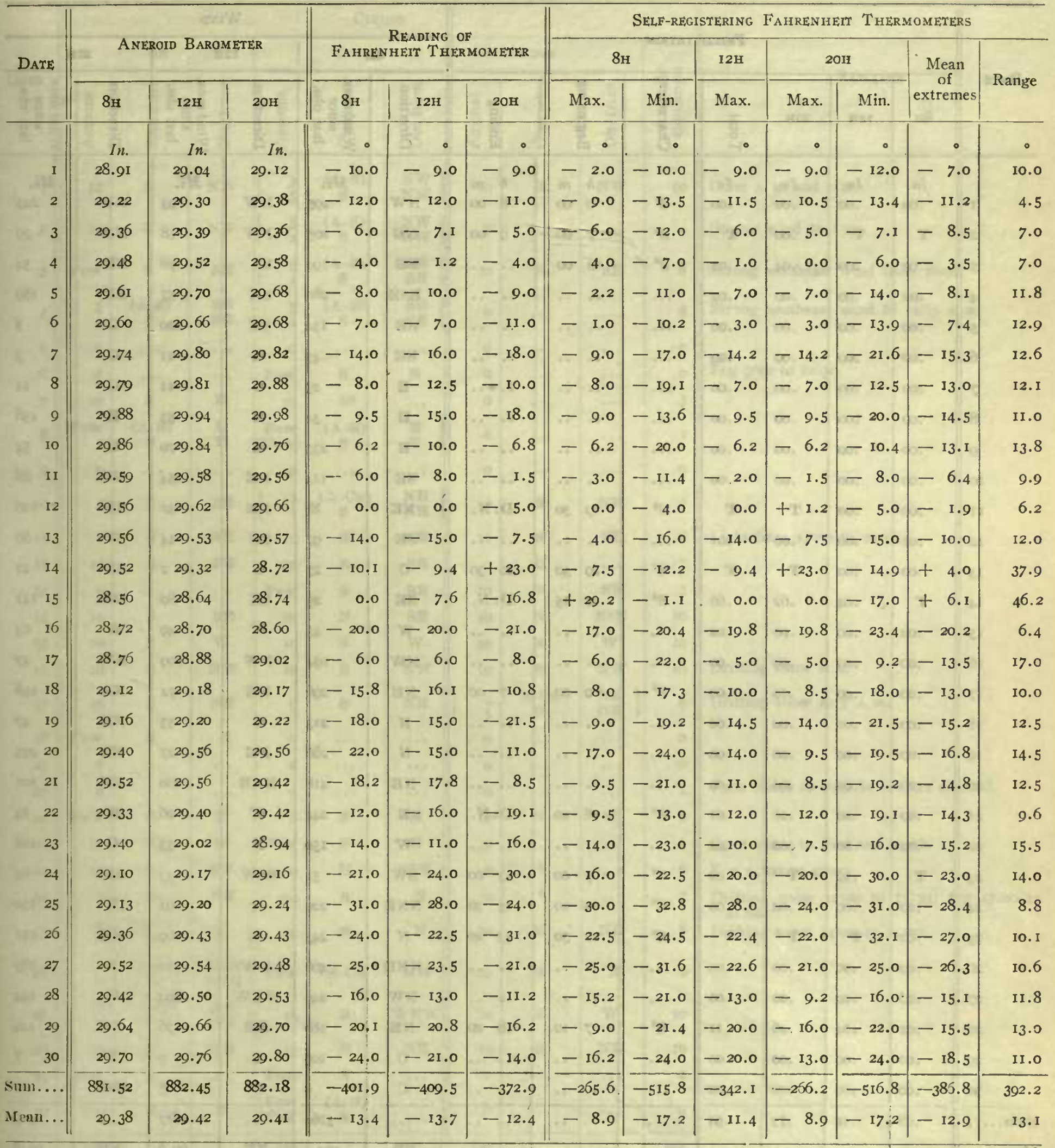


Tabulation of daily meteorological observations at Cape Flora during the month of November, I904-Continued Observer: FRANCIS LoNG

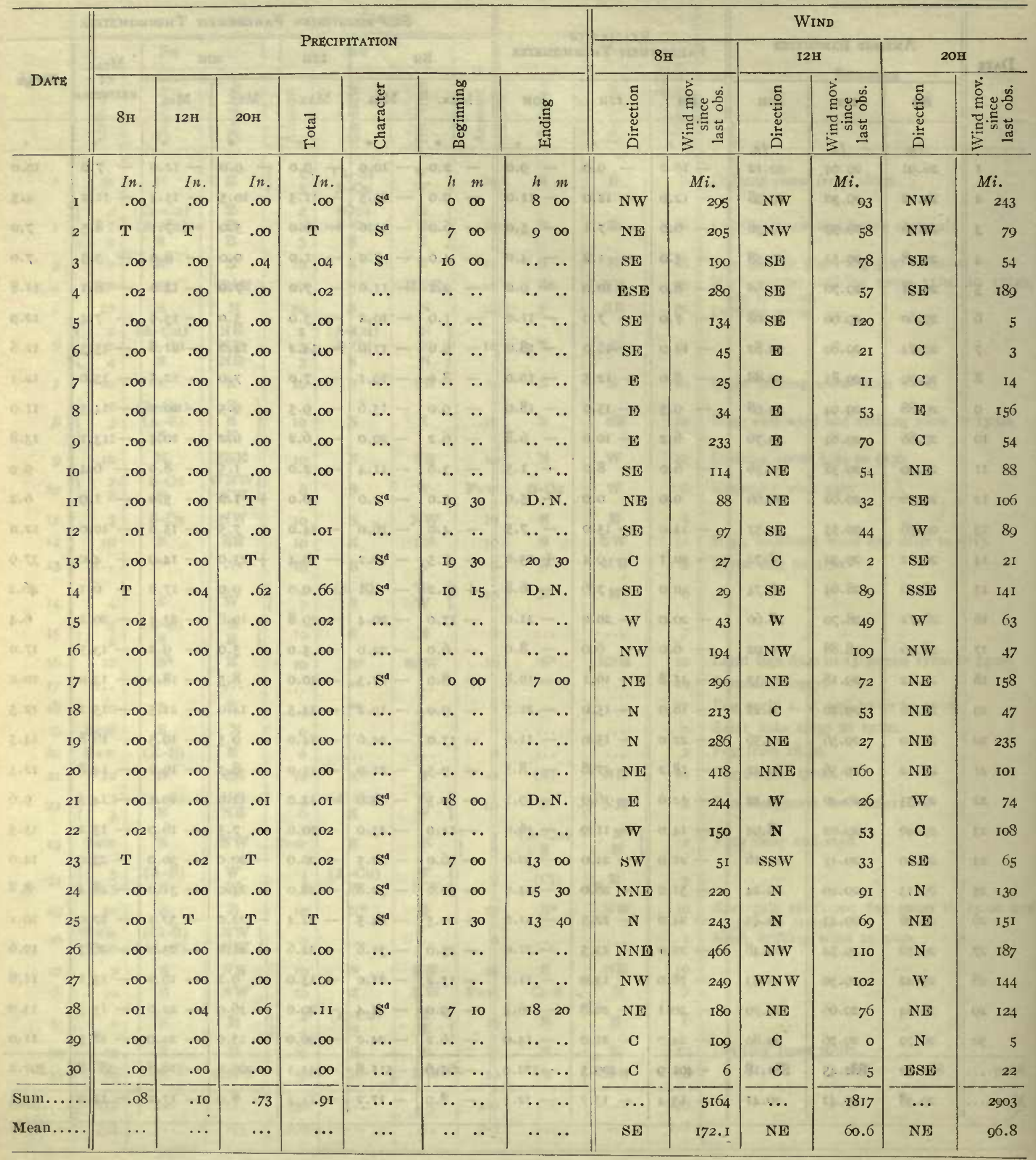


Tabulation of daily meteorological observations at Cape Flora during the month of November, 1904-Continued

Observer: Francis LoNG

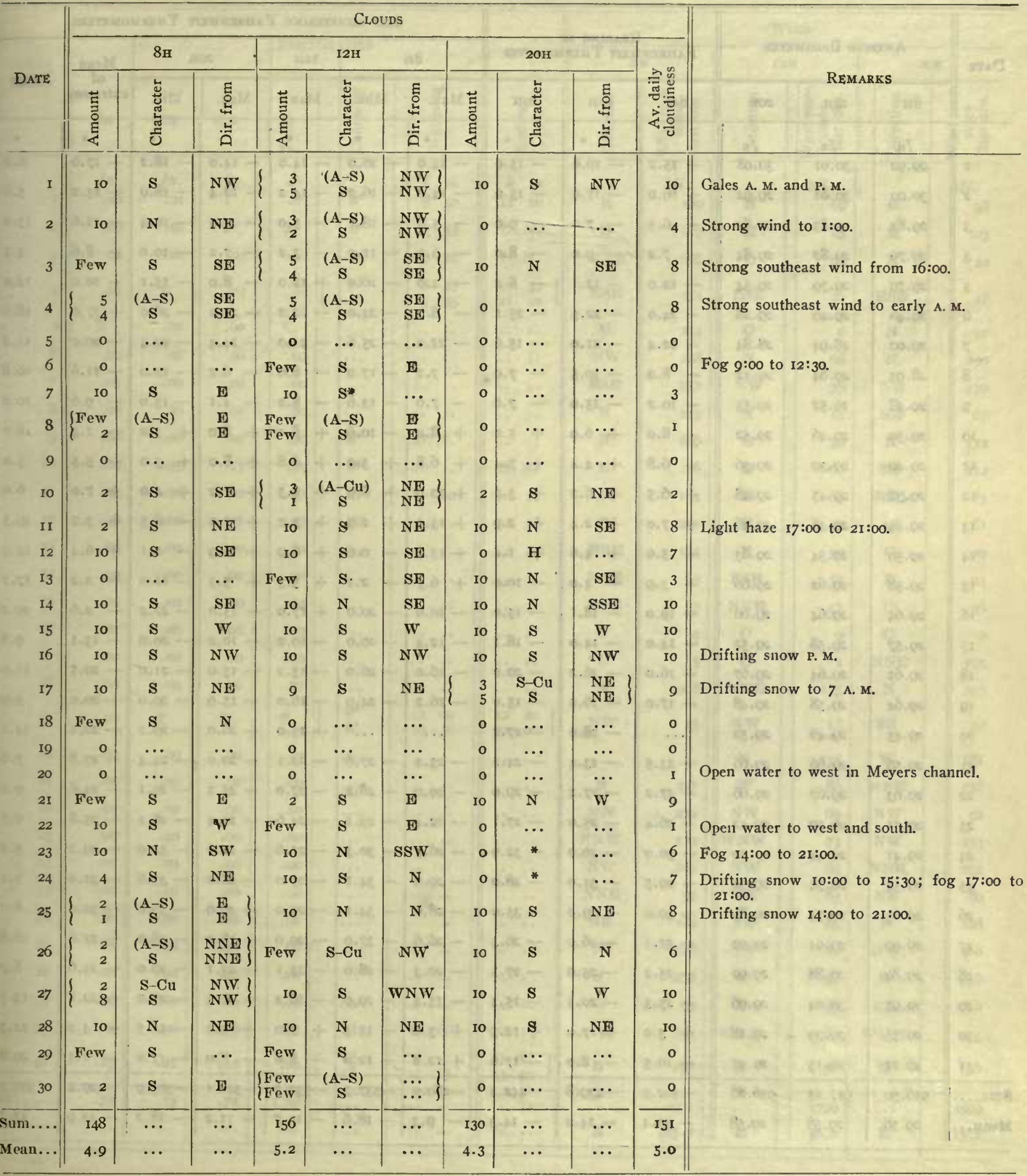


Tabulation of daily meteorolagical observations at Cape Flora during the manth of December, 1904 Observer: FRANCIS LONG

\begin{tabular}{|c|c|c|c|c|c|c|c|c|c|c|c|c|c|}
\hline \multirow{3}{*}{ DATE } & \multirow{2}{*}{\multicolumn{3}{|c|}{ ANEROID BAROMETER }} & \multirow{2}{*}{\multicolumn{3}{|c|}{$\begin{array}{c}\text { READING OF } \\
\text { FAHRENHEIT THERMOMETER }\end{array}$}} & \multicolumn{6}{|c|}{ SELF-REGISTERING FAHRENIEIT TIIERMOMETERS } & \multirow{3}{*}{ Range } \\
\hline & & & & & & & \multicolumn{2}{|c|}{$8 \mathrm{H}$} & \multirow{2}{*}{$\frac{\text { I2H }}{\text { Max. }}$} & \multicolumn{2}{|c|}{$2 \mathrm{OH}$} & \multirow{2}{*}{$\begin{array}{c}\text { Mean } \\
\text { of } \\
\text { extremes }\end{array}$} & \\
\hline & $8 \mathrm{H}$ & $12 \mathrm{H}$ & гон & $8 \mathrm{H}$ & $12 \mathrm{H}$ & $2 \mathrm{OH}$ & Max. & Min. & & Max. & Min. & & \\
\hline & In. & In. & In. & $\circ$ & E. & & 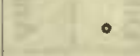 & $\circ$ & $\circ$ & $\circ$ & & & - \\
\hline I & 29.92 & 30.01 & 30.08 & -15.2 & -16.0 & -15.0 & -14.0 & -20.0 & -14.0 & -14.0 & -18.2 & -17.0 & 6.0 \\
\hline 2 & 30.02 & 30.01 & 29.92 & -16.0 & -17.0 & -15.0 & -14.0 & -16.2 & $-14 \cdot 2$ & -12.4 & -18.0 & -15.2 & 5.6 \\
\hline 3 & 29.83 & 29.83 & 1. 29.82 & -6.5 & -7.0 & -9.0 & -5.6 & -18.2 & -5.0 & -5.0 & -10.4 & 一 II.6 & 13.2 \\
\hline 4 & 29.79 & 29.82 & 29.84 & -7.2 & -9.0 & -8.0 & -6.1 & -11.0 & -7.2 & -7.2 & - го.0 & -8.6 & 4.9 \\
\hline 5 & 29.79 & 29.79 & 29.54 & -12.0 & -12.1 & -8.2 & -4.0 & $-\mathrm{x} 6.0$ & -12.0 & -8.0 & -15.1 & -10.0 & 12.0 \\
\hline 6 & 29.46 & 29.40 & 29.28 & -24.0 & -22.5 & -25.1 & -8.0 & -24.0 & -22.2 & -22.2 & -26.0 & -17.0 & 18.0 \\
\hline 7 & 29.00 & 28.94 & 28.84 & -22.4 & -21.0 & -15.6 & -22.0 & -25.6 & -21.0 & -14.4 & -22.4 & -20.0 & 11.2 \\
\hline 8 & 28.93 & 29.04 & 29.12 & -8.0 & -10.5 & -7.0 & -7.2 & -17.0 & -8.0 & -6.2 & -12.0 & 一 т1.6 & 10.8 \\
\hline 9 & 29.38 & 29.52 & 29.55 & -10.2 & -15.0 & -7.0 & -7.0 & $-\mathrm{I} 3.6$ & -8.2 & -4.1 & -15.0 & -9.6 & 10.9 \\
\hline 10 & 29.50 & 29.46 & $29 \cdot 52$ & +8.0 & +6.9 & +5.0 & +8.0 & - 10.6 & +8.0 & +8.0 & +3.0 & -1.3 & 18.6 \\
\hline II & 29.40 & 29.39 & 29.36 & +6.8 & +4.4 & +7.4 & +6.8 & +3.0 & +6.8 & +8.0 & +3.0 & +5.5 & 5.0 \\
\hline 12 & 29.36 & 29.45 & 29.48 & +6.5 & +6.2 & +5.0 & +10.0 & +6.9 & +6.5 & +7.2 & +4.0 & +7.0 & 6.0 \\
\hline 13 & 29.44 & 29.46 & 29.46 & +7.0 & +2.4 & +2.9 & +13.0 & +2.9 & +7.0 & +7.0 & -6.5 & +3.2 & 19.5 \\
\hline 14 & 29.50 & 29.54 & 29.63 & +5.0 & +4.6 & +1.4 & +13.8 & -0.6 & +6.9 & +6.9 & $-\mathrm{I} . \mathrm{I}$ & +6.4 & 14.9 \\
\hline I5 & 29.58 & 29.62 & 29.66 & +3.9 & -4.0 & -10.0 & +6.7 & -2.1 & +5.3 & +5.3 & -11.0 & -2.2 & 17.7 \\
\hline 16 & 29.64 & 29.64 & 29.66 & -19.0 & -18.1 & -15.0 & -10.0 & -20.0 & +17.0 & -15.0 & -22.2 & $-\quad 2.6$ & 39.2 \\
\hline 17 & 29.57 & 29.58 & 29.52 & -14.0 & -14.0 & -18.1 & -12.4 & -20.0 & -10.2 & -10.2 & -20.0 & $-\mathrm{I} 5 . \mathrm{I}$ & 9.8 \\
\hline 18 & $29.6 \mathrm{I}$ & 29.64 & 29.67 & -16.0 & -16.2 & -20.0 & -16.0 & -26.0 & -15.2 & -15.0 & -21.0 & -20.5 & II.O \\
\hline 19 & 29.64 & 29.58 & 29.48 & -17.0 & -16.0 & -15.0 & -16.2 & -24.9 & -16.0 & -15.0 & -20.0 & -20.0 & 0.9 \\
\hline 20 & 29.45 & 29.46 & 29.52 & $\ldots$ & -28.0 & $=27.2$ & $\ldots$ & ... & -15.0 & -26.0 & -29.2 & -22.1 & 14.2 \\
\hline $2 I$ & 29.56 & 29.66 & 29.66 & -23.5 & -23.4 & -24.2 & -23.4 & -27.6 & -22.1 & -20.0 & -24.4 & -23.8 & 7.6 \\
\hline 22 & 29.63 & 29.67 & 29.66 & -27.2 & $-27 \cdot 4$ & -29.0 & -20.0 & -28.2 & -27.0 & -25.2 & -29.1 & -24.6 & 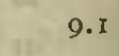 \\
\hline 23 & 29.51 & 29.50 & 29.42 & -26.4 & -25.0 & -27.1 & -22.4 & -29.1 & -24.6 & -22.4 & -28.2 & -25.8 & 6.7 \\
\hline 24 & $29.4 \mathrm{I}$ & 29.44 & 29.50 & -30.0 & -30.0 & -32.0 & -26.5 & -30.3 & -27.6 & -27.6 & $-32: 0$ & -29.2 & 5.5 \\
\hline 25 & 29.54 & 29.54 & 29.62 & -29.5 & -31.0 & -28.0 & -29.0 & -34 & -29.0 & -28.0 & -33.6 & $-3 \mathrm{I} .0$ & 6.1 \\
\hline 26 & 29.63 & 29.74 & 29.87 & -30.0 & -29.0 & -35.0 & -28.0 & -34.1 & -29.0 & -29.0 & -36.0 & -32.0 & 8.0 \\
\hline 27 & 29.90 & 29.94 & 29.90 & -27.1 & -26.0 & -26.3 & -26.6 & -37.0 & -20.0 & -18.2 & -28.0 & -27.6 & I8.8 \\
\hline 28 & 29.80 & 29.88 & 29.92 & -25.2 & -25.0 & -27.5 & -20.3 & -28.0 & -23.1 & -23.1 & -29.0 & -24.6 & 8.7 \\
\hline 29 & 29.95 & 30.04 & 29.96 & -25.5 & -20.1 & -15.3 & -15.4 & -29.0 & -18.2 & -15.3 & -26.0 & -22.2 & 13.7 \\
\hline 30 & 29.55 & 29.39 & 29. I8 & +3.0 & +7.0 & +12.5 & +3.0 & -15.3 & +7.0 & +3.0 & -12.5 & -4.2 & 22.3 \\
\hline $3 I$ & 29.21 & 29.15 & 29.22 & $-\mathrm{I0.5}$ & $\div 8.0$ & -17.0 & +12.8 & $-\mathrm{I} 2.0$ & -8.0 & -8.0 & -17.0 & -2.1 & 29.8 \\
\hline$\cdots$ & 916.50 & 917.13 & 916.86 & -402.2 & -439.8 & -442.4 & -280.0 & -557.7 & -332.3 & $-346 . I$ & -563.9 & -429.4 & 394.7 \\
\hline Mean... & 29.56 & 29.58 & 29.58 & -13.4 & -14.2 & -14.3 & -9.3 & -18.6 & -10.7 & -11.2 & $|-18.2|$ & -13.9 & 12.7 \\
\hline
\end{tabular}


Tabulation of daily metcorological obscrvations at Cape Flora during the month of Dccember, I904-Continued Observer: FRANCIS LONG

\begin{tabular}{|c|c|c|c|c|c|c|c|c|c|c|c|c|c|c|c|}
\hline \multirow[b]{3}{*}{ DATE } & \multirow{2}{*}{\multicolumn{5}{|c|}{ PRECIPITATION }} & \multirow{2}{*}{\multicolumn{4}{|c|}{ 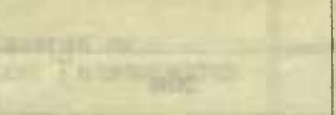 }} & \multicolumn{6}{|c|}{ Wind } \\
\hline & & & & & & & & & & = & 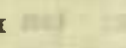 & & $\mathrm{H}$ & 20 & \\
\hline & $8 \pi$ & I 2 II & $2 \mathrm{OH}$ & $\begin{array}{c}\bar{\pi} \\
0 \\
\end{array}$ & 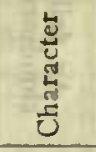 & \multicolumn{2}{|c|}{ 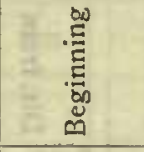 } & \multicolumn{2}{|c|}{ 密 } & 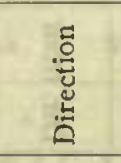 & 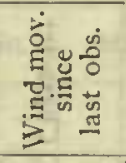 & 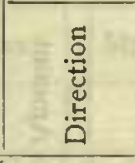 & 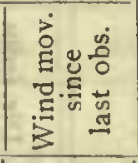 & 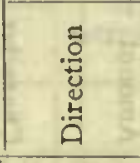 & 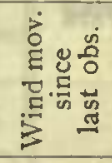 \\
\hline I & $\begin{array}{l}\text { In. } \\
. \infty\end{array}$ & $\begin{array}{l}\text { In. } \\
\text {.OI }\end{array}$ & $\begin{array}{l}\text { In } \\
\text { T }\end{array}$ & $\begin{array}{l}\text { In. } \\
.01\end{array}$ & $S^{n}$ & $\begin{array}{l}h \\
8\end{array}$ & $\begin{array}{l}m \\
50\end{array}$ & $\begin{array}{r}h \\
12\end{array}$ & $\begin{array}{l}m \\
20\end{array}$ & $\mathbf{E}$ & $\begin{array}{l}M i . \\
180\end{array}$ & E & Mi. & E & Mi. \\
\hline 2 & .00 & .00 & $\mathbf{T}$ & $\mathbf{T}$ & $\mathbf{S}^{d}$ & 7 & 55 & D. & N. & $\mathbf{E}$ & 167 & $\mathbf{E}$ & 65 & E & 103 \\
\hline 3 & .01 & .00 & .01 & .02 & $S^{\prime \prime}$ & I8 & IO & & $\mathbf{N}$ & ENE & 202 & E & 49 & E & \\
\hline 4 & .01 & $\mathbf{T}$ & .02 & .03 & $\mathbf{S}^{d}$ & $\begin{array}{r}8 \\
13\end{array}$ & $\begin{array}{l}25 \\
35\end{array}$ & $\begin{array}{l}10 \\
21\end{array}$ & $\left.\begin{array}{l}30 \\
10\end{array}\right\}$ & IESE & 174 & ESE & 26 & ESE & 47 \\
\hline 5 & $\mathbf{T}$ &.$\infty$ &.$\infty$ & $\mathrm{T}$ & $\cdots$ & $\cdot \cdots$ & .. & .. & . & 0 & 114 & C & o & $\mathbf{N}$ & 97 \\
\hline 6 & .00 & .00 &.$\infty$ & .00 & $\cdots$ & .. & . & .. & .. & NW & 258 & $\mathbf{N}$ & 68 & $\mathbf{N}$ & 190 \\
\hline 7 & .00 &.$\infty 0$ & .00 & .00 & $\cdots$ & $\cdots$ & .. & . & .. & RSE & 277 & $\mathrm{NW}$ & 78 & NE & 179 \\
\hline 8 & .00 &.$\infty$ & .00 & .00 & $\ldots$ & 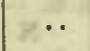 & .. & . & . & $\mathbf{N}$ & II5 & N & 12 & NE & 67 \\
\hline 9 & .00 & .00 &.$\infty$ & .00 & $S^{d}$ & D. & N. & $\cdots$ & $\cdots$ & $\mathbf{N}$ & 249 & C & 39 & $\mathbf{N}$ & 152 \\
\hline Io & .04 & .05 & .15 & .24 & $\mathbf{S}^{\mathrm{d}}$ & .. & .. & D. & N. & $\mathbf{E}$ & 256 & $\mathbf{E}$ & 68 & SE & 114 \\
\hline II & .01 & .00 & .02 & .03 & $\mathbf{S}^{\mathrm{m}}$ & 17 & 20 & .. & .. & $\mathbf{E}$ & I44 & $\mathbf{E}$ & 64 & ESE & 85 \\
\hline 12 & .40 & .06 & .01 & .47 & $S^{m}$ & $\cdots$ & . & 12 & 50 & $\mathbf{E}$ & 200 & $\mathbf{E}$ & 4 & 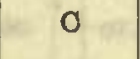 & $6 \mathrm{I}$ \\
\hline I 3 &.$\infty 0$ &.$\infty$ & .00 & .00 & .. & $\cdots$ & .. & . & .. & ENE & I64 & $\mathbf{E}$ & 50 & C & 29 \\
\hline 14 & .00 & .00 &.$\infty$ &.$\infty$ & .. & $\cdots$ & . & . & .. & ENE & 63 & RSA & 52 & E & 44 \\
\hline 15 & .00 & .00 &.$\infty$ & .00 & $\cdots$ & .. & . & . & .. & NNE & 14 57 & NNE & 47 & C & 46 \\
\hline 16 & .00 &.$\infty$ & .00 & .00 & $\cdots$ & .. & . & $\cdots$ & . & c & $\begin{array}{ll}71 & 4\end{array}$ & O & 9 & C & 0 \\
\hline 17 &.$\infty$ &.$\infty$ &.$\infty$ & .00 & $\cdots$ & . & $\because$ & . & . & C & 5 & C & I & NNE & 195 \\
\hline 18 & .00 & .00 &.$\infty 0$ & .00 & $\cdots$ & . & .. & . & . & $\mathbf{N}$ & 208 & NNE & 125 & $N$ & 327 \\
\hline 19 &.$\infty$ & 'T' & 1 & $\mathrm{~T}$ & $\begin{array}{l}\mathbf{S}^{d} \\
\mathbf{S}^{d}\end{array}$ & $\begin{array}{l}12 \\
19\end{array}$ & $\begin{array}{l}00 \\
30\end{array}$ & $\begin{array}{l}12 \\
22\end{array}$ & $\begin{array}{l}20 \\
\infty\end{array}$ & NW & 45 & NW & I5 & SE & 32 \\
\hline 20 &.$\infty$ & .00 &.$\infty 0$ & .00 & . & .. & .. & .. & . & $\cdots$ & $\ldots$ & $\mathbf{N}$ & 173 & $W$ & 181 \\
\hline 21 &.$\infty$ &.$\infty$ & .00 & .00 & . & $\therefore$ & .. & .. & $\cdots$ & $\mathbf{N}$ & 225 & WV & 86 & NNE & I56 \\
\hline 22 & .00 & .00 &.$\infty$ &.$\infty$ & $\cdots$ & $\quad \cdots$ & . & $\cdots$ & $\because$ & NW & 272 & N IV & 69 & NIV & 92 \\
\hline 23 & .00 & .00 & .00 & .00 & $\cdots$ & . & . & .. & . & NNT & 179 & WNW & 73 & NW & 153 \\
\hline 24 & .00 &.$\infty$ & .00 &.$\infty$ & .. & . & . & $\cdots$ & .. & NW & 269 & NTV & 64 & NNE & 192 \\
\hline 25 &.$\infty$ &.$\infty$ & .00 & .00 & $\cdots$ & . & . & . & . & NNE & 97 & NE & 63 & NE & 80 \\
\hline 26 &.$\infty$ & .00 & .00 &.$\infty$ & ... & . & .. & . & .. & NE & 184 & NE & 65 & E & I 38 \\
\hline 27 &.$\infty$ & .00 & .00 & .00 & $\cdots$ & $\cdots$ & . & . & . & $\mathbf{N}$ & 170 & NW & 40 & NW & 168 \\
\hline 28 & .00 &.$\infty$ & .00 &.$\infty 0$ & .. & $\cdots$ & .. & $\cdots$ & . & $\mathbf{N}$ & 250 & C & 95 & E & 116 \\
\hline 29 & .00 &.$\infty$ & .00 & .00 & .. & . & .. & . & . & WV & 93 & E & 33 & NE & 16 \\
\hline 30 & .12 & .10 & .40 & .62 & $S^{d}$ & D. & N. & . & . & NIV & 153 & IVNW & 84 & WNW & 195 \\
\hline 31 & .15 &. $\mathrm{OI}$ & .10 & .26 & $\begin{array}{l}S^{d} \\
S^{d}\end{array}$ & iI & $\ddot{15}$ & $\begin{array}{l}\text { D. } \\
\text { 13 }\end{array}$ & $\begin{array}{l}\text { N. } \\
0\end{array}$ & E & 197 & E & 72 & E & 136 \\
\hline Sum...... & .74 & .23 & .71 & 1.68 & ... & $\cdots$ & . & . & .. & $\cdots$ & 4971 & $\cdots$ & 1799 & $\cdots$ & 3509 \\
\hline Mean..... & $\cdots$ & $\cdots$ & $\cdots$ & $\cdots$ & ... & $\cdots$ & $\because$ & . & $\cdots$ & E & 165.7 & & 58.0 & $\mathbf{E}$ & 113.2 \\
\hline
\end{tabular}


Tabulation of daily metcorological observations at Cape Flora during the month of December, 1904-Continted Observer: FRANCIS LONG

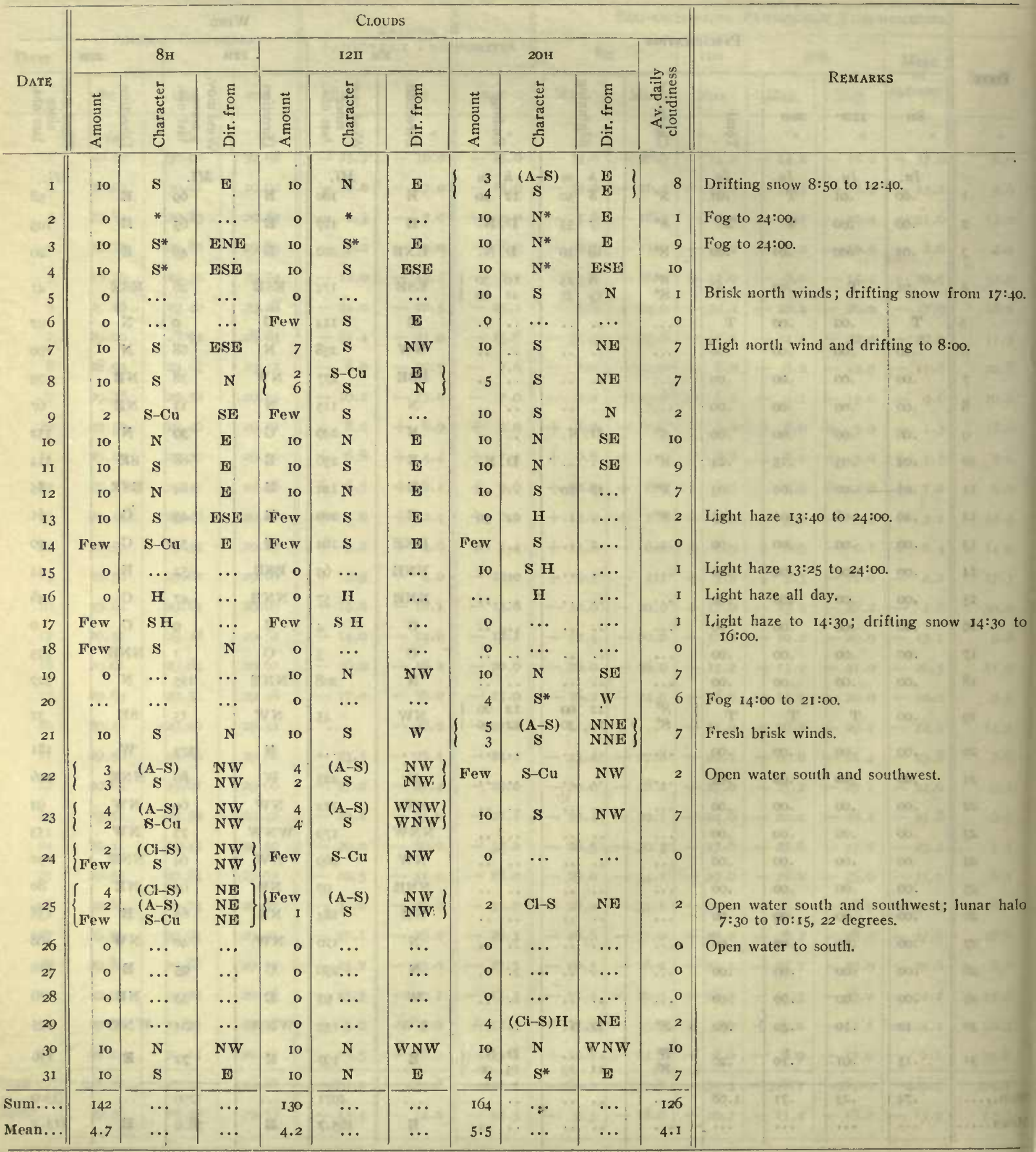


Tabulation of daily metcorological obscrvations at Cape Flora during the month of January, 1905

Observer: Francis LoNG

\begin{tabular}{|c|c|c|c|c|c|c|c|c|c|c|c|c|c|}
\hline \multirow{3}{*}{ DATE } & \multirow{2}{*}{\multicolumn{3}{|c|}{ ANEROID BAROMETER }} & \multirow{2}{*}{\multicolumn{3}{|c|}{$\begin{array}{c}\text { READING OF } \\
\text { FAHRENIIEIT T THERMOM ETER }\end{array}$}} & \multicolumn{7}{|c|}{ SELF-REGISTERING FAHREN HEIT THERMOMETERS } \\
\hline & & & & & & & \multicolumn{2}{|c|}{$8 \mathrm{H}$} & \multirow{2}{*}{$\begin{array}{l}\text { I2H } \\
\text { Max. }\end{array}$} & \multicolumn{2}{|c|}{$20 \mathrm{H}$} & \multirow{2}{*}{$\begin{array}{c}\text { Mean } \\
\text { of } \\
\text { extremes }\end{array}$} & \multirow{2}{*}{ Range } \\
\hline & $8 \mathbf{H}$ & $12 \mathrm{H}$ & $20 \mathrm{H}$ & $8 \mathrm{H}$ & $\mathrm{I} 2 \mathrm{II}$ & $20 \mathrm{H}$ & Max. & Min. & & Max. & Min. & & \\
\hline & In. & In. & In. & $=0$ & $=0$ & $\mathrm{P}^{2}$ & $2=0$ & • & $-\circ$ & • & - & - & ॰ \\
\hline I & 29.45 & 29.54 & 29.59 & -29.0 & -31.0 & -29.0 & -16.5 & -29.0 & -27.0 & -27.0 & -34.0 & -25.2 & 17.5 \\
\hline 2 & 29.44 & 29.42 & 29.26 & -30.0 & -29.0 & -28.0 & -29.0 & -30.0 & -28.0 & -28.0 & -30.0 & -29.0 & 2.0 \\
\hline 3 & 29.02 & 28.98 & 28.90 & -27.5 & -25.2 & -28.0 & -27.5 & -28.5 & -25.0 & -18.0 & -28.0 & -23.2 & 10.5 \\
\hline 4 & 28.93 & 28.98 & 29.00 & -38.0 & -34.0 & -33.4 & -26.4 & -41.0 & -34.0 & -30.0 & -38.0 & -33.7 & 14.6 \\
\hline 5 & 28.98 & 28.96 & 28.94 & -31.0 & -35.0 & $-34 \cdot I$ & -27.5 & -35.0 & -.31 .0 & -29.4 & -37.0 & -32.2 & 9.5 \\
\hline 6 & 28.86 & 28.87 & 28.90 & -20.0 & -17.0 & -19.1 & -20.0 & -39.0 & -17.0 & -14.5 & -23.2 & -26.8 & 24.5 \\
\hline 7 & 29.04 & 29.14 & 29.18 & -32.0 & -23.0 & -21.0 & - I9.I & -33.1 & -23.0 & -.19 .2 & -34.0 & -26.6 & I 4.9 \\
\hline 8 & 29.30 & 29.38 & $29.3^{8}$ & -30.0 & -32.0 & -31.0 & $-17 \cdot 3$ & -30.0 & -30.0 & -30.0 & -32.0 & -24.6 & 14.7 \\
\hline 9 & 29.24 & 29.24 & 29.06 & -30.0 & -28.0 & -27.0 & -29.0 & $-3 \mathrm{r} .5$ & -24.2 & -24.2 & -30.0 & -27.8 & $7 \cdot 3$ \\
\hline Io & 28.90 & 28.90 & 28.88 & -31.0 & -28.4 & -23.2 & -28.2 & -31.8 & -27.2 & -20.1 & $-3 \mathrm{I} .0$ & -26.0 & II.7 \\
\hline II & 28.88 & 28.98 & 28.98 & -20.0 & -22.0 & -22.2 & -14.2 & -28.0 & -16.2 & -15.4 & -22.2 & $-2 I . I$ & 13.8 \\
\hline 12 & $29 . \mathrm{i2}$ & 29.18 & 29.28 & -20.0 & -17.5 & -18.0 & -5.2 & -22.2 & -17.0 & -13.0 & -20.6 & $-13: 7$ & 17.0 \\
\hline I3 & $29 \cdot 32$ & 29.40 & 29.44 & -21.0 & -18.0 & -24.0 & $-\mathrm{I} 5 . \mathrm{I}$ & -21.0 & -18.0 & -18.0 & -24.0 & -19.6 & 8.9 \\
\hline I4 & 29.47 & 29.52 & 29.58 & -29.0 & -29.0 & -31.0 & -16.0 & -30.0 & -28.4 & -28.0 & -33.6 & -248 & 17.6 \\
\hline I5 & 29.64 & 29.68 & 29.48 & -32.0 & -27.0 & -30.0 & -31.0 & -38.0 & -27.0 & -27.0 & -34.0 & $-32: 5$ & 11.0 \\
\hline 16 & 29.22 & 29.35 & 29.64 & -18.2 & $-\mathrm{I} 6.5$ & -17.5 & - I8.0 & -32.4 & -12.6 & -12.6 & -21.0 & -22.5 & 19.8 \\
\hline 17 & 30.14 & 30.28 & 30.37 & -23.5 & -25.6 & -25.0 & -16.2 & -28.0 & -23.0 & -23.0 & -29.0 & -22.6 & 12.8 \\
\hline I8 & 30.00 & 29.85 & 29.67 & $-\mathrm{I} 3.0$ & -2.0 & +13.0 & -13.0 & -26.0 & -.2 .0 & +13.0 & -13.0 & -6.5 & 39.0 \\
\hline I9 & 29.62 & 29.60 & $29.6 \mathrm{I}$ & +19.0 & +16.0 & -1.5 & +21.0 & +12.0 & +19.0 & +20.0 & -1.5 & +9.8 & 22.5 \\
\hline 20 & 29.74 & 29.82 & 29.93 & -22.0 & -22.0 & -22.0 & -1.5 & -22.0 & -21.0 & -20.0 & -23.6 & -12.6 & 22.1 \\
\hline 21 & 29.76 & 29.66 & 29.42 & -26.0 & -16.0 & -12.0 & -17.5 & -28.0 & -16.0 & -12.0 & -26.0 & -20.0 & 16.0 \\
\hline 22 & 29.20 & 29.20 & 29.22 & -21.8 & -23.5 & -19.0 & -12.0 & -22.0 & -21.1 & -19.0 & -25.0 & $-\mathrm{I} 8.5$ & I3.0 \\
\hline 23 & 28.92 & 28.68 & 28. I9 & -11.5 & -9.8 & -5.0 & -11.0 & -20.0 & -9.0 & -3.8 & -11.5 & $-I I .9$ & 16.2 \\
\hline 24 & 28.10 & 28.28 & 28.56 & -18.0 & -26.0 & -28.0 & -5.0 & -18.0 & -18.0 & -18.0 & -28.0 & -16.5 & 23.0 \\
\hline 25 & 28.93 & 29.10 & 29.39 & -22.4 & -25.0 & -24.0 & -22.0 & -31.0 & -21.0 & -21.0 & -27.0 & -26.0 & 10.0 \\
\hline 26 & 29.55 & 29.66 & 29.82 & -25.6 & -27.0 & -28.0 & -23.8 & -26.6 & $-23 \cdot 3$ & -23.3 & -31.0 & -27.2 & 7.7 \\
\hline 27 & 29.83 & 29.85 & 29.68 & -23.5 & -27.0 & -21.0 & -18.5 & -32.0 & -23.5 & -21.0 & -27.8 & -25.2 & 13.5 \\
\hline 28 & 28.92 & 28.68 & 28.46 & -8.0 & 0.0 & +11.0 & -8.0 & -28.0 & 0.0 & +11.0 & -8.0 & -8.5 & 39.0 \\
\hline 29 & 28.46 & 28.51 & 28.52 & +8.0 & +8.1 & -4.0 & +11.0 & +8.0 & +9.0 & +9.0 & -4.0 & +3.5 & 15.0 \\
\hline 30 & 28.40 & 28.42 & 28.45 & $-\quad 5.0$ & -4.0 & -0.5 & +1.9 & -6.0 & -1.0 & +6.5 & -6.0 & +0.2 & 12.5 \\
\hline $3 I$ & 28.60 & 28.68 & $\cdots$ & -5.5 & -10.2 & $\cdots$ & -2.0 & -6.6 & -5.5 & $\cdots$ & & $-4 \cdot 3$ & 4.6 \\
\hline Sum & 904.98 & 905.79 & 876.78 & $-637 \cdot 5$ & -606.6 & -582.5 & -456.6 & $-774 \cdot 7$ & $-5+2.0$ & -456.0 & -734.0 & -595.6 & 482.2 \\
\hline Mean... & 29.19 & 29.22 & 29.23 & -20.6 & -19.6 & -19.4 & -14.7 & -25.0 & -17.5 & -15.2 & -24.5 & -19.2 & 15.6 \\
\hline
\end{tabular}


Tabulation of daily meteorological observations at Cape Flora during the month of Jamury, I905-Continued Observer: Francis LoNC

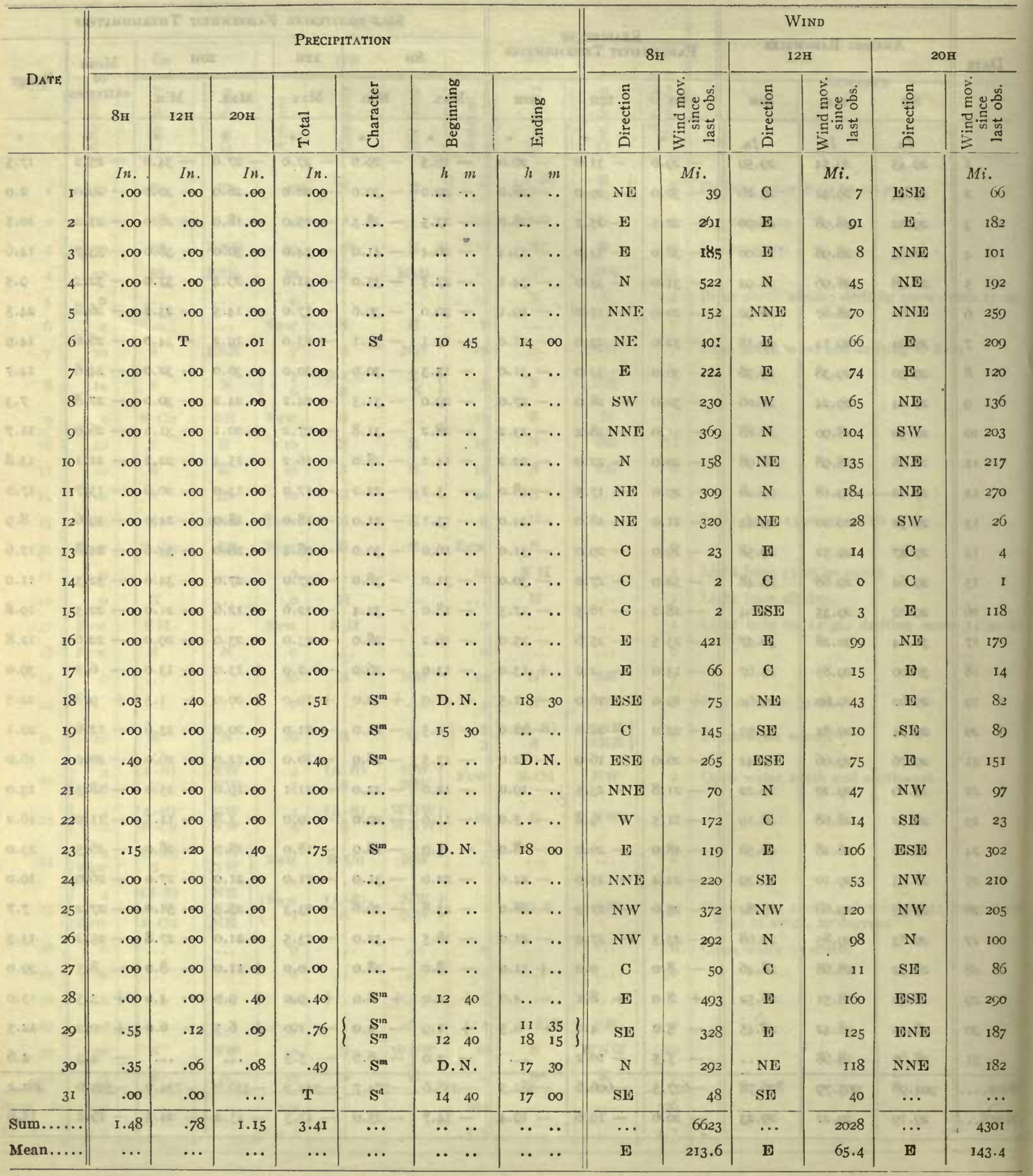


Tabulation of daily meteorological observations at Cape Flora during the month of January, 1905-Continued

Observer: Francis Long

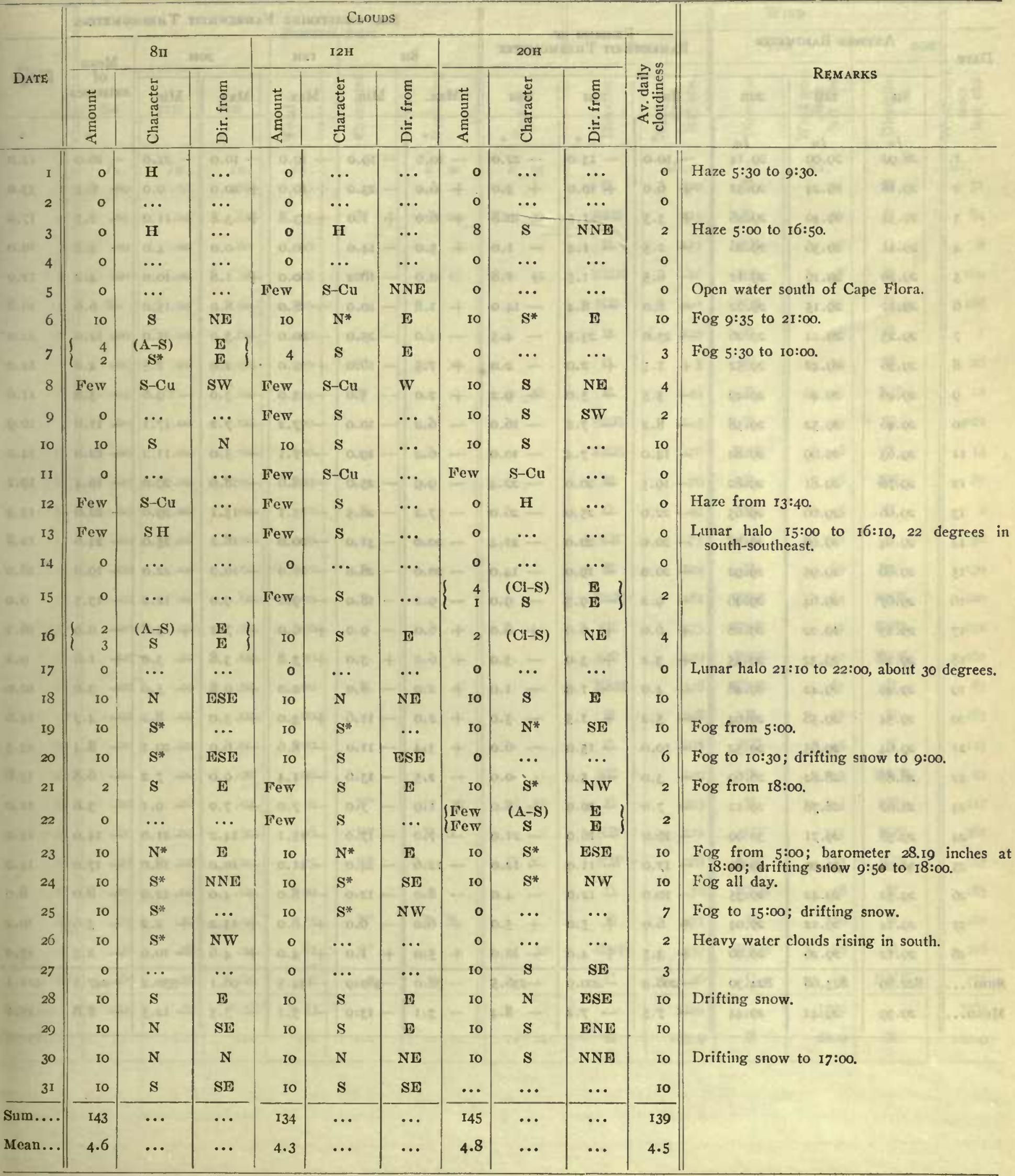


Tabulation of daily metcorological observations at Cape Flora during the month of February, 1905 Observer: Francis Long

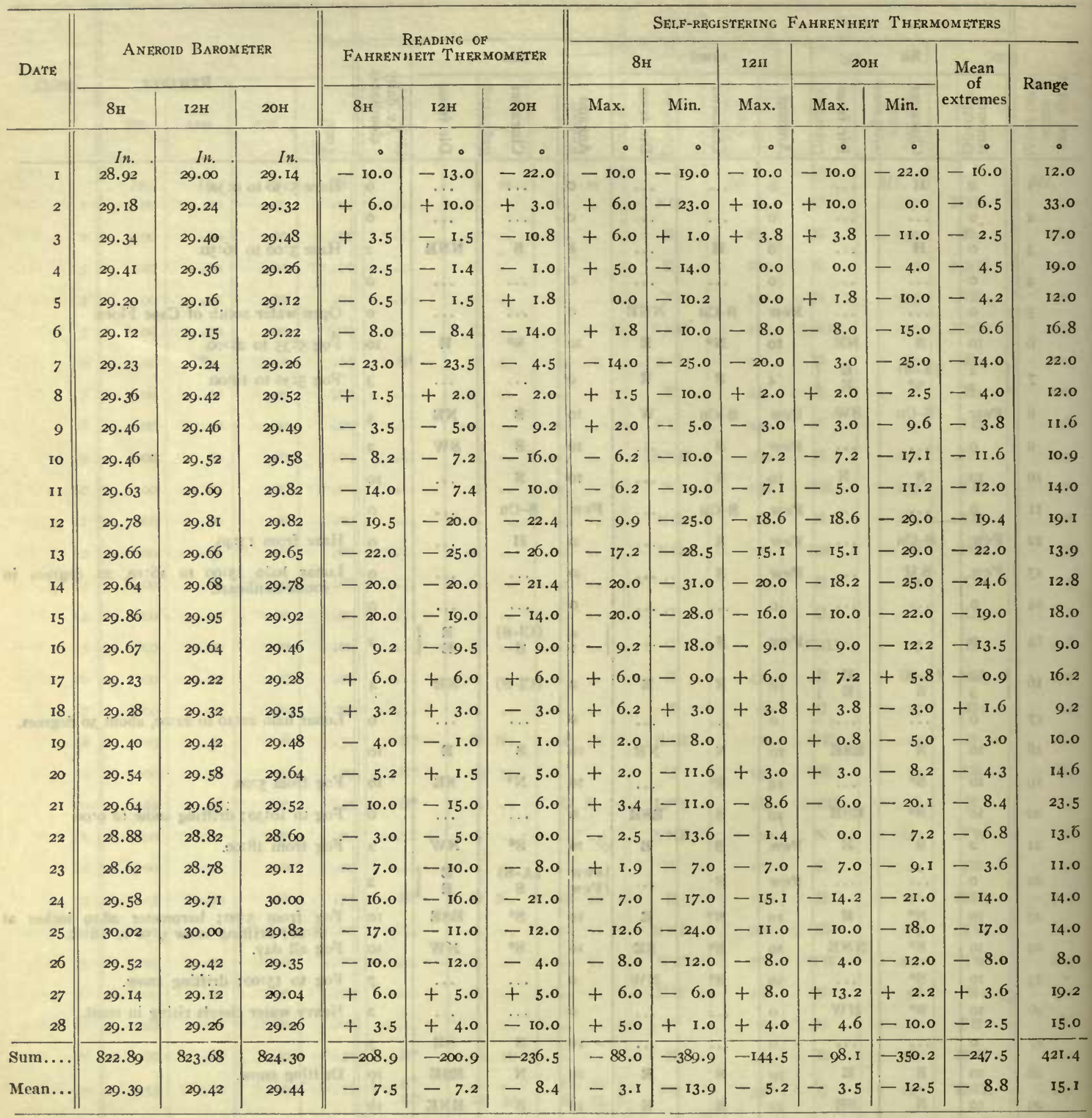


Tabulation of daily meteorological observations at Cape Flora during the month of February, Ig05-Continued Observer: FRANCIS LoNG

\begin{tabular}{|c|c|c|c|c|c|c|c|c|c|c|c|c|c|c|}
\hline \multirow[b]{3}{*}{ DATE } & \multirow{2}{*}{\multicolumn{8}{|c|}{ PRECIPITATtON }} & \multicolumn{6}{|c|}{ WIND } \\
\hline & & & & & & & & & \multicolumn{2}{|c|}{$8 \mathrm{H}$} & \multicolumn{2}{|c|}{$\mathrm{I} 2 \mathrm{H}$} & \multicolumn{2}{|c|}{$20 \mathrm{H}$} \\
\hline & $8 \mathrm{H}$ & $12 \mathrm{H}$ & 20H & 胥 & 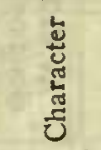 & \multicolumn{2}{|c|}{ 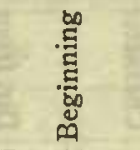 } & 莺 & 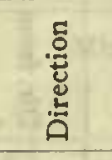 & 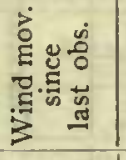 & 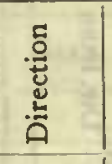 & 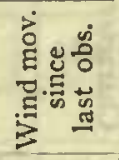 & 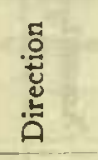 & 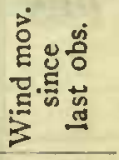 \\
\hline I & $\begin{array}{l}I n . \\
. \infty\end{array}$ & $\begin{array}{l}I n . \\
. \infty 0\end{array}$ & $\begin{array}{l}I n . \\
.00\end{array}$ & $\begin{array}{l}I n \\
. \infty\end{array}$ & ... & $\begin{array}{l}h \\
. .\end{array}$ & $\begin{array}{l}m \\
. .\end{array}$ & 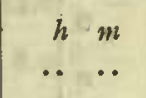 & $\mathbf{E}$ & $\begin{array}{l}M i . \\
\quad 270\end{array}$ & $\mathbf{E}$ & ${ }_{24}$ & $\mathbf{E}$ & $\begin{array}{l}M i . \\
5 I\end{array}$ \\
\hline 2 & .00 & .02 & .01 & .03 & $\mathbf{S}^{\mathrm{a}}$ & Io & $\infty$. & $13 \quad 30$ & ESE & 175 & E & 50 & $\mathbf{E}$ & 84 \\
\hline 3 &.$\infty$ &.$\infty$ &.$\infty$ &.$\infty$ & ... & .. & .. & .... & $\mathbf{E}$ & I5I & $\mathrm{Na}$ & 45 & C & 8 \\
\hline 4 &.$\infty$ &.$\infty$ & .18 & .18 & $\mathrm{~s}^{\mathrm{m}}$ & 13 & 25 & $20 \quad 15$ & FNE & 86 & NE & 48 & $\mathbf{E}$ & 160 \\
\hline 5 & $\mathbf{T}$ & .00 & .00 & .00 & ... & .. & .. & .. $\quad$.. & NE & . $\quad 203$ & NE & 55 & $\mathrm{~F}$ & 188 \\
\hline 6 & .00 & .00 & .00 & .00 & $\ldots$ & .. & .. & .. $\quad$. & $\mathbf{E}$ & 130 & E & 24 & C & I \\
\hline 7 & .00 & .00 & .00 &.$\infty$ & $S^{d}$ & 21 & 30 & .. & c & 5 & w & 14 & $\mathbf{E}$ & 28 \\
\hline 8 & .04 & $T$ & .00 & .04 & $S^{d}$ & .. & .. & 930 & $\mathbf{E}$ & 42 & $\mathbf{E}$ & 46 & NE & 12 \\
\hline 9 & .00 & .00 & .00 & .00 & $\ldots$ & .. & .. & .. $\quad$. & ESE & I 45 & $\mathbf{E}$ & 49 & E & 102 \\
\hline 10 & .00 &.$\infty$ & .00 &.$\infty$ & $\ldots$ & .. & .. & .. $\quad$. & ESE & 176 & ESE & 46 & c & 14 \\
\hline II & .00 &.$\infty$ &.$\infty 0$ & .00 & $\ldots$ & .. & .. & 6.. & E & 76 & D & 26 & SE & .87 \\
\hline I2 &.$\infty$ & .00 & .00 & .00 & ... & .. & .. & . $\quad$. & c & 49 & E & 5 & C & 2 \\
\hline 13 & .00 & .00 & .00 & .00 & ... & .. & .. & . $\quad$. & NE & 73 & NE & 100 & C & 52 \\
\hline $\mathrm{r} 4$ & .00 &.$\infty$ & .00 & .00 & ... & .. & .. & .. $\quad$. & $\mathbf{E}$ & 104 & D & 69 & SE & 130 \\
\hline I5 &.$\infty 0$ & .00 & .00 & .00 & $\ldots$ & $\cdot \cdot$ & .. & .. $\quad$. & ESE & $18 \mathrm{I}$ & ESE & 64 & ESE & 220 \\
\hline 16 & .00 & .00 & 2.00 & .00 & $\ldots$ & $\cdots$ & $\cdots$ & $\cdots$ & $S E$ & 373 & ESE & $13 \mathrm{I}$ & ESE & 252 \\
\hline I7 & .00 & .00 & .00 & .00 & .. & .. & .. & . $\quad \cdots$ & $\mathrm{SE}$ & 429 & ESE & 142 & ESE & 260 \\
\hline I8 &.$\infty$ &.$\infty$ &.$\infty$ & .00 & ... & .. & .. & . $\quad .$. & ESE & $3^{18}$ & ESE & 67 & $\mathbf{E}$ & 85 \\
\hline 19 &.$\infty$ &.$\infty$ & .00 &.$\infty$ & $\ldots$ & .. & .. & .. $\quad$. & $\mathbf{E}$ & 248 & $\mathrm{E}$ & 77 & E & 183 \\
\hline 20 &.$\infty$ & .00 &.$\infty$ &.$\infty$ & ... & .. & .. & .. $\quad$. & c & 195 & $\mathrm{NE}$ & 45 & N & 123 \\
\hline $2 I$ & .00 & .00 &.$\infty$ & .00 & .. & $\cdots$ & .. & . $\quad$. & $\mathrm{SE}$ & $\mathbf{1 4 9}$ & C & 3 & ENE & 49 \\
\hline 22 &.$\infty$ & .06 & .04 & .10 & $\mathrm{~S}^{\mathrm{m}}$ & I3 & 30. & 1955 & E & 303 & $\mathbf{E}$ & II 4 & SD & 142 \\
\hline 23 & .06 & .00 & .30 & .36 & $\mathrm{~S}^{\mathrm{m}}$ & D. & N. & .. $\quad .$. & NW & 122 & NW & III & NW & 361 \\
\hline 24 & .25 &.$\infty$ & .00 & .25 & $\mathrm{~S}^{\mathrm{m}}$ & .. & .. & D. N. & NIV & - $\quad 288$ & NW & 117 & NW & 187 \\
\hline 25 & .00 &.$\infty 0$ & .00 &.$\infty$ & ... & .. & .. & . $\quad \ldots$ & c & 78 & ENE & 18 & $\mathbf{E}$ & 183 \\
\hline 26 & .25 & .10 & .40 & .75 & $\mathrm{~S}^{\mathrm{m}}$ & D. & N. & .. $\quad .$. & D & 477 & E & I30 & D & 287 \\
\hline 27 & .30 & .08 & .24 & .62 & $\mathbf{S}^{m}$ & .. & .. & 1730 & ENE & 257 & MNE & 86 & $\mathbf{E}$ & 216 \\
\hline 28 &.$\infty$ &.$\infty$ & .00 &.$\infty$ & .. & .. & .. & .. $\quad$. & C & 46 & C & 3 & NNE & 115 \\
\hline uw & .90 & .26 & 1.17 & 2.33 & ... & .. & .. & $\quad . \quad \cdots$ & $\cdots$ & 5149 & $\cdots$ & 1709 & .. & 3582 \\
\hline fean... & & $\ldots$ & $\ldots$ & $\ldots$ & $\ldots$ & .. & .. & .. $\quad$. & $\mathbf{E}$ & 183.9 & E & 61.0 & II & 127.9 \\
\hline
\end{tabular}


Tabulation of daily meteorological observations at Cape Flora during the month of February, Igo5-Continued

Observer: FRANCIS LONG

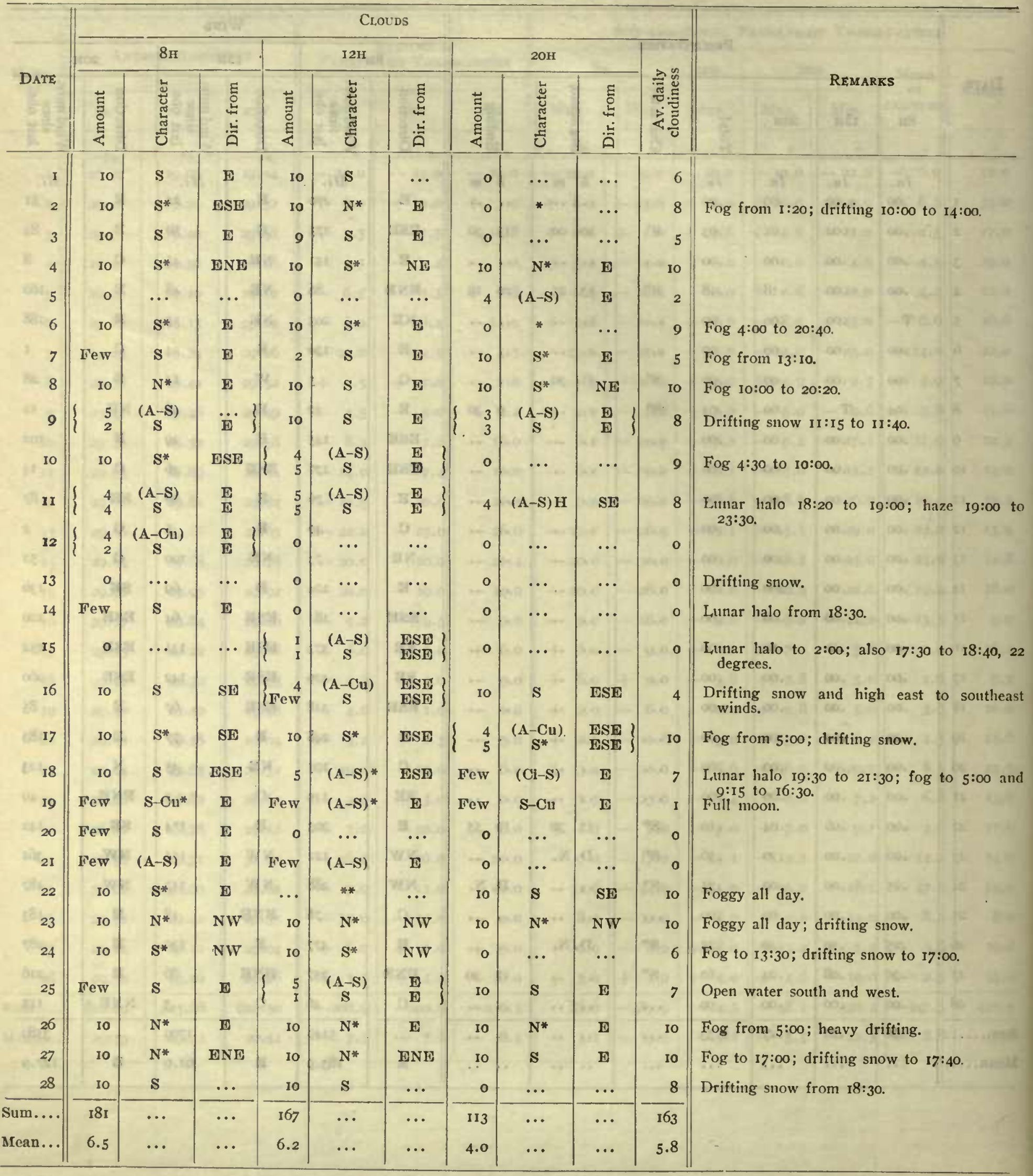


Tabulation of daily meteorological observations at Cape Flora during the month of March, I905

Observer: FRANCIS LONG

\begin{tabular}{|c|c|c|c|c|c|c|c|c|c|c|c|c|c|}
\hline \multirow{3}{*}{ DATE } & \multirow{2}{*}{\multicolumn{3}{|c|}{ ANEROID BAROMETER }} & \multirow{2}{*}{\multicolumn{3}{|c|}{$\begin{array}{c}\text { READING OF } \\
\text { FAHREN HEIT 'THERMOMETER }\end{array}$}} & \multicolumn{7}{|c|}{ SELF-REGISTERING FAHRENHEIT THERMOMETERS } \\
\hline & & & & & & & \multicolumn{2}{|c|}{$8 \mathrm{H}$} & \multirow{2}{*}{$\frac{\mathrm{I} 2 \mathrm{H}}{\mathrm{Max}}$} & \multicolumn{2}{|c|}{$20 \mathrm{H}$} & \multirow{2}{*}{$\begin{array}{c}\text { Mean } \\
\text { of } \\
\text { extremes }\end{array}$} & \multirow{2}{*}{ Range } \\
\hline & $8 \mathrm{n}$ & $12 \mathrm{H}$ & $20 \mathrm{H}$ & $8 \mathrm{H}$ & $\mathrm{I} 2 \mathrm{H}$ & $20 \mathrm{H}$ & Max. & Min. & & Max. & Min. & & \\
\hline & In. & $I n$. & In. & & & I & $\bullet$ & . & $\because$ & $1+2$ & $\bullet$ & $\bullet$ & • \\
\hline I & 29.62 & 29.74 & 29.84 & -20.0 & -18.0 & -22.0 & -9.6 & -21.0 & -18.0 & -15.6 & -25.0 & $-17 \cdot 3$ & 15.4 \\
\hline 2 & 29.64 & 29.54 & 29.40 & -16.5 & -12.0 & -8.0 & -16.5 & -23.2 & -12.0 & -8.0 & -17.2 & -15.6 & 15.2 \\
\hline 3 & 29.48 & 29.48 & 29.65 & -7.5 & -8.9 & -10.0 & $=4.0$ & -13.6 & -7.0 & -6.2 & -10.0 & -8.8 & $9 .($ \\
\hline 4 & 29.64 & 29.42 & 29.36 & +1.0 & +8.0 & -16.0 & +1.0 & -11.0 & +8.0 & +18.0 & -16.0 & +1.0 & 34. \\
\hline 5 & 29.41 & 29.50 & 29.62 & -17.0 & -13.0 & -14.0 & -16.0 & -24.0 & -12.9 & -12.2 & -18.0 & -18.1 & II. $\varepsilon$ \\
\hline 6 & 29.76 & 29.82 & 29.84 & -23.0 & -20.0 & $-I I .0$ & -14.0 & -26.0 & -19.8 & -11.0 & -23.0 & -18.5 & 15. \\
\hline 7 & 29.52 & 29.38 & 29.10 & -11.0 & -7.0 & -3.0 & -10.0 & -12.2 & -7.0 & -3.0 & -10.0 & -7.6 & .2 \\
\hline 8 & 29. I0 & 29.16 & 29.28 & -7.0 & -8.5 & -14.0 & -1.2 & -7.0 & -7.0 & -7.0 & -14.2 & -7.7 & $13 . \mathrm{C}$ \\
\hline 9 & 29.37 & 29.52 & 29.66 & $\therefore 22.2$ & -21.0 & -22.0 & -13.6 & -22.4 & -21.0 & -19.0 & -23.0 & -18.3 & 9.4 \\
\hline 10 & 29.76 & 29.84 & 29.76 & -26.0 & -25.0 & -24.2 & -21.0 & -30.0 & -22.0 & -21.0 & -29.0 & -25.5 & $.0 \mathrm{c}$ \\
\hline II & 29.52 & 29.58 & 29.58 & +4.0 & +5.2 & +9.9 & +4.0 & -25.0 & +6.1 & +10.0 & +3.1 & -7.5 & 5.0 \\
\hline 12 & $29.5 \mathrm{I}$ & 29.50 & 29.49 & +6.0 & +5.2 & +4.0 & +11.0 & +6.0 & +6.9 & +7.0 & +1.0 & +6.0 & \\
\hline 13 & & & & +5.0 & +1.0 & -1.0 & +7.9 & +1.0 & +5.2 & +5.2 & -3.9 & +2.0 & \\
\hline 14 & & 29.46 & 29.60 & +2.0 & +4.0 & -7.2 & +3.0 & -3.0 & +4.0 & +4.0 & -8.0 & -2.0 & \\
\hline 15 & 29.78 & 29.90 & 29.94 & -12.0 & -11.0 & -7.0 & -7.2 & $-\mathrm{I} 4 . \mathrm{I}$ & -11.0 & -6.0 & -12.0 & -10.0 & 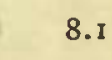 \\
\hline 16 & 29.95 & 30.02 & 30.09 & -1.0 & +4.0 & -1.0 & 0.0 & -10.0 & +4.1 & +4.0 & -5.0 & -3.0 & $4 \cdot 1$ \\
\hline 17 & 30.12 & 30.14 & 30.14 & 0.0 & -1.8 & -7.0 & +2.2 & -10.1 & +1.4 & +2.0 & -7.0 & -4.0 & 2.3 \\
\hline 18 & 30.03 & 30.08 & 30.09 & -17.0 & -16.0 & -19.0 & -5.2 & -20.0 & -12.3 & -12.3 & $-2 \mathrm{I} .0$ & -13.1 & 5. \\
\hline 19 & 30.06 & 30.08 & 30.02 & -12.8 & -12.0 & -8.2 & -12.5 & -20.2 & -10.0 & -8.2 & -12.8 & -14.2 & 2 \\
\hline 20 & 29.76 & 29.72 & 29.59 & $-\mathrm{II.O}$ & -9.2 & 0.0 & -7.6 & -12.4 & -9.0 & 0.0 & -13.0 & -6.5 & \\
\hline $2 I$ & 29.48 & 29.54 & 29.59 & +10.0 & +9.2 & -10.8 & +10.0 & -1.0 & +11.0 & +11.0 & -10.8 & +0.1 & 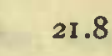 \\
\hline 22 & 29.62 & 29.69 & 29.78 & -2.5 & 0.0 & -3.0 & -2.5 & -14.6 & 0.0 & +1.2 & -3.0 & -6.7 & \\
\hline 23 & & & & +22.5 & +22.0 & +25.0 & +22.5 & -6.0 & +23.4 & +25.0 & +18.0 & + & \\
\hline 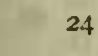 & & 29.54 & 29.43 & +24.0 & +32.0 & +33.0 & +26.0 & +21.0 & +32.0 & +33.0 & +23.0 & & 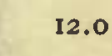 \\
\hline 25 & 29.62 & 29.57 & 29.46 & +26.0 & +25.0 & +27.2 & +33.0 & +18.0 & +26.5 & +27.2 & +23.0 & & 尚 \\
\hline 26 & & 29.52 & 29.54 & +14.9 & +11.1 & +3.0 & +27.2 & +12.1 & 7.0 & 7.0 & +3.0 & + & 24. \\
\hline 27 & 29.85 & 30.0 & 30.11 & -20.0 & -23.0 & -27.0 & +3.0 & -21.0 & -20.0 & -17.6 & -27.0 & -12.0 & 30. \\
\hline 28 & 30.00 & 29.9 & 29.8 & -17.5 & -14.2 & -14.8 & -17.5 & -27.0 & -14.0 & -10.0 & -18.0 & -18.5 & \\
\hline 29 & 29.62 & 29.66 & 29.7 & -5.2 & -7.8 & -24.0 & -5.2 & -17.2 & -4.5 & -4.5 & & & . \\
\hline 30 & & 29.77 & & -25.0 & -25.0 & -30.0 & -22.0 & -30.1 & -25.0 & -25.0 & & & 8.1 \\
\hline 31 & 29.78 & 29.78 & 29.72 & -27.0 & -20.0 & -29.0 & -27.0 & -36.2 & -20.0 & -14.4 & $-2 y .0$ & -25.3 & 21.8 \\
\hline & 919.19 & 919.97 & 920.11 & -185.8 & -146.7 & -231.1 & $-6 \mathrm{I} .8$ & -400.2 & -106.9 & -36.4 & -338.8 & -214.2 & 501.9 \\
\hline & 29.65 & 29.68 & 29.68 & -6.0 & -4.7 & -7.5 & -2.0 & -12.9 & -3.4 & -1.2 & -10.9 & -6.9 & 16.2 \\
\hline
\end{tabular}


Tabulation of daily mctcorological observations at Cape Flora during the month of March, Igos-Continued Observer: FRANCIS LoNG

\begin{tabular}{|c|c|c|c|c|c|c|c|c|c|c|c|c|c|c|}
\hline \multirow{3}{*}{\multicolumn{2}{|c|}{ DATE }} & \multicolumn{6}{|c|}{ 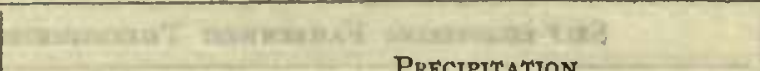 } & \multirow{3}{*}{ 诘 } & \multicolumn{6}{|c|}{ Wind } \\
\hline & & \multirow{2}{*}{$8 \mathbf{H}$} & \multicolumn{2}{|c|}{$x_{102}$} & \multicolumn{3}{|c|}{ PRECIPITATION } & & \multicolumn{2}{|c|}{$8 \mathbf{H}$} & \multicolumn{2}{|c|}{$12 \mathrm{H}$} & \multicolumn{2}{|c|}{$20 \mathrm{H}$} \\
\hline & & & $\mathrm{I2H}$ & $20 \mathrm{H}$ & $\begin{array}{c}7 \\
0 \\
6\end{array}$ & : & 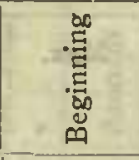 & & : & 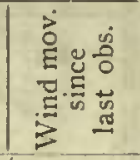 & 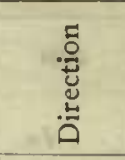 & 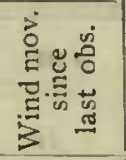 & 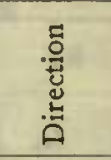 & 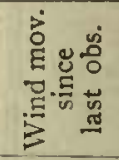 \\
\hline$\therefore 2$ & I & $\begin{array}{l}I n \\
. \infty\end{array}$ & $\begin{array}{c}I n . \\
. \infty\end{array}$ & $\begin{array}{l}\operatorname{In} . \\
. \infty\end{array}$ & In. & $=\ldots$ & $\begin{array}{cl}h & m \\
. . & . .\end{array}$ & $\begin{array}{ccc} & h & m \\
. & & \end{array}$ & Sw & $\begin{array}{r}\text { Mi. } \\
\quad 434\end{array}$ & $\mathrm{~N}$ & Mi. & $\mathrm{C}$ & Mi. ${ }_{56}$ \\
\hline 68 & 2 &.$\infty$ & .00 & 4.00 & .00 & $\cdots$ & .... & ... & $\mathbf{E}$ & 92 & E & 144 & ENE & 176 \\
\hline ate & 3 & $: . \infty$ & $\therefore .00$ & .00 & 9.1 .00 & $\ldots$ & .... & $\ldots \ldots$ & NE & 11204 & $\mathrm{C}$ & 6. 30 & $\mathrm{~W}$ & 98 \\
\hline$r$ & 4 &.$\infty$ & $\mathbf{T}$ & C.2.15 & $\ldots 15$ & $\mathrm{~S}^{\mathrm{m}}$ & II 30 & $16 \quad 30$ & NE & 77 & sw & 131 & NIV & 33 \\
\hline 0uas: & 5 & .00 & $10=.00$ & .11 .00 & $8 . .00$ & $\ldots$ & ... & .... & WNW & 333 & WNW & 102 & W & 220 \\
\hline e & 6 & .00 &.$\infty$ &.$\infty$ & .00 & $\cdots$ & o. . & ... & C & 17160 & Sw & 25 & ESE & 5 123 \\
\hline 4 & 7 & $\therefore \quad . \infty$ & 1.00 & 07.00 & .90 & $\cdots$ & 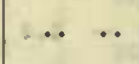 & ... & E & 468 & D & I70 & $\mathbf{E}$ & 340 \\
\hline-6 & 8 & .25 & .04 & $T$ & 14. .29 & $\mathbf{S}^{\mathbf{m}}$ & D. N. & .. $\quad .$. & SE & 320 & SE & 58 & $\mathbf{E}$ & 48 \\
\hline ale & 9- & $=. \infty$ &.$\infty$ & .00 & 6..$\infty$ & $\mathrm{S}^{\mathrm{m}}$ & ... & $\begin{array}{ll}0 & 40\end{array}$ & NE & 249 & $\mathrm{NE}$ & $\mathrm{I} 4 \mathrm{I}$ & NE & -48 \\
\hline $00 x$ & 10 &.$\infty$ &.$\infty$ &.$\infty$ & 11.00 & $\ldots$ & ... & .. & C & 18 & $\mathrm{C}$ & 13 & C & (4) 1 \\
\hline tor & II &.$\infty$ & .00 & .28 & 0.28 & $S^{1 m}$ & I3 40 & D.N. & ESE & 282 & ENE & 110 & $\mathbf{D}$ & 246 \\
\hline $8 \sin$ & 12 & .25 & .00 & $=.2 .00$ & .25 & $\ldots$ & .. & 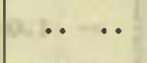 & E & 380 & $\mathrm{E}$ & II8 & $\mathbf{E}$ & 242 \\
\hline xat & 13. & $. \quad . \infty$ & .00 & 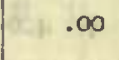 & .00 & $\ldots$ & $\therefore \quad \cdots$ & $\ldots \ldots$ & $\mathbf{E}$ & 314 & ENIE & Q1 110 & ESE & 17164 \\
\hline chet & 14 & .12 & .04 & .00 & c. .16 & $S^{m}$ & D. N. & II -30 & i1 $\mathrm{C}$ & $=40$ & $N$ & 8 & $N$ & 130 \\
\hline inyth & 15 &.$\infty$ & $\therefore .00$ & $=. \infty$ & .00 & $\cdots$ & .. $\quad$. & $\ldots \ldots$ & NW & 302 & NW & 40 & SSW & 33 \\
\hline tetis & 16 & .00 & .01 & .01 & .02 & $S^{m}$ & 10 40 & 1300 & c & 20 & WNW & +15 & $\mathrm{C}$ & 6) 35 \\
\hline Ay & 17 & .00 & 11: . & .00 &.$\infty$ & $\ldots$ &. & .. $\cdots$ & NE & 43 & NE & 82 & E & I30 \\
\hline wetr & 18 & .00 & .00 & .00 &.$\infty$ & $\ldots$ & .. & $\ldots$ & $=S$ & 48 & C & 12 & E & 6 \\
\hline biving & 19 & .00 & .00 & .03 & .03 & $S^{a}$ & 1730 & 2250 & NW & 2 & NW & 20 & WN W & 40 \\
\hline bite & 20. & .02 &.$\infty$ & .00 & 0.02 & . & ... & $\cdots \cdots$ & $\mathbf{C}$ & $5 I$ & IISW & 14: 14 & NE & is: 47 \\
\hline las & 21 & .45 & .or & .1 .00 & 2. .46 & $S^{a}$ & D. N. & $8-45$ & N W & 44 & NIV & Dif $3 \mathrm{I}$ & NIV & 59 \\
\hline $\log x$ & 22 & .04 & .00 &.. .0 & $: 04$ & $S^{d}$ & .50 & $.9-15$ & 25 & 16 & $\mathbf{E}$ & 32 & $\mathrm{C}$ & 7 \\
\hline .515 & 23 & .00 & .00 & .00 & .00 & $\ldots$ & $\therefore \quad$. & 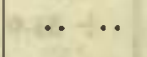 & $\mathbf{E}$ & I4I & $\mathbf{E}$ & 34 & ENE & s8 \\
\hline . $.81=$ & 24 & .25 & $\mathbf{T}$ & 1.02 & .27 & $\stackrel{\mathrm{S}^{\mathrm{m}}}{\mathrm{R}}$ & $\begin{array}{rr}4 & 00 \\
13 & 30\end{array}$ & $\begin{array}{rr}8 & 30 \\
21 & 30\end{array}$ & IENE & $8 \mathrm{I}$ & wsw & 29 & w & 04 \\
\hline $6-15$ & 25 & $\mathbf{T}$ & $\mathbf{T}$ & .04 & .04 & $S^{m}$ & II 30 & $\begin{array}{ll}18 & 30\end{array}$ & $\mathrm{NW}$ & 160 & NIV & 27 & NW & 50 \\
\hline 1,04 & 26 & .00 &.$\infty$ & .03 & .03 & $\mathrm{~S}^{\mathrm{m}}$ & 1630 & I9 40 & $\mathrm{NW}$ & I25 & C & $3^{6}$ & C & 14 \\
\hline 5 & 27 & .00 &.$\infty$ &.$\infty$ &.$\infty$ & 1. & .. .. & .... & NE & 243 & $\mathrm{NE}$ & 100 & $\mathbf{C}$ & $8 \mathrm{I}$ \\
\hline $2-4$ & 28 & .00 & $\begin{array}{ll}\cdots & .00\end{array}$ & .00 & .00 & $\ldots$ &. & .. & NWW & III & $\mathrm{N}$ & 16 & $\mathrm{C}$ & 24 \\
\hline $3^{8}$ & 29 &.$\infty$ &.$\infty$ &.$\infty$ & .00 & $\ldots$ & .. & .. .. & $\mathrm{N}$ & 42 & $\mathrm{~N}$ & 35 & NNW & 118 \\
\hline 8,12 & 30 & .00 &.$\infty$ & .00 & .00 & $\ldots$ & .. .. & .. .. & $\mathrm{N}$ & 130 & NIV & 57 & $\mathbf{N}$ & 100 \\
\hline$=$ & $3 I$ & .00 & .00 &.$\infty$ &.$\infty$ & $\ldots$ & $\because \quad \ldots$ & . & $\mathrm{C}$ & 25 & $\mathrm{~N}$ & $\begin{array}{l}. \quad 7 \\
\end{array}$ & w & 65 \\
\hline Sum... & $\cdots$ & I. 38 & .10 & .56 & 2.04 & $\ldots$ & $\therefore \quad$. & $\therefore \quad$. & $\cdots$ & 4955 & $\ldots$ & 1831 & $\therefore$ & I. 2948 \\
\hline Mean. . & ... & $\ldots$ & $\ldots$ & .. & .. & ... & .. $\quad$. & . $\quad$. & $\mathbf{E}$ & I59.8 & E & 59.1 & $\mathbf{E}$ & 95.I \\
\hline
\end{tabular}


Tabulations of daily meteorological observations at Cape Flora during the month of March, 1905-Continued Observer: FRANCIS LONG

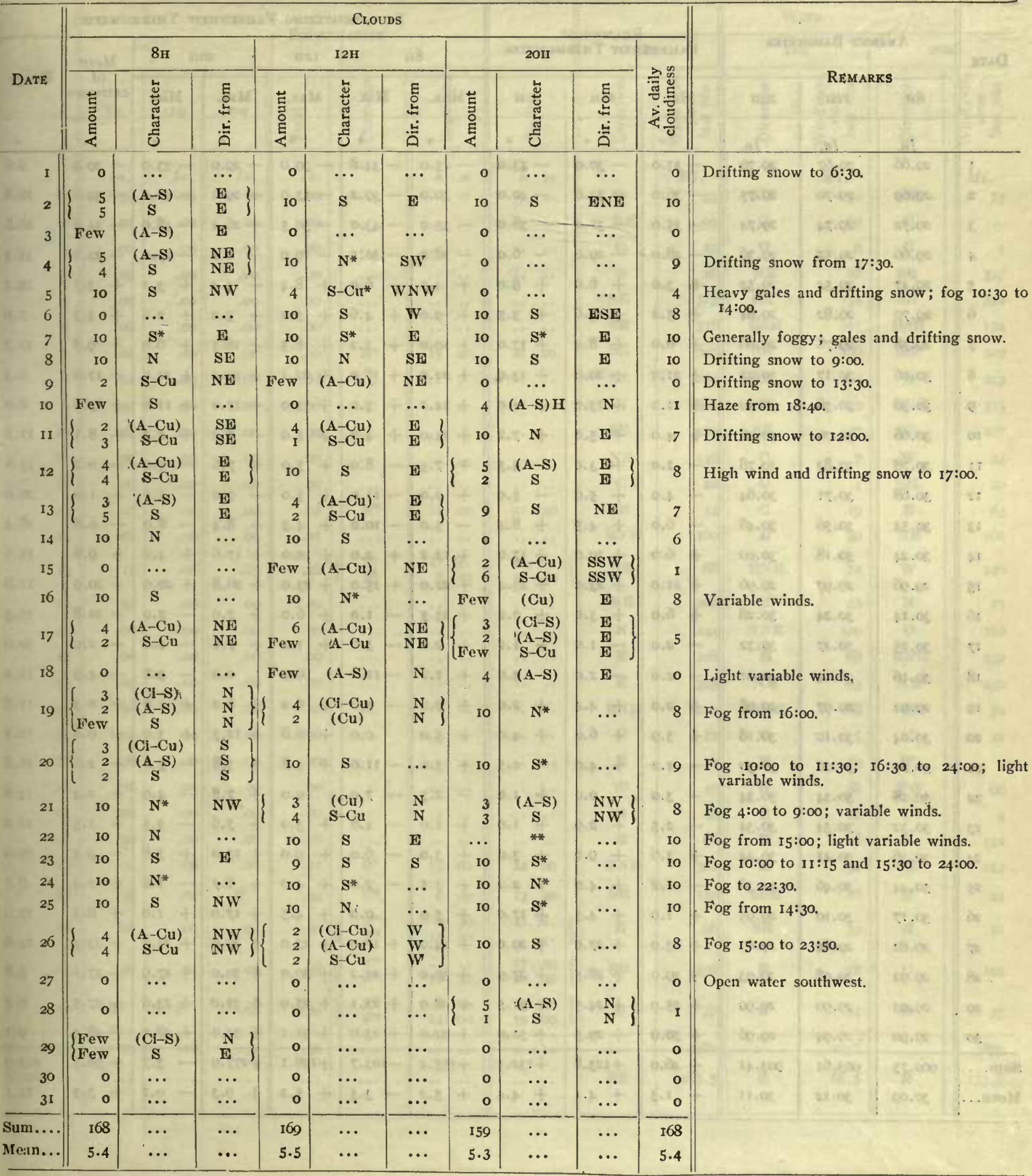


Tabulation of daily meteorological observations at Cape Flora during the manth of April, Igo5

Observer: FRANCIS LONC

\begin{tabular}{|c|c|c|c|c|c|c|c|c|c|c|c|c|c|}
\hline \multirow{3}{*}{ DATE } & \multirow{2}{*}{\multicolumn{3}{|c|}{ ANEROID BAROMETER }} & \multirow{2}{*}{\multicolumn{3}{|c|}{$\begin{array}{l}\text { READING OF } \\
\text { FAHRENHEIT THERMOMETER }\end{array}$}} & \multicolumn{7}{|c|}{ SELF-REGISTERING FAHRENHEIT THERMOMETERS } \\
\hline & & & & & & & \multicolumn{2}{|c|}{$8 \mathrm{H}$} & \multirow{2}{*}{$\frac{\text { I2II }}{\text { Max. }}$} & \multicolumn{2}{|c|}{$20 \mathrm{II}$} & \multirow{2}{*}{$\begin{array}{c}\text { Mean } \\
\text { of } \\
\text { extremes }\end{array}$} & \multirow{2}{*}{ Range } \\
\hline & $8 \mathbf{H}$ & $\mathrm{I} 2 \mathrm{H}$ & 2011 & $8 \mathrm{H}$ & $\mathrm{I} 2 \mathrm{H}$ & $20 \mathrm{H}$ & Max. & Min. & & Max. & Min. & & \\
\hline & In. & In. & In. & 。 & . & 。 & 。 & 。 & - & 。 & 。 & • & 。 \\
\hline I & 29.66 & 29.67 & 29.70 & -33.0 & -30.0 & -23.0 & -25.0 & -34.8 & -30.0 & -29.0 & -33.0 & -29.9 & 9.8 \\
\hline 2 & 29.69 & 29.70 & 29.75 & -36.0 & -33.0 & -40.0 & -30.0 & -39.2 & -33.0 & -29.2 & -40.0 & -34.6 & 10.8 \\
\hline 3 & 29.72 & 29.74 & 29.74 & -35.0 & -31.0 & -38.0 & -35.0 & -43.0 & -30.5 & -26.2 & -38.0 & -34.6 & I6.8 \\
\hline 4 & 29.66 & 29.52 & 29.36 & -28.0 & -29.0 & -6.0 & -28.0 & -40.5 & -19.0 & -6.0 & -29.0 & -23.2 & 34.5 \\
\hline 5 & 29.47 & 29.54 & 29.52 & +3.0 & +6.0 & +9.0 & +4.0 & -6.0 & +8.4 & +13.4 & +3.2 & +3.7 & I9.4 \\
\hline 6 & 29.70 & 29.82 & 29.84 & +5.2 & +4.6 & +3.5 & +9.0 & +4.6 & +5.2 & +5.2 & +2.0 & +5.5 & 7.0 \\
\hline 7 & 29.78 & 29.78 & 29.86 & +6.0 & +8.4 & +17.0 & + то. 0 & +1.2 & +8.5 & +18.5 & +6.0 & +9.8 & $17 \cdot 3$ \\
\hline 8 & 30.06 & 30.17 & 30.22 & +21.2 & +20.0 & +13.0 & +22.0 & +16.0 & +22.4 & +22.4 & +12.9 & +17.6 & 9.5 \\
\hline 9 & 30.30 & 30.38 & 30.50 & $+\mathrm{rI} .0$ & +13.0 & +14.0 & +14.2 & +9.0 & +13.0 & +17.0 & +11.0 & +13.0 & 8.0 \\
\hline I0 & 30.66 & 30.76 & 30.78 & +4.0 & +5.0 & +7.2 & +14.0 & +4.0 & +5.0 & +7.2 & +2.2 & $+8 . I$ & II. 8 \\
\hline II & 30.78 & 30.82 & 30.78 & -1.0 & +3.0 & -4.5 & +7.5 & -8.0 & +5.0 & +7.6 & -5.0 & -0.2 & 15.6 \\
\hline 12 & 30.68 & 30.71 & 30.64 & -4.0 & +5.0 & -5.0 & +2.0 & $-\mathbf{I I . 0}$ & +5.0 & +9.0 & -5.0 & -1.0 & 20.0 \\
\hline 13 & 30.54 & 30.56 & 30.48 & -6.0 & +4.5 & +8.4 & -5.0 & -10.0 & +4.5 & +8.4 & -8.0 & -0.8 & I8.4 \\
\hline 14 & 30.24 & 30.18 & 30.00 & +6.0 & +10.0 & +17.6 & +12.2 & +2.0 & +10.0 & +17.6 & +4.2 & +9.8 & 15.6 \\
\hline 15 & 29.96 & 29.97 & 29.96 & +21.0 & +23.0 & +23.4 & +22.0 & +15.0 & +23.0 & +26.8 & +20.0 & +20.9 & II 8 \\
\hline I6 & 30. 14 & 30.24 & 30.28 & +6.0 & +3.0 & +0.2 & +23.5 & $-\quad 1.0$ & +6.0 & +7.0 & -2.0 & +10.8 & 25.5 \\
\hline 17 & 30.25 & 30.27 & 30.32 & -2.0 & -1.0 & +1.2 & +2.6 & -3.0 & $+\mathrm{I} .2$ & +7.0 & -2.0 & +2.0 & I0.0 \\
\hline I8 & 30.16 & 30.14 & 30.01 & -2.0 & -2.0 & -3.0 & +1.2 & -11.0 & 0.0 & 0.0 & -3.0 & -4.9 & 12.2 \\
\hline I9 & 29.92 & 29.97 & 29.98 & +2.0 & +4.4 & +2.0 & +2.2 & -5.6 & +6.2 & +6.2 & +1.0 & +0.3 & II. 8 \\
\hline 20 & 30.04 & 30.10 & 30. 18 & +3.9 & +6.0 & +4.0 & +5.0 & 0.0 & +6.8 & +12.5 & +3.0 & +6.2 & I 2.5 \\
\hline $2 x$ & 30.20 & 30.26 & 30.30 & -1.2 & +4.0 & -2.5 & +3.0 & $-\mathrm{II} .0$ & +4.8 & +5.8 & -3.0 & -2.6 & I6.8 \\
\hline 22 & 30.28 & 30.34 & 30.34 & -3.0 & 0.0 & +1.0 & -2.5 & -7.0 & 0.0 & +7.8 & -4.0 & +0.4 & I4. 8 \\
\hline 23 & 30.32 & 30.34 & 30.34 & -2.5 & +2.9 & +1.0 & +1.0 & -4.6 & +3.0 & +7.6 & -2.5 & +1.5 & 12.2 \\
\hline 24 & $30.3^{6}$ & $30.4 \mathrm{I}$ & 30.44 & +3.6 & +9.0 & +3.0 & +3.0 & -6.0 & +9.0 & +13.0 & +3.6 & +3.5 & 19.0 \\
\hline 25 & 30.44 & 30.46 & 30.38 & -1.2 & +4.0 & +2.2 & +3.5 & -2.0 & +4.0 & +6.7 & -1.2 & +2.4 & 8.7 \\
\hline 26 & 30. 17 & 30.10 & 29.95 & +1.0 & +4.5 & +17.0 & +5.0 & 0.0 & +4.5 & +17.0 & +1.0 & +8.5 & 17.0 \\
\hline 27 & 29.82 & 29.84 & 29.87 & +23.0 & +27.0 & +29.0 & +24.0 & +17.0 & +27.0 & +29.6 & +23.0 & +23.3 & 12.6 \\
\hline 28 & 29.92 & 29.98 & 30.03 & +29.0 & +28.5 & +27.9 & +30.0 & +24.2 & +30.0 & +30.0 & +27.9 & $+27 . I$ & 5.8 \\
\hline 29 & 29.93 & 29.90 & 29.90 & +25.0 & +24.5 & +31.5 & +28.0 & $+23 . I$ & +25.0 & +32.0 & +23.0 & +27.5 & 9.0 \\
\hline 30 & 29.90 & 29.94 & 29.96 & +30.0 & +29.5 & +31.0 & +32.0 & +23.9 & +31.1 & +33.0 & +28.0 & +28.4 & 9. I \\
\hline 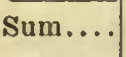 & 902.75 & $903.6 \mathrm{I}$ & 903.41 & +46.0 & +123.8 & +132.1 & +155.4 & -103.7 & +156.1 & +277.9 & -3.7 & +98.5 & $423 \cdot 3$ \\
\hline & 30.09 & 30.12 & 30. I I & +1.5 & +4.1 & +4.4 & +5.2 & -3.5 & +5.2 & +9.3 & - o.I & +3.3 & I4. I \\
\hline
\end{tabular}


Tabulation of daily meteorological observations at Cape Flora during the month of April, 1905-Continued Observer: FRANCIS LONG

\begin{tabular}{|c|c|c|c|c|c|c|c|c|c|c|c|c|c|c|}
\hline \multirow[b]{3}{*}{ DATE } & \multirow{2}{*}{\multicolumn{8}{|c|}{ Precipitation }} & \multicolumn{6}{|c|}{ WIND } \\
\hline & & & & & & & & & & a & 12 & 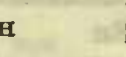 & & \\
\hline & $8 \mathrm{H}$ & $\mathrm{I} 2 \mathrm{H}$ & $20 \mathrm{H}$ & $\begin{array}{c}\frac{\pi}{0} \\
\text { [ } \\
\end{array}$ & 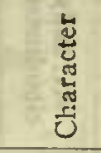 & \multicolumn{2}{|c|}{ 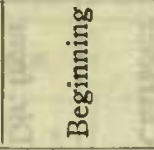 } & $\begin{array}{l}50 \\
\stackrel{5}{5} \\
\square\end{array}$ & 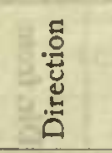 & 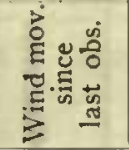 & 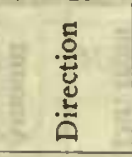 & 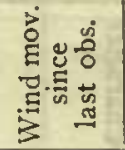 & 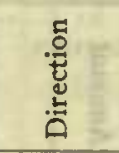 & 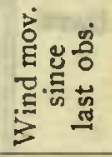 \\
\hline I & $\begin{array}{l}\text { In. } \\
.00\end{array}$ & $\begin{array}{l}I n . \\
.00\end{array}$ & $\begin{array}{l}\text { In. } \\
.00\end{array}$ & $\begin{array}{l}\text { In } \\
. \infty\end{array}$ & $\ldots$ & $\begin{array}{l}h \\
. .\end{array}$ & $\begin{array}{l}m \\
\cdots\end{array}$ & $\begin{array}{cc}h & m \\
. . & . .\end{array}$ & $w$ & Mi. & SIV & ${ }_{32}$ & NE & Mi. \\
\hline 2 & .00 & .00 & .00 & .00 & $\ldots$ & .. & .. & .. $\cdots$ & $\mathrm{NE}$ & $\cdots 95$ & $\mathrm{C}$ & 60 & c & $\ldots$ \\
\hline 3 &.$\infty 0$ &.$\infty$ & .00 & .00 & ... & .. & .. & .. $\quad$. & C & 13 & C & 0 & c & \\
\hline 4 & .00 & .00 &.$\infty$ &.$\infty$ & ... & .. & .. & . $\quad$. & $\mathbf{E}$ & 16 & NE & 88 & SE & 327 \\
\hline 5 &.$\infty$ &.$\infty$ &.$\infty$ & .00 & $\ldots$ & ... & .. & .. $\quad$. & SE & 323 & SE : & 154 & SE & 271 \\
\hline 6 & .00 & .00 &.$\infty 0$ &.$\infty$ & ... & $\because \cdot$ & .. & .... & $S E$ & 318 & ESE & 102 & ESE & 174 \\
\hline 7 &.$\infty$ &.$\infty$ &.$\infty$ & .00 & $\mathrm{~S}^{\mathrm{m}}$ & I5 & 40 & $21 \quad 40$ & SE & 393 & SE & 196 & $\mathrm{SE}$ & 329 \\
\hline 8 & .04 &.$\infty$ & .00 & .04 & $\ldots$ & .. & .. & .... & SE & 266 & SE & 100 & $\mathbf{E}$ & .223 \\
\hline 9 & .00 & .00 &.$\infty$ &.$\infty$ & $\ldots$ & .. & .. & .. $\quad$. & E & 380 & $\mathbf{E}$ & 99 & ENE & 315 \\
\hline 10 & .00 & .00 &.$\infty$ &.$\infty$ & $\ldots$ & .. &.. & . $\quad$. & SE & 423 & SE & 120 & SE & 237 \\
\hline II & .00 & .00 & .00 & .00 & .. & .. & .. & .. $\quad$. & $\mathrm{NE}$ & 146 & C & 7 & $\mathbf{w}$ & 24 \\
\hline 12 &.$\infty$ &.$\infty$ & .00 & .00 & $\ldots$ & .. & .. & .. $\quad$. & C & 22 & C & 4 & SE & 40 \\
\hline 13 & .00 & .00 & .02 & .02 & $\mathrm{~S}^{\mathrm{m}}$ & 12 & 40 & $21 \quad 30$ & SE & 54 & C & 19 & $\mathbf{E}$ & 63 \\
\hline I.4 & .or & .00 &.$\infty$ & .01 & .. & .. & .. & .. $\quad$. & ENE & 160 & E & 40 & NE & 162 \\
\hline I5 & .15 & .08 & .25 & .48 & $\left\{\begin{array}{l}\mathbf{S}^{a} \\
\mathbf{S}^{d}\end{array}\right.$ & D. & $\begin{array}{l}\mathrm{N} . \\
\mathrm{I}\end{array}$ & $\left.\begin{array}{l}\text { D. N. } \\
2 \mathrm{I}\end{array}\right\}$ & NE & 88 & ENE & Io & W & 146 \\
\hline 16 & .02 & .00 & .00 & .02 & .. & .. & .. & .. $\quad .$. & NE & 302 & $\mathbf{N}$ & 53 & $\mathbf{N}$ & 75 \\
\hline 17 &.$\infty$ & .00 &.$\infty$ & .00 & $\ldots$ & .. & $\cdot$. & .. $\quad$. & $N$ & i. 244 & NNE & 100 & c & 168 \\
\hline 18 & .00 & .00 &.$\infty$ & .00 & $\ldots$ & .. & .. & .. .. & E & 108 & E & 68 & ENE & 232 \\
\hline 19 & .00 & .00 & .00 & .00 & .. & .. & .. & .. $\quad$. & $\mathbf{E}$ & & $\mathrm{NE}$ & 93 & NE & 230 \\
\hline 20 & .00 & .00 & .00 & .00 & $\ldots$ & .. & .. & .. $\quad$. & ENE & 212 & ENE & 88 & E & 72 \\
\hline $2 \mathrm{I}$ &.$\infty$ & .00 & .00 & .00 & $\ldots$ & .. &.. & .. $\quad .$. & C & I2 & c & 5 & C & 81 \\
\hline 22 & .00 & .00 &.$\infty$ & .00 & $\cdots$ & .. &. & .. $\quad .$. & $\mathbf{E}$ & 12 & E & 19 & W & \\
\hline 23 & .00 & .00 & .00 & .00 & .. & .. & .. & .. $\quad$. & w & 47 & W & 68 & NIV & 66 \\
\hline 24 &.$\infty$ & .00 & .00 & .00 & $\ldots$ & .. & .. & . $\quad .$. & $\mathbf{N}$ & & c & 20 & C & 4 \\
\hline 25 &.$\infty$ & .00 & .00 & .00 & .. & .. & .. & $\quad$. $\quad .$. & $\mathbf{E}$ & 66 & E & 48 & $\mathbf{E}$ & 142 \\
\hline 26 & .00 & .00 & $.0 \mathrm{I}$ & .01 & $\mathrm{~S}^{a}$ & I9 & $\infty$ & 2200 & ENE & 322 & $\mathbf{E}$ & 130 & $S E$ & 254 \\
\hline 27 & .40 & .04 & $T$ & .44 & $\mathbf{S}^{m}$ & 0 & 30 & 1320 & SE & IIO & SSE & 86 & $\mathbf{E}$ & 44 \\
\hline 28 & . oI & .00 & .01 & .02 & $\left\{\begin{array}{l}\mathbf{S}^{m} \\
\mathbf{S}^{m}\end{array}\right.$ & $\begin{array}{r}0 \\
15\end{array}$ & $\begin{array}{l}30 \\
\infty\end{array}$ & $\left.\begin{array}{l}\text { D.N. } \\
\cdots\end{array}\right\}$ & $\mathbf{L}$ & & SE & 53 & $\mathbf{E}$ & $5 I$ \\
\hline 29 & .40 &.$a t$ & .04 & .48 & $\left\{\begin{array}{l}\mathbf{S}^{m} \\
\mathbf{S}^{\mathrm{m}}\end{array}\right.$ & ig & $\ddot{35}$ & $\left.\begin{array}{lll}17 & 30 \\
20 & 30\end{array}\right\}$ & SE & 196 & $\mathbf{E}$ & 72 & C & 100 \\
\hline 30 & $\mathbf{T}$ & $\mathbf{T}$ & .04 & .04 & $\left\{\begin{array}{l}\mathbf{S}^{\mathrm{m}} \\
\mathrm{S}^{\mathrm{m}}\end{array}\right.$ & $\begin{array}{r}9 \\
14\end{array}$ & $\begin{array}{l}\infty \\
\infty\end{array}$ & $\left.\begin{array}{ll}\text { II } & 30 \\
\text { I9 } & 00\end{array}\right\}$ & $s$ & $\begin{array}{r}37 \\
\end{array}$ & s & 24 & $\mathbf{E}$ & 35 \\
\hline Atiln... & I.03 & .16 & .37 & I. 56 & ... & .. &. & & & 4989 & $\cdots$ & 1958 & $\cdots$ & 3879 \\
\hline Mean. . & & $\ldots$ & $\ldots$ & $\ldots$ & & .. & .. & & SE & 166.3 & $\mathbf{E}$ & 65.3 & $\mathbf{E}$ & I33.8 \\
\hline
\end{tabular}


Tabulation of daily metcorological observations at Cape Flora during the month of April, I905-Continued

Observer: FRANCIS LONG

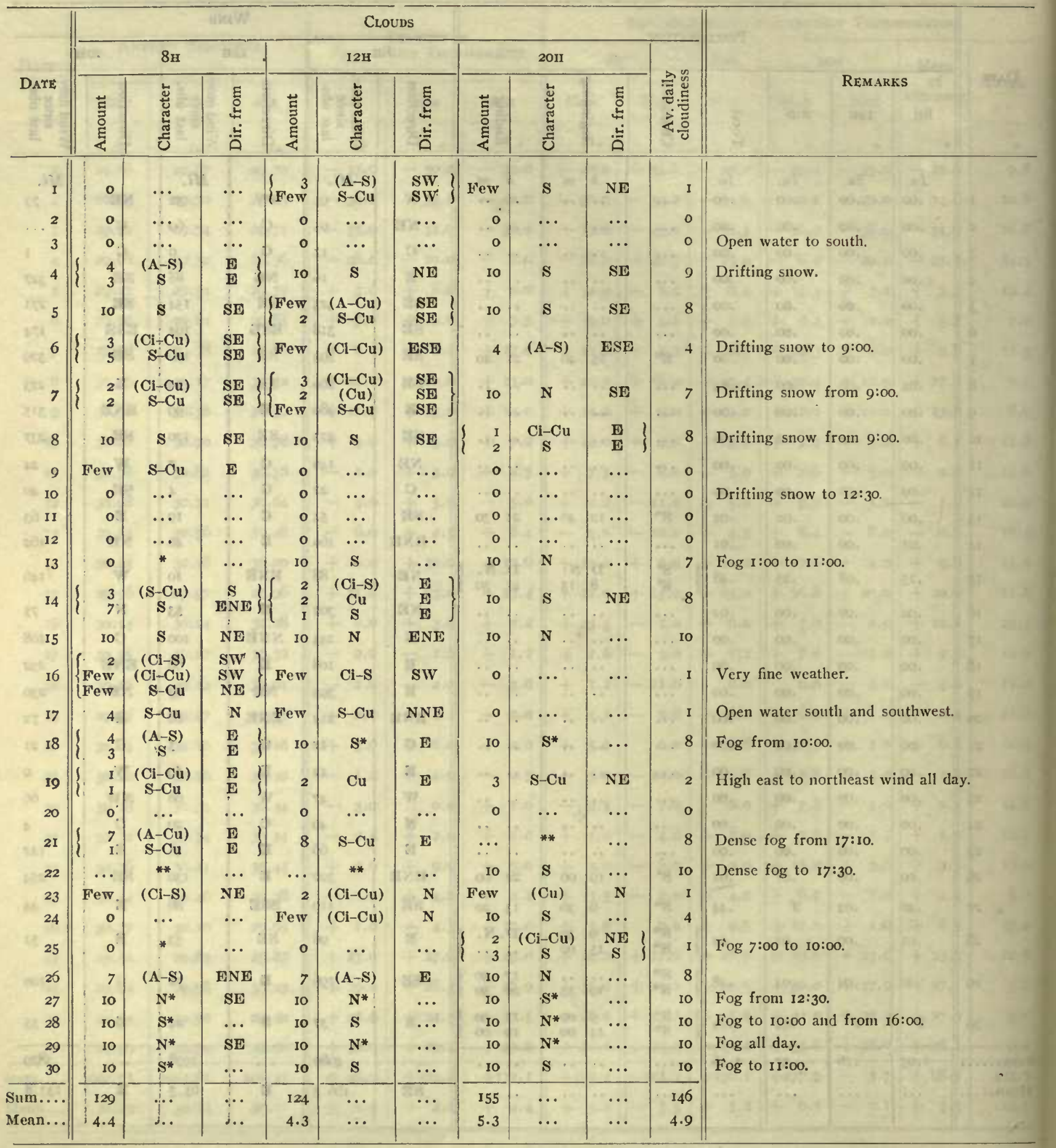


Tabulation of daily meteorological observations at Cape Flora during the month of May, 1905

Observer: Francis Long

\begin{tabular}{|c|c|c|c|c|c|c|c|c|c|c|c|c|c|}
\hline \multirow{3}{*}{ DATE } & \multirow{2}{*}{\multicolumn{3}{|c|}{$\cos =$}} & \multirow{2}{*}{\multicolumn{3}{|c|}{$\begin{array}{c}\text { READING OF } \\
\text { FAHRENHEIT THERMOMETER }\end{array}$}} & \multicolumn{7}{|c|}{ SELF-REGISTERING FAHRENHEIT THERMOMETERS } \\
\hline & & & & & & & \multicolumn{2}{|c|}{$8 \mathrm{H}$} & \multirow{2}{*}{$\begin{array}{l}\text { I21 } \\
\text { Max. }\end{array}$} & \multicolumn{2}{|c|}{2011} & \multirow{2}{*}{$\begin{array}{c}\text { Mean } \\
\text { of } \\
\text { extremes }\end{array}$} & \multirow{2}{*}{ Range } \\
\hline & $8 \mathrm{H}$ & $12 \mathrm{H}$ & $20 \mathrm{H}$ & $8 \mathbf{H}$ & $12 \mathrm{H}$ & $20 \mathrm{H}$ & Max. & Min. & & Max. & Min. & & \\
\hline & In. & ln. & In. & - & - & - & - & 。 & - & - & 。 & - & 。 \\
\hline I & 29.88 & 29.87 & 29.88 & +25.0 & +27.0 & +27.0 & +32.0 & +24.2 & +27.0 & +33.0 & +24.0 & +28.5 & 9.0 \\
\hline 2 & 29.74 & 29.78 & 29.78 & +29.5 & +31.4 & +32.0 & +30.0 & +24.6 & +31.0 & +32.0 & +28.0 & +28.3 & $7 \cdot 4$ \\
\hline 3 & 29.68 & 29.70 & 29.62 & +32.0 & +32.4 & +33.0 & +32.0 & +30.0 & +32.4 & +33.0 & $+3 I . I$ & $+3 \mathrm{I} \cdot 5$ & 3.0 \\
\hline 4 & 29.62 & 29.66 & 29.62 & +21.0 & +25.2 & +25.0 & +33.0 & +20.1 & +25.2 & +26.9 & +21.0 & +26.6 & 12.9 \\
\hline 5 & 29.44 & 29.39 & $29.4 \mathrm{I}$ & +26.0 & +21.4 & +18.8 & $+26,0$ & +24.0 & +26.0 & +26.0 & +.18 .0 & +22.0 & 8.0 \\
\hline 6 & $29 \cdot 48$ & 29.56 & 29.65 & +17.0 & $+\mathrm{I} 4.0$ & +15.9 & +18.8 & +14.2 & +17.0 & +22.0 & +.13 .6 & +17.8 & 8.4 \\
\hline 7 & 29.60 & 29.60 & 29.57 & +22.0 & +25.0 & +20.0 & +22.0 & +15.0 & +25.6 & +26.5 & +18.0 & +20.8 & 11.5 \\
\hline 8 & 29.22 & 29.23 & 29.08 & +17.0 & +23.0 & +32.0 & +20.0 & +14.0 & +23.0 & +32.0 & +17.0 & +23.0 & 18.0 \\
\hline 09 & 28.97 & 29.04 & 29.14 & +33.0 & +28.4 & $+23: 0$ & +33.0 & +30.0 & +35.0 & +35.0 & +22.0 & +28.5 & 13.0 \\
\hline 10 & 29. 19 & 29.28 & 29.40 & +24.0 & +25.0 & $+25: 8$ & $+\cdot 24.0$ & +22.0 & +25.6 & +29.0 & +23.4 & +25.5 & 7.0 \\
\hline II & $29.5^{8}$ & 29.67 & 29.82 & +16.0 & +15.4 & +13.0 & $+\cdot 25.0$ & +16.0 & +18.0 & +18.0 & +13.0 & +10.5 & 13.0 \\
\hline 12 & 29.92 & 30.00 & 30.08 & $+\mathrm{II} .0$ & +10.4 & +15.4 & +13.0 & + I0.0 & +14.0 & +24.0 & +10.0 & +17.0 & 14.0 \\
\hline 13 & 30.00 & 29.97 & 29.82 & +15.4 & +20.0 & +30.0 & +15.4 & +10.0 & +20.0 & +30.0 & +15.4 & +20.0 & 20.0 \\
\hline 14 & 29.66 & 29.62 & 29.46 & +31.0 & +31.0 & +30.0 & $+3^{\mathrm{I}} .0$ & +29.1 & $+3 i \cdot 4$ & +31.4 & +30.0. & +30.2 & 2.3 \\
\hline 15 & 29.28 & 29.31 & 29.28 & +33.0 & +33.0 & +33.0 & +33.0 & +29.0 & +33.4 & +34.0 & +30.0 & +31.5 & 5.0 \\
\hline 16 & 29.18 & 29. I9 & 29.24 & +33.0 & +36.0 & +33.5 & +35.0 & +31.0 & +37.0 & +38.8 & +32.0 & +34.9 & 7.8 \\
\hline 17 & 29.34 & 29.46 & 29.53 & +20.4 & +18.4 & +13.0 & +.33 .9 & +20.0 & +20.4 & +20.4 & +12.6 & +23.2 & 21.3 \\
\hline 18 & 29.54 & $29 \cdot 57$ & 29.60 & +11.5 & +11.5 & +11.0 & +13.0 & +10.0 & +13.2 & +14.0 & +.10 .0 & +12.0 & 4.0 \\
\hline 19 & 29.44 & 29.50 & 29.47 & +15.0 & $+2 I . I$ & +24.0 & +15.0 & +10.2 & +22.1 & +25.8 & +14.0 & $+\mathrm{I} 8.0$ & 15.6 \\
\hline 20 & $29.4^{\circ}$ & $29.5 \mathrm{I}$ & 29.59 & +25.0 & +26.5 & +28.5 & $+28: 5$ & $+24: 0$ & +26.6 & +29.0 & +25.0 & +26.5 & 5.0 \\
\hline 21 & 29.32 & 29.61 & 29.68 & +28.0 & +28.5 & +27.0 & +28.9 & +25.2 & +28.6 & +29.0 & +26.2 & +27.1 & 3.8 \\
\hline 22 & 29.68 & 29.71 & 29.70 & +29.0 & +28.5 & +24.0 & +29.0 & +26.0 & +29.0 & +29.8 & +24.0. & +26.9 & 5.8 \\
\hline 23 & 29.66 & 29.69 & 29.74 & +22.5 & +24.5 & +24.0 & +24.0 & +21.0 & +25.0 & +26.1 & +22.0 & +23.6 & 5.1 \\
\hline 24 & 29.69 & 29.78 & $29.8 \mathrm{I}$ & $+24.0^{\circ}$ & +24.0 & +20.0 & +24.0 & +21.0 & +25.0 & +25.1 & +20.0 & +22.6 & 5.1 \\
\hline 25 & 29.79 & 29.87 & 29.88 & +17.0 & +22.0 & +18.8 & +20.0 & +16.0 & +22.0 & +25.1 & +17.0 & +20.6 & 9.1 \\
\hline 26 & 29.88 & 29.92 & 29.97 & +21.0 & +21.5 & +20.0 & +23.0 & +15.0 & +25.0 & +34.1 & +20.0 & +24.6 & 19.1 \\
\hline 27 & 29.92 & 29.97 & 29.95 & +25.0 & +25.1 & $+25: 0$ & +25.0 & +17.1 & +27.1 & +35.0 & +24.6 & +26.0 & 17.9 \\
\hline 28 & 29.91 & 29.96 & 29.98 & +22.0 & +20.4 & +20.0 & $+25 . \mathrm{i}$ & +19.5 & +22.5 & +22.5 & +19.0 & +22.0 & 6.1 \\
\hline 29 & 29.97 & 30.01 & 30.00 & +22.0 & +23.0 & +24.0 & +22.0 & +18.0 & +23.0 & +24.0 & +20.0 & +21.0 & 6.0 \\
\hline 30 & 29.95 & 29.93 & 29.89 & +26.0 & +26.0 & +27.0 & $+^{\circ} 28.0$ & +23.1 & +26.4 & +32.2 & +24.1 & +27.6 & 0.I \\
\hline $3 \mathrm{I}$ & 29.95 & 29.96 & 30.08 & +24.0 & +28.0 & +27.0 & +27.8 & +23.8 & +28.8 & +35.0 & +23.8 & +29.4 & 11.2 \\
\hline & 917.88 & 919.32 & 919.72 & +718.3 & +747.6 & +740.7 & +791.4 & +633.1 & +786.3 & +878.7 & +648.8 & +756.7 & 304.5 \\
\hline 年 & $29.6 \mathrm{I}$ & 29.66 & 29.67 & +23.2 & +24.1 & +23.9 & +25.5 & +20.4 & +25.4 & +28.3 & +20.9 & $|+24.4|$ & 9.8 \\
\hline
\end{tabular}


Tabulation of daily meteorological observations at Cape Flora during the month of May, Igo5-Continued Observer: Francis Long

\begin{tabular}{|c|c|c|c|c|c|c|c|c|c|c|c|c|c|}
\hline \multirow[b]{3}{*}{ Dare } & \multicolumn{6}{|c|}{ 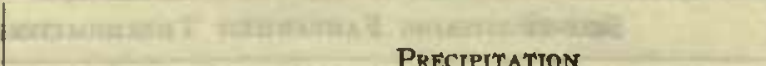 } & & \multicolumn{6}{|c|}{ WIND } \\
\hline & \multirow[b]{2}{*}{$8 \mathrm{n}$} & \multicolumn{2}{|c|}{$2=$} & \multicolumn{2}{|c|}{ 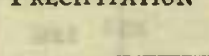 } & int & 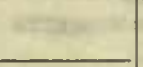 & \multicolumn{2}{|c|}{$8 \mathbf{H}$} & \multicolumn{2}{|c|}{ I2E } & \multicolumn{2}{|c|}{$20 \mathrm{H}$} \\
\hline & & $12 \mathrm{H}$ & $2 \mathrm{OH}$ & $\begin{array}{l}\overline{5} \\
\text { मे } \\
\end{array}$ & 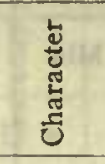 & 莺 & 芞 & 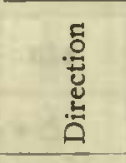 & 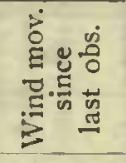 & 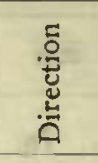 & 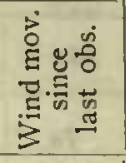 & 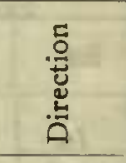 & 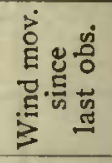 \\
\hline a.e & $\begin{array}{l}\text { In. } \\
.00\end{array}$ & $\begin{array}{l}I n . \\
.00\end{array}$ & $\begin{array}{l}\text { In. } \\
.00\end{array}$ & $\begin{array}{l}I n . \\
.00\end{array}$ & $\ldots$ & $\begin{array}{cc}h & m \\
. . & . .\end{array}$ & $\begin{array}{cc}h & m \\
. . & . .\end{array}$ & 1.87 & $\begin{array}{l}\text { Mi. } \\
\text { I00 }\end{array}$ & $\sqrt{325}$ & $\begin{array}{r}M i \\
78\end{array}$ & 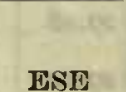 & $\begin{array}{l}M i . \\
\text { I07 }\end{array}$ \\
\hline 2 & .25 & .04 & .04 & .33 & $\mathrm{~s}^{\mathrm{m}}$ & D.N. & $21 \quad 29$ & ESE & 300 & $\mathbf{E}$ & 73 & ENE & II 2 \\
\hline Quer 3 & $.0 \mathrm{I}$ & $\mathbf{T}$ & .08 &.$\infty$ & $\begin{array}{l}\mathrm{S}^{\mathrm{m}} \\
\mathrm{S}^{\mathrm{m}}\end{array}$ & $\begin{array}{ll}10 & 30 \\
14 & 00\end{array}$ & $\left.\begin{array}{ll}\text { Io } & 45 \\
\text { I9 } & \infty\end{array}\right\}$ & ESE & 167 & ESE & 47 & $\mathrm{SE}$ & $5 \mathrm{I}$ \\
\hline weth 4 &.$\infty$ &.$\infty$ &.$\infty$ &.$\infty$ & ... & .... & .. $\quad .$. & w & 143 & W & $3 I$ & NW & $\ldots$ \\
\hline 5 & .04 & $\mathbf{T}$ & .00 & .04 & $\begin{array}{l}S^{4} \\
S^{d}\end{array}$ & $\begin{array}{l}\text { D. N. } \\
\text { I0 } 00\end{array}$ & $\left.\begin{array}{ll}\text { D. N. } \\
\text { II } & \infty\end{array}\right\}$ & sw & 37 & $\mathrm{~N}$ & 100 & $\mathrm{NW}$ & 134 \\
\hline 6 &.$\infty$ &.$\infty$ & .00 & .00 & ... & .. $\quad$. & .. $\quad .$. & NW & 195 & NIV & 49 & NW & 94 \\
\hline alit &.$\infty$ & .03 & .01 & .04 & $S^{m}$ & 10 26 & 1330 & C & 42 & SSE & 18 & E & 87 \\
\hline 0078 & .00 & .00 & .08 & .08 & $\mathrm{~S}^{\mathrm{m}}$ & 1430 & .. $\quad$. & $\mathbf{E}$ & 367 & $\mathbf{E}$ & 103 & $\mathbf{E}$ & 189 \\
\hline 2.7 9 & .10 & .02 & .02 & .14 & $S^{m}$ & .. . & 10 35 & C & 148 & SW & 38 & Sw & 90 \\
\hline ect 10 & .00 & .00 & .00 & .00 & .. & .. $\quad .$. & .. $\quad$. & ENE & 80 & E & 31 & C & 28 \\
\hline II &.$\infty$ & .00 & .00 & .00 & $\cdots$ & . $\quad .$. & .. $\quad$. & NE & 129 & NE & 103 & $\mathrm{NE}$ & 140 \\
\hline 12 &.$\infty$ & .00 & .00 & .00 & $\cdots$ & $\because \quad \cdots$ & .. & N & 189 & N & 59 & C & 13 \\
\hline 13 & $T$ & $\mathbf{T}$ & .04 & .04 & $\begin{array}{l}\mathrm{S}^{\mathrm{d}} \\
\mathrm{S}^{\mathrm{m}}\end{array}$ & $\begin{array}{l}\text { D. N. } \\
\text { II } 00\end{array}$ & $\begin{array}{lll}\text { D. N. } & \} \\
. . & . . & \}\end{array}$ & SSE & 44 & SE & & ssw & 78 \\
\hline 14 & .02 & $\mathbf{T}$ & .00 & .02 & $\mathrm{~s}^{\mathrm{m}}$ & . $\quad$. & $9 \quad 10$ & SSE & 80 & SE & 23 & SSIE & 96 \\
\hline A. 15 & .00 & .00 & .01 & .or & $\begin{array}{l}\mathrm{S}^{\mathrm{m}} \\
\mathrm{S}^{\mathrm{m}}\end{array}$ & $\begin{array}{ll}14 & 20 \\
18 & 26\end{array}$ & $\left.\begin{array}{l}\text { I4 } \\
\text { D. N. }\end{array}\right\}$ & SSW & 100 & SW & $4 \mathrm{I}$ & SSW & 50 \\
\hline 6.14 16 & .02 & .00 & .00 & .02 & $\cdots$ & .. $\quad .$. & .. $\quad$. & $\mathrm{C}$ & 30 & C & 4 & C & II \\
\hline 17 & .00 & $\mathbf{T}$ & .00 & $\mathrm{~T}$ & $\begin{array}{l}\mathbf{S}^{\mathbf{d}} \\
\mathbf{S}^{d} \\
\mathbf{S}^{d}\end{array}$ & $\begin{array}{rr}8 & 40 \\
12 & 14 \\
21 & 45\end{array}$ & $\left.\begin{array}{ll}10 & 35 \\
12 & 30 \\
D & \mathrm{~N} .\end{array}\right\}$ & NW & II5 & NW & & NW & 151 \\
\hline I8 & .02 &.$\infty$ & .00 & .02 & .. & .. $\quad$. & .. $\quad .$. & NW & 251 & Nw & 95 & Niv & 159 \\
\hline 19 & .00 & .02 & .08 & .10 & $S^{d}$ & 10 $\infty$ & 1700 & NE & 235 & $w$ & 106 & SSE & II 5 \\
\hline 20 & .30 & .04 & .00 & .34 & $\mathbf{S}^{\mathrm{m}}$ & D. N. & 1030 & E & 196 & E & 130 & ENE & 224 \\
\hline $2 \mathrm{I}$ & .00 & .00 &.$\infty$ & .00 & $\cdots$ & $\begin{array}{ll}. & .\end{array}$ & $\quad . \quad \quad$. & $\mathbf{E}$ & 295 & D & 89 & ENE & 176 \\
\hline 22 &.$\infty$ & .00 & .00 & .00 & ... & . $\quad$. & .. $\quad$. & $\mathrm{NE}$ & I9I & ENE & 65 & $\mathrm{NE}$ & I 28 \\
\hline 23 & .08 & $\mathbf{T}$ & .06 & .14 & $\begin{array}{l}\mathbf{S}^{\mathbf{a}} \\
\mathbf{S}^{\mathrm{d}}\end{array}$ & $\begin{array}{l}\text { D. N. } \\
\text { I5 } \infty\end{array}$ & $\left.\begin{array}{rr}8 & 30 \\
22 & 00\end{array}\right\}$ & $\mathrm{NE}$ & 206 & NE & 64 & $\mathrm{NE}$ & III \\
\hline $\begin{array}{r}24 \\
\text { bey }\end{array}$ & .04 & .02 & .00 & .06 & $\begin{array}{l}S^{d} \\
S^{d}\end{array}$ & $\begin{array}{ll}10 & 20 \\
22 & 35\end{array}$ & $\left.\begin{array}{ll}\text { II } & 30 \\
22 & 50\end{array}\right\}$ & IENE & 220 & ENE & 58 & NIV & 89 \\
\hline $687^{25}$ & $\mathbf{T}$ &.$\infty$ & .00 & $\mathrm{~T}$ & $\cdots$ &..$\quad$. & .. $\quad$. & NW & II 5 & $\mathrm{NE}$ & 56 & NW & I6! \\
\hline $\begin{array}{l}26 \\
-\quad 26\end{array}$ & .00 &.$\infty$ &.$\infty$ & .00 & $\cdots$ & . & .. $\quad$. & $\mathrm{NW}$ & 245 & $\mathrm{NE}$ & 36 & $\mathrm{~N}$ & 60 \\
\hline s.8. & .00 &.$\infty$ & .00 &.$\infty$ & $\cdots$ & .. $\quad$. & $\because \quad$. & $N$ & 82 & $N$ & 29 & $\mathbf{N}$ & 35 \\
\hline batil & .00 &.$\infty$ & $\mathbf{T}$ & $T$ & $\begin{array}{l}\mathbf{S}^{d} \\
\mathbf{S}^{d}\end{array}$ & $\begin{array}{ll}14 & 13 \\
19 & 10\end{array}$ & $\begin{array}{ll}14 & 23 \\
21 & 40\end{array}$ & NW & 108 & NW & 55 & NW & 76 \\
\hline 14 29 & $T$ & $\mathrm{~T}$ & .00 & $T$ & $S^{n}$ & 730 & 910 & w & 85 & wsw & 33 & IVSW & 73 \\
\hline 30 & .00 & $\mathbf{T}$ & .04 & .04 & $S^{d}$ & II $\infty$ & 1510 & $\mathbf{E}$ & 50 & $\mathbf{E}$ & 55 & w & 106 \\
\hline 31 & .00 & .00 & $\mathbf{T}$ & $\mathbf{T}$ & $S^{d}$ & $16 \quad 15$ & $17 \quad 30$ & W & 133 & $\mathrm{NW}$ & 39 & SIY & 50 \\
\hline Sum...... & .88 & .17 & .46 & I. $5 \mathrm{I}$ & ... & .. .. & .. . . & $\cdots$ & 4768 & $\ldots$ & 1798 & ... & 3084 \\
\hline Mean.... & $\cdots$ & ... & $\ldots$ & $\cdots$ & $\ldots$ & $\quad \cdots \quad$. & .. $\quad$. & NW & I53.8 & E & 58.0 & NW & 102.8 \\
\hline
\end{tabular}


Tabulation of daily meteorological observations of Cape Flora during the month of May, 1905-Continued

Observer: FRANCIS LONG

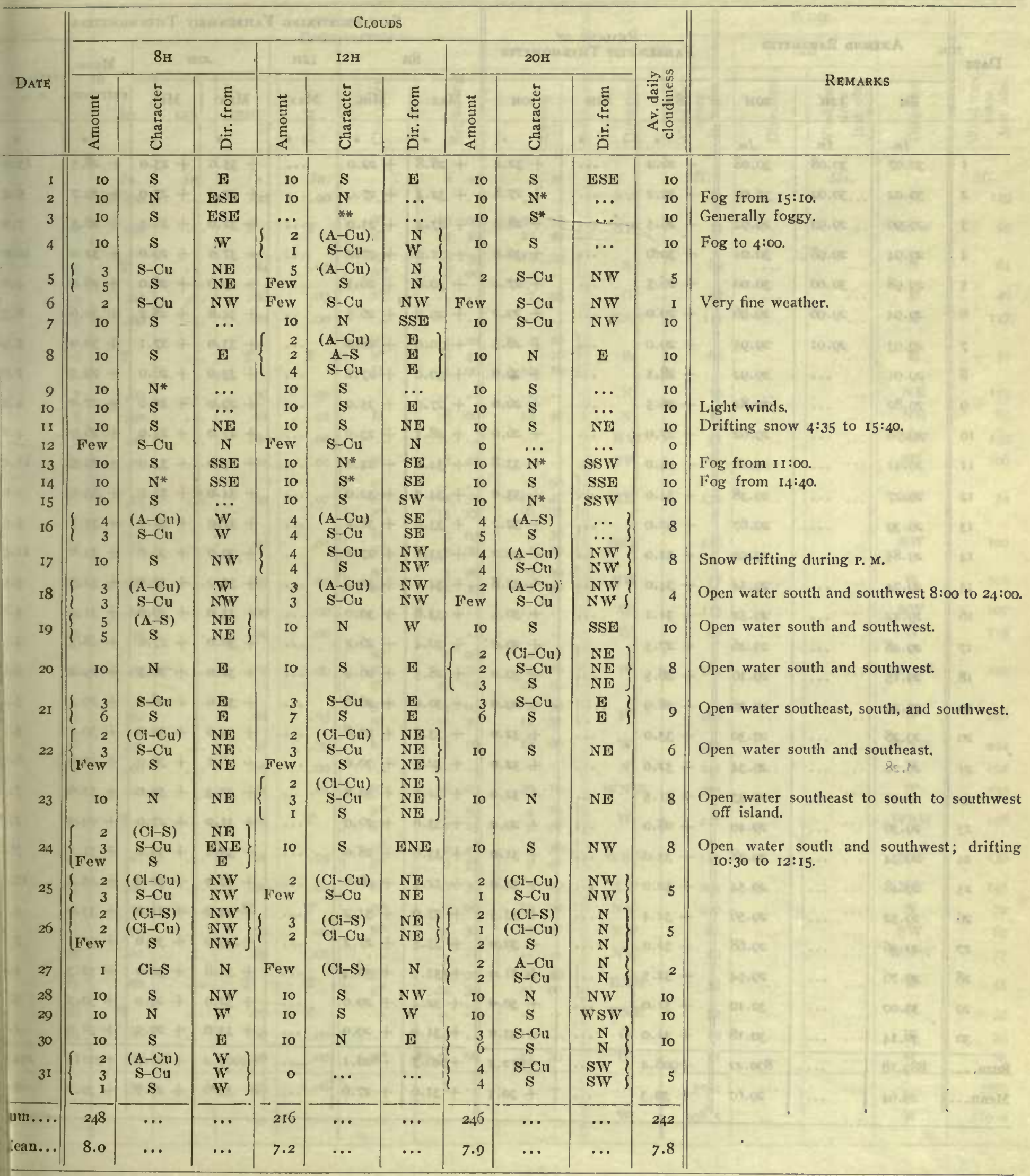


Tabulation of daily metcorological observations at Cape Flora during the month of June, 1905

Obscrver: Francis LoNG

\begin{tabular}{|c|c|c|c|c|c|c|c|c|c|c|c|c|c|c|}
\hline \multirow{3}{*}{ DATE } & \multirow{2}{*}{\multicolumn{4}{|c|}{ ANEROID BAROMETER }} & \multirow{2}{*}{\multicolumn{3}{|c|}{$\begin{array}{l}\text { READINC OF } \\
\text { FAHRENHEIT THERMOMETER }\end{array}$}} & \multicolumn{7}{|c|}{ SELF-REGISTERING FAIIRENIIFIT THERMOMETERS } \\
\hline & & & & & & & & \multicolumn{2}{|c|}{$8 \mathrm{H}$} & \multirow{2}{*}{$\frac{\text { I2II }}{\text { Max. }}$} & \multicolumn{2}{|c|}{2011} & \multirow{2}{*}{$\begin{array}{c}\text { Mean } \\
\text { of } \\
\text { extremes }\end{array}$} & \multirow{2}{*}{ Range } \\
\hline & $8 \mathrm{H}$ & 121 & $2 \mathrm{H}$ & $2 \mathrm{OH}$ & $8 \mathrm{H}$ & $\mathrm{I} 2 \mathrm{H}$ & $20 \mathrm{H}$ & Max. & Min. & & Max. & Min. & & \\
\hline & In. & & In. & In. & $=$ & $z^{\circ}$ & $\bullet$ & $\circ$ & $\circ$ & - & 0 & & - & • \\
\hline I & 30.07 & & .08 & 30.08 & +26.2 & $\cdots$ & +32.4 & +28.8 & +22.0 & $\cdots$ & +35.0 & +23.0 & +28.5 & 13.0 \\
\hline 2 & 30.02 & & .02 & 30.00 & +29.2 & $\ldots$ & +27.8 & +32.4 & +27.0 & ... & +29.4 & +27.0 & +29.7 & $5 \cdot 4$ \\
\hline 3 & 29.90 & & .92 & 29.92 & +26.5 & $\ldots$ & +28.0 & $+27 \cdot 4$ & +25.0 & . & +32.0 & +25.5 & +28.5 & 7.0 \\
\hline 4 & 29.94 & 29 . & .98 & 30.01 & +30.0 & & +30.0 & +31.0 & +27.0 & $\cdots$ & +37.0 & +29.0 & +32.0 & 10.0 \\
\hline 5 & 29.98 & 30. & .00 & 30.00 & +26.5 & & +27.4 & +30.0 & +26.1 & ... & +29.0 & +26.0 & +28.0 & 4.0 \\
\hline 6 & 29.94 & & .95 & 29.96 & +29.0 & $\ldots$ & +29.2 & +29.9 & +26.5 & .. & +34.8 & +28.2 & +30.6 & 8.3 \\
\hline 7 & 29.93 & & $.9 \mathrm{I}$ & 29.95 & +29.0 & $\cdots$ & +28.5 & +30.0 & +28.0 & $\ldots$ & +33.0 & +27.1 & +30.0 & 5.9 \\
\hline 8 & $29.9 \mathrm{I}$ & • & ... & 29.92 & $+28 . I$ & $\ldots$ & +26.0 & +30.0 & +27.2 & .. & +33.0 & +26.0 & +29.5 & 7.0 \\
\hline 9 & 29.82 & $n$ & ... & 29.82 & +26.5 & $\ldots$ & +26.0 & +27.9 & +25.0 & ... & +29.0 & +25.0 & +27.0 & 4.0 \\
\hline 10 & 29.78 & $t a$ & . & 29.62 & +27.0 & $\ldots$ & +26.0 & +28.1 & +23.9 & ... & +27.0 & +24.0 & +26.0 & 4.2 \\
\hline I I & $29.4 \mathrm{I}$ & & & 29.18 & +34.0 & $\ldots$ & +33.8 & +34.0 & +24.0 & ... & +35.0 & +33.0 & +29.5 & II.0 \\
\hline 12 & 29.27 & & $\cdots$ & 29.38 & +34.0 & $\ldots$ & +33.0 & +34.0 & +32.0 & $\cdots$ & +34.0 & +32.0 & +33.0 & 2.0 \\
\hline 13 & 29.39 & & $\ldots$ & 29.67 & +32.0 & 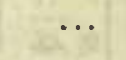 & +32.2 & +33.0 & +31.2 & $\cdots$ & +37.0 & +32.0 & $+34 \cdot I$ & 5.9 \\
\hline 14 & 29.85 & 43 & $\cdots$ & 29.92 & +32.0 & $\ldots$ & +32.2 & +33.0 & +27.1 & $\cdots$ & +39.0 & +30.0 & +33.0 & I1.9 \\
\hline 15 & 29.74 & 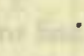 & $\cdots$ & $29 \cdot 54$ & +34.0 & $\cdots$ & +33.8 & +34.0 & +30.0 & ... & +35.0 & +32.0 & +32.5 & 5.0 \\
\hline I6 & 29.42 & . & $\cdots$ & 29.52 & $+3 I . I$ & ... & +30.4 & +33.8 & +30.0 & $\cdots$ & +33.0 & +29.0 & $+3 \mathrm{I} \cdot 4$ & 4.8 \\
\hline 17 & 29.48 & . & $\ldots$ & 29.46 & +27.5 & a $\cdots$ & +29.0 & +30.4 & +26.2 & $\ldots$ & +30.0 & +27.0 & +28.3 & 4.2 \\
\hline I8 & 29.15 & bous. & ... & 29. I6 & +28.5 & $\ldots$ & +30.0 & +28.5 & +26.2 & ... & +32.0 & +28.5 & +29.1 & 5.8 \\
\hline I9 & 29.24 & . & 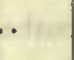 & 29.36 & +28.9 & $\ldots$ & +29.0 & +30.0 & +26.6 & $\cdots$ & +32.0 & +28.0 & +29.3 & 5.4 \\
\hline 20 & 29.28 & & & 29.30 & +33.0 & .. & +32.0 & +33.0 & +27.8 & $\ldots$ & +33.0 & +30.0 & +30.4 & 5.2 \\
\hline $2 I$ & 29.28 & 10 & $\ldots$ & 29.34 & +32.0 & $\ldots$ & +32.0 & +32.0 & +30.0 & $\ldots$ & +36.1 & +30.0 & +33.0 & 6.1 \\
\hline 22 & 29.22 & tar & ... & 29.33 & $+3 \mathrm{I} \cdot 5$ & $\ldots$ & +32.0 & +32.0 & +30.0 & $\cdots$ & +37.3 & +30.0 & +33.6 & $7 \cdot 3$ \\
\hline 23 & 29.36 & $a^{2}$ & ... & 29.40 & +28.0 & 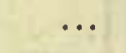 & +29.0 & +33.0 & +25.0 & $\cdots$ & +31.0 & +27.0 & +29.5 & 7.0 \\
\hline 24 & 29.34 & 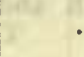 & ... & 29.38 & +33.0 & .. & +31.0 & +33.6 & +28.0 & $\ldots$ & +35.0 & +30.0 & +31.5 & 7.0 \\
\hline 25 & 29.48 & & ... & 29.54 & +32.0 & & +34.0 & +32.5 & +28.8 & $\cdots$ & +42.0 & +32.0 & +35.4 & 13.2 \\
\hline 26 & $29 \cdot 52$ & & ... & 29.56 & +32.4 & .. & +33.5 & +35.0 & +31.2 & ... & +34.0 & +31.0 & +33.0 & 4.0 \\
\hline 27 & 29.56 & & ... & 29.68 & +32.0 & & +30.0 & +33.5 & +27.2 & ... & +35.0 & +30.0 & +31.1 & 7.8 \\
\hline 28 & 29.76 & & ... & 29.94 & +32.5 & $\ldots$ & +31.0 & +32.5 & +30.1 & .. & +34.6 & +30.0 & +32.3 & 4.6 \\
\hline 29 & 30.00 & & $\cdots$ & 30.10 & +31.0 & $\ldots$ & +30.0 & +32.4 & +29.0 & & +32.0 & \pm 29.0 & +30.7 & 3.4 \\
\hline 30 & 30.14 & & $\cdots$ & 30.18 & +31.0 & & +33.0 & +31.0 & +29.0 & $\cdots$ & +33.0 & +29.0 & +31.0 & 4.0 \\
\hline Sum.... & 893.18 & & ... & 890.22 & +908.4 & $\ldots$ & +912.2 & +946.7 & +828.1 & & +1009.2 & $+85 \mathrm{I} \cdot 3$ & +921.5 & 194.4 \\
\hline Mean... & 29.64 & & & 29.67 & +30.3 & $\cdots$ & +30.4 & +31.6 & +27.6 & $\ldots$ & +33.6 & +28.7 & +30.7 & 6.5 \\
\hline
\end{tabular}


Tabulation of daily metcorological observations at Cape Flora during the month of June, rgo5-Continued

Observer: Francis LoNG

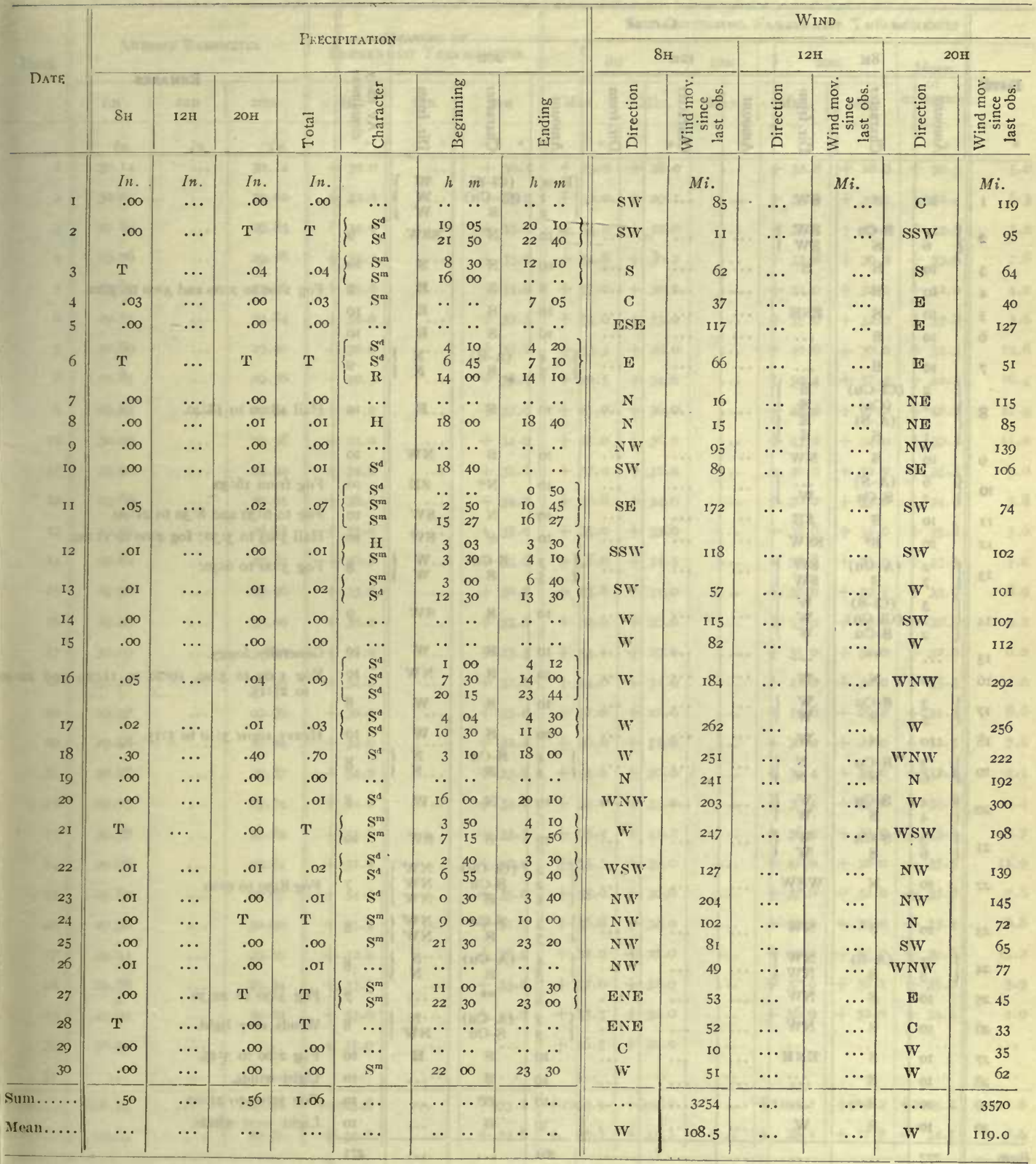


Tabulation of daily meteorological observations at Cape Flora during the month of June, 1905-Continued Observer: Francis LoNG

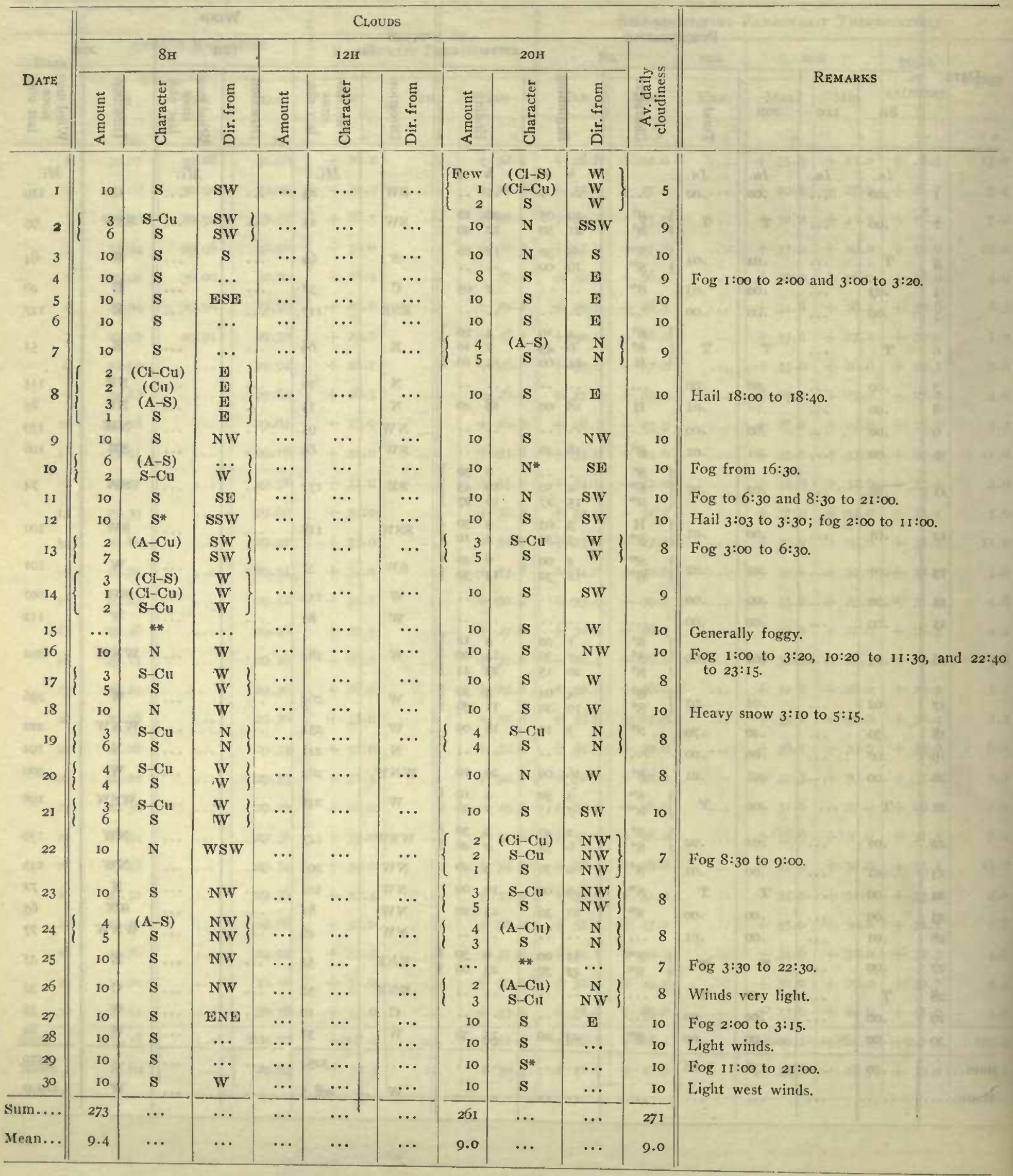


Tabulation of daily meteorological observations at Cape Flora during the month of July, 1905

Observer: FRANCIS LONG

\begin{tabular}{|c|c|c|c|c|c|c|c|c|c|c|c|c|c|}
\hline \multirow{3}{*}{ Date } & \multirow{2}{*}{\multicolumn{3}{|c|}{ ANEROID BAROMETER }} & \multirow{2}{*}{\multicolumn{3}{|c|}{$\begin{array}{c}\text { READING OF } \\
\text { FAHREN HEIT THERMOMETER }\end{array}$}} & \multicolumn{7}{|c|}{ SELF-REGISTERING FAHRENHEIT THERMOMETERS } \\
\hline & & & & & & & $8 \mathrm{H}$ & & $12 \mathrm{HI}$ & 201 & & & \\
\hline & $8 \mathrm{ri}$ & $12 \mathrm{H}$ & $20 \mathrm{H}$ & $8 \mathrm{H}$ & $\mathrm{I2H}$ & $20 \mathrm{H}$ & Max. & Min. & Max. & Max. & Min. & & thing \\
\hline & In. & In. & In. & & $\cdot$ & $=$ & 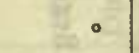 & $\circ$ & 。 & • & $\circ$ & - & - \\
\hline I & 30. I4 & ... & 30.12 & +30.0 & $\ldots$ & +30.2 & +33.0 & +28.0 & $\cdots$ & +32.8 & +28.0 & +30.5 & 5.0 \\
\hline 2 & 30.04 & & 30.00 & +32.0 & $\cdots$ & +33.0 & +32.0 & +29.1 & $\cdots$ & +35.6 & +30.0 & +32.4 & 6.5 \\
\hline 3 & 29.84 & & 29.83 & +34.0 & & +33.5 & +34.0 & +32.0 & $\ldots$ & +35.0 & +32.0 & +33.5 & 3.0 \\
\hline 4 & 29.76 & & 29.78 & +33.5 & 19te & +33.0 & +34.8 & +30.2 & $\ldots$ & +37.8 & +30.0 & +33.9 & 7.8 \\
\hline 5 & 29.67 & & 29.46 & +33.0 & w $\cdots$ & +34.0 & +33.0 & +30.0 & $\ldots$ & +34.0 & +32.0 & +32.0 & 4.0 \\
\hline 6 & 29.71 & & 29.84 & +34.0 & the & +33.3 & +34.0 & +33.0 & $\ldots$ & +35.6 & +32.0 & +33.8 & 3.6 \\
\hline 7 & 29.89 & & 29.90 & +30.0 & ... & +35.2 & +33.3 & +28.0 & $\ldots$ & +40.6 & +30.0 & +34.3 & 12.6 \\
\hline 8 & 29.85 & & 29.76 & +40.5 & $\ldots$ & +37.4 & +40.5 & +34.0 & $\ldots$ & +50.4 & +37.4 & +42.2 & 16.4 \\
\hline 9 & 29.83 & & 29.94 & $+4 \mathrm{I} .0$ & . & +37.0 & +41.0 & +36.0 & $\ldots$ & +48.9 & +37.0 & +42.4 & 12.9 \\
\hline 10 & 30.00 & & 29.98 & +42.0 & $\cdots$ & +34.0 & +45.0 & +36.0 & $\ldots$ & +43.2 & +34.0 & +39.5 & II.O \\
\hline II & 29.90 & & 29.92 & +34.0 & 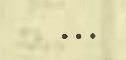 & +34.6 & +37.0 & +31.0 & $\ldots$ & +40.0 & +32.0 & +35.5 & 9.0 \\
\hline 12 & 29.86 & & 29.95 & +36.0 & $\ldots$ & +34.0 & +36.8 & +34.0 & $\ldots$ & +36.0 & +33.0 & +34.9 & 3.8 \\
\hline 13 & 29.93 & & 29.95 & +35.0 & . & +37.0 & +35.0 & +33.0 & $\ldots$ & +38.6 & +34.0 & +35.8 & 5.6 \\
\hline 14 & 29.82 & & 29.84 & +36.0 & at. & +33.6 & +38.0 & +34.5 & $\ldots$ & +36.8 & +33.0 & +35.5 & 5.0 \\
\hline 15 & 29.98 & & 29.96 & +33.0 & & +30.0 & +33.8 & +31.0 & $\ldots$ & +38.0 & +29.1 & +33.6 & 8.9 \\
\hline 16 & 29.95 & & 30.00 & +30.0 & & +33.0 & +30.0 & +26.0 & $\ldots$ & +35.0 & +29.0 & +30.5 & 9.0 \\
\hline 17 & 30.03 & & 30. 10 & +34.0 & & +33.5 & +34.0 & +29.0 & $\ldots$ & +35.0 & +32.0 & +32.0 & 6.0 \\
\hline 18 & 30.06 & & 30.06 & +34.0 & ... & +31.0 & +34.0 & +31.0 & $\because$ & $+4 \mathrm{r} .0$ & +30.0 & +35.5 & II.0 \\
\hline 19 & 29.97 & & 29.96 & +30.0 & $\ldots$ & +33.0 & +31.0 & +27.0 & $\ldots$ & +35.6 & +29.0 & $+3 \mathrm{I} \cdot 3$ & 8.6 \\
\hline 20 & 29.98 & & 30.04 & +34.0 & $\cdots$ & +32.4 & +36.0 & +31.0 & $\ldots$ & +36.0 & +29.0 & +32.5 & 7.0 \\
\hline 21 & 29.98 & & 29.87 & +34.0 & $\ldots$ & +33.0 & +34.0 & +30.0 & $\ldots$ & +34.4 & +32.0 & +32.2 & 4.4 \\
\hline 22 & 29.75 & & 29.74 & +34.0 & $\cdots$ & +34.0 & +34.0 & +33.0 & $\cdots$ & +36.4 & +33.6 & +34.7 & 3.4 \\
\hline 23 & 29.58 & & 29.82 & +35.0 & ... & +33.5 & +35.4 & +33.2 & $\ldots$ & +36.9 & +33.0 & +35.0 & 3.7 \\
\hline 24 & 29.96 & & 29.84 & +31.0 & $\ldots$ & +30.0 & +33.5 & +30.0 & $\cdots$ & +41.0 & +30.0 & +35.5 & 11.0 \\
\hline 25 & 29.60 & & 29.87 & +34.9 & $\ldots$ & +35.0 & +35.0 & +30.0 & $\ldots$ & +37.6 & +32.0 & +33.8 & 7.6 \\
\hline 26 & 29.90 & & 30.00 & +35.9 & $\ldots$ & +31.0 & +36.8 & +34.0 & $\ldots$ & +35.9 & +30.0 & +33.4 & 6.8 \\
\hline 27 & 29.94 & & 29.99 & +32.0 & & +36.9 & +32.0 & +27.0 & $\ldots$ & +42.2 & +32.0 & +39.6 & 15.2 \\
\hline 28 & 29.88 & & 29.94 & +33.0 & & +33.0 & +37.0 & +32.0 & $\cdots$ & +37.9 & +32.1 & +35.0 & 5.9 \\
\hline 29 & 29.92 & & 30.02 & +33.0 & $\cdots$ & +34.0 & +33.5 & +32.0 & $\cdots$ & +36.9 & +32.0 & +34.4 & 4.9 \\
\hline 30 & 30.01 & & $\ldots$ & +35.0 & $\cdots$ & .. & +36.5 & +29.0 & $\ldots$ & $\ldots$ & & & $\cdots$ \\
\hline 31 & $\ldots$ & & $\ldots$ & $\ldots$ & $\ldots$ & .. & $\cdots$ & $\ldots$ & $\cdots$ & $\cdots$ & $\cdots$ & $\cdots$ & $\cdots$ \\
\hline & 896.73 & $\ldots$ & 867.48 & +1023.8 & $\ldots$ & +973.1 & +1053.9 & +934.0 & $\ldots$ & +1105.1 & +919.2 & +1005.2 & 219.6 \\
\hline Mean... & 29.89 & & $29.9 \mathrm{I}$ & $+34 \cdot 1$ & $\cdots$ & +33.6 & +35.1 & +31.1 & $\cdots$ & $|+38.1|$ & +31.7 & +34.7 & 7.6 \\
\hline
\end{tabular}


Tabulation of daily meteorological observations at Cape Flora during the month of July, rgo5-Continued

Observer: FRANCIS LONG

\begin{tabular}{|c|c|c|c|c|c|c|c|c|c|c|c|c|c|}
\hline & $\|$ & Wow & Hensules & Certhor & reaste & & & & & & IND & & \\
\hline & follc & $\operatorname{3r} 2$ & $=$ & PRECII & PITATION & ee & & & & & & & wate \\
\hline DATE & $8 \mathrm{n}$ & 1218 & $20 \mathrm{H}$ & 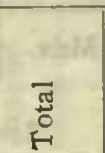 & 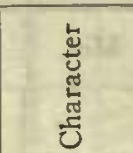 & 邑 & 章 & 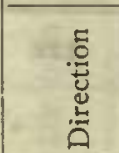 & 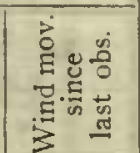 & 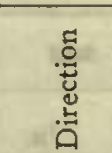 & 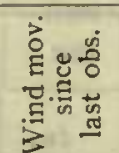 & 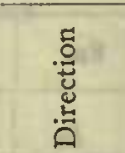 & 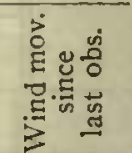 \\
\hline एक & 7 & ET & $y=-4$ & int & $-x$ & 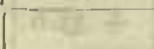 & 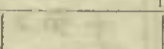 & & 2 & 40 & & $\sqrt{150}$ & \\
\hline 54. & $\begin{array}{l}\text { In. } \\
\text {.OI }\end{array}$ & $\begin{array}{l}\text { In. } \\
\ldots\end{array}$ & $\begin{array}{l}I n . \\
.02\end{array}$ & $\begin{array}{l}I n \\
.03\end{array}$ & $\mathrm{~S}^{\mathrm{m}}$ & & $\begin{array}{rr}h & m \\
\text { II } & 10\end{array}$ & C & Mi. & $\ldots$ & $M i$. & $s^{\infty}$ & Mi. \\
\hline 65 & $\mathrm{~T}$ & $\cdots$ & .02 & .02 & $\left\{\begin{array}{l}\mathbb{S}^{\mathrm{un}} \\
\mathbb{S}^{\mathrm{m}} \\
\mathbb{S}^{\mathrm{m}}\end{array}\right.$ & $\begin{array}{rr}7 & \infty \\
11 & 30 \\
20 & 40\end{array}$ & $\begin{array}{rr}8 & 50 \\
13 & 35 \\
. & .\end{array}$ & SSW & 92 & $\ldots$ & & $\mathrm{s}$ & 77 \\
\hline 3 & .02 & $\ldots$ & .09 &. .II & $\left\{\begin{array}{l}\mathbf{S}^{m} \\
\mathbf{R} \\
\mathbf{S}^{m}\end{array}\right.$ & $\begin{array}{rr}\ddot{8} & \ddot{45} \\
\text { II } & 0\end{array}$ & $\left.\begin{array}{rl}6 & 00 \\
\text { II } & 00 \\
\text { I5 } & 00\end{array}\right\}$ & $\mathbf{E}$ & 68 & $\ldots$ & & s & 25 \\
\hline 6. 4 & 10.00 & . & .02 & .02 & $\begin{array}{l}\mathbf{S}^{\mathrm{m}} \\
\mathbf{S}^{\mathrm{m}}\end{array}$ & I5 $\infty$ & $16 \quad 30$ & SE & 13 & $\ldots$ & $\cdots$ & Sw & 33 \\
\hline (1) 5 & 24.16 & $\ldots$ & $\cdot 30$ & .46 & $\left\{\begin{array}{l}S^{\operatorname{mm}} \\
\mathrm{R}\end{array}\right.$ & $\begin{array}{l}\mathrm{I} 4 \\
\mathrm{I} 6 \\
\mathrm{I}\end{array}$ & $\left.\begin{array}{ll}16 & 00 \\
20 & 30\end{array}\right\}$ & ESE & 73 & $\cdots$ & & $\mathbf{E}$ & 284 \\
\hline (xtr) & .01 & ... & .02 & .03 & $\left\{\begin{array}{l}\mathbf{S}^{\mathbf{m}} \\
\mathbf{R}\end{array}\right.$ & $\begin{array}{ll}\text { II } & 30 \\
19 & 00\end{array}$ & $\left.\begin{array}{ll}16 & 15 \\
20 & 50\end{array}\right\}$ & w & 76 & $\ldots$ & & $\mathbf{E}$ & 89 \\
\hline 112 & T & $\ldots$ &.$\infty$ & $\mathrm{T}$ & $\ldots$ & .. $\quad$. & .. $\quad .$. & C & 134 & .. & & C & 35 \\
\hline 8 & .00 & $\ldots$ &.$\infty$ & .00 & $\ldots$ & .. $\quad$. & .. $\quad$. & C & 8 & $\ldots$ & & $\mathrm{N}$ & 1763 \\
\hline 164 &.$\infty$ & $\ldots$ &.$\infty$ & .00 & ... & ... $\quad$. & .. $\quad$. & C & 62 & $\ldots$ & & $\mathrm{N}$ & $8 \mathrm{I}$ \\
\hline (172) 10 &.$\infty$ & $\ldots$ & 12.00 & .00 & $\ldots$ & .. $\quad$. & .. $\quad$. & C & 221 76 & $\ldots$ & & $\mathbf{E}$ & II 8 \\
\hline (4) II &.$\infty$ & $\ldots$ &.$\infty$ &.$\infty$ & $\ldots$ & .. $\quad$. & .. .. & $\mathbf{E}$ & 34 & $\ldots$ & & $\mathbf{E}$ & 237 \\
\hline 6) 12 & .02 & $\cdots$ & .01 & .03 & $\begin{array}{l}\mathbf{R} \\
\mathbf{R}\end{array}$ & $\begin{array}{l}30 \\
8 \quad 48\end{array}$ & $\left.\begin{array}{ll}4 & 30 \\
9 & 30\end{array}\right\}$ & E & $=54$ & $\cdots$ & & $\mathbf{E}$ & ${ }^{\mathrm{II} 2}$ \\
\hline De 13 &.$\infty$ & $\ldots$ & .00 &.$\infty$ & $\ldots$ & $\ldots$ & .. $\quad$. & C & 28 & $\ldots$ & & C & 12 \\
\hline 6.6. $\quad 14$ & 100 & $\cdots$ &.$\infty$ &.$\infty$ & $\ldots$ & .. $\quad$. & .. $\quad$. & C & 8 & ... & .. & NW & 68 \\
\hline 2.11 15 & .00 & $\ldots$ &.$\infty$ &.$\infty$ & $\cdots$ & $\quad \cdots \quad \cdots$ & . $\quad$. & NW & 135 & $\cdots$ & & W & 112 \\
\hline $4.8 \quad 16$ & $\mathbf{T}$ & & $T$ & $T$ & $\mathrm{~S}^{\mathrm{m}}$ & 730 & 840 & $w$ & $3 \mathrm{I}$ & $\ldots$ & & w & 62 \\
\hline 17 &.$\infty$ & $\ldots$ & .00 & .00 & $\cdots$ & . $\quad$. & .. & C & 39 & ... & & W & I8 \\
\hline I8 & .00 & $\ldots$ &.$\infty$ & .00 & $S^{i n}$ & $23 \infty$ & (6) & $\mathrm{C}$ & 37 & ... & & w & 27 \\
\hline 14 19 & $\mathrm{~T}$ & & $\mathrm{~T}$ & $\mathrm{~T}$ & $\left\{\begin{array}{l}\mathbf{S}^{\mathbf{m}} \\
\mathbf{S}^{\mathrm{m}}\end{array}\right.$ & $\ddot{\text { io }} \quad \ddot{15}$ & $\left.\begin{array}{rr}1 & 10 \\
10 & 35\end{array}\right\}$ & W & 14. 98 & $\ldots$ & & IVNIV & 170 \\
\hline 20 &.$\infty$ & $\ldots$ &.$\infty$ & .00 & $\mathbf{S}^{\mathrm{m}}$ & o 30 & 14 I0 & W & 122 & $\ldots$ & & W & I 50 \\
\hline $2 \mathrm{x}$ & .00 & $\ldots$ & .08 & .08 & $\mathbf{R}$ & $15 \quad 45$ & $20 \quad 50$ & w & 128 & $\ldots$ & & C & $1=50$ \\
\hline 22114 & .25 & $\ldots$ & 6. .04 & .29 & $\left\{\begin{array}{l}\mathbf{R} \\
\mathbf{R}\end{array}\right.$ & $\begin{array}{l}30 \\
600\end{array}$ & $\left.\begin{array}{rr}9 & 50 \\
. & . .\end{array}\right\}$ & $N W$ & 1460 & ... & & $\mathrm{N}$ & 68 \\
\hline 23 & .58 & $\ldots$ & .01 & .59 & li & .. $\quad$. & $9 \infty$ & W & 92 & ... & & W & 136 \\
\hline fis $\quad 24$ & $16 . \infty$ & $\ldots$ & .00 &.$\infty$ & ll & $22 \quad 25$ & .. $\quad$. & w & III & $\ldots$ & & E & 88 \\
\hline 단. 25 & .08 & $=\cdots$ & .00 & .08 & $\mathrm{R}$ & .. $\quad$. & D.N. & $\mathrm{NB}$ & 135 & $\ldots$ & & WNW & 107 \\
\hline 25 & .00 & $\ldots$ &.$\infty$ & .00 & $\cdots$ & .. $\quad$. & .. & $\mathrm{C}$ & 53 & $\cdots$ & & $\mathrm{SE}$ & 26 \\
\hline $6 \times \quad 27$ & 10.00 & $\ldots$ & .00 & .00 & $\ldots$ & $\therefore \quad$. & .. & $\mathbf{E}$ & 96 & ... & & $\mathbb{D}$ & 151 \\
\hline 28 & .15 & & .or & .16 & Ii & 200 & 850 & SE & 249 & $\ldots$ & & $\mathrm{SF}$ & 104 \\
\hline 29 & .00 & ... &.$\infty$ &.$\infty$ & ... & .. & .. $\quad$. & SE & II 7 & $\cdots$ & & $\mathrm{C}$ & 84 \\
\hline 30 & .00 & $\ldots$ & $\cdots$ & ... & $\ldots$ & .... & .. $\quad$. & $\cdots \mathrm{SIO}$ & 7 & $\cdots$ & & $\cdots$ & $\cdots$ \\
\hline $3 \mathrm{I}$ & $\ldots$ & $\ldots$ & $x+\cdots$ & $\ldots$ & $\cdots$ & . $\quad$. & $\begin{array}{lll} & . & \end{array}$ & $\ldots$ & . & $\ldots$ & & $\ldots$ & $\ldots$ \\
\hline Sum...... & 1.28 & $\ldots$ & 0.62 & 1.90 & $\ldots$ & ... $\quad$. & .. $\quad$. & $\ldots$ & 2602 & $\ldots$ & & $\cdots$ & 2648 \\
\hline $\operatorname{an} .$. & $\cdots$ & & $\cdots$ & .. & & .. & . $\quad$. & C & & & & E & 9ז.3 \\
\hline
\end{tabular}


Tabulation of daily meteorological observations at Cape Flora during the month of July, 1905-Continued Observer: Francis LONG

\begin{tabular}{|c|c|c|c|c|c|c|c|c|c|c|c|}
\hline \multirow[b]{3}{*}{ DATE } & \multicolumn{10}{|c|}{ Cloutos } & \multirow[b]{3}{*}{ REMARKS } \\
\hline & \multicolumn{3}{|c|}{$8 \mathrm{H}$} & \multicolumn{3}{|c|}{$12 \mathrm{H}$} & \multicolumn{3}{|c|}{$20 I 1$} & \multirow[b]{2}{*}{ 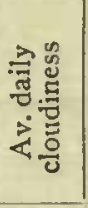 } & \\
\hline & $\begin{array}{l}\stackrel{E}{E} \\
\stackrel{\Xi}{E}\end{array}$ & 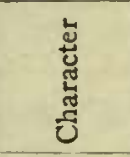 & $\begin{array}{l}E \\
\text { 누 } \\
.4 \\
0\end{array}$ & $\begin{array}{l}\text { E } \\
\text { हू } \\
\text { E }\end{array}$ & 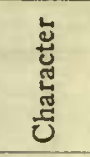 & 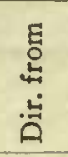 & 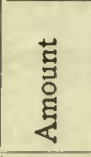 & 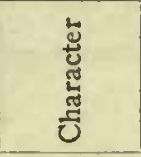 & 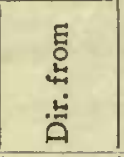 & & \\
\hline I & 10 & $\mathrm{N}^{*}$ & ... & .. & $\ldots$ & $\ldots$ & IO & $\mathbf{S}$ & $\mathbf{S}$ & Io & Fog $7: 00$ to $15: 00$ \\
\hline 2 & Io & $\mathbf{N}$ & SSW & .. & ... & ... & ro & $\mathbf{S}$ & $\mathbf{s}$ & 10 & Light south to southwest winds. \\
\hline 3 & 10 & $\mathbf{S}^{*}$ & $\ldots$ & $\ldots$ & ... & ... & ... & $* *$ & .. & Io & Generally foggy. \\
\hline 4 & 10 & $\mathbf{S}$ & $\ldots$ & ... & ... & ... & $\cdots$ & ** & ... & I0 & Fog from II: $\infty$ \\
\hline 5 & Io & $\mathbf{S}$ & ESE & $\ldots$ & $\cdots$ & $\cdots$ & Io & $\mathrm{N}^{*}$ & $\mathbf{E}$ & Io & Fog from Io:0o. \\
\hline 6 & $\left\{\begin{array}{l}3 \\
4\end{array}\right.$ & $\begin{array}{c}(\mathrm{A}-\mathrm{Cu}) \\
\mathrm{S}^{*}\end{array}$ & W & $\cdots$ & $\cdots$ & $\cdots$ & I0 & $N^{* *}$ & $\cdots$ & 7 & $\begin{array}{l}\text { Fog } 9: 30 \text { to } 11: 00,16: 00 \text { to } 17: 30 \text {, and } 5: 30 \text { to } \\
24: 00 \text {. }\end{array}$ \\
\hline 7 & $\left\{\begin{array}{r}\text { Few } \\
\text { Few } \\
8\end{array}\right.$ & $\begin{array}{c}(\mathrm{Cl}-\mathrm{S}) \\
(\mathrm{A}-\mathrm{Cu}) \\
\mathbf{S}\end{array}$ & $\ddot{N}$ & $\cdots$ & $\ldots$ & .. & $\left\{\begin{array}{l}4 \\
4\end{array}\right.$ & $\begin{array}{c}(\mathrm{A}-\mathrm{Cu}) \\
\mathrm{S}\end{array}$ & $\left.\begin{array}{l}\mathrm{E} \\
\ldots\end{array}\right\}$ & 8 & Fog $7: 00$ to $13: 30$ \\
\hline 8 & $\left\{\begin{array}{r}2 \\
I \\
2 \\
\text { Few }\end{array}\right.$ & $\begin{array}{c}(\mathrm{Ci}-\mathrm{S}) \\
(\mathrm{Ci}-\mathrm{Cu}) \\
(\mathrm{A}-\mathrm{S}) \\
\mathbf{S}\end{array}$ & $\begin{array}{l}\mathrm{E} \\
\mathrm{E} \\
\mathrm{E} \\
\cdots\end{array}$ & ... & $\cdots$ & $\cdots$ & $\left\{\begin{array}{r}\text { Few } \\
\text { Few } \\
2\end{array}\right.$ & $\begin{array}{c}(\mathrm{Ci}-\mathrm{S}) \\
(\mathrm{Ci}-\mathrm{Cu}) \\
\mathrm{S}-\mathrm{Cu}\end{array}$ & $\left.\begin{array}{l}\mathbf{N} \\
\mathbf{N} \\
\mathbf{N}\end{array}\right\}$ & 2 & \\
\hline 9 & 0 & $\ldots$ & ... & $\cdots$ & ... & $\cdots$ & Few & $(\mathrm{A}-\mathrm{Cu})^{*}$ & $\mathrm{~N}$ & I & Fog $18: 30$ to $22: 00$. \\
\hline Io & Few & $(\mathrm{Ci}-\mathrm{S})$ & $\mathbf{N}$ & $\ldots$ & $\ldots$ & $\ldots$ & Io & $\mathbf{S}^{*}$ & E & 6 & Fog from Io:00. \\
\hline II & $\ldots$ & * & $\ldots$ & $\ldots$ & ... & ... & Io & S & $\mathbf{E}$ & 7 & Fog to $15: 40$ \\
\hline I2 & Io & $\mathbf{s}$ & $\mathbf{E}$ & ... & ... & ... & $\cdots$ & ** & $\cdots$ & Io & Fog from I $5: 40$ \\
\hline I3 & Io & $\mathbf{S}^{*}$ & $\ldots$ & $\ldots$ & $\ldots$ & ... & I0 & $\mathbf{S}$ & ... & Io & Fog to $14: 30$ \\
\hline I4 & Io & $\mathbf{S}^{*}$ & $\ldots$ & $\ldots$ & ... & $\cdots$ & Io & $\mathbf{S}$ & W & 8 & Fog to $\mathrm{I}_{4}: 00$ \\
\hline 15 & I0 & $\mathbf{S}$ & NIV & $\ldots$ & .. & ... & ... & ** & ... & I0 & Dense fog from $5: 30$ \\
\hline I6 & IO & $\mathbf{N}^{*}$ & ... & ... & ... & ... & Io & $\mathbf{s}$ & $\cdots$ & 10 & Dense fog to $8: 40$ \\
\hline 17 & $\begin{array}{r}10 \\
2\end{array}$ & $\begin{array}{c}S \\
(\mathrm{Ci})\end{array}$ & $\ddot{N}$ & ... & $\cdots$ & $\cdots$ & $\left\{\begin{array}{l}4 \\
4\end{array}\right.$ & $\underset{\mathrm{S}}{\mathrm{S}-\mathrm{Cu}}$ & W $\quad ?$ & IO & Light winds. \\
\hline 18 & $\left\{\begin{array}{l}\text { Few } \\
\text { Few }\end{array}\right.$ & $\begin{array}{c}\text { S } \\
\text { S }\end{array}$ & $\mathrm{N}$ & $\cdots$ & ... & $\cdots$ & $\begin{array}{r}\text { IO } \\
2\end{array}$ & $\begin{array}{c}\mathrm{S} \\
(\mathrm{Ci}-\mathrm{S})\end{array}$ & $\begin{array}{l}\mathrm{W} \\
\mathrm{NW} \cdot \mathrm{l}\end{array}$ & 4 & Fog $20: 30$ to $21: 15$ \\
\hline I9 & I0 & $\mathbf{S}$ & W & $\ldots$ & $\cdots$ & $\cdots$ & $\begin{array}{l}3 \\
3\end{array}$ & $\begin{array}{l}\text { S } \\
\text { S-Cu }\end{array}$ & $\left.\begin{array}{l}\mathrm{NW} \\
\mathrm{NW}\end{array}\right\}$ & 9 & \\
\hline 20 & $\left\{\begin{array}{l}3 \\
6\end{array}\right.$ & $\begin{array}{l}\text { S-Cu } \\
\text { S }\end{array}$ & $\begin{array}{l}\text { W } \\
\text { W }\end{array}$ & $\cdots$ & $\cdots$ & $\cdots$ & Io & s & W & 8 & Fog to south all day. \\
\hline $2 I$ & 10 & $\mathbf{S}$ & W & .. & ... & $\cdots$ & 10 & $\mathrm{~N}^{*}$ & $\cdots$ & Io & Foggy; light variable winds. \\
\hline 22 & I0 & $N^{* *}$ & $\cdots$ & $\ldots$ & $\ldots$ & ... & Io & $N^{* *}$ & $\cdots$ & Io & Generally foggy. \\
\hline 23 & 10 & $\mathrm{N}^{* *}$ & W & $\cdots$ & $\cdots$ & $\cdots$ & Io & $\mathbf{S}$ & W & 8 & Fog to $13: 20$ and $16: 30$ to $16: 50$. \\
\hline 24 & $\cdots$ & $* *$ & $\cdots$ & $\ldots$ & $\ldots$ & $\ldots$ & 10 & $\mathbf{S}^{*}$ & $\mathbf{E}$ & 6 & Fog from $5: 00$ \\
\hline 25 & Io & $\mathbf{N}^{*}$ & $\cdots$ & $\cdots$ & $\cdots$ & $\cdots$ & $\begin{array}{l}4 \\
3\end{array}$ & $\underset{\mathrm{S}}{\mathrm{S}-\mathrm{Cul}}$ & $\left.\begin{array}{c}\mathrm{NW} \\
\mathrm{WNW}\end{array}\right\}$ & 8 & Fog to $18: 00$ \\
\hline 26 & 10 & $\mathbf{S}^{*}$ & $\ldots$ & ... & $\cdots$ & $\ldots$ & $\ldots$ & ** & $\ldots$ & 10 & Fog from $2: 00$ \\
\hline 27 & Io & $\mathbf{S}^{*}$ & $\mathrm{E}$ & ... & $\ldots$ & $\cdots$ & $\left\{\begin{array}{l}3 \\
1\end{array}\right.$ & $\begin{array}{l}(\mathrm{A}-\mathrm{Cu}) \\
\mathrm{S}\end{array}$ & $\begin{array}{ll}\mathbf{E} & \} \\
\mathbf{D} & \}\end{array}$ & 2 & Fog to Io:00. \\
\hline 28 & 10 & $\mathbf{N}^{*}$ & SE & $\cdots$ & $\cdots$ & ... & $\ldots$ & ** & $\cdots$ & 7 & Fog 8:00 to $19: 30$ \\
\hline 29 & $\cdots$ & ** & $\cdots$ & $\ldots$ & $\ldots$ & $\cdots$ & $\cdots$ & $* *$ & $\cdots$ & Io & Fog all day. \\
\hline 30 & 0 & $\cdots$ & $\cdots$ & $\cdots$ & $\cdots$ & $\cdots$ & $\cdots$ & $\cdots$ & $\cdots$ & $\cdots$ & Dense fog $\mathbf{A} . \mathbf{M}$. \\
\hline 31 & $\cdots$ & $\cdots$ & $\cdots$ & $\cdots$ & $\cdots$ & $\cdots$ & $\cdots$ & $\cdots$ & $\cdots$ & $\cdots$ & \\
\hline Sum.... & 221 & $\cdots$ & $\cdots$ & $\cdots$ & $\cdots$ & $\cdots$ & 187 & $\cdots$ & $\cdots$ & 231 & \\
\hline Mean... & 8.2 & $\cdots$ & $\cdots$ & $\cdots$ & $\cdots$ & $\cdots$ & 8.5 & $\cdots$ & $\cdots$ & 8.0 & \\
\hline
\end{tabular}

* Light fog. ** Dense fog.

Cloud characters enclosed in parentheses, thiss ( $\mathrm{Ci}-\mathrm{Cu}$ ), refer to upper clouds, all other references being to lower clouds. 


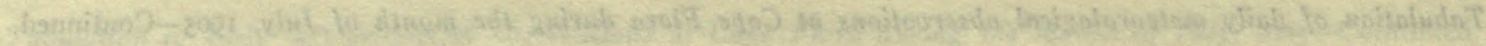

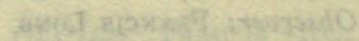

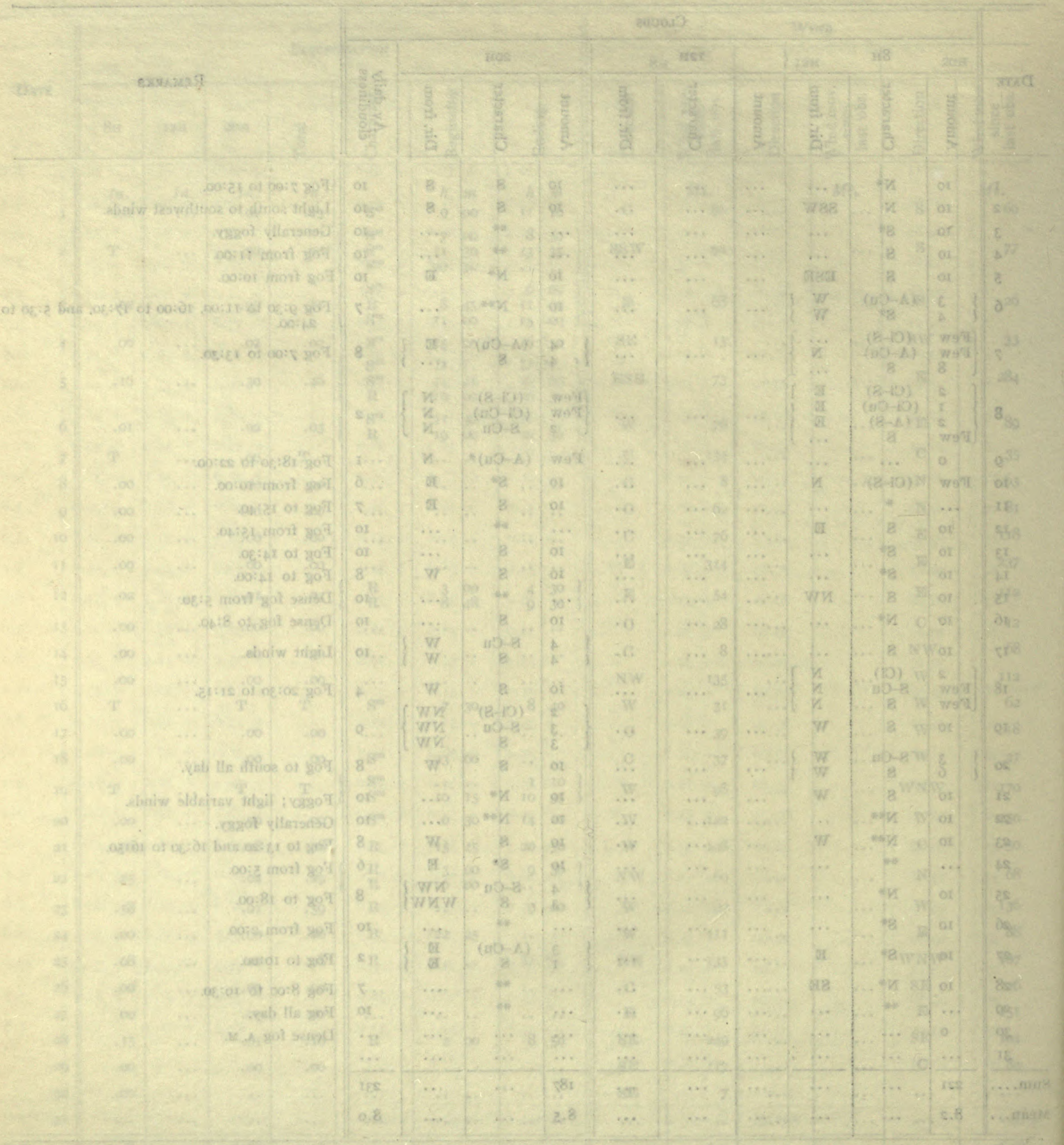




\title{
METEOROLOGICAL OBSERVATIONS
}

\section{TABULATION OF DAILY WIND RECORDS}

\author{
REGISTERED AT
}

TEPLITZ BAY STATION, RUDOLPH ISLAND

FRANZ JOSEF ARCHIPELAGO

SEPTEMBER 1, 1903, TO MAY 26, 1905

NORTH LaTITUDE: $81^{\circ} 47 .^{\prime} 6$

LONGITUDE EAST OF GREENWICH: $57^{\circ} 56^{\prime}$ 


\title{
2ИOITAVЯ3280 JADIDOJOЯOJTHM
}

\section{G90039 GMIW YIIAG 70 VIOITAJUAAT}

\author{
TA 939สT21038
}

QMAJ21 H9.OQUA ИOITAT2 YÅ STI.193T

ODA.139IHОЯA $7320 L$ ГИАЯन

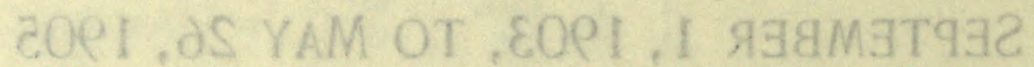

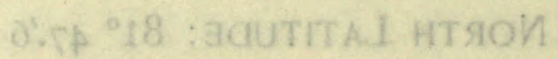

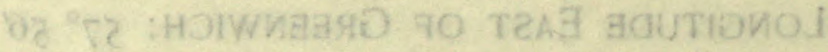




\section{WiNd MOVEMENT}

Mi. Mi. Mi. Mi. Mi. Mi. Mi. Mi. Mi. Mi. Mi. Mi. Mi. Mi. Mi. Mi. Mi. Mi. Mi. Mi. Mi. Mi. Mi. Mi. $\begin{array}{lllllllllllllllllllllllllll}1 & 9 & 13 & 11 & 10 & 9 & 8 & 20 & 17 & 23 & 26 & 27 & 21 & 18 & 14 & 12 & 11 & 6 & 21 & 11 & 10 & 15 & 18 & 18 & 13\end{array}$ \begin{tabular}{l|lllllllllllllllllllllllll}
2 & 22 & 25 & 13 & 8 & I0 & 19 & 17 & 26 & 32 & 28 & 41 & 43 & 32 & 28 & 31 & 36 & 32 & 35 & 38 & 51 & 51 & 41 & 36 & 34
\end{tabular} \begin{tabular}{l|llllllllllllllllllllllll}
3 & 32 & 29 & 25 & 27 & 23 & 24 & 27 & 29 & 22 & 23 & 26 & 27 & 28 & 25 & 19 & 22 & 22 & 21 & 21 & 19 & 15 & 17 & 16 & 9
\end{tabular} $\begin{array}{llllllllllllllllllllllllllll}4 & 7 & 7 & 8 & 9 & 6 & 6 & 6 & 4 & 7 & 10 & 12 & 13 & 15 & 12 & 9 & 8 & 9 & 7 & 5 & 7 & 7 & 6 & 5 & 11\end{array}$ 
Tabulation of hourly wind records at Teplitz Bay during the month of October, 1903

\section{WIND MOVEMENT}

Date

For the hour preceding

Daily

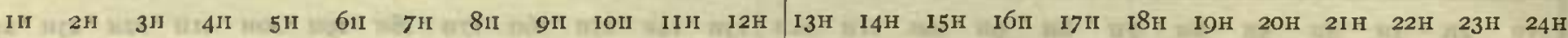

Mi. Mi. Mi. Mi. Mi. Mi. Mi. Mi. Mi. Mi. Mi. Mi. Mi. Mi. Mi. Mi. Mi. Mi. Mi. Mi. Mi. Mi. Mi. Mi. $\begin{array}{lllllllllllllllllllllll}23 & 22 & 25 & 30 & 4 \mathrm{I} & 50 & 47 & 50 & 44 & 13 & 24 & 29 & 28 & 28 & 24 & 22 & 20 & 17 & 14 & 16 & 15 & 15 & 15\end{array}$

$\begin{array}{llllllllllll}6 & 8 & 9 & 16 & 16 & 21 & 23 & 23 & 16 & 18 & 18 & 22\end{array}$

$\begin{array}{llllllllllll}12 & 2 I & 2 I & 5 & 2 & 7 & 4 & 6 & 8 & 5 & 7 & 5\end{array}$

$\begin{array}{llllllllllll}3 & 2 & 2 & 3 & 3 & 13 & 13 & 20 & 26 & 17 & 23 & 22\end{array}$

2I $16 \quad 15 \quad$ II

4

$\begin{array}{llllllllllll}6 & 5 & 4 & 6 & 5 & 6 & 6 & 15 & 13 & 6 & 4 & 4\end{array}$

367

$\begin{array}{llllllllllll}13 & 15 & 15 & 17 & 27 & 29 & 29 & 32 & 33 & 37 & 37 & 37\end{array}$

$\begin{array}{llllllllllll}33 & 36 & 36 & 35 & 35 & 31 & 28 & 33 & 34 & 29 & 23 & 15\end{array}$

$\begin{array}{llllllllllll}13 & \text { I5 } & 12 & 8 & 9 & 10 & 8 & 7 & 6 & 5 & 5 & 5\end{array}$

76

$\begin{array}{lllll}3 & 1 & 2 & 6 & 13\end{array}$

10

$\begin{array}{llllllllllll}3 & 2 & 3 & 4 & 3 & 6 & 5 & 10 & 9 & 11 & 21 & 29\end{array}$

$3 \quad 3 \quad 3$

I I I

$\begin{array}{llllllllllll}4 & 8 & 10 & 18 & 15 & 19 & 9 & 13 & 15 & 21 & 22 & 15\end{array}$

15

$$
\begin{array}{lllllllll}
5 & 16 & 18 & 20 & 19 & 20 & 17 & 18 & 14
\end{array}
$$

$$
\begin{array}{lllll}
6 & 6 & 8 & 12
\end{array}
$$




\section{WIND MOVEMENT}

Mi. Mi. Mi. Mi. Mi. Mi. Mi. Mi. Mi. Mi. Mi. Mi. Mi. Mi. Mi. Mi. Mi. Mi. Mi. Mi. Mi. Mi. Mi. Mi. Mi.

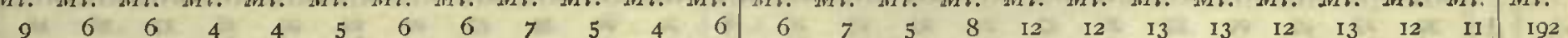

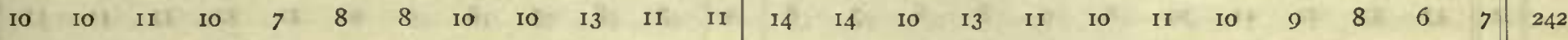
$\begin{array}{llllllllllllllllllllllllllll}8 & 5 & 6 & 8 & 8 & 6 & 6 & 9 & 8 & 7 & 8 & \text { 10 } & 12 & 13 & 10 & \text { 10 } & 9 & 6 & 4 & 4 & 7 & 7 & 9 & 9 & 189\end{array}$ $\begin{array}{lllllllllllllllllllllllll}13 & 12 & 10 & 6 & 5 & 5 & 5 & 4 & 5 & 1 & 2 & 1 & 3 & 5 & 5 & 6 & 8 & 10 & 10 & 4 & 7 & 8 & 9 & 1\end{array}$ 
Tabulation of hourly wind records at Teplitz Bay during the month of Deccmber, 1903

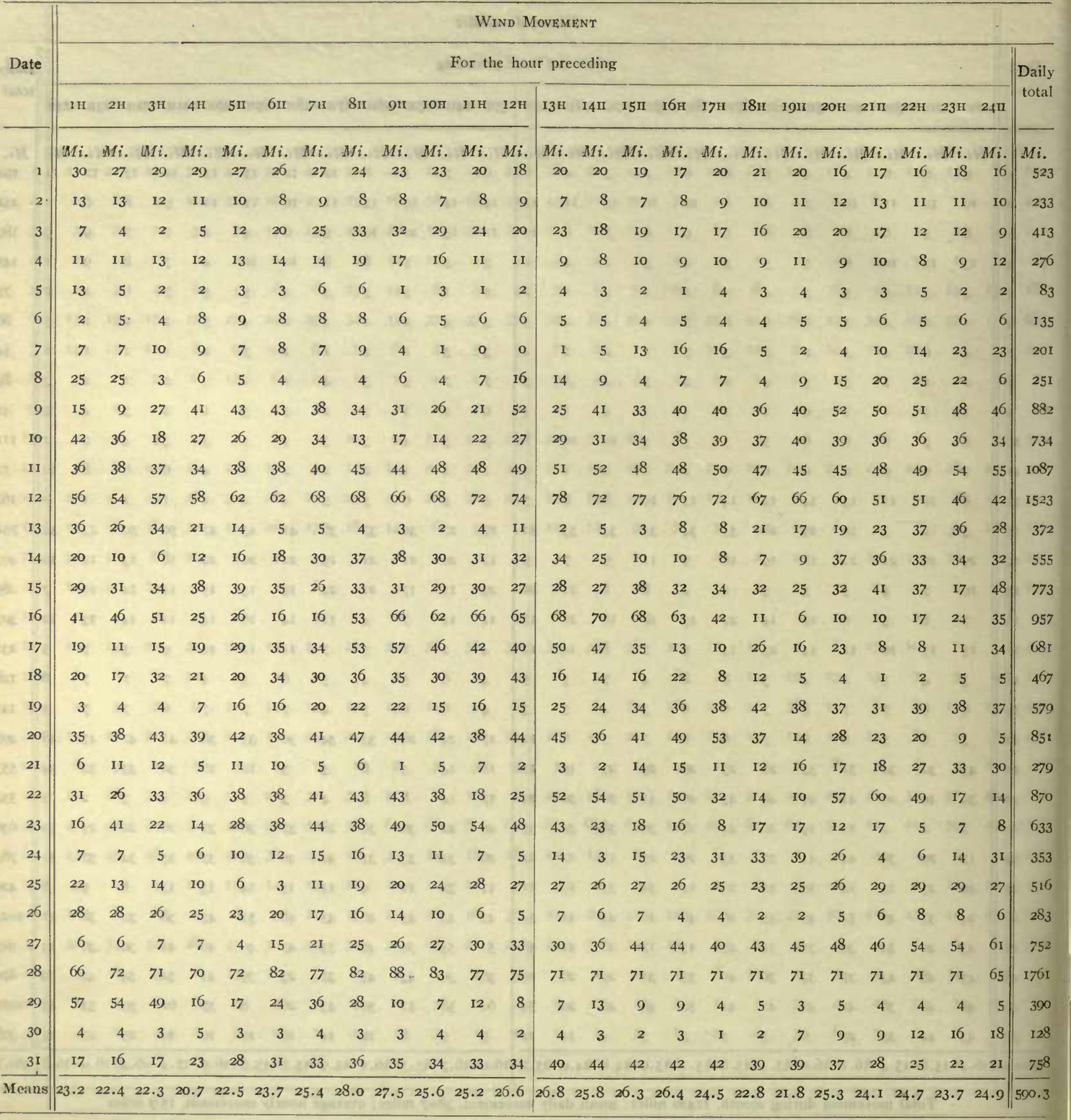

Total movement during month, 18299 miles; mean daily movement, 590.3 miles; average hourly movement, 24.6 miles 
Tabulation of hourly zvind records at Tepitz Bay during the month of January, 1904

\section{WIND MOVEMENT}

Mi. Mi. Mi. Mi. Mi. Mi. Mi. Mi. Mi. Mi. Mi. Mi. Mi. Mi. Mi. Mi. Mi. Mi. Mi. Mi. Mi. Mi. Mi. Mi.

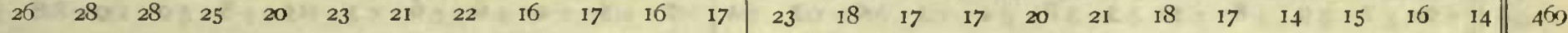


Tabulation of hourly zoind records at Teplitz Bay during the month of February, 1904

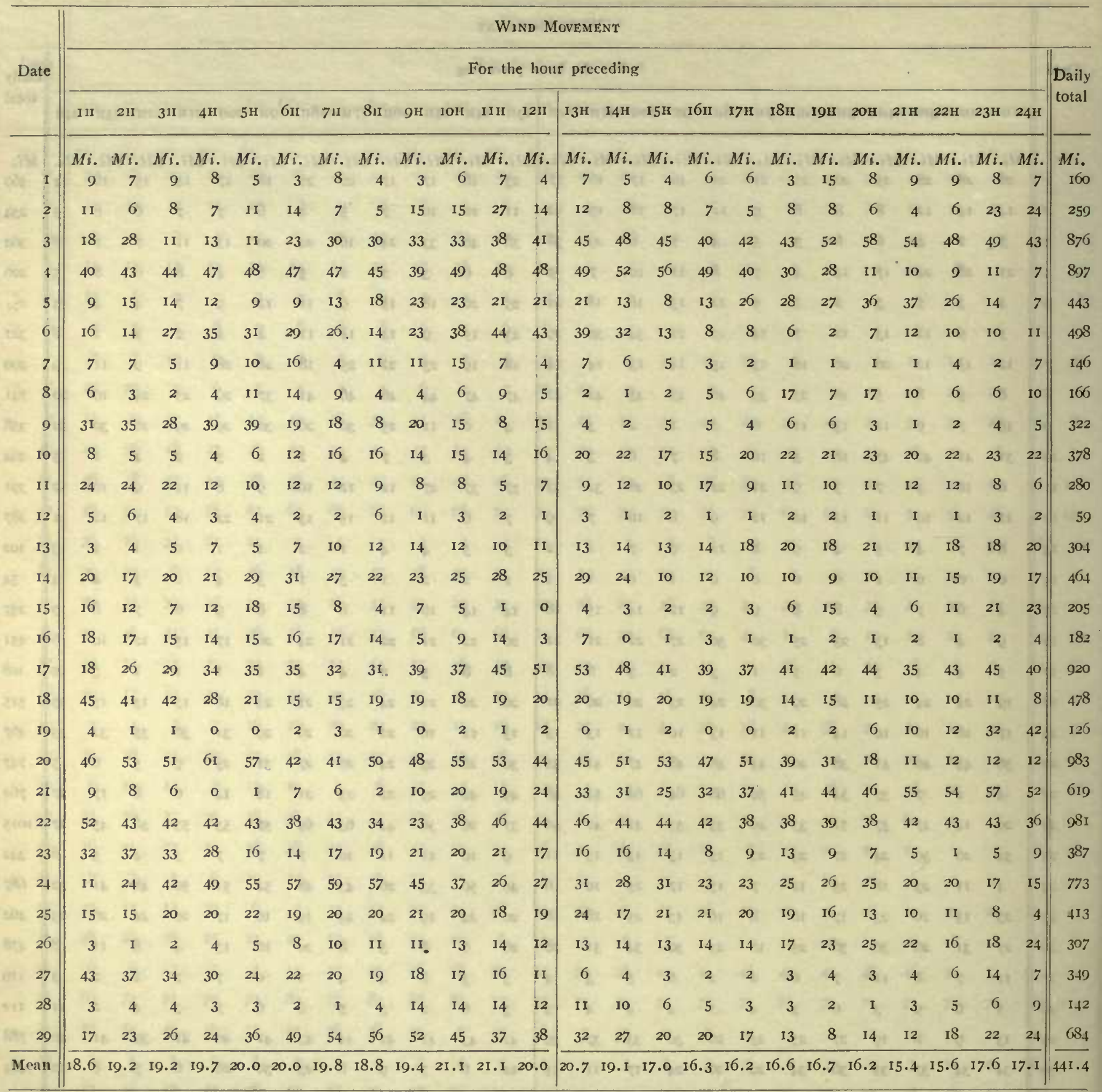


Tabulation of hourly roind records at Teplitz Bay during the month of March, 1904

WIND MOVEMENT

For the hour preceding

Daily

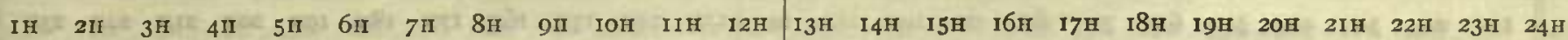
total

Mi. Mi. Mi. Mi. Mi. Mi. Mi. Mi. Mi. Mi. Mi. Mi. Mi. Mi. Mi. Mi. Mi. Mi. Mi. Mi. Mi. Mi. Mi. Mi. Mi.

$\begin{array}{lllllllllllll}\text { I } & 15 & 18 & 12 & 5 & 4 & 2 & 7 & 3 & 4 & 2 & 14 & 22\end{array}$

$\begin{array}{llllllllllll}2 \text { I I7 } & \text { I6 } & \text { II } & 9 & 8 & 6 & \text { I } & \text { I } & 8 & 3 & 8\end{array}$

217

2

12129 I0

9 II I0 99 I0 I7

$\begin{array}{llllllllllll}20 & \text { I9 } & \text { I2 } & \text { II } & \text { I9 } & 15 & \text { I4 } & \text { II } & 10 & 14 & 15 & 14\end{array}$

295

3

I5 10 $\begin{array}{llllllllllll}25 & 35 & 40 & 38 & 33 & 33 & 37 & 39 & 41 & 40 & 39 & 36\end{array}$ $\begin{array}{llllllllllll}6 & 7 & 2 & 22 & 43 & 58 & 61 & 65 & 54 & 32 & 19 & 20\end{array}$

\begin{tabular}{lllllllllllll|}
28 & I0 & 15 & 14 & 13 & 13 & 9 & 12 & 18 & 22 & 22 & 20 & 19
\end{tabular}

\begin{tabular}{l|llllllllllll|}
29 & 5 & 6 & 7 & 4 & 3 & 3 & 8 & 5 & 6 & 1 & 4 & 2
\end{tabular}

\begin{tabular}{l|llllllllllll|}
30 & 48 & 47 & 53 & 47 & 23 & 23 & 22 & 28 & 30 & 27 & 18 & 14
\end{tabular}

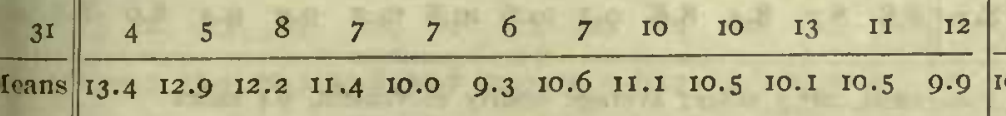

$\begin{array}{llllllllll}8 & 12 & 19 & 18 & 22 & 20 & 12 & 2 & \text { I } & 0\end{array}$

76

$2^{-1}$

3

$\begin{array}{ll}\text { I } & \text { I } \\ 5 & 6\end{array}$

$$
\begin{aligned}
& 1 \\
& 10 \\
& 2
\end{aligned}
$$$$
2
$$$$
\begin{array}{ll}
1 & 2 \\
1 & 1
\end{array}
$$$$
3
$$$$
\begin{array}{llllllllllll|l}
65 & 61 & 55 & 53 & 49 & 4 \mathrm{I} & 30 & 23 & 10 & 5 & 4 & 5 & 1093
\end{array}
$$$$
\begin{array}{llllllllllll||l}
\text { I8 } & 18 & 22 & 20 & 21 & 27 & 29 & 28 & 24 & 19 & 19 & 17 & 349
\end{array}
$$$$
\begin{array}{llllllllllll||l}
19 & 22 & 22 & 26 & 26 & 27 & 26 & 25 & 25 & 28 & 27 & 24 & 513
\end{array}
$$$$
\begin{array}{llllllllllll}
\text { I7 } & \text { I6 } & \text { I2 } & \text { II } & 10 & 9 & 8 & 4 & 4 & 5 & 5 & 4
\end{array}
$$ 
Tabulation of hourly zuind records at Teplitz Bay during the month of May, 1904

\section{WIND MOVEMENT}

Mi. Mi. Mi. Mi. Mi. Mi. Mi. Mi. Mi. Mi. Mi. Mi.

Mi. Mi. Mi. Mi. Mi. Mi. Mi. Mi. Mi. Mi. Mi. Mi. Mi. 
Tabulation of hourly wind records at Teplitz Bay during the month of June, 1904

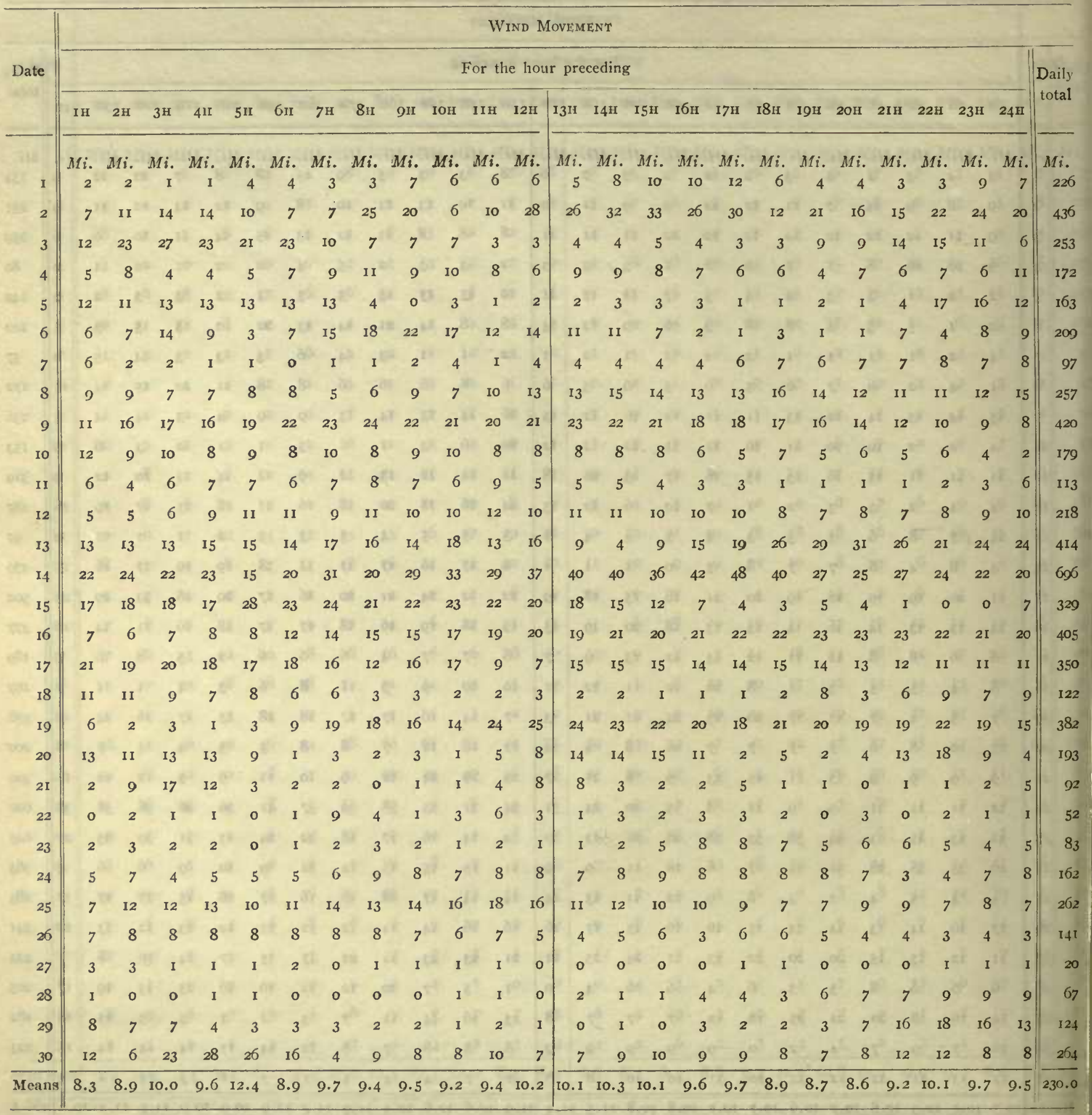

Total movement during month, 690I miles; mean daily movement, 230.0 miles; average hourly movement, 9.6 miles 
Tabulation of hourly wind records at Teplitz Bay during the month of July, Ig04

\section{WIND MONEMENT}

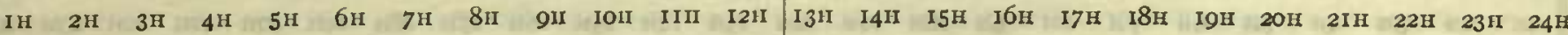

Mi. Mi. Mi. Mi. Mi. Mi. Mi. Mi. Mi. Mi. Mi. Mi.

Mi. Mi. Mi. Mi. Mi. Mi. Mi. Mi. Mi. Mi. Mi. Mi. Mi.

3

(1)

I

18

Ig

20

2 I

22

23

24

25

2

27

28

29

30 $\begin{array}{llllllllllll}5 & 3 & 2 & \mathrm{I} & 0 & \mathrm{I} & 4 & 6 & 8 & \text { 10 } & 12 & \text { IJ }\end{array}$ $\begin{array}{llllllllllll}7 & 13 & \text { II } & 9 & 7 & 8 & 7 & 9 & 7 & 6 & 8 & 6\end{array}$ $\begin{array}{llllllllllll}9 & 7 & 3 & 8 & 7 & 8 & 9 & 6 & 7 & 8 & 9 & 8\end{array}$ $\begin{array}{llllllllllll}5 & 5 & 6 & 5 & 3 & 6 & 5 & 8 & 9 & \text { IO } & \text { II } & \text { I3 }\end{array}$ $\begin{array}{llllllllllll}2 & 1 & 1 & 2 & 2 & 3 & 2 & 3 & 4 & 4 & 4 & 4\end{array}$

$\begin{array}{llllllllllll}\text { I3 } & 9 & 9 & 9 & 8 & \text { 10 } & 7 & 8 & 6 & 5 & 4 & 4\end{array}$ $\begin{array}{llllllllllll}7 & 8 & 23 & 23 & \text { I8 } & \text { II } & \text { I7 } & \text { I2 } & \text { I5 } & 9 & \text { 10 } & 8\end{array}$ $\begin{array}{llllllllllll}13 & 10 & 9 & 7 & 6 & 8 & \text { 10 } & \text { I3 } & \text { I } 8 & 5 & \text { II } & 10\end{array}$ $\begin{array}{llllllllllll}0 & \mathrm{I} & 0 & 2 & \mathrm{I} & 2 & 3 & 5 & 3 & 9 & 8 & 5\end{array}$

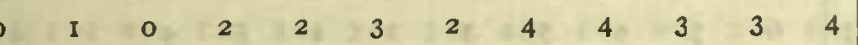
$\begin{array}{llllllllllll}2 & 0 & 0 & \text { I } & 4 & 5 & 7 & 9 & 8 & 9 & 7 & 5\end{array}$ $\begin{array}{llllllllllll}6 & 5 & 6 & 5 & 7 & 5 & 7 & 9 & 9 & 9 & 9 & \text { II }\end{array}$ $\begin{array}{llllllllllll}8 & 9 & 8 & 8 & 7 & 5 & 5 & 4 & 6 & 6 & 6 & 7\end{array}$ 5

$$
\text { (7) }
$$$$
5
$$$$
\text { I } 4
$$$$
343
$$$$
56
$$$$
3
$$$$
\text { I }
$$$$
\text { Io }
$$$$
5
$$$$
3
$$

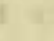$$
67
$$$$
6
$$$$
3
$$$$
2
$$$$
3
$$$$
\begin{array}{lllllllllll|l}
14 & 16 & 16 & 16 & 14 & 13 & 10 & 9 & 4 & 6 & 6 & 2
\end{array}
$$$$
\begin{array}{llllllllllll|l}
7 & 9 & 10 & 7 & 6 & 7 & 6 & 5 & 7 & 4 & 6 & 5 & 177
\end{array}
$$$$
\begin{array}{llllllllllll}
9 & \text { IO } 9 & \text { IO II } & \text { II } & \text { IO } & 8 & 8 & 8 & 5 & 6
\end{array}
$$$$
\text { II II II IO } 9 \quad 9 \quad \begin{array}{lllllll} 
& 7 & 4 & 5 & 6 & 5 & 3
\end{array}
$$$$
\begin{array}{llllllllllll}
4 & 5 & 7 & 8 & 7 & 9 & 9 & \text { II } & \text { II } & \text { II } & \text { I2 } & \text { I2 }
\end{array}
$$$$
2
$$$$
66
$$$$
\text { 10 } 8
$$$$
77
$$$$
45
$$$$
56
$$$$
\text { II }
$$$$
7
$$$$
\text { II II }
$$$$
3
$$$$
\text { . }
$$

$$
7
$$$$
5
$$$$
4
$$$$
\begin{array}{llllllll}
5 \mathrm{I} & 49 & 4 \mathrm{I} & 20 & 38 & 43 & 53 & 57
\end{array}
$$

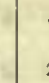$$
\begin{array}{llllllllllll}
57 & 54 & 50 & 35 & 40 & 47 & 51 & 46 & 45 & 47 & 40 & 3 \mathrm{I}
\end{array}
$$$$
28 \quad 29
$$$$
27
$$

$$
\begin{array}{lllllll}
3 & 4 & 4 & 3 & 3 & 3 & 1
\end{array}
$$

4

$$
4
$$$$
7 \mathrm{I}
$$$$
4
$$$$
4
$$$$
5
$$$$
9
$$$$
\begin{array}{rrr}
5 & 4 & 3 \\
4 & 4
\end{array}
$$

\section{$-$}

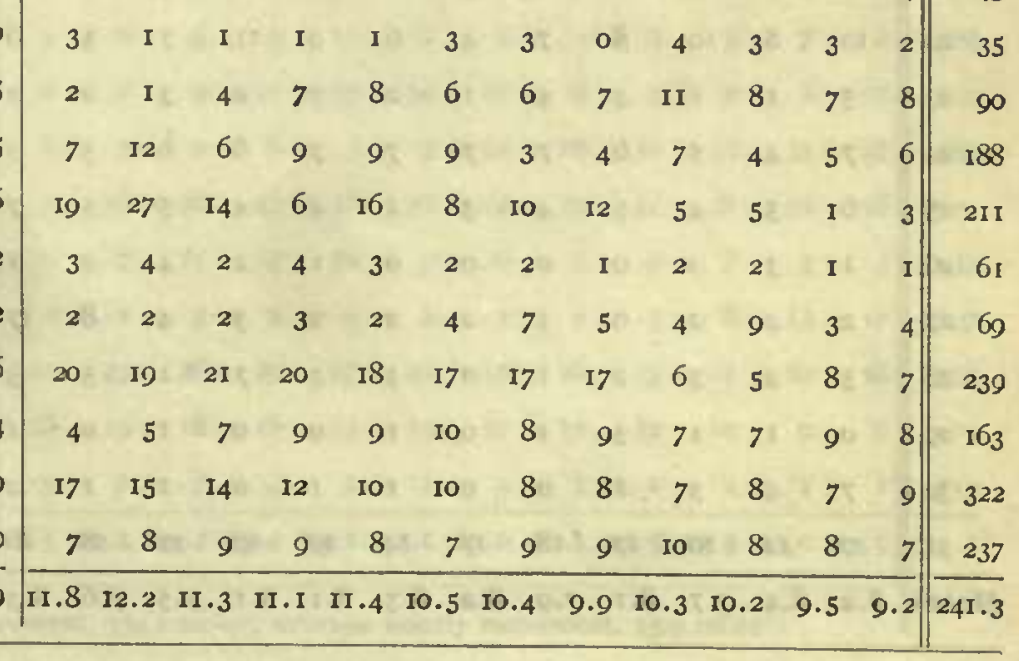

Total movement during month, 7480 niles; mean daily movement, $24 \mathrm{I} \cdot 3$ miles; average hourly movement, IO.I miles. 


\section{WIND MOVEMENT}

Mi. Mi. Mi. Mi. Mi. Mi. Mi. Mi. Mi. Mi. Mi. M. Mi. Mi. Mi. Mi. Mi. Mi. Mi. Mi. Mi. Mi. Mi. Mi. $\begin{array}{llllllllllllllllllllllll}6 & 6 & 4 & 6 & 5 & 6 & 12 & 12 & 11 & 15 & 10 & 8 & 11 & 12 & 13 & 7 & 6 & 6 & 6 & 7 & 7 & 5 & 4 & 3\end{array}$ \begin{tabular}{llllllllllll|llllllllllll}
0 & 4 & 5 & 2 & 2 & 7 & 9 & 8 & 6 & 9 & 12 & 11 & 10 & 8 & 14 & 17 & 17 & 16 & 17 & 20 & 17 & 15 & 15 & 15
\end{tabular} $\begin{array}{llllllllllll}15 & 16 & 14 & 15 & 21 & 20 & 17 & 19 & 17 & 12 & 17 & 17\end{array}$ $\begin{array}{llllllllllll}16 & 19 & 20 & 21 & 18 & 16 & 15 & 12 & 14 & 14 & 13 & 12\end{array}$ 
Tabulation of hourly wind records at Teplitz Bay during the month of September, 1904

\section{WiNd MOVEMEN'S}

IH $2 \mathrm{H} \quad 3 \mathrm{II} \quad 4 \mathrm{II} \quad 5 \mathrm{H} \quad 6 \mathrm{H} \quad 7 \mathrm{H} \quad 8 \mathrm{H}$ 9H IOH IIH I2H $13 \mathrm{II}$ I4H I5H I6II I7II ISII I9II $201 \mathrm{II}$ IH $221123 \mathrm{H} 24 \mathrm{II}$

Mi. Mi. Mi. Mi. Mi. Mi. Mi. Mi. Mi. Mi. Mi. Mi. Mi. Mi. Mi. Mi. Mi. Mi. Mi. Mi. Mi. Mi. Mi. Mi. $M i$.

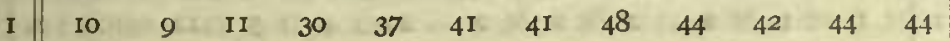

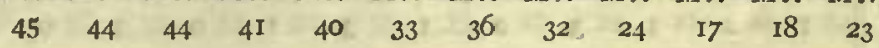

\begin{tabular}{l|llllllllllll}
23 & 24 & 26 & 29 & 27 & 19 & I8 & 23 & 24 & 24 & 29 & 26
\end{tabular}

20

23 $\begin{array}{rrrrrrrrrrrr}9 & 11 & 14 & 15 & \text { I6 } & \text { I4 } & 8 & 2 & 6 & 5 & 7 & 5 \\ & 9 & \text { I4 } & \text { I4 } & \text { I2 } & \text { I2 } & \text { 10 } & \text { I0 } & \text { I4 } & \text { I3 } & \text { II }\end{array}$

26 
Tabulation of hourly wind records at Teplitz Bay during the month of October, 1904

\section{Wind Movement}

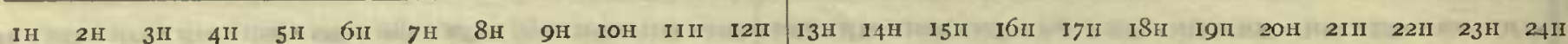

Mi. Mi. Mi. Mi. Mi. Mi. Mi. Mi. Mi. Mi. Mi. Mi. Mi. Mi. Mi. Mi. Mi. Mi. Mi. Mi. Mi. Mi. Mi. Mi.

I $\begin{array}{llllllllllll}I & 2 & I & 3 & 2 & \text { I } & 3 & \text { I } & 0 & 3 & 0 & \text { I }\end{array}$ 
Tabulation of hourly wind records at Teplitz Bay during the month of November, I904

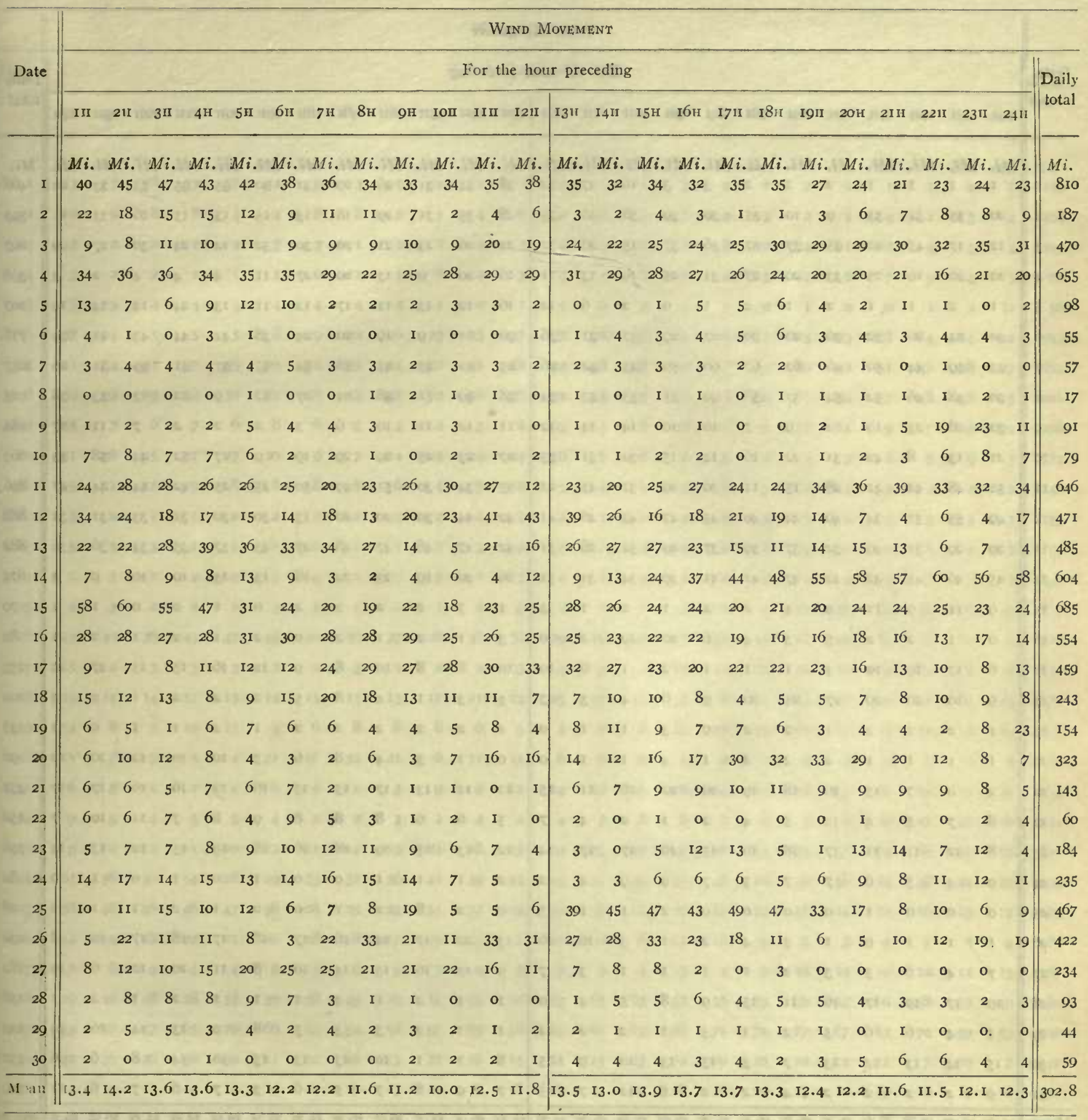

Total movement during month, 9084 miles; mean daily movement, 302.8 miles; average hourly movement, I2.6 miles 
Tabulation of hourly wind records at Teplitz Bay during the month of December, 1904

\section{Wind MOVEMENT}

Date

For the hour preceding

Daily

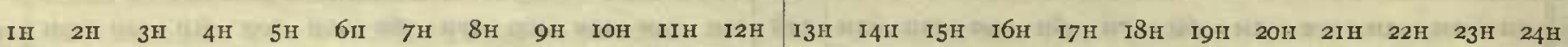

Mi. Mi. Mi. Mi. Mi. Mi. Mi. Mi. Mi. Mi. Mi. Mi. Mi. Mi. Mi. Mi. Mi. Mi. Mi. Mi. Mi. Mi. Mi. Mi.

$M i$.

$\begin{array}{lllllllllllllllllllllllll}\text { I } & \text { I } & \text { I } & \text { I } & 2 & 2 & 2 & 3 & 3 & \text { I } & 27 & 32 & 38 & 34 & 3 \mathrm{I} & 26 & 29 & 3 \mathrm{I} & 30 & 35 & 35 & 33 & 33 & 22\end{array}$ $\begin{array}{llllllllllll}20 & 35 & 34 & 33 & 9 & \text { 10 } & 35 & 30 & 39 & 38 & 40 & 35\end{array}$

$\begin{array}{llllllllllll}\text { I2 } & \text { I7 } & 45 & 42 & 35 & 37 & 37 & 36 & 37 & 15 & -26 & 32\end{array}$

$\begin{array}{llllllllllll}28 & 35 & 31 & 29 & 18 & \text { I3 } & \text { I5 } & \text { I3 } & \text { I3 } & \text { I6 } & \text { I3 } & \text { II }\end{array}$

408

3

$\begin{array}{llllllllllll}22 & 20 & 19 & 25 & 33 & 20 & 27 & 31 & 25 & 19 & 13 & \text { II }\end{array}$

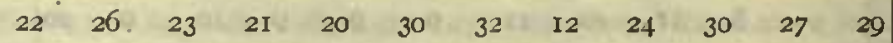

593

667

5

$\begin{array}{llllllllllll}\text { I } & 2 & \text { I } & \text { I } & 2 & \text { I } & 2 & \text { I } & 0 & 2 & 6 & \text { I2 }\end{array}$

$\begin{array}{llllllllllll}\text { I6 } & 20 & 9 & \text { I3 } & 20 & 27 & \text { II } & 4 & 4 & 4 & 2 & 3\end{array}$

398

6.

$\begin{array}{llllllllllll}30 & 24 & 26 & 32 & 36 & 20 & 30 & 27 & 29 & 27 & 31 & 36\end{array}$

$\begin{array}{llllllllllll}16 & 12 & I 3 & \text { II } & \text { I7 } & \text { I2 } & \text { II } & 13 & 24 & \text { I4 } & \text { I4 } & \text { I9 }\end{array}$

207

7.

$\begin{array}{llllllllllll}33 & 39 & 44 & 50 & 46 & 62 & 67 & 66 & 59 & 45 & 32 & 26\end{array}$

$\begin{array}{llllllllllll}30 & 48 & 46 & 52 & 54 & 57 & 58 & 49 & 55 & 55 & 53 & 34\end{array}$

$\begin{array}{llllllllllll}32 & 28 & 23 & \text { I9 } & 12 & 9 & 7 & \text { I0 } & \text { I0 } & \text { I4 } & \text { I4 } & 12\end{array}$

$\begin{array}{llllllllllll}21 & 13 & 8 & 42 & 31 & 22 & 16 & \text { I4 } & \text { I5 } & 32 & 3 \mathrm{I} & 33\end{array}$

$\begin{array}{llllllllllll}52 & 48 & 42 & 4 I & 28 & \text { I5 } & \text { II } & 36 & 29 & 5 \text { I } & 45 & 22\end{array}$

$\begin{array}{llllllllllll}49 & 55 & 5 \mathrm{I} & 34 & 40 & 44 & 40 & 45 & 45 & 43 & 46 & 4 \mathrm{I}\end{array}$

$\begin{array}{llllllllllll}29 & 29 & 31 & 29 & 32 & 37 & 39 & 37 & 29 & 31 & 28 & 31\end{array}$

$\begin{array}{llllllllllll}45 & 46 & 45 & 42 & 42 & 47 & 42 & 4 I & 39 & 34 & 34 & 31\end{array}$

$\begin{array}{llllllllllll}30 & 21 & 9 & \text { I9 } & 20 & 24 & 38 & 42 & 44 & 43 & 4 \mathrm{I} & 39\end{array}$

718

$\begin{array}{llllllllllll}25 & 42 & 45 & 45 & 58 & 54 & 55 & 37 & 31 & 39 & 31 & 26\end{array}$

$\begin{array}{llllllllllll}38 & 39 & 51 & 38 & 24 & 27 & \text { I2 } & 9 & \text { I2 } & \text { I3 } & 33 & 38\end{array}$

1057

$\begin{array}{llllllllllll}\text { II } & 12 & \text { II } & \text { IO } & 6 & 3 & 2 & 2 & 2 & 7 & \text { II } & \text { I7 }\end{array}$

925

284

$\begin{array}{llllllllllll}27 & 23 & 25 & 29 & 29 & 19 & 9 & 17 & 52 & 44 & 58 & 53\end{array}$

$\begin{array}{llllllllllll}35 & 34 & 30 & 5 I & 49 & 50 & 25 & 25 & 42 & 44 & 44 & 47\end{array}$

$\begin{array}{llllllllllll}42 & 44 & 30 & 20 & 20 & \text { I } 3 & 30 & 30 & 31 & 33 & 31 & 3 I\end{array}$

$\begin{array}{llllllllllll}42 & 47 & 48 & 47 & 48 & 47 & 43 & 37 & 35 & 34 & 36 & 36\end{array}$

14

$\begin{array}{llllllllllll}9 & 11 & 9 & \text { II. } & 9 & 4 & 2 & 2 & \text { I } & 2 & \text { I } & 3\end{array}$

16

o I.

2

17

$6 \quad 17$

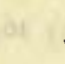

$\begin{array}{llll}4 & 6 & 20 & 19\end{array}$

2

18

10 20

10

I

I 2 .

610

19

2 I

$\begin{array}{llllllllllll}2 & 0 & 7 & 15 & 16 & \text { I8 } & \text { I9 } & 20 & 22 & \text { I6 } & \text { II } & 15\end{array}$

22

$8 \quad 7 \quad 5.4$

8 I2 II II

54

4

$9 \quad 4$

18.

25

26

- 0 o 1

7

$\begin{array}{llll}7 & 9 & 9 & 4\end{array}$

2
5

I2.

(1)

28

$\begin{array}{llll}7 & 4 & 6 & 5\end{array}$

$\begin{array}{rrrrrrrr}1 & 0 & 0 & 0 & 2 & I & I & 5 \\ 1 & 3 & 4 & 2 & 1 & 3 & 16 & 16\end{array}$

$\begin{array}{lllllllllll}0 . & 2 & 8 & 2 & I & 0 & 0 & I & 2 & I & I\end{array}$

$\begin{array}{lllllllllll}13 & 22 & 17 & 14 & 16 & \text { I7 } & \text { I8 } & \text { I7 } & \text { I8 } & 17 & \text { 10 }\end{array}$

$\begin{array}{llllll}9 . & 13 & 16 & 13 & 14 & \text { 10 }\end{array}$

8

1

12

663

896

888

882

674

79

82

235

200

35

96

335

I 54

$\begin{array}{lllllllllll}\text { I9 } & \text { I7 } & 19 & \text { I7 } & \text { I6 } & \text { II } & \text { I5 } & 9 & 8 & 5 & 4\end{array}$

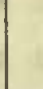

30

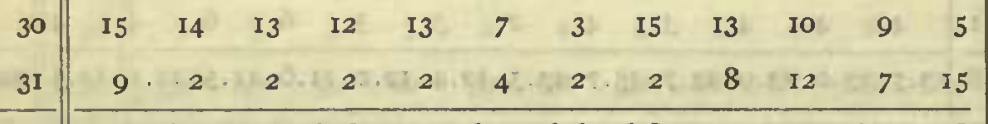

\begin{tabular}{l||llllllllllll} 
Means & 15.5 & 16.5 & 17.5 & 18.6 & 17.5 & 16.0 & 16.6 & 16.8 & 17.2 & 17.0 & 16.7 & 15.8
\end{tabular}

$\begin{array}{lllllllllll}8 & 1 & I & 10 & 13 & 13 & 13 & 10 & 14 & 8 & 6\end{array}$

Total movement during month, I2080 miles; mean daily movement, 389.7 miles; average hourly movement, 16.2 miles 
Tabulation of hourly wind records at Teplitz Bay during the month of January, 1905

\section{WIND MOVEMENT}

Date

For the hour preceding

Daily

\begin{tabular}{lllllllllll|lllllllllllll} 
IH & $2 \mathrm{II}$ & $3 \mathrm{H}$ & $4 \mathrm{H}$ & $5 \mathrm{II}$ & $6 \mathrm{II}$ & $7 \mathrm{II}$ & $8 \mathrm{H}$ & $9 \mathrm{H}$ & IOH & IIII & I2H & I3H & I4H & I5H & I6H & I7H & I $8 \mathrm{HI}$ & I9H & $2 \mathrm{H}$ & $2 \mathrm{III}$ & $22 \mathrm{H}$ & $23 \mathrm{H}$ & $24 \mathrm{H}$
\end{tabular}

Mi. Mi. Mi. Mi. Mi. Mi. Mi. Mi. Mi. Mi. Mi. Mi. Mi. Mi. Mi. Mi. Mi. Mi. Mi. Mi. Mi. Mi. Mi. Mi. Mi.

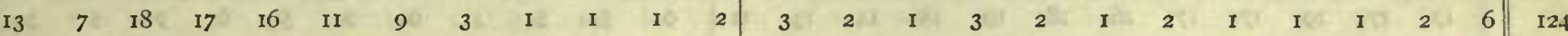

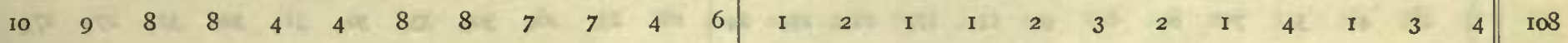

\begin{tabular}{llllllllllll|lllllllllllllllll}
3 & 2 & 2 & 2 & 2 & 3 & 3 & $\mathrm{I}$ & $\mathrm{I}$ & $\mathrm{I}$ & 2 & 3 & -4 & 2 & 4 & $\mathrm{I}$ & 8 & 10 & $\mathrm{I} 2$ & $\mathrm{II}$ & $\mathrm{I} 2$ & 9 & 5 & 4 & 107
\end{tabular}

\begin{tabular}{llllllllllll|lllllllllllllllll}
3 & 2 & 2 & $\mathrm{I}$ & $\mathrm{I}$ & $\mathrm{I}$ & $\mathrm{O}$ & $\mathrm{I}$ & $\mathrm{I}$ & 6 & 4 & 3 & 4 & 4 & 5 & 6 & 4 & 5 & 4 & 6 & 7 & 9 & 4 & 5 & 88
\end{tabular}

$\begin{array}{lllllllllllllllllllllllllllll}5 & 5 & 4 & 4 & 3 & 4 & 4 & 4 & 2 & \text { I } & 0 & \text { I } & \text { I } & 0 & 2 & \text { I } & \text { I } & 0 & 1 & 0 & 0 & \text { I } & \text { I } & \text { II } & 56\end{array}$

$\begin{array}{llllllllllll}27 & 24 & 5 & 5 & 5 & 8 & 19 & 22 & 26 & 25 & 33 & 47\end{array}$

$\begin{array}{llllllllllll}\text { II } & \text { I } 4 & \text { II } & 47 & 45 & 20 & \text { I2 } & 25 & 44 & 52 & 5 \text { I } & 52\end{array}$

$\begin{array}{llllllllllll}36 & 33 & 39 & 42 & 50 & 51 & 56 & 53 & 31 & 12 & 16 & 36\end{array}$

\begin{tabular}{llllllllllll|l}
27 & 18 & 39 & 27 & I8 & II & 25 & 23 & 28 & 14 & I4 & I2 & 502
\end{tabular}

\begin{tabular}{llllllllllll|l}
52 & 48 & 38 & 50 & 47 & 35 & 60 & 42 & 31 & 41 & 28 & 27 & 883
\end{tabular}

\begin{tabular}{llllllllllll|l}
42 & 48 & 47 & 55 & 56 & 50 & 51 & 54 & 57 & 58 & 38 & 15 & 1026
\end{tabular}

\begin{tabular}{l|llllllllllll}
9 & 15 & 7 & 6 & 7 & 6 & 8 & 6 & 5 & 4 & 4 & 3 & 2
\end{tabular}

IO $\quad$\begin{tabular}{llllllllllll|}
21 & 26 & 25 & 27 & 27 & 20 & 9 & 7 & 14 & 23 & 24 & 24
\end{tabular}

II

$\begin{array}{llllllllllll}\text { I5 II II } & \text { I2 } & 8 & 6 & 8 & 5 & 6 & 4 & 9 & 12\end{array}$

12

$\begin{array}{llllllllllll}3 & 2 & I & 1 & 2 & 2 & 2 & 2 & 2 & 2 & I & 2 .\end{array}$

13.

I5

I6

$\begin{array}{llllllllllll}43 & 40 & 36 & 43 & 47 & 45 & 50 & 46 & 37 & 28 & 38 & 39\end{array}$

I

I8

$\begin{array}{llllllllllll}\text { I4 } & \text { I5 } & \text { I4 } & \text { I7 } & \text { I5 } & \text { I4 } & \text { I5 } & \text { I3 } & \text { I3 } & \text { I7 } & 21 & 23\end{array}$

I9

20

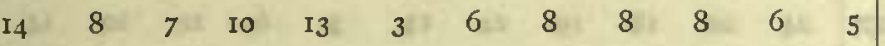

$\begin{array}{llllllllllll}4 & 5 & 8 & 8 & \text { 10 } & \text { 10 } & \text { II } & \text { II } & 9 & 7 & 18 & 24\end{array}$

Ig8

I0 $\quad$\begin{tabular}{lllllllllll|l}
7 & 6 & 4 & 3 & 2 & 2 & 3 & 6 & 8 & 9 & 5 & 3 I 2
\end{tabular}

$\begin{array}{llllllllllll}\text { II } & 7 & 9 & 5 & 4 & 2 \mathrm{I} & \text { I6 } & 32 & 2 \mathrm{I} & 6 & \text { II } & \text { II }\end{array}$

23

$\begin{array}{llllllllllll}6 & 5 & 4 & 2 & 4 & 6 & 5 & 2 & 3 & 9 & 9 & \text { I2 }\end{array}$

$$
\begin{array}{llll}
4 & 4 & 4 & 3 \\
3 & 3 & 2 & 2 \\
4 & 2 & 2 & 2
\end{array}
$$$$
3
$$

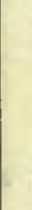

$$
\begin{array}{rrrrrrrrrrrr}
4 & 2 & 2 & 2 & 2 & \text { I } & 3 & 2 & 2 & 3 & 2 & 2 \\
2 & 1 & 2 & 2 & 2 & 2 & 2 & 3 & 3 & 2 & 2 & 2 \\
1 & 1 & 1 & 2 & 2 & 2 & 11 & 30 & 26 & 41 & 45 & 46 \\
29 & 29 & 19 & 17 & 31 & 11 & 12 & 21 & 16 & 9 & 10 & 5
\end{array}
$$$$
3
$$$$
\begin{array}{llll}
1 & 1 & 1 &
\end{array}
$$$$
\begin{array}{llllllllllll}
4 & 5 & 5 & \text { IO } & \text { 10 } & \text { 10 } & \text { IO } & \text { II } & \text { II } & 9 & 9
\end{array}
$$$$
\begin{array}{llllllllllll}
25 & \text { I7 } & \text { I3 } & 9 & 8 & \text { II } & \text { I5 } & \text { I7 } & \text { I9 } & 20 & \text { I7 } & \text { I4 }
\end{array}
$$$$
\begin{array}{llllllllllll}
4 & 6 & 7 & \text { I5 } & 27 & 33 & 22 & \text { II } & \text { II } & 20 & \text { I2 } & \text { II }
\end{array}
$$$$
62
$$$$
\text { I4 } 16 \quad 16 \quad 16 \quad 14 \text { I9 } 17 \quad \begin{array}{llllll} 
& 15 & 14 & \text { 10 } & \text { 10 } & 10
\end{array}
$$$$
\begin{array}{llllllllllll}
4 & 5 & 8 & 6 & 7 & 7 & 7 & 9 & \text { IO } & \text { II } & \text { II } & \text { I0 }
\end{array}
$$$$
\begin{array}{llllllllllll}
24 & 35 & 40 & 45 & 46 & 53 & 53 & 57 & 65 & 66 & 70 & 64
\end{array}
$$ 
For the hour preceding

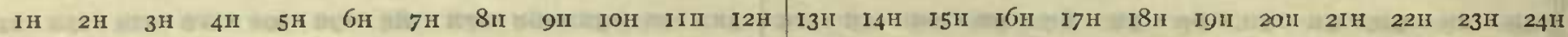

Mi. Mi. Mi. Mi. Mi. Mi. Mi. Mi. Mi. Mi. Mi. Mi. $\begin{array}{llllllllllll}17 & 17 & 19 & 17 & 17 & 16 & 18 & \text { I9 } & 18 & \text { I4 } & 13 & \text { II }\end{array}$ 2 $\begin{array}{llllllllllll}4 & 4 & 5 & 7 & 8 & 6 & 9 & 14 & 17 & 21 & 25 & 24\end{array}$ $\begin{array}{llllllllllll}25 & 27 & 22 & 23 & 22 & 22 & 19 & 19 & 19 & 20 & 19 & 14\end{array}$ $\begin{array}{llllllllllll}\text { I0 } & 4 & \text { I2 } & 16 & 5 & \text { 10 } & 8 & 6 & 8 & 17 & 20 & 22\end{array}$ $\begin{array}{llllllllllll}45 & 44 & 41 & 38 & 38 & 38 & 37 & 33 & 33 & 34 & 37 & 34\end{array}$ $\begin{array}{llllllllllll}38 & 44 & 42 & 40 & 43 & 46 & 41 & 33 & 29 & 24 & 23 & 24\end{array}$ $\begin{array}{llllllllllll}7 & 8 & 7 & 5 & 7 & 7 & 7 & 7 & \text { 10 } & 9 & 7 & 4\end{array}$ $\begin{array}{llllllllllll}5 & 3 & 4 & 5 & 3 & 3 & 4 & 5 & 6 & 4 & 4 & 5\end{array}$ $\begin{array}{llllllllllll}4 & 3 & 3 & 4 & 5 & 3 & 4 & 3 & 3 & 3 & 4 & 4\end{array}$ $\begin{array}{llllllllllll}30 & 33 & 26 & 8 & 12 & \text { I5 } & 42 & 41 & 38 & 30 & 29 & 25\end{array}$ $\begin{array}{llllllllllll}29 & 33 & 31 & 25 & 25 & 26 & 22 & 22 & 26 & 20 & 25 & 27\end{array}$ $\begin{array}{llllllllllll}10 & 4 & 4 & 1 & 4 & 8 & 5 & 4 & 0 & 3 & 5 & 12\end{array}$ $\begin{array}{llllllllllll}3 & 4 & 5 & 6 & \text { II } & \text { II } & \text { I3 } & \text { IO } & 9 & 3 & 6 & 6\end{array}$ $\begin{array}{llllllllllll}9 & 14 & 10 & 16 & 8 & 14 & 8 & 4 & 5 & 18 & \text { 10 } & 9\end{array}$ $\begin{array}{lllllllllllll}13 & 8 & 5 & 7 & 4 & 4 & 7 & 12 & 14 & 13 & 12 & 6\end{array}$ $\begin{array}{llllllllllll}26 & 27 & 30 & 29 & 26 & 24 & 27 & 34 & 39 & 43 & 46 & 47\end{array}$ $\begin{array}{llllllllllll}45 & 45 & 45 & 45 & 45 & 45 & 45 & 45 & 40 & 39 & 4 \mathrm{I} & 39\end{array}$ $\begin{array}{llllllllllll}27 & 25 & 28 & 26 & 27 & 24 & 28 & 35 & 34 & 36 & 38 & 27\end{array}$ $\begin{array}{llllllllllll}9 & \text { II } & \text { IO } & 2 & 5 & 5 & 4 & 7 & 9 & 6 & 9 & \text { I2 }\end{array}$ $\begin{array}{llllllllllll}32 & 26 & 42 & 54 & 51 & 49 & 50 & 53 & 49 & 33 & 30 & 42\end{array}$ $\begin{array}{llllllllllll}\text { I2 } & 13 & 8 & \text { II } & \text { I2 } & 6 & \text { I4 } & \text { I5 } & 9 & 5 & 3 & 4\end{array}$ $\begin{array}{llllllllllll}\text { I6 } & 30 & 36 & 44 & 50 & 55 & 62 & 61 & 59 & 62 & 61 & 59\end{array}$ \begin{tabular}{llllllllllll}
49 & 39 & $3 \mathrm{I}$ & 33 & 37 & 33 & 26 & 26 & 30 & 30 & 28 & 28 \\
\hline
\end{tabular}
Mi. Mi. Mi. Mi. Mi. Mi. Mi. Mi. Mi. Mi. Mi. Mi. $M i$. $\begin{array}{llllllllllll}40 & 44 & 45 & 45 & 43 & 47 & 49 & 47 & 58 & 62 & 56 & 44\end{array}$ $\begin{array}{llllllllllll}10 & 9 & 6 & 9 & 8 & 8 & 2 & 4 & 5 & 8 & 7 & 9\end{array}$ $\begin{array}{llllllllllll}30 & 32 & 33 & 39 & 35 & 32 & 33 & 29 & 21 & 33 & 38 & 39\end{array}$ $\begin{array}{llllllllllll}45 & 45 & 45 & 45 & 45 & 45 & 45 & 45 & 44 & 43 & 4 \mathrm{~T} & 39\end{array}$ $\begin{array}{llllllllllll}56 & 53 & 55 & 56 & 58 & 66 & 55 & 60 & 66 & 71 & 73 & 72\end{array}$ $\begin{array}{lllllllllllllll}3.1 & 23.2 & 23.2 & 23.4 & 23.4 & 23.9 & 24.4 & 24.8 & 24.9 & 25.1 & 25.4 & 24.6 & 25.6\end{array}$

Total movement during month, I6278 miles; mean daily movenent, 581.4 miles; average hourly movement, 24.2 miles.

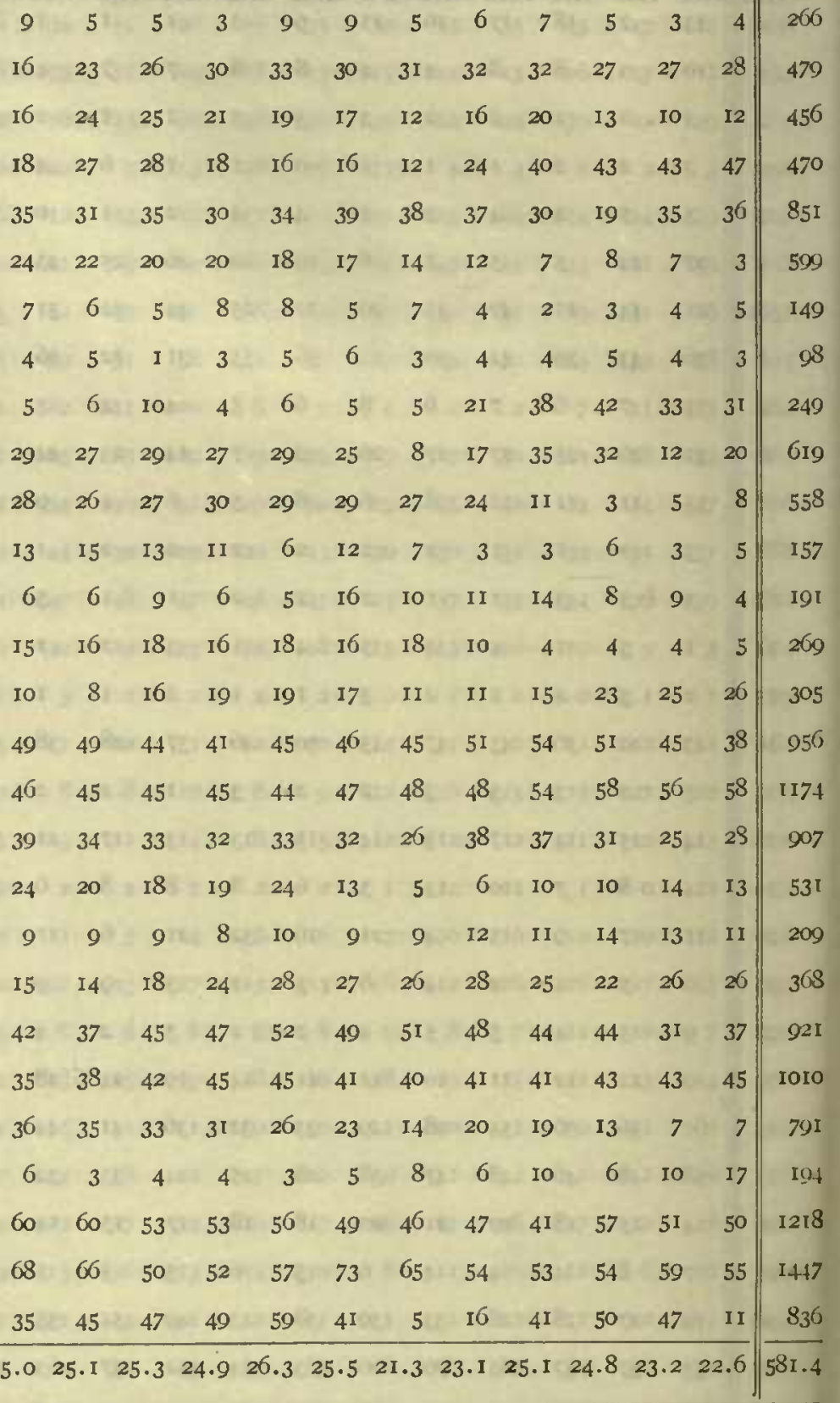




\section{Wind MOVEMENT}

Mi. Mi. Mi. Mi. Mi. Mi. Mi. Mi. Mi. Mi. Mi. Mi. Mi, Mi. Mi. Mi. Mi. Mi. Mi. Mi. Mi. Mi. Mi. Mi. 
Tabulation of hourly wind records at Teplitz Bay during the month of April, 1905

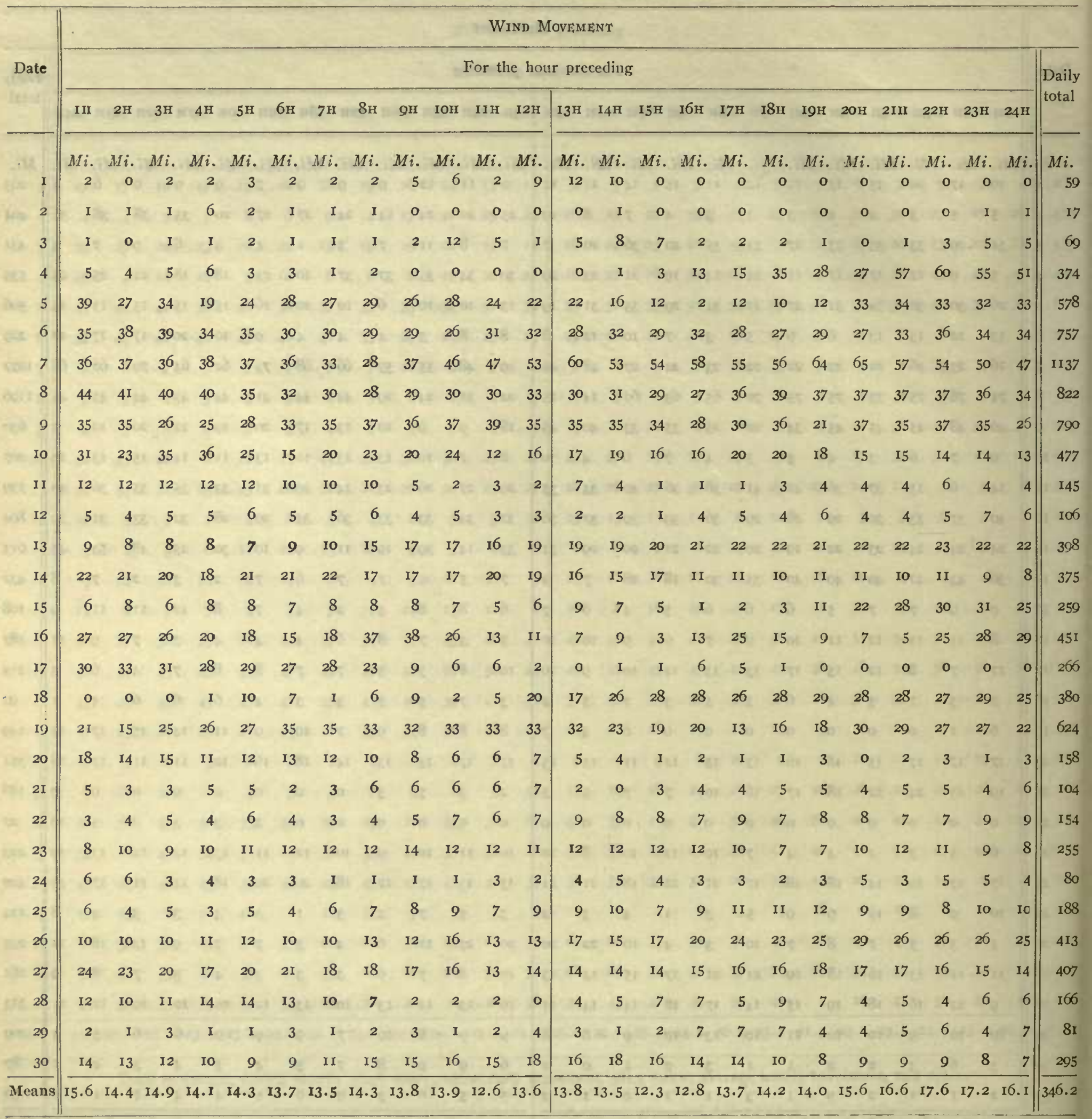

Total movement during month, I0385 miles; mean daily movement, 346.2 miles; average hourly movesnent, 14.4 miles. 
Tabulation of hourly acind records at Teplitz Bay during the month of May, Igo5

\section{WIND MOVEMENT}

III $2 \mathrm{H} \quad 3 \mathrm{H} \quad 4 \mathrm{II} \quad 5 \mathrm{II} \quad 6 \mathrm{H} \quad 7 \mathrm{H} \quad 8 \mathrm{H} \quad 9 \mathrm{H}$ IOH IIH I2H $\quad$ I3H I4H I5H I6H I7II I8H I9II $20 \mathrm{H} \quad 2 \mathrm{II} \quad 22 \mathrm{II} \quad 23 \mathrm{H} \quad 24 \mathrm{H}$

Mi. Mi. Mi. Mi. Mi. Mi. Mi. Mi. Mi. Mi. Mi. Mi.

Mi. Mi. Mi. Mi. Mi. Mi. Mi. Mi. Mi. Mi. Mi. Mi. Mi.

$\begin{array}{lllllllllllll}4 & 6 & 9 & 6 & 6 & 7 & 5 & 4 & \text { II } & \text { I3 } & \text { I3 } & 13\end{array}$

\begin{tabular}{llllllllllll||l}
15 & 20 & 15 & 21 & 25 & 26 & 27 & 26 & 26 & 26 & 25 & 26 & 375
\end{tabular}

I6

I

I8

I

\section{$\begin{array}{llllllllllll}8 & 23 & 23 & 21 & 21 & 19 & 24 & 26 & 26 & 24 & 28 & 29\end{array}$ \\ $\begin{array}{llllllllll}7 & \mathrm{II} & 5 & \mathrm{I} & 5 & \mathrm{I} & 6 & 3 & 2 & 6\end{array}$ \\ I9 $\begin{array}{llllllllllll}16 & \text { I5 } & \text { 12 } & 8 & 8 & 8 & 9 & \text { I0 } & \text { I0 } & \text { I3 } & \text { II }\end{array}$ \\ $\begin{array}{llllllllllll}4 & 3 & 4 & 6 & I 3 & I 8 & 19 & 20 & 2 I & 2 I & 2 I & 2 I\end{array}$ \\ $\begin{array}{llllllllllll}12 & 13 & 14 & 15 & 16 & 16 & 17 & 18 & 19 & 19 & 18 & 17\end{array}$ \\ $\begin{array}{llllllllllll}26 & 26 & 20 & \text { II } & 6 & 7 & 4 & 4 & 7 & 7 & 15 & 18\end{array}$ \\ $\begin{array}{llllllllllll}\text { IO } & 9 & 7 & \text { IO } & \text { I6 } & \text { I8 } & 20 & 24 & 25 & 23 & 22 & 23\end{array}$ \\ $\begin{array}{llllllllllll}18 & 19 & 20 & 23 & 23 & 22 & 20 & 18 & 21 & 17 & 15 & 13\end{array}$ \\ $\begin{array}{lllllllllll}\text { I4 II } & \text { II } & \text { I } & \text { I } & \text { I2 } & \text { I2 } & \text { I2 } & \text { I2 } & \text { II } & \text { IO } & 10\end{array}$}

$\begin{array}{rrrr}3 & I & 7 & 7\end{array}$

$\begin{array}{lllllllllll}9 & \text { II } & \text { II } & \text { I2 } & \text { I2 } & \text { I2 } & \text { I2 } & \text { I5 } & 14 & 16 & \text { I6 }\end{array}$

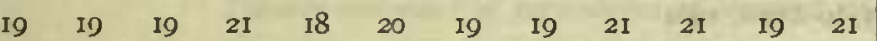

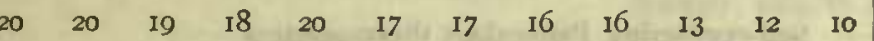

4 I3 I3

$9 \quad 12$

I

$25 \quad 24$

$27 \quad 33$

I8 26

$39 \quad 4 I \quad 35$

26

$34 \quad 35 \quad 32$

$\begin{array}{lllllllllllll}15.5 & 16.2 & 15.7 & 15.2 & 15.3 & 15.2 & 15.2 & 14.1 & 15.7 & 15.2 & 15.8 & 15.8\end{array}$

Total movement during 251/2 days, 9473 miles; mean daily movement, 371.5 miles; average hourly movement, 14.9 miles 
REDUCTION OF OBSERVATIONS AT TEPLITZ BAY

Summary of mean monthly daily records at Teplitz Bay

October, I903, to April, Igo4

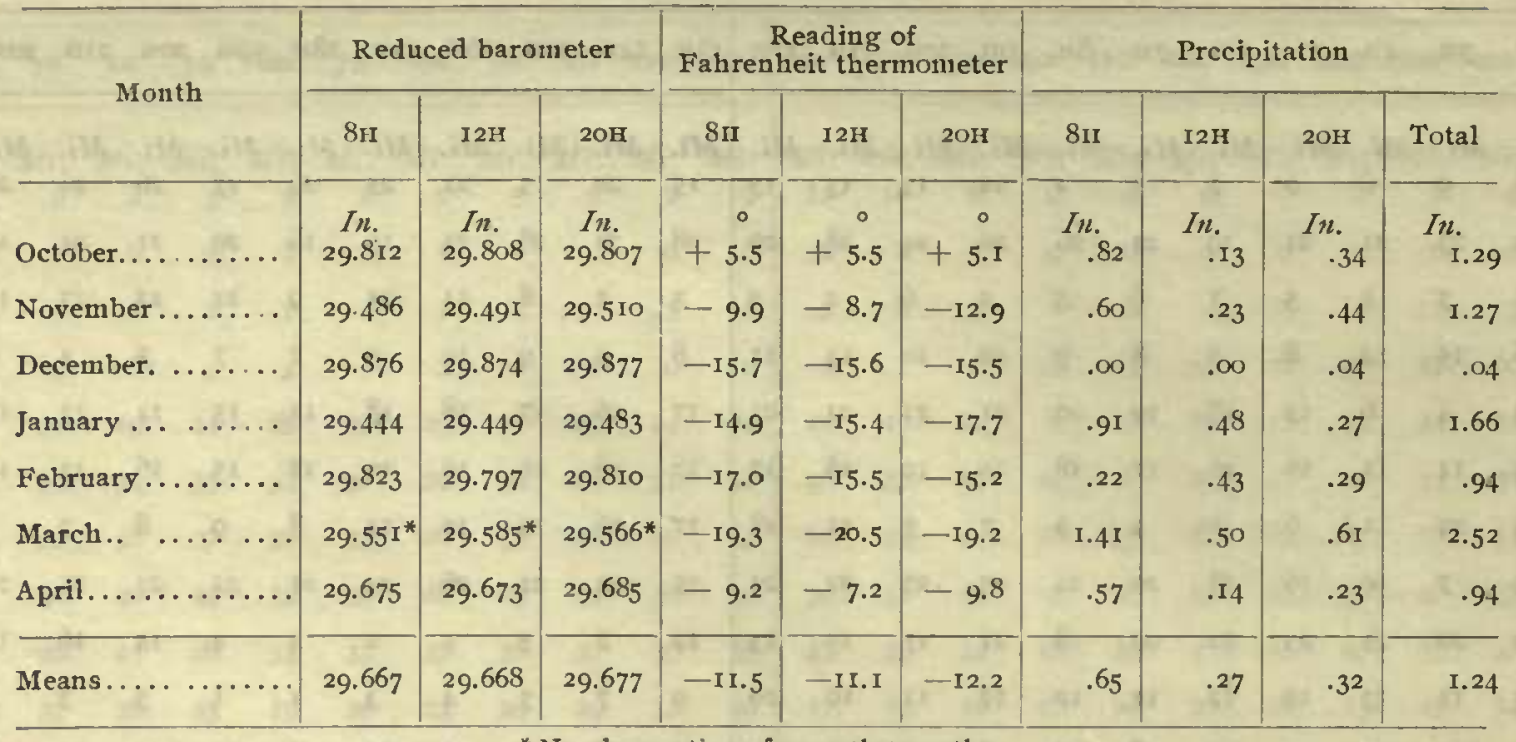

* No observatious from 7 th to I Ith.

Summary of mean monihly daily records al Teplilz Bay-Continued October, I903, to April, I904

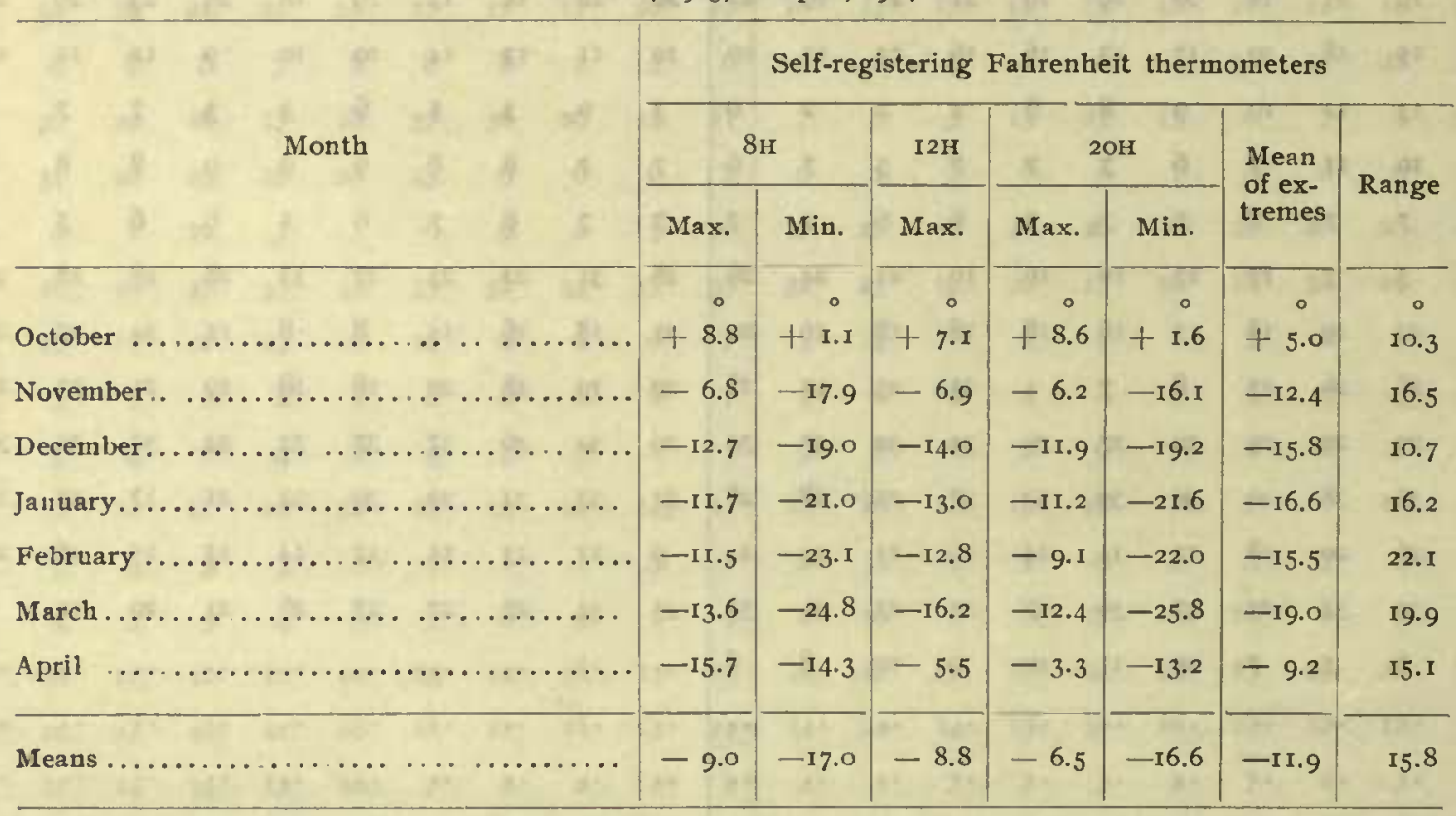


Tabutar summary of percentages of observed wind directions at Teplitz Bay

September, 1903, to December, 1903

\begin{tabular}{|c|c|c|c|c|c|c|c|c|c|c|c|c|c|c|c|c|c|c|c|c|c|c|c|c|}
\hline \multirow{3}{*}{ 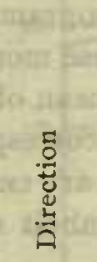 } & \multicolumn{6}{|c|}{ September, I903 } & \multicolumn{6}{|c|}{ October, I903 } & \multicolumn{6}{|c|}{ November, 1903} & \multicolumn{6}{|c|}{ December, 1903} \\
\hline & \multicolumn{2}{|c|}{$8 \mathrm{H}$} & \multicolumn{2}{|c|}{$\mathrm{I} 2 \mathrm{H}$} & \multicolumn{2}{|c|}{$2 \mathrm{OH}$} & \multicolumn{2}{|c|}{$8 \mathrm{H}$} & \multicolumn{2}{|c|}{$\mathrm{I} 2 \mathrm{H}$} & \multicolumn{2}{|c|}{$2 \mathrm{OH}$} & \multicolumn{2}{|c|}{$8 \mathrm{H}$} & \multicolumn{2}{|c|}{$12 \mathrm{H}$} & \multicolumn{2}{|c|}{2011} & \multicolumn{2}{|c|}{$8 \mathrm{H}$} & \multicolumn{2}{|c|}{$\mathrm{I}_{2} \mathrm{H}^{+}$} & \multicolumn{2}{|c|}{$2 \mathrm{OH}$} \\
\hline & : & \begin{tabular}{l}
$\dot{x}$ \\
\multirow{2}{*}{}
\end{tabular} & $\stackrel{\text { Dी }}{0}$ & 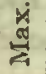 & $\stackrel{0}{0}$ & 党 & : & 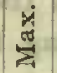 & $\stackrel{0}{0}^{\infty}$ & 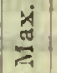 & $\tilde{0}_{0}^{\circ}$ & $\stackrel{\dot{\sigma}}{\sigma}$ & $\overbrace{0}^{80}$ & 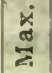 & $\stackrel{\dot{0}}{0}$ & 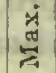 & مी & 兽 & : & 昶 & $\frac{0}{0}$ & 宷 & $\frac{1}{0}$ & $\stackrel{x}{\tilde{z}}$ \\
\hline & $\%$ & $\%$ & $\%$ & $\%$ & $\%$ & $\%$ & $\%$ & $\%$ & $\%$ & $\%$ & $\%$ & $\%$ & $\%$ & $\%$ & $\%$ & $\%$ & $\%$ & $\%$ & $\%$ & $\%$ & $\%$ & $\%$ & $\%$ & $\%$ \\
\hline NNE & & .. &. & . & . & . & $\because$ & $\begin{array}{c}3 \\
\cdots\end{array}$ & .. & $\begin{array}{l}3 \\
\cdots\end{array}$ & 10 & $\therefore$ & $\begin{array}{l}7 \\
. .\end{array}$ & 10 & $\because$ & $\because$. & $\begin{array}{l}13 \\
\ldots\end{array}$ & lo & 3 & .. & $\begin{array}{r}3 \\
\ldots\end{array}$ & 0 & 10 & \\
\hline & 7 & 7 & Io & 7 & 7 & 7 & 16 & 19 & 3 & $I_{3}$ & 23 & 19 & 23 & 20 & 24 & 21 & 7 & 3 & I0 & $\mathrm{I}_{3}$ & I3 & I3 & Io & Io \\
\hline & .. & .. & .. & . & .. & . & .. & $\therefore$ & .. & & & .. & .. & .. & .. & .. & .. & .. & & .. & $\ldots$ & $\ldots$ & .. & \\
\hline & 30 & 27 & I4 & $2 \mathrm{I}$ & 30 & 30 & 23 & 19 & 45 & 39 & 19 & 26 & 13 & Io & 14 & 24 & 13 & Io & 39 & 39 & 32 & 39 & 32 & 35 \\
\hline$E S$ & & &.. & & $\therefore$ & .. & .. & . & 3 & 3 & 3 & 3 & I3 & 17 & 10 & 3 & 7 & 7 & 6 & Io & 6 & 3 & 3 & \\
\hline & 27 & 33 & 34 & $3^{6}$ & 23 & 23 & 29 & 32 & I3 & I3 & 19 & 23 & 13 & 10 & 14 & 14 & 20 & 27 & I 6 & 13 & I9 & I9 & 19 & 16 \\
\hline SSE & $\therefore$ & & .. & .. & .. & . & $\therefore$ & 5 & 3 & 6 & .. & .. & 3 & 3 & 3 & 3 & 3 & 3 & 3 & 3 & .. & 3 & & \\
\hline & 13 & 10 & Io & 7 & I 3 & 13 & 3 & 3 & 6 & 3 & Io & 6 & 10 & 10 & 3 & 10 & Io & 7 & 3 & .. & 6 & 3 & 13 & I0 \\
\hline SSIV & & & .. & & 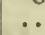 & .. & 3 & & .. &.. & .. & .. & 3 & 3 & .. & 3 & & 3 & .. & .. & &. & & 2 \\
\hline & Io & IO & I4 & I 8 & I3 & 13 & 10 & 16 & 3 & 3 & 3 & 6 & 3 & 3 & 7 & 3 & 3 & 3 & 6 & 3 & 3 & 3 & .. & 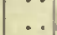 \\
\hline & & & .. & 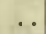 & & .. & & & & .. & .. & . & .. & .. & & $\therefore$ & & & . & & .. & & & \\
\hline & 7 & 7 & 7 & 3 & 3 & 3 & 13 & 3 & 10 & I 3 & 3 & 3 & 3 & 3 & 17 & 10 & 10 & I3 & 6 & IO & Io & Io & 13 & 13 \\
\hline & & & & & & & & 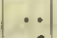 & &. & & & & 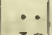 & & . & & & . & & .. & & & \\
\hline & 7 & 7 & 3 & . & .. & .. & 3 & 3 & 6 & 3 & & 3 & 7 & 10 & 7 & 7 & I 3 & I3 & 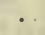 & .. & 3 & & & . \\
\hline & .. & & . & & - & . & & $\cdots$ & & & . & * & .. & .. & & & .. & & & . & .. & .. & . & . \\
\hline & & & .. & & & . & & $\cdots$ & & .. & 3 & & . & & & . & & & & & 3 & & & . \\
\hline
\end{tabular}

Tabular summary of percentages of observed wind directions at Teptitz Bay-Continued

January, I904, to April, I904

\begin{tabular}{|c|c|c|c|c|c|c|c|c|c|c|c|c|c|c|c|c|c|c|c|c|c|c|c|c|}
\hline \multirow{3}{*}{ 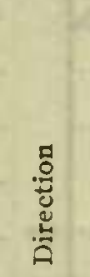 } & \multicolumn{6}{|c|}{ January, I904 } & \multicolumn{6}{|c|}{ February, 1904} & \multicolumn{6}{|c|}{ March, 1904} & \multicolumn{6}{|c|}{ April, I 904} \\
\hline & \multicolumn{2}{|c|}{$8 \mathbf{H}$} & \multicolumn{2}{|c|}{ I2H } & \multicolumn{2}{|c|}{$2 \mathrm{OH}$} & \multicolumn{2}{|c|}{$8 \mathbf{H}$} & \multicolumn{2}{|c|}{$\mathrm{I} 2 \mathrm{H}$} & \multicolumn{2}{|c|}{$2 \mathrm{OH}$} & \multicolumn{2}{|c|}{8} & \multicolumn{2}{|c|}{$\mathrm{I} 2 \mathrm{H}$} & \multicolumn{2}{|c|}{$20 \mathrm{H}$} & \multicolumn{2}{|c|}{$8 \mathrm{H}$} & \multicolumn{2}{|c|}{ I $2 \mathrm{H}$} & \multicolumn{2}{|c|}{$2 \mathrm{OH}$} \\
\hline & $\frac{\dot{0}}{0}$ & 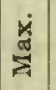 & हैं & $\frac{\pi}{2}$ & हैं & 荧 & $\frac{8}{0}$ & 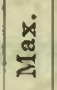 & $\frac{\dot{0}}{0}$ & 离 & ठิ & 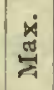 & $\frac{\text { ก }}{0}$ & 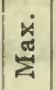 & $\frac{\dot{p}}{0}$ & $\frac{\stackrel{x}{\pi}}{\frac{\pi}{2}}$ & $\stackrel{0}{0}$ & 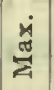 & ஜூ & 离 & है & 蒡 & : & 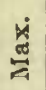 \\
\hline & $\%$ & $\%$ & $\%$ & $\%$ & $\%$ & $\%$ & $\%$ & $\%$ & $\%$ & $\%$ & $\%$ & $\%$ & $\%$ & $\%$ & $\%$ & $\%$ & $\%$ & $\%$ & $\%$ & $\%$ & $\%$ & $\%$ & $\%$ & $\%$ \\
\hline$\stackrel{N}{N}$ & 6 & I3 & 6 & 10 & 6 & 13 & I4 & I4 & 7 & 14 & IO & Io & I9 & 23 & 12 & 35 & I5 & 24 & 17 & 28 & 7 & I 7 & 28 & 25 \\
\hline & $\ddot{1} 6$ & $\ddot{\text { Io }}$ & i6 & $\ddot{13}$ & io & 13 & Io & $\ddot{3}$ & $\ddot{7}$ & $\begin{array}{l}3 \\
7\end{array}$ & ir & & ig & $\ddot{8}$ & i2 & $\ddot{15}$ & $\ddot{\text { II }}$ & 20 & 3 & $\begin{array}{r}3 \\
\text { I0 }\end{array}$ & $\begin{array}{r}3 \\
10\end{array}$ & & $\ddot{I}$ & $\ddot{4}$ \\
\hline ENE & & . & Io & 6 & .. & 3 & . & . . & 3 &.. & .. & 3 & . & . & 4 & $\begin{array}{l}15 \\
\cdots\end{array}$ & & . & $\begin{array}{l}14 \\
. .\end{array}$ & 7 & .. & & .. & $\begin{array}{l}4 \\
4\end{array}$ \\
\hline E & 29 & 29 & 13 & 19 & 39 & 26 & 21 & 28 & 24 & 24 & $2 \mathrm{I}$ & $3 \mathrm{I}$ & I I & 19 & 35 & 19 & 26 & 24 & 17 & 7 & $2 \mathrm{I}$ & I4 & Io & I I \\
\hline ESE & 3 & 6 & 13 & 10 & 3 & 10 & . & .. & &.. & .. & & $\because$ & $\therefore$ & $\cdots$ & $\therefore$ & $\cdots$ & $\therefore$ & 7 & 7 & & & 7 & 7 \\
\hline & I3 & 6 & 3 & $I_{3}$ & IO & I3 & Io & I4 & 17 & 17 & 14 & I7 & II & I2 & & 8 & 7 & 4 & IO & I0 & 7 & 17 & I0 & 14 \\
\hline SSE & 4 & .. & .. & & 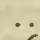 & 10 & 3 & 7 & .. & 3 & .. & 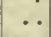 & 4 & 8 & 4 & 4 & 7 & 8 & 3 & 3 & 3 & & & 4 \\
\hline $\mathrm{S}$ & 23 & 23 & 23 . & 16 & 6 & Io & 17 & 14 & 7 & 3 & 14 & 3 & 7 & I 2 & 4 & 4 & Ii & 8 & 7 & 3 & 10 & 7 & .. & 4 \\
\hline SSW & & $\cdots$ & & & . & $\ddot{6}$ & 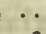 & & 3 & & $\because$ & 3 & .. & $\cdots$ & $\cdots$ & .. & $\cdots$ & .. & . & 3 & & & 3 & \\
\hline & 3 & 3 & 6 & 10 & & 6 & 3 & 3 & 3 & 3 & 7 & IO & . & & . . & .. & .. & .. & 7 & .. & 3 & 7 & 3 & 4 \\
\hline WSW & . & $\because$ & $\because$ & . & . & $\because$ & . & $\cdots$ & .. & $\cdots$ & .. & 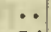 & . & $\because$ & $\because$ & $\cdots$ & .. & & $\because$ & 7 & .. & $\cdots$ & 3 & \\
\hline W & 3 & 6 & 6 & 3 & I9 & 6 & 14 & 17 & I4 & 17 & 14 & I4 & II & 8 & 8 & 12 & 4 & 4 & 7 & & 14 & 7 & I4 & 4 \\
\hline & . & $\because$ & . & . & & $\cdots$ & $\because$ & $\cdots$ & $\ddot{m}$ & & . & & . & & & . & & $\because$ & * & $\because$ & $\because$ & 3 & .. & 4 \\
\hline & .. & 3 & .. & .. & 6 & .. & 7 & .. & 7 & 7 & 3 & 7 & 4 & 8 & 8 & 4 & II & 8 & 3 & 3 & I4 & 7 & 3 & II \\
\hline & $\because$ & & * & . & .. & . & . & & $\because$ & & . & & & : & $\because$ & .. & .. & .. & . & & & & .. & 4 \\
\hline & 3 & & 3 & & & & - & & & & & & 15 & 4 & I5 & & & & & & & $\therefore$ & & \\
\hline
\end{tabular}

3 I 


\section{DIURNal, VARiation IN TEMPERATURE}

The thermograms obtained at Teplitz Bay from October 1, 1903, to April 30, 1904, at which latter date the thermograph failed, have been reduced to the standard of the thermometer used in the daily observations. By graphical methods the mean daily thermograms for each month of record have been deduced; the diurnal inequalities indicated by these montlly mean daily curves are shown in the following summary, values greater thran the mean of day being indicated by plus quantities, and vice versa. The mean monthly values for the corresponding period October, 1899 , to April, 1900 , as obtained by the Italian Expedition $*$ are entered herewith for the sake of comparison, the quantities having been reduced to Fahrenheit scale.

Summary of mean monthly diurnal variation in lemperature at Teptitz Bay

From thermograms October, Ig03, to A pril, Ig04

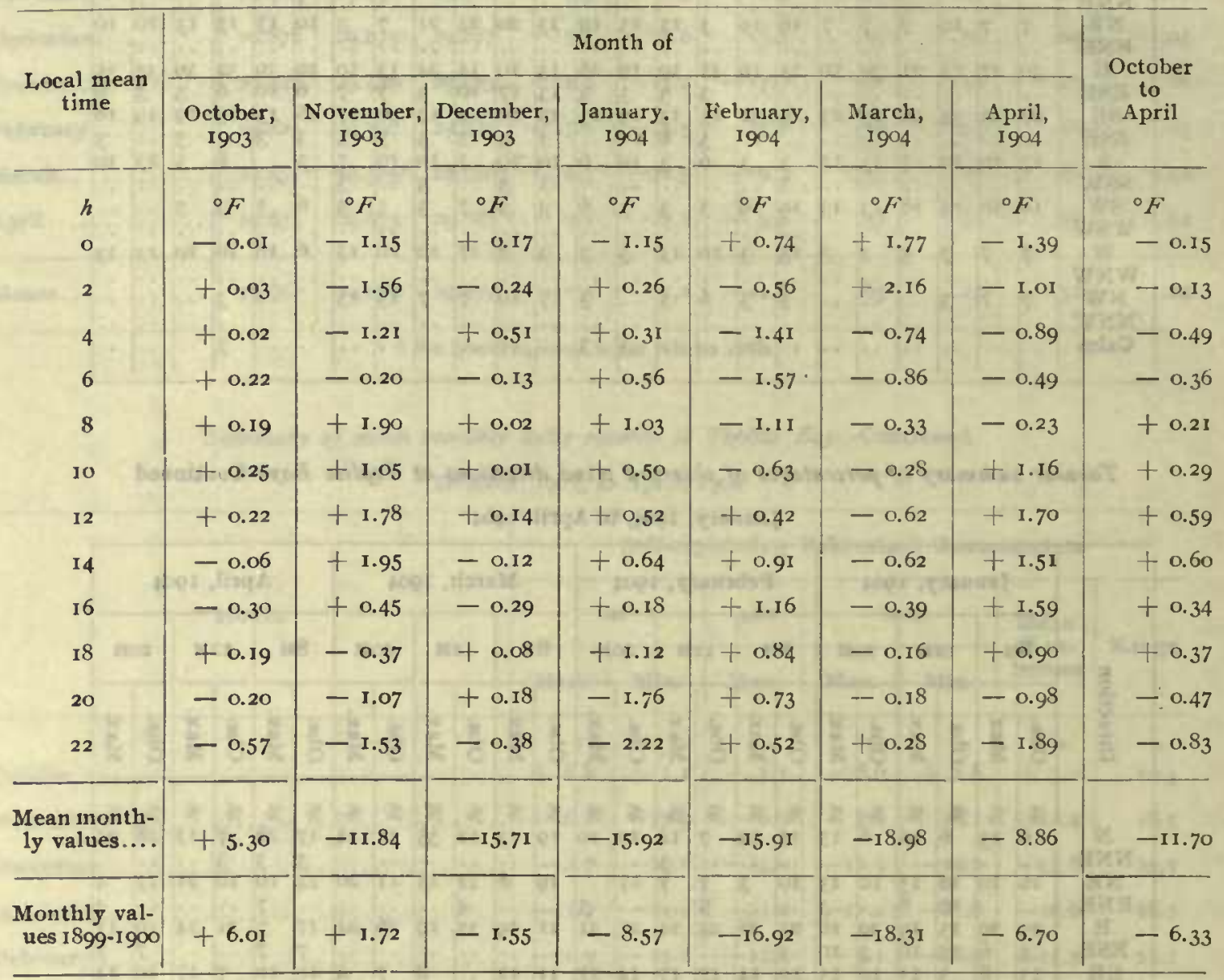

'The above series being of only seven months' duration, wo very elaborate reductions are possible. Analytical expressions representing the daily variation in temperature have been derived from the results by means of Bessel's periodic function (see page 289) to terms of the third order. The resulting amplitudes and phase angles are shown in the following tabulation, while the curves computed from the same are shown in figure 2.

* Osservazioni scientifiche esquite durante la spedizione Polare di S. A. R. Luigi Amedeo di Savoia, Duca degli Abruzzi, 1899-1900. Milan, I903. Pp. 33I-357. (Relazione sulle osservazioni meteorologiche fatta dal Professore Giovanni Battista Rizzo.) 
<smiles>C1CC2C3CC4CC3CC4C2C1</smiles> 


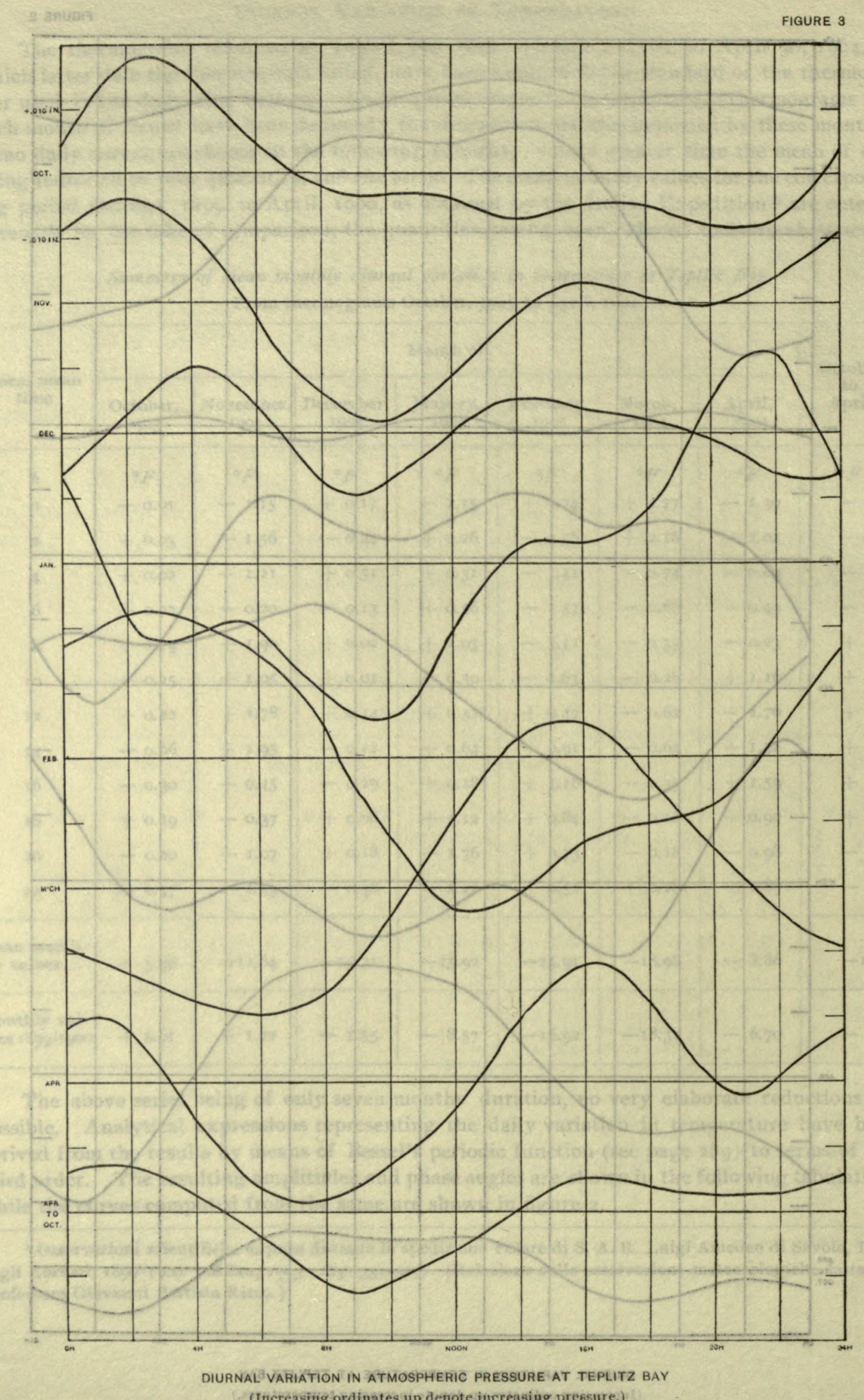




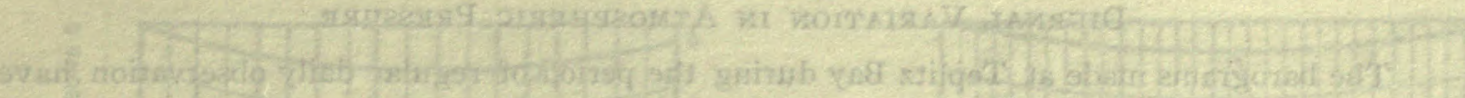

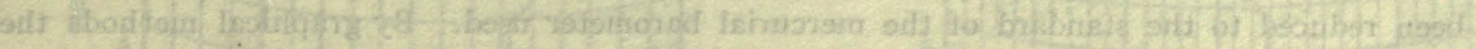

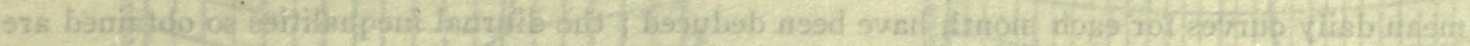

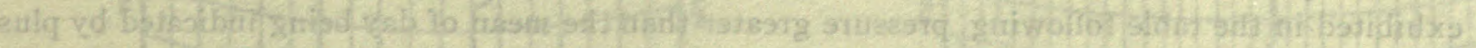

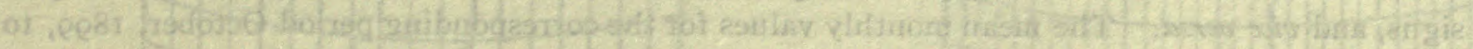

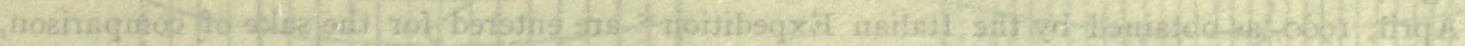

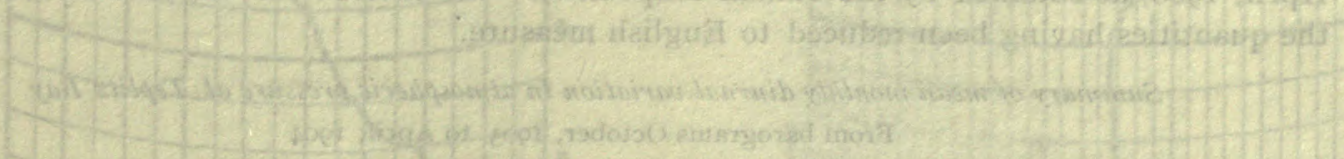

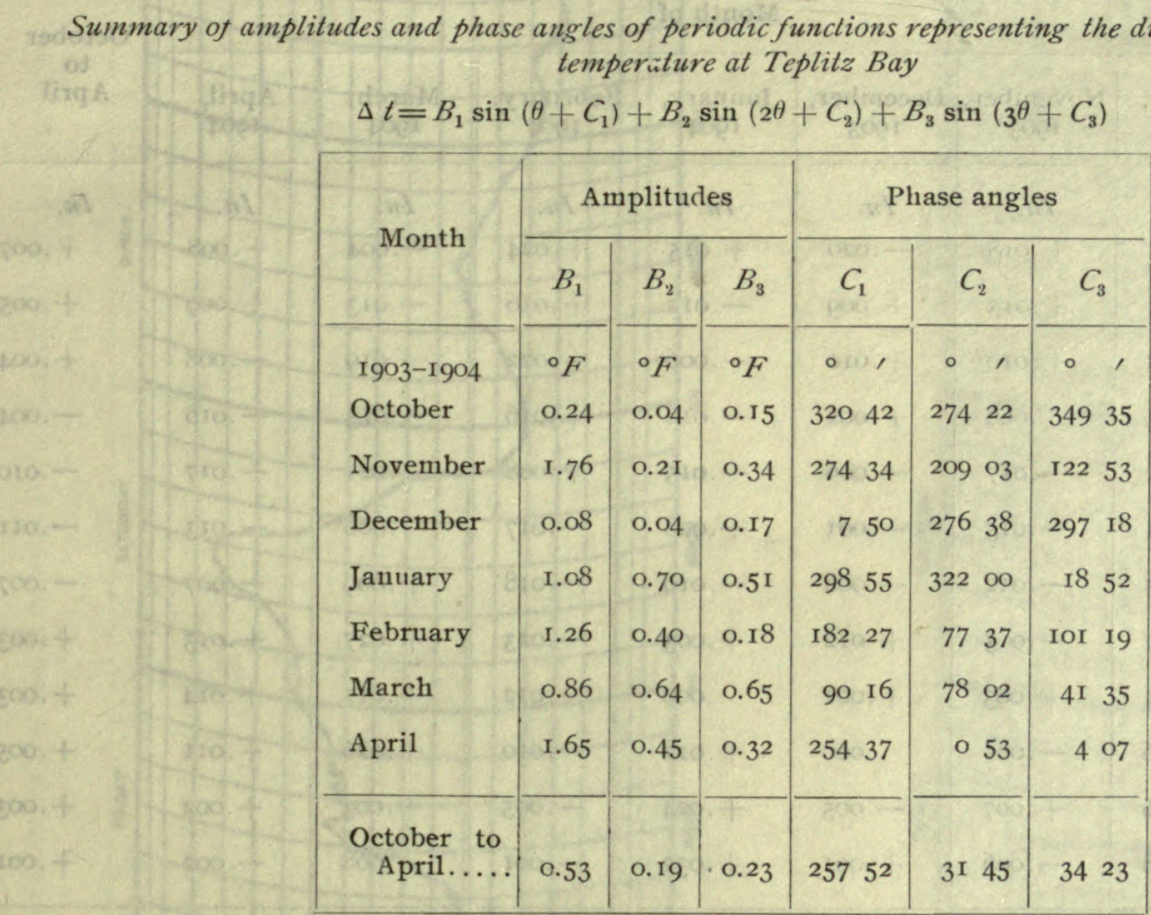

\footnotetext{
ero. ext

NoTF.-In these expressions the angle $\theta$ is to be reckoned from o hour A. M. as $0^{\circ}$.
}

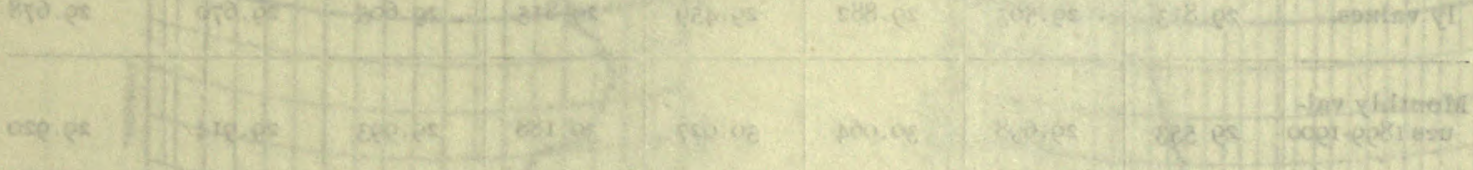

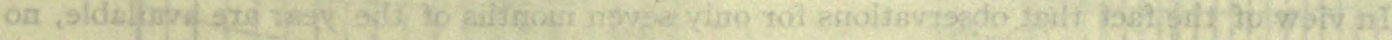

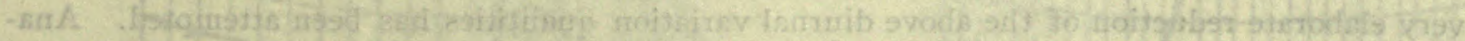

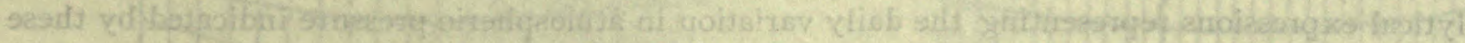

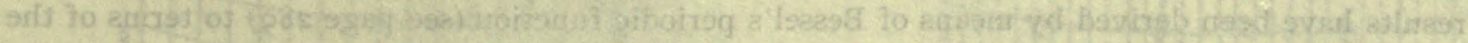

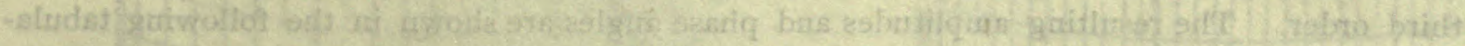

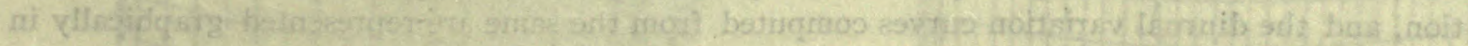




\section{Diurnal Variation in Atmospheric Pressure,}

The barograms made at Teplitz Bay during the period of regular daily observation have been reduced to the standard of the mercurial barometer used. By graphical methods the mean daily curves for each month have been dednced; the diurnal inequalities so obtained are exhibited in the table following, pressure greater than the mean of day being indicated by plus signs, and vice versa. The meau monthly values for the corresponding period October, 1899 , to April, 1900, as obtained by the Italian Expedition* are entered for the sake of comparison, the quantities having been reduced to English measure.

Summary of mean monthly dinrnat variation in almospheric pressure at Teplitz Bay

From barograms Octoher, 1903, to April, 1904

\begin{tabular}{|c|c|c|c|c|c|c|c|c|}
\hline \multirow{2}{*}{$\begin{array}{c}\text { I,ocal mean } \\
\text { time }\end{array}$} & \multicolumn{7}{|c|}{ Month of } & \multirow{2}{*}{$\begin{array}{l}\text { October } \\
\text { to } \\
\text { April }\end{array}$} \\
\hline & $\begin{array}{l}\text { October, } \\
\text { I } 903\end{array}$ & $\begin{array}{c}\text { Novemiber, } \\
1903\end{array}$ & $\begin{array}{c}\text { December, } \\
\text { I903 }\end{array}$ & $\begin{array}{c}\text { January, } \\
\text { Ig04 }\end{array}$ & $\begin{array}{l}\text { February, } \\
\text { I904 }\end{array}$ & $\begin{array}{l}\text { March, } \\
\text { Ig04 }\end{array}$ & $\begin{array}{l}\text { April, } \\
\text { I904 }\end{array}$ & \\
\hline$h$ & In. & $I n$ & $I n$ & $I n$ & $I n$ & $I n$. & $I n$ & $I n$. \\
\hline 0 & +.005 & +.018 & -.020 & +.815 & +.024 & -.004 & +.008 & +.007 \\
\hline 2 & +.015 & +.013 & +.009 & -.012 & +.016 & -.013 & +.009 & +.005 \\
\hline 4 & +.017 & +.oro & +.010 & -.006 & +.022 & -.019 & -.008 & +.004 \\
\hline 6 & +.003 & .000 & +.002 & -.016 & +.016 & -.015 & -.016 & -.004 \\
\hline 8 & -.001 & -.017 & -.006 & -.015 & +.008 & -.021 & -.017 & -.010 \\
\hline IO & -.001 & -.015 & $-.00 I$ & -.028 & -.017 & .000 & -.013 & -.011 \\
\hline 12 & -.005 & -.012 & -.008 & -.010 & -.018 & +.009 & -.007 & -.007 \\
\hline 14 & -.007 & -.005 & + or 2 & +.003 & -.023 & +.027 & +.013 & +.003 \\
\hline 16 & -.004 & +.003 & $+.00 \mathrm{I}$ & .000 & -.012 & +.017 & +.014 & +.003 \\
\hline 18 & -.006 &.$- \infty 1$ & .000 & +.012 & -.010 & +.026 & $+.0 I I$ & +.005 \\
\hline 20 & -.006 & $+\infty 7$ & -.005 & +.024 & -.005 & +.003 & +.002 & +.003 \\
\hline 22 & -.008 & -.006 & +.001 & +.032 & -.001 & -.008 & -.002 &.$+ \infty 01$ \\
\hline $\begin{array}{l}\text { Mean nronth- } \\
\text { ly values.. }\end{array}$ & 29.813 & $29 \cdot 503$ & 29.882 & $29 \cdot 4.59$ & 29.815 & 29.605 & 29.670 & 29.678 \\
\hline $\begin{array}{l}\text { Montbly val- } \\
\text { ues I } 899 \text {-I900 }\end{array}$ & 29.553 & 29.698 & 30.064 & 30.027 & 30.188 & 29.993 & 29.914 & 29.920 \\
\hline
\end{tabular}

In view of the fact that observations for only seven months of the year are available, no very elaborate rednction of the above diurnal variation quautities has been attempted. Analytical expressions representing the daily variation in atmospheric pressure indicated by these results have been derived by means of Bessel's periodic function (see page 289) to terms of the third order. The resulting amplitudes and phase angles are shown in the following tabulation, and the diurnal variation curves comptrted from the same are represented graphically in figure 3 .

* Osservazioni scientifiche esequite durante la spedizione Polare di S. A. R. Luigi Amedeo di Savoia, Duca degii Abruzzi, I899-Ig00. Milan, 1903. Pp. 331-357. (Relazione sulle osservazioni meteorologiche fatta dal Professore Giovanni Battista Rizzo.) 

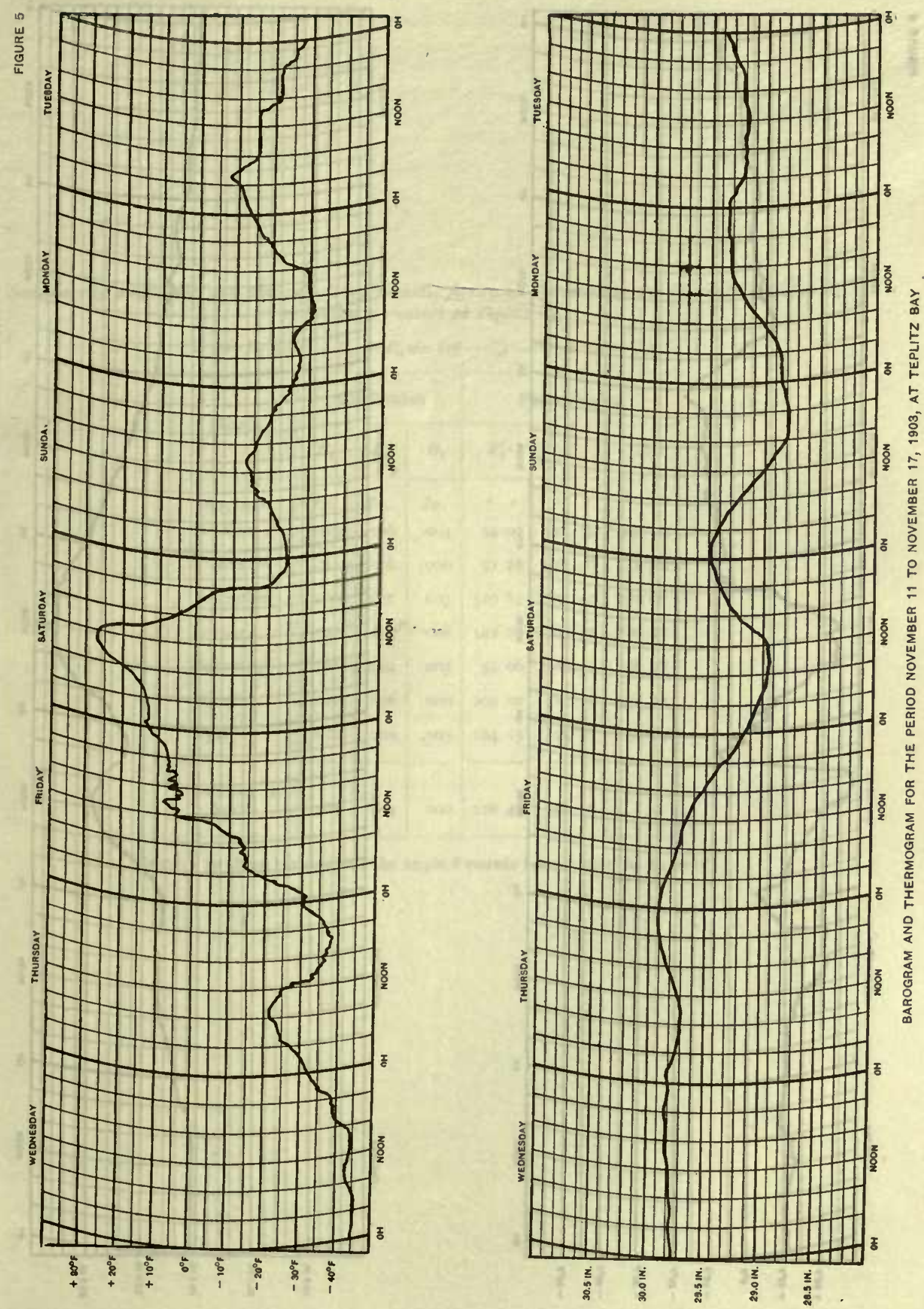

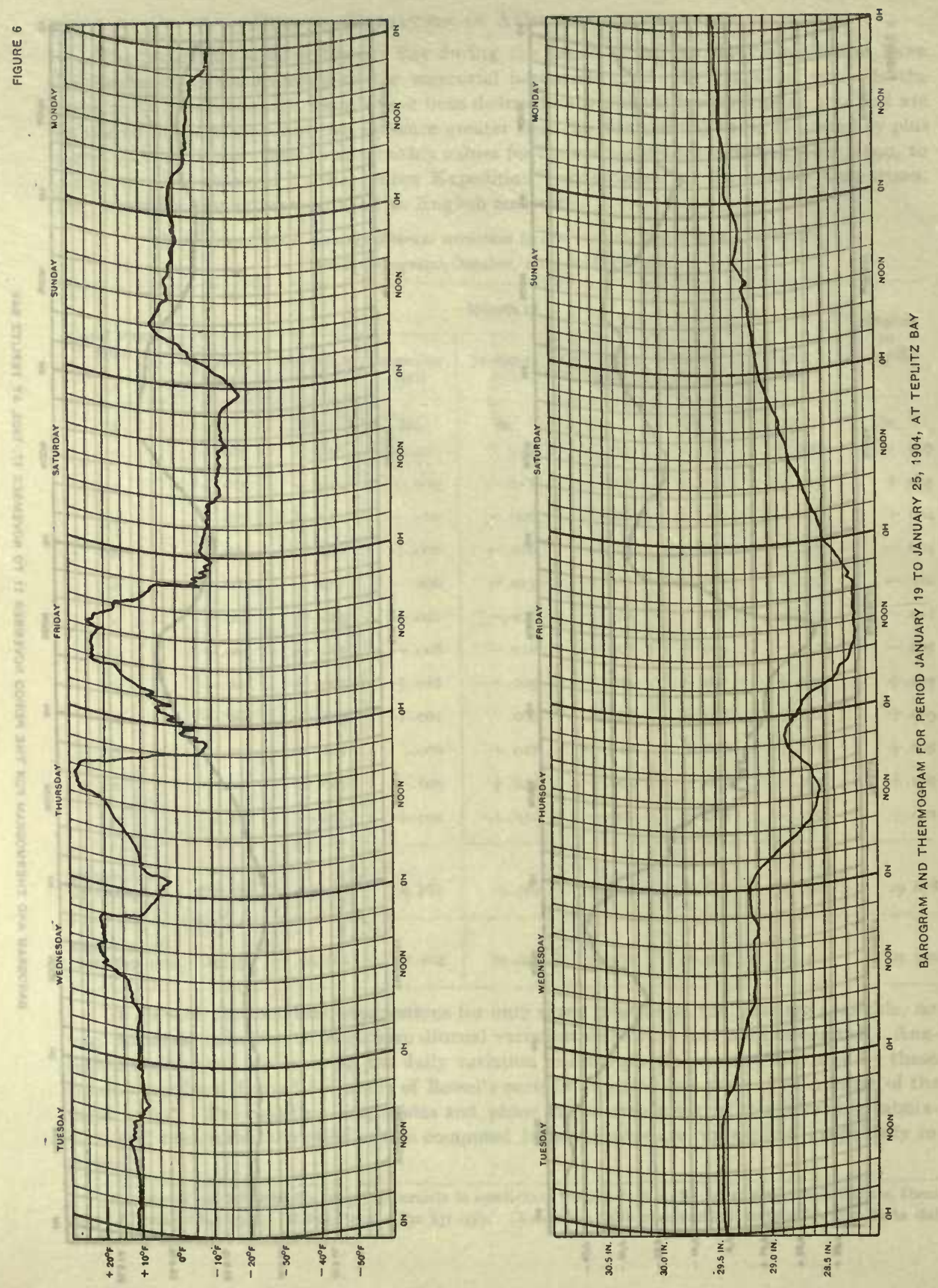


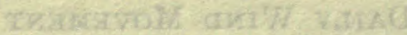

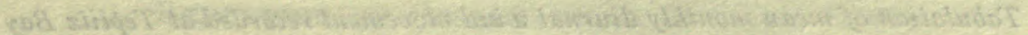

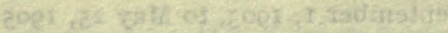

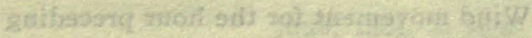

Summary of amplitudes and phase angles of periodic functions representing the diurnal variation in atmospheric pressure at Teplitz Bay

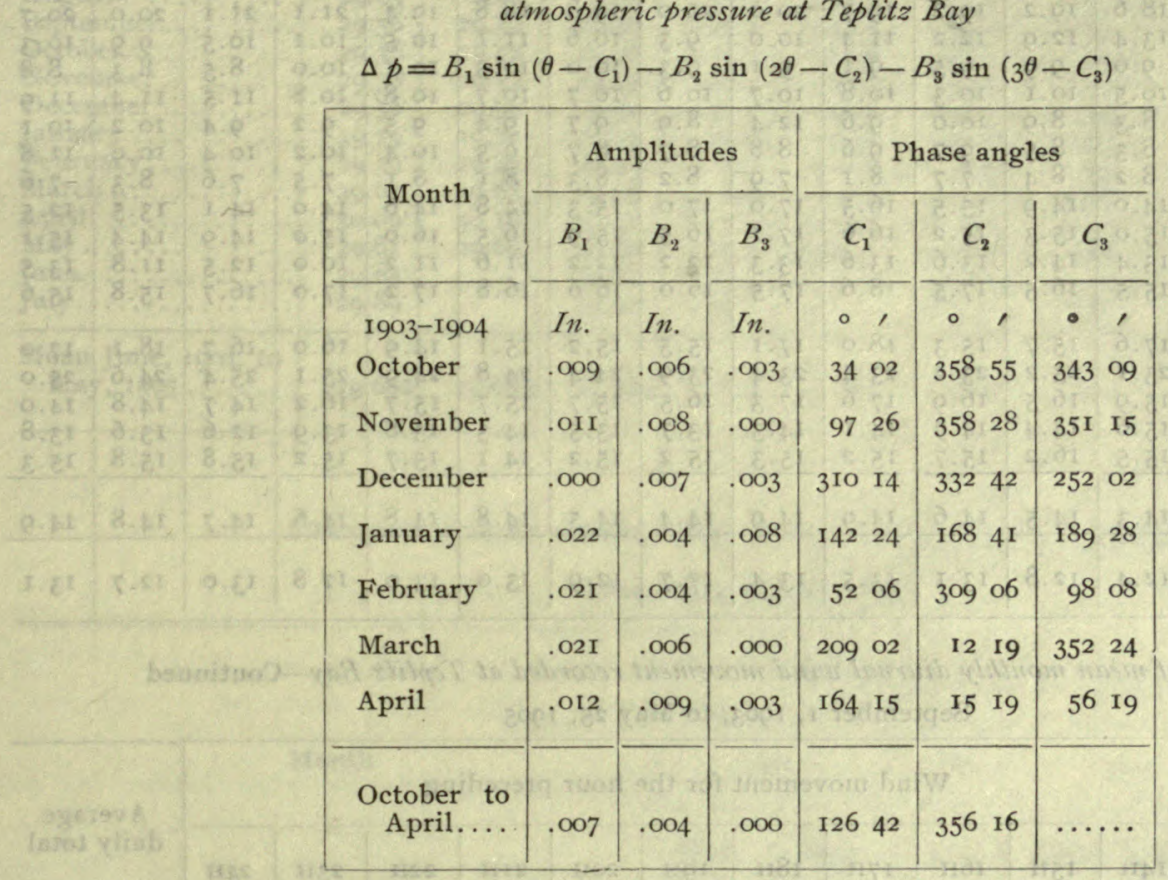

Nore.-In these expressions the angle $\theta$ counts from o hour A. M. as $0^{\circ}$. 


\section{DAILY WIND MOVEMENT}

Tabulation of mean monthly diurnal wind movement recorded at Teplitz Bay

September I, 1903, to May 25, I905

\begin{tabular}{|c|c|c|c|c|c|c|c|c|c|c|c|c|c|}
\hline \multirow{2}{*}{ Month } & \multicolumn{11}{|c|}{ Wind movement for tbe hour preceding } & \multirow[b]{2}{*}{$12 \mathrm{H}$} & \multirow[b]{2}{*}{ I3H } \\
\hline & IH & $2 \mathrm{H}$ & $3 \mathbf{H}$ & $4 \mathbf{H}$ & $5 \mathbf{H}$ & $6 \mathrm{HI}$ & $7 \mathrm{H}$ & $8 \mathrm{H}$ & $9 \mathrm{H}$ & IOH & I I H & & \\
\hline 1903 & $M i$. & $M i$ & $M i$. & $M I i$ & $M i$ & $M T i$ & $M i$ & $M i$ & $M i$ & $M i$. & $M i$ & $M / i$ & Mi. \\
\hline September .. & 12.2 & 12.9 & 12.2 & I I. 5 & & 10.3 & 10.3 & II. 5 & II. I & 12.2 & 13.8 & 14.0 & I2.I \\
\hline October........... & 12.4 & 13.2 & $13 \cdot 3$ & $4 \cdot 7$ & & 15.6 & 14.4 & 15.6 & $16 . t$ & 14. I & 14.5 & 14.6 & I5.0 \\
\hline November . ........ & 16.2 & 15.8 & 15.4 & I6. I & .0 & 15.1 & 15.8 & 15.1 & 15.0 & 14.8 & I 4.0 & 15.2 & 16.0 \\
\hline $\begin{array}{c}\text { December. . . . . . . . } \\
\text { I904 }\end{array}$ & 23.2 & 22.4 & 22.3 & 20.7 & 22.5 & 23.7 & 25.4 & 28.0 & $27 \cdot 5$ & 25.6 & 25.2 & 26.6 & 26.8 \\
\hline January........... & I4.I & I $4 \cdot 4$ & I6. 2 & 18.2 & I7. 4 & $15 \cdot 7$ & 17.2 & 18.2 & I8. 4 & 19. I & I8 5 & $18 \cdot 3$ & 19.7 \\
\hline February.. ........ & I8.6 & 19.2 & 19.2 & 19.7 & 0.0 & 20.0 & 19.8 & 18.8 & 19.4 & $2 I . I$ & $2 \mathrm{I} . \mathrm{I}$ & 20.0 & 20.7 \\
\hline 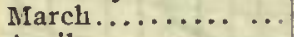 & 13.4 & 12.9 & 12.2 & I I. 4 & 10.0 & $9 \cdot 3$ & 10.6 & I I . I & 10.5 & IO. I & I0. 5 & 9.9 & IO. 3 \\
\hline April.............. & 9.0 & 9.9 & $9 \cdot 5$ & $9 . I$ & 9.1 & $9 \cdot 3$ & IO. 0 & IO. 5 & IO. I & IO. 0 & 8.5 & 8.3 & 8.8 \\
\hline May... ............ & 10.5 & IO. I & Io. 3 & I0. 8 & 10.7 & 10 6 & 10.7 & 10.7 & 10.8 & 10.8 & II. 5 & II. 4 & II .9 \\
\hline Juse.............. & 8.3 & 8.9 & 10.0 & 9.6 & 12.4 & 8.9 & $9 \cdot 7$ & $9 \cdot 4$ & $9 \cdot 5$ & 9.2 & $9 \cdot 4$ & 10.2 & IO. I \\
\hline 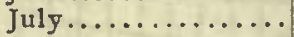 & $8 \cdot 3$ & 8.5 & 9.7 & 9.6 & 8.8 & 8.7 & 8.7 & 9.5 & 10.4 & I0. 2 & so. 4 & IO. 9 & II. 8 \\
\hline August........... & 8.2 & 8.4 & 7.7 & 8.1 & $7 \cdot 9$ & 8.2 & 8.3 & 8. I & 8. I & $7 \cdot 5$ & 7.6 & 8.3 & 7.6 \\
\hline September......... & 14.0 & 14.9 & $15 \cdot 5$ & 16.5 & 17.0 & 17.0 & $15 \cdot 3$ & 14.8 & I 4.6 & I 4.0 & 14.1 & 13.5 & 12.5 \\
\hline October. ......... & 15.0 & $15 \cdot 3$ & 15.2 & 16.6 & 17.1 & 16.5 & I5.9 & 16.5 & 16.0 & I 5.0 & 14.9 & 14.4 & I5. I \\
\hline November... ...... & 13.4 & 14.2 & 13.6 & 13.6 & 13.3 & 12.2 & 12.2 & I I. 6 & II. 2 & 10.0 & 12.5 & I 1.8 & I 3.5 \\
\hline December....... & I5. 5 & 16.5 & $17 \cdot 5$ & 18.6 & 17.5 & 16.0 & I6.6 & 16.8 & 17.2 & 17.0 & 16.7 & I5.8 & I5.6 \\
\hline $\begin{array}{r}1905 \\
\text { Ja11uary....... }\end{array}$ & I & 5.7 & I5. & 18.0 & & & I 5.2 & & & I6.0 & I6. 7 & IS. I & 17.0 \\
\hline February... & 23.1 & 23.2 & 23.2 & 23.4 & 23.4 & 23.9 & $24 \cdot 4$ & 24.8 & $24 \cdot 9$ & 25.1 & $25 \cdot 4$ & 24.6 & 25.0 \\
\hline Marcl ............ & 15.9 & 165 & 16.9 & 17.6 & $17 \cdot 3$ & 16.5 & $15 \cdot 7$ & I5.7 & 15.7 & 16.2 & 14.7 & 14.8 & 14.0 \\
\hline 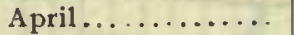 & 15.6 & 14.4 & 14.9 & I4. I & $14 \cdot 3$ & I3.7 & 13.5 & I $4 \cdot 3$ & 13.8 & I3.9 & 12.6 & $I_{3} .6$ & 13.8 \\
\hline May $\ldots \ldots \ldots \ldots$ & 15.5 & 16.2 & 15.7 & 15.2 & I5.3 & 15.2 & 15.2 & 14.1 & 15.7 & 15.2 & 15.8 & J 5.8 & 15.3 \\
\hline Mean of all......... & $14 \cdot 3$ & 14.5 & 14.6 & 14.9 & 14.9 & I 4.4 & $\mathrm{~T} 4.5$ & 14.8 & I 4.8 & $\mathrm{I} 4.6$ & 14.7 & $\mathrm{I} 4.8$ & 14.9 \\
\hline Mean year $1904 .$. & 12.4 & 12.8 & $13 . I$ & I3. 5 & I 3.4 & 12.7 & 12.9 & I3.0 & 13.0 & 12.8 & 13.0 & 12.7 & I3.I \\
\hline
\end{tabular}

Tabulation of mean monthly diurnal wind movement recorded at Teplitz Bay-Contiuued

September I, 1903, to May 25, 1905

\begin{tabular}{|c|c|c|c|c|c|c|c|c|c|c|c|c|}
\hline \multirow{2}{*}{ Moutb } & \multicolumn{11}{|c|}{ Wind movement for the bour preceding } & \multirow{2}{*}{$\begin{array}{l}\text { Average } \\
\text { daily total }\end{array}$} \\
\hline & $\mathrm{I} 4 \mathrm{H}$ & I5 H & I6u & I7H & I8II & 194 & $20 I I$ & 2 IH & $22 \mathrm{H}$ & $23 \mathrm{H}$ & $24 \mathrm{IH}$ & \\
\hline I903 & $M i$. & $M i$. & $M i$. & $M I i$. & $M i$. & $M i$. & $M i$. & $M / i$ & $M i$. & $M i$. & Mri. & $M i$. \\
\hline September . & II.3 & 12.3 & 12.6 & I 3.8 & 14.6 & 13.8 & 14.8 & $14 \cdot 3$ & $14 \cdot 5$ & 13.4 & I3.1 & 302.9 \\
\hline October. .......... & I5. I & 14. I & 12.2 & I I.O & 12.5 & I I. 4 & I I. 2 & 10.9 & I I. 2 & II 0 & II. 6 & 320.4 \\
\hline November........ & I6. 7 & 15.5 & I6. I & 15.9 & 5.6 & 15.9 & 16.9 & 17.4 & 16.4 & 16.9 & 16.8 & 380.7 \\
\hline Deceinber. ...... . & 25.8 & 26.3 & 26.4 & $24 \cdot 5$ & 22.8 & $2 \mathrm{I} .8$ & $25 \cdot 3$ & 24 . I & 24.7 & $23 \cdot 7$ & 24.9 & 590.3 \\
\hline 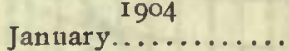 & 18.2 & 17.0 & I6. 6 & I7.8 & 18.3 & 18.4 & 170 & 13.9 & 13.5 & 13.4 & 14.4 & 403.8 \\
\hline February........... & I9. I & 17.0 & I6. 3 & 16.2 & 16.6 & I6.7 & 16.2 & I5. 4 & I5.6 & 17.6 & $\begin{array}{l}4.4 \\
17.1\end{array}$ & $\begin{array}{l}403.0 \\
441.4\end{array}$ \\
\hline March. . .......... & 10. I & 10.1 & I2. I & 12.9 & $14 \cdot 5$ & 14.0 & 13.0 & 13.0 & I3. I & I 2.3 & I 2.5 & 279.7 \\
\hline April. .. & 8.5 & 8.4 & 8.8 & 9.3 & 10.6 & 10. 8 & 10.2 & $9 \cdot 9$ & $9 \cdot 4$ & 8.6 & 8.8 & $225 \cdot 3$ \\
\hline May............. & 12.6 & 12.6 & 12.4 & II.9 & II $\cdot 3$ & II. 9 & I0.9 & 10.9 & I I. 5 & I I . 2 & 10. 6 & 268.5 \\
\hline June............. & 10.3 & IO. I & 9.6 & 9.7 & 8.9 & 8.7 & 8.6 & 9.2 & IO. I & 9.7 & 9.5 & 230.0 \\
\hline July ............ & 12.2 & II. 3 & I I. I & II. 4 & 10.5 & 10.4 & $9 \cdot 9$ & 10.3 & 10.2 & $9 \cdot 5$ & 9.2 & $241 \cdot 3$ \\
\hline A ugist............. & 7.8 & 8.I & 7.8 & $7 \cdot 3$ & 7.8 & 9.0 & 8.9 & 9.6 & 9.2 & 8. 0 & 8.6 & 196.0 \\
\hline September ...... & I 2.4 & 13.4 & 13.4 & I3. I & 12.1 & 12.0 & I 2.1 & 12.5 & 12.5 & I3.O & 13.9 & 334.1 \\
\hline October .......... & 16.8 & 16.7 & I 5.9 & 16.8 & 176 & 18.0 & 17.0 & 15.6 & I5. 4 & $15 \cdot 5$ & $15 \cdot 3$ & 384.2 \\
\hline November........ & 13.0 & 13.9 & 13.7 & 13.7 & $13 \cdot 3$ & 12.4 & 12.2 & II . 6 & I I. 5 & 12.1 & 12.3 & 302.8 \\
\hline Deceniber ......... & $\mathrm{I} 6.4$ & I5.5 & I6. I & 16.2 & I 5.5 & 14.2 & I 3.4 & I5.9 & 15.9 & 16.5 & I 6.4 & 389.7 \\
\hline January ......... & I6.8 & 16.9 & 17.0 & 17.8 & I6.7 & I8. 5 & I8. I & 17.8 & I 8.5 & I7. 4 & 16.4 & 404.0 \\
\hline February.. ........ & $25 . I$ & $25 \cdot 3$ & $24 \cdot 9$ & 26.3 & $25 \cdot 5$ & $2 I \cdot 3$ & $23 . I$ & $25 . I$ & 24.8 & 23.2 & 22.6 & $58 \mathrm{I} \cdot 4$ \\
\hline 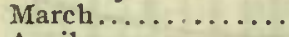 & 13.5 & 14.2 & I3. I & 13.0 & I4. I & I4. I & I 3.9 & 15.1 & I6. I & I 6.4 & I5. 7 & 366.8 \\
\hline A pril............ & 13.5 & $12 \cdot 3$ & 12.8 & I3.7 & $14 \cdot 2$ & 14.0 & $\mathrm{I} 5.6$ & 16.6 & 17.6 & 17.2 & I6. I & 346.2 \\
\hline 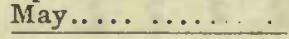 & I5.8 & I5.2 & 16.3 & 15.9 & 14.6 & 14.5 & 15.5 & I5.2 & I5.2 & I0.6 & 16.0 & 371.5 \\
\hline Mean of all ......... & 14.8 & 14.4 & 14.5 & I4. 7 & 14.6 & $14 \cdot 4$ & 14.5 & $14 \cdot 5$ & 14.6 & I 4.4 & 14.4 & 350.5 \\
\hline Mean year $1904 .$. & I3. I & 12.8 & 12.8 & 13.0 & I3 I & 130 & 12.4 & I2 3 & I 2.3 & I 2.3 & 12.4 & 308.1 \\
\hline
\end{tabular}




\section{Reduction of Observations at Cape Flora}

Summary of mean monthly daily records at Cape Flora

June, I904, to July, 1905

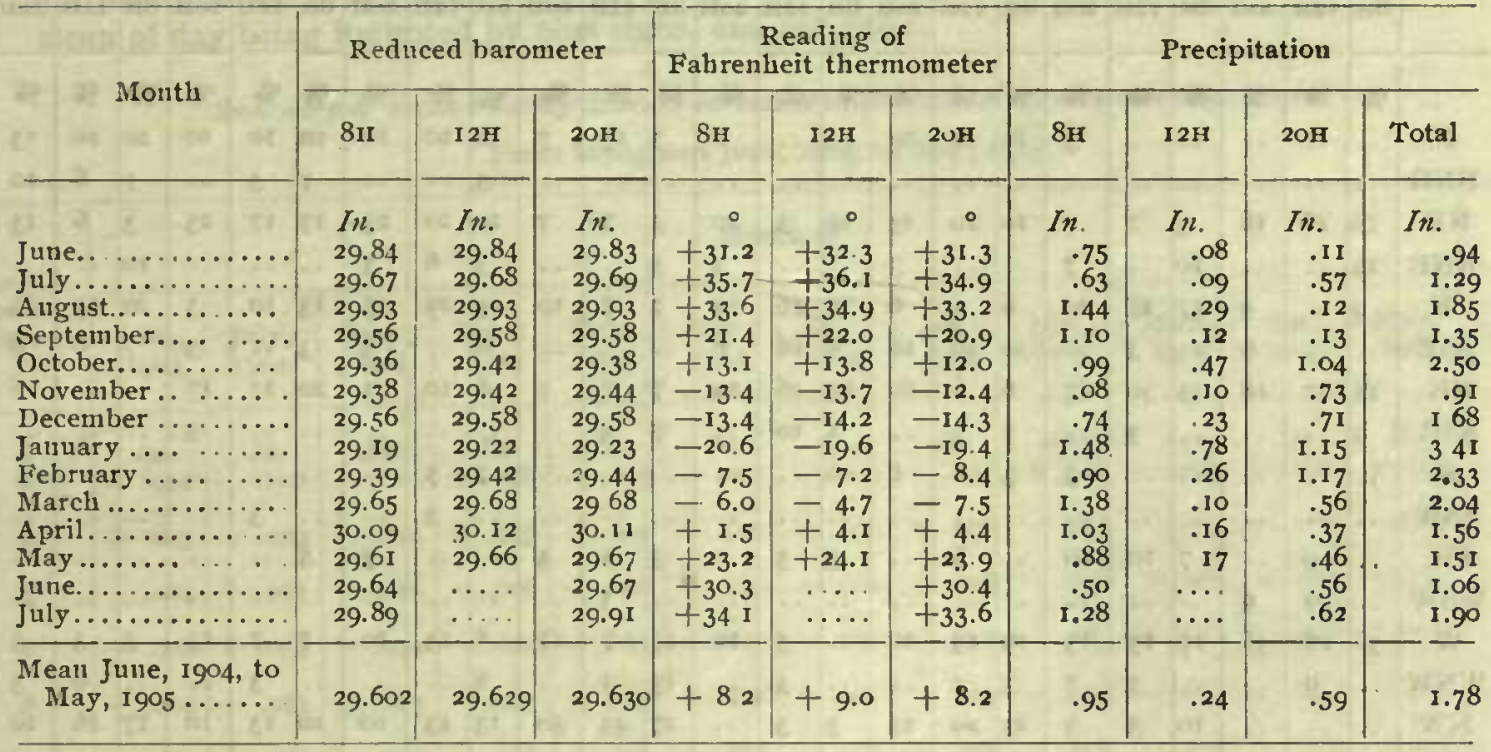

Summary of mean monthly daily records at Cape Flora-Continued

June, Igo4, to July, Igo5

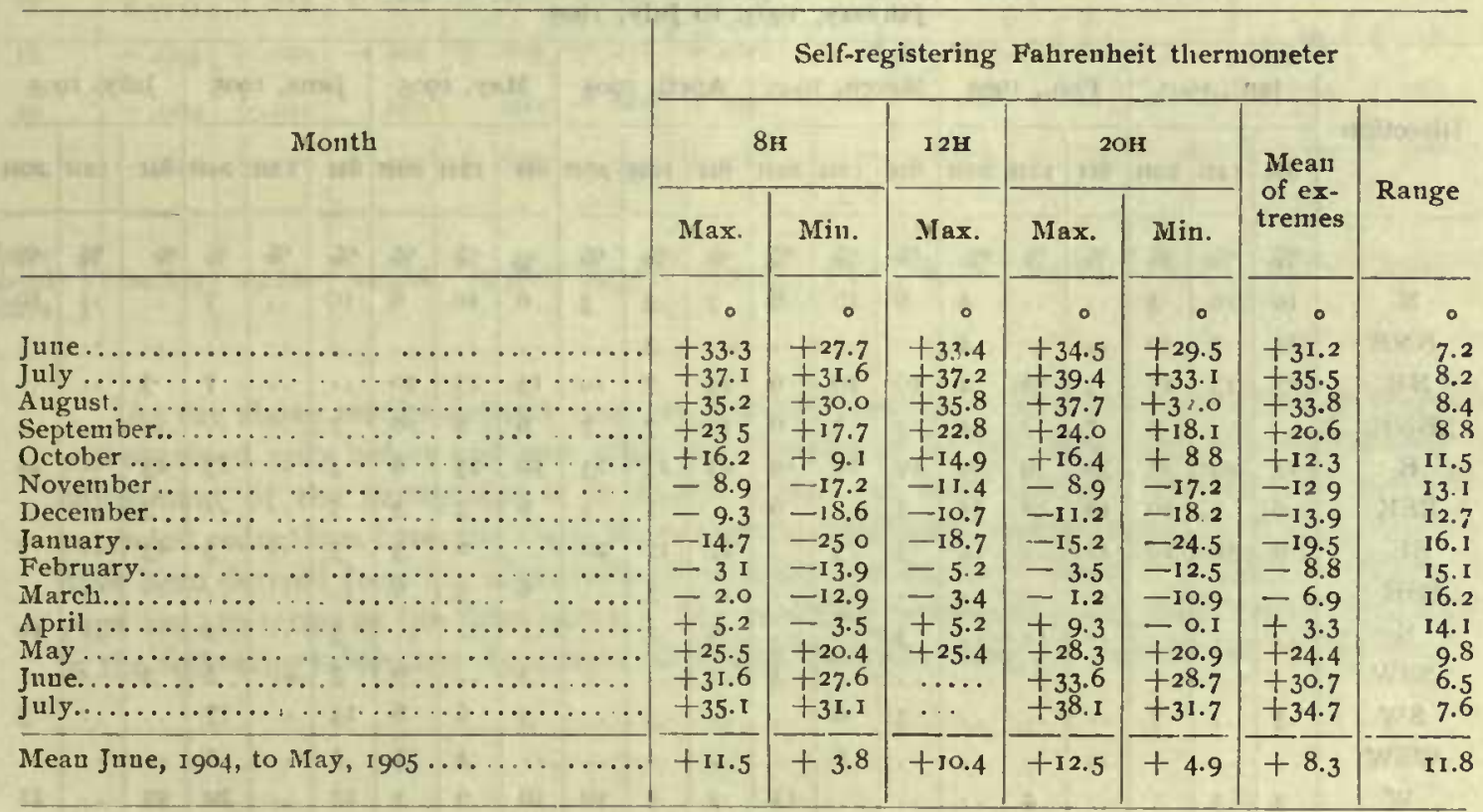


Tabular summary of percentages of observed wind diredions al Cape Flora

May, Ig04, to December, 1904

\begin{tabular}{|c|c|c|c|c|c|c|c|c|c|c|c|c|c|c|c|c|c|c|c|c|c|c|c|c|}
\hline \multirow{2}{*}{$\begin{array}{c}\text { Direc- } \\
\text { tion }\end{array}$} & \multicolumn{3}{|c|}{ May, 1904} & \multicolumn{3}{|c|}{ June, Igo4 } & \multicolumn{3}{|c|}{ July, 1904} & \multicolumn{3}{|c|}{ Aug., 1904} & \multicolumn{3}{|c|}{ Sept., 1904} & \multicolumn{3}{|c|}{ Oct., IgO4 } & \multicolumn{3}{|c|}{ Nov., 1904} & \multicolumn{3}{|c|}{ Dec., 1904} \\
\hline & 811 & $12 \mathrm{H}$ & $2 \mathrm{OH}$ & 811 & $\mathrm{I} 2 \mathrm{H}$ & $2 \mathrm{OH}$ & $3 \mathrm{~F}$ & $\mathrm{I} 2 \mathrm{H}$ & $2 \mathrm{OH}$ & $8 \mathrm{H}$ & $12 \mathrm{H}$ & $2 \mathrm{OH}$ & $\mathrm{SH}_{\mathrm{H}}$ & $12 \mathrm{H}$ & $2 \mathrm{OH}$ & $8 \mathrm{H}$ & $12 \mathrm{H}$ & $20 I I$ & $8 \mathrm{H}$ & $12 \mathrm{H}$ & 2011 & 811 & I $2 \mathrm{H}$ & $20 \mathrm{H}$ \\
\hline & $\%$ & $\%$ & $\%$ & $\%$ & $\%$ & $\%$ & $\%$ & $\%$ & $\%$ & $\%$ & $\%$ & $\%$ & $\%$ & $\%$ & $\%$ & $\%$ & $\%$ & $\%$ & $\%$ & $\%$ & $\%$ & $\%$ & $\%$ & $\%$ \\
\hline $\mathbf{N}$ & . & . & $\cdots$ & .. & . & 3 & 10 & . & I0 & .. & . & 6 & 7 & 13 & 7 & 10 & 10 & 10 & 10 & 10 & 10 & 20 & 10 & 13 \\
\hline NNE, & .. & .. & .. & .. & .. & .. & .. & .. & .. & .. & .. & .. & . & .. & .. & 3 & .. & . & 7 & 3 & 0 & 7 & 6 & 10 \\
\hline $\mathrm{NE}$ & 18 & 18 & 18 & 3 & 7 & 7 & 10 & 10 & I3 & 16 & 3 & Io & 3 & 7 & 7 & 10 & 10 & 23 & $\mathrm{I} 7$ & 17 & 23 & 3 & 6 & 13 \\
\hline ENE & I9 & $\therefore$ & . & 10 & .. & 7 & .. & . & 3 & .. & .. & 3 & 3 & . & . & 3 & 6 & 3 & . & . & .. & 10 & - & .. \\
\hline E & 9 & . & 9 & 17 & 27 & 20 & 6 & 3 & 6 & 29 & 26 & 19 & 7 & 7 & 10 & 29 & 29 & 35 & 13 & 10 & 3 & 20 & 29 & 23 \\
\hline ESE & & .. & 9 & . & 3 & .. & IO & 10 & I6 & 6 & 16 & 6 & .. & .. & .. & 6 & .. & 3 & 3 & . & 3 & 7 & 6 & 6 \\
\hline SE & 18 & 27 & 18 & 33 & 30 & 23 & 6 & 6 & Io & 29 & 26 & 29 & 7 & 7 & 7 & 6 & 10 & 3 & 20 & 17 & 17 & .. & . & 6 \\
\hline SSE & 9 & .. & 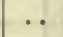 & .. & 3 & . & 3 & .. & .. & 3 & Io & .. & 7 & 3 & .. & .. & . & .. & .. & .. & 3 & $\cdots$ & $\cdots$ & . \\
\hline$s$ & .. & . & . & .. & . & 3 & 3 & 6 & 6 & 6 & . & .. & & .. & .. & 3 & 3 & .. & . & . & .. & .. & .. & .. \\
\hline Ssw & .. & .. & & $\therefore$ & .. & $\cdots$ & .. & .. & . & $\cdots$ & . & .. & - & . & . & .. & 3 & .. & .. & 3 & . & . & . & . \\
\hline SW & .. & 9 & •. & 7 & Io & 10 & .. & 6 & . & 3 & 3 & $\therefore$ & 3 & 3 & 3 & . & . & 3 & 3 & .. & $\cdots$ & 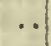 & . & .. \\
\hline WSW & .. & 9 & 9 & .. & . & $\cdots$ & . & . & .. & .. & .. & . & & 3 & . & . & .. & .. & . & . & $\cdots$ & $\cdots$ & $\cdots$ & . \\
\hline W & $3^{6}$ & 27 & $3^{6}$ & 17 & 13 & 13 & 19 & 19 & 10 & . & 3 & 10 & 13 & 7 & 17 & 6 & 13 & 10 & 7 & 7 & 13 & 3 & 3 & 3 \\
\hline WNW & .. & 9 & . & . & 3 & 7 & 3 & 3 & .. & .. & 3 & .. & $I_{3}$ & 3 & .. & 6 & .. & .. & - & 3 & .. & .. & 6 & 3 \\
\hline NW & .. & .. & .. & Io & 3 & 3 & 23 & 29 & 23 & 3 & 3 & . & 27 & 43 & 50 & I3 & I3 & 10 & Io & 13 & 10 & 17 & 16 & 10 \\
\hline NNW & .. & . & .. & .. & $\cdots$ & . & 3 & .. & $\cdots$ & .. & $\cdots$ & . & 3 & $\cdots$ & . & .. & $\cdots$ & $\cdots$ & . & $\cdots$ & .. & 3 & . & .. \\
\hline Calm & . & .. & .. & 3 & • & 3 & 3 & 6 & 3 & 3 & 6 & I6 & 7 & 3 & .. & 3 & 3 & .. & 10 & 17 & 17 & 10 & J6 & 13 \\
\hline
\end{tabular}

Tabular summary of percenlages of observed wind directions at Cape Flora January, I905, to July, 1905

\begin{tabular}{|c|c|c|c|c|c|c|c|c|c|c|c|c|c|c|c|c|c|c|c|c|c|}
\hline \multirow{3}{*}{ Direction } & \multicolumn{3}{|c|}{ Jan., 1905} & \multicolumn{6}{|c|}{\begin{tabular}{|l|l|} 
Feb., I905 & Marcli, 1905 \\
\end{tabular}} & \multicolumn{3}{|c|}{ April, I905 } & \multicolumn{3}{|c|}{ May, 1905} & \multicolumn{3}{|c|}{ June, 1905} & \multicolumn{3}{|c|}{ July, 1905} \\
\hline & & & & & & & & & & & & & & & & & & & & & \\
\hline & $8 \mathrm{H}$ & $12 \mathrm{H}$ & $2 \mathrm{OH}$ & $8 \mathbf{H}$ & $12 \mathrm{H}$ & $20 \mathrm{H}$ & $8 \mathrm{H}$ & $12 \mathrm{H}$ & $20 \mathrm{H}$ & $8 \mathrm{H}$ & $\mathrm{I2H}$ & $2 \mathrm{OH}$ & $8 \mathbf{H}$ & $12 \mathrm{~B}$ & $20 \mathrm{H}$ & $8 \mathbf{H}$ & I $2 \mathrm{H}$ & 2011 & $3 \mathbf{H}$ & $12 \mathrm{H}$ & $2 \mathrm{OH}$ \\
\hline & $\%$ & $\%$ & $\%$ & $\%$ & $\%$ & $\%$ & $\%$ & $\%$ & $\%$ & $\%$ & $\%$ & $\%$ & $\%$ & $\%$ & $\%$ & $\%$ & $\%$ & $\%$ & $\%$ & $\%$ & $\%$ \\
\hline $\mathbf{N}$ & 10 & I6 & 3 & . & . & 4 & 6 & 16 & 6 & 7 & 3 & 3 & 6 & Io & 6 & 10 & . & 7 & $\cdots$ & . & 10 \\
\hline NNE & I3 & 3 & 10 & . & . & 4 & . & $\cdots$ & . & . & 3 & & . & . & $\cdots$ & . & . & $\cdots$ & . & . & . \\
\hline NE & 13 & 13 & 17 & 7 & 18 & 4 & 16 & 10 & 6 & 13 & 7 & IO & 13 & 13 & 10 & .. & . & 7 & 3 & $\cdots$ & . \\
\hline FNE & .. & . & 3 & 7 & 7 & 4 & 3 & 6 & 6 & IO & 7 & 7 & 6 & 6 & I0 & 7 & . & . & . & . & .. \\
\hline$E$ & 23 & 29 & 23 & $3^{2}$ & 39 & 39 & I9 & I6 & 19 & 23 & 23 & 23 & 16 & 23 & 6 & 3 & . & 13 & 13 & . & 24 \\
\hline ESE & 6 & 6 & IO & I8 & 18 & 11 & 3 & $\cdots$ & 6 & $\cdots$ & 3 & 3 & 6 & 3 & 3 & 3 & $\cdots$ & $\cdots$ & 3 & . & $\cdots$ \\
\hline $\mathrm{SE}$ & 6 & 10 & 10 & II & $\cdots$ & II & 3 & 3 & .. & 27 & 17 & 20 & $\cdots$ & 6 & 3 & 3 & $\cdots$ & 3 & 13 & $\cdots$ & 7 \\
\hline SSE & . & . & $\cdots$ & $\because$ & . & $\because$ & $\cdots$ & $\cdots$ & & $\cdots$ & 3 & & 6 & 3 & 6 & . & $\cdots$ & $\cdots$ & . & .. & $\because$ \\
\hline$S$ & . & . & .. & .. & *. & $\cdots$ & 3 & $\cdots$ & . & 3 & 3 & . & .. & .. & .. & 3 & . & 3 & .. & .. & 10 \\
\hline SSW & - & . & $\therefore$ & . & . & . & . & . & 3 & . & . & . & 3 & . & 6 & 3 & $\cdots$ & 3 & 3 & .. & . \\
\hline SW & 3 & . & 7 & .. & . & .. & 3 & 6 & .. & .. & 3 & . & 3 & 6 & 6 & I3 & . & 17 & .. & . & 3 \\
\hline WSW & .. & .. & . & $\cdots$ & . & $\ldots$ & . & 6 & . & .. & .. & . & $\cdots$ & 3 & 3 & 3 & . & 3 & .. & . & .. \\
\hline W & 3 & 3 & . & . & 4 & .. & . & . & 13 & 7 & 3 & I0 & IO & 6 & 3 & 23 & . & 20 & 23 & .. & 21 \\
\hline WNW & . & $\cdots$ & $\cdots$ & . & .. & .. & 3 & 6 & 3 & $\cdots$ & . & . & $\cdots$ & $\cdots$ & . & 3 & $\cdots$ & Io & . & . & 7 \\
\hline NW & 6 & 3 & 10 & 7 & 7 & 7 & 19 & 16 & 10 & $\cdots$ & . & 3 & 19 & 16 & 26 & 17 & $\cdots$ & 7 & 7 & & 3 \\
\hline NNW & .. & . & .. & .. & . & . & .. & . & 3 & .. & . & . & . & . & . & . & . & . & .. & . & . \\
\hline Calm & 16 & 16 & 7 & 18 & 7 & 18 & I9 & 13 & 23 & 10 & 23 & 20 & 10 & 3 & 10 & 7 & . & 7 & 33 & . & I 4 \\
\hline
\end{tabular}




\section{DIURNal, VARIATION 1N AtMospheric Pressure}

The barograms at Cape Flora have been reduced to the aneroid barometer used at this station and the mean daily curves for each month deduced graphically. The values of the diurnal variation thus obtained are given in the following tabulation, pressures greater than mean of day being indicated by plus signs, and vice versa.

Summary of mean monthly diurnat variation in atmospheric pressure at Cape Flora

From barograms June, 1904, to May, 1905

\begin{tabular}{|c|c|c|c|c|c|c|c|c|c|c|c|c|c|}
\hline \multirow{2}{*}{$\begin{array}{c}\text { Local } \\
\text { meau } \\
\text { time }\end{array}$} & \multirow[b]{2}{*}{$\begin{array}{c}\text { June, } \\
\text { 1904 }\end{array}$} & \multirow[b]{2}{*}{$\begin{array}{l}\text { July, } \\
\text { I } 904\end{array}$} & \multirow[b]{2}{*}{$\begin{array}{l}\text { Aug., } \\
\text { 1904 }\end{array}$} & \multirow[b]{2}{*}{$\begin{array}{l}\text { Sept., } \\
1904\end{array}$} & \multirow[b]{2}{*}{$\begin{array}{l}\text { Oct., } \\
\text { I } 904\end{array}$} & \multicolumn{2}{|c|}{ Month of } & \multirow[b]{2}{*}{$\begin{array}{l}\text { Jaut, } \\
\text { I905 }\end{array}$} & \multirow[b]{2}{*}{$\begin{array}{l}\text { Feb., } \\
1905\end{array}$} & \multirow[b]{2}{*}{$\begin{array}{l}\text { M'ch, } \\
\text { I905 }\end{array}$} & \multirow[b]{2}{*}{$\begin{array}{l}\text { Apr., } \\
\text { I905 }\end{array}$} & \multirow[b]{2}{*}{$\begin{array}{l}\text { May, } \\
1905\end{array}$} & \multirow{2}{*}{$\frac{\text { Year }}{\begin{array}{c}\text { June, Ig04, } \\
\text { to May, } \\
\text { I905 }\end{array}}$} \\
\hline & & & & & & $\begin{array}{l}\text { Nov., } \\
\text { Igo04 }\end{array}$ & $\begin{array}{l}\text { Dec., } \\
1904\end{array}$ & & & & & & \\
\hline $\begin{array}{l}h \\
0\end{array}$ & $\begin{array}{c}\text { In. } \\
+. .002\end{array}$ & In. & $\begin{array}{l}I n . \\
-.00 \mathrm{~T}\end{array}$ & $\begin{array}{r}\text { In. } \\
+.018\end{array}$ & $\begin{array}{l}I n . \\
-.009\end{array}$ & $\begin{array}{c}I n . \\
-.001\end{array}$ & $\begin{aligned} & \text { In. } \\
+ & .009\end{aligned}$ & $\begin{array}{r}I n . \\
+.001\end{array}$ & $\begin{aligned} & \text { nn. } \\
+ & .008\end{aligned}$ & $\begin{array}{c}I n . \\
-.009\end{array}$ & $\begin{array}{c}I n . \\
-.010\end{array}$ & $\begin{aligned} & I n . \\
+ & .009\end{aligned}$ & $\begin{array}{l}I n . \\
+. .001\end{array}$ \\
\hline 2 & +.003 & -.009 & -.004 & +.007 & +.002 & -.020 & $+.00 I$ & +.008 & -.002 & -.004 & -007 & -.012 & -.003 \\
\hline 4 & +.004 & $-.0 I I$ & -.010 & -.006 & -.007 & -.019 & -.004 & +.002 & -.016 & +.002 & -.007 & -.014 & -.007 \\
\hline 6 & +.002 & -.014 & -.002 & -.014 & -.015 & -.022 & -.005 & -.002 & -.016 & -.oro & -.009 & -.031 & $-.01 \mathbf{I}$ \\
\hline 8 & +.004 & -.013 & +.003 & -.023 & -.027 & -.028 & -.011 & -.013 & -.030 & -.020 & -.013 & -.038 & -.017 \\
\hline Io & $-.00 \mathrm{I}$ & -.006 & -.005 & -.016 &.- .003 & -.015 & -.006 &.$+ \infty 01$ & -.017 & -.006 & -.005 & -.015 & -.008 \\
\hline 12 & $+.00 \mathrm{I}$ & -.002 & +.006 & -.004 & +.026 & +.003 & +.009 & +.013 & -.002 & +.006 & +.015 & +.008 & +.007 \\
\hline I4 & +.004 & +.007 & +.005 & +.008 & +.025 & +.016 & +.007 & +.004 & +.008 & +.015 &.+ oro & +.018 & +.011 \\
\hline 16 & +.005 & +.015 & +.006 & $+.0 I I$ & +.014 & +.015 & +.008 &.$+ \cos$ & $+.0 \mathrm{II}$ & +.009 & +.009 & +.021 & + .on \\
\hline I 8 & -.004 & +.010 & -.001 & +.009 & +.014 & +.023 & +.008 & -.003 & +.014 & +.007 & +.004 & +.023 & +.009 \\
\hline 20 & -.009 & +.011 & .000 & .000 & -.007 & +.027 & .000 & -.012 & +.020 & +.010 & +.009 & +.021 & +.006 \\
\hline 22 & -.010 & +.010 & +.001 & +.012 & -.009 & +.022 & -.015 & -.001 & +.023 & +.002 & .000 & +.008 & +.004 \\
\hline $\left.\begin{array}{c}\text { Meau } \\
\text { montlily } \\
\text { values }\end{array}\right\}$ & 29.839 & 29.680 & 29.926 & 29.581 & 29.391 & 29.412 & 29.576 & 29.206 & 29.419 & 29.671 & 30.105 & 29.647 & 29.621 \\
\hline
\end{tabular}

As the above results depend upon an aneroid barometer on which correction to standard was obtained only before and not after the completion of the work, and as the constancy of adjustment of the instrument is in doubt, it has not been thought advisable to make any extended reductions from the Cape Flora results. Formulæ representing the diurnal variation have been derived from the observations, as above, by means of Bessel's periodic function (see page 289) to terms of the third order. The resulting amplitudes and phase angles are shown in the following table, and the curves computed from the same are represented in figure 4 . 
Summary of amplitudes and phase angles of periodic functions representing the diumat varialion in atmospheric pressure at Cape Flora

$\Delta p=B_{1} \sin \left(\theta+C_{1}\right)+B_{2} \sin \left(2 \theta+C_{3}\right)+B_{3} \sin \left(3^{\theta}+C_{3}\right)$

\begin{tabular}{|c|c|c|c|c|c|c|}
\hline \multirow{2}{*}{ Month } & \multicolumn{3}{|c|}{ Amplitudes } & \multicolumn{3}{|c|}{ Phase angles } \\
\hline & $B_{1}$ & $B_{3}$ & $B_{3}$ & $C_{1}$ & $C_{2}$ & $C_{3}$ \\
\hline & & $\sqrt{5.12}$ & 4 & $\operatorname{lin}_{0}$ & $\sqrt{0}$ & 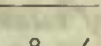 \\
\hline $1904-1905$ & $I n$. & $I n$. & $I n$. & & 01 & 01 \\
\hline June & .004 & .004 & .003 & 32447 & 229 & $83 \circ 9$ \\
\hline July & .014 & .000 & .000 & 17910 & $\ldots \ldots$ & 15142 \\
\hline August & .004 & .000 & .003 & 22407 & 10402 & 13859 \\
\hline September & .014 & 009 & .003 & 14559 & 3928 & 11019 \\
\hline October & .016 & .013 & .006 & 21937 & 2916 & 28920 \\
\hline November & .026 & .000 & 006 & 17724 & I 58 I2 & 22147 \\
\hline December & 006 & .006 & .003 & 20256 & 1721 & 4 I 4 \\
\hline January & 000 & .009 & .000 & 25913 & $3^{827}$ & $3034 \mathrm{I}$ \\
\hline February & .022 & .003 & .003 & ${ }_{15} 84$ & 7900 & 21343 \\
\hline March & . Oro & .006 & .006 & I9I 57 & 530 & 25454 \\
\hline April & $.01 \mathrm{I}$ & 000 & .006 & 20937 & 4022 & 25355 \\
\hline May & .027 & .007 & .003 & 17727 & 4245 & 273 or \\
\hline Year & . or I & .006 &.$c 00$ & 18431 & $334 \mathrm{I}$ & 24057 \\
\hline
\end{tabular}

NoTr. - In these expressions the angle $\theta$ connts from o hour A. M. as $o^{\circ}$.

\section{GeNeRAL ReMARKS}

It is of interest to note that the corrections necessary to the means of the daily observations made at $8 \mathrm{~A}$. M., noon, and $8 \mathrm{P}$. M. to obtain the mean results from continuous registration of atmospheric pressnre and temperature are small. Thus at Teplitz Bay this correction over the period of observation to reduce the mean of the three daily readings of thermometers to meall thermograin is $-0.10^{\circ} \mathrm{F}$; corresponding correction for atmospheric pressure is - .005 inch. At Cape Flora, where only barograms were made, the correction is -. 003 inch.

In connection with storm periods it was found that the temperature was even a better means of forecasting than the barometer, sudden and rapid rising of temperature being almost always accompanied by severe storms. Typical storm thermograns and barograms recorded at Teplitz Bay are shown in figures 4 and 5.

As will be seen from the tabulations of percentages of observed wind directions, the prevailing winds at Teplitz Bay are from the east; during September to December, 1903, in general from the east and southeast; during Jannary and Febrnary, 1904, in general from the east and south; during March and April, 1904, generally from the north and east. At Cape Flora, as already stated, the proximity of the high cliffs interferes with the winds; as recorded the prevailing winds during October to April are from the east and northeast, during May to July from the west and northwest, while during Angust and September they are variable with no very decided preponderance of direction. The summary of hourly wind movements at Teplitz Bay does not indicate any very characteristic dinrnal variation over the mean period of record. 
FIGURE 4

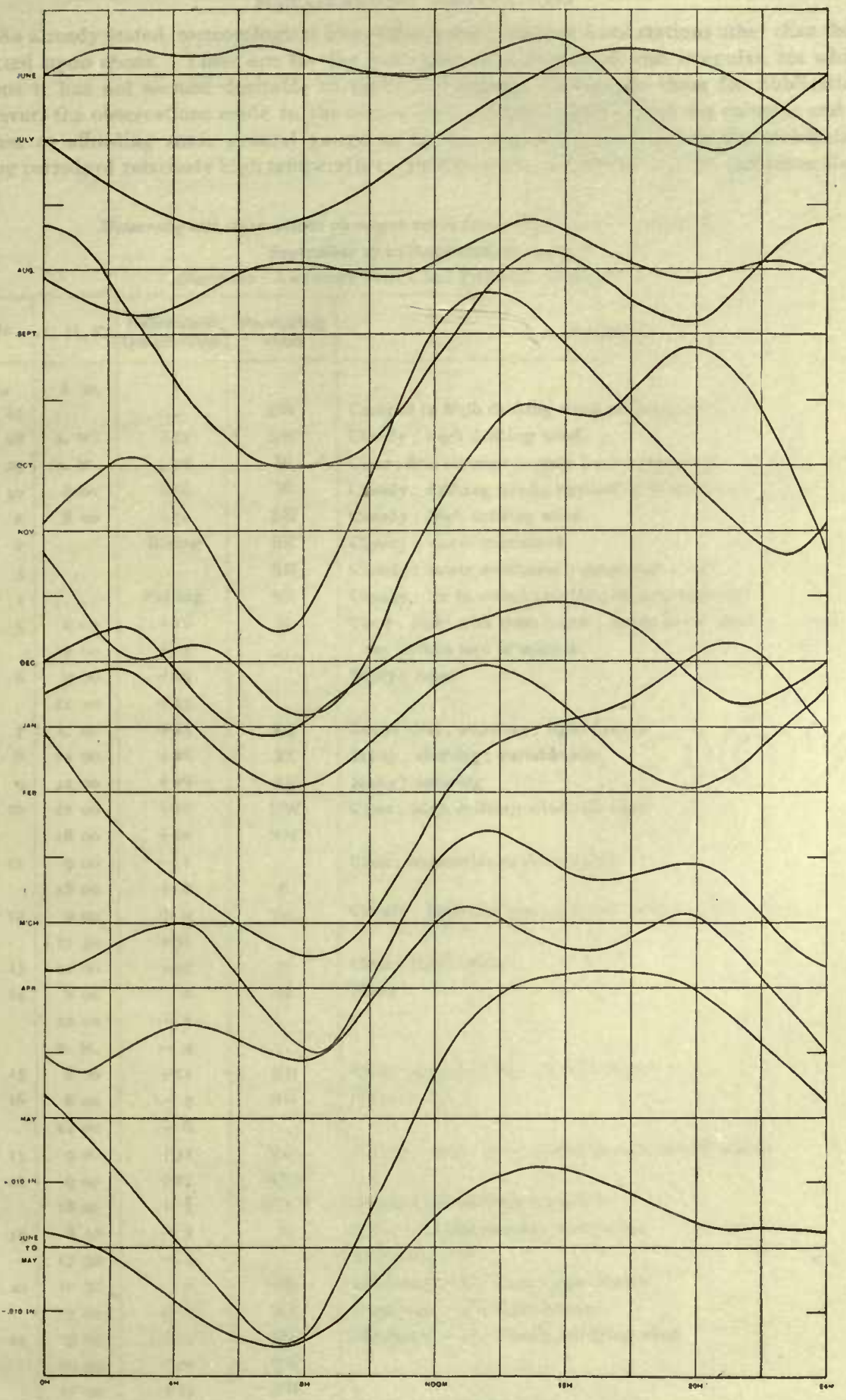

DIURNAL VARIATION IN ATMOSPHERIC PRESSURE AT CAPE FLORA

(Increasing ordinates up denote increasing pressures.) 



\section{MISCEILANEOUS OBSERVATIONS}

As already stated, meteorological observations were made at fixed stations other than those reported upon above. These are for the most part only occasional and irregular, for which reasons it has not seemed desirable to make any attenpt to compile them for publication. However, the observations made in the course of the several sledge trips are valuable and of interest as affording some general gauge as to the conditions of travel in the archipelago during periods of relatively high temperatures, such as were enconntered by the parties on these trips.

$$
\begin{aligned}
& \text { Meteorologicat observations on warch north from Cape Flora to Teplitz Bay } \\
& \text { September } 27 \text { to November 20, } 1904
\end{aligned}
$$

Observers: ANTHONY FIAI,A and CHARI, FS SEITZ, M. D.

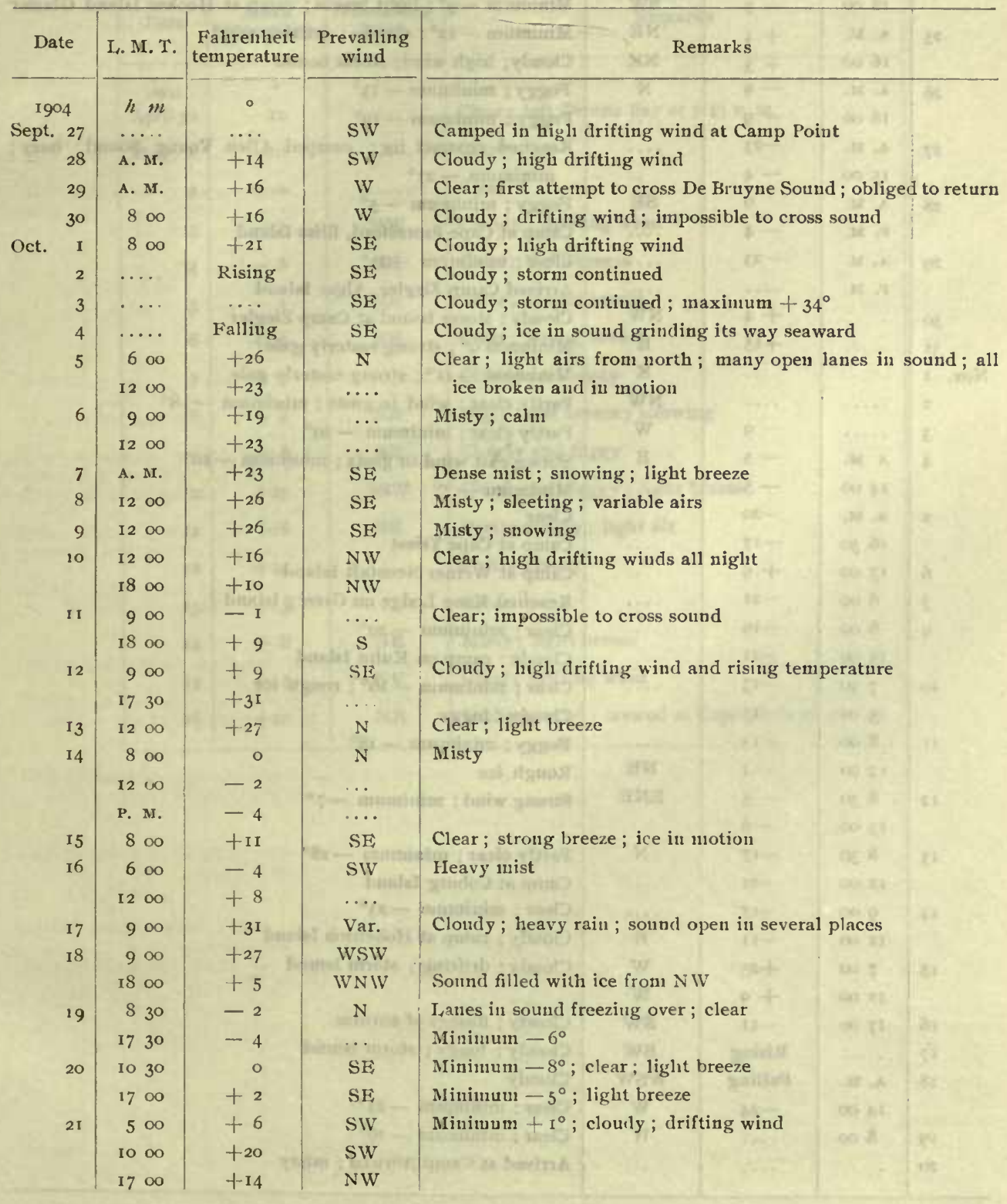


Meteorotogical observations on march north from Cape Flora to Teplitz Bay-Continued

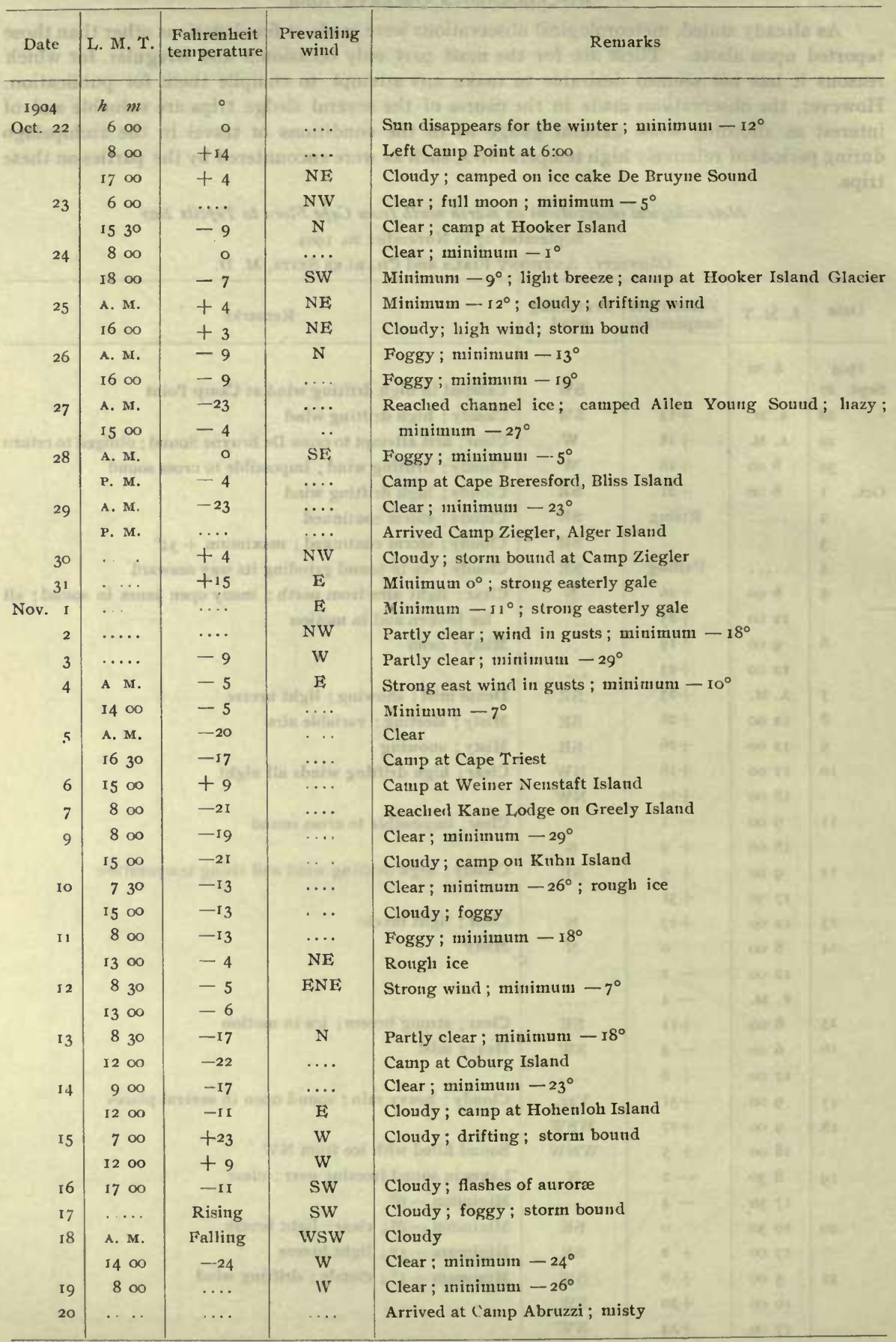




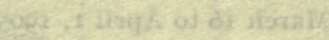

Meteorological observations on sledge trip south from Teplitz Bay to Cape Flora

April 30 to May 16, 1904

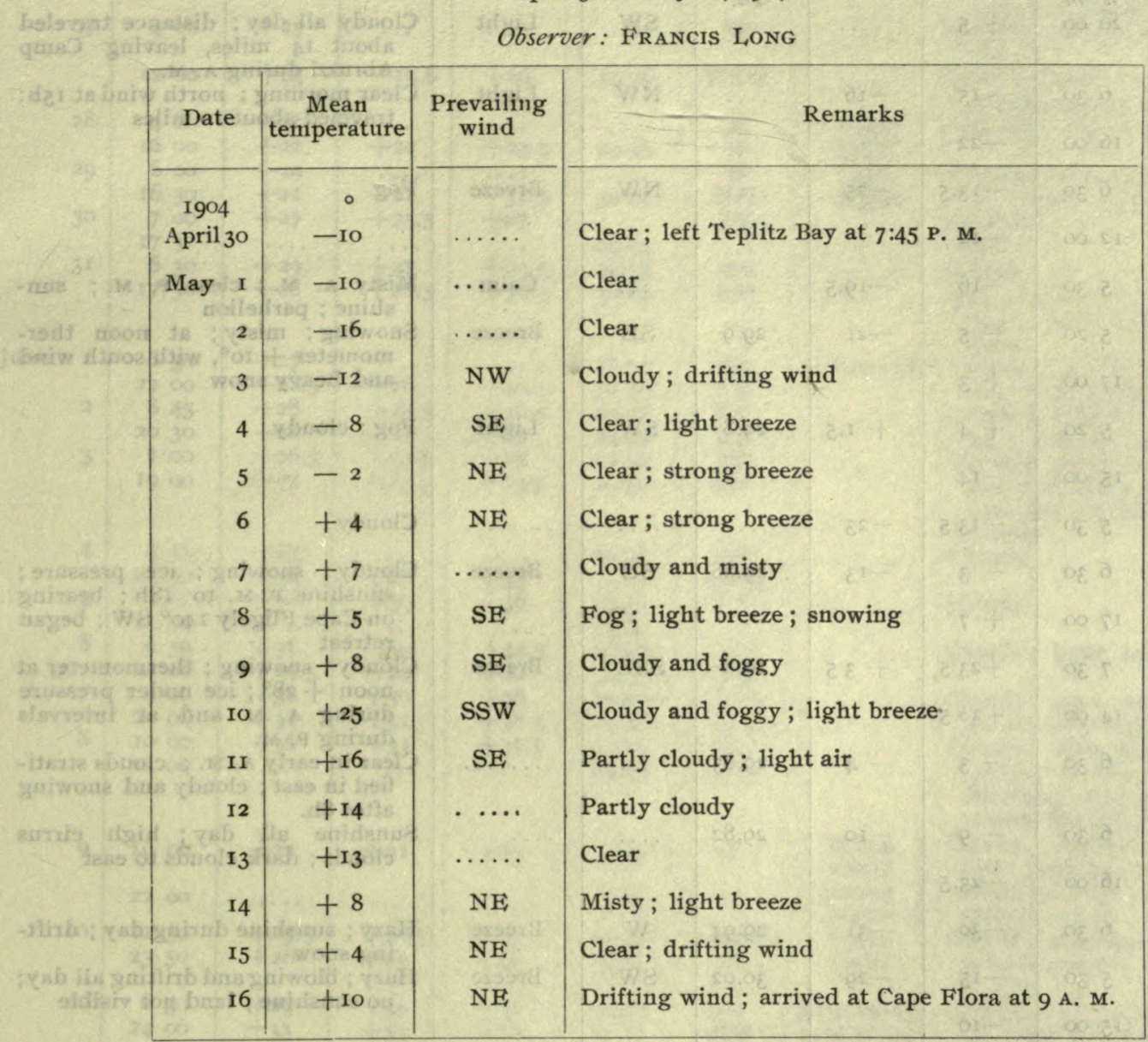

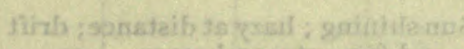

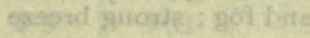

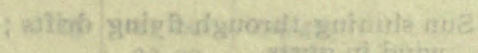

uasigg and finth 
Meleorologial observations on sledge trip north from Teplitz Bay

March 16 to April I, 1905

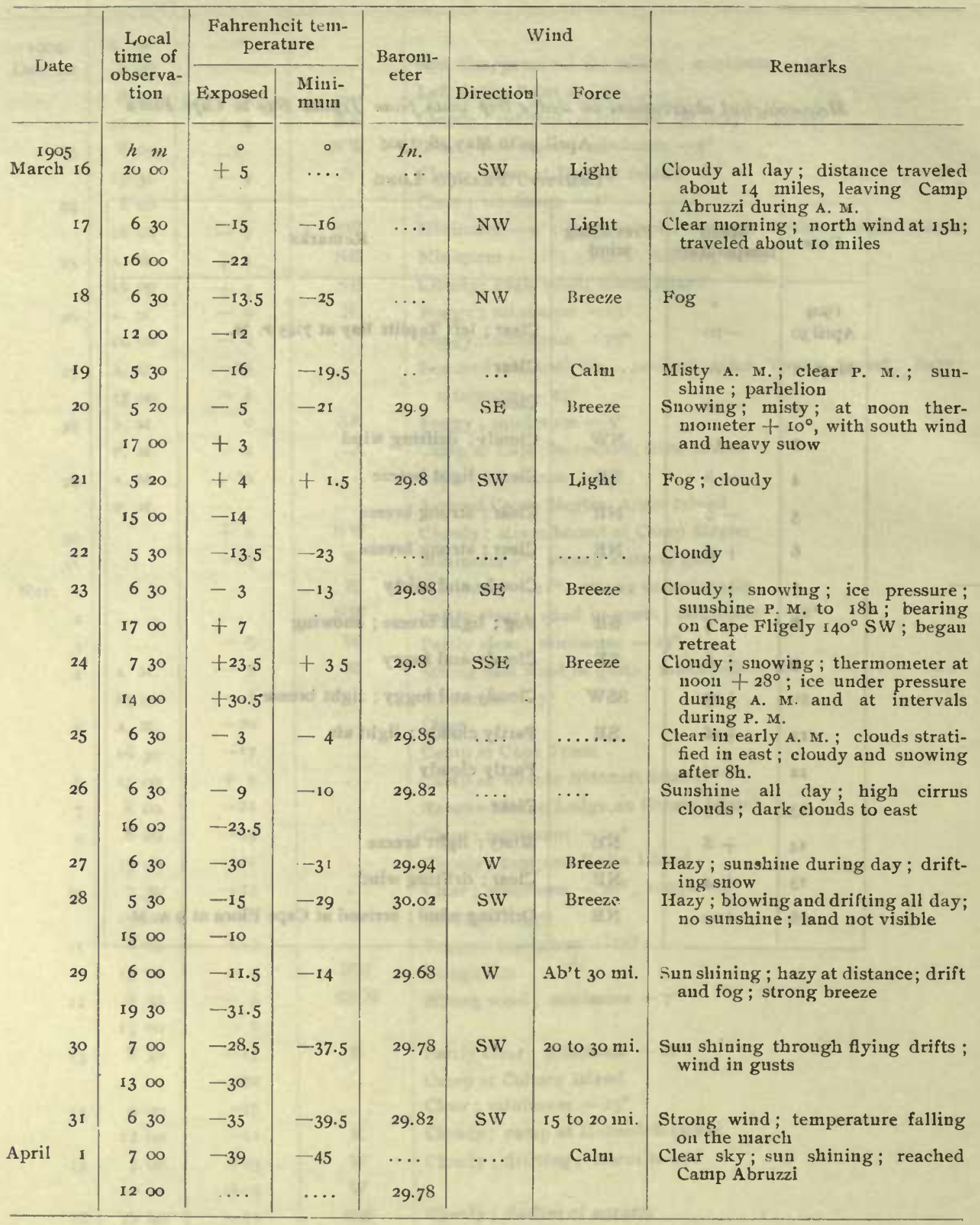


Mcteorologiaul observations on sledge trip soulh from Teplilz Bay to Alger Istand

May 26 to Jume 19, 1905

\begin{tabular}{|c|c|c|c|c|c|c|c|c|}
\hline \multirow{2}{*}{ Date } & \multirow{2}{*}{$\begin{array}{l}\text { Iocal } \\
\text { tine of } \\
\text { observa- } \\
\text { tion }\end{array}$} & \multicolumn{3}{|c|}{ Fahrenheit temperature } & \multirow{2}{*}{$\begin{array}{l}\text { Baronı- } \\
\text { eter }\end{array}$} & \multicolumn{2}{|c|}{ Wind } & \multirow{2}{*}{ Remarks } \\
\hline & & Exposed & $\begin{array}{l}\text { Mini- } \\
\text { mum }\end{array}$ & $\begin{array}{l}\text { Maxi- } \\
\text { muin }\end{array}$ & & Direction & Force & \\
\hline 1905 & $h m$ & $\circ$ & $\circ$ & $\circ$ & $I n$. & & & \\
\hline May 26 & $\begin{array}{r}900 \\
1200\end{array}$ & $\begin{array}{r}+18.8 \\
+19.2\end{array}$ & $\begin{array}{l}+11.5 \\
+16.5\end{array}$ & $\begin{array}{l}+25 \\
+20\end{array}$ & $29.9^{2}$ & $\stackrel{N}{N}$ & ....... & $\begin{array}{l}\text { Left Camp Abruzzi at } 16 \mathrm{~h} \\
45 \mathrm{~m} \text {; sun slining; alto- } \\
\text { cumulus clouds }\end{array}$ \\
\hline 27 & 400 & +25 & $\cdots$ & ... & $\cdots$ & W & $\ldots \ldots$ & $\begin{array}{l}\text { Cloudy; stratus clouds ho- } \\
\text { rizon }\end{array}$ \\
\hline & 1200 & +24 & +19.6 & +29 & 29.96 & WSW & $\cdots \cdots \cdots$ & $\begin{array}{l}\text { Alto-cumulus clouds; } \\
\text { shining overhead }\end{array}$ \\
\hline 28 & $\begin{array}{r}400 \\
1600\end{array}$ & $\begin{array}{l}+21 \\
+22\end{array}$ & +22 & $\ddot{+} \ddot{23.5}$ & 29.95 & W & $\begin{array}{l}\text { Light } \\
\text { Light }\end{array}$ & Cloudy; snowing \\
\hline 29 & 600 & +24 & & $\because \cdots$ & .... & W & Light & Cloudy; snowing \\
\hline 30 & $\begin{array}{rl}16 & 30 \\
7 & 00\end{array}$ & $\begin{array}{l}+21 \\
+27\end{array}$ & $\begin{array}{l}+21 \\
+25.5\end{array}$ & $\begin{array}{l}+31.5 \\
+27\end{array}$ & $\begin{array}{l}30.01 \\
\ldots\end{array}$ & $\begin{array}{l}\text { SW } \\
\text { SE }\end{array}$ & $\begin{array}{c}\text { Light } \\
\ldots \ldots \text {. }\end{array}$ & $\begin{array}{l}\text { Hazy; bad vision } \\
\text { Cloudy }\end{array}$ \\
\hline 3 & 1730 & & & & 29.94 & & ....... & Clear \\
\hline $3 \mathrm{I}$ & $\begin{array}{r}630 \\
1730\end{array}$ & $\begin{array}{l}+29 \\
+28\end{array}$ & $\begin{array}{l}+27 \\
+26.5\end{array}$ & $\begin{array}{l}+29.5 \\
+30.5\end{array}$ & $\begin{array}{l}29.93 \\
30.04\end{array}$ & $\begin{array}{l}\text { SE } \\
\text { SW }\end{array}$ & $\ldots \cdots \cdots$ & $\begin{array}{l}\text { Cloudy } \\
\text { Sun out at } 2 \mathrm{Ih} \text {; fine wavy } \\
\text { light cirrus clouds over- } \\
\text { liead }\end{array}$ \\
\hline Tune & $\begin{array}{rl}630 \\
22 & 00\end{array}$ & $\begin{array}{l}+27 \\
+28.5\end{array}$ & +26 & $\ddot{+} \ddot{30} .5$ & $\begin{array}{l}30.03 \\
30.30\end{array}$ & $\begin{array}{l}S E \\
S E\end{array}$ & 15 to $20 \mathrm{mil}$. & $\begin{array}{l}\text { Cloudy ; wind started at } 6 \mathrm{~h} \\
\text { from SE; drifting }\end{array}$ \\
\hline 2 & $\begin{array}{rl}845 \\
20 & 30\end{array}$ & $\begin{array}{l}+28 \\
+28\end{array}$ & $\begin{array}{l}+27.5 \\
+27\end{array}$ & $\begin{array}{l}+30.5 \\
+29\end{array}$ & $\begin{array}{l}29.98 \\
29.99\end{array}$ & $\begin{array}{l}\text { SW } \\
\text { SE }\end{array}$ & $\cdot \cdots \cdots$ & $\begin{array}{l}\text { Cloudy ; windy A. M. drift- } \\
\text { ing; wind shifting to SE }\end{array}$ \\
\hline 3 & $\begin{aligned} 700 \\
1900\end{aligned}$ & $\begin{array}{l}+26.5 \\
+27\end{array}$ & $\begin{array}{l}+25.5 \\
+25.5\end{array}$ & $\begin{array}{l}+29 \\
+29.5\end{array}$ & $\begin{array}{l}29.96 \\
29.97\end{array}$ & $\begin{array}{l}\text { SW } \\
\text { SW }\end{array}$ & $\ldots \ldots \ldots$ & $\begin{array}{l}\text { Driving wind from sw, } \\
\text { moist, at times filled with } \\
\text { hail that cut like a knife; } \\
\text { cloudy }\end{array}$ \\
\hline 4 & $\begin{array}{rr}4 & 15 \\
22 & 30\end{array}$ & $\begin{array}{l}+29 \\
+32\end{array}$ & +28 & $\ddot{+} 35$ & $\begin{array}{c}29.96 \\
\ldots \ldots\end{array}$ & $\begin{array}{c}\text { SW } \\
\text { Calin }\end{array}$ & $\ldots \ldots$ & Cloudy \\
\hline 5 & 900 & $+3^{n}$ & +30 & +36 & 30.12 & Calm & & Cloudy \\
\hline 6 & $\begin{aligned} 2100 \\
930\end{aligned}$ & +31 & $\begin{array}{r}7 \\
+30 \\
+20\end{array}$ & +44.5 & $\begin{array}{l}30.12 \\
30.03\end{array}$ & Calm & & Clottdy ; liglit snow \\
\hline 7 & $\begin{array}{r}2400 \\
900\end{array}$ & $\begin{array}{l}+29.5 \\
+32\end{array}$ & $\begin{array}{l}+29 \\
+30\end{array}$ & $\begin{array}{l}+40 \\
+36\end{array}$ & 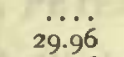 & $\begin{array}{l}\text { Calin } \\
\text { Calm }\end{array}$ & & Cloudy; open water holes \\
\hline & 2000 & +31 & $+3 i$ & +38.5 & 29.96 & Calnı & 10 & fill air with vapor \\
\hline 8 & $\begin{array}{l}1000 \\
2200\end{array}$ & $\begin{array}{l}+36 \\
\cdots\end{array}$ & $\begin{array}{l}+32 \\
\cdots\end{array}$ & $\begin{array}{c}+37 \cdot 5 \\
\ldots\end{array}$ & $\begin{array}{l}29.9^{8} \\
29.9^{8}\end{array}$ & $\begin{array}{l}\text { Calm } \\
\text { Calm }\end{array}$ & & $\begin{array}{l}\text { Cloudy; alto-cumulus at } \\
\text { zenith; stratus - nimbus, } \\
\text { denoting open water, all } \\
\text { around the horizon }\end{array}$ \\
\hline 9 & $\begin{array}{l}1000 \\
2200\end{array}$ & +27 & +21 & +29 & $\begin{array}{l}29.90 \\
29.86\end{array}$ & $\begin{array}{l}\mathbf{N} \\
\mathbf{N}\end{array}$ & $\left.\begin{array}{l}\text { Strong } \\
\text { breeze } \\
\text { Strong } \\
\text { breeze }\end{array}\right\}$ & $\begin{array}{l}\text { Cloudy; sunlight through } \\
\text { alto-cunulus and cirro- } \\
\text { cumulus from } 3 \text { h to } 6 \mathrm{~h} \text {; } \\
\text { cloudy at } 22 \mathrm{~h}\end{array}$ \\
\hline 10 & $\begin{array}{ll}11 & 30 \\
23 & 50\end{array}$ & $\begin{array}{l}+29.5 \\
+26\end{array}$ & $\begin{array}{l}+29.5 \\
+25\end{array}$ & $\begin{array}{l}+41.5 \\
+30\end{array}$ & $\begin{array}{l}29.94 \\
29.72\end{array}$ & $\begin{array}{l}\text { Variable } \\
\mathrm{SE}\end{array}$ & $\begin{array}{l}\text { I,ight } \\
\text { Light }\end{array}$ & $\begin{array}{l}\text { Cloudy; sunlight through } \\
\text { cunulo-stratus from } 6 \mathrm{~h} \\
\text { to } \mathrm{gh}\end{array}$ \\
\hline 11 & $\begin{array}{r}630 \\
2400\end{array}$ & $\begin{array}{l}+29 \\
+33\end{array}$ & $\begin{array}{l}+25 \\
+30\end{array}$ & $\begin{array}{l}+30 \\
+38\end{array}$ & $\begin{array}{l}29.58 \\
29.3^{8}\end{array}$ & $\begin{array}{l}\text { SE } \\
\text { SSW }\end{array}$ & $\begin{array}{l}20 \text { to } 40 \mathrm{mi} \\
20 \text { to } 30 \mathrm{mi} \text {. }\end{array}$ & $\begin{array}{l}\text { Cloudy; drifting wind from } \\
\text { SE, veering toward } 24 \mathrm{~h} \text { to } \\
\text { SSW, varied with down- } \\
\text { pours rain and hail }\end{array}$ \\
\hline 12 & $\begin{array}{rl}5 & 30 \\
21 & 00\end{array}$ & $\begin{array}{l}+33 \\
+33\end{array}$ & $\begin{array}{l}+32 \\
+32\end{array}$ & $\begin{array}{l}+34 \\
+36\end{array}$ & $\begin{array}{l}29.41 \\
29.48\end{array}$ & $\begin{array}{l}\text { SSW } \\
\text { SSW }\end{array}$ & 30 to $40 \mathrm{~m}$ ?. & $\begin{array}{l}\text { Cloudy; raining in squalls } \\
\text { during day; rain-soaked } \\
\text { snow makes wet traveling }\end{array}$ \\
\hline 13 & $\begin{array}{l}\text { Ir } \infty \\
2200\end{array}$ & $\begin{array}{l}+41 \\
+32\end{array}$ & $\begin{array}{l}\cdots \\
+31.5\end{array}$ & $\begin{array}{l}+44 \\
+43\end{array}$ & $\begin{array}{l}29.54 \\
29.74\end{array}$ & $\cdots$ & Light & Clondy \\
\hline 14 & $\begin{array}{l}100 \\
2100\end{array}$ & $\begin{array}{r}+34 \\
+33\end{array}$ & $\begin{array}{r}+30 \\
+33 \\
+33\end{array}$ & $\begin{array}{r}+35 \\
+35 \\
+43\end{array}$ & $\begin{array}{l}29.14 \\
29.94\end{array}$ & $\begin{array}{l}\text { S̈̈ } \\
\text { SE }\end{array}$ & I5 to $20 \mathrm{mi}$. & $\begin{array}{l}\text { Cloudy; sunshine intermit- } \\
\text { tently between } 7 \mathrm{~h} \text { 3om } \\
\text { and } 9 \mathrm{~h} ; \text { spots on sun; } \\
\text { strong SW breeze all } \mathrm{A} . \mathrm{M} \text {. }\end{array}$ \\
\hline 15 & $\begin{array}{l}1200 \\
2230\end{array}$ & $\begin{array}{l}+34 \\
+33.5\end{array}$ & $\begin{array}{l}+34 \\
+33\end{array}$ & $\begin{array}{l}+36 \\
+39\end{array}$ & $\begin{array}{l}29.78 \\
29.56\end{array}$ & $\begin{array}{l}\mathrm{SE} \\
\mathrm{SE}\end{array}$ & & $\begin{array}{l}\text { Cloudy; thick fog; bad } \\
\text { traveling }\end{array}$ \\
\hline 16 & $\cdots \cdots$ & & $\cdots$ & & $\ldots$ & SSW & 20 to $60 \mathrm{mi}$. & $\begin{array}{l}\text { Thermometers in canoe; } \\
\text { did not attempt to open } \\
\text { on account drift and rain; } \\
\text { cloudy; storm from SSW } \\
\text { irregular, from } 20 \text { to } 60 \\
\text { miles velocity; rain, hail, } \\
\text { sleet, snow; maximum } \\
\text { velocity about } 20 \text { h }\end{array}$ \\
\hline 17 & $\begin{array}{l}0030 \\
1900\end{array}$ & $\begin{array}{l}+30 \\
\cdots\end{array}$ & $\begin{array}{r}+30 \\
+26\end{array}$ & $\begin{array}{l}+36 \\
\cdots\end{array}$ & $\begin{array}{l}29.56 \\
26.56\end{array}$ & $\stackrel{S}{S W}$ & 25 to $50 \mathrm{mi}$. & $\begin{array}{l}\text { Cloudy; storm with lower } \\
\text { temperatures; wind vary- } \\
\text { ing from StoSW; drifting }\end{array}$ \\
\hline 18 & 600 & +28 & +27 & +30 & 29.28 & SSW & 40 to 60 mi. & Cloudy; drifting; storm \\
\hline 19 & $\begin{array}{r}1200 \\
300\end{array}$ & $\begin{array}{l}+31 \\
+28\end{array}$ & $\begin{array}{r}+31 \\
+27\end{array}$ & +27 & 29.23 & $\begin{array}{l}\mathrm{SW} \\
\mathrm{NE}\end{array}$ & & worst during early A. M. \\
\hline & 1200 & +31 & $\begin{array}{l}72 \\
+29.5\end{array}$ & +33.5 & $\begin{array}{l}29.20 \\
29.76\end{array}$ & NE & & $\begin{array}{l}\text { Cloudy; arrived at Alger } \\
\text { Island, Camp Ziegler. }\end{array}$ \\
\hline
\end{tabular}





\title{
SECTION D
}

\section{TIDAL OBSERVATIONS}

AND

\section{REDUCTIONS}

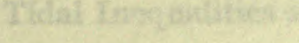

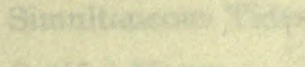

\author{
BY \\ W. J. PETERS \\ In Charge of Scientific Work of the Expedition
}

AND

L. P. SHIDY

Chief of Tidal Division, United States Coast and Geodetic Survey 


\section{a nolTo32}

\section{2ИOITAУЯG2УO JAQIT \\ GHA \\ 2ท01тวUазя}

Ya

$$
\text { 293739 .L.W }
$$

noisibsgaz ant to shoW setisnsize io syusto at

ahs

YaIHe $9 . .1$

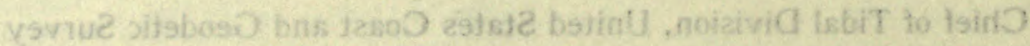




\section{CONTENTS}

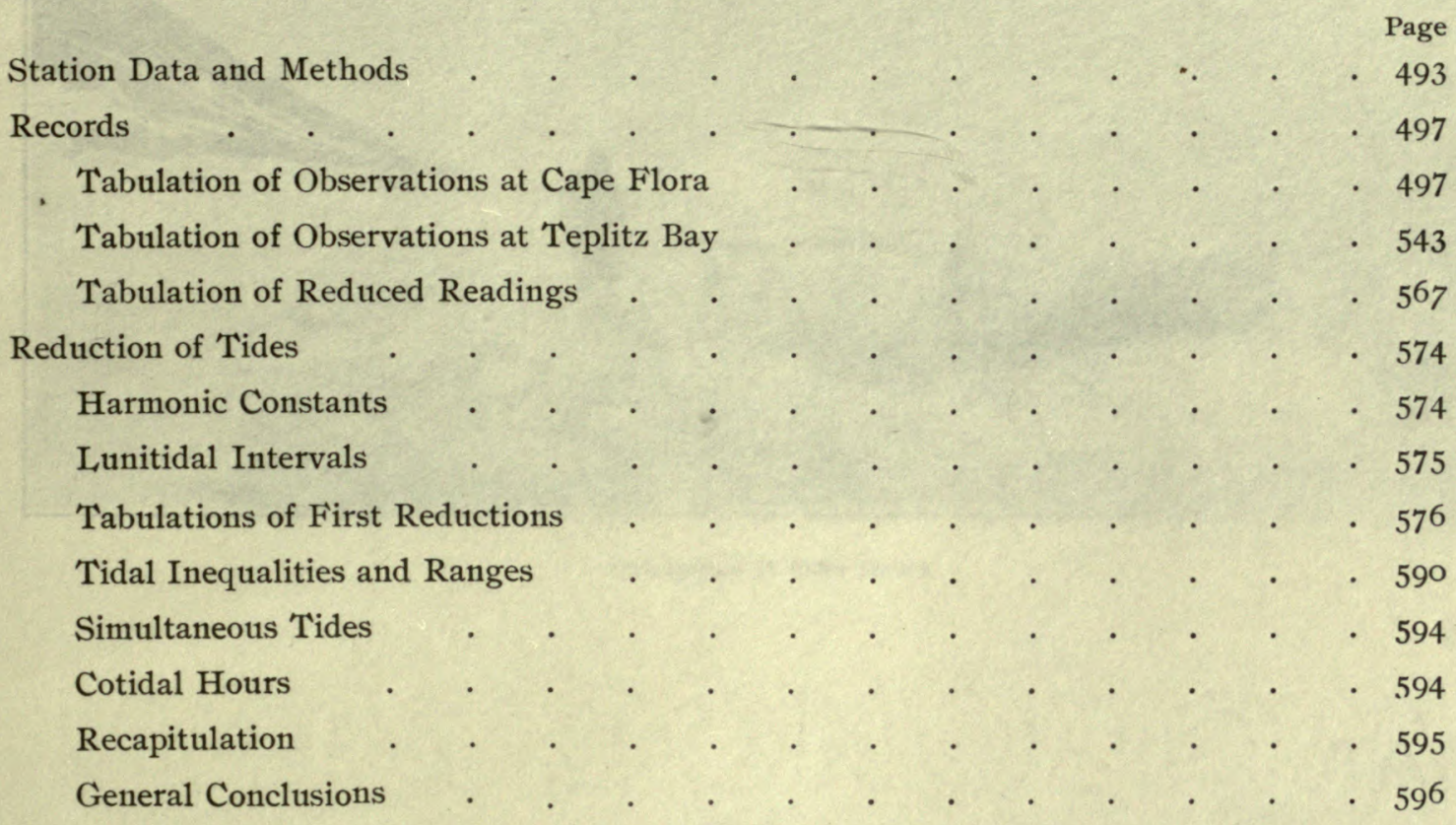

\section{ILLUSTRATIONS}

I Tide Gauge at Cape Flora

Opposite page

2 Diagram of Base Line and Bench Mark at Cape Flora . . . . . . 492

3 Tide Gauge at Teplitz Bay . . . . . . . . . . . 493

4 Outline of Coast Showing Location of Tide Gauge at Cape Flora . . . . 494

5 Outline of Coast Showing Location of Tide Gauge at Teplitz Bay . . . 495 

FIGURE 1

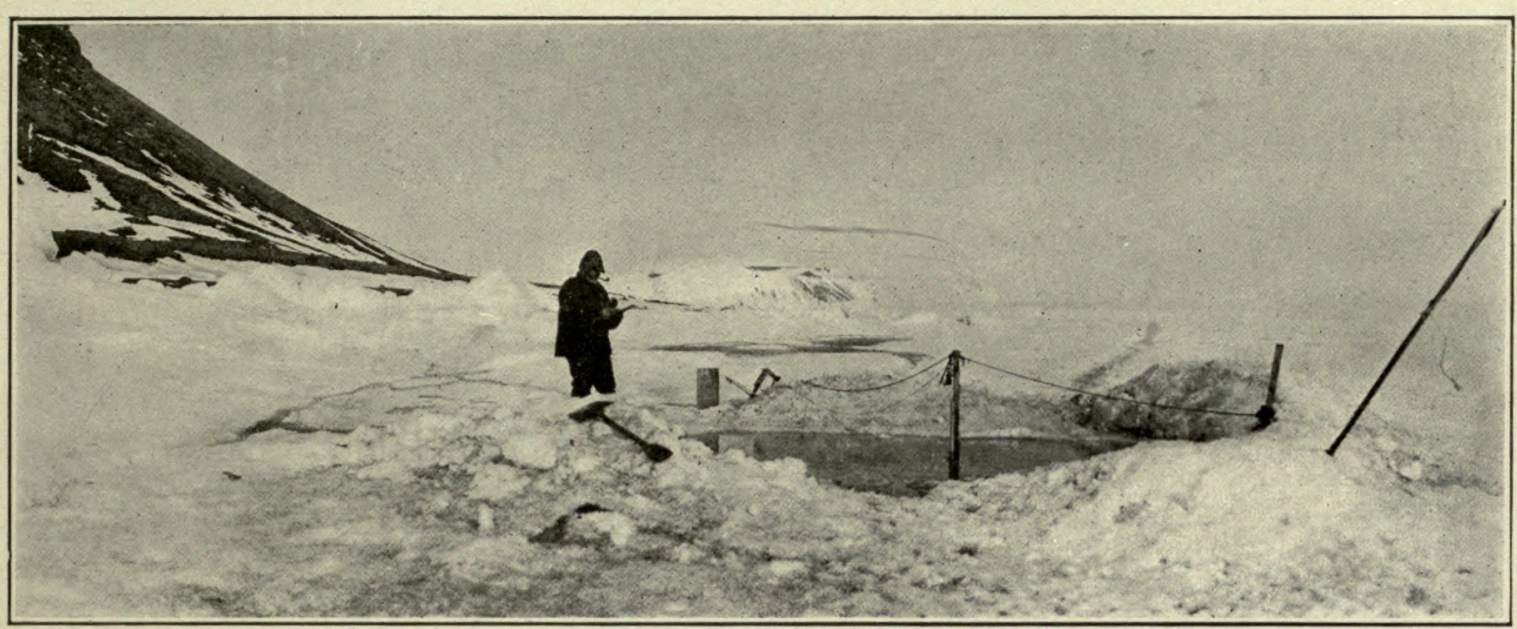

TIDE GAUGE AT CAPE FLORA

FICURE 2

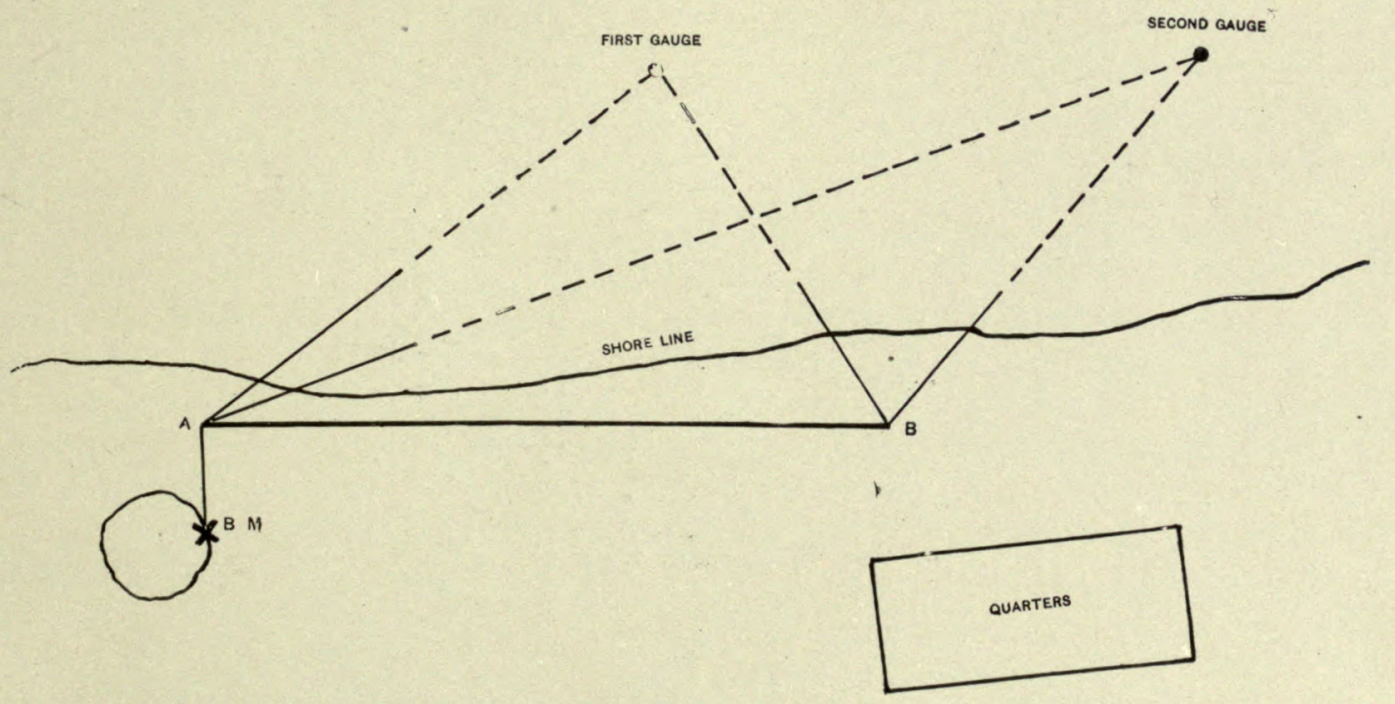

DIAGRAM OF BASE LINE AND BENCH MARK AT CAPE FLORA 
FIGURE 3

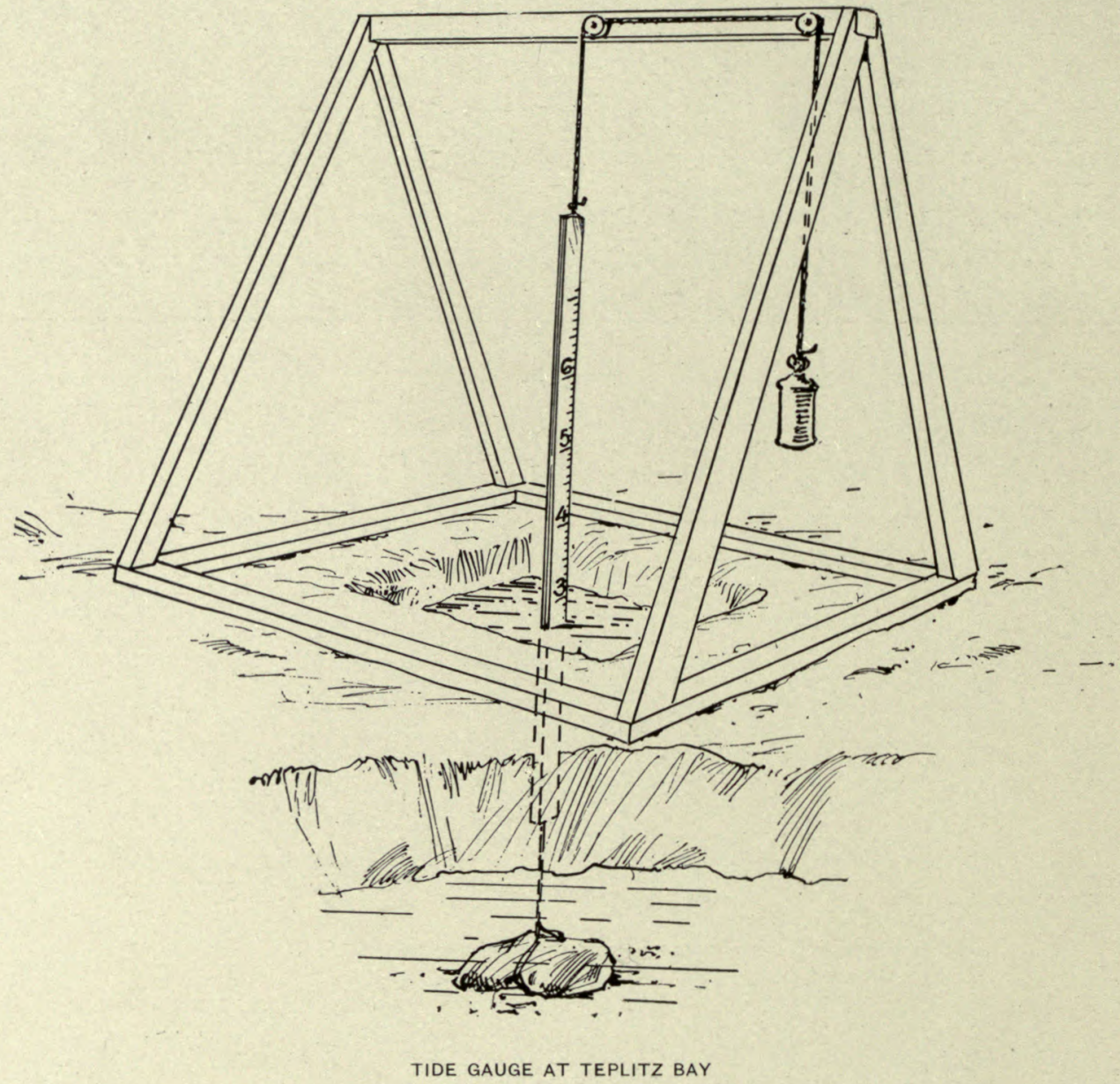




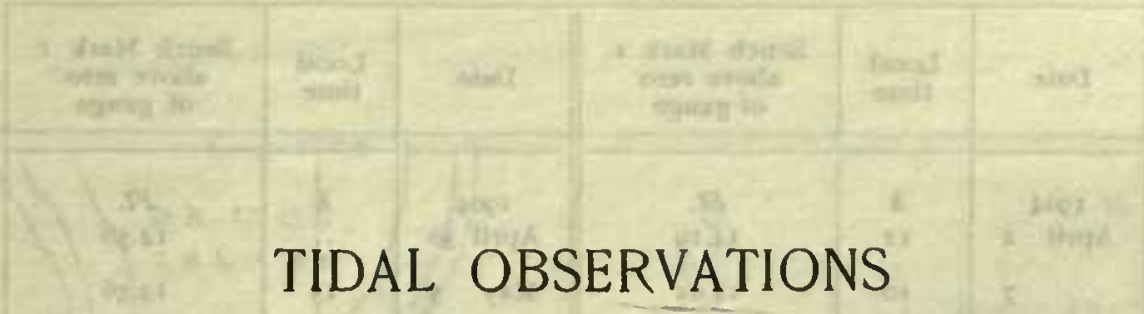

\section{STATION DATA AND METHODS}

During the Expedition two valnable series of tide observations were secured, one at Cape Flora, Northbrook Island, and the other at Teplitz Bay, Rudolph Island, in Franz Josef Arcliipelago. The tides at Cape Flora were observed from May 21 to August 3r, 1904, and at Teplitz Bay from April i to June 3, 1904.

The gauge at Cape Flora was a plain wooden staff, graduated to feet and tenths, which was wedged in between boulders on the shore (see figure 1 ). A gale having destroyed the gauge, a new one was set up in the same manner on July 18.

The gauge at Teplitz Bay (see figure 3) consisted of a heavy wooden framework supporting two pulleys; a wire, attached at one end to a lead weight of 149 pounds lying on basaltic rock at the bottom of the sea 35 feet below the surface, passed over the two pulleys and terminated in a counterpoise weighing 49 pounds. A light, graduated wooden rod six feet long was attaclred firmly to the wire to serve as a tide staff. The staff remained stationary, while the framework and ice on which it rested rose and fell with the tide.

At Cape Flora a bench mark was established on a large basaltic boulder near the shore and marked by a painted cross. The base line $A B=262.5$ feet in figure 2, and angles were measured to the bench mark and different positions of the tide gauge. The bench mark corresponds to a reading of 14.65 feet on first staff and to 14.70 feet on second staff. The series was all reduced to the first tide staff, on which mean sea level corresponds to a reading of 6.076 feet.

At Teplitz Bay two beuch marks were established. Bench Mark $r$ is on a boulder near the shore, and Bench Mark 2 is the top of the capstone of the astronomical brick pier. The latter is 50.99 feet above the former as determined by spirit levels on April 30, 1904. The relation of Bench Mark $r$ to tide staff was not constant, as the frame of the gauge slowly sank into the ice, and was considerably tilted at the close of the observations. The following table shows the results of various levels between tide staff and Bencli Mark I, only one station of the instrument being necessary. 


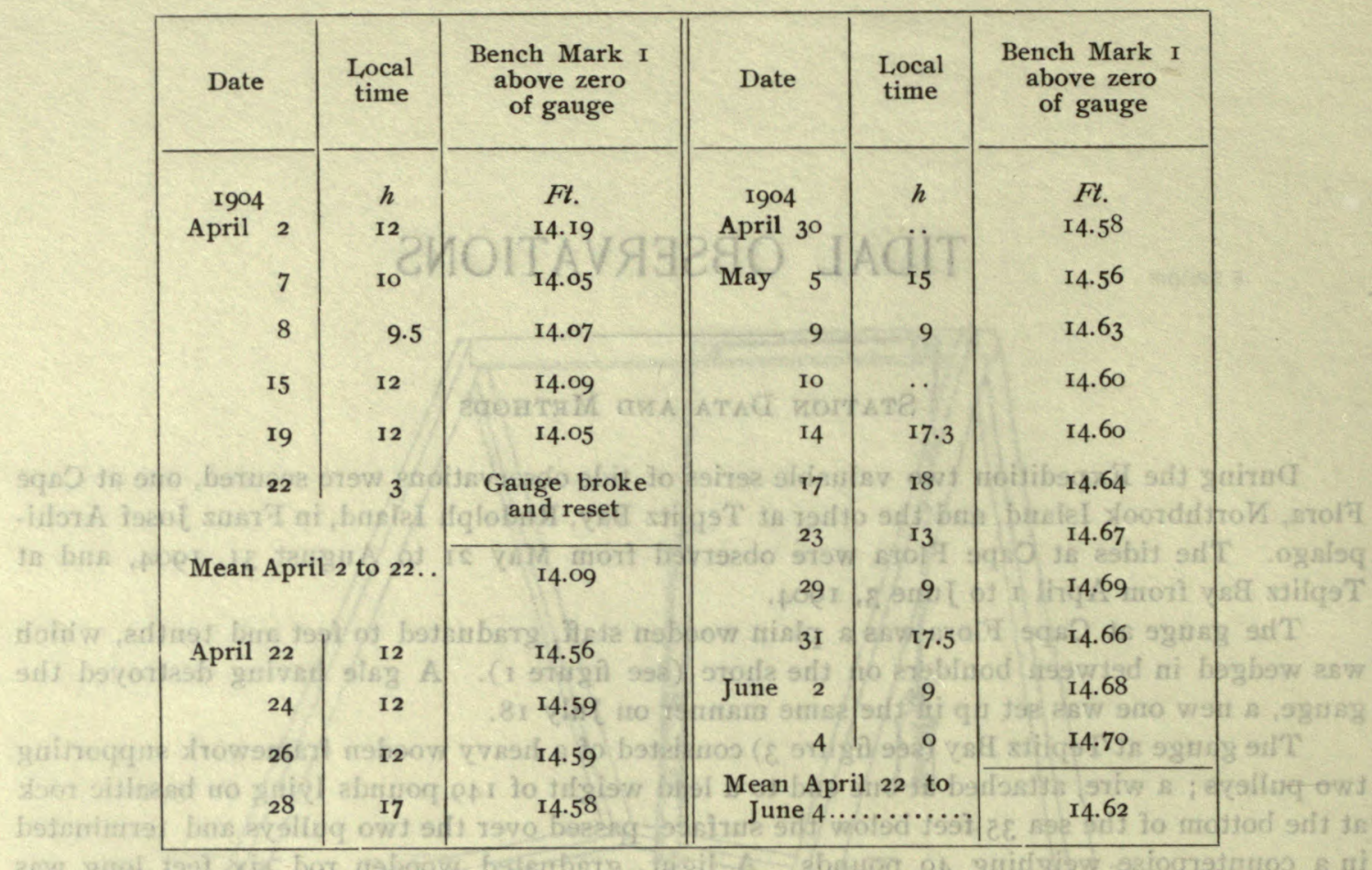

The heights of the whole series were reduced so as to make the mean difference between the zero of the corrected staff and Bench Mark I correspond to ${ }^{4} 4.62$ feet, which gives a mean sea-level reading of 4.133 feet on the corrected staff.

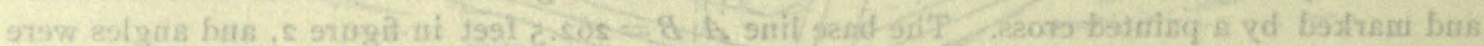

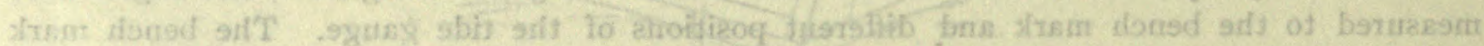

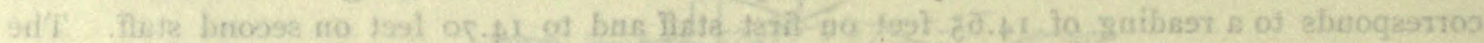

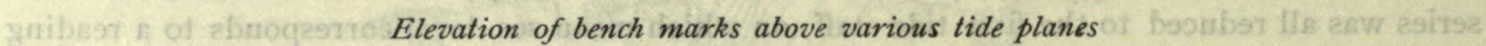

\begin{tabular}{|c|c|c|c|c|}
\hline \multirow{4}{*}{ 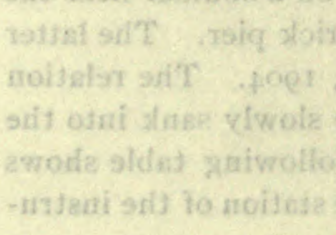 } & \multirow{2}{*}{ folizg/ Station } & \multirow{2}{*}{$\begin{array}{c}\text { Cape Flora } \\
\text { Bench } \\
\text { Mark }\end{array}$} & \multicolumn{2}{|c|}{ Teplitz Bay } \\
\hline & & & $\begin{array}{l}\text { Bench } \\
\text { Mark I }\end{array}$ & $\begin{array}{l}\text { Bench } \\
\text { Mark 2 }\end{array}$ \\
\hline & (1) & (2) 30 & $F_{t}$ & $F t$ \\
\hline & Highest tide observed. & 7.22 & $9 \cdot 31$ & 60.30 \\
\hline seel. & Mean high water... & 8.08 & 9.90 & 60.89 \\
\hline & Mean sea level .... & 8.57 & I0.49 & $6 \mathrm{r} .48$ \\
\hline & Mean low water....... & 9.05 & III.03 & 62.02 \\
\hline & Lowest tide observed .. & 10.02 & II. 76 & 62.75 \\
\hline
\end{tabular}




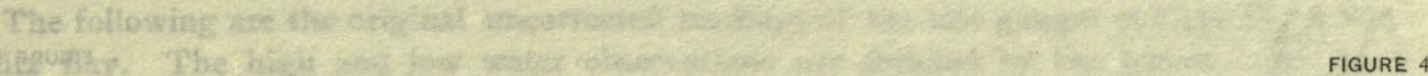

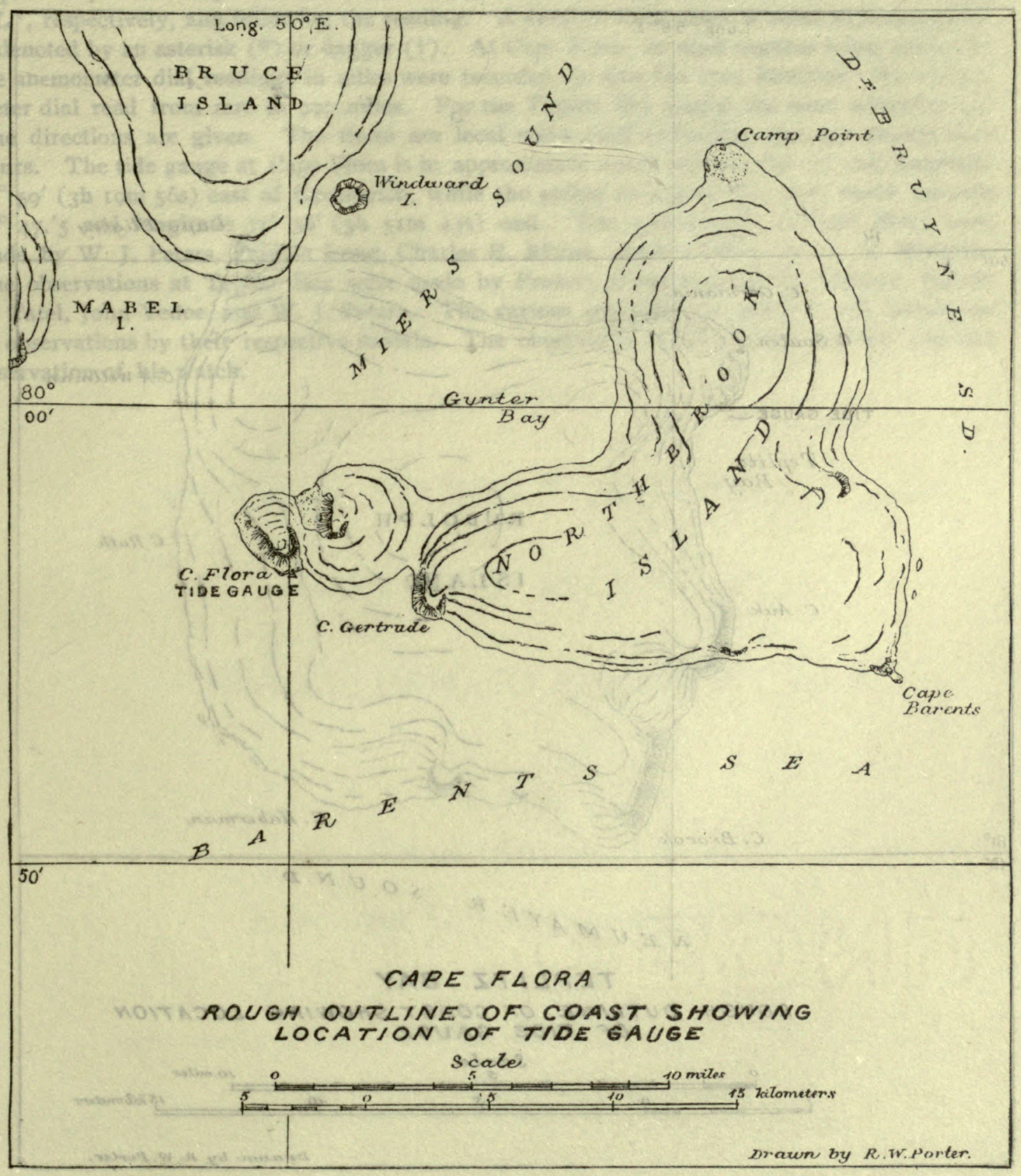




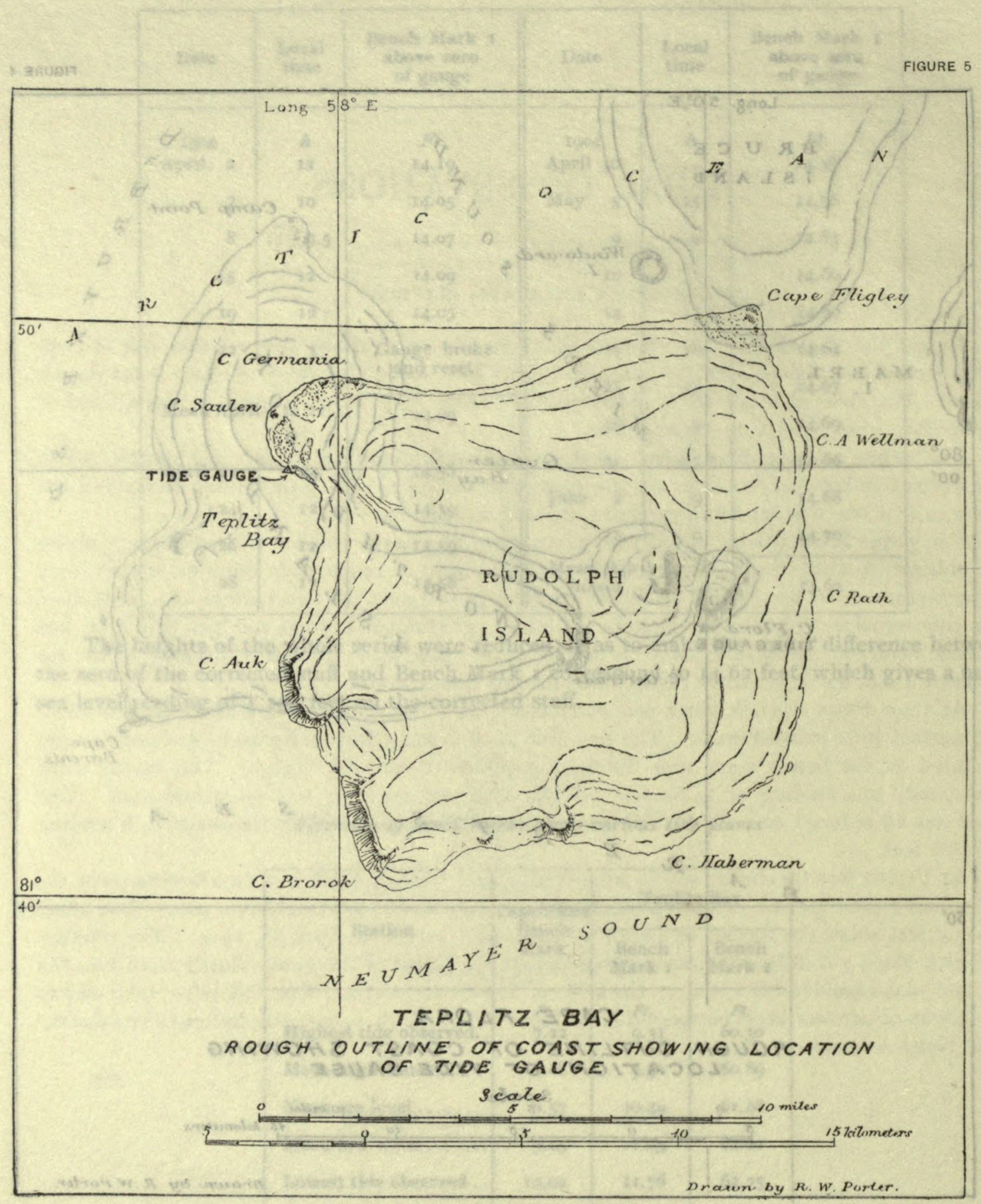




\section{RECORDS}

The following are the original uncorrected readings of the tide gatiges at Cape Flora and Teplitz Bay. The high and low water observations are denoted by the letters " $\mathrm{H}$ " and " $L$ ", respectively, and following the reading. A swell or light swell, if noted at observation, is denoted by an asterisk $(*)$ or dagger $(\dagger)$. At Cape Flora, no wind register being available, the anemometer dial readings in miles were recorded, as also the true direction; the anemometer dial read from zero to 990 miles. For the Teplitz Bay results the wind velocities and true directions are given. The times are local mean civil reckoning through twenty-four hours. The tide gauge at Cape Flora is in approximate north latitude $79^{\circ} 57^{\prime}$ and longitude $49^{\circ} 59^{\prime}$ (3 $19 \mathrm{~m} 56 \mathrm{~s}$ ) east of Greenwich, while the gauge at Teplitz Bay is in north latitude $8 \mathrm{r}^{\circ} 47^{\prime} 5$ and longitude $57^{\circ} 56^{\prime}\left(3^{\mathrm{h}} 5^{\mathrm{Im}} 43 \mathrm{~s}\right)$ east. The observations at Cape Flora were made by W. J. Peters, Francis Long, Charles E. Rilliet, Anton Vedoe, and J. E. Moulton. The observations at Teplitz Bay were made by Francis Long, Spencer W. Stewart, Robert R. Tafel, John Vedoe, and W. J. Peters. The various observers are noted in the tabulation of observations by their respective initials. The observer is noted only for the first and last observation of his watch. 



\title{
TIDAL OBSERVATIONS
}

\section{TABULATION OF TIDE GAUGE READINGS}

\author{
RECORDED AT
}

CAPE FLORA STATION, NORTHBROOK ISLAND

FRANZ JOSEF ARCHIPELAGO

MAY 21, 1904, TO AUGUST 31, 1904

NORTH LATITUDE: $79^{\circ} 57^{\prime}$

LONGITUDE EAST OF GREENWICH: $49^{\circ} 59^{\prime}$ 


\section{2ИOITAV9G280 JAQIT}

2OMICAGЯ JDUAD JUIT FO KOITAIUAAT

TA वзดя0องЯ

GИAJ21 XOOЯGHTЯOИ ИOITAT2 AЯOJ9 З9AD

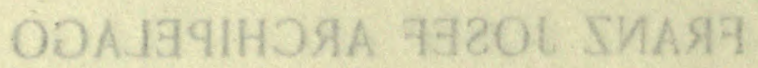

AOP1, IE TZUDUA OT, AOPI, IS YAM

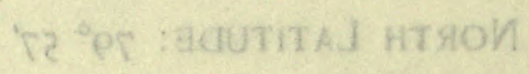

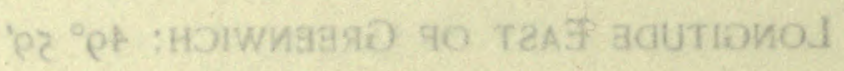


Tabulation of tidal observations at Cape Flora, Northbrook Island

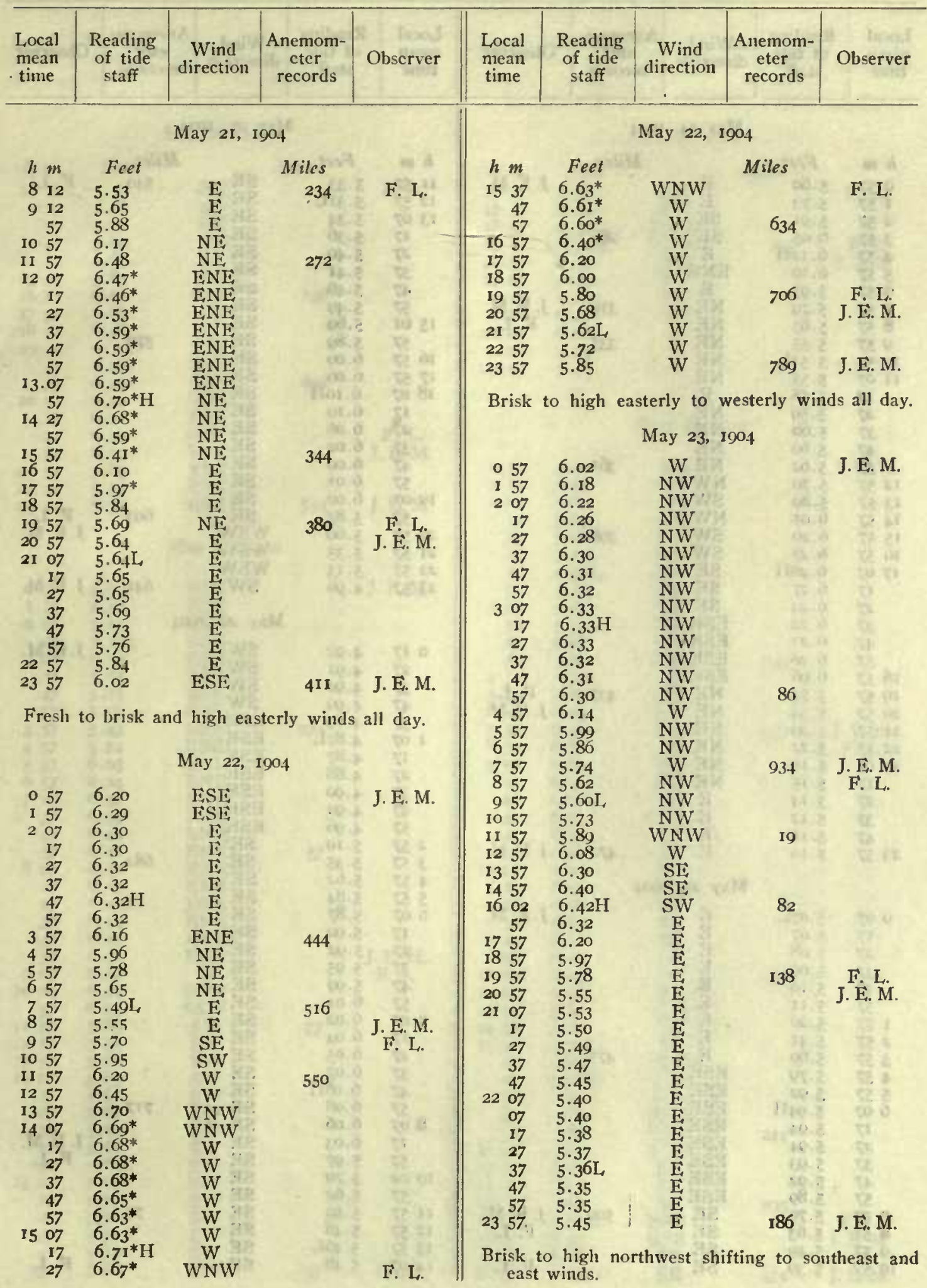


Tabulation of tidal observations at Cape Flora, Northbrook Island

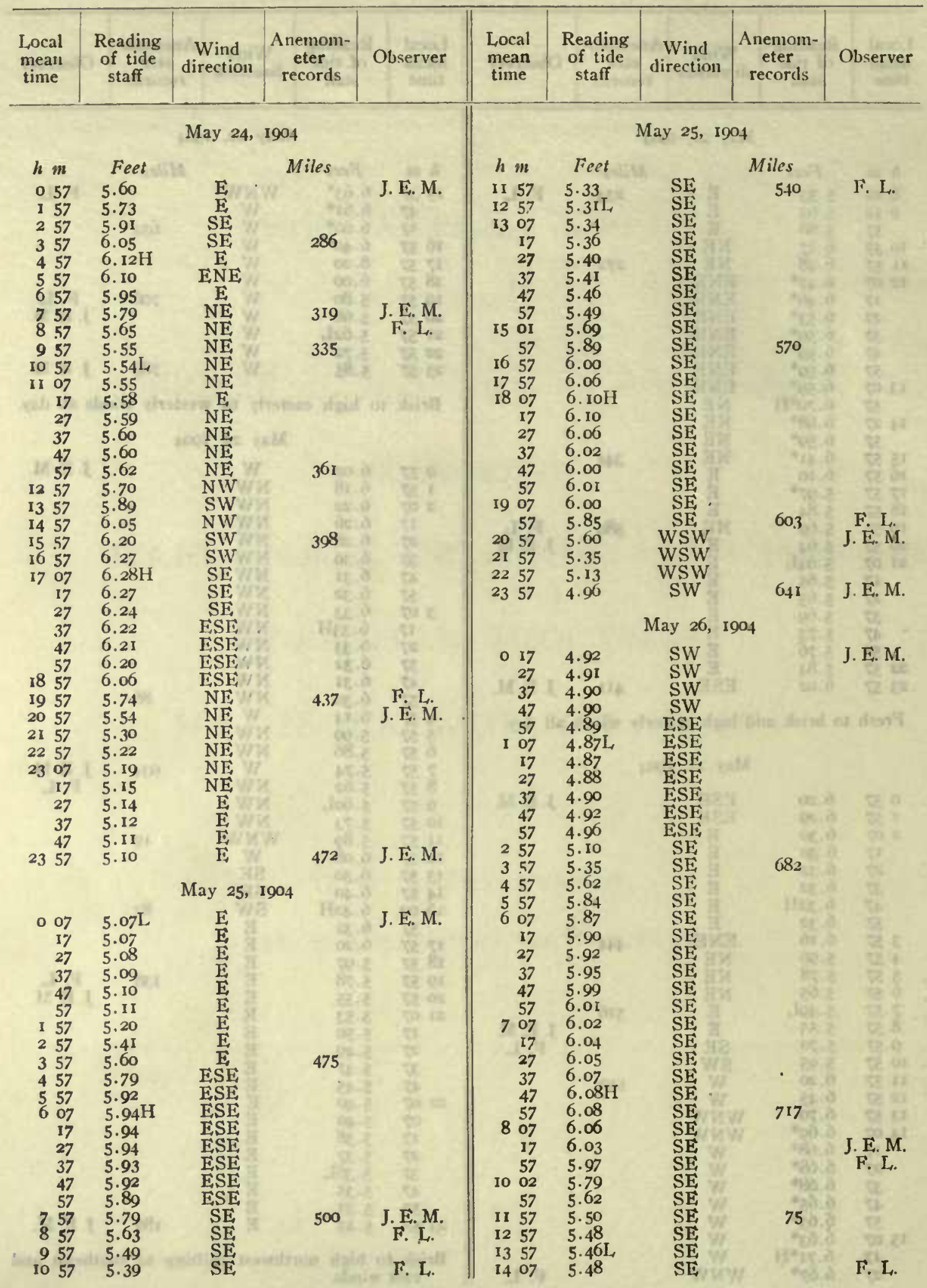


TIDAL, OBSERVA'IIONS

Tabulation of tidal obscrvations at Cape Flora, Northbrook Island

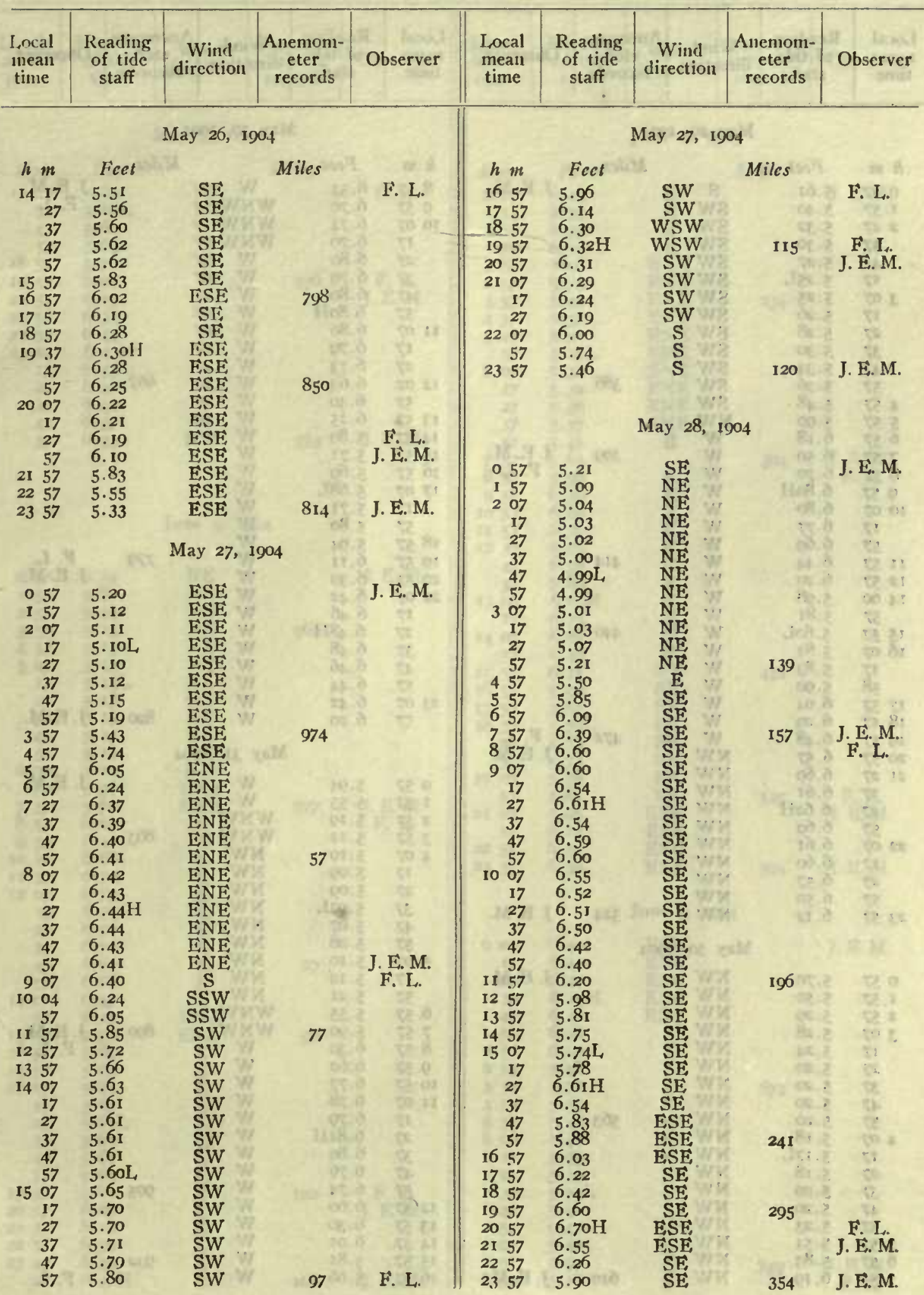


Tabulation of tidal obscrvations at Cape Flora, Northbrook Island

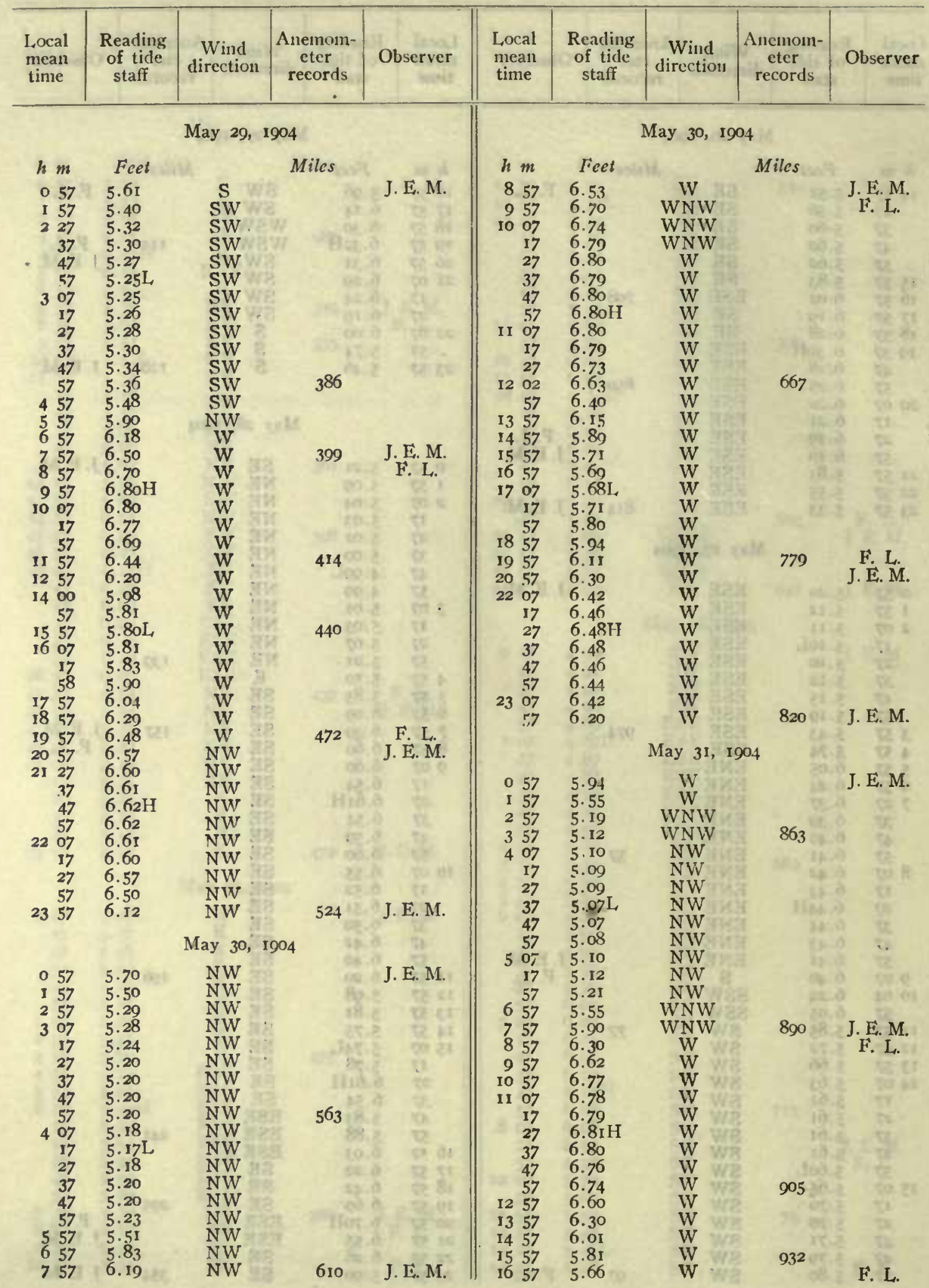


Tabulation of tidal obscrvations at Cape Flora, Northbrook Island

\begin{tabular}{|c|c|c|c|c|c|c|c|c|c|c|}
\hline $\begin{array}{c}\text { Local } \\
\text { mean } \\
\text { time }\end{array}$ & $\begin{array}{l}\text { Reading } \\
\text { of tide } \\
\text { staff }\end{array}$ & $\begin{array}{l}\text { Wind } \\
\text { direction }\end{array}$ & & $\begin{array}{l}\text { Anemom- } \\
\text { eter } \\
\text { records }\end{array}$ & Observer & $\begin{array}{c}\text { Local } \\
\text { mean } \\
\text { time }\end{array}$ & $\begin{array}{l}\text { Reading } \\
\text { of tide } \\
\text { staff }\end{array}$ & $\begin{array}{l}\text { Wind } \\
\text { direction }\end{array}$ & $\begin{array}{l}\text { Ancmom- } \\
\text { eter } \\
\text { records }\end{array}$ & Observer \\
\hline \multicolumn{6}{|c|}{ May 3I, I904 } & \multicolumn{5}{|c|}{ June 2, 1904} \\
\hline$h m$ & Fect & 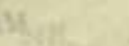 & & Miles & $\operatorname{set} 2 x$ & $h \mathrm{~m}$ & Feet & & Miles & wex $A$ \\
\hline 1727 & 5.62 & W & 푤 & ant tht & F. L. & 007 & 6.40 & E & 10.8 & J. E. M. \\
\hline 37 & $5.60 \mathrm{~L}$ & $W$ & 3 & 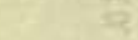 & -3 & 17 & 6.39 & $\vec{E}$ & stat & 10. \\
\hline 47 & 5.62 & W & 8 & at & tit & 27 & 6.35 & $\overrightarrow{\mathrm{E}}$ & a & $\mathrm{R}^{-}$ \\
\hline 85 & 5.69 & W & 8 & 32 & 87 & 57 & 6.24 & E & Howe & 5e 5 , \\
\hline $\begin{array}{l}1857 \\
1057\end{array}$ & 5.76 & W & G & & & I 57 & 6.08 & $E N E$ & w. & $50-84$ \\
\hline 1957 & 6.00 & W & 8 & 936 & F. L. & 257 & $5 \cdot 70$ & ENE & ye: & ta \\
\hline 20.57 & 6.19 & W & 要 & & J. E. M. & 357 & 5.49 & ENE. & 234 & 8 \\
\hline 2157 & 6.35 & W & & 26 & 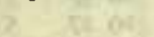 & 457 & 5.37 & ENE & -34 & to \\
\hline 2257 & 6.43 & W & I & ye & 28 & 547 & $5 \cdot 30$ & ENE & 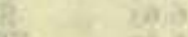 & 8 \\
\hline 2307 & $6.43 \mathrm{H}$ & W & 4 & $3 x$ & 87 & 57 & $5.29 \mathrm{~L}$ & ENE & sile & 官 \\
\hline 17 & 6.43 & $W$ & $4 n$ & 00 & 25 & 607 & 5.30 & ENE & 4. & 82 ar \\
\hline 27 & 6.42 & W & th & ant & wes & 17 & $5 \cdot 32$ & ENE & $\cos .8$ & $\frac{7 x}{3 x}$ \\
\hline 37 & 6.40 & W & & (x) & & 27 & $5 \cdot 36$ & ENE & $m: x^{2}$ & 32 of \\
\hline 47 & 6.37 & W & ary & & & 37 & 5.37 & ENE & . 1 & 851 \\
\hline 57 & 6.33 & NW & His & 953 & J. E. M. & 57 & 5.41 & $\mathbf{E}$ & 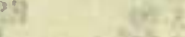 & -4 \\
\hline and & e & & 13 & a) & 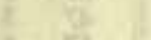 & 757 & 5.63 & & 304 & J. E. M. \\
\hline 8 & $=2$ & E. & & at & 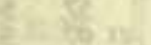 & $\begin{array}{l}857 \\
957\end{array}$ & $\begin{array}{l}5.98 \\
6.30\end{array}$ & $\underset{\mathrm{E}}{\mathbf{E}}$ & $\log 2$ & F. L. \\
\hline$\frac{1}{4} x$ & & June 1 , & 190 & 28 & t5: & 10 57 & 6.60 & $\mathbf{E}$ & 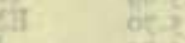 & ta \\
\hline & & & & dit & $78 x$ & $\begin{array}{l}1157 \\
1237\end{array}$ & $\begin{array}{l}6.70 \\
6.79\end{array}$ & $\overrightarrow{\mathrm{E}}$ & $\begin{array}{l}18.2 \\
10.3\end{array}$ & $\begin{array}{l}506 \\
815\end{array}$ \\
\hline 0 57 & 6.14 & $\mathrm{NE}$ & & In & J. E. M. & 47 & 6.76 & $\overrightarrow{\mathrm{E}}$ & 205 & 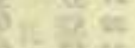 \\
\hline I 57 & 5.80 & $\mathrm{NE}$ & & & & 57 & 6.80 & $\frac{E}{E}$ & $\operatorname{cts} x$ & (e) $k$ \\
\hline 257 & 5.44 & $\mathrm{NE}$ & & & & 130 & $\begin{array}{l}0.80 \mathrm{H} \\
6.75\end{array}$ & E & & \\
\hline $\begin{array}{l}357 \\
457\end{array}$ & 5.25 & $\mathrm{NE}$ & & 963 & $(2) 10$ & 1407 & 6.61 & $\vec{E}$ & & \\
\hline $\begin{array}{l}457 \\
5\end{array}$ & 5.1 & $\begin{array}{l}\mathrm{NE} \\
\mathrm{NE}\end{array}$ & & (iif & 7). & 57 & 6.40 & $\vec{E}$ & 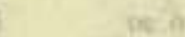 & ris: \\
\hline $\begin{array}{rl}5 & 07 \\
17\end{array}$ & $\begin{array}{l}5.13 \\
5.13 \mathrm{~L}\end{array}$ & NE & & Eim & (9) & 1557 & 6.14 & SE & 442 & $7 x$ \\
\hline 27 & 5.13 & $\mathrm{NE}$ & & 14 & (5x & I6 57 & 5.92 & SE & (2) & 6 \\
\hline 37 & 5.1 & $\mathrm{NE}$ & & pit & $\mathrm{X}$ & 1757 & $5.8 \mathrm{I}$ & SE & 1.0 & (3) \\
\hline 47 & 5.1 & $\mathrm{NE}$ & & a & 5 & $18 \quad 37$ & $5.72 \mathrm{~L}$ & SE & 263 & 8 \\
\hline 57 & 5.16 & $\mathrm{NE}$ & & set & 51 & 47 & 5.73 & SE & fites 4 & (8) 12 \\
\hline $\begin{array}{r}607 \\
57\end{array}$ & 5.20 & $\mathrm{NE}$ & & 3 & $\frac{92}{10}$ & $\begin{array}{r}57 \\
1907\end{array}$ & $\begin{array}{l}5.73 \\
5.78\end{array}$ & $\begin{array}{l}S E \\
S E\end{array}$ & ing & 78 \\
\hline $\begin{array}{r}57 \\
757\end{array}$ & $5 \cdot 3^{8}$ & ENE & & 0 & $T F M$ & 57 & 5.81 & SE & 525 & F. L. \\
\hline $\begin{array}{l}757 \\
857\end{array}$ & $\begin{array}{l}5.70 \\
6.04\end{array}$ & ENE & & 977 & J. E. M. & 2107 & 5.95 & ESE: & 525 & J. E. M. \\
\hline 957 & 6.4 & $\vec{E}$ & & $\%$ & & 57 & 6.12 & ESE & PQ. & $x^{2}=$ \\
\hline 1057 & 6.65 & E & & 4. & 605 & $\begin{array}{l}2257 \\
2357\end{array}$ & $\begin{array}{l}0.30 \\
6.39\end{array}$ & ESE & & J. E. M. \\
\hline II 57 & 6.7 & $\mathrm{E}$ & & 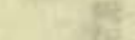 & $3 x$ & 238 & & ESE & 591 & J. L. 1 M. \\
\hline 1207 & $\begin{array}{l}6.73 \\
6.70 \mathrm{H}\end{array}$ & $\frac{E}{E}$ & & is & $\frac{2}{2}$ & $x_{1}$ & 8 & une 3 . & $\begin{array}{l}192 \\
4 y^{2}\end{array}$ & $\frac{18}{3}$ \\
\hline $\begin{array}{l}17 \\
27\end{array}$ & 6.76 & $\mathrm{E}$ & & If $x$ : & ts & 182 & & June 3 , & Exs & $\frac{710}{10}$ \\
\hline 37 & 6.70 & $\mathrm{E}$ & & IV & E & 007 & 6.40 & ESE & (A) & J. E. M. \\
\hline 47 & 6.69 & $\mathrm{E}$ & & 27 & 28 & I & 6.40 & ESE & $H$ & ter \\
\hline $\begin{array}{r}57 \\
14 \text { OI }\end{array}$ & 6.66 & $\mathrm{E}$ & & प्र & v & 27 & & ESE & 41 & $\frac{\mathrm{Z}}{5}=$ \\
\hline $\begin{array}{r}14 \text { OI } \\
57\end{array}$ & $\begin{array}{l}6.42 \\
6.10\end{array}$ & $\underset{\mathrm{ENE}}{\mathrm{E}}$ & & or & 38 & 37 & $\begin{array}{l}6.44 \mathrm{H} \\
6.42\end{array}$ & $\begin{array}{l}\text { ESE } \\
\text { ESE }\end{array}$ & 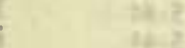 & $\frac{105}{51}$ \\
\hline $\begin{array}{r}57 \\
1557\end{array}$ & $\begin{array}{l}0.10 \\
5.90\end{array}$ & ESE & & 50 & $2 \mathrm{ni}$ & 57 & 6.38 & E & 4his? & $x^{2}$ \\
\hline 1657 & 5.69 & $\mathrm{E}$ & 17 & 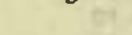 & $821 x$ & 157 & 6.2 & $\mathbf{E}$ & 02 & a \\
\hline 1757 & 5.63 & $\mathrm{E}$ & 4 & 5 & $Q=1$ & 257 & 5.9 & E & $-29 x^{2}$ & 81 \\
\hline 1807 & 5.61 & $\mathrm{E}$ & 12 & 77 & 30 & 357 & 5.71 & 1 & 672 & 8 \\
\hline 17 & 5.60 & E & $\mathrm{AM}$ & 15 & $60 \cdot 21$ & $4 \cdot 5$ & & $\mathbf{E}$ & -802 & 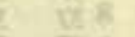 \\
\hline 27 & $5.60 \mathrm{~L}$ & $\mathrm{E}$ & 43 & we & 58 & 55 & 5.4 & E & 82 & $2:$ \\
\hline 37 & 5.60 & E & 23 & 14 & se & 60 & & $\mathbf{E}$ & 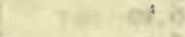 & 0.91 \\
\hline 47 & 5.61 & $\mathrm{E}$ & H & 1113. & ur & 17 & $5 \cdot 39$ & $\mathbf{E}$ & $\operatorname{ces} x$ & t) 11 \\
\hline 57 & 5.63 & E & 6 & in: & & 27 & & $\mathbf{E}$ & $a: p$ & tex: \\
\hline 1957 & 5.80 & $\mathrm{E}$ & W & 102 & $\vec{F}, L_{\text {. }}$ & 37 & $5.39 \mathrm{~L}$ & E & c: 2 & $70-0$ \\
\hline 2057 & 6.00 & E & 39: & It. & J. E. M. & 4 & & $\mathbf{E}$ & $n=?$ & 34 \\
\hline 2157 & 6.16 & $\bar{E}$ & & tit & & 57 & 5.42 & $\overline{\mathbf{E}}$ & $\cdots 3$ & $=$ \\
\hline 2257 & 6.32 & $\mathrm{E}$ & & 10 & & 707 & & $\overrightarrow{\mathrm{E}}$ & $=28$ & 35 \\
\hline 2347 & 6.40 & $E$ & 44 & & & 57 & 5. & $\vec{E}$ & 749 & J. E. M. \\
\hline 57 & $6.40 \mathrm{H}$ & $\mathbf{E}$ & an & 168 & J. E. M. & 857 & 5.78 & E & mitas at & F. L. \\
\hline
\end{tabular}


Tabulation of tidal observations at Cape Flora, Northbrook Island

\begin{tabular}{|c|c|c|c|c|c|c|c|c|c|}
\hline $\begin{array}{l}\text { Local } \\
\text { mean } \\
\text { time }\end{array}$ & $\begin{array}{l}\text { Reading } \\
\text { of tide } \\
\text { staff }\end{array}$ & $\begin{array}{l}\text { Wind } \\
\text { direction }\end{array}$ & $\begin{array}{c}\text { Anemom- } \\
\text { eter } \\
\text { records }\end{array}$ & Observer & $\begin{array}{c}\text { Local } \\
\text { time } \\
\text { mean }\end{array}$ & $\begin{array}{l}\text { Reading } \\
\text { of tide } \\
\text { staff }\end{array}$ & $\begin{array}{l}\text { Wind } \\
\text { direction }\end{array}$ & $\begin{array}{l}\text { Anemom- } \\
\text { eter } \\
\text { records }\end{array}$ & Observer \\
\hline \multicolumn{5}{|c|}{ Junc 3,1904} & \multicolumn{5}{|c|}{ June 4,1904} \\
\hline$h m$ & \multicolumn{2}{|c|}{ Feet $=1$} & Miles & \multirow{2}{*}{ F. L. } & $h m$ & \multicolumn{2}{|c|}{ Feet } & Miles & \multirow{2}{*}{ F. L. } \\
\hline 957 & 6.03 & ESE & $x^{2}=0$ & & 1407 & 6.54 & $\mathbf{E}$ & & \\
\hline 110 & & & & : & 17 & 6.52 & $\bar{E}$ & $\sin 2 x$ & ing \\
\hline & & ESP & 809 . & $\dot{8}$ & 57 & 6. & $\mathbf{E}$ & w. & 4 \\
\hline $\begin{array}{l}1257 \\
1307\end{array}$ & & ESE & $y=0$ & 3 & 1557 & 6.2 & $\vec{E}$ & 31 & Z: \\
\hline $\begin{array}{l}\text { o7 } \\
17\end{array}$ & $\begin{array}{l}6.67 \\
6.64\end{array}$ & ESE & $\operatorname{los}$ & $8:$ & I6 57 & $\begin{array}{l}6.02 \\
5.88\end{array}$ & $\frac{\mathrm{E}}{\mathrm{E}}$ & $\frac{04}{8}$ & to: \\
\hline 27 & & SE & 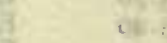 & a & 1857 & 5.70 & E & है। & पे बै \\
\hline 37 & & SE & 8 & 32t & 1927 & 5.6 & $\mathrm{E}$ & 28 & 2. \\
\hline 47 & & $\underset{\mathrm{ESE}}{\mathrm{SE}}$ & oge & (5) 8 & 37 & 5.6 & $\mathrm{E}$. & Hin & Dex \\
\hline 1457 & & ESE & $\sqrt{10}=$ & 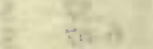 & 47 & 5. & EE & 52 & है. \\
\hline & & $\mathrm{SE}$ & 862 & $\therefore$ & $200^{5}$ & 5.8 & NE & 52 & $\ddot{z}$ \\
\hline & & ESE & 28 & $\approx$ & 17 & $\begin{array}{l}5.0 \\
5.6\end{array}$ & $\mathrm{NE}$ & 다 & 8 \\
\hline & 5.81 & $\underset{\mathrm{EF}}{\mathrm{E}}$ & $82-$ & 2 & 27 & $5.60 \mathrm{~L}$ & NE & $\therefore$ & 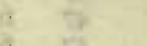 \\
\hline $\begin{array}{l}57 \\
07\end{array}$ & $\begin{array}{l}5.69 \\
5.65 \mathrm{~L}\end{array}$ & $\underset{\mathbf{E}}{\mathbf{E S E}}$ & 3 & $\frac{3}{3}-$ & 37 & & $N E$ & & 9. \\
\hline 17 & 5.68 & $\mathrm{NE}$ & . & \& 8 & $\begin{array}{l}47 \\
57\end{array}$ & 5.6 & $\mathrm{NE}$ & & \\
\hline 27 & & $\mathrm{NE}$ & 002 & $\therefore 8$ & 2107 & 5. & $\mathrm{NE}$ & & \\
\hline 2057 & 5.73 & $\mathbf{E}$ & $\begin{array}{l}902 \\
0.5\end{array}$ & F. L: & 7 & $\begin{array}{l}5.6 \\
5.6\end{array}$ & $\begin{array}{l}\text { NE } \\
\text { ENE }\end{array}$ & $\operatorname{xin}:$ & J. $\dot{\mathrm{E}} . \mathrm{M}$. \\
\hline & & E & & $1 F$ & 225 & 5.8 & ENE & & \\
\hline & & $\begin{array}{l}\mathbf{E} \\
\mathbf{E}\end{array}$ & 033 & J.E.M. & 2357 & 5.93 & $N W$ & 71 & J. E. M. \\
\hline & & & Hin & 4. 18 & 29 & & June 5 & 1904 & है है: \\
\hline & & June $4, \mathrm{I}$ & 904 & 5. & & & & & $\mathrm{~L} E \mathrm{M}$ \\
\hline 017 & 6.20 & E & & J. E. M. & $\begin{array}{l}057 \\
107 \\
107\end{array}$ & & $\frac{\mathrm{E}}{\mathrm{E}}$ & 25 & J.E. M. \\
\hline 27 & & & & & & & $\vec{E}$ & the & $\therefore$ \\
\hline 37 & & & $50:$ & 92 & 27 & & E & tit & . \\
\hline 47 & & & ise & 8 & 37 & & $\underset{\mathrm{F}}{\mathrm{E}}$ & it & E. \\
\hline $\begin{array}{r}57 \\
107\end{array}$ & $\begin{array}{l}6.25 \\
6.251\end{array}$ & E & 25 & 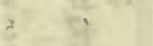 & $\begin{array}{l}47 \\
57\end{array}$ & $\begin{array}{l}6.0 \\
6.0\end{array}$ & $\mathrm{E}$ & & 3 \\
\hline 17 & 6.25 & E & & 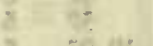 & 25 & 5. & $\mathrm{~N}$ & 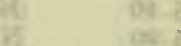 & ras \\
\hline 27 & & E & s. & 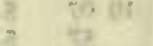 & 35 & & $\mathbf{N}$ & 97 & $\frac{1}{2}$ \\
\hline 37 & & $\mathrm{E}$ & $\geq 0$ & w) & 4. 5 & & $\begin{array}{l}N \\
N\end{array}$ & ast. & $\therefore$ \\
\hline 4 & & $\mathrm{E}$ & si. & 8 & 6 & & N & & 58 \\
\hline 25 & & ENE & at & & 7 & & $\mathrm{~N}$ & & \\
\hline & & ENE & 954 & $t$ & & & $\mathrm{~N}$ & & te : \\
\hline $\begin{array}{l}45 \\
5\end{array}$ & & $\frac{\mathbf{E}}{\mathbf{E}}$ & & & $\begin{array}{l}27 \\
37\end{array}$ & & $\mathrm{~N}$ & .3 & W. \\
\hline 6 & & $\mathbf{E}$ & & & & & $\mathrm{N}$ & $\operatorname{mos}^{2}$ & \\
\hline 3 & & $\overline{\mathrm{E}}$ & s. & $\infty \%$ & & & & 127 & $\ddot{x}$ \\
\hline 4 & & $\mathbf{E}$ & & : & 80 & & SEE & & I $M$ \\
\hline $\begin{array}{r}5 \\
70\end{array}$ & & E & & 5 & I & & SE & 2 & J.E. M. \\
\hline 70 & & $\underset{\mathbf{E}}{\mathrm{E}}$ & list. & ?. & & & $\frac{\mathrm{E}}{\mathrm{NE}}$ & 9 & $F, L$ \\
\hline & & $\mathrm{E}$ & a. & $\therefore$ & & & $\mathrm{NE}$ & & Fi \\
\hline 37 & & E & $\alpha$ & 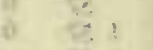 & & 6. & $N$ & 173 & $\therefore$ \\
\hline 47 & & $\mathbf{E}$ & 19 & & & & $N$ & t: & सें \\
\hline & & $\mathbf{E}$ & 972 & J. E. M. & & & $\mathrm{N}$ & & ए.: \\
\hline 8 & & ENE & 3 & F. L & 140 & & $\mathrm{~N}$ & & 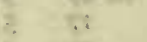 \\
\hline & & $\mathrm{E}$ & $1+18$ & & & & & in & \\
\hline & & E & & & & & & 0 & \\
\hline & & $\mathrm{E}$ & & & 3 & & & & \\
\hline & & $\mathrm{E}$ & & 5 & 4 & & & & \\
\hline & & E & is: & & & & & & \\
\hline I & & E & 7 & s. & & & $\mathrm{NE}$ & 233 & \\
\hline 2 & 6. & $\mathrm{E}$ & (it) & $\theta$ & & & $\mathbf{E}$ & & 3.1 \\
\hline & 6. & E & it. & 70 & & & $\mathbf{E}$ & Ba & te 1 \\
\hline 47 & & $\frac{\mathrm{E}}{\mathrm{E}}$ & & F L & 1857 & & $\begin{array}{l}\mathrm{NE} \\
\mathrm{NE}\end{array}$ & 8 & F. L. \\
\hline 57 & 0.5 & & & F. L. & 1957 & 5.63 & $\mathrm{NE}$ & 298. & F. L. \\
\hline
\end{tabular}


Tabulation of tidal observations at Cape Flora, Northbrook Island

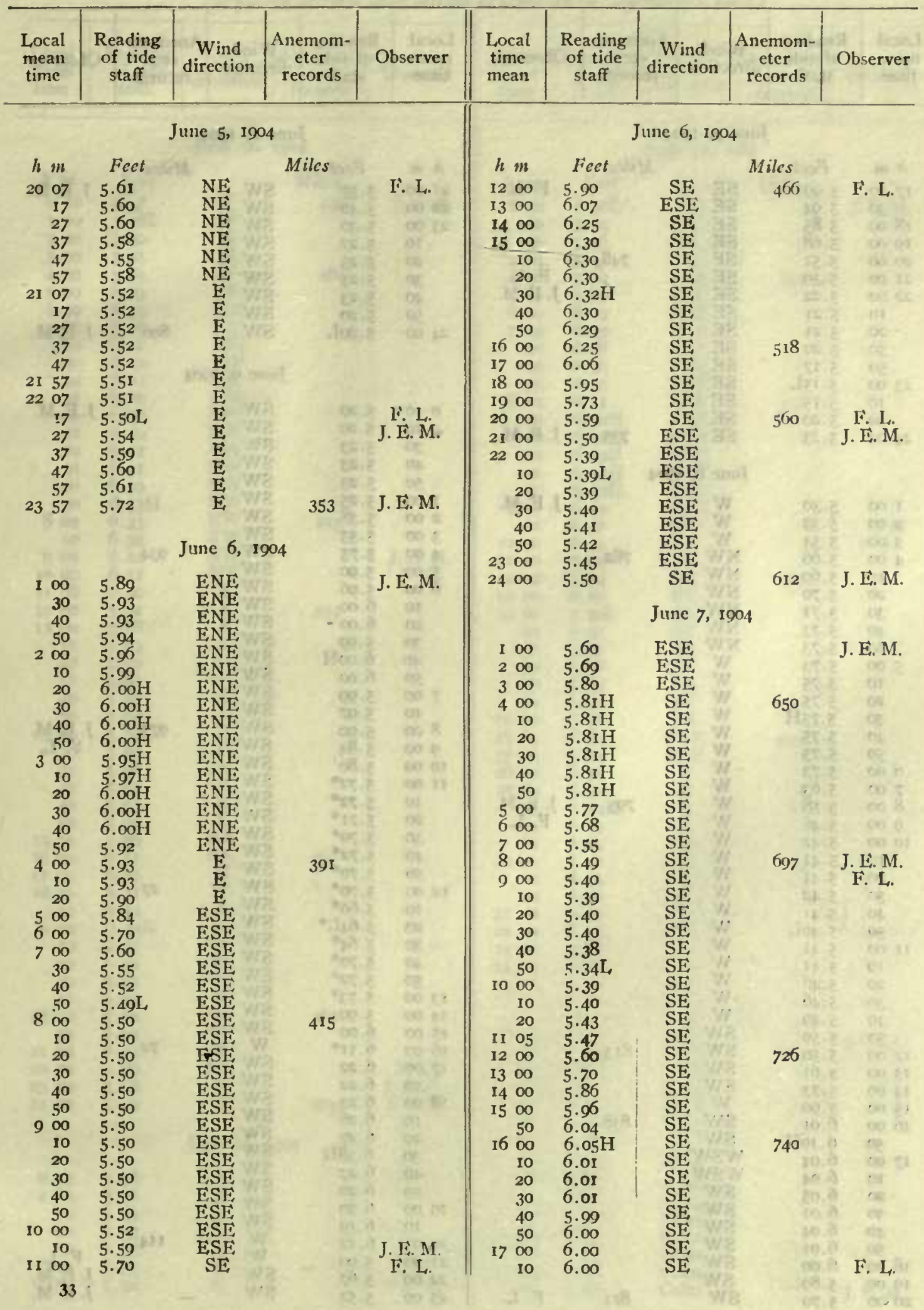


Tabulation of tidal observations at Cape Flora, Northbrook Island

\begin{tabular}{|c|c|c|c|c|c|c|c|c|c|c|c|}
\hline \multicolumn{2}{|c|}{$\begin{array}{l}\text { Local } \\
\text { mean } \\
\text { time }\end{array}$} & $\begin{array}{l}\text { Reading } \\
\text { of tide } \\
\text { staff }\end{array}$ & $\begin{array}{l}\text { Wind } \\
\text { direction }\end{array}$ & $\begin{array}{l}\text { Anemom- } \\
\text { eter } \\
\text { records }\end{array}$ & \multirow[t]{2}{*}{ Observer } & \multicolumn{2}{|c|}{$\begin{array}{l}\text { Local } \\
\text { mean } \\
\text { time }\end{array}$} & $\begin{array}{c}\text { Reading } \\
\text { of tide } \\
\text { staff }\end{array}$ & $\begin{array}{l}\text { Wind } \\
\text { direction }\end{array}$ & $\begin{array}{l}\text { Anemom- } \\
\text { eter } \\
\text { records }\end{array}$ & \multirow[t]{2}{*}{ Observer } \\
\hline \multicolumn{5}{|c|}{ June 7, I904 } & & & & & une 8 , I 9 & 4 & \\
\hline$h n$ & $m$ & Fect & 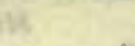 & Miles & 20 & h, & $m$ & Fect & 4 & Miles & $=4$ \\
\hline 172 & 20 & 5.99 & SE & 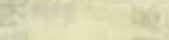 & F. L. & $21 \mathrm{c}$ & $\infty$ & $5 \cdot 57$ & SW & & T. E. M. \\
\hline & 30 & 5.94 & SE & 27 & 697 & $22 c$ & $\infty$ & 5.40 & SW & & \\
\hline 180 & & 5.85 & SE & 28. & $\infty: y$ & $23 c$ & & $5 \cdot 30$ & SW & 0 & to \\
\hline 190 & $\infty$ & 5.68 & SE & 10 & 00 ता & & 10 & 5.27 & SW & 8 & 4 \\
\hline 200 & $\infty$ & 5.51 & SE & 748 & $\ln ^{2}$ & & 20 & 5.25 & SW & 32 & a \\
\hline 210 & $\infty$ & 5.39 & SE & $x^{2}$ & F. L & & 30 & 5.24 & SW & $x$ & 2 \\
\hline 220 & $\infty$ & 5.22 & SE & $t=2$. & J. E. M. & & 40 & 5.23 & SW & 57 & $60 x$ \\
\hline & I0 & 5.21 & $\mathrm{SE}$ & $\ln$ & $a^{2}+x^{2}=$ & & 50 & 5.20 & SW & $=$ & str \\
\hline & 20 & 5.21 & SE & win & $\frac{25}{2}$ & 240 & $\infty$ & $5.20 \mathrm{~L}$ & SW & 896 & J. E. M. \\
\hline & 30 & 5.20 & SE & 25 & wercot & & & & & & $x^{2}+x^{2}$ \\
\hline & 50 & 5.17 & SE & be & 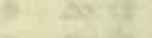 & to & & & June 9,1 & 904 & $3 \mathrm{r}$ \\
\hline 230 & $\infty$ & $5.15 \mathrm{~L}$ & SE & 3 & mar 8 & , & : & & & & $x^{2}+10$ \\
\hline 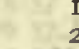 & $\begin{array}{l}10 . \\
20\end{array}$ & $\begin{array}{l}5.15 \\
5.20\end{array}$ & $\begin{array}{l}\text { SE } \\
\text { SE }\end{array}$ & 25 & wortests & 0 & 10 & 5.20 & SW & $\therefore$ & J. E. M. \\
\hline 240 & $\infty$ & 5.23 & $\mathrm{SE}$ & 735 & J. E. M. & & 20 & 5.20 & SW & & x \\
\hline & & & & pr & $c^{2}+20$ & & $\begin{array}{l}30 \\
40\end{array}$ & $\begin{array}{l}5.23 \\
5.23\end{array}$ & $\begin{array}{l}\text { SW } \\
\text { SW }\end{array}$ & & 8 \\
\hline & & & June 8 , & 1904 & ests & & $\begin{array}{l}40 \\
50\end{array}$ & 5.23 & SW & Bi) & a \\
\hline 10 & $\infty$ & $5 \cdot 30$ & W & 64 & J. E. M. & & $\infty$ & 5.25 & SW & & $y_{2}=$ \\
\hline 20 & $\infty$ & 5.39 & W & 11 & $z^{2}+x^{2}+x^{2}$ & & $\infty$ & $5 \cdot 38$ & SW & & \\
\hline 30 & $\infty$ & 5.54 & $W$ & 50 & Mx & & $\infty$ & 5.55 & SW & & te \\
\hline 40 & $\infty$ & 5.66 & NW & 782 & wi in & & $\infty$ & 5.75 & SW & 934 & \\
\hline 1 & 10 & 5.69 & NW & $x_{0}$ & 60072 & & $\infty$ & 5.90 & SW & (n) & Bs. \\
\hline 2 & 20 & 5.70 & NW & 2 & $x^{2}$ & 6 & & 5.96 & SW & is & e \\
\hline & 30 & $5.7 \mathrm{I}$ & NW & int: & te & & Io & 6.0 & SW & & 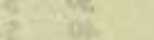 \\
\hline & 40 & 5.73 & NW & & & & 20 & $6.00=$ & SW & & 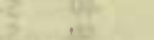 \\
\hline & 50 & 5.73 & NW & Bi). & be $\times 11$ & & 30 & 5.9 & Sy & s. & $10 x=$ \\
\hline 50 & & 5.73 & $W$ & $e^{3}$ & 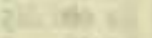 & & 4 & $6.00 \mathrm{H}$ & $\begin{array}{l}\text { SW } \\
\text { SW }\end{array}$ & 80 & $\frac{\pi}{4 \pi}$ \\
\hline & 10 & 5.75 & W & 8 & 60 & & 50 & 6.00 & $\begin{array}{l}\text { SW } \\
\text { SW }\end{array}$ & Hon & $\approx$ \\
\hline & 20 & 5.75 & $W$ & Hit? & $\infty$ & 7 & $\begin{array}{l}\infty \\
10\end{array}$ & $\begin{array}{l}5.99 \\
5.07\end{array}$ & $\begin{array}{l}\text { SW } \\
\text { SW }\end{array}$ & 300 & $y$ \\
\hline & $\begin{array}{l}30 \\
40\end{array}$ & $\begin{array}{l}5.75 \mathrm{H} \\
5.75\end{array}$ & $\underset{W}{W}$ & $\begin{array}{l}\text { Hats } \\
\text { prish }\end{array}$ & 4) & 8 & $\infty$ & $\begin{array}{l}5.97 \\
5.90\end{array}$ & SW & 973 & J. E. M. \\
\hline & $\begin{array}{l}40 \\
50\end{array}$ & $\begin{array}{l}5.75 \\
5.75\end{array}$ & W & मिए & or. & & $\infty$ & 5.84 & SV & I5s. & F. L. \\
\hline 60 & $\infty$ & & W & Dit? & a & Io & & 5.8 & SV & & $5=$ \\
\hline 70 & $\infty$ & 5.63 & W & Hile & & II & & $5.72 *$ & SV & tyos &. \\
\hline 80 & $\infty$ & 5.58 & W & 795 & J. E. M. & & IC & $5.72^{*}$ & SV & Ping & ie \\
\hline 90 & $\infty$ & 5.46 & W & - & F. L. & & 2 & $5.7 I^{*}$ & SV & fires. & sin \\
\hline I0 0 & $\infty$ & 5.42 & W & -2 & 00 & & 30 & $5 \cdot 70^{*}$ & SV & 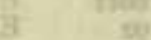 & 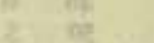 \\
\hline & IO & $5.4 \mathrm{I}$ & W & (4) & का & & 40 & $5.72^{*}$ & Sy & & \\
\hline & 20 . & 5.41 & W & 2 & ab. & & 50 & 5.70 & Sh & 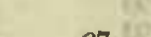 & 4 \\
\hline & 30 & 5.42 & W & est & a) & 12 & & 5.70 & sy & 27 & $\Leftrightarrow$ \\
\hline & 40 & 5.41 & W & 6 & $\alpha$ & & & 5.69 & Sy & XI & $m y$ \\
\hline & 50 & $5.40 \mathrm{~L}$ & W & c. & $\omega$ & & & & & we & $x_{n}$ \\
\hline 110 & $\infty$ & $5.4 \mathrm{I}$ & W & है, & 68 & & 3 & 5.64 & Sy & of & $\ln x$ \\
\hline & 10 & $5.4 \mathrm{I}$ & W & 332 & oge. & & & & & 27 & 97 \\
\hline & 20 & $5 \cdot 4^{6}$ & W & ac & $23=4$ & & 50 & $5.72^{*}$ & & 32 & 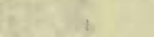 \\
\hline & 30 & 5.46 & W & of & 91 & 13 & & 5.7 & & Jes & 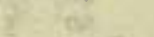 \\
\hline & 40 & & W & 31 & ec & I 4 & & 5.9 & & $\mathrm{n}$ & 6014 \\
\hline & 50 & 5.50 & SW & 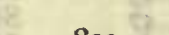 & 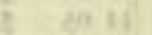 & 15 & & 6.0 & & mat & 69 \\
\hline 120 & $\infty 0$ & $5 \cdot 50$ & SW & $813 \ldots 0$ & $\cos x i$ & 16 & & 6.11 & W & 72 & $=$ \\
\hline 130 & $\infty$ & 5.61 & & Trt & $\cos <$ & 17 & $\infty$ & 6.2 & SW & $n^{n}$ & $\sigma$ \\
\hline 140 & $\infty$ & 5. & SW & (6). & 607 & & & 6.2 & & cet & - il \\
\hline 150 & & & W & & 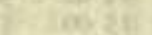 & 18 & $\infty$ & 6.2 & & as & 6 \\
\hline 160 & $\infty$ & 6.01 & SW & 838 & 어 & & & 6.26 & SV & Wr & 100 \\
\hline & 50 & $6.10 H$ & SW & 16rat & $x^{2}-81$ & & $2 c$ & 6.27 & & $\theta$ & ai \\
\hline 170 & 00 & 6.04 & WSW & 10. & ort & & 30 & $6.28 \mathrm{H}$ & & . & $\mathrm{mE}$ \\
\hline & 10 & 6.04 & WSW & 15 & es & & 40 & 6.27 & Sy & . & $\ldots$ \\
\hline 2 & 20 & 6.05 & SV & 10 & तr & & 50 & 6.2 & & co & . \\
\hline & 30 & 6.01 & SW & (c) & at & 19 & & 6.20 & & 18 & $\infty$ \\
\hline & 40 & 6.04 & SW & 2 & 62 & & 10 & 6.1 & sw & & as cis \\
\hline & 50 & 6.01 & SW & 0. & (1) & 20 & OI & 6.12 & W & $\mathrm{II}_{4}$ & E \\
\hline 180 & 00 & 6.00 & SW & $\infty$ & 6) & 21 & $\infty$ & 5.0 & & & \\
\hline 190 & $\infty$ & 5.89 & SW & & & 22 & & 5.67 & SW & & \\
\hline 200 & 00 & 5.70 & SW & 871 & F. L. & 23 & $\infty 0$ & $5 \cdot 52$ & SW & & J. Е. M. \\
\hline
\end{tabular}


Tabulation of tidal obscrvations of Capc Flora, Northbrook Island

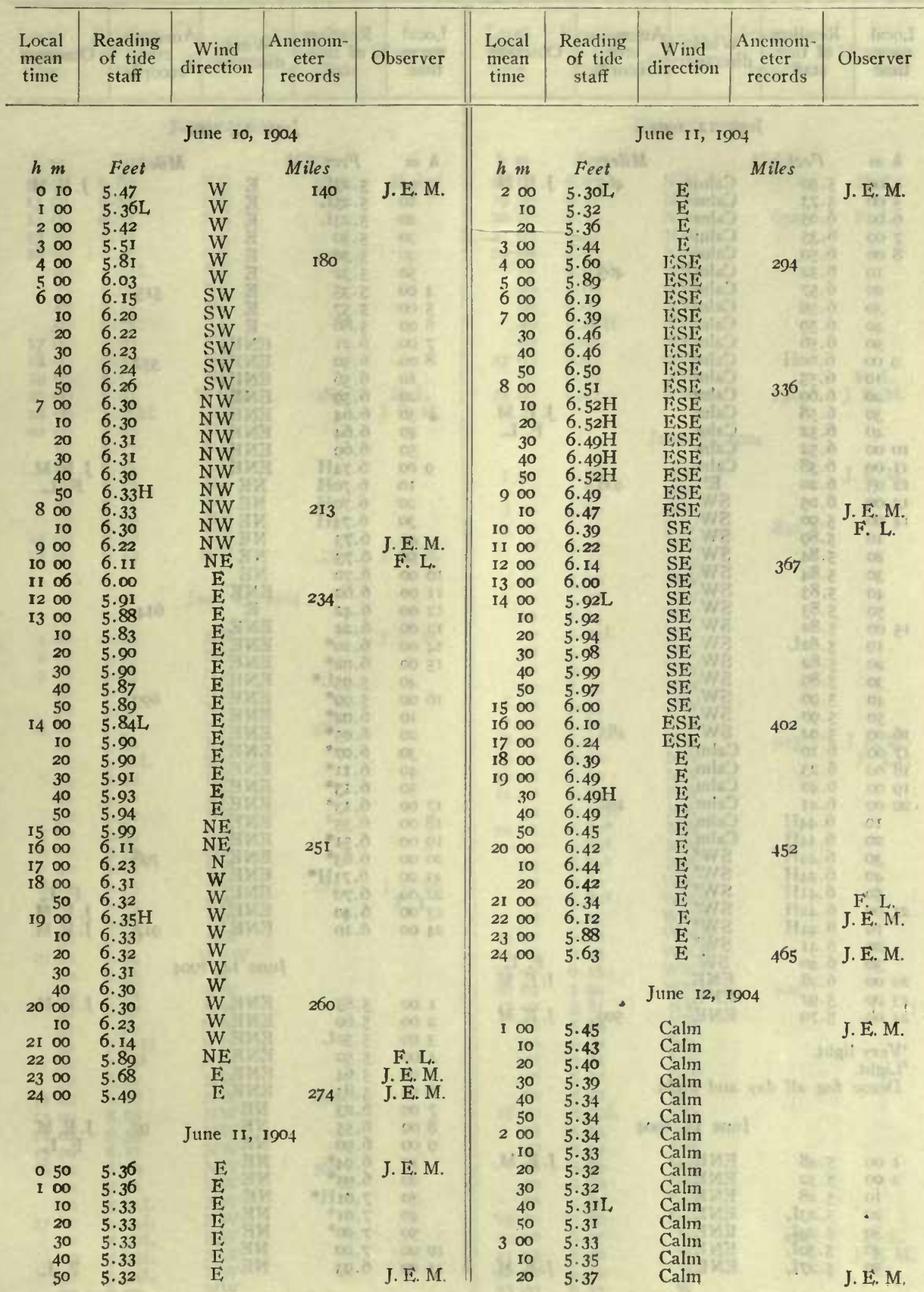


Tabulation of tidal observations at Capc Flora, Northbrook Island

\begin{tabular}{|c|c|c|c|c|c|c|c|c|c|}
\hline $\begin{array}{l}\text { Local } \\
\text { nuean1 } \\
\text { time }\end{array}$ & $\begin{array}{l}\text { Reading } \\
\text { of tide } \\
\text { staff }\end{array}$ & $\begin{array}{l}\text { Wind } \\
\text { direction }\end{array}$ & $\begin{array}{l}\text { Anemom- } \\
\text { eter } \\
\text { records }\end{array}$ & Observer & $\begin{array}{l}\text { Local } \\
\text { mean } \\
\text { time }\end{array}$ & $\begin{array}{l}\text { Reading } \\
\text { of tide } \\
\text { staff }\end{array}$ & $\begin{array}{l}\text { Wind } \\
\text { direction }\end{array}$ & $\begin{array}{c}\text { Ancmom- } \\
\text { eter } \\
\text { records }\end{array}$ & Observer \\
\hline \multicolumn{5}{|c|}{ June I2, I904 } & \multicolumn{5}{|c|}{ June 13,1904} \\
\hline$h m$ & Fect & \multirow{2}{*}{\multicolumn{2}{|c|}{ Miles }} & \multirow[b]{2}{*}{ J. E. M. } & \multicolumn{2}{|r|}{ Fect } & 4 & Miles & \multirow{2}{*}{ J. İ. M. } \\
\hline 400 & 5.50 & Calm & & & 300 & $5.27 \mathrm{~L}$ & $\mathbf{E}$ & $x^{2}=0$ & \\
\hline 500 & 5.73 & Calın. & - & tet & 10 & $5.25 \mathrm{~L}$ & E & 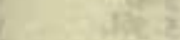 & ony \\
\hline 600 & 6.05 & Calm & aेt & et & & $5.23 \mathrm{~L}$ & E & 83 & 005 \\
\hline 700 & 6.35 & Calm & 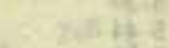 & as $t$. & 30 & $5 \cdot 30$ & E & Five & $00 x$ \\
\hline 800 & 6.50 & Calm & 465 & was. & 40 & $5 \cdot 30$ & $\mathrm{E}$ & ith & 001. \\
\hline 10 & 6.52 & Calm & 465 & 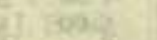 & 50 & $5 \cdot 32$ & E & 60 & 98 \\
\hline 20 & 6.57 & Calm & कर & $20 \%$ & 400 & 5.35 & E & 515 & na 3 \\
\hline 30 & 6.59 & Calm & 92 & an is & 500 & 5.57 & $\vec{E}$ & - & cash \\
\hline 40 & 6.59 & Calm & A & e. & 600 & 5.86 & $\mathrm{E}$ & $12 x$ & Getr \\
\hline 50 & $6.5^{6}$ & Calm & $8=$ & e. & 700 & 6.21 & ENE & & या \\
\hline 900 & $6.60 \mathrm{H}$ & Calı & ay & ox: & 800 & 6.50 & $\mathrm{ENE}$ & 552 & $\pi$ \\
\hline 10 & 6.55 & Calm & $x 2.8$ & bit & I0 & 6.56 & ENE & 30 & $\infty$. \\
\hline 20 & 6.60 & Calm & 78 ? & $I M$ & 20 & 6.59 & ENE & at & Pest \\
\hline 30 & 6.58 & Calm & (is) & & 30 & 6.64 & ENE & 68 & at \\
\hline 40 & $6.5^{2}$ & Calm & Files: & F. L. & 40 & 6.64 & ENE & 8 & 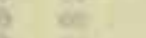 \\
\hline 1000 & 6.52 & Calın & Hests & (n) & 50 & 6.69 & ENE & ir. & $8 x \rightarrow$ \\
\hline II 00 & 6.38 & Calm & & ex & 900 & $6.74 \mathrm{H}$ & ENE & $=$ & J. E. M. \\
\hline 1203 & 6.20 & $E^{1}$ & 468 & tati & 10 & $6.7 \mathrm{oH}$ & $\mathrm{NE}$ & Hist & F. L. \\
\hline 1300 & 6.05 & SW & 5 5 & artat & 20 & $6.7 \mathrm{oH}$ & $\mathrm{NE}$ & Ut & 68 \\
\hline 1400 & 5.90 & SW & $\frac{d}{a}$ & ac xit & & 6.74 & $\mathrm{NE}$ & $\frac{a}{2}$ & fis \\
\hline I0 & 5.89 & SW & 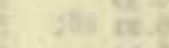 & of 17 & 40 & 6.73 & $\mathrm{NE}$ & 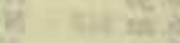 & on $\alpha=$ \\
\hline 20 & 5.84 & SW & ifis & ans ir & & 6.73 & $\mathrm{NE}$ & it. & $\infty x$ dx \\
\hline 30 & 5.84 & SW & कo. & 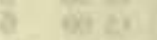 & Io $\infty$ & 6.70 & $\mathrm{NE}$ & os: & $-20 \pi$ \\
\hline 40 & 5.83 & SW & Whe z & sen $x$ & II $\infty$ & 6.62 & ENE & 30 & $109 \mathrm{si}$ \\
\hline 50 & 5.83 & SW & $\cos$ & (6) & $12 \infty$ & 6.42 & $\mathrm{E}$ & 614 & ne int \\
\hline 1500 & 5.82 & SW & 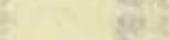 & es & 1300 & 6.24 & ENE & in & ait \\
\hline 10 & $5.82 \mathrm{~L}$ & SW & 89 & aes & 1400 & $6.10^{*}$ & ENE & has & 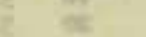 \\
\hline 20 & 5.82 & SW & 60 & of & 1500 & $6.02^{*}$ & ENE & $\frac{10}{6}$ & of \\
\hline 30 & 5.80 & SW & 70 & $a^{2}+x^{2}$ & 40 & 5.95 & ENE & & te. \\
\hline 40 & 5.90 & SW & oate & 00.42 & 1600 & $5.99^{*}$ & ENE & 699 & 33 \\
\hline 50 & 5.90 & SW & 483 & ho 71 & 10 & $6.02 *$ & ENE & ( 1 & $10 \%$ \\
\hline 1600 & 5.92 & SW & & 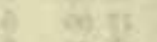 & 20 & $6.05^{*}$ & $\mathrm{ENE}$ & 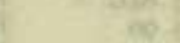 & of \\
\hline 1700 & 6.10 & Calm & 단 & 1003 & 30 & $6.07^{*}$ & ENE & 8 & 0 \\
\hline 1800 & 6.23 & Calm & (7) & nipit & 40 & $6 . \mathrm{II}^{*}$ & ENE & 10 & ax \\
\hline 1900 & 6.40 & Calnı & 402 & ext & 50 & $6.13^{*}$ & $\mathrm{ENE}$ & $10=$ & 8 \\
\hline 2000 & $6.4 \pi$ & Calm & 492 & 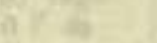 & 1700 & $6.13^{*}$ & $\mathrm{ENE}$ & $x^{2}$ & as \\
\hline 10 & $6.44 \mathrm{H}$ & Calm & $n$ & tor & 1800 & $6.35^{*}$ & $\mathrm{ENE}$ & 68 & $60+1$ \\
\hline 20 & $6.44 \mathrm{HI}$ & Calm & 4) & $\cos u$ & 1900 & 6.5 & ENE & & os dit \\
\hline 30 & $6.44 \mathrm{HI}$ & $\begin{array}{l}\mathrm{SW}^{2} \\
\mathrm{SW}^{2}\end{array}$ & 34 & oil & 2000 & 6.6 & ENE & 798 & 605 \\
\hline 40 & $6.44 \mathrm{H}$ & $\begin{array}{l}S W^{2} \\
\text { SW }^{2}\end{array}$ & 4.1 & $e c$ & 2100 & 6.7 & ENE & it & Fi \\
\hline 50 & $6.4 \mathrm{IH}$ & $\begin{array}{l}\mathrm{SW}^{2} \\
\mathrm{~S} W^{3}\end{array}$ & in: & $\cos x=$ & 2200 & 6.7 & ENE & 3 & F. L. \\
\hline 2100 & $6.42 \mathrm{H}$ & $\begin{array}{l}\mathrm{SW}^{2} \\
\mathrm{SW}\end{array}$ & int & $\infty e x$ & 2300 & $6.4 c$ & ENE & 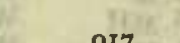 & J.E. M. \\
\hline I0 & $6.44 \mathrm{H}$ & $\begin{array}{l}\mathrm{SW}^{2} \\
\mathrm{SW}\end{array}$ & Fi: & no. 14 & 2400 & 6.10 & ENE & 917 & J. E. M. \\
\hline $\begin{array}{l}20 \\
30\end{array}$ & $\begin{array}{l}6.41 \\
6.40\end{array}$ & SW & 60 & F. L. & r & & & 잔 & es \\
\hline $22 \begin{array}{r}30 \\
00\end{array}$ & 6.28 & ENE & & J. $\dot{\mathrm{E}} . \dot{\mathrm{M}}$ & $x^{2}$ & & June I4, & 대을 & in \\
\hline 2300 & 5.97 & ENEE & & & I 00 & 5.85 & ENE, & 8 & J.E. M. \\
\hline 2400 & $5 \cdot 70$ & ENE & 500 & J. E. M. & 200 & 5.60 & ENE & $x^{2}$ & (2) \\
\hline${ }^{1} \mathrm{Ver}$ & iglit. & 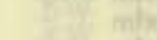 & 4 & nil & 300 & $5.50 \mathrm{~L}$ & ENE & 22 & $100 \pi$ \\
\hline${ }^{2}$ Light & & & 80 & is & 400 & $5 \cdot 50$ & ENE & 22 & $\cos 2=$ \\
\hline Dense & fog all $d$ & day and nig & hit. & 38 & 500 & 5.64 & ENE & $x^{3}$ & $\cos 1=$ \\
\hline (n) & - & & $\because$ & 67 & $\begin{array}{l}600 \\
700\end{array}$ & $\begin{array}{l}5.83 \\
6.23\end{array}$ & $\begin{array}{l}\mathrm{ENE} \\
\mathrm{NE}\end{array}$ & 58. & $00 \mathrm{ba}$ \\
\hline 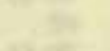 & & June 13 , & 1904 & 1006 & 800 & 6.55 & NE & 97 & J. E. M. \\
\hline I 00 & 5.48 & ENE & $\underline{H}$ & J. E. M. & $\begin{array}{l}900 \\
10\end{array}$ & $\begin{array}{l}6.90 \\
6.94^{*}\end{array}$ & NE & & F. L. \\
\hline 200 & & ENE & sis & ( & 20 & $6.95^{*}$ & $\mathrm{NE}$ & e & aㄹ 9 \\
\hline 10 & 5.28 & ENE & lin & 20 & 30 & $7.01 \mathrm{H}^{*}$ & $\mathrm{NE}$ & $x^{2}$ & net \\
\hline 20 & $5.23 \mathrm{~L}$ & ENE & at: & $\frac{3}{3} \quad 4$ & 40 & $7.01^{*}$ & NE & ift & ex \\
\hline 30 & $5.23 \mathrm{~L}$ & ENE & bre & $3 \quad 2007$ & & $7.00^{*}$ & $\mathrm{NE}$ & $14 y_{12}$ & $+x$ \\
\hline 40 & $5.30 \mathrm{~L}$ & $\begin{array}{l}\text { ENE } \\
\text { ENE }\end{array}$ & $7 x$ & J. E. M. & $\begin{array}{ll}\text { I0 } & 00 \\
\text { II } & 00\end{array}$ & $\begin{array}{l}7.00 \\
6.03\end{array}$ & NE & सt. & F. I \\
\hline 50 & $5.27 \mathrm{~L}$ & LAVI & & & II 00 & 0.93 & & ter & 1.4 \\
\hline
\end{tabular}


Tabulation of tidal observations of Cape Flora, Northbrook Island

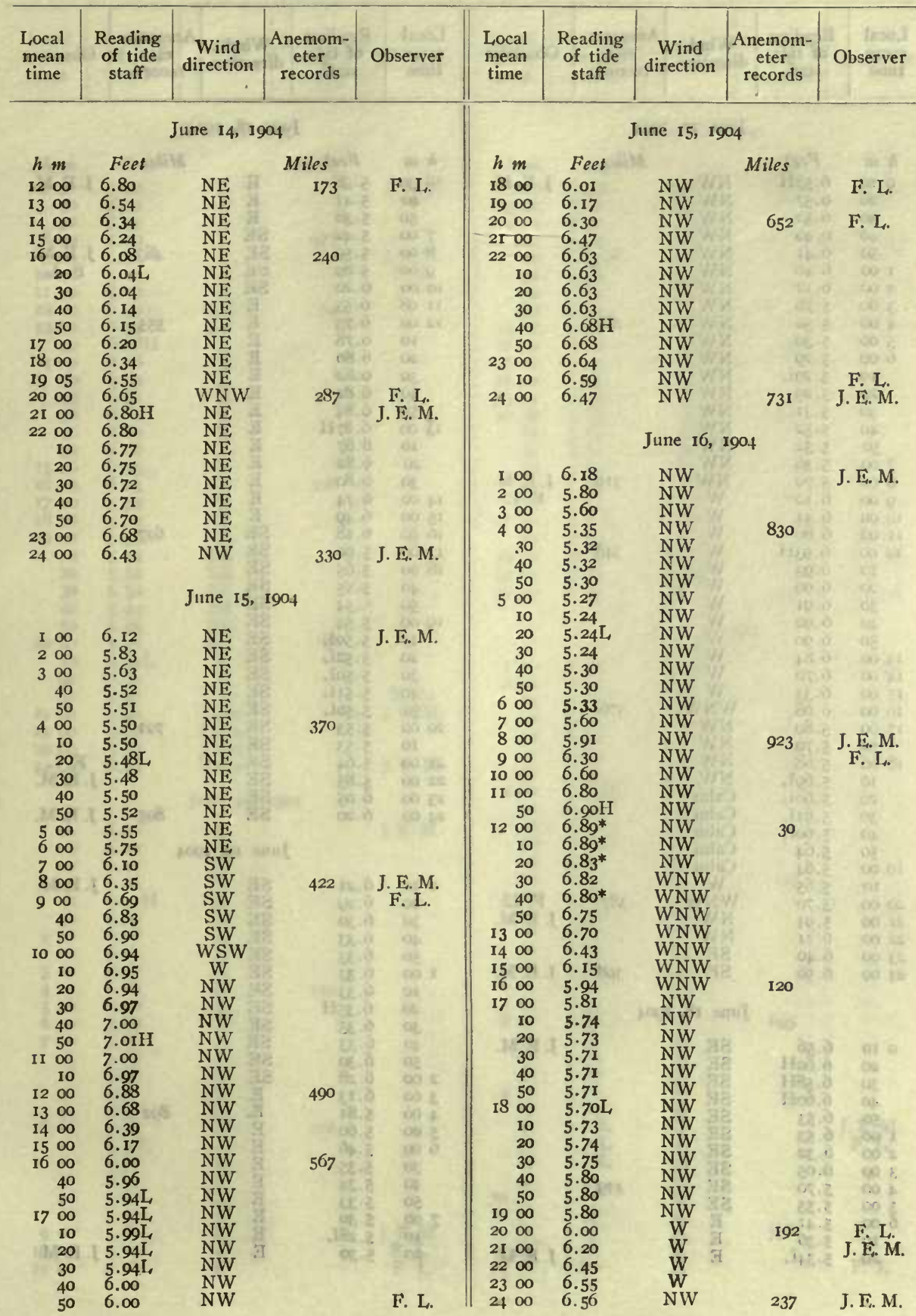


Tabulation of tidal observations at Cape Flora, Northbrook Island

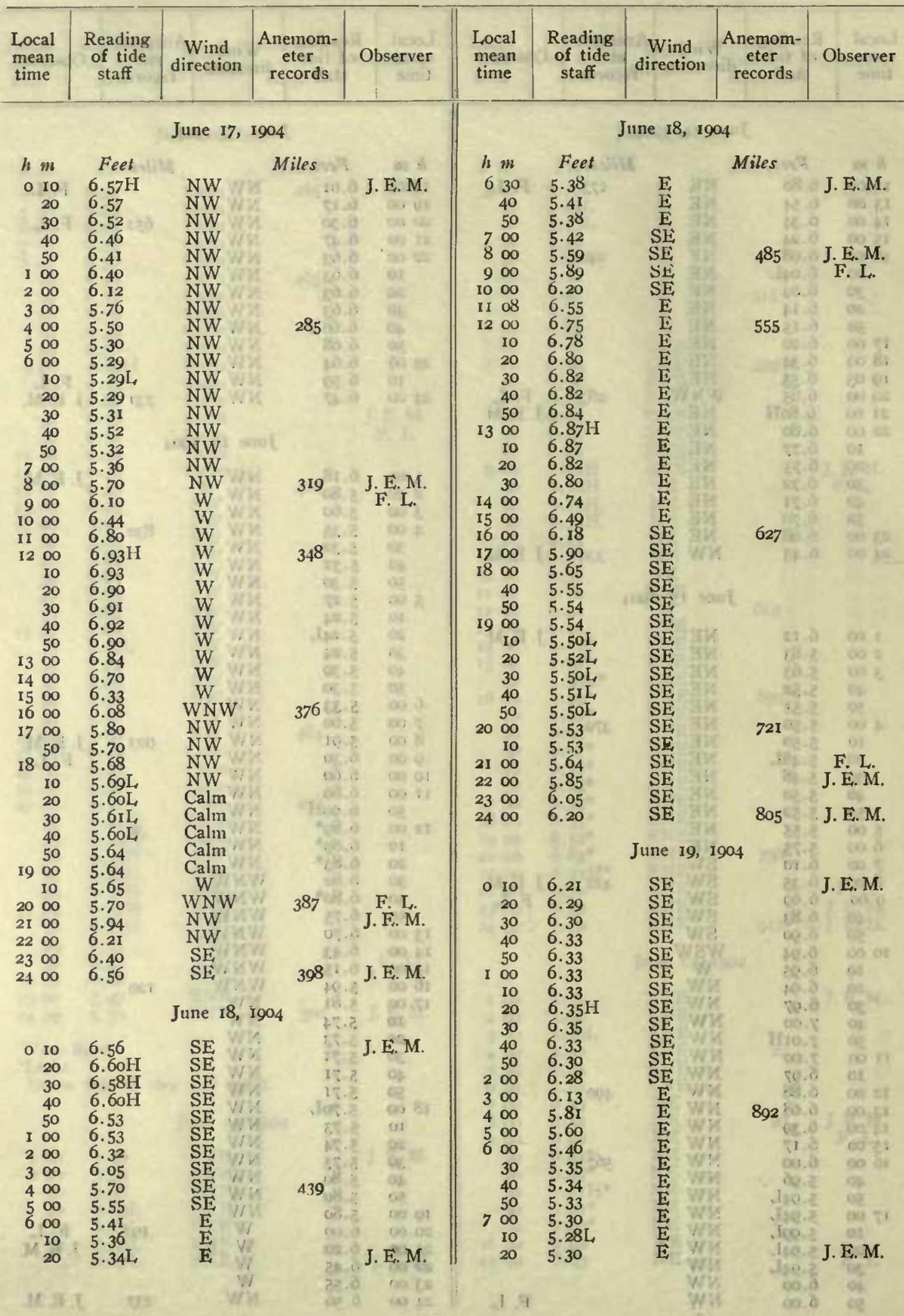


TIDAL, OBSERVATIONS

Tabulation of tidal observations at Cape Flora, Northbrook Island

\begin{tabular}{|c|c|c|c|c|c|c|c|c|c|}
\hline $\begin{array}{l}\text { Loca } \\
\text { mear } \\
\text { time }\end{array}$ & $\begin{array}{l}\text { Reading } \\
\text { of tide } \\
\text { staff }\end{array}$ & $\begin{array}{c}\text { Wind } \\
\text { direction }\end{array}$ & $\begin{array}{l}\text { Anemom- } \\
\text { eter } \\
\text { records }\end{array}$ & Observer & $\begin{array}{l}\text { Local } \\
\text { mean } \\
\text { time }\end{array}$ & $\begin{array}{l}\text { Reading } \\
\text { of tide } \\
\text { staff }\end{array}$ & $\begin{array}{l}\text { Wind } \\
\text { direction }\end{array}$ & $\begin{array}{c}\text { Anemom- } \\
\text { eter } \\
\text { records }\end{array}$ & Observer \\
\hline \multicolumn{5}{|c|}{ June 19,1904} & \multicolumn{5}{|c|}{ June 20,1904} \\
\hline$h$, & Feet & & Miles & 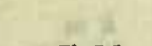 & $h m$ & Feet & & Miles & wit \\
\hline 73 & $5 \cdot 32$ & $\mathrm{E}$ & $x^{2}$ & J. E. M. & $12 \infty$ & 6.33 & SE & 392 & F. I. \\
\hline & 5.33 & $\mathrm{E}$ & E & $\sqrt{x+2}+2$ & 1300 & 6.60 & $\mathrm{SE}$ & ent 5 & -2 \\
\hline & 5.35 & $\mathrm{E}$ & 69 & - $x$ & 1400 & 6.71 & $\mathrm{SE}$ & 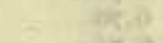 & 35 \\
\hline 80 & 5.39 & $\mathrm{E}$ & $97 \mathrm{I}$ & & Io & 6.73 & $\mathrm{SE}$ & & $\sqrt{2}=$ \\
\hline 90 & 5.50 & $\mathrm{E}$ & tat & J. L. M. & 20 & $6.75 \mathrm{H}$ & SE & 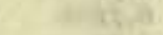 & 3. \\
\hline 100 & 5.80 & $\mathrm{E}$ & 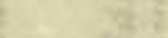 & F. L. & 30 & 6.75 & SE & Iरी & tor \\
\hline 110 & 6.10 & $\mathrm{E}$ & & 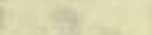 & 40 & 6.72 & $\mathrm{SE}$ & weste & $a^{2}=$ \\
\hline & 6.40 & $\mathrm{SE}$ & 62 & $\operatorname{sen} x$ & 50 & 6.74 & $\mathrm{SE}$ & 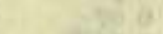 & 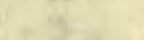 \\
\hline 130 & 6.59 & $S E$ & 단 & ox is & 1500 & 6.70 & SE & $4+50.3$ & $6 x^{2}$ \\
\hline & $6.68 \mathrm{H}$ & $\mathrm{E}$ & & & 1600 & 6.60 & SE & 452 & $a^{2}$ \\
\hline 140 & 6.68 & $\mathrm{E}$ & tri & & 1700 & 6.32 & $\mathrm{E}$ & $4 J^{2}-10$ & o. \\
\hline & 6.63 & $\mathrm{E}$ & & & 1800 & 6.04 & $\overrightarrow{\mathrm{E}}$ & 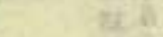 & $00 \times 1 /$ \\
\hline & 6.61 & $\mathrm{E}$ & bet ${ }^{2}$ & 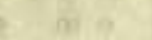 & 1914 & $5.8 \mathrm{I}$ & $\mathrm{E}$ & 150 & कences \\
\hline & $6.6 \mathrm{I}$ & $\mathbf{E}$ & $y_{-1}$ & war. & 2000 & 5.68 & $\mathrm{E}$ & 504 & $\omega=$ \\
\hline & 6.60 & $\overrightarrow{\mathrm{E}}$ & s. & thes & Io & 5.65 & $\mathrm{E}$ & $8=$ & 6075 \\
\hline & 6.60 & $\mathrm{E}$ & 35 & 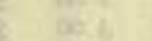 & 20 & 5.61 & $\mathrm{E}$ & nite & $602=$ \\
\hline 150 & 6.55 & $\vec{E}$ & & a. & 30 & 5.60 & $\vec{E}$ & 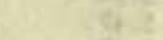 & $60 x=$ \\
\hline 160 & 6.24 & $\overline{\mathrm{E}}$ & 128 & $202^{2}$ & 40 & 5.60 & $\mathrm{E}$ & 25. 7 & Wr \\
\hline 170 & 5.94 & $\mathrm{E}$ & or 1 & $\ln x=$ & 50 & $5 \cdot 54$ & $\mathrm{E}$ & 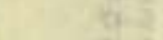 & 68 \\
\hline 180 & 5.70 & $\overrightarrow{\mathrm{E}}$ & 45 & is & 2100 & $5.5 \mathrm{IL}$ & E & $24=2$ & \\
\hline 190 & 5.59 & $\mathrm{~F}$ & 128 & ce & 10 & 5.53 & $\mathrm{E}$ & (6) 8 & F. L \\
\hline & 5.54 & $\mathrm{SE}$ & $\sqrt{52} e^{2}$ & $\theta$ & $22 \infty$ & 5.56 & ESE & $.16=$ & J. E. M \\
\hline & 5.52 & SE & 228 & 182 & 2300 & 5.63 & ESE & Due & 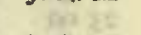 \\
\hline & $5 \cdot 50$ & SE & in. & 20 & 2400 & $5.8 \mathrm{I}$ & ESE & 577 & J. E. M. \\
\hline & 5.44 & SE & 6,4 & पt & \multirow{3}{*}{\multicolumn{5}{|c|}{ June 21,1904}} \\
\hline & 5.45 & $\mathrm{SE}$ & $-0^{3}$ & $n^{2}$ & & & & & \\
\hline 200 & 5.45 & SE & I82 & at & & & & & \\
\hline & $5.40 \mathrm{~L}$ & $\mathrm{E}$ & isiry & b) & 100 & 6.07 & ESE & & \multirow{2}{*}{ J. E. M. } \\
\hline & 5.40 & $\mathrm{E}$ & is & & 200 & 6.25 & $\mathrm{SE}$ & miter & \\
\hline & 5.47 & E & ces & F. L. & 10 & 6.27 & $\mathrm{SE}$ & $\tan 6$ & ar 4 \\
\hline & 5.42 & Calm & Exi. & J. E. M. & 20 & 6.33 & SE & 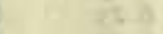 & $w i$ \\
\hline 220 & 5.60 & Calm & dest & a corit & 30 & 6.39 & $\mathrm{SE}$ & 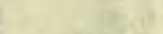 & coret \\
\hline 230 & 5.79 & Calm & 00.8 & & 40 & 6.39 & $\mathrm{SE}$ & then $x$ & 603 \\
\hline & 6.06 & Calm & os. & J. E. M. & 50 & 6.39 & $\mathrm{SE}$ & $y^{150}$ & 2018 \\
\hline 7 & & & 8 & 8 & 300 & $6.43 \mathrm{H}$ & $\mathrm{SE}$ & 0 or: & $(n) \mathrm{B}$ \\
\hline & & June 20 , & 1904 & oc & Io & $6.4 \mathrm{IH}$ & $\mathrm{SE}$ & $\cdots$ & $\omega x$ \\
\hline 10 & 6.20 & $\mathbf{E}$ & 35. & J. E. M. & 20 & $6.43 \mathrm{H}$ & SE & $a$ & 908 \\
\hline & 6.22 & $\overrightarrow{\mathrm{E}}$ & $05:$ & & 30 & $6.43 \mathrm{H}$ & $S E$ & ans & 6061 \\
\hline & 6.22 & E & $\sqrt{5} \mathrm{r}^{2}$ & fore is & 40 & $6.4 \mathrm{I}$ & $\mathrm{SE}$ & $x^{2-2}$ & 0911 \\
\hline & 6.26 & $\mathrm{E}$ & $c^{2}$. & of & 50 & 6.42 & $\mathrm{SE}$ & $64 \mathrm{I}$ & of \\
\hline & $6.3 \mathrm{IH}$ & $\mathrm{E}$ & 1082 & ex & 400 & 6.38 & $\mathrm{SE}$ & 72 & $\cos 2$ \\
\hline 5 & 6.29 & $\mathrm{E}$ & $168=$ & Q6. & I0 & 6.38 & $\mathrm{SE}$ & क. 8 & 6010 \\
\hline 20 & 6.28 & E & 682 & 9 & 20 & 6.32 & $\mathrm{SE}$ & ous , 8 & $00 \mathrm{ct}$ \\
\hline I & 6.24 & $\mathrm{E}$ & $\sqrt{2}$ & Q & 30 & 6.30 & $\mathrm{SE}$ & 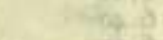 & .0 ir \\
\hline & 6.20 & $\mathrm{E}$ & 38.2 & $\omega_{0}=1$ & 500 & 6.26 & SE & 85 & 00121 \\
\hline 40 & 6.18 & $\mathbf{E}$ & 240 & ar & 60 & 6.11 & $\mathrm{SE}$ & 65,8 & of 1 \\
\hline 50 & $6 . \infty$ & $\mathrm{E}$ & -40 हो & $90 \mathrm{~b}$ & 700 & 5.94 & $\mathrm{SE}$ & 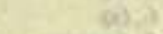 & 68 \\
\hline 60 & 5.82 & $\mathrm{E}$ & we & 603 & 800 & $5.8 \mathrm{I}$ & $\bar{E}$ & 695 & $\infty 0 \mathrm{By}$ \\
\hline 70 & 5.65 & $\mathrm{E}$ & 13 & 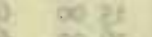 & 30 & 5.80 & $\mathrm{E}$ & 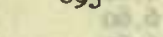 & er \\
\hline I & 5.62 & $\vec{E}$ & $8-1$ & 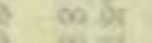 & 40 & 5.80 & $\overrightarrow{\mathrm{E}}$ & 00.0 & as \\
\hline 2 & 5.60 & $\overrightarrow{\mathrm{E}}$ & 1. & 007 & 50 & 5.80 & $\mathrm{E}$ & ty & $\pi$ \\
\hline 3 & 5.60 & $\mathrm{E}$ & 25. & 94 & 900 & 5.76 & VI: & $\cdot 0.0$ & 4 \\
\hline 4 & 5.55 & $\mathrm{E}$ & r. & ate. & 10 & 5.74 & $N 3$ & lixe & \\
\hline 80 & 5.55 & $\overrightarrow{\mathrm{E}}$ & 316 & $m 8$. & 20 & $5.73 \mathrm{~L}$ & $\mathbf{E}$ & 10. & J. E. M. \\
\hline & $5.50 \mathrm{~L}$ & $\overrightarrow{\mathrm{E}}$ & $30+4$ & at 1 & 30 & $5.74 \mathrm{~L}$ & $\mathbf{E}$ & 00.8 & F. L. \\
\hline 2 & 5.59 & $\overrightarrow{\mathrm{E}}$ & 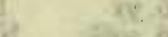 & 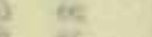 & 40 & $5.73 \mathrm{~L}$ & $\mathbf{E}$ & le 3 & (2) \\
\hline 3 & 5.59 & $E$ & 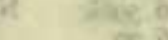 & $\Leftrightarrow$ & 50 & 5.75 & $\mathbf{E}$ & a. 0 & (c) $8 \mathrm{a}$ \\
\hline 4 & 5.54 & $\vec{E}$ & $\sigma$ & 94. & 100 & 5.75 & $\overrightarrow{\mathrm{E}}$ & $\operatorname{tin} 8$ & os of \\
\hline 5 & 5.59 & $\mathrm{E}$ & es-ct & f & $11 . \infty$ & 5.92 & $\mathbf{E}$ & $x_{0.2}$ & $\infty, 2$ \\
\hline 90 & 5.61 & $\overrightarrow{\mathrm{E}}$ & $2 x \cdot$ & J. E. M. & 1200 & 6.09 & $\overrightarrow{\mathrm{E}}$ & $75^{8}$ & $\alpha$, is \\
\hline 100 & 5.70 & E & $\cos 4$ & F. L. & 1300 & 6.41 & $\vec{E}$ & $x^{2}=2-2$ & -005 \\
\hline II 0 & 6.06 & $\mathrm{E}$ & fist & F. L. & 1400 & 6.60 & 32:= & Hais & F. L. \\
\hline
\end{tabular}


Tabulation of tidal observations at Cape Flora, Northbrook Island

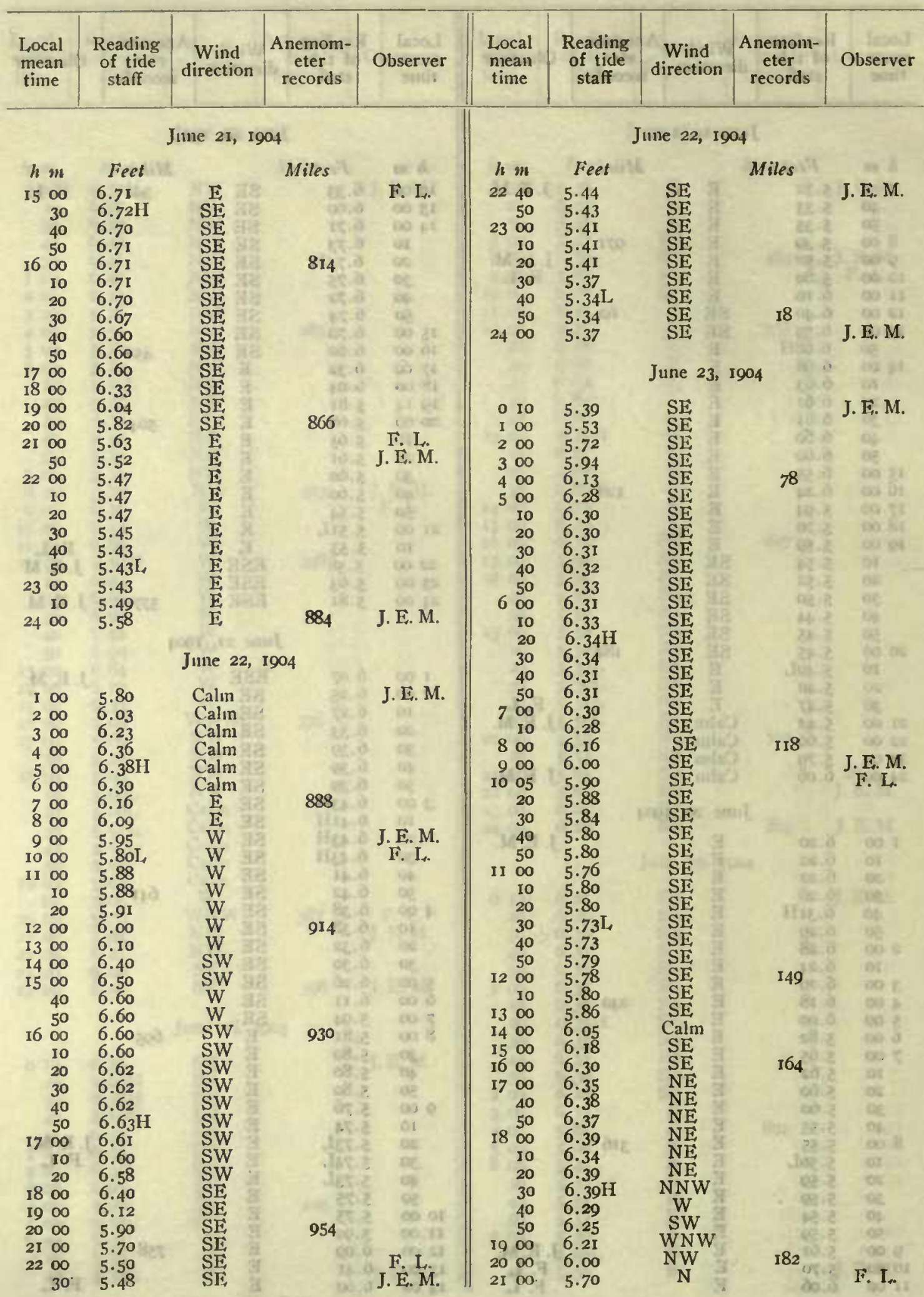


Tabulation of tidal observations at Cape Flora, Northbrook Island

\begin{tabular}{|c|c|c|c|c|c|c|c|c|c|c|}
\hline \multicolumn{2}{|c|}{$\begin{array}{l}\text { Local } \\
\text { mean } \\
\text { time }\end{array}$} & $\begin{array}{l}\text { Reading } \\
\text { of ticle } \\
\text { staff }\end{array}$ & $\begin{array}{l}\text { Wind } \\
\text { direction }\end{array}$ & $\begin{array}{l}\text { Anemom- } \\
\text { eter } \\
\text { records }\end{array}$ & Observer & $\begin{array}{l}\text { Local } \\
\text { time } \\
\text { mean }\end{array}$ & $\begin{array}{l}\text { Reading } \\
\text { of tide } \\
\text { staff }\end{array}$ & $\begin{array}{l}\text { Wind } \\
\text { direction }\end{array}$ & $\begin{array}{l}\text { Anemom- } \\
\text { eter } \\
\text { records }\end{array}$ & Observer \\
\hline \multicolumn{6}{|c|}{ June $23, \quad 1904$} & \multicolumn{5}{|c|}{ June 24,1904} \\
\hline$h$ & $m$ & Feet & H & Miles & $=4$ & $h m$ & Feet & $r$ & Milcs & $=4$ \\
\hline 22 & $\infty$ & 5.45 & $\mathrm{~N}$ & 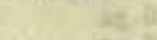 & J. E. M. & I9 20 & $6.27 \mathrm{H}$ & $\mathbf{E}$ & & F. L. \\
\hline 23 & & 5.26 & $N$ & 20.0 & $x \rightarrow 00$ - & 30 & $6.3 \mathrm{OH}$ & $\mathbf{E}$ & legat & 0.40 \\
\hline & 10 & 5.25 & $\mathrm{~N}$ & $6-1$ & $\cos 10$ & 40 & 6.22 & $\mathrm{E}$ & tat 8 & . \\
\hline & 20 & 5.25 & $\mathrm{~N}$. & 82 & 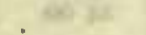 & & 6.20 & E & 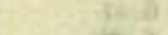 & -14 \\
\hline & 30 & 5.23 & $\mathrm{~N}$ & & & 2000 & 6.19 & $E$ & 403 & 00 \\
\hline & 40 & 5.23 & lave & & & 2100 & 5.90 & $\mathrm{E}$ & & F. L. \\
\hline & 50 & 5.19 & $N$ & & & 2200 & 5.64 & $\overrightarrow{\mathrm{E}}$ & is 3 & J.E. M. \\
\hline \multirow[t]{3}{*}{24} & $\infty$ & 5.17 & $\mathrm{~N}$ & I70 & J. E. M. & 2300 & 5.39 & E & & \\
\hline & Fer & $\sqrt{3}=0$ & Iune 24 & $\frac{0}{2} 2$ & $\frac{1}{4}$ & 2400 & 5.20 & E & 487 & J. E. M. \\
\hline & & 30 & June 24, & tail & $x^{2}+x$ & $12 \div 4$ & & June 25 , & 1904 & 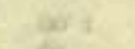 \\
\hline \multirow[t]{5}{*}{0} & 10 & 5.16 & $N$ & $\begin{array}{ll}x+3 \\
y\end{array}$ & J. E. M. & & & & $y=3$ & \\
\hline & 20 & 5.15 & $\mathrm{~N}$ & 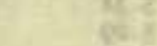 & 30 & $\begin{array}{rl}0 & 20 \\
30\end{array}$ & $\begin{array}{l}5.18 \\
5.16\end{array}$ & & ase & J.E. M. \\
\hline & $\begin{array}{l}30 \\
40\end{array}$ & $\begin{array}{l}5.13 \mathrm{~L} \\
5.28\end{array}$ & $\begin{array}{l}\mathbf{N} \\
\mathbf{N}\end{array}$ & sit. & $\sqrt{6 e_{0}} x^{2}$ & $\begin{array}{l}30 \\
40\end{array}$ & 5.14 & $\mathbf{E}$ & wisi? & 10 \\
\hline & $\begin{array}{l}40 \\
50\end{array}$ & $\begin{array}{l}3.20 \\
5.15\end{array}$ & $N$ & sit & 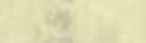 & 50 & 5.12 & $\mathrm{E}$ & - 0412 & nes \\
\hline & $\infty$ & 5.19 & SE & arite & ve & I Do & 5.10 & $\overline{\mathrm{SE}}$ & $13 \sqrt{2}$ & 00 is \\
\hline I & I0 & 5.19 & $S \overrightarrow{S E}$ & $x^{2}+x^{2}$ & $61 \mathrm{l}$ & IO & 5.10 & SL & 552 & Let \\
\hline & 20 & 5.22 & $\mathrm{SE}$ & thate & or & 20 & 5.13 & SE & fxiz & 0 \\
\hline 2 & $\infty$ & 5.30 & $\mathrm{SE}$ & toxis & as & 30 & $5.09 \mathrm{I}$ & SE & une & 48 \\
\hline 3 & $\infty$ & 5.50 & $\mathrm{SE}$ & 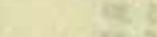 & o & 40 & 5.12 & SE & 0078 & 98 \\
\hline 4 & $\infty$ & 5.76 & SE & 207 & 68 & 50 & 5.12 & SE & pats $=$ & (6) \\
\hline \multirow{2}{*}{50} & $\infty$ & 6.00 & $\mathrm{SE}$ & w & Q & 20 & 5.15 & $\mathrm{SE}$ & 119 & 168 \\
\hline & Io & 6.03 & $\mathrm{SE}$ & 512 & wit & 300 & $5 \cdot 32$ & SE & 348 & Qwis \\
\hline & 20 & 6.07 & $\mathrm{SE}$ & Werta & 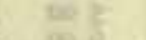 & 400 & 5.63 & ESE & 578 & 605 \\
\hline & 30 & 6.12 & $\mathrm{SE}$ & 10.7 & $92+2$ & 50 & 5.85 & ESE & 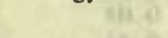 & 100 \\
\hline & 40 & 6.15 & SE & $x=$ & 005 & 600 & 6.10 & $\overline{\mathrm{E} S \mathrm{SE}}$ & 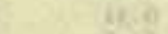 & 1005 \\
\hline & 50 & 6.20 & $\mathrm{SE}$ & $12 ; 0$ & os 2 & 50 & 6.23 & ESE & $D e-2$ & $10 \%$ \\
\hline 6 & $\infty$ & 6.19 & SE & orte & ese & 700 & 6.29 & $\overline{\mathrm{E}} \mathrm{SE}$ & 63.7 & ent \\
\hline & Io & 6.21 & SE & ste & is & 10 & $6.3^{\mathrm{I}}$ & ESE & 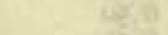 & 6 \\
\hline & 20 & 6.23 & $\mathrm{SE}$ & 15. & as & 20 & 6.31 & $\overrightarrow{E S E}$ & $182^{\circ}$ & ax \\
\hline & 30 & 6.25 & $S E$ & Mes at & is & 30 & 6.3 & ESE & 18.0 & 14: \\
\hline & 40 & 6.25 & SE & 15.9 & 09 & 40 & 6.3 & ESE & 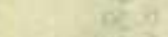 & we \\
\hline & 50 & 6.25 & $\mathrm{SE}$ & 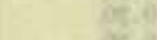 & ns. & 50 & 6.3 & ESE & $\log ^{2}-x^{2}$ & $60 \times 8$ \\
\hline \multirow{2}{*}{7} & $\infty$ & 6.27 & SE & Xit & (4), 여 & 800 & 6.39 & ESE & 666 & fit \\
\hline & 10 & 6.25 & $\mathrm{SE}$ & $\cos , 8$ & 31 & IO & 6.39 & ESE & $6 y^{2}=0$ & w \\
\hline & 20 & $6.28 \mathrm{H}$ & $\mathrm{SE}$ & 38. & be 71 & 20 & $6.40 \mathrm{H}$ & ESE & 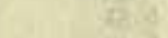 & 46 \\
\hline & 30 & 6.25 & $\mathrm{SE}$ & 6est & 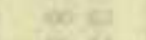 & 30 & 6.40 & ESE & Qy.p & of \\
\hline & 40 & 6.22 & SE & 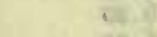 & 60.81 & 40 & 6.37 & ESE & $45-9$ & $\omega_{2}$ \\
\hline & 50 & 6.22 & $\mathrm{SE}$ & $29:$ & xi 4 at & 50 & 6.34 & $\mathrm{E}$ & 148 & (x) ty \\
\hline & & 6.18 & SE & 239 & on al & 900 & 6.32 & $\overline{\mathrm{E}}$ & de it & J. E. M. \\
\hline $\begin{array}{l}8 \\
9\end{array}$ & $\infty$ & 6.08 & $\mathrm{SE}$ & & J. IE. M. & Io $\infty$ & 6.20 & ESE & Bing & F. L. \\
\hline ro & $\infty$ & 5.94 & $\mathrm{E}$ & Wete & F. L & II oI & 6.05 & ESE & 79.2 & 60 \\
\hline I I & $\infty$ & 5.83 & $\bar{E}$ & 483 & - & 1200 & 5.84 & ESE & 747 & $100 \mathrm{Bi}$ \\
\hline 12 & $\infty$ & 5.75 & $\mathbf{E}$ & 290 & 4 & I3 00 & 5.74 & ESE & 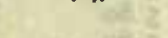 & 07 \\
\hline & 10 & 5.70 & SE & 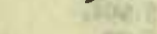 & 68 & 40 & 5.73 & ESE & $D E: 2$ & $\infty$ \\
\hline & 20 & 5.72 & SI & $68=$ & 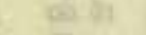 & 50 & $5.70 \mathrm{~L}$ & ESE & ins. & $\alpha$ \\
\hline & 30 & 5.72 & E & $x n=3$ & ont & 1400 & $5.72 \mathrm{~L}$ & SE & 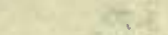 & 96 \\
\hline & 40 & 5.70 & $S E$ & 10. & esta & Io & $5.70 \mathrm{~L}$ & $\mathrm{SE}$ & 1188 & 68 \\
\hline & 50 & 5.70 & & 28 is & 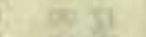 & 20 & 5.78 & ESE & $4+58$ & $002 x$ \\
\hline 13 & Do & 5.70 & $\mathrm{SE}$ & or 1 & $\infty 24$ & 30 & 5.74 & ESE & 85.2 & 01 \\
\hline & 05 & $5.67 \mathrm{~L}$ & SE & 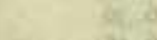 & $\cos 14$ & 40 & 5.80 & ESE & bi: है. & os, it \\
\hline & 10 & 5.71 & $\mathrm{SE}$ & . & 00 os & 50 & 5.81 & $E$ & 10.8 & 0051 \\
\hline & 20 & 5.74 & $\mathrm{SE}$ & Fiziz & $\infty \mathrm{it}$ & 1500 & 5.78 & $\bar{E}$ & 30.0 & $\infty 28$ \\
\hline 14 & $\infty$ & 5.80 & SE & $2 \pm$ & a & IO & 5.87 & ESE & & no 81 \\
\hline & & 5.99 & SE & fest & $=$ & 1600 & 6.00 & ESE & 814 & 00 os \\
\hline 16 & & 6.10 & SE & 343 & $\%$ & I7 $\infty 0$ & 6.11 & ESE. & 00 & of \\
\hline 17 & & 6.24 & $\mathrm{SE}$ &, 2 & 4 & I8 00 & 6.24 & $\mathrm{ESE}$ & Hoiso & os \\
\hline 18 & & 6.29 & SE & 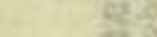 & 9 & 1900 & 6.34 & SE & 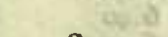 & 아. \\
\hline & 50 & $6.30 \mathrm{H}$ & $\mathrm{SE}$ & Bibs & 60 & 2000 & $6.34 \mathrm{H}$ & SE & 857 & of \\
\hline & $\infty$ & $6.30 \mathrm{H}$ & $E$ & 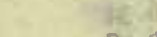 & 00 & IO & 6.34 & SE & $2(2,3)$ & $a^{6}$ \\
\hline & Io & $6.24 \mathrm{H}$ & E & 57 & F. It: & 20 & 6.30 & SE & 200 & F. Is. \\
\hline
\end{tabular}


Tabulation of tidal observations at Cape Flora, Northbrook Island

\begin{tabular}{|c|c|c|c|c|c|c|c|c|c|c|}
\hline \multicolumn{2}{|c|}{$\begin{array}{l}\text { Local } \\
\text { mean } \\
\text { time }\end{array}$} & $\begin{array}{l}\text { Reading } \\
\text { of tide } \\
\text { staff }\end{array}$ & $\begin{array}{l}\text { Wind } \\
\text { direction }\end{array}$ & \multirow[t]{2}{*}{$\begin{array}{c}\text { Anemom- } \\
\text { eter } \\
\text { records }\end{array}$} & \multirow[t]{2}{*}{ Observer } & $\begin{array}{c}\text { Local } \\
\text { mean } \\
\text { time }\end{array}$ & \multirow{2}{*}{$\begin{array}{c}\text { Reading } \\
\text { of tide } \\
\text { staff }\end{array}$} & \multirow{2}{*}{ 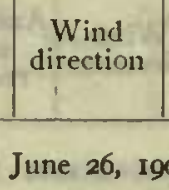 } & $\begin{array}{c}\text { Anemom- } \\
\text { eter } \\
\text { records }\end{array}$ & \multirow[t]{2}{*}{ Observer } \\
\hline \multicolumn{4}{|c|}{ June 25, 1904} & & & & & & 904 & \\
\hline$h$ & $m$ & Feet & & Miles & 64 & $h m$ & Feet & \multirow{6}{*}{$\begin{array}{c}\text { SW } \\
\text { SW } \\
\text { SW } \\
\text { SW }\end{array}$} & Miles & at \\
\hline 20 & 30 & 6.30 & SE & F(x)तो & F. L. & 21 IO & 6.32 & & $25-1$ & F. I. \\
\hline & 40 & 6.30 & $\mathrm{SE}$ & ifate & 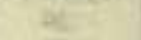 & 2200 & 6.23 & & Nit & J. E. M. \\
\hline & 50 & 6.21 & SE & 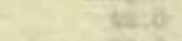 & & 2300 & 5.99 & & 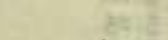 & \\
\hline $2 I$ & & 6.21 & SE & osia. & F. L. & 2400 & 5.67 & & 94 & J. E. M. \\
\hline 22 & & 5.98 & SE & lnosiat & J. E. M. & 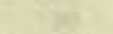 & 的 & & & $6 x^{2}$ \\
\hline 23 & $\infty 0$ & 5.67 & SE & 0 & & $y$ & 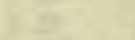 & June 27, & in: & 45: \\
\hline 24 & $\infty$ & 5.40 & E & 891 & J. E. M. & & & (2) $=8$ & $y^{4} z^{2}$ & $-\pi$ \\
\hline . & & & & 00.5 & 006 & $1 \infty$ & 5.50 & $W$ & xaik & J. E. M. \\
\hline & 35 & 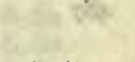 & June 26 , & I9O4 & $\cos \pi$ & $\begin{array}{l}30 \\
40\end{array}$ & $\begin{array}{l}5 \cdot 39 \\
5 \cdot 37\end{array}$ & $\begin{array}{l}W \\
W\end{array}$ & & \\
\hline & $\infty$ & 5.27 & ENE & E. & J. E. M. & 50 & 5.33 & W & & \\
\hline & 10 & 5.23 & ENE & & & 20 & 5.32 & W & orit & bi a \\
\hline $1 .$. & 20 & 5.23 & ENE & $\therefore: 2$ & 6. & I0 & 5.31 & $W$ & di ह & 28 \\
\hline & 30 & 5.20 & ENE & $8: 2$ & $a^{2}=$ & 20 & 5.29 & $W$ & dis. & as \\
\hline & 40 & 5.20 & ENE & :1. & 34. & 30 & 5.27 & W & $2 x^{2} y$ &. \\
\hline & 50 & 5.19 & ENE & 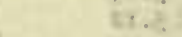 & 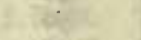 & 40 & 5.27 & W & win & $6=$ \\
\hline 2 & $\infty$ & 5. $17 \mathrm{~L}$ & ENE & $\mathrm{cos} .8$ & 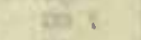 & 50 & 5.27 & W & $10=$ & wis $z$ \\
\hline & 10 & 5.17 & $\mathrm{E}$ & ar. & Us & 300 & 5.25 & $W$ & yris & os \\
\hline & 20 & 5.20 & E & :.... & $\approx$ & I0 & $5.24 \mathrm{~L}$ & W & 30 & $\cos$ \\
\hline & 30 & 5.20 & $\mathbf{E}$ & 200,3 & ac. & 20 & 5.27 & $W$ & G & का \\
\hline & 40 & 5.19 & $\mathrm{E}$ & 25.6 & D: & 30 & $5 \cdot 30$ & W & ars & $\cos 2$ \\
\hline & 50 & 5.20 & $\mathrm{E}:$ & .t.? & c: & 40 & $5 \cdot 32$ & W & 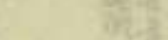 & an 3 \\
\hline 3 & $\infty$ & 5.21 & $\mathbf{E}$ & $2 \%$ & 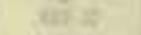 & 50 & $5 \cdot 32$ & W & & 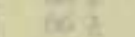 \\
\hline 4 & $\infty$ & 5.42 & E & 929 & 098 & $4 \infty$ & $5 \cdot 32$ & $W$ & 116 & $\Delta S^{2}$ \\
\hline 5 & $\infty$ & 5.70 & $\mathbf{E}$ & 8 8.7 & 85 & 500 & 5.59 & W & 00 & 6 \\
\hline 6 & $\infty$ & 6.01 & SE & $28 \%$ & $-\infty \pi$ & 600 & $5.9 I$ & W & 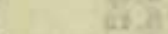 & int. \\
\hline 7 & $\infty$ & 6.31 & $\mathrm{SE}$ & if ${ }^{\circ}$ & J. L. M. & 700 & 6.20 & W & at & \\
\hline 8 & $\infty$ & 6.46 & SW & 968 & F. L. & 800 & 6.53 & $W$ & 132 & J.E. M. \\
\hline & 10 & 6.49 & SW & $\cos \theta$ & - & 905 & 6.70 & $W$ & air $=$ & F. L. \\
\hline & 20 & 6.50 & W & . 2.8 & 05 & Io & 6.72 & W & 32.8 & a) \\
\hline & 30 & 6.51 & W & 16.0 & 06 & 20 & 6.72 & W & $8 x .6$ & as \\
\hline & 40 & 6.50 & W & test & 8 & 30 & $6.78 \mathrm{H}$ & W & & ata \\
\hline & 50 & 6.50 & W & a.d & 68 & 40 & 6.74 & W & 28.8 & 64 \\
\hline 9 & $\infty$ & 6.50 & W & 10.0 & 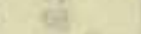 & 50 & 6.76 & W & & $a^{2}$ \\
\hline & IO & 6.51 & W & $\cos .6$ & $\cos \mathrm{e}^{2}$ & I0 00 & 6.76 & W & & $20 \%$ \\
\hline & 20 & $6.54 \mathrm{H}$ & W & xecis & ot & I0 & 6.72 & W & 37 & $61^{2}$ \\
\hline & 30 & $6.5 \mathrm{I}$ & W & Hag,o & $-x-t$ & II $\infty$ & 6.64 & W & 16 & oc \\
\hline & 40 & 6.49 & W & 매소 & 56 & 1200 & 6.43 & W & 148 & ait. \\
\hline & 50 & 6.44 & W & $8 x$ & 15 & 1300 & 6.20 & $W$ & 2x-7 & 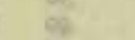 \\
\hline 10 & $\infty$ & 6.44 & $\mathbf{W}$ & 1. 8 & de & I 400 & 6.05 & $W$ & 28 & का \\
\hline II & $\infty$ & 6.30 & W & ti, a & $\sigma, 6$ & 1500 & 5.93 & $W$ & Li. & 60818 \\
\hline 12 & $\infty$ & 6.10 & SW & 3 & 60 ax & 10 & 5.91 & $W$ & 60 & ate \\
\hline 13 & $\infty$ & 5.94 & SW & 20.8 & .011 & 20 & $5.9 \mathrm{I}$ & $W$ & 10.2 & $0=8 t$ \\
\hline I4 & $\infty$ & 5.80 & SW & 25.2 & 0025 & 30 & 5.92 & $W$ & 285 & oy if \\
\hline & I0 & 5.80 & SW & 1,2 & $90 \mathrm{Br}$ & 40 & 5.90 & W & $\frac{1}{5} 3$ & $90+1$ \\
\hline & 20 & 5.80 & W & .253 & 48 & 50 & $5.90 \mathrm{~L}$ & W & & or \\
\hline & 30 & 5.80 & W & forea & de. & 1600 & 5.90 & $W$ & 165 & $\alpha=$ \\
\hline & 40 & 5.76 & W & 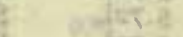 & $\cos 13$ & IO & 5.91 & $W$ & 58 & is. \\
\hline & 50 & $5.74 \mathrm{~L}$ & $W$ & $\sqrt{10 \%} . ?$ & 맘 & 20 & 5.92 & W & an: & \\
\hline 15 & $\infty$ & 5.74 & W & of 2 & as & I7 $\infty$ & 6.00 & W & $x=2$ & bi \\
\hline & 10 & 5.76 & SW & 45 & es & $18 \infty$ & 6.16 & W & 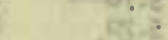 & on $n$ \\
\hline 16 & $\infty$ & 5.84 & SW & 42 & क & 1900 & 6.30 & $W$ & $4 y^{\circ}=$ & \\
\hline 17 & $\infty$ & 6.00 & SW & 18.2 & a & 2000 & 6.45 & $W$ & 186. & F. I \\
\hline 18 & $\infty$ & 6.14 & SW & this & $\infty 283$ & $21 \infty$ & $6.57 \mathrm{H}$ & W & Y & J.E. M. \\
\hline 19 & $\infty$ & 6.25 & $W$ & $48:$ & or & 10 & 6.55 & W & 0.3 & cot at \\
\hline 20 & 00 & 6.40 & W & 70 & $m$ dr & 20 & 6.54 & W & 603 & (1) 28 \\
\hline & Io & 6.40 & W & 13.9 & $\cos 58$ & 30 & 6.53 & $W$ & or. 8 & on है। \\
\hline se & 20 & $6.40 \mathrm{H}$ & W & is. & 67.4 & 40 & 6.53 & W & is. & ne $k$ tr. \\
\hline & 30 & 6.40 & SW & 68. & 0005 & 50 & 6.52 & W & & 608 \\
\hline & 40 & 6.40 & SW & $H_{12}=$ & wi es & $22 \infty$ & 6.52 & $W$ & Fic. & e. \\
\hline & 50 & 6.35 & SW & 10.2 & (1) & $23 \infty$ & 6.34 & $W$ & 1400 & \\
\hline 21 & $\infty$ & 6.34 & SW & Q. & F. I. & 2400 & 6.07 & W & 194 & J. E. M. \\
\hline
\end{tabular}


Tabulation of tidal observations at Cape Flora, Northbrook Island

\begin{tabular}{|c|c|c|c|c|c|c|c|c|c|}
\hline $\begin{array}{l}\text { Local } \\
\text { mean } \\
\text { time }\end{array}$ & $\begin{array}{l}\text { Reading } \\
\text { of tide } \\
\text { staff }\end{array}$ & \begin{tabular}{c|c} 
Wind \\
direction
\end{tabular} & $\begin{array}{l}\text { Anemom- } \\
\text { eter. } \\
\text { records }\end{array}$ & Observer & $\begin{array}{l}\text { Local } \\
\text { time } \\
\text { mean }\end{array}$ & $\begin{array}{l}\text { Reading } \\
\text { of tide. } \\
\text { staff }\end{array}$ & $\begin{array}{c}\text { Wind } \\
\text { direction }\end{array}$ & $\begin{array}{c}\text { Anemom- } \\
\text { eter } \\
\text { records } \\
\end{array}$ & Observer \\
\hline \multicolumn{5}{|c|}{ June 28,1904} & \multicolumn{5}{|c|}{ June 29,1904} \\
\hline$h m$ & Feet & & Miles & $=6$ & $h m$ & Feet & & Miles : & $\because i$ \\
\hline I 00 & 5.75 & W & ats & J. E. M. & 420 & 5.53 & $\mathrm{SE}$ & 18.2 & J. E. M. \\
\hline 200 & $5 \cdot 57$ & W & thins & elos & 30 & 5.54 & SE & Qs: & 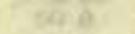 \\
\hline 300 & 5.42 & ESE & ots? & 200 & 40 & $5 \cdot 5^{2}$ & SE & $15 \%$ & $\omega$, \\
\hline IO & $5 \cdot 39$ & ESE & tyis & hr & $-50^{\circ}$ & 5.54 & SE & 28.8 & $\infty 8$ \\
\hline 20 & $5 \cdot 36$ & $\overline{\mathrm{ESE}}$ & 41.5 & 105 & 500 & 5.60 & SE & $\cos \theta$ & 000. \\
\hline 30 & 5.36 & ESE & 61,5 & .65 & 600 & 5.72 & SE & tyise & wex \\
\hline 40 & $5.35 \mathrm{~L}$ & $\overrightarrow{\mathrm{ESE}}$ & 00,1 & be & 700 & 6.03 & $\overline{\mathrm{SE}}$ & 37.8 & (1) \\
\hline 50 & 5.37 & ESE & 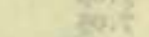 & be & 800 & 6.42 & SE & 534 & J. E. M. \\
\hline 400 & 5.40 & ESE & 215 & $\tan 15$ & 900 & 6.71 & $\mathrm{SE}$ & $e^{1}=$ & F. L. \\
\hline IO & 5.39 & ESE & 208 & cosit & Io 00 & 7.00 & $S E$. & ente & a) \\
\hline 20 & 5.40 & ESE & 400 & 1.65 .71 & 20 & 7.00 & SE & 624.2 & 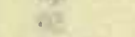 \\
\hline 30 & 5.42 & ESE & 400 & oe 5. & 30 & 7.03 & SE & or 7 & $-0 \leq 1$ \\
\hline 40 & 5.46 & $\overrightarrow{\mathrm{E}} \mathrm{SE}$ & (4)óld & $6 x$ & 40 & 7.05 & $\mathrm{SE}$ & se. & 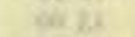 \\
\hline 500 & 5.53 & ESE & $\cdot 2$ & 1005 & 50 & 7.07 & SE. & x.6. & 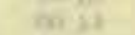 \\
\hline 600 & 5.75 & $\mathrm{ESE}$ & 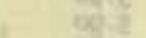 & en $=$ & 55 & 7. $\mathrm{IOH}$ & $\mathrm{SE}$ & int. & 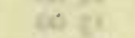 \\
\hline 700 & 6.11 & ESE & 24 & & II 00 & 7.09 & SE & Hed & $.0,4$ \\
\hline 800 & 6.44 & $\mathrm{SE}$ & 246 & J. E. M. & 10 & 7.06 & $\mathrm{SE}$ & $\ldots 5$ & 60 \\
\hline 900 & 6.75 & SE & 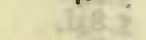 & F. L. & 20 & 7.04 & $\mathrm{~S}$. & 80 & $6 i$ \\
\hline 50 & 6.90 & $\mathrm{SE}$ & 24: & nes & 1200 & 7.01 & $\mathrm{SE}$ & 565 & $\infty$ \\
\hline IO 00 & 6.90 & SE & 60. & og es & I3 00 & 6.80 & $\mathrm{SE}$ & 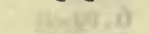 & 60 \\
\hline 10 & 6.90 & SE & 80,2 & 100.05 & I 400 & 6.53 & $\overrightarrow{\mathrm{SE}}$ & 05.9 & . \\
\hline 20 & $6.92 \mathrm{H}$ & $S E$ & ing & 60 is & I5 00 & 6.30 & $\mathrm{SE}$ & Aaro & से \\
\hline 30 & 6.92 & SE & 20.8 & sectus & 1600 & 6.15 & $\mathrm{NE}$ & 590 & एकi \\
\hline 40 & 6.91 & $\mathrm{SE}$ & inst & an ra & 40 & 6.10 & NE & 280 & 至 \\
\hline 50 & $6.9 I$ & $\mathrm{SE}$ & Ches & ovi is & 50 & $6.09 \mathrm{~L}$ & $\mathrm{NE}$ & aero & (6) $8 \mathrm{in}$ \\
\hline II 00 & 6.90 & $\mathrm{SE}$ & a.te & & I7 00 & 6.10 & $\mathrm{NE}$ & cas & of \\
\hline 1200 & 6.72 & $\mathrm{SE}$ & 296 & & 10 & 6.11 & $\mathrm{NI}$ & nx 0 & $\infty$ \\
\hline 1300 & 6.46 & $\mathrm{SE}$ & & & 20 & 6.11 & $\mathrm{NE}$ & as. & 6. \\
\hline I4 00 & 6.30 & $\mathrm{SE}$ & & & 30 & 6.1 & $\mathrm{SE}$ & the & कict \\
\hline 1500 & 6. 10 & $\overline{\mathrm{SE}}$ & me & 840 & 40 & 6.10 & SI & 40 & ing ief \\
\hline 50 & 6.01 & $\mathrm{SE}$ & $\cos 20$ & (es & 50 & 6.14 & SE & i. & se ils \\
\hline 1600 & 5.98 & $\overrightarrow{\mathrm{SE}}$ & 353 & 18 & I8 00 & 6.20 & $\mathrm{SE}$ & ixa & or bs \\
\hline I0 & 5.95 & $\mathrm{SE}$ & & 8 & I9 00 & 6.30 & $\mathrm{~S}$ & 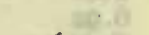 & $\alpha$ \\
\hline 20 & 5.95 & SE & gests & $Q$ & 2000 & 6.49 & SW & 607 & \\
\hline 30 & 5.98 & $\mathrm{SE}$ & 4.8 & Q 1 & 2100 & 6.65 & ENE & 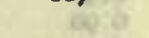 & F. L \\
\hline 40 & 5.98 & $\mathrm{SE}$ & 16.0 & be: & 2200 & 6.78 & ENE & -158 & J.E. M. \\
\hline 50 & 5.94 & $\mathrm{SE}$ & 00,0 & 1002 & Io & 6.80 & ENE & 76000 & - \\
\hline I7 00 & $5.94 \mathrm{I}$ & $\mathrm{SE}$ & 205.4 & 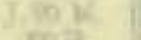 & 20 & 6.82 & ENE & 40.5 & 2 \\
\hline Io & 5.94 & $\mathrm{SE}$ & 00,2 & $\cos 2$ & 30 & 6.84 & ENE & me.e & 92. \\
\hline 20 & 6.00 & $\mathrm{SE}$ & 10.2 & 60 et & 40 & 6.80 & ENE & 60.0 & $\cos y=$ \\
\hline 1800 & 6.10 & SE & 0.2 & ot & 50 & $6.87 \mathrm{H}$ & ENE & & \\
\hline 1900 & 6.23 & SE & $y+5 e-2$ & 78- & 2300 & 6.84 & $\mathrm{E}$ & 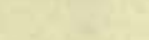 & 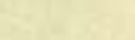 \\
\hline 2000 & 6.40 & SE & 401 & F. L. & Io & 6.80 & $\vec{E}$ & & \\
\hline 2100 & 6.58 & SE & & J. E. M. & 20 & $\cdot 6.78$ & $\mathbf{E}$ & & \\
\hline 2200 & 6.63 & SE & 1,2 & (5) & 2400 & 6.70 & $\overrightarrow{\mathrm{E}}$ & 682 & J. E. M. \\
\hline 20 & $6.641 \mathrm{I}$ & $\mathrm{SE}$ & 6.2 & $\frac{907}{31}$ & 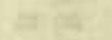 & 38 & The & an? & 6012 \\
\hline 30 & 6.63 & SE & its? & का है & 10 & & June 30 , 1904 & 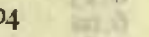 & $80 \%$ \\
\hline $\begin{array}{r}40 \\
50\end{array}$ & $\begin{array}{l}6.61 \\
6.39\end{array}$ & nvil & $\sin 5$ & $00 \mathrm{~g}$ & -1 & 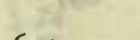 & $-W$ & 82 & 000 \\
\hline 2300 & $\begin{array}{l}0.39 \\
6.51\end{array}$ & SE & $28=9$ & 1603 & I 00 & 6.51 & E & 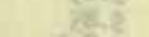 & J. E. M. \\
\hline 2400 & 6.32 & SE & 450 & J. E. M. & 200 & 6. I5 & NW & 1628 & $=0$ \\
\hline & & & 450 & & $\begin{array}{l}300 \\
400\end{array}$ & 6.01 & $\begin{array}{l}\text { NW } \\
\text { NW }\end{array}$ & 203 & $\mathrm{C}^{\circ}$ \\
\hline & & June 29,1904 & $\frac{13}{10} .8$ & 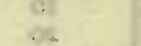 & 10 & $\begin{array}{l}5.75 \\
5.73\end{array}$ & $\begin{array}{l}\text { NW } \\
\text { NW }\end{array}$ & $721 \quad c$ & है \\
\hline I 00 & 6.07 & & 7.3 & $F \mathrm{M}$ & 20 & 5.70 & NW & $\cos$ & wo \\
\hline & $5.8 \mathrm{I}$ & & Hog. 8 & J.E. M. & 30 & 5.70 & NW & 8.8 & $00 \mathrm{~T}$ \\
\hline 300 & $\begin{array}{l}5.01 \\
5.66\end{array}$ & SE & 0.0 & D. & 40 & 5.72 & NW & is. 3 & 0.8 \\
\hline 30 & $\begin{array}{l}5.00 \\
5.56\end{array}$ & & 18.9 & 00 \& 1 & 50 & 5.72 & NW & 20.5 & $\cos 8$ \\
\hline 40 & $\begin{array}{l}2.50 \\
5.53\end{array}$ & $\mathrm{SE}$ & 40.3 & ot & 500 & & W & 28.2 & 0061 \\
\hline 50 & 5.52 & $\mathrm{SE}$ & $=.0$ & tis & I0 & $5.69 \mathrm{~L}$ & $W w y$ & $x, 5$ & 6211 \\
\hline 400 & 5.51 & & 8 & $60 \% 1$ & 20 & & W Wh & 10,5 & b: \\
\hline I0 & $5.50 \mathrm{~L}$ & SF & 490.0 & & 30 & $5.69 \mathrm{~L}$ & W . . . & 189.5 & 6 \\
\hline & 2.304 & $2 \pi$ & 78.9 & J. E. M. & $\sqrt{40}$ & 5.72 & W $4: 1$ & fois & J. E. M. \\
\hline
\end{tabular}


Tabulation of tidal observations at Cape Flora, Northbrook Island

\begin{tabular}{|c|c|c|c|c|c|c|c|c|c|}
\hline $\begin{array}{l}\text { Local } \\
\text { mean } \\
\text { time }\end{array}$ & $\begin{array}{l}\text { Reading } \\
\text { of tide } \\
\text { staff }\end{array}$ & $\begin{array}{c}\text { Wind } \\
\text { direction }\end{array}$ & $\begin{array}{l}\text { Anemom- } \\
\text { eter } \\
\text { records }\end{array}$ & Observer & $\begin{array}{l}\text { Local } \\
\text { mean } \\
\text { time }\end{array}$ & $\begin{array}{l}\text { Reading } \\
\text { of tide } \\
\text { staff }\end{array}$ & $\begin{array}{l}\text { Wind } \\
\text { direction }\end{array}$ & $\begin{array}{c}\text { Anemom- } \\
\text { eter } \\
\text { records }\end{array}$ & Observer \\
\hline \multicolumn{5}{|c|}{ June 30,1904} & \multicolumn{5}{|c|}{ July 1, 1904} \\
\hline$h m$ & Feet & & Miles & $\operatorname{lin} 8$ & $h m$ & Feet & & Miles & $=\mathbb{A}$ \\
\hline 550 & 5.73 & W & 122 & J. E. M. & II 40 & 7.12 & NW & & T. L. \\
\hline 600 & 5.80 & $\begin{array}{l}W \\
W\end{array}$ & M. & & 50 & $714 \mathrm{H} \dagger$ & NW & & $\cos ^{2}$ \\
\hline 800 & $\begin{array}{l}0.01 \\
6.32\end{array}$ & $w$ & 754 & J.E. M. & 12 10 & $\begin{array}{l}7.100 \\
7.12\end{array}$ & $\begin{array}{l}\text { NW } \\
\text { NW }\end{array}$ & 30 & औiin \\
\hline & 6.32 & W & 754 & F. L. & 20 & 7.12 & $\mathrm{NW}$ & $\sec$ & per \\
\hline I0 00 & 6.97 & $\dddot{W}$ & $\operatorname{arch} t$ & 200 & 30 & 7.10 & NW & $x: 2$ r & a \\
\hline II IO & 7.15 & $\underset{w}{W}$ & 10.5 & 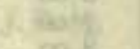 & 40 & 7.09 & NW & 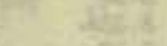 & $\approx$ \\
\hline $\begin{array}{l}20 \\
30\end{array}$ & $7.20 \mathrm{H}$ & W & स: 8 & 90 & 50 & 7.05 & NW & $8=$ & $82 \mathrm{se}$ \\
\hline $\begin{array}{l}30 \\
40\end{array}$ & 7.19 & $\begin{array}{l}\text { SW } \\
\text { sW }\end{array}$ & de & 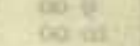 & 1300 & 7.04 & $\begin{array}{l}\text { NW } \\
\text { NNW }\end{array}$ & 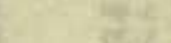 & wet \\
\hline $\begin{array}{l}40 \\
50\end{array}$ & $\begin{array}{l}7.19 \\
7.14\end{array}$ & SW & 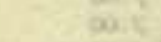 & $5=$ & 1400 & $\begin{array}{l}6.84^{*} \\
6.52^{*}\end{array}$ & $\begin{array}{l}\text { NNW } \\
\text { NNW }\end{array}$ & & 63 \\
\hline 1200 & $\begin{array}{l}7.14 \\
7.10\end{array}$ & SW & 790 & e. & $\begin{array}{l}15500 \\
1600\end{array}$ & $\begin{array}{l}0.50^{*} \\
6.30^{*}\end{array}$ & NN & 136 & 4 \\
\hline 1300 & 6.99 & SW & & 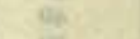 & 1700 & $6.06^{*}$ & NNW & & 4 \\
\hline 1400 & 6.77 & SW & 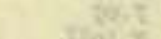 & 变 & 1800 & 5. & & & $0^{2}$ \\
\hline 1500 & 6.52 & SW & 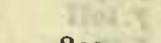 & 22. & I0 & & NNW & & ond \\
\hline 1600 & 6.34 & sw & 823 & $\infty \mathrm{n}$ & 20 & 5.86 & NNW & and & a. 5 \\
\hline 1700 & 6.24 & SW & & air & 30 & 5.84 & NNW & $4=$ & 008 \\
\hline 10 & 6.20 & SW & kis & 68 & 40 & $5.83 \mathrm{~L}$ & NNW & & ase \\
\hline 20 & 6.20 & SW & $\tan 5$ & $\infty \mathrm{kit}$ & 50 & & NN & 98.0 & esi \\
\hline 25 & 6.19 & $\mathrm{~s}$ & $06 \mathrm{~A}$ & 90 & 1900 & 5. & NNW & og a & os or \\
\hline 30 & & $\mathbf{S}$ & 280 & 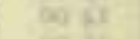 & 2000 & & N & & in \\
\hline 35 & $6.16 \mathrm{~L}$ & $\mathbf{S}$ & 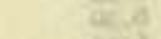 & 600 & 2100 & 6. & NI & 216 & F. L. \\
\hline 40 & 6.20 & s & $(x)$ & on at & 2200 & 6. & NI & & J. E. M. \\
\hline 50 & 6.20 & $\mathbf{S}$ & 653 & is & 2300 & 6. & NI & Wh & S. С. 1 . \\
\hline 1800 & & S & lien a & 6 & 2400 & 6.53 & NW & int & J. E. M. \\
\hline Io & 6.20 & $\mathrm{~S}$ & ointo & 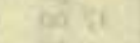 & the & (1) & No & 8 & \\
\hline 20 & 6.21 & S & 72 & stitit & $x^{2}$ & 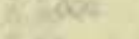 & July 2, & 1904 & wis \\
\hline 30 & 6.24 & S & vist. & 198. & $=0$ & & & & \\
\hline 2000 & $\begin{array}{l}0.24 \\
6.44\end{array}$ & S & 840 & 80 & 0 10 & 6.51 & NW & 257 & J. E. M. \\
\hline 2100 & 6.64 & s & 040 & 3 & 20 & & & & 16. \\
\hline 2200 & 6.8 & S & $\cos \theta$ & F. L. & 30 & 6.5 & $N V$ & $60 \geq$ & 50 at \\
\hline 50 & 6.9 & W & atent & J. E. M. & 40 & & NV & 625 & $x_{1}$ \\
\hline 2300 & & W & as a & 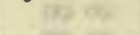 & $\begin{array}{l}50 \\
100\end{array}$ & $\begin{array}{l}6.50 \\
6.48\end{array}$ & NW & ह & in \\
\hline 10 & 6.9 & W & 300 & & & 6.48 & NW & siv & $\cos$ \\
\hline 20 & 6.93 & W & $5=8$ & $\approx+4$ & & 6. & NV & 823 & at \\
\hline 30 & $6.96 \mathrm{H}$ & W & $\% \circ$ & ot: & & & NV & & R' \\
\hline 40 & 6.92 & $W$ & 19. & ost & & & NV & 301 & $00 \div 51$ \\
\hline 50 & 6.92 & W & ted & & & & & 191 & 84 \\
\hline 2400 & 6.90 & W & 862 & J. E. M. & $\begin{array}{l}600 \\
10\end{array}$ & $\begin{array}{l}5.61 \\
5.60\end{array}$ & $\begin{array}{l}\text { NWW } \\
\text { NW }\end{array}$ & 00.9 & is in \\
\hline$x^{2}=$ & & & & $\infty 0^{2}$ & 20 & & NW & tas. & ser of \\
\hline & ? & July I, 190 & 80 & Sor & 30 & & $\mathrm{NV}$ & of $h$ & wis \\
\hline 100 & 6.75 & w & 2.8 & J. E. M. & $\begin{array}{l}40 \\
50\end{array}$ & 5.60 & NW & $63 x$ & 915 \\
\hline 200 & 6.50 & $w$ & & & 700 & & & thes & was \\
\hline $\begin{array}{l}300 \\
400\end{array}$ & $\begin{array}{l}6.23 \\
6.02\end{array}$ & $\underset{W}{W}=1$ & 800 & & 810 & & WN & 360 & I F M \\
\hline $\begin{array}{l}400 \\
500\end{array}$ & 5.89 & W & & & & $\begin{array}{l}5.7 \\
6.0\end{array}$ & WI & & F. L. \\
\hline 10 & 5.85 & $\ddot{W}$ & सहत? & 60 & & & WNW & $y 3$ & . 4. \\
\hline 20 & $5.83 \mathrm{~L}$ & W wW & & oo $\mathrm{k}$ & II 00 & & $\mathrm{NW}$ & 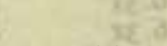 & $m_{i=1}$ \\
\hline 30 & 5.85 & W & 10,6 & $\cos 2$ & 1200 & & $N$ & 405 & \\
\hline $\begin{array}{l}40 \\
50\end{array}$ & $\begin{array}{l}5.87 \\
5.88\end{array}$ & $\mathrm{~W}$ & $\begin{array}{ll}20 \\
\text { in }\end{array}$ & ois & $\begin{array}{l}10 \\
20\end{array}$ & $\begin{array}{l}6.8 \\
6.8\end{array}$ & 100 & it. 620 & \\
\hline 600 & & & $x^{2} 3$ & $\approx$ & $\begin{array}{l}20 \\
30\end{array}$ & $\begin{array}{l}0.0 \\
6.8\end{array}$ & $w$ & -2 & $=5$ \\
\hline 700 & 5.5 & $\mathbf{W}$ & 205 & & 40 & & W & & \\
\hline 8 & 6.2 & $W$ & 934 & J. E. M. & 50 & & W & & $\infty i$. \\
\hline 9 & & & - & F. L. & 1300 & & $\mathrm{~W}$ & des & of \\
\hline $\begin{array}{l}1100 \\
1100\end{array}$ & & & (6) 2 & ला & & & $\begin{array}{l}W \\
W\end{array}$ & $z^{2}$ & to. \\
\hline Io & & & & se & $I_{4}$ & & $w$ & & \\
\hline 20 & 7.0 & & axc? & E & 15 & & $\dddot{W}$ & 12 & 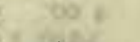 \\
\hline 1130 & $7.10 \dagger$ & NW ? & F. 3.3 & F. I & 1600 & 6.24 & $\ddot{W}$ & $43 I$ & Ji. $\mathrm{I}_{4}$ \\
\hline
\end{tabular}


Tabulation of tidal obscrvations at Cape Flora, Northbrook Island

\begin{tabular}{|c|c|c|c|c|c|c|c|c|c|c|}
\hline \multicolumn{2}{|c|}{$\begin{array}{l}\text { Local } \\
\text { mean } \\
\text { time }\end{array}$} & $\begin{array}{l}\text { Reading } \\
\text { of tide } \\
\text { staff }\end{array}$ & $\begin{array}{l}\text { Wind } \\
\text { direction }\end{array}$ & $\begin{array}{l}\text { Anemom- } \\
\text { eter } \\
\text { records }\end{array}$ & Observer & $\begin{array}{l}\text { Local } \\
\text { mean } \\
\text { time }\end{array}$ & $\begin{array}{l}\text { Reading } \\
\text { of tide } \\
\text { staff }\end{array}$ & $\begin{array}{l}\text { Wind } \\
\text { direction }\end{array}$ & $\begin{array}{c}\text { Anemom- } \\
\text { eter } \\
\text { records }\end{array}$ & Observer \\
\hline \multicolumn{6}{|c|}{ July 2, 1904} & \multicolumn{5}{|c|}{ July 4 , 1904 . } \\
\hline$h$ & $m$ & Feel & & Miles & $\mathrm{kn}^{2}=$ & $h m$ & Feet & & Miles & to is i \\
\hline 17 & $\infty$ & 6.00 & W & 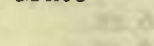 & I. L. & 100 & 6.40 & W & the 1 & J. E. M. \\
\hline & $\infty$ & 5.80 & W & 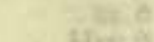 & 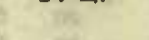 & Io & $6.4 I$ & W & $x^{2}$ in & Jo 280 \\
\hline & & 5.72 & W & Hrosill & 20 & 20 & 6.43 & W & 0287) & of \\
\hline & Io & 5.73 & W & 2528 & 18 & $30-$ & 6.40 & W & $\cos (5)$ & 78 \\
\hline & 20 & 5.75 & W & $\cos \lambda$ & os & 40 & $6.43 \mathrm{H}$ & W & $28 \pi$ & 98 \\
\hline & 30 & $5.70 \mathrm{~L}$ & W & is 8 & 60.6 & 50 & $6.40^{\circ}$ & W & $\cos 3$ & $\sin x$ \\
\hline & 40 & 5.70 & W & $15-4$ & $n$ & 200 & 6.37 & W & soo. & on 3 \\
\hline & 50 & 5.72 & W & 16r & we & 10 & 6.36 & W & no? & $100 \%$ \\
\hline & 00 & 5.74 & W & 472 & -1 & 20 & 6.35 & W & $12=$ & 97 \\
\hline $2 \mathrm{I}$ & & 5.90 & W & $4 / 2-1=$ & I. I. & 300 & 6.27 & W & Bt: $=$ & 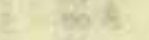 \\
\hline 22 & $\infty$ & 6.04 & W & wets & J. E. M. & 400 & 6. 12 & NW & 8,30 & 66 \\
\hline 23 & & 6.21 & W & 318 & & 500 & 5.93 & $\mathrm{NW}$ & $0,04=$ & in \\
\hline 24 & & 6.34 & W Wt & 521 & J. E. M. & 600 & 5.85 & NW & fas: & olt \\
\hline & & & 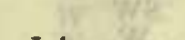 & $10=$ & ter $y$ & 700 & 5.78 & NW & Wre & 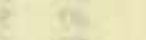 \\
\hline & nte & 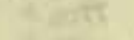 & July 3,1904 & -108 & 2008 & IO & 5.73 & NW & -28 & $\cos 1$ \\
\hline & & $y=2$ & a & 48 & & 20 & 5.75 & NW & $35=$ & $\cos 2$ \\
\hline o & 30 & 6.37 & NW & $125=$ & J. E. M. & $\begin{array}{l}30 \\
40\end{array}$ & 5.73 & $\begin{array}{l}\text { NW } \\
\text { NW }\end{array}$ & Whis & 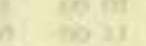 \\
\hline & 40 & 6.39 & NW & $\operatorname{lig}_{10}$ & 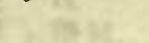 & $\begin{array}{l}40 \\
50\end{array}$ & $\begin{array}{l}5.73 \\
5.72 \mathrm{~L}\end{array}$ & $\begin{array}{l}\text { NW } \\
\text { NW }\end{array}$ & $e^{2}$ & .04 \\
\hline & 50 & 6.41 & NW & Jic: & $x_{3} x^{2}$ & $8 \stackrel{50}{00}$ & $\begin{array}{l}5.72 \mathrm{~L} \\
5.78\end{array}$ & NW & 870 & 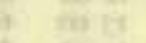 \\
\hline & $\infty$ & 6.40 & NW & hes: & 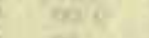 & IO & 5.80 & NW & 0 & $\ln 4 \mathrm{H}$ \\
\hline & Io & 6.36 & $\begin{array}{l}\text { NW } \\
\text { NW }\end{array}$ & 1S-2 & nets & 20 & 5.83 & NW & $=7$ & or \\
\hline & 20 & 6.40 & & 28,4 & $c=$ & 900 & 5.88 & NW & 165es & J. E. M. \\
\hline & 30 & $6.42 \mathrm{H}$ & & $y<\geq$ & dif & IO 00 & 6.12 & NW & 60.8 & F. L. \\
\hline & 40 & 6.39 & $\begin{array}{l}\text { NW } \\
\text { NW }\end{array}$ & Qe.te & at & II 00 & 6.34 & NW & 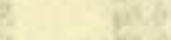 & L. \\
\hline & 50 & 6.39 & $\begin{array}{l}\text { NW } \\
\text { NW }\end{array}$ & 302 & 6 & 1200 & 6.54 & NW & 903 & $\sigma^{2}+2$ \\
\hline & 00 & 6.36 & & 165? & $\cos$ & I3 00 & 6.74 & NW & 300 & 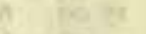 \\
\hline & $\infty$ & 6.19 & WWW & 504 & $\infty 0$ or & 10 & 6.72 & NW & 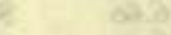 & 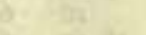 \\
\hline & $\infty$ & 5.94 & $\begin{array}{l}\text { WNW } \\
\text { WNW }\end{array}$ & 594 & $97 \div$ & 20 & 6.74 & NW & atsita & $\operatorname{mos}=4$ \\
\hline & $\infty$ & 5.81 & $\begin{array}{l}\text { WNW } \\
\text { WNW }\end{array}$ & 185 & 00 it & 30 & 6.77 & NW & 28.8 & 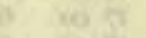 \\
\hline & $\infty$ & 5.67 & $\begin{array}{l}\text { WNW } \\
\text { NW }\end{array}$ & 6.8 & $\ln (1)$ & 40 & 6.79 & NW & wh & text \\
\hline & $\infty$ & $5.59 \mathrm{~L}$ & NW & colat & $00 \mathrm{gr}$ & 50 & $6.80 \mathrm{H}$ & NW & 10 & ace of \\
\hline & 10 & 5.63 & NW & $\cos \theta$ & er & 1400 & 6.75 & NW & ats & $10 \%$ \\
\hline & 20 & $\begin{array}{l}5.65 \\
5.60\end{array}$ & $\begin{array}{l}\text { NW } \\
\text { NW }\end{array}$ & mine & c5 & Io & 6.71 & NW & rys & or \\
\hline & 30 & $\begin{array}{l}5.69 \\
5.70\end{array}$ & $N W$ & $(10)=8$ & 매단. & 1500 & 6.69 & NW & $8 \mathrm{C}$ & 65 \\
\hline & 40 & $\begin{array}{l}5.70 \\
5.71\end{array}$ & NW & 164 & 5 & 1600 & 6.45 & NW & 924 & tos \\
\hline & 50 & $\begin{array}{l}5.71 \\
5.73\end{array}$ & $\mathrm{~N}$ & 588 & J. E. M. & $17 \infty$ & 6.25 & NW & & 애 \\
\hline 8 & $\infty$ & $\begin{array}{l}5.73 \\
5.92\end{array}$ & $\mathrm{~N}$ & 500 & F & I8 00 & 6.00 & NW & 185 & $78=$ \\
\hline & 02 & $\begin{array}{l}5.92 \\
6.20\end{array}$ & $\mathrm{~N}$ & Its & F. L. & 1900 & 5.83 & NW & $\sigma^{4}$ & 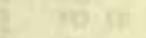 \\
\hline & $\infty$ & $\begin{array}{l}6.20 \\
6.50\end{array}$ & NW & test & 68 & 2000 & 5.74 & NW & 950 & ven \\
\hline II & $\infty$ & $\begin{array}{l}6.50 \\
6.74\end{array}$ & $\mathrm{NW}$ & 634 & es & I0 & 5.70 & W & ty & $x=$ \\
\hline 12 & $\infty$ & & NW & 034 & Ce. & 20 & 5.70 & W & S. & 248 \\
\hline I3 & 05 & $\begin{array}{l}6.90 \mathrm{H} \\
6.89\end{array}$ & NW & soll & $\cos 5$ & 30 & 5.70 & $w$ & $u^{3}$ & $0 s$ \\
\hline & 10 & $\begin{array}{l}6.89 \\
6.85\end{array}$ & NW & ofte & po ats & 40 & $5.69 \mathrm{~L}$ & W & $n=2$ & $\infty 8$ \\
\hline & $\begin{array}{l}20 \\
30\end{array}$ & $\begin{array}{l}0.05 \\
6.90\end{array}$ & $\mathrm{NW}$ & 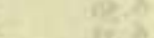 & 0051 & 50 & $5.7 \mathrm{I}$ & W & 100. & 600 \\
\hline & 40 & 6.84 & NW & $15: 8$ & on 64 & 2100 & 5.75 & W & & 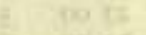 \\
\hline & 50 & 6.82 & NW & 412 & 9061 & IO & 5.72 & W & ise & 1619 \\
\hline I4 & $\infty$ & 6.82 & NW & 58 & 000 & 20 & 5.74 & W & & \\
\hline & 10 & 6.80 & NW & $3 \times 3$ & 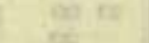 & & $5 \cdot 77$ & W & & F. L. \\
\hline 15 & $\infty$ & 6.65 & NW & 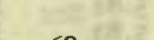 & $C^{2}-1$ & 2200 & 5.79 & W & & J. E. M. \\
\hline I6 & $\infty$ & 6.44 & W & 682 & $t^{4}+2$ & $23 \infty$ & 5.90 & $\underset{W}{W}$ & & \\
\hline 17 & $\infty$ & 6.15 & $\underset{N W}{W}$ & 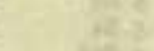 & 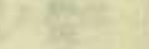 & 2400 & 6.03 & W & 960 & J.E. M. \\
\hline 18 & $\infty$ & 5.91 & $\begin{array}{l}\text { NW } \\
\text { NW }\end{array}$ & 165: & $60=2$ & & & July 5 , I & 904 & \\
\hline 19 & $\begin{array}{l}00 \\
10\end{array}$ & $\begin{array}{l}5.80 \\
5.76\end{array}$ & $\begin{array}{l}\text { NW } \\
\text { NW }\end{array}$ & whente & w & & & July $5, \mathbf{I}$ & 14 & \\
\hline & 20 & 5.80 & NW & 15.5 & 8 & 100 & 6.20 & NW & 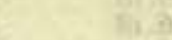 & J. E. M. \\
\hline & 30 & 5.76 & NW & 18:3 & 2 & 10 & 6.25 & NW & $7 x^{2}$ & 50 \\
\hline & 40 & 5.73 & NW & $3 x=8$ & 17 & 20 & 6.26 & NW & es. & of \\
\hline & 50 & 5.73 & NW & -1 & an & 30 & 6.25 & NW & 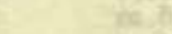 & 108 \\
\hline 20 & $\infty$ & $5.7 \mathrm{IL}$ & NW & 745 & 00 in & 40 & 6.28 & NW & sinit & $\infty \mathrm{t}$ \\
\hline & Io & 5.76 & $\begin{array}{l}\text { NW } \\
\text { NW }\end{array}$ & & & 50 & 6.30 & NW & & \\
\hline 21 & $\begin{array}{l}20 \\
00\end{array}$ & $\begin{array}{l}5.78 \\
5.80\end{array}$ & $\begin{array}{l}\text { NW } \\
\text { NW }\end{array}$ & & F. I. & $\begin{array}{r}200 \\
10\end{array}$ & $\begin{array}{l}6.30 \\
6.30\end{array}$ & $\begin{array}{l}\text { NW } \\
\text { NW }\end{array}$ & Whe & 25 \\
\hline 22 & $\infty$ & 5.93 & NW & & J. E. M. & 20 & $\begin{array}{l}0.30 \\
6.31\end{array}$ & NW & & \\
\hline 23 & $\mathbf{0 0}$ & 6.04 & NW & & & 30 & $6.33 \mathrm{H}$ & NW & & \\
\hline 24 & $\infty$ & 6.28 & WNW & 790 & J. E. M. & 40 & $6.31 \mathrm{H}$ & NW & & J. E. M. \\
\hline
\end{tabular}


Tabulation of tidal obscriations at Capc Flora, Northbronk Island

\begin{tabular}{|c|c|c|c|c|c|c|c|c|c|c|c|}
\hline \multicolumn{2}{|c|}{$\begin{array}{l}\text { Local } \\
\text { mean } \\
\text { time }\end{array}$} & $\begin{array}{l}\text { Reading } \\
\text { of tide } \\
\text { staff } \\
\end{array}$ & \multicolumn{2}{|c|}{$\begin{array}{l}\text { Wind } \\
\text { direction }\end{array}$} & $\begin{array}{c}\text { Anemom- } \\
\text { eter } \\
\text { records }\end{array}$ & \multirow[t]{2}{*}{ Observer } & $\begin{array}{l}\text { Loeal } \\
\text { time } \\
\text { mean }\end{array}$ & $\begin{array}{l}\text { Reading } \\
\text { of tide } \\
\text { staff }\end{array}$ & $\begin{array}{l}\text { Wind } \\
\text { direction }\end{array}$ & $\begin{array}{c}\text { Anemom- } \\
\text { eter } \\
\text { records }\end{array}$ & \multirow[t]{2}{*}{ Observer } \\
\hline \multicolumn{6}{|c|}{ July 5, 1904} & & \multicolumn{4}{|c|}{ July 6, 1904} & \\
\hline$h$, & $m$ & Feet & & & Miles & $x+1$ & $h m$ & Feet & 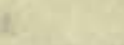 & Miles & int 1 \\
\hline & 50 & $6.33 \mathrm{H}$ & NW & 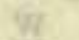 & ing & J. E. M. & 310 & 6.21 & $\mathrm{~S}$ & or & J. E. M. \\
\hline & oo & 6.30 & NW & $W$ & 16. & का & 20 & 6.22 & S & $2=$ & की \\
\hline & 10 & 6.29 & NW & $W$ & bis & 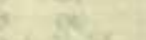 & 30 & $6.23 \mathrm{H}$ & S & 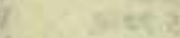 & wipt \\
\hline & 20 & 6.27 & NW & $W$ & jolat & tesil & 40 & 6.21 & S & 153 & vit \\
\hline & 30 & 6.25 & NW & W & Phats & ant & 50 & 6.20 & $\mathrm{~S}$ & r & ck \\
\hline & $\infty$ & 6.16 & NW & it & 971 & ay: & 400 & 6.20 & S & 66 & $6=$ \\
\hline & $\infty$ & 6.02 & NW & Wi & 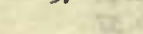 & 265 & IO & 6.21 & S & ex & nes \\
\hline & $\infty$ & 5.90 & NW & Wi & 21 & $6 f^{2}$ & 20 & $6.2 \mathrm{I}$ & $\mathrm{S}$ & $x=1$ & $\Leftrightarrow$ \\
\hline 7 & 00 & $5.8 \mathrm{I}$ & NW & 6 & $-2 y$ & es & 30 & 6.20 & S & $x_{5}$ & 60 es \\
\hline 8 & $\infty$ & 5.78 & NW & $w$ & 98.3 & onit. & 40 & 6.19 & S & or: & 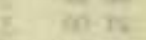 \\
\hline & 10 & 5.78 & NW & Wry & 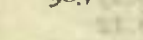 & 30 : & 50 & 6.16 & $\mathrm{~S}$ & $16: 2$ & bos. $3 x$ \\
\hline & 20 & $5.76 \mathrm{~L}$ & NW & wrat & $x+18$. & 000 & 500 & 6. I1 & $\mathrm{S}$ & tes? & 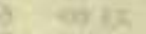 \\
\hline & 30 & 5.80 & NW & Wri & सी० & 00 尚 & 600 & 6.01 & SW & Met & $00 \mathrm{~F}=$ \\
\hline & 40 & 5.79 & NW & Whis & 65 & $\omega=$ & 700 & $5 \cdot 93$ & SW & & the \\
\hline & 50 & 5.82 & NW & $W K$ & $28 \%$ & & 800 & 5.92 & W & 77 & \\
\hline & oo & 5.83 & NW & Wh & Detsy & J. E. M. & 10 & 5.92 & W & & \\
\hline & oo & 5.96 & W & WK & is: & I. L. & 20 & 5.92 & w & & \\
\hline II. & $\infty$ & 6.14 & W & $w W$ & it: & i & 30 & $5.9 \mathrm{IL}$ & W & " & 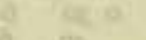 \\
\hline $12 c$ & $\infty$ & 6.39 & W & Whis & 7 & in: & 40 & $5.9 \mathrm{II}$ & $w$ & WE & 05 \\
\hline 13 & $\infty$ & 6.52 & W & Wh & 8 & (x) 1 & 50 & $5.91 \mathrm{I}$ & W & 48 & $\theta=$ \\
\hline 14 & $\infty$ & $6.6 \mathrm{I}$ & SW & Whis & (A) & bf & 900 & $5.9 \mathrm{IL}$ & W & nfl & 987 \\
\hline 24 & 10 & 6.62 & SW & $W$ & all & ot & 10 & 5.93 & W & te. & 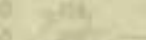 \\
\hline & 20 & $6.66 \mathrm{H}$ & SW & 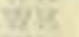 & (91) & 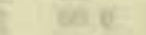 & 20 & 5.93 & $\mathbf{w}$ & 78 & tat \\
\hline & 30 & 6.63 & SW & wh & the & 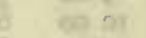 & 30 & 5.94 & W & mat & an: \\
\hline & 40 & 6.64 & SW & Wi: & IE. & is 11 & 35 & 5.90 & W & & 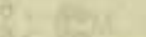 \\
\hline & 50 & 6.66 & SW & $1 \%$ & 72 & $\frac{6}{254}$ & 40 & 5.92 & W & 4 & $x^{2}$ \\
\hline 15 & oo & $6.6 i$ & SW & wit & X: & $60 \mathrm{ki}$ & 50 & 5.91 & W & & $80=5$ \\
\hline & 10 & 6.60 & SW & Witi & 9. & extor & 1000 & 5.92 & W & & J. E. M. \\
\hline 16 & $\infty$ & 6.50 & $\mathrm{~S}$ & Wh & 25 & ins & II $\infty$ & 6.13 & W & & F. L. \\
\hline 17 & $\infty$ & 6.25 & $\mathrm{~S}$ & 28 & The & $\theta$ & 1200 & 6.30 & W & 90 & 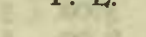 \\
\hline 18 & $\infty$ & 6.04 & $\mathbf{S}$ & whe & estat & an & 1300 & 6.46 & W & 80 & 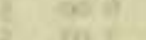 \\
\hline 19 & $\infty$ & 5.93 & $\mathrm{~S}$ & yits. & 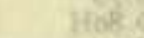 & 62 & 1400 & 6.60 & SSI: & 52 & 60 \\
\hline 20 & $\infty$ & 5.74 & $\mathrm{~S}$ & Whe & 38 & $\sin 6$ & 10 & 6.60 & SSE & 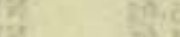 & et: \\
\hline & Io & 5.71 & S & $y=$ & 30 & 대 & 20 & 6.61 & SSI & t & in \\
\hline & 20 & 5.70 & $\mathrm{~S}$ & Whe & $\infty$ & was $y$ & 3 & 6.60 & SSE & 8 & $x$ \\
\hline & 30 & 5.70 & $\mathrm{~S}$ & Whis & 간. & 60 & 40 & $6.6 I$ & SSE & vis & 68 \\
\hline & 40 & 5.74 & $\mathrm{~S}$ & $63^{\circ}$ & as & 165 & 50 & $6.63 \mathrm{H}$ & SSE & 15 & $Q_{2}=$ \\
\hline & 50 & 5.72 & $\mathbf{S}$ & wis: & ins & $\log ^{2}$ ti & I5 Do & 6.62 & SSE & atas & 2006 \\
\hline 21 & Do & 5.70 & $\mathrm{~S}$ & 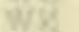 & is & $(x-1)$ & 10 & $6.6 \mathrm{I}$ & SSE & ats & 6036 \\
\hline & 10 & 5.64 & $S$ & 488 & & 80.72 & 20 & 6.62 & SSI & $\mathrm{ns}$ & 07.91 \\
\hline & 20 & $5.63 \mathrm{~L}$ & $\mathrm{~S}$ & $y$ & 22 & wir & 30 & 6.62 & SSE & $\theta$ & 00.45 \\
\hline & 30 & 5.66 & $\mathrm{~S}$ & & $9 t$ & te & 40 & 6.62 & SSE & 13. & 900 b? \\
\hline & 40 & 5.64 & S & 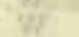 & $\sigma^{2}$ & sis & 50 & 6.62 & SSE. & Hog & and th \\
\hline & 50 & 5.63 & $\mathrm{~S}$ & W & dos: & exis & I6 o0 & 6.60 & SSE & 107 & ar \\
\hline 22 & $\infty$ & 5.64 & S & W & Fरs. & F. L. & 1700 & 6.50 & ENE & & $x=$ \\
\hline 23 & $\infty$ & 5.73 & $\mathrm{SE}$ & $w$ & จे & J. E. M. & 1800 & $6.3 \mathrm{I}$ & $\mathrm{ENE}$ & $a$ & is: \\
\hline 240 & $\infty$ & 5.86 & $\mathrm{SE}$ & wis & 56 & J. E. M. & 1900 & 6.14 & ENE & 18 & 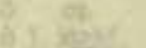 \\
\hline & & 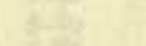 & ar & . & 17 & 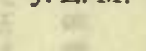 & 2000 & 5.98 & ENE & 120 & $\mathrm{Fi}$ \\
\hline & & & & & 52 & $\cos x$ & $21 \infty$ & 5.90 & ENE & t & J. $\dot{\mathrm{E}} . \dot{\mathrm{M}}$. \\
\hline & $x^{2}$ & & July 6 , & 5,190 & 8 & $m^{3}=$ & & $\begin{array}{l}5.84 \\
5.83\end{array}$ & ENE & 60 & J. L. M. \\
\hline I & $\infty$ & 5.99 & S & W & & J. E. M. & $\begin{array}{l}40 \\
50\end{array}$ & $\begin{array}{l}5.83 \\
5.8 \mathrm{I}\end{array}$ & $\begin{array}{l}\text { ENE } \\
\text { ENF }\end{array}$ & $27+1$ & 0851 \\
\hline & 50 & 6.13 & $\mathrm{~S}$ & & & & 2200 & $5.80 \mathrm{~L}$ & ENE & & 6041 \\
\hline 2 & $\infty$ & 6.15 & $\mathrm{~S}$ & $2 \pi$ & & & 10 & $5.80 \mathrm{~L}$ & ENE & $n^{2}$ & 1060 \\
\hline & 10 & 6.18 & $\mathbf{S}$ & & & & 20 & $5.8 \mathrm{I}$ & ENE & $6=$ & et \\
\hline & 20 & 6.18 & S & 32 & ast. & $\ln ^{3} 3$ & 30 & 5.83 & ENE & 085.7 & $\infty$ \\
\hline & 30 & 6. I7 & $\mathrm{S}$ & 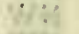 & 6 & E. & 40 & 5.83 & ENE & os -1 & se. \\
\hline & 40 & 6.20 & $\mathbf{S}$ & Wh & de & 15 & 50 & 5.84 & ENE & 85 & Fis \\
\hline & 50 & 6.20 & $\mathrm{~S}$ & $W:$ & 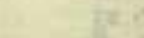 & 4 & 2300 & 5.84 & ENG & & \\
\hline 3 & $\infty$ & 6.20 & $\mathrm{~S}$ & in & As: & J. E. M. & 2400 & 5.93 & ENE & 127 & J. E. M. \\
\hline
\end{tabular}


Tabulation of tidal observations at Capc Flora, Northbrook Island

\begin{tabular}{|c|c|c|c|c|c|c|c|c|c|c|}
\hline $\begin{array}{l}\text { Loc } \\
\text { niea } \\
\text { tim }\end{array}$ & & $\begin{array}{l}\text { Reading } \\
\text { of tide } \\
\text { staff }\end{array}$ & $\begin{array}{l}\text { Wind } \\
\text { direction }\end{array}$ & $\begin{array}{l}\text { Anemom- } \\
\text { eter } \\
\text { records }\end{array}$ & Observer & $\begin{array}{c}\text { Local } \\
\text { mean } \\
\text { time }\end{array}$ & $\begin{array}{l}\text { Reading } \\
\text { of tide } \\
\text { staff }\end{array}$ & $\begin{array}{c}\text { Wind } \\
\text { direction }\end{array}$ & $\begin{array}{l}\text { Anemom- } \\
\text { eter } \\
\text { records }\end{array}$ & Observer \\
\hline \multicolumn{6}{|c|}{ July 7,1904} & \multicolumn{5}{|c|}{ July $7, \quad 1904$} \\
\hline$h$ & $m$ & \multicolumn{2}{|c|}{ Fect } & Miles & $=1$ & $h m$ & Feet & M & Miles & $x=1$ \\
\hline & $\infty$ & 6.09 & ENE & & \multirow[t]{2}{*}{ J. E. M. } & 2330 & $5.95 \mathrm{~L}$ & \multirow{4}{*}{$\begin{array}{l}\mathrm{NE} \\
\mathrm{NE} \\
\mathrm{NE} \\
\mathrm{NE}\end{array}$} & 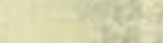 & \multirow{2}{*}{ J. E.M. } \\
\hline & 00 & 6.30 & ENE & & & 40 & $5.95 \mathrm{~L}$ & & Wh: & \\
\hline & $\infty$ & 6.43 & W & & 192 & 50 & $5.95 \mathrm{~L}$ & & \multirow{2}{*}{244} & -4 \\
\hline & Io & 6.43 & W & & $\infty+1$ & 240 & 5.98 & & & J. E. M. \\
\hline & 20 & 6.43 & $W$ & 10 & aris & & & & 4 & \\
\hline & .30 & 6.46 & W & 50 & wa & $7=$ & $2 y^{2}$ & & 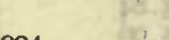 & 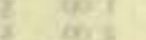 \\
\hline & 40 & 6.49 & W & 2 & 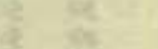 & s & & July 8,1 & 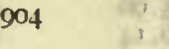 & 100 \\
\hline & 50 & 6.50 & $\underset{w}{W}$ & 77 & as & & $6 m$ & & sit & \\
\hline 4 & $\begin{array}{l}\infty \\
10\end{array}$ & $\begin{array}{l}6.50 \\
6.50\end{array}$ & $\begin{array}{l}W \\
W\end{array}$ & 137 & कon $x$ & 010 & 6.00 & $\mathrm{NE}$ & ge & J. E. M. \\
\hline & 20 & $6.53 \mathrm{H}$ & W & 8 & 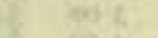 & $\begin{array}{lll}1 & 00 \\
2 & 00\end{array}$ & 6.04 & $\mathrm{E}$ & Bit & by \\
\hline & 30 & $6.53 \mathrm{LL}$ & W & 8 & 60.1 & 200 & 6.12 & $\mathrm{EF}$ & DS. & ex \\
\hline & 40 & 6.51 & W & 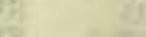 & an) 1 & 300 & $\begin{array}{l}0.38 \\
6.53\end{array}$ & SEF & (1) D. & of \\
\hline & 50 & 6.50 & W & 2 & $60 / 2$ & 400 & & SE & 264 & $0 \%$ \\
\hline 5 & $\infty$ & 6.49 & W & 9 & moर & 500 & 6.03 & SE & :ne. & $n$ \\
\hline & 10 & 6.49 & W & 1 & at & Io & $\begin{array}{l}6.66 \\
6.68\end{array}$ & SE & $102 x-4$ & 900 \\
\hline 6 & & 6.48 & W & at & 05 & 20 & $\begin{array}{l}6.08 \\
6.70 \mathrm{H}\end{array}$ & SE & ... & 대 \\
\hline 7 & $\infty$ & 6.40 & ESE & $2 x$ & Q5. & 30 & & $\begin{array}{l}\text { SE } \\
\text { SE }\end{array}$ & $85:$ & $\alpha$ \\
\hline 8 & $\infty$ & 6.37 & $\overrightarrow{\mathrm{ESE}}$ & I52 & J. E. M. & 40 & $\begin{array}{l}0.70 \mathrm{H} \\
6.70 \mathrm{H}\end{array}$ & SE & Quple & as: \\
\hline 9 & $\infty$ & 6.33 & ENE & 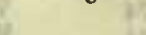 & F. L. & $6 \stackrel{50}{00}$ & $\begin{array}{l}0.70 \mathrm{H} \\
6.70 \mathrm{H}\end{array}$ & $\begin{array}{l}\text { SE } \\
\text { SE }\end{array}$ & $\therefore b$ & ae \\
\hline & IO & 6.32 & ENE & & (2) & Io & 6.60 & SE & beis & a \\
\hline & 20 & 6.31 & ENE & 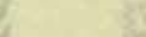 & art & $\begin{array}{l}10 \\
20\end{array}$ & $\begin{array}{l}0.09 \\
6.66\end{array}$ & SE & Hot. & $00: 5$ \\
\hline & 30 & 6.32 & $\mathrm{E}$ & 12 & or & 20 & 0.00 & SE & Finge.t. & os: \\
\hline & 40 & 6.30 & $\mathrm{E}$ & 107 & A6. & 30 & 6.63 & SE & 26. & 80 \\
\hline & 50 & 6.30 & $\vec{E}$ & et & o: & 40 & 6.62 & SE & 10. & ac: \\
\hline & 58 & $6.28 \mathrm{~L}$ & $\vec{E}$ & 8 & 00 & 700 & 6.59 & NW & 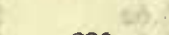 & T \\
\hline 10 & $\infty$ & 6.30 & $\mathrm{E}$ & ne & ne & 800 & 6.53 & NW & 293 & J. E. M. \\
\hline & 10 & 6.30 & E & $y$ & 009 & 900 & 6.50 & $W$ & nde & F. L. \\
\hline & 20 & 6.31 & $\mathrm{E}$ & S & $4=61$ & 1000 & 6.43 & SW & 26. & now 0 \\
\hline & 30 & 6.32 & & I & 78.34 & Io & 6.42 & SW & ote & on $\cos$ \\
\hline & 40 & 6.33 & E & e & as 51 & 20 & 6.41 & WNW & 14. & $160 \mathrm{it}$ \\
\hline & 50 & 6.37 & $\mathrm{E}$ & K & 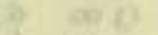 & 30 & 6.40 & WNW & 315. & $100 \leq 1$ \\
\hline II & $\infty$ & 6.40 & $\mathrm{E}$ & Dy & 3 of & 40 & 6.45 & WNW & inci & wit \\
\hline 12 & $\infty$ & 6.50 & ESE & 100 & as & 50 & 6.42 & WNW & .10 & $x_{x}$ \\
\hline 13 & $\infty$ & 6.63 & ESE & 190 & ie & II $\infty$ & 6.42 & NW & is & $n t$ \\
\hline I4 & $\infty$ & 6.79 & $\mathrm{NE}$ & 차 & 60 & IO & $6.38 \mathrm{~L}$ & NW & Iif & 6 \\
\hline 15 & 03 & 6.88 & $\mathrm{NE}$ & 18 & we & 20 & $6.38 \mathrm{~L}$ & NW & itris & $n=1$ \\
\hline & 30 & 6.90 & $\mathrm{NE}$ & $t w$ & Des 31 & 30 & 6.44 & NW & op $=$ & not $x+$ \\
\hline & 40 & 6.90 & $\mathrm{NE}$ & $\mathrm{f}$ & wi & 40 & 6.43 & NW & Mis & we \\
\hline & 50 & $6.9 \mathrm{IH}$ & $\mathrm{NE}$ & es & is: & 50 & 6.46 & NW & si & $60 \mathrm{b1}$ \\
\hline 16 & $\infty$ & $6.91 \mathrm{H}$ & $\mathrm{NE}$ & 212 & $y=3$ & 1200 & 6.47 & NW & 353 & ins 18 \\
\hline & IO & 6.90 & NE & 212 & $68 \times 4$ & 1300 & 6.56 & NW & 1001 & 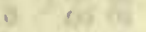 \\
\hline & 20 & 6.89 & $\mathrm{NE}$ & ta & 62 & 1400 & 6.64 & NW & 80 & $60 \mathrm{st}$ \\
\hline & 30 & 6.88 & $\mathrm{NE}$ & a & $\cos 8 t$ & 1500 & 6.75 & NW & H) & 3084 \\
\hline & 40 & 6.90 & $\mathrm{NE}$ & $\theta$ & $(10)(17$ & 1600 & 6.8 & NW & 389 & 200 \\
\hline & 50 & 6.89 & $\mathrm{NE}$ & is & $200 \times 5$ & IO & 6.8 & NW & Apose & $6 a$ \\
\hline 17 & $\infty$ & 6.84 & $\mathrm{NE}$ & 0 & 410801 & 20 & $6.84 \mathrm{H}$ & NW & & 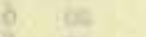 \\
\hline 18 & $\infty$ & $6.7 \mathrm{I}$ & $\mathrm{NE}$ & 0 & As & 30 & $6.84 \mathrm{~F}$ & NW & 20.8 & 9. \\
\hline 19 & $\infty$ & 6.50 & $\mathrm{NE}$ & & 68 & 40 & $6.84 \mathrm{H}$ & NW & it: & is \\
\hline 20 & I0 & 6.28 & $\mathrm{NE}$ & 228 & 59 es & 50 & 6.82 & NW & $\cos \theta$ & nes \\
\hline $2 I$ & $\infty$ & 6.05 & $\mathrm{NE}$ & 200 & F I & 1700 & 6.78 & NW & $x_{Z}$ & Do: (it) \\
\hline & 40 & 6.05 & $\mathrm{NE}$ & $M$ & $\overrightarrow{\mathrm{F}} \mathrm{M}$ & 1803 & 6.76 & NE & 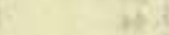 & 00 ine \\
\hline & 50 & 6.03 & $\mathrm{NE}$ & 10 & J. L. M. & 1900 & 6.65 & NE & $20=$ & 00 is \\
\hline 22 & 00 & 6.03 & $\mathrm{NE}$ & tes & Et & 2000 & 6.40 & NE & 440 & \\
\hline & Io & 6.02 & $\mathrm{NE}$ & 18 & Be & 2100 & 6.24 & SW & & F. I. \\
\hline & 20 & 6.01 & $\mathrm{NE}$ & 8 & as & 2200 & 6.07 & SW & $8 \mathrm{t}$ & J. E. M. \\
\hline & 30 & 6.00 & $\mathrm{NE}$ & C & mite & 2300 & 5.90 & SW & & \\
\hline & 40 & 6.00 & $\mathrm{NE}$ & ect & 10.13 & Io & 5.90 & SW & benter & $x^{2}$ \\
\hline & 50 & 5.97 & $\mathrm{NE}$ & & 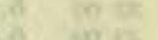 & 20 & 5.90 & SW & & \\
\hline 23 & $\infty$ & 5.97 & $N E$ & 3 & $\begin{array}{l}190 \\
y 3\end{array}$ & $\begin{array}{l}30 \\
40\end{array}$ & $\begin{array}{l}5.91 \\
5.80\end{array}$ & $\begin{array}{l}\text { SW } \\
\text { SW }\end{array}$ & ant & $x^{2}$ \\
\hline & Io & 5.99 & NE & & 4. & 50 & 5.84 & SW & & \\
\hline & 20 & $5.95 \mathrm{~L}$ & $\mathrm{NE}$ & & J. E. M. & 2400 & 5.80 & S & 449 & J. E. M. \\
\hline
\end{tabular}


Tabulation of tidal observations at Cape Flora, Northbrook Island

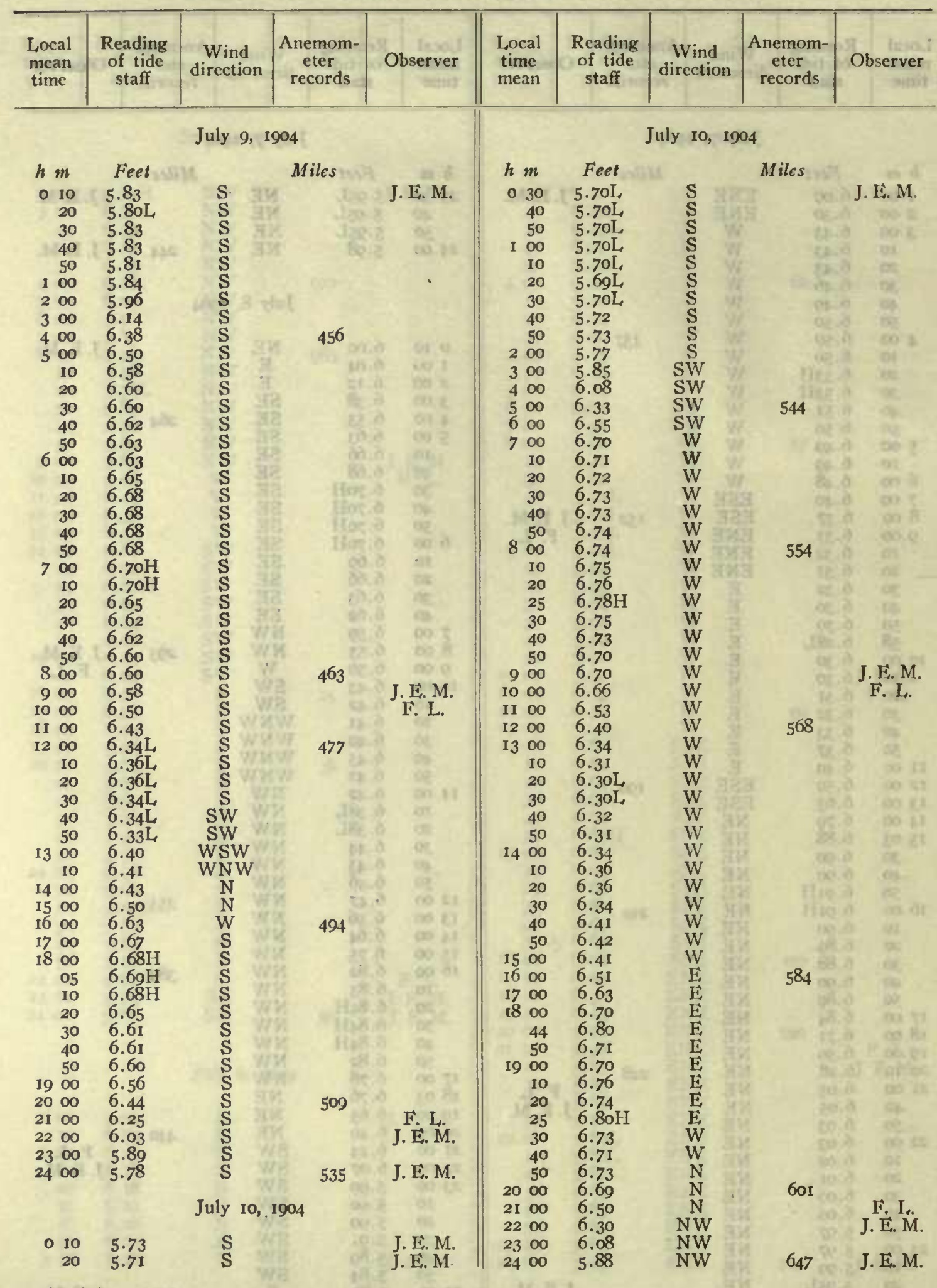


Tabulation of tidal observations at Cape Flora, Northbrook Island

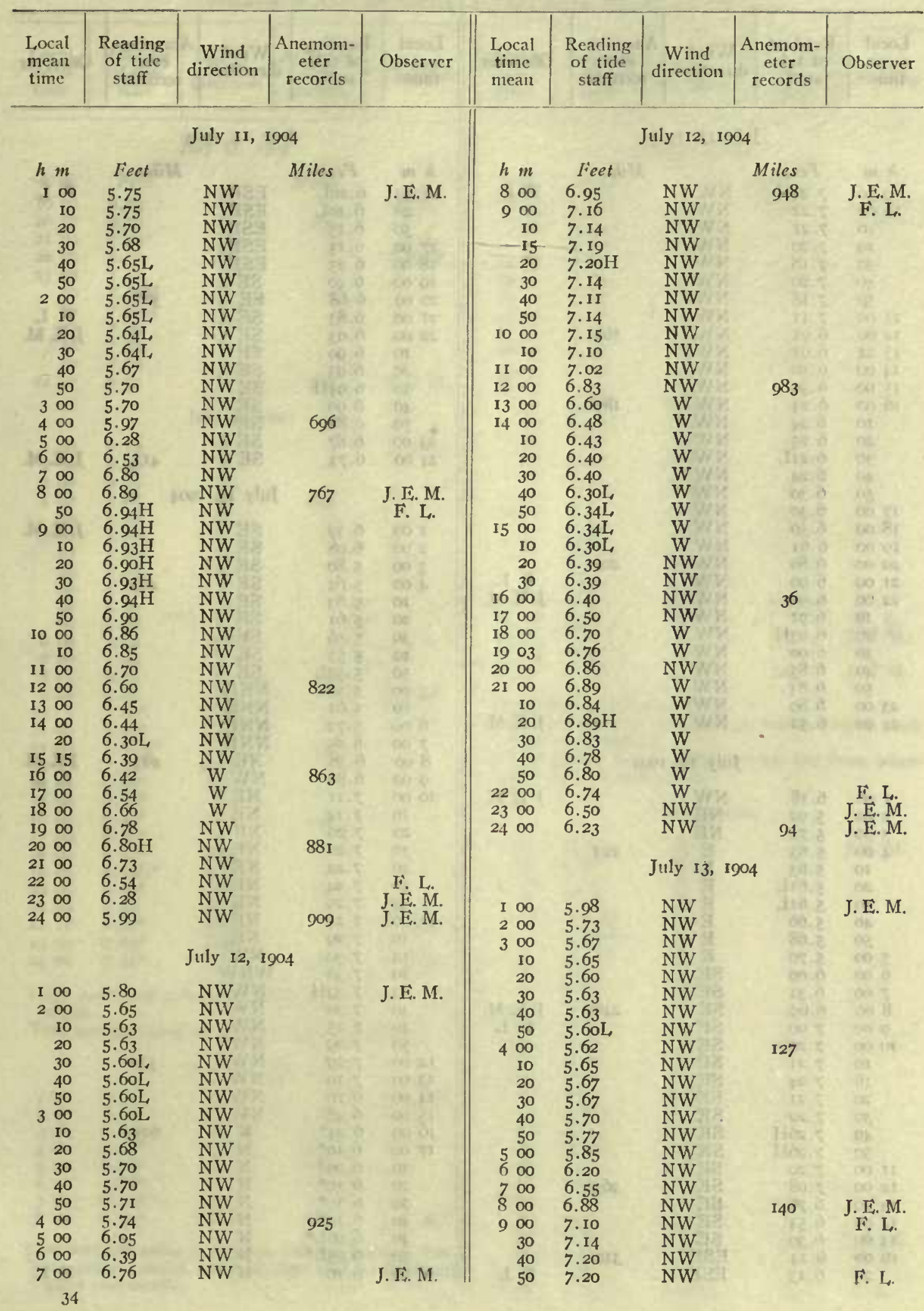


Tabulation of tidal observations at Cape Flora, Northbrook Island

\begin{tabular}{|c|c|c|c|c|c|c|c|c|c|}
\hline $\begin{array}{l}\text { Local } \\
\text { mean } \\
\text { time }\end{array}$ & $\begin{array}{l}\text { Reading } \\
\text { of tidc } \\
\text { staff }\end{array}$ & $\begin{array}{l}\text { Wind } \\
\text { direction }\end{array}$ & $\begin{array}{c}\text { Anemom- } \\
\text { eter } \\
\text { records }\end{array}$ & Observer & $\begin{array}{l}\text { Local } \\
\text { mean } \\
\text { time }\end{array}$ & $\begin{array}{l}\text { Reading } \\
\text { of tide } \\
\text { staff }\end{array}$ & $\begin{array}{l}\text { Wind } \\
\text { direction }\end{array}$ & $\begin{array}{c}\text { Anemom- } \\
\text { eter } \\
\text { records }\end{array}$ & Observer \\
\hline \multicolumn{5}{|c|}{ July 13,1904} & \multicolumn{5}{|c|}{ July I4, 1904} \\
\hline$h m$ & Feet & & Miles & $=$ if & $h m$ & Fieet & & Miles & $=x$ \\
\hline 956 & $7.24 \mathrm{H}$ & NW & & F. L. & 1630 & $6.10 \mathrm{~L}$ & ESE & & F. L. \\
\hline 1000 & 7.22 & NW & bits & 8 & 10 & $6.10 \mathrm{~L}$ & ESE & & F. L. \\
\hline 10 & 7.21 & NW & 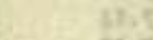 & of & 50 & 6.13 & ESE & & $=$ \\
\hline 20 & 7.20 & NW & er. & er & 1700 & 6.15 & ESE & & ec \\
\hline 30 & 7.18 & NW & Hase. & 2 & 1800 & 6.25 & ESE & & $E$ \\
\hline 40 & 7.20 & NW & pr. & ec & 1900 & 6.4 & & & की \\
\hline & 7.18 & NW & ix. & 48 & 2000 & 6.68 & $\mathrm{SE}$ & 366 & \\
\hline II 00 & 7.11 & NW & $i^{12}$ & 8 & 2100 & 6.83 & $\mathrm{SE}$ & & F. L. \\
\hline 1200 & 6.91 & NW & 165 & 80010 & 2200 & 6.93 & SE & & J. E. M. \\
\hline 1321 & $6.6 \mathrm{I}$ & NW & & ent & 10 & 6.90 & SE & is & \\
\hline 1400 & 6.50 & NW & wa: & $m=x$ & 20 & 6.93 & $\mathrm{SE}$ & & 8 \\
\hline 1500 & 6.30 & & & 6054 & 30 & $6.94 \mathrm{HH}$ & $S E$ & & ex \\
\hline 1600 & 6.24 & NW & 189 & $\cos$ & 40 & 6.90 & $\mathrm{SE}$ & iv 3 & aen it: \\
\hline 10 & 6.24 & $N W$ & 8 & 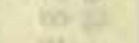 & 50 & 6.88 & $\mathrm{SE}$ & & $a \geqslant$ \\
\hline 20 & 6.25 & $N W$ & a. & $4{ }^{2}$ & 2300 & 6.87 & $\mathrm{SE}$ & & $\operatorname{can} 3$ \\
\hline 30 & $6.2 \mathrm{IL}$ & $N W$ & of & es & 2400 & 6.73 & $\mathrm{SE}$ & 413 & J. E. M. \\
\hline 40 & 6.24 & $\mathrm{NW}$ & a & Es & & & & & \\
\hline $\begin{array}{r}50 \\
1700\end{array}$ & $\begin{array}{l}6.30 \\
6.30\end{array}$ & $\begin{array}{l}\text { NW } \\
\text { NW }\end{array}$ & $\operatorname{tag} 2$ & 9 & monat & 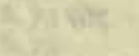 & July 15 , & 1904 & 60 \\
\hline 1800 & 6.50 & NW & $(3 x)$ & 10.0 & 100 & 6.39 & $S E$ & & J. E. M. \\
\hline 1900 & $6.6 \mathrm{I}$ & NW & $b^{2} e^{2}$ & at & 200 & 6.08 & & & \\
\hline 2000 & 6.80 & NW & 207 & $\Rightarrow$ & 300 & 5.8 & $\mathrm{SE}$ & 180 & 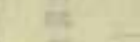 \\
\hline 2100 & 6.90 & NW & (1) & $\mathrm{F} . \mathrm{L}$ & 400 & 5.6 & $\mathrm{SE}$ & 460 & \& \\
\hline 2200 & 6.9 & NW & 30 & J.E. M. & 10 & $\begin{array}{l}5.04 \\
5.63\end{array}$ & $\mathrm{SE}$ & $4 \pi 0$ & 6 \\
\hline 10 & 6.9 & NW & 098 & 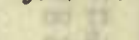 & 20 & $5.6 \mathrm{I}$ & $\mathrm{SE}$ & (2. A & 92 \\
\hline 20 & $6.93 \mathrm{H}$ & NW & 83 & 0521 & 30 & 5.5 & $\mathrm{SE}$ & 828 & 80 oi. \\
\hline 30 & 6.90 & NW & 84 & bost & 40 & 5.5 & $\mathrm{SE}$ & 280 & $e d$ \\
\hline 40 & 6.85 & NW & 1063. & $05=$ & 50 & $5 \cdot 5$ & $\mathrm{SE}$ & 050 & kolis \\
\hline 50 & 6.83 & NW & $\%$ & arose & 500 & 5.5 & NNE & 63 A & 90,4 \\
\hline 2300 & 6.80 & $N W$ & $x^{2}$ & & 10 & 5. & NNE & & $60 \mathrm{sr}$ \\
\hline \multirow[t]{3}{*}{2400} & 6.52 & NW & 212 & J. E. M. & 600 & 5. & NNE & & 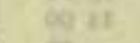 \\
\hline & & & & & & & NNE & ter & \\
\hline & & July 14, & 1904 & $=$ & 800 & 6.3 & NE & 485 & JE. M. \\
\hline 100 & 6.18 & NW & ex. & J. E. M. & $\begin{array}{r}900 \\
\text { 10 }\end{array}$ & $\begin{array}{l}0.80 \\
7.11\end{array}$ & NE & & 1. L. \\
\hline & 5.92 & $\mathrm{NE}$ & & & & & $\mathrm{NE}$ & hs de & $00 \mathrm{Rt}$ \\
\hline 30 & 5.7 & $\mathrm{NE}$ & $\theta=8$ & $00+4$ & 20 & & $\mathrm{NE}$ & & $\infty 0 . y x$ \\
\hline 400 & 5.63 & $E$ & 221 & & 30 & & $\mathrm{NE}$ & flod 5 & oc as \\
\hline I0 & 5.63 & E & & & 40 & & $\mathrm{NE}$ & 158 & $90 \mathrm{rt}$ \\
\hline 20 & $5.6 \mathrm{IL}$ & $\mathrm{E}$ & & & 5 & 7. & $\mathrm{~N}$ & & $\sigma E$ \\
\hline 30 & $5.6 \mathrm{IL}$ & E & he & 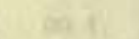 & 55 & & $\mathrm{~N}$ & 20 & on 09 \\
\hline & & $\mathrm{E}$ & & in & II 00 & 7.2 & N] & (v) 3 & orit: \\
\hline 50 & 5.68 & E & 25). & cost & 10 & 7.2 & NI & & \\
\hline 500 & & & & 81 & 14 & 7.3 & NV & & \\
\hline 600 & 6. & $\mathrm{SE}$ & 달 & 2 & 20 & & WI & & \\
\hline 700 & 6.31 & $\mathrm{SE}$ & & $\Rightarrow \rightarrow$ & 23 & & & $\infty$ & $\cos t$ \\
\hline 800 & 6.69 & $S E$ & 248 & J. E. M. & 30 & & & in & $\operatorname{met}=$ \\
\hline 900 & 7.00 & $\mathrm{SE}$ & 240 & F. L. & 40 & 7. & NI & E) & ex \\
\hline 1000 & 7.20 & $\mathrm{SE}$ & 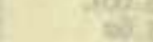 & 1.4 & 50 & 7. & N & 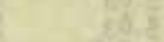 & 2 \\
\hline 10 & 7.21 & SE & 20 & tit & 1200 & 7.2 & NW & 532 & \\
\hline 16 & 7.24 & SE & 20 & te & 1300 & 7.1 & $\mathrm{NV}$ & 352 & 2 \\
\hline & 7.2 & SE & 45 & WE. & I4 00 & 6. & NV & $405=$ & 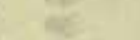 \\
\hline & 7.2 & SE & ar & 20: & 1500 & 6.4 & NW & 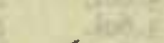 & $\infty 0$ \\
\hline 40 & 7.26 & $\mathrm{SE}$ & vit? & 64 & 1600 & 6.2 & $\mathrm{~N}$ & 60.4 & is: \\
\hline & & $\mathrm{SE}$ & 35 & & 1700 & & & & $\Leftrightarrow$ \\
\hline II 0 & & & & 3 & I & & & & \\
\hline 120 & & SI & 265 & & 2 & & & ind & 87 \\
\hline 130 & & SI & & कon iो & 30 & & & 557 & ef \\
\hline 140 & & & $\mathrm{get}$ & ind & 40 & & & 178 & wory \\
\hline $15 \mathrm{C}$ & & & & & 4 & & $\mathrm{~N}$ & 80.8 & $\operatorname{se} 3$ \\
\hline 1600 & & ESE & 310 & & 50 & & $\mathrm{~N}$ & bese & \\
\hline 20 & 6.13 & ESE & & F. L. & 1800 & 6.10 & $\mathrm{~N}$ & $20 \cos$ & F. L. \\
\hline
\end{tabular}


Tabulation of tidal observations at Cape Flora, Northbrook Island

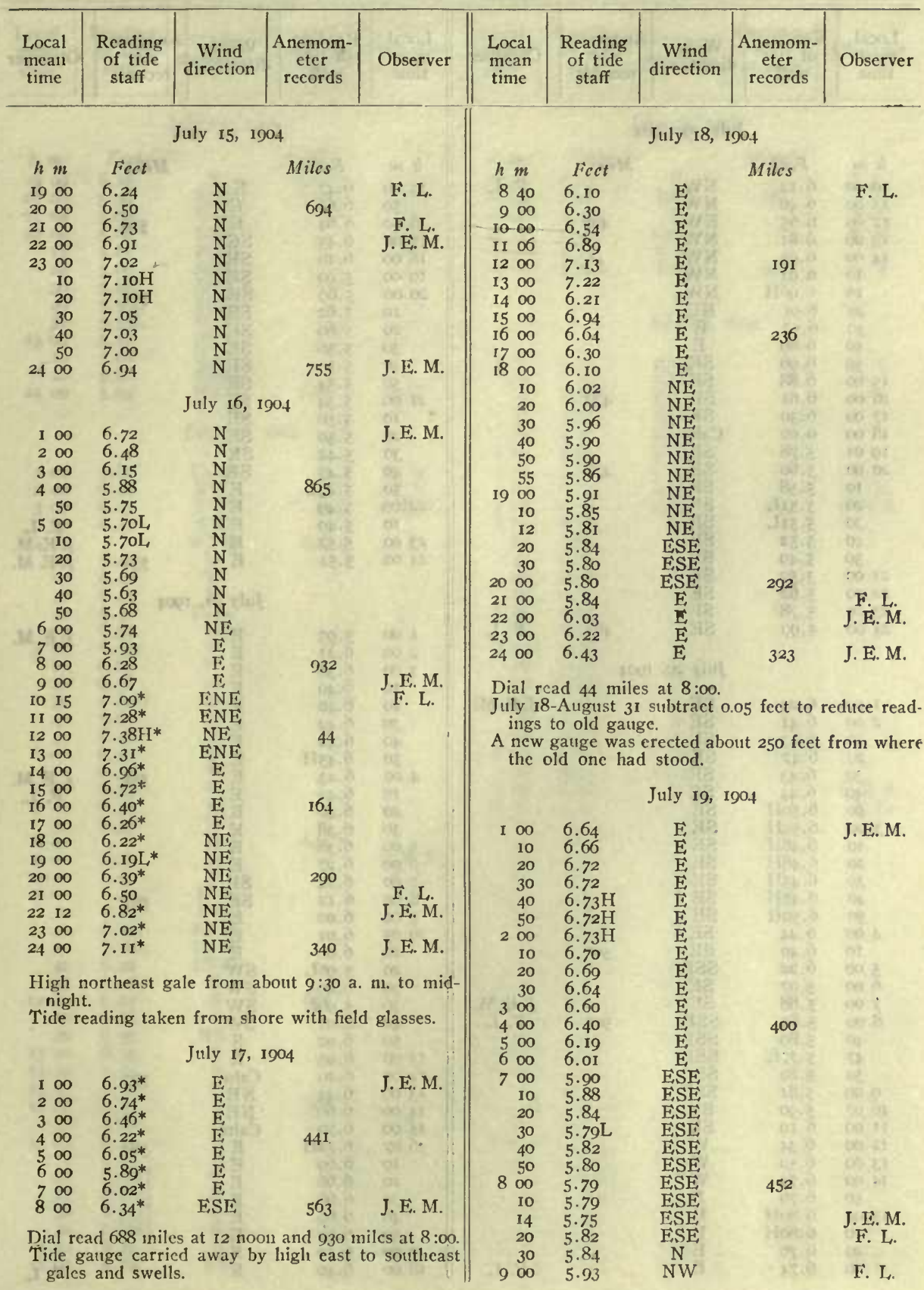


Tabulation of tidal observations at Cape Flora, Northbrook Island

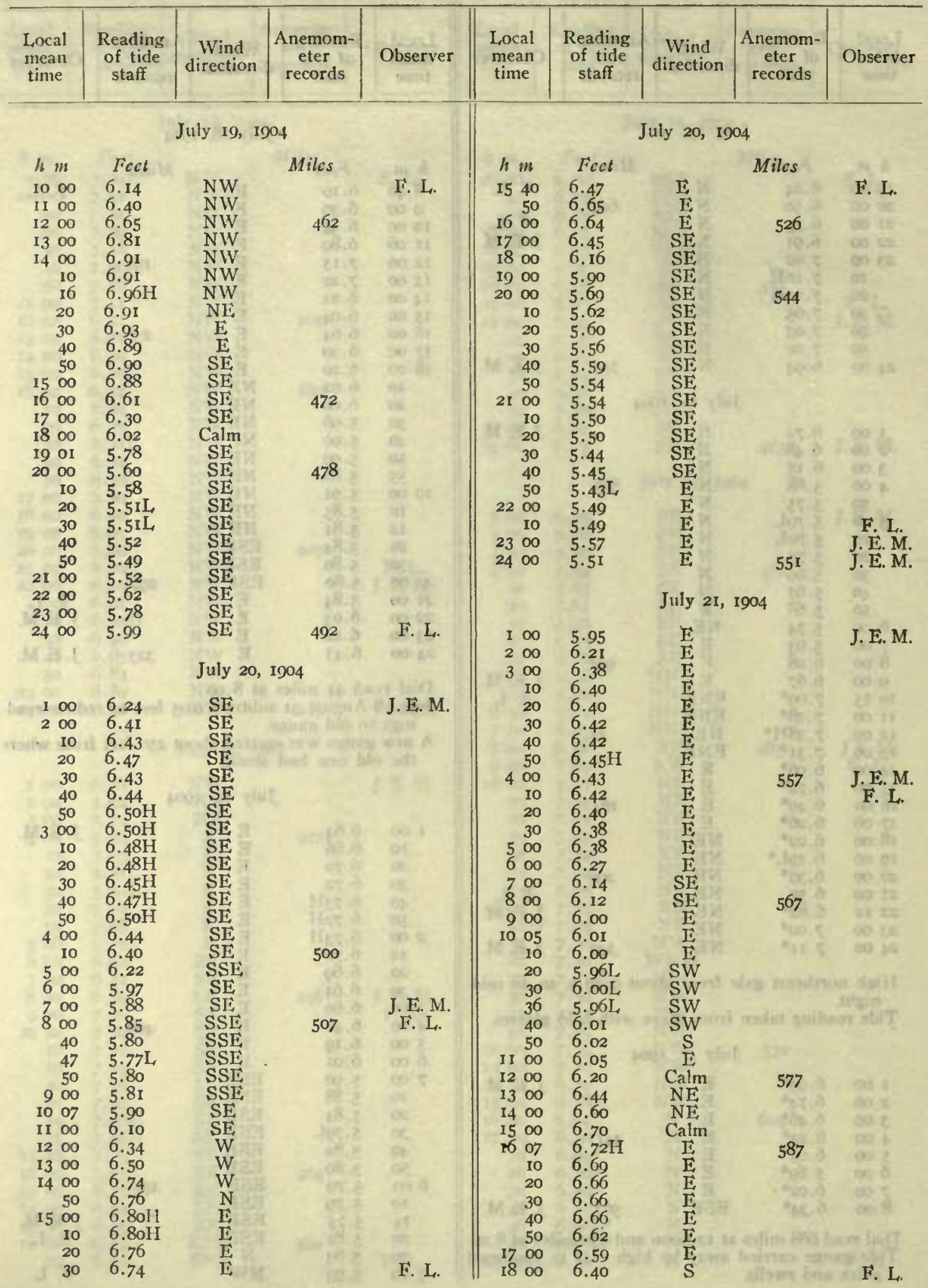


Tabulation of tidal observations at Cape Flora, Northbrook Island

\begin{tabular}{|c|c|c|c|c|c|c|c|c|c|}
\hline $\begin{array}{l}\text { Local } \\
\text { mean } \\
\text { time }\end{array}$ & $\begin{array}{l}\text { Reading } \\
\text { of tide } \\
\text { staff }\end{array}$ & $\begin{array}{l}\text { Wind } \\
\text { direction }\end{array}$ & $\begin{array}{l}\text { Anemom- } \\
\text { eter } \\
\text { records }\end{array}$ & Observer & $\begin{array}{l}\text { Local } \\
\text { mean } \\
\text { time }\end{array}$ & $\begin{array}{l}\text { Reading } \\
\text { of tide } \\
\text { staff }\end{array}$ & $\begin{array}{l}\text { Wind } \\
\text { direction }\end{array}$ & $\begin{array}{c}\text { Anemom- } \\
\text { eter } \\
\text { records }\end{array}$ & Observer \\
\hline \multicolumn{5}{|c|}{ July 21, 1904} & \multicolumn{5}{|c|}{ July 22, 1904} \\
\hline$h m$ & Feet & & Miles & 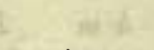 & $h m$ & Feet & & Miles & $y=\mathrm{z}$ \\
\hline 1900 & 6.18 & $\stackrel{S}{S}$ & & F. L. & $19 a_{4}$ & 6.55 & $\stackrel{E}{E}$ & & F. $\mathrm{L}$. \\
\hline $\begin{array}{ll}20 & 00 \\
21 & 00\end{array}$ & $\begin{array}{l}5.98 \\
5.80\end{array}$ & $\begin{array}{l}\text { SSE } \\
\text { SSE }\end{array}$ & 602 & F. Lt. & $\begin{array}{l}2000 \\
2100\end{array}$ & $\begin{array}{l}6.28 \\
6.10\end{array}$ & ESE & 684 & \\
\hline 2200 & 5.66 & SSE & 3 & A. V. & $2200^{-}$ & $\begin{array}{l}0.10 \\
5.93\end{array}$ & Calm & & A. $\mathrm{V}$ \\
\hline 17 & 5.61 & SSE & 4 & 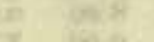 & 2300 & & ESE & $\cos ^{2}$ & \\
\hline 20 & 5.60 & SSE & the & $\operatorname{lin} 5$ & 2400 & $5.65 \mathrm{~L}$ & ESE & 696 & A. V. \\
\hline $\begin{array}{l}30 \\
40\end{array}$ & $\begin{array}{l}5.62 \\
5.55 \mathrm{~L}\end{array}$ & SSE & 50 & 2 & & & 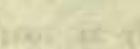 & & \\
\hline 50 & $\begin{array}{l}5.55 \mathrm{~L} \\
5.55 \mathrm{~L}\end{array}$ & SSE & th & Benter & & & July 23 , & 1904 & \\
\hline 2300 & 5.57 & SSE & $t^{2}$ & As it & $v_{i}$ & & & & phis \\
\hline 10 & $\begin{array}{l}5.60 \\
5.62\end{array}$ & $\begin{array}{l}\text { SSE } \\
\text { SSE }\end{array}$ & 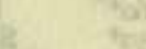 & $10=2$ & 0 10 & 5.70 & ESE & 18 & A. V. \\
\hline 2400 & $\begin{array}{l}5.62 \\
5.63\end{array}$ & $\begin{array}{l}\text { SSE } \\
\text { SW }\end{array}$ & 610 & A. V. & $\begin{array}{l}20 \\
30\end{array}$ & 5.68 & ESE & 38 & op \\
\hline 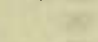 & & & & $5 \%$ & $\begin{array}{l}30 \\
40\end{array}$ & $\begin{array}{l}5.08 \\
5.70\end{array}$ & ESE & es & 68 \\
\hline 3 & 2 & July 22 , & 1904 & 60 & 50 & 5.72 & ESE & $\frac{10}{2}$ & 13: \\
\hline 100 & 5.82 & Calm & & A. V. & 200 & $\begin{array}{l}5.13 \\
5.84\end{array}$ & Calm & z & 38 \\
\hline 200 & 6.02 & SW & & A. $\mathrm{V}$ & 300 & 6.12 & $\mathrm{ESE}$ & & A. V. \\
\hline 300 & 6.27 & SW & & C. E. R. & 400 & 6.40 & Calm & 707 & C. E. R. \\
\hline 52 & 6.42 & SW & & & 500 & 6.58 & E & & \\
\hline 400 & & WSW & 627.5 & 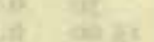 & 22 & 6.68 & $E$ & & tes? \\
\hline 10 & 6.48 & W & 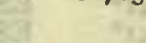 & 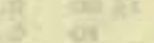 & 30 & 6.65 & $\bar{E}$ & & $\omega s^{2}$ \\
\hline 20 & 6.49 & $\mathrm{~W}$ & 35 & sin & 40 & 6.68 & $\mathrm{E}$ & 2 & es \\
\hline 30 & 6.49 & W & 40 & 20 & 650 & & $\bar{E}$ & at & an 1 \\
\hline 40 & 6.52 & Calm & 2 & 28 & 600 & 6. & F & iff & 20. \\
\hline 50 & 6.5 & $\underset{w}{W}$ & +2 & 12 & Io & 6.6 & E & thes & 아 \\
\hline 500 & 6.55 & W & 4 & 5674 & 700 & 6. & $\mathrm{E}$ & 67 & be: \\
\hline Io & $\begin{array}{l}6.60 \\
6=8\end{array}$ & W & th & 605 & 10 & 6.72 & E & the & 28 \\
\hline 20 & 6.58 & W & th & 160 & 20 & $6.75 \mathrm{H}$ & E & 24 & 28,0 \\
\hline 30 & $6.62 \mathrm{H}$ & W & 24 & $-\infty x^{3}$ & 30 & 6.76 & E & 10 & $8 x$ \\
\hline $\begin{array}{l}40 \\
50\end{array}$ & $\begin{array}{l}6.60 \\
6.58\end{array}$ & W & 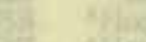 & 6072 & $\begin{array}{l}40 \\
50\end{array}$ & 6.6 & $\frac{E}{E}$ & 2. & anser \\
\hline $\begin{array}{l}50 \\
600\end{array}$ & $\begin{array}{l}0.58 \\
6.59\end{array}$ & W & tile & 68 & 850 & $\begin{array}{l}6.70 \\
6.67\end{array}$ & $\underset{\mathrm{Calm}}{\mathrm{E}}$ & 726 & (9) $: 1$ \\
\hline 10 & 6.57 & W & 7 & 12 & Io & 6.6 & Calm & & 4 \\
\hline 20 & 6.5 & W & $x^{2}$ & 4 & 900 & 6.5 & W & 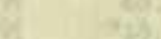 & C. E. R. \\
\hline 700 & 6.5 & W & t. & 3 & 1000 & 6.5 & $\mathrm{~S}$ & & F. L. \\
\hline 55 & 6.35 & $\mathrm{~W}$ & & Fe. & 1100 & 6.4 & E & nie & 102 \\
\hline 800 & & W & 641.7 & & 40 & 6.4 & $\mathrm{E}$ & $x$ & 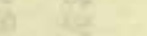 \\
\hline $\begin{array}{r}900 \\
1000\end{array}$ & $\begin{array}{l}0.27 \\
6.25\end{array}$ & W & $t$ & C. E.R. & $\begin{array}{l}46 \\
50\end{array}$ & 6.3 & E & $7 t$ & 1क्ष \\
\hline $\begin{array}{l}1000 \\
1100\end{array}$ & $\begin{array}{l}0.25 \\
6.24\end{array}$ & SW & & F. L. & $\begin{array}{l}50 \\
1200\end{array}$ & & $\underset{\mathrm{E}}{\mathrm{E}}$ & & wir \\
\hline Io & 6.22 & SW & & & 10 & $\begin{array}{l}0.34 \\
6.34\end{array}$ & $\mathbf{E}$ & 744 & res \\
\hline 20 & $6.20 \mathrm{~L}$ & SSW & & 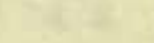 & 20 & $6.33 \mathrm{~L}$ & 焉 & thes & \\
\hline 30 & $6.20 \mathrm{I}$ & SSW & & & 3 & 6. & E & 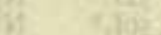 & se \\
\hline 40 & 6.22 & SSW & & $\cos \times$ & & & E & hos & \\
\hline 50 & 6.22 & $\mathbf{S}$ & & 18 & & & SW & 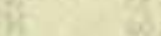 & \\
\hline 1200 & 6.2 & $\mathbf{s}$ & 658 & tat & 13 & & SW & 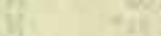 & \\
\hline 10 & & $\mathbf{S}$ & & $a$ & I. & 6. & $S$ & H. & \\
\hline 2 & & $\mathrm{NE}$ & & at & $\infty$ & 6. & Calm & & \\
\hline 1300 & & $\mathbf{S}$ & & $0 \%$ & 1600 & & $\mathrm{~S}$ & 761 & \\
\hline 1400 & & $\mathbf{S}$ & & xart & 1700 & & $\mathbf{S}$ & & \\
\hline 1500 & 6. & S & & iat & 30 & 6.8 & SW & 28 & \\
\hline 1600 & 6. & S & 668 & a & 40 & & & 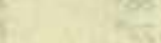 & \\
\hline 50 & 6.8 & $\mathbf{S}$ & & a & 50 & & & wa & \\
\hline 1700 & 6.7 & S & & 6 & 1800 & & SW & & \\
\hline I0 & 6.75 & $\mathbf{S}$ & & ar & I0 & $6.84 \mathrm{H}$ & Si & 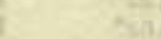 & 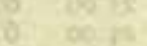 \\
\hline 20 & & $\mathbf{S}$ & & we & 20 & & SW & & \\
\hline 25 & $\begin{array}{l}6.80 \mathrm{H} \\
6.75 \mathrm{H}\end{array}$ & $\stackrel{S}{S}$ & 8 & dit & $\begin{array}{l}30 \\
40\end{array}$ & $\begin{array}{l}6.86 \mathrm{H} \\
6.82\end{array}$ & $\begin{array}{l}\text { SW } \\
\text { SW }\end{array}$ & & \\
\hline $\begin{array}{l}30 \\
40\end{array}$ & $\begin{array}{l}0.75 \mathrm{H} \\
6.8 \mathrm{H}\end{array}$ & $\stackrel{S}{S}$ & & 28 & $\begin{array}{l}40 \\
50\end{array}$ & $\begin{array}{l}0.8 \\
6.8\end{array}$ & SW & tift & \\
\hline 50 & 6.72 & $\mathbf{S}$ & & & $19 \infty$ & 6.80 & SW & & \\
\hline 1800 & 6.70 & $\mathrm{SE}$ & & F. I. & 10 & $6.8 \mathrm{I}$ & SW & & F. I. \\
\hline
\end{tabular}


Tabulation of tidal observations at Cape Flora, Northbrook Island

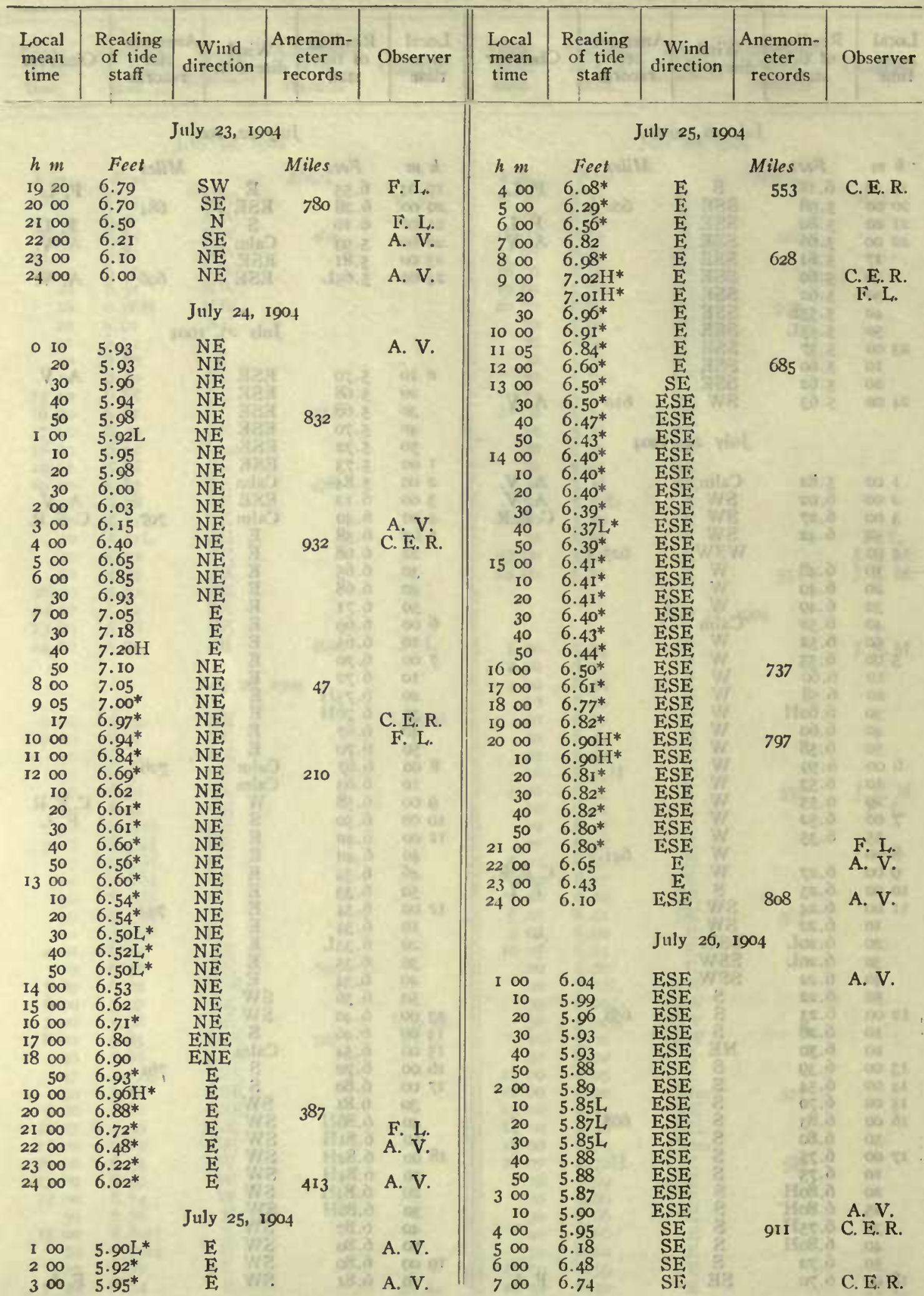


Tabulation of tidal observations at Cape Flora, Northbrook Island

\begin{tabular}{|c|c|c|c|c|c|c|c|c|c|c|}
\hline \multicolumn{2}{|c|}{$\begin{array}{l}\text { Local } \\
\text { meant } \\
\text { time }\end{array}$} & $\begin{array}{c}\text { Rcading } \\
\text { of tidc } \\
\text { staff } \\
\end{array}$ & $\begin{array}{l}\text { Wind } \\
\text { direction }\end{array}$ & \multirow[t]{2}{*}{$\begin{array}{l}\text { Anemom- } \\
\text { eter } \\
\text { records }\end{array}$} & \multirow[t]{2}{*}{ Observer } & $\begin{array}{l}\text { Local } \\
\text { time } \\
\text { mean }\end{array}$ & $\begin{array}{l}\text { Reading } \\
\text { of tide } \\
\text { staff }\end{array}$ & $\begin{array}{l}\text { Wind } \\
\text { direction }\end{array}$ & $\begin{array}{c}\text { Anemom- } \\
\text { eter } \\
\text { records }\end{array}$ & \multirow[t]{2}{*}{ Observer } \\
\hline \multicolumn{4}{|c|}{ July 26, 1904} & & & \multicolumn{4}{|c|}{ July 27, I904 } & \\
\hline$h$ & $m$ & \multicolumn{2}{|c|}{ Feet } & Miles & $\sqrt{2}+\frac{1}{2}$ & $h m$ & Feet & 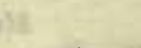 & Miles & 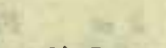 \\
\hline & $\infty$ & 7.00 & SE & 956 & C. E. R. & 940 & 7.12 & $\mathrm{NE}$ & & F. L. \\
\hline & $\infty$ & 7.12 & SE & 12 & C. E. R. & 50 & 7.12 & $\mathrm{NE}$ & & $-2=$ \\
\hline & 30 & $7.16^{*}$ & $\mathrm{E}$ & W. & F. L. & -1000 & 7.14 & $\mathrm{NE}$ & IS & art 0 \\
\hline & 35 & $7.20 \mathrm{H}$ & E & w. & $\sqrt{2}+x^{2}+2$ & 10 & 7.16 & NE & 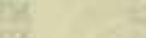 & in 8 \\
\hline & 40 & $7.20 \mathrm{H}$ & $\mathrm{E}$ & 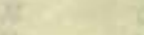 & Catige & 14 & $7.20 \mathrm{H}$ & $\mathrm{NE}$ & Ix & 045 학 \\
\hline & 50 & 7.15 & $\mathbf{E}$ & 4 & $\cos 64$ & 20 & $7.14 \mathrm{H}$ & NE & Ha & $(49=1)$ \\
\hline & $\infty$ & 7.15 & E & 11 & 06412 & 30 & $7.19 \mathrm{H}$ & $\mathrm{NE}$ & 35 & an: \\
\hline & 10 & 7.12 & $\mathbf{E}$ & E. & 64 & 40 & $7.20 \mathrm{H}$ & $\mathrm{NE}$ & 4 & 67 \\
\hline II $C$ & $\infty$ & 7.00 & ESE & $\mathrm{N}$ & nex & 50 & 7.10 & $\mathrm{NE}$ & in & 6 \\
\hline $12 c$ & $\infty$ & 6.80 & ESE & 27 & 6014 & II 00 & 7.10 & NE & II & kit TI \\
\hline I3 I & 14 & 6.60 & ESE & & & 1200 & 6.94 & $\mathrm{NE}$ & 212 & 445 \\
\hline $14 \mathrm{C}$ & $\infty$ & 6.46 & ESE & 1 & & 1300 & 6.70 & $\mathrm{NE}$ & arte & as \\
\hline & 10 & 6.44 & ESE & & & 1400 & 6.50 & $\mathrm{NE}$ & V & 68. \\
\hline & 20 & 6.40 & ESE & fi & 607 & 1500 & 6.31 & $\mathrm{NE}$ & 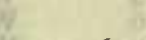 & $\cos x$ \\
\hline & 30 & 6.40 & ESE & 4 & whis & 1600 & $6.25 \mathrm{~L}$ & $\mathrm{NE}$ & 306 & as igit \\
\hline & 40 & 6.38 & ESE & 4 & osis. & 10 & $6.27 \mathrm{~L}$ & $\mathrm{NE}$ & 300 & 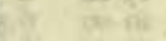 \\
\hline & 50 & 6.35 & ESE & W & $\cos 2$ & 20 & $6.25 \mathrm{~L}$ & $\mathrm{NE}$ & B & 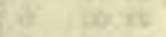 \\
\hline 150 & $\infty$ & 6.34 & ESE & Af & tors & 30 & $6.25 \mathrm{~L}$ & NE & 4 & retes \\
\hline & I0 & 6.34 & ESE & 167 & tist & 40 & $6.25 \mathrm{~L}$ & $\mathrm{NE}$ & +1 & we $=5$ \\
\hline & 20 & 6.33 & ESE & ex & 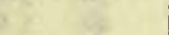 & 50 & 6.30 & $\mathrm{NE}$ & 14 & $43-$ \\
\hline & 30 & $6.30 \mathrm{~L}$ & ESE & 18 & 75 & 1700 & 6.30 & $\mathrm{NE}$ & We & $\cos 20$ \\
\hline & 40 & $6.29 \mathrm{~L}$ & ESE & 18 & $\cos 20$ & 1800 & $6.4 \mathrm{I}$ & E & V & or \\
\hline & 50 & $6.32 \mathrm{~L}$ & ESE & 13 & $x_{0}$ & 1900 & 6.54 & ENE & MI & 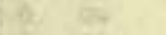 \\
\hline 160 & $\infty$ & $6.30 \mathrm{~L}$ & ESE & 72 & 12 & 2000 & 6.74 & $\mathrm{NE}$ & 366 & 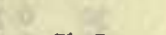 \\
\hline & 10 & $6.30 \mathrm{~L}$ & ESE & Fer & acese & 2100 & 6.82 & $\mathrm{NE}$ & 41 & F. L. \\
\hline & 20 & 6.32 & ESE & tit & wisir & 40 & $6.87 \mathrm{H}$ & Nli & H & A. V. \\
\hline 170 & $\infty$ & $6.3^{8}$ & SE & 2 & at is & 50 & $6.85 \mathrm{H}$ & $\mathrm{NE}$ & K & 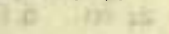 \\
\hline 180 & $\infty$ & 6.50 & SE & 4 & ath in & 2200 & $6.84 \mathrm{H}$ & $\mathrm{NE}$ & & \\
\hline 190 & $\infty$ & 6.64 & ESE & $\sqrt{60}$ & 6he & Io & $6.86 \mathrm{H}$ & $\mathrm{NE}$ & & \\
\hline 200 & $\infty$ & 6.76 & Calin & I 10 & atrines & 23 & 6.80 & $\mathrm{NE}$ & & \\
\hline 210 & 00 & $6.80 \mathrm{H}$ & Calm & 21 & $101 x^{2}=$ & 30 & 6.81 & $\mathrm{NE}$ & $5 \%$ & $80 \% 1$ \\
\hline & 10 & $6.8 \mathrm{IH}$ & Calm & 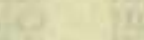 & 21 & 40 & $6.8 \mathrm{I}$ & $N E$ & ix & 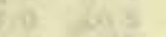 \\
\hline & 20 & $6.8 \mathrm{rH}$ & Calm & $(10)$ & inter & 50 & 6.79 & $N E_{i}$ & $r$ & $\cos 11$ \\
\hline & 30 & $6.79 \mathrm{H}$ & Calm & (n) & 14 & 2300 & 6.74 & SW & 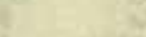 & \\
\hline & 40 & $6.80 \mathrm{H}$ & Calm & 40 & at & 2400 & $6.5 \mathrm{I}$ & SW & 400 & A. V. \\
\hline & 50 & 6.77 & Calm & 60 & $\Gamma \mathrm{T}$ & & & & $x^{2}$ & it \\
\hline 220 & $\infty 0$ & 6.70 & Calm & 20 & F. I. & & & July 28 & I904 & 4 \\
\hline 230 & $\infty$ & 6.50 & Calm & & A. V. & & & & & -2 \\
\hline 240 & $\infty$ & 6.24 & Calm & I16 & A. V. & I 00 & 6.12 & SW & & A. V. \\
\hline & 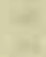 & & July 27, & 1904 & in & $\begin{array}{l}200 \\
3 \infty\end{array}$ & $\begin{array}{l}6.02 \\
5.92\end{array}$ & SW & & A. V. \\
\hline & $\infty$ & 6.03 & Calm & 12 & A. V. & & $\begin{array}{l}5.80 \\
5.75\end{array}$ & $\begin{array}{l}\text { W } \\
W\end{array}$ & 422 & C. E. R. \\
\hline & $\infty$ & 5.83 & Caln & & & 30 & $\begin{array}{l}5.75 \\
5.74\end{array}$ & $w$ & $y$ & 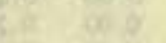 \\
\hline & 30 & 5.79 & Calin & & ate & 40 & $5.73 \mathrm{~L}^{*}$ & $w$ & & Do 801 \\
\hline & 40 & 5.79 & Calm & 3. & 3 & 50 & 5.74 & W & & 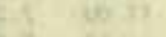 \\
\hline & 50 & 5.77 & Calm & 18 & 98 & 505 & 5.76 & W & $p$ & ar \\
\hline 30 & $\infty$ & 5.80 & Calm & & $n$ & 20 & 5.80 & W & 6 & 145 \\
\hline & 10 & 5.79 & SE & (2) & C4: & 30 & 5.85 & W & te & ats \\
\hline & 20 & $5.74 \mathrm{~L}$ & SE & 37 & Fin 87 & 600 & 6.02 & NW & te & (17) \\
\hline & 30 & $5.76 \mathrm{~L}$ & SE & IVI & weta & 700 & 6.35 & NW & & . \\
\hline & 40 & $5.76 \mathrm{~L}$ & Calm & IS & 12 & 800 & 6.65 & $\mathrm{~N}$ & 453 & C. E. R. \\
\hline & 50 & $5.73 \mathrm{~L}$ & Calm & & A. $V_{\dot{D}}$ & 900 & 6.96 & $\mathrm{~N}$ & & F. I. \\
\hline 40 & $\infty$ & 5.78 & Calm & 124 & C. E. R. & Io 00 & 7.12 & W & & - \\
\hline & 20 & $\begin{array}{l}5.82 \\
5.88\end{array}$ & $\underset{W}{\text { Calm }}$ & & & Io & 7.13 & $W$ & & 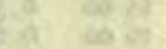 \\
\hline 50 & bo & $\begin{array}{l}5.00 \\
5.95\end{array}$ & $W$ & (1i) & & $\begin{array}{l}20 \\
30\end{array}$ & $\begin{array}{l}\text { 7.17 } \mathrm{H}^{*} \\
7.17 \mathrm{H}^{*}\end{array}$ & $\begin{array}{l}\mathrm{W} \\
\mathrm{W}\end{array}$ & & $\infty$ \\
\hline 60 & $\infty$ & 6.28 & W & & & 40 & 7. $8 \mathrm{H}^{*}$ & W & & 06.108 \\
\hline 70 & $\infty$ & 6.60 & W & - 1 th & 2379 & 50 & $7.18 \mathrm{H}$ & W & 15 & सम \\
\hline 80 & 05 & 6.88 & $\mathrm{NE}$ & 150 & & II $\infty$ & $7.16 \mathrm{H}$ & W & 7 & 16 \\
\hline 90 & $\infty$ & 7.02 & $N$ & 18 & C. E. R. & I0 & $7.17 \mathrm{H}$ & W & $\frac{5}{4}$ & 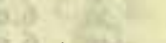 \\
\hline & 35 & 7.10 & NE & $n t$ & F. Ir. & 20 & $7.18 \mathrm{H}$ & W & th & F. I. \\
\hline
\end{tabular}


Tabulation of tidal observations at Cape Filora, Northbrook Island

\begin{tabular}{c|c|c|c|}
\hline $\begin{array}{c}\text { Local } \\
\text { mean } \\
\text { time }\end{array}$ & $\begin{array}{c}\text { Reading } \\
\text { of tide } \\
\text { staff }\end{array}$ & $\begin{array}{c}\text { Wind } \\
\text { direction }\end{array}$ & $\begin{array}{c}\text { Anemom- } \\
\text { eter } \\
\text { records }\end{array}$
\end{tabular} \mid Observer

\begin{tabular}{|c|c|c|c|c|}
$\begin{array}{c}\text { Local } \\
\text { time } \\
\text { mean }\end{array}$ & $\begin{array}{c}\text { Reading } \\
\text { of tide } \\
\text { staff }\end{array}$ & $\begin{array}{c}\text { Wind } \\
\text { direction }\end{array}$ & $\begin{array}{c}\text { Anemom- } \\
\text { eter } \\
\text { records }\end{array}$ & Observer \\
Jnly 29, 190.4
\end{tabular}

\begin{tabular}{|c|c|c|c|c|c|c|c|c|c|c|c|c|c|}
\hline$h m$ & Feet & & & Tiles & 1065 & $=18$ & $h m$ & Feet & & & Miles & 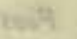 & at $\lambda$ \\
\hline II 30 & 7.12 & W & git & & wt: & F. L. & 1750 & $6.04 \mathrm{~L}$ & W & & & esest & F. I \\
\hline 1202 & 7.08 & $W$ & 35 & 473 & 73.5 & & 1800 & 6.05 & W & & & & \\
\hline 300 & $6.8 \mathrm{I}$ & $N W$ & 18 & & H. & of 91 & 1900 & 6.15 & W & & & Whe & Bh. \\
\hline 4 & 6.52 & NW & $3 x$ & & Br & of & $20 \infty$ & 6.35 & W & & 703 & & \\
\hline 500 & 6.30 & NW & 345 & & ton & I! & 2100 & 6.56 & W & & & & F. L. \\
\hline 600 & 6.15 & NW & 92 & 506 & by & & 2200 & 6.63 & W & & & 24 & A. V. \\
\hline Io & 6.20 & NW & in & & 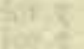 & $x^{3}$ & 2300 & $6.80 \mathrm{H}$ & W & & & & of \\
\hline $\begin{array}{l}40 \\
50\end{array}$ & 6.10L & W & 118 & & iff.? & ez & $\begin{array}{l}40 \\
50\end{array}$ & $\begin{array}{l}.74 \\
6.73\end{array}$ & $w$ & & & 2 & $\infty n$ \\
\hline 1700 & $6.10 \mathrm{~L}$ & W & 355 & & are & we is & 2400 & 6.71 & W & & $7 \mathrm{I} 3$ & $=$ & A. V. \\
\hline Io & 6. IoL, & W & 34 & & 30. & 2001 & & & & & & & \\
\hline 20 & $6.10 \mathrm{~L}$ & $W$ & 98 & & at & 99. & $x^{2}=$ & & July 30 , & 1904 & & & $\omega$ \\
\hline 1800 & $\begin{array}{l}0.13 \\
6.16\end{array}$ & $\underset{w}{W}$ & $y$ & 210 & the & 60 & & & W & 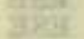 & & & A V \\
\hline 90 & 6.3 & W & Bas & & deate & $\infty \sin ^{2}$ & 200 & $\begin{array}{l}6.49 \\
6.12\end{array}$ & $w$ & & & & A. V. \\
\hline 2000 & 6.50 & NW & 35 & 545 & Iste & & 300 & 5.94 & W & & & & A. V. \\
\hline 2100 & $\begin{array}{l}6.70 \\
6.8 \mathrm{oH}\end{array}$ & $\begin{array}{l}\text { NW } \\
\text { NW }\end{array}$ & 98 & & 8 & F. $\mathrm{L}$. & 400 & 5.80 & NW & & 720 & & C. E. $\dot{R}$. \\
\hline 40 & $6.80 \mathrm{H}$ & NW & fin & & 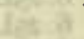 & & 500 & 5.70 & NW & 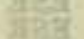 & & & $m$ \\
\hline & $6.8 \mathrm{IH}$ & NW & ath & & 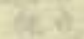 & (a) & $\begin{array}{l}30 \\
40\end{array}$ & $\begin{array}{l}5.00 \\
5.66 \mathrm{I}\end{array}$ & NW & & & & \\
\hline 2300 & 6.79 & NW & $3 n$ & (a) & es & wer & 50 & $\begin{array}{l}5.69 \\
5.69\end{array}$ & NW & & & & \\
\hline 10 & 6.78 & NW & $x$ & & Het & 67 & 600 & 5.75 & NW & & & & \\
\hline 20 & 6.74 & NW & 123 & & 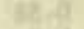 & too: & I0 & 5.80 & NW & 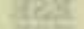 & & & \\
\hline 30 & 6.75 & NW & 18 & & 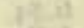 & 6010 & 700 & 5.92 & NW & & & & \\
\hline & $6.7 \mathrm{I}$ & NW & as & & 105 & $60 \mathrm{at}$ & 800 & 6.2 & NW & & 743 & 306 & \\
\hline 50 & 6.69 & NW & 764 & & vat. & & 900 & 6.5 & NW & & & ie & C.E \\
\hline 2400 & 6.64 & NW & ans & 590 & 18 & A. V. & 1004 & & W & & & & $\begin{array}{c}\text { C. E. R. } \\
\text { F. L }\end{array}$ \\
\hline 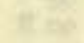 & $2=8$ & & 72 & & & & II 00 & 6.96 & W & & & & \\
\hline 96 & 36 & July 29 , & 1904 & & a d & ati & $\begin{array}{r}50 \\
1200\end{array}$ & $7.00 \mathrm{H}$ & Calm & 40 & & & wo \\
\hline 100 & 6.37 & NNW & 3 & & 4 & A. V & Io & $7.00 \mathrm{H}$ & Calm & & 132 & & 60 \\
\hline 200 & 6.15 & NW & 3 & & 15 & & 15 & $7.04 \mathrm{H}$ & Calm & & & & \\
\hline 300 & 5.93 & NW & 48 & & 920 & A. $V_{\dot{D}}$ & 20 & $7.00 \mathrm{H}$ & Calm & & & & \\
\hline 400 & 5.82 & $N$ & $y=$ & 627 & 1 & C. E. R. & 30 & 6.99 & Calm & & & 15 & \\
\hline 500 & $5.78 \mathrm{~L}$ & $\mathrm{~N}$ & 178 & & 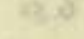 & & 40 & & Caln & & & & \\
\hline & 5.7 & $\mathrm{~N}$ & & & & & 50 & & Calm & 1020 & & t5 & \\
\hline & $5.77 \mathrm{I}$ & $\mathrm{N}$ & Xity & & & & 1300 & & Calm & & & os & \\
\hline & $5.78 \mathrm{I}$, & $\mathrm{N}$ & & & & & 1400 & 6.65 & $\mathrm{E}$ & aile? & & ce & \\
\hline 40 & $5.8 \mathrm{r}$ & $\mathrm{N}$ & whe & & ind & & 1500 & 6.34 & $\mathrm{NW}$ & intes & & Late & \\
\hline & 5.8 & $\mathrm{~N}$ & 123 & & & & 1600 & 6. 10 & NW & & 764 & & \\
\hline $6 \circ$ & & $\mathrm{N}$ & & & & & 1704 & $5.9 \mathrm{I}$ & W & & & & 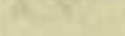 \\
\hline & 6.2 & $\mathrm{~N}$ & & & & & 45 & 5.85 & NW & & & & \\
\hline $8 c$ & 6.4 & $\mathrm{~N}$ & $y$ & 662 & & C. E. R. & & 5.89 & NW & 800 & & 127. & \\
\hline 9 & 6.7 & $\mathrm{NE}$ & $w$ & & is & F. L. & 1800 & $5.84 \mathrm{~L}$ & $\mathrm{~N}$ & & & & \\
\hline 100 & & SE & w & & $f=$ & & IO & & $\mathrm{N}$ & & & & \\
\hline II & & SE & Wr & & & & 2 & & & & & & \\
\hline & & $\mathrm{S}$ & w & & & & 3 & & & & & & \\
\hline & & S & w & & s. & & & & & & & & \\
\hline & & $\mathrm{S}$ & F & & & & & & & & & & \\
\hline & & $\mathbf{S}$ & wist & & & & $19 \mathrm{C}$ & & & & & & \\
\hline & & $\mathrm{S}$ & wive & & & & 20 & & & & 786 & & \\
\hline 1200 & 7.1 & $\mathrm{~S}$ & st & 677 & 36 & & $21 \mathrm{C}$ & & $\mathrm{N}$ & & & & \\
\hline $13 \mathrm{IS}$ & 6.8 & $\mathrm{C}$ & 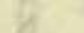 & & & & 2200 & & & & & & \\
\hline 1400 & & S & in & & tes & & 2300 & & N & & & & \\
\hline 1500 & & $\overline{\mathrm{S}}$ & 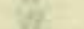 & & & . & 2400 & $6.52 \mathrm{H}$ & $\mathbf{N}$ & & 806 & & . \\
\hline 1600 & 6. & SW & $w$ & 701 & c & 3 & th & & & Vi & & s & ת. \\
\hline & & SW & $y$ & & & - & - & & July & & & & \\
\hline 1700 & & sw & & & & & & & & & & & \\
\hline IO & & W & $y$ & & 3 & c & O I5 & $6.53 \mathrm{H}$ & $\mathrm{N}$ & & & & A. V. \\
\hline 2 & & $y$ & WI & & & as & 20 & $6.52 \mathrm{H}$ & & & & & \\
\hline 31 & & 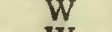 & 17 & & & & 30 & $6.5 \mathrm{I}$ & NW & 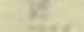 & & $\omega 0$ & \\
\hline 40 & $6.04 \mathrm{I}_{6}$ & W & $W$ & & & F. L & 40 & 6.50 & NW & Av & & 30 & \\
\hline
\end{tabular}


Tabulation of tidal observations at Cape Flora, Northbrook Island

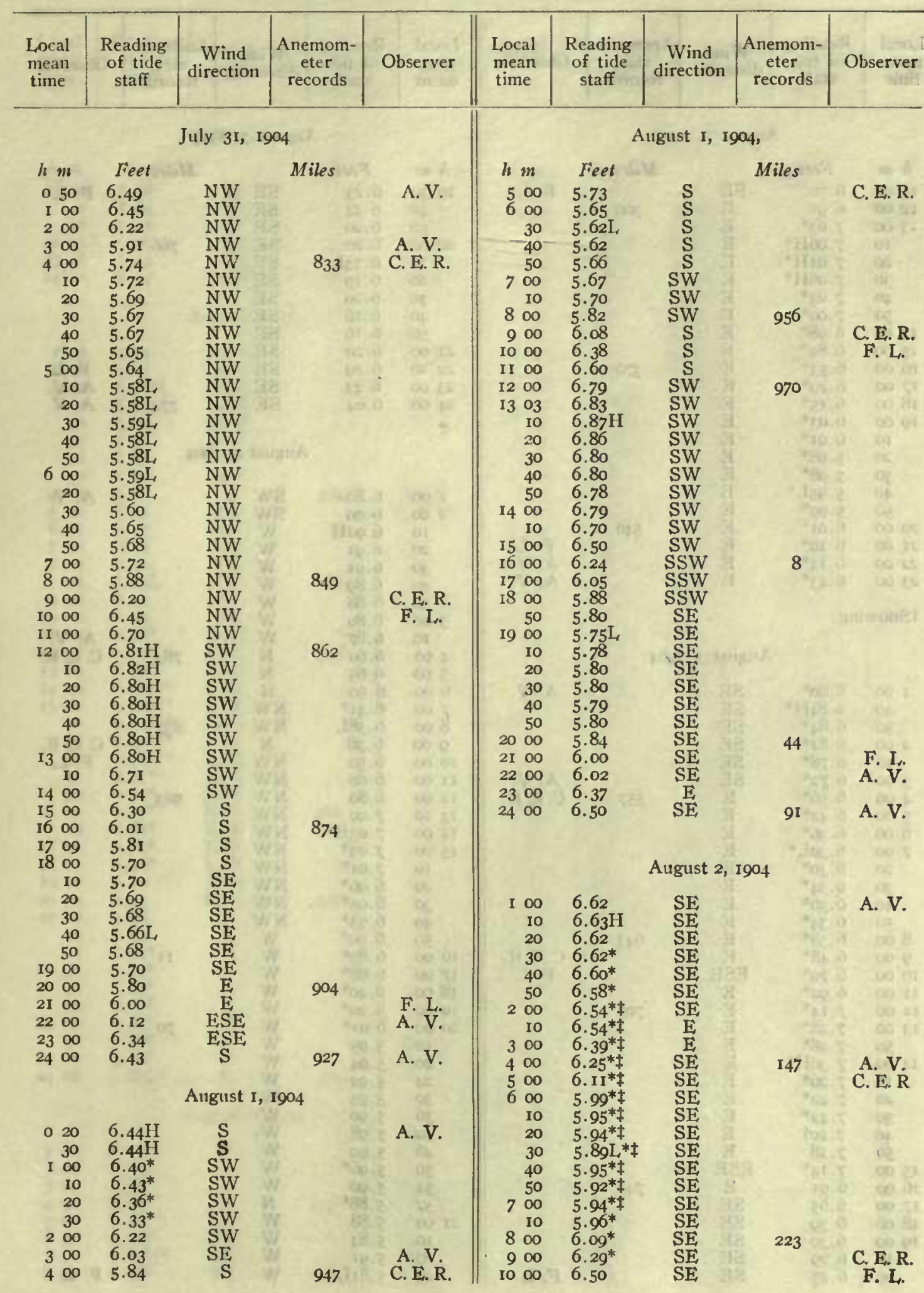


Tabulation of tidal abservations at Cape Flora, Northbroak Island

\begin{tabular}{c|c|c|c|c||}
\hline Local & $\begin{array}{c}\text { Reading } \\
\text { of tide } \\
\text { staff }\end{array}$ & $\begin{array}{c}\text { Wind } \\
\text { direction }\end{array}$ & $\begin{array}{c}\text { Anemon- } \\
\text { eter } \\
\text { records }\end{array}$ & Observer \\
&
\end{tabular}

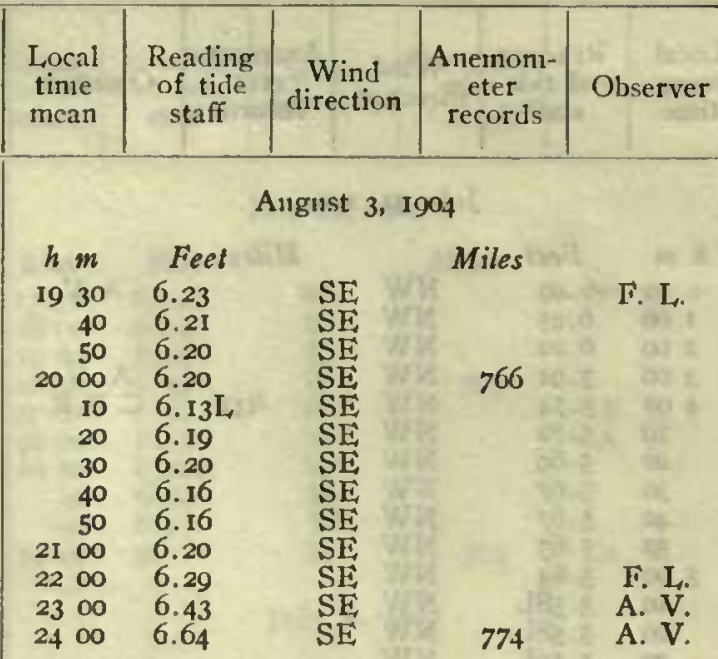

18 . $6.29^{*}$

$19006.03^{*}$

10 6.01*

$20 \quad 5.98^{*}$

$305.98 *$

40 5.95L*

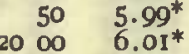

$21006.10^{*}$

$22006.14^{*}$

Angust 2, 1904

$23006.43^{*}$

SE
E
E
E
$E$
$E$
$E$
$E$
$E$
$E$
$E$
$E$
$E$
$E$
$E$
$E$
$E$
$E$
$E$
$E$
$E$
$E$
$E$

Miles

I

370

\$Snowing.

August 3, 1904

$\begin{array}{rll}\text { I } 00 & 6.80^{*} \\ 40 & 6.84^{*} \mathrm{I}^{*} \\ 50 & 6.84^{*} \\ 200 & 6.82^{*} \\ 10 & 6.78^{*} \\ 100 & 6.72^{*} \\ 300 & 6.56^{*} \\ 400 & 6.42^{*} \\ 500 & 6.36^{*} \\ 600 & 6.30 \\ 700 & 6.30 L^{*} \\ 20 & 6.30^{*} \\ 30 & 6.34^{*} \\ 40 & 6.36^{*} \\ 50 & 6.34^{*} \\ 800 & 6.38^{*} \\ 900 & 6.48^{*} \\ 1000 & 6.70^{*} \\ 1100 & 6.92^{*} \\ 1200 & 7.14^{*} \\ 1300 & 7.28 * \\ 50 & 7.28^{*} \\ 1400 & 7.30 H^{*} \\ 10 & 7.26^{*} \\ 20 & 7.22^{*} \\ 30 & 7.42^{*} \\ 40 & 7.20 \dagger \\ 50 & 7.18 \\ 1500 & 7.14^{*} \\ 1600 & 6.91 \\ 1700 & 6.65 \\ 1806 & 6.39 \\ 1900 & 6.30 \\ 10 & 6.29 \\ .20 & 6.25 \\ & & \\ 12 & \end{array}$

SE

A. V.

C. $\mathbf{E} . \dot{R}$.

F. L.

A. $\mathrm{V}$

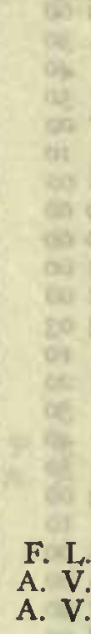

4

August 4, 1904

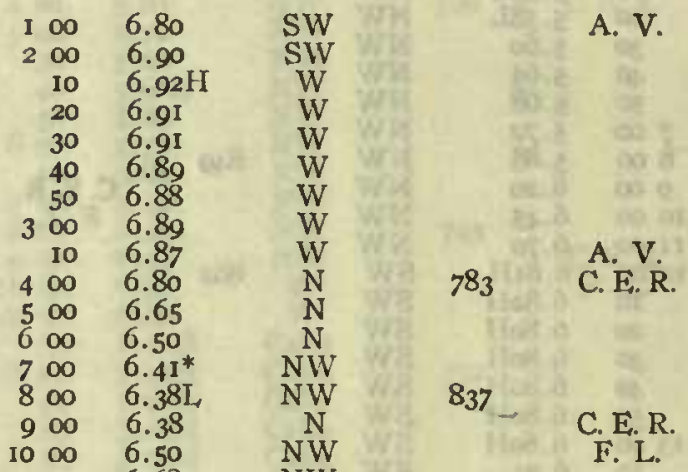

923

II

C. F. R.

II 006.68

NW

F. L.

643

13 10 7.00

NW

A.

$400-7.03 \mathrm{H}^{*} \quad \mathrm{NW}$

$15007.03^{*}$ NW

I0 $7.01^{*} \quad \mathrm{NW}$

$206.96^{*} \quad \mathrm{NW}$

30 6. $95^{*} \quad \mathrm{NW}$

$40 \quad 6.93^{*}$

16 00 $6.85^{*}$

$17006.68^{*}$

1800

707

1900
2000

$6.40^{*}$

I0 5.95

$20 \quad 5.94$

$24 \quad 5.92$

$30 \quad 5.95$

$\begin{array}{ll}36 & 5.90\end{array}$

$40 \quad 5.98$

$46 \quad 5.90$

50 5.96*

$54 \quad 5.90$

749

$2100 \quad 5.88$

I0 5.84

T. I.

$24 \quad 5.81 \mathrm{I}$

F. L. 
Tabulation of tidal observations at Cape Flora, Northbroak Island

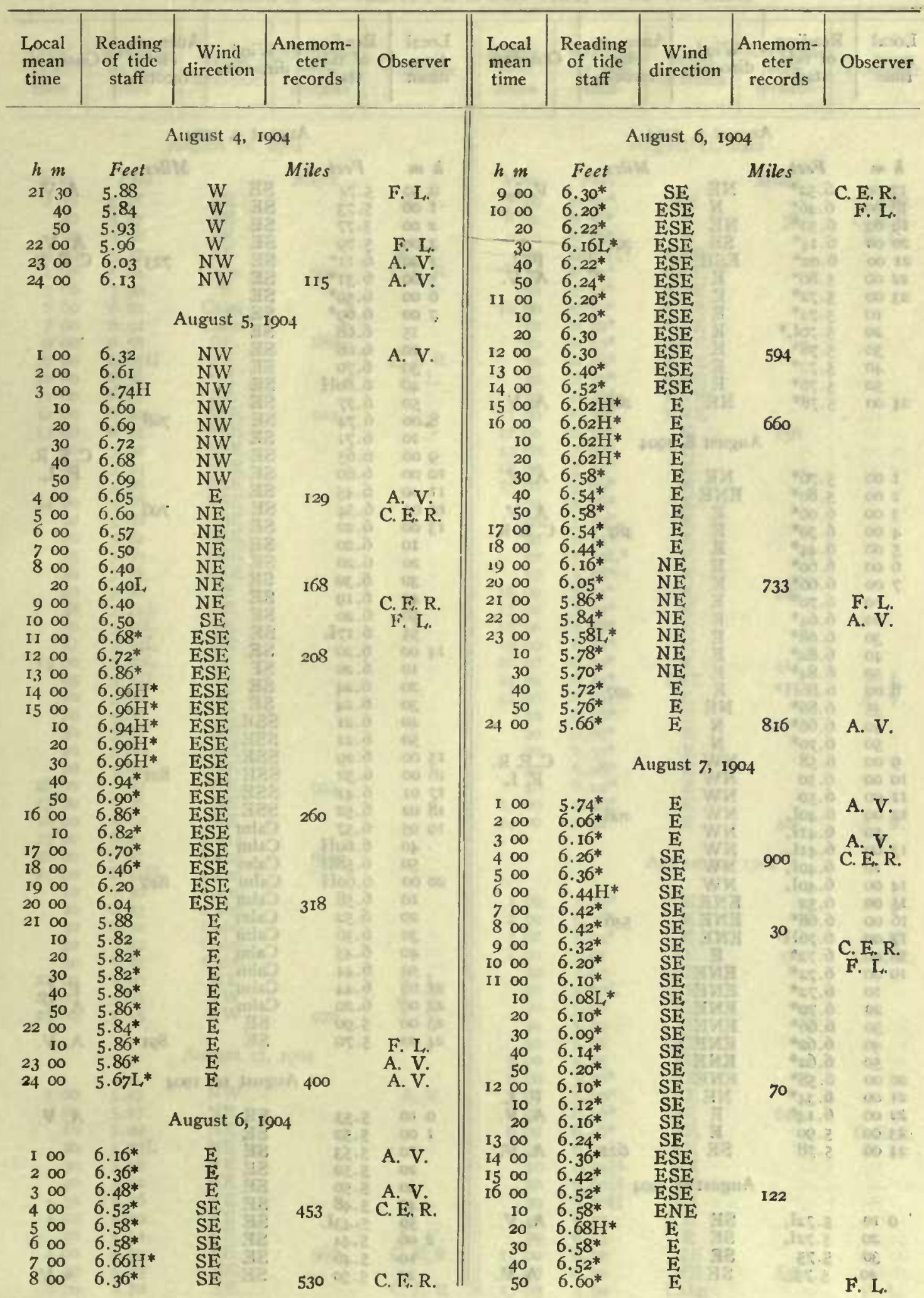


Tabulation of tidal observations at Cape Flora, Northbrook Island

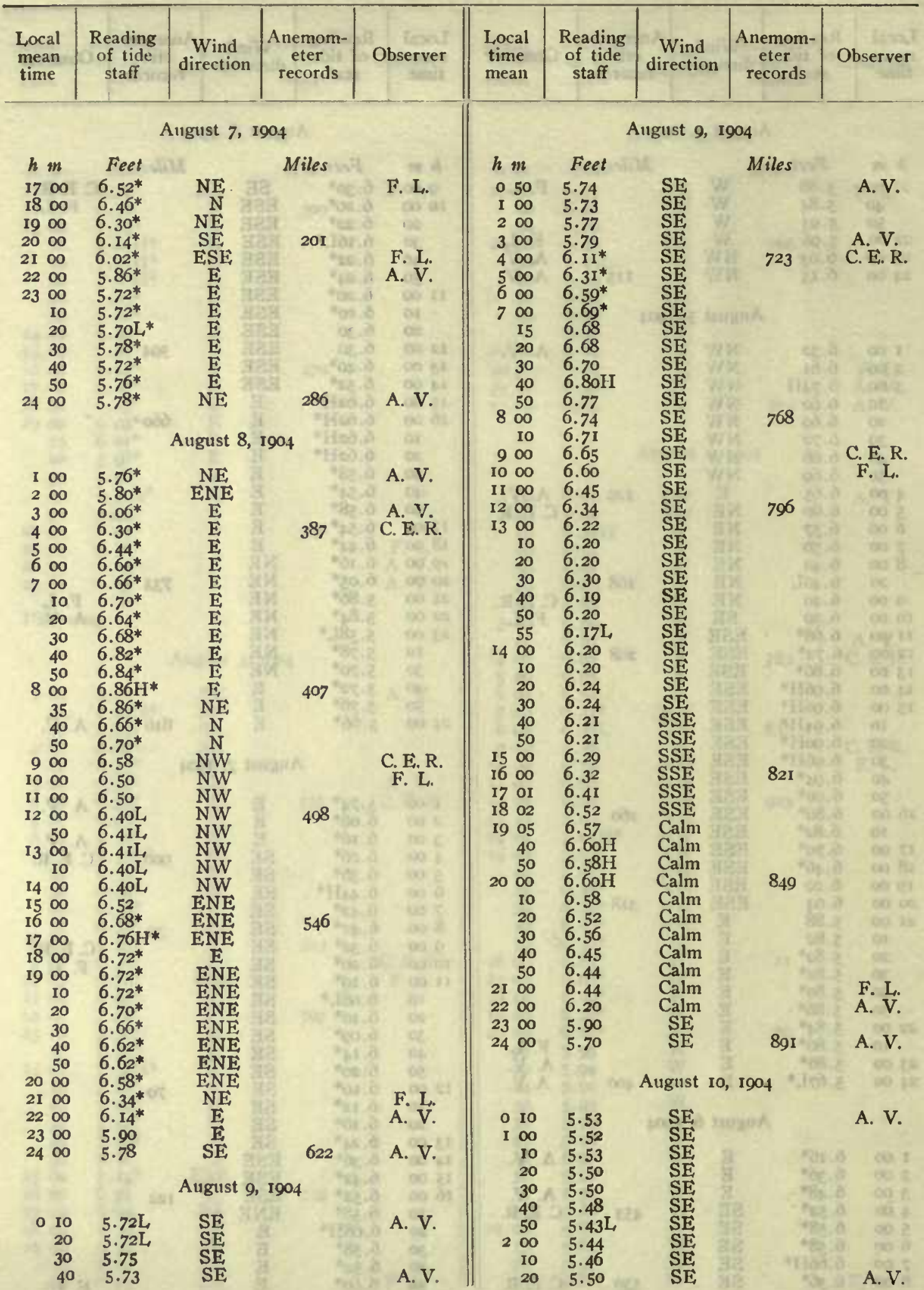


Tabulation of tidol observations at Cape Flora, Northbrook Island

\begin{tabular}{|c|c|c|c|c|c|c|c|c|c|}
\hline $\begin{array}{l}\text { Local } \\
\text { mean } \\
\text { time }\end{array}$ & $\begin{array}{l}\text { Reading } \\
\text { of tide } \\
\text { staff }\end{array}$ & $\begin{array}{l}\text { Wind } \\
\text { direction }\end{array}$ & $\begin{array}{l}\text { Anemom- } \\
\text { eter } \\
\text { records }\end{array}$ & Observer & $\begin{array}{l}\text { Local } \\
\text { time } \\
\text { mean }\end{array}$ & $\begin{array}{l}\text { Reading } \\
\text { of tide } \\
\text { staff }\end{array}$ & $\begin{array}{l}\text { Wind } \\
\text { direction }\end{array}$ & $\begin{array}{c}\text { Anemom- } \\
\text { eter } \\
\text { records }\end{array}$ & Observer \\
\hline \multicolumn{5}{|c|}{ August 10, 1904} & \multicolumn{5}{|c|}{ August II, I904 } \\
\hline$h m$ & \multicolumn{2}{|l|}{ Fect } & \multicolumn{2}{|l|}{ Miles } & $h m$ & \multicolumn{2}{|l|}{ Feet } & \multicolumn{2}{|l|}{ Miles } \\
\hline 230 & $5 \cdot 48$ & SE & & A. V. & 700 & $6.04^{*}$ & SE: & & C. E. R. \\
\hline $\begin{array}{l}40 \\
50\end{array}$ & 5.47 & $\begin{array}{l}\mathrm{SE} \\
\mathrm{SE}\end{array}$ & se 3 & $x=$ & $\begin{array}{r}800 \\
-900\end{array}$ & $\begin{array}{l}6.36^{*} \\
6.56^{*}\end{array}$ & $\mathrm{E}$ & 58 & C. E. R. \\
\hline 300 & 5.47 & $\mathrm{SE}$ & Thes & A. V. & 30 & $6.62 \mathrm{H}^{*}$ & ENE & $24=$ & F. L. \\
\hline 400 & 5.64 & Calm & 866 & C. E. R. & 40 & $6.62^{*}$ & ENE & $\operatorname{lng} 3$ & is \\
\hline 500 & 5.92 & $\mathrm{E}$ & & & 50 & $6.54^{*}$ & ENE & des & \&e \\
\hline 600 & 6.22 & Calm & & & I0 00 & $6.52^{*}$ & ENE & $14: 2$ & 24 \\
\hline $\begin{array}{l}700 \\
800\end{array}$ & 6.42 & Calm & 888 & & Io & $6.50^{*}$ & ENE & ettis & E \\
\hline $\begin{array}{l}800 \\
900\end{array}$ & $\begin{array}{l}6.58 \\
6.65 \mathrm{H}\end{array}$ & $\mathrm{SE}$ & 888 & C. E. R. & 20 & $6.50^{*}$ & ENE & 3:3 & $m a$ \\
\hline 1000 & 6.56 & $\mathrm{SE}$ & 23 & F. L. & 40 & $6.52^{*}$ & ENE & ins & is \\
\hline II 00 & $6.4 \mathrm{I}$ & $\underset{S C E}{S E}$ & & Dak & 50 & $6.46^{*}$ & ENE & 287 & $\infty 0$ in \\
\hline 1200 & 6.20 & SSE & 924 & 2012 & II oo & $6.36^{*}$ & ENE & a. & $60 \%$ \\
\hline 1306 & 6.01 & SSEE & & 100 & 1200 & 6. $12^{*}$ & $\mathrm{E}$ & 156 & 100 os \\
\hline 30 & 5.92 & SSE: & 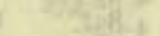 & $\omega^{2}$ & 1300 & $5.92 *$ & $\mathrm{E}$ & $-(x-1)$ & (no is \\
\hline 40 & 5.94 & SSE & 6 & $=$ & 1400 & $5.74^{*}$ & $\mathrm{E}$ & $\operatorname{sen} x$ & mess \\
\hline 50 & 5.86 & SSE & valis & $\omega$ & 1500 & $5.60^{*}$ & $\vec{E}$ & 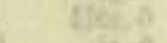 & 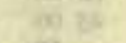 \\
\hline 1400 & 5.88 & SSE & (3) & 9 & I0 & $5.56^{*}$ & $\vec{E}$ & inge & or \\
\hline Io & 5.88 & SSE & 83 & se. & 20 & $5 \cdot 52^{*}$ & $\vec{E}$ & 364 & 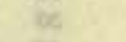 \\
\hline 20 & 5.82 & SSE & 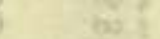 & es 8 & 30 & $5 \cdot 54^{*}$ & $\mathrm{E}$ & $n^{2}=0$ & 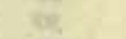 \\
\hline 25 & 5.80 & SSEE & $F_{1}=$ & $60 \mathrm{x}$ & 40 & $5 \cdot 5 \mathrm{LL}^{*}$ & $\mathrm{E}$ & estin & as \\
\hline 30 & 5.79 & SSE & $=5$ & os $\mathrm{k}$ & $5^{0}$ & $5 \cdot 58^{*}$ & $\mathrm{E}$ & & 08 is \\
\hline 40 & 5.80 & SE & $2 ?$ & $\log _{0}$ & 1600 & $5.54^{*}$ & $\mathrm{E}$ & 286 & \\
\hline $\begin{array}{l}48 \\
50\end{array}$ & $5.74 \mathrm{~L}$ & $\overline{\mathrm{SE}}$ & 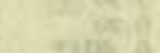 & 600 & 1700 & $5.72^{*}$ & E & & \\
\hline $\begin{array}{l}50 \\
57\end{array}$ & $\begin{array}{l}5.79 \mathrm{~L} \\
5.74 \mathrm{~L}\end{array}$ & SE & 7uls.9. & 6118 & $\begin{array}{l}1800 \\
1900\end{array}$ & $\begin{array}{l}5.88^{*} \\
6.12^{*}\end{array}$ & $\frac{\mathrm{E}}{\mathrm{E}}$ & & \\
\hline 1500 & $5.79 \mathrm{~L}$ & $S E$ & 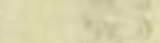 & 50 & 2000 & $6.30^{*}$ & 宸 & 414 & 91 \\
\hline 05 & $5.74 \mathrm{~L}$ & SE & $x_{x}=8$ & at & 2100 & $6.42^{*}$ & ESE & 标 & F. L. \\
\hline Io & 5.80 & $\underset{S E}{S E}$ & wis & 100.61 & 2200 & $6.46 \mathrm{H}^{*}$ & $\mathrm{E}$ & (5). & A. $\vec{V}$. \\
\hline $\begin{array}{l}20 \\
30\end{array}$ & $\begin{array}{l}5.81 \\
5.83\end{array}$ & NE & $\cos t$ & $\cos 79$ & $\begin{array}{l}10 \\
20\end{array}$ & $\begin{array}{l}6.38^{*} \\
6.20^{*}\end{array}$ & $\frac{E}{E}$ & tatifin & WE \\
\hline $\begin{array}{l}30 \\
40\end{array}$ & $\begin{array}{l}5.83 \\
5.80\end{array}$ & $\mathrm{NE}$ & 30 & sing & 30 & $\begin{array}{l}\text { b.20* } \\
6.30^{*}\end{array}$ & $\mathrm{E}$ & $3 x+1$ & wis \\
\hline 50 & 5.85 & $\mathrm{NE}$ & 60 & $\infty 8$ & 40 & $6.28 *$ & $\mathrm{E}$ & then & $\cos 5$ \\
\hline $16 \%$ & 5.89 & $\mathrm{NE}$ & 955 & gir & 50 & $6.24^{*}$ & $\vec{E}$ & in & 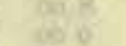 \\
\hline 1700 & 5.96 & $N E$ & 151 & Ex & 2300 & $6.22^{*}$ & E & $e^{2}$ & 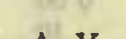 \\
\hline $18 \%$ & 6.10 & $\mathrm{NE}$ & E. & bets & 2400 & $5.92^{*}$ & $\mathrm{E}$ & 505 & A. V. \\
\hline 1900 & 6.22 & Calm & $x^{3}$ & atas & & & & & \\
\hline 2000 & 6.27 & Calm & 959 & Des & wh & W. & August I2, & 1904 & $\frac{2}{3}$ \\
\hline 2100 & $6.32 \mathrm{H}$ & Calm & & 60074 & 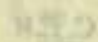 & & 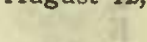 & 2004 & a \\
\hline 10 & 6.30 & Calm & is $-k$ & $\theta$ & & & $\mathrm{E}$ & $y=$ & A. $\mathrm{V}$. \\
\hline 20 & 6.23 & Calm & 65 & os & 200 & $\begin{array}{l}5.02 * \\
5.36^{*}\end{array}$ & $\mathrm{E}$ & 35: & A. V. \\
\hline 30 & 6.30 & Calm & Whe & Fis, 00 & 300 & $\begin{array}{l}5.304^{*} \\
5.04\end{array}$ & $\frac{\mathrm{E}}{\mathrm{E}}$ & vithes & A. $\mathrm{V}$. \\
\hline 40 & 6.22 & Calm & $6 x ?$ & 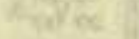 & 400 & $\begin{array}{l}5.04 \\
5.01\end{array}$ & $S_{S E}$ & $57 x$ & C. $\mathrm{E} \cdot \dot{\mathrm{R}}$ \\
\hline 50 & 6.20 & Calm & 60 & & 10 & $\begin{array}{l}5.01 \\
5.03\end{array}$ & $S E$ & 571 & C.E. R. \\
\hline 2200 & 6.20 & NE & $v^{2}$ & F. L. & 20 & $5.00 \mathrm{~L}$ & $\mathrm{SE}$ & & me \\
\hline 2300 & 5.83 & NW & & A. V. & 30 & $\begin{array}{l}5.001 \\
5.08\end{array}$ & $\mathrm{SE}$ & the & 9 \\
\hline 2400 & $5 \cdot 55$ & $N W$ & 970 & A. V. & 40 & 5.10 & SE & 468 & cos $x$ \\
\hline 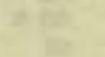 & $=$ & August II, & 1904 & & 500 & $\begin{array}{l}5.16 \\
5.25\end{array}$ & $\begin{array}{l}\mathrm{SE} \\
\mathrm{SE}\end{array}$ & 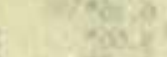 & bog \\
\hline 100 & 52 & NW & & $A \mathrm{~V}$ & & & SE & 483 & 87 \\
\hline 200 & 5.0 & NF & & A. $\mathrm{v}$. & 800 & $\begin{array}{l}5.90^{*} \\
6.16^{*}\end{array}$ & $\mathrm{SE}$ & 614 & \\
\hline I0 & 5.04 & $\mathrm{NI}$ & & (4) & 900 & $6.46^{*}$ & $\mathrm{SE}$ & $240=2$ & C. E. R. \\
\hline 20 & $5.03 \mathrm{~L}$ & & A & 601 & 1000 & 6.6 & $\mathrm{SE}$ & 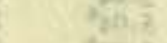 & F. L. \\
\hline 30 & 5.0 & & & & 10 & 6.5 & SE & $\tan 28$ & a \\
\hline $4^{\circ}$ & 5.12 & & 28 & & 20 & $6.60 \mathrm{H}$ & $\mathrm{SE}$ & thes & (3) \\
\hline 50 & 5.16 & & & 60 & 30 & $6.60 \mathrm{H}$ & $\mathrm{SE}$ & whit & ee \\
\hline 300 & 5. 10 & $\mathrm{NE}$ & & & 40 & 6.55 & $\mathbf{E}$ & 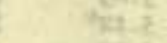 & 60 the \\
\hline IO & 5.14 & NE & & A. $\mathrm{V}$. & 50 & 6.53 & $\overrightarrow{\mathbf{E}}$ & 62 & $00 \mathrm{col}$ \\
\hline 400 & 5.20 & SE & 980 & C. E. R. & & 6.50 & NE & .003 & 6008 \\
\hline & & SE & & & 1200 & 6.3 & & & \\
\hline 00 & 5.70 & SE & 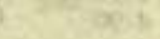 & C. E. R. & 1303 & 5.90 & Calm & $82=$ & F. $\mathrm{L}_{\text {. }}$ \\
\hline
\end{tabular}


Tabulation of tidal observations at Cape Flora, Northbrook Island

\begin{tabular}{|c|c|c|c|c|c|c|c|c|c|}
\hline $\begin{array}{l}\text { Local } \\
\text { mean } \\
\text { time }\end{array}$ & \multirow{2}{*}{$\begin{array}{c}\text { Reading } \\
\text { of tide } \\
\text { staff }\end{array}$} & \multirow{2}{*}{$\begin{array}{c}\begin{array}{c}\text { Wind } \\
\text { direction }\end{array} \\
\text { ugust } 12,1\end{array}$} & \multirow[t]{2}{*}{$\begin{array}{l}\text { Anemom- } \\
\text { eter } \\
\text { rccords }\end{array}$} & \multirow[t]{2}{*}{ Observer } & $\begin{array}{l}\text { Local } \\
\text { time } \\
\text { mean }\end{array}$ & $\begin{array}{l}\text { Reading } \\
\text { of tide } \\
\text { staff }\end{array}$ & \multirow{2}{*}{ ugust 13,19} & $\begin{array}{c}\text { Anemom- } \\
\text { eter } \\
\text { records }\end{array}$ & \multirow[t]{2}{*}{ Observer } \\
\hline & & & & & & & & 904 & \\
\hline$h m$ & Feet & & Miles & $=8$ & $h \mathrm{~m}$ & Fect & & Miles & $m i$ \\
\hline 1400 & 5.70 & $\mathrm{~N}$ & 30.3 & If. L. & 2300 & 5.99 & Calm, & $x^{2}+x_{0}$ & A. V. \\
\hline 1500 & $5 \cdot 40$ & $\mathrm{SE}$ & & (2) & 30 & 6.09 & SW & 782 & \\
\hline 1600 & $5 \cdot 31$ & SE & 667 & $\cos \theta$ & & $6.12 \mathrm{H}$ & SW & 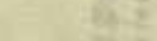 & 8 \\
\hline 10 & 5.33 & SE & $x^{-6}=0$ & retat & 50 & 6.11 & SW & है & \\
\hline 20 & $5.30 \mathrm{~L}$ & SE & $+\infty, 0,0$ & ife & 2400 & 6.08 & SW & 28 & A. V. \\
\hline 30 & 5.37 & $\mathrm{SE}$ & we, & 70 & & & & & \\
\hline 40 & 5.31 & SE & $3 \times 2.6$ & ong 04 & & & Angust I4, & $1=x$ & 008 \\
\hline 50 & 5.39 & SE & $\cos 21$ & at & & & & $4:$ & $\cos 5$ \\
\hline 1700 & 5.41 & SE & hes: & 205 & 0 I0 & 6.05 & Calm & 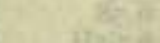 & A. V. \\
\hline ro & $5 \cdot 34$ & SE: & 7040 & 9 & 20 & 6.03 & Calnı & Hoiten & \\
\hline 20 & 5.41 & SE: & $\frac{5}{3} \times 20$ & 7it: & 100 & 5.81 & SW & 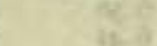 & $\cos ^{2}$ \\
\hline 1800 & $5 \cdot 52$ & SE & ates & 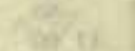 & 200 & 5.43 & SW & & \\
\hline 1900 & $5.76^{*}$ & $\mathrm{SE}$ & 700 & nes & 300 & 5.22 & SF & 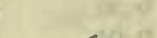 & A. V. \\
\hline 2000 & 6.08 & $\mathbf{N}$ & 709 & F. I. & 400 & 4.95 & W & 56 & C. E. R. \\
\hline $\begin{array}{l}2100 \\
2200\end{array}$ & $\begin{array}{l}6.25 \\
6.30\end{array}$ & $\begin{array}{l}\mathbf{N} \\
\mathrm{E}\end{array}$ & 4,5 & A. $\vec{V}$. & 500 & $4.85 \mathrm{~L}$ & Calm & & \\
\hline $\begin{array}{l}2200 \\
2300\end{array}$ & $\begin{array}{l}0.30 \\
6.40 \mathrm{H}\end{array}$ & NW & 400. & 3. & 10 & $4.88 \mathrm{I}$ & Calm & Q83 & 6 \\
\hline 2310 & 6.34 & Calm & Fotht? & $m$ & $\begin{array}{l}20 \\
30\end{array}$ & $\begin{array}{l}4.85 \mathrm{~L} \\
4.87^{2}\end{array}$ & Calm & 18 ह & 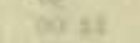 \\
\hline 20 & 6.34 & $\mathrm{SE}$ & +283 & 60 & $\begin{array}{l}30 \\
40\end{array}$ & $\begin{array}{l}4.87 \\
4.90\end{array}$ & $\begin{array}{l}\text { Calm } \\
\text { Calm }\end{array}$ & 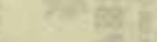 & (⿻) \\
\hline 30 & 6.30 & $\mathrm{SE}$ & tyz- तो & 2 & $6 \stackrel{40}{05}$ & $\begin{array}{l}4.00 \\
5.00\end{array}$ & Calm & te & cs \\
\hline 40 & 6.29 & SE & -102 & & 700 & 5.18 & E & 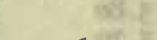 & 35 \\
\hline 2400 & 6.25 & Calm & 778 & A. V. & 800 & 5.52 & SE & 62 & $\operatorname{lig}_{\pi} x$ \\
\hline 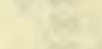 & & Alueust I2 & $45 x+3$ & onst & $\begin{array}{r}900 \\
1000\end{array}$ & $\begin{array}{l}5.90 \\
6.20\end{array}$ & $\begin{array}{l}\mathrm{SE} \\
\mathrm{SE}\end{array}$ & $\tan 2$ & C. E. R. \\
\hline $20=$ & $6 x^{4}$ & Aligust 13, & 38 & 00 aी & 11 IO & $6.38 \mathrm{H}^{*}$ & SE & 40. & F. L. \\
\hline 100 & 5.83 & $\mathbf{E}$ & $\operatorname{cis} 2$ & A. V. & 20 & $6.38^{*}$ & $\mathrm{SE}$ & ins 2 & 9 \\
\hline 200 & 5.55 & ESE & 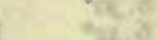 & & 1200 & $6.3^{6 *}$ & $S F$ & 139 & cos $2 x$ \\
\hline 300 & $5.24^{*}$ & $E$ & $\cos x=$ & A. V. & 10 & $6.30^{*}$ & $\mathrm{SF}$ & or & 20 \\
\hline 400 & $5.15^{*}$ & $\mathrm{E}$ & 800 & C. E. R. & 1300 & 6. $16^{*}$ & SE & 10. & 0 \\
\hline 500 & $5.14 \mathrm{I}^{*}$ & E & $4 x^{2}=3$ & axc & 1400 & $5.70^{*}$ & SE & t8:8 &. \\
\hline 600 & $5 \cdot 37^{*}$ & E & $407 .+2$ & ex & 1500 & $5 \cdot 3 \mathrm{I}$ & SE, & & \\
\hline 700 & $5.64^{*}$ & E & 00 & $x^{2}$ & 16 os & 5.0 .4 & SE & 196 & te? \\
\hline 800 & 5.98 & $\mathrm{E}$ & 883 & 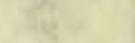 & 1700 & 4.80 & $\underset{S E}{S E}$ & 1725 & 20 br \\
\hline 900 & 6.28 & E & 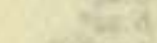 & oives & 10 & $4 \cdot 75$ & $\begin{array}{l}S E \\
\text { SE }\end{array}$ & tog & $\mathrm{crr} t \mathrm{tr}$ \\
\hline 10 & 6.34 & $\mathrm{E}$ & Whe & 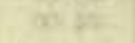 & 20 & 4.74 & $\begin{array}{l}\mathrm{SE} \\
\mathrm{SE}\end{array}$ & 418 & co itr \\
\hline 20 & 6.36 & $\mathbf{E}$ & & & 30 & 4.73 & $\begin{array}{l}\mathrm{SE} \\
\mathrm{SE}\end{array}$ & $x \leq 6$ & $26 \times 1$ \\
\hline 30 & 6.40 & $\vec{E}$ & & & 40 & 4.70 & $\begin{array}{l}\text { SE } \\
\text { SE }\end{array}$ & 100.9 & 00.05 \\
\hline 40 & 6.43 & $\underset{\mathbf{E}}{\mathbf{E}}$ & & C. F.R. & $\begin{array}{r}50 \\
800\end{array}$ & $4.68 \mathrm{~L}$ & SE & 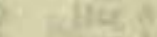 & on $\pi$ \\
\hline 50 & 6.50 & $\underset{\mathrm{E}}{\mathrm{E}}$ & 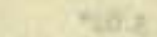 & F. L. & $\begin{array}{r}1800 \\
10\end{array}$ & $\begin{array}{l}4.70 \\
4.71\end{array}$ & $\mathrm{SE}$ & ices & At \\
\hline 100 & $6.53^{*}$ & $\underset{S S E}{E}$ & ther & 10. & $\begin{array}{l}10 \\
20\end{array}$ & $\begin{array}{l}4.71 \\
4.79\end{array}$ & $\mathrm{SE}$ & 25.8 & $=$ \\
\hline 1100 & $6.58^{*}$ & $\begin{array}{l}\text { SSE } \\
\text { SSE }\end{array}$ & Whis? & का ? & 1900 & $\begin{array}{l}4.79 \\
4.82\end{array}$ & SE & 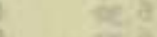 & nc. \\
\hline $\begin{array}{r}10 \\
20\end{array}$ & $\begin{array}{l}6.60 \mathrm{H}^{*} \\
6.58^{*}\end{array}$ & $\begin{array}{l}\text { SSE } \\
\text { SSE }\end{array}$ & to: 3 & on $\mathrm{x}$ & 2000 & 5.08 & SE & 2,31 & n. \\
\hline 30 & $6.53^{*}$ & SSE & 29.2 & in & 2100 & 5.40 & SF & $-1=$ & \\
\hline 40 & $6.52^{*}$ & SSE & $\operatorname{lin} 2 t$ & 105 & 2200 & 5.70 & $\mathrm{SE}$ & $x_{12}=0$ & F. L. \\
\hline 50 & $6.50^{*}$ & SSE & $16=3$ & ex & 2300 & 5.99 & Calm & & A. V. \\
\hline 1200 & $6.48^{*}$ & SSE & 930 & es & 2400 & 6.06 & NW & 251 & A. V. \\
\hline 1300 & $6.16^{*}$ & S & 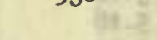 & 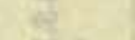 & (2) & & & & \\
\hline 1410 & $5.66^{*}$ & SW & $120 x^{3}$ & tac $\mathrm{E}$ & & & Auguist 15 , & 1904 & \\
\hline 1500 & $5 \cdot 3^{8 *}$ & SW & r. & theng & & & & & \\
\hline 1600 & $5.23^{*}$ & SW & 963 & 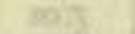 & 0.30 & 6.06 & SE & (2.) & A. V. \\
\hline $17 \infty 0$ & $5.08 *$ & SW & & boy & 40 & $6.07 \mathrm{H}$ & $\mathrm{SE}$ & piz & \\
\hline Io & $5.04^{*}$ & SW & $600 \times 0$ & $.00 \%$ & 50 & 6.0 .3 & SE & 30.8 & 01 \\
\hline 20 & $5.04^{*}$ & SW. & fitontat & insei & 100 & 5.98 & $\mathrm{SE}$ & $3 x-2$ & $\alpha$ \\
\hline 30 & $5.02^{*}$ & SW & 1102,3 & at & 10 & 5.97 & S & this 2 & at \\
\hline 40 & $4.92 \mathrm{~L}$ & SW & 1100,0 & $t=$ & 20 & 5.93 & Calm & this & e4 \\
\hline 50 & $5.09^{*}$ & SW & Hfor. 9 & ext & 200 & 5.74 & Calm & $712^{2}$ & $e$ \\
\hline 1800 & $5.12^{*}$ & SW & 20,3 & $x+1$ & 300 & 5.35 & ESE & 54 & A. V. \\
\hline 1900 & 5.30 & W & 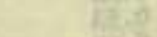 & 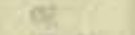 & 410 & 5.05 & Calm & 252 & C. E. R. \\
\hline 2000 & 5.60 & W & (a). & $\Rightarrow 11$ & 500 & 4.92 & Calm & 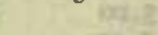 & (3) \\
\hline 2100 & 5.90 & WSW & $9 x^{2}$ & F. L. & 10 & 4.90 & Calm & $24 ?$ & $60^{2} 2$ \\
\hline 2200 & 5.97 & SW & we & A. V. & 20 & 4.90 & Calm & $\operatorname{crc} e$ & C. L. K. \\
\hline
\end{tabular}


Tabulation of tidal observations at Cape Flora, Northbrook Island

\begin{tabular}{|c|c|c|c|c|c|c|c|c|c|}
\hline $\begin{array}{l}\text { Local } \\
\text { mean } \\
\text { time }\end{array}$ & $\begin{array}{l}\text { Reading } \\
\text { of tide } \\
\text { staff }\end{array}$ & $\begin{array}{l}\text { Wind } \\
\text { direction }\end{array}$ & $\begin{array}{l}\text { Anemom- } \\
\text { eter } \\
\text { records }\end{array}$ & Observer & $\begin{array}{l}\text { Local } \\
\text { time } \\
\text { mean }\end{array}$ & $\begin{array}{l}\text { Reading } \\
\text { of tide } \\
\text { staff }\end{array}$ & $\begin{array}{l}\text { Wind } \\
\text { direction }\end{array}$ & $\begin{array}{l}\text { Anemom- } \\
\text { eter } \\
\text { records }\end{array}$ & Observer \\
\hline \multicolumn{5}{|c|}{ Al1gust 15, 1904} & \multicolumn{5}{|c|}{ August 16, I904 } \\
\hline$h m$ & Feet & & Miles & tis 8 & $h m$ & Feet & & Miles & $=4$ \\
\hline 530 & 4.88 & Calm & 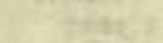 & C. E. R. & 900 & 5.48 & SW & 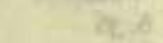 & C. E. R. \\
\hline 40 & 4.87 & Calm & Jing & (. 4.0 & I0 00 & 5.90 & $\mathrm{NE}$ & 68,0 & F. L. \\
\hline 50 & $4.82 \mathrm{I}$ & Calm & $y=$ & is & II 00 & 6.20 & $\mathrm{NE}$ & fese & 60 r \\
\hline 600 & 4.90 & Calm & xis & ins & $12 n 9$ & 6.38 & Calm & 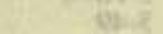 & ses 8 \\
\hline 10 & 4.90 & Calm & 6041 & $\sqrt{65}$ & I3 00 & 6.43 & Calm & 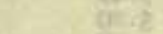 & 607 \\
\hline 20 & 4.92 & E & E. 3 & $\sqrt{602} x$ & 30 & $6.45 \mathrm{H}$ & Calm & Arize & of \\
\hline 30 & 4.92 & $\overrightarrow{\mathbf{E}}$ & $x^{4}+3$ & 65 is & 40 & $6.4 \mathrm{I}$ & Calm & we & $\alpha, \alpha$ \\
\hline 40 & 4.95 & $\mathbf{E}$ & 15.2 & 60035 & 50 & 6.36 & $\mathrm{NE}$ & $(2+4.5$ & by $y$ \\
\hline 50 & 4.97 & E & & & 1400 & 6.33 & $\mathrm{NE}$ & sistis & $\alpha$ \\
\hline 700 & 5.00 & $\vec{E}$ & & & 1500 & 6. 10 & $\mathrm{NE}$ & $c=8$ & as \\
\hline 800 & 5.28 & E & 264 & & I6 II & 5.60 & $\mathrm{NE}$ & 371 & né \\
\hline 900 & 5.60 & Calm & . & C. E. R. & 1700 & $5 \cdot 30$ & $\mathrm{NF}$ & as 2 & At \\
\hline 1000 & 6.00 & SE & $x^{2}=2$ & F. L. & 1800 & 5.10 & $\mathrm{NI}$ & $0=z^{2}$ & 0015 \\
\hline II 00 & 6.25 & SE & 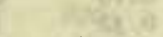 & 60 & 1908 & 4.94 & Calın & me $: 2$ & nis \\
\hline 1200 & 6.40 & $\mathrm{SE}$ & 272 & $28:$ & 10 & $4.90 \mathrm{~L}$ & Calm & 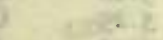 & trecta \\
\hline 10 & 6.40 & SE & so & (a) & 20 & $4.92 \mathrm{~L}$ & $\mathrm{NE}$ & i2: & $\operatorname{los} \pm 5$ \\
\hline 20 & 6.40 & SE, & Hetsin & as & 30 & $4.90 \mathrm{~L}$ & $\mathrm{NE}$ & 20.3 & $\infty: 0$ \\
\hline 30 & $6.4 \mathrm{IH}$ & SE & star & $x^{2}$ & 40 & 4.91 & $\mathrm{NE}$ & $(x), 3$ & 60 il \\
\hline 40 & 6.36 & $\mathrm{SE}$ & 203 & $6 x^{2}=$ & 50 & 4.97 & $\mathrm{NE}$ & $\cos ^{2}$ & \\
\hline 50 & $6.34 \dagger$ & SE & 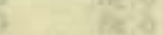 & in: & 2000 & 4.94 & $\mathrm{NE}$ & 377 & \\
\hline 1300 & $6.30^{\dagger}$ & $\mathrm{SE}$ & $\operatorname{cis} 5$ & $60 \%$ & 10 & 5.06 & $\mathrm{NE}$ & & \\
\hline 1400 & $6.04^{\dagger}$ & $\mathrm{SE}$ & ins & $x=$ & $2 \mathrm{I} 00$ & 5.12 & Calm & 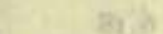 & F. L. \\
\hline 1500 & $5.65 t$ & SE & teon: & an है & 2200 & 5.40 & Calm & otsas & A. V. \\
\hline I6 03 & $5.26 \dagger$ & $\mathrm{SE}$ & 322 & $30 \%$ & 2300 & 5.76 & Calm & yth & \\
\hline 1700 & 5.04 & $\mathrm{SE}$ & ten & $x+5$ & 2400 & 6.02 & $\mathrm{~N}$ & $38 \mathrm{I}$ & A. V. \\
\hline 1800 & 4.85 & SF & Men & ar & -7. & . & $x^{2}+2$ & Hor & 60 \\
\hline 10 & 4.80 & SE & 19) & 5 & $12 x$ & 26xise & August 17 , & 1904 & $\alpha_{71}$ \\
\hline 20 & 4.80 & SE & tits & te & 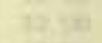 & siret & & $\cos 2$ & 04 \\
\hline 30 & $4.8 I$ & SE & 28.8 & 105 vi & & 6.15 & $\mathrm{NE}$ & xis. & A. $Y$ \\
\hline 40 & 4.77 & SE & 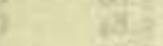 & af & $\begin{array}{l}100 \\
200\end{array}$ & 6.24 & Calm & it. & A. V. \\
\hline 50 & 4.79 & SE & sitte? & $80 x$ & I0 & $6.28 \mathrm{H}$ & $\mathrm{NF}$ & $\cos 0$ & ab \\
\hline 53 & $4.75 \mathrm{~L}$ & SE & ond & 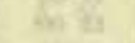 & 20 & 6.25 & $\mathrm{NE}$ & 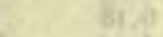 & (2) 5 \\
\hline 1900 & 4.80 & SE & riso & 100.18 & 30 & 6.22 & $\mathrm{NE}$ & 10.9 & $\cos 2$ \\
\hline 10 & 4.80 & $\mathrm{SE}$ & b & $\operatorname{los} x$ & 40 & 6.18 & Calm & 86.2 & 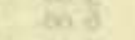 \\
\hline 2000 & 4.95 & Calm & 346 & 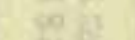 & 50 & 6.11 & Calm & esin & $00 \mathrm{k}$ \\
\hline 2100 & 5.22 & SE & (1) & & 300 & 6.12 & $\mathrm{NE}$. & $3(x), 2$ & A. V. \\
\hline 2200 & 5.49 & SE & Itsere & F. L. & 400 & 5.89 & $S$ & 392 & C. E. R. \\
\hline 2300 & 5.85 & Calm & ant & A. V. & 500 & 5.62 & S & 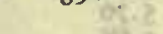 & \\
\hline 2400 & 6.02 & SW & 353 & A. V. & 600 & 5.55 & Calm & 40,2 & $n$ \\
\hline 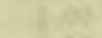 & 2.115 & & pe & ne & 45 & 5.42 & $\mathrm{~S}$ & 55:? & os \\
\hline AEt & (t) ong & August I 6 , & 1904 & 60104 & 50 & $5.40 \mathrm{~L}$ & Calm & 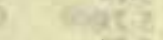 & 8 \\
\hline & & & te & & 700 & $5 \cdot 4^{2}$ & Calm & $0 \geq$ & 69 \\
\hline 100 & 6.10 & Calm & 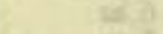 & A. V. & 15 & 5.42 & $\mathrm{Calm}$ & $\mathrm{Ex}: 3$ & es 1 \\
\hline 10 & 6.10 & NW & 60.3 & ans & 20 & 5.46 & Calm & 1872 & $\cos 2$ \\
\hline 20 & 6.08 & NW & as. ${ }^{2}$ & son 111 & 30 & 5.41 & Calm & Filit \& & 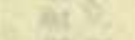 \\
\hline 30 & 6.07 & Calm & 25 & $\infty$ os of & 40 & $5.4 \mathrm{I}$ & Calm & 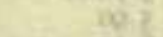 & 100 os \\
\hline 40 & $6.11 H$ & Calm & $\cos$ & $m$ is & 50 & $\begin{array}{l}2.41 \\
5.44\end{array}$ & Calm & ese pot id & 100 is \\
\hline 50 & 6.03 & Calm & byit & 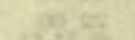 & 800 & $\begin{array}{l}3.44 \\
5.50\end{array}$ & Calm & 397 & $\log 5$ \\
\hline 200 & 6.02 & Calm & - ituent & cast & 900 & 5.60 & S & 398 & C. E. R. \\
\hline I0 & 5.97 & Calm & 58 & & I0 00 & 5.90 & S & 09,0 & F. L \\
\hline 300 & 5.70 & Calm & 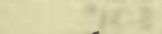 & A. V. & II 00 & 6. 15 & SSE & 68.0 & \\
\hline 400 & $5 \cdot 45$ & Calm & 356 & C. E. R. & 1200 & 6.35 & SSE & 411 & and $-2=$ \\
\hline 500 & $5 \cdot 30$ & SE & $x^{\prime}(x)=$ & का & 1300 & 6.52 & $\mathrm{~S}$ & 411 & as \\
\hline 600 & 5.15 & Calm & 74 & $E$ & 30 & 6.54 & 13 & CQSA & as \\
\hline 45 & 5.10 & Calm & 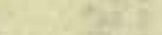 & is & 40 & $6.59 \mathrm{H}$ & S & 1230 & $100 \%$ \\
\hline 700 & 5.10 & Calm & towis. & 0231 & 50 & 6.53 & $\mathrm{~s}$ & os 0 & 60,16 \\
\hline 10 & $4.97 \mathrm{~L}$ & SE & & & 1400 & 6.51 & $\stackrel{\mathfrak{S}}{\mathbf{S}}$ & at; 0 & cossy \\
\hline 20 & 4.98 & Calm & & & 10 & 6.50 & Calm & 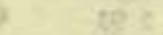 & 108,85 \\
\hline 30 & 5.08 & Calm & & & 20 & 6.50 & Calm & int.2. & mat \\
\hline 40 & 5.12 & Calm & 1xta & $\log 1$ & 30 & 6.45 & Calm. & 94.2 & 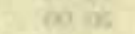 \\
\hline 50 & 5.18 & SE & $+20=$ & $100 x$ & 40 & 6.44 & Calm & 85 & ex \\
\hline 800 & 5.24 & Calm & 360 & C. E. K. & $\begin{array}{l}40 \\
50\end{array}$ & 6.41 & Calm & si: & F. L. \\
\hline
\end{tabular}


Tabulation of tidal obscrvations at Cape Flora, Northbrook Island

\begin{tabular}{|c|c|c|c|c|c|c|c|c|c|}
\hline $\begin{array}{l}\text { Local } \\
\text { mean } \\
\text { time }\end{array}$ & $\begin{array}{l}\text { Reading } \\
\text { of tide } \\
\text { staff }\end{array}$ & $\begin{array}{l}\text { Wind } \\
\text { direction }\end{array}$ & $\begin{array}{l}\text { Anemom- } \\
\text { eter } \\
\text { records }\end{array}$ & Observer & $\begin{array}{l}\text { Local } \\
\text { mean } \\
\text { timee }\end{array}$ & $\begin{array}{c}\text { Reading } \\
\text { of tide } \\
\text { staff }\end{array}$ & $\begin{array}{l}\text { Wind } \\
\text { direction }\end{array}$ & $\begin{array}{l}\text { Anemom- } \\
\text { eter } \\
\text { records }\end{array}$ & Observer \\
\hline \multicolumn{5}{|c|}{ August $\mathrm{I} 7, \mathrm{I} 904$} & \multicolumn{5}{|c|}{ August 18,1904} \\
\hline$h m$ & \multicolumn{2}{|c|}{ Ficet } & Miles & at $x$ & $n n$ & Feet & & Miles & ant $x$ \\
\hline 1500 & 6.36 & $\mathbf{S} W(x$ & & l. L. & 2110 & $5 \cdot 32$ & $\mathrm{SE}$ & 34 & I. L. \\
\hline 1600 & 6.10 & & 415 & & 20 & $5.3 \mathrm{IL}$ & $\mathrm{SE}$ & 30 & 80 \\
\hline I7 00 & 5.75 & $\mathbf{S}$ & $y=8$ & 0211 & 30 & 5 & SE & 2756 & $2 x$ \\
\hline 1800 & 5.49 & $\mathbf{S}$ & 8,8 & 0051 & 40 & $5 \cdot 36$ & SE & $100: 2$ & $\infty 0$ \\
\hline 1900 & 5.29 & & n:e & $\infty \pi$ & 50 & $5 \cdot 39$ & SE: & 000 & \\
\hline 50 & 5.18 & S & 17ove & As & 2200 & $5 \cdot 40$ & $\mathrm{SE}$ & $-40+$ & F. L. \\
\hline 2000 & 5.19 & Calm & 425 & 8 & 2300 & 5.52 & Calm & c5 & A. $\mathrm{V}$. \\
\hline Io & 5.18 & Calm & & e. & 2400 & 5.71 & ESE & 536 & A. V. \\
\hline 20 & $5.17 \mathrm{~L}$ & Calm & 12. & 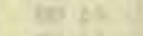 & & & \multirow{3}{*}{\multicolumn{2}{|c|}{ August 19,1904}} & \\
\hline 30 & 5.20 & Calm & Exte & not & 5 & 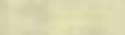 & & & \\
\hline 40 & 5.19 & Calm & Intze & notat & & $y=$ & & & an 8 \\
\hline 50 & 5.20 & Calm & 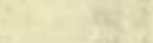 & 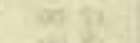 & 100 & 6.01 & Calm & $\infty, 2$ & A. $V$. \\
\hline 2100 & 5.20 & Calm & & 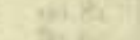 & & 6.19 & Calm & & 2. \\
\hline 10 & 5.20 & Calm & & ate the & 300 & 6.3 & $\mathrm{ES}$ & $=80$ & A. V. \\
\hline 20 & 5.25 & Calm & 60. & & 400 & 6.35 & $\mathrm{NE}$ & 541 & C. E. $\dot{R}$. \\
\hline & 5.35 & SSE & Esis & F. L. & 30 & 6.34 & Calm & 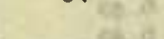 & (5) \\
\hline 2300 & & Cals & $\operatorname{lig} 15$ & A. V. & 40 & $6.36 \mathrm{H}$ & $\mathrm{Calm}$ & & 2 \\
\hline 2400 & 5.87 & SE & 430 & A. V. & 50 & 6.34 & Calm & $\operatorname{lng} 2$ & tas \\
\hline$x^{4}+1$ & sec & August 18 , & 1904 & og $\approx$ & $\begin{aligned} & 5 \infty \\
& 10\end{aligned}$ & $\begin{array}{l}6.30 \\
6.28\end{array}$ & $\begin{array}{l}\text { Calm } \\
\text { Calm }\end{array}$ & ne: & 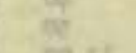 \\
\hline & & w & +3 & $\mathrm{y}$ & 600 & 6.12 & Calm & the & on is \\
\hline $\begin{array}{l}100 \\
200\end{array}$ & 6.18 & Calm & $(0,3)$ & A. V. & 700 & 6.00 & $\mathrm{NE}$ & $(52)=2$ & $\infty 2$ \\
\hline & $\begin{array}{l}0.3 \\
6.3\end{array}$ & $\mathrm{~W}$ & is & 100.85 & 900 & $\begin{array}{l}5.00 \\
5.80\end{array}$ & $\mathrm{Calm}$ & 543 & 605 \\
\hline 40 & 6.33 & Calm & 86 & 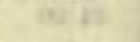 & 20 & 5.82 & Calm & $28 \%$ & obst \\
\hline 50 & $6.36 \mathrm{H}$ & W & & & 30 & $5.78 \mathrm{~L}$ & Calnt & & 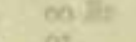 \\
\hline 300 & 6.36 & Calm & & 10 & 40 & & $\mathrm{Cal}$ & 05 & 97 \\
\hline & 6.30 & & & & 5 & 5.8 & Cal & $\sigma^{2}$ ? & $\infty$ \\
\hline 2 & 6.32 & $\mathrm{Cal}$ & & $0 x$ & 1000 & 5.8 & Cal & Tहै। & of \\
\hline $3 c$ & 6.3 & $\mathrm{Cal}$ & & & & 5.8 & Calm & 51 & C. E. R. \\
\hline 40 & 6.2 & Calt & the & A. V. & & 5.5 & $\mathrm{E}$ & $\infty+3$ & F. L. \\
\hline 415 & 6.18 & $\mathrm{SE}$ & 439 & C. E. R. & I2 & 6. & $\vec{E}$ & 551 & \\
\hline 500 & 6.0 & Calm & 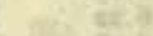 & at. & I3 & 6. & NE & 25 & sor \\
\hline 600 & 5.8 & $\mathrm{SE}$ & Anrte & sos & 14 & 6.3 & NE & in & \\
\hline 700 & 5.7 & S & in? & ie & 1500 & & $\mathrm{E}$ & $89=$ & \\
\hline 40 & 5.6 & & 51,2 & 60 & 10 & & $\mathrm{E}$ & $x^{2}$ & \\
\hline 50 & $5.7 c$ & Calm & & 90 & 20 & 6.4 & E & 92 & \\
\hline 800 & & E & 446 & 6 & 30 & 6.4 & $\bar{E}$ & 28 ? & 96 \\
\hline IO & & & & $\cos$ & 40 & 6. & $E$ & a.d & $\infty 0 \mathrm{in}$ \\
\hline 20 & 5.7 & Calm & 25 & & & 6. & $\mathrm{E}$ & & \\
\hline 30 & & & 16 & 를 & 1600 & 6. & $\overrightarrow{\mathrm{E}}$ & 560 & \\
\hline 40 & & $\mathrm{E}$ & 53 & 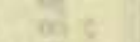 & Io & 6.3 & $\mathrm{SE}$ & & \\
\hline 50 & & Calin & 5 & $x$ & 17 & 6. & $\mathrm{SE}$ & & w 1 \\
\hline 900 & 5.70 & Calm & 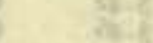 & 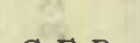 & 18 & 6.0 & SE & as 8 & \\
\hline 10 & 5.78 & $\mathrm{E}$ & & C. E. R. & 1900 & 5.7 & $\mathrm{SE}$ & $\sec 2$ & giv \\
\hline I0 0 & 5. & SE & $m$ & F. L. & 20 & 5 & $\mathrm{SE}$ & 598 & \\
\hline & 6. & SE & 5 & & & & $\mathrm{E}$ & & F. L. \\
\hline & 6.2 & SE & 477 & 20 & 220 & & $\overline{\mathbf{E}}$ & & A. V. \\
\hline 130 & & $\mathrm{NE}$ & 6 & 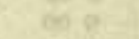 & 30 & 5.3 & $\bar{E}$ & (स) & \\
\hline 1407 & & & $0, ?$ & & & 5.3 & & 6.2 & of \\
\hline 20 & & & 810 & 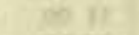 & ? & $5 \cdot 3$ & & (6) & on \\
\hline 30 & & $\mathrm{~N}$ & 721 & 100 & 2300 & 5. & & t2:- & \\
\hline 40 & & & at is & 69 & & 5. & & of. & on \\
\hline : & & $\mathrm{N}$ & 128 & ef & 20 & & & का है & $m$ \\
\hline 150 & & S & $15 x=$ & $a$ & 30 & $5.44^{*}$ & $\mathbf{E}$ & of & \\
\hline 160 & & & 500 & as & 2400 & $5.4^{*}$ & ESE & 662 & A. V. \\
\hline & 6. & & 18 & $\infty 1$ & & & & & \\
\hline $18 \mathrm{C}$ & 5. & Calm & 92 & & $y=$ & te & August 20, & 1904 & 80 \\
\hline 1900 & 5.6 & & & $\epsilon$ & & & & 80 & \\
\hline 2000 & 5.4 & SE & 516 & क & 10 & 5.7 & & ay $2=$ & A. V. \\
\hline & & & & & & & & 8.2 & $y$ \\
\hline 2100 & 5.32 & SE & & 1: L. & 300 & $6.05^{*}$ & $\mathrm{SE}$ & $y=2$ & A. $\mathrm{V}$. \\
\hline
\end{tabular}


Tabulation of tidal observations of Cape Flora, Northbrook Island

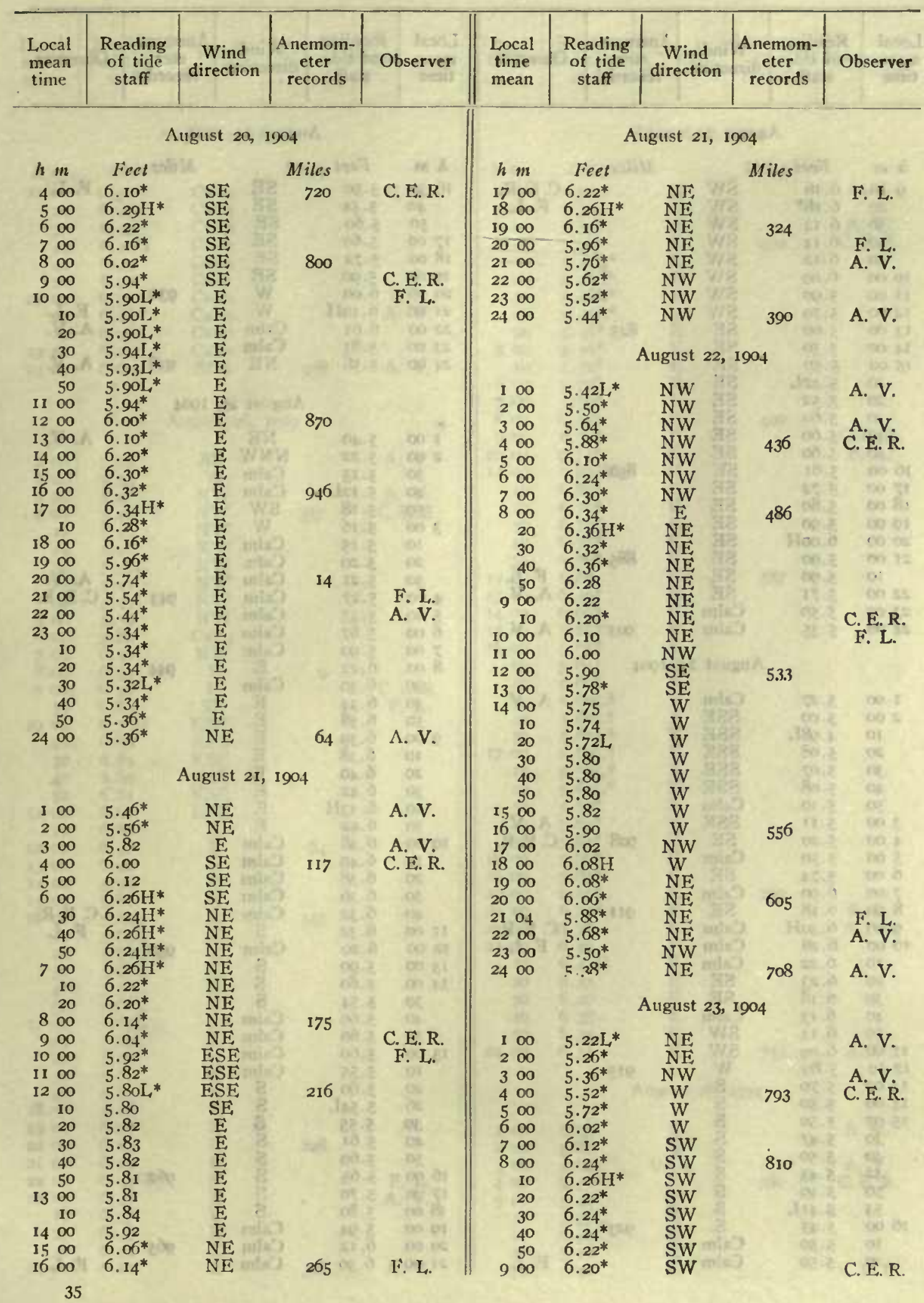


Tabulation of tidal observations at Cape Flora, Northbrook Island

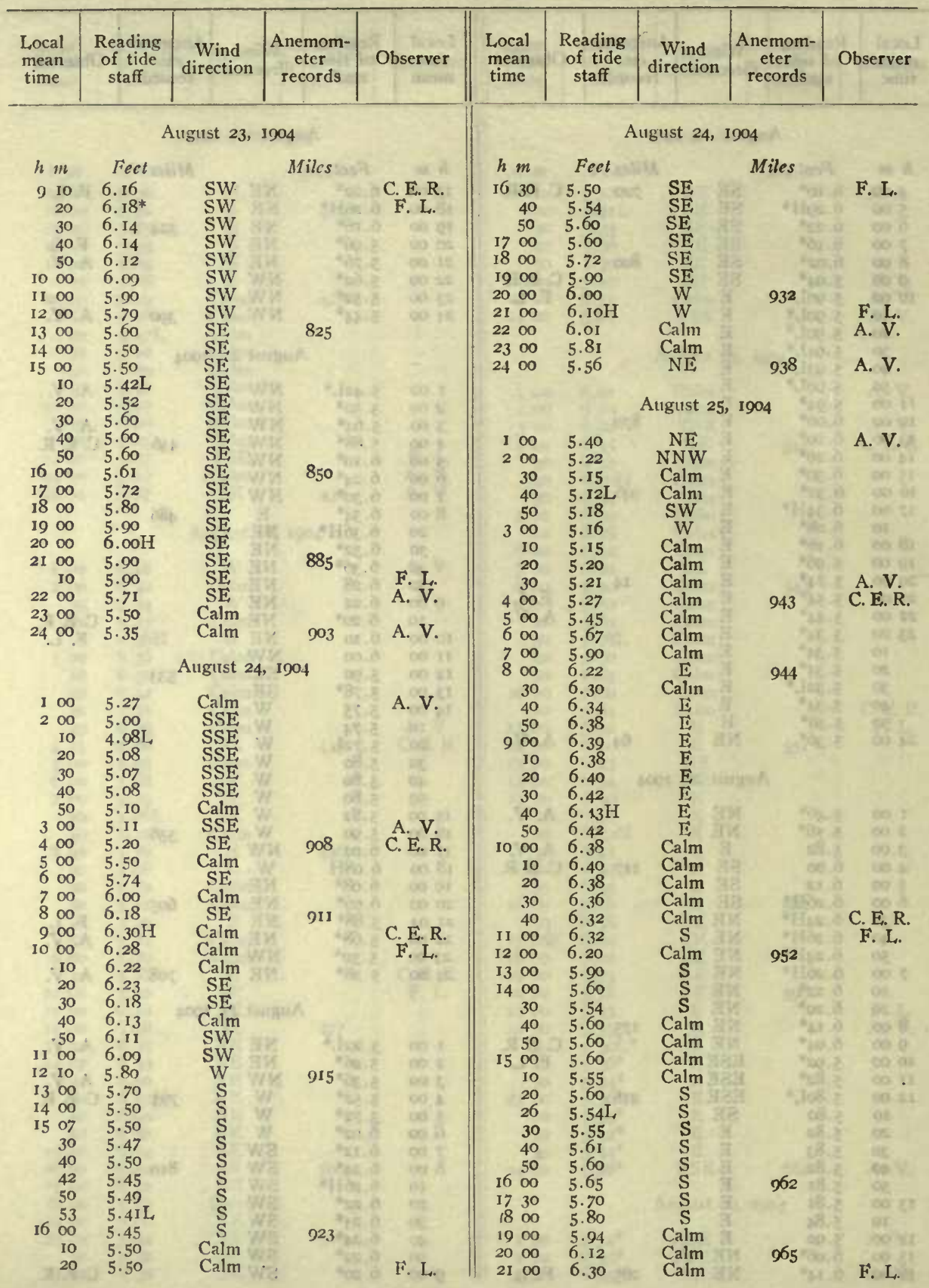


Tabulation of tidal observations at Cape Flora, Northbrook Island.

\begin{tabular}{|c|c|c|c|c|c|c|c|c|c|}
\hline $\begin{array}{l}\text { Local } \\
\text { mean } \\
\text { time }\end{array}$ & $\begin{array}{l}\text { Reading } \\
\text { of tide } \\
\text { staff }\end{array}$ & $\begin{array}{l}\text { Wind } \\
\text { direction }\end{array}$ & $\begin{array}{l}\text { Anemom- } \\
\text { eter } \\
\text { records }\end{array}$ & Observer & $\begin{array}{l}\text { Local } \\
\text { time } \\
\text { nean }\end{array}$ & $\begin{array}{l}\text { Reading } \\
\text { of tide } \\
\text { staff }\end{array}$ & $\begin{array}{l}\text { Wind } \\
\text { direction }\end{array}$ & $\begin{array}{c}\text { Anemom- } \\
\text { eter } \\
\text { records }\end{array}$ & Observer \\
\hline \multicolumn{5}{|c|}{ August 25,1904} & \multicolumn{5}{|c|}{ August 27,1904} \\
\hline$h m$ & Feet & & Miles & intar & $h m$ & Feet & & Miles & $\ln 6$ \\
\hline 2110 & 6.30 & Calm & 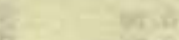 & I. L. & I 00 & 5.94 & $\mathrm{E}$ & $2045=$ & A. V. \\
\hline 20 & 6.35 & Calm & Bris & $-2+\frac{1}{x}$ & 200 & 5.72 & $\mathrm{E}$ & 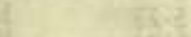 & \\
\hline 30 & 6.33 & Calm & 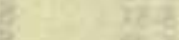 & censt & 300 & $5 \cdot 54^{*}$ & $\mathrm{E}$ & 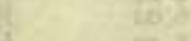 & A. V. \\
\hline 40 & $6.39 \mathrm{H}$ & Calm & 43 & 2684 & 4-00 & $5.46^{*}$ & $\mathrm{E}$ & 400 & C. E. R. \\
\hline 44 & 6.39 & Calm & $10+23$ & 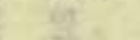 & 10 & $5.44 \mathrm{~L}^{*}$ & $\mathbf{E}$ & & \\
\hline 50 & 6.31 & Calm & is & $=0$ & 20 & $5.46 \mathrm{~L}^{*}$ & E & 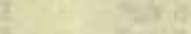 & 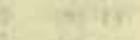 \\
\hline 2200 & 6.30 & Calm & 102 & F. L. & 30 & $5.46 \mathrm{~L}^{*}$ & $\overrightarrow{\mathrm{E}}$ & 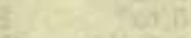 & fer: \\
\hline IO & 6.28 & Calm & $y=$ & A. V. & 40 & $5.44 \mathrm{~L}^{*}$ & $\mathrm{E}$ & Af pote & $\ln 5$ \\
\hline 20 & 6.26 & Calm & thentive & $x=2$ & 50 & $5.48^{*}$ & $\mathrm{E}$ & taxt? & 78 \\
\hline 2300 & 6.22 & Calm & 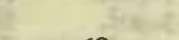 & 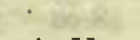 & 500 & $5.50^{*}$ & $\mathbf{E}$ & yeg it & \& \\
\hline 2400 & 6.00 & SSE & 968 & A. V. & 10 & $5 \cdot 52^{*}$ & $\mathrm{E}$ & 78 & 35 \\
\hline 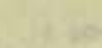 & 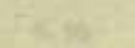 & & $x^{2}=$ & 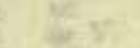 & $\begin{array}{l}600 \\
700\end{array}$ & $5.72^{*}$ & $\vec{E}$ & $\tan :$ & 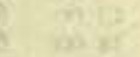 \\
\hline 340 & 5.45 & ugust 26 , & 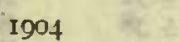 & Hist & $\begin{array}{l}700 \\
800\end{array}$ & $\begin{array}{l}5.90^{*} \\
6.24^{*}\end{array}$ & $\underset{\mathrm{E}}{\mathrm{E}}$ & $\operatorname{lon} x$ & $5 \operatorname{st}^{2}=$ \\
\hline 10 & 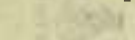 & & 58 & 308. & 900 & $6.50^{*}$ & $\mathrm{E}$ & $49992=$ & C. E. R. \\
\hline 100 & 5.74 & SE & $n=5$ & A. V. & 1000 & $6.72^{*}$ & $\mathrm{E}$ & ifnes & I. I. \\
\hline 200 & $5 \cdot 53$ & W & 10. & 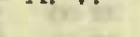 & 1100 & $6.82 \mathrm{H}^{*}$ & E & 4012 & - \\
\hline 300 & 5.47 & NW & mis & A. V. & Io & $6.80^{*}$ & $\vec{E}$ & द. & 8 \\
\hline 400 & 5.40 & NW & 977 & C. E. R. & 20 & $6.76^{*}$ & $\mathrm{E}$ & ar: & 28 \\
\hline 10 & 5.42 & NW & & & 30 & $6.72^{*}$ & $\overrightarrow{\mathrm{E}}$ & Det? & 13 \\
\hline 20 & 5.42 & NW & & & 40 & $6.72^{*}$ & E & i 23 & 或 \\
\hline 30 & $5.38 \mathrm{~L}$ & NW & & & 50 & $6.70^{*}$ & $\overrightarrow{\mathrm{E}}$ & $\sin ^{2}$ & ont 8 i \\
\hline 40 & 5.41 & NW & Heic io & ath is & 1200 & 6.62 & $\mathrm{E}$ & 587 & $\ln x$ \\
\hline 50 & 5.45 & NW & $x^{2}+1+3$ & ate & I3 00 & $6.400^{\dagger}$ & E & - & 12. \\
\hline 500 & 5.50 & NW & 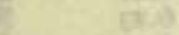 & 28 & 1400 & $6.06 t$ & E & $\frac{82}{2}$ & $\infty x^{2}, t$ \\
\hline 600 & 5.82 & Calm & 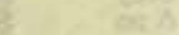 & 10 & 1500 & 5.8 & $\mathrm{E}$ & H & $\cos 2 x$ \\
\hline 700 & 6.10 & NW & $\cos \theta$ & ot: & 1600 & $5.65 t$ & $\mathrm{E}$ & 256 & coir $\mathrm{is}$ \\
\hline 800 & 6.33 & $\mathrm{E}$ & 9 & & IO & $5.64 t$ & $\overrightarrow{\mathrm{E}}$ & nas & mose \\
\hline 900 & 6.50 & $\vec{E}$ & 8 & C. E. R. & 20 & $5.62 t$ & $\overrightarrow{\mathrm{E}}$ & 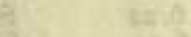 & or $t / 6$ \\
\hline 50 & 6.65 & $\mathrm{SE}$ & $\ln 3$ & F. L. & 30 & $5.60 \mathrm{Lt}$ & $\mathrm{E}$ & 160 & 66,14 \\
\hline 1000 & $6.7 \mathrm{oH}$ & ESE & ats & (2) & 40 & $5.60 \mathrm{~L} \uparrow$ & $\mathrm{E}$ & & \\
\hline 20 & 6.68 & ESE & 033 & (6): 1 & 50 & $5.6 \mathrm{IL}^{\dagger}$ & $\mathrm{E}$ & & \\
\hline 30 & 6.65 & ESE & 02 & 6012 & 1700 & 5.601 & E & & \\
\hline 40 & 6.60 & ESE & $77^{2}=$ & $\operatorname{lon} 2$ & IO & $5.58 \mathrm{~L}$ & $\mathrm{E}$ & $46 z-4$ & at $=$ \\
\hline 50 & $6.6 \mathrm{I}$ & ESE & 60 & $2 x^{2}$ & $2 c$ & $5.6 \% L^{*}$ & $\mathrm{E}$ & $\sec x$ & $\alpha$ \\
\hline II 00 & 6.61 & ESE & litb. & es & $3 c$ & $5.62^{*}$ & $\mathbf{E}$ & 152 & a \\
\hline I0 & 6.60 & E & 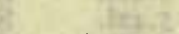 & ay & $4 c$ & $5.62^{*}$ & $\mathbf{E}$ & 14.8 & of \\
\hline 1200 & 6.42 & $\mathrm{E}$ & $64=$ & ab 0 & 50 & $5.64^{*}$ & $\mathrm{E}$ & z=ำ & 3 \\
\hline I3 00 & 6.20 & $E$ & t $10=0$ & $m$ & $18 \propto c$ & $5.66^{*}$ & $\mathbf{E}$ & $B_{3}=$ & $\infty, 1$ \\
\hline 1400 & 5.95 & E & in: 2 & it & 1900 & $5.82^{*}$ & E & in 7 & bis \\
\hline 1500 & 5.80 & $\vec{E}$ & in. & as & 2000 & $6.00^{*}$ & $\overline{\mathrm{E}}$ & 709 & \\
\hline 1600 & $5.64 \mathrm{~L}$ & $\mathrm{E}$ & 145 & 03 & 2100 & $6.22^{*}$ & $\mathrm{E}$ & 5 & F. L. \\
\hline 10 & $5.64 \mathrm{~L}$ & $\mathrm{E}$ & $-73-5=$ & wits & 2200 & 6.38 & $E$ & 86.2 & A. V. \\
\hline 20 & $5.60 \mathrm{~L}$ & $\overrightarrow{\mathrm{E}}$ & irs: & 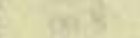 & 2300 & 6.40 & $\mathbf{E}$ & As. & 64 \\
\hline 30 & $5.68 \mathrm{~L}$ & $\mathrm{E}$ & 203 & ow & Io & $6.43 \mathrm{H}$ & $\mathrm{E}$ & sina & $\omega$ \\
\hline 40 & $5.66 \mathrm{~L}$ & $\overrightarrow{\mathrm{E}}$ & 2) & when & 20 & 6.43 & $\mathrm{E}$ & Din & $\infty$ \\
\hline 50 & $5.66 \mathrm{~L}$ & $\mathrm{E}$ & 8.8 & an in & 30 & 6.40 & $\mathrm{E}$ & E.2. & 17 \\
\hline 1700 & $5.64 \mathrm{~L}$ & $\mathrm{E}$ & 148 & $6 x+1$ & 40 & $6.3^{8}$ & $\mathbf{E}$ & 6his & $00 \pi$ \\
\hline I0 & $5.64 \mathrm{~L}$ & $\mathrm{E}$ & $\cos \theta$ & mit & 50 & 6.34 & $\mathbf{E}$ & 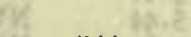 & \\
\hline 20 & 5.70 & $E$ & isth & is & 2400 & 6.31 & $\mathbf{E}$ & 755 & A. V. \\
\hline 30 & $5.7 \mathrm{I}$ & E & Init a & at & & th & & at & 008 \\
\hline 40 & 5.75 & $\mathrm{E}$ & $n$ i & dis: & 38713 & & August 28 & , I904 & $\operatorname{lin} 8$ \\
\hline 1800 & 5.76 & $\vec{E}$ & 2. 8 & er. & 18 & & & क. 8 & $\infty \omega$ \\
\hline 1900 & 6.00 & $\mathrm{E}$ & 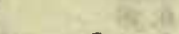 & $\cos t / 4$ & I $\infty$ & 6.03 & $\mathrm{E}$ & asede & A. V. \\
\hline 2000 & 6.14 & $E$ & 228 & one & & 5.80 & E & 19 & \\
\hline 2100 & 6.22 & $\mathrm{E}$ & 1the & 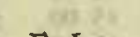 & 300 & 5.64 & Calm & 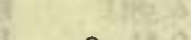 & A. V. \\
\hline 2200 & $6.44 \mathrm{H}$ & $\mathrm{E}$ & 42 & F. I. & 409 & 5.50 & $\mathrm{E}$ & 780 & C. E. R. \\
\hline 2300 & $6.4 \mathrm{I}$ & $\mathrm{E}$ & $\sqrt{3} 2$ & A. V. & 500 & 5.42 & $\vec{E}$ & 80 & \\
\hline Io & 6.37 & $E$ & cose & $\cos x^{2}$ & 10 & $5.39 \mathrm{~L}$ & $\overrightarrow{\mathrm{E}}$ & ars & ab \\
\hline $\begin{array}{l}20 \\
30\end{array}$ & $\begin{array}{l}6.33 \\
6.32\end{array}$ & $\stackrel{\mathrm{E}}{\mathrm{E}}$ & $2-\frac{4}{21}=3$ & 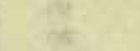 & $\begin{array}{l}20 \\
40\end{array}$ & $\begin{array}{l}5 \cdot 42 \\
5.40\end{array}$ & $\underset{\mathrm{E}}{\mathrm{E}}$ & 7.5 & $\cdot \quad+8$ \\
\hline 2400 & 6.21 & $\mathrm{E}$ & 308 & A. V. & 50 & 5.44 & $\mathrm{E}$ & 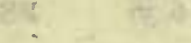 & C. E. R. \\
\hline
\end{tabular}


Tabulation of tidal observations at Cape Flora, Northbrook Island

\begin{tabular}{|c|c|c|c|c|c|c|c|c|c|c|c|}
\hline $\begin{array}{l}\text { Local } \\
\text { mean } \\
\text { time }\end{array}$ & $\begin{array}{l}\text { Reading } \\
\text { of tide } \\
\text { staff }\end{array}$ & $\begin{array}{l}\text { Wind } \\
\text { direction }\end{array}$ & $\begin{array}{l}\text { Anemom } \\
\text { eter } \\
\text { records }\end{array}$ & Observer & $\begin{array}{l}\text { Local } \\
\text { time } \\
\text { mean }\end{array}$ & & $\begin{array}{l}\text { Rcading } \\
\text { of tide } \\
\text { staff }\end{array}$ & $\begin{array}{c}\text { Wind } \\
\text { direction }\end{array}$ & $\begin{array}{l}\text { Anemom- } \\
\text { eter } \\
\text { records }\end{array}$ & & Observer \\
\hline \multicolumn{5}{|c|}{ August 28,1904} & \multicolumn{7}{|c|}{ Atrgust 29, 1904} \\
\hline$h m$ & \multicolumn{2}{|c|}{ Fect } & Miles & $\ln ^{2}+1$ & $h m$ & & Fect & 4 & \multicolumn{2}{|c|}{ Miles } & $3+4 x^{2}$ \\
\hline 600 & $5 \cdot 49$ & $\mathrm{E}$ & & C. E. R. & 140 & & 6.10 & SE & $\sqrt{2}-2 x$ & & F. L. \\
\hline 700 & 5.75 & $\mathrm{E}$ & & 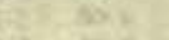 & 150 & & 5.73 & SE & & $d$ & at \\
\hline 800 & 6.02 & $\mathrm{E}$ & 809 & & 160 & & $5 \cdot 51$ & SE & 125 & $a^{t}$ & 25 \\
\hline 900 & 6.25 & $E$ & & C. E. R. & 170 & & $5 \cdot 32$ & SE & Hix & ati & ae. \\
\hline 1000 & 6.53 & $\mathrm{E}$ & & F. L. & & I0 & $5 \cdot 3 I$ & SE & & $=2$ & 24. \\
\hline II 00 & $6.62^{*}$ & $E$ & & $\alpha$ & & 20 & 5.31 & SE & & At & क \\
\hline 50 & $6.62^{*}$ & $\mathrm{E}$ & 8.0 & it. & & 30 & $5.30 \mathrm{~L}$ & SE & E & $y^{3}$ & 60 at \\
\hline 1200 & $6.64 \mathrm{H}^{*}$ & $\mathrm{SE}$ & 848 & (5) & & 40 & $5.30 \mathrm{~L}$ & SE & 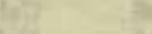 & $x^{2}=$ & $6 x$ \\
\hline IO & $6.60^{*}$ & $S E$ & $x^{4}$ & or & & 50 & 5.3oI, & SE & 15 & $23^{2}$ & ex: \\
\hline 20 & $6.5^{8 *}$ & SE & 4 & $m i z$ & 180 & & $5.30 \mathrm{~L}$ & SE & (4) & 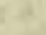 & Ift 64 \\
\hline 30 & $6.54^{*}$ & SI: & $\mathrm{n}$ & xiा & & 10 & $5.30 \mathrm{~L}$ & SE & 3 & 50 & ben ats \\
\hline 1300 & $6.40^{*}$ & SE & 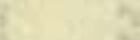 & $\log 0$ & & 20 & $5.30 \mathrm{~L}$ & SE & & & \\
\hline 1400 & $6.06^{*}$ & SE & 2 & $00 \mathrm{~F}$ & & 30 & $5 \cdot 32$ & SE & 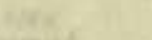 & & \\
\hline I5 00 & 5.75 & SE & 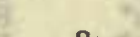 & wit & 191 & & 5.38 & SE & $=0$ & & \\
\hline I6 00 & $5 \cdot 55$ & SE & 899 & $00 \%$ & 200 & & 5.52 & SE & 167 & & $\rightarrow$ \\
\hline 1700 & $5.33 \mathrm{~L}$ & $S E$ & & $\cos \theta$ & 210 & & 5.72 & SE: & 32 & $i^{2}$ & F. L. \\
\hline Io & 5.40 & SE & "H। & $06 \mathrm{n}$ & $22 c$ & & 5.93 & $\mathrm{SE}$ & h & 5 & A. V. \\
\hline 20 & $5 \cdot 38$ & SE & 5 & wis & 230 & & 6.04 & Calm & tx & 51 & \\
\hline 30 & 5.40 & SE & 8 & 66 & 240 & & 6.22 & $\mathrm{SE}$ & 194 & 32 & A. $\mathrm{V}$. \\
\hline 40 & 5.40 & $\mathbf{E}$ & t. & 60 & (2) & & $1 x^{2}+x^{2}$ & & the & IE & 165. \\
\hline 50 & $5.4 \mathrm{I}$ & $\mathbf{E}$ & $\mathrm{z}$ & 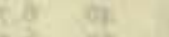 & 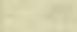 & & $x^{3}$ & August $3 c$ & 0,1904 & 6 है & is \\
\hline 1800 & 5.40 & $\mathbf{E}$ & 5 & (⿻) & 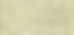 & & 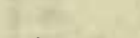 & & & 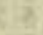 & 20 \\
\hline 10 & 5.43 & $\mathrm{E}$ & E & $60 \mathrm{k1}$ & 03 & & $6.24 \mathrm{H}$ & $\mathrm{SE}$ & 28 & & A. V. \\
\hline 20 & 5.45 & $\mathrm{E}$ & 1 & 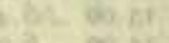 & & 40 & 6.21 & SE & IH & 12? & \\
\hline 1900 & $5 \cdot 52$ & E & & $\infty \times 1$ & & 50 & 6.23 & $\overline{\mathrm{SE}}$ & WS & 32 & 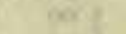 \\
\hline 2000 & 5.78 & $\mathbf{E}$ & 939 & 621 & & $\infty 0$ & 6.20 & SE & 40 & $3 z$ & 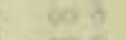 \\
\hline 2100 & 5.95 & SE & & 5 & & 10 & 6.09 & SE & 12 & t. & $\cos 5$ \\
\hline 2200 & 6.10 & SE & & F. L. & & 20 & 6.10 & SE & 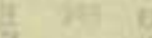 & $2 \geqslant$ & $\cos x$ \\
\hline 2300 & 6.22 & SE & & A. V. & & 30 & 6.03 & SE & 8 & $5 \geqslant$ & $\cos 6$ \\
\hline 2400 & 6.31 & SE & 988 & A. V. & & 40 & 5.97 & Calm & II & 10 & ats \\
\hline & & & & ce & 20 & & 5.86 & Calm & He & 50 & 4 \\
\hline . & & August 29 & & 80 & 3 & $\begin{array}{l}\infty \\
\infty\end{array}$ & $\begin{array}{l}5.69 \\
5.52\end{array}$ & $\begin{array}{l}\text { Calm } \\
\text { Calm }\end{array}$ & $2 \infty$ & 29 & A. V. \\
\hline 0 I0 & $6.33 \mathrm{H}$ & $\mathrm{SE}$ & $y$ & A. V. & 5 & $\infty$ & 5.40 & SE & 200 & 58 & C. E. R. \\
\hline 20 & 6.32 & SE & $t$ & tron & & 30 & $5.38 \mathrm{~L}$ & SE & & $x \rightarrow$ & w: \\
\hline 30 & 6.24 & SE & 년 & Be & & 40 & $5.4 \mathrm{IL}$ & SE & Bâ & 30 & $\cos x$ \\
\hline 40 & 6.21 & SE & \pm & 4 & & 50 & $5.42 \mathrm{~L}$ & SE & 3 & 200 & or \\
\hline 50 & 6.20 & $\mathrm{SE}$ & $m$ & 19. & 6 & $\infty$ & $5.38 \mathrm{~L}$ & $\mathrm{SE}$ & 3 & 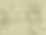 & $90 \leq 1$ \\
\hline 100 & 6.18 & $\mathrm{SE}$ & I] & $(06,21$ & & 10 & 5.40 & $\mathrm{SE}$ & 8 & 60 & soing \\
\hline 200 & 5.91 & SE & $=$ & 5001 & & 20 & 5.42 & Calm & 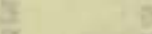 & $8 \%$ & $10 \mathrm{ht}$ \\
\hline 300 & 5.62 & Calin & 4 & A. V. & & 30 & $5.4 \mathrm{I}$ & E & & & 0021 \\
\hline 400 & 5.48 & $\mathrm{E}$ & 13 & C. E. R. & & 40 & 5.45 & E & 4) & 23 & $\%$ \\
\hline 500 & $5 \cdot 38$ & $N E$ & A & & 7 & $\infty$ & $5 \cdot 52$ & $\mathrm{E}$ & (1) & 22 & 6i) \\
\hline 10 & $5 \cdot 36$ & $N E$ & A & 000 & $8 c$ & $\infty$ & 5.70 & $\mathbf{E}$ & 243 & $a z$ & 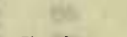 \\
\hline 20 & 5.37 & $\mathrm{NE}$ & B) & at & 9 & $\infty$ & 5.90 & E & -70 & & C. E. R. \\
\hline 30 & $5.35 \mathrm{~L}$ & $N$ & & 6.8 & Io & $\infty$ & 6.1 & $\underline{E}$ & $\frac{3}{2}$ & & F. L. \\
\hline 40 & 5.37 & $N$ & & 2.0 & II & $\infty$ & 6.3 & $\mathbf{E}$ & $\frac{1}{3}$ & 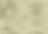 & \\
\hline 600 & 5.40 & NW & 5 & 5.8 & 12 & $\infty$ & 6.42 & E & 320 & & on 51 \\
\hline IO & 5.44 & NW & E & 60 & & 10 & 6.4 & $E$ & the & Af & ex/ \\
\hline 700 & 5.60 & NW & 9 & $\cos 36$ & & 20 & 6.43 & E & 3 & taE & $\sigma x$ \\
\hline 800 & 5.80 & SE & 28 & & & 30 & $6.44 \mathrm{H}$ & E & & the & or \\
\hline 900 & 6.05 & $\operatorname{arcs}$ & 4roph & C. E. R. & & 40 & 6.41 & $\overrightarrow{\mathrm{E}}$ & 푠 & E? & $9=$ \\
\hline 1000 & 6.30 & $\mathrm{E}$ & & F. L. & & 50 & 6.40 & E & & 42 & $008 t$ \\
\hline II 00 & 6.50 & $\mathrm{E}$ & If & on: & 13 & OO & 6.38 & E & 3 & wh & 60 of \\
\hline 50 & 6.52 & $\mathrm{E}$ & \pm & $6 y x$ & $14 c$ & $\infty 0$ & 6.11 & ESE & 3 & y.e & $00 \%$ \\
\hline 1200 & $6.58 \mathrm{H}$ & $\mathbf{E}$ & 76 & $a / c$ & I5 & $\infty$ & 5.81 & $\mathrm{SE}$ & 불 & 8.8 & coist \\
\hline 10 & 6.51 & E) & 3 & $\infty)^{\circ}$ i & 16 & 00 & $5 \cdot 54$ & $\mathrm{SE}$ & 383 & & $00 \leqslant$ \\
\hline 20 & 6.48 & $\mathrm{E}$ & if & PAt 2 & I7 & 00 & $5 \cdot 34$ & $\mathrm{SE}$ & ( & & Do its \\
\hline 30 & 6.45 & $\mathrm{E}$ & 3 & en & 18 & 00 & 5.20 & SE & 3 & 2,2 & d9 \\
\hline 40 & 6.43 & E: & 3 & $-n$ & & IO & $5.19 \mathrm{~L}$ & S & 쿨 & 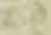 & 10 \\
\hline 50 & 6.40 & $\vec{E}$ & $\exists$ & $4 z$ & & 20 & 5.20 & SE & 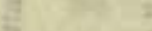 & 5.0 & flys. \\
\hline 1300 & 6.36 & ESE & 4 & F. L. & & 30 & 5.20 & SE & ב & 16.8 & F. L. \\
\hline
\end{tabular}


Tabulation of tidal observations at Cape Flora, Northbrook Island

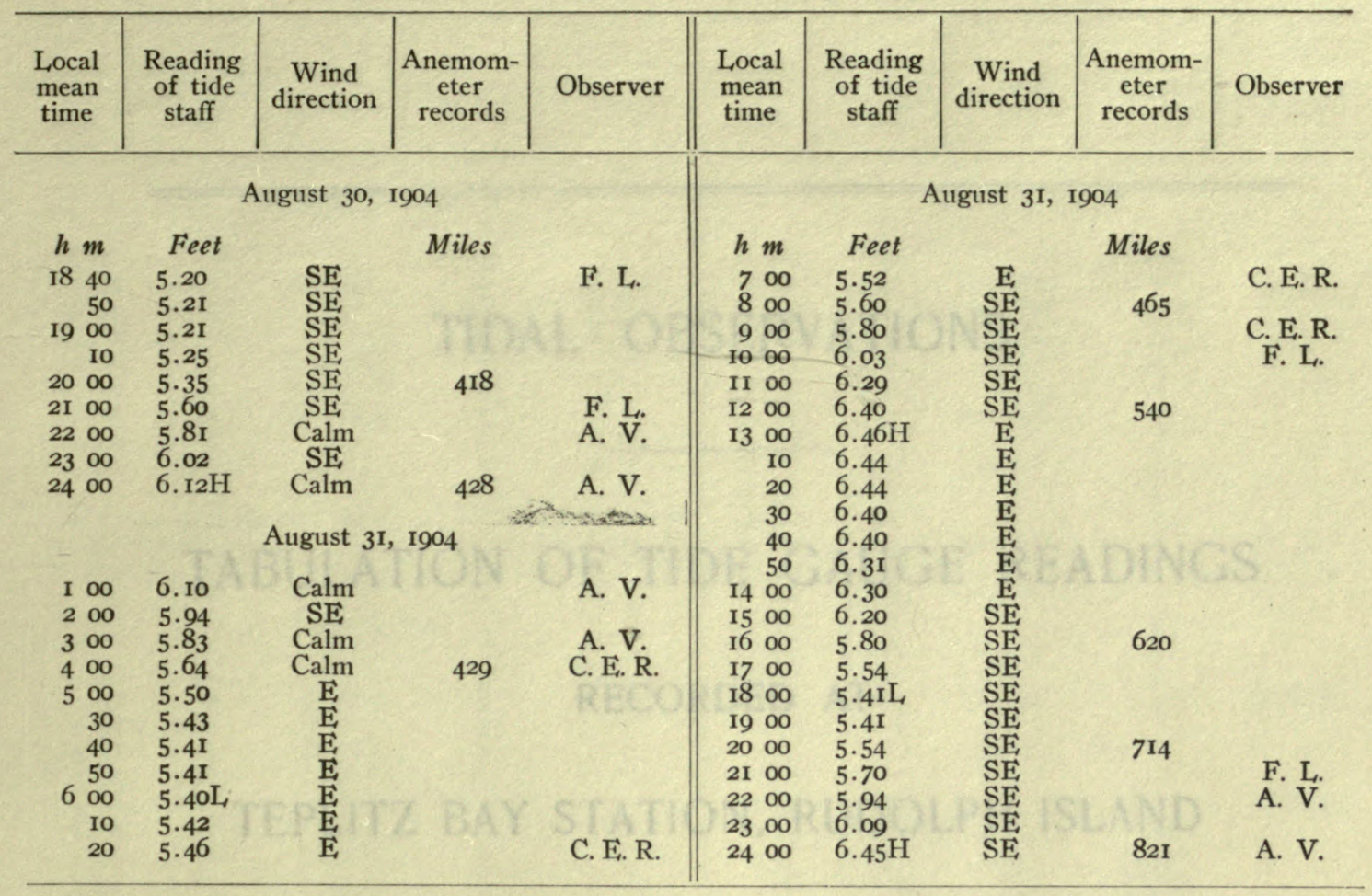




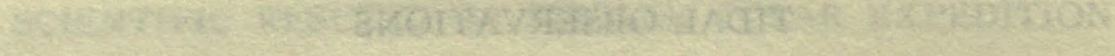

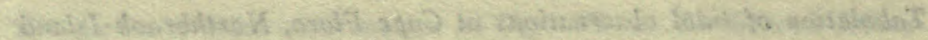

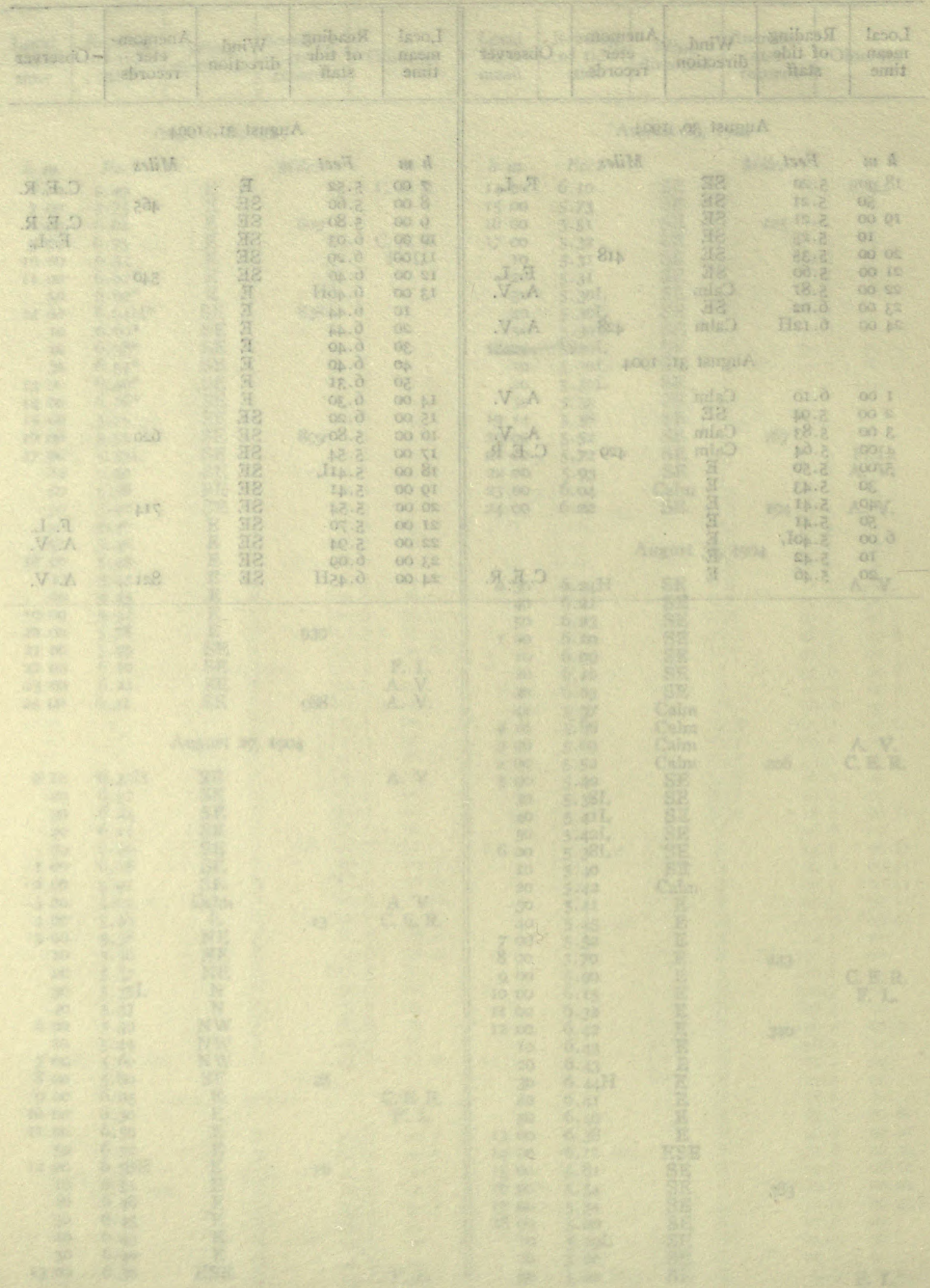




\title{
TIDAL OBSERVATIONS
}

\section{TABULATION OF TIDE GAUGE READINGS}

\author{
RECORDED AT
}

TEPLITZ BAY STATION, RUDOLPH ISLAND

FRANZ JOSEF ARCHIPELAGO

APRIL 1, 1904, TO JUNE 3, 1904

NORTH LaTITUDE: $81^{\circ} 47 \cdot 5$

LONGITUDE EAST OF GREENWICH: $57^{\circ} 56^{\prime}$ 


\title{
ZИOITAVЯG28O JAGIT
}

\section{OИIQAЭЯ GOUAD HAIT 70 ИOITAJUAAT}

\author{
TA 93990539
}

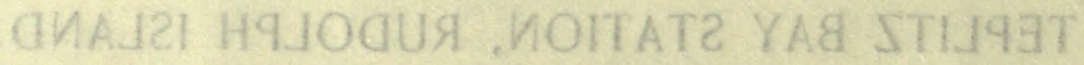

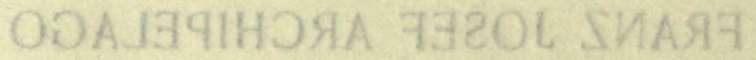

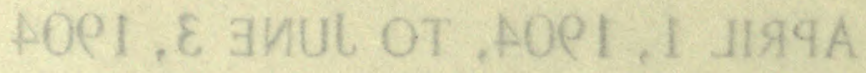

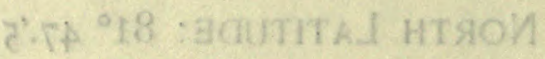 \\ Dy एद :HOH
}


Tabulation of tidal observations at Teplitz Bay, Rudolph Island

\begin{tabular}{|c|c|c|c|}
\hline $\begin{array}{l}\text { Local } \\
\text { mean } \\
\text { time }\end{array}$ & $\begin{array}{l}\text { Reading } \\
\text { of tide } \\
\text { staff }\end{array}$ & $\begin{array}{l}\text { Wind } \\
\text { direction }\end{array}$ & Velo \\
\hline & & April I, & I904 \\
\hline$h m$ & Feet & & Mil \\
\hline 17 00 & 4.05 & $S$ to $S E$ & \\
\hline I8 o0 & $4 \cdot 3^{I}$ & SW & \\
\hline 1900 & $4 \cdot 45 \mathrm{H}$ & W & \\
\hline 2000 & $4 \cdot 33$ & $W$ to $S W$ & \\
\hline 2100 & 4.19 & $W$ to $S W$ & \\
\hline 2200 & 3.55 & $\mathrm{~W}$ to $\mathrm{SW}$ & \\
\hline 2300 & 3.05 & $\mathrm{~S}$ & \\
\hline 2400 & 2.75 & SE & \\
\hline
\end{tabular}

At 19:00 grinding of ice could be heard for about one hour; direction was not discernible from the dwelling.

April 2, 1904

\begin{tabular}{|c|c|c|}
\hline ० 58 & $2.58 \mathrm{~L}$ & $\mathrm{SE}$ \\
\hline & 2.59 & SSW \\
\hline 258 & 2.82 & SW \\
\hline 358 & 3.23 & SSW \\
\hline 458 & 3.70 & SE \\
\hline 58 & 4.05 & SE \\
\hline & 4.27 & S \\
\hline & $4 \cdot 32 \mathrm{H}$ & S \\
\hline & $4 \cdot 14$ & $S$ \\
\hline $\begin{array}{l}50 \\
57\end{array}$ & 3.73 & SE \\
\hline $\begin{array}{l}57 \\
00\end{array}$ & $3 \cdot 3^{2}$ & S \\
\hline 57 & 3.00 & $\cos ^{5} \mathrm{~cm}$ \\
\hline & $2.8 \mathrm{I}$ & $S$ to $S W$ \\
\hline & 2.93 & $S$ \\
\hline 57 & 3.30 & $\mathrm{SE}$ \\
\hline & 3.72 & $\mathrm{~S}$ \\
\hline & 4.12 & $\mathrm{SE}$ \\
\hline & 4.40 & $S E$, to $S$ \\
\hline & $4.5 \mathrm{IH}$ & SE \\
\hline & $4 \cdot 47$ & SE \\
\hline & $4 \cdot 30$ & ESE \\
\hline & 4.00 & SSE \\
\hline & 3.52 & ESE \\
\hline & 3.13 & \\
\hline
\end{tabular}

Levels run at I2:00 from tide gauge to B. M. No. I. Tide gauge reading of $B$. M. No. I, 14.19 feet. Ice field extends to horizon.

\section{April 3, 1904}

$\begin{array}{llll}0 & 56 & 2.84 & \text { SE } \\ \text { I } 26 & 2.75 & \text { ESE } \\ 41 & 2.72 & \text { ESE } \\ & 56 & 2.70 & \text { ESE } \\ 2 & \text { II } & 2.69 \mathrm{~L} & \text { ESE } \\ & 26 & 2.71 & \text { ESE } \\ & 56 & 2.80 & \text { E } \\ 3 & 56 & 3.06 & \text { S } \\ 4 & 56 & 3.54 & \text { W } \\ 5 & 56 & 3.98 & \text { WSW } \\ 6 & 56 & 4.28 & \text { WSW } \\ 7 & 56 & 4.44 & \text { SW } \\ 8 & 26 & 4.46 \mathrm{H} & \text { SW } \\ 41 & 4.43 & \text { SW } \\ & 56 & 4.40 & \text { SW }\end{array}$

8 S.W. S.
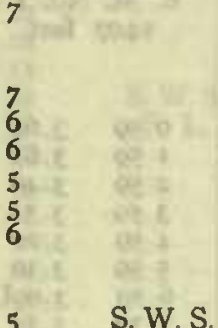

5
W. J. P.

S. W.

S. W. S.

S. W. L.

6

S.W. S.

\section{S.W.S.}

S. W. S.

Local
mean
time

\begin{tabular}{c|c|c|c}
$\begin{array}{c}\text { Reading } \\
\text { of tide } \\
\text { staff }\end{array}$ & $\begin{array}{c}\text { Wind } \\
\text { direction }\end{array}$ & Velocity & Observer \\
\hline
\end{tabular}

April 3, 1904

\begin{tabular}{|c|c|c|c|c|}
\hline$h m$ & Feet & & Miles & nis \\
\hline 955 & 4.12 & S to $S W$ & 2 & F. L. \\
\hline 1055 & 3.68 & S to SW & 2 & \\
\hline II 58 & 3.30 & $\mathrm{~S}$ to $\mathrm{SE}$ & 3 & \\
\hline 1255 & 3.02 & SE & 4 & \\
\hline I3 55 & $2.84 \mathrm{~L}$ & S & 4 & os \\
\hline 1455 & $2.90^{\circ}$ & $S$ to $S W$ & 4 & 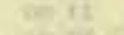 \\
\hline 1555 & 3.19 & S & 2 & wat \\
\hline 1655 & 3.53 & S to SW & 2 & 2007 \\
\hline I7 55 & 4.00 & $\mathrm{~S}$ & 3 & 10 \\
\hline 185 & 4.30 & $\mathrm{~S}$ & 4 & trit \\
\hline 195 & 4.54 & S to $S W$ & 4 & F. L \\
\hline 202 & 4.55 & s & & S. W. \\
\hline 45 & $4.57 \mathrm{H}$ & SSW & & \\
\hline & $4 \cdot 55$ & S & 5 & $\operatorname{lag} 25$ \\
\hline 21 & $\begin{array}{l}4.28 \\
3.87\end{array}$ & SSW & 3 & ser \\
\hline 235 & 3.46 & WSW & 4 & S. W. S \\
\hline
\end{tabular}

April 4, 1904

$\begin{array}{lllll}-55 & 3.15 & \mathrm{SE} & 3 & \text { S. W. S. }\end{array}$

I $55 \quad 2.89$ ESE

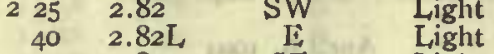

$\begin{array}{llll}55 & 2.83 & \mathrm{SE} & \text { Light }\end{array}$

$\begin{array}{lll}355 & 2.98 & \text { ESE }\end{array}$

455 3.30 W W W

$\begin{array}{llll}5 & 55 & 3.69 & \text { ESE }\end{array}$

$\begin{array}{llll}65 & 4.04 & \mathrm{ESE} & 2\end{array}$

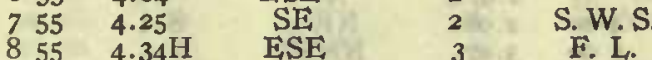

$925 \quad 4.31$

$\begin{array}{ll}55 & 4.21 \\ 5 & 3.90\end{array}$

$1055-3.90$

II $57 \quad 3.39$

$1300 \quad 3.17$

I4 $00 \quad 2.91$

$1500 \quad 2.80 \mathrm{~L}$

$\begin{array}{ll}16 & 00 \\ 2.97\end{array}$

$1700 \quad 3.24$

18003.62

$1900 \quad 4.04$

$2000 \quad 4.30$

$2 \mathrm{I} 00 \quad 4.43 \mathrm{H}$

$45 \quad 4.40$

$\begin{array}{lll}22 & 00 & 4.37\end{array}$

$2300 \quad 4.12$

E

$2400 \quad 3.28$
4
2
2
4
8
16
19
23
25
30
27
29
21
16
18

F. L.

S. W.S.

S. W. S.

Open water west to northwest at 10:00. Ice moving west out of bay within one-half mile off shore 18:30 to I9:00. Open water in sonth. Ice returning from southwest at 22:00.

April 5, 1904

$\begin{array}{lllll}100 & 3.42 & \text { ESE } & 18 & \text { S.W.S. } \\ 200 & 3.15 & \text { S } & 18 & \\ 300 & 3.01 L & S & 18 & \\ 30 & 3.03 & S & 18 & \\ 400 & 3.09 & S & 19 & \\ 500 & 3.30 & S & 17 & \text { S.W.S. }\end{array}$


Tabulation of tidal observations at Teplitz Bay, Rudolph Island

\begin{tabular}{|c|c|c|c|c|c|c|c|c|c|}
\hline $\begin{array}{l}\text { Local } \\
\text { mean } \\
\text { time }\end{array}$ & $\begin{array}{l}\text { Reading } \\
\text { of tide } \\
\text { staff }\end{array}$ & $\begin{array}{l}\text { Wind } \\
\text { direction }\end{array}$ & Velocity & Observer & $\begin{array}{c}\text { Local } \\
\text { mean } \\
\text { time }\end{array}$ & $\begin{array}{l}\text { Reading } \\
\text { of tide } \\
\text { staff }\end{array}$ & $\begin{array}{l}\text { Wind } \\
\text { direction }\end{array}$ & Velocity & Observer \\
\hline \multicolumn{5}{|c|}{ April 5, 1904} & \multicolumn{5}{|c|}{ April 7, 1904} \\
\hline$h m$ & Feet & & Miles & 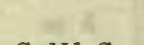 & $h m$ & Feet & & Miles & \\
\hline 600 & 3.66 & $\mathrm{SW}$ & 16 & S. W. S. & 700 & 3.20 & $\mathrm{NE}$ & 3 & S. W.S. \\
\hline 700 & 4.02 & SSW & 16 & & 800 & 3.45 & E & 2 & S. W. S. \\
\hline 800 & 4.35 & SW & 14 & S. W. S. & 900 & $3 \cdot 57$ & $\mathrm{NE}$ & I & F. L. \\
\hline 900 & $4.52 \mathrm{HI}$ & SW & 15 & li. L. & 1000 & 3.71 & $\mathrm{E}$ & 2 & 6as as \\
\hline Io 00 & 4.51 & WNW & 18 & $284 x$ & I I $\infty$ & $3.80 \mathrm{H}$ & $E$ & 3 & $\infty \pi$ \\
\hline 1100 & $4 \cdot 33$ & WNW & 12 & $2 \leq$ & 1202 & 3.79 & $\underline{E}$ & 2 & ocis: \\
\hline 1204 & 4.00 & NW & 13 & 282 & 1300 & 3.69 & $\mathrm{E}$ & I & $\cos 16$ \\
\hline 1300 & 3.69 & NW & II & 201 & 1400 & 3.49 & $N$ & 1 & $60 \%$ \\
\hline 1400 & 3.41 & NW & II & $23 x$ & 1500 & 3.29 & $E$ & 2 & \\
\hline 1502 & 3.21 & $N W$ & 13 & 2 & 1600 & 3.14 & NIV & 2 & or sh \\
\hline 1600 & $3.20 L$ & NW & I 5 & 204 & . 1700 & 3. $10 \mathrm{~L}$ & $\mathrm{NE}$ & I & . \\
\hline 17,00 & 3.36 & NW & 14 & dx ps & 1800 & 3.11 & $N$ & 2 & $\operatorname{sen} 6$ \\
\hline 1800 & 3.61 & NW & 15 & $2 x^{2}$ & 1900 & 3.29 & $\mathrm{~N}$ & 2 & \\
\hline 1900 & 3.90 & $\mathrm{~N}$ & 15 & & 2000 & 3.42 & $N$ & 4 & I. L. \\
\hline 2000 & 4.20 & $\mathrm{~N}$ & 16 & F. I. & 2100 & 3.61 & ENE & 2 & S. W. S. \\
\hline 2100 & $4.32^{\circ}$ & NNW & 15. & S. W. S. & 2200 & 3.74 & ENE & 3 & ate \\
\hline : 50 & $4.40 \mathrm{H}$ & $\mathrm{N}$ & & ats m & 2300 & $3.83 \mathrm{H}$ & ENE & 1 & \\
\hline 2200 & 4.39 & $N$ & 13 & & 2400 & 3.80 & NNW & 3 & S. W. S. \\
\hline
\end{tabular}

$2400 \quad 3.99$ NE

Ice came in at $5: 30$; pressure contiuned to $5: 45$ : 7:30 open water soutl of Cape Ank.

April 6, 1904

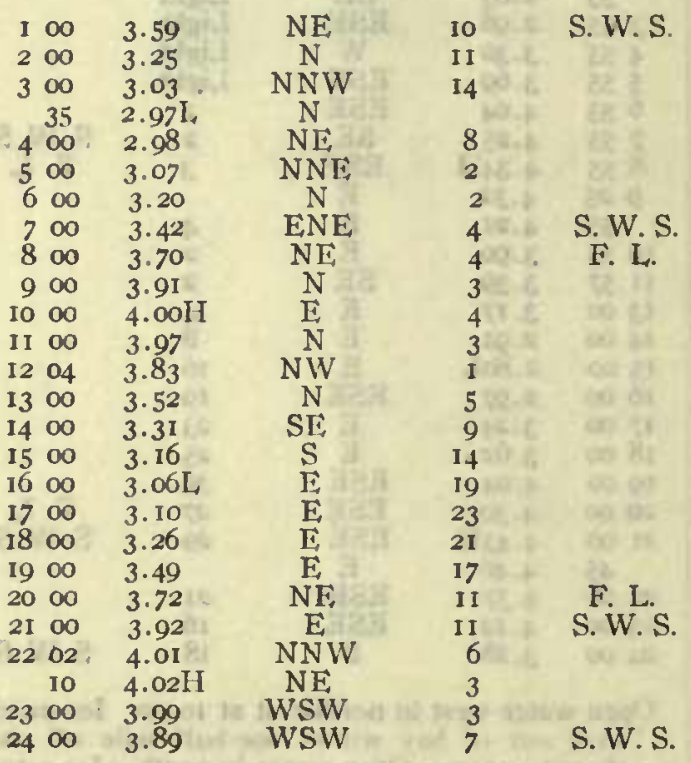

Ice moving out of bay in north at $8: 45$. Ice came in at $15: 10$. Ice moving out of bay in soutl at $18: 00$.

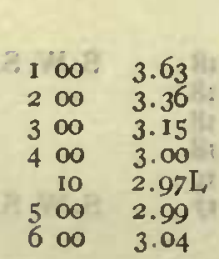
April 7, 1904

$\begin{array}{lll}\text { W } & \text { I } & \text { S. W.S. } \\ \text { W } & 5 & \\ \text { NNE } & 1 & \\ \text { W } & 2 & \\ \text { NNW } & & \\ \text { NNE } & 1 & \text { S. W.S. }\end{array}$

B. M. No. 1 corresponds to tide gange reading of 14.05 feet in morning.

Open water out of bay at 17:50 to 7:00 April 8.

A pril 8, 1904

$\begin{array}{cccccc}0 & 59 & 3.67 & \text { NNW } & 2 & \text { S. W. S. } \\ 1 & 59 & 3.48 & \text { ENE } & 3 & \\ 2 & 59 & 3.29 & \text { NNWW } & 3 & \\ 3 & 59 & 3.11 & \text { NWW } & 2 & \\ 459 & 3.02 & \text { NNW } & 2 & \\ 5 & 59 & 3.00 L & \text { NNWV } & 1 & \\ 6 & 59 & 3.10 & \text { WW } & 3 & \\ 7 & 59 & 3.20 & \text { NE. } & 1 & \text { S. IV. S. } \\ 8 & 59 & 3.39 & \text { NE } & 3 & \text { T. . L. }\end{array}$

$959 \quad 3.50$

10 $59 \quad 3.62$

$\begin{array}{ll}1203 & 3.68 \mathrm{H} \\ 59 & 3.66\end{array}$

$1359 \quad 3.69$

I4 $59 \quad 3.46$

$\begin{array}{ll}15 & 59 \\ 1.32\end{array}$

$1659 \quad 3.21$

$1759 \quad 3.15 \mathrm{~L}$

I8 $59 \quad 3.20$

$1959 \quad 3 \cdot 30$

$2059 \quad 3.40$

2I $59 \quad 3.54$

$\begin{array}{lll}22 & 59 & 3.63 \\ 23 & 39 & 3.68 \mathrm{H}\end{array}$

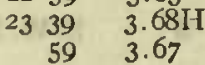

Calm

E

Calm

$\mathrm{NE}$

Calm

Calm

$\mathrm{NE}$

S. W. S

B. M. No. I corresponds to tide gauge reading of 14.07 feet.

April 9, 1904

$\begin{array}{llllll}0 & 59 & 3.65 & \text { NE } & 2 & \text { S. IV.S. } \\ \text { I } 59 & 3.61 & \text { NNW } & 4 & \\ 2 & 59 & 3.49 & \text { NNW } & 2 & \\ 3 & 59 & 3.34 & \text { Calm } & & \\ 4 & 59 & 3.20 & \text { NE } & 2 & \\ 5 & 59 & 3.10 & \text { Calm } & & \\ 6 & 59 & 3.09 \mathrm{I}, & \text { NNW } & 1 & \\ 7 & 59 & 3.13 & \text { NE } & 2 & \\ 8 & 59 & 3.22 & \text { N } & 2 & \text { S. W. S. }\end{array}$


Tabulation of tidal observations at Teplitz Bay, Rudolph Island

\begin{tabular}{|c|c|c|c|c|c|c|c|c|c|}
\hline $\begin{array}{l}\text { Local } \\
\text { nean } \\
\text { time }\end{array}$ & $\begin{array}{l}\text { Reading } \\
\text { of tide } \\
\text { staff }\end{array}$ & $\begin{array}{l}\text { Wind } \\
\text { direction }\end{array}$ & Velocity & Observer & $\begin{array}{l}\text { Local } \\
\text { mean } \\
\text { time }\end{array}$ & $\begin{array}{l}\text { Reading } \\
\text { of tide } \\
\text { staff }\end{array}$ & $\begin{array}{l}\text { Wind } \\
\text { direction }\end{array}$ & Velocity & Observer \\
\hline \multicolumn{5}{|c|}{ April 9, 1904} & \multicolumn{5}{|c|}{ April II, I904 } \\
\hline$h m$ & Feet & & Miles & an $A$ & $h m$ & Feet & & Miles & wi \\
\hline 959 & 3.35 & SE & I & I?. L. & $175^{8}$ & 3.96 & $N$ & 12 & F. It. \\
\hline 1059 & 3.49 & $\mathrm{SE}$ & 2 & 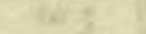 & 1858 & 3.80 & $N E$ & 7 & \\
\hline 1201 & 3.56 & $\mathrm{~N}$ & I $=1$ & $\cos \frac{1}{5}$ & 1958 & 3.61 & $\mathrm{NE}$ & 12 & F. L. \\
\hline 1259 & 3.66 & SE & 2 & tars & $-20 \quad 58$ & 3.47 & $\mathrm{NE}$ & 6 & S. W. S. \\
\hline 1359 & $3.66 \mathrm{H}$ & Calm & we & a & 2158 & $3.39 \mathrm{~L}$ & $\mathrm{NE}$ & 7 & (a) \\
\hline 1459 & 3.60 & $\mathrm{SE}$ & 2 & ges at & 2258 & $3.4 \mathrm{I}$ & NNE & 7 & \\
\hline I5 59 & 3.51 & $\mathrm{~N}$ & I 2,6 & S2: 11 & $235^{8}$ & 3.50 & $\mathrm{NE}$ & 9 & S. W.S. \\
\hline I6 59 & $3.4 \mathrm{I}$ & $\mathrm{NE}$ & I 5 & inger & \multirow{2}{*}{\multicolumn{5}{|c|}{ April 12, 1904}} \\
\hline 1759 & 3.30 & N & 2 & $\operatorname{sen} 2$ & 3.1 .2 & 31 & & & \\
\hline I8 59 & 3.23 & $N$ & 2 & $30-6 y$ & I Io & 3.69 & NNE & 4 & \multirow{2}{*}{ S. W. S. } \\
\hline I9 59 & $3.22 \mathrm{~L}$ & $\mathrm{NE}$ & I & & 58 & 3.78 & $\mathrm{NE}$ & 8 & \\
\hline 2103 & 3.29 & $N$ & $2=7$ & F. L. & 258 & 3.97 & $\mathrm{~N}$ & 8 & $\cos a \mathbf{a}$ \\
\hline-59 & 3.35 & ENE & $3+1$ & S. W. S. & 355 & 4.00 & $\mathrm{~N}$ & 10 & \\
\hline 2259 & 3.49 & NNE & 3 & & 458 & $4.03 \mathrm{H}$ & $\mathrm{N}$ & 14 & E2 $2 x$ \\
\hline 2359 & 3.55 & ENE & 4 & S. W. S. & 558 & 3.90 & $\mathrm{~N}$ & 10 & Resi \\
\hline G.WIR. & 6 & & 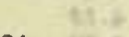 & cesces & 658 & $3 \cdot 74$ & $\mathrm{~N}$ & II & \\
\hline & $i i$ & April ro, Ig04 & 0,5 & 692 & 758 & 3.55 & $\mathbf{N}$ & Io & S. W. S. \\
\hline 059 & 3.62 & ENE: & 2 & S. W.S. & $\begin{array}{l}858 \\
0 \\
58\end{array}$ & $3 \cdot 39$ & $N$ & II & F. I \\
\hline J 59 & $3.65 \mathrm{HI}$ & NE & 3 & & $\begin{array}{r}950 \\
1058\end{array}$ & $\begin{array}{l}3.30 \\
3.29 \mathrm{~L}\end{array}$ & $\mathrm{~N}$ & $\begin{array}{r}10 \\
8\end{array}$ & Q \\
\hline 259 & 3.63 & $N$ & 2 & and sol & 1203 & & $\mathrm{NW}$ & 6 & 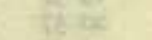 \\
\hline 359 & $3 \cdot 54$ & $\mathrm{E}$ & 3 & troves & 58 & 3 . & $N$ & 7 & 15 is \\
\hline 459 & 3.40 & SSF: & 2 & & 1358 & 3.78 & $\mathrm{~N}$ & 4 & bints \\
\hline 559 & $3 \cdot 31$ & $\mathrm{~N}$ & 2 & & 1458 & 3.95 & $\mathrm{~N}$ & 5 & $=3$ \\
\hline 659 & 3.23 & Calm & $20-1010$ & & 1558 & 4.10 & SE & 4 & 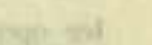 \\
\hline $\begin{array}{l}759 \\
859\end{array}$ & $\begin{array}{l}3.201 \\
3.20\end{array}$ & E & $\begin{array}{l}2 \\
2\end{array}$ & $\begin{array}{l}\text { S. W. S } \\
\text { F. L. }\end{array}$ & $\begin{array}{l}1658 \\
1758\end{array}$ & 4.1611 & E & 2 & tiluis \\
\hline 959 & 3.29 & $N$ & 2 & कo 4 & $\begin{array}{l}1858 \\
185\end{array}$ & $\begin{array}{l}4.04 \\
3.92\end{array}$ & W & 6 & \\
\hline I0 59 & $3 \cdot 42=$ & Calm & .et 2 & 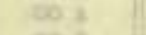 & 1958 & 3.71 & W & 7 & F. L. \\
\hline II 59 & $3.59=$ & $\mathrm{E}$ & I & $m 3$ & 2058 & 3.50 & W & 6 & S. W.S. \\
\hline 1300 & $3.7 \mathrm{I}$ & SE & I & $00 \%$ & 2158 & 3.40 & WSW & 9 & ? \\
\hline 59 & 3.82 & $\mathrm{SE}$ & 3 & $\cos : 5$ & 2258 & $3.39 \mathrm{~L}$ & WSW & 9 & \\
\hline I4 59 & 3.87 & $\mathrm{SE}_{\mathrm{S}}$ & 2 & $00:$ if & 2358 & 3.50 & SW & IO & S.W.S. \\
\hline $\begin{array}{l}1959 \\
1659\end{array}$ & $\begin{array}{l}3.59 \\
3.81\end{array}$ & $\stackrel{S}{S}$ & $\begin{array}{l}2 \\
2\end{array}$ & कè & \multirow{2}{*}{\multicolumn{5}{|c|}{ Ice drifting from the north at Cape Saule at I4:00. }} \\
\hline 1759 & 3.70 & $\mathrm{~N}$ & 3 & $60 \mathrm{in}$ & & & & & \\
\hline 1859 & 3.60 & $\mathrm{~N}$ & 6 & & \multicolumn{4}{|c|}{ April I3, 1904} & \\
\hline 1959 & 3.59 & $N$ & 2 & F. L. & - 58 & 3.65 & SW & 9 & \multirow{2}{*}{ S. W. S. } \\
\hline 2059 & 3.57 & N & 2 & S. W. S. & I 58 & 3.87 & SW & 9 & \\
\hline 2159 & $3.50 \mathrm{~L}$ & NNIV & 2 & 0031 & 258 & 4.06 & WSW & II & $6 \% 11$ \\
\hline 2259 & 3.56 & SW & 3 & & 35 & $4 \cdot 1$ & WSW & IO & $x=0$ \\
\hline 2359 & 3.69 & NW & 4 & S. W. S. & 458 & 4.25 & SW & I4 & $76 \mathrm{kr}$ \\
\hline Leave & open water & I I $: 00$ & 41 & tor 81 & 558 & $4 \cdot 32 \mathrm{H}$ & W & 15 & $60+3$ \\
\hline and & & . . & $\sin 3$ & $a-251$ & 658 & $4 \cdot 15$ & NNW & 15 & \\
\hline 8098 & $s$ & April II, I90 & $04=1$ & or is & $\begin{array}{ll}7 & 58 \\
858 & 58\end{array}$ & $\begin{array}{l}3.94 \\
3.70\end{array}$ & $\begin{array}{l}\text { W } \\
W\end{array}$ & $\begin{array}{l}15 \\
16\end{array}$ & S. W. S. \\
\hline 0 58 & 3.78 & $\mathrm{NE}$ & 5 & S. W. S. & 957 & 3.5 & NW & I7 & 20 \\
\hline I 58 & 3.88 & & 8 & & 1057 & 3.4 & W & I9 & tentest \\
\hline 258 & $3.96 \mathrm{H}$ & $\mathrm{NNE}$ & I2 & $\operatorname{sen}, 86$ & II 57 & $3 \cdot 5$ & W & $2 I$ & $200 \mathrm{kF}$ \\
\hline 358 & 3.94 & NNW & 6 & & 1251 & 3.6 & NW & 20 & 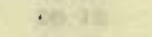 \\
\hline 458 & 3.86 & $\mathrm{NE}$ & 12 & $40>35$ & I3 57 & 3.80 & NW & 22 & 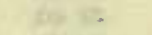 \\
\hline 558 & 3.71 & $\mathrm{NE}$ & 14 & eore & 1457 & 4. 10 & $W$ & $2 \mathrm{I}$ & 6018 \\
\hline 658 & $3 \cdot 58$ & SW & 13 & & I5 57 & $4 \cdot 3^{0}$ & IV .. & 20 & $a, 74$ \\
\hline $75^{8}$ & 3.52 & NE & 24 & S. W.S. & 165 & $4.43 \mathrm{H}$ & W & 18 & \\
\hline 858 & $3 \cdot 34 \mathrm{~L}$ & $N E$ & $3 \mathrm{I}$ & F. L. & 1757 & $4 \cdot 41$ & W & 18 & thest \\
\hline 958 & $3 \cdot 36$ & NE & 33 & $\cos 2$ & 1857 & $4 \cdot 30$ & $W$ & 18 & \\
\hline I0 58 & 3.42 & NW & I4 & $\infty \mathrm{f}$ & 1957 & 4.00 & WSW & 16 & F. L \\
\hline 1204 & $3 \cdot 5^{8}$ & NE & $2 \mathrm{I}$ & 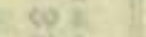 & 2057 & 3.71 & $\begin{array}{l}\text { WSW } \\
\text { WSW }\end{array}$ & 17 & S. W. S. \\
\hline 58 & $3.7^{\circ}$ & $\mathrm{N}$ & I0 & $05=$ & $\begin{array}{l}2157 \\
22\end{array}$ & 3.55 & WSw & 16 & $40:$ \\
\hline I3 58 & 3.89 . & $\mathrm{NE}$ & $17:$ & .00 & 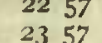 & $\begin{array}{l}3.40 \\
3.36 \mathrm{~L}\end{array}$ & W & $\begin{array}{l}10 \\
17\end{array}$ & \\
\hline I4 58 & 4.02 & SE & 9 & 60 & \multirow{2}{*}{\multicolumn{5}{|c|}{$\begin{array}{l}\text { At ol } 58 \mathrm{~m} \text { staff read } 3.60 \text { feet and } 3.70 \text { feet due to } \\
\text { swell. }\end{array}$}} \\
\hline $\begin{array}{l}15 \\
16 \\
16\end{array}$ & $\begin{array}{l}4.0911 \\
4.06\end{array}$ & $\stackrel{N}{N}$ & $\begin{array}{l}10 \\
14\end{array}$ & F. L. & & & & & \\
\hline
\end{tabular}


Tabulation of tidal observations at Teplitz Bay, Rudolph Island

\begin{tabular}{|c|c|c|c|c|c|c|c|c|c|}
\hline $\begin{array}{c}\text { Local } \\
\text { mean } \\
\text { time }\end{array}$ & $\begin{array}{l}\text { Reading } \\
\text { of tide } \\
\text { staff }\end{array}$ & $\begin{array}{l}\text { Wind } \\
\text { direction }\end{array}$ & Velocity & Observer & $\begin{array}{l}\text { Local } \\
\text { mean } \\
\text { time }\end{array}$ & $\begin{array}{l}\text { Reading } \\
\text { of tide } \\
\text { staff }\end{array}$ & $\begin{array}{l}\text { Wind } \\
\text { direction }\end{array}$ & Velocity & Observer \\
\hline
\end{tabular}

\begin{tabular}{|c|c|c|c|}
\hline$h m$ & Feet & 12 & Mile \\
\hline o 57 & 3.50 & WSW & 18 \\
\hline I 57 & $3.72^{*}$ & W & 17 \\
\hline 257 & 4.00 & W & \\
\hline 357 & 4.22 & WSW & 18 \\
\hline 457 & $4.50 \mathrm{H}$ & SW & \\
\hline 557 & 4.47 & WSW & 17 \\
\hline 657 & $4 \cdot 36$ & W & 18 \\
\hline 757 & 4.18 & W & 16 \\
\hline 857 & 3.89 & W & 14 \\
\hline 957 & 3.62 & W & \\
\hline 10 57 & 3.42 & W & \\
\hline 12 oI & $3.39 \mathrm{~L}$ & W & \\
\hline 57 & 3.49 & W & \\
\hline 1357 & 3.66 & W & \\
\hline 1457 & 3.99 & NW & \\
\hline 1557 & 4.21 & $\mathrm{~N}$ & \\
\hline 1657 & 4.40 & $\mathrm{~N}$ & \\
\hline 1757 & $4.48 \mathrm{H}$ & $\mathrm{NE}$ & \\
\hline 1857 & 4.40 & NW & \\
\hline I9 57 & 4.16 & $\mathrm{~N}$ & \\
\hline 2057 & 3.85 & SE & \\
\hline 2157 & 3.51 & $\mathbf{E}$ & \\
\hline $23 \mathrm{I} 4$ & 3.28 & $\mathbf{E}$ & \\
\hline 57 & $3.21 \mathrm{~L}$ & $\mathbf{E}$ & \\
\hline
\end{tabular}

S. W.S.

17
19

S. W.

$\begin{array}{ll}h m & \text { Feet } \\ 600 & 4.63 \\ 700 & 4.65 \mathrm{H} \\ 800 & 4.55 \\ 900 & 4.24 \\ 59 & 3.91 \\ 1059 & 3.58 \\ 1159 & 3.35 \\ 1259 & 3.25 \mathrm{~L} \\ 1359 & 3.36 \\ 1459 & 3.60 \\ 1559 & 3.97 \\ 1659 & 4.24 \\ 1759 & 4.42 \\ \text { I8 } 59 & 4.51 \mathrm{H} \\ 1959 & 4.4 \mathrm{I} \\ 2059 & 4.12 \\ 2159 & 3.70 \\ 2259 & 3.36 \\ 2359 & 3.07\end{array}$

$\begin{array}{cr} & \text { Miles } \\ \text { SW } & 9 \\ \text { S } & 7 \\ \text { S } & 4 \\ \text { SW } & 6 \\ \text { SW } & 12 \\ \text { W } & \text { II } \\ \text { W } & 14 \\ \text { SW } & \text { II } \\ \text { SE } & 6 \\ \text { SE } & 8 \\ \text { E } & 10 \\ \text { SW } & 15 \\ \text { SW } & 17 \\ \text { SW } & 17 \\ \text { SW } & 16 \\ \text { SW } & 13 \\ \text { WSW } & 11 \\ \text { WSW } & 10 \\ \text { SSE } & 76\end{array}$

S. W.S.

S.W.S

F. L.

S.W.S. Ice moving from southwest. Pressure in southwest. Ice pressure in west 7:00 to 8:15.

S. W. S.

Ice opened abont 50 yards wide, southwest, oneeight mile from shore.

\section{April 15, 1904}

$\begin{array}{lllll}0 & 57 & 3.30 & \mathrm{NE} & 3 \\ 1 & 57 & 3.50 & \mathrm{SE} & 3 \\ 2 & 57 & 3.83 & \mathrm{ENE} & 4 \\ 3 & 57 & 4.13 & \mathrm{E} & 2 \\ 4 & 57 & 4.39 & \mathrm{E} & 5 \\ 5 & 57 & 4.56 \mathrm{H} & \mathrm{E} & 2 \\ 6 & 57 & 4.51 & \mathrm{E} & 3 \\ 7 & 57 & 4.36 & \mathrm{E} & 4 \\ 900 & 4.02 & \mathrm{E} & 2 \\ 1000 & 3.70 & \mathrm{E} & 1 \\ 1100 & 3.48 & \mathrm{~N} & 6 \\ 1203 & 3.36 \mathrm{~L} & \mathrm{~N} & 9 \\ 1300 & 3.39 & \mathrm{~N} & 13 \\ 1400 & 3.59 & \mathrm{~N} & 9 \\ 1500 & 3.90 & \mathrm{NW} & 6 \\ 1600 & 4.20 & \mathrm{NW} & 3 \\ 1700 & 4.49 & \mathrm{NW} & 4 \\ 1800 & 4.68 & \mathrm{NW} & 3 \\ 1900 & 4.69 \mathrm{H} & \mathrm{W} & 4 \\ 2000 & 4.53 & \mathrm{SE} & 3 \\ 21 & 00 & 4.23 & \mathrm{SE} & 6 \\ 2200 & 3.85 & \mathrm{SE} & 7 \\ 2300 & 3.59 & \mathrm{ESE} & 7 \\ 2400 & 3.40 & \mathrm{SE} & 6\end{array}$

S. W. S.

\section{S. W. S. \\ F. L.}

S. W. L. S.

S. W.S.

Tide gauge reading of B. M. No. I, 14.09 feet at noon.

April 16, 1904

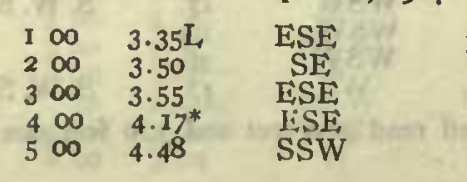

\section{S. W. S.}

S. W.S

\section{April 17, 1904}

$\begin{array}{rlrrr}100 & 2.97 \mathrm{~L} & \mathrm{ESE} & 7 & \text { S.W.S. } \\ 200 & 2.99 & \mathrm{ESE} & 6 & \\ 300 & 3.26 & \mathrm{E} & 3 & \\ 400 & 3.60 & \mathrm{E} & 12 & \\ 500 & 3.93 & \mathrm{NW} & 10 & \\ 600 & 4.28 & \mathrm{NW} & 8 & \\ 700 & 4.41 & \mathrm{NW} & 6 & \\ 800 & 4.50 \mathrm{H} & \mathrm{N} & 16 & \text { S. W.S. } \\ 900 & 4.30 & \mathrm{NE} & 6 & \mathrm{~F} . \mathrm{L} . \\ 1000 & 3.92 & \mathrm{NE} & 6 & \\ 1100 & 3.69 & \mathrm{NE} & 8 & \\ 1203 & 3.39 & \mathrm{NE} & 10 & \\ 1300 & 3.21 \mathrm{~L} & \mathrm{E} & 10 & \\ 1400 & 3.23 & \mathrm{NE} & 9 & \\ 1500 & 3.42 & \mathrm{NE} & 3 & \\ 1600 & 3.79 & \mathrm{NE} & 5 & \\ 1707 & 4.12 & \mathrm{NE} & 14 & \\ 1800 & 4.42 & \mathrm{NE} & 18 & \\ 1900 & 4.58 & \mathrm{NE} & 13 & \\ 2000 & 4.60 \mathrm{H} & \mathrm{W} & 18 & \text { F. L. } \\ 2100 & 4.45 & \mathrm{~N} & 4 & \text { S. W.S. } \\ 2200 & 4.10 & \mathrm{ENE} & 2 & \\ 2300 & 3.70 & \mathrm{SE} & 3 & \\ 2400 & 3.40 & \mathrm{ENE} & 9 & \text { S. W. S. }\end{array}$

Ice opened west $5: 00$. Ice moving northwest about 9:00.

\section{April 18, 1904}

$\begin{array}{lllll}100 & 3.12 & \text { ENE } & \text { II } & \text { S. .W S. } \\ 200 & 3.12 \mathrm{~L} & \text { ENE } & 17 & \\ 300 & 3.26 & \text { NE } & 20 & \\ 400 & 3.57 & \text { NE } & 18 & \\ 500 & 3.98 & \text { NE } & 18 & \\ 600 & 4.30 & \text { NE } & 18 & \\ 700 & 4.53 & \text { NNE } & 21 & \\ 800 & 4.64 \mathrm{H} & \text { NE } & 21 & \text { S. W. S. } \\ 901 & 4.53 & \text { N } & \text { I4 } & \text { F. L. }\end{array}$


'IIDAL, OBSERVATIONS

Tabulatian of tidal observatians at Teplitz Bay, Rudolph Island

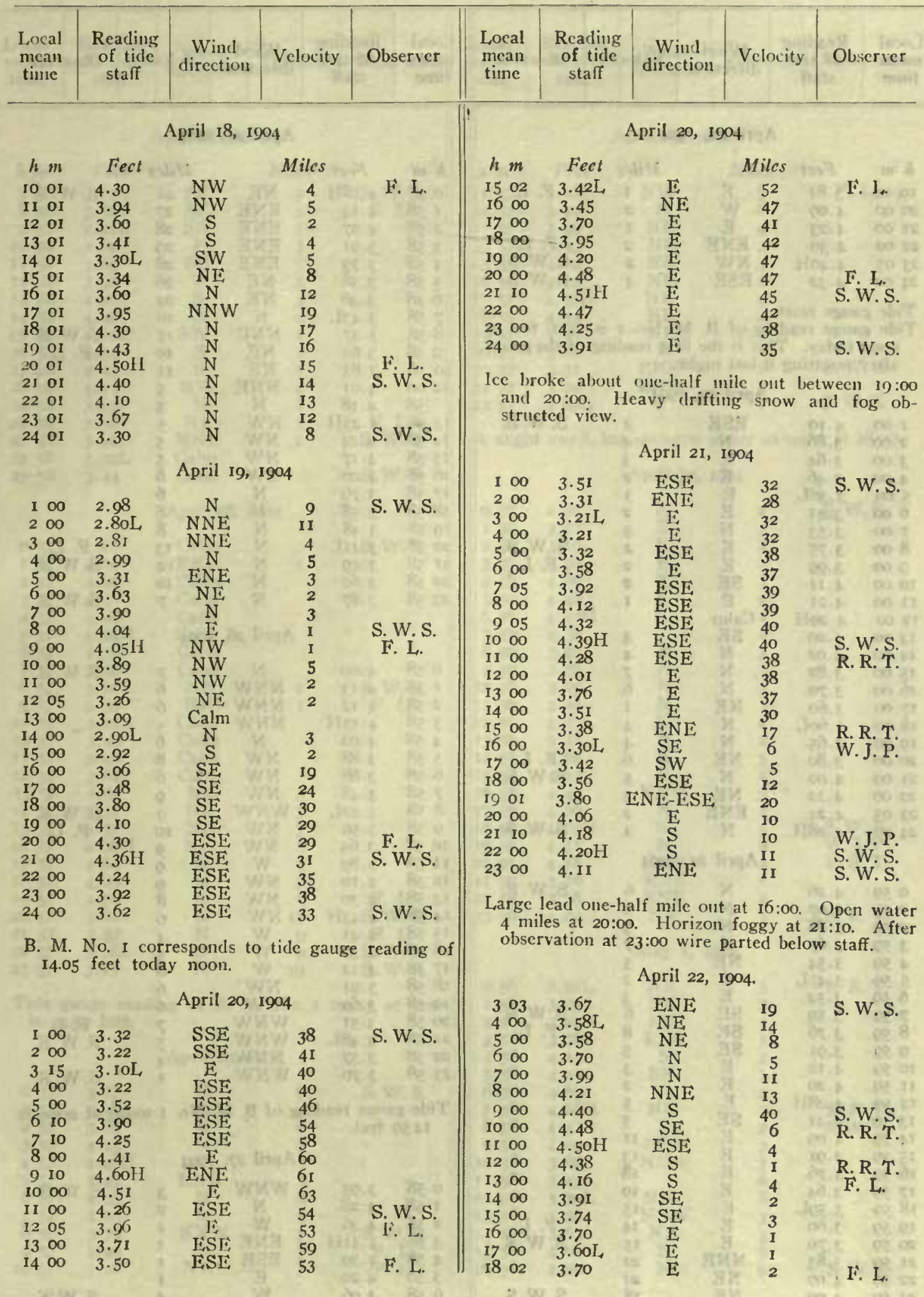


Tabulation of tidal observations at Teplitz Bay, Rudolph Island

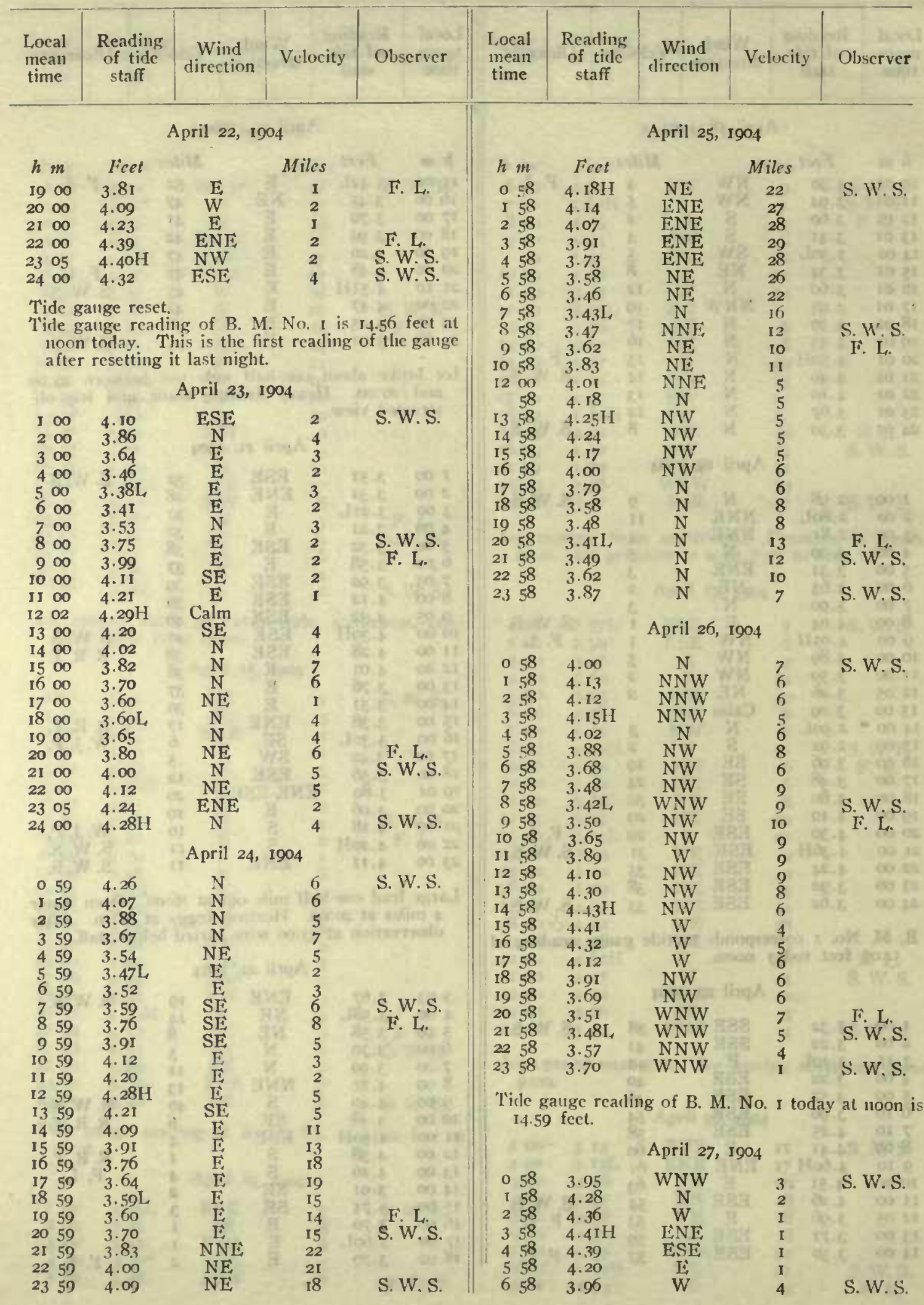


Tabulation of tidal observations at Teplitz Bay, Rudolph Island

\begin{tabular}{|c|c|c|c|c|c|c|c|c|c|c|c|}
\hline $\begin{array}{l}\text { Local } \\
\text { meall } \\
\text { time }\end{array}$ & $\begin{array}{l}\text { Reading } \\
\text { of tide } \\
\text { staff }\end{array}$ & $\begin{array}{l}\text { Wind } \\
\text { direction }\end{array}$ & Velocity & Observer & $\begin{array}{l}\text { Local } \\
\text { mean } \\
\text { time }\end{array}$ & $\begin{array}{l}\text { Reading } \\
\text { of tide } \\
\text { staff }\end{array}$ & $\begin{array}{l}\text { Wind } \\
\text { directiont }\end{array}$ & \multicolumn{2}{|c|}{ Velocity } & & Observer \\
\hline \multicolumn{5}{|c|}{ April 27, I904 } & \multicolumn{7}{|c|}{ April 29, I904 } \\
\hline$h m$ & Feet & 512 & Miles & $164 y^{4}$ & $h m$ & Feet & think: & \multicolumn{2}{|c|}{ Miles } & 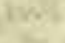 & $=8$ \\
\hline 758 & 3.73 & SE & 2 & S. W. S. & 1157 & $3.06 \mathrm{~L}$ & NW & \multirow{2}{*}{\multicolumn{2}{|c|}{5}} & & \multirow[t]{2}{*}{ F. L. } \\
\hline 858 & 3.54 & $\mathrm{SE}$ & 3 & & 1257 & 3.20 & $\mathrm{~N}$ & & & Afros & \\
\hline 958 & $3.4 \mathrm{IL}$ & SE & 3 & & 1357 & 3.49 & $\mathrm{NE}$ & & 4 & as & cas 31 \\
\hline 1058 & 3.50 & $\mathrm{E}$ & 3 & & 1457 & 3.83 & $\mathrm{NE}$ & $y$ & 3 & ax: & $18=0$ \\
\hline II 58 & 3.69 & SF & 5 & 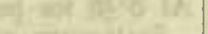 & I5 57 & 4.20 & NE & & 3 & ate & Le 1 \\
\hline 1258 & 3.91 & $S$ & 5 & rescthrs & 1657 & 4.42 & $\mathrm{NE}$ & 26 & 2 & 19 & $x=5$ \\
\hline 1358 & 4.21 & $\mathrm{~S}$ & 3 & at atull & I7 57 & $4.57 \mathrm{H}$ & Nli & Whe & 2 & Bite. & 20 \\
\hline 1458 & 4.48 & S & 2 & & 1857 & $4 \cdot 45$ & $\mathrm{NE}$ & & 2 & & \\
\hline 15.8 & $4.60 \mathrm{H}$ & SW & 2 & & 1957 & 4.16 & $\mathrm{NE}$ & Es & 2 & $=$ & 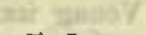 \\
\hline 1658 & 4.59 & $S$ & I & & 2057 & 3.80 & $\mathrm{~N}$ & & I & & F. L. \\
\hline 17.58 & 4.45 & S & I & 28.6 & 2157 & 3.31 & ENI: & & 2 & & S. W. S. \\
\hline 1858 & 4.19 & NW & I & 3 & 2257 & 3.02 & $\mathrm{ENE}$ & cats & 3 & & \\
\hline 1958 & 3.85 & $\mathrm{~N}$ & I & 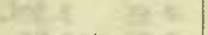 & 2357 & $2.82 \mathrm{~L}$ & $\mathrm{E}$ & & I & & S. W. S. \\
\hline 2058 & 3.59 & NE: & I & F. L & & & & & & & \\
\hline 2158 & 3.40 & NNE & i & S. W.S. & A sligh & t swell, b & out no open & water & er ill & sight & it at $18: 57$. \\
\hline $\begin{array}{l}2258 \\
2358\end{array}$ & $\begin{array}{l}3.32 \mathrm{~L} \\
3.44\end{array}$ & $\begin{array}{l}\text { Calm } \\
\text { Calm }\end{array}$ & & S. W. S. & & & April 30, & 1904 & & is. & 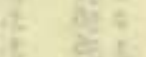 \\
\hline Ir: & & April 28, & 1904 & & o 56 & 2.89 & $\mathrm{E}$ & Ghe & 2 & 5 & S. W.S. \\
\hline o 57 & 3.67 & Calm & & S.W.S. & $\begin{array}{ll}1 & 56 \\
2 & 56\end{array}$ & $\begin{array}{l}3.13 \\
3.40\end{array}$ & (4) & Ku & $\begin{array}{l}3 \\
2\end{array}$ & (1). & i $\quad 85$ \\
\hline 157 & 4.00 & ENE & I & N. Pr.s. & 356 & $\begin{array}{l}3.49 \\
3.94\end{array}$ & $\mathbf{E}$ & $\frac{19}{4}$ & $\begin{array}{l}2 \\
2\end{array}$ & $\operatorname{ting} 2$ & 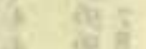 \\
\hline 257 & 4.29 & Calm & & 919 & 456 & 4.29 & $\mathbf{E}$ & sive & 3 & 106 & 98 \\
\hline 357 & $4.4 \mathrm{I}$ & Calm & & $\cos , \pi$ & 556 & 4.46 & $\overrightarrow{\mathrm{F}}$ & W & 4 & bes & 83 \\
\hline 457 & 4.48 & Calm & ty & noger & 656 & $4.49 \mathrm{H}$ & in & if & 2 & se: & $8 \pi$ \\
\hline 557 & $4.54 \mathrm{H}$ & Calm & t & $200=0$ & 756 & $4 \cdot 32$ & 47 & & 2 & $b^{2}$ & 68 \\
\hline 657 & $4 \cdot 34$ & Calm & 5 & no 84 & 856 & 3.95 & 64 & 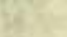 & 3 & tos. & 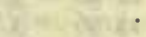 \\
\hline 757 & 3.08 & Calm & & 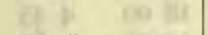 & 956 & 3.56 & $\mathrm{~N}$ & & 2 & ${ }^{2} \mathrm{~g}$ & at ith \\
\hline 857 & 3.70 & $\mathrm{NF}$ & I & S. W. S. & I0 56 & 3.29 & $N$ & $y$ & 3 & 2. & 2053 \\
\hline 957 & 3.50 & $\mathrm{NE}$ & I & F. L. & II 56 & 3.10 & $\mathrm{~N}$ & & 3 & ig. & 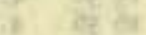 \\
\hline 1057 & $3.40 \mathrm{~L}$ & Calnı & & 15 phy & 1256 & $3.07 \mathrm{~L}$ & I: & $y$ & 4 & 20 & $20 \mathrm{ks}$ \\
\hline 1057 & 3.40 & Calm & & $2 x^{4}$ & 1356 & $3.4 \mathrm{I}$ & $\mathrm{N}$ & $\mathrm{y}$ & 2 & (6) & $5 \times$ \\
\hline 1157 & 3.46 & Calm & s & $29 \div$ & 1456 & 3.64 & $\mathrm{E}$ & 6: & 5 & fing & an of \\
\hline 1257 & 3.64 & Calm & & wext. & 1556 & 4.12 & $\mathrm{E}$ & mir & 2 & -0 & in \\
\hline 1357 & 4.00 & $\mathrm{NE}$ & 4 & 20918 & 1656 & 4.40 & NNW & Ex & 3 & & at: \\
\hline 1457 & 4.31 & $\mathrm{NE}$ & 7 & & 1756 & 4.60 & $\mathrm{~N}$ & a & 3 & 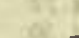 & b- \\
\hline 1557 & 4.51 & $\mathrm{NE}$ & 7 & hapt $\times 1$ & 1856 & $4.7 \mathrm{oH}$ & $\hat{N}$ & 3 & 5 & & S. W. S. \\
\hline 1657 & $4.68 \mathrm{H}$ & $\mathrm{NE}$ & 6 & & 1956 & 4.51 & $\mathrm{~N}$ & & 2 & & W. J. P. \\
\hline 1757 & 4.64 & $\mathrm{~N}$ & 5 & & 2056 & 4. 18 & ENE & & 2 & & R. R. T. \\
\hline 1857 & 4.40 & $\mathrm{~N}$ & 7 & & 2156 & 3.70 & ENE & 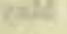 & 2 & & \\
\hline 1957 & 4.00 & $\mathrm{~N}$ & 9 & F. L. & 2256 & $3 \cdot 3$ & ENE & & 2 & & \\
\hline 2057 & 3.61 & $\mathrm{~N}$ & II & S.W.S. & 2356 & 3.02 & $\mathrm{NE}$ & AK & 3 & th & R.R.T. \\
\hline 2157 & 3.32 & $\mathrm{~N}$ & 9 & F. L. & & & 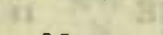 & & & 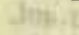 & \\
\hline 2257 & $3.08 \mathrm{~L}$ & $\mathrm{~N}$ & 9 & S.W.S. & te & & May I, I & 904 & & 3t: & sets \\
\hline 2357 & 3.13 & $\mathrm{~N}$ & 10 & S.W.S. & & & & & & & init \\
\hline & 10e $\pi$ & & & & 056 & $2.91 \mathrm{~L}$ & NE: & 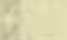 & 3 & 1 & R. R. T. \\
\hline $\begin{array}{l}\text { Tide } 8 \\
\text { is } 14\end{array}$ & $\begin{array}{l}\text { 1ge re } \\
3 \text { feet. }\end{array}$ & ing of $B$. & 1. No. I & today at $5: 00$ & $\begin{array}{l}156 \\
256\end{array}$ & 3.07 & $\underset{N E}{N E}$ & $2 x$ & 2 & $\frac{94}{65}:$ & a -18 \& \\
\hline 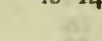 & & 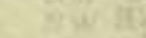 & & 28 & 356 & $\begin{array}{l}3.21 \\
3.76\end{array}$ & ENE & $4 \%$ & $\begin{array}{l}3 \\
1\end{array}$ & 25 & 300 \\
\hline & & April 29 & 1904 & 2,3 & 456 & 4.19 & $\mathrm{~N}$ & $4 \times 5$ & I & iilon & 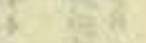 \\
\hline & & & & Wristas 50 & 556 & 4.45 & $\mathrm{SE}$ & & 3 & 154 & $A B$ \\
\hline 057 & 3.17 & $\mathrm{~N}$ & 8 & S. W. S. & 656 & 4.63 & $\mathrm{E}$ & & 2 & 601 & 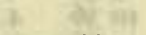 \\
\hline I 57 & & $\mathrm{~N}$ & 9 & & 756 & $4.68 \mathrm{H}$ & $\mathrm{NE}$ & $y$ & 2 & & R. R. T. \\
\hline 257 & 3.82 & $\mathrm{~N}$ & 6 & 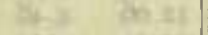 & 856 & 4.42 & $\mathrm{E}$ & 4 & 2 & 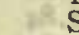 & S. W. S. \\
\hline 357 & 4.15 & $\mathrm{~N}$ & 7 & $\infty \mathrm{ur}$ & 956 & 4.02 & SSW & 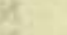 & & & \\
\hline 457 & $4.48 \mathrm{H}$ & $\mathrm{N}$ & 5 & $0 \cdot 17$ & 1056 & 3.66 & NNW & 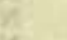 & 3 & Lakin & 243 \\
\hline 557 & 4.44 & ENE & 5 & as 31 & II 56 & 3.43 & 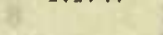 & 4 & 6 & 18.2 & $60 \mathrm{n}$ \\
\hline 657 & $4 \cdot 35$ & $\mathrm{~N}$ & 8 & 00 sit & 1256 & $3.30 \mathrm{~L}$ & NW & 4 & 8 & bits & Fe 53 \\
\hline 757 & 4.05 & $N$ & 9 & 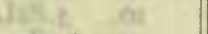 & 1356 & 3.36 & NNW & 5 & 3 & 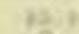 & the 51 \\
\hline 857 & $3 \cdot 70$ & NNW & 7 & S. W.S & 1456 & 3.63 & $\mathrm{NE}$ & 1 & 3 & 68.3 & 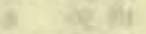 \\
\hline 957 & 3.40 & $N$ & 7 & F. L. & 1556 & 4.04 & $N$ & 6. & 5 & 001 & t. $\quad$ get of \\
\hline 1057 & 3.12 & NW & 7 & F. L. & 1656 & 4.50 & $\mathrm{~N}$ & An & 9 & fites 5 & S. W.S. \\
\hline
\end{tabular}


Tabulation of tidal obserzations at Teplits Bay, Rudolph Island

\begin{tabular}{|c|c|c|c|c|}
\hline $\begin{array}{l}\text { Local } \\
\text { mean } \\
\text { time }\end{array}$ & $\begin{array}{l}\text { Reading } \\
\text { of tide } \\
\text { staff }\end{array}$ & $\begin{array}{l}\text { Wind } \\
\text { direction }\end{array}$ & $\begin{array}{l}\text { Anemom- } \\
\text { eter } \\
\text { records }\end{array}$ & Observer \\
\hline \multicolumn{5}{|c|}{ May 1,1904} \\
\hline$h \mathrm{~m}$ & I'ect & $516-2$ & Milcs & \\
\hline 1756 & 4.78 & $\stackrel{\mathrm{N}}{\text { NNIV }}$ & $\begin{array}{r}15 \\
8\end{array}$ & S. W.S. \\
\hline 1956 & $\begin{array}{l}5.0311 \\
4.95\end{array}$ & NNW & 31 & S. W.S. \\
\hline 2056 & 4.79 & NW & 14 & V. \\
\hline $\begin{array}{l}2156 \\
2256\end{array}$ & $\begin{array}{l}4.36 \\
3.94\end{array}$ & $\begin{array}{l}\text { W } \\
\text { NNW }\end{array}$ & 12 & 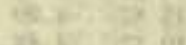 \\
\hline 2356 & $\begin{array}{l}3.94 \\
3.59\end{array}$ & NNIV & 13 & J. V. \\
\hline
\end{tabular}

Young ice opening one-half milc to the west at $16: 56$.

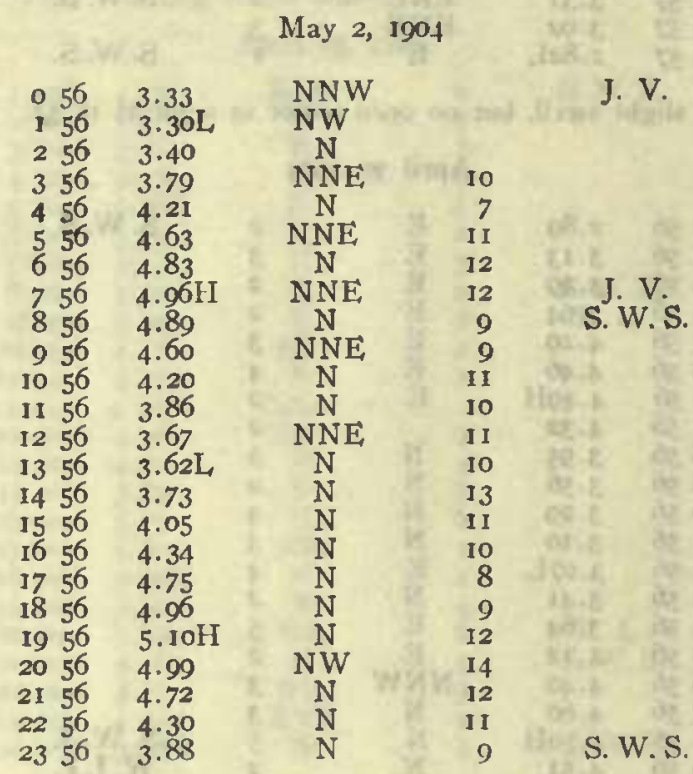

$\begin{array}{lll}0 & 56 & 3.61 \\ 1 & 56 & 3.40 \mathrm{~L} \\ 2 & 56 & 3.41 \\ 3 & 56 & 3.61 \\ 4 & 56 & 3.99 \\ 5 & 56 & 4.40 \\ 6 & 56 & 4.67 \\ 7 & 56 & 4.83 \\ 8 & 56 & 4.94 \mathrm{H} \\ 9 & 56 & 4.74 \\ 10 & 56 & 4.44 \\ 12 & 16 & 4.01 \\ & 56 & 3.84 \\ 13 & 56 & 3.69 \\ 14 & 56 & 3.66 \mathrm{~L} \\ 15 & 56 & 3.81 \\ 16 & 56 & 4.10 \\ 17 & 56 & 4.44 \\ 18 & 56 & 4.80 \\ 19 & 56 & 4.92 \\ 20 & 56 & 4.99 \mathrm{H}\end{array}$

May 3, 1904

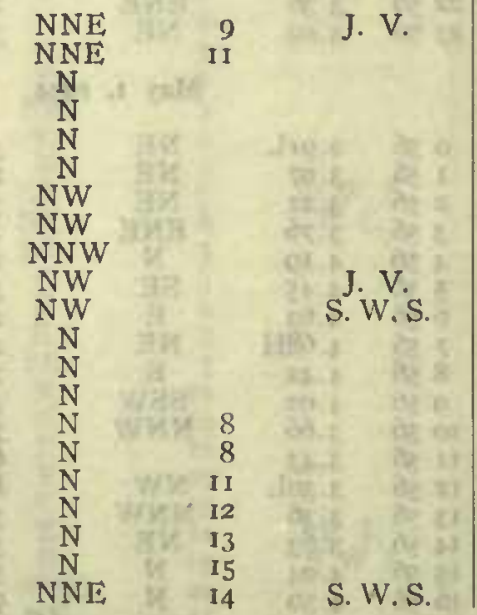

\begin{tabular}{|c|c|c|c|c|}
\hline $\begin{array}{l}\text { Local } \\
\text { time } \\
\text { meana }\end{array}$ & $\begin{array}{l}\text { Rearling } \\
\text { of tide } \\
\text { staff }\end{array}$ & $\begin{array}{l}\text { Wind } \\
\text { direction }\end{array}$ & $\begin{array}{l}\text { Anemom- } \\
\text { eter } \\
\text { records }\end{array}$ & Observer \\
\hline
\end{tabular}

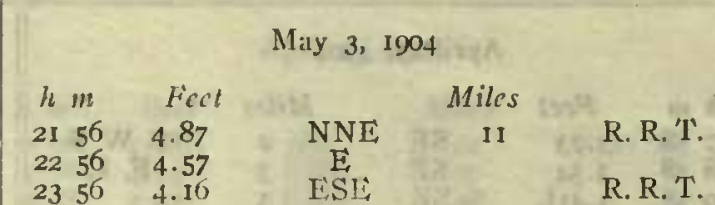

At $0: 56$ ice pack moving off in westerly direction. Stationtary at 12:16; has moved west about five miles.

\section{May 4, 1904}

$\begin{array}{rrl}0 & 55 & 3.82 \\ \text { I } & 55 & 3.61 \\ 2 & 55 & 3.50 I \\ 3 & 55 & 3.61 \\ 4 & 55 & 3.86 \\ 5 & 55 & 4.21 \\ 6 & 55 & 4.57 \\ 7 & 55 & 4.82 \\ 8 & 55 & 4.94 \mathrm{H} \\ 9 & 55 & 4.92 \\ 10 & 55 & 4.74 \\ 12 & 01 & 4.38 \\ & 55 & 4.19 \\ 13 & 59 & 3.94 \\ 15 & 00 & 3.82 \mathrm{~L} \\ 1600 & 3.91 \\ 1700 & 4.07 \\ 1800 & 4.45 \\ 19 & 00 & 4.65 \\ 20 & 04 & 4.87 \\ 21 & 00 & 4.95 \mathrm{H} \\ & 30 & 4.95 \\ 22 & 00 & 4.91 \\ 23 & 00 & 4.72 \\ 21 & 00 & 4.37\end{array}$

$\mathrm{NE}$

WSIV

ESE

$\mathrm{E}$

ENE

NW

ESE

S

N

SSE

SSE

SE

E

S. W. S.

J. V.

J. V.

Ice pack returning at $21: 30$.

\section{May 5, 1904}

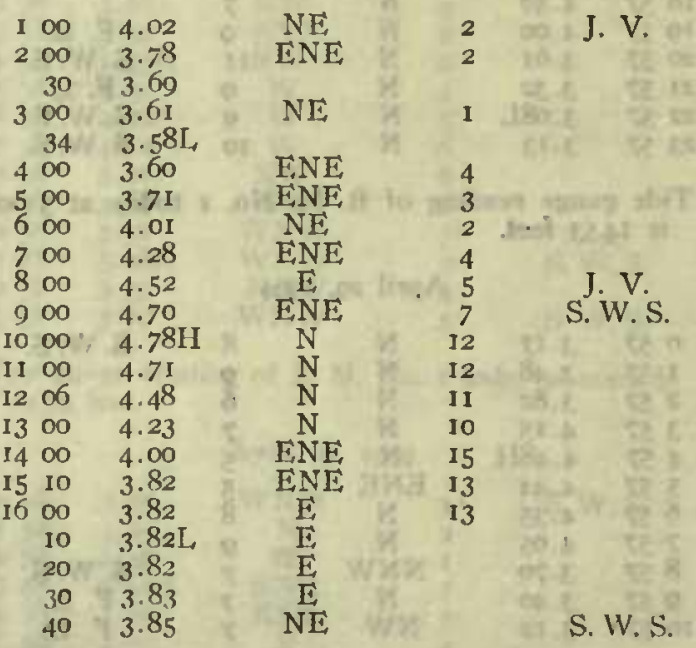


Tabulation of tidal observations at Teplitz Bay, Rudolph Island

\begin{tabular}{|c|c|c|c|c|c|c|c|c|c|c|c|}
\hline $\begin{array}{l}\text { Local } \\
\text { mean } \\
\text { time }\end{array}$ & $\begin{array}{l}\text { Reading } \\
\text { of tide } \\
\text { staff }\end{array}$ & $\begin{array}{l}\text { Wind } \\
\text { direction }\end{array}$ & Veloci & & Observer & $\begin{array}{l}\text { Local } \\
\text { mean } \\
\text { time }\end{array}$ & $\begin{array}{l}\text { Reading } \\
\text { of tide } \\
\text { staff }\end{array}$ & $\begin{array}{l}\text { Wind } \\
\text { direction }\end{array}$ & \multicolumn{2}{|c|}{ Velocity } & Observer \\
\hline \multicolumn{6}{|c|}{ May 5,1904} & \multicolumn{6}{|c|}{ May 6, 1904} \\
\hline$h m$ & \multicolumn{2}{|c|}{ Feet } & Miles & & \multirow{3}{*}{ S. W.S. } & $h m$ & Feet & & \multicolumn{2}{|l|}{ Miles } & ret \\
\hline 1700 & 3.90 & ESE & 5 & & & 1620 & 3.74 & & & & S. W. S. \\
\hline $\begin{array}{l}\text { I8 } 00 \\
\text { IO } 00\end{array}$ & & $\underset{\mathrm{E}}{\mathrm{NNW}}$ & 3 & & & 30 & $3.7 \mathrm{IL}$ & & & & (a) \\
\hline $\begin{array}{l}1900 \\
2000\end{array}$ & $\begin{array}{l}4 \cdot 28 \\
4 \cdot 5 t\end{array}$ & $\underset{N N E}{E}$ & $\begin{array}{l}9 \\
2\end{array}$ & & $\begin{array}{l}\text { S. W. S. } \\
\text { R. R. T. }\end{array}$ & $\begin{array}{r}40 \\
-50\end{array}$ & $\begin{array}{l}3.71 \\
3.73\end{array}$ & & & iny & $\mathrm{m}$ \\
\hline $\begin{array}{r}2000 \\
40\end{array}$ & 4.61 & $\mathbf{E}_{\mathbf{E}}$ & & & & 1700 & $\begin{array}{l}3.73 \\
3.73\end{array}$ & NNIV & 14 & & we \\
\hline 50 & 4.6 & $\vec{E}$ & & & & 10 & 3.74 & & & & int \\
\hline 2100 & 4.63 & $E \overline{N E}$ & 3 & & se & 20 & 3.76 & & & & 35 \\
\hline 10 & 4.64 & $\mathrm{NE}$ & & es & aif & 1800 & & $\mathrm{NE}$ & 13 & 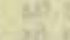 & 60 \\
\hline 20 & 4.6 & NNW & & sy & (111) & 1900 & & $\underset{\mathrm{E}}{\mathrm{N}}$ & 22 & & S W \\
\hline 30 & 4.69 & ENE: & & tis & tox yit & $\begin{array}{l}2000 \\
2100\end{array}$ & & $\underset{S E}{E}$ & 10 & $\sin$ & S. W.S. \\
\hline $\begin{array}{l}40 \\
50\end{array}$ & $\begin{array}{l}4.69 \mathrm{H} \\
4.69\end{array}$ & Calm & & & & 50 & & $\mathrm{NE}$ & 3 & the & R. R. T. \\
\hline 2200 & 4.68 & Calm & & $3^{3}$ & $3 x$ & 2200 & 4.3 & NNE & 5 & & aif \\
\hline 10 & 4.67 & $\mathrm{E} N \mathrm{NE}$ & & 29 & 58 & 10 & & & & 5019 & 9) \\
\hline 20 & 4.65 & $\mathrm{ENE}$ & & 70.1 & 100 & 20 & & ESE & & $\cos x$ & tas \\
\hline 2300 & 4.60 & $\mathrm{ENE}$ & & 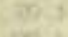 & $3=20$ & 30 & & $E$ & & $17)$ & Q: \\
\hline 2400 & $4 \cdot 39$ & $\mathrm{E}$ & 2 & go: & R. R. T. & 40 & $4.4 I$ & $N \overrightarrow{N E}$ & & & 62 \\
\hline \multirow{2}{*}{\multicolumn{3}{|c|}{ Ice opening in southwest }} & & & $=0$ & 2300 & $\begin{array}{l}4.4 \mathrm{I} \\
4.42 \mathrm{H}\end{array}$ & $\underset{\mathrm{E}}{\mathrm{S}}$ & 3 & & $\pi$ \\
\hline & & & & One- & 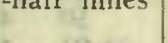 & 10 & 4.41 & ESE & 3 & & +6 \\
\hline \multirow{2}{*}{\multicolumn{6}{|c|}{ Tide reading of $B$. M. No. I at $15: 00$ is 14.565 feet. }} & 20 & $4 \cdot 40$ & ESE & & inis & Cas ist $y$ \\
\hline & & May 6.90 & & & & & 4.40 & ESE & & & 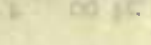 \\
\hline & & May 6, rgc & & & & $24 \stackrel{40}{00}$ & $\begin{array}{l}4.39 \\
4.33\end{array}$ & $\begin{array}{l}\mathrm{ESE} \\
\mathrm{NE}\end{array}$ & \multirow{2}{*}{\multicolumn{2}{|c|}{3}} & \multirow{2}{*}{ R. R. T. } \\
\hline $\begin{array}{ll}1 & 00 \\
2 & 00\end{array}$ & $4 \cdot 11$ & NNE & \multirow{2}{*}{\multicolumn{2}{|c|}{$\begin{array}{ll}2 & \text { R.R.T. } \\
\text { I }\end{array}$}} & \multirow{2}{*}{ R. R. T. } & & & & & & \\
\hline 200 & 3.81 & & & & & \multicolumn{6}{|c|}{ Ice closed at $3: 10$. Lead opened one mile in north } \\
\hline 300 & 3.63 & ENE & 5 & 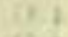 & 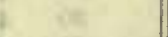 & wot & & at 16:10. $\mathrm{Op}_{\mathrm{p}}$ & Dpen w: & vater & two mile \\
\hline 10 & 3.61 & ENE & & a) & 85 & soutls & :00. & Ice closed so & south at & It $22:$ & 20.821 \\
\hline $\begin{array}{l}20 \\
30\end{array}$ & $\begin{array}{l}3.60 \\
3.58\end{array}$ & $\begin{array}{l}\text { WNW } \\
\text { WSW }\end{array}$ & & $\operatorname{lag}_{0}$ & . & & & & & & 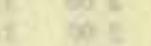 \\
\hline 40 & 3.56 & S & & (6) & in & & & May 7, 190 & & 6 & 201 \\
\hline 50 & $3 \cdot 52$ & SSE & & tore & mats & 100 & 4.13 & $\mathrm{NE}$ & & & R R T \\
\hline 400 & $3.5 \mathrm{IL}$ & $\mathbf{S E}$ & 3 & initi. & we & 200 & $\begin{array}{l}4 . \\
3 .\end{array}$ & $\mathrm{N}$ & $\begin{array}{l}4 \\
2\end{array}$ & & K. K. 1 . \\
\hline Io & $3 \cdot 52$ & ENE & & st: 1 & 89 & 300 & $3 . \%$ & SSW & I & 단. & $\propto$ \\
\hline 20 & $3 \cdot 52$ & $\mathrm{E}$ & & is. & 16 & 400 & & & I & 2 & w. \\
\hline 30 & $3 \cdot 5^{2}$ & & & $\cos 2$ & ex & 30 & 3.52 & EN & & 25 & (16) \\
\hline 40 & 3.53 & NNW & & 38 & 94 & 40 & & & & 3 & 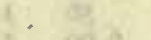 \\
\hline 500 & $3 \cdot 58$ & & 5 & $2, x$ & vil & 50 & & & & 블 & 200 \\
\hline 600 & 3.71 & & 8 & $\sqrt{x}=$ & $a=$ & 500 & 3. & & & $t_{10}=$ & \\
\hline 700 & 3.94 & ENE & 8 & (a) & & 10 & 3.5 & & & 10 & ce \\
\hline 800 & 4.21 & $\mathrm{NE}$ & 9 & $x^{5}$ & R. R. I & 20 & & & & 12. & iㅐㅇㅛ \\
\hline 900 & $4 \cdot 39$ & $\mathrm{E}$ & Ió & $x_{2}$ & S. W.S & 30 & 3.5 & NW & & $4=$ & 13 \\
\hline 20 & 4.43 & $\mathrm{NE}$ & & 8 & 20 & 40 & & & & 89 & $\infty$ \\
\hline 30 & 4.4 & N & & 10 & 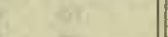 & 5 & & N. & & 38 & as \\
\hline 40 & & $\mathrm{~N}$ & & $\cos 2$ & F & 60 & & $\mathrm{E}$ & I & & $\pi$ \\
\hline 50 & $4 \cdot 53$ & $\mathrm{NE}$ & & & $x$ & 70 & & Cal & & 60 & \\
\hline 1000 & 4.54 & $\mathrm{NE}$ & 9 & 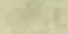 & 18 & 80 & 3.8 & ENE & 2 & $x^{2}$ & R. R. T. \\
\hline Io & 4.55 & NW & & Wo: & 50 & 90 & & $\mathbf{E}$ & 2 & (4) & S. W. S. \\
\hline 20 & $\begin{array}{l}4 \cdot 56 \mathrm{H} \\
4.56\end{array}$ & $\underset{E}{N W}$ & & coin & $(80)$ & 1000 & 4.1 & $\mathrm{E}$ & I & Fitis & by \\
\hline 30 & $\begin{array}{l}4 \cdot 56 \\
4 \cdot 55\end{array}$ & $\begin{array}{l}\mathrm{E} \\
\mathrm{NE}\end{array}$ & & $\begin{array}{l}\text { 2uly } \\
\text { his }\end{array}$ & 난 & 40 & & & & $2 x-1$ & ativ \\
\hline $\begin{array}{l}40 \\
50\end{array}$ & & ENE & & wist & 64 & 50 & & 18 & & Aat & (2) 4 \\
\hline 1100 & 4.54 & ENE & 12 & ce.9. & $\Delta$ & $\begin{array}{ll}\text { I I } & 00 \\
10\end{array}$ & $\begin{array}{l}4.2 \\
4.2\end{array}$ & $\mathbf{E}$ & 2 & 5t. 1 & $0 \pi$ \\
\hline 10 & & & & is:. & एक & 20 & $\begin{array}{l}4.2 \\
4.2\end{array}$ & & & 8 & $\leq$ \\
\hline 1200 & 4.47 & $\mathrm{~N}$ & 14 & $\operatorname{tin} 1$ & $r_{4}$ & 30 & & & & & ez \\
\hline I3 00 & & & 8 & 258 & 0 & 4 & & & & E & \\
\hline 1400 & 4.07 & NNE & 8 & xis) & in & 50 & 4.2 & & & & \\
\hline 1500 & 3.87 & $N$ & 14 & 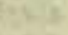 & $e x$ & 1200 & 4.2 & HSE & I & 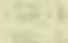 & $\frac{1}{x-1,2}$ \\
\hline 40 & 3.79 & $\mathrm{NE}$ & & 453 & 69 & 1300 & 4.22 & & 2 & we & 0024 \\
\hline 1650 & $\begin{array}{l}3.78 \\
3.77\end{array}$ & $\mathrm{NE}$ & $2 I$ & $18 y^{2}$ & 6 & 1400 & 4.05 & & 1 & 59 & 600 \\
\hline 10 & $\begin{array}{l}3.77 \\
3.76\end{array}$ & & & & S. W. S. & $\begin{array}{l}1500 \\
1600\end{array}$ & $\begin{array}{l}3.91 \\
3.76\end{array}$ & $\begin{array}{l}\mathrm{NE} \\
\mathrm{NE}\end{array}$ & $\begin{array}{l}I \\
3\end{array}$ & & S. W. S. \\
\hline 36 & & & & & & & & & & & \\
\hline
\end{tabular}


Tabulation of tidal observations at Teplitz Bay, Rudolph Island

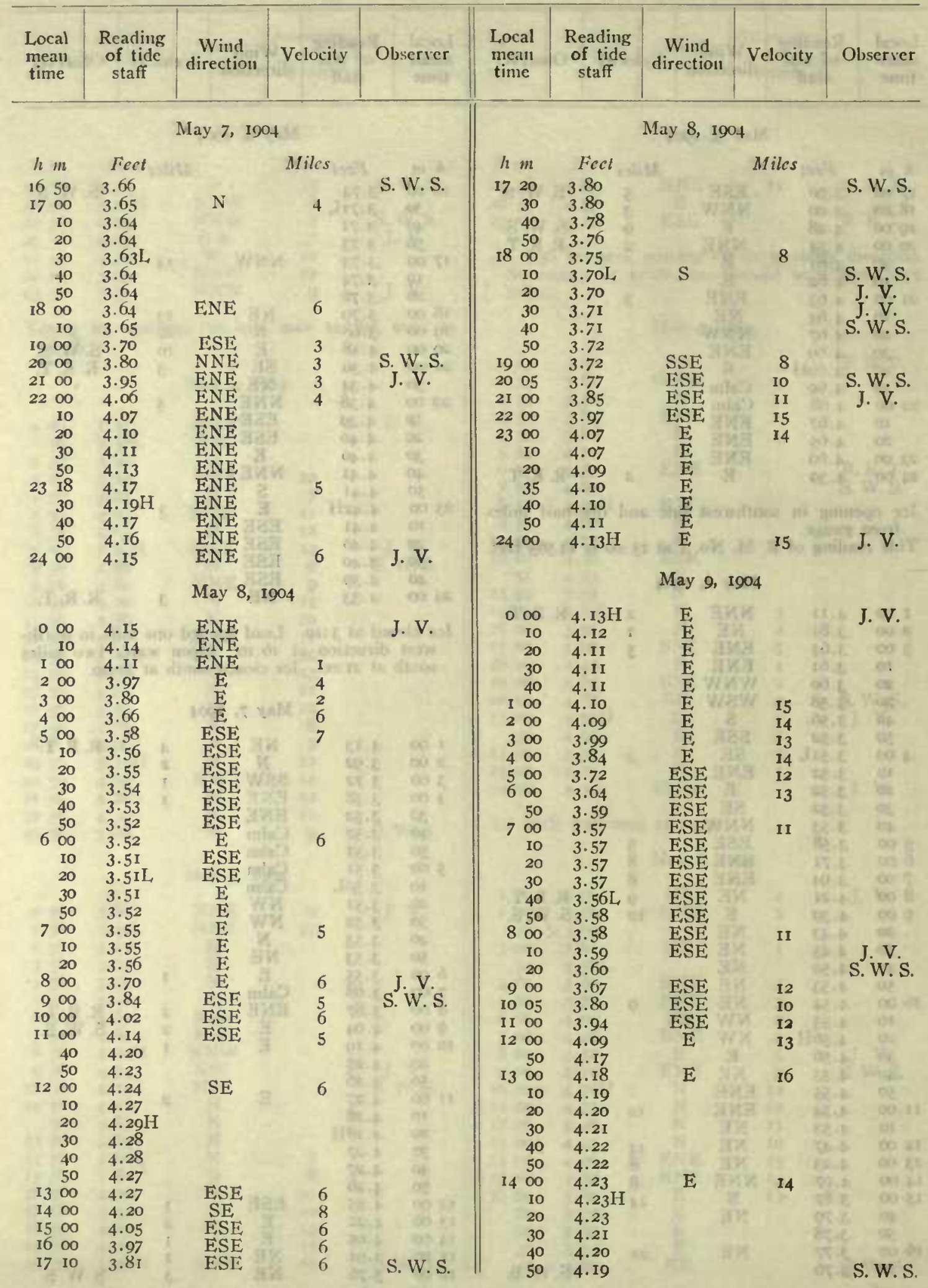


Tabulation of tidal observations at Teplitz Bay, Rudolph Island

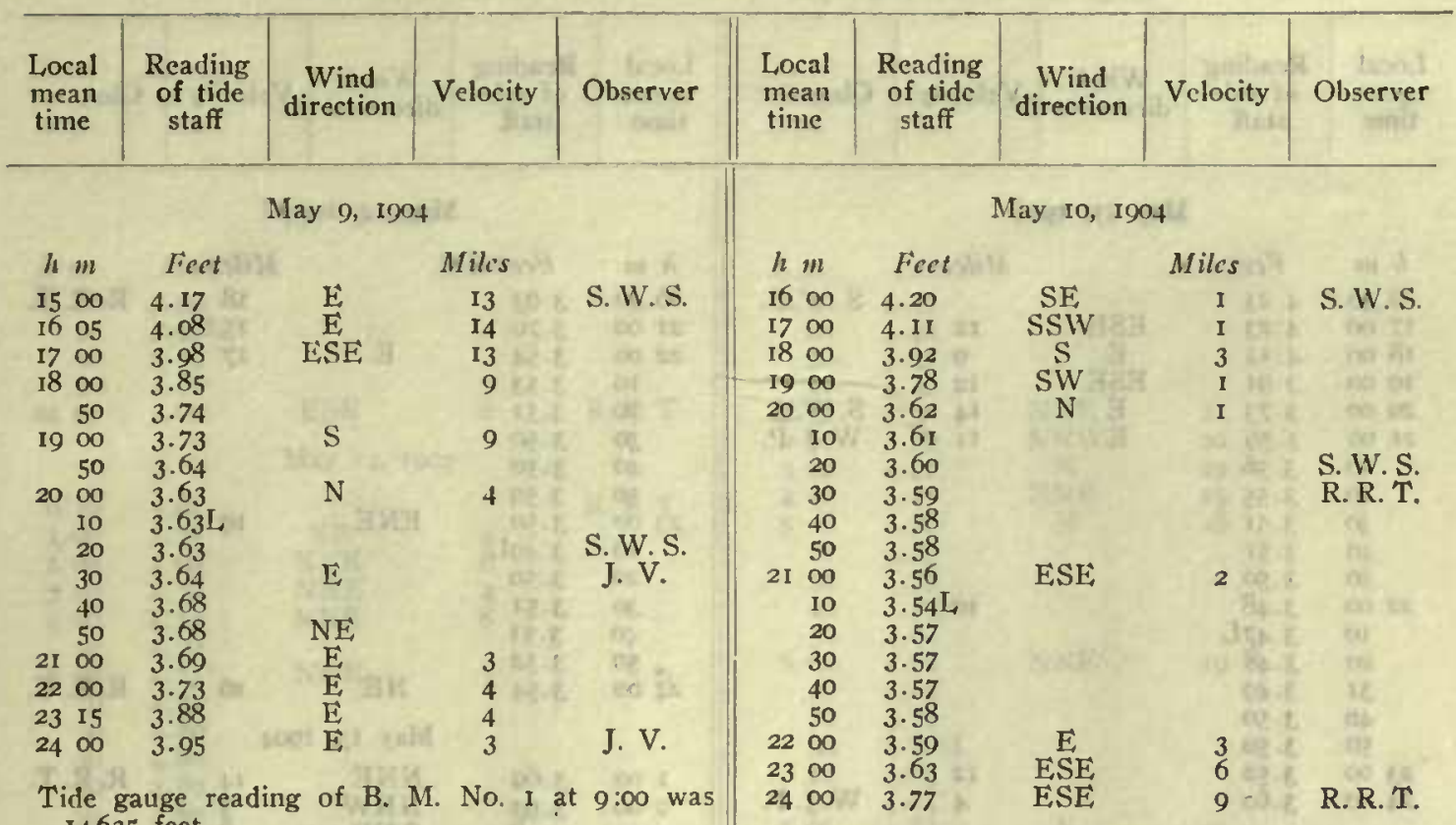

Tide gauge reading of B. M. No. I today is 14.60 feet.

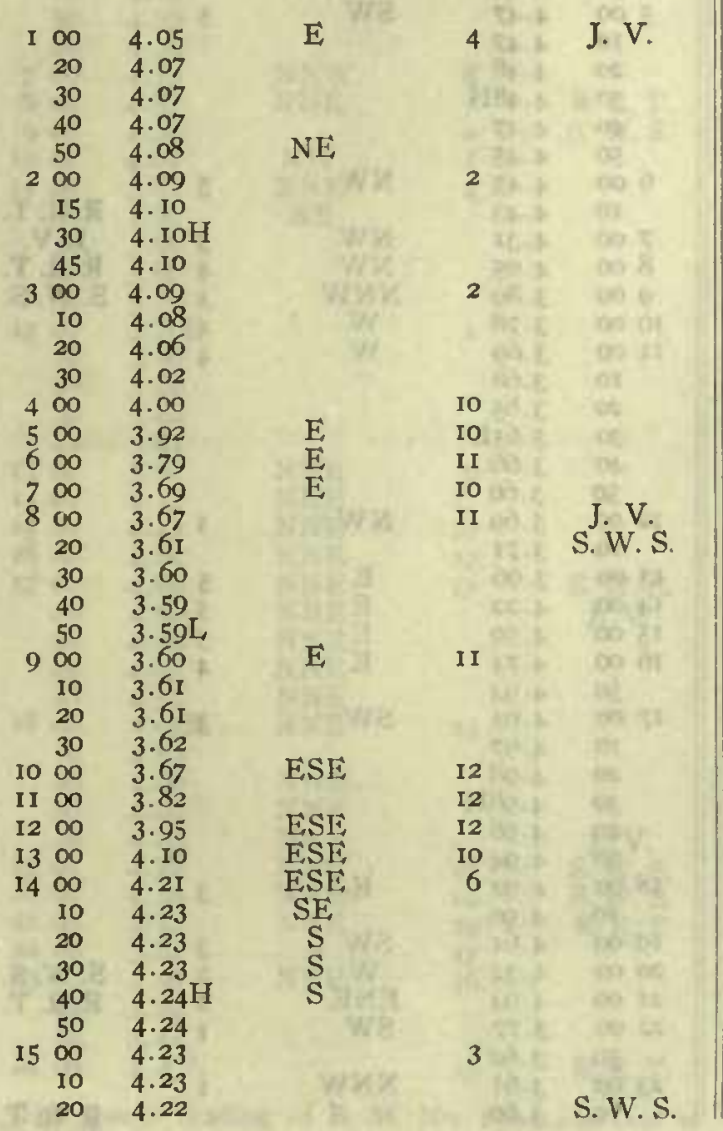

May ro, 1904

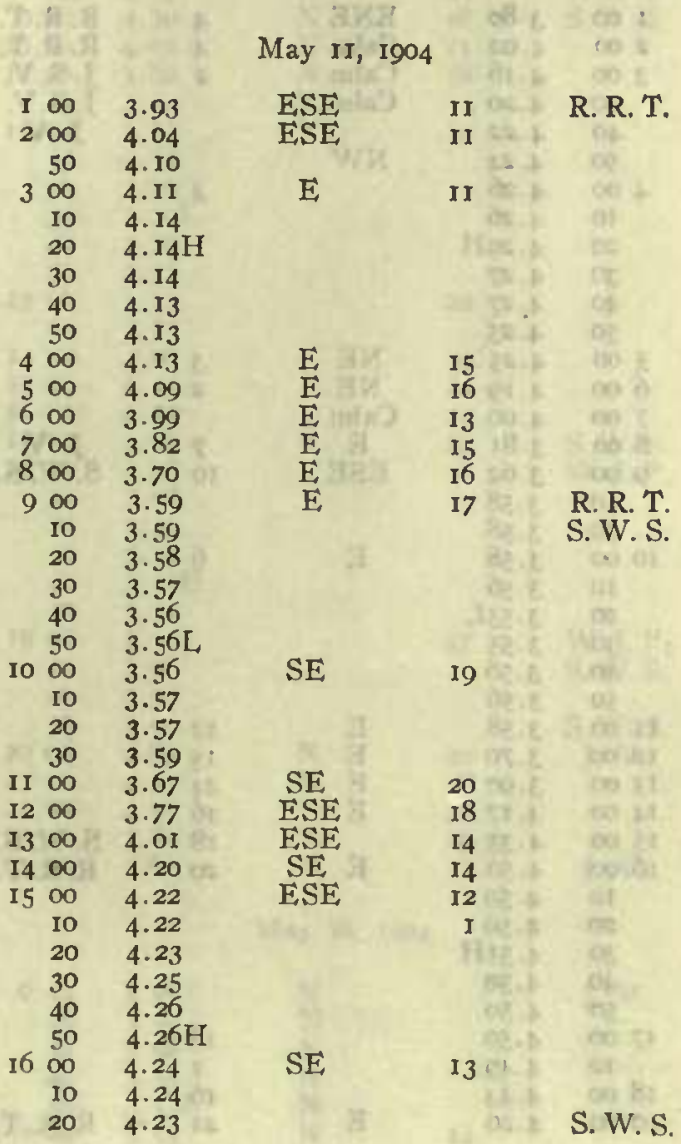


Tabulation of tidal observations at Teplitz Bay, Rudolph Island

\begin{tabular}{|c|c|c|c|c|c|c|c|c|c|c|c|c|}
\hline \multicolumn{2}{|c|}{$\begin{array}{l}\text { Local } \\
\text { meanl } \\
\text { time }\end{array}$} & $\begin{array}{l}\text { Reading } \\
\text { of tide } \\
\text { staff }\end{array}$ & \multirow{2}{*}{\begin{tabular}{|l}
$\mid \begin{array}{c}\text { Wind } \\
\text { direction }\end{array}$ \\
May II, 1904
\end{tabular}} & Velocity & \multirow{2}{*}{ Observer } & \multicolumn{2}{|c|}{$\begin{array}{l}\text { Local } \\
\text { mean } \\
\text { time }\end{array}$} & \multirow{2}{*}{$\begin{array}{c}\text { Reading } \\
\text { of tide } \\
\text { staff }\end{array}$} & \multirow{2}{*}{$\begin{array}{l}\left|\begin{array}{c}\text { Wind } \\
\text { directious }\end{array}\right| \\
\text { May } 12, \text { I904 }\end{array}$} & \multirow{2}{*}{\multicolumn{2}{|c|}{$\begin{array}{l}\text { Velocity } \\
04\end{array}$}} & \multirow[t]{2}{*}{ Observer } \\
\hline & & & & & & & & & & & & \\
\hline$h:$ & $m$ & Fect & & Miles & 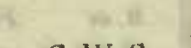 & $h n$ & & Fect & & Miles & & $\sqrt{2}$ \\
\hline 16 & 30 & 4.23 & & & S. W.S. & $20 \mathrm{c}$ & $\infty$ & 3.93 & & 18 & & R. R. T. \\
\hline 17 & $\infty 0$ & 4.23 & ESE & 12 & $7=0051$ & 21 & & 3.70 & & 15 & & 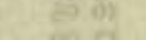 \\
\hline 18 & & $4 \cdot 14$ & E & 9 & og $x^{2}$ & 22 & & 3.54 & 1 & 17 & & 00,0 \\
\hline 19 & & 3.91 & ESE & 12 & $4+000$ & & 10 & 3.53 & & & 25 & 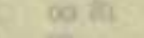 \\
\hline 20 & $\infty$ & 3.73 & $\mathrm{~F}$ & 14 & S. W.S. & & 20 & 3.51 & & w & 53 & 48. \\
\hline 21 & & 3.59 & $\mathrm{E}$ & II & W. J. P. & & 30 & $3 \cdot 50$ & & & 58 & 62 . \\
\hline & 20 & $\begin{array}{l}3.56 \\
3.55\end{array}$ & & 68 & 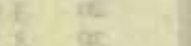 & & 40 & $\begin{array}{l}3.50 \\
3.50\end{array}$ & $1 \mathrm{k}$ & & 82 & teres \\
\hline & $\begin{array}{l}20 \\
.30\end{array}$ & $\begin{array}{l}3.55 \\
3.5 \mathrm{I}\end{array}$ & & है & is: & 23 & $\begin{array}{l}50 \\
00\end{array}$ & $\begin{array}{l}3.50 \\
3.50\end{array}$ & ENE & 19 & ate & er \\
\hline & 40 & 3.51 & & 3 & 28 & & 10 & $3.49 \mathrm{~L}$ & & & 3.2 & as \\
\hline & 50 & 3.50 & Hes. & 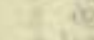 & son is & & 20 & 3.50 & & 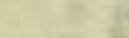 & 140 & $3^{2}$ \\
\hline 22 & $\infty$ & $3 \cdot 4^{8}$ & & 10 & bi & -2 & & $3.5 I$ & & a & 1,5 & at \\
\hline & 10 & $3.47 \mathrm{~L}$ & & $4: 3$ & at & & 40 & $3 \cdot 5 \mathrm{I}$ & & {$[1$} & are & Qits \\
\hline & 20 & 3.48 & & & ats & & 50 & 3.52 & & & & m \\
\hline & 31 & 3.49 & & 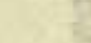 & 아. & 24 & & $3 \cdot 54$ & $\mathrm{NE}$ & 10 & s. & R. R. T. \\
\hline & $\begin{array}{l}40 \\
50\end{array}$ & $\begin{array}{l}3.50 \\
3.50\end{array}$ & 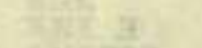 & & $x^{2} \leq 5$ & it & tit & $x^{2}+x^{2}$ & May I3, I & 1904 & 80 & 2014 \\
\hline 23 & $\infty$ & 3.52 & we तas & 12 & 16.95 & I & $\infty$ & 3.69 & NNE & 14 & & R. R. T. \\
\hline 24 & $\infty 0$ & 3.60 & 46fa & 4 & W. J. P. & & $\infty$ & 3.93 & NNW & $\begin{array}{r}14 \\
5\end{array}$ & & 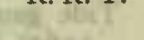 \\
\hline 068 & ant $x$ & abat of 1 & May 12, I904 & $\ln ^{2} x$ & 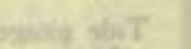 & & 00 & 4. 18 & SSW & 8 & 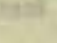 & $=8025$ \\
\hline sent & & & 17ady 12,8004 & & 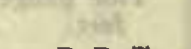 & 4 & $\begin{array}{l}00 \\
50\end{array}$ & $\begin{array}{l}4.35 \\
4.44\end{array}$ & WNW & ate & & \\
\hline I & $\infty 0$ & 3.80 & ENE & 4 & R. R. T. & 5 & $\infty$ & 4.47 & SW & 64 & & \\
\hline 2 & $\infty 0$ & 4.02 & Calm & 4 & R. R.T. & & 10 & 4.47 & & 3 & 20,8 & $a n t$ \\
\hline 3 & $\infty$ & 4. 16 & Calm & 2 & J. S. V. & & 20 & 4.48 & & & 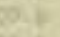 & $\cos =1$ \\
\hline 17 & 30 & 4.20 & Calm & & J.S. V. & & 30 & $4.48 \mathrm{H}$ & te & & 8.is & 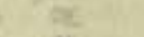 \\
\hline & 40 & 4.22 & $y^{2}=$ & & J. V. & & 40 & $4 \cdot 47$ & & & 808 & O4 \\
\hline & 50 & 4.24 & NW & & वe & & 50 & 4.45 & $4 x^{2}$ & & 6. I & Pis \\
\hline 4 & $\infty$ & 4.26 ty & $=8$ & 3 & $\cos 2$ & & $\infty$ & $4 \cdot 4$ & NW & 5 & wate & $\cos 2$ \\
\hline & IO & 4.26 & & $x^{2}$ & of & & 10 & $4 \cdot 43$ & & & wis & R. R. T. \\
\hline 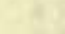 & 20 & $4.29 \mathrm{H}$ & & & $\alpha$ & & $\infty$ & $4 \cdot 31$ & NW & 3 & 206 & J. V. \\
\hline & 30 & 4.27 & & & $\infty$ & & $\infty$ & 4.0 & $N W$ & 4 & & R. R. T. \\
\hline & 40 & 4.27 & & & (19. & & $\infty$ & 3.89 & NNW & 3 & $x^{2}+2+2$ & S. W. S. \\
\hline & 50 & 4.25 & & & Q & Io & $\infty$ & 3.7 & IV & 4 & & \\
\hline 5 & 00 & 4.25 & $\mathrm{NE}$ & 3 & $09 \mathrm{~b}$ & II & oo & 3.69 & W & 4 & aif 1 & 05 \\
\hline 6 & 00 & 4.19 & $\mathrm{NE}$ & 2 & $00=$ & & 10 & 3.68 & & & 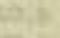 & ac. \\
\hline 7 & $\infty$ & 4.00 & Calm & & $80:$ & 1 & 20 & 3.65 & & 4 & wit & 001 \\
\hline 8 & $\infty$ & $3.8 \mathrm{r}$ & E & 7 & J. V. & & 30 & $3.65 \mathrm{~L}$ & & & 10,6 & 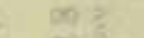 \\
\hline 9 & $\infty 0$ & 3.62 & ESE & Io & S.W.S. & & 40 & 3.66 & & & 6.18 & art \\
\hline & 40 & $3 \cdot 5^{8}$ & & & 1. 00.2 & & 50 & 3.66 & & & wis & os 5 \\
\hline C & 50 & 3.58 & & & 93 & 12 & 00 & 3.69 & NW & I & Q0. & $m: 8$ \\
\hline I0 & oo & 3.58 & $\mathrm{E}$ & 6 & 18 & 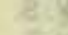 & 10 & 3.7 & & & $10, E$ & $\approx$ \\
\hline & I0 & 3.56 & & 5 & ot) & 13 & $\infty 0$ & 3.9 & $\mathrm{E}$ & 5 & 8. is & 8 \\
\hline & 20 & $3.55 \mathrm{~L}$ & 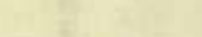 & 88 & 68 & 14 & oo & 4.22 & $\mathrm{E}$ & 5 & xy-6. & an: \\
\hline & 30 & 3.55 & & & os & 15 & $\infty 0$ & $4 \cdot 50$ & $\mathrm{E}$ & 5 & & vis 3 \\
\hline & 40 & $3 \cdot 56$ & 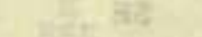 & & coor ar & 16 & $\infty$ & 4.74 & E & 4 & & 600 \\
\hline & 50 & $3 \cdot 56$ & & & exis: & te & 50 & 4.9 & & & & 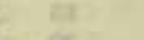 \\
\hline II & $\infty$ & 3.58 & E & I2 & as & 17 & oo & 4.95 & SW & 3 & 18. & cc \\
\hline 12 & $\infty$ & 3.70 & $\mathrm{E}$ & 15 & oc: & - & 10 & 4.9 & & & 20 & \\
\hline 13 & $\infty$ & 3.90 & $\mathbf{E}$ & 24 & 6. 18 & & 20 & & - 36 a & & 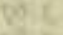 & coor \\
\hline 14 & $\infty$ & 4.17 & $E$ & 16 & $\tan 6 x$ & 265 & 30 & $4.08 \mathrm{H}$ & & 16 & s.te & $\cos i t$ \\
\hline 15 & $\infty$ & $4 \cdot 35$ & -37829 & 18 & S. W. S. & & 40 & 4.96 & प्रes & & $2 x+5$ & 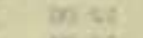 \\
\hline I6 & $\infty$ & $4 \cdot 50$ & $\mathrm{E}$ & 20 & R. R. T. & & 50 & 4.94 & & & osi h h & $\cos 4$. \\
\hline & Io & $4 \cdot 50$ & पर 39 & & की 2 & 18 & oo & 4.92 & $E$ & 3 & 15.3 & 00,11 \\
\hline & 20 & 4.50 & & & oir & & I0 & 4.90 & & & 224 & ot. \\
\hline & 30 & $4.51 \mathrm{H}$ & & & 2.1 & 19 & 00 & 4.61 & SW & 3 & 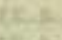 & 65 \\
\hline & 40 & $4 \cdot 50$ & & & 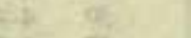 & 20 & 00 & $4 \cdot 3$ & - & 2 & & S. W.S. \\
\hline 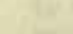 & 50 & $4 \cdot 50$ & & 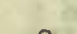 & Eit & 21 & oo & 4.0 .7 & $\mathrm{ENL}$ & 1 & 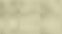 & R. R. T. \\
\hline 17 & $\infty$ & $4 \cdot 50$ & & 18 & $\lg$ & 22 & $\infty 0$ & 3.77 & SW & I & $13=$ & \\
\hline & 10 & 4.49 & 72 & I & $(102, \pi t$ & & 50 & 3.62 & & & & 00.81 \\
\hline 18 & $\infty$ & $4 \cdot 43$ & & 16 & $2 x$ & 23 & oo & 3.61 & NNW & I & $15 \%$ & की \\
\hline 19 & 00 & $4 \cdot 20$ & $\mathrm{E}$ & $2 \mathrm{I}$ & R. R. T. & & 10 & 3.60 & & & & R. R. T. \\
\hline
\end{tabular}


Tabulation of tidal observations at Teplitz Bay, Rudolph Island

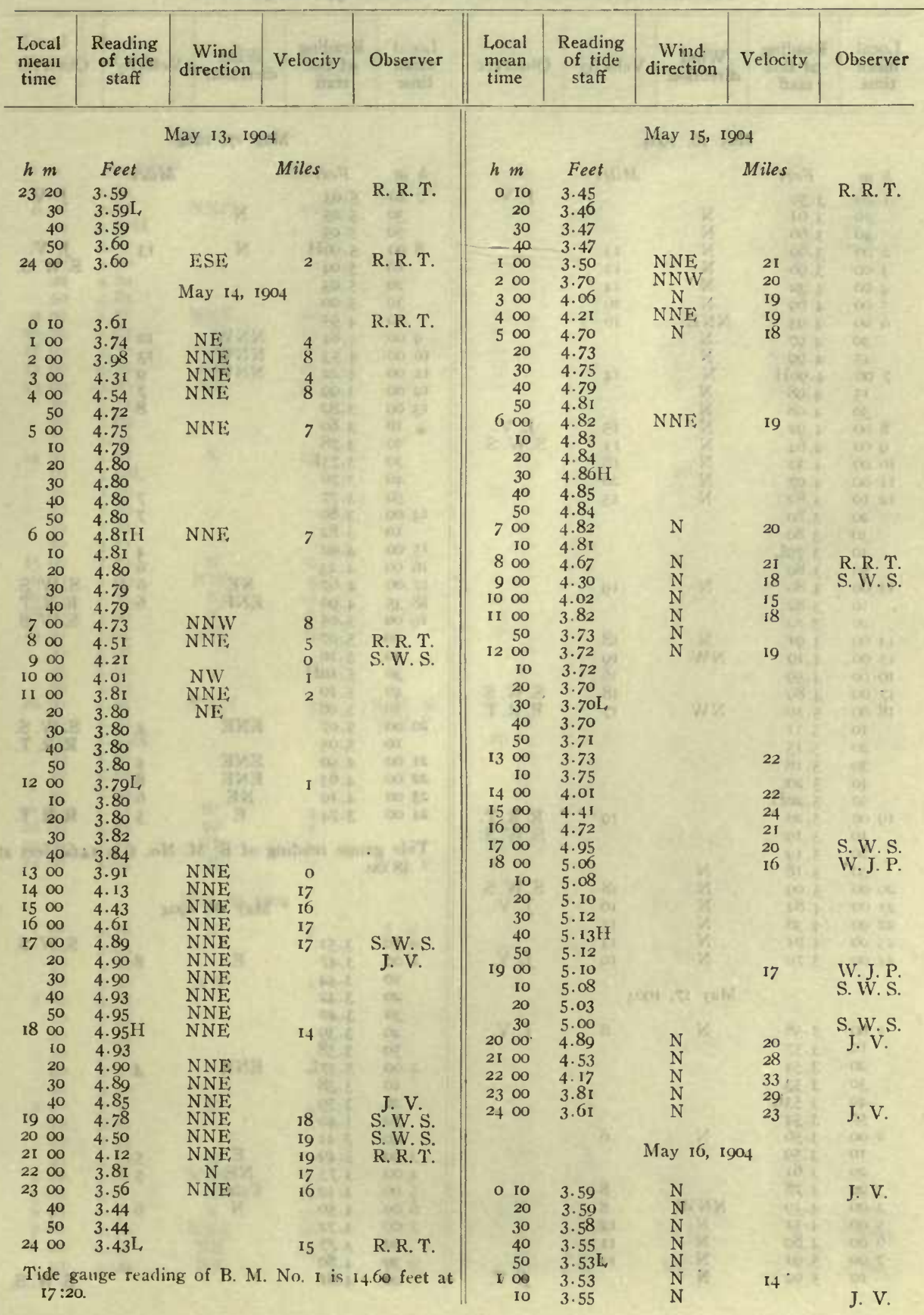


Tabulation of tidal observations at Teplitz Bay, Rudolph Island

\begin{tabular}{|c|c|c|c|c|c|c|c|c|c|c|c|}
\hline \multicolumn{2}{|c|}{$\begin{array}{l}\text { Local } \\
\text { mean } \\
\text { time }\end{array}$} & \multirow[t]{2}{*}{$\begin{array}{l}\text { Reading } \\
\text { of tide } \\
\text { staff }\end{array}$} & \multirow{2}{*}{$\begin{array}{l}\begin{array}{c}\text { Wind } \\
\text { direction }\end{array} \\
\text { May } 16,190\end{array}$} & \multicolumn{2}{|c|}{ Velocity } & \multirow{2}{*}{ Observer } & $\begin{array}{c}\text { Local } \\
\text { mean } \\
\text { time }\end{array}$ & \multirow[t]{2}{*}{$\begin{array}{l}\text { Reading } \\
\text { of tide } \\
\text { staff }\end{array}$} & \multirow{2}{*}{$\frac{\mid \begin{array}{c}\text { Wind } \\
\text { direction }\end{array}}{\text { May } 17,190}$} & \multirow[t]{2}{*}{ Velocity } & \multirow{2}{*}{ Observer } \\
\hline & & & & & & & & & & & \\
\hline & & Feet & & Miles & 77 & 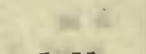 & $h m$ & Fect & a & Miles & min \\
\hline & 20 & 3.59 & & & E. & J. V. & 720 & 5.03 & & & J. V. \\
\hline & $\begin{array}{l}30 \\
40\end{array}$ & $\begin{array}{l}3.61 \\
3.66\end{array}$ & $\stackrel{N}{N}$ & & 5. & $9 \mathrm{~L}$ & $\begin{array}{l}30 \\
50\end{array}$ & $\begin{array}{l}5.05 \\
5.05\end{array}$ & $\mathrm{~N}$ & wros $x$ & $15=$ \\
\hline 2 & 00 & 3.69 & N & 13 & 72 & Q5. & 800 & $5.06 \mathrm{H}$ & $\mathrm{N}$ & 13 & J. V. \\
\hline 3 & $\infty$ & 3.96 & $\mathrm{~N}$ & 13 & 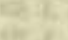 & $\operatorname{sen} 5$ & Io & 5.04 & & & S. W. S. \\
\hline 4 & $\infty$ & $4 \cdot 32$ & $N$ & I4 & $x^{2}-2$ & Do: & 20 & $\begin{array}{l}5.03 \\
5.00\end{array}$ & wayt \& th & & 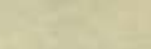 \\
\hline 5 & $\infty$ & 4.69 & NNE & $\begin{array}{l}16 \\
16\end{array}$ & $\cos$ & 62 & 30 & $\begin{array}{l}5.00 \\
4.95\end{array}$ & & & at 8 \\
\hline 6 & $\begin{array}{l}00 \\
30\end{array}$ & $\begin{array}{l}4.93 \\
4.97\end{array}$ & $\mathrm{~N}$ & & 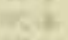 & 60,2 & $9 \stackrel{40}{90}$ & $\begin{array}{l}4.95 \\
4.84\end{array}$ & NNW & 12 & $00 ?$ \\
\hline & 45 & 4.99 & $\mathbf{N}$ & & 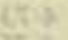 & us & 1000 & 4.53 & NNW & I3 & $\omega=$ \\
\hline 7 & $\infty$ & $4.90 \mathrm{H}$ & N & I4 & $25 x$ & at & II 00 & 4.22 & NNW & 9 & $\cos 2$ \\
\hline & I5 & 4.98 & $N$ & & 634 & $\frac{6}{4}$ & I2 00 & 3.99 & (x) & 7 & $\alpha z$ \\
\hline & 30 & 4.98 & N & & this & & 1300 & 3.82 & & 8 & ist \\
\hline & $\infty$ & 4.92 & $N$ & 15 & Diti & s. iv.s. & 10 & 3.80 & & $0^{2} 3$ & $60 \neq$ \\
\hline 9 & $\infty$ & 4.64 & $N$ & 13 & $y=3$ & S. W. S. & $\begin{array}{l}20 \\
30\end{array}$ & 3.78 & & $\sqrt{5}$ & $w^{2}$ \\
\hline 10 & $\infty$ & $4 \cdot 32$ & N & 18 & 965 & a & $\begin{array}{l}30 \\
40\end{array}$ & $\begin{array}{l}3.75 \mathrm{~L} \\
3.76\end{array}$ & 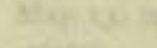 & & 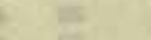 \\
\hline I I & $\infty$ & 4.07 & $N$ & 20 & 28 & 85 & $\begin{array}{l}40 \\
50\end{array}$ & $\begin{array}{l}3.76 \\
3.77\end{array}$ & & 7 & 5 \\
\hline 12 & $\begin{array}{l}10 \\
20\end{array}$ & $\begin{array}{l}3.82 \\
3.80\end{array}$ & $\mathrm{~N}$ & 15 & in: & wet & 1400 & $\begin{array}{l}3.77 \\
3.80\end{array}$ & & $\begin{array}{l}7 \\
7\end{array}$ & $45=$ \\
\hline & $\begin{array}{l}20 \\
30\end{array}$ & 3.80 & $Q$ & & 38.4 & & 10 & 3.82 & int & mine & 48 \\
\hline & 40 & $3.79 \mathrm{~L}$ & $7+2$ & & Ithit & 21 & 1500 & 4.02 & & 4 & at \\
\hline & 50 & 3.80 & & & 904 & $\log 0$ & 1600 & & & & \\
\hline 13 & $\infty$ & 3.80 & $\mathrm{~N}$ & 19 & noi: & 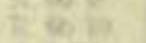 & $\begin{array}{l}1700 \\
1835\end{array}$ & 4.6 & NE & 6 & S. W.S. \\
\hline$x^{2}$ & $\begin{array}{l}10 \\
20\end{array}$ & $\begin{array}{l}3.82 \\
3.82\end{array}$ & A & 2 & 25 & nesirit & $\begin{array}{l}1835 \\
1900\end{array}$ & $\begin{array}{l}4.90 \\
5.05\end{array}$ & ENE & 6 & $\begin{array}{l}\text { R.R.T. } \\
\text { S. W. S. }\end{array}$ \\
\hline 14 & $\infty$ & 3.91 & $\mathrm{~N}$ & 18 & CS: 5 & te: & 10 & 5.07 & $7 x$ & is & De \\
\hline 15 & $\infty$ & 4.19 & NW & I9 & & onst & 20 & & & AE & as $x$ \\
\hline 16 & $\infty$ & 4.59 & & 16 & & & 30 & $5.10 \mathrm{H}$ & 30 & 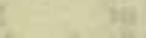 & povor: \\
\hline I7 & $\infty$ & 4.89 & & 18 & wate & S. W. S. & 40 & 5.10 & Whathe & 8 if & 60.13 \\
\hline 18 & $\infty$ & 5.10 & NW & I7 & 402 & R. R. T. & 50 & 5.0 & ENTE & a & \\
\hline & IO & 5.11 & & & it: & an & $\begin{array}{r}2000 \\
10\end{array}$ & $\begin{array}{l}5.0 \\
5.0\end{array}$ & ENE & 2 & S. W. S. \\
\hline & 20 & 5.15 & & & $\mathrm{cs}^{2} \mathrm{~T}$ & $\operatorname{lng} x 6$ & 2100 & $\begin{array}{l}5.05 \\
4.90\end{array}$ & ENE & 5 & R. R. T. \\
\hline & 30 & & & & whet & 64 & 2200 & $\begin{array}{l}4.90 \\
4.63\end{array}$ & ENE & 8 & $6, y^{4}$ \\
\hline & 40 & $\begin{array}{l}5.20 \\
5.20\end{array}$ & 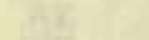 & 1 & 10.2 & 20041 & 2300 & 4.10 & $\mathrm{NE}$ & $\begin{array}{l}8 \\
6\end{array}$ & 0014 \\
\hline & 50 & $\begin{array}{l}5.2 C \\
5.2 C\end{array}$ & & 19 & the 4 & R R T & 2400 & 3.74 & $\mathrm{E}$ & & R. R. T. \\
\hline & $\begin{array}{l}\infty \\
10\end{array}$ & $\begin{array}{l}5.20 \\
5.19\end{array}$ & tent & & 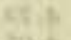 & S. W.S. & & & & & К. K. 1. \\
\hline
\end{tabular}

Tide gauge reading of $B$. M. No. I is 14.64 feet at 18:00.

\begin{tabular}{|c|c|}
\hline 30 & 5.18 \\
\hline 2000 & 5.09 \\
\hline $21 \infty$ & 4.82 \\
\hline 2200 & $4 \cdot 38$ \\
\hline 2300 & 4.01 \\
\hline 2400 & 3.70 \\
\hline
\end{tabular}

$\begin{array}{ll}\mathrm{N} & \\ \mathrm{N} & \mathrm{I} \\ \mathrm{N} & 16 \\ \mathrm{~N} & \mathrm{II} \\ \mathrm{N} & 14 \\ \mathrm{~N} & 10\end{array}$

May 18, 1904

May 17,1904

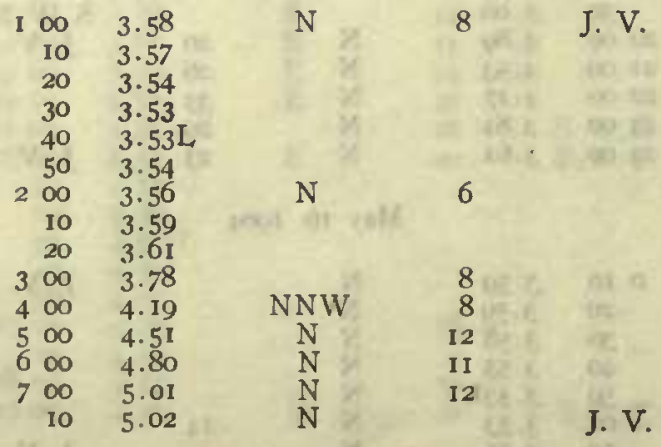

S. W. S.

J. V.

J. V.

J. V.

J. V.

\section{E}

$\begin{array}{ll}50 & 3.51 \\ \text { I } & 3.47\end{array}$

Io 3.44

$20 \quad 3.42$

$30 \quad 3.40$

$40 \quad 3.39$

50 3.38

$\begin{array}{ll}200 & 3.37 \mathrm{~L} \\ & 3.38\end{array}$

Io 3.38

30 3.40

$40 \quad 3.41$

$300 \quad 3.49$

$400 \quad 3.73$

$\begin{array}{lll}4 & 00 & 3.73 \\ 5 & 00 & 4.12\end{array}$

$600 \quad 4.50$

$\begin{array}{lll}700 \quad 4.72 & \end{array}$

$\begin{array}{ll}20 & 4.77 \\ 30 & 4.80\end{array}$

ENE

NE
S. W. S.

$\underset{N}{\text { Calm }}$
8

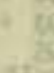

4

5

5 
Tabulation of tidal observations at Teplitz Bay, Rudolph Island

\begin{tabular}{|c|c|c|c|c|c|c|c|c|c|c|c|c|}
\hline $\begin{array}{l}\text { Local } \\
\text { mean } \\
\text { time }\end{array}$ & \multicolumn{2}{|c|}{$\begin{array}{l}\text { Reading } \\
\text { of tide } \\
\text { staff }\end{array}$} & \multirow{2}{*}{$\begin{array}{l}\left|\begin{array}{c}\text { Wind } \\
\text { direction }\end{array}\right| \\
\text { May } 18, \text { I904 }\end{array}$} & \multicolumn{2}{|c|}{ Velocity } & \multirow{2}{*}{ Observer } & $\begin{array}{l}\text { Local } \\
\text { mean } \\
\text { tinse }\end{array}$ & $\begin{array}{l}\text { Reading } \\
\text { of tide } \\
\text { staff }\end{array}$ & \multirow{2}{*}{$\frac{\begin{array}{c}\text { Wind } \\
\text { direction }\end{array} \mid}{\text { May } 19,190}$} & \multicolumn{2}{|c|}{ Velocity } & \multirow{2}{*}{ Observer } \\
\hline & & & & & & & & & & 94 & & \\
\hline$h m$ & Fieet & Wht & & Miles & & ati 4 & $h m$ & Feet & & Miles & b) 7 & w \\
\hline 750 & 4.83 & & & & & S. W. S. & 820 & 4.99 & & & & J. V. \\
\hline 800 & 4.87 & & NNE & 10 & & (en & 30 & 5.04 & & & & W. J.P. \\
\hline 20 & $\begin{array}{l}4.8711 \\
4.87\end{array}$ & & & & if & 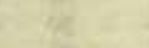 & $\begin{array}{r}40 \\
-\quad 50\end{array}$ & $\begin{array}{l}5.06 \mathrm{H} \\
5.04\end{array}$ & & & 22 & $\begin{array}{l}\text { W.J. P. } \\
\text { S. IV. S. }\end{array}$ \\
\hline 30 & 4.86 & & & & 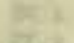 & 42 & 900 & 5.04 & NE: & 24 & hats & ( \\
\hline 40 & 4.84 & & ay & & $39:$ & 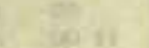 & I0 & 5.02 & 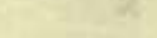 & & & 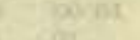 \\
\hline 50 & 4.83 & $\frac{n}{6}$ & NNE & I0 & 35 & weit & 20 & 5.00 & & & & 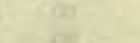 \\
\hline 900 & $4.8 \mathrm{I}$ & 8 & $\underset{N}{N N E}$ & $\begin{array}{l}\text { IO } \\
\text { II }\end{array}$ & 18.4 & $30 \mathrm{ki}$ & 10 00 & $4 \cdot 96$ & NE: & $2 I$ & & $a$ \\
\hline Io 00 & 4.63 & & NNE & I 2 & 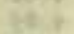 & (6) & II 00 & $4.6 \mathrm{I}$ & $\mathrm{NE}$ & $2 I$ & 2119 & n. \\
\hline II 00 & 4.27 & 2 & $\begin{array}{l}\mathrm{NNE} \\
\mathrm{NE}\end{array}$ & 12 & $\operatorname{sen}$ & $\begin{array}{l}0 \\
x^{2}\end{array}$ & 1200 & $4 \cdot 35$ & $\mathrm{NE}$ & 13 & 75. & (y) is \\
\hline 1200 & 4.00 & + & $\begin{array}{l}N E \\
N E\end{array}$ & II & 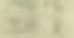 & at & 1305 & 4.06 & $\mathrm{~N}$ & 7 & theng & $\ln 2 \times 4$ \\
\hline 1300 & 3.80 & & $\mathrm{NE}$ & IO & 15. & 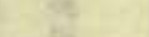 & I 400 & 3.90 & $\mathrm{~N}$ & I4 & 6os. & $x=14$ \\
\hline 30 & $3.7 \mathrm{I}$ & & & & 285 & 5 & 10 & 3.88 & 412 & & 20.1 & $60+7$ \\
\hline 40 & 3.69 & & 14 & & $60-5$ & & 20 & 3.86 & Ak & & [it] $\mathrm{t}$ & $\cos x$ \\
\hline 50 & 3.69 & th & in & & $y^{2}=2$ & cotos & 30 & 3.84 & & & & $\ln \mid=$ \\
\hline I4 00 & 3.68 & & $\mathrm{~N}$ & 10 & $25+2$ & 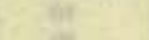 & 40 & 3.83 & & & & 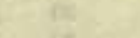 \\
\hline Jo & $3.68 \mathrm{~L}$ & & & & $\log 21$ & 28 & 50 & $3.83 \mathrm{~L}$ & $\mathbf{N}$ & & $\cos 1$ & ce \\
\hline 20 & 3.69 & & $x^{2}=$ & 14 & 545 & as & I5 00 & 3.83 & $\mathrm{~N}$ & 16 & STle & Wh \\
\hline 30 & 3.70 & & & 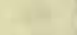 & 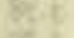 & 18 & 10 & 3.84 & & & & 03 \\
\hline 40 & 3.71 & & & 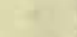 & 185 & $e^{3} 2$ & 20 & 3.85 & 412 & & 472 & 4081 \\
\hline 50 & 3.71 & e. & & 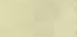 & 65.5 & $40-31$ & 30 & 3.85 & & & & 68 \\
\hline I5 00 & 3.72 & $6 ?$ & $\mathrm{~N}$ & 9 & 735 & 10050 & 1600 & 3.90 & $\mathrm{~N}$ & I7 & $4+46$ & 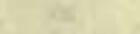 \\
\hline I6 00 & 3.99 & nit & NIV & 9 & 23.3 & 9855 & 1700 & $4 \cdot \mathrm{I}_{4}$ & $\mathrm{~N}$ & 17 & $=16$ & $\infty=$ \\
\hline 1705 & 4.35 & 6. & $\mathrm{N}$ & II & $2 x+$ & $y=6$ & 1800 & $4 \cdot 48$ & $\mathrm{~N}$ & I8 & $26+4$ & $\cos 10$ \\
\hline 1800 & 4.69 & & $N$ & 8 & Ket & 0014 & 1900 & $4 \cdot 76$ & $\mathrm{~N}$ & 18 & 083 & 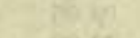 \\
\hline 1900 & 4.95 & 31 & NIV & 6 & $35 ?$ & ant $4=$ & 2000 & 4.92 & $\mathrm{~N}$ & 15 & $m+$ & 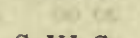 \\
\hline 30 & 4.98 & & & & 63 & ot & Io & $4 \cdot 93$ & & & $n^{3}+4$ & S. W.S. \\
\hline 40 & 5.00 & & $3^{3}$ & & gets & tons & 20 & 4.93 & & & 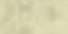 & R. R. T. \\
\hline 50 & 5.03 & & 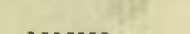 & & 54.3 & 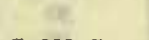 & 30 & 4.96 & & & aftot & $x^{5}$ \\
\hline 2000 & 5.05 & & NNW & 3 & 64 & S. W. S. & 40 & 4.99 & & & 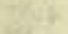 & at \\
\hline I0 & 5.06 & & & & $\ln 2$ & W. J. P. & 50 & 5.00 & & & (2) & (4t \\
\hline 20 & $5.09 \mathrm{H}$ & & wh & & $(152)$ & & 2100 & $5.01 \mathrm{H}$ & $\mathrm{N}$ & I7 & 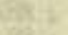 & B \\
\hline 30 & 5.08 & & & tat & $\$ 1$ & 09 & Io & 5.00 & IXe & & $153+$ & $60 \mathrm{se}$ \\
\hline 40 & 5.08 & & & & To. & 6 & 20 & 5.00 & & & $3 x^{2}$ & +14 \\
\hline 50 & 5.06 & & & & poin & 78 & 30 & 5.00 & & & itis & 12 \\
\hline 2100 & 5.04 & 31 & W & 4 & 203 & 0012 & 40 & 4.99 & & & 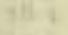 & $w$ \\
\hline 2200 & 4.82 & & W & I & & 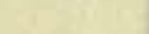 & 2200 & $4.9 \mathrm{I}$ & $\mathrm{N}$ & I6 & 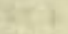 & 0068 \\
\hline 2300 & 4.42 & & $\mathrm{NE}$ & I & & & 2300 & 4.69 & $\mathrm{~N}$ & $I_{4}$ & 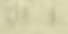 & 100 \\
\hline 2400 & 4.05 & & $\mathrm{~N}$ & 7 & & W. J. P. & 2400 & $4 \cdot 27$ & $\mathrm{~N}$ & 16 & & $\mathrm{R} R, \mathrm{~T}$. \\
\hline$y=$ & & $\frac{4}{71}$ & May I9, I904 & & ath & 605 & & & May zo, Ir & & & \\
\hline 100 & 3.76 & n & $\mathrm{N}$ & 7 & 805 & J. V. & & 3.02 & $\mathrm{~N}$ aris & & 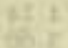 & -3 \\
\hline 30 & 3.63 & & - & 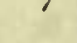 & inat: & J. V. & $\begin{array}{l}100 \\
200\end{array}$ & $\begin{array}{l}3.92 \\
3.6 .4\end{array}$ & NNE & $\begin{array}{l}\text { I3 } \\
\text { 10 }\end{array}$ & 49 & R. R. T. \\
\hline 40 & 3.60 & & & & 60.6 & 68 & 10 & 3.58 & & 8 & sols: & nt \\
\hline 50 & 3.58 & & NW & 5 & ta hit & 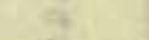 & 20 & 3.54 & & & 02 & 10. \\
\hline 200 & 3.58 & & & & 20.1 & 8. & 30 & 3.52 & & & gete & 34 \\
\hline IO & 3.55 & 4 & $3 \times 5$ & & as: 8 & 206 & 40 & $3 \cdot 50$ & & & & 14i \\
\hline 20 & 3.54 & & & & intat & 69 & 50 & 3.50 & NW & 6 & 69 & be \\
\hline 30 & $3 \cdot 5^{2}$ & & & & a. 5 & (85. & 300 & 3.49 & & & 4 & 201 \\
\hline 40 & 3. $50 \mathrm{~L}$ & & & 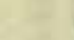 & ont: & $b^{4}$ & IO & $3.49 \mathrm{~L}$ & & & on & 16 \\
\hline 50 & $3 \cdot 50$ & & & & $\operatorname{san}$ & 64 & 20 & 3.49 & & & ta & es \\
\hline 300 & $3.5 \mathrm{I}$ & & $\mathrm{N}$ & 4 & 64. & ae & 30 & 3.50 & & & & 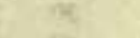 \\
\hline Io & $3 \cdot 52$ & a & 75 & 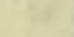 & ante & $\operatorname{lon}$ & 40 & $3 \cdot 50$ & & & & $\infty 0$ \\
\hline 20 & 3.53 & Ix & 1304 & 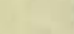 & wese & bes $=$ & 400 & $3.5 \mathrm{I}$ & NNE, & & (5) 1 & Q \\
\hline 30 & 3.59 & 87 & $N$ & 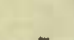 & itsin & $x^{2} x^{2}$ & 500 & 3.71 & NNW & 7 & 28 & pas it \\
\hline 400 & 3.68 & at & NNE: & 7 & se.i. & (a) $\mathrm{v}$. & 600 & 4.04 & NNW & 7 & or 1 & $\operatorname{ms} 2$ \\
\hline 500 & 4.01 & 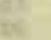 & $\mathrm{N}$ & II & 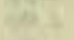 & we is & 700 & $4 \cdot 36$ & NW & 7 & 4. & 400 \\
\hline 600 & 4.37 & 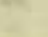 & $N$ & 17 & Solit. & 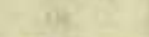 & 800 & 4.63 & $\mathrm{~N}$ & 7 & & R. R. T. \\
\hline 700 & 4.72 & & NNE & 20 & 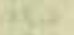 & (78) & 40 & 4.77 & NNW & & & S. W.S. \\
\hline 800 & 4.94 & & $\mathrm{NE}$ & 23 & $x^{2}+3$ & & 50 & 4.80 & & & & \\
\hline 10 & 4.99 & is & $\mathrm{NE}$ & & Whe & J. V. & 900 & 4.82 & $\mathrm{NE}$ & 7 & C & S. W. S. \\
\hline
\end{tabular}


Tabulation of tidal observations at Teplitz Bay, Rudolph Island

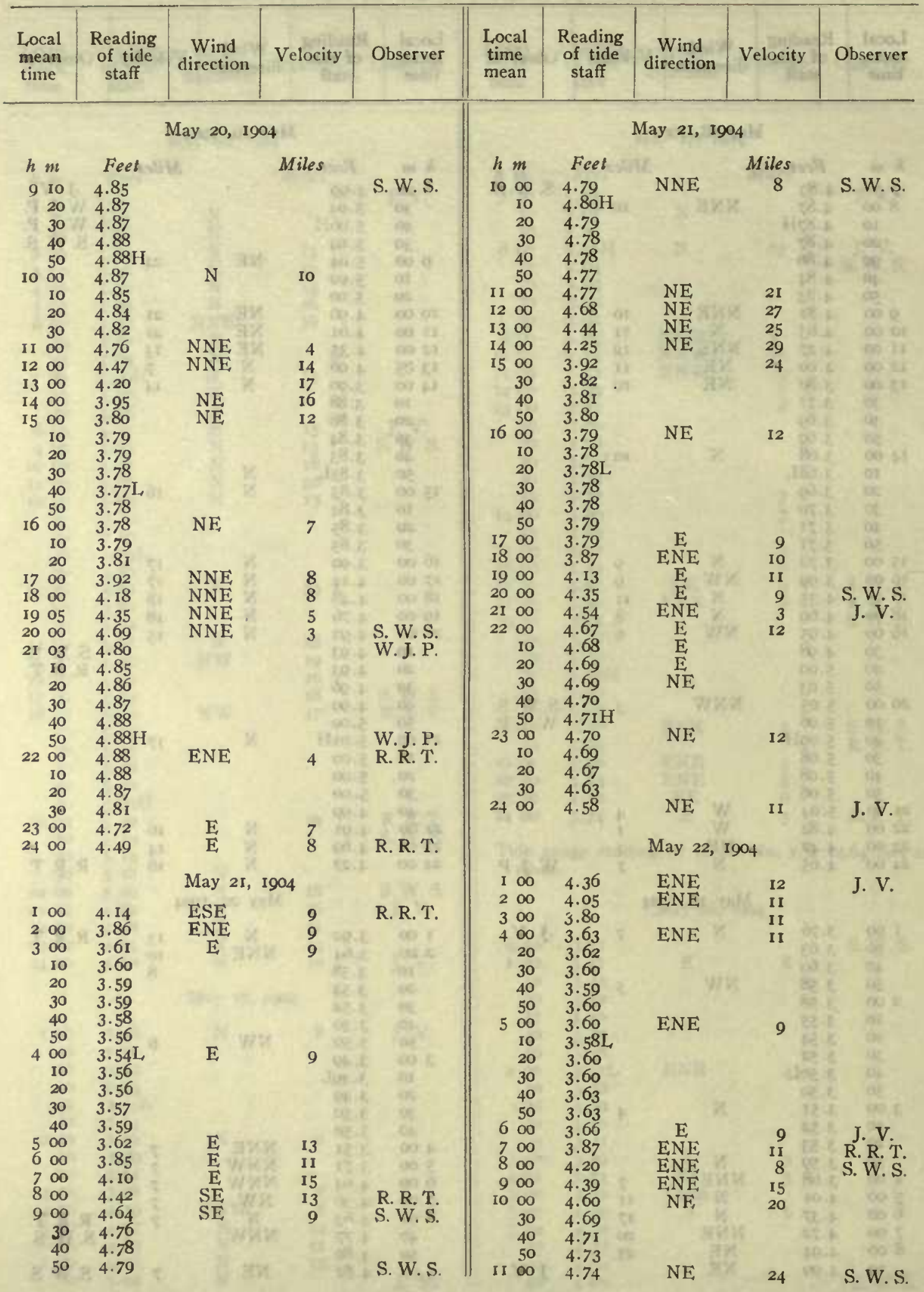




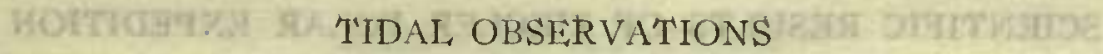

Tabulation of tidal observations at Teplitz Bay, Rudolph Island

\begin{tabular}{|c|c|c|c|c|c|c|c|c|c|c|c|}
\hline $\begin{array}{l}\text { Local } \\
\text { mean } \\
\text { time }\end{array}$ & $\begin{array}{l}\text { Reading } \\
\text { of tide } \\
\text { staff }\end{array}$ & $\begin{array}{l}\text { Wind } \\
\text { direction }\end{array}$ & \multicolumn{2}{|c|}{ Velocity } & \multirow{2}{*}{ Observer } & $\begin{array}{c}\text { Local } \\
\text { incair } \\
\text { time }\end{array}$ & \multirow[t]{2}{*}{$\begin{array}{l}\text { Reading } \\
\text { of tide } \\
\text { staft }\end{array}$} & \multirow{2}{*}{$\begin{array}{l}\begin{array}{c}\text { Wind } \\
\text { direction }\end{array} \mid \\
\text { May 23, I90 }\end{array}$} & \multicolumn{2}{|c|}{ Velocity } & \multirow[t]{2}{*}{ Observer } \\
\hline \multicolumn{5}{|c|}{ May 22, 1904} & & \multicolumn{3}{|r|}{ May 23, I90 } & & & \\
\hline$h m$ & Feet & 16. & Miles & 53 & 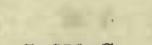 & $h m$ & Feet & & Miles & was: & the \\
\hline 1110 & 4.76 & & & the & S. W. S. & 1300 & 4.64 & NNE & 12 & & S. W. S. \\
\hline 20 & 4.77 & & & o: 1 & $x^{2}+x^{2}$ & 1400 & 4.58 & NNE & 14 & & tor \\
\hline 30 & $4.77 \mathrm{H}$ & & & (1) 5 & a. $=30$ & 1500 & 4.36 & NNE & 16 & & on $x_{1}$ \\
\hline 40 & 4.76 & & & $x 81$ & 20015 & 1600 & 4. I6 & NNE & 17 & 00.4 & $60 \times 1$ \\
\hline 50 & 4.76 & זיז & & 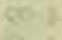 & $10 \times 14$ & 1700 & 3.96 & NNE & 18 & we.? & a. 86 \\
\hline 1200 & 4.74 & $N E$ & $3 I$ & 2.4 & $\ln x)$ & 10 & 3.94 & & & & OR \\
\hline I3 00 & 4.61 & $\mathrm{NE}$ & 34 & okis & 200151 & 20 & 3.90 & & & ante & \\
\hline 1400 & 4.42 & $\mathrm{NE}$ & $3 i$ & $4 k$ & wis & 30 & 3.88 & & & os. & S. W. S. \\
\hline 1500 & 4. 18 & $\mathrm{NE}$ & 35 & 54 & it & 40 & 3.83 & 935 & & bei. & R.R.T. \\
\hline 1600 & 3.98 & $\mathrm{NE}$ & 38 & ont & ach & 50 & 3.80 & - & & sube & (n) \\
\hline 1700 & 3.86 & $\mathrm{NE}$ & 39 & 84 & 6 & 1800 & 3.79 & NNE & I2 & (1) $x$ & 06 \\
\hline 10 & 3.84 & & & 1841 & $112:=$ & 10 & 3.79 & & & iset & we \\
\hline 20 & 3.82 & & & $7 x$ & was $\geq$ & 20 & 3.76 & & & & the \\
\hline 30 & $3.82 \mathrm{~L}$ & & & 37 & 41 & 30 & $3.72 \mathrm{~L}$ & & & $3 x y$ & wis $=$ \\
\hline 40 & 3.86 & & & 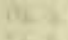 & $\alpha_{0}+$ & 40 & 3.73 & & & $n=1$ & ceices \\
\hline 50 & 3.86 & & & 145 & 68. & 50 & 3.73 & & & .2 & 046 \\
\hline 18 & 3.90 & $\mathrm{NF}$ & 37 & $1+1$ & anter & 1900 & 3.74 & $\mathrm{NE}$ & 24 & te & os \\
\hline 1900 & 3.98 & $\mathrm{NE}$ & $4 \mathrm{I}$ & 14.8 & was 58 & I0 & 3.75 & & & & \\
\hline 2000 & 4.21 . & & 39 & 39. & S. W.S. & 2000 & $3.8 \mathrm{I}$ & NNE & 23 & & R. R. T. \\
\hline 2100 & $4 \cdot 3^{8}$ & $\mathrm{NE}$ & 40 & 632 & J. V. & 2100. & 3.96 & NNE & 31 & & S.W.S. \\
\hline 2200 & $4 \cdot 54$ & $\mathrm{NE}$ & 38 & \& 1 & tay 2 & 2200 & 4.15 & NNE & 30 & $v_{5}$ & J. V. \\
\hline 2,300 & 4.60 & $\mathrm{NE}$ & 38 & $62=$ & $4 x^{2}$ & 2300 & 4.29 & NNE & 23 & (ve) 1 & \\
\hline $24 \begin{array}{r}50 \\
240\end{array}$ & $\begin{array}{l}4.62 \\
4.63\end{array}$ & & 38 & $\frac{4}{4}+\frac{1}{2}$ & J. V. & 2400 & $4 \cdot 44$ & & 27 & & J. V. \\
\hline
\end{tabular}

May 2.3, 1904

$13: 00$

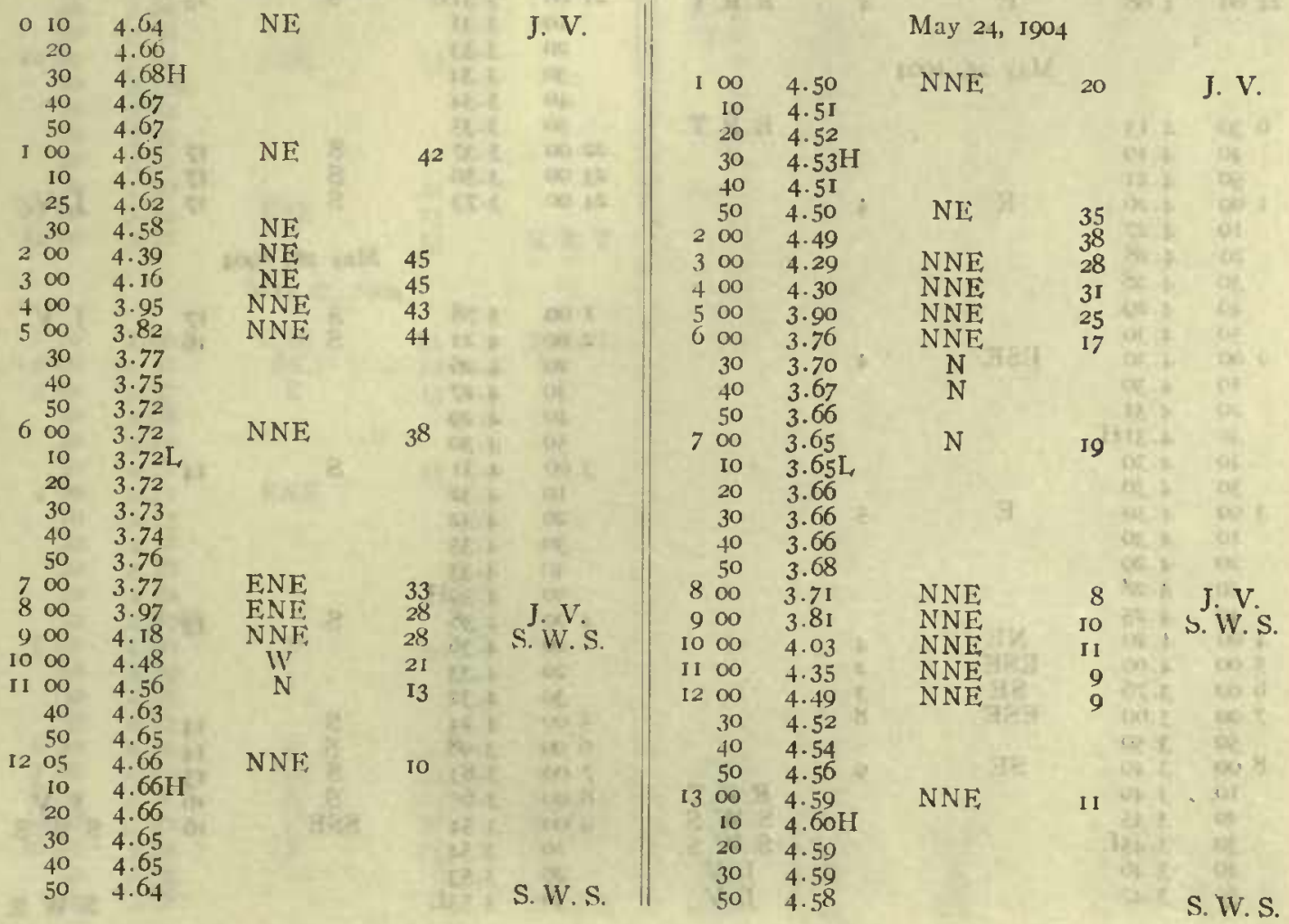


Tabulation of tidal observations at Teplitz Bay, Rudolph Island

\begin{tabular}{|c|c|c|c|c|c|c|c|c|c|c|c|c|}
\hline $\begin{array}{l}\text { Local } \\
\text { mean } \\
\text { time }\end{array}$ & $\begin{array}{l}\text { Reading } \\
\text { of tide } \\
\text { staff }\end{array}$ & $\begin{array}{l}\text { Wind } \\
\text { directio }\end{array}$ & & Velocit & & Observer & $\begin{array}{l}\text { Local } \\
\text { meanl } \\
\text { time }\end{array}$ & $\begin{array}{l}\text { Reading } \\
\text { of tide } \\
\text { staff }\end{array}$ & $\begin{array}{l}\text { Wind } \\
\text { direction }\end{array}$ & Velocit & & Observer \\
\hline \multicolumn{7}{|c|}{ May 24, 1904} & \multicolumn{6}{|c|}{ May 25, 1904} \\
\hline$h m$ & \multicolumn{2}{|c|}{ Feet } & \multicolumn{3}{|c|}{ Miles } & $=y$ & $h m$ & Feet & 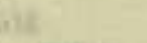 & \multicolumn{2}{|c|}{ Miles } & \\
\hline 1400 & 4.57 & $\mathrm{NE}$ & & 13 & & S. W. S. & 900 & 3.47 & ESE & 12 & & S.W.S. \\
\hline 150 & 4.51 & & 48 & 15 & & goner & & 3.49 & & & & en \\
\hline 160 & $4.3 \mathrm{I}$ & & 32. & 15 & c, & 002 & 1000 & 3.5 & SE & II & & ece. \\
\hline 1700 & 4.06 & & 68 & 12 & iili. & $00 \%$ & II 05 & 3.8 & Sh & 13 & & ist \\
\hline 1800 & 3.91 & & 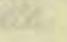 & 12 & w. & & & 4.09 & SE & 14 & & 3 \\
\hline 30 & 3.79 & $=$ & & & & S.W.S. & 1300 & 4.26 & $\mathrm{SE}$ & If & & $e^{2}$ \\
\hline 40 & 3.74 & 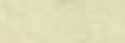 & & & 89 & W. J. P. & 1400 & $4 \cdot 39$ & $\mathrm{SE}$ & 14 & & \\
\hline 50 & 3.70 & & & & this & & 10 & $4.4 \mathrm{I}$ & & 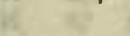 & & \\
\hline 1900 & 3.68 & $\mathrm{NE}$ & & & (8.) & AL & 20 & 4.43 & & 8 & & \\
\hline 10 & 3.67 & Calm & & & $\alpha$. & of & 30 & 4. & & en & & \\
\hline 20 & 3.65 & & 615 & xit & Qhat? & $102 x$ & 40 & & & as & at. & 8 \\
\hline 30 & 3.63 & & & $\frac{4}{7}$ & 20 & कi & 50 & $4.48 \mathrm{H}$ & & 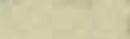 & 28 & wit. \\
\hline 40 & $3.6 \mathrm{I}$ & & & 36 & ots. & is & I5 00 & 4.47 & & 17 & & \\
\hline & 3.58 & & & $\sqrt{6}+3$ & Int? & es & & 4.4 & & & & Ee \\
\hline 2000 & 3.56 & & & Io & I3) & as & 20 & 4.2 & 36 & ter & & a \\
\hline Io & 3.56 & & & & 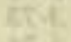 & Q. & & 4.4 & & & $\infty$ & \\
\hline 20 & & & 12 & & 25. & 0008 & I6 00 & 4.4 & SE & 18 & & $\infty$ \\
\hline 30 & $3.56 \mathrm{~L}$ & & & & & & & & SE & 16 & ant & \\
\hline 40 & 3.57 & & $6 \times$ & & 180 & W. J.P. & & & $\mathrm{SE}$ & 16 & & 6. \\
\hline 50 & $3 \cdot 57$ & & & & AN: & R. R. T. & & 3.6 & SSE & 17 & & 69 \\
\hline 2100 & 3.59 & $\mathrm{NE}$ & 68 & 9 & 27. & & 2000 & 3.4 & $\mathrm{~S}$ & 16 & & 69 \\
\hline 10 & 3. 59 & & $8 k$ & & 62 & $10 \mathrm{es}$ & I0 & 3. & & & & 6 \\
\hline 20 & 3.60 & & & 17 & 363 & $\infty 0$ is & 20 & & & 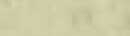 & & \\
\hline 30 & 3.61 & 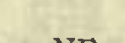 & & 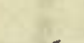 & & & 30 & 3.3 & & 3 & & S. W. \\
\hline 2200 & & $\mathrm{NE}$ & & 6 & $4=$ & $\min =27$ & 40 & 3.3 & & 3 & & J. V. \\
\hline$\infty$ & & ENE & & 6 & & & & & & sth & & \\
\hline 2400 & 4.08 & E & & 4 & & R.R.T. & 2100 & 3. $3 \mathrm{IL}$ & s & 15 & & \\
\hline & & $142+24=$ & & & & & $\begin{array}{l}10 \\
20\end{array}$ & $\begin{array}{l}3.3 \\
3.3\end{array}$ & +1 & & & 6) \\
\hline$y=$ & & May 25 & 1904 & & & osit & 30 & 3.3 & & & & $\alpha$ \\
\hline 030 & 4.13 & & & & 8 & $R, R, T$ & $\begin{array}{l}40 \\
50\end{array}$ & $3 \cdot 3$ & & & Q4 & ey \\
\hline 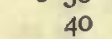 & & & & & & & 22 & & & & & $=0$ \\
\hline 50 & 4.2 & 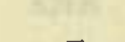 & & & 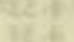 & Fist & 23 & & $\mathrm{~S}$ & 17 & & \\
\hline 100 & 4.26 & E & ik & 4 & ases & $e^{2}$ & $24 \infty 0$ & 3.73 & $\mathrm{~S}$ & 17 & & J. V. \\
\hline I0 & 4.27 & & & & & in & 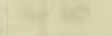 & & & 18 & & ( \\
\hline 20 & 4.28 & 4 & xis & 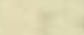 & 2 & & & $=$ & May 26 , & 1904 & & 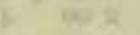 \\
\hline 30 & 4.28 & & Ex & 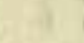 & & 186 & & & & & de & $10 \pi$ \\
\hline 40 & 4.29 & & 62 & & Q & & $1 \infty$ & 3.78 & $\mathrm{~S}$ & 17 & & J. V. \\
\hline 50 & 4.30 & & & & $\sqrt{5}$ & 608 & 200 & 4.21 & $\mathrm{~S}$ & 16 & & \\
\hline 200 & $4 \cdot 30$ & ESE & 6 & 4 & & $x^{2}$ & 20 & 4.26 & & & & a \\
\hline 10 & 4.30 & & & & & 642 & 30 & 4.2 & & 5 & & $=$ \\
\hline 20 & 4.3 & 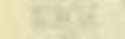 & & 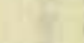 & of. & & 40 & 4.29 & & 13 & & $3=$ \\
\hline & $4.31 \mathrm{IH}$ & & $x$ & 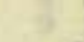 & 200 & ar & 50 & $4 \cdot 30$ & & Whe & & 000 \\
\hline 40 & 4. 30 & & & 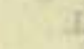 & 20 & of & 300 & 4.3 & $\mathrm{~S}$ & 14 & & 2 \\
\hline 300 & $4 \cdot 3$ & & & & 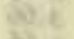 & 8 & I0 & 4. & 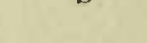 & & & $\approx$ \\
\hline 300 & $4 \cdot 3 c$ & $\mathrm{E}$ & & 5 & & t. & & & & & & \\
\hline $\begin{array}{l}10 \\
20\end{array}$ & $4 \cdot 3 c$ & & & & 92.6 & a & 30 & 4.3 & tithis & 8 & & 9 \\
\hline 20 & & & & & 20 & 68 & 40 & 4.35 & & 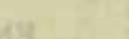 & & \\
\hline 30 & 4.28 & 17 & K* & 7 & $17 x$ & 897 & 50 & $4.39 \mathrm{H}$ & & & & 10 \\
\hline & 4.2 & & & & $10 y$ & & 400 & 4.38 & $\mathrm{~S}$ & 13 & & \\
\hline 45 & 4.2 & NE & & 4 & & $a$ & 1 & & & $y^{4}$ & & is \\
\hline 5 & & ESE & & 2 & 21 & 6 & & & & 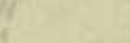 & 6 & 98 \\
\hline 60 & 3.7 & $\underset{S E}{S E}$ & & 3 & s. & In & & 2 & & 8 & & 9 \\
\hline 700 & 3.6 & ESE & & 8 & & set & & 4. & S & $I_{4}$ & & \\
\hline & & & & & & $\mathrm{c}$ & & & S & & & \\
\hline 800 & $\begin{array}{l}3.49 \\
3.10\end{array}$ & $\mathrm{SE}$ & & 9 & & & 7 & 3.8 & S & H & & 280 \\
\hline $\begin{array}{l}10 \\
20\end{array}$ & $\begin{array}{l}3.49 \\
3.45\end{array}$ & & $w 2$ & & & $\begin{array}{l}\text { R.R. R. } \\
\text { S.W. }\end{array}$ & 80 & 3. & $S$ & 16 & & \\
\hline 30 & $3.45 \mathrm{~L}$ & & & & & S.W.S. & 900 & 3. 54 & SSE & 16 & & S.W. S. \\
\hline 40 & 3.46 & & & & & J. V & & $\begin{array}{l}3.54 \\
3.53\end{array}$ & & & & 5 \\
\hline 50 & 3.47 & & & & & & 30 & 3.53L & & & & S.W s \\
\hline
\end{tabular}


Tabulation of tidal observations at Teplitz Bay, Rudolph Island

\begin{tabular}{|c|c|c|c|c|c|c|c|c|c|c|c|c|}
\hline \multicolumn{2}{|c|}{$\begin{array}{l}\text { Local } \\
\text { mean } \\
\text { tinee }\end{array}$} & $\begin{array}{l}\text { Reading } \\
\text { of tide } \\
\text { staff }\end{array}$ & \multicolumn{2}{|c|}{$\begin{array}{l}\text { Wind } \\
\text { direction }\end{array}$} & \multicolumn{2}{|c|}{ Velocity } & \multirow[t]{2}{*}{ Observer } & $\begin{array}{c}\text { Local } \\
\text { mean } \\
\text { time }\end{array}$ & $\begin{array}{l}\text { Reading } \\
\text { of tide } \\
\text { staff }\end{array}$ & $\begin{array}{l}\text { Wind } \\
\text { direction }\end{array}$ & Velocity & \multirow[t]{2}{*}{ Observer } \\
\hline \multicolumn{7}{|c|}{ May 26, 1904} & & & & May 27,19 & 04 & \\
\hline$h$ & & Ficet & & & Miles & wat & w 2 & $h m$ & Ficet & 1 & Miles & Wh \\
\hline & 40 & 3.55 & & $7 a^{2}$ & & 60 & S. IV. S. & 1030 & 3.78 & & & S. W. S. \\
\hline & 50 & 3.55 & & & & 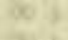 & ton -2 & 40 & $3.78 \mathrm{~L}$ & & & \\
\hline IO & oo & $3.5^{8}$ & SE & 18 & 15 & 345 & ay $w$ & 50 & 3.78 & & & 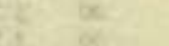 \\
\hline & 10 & 3. 59 & & & & Ave & (2) & I1 00 & 3.79 & $\mathrm{E}$ & 23 & or \\
\hline 11 & $\infty$ & 3.72 & $\mathrm{SE}$ & & 17 & 145 & as & 10 & 3.79 & & & 6s \\
\hline I 2 & 04 & 3.96 & SSE & Ne & 16 & We 1 & 10,5 & 20 & 3.8 & & & we \\
\hline I3 & oo & 4.24 & $\mathrm{SE}_{\mathrm{H}}$ & & 16 & ne 4 & G & 1206 & 4.00 & FiSE & 21 & wit \\
\hline 14 & 00 & $4 \cdot 5^{2}$ & $\mathrm{SE}$ & & 16 & Wer & $\infty$ & 1300 & & $\mathrm{E}$ & 21 & mit \\
\hline 15 & oo & 4.73 & SE & & I4 & wa & 48 & 1400 & $4 \cdot 56$ & $\vec{E}$ & 23 & $\varnothing$ \\
\hline & 10 & 4.75 & & & . & 195 & 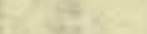 & 1500 & & $\mathrm{E}$ & 23 & as \\
\hline & 20 & 4.76 & 政 & & 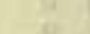 & 20 & -2 & 1600 & 4.99 & $\mathrm{E}$ & 22 & 00 \\
\hline 6. & 30 & 4.77 & Y & 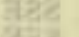 & at & ab.) & 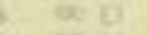 & 10 & 5.0 & & & $6+4$ \\
\hline 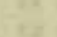 & 40 & 4.78 & ty & $3 E 0$ & 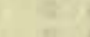 & 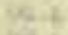 & 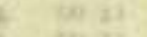 & 20 & 5.03 & & & 3036 \\
\hline & 50 & 4.79 & 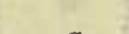 & 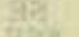 & (1) & ko. & 1024 & 3 & 5. & & & 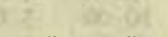 \\
\hline 16 & oo & $4.79 \mathrm{H}$ & $\mathbf{S}$ & 7824 & 14 & this & 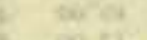 & 40 & 5.04 & & & S. W. S. \\
\hline & 10 & 4.79 & & & & $4 c$ & 2005 & 50 & 5.01 & & & J. V. \\
\hline 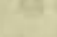 & 20 & 4.78 & & & we & 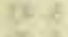 & as & I 700 & 5.05 & & 22 & W. J.P. \\
\hline & 30 & 4.78 & & & & $x+2$ & 6 & 10 & $5.05 \mathrm{H}$ & & & J. V. \\
\hline$=$ & 40 & 4.77 & SE & & 12 & $\sec 3$ & 6 & 20 & 5.0 & & & W. J.P. \\
\hline 17 & 00 & 4.73 & SE & & 12 & 0,7 & 蛋 & 30 & 5. & & II & T. V. \\
\hline 18 & of & 4.50 & $\mathrm{SE}$ & & & $2,-2$ & 68 & 4 & $0=$ & & & W. J.P. \\
\hline I9 & on & 4.20 & $\mathrm{SE}$ & 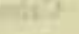 & I 3 & 82 & $\ln ^{2}$ & 5 & 4.98 & & & J. V. \\
\hline 20 & $\infty$ & 3.92 & SE & & 12 & $\theta_{2} 2$ & S. W.S. & 1800 & 4.95 & $\mathrm{E}$ & 17 & W. J.P. \\
\hline 21 & $\infty$ & 3.69 & SE & & 12 & 132 & R. R. T. & 10 & 4.91 & & & J. V. \\
\hline & 20 & 3.63 & & & a & $2 \pi \cdot 3$ & (5) & I9 oc & 4.64 & & 15 & S. W.S. \\
\hline & 30 & 3.61 & 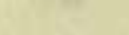 & & 10 & 20.4 & 05 & 2000 & 4.34 & & 17 & S. W.S. \\
\hline & 40 & 3.60 & & & & a. 3 & Tos & 2100 & 3.94 & & 14 & R. R. T. \\
\hline & 50 & 3.58 & & & & 1282 & 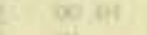 & 2200 & 3.69 & ESE & 10 & \\
\hline 22 & 00 & 3.54 & ESE & & 13 & $y=3$ & ati & 10 & 3.63 & & & as. $4=$ \\
\hline & 10 & $3.53 \mathrm{~L}$ & & & - & $x=2$ & as & 20 & $3.6 \mathrm{I}$ & & & 00028 \\
\hline & 22 & $3 \cdot 54$ & & & & 652 & $00 \mathrm{et}$ & 3 & 3.60 & . & & woy \\
\hline & 30 & 3.55 & & 24 & & 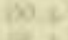 & 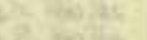 & 4 & 3.57 & & & yor \\
\hline & 40 & 3.57 & & 4 & & 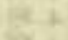 & Fit $\quad 60$ & 5 & 3. & & & 17. \\
\hline & 50 & 3.59 & & & 12 & 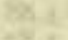 & $x^{2}=x_{2}$ & 2300 & $3 \cdot 52$ & SE & 8 & inte \\
\hline 23 & 00 & $3 \cdot 59$ & ESE & & 13 & 38.5 & 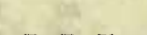 & 10 & $3.52 \mathrm{~L}$ & & & $\alpha$ \\
\hline 24 & $\infty$ & 3.71 & & & i 3 & $\cos x$ & R. R. T. & 20 & 3.53 & & 4 & Eve \\
\hline & ese & ry 64 & & & & & & 30 & 3.53 & & & 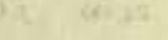 \\
\hline & & 1023 ing & May 27 & 7,190 & & 89 & 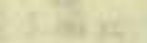 & $\begin{array}{l}40 \\
50\end{array}$ & $3 \cdot 53$ & & & \\
\hline 1 & oo & 4.00 & ESE & & 11 & & R. R. T. & 2700 & $\begin{array}{l}3 \cdot 50 \\
3 \cdot 59\end{array}$ & $\mathrm{SE}$ & 7 & R. R. T. \\
\hline 2 & $\infty 0$ & $4 \cdot 31$ & SE & & 12 & & 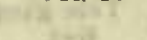 & & & & & \\
\hline 3 & oo & $\begin{array}{l}4.57 \\
1.68\end{array}$ & $\mathrm{E}$ & & 13 & & 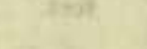 & & & May 28, & 1904 & ax \\
\hline f & $\begin{array}{l}30 \\
40\end{array}$ & $\begin{array}{l}4.08 \\
4.70\end{array}$ & inot la & $\mathrm{raH}$ & & & & & & & & $P \pi$ \\
\hline & 50 & 4.71 & & & 15 & & & $\begin{array}{l}100 \\
200\end{array}$ & 3.79 & $\underset{S S E}{S E}$ & 6 & R.R.T. \\
\hline 4 & $\infty$ & 4.71 & ENE & $y$ & & 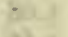 & $(6) 64$ & $\begin{array}{l}200 \\
300\end{array}$ & 4. 11 & SSE & $\begin{array}{l}6 \\
6\end{array}$ & print? \\
\hline 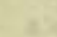 & 10 & 4.73 & $-5=2$. & & 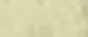 & 0256 & in & $\begin{array}{l}300 \\
400\end{array}$ & $\begin{array}{l}4.41 \\
4.60\end{array}$ & 3 & $\begin{array}{l}0 \\
8\end{array}$ & cosi \\
\hline & 20 & 4.78 & 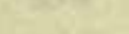 & & the & lain: & 35 & $\begin{array}{r}400 \\
30\end{array}$ & $\begin{array}{l}4.09 \\
4.80\end{array}$ & & & are \\
\hline & 30 & 4.78 & & & & Wat is & ax & 4 & 4.83 & & & $4 i$ \\
\hline & 40 & 4.80 & & & & 105. & 14 & 50 & 4.87 & & & 6 \\
\hline & 50 & $4.80 \mathrm{H}$ & & & & 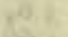 & 9 & 500 & 4.88 & SIV & 5 & 25 \\
\hline 5 & & 4.80 & E & 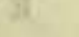 & 20 & king & 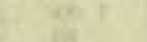 & I0 & 4.90 & & 3 & th \\
\hline & Io & 4.79 & & & & 504 & $\operatorname{lin}$ & 2 & $4.9 \mathrm{I}$ & & & 2 \\
\hline & 20 & 4.79 & & & & 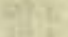 & 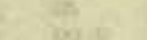 & 3 & 4.9 & & & $2001)$ \\
\hline & 30 & 4.77 & & & & 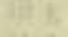 & & 4 & 4. & & & al \\
\hline 6 & $\infty$ & 4.70 & E & & 20 & 31 & (10) & 5 & 4.8 & & the & oc \\
\hline 7 & oo & $4 \cdot 42$ & $\mathrm{NE}$ & fif & 22 & 45 & 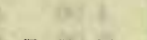 & 60 & 4.89 & & 3 & cen \\
\hline & $\infty 0$ & 4. 18 & $\mathrm{E}$ & & 23 & thet & R. R. T. & IC & 4.86 & & 3 & ws \\
\hline 9 & oo & 3.92 & $E$ & & 21 & 8) & S. W.S. & 700 & 4.74 & SSIV & 6 & Q \\
\hline 10 & $\infty$ & $3.8 \mathrm{I}$ & $E$ & Whe & 24 & & & 800 & 4.42 & $\mathrm{SE}$ & & \\
\hline & IO & 3.79 & & this? & & Whis & 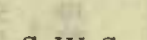 & 900 & 4.14 & $\mathrm{~S}$ & 6 & S.W.S. \\
\hline & 20 . & 3.78 & & & & $1 \times-2$ & S. WV. S. & 1000 & 3.92 & SE & 6 & S. W. S. \\
\hline
\end{tabular}


Tabulation of tidal abservations at Teplitz Bay, Rudolph Island

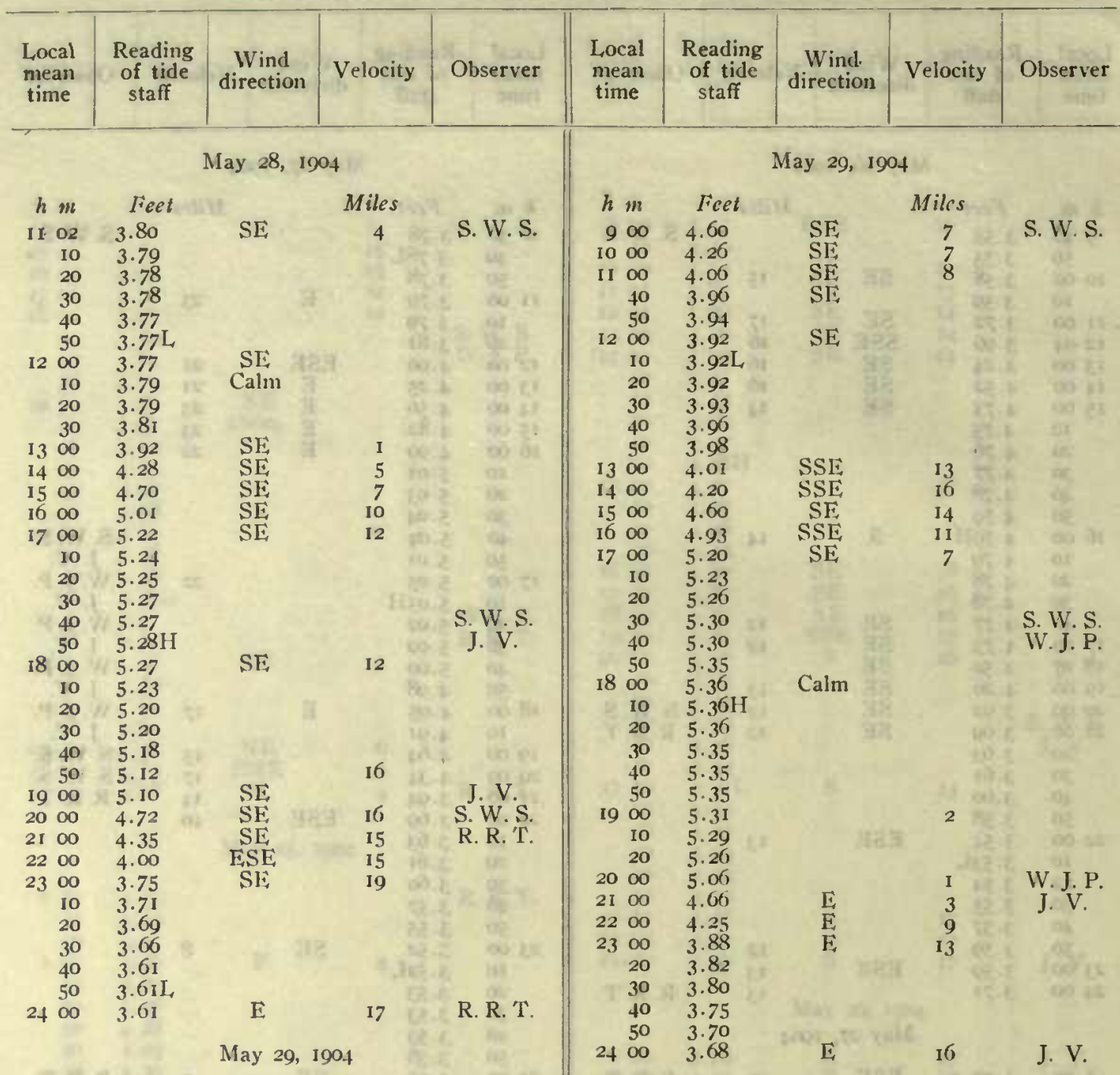

$\begin{array}{rrrrrr}010 & 3.61 & & & \text { R. R. T. } \\ 20 & 3.62 & & & \\ 30 & 3.65 & & & \\ 100 & 3.71 & \text { ESE } & 14 & \\ 200 & 4.00 & \text { E } & 16 & \\ 300 & 4.39 & \text { ESE } & 18 & \\ 400 & 4.78 & \text { E } & 20 & \\ 500 & 5.03 & \text { ENE } & 22 & \\ 10 & 5.08 & & & \\ 20 & 5.10 & & & \\ 30 & 5.13 & & & \\ 40 & 5.18 & & & \\ 50 & 5.20 & \text { ESE } & 25 & \\ 600 & 5.20 & & & \\ 10 & 5.20 & & & \\ 20 & 5.20 H & & & \\ 30 & 5.20 & & & \\ 40 & 5.20 & & & \\ 50 & 5.19 & & & \\ 700 & 5.17 & \text { E } & 18 & \\ 10 & 5.14 & & 12 & \text { R. R.T. } \\ 800 & 4.96 & \text { SE } & 7 & \end{array}$

Tide gange reading of $B$. M. No. I at 9:00 is 14.69 feet.

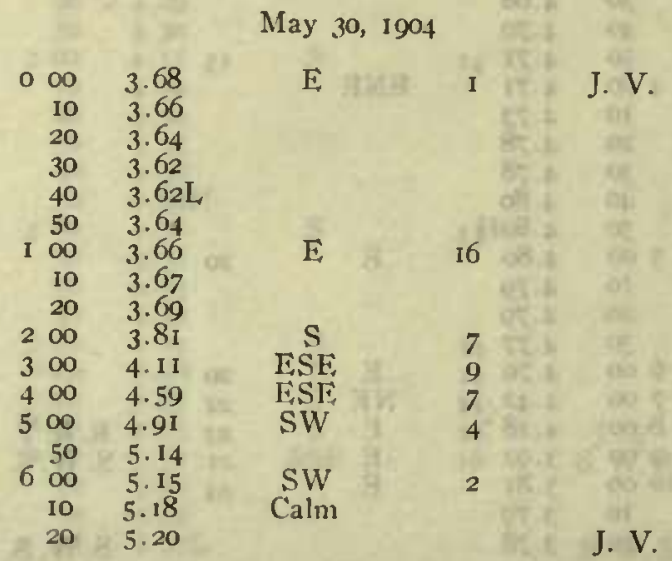


Tabulation of tidal observations at Teplitz Bay, Rudolph Island

\begin{tabular}{|c|c|c|c|c|c|c|c|c|c|c|c|}
\hline $\begin{array}{l}\text { Local } \\
\text { mean } \\
\text { time }\end{array}$ & $\begin{array}{l}\text { Reading } \\
\text { of tide } \\
\text { staff }\end{array}$ & \multirow{2}{*}{$\frac{\begin{array}{c}\text { Wind } \\
\text { direction }\end{array}}{\text { May } 30,190}$} & \multicolumn{2}{|c|}{ Velocity } & \multirow{2}{*}{ Observer } & \multirow[t]{2}{*}{$\begin{array}{c}\text { Local } \\
\text { mean } \\
\text { time }\end{array}$} & \multirow[t]{2}{*}{$\begin{array}{l}\text { Reading } \\
\text { of tide } \\
\text { staff }\end{array}$} & \multirow{2}{*}{\begin{tabular}{|c|}
$\begin{array}{c}\text { Wind } \\
\text { direction }\end{array}$ \\
May 31, 19
\end{tabular}} & \multicolumn{2}{|c|}{ Velocity } & \multirow[t]{2}{*}{ Observer } \\
\hline & & & 4 & & & & & & 04 & & \\
\hline$h m$ & \multicolumn{2}{|c|}{ Feet } & \multicolumn{2}{|c|}{ Miles } & $x^{2}$ & $h m$ & \multicolumn{2}{|c|}{ Feet } & Miles & $s$ & \\
\hline 63 & 5.22 & & & +8 & J. V. & $6 \mathrm{I}$ & 4.90 & & & $28 \div$ & J. V. \\
\hline 4 & 5.22 & & & & 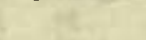 & 2 & 4.91 & & & दिश 3 & b. \\
\hline 5 & 5.23 & $\overrightarrow{E S E}$ & & 300 & sing & & 4.93 & & & 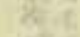 & $\omega$ \\
\hline 70 & 5.23 & & & bill & $146=$ & 4 & 4.98 & & & Ehis & $95 \mathrm{~h}$ \\
\hline I & 5.25 & & & 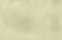 & 2084 & 5 & 5.00 & & & is: & $\operatorname{lin} 2$ \\
\hline 2 & 5.2711 & av & & vas: & why & 70 & 5.05 & $y$ & Io & 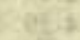 & $\infty$. \\
\hline 3 & 5.24 & & & ix: 1 & 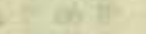 & 1 & 5.07 & (4) & & $158 \%$ & 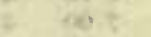 \\
\hline 4 & 5.22 & & & 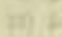 & $5+5 y^{2}$ & & 5.12 & & & $70 \%$ & of ? \\
\hline & 5.20 & & & 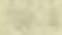 & & & $5.12 \mathrm{H}$ & & & D. & of \\
\hline 80 & 5.19 & WSW & 9 & $8=$ & J. V. & & 5.11 & & & -0.3 & $m^{2}$ \\
\hline $9 \alpha$ & 4.88 & IV & 9 & conit & S. W. S. & 5 & 5.10 & & & 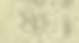 & 6011 \\
\hline $10 a$ & 4.49 & IV & 9 & aets & was & 80 & 5.00 & SSW & II & 68, & \\
\hline 110 & 4.20 & IV & 9 & 27 & ant 1 & & 5.07 & & & $400 . ?$ & J. V. \\
\hline 120 & 4.00 & WV & 9 & $N^{*}$ & 6Le. & 90 & 4.94 & SW & Io & 160 & S. W. S. \\
\hline & 3.92 & & & ASo & is: & Io 0 & $4.6 \mathrm{I}$ & SW & 10 & on: & t. \\
\hline 5 & 3.92 & IV & & 200 & Gornot & II 0 & $4 \cdot 30$ & SW & 8 & 625 & $\therefore$ \\
\hline $13 \alpha$ & 3.92 & 4 & 8 & tais & 109,1 & 120 & 4.04 & SIV & 6 & $3 x=4$ & 50 \\
\hline & $3.92 \mathrm{~L}$ & $x=5$ & $x^{2}$ & (4). & & 4 & 3.90 & 7i & & itis & (1) od \\
\hline 3 & 3.93 & I & a & ais & S. W.S. & 130 & 3.87 & 当 & & $\Rightarrow 3$ & 7) 11 \\
\hline 4 & 3.95 & & & mis & R. R. T. & I & 3.84 & & & 00,3 & 1008 \\
\hline 5 & 3.97 & & & $\frac{14}{818}$ & R. R. T. & 2 & 3.82 & 1 & & 312 & $x, k$ \\
\hline $14 \propto$ & 4.00 & W & 8 & 80.5 & S. W. S. & 3 & 3.82L & & & 25.1 & $\log 2$ \\
\hline $15 \alpha$ & 4.25 & SSW & 8 & ligit & 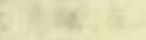 & 4 & 3.83 & $\approx$ & & 25.6 & 08 \\
\hline $16 \alpha$ & 4.67 & W & 7 & 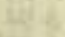 & as: & & 3.84 & & & ise & 60,21 \\
\hline $17 \propto$ & 5.02 & SW & 8 & $\operatorname{lon} x$ & ats & 140 & 3.85 & SSW & 7 & $35 \mathrm{E}$ & of \\
\hline $18 \alpha$ & $\begin{array}{l}5.25 \\
5.34\end{array}$ & SW & 12 & tiof & of 21 & 150 & $\begin{array}{l}3.86 \\
3.96\end{array}$ & & 9 & to: & 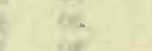 \\
\hline $\begin{array}{l}4 \\
5\end{array}$ & 5.36 & & & 168 & ant & 160 & $4 \cdot 32$ & SSE & 4 & $\therefore$ & 14: \\
\hline 19 o & $5 \cdot 36$ & IV & I4 & 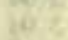 & ois & 170 & 4.69 & $\mathrm{~S}$ & 4 & 28.4 & $n$ \\
\hline If & $5 \cdot 36$ & . & & Anits & por at & 180 & 4.91 & SW & I & The & जो \\
\hline 2 & $5.36 \mathrm{H}$ & & & f 8 & 8 & 4 & 5.07 & $4 x$ & & ary & anthi \\
\hline 3 & $5 \cdot 34$ & & & 28.2 & S.W.S. & 5 & 5.09 & & & what & $(y)=5$ \\
\hline & 5.30 & & & ait: & R. R. T. & 190 & 5. 10 & SW & 3 & 65.4 & wisy \\
\hline & 5.28 & & & 60.5 & S.W.S. & & $5 \cdot 10$ & (1) & & $m$ & rapt \\
\hline $20 \propto$ & 5.25 & SW & 13 & 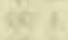 & & & 5.14 & & & $20 y$ & 64 \\
\hline 210 & 4.90 & SW & 14 & 60. & S. W. S. & 3 & $5 . \mathrm{I} 4 \mathrm{HI}$ & & & 50.3 & 82 \\
\hline 220 & 4.46 & $\mathrm{~S}$ & I4 & cores & J. V. & 4 & 5.13 & 47 & & 301 & 1000 \\
\hline 230 & 4.05 & $\mathrm{~S}$ & 14 & 102 & & $20 \stackrel{5}{\alpha}$ & $\begin{array}{r}5.13 \\
5.10\end{array}$ & $\mathrm{SSE}$ & 4 & fis is & ent \\
\hline 240 & $3 \cdot 73$ & 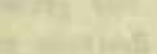 & 13 & 90. & J. V. & 200 & $\begin{array}{l}5.10 \\
5.10\end{array}$ & & 1 & $3 y^{4}$ & S. W. S. \\
\hline & 20 & & & & & 210 & 5.00 & & 3 & wint? & W. J. P. \\
\hline beros & $-4 t^{2}=$ & May 3I, I & & $y^{2}$ & $w_{0}$ & $22 \propto$ & 4.66 & & 4 & $\infty .2$ & (1) \\
\hline 02 & 3.65 & $18 x^{2}+5=$ & & wit & $\mathrm{V}$ & $\begin{array}{l}23 \alpha \\
24 \alpha\end{array}$ & 4.25 & $\mathrm{NF}$ & 2 & $80 .-2$ & W. J. P. \\
\hline & 3.62 & 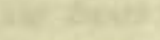 & & 289 & J. & & 3.97 & $\mathrm{NE}$ & 3 & iest & R. R. T. \\
\hline 4 & 3.60 & 4 & & ans & $6 n+x$ & At & o ice pres & sure about $t$ & hree-qua & arters & $s$ of a mile \\
\hline 5 & $3 \cdot 59$ & SCIV & & $106+$ & wire & & 1310 & utheast $\mathrm{t}$ & rthwest & it dire & ection. \\
\hline I $O$ & $3 \cdot 57$ & SSW & 12 & 21 & on in & V & & -13 & & 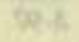 & $\infty$, is \\
\hline I & $\begin{array}{l}3 \cdot 54 \\
3 \cdot 53\end{array}$ & & & & & & & June $I$, & 904 & & \\
\hline 3 & 3.53L & 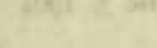 & & & & & & foxy a a & & & \\
\hline 4 & 3.53 & & & 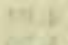 & cingat & o 5 & 3.63 & & & & R. R. T. \\
\hline $2 \stackrel{5}{\alpha}$ & $\begin{array}{l}3.55 \\
3.58\end{array}$ & ssw & & 354 & pil $*$ & I & 3.60 & Calm & & Xete & \\
\hline${ }_{1}^{2}$ & $\begin{array}{l}3.58 \\
3.60\end{array}$ & SSW & 13 & $3 \frac{1}{5}$ & ar & $\begin{array}{l}\text { I } \\
2\end{array}$ & $\begin{array}{l}3 \cdot 5^{8} \\
3.54\end{array}$ & $7 x$ & & ats & 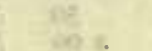 \\
\hline 2 & 3.61 & & & (52.). & $=2 x$ & 30 & $\begin{array}{l}3.54 \\
3.51\end{array}$ & & & 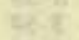 & or. \\
\hline 3 & 3.63 & Gover & $x^{2}$ & 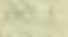 & 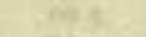 & 4 & $3.5 \mathrm{I}$ & & & nz: 4 & nt. \\
\hline $3 \mathrm{I}$ & 3.82 & SSW & I 1 & bitit & $3065=$ & 5 & 3.50 & & & $\operatorname{cec} 2$ & $n$ \\
\hline 40 & 4.12 & SSW & 12 & 0022 & $=$ & 20 & 3.49 & WNW & & $x^{3}+1$ & 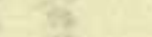 \\
\hline $5 \propto$ & $4 \cdot 56$ & SSW & I & 221 & 97 & IC & $3.49 \mathrm{~L}$ & & & $75-2$ & ee: \\
\hline 5 & $4.8 \mathrm{I}$ & & & est5 & 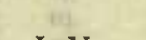 & 2 & 3.50 & 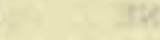 & & 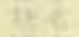 & we 2 \\
\hline 60 & 4.83 & SSW & 12 & whe & J. V. & 3 & $3.5 \mathrm{I}$ & & & test & R. R. 'T. \\
\hline
\end{tabular}


Tabulation of tidal observations at Teplitz Bay, Rudolph Island

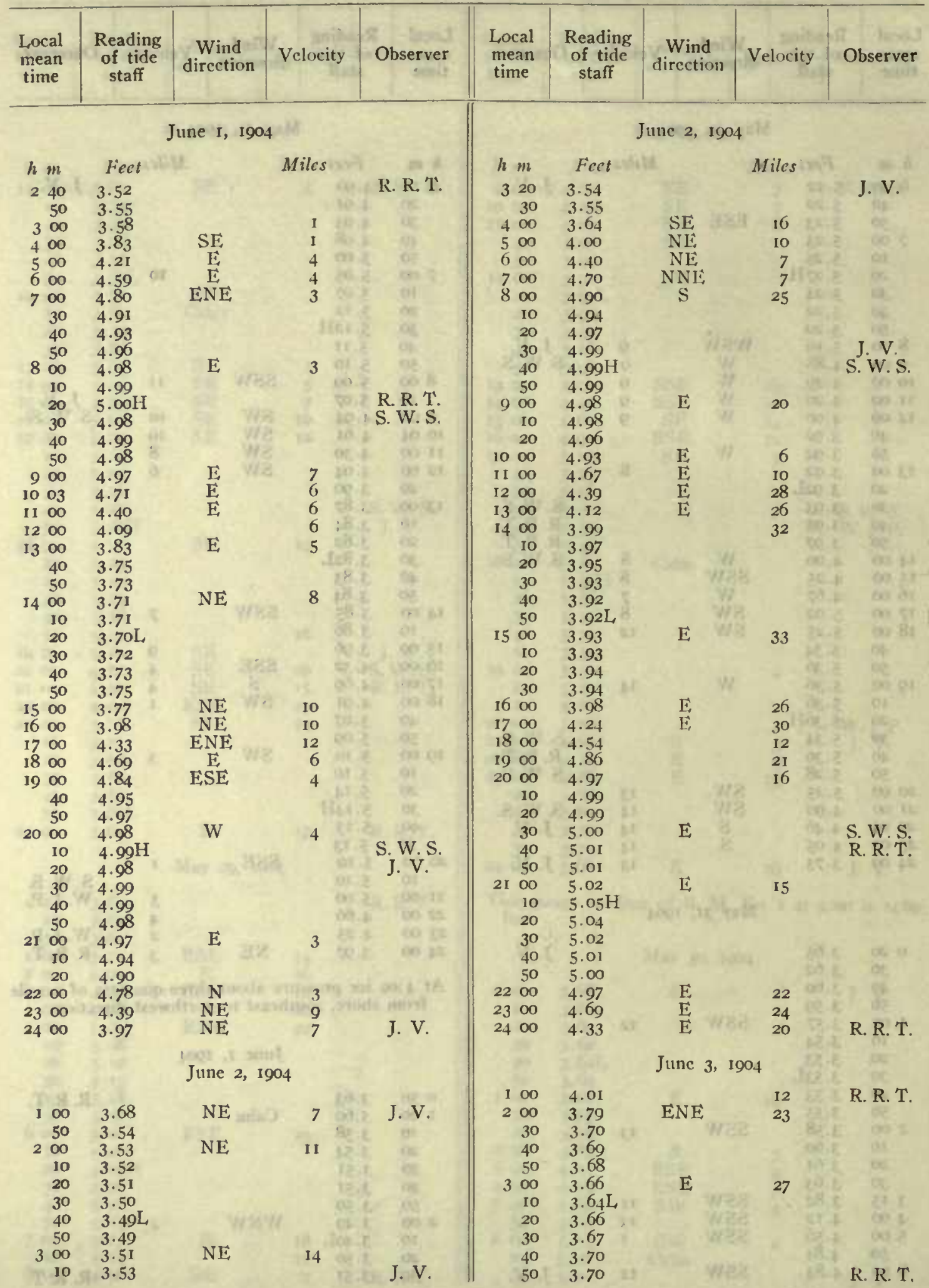


Tabulation of tidal observations at Tcplitz Bay, Rudolph Island

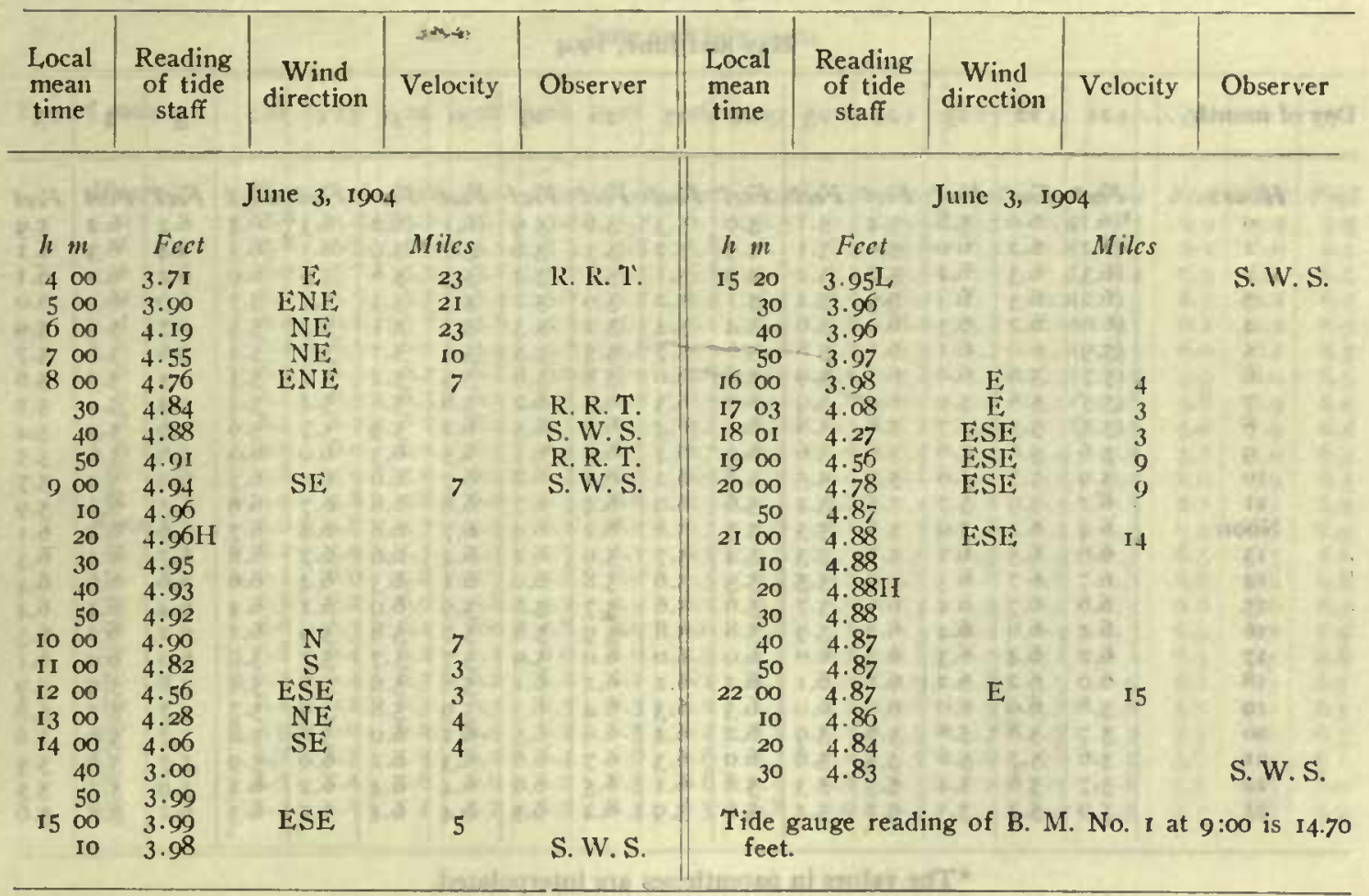

\section{RHDUCED READINGS}

After adjusting the foregoing original readings of the tide staves to a uniform datum, botli series were plotted on profile paper, and irregularities due to storms or mistakes were smoothed out. The smoothed curves were completed so as to fill small gaps in the record, and were then tabulated as hourly heights of the sea and also as high and low waters, the readings being cut down to tenths of feet, as shown in the following tables of hourly heights of the sea. 
Hourly heights of tide, Cape Ftora, Franz Josef Archipelago, Arctic Ocean

May and June, I904

\begin{tabular}{|c|c|c|c|c|c|c|c|c|c|c|c|c|c|c|c|c|}
\hline & 7 & & & & & & & & & & & & & & & \\
\hline Day of month. & 21 & 22 & 23 & 24 & 25 & 26 & 27 & $2 S$ & 29 & 30 & $3^{I}$ & I & 2 & 3 & 4 & 5 \\
\hline Hours & Feet & Feet & Fcet & Feet & Feet & Fect & Fect & Feet & Feet & Fect & Feet & Feet & Ficet & Fect & Feet & Feet \\
\hline o & ${ }^{*}(6.1)$ & 6.0 & 5.8 & 5.4 & $5 \cdot 1$ & 5.0 & $5 \cdot 3$ & 5.6 & 59 & 6.1 & 6.2 & 6.3 & 6.4 & 6.4 & 6.2 & $5 \cdot 9$ \\
\hline I & $(6.2)$ & 6.2 & 60 & 5.6 & 5.1 & 4.9 & 5.2 & $5 \cdot 3$ & 5.6 & 5.7 & 5.9 & 6.1 & 6.3 & & 6.3 & 6.1 \\
\hline 2 & (6.3) & 6.3 & 6.2 & 5.7 & 5.2 & 4.9 & 5.I & 5.1 & 5.4 & $5 \cdot 5$ & 56 & 5.8 & 6.0 & 6.2 & 6.2 & 6.1 \\
\hline 3 & $(6.2)$ & 6.3 & 6.3 & 5.9 & 5.4 & 5.1 & 5.2 & 5.0 & 5.2 & $5 \cdot 3$ & $5 \cdot 3$ & 5.4 & 5.7 & 6.0 & 6.1 & 6.0 \\
\hline 4 & $(6.0)$ & 6.2 & 6.3 & 6.0 & 5.6 & 5.4 & 5.4 & 5.2 & $5 \cdot 3$ & 5.2 & 5. I & $5 \cdot 3$ & 5.5 & 5.7 & 5.9 & 5.9 \\
\hline 5 & $(5.9)$ & 6.0 & 6.1 & 6.1 & 58 & 5.6 & 5.7 & $5 \cdot 5$ & 5.5 & 5.2 & 5.1 & 5.1 & 5.4 & 5.6 & 5.6 & 5.7 \\
\hline 6 & $(5.7)$ & 5.8 & 6.0 & 6. 5 & 5.9 & 5.8 & 6.0 & 58 & 5.8 & 5.5 & 5.2 & 52 & $5 \cdot 3$ & 5.4 & $5 \cdot 5$ & 5.6 \\
\hline 7 & $(5.5$ & 5.6 & 5.9 & 6.0 & 5.9 & 6.0 & 6.3 & 6.1 & 6.2 & 5.8 & 5.6 & 5.4 & 5.4 & 5.4 & 5.4 & $5 \cdot 5$ \\
\hline 8 & (5.5) & $5 \cdot 5$ & 5. & 5.8 & 5.8 & 6.I & f. 4 & 6.4 & 6.5 & 6.2 & 5.9 & 5 . & 5.6 & 5.6 & $5 \cdot 5$ & 5.4 \\
\hline 9 & 5.6 & 5. & 5. & 5.6 & 5.6 & 6.0 & 6.4 & 6. & 6.7 & 6.5 & 6.3 & 6.0 & 6.0 & 5.8 & 5.6 & 5.5 \\
\hline Ió & & 5 . & 5. & $5 \cdot 5$ & $5 \cdot 5$ & 5.8 & 6.2 & 6.6 & 6.8 & 67 & 6. & 6.4 & 6.3 & 6.0 & 5.9 & 5.7 \\
\hline I & 6.2 & 5. & 5 & 5.5 & 5.4 & 5.6 & 6.0 & 6.4 & 6.7 & 6.8 & 6.8 & 6.7 & 66 & 6.3 & 6.1 & 5.9 \\
\hline Noon & 64 & 6.2 & 5. & 5.6 & $5 \cdot 3$ & 55 & 5.8 & 6.2 & 6 & 6.7 & 6.8 & 6. & 6.7 & 6.6 & 6.3 & 6.1 \\
\hline I & 6.6 & 6.5 & 6. & 5.7 & $5 \cdot 3$ & 5.4 & $5 \cdot 7$ & 5. & 6.2 & 6.4 & 6.6 & 6.7 & 6.8 & 6.7 & 6.5 & 6.3 \\
\hline I & 6.7 & 6. & 6. & 5.9 & 5.5 & 5.5 & 5.6 & 5. & 6.0 & $6 . \mathrm{I}$ & 6. & 6 & 6. & 6.6 & 6.6 & 6.4 \\
\hline I & 6.6 & 6. & 6. & 6.0 & 5. & 5. & 5. & 5 & 5.8 & 5.9 & 60 & $6 . \mathrm{I}$ & 6.4 & 6.4 & 6.4 & 6.4 \\
\hline I & 6.4 & 6.6 & 6.4 & 6.2 & 5.9 & 5.8 & 5.8 & 5 & 5.8 & 5.7 & 58 & 5.9 & $6 . \mathrm{I}$ & 6.2 & 6.2 & 6.3 \\
\hline I & 6.2 & 6.4 & 6. & 6.3 & 6. & 6.0 & 6.0 & 6 & 5 & 5. & 5. & & & 6.0 & 6.0 & 6. I \\
\hline I & 6.0 & 6.2 & 6.2 & 6.2 & 6.1 & 6.2 & 6.I & 6.2 & 6.1 & 5.8 & 5. & 5.6 & 5.8 & 5.8 & 5.9 & 5.9 \\
\hline IS & 5.8 & 6.0 & 6.0 & 6.0 & 6.0 & 6.3 & 6.3 & 6 & 6.3 & 5.9 & 5 & 5. & 5. & 5.7 & & \\
\hline 20 & 5.7 & 5.8 & 5.8 & 5.8 & 5.9 & 6.2 & 6.4 & 6.6 & 6.5 & 6.1 & 6.0 & 5.8 & 5.8 & 5.7 & 5.6 & 5.6 \\
\hline 2I & 5. & .5 .7 & 5.6 & 5.5 & 5.6 & 60 & 6.3 & 6 & 6.6 & 6.3 & 6.2 & 6. & 5.9 & 5.7 & 5.6 & $5 \cdot 5$ \\
\hline 22 & 5.7 & 5.6 & 5.4 & $5 \cdot 3$ & $5 \cdot 3$ & 5.8 & 6.1 & 6. & 6.6 & 6.4 & 6.4 & 6.2 & 6.1 & 5.9 & 5.7 & 5.5 \\
\hline 23 & $5 \cdot 9$ & $5 \cdot 7$ & $5 \cdot 3$ & 5.2 & 5.1 & 5.6 & 5.9 & 6.2 & 6.5 & 6.4 & 6.4 & 6.3 & 6.3 & 6.I & 5.8 & 5.6 \\
\hline
\end{tabular}

* The values in parentheses are interpolated.

Hourly heights of tide, Cape Flora, Franz Josef Archipclago, Arctic Ocean-Continued

June, 1904

\begin{tabular}{|c|c|c|c|c|c|c|c|c|c|c|c|c|c|c|c|c|}
\hline Day of nionth.... & 6 & 7 & 8 & 9 & IO & II & 12 & 13 & I4 & I 5 & 16 & 17 & I 8 & 19 & 20 & $2 I$ \\
\hline Hours & Feet & Feet & Feet & Feet & Feet & Feet & Fect & Feet & Feet & Feet & Feet & Feet & Fcet & Fect & Fect & Fect \\
\hline 0 & $5 \cdot 7$ & $5 \cdot 5$ & 5.2 & 5.2 & $5 \cdot 4$ & $5 \cdot 5$ & 5.6 & 5.7 & 6.1 & 6.4 & 6.5 & 6.6 & 6.6 & 6.2 & 6.1 & 5.8 \\
\hline$I$ & 5.9 & 5.6 & $5 \cdot 3$ & 5.2 & 5.4 & 5.4 & $5 \cdot 5$ & $5 \cdot 5$ & 5.8 & 6. I & 6.2 & 6.4 & 6.5 & & 6.2 & 6.1 \\
\hline 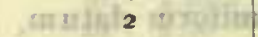 & 6.0 & $5 \cdot 7$ & $5 \cdot 4$ & 5.4 & 5.4 & $5 \cdot 3$ & 53 & 53 & 5.6 & 58 & 5.8 & 6.1 & 6.3 & 6. & 6.3 & 6.3 \\
\hline 3 & 6.0 & 58 & $5 \cdot 5$ & 5.6 & 5.6 & 5.4 & $5 \cdot 3$ & 5.2 & $5 \cdot 5$ & 5.6 & 56 & 58 & 6.0 & 6. I & 6.2 & 6.4 \\
\hline$\ddot{4}$ & 5.9 & 5.8 & 5.7 & 5.7 & 5.8 & 5.6 & $5 \cdot 5$ & $5 \cdot 4$ & 5.5 & 5.5 & 5.4 & 5.5 & 5.7 & 5.8 & 6.1 & 6.4 \\
\hline broos 5 stif net & 5.8 & 5.8 & $5 \cdot 7$ & 5.9 & 6.0 & 5.9 & 5.7 & 5.6 & 5.6 & .5 .5 & 5.2 & 5 . & 5 . 5 & 5 & 6.0 & 6.3 \\
\hline 6 & 5.7 & 5.7 & 5.7 & 6.0 & 6.2 & 6.2 & 60 & 5.8 & 5.9 & 5. & $5 \cdot 3$ & 5 . & 5.4 & 5 & 5.8 & 6.I \\
\hline & 5.6 & 5.6 & 5.6 & 6.0 & 6.3 & 6.4 & 6.3 & 6.2 & 6.2 & 6. I & 5.6 & 4 & 5.4 & & 5.6 & $5 \cdot 9$ \\
\hline astigis $\dot{8}$ frutoul & $5 \cdot 5$ & $5 \cdot 5$ & 5.6 & 59 & 6.3 & 6.5 & 6.5 & 6.5 & 6.6 & 6.4 & 59 & 5 . & 5. & & $5 \cdot 5$ & 5.8 \\
\hline 9 & $5 \cdot 5$ & 5.4 & $5 \cdot 5$ & 5.8 & 62 & 6.5 & 6.6 & 6.7 & 6.9 & 6.7 & 6.3 & & & & 5.6 & 5.7 \\
\hline 10 & 5.5 & 5.4 & 5. & 5.8 & 6.1 & 64 & 65 & 6.7 & 7.0 & 6. & 6. & 6. & 6. & & $5 \cdot 7$ & 5.8 \\
\hline I & 57 & 5 . & & 5 . & 6.0 & 6. & 6.4 & 6.6 & & 7. & 6. & & 6 & & & \\
\hline Noon & 5.9 & 5. & 5 & 5. & 5.9 & 6. & 6.2 & 64 & 6.8 & 6.9 & 69 & 6. & 6. & & 6.3 & 61 \\
\hline I & 6.1 & 5.7 & & 5 . & 5 . & 6.0 & 6.0 & 6.2 & 6.5 & 6. & 6 & & 6 & & & 6.4 \\
\hline I. & 6.2 & 5. & 5 . & 5. & & 5.9 & $5 \cdot 9$ & 6.1 & 6.3 & 6.4 & 6. & 6 & 6 & 7 & 67 & 6.6 \\
\hline I & 6.3 & 6.0 & & 6.0 & & 6. & 5.8 & 6. & 6.2 & 6. & 6.2 & & & & & 6.7 \\
\hline I6 & 6.2 & 6.0 & 6. & 6.1 & 6. I & 6.1 & 5.9 & 6.0 & 6.1 & 6.0 & 5 . & & 6. & & 6 & 6.7 \\
\hline $\mathbf{I}$ & 6.1 & 6.0 & 6.I & 6.2 & 6.2 & 6.2 & 6.1 & 6.1 & 6. I & 5.9 & 5 . & 5 . & 5 . & 5.9 & 6.3 & 6.6 \\
\hline I8 & $5 \cdot 9$ & 5.8 & 6.0 & 6.3 & 6.3 & 6.4 & 6.2 & 6.4 & & 6.0 & & & & & & 6.3 \\
\hline I9 & 5 . & 5.7 & 5.9 & 6.2 & 6.3 & 6.5 & 6.4 & 6.5 & 6.5 & 6.2 & 5. & 5.6 & 5.5 & & 5.8 & 6.0 \\
\hline 20 & 5.6 & 55 & & 6.1 & 6.3 & & 6.5 & 6.6 & 6.7 & 6.3 & 6.0 & & & & 5.6 & 5.8 \\
\hline 21 & 5.5 & 5.4 & 5.6 & 5.9 & 6.1 & 6.3 & 6.4 & 6.7 & 6.8 & 6.5 & 6.2 & & & & 5.5 & 56 \\
\hline 22 & $5 \cdot 4$ & 5.2 & 5.4 & 57 & 5.9 & $6 . I$ & 6.3 & 6.7 & 6.8 & 6.6 & 6.4 & 6.2 & 5 & & $5 \cdot 5$ & 5.5 \\
\hline 23 & 5.4 & 5.2 & $5 \cdot 3$ & 5.5 & 5.7 & 5.9 & 6.0 & 6.4 & 6.7 & 6.7 & 6.6 & 6.4 & 6.1 & 5.8 & 5.6 & 5.4 \\
\hline
\end{tabular}


Hourty heights of tide, Cape Flora, Franz Josef Archipelago, Arctic Ocean-Continued

June and July, 1904

\begin{tabular}{|c|c|c|c|c|c|c|c|c|c|c|c|c|c|c|c|c|}
\hline Day of month. & 22 & 23 & 24 & 25 & 26 & 27 & 28 & 29 & $3^{\circ}$ & I & 2 & 3 & 4 & 5 & 6 & 7 \\
\hline Hours & Feet & Feet & Ficet & Feet & Fect & Feet & Ficet & Feet & Feet & Feet & Feet & Fect & Ficet & Feet & Feet & Feet \\
\hline o & 5.6 & 5.4 & 5.2 & 5.2 & 5.4 & 5.7 & 6. I & 6.3 & 6.7 & 6.9 & 6.5 & 6.3 & 6.3 & 6.0 & 5.9 & 5.9 \\
\hline I & 5.8 & 5.5 & 5.2 & 5.1 & 5.3 & 5.5 & 5.8 & 6.1 & 6.4 & 6.8 & 6.5 & 6.4 & 6.4 & 6.2 & 6.0 & 6. I \\
\hline 2 & 6.0 & 5.7 & $5 \cdot 3$ & 5.1 & 5.2 & $5 \cdot 3$ & 5.6 & 5.8 & 6.2 & 6.5 & 6.3 & 6.4 & 6.4 & 6.3 & 6.I & 6.3 \\
\hline 3 & 6.2 & 5.9 & 55 & 5.3 & 5.2 & 5.2 & 5.4 & 5.7 & 6.0 & 6.2 & 6.I & 6.2 & 63 & 6.3 & 6.2 & 6.4 \\
\hline 4 & 6.4 & 6.1 & 5.8 & 5.6 & 5.4 & 5.3 & 5.4 & 5.5 & 5.8 & 6.0 & 5.8 & 6.0 & 6. I & 6.2 & 6.2 & 6.5 \\
\hline 5 & 6.4 & 6.3 & 6.0 & 5.9 & 5.7 & 5.6 & 5.5 & 5.6 & 5.7 & 59 & 5.7 & 5.8 & 5.9 & 6.0 & 6.1 & 6.5 \\
\hline 6 & 6.3 & 6.3 & 6.2 & $6: 1$ & 6.0 & 59 & 5.8 & 5.7 & 5.8 & 5.8 & 5.6 & 5.7 & 5.8 & 5.9 & 6.0 & 6.5 \\
\hline 7 & 6.2 & 6.3 & 6.3 & 6.3 & 6.3 & 6.2 & 6. I & 6.0 & 6.0 & 6.0 & 5.6 & 5.6 & 5.8 & 5.8 & 5.9 & 6.4 \\
\hline 8 & 6.1 & 6.2 & 6.2 & 6.4 & 6.5 & 6.4 & 6.4 & 6.4 & 6.3 & 6.2 & 5.7 & 5.7 & 5.7 & 5.8 & 5.9 & 6.4 \\
\hline 9 & 5.9 & 6.0 & 6.I & 63 & 6.5 & 6.7 & 6.7 & 6.7 & 6.6 & 6.5 & 6.1 & 5.9 & 5.9 & 5.8 & 5.9 & 6.3 \\
\hline Io & 5.8 & 5.9 & 5.9 & 6.2 & 6.4 & 6.8 & 6.9 & 7.0 & 6.9 & 6.8 & 6.4 & 6. & 6. I & 6.0 & 5.9 & 6.3 \\
\hline I I & 5.9 & 5.8 & 5.8 & 6.0 & 6.3 & 6.6 & 6.9 & 7. I & 7.2 & 7.1 & 6.6 & 6.5 & 6.3 & 6.1 & 6.1 & 6.4 \\
\hline Noon & 6.0 & 5.8 & $5 \cdot 7$ & $5 \cdot 9$ & 6.I & 6.4 & 6.7 & 7.0 & 7.1 & 7.1 & 6.8 & 6.7 & 6.5 & 6.4 & 6.3 & 6.5 \\
\hline I3 & 6.2 & 5.9 & 5.7 & 5.7 & 5.9 & 6.2 & 6.5 & 6.8 & 7.0 & 7.0 & 6.9 & 6. & 6.7 & 6.5 & 6.5 & 6.6 \\
\hline 14 & 6.4 & 6.0 & 5.8 & 5.7 & 5.8 & 6.0 & 6.3 & 6.5 & 6.8 & 6.8 & 6.8 & 6. & 6.8 & 6.6 & 6.6 & 6.8 \\
\hline 15 & 6.5 & 6.2 & 6.0 & 5.8 & 5. & 5.9 & 6.1 & 6.3 & 6.5 & 6.5 & 6.5 & 6.6 & 6.7 & 6.6 & 6.6 & 6.9 \\
\hline 16 & 6.6 & 6.3 & 6.1 & 6.0 & 5.8 & 5.9 & 6.0 & 6.2 & 6.3 & 6.3 & 6.2 & 6.4 & 6.5 & 6.5 & 6.6 & 6.9 \\
\hline 17 & 6.6 & 6.4 & 6.2 & 6.1 & 6.0 & 6.0 & 5.9 & 6.I & 6.2 & 6.I & 6.0 & $6 \mathrm{i}$ & 6.2 & 6.3 & 6.5 & 6.8 \\
\hline 18 & 6.4 & 64 & 6.3 & 6.2 & 6.2 & 6.2 & 6.1 & 6.1 & 6.2 & 5.9 & 5.8 & 5.9 & 6.0 & 6.1 & 6.3 & 6.7 \\
\hline 19 & $6 . \mathrm{I}$ & 6.2 & 6.3 & 6.3 & 6.3 & 6.3 & 6.2 & 6.3 & 6.2 & 5.8 & $5 \cdot 7$ & 5.8 & 5.8 & 5.9 & 6.2 & 6.5 \\
\hline 20 & 5.9 & 6.0 & 6.2 & 6.4 & 6.4 & 6.5 & 6.4 & 6.5 & 6.4 & 5.9 & 5.7 & 5.7 & 5.7 & 5.7 & 6.0 & 6.3 \\
\hline $2 \mathrm{I}$ & 5.7 & 5.7 & 5.9 & 6.2 & 6.4 & 6.6 & 6.6 & 6.7 & 6.6 & 6. I & & 5.8 & 5.7 & 5.6 & 5.9 & 6.1 \\
\hline 22 & $5 \cdot 5$ & 5.4 & 5.6 & 6.0 & 6.2 & 6.5 & 6.6 & 6.8 & 6.8 & 6.3 & 6.1 & 5.9 & 5.8 & 5.6 & 5.8 & 6.0 \\
\hline 23 & 5.4 & $5 \cdot 3$ & $5 \cdot 4$ & 5.7 & 6.0 & 63 & 6.5 & 6.8 & 6.9 & 6.4 & 6.2 & 6.0 & 5.9 & 5.7 & 5.8 & 5.9 \\
\hline
\end{tabular}

Hourly heights of tide, Cape Flora, Franz Josef Archipelugo, Arctic Ocean-Continued

July, 1904

\begin{tabular}{|c|c|c|c|c|c|c|c|c|c|c|c|c|c|c|c|c|c|}
\hline Day & of month. & 8 & 9 & Io & II & 12 & 13 & 14 & I5 & 16 & I7 & 18 & 19 & 20 & 2 I & 22 & 23 \\
\hline & Hours & Feet & Feet & Feet & Fieet & Feet & Feet & Feet & Feet & Feet & Feet & Feet & Fcet & Feet & Fect & Feet & Feet \\
\hline & 0 & 6.0 & 5.8 & 5.8 & $5 \cdot 9$ & 6.0 & 6.2 & 6.5 & 6.7 & 6.9 & 7.1 & (6.8) & 6.4 & 5.9 & 5.7 & 5.6 & 56 \\
\hline & $I$ & 6.0 & 5.8 & 5.7 & 5.8 & 5.8 & 6.0 & 6.2 & 6.4 & 6.7 & 6.9 & (6.8) & 6.6 & 6.2 & 5.9 & 5.8 & 5.6 \\
\hline & 2 & 6. I & 6.0 & 5.7 & 5.6 & 5.6 & 5.7 & 5.9 & 6.1 & 6.4 & 6.7 & $(6.6)$ & 6.7 & 6.4 & 6.2 & 6.0 & 5.8 \\
\hline & 3 & 6.4 & 6. I & 5.9 & 5.7 & 5.6 & 5.6 & 5.7 & 5.8 & 6.2 & 6.5 & $(6.5)$ & 6.5 & 6.4 & 6.3 & 6.2 & 6.I \\
\hline & 4 & 6.5 & 6.4 & 6.1 & 6.0 & 57 & 5.6 & 5.6 & 56 & $5 \cdot 9$ & 6.2 & $(6.3)$ & 6.3 & 6.4 & 6.4 & 6.4 & 6.4 \\
\hline & 5 & 6.6 & 6.5 & 6.3 & 6.3 & 6.0 & 5.8 & 5.7 & 5.6 & 5.7 & 6.0 & $(6 . I)$ & 6.1 & 6.2 & 6.3 & 6.5 & 6.5 \\
\hline & 6 & 6.7 & 6.6 & 6.6 & 6.5 & 6.4 & 6.2 & 6.0 & 5.7 & $5 \cdot 7$ & 5.9 & (5.9) & 6.0 & 5.9 & 6.2 & 6.5 & 6.6 \\
\hline & 7 & 6.6 & 6.7 & 6.7 & 6.8 & 6.8 & 65 & 6.3 & 6.0 & 5.9 & 6.0 & $(5.8)$ & 5.8 & 5.8 & 6.1 & 6.5 & 6.7 \\
\hline & 8 & 6.5 & 6.6 & 68 & 6.9 & 7.0 & $6 y$ & 6.7 & 6.4 & 6.3 & 6.3 & $(5.9)$ & $5 \cdot 7$ & 5.8 & 6.0 & 6.3 & 6.6 \\
\hline & 9 & 6.5 & 6.6 & 6.7 & 6.9 & 7.1 & 7.1 & 7.0 & 6.8 & 6.7 & *(6.6) & 6.2 & 5.9 & 5.7 & 6.0 & 6.2 & 6.5 \\
\hline & 10 & 6.4 & 6.5 & 6.7 & 6.9 & 7.2 & 7.2 & 7.2 & 7.1 & 7.0 & (6.9) & 6.5 & 6.1 & 5.8 & 5.9 & 6.2 & 6.4 \\
\hline & II & 6.4 & 6.4 & 6.5 & 6.7 & 7.0 & 7.2 & 7.2 & $7 \cdot 3$ & $7 \cdot 3$ & $(7.2)$ & 6.8 & 6.4 & 6.0 & 6.0 & 6.2 & 6.4 \\
\hline & Noon & 6.5 & 6.3 & 6.4 & 6.6 & 6.8 & 6.9 & 7.1 & 7.3 & 7.4 & $(7 \cdot 3)$ & 7.1 & 6.6 & 6.3 & 6.2 & 6.2 & 6.3 \\
\hline & I3 & 6.6 & 6.3 & 6.3 & 6.5 & 6.6 & 6.7 & 6.8 & 7.1 & $7 \cdot 3$ & $(7.4)$ & 7.2 & 6.8 & 6.5 & 6.4 & 6.3 & 6.3 \\
\hline & 14 & 6.7 & 6.4 & 6.3 & 6.4 & 6.4 & 6.5 & 6.5 & 6.8 & 7.0 & $(7.2)$ & 7.2 & 6.9 & 6.7 & 6.6 & 6.5 & 6.4 \\
\hline & I5 & 6.7 & 6.5 & 6.4 & 6.3 & 63 & 6.3 & 6.3 & 6.5 & 6.7 & $(6.9)$ & 6.9 & 6.8 & 6.8 & 6.7 & 6.7 & 6.5 \\
\hline & 16 & 6.8 & 6.6 & 6.5 & 6.4 & 6.4 & 6.2 & 6.1 & 6.2 & 6.4 & (6.6) & 6.6 & 6.0 & 6.6 & 6.7 & 6.8 & 6.6 \\
\hline & 17 & 6.8 & 6.7 & 6.6 & 6.5 & 6.5 & 6.3 & 6.1 & 6.1 & 6.3 & $(6.4)$ & 6.3 & 6.3 & 6.4 & 6.6 & 6.8 & 68 \\
\hline & I8 & 6.8 & 6.7 & 6.7 & 6.7 & 6.7 & 6.5 & 6.3 & 6.1 & 6.2 & $(6.2)$ & 6.0 & 6.0 & 6. I & 6.4 & 6.7 & 6.8 \\
\hline & I9 & 6.6 & 6.6 & 6.8 & 6.8 & 6.8 & 6.6 & 6.4 & 6.2 & 6.2 & (6.0) & 5.8 & 5.7 & 5.9 & 6.1 & 6.5 & 6.8 \\
\hline & 20 & 6.4 & 6.4 & 6.7 & 6.8 & 69 & 6.8 & 6.7 & 6.5 & 6.3 & (6.0) & 5.7 & 5.6 & 5.6 & & 6.2 & 6.6 \\
\hline & 21 & 6.2 & 6.2 & 6.5 & 6.7 & 6.9 & 6.9 & 6.8 & 6.7 & 6.5 & (6.I) & 5.8 & 5.4 & 5.5 & 5.8 & 6.0 & 6.4 \\
\hline & 22 & $6 . \mathrm{I}$ & 6.0 & 6.3 & 6.5 & 6.7 & 6.9 & 6.9 & 6.9 & 6.8 & $(6.3)$ & 6.0 & 5.6 & 5.4 & 5.6 & 5.9 & 6.2 \\
\hline & 23 & $5 \cdot 9$ & 5.9 & 6.I & 6.3 & 6.5 & 6.8 & 6.9 & 7.0 & 7.0 & $(6.6)$ & 6.2 & 5.7 & 5.5 & 5.5 & 5.8 & 6.0 \\
\hline
\end{tabular}

* The values in parentheses are interpolated. 
Hourly heights of tide, Cape Flora, Franz Josef Archipclago, Arctic Ocean-Continued

July and August, 1904

\begin{tabular}{|c|c|c|c|c|c|c|c|c|c|c|c|c|c|c|c|c|c|c|}
\hline Day & of 1110 & ith. & 2.4 & 25 & 26 & 27 & 28 & 29 & $3^{\circ}$ & 31 & I & 2 & 3 & 4 & 5 & 6 & 7 & 8 \\
\hline & llour & rs & Feet & Fect & Fect & Fect & Fect & Fect & Fect & Feet & Fect & Fect & Fied & Fect & Fect & Feet & Fect & Feet \\
\hline & & & 5.9 & 6.0 & 6.1 & 6.2 & 6.5 & 6.6 & 6.7 & 6.5 & 6.4 & 6.4 & 6.6 & 6.6 & 6.1 & 5.9 & 5.6 & 5.7 \\
\hline & I & ter & 5.9 & 5.8 & 5.9 & 6.0 & 6.2 & 6.3 & 6.4 & 6.4 & 6.4 & 6.6 & & 6.8 & 6.3 & & & \\
\hline & 2 & & 6.0 & 58 & 5.8 & 5.8 & 6.0 & 6.1 & 6.I & 6.2 & 6.2 & 65 & 6.8 & 6.9 & 6.6 & 6.3 & 6. & \\
\hline 8 & 3 & & 6.1 & 59 & 5.8 & 5.7 & 5.8 & 5.9 & $5 \cdot 9$ & 5.9 & 6.0 & 6.3 & 6.7 & 6.8 & 6.7 & 6.4 & 6.1 & \\
\hline & 4 & & 6.3 & 6.0 & 5.9 & 5.7 & 5.7 & 5.8 & 5.7 & 5.7 & 5.8 & 6.2 & 6.5 & 6.7 & 6.6 & 6.5 & 6.2 & \\
\hline 5 & 5 & & 6.6 & 6.2 & 6.1 & $5 \cdot 9$ & 5.7 & 5.7 & 5.6 & 5.6 & 5.7 & 6. I & 6.4 & 6. & 6.5 & & 6.3 & \\
\hline 8 & 6 & & 6.8 & 6.5 & 6.4 & 6.2 & 6.0 & 5.8 & 5.7 & 5.5 & 5.6 & 5.9 & 6.3 & 6.5 & 6.5 & 6.5 & 6.4 & \\
\hline 3 & 7 & & 7.0 & 6.8 & 6.7 & 6.5 & 6.3 & 6.1 & 5.9 & 5.6 & 5.6 & 5.9 & 6.2 & 6.4 & & & 6 & \\
\hline 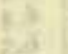 & 8 & 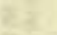 & 7.1 & 6.9 & 7.0 & 6.8 & 6.6 & 6.4 & 6.2 & 5.8 & 5.8 & 6.0 & 6.3 & 6.3 & 6. & 6.3 & 6 & \\
\hline$\frac{9}{8}$ & 9 & & 7.0 & 7.0 & 7.I & 7.0 & 6.9 & 6.7 & 6.5 & 6. I & 6.0 & 6.2 & 6.4 & 6.3 & 6.3 & & 6.3 & \\
\hline 8 & 10 & . & 6.9 & 6. & 7.1 & 7.1 & 7.1 & 7.0 & 6.8 & 6.4 & 6.3 & 6.5 & 6.7 & & & 6.2 & & \\
\hline & I & & 6.8 & 6. & 7.0 & 7.1 & 7.1 & 7.2 & 6.9 & 6.7 & 6.6 & 6.7 & 6.9 & 6.6 & 6.6 & 6.1 & 6.0 & \\
\hline 과 & Noon & & 6.6 & 6. & & 6.9 & 7.0 & 7.1 & 7. & 6.8 & 6.8 & 6.9 & 7.1 & 6.8 & & & 6.0 & \\
\hline 2 & 13 & & 6.5 & 6.4 & 6.6 & 6.7 & 6.8 & 6.9 & 6.8 & 6.7 & 6.8 & 7.0 & 7.2 & 6.9 & 6.8 & 6.4 & 6.2 & \\
\hline z & I4 & & 6.5 & 6.3 & 6.4 & 6.5 & 6.5 & 6.6 & 6.6 & 6.5 & 6.7 & 6.9 & 7.2 & 7.0 & 6.9 & & 6.3 & \\
\hline 간 & 15 & & 6.6 & 6 & 6. & 6.3 & 6.3 & 6.3 & 6.3 & 6.2 & 6. & 6.8 & $7 \cdot 1$ & & & & & \\
\hline 2 & 16 & & 6.7 & 6.4 & 6.2 & 6.2 & 6.I & 6.1 & 60 & 6.0 & 6.2 & 6.5 & 6.9 & 6. & 6.8 & 6.6 & 6.5 & \\
\hline 86 & 17 & & 6.8 & 6. & 6.3 & 6.2 & 6.0 & 6.0 & 5. & 5. & 6. & 6.2 & 6.6 & 6.6 & 6.7 & 6.5 & 5 & \\
\hline 2 & 18 & 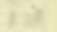 & 6.9 & 6.7 & 6.4 & 6.3 & 61 & 6.0 & 5. & 5.6 & 5.8 & 6.1 & 6.4 & 6.4 & 6.4 & 6.4 & 6.4 & \\
\hline (A) & 19 & & 6. & & 6.6 & 6.5 & 6.3 & 6.1 & 5.8 & 5.6 & 5. & 6.0 & 6.2 & & & & 6 & \\
\hline & 20 & & 6.8 & 6.8 & 6.7 & 6.7 & 6.5 & 6.3 & 6.0 & 5.8 & 5.8 & 5.9 & 6.1 & 6.8 & 6.0 & 60 & 6.1 & \\
\hline tie & $2 \mathrm{I}$ & & 6. & 6.8 & 6.8 & 6.8 & 6.6 & 6.5 & 6.2 & 5.9 & 5. & 6.0 & 6.1 & & & 5. & 6.0 & \\
\hline 6.6 & 22 & F & 6.4 & 6.6 & 6.7 & 6.8 & 6.7 & 6.6 & 6.4 & 6.1 & 6. I & 6.2 & 6.2 & 5.8 & 5. & 5.7 & 5.8 & 6. \\
\hline 122 & 23 & . & 6.2 & 6.4 & 6.4 & 6.7 & 6.8 & 6.7 & 6.5 & 6.3 & 6.3 & 6.4 & 6.4 & 6.0 & 5.8 & 5.6 & 57 & \\
\hline
\end{tabular}

Hourly heights of tidc, Cape Flora, Franz Josef Archipelago, Arctic Ocean--Continued

August, 1904

\begin{tabular}{|c|c|c|c|c|c|c|c|c|c|c|c|c|c|c|c|c|c|c|}
\hline Day & of 111011 & ith. & 9 & I0 & II & 12 & 13 & I 4 & 15 & 16 & 17 & 18 & 19 & 20 & 21 & 22 & 23 & 24 \\
\hline 3 & Hour. & rs & Ficet & Feet & Feet & Feet & Feet & Feet & Feet & Feel & Fect & Feet & Fect & Feet & Ficet & Feet & Feet & Feel \\
\hline $6 z$ & 0 & 12 & $5 \cdot 7$ & 5.7 & $5 \cdot 5$ & 5.9 & 6.2 & 60 & 6.0 & 60 & 6.0 & 5.8 & 5.7 & 5.4 & $5 \cdot 3$ & 5.4 & $5 \cdot 3$ & $5 \cdot 3$ \\
\hline 23 & I & 6 & & 55 & 5.2 & 5.6 & $5^{8}$ & 5.8 & 6.0 & 6.1 & 6.1 & 6.1 & 6.0 & 5.7 & 5.4 & 5.4 & 5.2 & 5.2 \\
\hline 12 & 2 & 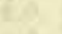 & $5 \cdot 7$ & 5.4 & 5.0 & 53 & $5 \cdot 5$ & 5.4 & $5 \cdot 7$ & 6.0 & 6.2 & 6.2 & 6.1 & 5.9 & $5 \cdot 5$ & $5 \cdot 5$ & 5.2 & 5.0 \\
\hline 7 & 3 & cat & 5.9 & 5.4 & 5.0 & 5.0 & 5.2 & 52 & $5 \cdot 3$ & 5.6 & $6, \mathbf{I}$ & 6.3 & 6.3 & 6.0 & 5.7 & 5.6 & $5 \cdot 3$ & 50 \\
\hline 8 & 4 & 20 & 6.1 & 5.6 & 5.2 & 4.9 & 5.1 & 4.9 & 5.0 & $5 \cdot 4$ & 5.8 & 6.2 & 6.3 & 6.1 & 5.9 & 5.8 & $5 \cdot 5$ & $5 \cdot 2$ \\
\hline t & 5 & $2=$ & 63 & 5.9 & $5 \cdot 4$ & 5.2 & $5 \cdot I$ & 4.8 & 4.9 & 5.2 & 5.6 & 6.0 & 6.3 & 6.2 & 6.1 & 60 & $5 \cdot 7$ & $5 \cdot 4$ \\
\hline $6=$ & 6 & $E^{*}$ & 6.5 & 6.2 & 5.6 & $5 \cdot 5$ & $5 \cdot 3$ & 4.9 & 4.8 & 5.1 & $5 \cdot 5$ & 5.8 & 6.I & 6.2 & 6.2 & 6.2 & 6.0 & 57 \\
\hline id & 7 & $1=$ & 6.6 & 6.4 & 6.0 & 5.8 & 5.6 & 5.0 & 5.0 & 5.0 & 5.4 & 5.7 & 5.9 & 6.1 & 6.2 & 6.3 & 6.I & 6.0 \\
\hline $\mathrm{rat}$ & 8 & ex- & 6.7 & 6.5 & 6.3 & 6.2 & 5.9 & 55 & 5.2 & $5 . I$ & $5 \cdot 4$ & 5.6 & 5.8 & 6.0 & 6.1 & 6.3 & 6.2 & 6. I \\
\hline 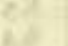 & 9 & 6 & 6.7 & 6.6 & 6.5 & 6.4 & 6.2 & 5.9 & 5.6 & 54 & 5.6 & 5.7 & 5.8 & 5.9 & 6.0 & 6.2 & 6.2 & 6.3 \\
\hline tat & 10 & $6^{2}$ & 6.6 & 6.5 & 6.6 & 6.6 & 6.5 & 6.2 & 5.9 & 5.8 & 58 & 58 & 5.8 & 5.8 & $5 \cdot 9$ & 6.0 & 6.0 & 6.2 \\
\hline & II & 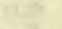 & 6.4 & 6.4 & 6.4 & 6.5 & 6.5 & 6.3 & 5.2 & 6.1 & 6. I & 6.0 & 5.9 & 5.9 & 5.8 & 5.9 & $5 \cdot 9$ & 6.0 \\
\hline 28 & Noon & Ir & 6.3 & 6.2 & 6.1 & 6.2 & 64 & 6.3 & 6.4 & 6.3 & 6.3 & 6.2 & 6.0 & 6.0 & 5.8 & 5.8 & 5.7 & 5.8 \\
\hline ent & 13 & 137 & 6.2 & 6.0 & 5.9 & 5.9 & 6.I & 6.1 & 6.3 & 6.4 & 6.5 & 64 & 6.2 & 6.I & 5.8 & $5 \cdot 7$ & 5.6 & 5.6 \\
\hline 7.7. & 14 & $3 x$ & 6. I & 5.8 & 5.7 & 5.6 & $5 \cdot 7$ & 5.7 & 6.0 & 6.3 & 6.5 & 6.5 & 6.3 & 6.2 & 5.9 & $5 \cdot 7$ & $5 \cdot 4$ & $5 \cdot 5$ \\
\hline 20 & I5 & 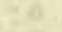 & 6.2 & 5.7 & 5.5 & 5.4 & 53 & $5 \cdot 3$ & 56 & 6.0 & 6.3 & 6.5 & 6.4 & 6.3 & 6.0 & 5.7 & 5.4 & 5.4 \\
\hline 81 & 16 & 54 & 6.3 & 5.8 & 5.5 & 5.2 & 5.2 & 5.0 & 5.2 & 5.6 & 6.0 & 6.3 & 6.3 & 6.3 & 6.1 & 5.8 & 5.5 & 5.4 \\
\hline 60 & 17 & 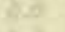 & 6.4 & 5.9 & 5.7 & $5 \cdot 3$ & 5.0 & 4.8 & 5.0 & 5.3 & 5.7 & 6.1 & 6.2 & 6.3 & 6.2 & 6.0 & 5.7 & 5.5 \\
\hline ntat & 18 & bet & 6.5 & 6.1 & 5.8 & $5 \cdot 5$ & 5.0 & 4.6 & 4.8 & 5.0 & $5 \cdot 4$ & 5.8 & 6.0 & $6 . \mathrm{I}$ & 6.2 & 6.0 & 5.8 & 5.7 \\
\hline 68 & 19 & $F=$ & 6.5 & 6.2 & 6.I & 5.7 & 5.2 & 4.8 & 4.7 & 4.9 & $5^{2}$ & 5.6 & 5.7 & 5.9 & 6,1 & 6.1 & 5.9 & 5.8 \\
\hline eAt & 20 & 8 & 85 & 6.2 & 6.3 & 6.0 & 5.5 & 5.0 & 4.9 & 4.9 & 5.1 & 5.4 & 5.5 & 5.7 & 5.9 & 6.0 & 6.0 & 0.0 \\
\hline 18 & 2 I & 15 & 6.4 & 6.2 & 6.4 & 6.2 & 5.8 & 5.3 & 5.2 & 5.1 & 5. I & 5.2 & 5.4 & 5.5 & 5.7 & 5.9 & 5.9 & 6.I \\
\hline 5. & 22 & 2,2 & 6.2 & $\begin{array}{l}6.1 \\
5.8\end{array}$ & 6.4 & $\begin{array}{l}6.3 \\
6.3\end{array}$ & 6.0 & 5.6 & 5.5 & 5.4 & 5.3 & 5.3 & $5 \cdot 3$ & 5.4 & 5.6 & 5.6 & 5.7 & 0.0 \\
\hline & 23 & 22 & $5 \cdot 9$ & 5.8 & 0.2 & 0.3 & 6.0 & 5.9 & 5.8 & 5.7 & 5.6 & 5.5 & $5 \cdot 3$ & $5 \cdot 3$ & 5.5 & 5.4 & 5.5 & 5.8 \\
\hline
\end{tabular}


Hourty heights of tide, Cape Ftora, Franz Josef Archipetago, Arctic Occan-Concluded Angust and September, Ig04

\begin{tabular}{|c|c|c|c|c|c|c|c|c|c|c|c|}
\hline \multicolumn{3}{|l|}{ 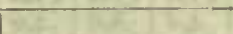 } & & & & & & & & & \\
\hline \multicolumn{3}{|c|}{ Day of month. } & 25 & 26 & 27 & 28 & 29 & 30 & $3 I$ & I & 2 \\
\hline \multicolumn{3}{|c|}{ Hours } & Feet & Feet & Feet & Feet & Feet & Feet & Feet & Feet & Feet \\
\hline & 0 & & 5.5 & 5.9 & 6.2 & 6.3 & -6.3 & 6.2 & 6.1 & 6.2 & $(6.0)$ \\
\hline & I & & $5 \cdot 3$ & 5.7 & 5.9 & 6.0 & 6. I & 6. I & 6. I & ${ }^{*}(6.2)$ & (6.2) \\
\hline 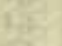 & 2 & 20 & 5.2 & 5.5 & 5.7 & 5.8 & 5.9 & $5 \cdot 9$ & 5.9 & (6.I) & (6.2) \\
\hline & 3 & 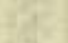 & 5. I & 5.4 & $5 \cdot 5$ & 5.6 & 5.6 & 5.6 & 5.8 & $(5.9)$ & $(6 . x)$ \\
\hline 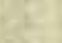 & 4 & Ris & 5.2 & $5 \cdot 3$ & 5.4 & 5.5 & 5.4 & 5.5 & 5.6 & $(5.7)$ & $(5.9)$ \\
\hline 4 & 5 & & 5.4 & 5.4 & 5.4 & 5.4 & $5 \cdot 3$ & 5.4 & 5.5 & $(5.5)$ & $(5.7)$ \\
\hline & 6 & 218 & 5.6 & 5.8 & 5.7 & 5.4 & 5.4 & $5 \cdot 3$ & 5.4 & $(5.4)$ & (5.5) \\
\hline 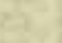 & 7 & 14. & 5.9 & 6. I & 5.9 & 5.7 & 5.6 & $5 \cdot 5$ & 5.4 & $(5.4)$ & (5.4) \\
\hline B & 8 & ets & 6.2 & 6.3 & 6.2 & 6.0 & 5.8 & 5.6 & 5.6 & $(5 \cdot 5)$ & $(5.4)$ \\
\hline & 9 & & 6.3 & 6.5 & 6.5 & 6.2 & 6.0 & 5.8 & 5.8 & $(5.7)$ & $(5 \cdot 5)$ \\
\hline & 10 & & 6.4 & 6.6 & 6.7 & 6.5 & 6.2 & 6.1 & 6.0 & $(5.9)$ & (5.7) \\
\hline & II & & 6.3 & 6.6 & 6.8 & 6.6 & 6.4 & 6.3 & 6.2 & $(6.0)$ & $(5.9)$ \\
\hline & Noon & & 6. I & 6.4 & 6.6 & 6.6 & 6.5 & 6.4 & 6.4 & $(6.2)$ & (6. I) \\
\hline & 13 & & 5.9 & 6.2 & 6.3 & 6.4 & 6.3 & 6.3 & 6.4 & $(6.4)$ & (6.2) \\
\hline if & 14 & t & 5.6 & $5 \cdot 9$ & 6.0 & 6.0 & 6.0 & 6.1 & 6.3 & $(6.4)$ & $(6.4)$ \\
\hline & 15 & - & $5 \cdot 5$ & 5.7 & -5.8 & 5.7 & $5 \cdot 7$ & 58 & 6.I & $(6.2)$ & $(6.4)$ \\
\hline 70 & 16 & 28 & $5 \cdot 5$ & 5.6 & 5.6 & $5 \cdot 5$ & 5.5 & 5.5 & 5.8 & $(6.0)$ & (6.I) \\
\hline 47 & 17 & I3 & 5.6 & 5.6 & 5.5 & 5.4 & 5.3 & $5 \cdot 3$ & 5.6 & $(57)$ & $(5.8)$ \\
\hline ins & 18 & 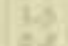 & 5.7 & 5.7 & 5.6 & $5 \cdot 3$ & 5.2 & 5.2 & 5.4 & $(5.4)$ & $(5.6)$ \\
\hline 2: & 19 & 1 & 5.9 & 5.9 & 5.8 & $5 \cdot 5$ & $5 \cdot 3$ & 5.2 & 5.4 & $(5 \cdot 3)$ & $(5.4)$ \\
\hline 83 & 20 & tit & 6.1 & 6. I & 6.0 & 5.7 & 5.5 & $5 \cdot 3$ & $5 \cdot 5$ & $(5 \cdot 3)$ & $(5 \cdot 3)$ \\
\hline-14 & $2 \mathrm{I}$ & 2 & 6.3 & 6.2 & 6.2 & 5.9 & 5.7 & 5.6 & 5.7 & $(5.5)$ & $(5.4)$ \\
\hline 3 & 22 & 4. & 6.3 & 6.4 & 6.3 & 6.0 & 5.9 & 5.8 & 5.9 & $(5.7)$ & $(5.5)$ \\
\hline 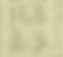 & 23 & 5 & 6.1 & 6.4 & 6.4 & 6.2 & 6.0 & 6.0 & 6.0 & $(5.9)$ & $(5.7)$ \\
\hline
\end{tabular}

* The values in parentheses are interpolated. 
Hourly heights of lide, Teplitz Bay, Franz Josef Archipclago, Arclic Ocean

April, I904

\begin{tabular}{|c|c|c|c|c|c|c|c|c|c|c|c|c|c|c|c|c|}
\hline Day of month.... & I & 2 & 3 & 4 & 5 & 6 & 7 & 8 & 9 & IO & I I & 12 & I3 & I4 & 15 & I6 \\
\hline Hous & Feel & Fiet & Fect & Feet & Feet & Feet & Feet & Feel & Feet & Fect & Fect & Fect & Fecl & Fect & Feet & Feet \\
\hline o & *(3.0) & 3.2 & 3.6 & 3.9 & 4.2 & 4.5 & 4.5 & 4.4 & 4.2 & 42 & $4 \cdot 2$ & $4 \cdot 0$ & 4.0 & 3.9 & 3.7 & 3.9 \\
\hline I & $(2.9)$ & 3.0 & $3 \cdot 3$ & 3.6 & 3.9 & 4.2 & 4.2 & 4.2 & 4.2 & $4 \cdot 3$ & $4 \cdot 3$ & 4.2 & 4.2 & 4.0 & 3.8 & 3.8 \\
\hline 2 & $(3.0)$ & 3.0 & 3.1 & 3.4 & 3.7 & 3.8 & 3.9 & 4.0 & 4. I & $4 \cdot 3$ & 4.4 & $4 \cdot 3$ & 4.4 & $4 \cdot 3$ & 4.0 & 3.9 \\
\hline 3 & $(3.4)$ & 3.3 & 3.3 & 3.3 & 3.5 & 3.6 & 3.7 & 3.8 & 4.0 & 4.2 & $4 \cdot 5$ & 4.5 & 4.6 & 4.5 & 4.4 & 4.2 \\
\hline 4 & $(3.8)$ & $3 \cdot 7$ & 3.5 & 3.5 & 3.6 & 3.5 & 3.6 & 3.7 & 3.9 & $4 . I$ & $4 \cdot 5$ & 4.6 & 4.7 & 4.8 & 4.7 & 4.7 \\
\hline 5 & $(4.2)$ & $4 . I$ & 4.0 & 3.8 & 3.8 & 3.6 & 3.6 & 3.6 & 3.7 & 3.9 & 4.4 & 4.6 & 4.8 & 5.0 & 4.9 & 5.0 \\
\hline 6 & $(4.5)$ & $4 \cdot 5$ & 4.4 & 4.2 & 4.2 & 3.8 & 3.6 & 3.5 & 3.6 & 3.8 & 4.2 & $4 \cdot 4$ & 4.8 & 5.0 & $5 . I$ & 5.2 \\
\hline 7 & $(4.7)$ & $4 \cdot 7$ & 4.7 & 4.5 & 4.6 & 4.0 & 3.8 & 3.6 & 3.6 & 3.8 & 4.1 & $4 \cdot 3$ & 4.6 & 4.9 & 5.0 & 5.2 \\
\hline 8 & $(4.7)$ & 4.7 & 4.9 & 4.7 & 4.9 & $4 \cdot 3$ & 4.0 & 3.8 & 3.7 & 3.7 & 4.0 & 4.1 & 4.5 & 4.7 & 4.9 & 5.I \\
\hline 9 & $(4.4)$ & 4.6 & 4.8 & 4.8 & 5.0 & 4.5 & 4. I & 3.9 & 3.8 & 3.8 & 3.9 & 3.9 & 4.2 & 4.4 & 4.6 & 4.8 \\
\hline IO & (4.I) & 42 & 4.5 & $4 \cdot 7$ & $4 \cdot 9$ & 4.6 & $4 \cdot 3$ & 4.1 & 3.9 & 3.8 & 3.9 & 3.8 & $4 . I$ & 4.I & 4.2 & 4.4 \\
\hline I I & $(3.8)$ & 3.7 & 4.I & 4.4 & 4.7 & $4 \cdot 5$ & 4.4 & 4.2 & 4.0 & 4.0 & 4.0 & 3.8 & 4.0 & 4.0 & 4.0 & 4. I \\
\hline Noon & $(3.5)$ & 3.4 & 3.7 & 4.I & $4 \cdot 3$ & 4.4 & 4.4 & 4.2 & 4. I & $4 . I$ & 4.1 & 3.9 & 4.0 & 3.9 & 3.9 & 3.9 \\
\hline 13 & $(3.2)$ & 3.2 & 3.5 & $3 \cdot 7$ & 4.0 & $4 . I$ & 4.2 & 4.2 & 4.2 & 4.3 & 4.2 & 4.1 & 4.I & 4.0 & 3.9 & 3.8 \\
\hline I4 & $(3.2)$ & 3.2 & 3.3 & $3 \cdot 4$ & 3.8 & 3.9 & 4.0 & 4.1 & 4.2 & 4.4 & 4.4 & $4 \cdot 3$ & $4 \cdot 3$ & 4.2 & 4.1 & 3.9 \\
\hline I5 & $(3.5)$ & 3.4 & 3.4 & 3.3 & 3.6 & 3.7 & 3.8 & 4.0 & 4.1 & 4.4 & 4.5 & $4 \cdot 5$ & 4.6 & 4.5 & 4.4 & 4.2 \\
\hline 16 & $(4.0)$ & 3.7 & 3.7 & 3.5 & 3.7 & 3.6 & 3.7 & 3.9 & 4.0 & 4.4 & 4.6 & 4.7 & 4.8 & $4 \cdot 7$ & 4.7 & 4.5 \\
\hline 17 & 4.5 & 4.2 & 4.0 & 3.7 & $3 \cdot 9$ & 3.7 & 3.6 & 3.8 & 3.9 & $4 \cdot 3$ & 4.6 & 4.7 & 4.9 & 4.9 & 5.0 & 4.8 \\
\hline I8 & 4.7 & 4.5 & 4.4 & 4.1 & 4.2 & 3.8 & 3.7 & 3.7 & 3.8 & 4.2 & 4.5 & 4.6 & 4.9 & 5.0 & 5.2 & 5.0 \\
\hline 19 & 4.8 & 4.8 & 4.8 & 4.5 & 4.5 & $4 . I$ & 38 & 3.8 & 3.8 & $4 . I$ & 4.3 & 4.4 & 4.8 & 4.9 & 5.2 & 5. I \\
\hline 20 & 4.8 & 4.9 & 5.0 & 4.8 & 4.8 & $4 \cdot 3$ & 40 & 3.9 & 3.8 & 4.0 & 4.I & 4.2 & 4.5 & 4.7 & 5.1 & 4.9 \\
\hline 2 I & 4.5 & $4 \cdot 7$ & 5.0 & 4.9 & 4.9 & 4.5 & 42 & 4.0 & 3.8 & 4.0 & 4.0 & 4.0 & 4.2 & 4.4 & 4.8 & 4.6 \\
\hline 22 & 4.0 & 4.4 & 4.7 & 4.9 & 4.9 & 4.6 & $4 \cdot 3$ & 4. I & 3.9 & 4.0 & 3.9 & $3 \cdot 9$ & $4 \mathrm{I}$ & 4.0 & 4.4 & 4.3 \\
\hline 23 & 3.5 & 3.9 & $4 \cdot 3$ & 4.6 & 4.8 & 4.6 & 4.4 & 4.2 & 4.0 & 4.I & 3.9 & 3.9 & 3.9 & 3.8 & 4. I & 3.9 \\
\hline
\end{tabular}

* The values in parentheses are interpolated.

Hourly heighls of tide, Teptilz Bay, Franz Josef Archipelago, Arctic Ocean-Continued

April, I904

\begin{tabular}{|c|c|c|c|c|c|c|c|c|c|c|c|c|c|c|}
\hline Day of month.... & I7 & 18 & I9 & 20 & $2 \mathrm{I}$ & 22 & 23 & 24 & 25 & 26 & 27 & 28 & 29 & 30 \\
\hline Hours & Feet & Feet & Feet & Feet & Feet & Feet & Feet & Feet & Feel & Feet & Feet & Feel & Feet & Feet \\
\hline o & 3.6 & 4.0 & 3.9 & 4.2 & 4.5 & 4.4 & 4.4 & $4 \cdot 3$ & 4. I & 3.9 & 3.7 & 3.5 & 3.2 & 2.9 \\
\hline I & 3.5 & 3.7 & 3.6 & 3.9 & 4. I & 4.I & 4. I & 4.2 & 4.2 & 4.0 & 4.0 & 3.7 & 3.2 & 2.9 \\
\hline 2 & 3.5 & 3.7 & 3.4 & 3.8 & 3.9 & 3.9 & 3.9 & 4. $\mathrm{I}$ & 4.2 & 4.2 & 4.2 & 4.0 & 3.5 & 3.2 \\
\hline 3 & 3.8 & 3.8 & 3.4 & 3.7 & 3.8 & 3.7 & 3.7 & 3.9 & 4.I & 4.2 & 4.4 & 4.3 & 3.9 & 3.5 \\
\hline 4 & 4.2 & 4. I & 3.6 & 3.8 & 3.8 & 3.6 & 3.5 & 3.7 & 3.9 & 4.2 & 4.4 & 4.4 & 4.2 & 4.0 \\
\hline 5 & 4.5 & 4.5 & 3.9 & $4 . I$ & 3.9 & 3.6 & 3.4 & 3.6 & 3.8 & $4 . I$ & 4.4 & 4.5 & 4.5 & 4.3 \\
\hline 6 & 4.8 & 4.9 & 4.2 & 4.4 & $4 \cdot 2$ & 3.8 & 3.4 & 3.5 & 3.6 & 3.9 & 4.2 & $4 \cdot 4$ & 4.4 & 4.5 \\
\hline 7 & 5.0 & 5. I & 4.5 & 4.8 & 4.5 & 4.0 & 3.6 & 3.5 & 3.5 & 3.7 & 4.0 & 4.3 & 4.3 & 4.5 \\
\hline 8 & 5.0 & 5.2 & 4.6 & 5.0 & 4.7 & $4 \cdot 3$ & 3.8 & 3.6 & 3.5 & 3.5 & 3.8 & 4.0 & 4.I & 4.4 \\
\hline 9 & 4.8 & 5.1 & 4.6 & 5.2 & 4.9 & 4.5 & 4.0 & 3.8 & 3.5 & 3.4 & 36 & 3.7 & 3.7 & 4.0 \\
\hline 10 & $4 \cdot 5$ & 4.9 & 4.5 & 5.1 & 5.0 & 4.5 & 4.2 & 4.0 & 3.7 & 3.5 & 3.4 & 3.5 & 3.4 & 3.6 \\
\hline I I & 4.2 & 4.5 & 4.2 & 4.8 & 4.8 & 4.6 & 4.3 & 4. I & 3.9 & 3.7 & 3.5 & 3.4 & 3.2 & 3.3 \\
\hline Noon & 4.0 & 4.2 & 3.9 & 4.5 & 4.6 & 4.4 & $4 \cdot 3$ & 4.2 & 4.0 & 3.9 & 3.7 & 3.5 & 3.1 & 3. I \\
\hline 13 & 3.8 & 4.0 & 3.6 & 4.3 & $4 \cdot 3$ & 4.2 & 4.2 & 4.3 & 4.2 & 4.1 & 4.0 & 3.7 & 3.2 & 3. I \\
\hline 14 & 3.8 & 3.9 & 3.5 & 4. I & 4. I & 4.0 & 4.I & 4.2 & 4.3 & 4.3 & 4.2 & 4.0 & 3.5 & 3.4 \\
\hline 15 & 4.0 & 3.9 & 3.5 & 4.0 & 3.9 & 3.8 & 3.9 & 4. I & 4.3 & 4.4 & 4.5 & $4 \cdot 3$ & 3.9 & 3.7 \\
\hline I6 & 4.3 & 4.2 & 3.6 & 4.0 & 3.9 & 3.7 & 3.7 & 3.9 & 4.2 & 4.4 & 4.6 & 4.6 & 4.2 & 4. I \\
\hline I 7 & 4.7 & 4.5 & 4.0 & 4.3 & $4 . c$ & 3.6 & 3.6 & 3.8 & 4.0 & 4.3 & 4.6 & 4.7 & 4.5 & 4.4 \\
\hline 18 & 5.0 & 4.8 & 4.4 & 4.5 & 4.2 & 3.7 & 3.6 & 3.7 & 3.8 & 4.2 & 4.5 & 4.7 & 4.6 & 4.6 \\
\hline I9 & 5.1 & 5.0 & 4.7 & 4.8 & 4.4 & 3.9 & 3.7 & 3.6 & 3.6 & 3.9 & 4.2 & 4.4 & 4.5 & 4.7 \\
\hline 20 & 5.2 & $5 \cdot \mathrm{I}$ & 4.9 & 5.0 & 4.6 & 4.I & 3.8 & 3.6 & 3.5 & 3.7 & 3.9 & 4.0 & 4.2 & 4.5 \\
\hline $2 \mathrm{I}$ & 5.0 & 5.0 & 4.9 & 5.I & 4.7 & 44 & 4.0 & 37 & 3.4 & 35 & 3.6 & 3.6 & 3.9 & 4.2 \\
\hline 22 & 4.6 & 4.7 & 4.8 & 5.0 & 4.8 & 4.5 & 4.2 & 3.9 & 3.5 & 3.5 & 3.4 & 3.4 & 3.4 & 3.8 \\
\hline 23 & $4 \cdot 3$ & 4.2 & 4.5 & 4.8 & 4.7 & 4.5 & 4.3 & 4.0 & 3.7 & 3.6 & 3.4 & 3.1 & 3.1 & 3.5 \\
\hline
\end{tabular}


Hourly heights of tide, Teplitz Bay, Franz Josef Archipelago, Arctic Ocean-Continued

May, 1904

\begin{tabular}{|c|c|c|c|c|c|c|c|c|c|c|c|c|c|c|c|c|}
\hline Day of month.... & I & 2 & 3 & 4 & 5 & 6 & 7 & 8 & 9 & so & I I & I 2 & 13 & I4 & I5 & 16 \\
\hline Hours & Feet & Feet & Fiet & Feet & Feet & Feet & Feet & Feet & Feet & Feet & Feet & Feet & Feet & Feet & Feet & Feet \\
\hline o & 3. I & 3.6 & 3.9 & 4.2 & 4.4 & 4.4 & 4.4 & 4.2 & 4.1 & 4.0 & 3.8 & 3.6 & 3.6 & 3.6 & 3.4 & 3.6 \\
\hline $\cos \mathbf{I}$ & 3.0 & 3.4 & 3.6 & $3 \cdot 9$ & 4. I & 4.2 & 4.2 & 4. 1 & 4.2 & 4. I & 4.0 & 3.8 & 3.7 & 3.8 & 3.5 & 3.5 \\
\hline $\begin{array}{r}2 \\
2\end{array}$ & 3. I & $3 \cdot 3$ & $3 \cdot 4$ & 3.7 & 3.8 & 3.9 & 3.9 & 4.0 & 4. 1 & 4. I & 4. I & 4.0 & 4.0 & 4.0 & 3.7 & 3.7 \\
\hline $\begin{array}{r}3 \\
4\end{array}$ & $3 \cdot 3$ & 3.5 & 3.5 & 3.6 & 3.7 & 3.7 & 3.7 & 3.8 & 4.0 & $4 . I$ & 4.1 & 4.2 & 4.2 & $4 \cdot 3$ & 4.0 & 4.0 \\
\hline $\begin{array}{l}4 \\
5\end{array}$ & 3.8 & 3.8 & 3.7 & 3.7 & 3.7 & 3.6 & 3.6 & 3.7 & 3.8 & 4.0 & 4.2 & $4 \cdot 3$ & 4.4 & 4.6 & $4 \cdot 3$ & $4 \cdot 3$ \\
\hline $\begin{array}{l}5 \\
6\end{array}$ & 4.2 & $4 \cdot 3$ & $4 \cdot I$ & $3 \cdot 9$ & 3.8 & 3.6 & $3 \cdot 5$ & 3.6 & 3.7 & 3.9 & 4.1 & $4 \cdot 3$ & 4.5 & 4.8 & 4.6 & 4.7 \\
\hline $\begin{array}{l}6 \\
7\end{array}$ & 4.5 & 4.6 & 4.5 & $4: 3$ & 4.1 & 3.8 & 3.6 & -3.5 & 3.6 & 3.8 & 4.0 & 4.2 & 4.5 & 4.8 & 4.8 & 4.9 \\
\hline 8 & 4.7 & 4.9 & $4 \cdot 7$ & 46 & $4 \cdot 3$ & 4.0 & 3.7 & 3.6 & 3.6 & 3.7 & 3.8 & 4.0 & $4 \cdot 3$ & 4.7 & 4.8 & 5.0 \\
\hline 13109010 & $\begin{array}{l}4.1 \\
4.4\end{array}$ & 5.0 & 4.9 & 4.9 & 4.6 & 4.2 & $3 \cdot 9$ & 3.7 & 3.6 & 3.6 & 3.7 & 3.8 & 4. I & $4 \cdot 5$ & 4.7 & 4.9 \\
\hline 10 & $\begin{array}{l}4.4 \\
4.1\end{array}$ & $\begin{array}{l}4.9 \\
4.5\end{array}$ & 4.8 & 5.0 & $\begin{array}{l}4.8 \\
4.8\end{array}$ & 4.4 & 4.I & 3.9 & 3.7 & 3.6 & 3.6 & 3.6 & 3.9 & 4.2 & $4 \cdot 3$ & 4.6 \\
\hline II & 3.7 & 4.2 & 45 & 4.8 & 4.8 & $\begin{array}{l}4.0 \\
4.6\end{array}$ & 4.2 & 4.0 & 3.8 & 3.7 & 3.6 & 3.6 & 3.8 & 4.0 & 4.0 & $4 \cdot 3$ \\
\hline Noon & 3.5 & 3.9 & 4. I & 4.5 & 4.6 & 4.5 & 4.3 & $\begin{array}{l}4.1 \\
4.2\end{array}$ & 3.9 & 3.8 & 3.7 & 3.6 & 3.7 & 3.9 & 3.8 & 4.I \\
\hline 13 & $3 \cdot 3$ & $3 \cdot 7$ & 3.9 & 4.2 & $4 \cdot 3$ & 4.3 & 4.2 & 4.3 & 4.2 & 4.0 & 3.0 & $3 \cdot 7$ & 3.1 & 3.0 & 3.7 & $\begin{array}{l}3.9 \\
3.8\end{array}$ \\
\hline 14 & 3.4 & 3.7 & 3.7 & 4.0 & $4 . I$ & 4.I & 4. I & 4.2 & 4.2 & 4.2 & 4.2 & 4.2 & 4.2 & 4.2 & 4.0 & 3.9 \\
\hline I5 & 3.7 & 3.8 & 3.7 & 3.9 & 3.9 & 3.9 & 3.9 & $4 . I$ & 4.2 & $4 \cdot 3$ & 4.3 & 4.4 & 4.5 & 4.4 & 4.4 & 4.2 \\
\hline 16 & 4. I & 4.1 & 3.9 & 4.0 & 3.8 & 3.8 & 3.8 & 4.0 & 4. I & 4.2 & $4 \cdot 3$ & 4.5 & 4.8 & 4.6 & 4.7 & 4.6 \\
\hline 17 & 4.5 & 4.4 & 4.2 & 42 & 4.0 & 3.7 & 3.7 & 3.8 & 4.0 & 4.I & 4.2 & 4.5 & 5.0 & 4.9 & 4.9 & 4.9 \\
\hline 18 & 4.8 & 4.7 & 4.5 & 4.4 & 4. I & 3.8 & 3.6 & 3.7 & 3.9 & 3.9 & 4. I & 4.4 & 4.9 & 5.0 & 5.1 & 5. I \\
\hline 19 & 5.0 & 5.0 & 48 & 4.7 & 43 & 4.0 & 3.7 & 3.7 & 3.7 & 3.8 & 3.9 & 4.2 & 4.6 & 4.8 & 5. I & $5 \cdot 3$ \\
\hline 20 & 5.0 & 5.1 & 5.0 & 4.9 & 4.5 & 4.2 & 3.8 & 3.8 & 3.6 & 3.6 & 3.7 & 4.0 & $4 \cdot 3$ & 4.5 & 4.9 & 5.I \\
\hline $2 I$ & 4.7 & 5.0 & 5.0 & 5.0 & 4.7 & 4.3 & 4.0 & 3.9 & 3.7 & 3.6 & 3.6 & 3.7 & $4 . I$ & 4.I & 45 & 4.8 \\
\hline 22 & 4.3 & 4.7 & 4.9 & 5.0 & 4.7 & 4.4 & 4. I & 4.0 & 3.8 & 3.6 & 3.5 & 3.6 & 3.8 & 3.8 & 4.2 & 4.4 \\
\hline 23 & 3.9 & 4.4 & 4.6 & 4.8 & 46 & 4.5 & 4.2 & 4.I & 3.9 & 3.7 & 3.5 & 3.5 & 3.6 & 3.6 & 3.8 & 4.0 \\
\hline
\end{tabular}

Hourly heights of tide, Teplitz Bay, Franz Josef Archipelago, Arclic Ocean-Concluded

May, 1904

\begin{tabular}{|c|c|c|c|c|c|c|c|c|c|c|c|c|c|c|c|}
\hline Day of month. & 17 & I8 & 19 & 20 & 21 & 22 & 23 & 24 & 25 & 26 & 27 & 28 & 29 & 30 & $3 \mathrm{I}$ \\
\hline Hours & Feet & Feet & Feet & Feet & Feet & Feet & Feet & Feet & Feet & Feet & Feet & Feet & Feet & Feet & Feet \\
\hline 0 & 3.7 & 3.7 & 4.0 & 42 & 4.4 & 4.5 & 4.6 & 4.4 & 4.0 & 3.7 & 3.6 & 3.4 & 3.5 & 3.6 & 3.7 \\
\hline I & 3.6 & 3.4 & 3.7 & 39 & $4 I$ & 4.3 & 4.6 & 4.5 & 4.2 & 3.9 & 3.9 & 3.7 & 3.6 & 3.6 & 3.5 \\
\hline 2 & 3.5 & $3 \cdot 3$ & 3.5 & 3.6 & 3.8 & 4.0 & 4.3 & 4.4 & 4.3 & 4.2 & 4.2 & 4.0 & 3.9 & 3.8 & 3.5 \\
\hline 3 & 3.8 & 3.5 & 3.5 & 3.4 & 36 & 3.7 & 4. I & 4.2 & 4.2 & 4.3 & 4.5 & $4 \cdot 3$ & 4.3 & $4 . I$ & 3.8 \\
\hline 4 & 4.2 & $3 \cdot 7$ & 3.7 & 3.5 & 3.5 & 3.6 & 3.9 & 4.0 & 4.I & 4.3 & 4.7 & 4.6 & 4.7 & 4.5 & 4. I \\
\hline 5 & 4.5 & 4.1 & 40 & 3.7 & 3.6 & 3.5 & 3.8 & 3.8 & 3.9 & 4.2 & 4.7 & 4.8 & 5.0 & 4.9 & 4.5 \\
\hline 6 & $4 . \overline{8}$ & 4.5 & $4 \cdot 3$ & 4.0 & 3.8 & 3.6 & 3.7 & 3.7 & $3 \cdot 7$ & 4.0 & 4.6 & 4.8 & 5.1 & 5.1 & 4.8 \\
\hline 7 & 5.0 & 4.7 & 4.7 & $4 \cdot 3$ & 4.0 & 3.8 & 3.7 & 3.6 & 3.5 & 3.8 & 4.4 & 4.7 & 5.1 & 5.2 & 5.0 \\
\hline 8 & 5.0 & 4.8 & 4.9 & 4.6 & 4.3 & 4.1 & 3.9 & 36 & 3.4 & 3.6 & $4 . I$ & 4.4 & 4.9 & 5.1 & 5.1 \\
\hline 9 & 4.8 & 4.8 & 5.0 & 4.8 & 4.6 & 4.3 & 4. I & 3.8 & 3.4 & 3.5 & 3.9 & $4 . I$ & 4.5 & 4.8 & 4.9 \\
\hline 10 & $4 \cdot 5$ & 4.6 & 4.9 & 4.8 & 4.7 & 4.6 & $4 \cdot 3$ & 4.0 & 3.5 & 3.5 & 3.7 & 3.9 & 4.2 & 4.5 & 4.6 \\
\hline II & 4.2 & 4.2 & 4.6 & 47 & 4.7 & 4.7 & 4.5 & 4.2 & 3.8 & 3.7 & 3.7 & 3.7 & 4.0 & 4. I & 4.3 \\
\hline Noon & 4.0 & 4.0 & 4.3 & 4.4 & 4.6 & 4.7 & 4.6 & 4.4 & 4.0 & 3.9 & 3.9 & 3.7 & 3.9 & 4.0 & 4.0 \\
\hline 13 & 3.8 & 3.8 & 4.0 & 4.2 & 4.4 & 4.6 & 4.6 & 4.5 & 4.2 & 4.2 & 4.2 & 3.8 & 3.9 & 3.9 & 3.8 \\
\hline 14 & 3.8 & 3.6 & 3.9 & 3.9 & 4.2 & 44 & 4.5 & 4.5 & $4 \cdot 3$ & 4.5 & 4.5 & 4.2 & 4.1 & 40 & 3.8 \\
\hline 15 & 4.0 & 3.7 & 3.8 & 3.8 & 3.9 & $4 . I$ & $4 \cdot 3$ & 4.4 & 4.4 & 4.7 & 4.8 & 4.6 & 4.5 & 4.2 & 3.9 \\
\hline 16 & 4.3 & 4.0 & 3.9 & 3.7 & 3.7 & 39 & 4.1 & 4.2 & $4 \cdot 3$ & 4.7 & 4.9 & 4.9 & 4.9 & 4.6 & 4.3 \\
\hline 17 & 4.5 & $4 \cdot 3$ & 4. I & 3.9 & 3.7 & 3.8 & 3.9 & 4.0 & $4 \cdot \mathrm{I}$ & 4.7 & 5.0 & 52 & 5.1 & 5.0 & 4.6 \\
\hline 18 & $4 . \overline{8}$ & 4.6 & 4.4 & 4. I & 3.8 & 3.8 & 3.7 & 3.8 & 3.9 & 4.5 & 4.8 & 5.2 & $5 \cdot 3$ & 5.2 & 4.9 \\
\hline 19 & 5.0 & 4.9 & 4.7 & 4.4 & 4.I & 3.9 & 3.7 & 3.6 & 3.6 & 4.2 & 4.6 & 5.0 & 5.2 & 5.3 & 5.1 \\
\hline 20 & 5.1 & 5.0 & 4.9 & 4.6 & $4 \cdot 3$ & 4.I I & 3.8 & 35 & 3.4 & 3.9 & 4.2 & 4.6 & 5.0 & 5.2 & 5.1 \\
\hline 2I & 4.9 & 5.0 & 5.0 & 4.8 & 4.5 & 4.3 & 3.9 & 3.5 & 3.2 & 3.6 & 3.9 & $4 \cdot 3$ & 4.6 & 4.9 & 5.0 \\
\hline 22 & 4.5 & 4.8 & 4.9 & 4.8 & 4.6 & 4.5 & $4 . I$ & 3.6 & 3.3 & 3.5 & 3.6 & 3.9 & 4.2 & 4.4 & 4.6 \\
\hline 23 & 4. I & 4.4 & 4.6 & 4.7 & 4.7 & 4.6 & 4.2 & 3.8 & 3.4 & 3.5 & 3.4 & 3.7 & 3.8 & 4.0 & 4.2 \\
\hline
\end{tabular}




\section{REDUC'TION OF TIDES}

The above hourly heights of the sea were discussed by the harmonic analysis, the process being essentially similar to that outlined by Prof. George $H$. Darwin, in the report of the British Association for the Advancement of Science, for the year $188_{3}$, and hence not necessary to reproduce here. The amplitudes $(H)$ or semi-ranges of the components expressed in feet, and their epochs $(\kappa)$ or component-tidal intervals expressed in degrees, as given in the table, have been corrected by a process for eliminating the small residual effect of one component upon another.

\section{HARMONic CONSTANTS}

Cape Fiora.-Results from $104 \frac{1}{2}$ days, May 21 , oh to September 2, II h, 1904, mean local civil time.

\begin{tabular}{|c|c|c|c|c|}
\hline Symbol & Naine of component & $\begin{array}{l}\text { Speed per } \\
\text { solar hour }\end{array}$ & $\begin{array}{c}\text { Ampli- } \\
\text { tude } \\
H\end{array}$ & $\begin{array}{l}\text { Epocli } \\
\kappa\end{array}$ \\
\hline T2 & 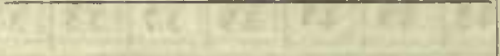 & 。 & Feet & 。 \\
\hline$A_{0}$ & Mean sea level on tide staff No. $1 .$. & $\ldots \ldots$ & 6.076 & .. \\
\hline $\mathrm{K}_{\mathrm{i}}$ & Luni-solar diurnal. . & 15.0410686 & 0.224 & 29.9 \\
\hline $\mathrm{K}_{2}$ & Luni-solar semidiurnal.... & 30.0821374 & 0.039 & $333 \cdot 3$ \\
\hline $\mathrm{L}_{2}$ & Smaller lunar elliptic semidinrnal. & 29.5284788 & 0.015 & 296.8 \\
\hline $\mathbf{M}_{2}$ & Principal lunar series..... & 28.9841042 & 0.435 & 278.8 \\
\hline$M_{4}$ & Principal lunar series...... & 57.9682084 & 0.006 & 189.3 \\
\hline $\mathrm{M}_{6}$ & Principai lunar series......... & 86.9523126 & 0.008 & 161.8 \\
\hline $\mathrm{N}_{2}$ & Larger lunar elliptic semidiurnal. & 28.4397296 & 0.083 & 245.1 \\
\hline $\mathrm{O}_{1}$ & Lunar diurnal...... & 13.9430356 & 0.073 & 47.3 \\
\hline$P_{1}$ & Solar diurnal ............ & 14.9589314 & 0.074 & 29.9 \\
\hline $\mathrm{S}_{2}$ & Principal solar semidiurnal & 30.0000000 & 0.145 & $333 \cdot 3$ \\
\hline$\mu_{3}$ & Variational............ & 27.9682084 & 0.010 & 224.4 \\
\hline$\nu_{2}$ & Larger lunar evectional.......... & 28.5125830 & 0.016 & 2496 \\
\hline
\end{tabular}


Tcplitz Bay.-Results from 58 days, April I, oh to May 28, 23h, 1904, mean local civil time, to which is added the results obtained by the expedition of the Duke of Abruzzi, 18991900 , as taken from the scientific restults of his polar expedition, published in Milan, 1903.

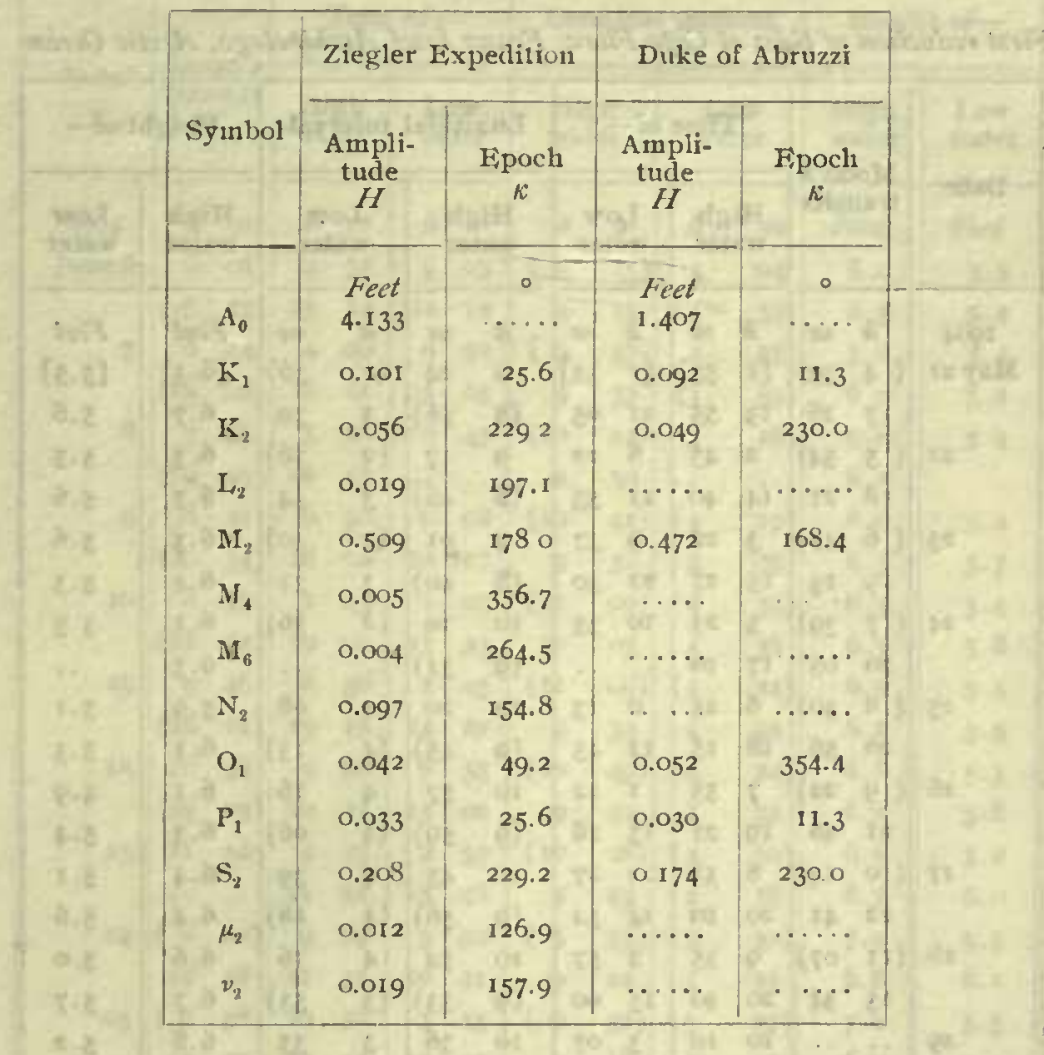

The tides discussed by the Duke of Abruzzi consisted of three short series-September is to October 17, 1899, March I6 to April 3, 1900, and June 3 to 27, 1900. The record was very defective, especially for the first series, where only a ferw readings were obtained on most days. The values given above are the corrected mean results, taken from his published report without change, other than converting meters into feet and minutes into tenths of degrees. For the most part there is a very satisfactory agreemeut between the results of the two analyses.

\section{LUNITIDAI, INTERVALS}

The tide follows the moon much more closely than it does the sun, so that there is a tendency for the tide to occur when the moon is in a given position in the heavens. The difference between the time of tide and the time of the moon's transit or meridian passage is called the lunitidal interval for the station. Both upper and lower transits of the moon are uswally compared with the time of the first high water and first low water which follows the given transit ; hence we may express the operation as follows:

High-water lunitidal interval $=\mathrm{HWI}=$ T'ime of HW. - D's transit

Low-water lunitidal interval $=\mathrm{LWI}=$ Time of LW - D's transit 
The purpose of the tabulation given below, called "First Reduction," is to compute the lunitidal intervals for high and low waters, and also to find the mean range of tide and mean half-tide level. In this work the moon's transits have been reduced to the meridians of the stations, so that all the work is expressed in local time.

First reduction of tides at Cape Flora, Franz Josef Archipelago, Arctic Ocean

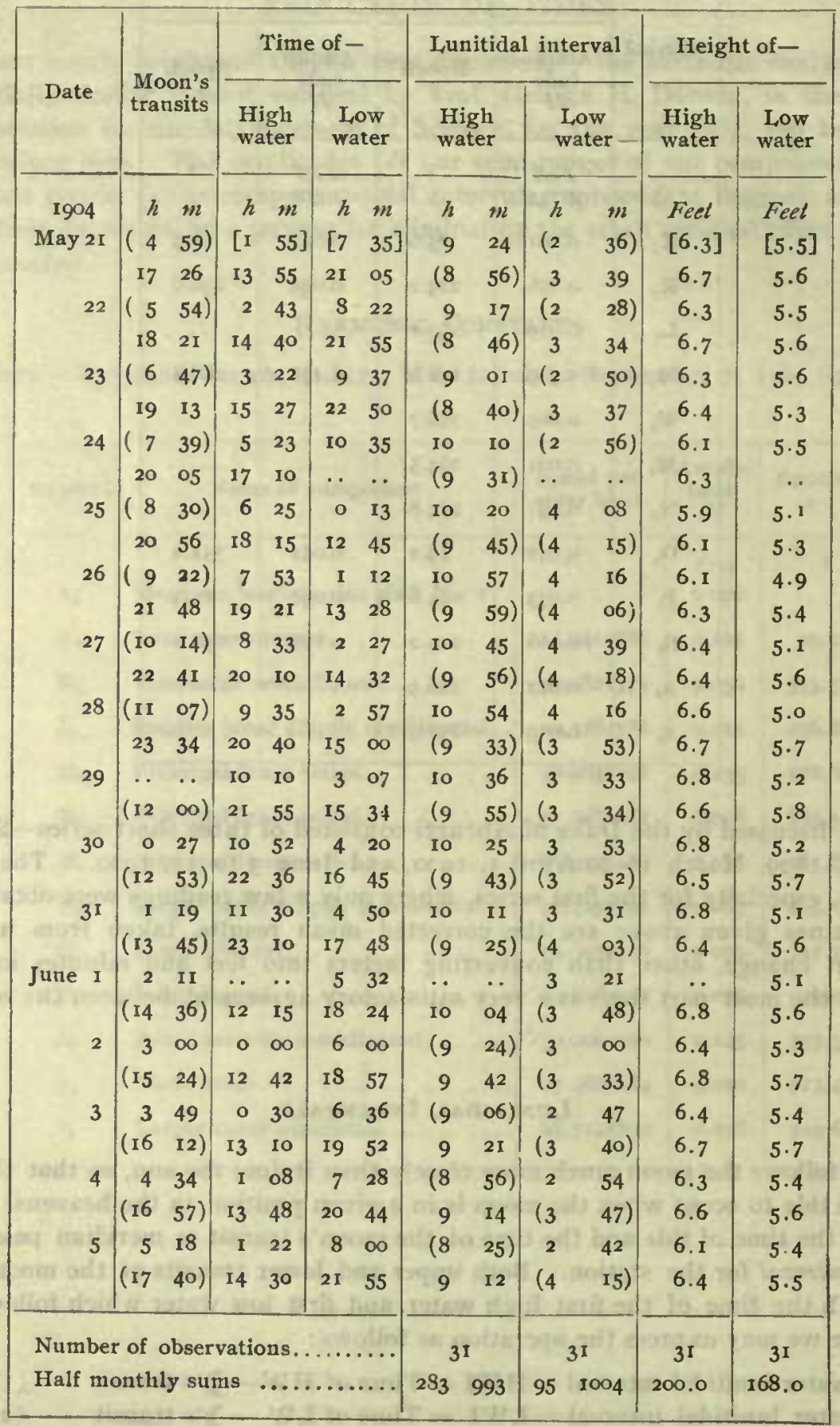


First reduction of tides at Cape Flora, Franz Josef Archipelago, Arctic Ocean-Continued

\begin{tabular}{|c|c|c|c|c|c|c|c|c|}
\hline \multirow[b]{2}{*}{ Date } & \multirow{2}{*}{$\begin{array}{c}\text { Moon's } \\
\text { transits }\end{array}$} & \multicolumn{3}{|c|}{ Time of - } & \multicolumn{2}{|c|}{ Lunitidal interval } & \multicolumn{2}{|c|}{ Height of- } \\
\hline & & \multicolumn{2}{|c|}{$\begin{array}{l}\text { High } \\
\text { water }\end{array}$} & $\begin{array}{l}\text { Low } \\
\text { water }\end{array}$ & $\begin{array}{l}\text { High } \\
\text { water }\end{array}$ & $\begin{array}{l}\text { Low } \\
\text { water }\end{array}$ & \multirow{2}{*}{$\begin{array}{c}\begin{array}{c}\text { High } \\
\text { water }\end{array} \\
\text { Feet }\end{array}$} & \multirow{2}{*}{$\frac{\begin{array}{c}\text { Low } \\
\text { water }\end{array}}{\text { Feet }}$} \\
\hline IgO4 & $h \quad m$ & $h$ & $m$ & $h m$ & $h \quad m$ & $h \quad m$ & & \\
\hline June 6 & 6 or & 2 & 55 & 905 & $\left(\begin{array}{ll}9 & 15\end{array}\right)$ & 04 & 6.0 & $5 \cdot 5$ \\
\hline 23 & $\left(\begin{array}{ll}\text { I8 } & 23\end{array}\right)$ & 15 & 12 & 2218 & 9 II & $\left(\begin{array}{ll}3 & 55\end{array}\right)$ & 6.3 & 5.4 \\
\hline 7 & $\begin{array}{ll}6 & 44\end{array}$ & 4 & $\infty$ & 941 & $\left(\begin{array}{ll}9 & 37\end{array}\right)$ & $2 \quad 57$ & 5.8 & $5 \cdot 4$ \\
\hline & $\left(\begin{array}{ll}19 & 06\end{array}\right)$ & I6 & 2 I & 2305 & $\begin{array}{ll}9 & 37\end{array}$ & (3 59$)$ & 6.0 & 5.2 \\
\hline 8 & $\begin{array}{ll}7 & 27\end{array}$ & 5 & 28 & Io 43 & (IO 22) & 316 & 5.8 & 5.4 \\
\hline 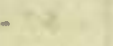 & $\left(\begin{array}{ll}19 & 49\end{array}\right)$ & 16 & 57 & . $\quad$. & 930 & .. $\quad \ldots$ & 6. I & .. \\
\hline 9 & $\begin{array}{ll}8 & \mathrm{I} 2\end{array}$ & 6 & 30 & $0 \quad 08$ & (ro $4 \mathrm{I})$ & $\left(\begin{array}{ll}4 & 19\end{array}\right)$ & 6.0 & 5.2 \\
\hline 72 & $\left(\begin{array}{ll}20 & 34\end{array}\right)$ & 18 & 02 & $12 \quad 17$ & 950 & $\begin{array}{ll}4 & 05\end{array}$ & 6.3 & $5 \cdot 7$ \\
\hline I0 & $\begin{array}{ll}8 & 57\end{array}$ & 7 & 43 & I 07 & $\left(\begin{array}{ll}\text { II } & \circ\end{array}\right)$ & $\left(\begin{array}{ll}4 & 33\end{array}\right)$ & 6.3 & 5.4 \\
\hline test & $\left(\begin{array}{ll}2 I & 2 I\end{array}\right)$ & 19 & $\infty$ & 1333 & I0 $\mathrm{O} 3$ & $4 \quad 36$ & 6.3 & 5.8 \\
\hline II & 946 & 8 & 30 & I 45 & $\left(\begin{array}{ll}\text { I } & 09\end{array}\right)$ & $\left(\begin{array}{ll}4 & 24\end{array}\right)$ & 6.5 & $5 \cdot 3$ \\
\hline$x \geq$ & $\left(\begin{array}{ll}22 & \text { II }\end{array}\right)$ & 19 & 25 & 1410 & $\begin{array}{ll}9 & 39\end{array}$ & $4 \quad 24$ & 6.5 & 5.9 \\
\hline 12 & Io $3^{8}$ & 9 & $\infty$ & $2 \quad 35$ & (10 49) & $\left(\begin{array}{ll}4 & 24\end{array}\right)$ & 6.6 & $5 \cdot 3$ \\
\hline 425 & $\left(\begin{array}{ll}23 & 05\end{array}\right)$ & 20 & 25 & I5 $\infty$ & $\begin{array}{ll}9 & 47\end{array}$ & $4 \quad 22$ & 6.5 & 5.8 \\
\hline $\mathrm{I}_{3}$ & II 32 & 9 & 33 & 255 & (1) 28 ) & $\left(\begin{array}{ll}3 & 50\end{array}\right)$ & 6.8 & 5.2 \\
\hline $8 a^{4}=$ & .. $\quad$. & $2 I$ & 2 I & $15 \quad 48$ & $9 \quad 49$ & $4 \quad 16$ & 6.7 & 6.0 \\
\hline I4 & $\left(\begin{array}{ll}0 & \infty\end{array}\right)$ & 9 & 59 & 330 & $\left(\begin{array}{ll}9 & 59\end{array}\right)$ & $\left(\begin{array}{ll}3 & 30\end{array}\right)$ & 7.0 & $5 \cdot 5$ \\
\hline $7 x$ & I2 28 & 2 I & 32 & I6 2I & $\begin{array}{ll}9 & 04\end{array}$ & 353 & 6.8 & 6.1 \\
\hline 15 & $\left(\begin{array}{ll}0 & 5^{8}\end{array}\right)$ & ro & 50 & $4 \quad 25$ & $\left(\begin{array}{ll}9 & 52\end{array}\right)$ & $\left(\begin{array}{ll}3 & 27\end{array}\right)$ & 7.0 & $5 \cdot 5$ \\
\hline Hal & I3 3 & 22 & 45 & $17 \quad 12$ & $\begin{array}{ll}9 & 18\end{array}$ & $3 \quad 45$ & 6.7 & 5.9 \\
\hline 16 & $\left(\begin{array}{ll}\text { I } & 5^{6}\end{array}\right)$ & I I & $5^{8}$ & 520 & (1) 1 (10 02$)$ & $\left(\begin{array}{ll}3 & 24\end{array}\right)$ & 6.9 & 5.2 \\
\hline & I $4 \quad 25$ & 23 & 33 & 1754 & $\begin{array}{ll}9 & 08\end{array}$ & $3 \quad 29$ & 6.6 & $5 \cdot 7$ \\
\hline I7 & $\left(\begin{array}{ll}2 & 53\end{array}\right)$ & . & .. & $5 \quad 5^{2}$ & . $\quad \ldots$ & $\left(\begin{array}{ll}2 & 59\end{array}\right)$ & . & $5 \cdot 3$ \\
\hline $7 z$ & 1521 & 12 & 18 & 1840 & $\left(\begin{array}{ll}9 & 25\end{array}\right)$ & $\begin{array}{ll}3 & 19\end{array}$ & 6.9 & 5.6 \\
\hline I8 & $\left(\begin{array}{ll}3 & 49\end{array}\right)$ & 0 & 30 & $\begin{array}{ll}6 & 35\end{array}$ & $9 \quad 09$ & $\left(\begin{array}{ll}2 & 46\end{array}\right)$ & 6.6 & $5 \cdot 4$ \\
\hline $7 x^{2}$ & I6 I6 & 13 & 05 & 1930 & $\left(\begin{array}{ll}9 & 16\end{array}\right)$ & $\begin{array}{ll}3 & 14\end{array}$ & 6.9 & 55 \\
\hline 19 & $\left(\begin{array}{ll}4 & 44\end{array}\right)$ & I & 14 & $7 \quad 32$ & $\begin{array}{ll}8 & 58\end{array}$ & $\left(\begin{array}{ll}2 & 48\end{array}\right)$ & 6.4 & $5 \cdot 3$ \\
\hline arta & I7 Io & 14 & $\infty$ & $20 \quad 35$ & $\left(\begin{array}{ll}9 & 16\end{array}\right)$ & $3 \quad 25$ & 6.7 & 5.4 \\
\hline 20 & $\left(\begin{array}{ll}5 & 36\end{array}\right)$ & 2 & 06 & 820 & 856 & $\left(\begin{array}{ll}2 & 44\end{array}\right)$ & 6.3 & $5 \cdot 5$ \\
\hline 7 & $\begin{array}{ll}18 & 02\end{array}$ & I4 & 30 & $21 \quad 25$ & $\left(\begin{array}{ll}8 & 54\end{array}\right)$ & $\begin{array}{ll}3 & 23\end{array}$ & 6.7 & 5.5 \\
\hline 21 & $\left(\begin{array}{ll}6 & 28\end{array}\right)$ & 3 & 25 & 918 & $9 \quad 23$ & $\left(\begin{array}{ll}2 & 50\end{array}\right)$ & 6.4 & $5 \cdot 7$ \\
\hline sut & I8 53 & I5 & 39 & $22 \quad 46$ & $\left(\begin{array}{ll}9 & \text { II }\end{array}\right)$ & $\begin{array}{ll}3 & 53\end{array}$ & 6.7 & $5 \cdot 4$ \\
\hline Numl & r of obse & $x_{0}$ & & & $3 I$ & $3 I$ & $3 I$ & $3 I$ \\
\hline Half & thlo & & & & $286 \quad 887$ & 96 I033 & 201 . I & I7I.o \\
\hline
\end{tabular}


lipst reduction of tides at Cape Flora, Frans Josef Archipclago, Arctic Occan-Continued

\begin{tabular}{|c|c|c|c|c|c|c|c|c|c|}
\hline \multirow{2}{*}{ Date } & \multirow{2}{*}{$\begin{array}{l}\text { Moon's } \\
\text { trausits }\end{array}$} & \multicolumn{3}{|c|}{ Time of- } & \multicolumn{3}{|c|}{ Lunitidal interval } & \multicolumn{2}{|c|}{ Height of-- } \\
\hline & & \multicolumn{2}{|c|}{$\begin{array}{l}\text { Highı } \\
\text { water }\end{array}$} & $\begin{array}{l}\text { Low } \\
\text { water }\end{array}$ & $\begin{array}{c}\text { High } \\
\text { water }\end{array}$ & \multicolumn{2}{|c|}{$\begin{array}{l}\text { Low } \\
\text { water }\end{array}$} & $\begin{array}{l}\text { High } \\
\text { water }\end{array}$ & $\begin{array}{l}\text { Low } \\
\text { water }\end{array}$ \\
\hline I904 & $h \quad m$ & $h$ & $m$ & $h m$ & $h-m$ & $h$ & $m$ & Feet & Feet \\
\hline June 22 & $\left(\begin{array}{ll}7 & 18\end{array}\right)$ & 4 & 45 & 10 15 & $9 \quad 5^{2}$ & $(2)$ & 57) & 6.4 & 5.8 \\
\hline$p ?$ & $\begin{array}{ll}19 & 44\end{array}$ & 16 & 22 & $23 \quad 43$ & $\left(\begin{array}{ll}9 & 04\end{array}\right)$ & 3 & 59 & 6.6 & 5.4 \\
\hline 23 & $\left(\begin{array}{ll}8 & 09\end{array}\right)$ & 6 & $\mathrm{r}_{3}$ & II $\quad 39$ & 10 1129 & $(3$ & 30) & 6.3 & 5.7 \\
\hline 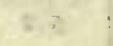 & $20 \quad 35$ & 17 & 42 & $\cdots \quad \cdots$ & $\left(\begin{array}{ll}9 & 33\end{array}\right)$ & $\cdots$ & $\because$ & 6.4 & . \\
\hline$\therefore \quad 24$ & $\left(\begin{array}{ll}9 & \text { or }\end{array}\right)$ & 7 & 04 & $0 \quad 29$ & 10 $\quad 29$ & 3 & 54 & 6.3 & 5. I \\
\hline & $21 \quad 27$ & 18 & 22 & 1252 & $(9 \quad 2 \mathrm{I})$ & $(3$ & $5 \mathrm{Ij}$ & 6.3 & $5 \cdot 7$ \\
\hline 25 & $\left(\begin{array}{ll}9 & 53\end{array}\right)$ & 8 & 17 & I 23 & $10 \quad 50$ & 3 & $5^{6}$ & 6.4 & 5.1 \\
\hline $6-2=$ & $22 \quad 19$ & 19 & 37 & 1358 & $\left(\begin{array}{ll}9 & 44\end{array}\right)$ & $(4$ & 05) & 6.4 & 5.7 \\
\hline$=26$ & (10 45) & 8 & 56 & $2 \quad 17$ & I0 $\quad 37$ & 3 & $5^{8}$ & 6.5 & 5.2 \\
\hline $7 x^{2}=$ & 23 II & 20 & 25 & $14 \quad 59$ & $(9 \quad 40)$ & $(4$ & 14) & 6.4 & 5.7 \\
\hline 27 & $\left(\begin{array}{ll}\text { I } & 37\end{array}\right)$ & $\cdot 9$ & 55 & 310 & Io 44 & 3 & 59 & 6.8 & 5.2 \\
\hline e.: & . $\quad \ldots$ & $2 I$ & I8 & $15 \quad 53$ & $\left(\begin{array}{ll}9 & 4 I\end{array}\right)$ & $(4$ & I6) & 6.6 & 5.9 \\
\hline 28 & $\begin{array}{lll}0 & 03\end{array}$ & 10 & 33 & 350 & 10 30 & 3 & 47 & 6.9 & $5 \cdot 3$ \\
\hline 8.7 & $\left(\begin{array}{ll}12 & 28\end{array}\right)$ & 22 & 07 & 1645 & $\left(\begin{array}{ll}9 & 39\end{array}\right)$ & $(4$ & I7) & 6.6 & 59 \\
\hline$=29$ & o 53 & II & 05 & $4 \quad \mathrm{I} 5$ & I0 $\quad 12$ & 3 & 22 & $=7.1$ & 5.5 \\
\hline $\cos 2$ & $\left(\begin{array}{ll}13 & 17\end{array}\right)$ & 22 & $5 \mathrm{I}$ & I7 09 & $\left(\begin{array}{ll}9 & 34\end{array}\right)$ & $(3$ & 52) & . 6.8 & 6. I \\
\hline 40 & $I=41$ & II & 28 & $5 \quad 16$ & $9 \quad 47$ & 3 & 35 & 7.2 & 57 \\
\hline 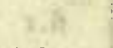 & $\left(\begin{array}{ll}14 & 05\end{array}\right)$ & 23 & 25 & I7 50 & $\left(\begin{array}{ll}9 & 20\end{array}\right)$ & 13 & 45) & 6.9 & 6.2 \\
\hline July I & $\begin{array}{ll}2 & 29\end{array}$ & .. & .. & $5 \quad 45$ & $\ldots \quad \ldots$ & 3 & I6 & $\ldots$ & 58 \\
\hline e.s & $\left(\begin{array}{ll}14 & 52\end{array}\right)$ & 12 & $\infty$ & $\begin{array}{ll}19 & 02\end{array}$ & $\begin{array}{ll}9 & 3 I\end{array}$ & $(4$ & 10) & 7.1 & 5.8 \\
\hline 122 & $\begin{array}{ll}3 & 14\end{array}$ & 0 & 20 & $6 \quad 34$ & $\left(\begin{array}{ll}9 & 28\end{array}\right)$ & 3 & 20 & 6.5 & 5.5 \\
\hline$\therefore 2$ & $\left(\begin{array}{ll}15 & 36\end{array}\right)$ & 12 & 55 & $19 \quad 22$ & $9 \quad 41$ & $(3$ & 46) & 6.9 & $5 \cdot 7$ \\
\hline$: 3$ & $\begin{array}{ll}3 & 57\end{array}$ & I & I 5 & 710 & $\left(\begin{array}{ll}9 & 39\end{array}\right)$ & 3 & 13 & 6.4 & 5.6 \\
\hline a. 8 & $\left(\begin{array}{ll}16 & \text { I }\end{array}\right)$ & I3 & 17 & $20 \quad 06$ & $9 \quad 20$ & $(3$ & $4^{8)}$ & 69 & 57 \\
\hline 644 & $4: 40$ & I & 32 & $\begin{array}{ll}7 & 49\end{array}$ & $\left(\begin{array}{ll}9 & 14\end{array}\right)$ & 3 & 09 & 6.4 & 5.7 \\
\hline 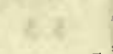 & $\left(\begin{array}{ll}17 & 0 I\end{array}\right)$ & 13 & 55 & $20 \quad 46$ & $9 \quad 15$ & $(3$ & 45) & 6.8 & $5 \cdot 7$ \\
\hline $6 \geq 5$ & $\begin{array}{ll}5 & 22 \\
\end{array}$ & 2 & 33 & $\begin{array}{ll}8 & 07\end{array}$ & $(9-32)$ & 2 & 45 & 6.3 & 5.8 \\
\hline 2.25 & $\left(\begin{array}{ll}17 & 44\end{array}\right)$ & 14 & 34 & $2 I \quad 39$ & $9 \quad 12$ & $(3$ & 55) & 6.6 & 5.6 \\
\hline 2. 6 & $\begin{array}{ll}6 & 05\end{array}$ & 3 & 35 & $8 \quad 5^{8}$ & $\left(\begin{array}{ll}9 & 5 \mathrm{I}\end{array}\right)$ & 2 & 53 & 6.2 & 5.9 \\
\hline ve & $\left(\begin{array}{ll}18 & 27\end{array}\right)$ & I5 & 15 & $22 \quad 16$ & $9=10$ & 13 & 49) & 66 & 5.8 \\
\hline$\therefore \geq 7$ & $\begin{array}{ll}6 & 50\end{array}$ & 4 & 25 & $9 \quad 57$ & $\left(\begin{array}{ll}9 & 5^{8}\end{array}\right)$ & 3 & 07 & 6.5 & 6.3 \\
\hline $4: 2$ & $\left(\begin{array}{ll}19 & 13\end{array}\right)$ & 16 & $\infty$ & $23 \quad 18$ & 9 ro & $(4$ & 05) & 6.9 & 5.9 \\
\hline \multirow{2}{*}{\multicolumn{4}{|c|}{$\begin{array}{l}\text { Number of observatic } \\
\text { Half montlily sums }\end{array}$}} & & $3 I$ & \multicolumn{2}{|c|}{$3^{1}$} & $3 I$ & 3I \\
\hline & & & & & $286 \quad 967$ & \multicolumn{2}{|c|}{$96 \quad \log 8$} & 205.0 & 176.0 \\
\hline
\end{tabular}


First reduction of tides at Cape Flora, Franz Josef Archipelago, Arctic Ocean-Continued

\begin{tabular}{|c|c|c|c|c|c|c|c|c|c|}
\hline \multirow{4}{*}{ 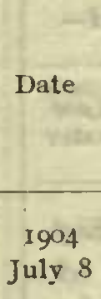 } & \multirow[b]{2}{*}{$\begin{array}{l}\text { Moou's } \\
\text { trausits }\end{array}$} & \multicolumn{2}{|c|}{ Time of - } & \multicolumn{4}{|c|}{ Irunitidal interval } & \multicolumn{2}{|c|}{ Height of - } \\
\hline & & $\begin{array}{l}\text { High } \\
\text { water }\end{array}$ & $\begin{array}{l}\text { Low } \\
\text { water }\end{array}$ & \multicolumn{2}{|c|}{$\begin{array}{l}\text { High } \\
\text { water }\end{array}$} & \multicolumn{2}{|c|}{$\begin{array}{l}\text { Low } \\
\text { water }\end{array}$} & \multirow{2}{*}{$\frac{\begin{array}{c}\text { High } \\
\text { water }\end{array}}{\text { Feet }}$} & \multirow{2}{*}{$\frac{\begin{array}{c}\text { Low } \\
\text { water }\end{array}}{\text { Feet }}$} \\
\hline & $h \quad m$ & $h m$ & $h m$ & $h$ & $m$ & $h$ & $m$ & & \\
\hline & 736 & $\begin{array}{ll}5 & 52\end{array}$ & II I 5 & (10 & $39)$ & 3 & 39 & 6.7 & 6.4 \\
\hline nat & $\left(\begin{array}{ll}20 & 00\end{array}\right)$ & $16 \quad 35$ & . . . & 8 & 59 & $\cdots$ & . & 6.8 & . \\
\hline 9 & $\begin{array}{ll}8 & 25\end{array}$ & $6 \quad 59$ & $\begin{array}{ll}0 & 15\end{array}$ & (Io & 591 & $(4$ & 15 & 6.7 & 5.8 \\
\hline cas & $\left(\begin{array}{ll}20 & 5 I\end{array}\right)$ & I7 $4 \mathrm{I}$ & 1233 & 9 & 16 & 4 & 08 & 6.7 & 6.3 \\
\hline 10 & $\begin{array}{ll}9 & 17\end{array}$ & $8 \mathrm{II}$ & I 02 & (II & 21) & $(4$ & II ) & 6.8 & 5.7 \\
\hline \pm 3 & $\left(\begin{array}{ll}2 I & 45\end{array}\right)$ & I8 44 & $13 \quad 35$ & 9 & 27 & 4 & 18 & 6.8 & 6.3 \\
\hline I I & 10 1.3 & 855 & $2 \quad 14$ & (I 1 & $10)$ & $(4)$ & $29)$ & 6.9 & 5.6 \\
\hline 2.85 & $\left(\begin{array}{ll}22 & 42\end{array}\right)$ & $19 \quad 56$ & I5 $\infty$ & 9 & 43 & 4 & 47 & 6.8 & 6.3 \\
\hline 12 & II 12 & 936 & 250 & (ró & $54)$ & 14 & o ) & 7.2 & 5.6 \\
\hline bes & $\left(\begin{array}{ll}23 & 41\end{array}\right)$ & $20 \quad 46$ & I5 $\quad 08$ & 9 & 34 & 3 & 56 & 6.9 & 6.3 \\
\hline I3 & . $\quad \cdots$ & 10 14 & $3 \quad 36$ & (10 & 33 ) & $(3$ & $55)$ & 7.2 & 5.6 \\
\hline 100 & 12 II & $21 \quad 54$ & $16 \quad 22$ & 9 & 43 & 4 & I I & 6.9 & 6.2 \\
\hline I4 & $\left(\begin{array}{ll}0 & 4 I\end{array}\right)$ & 10 37 & $\begin{array}{ll}4 & 23\end{array}$ & ( 9 & $56)$ & $(3$ & $42)$ & $7 \cdot 3$ & 5.6 \\
\hline 22 & 13 Io & $\begin{array}{ll}22 & 18\end{array}$ & $16 \quad 38$ & 9 & 108 & 3 & 28 & 6.9 & $6 . I$ \\
\hline 15 & $\left(\begin{array}{ll}\text { I } & 39\end{array}\right)$ & II 36 & $45^{2}$ & $(.9$ & $57)$ & ( 3 & $13)$ & $7 \cdot 3$ & 5.6 \\
\hline 72 & $\begin{array}{ll}14 & 08\end{array}$ & 2321 & I7 46 & 9 & 13 & 3 & $3^{8}$ & 7.1 & 6. I \\
\hline 16 & $\left(\begin{array}{ll}2 & 36\end{array}\right)$ & 1206 & $\begin{array}{ll}5 & 37\end{array}$ & $(9$ & $30)$ & 13 & or ) & 7.4 & 5.6 \\
\hline 54 & $\begin{array}{ll}15 & 03\end{array}$ & 2355 & $\begin{array}{ll}18 & 30\end{array}$ & 18 & $52)$ & $3:$ & 27 & 7.1 & 6.2 \\
\hline 17 & $\left(\begin{array}{ll}3 & 31\end{array}\right)$ & . & $\begin{array}{ll}6 & 18\end{array}$ & .. & $\cdots$ & $(3)$ & 471 & . & 5.9 \\
\hline 28 & $\begin{array}{ll}15 & 5^{8}\end{array}$ & {$\left[\begin{array}{ll}12 & 4^{8}\end{array}\right]$} & {$\left[\begin{array}{ll}19 & 37\end{array}\right]$} & ( [9 & 17]) & {$[3$} & 39] & {$[7.4]$} & [6. o] \\
\hline 18 & $\left(\begin{array}{ll}4 & 24\end{array}\right)$ & {$\left[\begin{array}{ll}0 & 27\end{array}\right]$} & {$\left[\begin{array}{ll}7 & 15\end{array}\right]$} & {$[8$} & 29] & $\left(\left[2^{\circ}\right.\right.$ & 51]) & [6.8] & {$[5.8]$} \\
\hline 18 & 1650 & $\begin{array}{ll}13 & 28\end{array}$ & $20 \quad 18$ & $(9$ & $04)$ & 3 & 28 & 7.2 & 5.7 \\
\hline 19 & $\left(\begin{array}{ll}5 & 16\end{array}\right)$ & I 50 & $\begin{array}{ll}8 & 04\end{array}$ & 9 & $\infty$ & $(2$ & $48)$ & 6.7 & 5.7 \\
\hline 4 & I7 4 II & I4 25 & $21 \quad 02$ & 19 & 091 & 3 & $2 I$ & 69 & 5.4 \\
\hline 20 & $\left(\begin{array}{ll}6 & 07\end{array}\right)$ & $\begin{array}{ll}3 & 12\end{array}$ & 840 & 9 & 31 & $(3$ & $33)$ & 6.4 & $5 \cdot 7$ \\
\hline $2=$ & $\begin{array}{ll}18 & 33\end{array}$ & $\begin{array}{ll}15 & 04\end{array}$ & 2150 & $(8$ & $57)$ & 3 & 17 & 6.8 & 5.4 \\
\hline 21 & $\left(\begin{array}{ll}6 & 5^{8}\end{array}\right)$ & 350 & $10 \quad 02$ & 9 & 17 & 13 & $04)$ & 6.4 & 5.9 \\
\hline $\mathrm{Ae}$ & $\begin{array}{ll}\text { I9 } & 24\end{array}$ & $15 \quad 42$ & $23 \quad 12$ & $(8$ & $44)$ & 3 & 48 & $6: 7$ & $5 \cdot 5$ \\
\hline 22 & $\left(\begin{array}{ll}7 & 49\end{array}\right)$ & 536 & II $D$ & Io & 12 & $(3$ & (I) & 6.6 & 6.2 \\
\hline$\{-2$ & $20 \quad 15$ & 1630 & . $\quad \ldots$ & $(8$ & $4 I 1$ & .. & .. & 6.8 & . \\
\hline 23 & $\left(\begin{array}{ll}8.4 & 1\end{array}\right)$ & $\begin{array}{ll}7 & 04\end{array}$ & $\begin{array}{ll}0 & 28\end{array}$ & Io & 49 & 4 & 13 & 6.7 & $5^{6}$ \\
\hline $16 \mathrm{~B}$ & $\begin{array}{ll}21 & 07\end{array}$ & $18 \cdot 12$ & $12 \quad 23$ & $(9$ & $3 I)($ & 3 & $42)$ & 6.8 & 6.3 \\
\hline Numbe & r of obse & alsons. & & & & 3 & 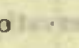 & $3 \mathrm{I}$ & . 30 \\
\hline Half $\mathrm{n}$ & onthly stu & ins.... & $\ldots ; \ldots$ & 283 & 995 & 97 & 848 & 213.7 & I 76.4 \\
\hline
\end{tabular}


First reduction of tides at Cape Flora, Franz Josef Archipelago, Arctic Ocean-Continued

\begin{tabular}{|c|c|c|c|c|c|c|c|c|c|c|c|}
\hline \multirow{2}{*}{\multicolumn{2}{|c|}{ Date }} & \multirow{2}{*}{\multicolumn{2}{|c|}{$\begin{array}{l}\text { Moon's } \\
\text { transits }\end{array}$}} & \multicolumn{3}{|c|}{ Time of- } & \multicolumn{3}{|c|}{ Lunitidal interval } & \multicolumn{2}{|c|}{ Height of- } \\
\hline & & & & & $\begin{array}{l}\text { ter } \\
\text { ter }\end{array}$ & $\begin{array}{l}\text { Low } \\
\text { water }\end{array}$ & $\begin{array}{l}\text { Hi } \\
\text { Wr }\end{array}$ & & $\begin{array}{l}\text { Low } \\
\text { water }\end{array}$ & $\begin{array}{l}\text { High } \\
\text { water }\end{array}$ & $\begin{array}{l}\text { Low } \\
\text { water }\end{array}$ \\
\hline \multirow{3}{*}{\multicolumn{2}{|c|}{$\begin{array}{c}\text { IgO4 } \\
\text { July } 24\end{array}$}} & $h$ & $m$ & $h$ & $m$ & $h \quad m$ & $h$ & $m$ & $h \quad m$ & Feet & Feet \\
\hline & & ( 9 & 32) & 7 & 50 & o 59 & 10 & 43 & 352 & 7.1 & 5.9 \\
\hline & & 21 & & I8 & 45 & 1346 & 19 & I3) & $\left(\begin{array}{ll}4 & I 4\end{array}\right)$ & 6.9 & 6.4 \\
\hline \multirow{2}{*}{\multicolumn{2}{|c|}{25}} & (10 & 23) & 8 & 59 & 128 & II & oI & 330 & 7.0 & 5.8 \\
\hline & & 22 & 48 & I9 & $5^{8}$ & 1451 & ( 9 & 35) & $\left(\begin{array}{ll}4 & 28\end{array}\right)$ & 6.8 & 6.3 \\
\hline \multirow{2}{*}{\multicolumn{2}{|c|}{26}} & (II & 12) & 9 & 32 & 245 & Io & 44 & $3 \quad 57$ & 7.2 & 5.8 \\
\hline & & 23 & 37 & $2 I$ & 12 & 1550 & (10 & 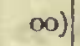 & $\left(\begin{array}{ll}4 & 3^{8}\end{array}\right)$ & 6.8 & 6.2 \\
\hline \multirow{2}{*}{\multicolumn{2}{|c|}{27}} & . & .. & 10 & 20 & $3 \quad 37$ & Io & 43 & $\begin{array}{ll}4 & 00\end{array}$ & 7.1 & 5.7 \\
\hline & & (12 & $\infty)$ & $2 I$ & 48 & 1630 & 19 & 48) & $\left(\begin{array}{ll}4 & 30\end{array}\right)$ & 6.8 & 6.2 \\
\hline \multirow{2}{*}{\multicolumn{2}{|c|}{28}} & 0 & 25 & ro & $5^{8}$ & 440 & Io & 33 & $4 \quad 15$ & 7.1 & 5.7 \\
\hline & & (12 & 48) & 22 & $3^{8}$ & 1705 & 19 & 50) & $\left(\begin{array}{ll}4 & 17\end{array}\right)$ & 6.8 & 6.0 \\
\hline \multirow{2}{*}{\multicolumn{2}{|c|}{29}} & I & 10 & II & $I_{3}$ & $5 \quad 05$ & 10 & 03 & 355 & 7.2 & $5 \cdot 7$ \\
\hline & & $(13$ & 32) & 23 & 18 & $17 \quad 34$ & 19 & 46) & $\left(\begin{array}{ll}4 & 02\end{array}\right)$ & 6.8 & 6.0 \\
\hline \multirow{2}{*}{\multicolumn{2}{|c|}{30}} & $I$ & 54 & II & $4 I$ & $\begin{array}{ll}5 & 29\end{array}$ & 9 & 47 & $3 \quad 35$ & 7.0 & 5.6 \\
\hline & & (14 & 16) & 23 & 52 & $\begin{array}{ll}18 & 13\end{array}$ & 19 & 36) & $\left(\begin{array}{ll}3 & 57\end{array}\right)$ & 6.5 & 5.8 \\
\hline \multirow{2}{*}{\multicolumn{2}{|c|}{31}} & 2 & 37 & .. & . & $5 \quad 54$ & .. & .. & $3 \quad 17$ & . & 5.5 \\
\hline & & (14 & $5^{8)}$ & 12 & 27 & I8 40 & 9 & 50 & $\left(\begin{array}{ll}3 & 42\end{array}\right)$ & 6.8 & 5.6 \\
\hline \multirow{2}{*}{\multicolumn{2}{|c|}{ Aug. }} & 3 & 19 & o & 30 & $\begin{array}{ll}6 & 34\end{array}$ & $(9$ & 32) & $3 \quad 15$ & 6.4 & 5.6 \\
\hline & & (15 & 40) & 12 & 54 & 1910 & 9 & 35 & $\left(\begin{array}{ll}3 & 30\end{array}\right)$ & 6.8 & 5.7 \\
\hline \multirow{2}{*}{$\log _{10}$} & 2 & 4 & 02 & I & 15 & $6 \quad 4^{8}$ & $(9$ & $35)$ & 246 & 6.6 & $5 \cdot 9$ \\
\hline & & (16 & 23) & I3 & I7 & I9 47 & 9 & 15 & (3 3 24) & 7.0 & 5.9 \\
\hline \multirow{2}{*}{$(4,1)$} & 3 & 4 & 45 & I & 40 & 705 & 19 & 17) & 220 & 6.8 & 6.2 \\
\hline & & (17) & o7) & I3 & 41 & $20 \quad 35$ & 8 & 56 & $\left(\begin{array}{ll}3 & 28\end{array}\right)$ & 7.2 & 6. I \\
\hline \multirow{2}{*}{$\sqrt{3}+2$} & 4 & 5 & 30 & 2 & 27 & 8 10 & $(9$ & 20) & 240 & 6.9 & 6.3 \\
\hline & & $(17$ & 53) & I4 & 20 & $21 \quad 24$ & 8 & 50 & $\left(\begin{array}{ll}3 & 3 I\end{array}\right)$ & 7.0 & 5.8 \\
\hline \multirow{2}{*}{$3 x$} & 5 & 6 & I6 & 3 & 20 & 835 & $(9$ & 27) & 219 & 6.7 & 6.3 \\
\hline & & (18 & 41) & I4 & $5^{6}$ & $22 \quad 14$ & 8 & 40 & $\left(\begin{array}{ll}3 & 33\end{array}\right)$ & 6.9 & 5.8 \\
\hline \multirow{2}{*}{42} & 6 & 7 & 06 & 5 & 16 & I0 44 & (10 & 35) & $3 \quad 3^{8}$ & 6.5 & 6.1 \\
\hline & & (19 & 32) & 15 & 42 & $23 \quad 15$ & 8 & 36 & $\left(\begin{array}{ll}3 & 43\end{array}\right)$ & 6.6 & 5.6 \\
\hline \multirow[t]{2}{*}{$y=$} & 7 & 7 & $5^{8}$ & 7 & $\infty$ & II 32 & (II & 28) & $3 \quad 34$ & 6.4 & 6.0 \\
\hline & & $(20$ & 26) & 16 & 30 & 2330 & 8 & $3^{2}$ & $\left(\begin{array}{ll}3 & 04\end{array}\right)$ & 6.5 & $5 \cdot 7$ \\
\hline \multirow{2}{*}{$\frac{02}{28}$} & 8 & 8 & & 7 & 44 & .. $\ldots$ & (II & 18) & $\ldots \quad \ldots$ & 6.7 & $\ldots$ \\
\hline & & & 23) & & 29 & 1307 & 8 & 35 & $\begin{array}{ll}4 & 13\end{array}$ & 6.7 & 6.3 \\
\hline \multirow{2}{*}{\multicolumn{4}{|c|}{$\begin{array}{l}\text { Number of observ } \\
\text { Half monthly sur }\end{array}$}} & vati & & $\cdots$ & \multicolumn{2}{|c|}{3 I } & 31 & 31 & $3^{I}$ \\
\hline & & & & ms. & & $5=$ & 286 & 1003 & $98 \quad 907$ & 211.6 & 183.5 \\
\hline
\end{tabular}


First reduction of tides at Cape Flora, Franz Josef Archipelago, Arctic Occan-Continued

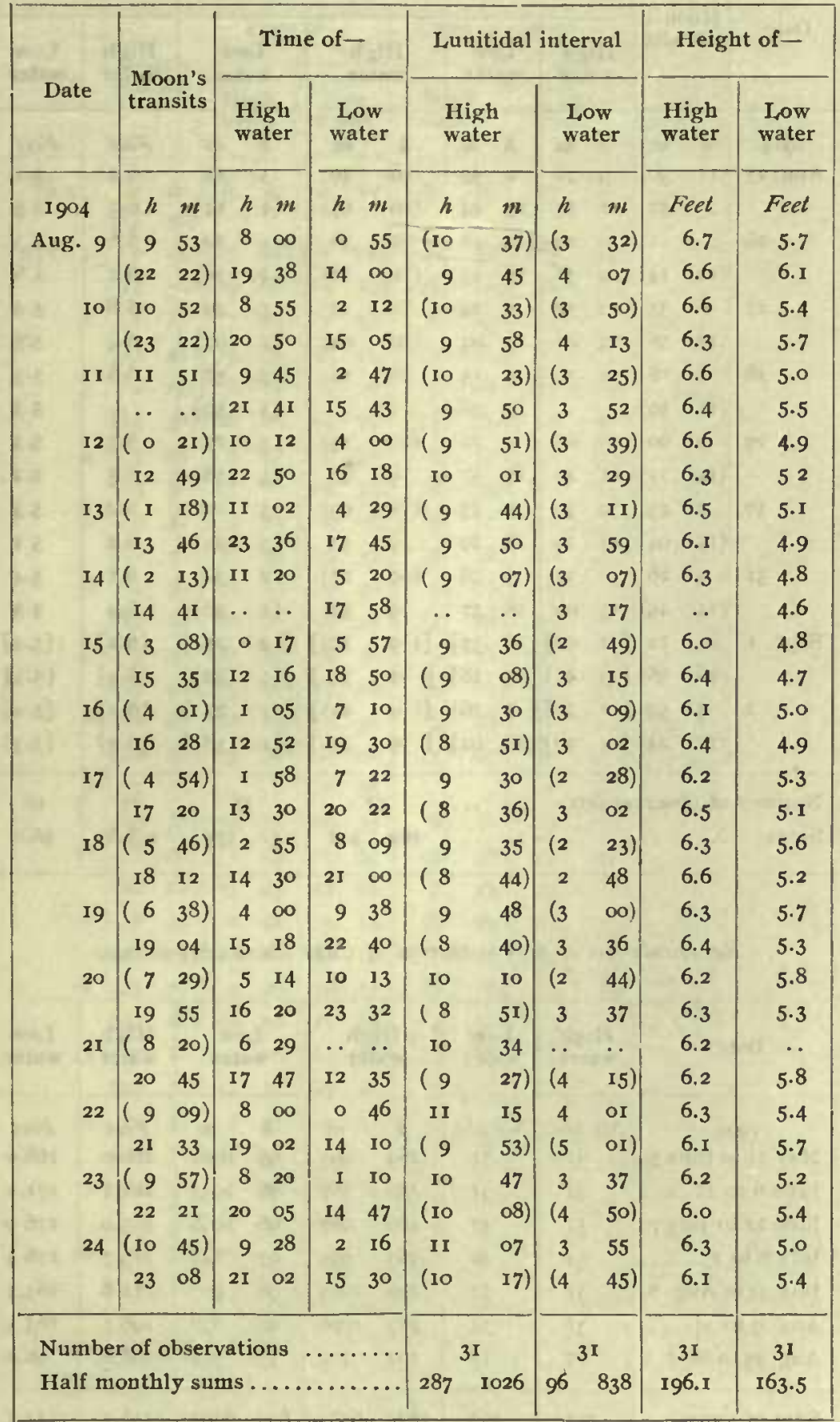


First reduction of tides at Cape Flora, Frans Joscf Archipclago, Arctic Occan-Concluded

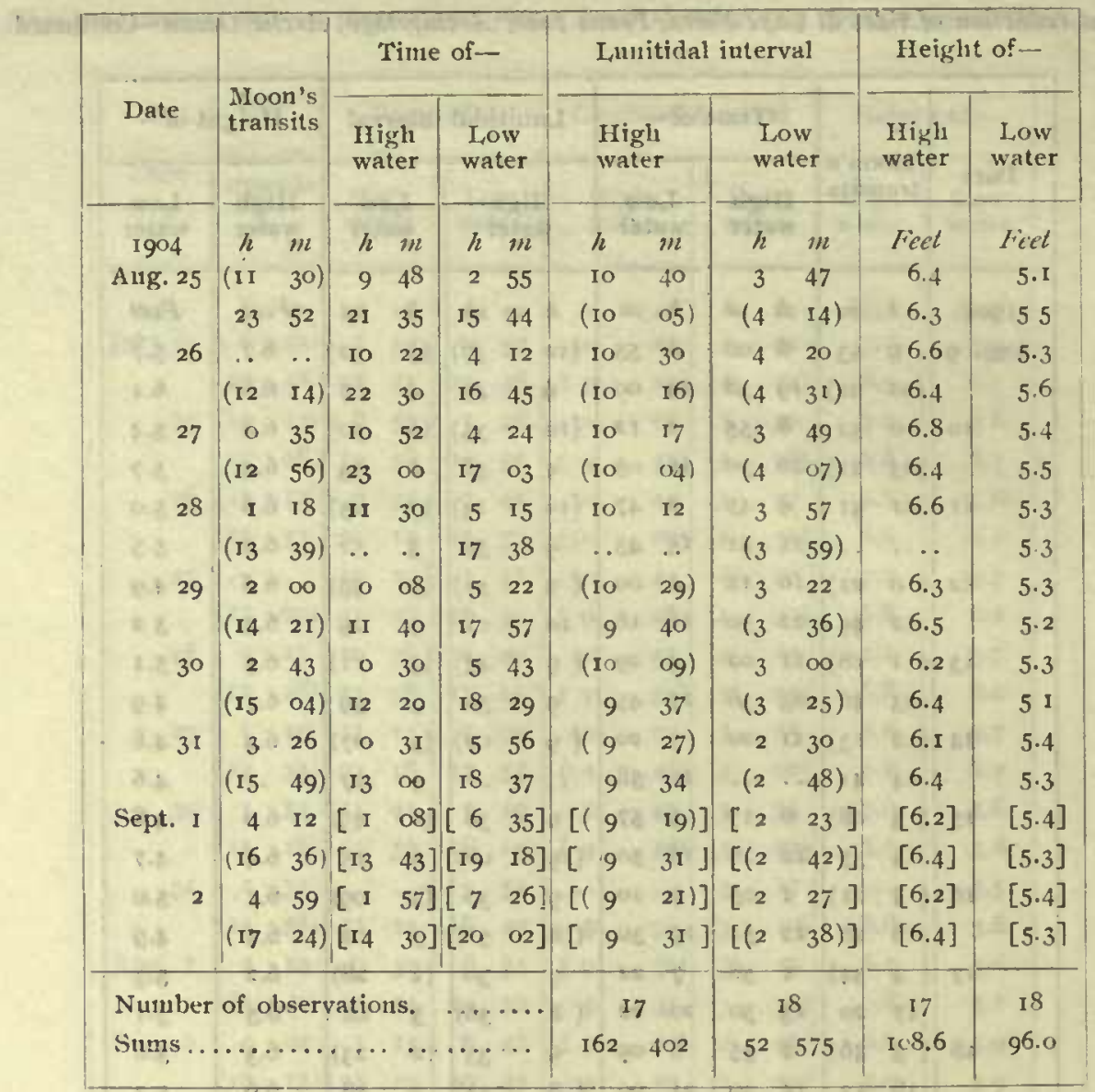

Recapitulation of first reduction of Cape Illora obscrations

\begin{tabular}{|c|c|c|c|c|c|c|}
\hline Date & $\begin{array}{l}\text { High } \\
\text { water }\end{array}$ & $\begin{array}{l}\text { Low } \\
\text { water }\end{array}$ & $\begin{array}{l}\text { High } \\
\text { water }\end{array}$ & $\begin{array}{l}\text { I.ow } \\
\text { water }\end{array}$ & $\begin{array}{l}\text { High } \\
\text { water }\end{array}$ & $\begin{array}{l}\text { Low } \\
\text { water }\end{array}$ \\
\hline 1904 & No.obs. & No.obs. & $m$ & $m$ & Feet & Feet \\
\hline May 2 I to June 5 . & 31 & $3 I$ & $283 \quad 993$ & $95 \quad 1004$ & 200.0 & I68 o \\
\hline June 6 to $21 . .$. & $3 I$ & $3 I$ & $286 \quad 887$ & $96 \quad 1033$ & 201.1 & 171.0 \\
\hline June 22 to July 7 & $3 I$ & $3 I$ & $286 \quad 967$ & 961098 & 205.0 & 1760 \\
\hline July 8 to $23 . . .$. & 31 & 30 & 283 & $97 \quad 848$ & 213.7 & 176.4 \\
\hline July 24 to Aug. 8. & $3 I$ & $3 I$ & $286 \quad \mathrm{IOO}_{3}$ & $98 \quad 907$ & 211.6 & 183.5 \\
\hline Aug 9 to 24 . & $3 I$ & $3 I$ & $287 \quad 1026$ & $96 \quad 8_{3} 8$ & J 96.1 & 163.5 \\
\hline Augr. 25 to Sept. 2. & 17 & 18 & 402 & $5^{2} \quad 575$ & I08.6 & 96.0 \\
\hline Sum of suns & 203 & 203 & I873 6273 & $630 \quad 6303$ & -1336.1 & 1134.4 \\
\hline Means ....... & $\ldots$ & $\ldots$ & 44.5 & 373 & $6.5^{8}$ & 5.:9 \\
\hline
\end{tabular}

Uncorrected mean range $=6.5^{8} \mathrm{ft} .-5.59 \mathrm{ft} .=0.99 \mathrm{ft}$. 
Iirst reduction of lides at Teplitz Bay, Franz Josef Archipclago, Arctic Ocean

\begin{tabular}{|c|c|c|c|c|c|c|c|c|c|c|c|c|}
\hline \multirow{2}{*}{\multicolumn{2}{|c|}{$7+1=1$}} & \multirow{2}{*}{\multicolumn{2}{|c|}{1}} & \multirow{2}{*}{\multicolumn{4}{|c|}{ Time of - }} & \multirow{3}{*}{\multicolumn{3}{|c|}{ I,unitidal interval }} & \multirow{2}{*}{\multicolumn{2}{|c|}{ Height of- }} \\
\hline & & & & & & & & & & & & \\
\hline \multirow{2}{*}{\multicolumn{2}{|c|}{ Date }} & \multirow{2}{*}{\multicolumn{2}{|c|}{$\begin{array}{l}\text { Moon's } \\
\text { transits }\end{array}$}} & \multirow{2}{*}{\multicolumn{2}{|c|}{$\begin{array}{l}\text { High } \\
\text { water }\end{array}$}} & \multirow{2}{*}{\multicolumn{2}{|c|}{$\begin{array}{l}\text { Low } \\
\text { water }\end{array}$}} & & & & \multirow{3}{*}{$\begin{array}{c}\begin{array}{c}\text { High } \\
\text { water }\end{array} \\
\text { Feet }\end{array}$} & \multirow[b]{2}{*}{$\begin{array}{l}\text { Low } \\
\text { water }\end{array}$} \\
\hline & & & & & & & & $\begin{array}{l}\text { IIigh } \\
\text { water }\end{array}$ & \multicolumn{2}{|c|}{$\begin{array}{l}\text { Low } \\
\text { water }\end{array}$} & & \\
\hline \multicolumn{2}{|c|}{1904} & $h$ & $m$ & $h$ & $m$ & $h$ & $m$ & $h \quad m$ & $h$ & $m$ & & Fett \\
\hline & & ten & 30 & s. & & & & & & & & \\
\hline Apr. & I & 0 & 23 & {$[7$} & 22] & {$[\mathbf{I}$} & 07] & 659 & (13 & 11) & {$[4.7]$} & {$[2.9]$} \\
\hline E4 & & ( 12 & 50) & 19 & 02 & {$[13$} & 297 & $\left(\begin{array}{ll}6 & \text { I }\end{array}\right)$ & 13 & 06 & 4.5 & {$[3.2]$} \\
\hline 6. & 2 & 1 & 17 & 7 & 44 & I & 33 & $\begin{array}{ll}6 & 27\end{array}$ & 112 & 43) & 4. 4.8 & 3.0 \\
\hline $\mathrm{r}:$ & & (13 & 44! & 19 & 46 & 13 & 52 & $\left(\begin{array}{ll}6 & 02\end{array}\right)$ & 12 & 35 & 4.9 & 3.2 \\
\hline 84 & 3 & 2 & II & 8 & 21 & 2 & 10 & 610 & $(12$ & 26) & 4.9 & 3.1 \\
\hline S4 & & (14 & 38) & 20 & 28 & 14 & 20 & $\left(\begin{array}{ll}5 & 50\end{array}\right)$ & 12 & $\infty$ & 5.0 & $3 \cdot 3$ \\
\hline 14 & 4 & 3 & 04 & 8 & 57 & 2 & 33 & $5 \quad 53$ & (II & 55) & 4.8 & $3 \cdot 3$ \\
\hline $5 x$ & & (15 & $3 I)$ & 21 & I0 & 14 & 53 & $\left(\begin{array}{ll}5 & 39\end{array}\right)$ & II & 49 & 4.9 & $3 \cdot 3$ \\
\hline esis & 5 & 3 & 57 & 9 & I 2 & 3 & 05 & $\begin{array}{ll}5 & 15\end{array}$ & (II & 34) & 5.0 & 3.5 \\
\hline 52 & & $(16$ & 23) & $2 t$ & 44 & 15 & 22 & $\left(\begin{array}{ll}5 & 21\end{array}\right)$ & II & 25 & 4.9 & 3.6 \\
\hline 64 & 6 & 4 & 49 & 10 & 12 & 3 & $3^{8}$ & $\begin{array}{ll}5 & 23\end{array}$ & (I1) & 15) & 4.6 & 3.5 \\
\hline $8 x$ & & $(17$ & 14) & 22 & 10 & 16 & 16 & $\left(\begin{array}{ll}4 & 56\end{array}\right)$ & II & 27 & 4.6 & 3.6 \\
\hline At & 7 & 5 & 39 & 11 & $2 I$ & 4 & 30 & $5 \quad 42$ & (II & 16) & $4: 4$ & 3.5 \\
\hline 1.8 & & $(18$ & 03) & 23 & I0 & 17 & 20 & $\left(\begin{array}{ll}5 & 07\end{array}\right)$ & II & $4 \mathrm{I}$ & 4.4 & 3.6 \\
\hline 92 & 8 & 6 & 27 & 12 & 15 & 5 & 35 & $5 \quad 48$ & (II & 32) & 4.2 & 3.5 \\
\hline 26 & & (IS & 5I) & 23 & $5^{8}$ & I8. & o8 & $\left(\begin{array}{ll}5 & 07\end{array}\right)$ & 11 & 41 & $4: 2$ & 3.7 \\
\hline$a 8$ & 9 & 7 & I5 & . & .. & 6 & 35 & $\ldots 21 \ldots$ & (I1) & 44) & Not & 3.6 \\
\hline is & & 119 & 37) & 13 & 25 & 19 & 35 & 6 10 & 12 & 20 & 4.2 & 3.7 \\
\hline$\sqrt{2}$ & IO & 8 & $\infty$ & I & 15 & 7 & 54 & $\left(\begin{array}{ll}5 & 38\end{array}\right)$ & (12 & 17) & 4.3 & 3.7 \\
\hline 47 & & 120 & 22) & 15 & 25 & 21 & 02 & $7 \quad 25$ & I3 & 02 & . 4.4 & 4.0 \\
\hline 24 & II & 8 & 44 & 2 & $5 \mathrm{I}$ & 9 & 15 & $\left(\begin{array}{ll}6 & 29\end{array}\right)$ & $(12$ & (3) & 4.5 & 3.9 \\
\hline $5+2$ & & i 21 & 05) & 16 & 10 & 22 & 15 & $7 \quad 26$ & 13 & $3 I$ & 4.6 & 3.9 \\
\hline 4ht2 & I 2 & 9 & 27 & 4 & 20 & IO & 25 & $\left(\begin{array}{ll}7 & 15\end{array}\right)$ & (13 & 20) & 4.6 & 3.8 \\
\hline $3 y$ & & ( 21 & 4) & 16 & 50 & 22 & 39 & $\begin{array}{ll}7 & 23\end{array}$ & 13 & 12 & 4.7 & $3 \cdot 9$ \\
\hline nat & I3 & 10 & 10 & 5 & $\infty$ & I 1 & 22 & $\left(\begin{array}{ll}7 & 12\end{array}\right)$ & (13 & 34) & 4.8 & 4.0 \\
\hline 12 & & $(22$ & 32) & I7 & 35 & 23 & 40 & $\begin{array}{ll}7 & 25\end{array}$ & I3 & 30 & 4.9 & 3.9 \\
\hline & 14 & 10 & 53 & 5 & 35 & II & 52 & $\left(\begin{array}{ll}7 & 03\end{array}\right)$ & $(13$ & 20) & 5.0 & $3 \cdot 9$ \\
\hline ex & & $(23$ & 16) & 18 & 05 & $\cdots$ & . & $\begin{array}{ll}7 & 12\end{array}$ & . & . & 5.0 & . \\
\hline ris & 15 & II & $3^{8}$ & 6 & 20 & 0 & I5 & $\left(\begin{array}{ll}7 & 04\end{array}\right)$ & 13 & 22 & 5.1 & 3.7 \\
\hline & & .. & . & 18 & 35 & 12 & 25 & $\begin{array}{ll}6 & 57\end{array}$ & (13 & og) & $5 \cdot 3$ & 3.9 \\
\hline 58 & 16 & 10 & o1) & 6 & 50 & 1 & I5 & $\left(\begin{array}{ll}6 & 49\end{array}\right)$ & 13 & 37 & 5.2 & 3.8 \\
\hline the & & 12 & 24 & 19 & 04 & 12 & 56 & $6 \quad 40$ & $(12$ & $55)$ & 5.1 & 3.8 \\
\hline Nur & alve & $r$ of 0 & iser & vatio & & & . & 3I & 31 & & 31 & 31 \\
\hline Hal & If $m$ & Ionth & ly sur & & & & .... & $182 \quad 839$ & 373 & 871 & 146.8 & I I I. 3 \\
\hline
\end{tabular}


First reduction of tides at Teplitz Bay, Frans Josef Archipelago, Arctic Ocean-Continued

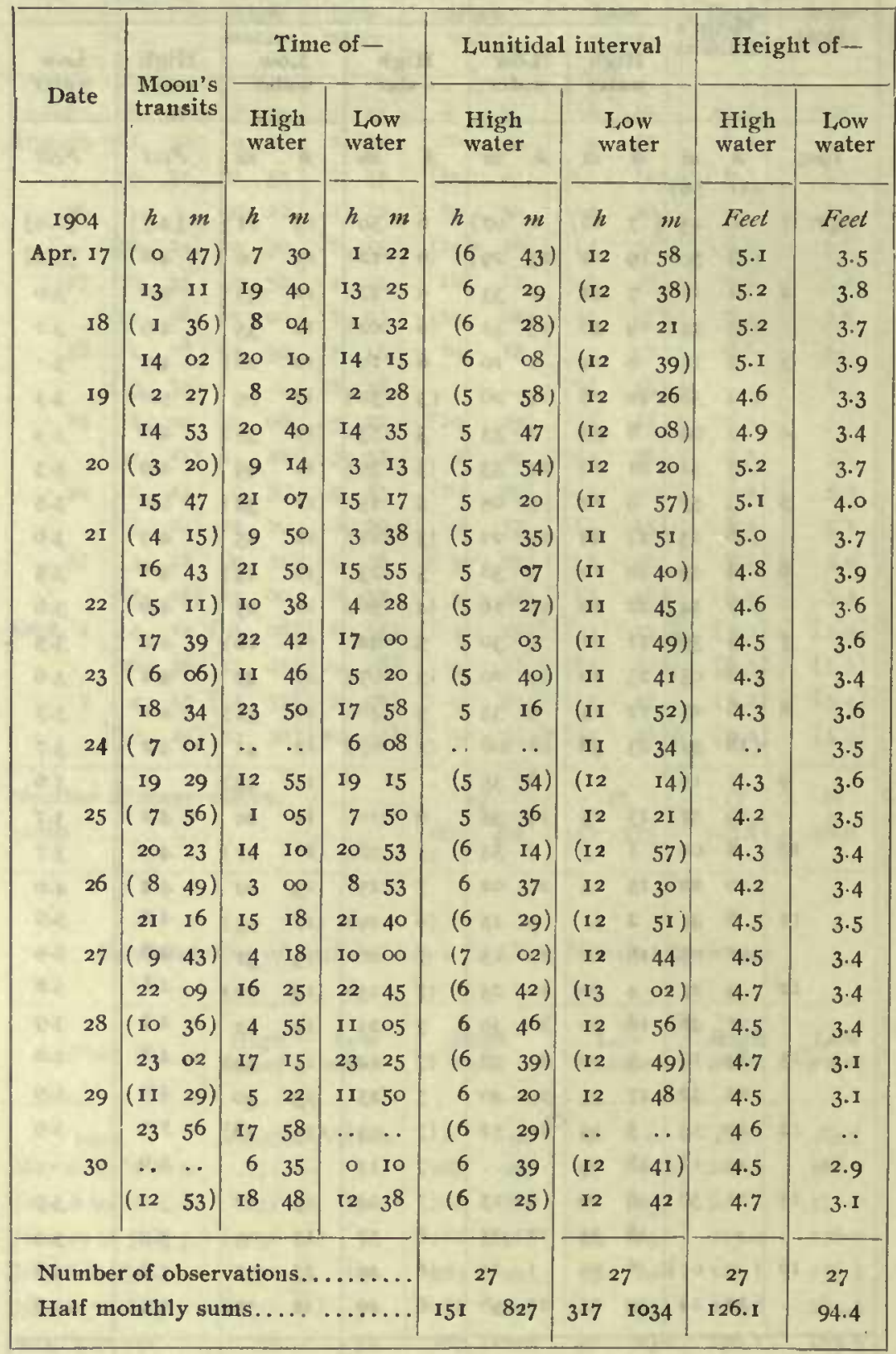


First reduction of tides at Teplitz Bay, Franz Josef Archipelago, Arctic Ocean-Continued

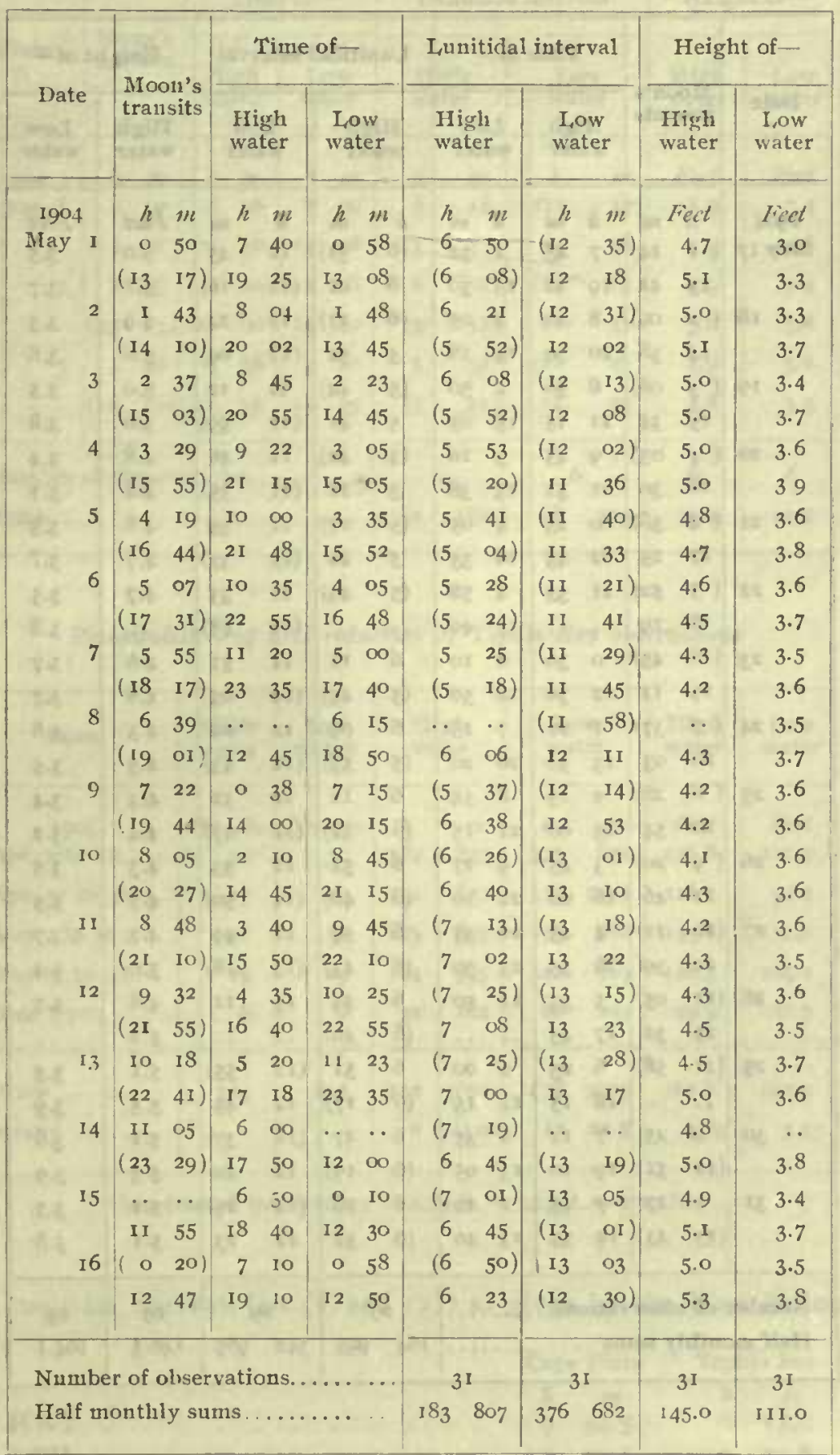


First reduction of tides at Teplitz Bay, Franz Josef Archipelago, Arctic Ocean-Continued

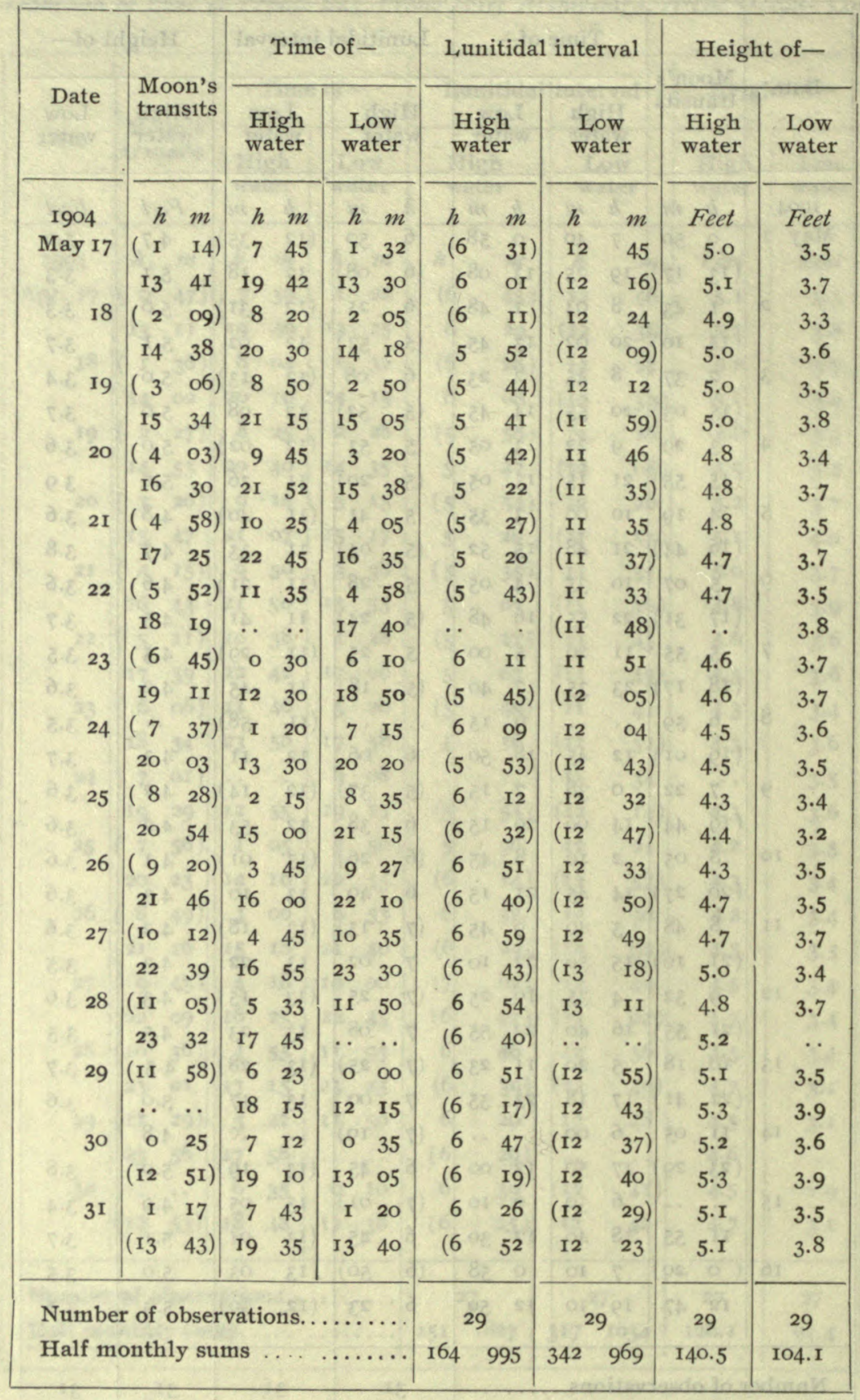


First reduction of tides at Teplitz Bay, Franz Josef Arclipelago, Arctic Ocean-Concluded

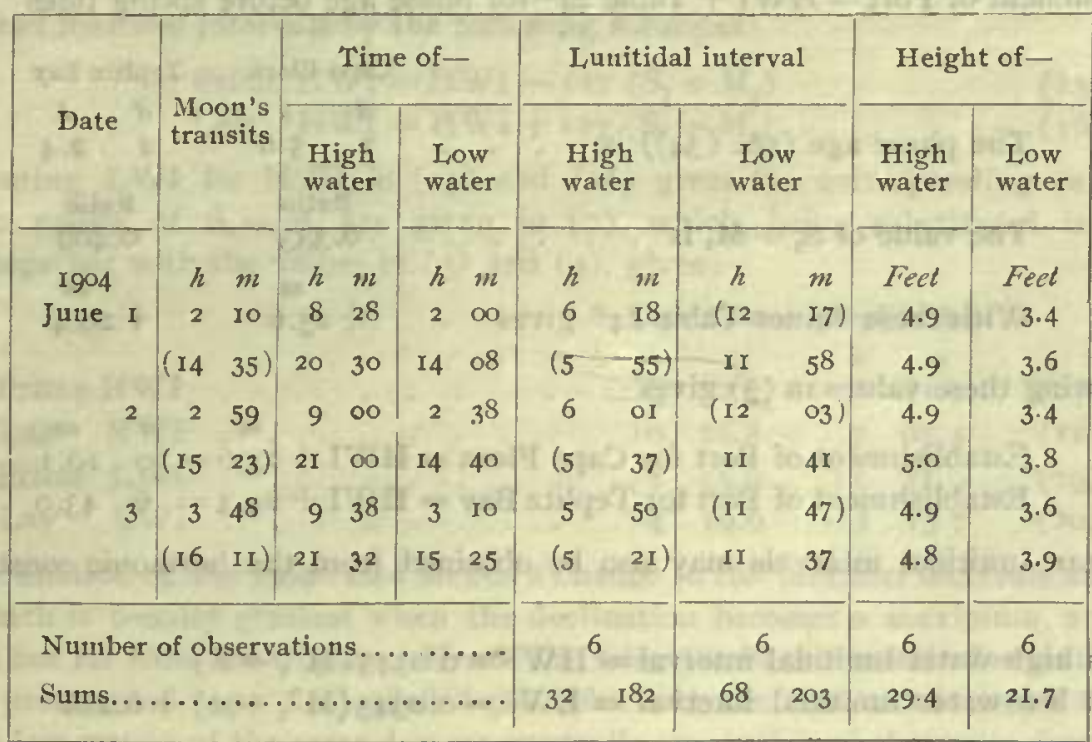

Recapitulation of first reduction of Teplitz Bay observations

\begin{tabular}{|c|c|c|c|c|c|c|c|}
\hline Date & $\begin{array}{l}\text { High } \\
\text { water }\end{array}$ & $\begin{array}{c}\text { Low } \\
\text { water }\end{array}$ & $\begin{array}{l}\text { I } \\
\text { W }\end{array}$ & igh & $\begin{array}{c}\text { Low } \\
\text { water }\end{array}$ & $\begin{array}{l}\text { High } \\
\text { water }\end{array}$ & $\begin{array}{c}\text { Low } \\
\text { water }\end{array}$ \\
\hline rgo4 & No.obs. & No.obs. & $h$ & $m$ & $h \quad m$ & Feel & Feet \\
\hline Apr. I to $16 \ldots$ & $3 I$ & $3 I$ & I 82 & 839 & $373 \quad 871$ & 146.8 & III.3 \\
\hline Apr. 17 to $30 . .$. & 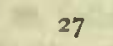 & 27 & I5I & 827 & $317 \quad 1034$ & I26. I & 94.4 \\
\hline May I to 16. & $3 I$ & $3 I$ & 183 & 807 & $376 \quad 682$ & 145.0 & I I I.O \\
\hline May 17 to $31 \ldots$ & 29 & 29 & I64 & 995 & $342 \quad 969$ & 140.5 & I04. I \\
\hline June $I$ to $3 \ldots$. & 6 & 6 & 32 & 182 & $68 \quad 203$ & 29.4 & 21.7 \\
\hline Sums .. & 124 & 124 & 712 & 3650 & $1476 \quad 3759$ & 587.8 & 442.5 \\
\hline Means & & & 6 & 14.0 & $12 \quad 24.5$ & 4.74 & 3.57 \\
\hline
\end{tabular}

Uncorrected mean range $=4.74 \mathrm{ft} .-3.57 \mathrm{ft} .=1.17 \mathrm{ft}$.

The mean lunitidal intervals (see (1) and (2)) as given by the First Reductions, are as follows :

$$
\begin{aligned}
& \text { Cape Flora Teplitz Bay } \\
& \text { HWI . . . . . . . . } \quad \begin{array}{cccc}
h & m & h & m \\
9 & 44.5 & 6 & \text { I } 3.6
\end{array} \\
& \text { LWI . . . . . . . } 337.3 \quad \text { I2 } 23.8
\end{aligned}
$$

The mean lunitidal interval for high water given in (3) is sometimes called the Correctcd Establishment of the Port, while the mean lunitidal interval for high water at full aud change (new) of the moon is called the Establishment of the Port. 
The Establishment of the Port may be derived from the mean lunitidal interval as follows:

Establishment of Port $=\mathrm{HWI}+$ Table $24^{*}$ for phase age before spring tides

\begin{tabular}{|c|c|c|c|}
\hline & & Cape Flora & Teplitz Bay \\
\hline & & $d \quad h$ & $d \quad h$ \\
\hline The phase age (see (34)) is & $\cdot \cdot$ & $2 \quad 5.6$ & $2 \quad 2.4$ \\
\hline & & Ratio & Ratio \\
\hline The value of $S_{2} \div M_{2}$ is & & & 0.409 \\
\hline Witl these values Table $24^{*} \mathrm{~g}$ & & +25.6 & +29.4 \\
\hline
\end{tabular}

and substituting these values in (5) gives

$\begin{array}{lcc}\text { Establishment of Port for Cape Flora }=\mathrm{HWI}+25.6=10 & m \\ \text { Establishment of Port for Teplitz Bay }=\text { HWI }+29.4=6 & 43.0\end{array}$

The mean lunitidal intervals may also be obtained from the liarmonic constants by the equations :

Mean high-water lunitidal interval $=\mathrm{HWI}=0.0345\left(\mathrm{M}_{2}^{\circ}-v\right)$

Mean low-water lunitidal interval $=\mathrm{LWI}=0.0345\left(\mathrm{M}^{\circ}{ }_{3}-w\right)+6.2 \mathrm{r} h$

Where $v$ and $w$ are such that

$$
\begin{aligned}
& \tan v=\frac{2 M_{4} \sin \left(2 M^{\circ}{ }_{2}-M^{\circ}{ }_{4}\right)+3 M_{6} \sin \left(3 M^{\circ}{ }_{2}-M^{\circ}{ }_{8}\right)+\ldots \ldots \ldots}{I^{2} M_{2}+2^{2} M_{4} \cos \left(2 M^{\circ}{ }_{2}-M^{\circ}{ }_{1}\right)+3^{3} M_{6} \cos \left(3 M^{\circ}{ }_{2}-M^{\circ}\right)+\ldots} \\
& \tan \tau=\frac{2 \mathrm{M}_{4} \sin \left(2 \mathrm{M}^{\circ}{ }_{3}-\mathrm{M}^{\circ}{ }_{4}\right)-3 \mathrm{M}_{6} \sin \left({ }_{3} \mathrm{M}_{3}^{\circ}-\mathrm{M}_{6}{ }_{6}\right)+\ldots \ldots}{-I^{2} \mathrm{M}_{2}+2^{2} \mathrm{M}_{4} \cos \left(2 \mathrm{M}_{2}^{\circ}-\mathrm{M}^{\circ}{ }_{4}\right)-3^{2} \mathrm{M}_{6} \cos \left(3 \mathrm{M}^{\circ}{ }_{2}-\mathrm{M}_{6}^{\circ}\right)+\ldots}
\end{aligned}
$$

From (II) and (12) we obtain :

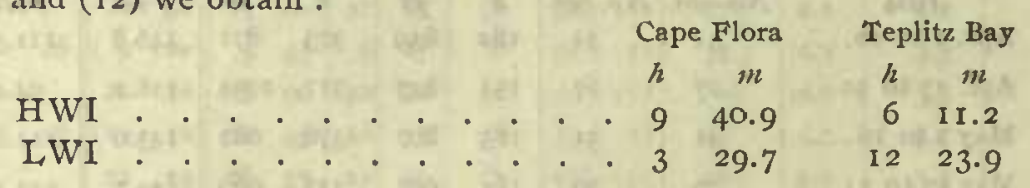

A comparison of these values with those of (3) and (4) indicates a fairly satisfactory agreement, especially if we take into account the great difference in the methods used to obtain the two sets of results.

The sun's effect upon the time of the tide is to disturb the mean time of its occurrence, making it alternately earlier and later, according to the inoon's phase.

The priming of the tide is the periodic acceleration of its time of occurrence, due to the sun's effect. At such times the lunitidal intervals are less than their mean, so that the tides occur earlier than the average. The priming of the tides occurs during the period between new or full moon and the following quadrature, beginning and ending at a time equal to the age of the phase inequality after these phases. It attains its maximum effect soon after the first and fifth octauts of the moon's phase.

The lagging of the tides is the corresponding retardation in the time of its occurrence, the greatest effect being soon after the third and seventh octants of the moon's phase.

* Whenever any table is referred to here by number, unless otherwise stated, it is contained in Appendix 7 , United States Coast and Geodetic Survey Report for IS94, for tables up to 35, and in Appendix 9 , Report for 1897 of the same Survey, for tables having numbers greater than 35 . 
The least and greatest lunitidal intervals due to prining and lagging of the tides may be desiguated as Prime HWI, Prime LWI, Lag HWI, and Lag LWI. They nay be derived from the mean lunitidal intervals by the following formulas :

$$
\begin{aligned}
& \text { Prime } \mathrm{HWI}=\mathrm{HWI}-127\left(\mathrm{~S}_{2} \div \mathrm{M}_{2}\right) \\
& \mathrm{L} \text { ag } \mathrm{HWI}=\mathrm{HWI}+127\left(\mathrm{~S}_{3} \div \mathrm{M}_{2}\right)
\end{aligned}
$$

Substituting I,WI for HWI in (15) and (16) gives the corresponding values for low water. The values of $S_{2} \div M_{2}$ are given iu (7), whicll, being substituted in the above equations, together with the values in (3) and (4), gives:

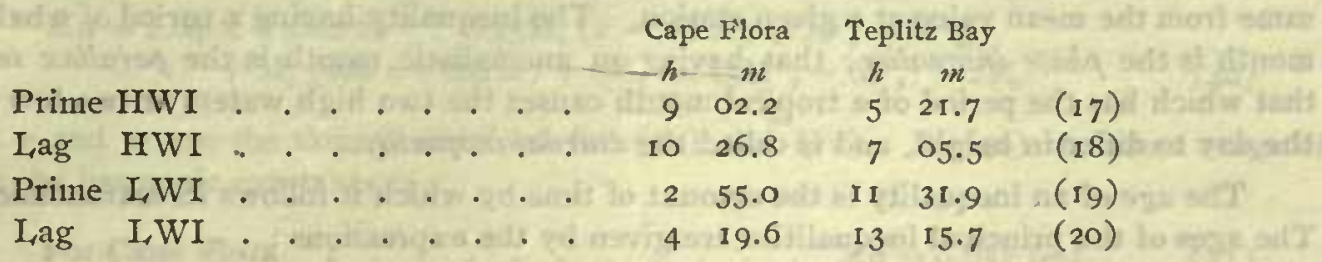

The declination of the moon also makes a cliange in the lunitidal intervals and heiglits of the tide, which is usually greatest when the declination becomes a maximum, at which time the moon is not far from the tropics. Hence the tides due to the moon's declination, when at their most pronounced type, are called tropic tides. At the time of the tropic tides tlie two high or two low waters of the same day are generally unequal, and the range from the higher ligh water to the lower low water is called the great tropic range.

The lunitidal intervals for the tropic tides may be obtained from the mean intervals by the following equations:

$$
\begin{aligned}
& \text { Tropic HHWI }=\mathrm{HWI}-2.07 \times \text { value from Table } 44^{*} \\
& \text { Tropic LHWI }=\mathrm{HWI}-2.07 \times \text { value from Table } 44 * \\
& \text { Tropic HLWI }=\text { LWI }-2.07 \times \text { value from Table } 44^{*} \\
& \text { Tropic LI,WI }=\text { I, WI }-2.07 \times \text { value from Table } 44^{*}
\end{aligned}
$$

In Table $44^{*}$ of these equations the arguments are different for each phase of tide, the corresponding intervals being as follows:

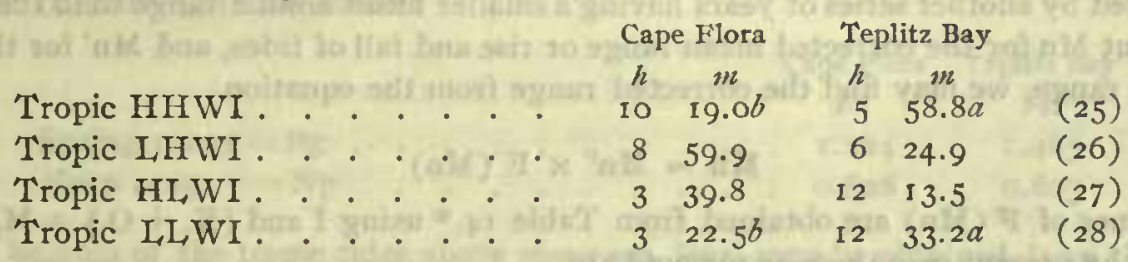

The tropic intervals for the higher high water and for the lower low water are marked by the letters $a$ and $b$ in order to enable one to obtain the approximate time of these tides by adding the interval to the upper or lower transit of the moon as explained below. When the tropic interval (HHWI or LLWI) is marked $a$ add the interval to the local time of the moon's upper
lower transit, or meridian passage, for ${ }_{\text {south }}^{\text {nortl }}$ declination of the moon; and when it is marked $b$ add the interval to the local time of the moon's $\begin{aligned} & \text { upper } \\ & \text { lower }\end{aligned}$

The tropic tides may be said to be formed by the combination of a semidiurnal wave witl a diurnal wave. The tropic lunitidal interval of the diurnal wave may be found by the equation

$$
\mathrm{D}_{1} \mathrm{HWI}=0.0345\left(\mathrm{~K}_{1}^{\circ}+\mathrm{O}_{1}^{\circ}\right) a
$$

where $D_{1}$ stands for diurnal. 
Substituting the values for $\mathrm{K}^{\circ}{ }_{1}$ and $\mathrm{O}^{\circ}{ }_{1}$ from the table of harmonic constants already given, we obtain :

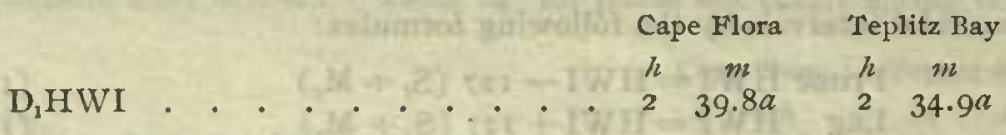

\section{TIUE INEQUALITILS AND RANGES}

An inequality in the interval, range, or height of tide is a systematic departure of the same from the mean value at a given station. The inequality having a period of a lialf sylodic month is the phase inequality; that having an anomalistic month is the parallax inequality; that which has the period of a tropical month causes the two high waters or two low waters of the day to differ in height, and is called the diumal inequality.

The age of an inequality is the amount of time by which it follows its astronomical cause. The ages of the principal inequalities are given by the expressions :

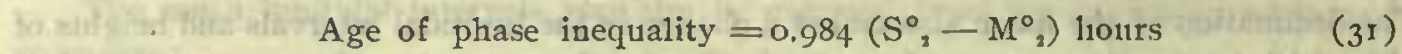

$$
\begin{aligned}
& \text { Age of parallax inequality }=\mathrm{r} .837\left(\mathrm{M}_{3}^{\circ}-\mathrm{N}_{2}^{\circ}\right) \text { hours } \\
& \text { Age of diurnal inequality }=0.91 \mathrm{I}\left(\mathrm{K}^{\circ}{ }_{1}-\mathrm{O}^{\circ}{ }_{1}\right) \text { hours }
\end{aligned}
$$

Substituting the values of the epochs or kappas giveu in the table of harmonic constants, we obtain :

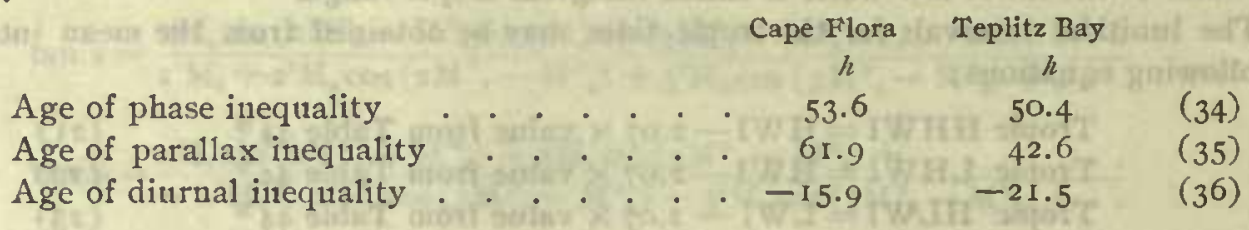

The mean range of tide, as given by the direct summation of higll and low waters, usually requires to be corrected for the longitude of the moon's ascending node, there being whole series of years during which the mean annual range is greater than an average for the lunar cycle, followed by another series of years having a smaller mean annual range than the average.

If we put $\mathrm{Mn}$ for the corrected mean range or rise and fall of tides, and $\mathrm{Mn}^{\prime}$ for the uncorrected mean range, we may find the corrected range from the equation

$$
\mathrm{Mn}=\mathrm{Mn}^{\prime} \times \mathrm{F}(\mathrm{Mn})
$$

The values of $F(M n)$ are obtained from Table $I_{4}$, $*$ using $I$ and $\left(K_{1}+O_{1}\right) \div M_{2}$ as argi1. ments. In the present case these arguments are

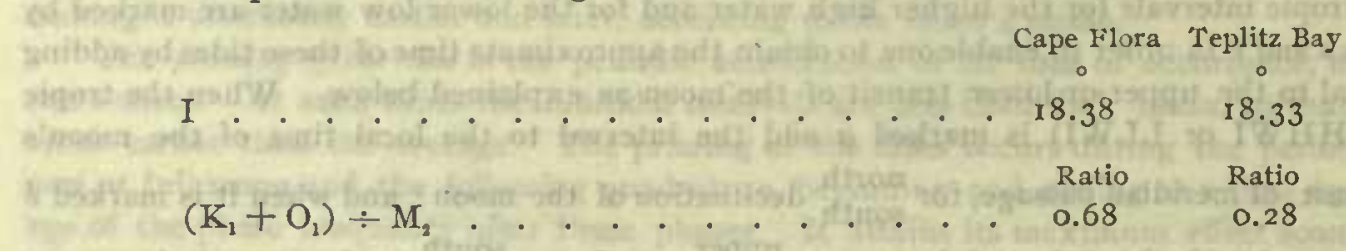

Entering Table $14^{*}$ with these arguments, we find

For Cape Flora . . . . . . $\mathrm{Mn}=0.99 \times 0.976=0.966$ feet

For Teplitz Bay . . . . . $M n=1.17 \times 0.972=1.138$ feet 
The mean range of tide may also be obtained from the harmonic constants by the formula

$$
\begin{aligned}
\mathrm{Mn}=2 \mathrm{M}_{2} & +\frac{\mathrm{I}}{2 \mathrm{M}_{2} m_{2}^{2}}\left[\mathrm{~S}_{2}^{2} s_{2}^{2}+\mathrm{N}_{2}{ }_{2} n^{2}+\ldots+\mathrm{K}_{1}{ }_{1} k_{1}{ }_{1}+\mathrm{O}_{1}^{2} o^{2}{ }_{1}+\ldots\right] \\
& +\mathrm{M}_{2}(\cos v+\cos w)+\frac{\pi}{\mathrm{r} 80} \times 2 \mathrm{M}_{4}(v-w) \sin \left(2 \mathrm{M}^{\circ}{ }_{2}-\mathrm{M}^{\circ}\right) \\
& +2 \mathrm{M}_{6} \cos \left(3 \mathrm{M}^{\circ}{ }_{2}-\mathrm{M}_{8}^{\circ}\right)-2 \mathrm{M}_{2}
\end{aligned}
$$

which by means of Table $22 *$, becomes

$$
\begin{aligned}
\mathrm{Mn}=2.04 & \times \text { Table } 22 *+.035 \mathrm{M}_{6}(v-v) \sin \left(2 \mathrm{M}^{\circ}{ }_{2}-\mathrm{M}^{\circ}\right) \\
& +\mathrm{M}_{2}(\cos v+\cos w)+2 \mathrm{M}_{6} \cos \left(3 \mathrm{M}^{\circ}{ }_{3}-\mathrm{M}^{\circ}{ }_{6}\right)-2 \mathrm{M}_{2}
\end{aligned}
$$

in which $v$ and $w$ are the same as obtained for (II) and (I2). By (4O) the neau range of tide from the harmonic constants is

$$
\begin{aligned}
& \text { For Cape Flora } \\
& \text { For Teplitz Bay . . . . . . . . . . . . . } \mathrm{Mn}=0.952 \text { feet } \\
& \mathrm{Mn}=1.100 \text { feet }
\end{aligned}
$$

which agrees fairly well with the values given in (38) and (39).

The spring and neap ranges of tide may be obtained from the harmonic constants by the formulas

$$
\begin{aligned}
& \mathrm{Sg}=\mathrm{Mn}-.536 \frac{\mathrm{S}_{2}^{2}}{\mathrm{M}_{2}}+\left[\mathrm{I} .96-.08\left(\frac{\mathrm{K}_{1}+\mathrm{O}_{1}}{\mathrm{M}_{2}}\right)^{2}\right] \\
& \quad \times\left[\mathrm{S}_{2}+\mu_{2} \cos \left(2 \mathrm{M}^{\circ}{ }_{2}-\mathrm{S}_{2}^{\circ}-\mu_{2}^{\circ}\right)\right] \\
& \mathrm{Np}=\mathrm{M} 11-.536 \frac{\mathrm{S}_{2}}{\mathrm{M}_{2}}-\left[\mathrm{I} .96-.08\left(\frac{\mathrm{K}_{1}+\mathrm{O}_{1}}{\mathrm{M}_{2}}\right)^{2}\right] \\
& \quad \times\left[\mathrm{S}_{2}+\mu_{2} \cos \left(2 \mathrm{M}^{\circ}{ }_{2}-\mathrm{S}_{2}^{\circ}-\mu_{2}^{\circ}\right)\right]
\end{aligned}
$$

in which the first and last letters of the words spring and neap are used as abbreviations.

From (43) and (44) we obtain:

$$
\begin{aligned}
& \text { Cape Flora Teplitz Bay } \\
& \text { Fi. Fi. }
\end{aligned}
$$

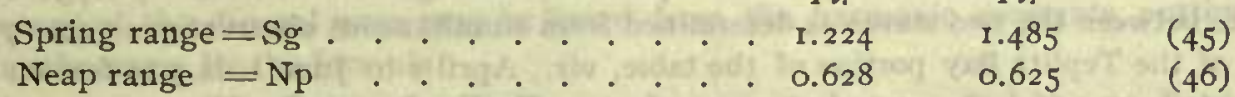

The heights of the tropic tides above mean sea level may be obtained from the harmonic constants by the following formulas :

Tropic HHW = $1.02 \Delta_{2} \times$ Table $45 \dagger$
Tropic LHW $=1.02 \Delta_{2} \times$ Table $45 \dagger$
Tropic HLW $=1.02 \Delta_{2} \times$ Table $45 \dagger$
Tropic LILW=1.02 $\Delta_{2} \times$ Table $45 \dagger$

where

$$
\Delta_{2}=\text { I.010 } \mathrm{M}_{2}+0.27\left(\mathrm{~S}_{2} \div \mathrm{M}_{2}\right)-\mathrm{K}_{2} \cos \left[\left(\mathrm{K}^{\circ},-\mathrm{O}^{\circ}\right) \sim\left(\mathrm{K}^{\circ}{ }_{2}-\mathrm{M}^{\circ}\right)\right]
$$


and different arguments are used for the various tides. From (47) to (50) we obtain the following values, the heights being reckoned from mean sea level:

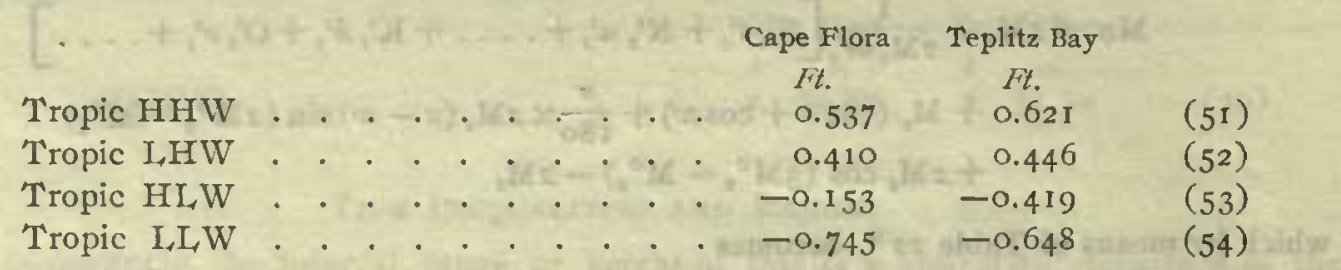

Mean sea level, as used above, is the mean of the hourly heights of the sea used for obtaining the harmonic constants, or

$$
\text { 10. } \text { MSLan }_{1}=\frac{1}{n} \dot{n}\left(h_{0}+h_{1}+h_{2}+\ldots \ldots h_{22}+h_{23}\right)
$$

in which $\Sigma h$ represents the sum of all the heights throughout the series for the hour designated by the subscript, and $n=24$ times the number of days in the series discussed. As there is usually a periodic variation in mean sea level from month to month, cliefly due to seasonal changes in the direction and velocity of winds, which ronghly complete their cycle in a year, it must be borne in mind that when less than a year of record is analyzed the resulting mean sea level is not a true mean for the station.

This will be more evident from a study of the following table of mean sea levels on the ist and i 6 th of each month during which observations were made:

\begin{tabular}{|c|c|c|c|c|c|c|c|}
\hline Date & Sea level & Date & Sea level & Date & Sea level & Date & Sea level \\
\hline \begin{tabular}{lr}
\multicolumn{2}{c}{1904} \\
April & I \\
April & I 6 \\
May & I
\end{tabular} & $\begin{array}{l}F t \\
5.9^{2} \\
5.85 \\
5.82\end{array}$ & \begin{tabular}{lr}
\multicolumn{2}{c}{ I904 } \\
May & 16 \\
June & 1 \\
June & 16
\end{tabular} & $\begin{array}{l}\text { Fit. } \\
5.83 \\
5.90 \\
6.03\end{array}$ & $\begin{array}{lr} & \text { I904 } \\
\text { July } & \text { I } \\
\text { July } & 16 \\
\text { Ang. } & \text { I }\end{array}$ & $\begin{array}{l}F t \\
6.20 \\
6.40 \\
6.30\end{array}$ & $\begin{array}{l}\text { 1904 } \\
\text { Aug. } 16 \\
\text { Sept. I } \\
. \quad\end{array}$ & $\begin{array}{c}\text { Ft. } \\
6.00 \\
5.72 \\
.\end{array}$ \\
\hline
\end{tabular}

In the above table the heights are all referred to the tide staff at Cape Flora, that portion which was obtained from the record at Teplitz Bay having been increased by 1.73 feet, the difference between the two staves as determined from simultaneous observations; see (77). The mean of the Teplitz Bay portion of the table, viz., April I to June 1, is 5.86 feet on the Cape Flora staff, or 5.86 feet -1.73 feet $=4.13$ feet on Teplitz Bay staff. The corresponding mean for Cape Flora, June I to September I, is 6.08 feet. The difference in the mean sea level for each of the two series is, therefore, 6.08 feet $-5.86 \mathrm{feet}=0.22 \mathrm{foot}$. The extreme difference in the half-monthly mean sea levels of the table is 6.40 feet -5.82 feet $=0.5^{8}$ foot, or about 7 inches, in less than three months.

Mean half-tide level is the mean of all the high and low waters for the period of observatiou. Abbreviating to initial letters, we have

$$
\mathrm{HTL}=\frac{\mathrm{I}}{2}(\mathrm{HW}+\mathrm{L} \mathrm{W})
$$

When the harnonic constants for the station are known, the approxinate value of mean half-tide level may be computed by the formula

$$
\mathrm{HTL}_{1}=\mathrm{MSL}+\mathrm{M}_{4} \cos \left(2 \mathrm{M}_{2}^{\circ}-\mathrm{M}^{\circ}\right)-0.04 \frac{\left(\mathrm{K}_{1}+\mathrm{O}_{1}\right)^{2}}{\mathrm{M}_{2}} \cos \left(\mathrm{M}_{3}^{\circ}{ }_{3}-\mathrm{K}_{1}^{\circ}{ }_{1}-\mathrm{O}^{\circ}{ }_{1}\right)
$$


The values obtained from (55), (56), and (57) are as follows :

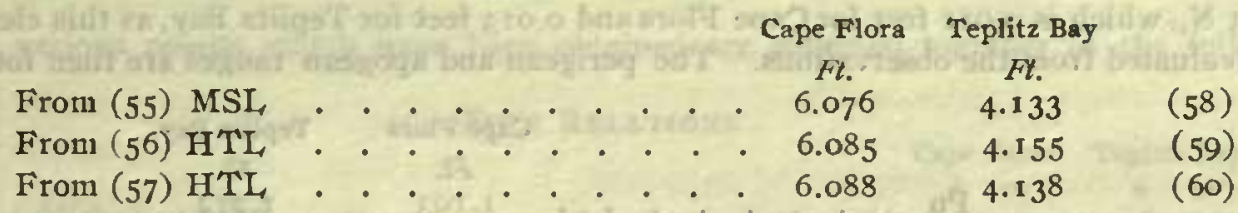

By adding the values of $(51),(52),(53)$, and (54) to those of $\left(5^{8}\right)$ we obtain the corresponding readings upon the tide staves, thus:

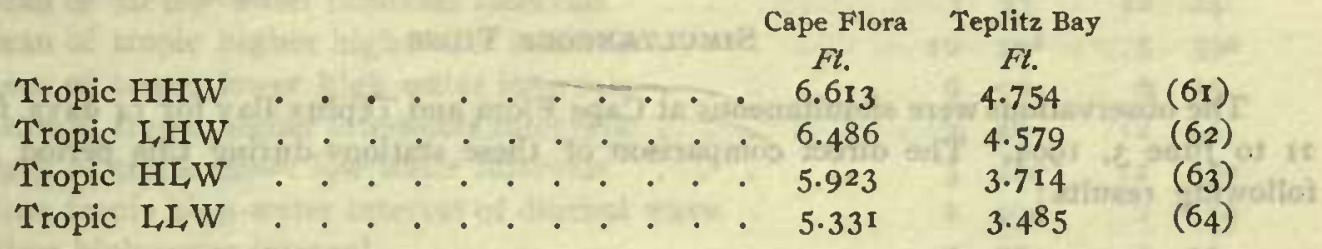

The difference between the two tropic high waters (HHW-LHW) is called the tropic high-water dinrnal inequality in height, abbreviated to tropic HWQ or often to HWQ alone, the word tropic being understood. In a similar way the tropic low-water diurnal inequality in heiglit (HLW-L,LW) is contracted to $I_{1} W Q$. The great tropic range $\left(H H W-L_{1}, W\right.$ ) is contracted to $\mathrm{Gc}$, while the small tropic range ( $\mathrm{LHW}-\mathrm{HLW}$ ) is represented by Sc. These values from (6I) to (64) are as follows:

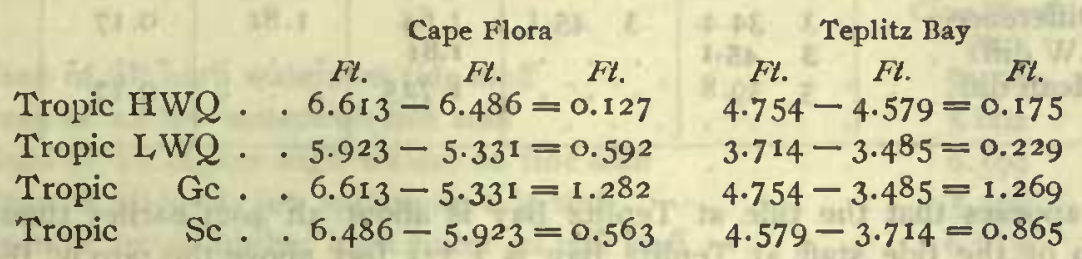

The difference between the mean of the higher high waters and the mean of the lower low waters for one or more months is called the great diurnal range, and is abbreviated to Gt. It may be computed as follows, when either tropic HWQ or LWQ is approximately as great as one-fourth of the mean range :

$$
\mathrm{Gt}=0.75 \mathrm{Gc}+0.25 \mathrm{Mn}
$$

The range of the diurnal wave may be found from the harmonic constants, putting $2 \mathrm{D}$ to represent the wave, by the formula

$$
2 \mathrm{D}_{1}=2.042\left(\mathrm{~K}_{1}+\mathrm{O}_{1}\right)
$$

Froin (69) and (7o) we obtain :

Cape Flora Teplitz Bay

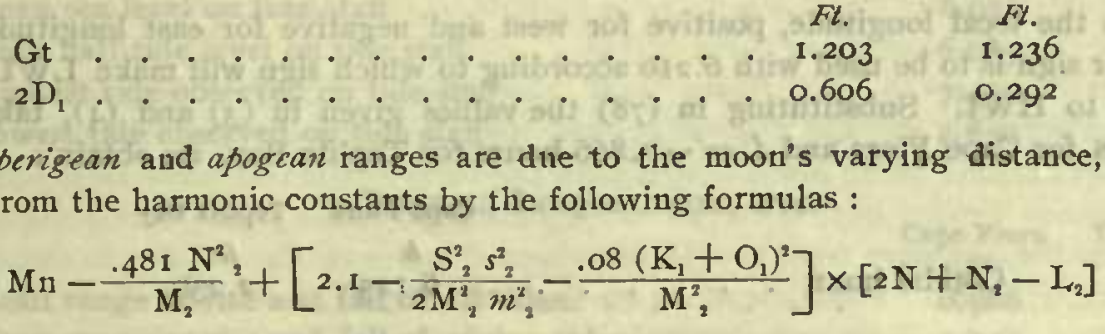

$$
\begin{aligned}
& \mathrm{A}_{11}=\mathrm{Mn}-\frac{.481 \mathrm{~N}^{2}}{\mathrm{M}_{2}}+\left[2.1-\frac{\mathrm{S}_{2}{ }_{2} s_{2}{ }_{2}}{2 \mathrm{M}_{2}^{2} m^{2}{ }_{2}}-\frac{.08\left(\mathrm{~K}_{1}+\mathrm{O}_{1}\right)^{2}}{\mathrm{M}_{2}{ }_{2}}\right] \times\left[2 \mathrm{~N}-\left(\mathrm{N}_{2}-\mathrm{L}_{2}\right)\right]
\end{aligned}
$$

in which the words perigean and apogean are abbreviated to their first and last letters. 
The values of the harmonic component $2 \mathrm{~N}$ in (73) and (74) must be estimated as about 0.I $33 \mathrm{~N}_{2}$, which is 0.01 I feet for Cape Flora and 0.013 feet for Teplitz Bay, as this element was not evaluated from the observations. The perigean and apogean ranges are then found to be

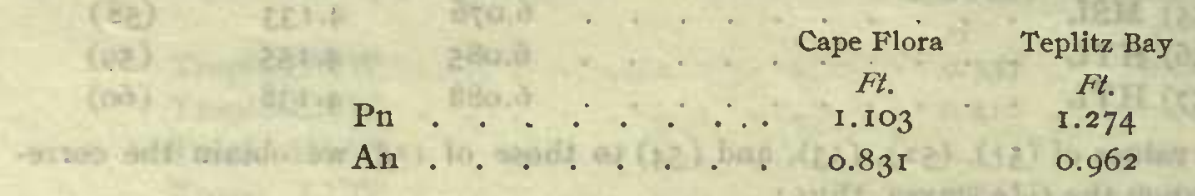

\section{Simultaneous Tides}

The observations were simultaneỏus at 'Cape Flora and Teplitz Bay for 14 days, from May 2 I to June 3, 1904. The direct comparison of these stations during this period gives the following results:

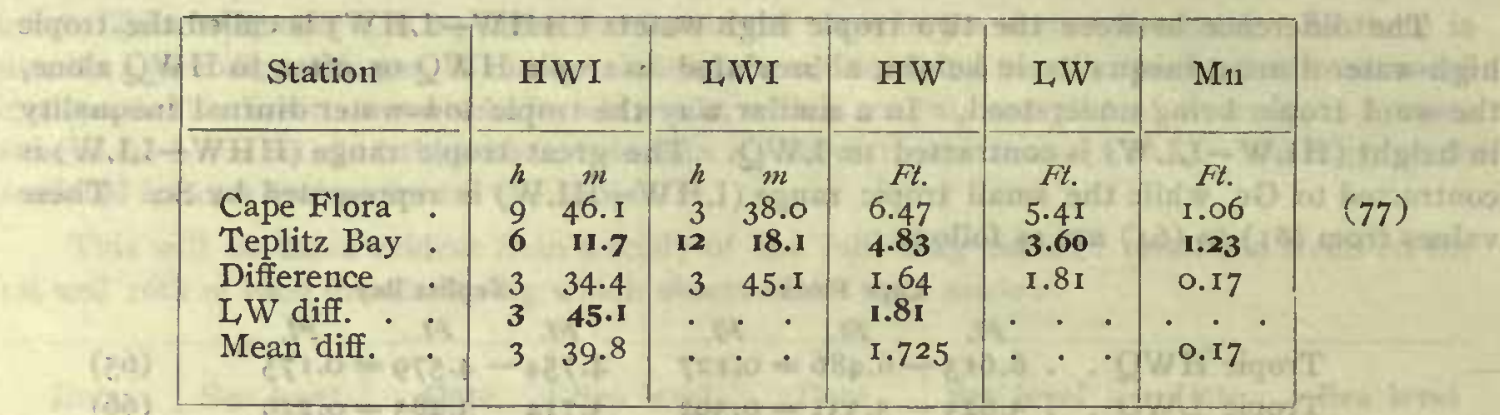

from which it appears that the tide at Teplitz Bay is about 3 h 4 oul earlier than at Cape Flora; the zero of the tide staff at Teplitz Bay is 1.725 feet above the zero of the staff at Cape Flora, and the mean rise and fall, or range, of tides is 0.17 feet greater at Teplitz Bay than at Cape Flora.

Nearly the same difference in time of tide at these two stations is obtained from the mean lunltidal intervals of (3) and (4), which makes Teplitz Bay about 3 h 3 rm earlier than Cape Flora.

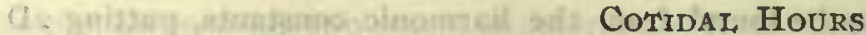

The cotidal lunar hour of a place may be found from the expression

$$
\text { Cotidal hour }=0.483(\mathrm{HWI}+\mathrm{LWI} \pm 6.2 \mathrm{IO}) \pm L
$$

in which $L$ is the local longitude, positive for west and negative for east longitudes. The upper or lower sign is to be used with 6.2 Io according to which sign will make L,WI approximately equal to HWI. Substitnting in (78) the values given in (3) and (4), taking $L=$ -3.332 . hours for Cape Flora and $L=-3.866$ hours for Teplitz Bay, we obtain :

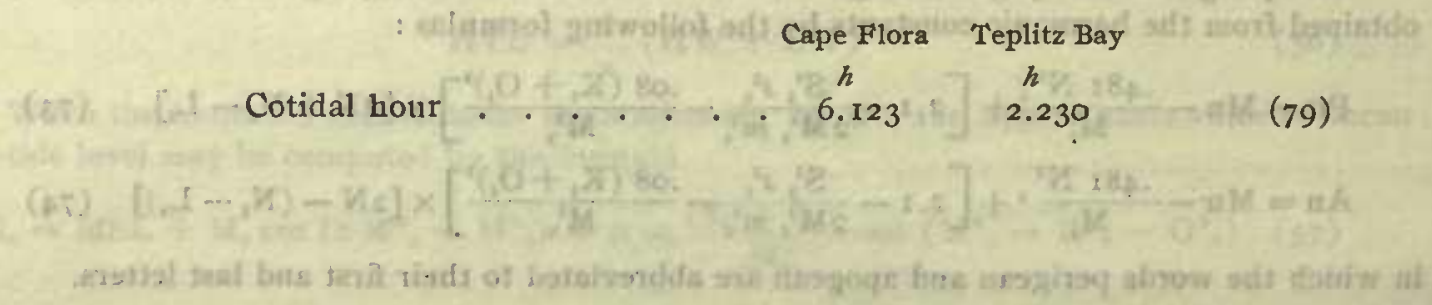


RECAPITULATION

The various results which liave been obtained are collected together in the following tables:

TIME RELATIONS

$\begin{array}{rlrl}\text { Cape Flora } & \text { Teplitz Bay } \\ h & m & h & m \\ 10 & 10 & 6 & 43 \\ 9 & 44 & 6 & 14 \\ 3 & 37 & 12 & 24 \\ 10 & 19 b & 5 & 59 a \\ 9 & 00 & 6 & 25 \\ 3 & 40 & 12 & 14 \\ 3 & 22 b & 12 & 33 a \\ 2 & 40 a & 2 & 35 a \\ 9 & 02 & 5 & 22 \\ 10 & 27 & 7 & 06 \\ 2 & 55 & 11 & 32 \\ 4 & 20 & 13 & 16 \\ 6 & 07 & 2 & 14\end{array}$

\section{HEIGH'T RELATIONS}

Mean of all high waters on tide staff ...... .

Cape Flora Teplitz Bay

Establishment of the port ............. . . 10 10 * 643

Mean of all high-water lunitidal intervals . . . . . $9944 \quad 6.14$

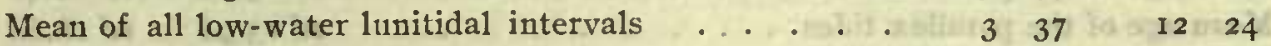

Mean of tropic higher high-water intervals ....... $10 \quad 19 b \quad 5 \quad 59 a$

Meall of tropic lower high-water intervals . . . . . $900 \quad 625$

Mean of tropic higher low-water intervals........ . . $340 \quad$ I2 14

Mean of tropic lower low-water intervals . . . . . $\begin{array}{lllll}3 & 22 b & 3_{2} & 33^{a}\end{array}$

Mean tropic high-water interval of diurnal wave. . . $2440 a \quad 2 \quad 35 a$

Prime high-water interval . . . . . . . . . $9 \begin{array}{lllll} & 9 & 02 & 5\end{array}$

Lag high-water interval . . . . . . . . . $1027 \quad$ o 2706

Prime low-water interval . . . . . . . . 255 II 32

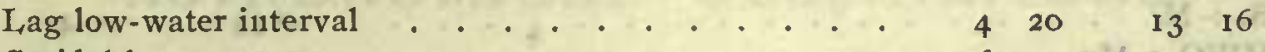

Cotidal hour . . . . . . . . . . . . . 6 o7 $14{ }^{2} 14$

Mean of all low waters on tide staff . . . . . . . . $5.602=3.586$

Meall of all higher high waters on tide staff . . . . $6.599 \quad 4.743$

Mean of all lower low waters on tide staff . . . . . $\quad 5.396 \quad 3.507$

Meall of tropic higher high water.on tide staff ...... 6.613 4.754

Mean of tropic lower higll water on tide staff . . . . $6.486 \quad 4.579$

Mean of tropic higher low water on tide staff . . . . . $5.923 \quad 3.714$

Mean of tropic lower low water on tide staff . . . . $5.331 \quad 3.4^{8} 5$

Mean of all spring high waters on tide staff . . . . 61697

Mean of all spring low waters on tide staff . . ... . 5.473 3.4 3

Mean of all neap high waters on tide staff . . . . . $6.399 \quad 4.467$

Mean of all neap low waters on tide staff . . . . $5.771 \quad 3.842$

Mean perigean high water on tide staff . . . . . $6.636 \quad 4.792$

Mean perigean low water on tide staff . . . . . . 5.533 3.518

Mean apogean high water on tide staff . . . . . 6.501 6 4.636

Mean apogean low water on tide staff . . . . . . 317570 3.674

Meari sea level on tide staff . . . . . . . . . . . $6.076=6.133$

Mean half-tide level on tide staff . . . . . . . . $6.085 \quad 4.155$

Highest tide observed on tide staff . . . ...... . . $7.430 \quad 5.310$

Lowest tide observed on tide staff . . . . . . . . $4.630 \quad 2.860$

RANGES, INEQUALITIES, FTC.

Mean range or rise and fall of all tides. . . . .

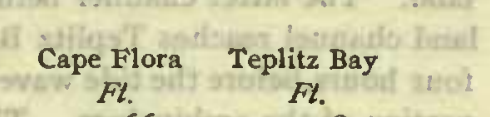

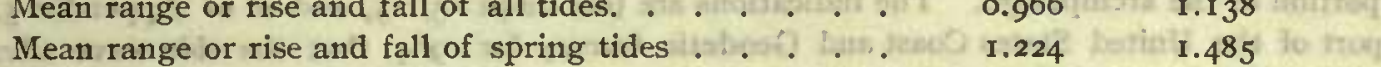

Mean range or rise and fall of neap tides . . . . . 0.628 thent 0.625

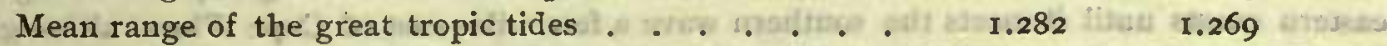

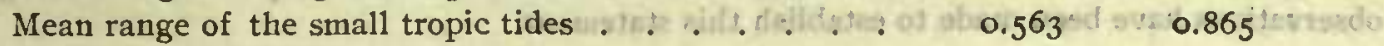




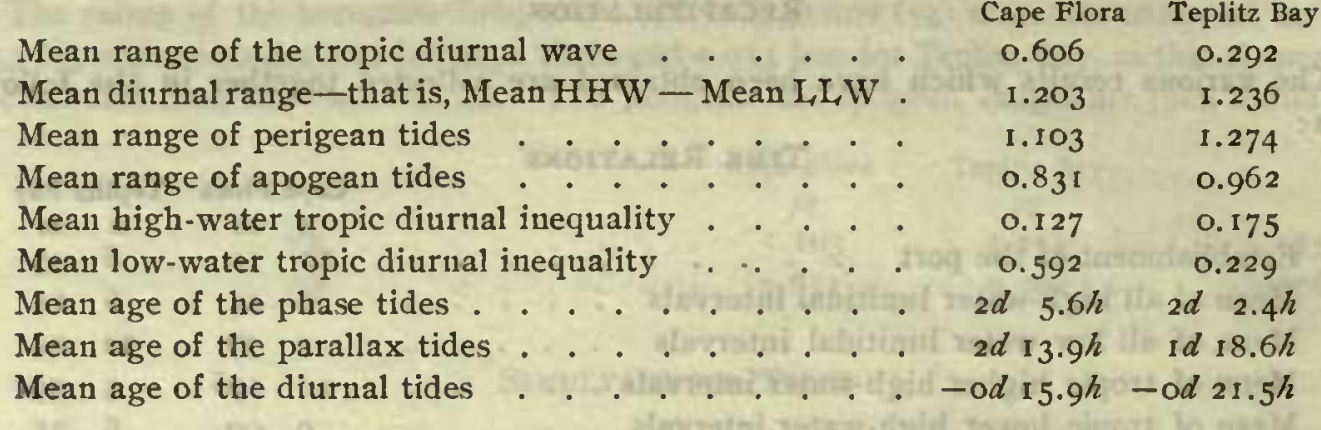

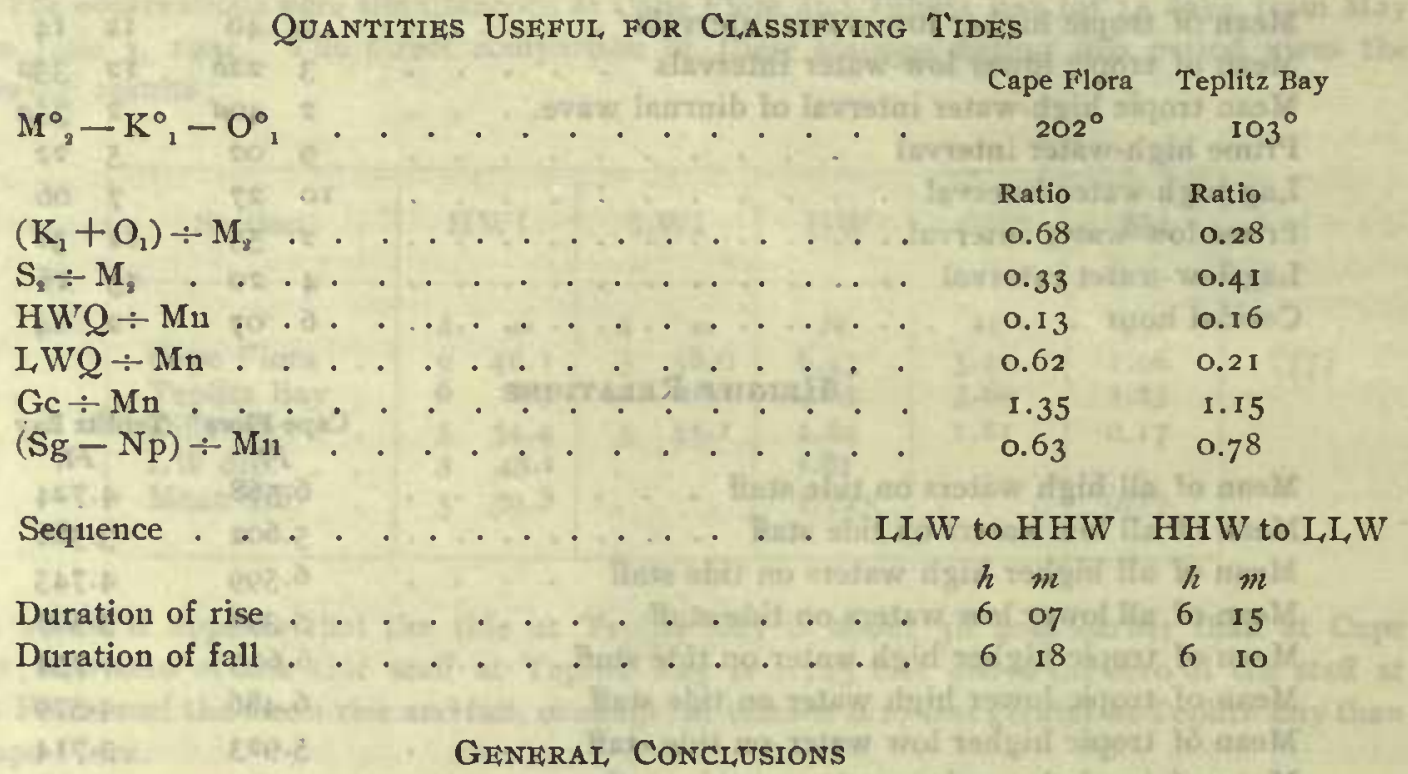

A comparison of the results obtained above indicates that the type of tide at Cape Flora is quite different from that at Teplitz Bay. It is interesting to trace out resemblances between the tides of Franz Josef Archipelago and those of more accessible portions of the earth. In some of the most prominent characteristics the tides of Cape Flora resemble those of Melbourne, Australia, while those of Teplitz Bay are in a similar way like those of Sitka, Alaska. It happens that both Melbourne and Sitka are in the Pacific Ocean, although widely separated, and some one might hastily conclude that the tides of Franz Josef Archipelago are derived from that ocean. But a very little consideration of the narrow and comparatively shallow opening at Bering Strait will convince one of the extreme improbability of the Arctic tides being derived from the Pacific Ocean to any appreciable extent.

The tide wave appears to reach Franz Josef Archipelago from the Atlantic Ocean by two channels, one between Norway and Spitzbergen and the other between Spitzbergen and Greenland. The latter channel being much deeper than the former, the tide wave from the Greenland channel reaches Teplitz Bay, in the northern portion of Franz Josef Archipelago, nearly four hours before the tide wave from the Norway channel arrives at Cape Flora, in the southern portion of the archipelago. The indications are (see maps 23, 25, and 26 of Appendix 5, Report of the United States Coast and Geodetic Survey for 1904) that the tide wave advances southerly through the channels between the various islands of the group and along their eastern coasts until it meets the southern wave a few miles east of Cape Flora, although no observations have been made to establish this statement. 


\title{
SECTION E
}

\section{ASTRONOMICAL OBSERVATIONS}

\author{
AND \\ REDUCTIONS
}

BY

\author{
W. J. PETERS \\ In Charge of Scientific Work of the Expedition
}

RUSSELL W. PORTER

First Assistant Scientist of the Expedition

AND

J. A. FLEMING

Department Terrestrial Magnetism, Carnegie Institution of Washington 


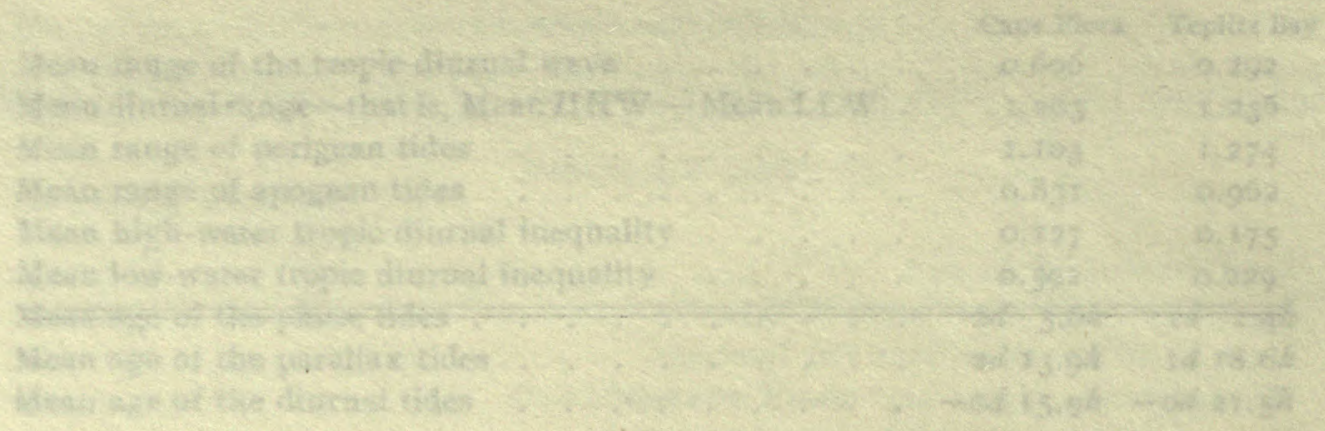

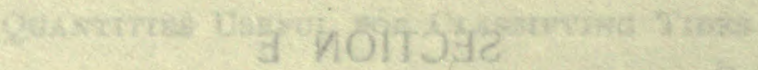

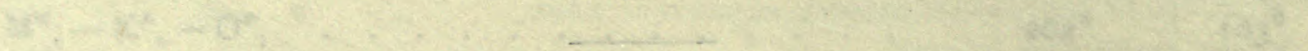

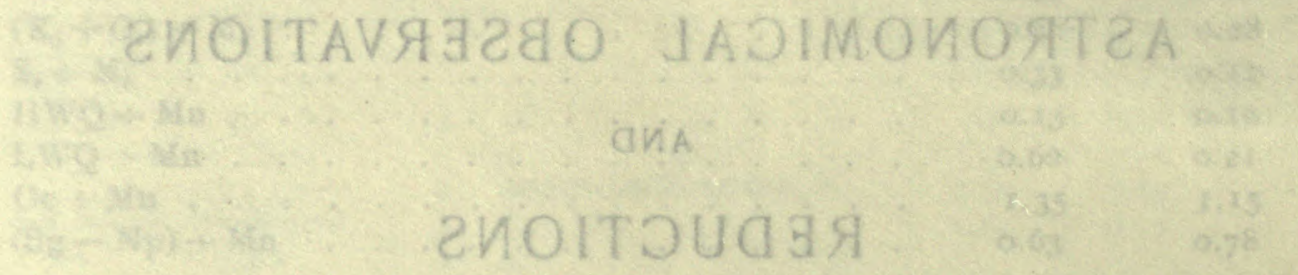

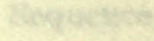

Diknestoms of rise

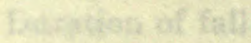

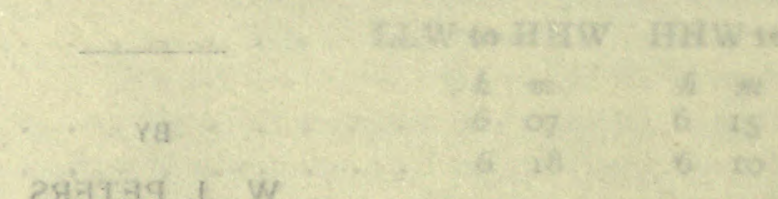

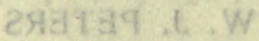

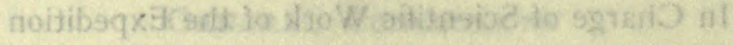

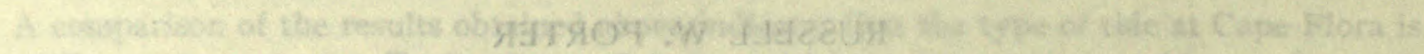

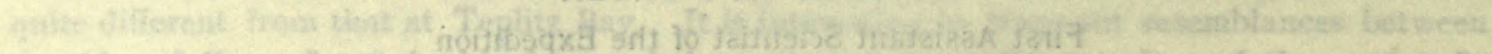

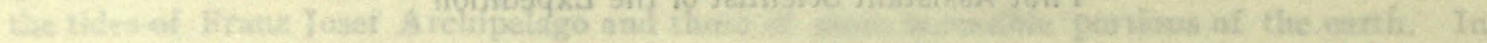

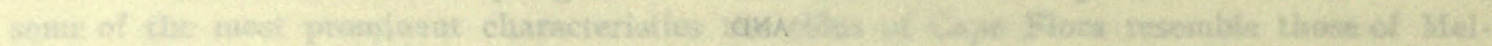

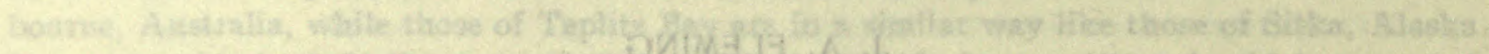

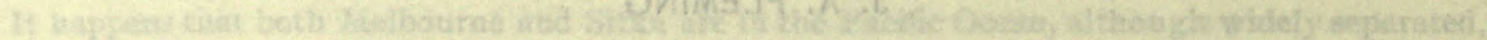

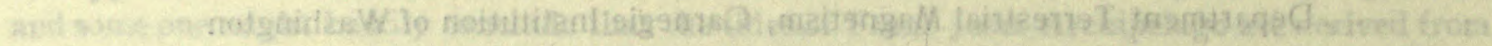

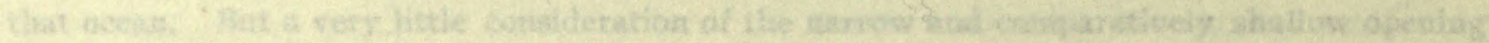
3to

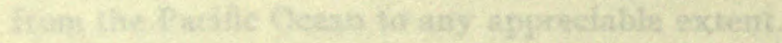

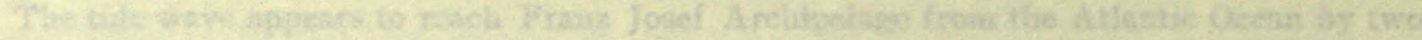
Chestre"

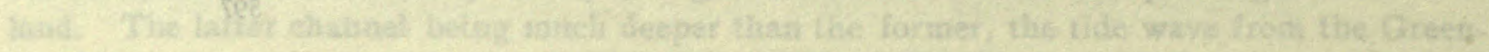

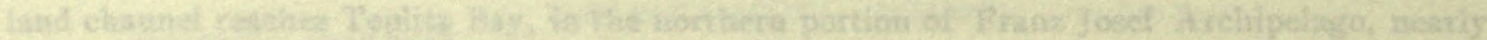

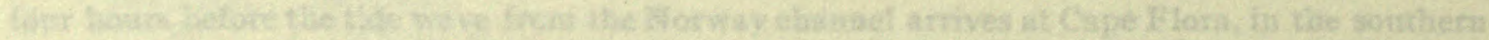

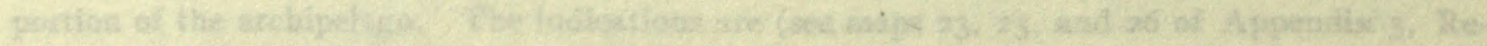

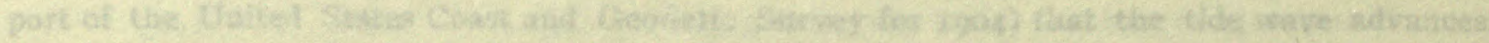

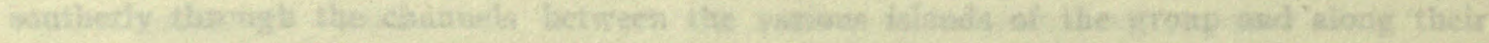

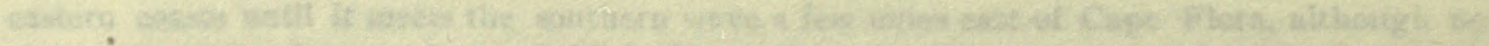

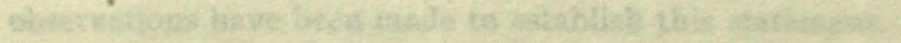




\section{CONTENTS}

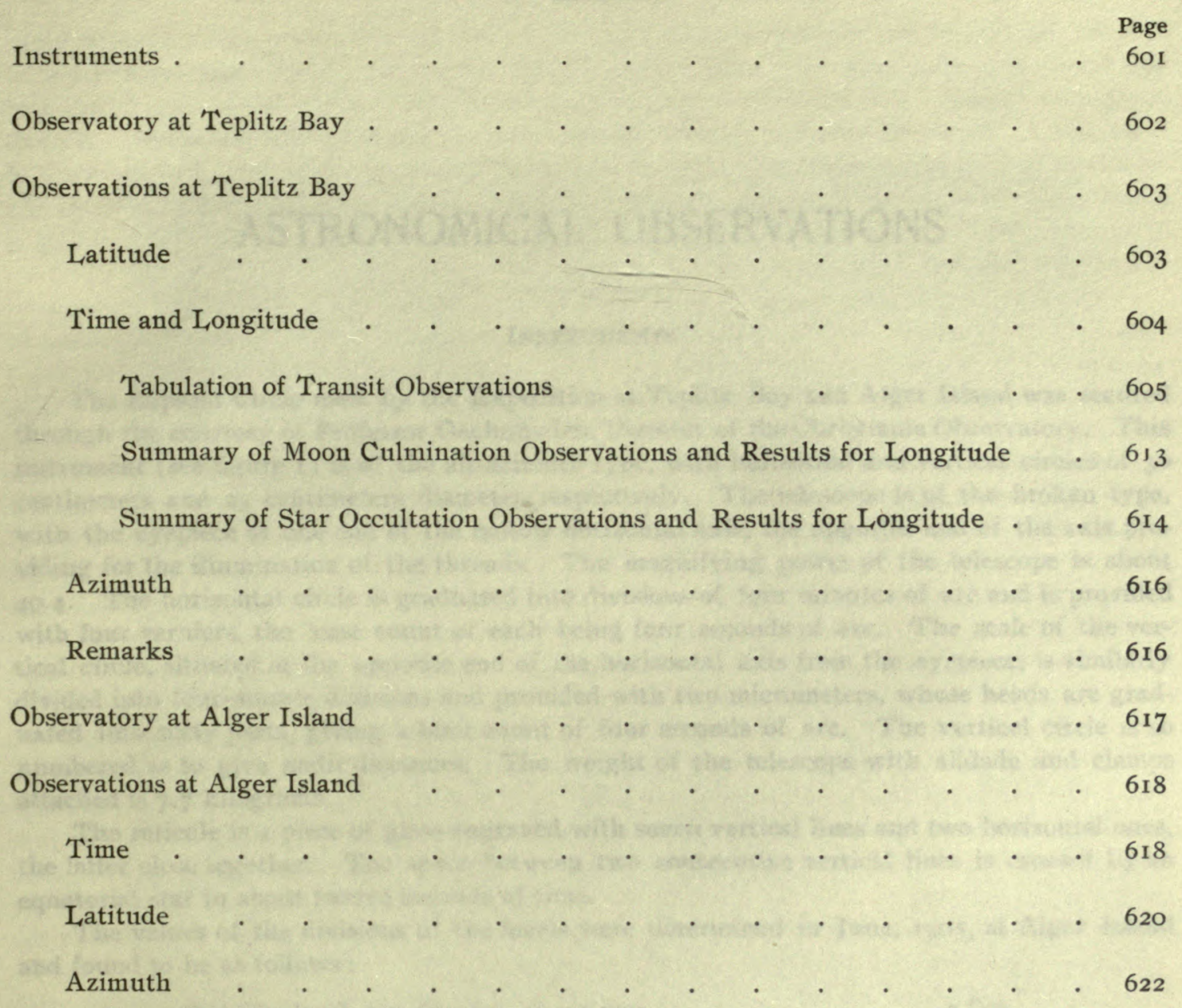

\section{ILLUSTRATIONS}

View of

I View of Interior of Observatory at Teplitz Bay, Showing Repsold Circle . . . 602

2 Io-Centimeter Alt-Azimuth Instrument . . . . . . . . . 603

3 View Showing Exterior of Observatory at Teplitz Bay . . . . . . 604 


\section{exkiankos}

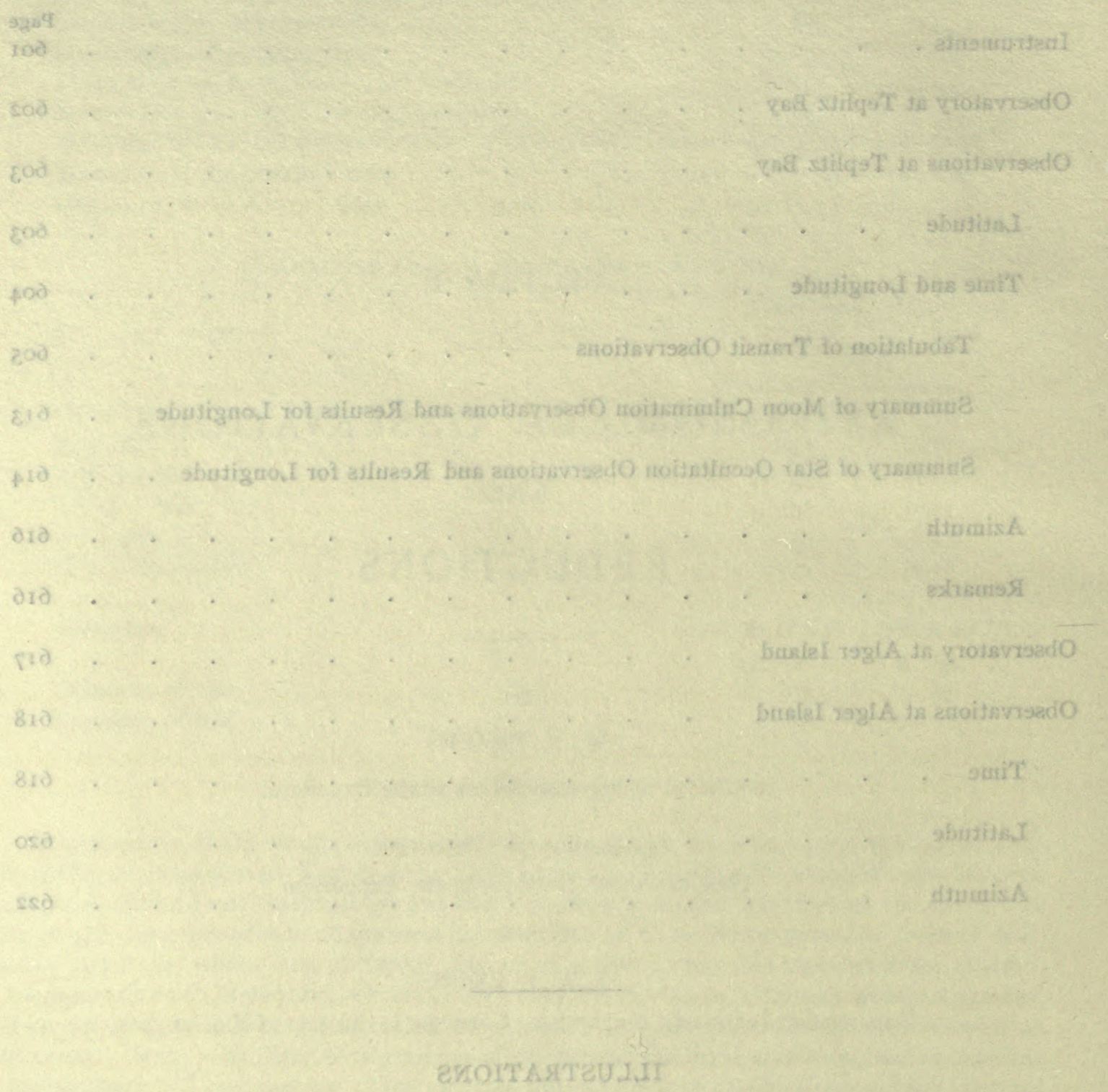

EИOITAXTEU,III

शुac stieoquO

gexis bloecs

s.or

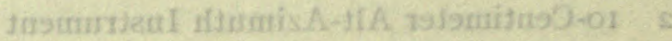

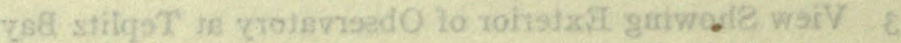




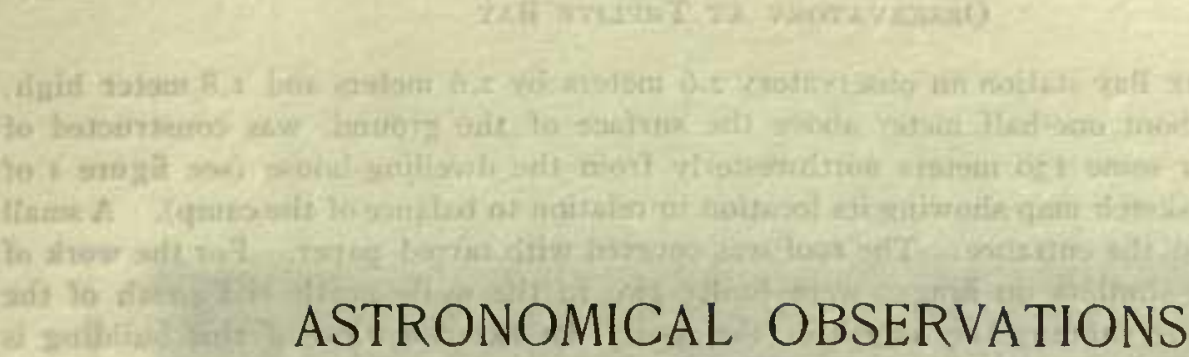

\section{INSTRUMENTS}

The Repsold Circle used by the Expedition at Teplitz Bay and Alger Island was secured through the conrtesy of Professor Geelnu1yden, Director of the Christiania Observatory. This instrument (see figure $\mathrm{I}$ ) is of the alt-azimuth type, with lorizontal and vertical circles of 30 centimeters and 25 centimeters diameter respectively. The telescope is of the broken type, with the eyepiece at one end of the hollow horizontal axis, the opposite end of the axis providing for the illumination of the threads. The magnifying power of the telescope is about 40.4. The horizontal circle is graduated into divisions of four minutes of arc and is provided with four veruiers, the least count of each being four seconds of arc. The scale of the vertical circle, situated at the opposite end of the horizontal axis from the eyepiece, is similarly divided into four-miutute divisions and provided with two micrometers, whose heads are graduated into sixty parts, giving a least count of four seconds of arc. The vertical circle is so numbered as to give nadir distances. The weight of the telescope with alidade and clamps attached is 7.7 kilograms.

The reticule is a piece of glass engraved with seven vertical lines and two lorizontal ones, the latter close together. The space between two consecutive vertical lines is crossed by an equatorial star in about twelve seconds of time.

The values of the divisions of the levels were determined in June, 1905, at Alger Island and found to be as follows:

Striding level, per division of $2.0 \mathrm{~mm}$. . . . . . . . 2." 98

Alidade level, per division of $1.8 \mathrm{~mm}$. . . . . . . 2." 16

Some of the secondary astronomical work was executed with two small ro-centimeter theodolites made by C. L. Berger and Sons, both vertical and horizontal circles being read to single minutes of arc by two verniers eacli. These were of the usual type of instrument (sec figure 2) made for finer grade field work by this firm, with the addition of several features to render them more convenient for use in extremely low temperatures. All of the tangent and leveling screw milled lieads had small ivory buttons set in the onter circumference about 3 millineters in diameter and projecting about the same distance beyond the heads. The compass needle was mounted in a closed tube attached to the under side of the telescope, the glass ends of this tube being engraved with collimating lines. These instruments, with cases, are very light and were found extremely useful and convenient, particularly so in the field work of the Expedition.

Only two chronometers were carricd; one, a mean time, and the other, a siderial time, both by Negus. 


\section{OBSERVATORY AT TEPLITZ BAY}

At the Teplitz. Bay station an observatory 2.6 meters by 2.6 meters and 1.8 meter high, the floor being about one-half meter above the surface of the ground, was constructed of undressed lumber some 130 meters northwesterly from the dwelling-house (see figure $I$ of "Section A" for sketch map showing its location in relation to balance of the camp). A small vestibulle protected the entrance. The roof was covered with tarred paper. For the work of observation three shitters on hinges were built; two in the walls 110 rth and south of the instrument and one running the length of the roof. An exterior view of this building is shown in figure 3 (also in figure 1 of "Section C").

The pier for the Repsold Circle was built upon a large basalt boulder in an ontcrop of rock and was composed of bricks laid in.clear cement and surmounted by a capstone some to centimeters thick and 60 centimeters square. The pier was approximately 1.3 meter high and 0.5 meter square. The elevation above mean sea level of the top of the capstone is 18.739 meters.

A collimator, used also as an approximate ineridian mark through the dark period, was placed in the meridian north of the instrument and abont 4.6 meters from it. This collimator consisted of one of the small theodolites by C. L. Berger and Sons mounted on a rock and cement pier. In the common focus of object glass and eyepiece were two closely spaced vertical threads. This space was bisected by the middle thread of the Repsold Circle at the beginning and middle of each time set. Illumination of the collimator was effected by a bull's-eye lantern placed just back of the eyepiece. The collimator was protected by a wood box resting on the basalt ledge, this box -being covered, when not in use, by a cloth bag to prevent the entry of the fine snow which was almost constantly driving during the winter.

On the return of dayliglit (spring of 1904) a meridian mark was set up on the brow of Cape Auk 6,640 meters south of the observatory. A bull's-eye lanterin at the cape was lined into the meridian in the evening by a prearranged system of rocket signals. During the first year this mark consisted of eight empty wood boxes set one on top of another and guyed with wire; snow was dug away to a rock foundation and a inilk tin embedded there to mark the point. Later the boxes were replaced by a heavy plank. These marks appeared throngh the telescope of the Repsold Circle silhouetted against the sky.

Chronometer time was obtained while observing by means of a sounder connected with the break-circuit siderial chronometer at the dwelling; a hack-watch-gave the hour and minute, while a stop-ivatch served to identify the second at any tine. Both mean and siderial chronometers were kept at the dwelling in an insulated box, and were wound and compared regularly each morning after breakfast.

The illumination of the Repsold Circle in the usual manner throngh the axis of the telescope was found to be defective. "Accordingly a reflector, similar to those used in small theodolites; was made of zinc; this received the rays of light from a lamp placed near the wall of the observatory and threw them into the tube of the telescope. For reversing the telescope in the wyes in low temperatures a lifting device consisting of a two-pronged hook at the end of a phosphor bronze wire operating over pulleys was resorted to. By this means it conld be suspended while the frost (condensation from the breath) conld be removed from the wyes and pivots.

When the instrument was not in use it was covered, first, with a cone of cloth suspended from the roof, and, later, by a box of heavy paper inverted over the instrument and resting on the capstone. 

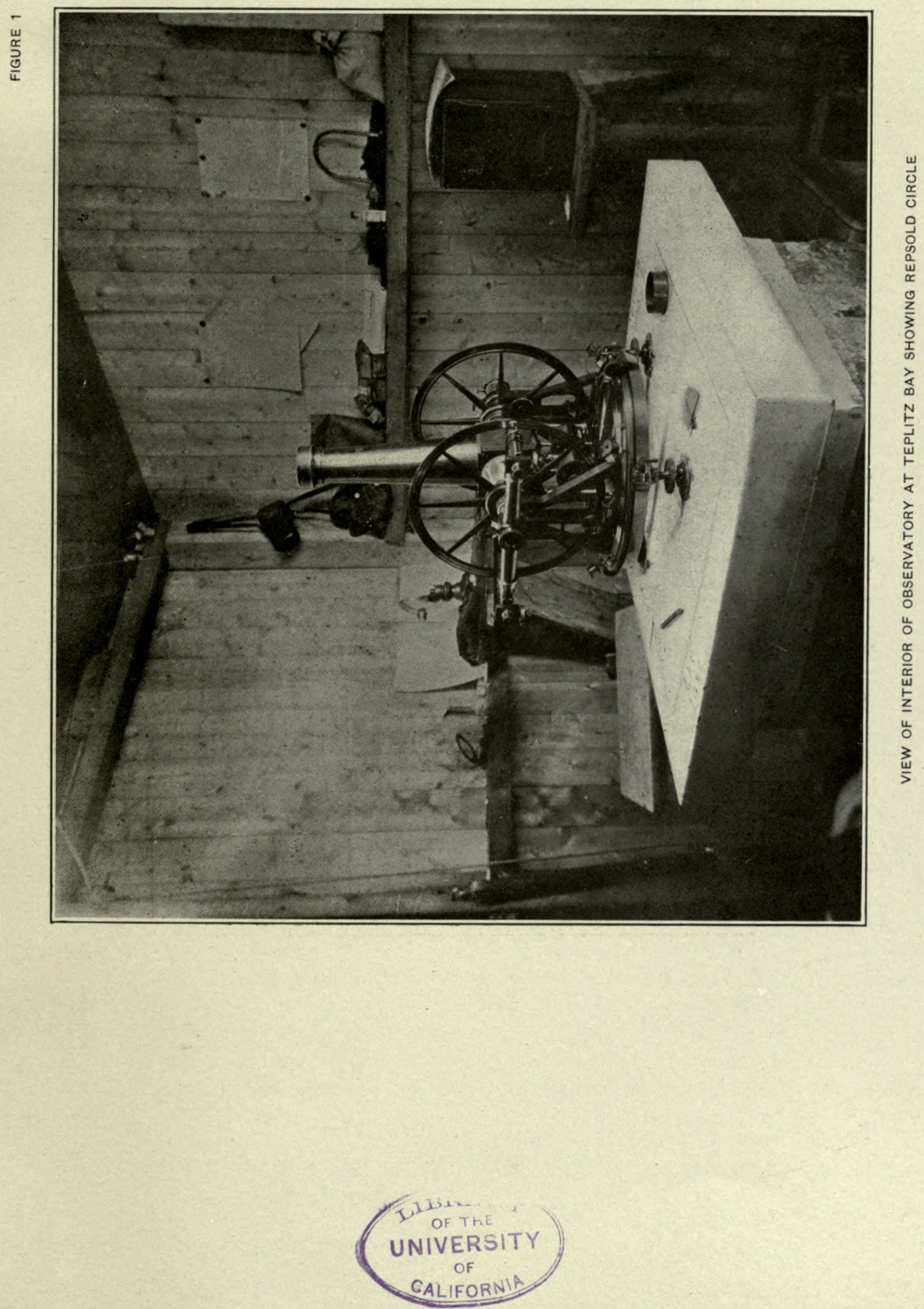
FIGURE 2

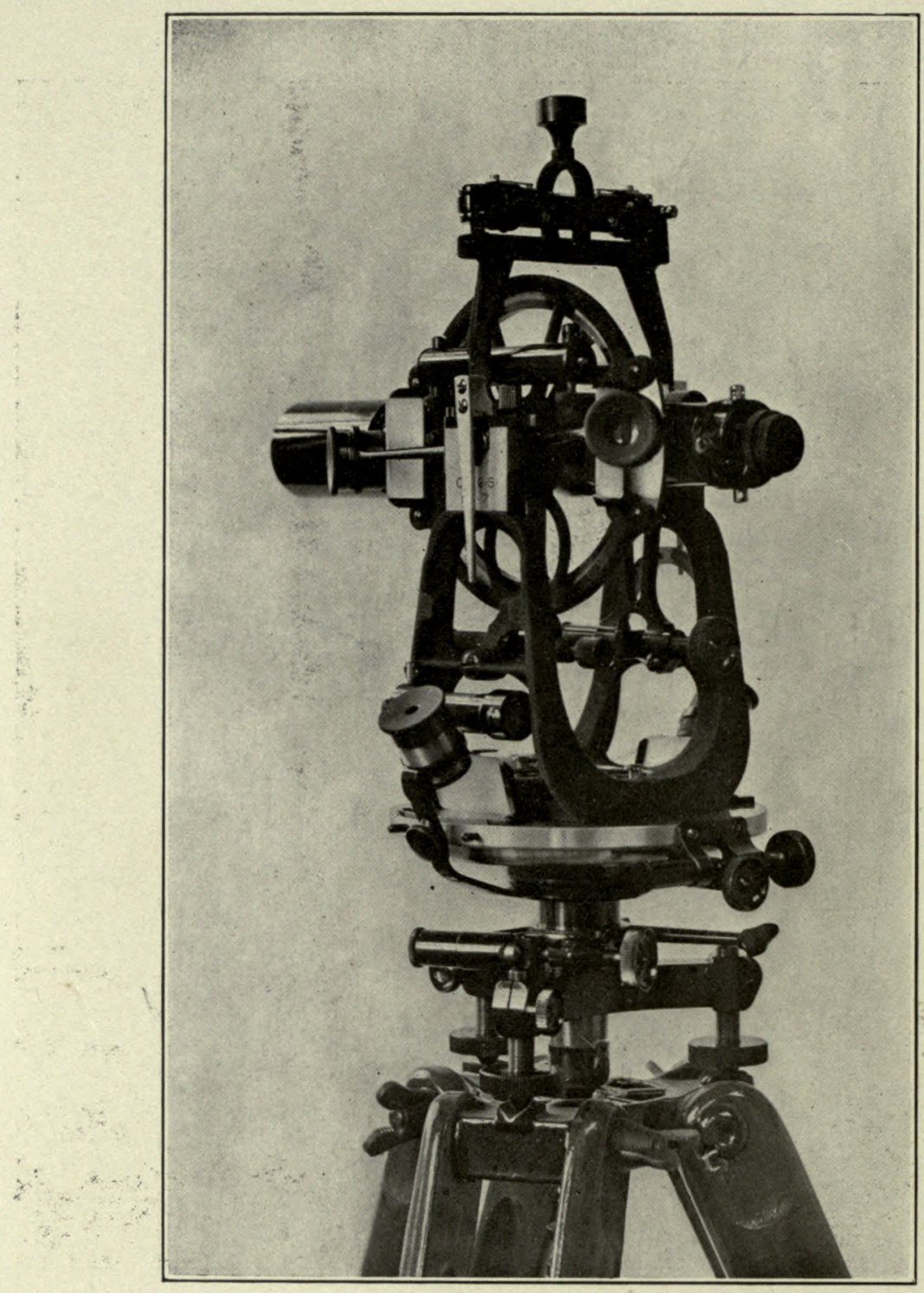

10-CENTIMETER ALT-AZIMUTH INSTRUMENT 


\section{OBSERVATIONS AT TEPIITZ BAY OBSERVATORY}

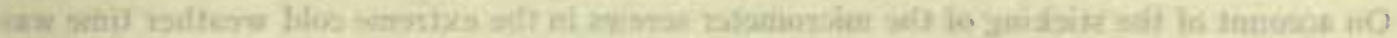 \\ LATITUDE}

Latitude was determined by observing star altitudes in the meridian. These observations and results are stummarized in the following tabulation :

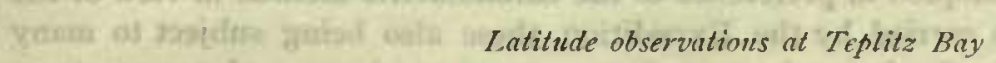

\begin{tabular}{|c|c|c|c|c|c|c|c|c|}
\hline $\begin{array}{l}\text { Iocal } \\
\text { mean date }\end{array}$ & $\begin{array}{c}\text { Siderial } \\
\text { time }\end{array}$ & Star & Circle & $\begin{array}{l}\text { Mean of verlical } \\
\text { circle readings } \\
\text { corrected for level }\end{array}$ & $\begin{array}{l}\text { Barom- } \\
\text { eter }\end{array}$ & $\begin{array}{l}\text { Attached } \\
\text { ther- } \\
\text { mometer }\end{array}$ & $\begin{array}{c}\text { Outside } \\
\text { ther- } \\
\text { mometer }\end{array}$ & $\begin{array}{l}\text { Resulting lati. } \\
\text { tude }\end{array}$ \\
\hline thith Th & Thing-nit & 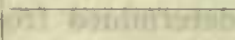 & जाता & 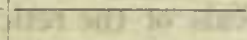 & Tा1त 7 & Bowntry & min & 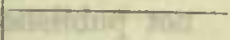 \\
\hline 1905 & $h \quad m s$ & (it) $x+[1]=6$ & ik uto & $\left.\ln x^{\circ}\right)^{\prime}, 11$ & $I n$ & ${ }^{\circ} \mathrm{F}$ & ${ }^{\circ} F$ & 0,11 \\
\hline \multirow[t]{19}{*}{ April 11.38} & 1242 or .5 & $\varepsilon$ Ursae Majoris & $1 \dot{1}$ & $15438 \quad 12.9$ & 30.805 & +54.5 & +4.0 & 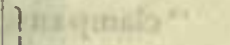 \\
\hline & 4722.0 & " & Fis & I54 $3^{8} \quad 35 \cdot 3$ & $\ldots \ldots \ldots$ & $\ldots \ldots \ldots$ & +4.0 & \\
\hline & 5432.0 & " " & W & 2051557.0 & $\ldots$ & .. . & +4.0 & $814734.4 \mathrm{~N}$ \\
\hline & 6018.0 & 1" & IV & $2051624 . I$ & $\cdots$ & $\ldots \ldots$ & +7.0 & \\
\hline & 132023.0 & Polaris & W & $170 \quad 3254.6$ & $\ldots \ldots$ & . $\ldots$. & +7.0 & \\
\hline & 2533.0 & " & W & $170 \quad 3254.4$ & ........ & $\ldots \ldots$ & +7.0 & \\
\hline & 2916.0 & 12: & W & $170 \quad 3253.8$ & ........ & $\ldots \ldots$ & +60 & $8,47,5=\mathrm{N}$ \\
\hline & 3936.5 & $4="$ & $\mathbf{E}$ & $\begin{array}{lll}189 & 2 \mathrm{I} & 13.4\end{array}$ & ....... & $\ldots \ldots$ & +6.0 & $014735.4 \mathrm{~N}$ \\
\hline & $45 \quad 14.0$ & wa & $\mathbf{E}$ & $\begin{array}{llll}\text { I } & 29 & 2 \text { I } & 0\end{array}$ & & 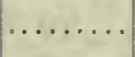 & +6.0 & \\
\hline & 4923.0 & any & I & 1892107.5 & & $\ldots \ldots \ldots$ & +6.0 & j \\
\hline & 135841.0 & a Bootis & $\mathbf{E}$ & II 75126.6 & $\ldots \ldots$ & . . & +6.0 & ! \\
\hline & 1403400 & a & $\mathrm{F}$ & I1 $751 \quad 39.7$ & & $\ldots \ldots$ & +6.0 & \\
\hline & 1427.0 & “ & W & $\begin{array}{llll}242 & 02 & 19.9\end{array}$ & 30.770 & +45.0 & +6.0 & $814733.3 \mathrm{~N}$ \\
\hline & 1954.0 & “ & W & $\begin{array}{lll}242 & 02 & 31.7\end{array}$ & & & +4.0 & j \\
\hline & $144^{8} 33 \circ$ & $\beta$ Ursie Minotis & w & $\begin{array}{lll}{ }_{1 S} 7 & 12 & 08.9\end{array}$ & ... & $\ldots \ldots$ & +4.0 & 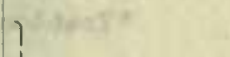 \\
\hline & 5227.0 & “ & W & $157 \quad 1205.6$ & ... & & +4.0 & Si \\
\hline & 5933.0 & $" ،$ & $\mathbf{E}$ & 1724133.5 & $\cdots \cdots$ & $\cdots \cdots$ & +4.0 & $514730.3 N$ \\
\hline & $6339 \cdot 5$ & " " & $\mathbf{E}$ & $172 \quad 4037 \cdot 7$ & 30.755 & +42.5 & +3.0 & \\
\hline & & & & Adopted mean va & & & & $814734.9 \mathrm{~N}$ \\
\hline
\end{tabular}

The mean value for latitude resulting agrees closely with that determined by the Italian Expedition. The Italian astronomical station was not relocated. Direct comparison of values may, however, be made by reference to the magnetic station of 1899 to 1900 which is 136.42 meters, or 4.4 seconds of arc, south of the Ziegler Expedition astronomical observatory and I.I second of arc south of the astronomical station observed at by the Italian party. Their finally adopted latitude was $81^{\circ} 47^{\prime} 26 .^{\prime \prime} 2$, * which referred to the station of 1905 would be $81^{\circ} 47^{\prime}$ $29 . " 5$; the two determinatious thus agree within less than six seconds of arc, the mean of the two being $8 r^{\circ} 47^{\prime} 32 . " 2$.

* Osservazioni scientifiche esequite durante la spedizione Polare di S, A. R. Luigi Aluedeo di Savoia, Duca degli Abruzzi. Milan, Igo3. Pp. Io5 and 447. 


\section{TIME AND LONGITUDE}

On account of the sticking of the micrometer screws in the extreme cold weather time was determined by star transits in preference to measuring star altitudes. These transits have been reduced by the usual method of least squares.* In connection with time observations, determinations of longitude were made during the winter of 1903 to 1904 by observing transits of the moon. This method was adopted in preference to the chronometric method in view of the small number of chronometers carried by the Expedition, these also being subject to many heavy shocks in navigating in the ice and exposed to very great clianges of temperature. Numerous other complete transit sets were observed and reduced, but in view of the fact that they have been used primarily in the cletermination of corrections simply to local time, they are not published. The equatorial intervals of the reticule as determined from observations and used in the reduction of the various transit observations are as follows, the signs applying for "clampeast":

\begin{tabular}{|c|c|c|c|c|c|}
\hline & $\begin{array}{c}\text { Period of } \\
\text { observation }\end{array}$ & $\begin{array}{c}\text { To } \\
\text { November } 2,1903\end{array}$ & $\begin{array}{l}\text { Noven ber } 3,1903 \text {, } \\
\text { to } \\
\text { January } 7,1904\end{array}$ & $\begin{array}{c}\text { January } 8, \text { I9 } \\
\text { to } \\
\text { February } 19,\end{array}$ & \\
\hline & Thread & $\begin{array}{c}s \\
-35 \cdot 378\end{array}$ & $\begin{array}{c}s \\
-35.778\end{array}$ & $\begin{array}{c}s \\
-35.267\end{array}$ & 7 \\
\hline & in & --23.638 & -23.865 & -23.504 & wis \\
\hline & III & $-\mathrm{II} .756$ & -11.904 & -11.862 & $2 \cdot 1$ \\
\hline & IV & -0.089 & +0.030 & -0.148 & a.) \\
\hline & V & $+I I .88 I$ & +11.895 & +11.821 & 9.4 \\
\hline & VI & +23.619 & +23.835 & +23.504 & \\
\hline $18 y_{1}$ & VII & +35.398 & $+35.7 S_{4}$ & +35.476 & \\
\hline
\end{tabular}

* Text-book of geodetic astronouny, by John F. Hayford. New York, 1898. 
FIGURE 3

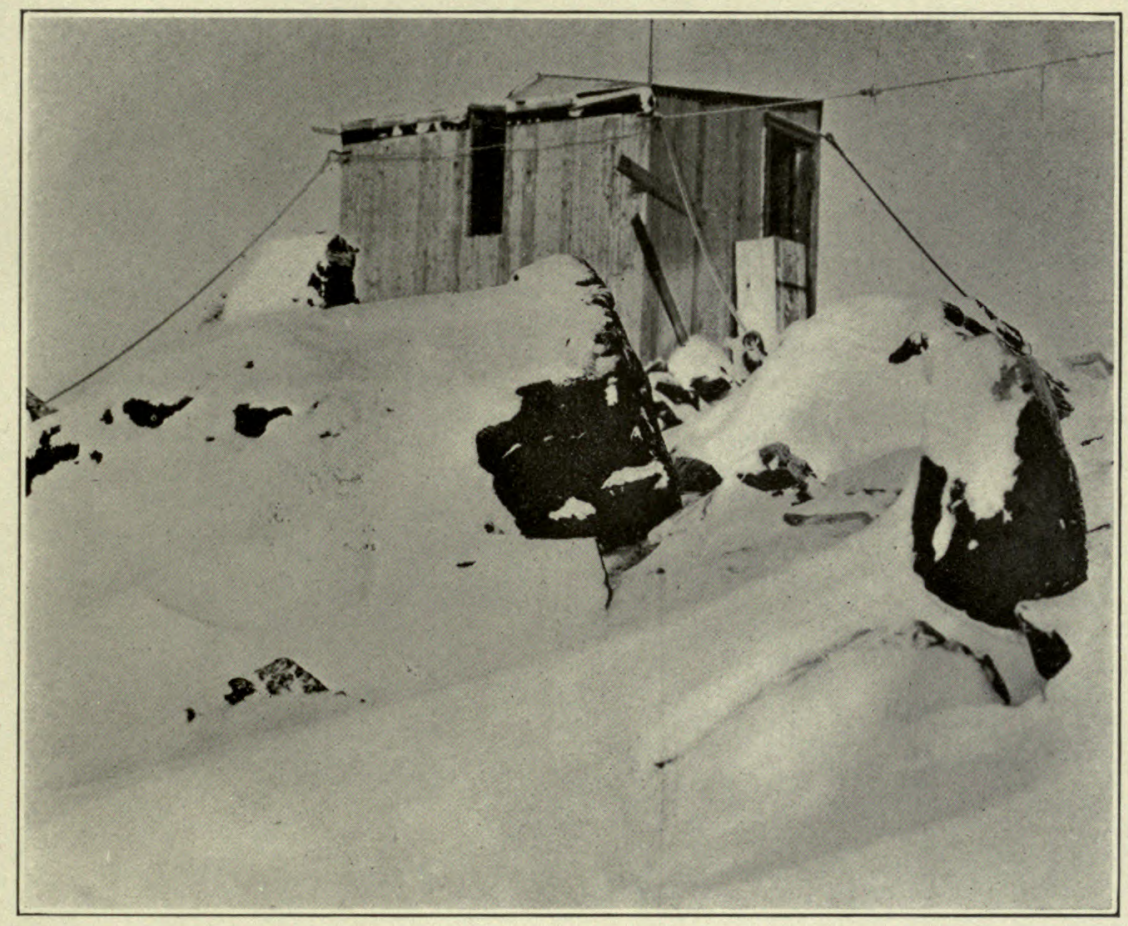

VIEW SHOWING EXTERIOR OF OBSERVATORY AT TEPLITZ BAY 

In the following tabulation of transit observations only those involving moon culminations are included :

Tabulation of transit observations at Teplitz Bay

November, 1903, to February, 1904

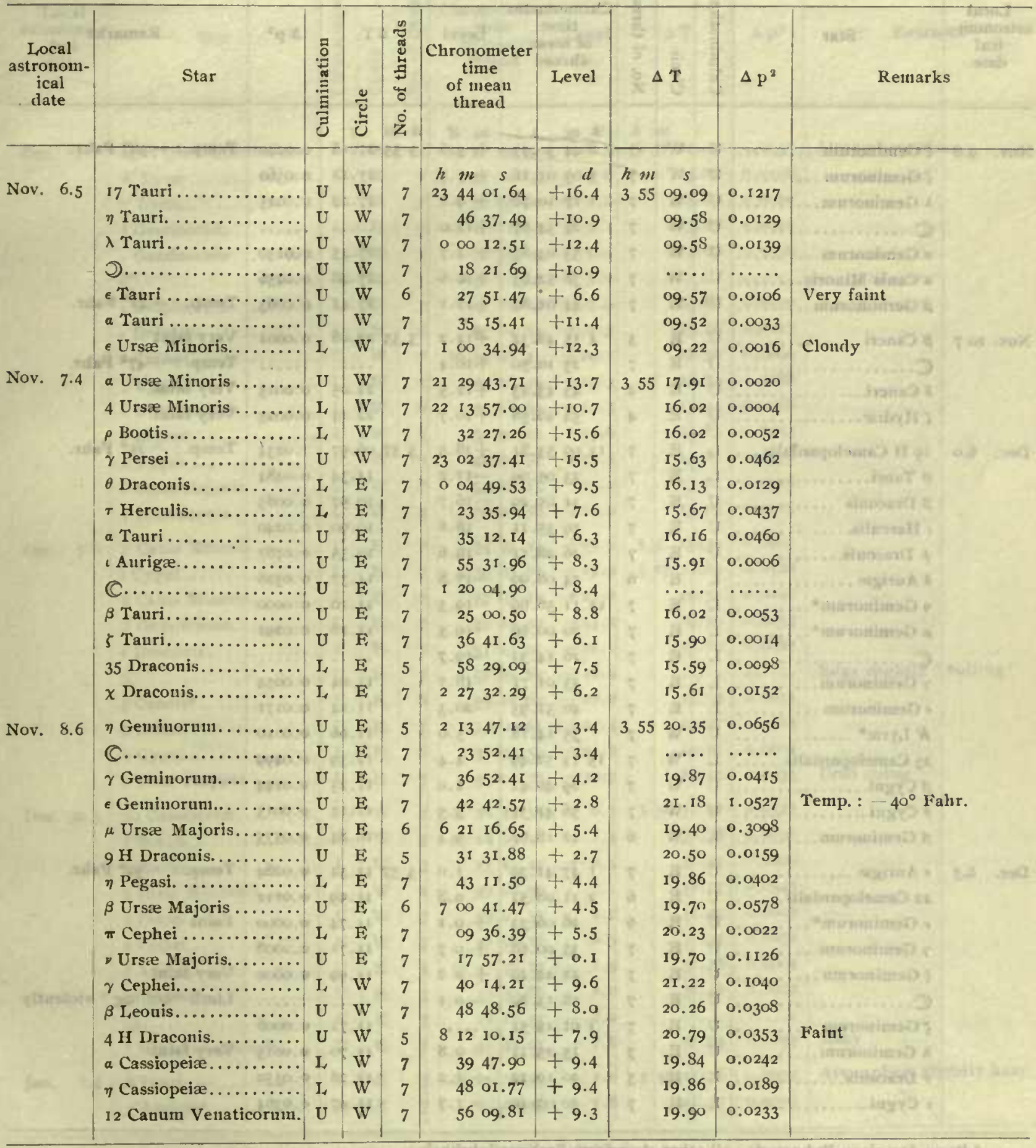


November, 1903, to February, I904

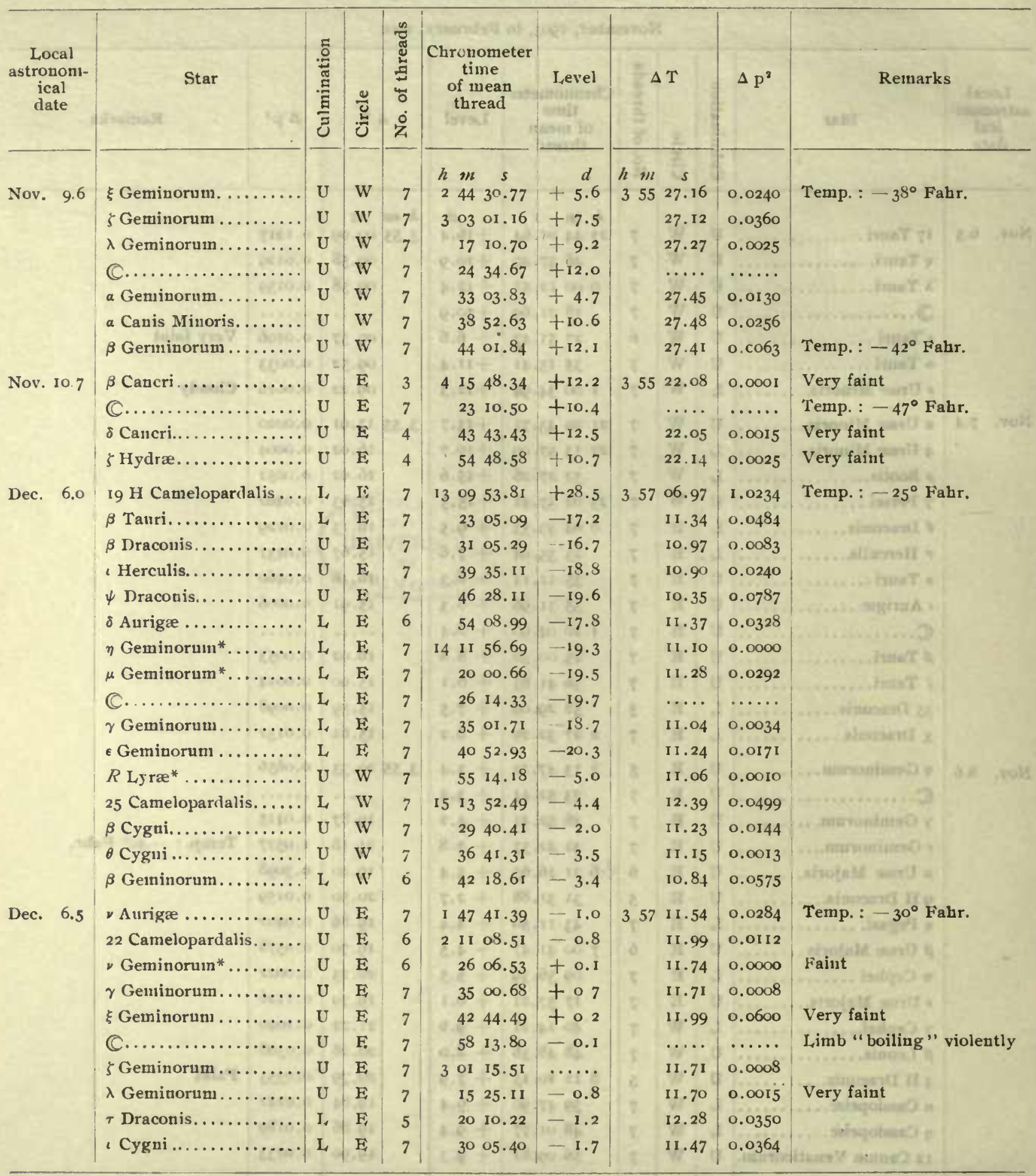

* From American Ephemeris; all other stars from Berliner Jahrbuch. 
Tabulation of transit abservations at Teplitz Bay-Continted

November, 1903, to February, 1904

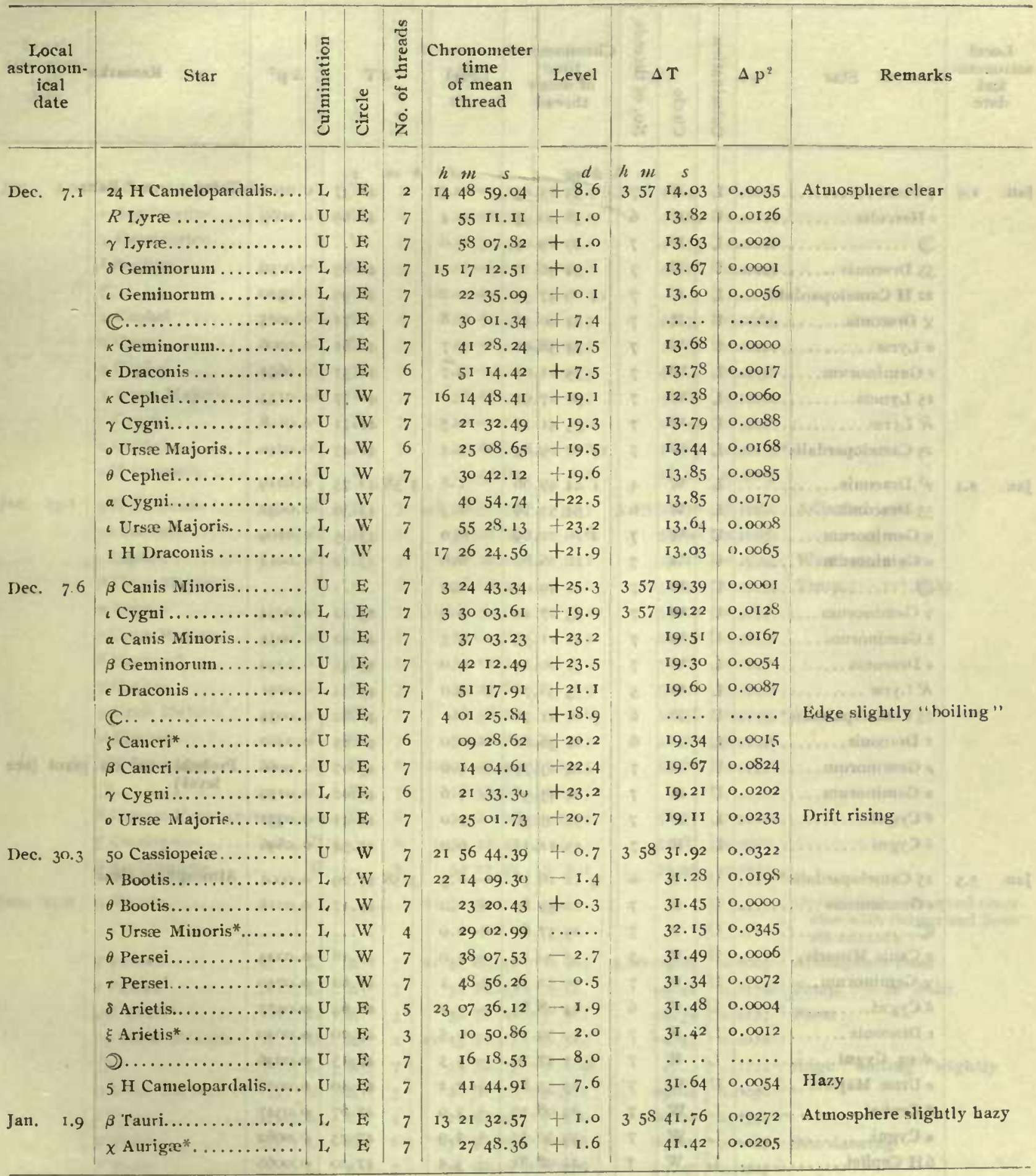

* From Anerican Ijphemeris ; all other stars from Berliner Jahrbuch. 
Tabulation of transit observations at Teplitz Bay-Continued

November, 1903, to February; 1904

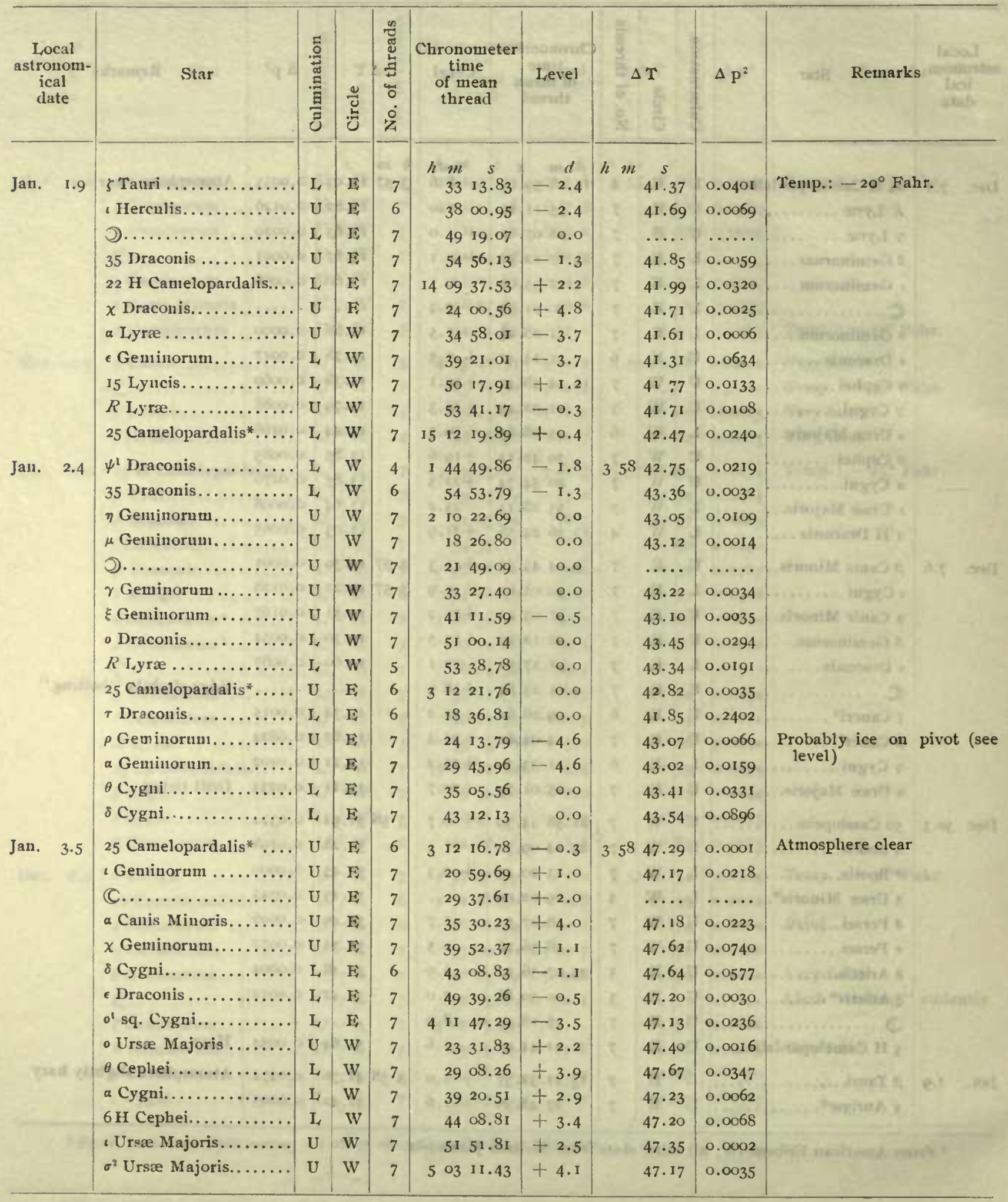

* From American Jiplıeneris ; all otler stars from Herliner Jalırbuclı. 
Tabulation of transit observations at Teplitz Bay-Contimued

November, 1903, to Febrnary, 1904

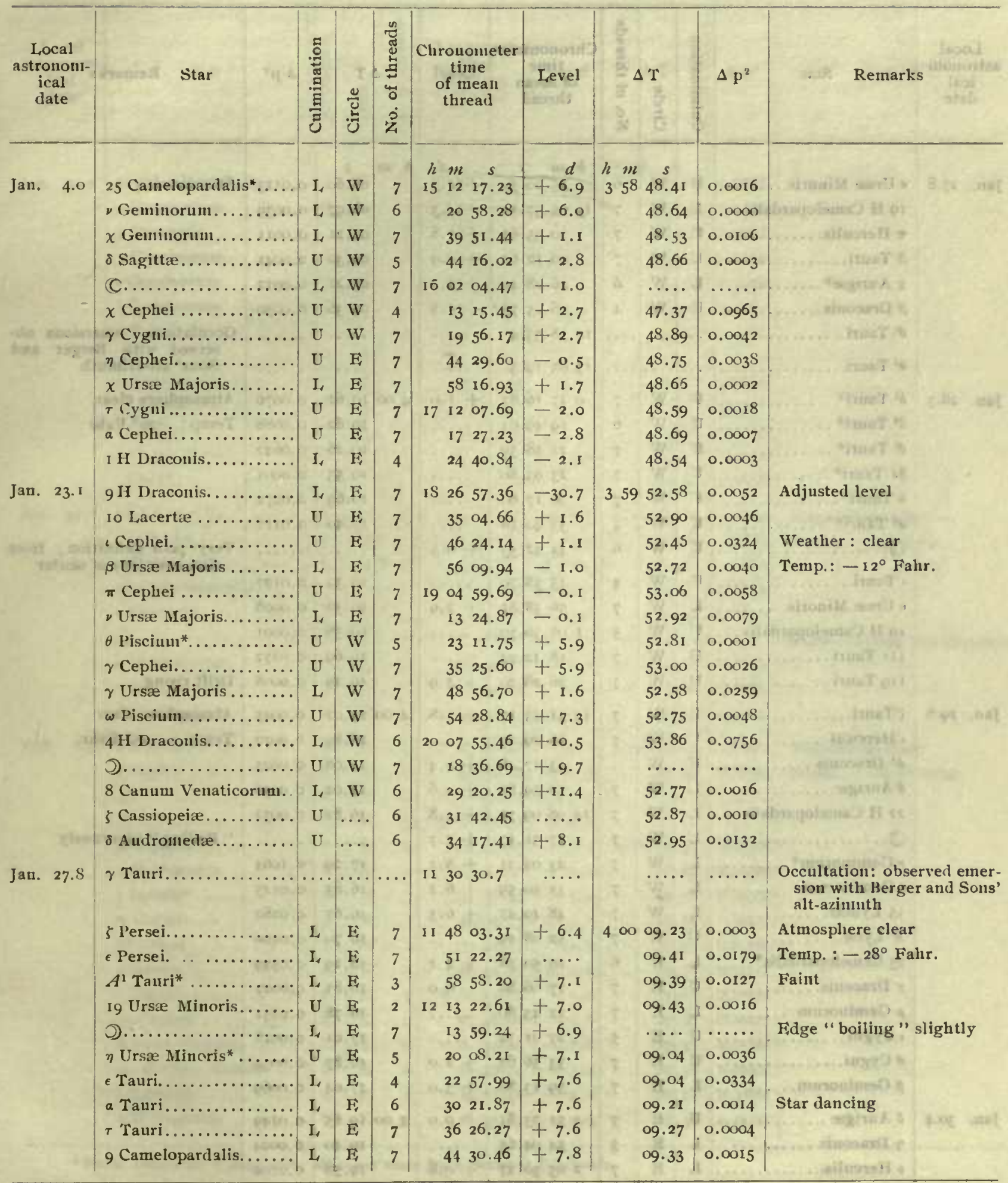

* From American Eplemeris; all other stars from lerliner Jahrhuch. 
Tabulation of transit observations at Teplitz Bay-Continued

November, I903, to February, 1904

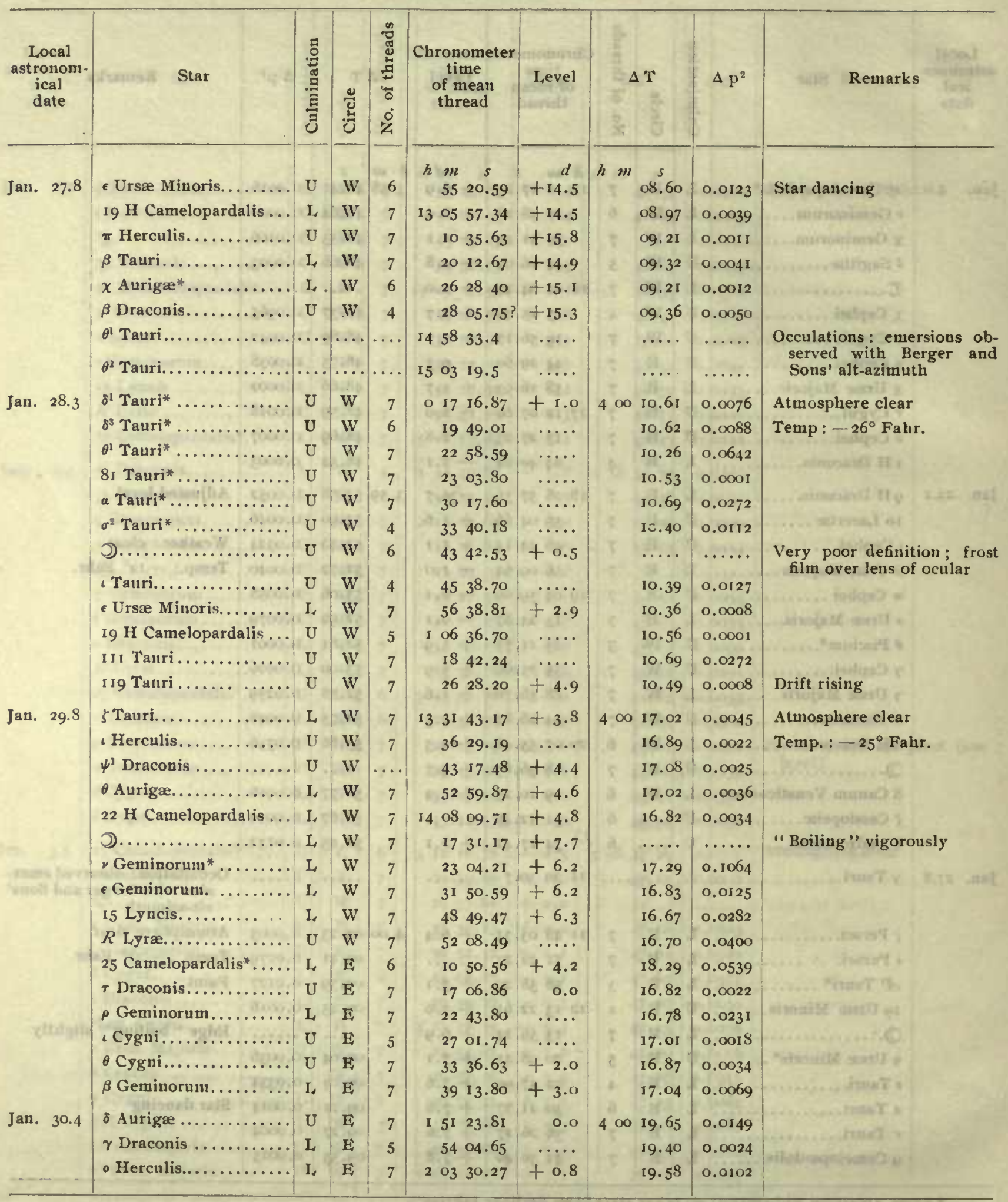

* From American Epphemeris ; all other stars from Berliner Jahrbuch. 
45 Tabulation of transit observations at Teplitz Bay-Continued

November, 1903, to February, I904

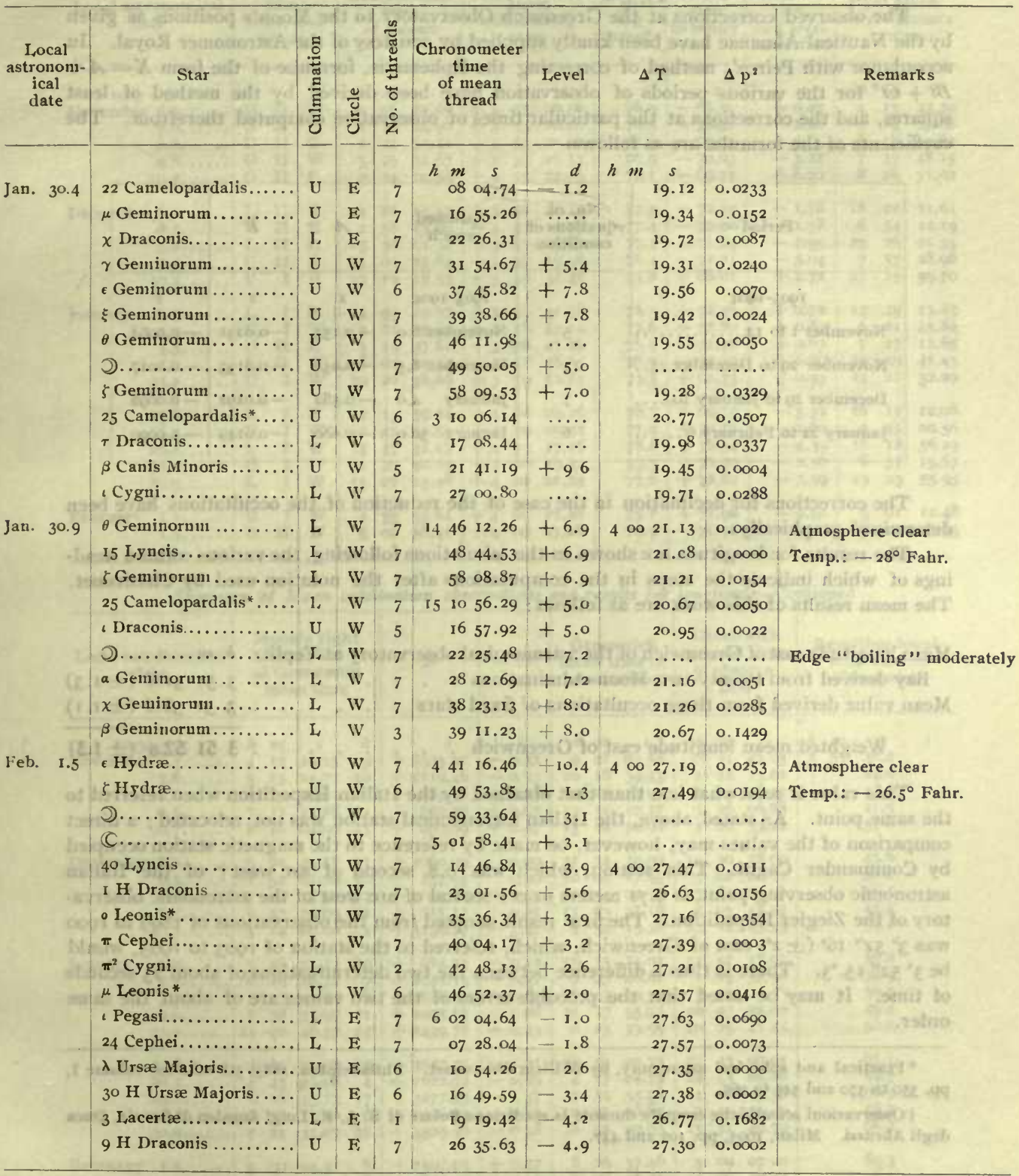

* From American Ephemeris ; all other stars from Berliner Jahrbuch. 
As will be noted from the above tabulation, twenty-two Moon culminations were observed, as also three occultations of fixed stars for the determination of longitude. These have been reduced by the methods developed by Clianvenet.*

The observed corrections at the Greenwich Observatory to the Moon's positions as given by the Nantical Almanac have been kindly supplied by courtesy of the Astronomer Royal. In accordance with Peirce's method of correcting the Ephemeris, formulæ of the form $X=A+$ $B t+C t^{2}$ for the various periods of observation have been derived by the method of least squares, and the corrections at the particular times of observation computed therefroin. The coefficients of the formulæ are as follows:

\begin{tabular}{|c|c|c|c|c|c|}
\hline 20 & करा & $x+y=0$ & & & 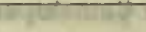 \\
\hline Period & $\begin{array}{l}\text { No. of } \\
\text { equations of } \\
\text { condition }\end{array}$ & $\begin{array}{l}\text { Assumed } \\
\text { epocli }\end{array}$ & $A$ & & $C$ \\
\hline Queser hase & $-1+2 \div$ & $210-1$ & 12 & & Exaci. \\
\hline $1903-1904$ & A. 70 & $1903-1904$ & $s$ & $s$ & $s$ \\
\hline November i to $14 \ldots \ldots \ldots \ldots$ & 5 & November 8.0 & -0.153 & -0.0231 & -0.0064 \\
\hline November 29 to Decemher $13 .$. & 11 & December 6.5 & -0.054 & -0.0193 & -0.0017 \\
\hline December 29 to Jaunary 10 & 7 & January 4.0 & $-0.5^{82}$ & -0.0192 & -0.0098 \\
\hline January 22 to February 6 & 16 & January 30.0 & -0.680 & -0.0149 & -0.0090 \\
\hline
\end{tabular}

The corrections for declination in the case of the reduction of the occultations have been determined graphically.

The results for longitude are shown in the tabulations following, the various column headings of which indicate the steps in the computations after the notation used by Chauvenet. The mean results of this work are as follows:

Mean longitude east of Greenwich of the astronomical observatory at Teplitz. $h m s \quad s$

Bay derived from twenty-two Moon culminations . . . . . . . 35 r 51.8 ( $\pm \mathrm{r} .3$ ) Mean value derived from three occultations of fixed stars . . . . . $35^{1} 58.6( \pm 1.1)$

Weighted mean longitude east of Greenwich . . . . . . . $35152.6( \pm 1.3)$

This value is somewhat less than that obtained by the Italian Expedition when referred to the sane point. As stated above, the Italian astronomical station was not relocated; a direct comparison of the values may, however, be made by reference to the magnetic station occupied by Commander Cagni. This station, which was 0.8 second of arc west $\dagger$ of the Italian astronomic observing point, is 6.71 meters or 0.1 second of arc west of the astronomic observatory of the Ziegler Expedition. The final result adopted from the observations of 1899 to 1900 was $3^{\mathrm{b}} 5^{\mathrm{m}^{\mathrm{m}}} 16^{\mathrm{a}}\left( \pm 2^{\mathrm{*}}\right.$ ) east of Greenwich, which, referred to the station of 1903 to 1905 , would be $3^{\mathrm{h}} 52^{\mathrm{m}} 15 \cdot^{\circ} 3$. There is thus a difference between the two determinations of about 23 seconds of time. It may be noted that the probable errors of the two values are of about the same order.

* Practical and spherical astronouy, by William Chauvenet. Philadelphia, 1885, 5th edition, volume I, pp. 350 to 370 and 549 to 565 .

† Osservazioni scientificlie esequite durante la spedizione Polare di S. A. R. Luigi Amedeo di Savoia, Duca degli Abruzzi. Milan, 1903, pp. Io5 and 447. 
Summary of Moon culmination observalions and results for longitude

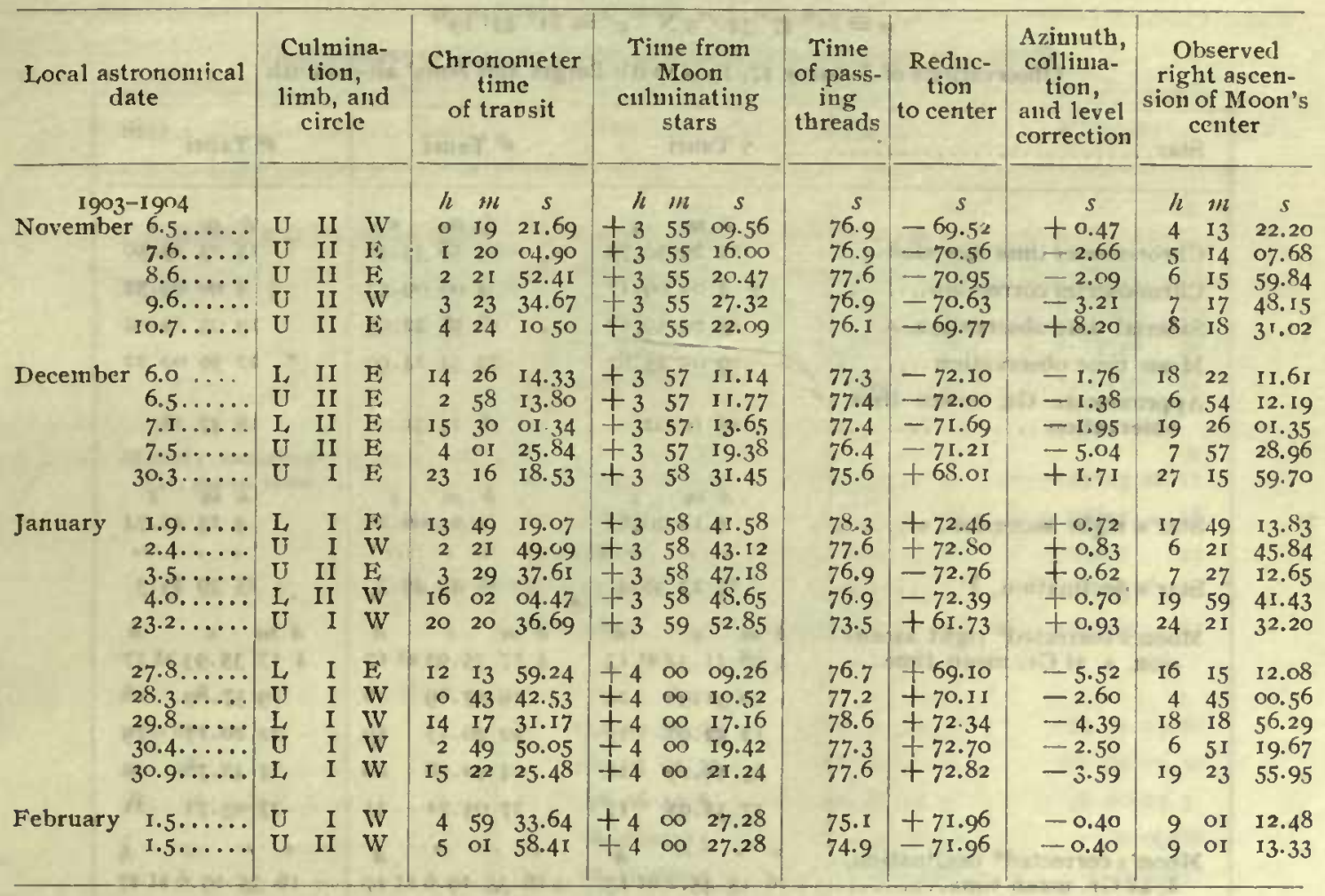

Summary of Moon culmination obscrvations and results for longtitude-Continued

\begin{tabular}{|c|c|c|c|c|c|c|c|c|c|c|c|c|c|c|c|c|}
\hline \multirow{3}{*}{\multicolumn{2}{|c|}{$\begin{array}{c}\begin{array}{c}\text { Local astronomical } \\
\text { date }\end{array} \\
\begin{array}{c}1903-1904 \\
\text { November } 6.5 \ldots . . . .\end{array}\end{array}$}} & \multicolumn{4}{|c|}{$\begin{array}{l}\text { Moon's right } \\
\text { ascension } \\
\text { at Greenwich } \\
\text { mean time }\end{array}$} & \multirow{2}{*}{$\frac{x^{\prime}}{s}$} & \multirow{2}{*}{$\frac{-x^{\prime \prime}}{s}$} & \multicolumn{3}{|c|}{$\begin{array}{l}\text { Greenwich } \\
\text { 111ean time of } \\
\text { observation }\end{array}$} & \multicolumn{3}{|c|}{$\begin{array}{c}\text { Greenwich } \\
\text { siderial time } \\
\text { of observa- } \\
\text { tion }\end{array}$} & \multicolumn{3}{|c|}{$\begin{array}{l}\text { Resulting longi- } \\
\text { tude east of } \\
\text { Greenwich }\end{array}$} \\
\hline & & $h$ & $m$ & $s$ & $h$ & & & $h$ & $m$ & $s$ & $h$ & & $s$ & $h m$ & $s$ & $s$ \\
\hline & & 4 & 12 & 29.25 & 9 & $+r 323.91$ & -0.33 & 9 & 22 & $03.5^{8}$ & 0 & & 42.03 & $35 \mathrm{I}$ & 40.2 & \\
\hline \multirow{4}{*}{ Noveniber } & $7.6 \ldots$ & & 13 & $21.7 \mathrm{I}$ & 10 & +1119.46 & -0.13 & 10 & 18 & 39.33 & I & 22 & 23.62 & & 44.0 & \\
\hline & & 6 & 15 & 19.01 & I I & +985.96 & +0.01 & II & 16 & 25.97 & 2 & 24 & 16.32 & & $43 \cdot 5$ & \\
\hline & $9.6 \ldots \ldots$ & 7 & 17 & 13.28 & 12 & +850.20 & +0.07 & 12 & I 4 & 10.27 & 3 & 26 & 06.68 & & $4 \mathrm{I} .5$ & \\
\hline & I0.7.... & 8 & 18 & 04.94 & 13 & +650.89 & +0.07 & I3 & Io & 50.96 & 4 & & 53.22 & & $37 \mathrm{~S}$ & \\
\hline \multirow[t]{5}{*}{ December } & 6.0 & 6 & 20 & 43.18 & $2 \mathrm{I}$ & + 2069.09 & 0.00 & 21 & 34 & 29.09 & I4. & 30 & 28.00 & & 43.6 & \\
\hline & 6.5 . & 6 & 54 & 01.20 & I0 & $+\quad 257.95$ & 0.00 & IO & 04 & 17.95 & & 02 & 20 ol & & 52.2 & \\
\hline & $7.1 \ldots \ldots$ & 7 & 24 & 34.96 & 22 & +2044.24 & +0.53 & 22 & 34 & 04.77 & 15 & 34 & 10.00 & & 51.4 & \\
\hline & $7.5 \ldots \ldots$ & 7 & 57 & 19.99 & II & +215.17 & +0.01 & II & 03 & 35.18 & 4 & 05 & 43.54 & & 45.4 & \\
\hline & $30.3 \ldots \ldots$ & 3 & 14 & 00.00 & 4 & +3138.77 & -3.41 & 4 & 52 & I5. $3^{6}$ & 23 & 24 & 03.53 & & 56.2 & \\
\hline \multirow[t]{10}{*}{ January } & I.9. . . & 5 & 48 & 34.42 & I9 & +914.13 & -0.12 & 19 & 15 & I4.0I & I3 & 57 & 17.07 & & 56.8 & \\
\hline & $2.4 \ldots \ldots$ & 6 & 19 & 46.32 & 7 & +2747.13 & -0.52 & 7 & 45 & 46.61 & 2 & 29 & 52.96 & & 52.9 & \\
\hline & $3.5 \ldots \ldots$ & 7 & 25 & 09.64 & 8 & -2828.59 & +0.60 & 8 & 47 & og. 19 & 3 & 35 & 22. 18 & & 50.5 & \\
\hline & $4.0 \ldots \ldots$ & 7 & $5^{8}$ & 55.97 & $2 I$ & +1055.65 & +0.08 & $2 I$ & 17 & 35.73 & 16 & o7 & 52.00 & & 49.4 & \\
\hline & $23.2 \ldots \ldots$ & 24 & 20 & 46.37 & 24 & +1439.76 & +0.23 & o & 23 & 59.99 & 20 & 29 & $4 \mathrm{I} .45$ & & 50.8 & \\
\hline & $27.8 \ldots \ldots$ & 4 & I 2 & $5^{2.98}$ & $\mathrm{I}_{5}$ & +3547.73 & $-4.0 \mathrm{r}$ & 15 & 59 & 03.72 & 12 & 23 & 05.01 & & 67.1 & \\
\hline & $28.3 \ldots \ldots$ & 4 & 43 & 55.08 & 4 & +1622.52 & -0.79 & 4 & 27 & 01.73 & 24 & 53 & 05.86 & & 54.7 & \\
\hline & $29.8 \ldots \ldots$ & 6 & 16 & 35.73 & 17 & +3277.55 & $-\mathrm{J} .57$ & 17 & 54 & 35.98 & 14 & 26 & 49.36 & & 66.9 & \\
\hline & $30.4 \ldots \ldots$ & 6 & 50 & 14.36 & 6 & +1506.99 & -0.17 & 6 & 25 & 06.82 & 2 & 59 & 23.49 & & 56.2 & \\
\hline & $30.9 \ldots \ldots$ & 7 & 21 & 31.07 & 18 & +3329.97 & -0.12 & I8 & 55 & 29.85 & I5 & $3 \mathrm{I}$ & 49.79 & & 66.2 & \\
\hline \multirow[t]{3}{*}{ February } & $1.5 \ldots \ldots$ & 9 & $\infty$ & 04.41 & 8 & +1597.09 & +0.37 & 8 & 26 & 37.46 & 5 & 09 & 07.20 & & $65 \cdot 3$ & \\
\hline & $1.5 \ldots \ldots$ & 9 & $\infty$ & 04.41 & 8 & +1617.04 & $+0.3^{8}$ & 8 & 26 & 57.42 & 5 & 09 & 27.22 & & 46.1 & \\
\hline & & \multicolumn{5}{|c|}{ Means of all. } & & & & & & & & & 51.8 & \\
\hline
\end{tabular}


Summary of star occultation obserzations and results for longilude al Teplitz Bay Observalory

$$
\varphi=81^{\circ} 47^{\prime} 34 .^{\prime \prime} 9 \mathrm{~N} \quad \varphi^{\prime}=81^{\circ} 44^{\prime} 19^{\prime \prime}
$$

Observations of January 27, 1904, with Berger and Sons' alt-azinuth

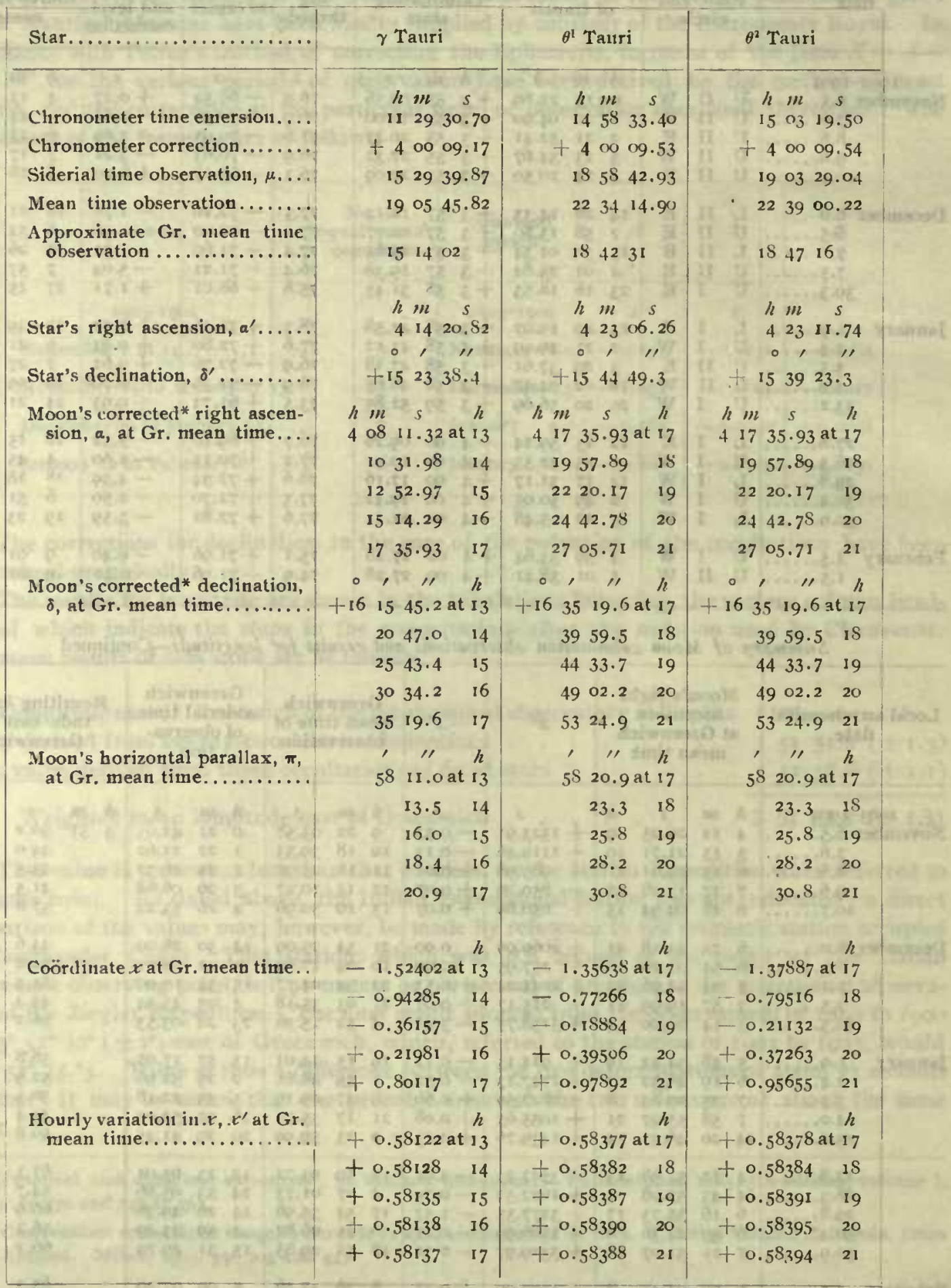

* See p. 612 for corrections to Nautical Almanac values. 
Summary of star occulation obserutions and results for longilude at Teplitz Bay Obscratory-Continued $\phi=8 r^{\circ} 47^{\prime} 34 .^{\prime \prime} 9 \mathrm{~N} \quad \phi^{\prime}=81^{\circ} 44^{\prime} 19^{\prime \prime}$

Observations of January 27 , I904, with Berger and Sous' alt-azimuti

\begin{tabular}{|c|c|c|c|}
\hline Star................... & $\gamma$ 'Tanri & $\theta^{1}$ Tauri & $\theta^{2}$ Tauri \\
\hline & $h$ & $h$ & $h$ \\
\hline Coördinate $y$ at Gr. mean time. & +0.90113 at 13 & +0.87001 at 17 & +0.96324 at 17 \\
\hline 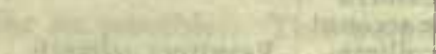 & $+0.98351 \quad 14$ & $+0.94632 \quad 18$ & $+1.03945 \quad 18$ \\
\hline 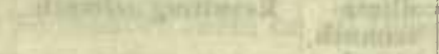 & $+1.06580 \quad 15$ & +1.02250 & $+1.11550 \quad 19$ \\
\hline 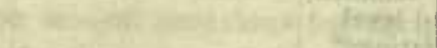 & $+1.14799 \quad 16$ & +1.09862 & $7+1.19149$ \\
\hline & $+1.23008 \quad 17$ & $+1.1745^{S}$ & +1.26730 \\
\hline $\begin{array}{l}\text { Houriy variation in } y_{1} y^{\prime} \text { at } \\
\text { Gr. urean time............... }\end{array}$ & $+0.0 \$ 234$ at $\begin{array}{l}h \\
13\end{array}$ & $\begin{array}{r}h \\
+0.07624 \text { at } 17\end{array}$ & $\begin{array}{r}h \\
+0.076 \mathrm{r}_{3} \text { at } 17\end{array}$ \\
\hline woe in mice fis and & $+0.08229 \quad 14$ & $+0.076 \mathrm{rs} \quad 18$ & $+0.07605 \quad$ IS \\
\hline & +0.08224 & +0.07616 & +0.07602 \\
\hline & $+0.08219 \quad 16$ & +0.07612 & $+0.07599 \quad 20$ \\
\hline & $+0.08214 \quad 17$ & +0.07604 & $+0.07590 \quad 21$ \\
\hline$\mu$ in $\operatorname{arc} . \ldots \ldots \ldots \ldots \ldots$ & 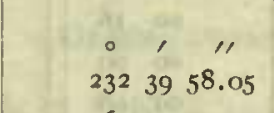 & $\begin{array}{ccc}\circ & , \quad 11 \\
284 & 40 & 43.95\end{array}$ & $\begin{array}{ccc}\circ & \prime \prime \\
285 & 52 & 15.60\end{array}$ \\
\hline$\mu-a^{\prime} \ldots \ldots \ldots \ldots \ldots \ldots \ldots$ & $16904 \quad 45.75$ & $21854 \quad 10.05$ & 220 o4 19.50 \\
\hline 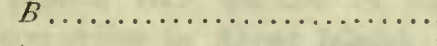 & 98 o6 49.6 & 962648.1 & 962024.3 \\
\hline$\xi \ldots \ldots \ldots \ldots \ldots \ldots \ldots \ldots \ldots \ldots$ & +0.02713 & 1 -0.08994 & -0.09220 \\
\hline$\eta \ldots \ldots \ldots \ldots \ldots \ldots \ldots \ldots, \ldots$ & +0.98834 & +0.97962 & +0.97937 \\
\hline$r_{0}$ assumed at $\ldots \ldots \ldots \ldots$ & $\stackrel{h}{h}$ & $\begin{array}{c}h \\
18.7\end{array}$ & $\begin{array}{c}h \\
18.8\end{array}$ \\
\hline$x_{0} \ldots \ldots \ldots \ldots \ldots \ldots \ldots \ldots$ & -0.21622 & -0.36398 & -0.32808 \\
\hline$y_{0} \ldots \ldots \ldots \ldots \ldots \ldots \ldots \ldots$ & +1.08634 & +0.99964 & +1.10029 \\
\hline$x_{0}^{\prime}, \ldots \ldots \ldots \ldots \ldots \ldots \ldots \ldots$ & $+0.5^{8136}$ & . $\quad+0.5^{8386}$ & $+0.5^{8393}$ \\
\hline$y_{0}^{\prime} \quad \ldots \ldots \ldots \ldots \ldots \ldots \ldots \ldots$ & $\begin{array}{c}+0.08223 \\
0,11\end{array}$ & $\begin{array}{r}+0.07617 \\
0,11\end{array}$ & $\begin{array}{c}+0.07603 \\
0,11\end{array}$ \\
\hline$\| \ldots \ldots \ldots \ldots \ldots \ldots \ldots \ldots$ & $291 \quad 5606.9$ & 2741042.0 & $\begin{array}{lll}297 & 08 & 28.3\end{array}$ \\
\hline$N_{\ldots} \ldots \ldots \ldots \ldots \ldots \ldots \ldots \ldots$ & 815657.3 & 823402.0 & 823452.3 \\
\hline$\psi \ldots \ldots \ldots \ldots \ldots \ldots \ldots \ldots \ldots$ & $2084454 \cdot 3$ & $19142 \mathrm{II} . \mathrm{I}$ & $2132 S \quad 15.2$ \\
\hline$T_{0} \ldots \ldots \ldots \ldots \ldots \ldots \ldots \ldots$ & $\begin{array}{ccc}h & m & s \\
15 & 15 & 00.00\end{array}$ & $\begin{array}{ccc}h & m i & s \\
18 & 42 & 00.00\end{array}$ & $\begin{array}{l}h \quad m 2 s \\
\text { I } 84800.00\end{array}$ \\
\hline Siderial time mean moon...... & $2021 \quad 23 \cdot 74$ & $\begin{array}{lll}20 & 21 & 23 \cdot 74\end{array}$ & $20 \quad 21 \quad 23.74$ \\
\hline Reduction.................. & +0220.31 & +0304.32 & $+\quad 0305.30$ \\
\hline$\mu_{0} \ldots \ldots \ldots \ldots \ldots \ldots \ldots \ldots \ldots$ & II 3854.05 & 150628.06 & 151229.04 \\
\hline$\mu \ldots \ldots \ldots \ldots \ldots \ldots \ldots \ldots \ldots$ & $152939.8_{7}$ & $18 \quad 5^{8} 42.93$ & ifracitit $1903 \quad 29.04$ \\
\hline$\mu_{0}-\mu \ldots \ldots \ldots \ldots \ldots \ldots \ldots$ & -35045.82 & -35214.87 & (1) $\rightarrow 35100.00$ \\
\hline$\tau \ldots \ldots \ldots \ldots \ldots \ldots \ldots \ldots \ldots$ & 175 $\quad$ or 12.44 & +0013.33 & - 00 oo 56.00 \\
\hline $\begin{array}{l}\text { Resulting longitude east of } \\
\text { Greenwich } \ldots \ldots \ldots \ldots \ldots \ldots \ldots\end{array}$ & 35158.3 & 352 or 5 & \\
\hline 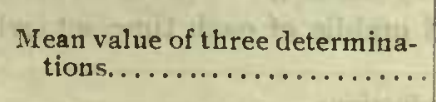 & 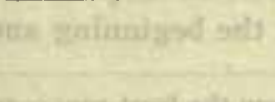 & 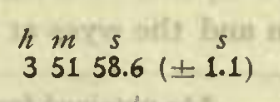 & 30,4 \\
\hline
\end{tabular}




\section{AzimuTH}

The star transit observations were also used in determining azimuths. The results are as follows :

Azimuth of magnclic hut from astronomical obscratory

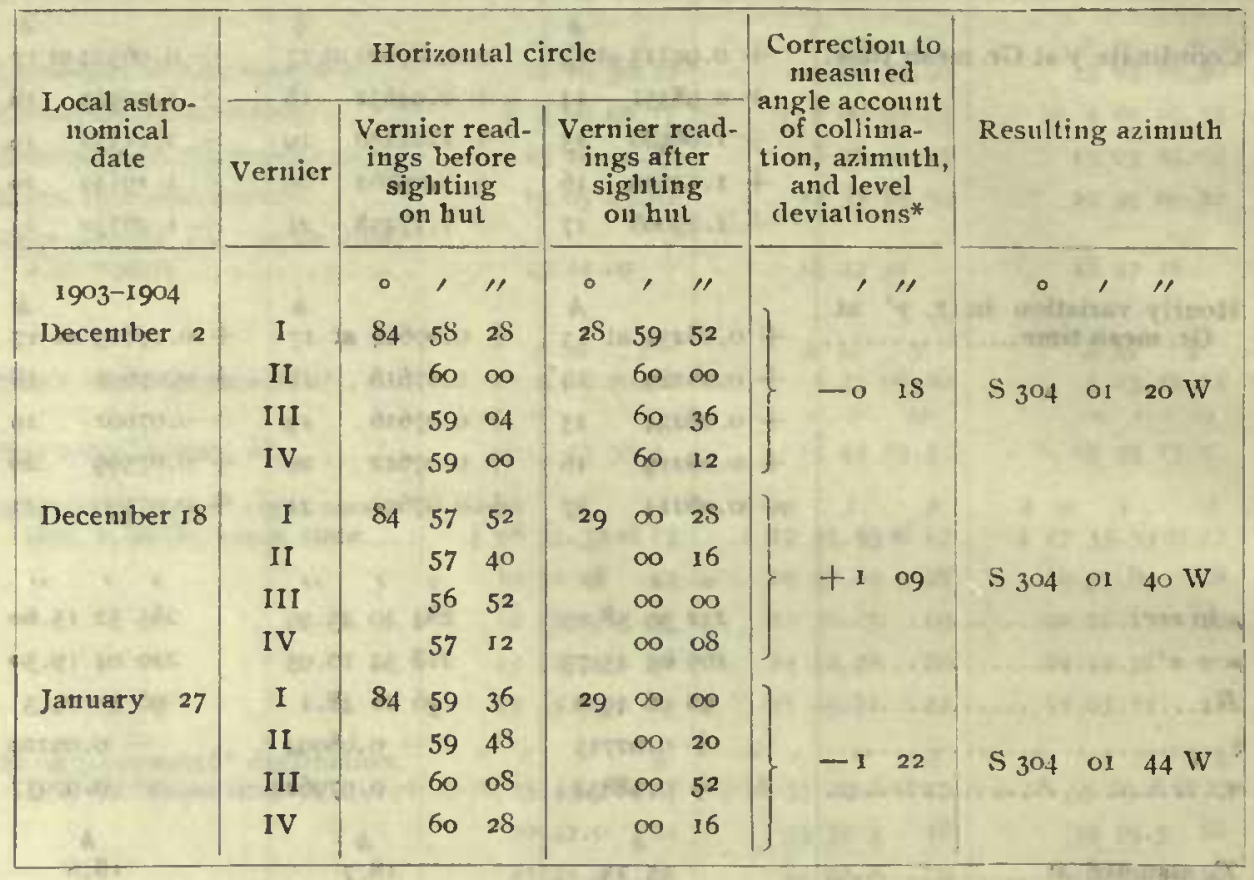

After the meridian mark at Cape Auk was established, February I 2, 1904, numerous measurements of the angle from it to the magnetic hut were made during the year 1904. The mean value from these observations of the included angle was $55^{\circ} 58^{\prime} 28^{\prime \prime}$. From the observations of the lower and upper culminations of the circumpolars $\varepsilon$ Ursæ Minoris and $19 \mathrm{H}$ Camel, respectively, on February 12, 1904, the correction to this angle on account of collimation, azimuth, and inclination of axis deviations was - $0 . " 1$. Hence the azimuth of magnetic hut from astronomic observatory by reference to the meridian mark on Cape Auk is S $304^{\circ}$ or $^{\prime} 32^{\prime \prime} \mathrm{W}$, a value agreeing very well with those obtained above. The resulting mean value adopted is $\mathrm{S} 304^{\circ} 01^{\prime} 34^{\prime \prime} \mathrm{W}$.

\section{RLMARKS}

One of the difficulties enconntered in observing at low temperatures was caused by the accumulation, due to the condensation from the breath and the proximity of a warm body, of small particles of frost over the different parts of the instrument. It is thought that the irregular readings of the striding level are due more to the presence of ice particles on the axis of the telescope than to any cliange in the inclination of the axis itself. This ice, which was being continually deposited, was removed as far as possible by dusting the pivots at each leveling and by wiping them and the wyes at the beginning and middle of each time set (when the 
telescope was raised out of its standards). Yet small pieces of ice or snow were bound to adhere to the pivots, and these could not be removed except by pressure sufficient to disturb the stability of the instrument. Condensation also collected on the object glass and eye piece, necessitating frequent cleaning. For the same reason as given above this could not be removed from the object glass except when the telescope was lifted out of the wyes; hence many fifth and sixth magnitude stars were lost in observing.

The strain to the body attendant on observing any length of time in low temperatures, especially if the surrounding air is at all in motion, necessitated shortening the period of time sets so far as possible. This accounts for many incomplete transits, stars coming too close together to observe them on all threads.

Upon several occasions fog accumulated in the observatory to such an extent as to effectually stop further observation (this with the shutters open). At other times, during temperatures between $-40^{\circ}$ and $-50^{\circ}$ Fahrenheit, the kerosene lamp refused to burn, and the siderial hack watch stopped soon after being exposed to the air.

\section{ALGER ISLAND STATION}

\section{OBSERVATORY}

When the retreat south was made in April of 1905 , the Repsold Circle was taken from its pier at Teplitz Bay, packed in its case, and, with the chronometers, transported by dog sledges 100 miles to Alger Island. At this station the observing hut was some $\mathbf{2 . 4}$ meters square and r. 8 meter high, with a flat roof, and built of wire netting stretched tightly over a wood frame and covered with a heavy roofing material called "rubberoid." Wall and roof shutters were placed in the plane of the meridian, and two trap-doors hing in the east and west walls for observations ont of the meridian. The pier at this point was made of an iron gasoline tank filled with sand and sunk about 0.3 meter in the frozen ground. So far as could be noted this seemed quite stable. The general location of the observing hut with reference to the balance of the camp is shown by the sketch map of figure 21 of Section A.

The south meridian mark was a tripod of oars firmly lashed together, the legs being embedded in stones, on a level ontcrop of basalt from the glacier of McClintock Island. The north mark was a tripod of light irou rods situated on the spur of the mountain immediately north of the station.

The chronometers were kept in a box inside an old hydrogen-generating tank about 1.2 meter in diameter and 1.5 meter higl, located 6 meters northwest of the observatory. This tank was banked up with sand and a small pyramid tent pitched over it. A manhole in the top permitted access to the interior of the tank. This arrangement gave very satisfactory temperature results in the chronometer box, the average daily range during the period May I to July 30,1905 , being only aboit $1.2^{\circ}$ centigrade. The siderial chronometer was connected with a sounder in the observatory. 


\section{OBSERVATIONS AT ALGER ISLAND}

Solar observations only were made at this station during the summer of 1905. The continuous daylight prohibited making trustworthy determinations for longitude. A value of longitude depending upon a rough survey beginning at Teplitz Bay Observatory and ending at Cape Flora, resting at the latter place on the determinations made by the Italian Expedition, of $3^{\mathrm{b}} 44^{\mathrm{m}} 22^{\mathrm{s}}$ east of Greenwich has been adopted.

\section{TIME}

Time observations at Alger Island

\begin{tabular}{|c|c|c|c|c|c|c|c|c|c|c|c|}
\hline \multirow{2}{*}{$\begin{array}{c}\begin{array}{c}\text { Greenwich } \\
\text { astronom- } \\
\text { ical date }\end{array} \\
\begin{array}{c}\text { I905 } \\
\text { June } 26.7\end{array}\end{array}$} & \multirow{2}{*}{$\begin{array}{c}\begin{array}{c}\text { Chronon- } \\
\text { eter No. }\end{array} \\
1809\end{array}$} & \multicolumn{3}{|c|}{$\begin{array}{l}\text { Mean of } \\
\text { clironometer } \\
\text { times }\end{array}$} & \multicolumn{3}{|c|}{$\begin{array}{c}\text { Mean of zenith } \\
\text { readings corrected } \\
\text { for level }\end{array}$} & \multirow{2}{*}{ 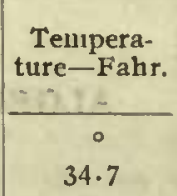 } & \multirow{2}{*}{$\begin{array}{c}\begin{array}{c}\text { Aneroid } \\
\text { barometer }\end{array} \\
\begin{array}{c}\text { In. } \\
30.03\end{array}\end{array}$} & \multirow{2}{*}{$\begin{array}{l}\text { A. M. } \\
\text { or } \\
\text { P. M. } \\
\text { A. M. }\end{array}$} & \multirow{2}{*}{$\begin{array}{c}\begin{array}{c}\text { No. of } \\
\text { point- } \\
\text { ings }\end{array} \\
3\end{array}$} \\
\hline & & $\begin{array}{l}h \\
17\end{array}$ & $\begin{array}{l}m \\
03\end{array}$ & $\stackrel{s}{\text { or. } 50}$ & 60 & 21 & $\begin{array}{c}\prime \prime \prime \\
42.65\end{array}$ & & & & \\
\hline 27.0 & ISO9 & 0 & 16 & 46.75 & 62 & Io & 32.50 & 36.7 & 30.09 & P. $\mathbf{M}$. & 6 \\
\hline$\cdots \quad 28.0$ & $\quad 1809$ & 10 & 24 & 22,80 & 62 & 29 & 40.60 & 28.7 & 30.29 & P. M. & 6 \\
\hline 28.7 & ISO9 & 15 & 38 & 10.50 & 63 & 35 & 26.30 & 34.0 & 30.40 & A. M. & 6 \\
\hline 29.0 & 1809 & 0 & 20 & II. 35 & 62 & 22 & I8.87 & 41.0 & 30.46 & P. $M$. & 6 \\
\hline July 2.0 & 1809 & 11/ 0 & 47 & 45.65 & 63 & 36 & II. 30 & 33.2 & 30.43 & P. $\mathbf{M}$. & 4 \\
\hline $\operatorname{lachin} 7.0$ & (1) 1809 & 0 & $\infty$ & 57.25 & 62 & 12 & 20.83 & 34.0 & 30.36 & P. M. & 6 \\
\hline Io.o & 1809 & 0 & 06 & 36.35 & 62 & 43 & 35.73 & 32.0 & 30.42 & P. M. & 6 \\
\hline \begin{tabular}{l|l}
10.7 & 10.7
\end{tabular} & 1764 & $1+3$ & 25 & 55.00 & 62 & $5 I$ & 06.65 & 36.0 & $30.4 \mathrm{I}$ & A. $M$. & 6 \\
\hline bhor II.0 & I809 & o & o7 & 20.17 & 62 & 52 & & 36.9 & 30.35 & P. M. & 6 \\
\hline (6) $\quad 15.7$ & I764 & 3 & I5 & $35 \cdot 75$ & 64 & 42 & 27.88 & 33.8 & 30.35 & A. $\mathrm{M}$. & 6 \\
\hline I6.7 & 1764 & 2 & 54 & I2.27 & 65 & 52 & 19.70 & 31.0 & 30.40 & A. $\mathbf{M}$. & 4 \\
\hline IS. 7 & 1764 & 3 & 05 & 29.60 & 66 & 04 & $25 \cdot 9.5$ & $35 \cdot 5$ & 30.34 & A. M. & 5 \\
\hline 19.7 & 1764 & 3 & 57 & 06.17 & 64 & 24 & $50.5^{8}$ & $34 \cdot 2$ & 30.34 & A. $\mathbf{M}$. & 6 \\
\hline 20.0 & I 764 & 12 & 05 & 47.27 & 65 & 06 & 37.95 & 34.7 & $3^{0} .38$ & P. M. & 6 \\
\hline 20.7 & 1764 & 3 & I I & $3^{6.72}$ & 66 & 30 & $23 \cdot 5^{2}$ & $35 \cdot 7$ & $30.3^{S}$ & A. $M$. & 6 \\
\hline 23.7 & 1764 & 3 & 32 & 50.13 & 66 & 42 & 48.80 & $35 \cdot 9$ & 30.35 & A. M. & 6 \\
\hline 24.0 & 1764 & I I & 50 & 51.89 & 64 & 44 & 38.90 & 32.0 & 30.34 & P. $\mathbf{M}$. & 6 \\
\hline 26.0 & 1764 & 12 & 22 & 48.63 & 66 & 03 & $17 \cdot 77$ & 36.2 & 30.36 & P. $\mathbf{M}$. & 6 \\
\hline$x=26.7$ & I 764 & 3 & $4 I$ & 49.62 & 67 & 27 & 14.27 & 32.2 & 30.42 & A. $\mathbf{M}$. & 6 \\
\hline $27 \cdot 7$ & I 764 & 3 & 39 & 49.89 & 67 & 54 & 38.83 & $35 \cdot 3$ & 30.37 & A. $\mathbf{M}$. & 6 \\
\hline e 28.0 & 1764 & 12 & 45 & 37.66 & 67 & 04 & 23.22 & 35.0 & 30.35 & P. $\mathbf{~ I . ~}$ & 6 \\
\hline
\end{tabular}


Time observations at Alger Island-Continued

\begin{tabular}{|c|c|c|c|c|c|c|c|}
\hline \multirow[t]{2}{*}{$\begin{array}{l}\text { Greenwich } \\
\text { astronom- } \\
\text { ical date }\end{array}$} & \multirow{2}{*}{\multicolumn{2}{|c|}{$\begin{array}{c}\text { Mean of } \\
\text { observed times, } \\
\text { corresponding } \\
\text { to mean } \\
\text { zenith distances }\end{array}$}} & \multirow{2}{*}{\multicolumn{2}{|c|}{$\begin{array}{l}\text { Time deduced } \\
\text { from } \\
\text { observed alti- } \\
\text { tudes }\end{array}$}} & \multicolumn{2}{|c|}{$\begin{array}{c}\text { Corrections of chronometers } \\
\text { to } \\
\text { local siderial time }\end{array}$} & \multirow{2}{*}{ 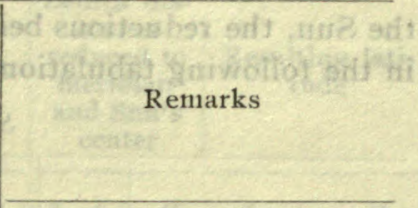 } \\
\hline & & & & & No. I8og & No. I 764 & \\
\hline $\begin{array}{c}\text { I905 } \\
\text { une } 26.7\end{array}$ & $\begin{array}{r}h \\
17\end{array}$ & $\begin{array}{cc}m & s \\
\mathrm{o} 3 & \mathrm{OI} \cdot 5 \mathrm{O}\end{array}$ & $\begin{array}{r}h \\
20\end{array}$ & $\begin{array}{c}s \\
12.67\end{array}$ & $\begin{array}{r}h \quad m s \\
+348 \text { II. } 77\end{array}$ & $\left.\begin{array}{lll}h & m & s \\
\cdots & \cdots & \cdots\end{array}\right]$ & abians \\
\hline 27.0 & o & I6 49.73 & 404 & 56.56 & $48 \quad 06.83$ & $\cdots \quad \cdots \quad \ldots \ldots$ & \\
\hline 28.0 & o & $24 \quad 24.63$ & $4 \quad 12$ & $29 \cdot 5 \mathrm{I}$ & $\begin{array}{lll}48 & 04.88\end{array}$ & $\cdots$ & \\
\hline 28.7 & I5 & $3^{8} \quad 07.05$ & I9 26 & 13.01 & $\begin{array}{ll}48 & 05.96\end{array}$ & $\cdots$ & 70017 \\
\hline 29.0 & o & $20 \quad 14.24$ & 408 & 15.08 & $48 \quad 00.84$ & 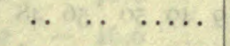 & anut \\
\hline July 2.0 & o & $47 \quad 47 \cdot 16$ & $4 \quad 35$ & 48.26 & 48 or. Io & $\ldots \quad \ldots \ldots$ & $(x+2) \quad$. \\
\hline 7.0 & o & or or 67 & 348 & 50.49 & $\begin{array}{ll}47 & 48.82\end{array}$ & $\cdots \quad \ldots \quad \ldots \ldots$ & Sun's edge "jumping", \\
\hline Io.o & o & $06 \quad 39.19$ & $3 \cdot 54$ & $2 \mathrm{I} \cdot 3 \mathrm{O}$ & 4742.11 & $\therefore \quad \cdots \quad \ldots \ldots$ & 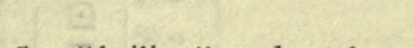 \\
\hline I0.7 & 3 & $25.50 .9 \mathrm{I}$ & $3 \cdot 28$ & 25.82 & & to $02 \quad 34.91$ & Sun "boiling" moderately \\
\hline II. O & o & 07 23.27 & 3.55 & $04 \cdot 71$ & $47 \quad 4 \mathrm{I} \cdot 44$ & $\ldots \quad \ldots+\ldots$ & "Boiling" violently \\
\hline $15 \cdot 7$ & 3 & I5 32.62 & 3 I8 & 15.26 & $\ldots 2 \quad \ldots \ldots$ & $02 \quad 42.64$ & Good definition \\
\hline 16.7 & 2 & 54 Io.I I & $2+56$ & 52.60 & & $02 \cdot 42 \cdot 49$ & Clouds \\
\hline 18.7 & 3 & $05 \quad 28.72$ & 308 & 17.88 & & $02 \quad 49.16$ & Drifting \\
\hline I9.7 & 3 & $\begin{array}{ll}57 & 03.08\end{array}$ & $3 \cdot 59$ & 54.29 & 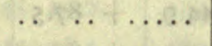 & $02 \quad 5 \mathrm{I} .2 \mathrm{I}$ & Goo \\
\hline 20.0 & 12 & $05 \quad 49 \cdot 57$ & I 208 & $41 \cdot 32$ & $\ldots \quad \ldots$ & $02 \quad 5 \mathrm{I} \cdot 75$ & Good \\
\hline 20.7 & 3 & II $\quad 35.44$ & $3 \quad \mathrm{I} 4$ & $29 \cdot 30$ & $\ldots \quad \ldots$ & O2 $\quad 53.86$ & Fair : \\
\hline $23 \cdot 7$ & 3 & $3^{2} \quad 4^{8} \cdot 53$ & 335 & 50.76 & $\cdots \quad \cdots$ & $03 \quad 02.23$ & Fair \\
\hline 24.0 & I I & $50 \quad 55.11$ & II 53 & $57 \cdot 43$ & $\cdots \quad \cdots$ & $03 \quad 02 \cdot 32$ & Good at phing \\
\hline 26.0 & 12 & $22 \cdot 5^{2} \cdot 5^{8}$ & I2 26 & $00 \cdot 39$ & & $0307.8 \mathrm{r}$ & Very good \\
\hline 26.7 & 3 & $4 I \quad 48 \cdot 37$ & 344. & 57.46 & $\cdots \quad \cdots$ & $03 \quad 09.09$ & Good \\
\hline $27 \cdot 7$ & 3 & $49 \quad 49.00$ & 342 & 59.28 & $\cdots \quad \cdots \quad \cdots \cdots$ & 0310.28 & Good \\
\hline 28.0 & 12 & $\begin{array}{ll}45 & 39.07\end{array}$ & $124^{8}$ & $53 \cdot 50$ & $\cdots \quad \cdots \quad \cdots \cdots$ & $\mathrm{O}_{3} \mathrm{I} 4.43$ & Good \\
\hline
\end{tabular}

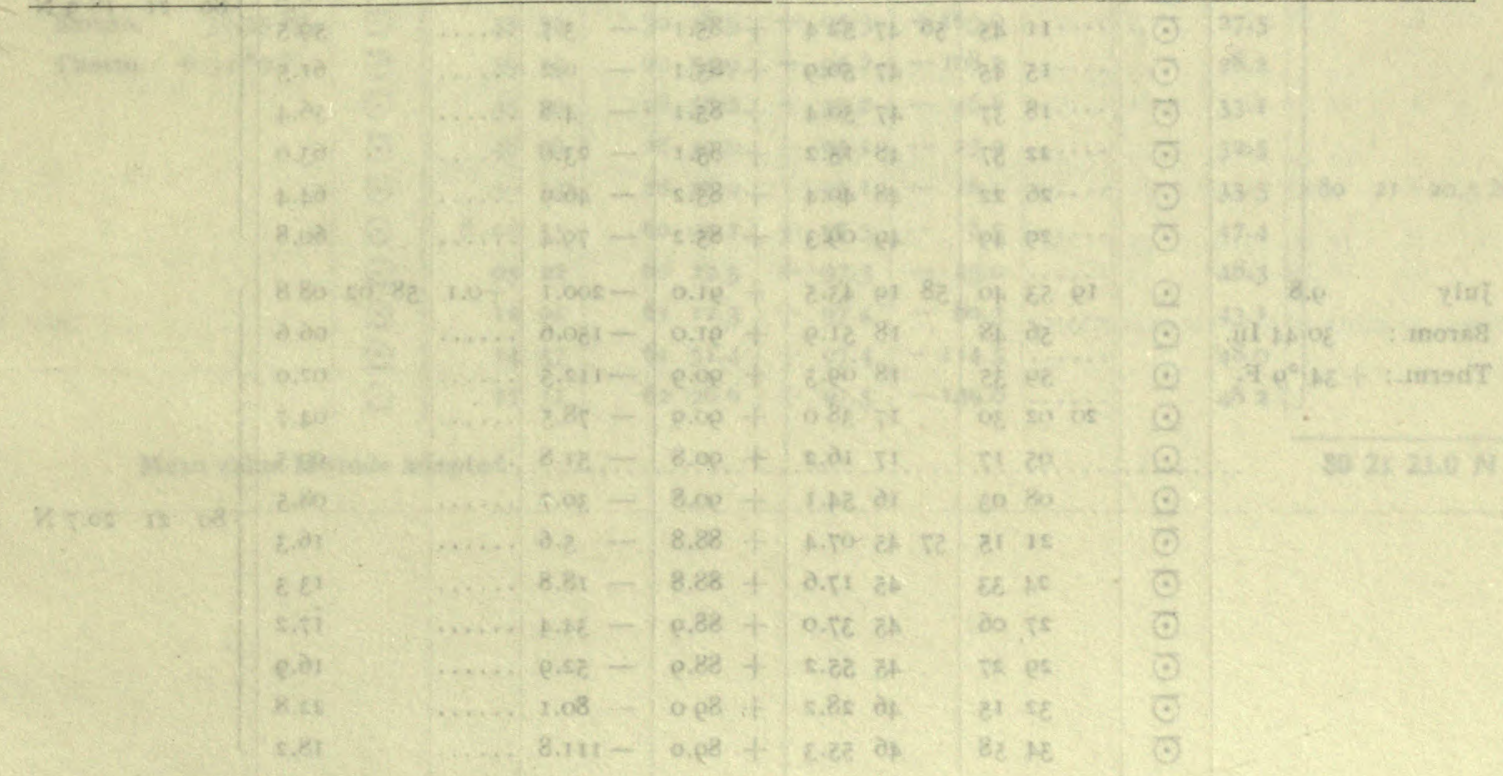




\section{LATITUDE}

Latitude was determined at Alger Island by the method of circummeridian observations of the Sun, the rednctions being carried ont in the usual method.* The results are summarized in the following tabulation:

Summary of tatitude observations at Alger Island.

June $\quad 28.8$

Barom.: $\quad 30.44 \mathrm{In}$.

Therm.: $+36 .{ }^{\circ} 6 \mathrm{~F}$.

July $\quad 9.8$

Barom: $\quad 3044$ I11.

Therni.: $-34^{\circ} 9 \mathrm{~F}$.

Therm.

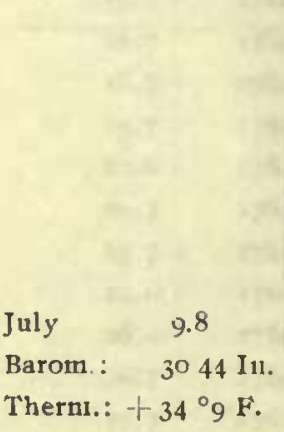

o

Barom.: $\quad 30.27$ In.

ThernI.: $+310^{\circ} \mathrm{OF}$.

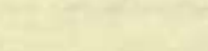

Obs'd
limb $\begin{gathered}\text { Chrononi. } \\ \text { No. } 1764 \\ \text { tine }\end{gathered}$

limb tine

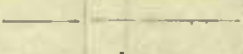

$\odot$

$\odot$

$\odot$

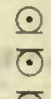

$\odot$

$\odot$

$\odot$

$\odot$

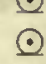

$\stackrel{\odot}{\odot}$

$\odot$

$\odot$

$\odot$

$\odot$

$\odot$

$\odot$

$\odot$

ఠ

ఠ h $m$ s

I9 4950

57 40

$20 \quad 0205$

$\begin{array}{ll}05 & 18\end{array}$

10 58

I6 51

$\begin{array}{lll}20 & 47\end{array}$

$26 \quad 21$

$\begin{array}{lll}30 & 14\end{array}$

$32 \quad 5^{8}$

$\begin{array}{lll}38 & 39\end{array}$

$43 \quad 30$

194930

53 og

$\begin{array}{lll}56 & 07\end{array}$

59 II

(2) $02 \quad 31$

$\begin{array}{ll}06 & 07\end{array}$

I1 45

1545

$18 \quad 37$

$\begin{array}{ll}22 & 57\end{array}$

$\begin{array}{ll}26 & 22\end{array}$

$29 \quad 49$

195340

$\begin{array}{ll}56 & 48\end{array}$

$\begin{array}{ll}59 & 35\end{array}$

$20 \quad 02 \quad 30$

O5 17

oS 03

$21 \quad 15$

2433

2706

$\begin{array}{ll}29 & 27\end{array}$

$\begin{array}{ll}32 & 15\end{array}$

$34 \quad 58$

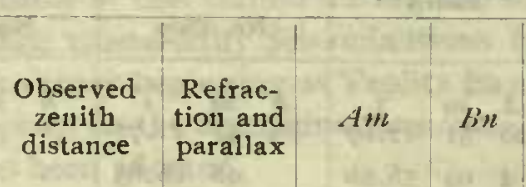

\begin{tabular}{|c|c}
\hline $\begin{array}{c}\text { Zenith dis- } \\
\text { tances } \\
\text { seduced to } \\
\text { meridian } \\
\text { and Sun's } \\
\text { center }\end{array}$ & $\begin{array}{c}\text { Resulting lati- } \\
\text { tude }\end{array}$ \\
\hline $0 \quad$, & \\
\hline $57 \quad 02$ & 17.6
\end{tabular}

$564849.4+85.8-2234$ to.1 $5702 \quad 17.6$

$7826.1+87.5-105.1 \ldots \ldots$

$7743.7+87.6-57.8 \ldots \ldots$

$7716.9+87.6-32.2 \ldots \ldots$

$4510.5+85.5-5.2 \ldots \ldots$

$4509.0+85.5-1.6 \ldots \ldots$

$4520.7+85.5-13.1 \ldots \ldots$

$7733.9+875-485 \ldots \ldots$

$7811.6+87.5-86.3 \ldots \ldots$

$7844.9+87.5-119.5 \ldots \ldots$

$4843.1+85.7-205.6+0.1$

50 $10.5+85.7-297.4+0.1$

$572325.4+87.3-233.0+0.1$

$2224.1+87.2-171.0 \ldots \ldots$

$2 \mathrm{t} 41.4+87.2-127.6 \ldots \ldots$

$2101.9+87.1-895 \ldots \ldots$

$2032.4+87.1-55.6 \ldots \ldots$

$2006.6+87.1-28.1 \ldots \ldots$

$5647524+85.1-3.7 \ldots \ldots$

$4750.9+85.1-0.2 \ldots \ldots$

$4750.4+85.1-4.8 \ldots \ldots$

$4815.2+85.1-23.0 \ldots \ldots$

$4840.4+85.2-46.9 \ldots \ldots$

$4909.3+85.2-79.4 \ldots \ldots$

22.8

27.8

26.6

I6. 5

18.6

$18.8\} \begin{array}{lll}80 & 2.1 & 19.1 \mathrm{~N}\end{array}$

27.2

27.0

27.2

28.9

24.5

$\begin{array}{lll}57 & 04 & 74.1\end{array}$

74.6

75.3

73.8

78.2

79.9

59.5

61.5

56.4

63.0

64.4

60.8

$581943.5+91.0-200.1+0.1 \quad 580208 \mathrm{~s}$

$1851.9+91.0-150.6 \ldots \ldots .66$

$1809.3+90.9-112.5 \ldots \ldots$

$17380+90.9-78.5 \ldots \ldots$

I7 $16.2+90.8-51.8 \ldots \ldots$

$1654.1+90.8-30.7 \ldots \ldots$

$574507.4+88.8-5.6 \ldots \ldots$

$4517.6+88.8-18.8 \ldots \ldots$

$4537.0+88.9-34.4 \cdots \cdots$

$4555.2+88.9-52.9 \ldots \ldots$

$4628.2+890-80.1 \ldots \ldots$

$4655.3+89.0-111.8 \ldots \ldots$

02.0

04.7

095

\begin{tabular}{l|lll}
08.5 & 80 & 21 & $20.7 \mathrm{~N}$
\end{tabular}

I 6.3

133

17.2

16.9

22.8

I 8.2

* Spherical aud practical astronony, by Willian Chanvenet. Philadelphia,1885, 5th edition. Pp. 233-253. 
Summary of lalitude observations al Alger Island-Continued

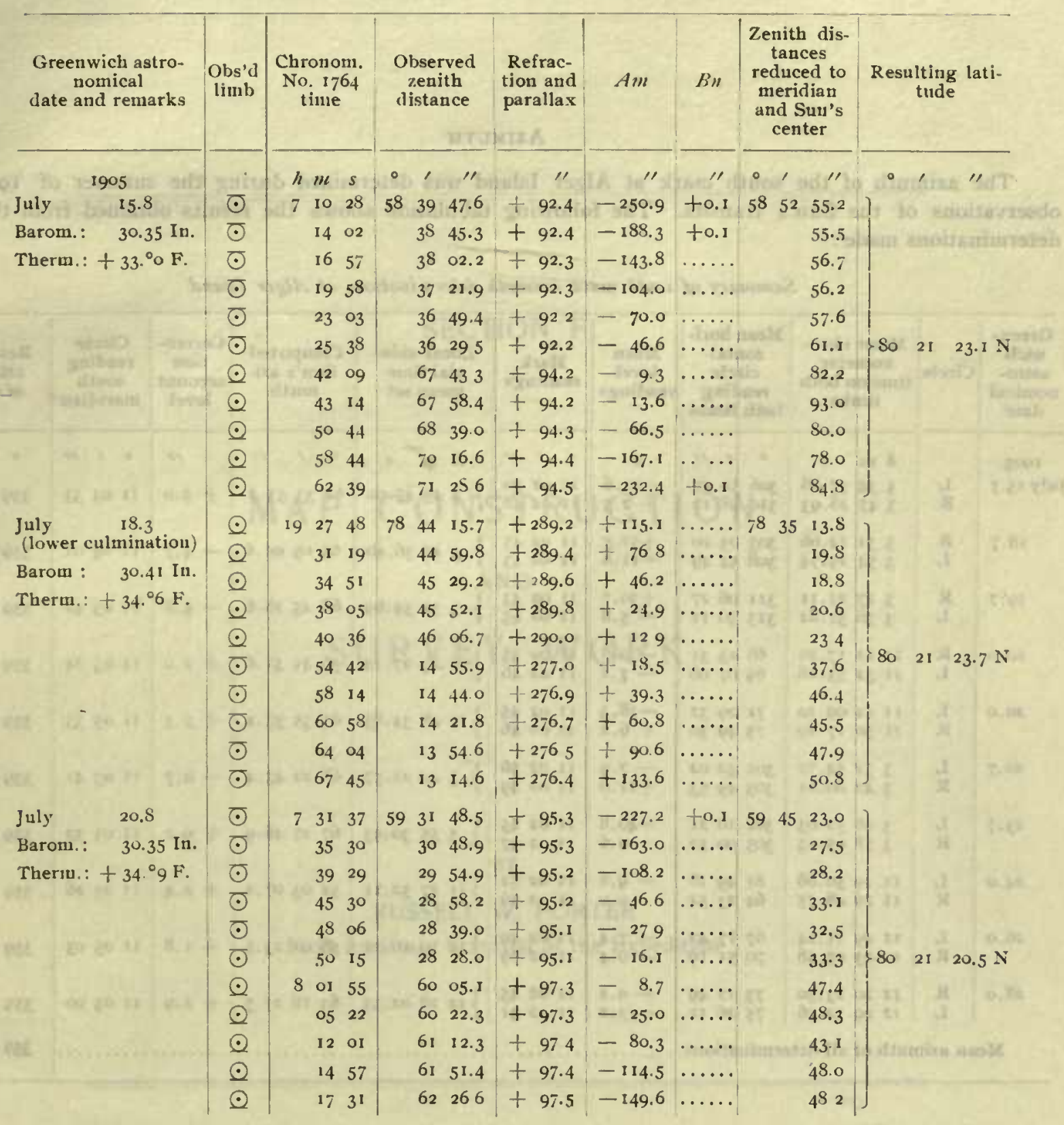

Mean value latitude adopter

$802121.0 \mathrm{~N}$ 

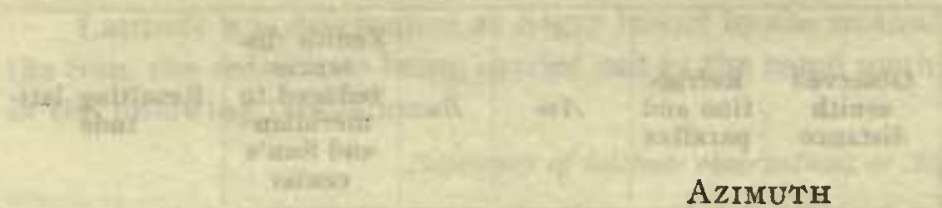

\section{AzIMUTH}

The azimuth of the south mark at Alger Island was determined during the summer of Igo5 by observations of the Sun's transits. The following tabulation shows the results obtained from the ten determinations made:

Summary of south mark azimuth determinations at Alger Island

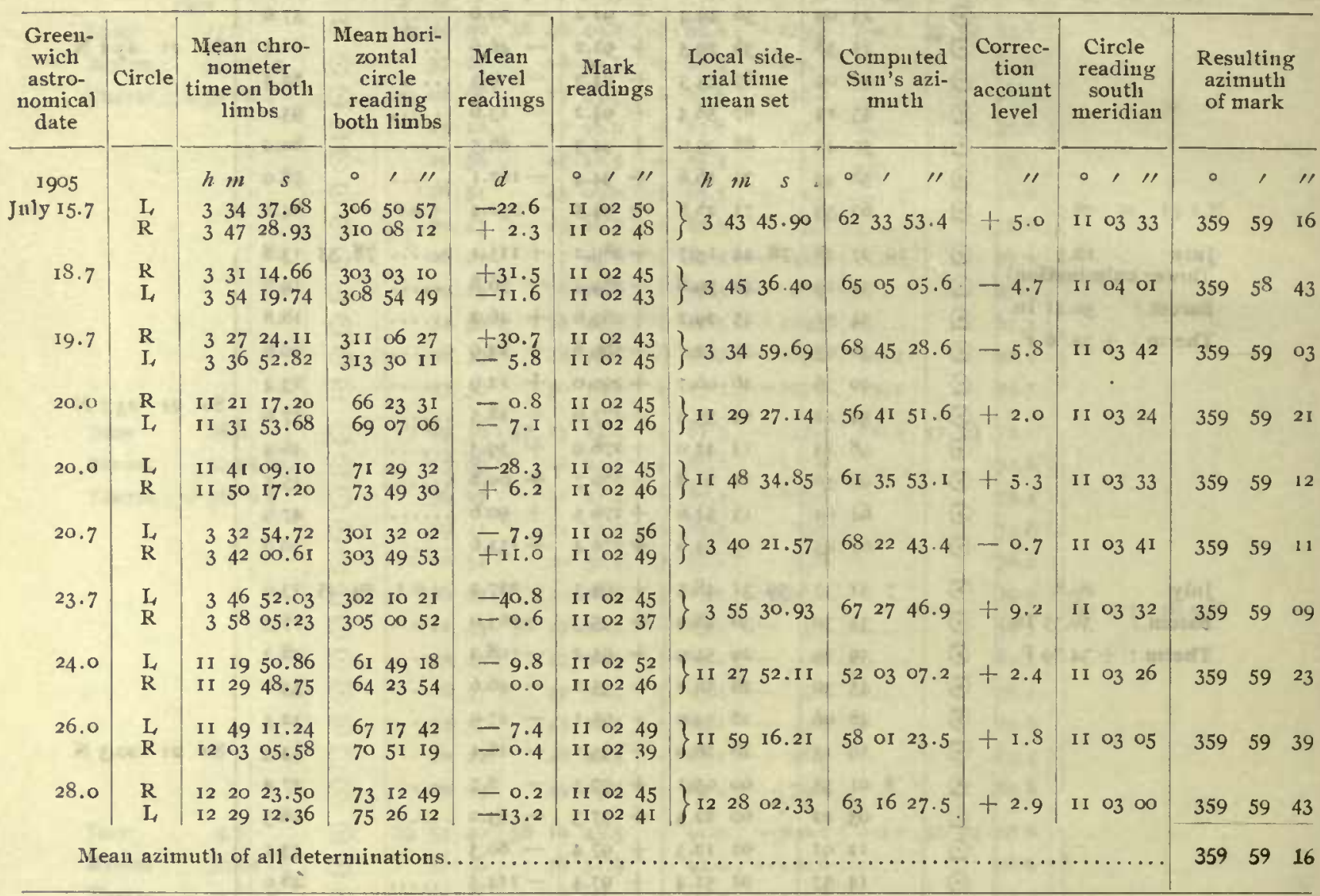




\section{SECTION F}

\section{MAP CONSTRUCTION \\ AND \\ SURVEY WORK}

BY

RUSSELL W. PORTER

First Assistant Scientist of the Expedition 


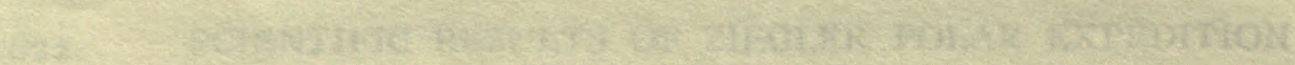

ationst

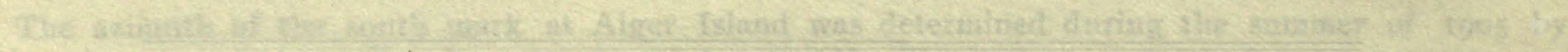

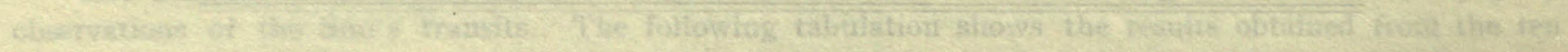

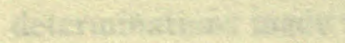

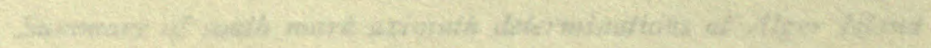

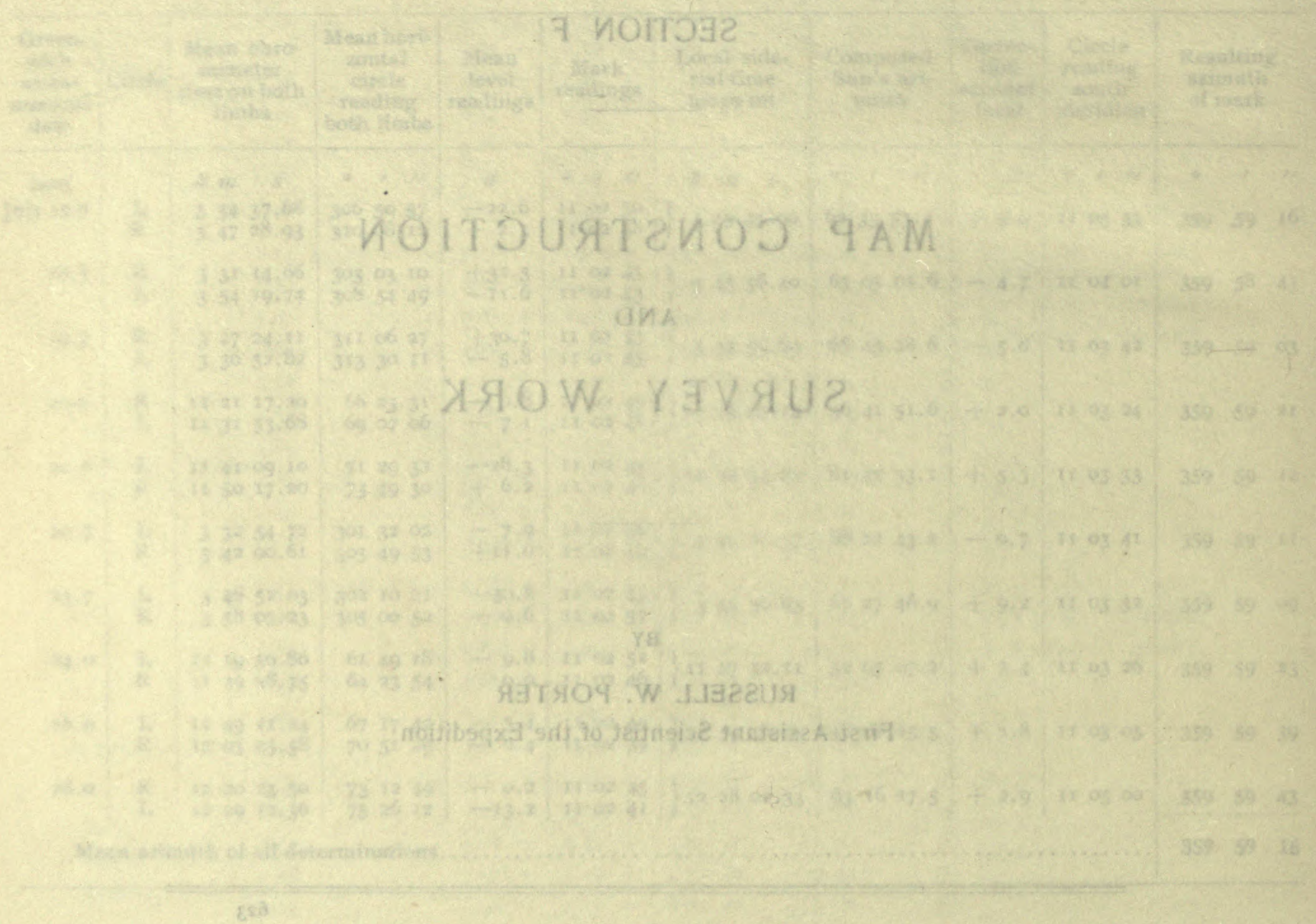




\section{CONTENTS}

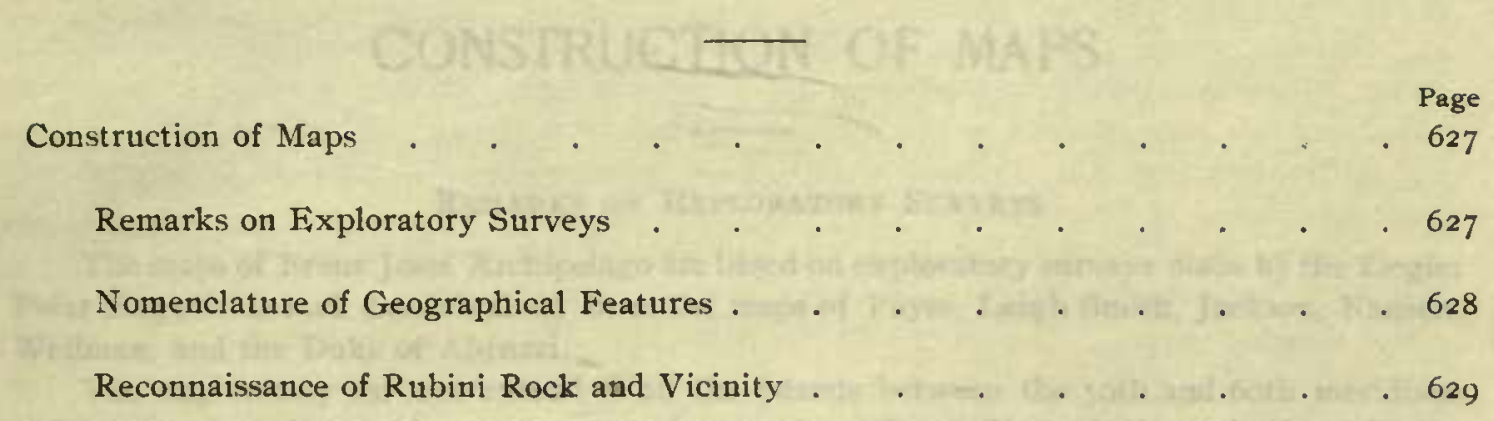

\section{MAPS}

I Sketch Map of Rubini Rock and Vicinity . . . . . Opposite page 6.30

A The North Polar Regions . . . . . . . . . . In pocket

B Franz Josef Archipelago . . . . . . . . . . In pocket

C Part of Franz Josef Archipelago Surveyed by the Ziegler Polar Expedition, 1 $903-4-5$

In pocket 


\section{етйти०O}

sga'

1.0

iso

850

$0 \times 3$

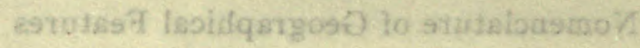

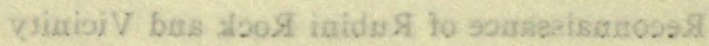

QQA.M.

Ofo sgisq stieogqo

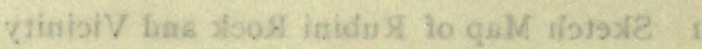

$19800 \mathrm{~g}$ aI

emoigsh 7 silo' droK sit

4.

$19 x \log n \mathrm{BI}$

ogniogitionk beot sast' \&

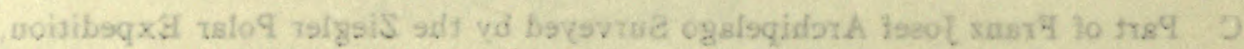

$18 \$ 200$ aI

$2+4-800 x$

zse 


\section{CONSTRUCTION OF MAPS}

\section{REMARKS ON EXPLORATORY SURVEYS}

The maps of Franz Josef Arclipelago are based on exploratory surveys made by the Ziegler Polar Expedition and data obtained from the maps of Payer, Leigh Smith, Jackson, Nansen, Wellman, and the Duke of Abruzzi.

The exploratory surveys extend to all the islands between the 5oth and 6oth meridians and consist of a plane-table traverse, run in 1904 from Camp Abruzzi, through Kane Lodge and Camp Ziegler, to Elmwood. Additional information was obtained from an earlier trip made the same year to Kane Lodge and to Nansen's hut and further plane-table work in I 905 in the region north of Markham Sound.

The longitude of the astronomic observatory at Camp Abruzzi was obtained by the methods of moon-star culminations and star occultations. Twenty-two moon-culmination and three staroccultation observations were made during the winter of $1903-4$. The resulting value, $3^{\mathrm{h}} 51^{\mathrm{m}}$ $52 . .^{\circ} 6\left(57^{\circ} 58^{\prime}\right.$ o9") east of Greenwich, is the one adopted in the map construction. Determinations resting on the chronometer alone were not used on account of the large variations in rate, supposed at the time to be due to jars caused by "bucking ice". Twenty-six out of the fiftyfour stations occupied were strengthened by latitude, azimuth, and time observations made with a Berger and Sons' especially constructed 4 -inch theodolite or alt-azimuth (see Section E for description). Two base lines were included, one at Camp Abruzzi, the other at Kane Lodge.

The longitudes of Camp Ziegler, Harmsworth House (Cape Tegetthoff), and Elmwood (Cape Flora), as determined by this traverse and referred to Camp Abruzzi, are:

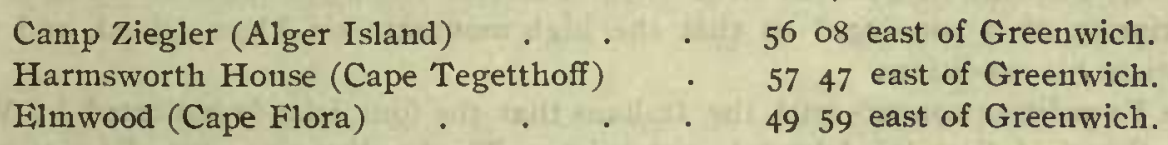

These values were adopted in the new map and required Jackson's work to be shifted 3.3 nautical miles to the east, Payer's 3.6 to the west, and Wellman's 0.5 to the west.

Heights are given in feet. Except in one or two instances, the brows of the cliffs are the points measured. With the exception of Stoliczka Island (by aneroid), all heights have been found by triangulation.

During the traverse of 1904 the party went into camp at Rubini Rock (Hooker Island) for ten days during the last of June. The surrounding region seemed peculiarly well adapted for offering a safe harbor to any ship intending to pass the winter in the Archipelago. As such a harbor has never been found heretofore in Franz Josef Arclipelago, where a ship can be sure of getting out the next year, a detailed map was therefore made of an area some 3 miles square (see figure 1 ). 


\section{Nomenclature of Geographical, Features}

In the nomenclature of the different geographical features, certain changes nave been made, as follows :

Backs Channel has been retained for that body of water separating Karl Alexander and Jackson Islands.

De Long Fjord (Nansen) proved to be a bay and not a channel separating Leigh Smith and Frederick Jackson Islands as Nansen supposed. The name of Jackson has been retained to the island which this bay indents.

Hofman Island was looked for on several occasious at a distance of some 20 miles under favorable atmospheric conditions but never was seen. It may be a low, snow-covered island and has been retained in the position ascribed by Wellman. Nansen dropped it from his preliminary map but Wellman's map shows his route so close to it as to preclude any reasonable doubt of its existence.

Freeden Island (Payer) has been retained on the map as the most southern island of Nansen's "Hvidtenland" because Payer saw au island in this neighborhood which he called Freeden Island. The identity of the island that Payer saw is a questiou that probably cannot be settled as it appeared in a direction where some islands are now known to exist. The name he gave should appear on some one of these and, as Nansen has suggested, one island of this group might very probably be the one Payer saw.

Booth, Rhodes, and Brown Fjords and the Ward Bay of Jackson have all been found to be channels running through to A ustria Sound and separating Payer's Zichey Land into several islands.

The group of small islands indicated on Wellman's map as lying south of Marklıam Sound and between Hooker and McClintock Islands have all been identified, with two exceptions, viz.: Simon Newcomb Islands and Willis Moore Islauds. When Jackson mapped this region lie passed through Hamilton Channel in thick. weather without seeing the channel which divides the land west of Hamilton Channel into two islands. We have placed Jackson's Bromwich on the northeru of these two islands and Wellman's Prichett on the southern.

La Ronciere Peninsula, Cape Berghaus, and Cape Littrow, all of Payer, were found by Wellman to be islands and were given new uames. The original proper names of Payer have been retained on the ground of priority.

Richthofen Pcak, seen by Payer from Cape Brunn, has been located on Alger Island and not where Jackson places it. Here the Expedition found a peak, or spur, some $\tau, 400$ feet high dominating the entire neighborhood, as Payer asserts. His wood cut illustrating the peak and lis description of it convinced us that the high mountain on Alger Island, and that only, could satisfy his conditions.

The Expedition concurs with the Italians that the four islands indicated by Wellman as lying northeast of Rudolph Island do not exist. The locality was crossed twice and no land found.

The word "land" has beell dropped entirely as being misguiding, now that the Archipelago is known to consist only of several comparatively small islands.

In the inap construction the last name only of proper names given to geographical feat. ures has been retained for the sake of brevity and clearness. The results of the survey work of the Expedition have all been made use of in constructing Maps B and C.

The map showing the Arctic regions (Map A) has been compiled by Mr. Gilbert $\mathrm{H}$. Grosvenor, Editor of the National Geographic Magazine. As will be readily noted, he has entered upon the same practically all data secured in the Arctic through the year I906. The Expedition is nnder great obligation to hin for the thorough execution of the laborious work of compilation of data and corrections necessary in the construction of this map. 


\section{Reconnaissanch, of Rubini Rock and Vicinity}

The traverse party crossed the ice-cap of Hooker Island the morning of June 21, 1904, and coasted down the glacier slopes in a xigzag course to Rubini Rock. The surroundings presented a far greater diversity of character as well as more vegetable and animal life than we had ever seen before in these Islands.

A good sized bay some three miles across from north to south was found here to indent the island from the British Channel. At the bottom of this bay a headland projected from the ice-cap, continuing as a low spit of land and terminating in a towering rock found later to rise almost sheer from the surface of the bay to a height of $5^{8} 7$ feet. Jackson mistook this rock for an island which error could easily be made in the spring when he visited it. This tongue of land, on which Rubini Rock is located, divides the bay into two smaller ones of nearly equal size and into which descend two glaciers from the ice-cap. The more northerly glacier showed almost no crevassing and had absolutely no face, its surface running imperceptibly into that of the bay ice.

The other glacier, however, immediately south of our camp, was the highly crevassed glacier (No. II on map) and showed signs of more activity than is usually met with among these Islands. Along its landward margin a lateral moraine had been formed in recent times; the detritus was fresh; the rocks angular and sharp and embedded in sand and clay. There were no signs of lichens. Between the moraine and the talus back of it flowed a good sized stream which expanded into two ponds some bundred feet wide before debouching into the bay.

The winter's ice was still in the two bays, its edge on June 20 being as indicated on the map. Outside of this line, and almost surrounding Keltie Island, lay open water between the headlands of the bay in which the broken floes moved back and forth with the tide. There were no bergs floating in the bay though we were constantly expecting them to be discharged from the larger glacier. An old beach raised 28 feet above the sea level was found on the spit of land uniting Rubini Rock with the island. On this beach a base line 600 feet long was measured twice, signals erected on the promineut headlands, and the triaugulation extended with the theodolite. With several points thus well determined the plane table was used to complete the map.

The inner side only of Rubini Rock retained a talus. After some search one spot was discovered where access could be had to the top. The table top of the rock towered a full hundred feet by measurement above the brows of the surrounding headlands. It was composed of sharp, angular blocks of basalt covered with a dense growth of spongy, black lichens resembling very coarse horsehair. This growth, of which there certainly was enough to last an expedition several years as fnel, was found to burn freely. Imbedded in these lichens was found part of a shed antler of an Arctic reindeer; he must have reached this plateau by some way other than the one we used.

The table top dipped toward the southwest like an amphitheater and then dropped vertically into the water. Under the southeastern cliffs the columnar structure of the basalt was very marked. And here thousands of little auks, loons, and sea gulls made their home. The slope of the talus under this rookery was covered with a luxuriant growth of grass whose roots ivere imbedded in ice and frozen earth.

Where the headlands and numataks protruded from the ice-sheet several acres of exposed table land were to be seen entirely free of ice and snow. They differ in elevation from 370 to 720 feet, but all are remarkably level; the basalt is weathered and crumbled to a very coarse sand or gravel. The writer examined the rock exposures of this vicinity for glacial markings and striæ, but found none. 
By the tinne the party was ready to leave on July i the accumulation of winter's snow had disappeared from the glaciers leaving their hard, blue surfaces exposed. A lake of some size had formed in one of the sags of Glacier II some 2,500 feet back from its face, and strean1s from the melting ice were furrowing the surface in every directions. On June 25 three points along the face of Glacier II were selected ( $a, b$, and $c$ of figure I) and their angular distances from a fixed mark on Nunatak B were determined on four different dates, the theodolite being set up at Station B. These measurements resulted as follows:

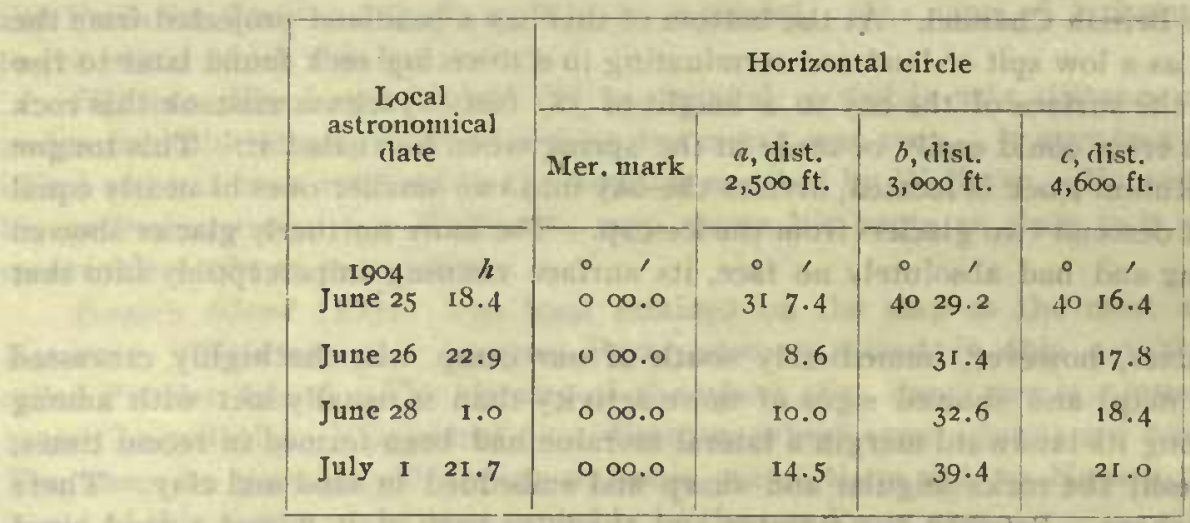

Assuming the movement to be approxinately normal to the lines $B a, B b, B c$, the above data gives the following daily rates of flow for the intervals observed:

\begin{tabular}{ll|llll} 
& $d$ & $h$ & $a$ & $b$ & $c$ \\
Ist interval & I & 4.5 & $0.64 \mathrm{ft}$. & $1.00 \mathrm{ft}$ & $\mathrm{I} .45 \mathrm{ft}$. \\
2d interval & I & 2.1 & 0.88 & 1.00 & 0.91 \\
3d interval & 3 & 20.7 & 0.70 & 0.75 & 1.33
\end{tabular}

and for the entire interval of 6 days 3.3 hours a mean daily movelnent of 0.74 foot, 0.92 foot, 1.23 foot for the three points selected. In other words, the face of this glacier was advancing into the bay at the rate of about a foot a day.

This result, meager enough, in that it represents an isolated case of a single glacier during a short interval of time is valuable as being the only definite information, so far as known, of ice movement in Franz Josef Archipelago. (Being inidsumner, with the temperature between +32 and +42 degrees Fahrenheit, the yearly novement was probably at its naximum.)

For future reference a substantial stone nark was erected on the moraine in line with the glacier face and the cliff on the farther side. Any subsequent change cant therefore be readily ascertained by a party visiting this locality again.

Although the ice still remained in the small bays on July $I$, it was disintegrating rapidly; a large water hole around Dundee Point had increased in size until it almost joined the open water in Mellenius Sound, while a few days later tle ice broke up in De Bruyne Sound. 


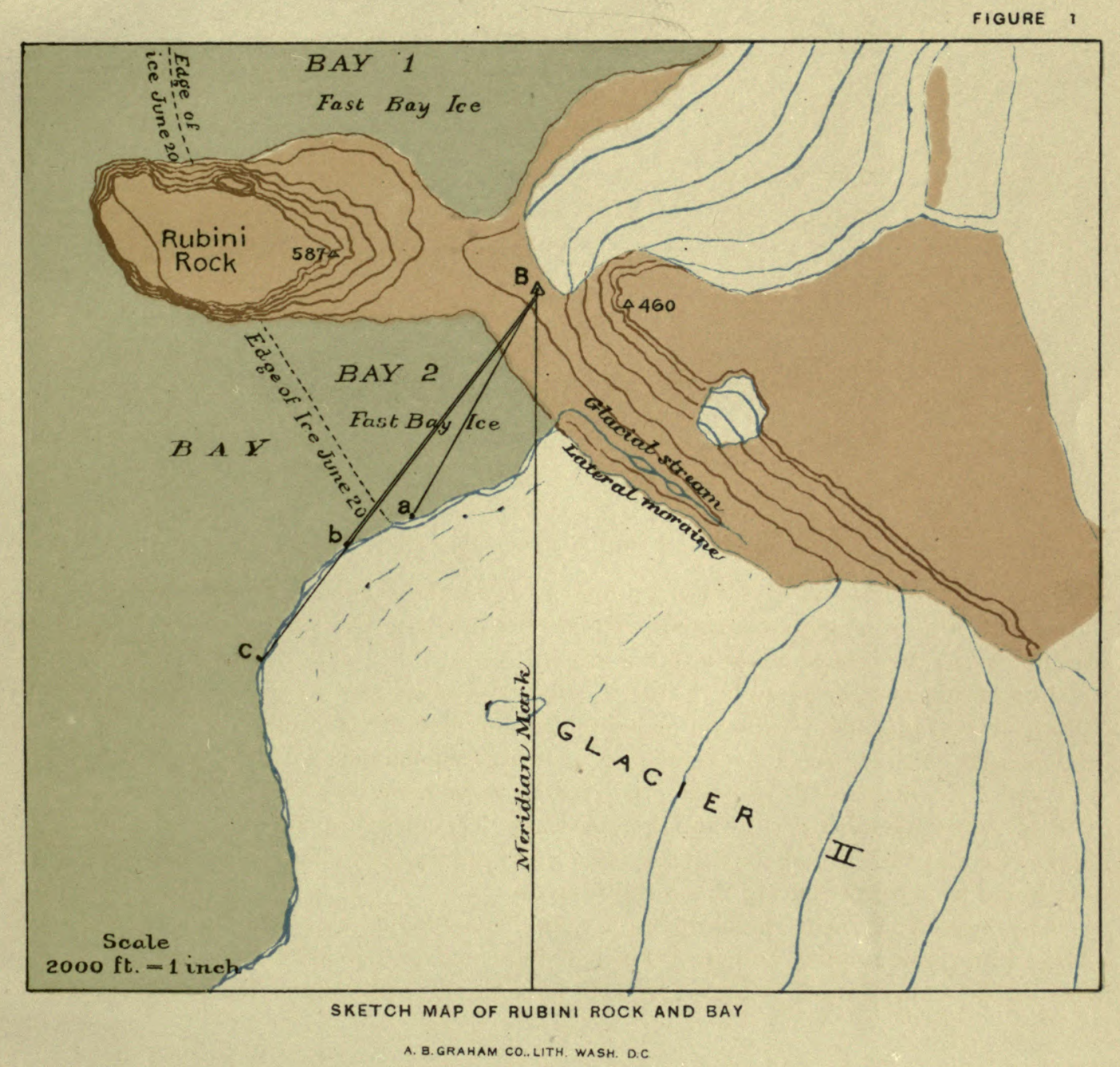







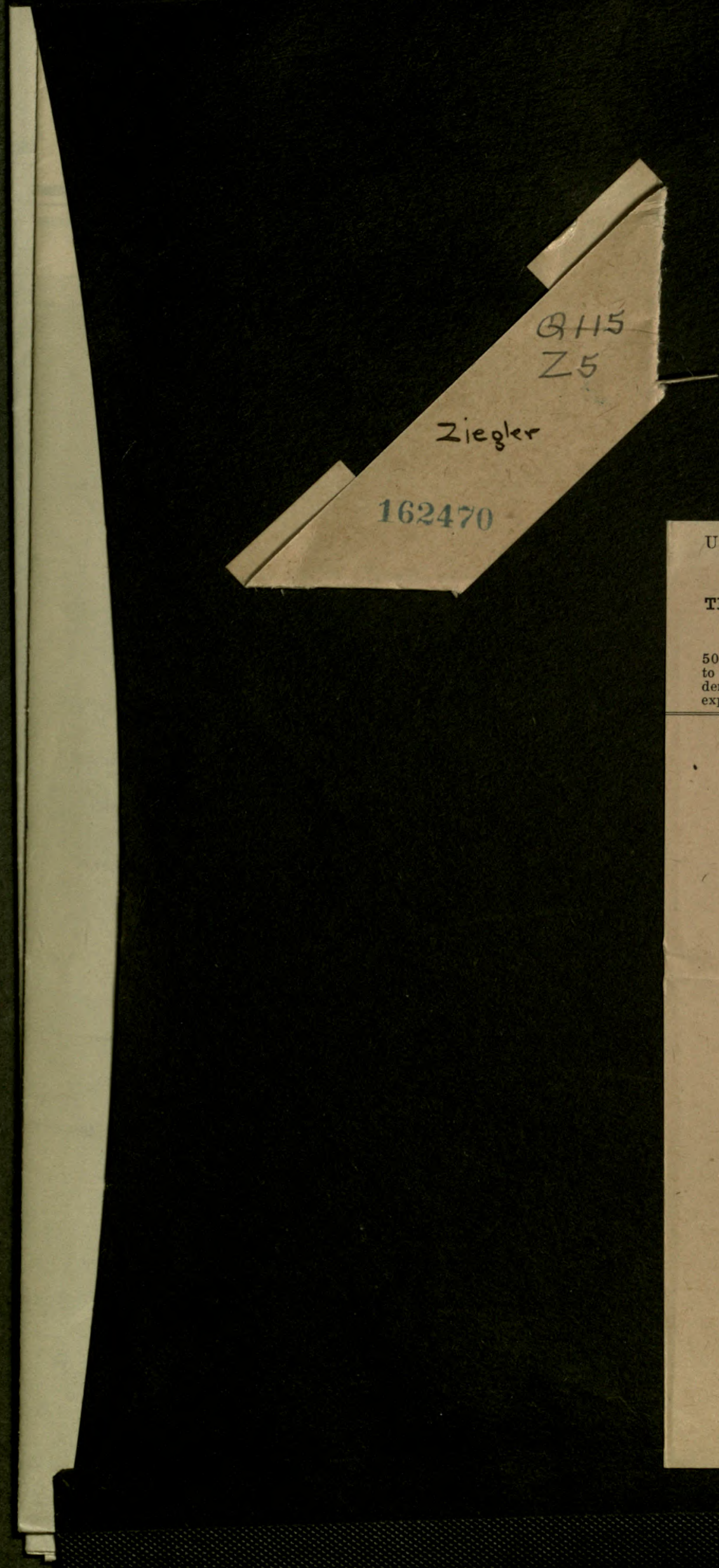

UNIVERSITY OF CALIFORNIA LIBRARY, BERKELEY

THIS BOOK IS DUE ON THE LAST DATE STAMPED BELOW

Books not returned on time are subject to a fine of $50 \mathrm{c}$ per volume after the third day overdue, increasing to $\$ 1.00$ per volume after the sixth day. Books not in demand may be renewed if application is made before expiration of loan period.

\section{SEP 231924}

$\operatorname{t26!} 6$ dis

SEF 91924

Srp $9 \quad 1924$

1 Sepsoan

LIBRAFV IIAE SEP 71950

11 Dec'53FF

MOV271953 Le 
